\title{
Estimation of the Genetic Substitution Rate of Hanwoo and Holstein Cattle Using Whole Genome Sequencing Data
}

\author{
Young-Sup Lee, Donghyun Shin* \\ Department of Animal Biotechnology, Chonbuk National University, Jeonju 54896, Korea
}

\begin{abstract}
Despite the importance of mutation rate, some difficulties exist in estimating it. Next-generation sequencing (NGS) data yields large numbers of single-nucleotide polymorphisms, which can make it feasible to estimate substitution rates. The genetic substitution rates of Hanwoo and Holstein cattle were estimated using NGS data. Our main findings was to calculate the gene's substitution rates. Through estimation of genetic substitution rates, we found: diving region of altered substitution density exists. This region may indicate a boundary between protected and unprotected genes. The protected region is mainly associated with the gene ontology terms of regulatory genes. The genes that distinguish Hanwoo from Holstein in terms of substitution rate predominantly have gene ontology terms related to blood and circulatory system. This might imply that Hanwoo and Holstein evolved with dissimilar mutation rates and processes after domestication. The difference in meat quality between Hanwoo and Holstein could originate from differential evolution of the genes related to these blood and circulatory system ontology terms.
\end{abstract}

Keywords: genetic substitution rate, Hanwoo, hidden substitution factor, Holstein, whole genome sequencing

\section{Introduction}

Mutation rates include genetic changes such as single nucleotide polymorphisms (SNPs), copy number variations (CNVs), insertion/deletions (indels), microsatellites and minisatellites. Specifically, the subset of the total mutation rate caused by SNPs is called the substitution rate. Rate of point mutation can be determined indirectly by estimating neutral substitution rates [1]. In spite of the importance of substitution rates, there have been few investigations to date. We estimated the substitution rates of Hanwoo and Holstein cattle using the concept of the hidden substitution factor (HSF). The vast number of SNPs in next-generation sequencing (NGS) require the use of HSF to estimate the substitution rate. We defined the HSF as an independent unit for substitution rate in any lineage. For instance, the initial founder number is the HSF if the effective population size has been maintained as a constant. Otherwise, HSF is related to both founder number and effective population size. Using HSF, we estimated the genetic substitution rates of the genes of the Hanwoo and Holstein breeds in Korea.

Kumar and Subramanian [1] estimated the substitution rate in the mammalian genes using amino acid degenerate sites and evolutionary distance. Lee et al. [2] obtained the nonsynonymous SNPs, splice-site variants and coding indels in the Bovine genome including Hanwoo. They used wholegenome resequencing. Melka et al. [3] used Bovine SNP 50 beadchip to identify genomic differences between Hanwoo and Holstein. In our analysis, we used whole-genome sequencing to estimate substitution rates of Hanwoo and Holstein and identified genomic differences between Hanwoo and Holstein using gene ontology (GO) analysis. Our novel approach was to use intraspecies substitution rate estimation by inserting HSF into the evolutionary distance. HSF of Hanwoo is closely related to the initial found number that was caused by migration into Korean peninsula. We hypothesized that initial founder number was more than 1 [1-3].

Hanwoo designates the native, taurine type of Korean cattle. The breed originated approximately 4,000 years ago. 
The production of Hanwoo as the main beef cattle has occurred since the 1960s with the rapid growth of the Korean economy. The tasty beef from Hanwoo cattle is popular among Koreans and foreigners. Holstein-Friesian cattle represents the famous milk producing breeds. The holstein was introduced to Korea in 1902, and the Korean cattle industry has developed rapidly since 1960 [4-6]. Hanwoo and Holstein are very important in cattle industry in Korea.

We estimated the evolutionary distance of Holstein and Hanwoo using the number of SNPs, and HSF. Evolutionary distance refers to the cumulative change between DNA or protein sequences that were derived from a common ancestor. There were various methods to estimate it such as JC69 model, Kimura-2-parameter model (K2P), F81 model, HKY85 model. K2P used transition/transversion ratio (tr/tv ratio) to estimate the evolutionary distance. It is moderately complex model [7-10]. We assumed that the evolutionary distance had a linear relationship to HSF. The generation times of Hanwoo and Holstein were set to be 5 years. Then, the genetic substitution rates of the genes were calculated and those features were surveyed.

\section{Methods}

\section{Data preparation}

In this study, we used a whole-genome sequence data set consisting of 23 Hanwoo and 10 Holstein from NCBI Sequence Read Archive database (PRJNA210523, PRJNA210521, and PRJNA210519). We used fastQC software to perform a quality check on the raw sequence data [11]. Using Trimmomatic- 0.32 , we removed potential adapter sequences before sequence alignment [11]. Paired-end sequence reads were mapped to the reference genome (UMD 3.1.75) from the Ensemble database using Bowtie2 with default settings [12]. For downstream processing and variant-calling, we used open-source software packages: Picard tools (http:// broadinstitute.github.io/picard/), SAMtools, and Genome Analysis Toolkit (GATK) $[13,14]$. The "CreateSequenceDictionary" and "MarkDuplicates" Picard command-line tools were used to read the reference FASTA sequence to write a bam file containing a sequence dictionary, and to filter potential PCR duplicates, respectively. Using SAMtools, we created index files for the reference and bam files. We then performed local realignment of sequence reads to correct misalignments due to the presence of small insertions and deletions using the GATK "Realigner-TargetCreator" and "IndelRealigner" arguments. Additionally, base quality score recalibration was performed to get accurate quality scores and to correct the variations in quality associated with machine cycle and sequence context. For calling variants, GATK "UnifiedGenotyper" and "SelectVariants" arguments were used with the following filtering criteria. All variants with (1) a Phred-scaled quality score of less than 30; (2) a read depth less than 5; (3) an MQ0 (total count across all samples of mapping quality zero reads) $>4$; or a (4) a Phred-scaled p-value using Fisher exact test of more than 200 were filtered out to reduce false-positive calls due to strand bias. We used the "vcf-merge" tools of VCFtools in order to merge all of the variant calling format files for the 33 samples [15].

The number of total SNPs was 37,484,886 and after using minor allele frequency; Holstein 0.1, Hanwoo 0.04) and Hardy-Weinberg equilibrium $(\mathrm{p}<0.0001)$, the number of SNPs left in Hanwoo and Holstein were 12,626,097 and $8,636,673$, respectively. The estimated transition/transversion ratio (tr/tv ratio) was calculated to be 2.24 using SnpEff [16].

\section{The relationship between evolutionary distance and founder number}

Evolutionary distance is linear to mutation rate and substitution rate $[17,18]$. Evolutionary distance is proportional to the harmonic number of of sample size $\left(a_{1}\right)$ because the sample size can increase the number of SNPs. Theta represents the population mutation parameter $(\theta=4 \mathrm{~N} \mu)$. While Watterson's theta estimator $\left(\hat{\theta}=\frac{\# \text { segregating sites }}{a_{1}}\right)$ have been used irrespective of HSF and general SNPchip did not require HSF, in the NGS data, substitution rate $(\mu)$ would be high without the concept of HSF. HSF concept assumes that the initial found number after migration would not be equal to 1 like SNPchips. Biologically, that initial migration number was not 1 , is very natural. $a_{1}$ is the harmonic number of sample size $n$. We defined the evolutionary distance as the following:

$$
\mathrm{d}=\mathrm{f} a_{1} \mu t
$$

, where $\mathrm{d}$ is evolutionary distance, $\mathrm{f}$ is the HSF, $a_{1}$ is the harmonic number of sample size, $\mu$ is the substitution rate, and $t$ is the divergence time between the species and reference species.

The evolutionary distance model was K2P [7, 19]. K2P assumes the transition/transversion ratio (tr/tv ratio). We set the tr/tv ratio to be 2.24 , and the divergence time to be 4,000 years ago $[4,20,21]$. The increase in the number of SNPs could be caused by sample size and HSF.

\section{HSF estimation via simulation}

To estimate HSF, we simulated the average substitution rate of SNP chip data. We assumed the SNP number of general SNP chip data. The SNP chip data have 30,00050,000 SNPs in total, and the founder number can be 
assumed to be close to " 1 ." We simulated using the following parameters: the number of SNPs would be 30,000-50,000 and the harmonic number of sample size would be $4-6$. Using Eq. (1), the expected value of the substitution rate was $3 \times 10^{-10}$ to $5 \times 10^{-10}(/ \mathrm{bp} / \mathrm{y})$. Using the result, we set the average substitution rate to be $4 \times 10^{-10}$. This was a similar result to Roach et al. [22] who estimated the average mutation rate of human as $10^{-8}$ order per generation.

The HSF of Hanwoo and Holstein was estimated using Eq. (1). The evolutionary distances of the Hanwoo and Holstein

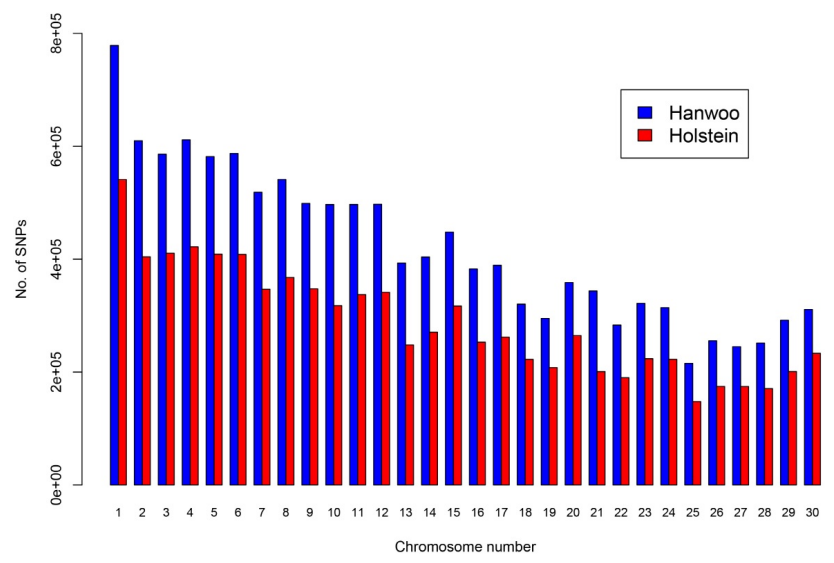

Fig. 1. The number of single nucleotide polymorphisms (SNPs) across chromosomes in Hanwoo and Holstein. The number of individuals of Hanwoo and Holstein was 23 and 10, respectively. Because the number of inidividuals in Hanwoo and Holstein differed, this barplot just showed the patterns of the number of SNPs in Hanwoo and Holstein. population across chromosomes were calculated and then average HSF of Hanwoo (23 individuals) and Holstein (10 individuals) were estimated using the substitution rates of each chromosome.

\section{Genetic substitution rate estimation of genes}

After estimating HSF, we calculated each gene's substitution rate in Hanwoo and Holstein populations. The bovine gene catalog was retrieved from the ensemble website (http://www.ensembl.org). The novel calculation was based on the number of SNPs inside each gene and used K2P model. We surveyed the genes that had the highest and lowest substitution rates. We compared the genetic substitution rates of Hanwoo with those of Holstein.

\section{Results \\ HSF estimation}

We estimated HSF of Hanwoo and Holstein. The HSF of Hanwoo and Holstein were $351 \pm 46$ (mean \pm SD) and $279 \pm$ 39 (mean \pm SD), respectively. The Holstein HSF was not greater than that of Hanwoo because the Holstein HSF might pertain only to the history of Korean Holsteins. Fig. 1 shows the number of SNPs across chromosomes in Hanwoo and Holstein. The difference in the number of SNPs across chromosomes might be mainly due to the number of individuals i.e., 23 and 10 in Hanwoo and Holstein, respectively.
(A)

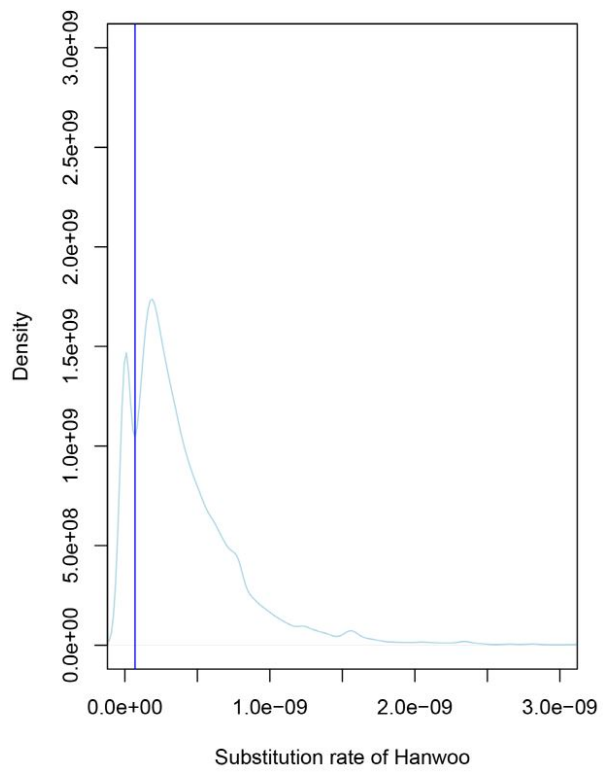

(B)

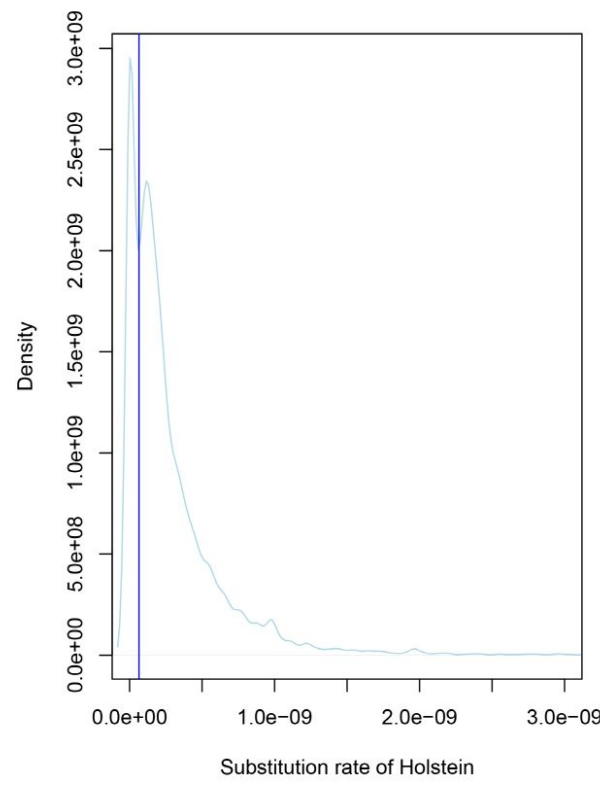

Fig. 2. Density plot of substitution rates. It indicates that diving region exists in both Hanwoo (A) and Holstein (B). The protected region (low substitution rates) and the unprotected region (high substitution rates) can be differentiated by the diving region. The dividing region's substitution rate (blue vertical line) was $7 \times 10^{-11}$ and $6.5 \times 10^{-11}$ in Hanwoo and Holstein, respectively. 

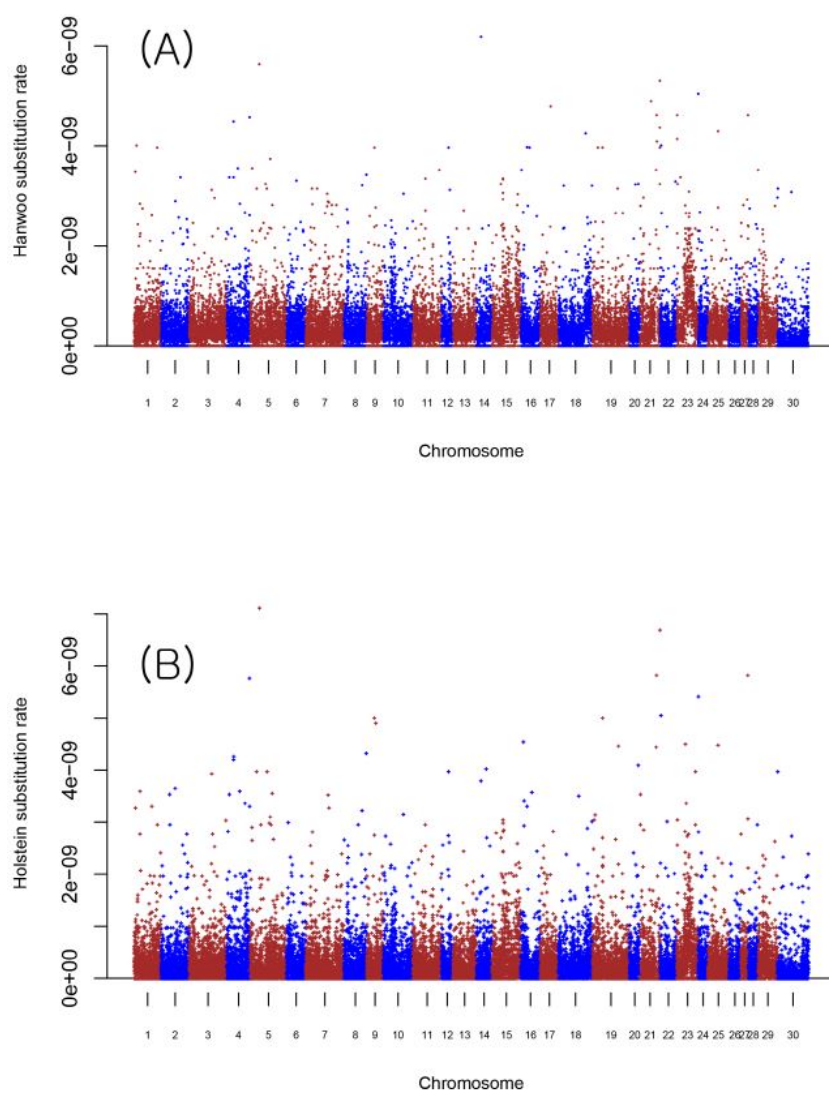

Fig. 3. Manhattan plot of Hanwoo (A) and Holstein (B) across chromosomes.

\section{Substitution rate}

Fig. 2 shows the density of Hanwoo (Fig. 2A) and Holstein (Fig. 2B) substitution rates. The density of substitution rates showed the presence of a region in which the density showed significant decrease and increase. We might consider that this diving region differentiated the protected regions from unprotected regions. The substitution rates of the boundary regions in Hanwoo and Holstein were $7 \times 10^{-11}$ and $6.5 \times$ $10^{-11}$, respectively $(/ \mathrm{bp} / \mathrm{y})$.

Fig. 3 shows Manhattan plot of substitution rates. It shows that the distribution of substitution rates between Hanwoo and Holstein was somewhat different across the genome. Fig. 4 shows the genetic substitution rate of Holstein against that of Hanwoo.

\section{GO in Hanwoo and Holstein}

Table 1 shows the results of a DAVID analysis of GO terms for genes with low substitution rates (Hanwoo $<7 \times 10^{-11}$; Holstein $<6.5 \times 10^{-11}$ ). The criteria was substitution rates below diving regions. Most terms were regulation. It is obvious that regulatory genes would have low substitution rates. Table 2 shows GO terms of genes with different substitution rates between Hanwoo and Holstein. The major $\mathrm{GO}$ terms were related to blood and circulatory system. The criteria of genes was an over 20-fold difference in substitution rate between Hanwoo and Holstein (Fig. 4). Supplementary Table 1 shows the total genetic substitution rates and those ratios between Hanwoo and Holstein for protein-coding genes. The averages of gene substitution rates in Hanwoo

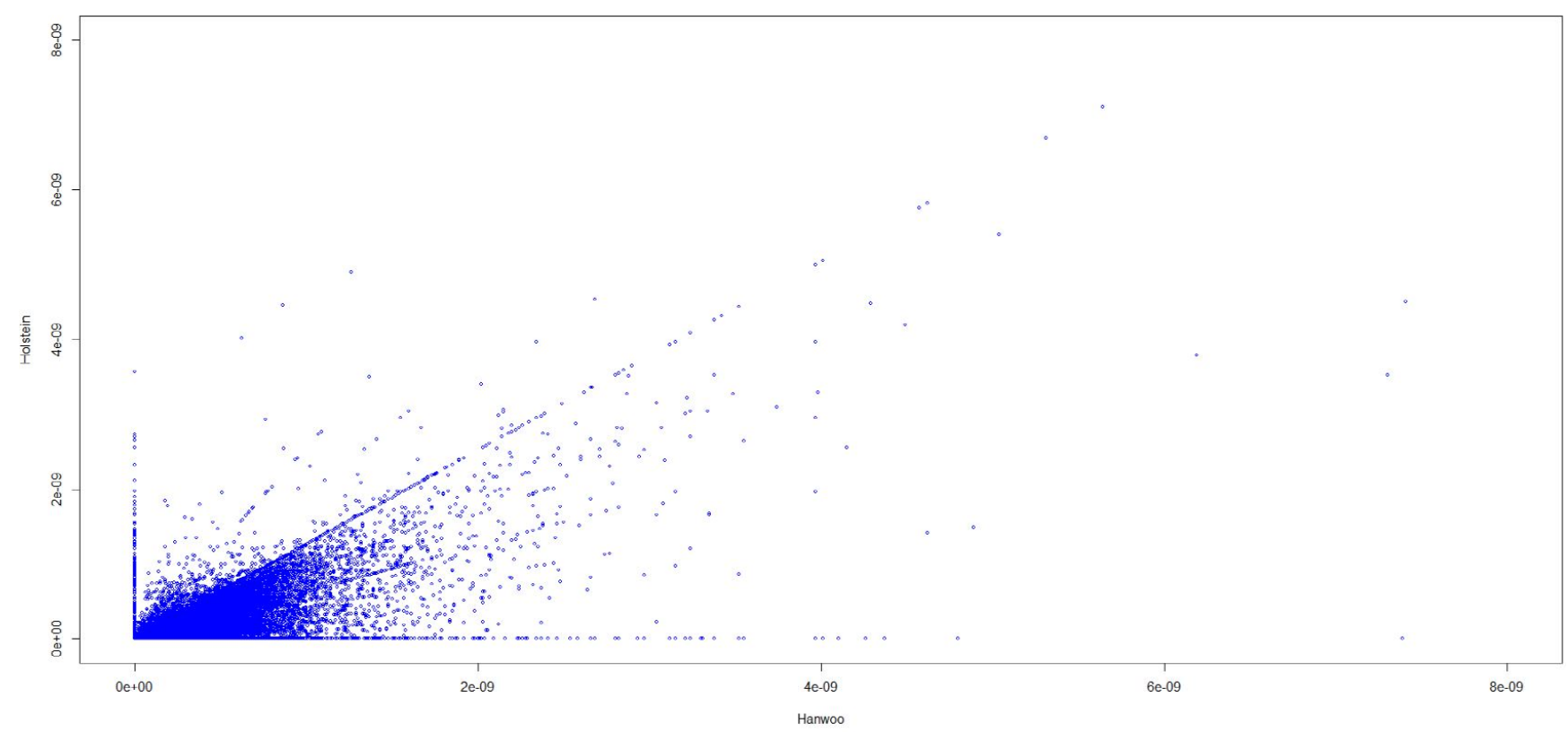

Fig. 4. The substitution rates of Holstein against Hanwoo. It showed the nonlinearlity of substitution rates between Hanwoo and Holstein. 
YS Lee and D Shin. Estimation of Genetic Substitution Rate Using Whole Genome Sequencing Data

Table 1. Gene ontology terms (GO terms) with low substitution rates of $7 \times 10^{-11}$ and $6.5 \times 10^{-11}$ in Hanwoo and Holstein, respectively

\begin{tabular}{lcc}
\hline \multicolumn{1}{c}{ Term } & Count & p-value \\
\hline GO:0009952; anterior/posterior pattern specification & 10 & $4.79 \mathrm{E}-05$ \\
GO:0006357; regulation of transcription from RNA polymerase II promoter & 18 & $1.23 \mathrm{E}-04$ \\
GO:0000122; negative regulation of transcription from RNA polymerase II promoter & 24 & $3.22 \mathrm{E}-04$ \\
GO:0006351; transcription, DNA-templated & 29 & 0.001 \\
GO:0045944; positive regulation of transcription from RNA polymerase II promoter & 27 & 0.002 \\
GO:0043518; negative regulation of DNA damage response, signal transduction by p53 class mediator & 4 & 0.003 \\
GO:0060828; regulation of canonical Wnt signaling pathway & 4 \\
GO:0045668; negative regulation of osteoblast differentiation & 0.004 \\
GO:0007264; small GTPase mediated signal transduction & 0.008 \\
GO:0032525; somite rostral/caudal axis specification & 12 \\
GO:0001764; neuron migration & 3 \\
GO:0048701; embryonic cranial skeleton morphogenesis & 0.0106 \\
GO:0007219; Notch signaling pathway & 0.015 \\
GO:0007519; skeletal muscle tissue development & 0.017 \\
GO:0007413; axonal fasciculation & 4 \\
GO:0045671; negative regulation of osteoclast differentiation & 6.031 \\
GO:0048704; embryonic skeletal system morphogenesis & 6 & 0.036 \\
GO:0021722; superior olivary nucleus maturation & 4 & 0.037 \\
GO:0050725; positive regulation of interleukin-1 beta biosynthetic process & 3 \\
GO:0032078; negative regulation of endodeoxyribonuclease activity & 0.039 \\
GO:0021568; rhombomere 2 development & 3 \\
GO:0061104; adrenal chromaffin cell differentiation & 4 & 0.039 \\
\hline The & 0.043 \\
\hline
\end{tabular}

The analysis shows that the regulatory genes have low substitution rates.

Table 2. GO of the genes based on Hanwoo/Holstein substitution rate ratio

\begin{tabular}{lcc}
\hline \multicolumn{1}{c}{ Term } & Count & p-value \\
\hline GO:0043933; macromolecular complex subunit organization & 18 & 0.002 \\
GO:0065003; macromolecular complex assembly & 17 & 0.003 \\
GO:0006461; protein complex assembly & 12 & 0.006 \\
GO:0070271; protein complex biogenesis & 12 & 0.006 \\
GO:0050880; regulation of blood vessel size & 5 & 0.007 \\
GO:0035150; regulation of tube size & 5 & 0.007 \\
GO:0003018; vascular process in circulatory system & 5 & 0.007 \\
GO:0003013; circulatory system process & 7 \\
GO:0008015; blood circulation & 0.009 \\
GO:0009303; rRNA transcription & 7 & 0.009 \\
GO:0008217; regulation of blood pressure & 3 & 0.012 \\
GO:0006351; transcription, DNA-dependent & 5 & 0.02 \\
GO:0006940; regulation of smooth muscle contraction & 6 & 0.03 \\
GO:0003044; regulation of systemic arterial blood pressure mediated by a chemical signal & 3 & 0.03 \\
GO:0048754; branching morphogenesis of a tube & 3 & 0.04 \\
GO:0032774; RNA biosynthetic process & 4 & 0.04 \\
GO:0006813; potassium ion transport & 6 & 0.04 \\
GO:0002035; brain renin-angiotensin system & 7 \\
GO:0002016; regulation of blood volume by renin-angiotensin & 0.04 \\
GO:0003072; renal control of peripheral vascular resistance involved in regulation of systemic arterial blood pressure & 2 \\
\hline The ratio was above 20-fold in Hanwoo compard to Holstin. & 0.05 \\
\hline
\end{tabular}

The ratio was above 20-fold in Hanwoo compared to Holstein. It is noteworthy that blood and circulatory system gene ontology (GO) terms were frequent. We suggest that these categories can differentiate the evolution of Hanwoo from that of Holstein. 
and Holstein were $4.1 \times 10^{-10}$ and $2.9 \times 10^{-10}$. This was concordant with the simulation study of substitution rates (see Materials and Methods). We used the pseudocount $10^{-13}$ where the substitution rate in Holstein was estimated at zero to calculate the ratios.

\section{Discussion}

\section{HSF estimation}

The HSF can be viewed as the number of individuals requiring to explain the substitution rates present in a population. In SNP chip data, the HSF can be as close as 1 . Founder effect can decrease nucleotide diversity and number of SNPs [23, 24]. Thus, smaller founder number can decrease the number of SNPs. Additionally, a population expansion after bottleneck events can increase the number of SNPs. Thus HSF should be estimated carefully, taking care of population history such as bottleneck event and population expansion.

The genes in Hanwoo and Holstein have average substitution rates on the order of $10^{-10}$. CNVs, microsatellites and minisatellites can also cause mutations. Therefore, it is very difficult to estimate the mutation rates with great accuracy. Instead, we focused on substitution rates which could be estimated using SNPs. The substitution rates on the order of $10^{-10}$ in Hanwoo and Holstein were similar to the human mutation rates [22].

The evolutionary distance can be assumed to be linear with HSF and the harmonic number of sample size $\left(a_{1}\right)$. Like Watterson's theta estimator, the number of SNPs increases with sample size and thus we assumed that the evolutionary distance was linear with the harmonic mean of sample size $\left(a_{1}\right)$. Because HSF represents the independent substitution unit in the population, thus is linear with the number of SNPs in the population as Eq. (1).

\section{Substitution diving region}

The gene ontology (GO terms) analysis for genes with the lowest substitution rates in Hanwoo and Holstein showed that regulatory genes belong to the low substitution rate gene set. Regulatory genes might be protected genes. The existence of boundaries between the highest and lowest substitution rates showed that there were gaps in substitution rate between protected genes (lowest substitution rates) and unprotected genes (highest substitution rates). Such diving regions might not be the special case in Hanwoo or Holstein. This could provide evidence of differentiation between protected genes and unprotected genes.

\section{GO results differentiating Hanwoo from Holstein}

The differentiation of Hanwoo from Holstein was identified by comparing the genetic substitution rate at the gene level. In GO analysis of differentially substituted genes between Hanwoo and Holstein, the blood and circulatory system terms were most common. The disparate evolutionary patterns after domestication between Hanwoo and Holstein could be caused by these genes. Hanwoo, which mainly provides meat, and Holstein, which mainly provides milk, might evolve differently, and blood and circulatory system-related GO genes could play some role in the evolution of Hanwoo and Holstein.

ORCID: Young-Sup Lee: http://orcid.org/0000-0002-08190553; Donghyun Shin: http://orcid.org/0000-0001-92670850

\section{Authors' contribution}

Conceptualization: YSL

Data curation: DS

Formal analysis: YSL

Methodology: YSL

Writing: YSL

Writing - review \& editing: YSL, DS

\section{Acknowledgments}

This work was supported by a grant from the Next Generation BioGreen21 project (No. PJ011044), Rural Development Administration, Republic of Korea. In addition, this research was supported by the Basic Science Research Program through the National Research Foundation of Korea (NRF) funded by the Ministry of Education (No.2017R 1A6A3A11033784).

\section{Supplementary material}

Supplementary data including one table can be found with this article online at http://www.genominfo.org/src/ sm/gni-16-14-s001.pdf.

\section{References}

1. Kumar S, Subramanian S. Mutation rates in mammalian genomes. Proc Natl Acad Sci U S A 2002;99:803-808.

2. Lee KT, Chung WH, Lee SY, Choi JW, Kim J, Lim D, et al. Whole-genome resequencing of Hanwoo (Korean cattle) and insight into regions of homozygosity. BMC Genomics 2013;14: 519.

3. Melka HD, Jeon EK, Kim SW, Han JB, Yoon D, Kim KS. Identification of genomic differences between Hanwoo and Holstein breeds using the Illumina Bovine SNP50 BeadChip. Genomics Inform 2011;9:69-73. 
4. Lee HJ, Kim J, Lee T, Son JK, Yoon HB, Baek KS, et al. Deciphering the genetic blueprint behind Holstein milk proteins and production. Genome Biol Evol 2014;6:1366-1374.

5. Rhee MS, Ryu YC, Imm JY, Kim BC. Combination of low voltage electrical stimulation and early postmortem temperature conditioning on degradation of myofibrillar proteins in Korean native cattle (Hanwoo). Meat Sci 2000;55:391-396.

6. Lee SH, Park BH, Sharma A, Dang CG, Lee SS, Choi TJ, et al. Hanwoo cattle: origin, domestication, breeding strategies and genomic selection. J Anim Sci Technol 2014;56:2.

7. Srivathsan A, Meier R. On the inappropriate use of Kimura2-parameter (K2P) divergences in the DNA-barcoding literature. Cladistics 2012;28:190-194.

8. Yang Z. Estimating the pattern of nucleotide substitution. $J$ Mol Evol 1994;39:105-111.

9. Yang Z. Statistical properties of the maximum likelihood method of phylogenetic estimation and comparison with distance matrix methods. Syst Biol 1994;43:329-342.

10. Steel MA, Fu YX. Classifying and counting linear phylogenetic invariants for the Jukes-Cantor model. J Comput Biol 1995;2: 39-47.

11. Bolger AM, Lohse M, Usadel B. Trimmomatic: a flexible trimmer for Illumina sequence data. Bioinformatics 2014;30:21142120.

12. Langmead B, Salzberg SL. Fast gapped-read alignment with Bowtie 2. Nat Methods 2012;9:357-359.

13. McKenna A, Hanna M, Banks E, Sivachenko A, Cibulskis K, Kernytsky A, et al. The Genome Analysis Toolkit: a MapReduce framework for analyzing next-generation DNA sequencing data. Genome Res 2010;20:1297-1303.

14. Li H, Handsaker B, Wysoker A, Fennell T, Ruan J, Homer N, et al. The sequence Alignment/Map format and SAMtools.
Bioinformatics 2009;25:2078-2079.

15. Danecek P, Auton A, Abecasis G, Albers CA, Banks E, DePristo $\mathrm{MA}$, et al. The variant call format and VCFtools. Bioinformatics 2011;27:2156-2158.

16. Cingolani P, Platts A, Wang le L, Coon M, Nguyen T, Wang L, et al. A program for annotating and predicting the effects of single nucleotide polymorphisms, SnpEff: SNPs in the genome of Drosophila melanogaster strain w1118; iso-2; iso-3. Fly (Austin) 2012;6:80-92.

17. Kimura M. A simple method for estimating evolutionary rates of base substitutions through comparative studies of nucleotide sequences. J Mol Evol 1980;16:111-120.

18. Tajima F, Nei M. Estimation of evolutionary distance between nucleotide sequences. Mol Biol Evol 1984;1:269-285.

19. Casanellas M, Fernández-Sánchez J. Geometry of the Kimura 3-parameter model. Adv Appl Math 2008;41:265-292.

20. Murray C, Huerta-Sanchez E, Casey F, Bradley DG. Cattle demographic history modelled from autosomal sequence variation. Philos Trans R Soc Lond B Biol Sci 2010;365:25312539.

21. Bovine HapMap Consortium, Gibbs RA, Taylor JF, Van Tassell CP, Barendse W, Eversole KA, et al. Genome-wide survey of SNP variation uncovers the genetic structure of cattle breeds. Science 2009;324:528-532.

22. Roach JC, Glusman G, Smit AF, Huff CD, Hubley R, Shannon $\mathrm{PT}$, et al. Analysis of genetic inheritance in a family quartet by whole-genome sequencing. Science 2010;328:636-639.

23. Nei M, Maruyama T, Chakraborty R. The bottleneck effect and genetic variability in populations. Evolution 1975;29:1-10.

24. Ladizinsky G. Founder effect in crop-plant evolution. Econ Bot 1985;39:191-199. 


\section{SUPPLEMENTARY INFORMATION}

\section{Estimation of the Genetic Substitution Rate of Hanwoo and}

Holstein Cattle Using Whole Genome Sequencing Data

\section{Young-Sup Lee, Donghyun Shin*}

Department of Animal Biotechnology, Chonbuk National University, Jeonju 54896, Korea 
Supplementary Table $\mathbf{1}$. The table of genetic substitution rates of protein coding genes

\begin{tabular}{|c|c|c|c|c|}
\hline Ensembl Gene ID & substitution rate of Hanwoo & substitution rate of Holstein & gene name & Hanwoo/Holstein substi ratio \\
\hline ENSBTAG00000045984 & 0 & $1.40 \mathrm{E}-10$ & & 7.14E-04 \\
\hline ENSBTAG00000047183 & 0 & $2.86 \mathrm{E}-10$ & & $3.50 \mathrm{E}-04$ \\
\hline ENSBTAG00000011631 & 0 & $6.65 \mathrm{E}-10$ & YWHAH & $1.50 \mathrm{E}-04$ \\
\hline ENSBTAG00000037547 & 0 & $1.24 \mathrm{E}-10$ & OLIG1 & $8.06 \mathrm{E}-04$ \\
\hline ENSBTAG00000046804 & 0 & $7.86 \mathrm{E}-10$ & KRTAP7-1 & $1.27 \mathrm{E}-04$ \\
\hline ENSBTAG00000037820 & 0 & $8.96 \mathrm{E}-10$ & & $1.12 \mathrm{E}-04$ \\
\hline ENSBTAG00000048225 & 0 & $1.47 \mathrm{E}-10$ & & $6.80 \mathrm{E}-04$ \\
\hline ENSBTAG00000015069 & $2.21 \mathrm{E}-11$ & $2.09 \mathrm{E}-10$ & PCNP & $1.06 \mathrm{E}-01$ \\
\hline ENSBTAG00000024027 & $3.87 E-11$ & $1.95 \mathrm{E}-10$ & TRMT10C & $1.99 \mathrm{E}-01$ \\
\hline ENSBTAG00000019514 & $9.71 \mathrm{E}-11$ & $3.70 \mathrm{E}-10$ & OPA1 & $2.63 \mathrm{E}-01$ \\
\hline ENSBTAG00000046790 & $5.23 \mathrm{E}-11$ & $1.98 \mathrm{E}-10$ & & 2.65E-01 \\
\hline ENSBTAG00000013460 & $5.85 \mathrm{E}-11$ & $2.21 \mathrm{E}-10$ & ZBTB11 & $2.65 \mathrm{E}-01$ \\
\hline ENSBTAG00000000605 & $5.60 \mathrm{E}-11$ & $2.12 \mathrm{E}-10$ & ATP5J & $2.64 \mathrm{E}-01$ \\
\hline ENSBTAG00000043957 & 4.64E-11 & $1.76 \mathrm{E}-10$ & TDRP & $2.64 \mathrm{E}-01$ \\
\hline ENSBTAG00000003064 & $4.74 \mathrm{E}-11$ & $1.60 \mathrm{E}-10$ & PAXBP1 & $2.96 \mathrm{E}-01$ \\
\hline ENSBTAG00000018278 & $2.01 \mathrm{E}-10$ & $6.19 \mathrm{E}-10$ & & $3.24 \mathrm{E}-01$ \\
\hline ENSBTAG00000012594 & $1.31 \mathrm{E}-10$ & $3.78 \mathrm{E}-10$ & MRPS6 & 3.47E-01 \\
\hline ENSBTAG00000018508 & $1.71 \mathrm{E}-10$ & $4.85 \mathrm{E}-10$ & TMEM108 & $3.54 \mathrm{E}-01$ \\
\hline ENSBTAG00000005203 & 2.77E-10 & $7.57 \mathrm{E}-10$ & C3orf33 & $3.66 \mathrm{E}-01$ \\
\hline ENSBTAG00000015212 & $1.09 \mathrm{E}-10$ & $2.96 \mathrm{E}-10$ & IFNAR2 & $3.68 \mathrm{E}-01$ \\
\hline ENSBTAG00000043993 & $3.38 \mathrm{E}-10$ & $8.66 \mathrm{E}-10$ & C21orf62 & $3.91 \mathrm{E}-01$ \\
\hline ENSBTAG00000034869 & $9.31 \mathrm{E}-11$ & $2.35 \mathrm{E}-10$ & KRTAP11-1 & $3.96 \mathrm{E}-01$ \\
\hline ENSBTAG00000011116 & 3.93E-11 & $9.92 \mathrm{E}-11$ & PAQR9 & $3.97 \mathrm{E}-01$ \\
\hline ENSBTAG00000030862 & $1.69 \mathrm{E}-10$ & $4.26 \mathrm{E}-10$ & & $3.96 \mathrm{E}-01$ \\
\hline ENSBTAG00000009622 & $5.38 \mathrm{E}-10$ & $1.27 \mathrm{E}-09$ & & 4.24E-01 \\
\hline ENSBTAG00000007068 & $1.35 \mathrm{E}-10$ & $3.02 \mathrm{E}-10$ & SH3BGR & 4.47E-01 \\
\hline ENSBTAG00000014233 & $1.25 \mathrm{E}-10$ & $2.78 \mathrm{E}-10$ & & $4.50 \mathrm{E}-01$ \\
\hline ENSBTAG00000017659 & $2.67 \mathrm{E}-10$ & $5.78 \mathrm{E}-10$ & BFSP2 & 4.62E-01 \\
\hline ENSBTAG00000016932 & $1.28 \mathrm{E}-10$ & $2.70 \mathrm{E}-10$ & SENP7 & 4.74E-01 \\
\hline ENSBTAG00000013464 & 8.07E-11 & $1.65 \mathrm{E}-10$ & CEP97 & $4.90 \mathrm{E}-01$ \\
\hline ENSBTAG00000013319 & $2.18 \mathrm{E}-10$ & 4.34E-10 & SMCO1 & $5.02 \mathrm{E}-01$ \\
\hline ENSBTAG00000014318 & $4.51 \mathrm{E}-10$ & $8.74 \mathrm{E}-10$ & SLC33A1 & $5.16 \mathrm{E}-01$ \\
\hline ENSBTAG00000046008 & $2.42 \mathrm{E}-10$ & $4.58 \mathrm{E}-10$ & & $5.28 \mathrm{E}-01$ \\
\hline ENSBTAG00000005540 & $3.01 \mathrm{E}-10$ & $5.69 \mathrm{E}-10$ & CBX3 & $5.28 \mathrm{E}-01$ \\
\hline ENSBTAG00000015662 & $1.43 \mathrm{E}-10$ & 2.63E-10 & IMPG2 & 5.44E-01 \\
\hline ENSBTAG00000026260 & $1.15 \mathrm{E}-10$ & $2.12 \mathrm{E}-10$ & KCNE2 & $5.45 \mathrm{E}-01$ \\
\hline ENSBTAG00000018025 & $2.66 \mathrm{E}-10$ & $4.83 \mathrm{E}-10$ & IQCG & $5.51 \mathrm{E}-01$ \\
\hline ENSBTAG00000013461 & $1.49 \mathrm{E}-10$ & $2.63 \mathrm{E}-10$ & RPL24 & 5.67E-01 \\
\hline ENSBTAG00000020122 & $9.19 \mathrm{E}-11$ & $1.62 \mathrm{E}-10$ & USP16 & $5.68 \mathrm{E}-01$ \\
\hline ENSBTAG00000018009 & $2.84 \mathrm{E}-10$ & $4.90 \mathrm{E}-10$ & & $5.80 \mathrm{E}-01$ \\
\hline ENSBTAG00000021490 & $3.59 \mathrm{E}-10$ & $6.08 \mathrm{E}-10$ & WDR4 & $5.91 \mathrm{E}-01$ \\
\hline ENSBTAG00000018749 & $1.58 \mathrm{E}-10$ & $2.65 \mathrm{E}-10$ & NUDT16 & $5.94 \mathrm{E}-01$ \\
\hline ENSBTAG00000017312 & $1.70 \mathrm{E}-10$ & $2.86 \mathrm{E}-10$ & SST & $5.96 \mathrm{E}-01$ \\
\hline ENSBTAG00000001497 & $3.50 \mathrm{E}-10$ & $5.69 \mathrm{E}-10$ & MRAS & $6.15 \mathrm{E}-01$ \\
\hline ENSBTAG00000018471 & $8.01 \mathrm{E}-11$ & $1.28 \mathrm{E}-10$ & SSR3 & $6.26 \mathrm{E}-01$ \\
\hline ENSBTAG00000035175 & $2.19 \mathrm{E}-10$ & $3.48 \mathrm{E}-10$ & DONSON & $6.31 \mathrm{E}-01$ \\
\hline ENSBTAG00000017310 & $3.22 \mathrm{E}-10$ & $5.11 \mathrm{E}-10$ & EVA1C & $6.30 \mathrm{E}-01$ \\
\hline ENSBTAG00000011986 & $2.86 \mathrm{E}-10$ & 4.39E-10 & PLSCR4 & $6.51 \mathrm{E}-01$ \\
\hline ENSBTAG00000011150 & $1.84 \mathrm{E}-10$ & $2.78 \mathrm{E}-10$ & PFN2 & $6.62 \mathrm{E}-01$ \\
\hline ENSBTAG00000009128 & $3.70 \mathrm{E}-10$ & $5.52 \mathrm{E}-10$ & POU1F1 & $6.71 \mathrm{E}-01$ \\
\hline ENSBTAG00000009187 & $3.19 \mathrm{E}-10$ & $4.68 \mathrm{E}-10$ & DNAJC28 & $6.81 \mathrm{E}-01$ \\
\hline ENSBTAG00000010448 & $4.90 \mathrm{E}-10$ & 7.15E-10 & & $6.85 \mathrm{E}-01$ \\
\hline ENSBTAG00000013841 & $5.65 \mathrm{E}-10$ & $8.14 \mathrm{E}-10$ & C21orf140 & $6.94 \mathrm{E}-01$ \\
\hline ENSBTAG00000045633 & $5.33 \mathrm{E}-10$ & $7.68 \mathrm{E}-10$ & AGTR1 & $6.94 \mathrm{E}-01$ \\
\hline ENSBTAG00000033437 & $6.10 \mathrm{E}-10$ & $8.79 \mathrm{E}-10$ & & $6.94 \mathrm{E}-01$ \\
\hline ENSBTAG00000011528 & $6.19 \mathrm{E}-10$ & $8.81 \mathrm{E}-10$ & SMIM11A & 7.03E-01 \\
\hline ENSBTAG00000032839 & $1.83 \mathrm{E}-10$ & $2.60 \mathrm{E}-10$ & $\mathrm{LRCH} 3$ & 7.04E-01 \\
\hline ENSBTAG00000018855 & $9.06 \mathrm{E}-11$ & $1.28 \mathrm{E}-10$ & SCAF4 & $7.08 \mathrm{E}-01$ \\
\hline ENSBTAG00000014874 & $1.99 \mathrm{E}-10$ & $2.78 \mathrm{E}-10$ & FYTTD1 & 7.15E-01 \\
\hline ENSBTAG00000019014 & 4.64E-10 & $6.50 \mathrm{E}-10$ & MAGEF1 & 7.14E-01 \\
\hline ENSBTAG00000003063 & $5.56 \mathrm{E}-11$ & $7.72 \mathrm{E}-11$ & SYNJ1 & $7.20 \mathrm{E}-01$ \\
\hline ENSBTAG00000005862 & $1.56 \mathrm{E}-10$ & $2.15 \mathrm{E}-10$ & SMC4 & 7.24E-01 \\
\hline ENSBTAG00000005313 & $1.13 \mathrm{E}-10$ & $1.54 \mathrm{E}-10$ & EPHB3 & 7.33E-01 \\
\hline ENSBTAG00000010874 & $3.49 \mathrm{E}-10$ & $4.71 \mathrm{E}-10$ & GHSR & 7.41E-01 \\
\hline ENSBTAG00000009263 & 3.33E-10 & $4.47 \mathrm{E}-10$ & MFSD1 & $7.45 \mathrm{E}-01$ \\
\hline ENSBTAG00000007939 & $3.07 E-10$ & $4.12 \mathrm{E}-10$ & $\mathrm{CHMP} 2 \mathrm{~B}$ & $7.46 \mathrm{E}-01$ \\
\hline ENSBTAG00000000448 & $3.07 E-10$ & 4.07E-10 & $\mathrm{BDH} 1$ & 7.53E-01 \\
\hline
\end{tabular}




\begin{tabular}{|c|c|c|c|c|}
\hline ENSBTAG00000019043 & $9.25 \mathrm{E}-11$ & $1.22 \mathrm{E}-10$ & GABPA & $7.59 \mathrm{E}-01$ \\
\hline ENSBTAG00000006909 & $3.76 \mathrm{E}-10$ & $4.95 \mathrm{E}-10$ & РIKЗСВ & 7.59E-01 \\
\hline ENSBTAG00000012164 & $6.91 \mathrm{E}-10$ & $9.03 \mathrm{E}-10$ & $\mathrm{CP}$ & 7.66E-01 \\
\hline ENSBTAG00000009188 & $1.90 \mathrm{E}-10$ & $2.48 \mathrm{E}-10$ & GART & 7.67E-01 \\
\hline ENSBTAG00000021139 & $8.56 \mathrm{E}-10$ & $1.11 \mathrm{E}-09$ & & $7.71 \mathrm{E}-01$ \\
\hline ENSBTAG00000021253 & $7.29 \mathrm{E}-10$ & $9.34 \mathrm{E}-10$ & PRDM15 & $7.80 \mathrm{E}-01$ \\
\hline ENSBTAG00000005066 & $1.52 \mathrm{E}-10$ & $1.93 \mathrm{E}-10$ & HSPBAP1 & 7.87E-01 \\
\hline ENSBTAG00000012899 & $4.24 \mathrm{E}-10$ & $5.39 \mathrm{E}-10$ & IFNGR2 & 7.87E-01 \\
\hline ENSBTAG00000008471 & $8.30 \mathrm{E}-10$ & 1.05E-09 & MX2 & $7.90 \mathrm{E}-01$ \\
\hline ENSBTAG00000025847 & $1.55 \mathrm{E}-10$ & $1.96 \mathrm{E}-10$ & & 7.93E-01 \\
\hline ENSBTAG00000011495 & $6.37 \mathrm{E}-10$ & $8.05 \mathrm{E}-10$ & DIP2A & $7.92 \mathrm{E}-01$ \\
\hline ENSBTAG00000047978 & $2.15 \mathrm{E}-10$ & $2.71 \mathrm{E}-10$ & & 7.94E-01 \\
\hline ENSBTAG00000019007 & 2.05E-10 & $2.59 \mathrm{E}-10$ & & $7.93 \mathrm{E}-01$ \\
\hline ENSBTAG00000014208 & $1.90 \mathrm{E}-10$ & $2.40 \mathrm{E}-10$ & & 7.94E-01 \\
\hline ENSBTAG00000006388 & $4.46 \mathrm{E}-10$ & $5.62 \mathrm{E}-10$ & FBXO45 & $7.94 \mathrm{E}-01$ \\
\hline ENSBTAG00000040155 & $5.27 \mathrm{E}-10$ & $6.64 \mathrm{E}-10$ & & $7.94 \mathrm{E}-01$ \\
\hline ENSBTAG00000002273 & $4.95 \mathrm{E}-10$ & $6.24 \mathrm{E}-10$ & ARGFX & 7.93E-01 \\
\hline ENSBTAG00000046620 & 1.17E-09 & 1.47E-09 & & 7.93E-01 \\
\hline ENSBTAG00000047076 & $2.01 \mathrm{E}-10$ & $2.53 \mathrm{E}-10$ & & 7.94E-01 \\
\hline ENSBTAG00000023593 & $9.54 \mathrm{E}-11$ & $1.20 \mathrm{E}-10$ & GMNC & 7.96E-01 \\
\hline ENSBTAG00000047095 & $7.09 \mathrm{E}-10$ & $8.93 \mathrm{E}-10$ & OR5AC2 & $7.94 \mathrm{E}-01$ \\
\hline ENSBTAG00000015812 & $8.59 \mathrm{E}-10$ & $1.08 \mathrm{E}-09$ & KRTAP8-1 & $7.96 \mathrm{E}-01$ \\
\hline ENSBTAG00000027213 & $8.02 \mathrm{E}-10$ & $1.01 \mathrm{E}-09$ & & $7.94 \mathrm{E}-01$ \\
\hline ENSBTAG00000018312 & $9.99 \mathrm{E}-10$ & $1.26 \mathrm{E}-09$ & & 7.93E-01 \\
\hline ENSBTAG00000048150 & $5.17 \mathrm{E}-10$ & $6.52 \mathrm{E}-10$ & & 7.93E-01 \\
\hline ENSBTAG00000027782 & $8.50 \mathrm{E}-11$ & $1.07 \mathrm{E}-10$ & & 7.95E-01 \\
\hline ENSBTAG00000010385 & $8.56 \mathrm{E}-11$ & $1.08 \mathrm{E}-10$ & & $7.93 \mathrm{E}-01$ \\
\hline ENSBTAG00000047371 & $8.11 \mathrm{E}-11$ & $1.02 \mathrm{E}-10$ & & $7.95 \mathrm{E}-01$ \\
\hline ENSBTAG00000030592 & $7.42 \mathrm{E}-10$ & $9.35 \mathrm{E}-10$ & & 7.94E-01 \\
\hline ENSBTAG00000030585 & $3.45 \mathrm{E}-10$ & 4.35E-10 & CLDN14 & 7.93E-01 \\
\hline ENSBTAG00000045590 & $1.01 \mathrm{E}-10$ & $1.27 \mathrm{E}-10$ & & $7.96 \mathrm{E}-01$ \\
\hline ENSBTAG00000046313 & $6.65 \mathrm{E}-11$ & $8.38 \mathrm{E}-11$ & CCDC54 & 7.94E-01 \\
\hline ENSBTAG00000038600 & $4.66 \mathrm{E}-11$ & $5.88 \mathrm{E}-11$ & LRRC15 & 7.94E-01 \\
\hline ENSBTAG00000019949 & $1.12 \mathrm{E}-10$ & $1.41 \mathrm{E}-10$ & DBR1 & 7.95E-01 \\
\hline ENSBTAG00000008359 & 1.17E-09 & $1.48 \mathrm{E}-09$ & & 7.93E-01 \\
\hline ENSBTAG00000008873 & $1.41 \mathrm{E}-10$ & $1.77 \mathrm{E}-10$ & NCBP2 & 7.95E-01 \\
\hline ENSBTAG00000008078 & $1.52 \mathrm{E}-10$ & $1.92 \mathrm{E}-10$ & TRIM59 & 7.94E-01 \\
\hline ENSBTAG00000009084 & $1.68 \mathrm{E}-10$ & $2.09 \mathrm{E}-10$ & ATG3 & $8.05 \mathrm{E}-01$ \\
\hline ENSBTAG00000036262 & 3.23E-10 & $3.99 \mathrm{E}-10$ & GBE1 & 8.09E-01 \\
\hline ENSBTAG00000008167 & $4.74 \mathrm{E}-10$ & $5.86 \mathrm{E}-10$ & TTC3 & 8.09E-01 \\
\hline ENSBTAG00000009012 & 4.34E-10 & $5.29 \mathrm{E}-10$ & PTX3 & 8.20E-01 \\
\hline ENSBTAG00000021997 & $5.05 \mathrm{E}-10$ & $6.16 \mathrm{E}-10$ & ITSN1 & $8.21 \mathrm{E}-01$ \\
\hline ENSBTAG00000018877 & $3.69 \mathrm{E}-10$ & $4.48 \mathrm{E}-10$ & PDIA5 & 8.23E-01 \\
\hline ENSBTAG00000002029 & $7.61 \mathrm{E}-10$ & $9.25 \mathrm{E}-10$ & IGSF5 & 8.23E-01 \\
\hline ENSBTAG00000023600 & $1.48 \mathrm{E}-10$ & $1.78 \mathrm{E}-10$ & APOD & $8.30 \mathrm{E}-01$ \\
\hline ENSBTAG00000011802 & $6.29 \mathrm{E}-10$ & $7.60 \mathrm{E}-10$ & COL6A1 & 8.27E-01 \\
\hline ENSBTAG00000023445 & $1.27 \mathrm{E}-10$ & $1.54 \mathrm{E}-10$ & $\mathrm{SIAH} 2$ & 8.23E- 01 \\
\hline ENSBTAG00000015010 & $1.79 \mathrm{E}-10$ & $2.14 \mathrm{E}-10$ & TBC1D23 & $8.34 \mathrm{E}-01$ \\
\hline ENSBTAG00000001150 & $5.06 \mathrm{E}-10$ & $6.06 \mathrm{E}-10$ & & $8.36 \mathrm{E}-01$ \\
\hline ENSBTAG00000001920 & $5.70 \mathrm{E}-10$ & $6.83 \mathrm{E}-10$ & POLQ & $8.35 \mathrm{E}-01$ \\
\hline ENSBTAG00000002395 & 4.64E-10 & $5.54 \mathrm{E}-10$ & HPS3 & $8.38 \mathrm{E}-01$ \\
\hline ENSBTAG00000001969 & $1.45 \mathrm{E}-10$ & $1.73 \mathrm{E}-10$ & CCDC39 & $8.41 \mathrm{E}-01$ \\
\hline ENSBTAG00000015895 & $4.18 \mathrm{E}-10$ & $4.98 \mathrm{E}-10$ & $\mathrm{HGD}$ & $8.40 \mathrm{E}-01$ \\
\hline ENSBTAG00000016026 & $4.79 \mathrm{E}-10$ & $5.70 \mathrm{E}-10$ & PCOLCE2 & $8.40 \mathrm{E}-01$ \\
\hline ENSBTAG00000048195 & 4.13E-10 & $4.92 \mathrm{E}-10$ & PIGX & $8.39 \mathrm{E}-01$ \\
\hline ENSBTAG00000018489 & $3.82 \mathrm{E}-10$ & $4.51 \mathrm{E}-10$ & OTOL1 & $8.46 \mathrm{E}-01$ \\
\hline ENSBTAG00000011512 & $5.11 \mathrm{E}-10$ & $6.03 \mathrm{E}-10$ & & $8.48 \mathrm{E}-01$ \\
\hline ENSBTAG00000032819 & $8.13 E-10$ & $9.56 \mathrm{E}-10$ & MUC20 & $8.51 \mathrm{E}-01$ \\
\hline ENSBTAG00000008307 & $4.25 \mathrm{E}-10$ & $5.00 \mathrm{E}-10$ & $\mathrm{PLCH} 1$ & 8.50E-01 \\
\hline ENSBTAG00000038069 & $2.14 \mathrm{E}-10$ & $2.51 \mathrm{E}-10$ & & $8.51 \mathrm{E}-01$ \\
\hline ENSBTAG00000012412 & $6.15 \mathrm{E}-10$ & $7.22 \mathrm{E}-10$ & URB1 & $8.52 \mathrm{E}-01$ \\
\hline ENSBTAG00000000526 & $7.90 \mathrm{E}-10$ & $9.27 \mathrm{E}-10$ & TOPBP1 & $8.52 \mathrm{E}-01$ \\
\hline ENSBTAG00000003851 & $2.65 \mathrm{E}-10$ & $3.10 \mathrm{E}-10$ & CCNL1 & 8.55E-01 \\
\hline ENSBTAG00000005071 & $1.35 \mathrm{E}-10$ & $1.57 \mathrm{E}-10$ & DIRC2 & $8.61 \mathrm{E}-01$ \\
\hline ENSBTAG00000021515 & $3.55 \mathrm{E}-10$ & 4.14E-10 & ZDHHC19 & 8.57E-01 \\
\hline ENSBTAG00000011423 & $5.34 \mathrm{E}-10$ & $6.21 \mathrm{E}-10$ & SERPINI2 & $8.60 \mathrm{E}-01$ \\
\hline ENSBTAG00000000603 & $5.29 \mathrm{E}-10$ & $6.15 \mathrm{E}-10$ & JAM2 & $8.60 \mathrm{E}-01$ \\
\hline ENSBTAG00000040393 & $2.59 \mathrm{E}-10$ & $3.02 \mathrm{E}-10$ & & $8.59 \mathrm{E}-01$ \\
\hline ENSBTAG00000001721 & 4.15E-10 & $4.81 \mathrm{E}-10$ & GYG1 & $8.63 \mathrm{E}-01$ \\
\hline ENSBTAG00000036019 & $4.86 \mathrm{E}-10$ & $5.64 \mathrm{E}-10$ & RIPPLY3 & $8.62 \mathrm{E}-01$ \\
\hline
\end{tabular}




\begin{tabular}{|c|c|c|c|c|}
\hline ENSBTAG00000000566 & $5.63 \mathrm{E}-10$ & $6.53 \mathrm{E}-10$ & CCDC14 & 8.63E-01 \\
\hline ENSBTAG00000006724 & $2.85 \mathrm{E}-10$ & $3.30 \mathrm{E}-10$ & & $8.63 \mathrm{E}-01$ \\
\hline ENSBTAG00000047856 & $3.06 \mathrm{E}-10$ & $3.54 \mathrm{E}-10$ & PAK2 & $8.66 \mathrm{E}-01$ \\
\hline ENSBTAG00000015056 & $4.77 \mathrm{E}-10$ & $5.51 \mathrm{E}-10$ & RBP2 & 8.66E-01 \\
\hline ENSBTAG00000004777 & $1.34 \mathrm{E}-10$ & $1.55 \mathrm{E}-10$ & S100B & 8.65E-01 \\
\hline ENSBTAG00000005604 & $6.62 \mathrm{E}-10$ & $7.58 \mathrm{E}-10$ & POPDC2 & $8.73 \mathrm{E}-01$ \\
\hline ENSBTAG00000014889 & $3.32 \mathrm{E}-10$ & $3.80 \mathrm{E}-10$ & DCBLD2 & 8.74E-01 \\
\hline ENSBTAG00000013909 & $1.44 \mathrm{E}-10$ & 1.65E-10 & $\mathrm{BOC}$ & 8.73E-01 \\
\hline ENSBTAG00000012798 & $5.21 \mathrm{E}-10$ & 5.94E-10 & $\mathrm{KCNH} 8$ & 8.77E-01 \\
\hline ENSBTAG00000023718 & $6.05 \mathrm{E}-10$ & $6.88 \mathrm{E}-10$ & MRAP & 8.79E-01 \\
\hline ENSBTAG00000004139 & $1.54 \mathrm{E}-10$ & $1.75 \mathrm{E}-10$ & $\mathrm{BACH} 1$ & $8.78 \mathrm{E}-01$ \\
\hline ENSBTAG00000023675 & $1.44 \mathrm{E}-10$ & $1.64 \mathrm{E}-10$ & & 8.77E-01 \\
\hline ENSBTAG00000014508 & $1.71 \mathrm{E}-10$ & $1.94 \mathrm{E}-10$ & $\mathrm{FBXO40}$ & $8.80 \mathrm{E}-01$ \\
\hline ENSBTAG00000031572 & $1.69 \mathrm{E}-10$ & $1.91 \mathrm{E}-10$ & & 8.83E-01 \\
\hline ENSBTAG00000030913 & $7.18 \mathrm{E}-10$ & $8.11 E-10$ & $M \times 1$ & $8.86 \mathrm{E}-01$ \\
\hline ENSBTAG00000008862 & $2.31 \mathrm{E}-10$ & $2.60 \mathrm{E}-10$ & GOLGB1 & 8.89E-01 \\
\hline ENSBTAG00000021364 & $3.03 \mathrm{E}-10$ & $3.41 \mathrm{E}-10$ & TNK2 & $8.88 \mathrm{E}-01$ \\
\hline ENSBTAG00000018397 & $1.44 \mathrm{E}-10$ & $1.62 \mathrm{E}-10$ & HHLA2 & $8.89 \mathrm{E}-01$ \\
\hline ENSBTAG00000021270 & $7.43 \mathrm{E}-10$ & 8.36E-10 & UMODL1 & $8.88 \mathrm{E}-01$ \\
\hline ENSBTAG00000024643 & $4.32 \mathrm{E}-10$ & $4.86 \mathrm{E}-10$ & VEPH1 & $8.90 \mathrm{E}-01$ \\
\hline ENSBTAG00000019704 & $3.42 \mathrm{E}-10$ & $3.83 \mathrm{E}-10$ & HLTF & $8.92 \mathrm{E}-01$ \\
\hline ENSBTAG00000001258 & $3.41 \mathrm{E}-10$ & $3.82 \mathrm{E}-10$ & TMEM50B & 8.93E-01 \\
\hline ENSBTAG00000046935 & $8.90 \mathrm{E}-10$ & $9.96 \mathrm{E}-10$ & & 8.93E-01 \\
\hline ENSBTAG00000032674 & $2.75 \mathrm{E}-10$ & $3.08 \mathrm{E}-10$ & SENP5 & 8.94E-01 \\
\hline ENSBTAG00000020378 & $1.54 \mathrm{E}-10$ & $1.72 \mathrm{E}-10$ & DLG1 & 8.93E-01 \\
\hline ENSBTAG00000000706 & $3.31 \mathrm{E}-10$ & $3.69 \mathrm{E}-10$ & ADAMTS1 & 8.97E-01 \\
\hline ENSBTAG00000018765 & $1.25 \mathrm{E}-10$ & $1.39 \mathrm{E}-10$ & SEMA5B & $9.02 \mathrm{E}-01$ \\
\hline ENSBTAG00000003865 & $3.44 \mathrm{E}-10$ & $3.82 \mathrm{E}-10$ & & $9.02 \mathrm{E}-01$ \\
\hline ENSBTAG00000017882 & $8.85 \mathrm{E}-10$ & $9.80 \mathrm{E}-10$ & LRRC3 & $9.03 \mathrm{E}-01$ \\
\hline ENSBTAG00000019542 & $1.72 \mathrm{E}-10$ & $1.90 \mathrm{E}-10$ & MRPL39 & $9.04 \mathrm{E}-01$ \\
\hline ENSBTAG00000031306 & 7.91E-10 & $8.74 \mathrm{E}-10$ & PLSCR1 & 9.05E-01 \\
\hline ENSBTAG00000008299 & $5.99 \mathrm{E}-10$ & $6.61 \mathrm{E}-10$ & IL2ORB & 9.07E-01 \\
\hline ENSBTAG00000046447 & $9.22 \mathrm{E}-10$ & 1.01E-09 & & $9.13 \mathrm{E}-01$ \\
\hline ENSBTAG00000014960 & $3.36 \mathrm{E}-10$ & $3.68 \mathrm{E}-10$ & & $9.12 \mathrm{E}-01$ \\
\hline ENSBTAG00000020035 & $4.55 \mathrm{E}-10$ & 4.99E-10 & RCAN1 & $9.12 \mathrm{E}-01$ \\
\hline ENSBTAG00000009848 & $2.89 \mathrm{E}-10$ & $3.16 \mathrm{E}-10$ & GPR171 & $9.13 \mathrm{E}-01$ \\
\hline ENSBTAG00000009214 & 7.74E-10 & 8.47E-10 & ETS2 & $9.14 \mathrm{E}-01$ \\
\hline ENSBTAG00000018346 & $7.55 \mathrm{E}-10$ & $8.25 \mathrm{E}-10$ & TRIM42 & $9.15 \mathrm{E}-01$ \\
\hline ENSBTAG00000030942 & $5.95 \mathrm{E}-10$ & $6.49 \mathrm{E}-10$ & MRPL3 & $9.17 \mathrm{E}-01$ \\
\hline ENSBTAG00000009115 & $5.52 \mathrm{E}-10$ & $6.00 \mathrm{E}-10$ & SPSB4 & $9.21 \mathrm{E}-01$ \\
\hline ENSBTAG00000039011 & $4.79 \mathrm{E}-10$ & $5.18 \mathrm{E}-10$ & HTR3D & $9.26 \mathrm{E}-01$ \\
\hline ENSBTAG00000020028 & $3.78 \mathrm{E}-10$ & 4.07E-10 & RBP1 & $9.28 \mathrm{E}-01$ \\
\hline ENSBTAG00000013602 & $1.24 \mathrm{E}-10$ & $1.33 \mathrm{E}-10$ & BRWD1 & $9.31 \mathrm{E}-01$ \\
\hline ENSBTAG00000015163 & $3.18 \mathrm{E}-10$ & $3.39 \mathrm{E}-10$ & TM4SF1 & 9.37E-01 \\
\hline ENSBTAG00000031618 & $2.99 \mathrm{E}-10$ & $3.20 \mathrm{E}-10$ & WDR49 & $9.36 \mathrm{E}-01$ \\
\hline ENSBTAG00000012850 & $2.16 \mathrm{E}-10$ & $2.31 \mathrm{E}-10$ & NME9 & 9.37E-01 \\
\hline ENSBTAG00000014797 & $4.28 \mathrm{E}-10$ & $4.55 \mathrm{E}-10$ & SEC22A & $9.40 \mathrm{E}-01$ \\
\hline ENSBTAG00000023429 & $3.52 \mathrm{E}-10$ & 3.73E-10 & PLS1 & $9.43 \mathrm{E}-01$ \\
\hline ENSBTAG00000023106 & $3.23 \mathrm{E}-10$ & $3.42 \mathrm{E}-10$ & & $9.44 \mathrm{E}-01$ \\
\hline ENSBTAG00000013013 & $8.41 \mathrm{E}-10$ & $8.92 \mathrm{E}-10$ & GMPS & $9.43 \mathrm{E}-01$ \\
\hline ENSBTAG00000014306 & $7.81 \mathrm{E}-10$ & $8.25 \mathrm{E}-10$ & DOPEY2 & 9.47E-01 \\
\hline ENSBTAG00000014660 & $3.23 \mathrm{E}-10$ & $3.42 \mathrm{E}-10$ & WDR53 & $9.45 \mathrm{E}-01$ \\
\hline ENSBTAG00000001535 & $3.91 \mathrm{E}-10$ & 4.13E-10 & LIPH & 9.47E-01 \\
\hline ENSBTAG00000001293 & $7.48 \mathrm{E}-10$ & 7.90E-10 & MAATS1 & $9.46 \mathrm{E}-01$ \\
\hline ENSBTAG00000030775 & $3.13 \mathrm{E}-10$ & 3.30E-10 & PCBP3 & $9.48 \mathrm{E}-01$ \\
\hline ENSBTAG00000006975 & $4.52 \mathrm{E}-10$ & $4.76 \mathrm{E}-10$ & SI & $9.51 \mathrm{E}-01$ \\
\hline ENSBTAG00000039151 & $4.48 \mathrm{E}-10$ & 4.70E-10 & HTR1F & $9.53 \mathrm{E}-01$ \\
\hline ENSBTAG00000011880 & $3.05 \mathrm{E}-10$ & 3.19E-10 & CHAF1B & $9.55 \mathrm{E}-01$ \\
\hline ENSBTAG00000013727 & $7.36 \mathrm{E}-10$ & 7.71E-10 & UMPS & 9.55E-01 \\
\hline ENSBTAG00000001395 & $1.85 \mathrm{E}-10$ & $1.93 \mathrm{E}-10$ & C21orf91 & $9.58 \mathrm{E}-01$ \\
\hline ENSBTAG00000020106 & $1.11 \mathrm{E}-10$ & $1.16 \mathrm{E}-10$ & AP2M1 & $9.54 \mathrm{E}-01$ \\
\hline ENSBTAG00000013755 & $6.33 \mathrm{E}-10$ & $6.61 E-10$ & ITGB5 & 9.57E-01 \\
\hline ENSBTAG00000008482 & $2.31 \mathrm{E}-10$ & $2.41 \mathrm{E}-10$ & SON & $9.60 \mathrm{E}-01$ \\
\hline ENSBTAG00000048276 & $9.74 \mathrm{E}-10$ & $1.02 \mathrm{E}-09$ & TFF1 & 9.55E-01 \\
\hline ENSBTAG00000012347 & $3.97 \mathrm{E}-10$ & 4.14E-10 & SLC51A & $9.60 \mathrm{E}-01$ \\
\hline ENSBTAG00000012463 & 4.04E-10 & $4.21 \mathrm{E}-10$ & ZMAT3 & $9.60 \mathrm{E}-01$ \\
\hline ENSBTAG00000013629 & $3.79 \mathrm{E}-10$ & $3.95 \mathrm{E}-10$ & WRB & $9.60 \mathrm{E}-01$ \\
\hline ENSBTAG00000030921 & 7.07E-10 & 7.35E-10 & FAM3B & 9.63E-01 \\
\hline ENSBTAG00000047147 & $4.90 \mathrm{E}-10$ & 5.10E-10 & & $9.61 \mathrm{E}-01$ \\
\hline ENSBTAG00000013920 & $3.60 \mathrm{E}-10$ & $3.73 E-10$ & & $9.64 \mathrm{E}-01$ \\
\hline
\end{tabular}




\begin{tabular}{|c|c|c|c|c|}
\hline ENSBTAG00000004154 & $4.12 \mathrm{E}-10$ & 4.27E-10 & SKIL & $9.65 \mathrm{E}-01$ \\
\hline ENSBTAG00000018768 & $3.61 \mathrm{E}-10$ & $3.73 \mathrm{E}-10$ & & $9.68 \mathrm{E}-01$ \\
\hline ENSBTAG00000004977 & $5.29 \mathrm{E}-10$ & $5.45 \mathrm{E}-10$ & QTRT2 & $9.71 \mathrm{E}-01$ \\
\hline ENSBTAG00000013600 & $1.43 \mathrm{E}-10$ & $1.47 \mathrm{E}-10$ & PSMG1 & $9.73 \mathrm{E}-01$ \\
\hline ENSBTAG00000022908 & $9.28 \mathrm{E}-10$ & $9.56 \mathrm{E}-10$ & & $9.71 \mathrm{E}-01$ \\
\hline ENSBTAG00000016979 & $2.11 \mathrm{E}-10$ & $2.16 \mathrm{E}-10$ & PCYT1A & $9.76 \mathrm{E}-01$ \\
\hline ENSBTAG00000009132 & $7.94 \mathrm{E}-10$ & 8.14E-10 & TMPRSS2 & 9.75E-01 \\
\hline ENSBTAG00000011267 & $6.96 \mathrm{E}-10$ & $7.12 \mathrm{E}-10$ & & $9.77 \mathrm{E}-01$ \\
\hline ENSBTAG00000014461 & 4.45E-10 & 4.56E-10 & ANAPC13 & $9.77 \mathrm{E}-01$ \\
\hline ENSBTAG00000000394 & $3.44 \mathrm{E}-10$ & $3.51 E-10$ & BACE2 & $9.80 \mathrm{E}-01$ \\
\hline ENSBTAG00000001412 & $6.84 \mathrm{E}-10$ & $6.95 \mathrm{E}-10$ & N6AMT1 & $9.84 \mathrm{E}-01$ \\
\hline ENSBTAG00000004833 & $3.19 E-10$ & 3.23E-10 & NIT2 & $9.87 \mathrm{E}-01$ \\
\hline ENSBTAG00000002394 & $1.61 \mathrm{E}-10$ & $1.62 \mathrm{E}-10$ & TMEM44 & $9.92 \mathrm{E}-01$ \\
\hline ENSBTAG00000012140 & $3.09 \mathrm{E}-10$ & $3.12 \mathrm{E}-10$ & ADGRG7 & $9.90 \mathrm{E}-01$ \\
\hline ENSBTAG00000011659 & $3.04 \mathrm{E}-10$ & $3.06 \mathrm{E}-10$ & PIGZ & $9.93 \mathrm{E}-01$ \\
\hline ENSBTAG00000010679 & 3.67E-10 & $3.70 \mathrm{E}-10$ & LRRC31 & $9.92 \mathrm{E}-01$ \\
\hline ENSBTAG00000009851 & $4.43 \mathrm{E}-10$ & 4.47E-10 & ROBO1 & $9.92 \mathrm{E}-01$ \\
\hline ENSBTAG00000000522 & $1.76 \mathrm{E}-10$ & $1.77 \mathrm{E}-10$ & AHSG & $9.95 \mathrm{E}-01$ \\
\hline ENSBTAG00000015150 & $1.83 \mathrm{E}-10$ & $1.85 \mathrm{E}-10$ & IL12A & $9.92 \mathrm{E}-01$ \\
\hline ENSBTAG00000013219 & $4.66 \mathrm{E}-10$ & 4.69E-10 & PEX5L & $9.94 \mathrm{E}-01$ \\
\hline ENSBTAG00000000496 & $2.37 \mathrm{E}-10$ & $2.38 \mathrm{E}-10$ & SLC12A8 & $9.96 \mathrm{E}-01$ \\
\hline ENSBTAG00000014843 & $3.96 \mathrm{E}-10$ & 3.97E-10 & COPB2 & $9.96 \mathrm{E}-01$ \\
\hline ENSBTAG00000006479 & $1.03 \mathrm{E}-09$ & $1.04 \mathrm{E}-09$ & CAPN7 & $9.94 \mathrm{E}-01$ \\
\hline ENSBTAG00000006142 & $6.16 \mathrm{E}-10$ & $6.19 \mathrm{E}-10$ & DHX36 & $9.95 \mathrm{E}-01$ \\
\hline ENSBTAG00000048157 & $5.52 \mathrm{E}-10$ & $5.52 \mathrm{E}-10$ & & $9.99 \mathrm{E}-01$ \\
\hline ENSBTAG00000000160 & $4.45 \mathrm{E}-10$ & $4.45 \mathrm{E}-10$ & & $1.00 \mathrm{E}+00$ \\
\hline ENSBTAG00000016656 & $1.21 \mathrm{E}-10$ & $1.21 \mathrm{E}-10$ & PARP14 & $9.97 \mathrm{E}-01$ \\
\hline ENSBTAG00000004061 & $3.87 \mathrm{E}-10$ & $3.86 \mathrm{E}-10$ & PP2D1 & $1.00 \mathrm{E}+00$ \\
\hline ENSBTAG00000001968 & $1.61 \mathrm{E}-10$ & $1.60 \mathrm{E}-10$ & & $1.00 \mathrm{E}+00$ \\
\hline ENSBTAG00000001009 & $1.63 \mathrm{E}-10$ & 1.63E-10 & HCLS1 & $1.00 \mathrm{E}+00$ \\
\hline ENSBTAG00000023384 & $6.40 \mathrm{E}-10$ & $6.37 \mathrm{E}-10$ & & $1.00 \mathrm{E}+00$ \\
\hline ENSBTAG00000019952 & $1.60 \mathrm{E}-10$ & $1.59 \mathrm{E}-10$ & ARMC8 & $1.01 \mathrm{E}+00$ \\
\hline ENSBTAG00000044111 & $4.05 \mathrm{E}-10$ & $4.02 \mathrm{E}-10$ & EPHA6 & $1.01 E+00$ \\
\hline ENSBTAG00000001890 & $3.54 \mathrm{E}-10$ & $3.52 \mathrm{E}-10$ & RUBCN & $1.01 E+00$ \\
\hline ENSBTAG00000009614 & $3.03 E-10$ & $3.01 \mathrm{E}-10$ & NRROS & $1.01 E+00$ \\
\hline ENSBTAG00000002640 & $5.93 \mathrm{E}-10$ & 5.87E-10 & KALRN & $1.01 \mathrm{E}+00$ \\
\hline ENSBTAG00000011524 & $1.12 \mathrm{E}-10$ & 1.10E-10 & XRN1 & $1.01 E+00$ \\
\hline ENSBTAG00000014721 & $6.21 \mathrm{E}-10$ & $6.13 \mathrm{E}-10$ & & $1.01 E+00$ \\
\hline ENSBTAG00000011634 & $5.07 \mathrm{E}-10$ & $5.01 \mathrm{E}-10$ & ACPP & $1.01 \mathrm{E}+00$ \\
\hline ENSBTAG00000010382 & $6.41 \mathrm{E}-10$ & $6.32 \mathrm{E}-10$ & MUC13 & $1.01 E+00$ \\
\hline ENSBTAG00000023652 & $7.93 \mathrm{E}-10$ & 7.80E-10 & PROS1 & $1.02 \mathrm{E}+00$ \\
\hline ENSBTAG00000009258 & $4.93 \mathrm{E}-10$ & $4.83 \mathrm{E}-10$ & NEK11 & $1.02 \mathrm{E}+00$ \\
\hline ENSBTAG00000038395 & $7.54 \mathrm{E}-10$ & $7.38 \mathrm{E}-10$ & & $1.02 \mathrm{E}+00$ \\
\hline ENSBTAG00000004471 & $2.36 \mathrm{E}-10$ & $2.30 \mathrm{E}-10$ & ST3GAL6 & $1.03 E+00$ \\
\hline ENSBTAG00000023375 & $9.03 \mathrm{E}-10$ & $8.82 \mathrm{E}-10$ & METTL6 & $1.02 \mathrm{E}+00$ \\
\hline ENSBTAG00000022227 & $4.99 \mathrm{E}-10$ & $4.86 \mathrm{E}-10$ & & $1.03 E+00$ \\
\hline ENSBTAG00000014302 & 3.07E-10 & 2.97E-10 & TOMM70 & $1.03 E+00$ \\
\hline ENSBTAG00000035556 & $2.25 \mathrm{E}-10$ & $2.18 \mathrm{E}-10$ & & $1.03 E+00$ \\
\hline ENSBTAG00000007814 & $2.02 \mathrm{E}-10$ & $1.95 \mathrm{E}-10$ & WWTR1 & $1.03 E+00$ \\
\hline ENSBTAG00000006471 & $1.36 \mathrm{E}-10$ & $1.30 \mathrm{E}-10$ & OSBPL11 & $1.05 E+00$ \\
\hline ENSBTAG00000016457 & $2.29 \mathrm{E}-10$ & 2.19E-10 & FXR1 & $1.05 E+00$ \\
\hline ENSBTAG00000006415 & $5.19 \mathrm{E}-10$ & $4.96 \mathrm{E}-10$ & LRRIQ4 & $1.05 \mathrm{E}+00$ \\
\hline ENSBTAG00000006147 & $7.89 \mathrm{E}-10$ & $7.55 \mathrm{E}-10$ & GPR149 & $1.04 \mathrm{E}+00$ \\
\hline ENSBTAG00000019404 & $7.72 \mathrm{E}-10$ & 7.35E-10 & & $1.05 \mathrm{E}+00$ \\
\hline ENSBTAG00000021259 & $2.23 \mathrm{E}-10$ & $2.13 \mathrm{E}-10$ & $\mathrm{C} 2 \mathrm{CD} 2$ & $1.05 E+00$ \\
\hline ENSBTAG00000017140 & $1.39 \mathrm{E}-10$ & $1.32 \mathrm{E}-10$ & KIAA1524 & $1.05 E+00$ \\
\hline ENSBTAG00000001918 & 4.64E-10 & $4.39 \mathrm{E}-10$ & STXBP5L & $1.06 \mathrm{E}+00$ \\
\hline ENSBTAG00000002232 & $1.99 \mathrm{E}-10$ & $1.88 \mathrm{E}-10$ & TRPC1 & $1.06 \mathrm{E}+00$ \\
\hline ENSBTAG00000031891 & $1.64 \mathrm{E}-10$ & $1.55 \mathrm{E}-10$ & NLGN1 & $1.06 \mathrm{E}+00$ \\
\hline ENSBTAG00000011143 & $7.64 \mathrm{E}-11$ & $7.22 \mathrm{E}-11$ & KPNA1 & $1.06 \mathrm{E}+00$ \\
\hline ENSBTAG00000010550 & $7.16 \mathrm{E}-10$ & $6.77 \mathrm{E}-10$ & EIF5A2 & $1.06 \mathrm{E}+00$ \\
\hline ENSBTAG00000018854 & $1.89 \mathrm{E}-10$ & $1.79 \mathrm{E}-10$ & & $1.06 \mathrm{E}+00$ \\
\hline ENSBTAG00000018399 & $3.72 \mathrm{E}-10$ & $3.52 \mathrm{E}-10$ & & $1.06 \mathrm{E}+00$ \\
\hline ENSBTAG00000020409 & 4.19E-10 & $3.95 \mathrm{E}-10$ & SPATC1L & $1.06 \mathrm{E}+00$ \\
\hline ENSBTAG00000005230 & $1.56 \mathrm{E}-10$ & 1.47E-10 & SHOX2 & $1.06 \mathrm{E}+00$ \\
\hline ENSBTAG00000045991 & $2.10 \mathrm{E}-09$ & 1.97E-09 & & $1.07 E+00$ \\
\hline ENSBTAG00000021025 & $2.72 \mathrm{E}-10$ & $2.56 \mathrm{E}-10$ & $\mathrm{P} 3 \mathrm{H} 2$ & $1.06 \mathrm{E}+00$ \\
\hline ENSBTAG00000038433 & $9.41 \mathrm{E}-10$ & 8.87E-10 & & $1.06 \mathrm{E}+00$ \\
\hline ENSBTAG00000020404 & $2.12 \mathrm{E}-10$ & $2.00 \mathrm{E}-10$ & FTCD & $1.06 \mathrm{E}+00$ \\
\hline ENSBTAG00000018402 & $1.65 \mathrm{E}-10$ & $1.56 \mathrm{E}-10$ & TSC22D2 & $1.06 \mathrm{E}+00$ \\
\hline
\end{tabular}




\begin{tabular}{|c|c|c|c|c|}
\hline ENSBTAG00000001416 & $4.51 \mathrm{E}-10$ & 4.24E-10 & GABRR3 & $1.06 \mathrm{E}+00$ \\
\hline ENSBTAG00000008346 & $3.10 \mathrm{E}-10$ & $2.91 \mathrm{E}-10$ & & $1.07 \mathrm{E}+00$ \\
\hline ENSBTAG00000021703 & $3.27 \mathrm{E}-10$ & 3.07E-10 & MED12L & $1.06 \mathrm{E}+00$ \\
\hline ENSBTAG00000011147 & 7.77E-10 & 7.29E-10 & RNF13 & $1.07 \mathrm{E}+00$ \\
\hline ENSBTAG00000011138 & $1.02 \mathrm{E}-10$ & $9.58 \mathrm{E}-11$ & CCDC58 & $1.07 E+00$ \\
\hline ENSBTAG00000008490 & $2.66 \mathrm{E}-10$ & $2.49 \mathrm{E}-10$ & CRYZL1 & $1.07 \mathrm{E}+00$ \\
\hline ENSBTAG00000001753 & $2.71 \mathrm{E}-10$ & $2.52 \mathrm{E}-10$ & & $1.07 \mathrm{E}+00$ \\
\hline ENSBTAG00000017115 & $8.84 \mathrm{E}-10$ & $8.24 \mathrm{E}-10$ & TMEM41A & $1.07 \mathrm{E}+00$ \\
\hline ENSBTAG00000013317 & $1.59 \mathrm{E}-10$ & $1.48 \mathrm{E}-10$ & & $1.08 \mathrm{E}+00$ \\
\hline ENSBTAG00000013070 & $9.56 \mathrm{E}-11$ & $8.90 \mathrm{E}-11$ & EAF2 & $1.07 \mathrm{E}+00$ \\
\hline ENSBTAG00000018704 & $2.64 \mathrm{E}-10$ & $2.45 \mathrm{E}-10$ & BTG3 & $1.08 \mathrm{E}+00$ \\
\hline ENSBTAG00000003161 & $3.66 \mathrm{E}-10$ & $3.39 \mathrm{E}-10$ & YBEY & $1.08 \mathrm{E}+00$ \\
\hline ENSBTAG00000003666 & $2.74 \mathrm{E}-10$ & $2.54 \mathrm{E}-10$ & ZPLD1 & $1.08 \mathrm{E}+00$ \\
\hline ENSBTAG00000031346 & $4.63 \mathrm{E}-10$ & 4.29E-10 & ANKUB1 & $1.08 \mathrm{E}+00$ \\
\hline ENSBTAG00000011427 & $3.72 \mathrm{E}-10$ & $3.44 \mathrm{E}-10$ & & $1.08 \mathrm{E}+00$ \\
\hline ENSBTAG00000011569 & $7.49 \mathrm{E}-11$ & $6.92 \mathrm{E}-11$ & AADAC & $1.08 \mathrm{E}+00$ \\
\hline ENSBTAG00000015065 & $6.84 \mathrm{E}-10$ & $6.31 \mathrm{E}-10$ & TMPRSS7 & $1.08 \mathrm{E}+00$ \\
\hline ENSBTAG00000019269 & $2.05 \mathrm{E}-10$ & $1.88 \mathrm{E}-10$ & COL6A2 & $1.09 \mathrm{E}+00$ \\
\hline ENSBTAG00000011921 & $2.24 \mathrm{E}-10$ & $2.05 \mathrm{E}-10$ & TIGIT & $1.09 \mathrm{E}+00$ \\
\hline ENSBTAG00000039597 & $5.29 \mathrm{E}-10$ & $4.84 \mathrm{E}-10$ & KRTAP27-1 & $1.09 \mathrm{E}+00$ \\
\hline ENSBTAG00000003148 & $2.83 \mathrm{E}-10$ & $2.59 \mathrm{E}-10$ & МСMЗАР & $1.09 \mathrm{E}+00$ \\
\hline ENSBTAG00000001749 & $4.04 \mathrm{E}-10$ & 3.67E-10 & SENP2 & $1.10 \mathrm{E}+00$ \\
\hline ENSBTAG00000018186 & $5.00 \mathrm{E}-10$ & 4.54E-10 & PDXK & $1.10 \mathrm{E}+00$ \\
\hline ENSBTAG00000005122 & $5.87 \mathrm{E}-10$ & 5.33E-10 & KNG1 & $1.10 \mathrm{E}+00$ \\
\hline ENSBTAG00000010793 & $3.46 \mathrm{E}-10$ & 3.13E-10 & CCDC80 & $1.10 E+00$ \\
\hline ENSBTAG00000005871 & $3.48 \mathrm{E}-10$ & $3.16 \mathrm{E}-10$ & MECOM & $1.10 \mathrm{E}+00$ \\
\hline ENSBTAG00000018465 & $3.99 \mathrm{E}-10$ & $3.61 E-10$ & KCNAB1 & $1.11 E+00$ \\
\hline ENSBTAG00000003301 & $5.03 \mathrm{E}-10$ & $4.55 \mathrm{E}-10$ & NCAM2 & $1.11 E+00$ \\
\hline ENSBTAG00000032719 & $2.95 \mathrm{E}-10$ & $2.66 \mathrm{E}-10$ & TFRC & $1.11 \mathrm{E}+00$ \\
\hline ENSBTAG00000046959 & $1.15 \mathrm{E}-10$ & $1.03 \mathrm{E}-10$ & & $1.12 \mathrm{E}+00$ \\
\hline ENSBTAG00000013205 & $3.27 \mathrm{E}-10$ & $2.93 \mathrm{E}-10$ & IL1RAP & $1.12 \mathrm{E}+00$ \\
\hline ENSBTAG00000016554 & $7.90 \mathrm{E}-10$ & 7.07E-10 & C3orf52 & $1.12 \mathrm{E}+00$ \\
\hline ENSBTAG00000006494 & $4.80 \mathrm{E}-10$ & $4.28 \mathrm{E}-10$ & CLDN16 & $1.12 \mathrm{E}+00$ \\
\hline ENSBTAG00000010987 & $1.77 \mathrm{E}-10$ & $1.58 \mathrm{E}-10$ & Mail & $1.12 \mathrm{E}+00$ \\
\hline ENSBTAG00000003140 & $2.42 \mathrm{E}-10$ & $2.15 \mathrm{E}-10$ & CRYGS & $1.12 \mathrm{E}+00$ \\
\hline ENSBTAG00000016363 & $2.67 \mathrm{E}-10$ & $2.36 \mathrm{E}-10$ & TFG & $1.13 \mathrm{E}+00$ \\
\hline ENSBTAG00000007039 & $2.78 \mathrm{E}-10$ & 2.47E-10 & FBXO25 & $1.13 \mathrm{E}+00$ \\
\hline ENSBTAG00000037965 & $1.39 \mathrm{E}-09$ & $1.23 \mathrm{E}-09$ & ZSCAN23 & $1.13 E+00$ \\
\hline ENSBTAG00000023814 & $2.01 \mathrm{E}-10$ & $1.78 \mathrm{E}-10$ & ECT2 & $1.13 \mathrm{E}+00$ \\
\hline ENSBTAG00000018727 & $3.50 \mathrm{E}-10$ & $3.09 \mathrm{E}-10$ & GOLIM4 & $1.13 E+00$ \\
\hline ENSBTAG00000026156 & $2.46 \mathrm{E}-10$ & $2.18 \mathrm{E}-10$ & VGLL3 & $1.13 E+00$ \\
\hline ENSBTAG00000013069 & $8.46 \mathrm{E}-11$ & $7.48 \mathrm{E}-11$ & IQCB1 & $1.13 \mathrm{E}+00$ \\
\hline ENSBTAG00000004975 & $2.55 \mathrm{E}-10$ & $2.26 \mathrm{E}-10$ & CCDC191 & $1.13 \mathrm{E}+00$ \\
\hline ENSBTAG00000017839 & $3.56 \mathrm{E}-10$ & $3.13 \mathrm{E}-10$ & TIAM1 & $1.14 \mathrm{E}+00$ \\
\hline ENSBTAG00000009942 & $4.92 \mathrm{E}-10$ & $4.33 \mathrm{E}-10$ & PLCL2 & $1.14 \mathrm{E}+00$ \\
\hline ENSBTAG00000023513 & $1.56 \mathrm{E}-10$ & 1.37E-10 & DNAJC19 & $1.14 \mathrm{E}+00$ \\
\hline ENSBTAG00000021447 & $1.74 \mathrm{E}-10$ & $1.53 \mathrm{E}-10$ & SIM2 & $1.14 \mathrm{E}+00$ \\
\hline ENSBTAG00000011377 & $3.96 \mathrm{E}-10$ & $3.48 \mathrm{E}-10$ & & $1.14 \mathrm{E}+00$ \\
\hline ENSBTAG00000019589 & $7.86 \mathrm{E}-10$ & $6.89 \mathrm{E}-10$ & C21orf2 & $1.14 \mathrm{E}+00$ \\
\hline ENSBTAG00000017753 & $6.23 \mathrm{E}-10$ & $5.45 \mathrm{E}-10$ & APP & $1.14 \mathrm{E}+00$ \\
\hline ENSBTAG00000003833 & $1.34 \mathrm{E}-10$ & 1.17E-10 & SLC35G2 & $1.15 \mathrm{E}+00$ \\
\hline ENSBTAG00000023668 & $6.78 \mathrm{E}-10$ & $5.90 \mathrm{E}-10$ & & $1.15 \mathrm{E}+00$ \\
\hline ENSBTAG00000015198 & $6.39 \mathrm{E}-10$ & $5.56 \mathrm{E}-10$ & DZIP1L & $1.15 E+00$ \\
\hline ENSBTAG00000019129 & $5.14 \mathrm{E}-10$ & 4.45E-10 & BTD & $1.16 \mathrm{E}+00$ \\
\hline ENSBTAG00000005155 & $1.77 \mathrm{E}-10$ & $1.54 \mathrm{E}-10$ & ARL13B & $1.15 E+00$ \\
\hline ENSBTAG00000008111 & $6.92 \mathrm{E}-10$ & $5.99 \mathrm{E}-10$ & ESYT3 & $1.16 \mathrm{E}+00$ \\
\hline ENSBTAG00000004564 & $3.04 \mathrm{E}-10$ & $2.62 \mathrm{E}-10$ & MBNL1 & $1.16 \mathrm{E}+00$ \\
\hline ENSBTAG00000021276 & $8.29 \mathrm{E}-10$ & 7.13E-10 & TFF3 & $1.16 \mathrm{E}+00$ \\
\hline ENSBTAG00000001274 & $3.13 \mathrm{E}-10$ & $2.68 \mathrm{E}-10$ & PPM1L & $1.17 \mathrm{E}+00$ \\
\hline ENSBTAG00000011876 & $3.05 \mathrm{E}-10$ & 2.60E-10 & MORC3 & $1.17 E+00$ \\
\hline ENSBTAG00000027795 & $5.10 \mathrm{E}-10$ & $4.35 \mathrm{E}-10$ & GFM1 & $1.17 \mathrm{E}+00$ \\
\hline ENSBTAG00000015837 & $2.98 \mathrm{E}-10$ & $2.53 \mathrm{E}-10$ & P2RY12 & $1.18 \mathrm{E}+00$ \\
\hline ENSBTAG00000004869 & $6.05 \mathrm{E}-10$ & $5.12 \mathrm{E}-10$ & & $1.18 \mathrm{E}+00$ \\
\hline ENSBTAG00000008714 & $6.09 \mathrm{E}-10$ & $5.13 \mathrm{E}-10$ & CLSTN2 & $1.19 E+00$ \\
\hline ENSBTAG00000016961 & $1.87 \mathrm{E}-10$ & $1.57 \mathrm{E}-10$ & RAP2B & $1.19 \mathrm{E}+00$ \\
\hline ENSBTAG00000001451 & 4.25E-10 & 3.56E-10 & A4GNT & $1.19 \mathrm{E}+00$ \\
\hline ENSBTAG00000003134 & $1.74 \mathrm{E}-10$ & $1.46 \mathrm{E}-10$ & & $1.19 E+00$ \\
\hline ENSBTAG00000008461 & $1.96 \mathrm{E}-10$ & $1.65 \mathrm{E}-10$ & LSG1 & $1.19 \mathrm{E}+00$ \\
\hline ENSBTAG00000034442 & $2.12 \mathrm{E}-10$ & $1.77 \mathrm{E}-10$ & LNP1 & $1.19 \mathrm{E}+00$ \\
\hline ENSBTAG00000020762 & $4.85 \mathrm{E}-10$ & 4.04E-10 & HUNK & $1.20 \mathrm{E}+00$ \\
\hline
\end{tabular}




\begin{tabular}{|c|c|c|c|c|}
\hline ENSBTAG00000021158 & $3.03 \mathrm{E}-10$ & $2.51 \mathrm{E}-10$ & SATB1 & $1.21 \mathrm{E}+00$ \\
\hline ENSBTAG00000004495 & $2.12 \mathrm{E}-10$ & $1.76 \mathrm{E}-10$ & UBA5 & $1.21 \mathrm{E}+00$ \\
\hline ENSBTAG00000019332 & $5.54 \mathrm{E}-10$ & 4.56E-10 & SLC9C1 & $1.21 \mathrm{E}+00$ \\
\hline ENSBTAG00000009014 & $5.33 \mathrm{E}-10$ & $4.39 \mathrm{E}-10$ & UPK1B & $1.21 \mathrm{E}+00$ \\
\hline ENSBTAG00000018427 & $1.68 \mathrm{E}-10$ & $1.38 \mathrm{E}-10$ & NCK1 & $1.21 E+00$ \\
\hline ENSBTAG00000001511 & $1.65 \mathrm{E}-10$ & $1.35 \mathrm{E}-10$ & $\mathrm{BCL6}$ & $1.22 \mathrm{E}+00$ \\
\hline ENSBTAG00000021984 & $3.71 \mathrm{E}-10$ & $3.05 \mathrm{E}-10$ & C21orf58 & $1.22 \mathrm{E}+00$ \\
\hline ENSBTAG00000047570 & $6.46 \mathrm{E}-10$ & $5.30 \mathrm{E}-10$ & & $1.22 \mathrm{E}+00$ \\
\hline ENSBTAG00000004974 & $1.59 \mathrm{E}-10$ & $1.30 \mathrm{E}-10$ & ZDHHC23 & $1.23 \mathrm{E}+00$ \\
\hline ENSBTAG00000016619 & $1.53 \mathrm{E}-10$ & $1.26 \mathrm{E}-10$ & MIS18A & $1.22 \mathrm{E}+00$ \\
\hline ENSBTAG00000002896 & $4.59 \mathrm{E}-10$ & $3.75 \mathrm{E}-10$ & KPNA6 & $1.22 \mathrm{E}+00$ \\
\hline ENSBTAG00000025146 & $6.06 \mathrm{E}-10$ & $4.94 \mathrm{E}-10$ & ZNF560 & $1.23 \mathrm{E}+00$ \\
\hline ENSBTAG00000000456 & $5.76 \mathrm{E}-10$ & $4.70 \mathrm{E}-10$ & $\mathrm{pCPB}$ & $1.22 \mathrm{E}+00$ \\
\hline ENSBTAG00000009085 & $2.59 \mathrm{E}-10$ & $2.11 \mathrm{E}-10$ & SLC35A5 & $1.23 E+00$ \\
\hline ENSBTAG00000038651 & 7.67E-10 & $6.24 \mathrm{E}-10$ & & $1.23 \mathrm{E}+00$ \\
\hline ENSBTAG00000035437 & $1.95 \mathrm{E}-10$ & $1.59 \mathrm{E}-10$ & STAG1 & $1.23 E+00$ \\
\hline ENSBTAG00000007462 & $2.52 \mathrm{E}-10$ & $2.04 \mathrm{E}-10$ & ASTE1 & $1.24 \mathrm{E}+00$ \\
\hline ENSBTAG00000015221 & $3.07 \mathrm{E}-10$ & $2.48 \mathrm{E}-10$ & PCCB & $1.24 \mathrm{E}+00$ \\
\hline ENSBTAG00000039820 & $5.79 \mathrm{E}-10$ & $4.68 \mathrm{E}-10$ & CLDN8 & $1.24 \mathrm{E}+00$ \\
\hline ENSBTAG00000011437 & $2.43 \mathrm{E}-10$ & $1.97 \mathrm{E}-10$ & & $1.23 E+00$ \\
\hline ENSBTAG00000011140 & $9.96 \mathrm{E}-11$ & $8.03 E-11$ & FAM162A & $1.24 \mathrm{E}+00$ \\
\hline ENSBTAG00000004754 & $5.77 \mathrm{E}-10$ & $4.64 \mathrm{E}-10$ & DSCAM & $1.24 \mathrm{E}+00$ \\
\hline ENSBTAG00000020456 & $4.01 \mathrm{E}-10$ & $3.23 \mathrm{E}-10$ & AGPAT3 & $1.24 \mathrm{E}+00$ \\
\hline ENSBTAG00000009219 & $5.82 \mathrm{E}-10$ & $4.67 \mathrm{E}-10$ & CADM2 & $1.25 \mathrm{E}+00$ \\
\hline ENSBTAG00000018777 & $2.40 \mathrm{E}-10$ & $1.92 \mathrm{E}-10$ & ADCY5 & $1.25 \mathrm{E}+00$ \\
\hline ENSBTAG00000010091 & $2.75 \mathrm{E}-10$ & $2.20 \mathrm{E}-10$ & ARL6 & $1.25 \mathrm{E}+00$ \\
\hline ENSBTAG00000000524 & $7.88 \mathrm{E}-10$ & $6.30 \mathrm{E}-10$ & & $1.25 E+00$ \\
\hline ENSBTAG00000015413 & $3.92 \mathrm{E}-10$ & $3.12 \mathrm{E}-10$ & RASA2 & $1.26 \mathrm{E}+00$ \\
\hline ENSBTAG00000012797 & $1.50 \mathrm{E}-10$ & $1.20 \mathrm{E}-10$ & DCUN1D1 & $1.25 \mathrm{E}+00$ \\
\hline ENSBTAG00000017321 & $1.63 \mathrm{E}-10$ & $1.30 \mathrm{E}-10$ & SUMO3 & $1.26 \mathrm{E}+00$ \\
\hline ENSBTAG00000002966 & $8.76 \mathrm{E}-10$ & $6.96 \mathrm{E}-10$ & DNAJC13 & $1.26 \mathrm{E}+00$ \\
\hline ENSBTAG00000000973 & 3.34E-10 & $2.66 \mathrm{E}-10$ & KCNJ15 & $1.26 \mathrm{E}+00$ \\
\hline ENSBTAG00000013663 & $2.64 \mathrm{E}-10$ & $2.10 \mathrm{E}-10$ & CMSS1 & $1.26 \mathrm{E}+00$ \\
\hline ENSBTAG00000018936 & $3.94 \mathrm{E}-10$ & $3.12 \mathrm{E}-10$ & LSS & $1.26 \mathrm{E}+00$ \\
\hline ENSBTAG00000023411 & $9.08 \mathrm{E}-10$ & $7.20 \mathrm{E}-10$ & & $1.26 \mathrm{E}+00$ \\
\hline ENSBTAG00000011871 & $3.79 \mathrm{E}-10$ & $3.00 \mathrm{E}-10$ & BTLA & $1.26 \mathrm{E}+00$ \\
\hline ENSBTAG00000007100 & $1.92 \mathrm{E}-10$ & $1.52 \mathrm{E}-10$ & TRAPPC10 & $1.26 \mathrm{E}+00$ \\
\hline ENSBTAG00000017407 & $3.09 \mathrm{E}-10$ & $2.44 \mathrm{E}-10$ & HSF2BP & $1.27 \mathrm{E}+00$ \\
\hline ENSBTAG00000003179 & $1.76 \mathrm{E}-10$ & $1.38 \mathrm{E}-10$ & RTP2 & $1.27 \mathrm{E}+00$ \\
\hline ENSBTAG00000007444 & $5.98 \mathrm{E}-10$ & $4.70 \mathrm{E}-10$ & MAP3K7CL & $1.27 \mathrm{E}+00$ \\
\hline ENSBTAG00000040061 & $1.81 \mathrm{E}-10$ & $1.43 \mathrm{E}-10$ & ZBTB38 & $1.27 \mathrm{E}+00$ \\
\hline ENSBTAG00000018538 & $5.87 \mathrm{E}-10$ & $4.58 \mathrm{E}-10$ & CEP70 & $1.28 \mathrm{E}+00$ \\
\hline ENSBTAG00000006158 & $2.09 \mathrm{E}-10$ & $1.63 \mathrm{E}-10$ & DZIP3 & $1.28 \mathrm{E}+00$ \\
\hline ENSBTAG00000020073 & $1.87 \mathrm{E}-10$ & $1.45 \mathrm{E}-10$ & NCEH 1 & $1.29 \mathrm{E}+00$ \\
\hline ENSBTAG00000021791 & $2.81 \mathrm{E}-10$ & $2.18 \mathrm{E}-10$ & PARP9 & $1.29 \mathrm{E}+00$ \\
\hline ENSBTAG00000032538 & 4.13E-10 & $3.21 \mathrm{E}-10$ & TPRG1 & $1.29 \mathrm{E}+00$ \\
\hline ENSBTAG00000046385 & $1.51 \mathrm{E}-10$ & $1.17 \mathrm{E}-10$ & RAB5A & $1.29 \mathrm{E}+00$ \\
\hline ENSBTAG00000022155 & $1.78 \mathrm{E}-10$ & $1.38 \mathrm{E}-10$ & FSTL1 & $1.29 \mathrm{E}+00$ \\
\hline ENSBTAG00000007577 & $2.99 \mathrm{E}-10$ & $2.30 \mathrm{E}-10$ & UBXN7 & $1.30 \mathrm{E}+00$ \\
\hline ENSBTAG00000014749 & $1.15 \mathrm{E}-10$ & $8.88 \mathrm{E}-11$ & ZIC4 & $1.30 \mathrm{E}+00$ \\
\hline ENSBTAG00000000201 & 2.73E-10 & $2.09 \mathrm{E}-10$ & LTN1 & $1.31 E+00$ \\
\hline ENSBTAG00000026836 & $3.82 \mathrm{E}-10$ & $2.92 \mathrm{E}-10$ & & $1.31 E+00$ \\
\hline ENSBTAG00000011529 & $1.73 \mathrm{E}-10$ & $1.32 \mathrm{E}-10$ & ATR & $1.31 E+00$ \\
\hline ENSBTAG00000003981 & $2.49 \mathrm{E}-10$ & $1.90 \mathrm{E}-10$ & RRP1 & $1.31 \mathrm{E}+00$ \\
\hline ENSBTAG00000007014 & $7.49 \mathrm{E}-11$ & $5.70 \mathrm{E}-11$ & CEP63 & $1.31 E+00$ \\
\hline ENSBTAG00000011305 & $1.59 \mathrm{E}-10$ & $1.21 \mathrm{E}-10$ & & $1.31 E+00$ \\
\hline ENSBTAG00000013192 & $2.18 \mathrm{E}-10$ & $1.65 E-10$ & CEP19 & $1.32 \mathrm{E}+00$ \\
\hline ENSBTAG00000013664 & $1.26 \mathrm{E}-10$ & $9.52 \mathrm{E}-11$ & FILIP1L & $1.32 \mathrm{E}+00$ \\
\hline ENSBTAG00000047943 & $5.03 \mathrm{E}-10$ & $3.79 \mathrm{E}-10$ & & $1.33 E+00$ \\
\hline ENSBTAG00000033170 & $1.73 \mathrm{E}-10$ & $1.30 \mathrm{E}-10$ & LRRC58 & $1.33 E+00$ \\
\hline ENSBTAG00000009336 & $3.40 \mathrm{E}-10$ & $2.54 \mathrm{E}-10$ & LXN & $1.34 \mathrm{E}+00$ \\
\hline ENSBTAG00000015546 & $2.30 \mathrm{E}-10$ & $1.71 \mathrm{E}-10$ & MINA & $1.35 \mathrm{E}+00$ \\
\hline ENSBTAG00000008562 & 4.39E-10 & $3.27 \mathrm{E}-10$ & POGLUT1 & $1.34 \mathrm{E}+00$ \\
\hline ENSBTAG00000005068 & $9.81 \mathrm{E}-10$ & $7.31 \mathrm{E}-10$ & COMMD2 & $1.34 \mathrm{E}+00$ \\
\hline ENSBTAG00000018138 & $2.47 \mathrm{E}-10$ & $1.84 \mathrm{E}-10$ & GK5 & $1.34 \mathrm{E}+00$ \\
\hline ENSBTAG00000020996 & $1.92 \mathrm{E}-10$ & $1.42 \mathrm{E}-10$ & CLDND1 & $1.35 E+00$ \\
\hline ENSBTAG00000021886 & 4.45E-10 & $3.28 \mathrm{E}-10$ & GRK7 & $1.36 \mathrm{E}+00$ \\
\hline ENSBTAG00000005742 & $3.25 \mathrm{E}-10$ & $2.40 \mathrm{E}-10$ & & $1.35 E+00$ \\
\hline ENSBTAG00000021251 & $2.57 \mathrm{E}-10$ & $1.89 \mathrm{E}-10$ & RIPK4 & $1.36 \mathrm{E}+00$ \\
\hline ENSBTAG00000015377 & 4.63E-10 & $3.41 \mathrm{E}-10$ & EPHB1 & $1.36 \mathrm{E}+00$ \\
\hline
\end{tabular}




\begin{tabular}{|c|c|c|c|c|}
\hline ENSBTAG00000033679 & $3.12 \mathrm{E}-10$ & $2.29 \mathrm{E}-10$ & HLCS & $1.36 \mathrm{E}+00$ \\
\hline ENSBTAG00000002998 & $1.48 \mathrm{E}-10$ & $1.08 \mathrm{E}-10$ & MELTF & $1.37 E+00$ \\
\hline ENSBTAG00000005946 & $2.81 \mathrm{E}-10$ & $2.06 \mathrm{E}-10$ & USP13 & $1.36 \mathrm{E}+00$ \\
\hline ENSBTAG00000012413 & $2.46 \mathrm{E}-10$ & $1.79 \mathrm{E}-10$ & FGF12 & $1.37 E+00$ \\
\hline ENSBTAG00000013662 & 4.69E-10 & $3.42 \mathrm{E}-10$ & COL8A1 & $1.37 \mathrm{E}+00$ \\
\hline ENSBTAG00000016323 & $4.58 \mathrm{E}-10$ & 3.33E-10 & SLC7A14 & $1.37 \mathrm{E}+00$ \\
\hline ENSBTAG00000014751 & $1.19 \mathrm{E}-10$ & 8.57E-11 & ZIC1 & $1.39 E+00$ \\
\hline ENSBTAG00000007970 & $1.92 \mathrm{E}-10$ & $1.38 \mathrm{E}-10$ & C3orf38 & $1.39 E+00$ \\
\hline ENSBTAG00000005212 & $2.86 \mathrm{E}-10$ & $2.05 \mathrm{E}-10$ & C3orf70 & $1.39 E+00$ \\
\hline ENSBTAG00000017490 & 4.15E-10 & 2.97E-10 & PLD1 & $1.40 E+00$ \\
\hline ENSBTAG00000011001 & 2.97E-10 & $2.12 \mathrm{E}-10$ & ERG & $1.40 \mathrm{E}+00$ \\
\hline ENSBTAG00000017486 & $6.85 \mathrm{E}-10$ & $4.88 \mathrm{E}-10$ & ADARB1 & $1.40 \mathrm{E}+00$ \\
\hline ENSBTAG00000032684 & $3.28 \mathrm{E}-10$ & $2.33 \mathrm{E}-10$ & & $1.41 \mathrm{E}+00$ \\
\hline ENSBTAG00000019376 & $2.37 \mathrm{E}-10$ & $1.66 \mathrm{E}-10$ & FNDC3B & $1.43 E+00$ \\
\hline ENSBTAG00000013937 & $3.17 \mathrm{E}-10$ & $2.22 \mathrm{E}-10$ & SPICE1 & $1.43 E+00$ \\
\hline ENSBTAG00000021491 & $6.90 \mathrm{E}-10$ & $4.84 \mathrm{E}-10$ & NDUFV3 & $1.43 E+00$ \\
\hline ENSBTAG00000005091 & $3.60 \mathrm{E}-10$ & $2.52 \mathrm{E}-10$ & DGKG & $1.43 \mathrm{E}+00$ \\
\hline ENSBTAG00000003059 & $1.27 \mathrm{E}-10$ & $8.88 \mathrm{E}-11$ & & $1.43 \mathrm{E}+00$ \\
\hline ENSBTAG00000046755 & $9.80 \mathrm{E}-10$ & $6.83 \mathrm{E}-10$ & & $1.43 E+00$ \\
\hline ENSBTAG00000017418 & $3.34 \mathrm{E}-10$ & $2.31 \mathrm{E}-10$ & RRP1B & $1.45 \mathrm{E}+00$ \\
\hline ENSBTAG00000021906 & $4.32 \mathrm{E}-10$ & $2.98 \mathrm{E}-10$ & & $1.45 \mathrm{E}+00$ \\
\hline ENSBTAG00000003718 & $6.35 E-10$ & $4.38 \mathrm{E}-10$ & HACL1 & $1.45 E+00$ \\
\hline ENSBTAG00000031704 & $2.63 \mathrm{E}-10$ & $1.81 \mathrm{E}-10$ & & $1.45 \mathrm{E}+00$ \\
\hline ENSBTAG00000005522 & 1.09E-09 & $7.46 \mathrm{E}-10$ & PLSCR5 & $1.46 \mathrm{E}+00$ \\
\hline ENSBTAG00000002623 & $3.49 \mathrm{E}-10$ & $2.38 \mathrm{E}-10$ & SAMSN1 & $1.46 \mathrm{E}+00$ \\
\hline ENSBTAG00000031561 & $2.82 \mathrm{E}-10$ & $1.92 \mathrm{E}-10$ & RSRC1 & $1.47 \mathrm{E}+00$ \\
\hline ENSBTAG00000015728 & $1.71 \mathrm{E}-10$ & $1.16 \mathrm{E}-10$ & NSUN3 & $1.48 \mathrm{E}+00$ \\
\hline ENSBTAG00000047608 & $3.06 \mathrm{E}-10$ & $2.08 \mathrm{E}-10$ & MRPS22 & $1.47 \mathrm{E}+00$ \\
\hline ENSBTAG00000000210 & $5.09 \mathrm{E}-10$ & $3.42 \mathrm{E}-10$ & ARHGAP31 & $1.49 E+00$ \\
\hline ENSBTAG00000023426 & $3.66 \mathrm{E}-10$ & $2.46 \mathrm{E}-10$ & FAIM & $1.49 E+00$ \\
\hline ENSBTAG00000044001 & $2.51 \mathrm{E}-10$ & $1.68 \mathrm{E}-10$ & LPP & $1.49 E+00$ \\
\hline ENSBTAG00000013667 & $1.76 \mathrm{E}-10$ & 1.17E-10 & & $1.50 \mathrm{E}+00$ \\
\hline ENSBTAG00000031802 & $6.59 \mathrm{E}-10$ & $4.36 \mathrm{E}-10$ & SPATA16 & $1.51 E+00$ \\
\hline ENSBTAG00000021000 & $8.50 \mathrm{E}-11$ & $5.61 \mathrm{E}-11$ & USF3 & $1.51 E+00$ \\
\hline ENSBTAG00000003143 & 4.67E-10 & $3.08 \mathrm{E}-10$ & TBCCD1 & $1.52 \mathrm{E}+00$ \\
\hline ENSBTAG00000038512 & 3.32E-10 & 2.17E-10 & & $1.53 E+00$ \\
\hline ENSBTAG00000031178 & $5.02 \mathrm{E}-10$ & $3.27 \mathrm{E}-10$ & SLC9A9 & $1.53 \mathrm{E}+00$ \\
\hline ENSBTAG00000007784 & $1.41 \mathrm{E}-10$ & $9.18 \mathrm{E}-11$ & NAA50 & $1.53 E+00$ \\
\hline ENSBTAG00000030993 & $1.63 \mathrm{E}-10$ & $1.06 \mathrm{E}-10$ & LCA5L & $1.54 \mathrm{E}+00$ \\
\hline ENSBTAG00000005787 & 4.54E-10 & $2.94 \mathrm{E}-10$ & IFT57 & $1.54 \mathrm{E}+00$ \\
\hline ENSBTAG00000014589 & $3.24 \mathrm{E}-10$ & $2.10 \mathrm{E}-10$ & CLDN18 & $1.54 \mathrm{E}+00$ \\
\hline ENSBTAG00000011742 & $6.03 \mathrm{E}-10$ & $3.88 \mathrm{E}-10$ & TNIK & $1.55 \mathrm{E}+00$ \\
\hline ENSBTAG00000013142 & $2.26 \mathrm{E}-10$ & $1.46 \mathrm{E}-10$ & MYNN & $1.55 \mathrm{E}+00$ \\
\hline ENSBTAG00000015898 & $2.06 \mathrm{E}-10$ & $1.32 \mathrm{E}-10$ & RABL3 & $1.56 \mathrm{E}+00$ \\
\hline ENSBTAG00000005503 & $5.68 \mathrm{E}-10$ & $3.63 \mathrm{E}-10$ & PRMT2 & $1.56 \mathrm{E}+00$ \\
\hline ENSBTAG00000013118 & $3.02 \mathrm{E}-10$ & $1.92 \mathrm{E}-10$ & CD86 & $1.58 \mathrm{E}+00$ \\
\hline ENSBTAG00000000905 & $1.02 \mathrm{E}-09$ & $6.44 \mathrm{E}-10$ & RAB6B & $1.58 \mathrm{E}+00$ \\
\hline ENSBTAG00000017180 & $1.11 \mathrm{E}-10$ & $7.01 \mathrm{E}-11$ & SLITRK3 & $1.59 E+00$ \\
\hline ENSBTAG00000039255 & $2.42 \mathrm{E}-10$ & $1.52 \mathrm{E}-10$ & CLDN17 & $1.59 E+00$ \\
\hline ENSBTAG00000016624 & $9.60 \mathrm{E}-11$ & $6.04 \mathrm{E}-11$ & & $1.59 \mathrm{E}+00$ \\
\hline ENSBTAG00000011645 & $1.52 \mathrm{E}-10$ & $9.55 \mathrm{E}-11$ & & $1.59 E+00$ \\
\hline ENSBTAG00000047293 & $4.72 \mathrm{E}-11$ & $2.97 \mathrm{E}-11$ & NRIP1 & $1.59 E+00$ \\
\hline ENSBTAG00000031397 & $1.79 \mathrm{E}-10$ & 1.13E-10 & P2RY13 & $1.59 E+00$ \\
\hline ENSBTAG00000026259 & $4.89 \mathrm{E}-10$ & $3.08 \mathrm{E}-10$ & & $1.59 \mathrm{E}+00$ \\
\hline ENSBTAG00000039334 & $6.03 \mathrm{E}-10$ & $3.79 \mathrm{E}-10$ & & $1.59 E+00$ \\
\hline ENSBTAG00000023447 & $2.65 \mathrm{E}-10$ & $1.67 \mathrm{E}-10$ & & $1.58 \mathrm{E}+00$ \\
\hline ENSBTAG00000004126 & $1.13 \mathrm{E}-10$ & 7.12E-11 & MLF1 & $1.59 E+00$ \\
\hline ENSBTAG00000013141 & $1.70 \mathrm{E}-10$ & $1.07 \mathrm{E}-10$ & ACTRT3 & $1.58 \mathrm{E}+00$ \\
\hline ENSBTAG00000037933 & $3.30 \mathrm{E}-10$ & 2.07E-10 & KRTAP13-3 & $1.59 E+00$ \\
\hline ENSBTAG00000046040 & $2.11 \mathrm{E}-10$ & $1.33 \mathrm{E}-10$ & & $1.59 E+00$ \\
\hline ENSBTAG00000038111 & $2.28 \mathrm{E}-10$ & $1.44 \mathrm{E}-10$ & & $1.58 \mathrm{E}+00$ \\
\hline ENSBTAG00000008343 & $1.79 \mathrm{E}-10$ & $1.13 \mathrm{E}-10$ & & $1.58 \mathrm{E}+00$ \\
\hline ENSBTAG00000045820 & $7.65 E-10$ & $4.81 \mathrm{E}-10$ & & $1.59 E+00$ \\
\hline ENSBTAG00000046015 & $1.33 \mathrm{E}-09$ & $8.30 \mathrm{E}-10$ & & $1.60 \mathrm{E}+00$ \\
\hline ENSBTAG00000006548 & $2.59 \mathrm{E}-10$ & $1.61 \mathrm{E}-10$ & MCCC1 & $1.61 E+00$ \\
\hline ENSBTAG00000023907 & $4.88 \mathrm{E}-10$ & $3.04 \mathrm{E}-10$ & COL18A1 & $1.60 E+00$ \\
\hline ENSBTAG00000012467 & $3.55 \mathrm{E}-10$ & $2.21 E-10$ & MASP1 & $1.61 E+00$ \\
\hline ENSBTAG00000020787 & $5.71 \mathrm{E}-10$ & 3.53E-10 & PIK3R4 & $1.62 E+00$ \\
\hline ENSBTAG00000048057 & $2.85 \mathrm{E}-10$ & $1.74 \mathrm{E}-10$ & & $1.64 \mathrm{E}+00$ \\
\hline ENSBTAG00000006222 & $2.76 \mathrm{E}-10$ & $1.68 \mathrm{E}-10$ & TFDP2 & $1.64 \mathrm{E}+00$ \\
\hline
\end{tabular}




\begin{tabular}{|c|c|c|c|c|}
\hline ENSBTAG00000045704 & $7.16 \mathrm{E}-10$ & $4.34 \mathrm{E}-10$ & IGSF11 & $1.65 E+00$ \\
\hline ENSBTAG00000023416 & $2.16 \mathrm{E}-10$ & $1.30 \mathrm{E}-10$ & PPP2R3A & $1.66 \mathrm{E}+00$ \\
\hline ENSBTAG00000012837 & $7.78 \mathrm{E}-10$ & $4.68 \mathrm{E}-10$ & COL6A6 & $1.66 \mathrm{E}+00$ \\
\hline ENSBTAG00000005162 & $2.14 \mathrm{E}-10$ & $1.28 \mathrm{E}-10$ & STX19 & $1.67 E+00$ \\
\hline ENSBTAG00000002462 & $5.59 \mathrm{E}-10$ & $3.33 \mathrm{E}-10$ & MRPL47 & $1.68 \mathrm{E}+00$ \\
\hline ENSBTAG00000001697 & $2.37 \mathrm{E}-10$ & $1.42 \mathrm{E}-10$ & TRA2B & $1.67 \mathrm{E}+00$ \\
\hline ENSBTAG00000000752 & $4.29 \mathrm{E}-10$ & $2.55 E-10$ & SGO1 & $1.68 \mathrm{E}+00$ \\
\hline ENSBTAG00000017416 & $1.19 \mathrm{E}-10$ & 7.04E-11 & KPNA4 & $1.68 \mathrm{E}+00$ \\
\hline ENSBTAG00000014567 & $2.59 \mathrm{E}-10$ & $1.52 \mathrm{E}-10$ & MYLK & $1.70 \mathrm{E}+00$ \\
\hline ENSBTAG00000007933 & $2.63 \mathrm{E}-10$ & $1.52 \mathrm{E}-10$ & C3orf58 & $1.73 E+00$ \\
\hline ENSBTAG00000019060 & $2.04 \mathrm{E}-10$ & $1.18 \mathrm{E}-10$ & CLDN11 & $1.72 \mathrm{E}+00$ \\
\hline ENSBTAG00000007273 & $5.76 \mathrm{E}-10$ & $3.31 \mathrm{E}-10$ & TF & $1.74 \mathrm{E}+00$ \\
\hline ENSBTAG00000007331 & $2.40 \mathrm{E}-10$ & $1.38 \mathrm{E}-10$ & PLOD2 & $1.74 \mathrm{E}+00$ \\
\hline ENSBTAG00000016999 & $3.01 \mathrm{E}-10$ & $1.72 \mathrm{E}-10$ & & $1.75 \mathrm{E}+00$ \\
\hline ENSBTAG00000018189 & $4.27 \mathrm{E}-10$ & $2.44 \mathrm{E}-10$ & & $1.75 E+00$ \\
\hline ENSBTAG00000004705 & $2.94 \mathrm{E}-10$ & $1.64 \mathrm{E}-10$ & ILDR1 & $1.80 \mathrm{E}+00$ \\
\hline ENSBTAG00000031435 & $4.74 \mathrm{E}-10$ & $2.62 \mathrm{E}-10$ & SELENOT & $1.81 E+00$ \\
\hline ENSBTAG00000000648 & $1.76 \mathrm{E}-10$ & $9.74 E-11$ & ADAMTS5 & $1.81 \mathrm{E}+00$ \\
\hline ENSBTAG00000019314 & $3.41 \mathrm{E}-10$ & $1.88 \mathrm{E}-10$ & USP25 & $1.81 \mathrm{E}+00$ \\
\hline ENSBTAG00000007394 & $9.43 \mathrm{E}-10$ & $5.20 \mathrm{E}-10$ & & $1.81 E+00$ \\
\hline ENSBTAG00000007666 & $2.04 \mathrm{E}-10$ & $1.12 \mathrm{E}-10$ & IGF2BP2 & $1.82 \mathrm{E}+00$ \\
\hline ENSBTAG00000000588 & $2.33 \mathrm{E}-10$ & $1.27 \mathrm{E}-10$ & CHODL & $1.83 \mathrm{E}+00$ \\
\hline ENSBTAG00000013465 & $2.42 \mathrm{E}-10$ & $1.31 \mathrm{E}-10$ & NXPE3 & $1.85 E+00$ \\
\hline ENSBTAG00000044075 & $6.15 \mathrm{E}-10$ & $3.34 \mathrm{E}-10$ & SRPRB & $1.84 \mathrm{E}+00$ \\
\hline ENSBTAG00000000746 & $4.39 \mathrm{E}-10$ & $2.38 \mathrm{E}-10$ & КАТ2B & $1.84 \mathrm{E}+00$ \\
\hline ENSBTAG00000006811 & $3.77 \mathrm{E}-10$ & $2.02 \mathrm{E}-10$ & EPHA3 & $1.87 \mathrm{E}+00$ \\
\hline ENSBTAG00000017531 & $2.52 \mathrm{E}-10$ & $1.35 \mathrm{E}-10$ & FETUB & $1.86 \mathrm{E}+00$ \\
\hline ENSBTAG00000015460 & $3.41 \mathrm{E}-10$ & $1.81 \mathrm{E}-10$ & TP63 & $1.88 \mathrm{E}+00$ \\
\hline ENSBTAG00000040161 & $3.20 \mathrm{E}-10$ & $1.69 \mathrm{E}-10$ & & $1.89 \mathrm{E}+00$ \\
\hline ENSBTAG00000021819 & $3.87 \mathrm{E}-10$ & 2.03E-10 & IFNAR1 & $1.91 E+00$ \\
\hline ENSBTAG00000040031 & $1.20 \mathrm{E}-10$ & $6.29 \mathrm{E}-11$ & ZNF148 & $1.90 \mathrm{E}+00$ \\
\hline ENSBTAG00000030581 & $2.56 \mathrm{E}-10$ & $1.34 \mathrm{E}-10$ & TBC1D5 & $1.91 \mathrm{E}+00$ \\
\hline ENSBTAG00000006409 & $1.55 \mathrm{E}-10$ & $8.12 \mathrm{E}-11$ & LRRC34 & $1.90 \mathrm{E}+00$ \\
\hline ENSBTAG00000002460 & $3.53 \mathrm{E}-10$ & $1.84 \mathrm{E}-10$ & ACTL6A & $1.92 E+00$ \\
\hline ENSBTAG00000004459 & $6.31 \mathrm{E}-10$ & $3.29 \mathrm{E}-10$ & & $1.92 \mathrm{E}+00$ \\
\hline ENSBTAG00000021987 & 1.14E-09 & $5.90 \mathrm{E}-10$ & GPX5 & $1.92 \mathrm{E}+00$ \\
\hline ENSBTAG00000033166 & $1.21 \mathrm{E}-10$ & $6.22 \mathrm{E}-11$ & SLC15A2 & $1.94 \mathrm{E}+00$ \\
\hline ENSBTAG00000014915 & $3.16 \mathrm{E}-10$ & $1.62 \mathrm{E}-10$ & ETV5 & $1.95 E+00$ \\
\hline ENSBTAG00000018657 & $2.65 \mathrm{E}-10$ & $1.36 \mathrm{E}-10$ & ATP13A5 & $1.95 \mathrm{E}+00$ \\
\hline ENSBTAG00000018994 & $3.93 \mathrm{E}-10$ & $2.02 \mathrm{E}-10$ & TNFSF10 & $1.95 \mathrm{E}+00$ \\
\hline ENSBTAG00000002182 & $7.49 \mathrm{E}-10$ & $3.84 \mathrm{E}-10$ & CoX17 & $1.95 \mathrm{E}+00$ \\
\hline ENSBTAG00000012867 & $2.29 \mathrm{E}-10$ & $1.18 \mathrm{E}-10$ & TXLNA & $1.94 \mathrm{E}+00$ \\
\hline ENSBTAG00000002463 & $3.48 \mathrm{E}-10$ & $1.78 \mathrm{E}-10$ & NDUFB5 & $1.95 \mathrm{E}+00$ \\
\hline ENSBTAG00000004791 & $3.95 \mathrm{E}-10$ & $2.02 \mathrm{E}-10$ & DSCR3 & $1.96 \mathrm{E}+00$ \\
\hline ENSBTAG00000047216 & $3.43 \mathrm{E}-10$ & $1.74 \mathrm{E}-10$ & NMNAT3 & $1.97 E+00$ \\
\hline ENSBTAG00000000569 & $1.28 \mathrm{E}-10$ & $6.47 E-11$ & HES1 & $1.98 \mathrm{E}+00$ \\
\hline ENSBTAG00000014441 & $9.03 \mathrm{E}-10$ & $4.56 \mathrm{E}-10$ & CD96 & $1.98 \mathrm{E}+00$ \\
\hline ENSBTAG00000005652 & $1.54 \mathrm{E}-10$ & $7.75 E-11$ & ALG3 & $1.98 \mathrm{E}+00$ \\
\hline ENSBTAG00000046637 & $6.26 \mathrm{E}-10$ & $3.15 \mathrm{E}-10$ & ZBED2 & $1.99 \mathrm{E}+00$ \\
\hline ENSBTAG00000002767 & $9.88 \mathrm{E}-10$ & 4.96E-10 & GNB4 & $1.99 \mathrm{E}+00$ \\
\hline ENSBTAG00000000750 & $1.75 \mathrm{E}-10$ & $8.73 E-11$ & MSL2 & $2.00 \mathrm{E}+00$ \\
\hline ENSBTAG00000014564 & $4.65 \mathrm{E}-10$ & $2.32 \mathrm{E}-10$ & XXYLT1 & $2.00 E+00$ \\
\hline ENSBTAG00000006947 & $2.24 \mathrm{E}-10$ & $1.12 \mathrm{E}-10$ & & $2.00 \mathrm{E}+00$ \\
\hline ENSBTAG00000013938 & $3.69 \mathrm{E}-10$ & $1.83 \mathrm{E}-10$ & PHC3 & $2.01 E+00$ \\
\hline ENSBTAG00000012646 & $8.72 \mathrm{E}-10$ & $4.32 \mathrm{E}-10$ & PLCXD2 & $2.02 \mathrm{E}+00$ \\
\hline ENSBTAG00000010357 & $5.91 \mathrm{E}-10$ & $2.92 \mathrm{E}-10$ & ST6GAL1 & $2.02 E+00$ \\
\hline ENSBTAG00000040193 & 1.07E-09 & $5.25 \mathrm{E}-10$ & COLQ & $2.04 E+00$ \\
\hline ENSBTAG00000002132 & $5.48 \mathrm{E}-10$ & $2.69 \mathrm{E}-10$ & DRD3 & $2.04 \mathrm{E}+00$ \\
\hline ENSBTAG00000011928 & $2.71 \mathrm{E}-10$ & $1.32 \mathrm{E}-10$ & ZBTB20 & $2.05 E+00$ \\
\hline ENSBTAG00000048090 & $2.39 \mathrm{E}-10$ & 1.17E-10 & UBE2G2 & $2.04 E+00$ \\
\hline ENSBTAG00000017103 & $3.59 \mathrm{E}-10$ & $1.75 \mathrm{E}-10$ & MAP3K13 & $2.05 E+00$ \\
\hline ENSBTAG00000000290 & $2.21 \mathrm{E}-10$ & $1.07 E-10$ & ATP11B & $2.06 \mathrm{E}+00$ \\
\hline ENSBTAG00000000476 & $2.23 \mathrm{E}-10$ & $1.08 \mathrm{E}-10$ & THPO & $2.07 E+00$ \\
\hline ENSBTAG00000003135 & $8.08 \mathrm{E}-10$ & $3.89 \mathrm{E}-10$ & IQCJ & $2.08 \mathrm{E}+00$ \\
\hline ENSBTAG00000009232 & $2.17 \mathrm{E}-10$ & $1.04 \mathrm{E}-10$ & PIK3CA & $2.08 \mathrm{E}+00$ \\
\hline ENSBTAG00000001403 & $5.31 \mathrm{E}-10$ & $2.54 \mathrm{E}-10$ & PRKCI & $2.09 E+00$ \\
\hline ENSBTAG00000035710 & $4.95 \mathrm{E}-10$ & $2.37 \mathrm{E}-10$ & ZBBX & $2.09 E+00$ \\
\hline ENSBTAG00000009106 & $4.73 \mathrm{E}-10$ & $2.25 \mathrm{E}-10$ & EIF2A & $2.10 \mathrm{E}+00$ \\
\hline ENSBTAG00000013439 & $7.42 \mathrm{E}-10$ & $3.54 \mathrm{E}-10$ & ARHGEF26 & $2.10 \mathrm{E}+00$ \\
\hline ENSBTAG00000019625 & $4.14 \mathrm{E}-10$ & $1.97 \mathrm{E}-10$ & EHHADH & $2.10 E+00$ \\
\hline
\end{tabular}




\begin{tabular}{|c|c|c|c|c|}
\hline ENSBTAG00000046051 & $1.42 \mathrm{E}-10$ & $6.69 \mathrm{E}-11$ & & $2.12 \mathrm{E}+00$ \\
\hline ENSBTAG00000010462 & 4.06E-10 & $1.90 \mathrm{E}-10$ & $\mathrm{ROBO} 2$ & $2.13 E+00$ \\
\hline ENSBTAG00000000597 & $5.93 \mathrm{E}-10$ & $2.77 \mathrm{E}-10$ & TMPRSS15 & $2.14 \mathrm{E}+00$ \\
\hline ENSBTAG00000013769 & 2.27E-10 & $1.05 \mathrm{E}-10$ & NECTIN3 & $2.16 \mathrm{E}+00$ \\
\hline ENSBTAG00000013203 & $3.71 \mathrm{E}-10$ & $1.72 \mathrm{E}-10$ & GP5 & $2.16 \mathrm{E}+00$ \\
\hline ENSBTAG00000034347 & $6.31 \mathrm{E}-10$ & $2.91 \mathrm{E}-10$ & RBM11 & $2.17 \mathrm{E}+00$ \\
\hline ENSBTAG00000039442 & $2.72 \mathrm{E}-10$ & 1.25E-10 & SEC62 & $2.18 \mathrm{E}+00$ \\
\hline ENSBTAG00000008240 & $7.42 \mathrm{E}-10$ & $3.40 \mathrm{E}-10$ & GALNT15 & $2.18 \mathrm{E}+00$ \\
\hline ENSBTAG00000021899 & $2.57 \mathrm{E}-10$ & 1.17E-10 & PDE9A & $2.20 \mathrm{E}+00$ \\
\hline ENSBTAG00000001464 & $2.62 \mathrm{E}-10$ & $1.19 \mathrm{E}-10$ & GPR156 & $2.20 E+00$ \\
\hline ENSBTAG00000016848 & $1.78 \mathrm{E}-10$ & $8.08 \mathrm{E}-11$ & GTF2E1 & $2.20 \mathrm{E}+00$ \\
\hline ENSBTAG00000040199 & $2.59 \mathrm{E}-10$ & $1.16 \mathrm{E}-10$ & POLR2H & $2.23 \mathrm{E}+00$ \\
\hline ENSBTAG00000006451 & 4.16E-10 & $1.86 \mathrm{E}-10$ & GAP43 & $2.24 \mathrm{E}+00$ \\
\hline ENSBTAG00000024162 & $3.15 \mathrm{E}-10$ & $1.41 \mathrm{E}-10$ & SLC19A1 & $2.23 \mathrm{E}+00$ \\
\hline ENSBTAG00000002075 & $5.71 \mathrm{E}-10$ & $2.54 \mathrm{E}-10$ & MME & $2.25 E+00$ \\
\hline ENSBTAG00000004742 & $3.92 \mathrm{E}-10$ & $1.74 \mathrm{E}-10$ & RUNX1 & $2.25 \mathrm{E}+00$ \\
\hline ENSBTAG00000003151 & $1.94 \mathrm{E}-10$ & $8.50 \mathrm{E}-11$ & & $2.28 \mathrm{E}+00$ \\
\hline ENSBTAG00000013378 & $1.46 \mathrm{E}-10$ & $6.38 \mathrm{E}-11$ & & $2.28 \mathrm{E}+00$ \\
\hline ENSBTAG00000018682 & $4.32 \mathrm{E}-10$ & $1.89 \mathrm{E}-10$ & SETD4 & $2.28 \mathrm{E}+00$ \\
\hline ENSBTAG00000003378 & $3.13 \mathrm{E}-10$ & $1.37 \mathrm{E}-10$ & ACAP2 & $2.29 E+00$ \\
\hline ENSBTAG00000044172 & $2.88 \mathrm{E}-10$ & $1.24 \mathrm{E}-10$ & LMLN & $2.32 \mathrm{E}+00$ \\
\hline ENSBTAG00000013148 & $6.50 \mathrm{E}-10$ & $2.79 \mathrm{E}-10$ & CLDN1 & $2.33 E+00$ \\
\hline ENSBTAG00000016556 & $6.58 \mathrm{E}-10$ & $2.80 \mathrm{E}-10$ & GCSAM & $2.35 \mathrm{E}+00$ \\
\hline ENSBTAG00000021771 & $4.01 \mathrm{E}-10$ & $1.70 \mathrm{E}-10$ & PTTG1IP & $2.36 \mathrm{E}+00$ \\
\hline ENSBTAG00000007818 & $2.72 \mathrm{E}-10$ & $1.14 \mathrm{E}-10$ & POFUT2 & $2.38 \mathrm{E}+00$ \\
\hline ENSBTAG00000046104 & $2.47 \mathrm{E}-10$ & $1.04 \mathrm{E}-10$ & B3GALNT1 & $2.37 E+00$ \\
\hline ENSBTAG00000019557 & $3.98 \mathrm{E}-10$ & $1.67 \mathrm{E}-10$ & NR112 & $2.38 \mathrm{E}+00$ \\
\hline ENSBTAG00000032265 & $2.86 \mathrm{E}-10$ & $1.20 \mathrm{E}-10$ & & $2.38 \mathrm{E}+00$ \\
\hline ENSBTAG00000011598 & $1.68 \mathrm{E}-10$ & 7.07E-11 & sox2 & $2.38 \mathrm{E}+00$ \\
\hline ENSBTAG00000022275 & $3.82 \mathrm{E}-10$ & $1.60 \mathrm{E}-10$ & & $2.39 E+00$ \\
\hline ENSBTAG00000040597 & $2.91 \mathrm{E}-10$ & $1.21 \mathrm{E}-10$ & & $2.41 E+00$ \\
\hline ENSBTAG00000016305 & $3.53 \mathrm{E}-10$ & $1.44 \mathrm{E}-10$ & ATP13A4 & $2.45 \mathrm{E}+00$ \\
\hline ENSBTAG00000001265 & $4.46 \mathrm{E}-10$ & $1.83 \mathrm{E}-10$ & HRG & $2.44 \mathrm{E}+00$ \\
\hline ENSBTAG00000004538 & $5.63 \mathrm{E}-10$ & $2.29 \mathrm{E}-10$ & ZNF596 & $2.46 \mathrm{E}+00$ \\
\hline ENSBTAG00000001771 & $3.63 \mathrm{E}-10$ & $1.46 \mathrm{E}-10$ & DYRK1A & $2.48 \mathrm{E}+00$ \\
\hline ENSBTAG00000030836 & 1.84E-10 & 7.34E-11 & ZBTB21 & $2.50 E+00$ \\
\hline ENSBTAG00000021910 & $3.76 \mathrm{E}-10$ & 1.49E-10 & TBL1XR1 & $2.52 E+00$ \\
\hline ENSBTAG00000023666 & $6.62 \mathrm{E}-10$ & $2.62 \mathrm{E}-10$ & & $2.53 E+00$ \\
\hline ENSBTAG00000031709 & $2.85 \mathrm{E}-10$ & $1.12 \mathrm{E}-10$ & PDCD10 & $2.55 \mathrm{E}+00$ \\
\hline ENSBTAG00000021901 & $3.89 \mathrm{E}-10$ & $1.53 \mathrm{E}-10$ & TRPM2 & $2.54 \mathrm{E}+00$ \\
\hline ENSBTAG00000013057 & $2.99 \mathrm{E}-10$ & $1.16 \mathrm{E}-10$ & CBLB & $2.58 \mathrm{E}+00$ \\
\hline ENSBTAG00000004891 & $8.08 \mathrm{E}-10$ & $3.14 \mathrm{E}-10$ & OXNAD1 & $2.57 E+00$ \\
\hline ENSBTAG00000013321 & $4.39 \mathrm{E}-10$ & $1.70 \mathrm{E}-10$ & & $2.58 \mathrm{E}+00$ \\
\hline ENSBTAG00000010688 & $9.79 \mathrm{E}-11$ & 3.79E-11 & CSTA & $2.58 \mathrm{E}+00$ \\
\hline ENSBTAG00000020990 & $5.91 \mathrm{E}-10$ & $2.29 \mathrm{E}-10$ & P2RY14 & $2.58 \mathrm{E}+00$ \\
\hline ENSBTAG00000010485 & $4.39 \mathrm{E}-10$ & $1.70 \mathrm{E}-10$ & MFN1 & $2.58 \mathrm{E}+00$ \\
\hline ENSBTAG00000004599 & $6.17 \mathrm{E}-10$ & $2.38 \mathrm{E}-10$ & TAGLN3 & $2.59 E+00$ \\
\hline ENSBTAG00000000088 & $3.88 \mathrm{E}-10$ & $1.49 \mathrm{E}-10$ & ALCAM & $2.60 \mathrm{E}+00$ \\
\hline ENSBTAG00000013292 & $4.52 \mathrm{E}-10$ & $1.72 \mathrm{E}-10$ & SIDT1 & $2.63 E+00$ \\
\hline ENSBTAG00000007105 & $4.69 \mathrm{E}-10$ & $1.78 \mathrm{E}-10$ & & $2.64 \mathrm{E}+00$ \\
\hline ENSBTAG00000046383 & 7.33E-10 & $2.73 \mathrm{E}-10$ & & $2.68 \mathrm{E}+00$ \\
\hline ENSBTAG00000023607 & $2.06 \mathrm{E}-10$ & $7.65 E-11$ & HACD2 & $2.69 E+00$ \\
\hline ENSBTAG00000030670 & $5.23 \mathrm{E}-10$ & $1.93 \mathrm{E}-10$ & PCNT & $2.71 E+00$ \\
\hline ENSBTAG00000015310 & $3.34 \mathrm{E}-10$ & $1.23 \mathrm{E}-10$ & SLC25A36 & $2.72 \mathrm{E}+00$ \\
\hline ENSBTAG00000010658 & $5.67 \mathrm{E}-10$ & $2.05 \mathrm{E}-10$ & PFKL & $2.76 \mathrm{E}+00$ \\
\hline ENSBTAG00000001324 & $4.32 \mathrm{E}-10$ & $1.57 \mathrm{E}-10$ & SLCO2A1 & $2.75 \mathrm{E}+00$ \\
\hline ENSBTAG00000011584 & $1.53 \mathrm{E}-10$ & $5.51 \mathrm{E}-11$ & $\mathrm{CHST} 2$ & $2.78 \mathrm{E}+00$ \\
\hline ENSBTAG00000011383 & $3.71 \mathrm{E}-10$ & $1.33 \mathrm{E}-10$ & SNX4 & $2.79 E+00$ \\
\hline ENSBTAG00000021396 & $3.18 \mathrm{E}-10$ & $1.14 \mathrm{E}-10$ & HEG1 & $2.78 \mathrm{E}+00$ \\
\hline ENSBTAG00000024815 & 4.77E-10 & $1.71 \mathrm{E}-10$ & ANKRD28 & $2.79 E+00$ \\
\hline ENSBTAG00000021272 & $5.06 \mathrm{E}-10$ & $1.81 \mathrm{E}-10$ & $A B C G 1$ & $2.80 \mathrm{E}+00$ \\
\hline ENSBTAG00000023377 & $8.85 \mathrm{E}-10$ & $3.11 \mathrm{E}-10$ & SH3BP5 & $2.85 \mathrm{E}+00$ \\
\hline ENSBTAG00000031010 & $5.08 \mathrm{E}-10$ & $1.79 \mathrm{E}-10$ & ACAD11 & $2.84 \mathrm{E}+00$ \\
\hline ENSBTAG00000018607 & 7.02E-10 & $2.45 \mathrm{E}-10$ & TSPEAR & $2.87 E+00$ \\
\hline ENSBTAG00000047341 & $3.25 \mathrm{E}-10$ & $1.14 \mathrm{E}-10$ & & $2.85 \mathrm{E}+00$ \\
\hline ENSBTAG00000014140 & $3.83 \mathrm{E}-10$ & $1.33 \mathrm{E}-10$ & ATP1B3 & $2.87 E+00$ \\
\hline ENSBTAG00000047093 & $1.13 \mathrm{E}-09$ & $3.93 \mathrm{E}-10$ & & $2.88 \mathrm{E}+00$ \\
\hline ENSBTAG00000000183 & $5.65 \mathrm{E}-10$ & $1.95 \mathrm{E}-10$ & TRAT1 & $2.90 E+00$ \\
\hline ENSBTAG00000032656 & $1.94 \mathrm{E}-10$ & $6.66 \mathrm{E}-11$ & CPN2 & $2.91 \mathrm{E}+00$ \\
\hline ENSBTAG00000021284 & $7.68 \mathrm{E}-10$ & $2.58 \mathrm{E}-10$ & RSPH1 & $2.98 \mathrm{E}+00$ \\
\hline
\end{tabular}




\begin{tabular}{|c|c|c|c|c|}
\hline ENSBTAG00000003916 & 7.73E-10 & $2.57 \mathrm{E}-10$ & DPH3 & $3.01 E+00$ \\
\hline ENSBTAG00000017060 & $5.02 \mathrm{E}-10$ & $1.67 \mathrm{E}-10$ & ITGB2 & $3.01 E+00$ \\
\hline ENSBTAG00000000213 & $3.28 \mathrm{E}-10$ & $1.07 \mathrm{E}-10$ & TMEM39A & $3.06 \mathrm{E}+00$ \\
\hline ENSBTAG00000009392 & 3.05E-10 & $9.85 \mathrm{E}-11$ & PLA1A & $3.10 \mathrm{E}+00$ \\
\hline ENSBTAG00000012881 & $2.20 \mathrm{E}-10$ & $7.08 \mathrm{E}-11$ & EIF4G1 & $3.11 \mathrm{E}+00$ \\
\hline ENSBTAG00000020121 & $6.63 \mathrm{E}-10$ & $2.12 \mathrm{E}-10$ & RWDD2B & $3.13 E+00$ \\
\hline ENSBTAG00000001442 & 4.74E-10 & $1.51 \mathrm{E}-10$ & & $3.14 E+00$ \\
\hline ENSBTAG00000013806 & $4.83 \mathrm{E}-10$ & $1.53 \mathrm{E}-10$ & EFHB & $3.15 E+00$ \\
\hline ENSBTAG00000009235 & $4.85 \mathrm{E}-10$ & $1.52 \mathrm{E}-10$ & KCNMB3 & $3.19 E+00$ \\
\hline ENSBTAG00000033319 & $3.39 \mathrm{E}-10$ & $1.05 \mathrm{E}-10$ & CD200 & $3.22 \mathrm{E}+00$ \\
\hline ENSBTAG00000021286 & $6.56 \mathrm{E}-10$ & $2.00 \mathrm{E}-10$ & SLC37A1 & $3.28 \mathrm{E}+00$ \\
\hline ENSBTAG00000001893 & $6.36 \mathrm{E}-10$ & $1.94 \mathrm{E}-10$ & TM4SF4 & $3.28 \mathrm{E}+00$ \\
\hline ENSBTAG00000006174 & 2.03E-10 & $6.18 \mathrm{E}-11$ & TMEM212 & $3.29 \mathrm{E}+00$ \\
\hline ENSBTAG00000012908 & $2.02 \mathrm{E}-10$ & $6.11 \mathrm{E}-11$ & HSPA13 & $3.31 E+00$ \\
\hline ENSBTAG00000012023 & $1.39 \mathrm{E}-09$ & $4.18 \mathrm{E}-10$ & & $3.32 E+00$ \\
\hline ENSBTAG00000001235 & 8.07E-10 & $2.40 \mathrm{E}-10$ & & $3.36 \mathrm{E}+00$ \\
\hline ENSBTAG00000006893 & $2.49 \mathrm{E}-10$ & 7.44E-11 & BBX & $3.35 E+00$ \\
\hline ENSBTAG00000011192 & $4.25 \mathrm{E}-10$ & $1.26 \mathrm{E}-10$ & GPR87 & $3.37 \mathrm{E}+00$ \\
\hline ENSBTAG00000003585 & $1.95 \mathrm{E}-10$ & $5.71 \mathrm{E}-11$ & CD47 & $3.42 E+00$ \\
\hline ENSBTAG00000016228 & $4.45 \mathrm{E}-10$ & $1.28 \mathrm{E}-10$ & NMD3 & $3.47 E+00$ \\
\hline ENSBTAG00000023393 & 2.37E-10 & $6.72 \mathrm{E}-11$ & AIRE & $3.52 E+00$ \\
\hline ENSBTAG00000000742 & $5.01 \mathrm{E}-10$ & $1.43 \mathrm{E}-10$ & AMOTL2 & $3.50 E+00$ \\
\hline ENSBTAG00000011414 & $1.62 \mathrm{E}-10$ & 4.54E-11 & EIF2B5 & $3.57 \mathrm{E}+00$ \\
\hline ENSBTAG00000002878 & $3.15 \mathrm{E}-10$ & $8.81 \mathrm{E}-11$ & CHRD & $3.58 \mathrm{E}+00$ \\
\hline ENSBTAG00000018059 & $3.89 \mathrm{E}-10$ & $1.08 \mathrm{E}-10$ & CD80 & $3.60 E+00$ \\
\hline ENSBTAG00000007106 & $5.54 \mathrm{E}-10$ & $1.53 \mathrm{E}-10$ & & $3.62 E+00$ \\
\hline ENSBTAG00000037662 & $1.16 \mathrm{E}-09$ & $3.22 \mathrm{E}-10$ & & $3.60 E+00$ \\
\hline ENSBTAG00000019336 & $1.93 \mathrm{E}-10$ & $5.32 \mathrm{E}-11$ & DVL3 & $3.63 E+00$ \\
\hline ENSBTAG00000004971 & $3.55 \mathrm{E}-10$ & $9.66 \mathrm{E}-11$ & GRAMD1C & $3.67 E+00$ \\
\hline ENSBTAG00000040144 & 7.51E-10 & $2.02 \mathrm{E}-10$ & & $3.72 E+00$ \\
\hline ENSBTAG00000011626 & $5.80 \mathrm{E}-10$ & $1.55 \mathrm{E}-10$ & ATP2C1 & $3.74 \mathrm{E}+00$ \\
\hline ENSBTAG00000035844 & 4.97E-10 & $1.31 \mathrm{E}-10$ & & $3.79 E+00$ \\
\hline ENSBTAG00000015008 & $3.99 \mathrm{E}-10$ & $1.03 \mathrm{E}-10$ & CPA3 & $3.87 E+00$ \\
\hline ENSBTAG00000010394 & $3.04 \mathrm{E}-10$ & $7.82 \mathrm{E}-11$ & MCF2L2 & $3.89 E+00$ \\
\hline ENSBTAG00000023407 & $2.02 \mathrm{E}-10$ & 5.09E-11 & B3GALT5 & $3.97 E+00$ \\
\hline ENSBTAG00000040304 & 2.34E-10 & $5.88 \mathrm{E}-11$ & HTR3E & $3.97 E+00$ \\
\hline ENSBTAG00000046854 & 7.34E-10 & $1.84 \mathrm{E}-10$ & & $3.99 \mathrm{E}+00$ \\
\hline ENSBTAG00000004124 & $5.31 \mathrm{E}-10$ & $1.30 \mathrm{E}-10$ & CPOX & $4.08 \mathrm{E}+00$ \\
\hline ENSBTAG00000005769 & $7.60 \mathrm{E}-10$ & $1.85 \mathrm{E}-10$ & & $4.11 \mathrm{E}+00$ \\
\hline ENSBTAG00000014880 & $2.84 \mathrm{E}-10$ & $6.87 \mathrm{E}-11$ & & $4.13 E+00$ \\
\hline ENSBTAG00000012581 & $5.19 \mathrm{E}-10$ & $1.22 \mathrm{E}-10$ & TIMMDC1 & $4.25 \mathrm{E}+00$ \\
\hline ENSBTAG00000005658 & $1.49 \mathrm{E}-10$ & $3.49 \mathrm{E}-11$ & ECE2 & $4.25 E+00$ \\
\hline ENSBTAG00000005209 & $5.16 \mathrm{E}-10$ & $1.18 \mathrm{E}-10$ & VPS8 & $4.37 \mathrm{E}+00$ \\
\hline ENSBTAG00000000502 & 1.20E-09 & $2.74 \mathrm{E}-10$ & & $4.39 E+00$ \\
\hline ENSBTAG00000005723 & $4.52 \mathrm{E}-10$ & $1.01 \mathrm{E}-10$ & NEPRO & $4.47 \mathrm{E}+00$ \\
\hline ENSBTAG00000040399 & $1.23 \mathrm{E}-10$ & $2.74 \mathrm{E}-11$ & VWA5B2 & $4.49 E+00$ \\
\hline ENSBTAG00000009154 & $5.42 \mathrm{E}-10$ & $1.17 \mathrm{E}-10$ & U2SURP & $4.63 E+00$ \\
\hline ENSBTAG00000014460 & $3.35 \mathrm{E}-10$ & $7.20 \mathrm{E}-11$ & PARL & $4.64 \mathrm{E}+00$ \\
\hline ENSBTAG00000012120 & $1.25 \mathrm{E}-10$ & $2.69 \mathrm{E}-11$ & TIPARP & $4.62 \mathrm{E}+00$ \\
\hline ENSBTAG00000019960 & $3.52 \mathrm{E}-10$ & $7.52 \mathrm{E}-11$ & TM4SF18 & $4.68 \mathrm{E}+00$ \\
\hline ENSBTAG00000009104 & $2.16 \mathrm{E}-10$ & $4.52 \mathrm{E}-11$ & SERP1 & $4.76 \mathrm{E}+00$ \\
\hline ENSBTAG00000046384 & $5.65 \mathrm{E}-10$ & $1.18 \mathrm{E}-10$ & KRTAP24-1 & $4.79 E+00$ \\
\hline ENSBTAG00000039602 & $5.84 \mathrm{E}-10$ & $1.22 \mathrm{E}-10$ & & $4.79 E+00$ \\
\hline ENSBTAG00000031795 & 8.07E-10 & $1.68 \mathrm{E}-10$ & & $4.80 E+00$ \\
\hline ENSBTAG00000014713 & $9.21 \mathrm{E}-10$ & $1.80 \mathrm{E}-10$ & RARRES1 & $5.12 \mathrm{E}+00$ \\
\hline ENSBTAG00000034854 & 7.04E-10 & $1.33 \mathrm{E}-10$ & GRIK1 & $5.29 E+00$ \\
\hline ENSBTAG00000006552 & $1.84 \mathrm{E}-10$ & $3.43 \mathrm{E}-11$ & LAMP3 & $5.36 \mathrm{E}+00$ \\
\hline ENSBTAG00000023398 & $3.88 \mathrm{E}-10$ & $7.15 E-11$ & UBASH3A & $5.43 E+00$ \\
\hline ENSBTAG00000017010 & $1.28 \mathrm{E}-09$ & $2.35 \mathrm{E}-10$ & FAM207A & $5.44 E+00$ \\
\hline ENSBTAG00000006920 & $1.46 \mathrm{E}-10$ & $2.63 \mathrm{E}-11$ & $\mathrm{ABCF} 3$ & $5.54 \mathrm{E}+00$ \\
\hline ENSBTAG00000009391 & $3.24 \mathrm{E}-10$ & $5.81 \mathrm{E}-11$ & ADPRH & $5.57 \mathrm{E}+00$ \\
\hline ENSBTAG00000012284 & $2.10 \mathrm{E}-10$ & 3.67E-11 & CLCN2 & $5.71 \mathrm{E}+00$ \\
\hline ENSBTAG00000015475 & $7.15 \mathrm{E}-10$ & $1.21 \mathrm{E}-10$ & MORC1 & $5.91 E+00$ \\
\hline ENSBTAG00000002185 & 4.29E-10 & $7.09 \mathrm{E}-11$ & RNF7 & $6.05 E+00$ \\
\hline ENSBTAG00000001390 & 4.96E-10 & $8.04 \mathrm{E}-11$ & B4GALT4 & $6.17 E+00$ \\
\hline ENSBTAG00000006534 & $2.80 \mathrm{E}-10$ & 4.49E-11 & GTPBP8 & $6.23 E+00$ \\
\hline ENSBTAG00000014034 & $6.28 \mathrm{E}-10$ & $9.91 \mathrm{E}-11$ & PXYLP1 & $6.33 E+00$ \\
\hline ENSBTAG00000011139 & $7.87 \mathrm{E}-10$ & $1.23 \mathrm{E}-10$ & $\mathrm{BCHE}$ & $6.40 E+00$ \\
\hline ENSBTAG00000048011 & $6.51 \mathrm{E}-10$ & $1.02 \mathrm{E}-10$ & GPR160 & $6.38 \mathrm{E}+00$ \\
\hline ENSBTAG00000021280 & 4.46E-10 & $6.87 \mathrm{E}-11$ & TMPRSS3 & $6.48 \mathrm{E}+00$ \\
\hline
\end{tabular}




\begin{tabular}{|c|c|c|c|c|}
\hline ENSBTAG00000030593 & $6.43 \mathrm{E}-10$ & $9.77 \mathrm{E}-11$ & & $6.57 \mathrm{E}+00$ \\
\hline ENSBTAG00000018228 & $8.25 \mathrm{E}-10$ & $1.24 \mathrm{E}-10$ & SAMD7 & $6.65 E+00$ \\
\hline ENSBTAG00000013204 & $2.01 \mathrm{E}-10$ & $3.01 \mathrm{E}-11$ & ATP13A3 & $6.65 \mathrm{E}+00$ \\
\hline ENSBTAG00000014157 & $4.73 \mathrm{E}-10$ & $6.85 \mathrm{E}-11$ & & $6.90 E+00$ \\
\hline ENSBTAG00000010397 & $1.85 \mathrm{E}-10$ & $2.64 \mathrm{E}-11$ & B3GNT5 & $6.99 \mathrm{E}+00$ \\
\hline ENSBTAG00000004601 & $2.81 \mathrm{E}-10$ & $3.93 \mathrm{E}-11$ & ABHD10 & $7.14 \mathrm{E}+00$ \\
\hline ENSBTAG00000011012 & $5.85 \mathrm{E}-10$ & $8.16 \mathrm{E}-11$ & & $7.17 E+00$ \\
\hline ENSBTAG00000017666 & $3.21 \mathrm{E}-10$ & 4.13E-11 & & $7.75 \mathrm{E}+00$ \\
\hline ENSBTAG00000002703 & $5.95 \mathrm{E}-10$ & $7.55 E-11$ & ATP6V1A & $7.87 \mathrm{E}+00$ \\
\hline ENSBTAG00000014724 & $1.30 \mathrm{E}-10$ & $1.63 \mathrm{E}-11$ & EIF4A2 & $7.92 \mathrm{E}+00$ \\
\hline ENSBTAG00000012273 & $1.28 \mathrm{E}-10$ & $1.61 \mathrm{E}-11$ & FAM131A & $7.90 E+00$ \\
\hline ENSBTAG00000006945 & $4.06 \mathrm{E}-10$ & 4.70E-11 & & $8.63 E+00$ \\
\hline ENSBTAG00000044148 & $3.87 \mathrm{E}-10$ & 4.32E-11 & UTS2B & $8.94 \mathrm{E}+00$ \\
\hline ENSBTAG00000030814 & $8.58 \mathrm{E}-10$ & $9.47 \mathrm{E}-11$ & TFF2 & $9.06 \mathrm{E}+00$ \\
\hline ENSBTAG00000005660 & $2.02 \mathrm{E}-10$ & $2.21 \mathrm{E}-11$ & PSMD2 & $9.10 \mathrm{E}+00$ \\
\hline ENSBTAG00000012708 & $3.36 \mathrm{E}-10$ & $3.61 \mathrm{E}-11$ & SERPINI1 & $9.27 \mathrm{E}+00$ \\
\hline ENSBTAG00000014727 & $3.48 \mathrm{E}-10$ & 3.64E-11 & RFC4 & $9.53 E+00$ \\
\hline ENSBTAG00000009933 & $1.21 \mathrm{E}-10$ & $1.27 \mathrm{E}-11$ & DTX3L & $9.49 \mathrm{E}+00$ \\
\hline ENSBTAG00000019202 & $8.76 \mathrm{E}-10$ & $9.13 \mathrm{E}-11$ & EAF1 & $9.59 E+00$ \\
\hline ENSBTAG00000001331 & $8.89 \mathrm{E}-10$ & $9.02 \mathrm{E}-11$ & ERICH6 & $9.84 \mathrm{E}+00$ \\
\hline ENSBTAG00000037393 & $6.10 \mathrm{E}-10$ & $5.88 \mathrm{E}-11$ & ZNF639 & $1.03 E+01$ \\
\hline ENSBTAG00000018688 & $5.63 \mathrm{E}-10$ & $5.27 \mathrm{E}-11$ & CBR3 & $1.07 \mathrm{E}+01$ \\
\hline ENSBTAG00000037951 & $2.56 \mathrm{E}-10$ & $2.35 \mathrm{E}-11$ & KLHL6 & $1.08 \mathrm{E}+01$ \\
\hline ENSBTAG00000018703 & $3.92 \mathrm{E}-10$ & $3.42 \mathrm{E}-11$ & OSTN & $1.14 \mathrm{E}+01$ \\
\hline ENSBTAG00000038103 & 7.11E-10 & 5.84E-11 & & $1.22 \mathrm{E}+01$ \\
\hline ENSBTAG00000001728 & $6.15 \mathrm{E}-10$ & 4.92E-11 & IGSF10 & $1.25 \mathrm{E}+01$ \\
\hline ENSBTAG00000040291 & $1.25 \mathrm{E}-09$ & $9.73 \mathrm{E}-11$ & & $1.28 \mathrm{E}+01$ \\
\hline ENSBTAG00000019813 & $4.56 \mathrm{E}-10$ & $3.13 \mathrm{E}-11$ & ADIPOQ & $1.45 \mathrm{E}+01$ \\
\hline ENSBTAG00000014882 & $6.78 \mathrm{E}-10$ & $4.28 \mathrm{E}-11$ & & $1.58 \mathrm{E}+01$ \\
\hline ENSBTAG00000013456 & $8.53 \mathrm{E}-10$ & $4.96 \mathrm{E}-11$ & TMEM207 & $1.72 \mathrm{E}+01$ \\
\hline ENSBTAG00000014451 & $4.03 E-10$ & $2.21 \mathrm{E}-11$ & YEATS2 & $1.82 \mathrm{E}+01$ \\
\hline ENSBTAG00000017450 & 4.33E-10 & $1.91 \mathrm{E}-11$ & KLHL24 & $2.26 \mathrm{E}+01$ \\
\hline ENSBTAG00000014153 & $2.61 \mathrm{E}-10$ & $9.95 \mathrm{E}-12$ & PKNOX1 & $2.60 \mathrm{E}+01$ \\
\hline ENSBTAG00000012706 & $9.62 \mathrm{E}-10$ & 3.64E-11 & RTP1 & $2.64 \mathrm{E}+01$ \\
\hline ENSBTAG00000005386 & $9.40 \mathrm{E}-10$ & $2.40 \mathrm{E}-11$ & SLC2A2 & $3.90 \mathrm{E}+01$ \\
\hline ENSBTAG00000038131 & $4.12 \mathrm{E}-10$ & 7.39E-12 & & $5.50 \mathrm{E}+01$ \\
\hline ENSBTAG00000001209 & $3.73 \mathrm{E}-10$ & $3.40 \mathrm{E}-12$ & PHLDB2 & $1.07 \mathrm{E}+02$ \\
\hline ENSBTAG00000039257 & 0 & 0 & & $1.00 \mathrm{E}+00$ \\
\hline ENSBTAG00000044154 & 0 & 0 & & $1.00 \mathrm{E}+00$ \\
\hline ENSBTAG00000045873 & $3.23 \mathrm{E}-10$ & 0 & & $3.23 E+03$ \\
\hline ENSBTAG00000024806 & $1.35 \mathrm{E}-10$ & 0 & & $1.35 \mathrm{E}+03$ \\
\hline ENSBTAG00000020460 & $2.14 \mathrm{E}-10$ & 0 & DPPA2 & $2.14 \mathrm{E}+03$ \\
\hline ENSBTAG00000045855 & $5.01 \mathrm{E}-10$ & 0 & & $5.01 E+03$ \\
\hline ENSBTAG00000015892 & $1.20 \mathrm{E}-10$ & 0 & & $1.20 \mathrm{E}+03$ \\
\hline ENSBTAG00000014133 & 0 & 0 & & $1.00 \mathrm{E}+00$ \\
\hline ENSBTAG00000005430 & $1.05 \mathrm{E}-10$ & 0 & MB21D2 & $1.05 E+03$ \\
\hline ENSBTAG00000047841 & 0 & 0 & & $1.00 \mathrm{E}+00$ \\
\hline ENSBTAG00000005657 & $3.80 \mathrm{E}-11$ & 0 & CAMK2N2 & $3.81 E+02$ \\
\hline ENSBTAG00000014459 & $3.07 \mathrm{E}-10$ & 0 & MAP6D1 & $3.07 \mathrm{E}+03$ \\
\hline ENSBTAG00000024623 & $1.30 \mathrm{E}-09$ & 0 & & $1.30 \mathrm{E}+04$ \\
\hline ENSBTAG00000045637 & 0 & 0 & & $1.00 \mathrm{E}+00$ \\
\hline ENSBTAG00000046781 & 0 & 0 & & $1.00 \mathrm{E}+00$ \\
\hline ENSBTAG00000016306 & 0 & 0 & PFDN4 & $1.00 \mathrm{E}+00$ \\
\hline ENSBTAG00000035701 & $8.69 \mathrm{E}-10$ & 0 & & $8.69 E+03$ \\
\hline ENSBTAG00000030629 & $2.77 \mathrm{E}-10$ & 0 & & $2.77 \mathrm{E}+03$ \\
\hline ENSBTAG00000047288 & 0 & 0 & & $1.00 \mathrm{E}+00$ \\
\hline ENSBTAG00000038900 & $4.02 \mathrm{E}-10$ & 0 & & $4.02 \mathrm{E}+03$ \\
\hline ENSBTAG00000040418 & 0 & 0 & PPP1CA & $1.00 \mathrm{E}+00$ \\
\hline ENSBTAG00000007296 & $6.64 \mathrm{E}-10$ & 0 & & $6.64 \mathrm{E}+03$ \\
\hline ENSBTAG00000027113 & $2.00 \mathrm{E}-09$ & 0 & & $2.00 E+04$ \\
\hline ENSBTAG00000014449 & $1.98 \mathrm{E}-10$ & 0 & & $1.98 \mathrm{E}+03$ \\
\hline ENSBTAG00000020997 & 0 & 0 & GPR15 & $1.00 \mathrm{E}+00$ \\
\hline ENSBTAG00000018731 & $7.73 \mathrm{E}-11$ & 0 & & $7.74 \mathrm{E}+02$ \\
\hline ENSBTAG00000047144 & 0 & 0 & & $1.00 \mathrm{E}+00$ \\
\hline ENSBTAG00000005414 & $2.70 \mathrm{E}-10$ & 0 & & $2.70 \mathrm{E}+03$ \\
\hline ENSBTAG00000047250 & 0 & 0 & SPTSSB & $1.00 \mathrm{E}+00$ \\
\hline ENSBTAG00000003383 & $2.71 \mathrm{E}-10$ & 0 & & $2.71 E+03$ \\
\hline ENSBTAG00000017322 & $1.42 \mathrm{E}-10$ & 0 & ARL14 & $1.42 \mathrm{E}+03$ \\
\hline ENSBTAG00000047247 & 0 & 0 & C3orf80 & $1.00 \mathrm{E}+00$ \\
\hline ENSBTAG00000008462 & 0 & 0 & FAM43A & $1.00 \mathrm{E}+00$ \\
\hline
\end{tabular}




\begin{tabular}{|c|c|c|c|c|}
\hline ENSBTAG00000001465 & $4.96 \mathrm{E}-11$ & 0 & P2RY1 & $4.97 \mathrm{E}+02$ \\
\hline ENSBTAG00000019670 & $1.73 \mathrm{E}-10$ & 0 & SUCNR1 & $1.73 E+03$ \\
\hline ENSBTAG00000046738 & $5.21 \mathrm{E}-10$ & 0 & & $5.21 E+03$ \\
\hline ENSBTAG00000031277 & 0 & 0 & FOXL2 & $1.00 \mathrm{E}+00$ \\
\hline ENSBTAG00000048305 & 0 & 0 & & $1.00 \mathrm{E}+00$ \\
\hline ENSBTAG00000035654 & $1.12 \mathrm{E}-10$ & 0 & & $1.12 \mathrm{E}+03$ \\
\hline ENSBTAG00000019577 & $2.84 \mathrm{E}-10$ & 0 & ACKR4 & $2.85 \mathrm{E}+03$ \\
\hline ENSBTAG00000045771 & $6.64 \mathrm{E}-10$ & 0 & KRTAP12-4 & $6.64 \mathrm{E}+03$ \\
\hline ENSBTAG00000046859 & $4.21 \mathrm{E}-10$ & 0 & & $4.21 E+03$ \\
\hline ENSBTAG00000018610 & $7.14 \mathrm{E}-10$ & 0 & & $7.14 \mathrm{E}+03$ \\
\hline ENSBTAG00000000972 & $8.91 \mathrm{E}-10$ & 0 & & $8.91 E+03$ \\
\hline ENSBTAG00000037616 & $8.83 E-11$ & 0 & KCNJ6 & $8.84 \mathrm{E}+02$ \\
\hline ENSBTAG00000039581 & 0 & 4.74E-11 & HOXD4 & $2.11 \mathrm{E}-03$ \\
\hline ENSBTAG00000004314 & 0 & $2.14 \mathrm{E}-10$ & HOXD12 & 4.67E-04 \\
\hline ENSBTAG00000012171 & 0 & $9.42 \mathrm{E}-11$ & & $1.06 \mathrm{E}-03$ \\
\hline ENSBTAG00000009223 & 0 & $7.68 \mathrm{E}-11$ & SFN & $1.30 \mathrm{E}-03$ \\
\hline ENSBTAG00000013170 & $4.55 \mathrm{E}-11$ & $2.68 \mathrm{E}-10$ & CTLA4 & $1.70 \mathrm{E}-01$ \\
\hline ENSBTAG00000030367 & $1.50 \mathrm{E}-10$ & $7.61 \mathrm{E}-10$ & RNF186 & $1.98 \mathrm{E}-01$ \\
\hline ENSBTAG00000012763 & 7.99E-11 & $3.02 \mathrm{E}-10$ & & $2.65 \mathrm{E}-01$ \\
\hline ENSBTAG00000012363 & $3.61 \mathrm{E}-10$ & $1.35 \mathrm{E}-09$ & & $2.68 \mathrm{E}-01$ \\
\hline ENSBTAG00000011909 & $1.14 \mathrm{E}-10$ & $3.91 \mathrm{E}-10$ & ACVR1 & $2.90 \mathrm{E}-01$ \\
\hline ENSBTAG00000015104 & $6.03 E-11$ & $2.03 \mathrm{E}-10$ & CCDC28B & $2.98 \mathrm{E}-01$ \\
\hline ENSBTAG00000009058 & $2.63 \mathrm{E}-10$ & $8.31 \mathrm{E}-10$ & TMEM177 & $3.16 \mathrm{E}-01$ \\
\hline ENSBTAG00000006136 & $2.43 \mathrm{E}-10$ & $5.99 \mathrm{E}-10$ & CDA & 4.06E-01 \\
\hline ENSBTAG00000009952 & $6.34 \mathrm{E}-11$ & $1.42 \mathrm{E}-10$ & C2orf69 & 4.47E-01 \\
\hline ENSBTAG00000044093 & $8.43 E-11$ & $1.86 \mathrm{E}-10$ & A3GALT2 & 4.53E-01 \\
\hline ENSBTAG00000016030 & $7.91 \mathrm{E}-11$ & $1.66 \mathrm{E}-10$ & HOXD10 & 4.77E-01 \\
\hline ENSBTAG00000000851 & $6.49 \mathrm{E}-11$ & $1.36 \mathrm{E}-10$ & CYP20A1 & 4.78E-01 \\
\hline ENSBTAG00000019614 & $3.00 \mathrm{E}-10$ & $6.11 \mathrm{E}-10$ & FAM76A & 4.91E-01 \\
\hline ENSBTAG00000004519 & $4.45 \mathrm{E}-10$ & $8.93 \mathrm{E}-10$ & ALPI & 4.99E-01 \\
\hline ENSBTAG00000012816 & $1.67 \mathrm{E}-10$ & $3.16 \mathrm{E}-10$ & & 5.29E-01 \\
\hline ENSBTAG00000047467 & $7.86 \mathrm{E}-11$ & $1.49 \mathrm{E}-10$ & & $5.28 \mathrm{E}-01$ \\
\hline ENSBTAG00000004267 & $1.15 \mathrm{E}-10$ & $2.04 \mathrm{E}-10$ & & $5.64 \mathrm{E}-01$ \\
\hline ENSBTAG00000047784 & $4.01 \mathrm{E}-10$ & $7.09 \mathrm{E}-10$ & C2orf57 & $5.66 \mathrm{E}-01$ \\
\hline ENSBTAG00000000266 & $1.45 \mathrm{E}-10$ & $2.56 \mathrm{E}-10$ & NAB1 & $5.68 \mathrm{E}-01$ \\
\hline ENSBTAG00000016895 & $8.48 \mathrm{E}-11$ & $1.48 \mathrm{E}-10$ & NIPA2 & 5.73E-01 \\
\hline ENSBTAG00000019231 & $1.13 \mathrm{E}-10$ & $1.97 \mathrm{E}-10$ & MAIP1 & $5.76 \mathrm{E}-01$ \\
\hline ENSBTAG00000011017 & $1.44 \mathrm{E}-10$ & $2.48 \mathrm{E}-10$ & $\mathrm{ABI} 2$ & $5.80 \mathrm{E}-01$ \\
\hline ENSBTAG00000002283 & $1.11 \mathrm{E}-10$ & $1.87 \mathrm{E}-10$ & FZD7 & $5.96 \mathrm{E}-01$ \\
\hline ENSBTAG00000005517 & $5.16 \mathrm{E}-11$ & $8.68 \mathrm{E}-11$ & NOP58 & $5.95 \mathrm{E}-01$ \\
\hline ENSBTAG00000010938 & $7.81 \mathrm{E}-11$ & $1.31 \mathrm{E}-10$ & $\mathrm{C} 2 \mathrm{H} 1$ orf64 & 5.97E-01 \\
\hline ENSBTAG00000019232 & $9.12 \mathrm{E}-11$ & $1.51 \mathrm{E}-10$ & TYW5 & $6.04 \mathrm{E}-01$ \\
\hline ENSBTAG00000015962 & $1.47 \mathrm{E}-10$ & $2.43 \mathrm{E}-10$ & MMADHC & $6.05 \mathrm{E}-01$ \\
\hline ENSBTAG00000015834 & $2.68 \mathrm{E}-10$ & $4.35 \mathrm{E}-10$ & ARHGEF4 & 6.17E-01 \\
\hline ENSBTAG00000012801 & $4.69 \mathrm{E}-10$ & $7.46 \mathrm{E}-10$ & UPP2 & $6.29 \mathrm{E}-01$ \\
\hline ENSBTAG00000014221 & $7.42 \mathrm{E}-11$ & $1.17 \mathrm{E}-10$ & RSG1 & $6.35 \mathrm{E}-01$ \\
\hline ENSBTAG00000039091 & $3.95 \mathrm{E}-10$ & $6.12 \mathrm{E}-10$ & & $6.45 \mathrm{E}-01$ \\
\hline ENSBTAG00000021526 & $5.11 \mathrm{E}-10$ & $7.88 \mathrm{E}-10$ & RPRM & $6.49 \mathrm{E}-01$ \\
\hline ENSBTAG00000011658 & $1.02 \mathrm{E}-10$ & $1.56 \mathrm{E}-10$ & PTPN18 & $6.56 \mathrm{E}-01$ \\
\hline ENSBTAG00000005629 & $4.27 \mathrm{E}-10$ & $6.51 \mathrm{E}-10$ & AIM1L & $6.56 \mathrm{E}-01$ \\
\hline ENSBTAG00000026994 & $3.28 \mathrm{E}-10$ & $4.99 \mathrm{E}-10$ & C2orf88 & 6.57E-01 \\
\hline ENSBTAG00000018199 & $2.33 \mathrm{E}-10$ & $3.53 \mathrm{E}-10$ & ZBTB8A & $6.59 \mathrm{E}-01$ \\
\hline ENSBTAG00000047806 & $2.81 \mathrm{E}-10$ & $4.15 \mathrm{E}-10$ & & $6.76 \mathrm{E}-01$ \\
\hline ENSBTAG00000007554 & 4.54E-10 & $6.58 \mathrm{E}-10$ & IFI6 & $6.90 \mathrm{E}-01$ \\
\hline ENSBTAG00000021653 & $8.79 E-11$ & $1.27 \mathrm{E}-10$ & TRIP12 & $6.92 \mathrm{E}-01$ \\
\hline ENSBTAG00000003403 & $4.80 \mathrm{E}-10$ & $6.92 \mathrm{E}-10$ & PADI2 & $6.94 \mathrm{E}-01$ \\
\hline ENSBTAG00000011971 & $2.68 \mathrm{E}-10$ & $3.85 \mathrm{E}-10$ & NRP2 & 6.97E-01 \\
\hline ENSBTAG00000031595 & $1.49 \mathrm{E}-10$ & $2.13 \mathrm{E}-10$ & & 6.99E-01 \\
\hline ENSBTAG00000005180 & $1.84 \mathrm{E}-10$ & $2.63 \mathrm{E}-10$ & RBMS1 & 7.00E-01 \\
\hline ENSBTAG00000026696 & $4.10 \mathrm{E}-10$ & $5.85 \mathrm{E}-10$ & $\mathrm{DCDC} 2 \mathrm{~B}$ & 7.01E-01 \\
\hline ENSBTAG00000008510 & $4.08 \mathrm{E}-10$ & $5.78 \mathrm{E}-10$ & $\mathrm{FBXO42}$ & 7.07E-01 \\
\hline ENSBTAG00000012629 & $8.80 \mathrm{E}-11$ & $1.23 \mathrm{E}-10$ & ZNF362 & 7.15E-01 \\
\hline ENSBTAG00000005353 & $1.16 \mathrm{E}-10$ & $1.61 \mathrm{E}-10$ & DES & 7.19E-01 \\
\hline ENSBTAG00000016894 & $3.87 \mathrm{E}-10$ & $5.29 \mathrm{E}-10$ & CYFIP1 & 7.31E-01 \\
\hline ENSBTAG00000009163 & $1.83 \mathrm{E}-10$ & $2.49 \mathrm{E}-10$ & TMEM234 & 7.33E-01 \\
\hline ENSBTAG00000018062 & $2.05 \mathrm{E}-10$ & $2.74 \mathrm{E}-10$ & KIAA1522 & 7.47E-01 \\
\hline ENSBTAG00000006654 & $1.41 \mathrm{E}-10$ & $1.86 \mathrm{E}-10$ & TANK & 7.59E-01 \\
\hline ENSBTAG00000003866 & $3.82 \mathrm{E}-10$ & $5.05 \mathrm{E}-10$ & PPP1R8 & 7.57E-01 \\
\hline ENSBTAG00000003253 & $3.73 \mathrm{E}-10$ & $4.92 \mathrm{E}-10$ & NPPC & 7.57E-01 \\
\hline ENSBTAG00000017437 & $3.08 \mathrm{E}-10$ & 4.07E-10 & TMEM237 & 7.57E-01 \\
\hline
\end{tabular}




\begin{tabular}{|c|c|c|c|c|}
\hline ENSBTAG00000010050 & $1.88 \mathrm{E}-10$ & $2.45 \mathrm{E}-10$ & COL16A1 & $7.68 \mathrm{E}-01$ \\
\hline ENSBTAG00000020855 & $1.20 \mathrm{E}-10$ & $1.54 \mathrm{E}-10$ & PTPN4 & $7.81 \mathrm{E}-01$ \\
\hline ENSBTAG00000034503 & $1.41 \mathrm{E}-10$ & $1.78 \mathrm{E}-10$ & & $7.94 \mathrm{E}-01$ \\
\hline ENSBTAG00000027694 & $2.79 \mathrm{E}-11$ & $3.52 \mathrm{E}-11$ & & $7.94 \mathrm{E}-01$ \\
\hline ENSBTAG00000021494 & $1.14 \mathrm{E}-10$ & $1.44 \mathrm{E}-10$ & EN1 & 7.95E-01 \\
\hline ENSBTAG00000012376 & $4.57 \mathrm{E}-10$ & $5.75 \mathrm{E}-10$ & & $7.94 \mathrm{E}-01$ \\
\hline ENSBTAG00000018282 & $8.16 \mathrm{E}-11$ & $1.03 \mathrm{E}-10$ & NIF3L1 & $7.92 \mathrm{E}-01$ \\
\hline ENSBTAG00000046044 & $5.01 \mathrm{E}-10$ & $6.32 \mathrm{E}-10$ & & 7.93E-01 \\
\hline ENSBTAG00000016033 & $4.88 \mathrm{E}-11$ & $6.15 E-11$ & HOXD9 & 7.95E-01 \\
\hline ENSBTAG00000005628 & $1.02 \mathrm{E}-10$ & $1.29 \mathrm{E}-10$ & CD52 & $7.94 \mathrm{E}-01$ \\
\hline ENSBTAG00000013282 & $4.46 \mathrm{E}-10$ & $5.62 \mathrm{E}-10$ & NECAP2 & 7.93E-01 \\
\hline ENSBTAG00000014949 & $1.00 \mathrm{E}-10$ & $1.26 \mathrm{E}-10$ & SP9 & 7.95E-01 \\
\hline ENSBTAG00000017855 & $4.08 \mathrm{E}-10$ & $5.14 \mathrm{E}-10$ & ITM2C & 7.94E-01 \\
\hline ENSBTAG00000046832 & $6.81 \mathrm{E}-10$ & $8.58 \mathrm{E}-10$ & & 7.93E-01 \\
\hline ENSBTAG00000006059 & $8.94 \mathrm{E}-11$ & $1.13 \mathrm{E}-10$ & TSN & 7.92E-01 \\
\hline ENSBTAG00000037902 & $8.62 \mathrm{E}-11$ & $1.09 \mathrm{E}-10$ & FAM229A & 7.91E-01 \\
\hline ENSBTAG00000033330 & 7.11E-11 & 8.96E-11 & HOXD11 & 7.94E-01 \\
\hline ENSBTAG00000015222 & $3.46 \mathrm{E}-10$ & $4.36 \mathrm{E}-10$ & RSRP1 & $7.94 \mathrm{E}-01$ \\
\hline ENSBTAG00000020540 & $9.65 \mathrm{E}-11$ & $1.22 \mathrm{E}-10$ & TMEM198 & $7.91 \mathrm{E}-01$ \\
\hline ENSBTAG00000005140 & $3.05 \mathrm{E}-10$ & $3.85 \mathrm{E}-10$ & & 7.93E-01 \\
\hline ENSBTAG00000000301 & $8.93 \mathrm{E}-11$ & $1.12 \mathrm{E}-10$ & & 7.97E-01 \\
\hline ENSBTAG00000007280 & $4.37 \mathrm{E}-10$ & $5.50 \mathrm{E}-10$ & & 7.94E-01 \\
\hline ENSBTAG00000015217 & $1.31 \mathrm{E}-10$ & $1.65 \mathrm{E}-10$ & DLX1 & 7.96E-01 \\
\hline ENSBTAG00000015054 & $3.99 \mathrm{E}-10$ & $5.03 E-10$ & CRYGD & 7.94E-01 \\
\hline ENSBTAG00000007580 & $1.40 \mathrm{E}-10$ & $1.76 \mathrm{E}-10$ & ZSWIM2 & $7.94 \mathrm{E}-01$ \\
\hline ENSBTAG00000012912 & $3.01 \mathrm{E}-10$ & $3.79 \mathrm{E}-10$ & ERMN & 7.94E-01 \\
\hline ENSBTAG00000003927 & $3.34 \mathrm{E}-11$ & 4.21E-11 & AAMP & 7.94E-01 \\
\hline ENSBTAG00000002056 & $1.83 \mathrm{E}-10$ & $2.31 \mathrm{E}-10$ & MARS2 & 7.94E-01 \\
\hline ENSBTAG00000017376 & $4.52 \mathrm{E}-10$ & $5.62 \mathrm{E}-10$ & GALNT5 & $8.05 \mathrm{E}-01$ \\
\hline ENSBTAG00000005207 & $2.66 \mathrm{E}-10$ & $3.31 \mathrm{E}-10$ & COPS7B & 8.03E-01 \\
\hline ENSBTAG00000032021 & $3.33 \mathrm{E}-10$ & 4.12E-10 & RALB & $8.08 \mathrm{E}-01$ \\
\hline ENSBTAG00000015715 & $1.56 \mathrm{E}-10$ & $1.93 \mathrm{E}-10$ & SPOCD1 & 8.07E-01 \\
\hline ENSBTAG00000039994 & $3.52 \mathrm{E}-10$ & $4.29 \mathrm{E}-10$ & & $8.21 \mathrm{E}-01$ \\
\hline ENSBTAG00000007830 & $4.38 \mathrm{E}-10$ & $5.33 \mathrm{E}-10$ & PLEKHM3 & $8.21 \mathrm{E}-01$ \\
\hline ENSBTAG00000032481 & $4.49 \mathrm{E}-10$ & $5.46 \mathrm{E}-10$ & DAPL1 & $8.22 \mathrm{E}-01$ \\
\hline ENSBTAG00000008329 & $4.01 \mathrm{E}-10$ & $4.87 \mathrm{E}-10$ & CYTIP & $8.23 \mathrm{E}-01$ \\
\hline ENSBTAG00000039346 & $8.73 \mathrm{E}-10$ & $1.06 \mathrm{E}-09$ & & 8.23E-01 \\
\hline ENSBTAG00000016193 & $5.47 \mathrm{E}-10$ & $6.64 \mathrm{E}-10$ & FASTKD2 & $8.24 \mathrm{E}-01$ \\
\hline ENSBTAG00000038992 & $5.75 \mathrm{E}-10$ & $6.96 \mathrm{E}-10$ & STK11IP & $8.26 \mathrm{E}-01$ \\
\hline ENSBTAG00000010378 & $2.35 \mathrm{E}-10$ & $2.84 \mathrm{E}-10$ & PEF1 & 8.27E-01 \\
\hline ENSBTAG00000021600 & $3.31 \mathrm{E}-10$ & $4.01 \mathrm{E}-10$ & KANSL1L & $8.26 \mathrm{E}-01$ \\
\hline ENSBTAG00000046649 & $5.36 \mathrm{E}-10$ & $6.47 \mathrm{E}-10$ & SGPP2 & $8.28 \mathrm{E}-01$ \\
\hline ENSBTAG00000010386 & $1.90 \mathrm{E}-10$ & $2.28 \mathrm{E}-10$ & ARPC2 & $8.33 \mathrm{E}-01$ \\
\hline ENSBTAG00000000815 & $1.37 \mathrm{E}-10$ & $1.65 \mathrm{E}-10$ & EPHA2 & $8.33 \mathrm{E}-01$ \\
\hline ENSBTAG00000031166 & $1.20 \mathrm{E}-10$ & $1.43 \mathrm{E}-10$ & ZDBF2 & 8.37E-01 \\
\hline ENSBTAG00000014819 & $4.81 \mathrm{E}-10$ & $5.74 \mathrm{E}-10$ & CRYBA2 & $8.38 \mathrm{E}-01$ \\
\hline ENSBTAG00000011582 & $5.30 \mathrm{E}-10$ & $6.32 \mathrm{E}-10$ & & $8.38 \mathrm{E}-01$ \\
\hline ENSBTAG00000014094 & $4.92 \mathrm{E}-10$ & $5.86 \mathrm{E}-10$ & & $8.40 \mathrm{E}-01$ \\
\hline ENSBTAG00000006349 & $3.31 \mathrm{E}-10$ & $3.93 E-10$ & EXTL1 & $8.42 \mathrm{E}-01$ \\
\hline ENSBTAG00000046940 & $5.63 \mathrm{E}-10$ & $6.68 \mathrm{E}-10$ & & 8.44E-01 \\
\hline ENSBTAG00000003645 & $3.40 \mathrm{E}-10$ & $4.02 \mathrm{E}-10$ & FSIP2 & $8.46 \mathrm{E}-01$ \\
\hline ENSBTAG00000013449 & $1.91 \mathrm{E}-10$ & $2.26 \mathrm{E}-10$ & RAB3GAP1 & $8.45 \mathrm{E}-01$ \\
\hline ENSBTAG00000008452 & $1.83 \mathrm{E}-10$ & $2.14 \mathrm{E}-10$ & $\mathrm{IHH}$ & 8.55E-01 \\
\hline ENSBTAG00000019480 & $1.82 \mathrm{E}-10$ & $2.13 \mathrm{E}-10$ & PDE6D & $8.55 \mathrm{E}-01$ \\
\hline ENSBTAG00000013916 & $3.45 \mathrm{E}-10$ & $3.99 \mathrm{E}-10$ & HERC2 & $8.66 \mathrm{E}-01$ \\
\hline ENSBTAG00000014158 & $1.53 \mathrm{E}-10$ & 1.77E-10 & CCNT2 & 8.67E-01 \\
\hline ENSBTAG00000009517 & $5.40 \mathrm{E}-10$ & $6.20 \mathrm{E}-10$ & & $8.71 E-01$ \\
\hline ENSBTAG00000031473 & $3.23 \mathrm{E}-10$ & $3.72 \mathrm{E}-10$ & ORC2 & $8.69 \mathrm{E}-01$ \\
\hline ENSBTAG00000026676 & $1.18 \mathrm{E}-10$ & $1.35 \mathrm{E}-10$ & SDC3 & $8.71 E-01$ \\
\hline ENSBTAG00000010598 & $3.59 \mathrm{E}-10$ & 4.11E-10 & EIF4E2 & $8.74 \mathrm{E}-01$ \\
\hline ENSBTAG00000018236 & $1.32 \mathrm{E}-10$ & $1.50 \mathrm{E}-10$ & EPB41L5 & $8.80 \mathrm{E}-01$ \\
\hline ENSBTAG00000001297 & $4.09 \mathrm{E}-10$ & $4.67 E-10$ & & $8.76 \mathrm{E}-01$ \\
\hline ENSBTAG00000021596 & $4.32 \mathrm{E}-10$ & $4.91 \mathrm{E}-10$ & ICOS & $8.81 \mathrm{E}-01$ \\
\hline ENSBTAG00000004835 & $2.00 \mathrm{E}-10$ & 2.27E-10 & HOXD3 & $8.81 \mathrm{E}-01$ \\
\hline ENSBTAG00000014390 & $1.15 \mathrm{E}-10$ & $1.31 \mathrm{E}-10$ & & $8.82 \mathrm{E}-01$ \\
\hline ENSBTAG00000026753 & $8.21 \mathrm{E}-10$ & $9.30 \mathrm{E}-10$ & & $8.82 \mathrm{E}-01$ \\
\hline ENSBTAG00000021787 & $1.49 \mathrm{E}-10$ & $1.69 \mathrm{E}-10$ & PAQR7 & 8.83E-01 \\
\hline ENSBTAG00000017108 & $7.89 \mathrm{E}-10$ & $8.93 E-10$ & OTUD3 & $8.84 \mathrm{E}-01$ \\
\hline ENSBTAG00000016817 & $2.03 \mathrm{E}-10$ & $2.28 \mathrm{E}-10$ & SNRNP40 & $8.92 \mathrm{E}-01$ \\
\hline ENSBTAG00000002316 & $5.49 \mathrm{E}-10$ & $6.16 \mathrm{E}-10$ & NIPA1 & $8.92 \mathrm{E}-01$ \\
\hline
\end{tabular}




\begin{tabular}{|c|c|c|c|c|}
\hline ENSBTAG00000021786 & $1.78 \mathrm{E}-10$ & $1.99 \mathrm{E}-10$ & AUNIP & $8.94 \mathrm{E}-01$ \\
\hline ENSBTAG00000000492 & $5.66 \mathrm{E}-10$ & $6.29 \mathrm{E}-10$ & ASNSD1 & $9.00 \mathrm{E}-01$ \\
\hline ENSBTAG00000017629 & $4.27 \mathrm{E}-10$ & $4.74 \mathrm{E}-10$ & & $9.01 \mathrm{E}-01$ \\
\hline ENSBTAG00000014863 & $3.59 \mathrm{E}-10$ & $3.98 \mathrm{E}-10$ & GYPC & $9.01 \mathrm{E}-01$ \\
\hline ENSBTAG00000010225 & $4.23 \mathrm{E}-10$ & 4.69E-10 & POLR2D & $9.01 \mathrm{E}-01$ \\
\hline ENSBTAG00000008800 & $5.59 \mathrm{E}-10$ & $6.16 \mathrm{E}-10$ & XKR8 & $9.08 \mathrm{E}-01$ \\
\hline ENSBTAG00000019337 & $1.87 \mathrm{E}-10$ & $2.06 \mathrm{E}-10$ & ACVR1C & $9.06 \mathrm{E}-01$ \\
\hline ENSBTAG00000018520 & $5.79 \mathrm{E}-10$ & 6.37E-10 & SCN1A & $9.09 \mathrm{E}-01$ \\
\hline ENSBTAG00000026851 & $2.73 \mathrm{E}-10$ & $3.00 \mathrm{E}-10$ & FMNL2 & $9.08 \mathrm{E}-01$ \\
\hline ENSBTAG00000009950 & $3.44 \mathrm{E}-10$ & $3.78 \mathrm{E}-10$ & PAX3 & $9.11 \mathrm{E}-01$ \\
\hline ENSBTAG00000015841 & $1.61 \mathrm{E}-10$ & 1.77E-10 & & $9.09 \mathrm{E}-01$ \\
\hline ENSBTAG00000006907 & $1.92 \mathrm{E}-10$ & $2.11 \mathrm{E}-10$ & NEB & $9.12 \mathrm{E}-01$ \\
\hline ENSBTAG00000027201 & $2.08 \mathrm{E}-10$ & $2.29 \mathrm{E}-10$ & RAPH1 & $9.09 \mathrm{E}-01$ \\
\hline ENSBTAG00000001651 & $3.78 \mathrm{E}-10$ & $4.14 \mathrm{E}-10$ & SYF2 & $9.13 \mathrm{E}-01$ \\
\hline ENSBTAG00000000176 & $1.30 \mathrm{E}-10$ & $1.42 \mathrm{E}-10$ & SP3 & $9.13 \mathrm{E}-01$ \\
\hline ENSBTAG00000045943 & $5.65 \mathrm{E}-10$ & $6.18 \mathrm{E}-10$ & CHRNG & $9.15 \mathrm{E}-01$ \\
\hline ENSBTAG00000018130 & $1.66 \mathrm{E}-10$ & $1.81 \mathrm{E}-10$ & MAP2 & $9.17 \mathrm{E}-01$ \\
\hline ENSBTAG00000047426 & $2.07 \mathrm{E}-10$ & $2.26 \mathrm{E}-10$ & LYPD6 & $9.17 \mathrm{E}-01$ \\
\hline ENSBTAG00000016063 & $3.72 \mathrm{E}-10$ & $4.05 \mathrm{E}-10$ & DNER & $9.19 \mathrm{E}-01$ \\
\hline ENSBTAG00000009579 & $2.43 \mathrm{E}-10$ & $2.64 \mathrm{E}-10$ & CEP85 & $9.21 \mathrm{E}-01$ \\
\hline ENSBTAG00000037640 & $3.71 \mathrm{E}-10$ & $4.02 \mathrm{E}-10$ & PID1 & $9.24 \mathrm{E}-01$ \\
\hline ENSBTAG00000015415 & $2.28 \mathrm{E}-10$ & $2.47 \mathrm{E}-10$ & UNC80 & $9.24 \mathrm{E}-01$ \\
\hline ENSBTAG00000007863 & $2.23 \mathrm{E}-10$ & $2.40 \mathrm{E}-10$ & GLS & $9.28 \mathrm{E}-01$ \\
\hline ENSBTAG00000003235 & $3.04 \mathrm{E}-10$ & $3.28 \mathrm{E}-10$ & DHDDS & $9.26 \mathrm{E}-01$ \\
\hline ENSBTAG00000012217 & $8.13 E-10$ & $8.78 \mathrm{E}-10$ & PLA2G2F & $9.26 \mathrm{E}-01$ \\
\hline ENSBTAG00000013782 & $7.88 \mathrm{E}-11$ & 8.51E-11 & DNAJB2 & $9.26 \mathrm{E}-01$ \\
\hline ENSBTAG00000046281 & $7.51 \mathrm{E}-10$ & $8.10 \mathrm{E}-10$ & & $9.27 \mathrm{E}-01$ \\
\hline ENSBTAG00000017797 & $1.91 \mathrm{E}-10$ & $2.06 \mathrm{E}-10$ & & 9.27E-01 \\
\hline ENSBTAG00000008766 & $3.95 \mathrm{E}-10$ & $4.26 \mathrm{E}-10$ & SLC9A1 & 9.27E-01 \\
\hline ENSBTAG00000012589 & $2.82 \mathrm{E}-10$ & $3.04 \mathrm{E}-10$ & HSPE1 & $9.26 \mathrm{E}-01$ \\
\hline ENSBTAG00000021781 & $2.24 \mathrm{E}-10$ & $2.41 \mathrm{E}-10$ & MTFR1L & $9.30 \mathrm{E}-01$ \\
\hline ENSBTAG00000038700 & 3.39E-10 & $3.63 \mathrm{E}-10$ & FAM124B & $9.33 \mathrm{E}-01$ \\
\hline ENSBTAG00000009631 & $6.81 \mathrm{E}-10$ & $7.30 \mathrm{E}-10$ & ZNF683 & $9.33 \mathrm{E}-01$ \\
\hline ENSBTAG00000008314 & $7.80 \mathrm{E}-10$ & 8.35E-10 & SDHB & $9.34 \mathrm{E}-01$ \\
\hline ENSBTAG00000002293 & $3.81 \mathrm{E}-10$ & 4.07E-10 & ARL5A & $9.35 \mathrm{E}-01$ \\
\hline ENSBTAG00000011390 & $3.73 E-10$ & $3.99 \mathrm{E}-10$ & CHRND & $9.36 \mathrm{E}-01$ \\
\hline ENSBTAG00000034674 & $1.36 \mathrm{E}-10$ & $1.46 \mathrm{E}-10$ & & $9.35 \mathrm{E}-01$ \\
\hline ENSBTAG00000001513 & $2.13 \mathrm{E}-10$ & $2.28 \mathrm{E}-10$ & PDIK1L & $9.35 \mathrm{E}-01$ \\
\hline ENSBTAG00000009057 & $4.48 \mathrm{E}-10$ & $4.78 \mathrm{E}-10$ & CFAP221 & $9.38 \mathrm{E}-01$ \\
\hline ENSBTAG00000010249 & $1.02 \mathrm{E}-10$ & $1.09 \mathrm{E}-10$ & TMEM222 & $9.40 \mathrm{E}-01$ \\
\hline ENSBTAG00000018653 & $4.21 \mathrm{E}-10$ & 4.47E-10 & NABP1 & $9.41 \mathrm{E}-01$ \\
\hline ENSBTAG00000021327 & $6.19 \mathrm{E}-10$ & $6.58 \mathrm{E}-10$ & DAW1 & $9.41 \mathrm{E}-01$ \\
\hline ENSBTAG00000006085 & $1.21 \mathrm{E}-09$ & $1.28 \mathrm{E}-09$ & DDOST & $9.43 \mathrm{E}-01$ \\
\hline ENSBTAG00000015718 & $2.39 \mathrm{E}-10$ & $2.53 \mathrm{E}-10$ & CASP8 & $9.45 \mathrm{E}-01$ \\
\hline ENSBTAG00000026812 & $4.01 \mathrm{E}-10$ & $4.23 \mathrm{E}-10$ & $\mathrm{AOX} 2$ & $9.47 \mathrm{E}-01$ \\
\hline ENSBTAG00000014407 & $2.85 \mathrm{E}-10$ & $3.01 E-10$ & INO80D & $9.48 \mathrm{E}-01$ \\
\hline ENSBTAG00000006261 & $3.26 \mathrm{E}-10$ & $3.43 \mathrm{E}-10$ & GPR17 & $9.52 \mathrm{E}-01$ \\
\hline ENSBTAG00000016217 & $3.00 \mathrm{E}-10$ & $3.14 \mathrm{E}-10$ & RBM43 & $9.54 \mathrm{E}-01$ \\
\hline ENSBTAG00000046032 & $6.39 \mathrm{E}-10$ & $6.70 \mathrm{E}-10$ & & $9.54 \mathrm{E}-01$ \\
\hline ENSBTAG00000001060 & $1.32 \mathrm{E}-10$ & $1.39 \mathrm{E}-10$ & CXCR4 & $9.52 \mathrm{E}-01$ \\
\hline ENSBTAG00000039437 & $3.74 \mathrm{E}-10$ & $3.92 \mathrm{E}-10$ & & $9.53 \mathrm{E}-01$ \\
\hline ENSBTAG00000005239 & 8.37E-10 & $8.78 \mathrm{E}-10$ & & $9.54 \mathrm{E}-01$ \\
\hline ENSBTAG00000007445 & $1.28 \mathrm{E}-10$ & $1.33 \mathrm{E}-10$ & $\mathrm{CD} 28$ & $9.59 \mathrm{E}-01$ \\
\hline ENSBTAG00000012100 & $3.09 \mathrm{E}-10$ & $3.24 \mathrm{E}-10$ & PDE1A & $9.55 \mathrm{E}-01$ \\
\hline ENSBTAG00000012334 & $4.41 E-10$ & $4.59 \mathrm{E}-10$ & ALS2CR12 & $9.60 \mathrm{E}-01$ \\
\hline ENSBTAG00000021103 & $1.94 \mathrm{E}-10$ & $2.02 \mathrm{E}-10$ & SLC35F5 & $9.60 \mathrm{E}-01$ \\
\hline ENSBTAG00000011133 & $1.96 \mathrm{E}-10$ & $2.04 \mathrm{E}-10$ & AP1S3 & $9.62 \mathrm{E}-01$ \\
\hline ENSBTAG00000009664 & $4.75 \mathrm{E}-10$ & $4.92 \mathrm{E}-10$ & & $9.66 \mathrm{E}-01$ \\
\hline ENSBTAG00000006003 & $2.56 \mathrm{E}-10$ & $2.64 \mathrm{E}-10$ & SAP130 & $9.69 \mathrm{E}-01$ \\
\hline ENSBTAG00000040055 & $2.85 \mathrm{E}-10$ & $2.94 \mathrm{E}-10$ & CAB39 & $9.70 \mathrm{E}-01$ \\
\hline ENSBTAG00000012604 & $8.51 \mathrm{E}-10$ & $8.75 \mathrm{E}-10$ & NCMAP & $9.72 \mathrm{E}-01$ \\
\hline ENSBTAG00000034949 & $2.11 \mathrm{E}-09$ & $2.16 \mathrm{E}-09$ & & $9.75 \mathrm{E}-01$ \\
\hline ENSBTAG00000010977 & $4.09 \mathrm{E}-10$ & $4.18 \mathrm{E}-10$ & FRZB & $9.79 \mathrm{E}-01$ \\
\hline ENSBTAG00000006225 & $5.30 \mathrm{E}-10$ & $5.41 \mathrm{E}-10$ & RPA2 & $9.80 \mathrm{E}-01$ \\
\hline ENSBTAG00000014933 & $2.95 \mathrm{E}-10$ & $3.00 \mathrm{E}-10$ & TRAK2 & $9.83 \mathrm{E}-01$ \\
\hline ENSBTAG00000048246 & $1.28 \mathrm{E}-10$ & $1.30 \mathrm{E}-10$ & & $9.86 \mathrm{E}-01$ \\
\hline ENSBTAG00000026751 & $1.47 \mathrm{E}-10$ & $1.50 \mathrm{E}-10$ & CFAP65 & $9.83 \mathrm{E}-01$ \\
\hline ENSBTAG00000009777 & $5.30 \mathrm{E}-10$ & 5.37E-10 & NUP35 & 9.87E-01 \\
\hline ENSBTAG00000016877 & $2.63 \mathrm{E}-10$ & $2.66 \mathrm{E}-10$ & PLA2G2C & $9.88 \mathrm{E}-01$ \\
\hline ENSBTAG00000002138 & $4.71 \mathrm{E}-10$ & 4.77E-10 & PADI1 & $9.88 \mathrm{E}-01$ \\
\hline
\end{tabular}




\begin{tabular}{|c|c|c|c|c|}
\hline ENSBTAG00000006262 & $4.06 \mathrm{E}-10$ & 4.11E-10 & LIMS2 & $9.89 \mathrm{E}-01$ \\
\hline ENSBTAG00000040338 & $1.67 \mathrm{E}-10$ & $1.68 \mathrm{E}-10$ & OBSL1 & 9.94E-01 \\
\hline ENSBTAG00000024632 & $5.20 \mathrm{E}-10$ & $5.25 \mathrm{E}-10$ & & $9.91 \mathrm{E}-01$ \\
\hline ENSBTAG00000007867 & 2.37E-10 & $2.39 \mathrm{E}-10$ & STAT1 & $9.91 \mathrm{E}-01$ \\
\hline ENSBTAG00000013131 & $1.34 \mathrm{E}-10$ & $1.35 \mathrm{E}-10$ & FAM110D & $9.89 \mathrm{E}-01$ \\
\hline ENSBTAG00000040547 & $4.86 \mathrm{E}-10$ & 4.89E-10 & UBXN10 & $9.94 \mathrm{E}-01$ \\
\hline ENSBTAG00000020499 & 1.73E-09 & 1.74E-09 & & 9.93E-01 \\
\hline ENSBTAG00000007996 & $6.56 \mathrm{E}-10$ & $6.59 \mathrm{E}-10$ & HTR6 & 9.96E-01 \\
\hline ENSBTAG00000038180 & 4.74E-10 & 4.76E-10 & SCN2A & 9.97E-01 \\
\hline ENSBTAG00000019800 & $5.22 \mathrm{E}-10$ & $5.21 \mathrm{E}-10$ & RUNX3 & $1.00 \mathrm{E}+00$ \\
\hline ENSBTAG00000034192 & $1.89 \mathrm{E}-10$ & $1.89 \mathrm{E}-10$ & & $1.00 \mathrm{E}+00$ \\
\hline ENSBTAG00000009080 & 4.27E-10 & $4.25 \mathrm{E}-10$ & ITGB6 & $1.00 \mathrm{E}+00$ \\
\hline ENSBTAG00000015066 & $2.66 \mathrm{E}-10$ & $2.65 \mathrm{E}-10$ & LANCL1 & $1.00 \mathrm{E}+00$ \\
\hline ENSBTAG00000017743 & $2.94 \mathrm{E}-10$ & $2.92 \mathrm{E}-10$ & XIRP2 & $1.01 E+00$ \\
\hline ENSBTAG00000011808 & $4.71 \mathrm{E}-10$ & $4.68 \mathrm{E}-10$ & MSTN & $1.01 E+00$ \\
\hline ENSBTAG00000026842 & $3.15 \mathrm{E}-10$ & 3.13E-10 & ZRANB3 & $1.01 E+00$ \\
\hline ENSBTAG00000020548 & $3.01 \mathrm{E}-10$ & $2.99 \mathrm{E}-10$ & AZIN2 & $1.01 E+00$ \\
\hline ENSBTAG00000012760 & $1.09 \mathrm{E}-10$ & $1.08 \mathrm{E}-10$ & NDUFB3 & $1.01 \mathrm{E}+00$ \\
\hline ENSBTAG00000014469 & $2.55 \mathrm{E}-10$ & $2.53 \mathrm{E}-10$ & NBEAL1 & $1.01 E+00$ \\
\hline ENSBTAG00000038710 & $4.54 \mathrm{E}-10$ & $4.50 \mathrm{E}-10$ & & $1.01 \mathrm{E}+00$ \\
\hline ENSBTAG00000014783 & $5.75 \mathrm{E}-10$ & $5.69 \mathrm{E}-10$ & CRYGC & $1.01 E+00$ \\
\hline ENSBTAG00000021770 & 5.19E-10 & $5.14 \mathrm{E}-10$ & CRYGB & $1.01 E+00$ \\
\hline ENSBTAG00000021762 & $1.99 \mathrm{E}-10$ & $1.96 \mathrm{E}-10$ & ZC3H15 & $1.01 \mathrm{E}+00$ \\
\hline ENSBTAG00000017258 & $1.82 \mathrm{E}-10$ & $1.79 \mathrm{E}-10$ & ACSL3 & $1.02 \mathrm{E}+00$ \\
\hline ENSBTAG00000026710 & $6.68 \mathrm{E}-10$ & $6.55 \mathrm{E}-10$ & C2orf72 & $1.02 \mathrm{E}+00$ \\
\hline ENSBTAG00000034680 & $1.04 \mathrm{E}-10$ & $1.02 \mathrm{E}-10$ & FAM131C & $1.02 \mathrm{E}+00$ \\
\hline ENSBTAG00000021020 & $1.78 \mathrm{E}-10$ & $1.75 \mathrm{E}-10$ & RIF1 & $1.02 E+00$ \\
\hline ENSBTAG00000006438 & $2.14 \mathrm{E}-10$ & $2.10 \mathrm{E}-10$ & ACTL8 & $1.02 \mathrm{E}+00$ \\
\hline ENSBTAG00000038042 & $2.41 \mathrm{E}-10$ & $2.34 \mathrm{E}-10$ & & $1.03 E+00$ \\
\hline ENSBTAG00000005759 & $2.28 \mathrm{E}-09$ & $2.22 \mathrm{E}-09$ & & $1.03 E+00$ \\
\hline ENSBTAG00000021832 & $1.76 \mathrm{E}-10$ & $1.71 \mathrm{E}-10$ & $\mathrm{HMGCL}$ & $1.03 E+00$ \\
\hline ENSBTAG00000014447 & $3.58 \mathrm{E}-10$ & 3.47E-10 & RPS6KA1 & $1.03 E+00$ \\
\hline ENSBTAG00000020537 & $1.42 \mathrm{E}-10$ & $1.37 \mathrm{E}-10$ & ASIC4 & $1.03 \mathrm{E}+00$ \\
\hline ENSBTAG00000011514 & $3.10 \mathrm{E}-10$ & $2.99 \mathrm{E}-10$ & MRPL44 & $1.04 \mathrm{E}+00$ \\
\hline ENSBTAG00000007998 & $6.84 \mathrm{E}-10$ & $6.58 \mathrm{E}-10$ & TMCO4 & $1.04 \mathrm{E}+00$ \\
\hline ENSBTAG00000008039 & 2.73E-10 & 2.63E-10 & ACMSD & $1.04 E+00$ \\
\hline ENSBTAG00000030718 & $2.47 \mathrm{E}-10$ & $2.37 \mathrm{E}-10$ & SPATA3 & $1.04 \mathrm{E}+00$ \\
\hline ENSBTAG00000001296 & $4.79 \mathrm{E}-10$ & $4.59 \mathrm{E}-10$ & TMEM50A & $1.04 \mathrm{E}+00$ \\
\hline ENSBTAG00000012888 & $6.53 \mathrm{E}-10$ & $6.23 \mathrm{E}-10$ & C2orf80 & $1.05 \mathrm{E}+00$ \\
\hline ENSBTAG00000007395 & $1.49 \mathrm{E}-10$ & $1.43 \mathrm{E}-10$ & ALS2 & $1.04 \mathrm{E}+00$ \\
\hline ENSBTAG00000019683 & $3.94 \mathrm{E}-10$ & $3.74 \mathrm{E}-10$ & LYPD6B & $1.05 \mathrm{E}+00$ \\
\hline ENSBTAG00000001899 & $2.42 \mathrm{E}-10$ & $2.30 \mathrm{E}-10$ & $\mathrm{KCNH7}$ & $1.05 \mathrm{E}+00$ \\
\hline ENSBTAG00000016819 & 4.07E-10 & $3.86 \mathrm{E}-10$ & FABP3 & $1.05 E+00$ \\
\hline ENSBTAG00000035081 & $1.78 \mathrm{E}-10$ & $1.68 \mathrm{E}-10$ & & $1.06 \mathrm{E}+00$ \\
\hline ENSBTAG00000012620 & $6.30 \mathrm{E}-11$ & $5.95 \mathrm{E}-11$ & FAM134A & $1.06 \mathrm{E}+00$ \\
\hline ENSBTAG00000005315 & $4.02 \mathrm{E}-10$ & $3.80 \mathrm{E}-10$ & RPS6 & $1.06 \mathrm{E}+00$ \\
\hline ENSBTAG00000033523 & $1.54 \mathrm{E}-10$ & $1.45 \mathrm{E}-10$ & & $1.06 \mathrm{E}+00$ \\
\hline ENSBTAG00000014849 & $9.69 \mathrm{E}-11$ & $9.15 \mathrm{E}-11$ & GPATCH3 & $1.06 \mathrm{E}+00$ \\
\hline ENSBTAG00000010366 & $1.27 \mathrm{E}-10$ & $1.20 \mathrm{E}-10$ & HCRTR1 & $1.06 \mathrm{E}+00$ \\
\hline ENSBTAG00000038318 & $5.43 \mathrm{E}-10$ & $5.12 \mathrm{E}-10$ & AMER3 & $1.06 \mathrm{E}+00$ \\
\hline ENSBTAG00000046176 & $2.37 \mathrm{E}-10$ & $2.24 \mathrm{E}-10$ & SPEG & $1.06 \mathrm{E}+00$ \\
\hline ENSBTAG00000010940 & $1.49 \mathrm{E}-10$ & $1.41 \mathrm{E}-10$ & HSPB7 & $1.06 \mathrm{E}+00$ \\
\hline ENSBTAG00000003207 & $1.24 \mathrm{E}-10$ & 1.17E-10 & SP5 & $1.06 \mathrm{E}+00$ \\
\hline ENSBTAG00000031918 & $2.30 \mathrm{E}-10$ & $2.17 \mathrm{E}-10$ & & $1.06 \mathrm{E}+00$ \\
\hline ENSBTAG00000039122 & $7.58 \mathrm{E}-10$ & $7.13 \mathrm{E}-10$ & PLA2G5 & $1.06 \mathrm{E}+00$ \\
\hline ENSBTAG00000021778 & 2.17E-10 & $2.03 \mathrm{E}-10$ & SELENON & $1.07 \mathrm{E}+00$ \\
\hline ENSBTAG00000001192 & $3.81 \mathrm{E}-10$ & $3.58 \mathrm{E}-10$ & STX12 & $1.06 \mathrm{E}+00$ \\
\hline ENSBTAG00000003650 & $1.85 \mathrm{E}-10$ & $1.73 \mathrm{E}-10$ & NR4A2 & $1.07 E+00$ \\
\hline ENSBTAG00000006159 & $3.91 \mathrm{E}-10$ & $3.64 \mathrm{E}-10$ & KCNJ3 & $1.07 \mathrm{E}+00$ \\
\hline ENSBTAG00000016951 & $4.63 \mathrm{E}-10$ & $4.31 \mathrm{E}-10$ & WDFY1 & $1.08 \mathrm{E}+00$ \\
\hline ENSBTAG00000004416 & 2.07E-10 & $1.92 \mathrm{E}-10$ & NUDC & $1.08 \mathrm{E}+00$ \\
\hline ENSBTAG00000015330 & $1.84 \mathrm{E}-10$ & $1.70 \mathrm{E}-10$ & & $1.08 \mathrm{E}+00$ \\
\hline ENSBTAG00000003846 & $4.71 \mathrm{E}-10$ & 4.34E-10 & RPL37A & $1.08 \mathrm{E}+00$ \\
\hline ENSBTAG00000019152 & $5.86 \mathrm{E}-10$ & $5.41 \mathrm{E}-10$ & NYAP2 & $1.08 \mathrm{E}+00$ \\
\hline ENSBTAG00000013039 & $2.18 \mathrm{E}-09$ & $2.00 \mathrm{E}-09$ & & $1.09 \mathrm{E}+00$ \\
\hline ENSBTAG00000009251 & $3.34 \mathrm{E}-10$ & $3.06 \mathrm{E}-10$ & SZRD1 & $1.09 \mathrm{E}+00$ \\
\hline ENSBTAG00000027051 & $3.12 \mathrm{E}-10$ & $2.86 \mathrm{E}-10$ & PTAFR & $1.09 \mathrm{E}+00$ \\
\hline ENSBTAG00000026684 & $3.84 \mathrm{E}-10$ & $3.51 \mathrm{E}-10$ & SYNC & $1.09 \mathrm{E}+00$ \\
\hline ENSBTAG00000011256 & $3.00 \mathrm{E}-10$ & $2.74 \mathrm{E}-10$ & MYO1B & $1.09 \mathrm{E}+00$ \\
\hline ENSBTAG00000016183 & $7.72 \mathrm{E}-10$ & 7.06E-10 & & $1.09 \mathrm{E}+00$ \\
\hline
\end{tabular}




\begin{tabular}{|c|c|c|c|c|}
\hline ENSBTAG000000027159 & $1.94 \mathrm{E}-10$ & $1.77 \mathrm{E}-10$ & ZBTB8OS & $1.10 \mathrm{E}+00$ \\
\hline ENSBTAG000000005501 & $2.17 \mathrm{E}-10$ & $1.98 \mathrm{E}-10$ & & $1.10 \mathrm{E}+00$ \\
\hline ENSBTAG000000011784 & $9.52 \mathrm{E}-11$ & $8.66 \mathrm{E}-11$ & FGR & $1.10 \mathrm{E}+00$ \\
\hline ENSBTAG000000003069 & $5.54 \mathrm{E}-10$ & $5.04 \mathrm{E}-10$ & MAN1C1 & $1.10 \mathrm{E}+00$ \\
\hline ENSBTAG000000002700 & 1.31E-09 & 1.19E-09 & & $1.10 \mathrm{E}+00$ \\
\hline ENSBTAG000000021636 & $4.83 \mathrm{E}-10$ & 4.39E-10 & GPR155 & $1.10 \mathrm{E}+00$ \\
\hline ENSBTAG000000012052 & $4.23 \mathrm{E}-10$ & 3.84E-10 & PADI4 & $1.10 \mathrm{E}+00$ \\
\hline ENSBTAG000000002549 & $1.13 \mathrm{E}-10$ & $1.02 \mathrm{E}-10$ & & $1.11 E+00$ \\
\hline ENSBTAG00000020961 & $2.09 \mathrm{E}-10$ & $1.88 \mathrm{E}-10$ & DUSP19 & $1.11 E+00$ \\
\hline ENSBTAG00000010599 & $6.89 \mathrm{E}-10$ & $6.19 \mathrm{E}-10$ & C2orf76 & $1.11 \mathrm{E}+00$ \\
\hline ENSBTAG000000038104 & $1.61 \mathrm{E}-10$ & $1.45 \mathrm{E}-10$ & & $1.11 E+00$ \\
\hline ENSBTAG000000019728 & $1.46 \mathrm{E}-10$ & $1.31 \mathrm{E}-10$ & CD164L2 & $1.11 E+00$ \\
\hline ENSBTAG00000019385 & $4.16 \mathrm{E}-10$ & $3.74 \mathrm{E}-10$ & SCN3A & $1.11 \mathrm{E}+00$ \\
\hline ENSBTAG000000003002 & $8.49 \mathrm{E}-10$ & 7.59E-10 & WDR75 & $1.12 \mathrm{E}+00$ \\
\hline ENSBTAG000000032277 & $4.23 \mathrm{E}-10$ & 3.77E-10 & KYNU & $1.12 \mathrm{E}+00$ \\
\hline ENSBTAG000000016818 & $2.28 \mathrm{E}-10$ & 2.03E-10 & & $1.12 \mathrm{E}+00$ \\
\hline ENSBTAG00000014714 & $2.42 \mathrm{E}-10$ & 2.17E-10 & TUBGCP5 & $1.12 \mathrm{E}+00$ \\
\hline ENSBTAG000000021691 & $1.98 \mathrm{E}-10$ & $1.76 \mathrm{E}-10$ & PSMD14 & $1.13 E+00$ \\
\hline ENSBTAG000000047837 & $2.24 \mathrm{E}-10$ & $1.99 \mathrm{E}-10$ & & $1.12 \mathrm{E}+00$ \\
\hline ENSBTAG000000014388 & $1.80 \mathrm{E}-10$ & $1.60 \mathrm{E}-10$ & EIF3I & $1.12 \mathrm{E}+00$ \\
\hline ENSBTAG000000008309 & $6.60 \mathrm{E}-10$ & $5.86 \mathrm{E}-10$ & ATP13A2 & $1.13 \mathrm{E}+00$ \\
\hline ENSBTAG000000008390 & 4.37E-10 & $3.88 \mathrm{E}-10$ & DNAJC10 & $1.13 E+00$ \\
\hline ENSBTAG000000004418 & $4.18 \mathrm{E}-10$ & $3.71 E-10$ & PLA2G2E & $1.13 E+00$ \\
\hline ENSBTAG00000013492 & $9.32 \mathrm{E}-10$ & 8.27E-10 & PRKAG3 & $1.13 \mathrm{E}+00$ \\
\hline ENSBTAG000000023963 & 3.17E-10 & $2.80 \mathrm{E}-10$ & RHBDD1 & $1.13 \mathrm{E}+00$ \\
\hline ENSBTAG000000005238 & $4.05 \mathrm{E}-10$ & $3.58 \mathrm{E}-10$ & NCKAP5 & $1.13 E+00$ \\
\hline ENSBTAG000000021466 & $1.76 \mathrm{E}-10$ & $1.55 \mathrm{E}-10$ & COL3A1 & $1.13 E+00$ \\
\hline ENSBTAG00000020191 & $2.21 \mathrm{E}-10$ & $1.95 \mathrm{E}-10$ & FEV & $1.13 \mathrm{E}+00$ \\
\hline ENSBTAG000000018065 & $2.17 \mathrm{E}-10$ & $1.91 \mathrm{E}-10$ & YARS & $1.14 \mathrm{E}+00$ \\
\hline ENSBTAG000000004216 & $3.06 \mathrm{E}-10$ & $2.69 \mathrm{E}-10$ & MAP3K2 & $1.14 \mathrm{E}+00$ \\
\hline ENSBTAG000000009859 & $2.95 \mathrm{E}-10$ & $2.58 \mathrm{E}-10$ & & $1.14 \mathrm{E}+00$ \\
\hline ENSBTAG000000020671 & $3.12 \mathrm{E}-10$ & $2.72 \mathrm{E}-10$ & TRIM62 & $1.15 \mathrm{E}+00$ \\
\hline ENSBTAG00000020159 & $2.11 \mathrm{E}-10$ & $1.84 \mathrm{E}-10$ & NKAIN1 & $1.14 \mathrm{E}+00$ \\
\hline ENSBTAG000000009770 & $1.98 \mathrm{E}-10$ & $1.72 \mathrm{E}-10$ & GPD2 & $1.15 E+00$ \\
\hline ENSBTAG000000021201 & $1.29 \mathrm{E}-10$ & $1.12 \mathrm{E}-10$ & FAM126B & $1.16 \mathrm{E}+00$ \\
\hline ENSBTAG00000002272 & $4.86 \mathrm{E}-10$ & $4.22 \mathrm{E}-10$ & KCTD18 & $1.15 E+00$ \\
\hline ENSBTAG00000021514 & $5.85 \mathrm{E}-10$ & $5.07 \mathrm{E}-10$ & DNPEP & $1.15 E+00$ \\
\hline ENSBTAG00000021323 & $2.62 \mathrm{E}-10$ & 2.27E-10 & AGFG1 & $1.15 E+00$ \\
\hline ENSBTAG00000001050 & $2.69 \mathrm{E}-10$ & $2.33 \mathrm{E}-10$ & LDLRAP1 & $1.15 \mathrm{E}+00$ \\
\hline ENSBTAG00000010293 & $6.10 \mathrm{E}-10$ & $5.26 \mathrm{E}-10$ & & $1.16 \mathrm{E}+00$ \\
\hline ENSBTAG00000005476 & $2.01 \mathrm{E}-10$ & $1.73 \mathrm{E}-10$ & METTL21A & $1.16 \mathrm{E}+00$ \\
\hline ENSBTAG00000015317 & $3.69 \mathrm{E}-10$ & $3.16 \mathrm{E}-10$ & SLC4A10 & $1.17 \mathrm{E}+00$ \\
\hline ENSBTAG00000003822 & $5.96 \mathrm{E}-10$ & $5.10 \mathrm{E}-10$ & CROCC & $1.17 \mathrm{E}+00$ \\
\hline ENSBTAG00000018147 & 4.67E-10 & $4.00 \mathrm{E}-10$ & $\mathrm{NXPH} 2$ & $1.17 \mathrm{E}+00$ \\
\hline ENSBTAG00000003936 & $1.35 \mathrm{E}-10$ & $1.15 \mathrm{E}-10$ & PNKD & $1.17 E+00$ \\
\hline ENSBTAG00000004184 & $2.00 \mathrm{E}-10$ & 1.70E-10 & WDSUB1 & $1.17 \mathrm{E}+00$ \\
\hline ENSBTAG00000015103 & $1.73 \mathrm{E}-10$ & $1.46 \mathrm{E}-10$ & IQCC & $1.19 \mathrm{E}+00$ \\
\hline ENSBTAG00000003867 & 4.30E-10 & $3.61 \mathrm{E}-10$ & & $1.19 E+00$ \\
\hline ENSBTAG00000047148 & $1.47 \mathrm{E}-10$ & $1.24 \mathrm{E}-10$ & & $1.19 E+00$ \\
\hline ENSBTAG00000020539 & $9.53 \mathrm{E}-11$ & $8.00 \mathrm{E}-11$ & CHPF & $1.19 \mathrm{E}+00$ \\
\hline ENSBTAG00000032405 & $2.32 \mathrm{E}-10$ & $1.95 \mathrm{E}-10$ & & $1.19 \mathrm{E}+00$ \\
\hline ENSBTAG00000003923 & $2.30 \mathrm{E}-10$ & $1.93 \mathrm{E}-10$ & GPBAR1 & $1.19 \mathrm{E}+00$ \\
\hline ENSBTAG00000012619 & $1.12 \mathrm{E}-10$ & $9.41 E-11$ & CNPPD1 & $1.19 \mathrm{E}+00$ \\
\hline ENSBTAG00000016512 & $5.39 \mathrm{E}-10$ & 4.53E-10 & TTC21B & $1.19 \mathrm{E}+00$ \\
\hline ENSBTAG00000017684 & $3.52 \mathrm{E}-10$ & $2.96 \mathrm{E}-10$ & NMUR1 & $1.19 \mathrm{E}+00$ \\
\hline ENSBTAG00000017214 & $6.67 \mathrm{E}-10$ & $5.59 \mathrm{E}-10$ & & $1.19 E+00$ \\
\hline ENSBTAG00000026882 & $7.04 \mathrm{E}-10$ & $5.90 \mathrm{E}-10$ & & $1.19 \mathrm{E}+00$ \\
\hline ENSBTAG00000004313 & $3.59 \mathrm{E}-10$ & 3.01E-10 & HOXD13 & $1.19 \mathrm{E}+00$ \\
\hline ENSBTAG00000020527 & $8.54 \mathrm{E}-10$ & $7.15 \mathrm{E}-10$ & IDH1 & $1.19 E+00$ \\
\hline ENSBTAG00000006223 & $5.98 \mathrm{E}-10$ & $5.01 \mathrm{E}-10$ & THEMIS2 & $1.19 E+00$ \\
\hline ENSBTAG00000014975 & $4.42 \mathrm{E}-10$ & $3.68 \mathrm{E}-10$ & SLC4A3 & $1.20 \mathrm{E}+00$ \\
\hline ENSBTAG00000012698 & $1.21 \mathrm{E}-10$ & $1.00 \mathrm{E}-10$ & HDAC1 & $1.21 \mathrm{E}+00$ \\
\hline ENSBTAG00000013281 & 4.19E-10 & $3.48 \mathrm{E}-10$ & & $1.20 \mathrm{E}+00$ \\
\hline ENSBTAG00000031041 & $1.63 \mathrm{E}-10$ & $1.36 \mathrm{E}-10$ & NHEJ1 & $1.20 \mathrm{E}+00$ \\
\hline ENSBTAG00000013773 & $3.13 \mathrm{E}-10$ & $2.60 \mathrm{E}-10$ & PKP4 & $1.20 \mathrm{E}+00$ \\
\hline ENSBTAG00000008717 & $5.00 \mathrm{E}-10$ & 4.14E-10 & SERPINE2 & $1.21 \mathrm{E}+00$ \\
\hline ENSBTAG00000010657 & $1.61 \mathrm{E}-10$ & $1.32 \mathrm{E}-10$ & ADGRB2 & $1.22 \mathrm{E}+00$ \\
\hline ENSBTAG00000009707 & $2.67 \mathrm{E}-10$ & $2.20 \mathrm{E}-10$ & MYL1 & $1.21 \mathrm{E}+00$ \\
\hline ENSBTAG00000013957 & $1.68 \mathrm{E}-10$ & $1.38 \mathrm{E}-10$ & GALNT3 & $1.22 \mathrm{E}+00$ \\
\hline ENSBTAG00000003832 & $4.24 \mathrm{E}-10$ & $3.48 \mathrm{E}-10$ & MFAP2 & $1.22 \mathrm{E}+00$ \\
\hline
\end{tabular}




\begin{tabular}{|c|c|c|c|c|}
\hline ENSBTAG00000013511 & $3.06 \mathrm{E}-10$ & $2.50 \mathrm{E}-10$ & VWA5B1 & $1.22 \mathrm{E}+00$ \\
\hline ENSBTAG00000021325 & $2.27 \mathrm{E}-10$ & $1.87 \mathrm{E}-10$ & SLC19A3 & $1.21 E+00$ \\
\hline ENSBTAG00000002678 & $2.94 \mathrm{E}-10$ & $2.41 \mathrm{E}-10$ & MED18 & $1.22 \mathrm{E}+00$ \\
\hline ENSBTAG00000009257 & $6.09 \mathrm{E}-10$ & $4.98 \mathrm{E}-10$ & CERKL & $1.22 \mathrm{E}+00$ \\
\hline ENSBTAG00000005474 & $3.18 \mathrm{E}-10$ & 2.60E-10 & CREB1 & $1.22 \mathrm{E}+00$ \\
\hline ENSBTAG00000004555 & 4.29E-10 & $3.49 \mathrm{E}-10$ & LRP2 & $1.23 E+00$ \\
\hline ENSBTAG00000003458 & $2.12 \mathrm{E}-10$ & $1.72 \mathrm{E}-10$ & CDCA7 & $1.23 \mathrm{E}+00$ \\
\hline ENSBTAG00000018446 & $2.06 \mathrm{E}-10$ & 1.67E-10 & GCA & $1.23 E+00$ \\
\hline ENSBTAG00000039803 & $6.62 \mathrm{E}-10$ & $5.39 \mathrm{E}-10$ & MYO7B & $1.23 \mathrm{E}+00$ \\
\hline ENSBTAG00000003202 & $3.09 \mathrm{E}-10$ & $2.51 \mathrm{E}-10$ & OPRD1 & $1.23 \mathrm{E}+00$ \\
\hline ENSBTAG00000004557 & $3.47 \mathrm{E}-10$ & $2.81 \mathrm{E}-10$ & DHRS9 & $1.24 \mathrm{E}+00$ \\
\hline ENSBTAG00000013235 & $2.36 \mathrm{E}-10$ & $1.91 \mathrm{E}-10$ & TINAGL1 & $1.24 \mathrm{E}+00$ \\
\hline ENSBTAG00000000684 & 8.07E-10 & $6.53 \mathrm{E}-10$ & ARHGEF10L & $1.24 \mathrm{E}+00$ \\
\hline ENSBTAG00000018125 & $4.58 \mathrm{E}-10$ & $3.70 \mathrm{E}-10$ & KIF5C & $1.24 \mathrm{E}+00$ \\
\hline ENSBTAG00000012695 & $9.78 \mathrm{E}-11$ & $7.88 \mathrm{E}-11$ & LCK & $1.24 \mathrm{E}+00$ \\
\hline ENSBTAG00000020777 & $4.36 \mathrm{E}-10$ & $3.51 E-10$ & ERCC3 & $1.24 \mathrm{E}+00$ \\
\hline ENSBTAG00000006693 & $3.47 \mathrm{E}-10$ & $2.79 \mathrm{E}-10$ & MTX2 & $1.24 \mathrm{E}+00$ \\
\hline ENSBTAG00000010897 & $1.63 \mathrm{E}-10$ & $1.30 \mathrm{E}-10$ & RESP18 & $1.25 \mathrm{E}+00$ \\
\hline ENSBTAG00000030435 & $2.23 \mathrm{E}-10$ & $1.79 \mathrm{E}-10$ & PNRC2 & $1.25 E+00$ \\
\hline ENSBTAG00000032369 & $7.38 \mathrm{E}-10$ & $5.91 \mathrm{E}-10$ & NMI & $1.25 \mathrm{E}+00$ \\
\hline ENSBTAG00000013489 & $4.41 \mathrm{E}-10$ & $3.52 \mathrm{E}-10$ & CYP27A1 & $1.25 \mathrm{E}+00$ \\
\hline ENSBTAG00000034978 & $5.40 \mathrm{E}-10$ & 4.30E-10 & FAM168B & $1.26 \mathrm{E}+00$ \\
\hline ENSBTAG00000031696 & $5.09 \mathrm{E}-10$ & $4.06 \mathrm{E}-10$ & & $1.25 \mathrm{E}+00$ \\
\hline ENSBTAG00000002177 & $4.38 \mathrm{E}-10$ & $3.49 \mathrm{E}-10$ & PIKFYVE & $1.25 \mathrm{E}+00$ \\
\hline ENSBTAG00000003327 & $2.59 \mathrm{E}-10$ & $2.05 \mathrm{E}-10$ & RUFY4 & $1.26 \mathrm{E}+00$ \\
\hline ENSBTAG00000013215 & $9.22 \mathrm{E}-10$ & 7.32E-10 & MREG & $1.26 \mathrm{E}+00$ \\
\hline ENSBTAG00000020655 & $1.54 \mathrm{E}-10$ & $1.22 \mathrm{E}-10$ & BSDC1 & $1.26 \mathrm{E}+00$ \\
\hline ENSBTAG00000018753 & $3.24 \mathrm{E}-10$ & $2.56 \mathrm{E}-10$ & TMEM163 & $1.26 \mathrm{E}+00$ \\
\hline ENSBTAG00000019399 & $1.36 \mathrm{E}-10$ & 1.07E-10 & & $1.27 \mathrm{E}+00$ \\
\hline ENSBTAG00000004603 & $1.63 \mathrm{E}-10$ & $1.29 \mathrm{E}-10$ & $\mathrm{PHC2}$ & $1.27 E+00$ \\
\hline ENSBTAG00000009897 & $3.04 \mathrm{E}-10$ & $2.40 \mathrm{E}-10$ & AGPS & $1.27 \mathrm{E}+00$ \\
\hline ENSBTAG00000008951 & $6.10 \mathrm{E}-10$ & $4.80 \mathrm{E}-10$ & ALPL & $1.27 \mathrm{E}+00$ \\
\hline ENSBTAG00000019592 & $1.13 \mathrm{E}-10$ & $8.90 \mathrm{E}-11$ & MBD5 & $1.27 \mathrm{E}+00$ \\
\hline ENSBTAG00000013650 & $7.41 \mathrm{E}-10$ & $5.80 \mathrm{E}-10$ & & $1.28 \mathrm{E}+00$ \\
\hline ENSBTAG00000044097 & $3.70 \mathrm{E}-10$ & $2.89 \mathrm{E}-10$ & KLF7 & $1.28 \mathrm{E}+00$ \\
\hline ENSBTAG00000008140 & 2.37E-10 & $1.85 \mathrm{E}-10$ & FAP & $1.28 \mathrm{E}+00$ \\
\hline ENSBTAG00000012615 & $1.94 \mathrm{E}-10$ & $1.52 \mathrm{E}-10$ & ZEB2 & $1.28 \mathrm{E}+00$ \\
\hline ENSBTAG00000005670 & $2.20 \mathrm{E}-10$ & $1.72 \mathrm{E}-10$ & ARHGEF19 & $1.28 \mathrm{E}+00$ \\
\hline ENSBTAG00000020102 & $9.67 \mathrm{E}-10$ & $7.55 \mathrm{E}-10$ & EPHA8 & $1.28 \mathrm{E}+00$ \\
\hline ENSBTAG00000006157 & $9.07 \mathrm{E}-10$ & 7.05E-10 & HS6ST1 & $1.29 E+00$ \\
\hline ENSBTAG00000003479 & $2.29 \mathrm{E}-10$ & $1.77 \mathrm{E}-10$ & MATN1 & $1.29 \mathrm{E}+00$ \\
\hline ENSBTAG00000003472 & $6.61 E-10$ & $5.12 \mathrm{E}-10$ & ABCA12 & $1.29 \mathrm{E}+00$ \\
\hline ENSBTAG00000015246 & $4.93 \mathrm{E}-10$ & $3.81 \mathrm{E}-10$ & TAS1R2 & $1.29 \mathrm{E}+00$ \\
\hline ENSBTAG00000032289 & $2.86 \mathrm{E}-10$ & $2.20 \mathrm{E}-10$ & ARHGAP15 & $1.30 \mathrm{E}+00$ \\
\hline ENSBTAG00000046699 & $3.78 \mathrm{E}-10$ & $2.91 \mathrm{E}-10$ & & $1.30 \mathrm{E}+00$ \\
\hline ENSBTAG00000006420 & $1.48 \mathrm{E}-10$ & $1.13 \mathrm{E}-10$ & BMPR2 & $1.31 E+00$ \\
\hline ENSBTAG00000046672 & $2.71 \mathrm{E}-10$ & 2.07E-10 & ZDHHC18 & $1.31 E+00$ \\
\hline ENSBTAG00000019177 & $2.62 \mathrm{E}-10$ & $2.00 \mathrm{E}-10$ & BIN1 & $1.31 E+00$ \\
\hline ENSBTAG00000021316 & $2.42 \mathrm{E}-10$ & $1.84 \mathrm{E}-10$ & COL4A3 & $1.32 \mathrm{E}+00$ \\
\hline ENSBTAG00000001755 & $1.74 \mathrm{E}-10$ & $1.31 \mathrm{E}-10$ & NEUROD1 & $1.32 \mathrm{E}+00$ \\
\hline ENSBTAG00000009580 & $2.53 \mathrm{E}-10$ & $1.91 \mathrm{E}-10$ & & $1.33 E+00$ \\
\hline ENSBTAG00000018473 & $1.69 \mathrm{E}-10$ & $1.27 \mathrm{E}-10$ & MARCO & $1.33 \mathrm{E}+00$ \\
\hline ENSBTAG00000007584 & $5.21 \mathrm{E}-10$ & 3.92E-10 & INPP1 & $1.33 \mathrm{E}+00$ \\
\hline ENSBTAG00000011950 & $2.86 \mathrm{E}-10$ & $2.14 \mathrm{E}-10$ & EMC1 & $1.33 \mathrm{E}+00$ \\
\hline ENSBTAG00000008952 & $5.13 \mathrm{E}-10$ & 3.83E-10 & RAP1GAP & $1.34 \mathrm{E}+00$ \\
\hline ENSBTAG00000014400 & $3.01 \mathrm{E}-10$ & $2.24 \mathrm{E}-10$ & E2F2 & $1.34 \mathrm{E}+00$ \\
\hline ENSBTAG00000044055 & $6.92 \mathrm{E}-10$ & $5.16 \mathrm{E}-10$ & THSD7B & $1.34 \mathrm{E}+00$ \\
\hline ENSBTAG00000016662 & $3.68 \mathrm{E}-10$ & $2.74 \mathrm{E}-10$ & CPS1 & $1.34 \mathrm{E}+00$ \\
\hline ENSBTAG00000031898 & $4.93 \mathrm{E}-10$ & 3.67E-10 & CNTNAP5 & $1.34 \mathrm{E}+00$ \\
\hline ENSBTAG00000007141 & $5.28 \mathrm{E}-10$ & 3.93E-10 & GULP1 & $1.34 \mathrm{E}+00$ \\
\hline ENSBTAG00000033690 & $4.23 \mathrm{E}-10$ & $3.14 \mathrm{E}-10$ & BARD1 & $1.35 \mathrm{E}+00$ \\
\hline ENSBTAG00000015169 & $1.80 \mathrm{E}-10$ & $1.34 \mathrm{E}-10$ & UBXN4 & $1.34 \mathrm{E}+00$ \\
\hline ENSBTAG00000018415 & $2.50 \mathrm{E}-10$ & $1.85 \mathrm{E}-10$ & TTLL4 & $1.35 \mathrm{E}+00$ \\
\hline ENSBTAG00000000581 & $6.53 \mathrm{E}-10$ & $4.83 \mathrm{E}-10$ & DOCK10 & $1.35 E+00$ \\
\hline ENSBTAG00000007787 & $4.16 \mathrm{E}-10$ & 3.07E-10 & $\mathrm{HIBCH}$ & $1.36 \mathrm{E}+00$ \\
\hline ENSBTAG00000020608 & $1.05 \mathrm{E}-10$ & $7.69 \mathrm{E}-11$ & ATG9A & $1.36 \mathrm{E}+00$ \\
\hline ENSBTAG00000020610 & $1.52 \mathrm{E}-10$ & $1.11 \mathrm{E}-10$ & ANKZF1 & $1.37 E+00$ \\
\hline ENSBTAG00000008483 & $3.48 \mathrm{E}-10$ & $2.54 \mathrm{E}-10$ & CALCRL & $1.37 E+00$ \\
\hline ENSBTAG00000016334 & $2.05 \mathrm{E}-10$ & $1.50 \mathrm{E}-10$ & SATB2 & $1.37 \mathrm{E}+00$ \\
\hline ENSBTAG00000038878 & $9.11 \mathrm{E}-10$ & $6.64 \mathrm{E}-10$ & AOX4 & $1.37 E+00$ \\
\hline
\end{tabular}




\begin{tabular}{|c|c|c|c|c|}
\hline ENSBTAG00000039731 & $1.98 \mathrm{E}-10$ & $1.44 \mathrm{E}-10$ & RND3 & $1.37 E+00$ \\
\hline ENSBTAG00000002425 & $2.89 \mathrm{E}-10$ & $2.10 \mathrm{E}-10$ & SCN9A & $1.38 \mathrm{E}+00$ \\
\hline ENSBTAG00000017605 & $2.12 \mathrm{E}-10$ & $1.53 \mathrm{E}-10$ & AK2 & $1.38 \mathrm{E}+00$ \\
\hline ENSBTAG00000007719 & $2.89 \mathrm{E}-10$ & $2.08 \mathrm{E}-10$ & MFSD6 & $1.39 E+00$ \\
\hline ENSBTAG00000002988 & $1.64 \mathrm{E}-10$ & $1.18 \mathrm{E}-10$ & TMEM57 & $1.39 E+00$ \\
\hline ENSBTAG00000026638 & $2.19 \mathrm{E}-10$ & 1.57E-10 & CATSPER4 & $1.39 \mathrm{E}+00$ \\
\hline ENSBTAG00000007652 & 4.17E-10 & $2.98 \mathrm{E}-10$ & RFTN2 & $1.40 \mathrm{E}+00$ \\
\hline ENSBTAG00000005444 & $4.02 \mathrm{E}-10$ & $2.86 \mathrm{E}-10$ & UGGT1 & $1.41 E+00$ \\
\hline ENSBTAG00000019387 & $1.25 \mathrm{E}-10$ & $8.89 \mathrm{E}-11$ & NIFK & $1.41 \mathrm{E}+00$ \\
\hline ENSBTAG00000018374 & $1.12 \mathrm{E}-10$ & $7.96 \mathrm{E}-11$ & WASF2 & $1.41 \mathrm{E}+00$ \\
\hline ENSBTAG00000008072 & $1.09 \mathrm{E}-10$ & $7.70 \mathrm{E}-11$ & SRSF10 & $1.41 E+00$ \\
\hline ENSBTAG00000003950 & $1.95 \mathrm{E}-10$ & $1.37 \mathrm{E}-10$ & PROC & $1.42 \mathrm{E}+00$ \\
\hline ENSBTAG00000013772 & $1.97 \mathrm{E}-10$ & $1.39 \mathrm{E}-10$ & SRRM1 & $1.42 \mathrm{E}+00$ \\
\hline ENSBTAG00000010254 & $1.14 \mathrm{E}-10$ & $8.00 \mathrm{E}-11$ & MAP3К6 & $1.43 \mathrm{E}+00$ \\
\hline ENSBTAG00000002995 & $1.06 \mathrm{E}-10$ & $7.39 \mathrm{E}-11$ & GMPPA & $1.43 \mathrm{E}+00$ \\
\hline ENSBTAG00000005416 & $9.77 \mathrm{E}-11$ & $6.84 \mathrm{E}-11$ & PPIL3 & $1.43 \mathrm{E}+00$ \\
\hline ENSBTAG00000030322 & $3.09 \mathrm{E}-10$ & $2.16 \mathrm{E}-10$ & KLHDC7A & $1.43 \mathrm{E}+00$ \\
\hline ENSBTAG00000016297 & $1.11 \mathrm{E}-10$ & $7.76 \mathrm{E}-11$ & CCNYL1 & $1.44 \mathrm{E}+00$ \\
\hline ENSBTAG00000001627 & $8.02 \mathrm{E}-10$ & $5.60 \mathrm{E}-10$ & & $1.43 E+00$ \\
\hline ENSBTAG00000009217 & 1.17E-10 & $8.19 \mathrm{E}-11$ & WNT10A & $1.43 E+00$ \\
\hline ENSBTAG00000002977 & $2.62 \mathrm{E}-10$ & $1.82 \mathrm{E}-10$ & ECE1 & $1.44 \mathrm{E}+00$ \\
\hline ENSBTAG00000019291 & $2.64 \mathrm{E}-10$ & $1.84 \mathrm{E}-10$ & GRB14 & $1.44 \mathrm{E}+00$ \\
\hline ENSBTAG00000004554 & $4.04 \mathrm{E}-10$ & $2.80 \mathrm{E}-10$ & CAPZB & $1.44 \mathrm{E}+00$ \\
\hline ENSBTAG00000015678 & $3.47 \mathrm{E}-10$ & $2.39 \mathrm{E}-10$ & NEMP2 & $1.45 \mathrm{E}+00$ \\
\hline ENSBTAG00000016430 & $4.29 \mathrm{E}-10$ & $2.94 \mathrm{E}-10$ & METTL8 & $1.46 \mathrm{E}+00$ \\
\hline ENSBTAG00000006970 & $2.24 \mathrm{E}-10$ & $1.54 \mathrm{E}-10$ & OLA1 & $1.46 \mathrm{E}+00$ \\
\hline ENSBTAG00000012966 & $2.65 \mathrm{E}-10$ & $1.81 \mathrm{E}-10$ & SCRN3 & $1.46 \mathrm{E}+00$ \\
\hline ENSBTAG00000016782 & $1.56 \mathrm{E}-10$ & 1.07E-10 & SLC39A10 & $1.46 \mathrm{E}+00$ \\
\hline ENSBTAG00000007111 & $7.91 \mathrm{E}-10$ & $5.34 \mathrm{E}-10$ & STEAP3 & $1.48 \mathrm{E}+00$ \\
\hline ENSBTAG00000012647 & $3.41 \mathrm{E}-10$ & $2.29 \mathrm{E}-10$ & & $1.49 \mathrm{E}+00$ \\
\hline ENSBTAG00000018067 & 1.17E-10 & $7.83 \mathrm{E}-11$ & S100PBP & $1.49 E+00$ \\
\hline ENSBTAG00000010413 & 5.19E-10 & $3.48 \mathrm{E}-10$ & TMEM169 & $1.49 \mathrm{E}+00$ \\
\hline ENSBTAG00000031614 & 2.69E-10 & $1.80 \mathrm{E}-10$ & BOLL & $1.50 \mathrm{E}+00$ \\
\hline ENSBTAG00000019781 & $2.50 \mathrm{E}-10$ & 1.67E-10 & CLASP1 & $1.50 \mathrm{E}+00$ \\
\hline ENSBTAG00000018114 & $1.71 \mathrm{E}-10$ & $1.14 \mathrm{E}-10$ & ACVR2A & $1.50 \mathrm{E}+00$ \\
\hline ENSBTAG00000018746 & $1.88 \mathrm{E}-10$ & $1.25 \mathrm{E}-10$ & & $1.50 \mathrm{E}+00$ \\
\hline ENSBTAG00000010579 & $3.85 \mathrm{E}-10$ & $2.55 \mathrm{E}-10$ & ACADL & $1.51 E+00$ \\
\hline ENSBTAG00000020654 & $1.90 \mathrm{E}-10$ & $1.26 \mathrm{E}-10$ & BAZ2B & $1.51 E+00$ \\
\hline ENSBTAG00000047133 & $2.84 \mathrm{E}-10$ & $1.87 \mathrm{E}-10$ & & $1.52 E+00$ \\
\hline ENSBTAG00000005987 & $2.36 \mathrm{E}-10$ & $1.55 \mathrm{E}-10$ & WDR33 & $1.53 E+00$ \\
\hline ENSBTAG00000005085 & $2.42 \mathrm{E}-10$ & $1.59 \mathrm{E}-10$ & TRIM63 & $1.52 E+00$ \\
\hline ENSBTAG00000007779 & $2.44 \mathrm{E}-10$ & $1.60 \mathrm{E}-10$ & GTF3C3 & $1.53 E+00$ \\
\hline ENSBTAG00000005105 & $2.01 \mathrm{E}-10$ & $1.31 \mathrm{E}-10$ & PAFAH2 & $1.53 E+00$ \\
\hline ENSBTAG00000018061 & $1.34 \mathrm{E}-10$ & $8.75 E-11$ & LNPK & $1.53 E+00$ \\
\hline ENSBTAG00000044009 & $6.24 \mathrm{E}-10$ & 4.05E-10 & PPP1R1C & $1.54 \mathrm{E}+00$ \\
\hline ENSBTAG00000021322 & $2.33 \mathrm{E}-10$ & $1.51 E-10$ & TM4SF20 & $1.54 \mathrm{E}+00$ \\
\hline ENSBTAG00000007650 & $2.02 \mathrm{E}-10$ & $1.31 E-10$ & SLC38A11 & $1.54 \mathrm{E}+00$ \\
\hline ENSBTAG00000021769 & $2.93 \mathrm{E}-10$ & $1.90 \mathrm{E}-10$ & CUL3 & $1.54 \mathrm{E}+00$ \\
\hline ENSBTAG00000005562 & $3.85 \mathrm{E}-10$ & $2.49 \mathrm{E}-10$ & GALNT13 & $1.54 \mathrm{E}+00$ \\
\hline ENSBTAG00000019694 & $3.76 \mathrm{E}-10$ & $2.43 \mathrm{E}-10$ & ADAM23 & $1.55 E+00$ \\
\hline ENSBTAG00000016000 & $1.62 \mathrm{E}-10$ & $1.04 \mathrm{E}-10$ & CARF & $1.56 \mathrm{E}+00$ \\
\hline ENSBTAG00000038195 & $1.44 \mathrm{E}-10$ & $9.27 \mathrm{E}-11$ & CD302 & $1.55 E+00$ \\
\hline ENSBTAG00000001024 & $1.90 \mathrm{E}-10$ & $1.22 \mathrm{E}-10$ & ARID1A & $1.56 \mathrm{E}+00$ \\
\hline ENSBTAG00000010498 & $7.29 \mathrm{E}-10$ & $4.66 \mathrm{E}-10$ & SLC40A1 & $1.56 \mathrm{E}+00$ \\
\hline ENSBTAG00000012848 & $2.77 \mathrm{E}-10$ & $1.76 \mathrm{E}-10$ & PTPRU & $1.57 \mathrm{E}+00$ \\
\hline ENSBTAG00000005235 & $3.35 \mathrm{E}-10$ & $2.12 \mathrm{E}-10$ & DPP10 & $1.58 \mathrm{E}+00$ \\
\hline ENSBTAG00000011964 & $1.60 \mathrm{E}-10$ & $1.01 \mathrm{E}-10$ & DIS3L2 & $1.58 \mathrm{E}+00$ \\
\hline ENSBTAG00000021525 & $1.24 \mathrm{E}-10$ & $7.83 \mathrm{E}-11$ & & $1.59 \mathrm{E}+00$ \\
\hline ENSBTAG00000015840 & $1.25 \mathrm{E}-10$ & $7.89 \mathrm{E}-11$ & HOXD1 & $1.59 E+00$ \\
\hline ENSBTAG00000014405 & $4.81 \mathrm{E}-10$ & 3.03E-10 & OSBPL6 & $1.59 \mathrm{E}+00$ \\
\hline ENSBTAG00000001357 & $1.37 \mathrm{E}-10$ & $8.62 \mathrm{E}-11$ & & $1.59 \mathrm{E}+00$ \\
\hline ENSBTAG00000021050 & $1.74 \mathrm{E}-10$ & $1.10 \mathrm{E}-10$ & ECEL1 & $1.58 \mathrm{E}+00$ \\
\hline ENSBTAG00000030425 & $1.02 \mathrm{E}-10$ & $6.45 \mathrm{E}-11$ & ID3 & $1.59 E+00$ \\
\hline ENSBTAG00000008704 & $7.20 \mathrm{E}-11$ & 4.54E-11 & FIGN & $1.59 \mathrm{E}+00$ \\
\hline ENSBTAG00000002027 & $1.03 \mathrm{E}-10$ & $6.49 \mathrm{E}-11$ & FAM167B & $1.59 \mathrm{E}+00$ \\
\hline ENSBTAG00000002363 & $2.36 \mathrm{E}-10$ & $1.49 \mathrm{E}-10$ & SESN2 & $1.59 E+00$ \\
\hline ENSBTAG00000020606 & $5.78 \mathrm{E}-11$ & 3.64E-11 & ZFAND2B & $1.59 \mathrm{E}+00$ \\
\hline ENSBTAG00000010253 & $9.13 \mathrm{E}-11$ & $5.75 \mathrm{E}-11$ & SYTL1 & $1.59 E+00$ \\
\hline ENSBTAG00000009562 & $1.52 \mathrm{E}-10$ & $9.55 \mathrm{E}-11$ & ZNF593 & $1.59 \mathrm{E}+00$ \\
\hline ENSBTAG00000018497 & $1.13 \mathrm{E}-10$ & $7.12 \mathrm{E}-11$ & SDPR & $1.59 E+00$ \\
\hline
\end{tabular}




\begin{tabular}{|c|c|c|c|c|}
\hline ENSBTAG00000038064 & $1.74 \mathrm{E}-10$ & $1.10 \mathrm{E}-10$ & & $1.58 \mathrm{E}+00$ \\
\hline ENSBTAG00000047783 & $3.79 \mathrm{E}-10$ & $2.39 \mathrm{E}-10$ & & $1.59 E+00$ \\
\hline ENSBTAG00000019003 & $2.78 \mathrm{E}-10$ & $1.75 \mathrm{E}-10$ & Tnp1 & $1.59 E+00$ \\
\hline ENSBTAG00000003771 & $3.40 \mathrm{E}-10$ & $2.14 \mathrm{E}-10$ & C1QL2 & $1.59 \mathrm{E}+00$ \\
\hline ENSBTAG00000047452 & $5.51 \mathrm{E}-10$ & $3.46 \mathrm{E}-10$ & & $1.59 \mathrm{E}+00$ \\
\hline ENSBTAG00000005986 & $2.47 \mathrm{E}-10$ & $1.56 \mathrm{E}-10$ & SFT2D3 & $1.59 E+00$ \\
\hline ENSBTAG00000038945 & $2.96 \mathrm{E}-10$ & $1.87 \mathrm{E}-10$ & PADI6 & $1.59 E+00$ \\
\hline ENSBTAG00000015291 & $2.01 \mathrm{E}-10$ & $1.26 \mathrm{E}-10$ & ORC4 & $1.59 E+00$ \\
\hline ENSBTAG00000008142 & $1.59 \mathrm{E}-10$ & $9.89 \mathrm{E}-11$ & IFIH1 & $1.60 \mathrm{E}+00$ \\
\hline ENSBTAG00000046977 & $1.12 \mathrm{E}-09$ & $6.93 \mathrm{E}-10$ & & $1.61 \mathrm{E}+00$ \\
\hline ENSBTAG00000001132 & $2.22 \mathrm{E}-10$ & $1.39 \mathrm{E}-10$ & GTDC1 & $1.60 \mathrm{E}+00$ \\
\hline ENSBTAG00000003819 & $2.22 \mathrm{E}-10$ & 1.37E-10 & STK36 & $1.62 \mathrm{E}+00$ \\
\hline ENSBTAG00000020296 & $4.66 \mathrm{E}-10$ & $2.89 \mathrm{E}-10$ & UBR3 & $1.61 E+00$ \\
\hline ENSBTAG00000003401 & $2.24 \mathrm{E}-10$ & $1.39 \mathrm{E}-10$ & ACTR3 & $1.61 E+00$ \\
\hline ENSBTAG00000004177 & $3.75 \mathrm{E}-10$ & $2.31 \mathrm{E}-10$ & TANC1 & $1.62 E+00$ \\
\hline ENSBTAG00000018552 & $5.16 \mathrm{E}-10$ & $3.14 \mathrm{E}-10$ & VWC2L & $1.64 \mathrm{E}+00$ \\
\hline ENSBTAG00000019116 & $2.18 \mathrm{E}-10$ & $1.32 \mathrm{E}-10$ & & $1.65 E+00$ \\
\hline ENSBTAG00000012289 & $5.70 \mathrm{E}-10$ & $3.44 \mathrm{E}-10$ & & $1.66 \mathrm{E}+00$ \\
\hline ENSBTAG00000010426 & $2.59 \mathrm{E}-10$ & $1.56 \mathrm{E}-10$ & XRCC5 & $1.66 \mathrm{E}+00$ \\
\hline ENSBTAG00000040497 & $1.38 \mathrm{E}-10$ & $8.25 \mathrm{E}-11$ & & $1.68 \mathrm{E}+00$ \\
\hline ENSBTAG00000003843 & $3.01 \mathrm{E}-10$ & $1.79 \mathrm{E}-10$ & SMARCAL1 & $1.68 \mathrm{E}+00$ \\
\hline ENSBTAG00000010051 & $2.02 \mathrm{E}-10$ & $1.20 \mathrm{E}-10$ & LGSN & $1.68 \mathrm{E}+00$ \\
\hline ENSBTAG00000010998 & $3.06 \mathrm{E}-10$ & $1.82 \mathrm{E}-10$ & CFLAR & $1.68 \mathrm{E}+00$ \\
\hline ENSBTAG00000013798 & 7.19E-11 & 4.26E-11 & PTPRN & $1.69 E+00$ \\
\hline ENSBTAG00000013196 & $1.36 \mathrm{E}-10$ & $8.02 E-11$ & STK17B & $1.69 \mathrm{E}+00$ \\
\hline ENSBTAG00000012554 & $3.51 \mathrm{E}-10$ & $2.06 \mathrm{E}-10$ & ANKAR & $1.70 \mathrm{E}+00$ \\
\hline ENSBTAG00000006821 & $5.24 \mathrm{E}-10$ & $3.04 \mathrm{E}-10$ & PECR & $1.72 \mathrm{E}+00$ \\
\hline ENSBTAG00000020416 & $1.60 \mathrm{E}-10$ & $9.26 \mathrm{E}-11$ & PGAP1 & $1.73 E+00$ \\
\hline ENSBTAG00000017436 & $7.51 \mathrm{E}-10$ & 4.34E-10 & ALS2CR11 & $1.73 E+00$ \\
\hline ENSBTAG00000018979 & $2.48 \mathrm{E}-10$ & $1.43 \mathrm{E}-10$ & PUM1 & $1.73 E+00$ \\
\hline ENSBTAG00000003935 & $5.13 \mathrm{E}-11$ & $2.94 \mathrm{E}-11$ & TMBIM1 & $1.74 \mathrm{E}+00$ \\
\hline ENSBTAG00000002485 & $1.90 \mathrm{E}-10$ & $1.09 \mathrm{E}-10$ & TNS1 & $1.74 \mathrm{E}+00$ \\
\hline ENSBTAG00000047036 & $6.48 \mathrm{E}-10$ & 3.70E-10 & GPR39 & $1.75 \mathrm{E}+00$ \\
\hline ENSBTAG00000018795 & $2.31 \mathrm{E}-10$ & $1.31 \mathrm{E}-10$ & PMS1 & $1.76 \mathrm{E}+00$ \\
\hline ENSBTAG00000005904 & $2.09 \mathrm{E}-10$ & $1.19 \mathrm{E}-10$ & RBBP4 & $1.76 \mathrm{E}+00$ \\
\hline ENSBTAG00000021580 & $3.01 \mathrm{E}-10$ & $1.70 \mathrm{E}-10$ & HNRNPA3 & $1.77 \mathrm{E}+00$ \\
\hline ENSBTAG00000000937 & $3.92 \mathrm{E}-10$ & $2.22 \mathrm{E}-10$ & SSFA2 & $1.77 \mathrm{E}+00$ \\
\hline ENSBTAG00000000730 & $2.29 \mathrm{E}-10$ & $1.29 \mathrm{E}-10$ & GCG & $1.78 \mathrm{E}+00$ \\
\hline ENSBTAG00000012586 & $2.29 \mathrm{E}-10$ & $1.29 \mathrm{E}-10$ & HSPD1 & $1.77 \mathrm{E}+00$ \\
\hline ENSBTAG00000018744 & $4.03 \mathrm{E}-10$ & 2.27E-10 & MGAT5 & $1.77 \mathrm{E}+00$ \\
\hline ENSBTAG00000037989 & 7.03E-10 & $3.95 \mathrm{E}-10$ & & $1.78 \mathrm{E}+00$ \\
\hline ENSBTAG00000004659 & $3.14 \mathrm{E}-10$ & $1.76 \mathrm{E}-10$ & COQ10B & $1.78 \mathrm{E}+00$ \\
\hline ENSBTAG00000018656 & $1.25 \mathrm{E}-10$ & 7.00E-11 & HP1BP3 & $1.78 \mathrm{E}+00$ \\
\hline ENSBTAG00000002646 & $1.35 \mathrm{E}-10$ & $7.53 \mathrm{E}-11$ & DYNC112 & $1.79 E+00$ \\
\hline ENSBTAG00000015752 & $2.67 \mathrm{E}-10$ & $1.49 \mathrm{E}-10$ & & $1.79 E+00$ \\
\hline ENSBTAG00000015170 & $3.08 \mathrm{E}-10$ & $1.72 \mathrm{E}-10$ & LCT & $1.79 E+00$ \\
\hline ENSBTAG00000019252 & $3.32 \mathrm{E}-10$ & $1.85 \mathrm{E}-10$ & KDF1 & $1.79 E+00$ \\
\hline ENSBTAG00000017452 & $4.59 \mathrm{E}-10$ & $2.55 \mathrm{E}-10$ & RCAN3 & $1.80 \mathrm{E}+00$ \\
\hline ENSBTAG00000009581 & $4.09 \mathrm{E}-10$ & 2.27E-10 & UBXN11 & $1.80 \mathrm{E}+00$ \\
\hline ENSBTAG00000026585 & $5.73 \mathrm{E}-10$ & $3.13 \mathrm{E}-10$ & TCEB3 & $1.83 \mathrm{E}+00$ \\
\hline ENSBTAG00000014409 & $4.61 \mathrm{E}-10$ & $2.52 \mathrm{E}-10$ & MAP3K19 & $1.83 E+00$ \\
\hline ENSBTAG00000013761 & $3.43 \mathrm{E}-10$ & $1.88 \mathrm{E}-10$ & & $1.82 \mathrm{E}+00$ \\
\hline ENSBTAG00000012561 & $4.74 \mathrm{E}-11$ & $2.56 \mathrm{E}-11$ & ORMDL1 & $1.85 \mathrm{E}+00$ \\
\hline ENSBTAG00000003225 & $1.15 \mathrm{E}-10$ & $6.22 \mathrm{E}-11$ & IMP4 & $1.85 \mathrm{E}+00$ \\
\hline ENSBTAG00000001861 & $7.41 \mathrm{E}-10$ & $3.99 \mathrm{E}-10$ & & $1.86 \mathrm{E}+00$ \\
\hline ENSBTAG00000006940 & $2.93 \mathrm{E}-10$ & $1.57 \mathrm{E}-10$ & USP48 & $1.86 \mathrm{E}+00$ \\
\hline ENSBTAG00000021726 & $1.51 \mathrm{E}-10$ & 7.99E-11 & CWC22 & $1.89 \mathrm{E}+00$ \\
\hline ENSBTAG00000016448 & $6.28 \mathrm{E}-10$ & 3.33E-10 & ZBTB40 & $1.89 E+00$ \\
\hline ENSBTAG00000026122 & $3.08 \mathrm{E}-10$ & $1.63 \mathrm{E}-10$ & ZNF804A & $1.89 E+00$ \\
\hline ENSBTAG00000006084 & $9.57 \mathrm{E}-10$ & $5.06 \mathrm{E}-10$ & PINK1 & $1.89 \mathrm{E}+00$ \\
\hline ENSBTAG00000009500 & $1.65 \mathrm{E}-10$ & $8.75 \mathrm{E}-11$ & KDM1A & $1.89 E+00$ \\
\hline ENSBTAG00000019274 & $6.81 \mathrm{E}-10$ & 3.60E-10 & ATIC & $1.89 E+00$ \\
\hline ENSBTAG00000013990 & $1.64 \mathrm{E}-10$ & $8.60 \mathrm{E}-11$ & WNT6 & $1.91 E+00$ \\
\hline ENSBTAG00000002297 & $6.10 \mathrm{E}-10$ & $3.20 \mathrm{E}-10$ & & $1.91 E+00$ \\
\hline ENSBTAG00000008716 & $2.46 \mathrm{E}-10$ & $1.29 \mathrm{E}-10$ & SSB & $1.91 \mathrm{E}+00$ \\
\hline ENSBTAG00000012557 & $4.70 \mathrm{E}-10$ & $2.46 \mathrm{E}-10$ & OSGEPL1 & $1.91 E+00$ \\
\hline ENSBTAG00000019371 & $3.33 \mathrm{E}-10$ & $1.74 \mathrm{E}-10$ & CNR2 & $1.91 E+00$ \\
\hline ENSBTAG00000010344 & $7.62 \mathrm{E}-10$ & 3.97E-10 & SLC16A14 & $1.92 \mathrm{E}+00$ \\
\hline ENSBTAG00000038275 & $5.75 \mathrm{E}-10$ & $2.97 \mathrm{E}-10$ & CYP27C1 & $1.94 \mathrm{E}+00$ \\
\hline ENSBTAG00000037804 & $2.54 \mathrm{E}-10$ & $1.30 \mathrm{E}-10$ & IKZF2 & $1.95 \mathrm{E}+00$ \\
\hline
\end{tabular}




\begin{tabular}{|c|c|c|c|c|}
\hline ENSBTAG00000047880 & $3.75 \mathrm{E}-10$ & $1.92 \mathrm{E}-10$ & & $1.95 \mathrm{E}+00$ \\
\hline ENSBTAG00000043989 & $5.44 \mathrm{E}-10$ & $2.78 \mathrm{E}-10$ & EYA3 & $1.96 \mathrm{E}+00$ \\
\hline ENSBTAG00000010030 & $2.92 \mathrm{E}-10$ & $1.50 \mathrm{E}-10$ & EPHA4 & $1.95 \mathrm{E}+00$ \\
\hline ENSBTAG00000032515 & $5.92 \mathrm{E}-10$ & $3.02 \mathrm{E}-10$ & PLA2R1 & $1.96 \mathrm{E}+00$ \\
\hline ENSBTAG00000020984 & $4.92 \mathrm{E}-10$ & $2.51 \mathrm{E}-10$ & RAPGEF4 & $1.96 \mathrm{E}+00$ \\
\hline ENSBTAG00000008755 & $2.13 \mathrm{E}-10$ & $1.08 \mathrm{E}-10$ & 07-Mar & $1.97 \mathrm{E}+00$ \\
\hline ENSBTAG00000018581 & $2.07 \mathrm{E}-10$ & $1.05 \mathrm{E}-10$ & EPC2 & $1.97 \mathrm{E}+00$ \\
\hline ENSBTAG00000014832 & $3.67 \mathrm{E}-10$ & $1.85 \mathrm{E}-10$ & TMEFF2 & $1.98 \mathrm{E}+00$ \\
\hline ENSBTAG00000040329 & $3.62 \mathrm{E}-10$ & $1.82 \mathrm{E}-10$ & HTR1D & $1.99 E+00$ \\
\hline ENSBTAG00000036258 & $6.65 \mathrm{E}-10$ & $3.34 \mathrm{E}-10$ & SMIM10L1 & $1.99 \mathrm{E}+00$ \\
\hline ENSBTAG00000047681 & $6.60 \mathrm{E}-10$ & 3.31E-10 & & $1.99 \mathrm{E}+00$ \\
\hline ENSBTAG00000011195 & $7.78 \mathrm{E}-10$ & $3.91 \mathrm{E}-10$ & & $1.99 \mathrm{E}+00$ \\
\hline ENSBTAG00000008579 & $3.44 \mathrm{E}-10$ & $1.71 \mathrm{E}-10$ & $\mathrm{RCC2}$ & $2.01 E+00$ \\
\hline ENSBTAG00000026758 & $3.06 \mathrm{E}-10$ & $1.51 \mathrm{E}-10$ & & $2.02 \mathrm{E}+00$ \\
\hline ENSBTAG00000030434 & $3.17 \mathrm{E}-10$ & $1.57 \mathrm{E}-10$ & FUCA1 & $2.02 \mathrm{E}+00$ \\
\hline ENSBTAG00000001585 & $3.56 \mathrm{E}-10$ & $1.76 \mathrm{E}-10$ & WIPF1 & $2.02 \mathrm{E}+00$ \\
\hline ENSBTAG00000011951 & $3.45 \mathrm{E}-10$ & $1.70 \mathrm{E}-10$ & MRTO4 & $2.03 E+00$ \\
\hline ENSBTAG00000009796 & $2.02 \mathrm{E}-10$ & $9.94 \mathrm{E}-11$ & PIGV & $2.03 E+00$ \\
\hline ENSBTAG00000044179 & $5.88 \mathrm{E}-10$ & $2.89 \mathrm{E}-10$ & CERS6 & $2.04 \mathrm{E}+00$ \\
\hline ENSBTAG00000048151 & 8.37E-11 & $4.11 E-11$ & PRPF40A & $2.03 E+00$ \\
\hline ENSBTAG00000037702 & $8.32 \mathrm{E}-10$ & $4.06 \mathrm{E}-10$ & & $2.05 E+00$ \\
\hline ENSBTAG00000016784 & $6.78 \mathrm{E}-10$ & $3.28 \mathrm{E}-10$ & DNAH7 & $2.07 E+00$ \\
\hline ENSBTAG00000002594 & $1.76 \mathrm{E}-10$ & $8.51 E-11$ & ZNF436 & $2.06 \mathrm{E}+00$ \\
\hline ENSBTAG00000016174 & $1.11 \mathrm{E}-10$ & $5.36 \mathrm{E}-11$ & & $2.06 \mathrm{E}+00$ \\
\hline ENSBTAG00000005477 & $2.80 \mathrm{E}-10$ & $1.34 \mathrm{E}-10$ & LAPTM5 & $2.09 \mathrm{E}+00$ \\
\hline ENSBTAG00000026986 & 4.14E-10 & $1.99 \mathrm{E}-10$ & & $2.08 \mathrm{E}+00$ \\
\hline ENSBTAG00000011682 & $1.86 \mathrm{E}-10$ & $8.91 \mathrm{E}-11$ & GLI2 & $2.08 \mathrm{E}+00$ \\
\hline ENSBTAG00000043956 & 4.07E-10 & $1.95 \mathrm{E}-10$ & UBE2E3 & $2.09 E+00$ \\
\hline ENSBTAG00000012263 & $3.99 \mathrm{E}-10$ & $1.91 \mathrm{E}-10$ & ASAP3 & $2.09 E+00$ \\
\hline ENSBTAG00000018413 & $8.78 \mathrm{E}-11$ & $4.14 \mathrm{E}-11$ & FAM46B & $2.12 \mathrm{E}+00$ \\
\hline ENSBTAG00000039121 & $1.34 \mathrm{E}-10$ & $6.35 \mathrm{E}-11$ & PTP4A2 & $2.11 E+00$ \\
\hline ENSBTAG00000008089 & $4.53 \mathrm{E}-10$ & $2.13 \mathrm{E}-10$ & AMMECR1L & $2.13 E+00$ \\
\hline ENSBTAG00000009256 & $6.12 \mathrm{E}-10$ & $2.87 \mathrm{E}-10$ & ITGA4 & $2.13 E+00$ \\
\hline ENSBTAG00000010278 & $7.13 \mathrm{E}-10$ & $3.33 \mathrm{E}-10$ & STPG1 & $2.14 E+00$ \\
\hline ENSBTAG00000030335 & $5.04 \mathrm{E}-10$ & $2.35 \mathrm{E}-10$ & ALDH4A1 & $2.14 \mathrm{E}+00$ \\
\hline ENSBTAG00000012997 & $7.48 \mathrm{E}-10$ & $3.48 \mathrm{E}-10$ & SMPDL3B & $2.15 E+00$ \\
\hline ENSBTAG00000007683 & $2.60 \mathrm{E}-10$ & $1.20 \mathrm{E}-10$ & DCAF17 & $2.17 \mathrm{E}+00$ \\
\hline ENSBTAG00000021588 & $1.64 \mathrm{E}-10$ & $7.49 \mathrm{E}-11$ & SCG2 & $2.18 \mathrm{E}+00$ \\
\hline ENSBTAG00000040215 & $2.44 \mathrm{E}-10$ & $1.11 \mathrm{E}-10$ & EIF4G3 & $2.20 E+00$ \\
\hline ENSBTAG00000008330 & $8.70 \mathrm{E}-11$ & $3.95 \mathrm{E}-11$ & RNF19B & $2.20 \mathrm{E}+00$ \\
\hline ENSBTAG00000001093 & $3.30 \mathrm{E}-10$ & $1.47 \mathrm{E}-10$ & KLHL23 & $2.25 \mathrm{E}+00$ \\
\hline ENSBTAG00000017695 & $3.20 \mathrm{E}-10$ & $1.42 \mathrm{E}-10$ & CCDC150 & $2.25 E+00$ \\
\hline ENSBTAG00000038865 & $2.99 \mathrm{E}-10$ & $1.32 \mathrm{E}-10$ & TCEA3 & $2.27 \mathrm{E}+00$ \\
\hline ENSBTAG00000011992 & 7.93E-10 & $3.46 \mathrm{E}-10$ & PTH2R & $2.29 E+00$ \\
\hline ENSBTAG00000007153 & $9.14 \mathrm{E}-10$ & $3.94 \mathrm{E}-10$ & C1QA & $2.32 \mathrm{E}+00$ \\
\hline ENSBTAG00000017253 & $2.16 \mathrm{E}-10$ & $9.15 E-11$ & MECR & $2.36 \mathrm{E}+00$ \\
\hline ENSBTAG00000005002 & $1.07 \mathrm{E}-10$ & $4.50 \mathrm{E}-11$ & GALE & $2.38 \mathrm{E}+00$ \\
\hline ENSBTAG00000030340 & $4.18 \mathrm{E}-10$ & $1.76 \mathrm{E}-10$ & & $2.37 E+00$ \\
\hline ENSBTAG00000005741 & $1.08 \mathrm{E}-10$ & $4.54 \mathrm{E}-11$ & DLX2 & $2.38 \mathrm{E}+00$ \\
\hline ENSBTAG00000001359 & $1.98 \mathrm{E}-10$ & $8.31 \mathrm{E}-11$ & B3GNT7 & $2.38 \mathrm{E}+00$ \\
\hline ENSBTAG00000006049 & $1.36 \mathrm{E}-10$ & $5.70 \mathrm{E}-11$ & BZW1 & $2.38 \mathrm{E}+00$ \\
\hline ENSBTAG00000026788 & $1.94 \mathrm{E}-10$ & $8.12 \mathrm{E}-11$ & SHOX & $2.38 \mathrm{E}+00$ \\
\hline ENSBTAG00000006342 & $2.19 \mathrm{E}-10$ & $9.20 \mathrm{E}-11$ & & $2.38 \mathrm{E}+00$ \\
\hline ENSBTAG00000037456 & $5.12 \mathrm{E}-11$ & $2.15 \mathrm{E}-11$ & AHDC1 & $2.38 \mathrm{E}+00$ \\
\hline ENSBTAG00000016748 & $3.30 \mathrm{E}-10$ & $1.38 \mathrm{E}-10$ & & $2.39 E+00$ \\
\hline ENSBTAG00000026822 & $2.25 \mathrm{E}-10$ & $9.45 E-11$ & & $2.38 \mathrm{E}+00$ \\
\hline ENSBTAG00000039046 & $1.36 \mathrm{E}-10$ & $5.72 \mathrm{E}-11$ & & $2.38 \mathrm{E}+00$ \\
\hline ENSBTAG00000014988 & $5.06 \mathrm{E}-10$ & $2.12 \mathrm{E}-10$ & & $2.39 E+00$ \\
\hline ENSBTAG00000046783 & $8.25 \mathrm{E}-10$ & $3.45 E-10$ & & $2.39 E+00$ \\
\hline ENSBTAG00000001101 & $8.94 \mathrm{E}-10$ & $3.72 \mathrm{E}-10$ & IFNLR1 & $2.40 E+00$ \\
\hline ENSBTAG00000007258 & $6.98 \mathrm{E}-10$ & $2.88 \mathrm{E}-10$ & GAD1 & $2.42 \mathrm{E}+00$ \\
\hline ENSBTAG00000013048 & $2.92 \mathrm{E}-10$ & $1.21 \mathrm{E}-10$ & NIPAL3 & $2.42 \mathrm{E}+00$ \\
\hline ENSBTAG00000009831 & $4.68 \mathrm{E}-10$ & $1.91 \mathrm{E}-10$ & OCA2 & $2.45 \mathrm{E}+00$ \\
\hline ENSBTAG00000045902 & $9.84 \mathrm{E}-10$ & $3.96 \mathrm{E}-10$ & EPHB2 & $2.49 E+00$ \\
\hline ENSBTAG00000015240 & $2.48 \mathrm{E}-10$ & $9.93 \mathrm{E}-11$ & UBR4 & $2.50 \mathrm{E}+00$ \\
\hline ENSBTAG00000010362 & $8.62 \mathrm{E}-10$ & $3.45 \mathrm{E}-10$ & NOSTRIN & $2.50 \mathrm{E}+00$ \\
\hline ENSBTAG00000007239 & $2.05 \mathrm{E}-10$ & $8.18 \mathrm{E}-11$ & TNFAIP6 & $2.50 \mathrm{E}+00$ \\
\hline ENSBTAG00000016092 & $2.53 \mathrm{E}-10$ & $1.01 \mathrm{E}-10$ & SPATS2L & $2.50 \mathrm{E}+00$ \\
\hline ENSBTAG00000008362 & $3.04 \mathrm{E}-10$ & $1.21 \mathrm{E}-10$ & CTDSP1 & $2.51 E+00$ \\
\hline ENSBTAG00000032846 & $6.54 \mathrm{E}-10$ & $2.58 \mathrm{E}-10$ & ERICH2 & $2.53 E+00$ \\
\hline
\end{tabular}




\begin{tabular}{|c|c|c|c|c|}
\hline ENSBTAG00000003120 & $4.57 \mathrm{E}-10$ & $1.79 \mathrm{E}-10$ & ZNF385B & $2.55 E+00$ \\
\hline ENSBTAG00000015520 & $2.17 \mathrm{E}-10$ & $8.49 \mathrm{E}-11$ & SLC11A1 & $2.56 \mathrm{E}+00$ \\
\hline ENSBTAG00000006355 & $3.25 \mathrm{E}-10$ & $1.27 \mathrm{E}-10$ & SLC30A2 & $2.56 \mathrm{E}+00$ \\
\hline ENSBTAG00000019255 & $2.95 \mathrm{E}-10$ & $1.14 \mathrm{E}-10$ & NFE2L2 & $2.59 \mathrm{E}+00$ \\
\hline ENSBTAG00000005234 & $3.70 \mathrm{E}-10$ & $1.41 \mathrm{E}-10$ & LYPD1 & $2.62 E+00$ \\
\hline ENSBTAG00000011649 & $3.90 \mathrm{E}-10$ & $1.48 \mathrm{E}-10$ & FARSB & $2.63 E+00$ \\
\hline ENSBTAG00000027789 & $1.87 \mathrm{E}-10$ & $7.09 \mathrm{E}-11$ & PPIG & $2.64 \mathrm{E}+00$ \\
\hline ENSBTAG00000014967 & 4.23E-10 & $1.60 \mathrm{E}-10$ & PAX7 & $2.64 \mathrm{E}+00$ \\
\hline ENSBTAG00000018502 & $4.68 \mathrm{E}-10$ & $1.77 \mathrm{E}-10$ & ARMC9 & $2.64 \mathrm{E}+00$ \\
\hline ENSBTAG00000016578 & $2.00 \mathrm{E}-10$ & $7.46 \mathrm{E}-11$ & HNRNPR & $2.68 \mathrm{E}+00$ \\
\hline ENSBTAG00000020696 & $2.18 \mathrm{E}-10$ & $8.13 \mathrm{E}-11$ & HECW2 & $2.68 \mathrm{E}+00$ \\
\hline ENSBTAG00000014432 & $2.18 \mathrm{E}-10$ & $8.04 \mathrm{E}-11$ & HNMT & $2.71 E+00$ \\
\hline ENSBTAG00000019298 & $6.32 \mathrm{E}-10$ & $2.33 \mathrm{E}-10$ & STRADB & $2.71 E+00$ \\
\hline ENSBTAG00000026885 & $3.94 \mathrm{E}-10$ & $1.45 \mathrm{E}-10$ & ABCB11 & $2.72 \mathrm{E}+00$ \\
\hline ENSBTAG00000000941 & $6.46 \mathrm{E}-10$ & $2.37 \mathrm{E}-10$ & PLEKHB2 & $2.73 E+00$ \\
\hline ENSBTAG00000021310 & $4.78 \mathrm{E}-10$ & $1.76 \mathrm{E}-10$ & COL4A4 & $2.72 E+00$ \\
\hline ENSBTAG00000007898 & $4.68 \mathrm{E}-10$ & $1.71 \mathrm{E}-10$ & CYBRD1 & $2.74 \mathrm{E}+00$ \\
\hline ENSBTAG00000038620 & $5.56 \mathrm{E}-10$ & $2.02 \mathrm{E}-10$ & CIR1 & $2.75 E+00$ \\
\hline ENSBTAG00000003626 & $7.00 \mathrm{E}-10$ & $2.53 \mathrm{E}-10$ & МYO3В & $2.77 \mathrm{E}+00$ \\
\hline ENSBTAG00000020905 & $1.37 \mathrm{E}-10$ & $4.92 \mathrm{E}-11$ & RPL11 & $2.78 \mathrm{E}+00$ \\
\hline ENSBTAG00000005408 & $7.92 \mathrm{E}-11$ & $2.85 \mathrm{E}-11$ & CLK1 & $2.77 \mathrm{E}+00$ \\
\hline ENSBTAG00000007765 & $1.17 \mathrm{E}-10$ & $4.18 \mathrm{E}-11$ & TFCP2L1 & $2.79 E+00$ \\
\hline ENSBTAG00000019791 & $8.47 \mathrm{E}-10$ & $3.01 \mathrm{E}-10$ & GRHL3 & $2.81 \mathrm{E}+00$ \\
\hline ENSBTAG00000001100 & 1.11E-09 & $3.91 \mathrm{E}-10$ & IL22RA1 & $2.83 \mathrm{E}+00$ \\
\hline ENSBTAG00000007635 & $5.15 \mathrm{E}-10$ & $1.80 \mathrm{E}-10$ & PLCL1 & $2.86 \mathrm{E}+00$ \\
\hline ENSBTAG00000001092 & $1.74 \mathrm{E}-10$ & $5.98 \mathrm{E}-11$ & PHOSPHO2 & $2.91 E+00$ \\
\hline ENSBTAG00000019190 & $4.62 \mathrm{E}-10$ & $1.55 \mathrm{E}-10$ & $\mathrm{CPO}$ & $2.98 \mathrm{E}+00$ \\
\hline ENSBTAG00000021328 & $4.94 \mathrm{E}-10$ & $1.66 \mathrm{E}-10$ & SPHKAP & $2.98 \mathrm{E}+00$ \\
\hline ENSBTAG00000019075 & $5.65 \mathrm{E}-10$ & $1.89 \mathrm{E}-10$ & METAP1D & $2.99 \mathrm{E}+00$ \\
\hline ENSBTAG00000046454 & $5.35 \mathrm{E}-10$ & $1.79 \mathrm{E}-10$ & DYTN & $2.99 \mathrm{E}+00$ \\
\hline ENSBTAG00000017644 & $3.73 \mathrm{E}-10$ & $1.24 \mathrm{E}-10$ & KIF17 & $3.01 E+00$ \\
\hline ENSBTAG00000008431 & $1.62 \mathrm{E}-10$ & $5.28 \mathrm{E}-11$ & MOGAT1 & $3.07 E+00$ \\
\hline ENSBTAG00000033662 & $2.74 \mathrm{E}-10$ & $8.74 \mathrm{E}-11$ & NCKAP1 & $3.14 \mathrm{E}+00$ \\
\hline ENSBTAG00000021172 & 2.27E-10 & 7.19E-11 & IWS1 & $3.15 E+00$ \\
\hline ENSBTAG00000012043 & $8.73 E-10$ & $2.76 \mathrm{E}-10$ & PADI3 & $3.16 \mathrm{E}+00$ \\
\hline ENSBTAG00000006432 & $2.06 \mathrm{E}-10$ & 6.47E-11 & KCNE4 & $3.17 E+00$ \\
\hline ENSBTAG00000020652 & $2.09 \mathrm{E}-10$ & $6.57 \mathrm{E}-11$ & TSSK3 & $3.17 E+00$ \\
\hline ENSBTAG00000020613 & $9.68 \mathrm{E}-11$ & 3.05E-11 & STK16 & $3.17 E+00$ \\
\hline ENSBTAG00000047490 & $9.99 \mathrm{E}-11$ & $3.15 \mathrm{E}-11$ & & $3.17 E+00$ \\
\hline ENSBTAG00000020607 & $8.59 \mathrm{E}-11$ & $2.70 \mathrm{E}-11$ & $\mathrm{ABCB} 6$ & $3.17 E+00$ \\
\hline ENSBTAG00000009502 & $1.06 \mathrm{E}-10$ & $3.33 \mathrm{E}-11$ & LUZP1 & $3.17 E+00$ \\
\hline ENSBTAG00000001784 & $1.97 \mathrm{E}-10$ & $6.21 \mathrm{E}-11$ & LDLRAD2 & $3.17 E+00$ \\
\hline ENSBTAG00000006918 & $2.89 \mathrm{E}-10$ & 8.97E-11 & TLK1 & $3.22 \mathrm{E}+00$ \\
\hline ENSBTAG00000015077 & $3.54 \mathrm{E}-10$ & $1.10 \mathrm{E}-10$ & & $3.22 \mathrm{E}+00$ \\
\hline ENSBTAG00000002295 & $1.90 \mathrm{E}-10$ & $5.84 \mathrm{E}-11$ & ATF2 & $3.25 E+00$ \\
\hline ENSBTAG00000033254 & $3.24 \mathrm{E}-10$ & $9.78 \mathrm{E}-11$ & DFNB59 & $3.31 E+00$ \\
\hline ENSBTAG00000017122 & $4.71 \mathrm{E}-10$ & $1.42 \mathrm{E}-10$ & HSPG2 & $3.32 E+00$ \\
\hline ENSBTAG00000045513 & $3.76 \mathrm{E}-10$ & 1.13E-10 & & $3.32 E+00$ \\
\hline ENSBTAG00000013619 & $6.44 \mathrm{E}-10$ & $1.93 \mathrm{E}-10$ & SCTR & $3.34 E+00$ \\
\hline ENSBTAG00000014885 & $8.61 \mathrm{E}-10$ & $2.56 \mathrm{E}-10$ & MYOM3 & $3.36 \mathrm{E}+00$ \\
\hline ENSBTAG00000001435 & $2.19 \mathrm{E}-10$ & $6.51 \mathrm{E}-11$ & ARL6IP6 & $3.36 \mathrm{E}+00$ \\
\hline ENSBTAG00000006667 & $2.27 \mathrm{E}-10$ & $6.71 E-11$ & EPB41 & $3.37 E+00$ \\
\hline ENSBTAG00000001083 & $2.57 \mathrm{E}-10$ & $7.49 E-11$ & ZAK & $3.43 E+00$ \\
\hline ENSBTAG00000019929 & 4.10E-10 & 1.17E-10 & ITGAV & $3.51 E+00$ \\
\hline ENSBTAG00000010736 & 4.23E-10 & $1.18 \mathrm{E}-10$ & & $3.58 \mathrm{E}+00$ \\
\hline ENSBTAG00000001090 & $1.69 \mathrm{E}-10$ & 4.67E-11 & CCDC173 & $3.60 E+00$ \\
\hline ENSBTAG00000026008 & $3.26 \mathrm{E}-10$ & $9.00 \mathrm{E}-11$ & METTL5 & $3.62 E+00$ \\
\hline ENSBTAG00000001683 & $6.36 \mathrm{E}-10$ & $1.74 \mathrm{E}-10$ & FAM171B & $3.65 E+00$ \\
\hline ENSBTAG00000027875 & $7.38 \mathrm{E}-10$ & $1.97 \mathrm{E}-10$ & CCDC141 & $3.75 E+00$ \\
\hline ENSBTAG00000008336 & $2.64 \mathrm{E}-10$ & $7.00 \mathrm{E}-11$ & HPCA & $3.77 E+00$ \\
\hline ENSBTAG00000008915 & $2.28 \mathrm{E}-10$ & $6.02 \mathrm{E}-11$ & SF3B1 & $3.79 E+00$ \\
\hline ENSBTAG00000003944 & 3.37E-10 & $8.82 \mathrm{E}-11$ & CATIP & $3.82 \mathrm{E}+00$ \\
\hline ENSBTAG00000004241 & 3.55E-10 & $9.23 \mathrm{E}-11$ & $\mathrm{G} 6 \mathrm{PC2}$ & $3.84 \mathrm{E}+00$ \\
\hline ENSBTAG00000027020 & $6.71 E-10$ & $1.72 \mathrm{E}-10$ & COL5A2 & $3.90 E+00$ \\
\hline ENSBTAG00000012618 & $1.99 \mathrm{E}-10$ & $5.01 \mathrm{E}-11$ & SLC23A3 & $3.97 \mathrm{E}+00$ \\
\hline ENSBTAG00000003813 & $1.03 \mathrm{E}-10$ & $2.60 \mathrm{E}-11$ & BCS1L & $3.97 E+00$ \\
\hline ENSBTAG00000030973 & $6.86 \mathrm{E}-11$ & $1.73 \mathrm{E}-11$ & & $3.95 E+00$ \\
\hline ENSBTAG00000002727 & $1.85 \mathrm{E}-10$ & $4.65 \mathrm{E}-11$ & PHACTR4 & $3.97 E+00$ \\
\hline ENSBTAG00000001097 & $2.66 \mathrm{E}-10$ & $6.68 \mathrm{E}-11$ & FKBP7 & $3.97 E+00$ \\
\hline ENSBTAG00000021319 & $6.14 \mathrm{E}-10$ & $1.53 \mathrm{E}-10$ & & $4.01 E+00$ \\
\hline
\end{tabular}




\begin{tabular}{|c|c|c|c|c|}
\hline ENSBTAG00000009470 & $2.31 \mathrm{E}-10$ & $5.76 \mathrm{E}-11$ & CLIC4 & $4.01 E+00$ \\
\hline ENSBTAG00000039276 & 4.59E-10 & $1.13 \mathrm{E}-10$ & & $4.06 \mathrm{E}+00$ \\
\hline ENSBTAG00000019137 & $6.90 \mathrm{E}-10$ & $1.70 \mathrm{E}-10$ & SCN7A & $4.05 E+00$ \\
\hline ENSBTAG00000005119 & $4.55 \mathrm{E}-10$ & $1.12 \mathrm{E}-10$ & PSMD1 & $4.06 \mathrm{E}+00$ \\
\hline ENSBTAG00000018385 & 2.09E-10 & $5.10 \mathrm{E}-11$ & DNAJC8 & $4.10 \mathrm{E}+00$ \\
\hline ENSBTAG00000039772 & $3.48 \mathrm{E}-10$ & 8.19E-11 & HTR2B & $4.24 E+00$ \\
\hline ENSBTAG00000001082 & $1.38 \mathrm{E}-10$ & 3.17E-11 & SH2D 5 & $4.35 E+00$ \\
\hline ENSBTAG00000017162 & $6.54 \mathrm{E}-10$ & $1.50 \mathrm{E}-10$ & & $4.36 \mathrm{E}+00$ \\
\hline ENSBTAG00000021692 & 1.17E-10 & $2.68 \mathrm{E}-11$ & TBR1 & $4.35 \mathrm{E}+00$ \\
\hline ENSBTAG00000026825 & $6.78 \mathrm{E}-10$ & $1.53 \mathrm{E}-10$ & TMEM37 & $4.43 \mathrm{E}+00$ \\
\hline ENSBTAG00000013455 & $4.71 \mathrm{E}-11$ & $1.06 \mathrm{E}-11$ & SPOPL & $4.41 E+00$ \\
\hline ENSBTAG00000011193 & $3.19 \mathrm{E}-10$ & $7.08 \mathrm{E}-11$ & C1QC & $4.50 \mathrm{E}+00$ \\
\hline ENSBTAG00000030413 & $8.88 \mathrm{E}-10$ & $1.91 \mathrm{E}-10$ & LACTBL1 & $4.65 \mathrm{E}+00$ \\
\hline ENSBTAG00000021976 & $1.48 \mathrm{E}-10$ & $3.15 \mathrm{E}-11$ & NDUFS1 & $4.67 E+00$ \\
\hline ENSBTAG00000021979 & $1.50 \mathrm{E}-10$ & 3.14E-11 & EEF1B2 & $4.76 \mathrm{E}+00$ \\
\hline ENSBTAG00000014848 & $2.81 \mathrm{E}-10$ & 5.90E-11 & NROB2 & $4.76 \mathrm{E}+00$ \\
\hline ENSBTAG00000009972 & $1.65 \mathrm{E}-10$ & 3.47E-11 & INHA & $4.76 \mathrm{E}+00$ \\
\hline ENSBTAG00000027696 & $1.87 \mathrm{E}-10$ & $3.91 \mathrm{E}-11$ & & $4.76 \mathrm{E}+00$ \\
\hline ENSBTAG00000009949 & $6.11 \mathrm{E}-10$ & $1.25 \mathrm{E}-10$ & DARS & $4.89 \mathrm{E}+00$ \\
\hline ENSBTAG00000013302 & $6.16 \mathrm{E}-10$ & $1.22 \mathrm{E}-10$ & R3HDM1 & $5.04 \mathrm{E}+00$ \\
\hline ENSBTAG00000008673 & $2.43 \mathrm{E}-10$ & $4.71 \mathrm{E}-11$ & ICA1L & $5.14 \mathrm{E}+00$ \\
\hline ENSBTAG00000010338 & 4.04E-10 & 7.76E-11 & FBXO36 & $5.20 E+00$ \\
\hline ENSBTAG00000021620 & $3.52 \mathrm{E}-10$ & $6.70 \mathrm{E}-11$ & PITHD1 & $5.25 \mathrm{E}+00$ \\
\hline ENSBTAG00000020850 & $4.31 \mathrm{E}-10$ & 8.13E-11 & MUL1 & $5.30 E+00$ \\
\hline ENSBTAG00000001096 & $6.32 \mathrm{E}-10$ & 1.17E-10 & PRKRA & $5.40 \mathrm{E}+00$ \\
\hline ENSBTAG00000020794 & $9.05 \mathrm{E}-10$ & $1.62 \mathrm{E}-10$ & & $5.59 \mathrm{E}+00$ \\
\hline ENSBTAG00000009937 & 1.20E-09 & $2.14 \mathrm{E}-10$ & & $5.62 \mathrm{E}+00$ \\
\hline ENSBTAG00000001098 & $4.35 \mathrm{E}-10$ & 7.70E-11 & PLEKHA3 & $5.65 \mathrm{E}+00$ \\
\hline ENSBTAG00000002112 & $4.99 \mathrm{E}-10$ & $8.51 E-11$ & INSIG2 & $5.86 \mathrm{E}+00$ \\
\hline ENSBTAG00000005596 & 5.64E-10 & $9.60 \mathrm{E}-11$ & IGFBP2 & $5.87 \mathrm{E}+00$ \\
\hline ENSBTAG00000018253 & $3.73 \mathrm{E}-10$ & $6.32 \mathrm{E}-11$ & CHRNA1 & $5.90 \mathrm{E}+00$ \\
\hline ENSBTAG00000017266 & $4.68 \mathrm{E}-10$ & $7.66 \mathrm{E}-11$ & ITGA6 & $6.10 E+00$ \\
\hline ENSBTAG00000016192 & 4.86E-10 & $7.84 \mathrm{E}-11$ & MDH1B & $6.19 E+00$ \\
\hline ENSBTAG00000018543 & $3.29 \mathrm{E}-10$ & $5.22 \mathrm{E}-11$ & VIL1 & $6.29 \mathrm{E}+00$ \\
\hline ENSBTAG00000020611 & $9.88 \mathrm{E}-11$ & $1.55 \mathrm{E}-11$ & GLB1L & $6.34 \mathrm{E}+00$ \\
\hline ENSBTAG00000010741 & $1.99 \mathrm{E}-10$ & $2.98 \mathrm{E}-11$ & KLHL41 & $6.66 \mathrm{E}+00$ \\
\hline ENSBTAG00000022671 & $3.20 \mathrm{E}-10$ & 4.67E-11 & WNT4 & $6.84 \mathrm{E}+00$ \\
\hline ENSBTAG00000019172 & 1.06E-09 & $1.51 \mathrm{E}-10$ & IGSF21 & $6.98 \mathrm{E}+00$ \\
\hline ENSBTAG00000006168 & $6.08 \mathrm{E}-10$ & $8.41 E-11$ & RBM45 & $7.23 E+00$ \\
\hline ENSBTAG00000016396 & $6.84 \mathrm{E}-10$ & $9.42 \mathrm{E}-11$ & PDE11A & $7.25 \mathrm{E}+00$ \\
\hline ENSBTAG00000015424 & $2.27 \mathrm{E}-10$ & $3.03 \mathrm{E}-11$ & WDR12 & $7.48 \mathrm{E}+00$ \\
\hline ENSBTAG00000014850 & $1.65 \mathrm{E}-10$ & $2.18 \mathrm{E}-11$ & GPN2 & $7.53 \mathrm{E}+00$ \\
\hline ENSBTAG00000008331 & 4.33E-10 & $5.73 \mathrm{E}-11$ & TMEM54 & $7.55 E+00$ \\
\hline ENSBTAG00000008300 & $5.21 \mathrm{E}-10$ & $6.86 \mathrm{E}-11$ & FN1 & $7.58 \mathrm{E}+00$ \\
\hline ENSBTAG00000001887 & $5.85 \mathrm{E}-10$ & $7.69 \mathrm{E}-11$ & STAM2 & $7.59 \mathrm{E}+00$ \\
\hline ENSBTAG00000016954 & $2.92 \mathrm{E}-10$ & $3.73 \mathrm{E}-11$ & TMEMЗ9B & $7.82 \mathrm{E}+00$ \\
\hline ENSBTAG00000016762 & $2.98 \mathrm{E}-10$ & $3.66 \mathrm{E}-11$ & SLC25A12 & $8.12 \mathrm{E}+00$ \\
\hline ENSBTAG00000008028 & $2.78 \mathrm{E}-10$ & $3.39 \mathrm{E}-11$ & CHN1 & $8.17 E+00$ \\
\hline ENSBTAG00000007062 & $4.40 \mathrm{E}-10$ & $4.98 \mathrm{E}-11$ & IGFBP5 & $8.83 \mathrm{E}+00$ \\
\hline ENSBTAG00000031579 & 4.87E-10 & $5.36 \mathrm{E}-11$ & $\mathrm{SGO} 2$ & $9.07 \mathrm{E}+00$ \\
\hline ENSBTAG00000016785 & $9.09 \mathrm{E}-10$ & $9.86 \mathrm{E}-11$ & & $9.21 \mathrm{E}+00$ \\
\hline ENSBTAG00000013218 & 8.37E-10 & 8.47E-11 & GORASP2 & $9.88 \mathrm{E}+00$ \\
\hline ENSBTAG00000013309 & $1.76 \mathrm{E}-10$ & $1.61 \mathrm{E}-11$ & SRSF4 & $1.09 \mathrm{E}+01$ \\
\hline ENSBTAG00000009725 & 7.14E-10 & $6.37 \mathrm{E}-11$ & AOX1 & $1.12 \mathrm{E}+01$ \\
\hline ENSBTAG00000016572 & $2.58 \mathrm{E}-10$ & $2.16 \mathrm{E}-11$ & USP37 & $1.19 \mathrm{E}+01$ \\
\hline ENSBTAG00000003809 & $6.20 \mathrm{E}-10$ & $5.00 \mathrm{E}-11$ & PLCD4 & $1.24 \mathrm{E}+01$ \\
\hline ENSBTAG00000001700 & $2.86 \mathrm{E}-10$ & $2.21 \mathrm{E}-11$ & $\mathrm{CDC} 42$ & $1.29 \mathrm{E}+01$ \\
\hline ENSBTAG00000012497 & $1.18 \mathrm{E}-10$ & 8.74E-12 & KHDRBS1 & $1.34 \mathrm{E}+01$ \\
\hline ENSBTAG00000010743 & $1.59 \mathrm{E}-10$ & $1.16 \mathrm{E}-11$ & FASTKD1 & $1.36 \mathrm{E}+01$ \\
\hline ENSBTAG00000026772 & $2.68 \mathrm{E}-10$ & $1.95 \mathrm{E}-11$ & ZNF142 & $1.37 \mathrm{E}+01$ \\
\hline ENSBTAG00000010048 & $7.59 \mathrm{E}-10$ & $5.00 \mathrm{E}-11$ & SPC25 & $1.51 E+01$ \\
\hline ENSBTAG00000040602 & $3.90 \mathrm{E}-10$ & $2.45 \mathrm{E}-11$ & & $1.59 \mathrm{E}+01$ \\
\hline ENSBTAG00000003807 & $2.64 \mathrm{E}-10$ & $1.55 \mathrm{E}-11$ & CNOT9 & $1.69 \mathrm{E}+01$ \\
\hline ENSBTAG00000010118 & $2.26 \mathrm{E}-10$ & $1.29 \mathrm{E}-11$ & HAT1 & $1.74 \mathrm{E}+01$ \\
\hline ENSBTAG00000013085 & $5.36 \mathrm{E}-10$ & $2.86 \mathrm{E}-11$ & & $1.87 \mathrm{E}+01$ \\
\hline ENSBTAG00000011196 & $8.81 \mathrm{E}-10$ & $4.59 \mathrm{E}-11$ & $\mathrm{C} 1 \mathrm{QB}$ & $1.92 \mathrm{E}+01$ \\
\hline ENSBTAG00000016836 & $2.54 \mathrm{E}-10$ & $1.29 \mathrm{E}-11$ & PDK1 & $1.96 \mathrm{E}+01$ \\
\hline ENSBTAG00000026660 & $1.85 \mathrm{E}-10$ & $8.62 E-12$ & & $2.12 \mathrm{E}+01$ \\
\hline ENSBTAG00000003817 & $2.39 \mathrm{E}-10$ & $1.11 \mathrm{E}-11$ & RNF25 & $2.14 \mathrm{E}+01$ \\
\hline ENSBTAG00000010221 & 4.69E-10 & $2.04 \mathrm{E}-11$ & CCDC93 & $2.29 \mathrm{E}+01$ \\
\hline
\end{tabular}




\begin{tabular}{|c|c|c|c|c|}
\hline ENSBTAG00000039522 & 7.67E-10 & $3.27 \mathrm{E}-11$ & & $2.34 \mathrm{E}+01$ \\
\hline ENSBTAG00000015771 & $2.23 \mathrm{E}-10$ & $7.00 \mathrm{E}-12$ & YTHDF2 & $3.14 E+01$ \\
\hline ENSBTAG00000015172 & $6.89 \mathrm{E}-10$ & $2.04 \mathrm{E}-11$ & MCM6 & $3.36 \mathrm{E}+01$ \\
\hline ENSBTAG00000018549 & $8.62 \mathrm{E}-10$ & 2.43E-11 & & $3.53 \mathrm{E}+01$ \\
\hline ENSBTAG00000010393 & $1.68 \mathrm{E}-10$ & $4.41 \mathrm{E}-12$ & TRNAU1AP & $3.74 E+01$ \\
\hline ENSBTAG00000002944 & $2.11 \mathrm{E}-10$ & 0 & ATP5G3 & $2.11 E+03$ \\
\hline ENSBTAG00000048306 & $2.32 \mathrm{E}-10$ & 0 & & $2.33 E+03$ \\
\hline ENSBTAG00000032017 & 0 & 0 & INHBB & $1.00 \mathrm{E}+00$ \\
\hline ENSBTAG00000047862 & 0 & 0 & & $1.00 \mathrm{E}+00$ \\
\hline ENSBTAG00000003731 & $2.65 \mathrm{E}-10$ & 0 & & $2.65 E+03$ \\
\hline ENSBTAG00000040367 & $9.18 \mathrm{E}-11$ & 0 & & $9.19 \mathrm{E}+02$ \\
\hline ENSBTAG00000048210 & $4.25 \mathrm{E}-10$ & 0 & & $4.25 \mathrm{E}+03$ \\
\hline ENSBTAG00000030974 & $1.29 \mathrm{E}-10$ & 0 & TUBA4A & $1.30 \mathrm{E}+03$ \\
\hline ENSBTAG00000021326 & $2.39 \mathrm{E}-10$ & 0 & CCL2O & $2.40 \mathrm{E}+03$ \\
\hline ENSBTAG00000002184 & $1.43 \mathrm{E}-10$ & 0 & FNDC5 & $1.43 E+03$ \\
\hline ENSBTAG00000046862 & $2.51 \mathrm{E}-11$ & 0 & MARCKSL1 & $2.52 \mathrm{E}+02$ \\
\hline ENSBTAG00000001160 & $9.80 \mathrm{E}-11$ & 0 & GMEB1 & $9.81 \mathrm{E}+02$ \\
\hline ENSBTAG00000048228 & $2.54 \mathrm{E}-10$ & 0 & & $2.55 E+03$ \\
\hline ENSBTAG00000003788 & $1.43 \mathrm{E}-10$ & 0 & RCC1 & $1.43 \mathrm{E}+03$ \\
\hline ENSBTAG00000011625 & $7.73 \mathrm{E}-11$ & 0 & & $7.74 \mathrm{E}+02$ \\
\hline ENSBTAG00000030269 & $6.39 \mathrm{E}-10$ & 0 & & $6.39 \mathrm{E}+03$ \\
\hline ENSBTAG00000009156 & $7.91 \mathrm{E}-11$ & 0 & CAMK2N1 & $7.92 E+02$ \\
\hline ENSBTAG00000031711 & $4.42 \mathrm{E}-10$ & 0 & & $4.42 \mathrm{E}+03$ \\
\hline ENSBTAG00000040494 & $3.70 \mathrm{E}-10$ & 0 & UBL5 & $3.70 \mathrm{E}+03$ \\
\hline ENSBTAG00000046878 & $4.60 \mathrm{E}-10$ & 0 & & $4.61 E+03$ \\
\hline ENSBTAG00000003164 & $8.35 \mathrm{E}-11$ & 0 & B3GALT1 & $8.36 \mathrm{E}+02$ \\
\hline ENSBTAG00000046347 & 0 & 0 & & $1.00 \mathrm{E}+00$ \\
\hline ENSBTAG00000016081 & $7.18 \mathrm{E}-10$ & 0 & & $7.18 \mathrm{E}+03$ \\
\hline ENSBTAG00000032264 & 0 & 0 & & $1.00 \mathrm{E}+00$ \\
\hline ENSBTAG00000047466 & 0 & 0 & & $1.00 \mathrm{E}+00$ \\
\hline ENSBTAG00000026829 & $7.75 \mathrm{E}-11$ & 0 & TMEM185B & $7.76 \mathrm{E}+02$ \\
\hline ENSBTAG00000037816 & 0 & 0 & & $1.00 \mathrm{E}+00$ \\
\hline ENSBTAG00000024003 & 0 & 0 & & $1.00 \mathrm{E}+00$ \\
\hline ENSBTAG00000046521 & $4.81 \mathrm{E}-10$ & 0 & & $4.81 E+03$ \\
\hline ENSBTAG00000046029 & 0 & 0 & & $1.00 \mathrm{E}+00$ \\
\hline ENSBTAG00000017043 & 0 & 0 & GPR1 & $1.00 \mathrm{E}+00$ \\
\hline ENSBTAG00000020957 & $4.66 \mathrm{E}-11$ & 0 & FZD5 & $4.67 E+02$ \\
\hline ENSBTAG00000048219 & 4.72E-10 & 0 & & $4.72 \mathrm{E}+03$ \\
\hline ENSBTAG00000031052 & 0 & 0 & CDK5R2 & $1.00 \mathrm{E}+00$ \\
\hline ENSBTAG00000021308 & $1.10 \mathrm{E}-10$ & 0 & IRS1 & $1.10 \mathrm{E}+03$ \\
\hline ENSBTAG00000023964 & 0 & 0 & & $1.00 \mathrm{E}+00$ \\
\hline ENSBTAG00000017431 & 0 & 0 & GPR55 & $1.00 \mathrm{E}+00$ \\
\hline ENSBTAG00000027930 & $3.77 \mathrm{E}-10$ & 0 & & $3.77 E+03$ \\
\hline ENSBTAG00000047524 & 0 & 0 & TMEM200B & $1.00 \mathrm{E}+00$ \\
\hline ENSBTAG00000003676 & $1.20 \mathrm{E}-09$ & 0 & & $1.20 \mathrm{E}+04$ \\
\hline ENSBTAG00000002505 & 0 & 0 & GPR3 & $1.00 \mathrm{E}+00$ \\
\hline ENSBTAG00000046988 & 0 & 0 & FAM43B & $1.00 \mathrm{E}+00$ \\
\hline ENSBTAG00000047162 & 0 & $1.63 \mathrm{E}-10$ & & $6.13 E-04$ \\
\hline ENSBTAG00000047468 & 0 & $2.79 \mathrm{E}-10$ & FAM78B & $3.58 \mathrm{E}-04$ \\
\hline ENSBTAG00000007446 & 0 & $1.16 \mathrm{E}-10$ & NGF & $8.61 \mathrm{E}-04$ \\
\hline ENSBTAG00000039951 & 0 & $7.59 \mathrm{E}-11$ & PRMT6 & $1.32 \mathrm{E}-03$ \\
\hline ENSBTAG00000030890 & 0 & $2.00 \mathrm{E}-10$ & & 5.00E-04 \\
\hline ENSBTAG00000046003 & 0 & $1.89 \mathrm{E}-10$ & $\mathrm{HIST} 2 \mathrm{H} 2 \mathrm{AC}$ & $5.29 \mathrm{E}-04$ \\
\hline ENSBTAG00000046915 & $8.76 \mathrm{E}-11$ & $5.54 \mathrm{E}-10$ & & $1.58 \mathrm{E}-01$ \\
\hline ENSBTAG00000003616 & $1.39 \mathrm{E}-11$ & $5.27 \mathrm{E}-11$ & DCLRE1B & $2.66 \mathrm{E}-01$ \\
\hline ENSBTAG00000037546 & $8.58 \mathrm{E}-11$ & $3.25 \mathrm{E}-10$ & & 2.64E-01 \\
\hline ENSBTAG00000005149 & $2.90 \mathrm{E}-11$ & 1.10E-10 & AQP10 & 2.64E-01 \\
\hline ENSBTAG00000015060 & 4.54E-10 & $1.56 \mathrm{E}-09$ & GBP5 & 2.91E-01 \\
\hline ENSBTAG00000015329 & $1.06 \mathrm{E}-10$ & $3.35 \mathrm{E}-10$ & FAM63A & 3.17E-01 \\
\hline ENSBTAG00000010033 & $2.71 \mathrm{E}-10$ & $8.39 \mathrm{E}-10$ & & 3.23E-01 \\
\hline ENSBTAG00000015776 & $2.49 \mathrm{E}-10$ & $7.35 \mathrm{E}-10$ & MFSD14A & 3.40E-01 \\
\hline ENSBTAG00000017564 & $2.73 \mathrm{E}-10$ & 7.17E-10 & CGN & $3.81 \mathrm{E}-01$ \\
\hline ENSBTAG00000020356 & $1.38 \mathrm{E}-10$ & $3.61 \mathrm{E}-10$ & & 3.82E-01 \\
\hline ENSBTAG00000000098 & 1.37E-10 & $3.56 \mathrm{E}-10$ & SETDB1 & $3.86 \mathrm{E}-01$ \\
\hline ENSBTAG00000015304 & $2.33 \mathrm{E}-10$ & $5.98 \mathrm{E}-10$ & ANXA9 & 3.89E-01 \\
\hline ENSBTAG00000000963 & $2.66 \mathrm{E}-10$ & $6.73 \mathrm{E}-10$ & & 3.96E-01 \\
\hline ENSBTAG00000038604 & $1.08 \mathrm{E}-10$ & $2.73 \mathrm{E}-10$ & & $3.96 \mathrm{E}-01$ \\
\hline ENSBTAG00000026613 & $7.85 \mathrm{E}-11$ & $1.98 \mathrm{E}-10$ & MSTO1 & 3.97E-01 \\
\hline ENSBTAG00000014358 & $9.40 \mathrm{E}-11$ & 2.37E-10 & EVA1B & 3.97E-01 \\
\hline ENSBTAG00000010814 & $2.19 \mathrm{E}-10$ & $5.52 \mathrm{E}-10$ & & $3.96 \mathrm{E}-01$ \\
\hline
\end{tabular}




\begin{tabular}{|c|c|c|c|c|}
\hline ENSBTAG00000012061 & $6.51 \mathrm{E}-11$ & $1.64 \mathrm{E}-10$ & POU3F1 & $3.98 \mathrm{E}-01$ \\
\hline ENSBTAG00000011284 & $2.03 \mathrm{E}-11$ & $5.12 \mathrm{E}-11$ & SLC39A1 & $3.98 \mathrm{E}-01$ \\
\hline ENSBTAG00000007642 & 4.26E-11 & 1.07E-10 & ADORA3 & $3.99 \mathrm{E}-01$ \\
\hline ENSBTAG00000003948 & $1.78 \mathrm{E}-10$ & 4.27E-10 & & 4.17E-01 \\
\hline ENSBTAG00000020037 & $3.43 \mathrm{E}-10$ & 8.06E-10 & & 4.26E-01 \\
\hline ENSBTAG00000005029 & $1.19 \mathrm{E}-10$ & $2.75 \mathrm{E}-10$ & TAL1 & 4.32E-01 \\
\hline ENSBTAG00000007333 & $3.21 \mathrm{E}-10$ & $7.36 \mathrm{E}-10$ & & 4.36E-01 \\
\hline ENSBTAG00000045596 & $3.81 \mathrm{E}-10$ & 8.53E-10 & & 4.47E-01 \\
\hline ENSBTAG00000001538 & $5.82 \mathrm{E}-10$ & 1.29E-09 & & 4.52E-01 \\
\hline ENSBTAG00000003954 & $1.35 \mathrm{E}-10$ & $2.93 \mathrm{E}-10$ & ASH1L & 4.59E-01 \\
\hline ENSBTAG00000008711 & $2.04 \mathrm{E}-10$ & $4.32 \mathrm{E}-10$ & BOK & 4.72E-01 \\
\hline ENSBTAG00000001343 & $1.51 \mathrm{E}-10$ & $3.09 \mathrm{E}-10$ & DEPDC1 & 4.89E-01 \\
\hline ENSBTAG00000000108 & 4.09E-10 & $8.35 \mathrm{E}-10$ & KIAA0907 & $4.90 \mathrm{E}-01$ \\
\hline ENSBTAG00000013881 & $4.63 \mathrm{E}-10$ & $9.35 \mathrm{E}-10$ & GJA4 & 4.95E-01 \\
\hline ENSBTAG00000000406 & $5.65 E-10$ & 1.14E-09 & GPATCH4 & 4.96E-01 \\
\hline ENSBTAG00000030385 & $1.75 \mathrm{E}-10$ & 3.30E-10 & & 5.29E-01 \\
\hline ENSBTAG00000047528 & $1.57 \mathrm{E}-10$ & $2.98 \mathrm{E}-10$ & C2CD4D & 5.29E-01 \\
\hline ENSBTAG00000037807 & $2.64 \mathrm{E}-10$ & $4.99 \mathrm{E}-10$ & & 5.29E-01 \\
\hline ENSBTAG00000012470 & $9.72 \mathrm{E}-11$ & $1.84 \mathrm{E}-10$ & SNAPIN & 5.29E-01 \\
\hline ENSBTAG00000019093 & $2.10 \mathrm{E}-10$ & 3.97E-10 & & 5.29E-01 \\
\hline ENSBTAG00000006441 & $3.16 \mathrm{E}-10$ & $5.88 \mathrm{E}-10$ & ATP5F1 & 5.37E-01 \\
\hline ENSBTAG00000000099 & $1.62 \mathrm{E}-10$ & 3.00E-10 & CERS2 & 5.39E-01 \\
\hline ENSBTAG00000001199 & $1.94 \mathrm{E}-10$ & $3.58 \mathrm{E}-10$ & DAP3 & $5.41 \mathrm{E}-01$ \\
\hline ENSBTAG00000031012 & $3.88 \mathrm{E}-10$ & 7.01E-10 & PIGK & $5.54 \mathrm{E}-01$ \\
\hline ENSBTAG00000016389 & $2.54 \mathrm{E}-10$ & 4.49E-10 & UBL4B & $5.66 \mathrm{E}-01$ \\
\hline ENSBTAG00000011209 & $2.84 \mathrm{E}-10$ & $4.91 \mathrm{E}-10$ & SYT11 & $5.78 \mathrm{E}-01$ \\
\hline ENSBTAG00000045987 & 7.17E-10 & 1.21E-09 & & 5.93E-01 \\
\hline ENSBTAG00000017357 & 4.77E-10 & 7.99E-10 & CLCA4 & 5.97E-01 \\
\hline ENSBTAG00000020964 & $6.29 \mathrm{E}-11$ & $1.04 \mathrm{E}-10$ & STRIP1 & 6.05E-01 \\
\hline ENSBTAG00000016990 & $2.35 \mathrm{E}-10$ & $3.85 \mathrm{E}-10$ & USP24 & 6.10E-01 \\
\hline ENSBTAG00000017055 & $1.55 \mathrm{E}-10$ & $2.54 \mathrm{E}-10$ & HCN3 & $6.12 \mathrm{E}-01$ \\
\hline ENSBTAG00000018058 & $1.48 \mathrm{E}-10$ & $2.39 \mathrm{E}-10$ & C2orf82 & $6.17 \mathrm{E}-01$ \\
\hline ENSBTAG00000014289 & $1.58 \mathrm{E}-10$ & $2.56 \mathrm{E}-10$ & ZFYVE9 & $6.19 \mathrm{E}-01$ \\
\hline ENSBTAG00000002555 & $2.70 \mathrm{E}-10$ & 4.35E-10 & PDZK1IP1 & $6.21 \mathrm{E}-01$ \\
\hline ENSBTAG00000009421 & $4.71 \mathrm{E}-10$ & $7.42 \mathrm{E}-10$ & CD1E & $6.34 \mathrm{E}-01$ \\
\hline ENSBTAG00000046656 & $3.61 \mathrm{E}-10$ & $5.68 \mathrm{E}-10$ & & $6.35 \mathrm{E}-01$ \\
\hline ENSBTAG00000001842 & $1.18 \mathrm{E}-10$ & $1.86 \mathrm{E}-10$ & GSTM3 & $6.35 \mathrm{E}-01$ \\
\hline ENSBTAG00000012584 & $3.04 \mathrm{E}-10$ & 4.75E-10 & GJB3 & $6.40 \mathrm{E}-01$ \\
\hline ENSBTAG00000002583 & $8.60 \mathrm{E}-11$ & $1.32 \mathrm{E}-10$ & DNALI1 & $6.52 \mathrm{E}-01$ \\
\hline ENSBTAG00000010265 & $5.14 \mathrm{E}-10$ & 7.79E-10 & MGST3 & 6.60E-01 \\
\hline ENSBTAG00000005858 & $5.72 \mathrm{E}-11$ & $8.65 \mathrm{E}-11$ & KCNJ13 & $6.61 \mathrm{E}-01$ \\
\hline ENSBTAG00000009062 & $1.30 \mathrm{E}-10$ & $1.94 \mathrm{E}-10$ & DMRTB1 & $6.72 \mathrm{E}-01$ \\
\hline ENSBTAG00000024086 & $1.53 \mathrm{E}-10$ & $2.25 \mathrm{E}-10$ & TMEM35B & $6.81 \mathrm{E}-01$ \\
\hline ENSBTAG00000012692 & $5.45 \mathrm{E}-11$ & 8.01E-11 & & $6.81 \mathrm{E}-01$ \\
\hline ENSBTAG00000020244 & $1.86 \mathrm{E}-10$ & $2.66 \mathrm{E}-10$ & EFNA1 & 7.01E-01 \\
\hline ENSBTAG00000019091 & $2.01 \mathrm{E}-10$ & 2.87E-10 & RNPC3 & $6.99 \mathrm{E}-01$ \\
\hline ENSBTAG00000024255 & $2.34 \mathrm{E}-10$ & $3.33 \mathrm{E}-10$ & & 7.01E-01 \\
\hline ENSBTAG00000047047 & 7.07E-10 & 1.00E-09 & & 7.07E-01 \\
\hline ENSBTAG00000038082 & $2.15 \mathrm{E}-09$ & 3.03E-09 & & 7.09E-01 \\
\hline ENSBTAG00000014857 & $4.73 \mathrm{E}-10$ & $6.67 \mathrm{E}-10$ & & 7.10E-01 \\
\hline ENSBTAG00000012907 & $3.76 \mathrm{E}-10$ & 5.29E-10 & ODF2L & 7.11E-01 \\
\hline ENSBTAG00000020958 & $2.82 \mathrm{E}-10$ & $3.95 \mathrm{E}-10$ & LRRC8D & 7.13E-01 \\
\hline ENSBTAG00000008652 & $6.56 \mathrm{E}-10$ & 9.17E-10 & DUSP27 & 7.16E-01 \\
\hline ENSBTAG00000046231 & 4.17E-10 & $5.78 \mathrm{E}-10$ & & 7.21E-01 \\
\hline ENSBTAG00000014079 & $1.34 \mathrm{E}-10$ & $1.82 \mathrm{E}-10$ & GIGYF2 & 7.39E-01 \\
\hline ENSBTAG00000020494 & $1.10 \mathrm{E}-10$ & $1.48 \mathrm{E}-10$ & RPE65 & 7.44E-01 \\
\hline ENSBTAG00000006320 & $3.46 \mathrm{E}-10$ & 4.66E-10 & DBT & 7.42E-01 \\
\hline ENSBTAG00000046294 & $4.80 \mathrm{E}-10$ & $6.43 \mathrm{E}-10$ & & 7.46E-01 \\
\hline ENSBTAG00000046766 & $9.52 \mathrm{E}-10$ & 1.27E-09 & & 7.49E-01 \\
\hline ENSBTAG00000021434 & $5.13 \mathrm{E}-10$ & $6.82 \mathrm{E}-10$ & EDN2 & 7.53E-01 \\
\hline ENSBTAG00000012312 & $2.35 \mathrm{E}-10$ & $3.08 \mathrm{E}-10$ & ROR1 & 7.62E-01 \\
\hline ENSBTAG00000018942 & $1.67 \mathrm{E}-10$ & $2.20 \mathrm{E}-10$ & SSX2IP & 7.61E-01 \\
\hline ENSBTAG00000011322 & $1.30 \mathrm{E}-10$ & $1.68 \mathrm{E}-10$ & HIPK1 & 7.74E-01 \\
\hline ENSBTAG00000017566 & $3.68 \mathrm{E}-10$ & $4.72 \mathrm{E}-10$ & TUFT1 & 7.80E-01 \\
\hline ENSBTAG00000010715 & $6.05 \mathrm{E}-10$ & 7.74E-10 & & 7.82E-01 \\
\hline ENSBTAG00000006882 & $2.91 \mathrm{E}-10$ & $3.72 \mathrm{E}-10$ & IQGAP3 & 7.82E-01 \\
\hline ENSBTAG00000011953 & $2.49 \mathrm{E}-10$ & $3.19 \mathrm{E}-10$ & PRKACB & 7.82E-01 \\
\hline ENSBTAG00000016028 & $1.22 \mathrm{E}-10$ & $1.54 \mathrm{E}-10$ & ZNF691 & 7.94E-01 \\
\hline ENSBTAG00000010617 & $1.86 \mathrm{E}-10$ & $2.35 \mathrm{E}-10$ & KRTCAP2 & 7.93E-01 \\
\hline ENSBTAG00000009148 & $6.16 \mathrm{E}-10$ & $7.78 \mathrm{E}-10$ & GPA33 & 7.92E-01 \\
\hline
\end{tabular}




\begin{tabular}{|c|c|c|c|c|}
\hline ENSBTAG00000003083 & $2.65 \mathrm{E}-10$ & $3.35 \mathrm{E}-10$ & SLC50A1 & 7.93E-01 \\
\hline ENSBTAG00000003604 & $1.64 \mathrm{E}-10$ & $2.06 \mathrm{E}-10$ & ADAMTSL4 & $7.95 \mathrm{E}-01$ \\
\hline ENSBTAG00000010414 & $1.27 \mathrm{E}-10$ & $1.60 \mathrm{E}-10$ & DMRTA2 & 7.96E-01 \\
\hline ENSBTAG00000019709 & $1.64 \mathrm{E}-10$ & $2.06 \mathrm{E}-10$ & & $7.94 \mathrm{E}-01$ \\
\hline ENSBTAG00000024201 & $6.78 \mathrm{E}-10$ & $8.54 \mathrm{E}-10$ & & 7.94E-01 \\
\hline ENSBTAG00000009888 & $1.99 \mathrm{E}-10$ & $2.51 \mathrm{E}-10$ & DRAM2 & $7.92 \mathrm{E}-01$ \\
\hline ENSBTAG00000017765 & $9.96 \mathrm{E}-11$ & $1.26 \mathrm{E}-10$ & & $7.91 \mathrm{E}-01$ \\
\hline ENSBTAG00000018893 & $1.27 \mathrm{E}-10$ & $1.60 \mathrm{E}-10$ & AHCYL1 & 7.95E-01 \\
\hline ENSBTAG00000003096 & $7.80 \mathrm{E}-10$ & $9.83 \mathrm{E}-10$ & OR6K3 & 7.94E-01 \\
\hline ENSBTAG00000021481 & $4.56 \mathrm{E}-11$ & $5.75 \mathrm{E}-11$ & CA14 & $7.94 \mathrm{E}-01$ \\
\hline ENSBTAG00000046450 & $1.24 \mathrm{E}-10$ & $1.56 \mathrm{E}-10$ & & 7.95E-01 \\
\hline ENSBTAG00000020672 & $7.87 \mathrm{E}-10$ & $9.92 \mathrm{E}-10$ & NEU2 & 7.94E-01 \\
\hline ENSBTAG00000045912 & $6.49 \mathrm{E}-10$ & $8.18 \mathrm{E}-10$ & & 7.94E-01 \\
\hline ENSBTAG00000040435 & $8.76 \mathrm{E}-10$ & $1.10 \mathrm{E}-09$ & & 7.96E-01 \\
\hline ENSBTAG00000046138 & $1.04 \mathrm{E}-10$ & $1.31 \mathrm{E}-10$ & C1orf68 & 7.93E-01 \\
\hline ENSBTAG00000005938 & $3.55 \mathrm{E}-11$ & $4.47 \mathrm{E}-11$ & BARHL2 & 7.94E-01 \\
\hline ENSBTAG00000047063 & $7.98 \mathrm{E}-10$ & $1.01 \mathrm{E}-09$ & OR10J1 & 7.90E-01 \\
\hline ENSBTAG00000007627 & $6.08 \mathrm{E}-10$ & $7.66 \mathrm{E}-10$ & & 7.93E-01 \\
\hline ENSBTAG00000015459 & $9.39 \mathrm{E}-11$ & $1.18 \mathrm{E}-10$ & KCNA2 & 7.96E-01 \\
\hline ENSBTAG00000046242 & $1.75 \mathrm{E}-10$ & $2.21 \mathrm{E}-10$ & & 7.93E-01 \\
\hline ENSBTAG00000019618 & $1.75 \mathrm{E}-10$ & $2.21 \mathrm{E}-10$ & & 7.93E-01 \\
\hline ENSBTAG00000007297 & $4.29 \mathrm{E}-10$ & $5.41 \mathrm{E}-10$ & OR10T2 & 7.93E-01 \\
\hline ENSBTAG00000021862 & $9.11 \mathrm{E}-11$ & $1.15 \mathrm{E}-10$ & SLC27A3 & 7.92E-01 \\
\hline ENSBTAG00000033837 & $2.85 \mathrm{E}-10$ & $3.59 \mathrm{E}-10$ & & 7.94E-01 \\
\hline ENSBTAG00000046315 & $2.80 \mathrm{E}-10$ & $3.52 \mathrm{E}-10$ & & 7.95E-01 \\
\hline ENSBTAG00000011059 & $1.30 \mathrm{E}-10$ & $1.64 \mathrm{E}-10$ & CDKN2C & 7.94E-01 \\
\hline ENSBTAG00000047879 & $4.73 E-11$ & $5.96 \mathrm{E}-11$ & KCNA3 & 7.94E-01 \\
\hline ENSBTAG00000031656 & $1.64 \mathrm{E}-10$ & $2.07 \mathrm{E}-10$ & TAF13 & 7.94E-01 \\
\hline ENSBTAG00000024195 & $1.48 \mathrm{E}-10$ & $1.87 \mathrm{E}-10$ & LDLRAD1 & $7.94 \mathrm{E}-01$ \\
\hline ENSBTAG00000000335 & $2.26 \mathrm{E}-10$ & $2.85 \mathrm{E}-10$ & HMGB4 & 7.94E-01 \\
\hline ENSBTAG00000030532 & $1.42 \mathrm{E}-10$ & $1.79 \mathrm{E}-10$ & KNCN & 7.93E-01 \\
\hline ENSBTAG00000018756 & $3.00 \mathrm{E}-10$ & $3.78 \mathrm{E}-10$ & & 7.93E-01 \\
\hline ENSBTAG00000040098 & $1.29 \mathrm{E}-10$ & $1.63 \mathrm{E}-10$ & & $7.92 \mathrm{E}-01$ \\
\hline ENSBTAG00000031082 & $1.75 \mathrm{E}-09$ & $2.20 \mathrm{E}-09$ & & 7.95E-01 \\
\hline ENSBTAG00000046509 & $1.19 \mathrm{E}-10$ & $1.50 \mathrm{E}-10$ & FAM46C & 7.96E-01 \\
\hline ENSBTAG00000031788 & $3.84 \mathrm{E}-10$ & $4.79 \mathrm{E}-10$ & & 8.03E-01 \\
\hline ENSBTAG00000006235 & $2.79 \mathrm{E}-10$ & $3.47 \mathrm{E}-10$ & INTS3 & 8.05E-01 \\
\hline ENSBTAG00000012305 & $2.91 \mathrm{E}-10$ & $3.58 \mathrm{E}-10$ & ADGRL2 & $8.12 \mathrm{E}-01$ \\
\hline ENSBTAG00000040268 & $4.33 \mathrm{E}-10$ & $5.30 \mathrm{E}-10$ & UBE2U & 8.17E-01 \\
\hline ENSBTAG00000047871 & $5.89 \mathrm{E}-10$ & $7.20 \mathrm{E}-10$ & & 8.17E-01 \\
\hline ENSBTAG00000013481 & $3.27 \mathrm{E}-10$ & $3.98 \mathrm{E}-10$ & CYP4A22 & $8.21 \mathrm{E}-01$ \\
\hline ENSBTAG00000000105 & $2.32 \mathrm{E}-10$ & $2.81 \mathrm{E}-10$ & RIT1 & $8.25 \mathrm{E}-01$ \\
\hline ENSBTAG00000019937 & $8.31 \mathrm{E}-10$ & $1.00 \mathrm{E}-09$ & WDR3 & $8.31 \mathrm{E}-01$ \\
\hline ENSBTAG00000038576 & $6.51 \mathrm{E}-10$ & $7.81 \mathrm{E}-10$ & & 8.33E-01 \\
\hline ENSBTAG00000000401 & $4.75 \mathrm{E}-10$ & $5.68 \mathrm{E}-10$ & TTC24 & $8.36 \mathrm{E}-01$ \\
\hline ENSBTAG00000015647 & $2.21 \mathrm{E}-10$ & 2.64E-10 & CYP4X1 & 8.37E-01 \\
\hline ENSBTAG00000019031 & $6.88 \mathrm{E}-10$ & $8.19 \mathrm{E}-10$ & PLPPR4 & $8.40 \mathrm{E}-01$ \\
\hline ENSBTAG00000002952 & $4.28 \mathrm{E}-10$ & $5.08 \mathrm{E}-10$ & TBX19 & $8.43 \mathrm{E}-01$ \\
\hline ENSBTAG00000012194 & $1.41 \mathrm{E}-10$ & $1.67 \mathrm{E}-10$ & DENND2C & $8.42 \mathrm{E}-01$ \\
\hline ENSBTAG00000008942 & $3.68 \mathrm{E}-10$ & $4.35 \mathrm{E}-10$ & NGEF & $8.46 \mathrm{E}-01$ \\
\hline ENSBTAG00000021754 & $6.57 \mathrm{E}-10$ & $7.76 \mathrm{E}-10$ & DUSP12 & 8.47E-01 \\
\hline ENSBTAG00000012976 & $1.65 \mathrm{E}-10$ & $1.94 \mathrm{E}-10$ & WLS & $8.49 \mathrm{E}-01$ \\
\hline ENSBTAG00000017056 & $4.09 \mathrm{E}-10$ & $4.82 \mathrm{E}-10$ & PKLR & $8.48 \mathrm{E}-01$ \\
\hline ENSBTAG00000034159 & $5.82 \mathrm{E}-10$ & $6.85 \mathrm{E}-10$ & FCRLA & $8.50 \mathrm{E}-01$ \\
\hline ENSBTAG00000000258 & $3.94 \mathrm{E}-10$ & $4.61 \mathrm{E}-10$ & & $8.54 \mathrm{E}-01$ \\
\hline ENSBTAG00000002809 & $1.54 \mathrm{E}-10$ & $1.80 \mathrm{E}-10$ & CAPN10 & $8.55 \mathrm{E}-01$ \\
\hline ENSBTAG00000021443 & $6.71 \mathrm{E}-10$ & $7.85 E-10$ & PGLYRP3 & 8.55E-01 \\
\hline ENSBTAG00000019038 & 1.16E-09 & $1.34 \mathrm{E}-09$ & OR10J5 & $8.63 \mathrm{E}-01$ \\
\hline ENSBTAG00000020036 & $3.58 \mathrm{E}-10$ & $4.16 \mathrm{E}-10$ & $\mathrm{CDCP} 2$ & 8.62E-01 \\
\hline ENSBTAG00000011976 & $3.65 E-10$ & $4.24 \mathrm{E}-10$ & CYP4B1 & $8.62 \mathrm{E}-01$ \\
\hline ENSBTAG00000009819 & $2.74 \mathrm{E}-10$ & $3.19 \mathrm{E}-10$ & $\mathrm{CDC20}$ & $8.60 \mathrm{E}-01$ \\
\hline ENSBTAG00000024726 & $1.91 \mathrm{E}-10$ & $2.22 \mathrm{E}-10$ & HJURP & $8.63 \mathrm{E}-01$ \\
\hline ENSBTAG00000015786 & $2.93 \mathrm{E}-10$ & $3.40 \mathrm{E}-10$ & LRRC39 & 8.63E-01 \\
\hline ENSBTAG00000015778 & $2.29 \mathrm{E}-10$ & $2.65 E-10$ & SASS6 & 8.63E-01 \\
\hline ENSBTAG00000000113 & $3.66 \mathrm{E}-10$ & $4.23 \mathrm{E}-10$ & ARHGEF2 & $8.66 \mathrm{E}-01$ \\
\hline ENSBTAG00000017381 & $7.83 \mathrm{E}-10$ & $9.01 \mathrm{E}-10$ & CLDN19 & $8.70 \mathrm{E}-01$ \\
\hline ENSBTAG00000024849 & $9.35 \mathrm{E}-10$ & 1.07E-09 & & $8.74 \mathrm{E}-01$ \\
\hline ENSBTAG00000046277 & $5.19 \mathrm{E}-10$ & $5.96 \mathrm{E}-10$ & RGS4 & $8.70 \mathrm{E}-01$ \\
\hline ENSBTAG00000003484 & $2.70 \mathrm{E}-10$ & $3.10 \mathrm{E}-10$ & C1orf123 & $8.71 E-01$ \\
\hline ENSBTAG00000003485 & $1.95 \mathrm{E}-10$ & $2.23 \mathrm{E}-10$ & MAGOH & $8.73 \mathrm{E}-01$ \\
\hline
\end{tabular}




\begin{tabular}{|c|c|c|c|c|}
\hline ENSBTAG00000003444 & $2.80 \mathrm{E}-10$ & $3.20 \mathrm{E}-10$ & SYDE2 & $8.76 \mathrm{E}-01$ \\
\hline ENSBTAG00000001686 & $4.02 \mathrm{E}-10$ & 4.59E-10 & LRRC52 & $8.76 \mathrm{E}-01$ \\
\hline ENSBTAG00000011242 & 4.12E-10 & $4.71 \mathrm{E}-10$ & MUTYH & $8.76 \mathrm{E}-01$ \\
\hline ENSBTAG00000003850 & 4.96E-10 & $5.66 \mathrm{E}-10$ & INSL5 & 8.77E-01 \\
\hline ENSBTAG00000038085 & $5.98 \mathrm{E}-10$ & $6.83 \mathrm{E}-10$ & & $8.75 \mathrm{E}-01$ \\
\hline ENSBTAG00000019198 & $2.93 \mathrm{E}-10$ & $3.35 \mathrm{E}-10$ & LRRC42 & $8.76 \mathrm{E}-01$ \\
\hline ENSBTAG00000010775 & $5.44 \mathrm{E}-10$ & $6.20 \mathrm{E}-10$ & POGK & 8.77E-01 \\
\hline ENSBTAG00000046583 & $8.60 \mathrm{E}-10$ & 9.77E-10 & TMEM61 & $8.81 \mathrm{E}-01$ \\
\hline ENSBTAG00000008931 & $5.93 \mathrm{E}-10$ & $6.73 \mathrm{E}-10$ & CREG1 & $8.80 \mathrm{E}-01$ \\
\hline ENSBTAG00000038669 & $8.46 \mathrm{E}-10$ & $9.59 \mathrm{E}-10$ & & $8.82 \mathrm{E}-01$ \\
\hline ENSBTAG00000014132 & $1.41 \mathrm{E}-10$ & $1.59 \mathrm{E}-10$ & SNED1 & $8.88 \mathrm{E}-01$ \\
\hline ENSBTAG00000024288 & $3.65 \mathrm{E}-10$ & 4.12E-10 & & $8.85 \mathrm{E}-01$ \\
\hline ENSBTAG00000010663 & $3.25 \mathrm{E}-10$ & 3.67E-10 & ADAM15 & $8.85 \mathrm{E}-01$ \\
\hline ENSBTAG00000010910 & $3.39 \mathrm{E}-10$ & $3.82 \mathrm{E}-10$ & & $8.88 \mathrm{E}-01$ \\
\hline ENSBTAG00000013241 & $1.02 \mathrm{E}-09$ & 1.15E-09 & BSND & $8.88 \mathrm{E}-01$ \\
\hline ENSBTAG00000020384 & $2.50 \mathrm{E}-10$ & $2.82 \mathrm{E}-10$ & WDR47 & $8.85 \mathrm{E}-01$ \\
\hline ENSBTAG00000002719 & $9.88 \mathrm{E}-11$ & $1.11 \mathrm{E}-10$ & ORC1 & $8.90 \mathrm{E}-01$ \\
\hline ENSBTAG00000013851 & $1.64 \mathrm{E}-10$ & $1.85 \mathrm{E}-10$ & BCL10 & 8.89E-01 \\
\hline ENSBTAG00000012722 & $5.71 \mathrm{E}-10$ & $6.42 \mathrm{E}-10$ & & 8.89E-01 \\
\hline ENSBTAG00000015854 & $1.66 \mathrm{E}-10$ & $1.86 \mathrm{E}-10$ & TCHHL1 & 8.93E-01 \\
\hline ENSBTAG00000003898 & $6.53 \mathrm{E}-10$ & 7.31E-10 & HMGCS2 & 8.93E-01 \\
\hline ENSBTAG00000017132 & $7.59 \mathrm{E}-10$ & $8.49 \mathrm{E}-10$ & TTC22 & 8.94E-01 \\
\hline ENSBTAG00000009844 & $2.54 \mathrm{E}-10$ & $2.84 \mathrm{E}-10$ & CYR61 & $8.94 \mathrm{E}-01$ \\
\hline ENSBTAG00000044141 & $3.00 \mathrm{E}-10$ & 3.36E-10 & & $8.93 \mathrm{E}-01$ \\
\hline ENSBTAG00000005337 & $1.79 \mathrm{E}-10$ & $1.99 \mathrm{E}-10$ & RAB3B & 8.97E-01 \\
\hline ENSBTAG00000001748 & $3.95 \mathrm{E}-10$ & $4.40 \mathrm{E}-10$ & TRIM45 & $8.98 \mathrm{E}-01$ \\
\hline ENSBTAG00000017875 & $5.55 \mathrm{E}-10$ & $6.16 \mathrm{E}-10$ & ARHGAP30 & $9.01 \mathrm{E}-01$ \\
\hline ENSBTAG00000022580 & $2.46 \mathrm{E}-10$ & $2.72 \mathrm{E}-10$ & FAM212B & 9.05E-01 \\
\hline ENSBTAG00000038025 & $2.83 \mathrm{E}-10$ & $3.11 \mathrm{E}-10$ & & $9.09 \mathrm{E}-01$ \\
\hline ENSBTAG00000017129 & $1.58 \mathrm{E}-10$ & $1.75 \mathrm{E}-10$ & CLCC1 & 9.05E-01 \\
\hline ENSBTAG00000047314 & $1.03 \mathrm{E}-09$ & 1.13E-09 & PPIE & $9.09 \mathrm{E}-01$ \\
\hline ENSBTAG00000005442 & $5.18 \mathrm{E}-10$ & $5.71 \mathrm{E}-10$ & WDR78 & 9.07E-01 \\
\hline ENSBTAG00000038979 & $1.34 \mathrm{E}-10$ & $1.48 \mathrm{E}-10$ & TMEM167B & 9.07E-01 \\
\hline ENSBTAG00000002720 & $3.23 \mathrm{E}-10$ & $3.54 \mathrm{E}-10$ & PRPF38A & $9.13 \mathrm{E}-01$ \\
\hline ENSBTAG00000012884 & $1.18 \mathrm{E}-09$ & 1.30E-09 & & 9.09E-01 \\
\hline ENSBTAG00000001406 & $6.51 \mathrm{E}-10$ & 7.14E-10 & MAB21L3 & $9.12 \mathrm{E}-01$ \\
\hline ENSBTAG00000017786 & $2.00 \mathrm{E}-10$ & $2.19 \mathrm{E}-10$ & FCRL6 & $9.12 \mathrm{E}-01$ \\
\hline ENSBTAG00000006625 & $2.74 \mathrm{E}-10$ & $3.01 E-10$ & NDC1 & $9.12 \mathrm{E}-01$ \\
\hline ENSBTAG00000014387 & $3.85 \mathrm{E}-10$ & $4.21 \mathrm{E}-10$ & PRKAB2 & $9.14 \mathrm{E}-01$ \\
\hline ENSBTAG00000038215 & $1.04 \mathrm{E}-09$ & $1.14 \mathrm{E}-09$ & CLCA2 & $9.11 \mathrm{E}-01$ \\
\hline ENSBTAG00000018881 & $1.79 \mathrm{E}-10$ & $1.96 \mathrm{E}-10$ & SYT6 & $9.14 \mathrm{E}-01$ \\
\hline ENSBTAG00000047731 & $3.76 \mathrm{E}-10$ & 4.09E-10 & FAM189B & $9.20 \mathrm{E}-01$ \\
\hline ENSBTAG00000007330 & $1.64 \mathrm{E}-10$ & $1.78 \mathrm{E}-10$ & STXBP3 & $9.24 \mathrm{E}-01$ \\
\hline ENSBTAG00000017124 & $4.92 \mathrm{E}-10$ & $5.33 \mathrm{E}-10$ & GPSM2 & $9.24 \mathrm{E}-01$ \\
\hline ENSBTAG00000020060 & $1.38 \mathrm{E}-10$ & $1.49 \mathrm{E}-10$ & TXNIP & 9.27E-01 \\
\hline ENSBTAG00000047874 & $7.35 \mathrm{E}-11$ & 7.94E-11 & PRPF38B & $9.26 \mathrm{E}-01$ \\
\hline ENSBTAG00000021544 & 4.16E-10 & $4.49 \mathrm{E}-10$ & EBNA1BP2 & $9.26 \mathrm{E}-01$ \\
\hline ENSBTAG00000017388 & $1.77 \mathrm{E}-10$ & $1.91 \mathrm{E}-10$ & SVBP & $9.28 \mathrm{E}-01$ \\
\hline ENSBTAG00000046353 & $5.79 \mathrm{E}-10$ & $6.25 \mathrm{E}-10$ & & $9.26 \mathrm{E}-01$ \\
\hline ENSBTAG00000012234 & $3.29 \mathrm{E}-10$ & $3.55 \mathrm{E}-10$ & RHBG & $9.28 \mathrm{E}-01$ \\
\hline ENSBTAG00000014710 & $2.51 \mathrm{E}-10$ & $2.70 \mathrm{E}-10$ & RAP1A & $9.30 \mathrm{E}-01$ \\
\hline ENSBTAG00000031439 & $3.56 \mathrm{E}-10$ & $3.84 \mathrm{E}-10$ & CDC14A & $9.28 \mathrm{E}-01$ \\
\hline ENSBTAG00000005227 & $3.62 \mathrm{E}-10$ & 3.87E-10 & ATF6 & $9.34 \mathrm{E}-01$ \\
\hline ENSBTAG00000033007 & $8.02 \mathrm{E}-10$ & $8.60 \mathrm{E}-10$ & & $9.33 \mathrm{E}-01$ \\
\hline ENSBTAG00000003791 & 4.12E-10 & $4.41 \mathrm{E}-10$ & LPAR3 & $9.33 \mathrm{E}-01$ \\
\hline ENSBTAG00000020798 & $6.38 \mathrm{E}-10$ & $6.84 \mathrm{E}-10$ & Clorf94 & 9.33E-01 \\
\hline ENSBTAG00000000845 & 5.39E-10 & $5.76 \mathrm{E}-10$ & OLFM3 & $9.35 \mathrm{E}-01$ \\
\hline ENSBTAG00000013910 & $1.11 \mathrm{E}-10$ & $1.18 \mathrm{E}-10$ & & 9.37E-01 \\
\hline ENSBTAG00000037555 & $3.78 \mathrm{E}-10$ & $4.03 E-10$ & FAM183A & $9.38 \mathrm{E}-01$ \\
\hline ENSBTAG00000008161 & $6.31 \mathrm{E}-10$ & $6.72 \mathrm{E}-10$ & CLCA1 & $9.39 \mathrm{E}-01$ \\
\hline ENSBTAG00000021547 & $4.69 \mathrm{E}-10$ & 4.99E-10 & CFAP57 & $9.40 \mathrm{E}-01$ \\
\hline ENSBTAG00000013907 & $1.02 \mathrm{E}-09$ & $1.09 \mathrm{E}-09$ & CRP & $9.38 \mathrm{E}-01$ \\
\hline ENSBTAG00000012516 & $2.23 \mathrm{E}-10$ & 2.37E-10 & SLC1A7 & 9.43E-01 \\
\hline ENSBTAG00000039702 & 7.34E-10 & 7.79E-10 & FCRL3 & 9.43E-01 \\
\hline ENSBTAG00000007309 & $2.69 \mathrm{E}-10$ & $2.85 \mathrm{E}-10$ & ST6GALNAC5 & $9.43 \mathrm{E}-01$ \\
\hline ENSBTAG00000001052 & $3.35 \mathrm{E}-10$ & $3.55 \mathrm{E}-10$ & ADCY10 & 9.43E-01 \\
\hline ENSBTAG00000024437 & $5.82 \mathrm{E}-10$ & $6.17 \mathrm{E}-10$ & & $9.43 \mathrm{E}-01$ \\
\hline ENSBTAG00000014102 & $2.15 E-10$ & $2.28 \mathrm{E}-10$ & WDR77 & $9.43 \mathrm{E}-01$ \\
\hline ENSBTAG00000000616 & $2.75 \mathrm{E}-10$ & $2.92 \mathrm{E}-10$ & GPR161 & $9.43 \mathrm{E}-01$ \\
\hline ENSBTAG00000003389 & $2.50 \mathrm{E}-10$ & $2.65 \mathrm{E}-10$ & & 9.43E-01 \\
\hline
\end{tabular}




\begin{tabular}{|c|c|c|c|c|}
\hline ENSBTAG00000014596 & $3.50 \mathrm{E}-10$ & $3.71 \mathrm{E}-10$ & EFHD1 & $9.44 \mathrm{E}-01$ \\
\hline ENSBTAG00000037819 & $3.43 \mathrm{E}-10$ & $3.63 \mathrm{E}-10$ & SFT2D2 & $9.44 \mathrm{E}-01$ \\
\hline ENSBTAG00000025621 & $1.60 \mathrm{E}-09$ & $1.69 \mathrm{E}-09$ & & $9.46 \mathrm{E}-01$ \\
\hline ENSBTAG00000048275 & $1.29 \mathrm{E}-09$ & $1.36 \mathrm{E}-09$ & & $9.50 \mathrm{E}-01$ \\
\hline ENSBTAG00000008525 & $4.73 \mathrm{E}-10$ & 4.99E-10 & LRIG2 & $9.48 \mathrm{E}-01$ \\
\hline ENSBTAG00000039738 & $2.48 \mathrm{E}-10$ & $2.61 E-10$ & & $9.51 \mathrm{E}-01$ \\
\hline ENSBTAG00000038124 & $6.79 \mathrm{E}-10$ & $7.15 \mathrm{E}-10$ & & $9.49 \mathrm{E}-01$ \\
\hline ENSBTAG00000014326 & $3.65 \mathrm{E}-10$ & $3.84 \mathrm{E}-10$ & CDCA8 & $9.51 \mathrm{E}-01$ \\
\hline ENSBTAG00000039366 & $1.66 \mathrm{E}-10$ & $1.74 \mathrm{E}-10$ & & $9.52 \mathrm{E}-01$ \\
\hline ENSBTAG00000023535 & $7.29 \mathrm{E}-10$ & $7.66 \mathrm{E}-10$ & & $9.52 \mathrm{E}-01$ \\
\hline ENSBTAG00000001135 & $1.16 \mathrm{E}-10$ & $1.22 \mathrm{E}-10$ & C1orf194 & $9.55 \mathrm{E}-01$ \\
\hline ENSBTAG00000048312 & $2.88 \mathrm{E}-10$ & $3.03 E-10$ & & $9.52 \mathrm{E}-01$ \\
\hline ENSBTAG00000039540 & $2.07 \mathrm{E}-10$ & $2.18 \mathrm{E}-10$ & & $9.51 \mathrm{E}-01$ \\
\hline ENSBTAG00000048292 & $5.22 \mathrm{E}-10$ & $5.48 \mathrm{E}-10$ & & $9.53 \mathrm{E}-01$ \\
\hline ENSBTAG00000012495 & $2.68 \mathrm{E}-10$ & $2.81 \mathrm{E}-10$ & & $9.53 \mathrm{E}-01$ \\
\hline ENSBTAG00000030386 & $1.06 \mathrm{E}-09$ & $1.11 \mathrm{E}-09$ & & $9.51 \mathrm{E}-01$ \\
\hline ENSBTAG00000031940 & $2.47 \mathrm{E}-10$ & $2.58 \mathrm{E}-10$ & RSBN1 & 9.57E-01 \\
\hline ENSBTAG00000037565 & $3.48 \mathrm{E}-10$ & $3.64 \mathrm{E}-10$ & & 9.57E-01 \\
\hline ENSBTAG00000003752 & $8.75 \mathrm{E}-10$ & $9.15 \mathrm{E}-10$ & SLC25A24 & $9.56 \mathrm{E}-01$ \\
\hline ENSBTAG00000003322 & $6.35 \mathrm{E}-10$ & $6.64 \mathrm{E}-10$ & TTLL7 & 9.57E-01 \\
\hline ENSBTAG00000024144 & $5.73 \mathrm{E}-10$ & $5.96 \mathrm{E}-10$ & LURAP1 & $9.61 \mathrm{E}-01$ \\
\hline ENSBTAG00000001564 & $3.78 \mathrm{E}-10$ & $3.93 E-10$ & PDE4DIP & $9.61 \mathrm{E}-01$ \\
\hline ENSBTAG00000017204 & $8.34 \mathrm{E}-10$ & $8.67 E-10$ & & $9.62 \mathrm{E}-01$ \\
\hline ENSBTAG00000006511 & $1.76 \mathrm{E}-10$ & $1.83 \mathrm{E}-10$ & MTF1 & $9.62 \mathrm{E}-01$ \\
\hline ENSBTAG00000008467 & $1.29 \mathrm{E}-10$ & $1.33 \mathrm{E}-10$ & HENMT1 & 9.67E-01 \\
\hline ENSBTAG00000011733 & $3.38 \mathrm{E}-10$ & $3.49 \mathrm{E}-10$ & GIPC2 & $9.69 \mathrm{E}-01$ \\
\hline ENSBTAG00000021591 & $2.94 \mathrm{E}-10$ & $3.03 E-10$ & OAZ3 & $9.70 \mathrm{E}-01$ \\
\hline ENSBTAG00000024378 & $6.98 \mathrm{E}-11$ & 7.19E-11 & MRPS21 & $9.70 \mathrm{E}-01$ \\
\hline ENSBTAG00000006505 & $3.24 \mathrm{E}-10$ & $3.34 \mathrm{E}-10$ & S100A9 & $9.69 \mathrm{E}-01$ \\
\hline ENSBTAG00000020831 & $6.02 \mathrm{E}-10$ & $6.20 \mathrm{E}-10$ & CHD1L & $9.71 \mathrm{E}-01$ \\
\hline ENSBTAG00000002422 & $2.54 \mathrm{E}-10$ & $2.62 \mathrm{E}-10$ & BCL9 & $9.70 \mathrm{E}-01$ \\
\hline ENSBTAG00000035697 & $1.88 \mathrm{E}-10$ & $1.93 \mathrm{E}-10$ & GNG12 & $9.75 \mathrm{E}-01$ \\
\hline ENSBTAG00000014652 & $5.18 \mathrm{E}-10$ & $5.30 \mathrm{E}-10$ & TRPM8 & $9.76 \mathrm{E}-01$ \\
\hline ENSBTAG00000000259 & $5.16 \mathrm{E}-10$ & $5.29 \mathrm{E}-10$ & CHIA & $9.74 \mathrm{E}-01$ \\
\hline ENSBTAG00000019933 & $2.25 \mathrm{E}-10$ & $2.30 \mathrm{E}-10$ & MKNK1 & $9.77 \mathrm{E}-01$ \\
\hline ENSBTAG00000021602 & $1.31 \mathrm{E}-10$ & $1.33 \mathrm{E}-10$ & CTTNBP2NL & $9.84 \mathrm{E}-01$ \\
\hline ENSBTAG00000019401 & $1.65 \mathrm{E}-10$ & $1.68 \mathrm{E}-10$ & & $9.81 \mathrm{E}-01$ \\
\hline ENSBTAG00000005910 & $1.87 \mathrm{E}-10$ & $1.91 \mathrm{E}-10$ & LEPR & $9.80 \mathrm{E}-01$ \\
\hline ENSBTAG00000019033 & 4.77E-10 & $4.85 \mathrm{E}-10$ & $\mathrm{CD} 84$ & $9.83 \mathrm{E}-01$ \\
\hline ENSBTAG00000018807 & $3.54 \mathrm{E}-10$ & $3.61 \mathrm{E}-10$ & MYBPHL & $9.81 \mathrm{E}-01$ \\
\hline ENSBTAG00000009676 & $6.03 E-10$ & $6.14 \mathrm{E}-10$ & STK25 & $9.82 \mathrm{E}-01$ \\
\hline ENSBTAG00000010655 & $4.15 \mathrm{E}-10$ & 4.22E-10 & DCST1 & $9.84 \mathrm{E}-01$ \\
\hline ENSBTAG00000003128 & $8.13 E-10$ & $8.26 \mathrm{E}-10$ & SPATA6 & $9.85 \mathrm{E}-01$ \\
\hline ENSBTAG00000009603 & $3.16 \mathrm{E}-10$ & $3.20 \mathrm{E}-10$ & & $9.86 \mathrm{E}-01$ \\
\hline ENSBTAG00000014393 & $2.85 \mathrm{E}-10$ & $2.90 \mathrm{E}-10$ & CLK2 & $9.84 \mathrm{E}-01$ \\
\hline ENSBTAG00000021501 & $6.40 \mathrm{E}-10$ & $6.49 \mathrm{E}-10$ & & $9.86 \mathrm{E}-01$ \\
\hline ENSBTAG00000000505 & $5.85 \mathrm{E}-10$ & $5.94 \mathrm{E}-10$ & KYAT3 & $9.85 \mathrm{E}-01$ \\
\hline ENSBTAG00000005393 & $2.14 \mathrm{E}-10$ & $2.16 \mathrm{E}-10$ & TIPRL & $9.89 \mathrm{E}-01$ \\
\hline ENSBTAG00000018806 & $1.15 \mathrm{E}-10$ & $1.16 \mathrm{E}-10$ & PSRC1 & $9.89 \mathrm{E}-01$ \\
\hline ENSBTAG00000040437 & $5.30 \mathrm{E}-10$ & $5.36 \mathrm{E}-10$ & & $9.89 \mathrm{E}-01$ \\
\hline ENSBTAG00000009625 & $1.46 \mathrm{E}-10$ & $1.47 \mathrm{E}-10$ & DMBX1 & $9.92 \mathrm{E}-01$ \\
\hline ENSBTAG00000014841 & 4.19E-10 & 4.23E-10 & GBA & $9.90 \mathrm{E}-01$ \\
\hline ENSBTAG00000021482 & $1.12 \mathrm{E}-10$ & $1.13 \mathrm{E}-10$ & $\mathrm{APH} 1 \mathrm{~A}$ & $9.94 \mathrm{E}-01$ \\
\hline ENSBTAG00000003799 & $7.04 \mathrm{E}-11$ & $7.10 \mathrm{E}-11$ & TARS2 & $9.92 \mathrm{E}-01$ \\
\hline ENSBTAG00000039494 & $4.31 \mathrm{E}-10$ & $4.34 \mathrm{E}-10$ & OR6P1 & $9.92 \mathrm{E}-01$ \\
\hline ENSBTAG00000037810 & $8.90 \mathrm{E}-10$ & $8.96 \mathrm{E}-10$ & & $9.93 \mathrm{E}-01$ \\
\hline ENSBTAG00000012886 & $8.76 \mathrm{E}-10$ & $8.81 \mathrm{E}-10$ & OR10J3 & $9.94 \mathrm{E}-01$ \\
\hline ENSBTAG00000022954 & $7.60 \mathrm{E}-10$ & $7.65 E-10$ & & $9.94 \mathrm{E}-01$ \\
\hline ENSBTAG00000004381 & $4.28 \mathrm{E}-10$ & $4.31 \mathrm{E}-10$ & TACSTD2 & $9.93 \mathrm{E}-01$ \\
\hline ENSBTAG00000007215 & $2.22 \mathrm{E}-10$ & $2.23 E-10$ & RNPEPL1 & $9.95 \mathrm{E}-01$ \\
\hline ENSBTAG00000000736 & $2.72 \mathrm{E}-10$ & $2.74 \mathrm{E}-10$ & LRP8 & $9.94 \mathrm{E}-01$ \\
\hline ENSBTAG00000017145 & $5.23 \mathrm{E}-10$ & $5.26 \mathrm{E}-10$ & LEXM & $9.95 \mathrm{E}-01$ \\
\hline ENSBTAG00000014482 & $2.46 \mathrm{E}-10$ & $2.47 E-10$ & FAF1 & 9.97E-01 \\
\hline ENSBTAG00000020968 & $2.91 \mathrm{E}-10$ & $2.93 E-10$ & MPC2 & $9.94 \mathrm{E}-01$ \\
\hline ENSBTAG00000016504 & $4.74 \mathrm{E}-10$ & $4.76 \mathrm{E}-10$ & AGAP1 & $9.96 \mathrm{E}-01$ \\
\hline ENSBTAG00000024097 & $7.09 \mathrm{E}-10$ & $7.10 \mathrm{E}-10$ & & $9.98 \mathrm{E}-01$ \\
\hline ENSBTAG00000022937 & $1.18 \mathrm{E}-09$ & 1.19E-09 & & $9.95 \mathrm{E}-01$ \\
\hline ENSBTAG00000011640 & $2.28 \mathrm{E}-10$ & $2.28 \mathrm{E}-10$ & PLPP3 & $9.99 \mathrm{E}-01$ \\
\hline ENSBTAG00000007507 & $3.05 \mathrm{E}-10$ & $3.06 \mathrm{E}-10$ & FAAH & $9.98 \mathrm{E}-01$ \\
\hline ENSBTAG00000015896 & $4.20 \mathrm{E}-10$ & 4.20E-10 & CC2D1B & $1.00 \mathrm{E}+00$ \\
\hline
\end{tabular}




\begin{tabular}{|c|c|c|c|c|}
\hline ENSBTAG00000013322 & $5.86 \mathrm{E}-10$ & $5.85 \mathrm{E}-10$ & POMGNT1 & $1.00 \mathrm{E}+00$ \\
\hline ENSBTAG00000000305 & $3.16 \mathrm{E}-10$ & $3.15 \mathrm{E}-10$ & LMO4 & $1.00 \mathrm{E}+00$ \\
\hline ENSBTAG00000017125 & $5.57 \mathrm{E}-10$ & $5.55 \mathrm{E}-10$ & PABPC4 & $1.00 \mathrm{E}+00$ \\
\hline ENSBTAG00000010506 & $1.92 \mathrm{E}-10$ & $1.91 \mathrm{E}-10$ & MEAF6 & $1.00 \mathrm{E}+00$ \\
\hline ENSBTAG00000015931 & $3.56 \mathrm{E}-10$ & $3.53 \mathrm{E}-10$ & TTC4 & $1.01 \mathrm{E}+00$ \\
\hline ENSBTAG00000002823 & $6.67 \mathrm{E}-10$ & $6.62 \mathrm{E}-10$ & MPZL1 & $1.01 \mathrm{E}+00$ \\
\hline ENSBTAG00000009338 & 4.59E-10 & $4.56 \mathrm{E}-10$ & LRRC7 & $1.01 \mathrm{E}+00$ \\
\hline ENSBTAG00000002721 & $2.32 \mathrm{E}-10$ & $2.30 \mathrm{E}-10$ & ZCCHC11 & $1.01 \mathrm{E}+00$ \\
\hline ENSBTAG00000017986 & 4.09E-10 & $4.05 \mathrm{E}-10$ & GLIS1 & $1.01 E+00$ \\
\hline ENSBTAG00000038237 & $6.66 \mathrm{E}-10$ & $6.58 \mathrm{E}-10$ & KPRP & $1.01 \mathrm{E}+00$ \\
\hline ENSBTAG00000018887 & $3.14 \mathrm{E}-10$ & $3.11 \mathrm{E}-10$ & IPO13 & $1.01 E+00$ \\
\hline ENSBTAG00000045948 & $1.22 \mathrm{E}-09$ & $1.20 \mathrm{E}-09$ & CSF2RA & $1.01 E+00$ \\
\hline ENSBTAG00000011200 & $4.96 \mathrm{E}-10$ & $4.89 \mathrm{E}-10$ & ZNF644 & $1.01 E+00$ \\
\hline ENSBTAG00000013110 & $5.53 \mathrm{E}-10$ & $5.44 \mathrm{E}-10$ & & $1.02 \mathrm{E}+00$ \\
\hline ENSBTAG00000017595 & $8.87 \mathrm{E}-10$ & $8.71 E-10$ & & $1.02 \mathrm{E}+00$ \\
\hline ENSBTAG00000015380 & $1.96 \mathrm{E}-10$ & $1.93 \mathrm{E}-10$ & SLC16A4 & $1.02 \mathrm{E}+00$ \\
\hline ENSBTAG00000021637 & $2.21 \mathrm{E}-10$ & $2.17 \mathrm{E}-10$ & POLR3C & $1.02 \mathrm{E}+00$ \\
\hline ENSBTAG00000014538 & $4.96 \mathrm{E}-10$ & $4.86 \mathrm{E}-10$ & HPCAL4 & $1.02 E+00$ \\
\hline ENSBTAG00000000797 & $2.09 \mathrm{E}-10$ & $2.05 \mathrm{E}-10$ & MRPL9 & $1.02 \mathrm{E}+00$ \\
\hline ENSBTAG00000030623 & $5.36 \mathrm{E}-10$ & $5.24 \mathrm{E}-10$ & & $1.02 \mathrm{E}+00$ \\
\hline ENSBTAG00000005437 & $1.42 \mathrm{E}-10$ & $1.39 \mathrm{E}-10$ & SMCP & $1.02 E+00$ \\
\hline ENSBTAG00000038263 & $2.02 \mathrm{E}-09$ & 1.97E-09 & & $1.03 \mathrm{E}+00$ \\
\hline ENSBTAG00000007712 & $1.16 \mathrm{E}-10$ & $1.14 \mathrm{E}-10$ & SEC22B & $1.02 \mathrm{E}+00$ \\
\hline ENSBTAG00000004591 & $5.92 \mathrm{E}-10$ & $5.78 \mathrm{E}-10$ & PLPPR5 & $1.02 E+00$ \\
\hline ENSBTAG00000005784 & $5.31 \mathrm{E}-10$ & 5.17E-10 & CSMD2 & $1.03 E+00$ \\
\hline ENSBTAG00000011097 & $5.51 \mathrm{E}-10$ & $5.36 \mathrm{E}-10$ & TCTEX1D1 & $1.03 E+00$ \\
\hline ENSBTAG00000003849 & $1.62 \mathrm{E}-10$ & $1.58 \mathrm{E}-10$ & GNL2 & $1.03 E+00$ \\
\hline ENSBTAG00000017387 & $1.78 \mathrm{E}-10$ & $1.73 \mathrm{E}-10$ & & $1.03 E+00$ \\
\hline ENSBTAG00000012496 & $1.34 \mathrm{E}-10$ & $1.29 \mathrm{E}-10$ & MTMR11 & $1.04 \mathrm{E}+00$ \\
\hline ENSBTAG00000008238 & $2.77 \mathrm{E}-10$ & $2.69 \mathrm{E}-10$ & & $1.03 E+00$ \\
\hline ENSBTAG00000007112 & 1.57E-10 & $1.52 \mathrm{E}-10$ & HSPB11 & $1.04 \mathrm{E}+00$ \\
\hline ENSBTAG00000027172 & $1.87 \mathrm{E}-09$ & $1.80 \mathrm{E}-09$ & & $1.04 \mathrm{E}+00$ \\
\hline ENSBTAG00000000533 & 1.09E-09 & $1.05 \mathrm{E}-09$ & ZNF684 & $1.03 E+00$ \\
\hline ENSBTAG00000030190 & $3.85 \mathrm{E}-10$ & $3.70 \mathrm{E}-10$ & COL6A3 & $1.04 \mathrm{E}+00$ \\
\hline ENSBTAG00000017852 & $5.46 \mathrm{E}-10$ & $5.24 \mathrm{E}-10$ & ALDH9A1 & $1.04 \mathrm{E}+00$ \\
\hline ENSBTAG00000007352 & $2.63 \mathrm{E}-10$ & $2.52 \mathrm{E}-10$ & KCNC4 & $1.04 \mathrm{E}+00$ \\
\hline ENSBTAG00000013961 & $1.58 \mathrm{E}-10$ & $1.51 \mathrm{E}-10$ & MYSM1 & $1.05 E+00$ \\
\hline ENSBTAG00000031171 & $5.87 \mathrm{E}-10$ & $5.62 \mathrm{E}-10$ & SH3GLB1 & $1.04 \mathrm{E}+00$ \\
\hline ENSBTAG00000002026 & $2.39 \mathrm{E}-10$ & $2.29 \mathrm{E}-10$ & & $1.04 \mathrm{E}+00$ \\
\hline ENSBTAG00000021217 & $4.86 \mathrm{E}-10$ & $4.63 \mathrm{E}-10$ & COL11A1 & $1.05 E+00$ \\
\hline ENSBTAG00000002644 & $4.32 \mathrm{E}-10$ & 4.12E-10 & KCNQ4 & $1.05 E+00$ \\
\hline ENSBTAG00000005168 & $2.68 \mathrm{E}-10$ & $2.55 \mathrm{E}-10$ & CROCC2 & $1.05 E+00$ \\
\hline ENSBTAG00000024107 & $2.76 \mathrm{E}-10$ & $2.62 \mathrm{E}-10$ & INPP5B & $1.05 E+00$ \\
\hline ENSBTAG00000003162 & $6.51 \mathrm{E}-10$ & $6.19 \mathrm{E}-10$ & CRYZ & $1.05 E+00$ \\
\hline ENSBTAG00000002489 & $7.69 \mathrm{E}-10$ & 7.30E-10 & PIFO & $1.05 E+00$ \\
\hline ENSBTAG00000003586 & $5.88 \mathrm{E}-10$ & 5.57E-10 & MAN1A2 & $1.06 \mathrm{E}+00$ \\
\hline ENSBTAG00000001136 & $2.17 \mathrm{E}-10$ & $2.05 \mathrm{E}-10$ & KIAA1324 & $1.06 \mathrm{E}+00$ \\
\hline ENSBTAG00000012094 & $2.28 \mathrm{E}-10$ & $2.15 \mathrm{E}-10$ & SCAMP3 & $1.06 \mathrm{E}+00$ \\
\hline ENSBTAG00000008849 & $2.18 \mathrm{E}-10$ & $2.06 \mathrm{E}-10$ & SORT1 & $1.06 \mathrm{E}+00$ \\
\hline ENSBTAG00000007773 & $6.98 \mathrm{E}-10$ & $6.59 \mathrm{E}-10$ & & $1.06 \mathrm{E}+00$ \\
\hline ENSBTAG00000013860 & $1.05 \mathrm{E}-10$ & $9.94 \mathrm{E}-11$ & GADD45A & $1.06 \mathrm{E}+00$ \\
\hline ENSBTAG00000017604 & $8.02 \mathrm{E}-11$ & $7.58 \mathrm{E}-11$ & RAB13 & $1.06 \mathrm{E}+00$ \\
\hline ENSBTAG00000012437 & $7.40 \mathrm{E}-10$ & $6.98 \mathrm{E}-10$ & OR6N2 & $1.06 \mathrm{E}+00$ \\
\hline ENSBTAG00000011243 & $2.70 \mathrm{E}-10$ & $2.55 \mathrm{E}-10$ & TOE1 & $1.06 \mathrm{E}+00$ \\
\hline ENSBTAG00000009212 & $5.25 \mathrm{E}-10$ & $4.95 \mathrm{E}-10$ & APOA2 & $1.06 \mathrm{E}+00$ \\
\hline ENSBTAG00000021101 & $2.85 \mathrm{E}-10$ & $2.69 \mathrm{E}-10$ & GPR88 & $1.06 \mathrm{E}+00$ \\
\hline ENSBTAG00000039653 & $9.14 \mathrm{E}-10$ & $8.62 \mathrm{E}-10$ & & $1.06 \mathrm{E}+00$ \\
\hline ENSBTAG00000031358 & $4.82 \mathrm{E}-10$ & 4.54E-10 & DPYD & $1.06 \mathrm{E}+00$ \\
\hline ENSBTAG00000038625 & $6.32 \mathrm{E}-10$ & $5.95 \mathrm{E}-10$ & & $1.06 \mathrm{E}+00$ \\
\hline ENSBTAG00000012355 & 4.13E-10 & $3.87 \mathrm{E}-10$ & RNF220 & $1.07 \mathrm{E}+00$ \\
\hline ENSBTAG00000014791 & $3.88 \mathrm{E}-10$ & $3.63 \mathrm{E}-10$ & $\mathrm{CTH}$ & $1.07 \mathrm{E}+00$ \\
\hline ENSBTAG00000017161 & $2.68 \mathrm{E}-10$ & $2.50 \mathrm{E}-10$ & TM2D1 & $1.07 \mathrm{E}+00$ \\
\hline ENSBTAG00000011024 & $1.78 \mathrm{E}-10$ & $1.66 \mathrm{E}-10$ & TXNDC12 & $1.07 \mathrm{E}+00$ \\
\hline ENSBTAG00000016341 & $6.59 \mathrm{E}-10$ & $6.14 \mathrm{E}-10$ & RGS5 & $1.07 \mathrm{E}+00$ \\
\hline ENSBTAG00000013253 & $3.21 \mathrm{E}-10$ & $2.98 \mathrm{E}-10$ & THAP4 & $1.08 \mathrm{E}+00$ \\
\hline ENSBTAG00000017386 & $2.11 E-10$ & $1.96 \mathrm{E}-10$ & C1orf50 & $1.08 \mathrm{E}+00$ \\
\hline ENSBTAG00000003774 & $4.51 \mathrm{E}-10$ & $4.18 \mathrm{E}-10$ & $\mathrm{C} 8 \mathrm{~A}$ & $1.08 \mathrm{E}+00$ \\
\hline ENSBTAG00000046293 & 7.07E-10 & $6.55 \mathrm{E}-10$ & & $1.08 \mathrm{E}+00$ \\
\hline ENSBTAG00000005809 & $1.30 \mathrm{E}-10$ & $1.21 \mathrm{E}-10$ & GPR89A & $1.07 \mathrm{E}+00$ \\
\hline ENSBTAG00000038480 & $1.51 \mathrm{E}-10$ & $1.40 \mathrm{E}-10$ & FPGT & $1.08 \mathrm{E}+00$ \\
\hline
\end{tabular}




\begin{tabular}{|c|c|c|c|c|}
\hline ENSBTAG00000017778 & $2.81 \mathrm{E}-10$ & $2.58 \mathrm{E}-10$ & OR10R2 & $1.09 \mathrm{E}+00$ \\
\hline ENSBTAG00000005064 & $2.00 \mathrm{E}-10$ & $1.83 \mathrm{E}-10$ & WARS2 & $1.09 E+00$ \\
\hline ENSBTAG00000005445 & $2.22 \mathrm{E}-10$ & $2.03 \mathrm{E}-10$ & SLC35D1 & $1.10 \mathrm{E}+00$ \\
\hline ENSBTAG00000017670 & $8.46 \mathrm{E}-10$ & $7.69 \mathrm{E}-10$ & & $1.10 \mathrm{E}+00$ \\
\hline ENSBTAG00000011025 & $1.88 \mathrm{E}-10$ & 1.70E-10 & BTF3L4 & $1.11 \mathrm{E}+00$ \\
\hline ENSBTAG00000012962 & $1.78 \mathrm{E}-10$ & $1.62 \mathrm{E}-10$ & SARS & $1.10 \mathrm{E}+00$ \\
\hline ENSBTAG00000031461 & $3.73 \mathrm{E}-10$ & 3.37E-10 & SNX7 & $1.11 \mathrm{E}+00$ \\
\hline ENSBTAG00000021037 & $2.28 \mathrm{E}-10$ & $2.06 \mathrm{E}-10$ & ARNT & $1.11 \mathrm{E}+00$ \\
\hline ENSBTAG00000014294 & $1.78 \mathrm{E}-10$ & $1.60 \mathrm{E}-10$ & ST7L & $1.11 \mathrm{E}+00$ \\
\hline ENSBTAG00000007281 & 1.10E-10 & $9.91 \mathrm{E}-11$ & SEMA6C & $1.11 E+00$ \\
\hline ENSBTAG00000013014 & $1.73 \mathrm{E}-10$ & $1.56 \mathrm{E}-10$ & GPR61 & $1.11 E+00$ \\
\hline ENSBTAG00000004333 & $1.25 \mathrm{E}-10$ & $1.12 \mathrm{E}-10$ & COPA & $1.11 E+00$ \\
\hline ENSBTAG00000005719 & $4.30 \mathrm{E}-10$ & 3.87E-10 & GJB4 & $1.11 E+00$ \\
\hline ENSBTAG00000048179 & $1.46 \mathrm{E}-09$ & 1.30E-09 & & $1.12 \mathrm{E}+00$ \\
\hline ENSBTAG00000009674 & $2.32 \mathrm{E}-10$ & $2.08 \mathrm{E}-10$ & $\mathrm{C} 3 \mathrm{H} 1$ orf185 & $1.12 \mathrm{E}+00$ \\
\hline ENSBTAG00000030553 & $2.71 \mathrm{E}-10$ & $2.43 \mathrm{E}-10$ & & $1.11 \mathrm{E}+00$ \\
\hline ENSBTAG00000026181 & $3.72 \mathrm{E}-10$ & 3.33E-10 & UGT1A1 & $1.12 \mathrm{E}+00$ \\
\hline ENSBTAG00000001981 & $3.35 \mathrm{E}-10$ & $3.00 \mathrm{E}-10$ & SLC6A17 & $1.12 \mathrm{E}+00$ \\
\hline ENSBTAG00000014297 & $1.65 \mathrm{E}-10$ & 1.47E-10 & MOV10 & $1.12 \mathrm{E}+00$ \\
\hline ENSBTAG00000024240 & $2.05 \mathrm{E}-10$ & $1.83 \mathrm{E}-10$ & ACADM & $1.12 \mathrm{E}+00$ \\
\hline ENSBTAG00000001246 & $3.46 \mathrm{E}-10$ & 3.09E-10 & ATP1A1 & $1.12 \mathrm{E}+00$ \\
\hline ENSBTAG00000017846 & $5.12 \mathrm{E}-10$ & $4.55 \mathrm{E}-10$ & F11R & $1.13 \mathrm{E}+00$ \\
\hline ENSBTAG00000007247 & $2.85 \mathrm{E}-10$ & $2.53 \mathrm{E}-10$ & NUF2 & $1.13 E+00$ \\
\hline ENSBTAG00000020635 & $3.34 \mathrm{E}-10$ & $2.96 \mathrm{E}-10$ & VPS45 & $1.13 E+00$ \\
\hline ENSBTAG00000005810 & $2.90 \mathrm{E}-10$ & $2.58 \mathrm{E}-10$ & PDZK1 & $1.12 \mathrm{E}+00$ \\
\hline ENSBTAG00000009481 & $4.49 \mathrm{E}-10$ & 3.97E-10 & C3H1orf87 & $1.13 E+00$ \\
\hline ENSBTAG00000012419 & $3.39 \mathrm{E}-10$ & $2.99 \mathrm{E}-10$ & BCL2L15 & $1.13 \mathrm{E}+00$ \\
\hline ENSBTAG00000037575 & $5.81 \mathrm{E}-10$ & $5.11 \mathrm{E}-10$ & & $1.14 \mathrm{E}+00$ \\
\hline ENSBTAG00000006600 & $5.15 E-10$ & $4.54 \mathrm{E}-10$ & & $1.13 E+00$ \\
\hline ENSBTAG00000007842 & $3.38 \mathrm{E}-10$ & 2.97E-10 & GCLM & $1.14 \mathrm{E}+00$ \\
\hline ENSBTAG00000018889 & $2.19 \mathrm{E}-10$ & $1.93 \mathrm{E}-10$ & ATP6V0B & $1.13 \mathrm{E}+00$ \\
\hline ENSBTAG00000001499 & $2.77 \mathrm{E}-10$ & $2.44 \mathrm{E}-10$ & TRIM33 & $1.14 \mathrm{E}+00$ \\
\hline ENSBTAG00000008592 & $2.11 \mathrm{E}-10$ & $1.85 \mathrm{E}-10$ & & $1.14 \mathrm{E}+00$ \\
\hline ENSBTAG00000019901 & $2.39 \mathrm{E}-10$ & $2.08 \mathrm{E}-10$ & RAD54L & $1.15 \mathrm{E}+00$ \\
\hline ENSBTAG00000039487 & $4.04 \mathrm{E}-10$ & $3.53 \mathrm{E}-10$ & & $1.14 \mathrm{E}+00$ \\
\hline ENSBTAG00000019904 & $2.55 \mathrm{E}-10$ & $2.22 \mathrm{E}-10$ & & $1.15 E+00$ \\
\hline ENSBTAG00000014295 & $1.99 \mathrm{E}-10$ & $1.73 \mathrm{E}-10$ & CAPZA1 & $1.15 \mathrm{E}+00$ \\
\hline ENSBTAG00000015336 & $2.00 \mathrm{E}-10$ & $1.74 \mathrm{E}-10$ & PRUNE1 & $1.15 \mathrm{E}+00$ \\
\hline ENSBTAG00000001942 & $3.41 \mathrm{E}-10$ & $2.96 \mathrm{E}-10$ & SNX27 & $1.15 \mathrm{E}+00$ \\
\hline ENSBTAG00000009042 & $1.51 \mathrm{E}-10$ & $1.31 \mathrm{E}-10$ & ATXN7L2 & $1.15 E+00$ \\
\hline ENSBTAG00000030710 & $1.81 \mathrm{E}-10$ & $1.56 \mathrm{E}-10$ & ITGB3BP & $1.16 \mathrm{E}+00$ \\
\hline ENSBTAG00000020969 & $2.39 \mathrm{E}-10$ & $2.06 \mathrm{E}-10$ & DCAF6 & $1.16 \mathrm{E}+00$ \\
\hline ENSBTAG00000033835 & $2.70 \mathrm{E}-10$ & $2.34 \mathrm{E}-10$ & MPZ & $1.16 \mathrm{E}+00$ \\
\hline ENSBTAG00000006466 & $2.87 \mathrm{E}-10$ & $2.47 \mathrm{E}-10$ & CD53 & $1.16 \mathrm{E}+00$ \\
\hline ENSBTAG00000016326 & $2.15 \mathrm{E}-10$ & $1.85 \mathrm{E}-10$ & C1orf52 & $1.16 \mathrm{E}+00$ \\
\hline ENSBTAG00000002600 & $1.80 \mathrm{E}-10$ & $1.55 \mathrm{E}-10$ & EFCAB7 & $1.16 \mathrm{E}+00$ \\
\hline ENSBTAG00000024095 & 4.64E-10 & $3.98 \mathrm{E}-10$ & THRAP3 & $1.17 \mathrm{E}+00$ \\
\hline ENSBTAG00000000888 & $1.26 \mathrm{E}-09$ & $1.08 \mathrm{E}-09$ & SPTA1 & $1.17 E+00$ \\
\hline ENSBTAG00000009123 & $9.75 \mathrm{E}-10$ & $8.34 \mathrm{E}-10$ & ING5 & $1.17 E+00$ \\
\hline ENSBTAG00000016712 & $3.92 \mathrm{E}-10$ & 3.35E-10 & & $1.17 \mathrm{E}+00$ \\
\hline ENSBTAG00000030671 & $3.12 \mathrm{E}-10$ & $2.67 \mathrm{E}-10$ & HOOK1 & $1.17 \mathrm{E}+00$ \\
\hline ENSBTAG00000032071 & $2.85 \mathrm{E}-10$ & $2.44 \mathrm{E}-10$ & SYCP1 & $1.17 E+00$ \\
\hline ENSBTAG00000019497 & $2.04 \mathrm{E}-10$ & $1.74 \mathrm{E}-10$ & ILDR2 & $1.17 \mathrm{E}+00$ \\
\hline ENSBTAG00000030669 & $3.08 \mathrm{E}-10$ & $2.62 \mathrm{E}-10$ & FGGY & $1.17 \mathrm{E}+00$ \\
\hline ENSBTAG00000046807 & $6.30 \mathrm{E}-10$ & 5.37E-10 & & $1.17 \mathrm{E}+00$ \\
\hline ENSBTAG00000021842 & $3.08 \mathrm{E}-10$ & 2.63E-10 & & $1.17 \mathrm{E}+00$ \\
\hline ENSBTAG00000015371 & $1.75 \mathrm{E}-10$ & $1.49 \mathrm{E}-10$ & GABPB2 & $1.17 \mathrm{E}+00$ \\
\hline ENSBTAG00000040131 & $2.51 \mathrm{E}-10$ & $2.13 \mathrm{E}-10$ & CD58 & $1.18 \mathrm{E}+00$ \\
\hline ENSBTAG00000021948 & $2.46 \mathrm{E}-10$ & $2.08 \mathrm{E}-10$ & & $1.18 \mathrm{E}+00$ \\
\hline ENSBTAG00000021023 & $2.99 \mathrm{E}-10$ & $2.53 \mathrm{E}-10$ & ZMYM4 & $1.18 \mathrm{E}+00$ \\
\hline ENSBTAG00000018717 & $1.17 \mathrm{E}-10$ & $9.82 \mathrm{E}-11$ & DDX20 & $1.19 E+00$ \\
\hline ENSBTAG00000004037 & $1.20 \mathrm{E}-10$ & $1.01 \mathrm{E}-10$ & JUN & $1.19 E+00$ \\
\hline ENSBTAG00000012996 & $1.09 \mathrm{E}-10$ & $9.18 \mathrm{E}-11$ & RNF11 & $1.19 E+00$ \\
\hline ENSBTAG00000021897 & $1.48 \mathrm{E}-10$ & $1.24 \mathrm{E}-10$ & B4GALT3 & $1.19 \mathrm{E}+00$ \\
\hline ENSBTAG00000011879 & $2.18 \mathrm{E}-10$ & $1.83 \mathrm{E}-10$ & B4GALT2 & $1.19 E+00$ \\
\hline ENSBTAG00000040060 & $2.13 \mathrm{E}-10$ & $1.79 \mathrm{E}-10$ & ARTN & $1.19 E+00$ \\
\hline ENSBTAG00000017434 & $2.43 \mathrm{E}-10$ & $2.04 \mathrm{E}-10$ & $\mathrm{MROH} 2 \mathrm{~A}$ & $1.19 E+00$ \\
\hline ENSBTAG00000015180 & $1.75 \mathrm{E}-10$ & 1.47E-10 & & $1.19 E+00$ \\
\hline ENSBTAG00000024115 & $1.78 \mathrm{E}-10$ & $1.50 \mathrm{E}-10$ & FOXO6 & $1.19 E+00$ \\
\hline ENSBTAG00000000574 & $1.32 \mathrm{E}-09$ & 1.10E-09 & OR10Z1 & $1.20 \mathrm{E}+00$ \\
\hline
\end{tabular}




\begin{tabular}{|c|c|c|c|c|}
\hline ENSBTAG00000014235 & $1.15 \mathrm{E}-10$ & $9.69 \mathrm{E}-11$ & SMIM12 & $1.19 \mathrm{E}+00$ \\
\hline ENSBTAG00000040596 & $1.36 \mathrm{E}-10$ & $1.14 \mathrm{E}-10$ & LINGO4 & $1.19 E+00$ \\
\hline ENSBTAG00000046002 & $5.24 \mathrm{E}-10$ & $4.39 \mathrm{E}-10$ & & $1.19 E+00$ \\
\hline ENSBTAG00000001599 & $3.21 \mathrm{E}-10$ & $2.70 \mathrm{E}-10$ & SV2A & $1.19 \mathrm{E}+00$ \\
\hline ENSBTAG00000039566 & $5.14 \mathrm{E}-10$ & $4.31 \mathrm{E}-10$ & & $1.19 E+00$ \\
\hline ENSBTAG00000023551 & $4.61 \mathrm{E}-10$ & 3.87E-10 & МОВ3С & $1.19 E+00$ \\
\hline ENSBTAG00000005143 & $1.63 \mathrm{E}-10$ & 1.37E-10 & & $1.19 \mathrm{E}+00$ \\
\hline ENSBTAG00000037432 & 7.83E-10 & $6.56 \mathrm{E}-10$ & & $1.19 E+00$ \\
\hline ENSBTAG00000006081 & $1.32 \mathrm{E}-10$ & $1.11 \mathrm{E}-10$ & PPM1J & $1.19 \mathrm{E}+00$ \\
\hline ENSBTAG00000045911 & $2.65 \mathrm{E}-10$ & $2.22 \mathrm{E}-10$ & & $1.19 E+00$ \\
\hline ENSBTAG00000047248 & $7.04 \mathrm{E}-10$ & $5.90 \mathrm{E}-10$ & & $1.19 \mathrm{E}+00$ \\
\hline ENSBTAG00000012514 & $1.52 \mathrm{E}-10$ & $1.28 \mathrm{E}-10$ & PODN & $1.19 \mathrm{E}+00$ \\
\hline ENSBTAG00000014299 & $1.51 \mathrm{E}-10$ & $1.27 \mathrm{E}-10$ & $\mathrm{RHOC}$ & $1.19 \mathrm{E}+00$ \\
\hline ENSBTAG00000009455 & $3.41 \mathrm{E}-10$ & $2.84 \mathrm{E}-10$ & IL12RB2 & $1.20 \mathrm{E}+00$ \\
\hline ENSBTAG00000015392 & $8.21 \mathrm{E}-10$ & $6.81 \mathrm{E}-10$ & TTF2 & $1.20 \mathrm{E}+00$ \\
\hline ENSBTAG00000011543 & $3.39 \mathrm{E}-10$ & $2.81 \mathrm{E}-10$ & PDCD1 & $1.21 \mathrm{E}+00$ \\
\hline ENSBTAG00000038502 & $9.94 \mathrm{E}-10$ & 8.23E-10 & & $1.21 \mathrm{E}+00$ \\
\hline ENSBTAG00000005161 & $1.23 \mathrm{E}-10$ & $1.02 \mathrm{E}-10$ & ATP8B2 & $1.21 E+00$ \\
\hline ENSBTAG00000007519 & $2.70 \mathrm{E}-10$ & $2.22 \mathrm{E}-10$ & ADAR & $1.22 \mathrm{E}+00$ \\
\hline ENSBTAG00000020641 & $2.34 \mathrm{E}-10$ & $1.91 \mathrm{E}-10$ & PSMA5 & $1.22 \mathrm{E}+00$ \\
\hline ENSBTAG00000013320 & $3.22 \mathrm{E}-10$ & $2.64 \mathrm{E}-10$ & TSPAN1 & $1.22 \mathrm{E}+00$ \\
\hline ENSBTAG00000019733 & $2.82 \mathrm{E}-10$ & 2.30E-10 & ADGRL4 & $1.23 \mathrm{E}+00$ \\
\hline ENSBTAG00000006720 & $5.69 \mathrm{E}-10$ & $4.64 \mathrm{E}-10$ & GJA5 & $1.23 \mathrm{E}+00$ \\
\hline ENSBTAG00000010638 & $2.39 \mathrm{E}-10$ & $1.95 \mathrm{E}-10$ & DCST2 & $1.23 E+00$ \\
\hline ENSBTAG00000015973 & $7.23 \mathrm{E}-10$ & 5.87E-10 & TRAF3IP1 & $1.23 E+00$ \\
\hline ENSBTAG00000012025 & $5.22 \mathrm{E}-10$ & $4.25 \mathrm{E}-10$ & LMX1A & $1.23 E+00$ \\
\hline ENSBTAG00000017502 & $2.63 \mathrm{E}-10$ & $2.14 \mathrm{E}-10$ & RIMKLA & $1.23 \mathrm{E}+00$ \\
\hline ENSBTAG00000010124 & $3.23 \mathrm{E}-10$ & $2.62 \mathrm{E}-10$ & ATG4C & $1.23 \mathrm{E}+00$ \\
\hline ENSBTAG00000005854 & $4.60 \mathrm{E}-10$ & $3.72 \mathrm{E}-10$ & COPS8 & $1.24 \mathrm{E}+00$ \\
\hline ENSBTAG00000003775 & $3.82 \mathrm{E}-10$ & $3.09 \mathrm{E}-10$ & $\mathrm{C} 8 \mathrm{~B}$ & $1.24 \mathrm{E}+00$ \\
\hline ENSBTAG00000016730 & $9.50 \mathrm{E}-11$ & 7.69E-11 & ANP32E & $1.24 \mathrm{E}+00$ \\
\hline ENSBTAG00000005799 & $3.46 \mathrm{E}-10$ & $2.79 \mathrm{E}-10$ & KIRREL & $1.24 \mathrm{E}+00$ \\
\hline ENSBTAG00000021512 & $1.46 \mathrm{E}-10$ & $1.18 \mathrm{E}-10$ & ZBTB7B & $1.24 \mathrm{E}+00$ \\
\hline ENSBTAG00000016643 & $1.18 \mathrm{E}-10$ & $9.44 \mathrm{E}-11$ & CYB561D1 & $1.25 \mathrm{E}+00$ \\
\hline ENSBTAG00000001080 & $3.35 \mathrm{E}-10$ & $2.68 \mathrm{E}-10$ & SPAG17 & $1.25 \mathrm{E}+00$ \\
\hline ENSBTAG00000021485 & $1.31 \mathrm{E}-10$ & $1.05 \mathrm{E}-10$ & C1orf54 & $1.25 \mathrm{E}+00$ \\
\hline ENSBTAG00000001629 & $2.45 \mathrm{E}-10$ & $1.96 \mathrm{E}-10$ & SCMH1 & $1.25 \mathrm{E}+00$ \\
\hline ENSBTAG00000019695 & $4.22 \mathrm{E}-10$ & $3.38 \mathrm{E}-10$ & RLF & $1.25 \mathrm{E}+00$ \\
\hline ENSBTAG00000047541 & $8.92 \mathrm{E}-10$ & 7.13E-10 & & $1.25 \mathrm{E}+00$ \\
\hline ENSBTAG00000021635 & $1.70 \mathrm{E}-10$ & $1.37 \mathrm{E}-10$ & NUDT17 & $1.24 \mathrm{E}+00$ \\
\hline ENSBTAG00000022741 & $6.52 \mathrm{E}-10$ & $5.21 \mathrm{E}-10$ & MGC134040 & $1.25 \mathrm{E}+00$ \\
\hline ENSBTAG00000001845 & $1.90 \mathrm{E}-10$ & $1.51 \mathrm{E}-10$ & & $1.26 \mathrm{E}+00$ \\
\hline ENSBTAG00000000900 & $4.30 \mathrm{E}-10$ & $3.42 \mathrm{E}-10$ & LRRC8C & $1.26 \mathrm{E}+00$ \\
\hline ENSBTAG00000004190 & $3.66 \mathrm{E}-10$ & $2.90 \mathrm{E}-10$ & ARHGAP29 & $1.26 \mathrm{E}+00$ \\
\hline ENSBTAG00000003806 & $2.88 \mathrm{E}-10$ & $2.29 \mathrm{E}-10$ & ECM1 & $1.26 \mathrm{E}+00$ \\
\hline ENSBTAG00000003928 & $2.55 \mathrm{E}-10$ & 2.03E-10 & ATG16L1 & $1.26 \mathrm{E}+00$ \\
\hline ENSBTAG00000015902 & $1.67 \mathrm{E}-10$ & $1.33 \mathrm{E}-10$ & PTGFR & $1.26 \mathrm{E}+00$ \\
\hline ENSBTAG00000005439 & $4.25 \mathrm{E}-10$ & 3.37E-10 & FAM102B & $1.26 \mathrm{E}+00$ \\
\hline ENSBTAG00000044107 & 4.05E-10 & $3.21 \mathrm{E}-10$ & FAM159A & $1.26 \mathrm{E}+00$ \\
\hline ENSBTAG00000020745 & $3.53 \mathrm{E}-10$ & $2.79 \mathrm{E}-10$ & HIVEP3 & $1.27 \mathrm{E}+00$ \\
\hline ENSBTAG00000012405 & $3.52 \mathrm{E}-10$ & $2.79 \mathrm{E}-10$ & PEAR1 & $1.26 \mathrm{E}+00$ \\
\hline ENSBTAG00000011822 & $4.73 \mathrm{E}-10$ & $3.75 \mathrm{E}-10$ & C1orf228 & $1.26 \mathrm{E}+00$ \\
\hline ENSBTAG00000046989 & $1.10 \mathrm{E}-10$ & $8.69 \mathrm{E}-11$ & MANEAL & $1.27 \mathrm{E}+00$ \\
\hline ENSBTAG00000002024 & $3.65 E-10$ & $2.88 \mathrm{E}-10$ & ASB18 & $1.27 \mathrm{E}+00$ \\
\hline ENSBTAG00000019029 & $4.36 \mathrm{E}-10$ & $3.44 \mathrm{E}-10$ & DPH5 & $1.27 \mathrm{E}+00$ \\
\hline ENSBTAG00000015853 & $3.73 \mathrm{E}-10$ & $2.93 \mathrm{E}-10$ & SDHC & $1.27 \mathrm{E}+00$ \\
\hline ENSBTAG00000021900 & $5.77 \mathrm{E}-10$ & 4.54E-10 & PKN2 & $1.27 \mathrm{E}+00$ \\
\hline ENSBTAG00000010560 & $1.31 \mathrm{E}-10$ & $1.03 \mathrm{E}-10$ & PROK1 & $1.27 \mathrm{E}+00$ \\
\hline ENSBTAG00000006491 & $9.08 \mathrm{E}-10$ & 7.13E-10 & & $1.27 \mathrm{E}+00$ \\
\hline ENSBTAG00000002030 & $7.38 \mathrm{E}-10$ & $5.74 \mathrm{E}-10$ & SPP2 & $1.29 E+00$ \\
\hline ENSBTAG00000013505 & $4.42 \mathrm{E}-10$ & $3.43 \mathrm{E}-10$ & IQCA1 & $1.29 E+00$ \\
\hline ENSBTAG00000001879 & $4.66 \mathrm{E}-10$ & $3.61 E-10$ & & $1.29 E+00$ \\
\hline ENSBTAG00000031575 & $4.83 \mathrm{E}-10$ & $3.74 \mathrm{E}-10$ & VAV3 & $1.29 E+00$ \\
\hline ENSBTAG00000020765 & $7.05 \mathrm{E}-10$ & $5.46 \mathrm{E}-10$ & & $1.29 \mathrm{E}+00$ \\
\hline ENSBTAG00000038961 & 8.06E-10 & $6.23 \mathrm{E}-10$ & & $1.29 E+00$ \\
\hline ENSBTAG00000003614 & $2.47 \mathrm{E}-10$ & $1.91 \mathrm{E}-10$ & AP4B1 & $1.29 E+00$ \\
\hline ENSBTAG00000021594 & $1.54 \mathrm{E}-10$ & $1.20 \mathrm{E}-10$ & TDRKH & $1.29 E+00$ \\
\hline ENSBTAG00000005864 & $1.66 \mathrm{E}-10$ & $1.28 \mathrm{E}-10$ & ASB17 & $1.30 \mathrm{E}+00$ \\
\hline ENSBTAG00000011316 & $2.71 \mathrm{E}-10$ & $2.09 \mathrm{E}-10$ & ZC $3 \mathrm{H} 12 \mathrm{~A}$ & $1.30 \mathrm{E}+00$ \\
\hline ENSBTAG00000008131 & $2.79 \mathrm{E}-10$ & $2.14 \mathrm{E}-10$ & RNF115 & $1.30 \mathrm{E}+00$ \\
\hline
\end{tabular}




\begin{tabular}{|c|c|c|c|c|}
\hline ENSBTAG00000015781 & $1.65 \mathrm{E}-10$ & $1.27 \mathrm{E}-10$ & TRMT13 & $1.30 \mathrm{E}+00$ \\
\hline ENSBTAG00000020838 & $1.58 \mathrm{E}-09$ & $1.21 \mathrm{E}-09$ & & $1.31 E+00$ \\
\hline ENSBTAG00000012372 & $2.13 \mathrm{E}-10$ & $1.64 \mathrm{E}-10$ & TMEM53 & $1.30 \mathrm{E}+00$ \\
\hline ENSBTAG00000018249 & $3.49 \mathrm{E}-10$ & $2.67 \mathrm{E}-10$ & KCNN3 & $1.31 \mathrm{E}+00$ \\
\hline ENSBTAG00000030809 & 8.69E-10 & $6.65 \mathrm{E}-10$ & Clorf141 & $1.31 E+00$ \\
\hline ENSBTAG00000038617 & $5.47 \mathrm{E}-10$ & 4.19E-10 & SH3D21 & $1.31 \mathrm{E}+00$ \\
\hline ENSBTAG00000013578 & $3.05 \mathrm{E}-10$ & 2.33E-10 & CHI3L2 & $1.31 \mathrm{E}+00$ \\
\hline ENSBTAG00000013778 & $1.93 \mathrm{E}-10$ & 1.47E-10 & WDR63 & $1.31 \mathrm{E}+00$ \\
\hline ENSBTAG00000005909 & $2.53 \mathrm{E}-10$ & $1.93 \mathrm{E}-10$ & LEPROT & $1.31 E+00$ \\
\hline ENSBTAG00000020622 & $6.09 \mathrm{E}-10$ & 4.64E-10 & COL9A2 & $1.31 E+00$ \\
\hline ENSBTAG00000037890 & $4.42 \mathrm{E}-10$ & $3.37 \mathrm{E}-10$ & & $1.31 E+00$ \\
\hline ENSBTAG00000013802 & $3.68 \mathrm{E}-10$ & $2.79 \mathrm{E}-10$ & DAB1 & $1.32 \mathrm{E}+00$ \\
\hline ENSBTAG00000044125 & $2.26 \mathrm{E}-10$ & $1.72 \mathrm{E}-10$ & & $1.32 \mathrm{E}+00$ \\
\hline ENSBTAG00000002983 & $2.57 \mathrm{E}-10$ & $1.95 \mathrm{E}-10$ & NT5C1A & $1.32 \mathrm{E}+00$ \\
\hline ENSBTAG00000019680 & $3.69 \mathrm{E}-10$ & $2.80 \mathrm{E}-10$ & ZYG11B & $1.32 \mathrm{E}+00$ \\
\hline ENSBTAG00000002416 & $6.69 \mathrm{E}-10$ & 5.07E-10 & & $1.32 \mathrm{E}+00$ \\
\hline ENSBTAG00000011548 & $1.41 \mathrm{E}-10$ & $1.07 \mathrm{E}-10$ & AMPD1 & $1.32 \mathrm{E}+00$ \\
\hline ENSBTAG00000019026 & $5.69 \mathrm{E}-10$ & $4.31 \mathrm{E}-10$ & EXTL2 & $1.32 \mathrm{E}+00$ \\
\hline ENSBTAG00000015154 & $8.83 E-11$ & $6.68 \mathrm{E}-11$ & & $1.32 \mathrm{E}+00$ \\
\hline ENSBTAG00000039357 & $6.18 \mathrm{E}-10$ & $4.66 \mathrm{E}-10$ & & $1.33 \mathrm{E}+00$ \\
\hline ENSBTAG00000024272 & $1.02 \mathrm{E}-09$ & $7.66 \mathrm{E}-10$ & & $1.33 \mathrm{E}+00$ \\
\hline ENSBTAG00000022514 & $6.34 \mathrm{E}-10$ & $4.78 \mathrm{E}-10$ & CD5L & $1.33 \mathrm{E}+00$ \\
\hline ENSBTAG00000046773 & $2.70 \mathrm{E}-10$ & $2.04 \mathrm{E}-10$ & MCOLN2 & $1.32 \mathrm{E}+00$ \\
\hline ENSBTAG00000027775 & $7.41 \mathrm{E}-10$ & $5.58 \mathrm{E}-10$ & & $1.33 \mathrm{E}+00$ \\
\hline ENSBTAG00000039189 & $1.23 \mathrm{E}-09$ & $9.23 \mathrm{E}-10$ & & $1.33 \mathrm{E}+00$ \\
\hline ENSBTAG00000021046 & $3.00 \mathrm{E}-10$ & $2.25 \mathrm{E}-10$ & ELAVL4 & $1.33 E+00$ \\
\hline ENSBTAG00000002847 & $5.70 \mathrm{E}-10$ & 4.26E-10 & $\mathrm{D} 2 \mathrm{HGDH}$ & $1.34 \mathrm{E}+00$ \\
\hline ENSBTAG00000012454 & $2.58 \mathrm{E}-10$ & $1.93 \mathrm{E}-10$ & SLC35A3 & $1.34 \mathrm{E}+00$ \\
\hline ENSBTAG00000008809 & $3.13 \mathrm{E}-10$ & $2.33 \mathrm{E}-10$ & & $1.34 \mathrm{E}+00$ \\
\hline ENSBTAG00000018711 & $1.41 \mathrm{E}-10$ & $1.05 \mathrm{E}-10$ & DENND4B & $1.34 \mathrm{E}+00$ \\
\hline ENSBTAG00000015996 & $3.98 \mathrm{E}-10$ & $2.95 \mathrm{E}-10$ & GPC1 & $1.35 E+00$ \\
\hline ENSBTAG00000021051 & $2.42 \mathrm{E}-10$ & $1.79 \mathrm{E}-10$ & ZNHIT6 & $1.35 \mathrm{E}+00$ \\
\hline ENSBTAG00000015470 & $1.85 \mathrm{E}-10$ & $1.37 \mathrm{E}-10$ & SYPL2 & $1.35 \mathrm{E}+00$ \\
\hline ENSBTAG00000019010 & $3.25 \mathrm{E}-10$ & $2.40 \mathrm{E}-10$ & EIF2B3 & $1.36 \mathrm{E}+00$ \\
\hline ENSBTAG00000021170 & $1.58 \mathrm{E}-10$ & 1.17E-10 & OSBPL9 & $1.35 \mathrm{E}+00$ \\
\hline ENSBTAG00000032193 & $7.50 \mathrm{E}-10$ & $5.53 \mathrm{E}-10$ & REG4 & $1.36 \mathrm{E}+00$ \\
\hline ENSBTAG00000005542 & $1.67 \mathrm{E}-10$ & $1.23 \mathrm{E}-10$ & EPS15 & $1.36 \mathrm{E}+00$ \\
\hline ENSBTAG00000005716 & $1.79 \mathrm{E}-10$ & $1.31 \mathrm{E}-10$ & CRABP2 & $1.36 \mathrm{E}+00$ \\
\hline ENSBTAG00000017326 & $2.93 \mathrm{E}-10$ & $2.15 \mathrm{E}-10$ & OMA1 & $1.36 \mathrm{E}+00$ \\
\hline ENSBTAG00000026032 & $5.28 \mathrm{E}-10$ & $3.88 \mathrm{E}-10$ & ZYG11A & $1.36 \mathrm{E}+00$ \\
\hline ENSBTAG00000003746 & $3.43 \mathrm{E}-10$ & $2.52 \mathrm{E}-10$ & SCP2 & $1.36 \mathrm{E}+00$ \\
\hline ENSBTAG00000003220 & $6.53 \mathrm{E}-10$ & $4.78 \mathrm{E}-10$ & ACKR1 & $1.37 \mathrm{E}+00$ \\
\hline ENSBTAG00000013367 & $1.06 \mathrm{E}-09$ & 7.76E-10 & PPT1 & $1.36 \mathrm{E}+00$ \\
\hline ENSBTAG00000012659 & $2.07 \mathrm{E}-10$ & $1.51 \mathrm{E}-10$ & LRIF1 & $1.37 E+00$ \\
\hline ENSBTAG00000017877 & $5.01 \mathrm{E}-10$ & $3.66 \mathrm{E}-10$ & NECTIN4 & $1.37 E+00$ \\
\hline ENSBTAG00000008688 & 3.39E-10 & $2.46 \mathrm{E}-10$ & HEYL & $1.38 \mathrm{E}+00$ \\
\hline ENSBTAG00000020152 & $1.78 \mathrm{E}-10$ & $1.30 \mathrm{E}-10$ & & $1.37 E+00$ \\
\hline ENSBTAG00000013016 & $2.09 \mathrm{E}-10$ & $1.51 \mathrm{E}-10$ & GNAI3 & $1.38 \mathrm{E}+00$ \\
\hline ENSBTAG00000001481 & 3.13E-10 & $2.27 \mathrm{E}-10$ & IGSF9 & $1.38 \mathrm{E}+00$ \\
\hline ENSBTAG00000000965 & $5.26 \mathrm{E}-10$ & $3.80 \mathrm{E}-10$ & TNNI3K & $1.38 \mathrm{E}+00$ \\
\hline ENSBTAG00000013867 & $3.29 \mathrm{E}-10$ & $2.38 \mathrm{E}-10$ & DLGAP3 & $1.38 \mathrm{E}+00$ \\
\hline ENSBTAG00000019158 & $1.14 \mathrm{E}-10$ & $8.18 \mathrm{E}-11$ & MEX3A & $1.39 \mathrm{E}+00$ \\
\hline ENSBTAG00000019615 & 5.19E-10 & $3.74 \mathrm{E}-10$ & PHTF1 & $1.39 E+00$ \\
\hline ENSBTAG00000004849 & $4.94 \mathrm{E}-10$ & $3.57 \mathrm{E}-10$ & IGSF3 & $1.38 \mathrm{E}+00$ \\
\hline ENSBTAG00000020173 & $3.96 \mathrm{E}-10$ & $2.85 \mathrm{E}-10$ & INPP5D & $1.39 E+00$ \\
\hline ENSBTAG00000037770 & $6.08 \mathrm{E}-10$ & $4.36 \mathrm{E}-10$ & & $1.39 \mathrm{E}+00$ \\
\hline ENSBTAG00000046650 & $6.10 \mathrm{E}-10$ & $4.38 \mathrm{E}-10$ & OR6K2 & $1.39 E+00$ \\
\hline ENSBTAG00000021487 & $1.73 \mathrm{E}-10$ & $1.24 \mathrm{E}-10$ & CIART & $1.39 \mathrm{E}+00$ \\
\hline ENSBTAG00000005717 & $7.01 \mathrm{E}-10$ & $5.04 \mathrm{E}-10$ & GJB5 & $1.39 \mathrm{E}+00$ \\
\hline ENSBTAG00000024534 & 3.17E-10 & $2.27 \mathrm{E}-10$ & POU2F1 & $1.39 E+00$ \\
\hline ENSBTAG00000005891 & $3.68 \mathrm{E}-10$ & $2.63 \mathrm{E}-10$ & FCRL1 & $1.40 \mathrm{E}+00$ \\
\hline ENSBTAG00000003376 & 4.37E-10 & $3.13 \mathrm{E}-10$ & ASB1 & $1.40 \mathrm{E}+00$ \\
\hline ENSBTAG00000012374 & 4.13E-10 & $2.95 \mathrm{E}-10$ & C2orf54 & $1.40 \mathrm{E}+00$ \\
\hline ENSBTAG00000003837 & $2.38 \mathrm{E}-10$ & $1.70 \mathrm{E}-10$ & RSPO1 & $1.40 \mathrm{E}+00$ \\
\hline ENSBTAG00000004383 & $2.50 \mathrm{E}-10$ & $1.78 \mathrm{E}-10$ & FNBP1L & $1.40 \mathrm{E}+00$ \\
\hline ENSBTAG00000000584 & $5.31 \mathrm{E}-10$ & $3.78 \mathrm{E}-10$ & & $1.40 \mathrm{E}+00$ \\
\hline ENSBTAG00000014225 & 3.34E-10 & $2.38 \mathrm{E}-10$ & SLC5A9 & $1.40 \mathrm{E}+00$ \\
\hline ENSBTAG00000012700 & $5.11 \mathrm{E}-10$ & $3.63 \mathrm{E}-10$ & CD247 & $1.41 E+00$ \\
\hline ENSBTAG00000020966 & $1.41 \mathrm{E}-10$ & $1.00 \mathrm{E}-10$ & ALX3 & $1.41 \mathrm{E}+00$ \\
\hline ENSBTAG00000001586 & $2.41 \mathrm{E}-10$ & $1.71 \mathrm{E}-10$ & OTUD7B & $1.41 E+00$ \\
\hline
\end{tabular}




\begin{tabular}{|c|c|c|c|c|}
\hline ENSBTAG00000016550 & $1.86 \mathrm{E}-10$ & $1.32 \mathrm{E}-10$ & MAEL & $1.41 \mathrm{E}+00$ \\
\hline ENSBTAG00000020180 & $2.13 \mathrm{E}-10$ & $1.51 \mathrm{E}-10$ & TMED5 & $1.41 E+00$ \\
\hline ENSBTAG00000013334 & $5.48 \mathrm{E}-10$ & $3.88 \mathrm{E}-10$ & CSF3R & $1.41 E+00$ \\
\hline ENSBTAG00000014984 & $9.67 \mathrm{E}-11$ & $6.83 \mathrm{E}-11$ & RPRD2 & $1.42 \mathrm{E}+00$ \\
\hline ENSBTAG00000009813 & $4.32 \mathrm{E}-10$ & $3.04 \mathrm{E}-10$ & ELOVL1 & $1.42 \mathrm{E}+00$ \\
\hline ENSBTAG00000017411 & $6.62 \mathrm{E}-10$ & 4.65E-10 & AK5 & $1.42 \mathrm{E}+00$ \\
\hline ENSBTAG00000012957 & $1.93 \mathrm{E}-10$ & $1.36 \mathrm{E}-10$ & SERBP1 & $1.42 \mathrm{E}+00$ \\
\hline ENSBTAG00000039719 & $6.15 \mathrm{E}-10$ & $4.31 \mathrm{E}-10$ & PHGDH & $1.43 \mathrm{E}+00$ \\
\hline ENSBTAG00000001519 & $2.12 \mathrm{E}-10$ & $1.48 \mathrm{E}-10$ & RRNAD1 & $1.43 \mathrm{E}+00$ \\
\hline ENSBTAG00000039686 & $3.79 \mathrm{E}-10$ & $2.65 \mathrm{E}-10$ & АCOT11 & $1.43 E+00$ \\
\hline ENSBTAG00000007537 & $1.36 \mathrm{E}-10$ & $9.48 \mathrm{E}-11$ & PEX19 & $1.43 E+00$ \\
\hline ENSBTAG00000010456 & $7.90 \mathrm{E}-10$ & $5.52 \mathrm{E}-10$ & TADA1 & $1.43 E+00$ \\
\hline ENSBTAG00000000229 & 3.35E-10 & $2.34 \mathrm{E}-10$ & & $1.43 \mathrm{E}+00$ \\
\hline ENSBTAG00000047679 & $3.92 \mathrm{E}-10$ & $2.74 \mathrm{E}-10$ & DNTTIP2 & $1.43 E+00$ \\
\hline ENSBTAG00000006555 & $2.36 \mathrm{E}-10$ & $1.65 \mathrm{E}-10$ & ZMYM1 & $1.43 E+00$ \\
\hline ENSBTAG00000006321 & 7.19E-10 & $5.01 \mathrm{E}-10$ & RTCA & $1.43 E+00$ \\
\hline ENSBTAG00000015107 & $2.12 \mathrm{E}-10$ & $1.47 \mathrm{E}-10$ & SLC16A1 & $1.44 \mathrm{E}+00$ \\
\hline ENSBTAG00000004852 & $3.63 \mathrm{E}-10$ & $2.51 \mathrm{E}-10$ & PLEKHO1 & $1.44 \mathrm{E}+00$ \\
\hline ENSBTAG00000016801 & $3.15 \mathrm{E}-10$ & $2.18 \mathrm{E}-10$ & RXRG & $1.44 \mathrm{E}+00$ \\
\hline ENSBTAG00000016387 & $5.79 \mathrm{E}-10$ & $4.00 \mathrm{E}-10$ & GDAP2 & $1.45 \mathrm{E}+00$ \\
\hline ENSBTAG00000000283 & $1.30 \mathrm{E}-10$ & $8.91 \mathrm{E}-11$ & CSF1 & $1.46 \mathrm{E}+00$ \\
\hline ENSBTAG00000044150 & $4.34 \mathrm{E}-10$ & $2.98 \mathrm{E}-10$ & UCK2 & $1.46 \mathrm{E}+00$ \\
\hline ENSBTAG00000001628 & $2.55 \mathrm{E}-10$ & $1.75 \mathrm{E}-10$ & SLFNL1 & $1.46 \mathrm{E}+00$ \\
\hline ENSBTAG00000017382 & $1.86 \mathrm{E}-10$ & $1.28 \mathrm{E}-10$ & P3H1 & $1.45 \mathrm{E}+00$ \\
\hline ENSBTAG00000009093 & 3.03E-10 & $2.08 \mathrm{E}-10$ & GAL3ST2 & $1.46 \mathrm{E}+00$ \\
\hline ENSBTAG00000012361 & 5.17E-10 & $3.53 \mathrm{E}-10$ & ARHGEF11 & $1.46 \mathrm{E}+00$ \\
\hline ENSBTAG00000009314 & $5.14 \mathrm{E}-10$ & $3.51 \mathrm{E}-10$ & C1orf168 & $1.46 \mathrm{E}+00$ \\
\hline ENSBTAG00000014083 & $1.94 \mathrm{E}-10$ & $1.31 \mathrm{E}-10$ & AMIGO1 & $1.48 \mathrm{E}+00$ \\
\hline ENSBTAG00000002451 & $2.15 \mathrm{E}-10$ & $1.45 \mathrm{E}-10$ & ТTC39A & $1.48 \mathrm{E}+00$ \\
\hline ENSBTAG00000034776 & $2.04 \mathrm{E}-10$ & $1.38 \mathrm{E}-10$ & DDAH1 & $1.48 \mathrm{E}+00$ \\
\hline ENSBTAG00000045685 & 8.17E-10 & $5.51 \mathrm{E}-10$ & & $1.48 \mathrm{E}+00$ \\
\hline ENSBTAG00000045664 & $3.06 \mathrm{E}-10$ & 2.07E-10 & LRRC41 & $1.48 \mathrm{E}+00$ \\
\hline ENSBTAG00000008023 & $5.99 \mathrm{E}-10$ & 4.03E-10 & RHBDL2 & $1.49 E+00$ \\
\hline ENSBTAG00000040084 & $1.18 \mathrm{E}-09$ & 7.99E-10 & & $1.48 \mathrm{E}+00$ \\
\hline ENSBTAG00000016852 & $2.43 \mathrm{E}-10$ & $1.63 \mathrm{E}-10$ & SNIP1 & $1.49 E+00$ \\
\hline ENSBTAG00000015961 & $2.19 \mathrm{E}-10$ & 1.47E-10 & TSPAN2 & $1.49 E+00$ \\
\hline ENSBTAG00000010676 & $6.59 \mathrm{E}-10$ & $4.42 \mathrm{E}-10$ & RBM44 & $1.49 E+00$ \\
\hline ENSBTAG00000004585 & $3.41 \mathrm{E}-10$ & $2.29 \mathrm{E}-10$ & CCDC30 & $1.49 E+00$ \\
\hline ENSBTAG00000015474 & $2.18 \mathrm{E}-10$ & $1.47 \mathrm{E}-10$ & SRSF11 & $1.49 \mathrm{E}+00$ \\
\hline ENSBTAG00000038362 & 1.31E-09 & $8.79 \mathrm{E}-10$ & & $1.50 \mathrm{E}+00$ \\
\hline ENSBTAG00000014530 & $2.96 \mathrm{E}-10$ & 1.97E-10 & NTNG1 & $1.50 \mathrm{E}+00$ \\
\hline ENSBTAG00000021174 & $1.62 \mathrm{E}-10$ & $1.07 \mathrm{E}-10$ & NRDC & $1.51 E+00$ \\
\hline ENSBTAG00000005796 & $1.61 \mathrm{E}-10$ & $1.07 \mathrm{E}-10$ & & $1.50 \mathrm{E}+00$ \\
\hline ENSBTAG00000015784 & $2.66 \mathrm{E}-10$ & $1.77 \mathrm{E}-10$ & HAPLN2 & $1.50 \mathrm{E}+00$ \\
\hline ENSBTAG00000016696 & $1.78 \mathrm{E}-10$ & $1.18 \mathrm{E}-10$ & NCDN & $1.50 \mathrm{E}+00$ \\
\hline ENSBTAG00000018010 & $6.93 \mathrm{E}-10$ & $4.60 \mathrm{E}-10$ & ABCA4 & $1.51 E+00$ \\
\hline ENSBTAG00000018739 & 3.54E-10 & $2.35 \mathrm{E}-10$ & RPAP2 & $1.51 E+00$ \\
\hline ENSBTAG00000009213 & $3.47 \mathrm{E}-10$ & $2.30 \mathrm{E}-10$ & Tom40B & $1.51 E+00$ \\
\hline ENSBTAG00000007994 & $7.37 \mathrm{E}-10$ & $4.86 \mathrm{E}-10$ & OSCP1 & $1.52 \mathrm{E}+00$ \\
\hline ENSBTAG00000037490 & $7.10 \mathrm{E}-10$ & $4.69 \mathrm{E}-10$ & & $1.51 \mathrm{E}+00$ \\
\hline ENSBTAG00000006769 & $1.74 \mathrm{E}-10$ & $1.15 \mathrm{E}-10$ & & $1.51 E+00$ \\
\hline ENSBTAG00000017764 & $5.15 \mathrm{E}-10$ & $3.39 \mathrm{E}-10$ & HDAC4 & $1.52 \mathrm{E}+00$ \\
\hline ENSBTAG00000040598 & $4.68 \mathrm{E}-10$ & $3.08 \mathrm{E}-10$ & & $1.52 \mathrm{E}+00$ \\
\hline ENSBTAG00000009215 & $3.51 \mathrm{E}-10$ & $2.31 \mathrm{E}-10$ & NR1I3 & $1.52 E+00$ \\
\hline ENSBTAG00000027936 & $3.14 \mathrm{E}-10$ & $2.06 \mathrm{E}-10$ & Clorf146 & $1.53 E+00$ \\
\hline ENSBTAG00000005354 & $6.88 \mathrm{E}-10$ & $4.52 \mathrm{E}-10$ & & $1.52 \mathrm{E}+00$ \\
\hline ENSBTAG00000020223 & $1.95 \mathrm{E}-10$ & $1.28 \mathrm{E}-10$ & CASQ1 & $1.53 \mathrm{E}+00$ \\
\hline ENSBTAG00000007767 & $2.14 \mathrm{E}-10$ & $1.40 \mathrm{E}-10$ & TBX15 & $1.53 \mathrm{E}+00$ \\
\hline ENSBTAG00000021104 & $7.81 \mathrm{E}-10$ & $5.12 \mathrm{E}-10$ & & $1.53 \mathrm{E}+00$ \\
\hline ENSBTAG00000010622 & $2.10 \mathrm{E}-10$ & $1.38 \mathrm{E}-10$ & TRIM46 & $1.52 \mathrm{E}+00$ \\
\hline ENSBTAG00000019192 & $1.58 \mathrm{E}-10$ & $1.03 \mathrm{E}-10$ & LHX8 & $1.53 \mathrm{E}+00$ \\
\hline ENSBTAG00000005782 & $1.48 \mathrm{E}-10$ & $9.71 \mathrm{E}-11$ & CTBS & $1.53 E+00$ \\
\hline ENSBTAG00000038500 & $4.68 \mathrm{E}-10$ & $3.06 \mathrm{E}-10$ & & $1.53 \mathrm{E}+00$ \\
\hline ENSBTAG00000015891 & $2.98 \mathrm{E}-10$ & $1.94 \mathrm{E}-10$ & NSUN4 & $1.53 E+00$ \\
\hline ENSBTAG00000027321 & $1.48 \mathrm{E}-10$ & $9.68 \mathrm{E}-11$ & CCDC18 & $1.53 E+00$ \\
\hline ENSBTAG00000030852 & $4.53 \mathrm{E}-10$ & $2.95 \mathrm{E}-10$ & & $1.54 \mathrm{E}+00$ \\
\hline ENSBTAG00000014649 & 1.19E-10 & 7.71E-11 & CPT2 & $1.54 \mathrm{E}+00$ \\
\hline ENSBTAG00000022504 & $9.68 \mathrm{E}-10$ & $6.29 \mathrm{E}-10$ & & $1.54 \mathrm{E}+00$ \\
\hline ENSBTAG00000011538 & 3.63E-10 & $2.36 \mathrm{E}-10$ & KIF1A & $1.54 \mathrm{E}+00$ \\
\hline ENSBTAG00000008649 & $3.03 E-10$ & $1.96 \mathrm{E}-10$ & ETV3 & $1.54 \mathrm{E}+00$ \\
\hline
\end{tabular}




\begin{tabular}{|c|c|c|c|c|}
\hline ENSBTAG000000001959 & $1.91 \mathrm{E}-10$ & $1.24 \mathrm{E}-10$ & TFAP2E & $1.54 \mathrm{E}+00$ \\
\hline ENSBTAG00000037539 & $4.98 \mathrm{E}-10$ & $3.22 \mathrm{E}-10$ & & $1.55 \mathrm{E}+00$ \\
\hline ENSBTAG000000017655 & 7.19E-10 & 4.62E-10 & PALMD & $1.56 \mathrm{E}+00$ \\
\hline ENSBTAG000000017405 & $1.61 \mathrm{E}-10$ & $1.03 \mathrm{E}-10$ & RORC & $1.56 \mathrm{E}+00$ \\
\hline ENSBTAG000000018804 & $1.78 \mathrm{E}-10$ & $1.14 \mathrm{E}-10$ & CELSR2 & $1.56 \mathrm{E}+00$ \\
\hline ENSBTAG000000019027 & $5.34 \mathrm{E}-10$ & $3.42 \mathrm{E}-10$ & SLC30A7 & $1.56 \mathrm{E}+00$ \\
\hline ENSBTAG000000009269 & $3.50 \mathrm{E}-10$ & $2.24 \mathrm{E}-10$ & GRIK3 & $1.56 \mathrm{E}+00$ \\
\hline ENSBTAG000000002028 & $2.91 \mathrm{E}-10$ & $1.86 \mathrm{E}-10$ & FAM69A & $1.57 E+00$ \\
\hline ENSBTAG00000019956 & $1.92 \mathrm{E}-10$ & $1.23 \mathrm{E}-10$ & CMPK1 & $1.56 \mathrm{E}+00$ \\
\hline ENSBTAG000000019877 & $3.24 \mathrm{E}-10$ & $2.06 \mathrm{E}-10$ & DOCK7 & $1.57 \mathrm{E}+00$ \\
\hline ENSBTAG000000014983 & $5.03 \mathrm{E}-10$ & $3.20 \mathrm{E}-10$ & IL23R & $1.57 E+00$ \\
\hline ENSBTAG000000005732 & $6.53 \mathrm{E}-10$ & $4.14 \mathrm{E}-10$ & TRAPPC3 & $1.58 \mathrm{E}+00$ \\
\hline ENSBTAG00000014368 & $5.85 \mathrm{E}-10$ & $3.70 \mathrm{E}-10$ & SLAMF6 & $1.58 \mathrm{E}+00$ \\
\hline ENSBTAG000000002414 & $7.08 \mathrm{E}-10$ & 4.47E-10 & KCNJ10 & $1.58 \mathrm{E}+00$ \\
\hline ENSBTAG000000013025 & $1.26 \mathrm{E}-10$ & $7.96 \mathrm{E}-11$ & HAX1 & $1.59 \mathrm{E}+00$ \\
\hline ENSBTAG000000001520 & $2.29 \mathrm{E}-10$ & $1.44 \mathrm{E}-10$ & MRPL24 & $1.59 E+00$ \\
\hline ENSBTAG000000008370 & $1.47 \mathrm{E}-10$ & $9.23 \mathrm{E}-11$ & & $1.59 \mathrm{E}+00$ \\
\hline ENSBTAG000000045967 & $2.34 \mathrm{E}-10$ & $1.47 \mathrm{E}-10$ & LENEP & $1.59 \mathrm{E}+00$ \\
\hline ENSBTAG000000012644 & $3.00 \mathrm{E}-10$ & $1.89 \mathrm{E}-10$ & UROD & $1.59 E+00$ \\
\hline ENSBTAG000000047910 & $1.76 \mathrm{E}-10$ & $1.11 \mathrm{E}-10$ & & $1.59 \mathrm{E}+00$ \\
\hline ENSBTAG000000021288 & $2.08 \mathrm{E}-10$ & $1.31 \mathrm{E}-10$ & PSMB4 & $1.59 \mathrm{E}+00$ \\
\hline ENSBTAG000000020592 & $1.94 \mathrm{E}-10$ & $1.22 \mathrm{E}-10$ & отоS & $1.59 \mathrm{E}+00$ \\
\hline ENSBTAG000000021833 & $2.48 \mathrm{E}-10$ & $1.56 \mathrm{E}-10$ & GJA8 & $1.59 \mathrm{E}+00$ \\
\hline ENSBTAG000000005793 & $2.08 \mathrm{E}-10$ & $1.31 \mathrm{E}-10$ & PEA15 & $1.59 \mathrm{E}+00$ \\
\hline ENSBTAG000000006050 & $3.72 \mathrm{E}-10$ & $2.34 \mathrm{E}-10$ & BOLA1 & $1.59 \mathrm{E}+00$ \\
\hline ENSBTAG000000001397 & $3.46 \mathrm{E}-10$ & $2.18 \mathrm{E}-10$ & & $1.59 E+00$ \\
\hline ENSBTAG000000000784 & $2.62 \mathrm{E}-10$ & $1.65 \mathrm{E}-10$ & MTERF4 & $1.59 E+00$ \\
\hline ENSBTAG000000001518 & $1.61 \mathrm{E}-10$ & $1.02 \mathrm{E}-10$ & ISG2OL2 & $1.58 \mathrm{E}+00$ \\
\hline ENSBTAG000000005797 & $4.08 \mathrm{E}-10$ & $2.57 \mathrm{E}-10$ & AGXT & $1.59 E+00$ \\
\hline ENSBTAG000000047830 & $2.30 \mathrm{E}-10$ & $1.44 \mathrm{E}-10$ & & $1.59 E+00$ \\
\hline ENSBTAG00000015113 & 3.67E-10 & $2.31 \mathrm{E}-10$ & CITED4 & $1.59 \mathrm{E}+00$ \\
\hline ENSBTAG000000021649 & $3.21 \mathrm{E}-10$ & $2.02 \mathrm{E}-10$ & & $1.59 \mathrm{E}+00$ \\
\hline ENSBTAG000000000404 & $5.12 \mathrm{E}-10$ & $3.22 \mathrm{E}-10$ & NAXE & $1.59 E+00$ \\
\hline ENSBTAG000000014840 & 5.07E-10 & $3.19 \mathrm{E}-10$ & MTX1 & $1.59 E+00$ \\
\hline ENSBTAG00000047496 & $2.05 \mathrm{E}-10$ & $1.29 \mathrm{E}-10$ & & $1.59 \mathrm{E}+00$ \\
\hline ENSBTAG00000032451 & $6.71 \mathrm{E}-10$ & $4.21 \mathrm{E}-10$ & & $1.59 \mathrm{E}+00$ \\
\hline ENSBTAG00000000253 & $3.12 \mathrm{E}-10$ & $1.96 \mathrm{E}-10$ & PTPRF & $1.59 \mathrm{E}+00$ \\
\hline ENSBTAG00000021082 & $8.00 \mathrm{E}-10$ & $5.02 \mathrm{E}-10$ & TMEM125 & $1.59 \mathrm{E}+00$ \\
\hline ENSBTAG00000013801 & $3.47 \mathrm{E}-10$ & $2.18 \mathrm{E}-10$ & PBX1 & $1.59 \mathrm{E}+00$ \\
\hline ENSBTAG00000020542 & $4.83 \mathrm{E}-10$ & $2.98 \mathrm{E}-10$ & SHE & $1.62 E+00$ \\
\hline ENSBTAG00000037634 & 1.00E-09 & $6.18 \mathrm{E}-10$ & & $1.62 E+00$ \\
\hline ENSBTAG00000010841 & $1.56 \mathrm{E}-10$ & $9.58 \mathrm{E}-11$ & FMO5 & $1.63 E+00$ \\
\hline ENSBTAG00000009368 & $2.10 \mathrm{E}-10$ & $1.29 \mathrm{E}-10$ & RRAGC & $1.63 E+00$ \\
\hline ENSBTAG00000005788 & $9.43 \mathrm{E}-10$ & $5.76 \mathrm{E}-10$ & VANGL1 & $1.64 E+00$ \\
\hline ENSBTAG00000015789 & $3.38 \mathrm{E}-10$ & 2.07E-10 & BCAN & $1.63 E+00$ \\
\hline ENSBTAG00000015482 & $2.59 \mathrm{E}-10$ & $1.58 \mathrm{E}-10$ & ANKRD13C & $1.64 \mathrm{E}+00$ \\
\hline ENSBTAG00000013832 & $8.04 \mathrm{E}-10$ & $4.91 \mathrm{E}-10$ & TRIT1 & $1.64 \mathrm{E}+00$ \\
\hline ENSBTAG00000021480 & $3.62 \mathrm{E}-10$ & $2.21 \mathrm{E}-10$ & SAG & $1.64 \mathrm{E}+00$ \\
\hline ENSBTAG00000015559 & $3.31 \mathrm{E}-10$ & $2.01 E-10$ & ERI3 & $1.64 \mathrm{E}+00$ \\
\hline ENSBTAG00000010176 & 5.53E-10 & 3.37E-10 & ILKAP & $1.64 \mathrm{E}+00$ \\
\hline ENSBTAG00000020616 & $3.10 \mathrm{E}-10$ & $1.88 \mathrm{E}-10$ & DGKD & $1.65 E+00$ \\
\hline ENSBTAG00000031654 & $2.53 \mathrm{E}-10$ & $1.53 \mathrm{E}-10$ & KCND3 & $1.65 E+00$ \\
\hline ENSBTAG00000011500 & $6.24 \mathrm{E}-10$ & $3.78 \mathrm{E}-10$ & CASQ2 & $1.65 E+00$ \\
\hline ENSBTAG00000019555 & $1.90 \mathrm{E}-10$ & $1.15 \mathrm{E}-10$ & ZSCAN20 & $1.65 E+00$ \\
\hline ENSBTAG00000018448 & $2.70 \mathrm{E}-10$ & $1.63 \mathrm{E}-10$ & $\mathrm{MSH} 4$ & $1.66 \mathrm{E}+00$ \\
\hline ENSBTAG00000000057 & $2.02 \mathrm{E}-10$ & $1.22 \mathrm{E}-10$ & THBS3 & $1.66 \mathrm{E}+00$ \\
\hline ENSBTAG00000016328 & $2.44 \mathrm{E}-10$ & $1.47 \mathrm{E}-10$ & SFPQ & $1.66 \mathrm{E}+00$ \\
\hline ENSBTAG00000011756 & $5.93 \mathrm{E}-10$ & 3.56E-10 & & $1.66 \mathrm{E}+00$ \\
\hline ENSBTAG00000016472 & $1.49 \mathrm{E}-10$ & 8.87E-11 & & $1.68 \mathrm{E}+00$ \\
\hline ENSBTAG00000010164 & $3.68 \mathrm{E}-10$ & $2.20 \mathrm{E}-10$ & ESPNL & $1.67 E+00$ \\
\hline ENSBTAG00000030749 & $6.89 \mathrm{E}-10$ & $4.11 \mathrm{E}-10$ & DNAJC6 & $1.68 \mathrm{E}+00$ \\
\hline ENSBTAG00000034332 & $9.52 \mathrm{E}-10$ & $5.66 \mathrm{E}-10$ & & $1.68 \mathrm{E}+00$ \\
\hline ENSBTAG00000048203 & $1.29 \mathrm{E}-10$ & $7.68 \mathrm{E}-11$ & SAMD13 & $1.68 \mathrm{E}+00$ \\
\hline ENSBTAG00000010565 & $2.43 \mathrm{E}-10$ & $1.43 \mathrm{E}-10$ & & $1.70 \mathrm{E}+00$ \\
\hline ENSBTAG00000008118 & $1.59 \mathrm{E}-10$ & $9.40 \mathrm{E}-11$ & RPF1 & $1.69 \mathrm{E}+00$ \\
\hline ENSBTAG00000000964 & $5.11 \mathrm{E}-10$ & $3.02 \mathrm{E}-10$ & FCRL5 & $1.69 E+00$ \\
\hline ENSBTAG00000005912 & $2.86 \mathrm{E}-10$ & $1.68 \mathrm{E}-10$ & DMAP1 & $1.70 \mathrm{E}+00$ \\
\hline ENSBTAG00000000074 & $1.85 \mathrm{E}-10$ & $1.09 \mathrm{E}-10$ & NFIA & $1.69 E+00$ \\
\hline ENSBTAG00000007909 & $3.45 \mathrm{E}-10$ & 2.03E-10 & $\mathrm{NOTCH} 2$ & $1.70 \mathrm{E}+00$ \\
\hline ENSBTAG00000004422 & $5.42 \mathrm{E}-10$ & $3.19 \mathrm{E}-10$ & ALG14 & $1.70 \mathrm{E}+00$ \\
\hline
\end{tabular}




\begin{tabular}{|c|c|c|c|c|}
\hline ENSBTAG00000013946 & $6.59 \mathrm{E}-11$ & $3.88 \mathrm{E}-11$ & PRPF3 & $1.70 \mathrm{E}+00$ \\
\hline ENSBTAG00000011888 & $3.31 \mathrm{E}-10$ & $1.94 \mathrm{E}-10$ & CCDC24 & $1.70 \mathrm{E}+00$ \\
\hline ENSBTAG00000013018 & $2.06 \mathrm{E}-10$ & $1.21 \mathrm{E}-10$ & AMPD2 & $1.70 \mathrm{E}+00$ \\
\hline ENSBTAG00000030686 & $9.20 \mathrm{E}-10$ & $5.39 \mathrm{E}-10$ & RAVER2 & $1.71 \mathrm{E}+00$ \\
\hline ENSBTAG00000017892 & $6.03 \mathrm{E}-10$ & $3.53 \mathrm{E}-10$ & & $1.71 E+00$ \\
\hline ENSBTAG00000017844 & $1.64 \mathrm{E}-10$ & $9.63 \mathrm{E}-11$ & STIL & $1.71 E+00$ \\
\hline ENSBTAG00000002979 & $5.80 \mathrm{E}-10$ & $3.40 \mathrm{E}-10$ & PIK3R3 & $1.71 \mathrm{E}+00$ \\
\hline ENSBTAG00000018424 & $9.86 \mathrm{E}-10$ & $5.76 \mathrm{E}-10$ & ACKR3 & $1.71 E+00$ \\
\hline ENSBTAG00000030301 & $3.49 \mathrm{E}-10$ & $2.04 \mathrm{E}-10$ & MAP7D1 & $1.71 E+00$ \\
\hline ENSBTAG00000021975 & $5.12 \mathrm{E}-10$ & $2.96 \mathrm{E}-10$ & PATJ & $1.73 E+00$ \\
\hline ENSBTAG00000024153 & $2.11 \mathrm{E}-10$ & $1.22 \mathrm{E}-10$ & & $1.73 \mathrm{E}+00$ \\
\hline ENSBTAG00000000149 & $3.17 \mathrm{E}-10$ & $1.80 \mathrm{E}-10$ & USP40 & $1.76 \mathrm{E}+00$ \\
\hline ENSBTAG00000009527 & $2.52 \mathrm{E}-10$ & $1.43 \mathrm{E}-10$ & TCEANC2 & $1.76 \mathrm{E}+00$ \\
\hline ENSBTAG00000007101 & $4.53 \mathrm{E}-10$ & $2.56 \mathrm{E}-10$ & F3 & $1.77 \mathrm{E}+00$ \\
\hline ENSBTAG00000009209 & $3.04 \mathrm{E}-10$ & $1.72 \mathrm{E}-10$ & BEND5 & $1.77 \mathrm{E}+00$ \\
\hline ENSBTAG00000008122 & $2.01 \mathrm{E}-10$ & $1.14 \mathrm{E}-10$ & & $1.77 E+00$ \\
\hline ENSBTAG00000002078 & $2.02 \mathrm{E}-10$ & $1.13 \mathrm{E}-10$ & KDM4A & $1.79 \mathrm{E}+00$ \\
\hline ENSBTAG00000013507 & $4.06 \mathrm{E}-10$ & $2.27 \mathrm{E}-10$ & SPRR3 & $1.79 \mathrm{E}+00$ \\
\hline ENSBTAG00000014970 & $1.70 \mathrm{E}-10$ & $9.54 \mathrm{E}-11$ & & $1.79 \mathrm{E}+00$ \\
\hline ENSBTAG00000003281 & $7.90 \mathrm{E}-10$ & $4.41 \mathrm{E}-10$ & & $1.79 \mathrm{E}+00$ \\
\hline ENSBTAG00000018074 & $2.36 \mathrm{E}-10$ & $1.31 \mathrm{E}-10$ & VPS72 & $1.80 \mathrm{E}+00$ \\
\hline ENSBTAG00000015147 & $1.87 \mathrm{E}-10$ & $1.04 \mathrm{E}-10$ & S100A10 & $1.80 \mathrm{E}+00$ \\
\hline ENSBTAG00000015794 & $3.09 \mathrm{E}-10$ & $1.71 \mathrm{E}-10$ & NES & $1.81 E+00$ \\
\hline ENSBTAG00000031044 & $7.12 \mathrm{E}-10$ & $3.95 \mathrm{E}-10$ & MIGA1 & $1.80 \mathrm{E}+00$ \\
\hline ENSBTAG00000009192 & $2.78 \mathrm{E}-10$ & $1.54 \mathrm{E}-10$ & & $1.80 \mathrm{E}+00$ \\
\hline ENSBTAG00000020154 & $1.29 \mathrm{E}-10$ & $7.08 \mathrm{E}-11$ & DEDD & $1.81 \mathrm{E}+00$ \\
\hline ENSBTAG00000013210 & $3.20 \mathrm{E}-10$ & $1.76 \mathrm{E}-10$ & ADAMTS4 & $1.82 \mathrm{E}+00$ \\
\hline ENSBTAG00000020010 & $1.97 \mathrm{E}-10$ & $1.09 \mathrm{E}-10$ & & $1.81 E+00$ \\
\hline ENSBTAG00000039793 & $2.16 \mathrm{E}-10$ & $1.18 \mathrm{E}-10$ & HDGF & $1.83 \mathrm{E}+00$ \\
\hline ENSBTAG00000022893 & 1.36E-09 & $7.43 \mathrm{E}-10$ & & $1.83 E+00$ \\
\hline ENSBTAG00000007517 & $2.34 \mathrm{E}-10$ & $1.28 \mathrm{E}-10$ & CHRNB2 & $1.82 \mathrm{E}+00$ \\
\hline ENSBTAG00000003245 & $6.39 \mathrm{E}-10$ & $3.47 \mathrm{E}-10$ & BCAR3 & $1.84 \mathrm{E}+00$ \\
\hline ENSBTAG00000014529 & $8.58 \mathrm{E}-10$ & 4.64E-10 & & $1.85 E+00$ \\
\hline ENSBTAG00000009870 & $2.76 \mathrm{E}-10$ & $1.49 \mathrm{E}-10$ & HES6 & $1.85 E+00$ \\
\hline ENSBTAG00000032453 & $2.79 \mathrm{E}-10$ & $1.50 \mathrm{E}-10$ & & $1.86 \mathrm{E}+00$ \\
\hline ENSBTAG00000000186 & $9.63 \mathrm{E}-11$ & $5.20 \mathrm{E}-11$ & FLAD1 & $1.85 \mathrm{E}+00$ \\
\hline ENSBTAG00000015145 & $3.55 \mathrm{E}-10$ & $1.91 \mathrm{E}-10$ & S100A11 & $1.86 \mathrm{E}+00$ \\
\hline ENSBTAG00000011327 & $1.91 \mathrm{E}-10$ & $1.03 \mathrm{E}-10$ & OLFML3 & $1.85 E+00$ \\
\hline ENSBTAG00000007241 & $1.85 \mathrm{E}-10$ & $9.95 \mathrm{E}-11$ & SLAMF9 & $1.85 \mathrm{E}+00$ \\
\hline ENSBTAG00000008322 & $3.74 \mathrm{E}-10$ & $2.01 \mathrm{E}-10$ & KCNA10 & $1.86 \mathrm{E}+00$ \\
\hline ENSBTAG00000016698 & $5.37 \mathrm{E}-10$ & $2.89 \mathrm{E}-10$ & SLAMF8 & $1.86 \mathrm{E}+00$ \\
\hline ENSBTAG00000012228 & $4.24 \mathrm{E}-10$ & $2.28 \mathrm{E}-10$ & SEMA4A & $1.86 \mathrm{E}+00$ \\
\hline ENSBTAG00000000678 & $1.70 \mathrm{E}-10$ & $9.07 \mathrm{E}-11$ & CSDE1 & $1.87 \mathrm{E}+00$ \\
\hline ENSBTAG00000004688 & $2.84 \mathrm{E}-10$ & $1.52 \mathrm{E}-10$ & DHCR24 & $1.87 \mathrm{E}+00$ \\
\hline ENSBTAG00000015856 & $5.48 \mathrm{E}-10$ & $2.92 \mathrm{E}-10$ & CFAP126 & $1.88 \mathrm{E}+00$ \\
\hline ENSBTAG00000000664 & $4.16 \mathrm{E}-10$ & $2.21 \mathrm{E}-10$ & SLC22A15 & $1.88 \mathrm{E}+00$ \\
\hline ENSBTAG00000006101 & $1.71 \mathrm{E}-10$ & $9.07 \mathrm{E}-11$ & PSMD4 & $1.89 \mathrm{E}+00$ \\
\hline ENSBTAG00000001326 & $6.90 \mathrm{E}-10$ & $3.66 \mathrm{E}-10$ & SLC44A5 & $1.89 \mathrm{E}+00$ \\
\hline ENSBTAG00000004885 & $9.20 \mathrm{E}-10$ & $4.87 \mathrm{E}-10$ & DDR2 & $1.89 \mathrm{E}+00$ \\
\hline ENSBTAG00000016705 & $2.07 \mathrm{E}-10$ & $1.08 \mathrm{E}-10$ & & $1.91 \mathrm{E}+00$ \\
\hline ENSBTAG00000018726 & $6.47 \mathrm{E}-10$ & $3.38 \mathrm{E}-10$ & RIMS3 & $1.91 E+00$ \\
\hline ENSBTAG00000002419 & $6.96 \mathrm{E}-10$ & $3.61 \mathrm{E}-10$ & PIGM & $1.93 \mathrm{E}+00$ \\
\hline ENSBTAG00000003279 & $8.86 \mathrm{E}-10$ & $4.61 E-10$ & NDUFA10 & $1.92 \mathrm{E}+00$ \\
\hline ENSBTAG00000019230 & $2.27 \mathrm{E}-10$ & $1.18 \mathrm{E}-10$ & PTGER3 & $1.92 \mathrm{E}+00$ \\
\hline ENSBTAG00000019133 & $2.32 \mathrm{E}-10$ & $1.20 \mathrm{E}-10$ & ZNF326 & $1.93 E+00$ \\
\hline ENSBTAG00000008737 & $2.08 \mathrm{E}-10$ & $1.07 \mathrm{E}-10$ & DR1 & $1.94 \mathrm{E}+00$ \\
\hline ENSBTAG00000039993 & $4.38 \mathrm{E}-10$ & $2.27 \mathrm{E}-10$ & & $1.93 E+00$ \\
\hline ENSBTAG00000012979 & $2.84 \mathrm{E}-10$ & $1.46 \mathrm{E}-10$ & ZMYND12 & $1.95 E+00$ \\
\hline ENSBTAG00000010158 & $1.11 \mathrm{E}-09$ & $5.71 \mathrm{E}-10$ & & $1.94 \mathrm{E}+00$ \\
\hline ENSBTAG00000018474 & $3.84 \mathrm{E}-10$ & $1.97 \mathrm{E}-10$ & IL6R & $1.95 E+00$ \\
\hline ENSBTAG00000011244 & $3.69 \mathrm{E}-10$ & $1.89 \mathrm{E}-10$ & TESK2 & $1.95 E+00$ \\
\hline ENSBTAG00000027425 & $4.42 \mathrm{E}-10$ & $2.25 \mathrm{E}-10$ & CCDC190 & $1.97 \mathrm{E}+00$ \\
\hline ENSBTAG00000001626 & $3.06 \mathrm{E}-10$ & $1.54 \mathrm{E}-10$ & CTPS1 & $1.98 \mathrm{E}+00$ \\
\hline ENSBTAG00000020444 & $5.35 \mathrm{E}-10$ & $2.70 \mathrm{E}-10$ & MAST2 & $1.98 \mathrm{E}+00$ \\
\hline ENSBTAG00000018447 & $1.20 \mathrm{E}-10$ & $6.03 E-11$ & RABGGTB & $1.98 \mathrm{E}+00$ \\
\hline ENSBTAG00000017942 & $1.29 \mathrm{E}-10$ & $6.52 \mathrm{E}-11$ & & $1.98 \mathrm{E}+00$ \\
\hline ENSBTAG00000005990 & $8.80 \mathrm{E}-11$ & $4.43 \mathrm{E}-11$ & S1PR1 & $1.98 \mathrm{E}+00$ \\
\hline ENSBTAG00000017104 & $3.02 \mathrm{E}-10$ & $1.52 \mathrm{E}-10$ & MUC1 & $1.98 \mathrm{E}+00$ \\
\hline ENSBTAG00000003781 & $3.58 \mathrm{E}-10$ & $1.80 \mathrm{E}-10$ & MPL & $1.99 \mathrm{E}+00$ \\
\hline ENSBTAG00000019013 & $2.06 \mathrm{E}-10$ & $1.04 \mathrm{E}-10$ & HECTD3 & $1.98 \mathrm{E}+00$ \\
\hline
\end{tabular}




\begin{tabular}{|c|c|c|c|c|}
\hline ENSBTAG00000045833 & $4.33 \mathrm{E}-10$ & $2.18 \mathrm{E}-10$ & & $1.99 \mathrm{E}+00$ \\
\hline ENSBTAG00000030674 & 4.15E-10 & $2.08 \mathrm{E}-10$ & AK4 & $1.99 \mathrm{E}+00$ \\
\hline ENSBTAG00000017827 & $9.98 \mathrm{E}-10$ & $5.00 \mathrm{E}-10$ & & $1.99 \mathrm{E}+00$ \\
\hline ENSBTAG00000019801 & $3.98 \mathrm{E}-10$ & $1.97 \mathrm{E}-10$ & FAM151A & $2.02 \mathrm{E}+00$ \\
\hline ENSBTAG00000017316 & $9.96 \mathrm{E}-10$ & $4.93 \mathrm{E}-10$ & & $2.02 \mathrm{E}+00$ \\
\hline ENSBTAG00000010717 & $3.03 \mathrm{E}-10$ & $1.50 \mathrm{E}-10$ & SSBP3 & $2.02 \mathrm{E}+00$ \\
\hline ENSBTAG00000021289 & $1.68 \mathrm{E}-10$ & 8.30E-11 & POGZ & $2.02 E+00$ \\
\hline ENSBTAG00000020761 & $3.61 \mathrm{E}-10$ & $1.78 \mathrm{E}-10$ & USP33 & $2.03 E+00$ \\
\hline ENSBTAG00000017819 & $2.35 \mathrm{E}-10$ & $1.16 \mathrm{E}-10$ & PMVK & $2.02 \mathrm{E}+00$ \\
\hline ENSBTAG00000024269 & $4.20 \mathrm{E}-10$ & $2.06 \mathrm{E}-10$ & TGFBR3 & $2.04 E+00$ \\
\hline ENSBTAG00000047375 & $7.11 \mathrm{E}-10$ & $3.45 \mathrm{E}-10$ & & $2.06 \mathrm{E}+00$ \\
\hline ENSBTAG00000018071 & $2.38 \mathrm{E}-10$ & $1.15 \mathrm{E}-10$ & TMOD4 & $2.07 E+00$ \\
\hline ENSBTAG00000001393 & 2.44E-10 & $1.18 \mathrm{E}-10$ & MTF2 & $2.06 \mathrm{E}+00$ \\
\hline ENSBTAG00000040512 & 8.33E-10 & $4.02 \mathrm{E}-10$ & PCP4L1 & $2.07 E+00$ \\
\hline ENSBTAG00000008636 & $3.60 \mathrm{E}-10$ & $1.73 \mathrm{E}-10$ & PDE4B & $2.08 \mathrm{E}+00$ \\
\hline ENSBTAG00000020172 & $2.61 \mathrm{E}-10$ & $1.25 \mathrm{E}-10$ & DENND2D & $2.09 \mathrm{E}+00$ \\
\hline ENSBTAG00000000861 & $4.75 \mathrm{E}-10$ & $2.26 \mathrm{E}-10$ & ETV3L & $2.10 E+00$ \\
\hline ENSBTAG00000017051 & $2.08 \mathrm{E}-10$ & $9.87 \mathrm{E}-11$ & ACP6 & $2.10 \mathrm{E}+00$ \\
\hline ENSBTAG00000022938 & $7.85 \mathrm{E}-10$ & $3.72 \mathrm{E}-10$ & & $2.11 E+00$ \\
\hline ENSBTAG00000021024 & $4.65 \mathrm{E}-10$ & $2.20 \mathrm{E}-10$ & MACF1 & $2.11 E+00$ \\
\hline ENSBTAG00000004987 & $1.34 \mathrm{E}-10$ & $6.36 \mathrm{E}-11$ & NUP210L & $2.11 E+00$ \\
\hline ENSBTAG00000003054 & $2.09 \mathrm{E}-10$ & $9.88 \mathrm{E}-11$ & INSRR & $2.12 \mathrm{E}+00$ \\
\hline ENSBTAG00000030706 & $2.70 \mathrm{E}-10$ & $1.27 \mathrm{E}-10$ & ALG6 & $2.13 E+00$ \\
\hline ENSBTAG00000017826 & 4.69E-10 & $2.21 \mathrm{E}-10$ & SCLY & $2.12 \mathrm{E}+00$ \\
\hline ENSBTAG00000031186 & $9.26 \mathrm{E}-10$ & 4.33E-10 & & $2.14 \mathrm{E}+00$ \\
\hline ENSBTAG00000014151 & $4.48 \mathrm{E}-10$ & $2.09 \mathrm{E}-10$ & RCSD1 & $2.14 \mathrm{E}+00$ \\
\hline ENSBTAG00000009434 & $1.99 \mathrm{E}-10$ & $9.11 \mathrm{E}-11$ & PAQR6 & $2.18 \mathrm{E}+00$ \\
\hline ENSBTAG00000015969 & $6.27 \mathrm{E}-10$ & $2.87 \mathrm{E}-10$ & STK40 & $2.18 \mathrm{E}+00$ \\
\hline ENSBTAG00000018203 & $3.03 \mathrm{E}-10$ & $1.38 \mathrm{E}-10$ & CD101 & $2.20 E+00$ \\
\hline ENSBTAG00000044178 & $7.05 \mathrm{E}-10$ & $3.18 \mathrm{E}-10$ & ZMPSTE24 & $2.22 \mathrm{E}+00$ \\
\hline ENSBTAG00000008367 & $1.50 \mathrm{E}-10$ & $6.68 \mathrm{E}-11$ & LIX1L & $2.24 \mathrm{E}+00$ \\
\hline ENSBTAG00000006099 & $2.60 \mathrm{E}-10$ & $1.16 \mathrm{E}-10$ & PIP5K1A & $2.24 E+00$ \\
\hline ENSBTAG00000010009 & $3.55 \mathrm{E}-10$ & $1.59 \mathrm{E}-10$ & TMCO1 & $2.23 \mathrm{E}+00$ \\
\hline ENSBTAG00000038233 & $5.98 \mathrm{E}-10$ & $2.66 \mathrm{E}-10$ & & $2.25 \mathrm{E}+00$ \\
\hline ENSBTAG00000034147 & $3.25 \mathrm{E}-10$ & $1.45 \mathrm{E}-10$ & OLFML2B & $2.24 \mathrm{E}+00$ \\
\hline ENSBTAG00000017419 & 3.30E-10 & $1.47 \mathrm{E}-10$ & ZZZ3 & $2.25 E+00$ \\
\hline ENSBTAG00000015345 & $1.99 \mathrm{E}-10$ & 8.76E-11 & BNIPL & $2.27 \mathrm{E}+00$ \\
\hline ENSBTAG00000010670 & $4.36 \mathrm{E}-10$ & $1.92 \mathrm{E}-10$ & VSIG8 & $2.27 E+00$ \\
\hline ENSBTAG00000038844 & $1.13 \mathrm{E}-10$ & 4.97E-11 & ANKRD35 & $2.28 \mathrm{E}+00$ \\
\hline ENSBTAG00000019131 & $2.41 \mathrm{E}-10$ & $1.06 \mathrm{E}-10$ & CELF3 & $2.28 \mathrm{E}+00$ \\
\hline ENSBTAG00000046545 & $1.68 \mathrm{E}-10$ & $7.35 \mathrm{E}-11$ & MEF2D & $2.28 \mathrm{E}+00$ \\
\hline ENSBTAG00000000504 & $5.36 \mathrm{E}-10$ & $2.33 \mathrm{E}-10$ & GTF2B & $2.30 \mathrm{E}+00$ \\
\hline ENSBTAG00000016694 & $1.36 \mathrm{E}-10$ & $5.83 \mathrm{E}-11$ & KIAA0319L & $2.33 \mathrm{E}+00$ \\
\hline ENSBTAG00000019011 & $3.20 \mathrm{E}-10$ & $1.37 \mathrm{E}-10$ & PGM1 & $2.33 E+00$ \\
\hline ENSBTAG00000032733 & $5.61 \mathrm{E}-10$ & $2.39 \mathrm{E}-10$ & RIIAD1 & $2.35 \mathrm{E}+00$ \\
\hline ENSBTAG00000015887 & $2.95 \mathrm{E}-10$ & $1.26 \mathrm{E}-10$ & FOXJ3 & $2.34 \mathrm{E}+00$ \\
\hline ENSBTAG00000006510 & $1.23 \mathrm{E}-10$ & $5.18 \mathrm{E}-11$ & YRDC & $2.38 \mathrm{E}+00$ \\
\hline ENSBTAG00000008372 & 4.41E-11 & $1.85 \mathrm{E}-11$ & PEX11B & $2.37 \mathrm{E}+00$ \\
\hline ENSBTAG00000008501 & $1.70 \mathrm{E}-10$ & 7.13E-11 & FOXD3 & $2.38 \mathrm{E}+00$ \\
\hline ENSBTAG00000033217 & $1.35 \mathrm{E}-10$ & $5.65 \mathrm{E}-11$ & TPM1 & $2.38 \mathrm{E}+00$ \\
\hline ENSBTAG00000020153 & $8.44 \mathrm{E}-11$ & $3.54 \mathrm{E}-11$ & NIT1 & $2.38 \mathrm{E}+00$ \\
\hline ENSBTAG00000021634 & $7.85 \mathrm{E}-11$ & $3.29 \mathrm{E}-11$ & PIAS3 & $2.38 \mathrm{E}+00$ \\
\hline ENSBTAG00000015346 & $2.64 \mathrm{E}-10$ & $1.11 \mathrm{E}-10$ & NASP & $2.37 E+00$ \\
\hline ENSBTAG00000031088 & $4.05 \mathrm{E}-10$ & $1.70 \mathrm{E}-10$ & & $2.38 \mathrm{E}+00$ \\
\hline ENSBTAG00000017031 & 4.57E-10 & $1.92 \mathrm{E}-10$ & & $2.38 \mathrm{E}+00$ \\
\hline ENSBTAG00000017029 & $2.59 \mathrm{E}-10$ & $1.09 \mathrm{E}-10$ & & $2.38 \mathrm{E}+00$ \\
\hline ENSBTAG00000017873 & $2.46 \mathrm{E}-10$ & $1.03 \mathrm{E}-10$ & USF1 & $2.39 E+00$ \\
\hline ENSBTAG00000032452 & $5.96 \mathrm{E}-10$ & $2.50 \mathrm{E}-10$ & HIST2H3A & $2.38 \mathrm{E}+00$ \\
\hline ENSBTAG00000020169 & $1.82 \mathrm{E}-10$ & $7.65 \mathrm{E}-11$ & CEPT1 & $2.38 \mathrm{E}+00$ \\
\hline ENSBTAG00000034601 & $5.25 \mathrm{E}-10$ & $2.20 \mathrm{E}-10$ & OR6N1 & $2.39 E+00$ \\
\hline ENSBTAG00000012640 & $5.03 \mathrm{E}-10$ & $2.11 \mathrm{E}-10$ & S100A8 & $2.38 \mathrm{E}+00$ \\
\hline ENSBTAG00000038894 & $7.58 \mathrm{E}-10$ & $3.17 \mathrm{E}-10$ & OR6Y1 & $2.39 E+00$ \\
\hline ENSBTAG00000040217 & $1.05 \mathrm{E}-09$ & $4.40 \mathrm{E}-10$ & & $2.39 E+00$ \\
\hline ENSBTAG00000008022 & $5.27 \mathrm{E}-10$ & $2.19 \mathrm{E}-10$ & PTGFRN & $2.41 E+00$ \\
\hline ENSBTAG00000020510 & $2.44 \mathrm{E}-10$ & $1.02 \mathrm{E}-10$ & EPHA10 & $2.39 E+00$ \\
\hline ENSBTAG00000011534 & $6.42 \mathrm{E}-10$ & $2.66 \mathrm{E}-10$ & RAMP1 & $2.41 E+00$ \\
\hline ENSBTAG00000013693 & $8.99 \mathrm{E}-10$ & $3.72 \mathrm{E}-10$ & & $2.42 \mathrm{E}+00$ \\
\hline ENSBTAG00000000634 & $8.66 \mathrm{E}-10$ & $3.58 \mathrm{E}-10$ & MLPH & $2.42 \mathrm{E}+00$ \\
\hline ENSBTAG00000002377 & $4.62 \mathrm{E}-10$ & $1.91 \mathrm{E}-10$ & PSMB2 & $2.42 \mathrm{E}+00$ \\
\hline ENSBTAG00000006370 & $3.61 \mathrm{E}-10$ & $1.49 \mathrm{E}-10$ & ССТ3 & $2.42 E+00$ \\
\hline
\end{tabular}




\begin{tabular}{|c|c|c|c|c|}
\hline ENSBTAG00000008243 & $3.19 \mathrm{E}-10$ & $1.31 \mathrm{E}-10$ & LRRC40 & $2.43 \mathrm{E}+00$ \\
\hline ENSBTAG00000008690 & $3.11 \mathrm{E}-10$ & $1.27 \mathrm{E}-10$ & & $2.45 E+00$ \\
\hline ENSBTAG00000009698 & $5.59 \mathrm{E}-10$ & $2.29 \mathrm{E}-10$ & $A B C D 3$ & $2.44 E+00$ \\
\hline ENSBTAG00000030932 & $6.26 \mathrm{E}-10$ & $2.55 \mathrm{E}-10$ & IFI44L & $2.45 E+00$ \\
\hline ENSBTAG00000014291 & $1.91 \mathrm{E}-10$ & 7.79E-11 & WNT2B & $2.46 \mathrm{E}+00$ \\
\hline ENSBTAG00000002627 & $1.63 \mathrm{E}-10$ & $6.62 \mathrm{E}-11$ & ZRANB2 & $2.46 \mathrm{E}+00$ \\
\hline ENSBTAG00000019617 & 4.25E-10 & $1.72 \mathrm{E}-10$ & PTPN22 & $2.47 \mathrm{E}+00$ \\
\hline ENSBTAG00000047988 & $5.30 \mathrm{E}-10$ & $2.12 \mathrm{E}-10$ & & $2.50 \mathrm{E}+00$ \\
\hline ENSBTAG00000006234 & $1.33 \mathrm{E}-10$ & $5.34 \mathrm{E}-11$ & NPR1 & $2.49 E+00$ \\
\hline ENSBTAG00000001143 & 7.47E-10 & $2.98 \mathrm{E}-10$ & & $2.51 E+00$ \\
\hline ENSBTAG00000046771 & $4.48 \mathrm{E}-10$ & $1.78 \mathrm{E}-10$ & FNDC7 & $2.52 E+00$ \\
\hline ENSBTAG00000002608 & $5.41 \mathrm{E}-10$ & $2.14 \mathrm{E}-10$ & 02-Sep & $2.53 E+00$ \\
\hline ENSBTAG00000015067 & $5.01 \mathrm{E}-10$ & $1.99 \mathrm{E}-10$ & NHLH2 & $2.52 E+00$ \\
\hline ENSBTAG00000004334 & $1.58 \mathrm{E}-10$ & $6.18 \mathrm{E}-11$ & NCSTN & $2.56 \mathrm{E}+00$ \\
\hline ENSBTAG00000021444 & $3.59 \mathrm{E}-10$ & $1.40 \mathrm{E}-10$ & TWIST2 & $2.56 \mathrm{E}+00$ \\
\hline ENSBTAG00000024137 & $5.01 \mathrm{E}-10$ & $1.95 \mathrm{E}-10$ & PTCH2 & $2.57 E+00$ \\
\hline ENSBTAG00000004772 & 3.30E-10 & $1.28 \mathrm{E}-10$ & THEM4 & $2.58 \mathrm{E}+00$ \\
\hline ENSBTAG00000001212 & $3.25 \mathrm{E}-10$ & $1.26 \mathrm{E}-10$ & SSR2 & $2.58 \mathrm{E}+00$ \\
\hline ENSBTAG00000016307 & $2.03 \mathrm{E}-10$ & $7.82 \mathrm{E}-11$ & $\mathrm{CDC7}$ & $2.59 E+00$ \\
\hline ENSBTAG00000012502 & $1.24 \mathrm{E}-09$ & 4.77E-10 & & $2.60 E+00$ \\
\hline ENSBTAG00000002947 & $3.54 \mathrm{E}-10$ & $1.36 \mathrm{E}-10$ & LY9 & $2.60 \mathrm{E}+00$ \\
\hline ENSBTAG00000012348 & $2.84 \mathrm{E}-10$ & $1.08 \mathrm{E}-10$ & LRRC71 & $2.63 \mathrm{E}+00$ \\
\hline ENSBTAG00000007299 & $2.90 \mathrm{E}-10$ & $1.10 \mathrm{E}-10$ & SF3A3 & $2.64 \mathrm{E}+00$ \\
\hline ENSBTAG00000020856 & $4.27 \mathrm{E}-10$ & $1.62 \mathrm{E}-10$ & HFM1 & $2.64 \mathrm{E}+00$ \\
\hline ENSBTAG00000018888 & $2.31 \mathrm{E}-10$ & $8.71 E-11$ & $\mathrm{DPH} 2$ & $2.65 E+00$ \\
\hline ENSBTAG00000015977 & $1.40 \mathrm{E}-10$ & 5.27E-11 & SIKE1 & $2.64 \mathrm{E}+00$ \\
\hline ENSBTAG00000017856 & $5.64 \mathrm{E}-10$ & $2.12 \mathrm{E}-10$ & TSTD1 & $2.66 \mathrm{E}+00$ \\
\hline ENSBTAG00000037673 & $1.25 \mathrm{E}-10$ & $4.70 \mathrm{E}-11$ & & $2.65 E+00$ \\
\hline ENSBTAG00000011363 & $3.50 \mathrm{E}-10$ & $1.32 \mathrm{E}-10$ & EVI5 & $2.65 E+00$ \\
\hline ENSBTAG00000046885 & $1.05 \mathrm{E}-09$ & $3.95 \mathrm{E}-10$ & RXFP4 & $2.66 \mathrm{E}+00$ \\
\hline ENSBTAG00000006560 & $1.47 \mathrm{E}-10$ & $5.45 \mathrm{E}-11$ & VANGL2 & $2.69 E+00$ \\
\hline ENSBTAG00000002096 & $5.10 \mathrm{E}-10$ & $1.90 \mathrm{E}-10$ & & $2.68 \mathrm{E}+00$ \\
\hline ENSBTAG00000015280 & $2.23 \mathrm{E}-10$ & $8.26 \mathrm{E}-11$ & $\mathrm{KIF} 2 \mathrm{C}$ & $2.70 \mathrm{E}+00$ \\
\hline ENSBTAG00000021922 & $5.01 \mathrm{E}-10$ & $1.84 \mathrm{E}-10$ & SMAP2 & $2.72 \mathrm{E}+00$ \\
\hline ENSBTAG00000006167 & $5.69 \mathrm{E}-10$ & $2.09 \mathrm{E}-10$ & KIAA1107 & $2.72 \mathrm{E}+00$ \\
\hline ENSBTAG00000004315 & $7.30 \mathrm{E}-10$ & $2.67 \mathrm{E}-10$ & HDLBP & $2.73 E+00$ \\
\hline ENSBTAG00000000532 & $9.66 \mathrm{E}-10$ & $3.51 \mathrm{E}-10$ & EXO5 & $2.75 \mathrm{E}+00$ \\
\hline ENSBTAG00000008091 & $1.31 \mathrm{E}-10$ & 4.70E-11 & SELENBP1 & $2.78 \mathrm{E}+00$ \\
\hline ENSBTAG00000021752 & $1.97 \mathrm{E}-10$ & $7.08 \mathrm{E}-11$ & DNAJB4 & $2.78 \mathrm{E}+00$ \\
\hline ENSBTAG00000014694 & $2.34 \mathrm{E}-10$ & $8.41 E-11$ & JTB & $2.78 \mathrm{E}+00$ \\
\hline ENSBTAG00000010175 & $2.11 \mathrm{E}-10$ & $7.60 \mathrm{E}-11$ & COL8A2 & $2.78 \mathrm{E}+00$ \\
\hline ENSBTAG00000003642 & $3.74 \mathrm{E}-10$ & $1.34 \mathrm{E}-10$ & PRDX1 & $2.79 E+00$ \\
\hline ENSBTAG00000015369 & $1.13 \mathrm{E}-10$ & 4.07E-11 & MLLT11 & $2.77 E+00$ \\
\hline ENSBTAG00000011091 & $3.01 \mathrm{E}-10$ & $1.08 \mathrm{E}-10$ & SGIP1 & $2.79 E+00$ \\
\hline ENSBTAG00000011241 & $3.48 \mathrm{E}-10$ & $1.25 \mathrm{E}-10$ & HPDL & $2.78 \mathrm{E}+00$ \\
\hline ENSBTAG00000032997 & $8.89 \mathrm{E}-10$ & $3.18 \mathrm{E}-10$ & & $2.79 E+00$ \\
\hline ENSBTAG00000016982 & $7.13 \mathrm{E}-10$ & $2.55 \mathrm{E}-10$ & MCOLN3 & $2.79 E+00$ \\
\hline ENSBTAG00000002068 & $4.10 \mathrm{E}-10$ & $1.45 \mathrm{E}-10$ & TAGLN2 & $2.83 E+00$ \\
\hline ENSBTAG00000005976 & $8.02 \mathrm{E}-10$ & $2.84 \mathrm{E}-10$ & HSD17B7 & $2.82 \mathrm{E}+00$ \\
\hline ENSBTAG00000037858 & $1.36 \mathrm{E}-10$ & $4.78 \mathrm{E}-11$ & & $2.83 E+00$ \\
\hline ENSBTAG00000003593 & $1.70 \mathrm{E}-10$ & $5.95 \mathrm{E}-11$ & ANKMY1 & $2.86 \mathrm{E}+00$ \\
\hline ENSBTAG00000002951 & $2.24 \mathrm{E}-10$ & $7.81 \mathrm{E}-11$ & $\mathrm{CD} 244$ & $2.86 \mathrm{E}+00$ \\
\hline ENSBTAG00000021537 & $4.25 \mathrm{E}-10$ & $1.48 \mathrm{E}-10$ & RWDD3 & $2.87 \mathrm{E}+00$ \\
\hline ENSBTAG00000021705 & $2.44 \mathrm{E}-10$ & 8.37E-11 & UFC1 & $2.91 E+00$ \\
\hline ENSBTAG00000012982 & $2.43 \mathrm{E}-10$ & $8.33 \mathrm{E}-11$ & PPCS & $2.91 E+00$ \\
\hline ENSBTAG00000047776 & $2.64 \mathrm{E}-10$ & $9.05 \mathrm{E}-11$ & & $2.91 E+00$ \\
\hline ENSBTAG00000007318 & $1.83 \mathrm{E}-10$ & $6.30 \mathrm{E}-11$ & ZNF687 & $2.91 \mathrm{E}+00$ \\
\hline ENSBTAG00000012638 & $6.27 \mathrm{E}-10$ & $2.15 E-10$ & $\mathrm{~S} 100 \mathrm{~A} 12$ & $2.92 \mathrm{E}+00$ \\
\hline ENSBTAG00000003060 & $2.67 \mathrm{E}-10$ & $9.11 \mathrm{E}-11$ & NTRK1 & $2.93 E+00$ \\
\hline ENSBTAG00000018747 & $1.39 \mathrm{E}-10$ & $4.74 \mathrm{E}-11$ & PRKAA2 & $2.93 E+00$ \\
\hline ENSBTAG00000019611 & $3.41 \mathrm{E}-10$ & $1.16 \mathrm{E}-10$ & MAGI3 & $2.94 \mathrm{E}+00$ \\
\hline ENSBTAG00000009432 & $4.34 \mathrm{E}-10$ & 1.47E-10 & PMF1 & $2.95 E+00$ \\
\hline ENSBTAG00000047685 & $4.78 \mathrm{E}-10$ & $1.62 \mathrm{E}-10$ & & $2.95 E+00$ \\
\hline ENSBTAG00000012648 & $2.69 \mathrm{E}-10$ & $9.05 \mathrm{E}-11$ & ZSWIM5 & $2.97 E+00$ \\
\hline ENSBTAG00000014382 & $2.78 \mathrm{E}-10$ & 9.37E-11 & & $2.97 \mathrm{E}+00$ \\
\hline ENSBTAG00000018737 & $2.54 \mathrm{E}-10$ & $8.51 E-11$ & GLMN & $2.98 \mathrm{E}+00$ \\
\hline ENSBTAG00000012471 & $5.86 \mathrm{E}-10$ & $1.96 \mathrm{E}-10$ & ILF2 & $2.99 \mathrm{E}+00$ \\
\hline ENSBTAG00000013017 & $1.31 \mathrm{E}-10$ & 4.40E-11 & GNAT2 & $2.97 E+00$ \\
\hline ENSBTAG00000021943 & $3.32 \mathrm{E}-10$ & $1.10 \mathrm{E}-10$ & NFYC & $3.02 E+00$ \\
\hline ENSBTAG00000017135 & $2.58 \mathrm{E}-10$ & $8.55 \mathrm{E}-11$ & CTSS & $3.01 E+00$ \\
\hline
\end{tabular}




\begin{tabular}{|c|c|c|c|c|}
\hline ENSBTAG00000020026 & $1.85 \mathrm{E}-10$ & $6.13 \mathrm{E}-11$ & & $3.02 E+00$ \\
\hline ENSBTAG00000008921 & $3.21 \mathrm{E}-10$ & $1.05 \mathrm{E}-10$ & NEXN & $3.05 E+00$ \\
\hline ENSBTAG00000013789 & $1.67 \mathrm{E}-09$ & $5.46 \mathrm{E}-10$ & OR6K6 & $3.05 E+00$ \\
\hline ENSBTAG00000013869 & $8.71 \mathrm{E}-10$ & $2.82 \mathrm{E}-10$ & SH3BP4 & $3.09 E+00$ \\
\hline ENSBTAG00000002910 & $5.60 \mathrm{E}-10$ & $1.80 \mathrm{E}-10$ & ECHDC2 & $3.11 \mathrm{E}+00$ \\
\hline ENSBTAG00000011511 & $2.88 \mathrm{E}-10$ & $9.14 \mathrm{E}-11$ & & $3.15 E+00$ \\
\hline ENSBTAG00000047351 & 1.67E-10 & $5.25 \mathrm{E}-11$ & & $3.18 \mathrm{E}+00$ \\
\hline ENSBTAG00000024476 & $9.36 \mathrm{E}-11$ & $2.94 \mathrm{E}-11$ & CKS1B & $3.17 E+00$ \\
\hline ENSBTAG00000048212 & $3.79 \mathrm{E}-10$ & 1.19E-10 & & $3.19 E+00$ \\
\hline ENSBTAG00000021714 & $4.48 \mathrm{E}-10$ & $1.41 \mathrm{E}-10$ & TEKT2 & $3.17 E+00$ \\
\hline ENSBTAG00000045889 & $1.28 \mathrm{E}-10$ & $4.03 \mathrm{E}-11$ & & $3.17 E+00$ \\
\hline ENSBTAG00000000608 & $3.32 \mathrm{E}-10$ & $1.04 \mathrm{E}-10$ & PRCC & $3.19 E+00$ \\
\hline ENSBTAG00000023247 & $6.80 \mathrm{E}-10$ & $2.13 \mathrm{E}-10$ & & $3.19 E+00$ \\
\hline ENSBTAG00000017615 & $1.29 \mathrm{E}-09$ & 4.03E-10 & PRLH & $3.21 E+00$ \\
\hline ENSBTAG00000020214 & $9.83 \mathrm{E}-10$ & $3.02 \mathrm{E}-10$ & LRRC8B & $3.25 E+00$ \\
\hline ENSBTAG00000015408 & $3.94 \mathrm{E}-10$ & $1.21 \mathrm{E}-10$ & DTYMK & $3.26 \mathrm{E}+00$ \\
\hline ENSBTAG00000003724 & $2.60 \mathrm{E}-10$ & 7.91E-11 & & $3.28 \mathrm{E}+00$ \\
\hline ENSBTAG00000038938 & $1.42 \mathrm{E}-09$ & $4.31 \mathrm{E}-10$ & & $3.30 \mathrm{E}+00$ \\
\hline ENSBTAG00000020395 & $2.41 \mathrm{E}-10$ & $7.26 \mathrm{E}-11$ & LYSMD1 & $3.31 E+00$ \\
\hline ENSBTAG00000016723 & $2.49 \mathrm{E}-10$ & $7.51 \mathrm{E}-11$ & GFI1 & $3.31 E+00$ \\
\hline ENSBTAG00000018281 & 2.24E-10 & $6.70 \mathrm{E}-11$ & GPX7 & $3.34 \mathrm{E}+00$ \\
\hline ENSBTAG00000003217 & $6.25 \mathrm{E}-10$ & $1.87 \mathrm{E}-10$ & CADM3 & $3.34 \mathrm{E}+00$ \\
\hline ENSBTAG00000006364 & $4.90 \mathrm{E}-10$ & $1.45 \mathrm{E}-10$ & GLMP & $3.38 \mathrm{E}+00$ \\
\hline ENSBTAG00000030208 & $8.92 \mathrm{E}-10$ & $2.58 \mathrm{E}-10$ & ERFE & $3.46 E+00$ \\
\hline ENSBTAG00000012694 & $1.01 \mathrm{E}-09$ & $2.87 \mathrm{E}-10$ & UHMK1 & $3.53 E+00$ \\
\hline ENSBTAG00000013054 & $5.13 \mathrm{E}-10$ & $1.45 \mathrm{E}-10$ & MFSD2A & $3.53 E+00$ \\
\hline ENSBTAG00000017656 & $5.98 \mathrm{E}-10$ & $1.69 \mathrm{E}-10$ & & $3.54 \mathrm{E}+00$ \\
\hline ENSBTAG00000014204 & $2.14 \mathrm{E}-10$ & 5.97E-11 & S100A16 & $3.57 E+00$ \\
\hline ENSBTAG00000006295 & $1.41 \mathrm{E}-10$ & $3.94 \mathrm{E}-11$ & TSHB & $3.57 E+00$ \\
\hline ENSBTAG00000000094 & 7.39E-10 & $2.06 \mathrm{E}-10$ & ATPAF1 & $3.58 \mathrm{E}+00$ \\
\hline ENSBTAG00000045970 & $5.98 \mathrm{E}-10$ & 1.67E-10 & & $3.58 \mathrm{E}+00$ \\
\hline ENSBTAG00000040248 & $4.76 \mathrm{E}-10$ & $1.33 \mathrm{E}-10$ & & $3.58 \mathrm{E}+00$ \\
\hline ENSBTAG00000010445 & $5.99 \mathrm{E}-10$ & $1.66 \mathrm{E}-10$ & DNASE2B & $3.61 E+00$ \\
\hline ENSBTAG00000007515 & $3.19 \mathrm{E}-10$ & 8.77E-11 & UBE2Q1 & $3.63 E+00$ \\
\hline ENSBTAG00000008926 & $2.84 \mathrm{E}-10$ & $7.74 \mathrm{E}-11$ & FUBP1 & $3.67 E+00$ \\
\hline ENSBTAG00000000123 & 2.32E-10 & $6.29 \mathrm{E}-11$ & $\mathrm{HAO} 2$ & $3.68 \mathrm{E}+00$ \\
\hline ENSBTAG00000034841 & $1.40 \mathrm{E}-09$ & $3.79 \mathrm{E}-10$ & & $3.69 E+00$ \\
\hline ENSBTAG00000002306 & $3.86 \mathrm{E}-10$ & $1.04 \mathrm{E}-10$ & UTP11 & $3.71 E+00$ \\
\hline ENSBTAG00000000785 & $6.27 \mathrm{E}-10$ & 1.69E-10 & PASK & $3.71 \mathrm{E}+00$ \\
\hline ENSBTAG00000012887 & 5.57E-10 & $1.50 \mathrm{E}-10$ & FCER1A & $3.71 E+00$ \\
\hline ENSBTAG00000007320 & $2.02 \mathrm{E}-10$ & $5.39 \mathrm{E}-11$ & PI4KB & $3.75 \mathrm{E}+00$ \\
\hline ENSBTAG00000024470 & $1.99 \mathrm{E}-10$ & $5.21 \mathrm{E}-11$ & $\mathrm{SH} 2 \mathrm{D} 2 \mathrm{~A}$ & $3.81 E+00$ \\
\hline ENSBTAG00000000497 & $7.23 \mathrm{E}-10$ & $1.85 \mathrm{E}-10$ & AKR1A1 & $3.91 E+00$ \\
\hline ENSBTAG00000000097 & $6.45 \mathrm{E}-10$ & $1.64 \mathrm{E}-10$ & EFCAB14 & $3.93 E+00$ \\
\hline ENSBTAG00000008373 & $7.86 \mathrm{E}-11$ & $1.98 \mathrm{E}-11$ & ITGA10 & $3.96 \mathrm{E}+00$ \\
\hline ENSBTAG00000019698 & $3.11 \mathrm{E}-10$ & 7.82E-11 & TMCO2 & $3.98 \mathrm{E}+00$ \\
\hline ENSBTAG00000022528 & $4.25 \mathrm{E}-10$ & $1.07 \mathrm{E}-10$ & & $3.97 \mathrm{E}+00$ \\
\hline ENSBTAG00000023748 & 4.35E-10 & $1.09 \mathrm{E}-10$ & & $3.99 E+00$ \\
\hline ENSBTAG00000032187 & $4.43 \mathrm{E}-10$ & $1.11 \mathrm{E}-10$ & ZNF697 & $3.99 E+00$ \\
\hline ENSBTAG00000024096 & $6.70 \mathrm{E}-10$ & $1.68 \mathrm{E}-10$ & LSM10 & $3.99 E+00$ \\
\hline ENSBTAG00000003777 & $4.88 \mathrm{E}-10$ & $1.22 \mathrm{E}-10$ & TIE1 & $4.00 E+00$ \\
\hline ENSBTAG00000002050 & $2.96 \mathrm{E}-10$ & 7.34E-11 & COA7 & $4.03 E+00$ \\
\hline ENSBTAG00000015220 & 3.27E-10 & $8.08 \mathrm{E}-11$ & AGO4 & $4.05 E+00$ \\
\hline ENSBTAG00000020451 & $2.26 \mathrm{E}-10$ & $5.50 \mathrm{E}-11$ & USP1 & $4.10 E+00$ \\
\hline ENSBTAG00000016899 & $4.90 \mathrm{E}-10$ & 1.19E-10 & MED8 & $4.11 E+00$ \\
\hline ENSBTAG00000006373 & $3.15 \mathrm{E}-10$ & $7.61 \mathrm{E}-11$ & TSACC & $4.13 E+00$ \\
\hline ENSBTAG00000019540 & $6.10 \mathrm{E}-10$ & 1.47E-10 & CRNN & $4.15 E+00$ \\
\hline ENSBTAG00000014325 & $2.08 \mathrm{E}-10$ & 5.00E-11 & C1orf109 & $4.16 \mathrm{E}+00$ \\
\hline ENSBTAG00000009526 & $5.50 \mathrm{E}-10$ & $1.31 \mathrm{E}-10$ & TMEM59 & $4.20 E+00$ \\
\hline ENSBTAG00000009435 & $2.99 \mathrm{E}-10$ & $7.08 \mathrm{E}-11$ & SMG5 & $4.23 E+00$ \\
\hline ENSBTAG00000016455 & $4.48 \mathrm{E}-10$ & $1.05 \mathrm{E}-10$ & CACHD1 & $4.26 \mathrm{E}+00$ \\
\hline ENSBTAG00000016377 & $5.06 \mathrm{E}-10$ & 1.17E-10 & IPP & $4.32 \mathrm{E}+00$ \\
\hline ENSBTAG00000017256 & $3.40 \mathrm{E}-10$ & $7.84 \mathrm{E}-11$ & CD2 & $4.33 E+00$ \\
\hline ENSBTAG00000017624 & 4.70E-10 & $1.08 \mathrm{E}-10$ & RAB17 & $4.35 E+00$ \\
\hline ENSBTAG00000021894 & $2.44 \mathrm{E}-10$ & 5.57E-11 & PPOX & $4.37 E+00$ \\
\hline ENSBTAG00000046812 & $5.11 \mathrm{E}-10$ & 1.17E-10 & TEX38 & $4.36 \mathrm{E}+00$ \\
\hline ENSBTAG00000030193 & $6.75 \mathrm{E}-10$ & $1.54 \mathrm{E}-10$ & GPR35 & $4.38 \mathrm{E}+00$ \\
\hline ENSBTAG00000039281 & 9.64E-10 & 2.19E-10 & & $4.40 E+00$ \\
\hline ENSBTAG00000033423 & $2.63 \mathrm{E}-10$ & $5.91 \mathrm{E}-11$ & & $4.45 \mathrm{E}+00$ \\
\hline ENSBTAG00000012414 & $5.56 \mathrm{E}-10$ & $1.24 \mathrm{E}-10$ & & $4.48 \mathrm{E}+00$ \\
\hline
\end{tabular}




\begin{tabular}{|c|c|c|c|c|}
\hline ENSBTAG00000026114 & $6.10 \mathrm{E}-10$ & $1.36 \mathrm{E}-10$ & & $4.48 \mathrm{E}+00$ \\
\hline ENSBTAG00000007300 & $1.83 \mathrm{E}-10$ & 4.06E-11 & FHL3 & $4.50 \mathrm{E}+00$ \\
\hline ENSBTAG00000006903 & $6.13 \mathrm{E}-10$ & $1.36 \mathrm{E}-10$ & & $4.51 E+00$ \\
\hline ENSBTAG00000015285 & $6.62 \mathrm{E}-10$ & $1.47 \mathrm{E}-10$ & RPS8 & $4.50 \mathrm{E}+00$ \\
\hline ENSBTAG00000005805 & $6.88 \mathrm{E}-10$ & $1.51 \mathrm{E}-10$ & EPHX4 & $4.55 \mathrm{E}+00$ \\
\hline ENSBTAG00000017368 & $2.72 \mathrm{E}-10$ & $5.96 \mathrm{E}-11$ & YBX1 & $4.57 \mathrm{E}+00$ \\
\hline ENSBTAG00000021386 & 5.10E-10 & $1.10 \mathrm{E}-10$ & ANGPTL3 & $4.64 E+00$ \\
\hline ENSBTAG00000004271 & $1.68 \mathrm{E}-10$ & 3.60E-11 & UBAP2L & $4.65 E+00$ \\
\hline ENSBTAG00000018487 & $5.97 \mathrm{E}-10$ & $1.27 \mathrm{E}-10$ & CFAP45 & $4.70 E+00$ \\
\hline ENSBTAG00000000789 & $5.18 \mathrm{E}-10$ & $1.09 \mathrm{E}-10$ & PPP1R7 & $4.75 E+00$ \\
\hline ENSBTAG00000014466 & 4.14E-10 & $8.72 \mathrm{E}-11$ & VTCN1 & $4.74 \mathrm{E}+00$ \\
\hline ENSBTAG00000006678 & $1.41 \mathrm{E}-10$ & $2.96 \mathrm{E}-11$ & GATAD2B & $4.76 \mathrm{E}+00$ \\
\hline ENSBTAG00000018070 & $1.93 \mathrm{E}-10$ & 4.05E-11 & SCNM1 & $4.76 \mathrm{E}+00$ \\
\hline ENSBTAG00000009436 & 4.07E-10 & 8.53E-11 & TMEM79 & $4.77 E+00$ \\
\hline ENSBTAG00000015349 & $2.29 \mathrm{E}-10$ & 4.81E-11 & Clorf56 & $4.76 E+00$ \\
\hline ENSBTAG00000021079 & $4.85 \mathrm{E}-10$ & $1.02 \mathrm{E}-10$ & C1orf210 & $4.76 \mathrm{E}+00$ \\
\hline ENSBTAG00000046792 & $1.02 \mathrm{E}-09$ & $2.13 \mathrm{E}-10$ & & $4.80 E+00$ \\
\hline ENSBTAG00000013363 & $6.31 \mathrm{E}-10$ & $1.31 \mathrm{E}-10$ & CAP1 & $4.81 E+00$ \\
\hline ENSBTAG00000011881 & $4.58 \mathrm{E}-10$ & $9.20 \mathrm{E}-11$ & TDRD10 & $4.98 \mathrm{E}+00$ \\
\hline ENSBTAG00000014692 & $3.13 \mathrm{E}-10$ & $6.21 \mathrm{E}-11$ & CREB3L4 & $5.03 E+00$ \\
\hline ENSBTAG00000021893 & 2.53E-10 & $5.02 \mathrm{E}-11$ & USP21 & $5.03 E+00$ \\
\hline ENSBTAG00000006125 & 2.19E-10 & 4.23E-11 & PLK3 & $5.16 \mathrm{E}+00$ \\
\hline ENSBTAG00000015363 & $2.80 \mathrm{E}-10$ & $5.41 \mathrm{E}-11$ & CDC42SE1 & $5.16 \mathrm{E}+00$ \\
\hline ENSBTAG00000048081 & $4.46 \mathrm{E}-10$ & $8.62 \mathrm{E}-11$ & TOMM20 & $5.17 E+00$ \\
\hline ENSBTAG00000021035 & $4.15 \mathrm{E}-10$ & $8.02 \mathrm{E}-11$ & CTSK & $5.17 \mathrm{E}+00$ \\
\hline ENSBTAG00000008810 & $5.52 \mathrm{E}-10$ & $1.05 \mathrm{E}-10$ & & $5.26 \mathrm{E}+00$ \\
\hline ENSBTAG00000005973 & $8.14 \mathrm{E}-10$ & $1.47 \mathrm{E}-10$ & & $5.54 \mathrm{E}+00$ \\
\hline ENSBTAG00000017027 & $1.15 \mathrm{E}-10$ & $2.06 \mathrm{E}-11$ & MMACHC & $5.54 \mathrm{E}+00$ \\
\hline ENSBTAG00000010673 & 4.19E-10 & $7.52 \mathrm{E}-11$ & EFNA4 & $5.57 E+00$ \\
\hline ENSBTAG00000008808 & 4.34E-10 & 7.79E-11 & CCDC17 & $5.57 \mathrm{E}+00$ \\
\hline ENSBTAG00000007724 & $2.28 \mathrm{E}-10$ & 4.10E-11 & & $5.55 \mathrm{E}+00$ \\
\hline ENSBTAG00000039529 & $5.30 \mathrm{E}-10$ & $9.50 \mathrm{E}-11$ & BTBD19 & $5.57 E+00$ \\
\hline ENSBTAG00000010174 & $8.35 \mathrm{E}-10$ & $1.49 \mathrm{E}-10$ & C1orf111 & $5.60 \mathrm{E}+00$ \\
\hline ENSBTAG00000046632 & $1.15 \mathrm{E}-09$ & $2.05 \mathrm{E}-10$ & & $5.61 E+00$ \\
\hline ENSBTAG00000010206 & $7.52 \mathrm{E}-10$ & $1.33 \mathrm{E}-10$ & UAP1 & $5.65 E+00$ \\
\hline ENSBTAG00000007123 & $3.41 \mathrm{E}-10$ & $5.83 \mathrm{E}-11$ & ENSA & $5.83 \mathrm{E}+00$ \\
\hline ENSBTAG00000000690 & $5.51 \mathrm{E}-10$ & $9.42 \mathrm{E}-11$ & THEM5 & $5.84 \mathrm{E}+00$ \\
\hline ENSBTAG00000011238 & $2.61 \mathrm{E}-10$ & 4.40E-11 & CD48 & $5.92 \mathrm{E}+00$ \\
\hline ENSBTAG00000005443 & $2.08 \mathrm{E}-10$ & $3.50 \mathrm{E}-11$ & MIER1 & $5.93 E+00$ \\
\hline ENSBTAG00000003440 & $7.95 \mathrm{E}-10$ & $1.33 \mathrm{E}-10$ & & $5.98 \mathrm{E}+00$ \\
\hline ENSBTAG00000004715 & $4.66 \mathrm{E}-10$ & 7.63E-11 & AKIRIN1 & $6.10 \mathrm{E}+00$ \\
\hline ENSBTAG00000038523 & $3.14 \mathrm{E}-10$ & $5.13 \mathrm{E}-11$ & ATP1A4 & $6.11 E+00$ \\
\hline ENSBTAG00000019838 & $1.88 \mathrm{E}-10$ & 2.96E-11 & $\mathrm{SHCl}$ & $6.33 E+00$ \\
\hline ENSBTAG00000024503 & $2.03 \mathrm{E}-10$ & 3.19E-11 & FCER1G & $6.34 \mathrm{E}+00$ \\
\hline ENSBTAG00000046720 & $3.98 \mathrm{E}-10$ & $6.26 \mathrm{E}-11$ & & $6.36 \mathrm{E}+00$ \\
\hline ENSBTAG00000002724 & $5.30 \mathrm{E}-10$ & $8.31 \mathrm{E}-11$ & HORMAD1 & $6.37 E+00$ \\
\hline ENSBTAG00000023556 & $7.02 \mathrm{E}-10$ & $1.10 \mathrm{E}-10$ & OR10K2 & $6.37 \mathrm{E}+00$ \\
\hline ENSBTAG00000020345 & 2.67E-10 & 4.10E-11 & & $6.49 E+00$ \\
\hline ENSBTAG00000015401 & $3.80 \mathrm{E}-10$ & $5.83 \mathrm{E}-11$ & ATG4B & $6.52 \mathrm{E}+00$ \\
\hline ENSBTAG00000024960 & $9.03 \mathrm{E}-10$ & $1.38 \mathrm{E}-10$ & & $6.54 \mathrm{E}+00$ \\
\hline ENSBTAG00000002203 & $4.31 \mathrm{E}-10$ & $6.50 \mathrm{E}-11$ & NDUFS2 & $6.63 E+00$ \\
\hline ENSBTAG00000030337 & $2.39 \mathrm{E}-10$ & $3.54 \mathrm{E}-11$ & & $6.73 E+00$ \\
\hline ENSBTAG00000039287 & $5.72 \mathrm{E}-10$ & 8.04E-11 & ZFP69 & $7.10 \mathrm{E}+00$ \\
\hline ENSBTAG00000010458 & $2.19 \mathrm{E}-10$ & 3.07E-11 & & $7.13 E+00$ \\
\hline ENSBTAG00000032995 & $3.12 \mathrm{E}-10$ & 4.36E-11 & LELP1 & $7.14 \mathrm{E}+00$ \\
\hline ENSBTAG00000004776 & $5.25 \mathrm{E}-10$ & 7.32E-11 & DUSP23 & $7.17 \mathrm{E}+00$ \\
\hline ENSBTAG00000021715 & $7.85 \mathrm{E}-10$ & $1.09 \mathrm{E}-10$ & ADPRHL2 & $7.20 \mathrm{E}+00$ \\
\hline ENSBTAG00000019616 & $7.14 \mathrm{E}-10$ & $9.94 \mathrm{E}-11$ & APCS & $7.18 \mathrm{E}+00$ \\
\hline ENSBTAG00000020344 & $6.83 \mathrm{E}-10$ & $9.44 \mathrm{E}-11$ & SLC44A3 & $7.23 E+00$ \\
\hline ENSBTAG00000009431 & $4.00 \mathrm{E}-10$ & $5.51 \mathrm{E}-11$ & SLC25A44 & $7.24 \mathrm{E}+00$ \\
\hline ENSBTAG00000003147 & $5.02 \mathrm{E}-10$ & $6.55 \mathrm{E}-11$ & JAK1 & $7.66 \mathrm{E}+00$ \\
\hline ENSBTAG00000017574 & 4.17E-10 & $5.40 \mathrm{E}-11$ & LMNA & $7.71 E+00$ \\
\hline ENSBTAG00000015288 & $7.84 \mathrm{E}-10$ & $1.01 \mathrm{E}-10$ & BEST4 & $7.76 \mathrm{E}+00$ \\
\hline ENSBTAG00000001197 & $2.66 \mathrm{E}-10$ & $3.22 \mathrm{E}-11$ & SLAMF7 & $8.25 E+00$ \\
\hline ENSBTAG00000014580 & $8.66 \mathrm{E}-10$ & $1.04 \mathrm{E}-10$ & FCRLB & $8.32 \mathrm{E}+00$ \\
\hline ENSBTAG00000046797 & 1.74E-10 & $2.08 \mathrm{E}-11$ & NRAS & $8.33 \mathrm{E}+00$ \\
\hline ENSBTAG00000020767 & $9.85 \mathrm{E}-10$ & $1.16 \mathrm{E}-10$ & & $8.48 \mathrm{E}+00$ \\
\hline ENSBTAG00000046220 & $4.93 \mathrm{E}-10$ & $5.79 \mathrm{E}-11$ & & $8.49 E+00$ \\
\hline ENSBTAG00000010232 & $8.10 \mathrm{E}-10$ & $9.39 \mathrm{E}-11$ & NDUFS5 & $8.62 \mathrm{E}+00$ \\
\hline ENSBTAG00000017580 & $1.84 \mathrm{E}-10$ & $2.10 \mathrm{E}-11$ & RFX5 & $8.72 E+00$ \\
\hline
\end{tabular}




\begin{tabular}{|c|c|c|c|c|}
\hline ENSBTAG00000021823 & $2.42 \mathrm{E}-10$ & $2.77 \mathrm{E}-11$ & HFE2 & $8.72 \mathrm{E}+00$ \\
\hline ENSBTAG00000046175 & $4.26 \mathrm{E}-10$ & $4.87 \mathrm{E}-11$ & & $8.74 E+00$ \\
\hline ENSBTAG00000018324 & 4.16E-10 & $4.65 \mathrm{E}-11$ & AGO3 & $8.92 \mathrm{E}+00$ \\
\hline ENSBTAG00000002826 & $2.59 \mathrm{E}-10$ & $2.74 \mathrm{E}-11$ & CLSPN & $9.41 \mathrm{E}+00$ \\
\hline ENSBTAG00000034349 & $1.99 \mathrm{E}-10$ & $2.09 \mathrm{E}-11$ & IFI44 & $9.48 \mathrm{E}+00$ \\
\hline ENSBTAG00000009617 & $3.17 \mathrm{E}-10$ & $3.29 \mathrm{E}-11$ & SLC2A1 & $9.61 \mathrm{E}+00$ \\
\hline ENSBTAG00000047038 & $6.46 \mathrm{E}-10$ & $6.23 \mathrm{E}-11$ & & $1.04 \mathrm{E}+01$ \\
\hline ENSBTAG00000039260 & $7.24 \mathrm{E}-10$ & $6.98 \mathrm{E}-11$ & RPTN & $1.04 \mathrm{E}+01$ \\
\hline ENSBTAG00000045515 & $9.59 \mathrm{E}-10$ & $9.22 \mathrm{E}-11$ & & $1.04 \mathrm{E}+01$ \\
\hline ENSBTAG00000007194 & $6.82 \mathrm{E}-10$ & $6.33 \mathrm{E}-11$ & GUCA2B & $1.08 \mathrm{E}+01$ \\
\hline ENSBTAG00000020357 & $5.67 \mathrm{E}-10$ & $5.11 \mathrm{E}-11$ & GOLPH3L & $1.11 E+01$ \\
\hline ENSBTAG00000004331 & $2.73 E-10$ & $2.46 \mathrm{E}-11$ & PYGO2 & $1.11 \mathrm{E}+01$ \\
\hline ENSBTAG00000005580 & $5.71 \mathrm{E}-10$ & $4.80 \mathrm{E}-11$ & SZT2 & $1.19 \mathrm{E}+01$ \\
\hline ENSBTAG00000012253 & $1.47 \mathrm{E}-10$ & $1.20 \mathrm{E}-11$ & AGO1 & $1.21 E+01$ \\
\hline ENSBTAG00000047443 & $6.76 \mathrm{E}-10$ & $5.49 \mathrm{E}-11$ & & $1.23 \mathrm{E}+01$ \\
\hline ENSBTAG00000005196 & $5.56 \mathrm{E}-10$ & 4.13E-11 & TYW3 & $1.34 \mathrm{E}+01$ \\
\hline ENSBTAG00000018914 & $1.97 \mathrm{E}-10$ & $1.46 \mathrm{E}-11$ & RAB25 & $1.34 \mathrm{E}+01$ \\
\hline ENSBTAG00000006338 & $3.40 \mathrm{E}-10$ & $2.51 \mathrm{E}-11$ & $\mathrm{MYCL}$ & $1.35 E+01$ \\
\hline ENSBTAG00000048022 & $6.36 \mathrm{E}-10$ & 4.19E-11 & & $1.51 E+01$ \\
\hline ENSBTAG00000023986 & $7.46 \mathrm{E}-10$ & $3.81 \mathrm{E}-11$ & & $1.95 \mathrm{E}+01$ \\
\hline ENSBTAG00000005166 & $2.35 \mathrm{E}-10$ & $1.09 \mathrm{E}-11$ & CHTOP & $2.14 \mathrm{E}+01$ \\
\hline ENSBTAG00000005583 & $9.40 \mathrm{E}-10$ & $3.91 \mathrm{E}-11$ & HYI & $2.40 \mathrm{E}+01$ \\
\hline ENSBTAG00000007927 & $2.94 \mathrm{E}-10$ & $1.06 \mathrm{E}-11$ & SLAMF1 & $2.75 \mathrm{E}+01$ \\
\hline ENSBTAG00000009982 & 4.10E-10 & $1.47 \mathrm{E}-11$ & IGSF8 & $2.77 E+01$ \\
\hline ENSBTAG00000004590 & $1.53 \mathrm{E}-10$ & $5.21 \mathrm{E}-12$ & $\mathrm{PPIH}$ & $2.89 \mathrm{E}+01$ \\
\hline ENSBTAG00000010551 & $3.67 \mathrm{E}-10$ & 8.23E-12 & ATP1A2 & $4.41 E+01$ \\
\hline ENSBTAG00000003249 & $1.30 \mathrm{E}-09$ & 0 & & $1.30 \mathrm{E}+04$ \\
\hline ENSBTAG00000020150 & $1.35 \mathrm{E}-10$ & 0 & KLHDC9 & $1.35 E+03$ \\
\hline ENSBTAG00000024507 & $3.20 \mathrm{E}-10$ & 0 & KCNJ9 & $3.20 E+03$ \\
\hline ENSBTAG00000038426 & $9.40 \mathrm{E}-11$ & 0 & & $9.41 E+02$ \\
\hline ENSBTAG00000038366 & 0 & 0 & & $1.00 \mathrm{E}+00$ \\
\hline ENSBTAG00000026180 & $1.21 \mathrm{E}-10$ & 0 & & $1.21 E+03$ \\
\hline ENSBTAG00000009433 & $1.56 \mathrm{E}-10$ & 0 & BGLAP & $1.56 \mathrm{E}+03$ \\
\hline ENSBTAG00000011754 & $2.13 \mathrm{E}-10$ & 0 & LAMTOR2 & $2.13 E+03$ \\
\hline ENSBTAG00000001548 & $2.98 \mathrm{E}-10$ & 0 & UBQLN4 & $2.98 \mathrm{E}+03$ \\
\hline ENSBTAG00000003535 & 0 & 0 & DPM3 & $1.00 \mathrm{E}+00$ \\
\hline ENSBTAG00000004552 & $3.52 \mathrm{E}-10$ & 0 & PBXIP1 & $3.52 E+03$ \\
\hline ENSBTAG00000033220 & $1.78 \mathrm{E}-10$ & 0 & C1orf189 & $1.78 \mathrm{E}+03$ \\
\hline ENSBTAG00000013866 & $1.08 \mathrm{E}-10$ & 0 & & $1.08 \mathrm{E}+03$ \\
\hline ENSBTAG00000005163 & $2.67 \mathrm{E}-10$ & 0 & & $2.68 \mathrm{E}+03$ \\
\hline ENSBTAG00000021378 & $1.58 \mathrm{E}-10$ & 0 & S100A13 & $1.58 \mathrm{E}+03$ \\
\hline ENSBTAG00000021377 & 7.97E-11 & 0 & S100A14 & $7.98 \mathrm{E}+02$ \\
\hline ENSBTAG00000037651 & 7.37E-11 & 0 & S100A2 & $7.38 \mathrm{E}+02$ \\
\hline ENSBTAG00000039105 & $5.94 \mathrm{E}-11$ & 0 & S100A3 & $5.95 E+02$ \\
\hline ENSBTAG00000019203 & $5.57 \mathrm{E}-11$ & 0 & S100A4 & $5.58 \mathrm{E}+02$ \\
\hline ENSBTAG00000000644 & $1.21 \mathrm{E}-10$ & 0 & S100A5 & $1.21 \mathrm{E}+03$ \\
\hline ENSBTAG00000008700 & $6.86 \mathrm{E}-10$ & 0 & & $6.86 E+03$ \\
\hline ENSBTAG00000020394 & $4.67 \mathrm{E}-10$ & 0 & TNFAIP8L2 & $4.67 E+03$ \\
\hline ENSBTAG00000008369 & $1.23 \mathrm{E}-10$ & 0 & RBM8A & $1.23 \mathrm{E}+03$ \\
\hline ENSBTAG00000001873 & $2.35 \mathrm{E}-10$ & 0 & & $2.35 \mathrm{E}+03$ \\
\hline ENSBTAG00000012182 & 0 & 0 & DIRAS3 & $1.00 \mathrm{E}+00$ \\
\hline ENSBTAG00000045643 & $7.78 \mathrm{E}-11$ & 0 & FOXD2 & $7.79 \mathrm{E}+02$ \\
\hline ENSBTAG00000007195 & $2.05 \mathrm{E}-10$ & 0 & GUCA2A & $2.05 E+03$ \\
\hline ENSBTAG00000045776 & $1.54 \mathrm{E}-10$ & 0 & C1orf122 & $1.54 \mathrm{E}+03$ \\
\hline ENSBTAG00000030239 & $1.76 \mathrm{E}-10$ & 0 & & $1.76 \mathrm{E}+03$ \\
\hline ENSBTAG00000008898 & $3.90 \mathrm{E}-11$ & 0 & GBX2 & $3.91 E+02$ \\
\hline ENSBTAG00000030221 & $2.08 \mathrm{E}-10$ & 0 & & $2.09 \mathrm{E}+03$ \\
\hline ENSBTAG00000047813 & $1.61 \mathrm{E}-09$ & 0 & & $1.61 E+04$ \\
\hline ENSBTAG00000014833 & $1.72 \mathrm{E}-10$ & 0 & NEU4 & $1.72 \mathrm{E}+03$ \\
\hline ENSBTAG00000045647 & $1.42 \mathrm{E}-10$ & 0 & ARL4C & $1.42 \mathrm{E}+03$ \\
\hline ENSBTAG00000048084 & $9.74 \mathrm{E}-10$ & 0 & & $9.74 E+03$ \\
\hline ENSBTAG00000040382 & $5.93 \mathrm{E}-10$ & 0 & & $5.93 E+03$ \\
\hline ENSBTAG00000046872 & $1.78 \mathrm{E}-10$ & 0 & & $1.78 \mathrm{E}+03$ \\
\hline ENSBTAG00000032057 & 0 & 0 & & $1.00 \mathrm{E}+00$ \\
\hline ENSBTAG00000012555 & $2.98 \mathrm{E}-11$ & 0 & RBM15 & $2.99 \mathrm{E}+02$ \\
\hline ENSBTAG00000046077 & 0 & 0 & & $1.00 \mathrm{E}+00$ \\
\hline ENSBTAG00000040313 & $2.30 \mathrm{E}-10$ & 0 & PARS2 & $2.30 \mathrm{E}+03$ \\
\hline ENSBTAG00000006128 & $3.73 \mathrm{E}-10$ & 0 & TCTEX1D4 & $3.73 E+03$ \\
\hline ENSBTAG00000030338 & $6.41 \mathrm{E}-10$ & 0 & GJA9 & $6.41 E+03$ \\
\hline ENSBTAG00000038292 & $4.69 \mathrm{E}-10$ & 0 & C1orf216 & $4.69 E+03$ \\
\hline
\end{tabular}




\begin{tabular}{|c|c|c|c|c|}
\hline ENSBTAG00000034449 & $1.37 \mathrm{E}-10$ & 0 & & $1.38 \mathrm{E}+03$ \\
\hline ENSBTAG00000039035 & $8.48 \mathrm{E}-11$ & 0 & HSPA6 & $8.49 E+02$ \\
\hline ENSBTAG00000020027 & 0 & 0 & NHLH1 & $1.00 \mathrm{E}+00$ \\
\hline ENSBTAG00000022556 & $6.12 \mathrm{E}-10$ & 0 & & $6.12 \mathrm{E}+03$ \\
\hline ENSBTAG00000045672 & 0 & 0 & & $1.00 \mathrm{E}+00$ \\
\hline ENSBTAG00000018987 & 0 & 0 & & $1.00 \mathrm{E}+00$ \\
\hline ENSBTAG00000032446 & 0 & 0 & & $1.00 \mathrm{E}+00$ \\
\hline ENSBTAG00000038476 & $1.21 \mathrm{E}-09$ & 0 & $\mathrm{HIST} 2 \mathrm{H} 2 \mathrm{AB}$ & $1.21 E+04$ \\
\hline ENSBTAG00000032458 & 0 & 0 & & $1.00 \mathrm{E}+00$ \\
\hline ENSBTAG00000032456 & 0 & 0 & $\mathrm{HIST} 2 \mathrm{H} 2 \mathrm{AC}$ & $1.00 \mathrm{E}+00$ \\
\hline ENSBTAG00000032455 & $4.31 \mathrm{E}-10$ & 0 & & $4.31 \mathrm{E}+03$ \\
\hline ENSBTAG00000046368 & 0 & 0 & & $1.00 \mathrm{E}+00$ \\
\hline ENSBTAG00000008365 & 0 & 0 & ANKRD34A & $1.00 \mathrm{E}+00$ \\
\hline ENSBTAG00000031965 & 0 & 4.40E-10 & TRGV11 & 2.27E-04 \\
\hline ENSBTAG00000047459 & 0 & $6.17 \mathrm{E}-10$ & & $1.62 \mathrm{E}-04$ \\
\hline ENSBTAG00000020004 & $7.35 \mathrm{E}-11$ & $5.63 \mathrm{E}-10$ & ZNF800 & $1.31 \mathrm{E}-01$ \\
\hline ENSBTAG00000007400 & $1.76 \mathrm{E}-10$ & $1.23 \mathrm{E}-09$ & OR2A5 & 1.43E-01 \\
\hline ENSBTAG00000022613 & $2.05 \mathrm{E}-10$ & 7.03E-10 & & $2.91 \mathrm{E}-01$ \\
\hline ENSBTAG00000020448 & $2.32 \mathrm{E}-10$ & $7.52 \mathrm{E}-10$ & ZNF282 & $3.08 \mathrm{E}-01$ \\
\hline ENSBTAG00000017866 & $1.52 \mathrm{E}-10$ & $4.80 \mathrm{E}-10$ & & 3.17E-01 \\
\hline ENSBTAG00000046648 & $4.50 \mathrm{E}-11$ & $1.18 \mathrm{E}-10$ & CPA4 & 3.81E-01 \\
\hline ENSBTAG00000014532 & $9.45 \mathrm{E}-11$ & $2.38 \mathrm{E}-10$ & & 3.97E-01 \\
\hline ENSBTAG00000038030 & $9.35 \mathrm{E}-11$ & $2.36 \mathrm{E}-10$ & & 3.96E-01 \\
\hline ENSBTAG00000038201 & $8.69 \mathrm{E}-11$ & 2.19E-10 & OR2A12 & 3.97E-01 \\
\hline ENSBTAG00000013473 & $5.92 \mathrm{E}-11$ & 1.49E-10 & SSMEM1 & 3.97E-01 \\
\hline ENSBTAG00000004995 & $4.31 \mathrm{E}-10$ & $9.95 \mathrm{E}-10$ & C1GALT1 & 4.33E-01 \\
\hline ENSBTAG00000016766 & $2.49 \mathrm{E}-10$ & $5.23 \mathrm{E}-10$ & TMEM176B & 4.76E-01 \\
\hline ENSBTAG00000001822 & $1.98 \mathrm{E}-10$ & 4.04E-10 & SNX10 & 4.89E-01 \\
\hline ENSBTAG00000031106 & $2.20 \mathrm{E}-10$ & $4.48 \mathrm{E}-10$ & ZNF746 & 4.90E-01 \\
\hline ENSBTAG00000002810 & $1.53 \mathrm{E}-10$ & $2.70 \mathrm{E}-10$ & ZNF777 & 5.65E-01 \\
\hline ENSBTAG00000019447 & $3.18 \mathrm{E}-10$ & $5.55 E-10$ & NPVF & 5.73E-01 \\
\hline ENSBTAG00000031958 & $3.64 \mathrm{E}-10$ & $6.13 \mathrm{E}-10$ & & 5.94E-01 \\
\hline ENSBTAG00000003825 & $9.39 \mathrm{E}-11$ & $1.53 \mathrm{E}-10$ & PTPN12 & $6.14 \mathrm{E}-01$ \\
\hline ENSBTAG00000001592 & $3.54 \mathrm{E}-10$ & $5.64 \mathrm{E}-10$ & INSIG1 & $6.28 \mathrm{E}-01$ \\
\hline ENSBTAG00000005102 & $1.45 \mathrm{E}-10$ & $2.31 \mathrm{E}-10$ & PHTF2 & $6.28 \mathrm{E}-01$ \\
\hline ENSBTAG00000045673 & 7.23E-10 & $1.14 \mathrm{E}-09$ & & $6.34 \mathrm{E}-01$ \\
\hline ENSBTAG00000037927 & $3.52 \mathrm{E}-10$ & $5.55 \mathrm{E}-10$ & & 6.34E-01 \\
\hline ENSBTAG00000002445 & $1.43 \mathrm{E}-10$ & $2.25 \mathrm{E}-10$ & ZNF783 & $6.36 \mathrm{E}-01$ \\
\hline ENSBTAG00000000911 & $8.01 \mathrm{E}-11$ & 1.22E-10 & UBE2H & 6.57E-01 \\
\hline ENSBTAG00000024552 & $1.52 \mathrm{E}-10$ & $2.25 \mathrm{E}-10$ & $\mathrm{SHH}$ & $6.76 \mathrm{E}-01$ \\
\hline ENSBTAG00000003372 & 4.10E-10 & $6.05 \mathrm{E}-10$ & SLC35B4 & 6.77E-01 \\
\hline ENSBTAG00000020364 & $1.05 \mathrm{E}-09$ & $1.55 \mathrm{E}-09$ & & 6.77E-01 \\
\hline ENSBTAG00000006022 & $6.87 \mathrm{E}-10$ & $1.01 \mathrm{E}-09$ & & $6.80 \mathrm{E}-01$ \\
\hline ENSBTAG00000019445 & $5.68 \mathrm{E}-10$ & $8.32 \mathrm{E}-10$ & C7orf31 & $6.83 \mathrm{E}-01$ \\
\hline ENSBTAG00000032650 & $5.76 \mathrm{E}-10$ & $8.32 \mathrm{E}-10$ & DPY19L2 & $6.93 \mathrm{E}-01$ \\
\hline ENSBTAG00000034645 & $2.32 \mathrm{E}-10$ & $3.33 \mathrm{E}-10$ & PON3 & 6.96E-01 \\
\hline ENSBTAG00000045567 & $1.96 \mathrm{E}-10$ & $2.77 \mathrm{E}-10$ & PRR15 & 7.07E-01 \\
\hline ENSBTAG00000039614 & $1.05 \mathrm{E}-09$ & $1.44 \mathrm{E}-09$ & & 7.31E-01 \\
\hline ENSBTAG00000039594 & $4.31 \mathrm{E}-10$ & $5.88 \mathrm{E}-10$ & ZNF862 & 7.33E-01 \\
\hline ENSBTAG00000007680 & $3.42 \mathrm{E}-10$ & 4.57E-10 & THSD7A & 7.48E-01 \\
\hline ENSBTAG00000013768 & 4.07E-10 & $5.43 \mathrm{E}-10$ & NPSR1 & 7.49E-01 \\
\hline ENSBTAG00000019794 & $2.55 \mathrm{E}-10$ & $3.35 E-10$ & SYPL1 & 7.60E-01 \\
\hline ENSBTAG00000009832 & $5.23 \mathrm{E}-10$ & $6.78 \mathrm{E}-10$ & NOBOX & 7.71E-01 \\
\hline ENSBTAG00000001815 & 7.01E-10 & $9.02 \mathrm{E}-10$ & ARHGEF5 & 7.77E-01 \\
\hline ENSBTAG00000007746 & 2.13E-10 & $2.71 \mathrm{E}-10$ & & 7.86E-01 \\
\hline ENSBTAG00000011191 & $1.73 \mathrm{E}-10$ & $2.19 \mathrm{E}-10$ & & 7.92E-01 \\
\hline ENSBTAG00000047193 & $1.94 \mathrm{E}-10$ & 2.44E-10 & & 7.94E-01 \\
\hline ENSBTAG00000047555 & $2.15 \mathrm{E}-10$ & $2.71 \mathrm{E}-10$ & & 7.94E-01 \\
\hline ENSBTAG00000046817 & $1.77 \mathrm{E}-10$ & $2.23 \mathrm{E}-10$ & & 7.92E-01 \\
\hline ENSBTAG00000024242 & $1.55 \mathrm{E}-09$ & 1.96E-09 & TRBV16 & 7.92E-01 \\
\hline ENSBTAG00000047722 & $1.76 \mathrm{E}-10$ & $2.21 \mathrm{E}-10$ & & 7.94E-01 \\
\hline ENSBTAG00000038960 & $3.02 \mathrm{E}-10$ & $3.80 \mathrm{E}-10$ & & 7.94E-01 \\
\hline ENSBTAG00000009717 & $3.31 \mathrm{E}-10$ & $4.18 \mathrm{E}-10$ & FGL2 & 7.93E-01 \\
\hline ENSBTAG00000008138 & 4.17E-11 & $5.25 \mathrm{E}-11$ & HOXA2 & 7.95E-01 \\
\hline ENSBTAG00000001063 & $3.61 \mathrm{E}-11$ & $4.55 \mathrm{E}-11$ & HOXA4 & 7.95E-01 \\
\hline ENSBTAG00000015844 & 7.61E-11 & $9.59 \mathrm{E}-11$ & TFPI2 & 7.94E-01 \\
\hline ENSBTAG00000033290 & $1.06 \mathrm{E}-10$ & $1.34 \mathrm{E}-10$ & & 7.94E-01 \\
\hline ENSBTAG00000001448 & 4.57E-10 & $5.76 \mathrm{E}-10$ & & 7.94E-01 \\
\hline ENSBTAG00000024199 & $2.96 \mathrm{E}-10$ & $3.73 \mathrm{E}-10$ & KMT2C & 7.92E-01 \\
\hline ENSBTAG00000046275 & $5.84 \mathrm{E}-10$ & 7.35E-10 & & 7.94E-01 \\
\hline
\end{tabular}




\begin{tabular}{|c|c|c|c|c|}
\hline ENSBTAG00000046823 & $4.52 \mathrm{E}-11$ & $5.70 \mathrm{E}-11$ & LINC00998 & 7.93E-01 \\
\hline ENSBTAG00000039401 & $7.15 \mathrm{E}-10$ & $9.01 \mathrm{E}-10$ & TRBV15 & 7.93E-01 \\
\hline ENSBTAG00000045576 & $7.78 \mathrm{E}-10$ & $9.80 \mathrm{E}-10$ & & 7.94E-01 \\
\hline ENSBTAG00000039956 & $3.19 \mathrm{E}-10$ & $4.02 \mathrm{E}-10$ & & 7.94E-01 \\
\hline ENSBTAG00000030876 & $8.20 \mathrm{E}-11$ & $1.03 \mathrm{E}-10$ & & 7.96E-01 \\
\hline ENSBTAG00000000397 & 7.10E-10 & $8.95 \mathrm{E}-10$ & CNPY1 & 7.93E-01 \\
\hline ENSBTAG00000040130 & $6.00 \mathrm{E}-10$ & $7.56 \mathrm{E}-10$ & OR6B1 & 7.94E-01 \\
\hline ENSBTAG00000040387 & $1.45 \mathrm{E}-09$ & $1.83 \mathrm{E}-09$ & & 7.93E-01 \\
\hline ENSBTAG00000047922 & $3.94 \mathrm{E}-10$ & 4.96E-10 & & 7.94E-01 \\
\hline ENSBTAG00000012894 & $1.29 \mathrm{E}-09$ & 1.63E-09 & SAMD9 & 7.92E-01 \\
\hline ENSBTAG00000039674 & $1.11 \mathrm{E}-09$ & $1.40 \mathrm{E}-09$ & & 7.93E-01 \\
\hline ENSBTAG00000048097 & $4.28 \mathrm{E}-10$ & $5.39 \mathrm{E}-10$ & & 7.94E-01 \\
\hline ENSBTAG00000046588 & $2.84 \mathrm{E}-11$ & $3.58 \mathrm{E}-11$ & DNAJB9 & 7.93E-01 \\
\hline ENSBTAG00000047294 & $3.55 \mathrm{E}-11$ & $4.48 \mathrm{E}-11$ & CUL1 & 7.94E-01 \\
\hline ENSBTAG00000040261 & $3.43 \mathrm{E}-10$ & $4.33 \mathrm{E}-10$ & & 7.93E-01 \\
\hline ENSBTAG00000048016 & 2.67E-09 & 3.36E-09 & & 7.93E-01 \\
\hline ENSBTAG00000031216 & $1.90 \mathrm{E}-10$ & $2.39 \mathrm{E}-10$ & & 7.94E-01 \\
\hline ENSBTAG00000045648 & $1.43 \mathrm{E}-10$ & $1.80 \mathrm{E}-10$ & & 7.93E-01 \\
\hline ENSBTAG00000008025 & $2.58 \mathrm{E}-10$ & $3.24 \mathrm{E}-10$ & UBE3C & 7.98E-01 \\
\hline ENSBTAG00000013226 & $9.03 \mathrm{E}-10$ & 1.13E-09 & HUS1 & 7.99E-01 \\
\hline ENSBTAG00000027341 & 7.36E-10 & $9.17 \mathrm{E}-10$ & & 8.03E-01 \\
\hline ENSBTAG00000015753 & $1.73 \mathrm{E}-10$ & $2.14 \mathrm{E}-10$ & RSBN1L & 8.07E-01 \\
\hline ENSBTAG00000008361 & $3.28 \mathrm{E}-10$ & 4.05E-10 & PON2 & 8.09E-01 \\
\hline ENSBTAG00000017676 & $4.91 \mathrm{E}-10$ & $6.06 \mathrm{E}-10$ & TPK1 & $8.11 \mathrm{E}-01$ \\
\hline ENSBTAG00000002912 & $1.71 \mathrm{E}-10$ & 2.07E-10 & INHBA & 8.26E-01 \\
\hline ENSBTAG00000005785 & $6.63 \mathrm{E}-10$ & $8.01 \mathrm{E}-10$ & DPY19L1 & $8.28 \mathrm{E}-01$ \\
\hline ENSBTAG00000015800 & $7.00 \mathrm{E}-10$ & 8.44E-10 & & 8.30E-01 \\
\hline ENSBTAG00000003424 & 7.61E-10 & $9.08 \mathrm{E}-10$ & BET1 & $8.38 \mathrm{E}-01$ \\
\hline ENSBTAG00000005475 & $4.45 \mathrm{E}-10$ & $5.31 \mathrm{E}-10$ & & $8.38 \mathrm{E}-01$ \\
\hline ENSBTAG00000014381 & $6.32 \mathrm{E}-10$ & 7.51E-10 & CRHR2 & $8.41 \mathrm{E}-01$ \\
\hline ENSBTAG00000003508 & $4.42 \mathrm{E}-10$ & $5.21 \mathrm{E}-10$ & CFAP69 & $8.49 \mathrm{E}-01$ \\
\hline ENSBTAG00000013168 & $3.85 \mathrm{E}-10$ & 4.53E-10 & RPA3 & $8.50 \mathrm{E}-01$ \\
\hline ENSBTAG00000019844 & $1.90 \mathrm{E}-10$ & $2.22 \mathrm{E}-10$ & ZNF467 & $8.55 \mathrm{E}-01$ \\
\hline ENSBTAG00000004640 & $8.69 \mathrm{E}-10$ & 1.00E-09 & & 8.69E-01 \\
\hline ENSBTAG00000011145 & $5.33 \mathrm{E}-10$ & $6.15 \mathrm{E}-10$ & NDUFA4 & $8.66 \mathrm{E}-01$ \\
\hline ENSBTAG00000030897 & $2.51 \mathrm{E}-10$ & $2.88 \mathrm{E}-10$ & XRCC2 & $8.73 \mathrm{E}-01$ \\
\hline ENSBTAG00000006721 & $4.85 \mathrm{E}-10$ & $5.53 \mathrm{E}-10$ & TWISTNB & 8.77E-01 \\
\hline ENSBTAG00000019342 & $6.76 \mathrm{E}-10$ & $7.68 \mathrm{E}-10$ & OSBPL3 & $8.80 \mathrm{E}-01$ \\
\hline ENSBTAG00000001502 & $8.27 \mathrm{E}-10$ & $9.37 \mathrm{E}-10$ & & $8.83 \mathrm{E}-01$ \\
\hline ENSBTAG00000006161 & $2.96 \mathrm{E}-10$ & $3.34 \mathrm{E}-10$ & MET & $8.86 \mathrm{E}-01$ \\
\hline ENSBTAG00000002714 & $5.14 \mathrm{E}-10$ & 5.77E-10 & GNAI1 & $8.91 \mathrm{E}-01$ \\
\hline ENSBTAG00000017680 & $9.95 \mathrm{E}-10$ & $1.12 \mathrm{E}-09$ & NOS3 & $8.88 \mathrm{E}-01$ \\
\hline ENSBTAG00000014074 & $1.02 \mathrm{E}-10$ & $1.15 \mathrm{E}-10$ & & $8.89 \mathrm{E}-01$ \\
\hline ENSBTAG00000003628 & $3.61 E-10$ & 4.04E-10 & PRSS58 & 8.94E-01 \\
\hline ENSBTAG00000011120 & $5.20 \mathrm{E}-10$ & $5.81 \mathrm{E}-10$ & GBX1 & 8.95E-01 \\
\hline ENSBTAG00000021967 & $4.02 \mathrm{E}-10$ & 4.47E-10 & TMEM168 & $9.00 \mathrm{E}-01$ \\
\hline ENSBTAG00000048115 & $1.48 \mathrm{E}-09$ & 1.64E-09 & & $9.01 \mathrm{E}-01$ \\
\hline ENSBTAG00000004078 & $6.48 \mathrm{E}-10$ & 7.20E-10 & $\mathrm{KCNH} 2$ & $9.00 \mathrm{E}-01$ \\
\hline ENSBTAG00000019403 & $1.03 \mathrm{E}-09$ & 1.14E-09 & MALSU1 & 9.03E-01 \\
\hline ENSBTAG00000018064 & $1.69 \mathrm{E}-10$ & $1.87 \mathrm{E}-10$ & FAM221A & $9.05 \mathrm{E}-01$ \\
\hline ENSBTAG00000012119 & 4.14E-10 & 4.59E-10 & PTPRZ1 & $9.01 \mathrm{E}-01$ \\
\hline ENSBTAG00000044012 & $1.14 \mathrm{E}-10$ & $1.26 \mathrm{E}-10$ & TMEM196 & 9.07E-01 \\
\hline ENSBTAG00000038934 & $1.58 \mathrm{E}-09$ & 1.74E-09 & & $9.09 \mathrm{E}-01$ \\
\hline ENSBTAG00000011246 & $2.12 \mathrm{E}-10$ & $2.33 \mathrm{E}-10$ & FOXP2 & $9.08 \mathrm{E}-01$ \\
\hline ENSBTAG00000015802 & $4.68 \mathrm{E}-10$ & $5.12 \mathrm{E}-10$ & CREB3L2 & $9.14 \mathrm{E}-01$ \\
\hline ENSBTAG00000013476 & $4.52 \mathrm{E}-11$ & 4.94E-11 & CPA5 & $9.16 \mathrm{E}-01$ \\
\hline ENSBTAG00000004215 & $3.99 \mathrm{E}-10$ & 4.35E-10 & RARRES2 & $9.17 \mathrm{E}-01$ \\
\hline ENSBTAG00000000144 & $1.43 \mathrm{E}-09$ & $1.56 \mathrm{E}-09$ & & $9.19 \mathrm{E}-01$ \\
\hline ENSBTAG00000008032 & $2.78 \mathrm{E}-10$ & $3.01 E-10$ & ACTR3B & $9.24 \mathrm{E}-01$ \\
\hline ENSBTAG00000008895 & $1.44 \mathrm{E}-10$ & $1.55 \mathrm{E}-10$ & & 9.27E-01 \\
\hline ENSBTAG00000046461 & $5.90 \mathrm{E}-10$ & $6.37 \mathrm{E}-10$ & & $9.26 \mathrm{E}-01$ \\
\hline ENSBTAG00000014674 & $3.90 \mathrm{E}-10$ & 4.21E-10 & CHRM2 & $9.26 \mathrm{E}-01$ \\
\hline ENSBTAG00000021996 & 7.77E-10 & $8.38 \mathrm{E}-10$ & GATAD1 & 9.27E-01 \\
\hline ENSBTAG00000006674 & 4.67E-10 & 5.01E-10 & SLC13A1 & $9.33 \mathrm{E}-01$ \\
\hline ENSBTAG00000046169 & $2.47 \mathrm{E}-10$ & $2.65 \mathrm{E}-10$ & CPA1 & $9.33 \mathrm{E}-01$ \\
\hline ENSBTAG00000000604 & $3.97 \mathrm{E}-10$ & 4.25E-10 & GPNMB & $9.35 \mathrm{E}-01$ \\
\hline ENSBTAG00000007806 & $7.06 \mathrm{E}-10$ & $7.55 \mathrm{E}-10$ & MTPN & $9.36 \mathrm{E}-01$ \\
\hline ENSBTAG00000018972 & $2.24 \mathrm{E}-10$ & $2.39 \mathrm{E}-10$ & GARS & 9.37E-01 \\
\hline ENSBTAG00000047979 & $7.51 \mathrm{E}-10$ & 8.02E-10 & & $9.36 \mathrm{E}-01$ \\
\hline ENSBTAG00000024420 & $7.13 \mathrm{E}-10$ & $7.60 \mathrm{E}-10$ & COL28A1 & $9.38 \mathrm{E}-01$ \\
\hline
\end{tabular}




\begin{tabular}{|c|c|c|c|c|}
\hline ENSBTAG00000000548 & $7.84 \mathrm{E}-11$ & $8.34 \mathrm{E}-11$ & KLHDC10 & $9.40 \mathrm{E}-01$ \\
\hline ENSBTAG00000000296 & $1.18 \mathrm{E}-10$ & $1.25 \mathrm{E}-10$ & IMPDH1 & $9.44 \mathrm{E}-01$ \\
\hline ENSBTAG00000007305 & $3.37 \mathrm{E}-10$ & $3.57 \mathrm{E}-10$ & VPS41 & $9.45 \mathrm{E}-01$ \\
\hline ENSBTAG00000018185 & $5.36 \mathrm{E}-10$ & $5.67 \mathrm{E}-10$ & ASB4 & $9.46 \mathrm{E}-01$ \\
\hline ENSBTAG00000004136 & $2.48 \mathrm{E}-10$ & $2.62 \mathrm{E}-10$ & NFE2L3 & $9.47 \mathrm{E}-01$ \\
\hline ENSBTAG00000032288 & $6.94 \mathrm{E}-10$ & $7.30 \mathrm{E}-10$ & GCK & $9.51 \mathrm{E}-01$ \\
\hline ENSBTAG00000039231 & $1.97 \mathrm{E}-10$ & $2.08 \mathrm{E}-10$ & MTURN & $9.49 \mathrm{E}-01$ \\
\hline ENSBTAG00000018917 & $2.37 \mathrm{E}-10$ & 2.49E-10 & & $9.52 \mathrm{E}-01$ \\
\hline ENSBTAG00000003808 & $3.64 \mathrm{E}-10$ & $3.81 \mathrm{E}-10$ & HDAC9 & $9.56 \mathrm{E}-01$ \\
\hline ENSBTAG00000015749 & $2.25 \mathrm{E}-10$ & $2.35 \mathrm{E}-10$ & STEAP1 & 9.59E-01 \\
\hline ENSBTAG00000016879 & $2.02 \mathrm{E}-10$ & $2.10 \mathrm{E}-10$ & KIAA1147 & $9.61 \mathrm{E}-01$ \\
\hline ENSBTAG00000019980 & $5.15 \mathrm{E}-10$ & $5.36 \mathrm{E}-10$ & DGKI & $9.61 \mathrm{E}-01$ \\
\hline ENSBTAG00000017427 & 3.13E-10 & $3.25 \mathrm{E}-10$ & FAM180A & $9.64 \mathrm{E}-01$ \\
\hline ENSBTAG00000043955 & $5.07 \mathrm{E}-10$ & $5.24 \mathrm{E}-10$ & C7orf72 & $9.67 \mathrm{E}-01$ \\
\hline ENSBTAG00000017541 & $6.38 \mathrm{E}-10$ & $6.58 \mathrm{E}-10$ & TBX20 & $9.70 \mathrm{E}-01$ \\
\hline ENSBTAG00000009218 & $3.35 \mathrm{E}-10$ & $3.46 \mathrm{E}-10$ & ANLN & 9.69E-01 \\
\hline ENSBTAG00000046433 & $1.34 \mathrm{E}-09$ & 1.39E-09 & & 9.67E-01 \\
\hline ENSBTAG00000047657 & $1.95 \mathrm{E}-09$ & 2.00E-09 & & $9.75 \mathrm{E}-01$ \\
\hline ENSBTAG00000038690 & $1.95 \mathrm{E}-09$ & 2.00E-09 & & $9.75 \mathrm{E}-01$ \\
\hline ENSBTAG00000020931 & $3.99 \mathrm{E}-10$ & $4.08 \mathrm{E}-10$ & $\mathrm{CHN} 2$ & $9.78 \mathrm{E}-01$ \\
\hline ENSBTAG00000004575 & $2.75 \mathrm{E}-10$ & $2.81 \mathrm{E}-10$ & FAM126A & $9.80 \mathrm{E}-01$ \\
\hline ENSBTAG00000004958 & $3.01 \mathrm{E}-10$ & $3.06 \mathrm{E}-10$ & & $9.84 \mathrm{E}-01$ \\
\hline ENSBTAG00000021941 & $5.64 \mathrm{E}-10$ & $5.73 \mathrm{E}-10$ & & 9.84E-01 \\
\hline ENSBTAG00000031866 & $1.48 \mathrm{E}-10$ & $1.49 \mathrm{E}-10$ & & $9.93 \mathrm{E}-01$ \\
\hline ENSBTAG00000021073 & 4.54E-10 & $4.58 \mathrm{E}-10$ & KIAA1549 & $9.92 \mathrm{E}-01$ \\
\hline ENSBTAG00000017223 & $3.22 \mathrm{E}-11$ & $3.24 \mathrm{E}-11$ & MEST & $9.93 \mathrm{E}-01$ \\
\hline ENSBTAG00000001198 & $4.60 \mathrm{E}-10$ & 4.63E-10 & & $9.93 \mathrm{E}-01$ \\
\hline ENSBTAG00000002154 & $4.76 \mathrm{E}-10$ & 4.79E-10 & GPR141 & $9.93 \mathrm{E}-01$ \\
\hline ENSBTAG00000000991 & $2.41 \mathrm{E}-10$ & $2.43 E-10$ & MRPL32 & $9.93 \mathrm{E}-01$ \\
\hline ENSBTAG00000002107 & $1.97 \mathrm{E}-10$ & $1.99 \mathrm{E}-10$ & FZD1 & $9.92 \mathrm{E}-01$ \\
\hline ENSBTAG00000034154 & $4.39 \mathrm{E}-10$ & $4.41 \mathrm{E}-10$ & AGMO & $9.95 \mathrm{E}-01$ \\
\hline ENSBTAG00000021260 & $1.16 \mathrm{E}-10$ & 1.17E-10 & GALNT11 & $9.91 \mathrm{E}-01$ \\
\hline ENSBTAG00000008468 & $5.04 \mathrm{E}-10$ & $5.06 \mathrm{E}-10$ & AGAP3 & 9.97E-01 \\
\hline ENSBTAG00000013781 & $2.41 \mathrm{E}-10$ & $2.42 \mathrm{E}-10$ & MIOS & $9.96 \mathrm{E}-01$ \\
\hline ENSBTAG00000032077 & $3.35 \mathrm{E}-10$ & $3.34 \mathrm{E}-10$ & KCND2 & $1.00 \mathrm{E}+00$ \\
\hline ENSBTAG00000018838 & $8.18 \mathrm{E}-10$ & $8.14 \mathrm{E}-10$ & IQCA1L & $1.00 \mathrm{E}+00$ \\
\hline ENSBTAG00000004643 & $3.37 \mathrm{E}-10$ & $3.35 \mathrm{E}-10$ & WASL & $1.01 E+00$ \\
\hline ENSBTAG00000007762 & $7.91 \mathrm{E}-10$ & $7.85 \mathrm{E}-10$ & ASIC3 & $1.01 \mathrm{E}+00$ \\
\hline ENSBTAG00000002332 & $1.55 \mathrm{E}-09$ & $1.54 \mathrm{E}-09$ & LSM5 & $1.00 \mathrm{E}+00$ \\
\hline ENSBTAG00000010062 & $3.41 \mathrm{E}-10$ & $3.37 \mathrm{E}-10$ & SLC26A4 & $1.01 \mathrm{E}+00$ \\
\hline ENSBTAG00000018442 & $7.79 \mathrm{E}-10$ & $7.70 \mathrm{E}-10$ & PRSS37 & $1.01 \mathrm{E}+00$ \\
\hline ENSBTAG00000016435 & $3.06 \mathrm{E}-10$ & $3.02 \mathrm{E}-10$ & NOM1 & $1.01 E+00$ \\
\hline ENSBTAG00000027832 & $6.65 \mathrm{E}-10$ & $6.54 \mathrm{E}-10$ & & $1.02 \mathrm{E}+00$ \\
\hline ENSBTAG00000016707 & $5.77 \mathrm{E}-10$ & $5.65 \mathrm{E}-10$ & FKBP9 & $1.02 \mathrm{E}+00$ \\
\hline ENSBTAG00000039093 & $8.85 \mathrm{E}-10$ & $8.66 \mathrm{E}-10$ & & $1.02 \mathrm{E}+00$ \\
\hline ENSBTAG00000016014 & 7.17E-10 & 7.02E-10 & NUPL2 & $1.02 \mathrm{E}+00$ \\
\hline ENSBTAG00000000389 & $2.26 \mathrm{E}-10$ & $2.21 \mathrm{E}-10$ & ZNF212 & $1.02 \mathrm{E}+00$ \\
\hline ENSBTAG00000013673 & $1.60 \mathrm{E}-09$ & 1.57E-09 & & $1.02 \mathrm{E}+00$ \\
\hline ENSBTAG00000017245 & $1.18 \mathrm{E}-10$ & $1.16 \mathrm{E}-10$ & COPG2 & $1.02 \mathrm{E}+00$ \\
\hline ENSBTAG00000009426 & $1.19 \mathrm{E}-10$ & $1.15 \mathrm{E}-10$ & EZH2 & $1.03 E+00$ \\
\hline ENSBTAG00000012456 & $7.88 \mathrm{E}-10$ & 7.67E-10 & HEPACAM2 & $1.03 E+00$ \\
\hline ENSBTAG00000024431 & $8.05 \mathrm{E}-10$ & 7.81E-10 & PEX1 & $1.03 E+00$ \\
\hline ENSBTAG00000046257 & $2.84 \mathrm{E}-10$ & $2.75 \mathrm{E}-10$ & & $1.03 E+00$ \\
\hline ENSBTAG00000031212 & $3.55 \mathrm{E}-10$ & $3.44 \mathrm{E}-10$ & & $1.03 E+00$ \\
\hline ENSBTAG00000016220 & $2.05 \mathrm{E}-10$ & $1.96 \mathrm{E}-10$ & WIPF3 & $1.04 E+00$ \\
\hline ENSBTAG00000000799 & $4.88 \mathrm{E}-10$ & 4.69E-10 & ICA1 & $1.04 E+00$ \\
\hline ENSBTAG00000001908 & $4.36 \mathrm{E}-10$ & 4.18E-10 & DLD & $1.04 \mathrm{E}+00$ \\
\hline ENSBTAG00000004799 & $1.55 \mathrm{E}-10$ & $1.48 \mathrm{E}-10$ & DENND2A & $1.05 E+00$ \\
\hline ENSBTAG00000014879 & $5.05 \mathrm{E}-10$ & $4.82 \mathrm{E}-10$ & FAM185A & $1.05 E+00$ \\
\hline ENSBTAG00000018909 & $2.25 \mathrm{E}-10$ & $2.15 \mathrm{E}-10$ & & $1.05 E+00$ \\
\hline ENSBTAG00000024340 & $1.35 \mathrm{E}-10$ & $1.27 E-10$ & TRIL & $1.06 \mathrm{E}+00$ \\
\hline ENSBTAG00000038127 & 7.74E-10 & 7.30E-10 & & $1.06 \mathrm{E}+00$ \\
\hline ENSBTAG00000046020 & $1.39 \mathrm{E}-09$ & $1.31 \mathrm{E}-09$ & & $1.06 \mathrm{E}+00$ \\
\hline ENSBTAG00000024230 & $1.05 \mathrm{E}-09$ & $9.89 \mathrm{E}-10$ & & $1.06 \mathrm{E}+00$ \\
\hline ENSBTAG00000014372 & $8.33 \mathrm{E}-10$ & 7.84E-10 & SMARCD3 & $1.06 \mathrm{E}+00$ \\
\hline ENSBTAG00000001582 & $6.92 \mathrm{E}-10$ & $6.50 \mathrm{E}-10$ & & $1.07 E+00$ \\
\hline ENSBTAG00000039588 & $1.03 \mathrm{E}-09$ & $9.65 \mathrm{E}-10$ & & $1.07 E+00$ \\
\hline ENSBTAG00000021851 & $3.88 \mathrm{E}-10$ & $3.63 \mathrm{E}-10$ & BCAP29 & $1.07 E+00$ \\
\hline ENSBTAG00000012078 & $7.95 \mathrm{E}-10$ & 7.42E-10 & TMEM176A & $1.07 E+00$ \\
\hline ENSBTAG00000035945 & $1.74 \mathrm{E}-10$ & $1.62 \mathrm{E}-10$ & & $1.07 E+00$ \\
\hline
\end{tabular}




\begin{tabular}{|c|c|c|c|c|}
\hline ENSBTAG000000011226 & $5.81 \mathrm{E}-10$ & $5.42 \mathrm{E}-10$ & SLC4A2 & $1.07 \mathrm{E}+00$ \\
\hline ENSBTAG000000037510 & $7.84 \mathrm{E}-10$ & 7.28E-10 & & $1.08 \mathrm{E}+00$ \\
\hline ENSBTAG00000040331 & 1.00E-09 & $9.29 \mathrm{E}-10$ & & $1.08 \mathrm{E}+00$ \\
\hline ENSBTAG000000024406 & $6.49 \mathrm{E}-10$ & $5.98 \mathrm{E}-10$ & AGR2 & $1.09 E+00$ \\
\hline ENSBTAG000000001036 & $3.24 \mathrm{E}-10$ & $2.97 \mathrm{E}-10$ & HIBADH & $1.09 \mathrm{E}+00$ \\
\hline ENSBTAG00000011247 & $5.54 \mathrm{E}-11$ & $5.08 \mathrm{E}-11$ & $\mathrm{ZC} 3 \mathrm{HCl}$ & $1.09 \mathrm{E}+00$ \\
\hline ENSBTAG00000010300 & 1.64E-09 & 1.50E-09 & & $1.09 \mathrm{E}+00$ \\
\hline ENSBTAG000000003773 & $2.76 \mathrm{E}-10$ & 2.53E-10 & SLC25A13 & $1.09 \mathrm{E}+00$ \\
\hline ENSBTAG00000000189 & $4.16 \mathrm{E}-10$ & $3.81 E-10$ & LHFPL3 & $1.09 \mathrm{E}+00$ \\
\hline ENSBTAG000000015981 & $2.64 \mathrm{E}-10$ & $2.41 E-10$ & ETV1 & $1.10 \mathrm{E}+00$ \\
\hline ENSBTAG000000019486 & $1.06 \mathrm{E}-09$ & $9.69 \mathrm{E}-10$ & CLDN12 & $1.09 E+00$ \\
\hline ENSBTAG000000018119 & $4.30 \mathrm{E}-10$ & $3.91 \mathrm{E}-10$ & $\mathrm{AOAH}$ & $1.10 \mathrm{E}+00$ \\
\hline ENSBTAG00000047605 & $1.26 \mathrm{E}-09$ & $1.15 \mathrm{E}-09$ & & $1.10 \mathrm{E}+00$ \\
\hline ENSBTAG000000015773 & $5.10 \mathrm{E}-10$ & 4.63E-10 & LRGUK & $1.10 \mathrm{E}+00$ \\
\hline ENSBTAG000000012805 & $3.08 \mathrm{E}-10$ & $2.79 \mathrm{E}-10$ & TSPAN13 & $1.10 \mathrm{E}+00$ \\
\hline ENSBTAG000000019750 & $4.29 E-10$ & $3.89 \mathrm{E}-10$ & TMEM106B & $1.10 \mathrm{E}+00$ \\
\hline ENSBTAG00000013180 & $7.40 \mathrm{E}-10$ & $6.69 \mathrm{E}-10$ & РОT1 & $1.11 E+00$ \\
\hline ENSBTAG000000036154 & 1.11E-09 & $1.00 \mathrm{E}-09$ & TARP & $1.11 E+00$ \\
\hline ENSBTAG000000040388 & $1.12 \mathrm{E}-09$ & 1.01E-09 & & $1.11 E+00$ \\
\hline ENSBTAG000000009655 & $2.73 \mathrm{E}-10$ & $2.46 \mathrm{E}-10$ & TNS3 & $1.11 E+00$ \\
\hline ENSBTAG000000008736 & $3.22 \mathrm{E}-10$ & $2.91 \mathrm{E}-10$ & & $1.11 E+00$ \\
\hline ENSBTAG00000032844 & $1.39 \mathrm{E}-10$ & $1.25 \mathrm{E}-10$ & GPR85 & $1.11 E+00$ \\
\hline ENSBTAG000000024157 & $3.18 \mathrm{E}-10$ & $2.84 \mathrm{E}-10$ & WDR60 & $1.12 \mathrm{E}+00$ \\
\hline ENSBTAG000000009850 & 1.09E-09 & $9.75 \mathrm{E}-10$ & & $1.12 \mathrm{E}+00$ \\
\hline ENSBTAG00000047725 & 1.17E-09 & $1.05 \mathrm{E}-09$ & & $1.12 \mathrm{E}+00$ \\
\hline ENSBTAG000000010390 & $1.65 \mathrm{E}-10$ & $1.48 \mathrm{E}-10$ & SRI & $1.12 \mathrm{E}+00$ \\
\hline ENSBTAG000000047844 & $1.38 \mathrm{E}-09$ & $1.24 \mathrm{E}-09$ & & $1.12 \mathrm{E}+00$ \\
\hline ENSBTAG000000021905 & 4.65E-10 & $4.13 \mathrm{E}-10$ & DGKB & $1.13 \mathrm{E}+00$ \\
\hline ENSBTAG00000006138 & $3.32 \mathrm{E}-10$ & $2.95 \mathrm{E}-10$ & SEMA3C & $1.12 \mathrm{E}+00$ \\
\hline ENSBTAG000000024245 & $3.61 \mathrm{E}-10$ & $3.21 \mathrm{E}-10$ & & $1.12 \mathrm{E}+00$ \\
\hline ENSBTAG00000014112 & $3.93 \mathrm{E}-10$ & $3.49 \mathrm{E}-10$ & EXOC4 & $1.13 \mathrm{E}+00$ \\
\hline ENSBTAG000000037649 & $2.77 \mathrm{E}-10$ & $2.45 \mathrm{E}-10$ & VIPR2 & $1.13 \mathrm{E}+00$ \\
\hline ENSBTAG00000024219 & $6.16 \mathrm{E}-10$ & $5.43 \mathrm{E}-10$ & TRPV6 & $1.13 \mathrm{E}+00$ \\
\hline ENSBTAG000000013472 & $1.94 \mathrm{E}-10$ & $1.72 \mathrm{E}-10$ & COL1A2 & $1.13 E+00$ \\
\hline ENSBTAG00000012031 & $4.48 \mathrm{E}-10$ & $3.95 \mathrm{E}-10$ & ATG9B & $1.13 \mathrm{E}+00$ \\
\hline ENSBTAG00000027134 & $4.46 \mathrm{E}-10$ & $3.91 \mathrm{E}-10$ & DYNC1I1 & $1.14 \mathrm{E}+00$ \\
\hline ENSBTAG00000009341 & $4.38 \mathrm{E}-10$ & $3.84 \mathrm{E}-10$ & CCDC146 & $1.14 \mathrm{E}+00$ \\
\hline ENSBTAG00000005499 & $4.20 \mathrm{E}-10$ & $3.68 \mathrm{E}-10$ & ZPBP & $1.14 E+00$ \\
\hline ENSBTAG00000017664 & $2.58 \mathrm{E}-10$ & $2.26 \mathrm{E}-10$ & HGF & $1.14 \mathrm{E}+00$ \\
\hline ENSBTAG00000003102 & $6.30 \mathrm{E}-10$ & $5.48 \mathrm{E}-10$ & GCC1 & $1.15 E+00$ \\
\hline ENSBTAG00000014273 & $2.42 \mathrm{E}-10$ & $2.11 \mathrm{E}-10$ & NRF1 & $1.15 \mathrm{E}+00$ \\
\hline ENSBTAG00000001658 & $1.76 \mathrm{E}-10$ & $1.53 \mathrm{E}-10$ & AKR1D1 & $1.15 \mathrm{E}+00$ \\
\hline ENSBTAG00000033169 & $6.92 \mathrm{E}-10$ & $6.00 \mathrm{E}-10$ & LAMB4 & $1.15 \mathrm{E}+00$ \\
\hline ENSBTAG00000024426 & $4.08 \mathrm{E}-10$ & 3.53E-10 & PPP1R9A & $1.16 \mathrm{E}+00$ \\
\hline ENSBTAG00000014533 & $2.97 \mathrm{E}-10$ & $2.56 \mathrm{E}-10$ & KEL & $1.16 \mathrm{E}+00$ \\
\hline ENSBTAG00000000771 & $2.92 \mathrm{E}-10$ & $2.51 E-10$ & & $1.16 \mathrm{E}+00$ \\
\hline ENSBTAG00000013648 & $5.08 \mathrm{E}-10$ & $4.38 \mathrm{E}-10$ & POU6F2 & $1.16 \mathrm{E}+00$ \\
\hline ENSBTAG00000007146 & $2.93 \mathrm{E}-10$ & $2.53 \mathrm{E}-10$ & CPVL & $1.16 \mathrm{E}+00$ \\
\hline ENSBTAG00000045617 & $5.14 \mathrm{E}-10$ & $4.42 \mathrm{E}-10$ & MTERF1 & $1.16 \mathrm{E}+00$ \\
\hline ENSBTAG00000038235 & 4.53E-10 & $3.89 \mathrm{E}-10$ & NOD1 & $1.16 \mathrm{E}+00$ \\
\hline ENSBTAG00000046152 & $1.35 \mathrm{E}-09$ & $1.16 \mathrm{E}-09$ & MGAM & $1.17 E+00$ \\
\hline ENSBTAG00000015547 & $4.53 \mathrm{E}-10$ & $3.88 \mathrm{E}-10$ & SLC26A3 & $1.17 E+00$ \\
\hline ENSBTAG00000038795 & $2.05 \mathrm{E}-10$ & $1.75 \mathrm{E}-10$ & STARD3NL & $1.17 E+00$ \\
\hline ENSBTAG00000020445 & $3.47 \mathrm{E}-10$ & $2.94 \mathrm{E}-10$ & ZNF398 & $1.18 \mathrm{E}+00$ \\
\hline ENSBTAG00000015073 & $4.97 \mathrm{E}-10$ & 4.20E-10 & ISPD & $1.18 \mathrm{E}+00$ \\
\hline ENSBTAG00000031548 & $9.61 \mathrm{E}-10$ & $8.08 \mathrm{E}-10$ & & $1.19 E+00$ \\
\hline ENSBTAG00000014543 & $5.15 \mathrm{E}-10$ & $4.33 \mathrm{E}-10$ & PLXNA4 & $1.19 \mathrm{E}+00$ \\
\hline ENSBTAG00000005483 & $2.84 \mathrm{E}-10$ & $2.39 \mathrm{E}-10$ & ESYT2 & $1.19 \mathrm{E}+00$ \\
\hline ENSBTAG00000012003 & $1.99 \mathrm{E}-10$ & $1.67 \mathrm{E}-10$ & & $1.19 E+00$ \\
\hline ENSBTAG00000014795 & $2.79 \mathrm{E}-10$ & $2.34 \mathrm{E}-10$ & & $1.19 E+00$ \\
\hline ENSBTAG00000012211 & $9.21 \mathrm{E}-11$ & $7.73 \mathrm{E}-11$ & HOXA5 & $1.19 \mathrm{E}+00$ \\
\hline ENSBTAG00000031232 & $4.85 \mathrm{E}-10$ & 4.07E-10 & & $1.19 E+00$ \\
\hline ENSBTAG00000016485 & $7.44 \mathrm{E}-10$ & $6.24 \mathrm{E}-10$ & OPN1SW & $1.19 \mathrm{E}+00$ \\
\hline ENSBTAG00000007759 & $6.11 E-10$ & $5.12 \mathrm{E}-10$ & $A B C B 8$ & $1.19 E+00$ \\
\hline ENSBTAG00000024204 & $8.80 \mathrm{E}-10$ & 7.37E-10 & & $1.19 \mathrm{E}+00$ \\
\hline ENSBTAG00000014871 & $1.61 \mathrm{E}-10$ & $1.35 \mathrm{E}-10$ & ССT8L2 & $1.19 \mathrm{E}+00$ \\
\hline ENSBTAG00000031059 & $3.82 \mathrm{E}-10$ & 3.20E-10 & LRRC61 & $1.19 E+00$ \\
\hline ENSBTAG00000010452 & 1.15E-09 & 9.65E-10 & PODXL & $1.19 \mathrm{E}+00$ \\
\hline ENSBTAG00000015654 & 5.19E-10 & 4.30E-10 & PON1 & $1.21 \mathrm{E}+00$ \\
\hline ENSBTAG00000016752 & $3.98 \mathrm{E}-10$ & $3.28 \mathrm{E}-10$ & ASB10 & $1.21 E+00$ \\
\hline
\end{tabular}




\begin{tabular}{|c|c|c|c|c|}
\hline ENSBTAG00000018363 & $4.86 \mathrm{E}-10$ & $3.96 \mathrm{E}-10$ & RBM48 & $1.23 \mathrm{E}+00$ \\
\hline ENSBTAG00000004989 & $5.75 \mathrm{E}-10$ & $4.68 \mathrm{E}-10$ & IRF5 & $1.23 \mathrm{E}+00$ \\
\hline ENSBTAG00000003490 & $3.97 \mathrm{E}-10$ & $3.22 \mathrm{E}-10$ & ELMO1 & $1.23 \mathrm{E}+00$ \\
\hline ENSBTAG00000006589 & 4.32E-10 & $3.50 \mathrm{E}-10$ & CFTR & $1.24 \mathrm{E}+00$ \\
\hline ENSBTAG00000014262 & $2.19 \mathrm{E}-10$ & $1.77 \mathrm{E}-10$ & BZW2 & $1.24 \mathrm{E}+00$ \\
\hline ENSBTAG00000007413 & $9.10 \mathrm{E}-11$ & $7.36 \mathrm{E}-11$ & CEP41 & $1.24 \mathrm{E}+00$ \\
\hline ENSBTAG00000000607 & $6.50 \mathrm{E}-10$ & $5.21 \mathrm{E}-10$ & $A B C F 2$ & $1.25 \mathrm{E}+00$ \\
\hline ENSBTAG00000000260 & $2.02 \mathrm{E}-10$ & $1.62 \mathrm{E}-10$ & ZNRF2 & $1.25 E+00$ \\
\hline ENSBTAG00000030940 & $2.91 \mathrm{E}-10$ & $2.32 \mathrm{E}-10$ & & $1.25 \mathrm{E}+00$ \\
\hline ENSBTAG00000014402 & $1.25 \mathrm{E}-09$ & $9.94 \mathrm{E}-10$ & GIMAP8 & $1.25 \mathrm{E}+00$ \\
\hline ENSBTAG00000015366 & $1.47 \mathrm{E}-10$ & 1.17E-10 & SFRP4 & $1.26 \mathrm{E}+00$ \\
\hline ENSBTAG00000004992 & $2.49 \mathrm{E}-10$ & $1.97 \mathrm{E}-10$ & TNPO3 & $1.26 \mathrm{E}+00$ \\
\hline ENSBTAG00000038649 & $1.30 \mathrm{E}-09$ & $1.03 \mathrm{E}-09$ & & $1.26 \mathrm{E}+00$ \\
\hline ENSBTAG00000005419 & $4.59 \mathrm{E}-10$ & $3.63 \mathrm{E}-10$ & $\mathrm{AOC1}$ & $1.26 \mathrm{E}+00$ \\
\hline ENSBTAG00000008306 & $2.26 \mathrm{E}-10$ & $1.78 \mathrm{E}-10$ & MKRN1 & $1.27 \mathrm{E}+00$ \\
\hline ENSBTAG00000008542 & $4.94 \mathrm{E}-10$ & $3.89 \mathrm{E}-10$ & SSPO & $1.27 \mathrm{E}+00$ \\
\hline ENSBTAG00000005548 & 8.67E-10 & $6.82 \mathrm{E}-10$ & HYAL4 & $1.27 \mathrm{E}+00$ \\
\hline ENSBTAG00000024231 & $1.40 \mathrm{E}-09$ & 1.10E-09 & & $1.28 \mathrm{E}+00$ \\
\hline ENSBTAG00000001500 & $3.20 \mathrm{E}-10$ & $2.52 \mathrm{E}-10$ & FIGNL1 & $1.27 E+00$ \\
\hline ENSBTAG00000011819 & $2.28 \mathrm{E}-10$ & $1.79 \mathrm{E}-10$ & PHF14 & $1.27 \mathrm{E}+00$ \\
\hline ENSBTAG00000018159 & $3.63 \mathrm{E}-10$ & $2.84 \mathrm{E}-10$ & CASP2 & $1.28 \mathrm{E}+00$ \\
\hline ENSBTAG00000008533 & $1.57 \mathrm{E}-10$ & $1.23 \mathrm{E}-10$ & GLCCI1 & $1.28 \mathrm{E}+00$ \\
\hline ENSBTAG00000001571 & $5.36 \mathrm{E}-10$ & 4.17E-10 & LRRD1 & $1.28 \mathrm{E}+00$ \\
\hline ENSBTAG00000007206 & $5.20 \mathrm{E}-10$ & 4.04E-10 & STRIP2 & $1.29 \mathrm{E}+00$ \\
\hline ENSBTAG00000015137 & $1.14 \mathrm{E}-09$ & $8.79 \mathrm{E}-10$ & OR9A4 & $1.29 \mathrm{E}+00$ \\
\hline ENSBTAG00000009182 & $5.64 \mathrm{E}-10$ & $4.35 \mathrm{E}-10$ & CLCN1 & $1.30 \mathrm{E}+00$ \\
\hline ENSBTAG00000021565 & $6.76 \mathrm{E}-10$ & $5.21 \mathrm{E}-10$ & & $1.30 \mathrm{E}+00$ \\
\hline ENSBTAG00000016131 & $2.54 \mathrm{E}-10$ & $1.95 \mathrm{E}-10$ & NCAPG2 & $1.30 \mathrm{E}+00$ \\
\hline ENSBTAG00000002938 & $5.53 \mathrm{E}-10$ & 4.25E-10 & CPED1 & $1.30 \mathrm{E}+00$ \\
\hline ENSBTAG00000007766 & $4.39 \mathrm{E}-10$ & $3.36 \mathrm{E}-10$ & CDK5 & $1.31 E+00$ \\
\hline ENSBTAG00000000990 & $1.77 \mathrm{E}-10$ & $1.36 \mathrm{E}-10$ & & $1.30 \mathrm{E}+00$ \\
\hline ENSBTAG00000010980 & $1.53 \mathrm{E}-10$ & 1.17E-10 & ANKMY2 & $1.31 \mathrm{E}+00$ \\
\hline ENSBTAG00000017505 & 7.65E-10 & $5.83 \mathrm{E}-10$ & PAXIP1 & $1.31 E+00$ \\
\hline ENSBTAG00000010219 & $4.10 \mathrm{E}-10$ & $3.12 \mathrm{E}-10$ & KRBA1 & $1.31 E+00$ \\
\hline ENSBTAG00000004540 & $4.38 \mathrm{E}-10$ & $3.33 \mathrm{E}-10$ & NUB1 & $1.32 \mathrm{E}+00$ \\
\hline ENSBTAG00000003222 & $2.36 \mathrm{E}-10$ & $1.79 \mathrm{E}-10$ & ASNS & $1.32 \mathrm{E}+00$ \\
\hline ENSBTAG00000006528 & $3.32 \mathrm{E}-10$ & $2.52 \mathrm{E}-10$ & BBS9 & $1.32 \mathrm{E}+00$ \\
\hline ENSBTAG00000002604 & $1.71 \mathrm{E}-10$ & $1.30 \mathrm{E}-10$ & SP8 & $1.32 \mathrm{E}+00$ \\
\hline ENSBTAG00000004263 & $2.11 \mathrm{E}-10$ & $1.59 \mathrm{E}-10$ & ATP6V0A4 & $1.32 \mathrm{E}+00$ \\
\hline ENSBTAG00000006876 & $6.28 \mathrm{E}-10$ & $4.75 \mathrm{E}-10$ & PMPCB & $1.32 \mathrm{E}+00$ \\
\hline ENSBTAG00000019065 & $6.15 \mathrm{E}-10$ & 4.65E-10 & EEPD1 & $1.32 \mathrm{E}+00$ \\
\hline ENSBTAG00000001892 & $2.92 \mathrm{E}-10$ & $2.20 \mathrm{E}-10$ & YAE1D1 & $1.33 \mathrm{E}+00$ \\
\hline ENSBTAG00000021458 & $8.06 \mathrm{E}-11$ & $6.09 \mathrm{E}-11$ & DLX6 & $1.32 \mathrm{E}+00$ \\
\hline ENSBTAG00000020335 & $6.89 \mathrm{E}-10$ & $5.20 \mathrm{E}-10$ & RNF32 & $1.32 \mathrm{E}+00$ \\
\hline ENSBTAG00000013248 & $1.33 \mathrm{E}-09$ & $9.99 \mathrm{E}-10$ & & $1.33 \mathrm{E}+00$ \\
\hline ENSBTAG00000032049 & $9.11 \mathrm{E}-10$ & $6.86 \mathrm{E}-10$ & TAS2R16 & $1.33 \mathrm{E}+00$ \\
\hline ENSBTAG00000035660 & $1.00 \mathrm{E}-09$ & 7.57E-10 & RPL36AL & $1.33 E+00$ \\
\hline ENSBTAG00000017086 & $5.75 \mathrm{E}-10$ & 4.32E-10 & GRB10 & $1.33 E+00$ \\
\hline ENSBTAG00000024723 & $2.46 \mathrm{E}-10$ & $1.85 \mathrm{E}-10$ & $\mathrm{CHCHD} 3$ & $1.33 E+00$ \\
\hline ENSBTAG00000031234 & $1.60 \mathrm{E}-09$ & $1.20 \mathrm{E}-09$ & & $1.33 E+00$ \\
\hline ENSBTAG00000002554 & $6.81 \mathrm{E}-10$ & 5.10E-10 & TCAF1 & $1.34 \mathrm{E}+00$ \\
\hline ENSBTAG00000001912 & $2.43 \mathrm{E}-10$ & $1.82 \mathrm{E}-10$ & TRPV5 & $1.34 \mathrm{E}+00$ \\
\hline ENSBTAG00000011228 & $4.93 \mathrm{E}-10$ & $3.68 \mathrm{E}-10$ & FASTK & $1.34 \mathrm{E}+00$ \\
\hline ENSBTAG00000000274 & $2.31 \mathrm{E}-10$ & $1.72 \mathrm{E}-10$ & YKT6 & $1.34 \mathrm{E}+00$ \\
\hline ENSBTAG00000017350 & $4.45 \mathrm{E}-10$ & $3.29 \mathrm{E}-10$ & PCLO & $1.35 E+00$ \\
\hline ENSBTAG00000008641 & 7.74E-10 & $5.71 \mathrm{E}-10$ & GNAT3 & $1.36 \mathrm{E}+00$ \\
\hline ENSBTAG00000008550 & 1.76E-09 & 1.30E-09 & & $1.35 E+00$ \\
\hline ENSBTAG00000007976 & $3.23 \mathrm{E}-10$ & 2.37E-10 & FАMЗC & $1.36 \mathrm{E}+00$ \\
\hline ENSBTAG00000016332 & $3.02 \mathrm{E}-10$ & $2.22 \mathrm{E}-10$ & ING3 & $1.36 \mathrm{E}+00$ \\
\hline ENSBTAG00000016975 & $1.66 \mathrm{E}-10$ & $1.22 \mathrm{E}-10$ & $\mathrm{H} 2 \mathrm{AFZ}$ & $1.36 \mathrm{E}+00$ \\
\hline ENSBTAG00000021849 & $2.14 \mathrm{E}-10$ & $1.57 \mathrm{E}-10$ & DUS4L & $1.36 \mathrm{E}+00$ \\
\hline ENSBTAG00000032598 & $1.31 \mathrm{E}-10$ & $9.57 \mathrm{E}-11$ & RP9 & $1.37 \mathrm{E}+00$ \\
\hline ENSBTAG00000015353 & $3.20 \mathrm{E}-10$ & $2.32 \mathrm{E}-10$ & NME8 & $1.38 \mathrm{E}+00$ \\
\hline ENSBTAG00000031967 & $5.46 \mathrm{E}-10$ & $3.95 \mathrm{E}-10$ & AMPH & $1.38 \mathrm{E}+00$ \\
\hline ENSBTAG00000017770 & $2.20 \mathrm{E}-10$ & $1.59 \mathrm{E}-10$ & & $1.38 \mathrm{E}+00$ \\
\hline ENSBTAG00000008302 & $6.62 \mathrm{E}-10$ & 4.77E-10 & RINT1 & $1.39 E+00$ \\
\hline ENSBTAG00000005526 & $2.78 \mathrm{E}-10$ & $2.01 \mathrm{E}-10$ & COG5 & $1.39 E+00$ \\
\hline ENSBTAG00000047072 & $1.23 \mathrm{E}-09$ & $8.83 \mathrm{E}-10$ & & $1.40 \mathrm{E}+00$ \\
\hline ENSBTAG00000039005 & $3.82 \mathrm{E}-10$ & $2.75 \mathrm{E}-10$ & OR2A2 & $1.39 \mathrm{E}+00$ \\
\hline ENSBTAG00000024361 & 4.14E-10 & $2.98 \mathrm{E}-10$ & & $1.39 E+00$ \\
\hline
\end{tabular}




\begin{tabular}{|c|c|c|c|c|}
\hline ENSBTAG00000016481 & $2.94 \mathrm{E}-10$ & $2.11 E-10$ & CALU & $1.39 E+00$ \\
\hline ENSBTAG00000009002 & $8.62 \mathrm{E}-10$ & $6.18 \mathrm{E}-10$ & & $1.39 E+00$ \\
\hline ENSBTAG00000002011 & $5.37 \mathrm{E}-10$ & $3.84 \mathrm{E}-10$ & KIAA0895 & $1.40 \mathrm{E}+00$ \\
\hline ENSBTAG00000005726 & 2.09E-10 & $1.49 \mathrm{E}-10$ & HNRNPA2B1 & $1.40 \mathrm{E}+00$ \\
\hline ENSBTAG00000009520 & $7.72 \mathrm{E}-10$ & $5.50 \mathrm{E}-10$ & $A D C Y 1$ & $1.40 \mathrm{E}+00$ \\
\hline ENSBTAG00000003658 & 4.67E-10 & 3.32E-10 & RELN & $1.41 E+00$ \\
\hline ENSBTAG00000009254 & $7.51 \mathrm{E}-10$ & $5.34 \mathrm{E}-10$ & CCDC129 & $1.41 \mathrm{E}+00$ \\
\hline ENSBTAG00000001527 & $3.03 \mathrm{E}-10$ & $2.16 \mathrm{E}-10$ & TMEM213 & $1.40 \mathrm{E}+00$ \\
\hline ENSBTAG00000014228 & $5.13 \mathrm{E}-10$ & $3.64 \mathrm{E}-10$ & TFEC & $1.41 \mathrm{E}+00$ \\
\hline ENSBTAG00000002859 & $1.55 \mathrm{E}-09$ & 1.10E-09 & & $1.41 \mathrm{E}+00$ \\
\hline ENSBTAG00000017860 & $4.21 \mathrm{E}-10$ & $2.98 \mathrm{E}-10$ & HIPK2 & $1.41 \mathrm{E}+00$ \\
\hline ENSBTAG00000017001 & 4.27E-10 & $3.00 \mathrm{E}-10$ & RBM28 & $1.42 \mathrm{E}+00$ \\
\hline ENSBTAG00000003633 & $3.36 \mathrm{E}-10$ & $2.35 \mathrm{E}-10$ & & $1.43 \mathrm{E}+00$ \\
\hline ENSBTAG00000003238 & $3.92 \mathrm{E}-10$ & $2.74 \mathrm{E}-10$ & MEOX2 & $1.43 \mathrm{E}+00$ \\
\hline ENSBTAG00000005110 & $2.53 \mathrm{E}-10$ & 1.77E-10 & CADPS2 & $1.43 \mathrm{E}+00$ \\
\hline ENSBTAG00000046308 & $2.33 \mathrm{E}-10$ & $1.62 \mathrm{E}-10$ & & $1.44 \mathrm{E}+00$ \\
\hline ENSBTAG00000046341 & $7.53 \mathrm{E}-10$ & $5.24 \mathrm{E}-10$ & & $1.44 \mathrm{E}+00$ \\
\hline ENSBTAG00000021617 & $3.72 \mathrm{E}-10$ & $2.58 \mathrm{E}-10$ & ZC3HAV1 & $1.44 \mathrm{E}+00$ \\
\hline ENSBTAG00000010243 & $5.39 \mathrm{E}-10$ & $3.69 \mathrm{E}-10$ & RBM33 & $1.46 \mathrm{E}+00$ \\
\hline ENSBTAG00000016373 & $1.23 \mathrm{E}-10$ & $8.38 \mathrm{E}-11$ & TTC26 & $1.46 \mathrm{E}+00$ \\
\hline ENSBTAG00000034091 & $1.46 \mathrm{E}-10$ & 9.97E-11 & LRRC72 & $1.46 \mathrm{E}+00$ \\
\hline ENSBTAG00000015925 & $4.39 \mathrm{E}-10$ & $2.98 \mathrm{E}-10$ & SDHAF3 & $1.47 \mathrm{E}+00$ \\
\hline ENSBTAG00000004398 & $5.37 \mathrm{E}-10$ & 3.62E-10 & IMMP2L & $1.48 \mathrm{E}+00$ \\
\hline ENSBTAG00000003784 & 5.87E-10 & $3.94 \mathrm{E}-10$ & DNAJC2 & $1.49 \mathrm{E}+00$ \\
\hline ENSBTAG00000016324 & $6.51 \mathrm{E}-10$ & $4.38 \mathrm{E}-10$ & FBXL13 & $1.49 \mathrm{E}+00$ \\
\hline ENSBTAG00000005524 & $2.05 \mathrm{E}-10$ & $1.38 \mathrm{E}-10$ & HBP1 & $1.49 \mathrm{E}+00$ \\
\hline ENSBTAG00000017905 & $3.95 \mathrm{E}-10$ & $2.65 \mathrm{E}-10$ & WDR91 & $1.49 E+00$ \\
\hline ENSBTAG00000014920 & $5.10 \mathrm{E}-10$ & $3.41 \mathrm{E}-10$ & SEMA3E & $1.50 E+00$ \\
\hline ENSBTAG00000044023 & $5.74 \mathrm{E}-10$ & $3.83 \mathrm{E}-10$ & CDK6 & $1.50 \mathrm{E}+00$ \\
\hline ENSBTAG00000024394 & $3.42 \mathrm{E}-10$ & $2.28 \mathrm{E}-10$ & SEMA3D & $1.50 \mathrm{E}+00$ \\
\hline ENSBTAG00000011412 & 7.33E-10 & $4.88 \mathrm{E}-10$ & LAMB1 & $1.50 \mathrm{E}+00$ \\
\hline ENSBTAG00000013414 & $2.22 \mathrm{E}-10$ & $1.48 \mathrm{E}-10$ & BLVRA & $1.50 \mathrm{E}+00$ \\
\hline ENSBTAG00000015510 & $3.80 \mathrm{E}-10$ & $2.52 \mathrm{E}-10$ & EPHB6 & $1.51 E+00$ \\
\hline ENSBTAG00000032121 & $3.74 \mathrm{E}-10$ & 2.47E-10 & SUGCT & $1.52 E+00$ \\
\hline ENSBTAG00000020183 & $7.06 \mathrm{E}-11$ & $4.66 \mathrm{E}-11$ & TSGA13 & $1.51 E+00$ \\
\hline ENSBTAG00000045535 & $6.17 \mathrm{E}-10$ & $4.06 \mathrm{E}-10$ & GALNTL5 & $1.52 E+00$ \\
\hline ENSBTAG00000023806 & $5.45 \mathrm{E}-10$ & $3.58 \mathrm{E}-10$ & & $1.52 \mathrm{E}+00$ \\
\hline ENSBTAG00000010866 & $3.08 \mathrm{E}-10$ & $2.02 \mathrm{E}-10$ & BMPER & $1.52 \mathrm{E}+00$ \\
\hline ENSBTAG00000038854 & $2.23 \mathrm{E}-10$ & $1.46 \mathrm{E}-10$ & CLEC2L & $1.53 \mathrm{E}+00$ \\
\hline ENSBTAG00000008139 & $6.75 \mathrm{E}-11$ & $4.41 \mathrm{E}-11$ & HOXA3 & $1.53 E+00$ \\
\hline ENSBTAG00000010756 & $2.12 \mathrm{E}-10$ & $1.38 \mathrm{E}-10$ & ST7 & $1.53 \mathrm{E}+00$ \\
\hline ENSBTAG00000008097 & $2.60 \mathrm{E}-10$ & $1.69 \mathrm{E}-10$ & WNT2 & $1.54 \mathrm{E}+00$ \\
\hline ENSBTAG00000007608 & $1.24 \mathrm{E}-10$ & $8.08 \mathrm{E}-11$ & ВMT2 & $1.53 E+00$ \\
\hline ENSBTAG00000046616 & $4.06 \mathrm{E}-10$ & $2.63 \mathrm{E}-10$ & & $1.54 \mathrm{E}+00$ \\
\hline ENSBTAG00000014250 & $2.50 \mathrm{E}-10$ & $1.62 \mathrm{E}-10$ & ZC3HAV1L & $1.54 \mathrm{E}+00$ \\
\hline ENSBTAG00000009296 & $6.45 \mathrm{E}-10$ & $4.18 \mathrm{E}-10$ & SLC26A5 & $1.54 \mathrm{E}+00$ \\
\hline ENSBTAG00000012261 & $2.18 \mathrm{E}-10$ & $1.41 \mathrm{E}-10$ & RAB19 & $1.55 \mathrm{E}+00$ \\
\hline ENSBTAG00000014161 & $7.69 \mathrm{E}-10$ & 4.97E-10 & ARMC10 & $1.55 E+00$ \\
\hline ENSBTAG00000017970 & $3.38 \mathrm{E}-10$ & $2.19 \mathrm{E}-10$ & $Z Y X$ & $1.54 \mathrm{E}+00$ \\
\hline ENSBTAG00000002157 & $3.72 \mathrm{E}-10$ & $2.40 \mathrm{E}-10$ & LMOD2 & $1.55 \mathrm{E}+00$ \\
\hline ENSBTAG00000047919 & $5.35 \mathrm{E}-10$ & $3.40 \mathrm{E}-10$ & & $1.57 \mathrm{E}+00$ \\
\hline ENSBTAG00000017458 & $5.87 \mathrm{E}-10$ & 3.73E-10 & CALCR & $1.57 E+00$ \\
\hline ENSBTAG00000006732 & $3.58 \mathrm{E}-10$ & 2.27E-10 & NRCAM & $1.58 \mathrm{E}+00$ \\
\hline ENSBTAG00000014735 & $9.85 \mathrm{E}-11$ & $6.20 \mathrm{E}-11$ & HOXA13 & $1.59 E+00$ \\
\hline ENSBTAG00000024341 & $2.31 \mathrm{E}-10$ & $1.45 \mathrm{E}-10$ & HOXA6 & $1.59 E+00$ \\
\hline ENSBTAG00000048012 & $2.65 \mathrm{E}-10$ & $1.67 \mathrm{E}-10$ & & $1.59 E+00$ \\
\hline ENSBTAG00000046917 & $7.45 \mathrm{E}-11$ & 4.69E-11 & GPR22 & $1.59 E+00$ \\
\hline ENSBTAG00000047188 & $3.44 \mathrm{E}-10$ & 2.17E-10 & & $1.59 E+00$ \\
\hline ENSBTAG00000012239 & $3.63 \mathrm{E}-10$ & $2.29 \mathrm{E}-10$ & SLC37A3 & $1.59 E+00$ \\
\hline ENSBTAG00000039559 & $2.82 \mathrm{E}-10$ & 1.77E-10 & & $1.59 E+00$ \\
\hline ENSBTAG00000048186 & $4.66 \mathrm{E}-10$ & $2.93 \mathrm{E}-10$ & & $1.59 E+00$ \\
\hline ENSBTAG00000039928 & $5.07 \mathrm{E}-10$ & $3.19 \mathrm{E}-10$ & & $1.59 E+00$ \\
\hline ENSBTAG00000002066 & $1.57 \mathrm{E}-10$ & $9.86 \mathrm{E}-11$ & MYL7 & $1.59 E+00$ \\
\hline ENSBTAG00000040040 & $1.19 \mathrm{E}-10$ & $7.51 \mathrm{E}-11$ & TAS2R40 & $1.59 E+00$ \\
\hline ENSBTAG00000012081 & $8.43 \mathrm{E}-10$ & $5.29 \mathrm{E}-10$ & & $1.59 E+00$ \\
\hline ENSBTAG00000038605 & $7.65 E-10$ & $4.81 \mathrm{E}-10$ & & $1.59 E+00$ \\
\hline ENSBTAG00000047562 & $1.18 \mathrm{E}-09$ & 7.37E-10 & & $1.60 E+00$ \\
\hline ENSBTAG00000021282 & $1.56 \mathrm{E}-10$ & $9.70 \mathrm{E}-11$ & SGCE & $1.61 E+00$ \\
\hline ENSBTAG00000003621 & $5.17 \mathrm{E}-10$ & $3.18 \mathrm{E}-10$ & & $1.63 E+00$ \\
\hline ENSBTAG00000033197 & $2.04 \mathrm{E}-10$ & $1.26 \mathrm{E}-10$ & EPDR1 & $1.62 \mathrm{E}+00$ \\
\hline
\end{tabular}




\begin{tabular}{|c|c|c|c|c|}
\hline ENSBTAG00000001976 & 7.79E-10 & $4.79 \mathrm{E}-10$ & PPP1R17 & $1.63 \mathrm{E}+00$ \\
\hline ENSBTAG00000013732 & $1.58 \mathrm{E}-10$ & $9.68 \mathrm{E}-11$ & GPR37 & $1.63 \mathrm{E}+00$ \\
\hline ENSBTAG00000027204 & $8.56 \mathrm{E}-10$ & $5.24 \mathrm{E}-10$ & & $1.63 \mathrm{E}+00$ \\
\hline ENSBTAG00000010671 & 3.17E-10 & $1.94 \mathrm{E}-10$ & GLI3 & $1.63 \mathrm{E}+00$ \\
\hline ENSBTAG00000014021 & $2.73 \mathrm{E}-10$ & $1.67 \mathrm{E}-10$ & UBN2 & $1.63 \mathrm{E}+00$ \\
\hline ENSBTAG00000003114 & $2.21 \mathrm{E}-10$ & $1.35 \mathrm{E}-10$ & AVL9 & $1.64 \mathrm{E}+00$ \\
\hline ENSBTAG00000019265 & 2.65E-10 & $1.61 \mathrm{E}-10$ & AGK & $1.64 E+00$ \\
\hline ENSBTAG00000005650 & $2.28 \mathrm{E}-10$ & $1.38 \mathrm{E}-10$ & SKAP2 & $1.65 E+00$ \\
\hline ENSBTAG00000014016 & $2.06 \mathrm{E}-10$ & $1.24 \mathrm{E}-10$ & IKZF1 & $1.66 \mathrm{E}+00$ \\
\hline ENSBTAG00000017919 & $3.98 \mathrm{E}-10$ & $2.38 \mathrm{E}-10$ & CNOT4 & $1.67 E+00$ \\
\hline ENSBTAG00000005997 & $1.23 \mathrm{E}-09$ & $7.36 \mathrm{E}-10$ & & $1.67 \mathrm{E}+00$ \\
\hline ENSBTAG00000004023 & $7.48 \mathrm{E}-10$ & 4.47E-10 & KIAA1324L & $1.67 \mathrm{E}+00$ \\
\hline ENSBTAG00000002317 & $2.53 \mathrm{E}-10$ & $1.51 \mathrm{E}-10$ & PTN & $1.68 \mathrm{E}+00$ \\
\hline ENSBTAG00000046491 & 2.20E-09 & $1.31 \mathrm{E}-09$ & & $1.68 \mathrm{E}+00$ \\
\hline ENSBTAG00000034609 & $8.70 \mathrm{E}-10$ & $5.14 \mathrm{E}-10$ & & $1.69 E+00$ \\
\hline ENSBTAG00000008155 & 1.14E-09 & $6.74 \mathrm{E}-10$ & SUN3 & $1.69 \mathrm{E}+00$ \\
\hline ENSBTAG00000019406 & $4.20 \mathrm{E}-10$ & $2.47 \mathrm{E}-10$ & IGF2BP3 & $1.70 \mathrm{E}+00$ \\
\hline ENSBTAG00000002891 & $6.24 \mathrm{E}-10$ & $3.65 \mathrm{E}-10$ & HTR5A & $1.71 \mathrm{E}+00$ \\
\hline ENSBTAG00000014389 & $3.86 \mathrm{E}-10$ & $2.24 \mathrm{E}-10$ & SP4 & $1.72 \mathrm{E}+00$ \\
\hline ENSBTAG00000021204 & $1.85 \mathrm{E}-10$ & $1.07 \mathrm{E}-10$ & TES & $1.73 \mathrm{E}+00$ \\
\hline ENSBTAG00000031236 & $3.96 \mathrm{E}-10$ & $2.29 \mathrm{E}-10$ & & $1.73 \mathrm{E}+00$ \\
\hline ENSBTAG00000003506 & $1.50 \mathrm{E}-10$ & $8.60 \mathrm{E}-11$ & STEAP2 & $1.74 \mathrm{E}+00$ \\
\hline ENSBTAG00000024379 & $7.95 \mathrm{E}-10$ & 4.57E-10 & LRRC17 & $1.74 \mathrm{E}+00$ \\
\hline ENSBTAG00000014768 & $5.47 \mathrm{E}-10$ & $3.14 \mathrm{E}-10$ & ZNF786 & $1.74 \mathrm{E}+00$ \\
\hline ENSBTAG00000038093 & $1.92 \mathrm{E}-10$ & $1.10 \mathrm{E}-10$ & PEG10 & $1.74 \mathrm{E}+00$ \\
\hline ENSBTAG00000019020 & $2.55 \mathrm{E}-10$ & $1.45 \mathrm{E}-10$ & TAX1BP1 & $1.76 \mathrm{E}+00$ \\
\hline ENSBTAG00000014371 & $3.81 \mathrm{E}-10$ & $2.16 \mathrm{E}-10$ & CHPF2 & $1.77 \mathrm{E}+00$ \\
\hline ENSBTAG00000003625 & $3.97 \mathrm{E}-10$ & $2.24 \mathrm{E}-10$ & VSTM2A & $1.77 \mathrm{E}+00$ \\
\hline ENSBTAG00000016223 & $4.61 \mathrm{E}-10$ & $2.60 \mathrm{E}-10$ & SCRN1 & $1.77 E+00$ \\
\hline ENSBTAG00000003531 & $8.89 \mathrm{E}-10$ & $5.01 \mathrm{E}-10$ & ABCA13 & $1.77 \mathrm{E}+00$ \\
\hline ENSBTAG00000018989 & $8.60 \mathrm{E}-10$ & $4.83 \mathrm{E}-10$ & GRM3 & $1.78 \mathrm{E}+00$ \\
\hline ENSBTAG00000016010 & $4.82 \mathrm{E}-10$ & $2.71 \mathrm{E}-10$ & KLHL7 & $1.78 \mathrm{E}+00$ \\
\hline ENSBTAG00000012649 & $6.17 \mathrm{E}-10$ & $3.46 \mathrm{E}-10$ & LSM8 & $1.78 \mathrm{E}+00$ \\
\hline ENSBTAG00000000715 & $9.60 \mathrm{E}-10$ & $5.36 \mathrm{E}-10$ & & $1.79 E+00$ \\
\hline ENSBTAG00000037918 & 7.93E-10 & $4.42 \mathrm{E}-10$ & & $1.79 E+00$ \\
\hline ENSBTAG00000020848 & 3.13E-10 & $1.74 \mathrm{E}-10$ & PIK3CG & $1.80 \mathrm{E}+00$ \\
\hline ENSBTAG00000012653 & $3.52 \mathrm{E}-10$ & $1.95 \mathrm{E}-10$ & CAMK2B & $1.81 \mathrm{E}+00$ \\
\hline ENSBTAG00000004072 & $4.02 \mathrm{E}-10$ & $2.22 \mathrm{E}-10$ & CAPZA2 & $1.81 \mathrm{E}+00$ \\
\hline ENSBTAG00000030817 & $3.04 \mathrm{E}-10$ & $1.67 \mathrm{E}-10$ & LMBR1 & $1.82 \mathrm{E}+00$ \\
\hline ENSBTAG00000004894 & $1.60 \mathrm{E}-09$ & $8.75 \mathrm{E}-10$ & & $1.83 \mathrm{E}+00$ \\
\hline ENSBTAG00000031265 & $4.85 \mathrm{E}-10$ & $2.66 \mathrm{E}-10$ & SVOPL & $1.82 \mathrm{E}+00$ \\
\hline ENSBTAG00000009109 & $1.86 \mathrm{E}-10$ & $1.00 \mathrm{E}-10$ & CASD1 & $1.86 \mathrm{E}+00$ \\
\hline ENSBTAG00000010609 & $1.91 \mathrm{E}-10$ & $1.02 \mathrm{E}-10$ & STK31 & $1.87 \mathrm{E}+00$ \\
\hline ENSBTAG00000040082 & $1.52 \mathrm{E}-10$ & $8.22 \mathrm{E}-11$ & HOXA10 & $1.85 \mathrm{E}+00$ \\
\hline ENSBTAG00000017143 & $3.88 \mathrm{E}-10$ & $2.07 \mathrm{E}-10$ & PDIA4 & $1.87 \mathrm{E}+00$ \\
\hline ENSBTAG00000000820 & $5.08 \mathrm{E}-10$ & $2.68 \mathrm{E}-10$ & & $1.89 E+00$ \\
\hline ENSBTAG00000013050 & $3.87 \mathrm{E}-10$ & $2.03 \mathrm{E}-10$ & ZNF277 & $1.91 \mathrm{E}+00$ \\
\hline ENSBTAG00000046793 & $1.20 \mathrm{E}-09$ & $6.26 \mathrm{E}-10$ & & $1.92 \mathrm{E}+00$ \\
\hline ENSBTAG00000014911 & $7.13 \mathrm{E}-10$ & $3.70 \mathrm{E}-10$ & LEP & $1.93 \mathrm{E}+00$ \\
\hline ENSBTAG00000002151 & $2.05 \mathrm{E}-10$ & $1.07 \mathrm{E}-10$ & ASB15 & $1.92 \mathrm{E}+00$ \\
\hline ENSBTAG00000006232 & $1.06 \mathrm{E}-09$ & $5.50 \mathrm{E}-10$ & WDR86 & $1.93 \mathrm{E}+00$ \\
\hline ENSBTAG00000008428 & $4.93 \mathrm{E}-10$ & $2.54 \mathrm{E}-10$ & UPP1 & $1.94 \mathrm{E}+00$ \\
\hline ENSBTAG00000013078 & 4.45E-10 & $2.29 \mathrm{E}-10$ & DNAH11 & $1.94 \mathrm{E}+00$ \\
\hline ENSBTAG00000021365 & $3.50 \mathrm{E}-10$ & $1.80 \mathrm{E}-10$ & CDHR3 & $1.95 \mathrm{E}+00$ \\
\hline ENSBTAG00000004399 & $1.93 \mathrm{E}-10$ & $9.70 \mathrm{E}-11$ & LRRN3 & $1.99 \mathrm{E}+00$ \\
\hline ENSBTAG00000013263 & $2.73 \mathrm{E}-10$ & $1.37 \mathrm{E}-10$ & HOXA1 & $1.99 \mathrm{E}+00$ \\
\hline ENSBTAG00000018645 & $9.59 \mathrm{E}-11$ & $4.83 \mathrm{E}-11$ & DLX5 & $1.98 \mathrm{E}+00$ \\
\hline ENSBTAG00000000558 & $1.28 \mathrm{E}-10$ & $6.46 \mathrm{E}-11$ & NEUROD6 & $1.98 \mathrm{E}+00$ \\
\hline ENSBTAG00000016546 & $3.95 \mathrm{E}-10$ & $1.99 \mathrm{E}-10$ & PARP12 & $1.98 \mathrm{E}+00$ \\
\hline ENSBTAG00000014958 & $2.26 \mathrm{E}-10$ & $1.14 \mathrm{E}-10$ & PRKAR2B & $1.98 \mathrm{E}+00$ \\
\hline ENSBTAG00000002917 & $5.13 \mathrm{E}-10$ & $2.58 \mathrm{E}-10$ & & $1.99 E+00$ \\
\hline ENSBTAG00000009418 & $3.91 \mathrm{E}-10$ & $1.97 \mathrm{E}-10$ & FAM131B & $1.98 \mathrm{E}+00$ \\
\hline ENSBTAG00000009472 & $1.54 \mathrm{E}-10$ & 7.73E-11 & C7orf25 & $1.99 \mathrm{E}+00$ \\
\hline ENSBTAG00000020484 & $1.66 \mathrm{E}-10$ & $8.37 E-11$ & ADCK2 & $1.98 \mathrm{E}+00$ \\
\hline ENSBTAG00000046164 & $8.38 \mathrm{E}-10$ & $4.20 \mathrm{E}-10$ & & $2.00 \mathrm{E}+00$ \\
\hline ENSBTAG00000020225 & $5.52 \mathrm{E}-10$ & $2.71 \mathrm{E}-10$ & TBXAS1 & $2.04 \mathrm{E}+00$ \\
\hline ENSBTAG00000021469 & $4.15 \mathrm{E}-10$ & $2.03 \mathrm{E}-10$ & CTTNBP2 & $2.04 \mathrm{E}+00$ \\
\hline ENSBTAG00000002175 & 3.57E-10 & $1.75 \mathrm{E}-10$ & SRPK2 & $2.04 \mathrm{E}+00$ \\
\hline ENSBTAG00000045794 & $3.50 \mathrm{E}-10$ & $1.71 \mathrm{E}-10$ & C7orf73 & $2.05 \mathrm{E}+00$ \\
\hline ENSBTAG00000015666 & 1.15E-09 & $5.58 \mathrm{E}-10$ & & $2.05 E+00$ \\
\hline
\end{tabular}




\begin{tabular}{|c|c|c|c|c|}
\hline ENSBTAG00000047482 & $6.47 \mathrm{E}-10$ & $3.14 \mathrm{E}-10$ & PRRT4 & $2.06 \mathrm{E}+00$ \\
\hline ENSBTAG00000001754 & 4.15E-10 & $2.01 \mathrm{E}-10$ & AHCYL2 & $2.06 E+00$ \\
\hline ENSBTAG00000003495 & $2.40 \mathrm{E}-10$ & $1.16 \mathrm{E}-10$ & KDM7A & $2.07 E+00$ \\
\hline ENSBTAG00000006733 & $1.03 \mathrm{E}-10$ & 4.94E-11 & PPP1R3A & $2.09 E+00$ \\
\hline ENSBTAG00000006359 & 3.77E-10 & $1.80 \mathrm{E}-10$ & EPHA1 & $2.10 \mathrm{E}+00$ \\
\hline ENSBTAG00000003709 & 4.34E-10 & $2.05 \mathrm{E}-10$ & ITGB8 & $2.11 E+00$ \\
\hline ENSBTAG00000003994 & 3.45E-10 & $1.63 \mathrm{E}-10$ & IgFBp3 & $2.12 \mathrm{E}+00$ \\
\hline ENSBTAG00000002595 & $6.41 \mathrm{E}-10$ & $3.02 \mathrm{E}-10$ & ABCB5 & $2.12 \mathrm{E}+00$ \\
\hline ENSBTAG00000012241 & $1.66 \mathrm{E}-10$ & $7.85 E-11$ & POLD2 & $2.12 \mathrm{E}+00$ \\
\hline ENSBTAG00000046712 & $4.19 \mathrm{E}-10$ & $1.96 \mathrm{E}-10$ & PSMC2 & $2.14 E+00$ \\
\hline ENSBTAG00000007136 & $3.64 \mathrm{E}-10$ & $1.70 \mathrm{E}-10$ & ADAM22 & $2.14 \mathrm{E}+00$ \\
\hline ENSBTAG00000014220 & 4.79E-10 & $2.23 \mathrm{E}-10$ & & $2.15 \mathrm{E}+00$ \\
\hline ENSBTAG00000021535 & 5.13E-10 & $2.38 \mathrm{E}-10$ & CROT & $2.15 \mathrm{E}+00$ \\
\hline ENSBTAG00000002739 & $3.74 \mathrm{E}-10$ & $1.73 \mathrm{E}-10$ & PDE1C & $2.16 \mathrm{E}+00$ \\
\hline ENSBTAG00000033359 & $6.32 \mathrm{E}-10$ & $2.89 \mathrm{E}-10$ & & $2.19 E+00$ \\
\hline ENSBTAG00000013976 & 3.27E-10 & $1.49 \mathrm{E}-10$ & AGBL3 & $2.19 E+00$ \\
\hline ENSBTAG00000010692 & $5.62 \mathrm{E}-10$ & $2.53 \mathrm{E}-10$ & SND1 & $2.22 \mathrm{E}+00$ \\
\hline ENSBTAG00000012128 & $2.51 \mathrm{E}-10$ & $1.12 \mathrm{E}-10$ & AASS & $2.24 \mathrm{E}+00$ \\
\hline ENSBTAG00000018133 & $5.59 \mathrm{E}-10$ & $2.45 \mathrm{E}-10$ & SEMA3A & $2.28 \mathrm{E}+00$ \\
\hline ENSBTAG00000011127 & $3.39 \mathrm{E}-10$ & $1.47 \mathrm{E}-10$ & NUP205 & $2.31 E+00$ \\
\hline ENSBTAG00000010437 & $4.25 \mathrm{E}-10$ & $1.82 \mathrm{E}-10$ & MKLN1 & $2.33 \mathrm{E}+00$ \\
\hline ENSBTAG00000016709 & 2.13E-10 & $9.12 \mathrm{E}-11$ & NT5C3A & $2.34 \mathrm{E}+00$ \\
\hline ENSBTAG00000015509 & $4.52 \mathrm{E}-10$ & $1.93 \mathrm{E}-10$ & NAMPT & $2.34 \mathrm{E}+00$ \\
\hline ENSBTAG00000009207 & $3.01 \mathrm{E}-10$ & $1.27 \mathrm{E}-10$ & KMT2E & $2.37 E+00$ \\
\hline ENSBTAG00000033449 & $1.12 \mathrm{E}-09$ & $4.73 \mathrm{E}-10$ & SLC25A40 & $2.36 \mathrm{E}+00$ \\
\hline ENSBTAG00000003115 & $9.56 \mathrm{E}-11$ & 4.01E-11 & KBTBD2 & $2.38 \mathrm{E}+00$ \\
\hline ENSBTAG00000009366 & $2.48 \mathrm{E}-10$ & $1.04 \mathrm{E}-10$ & ARF5 & $2.38 \mathrm{E}+00$ \\
\hline ENSBTAG00000046211 & $4.80 \mathrm{E}-10$ & $2.01 \mathrm{E}-10$ & & $2.39 E+00$ \\
\hline ENSBTAG00000022498 & 4.69E-10 & $1.96 \mathrm{E}-10$ & & $2.39 E+00$ \\
\hline ENSBTAG00000018430 & $2.92 \mathrm{E}-10$ & $1.23 \mathrm{E}-10$ & GSAP & $2.37 E+00$ \\
\hline ENSBTAG00000046586 & $7.08 \mathrm{E}-10$ & $2.96 \mathrm{E}-10$ & & $2.39 E+00$ \\
\hline ENSBTAG00000038385 & $1.02 \mathrm{E}-09$ & 4.24E-10 & & $2.40 E+00$ \\
\hline ENSBTAG00000045978 & $9.93 \mathrm{E}-10$ & 4.15E-10 & & $2.39 E+00$ \\
\hline ENSBTAG00000015337 & $5.53 \mathrm{E}-10$ & $2.29 \mathrm{E}-10$ & TSPAN12 & $2.41 E+00$ \\
\hline ENSBTAG00000021313 & $9.32 \mathrm{E}-10$ & $3.84 \mathrm{E}-10$ & RUNDC3B & $2.43 \mathrm{E}+00$ \\
\hline ENSBTAG00000021543 & $2.92 \mathrm{E}-10$ & $1.20 \mathrm{E}-10$ & MDFIC & $2.43 E+00$ \\
\hline ENSBTAG00000019024 & 3.93E-10 & $1.60 \mathrm{E}-10$ & JAZF1 & $2.46 \mathrm{E}+00$ \\
\hline ENSBTAG00000046430 & $5.22 \mathrm{E}-10$ & $2.12 \mathrm{E}-10$ & ZNF804B & $2.46 E+00$ \\
\hline ENSBTAG00000044068 & $5.84 \mathrm{E}-10$ & $2.37 \mathrm{E}-10$ & VWC2 & $2.46 \mathrm{E}+00$ \\
\hline ENSBTAG00000021182 & $3.45 \mathrm{E}-10$ & $1.39 \mathrm{E}-10$ & DOCK4 & $2.48 \mathrm{E}+00$ \\
\hline ENSBTAG00000002940 & $1.60 \mathrm{E}-10$ & $6.41 \mathrm{E}-11$ & WNT16 & $2.49 \mathrm{E}+00$ \\
\hline ENSBTAG00000018158 & $5.81 \mathrm{E}-10$ & $2.30 \mathrm{E}-10$ & TMEM139 & $2.53 E+00$ \\
\hline ENSBTAG00000001182 & $2.11 \mathrm{E}-10$ & $8.26 \mathrm{E}-11$ & 07-Sep & $2.55 \mathrm{E}+00$ \\
\hline ENSBTAG00000000169 & $3.41 \mathrm{E}-10$ & $1.33 \mathrm{E}-10$ & ASZ1 & $2.56 \mathrm{E}+00$ \\
\hline ENSBTAG00000018348 & $3.43 \mathrm{E}-10$ & $1.34 \mathrm{E}-10$ & CBLL1 & $2.55 E+00$ \\
\hline ENSBTAG00000010597 & $1.13 \mathrm{E}-10$ & 4.37E-11 & & $2.58 \mathrm{E}+00$ \\
\hline ENSBTAG00000020704 & $1.29 \mathrm{E}-10$ & 4.97E-11 & RAMP3 & $2.60 \mathrm{E}+00$ \\
\hline ENSBTAG00000020453 & $1.14 \mathrm{E}-09$ & $4.31 \mathrm{E}-10$ & CLEC5A & $2.63 \mathrm{E}+00$ \\
\hline ENSBTAG00000010168 & $4.39 \mathrm{E}-10$ & $1.66 \mathrm{E}-10$ & & $2.64 \mathrm{E}+00$ \\
\hline ENSBTAG00000033954 & $7.34 \mathrm{E}-10$ & $2.76 \mathrm{E}-10$ & PRPS1L1 & $2.66 \mathrm{E}+00$ \\
\hline ENSBTAG00000032166 & $3.64 \mathrm{E}-10$ & $1.36 \mathrm{E}-10$ & GTPBP10 & $2.68 \mathrm{E}+00$ \\
\hline ENSBTAG00000040058 & $7.08 \mathrm{E}-10$ & $2.62 \mathrm{E}-10$ & ORC5 & $2.70 E+00$ \\
\hline ENSBTAG00000012622 & $4.39 \mathrm{E}-10$ & $1.61 \mathrm{E}-10$ & TRA2A & $2.72 \mathrm{E}+00$ \\
\hline ENSBTAG00000013953 & $3.78 \mathrm{E}-10$ & $1.38 \mathrm{E}-10$ & CALD1 & $2.74 \mathrm{E}+00$ \\
\hline ENSBTAG00000014069 & $4.02 \mathrm{E}-10$ & $1.46 \mathrm{E}-10$ & PDK4 & $2.75 \mathrm{E}+00$ \\
\hline ENSBTAG00000010612 & 2.07E-10 & 7.43E-11 & TMED4 & $2.78 \mathrm{E}+00$ \\
\hline ENSBTAG00000020919 & $1.70 \mathrm{E}-10$ & $6.11 \mathrm{E}-11$ & EVX1 & $2.78 \mathrm{E}+00$ \\
\hline ENSBTAG00000011229 & $2.37 \mathrm{E}-10$ & $8.51 E-11$ & TMUB1 & $2.78 \mathrm{E}+00$ \\
\hline ENSBTAG00000003864 & $5.20 \mathrm{E}-10$ & $1.86 \mathrm{E}-10$ & SLC13A4 & $2.79 E+00$ \\
\hline ENSBTAG00000001346 & $5.50 \mathrm{E}-10$ & $1.97 \mathrm{E}-10$ & STRA8 & $2.79 E+00$ \\
\hline ENSBTAG00000002431 & $5.03 \mathrm{E}-10$ & $1.80 \mathrm{E}-10$ & TRIM24 & $2.80 \mathrm{E}+00$ \\
\hline ENSBTAG00000008090 & $2.23 \mathrm{E}-10$ & 7.87E-11 & $\mathrm{CCM} 2$ & $2.83 E+00$ \\
\hline ENSBTAG00000020869 & $6.46 \mathrm{E}-10$ & $2.27 \mathrm{E}-10$ & DDC & $2.85 \mathrm{E}+00$ \\
\hline ENSBTAG00000003019 & $9.98 \mathrm{E}-10$ & $3.50 \mathrm{E}-10$ & CRYGN & $2.85 \mathrm{E}+00$ \\
\hline ENSBTAG00000007796 & $1.82 \mathrm{E}-10$ & $6.38 \mathrm{E}-11$ & POLM & $2.86 \mathrm{E}+00$ \\
\hline ENSBTAG00000015539 & $5.98 \mathrm{E}-10$ & $2.08 \mathrm{E}-10$ & RAPGEF5 & $2.87 E+00$ \\
\hline ENSBTAG00000000719 & 8.04E-10 & $2.76 \mathrm{E}-10$ & & $2.91 E+00$ \\
\hline ENSBTAG00000015326 & $6.99 \mathrm{E}-10$ & $2.40 \mathrm{E}-10$ & DFNA5 & $2.91 E+00$ \\
\hline ENSBTAG00000004658 & $9.34 \mathrm{E}-10$ & $3.17 \mathrm{E}-10$ & WEE2 & $2.94 \mathrm{E}+00$ \\
\hline ENSBTAG00000020569 & $3.79 \mathrm{E}-10$ & $1.28 \mathrm{E}-10$ & CACNA2D1 & $2.96 \mathrm{E}+00$ \\
\hline
\end{tabular}




\begin{tabular}{|c|c|c|c|c|}
\hline ENSBTAG00000031861 & $5.14 \mathrm{E}-10$ & $1.72 \mathrm{E}-10$ & RHEB & $2.99 \mathrm{E}+00$ \\
\hline ENSBTAG00000024065 & 1.37E-09 & $4.55 \mathrm{E}-10$ & TAS2R38 & $3.01 E+00$ \\
\hline ENSBTAG00000002259 & $7.23 \mathrm{E}-10$ & $2.39 \mathrm{E}-10$ & & $3.02 E+00$ \\
\hline ENSBTAG00000004503 & $3.57 \mathrm{E}-10$ & $1.16 \mathrm{E}-10$ & NPY & $3.08 \mathrm{E}+00$ \\
\hline ENSBTAG00000009390 & $5.48 \mathrm{E}-10$ & $1.78 \mathrm{E}-10$ & FAM188B & $3.08 \mathrm{E}+00$ \\
\hline ENSBTAG00000007870 & $4.50 \mathrm{E}-10$ & $1.46 \mathrm{E}-10$ & FKBP14 & $3.08 \mathrm{E}+00$ \\
\hline ENSBTAG00000002037 & $5.34 \mathrm{E}-10$ & $1.72 \mathrm{E}-10$ & ATXN7L1 & $3.10 \mathrm{E}+00$ \\
\hline ENSBTAG00000010704 & $4.86 \mathrm{E}-10$ & $1.55 \mathrm{E}-10$ & LRRC4 & $3.13 E+00$ \\
\hline ENSBTAG00000000521 & 3.07E-10 & $9.77 \mathrm{E}-11$ & PLEKHA8 & $3.14 \mathrm{E}+00$ \\
\hline ENSBTAG00000004976 & $5.06 \mathrm{E}-10$ & $1.60 \mathrm{E}-10$ & CDCA7L & $3.16 \mathrm{E}+00$ \\
\hline ENSBTAG00000005103 & $1.43 \mathrm{E}-10$ & $4.48 \mathrm{E}-11$ & FEZF1 & $3.18 \mathrm{E}+00$ \\
\hline ENSBTAG00000009902 & $1.56 \mathrm{E}-10$ & $4.91 \mathrm{E}-11$ & & $3.17 E+00$ \\
\hline ENSBTAG00000003664 & $3.48 \mathrm{E}-10$ & $1.09 \mathrm{E}-10$ & & $3.19 E+00$ \\
\hline ENSBTAG00000045592 & $3.88 \mathrm{E}-10$ & $1.22 \mathrm{E}-10$ & & $3.18 \mathrm{E}+00$ \\
\hline ENSBTAG00000038241 & $1.95 \mathrm{E}-10$ & $6.14 \mathrm{E}-11$ & REPIN1 & $3.18 \mathrm{E}+00$ \\
\hline ENSBTAG00000047684 & $1.20 \mathrm{E}-09$ & $3.75 \mathrm{E}-10$ & & $3.20 \mathrm{E}+00$ \\
\hline ENSBTAG00000002869 & $6.42 \mathrm{E}-10$ & $2.01 \mathrm{E}-10$ & ARL4A & $3.19 E+00$ \\
\hline ENSBTAG00000034905 & $5.83 \mathrm{E}-10$ & $1.81 \mathrm{E}-10$ & & $3.22 \mathrm{E}+00$ \\
\hline ENSBTAG00000005653 & $3.74 \mathrm{E}-10$ & $1.14 \mathrm{E}-10$ & $\mathrm{ABCB} 4$ & $3.28 \mathrm{E}+00$ \\
\hline ENSBTAG00000006377 & $1.50 \mathrm{E}-10$ & 4.54E-11 & MYO1G & $3.29 E+00$ \\
\hline ENSBTAG00000003810 & $3.99 \mathrm{E}-10$ & $1.21 \mathrm{E}-10$ & & $3.29 E+00$ \\
\hline ENSBTAG00000013010 & 8.33E-10 & $2.52 \mathrm{E}-10$ & CCDC126 & $3.30 \mathrm{E}+00$ \\
\hline ENSBTAG00000005285 & 4.37E-10 & $1.31 \mathrm{E}-10$ & MRPS33 & $3.33 E+00$ \\
\hline ENSBTAG00000047646 & $4.66 \mathrm{E}-10$ & $1.38 \mathrm{E}-10$ & & $3.38 \mathrm{E}+00$ \\
\hline ENSBTAG00000001528 & $1.85 \mathrm{E}-10$ & $5.46 \mathrm{E}-11$ & CDK13 & $3.39 E+00$ \\
\hline ENSBTAG00000007253 & $1.94 \mathrm{E}-10$ & $5.72 \mathrm{E}-11$ & TSPAN33 & $3.39 E+00$ \\
\hline ENSBTAG00000021761 & $2.16 \mathrm{E}-10$ & $6.35 \mathrm{E}-11$ & BRAF & $3.40 E+00$ \\
\hline ENSBTAG00000034936 & 4.07E-10 & $1.18 \mathrm{E}-10$ & ANKIB1 & $3.44 E+00$ \\
\hline ENSBTAG00000020878 & $3.19 \mathrm{E}-10$ & $9.10 \mathrm{E}-11$ & DMTF1 & $3.50 E+00$ \\
\hline ENSBTAG00000001576 & $3.14 \mathrm{E}-10$ & $8.78 \mathrm{E}-11$ & TMEM140 & $3.58 \mathrm{E}+00$ \\
\hline ENSBTAG00000038283 & $4.88 \mathrm{E}-10$ & $1.34 \mathrm{E}-10$ & TMEM243 & $3.64 E+00$ \\
\hline ENSBTAG00000013287 & $3.48 \mathrm{E}-10$ & 9.34E-11 & SMO & $3.72 E+00$ \\
\hline ENSBTAG00000008093 & $4.84 \mathrm{E}-10$ & $1.29 \mathrm{E}-10$ & NACAD & $3.75 E+00$ \\
\hline ENSBTAG00000011002 & $3.96 \mathrm{E}-10$ & $1.03 \mathrm{E}-10$ & CCDC136 & $3.84 \mathrm{E}+00$ \\
\hline ENSBTAG00000035064 & $9.83 \mathrm{E}-10$ & $2.52 \mathrm{E}-10$ & C7orf57 & $3.90 E+00$ \\
\hline ENSBTAG00000007743 & $5.94 \mathrm{E}-10$ & $1.50 \mathrm{E}-10$ & PUS7 & $3.96 \mathrm{E}+00$ \\
\hline ENSBTAG00000012237 & $1.25 \mathrm{E}-10$ & $3.16 \mathrm{E}-11$ & AEBP1 & $3.96 \mathrm{E}+00$ \\
\hline ENSBTAG00000011997 & $3.04 \mathrm{E}-10$ & $7.65 \mathrm{E}-11$ & ZMIZ2 & $3.97 E+00$ \\
\hline ENSBTAG00000045677 & $1.69 \mathrm{E}-10$ & 4.26E-11 & & $3.97 \mathrm{E}+00$ \\
\hline ENSBTAG00000015303 & $4.39 \mathrm{E}-10$ & $1.09 \mathrm{E}-10$ & MPP6 & $4.02 E+00$ \\
\hline ENSBTAG00000009867 & $5.48 \mathrm{E}-10$ & $1.32 \mathrm{E}-10$ & FAM71F1 & $4.15 E+00$ \\
\hline ENSBTAG00000014711 & $7.13 \mathrm{E}-10$ & $1.64 \mathrm{E}-10$ & DBF4 & $4.35 E+00$ \\
\hline ENSBTAG00000006029 & $1.49 \mathrm{E}-10$ & 3.25E-11 & $\mathrm{OGDH}$ & $4.57 E+00$ \\
\hline ENSBTAG00000001773 & $4.61 E-10$ & $9.97 \mathrm{E}-11$ & C7orf49 & $4.62 E+00$ \\
\hline ENSBTAG00000009334 & $2.13 \mathrm{E}-10$ & 4.57E-11 & NDUFA5 & $4.64 E+00$ \\
\hline ENSBTAG00000000410 & $5.77 \mathrm{E}-10$ & $1.21 \mathrm{E}-10$ & PAX4 & $4.76 \mathrm{E}+00$ \\
\hline ENSBTAG00000010602 & $1.14 \mathrm{E}-10$ & $2.20 \mathrm{E}-11$ & DDX56 & $5.15 E+00$ \\
\hline ENSBTAG00000002340 & $4.95 \mathrm{E}-10$ & $9.40 \mathrm{E}-11$ & STEAP4 & $5.26 \mathrm{E}+00$ \\
\hline ENSBTAG00000007442 & $6.60 \mathrm{E}-10$ & $1.25 \mathrm{E}-10$ & AKAP9 & $5.28 \mathrm{E}+00$ \\
\hline ENSBTAG00000009558 & $1.71 \mathrm{E}-09$ & $3.19 \mathrm{E}-10$ & TRBV30 & $5.37 E+00$ \\
\hline ENSBTAG00000009786 & 7.07E-10 & $1.32 \mathrm{E}-10$ & SCIN & $5.35 E+00$ \\
\hline ENSBTAG00000006253 & $2.44 \mathrm{E}-10$ & $4.55 \mathrm{E}-11$ & FLNC & $5.36 \mathrm{E}+00$ \\
\hline ENSBTAG00000021216 & $6.22 \mathrm{E}-10$ & 1.15E-10 & HECW1 & $5.41 \mathrm{E}+00$ \\
\hline ENSBTAG00000003449 & $4.02 \mathrm{E}-10$ & $7.45 \mathrm{E}-11$ & KCP & $5.39 E+00$ \\
\hline ENSBTAG00000004405 & $2.47 \mathrm{E}-10$ & $4.56 \mathrm{E}-11$ & IQUB & $5.41 E+00$ \\
\hline ENSBTAG00000002674 & $3.26 \mathrm{E}-10$ & $5.86 \mathrm{E}-11$ & GNGT1 & $5.56 \mathrm{E}+00$ \\
\hline ENSBTAG00000046334 & $3.27 \mathrm{E}-10$ & 5.87E-11 & & $5.57 \mathrm{E}+00$ \\
\hline ENSBTAG00000021869 & $1.89 \mathrm{E}-10$ & $3.39 \mathrm{E}-11$ & THAP5 & $5.55 E+00$ \\
\hline ENSBTAG00000009839 & $5.64 \mathrm{E}-10$ & $1.01 \mathrm{E}-10$ & GSTK1 & $5.58 \mathrm{E}+00$ \\
\hline ENSBTAG00000014171 & $6.10 \mathrm{E}-10$ & 1.07E-10 & NAPEPLD & $5.69 E+00$ \\
\hline ENSBTAG00000015588 & $2.44 \mathrm{E}-10$ & 4.09E-11 & TMEM209 & $5.96 \mathrm{E}+00$ \\
\hline ENSBTAG00000017793 & $9.78 \mathrm{E}-10$ & $1.59 \mathrm{E}-10$ & KLRG2 & $6.15 E+00$ \\
\hline ENSBTAG00000020247 & $4.20 \mathrm{E}-10$ & $6.67 \mathrm{E}-11$ & ADCYAP1R1 & $6.28 \mathrm{E}+00$ \\
\hline ENSBTAG00000024387 & $7.02 \mathrm{E}-10$ & $1.11 \mathrm{E}-10$ & PNPLA8 & $6.32 E+00$ \\
\hline ENSBTAG00000014921 & $1.50 \mathrm{E}-10$ & $2.37 \mathrm{E}-11$ & IL6 & $6.33 E+00$ \\
\hline ENSBTAG00000002750 & $6.80 \mathrm{E}-10$ & $1.06 \mathrm{E}-10$ & KRIT1 & $6.41 E+00$ \\
\hline ENSBTAG00000006661 & $7.09 \mathrm{E}-10$ & $1.11 \mathrm{E}-10$ & RALA & $6.38 \mathrm{E}+00$ \\
\hline ENSBTAG00000040349 & 5.57E-10 & $8.65 \mathrm{E}-11$ & & $6.43 E+00$ \\
\hline ENSBTAG00000001992 & $6.36 \mathrm{E}-10$ & $9.80 \mathrm{E}-11$ & CYP51A1 & $6.48 \mathrm{E}+00$ \\
\hline ENSBTAG00000045578 & $7.35 \mathrm{E}-10$ & $1.12 \mathrm{E}-10$ & AGR3 & $6.56 \mathrm{E}+00$ \\
\hline
\end{tabular}




\begin{tabular}{|c|c|c|c|c|}
\hline ENSBTAG00000021759 & $2.48 \mathrm{E}-10$ & 3.67E-11 & NDUFB2 & $6.75 E+00$ \\
\hline ENSBTAG00000015356 & $8.41 \mathrm{E}-11$ & $1.18 \mathrm{E}-11$ & TAC1 & $7.07 E+00$ \\
\hline ENSBTAG00000024291 & $3.95 \mathrm{E}-10$ & $4.51 \mathrm{E}-11$ & & $8.74 E+00$ \\
\hline ENSBTAG00000009365 & $7.20 \mathrm{E}-10$ & 7.62E-11 & FSCN3 & $9.44 E+00$ \\
\hline ENSBTAG00000010504 & $2.73 \mathrm{E}-10$ & $2.86 \mathrm{E}-11$ & TBRG4 & $9.51 E+00$ \\
\hline ENSBTAG00000014255 & $6.84 \mathrm{E}-10$ & $5.86 \mathrm{E}-11$ & VPS50 & $1.16 \mathrm{E}+01$ \\
\hline ENSBTAG00000003751 & $9.24 \mathrm{E}-10$ & $7.62 \mathrm{E}-11$ & MACC1 & $1.21 E+01$ \\
\hline ENSBTAG00000045645 & $6.77 \mathrm{E}-10$ & $4.99 \mathrm{E}-11$ & & $1.35 E+01$ \\
\hline ENSBTAG00000015105 & $5.45 \mathrm{E}-10$ & $4.01 E-11$ & HERPUD2 & $1.36 \mathrm{E}+01$ \\
\hline ENSBTAG00000010931 & $6.76 \mathrm{E}-10$ & $4.81 \mathrm{E}-11$ & SSBP1 & $1.40 \mathrm{E}+01$ \\
\hline ENSBTAG00000017869 & $6.13 \mathrm{E}-10$ & $2.61 \mathrm{E}-11$ & CAV1 & $2.34 \mathrm{E}+01$ \\
\hline ENSBTAG00000010549 & 4.37E-10 & $1.74 \mathrm{E}-11$ & IFRD1 & $2.50 \mathrm{E}+01$ \\
\hline ENSBTAG00000045584 & $5.81 \mathrm{E}-10$ & $1.97 \mathrm{E}-11$ & CAV2 & $2.93 \mathrm{E}+01$ \\
\hline ENSBTAG00000000745 & $5.45 \mathrm{E}-10$ & $1.45 \mathrm{E}-11$ & & $3.73 E+01$ \\
\hline ENSBTAG00000006325 & $3.10 \mathrm{E}-10$ & $5.76 \mathrm{E}-12$ & NUDCD3 & $5.29 \mathrm{E}+01$ \\
\hline ENSBTAG00000008143 & $7.78 \mathrm{E}-10$ & $1.44 \mathrm{E}-11$ & STK17A & $5.36 \mathrm{E}+01$ \\
\hline ENSBTAG00000047599 & 7.67E-10 & $1.30 \mathrm{E}-11$ & GHRHR & $5.86 \mathrm{E}+01$ \\
\hline ENSBTAG00000027319 & 0 & 0 & & $1.00 \mathrm{E}+00$ \\
\hline ENSBTAG00000020550 & $1.26 \mathrm{E}-10$ & 0 & RPL7 & $1.27 \mathrm{E}+03$ \\
\hline ENSBTAG00000006924 & $2.48 \mathrm{E}-10$ & 0 & NXPH1 & $2.48 \mathrm{E}+03$ \\
\hline ENSBTAG00000005377 & $5.79 \mathrm{E}-11$ & 0 & SOSTDC1 & $5.80 \mathrm{E}+02$ \\
\hline ENSBTAG00000046905 & 0 & 0 & & $1.00 \mathrm{E}+00$ \\
\hline ENSBTAG00000010555 & $5.58 \mathrm{E}-10$ & 0 & LSMEM1 & $5.58 \mathrm{E}+03$ \\
\hline ENSBTAG00000014738 & $9.35 E-11$ & 0 & HOXA11 & $9.36 \mathrm{E}+02$ \\
\hline ENSBTAG00000001455 & $9.38 \mathrm{E}-11$ & 0 & HOXA7 & $9.39 \mathrm{E}+02$ \\
\hline ENSBTAG00000046457 & 0 & 0 & & $1.00 \mathrm{E}+00$ \\
\hline ENSBTAG00000046768 & $8.59 \mathrm{E}-10$ & 0 & IGFBP1 & $8.59 \mathrm{E}+03$ \\
\hline ENSBTAG00000044146 & $2.85 E-10$ & 0 & NPC1L1 & $2.85 E+03$ \\
\hline ENSBTAG00000012968 & $5.54 \mathrm{E}-10$ & 0 & COA1 & $5.54 \mathrm{E}+03$ \\
\hline ENSBTAG00000018715 & 0 & 0 & MPLKIP & $1.00 \mathrm{E}+00$ \\
\hline ENSBTAG00000031863 & $4.38 \mathrm{E}-10$ & 0 & & $4.38 \mathrm{E}+03$ \\
\hline ENSBTAG00000007572 & $5.21 \mathrm{E}-10$ & 0 & & $5.21 \mathrm{E}+03$ \\
\hline ENSBTAG00000046660 & $1.63 \mathrm{E}-10$ & 0 & & $1.63 E+03$ \\
\hline ENSBTAG00000040281 & 0 & 0 & & $1.00 \mathrm{E}+00$ \\
\hline ENSBTAG00000046496 & 0 & 0 & & $1.00 \mathrm{E}+00$ \\
\hline ENSBTAG00000038943 & $1.60 \mathrm{E}-10$ & 0 & & $1.60 \mathrm{E}+03$ \\
\hline ENSBTAG00000038307 & 0 & 0 & & $1.00 \mathrm{E}+00$ \\
\hline ENSBTAG00000048189 & 0 & 0 & & $1.00 \mathrm{E}+00$ \\
\hline ENSBTAG00000006655 & $5.89 \mathrm{E}-10$ & 0 & & $5.89 \mathrm{E}+03$ \\
\hline ENSBTAG00000007983 & $1.73 \mathrm{E}-10$ & 0 & & $1.74 \mathrm{E}+03$ \\
\hline ENSBTAG00000048169 & $2.09 \mathrm{E}-10$ & 0 & & $2.09 \mathrm{E}+03$ \\
\hline ENSBTAG00000020685 & $1.39 \mathrm{E}-10$ & 0 & & $1.39 \mathrm{E}+03$ \\
\hline ENSBTAG00000038527 & $2.71 \mathrm{E}-10$ & 0 & & $2.71 \mathrm{E}+03$ \\
\hline ENSBTAG00000046922 & 0 & 0 & TWIST1 & $1.00 \mathrm{E}+00$ \\
\hline ENSBTAG00000039955 & 0 & 0 & FERD3L & $1.00 \mathrm{E}+00$ \\
\hline ENSBTAG00000033806 & $5.51 \mathrm{E}-10$ & 0 & & $5.51 E+03$ \\
\hline ENSBTAG00000011476 & $5.22 \mathrm{E}-11$ & 0 & & $5.23 E+02$ \\
\hline ENSBTAG00000032393 & $3.24 \mathrm{E}-10$ & 0 & C7orf62 & $3.25 E+03$ \\
\hline ENSBTAG00000047435 & $3.14 \mathrm{E}-10$ & 0 & & $3.15 E+03$ \\
\hline ENSBTAG00000046625 & 8.95E-11 & 0 & PURB & $8.96 \mathrm{E}+02$ \\
\hline ENSBTAG00000047845 & 0 & 0 & & $1.00 \mathrm{E}+00$ \\
\hline ENSBTAG00000038448 & 0 & 0 & & $1.00 \mathrm{E}+00$ \\
\hline ENSBTAG00000016880 & $8.67 \mathrm{E}-10$ & 0 & TAS2R3 & $8.68 \mathrm{E}+03$ \\
\hline ENSBTAG00000018440 & $6.47 \mathrm{E}-10$ & 0 & TAS2R4 & $6.47 E+03$ \\
\hline ENSBTAG00000047873 & 0 & 0 & & $1.00 \mathrm{E}+00$ \\
\hline ENSBTAG00000045871 & 0 & 0 & & $1.00 \mathrm{E}+00$ \\
\hline ENSBTAG00000006879 & 0 & 4.17E-11 & HoXC11 & $2.39 \mathrm{E}-03$ \\
\hline ENSBTAG00000012503 & 0 & $6.40 \mathrm{E}-11$ & MGAT3 & $1.56 \mathrm{E}-03$ \\
\hline ENSBTAG00000006313 & 0 & $1.07 \mathrm{E}-10$ & OR6C76 & $9.34 \mathrm{E}-04$ \\
\hline ENSBTAG00000011153 & $9.82 \mathrm{E}-11$ & $6.02 \mathrm{E}-10$ & MGAT4C & 1.63E-01 \\
\hline ENSBTAG00000017462 & $3.91 \mathrm{E}-11$ & $1.97 \mathrm{E}-10$ & ATF4 & $1.99 \mathrm{E}-01$ \\
\hline ENSBTAG00000026467 & 5.17E-11 & $2.61 \mathrm{E}-10$ & & $1.98 \mathrm{E}-01$ \\
\hline ENSBTAG00000014237 & $1.84 \mathrm{E}-10$ & 7.37E-10 & CYTH4 & 2.49E-01 \\
\hline ENSBTAG00000019777 & $3.65 E-11$ & $1.38 \mathrm{E}-10$ & CDCA3 & 2.65E-01 \\
\hline ENSBTAG00000009887 & $1.30 \mathrm{E}-10$ & 4.15E-10 & DDX17 & 3.14E-01 \\
\hline ENSBTAG00000005936 & $1.90 \mathrm{E}-10$ & $5.85 \mathrm{E}-10$ & DMC1 & 3.24E-01 \\
\hline ENSBTAG00000030423 & $1.42 \mathrm{E}-10$ & $3.93 \mathrm{E}-10$ & CLEC1B & 3.62E-01 \\
\hline ENSBTAG00000012267 & $1.73 \mathrm{E}-10$ & $4.50 \mathrm{E}-10$ & ZFC3H1 & 3.84E-01 \\
\hline ENSBTAG00000003505 & $3.34 \mathrm{E}-10$ & $8.52 \mathrm{E}-10$ & $\mathrm{DCN}$ & $3.92 \mathrm{E}-01$ \\
\hline ENSBTAG00000016904 & $2.61 \mathrm{E}-11$ & $6.57 \mathrm{E}-11$ & 09-Mar & $3.98 \mathrm{E}-01$ \\
\hline
\end{tabular}




\begin{tabular}{|c|c|c|c|c|}
\hline ENSBTAG00000004654 & 4.06E-10 & $1.02 \mathrm{E}-09$ & PWP1 & $3.98 \mathrm{E}-01$ \\
\hline ENSBTAG00000017463 & $2.26 \mathrm{E}-10$ & $5.42 \mathrm{E}-10$ & RPS19BP1 & 4.17E-01 \\
\hline ENSBTAG00000047347 & $2.32 \mathrm{E}-11$ & $5.26 \mathrm{E}-11$ & & 4.42E-01 \\
\hline ENSBTAG00000030255 & $1.14 \mathrm{E}-10$ & $2.51 \mathrm{E}-10$ & PDXP & 4.53E-01 \\
\hline ENSBTAG00000031097 & $3.50 \mathrm{E}-10$ & $7.73 \mathrm{E}-10$ & & 4.53E-01 \\
\hline ENSBTAG00000014683 & $5.95 \mathrm{E}-11$ & $1.31 \mathrm{E}-10$ & APOBEC1 & 4.54E-01 \\
\hline ENSBTAG00000001745 & $1.14 \mathrm{E}-10$ & $2.43 \mathrm{E}-10$ & LUM & 4.67E-01 \\
\hline ENSBTAG00000009913 & $3.62 \mathrm{E}-10$ & $7.78 \mathrm{E}-10$ & UPK3A & 4.65E-01 \\
\hline ENSBTAG00000024691 & $2.63 \mathrm{E}-10$ & $5.53 \mathrm{E}-10$ & OR6C1 & 4.76E-01 \\
\hline ENSBTAG00000006635 & $1.26 \mathrm{E}-10$ & $2.53 \mathrm{E}-10$ & $\mathrm{DBX} 2$ & 4.97E-01 \\
\hline ENSBTAG00000007259 & $2.32 \mathrm{E}-10$ & 4.60E-10 & ELFN2 & 5.04E-01 \\
\hline ENSBTAG00000037799 & $5.67 \mathrm{E}-10$ & 1.09E-09 & & 5.20E-01 \\
\hline ENSBTAG00000014609 & $1.24 \mathrm{E}-10$ & $2.36 \mathrm{E}-10$ & PPP1R12A & $5.25 \mathrm{E}-01$ \\
\hline ENSBTAG00000039756 & $1.72 \mathrm{E}-10$ & $3.26 \mathrm{E}-10$ & OR10A7 & 5.29E-01 \\
\hline ENSBTAG00000002673 & $7.53 \mathrm{E}-11$ & $1.42 \mathrm{E}-10$ & FIGNL2 & 5.31E-01 \\
\hline ENSBTAG00000048162 & $3.06 \mathrm{E}-11$ & $5.78 \mathrm{E}-11$ & & 5.30E-01 \\
\hline ENSBTAG00000018849 & $7.56 \mathrm{E}-11$ & $1.43 \mathrm{E}-10$ & AICDA & 5.29E-01 \\
\hline ENSBTAG00000047890 & $1.75 \mathrm{E}-10$ & $3.31 \mathrm{E}-10$ & & 5.29E-01 \\
\hline ENSBTAG00000040065 & $1.85 \mathrm{E}-11$ & $3.49 \mathrm{E}-11$ & & 5.30E-01 \\
\hline ENSBTAG00000002651 & $2.03 \mathrm{E}-10$ & $3.61 E-10$ & PKP2 & 5.63E-01 \\
\hline ENSBTAG00000048271 & 1.54E-10 & $2.74 \mathrm{E}-10$ & MICAL3 & $5.62 \mathrm{E}-01$ \\
\hline ENSBTAG00000005859 & $2.03 \mathrm{E}-10$ & $3.58 \mathrm{E}-10$ & LALBA & 5.66E-01 \\
\hline ENSBTAG00000046519 & $3.50 \mathrm{E}-11$ & $6.06 \mathrm{E}-11$ & & 5.77E-01 \\
\hline ENSBTAG00000001673 & $2.12 \mathrm{E}-10$ & $3.65 \mathrm{E}-10$ & C2CD5 & $5.80 \mathrm{E}-01$ \\
\hline ENSBTAG00000045610 & $3.51 \mathrm{E}-10$ & $5.99 \mathrm{E}-10$ & CACNA1I & 5.85E-01 \\
\hline ENSBTAG00000002227 & $1.75 \mathrm{E}-10$ & $2.98 \mathrm{E}-10$ & LRIG3 & $5.88 \mathrm{E}-01$ \\
\hline ENSBTAG00000034985 & $1.24 \mathrm{E}-10$ & $2.09 \mathrm{E}-10$ & & $5.94 \mathrm{E}-01$ \\
\hline ENSBTAG00000031096 & $2.62 \mathrm{E}-10$ & $4.41 \mathrm{E}-10$ & & $5.94 \mathrm{E}-01$ \\
\hline ENSBTAG00000001517 & $6.73 \mathrm{E}-11$ & $1.13 \mathrm{E}-10$ & KRT18 & 5.96E-01 \\
\hline ENSBTAG00000030632 & $9.19 \mathrm{E}-11$ & $1.49 \mathrm{E}-10$ & ALG10 & 6.17E-01 \\
\hline ENSBTAG00000025837 & $1.20 \mathrm{E}-10$ & $1.93 \mathrm{E}-10$ & RFX4 & $6.20 \mathrm{E}-01$ \\
\hline ENSBTAG00000003221 & $6.21 \mathrm{E}-11$ & 9.97E-11 & ATF7IP & $6.24 \mathrm{E}-01$ \\
\hline ENSBTAG00000009886 & $3.30 \mathrm{E}-10$ & $5.27 \mathrm{E}-10$ & KDELR3 & 6.27E-01 \\
\hline ENSBTAG00000026972 & $1.01 \mathrm{E}-10$ & $1.60 \mathrm{E}-10$ & MYF5 & 6.33E-01 \\
\hline ENSBTAG00000016345 & $4.91 \mathrm{E}-10$ & $7.66 \mathrm{E}-10$ & IL2RB & $6.42 \mathrm{E}-01$ \\
\hline ENSBTAG00000009537 & $5.15 \mathrm{E}-10$ & $7.90 \mathrm{E}-10$ & CLEC9A & $6.52 \mathrm{E}-01$ \\
\hline ENSBTAG00000020916 & $7.94 \mathrm{E}-11$ & $1.20 \mathrm{E}-10$ & & $6.62 \mathrm{E}-01$ \\
\hline ENSBTAG00000047048 & $2.71 \mathrm{E}-10$ & 4.10E-10 & & $6.61 \mathrm{E}-01$ \\
\hline ENSBTAG00000010763 & $1.73 \mathrm{E}-10$ & $2.60 \mathrm{E}-10$ & DUSP16 & 6.67E-01 \\
\hline ENSBTAG00000006160 & $1.15 \mathrm{E}-10$ & $1.69 \mathrm{E}-10$ & SUOX & $6.81 \mathrm{E}-01$ \\
\hline ENSBTAG00000013256 & $3.64 \mathrm{E}-10$ & $5.32 \mathrm{E}-10$ & LGR5 & 6.83E-01 \\
\hline ENSBTAG00000006883 & $1.56 \mathrm{E}-10$ & $2.26 \mathrm{E}-10$ & EIF4B & 6.89E-01 \\
\hline ENSBTAG00000015268 & $1.31 \mathrm{E}-10$ & $1.90 \mathrm{E}-10$ & PICK1 & $6.90 \mathrm{E}-01$ \\
\hline ENSBTAG00000016589 & $8.34 \mathrm{E}-11$ & $1.20 \mathrm{E}-10$ & PRPF40B & 6.95E-01 \\
\hline ENSBTAG00000014238 & $1.38 \mathrm{E}-10$ & $1.97 \mathrm{E}-10$ & ATP6V1E1 & 7.01E-01 \\
\hline ENSBTAG00000018743 & $1.57 \mathrm{E}-10$ & $2.19 \mathrm{E}-10$ & C12orf29 & $7.18 \mathrm{E}-01$ \\
\hline ENSBTAG00000010171 & $2.15 E-10$ & $3.00 \mathrm{E}-10$ & JOSD1 & 7.16E-01 \\
\hline ENSBTAG00000008541 & $6.66 \mathrm{E}-10$ & $9.24 \mathrm{E}-10$ & MGST1 & 7.21E-01 \\
\hline ENSBTAG00000001553 & $1.82 \mathrm{E}-10$ & $2.52 \mathrm{E}-10$ & HNRNPA1 & 7.21E-01 \\
\hline ENSBTAG00000015407 & 1.10E-09 & $1.52 \mathrm{E}-09$ & IL22 & 7.23E-01 \\
\hline ENSBTAG00000004832 & $3.00 \mathrm{E}-10$ & $4.01 \mathrm{E}-10$ & KIF21A & 7.49E-01 \\
\hline ENSBTAG00000012370 & $4.18 \mathrm{E}-10$ & $5.56 \mathrm{E}-10$ & & 7.51E-01 \\
\hline ENSBTAG00000002135 & $2.19 \mathrm{E}-10$ & $2.91 \mathrm{E}-10$ & CD69 & 7.54E-01 \\
\hline ENSBTAG00000000014 & $1.68 \mathrm{E}-10$ & $2.22 \mathrm{E}-10$ & TXN2 & $7.58 \mathrm{E}-01$ \\
\hline ENSBTAG00000006039 & $2.21 \mathrm{E}-10$ & $2.90 \mathrm{E}-10$ & ARHGDIB & 7.63E-01 \\
\hline ENSBTAG00000010324 & $1.87 \mathrm{E}-10$ & $2.45 \mathrm{E}-10$ & GTPBP1 & 7.63E-01 \\
\hline ENSBTAG00000013541 & $2.06 \mathrm{E}-10$ & $2.69 \mathrm{E}-10$ & LMO3 & 7.66E-01 \\
\hline ENSBTAG00000001341 & $2.09 \mathrm{E}-10$ & $2.72 \mathrm{E}-10$ & WNT5B & 7.70E-01 \\
\hline ENSBTAG00000000818 & $4.90 \mathrm{E}-10$ & $6.35 \mathrm{E}-10$ & PLEKHG7 & 7.71E-01 \\
\hline ENSBTAG00000026666 & $6.88 \mathrm{E}-10$ & $8.86 \mathrm{E}-10$ & & 7.77E-01 \\
\hline ENSBTAG00000019608 & $7.90 \mathrm{E}-10$ & 1.01E-09 & DDX47 & 7.82E-01 \\
\hline ENSBTAG00000019738 & $3.88 \mathrm{E}-10$ & $4.96 \mathrm{E}-10$ & MDM1 & 7.82E-01 \\
\hline ENSBTAG00000011758 & $1.49 \mathrm{E}-10$ & $1.88 \mathrm{E}-10$ & & 7.93E-01 \\
\hline ENSBTAG00000008352 & $1.66 \mathrm{E}-10$ & $2.09 \mathrm{E}-10$ & ART4 & 7.94E-01 \\
\hline ENSBTAG00000013207 & $1.51 \mathrm{E}-10$ & $1.90 \mathrm{E}-10$ & CAPZA3 & 7.94E-01 \\
\hline ENSBTAG00000048224 & $2.62 \mathrm{E}-10$ & $3.30 \mathrm{E}-10$ & & 7.94E-01 \\
\hline ENSBTAG00000038290 & $3.51 \mathrm{E}-10$ & $4.42 \mathrm{E}-10$ & & 7.94E-01 \\
\hline ENSBTAG00000031099 & $3.50 \mathrm{E}-10$ & $4.41 \mathrm{E}-10$ & & 7.93E-01 \\
\hline ENSBTAG00000046392 & 3.97E-10 & $5.00 \mathrm{E}-10$ & & 7.94E-01 \\
\hline ENSBTAG00000024477 & $3.83 \mathrm{E}-10$ & $4.82 \mathrm{E}-10$ & & 7.94E-01 \\
\hline
\end{tabular}




\begin{tabular}{|c|c|c|c|c|}
\hline ENSBTAG00000000924 & $5.14 \mathrm{E}-11$ & $6.48 \mathrm{E}-11$ & $\mathrm{HOXC12}$ & 7.94E-01 \\
\hline ENSBTAG00000039435 & $1.95 \mathrm{E}-10$ & $2.45 \mathrm{E}-10$ & LEMD3 & $7.94 \mathrm{E}-01$ \\
\hline ENSBTAG00000032429 & $6.91 \mathrm{E}-10$ & 8.70E-10 & OR10AD1 & $7.94 \mathrm{E}-01$ \\
\hline ENSBTAG00000026249 & $2.29 \mathrm{E}-10$ & $2.88 \mathrm{E}-10$ & & $7.94 \mathrm{E}-01$ \\
\hline ENSBTAG00000030468 & $8.81 \mathrm{E}-11$ & $1.11 \mathrm{E}-10$ & & $7.94 \mathrm{E}-01$ \\
\hline ENSBTAG00000014987 & $1.94 \mathrm{E}-10$ & $2.45 \mathrm{E}-10$ & & 7.94E-01 \\
\hline ENSBTAG00000037791 & $2.58 \mathrm{E}-10$ & $3.25 \mathrm{E}-10$ & & 7.93E-01 \\
\hline ENSBTAG00000046721 & $1.44 \mathrm{E}-10$ & $1.82 \mathrm{E}-10$ & & 7.93E-01 \\
\hline ENSBTAG00000046402 & 3.24E-10 & $4.08 \mathrm{E}-10$ & ASCL4 & 7.94E-01 \\
\hline ENSBTAG00000005107 & $1.01 \mathrm{E}-10$ & $1.28 \mathrm{E}-10$ & & 7.93E-01 \\
\hline ENSBTAG00000038384 & $1.70 \mathrm{E}-10$ & $2.14 \mathrm{E}-10$ & KRT5 & $7.94 \mathrm{E}-01$ \\
\hline ENSBTAG00000013759 & $2.02 \mathrm{E}-10$ & $2.54 \mathrm{E}-10$ & TSPAN9 & $7.95 \mathrm{E}-01$ \\
\hline ENSBTAG00000017781 & 8.96E-11 & 1.13E-10 & BCDIN3D & 7.93E-01 \\
\hline ENSBTAG00000047967 & $4.35 \mathrm{E}-10$ & $5.48 \mathrm{E}-10$ & & 7.93E-01 \\
\hline ENSBTAG00000033884 & $8.46 \mathrm{E}-10$ & 1.07E-09 & TMA7 & $7.91 \mathrm{E}-01$ \\
\hline ENSBTAG00000006557 & 4.75E-11 & 5.99E-11 & RHEBL1 & $7.94 \mathrm{E}-01$ \\
\hline ENSBTAG00000030652 & $1.88 \mathrm{E}-10$ & 2.37E-10 & TEX33 & 7.93E-01 \\
\hline ENSBTAG00000006599 & $1.57 \mathrm{E}-10$ & $1.98 \mathrm{E}-10$ & INHBE & 7.93E-01 \\
\hline ENSBTAG00000048295 & $1.75 \mathrm{E}-10$ & $2.21 \mathrm{E}-10$ & & $7.93 \mathrm{E}-01$ \\
\hline ENSBTAG00000026624 & $8.32 \mathrm{E}-11$ & $1.05 \mathrm{E}-10$ & ETFRF1 & 7.92E-01 \\
\hline ENSBTAG00000009238 & $5.17 \mathrm{E}-11$ & $6.51 \mathrm{E}-11$ & HOXC6 & 7.94E-01 \\
\hline ENSBTAG000000008020 & $3.01 \mathrm{E}-10$ & $3.79 \mathrm{E}-10$ & & 7.95E-01 \\
\hline ENSBTAG00000026813 & $2.11 \mathrm{E}-11$ & $2.66 \mathrm{E}-11$ & AQP5 & 7.96E-01 \\
\hline ENSBTAG00000039524 & $8.75 \mathrm{E}-10$ & 1.10E-09 & & 7.95E-01 \\
\hline ENSBTAG00000012529 & $2.04 \mathrm{E}-10$ & 2.57E-10 & IFNG & 7.94E-01 \\
\hline ENSBTAG00000045922 & $8.48 \mathrm{E}-11$ & 1.07E-10 & & $7.93 \mathrm{E}-01$ \\
\hline ENSBTAG00000033531 & $6.66 \mathrm{E}-11$ & 8.39E-11 & & 7.94E-01 \\
\hline ENSBTAG00000021974 & $6.73 \mathrm{E}-11$ & 8.47E-11 & SMUG1 & 7.94E-01 \\
\hline ENSBTAG00000018451 & $1.31 \mathrm{E}-10$ & $1.65 \mathrm{E}-10$ & PTMS & 7.96E-01 \\
\hline ENSBTAG00000037629 & $4.33 \mathrm{E}-10$ & $5.46 \mathrm{E}-10$ & & 7.94E-01 \\
\hline ENSBTAG00000023471 & $2.08 \mathrm{E}-10$ & $2.62 \mathrm{E}-10$ & RPL36 & $7.94 \mathrm{E}-01$ \\
\hline ENSBTAG00000000923 & 3.84E-11 & 4.84E-11 & $\mathrm{HOXC13}$ & 7.94E-01 \\
\hline ENSBTAG00000026497 & $2.97 \mathrm{E}-10$ & $3.74 \mathrm{E}-10$ & A4GALT & 7.93E-01 \\
\hline ENSBTAG00000040344 & $1.52 \mathrm{E}-09$ & 1.92E-09 & & 7.94E-01 \\
\hline ENSBTAG00000038351 & $1.22 \mathrm{E}-10$ & $1.53 \mathrm{E}-10$ & & 7.95E-01 \\
\hline ENSBTAG000000000454 & $2.40 \mathrm{E}-10$ & $3.02 \mathrm{E}-10$ & TMEM106C & 7.94E-01 \\
\hline ENSBTAG00000031503 & $1.78 \mathrm{E}-10$ & $2.24 \mathrm{E}-10$ & NDUFA4L2 & 7.95E-01 \\
\hline ENSBTAG00000039041 & $1.22 \mathrm{E}-09$ & $1.52 \mathrm{E}-09$ & & 8.00E-01 \\
\hline ENSBTAG00000019828 & $6.12 \mathrm{E}-10$ & $7.62 \mathrm{E}-10$ & TAB1 & 8.03E-01 \\
\hline ENSBTAG00000005833 & $1.11 \mathrm{E}-10$ & $1.38 \mathrm{E}-10$ & ETNK1 & 8.07E-01 \\
\hline ENSBTAG00000002127 & $3.30 \mathrm{E}-10$ & $4.08 \mathrm{E}-10$ & TCP11L2 & $8.09 \mathrm{E}-01$ \\
\hline ENSBTAG00000012272 & $6.91 \mathrm{E}-10$ & 8.53E-10 & TMEM19 & 8.10E-01 \\
\hline ENSBTAG00000009064 & $5.59 \mathrm{E}-10$ & $6.89 \mathrm{E}-10$ & CSF2RB & $8.11 \mathrm{E}-01$ \\
\hline ENSBTAG00000009663 & 4.99E-10 & $6.12 \mathrm{E}-10$ & YBX3 & 8.15E-01 \\
\hline ENSBTAG00000017439 & $1.92 \mathrm{E}-10$ & $2.34 \mathrm{E}-10$ & MIEF1 & 8.19E-01 \\
\hline ENSBTAG00000013202 & $7.80 \mathrm{E}-10$ & $9.55 \mathrm{E}-10$ & PLCZ1 & 8.17E-01 \\
\hline ENSBTAG00000016165 & $6.16 \mathrm{E}-10$ & 7.53E-10 & KRT7 & $8.18 \mathrm{E}-01$ \\
\hline ENSBTAG00000020715 & $4.92 \mathrm{E}-10$ & $6.03 \mathrm{E}-10$ & PIK3C2G & 8.16E-01 \\
\hline ENSBTAG00000012828 & $6.13 \mathrm{E}-10$ & $7.50 \mathrm{E}-10$ & TBC1D15 & 8.17E-01 \\
\hline ENSBTAG00000005305 & $2.32 \mathrm{E}-10$ & $2.83 \mathrm{E}-10$ & NTS & 8.19E-01 \\
\hline ENSBTAG00000013956 & $1.44 \mathrm{E}-10$ & $1.76 \mathrm{E}-10$ & & 8.20E-01 \\
\hline ENSBTAG00000039980 & $6.33 \mathrm{E}-10$ & 7.67E-10 & & 8.26E-01 \\
\hline ENSBTAG00000002902 & $1.33 \mathrm{E}-10$ & 1.60E-10 & ANO6 & $8.30 \mathrm{E}-01$ \\
\hline ENSBTAG00000026962 & $3.18 \mathrm{E}-10$ & 3.84E-10 & XРОT & $8.29 \mathrm{E}-01$ \\
\hline ENSBTAG00000000015 & $4.92 \mathrm{E}-10$ & $5.89 \mathrm{E}-10$ & FOXRED2 & $8.36 \mathrm{E}-01$ \\
\hline ENSBTAG00000009252 & $1.14 \mathrm{E}-09$ & 1.36E-09 & & $8.42 \mathrm{E}-01$ \\
\hline ENSBTAG00000021658 & $1.89 \mathrm{E}-10$ & $2.25 \mathrm{E}-10$ & GRASP & 8.40E-01 \\
\hline ENSBTAG00000002775 & $9.77 \mathrm{E}-10$ & 1.16E-09 & C12orf71 & $8.42 \mathrm{E}-01$ \\
\hline ENSBTAG000000000147 & $1.82 \mathrm{E}-10$ & 2.17E-10 & RIC8B & $8.40 \mathrm{E}-01$ \\
\hline ENSBTAG000000000421 & $3.57 \mathrm{E}-10$ & 4.24E-10 & EEA1 & $8.42 \mathrm{E}-01$ \\
\hline ENSBTAG00000005225 & $2.87 \mathrm{E}-10$ & $3.42 \mathrm{E}-10$ & RAD52 & 8.40E-01 \\
\hline ENSBTAG00000013988 & $2.34 \mathrm{E}-10$ & 2.77E-10 & BID & $8.46 \mathrm{E}-01$ \\
\hline ENSBTAG000000003574 & $9.27 \mathrm{E}-11$ & $1.10 \mathrm{E}-10$ & KIAA1644 & 8.43E-01 \\
\hline ENSBTAG00000008897 & $3.85 \mathrm{E}-10$ & 4.54E-10 & BIN2 & 8.47E-01 \\
\hline ENSBTAG00000030493 & $5.88 \mathrm{E}-10$ & $6.92 \mathrm{E}-10$ & RERGL & 8.49E-01 \\
\hline ENSBTAG00000030189 & $4.52 \mathrm{E}-10$ & 5.31E-10 & EFCAB6 & $8.51 \mathrm{E}-01$ \\
\hline ENSBTAG000000007228 & $2.07 \mathrm{E}-10$ & $2.42 \mathrm{E}-10$ & CBX7 & 8.56E-01 \\
\hline ENSBTAG00000033671 & $9.70 \mathrm{E}-10$ & 1.13E-09 & & $8.59 \mathrm{E}-01$ \\
\hline ENSBTAG00000009704 & $3.14 \mathrm{E}-10$ & $3.66 \mathrm{E}-10$ & CECR2 & $8.58 \mathrm{E}-01$ \\
\hline ENSBTAG00000016121 & 4.25E-10 & $4.95 \mathrm{E}-10$ & KRT75 & 8.59E-01 \\
\hline
\end{tabular}




\begin{tabular}{|c|c|c|c|c|}
\hline ENSBTAG00000004781 & $1.16 \mathrm{E}-10$ & $1.35 \mathrm{E}-10$ & POLR3B & $8.59 \mathrm{E}-01$ \\
\hline ENSBTAG00000004569 & $7.68 \mathrm{E}-10$ & $8.90 \mathrm{E}-10$ & GLIPR1L1 & 8.62E-01 \\
\hline ENSBTAG00000010144 & $1.76 \mathrm{E}-10$ & $2.04 \mathrm{E}-10$ & MTERF2 & $8.65 \mathrm{E}-01$ \\
\hline ENSBTAG00000017313 & $1.57 \mathrm{E}-10$ & $1.81 \mathrm{E}-10$ & CECR1 & $8.69 \mathrm{E}-01$ \\
\hline ENSBTAG00000001983 & $5.90 \mathrm{E}-10$ & $6.79 \mathrm{E}-10$ & SMAGP & $8.69 \mathrm{E}-01$ \\
\hline ENSBTAG00000044118 & $3.48 \mathrm{E}-10$ & $3.99 \mathrm{E}-10$ & HMGA2 & $8.72 \mathrm{E}-01$ \\
\hline ENSBTAG00000008935 & $2.20 \mathrm{E}-10$ & $2.52 \mathrm{E}-10$ & PFDN5 & $8.74 \mathrm{E}-01$ \\
\hline ENSBTAG00000019373 & $2.74 \mathrm{E}-10$ & 3.13E-10 & CAPRIN2 & $8.75 \mathrm{E}-01$ \\
\hline ENSBTAG00000013173 & $5.54 \mathrm{E}-10$ & $6.34 \mathrm{E}-10$ & 03-Sep & $8.73 \mathrm{E}-01$ \\
\hline ENSBTAG00000032902 & $5.70 \mathrm{E}-10$ & $6.53 \mathrm{E}-10$ & & $8.73 \mathrm{E}-01$ \\
\hline ENSBTAG00000008910 & $7.21 \mathrm{E}-10$ & $8.25 \mathrm{E}-10$ & SSTR3 & $8.74 \mathrm{E}-01$ \\
\hline ENSBTAG00000008967 & 4.63E-10 & $5.26 \mathrm{E}-10$ & RAP1B & $8.80 \mathrm{E}-01$ \\
\hline ENSBTAG00000017943 & $1.23 \mathrm{E}-10$ & $1.40 \mathrm{E}-10$ & GALNT6 & $8.81 \mathrm{E}-01$ \\
\hline ENSBTAG00000004283 & $5.22 \mathrm{E}-10$ & $5.93 \mathrm{E}-10$ & PPFIBP1 & $8.81 \mathrm{E}-01$ \\
\hline ENSBTAG00000010790 & $3.89 \mathrm{E}-10$ & $4.41 \mathrm{E}-10$ & EIF3L & $8.82 \mathrm{E}-01$ \\
\hline ENSBTAG00000027900 & $2.25 \mathrm{E}-10$ & $2.55 \mathrm{E}-10$ & LRRIQ1 & $8.84 \mathrm{E}-01$ \\
\hline ENSBTAG00000031919 & $5.66 \mathrm{E}-10$ & $6.40 \mathrm{E}-10$ & SLC35E3 & $8.84 \mathrm{E}-01$ \\
\hline ENSBTAG00000001988 & $3.36 \mathrm{E}-10$ & $3.79 \mathrm{E}-10$ & EIF3D & $8.86 \mathrm{E}-01$ \\
\hline ENSBTAG00000010590 & $2.39 \mathrm{E}-10$ & $2.70 \mathrm{E}-10$ & FAM118A & $8.86 \mathrm{E}-01$ \\
\hline ENSBTAG00000019155 & $4.08 \mathrm{E}-10$ & 4.61E-10 & FRS2 & $8.85 \mathrm{E}-01$ \\
\hline ENSBTAG00000011399 & 4.44E-10 & $5.01 \mathrm{E}-10$ & YARS2 & $8.86 \mathrm{E}-01$ \\
\hline ENSBTAG00000020315 & $5.47 \mathrm{E}-10$ & $6.17 \mathrm{E}-10$ & DENND5B & $8.87 \mathrm{E}-01$ \\
\hline ENSBTAG00000007904 & $1.26 \mathrm{E}-10$ & $1.42 \mathrm{E}-10$ & KRT72 & $8.85 \mathrm{E}-01$ \\
\hline ENSBTAG00000005868 & $8.47 \mathrm{E}-10$ & $9.47 \mathrm{E}-10$ & & 8.95E-01 \\
\hline ENSBTAG00000012815 & 1.13E-09 & $1.26 \mathrm{E}-09$ & CLEC12A & 8.97E-01 \\
\hline ENSBTAG00000011395 & $7.34 \mathrm{E}-10$ & $8.18 \mathrm{E}-10$ & DNM1L & $8.98 \mathrm{E}-01$ \\
\hline ENSBTAG00000006369 & $4.56 \mathrm{E}-10$ & $5.08 \mathrm{E}-10$ & MRPS35 & $8.98 \mathrm{E}-01$ \\
\hline ENSBTAG00000016414 & $1.72 \mathrm{E}-10$ & $1.91 \mathrm{E}-10$ & VDR & $9.03 \mathrm{E}-01$ \\
\hline ENSBTAG00000002490 & $6.17 \mathrm{E}-10$ & $6.86 \mathrm{E}-10$ & CHPT1 & $9.00 \mathrm{E}-01$ \\
\hline ENSBTAG00000004721 & $4.60 \mathrm{E}-10$ & $5.10 \mathrm{E}-10$ & GSG1 & $9.02 \mathrm{E}-01$ \\
\hline ENSBTAG00000037783 & $4.73 \mathrm{E}-10$ & $5.23 \mathrm{E}-10$ & & $9.05 \mathrm{E}-01$ \\
\hline ENSBTAG00000000310 & $2.30 \mathrm{E}-10$ & $2.55 \mathrm{E}-10$ & MFAP5 & $9.03 \mathrm{E}-01$ \\
\hline ENSBTAG00000036078 & 4.09E-10 & $4.52 \mathrm{E}-10$ & EMP1 & $9.05 \mathrm{E}-01$ \\
\hline ENSBTAG00000008074 & $5.54 \mathrm{E}-10$ & $6.12 \mathrm{E}-10$ & C1QTNF6 & $9.06 \mathrm{E}-01$ \\
\hline ENSBTAG00000004876 & 3.25E-10 & $3.59 \mathrm{E}-10$ & & $9.05 \mathrm{E}-01$ \\
\hline ENSBTAG00000031031 & $7.02 \mathrm{E}-10$ & 7.73E-10 & OR10P1 & $9.08 \mathrm{E}-01$ \\
\hline ENSBTAG00000026792 & $4.80 \mathrm{E}-10$ & $5.28 \mathrm{E}-10$ & & $9.09 \mathrm{E}-01$ \\
\hline ENSBTAG00000018861 & $3.88 \mathrm{E}-10$ & $4.26 \mathrm{E}-10$ & C12orf56 & $9.11 \mathrm{E}-01$ \\
\hline ENSBTAG00000000619 & $5.31 \mathrm{E}-10$ & $5.84 \mathrm{E}-10$ & KRT74 & $9.09 \mathrm{E}-01$ \\
\hline ENSBTAG00000037703 & $2.30 \mathrm{E}-10$ & $2.52 \mathrm{E}-10$ & MICALL1 & $9.13 \mathrm{E}-01$ \\
\hline ENSBTAG00000014733 & $4.31 \mathrm{E}-10$ & $4.72 \mathrm{E}-10$ & NOP2 & $9.13 \mathrm{E}-01$ \\
\hline ENSBTAG00000007447 & $7.49 \mathrm{E}-11$ & $8.18 \mathrm{E}-11$ & & $9.16 \mathrm{E}-01$ \\
\hline ENSBTAG00000002492 & $6.02 \mathrm{E}-10$ & $6.56 \mathrm{E}-10$ & SYCP3 & $9.18 \mathrm{E}-01$ \\
\hline ENSBTAG00000030257 & $2.44 \mathrm{E}-10$ & $2.66 \mathrm{E}-10$ & SH3BP1 & $9.18 \mathrm{E}-01$ \\
\hline ENSBTAG00000007786 & $2.04 \mathrm{E}-10$ & $2.20 \mathrm{E}-10$ & FBXL14 & $9.28 \mathrm{E}-01$ \\
\hline ENSBTAG00000045684 & $6.16 \mathrm{E}-10$ & $6.64 \mathrm{E}-10$ & & 9.27E-01 \\
\hline ENSBTAG00000010457 & $3.41 \mathrm{E}-10$ & $3.68 \mathrm{E}-10$ & NUAK1 & $9.26 \mathrm{E}-01$ \\
\hline ENSBTAG00000030994 & $3.78 \mathrm{E}-10$ & $4.08 \mathrm{E}-10$ & ANKS1B & $9.27 \mathrm{E}-01$ \\
\hline ENSBTAG00000047733 & $6.20 \mathrm{E}-10$ & $6.69 \mathrm{E}-10$ & & $9.26 \mathrm{E}-01$ \\
\hline ENSBTAG00000006588 & $4.20 \mathrm{E}-10$ & $4.52 \mathrm{E}-10$ & CBY1 & $9.29 \mathrm{E}-01$ \\
\hline ENSBTAG00000012676 & $5.30 \mathrm{E}-10$ & $5.69 \mathrm{E}-10$ & KRT79 & $9.32 \mathrm{E}-01$ \\
\hline ENSBTAG00000006973 & $6.93 \mathrm{E}-10$ & $7.43 \mathrm{E}-10$ & PHF21B & $9.32 \mathrm{E}-01$ \\
\hline ENSBTAG00000017378 & $2.49 \mathrm{E}-10$ & $2.68 \mathrm{E}-10$ & GALNT8 & $9.31 \mathrm{E}-01$ \\
\hline ENSBTAG00000015024 & $2.62 \mathrm{E}-10$ & $2.81 \mathrm{E}-10$ & GXYLT1 & $9.31 \mathrm{E}-01$ \\
\hline ENSBTAG00000045662 & $3.27 \mathrm{E}-10$ & $3.50 \mathrm{E}-10$ & KRT3 & $9.33 \mathrm{E}-01$ \\
\hline ENSBTAG00000005765 & $3.20 \mathrm{E}-10$ & $3.43 \mathrm{E}-10$ & SYNGR1 & $9.33 \mathrm{E}-01$ \\
\hline ENSBTAG00000008858 & $5.15 \mathrm{E}-10$ & $5.51 \mathrm{E}-10$ & & $9.34 \mathrm{E}-01$ \\
\hline ENSBTAG00000017116 & $4.80 \mathrm{E}-10$ & $5.13 \mathrm{E}-10$ & RASD2 & $9.36 \mathrm{E}-01$ \\
\hline ENSBTAG00000009552 & $4.68 \mathrm{E}-10$ & $4.98 \mathrm{E}-10$ & ATP2B1 & $9.39 \mathrm{E}-01$ \\
\hline ENSBTAG00000011068 & $5.10 \mathrm{E}-10$ & $5.40 \mathrm{E}-10$ & MON2 & $9.44 \mathrm{E}-01$ \\
\hline ENSBTAG00000006273 & $5.62 \mathrm{E}-10$ & $5.95 \mathrm{E}-10$ & SULT4A1 & $9.44 \mathrm{E}-01$ \\
\hline ENSBTAG00000030621 & $4.63 \mathrm{E}-10$ & $4.89 \mathrm{E}-10$ & SMCO2 & $9.47 \mathrm{E}-01$ \\
\hline ENSBTAG00000004547 & $6.33 \mathrm{E}-10$ & $6.69 \mathrm{E}-10$ & OLR1 & $9.47 \mathrm{E}-01$ \\
\hline ENSBTAG00000047203 & $1.85 \mathrm{E}-10$ & $1.95 \mathrm{E}-10$ & TSPAN19 & $9.49 \mathrm{E}-01$ \\
\hline ENSBTAG00000007531 & $6.21 \mathrm{E}-10$ & $6.53 \mathrm{E}-10$ & NCF4 & $9.51 \mathrm{E}-01$ \\
\hline ENSBTAG00000047629 & $1.74 \mathrm{E}-10$ & $1.83 \mathrm{E}-10$ & C22orf46 & $9.53 \mathrm{E}-01$ \\
\hline ENSBTAG00000047983 & $2.14 \mathrm{E}-10$ & $2.24 \mathrm{E}-10$ & & $9.55 \mathrm{E}-01$ \\
\hline ENSBTAG00000003228 & $3.14 \mathrm{E}-10$ & $3.30 \mathrm{E}-10$ & & $9.52 \mathrm{E}-01$ \\
\hline ENSBTAG00000008922 & $2.48 \mathrm{E}-10$ & $2.60 \mathrm{E}-10$ & APOL6 & $9.54 \mathrm{E}-01$ \\
\hline ENSBTAG00000008989 & $2.95 \mathrm{E}-10$ & $3.09 \mathrm{E}-10$ & BORCS5 & $9.56 \mathrm{E}-01$ \\
\hline
\end{tabular}




\begin{tabular}{|c|c|c|c|c|}
\hline ENSBTAG00000011023 & $4.45 \mathrm{E}-10$ & 4.67E-10 & TMEM52B & 9.54E-01 \\
\hline ENSBTAG00000030622 & $3.25 \mathrm{E}-10$ & $3.42 \mathrm{E}-10$ & MANSC4 & $9.52 \mathrm{E}-01$ \\
\hline ENSBTAG00000030683 & $1.61 \mathrm{E}-10$ & $1.69 \mathrm{E}-10$ & & $9.53 \mathrm{E}-01$ \\
\hline ENSBTAG00000016995 & $3.64 \mathrm{E}-10$ & $3.82 \mathrm{E}-10$ & CECR5 & 9.54E-01 \\
\hline ENSBTAG00000017549 & $2.07 \mathrm{E}-10$ & 2.17E-10 & KITLG & 9.53E-01 \\
\hline ENSBTAG00000007720 & $2.16 \mathrm{E}-10$ & $2.27 \mathrm{E}-10$ & & $9.53 \mathrm{E}-01$ \\
\hline ENSBTAG00000013843 & $3.23 \mathrm{E}-10$ & 3.37E-10 & ACVRL1 & $9.58 \mathrm{E}-01$ \\
\hline ENSBTAG00000004293 & 4.54E-10 & $4.73 \mathrm{E}-10$ & CLEC12B & $9.59 \mathrm{E}-01$ \\
\hline ENSBTAG00000000109 & $2.17 \mathrm{E}-10$ & $2.26 \mathrm{E}-10$ & NPTXR & $9.61 \mathrm{E}-01$ \\
\hline ENSBTAG00000030371 & $8.92 \mathrm{E}-10$ & $9.28 \mathrm{E}-10$ & & $9.61 \mathrm{E}-01$ \\
\hline ENSBTAG00000000380 & $6.70 \mathrm{E}-10$ & $6.94 \mathrm{E}-10$ & SPX & $9.65 \mathrm{E}-01$ \\
\hline ENSBTAG00000009023 & $1.36 \mathrm{E}-10$ & $1.40 \mathrm{E}-10$ & TMTC3 & $9.72 \mathrm{E}-01$ \\
\hline ENSBTAG00000006800 & $8.51 \mathrm{E}-10$ & $8.81 \mathrm{E}-10$ & FGF6 & $9.66 \mathrm{E}-01$ \\
\hline ENSBTAG00000046980 & $8.52 \mathrm{E}-10$ & $8.80 \mathrm{E}-10$ & KLRF2 & $9.68 \mathrm{E}-01$ \\
\hline ENSBTAG00000021078 & $3.52 \mathrm{E}-10$ & $3.61 \mathrm{E}-10$ & PYROXD1 & $9.75 \mathrm{E}-01$ \\
\hline ENSBTAG00000015290 & 4.05E-10 & $4.16 \mathrm{E}-10$ & BAIAP2L2 & $9.75 \mathrm{E}-01$ \\
\hline ENSBTAG00000008934 & $1.39 \mathrm{E}-10$ & $1.42 \mathrm{E}-10$ & ESPL1 & $9.81 \mathrm{E}-01$ \\
\hline ENSBTAG00000003693 & $4.98 \mathrm{E}-10$ & 5.09E-10 & SUN2 & $9.79 \mathrm{E}-01$ \\
\hline ENSBTAG00000000326 & $2.25 \mathrm{E}-10$ & $2.30 \mathrm{E}-10$ & ENTHD1 & $9.78 \mathrm{E}-01$ \\
\hline ENSBTAG00000026966 & $1.29 \mathrm{E}-10$ & $1.32 \mathrm{E}-10$ & RASSF9 & $9.79 \mathrm{E}-01$ \\
\hline ENSBTAG00000044048 & $1.46 \mathrm{E}-10$ & $1.48 \mathrm{E}-10$ & LMBR1L & $9.84 \mathrm{E}-01$ \\
\hline ENSBTAG00000019425 & $5.76 \mathrm{E}-10$ & $5.88 \mathrm{E}-10$ & TSPAN8 & $9.79 \mathrm{E}-01$ \\
\hline ENSBTAG00000010149 & $2.18 \mathrm{E}-10$ & $2.22 \mathrm{E}-10$ & CRY1 & $9.81 \mathrm{E}-01$ \\
\hline ENSBTAG00000007990 & $3.71 \mathrm{E}-10$ & 3.77E-10 & EPYC & $9.83 \mathrm{E}-01$ \\
\hline ENSBTAG00000014759 & $1.63 \mathrm{E}-10$ & $1.66 \mathrm{E}-10$ & WBP11 & $9.85 \mathrm{E}-01$ \\
\hline ENSBTAG00000011798 & $3.72 \mathrm{E}-10$ & 3.77E-10 & STK38L & 9.87E-01 \\
\hline ENSBTAG00000021339 & $3.26 \mathrm{E}-10$ & 3.30E-10 & SCN8A & $9.89 \mathrm{E}-01$ \\
\hline ENSBTAG00000013174 & $1.27 \mathrm{E}-10$ & $1.29 \mathrm{E}-10$ & WBP2NL & $9.84 \mathrm{E}-01$ \\
\hline ENSBTAG00000019156 & $5.46 \mathrm{E}-10$ & $5.50 \mathrm{E}-10$ & ССТ2 & $9.94 \mathrm{E}-01$ \\
\hline ENSBTAG00000020177 & $1.66 \mathrm{E}-10$ & 1.67E-10 & C12orf10 & $9.91 \mathrm{E}-01$ \\
\hline ENSBTAG00000004048 & $3.55 \mathrm{E}-10$ & 3.57E-10 & MCHR1 & $9.93 \mathrm{E}-01$ \\
\hline ENSBTAG00000008351 & $3.80 \mathrm{E}-10$ & 3.83E-10 & C12orf60 & $9.93 \mathrm{E}-01$ \\
\hline ENSBTAG00000047259 & $2.83 \mathrm{E}-10$ & $2.85 \mathrm{E}-10$ & & $9.93 \mathrm{E}-01$ \\
\hline ENSBTAG00000002799 & $1.54 \mathrm{E}-10$ & $1.55 \mathrm{E}-10$ & SLC39A5 & $9.92 \mathrm{E}-01$ \\
\hline ENSBTAG00000019235 & $7.86 \mathrm{E}-10$ & $7.92 \mathrm{E}-10$ & MAGOHB & $9.93 \mathrm{E}-01$ \\
\hline ENSBTAG00000018131 & $2.74 \mathrm{E}-10$ & $2.76 \mathrm{E}-10$ & ATF1 & $9.94 \mathrm{E}-01$ \\
\hline ENSBTAG00000031217 & $1.51 \mathrm{E}-10$ & $1.53 \mathrm{E}-10$ & MYL6B & $9.90 \mathrm{E}-01$ \\
\hline ENSBTAG00000010928 & $1.40 \mathrm{E}-10$ & $1.41 \mathrm{E}-10$ & CSRNP2 & $9.91 \mathrm{E}-01$ \\
\hline ENSBTAG00000026078 & $7.78 \mathrm{E}-10$ & $7.83 \mathrm{E}-10$ & & $9.94 \mathrm{E}-01$ \\
\hline ENSBTAG00000026531 & 1.57E-09 & $1.58 \mathrm{E}-09$ & & 9.93E-01 \\
\hline ENSBTAG00000015025 & $1.17 \mathrm{E}-10$ & $1.18 \mathrm{E}-10$ & YAF2 & $9.92 \mathrm{E}-01$ \\
\hline ENSBTAG00000008066 & $8.64 \mathrm{E}-10$ & 8.64E-10 & PKDREJ & $1.00 E+00$ \\
\hline ENSBTAG00000030369 & $4.60 \mathrm{E}-10$ & 4.60E-10 & IFFO1 & $1.00 E+00$ \\
\hline ENSBTAG00000024318 & $6.42 \mathrm{E}-10$ & $6.42 \mathrm{E}-10$ & & $1.00 E+00$ \\
\hline ENSBTAG00000000077 & $1.87 \mathrm{E}-10$ & $1.86 \mathrm{E}-10$ & & $1.01 E+00$ \\
\hline ENSBTAG00000015878 & $8.88 \mathrm{E}-10$ & $8.80 \mathrm{E}-10$ & RHNO1 & $1.01 \mathrm{E}+00$ \\
\hline ENSBTAG00000011590 & $5.65 \mathrm{E}-10$ & $5.60 \mathrm{E}-10$ & GLIPR1 & $1.01 \mathrm{E}+00$ \\
\hline ENSBTAG00000006246 & $1.57 \mathrm{E}-10$ & $1.56 \mathrm{E}-10$ & CBX5 & $1.01 E+00$ \\
\hline ENSBTAG00000004999 & $2.81 \mathrm{E}-10$ & $2.78 \mathrm{E}-10$ & KIAA1551 & $1.01 \mathrm{E}+00$ \\
\hline ENSBTAG00000004822 & $1.17 \mathrm{E}-10$ & $1.16 \mathrm{E}-10$ & sox10 & $1.01 E+00$ \\
\hline ENSBTAG00000000219 & $4.88 \mathrm{E}-10$ & 4.80E-10 & GRIN2B & $1.02 \mathrm{E}+00$ \\
\hline ENSBTAG00000019409 & $3.14 \mathrm{E}-10$ & $3.09 \mathrm{E}-10$ & GNPTAB & $1.02 \mathrm{E}+00$ \\
\hline ENSBTAG00000014977 & $1.69 \mathrm{E}-10$ & $1.65 \mathrm{E}-10$ & ALX1 & $1.02 \mathrm{E}+00$ \\
\hline ENSBTAG00000012448 & $1.52 \mathrm{E}-10$ & $1.50 \mathrm{E}-10$ & CCDC91 & $1.02 \mathrm{E}+00$ \\
\hline ENSBTAG00000016275 & 8.63E-10 & $8.46 \mathrm{E}-10$ & AMDHD1 & $1.02 \mathrm{E}+00$ \\
\hline ENSBTAG00000047619 & $7.47 \mathrm{E}-10$ & 7.31E-10 & & $1.02 \mathrm{E}+00$ \\
\hline ENSBTAG00000010490 & $2.74 \mathrm{E}-10$ & $2.68 \mathrm{E}-10$ & PEX5 & $1.02 \mathrm{E}+00$ \\
\hline ENSBTAG00000015562 & $1.65 \mathrm{E}-10$ & $1.62 \mathrm{E}-10$ & PLBD1 & $1.02 \mathrm{E}+00$ \\
\hline ENSBTAG00000030424 & $3.98 \mathrm{E}-10$ & $3.90 \mathrm{E}-10$ & CLEC1A & $1.02 \mathrm{E}+00$ \\
\hline ENSBTAG00000002539 & $2.84 \mathrm{E}-10$ & $2.78 \mathrm{E}-10$ & TRIOBP & $1.02 \mathrm{E}+00$ \\
\hline ENSBTAG00000008036 & $5.97 \mathrm{E}-10$ & $5.83 \mathrm{E}-10$ & & $1.02 \mathrm{E}+00$ \\
\hline ENSBTAG00000039992 & $8.36 \mathrm{E}-10$ & $8.16 \mathrm{E}-10$ & & $1.02 \mathrm{E}+00$ \\
\hline ENSBTAG00000047872 & $1.08 \mathrm{E}-10$ & $1.06 \mathrm{E}-10$ & KRT84 & $1.02 \mathrm{E}+00$ \\
\hline ENSBTAG00000009852 & $3.45 \mathrm{E}-10$ & $3.36 \mathrm{E}-10$ & NAV3 & $1.03 E+00$ \\
\hline ENSBTAG00000000198 & $9.49 \mathrm{E}-10$ & 9.23E-10 & & $1.03 E+00$ \\
\hline ENSBTAG00000004753 & $1.37 \mathrm{E}-10$ & $1.34 \mathrm{E}-10$ & TMEM5 & $1.03 E+00$ \\
\hline ENSBTAG00000007204 & $1.93 \mathrm{E}-10$ & $1.86 \mathrm{E}-10$ & KRT80 & $1.04 E+00$ \\
\hline ENSBTAG00000018745 & $1.47 \mathrm{E}-10$ & $1.42 \mathrm{E}-10$ & CEP290 & $1.03 E+00$ \\
\hline ENSBTAG00000000286 & $2.04 \mathrm{E}-10$ & $1.98 \mathrm{E}-10$ & PFKM & $1.03 E+00$ \\
\hline ENSBTAG00000039817 & $1.05 \mathrm{E}-09$ & $1.02 \mathrm{E}-09$ & & $1.03 E+00$ \\
\hline
\end{tabular}




\begin{tabular}{|c|c|c|c|c|}
\hline ENSBTAG00000016276 & $4.12 \mathrm{E}-10$ & $3.99 \mathrm{E}-10$ & HAL & $1.03 E+00$ \\
\hline ENSBTAG00000013496 & $4.91 \mathrm{E}-10$ & $4.73 \mathrm{E}-10$ & CPM & $1.04 \mathrm{E}+00$ \\
\hline ENSBTAG00000003239 & $2.77 \mathrm{E}-10$ & $2.67 \mathrm{E}-10$ & UTP20 & $1.04 \mathrm{E}+00$ \\
\hline ENSBTAG00000014546 & $2.36 \mathrm{E}-10$ & 2.27E-10 & CLEC7A & $1.04 \mathrm{E}+00$ \\
\hline ENSBTAG00000005221 & $1.80 \mathrm{E}-10$ & $1.72 \mathrm{E}-10$ & WNK1 & $1.05 \mathrm{E}+00$ \\
\hline ENSBTAG00000039256 & $9.94 \mathrm{E}-10$ & $9.54 \mathrm{E}-10$ & & $1.04 \mathrm{E}+00$ \\
\hline ENSBTAG00000045742 & $2.25 \mathrm{E}-10$ & $2.16 \mathrm{E}-10$ & C12orf75 & $1.04 E+00$ \\
\hline ENSBTAG00000017260 & $3.65 \mathrm{E}-10$ & $3.50 \mathrm{E}-10$ & PDE3A & $1.04 E+00$ \\
\hline ENSBTAG00000013555 & $1.30 \mathrm{E}-10$ & $1.24 \mathrm{E}-10$ & ACVR1B & $1.04 \mathrm{E}+00$ \\
\hline ENSBTAG00000003282 & $3.24 \mathrm{E}-10$ & $3.11 \mathrm{E}-10$ & SLCO1A2 & $1.04 E+00$ \\
\hline ENSBTAG00000011913 & $2.29 \mathrm{E}-10$ & $2.20 \mathrm{E}-10$ & CKAP4 & $1.04 \mathrm{E}+00$ \\
\hline ENSBTAG00000048175 & $5.03 \mathrm{E}-10$ & $4.81 \mathrm{E}-10$ & & $1.05 \mathrm{E}+00$ \\
\hline ENSBTAG00000014106 & $2.61 \mathrm{E}-10$ & 2.49E-10 & IPO8 & $1.05 \mathrm{E}+00$ \\
\hline ENSBTAG00000006372 & 4.40E-10 & 4.20E-10 & KLHL42 & $1.05 \mathrm{E}+00$ \\
\hline ENSBTAG00000004851 & $2.39 \mathrm{E}-10$ & $2.28 \mathrm{E}-10$ & NCKAP5L & $1.05 E+00$ \\
\hline ENSBTAG00000004240 & $1.05 \mathrm{E}-10$ & $1.01 \mathrm{E}-10$ & & $1.04 \mathrm{E}+00$ \\
\hline ENSBTAG00000011070 & $4.93 \mathrm{E}-10$ & $4.70 \mathrm{E}-10$ & RTCB & $1.05 E+00$ \\
\hline ENSBTAG00000037534 & $2.32 \mathrm{E}-10$ & $2.22 \mathrm{E}-10$ & KLRF1 & $1.05 \mathrm{E}+00$ \\
\hline ENSBTAG00000037552 & $6.69 \mathrm{E}-10$ & $6.37 \mathrm{E}-10$ & & $1.05 \mathrm{E}+00$ \\
\hline ENSBTAG00000038490 & $2.36 \mathrm{E}-10$ & $2.25 \mathrm{E}-10$ & CLEC4A & $1.05 \mathrm{E}+00$ \\
\hline ENSBTAG00000024132 & $1.91 \mathrm{E}-10$ & $1.81 \mathrm{E}-10$ & SMC1B & $1.05 \mathrm{E}+00$ \\
\hline ENSBTAG00000016646 & $2.20 \mathrm{E}-10$ & $2.08 \mathrm{E}-10$ & KCNA1 & $1.06 \mathrm{E}+00$ \\
\hline ENSBTAG00000005626 & $3.31 \mathrm{E}-10$ & $3.12 \mathrm{E}-10$ & NEUROD4 & $1.06 \mathrm{E}+00$ \\
\hline ENSBTAG00000026030 & $3.50 \mathrm{E}-10$ & 3.30E-10 & & $1.06 \mathrm{E}+00$ \\
\hline ENSBTAG00000010818 & $3.85 \mathrm{E}-10$ & $3.63 \mathrm{E}-10$ & AEBP2 & $1.06 \mathrm{E}+00$ \\
\hline ENSBTAG00000047818 & 2.77E-10 & $2.61 \mathrm{E}-10$ & FAM19A2 & $1.06 \mathrm{E}+00$ \\
\hline ENSBTAG00000012890 & $5.29 \mathrm{E}-11$ & $5.00 \mathrm{E}-11$ & SLC25A3 & $1.06 \mathrm{E}+00$ \\
\hline ENSBTAG00000000289 & $3.11 \mathrm{E}-10$ & $2.94 \mathrm{E}-10$ & ASB8 & $1.06 \mathrm{E}+00$ \\
\hline ENSBTAG00000010660 & 4.27E-10 & 4.03E-10 & & $1.06 \mathrm{E}+00$ \\
\hline ENSBTAG00000012271 & $5.01 \mathrm{E}-10$ & $4.73 \mathrm{E}-10$ & & $1.06 \mathrm{E}+00$ \\
\hline ENSBTAG00000033594 & 2.83E-10 & 2.67E-10 & C12orf74 & $1.06 \mathrm{E}+00$ \\
\hline ENSBTAG00000000124 & $1.32 \mathrm{E}-10$ & $1.25 \mathrm{E}-10$ & $\mathrm{DHH}$ & $1.06 \mathrm{E}+00$ \\
\hline ENSBTAG00000017113 & $1.25 \mathrm{E}-10$ & $1.18 \mathrm{E}-10$ & ANKRD33 & $1.06 \mathrm{E}+00$ \\
\hline ENSBTAG00000013756 & $1.26 \mathrm{E}-10$ & $1.19 \mathrm{E}-10$ & & $1.06 \mathrm{E}+00$ \\
\hline ENSBTAG00000014044 & $5.54 \mathrm{E}-10$ & $5.21 \mathrm{E}-10$ & VEZT & $1.06 \mathrm{E}+00$ \\
\hline ENSBTAG00000017571 & 4.19E-10 & $3.94 \mathrm{E}-10$ & PLEKHA5 & $1.06 \mathrm{E}+00$ \\
\hline ENSBTAG00000012999 & 2.67E-10 & $2.51 \mathrm{E}-10$ & PRDM4 & $1.06 \mathrm{E}+00$ \\
\hline ENSBTAG00000020125 & $1.82 \mathrm{E}-10$ & $1.71 \mathrm{E}-10$ & RBFOX2 & $1.06 \mathrm{E}+00$ \\
\hline ENSBTAG00000009444 & $6.92 \mathrm{E}-10$ & $6.48 \mathrm{E}-10$ & SLC15A5 & $1.07 \mathrm{E}+00$ \\
\hline ENSBTAG00000019722 & $1.78 \mathrm{E}-10$ & $1.67 \mathrm{E}-10$ & GUCY2C & $1.07 \mathrm{E}+00$ \\
\hline ENSBTAG00000000662 & $5.84 \mathrm{E}-10$ & $5.46 \mathrm{E}-10$ & TCF20 & $1.07 \mathrm{E}+00$ \\
\hline ENSBTAG00000015351 & $1.09 \mathrm{E}-09$ & $1.02 \mathrm{E}-09$ & STYK1 & $1.06 \mathrm{E}+00$ \\
\hline ENSBTAG00000026977 & $5.36 \mathrm{E}-10$ & $5.00 \mathrm{E}-10$ & PTPRQ & $1.07 \mathrm{E}+00$ \\
\hline ENSBTAG00000014859 & $4.71 \mathrm{E}-10$ & $4.38 \mathrm{E}-10$ & CAPS2 & $1.07 E+00$ \\
\hline ENSBTAG00000000275 & $5.93 \mathrm{E}-10$ & $5.51 \mathrm{E}-10$ & PARP11 & $1.08 \mathrm{E}+00$ \\
\hline ENSBTAG00000046609 & $2.00 \mathrm{E}-10$ & $1.85 \mathrm{E}-10$ & & $1.08 \mathrm{E}+00$ \\
\hline ENSBTAG00000030744 & $2.58 \mathrm{E}-10$ & $2.39 \mathrm{E}-10$ & NFYB & $1.08 \mathrm{E}+00$ \\
\hline ENSBTAG00000019157 & $5.07 \mathrm{E}-10$ & $4.70 \mathrm{E}-10$ & LRRC10 & $1.08 \mathrm{E}+00$ \\
\hline ENSBTAG00000015296 & $2.15 \mathrm{E}-10$ & $1.99 \mathrm{E}-10$ & PTPRB & $1.08 \mathrm{E}+00$ \\
\hline ENSBTAG00000003598 & $2.15 \mathrm{E}-10$ & $1.99 \mathrm{E}-10$ & P3H3 & $1.08 \mathrm{E}+00$ \\
\hline ENSBTAG00000014426 & $1.20 \mathrm{E}-10$ & $1.11 \mathrm{E}-10$ & PRKAG1 & $1.08 \mathrm{E}+00$ \\
\hline ENSBTAG00000021953 & $5.66 \mathrm{E}-10$ & $5.23 \mathrm{E}-10$ & LARGE1 & $1.08 \mathrm{E}+00$ \\
\hline ENSBTAG00000010924 & $1.60 \mathrm{E}-10$ & $1.48 \mathrm{E}-10$ & LETMD1 & $1.08 \mathrm{E}+00$ \\
\hline ENSBTAG00000006851 & $5.06 \mathrm{E}-10$ & 4.67E-10 & & $1.08 \mathrm{E}+00$ \\
\hline ENSBTAG00000009506 & $2.61 \mathrm{E}-10$ & $2.40 \mathrm{E}-10$ & NAGA & $1.09 \mathrm{E}+00$ \\
\hline ENSBTAG00000013635 & $2.15 \mathrm{E}-10$ & $1.98 \mathrm{E}-10$ & PDE6H & $1.08 \mathrm{E}+00$ \\
\hline ENSBTAG00000019563 & $3.88 \mathrm{E}-10$ & $3.56 \mathrm{E}-10$ & $\mathrm{C} 5 \mathrm{H} 22$ orf 23 & $1.09 \mathrm{E}+00$ \\
\hline ENSBTAG00000012423 & $1.84 \mathrm{E}-10$ & $1.68 \mathrm{E}-10$ & SENP1 & $1.09 E+00$ \\
\hline ENSBTAG00000004917 & $7.66 \mathrm{E}-10$ & $7.01 \mathrm{E}-10$ & KLRK1 & $1.09 E+00$ \\
\hline ENSBTAG00000013825 & $5.96 \mathrm{E}-10$ & $5.44 \mathrm{E}-10$ & BCAT1 & $1.10 \mathrm{E}+00$ \\
\hline ENSBTAG00000012007 & $4.89 \mathrm{E}-10$ & $4.45 \mathrm{E}-10$ & SOCS2 & $1.10 \mathrm{E}+00$ \\
\hline ENSBTAG00000027064 & 4.36E-10 & $3.96 \mathrm{E}-10$ & BTBD11 & $1.10 \mathrm{E}+00$ \\
\hline ENSBTAG00000016415 & $6.77 \mathrm{E}-10$ & $6.14 \mathrm{E}-10$ & LTA4H & $1.10 \mathrm{E}+00$ \\
\hline ENSBTAG00000017504 & $5.03 \mathrm{E}-10$ & $4.56 \mathrm{E}-10$ & FAIM2 & $1.10 \mathrm{E}+00$ \\
\hline ENSBTAG00000009624 & $2.65 \mathrm{E}-10$ & $2.40 \mathrm{E}-10$ & SGSM3 & $1.11 \mathrm{E}+00$ \\
\hline ENSBTAG00000018432 & $2.49 \mathrm{E}-10$ & $2.25 \mathrm{E}-10$ & SLC6A15 & $1.11 E+00$ \\
\hline ENSBTAG00000014734 & $1.93 \mathrm{E}-10$ & $1.74 \mathrm{E}-10$ & $\mathrm{CHD} 4$ & $1.11 \mathrm{E}+00$ \\
\hline ENSBTAG00000020757 & $1.36 \mathrm{E}-10$ & $1.22 \mathrm{E}-10$ & PCBP2 & $1.12 \mathrm{E}+00$ \\
\hline ENSBTAG00000045957 & $9.74 \mathrm{E}-10$ & $8.79 \mathrm{E}-10$ & SAMM50 & $1.11 \mathrm{E}+00$ \\
\hline ENSBTAG00000011927 & $1.51 \mathrm{E}-10$ & $1.36 \mathrm{E}-10$ & RDH5 & $1.11 \mathrm{E}+00$ \\
\hline
\end{tabular}




\begin{tabular}{|c|c|c|c|c|}
\hline ENSBTAG00000000859 & $3.36 \mathrm{E}-10$ & $3.02 \mathrm{E}-10$ & SLC38A1 & $1.11 E+00$ \\
\hline ENSBTAG00000015825 & $1.40 \mathrm{E}-10$ & $1.26 \mathrm{E}-10$ & CCDC65 & $1.11 E+00$ \\
\hline ENSBTAG00000045842 & $1.21 \mathrm{E}-09$ & 1.09E-09 & & $1.11 \mathrm{E}+00$ \\
\hline ENSBTAG00000026323 & $6.63 \mathrm{E}-10$ & $5.93 \mathrm{E}-10$ & & $1.12 \mathrm{E}+00$ \\
\hline ENSBTAG00000046778 & $1.23 \mathrm{E}-09$ & 1.11E-09 & & $1.11 \mathrm{E}+00$ \\
\hline ENSBTAG00000039347 & $2.41 \mathrm{E}-10$ & $2.15 \mathrm{E}-10$ & CLEC4D & $1.12 \mathrm{E}+00$ \\
\hline ENSBTAG00000020908 & $2.31 \mathrm{E}-10$ & $2.05 \mathrm{E}-10$ & & $1.13 \mathrm{E}+00$ \\
\hline ENSBTAG00000019294 & $4.04 \mathrm{E}-10$ & 3.60E-10 & $A B C C 9$ & $1.12 \mathrm{E}+00$ \\
\hline ENSBTAG00000039509 & $2.52 \mathrm{E}-10$ & $2.24 \mathrm{E}-10$ & GPRC5A & $1.13 \mathrm{E}+00$ \\
\hline ENSBTAG00000013155 & $1.24 \mathrm{E}-10$ & $1.10 \mathrm{E}-10$ & COL2A1 & $1.13 E+00$ \\
\hline ENSBTAG00000020792 & $4.66 \mathrm{E}-10$ & 4.13E-10 & TPH2 & $1.13 \mathrm{E}+00$ \\
\hline ENSBTAG00000010422 & $2.16 \mathrm{E}-10$ & $1.91 \mathrm{E}-10$ & MDM2 & $1.13 \mathrm{E}+00$ \\
\hline ENSBTAG00000016296 & $1.47 \mathrm{E}-10$ & $1.29 \mathrm{E}-10$ & GPD1 & $1.14 \mathrm{E}+00$ \\
\hline ENSBTAG00000004097 & $6.73 \mathrm{E}-10$ & $5.94 \mathrm{E}-10$ & A2ML1 & $1.13 E+00$ \\
\hline ENSBTAG00000000049 & $1.75 \mathrm{E}-10$ & $1.55 \mathrm{E}-10$ & CCDC77 & $1.13 \mathrm{E}+00$ \\
\hline ENSBTAG00000007035 & $4.55 \mathrm{E}-10$ & $4.01 E-10$ & CCER1 & $1.14 \mathrm{E}+00$ \\
\hline ENSBTAG00000014411 & $2.51 \mathrm{E}-10$ & $2.21 \mathrm{E}-10$ & TMEM263 & $1.14 \mathrm{E}+00$ \\
\hline ENSBTAG00000003594 & $1.51 \mathrm{E}-10$ & $1.33 \mathrm{E}-10$ & RIBC2 & $1.14 \mathrm{E}+00$ \\
\hline ENSBTAG00000016661 & $3.14 \mathrm{E}-10$ & $2.76 \mathrm{E}-10$ & & $1.14 \mathrm{E}+00$ \\
\hline ENSBTAG00000002551 & $1.08 \mathrm{E}-10$ & $9.48 \mathrm{E}-11$ & KCNJ8 & $1.13 \mathrm{E}+00$ \\
\hline ENSBTAG00000026779 & $2.14 \mathrm{E}-10$ & $1.88 \mathrm{E}-10$ & LYZ & $1.14 \mathrm{E}+00$ \\
\hline ENSBTAG00000021767 & $2.37 \mathrm{E}-10$ & $2.08 \mathrm{E}-10$ & UBE2N & $1.14 \mathrm{E}+00$ \\
\hline ENSBTAG00000002355 & 7.37E-10 & $6.47 \mathrm{E}-10$ & & $1.14 \mathrm{E}+00$ \\
\hline ENSBTAG00000012265 & $6.95 \mathrm{E}-10$ & $6.09 \mathrm{E}-10$ & VWF & $1.14 \mathrm{E}+00$ \\
\hline ENSBTAG00000015311 & $4.70 \mathrm{E}-10$ & 4.12E-10 & PTPRR & $1.14 \mathrm{E}+00$ \\
\hline ENSBTAG00000006605 & $1.12 \mathrm{E}-09$ & $9.71 \mathrm{E}-10$ & & $1.15 \mathrm{E}+00$ \\
\hline ENSBTAG00000014931 & $4.38 \mathrm{E}-10$ & $3.82 \mathrm{E}-10$ & MLF2 & $1.15 \mathrm{E}+00$ \\
\hline ENSBTAG00000011367 & $3.40 \mathrm{E}-10$ & $2.96 \mathrm{E}-10$ & LIN7A & $1.15 \mathrm{E}+00$ \\
\hline ENSBTAG00000007755 & 4.04E-10 & $3.50 \mathrm{E}-10$ & & $1.15 \mathrm{E}+00$ \\
\hline ENSBTAG00000034693 & 4.97E-10 & $4.30 \mathrm{E}-10$ & SYT1 & $1.16 \mathrm{E}+00$ \\
\hline ENSBTAG00000023192 & $1.98 \mathrm{E}-10$ & $1.72 \mathrm{E}-10$ & & $1.15 E+00$ \\
\hline ENSBTAG00000015109 & $1.55 \mathrm{E}-10$ & $1.34 \mathrm{E}-10$ & TOB2 & $1.16 \mathrm{E}+00$ \\
\hline ENSBTAG00000011336 & $5.52 \mathrm{E}-10$ & $4.75 \mathrm{E}-10$ & OTOGL & $1.16 \mathrm{E}+00$ \\
\hline ENSBTAG00000004371 & $2.75 \mathrm{E}-10$ & $2.36 \mathrm{E}-10$ & CS & $1.16 \mathrm{E}+00$ \\
\hline ENSBTAG00000008553 & $6.66 \mathrm{E}-10$ & $5.73 \mathrm{E}-10$ & B4GALNT3 & $1.16 \mathrm{E}+00$ \\
\hline ENSBTAG00000001810 & $1.35 \mathrm{E}-10$ & $1.16 \mathrm{E}-10$ & SCAF11 & $1.17 \mathrm{E}+00$ \\
\hline ENSBTAG00000014015 & $1.33 \mathrm{E}-10$ & $1.14 \mathrm{E}-10$ & PLA2G6 & $1.16 \mathrm{E}+00$ \\
\hline ENSBTAG00000015092 & $3.60 \mathrm{E}-10$ & $3.09 \mathrm{E}-10$ & & $1.16 \mathrm{E}+00$ \\
\hline ENSBTAG00000007102 & $5.69 \mathrm{E}-10$ & 4.86E-10 & GTSE1 & $1.17 \mathrm{E}+00$ \\
\hline ENSBTAG00000000877 & $2.67 \mathrm{E}-10$ & $2.28 \mathrm{E}-10$ & ETFBKMT & $1.17 \mathrm{E}+00$ \\
\hline ENSBTAG00000003255 & $4.32 \mathrm{E}-10$ & 3.69E-10 & CD4 & $1.17 \mathrm{E}+00$ \\
\hline ENSBTAG00000030325 & $7.48 \mathrm{E}-10$ & 6.37E-10 & & $1.17 \mathrm{E}+00$ \\
\hline ENSBTAG00000018588 & $1.98 \mathrm{E}-10$ & $1.68 \mathrm{E}-10$ & TMBIM6 & $1.18 \mathrm{E}+00$ \\
\hline ENSBTAG00000005120 & $4.56 \mathrm{E}-10$ & 3.87E-10 & TESPA1 & $1.18 \mathrm{E}+00$ \\
\hline ENSBTAG00000003749 & $4.35 \mathrm{E}-10$ & $3.68 \mathrm{E}-10$ & KCNMB4 & $1.18 \mathrm{E}+00$ \\
\hline ENSBTAG00000012149 & $1.09 \mathrm{E}-10$ & $9.19 \mathrm{E}-11$ & HOXC8 & $1.19 \mathrm{E}+00$ \\
\hline ENSBTAG00000013640 & $9.25 \mathrm{E}-10$ & $7.78 \mathrm{E}-10$ & KLRG1 & $1.19 E+00$ \\
\hline ENSBTAG00000011273 & $2.30 \mathrm{E}-10$ & $1.93 \mathrm{E}-10$ & LRRC23 & $1.19 E+00$ \\
\hline ENSBTAG00000016593 & $2.20 \mathrm{E}-10$ & $1.85 \mathrm{E}-10$ & FMNL3 & $1.19 E+00$ \\
\hline ENSBTAG00000012244 & $1.37 \mathrm{E}-10$ & $1.15 \mathrm{E}-10$ & TUBA1B & $1.19 E+00$ \\
\hline ENSBTAG00000030258 & $6.28 \mathrm{E}-11$ & $5.27 \mathrm{E}-11$ & CDC42EP1 & $1.19 E+00$ \\
\hline ENSBTAG00000046446 & $2.66 \mathrm{E}-10$ & $2.24 \mathrm{E}-10$ & & $1.19 E+00$ \\
\hline ENSBTAG00000015828 & 7.10E-11 & $5.96 \mathrm{E}-11$ & FKBP11 & $1.19 E+00$ \\
\hline ENSBTAG00000007323 & $1.67 \mathrm{E}-10$ & $1.40 \mathrm{E}-10$ & CPSF6 & $1.19 E+00$ \\
\hline ENSBTAG00000021803 & $2.52 \mathrm{E}-10$ & $2.12 \mathrm{E}-10$ & GRAMD4 & $1.19 E+00$ \\
\hline ENSBTAG00000018524 & $8.31 \mathrm{E}-11$ & $6.98 \mathrm{E}-11$ & AMHR2 & $1.19 E+00$ \\
\hline ENSBTAG00000014129 & $7.65 \mathrm{E}-11$ & $6.42 \mathrm{E}-11$ & RAB5B & $1.19 E+00$ \\
\hline ENSBTAG00000035083 & $3.96 \mathrm{E}-10$ & 3.32E-10 & ATXN7L3B & $1.19 E+00$ \\
\hline ENSBTAG00000007611 & $5.15 E-10$ & $4.32 \mathrm{E}-10$ & NRIP2 & $1.19 E+00$ \\
\hline ENSBTAG00000012291 & $6.84 \mathrm{E}-10$ & $5.74 \mathrm{E}-10$ & TBC1D22A & $1.19 E+00$ \\
\hline ENSBTAG00000047080 & $4.63 \mathrm{E}-10$ & $3.89 \mathrm{E}-10$ & & $1.19 E+00$ \\
\hline ENSBTAG00000009508 & $1.99 \mathrm{E}-10$ & 1.67E-10 & SMDT1 & $1.19 E+00$ \\
\hline ENSBTAG00000000966 & $6.57 \mathrm{E}-10$ & $5.50 \mathrm{E}-10$ & & $1.19 E+00$ \\
\hline ENSBTAG00000008900 & $2.31 \mathrm{E}-10$ & $1.94 \mathrm{E}-10$ & CELA1 & $1.19 \mathrm{E}+00$ \\
\hline ENSBTAG00000011892 & $3.10 \mathrm{E}-10$ & 2.57E-10 & SRGAP1 & $1.21 \mathrm{E}+00$ \\
\hline ENSBTAG00000011096 & $1.76 \mathrm{E}-10$ & $1.45 \mathrm{E}-10$ & ERGIC2 & $1.21 E+00$ \\
\hline ENSBTAG00000011957 & $1.70 \mathrm{E}-10$ & $1.40 \mathrm{E}-10$ & CREBL2 & $1.21 \mathrm{E}+00$ \\
\hline ENSBTAG00000011095 & $1.43 \mathrm{E}-10$ & $1.18 \mathrm{E}-10$ & FAR2 & $1.21 \mathrm{E}+00$ \\
\hline ENSBTAG00000019788 & 4.23E-10 & 3.47E-10 & TEAD4 & $1.22 \mathrm{E}+00$ \\
\hline ENSBTAG00000013745 & $3.47 \mathrm{E}-10$ & $2.85 \mathrm{E}-10$ & ITGA5 & $1.22 \mathrm{E}+00$ \\
\hline
\end{tabular}




\begin{tabular}{|c|c|c|c|c|}
\hline ENSBTAG000000047582 & 4.57E-10 & $3.74 \mathrm{E}-10$ & & $1.22 \mathrm{E}+00$ \\
\hline ENSBTAG00000022329 & 4.03E-10 & $3.29 \mathrm{E}-10$ & & $1.22 \mathrm{E}+00$ \\
\hline ENSBTAG00000011883 & $4.71 \mathrm{E}-10$ & $3.84 \mathrm{E}-10$ & ARL1 & $1.23 \mathrm{E}+00$ \\
\hline ENSBTAG000000011525 & $1.61 \mathrm{E}-10$ & $1.31 \mathrm{E}-10$ & SLC5A8 & $1.23 E+00$ \\
\hline ENSBTAG000000014247 & $2.87 \mathrm{E}-10$ & $2.35 \mathrm{E}-10$ & FAM83F & $1.22 \mathrm{E}+00$ \\
\hline ENSBTAG00000019260 & $8.09 \mathrm{E}-10$ & $6.60 \mathrm{E}-10$ & GLIPR1L2 & $1.23 E+00$ \\
\hline ENSBTAG000000009904 & $4.69 \mathrm{E}-10$ & $3.83 \mathrm{E}-10$ & SPIC & $1.22 \mathrm{E}+00$ \\
\hline ENSBTAG000000012026 & $5.85 \mathrm{E}-10$ & $4.78 \mathrm{E}-10$ & & $1.22 \mathrm{E}+00$ \\
\hline ENSBTAG000000007144 & $2.01 \mathrm{E}-10$ & $1.64 \mathrm{E}-10$ & & $1.23 \mathrm{E}+00$ \\
\hline ENSBTAG000000045542 & $7.64 \mathrm{E}-10$ & $6.23 \mathrm{E}-10$ & & $1.23 \mathrm{E}+00$ \\
\hline ENSBTAG000000015016 & $1.97 \mathrm{E}-10$ & $1.60 \mathrm{E}-10$ & CALCOCO1 & $1.23 E+00$ \\
\hline ENSBTAG000000010182 & $3.79 \mathrm{E}-10$ & $3.08 \mathrm{E}-10$ & $A C R$ & $1.23 \mathrm{E}+00$ \\
\hline ENSBTAG00000027770 & $4.76 \mathrm{E}-10$ & 3.87E-10 & & $1.23 \mathrm{E}+00$ \\
\hline ENSBTAG000000005465 & $1.80 \mathrm{E}-10$ & $1.47 \mathrm{E}-10$ & & $1.23 E+00$ \\
\hline ENSBTAG000000043962 & $3.02 \mathrm{E}-10$ & $2.46 \mathrm{E}-10$ & SLC2A13 & $1.23 \mathrm{E}+00$ \\
\hline ENSBTAG000000044176 & 7.07E-10 & $5.73 \mathrm{E}-10$ & C12orf40 & $1.23 E+00$ \\
\hline ENSBTAG000000004820 & $1.39 \mathrm{E}-10$ & $1.12 \mathrm{E}-10$ & POLR2F & $1.24 \mathrm{E}+00$ \\
\hline ENSBTAG000000030179 & $3.15 \mathrm{E}-10$ & $2.54 \mathrm{E}-10$ & MAPK8IP2 & $1.24 \mathrm{E}+00$ \\
\hline ENSBTAG000000007256 & $1.97 \mathrm{E}-10$ & $1.58 \mathrm{E}-10$ & DYRK2 & $1.24 \mathrm{E}+00$ \\
\hline ENSBTAG000000013658 & $2.45 \mathrm{E}-10$ & 1.97E-10 & ERC1 & $1.24 \mathrm{E}+00$ \\
\hline ENSBTAG000000045785 & $4.70 \mathrm{E}-10$ & 3.77E-10 & TOM1 & $1.25 \mathrm{E}+00$ \\
\hline ENSBTAG00000026916 & $5.58 \mathrm{E}-10$ & $4.46 \mathrm{E}-10$ & PRR13 & $1.25 \mathrm{E}+00$ \\
\hline ENSBTAG000000037842 & $9.70 \mathrm{E}-10$ & $7.76 \mathrm{E}-10$ & & $1.25 E+00$ \\
\hline ENSBTAG000000000022 & $1.76 \mathrm{E}-10$ & $1.41 \mathrm{E}-10$ & NUP37 & $1.25 \mathrm{E}+00$ \\
\hline ENSBTAG00000008596 & $3.65 E-10$ & $2.91 E-10$ & PRICKLE1 & $1.25 \mathrm{E}+00$ \\
\hline ENSBTAG000000019119 & $3.31 \mathrm{E}-10$ & $2.64 \mathrm{E}-10$ & ZCRB1 & $1.25 E+00$ \\
\hline ENSBTAG000000002313 & $3.72 \mathrm{E}-10$ & $2.95 \mathrm{E}-10$ & ITPR2 & $1.26 \mathrm{E}+00$ \\
\hline ENSBTAG000000000606 & $3.10 \mathrm{E}-10$ & $2.46 \mathrm{E}-10$ & SYT10 & $1.26 \mathrm{E}+00$ \\
\hline ENSBTAG000000021980 & $8.77 \mathrm{E}-10$ & $6.95 \mathrm{E}-10$ & RASSF8 & $1.26 \mathrm{E}+00$ \\
\hline ENSBTAG000000022986 & $4.78 \mathrm{E}-10$ & 3.77E-10 & FAM19A5 & $1.27 \mathrm{E}+00$ \\
\hline ENSBTAG000000021173 & $8.63 \mathrm{E}-11$ & $6.79 \mathrm{E}-11$ & CCDC134 & $1.27 \mathrm{E}+00$ \\
\hline ENSBTAG00000015792 & $6.55 \mathrm{E}-10$ & $5.15 \mathrm{E}-10$ & & $1.27 \mathrm{E}+00$ \\
\hline ENSBTAG000000003021 & $1.80 \mathrm{E}-10$ & $1.41 \mathrm{E}-10$ & SP1 & $1.28 \mathrm{E}+00$ \\
\hline ENSBTAG000000014424 & $1.33 \mathrm{E}-10$ & $1.05 \mathrm{E}-10$ & DDN & $1.27 E+00$ \\
\hline ENSBTAG00000010644 & $4.08 \mathrm{E}-10$ & $3.19 \mathrm{E}-10$ & CHST11 & $1.28 \mathrm{E}+00$ \\
\hline ENSBTAG00000009668 & $1.47 \mathrm{E}-10$ & $1.15 \mathrm{E}-10$ & TMEM184B & $1.28 \mathrm{E}+00$ \\
\hline ENSBTAG00000021435 & $1.90 \mathrm{E}-10$ & $1.48 \mathrm{E}-10$ & MAFF & $1.28 \mathrm{E}+00$ \\
\hline ENSBTAG00000005384 & $1.44 \mathrm{E}-10$ & $1.12 \mathrm{E}-10$ & COPZ1 & $1.29 \mathrm{E}+00$ \\
\hline ENSBTAG00000012030 & 8.07E-10 & $6.28 \mathrm{E}-10$ & TTLL1 & $1.28 \mathrm{E}+00$ \\
\hline ENSBTAG00000002174 & $3.14 \mathrm{E}-10$ & 2.44E-10 & RERG & $1.29 E+00$ \\
\hline ENSBTAG00000017164 & $1.64 \mathrm{E}-10$ & $1.28 \mathrm{E}-10$ & LARP4 & $1.28 \mathrm{E}+00$ \\
\hline ENSBTAG00000039891 & $5.91 \mathrm{E}-10$ & $4.58 \mathrm{E}-10$ & KRT71 & $1.29 \mathrm{E}+00$ \\
\hline ENSBTAG00000006013 & $9.68 \mathrm{E}-10$ & 7.50E-10 & NT5DC3 & $1.29 \mathrm{E}+00$ \\
\hline ENSBTAG00000004208 & $2.78 \mathrm{E}-10$ & $2.16 \mathrm{E}-10$ & PLEKHG6 & $1.29 E+00$ \\
\hline ENSBTAG00000046389 & $8.23 \mathrm{E}-10$ & $6.36 \mathrm{E}-10$ & & $1.29 \mathrm{E}+00$ \\
\hline ENSBTAG00000034385 & $1.82 \mathrm{E}-10$ & $1.41 \mathrm{E}-10$ & GPR162 & $1.29 \mathrm{E}+00$ \\
\hline ENSBTAG00000011591 & 2.77E-10 & $2.14 \mathrm{E}-10$ & KRR1 & $1.30 \mathrm{E}+00$ \\
\hline ENSBTAG00000000925 & $6.61 \mathrm{E}-10$ & $5.08 \mathrm{E}-10$ & GLT8D2 & $1.30 \mathrm{E}+00$ \\
\hline ENSBTAG00000005863 & $1.20 \mathrm{E}-10$ & $9.28 \mathrm{E}-11$ & & $1.30 \mathrm{E}+00$ \\
\hline ENSBTAG00000010865 & 4.19E-10 & $3.22 \mathrm{E}-10$ & CACNG2 & $1.30 \mathrm{E}+00$ \\
\hline ENSBTAG00000008595 & $3.74 \mathrm{E}-10$ & $2.87 \mathrm{E}-10$ & & $1.30 \mathrm{E}+00$ \\
\hline ENSBTAG00000011392 & $2.15 \mathrm{E}-10$ & $1.65 \mathrm{E}-10$ & MYBPC1 & $1.30 \mathrm{E}+00$ \\
\hline ENSBTAG00000019312 & $3.23 \mathrm{E}-10$ & $2.48 \mathrm{E}-10$ & TFCP2 & $1.30 \mathrm{E}+00$ \\
\hline ENSBTAG00000046511 & $2.96 \mathrm{E}-10$ & $2.26 \mathrm{E}-10$ & & $1.31 \mathrm{E}+00$ \\
\hline ENSBTAG00000016511 & $2.62 \mathrm{E}-10$ & $2.01 E-10$ & POLDIP3 & $1.31 E+00$ \\
\hline ENSBTAG00000006464 & $1.63 \mathrm{E}-10$ & $1.24 \mathrm{E}-10$ & GGA1 & $1.31 E+00$ \\
\hline ENSBTAG00000004211 & $1.79 \mathrm{E}-10$ & 1.37E-10 & TNFRSF1A & $1.31 E+00$ \\
\hline ENSBTAG00000000395 & $1.99 \mathrm{E}-10$ & $1.51 E-10$ & ADAMTS20 & $1.32 \mathrm{E}+00$ \\
\hline ENSBTAG00000004004 & $4.20 \mathrm{E}-10$ & $3.20 \mathrm{E}-10$ & $\operatorname{CSDC2}$ & $1.31 E+00$ \\
\hline ENSBTAG00000011838 & $3.20 \mathrm{E}-10$ & $2.44 \mathrm{E}-10$ & PCED1B & $1.31 E+00$ \\
\hline ENSBTAG00000021660 & $1.48 \mathrm{E}-10$ & $1.13 \mathrm{E}-10$ & & $1.31 E+00$ \\
\hline ENSBTAG00000017864 & $1.14 \mathrm{E}-10$ & $8.59 \mathrm{E}-11$ & PRPH & $1.32 \mathrm{E}+00$ \\
\hline ENSBTAG00000004374 & $1.36 \mathrm{E}-10$ & $1.03 \mathrm{E}-10$ & & $1.32 \mathrm{E}+00$ \\
\hline ENSBTAG00000010127 & $1.20 \mathrm{E}-10$ & 9.07E-11 & MIP & $1.32 \mathrm{E}+00$ \\
\hline ENSBTAG00000009297 & $2.18 \mathrm{E}-10$ & $1.65 \mathrm{E}-10$ & PANX2 & $1.32 \mathrm{E}+00$ \\
\hline ENSBTAG00000011067 & $2.59 \mathrm{E}-10$ & $1.95 \mathrm{E}-10$ & IL17RA & $1.33 \mathrm{E}+00$ \\
\hline ENSBTAG00000007546 & $4.14 \mathrm{E}-10$ & $3.12 \mathrm{E}-10$ & & $1.33 \mathrm{E}+00$ \\
\hline ENSBTAG00000024771 & 2.69E-10 & 2.03E-10 & & $1.33 E+00$ \\
\hline ENSBTAG00000012624 & 4.33E-10 & $3.27 \mathrm{E}-10$ & & $1.32 \mathrm{E}+00$ \\
\hline ENSBTAG00000026322 & $4.03 \mathrm{E}-10$ & $3.04 \mathrm{E}-10$ & & $1.32 \mathrm{E}+00$ \\
\hline
\end{tabular}




\begin{tabular}{|c|c|c|c|c|}
\hline ENSBTAG00000007175 & $2.36 \mathrm{E}-10$ & $1.78 \mathrm{E}-10$ & AVPR1A & $1.32 \mathrm{E}+00$ \\
\hline ENSBTAG00000021460 & $4.39 \mathrm{E}-10$ & $3.31 \mathrm{E}-10$ & & $1.33 E+00$ \\
\hline ENSBTAG00000026385 & $4.01 \mathrm{E}-10$ & $3.03 \mathrm{E}-10$ & & $1.32 \mathrm{E}+00$ \\
\hline ENSBTAG00000018358 & $2.01 \mathrm{E}-10$ & $1.52 \mathrm{E}-10$ & STAC3 & $1.32 \mathrm{E}+00$ \\
\hline ENSBTAG00000046628 & $2.93 \mathrm{E}-10$ & $2.22 \mathrm{E}-10$ & & $1.32 E+00$ \\
\hline ENSBTAG00000002390 & $2.19 \mathrm{E}-10$ & $1.65 \mathrm{E}-10$ & CCDC184 & $1.32 \mathrm{E}+00$ \\
\hline ENSBTAG00000009351 & $3.85 \mathrm{E}-10$ & $2.90 \mathrm{E}-10$ & ATXN10 & $1.33 E+00$ \\
\hline ENSBTAG00000021076 & $1.66 \mathrm{E}-10$ & $1.24 \mathrm{E}-10$ & TNRC6B & $1.34 \mathrm{E}+00$ \\
\hline ENSBTAG00000020638 & $3.49 \mathrm{E}-10$ & 2.62E-10 & TIMP3 & $1.33 E+00$ \\
\hline ENSBTAG00000007636 & $1.66 \mathrm{E}-10$ & $1.24 \mathrm{E}-10$ & IRAK3 & $1.34 \mathrm{E}+00$ \\
\hline ENSBTAG00000021287 & 3.57E-10 & $2.66 \mathrm{E}-10$ & SLC16A7 & $1.34 \mathrm{E}+00$ \\
\hline ENSBTAG00000014288 & $3.09 \mathrm{E}-10$ & $2.31 \mathrm{E}-10$ & GPRC5D & $1.34 \mathrm{E}+00$ \\
\hline ENSBTAG00000017287 & 4.07E-10 & $3.03 \mathrm{E}-10$ & DRAM1 & $1.34 \mathrm{E}+00$ \\
\hline ENSBTAG00000007417 & $1.45 \mathrm{E}-10$ & $1.06 \mathrm{E}-10$ & GDF11 & $1.37 E+00$ \\
\hline ENSBTAG00000020933 & $3.25 \mathrm{E}-10$ & $2.39 \mathrm{E}-10$ & HCFC2 & $1.36 \mathrm{E}+00$ \\
\hline ENSBTAG00000007145 & $1.49 \mathrm{E}-10$ & $1.10 \mathrm{E}-10$ & KRT82 & $1.36 \mathrm{E}+00$ \\
\hline ENSBTAG00000006324 & $1.64 \mathrm{E}-10$ & $1.21 \mathrm{E}-10$ & NAB2 & $1.36 \mathrm{E}+00$ \\
\hline ENSBTAG00000019944 & $1.60 \mathrm{E}-10$ & $1.18 \mathrm{E}-10$ & METAP2 & $1.36 \mathrm{E}+00$ \\
\hline ENSBTAG00000011071 & $4.18 \mathrm{E}-10$ & 3.06E-10 & BPIFC & $1.36 \mathrm{E}+00$ \\
\hline ENSBTAG00000007152 & $1.08 \mathrm{E}-10$ & 7.84E-11 & OS9 & $1.37 \mathrm{E}+00$ \\
\hline ENSBTAG00000005018 & $2.58 \mathrm{E}-10$ & $1.87 \mathrm{E}-10$ & NFAM1 & $1.38 \mathrm{E}+00$ \\
\hline ENSBTAG00000022861 & $4.76 \mathrm{E}-10$ & $3.45 \mathrm{E}-10$ & & $1.38 \mathrm{E}+00$ \\
\hline ENSBTAG00000032914 & $1.45 \mathrm{E}-10$ & $1.05 \mathrm{E}-10$ & & $1.38 \mathrm{E}+00$ \\
\hline ENSBTAG00000014175 & $1.78 \mathrm{E}-10$ & $1.29 \mathrm{E}-10$ & STRAP & $1.38 \mathrm{E}+00$ \\
\hline ENSBTAG00000016516 & $2.90 \mathrm{E}-10$ & $2.09 \mathrm{E}-10$ & CYB5R3 & $1.39 E+00$ \\
\hline ENSBTAG00000002251 & $4.96 \mathrm{E}-10$ & $3.58 \mathrm{E}-10$ & & $1.39 E+00$ \\
\hline ENSBTAG00000034295 & $1.61 \mathrm{E}-10$ & $1.16 \mathrm{E}-10$ & LAG3 & $1.38 \mathrm{E}+00$ \\
\hline ENSBTAG00000016271 & $7.49 \mathrm{E}-10$ & $5.40 \mathrm{E}-10$ & SNRPF & $1.39 \mathrm{E}+00$ \\
\hline ENSBTAG00000019780 & $2.55 \mathrm{E}-10$ & $1.83 \mathrm{E}-10$ & USP5 & $1.39 \mathrm{E}+00$ \\
\hline ENSBTAG00000015364 & $1.86 \mathrm{E}-10$ & $1.34 \mathrm{E}-10$ & WNT1 & $1.39 E+00$ \\
\hline ENSBTAG00000020758 & $9.39 \mathrm{E}-11$ & $6.76 \mathrm{E}-11$ & MAP3K12 & $1.39 \mathrm{E}+00$ \\
\hline ENSBTAG00000045746 & $1.02 \mathrm{E}-09$ & 7.34E-10 & & $1.39 \mathrm{E}+00$ \\
\hline ENSBTAG00000008721 & $3.76 \mathrm{E}-10$ & $2.71 E-10$ & MANSC1 & $1.39 E+00$ \\
\hline ENSBTAG00000039380 & $8.14 \mathrm{E}-10$ & $5.85 \mathrm{E}-10$ & & $1.39 E+00$ \\
\hline ENSBTAG00000024798 & $6.16 \mathrm{E}-10$ & $4.42 \mathrm{E}-10$ & & $1.39 \mathrm{E}+00$ \\
\hline ENSBTAG00000030180 & $2.91 \mathrm{E}-10$ & $2.09 \mathrm{E}-10$ & SHANK3 & $1.39 E+00$ \\
\hline ENSBTAG00000016050 & $1.74 \mathrm{E}-10$ & $1.25 \mathrm{E}-10$ & & $1.39 E+00$ \\
\hline ENSBTAG00000018002 & $1.62 \mathrm{E}-10$ & 1.17E-10 & DTX3 & $1.38 \mathrm{E}+00$ \\
\hline ENSBTAG00000047109 & $6.24 \mathrm{E}-10$ & $4.48 \mathrm{E}-10$ & & $1.39 \mathrm{E}+00$ \\
\hline ENSBTAG00000015275 & $1.23 \mathrm{E}-10$ & $8.88 \mathrm{E}-11$ & SLC16A8 & $1.39 E+00$ \\
\hline ENSBTAG00000020679 & 4.57E-10 & $3.27 \mathrm{E}-10$ & CNTN1 & $1.40 \mathrm{E}+00$ \\
\hline ENSBTAG00000005082 & $3.11 E-10$ & $2.22 \mathrm{E}-10$ & MED21 & $1.40 \mathrm{E}+00$ \\
\hline ENSBTAG00000010444 & $1.80 \mathrm{E}-10$ & $1.28 \mathrm{E}-10$ & ERBB3 & $1.41 E+00$ \\
\hline ENSBTAG00000037800 & 1.16E-09 & $8.21 \mathrm{E}-10$ & & $1.41 E+00$ \\
\hline ENSBTAG00000047225 & $2.88 \mathrm{E}-10$ & $2.04 \mathrm{E}-10$ & BCL2L14 & $1.41 E+00$ \\
\hline ENSBTAG00000039470 & $8.56 \mathrm{E}-10$ & $6.08 \mathrm{E}-10$ & & $1.41 \mathrm{E}+00$ \\
\hline ENSBTAG00000000593 & $2.20 \mathrm{E}-10$ & $1.56 \mathrm{E}-10$ & ST8SIA1 & $1.41 E+00$ \\
\hline ENSBTAG00000001020 & $2.06 \mathrm{E}-10$ & $1.46 \mathrm{E}-10$ & AKAP3 & $1.41 E+00$ \\
\hline ENSBTAG00000011765 & $4.76 \mathrm{E}-10$ & $3.36 \mathrm{E}-10$ & GABARAPL1 & $1.42 \mathrm{E}+00$ \\
\hline ENSBTAG00000044017 & $2.16 \mathrm{E}-10$ & $1.52 \mathrm{E}-10$ & MSRB3 & $1.42 \mathrm{E}+00$ \\
\hline ENSBTAG00000016496 & $3.64 \mathrm{E}-10$ & $2.57 \mathrm{E}-10$ & ZNF641 & $1.42 \mathrm{E}+00$ \\
\hline ENSBTAG00000016931 & $3.69 \mathrm{E}-10$ & $2.60 \mathrm{E}-10$ & E2F7 & $1.42 \mathrm{E}+00$ \\
\hline ENSBTAG00000014730 & $6.24 \mathrm{E}-10$ & $4.38 \mathrm{E}-10$ & NCAPD2 & $1.42 \mathrm{E}+00$ \\
\hline ENSBTAG00000046324 & $4.68 \mathrm{E}-10$ & $3.29 \mathrm{E}-10$ & & $1.42 \mathrm{E}+00$ \\
\hline ENSBTAG00000012034 & $5.11 \mathrm{E}-10$ & $3.59 \mathrm{E}-10$ & KRT4 & $1.42 \mathrm{E}+00$ \\
\hline ENSBTAG00000015875 & $7.69 \mathrm{E}-10$ & $5.39 \mathrm{E}-10$ & FOXM1 & $1.43 E+00$ \\
\hline ENSBTAG00000023546 & $1.47 \mathrm{E}-10$ & $1.03 \mathrm{E}-10$ & FAM186A & $1.43 \mathrm{E}+00$ \\
\hline ENSBTAG00000005243 & $5.25 \mathrm{E}-10$ & 3.67E-10 & ovos2 & $1.43 \mathrm{E}+00$ \\
\hline ENSBTAG00000002972 & $2.91 \mathrm{E}-10$ & $2.03 \mathrm{E}-10$ & CASC1 & $1.43 E+00$ \\
\hline ENSBTAG00000008129 & $3.32 \mathrm{E}-10$ & $2.31 \mathrm{E}-10$ & CLSTN3 & $1.44 \mathrm{E}+00$ \\
\hline ENSBTAG00000030650 & $2.49 \mathrm{E}-10$ & $1.73 \mathrm{E}-10$ & TST & $1.44 \mathrm{E}+00$ \\
\hline ENSBTAG00000004556 & $1.97 \mathrm{E}-10$ & $1.36 \mathrm{E}-10$ & & $1.45 \mathrm{E}+00$ \\
\hline ENSBTAG00000002630 & $2.75 \mathrm{E}-10$ & $1.89 \mathrm{E}-10$ & MKL1 & $1.45 \mathrm{E}+00$ \\
\hline ENSBTAG00000018137 & $4.20 \mathrm{E}-10$ & $2.88 \mathrm{E}-10$ & $\mathrm{~A} 2 \mathrm{M}$ & $1.46 \mathrm{E}+00$ \\
\hline ENSBTAG00000000836 & $3.87 \mathrm{E}-10$ & 2.64E-10 & KRT8 & $1.47 \mathrm{E}+00$ \\
\hline ENSBTAG00000026611 & $4.84 \mathrm{E}-10$ & $3.30 \mathrm{E}-10$ & & $1.47 E+00$ \\
\hline ENSBTAG00000020237 & $2.44 \mathrm{E}-10$ & 1.67E-10 & NECAP1 & $1.46 \mathrm{E}+00$ \\
\hline ENSBTAG00000011082 & $1.64 \mathrm{E}-10$ & $1.12 \mathrm{E}-10$ & IGF-I & $1.47 E+00$ \\
\hline ENSBTAG00000006734 & $1.94 \mathrm{E}-10$ & $1.32 \mathrm{E}-10$ & BICD1 & $1.47 \mathrm{E}+00$ \\
\hline ENSBTAG00000002631 & $3.15 \mathrm{E}-10$ & $2.14 \mathrm{E}-10$ & SCNN1A & $1.47 \mathrm{E}+00$ \\
\hline
\end{tabular}




\begin{tabular}{|c|c|c|c|c|}
\hline ENSBTAG00000021621 & $1.12 \mathrm{E}-09$ & $7.54 \mathrm{E}-10$ & & $1.48 \mathrm{E}+00$ \\
\hline ENSBTAG00000018940 & $4.58 \mathrm{E}-10$ & $3.11 \mathrm{E}-10$ & & $1.47 \mathrm{E}+00$ \\
\hline ENSBTAG00000016198 & $3.09 \mathrm{E}-10$ & $2.09 \mathrm{E}-10$ & EP300 & $1.48 \mathrm{E}+00$ \\
\hline ENSBTAG00000012342 & $2.10 \mathrm{E}-10$ & $1.42 \mathrm{E}-10$ & LIMA1 & $1.48 \mathrm{E}+00$ \\
\hline ENSBTAG00000015157 & $2.27 \mathrm{E}-10$ & $1.54 \mathrm{E}-10$ & GRAP2 & $1.48 \mathrm{E}+00$ \\
\hline ENSBTAG00000003748 & $1.92 \mathrm{E}-10$ & $1.29 \mathrm{E}-10$ & CNOT2 & $1.49 \mathrm{E}+00$ \\
\hline ENSBTAG00000015044 & $5.90 \mathrm{E}-10$ & 3.97E-10 & CARD10 & $1.49 E+00$ \\
\hline ENSBTAG00000014332 & 1.36E-09 & $9.20 \mathrm{E}-10$ & & $1.48 \mathrm{E}+00$ \\
\hline ENSBTAG00000047650 & $2.78 \mathrm{E}-10$ & $1.87 \mathrm{E}-10$ & NXPH4 & $1.49 \mathrm{E}+00$ \\
\hline ENSBTAG00000020699 & $4.81 \mathrm{E}-10$ & 3.23E-10 & TSPAN11 & $1.49 E+00$ \\
\hline ENSBTAG00000016910 & $1.63 \mathrm{E}-10$ & $1.10 \mathrm{E}-10$ & METTL21B & $1.48 \mathrm{E}+00$ \\
\hline ENSBTAG00000009543 & $1.75 \mathrm{E}-10$ & $1.17 \mathrm{E}-10$ & ESYT1 & $1.49 \mathrm{E}+00$ \\
\hline ENSBTAG00000009956 & 2.17E-10 & $1.45 \mathrm{E}-10$ & CENPM & $1.50 \mathrm{E}+00$ \\
\hline ENSBTAG00000021994 & $3.26 \mathrm{E}-10$ & $2.18 \mathrm{E}-10$ & CACNA2D4 & $1.49 \mathrm{E}+00$ \\
\hline ENSBTAG00000032183 & $3.21 \mathrm{E}-10$ & $2.14 \mathrm{E}-10$ & NELL2 & $1.50 \mathrm{E}+00$ \\
\hline ENSBTAG00000013859 & 7.73E-10 & $5.16 \mathrm{E}-10$ & SCYL2 & $1.50 \mathrm{E}+00$ \\
\hline ENSBTAG00000021697 & $6.85 \mathrm{E}-11$ & 4.57E-11 & PDGFB & $1.50 \mathrm{E}+00$ \\
\hline ENSBTAG00000016274 & $7.85 \mathrm{E}-10$ & $5.24 \mathrm{E}-10$ & CCDC38 & $1.50 \mathrm{E}+00$ \\
\hline ENSBTAG00000009778 & $1.42 \mathrm{E}-10$ & $9.46 \mathrm{E}-11$ & KRAS & $1.50 \mathrm{E}+00$ \\
\hline ENSBTAG00000021978 & $8.85 \mathrm{E}-10$ & $5.89 \mathrm{E}-10$ & PARVB & $1.50 \mathrm{E}+00$ \\
\hline ENSBTAG00000017652 & $3.38 \mathrm{E}-10$ & $2.24 \mathrm{E}-10$ & NUP50 & $1.51 E+00$ \\
\hline ENSBTAG00000046760 & 3.96E-10 & $2.62 \mathrm{E}-10$ & BRD1 & $1.51 E+00$ \\
\hline ENSBTAG00000048233 & $1.49 \mathrm{E}-09$ & $9.80 \mathrm{E}-10$ & & $1.52 \mathrm{E}+00$ \\
\hline ENSBTAG00000004019 & $2.12 \mathrm{E}-10$ & $1.40 \mathrm{E}-10$ & PMEL & $1.52 \mathrm{E}+00$ \\
\hline ENSBTAG00000019711 & $1.39 \mathrm{E}-10$ & $9.13 \mathrm{E}-11$ & LRMP & $1.52 \mathrm{E}+00$ \\
\hline ENSBTAG00000046089 & $3.40 \mathrm{E}-10$ & $2.23 \mathrm{E}-10$ & KRT1 & $1.52 \mathrm{E}+00$ \\
\hline ENSBTAG00000038461 & $6.66 \mathrm{E}-10$ & $4.36 \mathrm{E}-10$ & & $1.53 \mathrm{E}+00$ \\
\hline ENSBTAG00000006036 & $2.51 \mathrm{E}-10$ & $1.64 \mathrm{E}-10$ & ERP27 & $1.53 E+00$ \\
\hline ENSBTAG00000019290 & 8.34E-10 & $5.44 \mathrm{E}-10$ & PACSIN2 & $1.53 \mathrm{E}+00$ \\
\hline ENSBTAG00000018575 & 7.06E-10 & $4.58 \mathrm{E}-10$ & TRHDE & $1.54 \mathrm{E}+00$ \\
\hline ENSBTAG00000021661 & $2.08 \mathrm{E}-10$ & $1.35 \mathrm{E}-10$ & APAF1 & $1.54 \mathrm{E}+00$ \\
\hline ENSBTAG00000014829 & $5.00 \mathrm{E}-10$ & $3.24 \mathrm{E}-10$ & MYRFL & $1.54 \mathrm{E}+00$ \\
\hline ENSBTAG00000020564 & $6.95 \mathrm{E}-10$ & 4.49E-10 & & $1.55 \mathrm{E}+00$ \\
\hline ENSBTAG00000005370 & $2.68 \mathrm{E}-10$ & $1.72 \mathrm{E}-10$ & TMTC1 & $1.56 \mathrm{E}+00$ \\
\hline ENSBTAG00000019058 & $5.21 \mathrm{E}-10$ & $3.36 \mathrm{E}-10$ & IQSEC3 & $1.55 \mathrm{E}+00$ \\
\hline ENSBTAG00000016362 & $5.49 \mathrm{E}-10$ & $3.52 \mathrm{E}-10$ & & $1.56 \mathrm{E}+00$ \\
\hline ENSBTAG00000001708 & $3.01 \mathrm{E}-10$ & $1.93 \mathrm{E}-10$ & TTLL12 & $1.56 \mathrm{E}+00$ \\
\hline ENSBTAG00000015879 & $2.45 \mathrm{E}-10$ & $1.56 \mathrm{E}-10$ & TULP3 & $1.57 E+00$ \\
\hline ENSBTAG00000012784 & $1.26 \mathrm{E}-10$ & 7.96E-11 & RACGAP1 & $1.59 \mathrm{E}+00$ \\
\hline ENSBTAG00000014003 & $1.08 \mathrm{E}-10$ & $6.81 \mathrm{E}-11$ & MYF6 & $1.59 E+00$ \\
\hline ENSBTAG00000000435 & $1.23 \mathrm{E}-10$ & $7.75 \mathrm{E}-11$ & TARBP2 & $1.59 \mathrm{E}+00$ \\
\hline ENSBTAG00000008933 & $1.45 \mathrm{E}-10$ & $9.10 \mathrm{E}-11$ & MFSD5 & $1.59 E+00$ \\
\hline ENSBTAG00000015347 & $1.22 \mathrm{E}-10$ & $7.68 \mathrm{E}-11$ & & $1.59 E+00$ \\
\hline ENSBTAG00000003278 & $3.91 \mathrm{E}-11$ & $2.47 \mathrm{E}-11$ & HOXC10 & $1.58 \mathrm{E}+00$ \\
\hline ENSBTAG00000006335 & $1.54 \mathrm{E}-10$ & $9.70 \mathrm{E}-11$ & STAT6 & $1.59 \mathrm{E}+00$ \\
\hline ENSBTAG00000008857 & $1.73 \mathrm{E}-10$ & 1.09E-10 & GALR3 & $1.59 E+00$ \\
\hline ENSBTAG00000008434 & $4.93 \mathrm{E}-11$ & $3.10 \mathrm{E}-11$ & GCAT & $1.59 \mathrm{E}+00$ \\
\hline ENSBTAG00000026993 & $6.99 \mathrm{E}-11$ & $4.40 \mathrm{E}-11$ & & $1.59 \mathrm{E}+00$ \\
\hline ENSBTAG00000046800 & $2.78 \mathrm{E}-10$ & $1.75 \mathrm{E}-10$ & KLHDC7B & $1.59 \mathrm{E}+00$ \\
\hline ENSBTAG00000014731 & $3.22 \mathrm{E}-10$ & $2.03 E-10$ & & $1.59 E+00$ \\
\hline ENSBTAG00000012782 & $1.28 \mathrm{E}-10$ & $8.08 \mathrm{E}-11$ & AQP6 & $1.59 E+00$ \\
\hline ENSBTAG00000015089 & $9.82 \mathrm{E}-11$ & $6.18 \mathrm{E}-11$ & LGALS1 & $1.59 \mathrm{E}+00$ \\
\hline ENSBTAG00000021122 & $3.51 \mathrm{E}-10$ & $2.21 \mathrm{E}-10$ & & $1.59 \mathrm{E}+00$ \\
\hline ENSBTAG00000045752 & $3.50 \mathrm{E}-10$ & $2.20 \mathrm{E}-10$ & & $1.59 \mathrm{E}+00$ \\
\hline ENSBTAG00000006631 & $1.42 \mathrm{E}-10$ & $8.95 \mathrm{E}-11$ & GLI1 & $1.59 \mathrm{E}+00$ \\
\hline ENSBTAG00000039152 & $1.76 \mathrm{E}-10$ & $1.11 \mathrm{E}-10$ & & $1.59 \mathrm{E}+00$ \\
\hline ENSBTAG00000027899 & $9.08 \mathrm{E}-10$ & $5.71 \mathrm{E}-10$ & & $1.59 E+00$ \\
\hline ENSBTAG00000011000 & $3.34 \mathrm{E}-10$ & $2.10 \mathrm{E}-10$ & HDAC10 & $1.59 E+00$ \\
\hline ENSBTAG00000009634 & $1.13 \mathrm{E}-10$ & 7.12E-11 & HOXC5 & $1.59 \mathrm{E}+00$ \\
\hline ENSBTAG00000045919 & $4.35 \mathrm{E}-10$ & $2.73 \mathrm{E}-10$ & & $1.59 E+00$ \\
\hline ENSBTAG00000002004 & $4.11 \mathrm{E}-10$ & $2.58 \mathrm{E}-10$ & LLPH & $1.59 E+00$ \\
\hline ENSBTAG00000022083 & $2.35 \mathrm{E}-10$ & $1.48 \mathrm{E}-10$ & REP15 & $1.59 \mathrm{E}+00$ \\
\hline ENSBTAG00000047931 & $5.25 \mathrm{E}-10$ & $3.30 \mathrm{E}-10$ & & $1.59 \mathrm{E}+00$ \\
\hline ENSBTAG00000002913 & $5.25 \mathrm{E}-10$ & $3.30 \mathrm{E}-10$ & & $1.59 E+00$ \\
\hline ENSBTAG00000047980 & $3.54 \mathrm{E}-10$ & $2.23 \mathrm{E}-10$ & & $1.59 \mathrm{E}+00$ \\
\hline ENSBTAG00000030185 & 7.74E-10 & $4.86 \mathrm{E}-10$ & & $1.59 E+00$ \\
\hline ENSBTAG00000026489 & $2.18 \mathrm{E}-10$ & $1.37 \mathrm{E}-10$ & TNFRSF13C & $1.59 \mathrm{E}+00$ \\
\hline ENSBTAG00000004181 & $1.78 \mathrm{E}-10$ & $1.12 \mathrm{E}-10$ & & $1.59 E+00$ \\
\hline ENSBTAG00000032428 & $4.95 \mathrm{E}-10$ & $3.11 \mathrm{E}-10$ & & $1.59 \mathrm{E}+00$ \\
\hline ENSBTAG00000020664 & $1.18 \mathrm{E}-10$ & $7.45 \mathrm{E}-11$ & & $1.59 \mathrm{E}+00$ \\
\hline
\end{tabular}




\begin{tabular}{|c|c|c|c|c|}
\hline ENSBTAG00000038567 & $8.23 \mathrm{E}-10$ & $5.16 \mathrm{E}-10$ & & $1.59 E+00$ \\
\hline ENSBTAG00000031786 & 7.07E-10 & 4.44E-10 & FAU & $1.59 E+00$ \\
\hline ENSBTAG00000045487 & $1.73 \mathrm{E}-10$ & $1.09 \mathrm{E}-10$ & & $1.58 \mathrm{E}+00$ \\
\hline ENSBTAG00000012192 & 7.16E-10 & $4.50 \mathrm{E}-10$ & & $1.59 \mathrm{E}+00$ \\
\hline ENSBTAG00000002362 & $1.27 \mathrm{E}-10$ & $7.98 \mathrm{E}-11$ & APOLD1 & $1.59 \mathrm{E}+00$ \\
\hline ENSBTAG00000046599 & $8.46 \mathrm{E}-10$ & $5.31 \mathrm{E}-10$ & & $1.59 E+00$ \\
\hline ENSBTAG00000023670 & $9.20 \mathrm{E}-10$ & $5.77 \mathrm{E}-10$ & & $1.59 E+00$ \\
\hline ENSBTAG00000048277 & $1.04 \mathrm{E}-09$ & $6.52 \mathrm{E}-10$ & & $1.59 E+00$ \\
\hline ENSBTAG00000017950 & $3.21 \mathrm{E}-10$ & $2.00 \mathrm{E}-10$ & SLC4A8 & $1.60 \mathrm{E}+00$ \\
\hline ENSBTAG00000031950 & $6.47 \mathrm{E}-10$ & 4.03E-10 & & $1.60 E+00$ \\
\hline ENSBTAG00000010428 & $2.21 \mathrm{E}-10$ & 1.37E-10 & USP15 & $1.62 \mathrm{E}+00$ \\
\hline ENSBTAG00000013406 & $8.88 \mathrm{E}-10$ & $5.51 \mathrm{E}-10$ & CSRP2 & $1.61 \mathrm{E}+00$ \\
\hline ENSBTAG00000011804 & $5.29 \mathrm{E}-10$ & 3.27E-10 & ARNTL2 & $1.62 \mathrm{E}+00$ \\
\hline ENSBTAG00000003183 & $5.63 \mathrm{E}-10$ & $3.48 \mathrm{E}-10$ & NTN4 & $1.62 E+00$ \\
\hline ENSBTAG00000017967 & $1.83 \mathrm{E}-10$ & $1.12 \mathrm{E}-10$ & PTGES3 & $1.63 E+00$ \\
\hline ENSBTAG00000014697 & $2.00 \mathrm{E}-10$ & $1.23 \mathrm{E}-10$ & SMARCC2 & $1.63 E+00$ \\
\hline ENSBTAG00000014340 & $3.99 \mathrm{E}-10$ & $2.44 \mathrm{E}-10$ & KERA & $1.64 \mathrm{E}+00$ \\
\hline ENSBTAG00000019283 & $2.28 \mathrm{E}-10$ & $1.39 \mathrm{E}-10$ & FAM186B & $1.64 E+00$ \\
\hline ENSBTAG00000030784 & $6.08 \mathrm{E}-10$ & $3.71 \mathrm{E}-10$ & APPL2 & $1.64 \mathrm{E}+00$ \\
\hline ENSBTAG00000018690 & $2.81 \mathrm{E}-10$ & $1.71 \mathrm{E}-10$ & RPAP3 & $1.64 \mathrm{E}+00$ \\
\hline ENSBTAG00000017395 & $2.65 \mathrm{E}-10$ & $1.60 \mathrm{E}-10$ & CERS5 & $1.65 \mathrm{E}+00$ \\
\hline ENSBTAG00000018085 & $2.39 \mathrm{E}-10$ & $1.43 \mathrm{E}-10$ & GTSF1 & $1.67 E+00$ \\
\hline ENSBTAG00000012088 & $5.56 \mathrm{E}-10$ & 3.33E-10 & FBLN1 & $1.67 E+00$ \\
\hline ENSBTAG00000000687 & $3.93 \mathrm{E}-10$ & $2.35 \mathrm{E}-10$ & POC1B & $1.67 E+00$ \\
\hline ENSBTAG00000014154 & $7.29 \mathrm{E}-10$ & 4.35E-10 & & $1.67 \mathrm{E}+00$ \\
\hline ENSBTAG00000020459 & $1.90 \mathrm{E}-10$ & 1.13E-10 & TIMELESS & $1.68 \mathrm{E}+00$ \\
\hline ENSBTAG00000011761 & 2.06E-10 & $1.23 \mathrm{E}-10$ & LRP6 & $1.67 \mathrm{E}+00$ \\
\hline ENSBTAG00000014375 & 7.02E-10 & 4.17E-10 & TMCC3 & $1.68 \mathrm{E}+00$ \\
\hline ENSBTAG00000006204 & $4.96 \mathrm{E}-10$ & $2.95 \mathrm{E}-10$ & XPNPEP3 & $1.68 \mathrm{E}+00$ \\
\hline ENSBTAG00000013822 & $1.76 \mathrm{E}-10$ & $1.04 \mathrm{E}-10$ & GAS2L3 & $1.69 E+00$ \\
\hline ENSBTAG00000014659 & $3.31 \mathrm{E}-10$ & $1.95 \mathrm{E}-10$ & NEMP1 & $1.70 \mathrm{E}+00$ \\
\hline ENSBTAG00000014758 & $2.45 \mathrm{E}-10$ & $1.44 \mathrm{E}-10$ & WIF1 & $1.70 \mathrm{E}+00$ \\
\hline ENSBTAG00000043949 & $2.51 \mathrm{E}-10$ & $1.48 \mathrm{E}-10$ & & $1.70 \mathrm{E}+00$ \\
\hline ENSBTAG00000020341 & $3.70 \mathrm{E}-10$ & 2.17E-10 & MOV10L1 & $1.70 E+00$ \\
\hline ENSBTAG00000030426 & $1.18 \mathrm{E}-09$ & $6.87 \mathrm{E}-10$ & & $1.71 E+00$ \\
\hline ENSBTAG00000004660 & $2.60 \mathrm{E}-10$ & $1.52 \mathrm{E}-10$ & & $1.71 E+00$ \\
\hline ENSBTAG00000000393 & $1.81 \mathrm{E}-10$ & $1.05 \mathrm{E}-10$ & SSPN & $1.72 \mathrm{E}+00$ \\
\hline ENSBTAG00000047358 & $3.47 \mathrm{E}-10$ & $2.01 E-10$ & HIGD1C & $1.72 \mathrm{E}+00$ \\
\hline ENSBTAG00000020472 & $2.45 \mathrm{E}-10$ & $1.42 \mathrm{E}-10$ & KDM5A & $1.72 \mathrm{E}+00$ \\
\hline ENSBTAG00000006985 & $2.72 \mathrm{E}-10$ & $1.58 \mathrm{E}-10$ & TM7SF3 & $1.72 \mathrm{E}+00$ \\
\hline ENSBTAG00000006202 & $3.53 \mathrm{E}-10$ & $2.05 \mathrm{E}-10$ & & $1.72 \mathrm{E}+00$ \\
\hline ENSBTAG00000000650 & 4.30E-10 & $2.49 \mathrm{E}-10$ & TUBGCP6 & $1.73 E+00$ \\
\hline ENSBTAG00000018180 & $1.39 \mathrm{E}-10$ & 8.04E-11 & $\mathrm{C} 5 \mathrm{H} 12 \mathrm{orf} 50$ & $1.73 E+00$ \\
\hline ENSBTAG00000015604 & $1.25 \mathrm{E}-10$ & $7.23 \mathrm{E}-11$ & ZNF385A & $1.73 E+00$ \\
\hline ENSBTAG00000006828 & $1.41 \mathrm{E}-10$ & $8.14 \mathrm{E}-11$ & RAPGEF3 & $1.73 E+00$ \\
\hline ENSBTAG00000009542 & $1.69 \mathrm{E}-10$ & 9.67E-11 & $\mathrm{ZC} 3 \mathrm{H} 10$ & $1.75 \mathrm{E}+00$ \\
\hline ENSBTAG00000018556 & $3.38 \mathrm{E}-10$ & $1.93 \mathrm{E}-10$ & & $1.75 \mathrm{E}+00$ \\
\hline ENSBTAG00000001489 & $2.03 \mathrm{E}-10$ & $1.16 \mathrm{E}-10$ & TUBA1A & $1.75 \mathrm{E}+00$ \\
\hline ENSBTAG00000046742 & $7.61 E-10$ & $4.34 \mathrm{E}-10$ & CLEC2A & $1.75 E+00$ \\
\hline ENSBTAG00000009509 & $2.31 \mathrm{E}-10$ & $1.31 \mathrm{E}-10$ & NDUFA6 & $1.76 \mathrm{E}+00$ \\
\hline ENSBTAG00000006911 & $3.05 \mathrm{E}-10$ & $1.74 \mathrm{E}-10$ & NUP107 & $1.75 E+00$ \\
\hline ENSBTAG00000005246 & $3.17 \mathrm{E}-10$ & $1.80 \mathrm{E}-10$ & DIP2B & $1.76 \mathrm{E}+00$ \\
\hline ENSBTAG00000003017 & $7.53 \mathrm{E}-10$ & $4.28 \mathrm{E}-10$ & TTC38 & $1.76 \mathrm{E}+00$ \\
\hline ENSBTAG00000009050 & $3.83 \mathrm{E}-10$ & 2.17E-10 & ADIPOR2 & $1.76 \mathrm{E}+00$ \\
\hline ENSBTAG00000011857 & $4.28 \mathrm{E}-10$ & 2.42E-10 & PPM1H & $1.77 \mathrm{E}+00$ \\
\hline ENSBTAG00000046268 & $1.04 \mathrm{E}-09$ & $5.82 \mathrm{E}-10$ & & $1.78 \mathrm{E}+00$ \\
\hline ENSBTAG00000017072 & $2.34 \mathrm{E}-10$ & $1.31 \mathrm{E}-10$ & ZNF384 & $1.78 \mathrm{E}+00$ \\
\hline ENSBTAG00000002888 & $6.32 \mathrm{E}-10$ & $3.54 \mathrm{E}-10$ & TMTC2 & $1.78 \mathrm{E}+00$ \\
\hline ENSBTAG00000011959 & $1.93 \mathrm{E}-10$ & $1.08 \mathrm{E}-10$ & GPR19 & $1.79 E+00$ \\
\hline ENSBTAG00000005333 & $1.37 \mathrm{E}-10$ & $7.65 E-11$ & $M B$ & $1.79 E+00$ \\
\hline ENSBTAG00000017064 & $2.05 \mathrm{E}-10$ & $1.15 \mathrm{E}-10$ & ING4 & $1.78 \mathrm{E}+00$ \\
\hline ENSBTAG00000010451 & $1.10 \mathrm{E}-10$ & $6.15 E-11$ & PA2G4 & $1.79 E+00$ \\
\hline ENSBTAG00000045808 & $2.24 \mathrm{E}-10$ & $1.25 \mathrm{E}-10$ & IL17REL & $1.79 E+00$ \\
\hline ENSBTAG00000046994 & $7.90 \mathrm{E}-10$ & $4.41 \mathrm{E}-10$ & & $1.79 \mathrm{E}+00$ \\
\hline ENSBTAG00000046298 & $2.84 \mathrm{E}-10$ & $1.58 \mathrm{E}-10$ & SYCE3 & $1.80 \mathrm{E}+00$ \\
\hline ENSBTAG00000001931 & $3.80 \mathrm{E}-10$ & $2.11 E-10$ & TRABD & $1.80 \mathrm{E}+00$ \\
\hline ENSBTAG00000011826 & $6.76 \mathrm{E}-10$ & $3.75 \mathrm{E}-10$ & PEX26 & $1.80 \mathrm{E}+00$ \\
\hline ENSBTAG00000004145 & $6.79 \mathrm{E}-10$ & $3.76 \mathrm{E}-10$ & ANO4 & $1.81 \mathrm{E}+00$ \\
\hline ENSBTAG00000045786 & $4.78 \mathrm{E}-10$ & 2.64E-10 & & $1.81 \mathrm{E}+00$ \\
\hline ENSBTAG00000012246 & $4.63 \mathrm{E}-10$ & $2.55 \mathrm{E}-10$ & ANO2 & $1.81 \mathrm{E}+00$ \\
\hline
\end{tabular}




\begin{tabular}{|c|c|c|c|c|}
\hline ENSBTAG00000016996 & $3.30 \mathrm{E}-10$ & $1.82 \mathrm{E}-10$ & $\mathrm{C} 5 \mathrm{H} 12$ orf 54 & $1.81 \mathrm{E}+00$ \\
\hline ENSBTAG00000008554 & $6.81 \mathrm{E}-10$ & $3.75 \mathrm{E}-10$ & NINJ2 & $1.82 \mathrm{E}+00$ \\
\hline ENSBTAG00000047801 & $8.41 \mathrm{E}-10$ & $4.60 \mathrm{E}-10$ & CRELD2 & $1.83 \mathrm{E}+00$ \\
\hline ENSBTAG00000000647 & $5.47 \mathrm{E}-10$ & $2.98 \mathrm{E}-10$ & SELENOO & $1.84 \mathrm{E}+00$ \\
\hline ENSBTAG00000021626 & $6.12 \mathrm{E}-10$ & $3.34 \mathrm{E}-10$ & MLC1 & $1.83 E+00$ \\
\hline ENSBTAG00000021756 & $4.58 \mathrm{E}-10$ & $2.49 \mathrm{E}-10$ & ZDHHC17 & $1.84 \mathrm{E}+00$ \\
\hline ENSBTAG00000016048 & $2.96 \mathrm{E}-10$ & $1.61 \mathrm{E}-10$ & CPT1B & $1.84 \mathrm{E}+00$ \\
\hline ENSBTAG00000012185 & $3.43 \mathrm{E}-10$ & $1.86 \mathrm{E}-10$ & CLEC4E & $1.84 \mathrm{E}+00$ \\
\hline ENSBTAG00000000970 & $2.76 \mathrm{E}-10$ & $1.50 \mathrm{E}-10$ & ASIC1 & $1.84 \mathrm{E}+00$ \\
\hline ENSBTAG00000037638 & $5.47 \mathrm{E}-11$ & $2.96 \mathrm{E}-11$ & KRT73 & $1.85 \mathrm{E}+00$ \\
\hline ENSBTAG00000000510 & $9.88 \mathrm{E}-11$ & $5.33 \mathrm{E}-11$ & ATG101 & $1.85 \mathrm{E}+00$ \\
\hline ENSBTAG00000003036 & $4.45 \mathrm{E}-10$ & $2.40 \mathrm{E}-10$ & PPFIA2 & $1.85 \mathrm{E}+00$ \\
\hline ENSBTAG00000045904 & $1.77 \mathrm{E}-10$ & $9.57 \mathrm{E}-11$ & PIM3 & $1.85 \mathrm{E}+00$ \\
\hline ENSBTAG00000011111 & $4.60 \mathrm{E}-10$ & $2.48 \mathrm{E}-10$ & COPS7A & $1.85 \mathrm{E}+00$ \\
\hline ENSBTAG00000013126 & $4.04 \mathrm{E}-10$ & $2.18 \mathrm{E}-10$ & & $1.85 \mathrm{E}+00$ \\
\hline ENSBTAG00000010157 & $2.91 \mathrm{E}-10$ & 1.57E-10 & Tom22 & $1.86 \mathrm{E}+00$ \\
\hline ENSBTAG00000045816 & $6.16 \mathrm{E}-10$ & $3.31 \mathrm{E}-10$ & & $1.86 \mathrm{E}+00$ \\
\hline ENSBTAG00000033600 & $8.57 \mathrm{E}-10$ & $4.61 \mathrm{E}-10$ & & $1.86 \mathrm{E}+00$ \\
\hline ENSBTAG00000008063 & $5.25 \mathrm{E}-10$ & $2.82 \mathrm{E}-10$ & PPARA & $1.86 \mathrm{E}+00$ \\
\hline ENSBTAG00000011275 & $3.97 \mathrm{E}-10$ & $2.13 \mathrm{E}-10$ & SCUBE1 & $1.86 \mathrm{E}+00$ \\
\hline ENSBTAG00000038043 & $4.79 \mathrm{E}-10$ & $2.57 \mathrm{E}-10$ & $A B C D 2$ & $1.86 \mathrm{E}+00$ \\
\hline ENSBTAG00000020806 & $1.73 \mathrm{E}-10$ & $9.25 \mathrm{E}-11$ & HELB & $1.87 E+00$ \\
\hline ENSBTAG00000005595 & $2.77 \mathrm{E}-10$ & $1.47 \mathrm{E}-10$ & TRMU & $1.88 \mathrm{E}+00$ \\
\hline ENSBTAG00000000400 & $2.07 \mathrm{E}-10$ & 1.10E-10 & KRT85 & $1.88 \mathrm{E}+00$ \\
\hline ENSBTAG00000012275 & $2.64 \mathrm{E}-10$ & $1.39 \mathrm{E}-10$ & ENDOU & $1.90 \mathrm{E}+00$ \\
\hline ENSBTAG00000009049 & $2.26 \mathrm{E}-10$ & $1.18 \mathrm{E}-10$ & & $1.91 \mathrm{E}+00$ \\
\hline ENSBTAG00000010616 & $1.73 \mathrm{E}-10$ & $9.08 \mathrm{E}-11$ & MBD6 & $1.91 E+00$ \\
\hline ENSBTAG00000013596 & $3.73 \mathrm{E}-10$ & $1.95 \mathrm{E}-10$ & $\mathrm{NR} 1 \mathrm{H} 4$ & $1.91 E+00$ \\
\hline ENSBTAG00000038652 & $9.13 \mathrm{E}-10$ & $4.75 \mathrm{E}-10$ & & $1.92 \mathrm{E}+00$ \\
\hline ENSBTAG00000022360 & 4.07E-10 & $2.12 \mathrm{E}-10$ & SOX5 & $1.92 \mathrm{E}+00$ \\
\hline ENSBTAG00000002413 & $3.61 E-10$ & $1.87 \mathrm{E}-10$ & MCAT & $1.93 E+00$ \\
\hline ENSBTAG00000032148 & $4.08 \mathrm{E}-10$ & $2.10 \mathrm{E}-10$ & TMEM117 & $1.94 \mathrm{E}+00$ \\
\hline ENSBTAG00000011105 & $1.25 \mathrm{E}-10$ & $6.43 \mathrm{E}-11$ & SLC38A2 & $1.94 \mathrm{E}+00$ \\
\hline ENSBTAG00000011762 & $1.89 \mathrm{E}-10$ & 9.67E-11 & ANKRD52 & $1.95 E+00$ \\
\hline ENSBTAG00000010136 & $1.69 \mathrm{E}-10$ & $8.65 \mathrm{E}-11$ & CMAS & $1.95 \mathrm{E}+00$ \\
\hline ENSBTAG00000039784 & $1.94 \mathrm{E}-10$ & $9.94 \mathrm{E}-11$ & PRR5 & $1.96 \mathrm{E}+00$ \\
\hline ENSBTAG00000005861 & $8.38 \mathrm{E}-11$ & $4.28 \mathrm{E}-11$ & CCNT1 & $1.96 \mathrm{E}+00$ \\
\hline ENSBTAG00000026501 & $6.05 \mathrm{E}-10$ & $3.08 \mathrm{E}-10$ & MGC127055 & $1.96 \mathrm{E}+00$ \\
\hline ENSBTAG00000018073 & $2.73 \mathrm{E}-10$ & $1.39 \mathrm{E}-10$ & TSPO & $1.96 \mathrm{E}+00$ \\
\hline ENSBTAG00000010789 & $7.84 \mathrm{E}-10$ & $3.99 \mathrm{E}-10$ & ANKRD54 & $1.97 \mathrm{E}+00$ \\
\hline ENSBTAG00000006103 & 4.14E-10 & 2.09E-10 & XRCC6 & $1.98 \mathrm{E}+00$ \\
\hline ENSBTAG00000006739 & $6.96 \mathrm{E}-11$ & $3.50 \mathrm{E}-11$ & & $1.98 \mathrm{E}+00$ \\
\hline ENSBTAG00000009507 & $8.78 \mathrm{E}-11$ & $4.42 \mathrm{E}-11$ & FAM109B & $1.99 E+00$ \\
\hline ENSBTAG00000006205 & $3.87 \mathrm{E}-10$ & $1.95 \mathrm{E}-10$ & DNAJB7 & $1.98 \mathrm{E}+00$ \\
\hline ENSBTAG00000047886 & $4.21 \mathrm{E}-10$ & $2.12 \mathrm{E}-10$ & & $1.99 E+00$ \\
\hline ENSBTAG00000021337 & $3.63 \mathrm{E}-10$ & $1.83 \mathrm{E}-10$ & SBF1 & $1.99 \mathrm{E}+00$ \\
\hline ENSBTAG00000047825 & 4.31E-10 & 2.17E-10 & & $1.98 \mathrm{E}+00$ \\
\hline ENSBTAG00000045722 & $3.60 \mathrm{E}-10$ & $1.81 \mathrm{E}-10$ & & $1.99 E+00$ \\
\hline ENSBTAG00000019084 & $1.97 \mathrm{E}-10$ & $9.93 \mathrm{E}-11$ & INHBC & $1.99 E+00$ \\
\hline ENSBTAG00000007553 & $1.86 \mathrm{E}-10$ & $9.39 \mathrm{E}-11$ & KCNA5 & $1.98 \mathrm{E}+00$ \\
\hline ENSBTAG00000011918 & $1.20 \mathrm{E}-10$ & $6.06 \mathrm{E}-11$ & & $1.98 \mathrm{E}+00$ \\
\hline ENSBTAG00000006256 & $2.96 \mathrm{E}-10$ & $1.48 \mathrm{E}-10$ & PTPRO & $2.00 E+00$ \\
\hline ENSBTAG00000019602 & $4.64 \mathrm{E}-10$ & $2.31 E-10$ & SLCO1C1 & $2.01 E+00$ \\
\hline ENSBTAG00000016043 & $5.49 \mathrm{E}-10$ & $2.72 \mathrm{E}-10$ & GNB3 & $2.02 \mathrm{E}+00$ \\
\hline ENSBTAG00000048280 & $4.55 \mathrm{E}-10$ & $2.26 \mathrm{E}-10$ & C12orf73 & $2.02 \mathrm{E}+00$ \\
\hline ENSBTAG00000025005 & $5.65 E-10$ & $2.80 \mathrm{E}-10$ & & $2.02 E+00$ \\
\hline ENSBTAG00000001510 & $3.51 \mathrm{E}-10$ & $1.74 \mathrm{E}-10$ & CDK17 & $2.01 E+00$ \\
\hline ENSBTAG00000017840 & $1.68 \mathrm{E}-10$ & 8.34E-11 & BAZ2A & $2.02 \mathrm{E}+00$ \\
\hline ENSBTAG00000030343 & $2.68 \mathrm{E}-10$ & $1.32 \mathrm{E}-10$ & FGF23 & $2.03 E+00$ \\
\hline ENSBTAG00000034246 & $3.01 \mathrm{E}-10$ & $1.49 \mathrm{E}-10$ & ACRBP & $2.02 E+00$ \\
\hline ENSBTAG00000046173 & $6.89 \mathrm{E}-10$ & $3.39 \mathrm{E}-10$ & ALG12 & $2.03 E+00$ \\
\hline ENSBTAG00000006010 & 4.30E-10 & $2.11 \mathrm{E}-10$ & STAB2 & $2.04 \mathrm{E}+00$ \\
\hline ENSBTAG00000010402 & $6.46 \mathrm{E}-10$ & $3.15 \mathrm{E}-10$ & MYH9 & $2.05 E+00$ \\
\hline ENSBTAG00000016510 & $5.05 \mathrm{E}-10$ & $2.46 \mathrm{E}-10$ & & $2.05 E+00$ \\
\hline ENSBTAG00000011087 & $1.94 \mathrm{E}-10$ & $9.45 \mathrm{E}-11$ & ARID2 & $2.05 E+00$ \\
\hline ENSBTAG00000000808 & $2.48 \mathrm{E}-10$ & $1.20 \mathrm{E}-10$ & cox14 & $2.07 E+00$ \\
\hline ENSBTAG00000009447 & $3.78 \mathrm{E}-10$ & $1.83 \mathrm{E}-10$ & PRMT8 & $2.06 \mathrm{E}+00$ \\
\hline ENSBTAG00000018405 & $1.48 \mathrm{E}-10$ & 7.15E-11 & MARS & $2.07 E+00$ \\
\hline ENSBTAG00000020914 & $7.89 \mathrm{E}-10$ & $3.78 \mathrm{E}-10$ & CPNE8 & $2.09 E+00$ \\
\hline ENSBTAG00000021995 & $3.04 \mathrm{E}-10$ & $1.46 \mathrm{E}-10$ & LRTM2 & $2.08 \mathrm{E}+00$ \\
\hline
\end{tabular}




\begin{tabular}{|c|c|c|c|c|}
\hline ENSBTAG00000019741 & $2.55 \mathrm{E}-10$ & $1.22 \mathrm{E}-10$ & C3AR1 & $2.09 E+00$ \\
\hline ENSBTAG00000039709 & $2.28 \mathrm{E}-10$ & $1.09 \mathrm{E}-10$ & PARPBP & $2.09 \mathrm{E}+00$ \\
\hline ENSBTAG00000013842 & $7.91 \mathrm{E}-10$ & $3.76 \mathrm{E}-10$ & ACTR6 & $2.10 \mathrm{E}+00$ \\
\hline ENSBTAG00000017830 & $1.86 \mathrm{E}-10$ & $8.79 \mathrm{E}-11$ & RBMS2 & $2.12 \mathrm{E}+00$ \\
\hline ENSBTAG00000003291 & $2.04 \mathrm{E}-10$ & $9.65 E-11$ & RIMKLB & $2.11 E+00$ \\
\hline ENSBTAG00000046724 & $8.10 \mathrm{E}-11$ & $3.83 \mathrm{E}-11$ & $\mathrm{CBX} 6$ & $2.11 E+00$ \\
\hline ENSBTAG00000012896 & $3.01 \mathrm{E}-10$ & $1.42 \mathrm{E}-10$ & METTL7B & $2.12 E+00$ \\
\hline ENSBTAG00000019946 & $2.30 \mathrm{E}-10$ & $1.08 \mathrm{E}-10$ & USP44 & $2.13 E+00$ \\
\hline ENSBTAG00000020292 & $3.57 \mathrm{E}-10$ & $1.68 \mathrm{E}-10$ & $\mathrm{C} 5 \mathrm{H} 12$ orf57 & $2.12 \mathrm{E}+00$ \\
\hline ENSBTAG00000047374 & $2.55 \mathrm{E}-10$ & $1.20 \mathrm{E}-10$ & & $2.12 \mathrm{E}+00$ \\
\hline ENSBTAG00000010830 & $2.64 \mathrm{E}-10$ & $1.24 \mathrm{E}-10$ & LRP1 & $2.13 E+00$ \\
\hline ENSBTAG00000020330 & $2.42 \mathrm{E}-10$ & $1.14 \mathrm{E}-10$ & BTG1 & $2.12 \mathrm{E}+00$ \\
\hline ENSBTAG00000006040 & $7.24 \mathrm{E}-10$ & $3.41 \mathrm{E}-10$ & UHRF1BP1L & $2.12 \mathrm{E}+00$ \\
\hline ENSBTAG00000038521 & $7.04 \mathrm{E}-10$ & $3.31 \mathrm{E}-10$ & & $2.13 E+00$ \\
\hline ENSBTAG00000013515 & $1.35 \mathrm{E}-10$ & $6.33 \mathrm{E}-11$ & ASUN & $2.13 E+00$ \\
\hline ENSBTAG00000038843 & $9.47 \mathrm{E}-10$ & $4.45 E-10$ & & $2.13 E+00$ \\
\hline ENSBTAG00000014197 & $4.79 \mathrm{E}-10$ & $2.23 \mathrm{E}-10$ & SLC38A4 & $2.15 E+00$ \\
\hline ENSBTAG00000001088 & $4.68 \mathrm{E}-10$ & $2.18 \mathrm{E}-10$ & SLC17A8 & $2.15 \mathrm{E}+00$ \\
\hline ENSBTAG00000033726 & $5.35 \mathrm{E}-10$ & $2.49 \mathrm{E}-10$ & GRIP1 & $2.15 \mathrm{E}+00$ \\
\hline ENSBTAG00000022167 & $3.41 \mathrm{E}-10$ & $1.58 \mathrm{E}-10$ & DERA & $2.16 \mathrm{E}+00$ \\
\hline ENSBTAG00000017543 & $2.87 \mathrm{E}-10$ & $1.32 \mathrm{E}-10$ & ATP23 & $2.17 E+00$ \\
\hline ENSBTAG00000011720 & $1.83 \mathrm{E}-10$ & $8.48 \mathrm{E}-11$ & HSD17B6 & $2.16 \mathrm{E}+00$ \\
\hline ENSBTAG00000001894 & $2.16 \mathrm{E}-10$ & $9.92 \mathrm{E}-11$ & NEDD1 & $2.18 \mathrm{E}+00$ \\
\hline ENSBTAG00000005453 & $2.43 \mathrm{E}-10$ & $1.12 \mathrm{E}-10$ & FGD4 & $2.17 E+00$ \\
\hline ENSBTAG00000016908 & $2.50 \mathrm{E}-10$ & $1.15 \mathrm{E}-10$ & METTL1 & $2.18 \mathrm{E}+00$ \\
\hline ENSBTAG00000031500 & $1.71 \mathrm{E}-10$ & $7.83 \mathrm{E}-11$ & SHMT2 & $2.18 \mathrm{E}+00$ \\
\hline ENSBTAG00000007592 & 4.37E-11 & $2.00 \mathrm{E}-11$ & RARG & $2.18 \mathrm{E}+00$ \\
\hline ENSBTAG00000014429 & $1.48 \mathrm{E}-10$ & $6.74 \mathrm{E}-11$ & KMT2D & $2.19 E+00$ \\
\hline ENSBTAG00000021615 & $1.44 \mathrm{E}-10$ & $6.59 \mathrm{E}-11$ & LGALS2 & $2.18 \mathrm{E}+00$ \\
\hline ENSBTAG00000030648 & $1.93 \mathrm{E}-10$ & $8.83 \mathrm{E}-11$ & MPST & $2.18 \mathrm{E}+00$ \\
\hline ENSBTAG00000032152 & $2.16 \mathrm{E}-10$ & $9.90 \mathrm{E}-11$ & TMPRSS6 & $2.18 \mathrm{E}+00$ \\
\hline ENSBTAG00000011902 & 3.57E-10 & $1.63 \mathrm{E}-10$ & & $2.19 E+00$ \\
\hline ENSBTAG00000015582 & $2.90 \mathrm{E}-10$ & $1.31 \mathrm{E}-10$ & HMOX1 & $2.22 \mathrm{E}+00$ \\
\hline ENSBTAG00000016508 & $2.57 \mathrm{E}-10$ & $1.16 \mathrm{E}-10$ & CTDSP2 & $2.21 E+00$ \\
\hline ENSBTAG00000011098 & $6.84 \mathrm{E}-10$ & $3.09 \mathrm{E}-10$ & TTLL8 & $2.21 E+00$ \\
\hline ENSBTAG00000033735 & $3.85 \mathrm{E}-10$ & $1.73 \mathrm{E}-10$ & KIAA1033 & $2.23 E+00$ \\
\hline ENSBTAG00000005735 & $3.69 \mathrm{E}-10$ & $1.64 \mathrm{E}-10$ & ATP5G2 & $2.25 \mathrm{E}+00$ \\
\hline ENSBTAG00000037935 & $1.31 \mathrm{E}-10$ & $5.82 \mathrm{E}-11$ & SMARCD1 & $2.25 E+00$ \\
\hline ENSBTAG00000014605 & $3.24 \mathrm{E}-10$ & $1.43 \mathrm{E}-10$ & ETV6 & $2.27 \mathrm{E}+00$ \\
\hline ENSBTAG00000012636 & $2.95 \mathrm{E}-10$ & $1.31 \mathrm{E}-10$ & IKZF4 & $2.25 E+00$ \\
\hline ENSBTAG00000018660 & $3.68 \mathrm{E}-10$ & $1.61 \mathrm{E}-10$ & PPP6R2 & $2.28 \mathrm{E}+00$ \\
\hline ENSBTAG00000014729 & $5.71 \mathrm{E}-10$ & $2.49 \mathrm{E}-10$ & MRPL51 & $2.29 \mathrm{E}+00$ \\
\hline ENSBTAG00000010701 & $2.16 \mathrm{E}-10$ & $9.38 \mathrm{E}-11$ & & $2.30 \mathrm{E}+00$ \\
\hline ENSBTAG00000039847 & $4.90 \mathrm{E}-10$ & $2.12 \mathrm{E}-10$ & & $2.31 E+00$ \\
\hline ENSBTAG00000012607 & $2.88 \mathrm{E}-10$ & $1.24 \mathrm{E}-10$ & $\mathrm{NCAPH} 2$ & $2.32 \mathrm{E}+00$ \\
\hline ENSBTAG00000002931 & $8.80 \mathrm{E}-10$ & $3.79 \mathrm{E}-10$ & BEST3 & $2.32 \mathrm{E}+00$ \\
\hline ENSBTAG00000003367 & $7.93 \mathrm{E}-10$ & $3.42 \mathrm{E}-10$ & & $2.32 \mathrm{E}+00$ \\
\hline ENSBTAG00000004488 & $3.18 \mathrm{E}-10$ & $1.37 \mathrm{E}-10$ & MEI1 & $2.32 \mathrm{E}+00$ \\
\hline ENSBTAG00000018694 & $4.79 \mathrm{E}-10$ & $2.05 E-10$ & ACSS3 & $2.34 \mathrm{E}+00$ \\
\hline ENSBTAG00000018254 & $1.42 \mathrm{E}-10$ & $5.97 \mathrm{E}-11$ & CDKN1B & $2.38 \mathrm{E}+00$ \\
\hline ENSBTAG00000016053 & $7.83 \mathrm{E}-11$ & $3.29 \mathrm{E}-11$ & ARSA & $2.38 \mathrm{E}+00$ \\
\hline ENSBTAG00000044000 & $2.07 \mathrm{E}-10$ & $8.70 \mathrm{E}-11$ & PRIM1 & $2.38 \mathrm{E}+00$ \\
\hline ENSBTAG00000007160 & $8.28 \mathrm{E}-11$ & $3.47 E-11$ & & $2.38 \mathrm{E}+00$ \\
\hline ENSBTAG00000046761 & $4.49 \mathrm{E}-10$ & $1.88 \mathrm{E}-10$ & & $2.39 \mathrm{E}+00$ \\
\hline ENSBTAG00000017062 & $4.29 \mathrm{E}-10$ & $1.80 \mathrm{E}-10$ & ADM2 & $2.38 \mathrm{E}+00$ \\
\hline ENSBTAG00000002798 & $2.01 \mathrm{E}-10$ & $8.42 \mathrm{E}-11$ & NABP2 & $2.38 \mathrm{E}+00$ \\
\hline ENSBTAG00000038439 & $2.23 \mathrm{E}-10$ & $9.35 E-11$ & H1F0 & $2.38 \mathrm{E}+00$ \\
\hline ENSBTAG00000046108 & $5.08 \mathrm{E}-10$ & $2.12 \mathrm{E}-10$ & & $2.39 E+00$ \\
\hline ENSBTAG00000026495 & $1.44 \mathrm{E}-10$ & $6.04 \mathrm{E}-11$ & & $2.38 \mathrm{E}+00$ \\
\hline ENSBTAG00000037758 & $5.25 \mathrm{E}-10$ & $2.20 \mathrm{E}-10$ & & $2.39 E+00$ \\
\hline ENSBTAG00000023274 & $4.83 \mathrm{E}-10$ & $2.02 \mathrm{E}-10$ & & $2.39 E+00$ \\
\hline ENSBTAG00000018828 & $1.74 \mathrm{E}-10$ & $7.28 \mathrm{E}-11$ & ATN1 & $2.38 \mathrm{E}+00$ \\
\hline ENSBTAG00000038027 & $8.42 \mathrm{E}-10$ & $3.52 \mathrm{E}-10$ & & $2.39 E+00$ \\
\hline ENSBTAG00000038893 & $7.91 \mathrm{E}-10$ & $3.31 E-10$ & & $2.39 E+00$ \\
\hline ENSBTAG00000012794 & $3.43 \mathrm{E}-10$ & $1.43 \mathrm{E}-10$ & $\mathrm{PAH}$ & $2.40 \mathrm{E}+00$ \\
\hline ENSBTAG00000004427 & $2.53 \mathrm{E}-10$ & $1.04 \mathrm{E}-10$ & OSBPL8 & $2.43 E+00$ \\
\hline ENSBTAG00000004484 & $2.76 \mathrm{E}-10$ & $1.14 \mathrm{E}-10$ & CEP83 & $2.42 \mathrm{E}+00$ \\
\hline ENSBTAG00000020294 & $3.72 \mathrm{E}-10$ & $1.52 \mathrm{E}-10$ & PTPN6 & $2.45 E+00$ \\
\hline ENSBTAG00000026754 & $2.45 \mathrm{E}-10$ & $9.99 \mathrm{E}-11$ & RNF41 & $2.45 E+00$ \\
\hline ENSBTAG00000006279 & $6.68 \mathrm{E}-10$ & $2.72 \mathrm{E}-10$ & PNPLA5 & $2.45 E+00$ \\
\hline
\end{tabular}




\begin{tabular}{|c|c|c|c|c|}
\hline ENSBTAG00000018361 & $1.82 \mathrm{E}-10$ & 7.39E-11 & R3HDM2 & $2.46 \mathrm{E}+00$ \\
\hline ENSBTAG00000016650 & $3.57 \mathrm{E}-10$ & $1.45 \mathrm{E}-10$ & TIGAR & $2.46 \mathrm{E}+00$ \\
\hline ENSBTAG00000045768 & $3.47 \mathrm{E}-10$ & $1.40 \mathrm{E}-10$ & & $2.48 \mathrm{E}+00$ \\
\hline ENSBTAG00000016166 & $6.43 \mathrm{E}-10$ & $2.60 \mathrm{E}-10$ & & $2.47 E+00$ \\
\hline ENSBTAG00000013127 & $3.44 \mathrm{E}-10$ & $1.38 \mathrm{E}-10$ & LPCAT3 & $2.49 E+00$ \\
\hline ENSBTAG00000001509 & $3.47 \mathrm{E}-10$ & $1.39 \mathrm{E}-10$ & ELK3 & $2.50 \mathrm{E}+00$ \\
\hline ENSBTAG00000034678 & $6.50 \mathrm{E}-10$ & $2.61 \mathrm{E}-10$ & & $2.49 E+00$ \\
\hline ENSBTAG00000019603 & $6.84 \mathrm{E}-10$ & $2.73 \mathrm{E}-10$ & LDHB & $2.51 \mathrm{E}+00$ \\
\hline ENSBTAG00000000667 & $6.24 \mathrm{E}-10$ & $2.49 \mathrm{E}-10$ & & $2.50 \mathrm{E}+00$ \\
\hline ENSBTAG00000011043 & $6.00 \mathrm{E}-10$ & $2.39 \mathrm{E}-10$ & RAC2 & $2.51 E+00$ \\
\hline ENSBTAG00000014265 & $7.74 \mathrm{E}-11$ & $3.06 \mathrm{E}-11$ & SREBF2 & $2.52 E+00$ \\
\hline ENSBTAG00000006423 & $2.91 \mathrm{E}-10$ & $1.15 \mathrm{E}-10$ & PHF5A & $2.53 E+00$ \\
\hline ENSBTAG00000000369 & $3.04 \mathrm{E}-10$ & 1.19E-10 & EPS8 & $2.55 \mathrm{E}+00$ \\
\hline ENSBTAG00000020636 & $7.09 \mathrm{E}-10$ & $2.78 \mathrm{E}-10$ & SYN3 & $2.55 \mathrm{E}+00$ \\
\hline ENSBTAG00000001057 & $6.66 \mathrm{E}-10$ & $2.61 \mathrm{E}-10$ & ARFGAP3 & $2.55 \mathrm{E}+00$ \\
\hline ENSBTAG00000016580 & $2.15 \mathrm{E}-10$ & $8.31 E-11$ & TEF & $2.58 \mathrm{E}+00$ \\
\hline ENSBTAG00000006429 & $4.73 \mathrm{E}-10$ & $1.81 \mathrm{E}-10$ & $\mathrm{ACO} 2$ & $2.61 E+00$ \\
\hline ENSBTAG00000002256 & $3.08 \mathrm{E}-10$ & $1.18 \mathrm{E}-10$ & YEATS4 & $2.61 E+00$ \\
\hline ENSBTAG00000016649 & $2.25 \mathrm{E}-10$ & $8.56 \mathrm{E}-11$ & CCND2 & $2.63 E+00$ \\
\hline ENSBTAG00000009281 & $3.96 \mathrm{E}-10$ & $1.49 \mathrm{E}-10$ & SPRYD4 & $2.66 \mathrm{E}+00$ \\
\hline ENSBTAG00000004541 & $1.64 \mathrm{E}-10$ & $6.20 \mathrm{E}-11$ & LPAR5 & $2.64 \mathrm{E}+00$ \\
\hline ENSBTAG00000010624 & $1.63 \mathrm{E}-10$ & $6.08 \mathrm{E}-11$ & DCTN2 & $2.68 \mathrm{E}+00$ \\
\hline ENSBTAG00000014030 & $4.58 \mathrm{E}-10$ & $1.71 \mathrm{E}-10$ & FGD6 & $2.68 \mathrm{E}+00$ \\
\hline ENSBTAG00000011245 & $3.73 \mathrm{E}-10$ & 1.37E-10 & PLXNC1 & $2.72 \mathrm{E}+00$ \\
\hline ENSBTAG00000026502 & 4.34E-10 & $1.59 \mathrm{E}-10$ & & $2.73 E+00$ \\
\hline ENSBTAG00000021105 & $9.05 \mathrm{E}-11$ & 3.31E-11 & IRAK4 & $2.73 E+00$ \\
\hline ENSBTAG00000000507 & $1.60 \mathrm{E}-10$ & $5.85 \mathrm{E}-11$ & NR4A1 & $2.73 E+00$ \\
\hline ENSBTAG00000008057 & $8.83 \mathrm{E}-10$ & $3.23 \mathrm{E}-10$ & PARVG & $2.73 E+00$ \\
\hline ENSBTAG00000015043 & $3.43 \mathrm{E}-10$ & $1.25 \mathrm{E}-10$ & MFNG & $2.74 \mathrm{E}+00$ \\
\hline ENSBTAG00000040244 & $6.68 \mathrm{E}-10$ & $2.42 \mathrm{E}-10$ & & $2.76 \mathrm{E}+00$ \\
\hline ENSBTAG00000023635 & 4.64E-10 & $1.68 \mathrm{E}-10$ & CLEC6A & $2.76 \mathrm{E}+00$ \\
\hline ENSBTAG00000038146 & $2.69 \mathrm{E}-10$ & $9.68 \mathrm{E}-11$ & & $2.78 \mathrm{E}+00$ \\
\hline ENSBTAG00000004367 & $1.70 \mathrm{E}-10$ & $6.10 \mathrm{E}-11$ & COQ10A & $2.78 \mathrm{E}+00$ \\
\hline ENSBTAG00000007605 & $5.09 \mathrm{E}-10$ & $1.82 \mathrm{E}-10$ & FKBP4 & $2.79 E+00$ \\
\hline ENSBTAG00000001099 & $5.06 \mathrm{E}-10$ & $1.82 \mathrm{E}-10$ & PIANP & $2.78 \mathrm{E}+00$ \\
\hline ENSBTAG00000046639 & $5.91 \mathrm{E}-10$ & $2.12 \mathrm{E}-10$ & & $2.79 E+00$ \\
\hline ENSBTAG00000016905 & $6.20 \mathrm{E}-10$ & $2.22 \mathrm{E}-10$ & & $2.79 E+00$ \\
\hline ENSBTAG00000018506 & $1.02 \mathrm{E}-09$ & $3.65 \mathrm{E}-10$ & & $2.80 \mathrm{E}+00$ \\
\hline ENSBTAG00000023258 & 1.97E-09 & 7.00E-10 & & $2.82 \mathrm{E}+00$ \\
\hline ENSBTAG00000006326 & $4.92 \mathrm{E}-10$ & $1.74 \mathrm{E}-10$ & ALDH1L2 & $2.83 E+00$ \\
\hline ENSBTAG00000006538 & $2.13 \mathrm{E}-10$ & 7.53E-11 & PTHLH & $2.82 \mathrm{E}+00$ \\
\hline ENSBTAG00000037632 & 1.03E-09 & $3.63 \mathrm{E}-10$ & & $2.84 \mathrm{E}+00$ \\
\hline ENSBTAG00000008127 & $2.80 \mathrm{E}-10$ & $9.80 \mathrm{E}-11$ & RBP5 & $2.86 \mathrm{E}+00$ \\
\hline ENSBTAG00000007609 & $6.56 \mathrm{E}-10$ & $2.28 \mathrm{E}-10$ & ITFG2 & $2.88 \mathrm{E}+00$ \\
\hline ENSBTAG00000021336 & $2.07 \mathrm{E}-10$ & $7.21 \mathrm{E}-11$ & KIF5A & $2.87 E+00$ \\
\hline ENSBTAG00000018205 & $3.91 \mathrm{E}-10$ & $1.35 \mathrm{E}-10$ & PHC1 & $2.89 \mathrm{E}+00$ \\
\hline ENSBTAG00000003942 & $1.22 \mathrm{E}-10$ & 4.19E-11 & PIP4K2C & $2.91 E+00$ \\
\hline ENSBTAG00000019783 & $3.74 \mathrm{E}-10$ & $1.28 \mathrm{E}-10$ & SPSB2 & $2.92 \mathrm{E}+00$ \\
\hline ENSBTAG00000013123 & $2.13 \mathrm{E}-10$ & $7.32 \mathrm{E}-11$ & PHB2 & $2.91 E+00$ \\
\hline ENSBTAG00000031544 & $2.14 \mathrm{E}-10$ & $7.36 \mathrm{E}-11$ & DDIT3 & $2.91 \mathrm{E}+00$ \\
\hline ENSBTAG00000004425 & $3.29 \mathrm{E}-10$ & $1.13 \mathrm{E}-10$ & BBS10 & $2.91 E+00$ \\
\hline ENSBTAG00000031111 & $8.68 \mathrm{E}-10$ & $2.97 \mathrm{E}-10$ & & $2.92 \mathrm{E}+00$ \\
\hline ENSBTAG00000030646 & $3.43 \mathrm{E}-10$ & 1.17E-10 & KCTD17 & $2.93 E+00$ \\
\hline ENSBTAG00000007674 & $8.59 \mathrm{E}-10$ & $2.93 \mathrm{E}-10$ & ISX & $2.93 E+00$ \\
\hline ENSBTAG00000006776 & $3.82 \mathrm{E}-10$ & $1.30 \mathrm{E}-10$ & KIAA0930 & $2.93 E+00$ \\
\hline ENSBTAG00000007093 & $3.46 \mathrm{E}-10$ & $1.16 \mathrm{E}-10$ & DDX11 & $2.98 \mathrm{E}+00$ \\
\hline ENSBTAG00000024756 & $7.00 \mathrm{E}-10$ & $2.34 \mathrm{E}-10$ & DENND6B & $2.99 \mathrm{E}+00$ \\
\hline ENSBTAG00000016398 & $5.16 \mathrm{E}-10$ & $1.71 \mathrm{E}-10$ & DNAJC22 & $3.02 \mathrm{E}+00$ \\
\hline ENSBTAG00000011274 & $2.25 \mathrm{E}-10$ & $7.45 \mathrm{E}-11$ & ENO2 & $3.02 E+00$ \\
\hline ENSBTAG00000001392 & 3.19E-10 & $1.05 \mathrm{E}-10$ & $\mathrm{RDH} 16$ & $3.04 E+00$ \\
\hline ENSBTAG00000015312 & $2.43 \mathrm{E}-10$ & $7.98 \mathrm{E}-11$ & LTBR & $3.04 \mathrm{E}+00$ \\
\hline ENSBTAG00000005311 & $7.36 \mathrm{E}-10$ & $2.39 \mathrm{E}-10$ & POLR3H & $3.08 \mathrm{E}+00$ \\
\hline ENSBTAG00000016260 & $3.73 \mathrm{E}-10$ & $1.21 \mathrm{E}-10$ & LRRK2 & $3.08 \mathrm{E}+00$ \\
\hline ENSBTAG00000019574 & $4.04 \mathrm{E}-10$ & $1.30 \mathrm{E}-10$ & MAPK12 & $3.11 E+00$ \\
\hline ENSBTAG00000012897 & $3.19 \mathrm{E}-10$ & $1.01 \mathrm{E}-10$ & ITGA7 & $3.15 E+00$ \\
\hline ENSBTAG00000013912 & $6.50 \mathrm{E}-10$ & $2.06 \mathrm{E}-10$ & TXNRD1 & $3.15 E+00$ \\
\hline ENSBTAG00000047191 & $2.07 \mathrm{E}-10$ & $6.50 \mathrm{E}-11$ & KCNA6 & $3.18 \mathrm{E}+00$ \\
\hline ENSBTAG00000014725 & $1.18 \mathrm{E}-10$ & $3.70 \mathrm{E}-11$ & $\mathrm{CD} 27$ & $3.17 E+00$ \\
\hline ENSBTAG00000000379 & $1.41 \mathrm{E}-10$ & 4.44E-11 & GOLT1B & $3.17 \mathrm{E}+00$ \\
\hline ENSBTAG00000004587 & $8.90 \mathrm{E}-11$ & $2.80 \mathrm{E}-11$ & DUSP6 & $3.17 E+00$ \\
\hline
\end{tabular}




\begin{tabular}{|c|c|c|c|c|}
\hline ENSBTAG00000004021 & $1.40 \mathrm{E}-10$ & $4.40 \mathrm{E}-11$ & CDK2 & $3.18 \mathrm{E}+00$ \\
\hline ENSBTAG00000032331 & $3.70 \mathrm{E}-11$ & 1.17E-11 & SLC48A1 & $3.15 E+00$ \\
\hline ENSBTAG00000045982 & $3.49 \mathrm{E}-10$ & 1.10E-10 & & $3.17 E+00$ \\
\hline ENSBTAG00000012425 & 4.57E-10 & $1.43 \mathrm{E}-10$ & LDOC1L & $3.19 E+00$ \\
\hline ENSBTAG00000047741 & $2.91 \mathrm{E}-10$ & $9.16 \mathrm{E}-11$ & & $3.18 \mathrm{E}+00$ \\
\hline ENSBTAG00000000280 & $3.53 \mathrm{E}-10$ & $1.11 \mathrm{E}-10$ & & $3.18 \mathrm{E}+00$ \\
\hline ENSBTAG00000002693 & $1.27 \mathrm{E}-10$ & $3.99 \mathrm{E}-11$ & KANSL2 & $3.17 E+00$ \\
\hline ENSBTAG00000001336 & $7.18 \mathrm{E}-10$ & $2.25 \mathrm{E}-10$ & & $3.19 E+00$ \\
\hline ENSBTAG00000015646 & $7.35 \mathrm{E}-10$ & $2.30 \mathrm{E}-10$ & & $3.19 E+00$ \\
\hline ENSBTAG00000012481 & $5.66 \mathrm{E}-10$ & $1.77 \mathrm{E}-10$ & RANGAP1 & $3.19 E+00$ \\
\hline ENSBTAG00000022971 & $1.22 \mathrm{E}-09$ & $3.76 \mathrm{E}-10$ & & $3.25 E+00$ \\
\hline ENSBTAG00000004018 & $2.51 \mathrm{E}-10$ & $7.66 \mathrm{E}-11$ & DGKA & $3.28 \mathrm{E}+00$ \\
\hline ENSBTAG00000001161 & $7.84 \mathrm{E}-11$ & $2.38 \mathrm{E}-11$ & FAM60A & $3.28 \mathrm{E}+00$ \\
\hline ENSBTAG00000006200 & $4.23 \mathrm{E}-10$ & $1.28 \mathrm{E}-10$ & SLC25A17 & $3.30 E+00$ \\
\hline ENSBTAG00000016913 & $1.48 \mathrm{E}-10$ & $4.51 \mathrm{E}-11$ & AVIL & $3.28 \mathrm{E}+00$ \\
\hline ENSBTAG00000014322 & $4.09 \mathrm{E}-10$ & $1.24 \mathrm{E}-10$ & FAM234B & $3.30 E+00$ \\
\hline ENSBTAG00000026880 & $6.65 \mathrm{E}-10$ & $1.99 \mathrm{E}-10$ & & $3.34 \mathrm{E}+00$ \\
\hline ENSBTAG00000013486 & $8.54 \mathrm{E}-10$ & $2.53 \mathrm{E}-10$ & C12orf66 & $3.37 E+00$ \\
\hline ENSBTAG00000014966 & $5.71 \mathrm{E}-10$ & $1.70 \mathrm{E}-10$ & PLXNB2 & $3.36 \mathrm{E}+00$ \\
\hline ENSBTAG00000017401 & $3.79 \mathrm{E}-10$ & $1.12 \mathrm{E}-10$ & TBK1 & $3.38 \mathrm{E}+00$ \\
\hline ENSBTAG00000000749 & $1.80 \mathrm{E}-10$ & $5.29 \mathrm{E}-11$ & LMNTD1 & $3.40 \mathrm{E}+00$ \\
\hline ENSBTAG00000030285 & $1.76 \mathrm{E}-10$ & $5.10 \mathrm{E}-11$ & DCP1B & $3.44 E+00$ \\
\hline ENSBTAG00000017925 & $2.90 \mathrm{E}-10$ & $8.41 \mathrm{E}-11$ & LMF2 & $3.44 \mathrm{E}+00$ \\
\hline ENSBTAG00000010530 & $1.03 \mathrm{E}-09$ & $2.98 \mathrm{E}-10$ & IAPP & $3.45 \mathrm{E}+00$ \\
\hline ENSBTAG00000023289 & $2.23 \mathrm{E}-10$ & $6.47 \mathrm{E}-11$ & & $3.44 E+00$ \\
\hline ENSBTAG00000014655 & $6.60 \mathrm{E}-10$ & $1.87 \mathrm{E}-10$ & MYO1A & $3.53 E+00$ \\
\hline ENSBTAG00000039261 & $9.67 \mathrm{E}-11$ & $2.71 E-11$ & KRT2 & $3.56 E+00$ \\
\hline ENSBTAG00000030466 & $7.95 \mathrm{E}-10$ & $2.21 \mathrm{E}-10$ & & $3.60 E+00$ \\
\hline ENSBTAG00000002708 & $3.31 \mathrm{E}-10$ & $9.07 \mathrm{E}-11$ & MRPL42 & $3.65 E+00$ \\
\hline ENSBTAG00000010573 & $4.93 \mathrm{E}-10$ & $1.32 \mathrm{E}-10$ & FAM227A & $3.73 E+00$ \\
\hline ENSBTAG00000009939 & $1.70 \mathrm{E}-10$ & $4.51 E-11$ & CAND1 & $3.76 \mathrm{E}+00$ \\
\hline ENSBTAG00000026088 & $2.71 \mathrm{E}-10$ & $7.18 \mathrm{E}-11$ & & $3.77 \mathrm{E}+00$ \\
\hline ENSBTAG00000020634 & 4.47E-10 & $1.17 \mathrm{E}-10$ & FBXO7 & $3.82 \mathrm{E}+00$ \\
\hline ENSBTAG00000009051 & $3.22 \mathrm{E}-10$ & 8.44E-11 & MMP19 & $3.82 \mathrm{E}+00$ \\
\hline ENSBTAG00000026522 & $8.47 \mathrm{E}-10$ & $2.19 \mathrm{E}-10$ & & $3.87 E+00$ \\
\hline ENSBTAG00000016507 & $6.80 \mathrm{E}-10$ & $1.75 \mathrm{E}-10$ & SERHL2 & $3.88 \mathrm{E}+00$ \\
\hline ENSBTAG00000014764 & $3.16 \mathrm{E}-10$ & $8.12 \mathrm{E}-11$ & CD9 & $3.89 E+00$ \\
\hline ENSBTAG00000006904 & $1.22 \mathrm{E}-10$ & $3.08 \mathrm{E}-11$ & TNS2 & $3.96 \mathrm{E}+00$ \\
\hline ENSBTAG00000008374 & $1.50 \mathrm{E}-10$ & 3.77E-11 & AQP2 & $3.96 \mathrm{E}+00$ \\
\hline ENSBTAG00000015592 & 4.27E-10 & $1.07 \mathrm{E}-10$ & GPR84 & $3.99 E+00$ \\
\hline ENSBTAG00000038498 & $1.93 \mathrm{E}-10$ & $4.86 \mathrm{E}-11$ & ZВTB39 & $3.96 \mathrm{E}+00$ \\
\hline ENSBTAG00000030182 & $4.27 \mathrm{E}-10$ & $1.07 \mathrm{E}-10$ & MAPK11 & $3.99 \mathrm{E}+00$ \\
\hline ENSBTAG00000019281 & $3.13 \mathrm{E}-10$ & $7.86 \mathrm{E}-11$ & MCRS1 & $3.97 \mathrm{E}+00$ \\
\hline ENSBTAG00000030575 & $1.28 \mathrm{E}-10$ & $3.22 \mathrm{E}-11$ & BHLHE41 & $3.96 \mathrm{E}+00$ \\
\hline ENSBTAG00000018403 & $1.68 \mathrm{E}-10$ & $4.24 \mathrm{E}-11$ & ARHGAP9 & $3.96 \mathrm{E}+00$ \\
\hline ENSBTAG00000021083 & $3.12 \mathrm{E}-10$ & $7.79 E-11$ & RECQL & $4.01 E+00$ \\
\hline ENSBTAG00000047942 & $4.94 \mathrm{E}-10$ & $1.22 \mathrm{E}-10$ & DESI1 & $4.04 E+00$ \\
\hline ENSBTAG00000014525 & $2.68 \mathrm{E}-10$ & $6.51 E-11$ & SLC6A13 & $4.11 E+00$ \\
\hline ENSBTAG00000021807 & $7.02 \mathrm{E}-10$ & $1.70 \mathrm{E}-10$ & TAC3 & $4.13 E+00$ \\
\hline ENSBTAG00000004376 & $1.54 \mathrm{E}-10$ & $3.72 \mathrm{E}-11$ & PAN2 & $4.13 E+00$ \\
\hline ENSBTAG00000000021 & $1.98 \mathrm{E}-10$ & 4.77E-11 & & $4.14 \mathrm{E}+00$ \\
\hline ENSBTAG00000008612 & $5.16 \mathrm{E}-10$ & $1.24 \mathrm{E}-10$ & $\mathrm{C} 1 \mathrm{R}$ & $4.16 \mathrm{E}+00$ \\
\hline ENSBTAG00000020662 & $2.88 \mathrm{E}-10$ & $6.91 \mathrm{E}-11$ & & $4.17 E+00$ \\
\hline ENSBTAG00000036282 & $2.68 \mathrm{E}-10$ & $6.42 \mathrm{E}-11$ & CCDC59 & $4.17 E+00$ \\
\hline ENSBTAG00000015595 & $3.74 \mathrm{E}-10$ & $8.94 \mathrm{E}-11$ & MCM5 & $4.17 \mathrm{E}+00$ \\
\hline ENSBTAG00000014156 & $6.66 \mathrm{E}-10$ & $1.59 \mathrm{E}-10$ & AMN1 & $4.19 E+00$ \\
\hline ENSBTAG00000008065 & $6.20 \mathrm{E}-10$ & $1.48 \mathrm{E}-10$ & CDPF1 & $4.19 E+00$ \\
\hline ENSBTAG00000030225 & $5.26 \mathrm{E}-10$ & $1.25 \mathrm{E}-10$ & & $4.20 \mathrm{E}+00$ \\
\hline ENSBTAG00000004009 & $4.62 \mathrm{E}-10$ & $1.10 \mathrm{E}-10$ & PMM1 & $4.19 E+00$ \\
\hline ENSBTAG00000018530 & $5.90 \mathrm{E}-10$ & $1.39 \mathrm{E}-10$ & TUBA8 & $4.24 \mathrm{E}+00$ \\
\hline ENSBTAG00000030476 & $4.58 \mathrm{E}-10$ & $1.08 \mathrm{E}-10$ & & $4.23 E+00$ \\
\hline ENSBTAG00000026657 & $7.82 \mathrm{E}-10$ & $1.82 \mathrm{E}-10$ & & $4.30 E+00$ \\
\hline ENSBTAG00000019277 & $2.86 \mathrm{E}-10$ & $6.64 \mathrm{E}-11$ & $\mathrm{KCNH3}$ & $4.30 E+00$ \\
\hline ENSBTAG00000039170 & $9.72 \mathrm{E}-10$ & $2.23 \mathrm{E}-10$ & & $4.36 \mathrm{E}+00$ \\
\hline ENSBTAG00000013315 & $1.68 \mathrm{E}-10$ & $3.84 \mathrm{E}-11$ & ATP5B & $4.36 \mathrm{E}+00$ \\
\hline ENSBTAG00000018003 & $1.25 \mathrm{E}-10$ & $2.86 \mathrm{E}-11$ & ARHGEF25 & $4.35 E+00$ \\
\hline ENSBTAG00000020663 & $2.67 \mathrm{E}-10$ & $6.09 \mathrm{E}-11$ & ORMDL2 & $4.37 E+00$ \\
\hline ENSBTAG00000005464 & $1.16 \mathrm{E}-10$ & $2.66 \mathrm{E}-11$ & ADCY6 & $4.35 E+00$ \\
\hline ENSBTAG00000037775 & 3.07E-10 & $6.92 \mathrm{E}-11$ & & $4.43 E+00$ \\
\hline ENSBTAG00000020938 & $1.22 \mathrm{E}-10$ & $2.71 \mathrm{E}-11$ & TWF1 & $4.50 E+00$ \\
\hline
\end{tabular}




\begin{tabular}{|c|c|c|c|c|}
\hline ENSBTAG00000019742 & $2.45 \mathrm{E}-10$ & $5.40 \mathrm{E}-11$ & FOXJ2 & $4.52 \mathrm{E}+00$ \\
\hline ENSBTAG00000004384 & $3.11 \mathrm{E}-10$ & $6.87 \mathrm{E}-11$ & APOF & $4.53 E+00$ \\
\hline ENSBTAG00000020179 & $1.48 \mathrm{E}-10$ & $3.22 \mathrm{E}-11$ & AAAS & $4.60 \mathrm{E}+00$ \\
\hline ENSBTAG00000019669 & $2.35 \mathrm{E}-10$ & $5.08 \mathrm{E}-11$ & CD163 & $4.61 E+00$ \\
\hline ENSBTAG00000003362 & $5.13 \mathrm{E}-10$ & 1.10E-10 & HSP90B1 & $4.66 \mathrm{E}+00$ \\
\hline ENSBTAG00000004349 & 1.17E-10 & $2.45 \mathrm{E}-11$ & DAZAP2 & $4.75 E+00$ \\
\hline ENSBTAG00000011931 & $1.53 \mathrm{E}-10$ & $3.21 \mathrm{E}-11$ & CD63 & $4.76 \mathrm{E}+00$ \\
\hline ENSBTAG00000004494 & $1.42 \mathrm{E}-10$ & $2.99 \mathrm{E}-11$ & B4GALNT1 & $4.75 E+00$ \\
\hline ENSBTAG00000026819 & $7.78 \mathrm{E}-11$ & $1.63 \mathrm{E}-11$ & HDAC7 & $4.75 E+00$ \\
\hline ENSBTAG00000004532 & $4.09 \mathrm{E}-10$ & $8.55 \mathrm{E}-11$ & FGFR1OP2 & $4.77 \mathrm{E}+00$ \\
\hline ENSBTAG00000007706 & 1.07E-09 & $2.23 \mathrm{E}-10$ & & $4.80 E+00$ \\
\hline ENSBTAG00000046250 & $9.27 \mathrm{E}-10$ & $1.93 \mathrm{E}-10$ & & $4.80 \mathrm{E}+00$ \\
\hline ENSBTAG00000031146 & 4.87E-10 & $1.00 \mathrm{E}-10$ & PYM1 & $4.86 \mathrm{E}+00$ \\
\hline ENSBTAG00000030222 & $4.92 \mathrm{E}-10$ & $9.98 \mathrm{E}-11$ & L3MBTL2 & $4.93 E+00$ \\
\hline ENSBTAG00000013443 & $2.16 \mathrm{E}-10$ & 4.35E-11 & SDR9C7 & $4.96 \mathrm{E}+00$ \\
\hline ENSBTAG00000010533 & $4.93 \mathrm{E}-10$ & $9.79 \mathrm{E}-11$ & HMGXB4 & $5.03 E+00$ \\
\hline ENSBTAG00000040106 & $5.45 \mathrm{E}-10$ & $1.06 \mathrm{E}-10$ & CHADL & $5.13 E+00$ \\
\hline ENSBTAG00000021464 & $8.06 \mathrm{E}-11$ & $1.56 \mathrm{E}-11$ & SPRYD3 & $5.14 \mathrm{E}+00$ \\
\hline ENSBTAG00000037743 & $9.12 \mathrm{E}-10$ & $1.75 \mathrm{E}-10$ & & $5.21 E+00$ \\
\hline ENSBTAG00000011941 & $6.64 \mathrm{E}-10$ & $1.21 \mathrm{E}-10$ & & $5.48 \mathrm{E}+00$ \\
\hline ENSBTAG00000013979 & $6.90 \mathrm{E}-10$ & $1.25 \mathrm{E}-10$ & & $5.52 E+00$ \\
\hline ENSBTAG00000033292 & 7.47E-11 & $1.34 \mathrm{E}-11$ & SP7 & $5.54 \mathrm{E}+00$ \\
\hline ENSBTAG00000019686 & $2.79 \mathrm{E}-10$ & $5.01 \mathrm{E}-11$ & NCKAP1L & $5.56 \mathrm{E}+00$ \\
\hline ENSBTAG00000008499 & $3.42 \mathrm{E}-10$ & $6.15 \mathrm{E}-11$ & TROAP & $5.56 \mathrm{E}+00$ \\
\hline ENSBTAG00000030472 & $6.14 \mathrm{E}-10$ & $1.10 \mathrm{E}-10$ & & $5.57 E+00$ \\
\hline ENSBTAG00000027974 & $6.28 \mathrm{E}-10$ & $1.12 \mathrm{E}-10$ & & $5.60 \mathrm{E}+00$ \\
\hline ENSBTAG00000008382 & $3.19 E-10$ & $5.66 \mathrm{E}-11$ & METTL25 & $5.63 E+00$ \\
\hline ENSBTAG00000018207 & $5.12 \mathrm{E}-10$ & $9.06 \mathrm{E}-11$ & M6PR & $5.64 \mathrm{E}+00$ \\
\hline ENSBTAG00000046654 & $5.61 \mathrm{E}-10$ & $9.71 \mathrm{E}-11$ & CERK & $5.77 \mathrm{E}+00$ \\
\hline ENSBTAG00000013333 & $7.70 \mathrm{E}-10$ & $1.32 \mathrm{E}-10$ & GYS2 & $5.83 E+00$ \\
\hline ENSBTAG00000040019 & $1.01 \mathrm{E}-09$ & $1.70 \mathrm{E}-10$ & & $5.91 E+00$ \\
\hline ENSBTAG00000013720 & $1.78 \mathrm{E}-10$ & $2.99 \mathrm{E}-11$ & & $5.94 E+00$ \\
\hline ENSBTAG00000012029 & $3.96 \mathrm{E}-10$ & $6.64 \mathrm{E}-11$ & KLRB1 & $5.96 \mathrm{E}+00$ \\
\hline ENSBTAG00000007873 & $1.32 \mathrm{E}-09$ & $2.20 \mathrm{E}-10$ & & $6.01 E+00$ \\
\hline ENSBTAG00000038896 & $6.79 \mathrm{E}-10$ & $1.11 \mathrm{E}-10$ & RPS26 & $6.11 E+00$ \\
\hline ENSBTAG00000016204 & $6.91 \mathrm{E}-10$ & $1.10 \mathrm{E}-10$ & C1RL & $6.28 \mathrm{E}+00$ \\
\hline ENSBTAG00000019159 & $7.98 \mathrm{E}-10$ & $1.26 \mathrm{E}-10$ & PVALB & $6.33 E+00$ \\
\hline ENSBTAG00000002782 & $6.83 \mathrm{E}-10$ & $1.08 \mathrm{E}-10$ & ZC3H7B & $6.32 E+00$ \\
\hline ENSBTAG00000038415 & $5.62 \mathrm{E}-10$ & $8.88 \mathrm{E}-11$ & SLC6A12 & $6.32 E+00$ \\
\hline ENSBTAG00000021808 & $7.22 \mathrm{E}-11$ & $1.14 \mathrm{E}-11$ & CACNB3 & $6.29 E+00$ \\
\hline ENSBTAG00000018773 & $9.45 \mathrm{E}-11$ & $1.49 \mathrm{E}-11$ & RND1 & $6.31 E+00$ \\
\hline ENSBTAG00000032899 & $6.12 \mathrm{E}-10$ & $9.60 \mathrm{E}-11$ & & $6.37 E+00$ \\
\hline ENSBTAG00000014748 & $7.09 \mathrm{E}-10$ & $1.11 \mathrm{E}-10$ & & $6.38 \mathrm{E}+00$ \\
\hline ENSBTAG00000004337 & $3.28 \mathrm{E}-10$ & $5.05 \mathrm{E}-11$ & PDE1B & $6.48 \mathrm{E}+00$ \\
\hline ENSBTAG00000007556 & $5.82 \mathrm{E}-10$ & 8.87E-11 & DYRK4 & $6.56 \mathrm{E}+00$ \\
\hline ENSBTAG00000039157 & $6.70 \mathrm{E}-10$ & $9.87 \mathrm{E}-11$ & GPR182 & $6.78 \mathrm{E}+00$ \\
\hline ENSBTAG00000017465 & 8.25E-10 & $1.16 \mathrm{E}-10$ & GNS & $7.10 \mathrm{E}+00$ \\
\hline ENSBTAG00000001562 & $1.05 \mathrm{E}-10$ & $1.46 \mathrm{E}-11$ & NFE2 & $7.12 \mathrm{E}+00$ \\
\hline ENSBTAG00000010711 & $1.33 \mathrm{E}-09$ & $1.85 \mathrm{E}-10$ & & $7.21 \mathrm{E}+00$ \\
\hline ENSBTAG00000005365 & $7.72 \mathrm{E}-10$ & $1.04 \mathrm{E}-10$ & $\mathrm{OVCH} 1$ & $7.42 \mathrm{E}+00$ \\
\hline ENSBTAG00000007156 & $1.36 \mathrm{E}-10$ & $1.80 \mathrm{E}-11$ & AGAP2 & $7.51 E+00$ \\
\hline ENSBTAG00000006104 & 7.97E-10 & $1.06 \mathrm{E}-10$ & SNU13 & $7.51 E+00$ \\
\hline ENSBTAG00000021633 & $4.50 \mathrm{E}-10$ & $5.94 \mathrm{E}-11$ & TDG & $7.56 \mathrm{E}+00$ \\
\hline ENSBTAG00000016906 & $1.66 \mathrm{E}-10$ & 2.09E-11 & CYP27B1 & $7.93 E+00$ \\
\hline ENSBTAG00000009284 & 4.07E-10 & 4.94E-11 & GLS2 & $8.22 \mathrm{E}+00$ \\
\hline ENSBTAG00000021196 & $7.47 \mathrm{E}-10$ & $8.80 \mathrm{E}-11$ & C12orf45 & $8.48 \mathrm{E}+00$ \\
\hline ENSBTAG00000019782 & $2.69 \mathrm{E}-10$ & 3.07E-11 & TPI1 & $8.73 E+00$ \\
\hline ENSBTAG00000004840 & $5.07 \mathrm{E}-10$ & $5.53 \mathrm{E}-11$ & C1S & $9.16 \mathrm{E}+00$ \\
\hline ENSBTAG00000032567 & $6.26 \mathrm{E}-10$ & $6.83 \mathrm{E}-11$ & C1QL4 & $9.16 \mathrm{E}+00$ \\
\hline ENSBTAG00000016912 & $1.22 \mathrm{E}-10$ & $1.28 \mathrm{E}-11$ & TSFM & $9.48 \mathrm{E}+00$ \\
\hline ENSBTAG00000014603 & $4.26 \mathrm{E}-10$ & 4.46E-11 & VAMP1 & $9.54 \mathrm{E}+00$ \\
\hline ENSBTAG00000030471 & $1.08 \mathrm{E}-09$ & $1.12 \mathrm{E}-10$ & & $9.64 \mathrm{E}+00$ \\
\hline ENSBTAG00000011352 & $6.26 \mathrm{E}-10$ & $6.31 \mathrm{E}-11$ & TBC1D30 & $9.90 E+00$ \\
\hline ENSBTAG00000013417 & $3.68 \mathrm{E}-10$ & $3.56 \mathrm{E}-11$ & & $1.03 \mathrm{E}+01$ \\
\hline ENSBTAG00000039967 & 4.23E-10 & $3.88 \mathrm{E}-11$ & KRT78 & $1.09 \mathrm{E}+01$ \\
\hline ENSBTAG00000021467 & $2.99 \mathrm{E}-10$ & $2.68 \mathrm{E}-11$ & IGFBP6 & $1.11 E+01$ \\
\hline ENSBTAG00000038670 & $1.23 \mathrm{E}-09$ & $1.10 \mathrm{E}-10$ & OR8S1 & $1.12 \mathrm{E}+01$ \\
\hline ENSBTAG00000045770 & $6.70 \mathrm{E}-10$ & $5.82 \mathrm{E}-11$ & KRT77 & $1.15 \mathrm{E}+01$ \\
\hline ENSBTAG00000004718 & 1.09E-09 & $9.41 \mathrm{E}-11$ & PUS7L & $1.16 \mathrm{E}+01$ \\
\hline ENSBTAG00000017729 & $3.49 \mathrm{E}-10$ & $2.93 \mathrm{E}-11$ & $\mathrm{NR} 2 \mathrm{C} 1$ & $1.19 \mathrm{E}+01$ \\
\hline
\end{tabular}




\begin{tabular}{|c|c|c|c|c|}
\hline ENSBTAG00000039425 & $2.66 \mathrm{E}-10$ & $1.96 \mathrm{E}-11$ & & $1.35 \mathrm{E}+01$ \\
\hline ENSBTAG00000038033 & $5.13 \mathrm{E}-10$ & $3.79 \mathrm{E}-11$ & & $1.35 \mathrm{E}+01$ \\
\hline ENSBTAG00000004380 & $4.90 \mathrm{E}-10$ & $3.54 \mathrm{E}-11$ & STAT2 & $1.38 \mathrm{E}+01$ \\
\hline ENSBTAG00000010223 & $6.02 \mathrm{E}-10$ & 4.13E-11 & NTF3 & $1.46 \mathrm{E}+01$ \\
\hline ENSBTAG00000009532 & $2.36 \mathrm{E}-10$ & $1.56 \mathrm{E}-11$ & WNT7B & $1.50 \mathrm{E}+01$ \\
\hline ENSBTAG00000014728 & 2.17E-10 & $1.36 \mathrm{E}-11$ & TAPBPL & $1.58 \mathrm{E}+01$ \\
\hline ENSBTAG00000021810 & $1.24 \mathrm{E}-10$ & 7.07E-12 & DDX23 & $1.72 \mathrm{E}+01$ \\
\hline ENSBTAG00000005623 & $1.82 \mathrm{E}-10$ & 9.53E-12 & SOAT2 & $1.89 \mathrm{E}+01$ \\
\hline ENSBTAG00000021875 & $4.87 \mathrm{E}-10$ & $2.02 \mathrm{E}-11$ & SLC41A2 & $2.40 E+01$ \\
\hline ENSBTAG00000014669 & $6.80 \mathrm{E}-10$ & $1.53 \mathrm{E}-11$ & NDUFA12 & $4.41 \mathrm{E}+01$ \\
\hline ENSBTAG00000010907 & 9.77E-11 & 0 & PPP1R1A & $9.78 \mathrm{E}+02$ \\
\hline ENSBTAG00000003304 & $7.69 \mathrm{E}-11$ & 0 & HOXC4 & $7.70 \mathrm{E}+02$ \\
\hline ENSBTAG00000005606 & 0 & 0 & HOXC9 & $1.00 \mathrm{E}+00$ \\
\hline ENSBTAG00000002401 & $2.08 \mathrm{E}-10$ & 0 & NPFF & $2.08 \mathrm{E}+03$ \\
\hline ENSBTAG00000018993 & $1.55 \mathrm{E}-10$ & 0 & ITGB7 & $1.55 E+03$ \\
\hline ENSBTAG00000046481 & $2.58 \mathrm{E}-10$ & 0 & & $2.59 E+03$ \\
\hline ENSBTAG00000007829 & $2.00 \mathrm{E}-10$ & 0 & CSAD & $2.01 E+03$ \\
\hline ENSBTAG00000047199 & 0 & 0 & & $1.00 \mathrm{E}+00$ \\
\hline ENSBTAG00000046601 & $3.34 \mathrm{E}-10$ & 0 & & $3.34 \mathrm{E}+03$ \\
\hline ENSBTAG00000007158 & $9.10 \mathrm{E}-11$ & 0 & TSPAN31 & $9.11 \mathrm{E}+02$ \\
\hline ENSBTAG00000045969 & $1.78 \mathrm{E}-10$ & 0 & & $1.78 \mathrm{E}+03$ \\
\hline ENSBTAG00000004378 & $3.14 \mathrm{E}-10$ & 0 & IL23A & $3.14 E+03$ \\
\hline ENSBTAG00000010799 & $1.58 \mathrm{E}-10$ & 0 & MYL6 & $1.58 \mathrm{E}+03$ \\
\hline ENSBTAG00000046610 & $5.36 \mathrm{E}-10$ & 0 & & $5.36 \mathrm{E}+03$ \\
\hline ENSBTAG00000017385 & $1.96 \mathrm{E}-10$ & 0 & & $1.96 \mathrm{E}+03$ \\
\hline ENSBTAG00000013008 & 0 & 0 & $\mathrm{PMCH}$ & $1.00 \mathrm{E}+00$ \\
\hline ENSBTAG00000016876 & 3.13E-10 & 0 & & $3.13 \mathrm{E}+03$ \\
\hline ENSBTAG00000002559 & $4.50 \mathrm{E}-11$ & 0 & & $4.51 E+02$ \\
\hline ENSBTAG00000012609 & $3.61 \mathrm{E}-10$ & 0 & $\mathrm{SCO} 2$ & $3.61 E+03$ \\
\hline ENSBTAG00000032790 & $1.06 \mathrm{E}-09$ & 0 & & $1.06 \mathrm{E}+04$ \\
\hline ENSBTAG00000016317 & $6.34 \mathrm{E}-10$ & 0 & & $6.34 \mathrm{E}+03$ \\
\hline ENSBTAG00000005054 & $1.77 \mathrm{E}-10$ & 0 & & $1.78 \mathrm{E}+03$ \\
\hline ENSBTAG00000002025 & 0 & 0 & AMIGO2 & $1.00 \mathrm{E}+00$ \\
\hline ENSBTAG00000000237 & 8.94E-10 & 0 & & $8.94 E+03$ \\
\hline ENSBTAG00000047920 & $2.16 \mathrm{E}-09$ & 0 & OR2AP1 & $2.16 \mathrm{E}+04$ \\
\hline ENSBTAG00000008137 & 0 & 0 & & $1.00 E+00$ \\
\hline ENSBTAG00000023487 & $1.71 \mathrm{E}-10$ & 0 & Cox6B1 & $1.71 E+03$ \\
\hline ENSBTAG00000026610 & 0 & 0 & SMCO3 & $1.00 \mathrm{E}+00$ \\
\hline ENSBTAG00000030503 & 0 & 0 & H2AFJ & $1.00 \mathrm{E}+00$ \\
\hline ENSBTAG00000030504 & 0 & 0 & & $1.00 \mathrm{E}+00$ \\
\hline ENSBTAG00000039496 & 0 & 0 & & $1.00 \mathrm{E}+00$ \\
\hline ENSBTAG00000030463 & $2.73 \mathrm{E}-10$ & 0 & & $2.74 E+03$ \\
\hline ENSBTAG00000030461 & $2.65 \mathrm{E}-10$ & 0 & & $2.65 E+03$ \\
\hline ENSBTAG00000046927 & $1.20 \mathrm{E}-10$ & 0 & & $1.20 E+03$ \\
\hline ENSBTAG00000030199 & $5.96 \mathrm{E}-10$ & 0 & & $5.96 \mathrm{E}+03$ \\
\hline ENSBTAG00000047292 & $5.25 \mathrm{E}-10$ & 0 & & $5.26 \mathrm{E}+03$ \\
\hline ENSBTAG00000024607 & $8.72 \mathrm{E}-11$ & 0 & OR6C75 & $8.73 E+02$ \\
\hline ENSBTAG00000046016 & $8.75 \mathrm{E}-11$ & 0 & & $8.76 E+02$ \\
\hline ENSBTAG00000047056 & $2.63 \mathrm{E}-10$ & 0 & & $2.63 E+03$ \\
\hline ENSBTAG00000046317 & $5.78 \mathrm{E}-10$ & 0 & & $5.78 \mathrm{E}+03$ \\
\hline ENSBTAG00000046884 & $4.38 \mathrm{E}-10$ & 0 & & $4.38 \mathrm{E}+03$ \\
\hline ENSBTAG00000045807 & $4.36 \mathrm{E}-10$ & 0 & & $4.36 \mathrm{E}+03$ \\
\hline ENSBTAG00000047631 & $3.55 \mathrm{E}-10$ & 0 & & $3.56 E+03$ \\
\hline ENSBTAG00000016227 & 0 & 0 & ASCL1 & $1.00 E+00$ \\
\hline ENSBTAG00000024874 & 0 & 4.73E-11 & & $2.11 \mathrm{E}-03$ \\
\hline ENSBTAG00000001751 & 0 & $9.50 \mathrm{E}-11$ & & 1.05E-03 \\
\hline ENSBTAG00000003766 & $5.50 \mathrm{E}-11$ & $2.08 \mathrm{E}-10$ & BLOC1S4 & $2.65 \mathrm{E}-01$ \\
\hline ENSBTAG00000046671 & $1.22 \mathrm{E}-10$ & $4.58 \mathrm{E}-10$ & WFS1 & 2.67E-01 \\
\hline ENSBTAG00000000073 & $3.06 \mathrm{E}-10$ & $1.08 \mathrm{E}-09$ & LRPAP1 & $2.84 \mathrm{E}-01$ \\
\hline ENSBTAG00000047977 & $7.88 \mathrm{E}-11$ & $2.73 E-10$ & & 2.89E-01 \\
\hline ENSBTAG00000007923 & $8.62 \mathrm{E}-11$ & $2.90 \mathrm{E}-10$ & LAMTOR3 & $2.98 \mathrm{E}-01$ \\
\hline ENSBTAG00000039222 & $4.83 \mathrm{E}-10$ & $1.47 \mathrm{E}-09$ & & 3.29E-01 \\
\hline ENSBTAG00000004426 & $8.60 \mathrm{E}-11$ & $2.58 \mathrm{E}-10$ & DNAJB14 & $3.34 \mathrm{E}-01$ \\
\hline ENSBTAG00000024080 & 2.43E-10 & $6.15 \mathrm{E}-10$ & & 3.96E-01 \\
\hline ENSBTAG00000027531 & $4.38 \mathrm{E}-11$ & $1.11 \mathrm{E}-10$ & & 3.95E-01 \\
\hline ENSBTAG00000010185 & $8.01 E-11$ & $2.02 \mathrm{E}-10$ & TADA2B & 3.97E-01 \\
\hline ENSBTAG00000017335 & $9.16 \mathrm{E}-11$ & $2.31 \mathrm{E}-10$ & & 3.97E-01 \\
\hline ENSBTAG00000009059 & $6.16 \mathrm{E}-11$ & $1.55 \mathrm{E}-10$ & PITX2 & $3.98 \mathrm{E}-01$ \\
\hline ENSBTAG00000021963 & $5.17 \mathrm{E}-11$ & $1.03 \mathrm{E}-10$ & SLAIN2 & 5.02E-01 \\
\hline ENSBTAG00000033648 & 4.47E-10 & 8.66E-10 & INTS12 & 5.16E-01 \\
\hline
\end{tabular}




\begin{tabular}{|c|c|c|c|c|}
\hline ENSBTAG00000002333 & $5.26 \mathrm{E}-10$ & 9.97E-10 & & $5.28 \mathrm{E}-01$ \\
\hline ENSBTAG00000014042 & $4.68 \mathrm{E}-11$ & $8.86 \mathrm{E}-11$ & TLR10 & 5.29E-01 \\
\hline ENSBTAG00000038659 & $1.77 \mathrm{E}-10$ & 3.36E-10 & & $5.28 \mathrm{E}-01$ \\
\hline ENSBTAG00000015259 & $1.10 \mathrm{E}-10$ & $2.08 \mathrm{E}-10$ & NEUROG2 & $5.28 \mathrm{E}-01$ \\
\hline ENSBTAG00000014680 & $3.14 \mathrm{E}-10$ & $5.75 \mathrm{E}-10$ & ZBTB49 & $5.46 \mathrm{E}-01$ \\
\hline ENSBTAG00000013699 & $1.52 \mathrm{E}-10$ & $2.74 \mathrm{E}-10$ & TBC1D1 & $5.55 \mathrm{E}-01$ \\
\hline ENSBTAG00000015729 & $6.85 \mathrm{E}-10$ & $1.21 \mathrm{E}-09$ & ADH7 & $5.66 \mathrm{E}-01$ \\
\hline ENSBTAG00000018360 & $1.20 \mathrm{E}-10$ & 2.07E-10 & MRPL1 & $5.80 \mathrm{E}-01$ \\
\hline ENSBTAG00000015649 & $1.25 \mathrm{E}-10$ & $2.11 \mathrm{E}-10$ & TMEM156 & $5.94 \mathrm{E}-01$ \\
\hline ENSBTAG00000033160 & $4.08 \mathrm{E}-10$ & $6.00 \mathrm{E}-10$ & FAM114A1 & $6.80 \mathrm{E}-01$ \\
\hline ENSBTAG00000018638 & $1.08 \mathrm{E}-10$ & $1.55 \mathrm{E}-10$ & CC2D2A & $6.97 \mathrm{E}-01$ \\
\hline ENSBTAG00000033214 & $3.46 \mathrm{E}-10$ & $4.93 \mathrm{E}-10$ & TBC1D19 & 7.01E-01 \\
\hline ENSBTAG00000039213 & $7.51 \mathrm{E}-10$ & 1.07E-09 & & $7.02 \mathrm{E}-01$ \\
\hline ENSBTAG00000002518 & $1.48 \mathrm{E}-10$ & 2.07E-10 & NKX6-1 & $7.13 \mathrm{E}-01$ \\
\hline ENSBTAG00000031197 & $6.02 \mathrm{E}-10$ & 8.37E-10 & HMX1 & 7.19E-01 \\
\hline ENSBTAG00000039871 & $3.53 \mathrm{E}-10$ & $4.85 \mathrm{E}-10$ & & 7.27E-01 \\
\hline ENSBTAG00000027453 & $1.42 \mathrm{E}-10$ & $1.94 \mathrm{E}-10$ & RNF4 & 7.31E-01 \\
\hline ENSBTAG00000010610 & $3.15 \mathrm{E}-10$ & 4.17E-10 & DDIT4L & $7.54 \mathrm{E}-01$ \\
\hline ENSBTAG00000019429 & $2.00 \mathrm{E}-10$ & $2.64 \mathrm{E}-10$ & C4orf17 & $7.58 \mathrm{E}-01$ \\
\hline ENSBTAG00000003602 & 1.17E-10 & $1.54 \mathrm{E}-10$ & RBPJ & 7.59E-01 \\
\hline ENSBTAG00000002205 & $4.92 \mathrm{E}-10$ & 6.47E-10 & CNGA1 & 7.60E-01 \\
\hline ENSBTAG00000003218 & $2.52 \mathrm{E}-10$ & $3.31 \mathrm{E}-10$ & RAB28 & $7.61 \mathrm{E}-01$ \\
\hline ENSBTAG00000006991 & $4.12 \mathrm{E}-10$ & 5.39E-10 & $\mathrm{ADH} 6$ & 7.64E-01 \\
\hline ENSBTAG00000001348 & $1.26 \mathrm{E}-10$ & $1.59 \mathrm{E}-10$ & COMMD8 & 7.95E-01 \\
\hline ENSBTAG00000045570 & 1.27E-09 & $1.61 E-09$ & & 7.91E-01 \\
\hline ENSBTAG00000001328 & $1.64 \mathrm{E}-10$ & $2.06 \mathrm{E}-10$ & SLBP & $7.95 \mathrm{E}-01$ \\
\hline ENSBTAG00000034514 & $3.76 \mathrm{E}-10$ & $4.74 \mathrm{E}-10$ & & $7.94 \mathrm{E}-01$ \\
\hline ENSBTAG00000024125 & $6.46 \mathrm{E}-10$ & 8.14E-10 & RPS27 & 7.93E-01 \\
\hline ENSBTAG00000045966 & $3.50 \mathrm{E}-10$ & 4.42E-10 & GPRIN3 & 7.93E-01 \\
\hline ENSBTAG00000008947 & $9.55 \mathrm{E}-11$ & $1.20 \mathrm{E}-10$ & TKTL2 & 7.96E-01 \\
\hline ENSBTAG00000046413 & $4.41 \mathrm{E}-10$ & $5.56 \mathrm{E}-10$ & & $7.93 \mathrm{E}-01$ \\
\hline ENSBTAG00000044166 & $5.38 \mathrm{E}-11$ & $6.78 \mathrm{E}-11$ & PHOX2B & 7.94E-01 \\
\hline ENSBTAG00000002895 & $3.78 \mathrm{E}-11$ & $4.76 \mathrm{E}-11$ & ATOH1 & 7.94E-01 \\
\hline ENSBTAG00000032425 & $2.51 \mathrm{E}-10$ & $3.16 \mathrm{E}-10$ & PPBP & 7.94E-01 \\
\hline ENSBTAG00000046473 & $2.18 \mathrm{E}-10$ & $2.74 \mathrm{E}-10$ & & 7.94E-01 \\
\hline ENSBTAG00000033351 & $1.27 \mathrm{E}-10$ & $1.60 \mathrm{E}-10$ & PACRGL & 7.92E-01 \\
\hline ENSBTAG00000048119 & $1.59 \mathrm{E}-10$ & 2.00E-10 & & 7.93E-01 \\
\hline ENSBTAG00000017829 & $3.21 \mathrm{E}-10$ & 4.02E-10 & & 8.00E-01 \\
\hline ENSBTAG00000005055 & $1.85 \mathrm{E}-10$ & $2.30 \mathrm{E}-10$ & TXK & $8.03 \mathrm{E}-01$ \\
\hline ENSBTAG00000000748 & $6.71 \mathrm{E}-10$ & $8.32 \mathrm{E}-10$ & GAR1 & $8.06 \mathrm{E}-01$ \\
\hline ENSBTAG00000000646 & $6.86 \mathrm{E}-10$ & $8.43 \mathrm{E}-10$ & ANAPC4 & $8.14 \mathrm{E}-01$ \\
\hline ENSBTAG00000011944 & $3.15 \mathrm{E}-10$ & 3.77E-10 & MTTP & $8.36 \mathrm{E}-01$ \\
\hline ENSBTAG00000017045 & $1.21 \mathrm{E}-09$ & $1.43 \mathrm{E}-09$ & FABP2 & 8.44E-01 \\
\hline ENSBTAG00000012458 & $9.32 \mathrm{E}-10$ & 1.09E-09 & PSAPL1 & 8.55E-01 \\
\hline ENSBTAG00000000751 & $5.22 \mathrm{E}-10$ & $6.10 \mathrm{E}-10$ & RRH & 8.56E-01 \\
\hline ENSBTAG00000017073 & $3.73 \mathrm{E}-10$ & 4.32E-10 & HPGDS & 8.63E-01 \\
\hline ENSBTAG00000047833 & $2.82 \mathrm{E}-10$ & $3.25 \mathrm{E}-10$ & AFM & 8.67E-01 \\
\hline ENSBTAG00000005773 & $5.11 \mathrm{E}-10$ & $5.89 \mathrm{E}-10$ & PGM2 & $8.68 \mathrm{E}-01$ \\
\hline ENSBTAG00000047900 & $3.84 \mathrm{E}-10$ & $4.41 \mathrm{E}-10$ & & $8.70 \mathrm{E}-01$ \\
\hline ENSBTAG00000008887 & $5.28 \mathrm{E}-10$ & $6.02 \mathrm{E}-10$ & PAQR3 & 8.77E-01 \\
\hline ENSBTAG00000016645 & $3.36 \mathrm{E}-10$ & $3.83 \mathrm{E}-10$ & GABRA4 & $8.78 \mathrm{E}-01$ \\
\hline ENSBTAG00000004094 & $1.57 \mathrm{E}-10$ & $1.79 \mathrm{E}-10$ & SPARCL1 & $8.80 \mathrm{E}-01$ \\
\hline ENSBTAG00000006044 & 5.69E-10 & $6.43 \mathrm{E}-10$ & KLHL5 & $8.84 \mathrm{E}-01$ \\
\hline ENSBTAG00000019716 & $6.34 \mathrm{E}-10$ & $7.16 \mathrm{E}-10$ & IL-8 & 8.85E-01 \\
\hline ENSBTAG00000004292 & 4.67E-10 & $5.25 \mathrm{E}-10$ & CRMP1 & $8.90 \mathrm{E}-01$ \\
\hline ENSBTAG00000002428 & $3.75 \mathrm{E}-10$ & $4.21 \mathrm{E}-10$ & PPA2 & $8.92 \mathrm{E}-01$ \\
\hline ENSBTAG00000027513 & $3.58 \mathrm{E}-10$ & $4.01 \mathrm{E}-10$ & & 8.93E-01 \\
\hline ENSBTAG00000004227 & $5.24 \mathrm{E}-10$ & $5.86 \mathrm{E}-10$ & TBCK & 8.94E-01 \\
\hline ENSBTAG00000017061 & $2.38 \mathrm{E}-10$ & 2.67E-10 & SMARCAD1 & 8.93E-01 \\
\hline ENSBTAG00000013569 & $9.76 \mathrm{E}-10$ & 1.09E-09 & CD38 & 8.96E-01 \\
\hline ENSBTAG00000008255 & $5.42 \mathrm{E}-10$ & $6.02 \mathrm{E}-10$ & QRFPR & 9.00E-01 \\
\hline ENSBTAG00000005754 & $4.43 \mathrm{E}-10$ & 4.93E-10 & PPM1K & 8.99E-01 \\
\hline ENSBTAG00000002356 & $1.45 \mathrm{E}-10$ & $1.60 \mathrm{E}-10$ & RBM47 & $9.04 \mathrm{E}-01$ \\
\hline ENSBTAG00000035129 & $6.69 \mathrm{E}-11$ & $7.38 \mathrm{E}-11$ & & 9.07E-01 \\
\hline ENSBTAG00000027655 & $4.89 \mathrm{E}-10$ & $5.36 \mathrm{E}-10$ & TIFA & $9.13 \mathrm{E}-01$ \\
\hline ENSBTAG00000019633 & $3.85 \mathrm{E}-10$ & $4.18 \mathrm{E}-10$ & SHROOM3 & $9.21 \mathrm{E}-01$ \\
\hline ENSBTAG00000007639 & $7.18 \mathrm{E}-10$ & $7.79 \mathrm{E}-10$ & SDAD1 & $9.21 \mathrm{E}-01$ \\
\hline ENSBTAG00000000962 & $2.85 \mathrm{E}-10$ & $3.08 \mathrm{E}-10$ & STAP1 & $9.26 \mathrm{E}-01$ \\
\hline ENSBTAG00000005062 & $3.52 \mathrm{E}-10$ & $3.80 \mathrm{E}-10$ & TEC & 9.27E-01 \\
\hline ENSBTAG00000027464 & $2.04 \mathrm{E}-10$ & $2.20 \mathrm{E}-10$ & FGFRL1 & $9.26 \mathrm{E}-01$ \\
\hline
\end{tabular}




\begin{tabular}{|c|c|c|c|c|}
\hline ENSBTAG00000014463 & $4.46 \mathrm{E}-10$ & $4.80 \mathrm{E}-10$ & CAMK2D & $9.29 \mathrm{E}-01$ \\
\hline ENSBTAG00000034206 & $5.23 \mathrm{E}-10$ & $5.63 \mathrm{E}-10$ & EMCN & $9.29 \mathrm{E}-01$ \\
\hline ENSBTAG00000016007 & $1.88 \mathrm{E}-10$ & $2.02 \mathrm{E}-10$ & $\mathrm{ADH} 5$ & $9.30 \mathrm{E}-01$ \\
\hline ENSBTAG00000009423 & $3.66 \mathrm{E}-10$ & $3.92 \mathrm{E}-10$ & NIPAL1 & $9.34 \mathrm{E}-01$ \\
\hline ENSBTAG00000019847 & $1.46 \mathrm{E}-10$ & $1.55 \mathrm{E}-10$ & & $9.40 \mathrm{E}-01$ \\
\hline ENSBTAG00000004287 & $2.76 \mathrm{E}-10$ & $2.95 \mathrm{E}-10$ & EVC & $9.35 \mathrm{E}-01$ \\
\hline ENSBTAG00000020705 & 4.44E-10 & $4.75 \mathrm{E}-10$ & PRSS12 & $9.36 \mathrm{E}-01$ \\
\hline ENSBTAG00000009522 & $3.07 \mathrm{E}-10$ & $3.27 E-10$ & EIF4E & $9.38 \mathrm{E}-01$ \\
\hline ENSBTAG00000013736 & $6.58 \mathrm{E}-10$ & $7.02 \mathrm{E}-10$ & PROM1 & $9.37 \mathrm{E}-01$ \\
\hline ENSBTAG00000017375 & $2.09 \mathrm{E}-10$ & $2.23 \mathrm{E}-10$ & C4orf32 & $9.39 \mathrm{E}-01$ \\
\hline ENSBTAG00000004237 & $5.03 \mathrm{E}-10$ & $5.34 \mathrm{E}-10$ & BTC & $9.42 \mathrm{E}-01$ \\
\hline ENSBTAG00000009744 & $2.02 \mathrm{E}-10$ & $2.13 \mathrm{E}-10$ & FAM193A & $9.49 \mathrm{E}-01$ \\
\hline ENSBTAG00000034189 & $3.76 \mathrm{E}-10$ & $3.97 \mathrm{E}-10$ & & $9.48 \mathrm{E}-01$ \\
\hline ENSBTAG00000012296 & $5.35 \mathrm{E}-10$ & $5.63 \mathrm{E}-10$ & GABRG1 & $9.49 \mathrm{E}-01$ \\
\hline ENSBTAG00000007019 & $3.15 \mathrm{E}-10$ & $3.32 \mathrm{E}-10$ & LRIT3 & $9.49 \mathrm{E}-01$ \\
\hline ENSBTAG00000008797 & $5.10 \mathrm{E}-10$ & $5.37 \mathrm{E}-10$ & & $9.51 \mathrm{E}-01$ \\
\hline ENSBTAG00000027434 & $7.00 \mathrm{E}-10$ & $7.35 E-10$ & & $9.52 \mathrm{E}-01$ \\
\hline ENSBTAG00000004653 & $4.70 \mathrm{E}-10$ & $4.91 \mathrm{E}-10$ & ADGRA3 & $9.56 \mathrm{E}-01$ \\
\hline ENSBTAG00000003619 & $5.42 \mathrm{E}-10$ & $5.67 E-10$ & SEC24D & $9.57 \mathrm{E}-01$ \\
\hline ENSBTAG00000038648 & $2.28 \mathrm{E}-10$ & $2.38 \mathrm{E}-10$ & & $9.59 \mathrm{E}-01$ \\
\hline ENSBTAG00000000197 & $1.96 \mathrm{E}-10$ & $2.03 E-10$ & TRMT10A & $9.66 \mathrm{E}-01$ \\
\hline ENSBTAG00000001505 & $2.61 \mathrm{E}-10$ & $2.71 \mathrm{E}-10$ & GRK4 & $9.65 \mathrm{E}-01$ \\
\hline ENSBTAG00000003651 & 7.33E-10 & $7.54 \mathrm{E}-10$ & OTOP1 & $9.73 \mathrm{E}-01$ \\
\hline ENSBTAG00000000599 & $5.75 \mathrm{E}-10$ & $5.91 \mathrm{E}-10$ & CCNI & $9.73 \mathrm{E}-01$ \\
\hline ENSBTAG00000024787 & $4.13 \mathrm{E}-10$ & 4.25E-10 & HAUS3 & $9.73 \mathrm{E}-01$ \\
\hline ENSBTAG00000014080 & 7.04E-10 & 7.23E-10 & METTL14 & $9.74 \mathrm{E}-01$ \\
\hline ENSBTAG00000014038 & $3.80 \mathrm{E}-10$ & $3.90 \mathrm{E}-10$ & CDKL2 & $9.74 \mathrm{E}-01$ \\
\hline ENSBTAG00000006307 & $6.25 \mathrm{E}-10$ & $6.38 \mathrm{E}-10$ & HSD17B11 & $9.79 \mathrm{E}-01$ \\
\hline ENSBTAG00000007522 & $2.14 \mathrm{E}-10$ & $2.18 \mathrm{E}-10$ & RAP1GDS1 & $9.80 \mathrm{E}-01$ \\
\hline ENSBTAG00000019427 & $3.31 \mathrm{E}-10$ & $3.37 \mathrm{E}-10$ & KIAA0232 & $9.83 \mathrm{E}-01$ \\
\hline ENSBTAG00000039384 & $1.22 \mathrm{E}-10$ & $1.24 \mathrm{E}-10$ & & $9.87 \mathrm{E}-01$ \\
\hline ENSBTAG00000046333 & $7.62 \mathrm{E}-11$ & $7.68 \mathrm{E}-11$ & & $9.92 \mathrm{E}-01$ \\
\hline ENSBTAG00000038606 & $2.89 \mathrm{E}-10$ & $2.91 \mathrm{E}-10$ & DRD5 & $9.91 \mathrm{E}-01$ \\
\hline ENSBTAG00000011961 & $4.24 \mathrm{E}-10$ & 4.27E-10 & & $9.93 \mathrm{E}-01$ \\
\hline ENSBTAG00000037426 & $2.86 \mathrm{E}-10$ & $2.88 \mathrm{E}-10$ & & $9.93 \mathrm{E}-01$ \\
\hline ENSBTAG00000017704 & $6.33 \mathrm{E}-10$ & $6.38 \mathrm{E}-10$ & $A B C G 2$ & $9.93 \mathrm{E}-01$ \\
\hline ENSBTAG00000021582 & $4.21 \mathrm{E}-10$ & 4.22E-10 & NCAPG & $9.97 \mathrm{E}-01$ \\
\hline ENSBTAG00000034875 & $5.20 \mathrm{E}-10$ & $5.21 \mathrm{E}-10$ & ALPK1 & $9.98 \mathrm{E}-01$ \\
\hline ENSBTAG00000004277 & 4.59E-10 & $4.60 \mathrm{E}-10$ & EVC2 & $9.99 \mathrm{E}-01$ \\
\hline ENSBTAG00000005932 & 3.36E-10 & $3.35 \mathrm{E}-10$ & FAM184B & $1.00 \mathrm{E}+00$ \\
\hline ENSBTAG00000043960 & $4.26 \mathrm{E}-10$ & 4.24E-10 & MTHFD2L & $1.01 E+00$ \\
\hline ENSBTAG00000012658 & $4.18 \mathrm{E}-10$ & 4.16E-10 & TMA16 & $1.01 E+00$ \\
\hline ENSBTAG00000010350 & $9.03 \mathrm{E}-10$ & $8.98 \mathrm{E}-10$ & USP53 & $1.01 E+00$ \\
\hline ENSBTAG00000005493 & $5.21 \mathrm{E}-10$ & $5.17 \mathrm{E}-10$ & TBC1D14 & $1.01 E+00$ \\
\hline ENSBTAG00000040228 & $5.24 \mathrm{E}-10$ & $5.18 \mathrm{E}-10$ & & $1.01 E+00$ \\
\hline ENSBTAG00000019441 & $5.81 \mathrm{E}-10$ & $5.70 \mathrm{E}-10$ & MED28 & $1.02 \mathrm{E}+00$ \\
\hline ENSBTAG00000011163 & $1.68 \mathrm{E}-10$ & $1.64 \mathrm{E}-10$ & CYTL1 & $1.02 \mathrm{E}+00$ \\
\hline ENSBTAG00000039645 & $4.08 \mathrm{E}-10$ & 4.00E-10 & & $1.02 \mathrm{E}+00$ \\
\hline ENSBTAG00000016714 & $3.61 \mathrm{E}-10$ & $3.53 \mathrm{E}-10$ & & $1.02 \mathrm{E}+00$ \\
\hline ENSBTAG00000020598 & $2.17 \mathrm{E}-10$ & $2.12 \mathrm{E}-10$ & PPP2R2C & $1.02 \mathrm{E}+00$ \\
\hline ENSBTAG00000004988 & $1.61 \mathrm{E}-10$ & $1.56 \mathrm{E}-10$ & CCKAR & $1.03 E+00$ \\
\hline ENSBTAG00000035764 & $3.24 \mathrm{E}-10$ & $3.16 \mathrm{E}-10$ & & $1.03 E+00$ \\
\hline ENSBTAG00000014031 & $6.05 \mathrm{E}-10$ & $5.91 \mathrm{E}-10$ & TLR6 & $1.02 \mathrm{E}+00$ \\
\hline ENSBTAG00000006643 & $3.25 \mathrm{E}-10$ & $3.17 \mathrm{E}-10$ & C4orf19 & $1.03 E+00$ \\
\hline ENSBTAG00000005989 & $5.30 \mathrm{E}-10$ & $5.17 E-10$ & LAP3 & $1.03 E+00$ \\
\hline ENSBTAG00000002574 & $2.52 \mathrm{E}-10$ & $2.45 \mathrm{E}-10$ & MYOZ2 & $1.03 E+00$ \\
\hline ENSBTAG00000032947 & $3.97 \mathrm{E}-10$ & $3.85 \mathrm{E}-10$ & GRXCR1 & $1.03 E+00$ \\
\hline ENSBTAG00000021347 & $4.38 \mathrm{E}-10$ & 4.24E-10 & $\mathrm{CWH} 43$ & $1.03 \mathrm{E}+00$ \\
\hline ENSBTAG00000015919 & $2.10 \mathrm{E}-10$ & $2.02 \mathrm{E}-10$ & PARM1 & $1.04 \mathrm{E}+00$ \\
\hline ENSBTAG00000006561 & $5.76 \mathrm{E}-10$ & $5.55 \mathrm{E}-10$ & RASGEF1B & $1.04 \mathrm{E}+00$ \\
\hline ENSBTAG00000017808 & $6.72 \mathrm{E}-10$ & $6.46 \mathrm{E}-10$ & ARSJ & $1.04 \mathrm{E}+00$ \\
\hline ENSBTAG00000010389 & $3.87 \mathrm{E}-10$ & $3.73 E-10$ & STBD1 & $1.04 \mathrm{E}+00$ \\
\hline ENSBTAG00000046561 & $3.10 \mathrm{E}-10$ & $2.99 \mathrm{E}-10$ & LCORL & $1.04 \mathrm{E}+00$ \\
\hline ENSBTAG00000035319 & $1.05 \mathrm{E}-09$ & $1.01 \mathrm{E}-09$ & MAD2L1 & $1.04 \mathrm{E}+00$ \\
\hline ENSBTAG00000008332 & $2.18 \mathrm{E}-10$ & 2.09E-10 & ENPEP & $1.04 \mathrm{E}+00$ \\
\hline ENSBTAG00000044082 & $4.52 \mathrm{E}-10$ & $4.32 \mathrm{E}-10$ & 01-Mar & $1.05 E+00$ \\
\hline ENSBTAG00000047743 & 4.55E-10 & $4.32 \mathrm{E}-10$ & KCNIP4 & $1.05 E+00$ \\
\hline ENSBTAG00000015230 & $5.38 \mathrm{E}-10$ & $5.12 \mathrm{E}-10$ & PLA2G12A & $1.05 \mathrm{E}+00$ \\
\hline ENSBTAG00000011170 & 4.05E-10 & $3.84 \mathrm{E}-10$ & JAKMIP1 & $1.05 \mathrm{E}+00$ \\
\hline ENSBTAG00000031395 & $2.47 \mathrm{E}-10$ & $2.33 \mathrm{E}-10$ & C4orf48 & $1.06 \mathrm{E}+00$ \\
\hline
\end{tabular}




\begin{tabular}{|c|c|c|c|c|}
\hline ENSBTAG00000023000 & 4.10E-10 & $3.87 \mathrm{E}-10$ & & $1.06 \mathrm{E}+00$ \\
\hline ENSBTAG00000033327 & $6.56 \mathrm{E}-10$ & $6.19 \mathrm{E}-10$ & & $1.06 \mathrm{E}+00$ \\
\hline ENSBTAG00000024888 & $5.20 \mathrm{E}-10$ & $4.91 \mathrm{E}-10$ & PDE5A & $1.06 \mathrm{E}+00$ \\
\hline ENSBTAG00000003653 & $3.14 \mathrm{E}-10$ & $2.96 \mathrm{E}-10$ & LYAR & $1.06 \mathrm{E}+00$ \\
\hline ENSBTAG00000010564 & $2.94 \mathrm{E}-10$ & $2.76 \mathrm{E}-10$ & ELOVL6 & $1.07 \mathrm{E}+00$ \\
\hline ENSBTAG00000020363 & 3.17E-10 & $2.97 \mathrm{E}-10$ & GBA3 & $1.07 \mathrm{E}+00$ \\
\hline ENSBTAG00000020525 & $1.88 \mathrm{E}-10$ & $1.76 \mathrm{E}-10$ & SEC31A & $1.07 \mathrm{E}+00$ \\
\hline ENSBTAG00000002084 & $4.92 \mathrm{E}-10$ & $4.61 \mathrm{E}-10$ & UNC5C & $1.07 E+00$ \\
\hline ENSBTAG00000000438 & $5.33 \mathrm{E}-10$ & $4.98 \mathrm{E}-10$ & GNRHR & $1.07 \mathrm{E}+00$ \\
\hline ENSBTAG00000001491 & $4.11 \mathrm{E}-10$ & $3.84 \mathrm{E}-10$ & NWD2 & $1.07 E+00$ \\
\hline ENSBTAG00000010343 & $1.78 \mathrm{E}-10$ & $1.66 \mathrm{E}-10$ & C1QTNF7 & $1.07 \mathrm{E}+00$ \\
\hline ENSBTAG00000017296 & $6.94 \mathrm{E}-10$ & $6.45 E-10$ & POLN & $1.08 \mathrm{E}+00$ \\
\hline ENSBTAG00000022205 & $9.28 \mathrm{E}-10$ & $8.62 E-10$ & & $1.08 \mathrm{E}+00$ \\
\hline ENSBTAG00000038340 & $6.24 \mathrm{E}-10$ & $5.79 \mathrm{E}-10$ & CLNK & $1.08 \mathrm{E}+00$ \\
\hline ENSBTAG00000008896 & $1.49 \mathrm{E}-10$ & $1.38 \mathrm{E}-10$ & DHX15 & $1.08 \mathrm{E}+00$ \\
\hline ENSBTAG00000004921 & $6.71 E-10$ & $6.21 \mathrm{E}-10$ & & $1.08 \mathrm{E}+00$ \\
\hline ENSBTAG00000017390 & $1.10 \mathrm{E}-10$ & $1.02 \mathrm{E}-10$ & LGI2 & $1.07 E+00$ \\
\hline ENSBTAG00000017682 & $2.35 \mathrm{E}-10$ & $2.17 \mathrm{E}-10$ & TET2 & $1.08 \mathrm{E}+00$ \\
\hline ENSBTAG00000012537 & $2.20 \mathrm{E}-10$ & $2.03 \mathrm{E}-10$ & CASP6 & $1.08 \mathrm{E}+00$ \\
\hline ENSBTAG00000010677 & $3.56 \mathrm{E}-10$ & $3.28 \mathrm{E}-10$ & LIMCH1 & $1.08 \mathrm{E}+00$ \\
\hline ENSBTAG00000021214 & $2.14 \mathrm{E}-10$ & $1.96 \mathrm{E}-10$ & FBXL5 & $1.09 \mathrm{E}+00$ \\
\hline ENSBTAG00000007921 & $2.34 \mathrm{E}-10$ & $2.15 \mathrm{E}-10$ & DAPP1 & $1.09 \mathrm{E}+00$ \\
\hline ENSBTAG00000004329 & $2.94 \mathrm{E}-10$ & $2.69 \mathrm{E}-10$ & RELL1 & $1.09 \mathrm{E}+00$ \\
\hline ENSBTAG00000021746 & $1.01 \mathrm{E}-09$ & $9.18 \mathrm{E}-10$ & ANXA5 & $1.10 \mathrm{E}+00$ \\
\hline ENSBTAG00000001506 & $3.68 \mathrm{E}-10$ & $3.35 \mathrm{E}-10$ & HTT & $1.10 E+00$ \\
\hline ENSBTAG00000019451 & $1.58 \mathrm{E}-10$ & $1.43 \mathrm{E}-10$ & TAPT1 & $1.10 \mathrm{E}+00$ \\
\hline ENSBTAG00000019808 & $4.21 \mathrm{E}-10$ & $3.81 E-10$ & CCSER1 & $1.11 E+00$ \\
\hline ENSBTAG00000015297 & $4.28 \mathrm{E}-10$ & $3.85 E-10$ & & $1.11 E+00$ \\
\hline ENSBTAG00000011156 & $3.87 \mathrm{E}-10$ & $3.48 \mathrm{E}-10$ & ATP8A1 & $1.11 \mathrm{E}+00$ \\
\hline ENSBTAG00000034139 & $6.35 \mathrm{E}-10$ & $5.71 \mathrm{E}-10$ & $\mathrm{ADH} 4$ & $1.11 E+00$ \\
\hline ENSBTAG00000017028 & $2.19 E-10$ & $1.96 \mathrm{E}-10$ & USO1 & $1.12 \mathrm{E}+00$ \\
\hline ENSBTAG00000017024 & $2.90 \mathrm{E}-10$ & $2.59 \mathrm{E}-10$ & PPARGC1A & $1.12 \mathrm{E}+00$ \\
\hline ENSBTAG00000004230 & $1.24 \mathrm{E}-09$ & $1.11 \mathrm{E}-09$ & AIMP1 & $1.12 \mathrm{E}+00$ \\
\hline ENSBTAG00000013198 & $1.27 \mathrm{E}-10$ & $1.13 \mathrm{E}-10$ & & $1.13 \mathrm{E}+00$ \\
\hline ENSBTAG00000003131 & $6.16 \mathrm{E}-10$ & $5.48 \mathrm{E}-10$ & NDST4 & $1.12 \mathrm{E}+00$ \\
\hline ENSBTAG00000013980 & $4.28 \mathrm{E}-10$ & $3.80 \mathrm{E}-10$ & SOD3 & $1.13 E+00$ \\
\hline ENSBTAG00000004945 & $5.93 \mathrm{E}-10$ & $5.26 \mathrm{E}-10$ & BBS7 & $1.13 \mathrm{E}+00$ \\
\hline ENSBTAG00000009632 & $1.81 \mathrm{E}-10$ & $1.60 \mathrm{E}-10$ & LARP7 & $1.13 E+00$ \\
\hline ENSBTAG00000012464 & $4.85 \mathrm{E}-10$ & $4.29 \mathrm{E}-10$ & AFAP1 & $1.13 \mathrm{E}+00$ \\
\hline ENSBTAG00000007189 & $2.28 \mathrm{E}-10$ & $2.00 \mathrm{E}-10$ & RCHY1 & $1.14 \mathrm{E}+00$ \\
\hline ENSBTAG00000004052 & $2.97 \mathrm{E}-10$ & $2.57 \mathrm{E}-10$ & EPGN & $1.16 \mathrm{E}+00$ \\
\hline ENSBTAG00000007568 & $2.52 \mathrm{E}-10$ & $2.18 \mathrm{E}-10$ & MEPE & $1.16 \mathrm{E}+00$ \\
\hline ENSBTAG00000004574 & 4.67E-10 & $4.01 \mathrm{E}-10$ & UGT8 & $1.16 \mathrm{E}+00$ \\
\hline ENSBTAG00000002201 & $1.90 \mathrm{E}-10$ & $1.63 \mathrm{E}-10$ & NFXL1 & $1.17 \mathrm{E}+00$ \\
\hline ENSBTAG00000026936 & $2.14 \mathrm{E}-10$ & $1.82 \mathrm{E}-10$ & & $1.17 E+00$ \\
\hline ENSBTAG00000010945 & $2.44 \mathrm{E}-10$ & $2.08 \mathrm{E}-10$ & SEC24B & $1.17 \mathrm{E}+00$ \\
\hline ENSBTAG00000008479 & 4.74E-10 & $4.03 \mathrm{E}-10$ & CXCL13 & $1.18 \mathrm{E}+00$ \\
\hline ENSBTAG00000015926 & $5.53 \mathrm{E}-10$ & $4.69 \mathrm{E}-10$ & ABLIM2 & $1.18 \mathrm{E}+00$ \\
\hline ENSBTAG00000019353 & $1.24 \mathrm{E}-10$ & $1.05 \mathrm{E}-10$ & STIM2 & $1.18 \mathrm{E}+00$ \\
\hline ENSBTAG00000006434 & $3.90 \mathrm{E}-10$ & $3.30 \mathrm{E}-10$ & SYNPO2 & $1.18 \mathrm{E}+00$ \\
\hline ENSBTAG00000019478 & $7.42 \mathrm{E}-10$ & $6.25 \mathrm{E}-10$ & NAAA & $1.19 \mathrm{E}+00$ \\
\hline ENSBTAG00000015252 & $9.18 \mathrm{E}-11$ & $7.71 \mathrm{E}-11$ & CHRNA9 & $1.19 \mathrm{E}+00$ \\
\hline ENSBTAG00000013996 & $2.88 \mathrm{E}-10$ & $2.41 \mathrm{E}-10$ & SH3BP2 & $1.19 \mathrm{E}+00$ \\
\hline ENSBTAG00000002330 & $5.20 \mathrm{E}-10$ & $4.36 \mathrm{E}-10$ & & $1.19 \mathrm{E}+00$ \\
\hline ENSBTAG00000047891 & $6.36 \mathrm{E}-10$ & $5.30 \mathrm{E}-10$ & GIMD1 & $1.20 \mathrm{E}+00$ \\
\hline ENSBTAG00000010956 & $8.17 \mathrm{E}-10$ & $6.81 E-10$ & SCARB2 & $1.20 \mathrm{E}+00$ \\
\hline ENSBTAG00000019359 & $3.40 \mathrm{E}-10$ & $2.82 \mathrm{E}-10$ & & $1.21 \mathrm{E}+00$ \\
\hline ENSBTAG00000014947 & $4.41 \mathrm{E}-10$ & $3.66 \mathrm{E}-10$ & PTPN13 & $1.21 \mathrm{E}+00$ \\
\hline ENSBTAG00000009673 & $6.36 \mathrm{E}-10$ & $5.24 \mathrm{E}-10$ & SLC9B1 & $1.21 \mathrm{E}+00$ \\
\hline ENSBTAG00000006986 & $6.93 \mathrm{E}-10$ & $5.70 \mathrm{E}-10$ & & $1.22 \mathrm{E}+00$ \\
\hline ENSBTAG00000018106 & $1.96 \mathrm{E}-10$ & $1.61 \mathrm{E}-10$ & SPATA18 & $1.22 \mathrm{E}+00$ \\
\hline ENSBTAG00000001126 & $3.48 \mathrm{E}-10$ & $2.86 \mathrm{E}-10$ & & $1.22 \mathrm{E}+00$ \\
\hline ENSBTAG00000000175 & $2.34 \mathrm{E}-10$ & $1.92 \mathrm{E}-10$ & N4BP2 & $1.22 \mathrm{E}+00$ \\
\hline ENSBTAG00000018134 & $2.70 \mathrm{E}-10$ & $2.21 \mathrm{E}-10$ & AREG & $1.22 \mathrm{E}+00$ \\
\hline ENSBTAG00000011187 & $2.65 \mathrm{E}-10$ & $2.17 \mathrm{E}-10$ & FAM13A & $1.22 \mathrm{E}+00$ \\
\hline ENSBTAG00000006686 & $4.44 \mathrm{E}-10$ & $3.62 \mathrm{E}-10$ & NPNT & $1.23 E+00$ \\
\hline ENSBTAG00000007449 & $2.92 \mathrm{E}-10$ & $2.39 \mathrm{E}-10$ & HS3ST1 & $1.22 \mathrm{E}+00$ \\
\hline ENSBTAG00000006492 & $2.11 E-10$ & $1.71 \mathrm{E}-10$ & & $1.23 \mathrm{E}+00$ \\
\hline ENSBTAG00000020538 & $2.47 \mathrm{E}-10$ & $2.00 \mathrm{E}-10$ & HERC5 & $1.24 \mathrm{E}+00$ \\
\hline ENSBTAG00000010611 & $2.50 \mathrm{E}-10$ & $2.03 \mathrm{E}-10$ & OCIAD1 & $1.23 \mathrm{E}+00$ \\
\hline
\end{tabular}




\begin{tabular}{|c|c|c|c|c|}
\hline ENSBTAG000000005712 & $3.42 \mathrm{E}-10$ & $2.76 \mathrm{E}-10$ & STX18 & $1.24 \mathrm{E}+00$ \\
\hline ENSBTAG00000019188 & $3.95 \mathrm{E}-10$ & 3.17E-10 & GAK & $1.25 \mathrm{E}+00$ \\
\hline ENSBTAG000000004943 & $1.48 \mathrm{E}-10$ & $1.18 \mathrm{E}-10$ & CCNA2 & $1.25 \mathrm{E}+00$ \\
\hline ENSBTAG000000000137 & $1.46 \mathrm{E}-10$ & 1.17E-10 & FRYL & $1.25 E+00$ \\
\hline ENSBTAG000000015409 & $2.58 \mathrm{E}-10$ & $2.06 \mathrm{E}-10$ & & $1.25 E+00$ \\
\hline ENSBTAG000000010284 & $1.91 \mathrm{E}-10$ & $1.53 \mathrm{E}-10$ & ETNPPL & $1.25 E+00$ \\
\hline ENSBTAG000000047876 & $9.21 \mathrm{E}-10$ & 7.35E-10 & SH3TC1 & $1.25 \mathrm{E}+00$ \\
\hline ENSBTAG000000012995 & 4.35E-10 & $3.47 \mathrm{E}-10$ & MCUB & $1.25 E+00$ \\
\hline ENSBTAG000000020175 & $1.44 \mathrm{E}-10$ & $1.14 \mathrm{E}-10$ & UBE2K & $1.26 \mathrm{E}+00$ \\
\hline ENSBTAG00000014522 & $3.74 \mathrm{E}-10$ & $2.96 \mathrm{E}-10$ & SMIM14 & $1.26 \mathrm{E}+00$ \\
\hline ENSBTAG000000004040 & 1.01E-09 & $8.01 E-10$ & UGT2A3 & $1.26 \mathrm{E}+00$ \\
\hline ENSBTAG000000006508 & $8.19 E-10$ & $6.48 \mathrm{E}-10$ & & $1.26 \mathrm{E}+00$ \\
\hline ENSBTAG000000001545 & $5.08 \mathrm{E}-10$ & $4.02 \mathrm{E}-10$ & SLC34A2 & $1.26 \mathrm{E}+00$ \\
\hline ENSBTAG000000014058 & $5.35 \mathrm{E}-10$ & 4.23E-10 & LDB2 & $1.26 \mathrm{E}+00$ \\
\hline ENSBTAG00000015209 & $1.15 \mathrm{E}-10$ & $9.05 \mathrm{E}-11$ & MXD4 & $1.27 \mathrm{E}+00$ \\
\hline ENSBTAG000000020108 & $3.50 \mathrm{E}-10$ & $2.73 \mathrm{E}-10$ & LETM1 & $1.28 \mathrm{E}+00$ \\
\hline ENSBTAG000000004353 & $1.86 \mathrm{E}-10$ & $1.45 \mathrm{E}-10$ & CNOT6L & $1.28 \mathrm{E}+00$ \\
\hline ENSBTAG000000002392 & $1.57 \mathrm{E}-10$ & $1.22 \mathrm{E}-10$ & & $1.29 \mathrm{E}+00$ \\
\hline ENSBTAG000000011817 & $4.02 \mathrm{E}-10$ & $3.11 \mathrm{E}-10$ & & $1.29 E+00$ \\
\hline ENSBTAG000000010716 & $4.36 \mathrm{E}-10$ & 3.37E-10 & FRAS1 & $1.29 \mathrm{E}+00$ \\
\hline ENSBTAG000000010153 & $3.29 \mathrm{E}-10$ & $2.54 \mathrm{E}-10$ & ANXA3 & $1.29 \mathrm{E}+00$ \\
\hline ENSBTAG000000012818 & $3.69 \mathrm{E}-10$ & $2.84 \mathrm{E}-10$ & PDLIM5 & $1.30 \mathrm{E}+00$ \\
\hline ENSBTAG000000006844 & $2.58 \mathrm{E}-10$ & $1.98 \mathrm{E}-10$ & LEF1 & $1.30 \mathrm{E}+00$ \\
\hline ENSBTAG000000047514 & $4.94 \mathrm{E}-10$ & $3.80 \mathrm{E}-10$ & & $1.30 \mathrm{E}+00$ \\
\hline ENSBTAG000000017480 & $1.96 \mathrm{E}-10$ & $1.50 \mathrm{E}-10$ & PDE6B & $1.30 \mathrm{E}+00$ \\
\hline ENSBTAG000000027630 & $3.46 \mathrm{E}-10$ & $2.65 \mathrm{E}-10$ & CISD2 & $1.31 E+00$ \\
\hline ENSBTAG000000017282 & $2.36 \mathrm{E}-10$ & $1.80 \mathrm{E}-10$ & COL25A1 & $1.31 E+00$ \\
\hline ENSBTAG000000016005 & $3.29 \mathrm{E}-10$ & $2.50 \mathrm{E}-10$ & РPP3СA & $1.32 \mathrm{E}+00$ \\
\hline ENSBTAG000000002199 & $2.91 \mathrm{E}-10$ & $2.21 \mathrm{E}-10$ & CORIN & $1.32 \mathrm{E}+00$ \\
\hline ENSBTAG000000016004 & $1.34 \mathrm{E}-10$ & $1.02 \mathrm{E}-10$ & METAP1 & $1.32 \mathrm{E}+00$ \\
\hline ENSBTAG00000019362 & $1.99 \mathrm{E}-10$ & $1.50 \mathrm{E}-10$ & NOA1 & $1.33 \mathrm{E}+00$ \\
\hline ENSBTAG000000010273 & $1.99 \mathrm{E}-10$ & $1.50 \mathrm{E}-10$ & EREG & $1.33 \mathrm{E}+00$ \\
\hline ENSBTAG00000021130 & $2.12 \mathrm{E}-10$ & $1.60 \mathrm{E}-10$ & MFSD10 & $1.32 \mathrm{E}+00$ \\
\hline ENSBTAG000000010875 & $9.53 \mathrm{E}-11$ & 7.20E-11 & MSX1 & $1.32 E+00$ \\
\hline ENSBTAG00000031497 & $1.38 \mathrm{E}-10$ & $1.04 \mathrm{E}-10$ & FGFBP1 & $1.33 \mathrm{E}+00$ \\
\hline ENSBTAG00000001504 & 4.65E-10 & $3.51 \mathrm{E}-10$ & NOP14 & $1.32 \mathrm{E}+00$ \\
\hline ENSBTAG00000005108 & $2.68 \mathrm{E}-10$ & $2.02 \mathrm{E}-10$ & SLIT2 & $1.33 \mathrm{E}+00$ \\
\hline ENSBTAG00000013927 & 3.53E-10 & 2.63E-10 & GSTCD & $1.34 \mathrm{E}+00$ \\
\hline ENSBTAG00000000470 & $1.61 \mathrm{E}-10$ & $1.20 \mathrm{E}-10$ & IBSP & $1.34 \mathrm{E}+00$ \\
\hline ENSBTAG00000033643 & 7.64E-10 & 5.67E-10 & & $1.35 E+00$ \\
\hline ENSBTAG00000000061 & $4.96 \mathrm{E}-10$ & $3.65 \mathrm{E}-10$ & $\mathrm{PCDH7}$ & $1.36 \mathrm{E}+00$ \\
\hline ENSBTAG00000014226 & $2.15 \mathrm{E}-10$ & $1.58 \mathrm{E}-10$ & RPL34 & $1.36 \mathrm{E}+00$ \\
\hline ENSBTAG00000024683 & $2.10 \mathrm{E}-10$ & $1.55 \mathrm{E}-10$ & & $1.36 \mathrm{E}+00$ \\
\hline ENSBTAG00000010120 & $2.86 \mathrm{E}-10$ & $2.10 \mathrm{E}-10$ & HERC3 & $1.36 \mathrm{E}+00$ \\
\hline ENSBTAG00000014521 & $4.78 \mathrm{E}-10$ & $3.51 \mathrm{E}-10$ & UGDH & $1.36 \mathrm{E}+00$ \\
\hline ENSBTAG00000010546 & $8.70 \mathrm{E}-11$ & $6.35 \mathrm{E}-11$ & LIN54 & $1.37 \mathrm{E}+00$ \\
\hline ENSBTAG00000003941 & $6.88 \mathrm{E}-10$ & $5.02 \mathrm{E}-10$ & AP1AR & $1.37 \mathrm{E}+00$ \\
\hline ENSBTAG00000045787 & $2.66 \mathrm{E}-10$ & $1.94 \mathrm{E}-10$ & CDS1 & $1.37 E+00$ \\
\hline ENSBTAG00000034700 & $2.95 \mathrm{E}-10$ & $2.14 \mathrm{E}-10$ & NAF1 & $1.38 \mathrm{E}+00$ \\
\hline ENSBTAG00000014515 & 4.10E-10 & $2.97 \mathrm{E}-10$ & & $1.38 \mathrm{E}+00$ \\
\hline ENSBTAG00000010571 & $4.51 \mathrm{E}-10$ & $3.28 \mathrm{E}-10$ & PPAT & $1.38 \mathrm{E}+00$ \\
\hline ENSBTAG00000043958 & $3.76 \mathrm{E}-10$ & $2.72 \mathrm{E}-10$ & TMEM33 & $1.38 \mathrm{E}+00$ \\
\hline ENSBTAG00000038148 & $2.46 \mathrm{E}-10$ & $1.77 \mathrm{E}-10$ & SOWAHB & $1.39 E+00$ \\
\hline ENSBTAG00000007316 & $2.26 \mathrm{E}-10$ & $1.62 \mathrm{E}-10$ & NDST3 & $1.39 \mathrm{E}+00$ \\
\hline ENSBTAG00000014520 & $1.45 \mathrm{E}-10$ & $1.04 \mathrm{E}-10$ & LIAS & $1.39 E+00$ \\
\hline ENSBTAG00000007190 & $2.47 \mathrm{E}-10$ & $1.76 \mathrm{E}-10$ & THAP6 & $1.40 \mathrm{E}+00$ \\
\hline ENSBTAG00000005711 & $6.34 \mathrm{E}-10$ & $4.46 \mathrm{E}-10$ & & $1.42 \mathrm{E}+00$ \\
\hline ENSBTAG00000006507 & $5.43 \mathrm{E}-10$ & $3.81 E-10$ & ADAMTS3 & $1.43 E+00$ \\
\hline ENSBTAG00000037558 & $3.53 \mathrm{E}-10$ & $2.47 \mathrm{E}-10$ & & $1.43 \mathrm{E}+00$ \\
\hline ENSBTAG00000001725 & $3.14 \mathrm{E}-10$ & $2.20 \mathrm{E}-10$ & CXCL10 & $1.43 E+00$ \\
\hline ENSBTAG00000009833 & $5.98 \mathrm{E}-10$ & $4.18 \mathrm{E}-10$ & DTHD1 & $1.43 E+00$ \\
\hline ENSBTAG00000020647 & $1.65 \mathrm{E}-10$ & $1.16 \mathrm{E}-10$ & RASL11B & $1.42 \mathrm{E}+00$ \\
\hline ENSBTAG00000017496 & $4.79 \mathrm{E}-10$ & $3.35 \mathrm{E}-10$ & & $1.43 \mathrm{E}+00$ \\
\hline ENSBTAG00000014616 & $1.62 \mathrm{E}-10$ & $1.13 \mathrm{E}-10$ & SEPSECS & $1.44 \mathrm{E}+00$ \\
\hline ENSBTAG00000048273 & $3.24 \mathrm{E}-10$ & $2.24 \mathrm{E}-10$ & & $1.45 \mathrm{E}+00$ \\
\hline ENSBTAG00000012157 & $5.43 \mathrm{E}-10$ & 3.75E-10 & CCDC158 & $1.45 E+00$ \\
\hline ENSBTAG00000022449 & $5.79 \mathrm{E}-10$ & $3.99 \mathrm{E}-10$ & SCD5 & $1.45 E+00$ \\
\hline ENSBTAG00000003959 & $2.94 \mathrm{E}-10$ & $2.02 \mathrm{E}-10$ & ARHGAP24 & $1.45 E+00$ \\
\hline ENSBTAG00000001839 & $4.59 \mathrm{E}-10$ & $3.15 \mathrm{E}-10$ & OCIAD2 & $1.46 \mathrm{E}+00$ \\
\hline ENSBTAG00000009035 & $9.45 \mathrm{E}-10$ & $6.47 \mathrm{E}-10$ & CENPE & $1.46 \mathrm{E}+00$ \\
\hline
\end{tabular}




\begin{tabular}{|c|c|c|c|c|}
\hline ENSBTAG00000021729 & $1.03 \mathrm{E}-09$ & $7.01 E-10$ & & $1.46 \mathrm{E}+00$ \\
\hline ENSBTAG00000018488 & $4.89 \mathrm{E}-10$ & $3.33 \mathrm{E}-10$ & AFF1 & $1.47 E+00$ \\
\hline ENSBTAG00000004893 & $7.31 \mathrm{E}-10$ & $4.95 \mathrm{E}-10$ & ACOX3 & $1.48 \mathrm{E}+00$ \\
\hline ENSBTAG00000017240 & $2.78 \mathrm{E}-10$ & $1.88 \mathrm{E}-10$ & DGKQ & $1.48 \mathrm{E}+00$ \\
\hline ENSBTAG00000021768 & $2.04 \mathrm{E}-10$ & $1.38 \mathrm{E}-10$ & CCNG2 & $1.48 \mathrm{E}+00$ \\
\hline ENSBTAG00000009070 & $1.72 \mathrm{E}-10$ & $1.16 \mathrm{E}-10$ & NPFFR2 & $1.48 \mathrm{E}+00$ \\
\hline ENSBTAG00000005745 & 8.13E-10 & $5.43 \mathrm{E}-10$ & HPSE & $1.50 \mathrm{E}+00$ \\
\hline ENSBTAG00000015126 & 7.24E-10 & $4.83 \mathrm{E}-10$ & TNIP2 & $1.50 \mathrm{E}+00$ \\
\hline ENSBTAG00000000473 & $2.89 \mathrm{E}-10$ & $1.92 \mathrm{E}-10$ & ATP10D & $1.51 \mathrm{E}+00$ \\
\hline ENSBTAG00000010954 & $3.59 \mathrm{E}-10$ & $2.38 \mathrm{E}-10$ & ART3 & $1.51 \mathrm{E}+00$ \\
\hline ENSBTAG00000018531 & $1.66 \mathrm{E}-10$ & $1.10 \mathrm{E}-10$ & JCHAIN & $1.51 \mathrm{E}+00$ \\
\hline ENSBTAG00000010285 & 3.37E-10 & $2.23 \mathrm{E}-10$ & MMRN1 & $1.51 \mathrm{E}+00$ \\
\hline ENSBTAG00000014076 & $3.69 \mathrm{E}-10$ & $2.44 \mathrm{E}-10$ & FAM175A & $1.51 E+00$ \\
\hline ENSBTAG00000039197 & $8.40 \mathrm{E}-10$ & $5.53 \mathrm{E}-10$ & SLC2A9 & $1.52 E+00$ \\
\hline ENSBTAG00000014324 & $2.28 \mathrm{E}-10$ & $1.50 \mathrm{E}-10$ & ANTXR2 & $1.52 E+00$ \\
\hline ENSBTAG00000009438 & $4.08 \mathrm{E}-10$ & $2.68 \mathrm{E}-10$ & EPHA5 & $1.52 \mathrm{E}+00$ \\
\hline ENSBTAG00000020536 & $4.56 \mathrm{E}-10$ & 2.97E-10 & HERC6 & $1.53 E+00$ \\
\hline ENSBTAG00000034501 & $3.30 \mathrm{E}-10$ & $2.13 \mathrm{E}-10$ & CFI & $1.55 \mathrm{E}+00$ \\
\hline ENSBTAG00000016805 & $7.08 \mathrm{E}-10$ & 4.59E-10 & SGMS2 & $1.54 \mathrm{E}+00$ \\
\hline ENSBTAG00000010346 & $1.56 \mathrm{E}-10$ & $1.01 \mathrm{E}-10$ & ENAM & $1.54 \mathrm{E}+00$ \\
\hline ENSBTAG00000047613 & $6.81 \mathrm{E}-10$ & 4.40E-10 & HTRA3 & $1.55 \mathrm{E}+00$ \\
\hline ENSBTAG00000040088 & $8.75 \mathrm{E}-10$ & $5.64 \mathrm{E}-10$ & SLC9B2 & $1.55 E+00$ \\
\hline ENSBTAG00000005964 & $2.70 \mathrm{E}-10$ & $1.74 \mathrm{E}-10$ & PIGG & $1.55 E+00$ \\
\hline ENSBTAG00000013952 & $2.34 \mathrm{E}-10$ & $1.50 \mathrm{E}-10$ & HNRNPD & $1.56 \mathrm{E}+00$ \\
\hline ENSBTAG00000020583 & $2.94 \mathrm{E}-10$ & $1.89 \mathrm{E}-10$ & AASDH & $1.56 \mathrm{E}+00$ \\
\hline ENSBTAG00000027569 & $3.74 \mathrm{E}-10$ & $2.38 \mathrm{E}-10$ & APBB2 & $1.57 \mathrm{E}+00$ \\
\hline ENSBTAG00000047255 & $3.91 \mathrm{E}-10$ & $2.48 \mathrm{E}-10$ & & $1.58 \mathrm{E}+00$ \\
\hline ENSBTAG00000011935 & $3.72 \mathrm{E}-10$ & $2.36 \mathrm{E}-10$ & RASSF6 & $1.57 \mathrm{E}+00$ \\
\hline ENSBTAG00000003776 & $1.38 \mathrm{E}-10$ & $8.71 E-11$ & MSANTD1 & $1.59 E+00$ \\
\hline ENSBTAG00000047586 & $1.92 \mathrm{E}-10$ & $1.21 \mathrm{E}-10$ & NPY1R & $1.58 \mathrm{E}+00$ \\
\hline ENSBTAG00000021372 & $5.30 \mathrm{E}-10$ & $3.34 \mathrm{E}-10$ & 11-Sep & $1.59 E+00$ \\
\hline ENSBTAG00000015265 & $1.04 \mathrm{E}-10$ & $6.54 \mathrm{E}-11$ & TIGD2 & $1.59 \mathrm{E}+00$ \\
\hline ENSBTAG00000010128 & $2.55 \mathrm{E}-10$ & $1.61 \mathrm{E}-10$ & NAP1L5 & $1.59 E+00$ \\
\hline ENSBTAG00000009812 & $7.75 \mathrm{E}-11$ & $4.88 \mathrm{E}-11$ & & $1.59 E+00$ \\
\hline ENSBTAG00000047198 & $4.82 \mathrm{E}-10$ & $3.03 E-10$ & & $1.59 E+00$ \\
\hline ENSBTAG00000046440 & $1.44 \mathrm{E}-10$ & $9.07 \mathrm{E}-11$ & TRAM1L1 & $1.59 E+00$ \\
\hline ENSBTAG00000004888 & $2.73 \mathrm{E}-11$ & $1.72 \mathrm{E}-11$ & SLC10A4 & $1.58 \mathrm{E}+00$ \\
\hline ENSBTAG00000005603 & $2.58 \mathrm{E}-10$ & 1.63E-10 & CXCL11 & $1.58 \mathrm{E}+00$ \\
\hline ENSBTAG00000045839 & $5.96 \mathrm{E}-10$ & $3.75 \mathrm{E}-10$ & & $1.59 E+00$ \\
\hline ENSBTAG00000048196 & $1.42 \mathrm{E}-10$ & 8.93E-11 & & $1.59 E+00$ \\
\hline ENSBTAG00000035776 & $4.95 \mathrm{E}-10$ & $3.11 E-10$ & C4orf22 & $1.59 \mathrm{E}+00$ \\
\hline ENSBTAG00000012575 & $2.33 \mathrm{E}-10$ & 1.47E-10 & MAEA & $1.59 E+00$ \\
\hline ENSBTAG00000026250 & $1.23 \mathrm{E}-10$ & 7.77E-11 & PDHA2 & $1.59 E+00$ \\
\hline ENSBTAG00000006837 & $2.32 \mathrm{E}-10$ & $1.44 \mathrm{E}-10$ & UBA6 & $1.61 E+00$ \\
\hline ENSBTAG00000020048 & $6.53 \mathrm{E}-10$ & $4.01 \mathrm{E}-10$ & MAPK10 & $1.63 E+00$ \\
\hline ENSBTAG00000010181 & $2.16 \mathrm{E}-10$ & $1.32 \mathrm{E}-10$ & GRPEL1 & $1.63 E+00$ \\
\hline ENSBTAG00000033346 & $9.61 \mathrm{E}-11$ & 5.87E-11 & & $1.64 \mathrm{E}+00$ \\
\hline ENSBTAG00000005567 & $7.10 \mathrm{E}-11$ & $4.29 \mathrm{E}-11$ & BEND4 & $1.65 E+00$ \\
\hline ENSBTAG00000005668 & $4.38 \mathrm{E}-10$ & $2.61 \mathrm{E}-10$ & SLC39A8 & $1.68 \mathrm{E}+00$ \\
\hline ENSBTAG00000045714 & $2.78 \mathrm{E}-10$ & $1.64 \mathrm{E}-10$ & & $1.69 E+00$ \\
\hline ENSBTAG00000006156 & $6.49 \mathrm{E}-10$ & 3.80E-10 & BST1 & $1.71 E+00$ \\
\hline ENSBTAG00000020031 & $1.89 \mathrm{E}-10$ & $1.11 \mathrm{E}-10$ & PKD2 & $1.70 E+00$ \\
\hline ENSBTAG00000003652 & $2.31 \mathrm{E}-10$ & $1.35 \mathrm{E}-10$ & TMEM128 & $1.71 E+00$ \\
\hline ENSBTAG00000015047 & $8.06 \mathrm{E}-10$ & 4.70E-10 & & $1.72 \mathrm{E}+00$ \\
\hline ENSBTAG00000007986 & $2.89 \mathrm{E}-10$ & $1.68 \mathrm{E}-10$ & WHSC1 & $1.72 \mathrm{E}+00$ \\
\hline ENSBTAG00000034411 & $2.99 \mathrm{E}-10$ & $1.74 \mathrm{E}-10$ & SHISA3 & $1.72 \mathrm{E}+00$ \\
\hline ENSBTAG00000001600 & $2.24 \mathrm{E}-10$ & 1.30E-10 & & $1.72 \mathrm{E}+00$ \\
\hline ENSBTAG00000016795 & $2.43 \mathrm{E}-10$ & $1.40 \mathrm{E}-10$ & RUFY3 & $1.73 E+00$ \\
\hline ENSBTAG00000002049 & $5.61 \mathrm{E}-10$ & $3.24 \mathrm{E}-10$ & $\mathrm{HADH}$ & $1.73 E+00$ \\
\hline ENSBTAG00000039991 & $5.27 \mathrm{E}-10$ & $3.02 \mathrm{E}-10$ & & $1.74 \mathrm{E}+00$ \\
\hline ENSBTAG00000012397 & $1.76 \mathrm{E}-10$ & $9.95 \mathrm{E}-11$ & DCK & $1.77 \mathrm{E}+00$ \\
\hline ENSBTAG00000017921 & $3.49 \mathrm{E}-10$ & $1.98 \mathrm{E}-10$ & NELFA & $1.76 \mathrm{E}+00$ \\
\hline ENSBTAG00000031750 & $2.31 \mathrm{E}-10$ & $1.31 \mathrm{E}-10$ & PLAC8 & $1.76 \mathrm{E}+00$ \\
\hline ENSBTAG00000039362 & $5.20 \mathrm{E}-10$ & $2.93 \mathrm{E}-10$ & & $1.78 \mathrm{E}+00$ \\
\hline ENSBTAG00000031364 & $3.10 \mathrm{E}-10$ & $1.74 \mathrm{E}-10$ & & $1.78 \mathrm{E}+00$ \\
\hline ENSBTAG00000014512 & $3.59 \mathrm{E}-10$ & 2.00E-10 & WDR19 & $1.79 E+00$ \\
\hline ENSBTAG00000013451 & $2.51 \mathrm{E}-10$ & $1.39 \mathrm{E}-10$ & $\mathrm{RHOH}$ & $1.80 \mathrm{E}+00$ \\
\hline ENSBTAG00000014041 & $8.50 \mathrm{E}-11$ & 4.63E-11 & G3BP2 & $1.83 E+00$ \\
\hline ENSBTAG00000011042 & $1.31 \mathrm{E}-10$ & $7.05 \mathrm{E}-11$ & TMEM129 & $1.85 \mathrm{E}+00$ \\
\hline ENSBTAG00000012343 & $4.95 \mathrm{E}-10$ & $2.67 \mathrm{E}-10$ & TSPAN5 & $1.85 \mathrm{E}+00$ \\
\hline
\end{tabular}




\begin{tabular}{|c|c|c|c|c|}
\hline ENSBTAG00000001603 & $5.41 \mathrm{E}-10$ & $2.91 \mathrm{E}-10$ & YIPF7 & $1.86 \mathrm{E}+00$ \\
\hline ENSBTAG00000011611 & $6.27 \mathrm{E}-10$ & $3.36 \mathrm{E}-10$ & OSTC & $1.87 \mathrm{E}+00$ \\
\hline ENSBTAG00000038421 & $7.52 \mathrm{E}-10$ & $4.01 \mathrm{E}-10$ & NPY5R & $1.88 \mathrm{E}+00$ \\
\hline ENSBTAG00000012111 & $4.22 \mathrm{E}-10$ & $2.25 \mathrm{E}-10$ & PRDM5 & $1.87 \mathrm{E}+00$ \\
\hline ENSBTAG00000006683 & $3.97 \mathrm{E}-10$ & $2.10 \mathrm{E}-10$ & ZFYVE28 & $1.89 \mathrm{E}+00$ \\
\hline ENSBTAG00000002081 & $4.51 \mathrm{E}-10$ & $2.39 \mathrm{E}-10$ & BMPR1B & $1.89 \mathrm{E}+00$ \\
\hline ENSBTAG00000015449 & $6.07 \mathrm{E}-10$ & $3.21 \mathrm{E}-10$ & PPEF2 & $1.89 \mathrm{E}+00$ \\
\hline ENSBTAG00000003443 & $1.16 \mathrm{E}-10$ & $6.11 \mathrm{E}-11$ & USP46 & $1.90 \mathrm{E}+00$ \\
\hline ENSBTAG00000007001 & $3.87 \mathrm{E}-10$ & $2.03 \mathrm{E}-10$ & SLC26A1 & $1.91 E+00$ \\
\hline ENSBTAG00000017592 & $6.23 \mathrm{E}-10$ & $3.23 \mathrm{E}-10$ & GPAT3 & $1.93 \mathrm{E}+00$ \\
\hline ENSBTAG00000019366 & $6.26 \mathrm{E}-10$ & $3.22 \mathrm{E}-10$ & POLR2B & $1.94 \mathrm{E}+00$ \\
\hline ENSBTAG00000017131 & 1.67E-10 & $8.62 \mathrm{E}-11$ & AFP & $1.94 \mathrm{E}+00$ \\
\hline ENSBTAG00000002928 & $4.30 \mathrm{E}-10$ & $2.20 \mathrm{E}-10$ & AMTN & $1.95 \mathrm{E}+00$ \\
\hline ENSBTAG00000011952 & $5.46 \mathrm{E}-10$ & $2.77 \mathrm{E}-10$ & SULT1E1 & $1.97 \mathrm{E}+00$ \\
\hline ENSBTAG00000001323 & $2.60 \mathrm{E}-10$ & $1.30 \mathrm{E}-10$ & CENPC & $2.00 \mathrm{E}+00$ \\
\hline ENSBTAG00000003897 & $2.77 \mathrm{E}-10$ & $1.38 \mathrm{E}-10$ & & $2.00 E+00$ \\
\hline ENSBTAG00000017121 & $1.70 \mathrm{E}-10$ & $8.43 \mathrm{E}-11$ & ALB & $2.01 E+00$ \\
\hline ENSBTAG00000017837 & $3.70 \mathrm{E}-10$ & $1.83 \mathrm{E}-10$ & GABRB1 & $2.02 \mathrm{E}+00$ \\
\hline ENSBTAG00000013495 & $1.85 \mathrm{E}-10$ & 8.92E-11 & WDFY3 & $2.07 E+00$ \\
\hline ENSBTAG00000002526 & $8.64 \mathrm{E}-10$ & 4.15E-10 & $\mathrm{BDH} 2$ & $2.08 \mathrm{E}+00$ \\
\hline ENSBTAG00000038520 & $6.28 \mathrm{E}-10$ & $3.02 \mathrm{E}-10$ & & $2.08 \mathrm{E}+00$ \\
\hline ENSBTAG00000002455 & $1.86 \mathrm{E}-10$ & 8.93E-11 & KLHL8 & $2.09 \mathrm{E}+00$ \\
\hline ENSBTAG00000038639 & $1.41 \mathrm{E}-10$ & $6.67 \mathrm{E}-11$ & CXCL9 & $2.12 \mathrm{E}+00$ \\
\hline ENSBTAG00000020658 & $6.62 \mathrm{E}-10$ & $3.13 \mathrm{E}-10$ & LNX1 & $2.11 E+00$ \\
\hline ENSBTAG00000002978 & $9.48 \mathrm{E}-10$ & $4.48 \mathrm{E}-10$ & PRKG2 & $2.12 \mathrm{E}+00$ \\
\hline ENSBTAG00000013918 & $3.44 \mathrm{E}-10$ & $1.62 \mathrm{E}-10$ & ADGRL3 & $2.12 \mathrm{E}+00$ \\
\hline ENSBTAG00000011044 & $2.40 \mathrm{E}-10$ & $1.12 \mathrm{E}-10$ & & $2.14 \mathrm{E}+00$ \\
\hline ENSBTAG00000017809 & $1.10 \mathrm{E}-10$ & $5.05 \mathrm{E}-11$ & PDS5A & $2.17 \mathrm{E}+00$ \\
\hline ENSBTAG00000007164 & $2.73 \mathrm{E}-10$ & $1.25 \mathrm{E}-10$ & FGFR3 & $2.18 \mathrm{E}+00$ \\
\hline ENSBTAG00000039245 & $5.45 \mathrm{E}-10$ & $2.49 \mathrm{E}-10$ & GK2 & $2.19 E+00$ \\
\hline ENSBTAG00000011789 & $2.86 \mathrm{E}-10$ & $1.28 \mathrm{E}-10$ & & $2.24 \mathrm{E}+00$ \\
\hline ENSBTAG00000016247 & $5.63 \mathrm{E}-10$ & $2.51 \mathrm{E}-10$ & TACR3 & $2.24 \mathrm{E}+00$ \\
\hline ENSBTAG00000003357 & $2.18 \mathrm{E}-10$ & $9.51 E-11$ & CLRN2 & $2.29 E+00$ \\
\hline ENSBTAG00000024957 & $4.36 \mathrm{E}-10$ & $1.89 \mathrm{E}-10$ & SNCA & $2.30 \mathrm{E}+00$ \\
\hline ENSBTAG00000001572 & $1.47 \mathrm{E}-10$ & $6.34 \mathrm{E}-11$ & NSUN7 & $2.32 E+00$ \\
\hline ENSBTAG00000020648 & $4.18 \mathrm{E}-10$ & $1.78 \mathrm{E}-10$ & SCFD2 & $2.35 E+00$ \\
\hline ENSBTAG00000005260 & $1.40 \mathrm{E}-10$ & $5.89 E-11$ & SPP1 & $2.38 \mathrm{E}+00$ \\
\hline ENSBTAG00000010896 & $1.97 \mathrm{E}-10$ & $8.25 \mathrm{E}-11$ & NKX3-2 & $2.38 \mathrm{E}+00$ \\
\hline ENSBTAG00000018155 & $3.97 \mathrm{E}-10$ & $1.66 \mathrm{E}-10$ & MRPS18C & $2.39 E+00$ \\
\hline ENSBTAG00000005005 & $2.80 \mathrm{E}-10$ & 1.17E-10 & & $2.39 E+00$ \\
\hline ENSBTAG00000018379 & $3.71 E-10$ & $1.56 \mathrm{E}-10$ & TMEM175 & $2.38 \mathrm{E}+00$ \\
\hline ENSBTAG00000019490 & $3.30 \mathrm{E}-10$ & $1.38 \mathrm{E}-10$ & & $2.39 E+00$ \\
\hline ENSBTAG00000012969 & $3.64 \mathrm{E}-10$ & $1.52 \mathrm{E}-10$ & DKK2 & $2.39 E+00$ \\
\hline ENSBTAG00000019761 & $6.35 \mathrm{E}-10$ & $2.64 \mathrm{E}-10$ & MANBA & $2.40 \mathrm{E}+00$ \\
\hline ENSBTAG00000014803 & $5.00 \mathrm{E}-10$ & $2.08 \mathrm{E}-10$ & $\mathrm{CPZ}$ & $2.40 \mathrm{E}+00$ \\
\hline ENSBTAG00000009742 & $3.63 \mathrm{E}-10$ & $1.50 \mathrm{E}-10$ & DOK7 & $2.42 \mathrm{E}+00$ \\
\hline ENSBTAG00000032588 & $9.09 \mathrm{E}-10$ & $3.73 \mathrm{E}-10$ & TMEM150C & $2.44 E+00$ \\
\hline ENSBTAG00000005744 & $9.89 \mathrm{E}-10$ & $4.04 \mathrm{E}-10$ & COQ2 & $2.45 E+00$ \\
\hline ENSBTAG00000002348 & $3.41 \mathrm{E}-10$ & $1.39 \mathrm{E}-10$ & SLC4A4 & $2.45 \mathrm{E}+00$ \\
\hline ENSBTAG00000012577 & $4.39 \mathrm{E}-10$ & $1.79 \mathrm{E}-10$ & UVSSA & $2.45 E+00$ \\
\hline ENSBTAG00000007240 & $3.41 \mathrm{E}-10$ & $1.39 \mathrm{E}-10$ & $\mathrm{ZCCHC4}$ & $2.45 E+00$ \\
\hline ENSBTAG00000020298 & $2.03 \mathrm{E}-10$ & $8.24 \mathrm{E}-11$ & TMPRSS11A & $2.46 \mathrm{E}+00$ \\
\hline ENSBTAG00000014517 & $3.06 \mathrm{E}-10$ & $1.24 \mathrm{E}-10$ & KLB & $2.47 \mathrm{E}+00$ \\
\hline ENSBTAG00000004316 & $3.35 \mathrm{E}-10$ & $1.33 \mathrm{E}-10$ & BOD1L1 & $2.52 \mathrm{E}+00$ \\
\hline ENSBTAG00000000782 & $3.53 \mathrm{E}-10$ & $1.40 \mathrm{E}-10$ & KDR & $2.52 \mathrm{E}+00$ \\
\hline ENSBTAG00000014518 & $2.76 \mathrm{E}-10$ & $1.08 \mathrm{E}-10$ & & $2.55 E+00$ \\
\hline ENSBTAG00000013718 & $6.66 \mathrm{E}-10$ & $2.60 \mathrm{E}-10$ & GC & $2.56 \mathrm{E}+00$ \\
\hline ENSBTAG00000021940 & $3.19 \mathrm{E}-10$ & $1.24 \mathrm{E}-10$ & CCDC149 & $2.57 E+00$ \\
\hline ENSBTAG00000010577 & $6.31 \mathrm{E}-10$ & $2.45 \mathrm{E}-10$ & & $2.58 \mathrm{E}+00$ \\
\hline ENSBTAG00000002299 & $5.01 \mathrm{E}-10$ & $1.94 \mathrm{E}-10$ & SEL1L3 & $2.58 \mathrm{E}+00$ \\
\hline ENSBTAG00000016290 & $2.49 \mathrm{E}-10$ & $9.42 \mathrm{E}-11$ & MOB1B & $2.64 \mathrm{E}+00$ \\
\hline ENSBTAG00000001925 & $6.23 \mathrm{E}-10$ & $2.35 \mathrm{E}-10$ & TMPRSS11D & $2.65 E+00$ \\
\hline ENSBTAG00000007002 & $3.90 \mathrm{E}-10$ & $1.46 \mathrm{E}-10$ & IDUA & $2.67 E+00$ \\
\hline ENSBTAG00000021128 & $5.56 \mathrm{E}-10$ & $2.08 \mathrm{E}-10$ & ADD1 & $2.67 E+00$ \\
\hline ENSBTAG00000014017 & $2.90 \mathrm{E}-10$ & $1.08 \mathrm{E}-10$ & & $2.68 \mathrm{E}+00$ \\
\hline ENSBTAG00000017488 & $1.65 \mathrm{E}-10$ & $6.11 E-11$ & KLF3 & $2.70 E+00$ \\
\hline ENSBTAG00000044161 & $8.45 \mathrm{E}-10$ & $3.08 \mathrm{E}-10$ & NMU & $2.74 \mathrm{E}+00$ \\
\hline ENSBTAG00000020541 & $2.05 \mathrm{E}-10$ & 7.37E-11 & PYURF & $2.78 \mathrm{E}+00$ \\
\hline ENSBTAG00000019849 & $2.74 \mathrm{E}-10$ & $9.86 \mathrm{E}-11$ & CABS1 & $2.78 \mathrm{E}+00$ \\
\hline ENSBTAG00000019368 & $4.27 \mathrm{E}-10$ & $1.53 \mathrm{E}-10$ & IGFBP7 & $2.79 E+00$ \\
\hline
\end{tabular}




\begin{tabular}{|c|c|c|c|c|}
\hline ENSBTAG00000020270 & 4.87E-10 & $1.75 \mathrm{E}-10$ & NFKB1 & $2.78 \mathrm{E}+00$ \\
\hline ENSBTAG00000004942 & $2.25 \mathrm{E}-10$ & $8.09 \mathrm{E}-11$ & EXOSC9 & $2.78 \mathrm{E}+00$ \\
\hline ENSBTAG00000004412 & $6.50 \mathrm{E}-10$ & $2.32 \mathrm{E}-10$ & TMPRSS11F & $2.80 \mathrm{E}+00$ \\
\hline ENSBTAG00000020653 & $1.83 \mathrm{E}-10$ & $6.48 \mathrm{E}-11$ & FIP1L1 & $2.82 \mathrm{E}+00$ \\
\hline ENSBTAG00000022450 & $2.03 \mathrm{E}-10$ & $6.86 \mathrm{E}-11$ & THAP9 & $2.95 \mathrm{E}+00$ \\
\hline ENSBTAG00000010955 & $1.81 \mathrm{E}-10$ & 6.07E-11 & NUP54 & $2.98 \mathrm{E}+00$ \\
\hline ENSBTAG00000031553 & $3.28 \mathrm{E}-10$ & $1.06 \mathrm{E}-10$ & MFSD7 & $3.09 E+00$ \\
\hline ENSBTAG00000012961 & 3.93E-10 & 1.27E-10 & & $3.10 \mathrm{E}+00$ \\
\hline ENSBTAG00000032350 & $5.52 \mathrm{E}-10$ & $1.78 \mathrm{E}-10$ & & $3.10 \mathrm{E}+00$ \\
\hline ENSBTAG00000002247 & 2.22E-10 & 7.06E-11 & & $3.14 E+00$ \\
\hline ENSBTAG00000011354 & $2.17 \mathrm{E}-10$ & $6.91 \mathrm{E}-11$ & NUDT9 & $3.14 E+00$ \\
\hline ENSBTAG00000019584 & $6.58 \mathrm{E}-10$ & $2.08 \mathrm{E}-10$ & & $3.16 \mathrm{E}+00$ \\
\hline ENSBTAG00000009310 & $2.30 \mathrm{E}-10$ & $7.24 \mathrm{E}-11$ & UTP3 & $3.18 \mathrm{E}+00$ \\
\hline ENSBTAG00000046936 & $1.56 \mathrm{E}-10$ & $4.91 \mathrm{E}-11$ & MRFAP1 & $3.17 E+00$ \\
\hline ENSBTAG00000010634 & $4.09 \mathrm{E}-10$ & $1.29 \mathrm{E}-10$ & NDNF & $3.17 \mathrm{E}+00$ \\
\hline ENSBTAG00000040324 & $2.74 \mathrm{E}-10$ & 8.62E-11 & & $3.18 \mathrm{E}+00$ \\
\hline ENSBTAG00000038232 & $2.24 \mathrm{E}-09$ & $6.94 \mathrm{E}-10$ & & $3.22 \mathrm{E}+00$ \\
\hline ENSBTAG00000013607 & $3.77 \mathrm{E}-10$ & 1.17E-10 & WDR1 & $3.22 \mathrm{E}+00$ \\
\hline ENSBTAG00000000285 & $3.21 \mathrm{E}-10$ & $9.67 \mathrm{E}-11$ & GUF1 & $3.32 \mathrm{E}+00$ \\
\hline ENSBTAG00000015572 & $2.09 \mathrm{E}-10$ & $6.23 \mathrm{E}-11$ & YTHDC1 & $3.35 E+00$ \\
\hline ENSBTAG00000012972 & 3.93E-10 & 1.15E-10 & CYP2U1 & $3.42 \mathrm{E}+00$ \\
\hline ENSBTAG00000000287 & $1.78 \mathrm{E}-10$ & $5.09 \mathrm{E}-11$ & GNPDA2 & $3.49 \mathrm{E}+00$ \\
\hline ENSBTAG00000002632 & $6.37 \mathrm{E}-10$ & $1.82 \mathrm{E}-10$ & CSN2 & $3.50 \mathrm{E}+00$ \\
\hline ENSBTAG00000008577 & $3.43 \mathrm{E}-10$ & $9.40 \mathrm{E}-11$ & GRSF1 & $3.65 E+00$ \\
\hline ENSBTAG00000006950 & $3.15 \mathrm{E}-10$ & $8.55 \mathrm{E}-11$ & COPS4 & $3.68 \mathrm{E}+00$ \\
\hline ENSBTAG00000014913 & $5.86 \mathrm{E}-10$ & $1.55 \mathrm{E}-10$ & SRD5A3 & $3.78 \mathrm{E}+00$ \\
\hline ENSBTAG00000010593 & $6.83 \mathrm{E}-10$ & $1.81 \mathrm{E}-10$ & SRP72 & $3.77 \mathrm{E}+00$ \\
\hline ENSBTAG00000027825 & 7.33E-10 & $1.90 \mathrm{E}-10$ & & $3.86 \mathrm{E}+00$ \\
\hline ENSBTAG00000014601 & $1.79 \mathrm{E}-10$ & $4.58 \mathrm{E}-11$ & SGCB & $3.90 \mathrm{E}+00$ \\
\hline ENSBTAG00000004428 & $3.10 \mathrm{E}-10$ & $7.80 \mathrm{E}-11$ & $\mathrm{H} 2 \mathrm{AFZ}$ & $3.97 \mathrm{E}+00$ \\
\hline ENSBTAG00000019132 & $5.63 \mathrm{E}-10$ & $1.41 \mathrm{E}-10$ & DMP1 & $3.99 E+00$ \\
\hline ENSBTAG00000004793 & $1.45 \mathrm{E}-10$ & $3.65 \mathrm{E}-11$ & AMBN & $3.96 \mathrm{E}+00$ \\
\hline ENSBTAG00000009849 & 4.20E-10 & $1.04 \mathrm{E}-10$ & & $4.03 E+00$ \\
\hline ENSBTAG00000024826 & $7.26 \mathrm{E}-10$ & $1.79 \mathrm{E}-10$ & TECRL & $4.05 E+00$ \\
\hline ENSBTAG00000040398 & $6.78 \mathrm{E}-10$ & $1.67 \mathrm{E}-10$ & MGC148692 & $4.06 \mathrm{E}+00$ \\
\hline ENSBTAG00000004912 & $1.25 \mathrm{E}-10$ & $2.96 \mathrm{E}-11$ & ANKRD17 & $4.20 \mathrm{E}+00$ \\
\hline ENSBTAG00000032560 & $9.08 \mathrm{E}-10$ & $2.10 \mathrm{E}-10$ & & $4.32 E+00$ \\
\hline ENSBTAG00000039922 & $4.61 \mathrm{E}-10$ & $1.02 \mathrm{E}-10$ & ARAP2 & $4.52 \mathrm{E}+00$ \\
\hline ENSBTAG00000044044 & $3.04 \mathrm{E}-10$ & $6.67 \mathrm{E}-11$ & CLOCK & $4.56 \mathrm{E}+00$ \\
\hline ENSBTAG00000031679 & $4.55 \mathrm{E}-10$ & $9.95 \mathrm{E}-11$ & HSD17B13 & $4.57 E+00$ \\
\hline ENSBTAG00000004797 & $8.70 \mathrm{E}-10$ & $1.82 \mathrm{E}-10$ & TRMT44 & $4.78 \mathrm{E}+00$ \\
\hline ENSBTAG00000045812 & $6.17 \mathrm{E}-10$ & $1.29 \mathrm{E}-10$ & GSX2 & $4.78 \mathrm{E}+00$ \\
\hline ENSBTAG00000020367 & $2.58 \mathrm{E}-10$ & $5.32 \mathrm{E}-11$ & SLC30A9 & $4.83 E+00$ \\
\hline ENSBTAG00000009471 & $8.19 \mathrm{E}-10$ & $1.57 \mathrm{E}-10$ & CEP135 & $5.21 E+00$ \\
\hline ENSBTAG00000005394 & $1.56 \mathrm{E}-10$ & $2.95 \mathrm{E}-11$ & $\operatorname{cox} 18$ & $5.28 \mathrm{E}+00$ \\
\hline ENSBTAG00000020570 & $2.38 \mathrm{E}-10$ & 4.49E-11 & & $5.29 E+00$ \\
\hline ENSBTAG00000008480 & $2.83 \mathrm{E}-10$ & 5.27E-11 & СТВP1 & $5.36 \mathrm{E}+00$ \\
\hline ENSBTAG00000018151 & $3.39 \mathrm{E}-10$ & $6.08 \mathrm{E}-11$ & & $5.56 \mathrm{E}+00$ \\
\hline ENSBTAG00000032660 & $5.29 \mathrm{E}-10$ & $9.20 \mathrm{E}-11$ & $\mathrm{CHIC2}$ & $5.74 \mathrm{E}+00$ \\
\hline ENSBTAG00000002699 & $5.73 \mathrm{E}-10$ & $9.29 \mathrm{E}-11$ & KIT & $6.17 E+00$ \\
\hline ENSBTAG00000001249 & $6.66 \mathrm{E}-10$ & $1.07 \mathrm{E}-10$ & SULT1B1 & $6.22 \mathrm{E}+00$ \\
\hline ENSBTAG00000001269 & $3.44 \mathrm{E}-10$ & $5.50 \mathrm{E}-11$ & TMEM165 & $6.24 \mathrm{E}+00$ \\
\hline ENSBTAG00000001772 & $5.09 \mathrm{E}-10$ & $8.09 \mathrm{E}-11$ & PDCL2 & $6.28 \mathrm{E}+00$ \\
\hline ENSBTAG00000048013 & $3.06 \mathrm{E}-10$ & $4.68 \mathrm{E}-11$ & & $6.52 E+00$ \\
\hline ENSBTAG00000010615 & $7.46 \mathrm{E}-10$ & $1.14 \mathrm{E}-10$ & & $6.54 E+00$ \\
\hline ENSBTAG00000017642 & $6.17 \mathrm{E}-10$ & $9.38 \mathrm{E}-11$ & BMP3 & $6.57 E+00$ \\
\hline ENSBTAG00000040337 & $5.65 \mathrm{E}-10$ & 8.47E-11 & & $6.67 \mathrm{E}+00$ \\
\hline ENSBTAG00000017348 & $1.83 \mathrm{E}-10$ & $2.71 \mathrm{E}-11$ & FGF5 & $6.75 E+00$ \\
\hline ENSBTAG00000045602 & $4.26 \mathrm{E}-10$ & $6.11 E-11$ & & $6.96 \mathrm{E}+00$ \\
\hline ENSBTAG00000048250 & $8.53 \mathrm{E}-10$ & $1.19 \mathrm{E}-10$ & & $7.16 \mathrm{E}+00$ \\
\hline ENSBTAG00000037613 & 5.37E-10 & $6.93 \mathrm{E}-11$ & & $7.74 \mathrm{E}+00$ \\
\hline ENSBTAG00000000872 & 4.70E-10 & $5.90 \mathrm{E}-11$ & & $7.96 \mathrm{E}+00$ \\
\hline ENSBTAG00000005078 & $6.34 \mathrm{E}-10$ & 7.94E-11 & UCHL1 & $7.97 \mathrm{E}+00$ \\
\hline ENSBTAG00000015464 & $1.19 \mathrm{E}-09$ & $1.23 \mathrm{E}-10$ & & $9.64 E+00$ \\
\hline ENSBTAG00000007695 & $7.42 \mathrm{E}-10$ & $7.65 \mathrm{E}-11$ & CSN1S1 & $9.69 E+00$ \\
\hline ENSBTAG00000037778 & $5.71 \mathrm{E}-10$ & 5.11E-11 & & $1.11 \mathrm{E}+01$ \\
\hline ENSBTAG00000045917 & $9.12 \mathrm{E}-10$ & $7.60 \mathrm{E}-11$ & & $1.20 \mathrm{E}+01$ \\
\hline ENSBTAG00000014805 & $9.40 \mathrm{E}-10$ & 7.76E-11 & ENOPH1 & $1.21 \mathrm{E}+01$ \\
\hline ENSBTAG00000007173 & $6.74 \mathrm{E}-10$ & $5.40 \mathrm{E}-11$ & PDGFRA & $1.25 \mathrm{E}+01$ \\
\hline ENSBTAG00000039787 & $6.83 \mathrm{E}-10$ & $5.03 \mathrm{E}-11$ & CSN3 & $1.35 E+01$ \\
\hline
\end{tabular}




\begin{tabular}{|c|c|c|c|c|}
\hline ENSBTAG00000003523 & $8.16 \mathrm{E}-10$ & $5.58 \mathrm{E}-11$ & & $1.46 \mathrm{E}+01$ \\
\hline ENSBTAG00000039647 & $6.61 \mathrm{E}-10$ & $4.26 \mathrm{E}-11$ & & $1.55 \mathrm{E}+01$ \\
\hline ENSBTAG00000032637 & $9.50 \mathrm{E}-10$ & $6.07 \mathrm{E}-11$ & EXOC1 & $1.56 \mathrm{E}+01$ \\
\hline ENSBTAG00000040333 & $6.95 \mathrm{E}-10$ & $3.81 E-11$ & QDPR & $1.82 \mathrm{E}+01$ \\
\hline ENSBTAG00000014804 & $6.01 \mathrm{E}-10$ & $3.14 \mathrm{E}-11$ & HNRNPDL & $1.91 \mathrm{E}+01$ \\
\hline ENSBTAG00000014599 & $6.20 \mathrm{E}-10$ & $2.43 \mathrm{E}-11$ & LRRC66 & $2.54 \mathrm{E}+01$ \\
\hline ENSBTAG00000025920 & $3.69 \mathrm{E}-10$ & $1.23 \mathrm{E}-11$ & & $2.98 \mathrm{E}+01$ \\
\hline ENSBTAG00000038214 & $7.61 \mathrm{E}-10$ & $1.97 \mathrm{E}-11$ & & $3.84 \mathrm{E}+01$ \\
\hline ENSBTAG00000004886 & 7.93E-11 & 0 & ZAR1 & $7.94 E+02$ \\
\hline ENSBTAG00000006810 & $5.67 \mathrm{E}-10$ & 0 & ODAM & $5.67 E+03$ \\
\hline ENSBTAG00000004261 & $2.27 E-10$ & 0 & SPON2 & $2.27 E+03$ \\
\hline ENSBTAG00000047810 & $6.40 \mathrm{E}-11$ & 0 & CCDC96 & $6.41 E+02$ \\
\hline ENSBTAG00000034844 & 0 & 0 & RPL39 & $1.00 \mathrm{E}+00$ \\
\hline ENSBTAG00000034849 & $1.08 \mathrm{E}-09$ & 0 & & $1.08 \mathrm{E}+04$ \\
\hline ENSBTAG00000038368 & $3.55 E-10$ & 0 & SNRPG & $3.56 \mathrm{E}+03$ \\
\hline ENSBTAG00000046949 & 0 & 0 & & $1.00 E+00$ \\
\hline ENSBTAG00000047821 & 0 & 0 & & $1.00 E+00$ \\
\hline ENSBTAG00000011973 & 0 & 0 & DCAF16 & $1.00 \mathrm{E}+00$ \\
\hline ENSBTAG00000032022 & 0 & 0 & & $1.00 E+00$ \\
\hline ENSBTAG00000013149 & $2.38 \mathrm{E}-10$ & 0 & NAA11 & $2.38 \mathrm{E}+03$ \\
\hline ENSBTAG00000032034 & $6.10 \mathrm{E}-10$ & 0 & & $6.10 E+03$ \\
\hline ENSBTAG00000034901 & 0 & $1.39 \mathrm{E}-10$ & HIST3H2BB & 7.19E-04 \\
\hline ENSBTAG00000047589 & 0 & $8.46 \mathrm{E}-11$ & & $1.18 \mathrm{E}-03$ \\
\hline ENSBTAG00000011398 & 0 & $1.00 \mathrm{E}-10$ & PRR7 & 9.99E-04 \\
\hline ENSBTAG00000025401 & 0 & $2.03 \mathrm{E}-11$ & DOK3 & 4.90E-03 \\
\hline ENSBTAG00000046245 & 0 & $2.24 \mathrm{E}-10$ & & $4.46 \mathrm{E}-04$ \\
\hline ENSBTAG00000006662 & 0 & $8.35 \mathrm{E}-11$ & & $1.20 \mathrm{E}-03$ \\
\hline ENSBTAG00000012046 & 0 & $1.14 \mathrm{E}-10$ & JUNB & $8.76 \mathrm{E}-04$ \\
\hline ENSBTAG00000004714 & $7.08 \mathrm{E}-11$ & $4.02 \mathrm{E}-10$ & MCEMP1 & $1.76 \mathrm{E}-01$ \\
\hline ENSBTAG00000016721 & $1.03 \mathrm{E}-10$ & $4.34 \mathrm{E}-10$ & ZNF791 & $2.38 \mathrm{E}-01$ \\
\hline ENSBTAG00000017912 & $8.50 \mathrm{E}-11$ & $3.22 \mathrm{E}-10$ & & 2.64E-01 \\
\hline ENSBTAG00000019909 & $9.94 \mathrm{E}-12$ & $3.76 \mathrm{E}-11$ & SIRT6 & $2.66 \mathrm{E}-01$ \\
\hline ENSBTAG00000009737 & $2.81 \mathrm{E}-11$ & $1.06 \mathrm{E}-10$ & LTC4S & $2.66 \mathrm{E}-01$ \\
\hline ENSBTAG00000035966 & $1.68 \mathrm{E}-10$ & $6.21 \mathrm{E}-10$ & GRXCR2 & 2.70E-01 \\
\hline ENSBTAG00000014835 & $3.41 \mathrm{E}-10$ & 1.12E-09 & SPARC & 3.04E-01 \\
\hline ENSBTAG00000000595 & $1.58 \mathrm{E}-10$ & $4.89 \mathrm{E}-10$ & FBN3 & $3.22 \mathrm{E}-01$ \\
\hline ENSBTAG00000040028 & $3.42 \mathrm{E}-10$ & $1.05 \mathrm{E}-09$ & & $3.26 \mathrm{E}-01$ \\
\hline ENSBTAG00000047519 & $4.42 \mathrm{E}-10$ & $1.23 \mathrm{E}-09$ & & 3.59E-01 \\
\hline ENSBTAG00000007350 & $3.18 \mathrm{E}-10$ & $8.85 \mathrm{E}-10$ & & 3.59E-01 \\
\hline ENSBTAG00000019950 & $2.74 \mathrm{E}-11$ & $6.90 \mathrm{E}-11$ & & $3.98 \mathrm{E}-01$ \\
\hline ENSBTAG00000024653 & 7.44E-11 & $1.88 \mathrm{E}-10$ & OR2T1 & $3.96 \mathrm{E}-01$ \\
\hline ENSBTAG00000048015 & $8.48 \mathrm{E}-11$ & $2.14 \mathrm{E}-10$ & & $3.96 \mathrm{E}-01$ \\
\hline ENSBTAG00000047507 & $3.73 \mathrm{E}-11$ & $9.40 \mathrm{E}-11$ & & 3.97E-01 \\
\hline ENSBTAG00000015144 & $6.73 E-11$ & $1.70 \mathrm{E}-10$ & GMIP & 3.96E-01 \\
\hline ENSBTAG00000016350 & 7.27E-11 & $1.65 \mathrm{E}-10$ & NACC1 & 4.41E-01 \\
\hline ENSBTAG00000046200 & $2.69 \mathrm{E}-10$ & $5.66 \mathrm{E}-10$ & & 4.75E-01 \\
\hline ENSBTAG00000006083 & $1.33 \mathrm{E}-10$ & $2.64 \mathrm{E}-10$ & KLF1 & 5.05E-01 \\
\hline ENSBTAG00000019951 & 7.36E-11 & $1.43 \mathrm{E}-10$ & PCYOX1L & 5.15E-01 \\
\hline ENSBTAG00000035777 & $2.36 \mathrm{E}-10$ & $4.46 \mathrm{E}-10$ & & 5.29E-01 \\
\hline ENSBTAG00000006082 & $6.79 \mathrm{E}-11$ & $1.28 \mathrm{E}-10$ & DNASE2 & 5.31E-01 \\
\hline ENSBTAG00000017482 & $5.12 \mathrm{E}-11$ & $9.69 \mathrm{E}-11$ & ISYNA1 & 5.29E-01 \\
\hline ENSBTAG00000016841 & $2.68 \mathrm{E}-10$ & $5.04 \mathrm{E}-10$ & ATP10B & 5.33E-01 \\
\hline ENSBTAG00000018229 & $1.05 \mathrm{E}-10$ & $1.89 \mathrm{E}-10$ & NFIX & 5.55E-01 \\
\hline ENSBTAG00000006241 & $1.12 \mathrm{E}-10$ & $2.02 \mathrm{E}-10$ & MAN2B1 & $5.56 \mathrm{E}-01$ \\
\hline ENSBTAG00000017362 & $1.29 \mathrm{E}-10$ & $2.31 \mathrm{E}-10$ & CNOT6 & 5.59E-01 \\
\hline ENSBTAG00000045615 & $1.23 \mathrm{E}-10$ & $2.18 \mathrm{E}-10$ & & $5.66 \mathrm{E}-01$ \\
\hline ENSBTAG00000013112 & $2.40 \mathrm{E}-10$ & $4.23 \mathrm{E}-10$ & C5orf15 & $5.68 \mathrm{E}-01$ \\
\hline ENSBTAG00000002224 & $1.70 \mathrm{E}-10$ & $2.92 \mathrm{E}-10$ & UHRF1 & $5.83 \mathrm{E}-01$ \\
\hline ENSBTAG00000045858 & $3.45 \mathrm{E}-10$ & $5.80 \mathrm{E}-10$ & DNAJB1 & 5.95E-01 \\
\hline ENSBTAG00000009478 & $8.36 \mathrm{E}-11$ & $1.40 \mathrm{E}-10$ & GDF9 & 5.97E-01 \\
\hline ENSBTAG00000010316 & $1.10 \mathrm{E}-10$ & $1.84 \mathrm{E}-10$ & ICAM5 & 5.97E-01 \\
\hline ENSBTAG00000010578 & $1.44 \mathrm{E}-10$ & $2.33 \mathrm{E}-10$ & IL17B & $6.16 \mathrm{E}-01$ \\
\hline ENSBTAG00000013113 & $2.10 \mathrm{E}-10$ & $3.38 \mathrm{E}-10$ & VDAC1 & $6.23 \mathrm{E}-01$ \\
\hline ENSBTAG00000016354 & $1.85 \mathrm{E}-10$ & $2.92 \mathrm{E}-10$ & IER2 & 6.34E-01 \\
\hline ENSBTAG00000030529 & $3.49 \mathrm{E}-11$ & 5.50E-11 & FAM53C & $6.36 \mathrm{E}-01$ \\
\hline ENSBTAG00000016213 & $1.21 \mathrm{E}-10$ & $1.89 \mathrm{E}-10$ & SYCE2 & 6.39E-01 \\
\hline ENSBTAG00000014615 & $1.11 \mathrm{E}-10$ & $1.68 \mathrm{E}-10$ & SLC26A2 & $6.62 \mathrm{E}-01$ \\
\hline ENSBTAG00000020776 & $9.20 \mathrm{E}-11$ & $1.39 \mathrm{E}-10$ & POLR2E & $6.62 \mathrm{E}-01$ \\
\hline ENSBTAG00000047612 & $1.25 \mathrm{E}-10$ & $1.88 \mathrm{E}-10$ & & $6.62 \mathrm{E}-01$ \\
\hline ENSBTAG00000046262 & $5.51 \mathrm{E}-10$ & $8.25 \mathrm{E}-10$ & PCDHB7 & $6.68 \mathrm{E}-01$ \\
\hline
\end{tabular}




\begin{tabular}{|c|c|c|c|c|}
\hline ENSBTAG00000008654 & $8.46 \mathrm{E}-11$ & $1.26 \mathrm{E}-10$ & HOOK2 & $6.72 \mathrm{E}-01$ \\
\hline ENSBTAG00000045561 & $5.32 \mathrm{E}-10$ & $7.83 \mathrm{E}-10$ & & $6.80 \mathrm{E}-01$ \\
\hline ENSBTAG00000039422 & 4.89E-10 & $7.20 \mathrm{E}-10$ & OR2B11 & $6.80 \mathrm{E}-01$ \\
\hline ENSBTAG00000012829 & $6.83 \mathrm{E}-11$ & $1.00 \mathrm{E}-10$ & EBI3 & $6.83 \mathrm{E}-01$ \\
\hline ENSBTAG00000030929 & $1.34 \mathrm{E}-10$ & $1.95 \mathrm{E}-10$ & ZNF558 & $6.86 \mathrm{E}-01$ \\
\hline ENSBTAG00000015192 & $5.05 \mathrm{E}-11$ & 7.37E-11 & FAM193B & $6.86 \mathrm{E}-01$ \\
\hline ENSBTAG00000006374 & $1.67 \mathrm{E}-10$ & $2.37 \mathrm{E}-10$ & NHP2 & 7.05E-01 \\
\hline ENSBTAG00000015831 & $3.87 \mathrm{E}-10$ & $5.49 \mathrm{E}-10$ & RPL18A & 7.05E-01 \\
\hline ENSBTAG00000004856 & $3.06 \mathrm{E}-10$ & $4.35 \mathrm{E}-10$ & RNF130 & 7.05E-01 \\
\hline ENSBTAG00000031658 & $5.91 \mathrm{E}-11$ & $8.27 \mathrm{E}-11$ & SEMA6B & 7.14E-01 \\
\hline ENSBTAG00000039555 & $7.56 \mathrm{E}-10$ & $1.05 \mathrm{E}-09$ & & 7.20E-01 \\
\hline ENSBTAG00000039433 & $3.43 \mathrm{E}-10$ & $4.75 \mathrm{E}-10$ & & 7.22E-01 \\
\hline ENSBTAG00000007231 & $9.15 \mathrm{E}-11$ & $1.27 \mathrm{E}-10$ & SPATA9 & 7.21E-01 \\
\hline ENSBTAG00000020267 & $2.19 \mathrm{E}-10$ & $3.01 E-10$ & WNT9A & 7.27E-01 \\
\hline ENSBTAG00000039246 & $2.40 \mathrm{E}-10$ & $3.28 \mathrm{E}-10$ & TRIM11 & 7.30E-01 \\
\hline ENSBTAG00000020213 & $9.51 \mathrm{E}-11$ & $1.30 \mathrm{E}-10$ & NIPAL4 & 7.32E-01 \\
\hline ENSBTAG00000045859 & 4.54E-10 & 6.17E-10 & & 7.36E-01 \\
\hline ENSBTAG00000012771 & $1.43 \mathrm{E}-10$ & $1.93 \mathrm{E}-10$ & CSF1R & 7.41E-01 \\
\hline ENSBTAG00000045603 & $4.81 \mathrm{E}-10$ & $6.50 \mathrm{E}-10$ & PCDHB4 & 7.41E-01 \\
\hline ENSBTAG00000003987 & $2.64 \mathrm{E}-10$ & $3.55 \mathrm{E}-10$ & & 7.43E-01 \\
\hline ENSBTAG00000008457 & $1.60 \mathrm{E}-10$ & $2.15 \mathrm{E}-10$ & SLU7 & 7.44E-01 \\
\hline ENSBTAG00000020303 & $1.03 \mathrm{E}-10$ & $1.37 \mathrm{E}-10$ & $\mathrm{FBXO38}$ & 7.49E-01 \\
\hline ENSBTAG00000015591 & $2.93 \mathrm{E}-10$ & $3.87 \mathrm{E}-10$ & SQSTM1 & 7.57E-01 \\
\hline ENSBTAG00000037508 & $1.54 \mathrm{E}-10$ & $2.03 \mathrm{E}-10$ & EBF1 & 7.57E-01 \\
\hline ENSBTAG00000011110 & $5.15 \mathrm{E}-10$ & $6.51 \mathrm{E}-10$ & ACOT12 & 7.91E-01 \\
\hline ENSBTAG00000007633 & 2.67E-10 & $3.36 \mathrm{E}-10$ & ANKRD34B & 7.93E-01 \\
\hline ENSBTAG00000021616 & $1.32 \mathrm{E}-10$ & $1.66 \mathrm{E}-10$ & PRSS57 & 7.93E-01 \\
\hline ENSBTAG00000037885 & $3.01 \mathrm{E}-10$ & $3.79 \mathrm{E}-10$ & PCDHB1 & 7.93E-01 \\
\hline ENSBTAG00000004652 & $1.42 \mathrm{E}-10$ & $1.79 \mathrm{E}-10$ & LPAR2 & 7.95E-01 \\
\hline ENSBTAG00000004200 & $1.26 \mathrm{E}-10$ & $1.59 \mathrm{E}-10$ & GTPBP3 & 7.94E-01 \\
\hline ENSBTAG00000045981 & $2.75 \mathrm{E}-10$ & $3.46 \mathrm{E}-10$ & DIRAS1 & 7.94E-01 \\
\hline ENSBTAG00000025191 & 4.07E-11 & $5.13 \mathrm{E}-11$ & SKP1 & 7.95E-01 \\
\hline ENSBTAG00000001792 & $5.28 \mathrm{E}-11$ & $6.66 \mathrm{E}-11$ & C19orf70 & 7.93E-01 \\
\hline ENSBTAG00000020269 & $6.59 \mathrm{E}-11$ & $8.30 \mathrm{E}-11$ & MISP & 7.94E-01 \\
\hline ENSBTAG00000009285 & $3.46 \mathrm{E}-10$ & $4.36 \mathrm{E}-10$ & & 7.95E-01 \\
\hline ENSBTAG00000014918 & $1.68 \mathrm{E}-10$ & $2.11 \mathrm{E}-10$ & IBA57 & 7.96E-01 \\
\hline ENSBTAG00000000550 & $7.54 \mathrm{E}-11$ & $9.50 \mathrm{E}-11$ & ATP5D & 7.94E-01 \\
\hline ENSBTAG00000018817 & $6.15 \mathrm{E}-11$ & $7.75 \mathrm{E}-11$ & POU4F3 & 7.93E-01 \\
\hline ENSBTAG00000039696 & $8.23 \mathrm{E}-11$ & $1.04 \mathrm{E}-10$ & SHD & $7.92 \mathrm{E}-01$ \\
\hline ENSBTAG00000046233 & $6.14 \mathrm{E}-10$ & $7.73 \mathrm{E}-10$ & & 7.94E-01 \\
\hline ENSBTAG00000040610 & $8.72 \mathrm{E}-11$ & $1.10 \mathrm{E}-10$ & & 7.93E-01 \\
\hline ENSBTAG00000045691 & $5.32 \mathrm{E}-10$ & $6.71 \mathrm{E}-10$ & & 7.93E-01 \\
\hline ENSBTAG00000046542 & $5.74 \mathrm{E}-11$ & $7.23 \mathrm{E}-11$ & C19orf24 & 7.94E-01 \\
\hline ENSBTAG00000002335 & 4.94E-11 & $6.22 \mathrm{E}-11$ & HAND1 & 7.94E-01 \\
\hline ENSBTAG00000039804 & $1.77 \mathrm{E}-10$ & $2.24 \mathrm{E}-10$ & & 7.92E-01 \\
\hline ENSBTAG00000010283 & 7.17E-11 & $9.03 \mathrm{E}-11$ & & 7.94E-01 \\
\hline ENSBTAG00000001647 & $9.33 \mathrm{E}-11$ & $1.18 \mathrm{E}-10$ & & 7.91E-01 \\
\hline ENSBTAG00000046963 & $8.75 \mathrm{E}-11$ & $1.10 \mathrm{E}-10$ & & 7.96E-01 \\
\hline ENSBTAG00000002284 & $6.22 \mathrm{E}-10$ & $7.83 \mathrm{E}-10$ & & 7.94E-01 \\
\hline ENSBTAG00000039484 & $1.76 \mathrm{E}-10$ & $2.22 \mathrm{E}-10$ & & 7.94E-01 \\
\hline ENSBTAG00000020923 & $9.08 \mathrm{E}-11$ & $1.14 \mathrm{E}-10$ & & 7.97E-01 \\
\hline ENSBTAG00000025788 & $7.82 \mathrm{E}-11$ & $9.85 \mathrm{E}-11$ & MPV17L2 & 7.94E-01 \\
\hline ENSBTAG00000000381 & $7.07 \mathrm{E}-11$ & $8.91 E-11$ & S1PR4 & 7.94E-01 \\
\hline ENSBTAG00000023507 & $5.69 \mathrm{E}-10$ & 7.17E-10 & & 7.94E-01 \\
\hline ENSBTAG00000030704 & $7.66 \mathrm{E}-10$ & $9.65 \mathrm{E}-10$ & & 7.94E-01 \\
\hline ENSBTAG00000047415 & $8.62 \mathrm{E}-10$ & 1.09E-09 & & 7.91E-01 \\
\hline ENSBTAG00000048315 & $2.56 \mathrm{E}-10$ & $3.23 \mathrm{E}-10$ & & 7.94E-01 \\
\hline ENSBTAG00000047927 & $9.58 \mathrm{E}-10$ & $1.21 \mathrm{E}-09$ & & 7.92E-01 \\
\hline ENSBTAG00000027357 & $8.89 \mathrm{E}-11$ & $1.12 \mathrm{E}-10$ & FGF22 & 7.94E-01 \\
\hline ENSBTAG00000039067 & 4.13E-10 & $5.21 \mathrm{E}-10$ & PCDHB15 & 7.93E-01 \\
\hline ENSBTAG00000007349 & $5.16 \mathrm{E}-10$ & $6.50 \mathrm{E}-10$ & PCDHB14 & 7.94E-01 \\
\hline ENSBTAG00000047539 & 4.43E-10 & $5.58 \mathrm{E}-10$ & & 7.94E-01 \\
\hline ENSBTAG00000036175 & $8.86 \mathrm{E}-11$ & $1.12 \mathrm{E}-10$ & & 7.92E-01 \\
\hline ENSBTAG00000047660 & 7.10E-10 & $8.95 \mathrm{E}-10$ & & 7.93E-01 \\
\hline ENSBTAG00000038533 & 4.29E-10 & $5.41 \mathrm{E}-10$ & & 7.93E-01 \\
\hline ENSBTAG00000016648 & 1.17E-10 & $1.48 \mathrm{E}-10$ & BSG & 7.94E-01 \\
\hline ENSBTAG00000038437 & 4.60E-11 & $5.80 \mathrm{E}-11$ & RLN3 & 7.94E-01 \\
\hline ENSBTAG00000045829 & $2.83 \mathrm{E}-11$ & $3.57 \mathrm{E}-11$ & AZU1 & 7.93E-01 \\
\hline ENSBTAG00000018349 & 7.31E-11 & $9.22 \mathrm{E}-11$ & IFI30 & 7.93E-01 \\
\hline ENSBTAG00000036242 & $3.25 \mathrm{E}-11$ & 4.09E-11 & RFESD & 7.94E-01 \\
\hline
\end{tabular}




\begin{tabular}{|c|c|c|c|c|}
\hline ENSBTAG00000030735 & $3.86 \mathrm{E}-10$ & 4.87E-10 & & 7.94E-01 \\
\hline ENSBTAG00000039797 & $1.74 \mathrm{E}-10$ & $2.19 \mathrm{E}-10$ & & 7.95E-01 \\
\hline ENSBTAG00000033055 & $2.28 \mathrm{E}-10$ & $2.88 \mathrm{E}-10$ & & 7.93E-01 \\
\hline ENSBTAG00000011419 & $3.75 \mathrm{E}-11$ & $4.72 \mathrm{E}-11$ & HSPA9 & 7.94E-01 \\
\hline ENSBTAG00000047190 & $3.48 \mathrm{E}-10$ & $4.38 \mathrm{E}-10$ & & 7.94E-01 \\
\hline ENSBTAG00000008692 & $2.53 \mathrm{E}-10$ & $3.18 \mathrm{E}-10$ & & 7.95E-01 \\
\hline ENSBTAG00000046984 & $1.76 \mathrm{E}-10$ & $2.22 \mathrm{E}-10$ & ZNF672 & 7.92E-01 \\
\hline ENSBTAG00000015032 & $1.14 \mathrm{E}-10$ & $1.44 \mathrm{E}-10$ & CD14 & 7.92E-01 \\
\hline ENSBTAG00000039930 & $1.21 \mathrm{E}-09$ & $1.53 \mathrm{E}-09$ & OR2G2 & 7.94E-01 \\
\hline ENSBTAG00000013358 & $1.43 \mathrm{E}-09$ & 1.80E-09 & & 7.93E-01 \\
\hline ENSBTAG00000013429 & $1.34 \mathrm{E}-10$ & $1.69 \mathrm{E}-10$ & CLEC4G & $7.94 \mathrm{E}-01$ \\
\hline ENSBTAG00000015721 & 3.07E-10 & $3.80 \mathrm{E}-10$ & RAVER1 & $8.09 \mathrm{E}-01$ \\
\hline ENSBTAG00000007865 & $6.27 \mathrm{E}-10$ & 7.72E-10 & GNPDA1 & 8.13E-01 \\
\hline ENSBTAG00000018585 & $1.92 \mathrm{E}-10$ & $2.34 \mathrm{E}-10$ & GABRB2 & 8.19E-01 \\
\hline ENSBTAG00000001761 & $2.88 \mathrm{E}-09$ & $3.52 \mathrm{E}-09$ & & 8.19E-01 \\
\hline ENSBTAG00000047835 & $1.23 \mathrm{E}-09$ & $1.50 \mathrm{E}-09$ & & 8.19E-01 \\
\hline ENSBTAG00000005592 & $4.83 \mathrm{E}-10$ & $5.85 \mathrm{E}-10$ & MCOLN1 & 8.26E-01 \\
\hline ENSBTAG00000037693 & $7.29 \mathrm{E}-10$ & $8.81 E-10$ & & 8.27E-01 \\
\hline ENSBTAG00000018673 & $7.94 \mathrm{E}-10$ & $9.59 \mathrm{E}-10$ & TRIM17 & $8.28 \mathrm{E}-01$ \\
\hline ENSBTAG00000016077 & $6.29 \mathrm{E}-10$ & 7.60E-10 & ZNF317 & $8.28 \mathrm{E}-01$ \\
\hline ENSBTAG00000000626 & $3.52 \mathrm{E}-10$ & 4.25E-10 & STK32A & 8.27E-01 \\
\hline ENSBTAG00000039983 & $2.11 \mathrm{E}-09$ & 2.55E-09 & & $8.29 \mathrm{E}-01$ \\
\hline ENSBTAG00000014395 & $2.87 \mathrm{E}-10$ & $3.46 \mathrm{E}-10$ & GLRA1 & $8.31 \mathrm{E}-01$ \\
\hline ENSBTAG00000048257 & $2.15 \mathrm{E}-10$ & $2.58 \mathrm{E}-10$ & & 8.34E-01 \\
\hline ENSBTAG00000046343 & $5.97 \mathrm{E}-10$ & 7.16E-10 & CCNJL & $8.34 \mathrm{E}-01$ \\
\hline ENSBTAG00000015487 & $2.32 \mathrm{E}-10$ & $2.78 \mathrm{E}-10$ & KDM4B & $8.34 \mathrm{E}-01$ \\
\hline ENSBTAG00000047420 & $2.46 \mathrm{E}-10$ & $2.95 \mathrm{E}-10$ & & $8.34 \mathrm{E}-01$ \\
\hline ENSBTAG00000012350 & $1.06 \mathrm{E}-10$ & $1.27 \mathrm{E}-10$ & МАT2B & 8.33E-01 \\
\hline ENSBTAG00000020578 & $4.93 \mathrm{E}-10$ & $5.91 \mathrm{E}-10$ & PRDM6 & 8.35E-01 \\
\hline ENSBTAG00000037941 & $4.04 \mathrm{E}-10$ & $4.83 \mathrm{E}-10$ & QTRT1 & 8.37E-01 \\
\hline ENSBTAG00000015602 & $6.91 \mathrm{E}-10$ & $8.22 \mathrm{E}-10$ & MRNIP & $8.40 \mathrm{E}-01$ \\
\hline ENSBTAG00000040253 & $2.34 \mathrm{E}-10$ & $2.78 \mathrm{E}-10$ & ADRA1B & $8.41 \mathrm{E}-01$ \\
\hline ENSBTAG00000047478 & $5.86 \mathrm{E}-10$ & $6.94 \mathrm{E}-10$ & PCDHB6 & 8.44E-01 \\
\hline ENSBTAG00000014712 & $2.67 \mathrm{E}-10$ & $3.16 \mathrm{E}-10$ & CLPP & 8.45E-01 \\
\hline ENSBTAG00000011135 & $6.74 \mathrm{E}-10$ & 7.99E-10 & & 8.43E-01 \\
\hline ENSBTAG00000008201 & $5.01 \mathrm{E}-10$ & $5.92 \mathrm{E}-10$ & CCDC151 & 8.46E-01 \\
\hline ENSBTAG00000003914 & $6.48 \mathrm{E}-10$ & 7.65E-10 & MVB12A & 8.47E-01 \\
\hline ENSBTAG00000004153 & $9.23 \mathrm{E}-10$ & 1.09E-09 & SCGB3A2 & 8.47E-01 \\
\hline ENSBTAG00000002143 & $5.69 \mathrm{E}-10$ & $6.70 \mathrm{E}-10$ & DTWD2 & 8.49E-01 \\
\hline ENSBTAG00000006005 & $1.32 \mathrm{E}-10$ & $1.56 \mathrm{E}-10$ & MPND & $8.46 \mathrm{E}-01$ \\
\hline ENSBTAG00000011134 & $4.22 \mathrm{E}-10$ & 4.97E-10 & & 8.50E-01 \\
\hline ENSBTAG00000046808 & $9.03 \mathrm{E}-10$ & 1.06E-09 & & $8.52 \mathrm{E}-01$ \\
\hline ENSBTAG00000044169 & $3.10 \mathrm{E}-10$ & $3.65 \mathrm{E}-10$ & PRELID2 & 8.50E-01 \\
\hline ENSBTAG00000021374 & $1.22 \mathrm{E}-10$ & $1.43 \mathrm{E}-10$ & FARSA & $8.51 \mathrm{E}-01$ \\
\hline ENSBTAG00000021529 & $6.70 \mathrm{E}-10$ & $7.86 \mathrm{E}-10$ & & 8.53E-01 \\
\hline ENSBTAG00000002734 & $2.48 \mathrm{E}-10$ & $2.90 \mathrm{E}-10$ & EIF3G & $8.56 \mathrm{E}-01$ \\
\hline ENSBTAG00000013184 & 4.42E-10 & $5.18 \mathrm{E}-10$ & CEP120 & 8.54E-01 \\
\hline ENSBTAG00000045743 & $4.86 \mathrm{E}-10$ & $5.68 \mathrm{E}-10$ & & 8.55E-01 \\
\hline ENSBTAG00000009069 & $1.20 \mathrm{E}-09$ & 1.40E-09 & & $8.54 \mathrm{E}-01$ \\
\hline ENSBTAG00000025767 & $5.69 \mathrm{E}-10$ & $6.64 \mathrm{E}-10$ & & $8.56 \mathrm{E}-01$ \\
\hline ENSBTAG00000025293 & 1.14E-09 & $1.32 \mathrm{E}-09$ & & 8.62E-01 \\
\hline ENSBTAG00000030227 & $3.01 \mathrm{E}-10$ & $3.48 \mathrm{E}-10$ & PCDHA13 & 8.64E-01 \\
\hline ENSBTAG00000033470 & $3.75 \mathrm{E}-10$ & 4.33E-10 & SPINK6 & 8.67E-01 \\
\hline ENSBTAG00000004413 & $3.24 \mathrm{E}-10$ & $3.75 \mathrm{E}-10$ & RHOBTB3 & 8.65E-01 \\
\hline ENSBTAG00000019881 & $3.34 \mathrm{E}-10$ & $3.84 \mathrm{E}-10$ & & 8.69E-01 \\
\hline ENSBTAG00000019071 & $4.41 \mathrm{E}-10$ & $5.08 \mathrm{E}-10$ & COMMD10 & $8.68 \mathrm{E}-01$ \\
\hline ENSBTAG00000010837 & $2.30 \mathrm{E}-10$ & $2.65 \mathrm{E}-10$ & HTR4 & 8.66E-01 \\
\hline ENSBTAG00000010450 & $1.18 \mathrm{E}-10$ & $1.37 \mathrm{E}-10$ & WIZ & 8.63E-01 \\
\hline ENSBTAG00000007366 & $2.91 \mathrm{E}-10$ & $3.33 \mathrm{E}-10$ & HAUS8 & 8.73E-01 \\
\hline ENSBTAG00000039302 & $2.37 \mathrm{E}-10$ & $2.72 \mathrm{E}-10$ & ANGPTL6 & 8.73E-01 \\
\hline ENSBTAG00000006001 & $7.70 \mathrm{E}-11$ & $8.82 \mathrm{E}-11$ & STAP2 & $8.74 \mathrm{E}-01$ \\
\hline ENSBTAG00000027911 & 7.50E-10 & $8.58 \mathrm{E}-10$ & OR2G3 & $8.74 \mathrm{E}-01$ \\
\hline ENSBTAG00000045623 & $8.42 \mathrm{E}-10$ & $9.63 \mathrm{E}-10$ & & $8.74 \mathrm{E}-01$ \\
\hline ENSBTAG00000025358 & $6.44 \mathrm{E}-10$ & 7.34E-10 & SNX2 & 8.77E-01 \\
\hline ENSBTAG00000038722 & 2.87E-09 & 3.27E-09 & & $8.79 \mathrm{E}-01$ \\
\hline ENSBTAG00000014809 & $5.49 \mathrm{E}-10$ & $6.25 \mathrm{E}-10$ & ANXA6 & $8.79 \mathrm{E}-01$ \\
\hline ENSBTAG00000011602 & $2.07 \mathrm{E}-10$ & $2.36 \mathrm{E}-10$ & RASAL3 & $8.78 \mathrm{E}-01$ \\
\hline ENSBTAG00000047796 & $8.46 \mathrm{E}-10$ & $9.59 \mathrm{E}-10$ & & $8.82 \mathrm{E}-01$ \\
\hline ENSBTAG00000046914 & $1.27 \mathrm{E}-09$ & 1.44E-09 & & $8.80 \mathrm{E}-01$ \\
\hline ENSBTAG00000017744 & $3.72 \mathrm{E}-10$ & 4.22E-10 & FAF2 & 8.83E-01 \\
\hline
\end{tabular}




\begin{tabular}{|c|c|c|c|c|}
\hline ENSBTAG00000025148 & $1.21 \mathrm{E}-10$ & $1.38 \mathrm{E}-10$ & FBXL12 & $8.80 \mathrm{E}-01$ \\
\hline ENSBTAG00000015186 & $3.36 \mathrm{E}-10$ & $3.80 \mathrm{E}-10$ & ILVBL & $8.86 \mathrm{E}-01$ \\
\hline ENSBTAG00000004270 & $1.42 \mathrm{E}-10$ & $1.60 \mathrm{E}-10$ & FCER2 & $8.88 \mathrm{E}-01$ \\
\hline ENSBTAG00000010543 & $1.37 \mathrm{E}-10$ & $1.54 \mathrm{E}-10$ & FGFR4 & $8.91 \mathrm{E}-01$ \\
\hline ENSBTAG00000010635 & $1.30 \mathrm{E}-10$ & $1.45 \mathrm{E}-10$ & RAB3A & 8.94E-01 \\
\hline ENSBTAG00000016211 & $2.17 \mathrm{E}-10$ & $2.43 \mathrm{E}-10$ & $\mathrm{GCDH}$ & $8.94 \mathrm{E}-01$ \\
\hline ENSBTAG00000019414 & $4.49 \mathrm{E}-10$ & $5.04 \mathrm{E}-10$ & CLK4 & $8.91 \mathrm{E}-01$ \\
\hline ENSBTAG00000025775 & $4.68 \mathrm{E}-10$ & $5.24 \mathrm{E}-10$ & INSL3 & $8.93 \mathrm{E}-01$ \\
\hline ENSBTAG00000037964 & 4.90E-10 & $5.49 \mathrm{E}-10$ & HIST3H2A & 8.93E-01 \\
\hline ENSBTAG00000019457 & $5.34 \mathrm{E}-10$ & $5.96 \mathrm{E}-10$ & MRPL22 & $8.95 \mathrm{E}-01$ \\
\hline ENSBTAG00000004224 & $2.22 \mathrm{E}-10$ & $2.48 \mathrm{E}-10$ & C19orf38 & 8.94E-01 \\
\hline ENSBTAG00000034973 & $1.27 \mathrm{E}-09$ & $1.42 \mathrm{E}-09$ & & 8.93E-01 \\
\hline ENSBTAG00000013262 & $2.08 \mathrm{E}-10$ & $2.32 \mathrm{E}-10$ & GABRA6 & $8.98 \mathrm{E}-01$ \\
\hline ENSBTAG00000014974 & $3.77 \mathrm{E}-10$ & $4.20 \mathrm{E}-10$ & GRAMD3 & 8.97E-01 \\
\hline ENSBTAG00000002834 & $4.41 \mathrm{E}-10$ & $4.91 \mathrm{E}-10$ & CCDC69 & $8.98 \mathrm{E}-01$ \\
\hline ENSBTAG00000000341 & $2.38 \mathrm{E}-10$ & $2.64 \mathrm{E}-10$ & ISOC1 & $9.00 \mathrm{E}-01$ \\
\hline ENSBTAG00000015558 & $4.92 \mathrm{E}-10$ & $5.46 \mathrm{E}-10$ & SPINK1 & $9.01 \mathrm{E}-01$ \\
\hline ENSBTAG00000044183 & $3.44 \mathrm{E}-10$ & $3.82 \mathrm{E}-10$ & SPINK7 & $9.00 \mathrm{E}-01$ \\
\hline ENSBTAG00000011136 & $4.09 \mathrm{E}-10$ & 4.54E-10 & NR2F6 & $9.00 \mathrm{E}-01$ \\
\hline ENSBTAG00000013557 & $9.07 \mathrm{E}-10$ & $1.01 \mathrm{E}-09$ & ERAP1 & $8.98 \mathrm{E}-01$ \\
\hline ENSBTAG00000015108 & $2.18 \mathrm{E}-10$ & $2.41 \mathrm{E}-10$ & USHBP1 & $9.06 \mathrm{E}-01$ \\
\hline ENSBTAG00000001793 & $1.36 \mathrm{E}-10$ & $1.50 \mathrm{E}-10$ & HSD11B1L & $9.04 \mathrm{E}-01$ \\
\hline ENSBTAG00000017371 & $1.78 \mathrm{E}-10$ & $1.97 \mathrm{E}-10$ & RELL2 & 9.05E-01 \\
\hline ENSBTAG00000048069 & $6.97 \mathrm{E}-10$ & $7.68 \mathrm{E}-10$ & & $9.08 \mathrm{E}-01$ \\
\hline ENSBTAG00000030616 & $5.67 \mathrm{E}-10$ & $6.24 \mathrm{E}-10$ & $\mathrm{ZCCHC10}$ & $9.09 \mathrm{E}-01$ \\
\hline ENSBTAG00000012048 & $3.66 \mathrm{E}-10$ & 4.03E-10 & CARM1 & $9.08 \mathrm{E}-01$ \\
\hline ENSBTAG00000006624 & $6.73 \mathrm{E}-10$ & $7.39 \mathrm{E}-10$ & SLC36A3 & $9.10 \mathrm{E}-01$ \\
\hline ENSBTAG00000002350 & $1.49 \mathrm{E}-10$ & $1.64 \mathrm{E}-10$ & & $9.11 \mathrm{E}-01$ \\
\hline ENSBTAG00000004896 & $2.65 \mathrm{E}-10$ & $2.89 \mathrm{E}-10$ & ABLIM3 & $9.16 \mathrm{E}-01$ \\
\hline ENSBTAG00000046750 & $6.16 \mathrm{E}-11$ & $6.73 \mathrm{E}-11$ & B3GNT3 & $9.16 \mathrm{E}-01$ \\
\hline ENSBTAG00000012518 & $4.39 \mathrm{E}-10$ & $4.79 \mathrm{E}-10$ & PCDH1 & $9.17 \mathrm{E}-01$ \\
\hline ENSBTAG00000004936 & $1.37 \mathrm{E}-10$ & $1.49 \mathrm{E}-10$ & TMIGD2 & $9.16 \mathrm{E}-01$ \\
\hline ENSBTAG00000012768 & $2.92 \mathrm{E}-10$ & $3.18 \mathrm{E}-10$ & HMGXB3 & $9.17 \mathrm{E}-01$ \\
\hline ENSBTAG00000021115 & $6.56 \mathrm{E}-10$ & $7.13 \mathrm{E}-10$ & ZNF692 & $9.20 \mathrm{E}-01$ \\
\hline ENSBTAG00000019012 & $3.76 \mathrm{E}-10$ & $4.09 \mathrm{E}-10$ & KCTD16 & $9.19 \mathrm{E}-01$ \\
\hline ENSBTAG00000003034 & 3.37E-10 & $3.65 E-10$ & GATAD2A & $9.22 \mathrm{E}-01$ \\
\hline ENSBTAG00000033759 & $6.97 \mathrm{E}-10$ & $7.56 \mathrm{E}-10$ & & $9.22 \mathrm{E}-01$ \\
\hline ENSBTAG00000019419 & $2.79 \mathrm{E}-10$ & $3.02 \mathrm{E}-10$ & NDUFS7 & $9.25 \mathrm{E}-01$ \\
\hline ENSBTAG00000000874 & $8.41 \mathrm{E}-10$ & $8.99 \mathrm{E}-10$ & CAST & $9.36 \mathrm{E}-01$ \\
\hline ENSBTAG00000030259 & $4.97 \mathrm{E}-10$ & $5.29 \mathrm{E}-10$ & RASGRF2 & $9.39 \mathrm{E}-01$ \\
\hline ENSBTAG00000001762 & $6.22 \mathrm{E}-10$ & $6.63 \mathrm{E}-10$ & RFXANK & $9.38 \mathrm{E}-01$ \\
\hline ENSBTAG00000032137 & $5.70 \mathrm{E}-10$ & $6.07 \mathrm{E}-10$ & PNPLA6 & $9.39 \mathrm{E}-01$ \\
\hline ENSBTAG00000046423 & 2.07E-09 & $2.21 \mathrm{E}-09$ & & 9.37E-01 \\
\hline ENSBTAG00000006007 & $1.95 \mathrm{E}-10$ & $2.08 \mathrm{E}-10$ & SH3GL1 & $9.39 \mathrm{E}-01$ \\
\hline ENSBTAG00000014626 & $8.69 \mathrm{E}-10$ & $9.23 \mathrm{E}-10$ & RARS & $9.41 \mathrm{E}-01$ \\
\hline ENSBTAG00000006762 & $4.41 \mathrm{E}-10$ & 4.67E-10 & SH3RF2 & $9.45 \mathrm{E}-01$ \\
\hline ENSBTAG00000000682 & $2.02 \mathrm{E}-10$ & $2.13 \mathrm{E}-10$ & & $9.47 \mathrm{E}-01$ \\
\hline ENSBTAG00000010359 & $1.42 \mathrm{E}-10$ & $1.50 \mathrm{E}-10$ & THG1L & $9.50 \mathrm{E}-01$ \\
\hline ENSBTAG00000013362 & $4.11 \mathrm{E}-10$ & $4.33 \mathrm{E}-10$ & DNM2 & $9.49 \mathrm{E}-01$ \\
\hline ENSBTAG00000044067 & $4.31 \mathrm{E}-10$ & $4.53 \mathrm{E}-10$ & PLAC8L1 & $9.51 \mathrm{E}-01$ \\
\hline ENSBTAG00000012172 & $8.07 \mathrm{E}-11$ & $8.47 E-11$ & MIDN & $9.53 \mathrm{E}-01$ \\
\hline ENSBTAG00000015453 & 4.23E-10 & $4.44 \mathrm{E}-10$ & PCDHB16 & $9.54 \mathrm{E}-01$ \\
\hline ENSBTAG00000015116 & $8.14 \mathrm{E}-11$ & $8.55 E-11$ & RAD23A & $9.52 \mathrm{E}-01$ \\
\hline ENSBTAG00000030589 & $8.48 \mathrm{E}-11$ & $8.91 \mathrm{E}-11$ & TMCO6 & $9.52 \mathrm{E}-01$ \\
\hline ENSBTAG00000038629 & $5.25 \mathrm{E}-10$ & $5.51 \mathrm{E}-10$ & OR2Y1 & $9.54 \mathrm{E}-01$ \\
\hline ENSBTAG00000006185 & $2.43 \mathrm{E}-10$ & $2.55 E-10$ & SPC24 & $9.54 \mathrm{E}-01$ \\
\hline ENSBTAG00000025908 & $4.06 \mathrm{E}-10$ & $4.26 \mathrm{E}-10$ & & $9.52 \mathrm{E}-01$ \\
\hline ENSBTAG00000046297 & $1.04 \mathrm{E}-09$ & 1.09E-09 & & $9.54 \mathrm{E}-01$ \\
\hline ENSBTAG00000019814 & $2.76 \mathrm{E}-10$ & $2.90 \mathrm{E}-10$ & PGBD2 & $9.53 \mathrm{E}-01$ \\
\hline ENSBTAG00000002628 & $3.97 \mathrm{E}-10$ & $4.15 E-10$ & SLC44A2 & $9.56 \mathrm{E}-01$ \\
\hline ENSBTAG00000002140 & $2.24 \mathrm{E}-10$ & $2.35 \mathrm{E}-10$ & & $9.53 \mathrm{E}-01$ \\
\hline ENSBTAG00000013536 & $2.10 \mathrm{E}-10$ & $2.20 \mathrm{E}-10$ & P2RY11 & $9.54 \mathrm{E}-01$ \\
\hline ENSBTAG00000006110 & $7.12 \mathrm{E}-10$ & $7.45 \mathrm{E}-10$ & & $9.55 \mathrm{E}-01$ \\
\hline ENSBTAG00000007312 & $1.69 \mathrm{E}-10$ & $1.78 \mathrm{E}-10$ & & $9.51 \mathrm{E}-01$ \\
\hline ENSBTAG00000003551 & $1.08 \mathrm{E}-09$ & 1.13E-09 & OR2T11 & $9.53 \mathrm{E}-01$ \\
\hline ENSBTAG00000021577 & $4.58 \mathrm{E}-10$ & $4.78 \mathrm{E}-10$ & ZFYVE16 & $9.58 \mathrm{E}-01$ \\
\hline ENSBTAG00000006748 & $1.59 \mathrm{E}-10$ & $1.66 \mathrm{E}-10$ & DMXL1 & 9.57E-01 \\
\hline ENSBTAG00000010979 & $6.00 \mathrm{E}-11$ & $6.25 E-11$ & CDKL3 & $9.61 \mathrm{E}-01$ \\
\hline ENSBTAG00000003349 & $4.85 \mathrm{E}-10$ & $5.05 \mathrm{E}-10$ & JAKMIP2 & $9.60 \mathrm{E}-01$ \\
\hline ENSBTAG00000002804 & $2.65 \mathrm{E}-10$ & $2.75 \mathrm{E}-10$ & PDGFRB & $9.65 \mathrm{E}-01$ \\
\hline
\end{tabular}




\begin{tabular}{|c|c|c|c|c|}
\hline ENSBTAG00000046813 & $1.63 \mathrm{E}-09$ & 1.69E-09 & & $9.66 \mathrm{E}-01$ \\
\hline ENSBTAG00000007812 & $1.09 \mathrm{E}-10$ & $1.13 \mathrm{E}-10$ & NDUFA13 & $9.68 \mathrm{E}-01$ \\
\hline ENSBTAG00000016797 & 4.19E-10 & $4.32 \mathrm{E}-10$ & CSNK1G2 & $9.70 \mathrm{E}-01$ \\
\hline ENSBTAG00000014969 & $9.99 \mathrm{E}-11$ & $1.03 \mathrm{E}-10$ & CILP2 & $9.70 \mathrm{E}-01$ \\
\hline ENSBTAG00000008034 & $5.74 \mathrm{E}-10$ & $5.92 \mathrm{E}-10$ & LMAN2 & $9.70 \mathrm{E}-01$ \\
\hline ENSBTAG00000025233 & $1.02 \mathrm{E}-10$ & $1.05 \mathrm{E}-10$ & & $9.72 \mathrm{E}-01$ \\
\hline ENSBTAG00000027699 & $9.70 \mathrm{E}-10$ & $9.99 \mathrm{E}-10$ & & $9.71 \mathrm{E}-01$ \\
\hline ENSBTAG00000021977 & 7.14E-10 & 7.35E-10 & PRRC1 & $9.71 \mathrm{E}-01$ \\
\hline ENSBTAG00000001367 & $1.63 \mathrm{E}-10$ & $1.68 \mathrm{E}-10$ & C1QTNF2 & $9.71 \mathrm{E}-01$ \\
\hline ENSBTAG00000013623 & $1.13 \mathrm{E}-09$ & 1.15E-09 & & $9.80 \mathrm{E}-01$ \\
\hline ENSBTAG00000006488 & $1.40 \mathrm{E}-09$ & $1.43 \mathrm{E}-09$ & & $9.78 \mathrm{E}-01$ \\
\hline ENSBTAG00000008192 & $2.11 \mathrm{E}-10$ & $2.15 \mathrm{E}-10$ & PLPPR2 & $9.80 \mathrm{E}-01$ \\
\hline ENSBTAG00000008626 & $1.33 \mathrm{E}-10$ & $1.36 \mathrm{E}-10$ & KCNN1 & $9.80 \mathrm{E}-01$ \\
\hline ENSBTAG00000007785 & $1.90 \mathrm{E}-10$ & $1.94 \mathrm{E}-10$ & PKD2L2 & $9.80 \mathrm{E}-01$ \\
\hline ENSBTAG00000031069 & $5.39 \mathrm{E}-10$ & $5.49 \mathrm{E}-10$ & SNX24 & $9.81 \mathrm{E}-01$ \\
\hline ENSBTAG00000015611 & $5.21 \mathrm{E}-10$ & $5.30 \mathrm{E}-10$ & TBC1D9B & $9.83 \mathrm{E}-01$ \\
\hline ENSBTAG00000001568 & $1.84 \mathrm{E}-10$ & $1.87 \mathrm{E}-10$ & PPIC & $9.85 \mathrm{E}-01$ \\
\hline ENSBTAG00000008854 & $1.10 \mathrm{E}-10$ & $1.12 \mathrm{E}-10$ & RFX1 & $9.85 \mathrm{E}-01$ \\
\hline ENSBTAG00000038117 & $2.64 \mathrm{E}-10$ & $2.68 \mathrm{E}-10$ & ATG10 & $9.85 \mathrm{E}-01$ \\
\hline ENSBTAG00000014551 & 4.44E-10 & $4.52 \mathrm{E}-10$ & TNFAIP8 & $9.83 \mathrm{E}-01$ \\
\hline ENSBTAG00000015465 & $5.76 \mathrm{E}-10$ & $5.85 \mathrm{E}-10$ & C5orf46 & $9.84 \mathrm{E}-01$ \\
\hline ENSBTAG00000007122 & $2.34 \mathrm{E}-10$ & 2.37E-10 & ZFP2 & $9.89 \mathrm{E}-01$ \\
\hline ENSBTAG00000014897 & 2.73E-10 & $2.77 \mathrm{E}-10$ & UNC5A & $9.87 \mathrm{E}-01$ \\
\hline ENSBTAG00000019329 & $1.92 \mathrm{E}-10$ & $1.93 \mathrm{E}-10$ & LYL1 & $9.93 \mathrm{E}-01$ \\
\hline ENSBTAG00000001707 & $2.61 \mathrm{E}-10$ & 2.64E-10 & UIMC1 & $9.90 \mathrm{E}-01$ \\
\hline ENSBTAG00000003476 & $1.14 \mathrm{E}-10$ & $1.15 \mathrm{E}-10$ & FEM1A & $9.95 \mathrm{E}-01$ \\
\hline ENSBTAG00000011639 & 5.57E-11 & $5.61 \mathrm{E}-11$ & STK11 & $9.92 \mathrm{E}-01$ \\
\hline ENSBTAG00000043994 & 8.93E-11 & $9.00 \mathrm{E}-11$ & GRK6 & $9.92 \mathrm{E}-01$ \\
\hline ENSBTAG00000048072 & $4.39 \mathrm{E}-10$ & $4.42 \mathrm{E}-10$ & & 9.93E-01 \\
\hline ENSBTAG00000008181 & $1.47 \mathrm{E}-10$ & $1.48 \mathrm{E}-10$ & CHAF1A & $9.95 \mathrm{E}-01$ \\
\hline ENSBTAG00000046696 & $6.76 \mathrm{E}-10$ & $6.81 \mathrm{E}-10$ & & 9.93E-01 \\
\hline ENSBTAG00000015049 & $1.74 \mathrm{E}-10$ & $1.75 \mathrm{E}-10$ & ECSIT & $9.93 \mathrm{E}-01$ \\
\hline ENSBTAG00000040153 & $1.56 \mathrm{E}-10$ & $1.57 \mathrm{E}-10$ & & $9.91 \mathrm{E}-01$ \\
\hline ENSBTAG00000004860 & 4.64E-10 & $4.66 \mathrm{E}-10$ & SLC27A6 & $9.95 \mathrm{E}-01$ \\
\hline ENSBTAG00000003086 & $1.88 \mathrm{E}-09$ & $1.89 \mathrm{E}-09$ & RPLP2 & $9.95 \mathrm{E}-01$ \\
\hline ENSBTAG00000030499 & $1.71 \mathrm{E}-10$ & $1.71 \mathrm{E}-10$ & FCHSD1 & $9.98 \mathrm{E}-01$ \\
\hline ENSBTAG00000014774 & $2.08 \mathrm{E}-10$ & $2.09 \mathrm{E}-10$ & ANKRD24 & $9.95 \mathrm{E}-01$ \\
\hline ENSBTAG00000004815 & $7.89 \mathrm{E}-10$ & 7.90E-10 & & $9.99 \mathrm{E}-01$ \\
\hline ENSBTAG00000012314 & $2.46 \mathrm{E}-10$ & $2.45 \mathrm{E}-10$ & LDLR & $1.00 \mathrm{E}+00$ \\
\hline ENSBTAG00000020491 & $1.97 \mathrm{E}-10$ & $1.96 \mathrm{E}-10$ & sox30 & $1.01 E+00$ \\
\hline ENSBTAG00000016988 & $1.87 \mathrm{E}-10$ & $1.85 \mathrm{E}-10$ & PIN1 & $1.01 \mathrm{E}+00$ \\
\hline ENSBTAG00000030839 & $2.53 \mathrm{E}-10$ & $2.51 \mathrm{E}-10$ & MUM1 & $1.01 E+00$ \\
\hline ENSBTAG00000010661 & $2.91 \mathrm{E}-10$ & $2.87 \mathrm{E}-10$ & DPYSL3 & $1.01 E+00$ \\
\hline ENSBTAG00000034917 & $2.84 \mathrm{E}-09$ & 2.81E-09 & & $1.01 E+00$ \\
\hline ENSBTAG00000032125 & $4.62 \mathrm{E}-10$ & $4.55 \mathrm{E}-10$ & & $1.01 \mathrm{E}+00$ \\
\hline ENSBTAG00000001003 & $1.62 \mathrm{E}-10$ & $1.59 \mathrm{E}-10$ & CKMT2 & $1.02 \mathrm{E}+00$ \\
\hline ENSBTAG00000015051 & $2.27 \mathrm{E}-10$ & $2.24 \mathrm{E}-10$ & POLRMT & $1.01 \mathrm{E}+00$ \\
\hline ENSBTAG00000010632 & $5.57 \mathrm{E}-10$ & 5.47E-10 & FABP6 & $1.02 \mathrm{E}+00$ \\
\hline ENSBTAG00000025652 & $1.37 \mathrm{E}-10$ & $1.34 \mathrm{E}-10$ & BEST2 & $1.02 \mathrm{E}+00$ \\
\hline ENSBTAG00000033092 & $7.83 \mathrm{E}-10$ & $7.66 \mathrm{E}-10$ & & $1.02 \mathrm{E}+00$ \\
\hline ENSBTAG00000010689 & $1.44 \mathrm{E}-10$ & $1.41 \mathrm{E}-10$ & NRG2 & $1.02 \mathrm{E}+00$ \\
\hline ENSBTAG00000010959 & $2.73 \mathrm{E}-10$ & $2.66 \mathrm{E}-10$ & HINT1 & $1.03 E+00$ \\
\hline ENSBTAG00000044033 & $3.70 \mathrm{E}-10$ & $3.58 \mathrm{E}-10$ & EDIL3 & $1.03 E+00$ \\
\hline ENSBTAG00000016187 & $3.70 \mathrm{E}-10$ & $3.58 \mathrm{E}-10$ & GRM6 & $1.03 E+00$ \\
\hline ENSBTAG00000046982 & $1.12 \mathrm{E}-09$ & 1.09E-09 & & $1.03 E+00$ \\
\hline ENSBTAG00000009975 & $7.16 \mathrm{E}-10$ & $6.92 \mathrm{E}-10$ & PBX4 & $1.03 E+00$ \\
\hline ENSBTAG00000004262 & $2.40 \mathrm{E}-10$ & $2.32 \mathrm{E}-10$ & ZNF454 & $1.03 E+00$ \\
\hline ENSBTAG00000010341 & $2.42 \mathrm{E}-10$ & $2.32 \mathrm{E}-10$ & PWWP2A & $1.04 \mathrm{E}+00$ \\
\hline ENSBTAG00000034113 & $2.88 \mathrm{E}-10$ & $2.76 \mathrm{E}-10$ & GALNT10 & $1.04 \mathrm{E}+00$ \\
\hline ENSBTAG00000032292 & $5.43 \mathrm{E}-10$ & 5.17E-10 & CCDC159 & $1.05 \mathrm{E}+00$ \\
\hline ENSBTAG00000000613 & $9.12 \mathrm{E}-11$ & $8.69 \mathrm{E}-11$ & ELAVL1 & $1.05 \mathrm{E}+00$ \\
\hline ENSBTAG00000015938 & $1.54 \mathrm{E}-10$ & $1.46 \mathrm{E}-10$ & ARAP3 & $1.05 E+00$ \\
\hline ENSBTAG00000013624 & $6.11 \mathrm{E}-10$ & $5.81 E-10$ & LMNB2 & $1.05 E+00$ \\
\hline ENSBTAG00000035584 & $2.06 \mathrm{E}-10$ & $1.95 \mathrm{E}-10$ & & $1.06 \mathrm{E}+00$ \\
\hline ENSBTAG00000008198 & $1.40 \mathrm{E}-10$ & $1.33 \mathrm{E}-10$ & RGL3 & $1.06 \mathrm{E}+00$ \\
\hline ENSBTAG00000015041 & $1.41 \mathrm{E}-10$ & $1.33 \mathrm{E}-10$ & & $1.06 \mathrm{E}+00$ \\
\hline ENSBTAG00000014906 & $2.70 \mathrm{E}-10$ & $2.56 \mathrm{E}-10$ & VCAN & $1.06 \mathrm{E}+00$ \\
\hline ENSBTAG00000046263 & $9.87 \mathrm{E}-11$ & $9.32 \mathrm{E}-11$ & & $1.06 \mathrm{E}+00$ \\
\hline ENSBTAG00000015742 & $3.16 \mathrm{E}-10$ & $2.99 \mathrm{E}-10$ & ICAM3 & $1.06 \mathrm{E}+00$ \\
\hline ENSBTAG00000004300 & $9.53 \mathrm{E}-11$ & $9.00 \mathrm{E}-11$ & LRRC8E & $1.06 \mathrm{E}+00$ \\
\hline
\end{tabular}




\begin{tabular}{|c|c|c|c|c|}
\hline ENSBTAG00000038980 & $3.45 \mathrm{E}-10$ & $3.26 \mathrm{E}-10$ & & $1.06 \mathrm{E}+00$ \\
\hline ENSBTAG00000030474 & $4.50 \mathrm{E}-10$ & 4.25E-10 & & $1.06 \mathrm{E}+00$ \\
\hline ENSBTAG00000046034 & $2.37 \mathrm{E}-10$ & $2.24 \mathrm{E}-10$ & EEF1A1 & $1.06 \mathrm{E}+00$ \\
\hline ENSBTAG00000001449 & $5.49 \mathrm{E}-11$ & 5.19E-11 & SRA1 & $1.06 \mathrm{E}+00$ \\
\hline ENSBTAG00000045638 & $2.81 \mathrm{E}-10$ & $2.65 \mathrm{E}-10$ & & $1.06 \mathrm{E}+00$ \\
\hline ENSBTAG00000003018 & 5.17E-11 & 4.88E-11 & FSTL3 & $1.06 \mathrm{E}+00$ \\
\hline ENSBTAG00000046163 & $6.86 \mathrm{E}-10$ & $6.48 \mathrm{E}-10$ & & $1.06 \mathrm{E}+00$ \\
\hline ENSBTAG00000045893 & $7.21 \mathrm{E}-10$ & $6.80 \mathrm{E}-10$ & TIMM29 & $1.06 \mathrm{E}+00$ \\
\hline ENSBTAG00000017706 & $2.94 \mathrm{E}-10$ & $2.78 \mathrm{E}-10$ & SLC39A3 & $1.06 \mathrm{E}+00$ \\
\hline ENSBTAG00000037611 & $6.95 \mathrm{E}-10$ & $6.56 \mathrm{E}-10$ & & $1.06 \mathrm{E}+00$ \\
\hline ENSBTAG00000047082 & $6.95 \mathrm{E}-10$ & $6.56 \mathrm{E}-10$ & & $1.06 \mathrm{E}+00$ \\
\hline ENSBTAG00000039805 & $2.94 \mathrm{E}-10$ & $2.78 \mathrm{E}-10$ & & $1.06 \mathrm{E}+00$ \\
\hline ENSBTAG00000046349 & $8.66 \mathrm{E}-10$ & 8.17E-10 & & $1.06 \mathrm{E}+00$ \\
\hline ENSBTAG00000025136 & $1.74 \mathrm{E}-10$ & $1.64 \mathrm{E}-10$ & MYOZ3 & $1.06 \mathrm{E}+00$ \\
\hline ENSBTAG00000031647 & $1.11 \mathrm{E}-10$ & $1.05 \mathrm{E}-10$ & LRG1 & $1.05 E+00$ \\
\hline ENSBTAG00000017105 & $3.05 \mathrm{E}-10$ & $2.87 \mathrm{E}-10$ & FSTL4 & $1.06 \mathrm{E}+00$ \\
\hline ENSBTAG00000013856 & $3.24 \mathrm{E}-10$ & $3.05 \mathrm{E}-10$ & SSBP2 & $1.06 \mathrm{E}+00$ \\
\hline ENSBTAG00000007901 & $5.51 \mathrm{E}-10$ & $5.16 \mathrm{E}-10$ & ADGRE1 & $1.07 \mathrm{E}+00$ \\
\hline ENSBTAG00000046265 & $5.94 \mathrm{E}-10$ & $5.55 \mathrm{E}-10$ & & $1.07 \mathrm{E}+00$ \\
\hline ENSBTAG00000038464 & $1.61 \mathrm{E}-10$ & $1.50 \mathrm{E}-10$ & PLIN5 & $1.07 \mathrm{E}+00$ \\
\hline ENSBTAG00000015727 & $9.58 \mathrm{E}-10$ & $8.92 \mathrm{E}-10$ & & $1.07 \mathrm{E}+00$ \\
\hline ENSBTAG00000007632 & $6.36 \mathrm{E}-10$ & $5.91 \mathrm{E}-10$ & FAM151B & $1.08 \mathrm{E}+00$ \\
\hline ENSBTAG00000040076 & $3.20 \mathrm{E}-10$ & $2.97 \mathrm{E}-10$ & ILF3 & $1.08 \mathrm{E}+00$ \\
\hline ENSBTAG00000025817 & $5.07 \mathrm{E}-10$ & $4.71 \mathrm{E}-10$ & & $1.08 \mathrm{E}+00$ \\
\hline ENSBTAG00000019771 & $9.89 \mathrm{E}-11$ & $9.14 \mathrm{E}-11$ & PALM & $1.08 \mathrm{E}+00$ \\
\hline ENSBTAG00000008437 & $9.86 \mathrm{E}-11$ & $9.11 \mathrm{E}-11$ & PCDH12 & $1.08 \mathrm{E}+00$ \\
\hline ENSBTAG00000016773 & $2.62 \mathrm{E}-10$ & $2.42 \mathrm{E}-10$ & NXNL1 & $1.08 \mathrm{E}+00$ \\
\hline ENSBTAG00000038151 & $1.47 \mathrm{E}-10$ & $1.34 \mathrm{E}-10$ & ELOF1 & $1.09 E+00$ \\
\hline ENSBTAG00000039591 & $1.96 \mathrm{E}-10$ & $1.80 \mathrm{E}-10$ & TMEM167A & $1.09 \mathrm{E}+00$ \\
\hline ENSBTAG00000003144 & $4.32 \mathrm{E}-10$ & $3.95 \mathrm{E}-10$ & & $1.09 E+00$ \\
\hline ENSBTAG00000007359 & 2.24E-10 & $2.05 \mathrm{E}-10$ & PROP1 & $1.09 \mathrm{E}+00$ \\
\hline ENSBTAG00000033146 & $1.93 \mathrm{E}-09$ & 1.76E-09 & & $1.10 \mathrm{E}+00$ \\
\hline ENSBTAG00000011481 & $5.28 \mathrm{E}-10$ & $4.82 \mathrm{E}-10$ & IL12RB1 & $1.10 \mathrm{E}+00$ \\
\hline ENSBTAG00000007128 & $4.01 \mathrm{E}-10$ & $3.65 \mathrm{E}-10$ & ATP13A1 & $1.10 \mathrm{E}+00$ \\
\hline ENSBTAG00000000910 & $1.55 \mathrm{E}-10$ & $1.41 \mathrm{E}-10$ & SLC6A7 & $1.10 \mathrm{E}+00$ \\
\hline ENSBTAG00000006031 & $5.61 \mathrm{E}-10$ & $5.11 \mathrm{E}-10$ & ADGRV1 & $1.10 \mathrm{E}+00$ \\
\hline ENSBTAG00000009331 & $5.24 \mathrm{E}-10$ & 4.77E-10 & CPAMD8 & $1.10 \mathrm{E}+00$ \\
\hline ENSBTAG00000017550 & $2.25 \mathrm{E}-10$ & $2.04 \mathrm{E}-10$ & CSNK1G3 & $1.10 E+00$ \\
\hline ENSBTAG00000039493 & $8.76 \mathrm{E}-10$ & $7.95 \mathrm{E}-10$ & & $1.10 \mathrm{E}+00$ \\
\hline ENSBTAG00000012184 & $1.92 \mathrm{E}-10$ & $1.75 \mathrm{E}-10$ & & $1.10 \mathrm{E}+00$ \\
\hline ENSBTAG00000014979 & $3.71 \mathrm{E}-10$ & $3.36 \mathrm{E}-10$ & MFSD12 & $1.10 \mathrm{E}+00$ \\
\hline ENSBTAG00000006765 & $3.58 \mathrm{E}-10$ & $3.24 \mathrm{E}-10$ & LARS & $1.10 \mathrm{E}+00$ \\
\hline ENSBTAG00000016199 & $1.62 \mathrm{E}-10$ & $1.46 \mathrm{E}-10$ & CLINT1 & $1.11 E+00$ \\
\hline ENSBTAG00000039335 & $1.27 \mathrm{E}-10$ & $1.15 \mathrm{E}-10$ & ARRDC2 & $1.11 E+00$ \\
\hline ENSBTAG00000030282 & $1.01 \mathrm{E}-10$ & $9.05 \mathrm{E}-11$ & ZBED8 & $1.11 \mathrm{E}+00$ \\
\hline ENSBTAG00000046564 & $6.14 \mathrm{E}-10$ & $5.51 \mathrm{E}-10$ & & $1.11 \mathrm{E}+00$ \\
\hline ENSBTAG00000021016 & $4.82 \mathrm{E}-10$ & 4.33E-10 & & $1.11 \mathrm{E}+00$ \\
\hline ENSBTAG00000015953 & $2.87 \mathrm{E}-10$ & $2.58 \mathrm{E}-10$ & IL13 & $1.11 \mathrm{E}+00$ \\
\hline ENSBTAG00000017599 & $1.21 \mathrm{E}-10$ & $1.08 \mathrm{E}-10$ & NR2F1 & $1.12 \mathrm{E}+00$ \\
\hline ENSBTAG00000008335 & $6.10 \mathrm{E}-10$ & $5.48 \mathrm{E}-10$ & & $1.11 \mathrm{E}+00$ \\
\hline ENSBTAG00000019472 & $1.43 \mathrm{E}-10$ & $1.28 \mathrm{E}-10$ & NR3C1 & $1.12 \mathrm{E}+00$ \\
\hline ENSBTAG00000005326 & $1.67 \mathrm{E}-10$ & $1.50 \mathrm{E}-10$ & & $1.12 \mathrm{E}+00$ \\
\hline ENSBTAG00000047414 & $1.25 \mathrm{E}-09$ & 1.12E-09 & & $1.12 \mathrm{E}+00$ \\
\hline ENSBTAG00000002148 & $4.71 \mathrm{E}-10$ & 4.22E-10 & RAB3D & $1.12 \mathrm{E}+00$ \\
\hline ENSBTAG00000013614 & $5.79 \mathrm{E}-10$ & $5.18 \mathrm{E}-10$ & TMEM38A & $1.12 \mathrm{E}+00$ \\
\hline ENSBTAG00000000813 & $2.22 \mathrm{E}-10$ & $1.97 \mathrm{E}-10$ & HOMER3 & $1.12 \mathrm{E}+00$ \\
\hline ENSBTAG00000004630 & $1.84 \mathrm{E}-10$ & $1.64 \mathrm{E}-10$ & COMP & $1.12 \mathrm{E}+00$ \\
\hline ENSBTAG00000002215 & $2.27 \mathrm{E}-10$ & $2.01 \mathrm{E}-10$ & GFPT2 & $1.13 E+00$ \\
\hline ENSBTAG00000007782 & $8.01 \mathrm{E}-11$ & 7.12E-11 & MYOT & $1.12 \mathrm{E}+00$ \\
\hline ENSBTAG00000009772 & $4.03 \mathrm{E}-10$ & $3.57 \mathrm{E}-10$ & LIX1 & $1.13 E+00$ \\
\hline ENSBTAG00000015050 & $2.28 \mathrm{E}-10$ & $2.02 \mathrm{E}-10$ & $\mathrm{HCN} 2$ & $1.13 \mathrm{E}+00$ \\
\hline ENSBTAG00000008250 & $1.20 \mathrm{E}-10$ & $1.07 \mathrm{E}-10$ & SPRY4 & $1.12 \mathrm{E}+00$ \\
\hline ENSBTAG00000014956 & $2.11 \mathrm{E}-10$ & $1.86 \mathrm{E}-10$ & JSRP1 & $1.13 \mathrm{E}+00$ \\
\hline ENSBTAG00000018872 & $1.98 \mathrm{E}-10$ & $1.74 \mathrm{E}-10$ & F12 & $1.14 \mathrm{E}+00$ \\
\hline ENSBTAG00000015307 & $3.26 \mathrm{E}-10$ & $2.87 \mathrm{E}-10$ & FBN2 & $1.14 \mathrm{E}+00$ \\
\hline ENSBTAG00000035241 & $8.16 \mathrm{E}-10$ & $7.18 \mathrm{E}-10$ & & $1.14 \mathrm{E}+00$ \\
\hline ENSBTAG00000004268 & $3.67 \mathrm{E}-10$ & $3.24 \mathrm{E}-10$ & TRAPPC5 & $1.13 \mathrm{E}+00$ \\
\hline ENSBTAG00000045927 & 7.93E-10 & $6.98 \mathrm{E}-10$ & & $1.14 \mathrm{E}+00$ \\
\hline ENSBTAG00000010961 & $3.76 \mathrm{E}-10$ & $3.30 \mathrm{E}-10$ & LYRM7 & $1.14 \mathrm{E}+00$ \\
\hline ENSBTAG00000002347 & $3.76 \mathrm{E}-10$ & $3.30 \mathrm{E}-10$ & MAST3 & $1.14 \mathrm{E}+00$ \\
\hline
\end{tabular}




\begin{tabular}{|c|c|c|c|c|}
\hline ENSBTAG000000012830 & $7.61 \mathrm{E}-11$ & $6.63 \mathrm{E}-11$ & CCDC94 & $1.15 E+00$ \\
\hline ENSBTAG00000010982 & 8.44E-11 & 7.36E-11 & UBE2B & $1.15 \mathrm{E}+00$ \\
\hline ENSBTAG000000017291 & $4.04 \mathrm{E}-10$ & $3.52 \mathrm{E}-10$ & GPR108 & $1.15 \mathrm{E}+00$ \\
\hline ENSBTAG000000038186 & $4.58 \mathrm{E}-10$ & $3.98 \mathrm{E}-10$ & & $1.15 E+00$ \\
\hline ENSBTAG000000020309 & $3.97 \mathrm{E}-10$ & $3.45 \mathrm{E}-10$ & G3BP1 & $1.15 E+00$ \\
\hline ENSBTAG00000019948 & $1.73 \mathrm{E}-10$ & $1.50 \mathrm{E}-10$ & AFAP1L1 & $1.15 E+00$ \\
\hline ENSBTAG00000011698 & 4.47E-10 & $3.88 \mathrm{E}-10$ & TYK2 & $1.15 \mathrm{E}+00$ \\
\hline ENSBTAG000000012587 & $2.25 \mathrm{E}-10$ & $1.95 \mathrm{E}-10$ & & $1.15 E+00$ \\
\hline ENSBTAG000000009647 & $2.31 \mathrm{E}-10$ & $1.99 \mathrm{E}-10$ & PHAX & $1.16 \mathrm{E}+00$ \\
\hline ENSBTAG000000010321 & $2.70 \mathrm{E}-10$ & $2.32 \mathrm{E}-10$ & TTC1 & $1.16 \mathrm{E}+00$ \\
\hline ENSBTAG000000019708 & $4.02 \mathrm{E}-10$ & $3.45 \mathrm{E}-10$ & & $1.16 \mathrm{E}+00$ \\
\hline ENSBTAG000000039523 & $2.40 \mathrm{E}-10$ & $2.06 \mathrm{E}-10$ & & $1.17 E+00$ \\
\hline ENSBTAG00000008186 & $1.90 \mathrm{E}-10$ & $1.63 \mathrm{E}-10$ & UBXN6 & $1.17 \mathrm{E}+00$ \\
\hline ENSBTAG000000024904 & $7.79 \mathrm{E}-10$ & $6.64 \mathrm{E}-10$ & KIAA1683 & $1.17 \mathrm{E}+00$ \\
\hline ENSBTAG000000000306 & $5.34 \mathrm{E}-10$ & $4.55 \mathrm{E}-10$ & ITK & $1.17 \mathrm{E}+00$ \\
\hline ENSBTAG000000007681 & $2.57 \mathrm{E}-10$ & $2.18 \mathrm{E}-10$ & DHFR & $1.18 \mathrm{E}+00$ \\
\hline ENSBTAG000000010740 & $5.87 \mathrm{E}-10$ & $4.95 \mathrm{E}-10$ & CLTB & $1.19 \mathrm{E}+00$ \\
\hline ENSBTAG000000019718 & $1.46 \mathrm{E}-10$ & $1.22 \mathrm{E}-10$ & RPS15 & $1.19 \mathrm{E}+00$ \\
\hline ENSBTAG000000047127 & $1.90 \mathrm{E}-10$ & $1.59 \mathrm{E}-10$ & C19orf25 & $1.19 E+00$ \\
\hline ENSBTAG000000020090 & $1.55 \mathrm{E}-10$ & $1.30 \mathrm{E}-10$ & EPHX3 & $1.19 \mathrm{E}+00$ \\
\hline ENSBTAG000000040305 & $1.56 \mathrm{E}-10$ & $1.31 \mathrm{E}-10$ & & $1.19 \mathrm{E}+00$ \\
\hline ENSBTAG000000010968 & $1.50 \mathrm{E}-10$ & $1.26 \mathrm{E}-10$ & TMED1 & $1.19 \mathrm{E}+00$ \\
\hline ENSBTAG000000044018 & $2.55 \mathrm{E}-10$ & $2.14 \mathrm{E}-10$ & & $1.19 \mathrm{E}+00$ \\
\hline ENSBTAG000000004000 & $5.95 \mathrm{E}-11$ & $5.00 \mathrm{E}-11$ & SAP3OL & $1.19 \mathrm{E}+00$ \\
\hline ENSBTAG000000005581 & $7.06 \mathrm{E}-11$ & $5.92 \mathrm{E}-11$ & $\mathrm{CCL} 25$ & $1.19 \mathrm{E}+00$ \\
\hline ENSBTAG000000014926 & 8.67E-11 & $7.28 \mathrm{E}-11$ & SNAPC2 & $1.19 E+00$ \\
\hline ENSBTAG000000014955 & $8.57 \mathrm{E}-11$ & $7.20 \mathrm{E}-11$ & $\mathrm{AMH}$ & $1.19 E+00$ \\
\hline ENSBTAG000000010069 & $6.51 \mathrm{E}-11$ & $5.47 \mathrm{E}-11$ & EGR1 & $1.19 \mathrm{E}+00$ \\
\hline ENSBTAG00000006077 & $1.92 \mathrm{E}-10$ & $1.62 \mathrm{E}-10$ & RTBDN & $1.19 \mathrm{E}+00$ \\
\hline ENSBTAG000000007739 & $9.29 \mathrm{E}-11$ & $7.80 \mathrm{E}-11$ & C19orf60 & $1.19 E+00$ \\
\hline ENSBTAG000000011400 & $1.06 \mathrm{E}-10$ & 8.94E-11 & DBN1 & $1.19 \mathrm{E}+00$ \\
\hline ENSBTAG000000025425 & $2.59 \mathrm{E}-10$ & 2.17E-10 & RAB24 & $1.19 \mathrm{E}+00$ \\
\hline ENSBTAG00000011351 & $8.33 \mathrm{E}-11$ & 7.00E-11 & TMEM259 & $1.19 \mathrm{E}+00$ \\
\hline ENSBTAG000000009752 & $2.13 \mathrm{E}-10$ & $1.78 \mathrm{E}-10$ & CD70 & $1.19 E+00$ \\
\hline ENSBTAG00000014968 & $1.36 \mathrm{E}-10$ & $1.14 \mathrm{E}-10$ & YJEFN3 & $1.19 \mathrm{E}+00$ \\
\hline ENSBTAG00000047433 & 4.10E-10 & $3.44 \mathrm{E}-10$ & & $1.19 \mathrm{E}+00$ \\
\hline ENSBTAG00000009273 & $3.72 \mathrm{E}-10$ & $3.12 \mathrm{E}-10$ & & $1.19 \mathrm{E}+00$ \\
\hline ENSBTAG00000010984 & $2.55 \mathrm{E}-10$ & $2.14 \mathrm{E}-10$ & CDKN2AIPNL & $1.19 \mathrm{E}+00$ \\
\hline ENSBTAG00000046513 & $5.32 \mathrm{E}-10$ & $4.46 \mathrm{E}-10$ & & $1.19 \mathrm{E}+00$ \\
\hline ENSBTAG00000001794 & $1.96 \mathrm{E}-10$ & $1.65 \mathrm{E}-10$ & & $1.19 E+00$ \\
\hline ENSBTAG00000032142 & $5.38 \mathrm{E}-10$ & $4.51 \mathrm{E}-10$ & & $1.19 \mathrm{E}+00$ \\
\hline ENSBTAG00000014916 & $1.28 \mathrm{E}-10$ & $1.08 \mathrm{E}-10$ & GJC2 & $1.19 \mathrm{E}+00$ \\
\hline ENSBTAG00000031412 & 5.17E-10 & $4.34 \mathrm{E}-10$ & OR6F1 & $1.19 \mathrm{E}+00$ \\
\hline ENSBTAG00000016936 & $1.30 \mathrm{E}-10$ & $1.09 \mathrm{E}-10$ & & $1.19 E+00$ \\
\hline ENSBTAG00000004112 & $2.45 \mathrm{E}-10$ & $2.06 \mathrm{E}-10$ & GAMT & $1.19 E+00$ \\
\hline ENSBTAG00000009568 & $2.85 \mathrm{E}-10$ & $2.39 \mathrm{E}-10$ & KANK2 & $1.19 \mathrm{E}+00$ \\
\hline ENSBTAG00000048056 & $5.00 \mathrm{E}-10$ & 4.19E-10 & & $1.19 \mathrm{E}+00$ \\
\hline ENSBTAG00000015618 & $5.00 \mathrm{E}-10$ & 4.19E-10 & GDF15 & $1.19 E+00$ \\
\hline ENSBTAG00000046953 & $2.61 \mathrm{E}-10$ & $2.19 \mathrm{E}-10$ & & $1.19 E+00$ \\
\hline ENSBTAG00000002434 & $1.36 \mathrm{E}-10$ & $1.15 \mathrm{E}-10$ & WDR18 & $1.19 \mathrm{E}+00$ \\
\hline ENSBTAG00000011254 & $1.05 \mathrm{E}-09$ & $8.81 \mathrm{E}-10$ & OR2Z1 & $1.19 \mathrm{E}+00$ \\
\hline ENSBTAG00000016429 & $1.83 \mathrm{E}-10$ & $1.54 \mathrm{E}-10$ & TMEM205 & $1.19 \mathrm{E}+00$ \\
\hline ENSBTAG00000023623 & $7.90 \mathrm{E}-10$ & $6.62 \mathrm{E}-10$ & & $1.19 \mathrm{E}+00$ \\
\hline ENSBTAG00000019966 & $9.69 \mathrm{E}-10$ & $8.11 E-10$ & TICAM1 & $1.19 E+00$ \\
\hline ENSBTAG00000003434 & $2.84 \mathrm{E}-10$ & 2.37E-10 & CERS4 & $1.20 \mathrm{E}+00$ \\
\hline ENSBTAG00000045733 & $1.06 \mathrm{E}-09$ & $8.84 \mathrm{E}-10$ & & $1.19 E+00$ \\
\hline ENSBTAG00000010871 & $1.23 \mathrm{E}-10$ & $1.02 \mathrm{E}-10$ & & $1.20 \mathrm{E}+00$ \\
\hline ENSBTAG00000019900 & 4.39E-10 & 3.65E-10 & LNPEP & $1.20 \mathrm{E}+00$ \\
\hline ENSBTAG00000007644 & $2.15 \mathrm{E}-10$ & $1.78 \mathrm{E}-10$ & GNG7 & $1.21 E+00$ \\
\hline ENSBTAG00000008695 & $2.16 \mathrm{E}-10$ & $1.79 \mathrm{E}-10$ & TCF3 & $1.21 E+00$ \\
\hline ENSBTAG00000014365 & $3.54 \mathrm{E}-10$ & $2.93 \mathrm{E}-10$ & & $1.21 \mathrm{E}+00$ \\
\hline ENSBTAG00000031673 & $2.02 \mathrm{E}-10$ & $1.67 \mathrm{E}-10$ & ARRDC5 & $1.21 \mathrm{E}+00$ \\
\hline ENSBTAG00000031352 & $2.23 \mathrm{E}-10$ & $1.84 \mathrm{E}-10$ & ZNF554 & $1.21 E+00$ \\
\hline ENSBTAG00000018287 & $5.90 \mathrm{E}-10$ & $4.86 \mathrm{E}-10$ & & $1.21 E+00$ \\
\hline ENSBTAG00000025443 & $1.23 \mathrm{E}-10$ & $1.01 \mathrm{E}-10$ & FNIP1 & $1.22 \mathrm{E}+00$ \\
\hline ENSBTAG00000005265 & $2.22 \mathrm{E}-10$ & $1.82 \mathrm{E}-10$ & & $1.22 \mathrm{E}+00$ \\
\hline ENSBTAG00000025606 & $3.83 \mathrm{E}-10$ & $3.15 E-10$ & YIPF2 & $1.22 \mathrm{E}+00$ \\
\hline ENSBTAG00000020922 & $2.23 \mathrm{E}-10$ & $1.83 \mathrm{E}-10$ & UPF1 & $1.22 \mathrm{E}+00$ \\
\hline ENSBTAG00000024450 & $8.73 \mathrm{E}-11$ & 7.17E-11 & & $1.22 \mathrm{E}+00$ \\
\hline ENSBTAG00000006371 & $2.05 \mathrm{E}-10$ & $1.68 \mathrm{E}-10$ & RMND5B & $1.22 \mathrm{E}+00$ \\
\hline
\end{tabular}




\begin{tabular}{|c|c|c|c|c|}
\hline ENSBTAG00000005999 & $1.18 \mathrm{E}-10$ & $9.62 \mathrm{E}-11$ & FSD1 & $1.23 \mathrm{E}+00$ \\
\hline ENSBTAG00000008435 & $9.29 \mathrm{E}-11$ & 7.57E-11 & KIAA0141 & $1.23 E+00$ \\
\hline ENSBTAG00000010487 & $4.09 \mathrm{E}-10$ & $3.32 \mathrm{E}-10$ & SMIM3 & $1.23 E+00$ \\
\hline ENSBTAG00000010639 & $1.20 \mathrm{E}-10$ & $9.73 \mathrm{E}-11$ & MAP2K7 & $1.23 \mathrm{E}+00$ \\
\hline ENSBTAG00000047215 & $1.49 \mathrm{E}-09$ & $1.21 \mathrm{E}-09$ & & $1.23 E+00$ \\
\hline ENSBTAG00000019868 & $3.10 \mathrm{E}-10$ & $2.52 \mathrm{E}-10$ & & $1.23 E+00$ \\
\hline ENSBTAG00000047921 & $1.24 \mathrm{E}-09$ & $1.00 \mathrm{E}-09$ & & $1.24 \mathrm{E}+00$ \\
\hline ENSBTAG00000021445 & $4.65 \mathrm{E}-10$ & $3.75 \mathrm{E}-10$ & ST8SIA4 & $1.24 \mathrm{E}+00$ \\
\hline ENSBTAG00000007343 & $1.02 \mathrm{E}-10$ & $8.22 \mathrm{E}-11$ & PDLIM7 & $1.24 \mathrm{E}+00$ \\
\hline ENSBTAG00000006978 & $6.37 \mathrm{E}-10$ & $5.13 \mathrm{E}-10$ & HSD17B4 & $1.24 \mathrm{E}+00$ \\
\hline ENSBTAG00000048193 & $1.38 \mathrm{E}-09$ & $1.11 \mathrm{E}-09$ & & $1.24 \mathrm{E}+00$ \\
\hline ENSBTAG00000033886 & $5.94 \mathrm{E}-10$ & 4.77E-10 & & $1.24 \mathrm{E}+00$ \\
\hline ENSBTAG00000010379 & $3.11 E-10$ & $2.50 \mathrm{E}-10$ & CRTC1 & $1.24 \mathrm{E}+00$ \\
\hline ENSBTAG00000011415 & $3.23 \mathrm{E}-11$ & $2.59 \mathrm{E}-11$ & ETF1 & $1.24 \mathrm{E}+00$ \\
\hline ENSBTAG00000013450 & $3.39 \mathrm{E}-10$ & $2.71 \mathrm{E}-10$ & FAM71B & $1.25 E+00$ \\
\hline ENSBTAG00000043971 & $2.39 \mathrm{E}-10$ & $1.91 \mathrm{E}-10$ & NOTCH3 & $1.25 \mathrm{E}+00$ \\
\hline ENSBTAG00000010318 & $4.10 \mathrm{E}-10$ & $3.28 \mathrm{E}-10$ & FDX2 & $1.25 \mathrm{E}+00$ \\
\hline ENSBTAG00000020229 & $1.93 \mathrm{E}-10$ & $1.55 \mathrm{E}-10$ & ATP8B3 & $1.25 \mathrm{E}+00$ \\
\hline ENSBTAG00000012682 & $2.45 \mathrm{E}-10$ & $1.95 \mathrm{E}-10$ & UNC13A & $1.26 \mathrm{E}+00$ \\
\hline ENSBTAG00000024809 & 5.67E-10 & $4.52 \mathrm{E}-10$ & & $1.25 \mathrm{E}+00$ \\
\hline ENSBTAG00000012671 & $4.54 \mathrm{E}-10$ & $3.61 E-10$ & TNIP1 & $1.26 \mathrm{E}+00$ \\
\hline ENSBTAG00000038981 & $8.27 \mathrm{E}-10$ & $6.59 \mathrm{E}-10$ & & $1.26 \mathrm{E}+00$ \\
\hline ENSBTAG00000015034 & $1.81 \mathrm{E}-10$ & $1.44 \mathrm{E}-10$ & IK & $1.26 \mathrm{E}+00$ \\
\hline ENSBTAG00000008916 & $5.20 \mathrm{E}-10$ & 4.13E-10 & CYFIP2 & $1.26 \mathrm{E}+00$ \\
\hline ENSBTAG00000021360 & $3.40 \mathrm{E}-10$ & 2.70E-10 & RASGEF1C & $1.26 \mathrm{E}+00$ \\
\hline ENSBTAG00000040157 & $6.55 \mathrm{E}-10$ & $5.20 \mathrm{E}-10$ & & $1.26 \mathrm{E}+00$ \\
\hline ENSBTAG00000020860 & $2.46 \mathrm{E}-10$ & $1.95 \mathrm{E}-10$ & $\mathrm{CHD} 1$ & $1.26 \mathrm{E}+00$ \\
\hline ENSBTAG00000000717 & $2.21 \mathrm{E}-10$ & $1.75 \mathrm{E}-10$ & PLPP2 & $1.26 \mathrm{E}+00$ \\
\hline ENSBTAG00000020780 & $9.11 \mathrm{E}-11$ & $7.21 \mathrm{E}-11$ & SBNO2 & $1.26 \mathrm{E}+00$ \\
\hline ENSBTAG00000009482 & $1.70 \mathrm{E}-10$ & $1.34 \mathrm{E}-10$ & AFF4 & $1.27 E+00$ \\
\hline ENSBTAG00000008753 & $7.00 \mathrm{E}-11$ & $5.51 \mathrm{E}-11$ & BRD8 & $1.27 \mathrm{E}+00$ \\
\hline ENSBTAG00000047244 & $6.88 \mathrm{E}-10$ & $5.41 \mathrm{E}-10$ & & $1.27 \mathrm{E}+00$ \\
\hline ENSBTAG00000047668 & $2.82 \mathrm{E}-10$ & $2.22 \mathrm{E}-10$ & & $1.27 \mathrm{E}+00$ \\
\hline ENSBTAG00000007875 & 2.29E-10 & $1.80 \mathrm{E}-10$ & SAFB & $1.27 \mathrm{E}+00$ \\
\hline ENSBTAG00000035399 & $7.00 \mathrm{E}-10$ & $5.50 \mathrm{E}-10$ & OR1M1 & $1.27 \mathrm{E}+00$ \\
\hline ENSBTAG00000000399 & $2.49 \mathrm{E}-10$ & $1.96 \mathrm{E}-10$ & NANOS3 & $1.27 \mathrm{E}+00$ \\
\hline ENSBTAG00000013857 & $6.91 \mathrm{E}-10$ & $5.40 \mathrm{E}-10$ & $\mathrm{SH} 2 \mathrm{D} 3 \mathrm{~A}$ & $1.28 \mathrm{E}+00$ \\
\hline ENSBTAG00000013291 & $1.91 \mathrm{E}-10$ & $1.49 \mathrm{E}-10$ & ARMC6 & $1.28 \mathrm{E}+00$ \\
\hline ENSBTAG00000001604 & $7.61 \mathrm{E}-10$ & $5.95 \mathrm{E}-10$ & & $1.28 \mathrm{E}+00$ \\
\hline ENSBTAG00000002566 & $5.71 \mathrm{E}-10$ & 4.45E-10 & PGPEP1 & $1.28 \mathrm{E}+00$ \\
\hline ENSBTAG00000032364 & $5.01 \mathrm{E}-10$ & $3.91 \mathrm{E}-10$ & & $1.28 \mathrm{E}+00$ \\
\hline ENSBTAG00000008607 & $7.32 \mathrm{E}-11$ & $5.68 \mathrm{E}-11$ & ARID3A & $1.29 \mathrm{E}+00$ \\
\hline ENSBTAG00000039160 & $3.54 \mathrm{E}-10$ & $2.74 \mathrm{E}-10$ & VAV1 & $1.29 \mathrm{E}+00$ \\
\hline ENSBTAG00000014828 & $4.99 \mathrm{E}-10$ & $3.86 \mathrm{E}-10$ & CACNA1A & $1.29 \mathrm{E}+00$ \\
\hline ENSBTAG00000007550 & $1.60 \mathrm{E}-10$ & $1.24 \mathrm{E}-10$ & OLFM2 & $1.29 \mathrm{E}+00$ \\
\hline ENSBTAG00000001002 & $7.74 \mathrm{E}-11$ & $5.98 \mathrm{E}-11$ & TCF7 & $1.29 \mathrm{E}+00$ \\
\hline ENSBTAG00000017273 & $3.34 \mathrm{E}-10$ & $2.57 \mathrm{E}-10$ & LRRC25 & $1.30 \mathrm{E}+00$ \\
\hline ENSBTAG00000015433 & $1.47 \mathrm{E}-10$ & $1.14 \mathrm{E}-10$ & APC2 & $1.29 E+00$ \\
\hline ENSBTAG00000010727 & 7.77E-10 & $5.98 \mathrm{E}-10$ & ATG4D & $1.30 \mathrm{E}+00$ \\
\hline ENSBTAG00000003498 & $3.16 \mathrm{E}-10$ & $2.41 E-10$ & FAT2 & $1.31 \mathrm{E}+00$ \\
\hline ENSBTAG00000004709 & $2.31 \mathrm{E}-10$ & $1.76 \mathrm{E}-10$ & MAPK9 & $1.31 E+00$ \\
\hline ENSBTAG00000001153 & $5.71 \mathrm{E}-10$ & $4.33 \mathrm{E}-10$ & TLE2 & $1.32 \mathrm{E}+00$ \\
\hline ENSBTAG00000014349 & $5.18 \mathrm{E}-11$ & $3.91 E-11$ & RNF126 & $1.32 \mathrm{E}+00$ \\
\hline ENSBTAG00000025540 & $2.56 \mathrm{E}-10$ & $1.93 \mathrm{E}-10$ & ALKBH7 & $1.32 \mathrm{E}+00$ \\
\hline ENSBTAG00000025597 & $7.38 \mathrm{E}-11$ & $5.58 \mathrm{E}-11$ & GRIN3B & $1.32 \mathrm{E}+00$ \\
\hline ENSBTAG00000015090 & $2.27 \mathrm{E}-10$ & $1.72 \mathrm{E}-10$ & SGTA & $1.32 \mathrm{E}+00$ \\
\hline ENSBTAG00000005009 & $1.15 \mathrm{E}-10$ & $8.71 E-11$ & PRELID1 & $1.32 \mathrm{E}+00$ \\
\hline ENSBTAG00000046397 & $3.88 \mathrm{E}-10$ & $2.93 \mathrm{E}-10$ & & $1.32 \mathrm{E}+00$ \\
\hline ENSBTAG00000020709 & $2.44 \mathrm{E}-10$ & $1.85 \mathrm{E}-10$ & MAP1S & $1.32 \mathrm{E}+00$ \\
\hline ENSBTAG00000003587 & $2.06 \mathrm{E}-10$ & $1.56 \mathrm{E}-10$ & ELL & $1.32 \mathrm{E}+00$ \\
\hline ENSBTAG00000013613 & $5.46 \mathrm{E}-10$ & $4.12 \mathrm{E}-10$ & SMIM7 & $1.32 \mathrm{E}+00$ \\
\hline ENSBTAG00000038409 & $3.49 \mathrm{E}-10$ & 2.63E-10 & MBD3 & $1.33 \mathrm{E}+00$ \\
\hline ENSBTAG00000005441 & $7.28 \mathrm{E}-10$ & $5.49 \mathrm{E}-10$ & ZMAT2 & $1.33 \mathrm{E}+00$ \\
\hline ENSBTAG00000020843 & $7.18 \mathrm{E}-10$ & $5.41 \mathrm{E}-10$ & PCSK1 & $1.33 E+00$ \\
\hline ENSBTAG00000019714 & $1.37 \mathrm{E}-10$ & $1.04 \mathrm{E}-10$ & DAZAP1 & $1.32 \mathrm{E}+00$ \\
\hline ENSBTAG00000002686 & $8.76 \mathrm{E}-10$ & $6.60 \mathrm{E}-10$ & & $1.33 E+00$ \\
\hline ENSBTAG00000040482 & $8.76 \mathrm{E}-10$ & $6.60 \mathrm{E}-10$ & & $1.33 E+00$ \\
\hline ENSBTAG00000020764 & $1.38 \mathrm{E}-10$ & $1.05 \mathrm{E}-10$ & CNN2 & $1.32 \mathrm{E}+00$ \\
\hline ENSBTAG00000009328 & $4.79 \mathrm{E}-10$ & 3.60E-10 & NWD1 & $1.33 \mathrm{E}+00$ \\
\hline ENSBTAG00000012758 & $2.71 \mathrm{E}-10$ & $2.03 \mathrm{E}-10$ & YIPF5 & $1.33 E+00$ \\
\hline
\end{tabular}




\begin{tabular}{|c|c|c|c|c|}
\hline ENSBTAG00000005249 & $1.20 \mathrm{E}-10$ & 8.97E-11 & RNF14 & $1.34 \mathrm{E}+00$ \\
\hline ENSBTAG00000020489 & $1.94 \mathrm{E}-10$ & $1.45 \mathrm{E}-10$ & SEMA6A & $1.34 \mathrm{E}+00$ \\
\hline ENSBTAG00000046424 & 2.79E-09 & 2.07E-09 & & $1.35 \mathrm{E}+00$ \\
\hline ENSBTAG00000014415 & $1.18 \mathrm{E}-10$ & $8.79 E-11$ & ZNF653 & $1.34 \mathrm{E}+00$ \\
\hline ENSBTAG00000001816 & $1.32 \mathrm{E}-10$ & $9.81 \mathrm{E}-11$ & SLC25A42 & $1.35 \mathrm{E}+00$ \\
\hline ENSBTAG00000025340 & $4.85 \mathrm{E}-10$ & $3.59 \mathrm{E}-10$ & CDHR2 & $1.35 E+00$ \\
\hline ENSBTAG00000015557 & $5.13 \mathrm{E}-10$ & $3.80 \mathrm{E}-10$ & & $1.35 \mathrm{E}+00$ \\
\hline ENSBTAG00000010188 & $4.32 \mathrm{E}-10$ & $3.19 \mathrm{E}-10$ & $\mathrm{RDH} 8$ & $1.35 E+00$ \\
\hline ENSBTAG00000001763 & $6.34 \mathrm{E}-10$ & 4.69E-10 & NR2C2AP & $1.35 E+00$ \\
\hline ENSBTAG00000000414 & $7.80 \mathrm{E}-10$ & $5.75 \mathrm{E}-10$ & & $1.36 \mathrm{E}+00$ \\
\hline ENSBTAG00000036102 & $1.03 \mathrm{E}-09$ & $7.61 \mathrm{E}-10$ & & $1.36 \mathrm{E}+00$ \\
\hline ENSBTAG00000031205 & $1.25 \mathrm{E}-09$ & $9.18 \mathrm{E}-10$ & & $1.37 \mathrm{E}+00$ \\
\hline ENSBTAG00000003836 & $3.63 \mathrm{E}-10$ & $2.66 \mathrm{E}-10$ & ADAM19 & $1.36 \mathrm{E}+00$ \\
\hline ENSBTAG00000017661 & $5.91 \mathrm{E}-10$ & $4.33 \mathrm{E}-10$ & RFX2 & $1.37 \mathrm{E}+00$ \\
\hline ENSBTAG00000024275 & $2.34 \mathrm{E}-10$ & $1.71 \mathrm{E}-10$ & XRCC4 & $1.37 \mathrm{E}+00$ \\
\hline ENSBTAG00000004369 & $8.51 \mathrm{E}-10$ & $6.19 \mathrm{E}-10$ & & $1.37 E+00$ \\
\hline ENSBTAG00000001546 & $1.23 \mathrm{E}-10$ & 8.93E-11 & MGAT1 & $1.38 \mathrm{E}+00$ \\
\hline ENSBTAG00000008752 & $9.47 \mathrm{E}-11$ & $6.87 E-11$ & NME5 & $1.38 \mathrm{E}+00$ \\
\hline ENSBTAG00000047300 & $6.50 \mathrm{E}-10$ & $4.71 \mathrm{E}-10$ & & $1.38 \mathrm{E}+00$ \\
\hline ENSBTAG00000037602 & $2.23 \mathrm{E}-10$ & $1.62 \mathrm{E}-10$ & & $1.38 \mathrm{E}+00$ \\
\hline ENSBTAG00000012868 & $4.87 \mathrm{E}-10$ & $3.51 \mathrm{E}-10$ & ZNF77 & $1.39 \mathrm{E}+00$ \\
\hline ENSBTAG00000002473 & $8.11 \mathrm{E}-11$ & $5.84 \mathrm{E}-11$ & ANGPTL4 & $1.39 E+00$ \\
\hline ENSBTAG00000001450 & $9.20 \mathrm{E}-11$ & $6.62 \mathrm{E}-11$ & APBB3 & $1.39 E+00$ \\
\hline ENSBTAG00000037764 & $3.82 \mathrm{E}-10$ & $2.75 \mathrm{E}-10$ & ZNRF4 & $1.39 \mathrm{E}+00$ \\
\hline ENSBTAG00000004521 & $2.43 \mathrm{E}-10$ & $1.75 \mathrm{E}-10$ & LSM7 & $1.39 E+00$ \\
\hline ENSBTAG00000014676 & $1.57 \mathrm{E}-10$ & $1.13 \mathrm{E}-10$ & SHROOM1 & $1.39 \mathrm{E}+00$ \\
\hline ENSBTAG00000020446 & $1.77 \mathrm{E}-10$ & $1.27 E-10$ & THOP1 & $1.40 \mathrm{E}+00$ \\
\hline ENSBTAG00000021775 & $1.35 \mathrm{E}-10$ & $9.58 \mathrm{E}-11$ & SLC4A9 & $1.40 \mathrm{E}+00$ \\
\hline ENSBTAG00000017068 & $1.34 \mathrm{E}-10$ & $9.49 \mathrm{E}-11$ & & $1.41 \mathrm{E}+00$ \\
\hline ENSBTAG00000000329 & $5.36 \mathrm{E}-10$ & $3.81 \mathrm{E}-10$ & MEGF10 & $1.41 E+00$ \\
\hline ENSBTAG00000020178 & $2.22 \mathrm{E}-10$ & $1.58 \mathrm{E}-10$ & ARSK & $1.41 E+00$ \\
\hline ENSBTAG00000017079 & $1.77 \mathrm{E}-10$ & $1.25 \mathrm{E}-10$ & MFAP3 & $1.41 \mathrm{E}+00$ \\
\hline ENSBTAG00000001795 & $2.06 \mathrm{E}-10$ & $1.46 \mathrm{E}-10$ & LONP1 & $1.41 E+00$ \\
\hline ENSBTAG00000010541 & $5.27 \mathrm{E}-11$ & $3.73 E-11$ & ZNF346 & $1.41 E+00$ \\
\hline ENSBTAG00000000238 & $1.25 \mathrm{E}-10$ & $8.84 \mathrm{E}-11$ & PSD2 & $1.41 E+00$ \\
\hline ENSBTAG00000011837 & $1.62 \mathrm{E}-10$ & $1.15 \mathrm{E}-10$ & ASNA1 & $1.41 E+00$ \\
\hline ENSBTAG00000010179 & $4.86 \mathrm{E}-10$ & $3.44 \mathrm{E}-10$ & COL5A3 & $1.41 E+00$ \\
\hline ENSBTAG00000047578 & $1.43 \mathrm{E}-10$ & $1.01 \mathrm{E}-10$ & & $1.41 E+00$ \\
\hline ENSBTAG00000015625 & $2.27 \mathrm{E}-10$ & $1.59 \mathrm{E}-10$ & DCTN4 & $1.43 E+00$ \\
\hline ENSBTAG00000018522 & $2.09 \mathrm{E}-10$ & $1.46 \mathrm{E}-10$ & OAZ1 & $1.43 \mathrm{E}+00$ \\
\hline ENSBTAG00000015114 & $1.93 \mathrm{E}-10$ & $1.35 \mathrm{E}-10$ & CALR & $1.43 \mathrm{E}+00$ \\
\hline ENSBTAG00000020772 & 7.91E-11 & $5.54 \mathrm{E}-11$ & ARHGAP45 & $1.43 E+00$ \\
\hline ENSBTAG00000014114 & $1.73 \mathrm{E}-10$ & $1.21 \mathrm{E}-10$ & KLHL26 & $1.43 \mathrm{E}+00$ \\
\hline ENSBTAG00000001692 & $1.68 \mathrm{E}-10$ & $1.17 \mathrm{E}-10$ & NDST1 & $1.43 \mathrm{E}+00$ \\
\hline ENSBTAG00000031387 & $3.49 \mathrm{E}-10$ & $2.43 \mathrm{E}-10$ & FZR1 & $1.43 \mathrm{E}+00$ \\
\hline ENSBTAG00000047696 & $7.93 \mathrm{E}-10$ & $5.53 \mathrm{E}-10$ & & $1.43 E+00$ \\
\hline ENSBTAG00000008202 & $3.42 \mathrm{E}-10$ & $2.38 \mathrm{E}-10$ & PRKCSH & $1.44 \mathrm{E}+00$ \\
\hline ENSBTAG00000008609 & $1.64 \mathrm{E}-10$ & $1.14 \mathrm{E}-10$ & 08-Sep & $1.44 \mathrm{E}+00$ \\
\hline ENSBTAG00000019767 & $2.92 \mathrm{E}-10$ & $2.04 \mathrm{E}-10$ & MIER2 & $1.43 E+00$ \\
\hline ENSBTAG00000018049 & $1.71 \mathrm{E}-10$ & $1.19 \mathrm{E}-10$ & MKNK2 & $1.44 \mathrm{E}+00$ \\
\hline ENSBTAG00000013251 & $1.82 \mathrm{E}-10$ & $1.26 \mathrm{E}-10$ & & $1.44 \mathrm{E}+00$ \\
\hline ENSBTAG00000012150 & $1.33 \mathrm{E}-10$ & $9.13 \mathrm{E}-11$ & & $1.46 \mathrm{E}+00$ \\
\hline ENSBTAG00000007661 & $2.36 \mathrm{E}-10$ & $1.62 \mathrm{E}-10$ & MYO1F & $1.45 E+00$ \\
\hline ENSBTAG00000031135 & $2.93 \mathrm{E}-10$ & $2.01 \mathrm{E}-10$ & CTXN3 & $1.46 \mathrm{E}+00$ \\
\hline ENSBTAG00000011255 & $6.29 \mathrm{E}-10$ & $4.31 \mathrm{E}-10$ & ACTL9 & $1.46 \mathrm{E}+00$ \\
\hline ENSBTAG00000012280 & $1.78 \mathrm{E}-10$ & $1.22 \mathrm{E}-10$ & PGLYRP2 & $1.46 \mathrm{E}+00$ \\
\hline ENSBTAG00000020759 & $3.01 \mathrm{E}-10$ & $2.06 \mathrm{E}-10$ & NCLN & $1.46 \mathrm{E}+00$ \\
\hline ENSBTAG00000005520 & $9.43 \mathrm{E}-10$ & $6.45 \mathrm{E}-10$ & OR7C2 & $1.46 \mathrm{E}+00$ \\
\hline ENSBTAG00000001790 & $1.69 \mathrm{E}-10$ & $1.15 \mathrm{E}-10$ & SAFB2 & $1.47 \mathrm{E}+00$ \\
\hline ENSBTAG00000045762 & $2.80 \mathrm{E}-10$ & $1.91 \mathrm{E}-10$ & SCAMP4 & $1.46 \mathrm{E}+00$ \\
\hline ENSBTAG00000017074 & $2.11 \mathrm{E}-10$ & $1.43 \mathrm{E}-10$ & ANO8 & $1.47 \mathrm{E}+00$ \\
\hline ENSBTAG00000013627 & $1.82 \mathrm{E}-10$ & $1.23 \mathrm{E}-10$ & HNRNPAB & $1.48 \mathrm{E}+00$ \\
\hline ENSBTAG00000008095 & $4.68 \mathrm{E}-10$ & $3.16 \mathrm{E}-10$ & ACER1 & $1.48 \mathrm{E}+00$ \\
\hline ENSBTAG00000013329 & $4.02 \mathrm{E}-10$ & $2.72 \mathrm{E}-10$ & AP1M1 & $1.48 \mathrm{E}+00$ \\
\hline ENSBTAG00000004188 & $7.22 \mathrm{E}-10$ & $4.88 \mathrm{E}-10$ & SNAP47 & $1.48 \mathrm{E}+00$ \\
\hline ENSBTAG00000043553 & $5.64 \mathrm{E}-10$ & $3.80 \mathrm{E}-10$ & GPX3 & $1.48 \mathrm{E}+00$ \\
\hline ENSBTAG00000027151 & $2.03 \mathrm{E}-10$ & $1.36 \mathrm{E}-10$ & ARHGAP26 & $1.49 E+00$ \\
\hline ENSBTAG00000039809 & $5.38 \mathrm{E}-10$ & $3.60 \mathrm{E}-10$ & & $1.49 \mathrm{E}+00$ \\
\hline ENSBTAG00000037729 & $3.54 \mathrm{E}-10$ & $2.37 \mathrm{E}-10$ & & $1.49 \mathrm{E}+00$ \\
\hline ENSBTAG00000016264 & $1.78 \mathrm{E}-10$ & $1.18 \mathrm{E}-10$ & & $1.50 \mathrm{E}+00$ \\
\hline
\end{tabular}




\begin{tabular}{|c|c|c|c|c|}
\hline ENSBTAG000000013502 & $2.96 \mathrm{E}-10$ & $1.95 \mathrm{E}-10$ & SLC1A6 & $1.52 \mathrm{E}+00$ \\
\hline ENSBTAG000000005288 & $3.59 \mathrm{E}-10$ & 2.37E-10 & GFRA3 & $1.52 E+00$ \\
\hline ENSBTAG000000039130 & 7.57E-10 & $4.98 \mathrm{E}-10$ & & $1.52 E+00$ \\
\hline ENSBTAG000000020904 & $1.41 \mathrm{E}-10$ & $9.27 \mathrm{E}-11$ & JAK3 & $1.52 E+00$ \\
\hline ENSBTAG000000008841 & $2.04 \mathrm{E}-10$ & $1.34 \mathrm{E}-10$ & DCAF15 & $1.52 \mathrm{E}+00$ \\
\hline ENSBTAG00000006619 & $5.69 \mathrm{E}-10$ & $3.71 \mathrm{E}-10$ & MATK & $1.53 E+00$ \\
\hline ENSBTAG00000011811 & $1.31 \mathrm{E}-10$ & $8.55 \mathrm{E}-11$ & TNPO2 & $1.53 \mathrm{E}+00$ \\
\hline ENSBTAG000000015683 & 4.19E-10 & $2.72 \mathrm{E}-10$ & HSPA4 & $1.54 \mathrm{E}+00$ \\
\hline ENSBTAG000000030366 & $2.50 \mathrm{E}-10$ & $1.62 \mathrm{E}-10$ & RBM22 & $1.54 \mathrm{E}+00$ \\
\hline ENSBTAG000000020954 & $2.07 \mathrm{E}-10$ & $1.34 \mathrm{E}-10$ & TRIM7 & $1.54 \mathrm{E}+00$ \\
\hline ENSBTAG000000038541 & $1.99 \mathrm{E}-10$ & $1.29 \mathrm{E}-10$ & ZNF879 & $1.54 \mathrm{E}+00$ \\
\hline ENSBTAG000000045972 & $2.38 \mathrm{E}-09$ & $1.54 \mathrm{E}-09$ & & $1.54 \mathrm{E}+00$ \\
\hline ENSBTAG00000031246 & $4.78 \mathrm{E}-10$ & $3.10 \mathrm{E}-10$ & CCNI2 & $1.54 \mathrm{E}+00$ \\
\hline ENSBTAG000000016094 & $6.00 \mathrm{E}-10$ & $3.88 \mathrm{E}-10$ & SLC36A1 & $1.55 \mathrm{E}+00$ \\
\hline ENSBTAG000000000810 & $3.26 \mathrm{E}-10$ & $2.11 E-10$ & COPE & $1.54 \mathrm{E}+00$ \\
\hline ENSBTAG000000006068 & $3.14 \mathrm{E}-10$ & 2.03E-10 & CC2D1A & $1.54 \mathrm{E}+00$ \\
\hline ENSBTAG000000007116 & $2.65 \mathrm{E}-10$ & $1.71 \mathrm{E}-10$ & ARRDC3 & $1.55 E+00$ \\
\hline ENSBTAG000000001764 & 5.63E-10 & 3.62E-10 & NCAN & $1.56 \mathrm{E}+00$ \\
\hline ENSBTAG000000045588 & 1.53E-09 & $9.86 \mathrm{E}-10$ & & $1.56 \mathrm{E}+00$ \\
\hline ENSBTAG000000030210 & $5.73 \mathrm{E}-10$ & $3.66 \mathrm{E}-10$ & SLCO6A1 & $1.56 \mathrm{E}+00$ \\
\hline ENSBTAG000000014746 & $4.48 \mathrm{E}-10$ & 2.87E-10 & CATSPER3 & $1.56 \mathrm{E}+00$ \\
\hline ENSBTAG000000001462 & $1.66 \mathrm{E}-10$ & $1.06 \mathrm{E}-10$ & FLT4 & $1.57 E+00$ \\
\hline ENSBTAG000000009330 & 8.93E-10 & 5.70E-10 & SIN3B & $1.57 \mathrm{E}+00$ \\
\hline ENSBTAG000000003502 & $5.97 \mathrm{E}-10$ & $3.80 \mathrm{E}-10$ & & $1.57 \mathrm{E}+00$ \\
\hline ENSBTAG000000006080 & $3.61 \mathrm{E}-10$ & 2.29E-10 & MAST1 & $1.57 \mathrm{E}+00$ \\
\hline ENSBTAG00000009479 & $1.35 \mathrm{E}-10$ & $8.51 \mathrm{E}-11$ & UQCRQ & $1.59 \mathrm{E}+00$ \\
\hline ENSBTAG000000046718 & $2.06 \mathrm{E}-10$ & $1.29 \mathrm{E}-10$ & TEX43 & $1.59 E+00$ \\
\hline ENSBTAG000000025452 & $1.38 \mathrm{E}-10$ & $8.69 \mathrm{E}-11$ & SF3A2 & $1.59 \mathrm{E}+00$ \\
\hline ENSBTAG000000013628 & $2.33 \mathrm{E}-10$ & 1.47E-10 & PHYKPL & $1.59 E+00$ \\
\hline ENSBTAG000000010363 & $1.81 \mathrm{E}-10$ & $1.14 \mathrm{E}-10$ & LSM11 & $1.59 E+00$ \\
\hline ENSBTAG000000019325 & $1.26 \mathrm{E}-10$ & $7.95 \mathrm{E}-11$ & TRMT1 & $1.59 \mathrm{E}+00$ \\
\hline ENSBTAG00000011207 & $6.28 \mathrm{E}-11$ & $3.95 \mathrm{E}-11$ & CNN1 & $1.59 \mathrm{E}+00$ \\
\hline ENSBTAG000000008789 & $8.63 \mathrm{E}-11$ & $5.43 \mathrm{E}-11$ & & $1.59 E+00$ \\
\hline ENSBTAG000000017748 & $2.97 \mathrm{E}-10$ & 1.87E-10 & RNF44 & $1.59 E+00$ \\
\hline ENSBTAG00000046188 & $1.05 \mathrm{E}-10$ & $6.60 \mathrm{E}-11$ & ELANE & $1.59 \mathrm{E}+00$ \\
\hline ENSBTAG00000005570 & $1.33 \mathrm{E}-10$ & 8.35E-11 & WNT8A & $1.59 \mathrm{E}+00$ \\
\hline ENSBTAG00000003947 & $1.67 \mathrm{E}-10$ & $1.05 \mathrm{E}-10$ & SSBP4 & $1.59 \mathrm{E}+00$ \\
\hline ENSBTAG00000047576 & 3.55E-10 & 2.23E-10 & & $1.59 \mathrm{E}+00$ \\
\hline ENSBTAG00000039711 & $6.02 \mathrm{E}-11$ & $3.79 \mathrm{E}-11$ & ZFP62 & $1.59 \mathrm{E}+00$ \\
\hline ENSBTAG00000002525 & $1.21 \mathrm{E}-10$ & 7.60E-11 & & $1.59 E+00$ \\
\hline ENSBTAG00000012014 & $1.01 \mathrm{E}-10$ & $6.39 \mathrm{E}-11$ & & $1.59 E+00$ \\
\hline ENSBTAG00000016477 & $1.88 \mathrm{E}-10$ & $1.18 \mathrm{E}-10$ & C19orf35 & $1.59 \mathrm{E}+00$ \\
\hline ENSBTAG00000015187 & $8.81 \mathrm{E}-11$ & $5.55 \mathrm{E}-11$ & DDX41 & $1.59 \mathrm{E}+00$ \\
\hline ENSBTAG00000047217 & $8.86 \mathrm{E}-11$ & $5.58 \mathrm{E}-11$ & MED16 & $1.59 E+00$ \\
\hline ENSBTAG00000002100 & $2.68 \mathrm{E}-10$ & $1.69 \mathrm{E}-10$ & GZMM & $1.59 E+00$ \\
\hline ENSBTAG00000008395 & $2.21 \mathrm{E}-10$ & $1.39 \mathrm{E}-10$ & AРBA3 & $1.59 \mathrm{E}+00$ \\
\hline ENSBTAG00000030517 & $9.15 \mathrm{E}-11$ & $5.76 \mathrm{E}-11$ & & $1.59 \mathrm{E}+00$ \\
\hline ENSBTAG00000014982 & $4.03 \mathrm{E}-10$ & $2.54 \mathrm{E}-10$ & C19orf71 & $1.59 E+00$ \\
\hline ENSBTAG00000017957 & $6.34 \mathrm{E}-11$ & $3.99 \mathrm{E}-11$ & SPATA24 & $1.59 E+00$ \\
\hline ENSBTAG00000015830 & $1.28 \mathrm{E}-10$ & 8.07E-11 & SLC5A5 & $1.59 \mathrm{E}+00$ \\
\hline ENSBTAG00000006368 & $2.72 \mathrm{E}-10$ & $1.71 \mathrm{E}-10$ & N4BP3 & $1.59 \mathrm{E}+00$ \\
\hline ENSBTAG00000048170 & 3.39E-10 & 2.13E-10 & PCDHA11 & $1.59 \mathrm{E}+00$ \\
\hline ENSBTAG00000020353 & $3.00 \mathrm{E}-10$ & $1.89 \mathrm{E}-10$ & PFN3 & $1.59 \mathrm{E}+00$ \\
\hline ENSBTAG00000012223 & $1.16 \mathrm{E}-10$ & $7.32 \mathrm{E}-11$ & TNFSF14 & $1.59 \mathrm{E}+00$ \\
\hline ENSBTAG00000034743 & $6.76 \mathrm{E}-10$ & 4.24E-10 & & $1.59 E+00$ \\
\hline ENSBTAG00000047180 & $5.25 \mathrm{E}-10$ & $3.30 \mathrm{E}-10$ & & $1.59 \mathrm{E}+00$ \\
\hline ENSBTAG00000047182 & $5.25 \mathrm{E}-10$ & 3.30E-10 & & $1.59 \mathrm{E}+00$ \\
\hline ENSBTAG00000010314 & 4.67E-10 & 2.93E-10 & & $1.59 \mathrm{E}+00$ \\
\hline ENSBTAG00000006216 & $7.06 \mathrm{E}-10$ & $4.44 \mathrm{E}-10$ & & $1.59 \mathrm{E}+00$ \\
\hline ENSBTAG00000005434 & $6.54 \mathrm{E}-10$ & $4.11 E-10$ & PLVAP & $1.59 E+00$ \\
\hline ENSBTAG00000048025 & $5.31 \mathrm{E}-10$ & $3.33 \mathrm{E}-10$ & & $1.59 \mathrm{E}+00$ \\
\hline ENSBTAG00000012062 & $1.87 \mathrm{E}-10$ & 1.17E-10 & PRDX2 & $1.59 \mathrm{E}+00$ \\
\hline ENSBTAG00000017360 & $1.27 \mathrm{E}-10$ & $7.98 \mathrm{E}-11$ & & $1.59 \mathrm{E}+00$ \\
\hline ENSBTAG00000039533 & $7.02 \mathrm{E}-10$ & $4.41 E-10$ & & $1.59 E+00$ \\
\hline ENSBTAG00000011216 & $1.64 \mathrm{E}-10$ & $1.03 \mathrm{E}-10$ & & $1.59 \mathrm{E}+00$ \\
\hline ENSBTAG00000048033 & $7.42 \mathrm{E}-10$ & 4.66E-10 & SMIM15 & $1.59 \mathrm{E}+00$ \\
\hline ENSBTAG00000016065 & $6.93 \mathrm{E}-10$ & $4.35 \mathrm{E}-10$ & & $1.59 \mathrm{E}+00$ \\
\hline ENSBTAG00000046417 & 8.57E-10 & 5.37E-10 & & $1.59 \mathrm{E}+00$ \\
\hline ENSBTAG00000036331 & $8.76 \mathrm{E}-10$ & 5.50E-10 & & $1.59 \mathrm{E}+00$ \\
\hline ENSBTAG00000013880 & $8.09 \mathrm{E}-10$ & $5.06 \mathrm{E}-10$ & WWC1 & $1.60 \mathrm{E}+00$ \\
\hline
\end{tabular}




\begin{tabular}{|c|c|c|c|c|}
\hline ENSBTAG00000009071 & $6.22 \mathrm{E}-10$ & $3.88 \mathrm{E}-10$ & ZNF699 & $1.60 \mathrm{E}+00$ \\
\hline ENSBTAG00000030286 & $2.66 \mathrm{E}-10$ & $1.66 \mathrm{E}-10$ & GABRA1 & $1.60 \mathrm{E}+00$ \\
\hline ENSBTAG00000013183 & $2.69 \mathrm{E}-10$ & $1.68 \mathrm{E}-10$ & COL23A1 & $1.60 \mathrm{E}+00$ \\
\hline ENSBTAG00000011125 & $4.00 \mathrm{E}-10$ & $2.48 \mathrm{E}-10$ & MYO9B & $1.61 E+00$ \\
\hline ENSBTAG00000004294 & $1.34 \mathrm{E}-10$ & $8.25 \mathrm{E}-11$ & EVI5L & $1.62 E+00$ \\
\hline ENSBTAG00000006756 & $1.62 \mathrm{E}-10$ & $1.00 \mathrm{E}-10$ & & $1.62 \mathrm{E}+00$ \\
\hline ENSBTAG00000001862 & $3.40 \mathrm{E}-10$ & $2.09 \mathrm{E}-10$ & PPP2R2B & $1.62 E+00$ \\
\hline ENSBTAG00000008570 & $2.39 \mathrm{E}-10$ & 1.47E-10 & & $1.62 \mathrm{E}+00$ \\
\hline ENSBTAG00000012095 & $2.82 \mathrm{E}-10$ & $1.73 \mathrm{E}-10$ & GEMIN5 & $1.63 \mathrm{E}+00$ \\
\hline ENSBTAG00000010251 & $2.60 \mathrm{E}-10$ & $1.59 \mathrm{E}-10$ & GABRG2 & $1.63 \mathrm{E}+00$ \\
\hline ENSBTAG00000010303 & $2.91 \mathrm{E}-10$ & $1.77 \mathrm{E}-10$ & ICAM1 & $1.64 \mathrm{E}+00$ \\
\hline ENSBTAG00000013288 & $1.90 \mathrm{E}-10$ & $1.16 \mathrm{E}-10$ & SUGP2 & $1.64 \mathrm{E}+00$ \\
\hline ENSBTAG00000020809 & $6.66 \mathrm{E}-10$ & 4.07E-10 & SLC36A2 & $1.64 \mathrm{E}+00$ \\
\hline ENSBTAG00000012687 & $3.58 \mathrm{E}-10$ & $2.18 \mathrm{E}-10$ & INSR & $1.64 \mathrm{E}+00$ \\
\hline ENSBTAG00000021675 & $2.19 \mathrm{E}-10$ & $1.33 \mathrm{E}-10$ & PJA2 & $1.65 E+00$ \\
\hline ENSBTAG00000021471 & $1.98 \mathrm{E}-10$ & $1.20 \mathrm{E}-10$ & GIN1 & $1.65 E+00$ \\
\hline ENSBTAG00000013185 & $9.94 \mathrm{E}-10$ & $5.98 \mathrm{E}-10$ & TIMD4 & $1.66 \mathrm{E}+00$ \\
\hline ENSBTAG00000020766 & $9.43 \mathrm{E}-11$ & $5.63 \mathrm{E}-11$ & $A B C A 7$ & $1.67 \mathrm{E}+00$ \\
\hline ENSBTAG00000039319 & $1.94 \mathrm{E}-10$ & $1.16 \mathrm{E}-10$ & & $1.67 E+00$ \\
\hline ENSBTAG00000017451 & $3.56 \mathrm{E}-10$ & $2.12 \mathrm{E}-10$ & TSPAN17 & $1.68 \mathrm{E}+00$ \\
\hline ENSBTAG00000037968 & $6.10 \mathrm{E}-10$ & $3.63 \mathrm{E}-10$ & PCDHGB1 & $1.68 \mathrm{E}+00$ \\
\hline ENSBTAG00000011642 & $2.37 \mathrm{E}-10$ & $1.41 \mathrm{E}-10$ & & $1.68 \mathrm{E}+00$ \\
\hline ENSBTAG00000021829 & $4.75 \mathrm{E}-10$ & 2.82E-10 & GM2A & $1.69 \mathrm{E}+00$ \\
\hline ENSBTAG00000014320 & $4.11 \mathrm{E}-10$ & $2.43 \mathrm{E}-10$ & SRFBP1 & $1.69 \mathrm{E}+00$ \\
\hline ENSBTAG00000002736 & 3.17E-10 & $1.88 \mathrm{E}-10$ & DNMT1 & $1.69 E+00$ \\
\hline ENSBTAG00000025748 & $1.75 \mathrm{E}-10$ & $1.04 \mathrm{E}-10$ & & $1.68 \mathrm{E}+00$ \\
\hline ENSBTAG00000019220 & $2.17 \mathrm{E}-10$ & $1.28 \mathrm{E}-10$ & SMARCA4 & $1.70 \mathrm{E}+00$ \\
\hline ENSBTAG00000014898 & $5.02 \mathrm{E}-10$ & $2.96 \mathrm{E}-10$ & HK3 & $1.69 E+00$ \\
\hline ENSBTAG00000046406 & $1.24 \mathrm{E}-10$ & $7.28 \mathrm{E}-11$ & R3HDM4 & $1.70 \mathrm{E}+00$ \\
\hline ENSBTAG00000005800 & $4.87 \mathrm{E}-10$ & $2.86 \mathrm{E}-10$ & GRIA1 & $1.70 \mathrm{E}+00$ \\
\hline ENSBTAG00000008578 & $3.80 \mathrm{E}-10$ & $2.22 \mathrm{E}-10$ & LSM4 & $1.71 E+00$ \\
\hline ENSBTAG00000014775 & $2.23 \mathrm{E}-10$ & $1.30 \mathrm{E}-10$ & GUK1 & $1.71 \mathrm{E}+00$ \\
\hline ENSBTAG00000016775 & 7.19E-10 & 4.20E-10 & SLC27A1 & $1.71 \mathrm{E}+00$ \\
\hline ENSBTAG00000002937 & $6.53 \mathrm{E}-10$ & $3.80 \mathrm{E}-10$ & ADGRE3 & $1.72 \mathrm{E}+00$ \\
\hline ENSBTAG00000002524 & $1.66 \mathrm{E}-10$ & $9.65 \mathrm{E}-11$ & MRI1 & $1.72 \mathrm{E}+00$ \\
\hline ENSBTAG00000000469 & $8.60 \mathrm{E}-11$ & $5.00 \mathrm{E}-11$ & PPP2CA & $1.72 \mathrm{E}+00$ \\
\hline ENSBTAG00000009178 & $2.34 \mathrm{E}-10$ & $1.36 \mathrm{E}-10$ & STXBP2 & $1.72 \mathrm{E}+00$ \\
\hline ENSBTAG00000004524 & $2.28 \mathrm{E}-10$ & $1.32 \mathrm{E}-10$ & SPPL2B & $1.73 \mathrm{E}+00$ \\
\hline ENSBTAG00000038055 & $1.69 \mathrm{E}-10$ & $9.74 \mathrm{E}-11$ & KLF16 & $1.73 \mathrm{E}+00$ \\
\hline ENSBTAG00000009569 & $7.08 \mathrm{E}-10$ & $4.08 \mathrm{E}-10$ & DOCK6 & $1.74 \mathrm{E}+00$ \\
\hline ENSBTAG00000038976 & 3.07E-10 & $1.76 \mathrm{E}-10$ & ZGLP1 & $1.74 \mathrm{E}+00$ \\
\hline ENSBTAG00000008340 & $5.41 \mathrm{E}-10$ & $3.09 \mathrm{E}-10$ & ATOX1 & $1.75 E+00$ \\
\hline ENSBTAG00000044035 & $3.12 \mathrm{E}-10$ & $1.79 \mathrm{E}-10$ & GPRIN1 & $1.74 \mathrm{E}+00$ \\
\hline ENSBTAG00000005010 & $2.08 \mathrm{E}-10$ & $1.19 \mathrm{E}-10$ & MXD3 & $1.75 E+00$ \\
\hline ENSBTAG00000009034 & $2.00 \mathrm{E}-10$ & $1.14 \mathrm{E}-10$ & AP3D1 & $1.76 \mathrm{E}+00$ \\
\hline ENSBTAG00000002277 & $3.03 \mathrm{E}-10$ & $1.72 \mathrm{E}-10$ & MLLT1 & $1.76 \mathrm{E}+00$ \\
\hline ENSBTAG00000031363 & $2.59 \mathrm{E}-10$ & $1.47 \mathrm{E}-10$ & AES & $1.76 \mathrm{E}+00$ \\
\hline ENSBTAG00000003528 & $1.10 \mathrm{E}-09$ & $6.21 \mathrm{E}-10$ & & $1.78 \mathrm{E}+00$ \\
\hline ENSBTAG00000025426 & $1.47 \mathrm{E}-10$ & $8.31 E-11$ & NSD1 & $1.77 \mathrm{E}+00$ \\
\hline ENSBTAG00000009990 & $4.99 \mathrm{E}-10$ & $2.81 \mathrm{E}-10$ & EPS15L1 & $1.78 \mathrm{E}+00$ \\
\hline ENSBTAG00000012634 & $3.37 \mathrm{E}-10$ & $1.88 \mathrm{E}-10$ & NDUFB7 & $1.79 E+00$ \\
\hline ENSBTAG00000038549 & $1.93 \mathrm{E}-10$ & $1.08 \mathrm{E}-10$ & & $1.79 \mathrm{E}+00$ \\
\hline ENSBTAG00000005961 & $3.64 \mathrm{E}-10$ & $2.03 \mathrm{E}-10$ & CDC42SE2 & $1.79 E+00$ \\
\hline ENSBTAG00000002475 & $1.54 \mathrm{E}-10$ & $8.59 \mathrm{E}-11$ & RAB11B & $1.79 E+00$ \\
\hline ENSBTAG00000006124 & $1.65 \mathrm{E}-10$ & $9.24 \mathrm{E}-11$ & MAU2 & $1.79 \mathrm{E}+00$ \\
\hline ENSBTAG00000034909 & 7.63E-10 & $4.26 \mathrm{E}-10$ & & $1.79 E+00$ \\
\hline ENSBTAG00000017280 & $1.44 \mathrm{E}-10$ & $8.05 \mathrm{E}-11$ & $\mathrm{C} 3$ & $1.79 E+00$ \\
\hline ENSBTAG00000030297 & $7.05 \mathrm{E}-10$ & $3.93 \mathrm{E}-10$ & & $1.79 \mathrm{E}+00$ \\
\hline ENSBTAG00000013977 & $4.73 \mathrm{E}-10$ & $2.63 \mathrm{E}-10$ & PRSS38 & $1.80 \mathrm{E}+00$ \\
\hline ENSBTAG00000005198 & $2.89 \mathrm{E}-10$ & $1.61 \mathrm{E}-10$ & FGF1 & $1.80 E+00$ \\
\hline ENSBTAG00000000718 & $4.82 \mathrm{E}-10$ & 2.67E-10 & TMPRSS9 & $1.81 E+00$ \\
\hline ENSBTAG00000001817 & $3.42 \mathrm{E}-10$ & $1.89 \mathrm{E}-10$ & TMEM161A & $1.81 \mathrm{E}+00$ \\
\hline ENSBTAG00000004604 & $3.86 \mathrm{E}-10$ & $2.14 \mathrm{E}-10$ & CCDC124 & $1.80 \mathrm{E}+00$ \\
\hline ENSBTAG00000039355 & 4.39E-10 & $2.43 \mathrm{E}-10$ & MADCAM1 & $1.81 E+00$ \\
\hline ENSBTAG00000002495 & $1.63 \mathrm{E}-10$ & $8.96 \mathrm{E}-11$ & BRD4 & $1.81 \mathrm{E}+00$ \\
\hline ENSBTAG00000045909 & $9.19 E-10$ & 5.07E-10 & FAM174A & $1.81 E+00$ \\
\hline ENSBTAG00000016137 & $1.42 \mathrm{E}-10$ & $7.81 \mathrm{E}-11$ & ZNF608 & $1.82 \mathrm{E}+00$ \\
\hline ENSBTAG00000012181 & $4.00 \mathrm{E}-10$ & $2.19 \mathrm{E}-10$ & GNA11 & $1.83 E+00$ \\
\hline ENSBTAG00000007660 & $1.56 \mathrm{E}-10$ & $8.44 \mathrm{E}-11$ & ZNF414 & $1.85 \mathrm{E}+00$ \\
\hline ENSBTAG00000020924 & 1.17E-10 & $6.29 \mathrm{E}-11$ & FBXW9 & $1.85 \mathrm{E}+00$ \\
\hline
\end{tabular}




\begin{tabular}{|c|c|c|c|c|}
\hline ENSBTAG00000009661 & $1.66 \mathrm{E}-10$ & $8.94 \mathrm{E}-11$ & RNASEH $2 \mathrm{~A}$ & $1.85 \mathrm{E}+00$ \\
\hline ENSBTAG00000021766 & $1.03 \mathrm{E}-10$ & $5.53 \mathrm{E}-11$ & HBEGF & $1.85 \mathrm{E}+00$ \\
\hline ENSBTAG00000011699 & $1.54 \mathrm{E}-10$ & $8.30 \mathrm{E}-11$ & CDC37 & $1.85 \mathrm{E}+00$ \\
\hline ENSBTAG00000003103 & $2.05 \mathrm{E}-10$ & $1.11 \mathrm{E}-10$ & EIF4E1B & $1.85 \mathrm{E}+00$ \\
\hline ENSBTAG00000039986 & $6.14 \mathrm{E}-10$ & $3.30 \mathrm{E}-10$ & & $1.86 \mathrm{E}+00$ \\
\hline ENSBTAG00000018845 & $2.11 \mathrm{E}-10$ & $1.14 \mathrm{E}-10$ & DND1 & $1.85 \mathrm{E}+00$ \\
\hline ENSBTAG00000007557 & $6.20 \mathrm{E}-10$ & 3.33E-10 & OR2AK2 & $1.86 \mathrm{E}+00$ \\
\hline ENSBTAG00000009565 & $2.33 \mathrm{E}-10$ & $1.25 \mathrm{E}-10$ & RASA1 & $1.86 \mathrm{E}+00$ \\
\hline ENSBTAG00000045589 & $6.24 \mathrm{E}-10$ & $3.36 \mathrm{E}-10$ & & $1.86 \mathrm{E}+00$ \\
\hline ENSBTAG00000017835 & $7.11 \mathrm{E}-10$ & $3.83 \mathrm{E}-10$ & C5orf63 & $1.86 \mathrm{E}+00$ \\
\hline ENSBTAG00000000755 & $2.37 \mathrm{E}-10$ & $1.27 \mathrm{E}-10$ & ZFR2 & $1.86 \mathrm{E}+00$ \\
\hline ENSBTAG00000006428 & $2.25 \mathrm{E}-10$ & $1.21 \mathrm{E}-10$ & ZNF354A & $1.86 \mathrm{E}+00$ \\
\hline ENSBTAG00000016145 & $4.04 \mathrm{E}-10$ & 2.17E-10 & ADAMTS19 & $1.86 \mathrm{E}+00$ \\
\hline ENSBTAG00000033061 & $1.61 E-09$ & 8.64E-10 & & $1.87 \mathrm{E}+00$ \\
\hline ENSBTAG00000027728 & $1.52 \mathrm{E}-10$ & 8.09E-11 & NUDT12 & $1.88 \mathrm{E}+00$ \\
\hline ENSBTAG00000006797 & $6.51 \mathrm{E}-10$ & $3.43 \mathrm{E}-10$ & 03-Mar & $1.90 \mathrm{E}+00$ \\
\hline ENSBTAG00000014772 & $1.66 \mathrm{E}-10$ & $8.70 \mathrm{E}-11$ & NUDCD2 & $1.90 \mathrm{E}+00$ \\
\hline ENSBTAG00000002311 & $1.80 \mathrm{E}-10$ & $9.46 \mathrm{E}-11$ & REEP6 & $1.91 \mathrm{E}+00$ \\
\hline ENSBTAG00000046837 & $5.87 \mathrm{E}-10$ & 3.07E-10 & ZNF358 & $1.91 \mathrm{E}+00$ \\
\hline ENSBTAG00000010439 & $1.33 \mathrm{E}-10$ & $6.93 \mathrm{E}-11$ & AKAP8L & $1.91 E+00$ \\
\hline ENSBTAG00000025071 & $3.93 \mathrm{E}-10$ & $2.05 \mathrm{E}-10$ & TENM2 & $1.92 \mathrm{E}+00$ \\
\hline ENSBTAG00000016108 & $3.32 \mathrm{E}-10$ & $1.72 \mathrm{E}-10$ & BTBD2 & $1.93 E+00$ \\
\hline ENSBTAG00000047424 & $4.35 \mathrm{E}-10$ & $2.25 \mathrm{E}-10$ & & $1.93 E+00$ \\
\hline ENSBTAG00000000413 & $3.83 \mathrm{E}-10$ & $1.98 \mathrm{E}-10$ & NRTN & $1.94 \mathrm{E}+00$ \\
\hline ENSBTAG00000004191 & $2.68 \mathrm{E}-10$ & $1.39 \mathrm{E}-10$ & JMJD4 & $1.92 \mathrm{E}+00$ \\
\hline ENSBTAG00000012678 & 8.06E-10 & $4.15 E-10$ & COLGALT1 & $1.94 \mathrm{E}+00$ \\
\hline ENSBTAG00000015957 & $2.29 \mathrm{E}-10$ & $1.18 \mathrm{E}-10$ & IL4 & $1.94 \mathrm{E}+00$ \\
\hline ENSBTAG00000009996 & $2.06 \mathrm{E}-10$ & $1.06 \mathrm{E}-10$ & DOT1L & $1.94 \mathrm{E}+00$ \\
\hline ENSBTAG00000011763 & $3.89 \mathrm{E}-10$ & $1.99 \mathrm{E}-10$ & RIOK2 & $1.95 \mathrm{E}+00$ \\
\hline ENSBTAG00000000495 & $8.30 \mathrm{E}-10$ & $4.24 \mathrm{E}-10$ & & $1.96 \mathrm{E}+00$ \\
\hline ENSBTAG00000008520 & $3.16 \mathrm{E}-10$ & $1.61 \mathrm{E}-10$ & NFIC & $1.96 \mathrm{E}+00$ \\
\hline ENSBTAG00000019285 & $4.53 \mathrm{E}-10$ & $2.30 \mathrm{E}-10$ & & $1.97 \mathrm{E}+00$ \\
\hline ENSBTAG00000011252 & $5.91 \mathrm{E}-10$ & $3.00 \mathrm{E}-10$ & RAD50 & $1.97 \mathrm{E}+00$ \\
\hline ENSBTAG00000048107 & $1.45 \mathrm{E}-10$ & $7.38 \mathrm{E}-11$ & CANX & $1.97 E+00$ \\
\hline ENSBTAG00000017078 & $1.79 \mathrm{E}-10$ & $9.00 \mathrm{E}-11$ & FAM114A2 & $1.99 E+00$ \\
\hline ENSBTAG00000009738 & 7.59E-11 & $3.82 \mathrm{E}-11$ & MGAT4B & $1.98 \mathrm{E}+00$ \\
\hline ENSBTAG00000048053 & $9.68 \mathrm{E}-11$ & 4.87E-11 & SLBP2 & $1.99 \mathrm{E}+00$ \\
\hline ENSBTAG00000002796 & $2.12 \mathrm{E}-10$ & 1.07E-10 & KLHL3 & $1.98 \mathrm{E}+00$ \\
\hline ENSBTAG00000047377 & $3.15 \mathrm{E}-10$ & $1.59 \mathrm{E}-10$ & & $1.98 \mathrm{E}+00$ \\
\hline ENSBTAG00000015228 & $5.27 \mathrm{E}-11$ & $2.66 \mathrm{E}-11$ & $\mathrm{CD} 74$ & $1.98 \mathrm{E}+00$ \\
\hline ENSBTAG00000030518 & 5.07E-11 & $2.55 \mathrm{E}-11$ & ECSCR & $1.98 \mathrm{E}+00$ \\
\hline ENSBTAG00000045644 & 4.32E-10 & 2.17E-10 & OR2T6 & $1.99 E+00$ \\
\hline ENSBTAG00000030584 & $2.19 \mathrm{E}-10$ & 1.10E-10 & SLC35A4 & $1.99 E+00$ \\
\hline ENSBTAG00000031383 & $2.40 \mathrm{E}-10$ & $1.21 \mathrm{E}-10$ & SMIM24 & $1.98 \mathrm{E}+00$ \\
\hline ENSBTAG00000004602 & $2.37 \mathrm{E}-10$ & $1.19 \mathrm{E}-10$ & PITX1 & $1.99 E+00$ \\
\hline ENSBTAG00000048017 & $5.42 \mathrm{E}-10$ & $2.72 \mathrm{E}-10$ & & $1.99 \mathrm{E}+00$ \\
\hline ENSBTAG00000025448 & $1.33 \mathrm{E}-10$ & $6.71 \mathrm{E}-11$ & IZUMO4 & $1.98 \mathrm{E}+00$ \\
\hline ENSBTAG00000039337 & $5.57 \mathrm{E}-10$ & $2.80 \mathrm{E}-10$ & & $1.99 E+00$ \\
\hline ENSBTAG00000039921 & $1.31 E-09$ & $6.54 \mathrm{E}-10$ & & $2.00 E+00$ \\
\hline ENSBTAG00000019287 & $4.21 \mathrm{E}-10$ & $2.06 \mathrm{E}-10$ & FAM81B & $2.04 E+00$ \\
\hline ENSBTAG00000021036 & $2.75 \mathrm{E}-10$ & $1.34 \mathrm{E}-10$ & 02-Mar & $2.05 E+00$ \\
\hline ENSBTAG00000047888 & $1.55 \mathrm{E}-09$ & $7.51 \mathrm{E}-10$ & & $2.06 \mathrm{E}+00$ \\
\hline ENSBTAG00000008224 & $6.03 \mathrm{E}-11$ & $2.92 \mathrm{E}-11$ & PAIP2 & $2.06 \mathrm{E}+00$ \\
\hline ENSBTAG00000012943 & $2.77 \mathrm{E}-10$ & $1.34 \mathrm{E}-10$ & PPARGC1B & $2.07 E+00$ \\
\hline ENSBTAG00000020041 & $1.06 \mathrm{E}-10$ & $5.09 \mathrm{E}-11$ & FAM13B & $2.07 \mathrm{E}+00$ \\
\hline ENSBTAG00000000710 & $3.12 \mathrm{E}-10$ & $1.50 \mathrm{E}-10$ & PIP5K1C & $2.08 \mathrm{E}+00$ \\
\hline ENSBTAG00000014665 & $4.26 \mathrm{E}-10$ & $2.05 E-10$ & ADAMTS2 & $2.08 \mathrm{E}+00$ \\
\hline ENSBTAG00000045860 & $1.09 \mathrm{E}-09$ & $5.23 \mathrm{E}-10$ & & $2.08 \mathrm{E}+00$ \\
\hline ENSBTAG00000012994 & $4.82 \mathrm{E}-10$ & $2.30 \mathrm{E}-10$ & LOX & $2.10 \mathrm{E}+00$ \\
\hline ENSBTAG00000020087 & $1.62 \mathrm{E}-10$ & $7.73 \mathrm{E}-11$ & CAMK2A & $2.10 E+00$ \\
\hline ENSBTAG00000047933 & $2.50 \mathrm{E}-10$ & $1.18 \mathrm{E}-10$ & CYP4F22 & $2.12 \mathrm{E}+00$ \\
\hline ENSBTAG00000009103 & $6.54 \mathrm{E}-11$ & $3.09 \mathrm{E}-11$ & UBLCP1 & $2.11 E+00$ \\
\hline ENSBTAG00000025809 & $9.42 \mathrm{E}-11$ & 4.45E-11 & ABHD8 & $2.11 E+00$ \\
\hline ENSBTAG00000015184 & $1.46 \mathrm{E}-10$ & $6.91 \mathrm{E}-11$ & SYDE1 & $2.12 \mathrm{E}+00$ \\
\hline ENSBTAG00000008197 & $1.25 \mathrm{E}-10$ & $5.89 \mathrm{E}-11$ & EPOR & $2.12 \mathrm{E}+00$ \\
\hline ENSBTAG00000012834 & $1.18 \mathrm{E}-10$ & $5.58 \mathrm{E}-11$ & ARSI & $2.12 \mathrm{E}+00$ \\
\hline ENSBTAG00000046318 & $7.02 \mathrm{E}-10$ & $3.30 \mathrm{E}-10$ & & $2.13 E+00$ \\
\hline ENSBTAG00000044046 & $7.56 \mathrm{E}-10$ & 3.55E-10 & SGCD & $2.13 E+00$ \\
\hline ENSBTAG00000046249 & $1.39 \mathrm{E}-09$ & $6.50 \mathrm{E}-10$ & & $2.13 E+00$ \\
\hline ENSBTAG00000026148 & $9.32 \mathrm{E}-10$ & 4.36E-10 & & $2.14 \mathrm{E}+00$ \\
\hline
\end{tabular}




\begin{tabular}{|c|c|c|c|c|}
\hline ENSBTAG00000006064 & $2.74 \mathrm{E}-10$ & $1.27 \mathrm{E}-10$ & C19orf57 & $2.15 E+00$ \\
\hline ENSBTAG00000046717 & $9.40 \mathrm{E}-10$ & 4.36E-10 & & $2.16 \mathrm{E}+00$ \\
\hline ENSBTAG00000021519 & $4.02 \mathrm{E}-10$ & $1.86 \mathrm{E}-10$ & CAMLG & $2.16 \mathrm{E}+00$ \\
\hline ENSBTAG00000014207 & $3.21 \mathrm{E}-10$ & $1.49 \mathrm{E}-10$ & ADAMTS10 & $2.16 \mathrm{E}+00$ \\
\hline ENSBTAG00000010545 & $1.21 \mathrm{E}-10$ & $5.53 E-11$ & KXD1 & $2.18 \mathrm{E}+00$ \\
\hline ENSBTAG00000010772 & $2.43 \mathrm{E}-10$ & $1.11 \mathrm{E}-10$ & TPGS1 & $2.19 \mathrm{E}+00$ \\
\hline ENSBTAG00000046688 & $6.52 \mathrm{E}-10$ & $2.98 \mathrm{E}-10$ & & $2.19 \mathrm{E}+00$ \\
\hline ENSBTAG00000020701 & $2.27 \mathrm{E}-10$ & $1.03 E-10$ & MEF2C & $2.21 E+00$ \\
\hline ENSBTAG00000014412 & $2.57 \mathrm{E}-10$ & $1.16 \mathrm{E}-10$ & ELAVL3 & $2.21 E+00$ \\
\hline ENSBTAG00000027727 & $3.83 \mathrm{E}-10$ & $1.73 \mathrm{E}-10$ & & $2.21 \mathrm{E}+00$ \\
\hline ENSBTAG00000005295 & $1.42 \mathrm{E}-10$ & $6.36 \mathrm{E}-11$ & SLC35E1 & $2.22 \mathrm{E}+00$ \\
\hline ENSBTAG00000001247 & $1.95 \mathrm{E}-10$ & $8.75 E-11$ & LECT2 & $2.22 \mathrm{E}+00$ \\
\hline ENSBTAG00000020232 & $3.26 \mathrm{E}-10$ & $1.45 \mathrm{E}-10$ & REXO1 & $2.25 E+00$ \\
\hline ENSBTAG00000012387 & $1.90 \mathrm{E}-10$ & $8.49 \mathrm{E}-11$ & PAM & $2.24 E+00$ \\
\hline ENSBTAG00000046744 & $1.55 \mathrm{E}-10$ & $6.89 \mathrm{E}-11$ & PALM3 & $2.25 E+00$ \\
\hline ENSBTAG00000014661 & $6.20 \mathrm{E}-10$ & $2.76 \mathrm{E}-10$ & $\mathrm{CHSY} 3$ & $2.25 E+00$ \\
\hline ENSBTAG00000000811 & $3.60 \mathrm{E}-10$ & $1.60 \mathrm{E}-10$ & DDX49 & $2.25 E+00$ \\
\hline ENSBTAG00000002882 & $5.35 \mathrm{E}-10$ & $2.37 \mathrm{E}-10$ & LMNB1 & $2.26 \mathrm{E}+00$ \\
\hline ENSBTAG00000047177 & $7.23 \mathrm{E}-10$ & $3.18 \mathrm{E}-10$ & & $2.27 \mathrm{E}+00$ \\
\hline ENSBTAG00000025029 & $4.21 \mathrm{E}-10$ & $1.85 \mathrm{E}-10$ & MAN2A1 & $2.28 \mathrm{E}+00$ \\
\hline ENSBTAG00000013744 & $2.37 \mathrm{E}-10$ & $1.04 \mathrm{E}-10$ & SYNPO & $2.28 \mathrm{E}+00$ \\
\hline ENSBTAG00000008497 & $4.11 \mathrm{E}-10$ & $1.80 \mathrm{E}-10$ & RGS14 & $2.29 E+00$ \\
\hline ENSBTAG00000027241 & $1.78 \mathrm{E}-09$ & $7.76 \mathrm{E}-10$ & & $2.29 E+00$ \\
\hline ENSBTAG00000009646 & $3.27 \mathrm{E}-10$ & $1.42 \mathrm{E}-10$ & ALDH7A1 & $2.30 \mathrm{E}+00$ \\
\hline ENSBTAG00000019140 & $4.74 \mathrm{E}-10$ & $2.05 E-10$ & SPINK5 & $2.31 \mathrm{E}+00$ \\
\hline ENSBTAG00000011356 & $2.05 \mathrm{E}-10$ & $8.82 \mathrm{E}-11$ & CAMSAP3 & $2.32 \mathrm{E}+00$ \\
\hline ENSBTAG00000012411 & $3.16 \mathrm{E}-10$ & $1.34 \mathrm{E}-10$ & HAPLN1 & $2.36 \mathrm{E}+00$ \\
\hline ENSBTAG00000009105 & $5.30 \mathrm{E}-10$ & $2.24 \mathrm{E}-10$ & ACSBG2 & $2.36 \mathrm{E}+00$ \\
\hline ENSBTAG00000016515 & $6.42 \mathrm{E}-10$ & $2.70 \mathrm{E}-10$ & EFNA5 & $2.38 \mathrm{E}+00$ \\
\hline ENSBTAG00000001152 & $1.90 \mathrm{E}-10$ & 7.95E-11 & TLE6 & $2.38 \mathrm{E}+00$ \\
\hline ENSBTAG00000009131 & $2.76 \mathrm{E}-10$ & $1.16 \mathrm{E}-10$ & TTC37 & $2.38 \mathrm{E}+00$ \\
\hline ENSBTAG00000045828 & $6.90 \mathrm{E}-11$ & 2.90E-11 & PTBP1 & $2.38 \mathrm{E}+00$ \\
\hline ENSBTAG00000005289 & $1.91 \mathrm{E}-10$ & $8.03 E-11$ & CHERP & $2.38 \mathrm{E}+00$ \\
\hline ENSBTAG00000012592 & $6.33 E-11$ & $2.66 \mathrm{E}-11$ & ANKLE1 & $2.38 \mathrm{E}+00$ \\
\hline ENSBTAG00000016933 & $1.44 \mathrm{E}-10$ & $6.05 E-11$ & IL27RA & $2.38 \mathrm{E}+00$ \\
\hline ENSBTAG00000010215 & $6.42 \mathrm{E}-11$ & 2.69E-11 & CREB3L3 & $2.38 \mathrm{E}+00$ \\
\hline ENSBTAG00000010735 & $2.50 \mathrm{E}-10$ & $1.05 \mathrm{E}-10$ & & $2.38 \mathrm{E}+00$ \\
\hline ENSBTAG00000010068 & $4.10 \mathrm{E}-11$ & $1.72 \mathrm{E}-11$ & REEP2 & $2.37 E+00$ \\
\hline ENSBTAG00000012745 & $2.46 \mathrm{E}-10$ & $1.03 E-10$ & & $2.38 \mathrm{E}+00$ \\
\hline ENSBTAG00000001818 & 4.27E-10 & $1.79 \mathrm{E}-10$ & MEF2B & $2.38 \mathrm{E}+00$ \\
\hline ENSBTAG00000011496 & $2.51 \mathrm{E}-10$ & $1.05 \mathrm{E}-10$ & & $2.39 E+00$ \\
\hline ENSBTAG00000006607 & $1.35 \mathrm{E}-10$ & $5.68 \mathrm{E}-11$ & CCNG1 & $2.38 \mathrm{E}+00$ \\
\hline ENSBTAG00000019988 & $5.43 \mathrm{E}-10$ & $2.27 E-10$ & GNA15 & $2.39 \mathrm{E}+00$ \\
\hline ENSBTAG00000001084 & $7.83 \mathrm{E}-10$ & $3.27 E-10$ & & $2.39 E+00$ \\
\hline ENSBTAG00000022640 & $5.12 \mathrm{E}-10$ & $2.14 \mathrm{E}-10$ & & $2.39 E+00$ \\
\hline ENSBTAG00000046873 & $9.53 \mathrm{E}-10$ & $3.98 \mathrm{E}-10$ & & $2.39 E+00$ \\
\hline ENSBTAG00000017648 & $1.03 \mathrm{E}-09$ & $4.28 \mathrm{E}-10$ & TIFAB & $2.40 \mathrm{E}+00$ \\
\hline ENSBTAG00000030198 & $4.61 \mathrm{E}-10$ & $1.91 \mathrm{E}-10$ & TMEM232 & $2.41 E+00$ \\
\hline ENSBTAG00000012632 & $3.76 \mathrm{E}-10$ & $1.54 \mathrm{E}-10$ & TECR & $2.44 \mathrm{E}+00$ \\
\hline ENSBTAG00000016596 & $1.30 \mathrm{E}-10$ & $5.32 \mathrm{E}-11$ & PFDN1 & $2.44 \mathrm{E}+00$ \\
\hline ENSBTAG00000016105 & $2.93 \mathrm{E}-10$ & 1.19E-10 & H2AFY & $2.46 \mathrm{E}+00$ \\
\hline ENSBTAG00000018894 & $2.44 \mathrm{E}-10$ & $9.89 E-11$ & PEX11G & $2.46 \mathrm{E}+00$ \\
\hline ENSBTAG00000015690 & $2.26 \mathrm{E}-10$ & $9.14 \mathrm{E}-11$ & PLIN4 & $2.47 E+00$ \\
\hline ENSBTAG00000015419 & $6.37 \mathrm{E}-10$ & $2.56 \mathrm{E}-10$ & ARHGEF37 & $2.49 E+00$ \\
\hline ENSBTAG00000017151 & $1.97 \mathrm{E}-10$ & $7.88 \mathrm{E}-11$ & SH3TC2 & $2.50 \mathrm{E}+00$ \\
\hline ENSBTAG00000047747 & $2.08 \mathrm{E}-10$ & $8.28 \mathrm{E}-11$ & NDFIP1 & $2.51 E+00$ \\
\hline ENSBTAG00000001485 & $1.59 \mathrm{E}-10$ & $6.32 \mathrm{E}-11$ & PPIP5K2 & $2.52 E+00$ \\
\hline ENSBTAG00000001807 & $1.46 \mathrm{E}-10$ & $5.73 E-11$ & AKAP8 & $2.54 \mathrm{E}+00$ \\
\hline ENSBTAG00000038284 & $6.60 \mathrm{E}-10$ & $2.60 \mathrm{E}-10$ & ZNF496 & $2.54 \mathrm{E}+00$ \\
\hline ENSBTAG00000006694 & $3.14 \mathrm{E}-10$ & $1.23 \mathrm{E}-10$ & CXCL14 & $2.55 E+00$ \\
\hline ENSBTAG00000002645 & $4.95 \mathrm{E}-10$ & $1.94 \mathrm{E}-10$ & SH3BP5L & $2.55 E+00$ \\
\hline ENSBTAG00000003259 & $2.22 \mathrm{E}-10$ & $8.72 \mathrm{E}-11$ & TCERG1 & $2.55 E+00$ \\
\hline ENSBTAG00000038696 & $2.49 \mathrm{E}-10$ & $9.74 E-11$ & RAB8A & $2.55 E+00$ \\
\hline ENSBTAG00000046319 & 3.27E-10 & $1.28 \mathrm{E}-10$ & & $2.55 E+00$ \\
\hline ENSBTAG00000001796 & $2.96 \mathrm{E}-10$ & $1.15 \mathrm{E}-10$ & CATSPERD & $2.58 \mathrm{E}+00$ \\
\hline ENSBTAG00000003986 & $1.34 \mathrm{E}-10$ & $5.18 \mathrm{E}-11$ & CXXC5 & $2.58 \mathrm{E}+00$ \\
\hline ENSBTAG00000015042 & $2.17 \mathrm{E}-10$ & $8.41 \mathrm{E}-11$ & WDR55 & $2.58 \mathrm{E}+00$ \\
\hline ENSBTAG00000021971 & $3.22 \mathrm{E}-10$ & $1.25 \mathrm{E}-10$ & SNCAIP & $2.58 \mathrm{E}+00$ \\
\hline ENSBTAG00000006069 & $6.98 \mathrm{E}-10$ & $2.69 \mathrm{E}-10$ & CAPS & $2.59 \mathrm{E}+00$ \\
\hline ENSBTAG00000011805 & $2.76 \mathrm{E}-10$ & $1.05 E-10$ & PLIN3 & $2.62 E+00$ \\
\hline
\end{tabular}




\begin{tabular}{|c|c|c|c|c|}
\hline ENSBTAG00000002527 & $1.52 \mathrm{E}-10$ & $5.80 \mathrm{E}-11$ & ZSWIM4 & $2.62 \mathrm{E}+00$ \\
\hline ENSBTAG00000002516 & $1.82 \mathrm{E}-10$ & $6.87 \mathrm{E}-11$ & CCDC130 & $2.65 E+00$ \\
\hline ENSBTAG00000006244 & $3.13 \mathrm{E}-10$ & $1.18 \mathrm{E}-10$ & WDR83OS & $2.65 E+00$ \\
\hline ENSBTAG00000019925 & $4.92 \mathrm{E}-10$ & $1.85 \mathrm{E}-10$ & & $2.66 \mathrm{E}+00$ \\
\hline ENSBTAG00000010063 & $6.78 \mathrm{E}-11$ & $2.55 \mathrm{E}-11$ & KDM3B & $2.65 \mathrm{E}+00$ \\
\hline ENSBTAG00000018342 & 4.46E-10 & $1.65 \mathrm{E}-10$ & TJP3 & $2.70 E+00$ \\
\hline ENSBTAG00000003051 & $4.26 \mathrm{E}-10$ & $1.54 \mathrm{E}-10$ & FER & $2.77 \mathrm{E}+00$ \\
\hline ENSBTAG00000020117 & $1.80 \mathrm{E}-10$ & $6.46 \mathrm{E}-11$ & ZBTB7A & $2.78 \mathrm{E}+00$ \\
\hline ENSBTAG00000015567 & $1.32 \mathrm{E}-10$ & $4.76 \mathrm{E}-11$ & TIMM44 & $2.78 \mathrm{E}+00$ \\
\hline ENSBTAG00000046663 & $2.96 \mathrm{E}-10$ & $1.06 \mathrm{E}-10$ & CBY3 & $2.79 E+00$ \\
\hline ENSBTAG00000025124 & $1.80 \mathrm{E}-10$ & $6.49 \mathrm{E}-11$ & & $2.78 \mathrm{E}+00$ \\
\hline ENSBTAG00000046115 & $6.12 \mathrm{E}-10$ & $2.19 \mathrm{E}-10$ & & $2.79 E+00$ \\
\hline ENSBTAG00000035396 & $6.14 \mathrm{E}-10$ & $2.20 \mathrm{E}-10$ & OR7D2 & $2.79 \mathrm{E}+00$ \\
\hline ENSBTAG00000025462 & $2.72 \mathrm{E}-10$ & $9.77 \mathrm{E}-11$ & GADD45B & $2.78 \mathrm{E}+00$ \\
\hline ENSBTAG00000013834 & $1.35 \mathrm{E}-10$ & $4.86 \mathrm{E}-11$ & & $2.78 \mathrm{E}+00$ \\
\hline ENSBTAG00000020351 & $1.96 \mathrm{E}-10$ & $6.93 \mathrm{E}-11$ & SLC34A1 & $2.82 \mathrm{E}+00$ \\
\hline ENSBTAG00000002469 & $3.03 \mathrm{E}-10$ & $1.08 \mathrm{E}-10$ & KANK3 & $2.81 E+00$ \\
\hline ENSBTAG00000025182 & $3.00 \mathrm{E}-10$ & $1.05 \mathrm{E}-10$ & & $2.85 E+00$ \\
\hline ENSBTAG00000009667 & $4.10 \mathrm{E}-10$ & $1.44 \mathrm{E}-10$ & SLC25A46 & $2.85 E+00$ \\
\hline ENSBTAG00000009637 & 5.97E-10 & $2.08 \mathrm{E}-10$ & SLC12A2 & $2.87 \mathrm{E}+00$ \\
\hline ENSBTAG00000047817 & $9.86 \mathrm{E}-10$ & 3.37E-10 & & $2.93 \mathrm{E}+00$ \\
\hline ENSBTAG00000014773 & $2.69 \mathrm{E}-10$ & $9.19 \mathrm{E}-11$ & HMMR & $2.93 \mathrm{E}+00$ \\
\hline ENSBTAG00000018847 & $3.70 \mathrm{E}-10$ & $1.26 \mathrm{E}-10$ & HARS & $2.93 E+00$ \\
\hline ENSBTAG00000007741 & $1.54 \mathrm{E}-10$ & $5.18 \mathrm{E}-11$ & CRLF1 & $2.97 E+00$ \\
\hline ENSBTAG00000021772 & $1.92 \mathrm{E}-10$ & $6.46 \mathrm{E}-11$ & SMAD5 & $2.97 E+00$ \\
\hline ENSBTAG00000003544 & $3.00 \mathrm{E}-10$ & $1.01 \mathrm{E}-10$ & S1PR5 & $2.97 \mathrm{E}+00$ \\
\hline ENSBTAG00000002738 & $3.60 \mathrm{E}-10$ & $1.20 \mathrm{E}-10$ & S1PR2 & $3.00 \mathrm{E}+00$ \\
\hline ENSBTAG00000015735 & $3.93 \mathrm{E}-10$ & $1.30 \mathrm{E}-10$ & & $3.02 \mathrm{E}+00$ \\
\hline ENSBTAG00000008396 & $5.00 \mathrm{E}-10$ & $1.65 \mathrm{E}-10$ & MRPL54 & $3.03 E+00$ \\
\hline ENSBTAG00000014526 & $2.03 \mathrm{E}-10$ & $6.66 \mathrm{E}-11$ & MOB3A & $3.05 E+00$ \\
\hline ENSBTAG00000011131 & $2.69 \mathrm{E}-10$ & $8.76 \mathrm{E}-11$ & NMUR2 & $3.07 E+00$ \\
\hline ENSBTAG00000009513 & $2.56 \mathrm{E}-10$ & $8.25 \mathrm{E}-11$ & TGFBI & $3.09 E+00$ \\
\hline ENSBTAG00000009389 & $1.46 \mathrm{E}-10$ & $4.58 \mathrm{E}-11$ & HNRNPH1 & $3.18 \mathrm{E}+00$ \\
\hline ENSBTAG00000015117 & $1.33 \mathrm{E}-10$ & $4.18 \mathrm{E}-11$ & GADD45GIP1 & $3.17 E+00$ \\
\hline ENSBTAG00000008906 & $2.24 \mathrm{E}-10$ & 7.03E-11 & PCP2 & $3.18 \mathrm{E}+00$ \\
\hline ENSBTAG00000016352 & $9.45 \mathrm{E}-11$ & 2.97E-11 & STX10 & $3.17 E+00$ \\
\hline ENSBTAG00000020720 & $2.91 \mathrm{E}-10$ & $9.15 \mathrm{E}-11$ & C1orf35 & $3.18 \mathrm{E}+00$ \\
\hline ENSBTAG00000001767 & $7.73 \mathrm{E}-11$ & $2.43 \mathrm{E}-11$ & B4GALT7 & $3.17 E+00$ \\
\hline ENSBTAG00000004826 & $1.01 \mathrm{E}-10$ & $3.17 \mathrm{E}-11$ & ACP5 & $3.17 E+00$ \\
\hline ENSBTAG00000038510 & $3.52 \mathrm{E}-10$ & $1.11 \mathrm{E}-10$ & & $3.17 E+00$ \\
\hline ENSBTAG00000004657 & 3.33E-10 & $1.05 \mathrm{E}-10$ & FBLL1 & $3.17 E+00$ \\
\hline ENSBTAG00000045800 & 3.37E-10 & $1.06 \mathrm{E}-10$ & & $3.18 \mathrm{E}+00$ \\
\hline ENSBTAG00000040109 & $3.39 \mathrm{E}-10$ & $1.06 \mathrm{E}-10$ & & $3.19 E+00$ \\
\hline ENSBTAG00000031235 & $4.11 \mathrm{E}-10$ & $1.29 \mathrm{E}-10$ & IL5 & $3.18 \mathrm{E}+00$ \\
\hline ENSBTAG00000047016 & $2.98 \mathrm{E}-10$ & $9.36 \mathrm{E}-11$ & & $3.18 \mathrm{E}+00$ \\
\hline ENSBTAG00000016478 & $1.78 \mathrm{E}-10$ & $5.60 \mathrm{E}-11$ & LINGO3 & $3.17 \mathrm{E}+00$ \\
\hline ENSBTAG00000006073 & $2.61 \mathrm{E}-10$ & $8.21 \mathrm{E}-11$ & PODNL1 & $3.18 \mathrm{E}+00$ \\
\hline ENSBTAG00000047695 & $5.54 \mathrm{E}-10$ & $1.74 \mathrm{E}-10$ & & $3.18 \mathrm{E}+00$ \\
\hline ENSBTAG00000030722 & $4.84 \mathrm{E}-10$ & $1.52 \mathrm{E}-10$ & OR2M4 & $3.18 \mathrm{E}+00$ \\
\hline ENSBTAG00000037458 & $6.69 \mathrm{E}-10$ & $2.10 \mathrm{E}-10$ & & $3.19 \mathrm{E}+00$ \\
\hline ENSBTAG00000038623 & 7.13E-10 & $2.24 \mathrm{E}-10$ & & $3.18 \mathrm{E}+00$ \\
\hline ENSBTAG00000021774 & $2.41 \mathrm{E}-10$ & $7.48 \mathrm{E}-11$ & CELF5 & $3.22 \mathrm{E}+00$ \\
\hline ENSBTAG00000018946 & $6.84 \mathrm{E}-10$ & $2.12 \mathrm{E}-10$ & FAM129C & $3.22 \mathrm{E}+00$ \\
\hline ENSBTAG00000006107 & $1.82 \mathrm{E}-10$ & $5.62 \mathrm{E}-11$ & SLF1 & $3.23 E+00$ \\
\hline ENSBTAG00000016595 & $2.49 \mathrm{E}-10$ & 7.74E-11 & CYSTM1 & $3.22 \mathrm{E}+00$ \\
\hline ENSBTAG00000012032 & $4.94 \mathrm{E}-10$ & $1.52 \mathrm{E}-10$ & PDE4A & $3.25 E+00$ \\
\hline ENSBTAG00000025099 & $1.45 \mathrm{E}-10$ & 4.44E-11 & RNF145 & $3.26 \mathrm{E}+00$ \\
\hline ENSBTAG00000016874 & $3.79 \mathrm{E}-10$ & $1.12 \mathrm{E}-10$ & & $3.39 E+00$ \\
\hline ENSBTAG00000018322 & $3.58 \mathrm{E}-10$ & $1.06 \mathrm{E}-10$ & SEC24A & $3.38 \mathrm{E}+00$ \\
\hline ENSBTAG00000001273 & 4.63E-10 & $1.36 \mathrm{E}-10$ & NLRP3 & $3.40 \mathrm{E}+00$ \\
\hline ENSBTAG00000021018 & $3.67 \mathrm{E}-10$ & $1.08 \mathrm{E}-10$ & KHSRP & $3.40 E+00$ \\
\hline ENSBTAG00000018375 & $6.49 \mathrm{E}-10$ & $1.89 \mathrm{E}-10$ & ATCAY & $3.43 E+00$ \\
\hline ENSBTAG00000008397 & $3.82 \mathrm{E}-10$ & $1.11 \mathrm{E}-10$ & ZNF555 & $3.44 \mathrm{E}+00$ \\
\hline ENSBTAG00000001765 & $3.69 \mathrm{E}-10$ & $1.07 \mathrm{E}-10$ & HAPLN4 & $3.44 E+00$ \\
\hline ENSBTAG00000047799 & $5.38 \mathrm{E}-10$ & $1.56 \mathrm{E}-10$ & & $3.45 E+00$ \\
\hline ENSBTAG00000010731 & $4.31 \mathrm{E}-10$ & $1.25 \mathrm{E}-10$ & CDKN2D & $3.44 E+00$ \\
\hline ENSBTAG00000025345 & $7.25 \mathrm{E}-10$ & $2.10 \mathrm{E}-10$ & ARL10 & $3.45 E+00$ \\
\hline ENSBTAG00000002136 & $4.89 \mathrm{E}-10$ & $1.41 \mathrm{E}-10$ & $\mathrm{FCHO} 1$ & $3.47 E+00$ \\
\hline ENSBTAG00000006116 & $5.62 \mathrm{E}-10$ & $1.61 \mathrm{E}-10$ & SUGP1 & $3.49 \mathrm{E}+00$ \\
\hline ENSBTAG00000045941 & $3.09 \mathrm{E}-10$ & 8.87E-11 & C5orf30 & $3.49 \mathrm{E}+00$ \\
\hline
\end{tabular}




\begin{tabular}{|c|c|c|c|c|}
\hline ENSBTAG00000021226 & $1.55 \mathrm{E}-10$ & 4.44E-11 & SAR1B & $3.48 \mathrm{E}+00$ \\
\hline ENSBTAG00000019651 & $4.76 \mathrm{E}-10$ & $1.35 \mathrm{E}-10$ & TRIM52 & $3.52 E+00$ \\
\hline ENSBTAG00000047625 & $4.43 \mathrm{E}-10$ & $1.24 \mathrm{E}-10$ & & $3.57 E+00$ \\
\hline ENSBTAG00000012086 & $1.68 \mathrm{E}-10$ & $4.69 \mathrm{E}-11$ & CNOT8 & $3.57 E+00$ \\
\hline ENSBTAG00000011864 & $2.16 \mathrm{E}-10$ & $6.02 \mathrm{E}-11$ & RGMB & $3.58 \mathrm{E}+00$ \\
\hline ENSBTAG00000010734 & $3.11 \mathrm{E}-10$ & $8.68 \mathrm{E}-11$ & NOP16 & $3.58 \mathrm{E}+00$ \\
\hline ENSBTAG00000039275 & 7.77E-10 & $2.16 \mathrm{E}-10$ & ERAP2 & $3.59 E+00$ \\
\hline ENSBTAG00000016072 & $2.10 \mathrm{E}-10$ & $5.84 \mathrm{E}-11$ & $\mathrm{SHC2}$ & $3.60 E+00$ \\
\hline ENSBTAG00000005293 & $1.10 \mathrm{E}-10$ & $3.03 \mathrm{E}-11$ & $\mathrm{CDC} 25 \mathrm{C}$ & $3.61 E+00$ \\
\hline ENSBTAG00000011360 & $3.00 \mathrm{E}-10$ & $8.26 \mathrm{E}-11$ & XAB2 & $3.63 E+00$ \\
\hline ENSBTAG00000032293 & $8.50 \mathrm{E}-10$ & $2.30 \mathrm{E}-10$ & TSPAN16 & $3.69 \mathrm{E}+00$ \\
\hline ENSBTAG00000046139 & 2.02E-09 & $5.46 \mathrm{E}-10$ & & $3.70 \mathrm{E}+00$ \\
\hline ENSBTAG00000038154 & $3.70 \mathrm{E}-10$ & $9.78 \mathrm{E}-11$ & $\mathrm{HSH} 2 \mathrm{D}$ & $3.78 \mathrm{E}+00$ \\
\hline ENSBTAG00000025477 & $6.26 \mathrm{E}-10$ & $1.64 \mathrm{E}-10$ & KIF3A & $3.81 E+00$ \\
\hline ENSBTAG00000019648 & $4.11 \mathrm{E}-10$ & $1.08 \mathrm{E}-10$ & RACK1 & $3.81 E+00$ \\
\hline ENSBTAG00000014373 & $1.36 \mathrm{E}-10$ & $3.54 \mathrm{E}-11$ & CETN3 & $3.83 E+00$ \\
\hline ENSBTAG00000003047 & 7.10E-10 & $1.83 \mathrm{E}-10$ & FAM170A & $3.88 \mathrm{E}+00$ \\
\hline ENSBTAG00000040507 & $5.04 \mathrm{E}-10$ & $1.30 \mathrm{E}-10$ & & $3.87 E+00$ \\
\hline ENSBTAG00000040485 & $8.69 \mathrm{E}-10$ & $2.24 \mathrm{E}-10$ & THEG & $3.88 \mathrm{E}+00$ \\
\hline ENSBTAG00000046105 & $1.52 \mathrm{E}-10$ & $3.83 \mathrm{E}-11$ & PRTN3 & $3.97 E+00$ \\
\hline ENSBTAG00000001419 & $1.80 \mathrm{E}-10$ & $4.53 \mathrm{E}-11$ & MRPL4 & $3.97 E+00$ \\
\hline ENSBTAG00000011842 & $1.67 \mathrm{E}-10$ & $4.20 \mathrm{E}-11$ & & $3.97 E+00$ \\
\hline ENSBTAG00000039372 & $9.21 \mathrm{E}-11$ & $2.32 \mathrm{E}-11$ & ODF3L2 & $3.96 \mathrm{E}+00$ \\
\hline ENSBTAG00000038221 & $1.69 \mathrm{E}-10$ & 4.25E-11 & EFNA2 & $3.97 E+00$ \\
\hline ENSBTAG00000002468 & $3.62 \mathrm{E}-10$ & $9.11 \mathrm{E}-11$ & RPS28 & $3.97 \mathrm{E}+00$ \\
\hline ENSBTAG00000009570 & $4.39 \mathrm{E}-10$ & $1.10 \mathrm{E}-10$ & ANGPTL8 & $3.99 E+00$ \\
\hline ENSBTAG00000018503 & $1.17 \mathrm{E}-10$ & $2.95 \mathrm{E}-11$ & & $3.97 E+00$ \\
\hline ENSBTAG00000020017 & $4.21 \mathrm{E}-10$ & $1.06 \mathrm{E}-10$ & SLC25A41 & $3.97 E+00$ \\
\hline ENSBTAG00000021098 & $4.31 \mathrm{E}-10$ & $1.08 \mathrm{E}-10$ & & $3.98 \mathrm{E}+00$ \\
\hline ENSBTAG00000004741 & $1.58 \mathrm{E}-10$ & 3.97E-11 & IL12B & $3.96 \mathrm{E}+00$ \\
\hline ENSBTAG00000005882 & $5.77 \mathrm{E}-10$ & $1.44 \mathrm{E}-10$ & ZNF333 & $4.00 E+00$ \\
\hline ENSBTAG00000048066 & $1.54 \mathrm{E}-09$ & $3.83 \mathrm{E}-10$ & & $4.01 E+00$ \\
\hline ENSBTAG00000021134 & $2.33 \mathrm{E}-10$ & $5.79 \mathrm{E}-11$ & DPP9 & $4.02 \mathrm{E}+00$ \\
\hline ENSBTAG00000014182 & $2.18 \mathrm{E}-10$ & $5.41 \mathrm{E}-11$ & CTNNA1 & $4.03 E+00$ \\
\hline ENSBTAG00000046234 & 2.64E-09 & $6.52 \mathrm{E}-10$ & & $4.04 E+00$ \\
\hline ENSBTAG00000025212 & $4.83 \mathrm{E}-10$ & $1.18 \mathrm{E}-10$ & HARS2 & $4.09 E+00$ \\
\hline ENSBTAG00000010652 & $5.28 \mathrm{E}-10$ & $1.29 \mathrm{E}-10$ & PDE4C & $4.09 E+00$ \\
\hline ENSBTAG00000014638 & $7.02 \mathrm{E}-10$ & $1.71 \mathrm{E}-10$ & TRPC7 & $4.10 E+00$ \\
\hline ENSBTAG00000006247 & $5.47 \mathrm{E}-10$ & $1.32 \mathrm{E}-10$ & DHPS & $4.14 \mathrm{E}+00$ \\
\hline ENSBTAG00000027713 & $3.14 \mathrm{E}-10$ & $7.52 \mathrm{E}-11$ & LYSMD3 & $4.17 E+00$ \\
\hline ENSBTAG00000021039 & $2.47 \mathrm{E}-10$ & $5.85 \mathrm{E}-11$ & HNRNPM & $4.21 E+00$ \\
\hline ENSBTAG00000005312 & $2.58 \mathrm{E}-10$ & $6.09 \mathrm{E}-11$ & & $4.24 E+00$ \\
\hline ENSBTAG00000045683 & $5.51 \mathrm{E}-10$ & $1.30 \mathrm{E}-10$ & PCDHGA5 & $4.24 E+00$ \\
\hline ENSBTAG00000046798 & $1.36 \mathrm{E}-09$ & 3.17E-10 & & $4.28 \mathrm{E}+00$ \\
\hline ENSBTAG00000017349 & $3.67 \mathrm{E}-10$ & $8.53 \mathrm{E}-11$ & PCDHGC5 & $4.30 E+00$ \\
\hline ENSBTAG00000033747 & $1.31 \mathrm{E}-10$ & $3.02 \mathrm{E}-11$ & SIL1 & $4.32 \mathrm{E}+00$ \\
\hline ENSBTAG00000002447 & $4.12 \mathrm{E}-10$ & $9.55 \mathrm{E}-11$ & & $4.31 E+00$ \\
\hline ENSBTAG00000031573 & $2.67 \mathrm{E}-10$ & $6.11 \mathrm{E}-11$ & & $4.37 E+00$ \\
\hline ENSBTAG00000037717 & $5.76 \mathrm{E}-10$ & $1.27 \mathrm{E}-10$ & PCDHGA7 & $4.53 E+00$ \\
\hline ENSBTAG00000007684 & $6.33 \mathrm{E}-10$ & $1.40 \mathrm{E}-10$ & MSH3 & $4.52 \mathrm{E}+00$ \\
\hline ENSBTAG00000015426 & $5.94 \mathrm{E}-10$ & $1.29 \mathrm{E}-10$ & PDLIM4 & $4.60 E+00$ \\
\hline ENSBTAG00000008759 & $1.18 \mathrm{E}-10$ & $2.57 \mathrm{E}-11$ & $\mathrm{CDC} 23$ & $4.59 E+00$ \\
\hline ENSBTAG00000006070 & $4.61 \mathrm{E}-10$ & $9.95 \mathrm{E}-11$ & RANBP3 & $4.63 E+00$ \\
\hline ENSBTAG00000031209 & $5.36 \mathrm{E}-10$ & $1.14 \mathrm{E}-10$ & SLC22A4 & $4.69 E+00$ \\
\hline ENSBTAG00000047633 & $1.24 \mathrm{E}-10$ & $2.60 \mathrm{E}-11$ & & $4.75 E+00$ \\
\hline ENSBTAG00000006245 & $3.20 \mathrm{E}-10$ & $6.70 \mathrm{E}-11$ & WDR83 & $4.77 E+00$ \\
\hline ENSBTAG00000002305 & 8.77E-11 & $1.84 \mathrm{E}-11$ & PCSK4 & $4.74 E+00$ \\
\hline ENSBTAG00000003260 & $3.90 \mathrm{E}-10$ & 8.17E-11 & GPR151 & $4.77 \mathrm{E}+00$ \\
\hline ENSBTAG00000021687 & $2.04 \mathrm{E}-10$ & $4.23 \mathrm{E}-11$ & JADE2 & $4.81 E+00$ \\
\hline ENSBTAG00000003491 & $4.40 \mathrm{E}-10$ & $8.98 \mathrm{E}-11$ & SLC25A23 & $4.89 E+00$ \\
\hline ENSBTAG00000008484 & $2.20 \mathrm{E}-10$ & 4.47E-11 & TMEM161B & $4.91 E+00$ \\
\hline ENSBTAG00000008021 & $8.28 \mathrm{E}-10$ & $1.66 \mathrm{E}-10$ & & $4.98 \mathrm{E}+00$ \\
\hline ENSBTAG00000004403 & $3.31 \mathrm{E}-10$ & $6.56 \mathrm{E}-11$ & CD320 & $5.03 E+00$ \\
\hline ENSBTAG00000018052 & $3.13 \mathrm{E}-10$ & $6.21 \mathrm{E}-11$ & & $5.03 E+00$ \\
\hline ENSBTAG00000009750 & $5.53 \mathrm{E}-10$ & 1.10E-10 & RAX2 & $5.03 E+00$ \\
\hline ENSBTAG00000000668 & $4.41 \mathrm{E}-10$ & $8.64 \mathrm{E}-11$ & SLC22A5 & $5.10 E+00$ \\
\hline ENSBTAG00000021013 & $6.14 \mathrm{E}-10$ & $1.20 \mathrm{E}-10$ & TUBB4A & $5.11 E+00$ \\
\hline ENSBTAG00000002098 & $3.66 \mathrm{E}-10$ & 7.07E-11 & $\mathrm{CDC} 34$ & $5.16 \mathrm{E}+00$ \\
\hline ENSBTAG00000015224 & $2.09 \mathrm{E}-10$ & $4.02 \mathrm{E}-11$ & TCOF1 & $5.19 E+00$ \\
\hline ENSBTAG00000002286 & $8.44 \mathrm{E}-11$ & $1.61 \mathrm{E}-11$ & DNAJC18 & $5.22 \mathrm{E}+00$ \\
\hline
\end{tabular}




\begin{tabular}{|c|c|c|c|c|}
\hline ENSBTAG00000007698 & $2.79 \mathrm{E}-10$ & $5.27 \mathrm{E}-11$ & TMEM59L & $5.30 \mathrm{E}+00$ \\
\hline ENSBTAG00000033278 & $8.81 \mathrm{E}-10$ & $1.65 \mathrm{E}-10$ & F2RL3 & $5.33 E+00$ \\
\hline ENSBTAG00000004161 & $9.23 \mathrm{E}-11$ & $1.70 \mathrm{E}-11$ & UBE2D3 & $5.40 E+00$ \\
\hline ENSBTAG00000019646 & $5.33 \mathrm{E}-10$ & $9.73 \mathrm{E}-11$ & TRIM41 & $5.47 E+00$ \\
\hline ENSBTAG00000007725 & $5.33 \mathrm{E}-10$ & $9.71 \mathrm{E}-11$ & ARF3 & $5.48 \mathrm{E}+00$ \\
\hline ENSBTAG00000048309 & $4.82 \mathrm{E}-10$ & $8.74 \mathrm{E}-11$ & KIAA1024L & $5.51 \mathrm{E}+00$ \\
\hline ENSBTAG00000019667 & $1.65 \mathrm{E}-10$ & $2.96 \mathrm{E}-11$ & RNF187 & $5.55 \mathrm{E}+00$ \\
\hline ENSBTAG00000010798 & $4.48 \mathrm{E}-11$ & $8.06 \mathrm{E}-12$ & SLC23A1 & $5.50 \mathrm{E}+00$ \\
\hline ENSBTAG00000009566 & $6.02 \mathrm{E}-10$ & $1.08 \mathrm{E}-10$ & & $5.57 \mathrm{E}+00$ \\
\hline ENSBTAG00000047429 & $5.94 \mathrm{E}-10$ & 1.06E-10 & & $5.60 \mathrm{E}+00$ \\
\hline ENSBTAG00000046867 & $6.09 \mathrm{E}-10$ & $1.09 \mathrm{E}-10$ & & $5.58 \mathrm{E}+00$ \\
\hline ENSBTAG00000047546 & $1.52 \mathrm{E}-09$ & $2.71 \mathrm{E}-10$ & & $5.63 E+00$ \\
\hline ENSBTAG00000001116 & $6.39 \mathrm{E}-10$ & $1.09 \mathrm{E}-10$ & P4HA2 & $5.85 \mathrm{E}+00$ \\
\hline ENSBTAG00000019025 & $3.58 \mathrm{E}-10$ & $6.13 \mathrm{E}-11$ & & $5.83 E+00$ \\
\hline ENSBTAG00000009803 & $6.09 \mathrm{E}-10$ & $1.04 \mathrm{E}-10$ & SNCB & $5.85 \mathrm{E}+00$ \\
\hline ENSBTAG00000012945 & $3.01 \mathrm{E}-10$ & $5.11 \mathrm{E}-11$ & PDE6A & $5.89 E+00$ \\
\hline ENSBTAG00000004716 & 8.92E-10 & $1.49 \mathrm{E}-10$ & RETN & $5.98 \mathrm{E}+00$ \\
\hline ENSBTAG00000003675 & $1.03 \mathrm{E}-10$ & $1.71 \mathrm{E}-11$ & ADGRL1 & $6.02 E+00$ \\
\hline ENSBTAG00000046846 & $9.23 \mathrm{E}-10$ & $1.52 \mathrm{E}-10$ & & $6.07 E+00$ \\
\hline ENSBTAG00000013612 & $3.59 \mathrm{E}-10$ & $5.89 \mathrm{E}-11$ & MBLAC2 & $6.08 \mathrm{E}+00$ \\
\hline ENSBTAG00000031809 & $6.06 \mathrm{E}-10$ & $9.92 \mathrm{E}-11$ & DENND1C & $6.11 \mathrm{E}+00$ \\
\hline ENSBTAG00000015636 & $1.51 \mathrm{E}-10$ & 2.37E-11 & C19orf66 & $6.34 \mathrm{E}+00$ \\
\hline ENSBTAG00000025277 & $2.40 \mathrm{E}-10$ & 3.77E-11 & ABHD17A & $6.34 \mathrm{E}+00$ \\
\hline ENSBTAG00000006642 & 7.54E-11 & 1.19E-11 & PRKACA & $6.29 E+00$ \\
\hline ENSBTAG00000008758 & $7.80 \mathrm{E}-11$ & $1.23 \mathrm{E}-11$ & KIF20A & $6.30 E+00$ \\
\hline ENSBTAG00000038564 & $6.84 \mathrm{E}-10$ & 1.07E-10 & & $6.39 E+00$ \\
\hline ENSBTAG00000048208 & $6.95 \mathrm{E}-10$ & $1.09 \mathrm{E}-10$ & & $6.37 E+00$ \\
\hline ENSBTAG00000046474 & $1.41 \mathrm{E}-09$ & $2.20 \mathrm{E}-10$ & & $6.42 E+00$ \\
\hline ENSBTAG00000046865 & $1.75 \mathrm{E}-09$ & $2.71 \mathrm{E}-10$ & & $6.44 E+00$ \\
\hline ENSBTAG00000021520 & $4.82 \mathrm{E}-10$ & $7.26 \mathrm{E}-11$ & DDX46 & $6.63 E+00$ \\
\hline ENSBTAG00000037765 & $2.54 \mathrm{E}-10$ & $3.78 \mathrm{E}-11$ & TNFAIP8L1 & $6.70 E+00$ \\
\hline ENSBTAG00000039877 & $9.77 \mathrm{E}-10$ & $1.44 \mathrm{E}-10$ & & $6.78 \mathrm{E}+00$ \\
\hline ENSBTAG00000016042 & $4.30 \mathrm{E}-10$ & $6.00 \mathrm{E}-11$ & TM6SF2 & $7.15 E+00$ \\
\hline ENSBTAG00000039162 & 4.64E-10 & $6.47 \mathrm{E}-11$ & PCDHGA2 & $7.16 \mathrm{E}+00$ \\
\hline ENSBTAG00000010373 & 4.57E-10 & $6.38 \mathrm{E}-11$ & CACTIN & $7.16 \mathrm{E}+00$ \\
\hline ENSBTAG00000046958 & $5.48 \mathrm{E}-10$ & $7.64 \mathrm{E}-11$ & & $7.17 \mathrm{E}+00$ \\
\hline ENSBTAG00000000753 & $2.26 \mathrm{E}-10$ & $3.10 \mathrm{E}-11$ & PIAS4 & $7.26 \mathrm{E}+00$ \\
\hline ENSBTAG00000033222 & $2.65 \mathrm{E}-10$ & $3.61 \mathrm{E}-11$ & CALR3 & $7.31 E+00$ \\
\hline ENSBTAG00000020417 & $1.79 \mathrm{E}-10$ & $2.34 \mathrm{E}-11$ & DAPK3 & $7.64 E+00$ \\
\hline ENSBTAG00000003543 & $6.80 \mathrm{E}-10$ & 8.77E-11 & KEAP1 & $7.75 E+00$ \\
\hline ENSBTAG00000007214 & $8.98 \mathrm{E}-11$ & $1.13 \mathrm{E}-11$ & ELL2 & $7.89 E+00$ \\
\hline ENSBTAG00000039706 & $6.95 \mathrm{E}-10$ & 8.70E-11 & & $7.98 \mathrm{E}+00$ \\
\hline ENSBTAG00000006067 & $7.84 \mathrm{E}-10$ & $9.81 \mathrm{E}-11$ & VMAC & $7.99 E+00$ \\
\hline ENSBTAG00000009497 & $8.81 E-10$ & $1.10 \mathrm{E}-10$ & & $8.01 E+00$ \\
\hline ENSBTAG00000021820 & $2.01 \mathrm{E}-10$ & $2.41 \mathrm{E}-11$ & DDX39A & $8.32 \mathrm{E}+00$ \\
\hline ENSBTAG00000002629 & $2.87 \mathrm{E}-10$ & $3.44 \mathrm{E}-11$ & C19orf45 & $8.32 E+00$ \\
\hline ENSBTAG00000046190 & $1.22 \mathrm{E}-09$ & $1.45 \mathrm{E}-10$ & PCDHA3 & $8.43 E+00$ \\
\hline ENSBTAG00000004553 & $2.48 \mathrm{E}-10$ & 2.87E-11 & TPM4 & $8.62 E+00$ \\
\hline ENSBTAG00000008443 & $1.41 \mathrm{E}-10$ & $1.61 \mathrm{E}-11$ & LARP1 & $8.71 E+00$ \\
\hline ENSBTAG00000003671 & $5.33 \mathrm{E}-10$ & $6.08 \mathrm{E}-11$ & SOWAHA & $8.75 E+00$ \\
\hline ENSBTAG00000007470 & $3.84 \mathrm{E}-10$ & 4.19E-11 & TRIM58 & $9.13 \mathrm{E}+00$ \\
\hline ENSBTAG00000005272 & $6.90 \mathrm{E}-10$ & 7.52E-11 & $\mathrm{DOHH}$ & $9.17 E+00$ \\
\hline ENSBTAG00000020615 & $7.02 \mathrm{E}-10$ & $7.51 \mathrm{E}-11$ & CCDC105 & $9.34 \mathrm{E}+00$ \\
\hline ENSBTAG00000039397 & $2.23 \mathrm{E}-10$ & $2.34 \mathrm{E}-11$ & WNT3A & $9.51 E+00$ \\
\hline ENSBTAG00000000764 & $5.04 \mathrm{E}-10$ & $5.27 \mathrm{E}-11$ & GIPC1 & $9.54 \mathrm{E}+00$ \\
\hline ENSBTAG00000021818 & $2.24 \mathrm{E}-10$ & $2.30 \mathrm{E}-11$ & ADGRE5 & $9.72 \mathrm{E}+00$ \\
\hline ENSBTAG00000002281 & $6.87 \mathrm{E}-10$ & $6.89 \mathrm{E}-11$ & ZNF354C & $9.96 \mathrm{E}+00$ \\
\hline ENSBTAG00000010729 & $3.34 \mathrm{E}-10$ & $3.23 \mathrm{E}-11$ & KRI1 & $1.03 E+01$ \\
\hline ENSBTAG00000015439 & $1.43 \mathrm{E}-09$ & $1.30 \mathrm{E}-10$ & & $1.10 \mathrm{E}+01$ \\
\hline ENSBTAG00000013691 & $1.24 \mathrm{E}-09$ & $1.10 \mathrm{E}-10$ & OR2L13 & $1.12 \mathrm{E}+01$ \\
\hline ENSBTAG00000014944 & $6.91 \mathrm{E}-10$ & 5.92E-11 & TBXA2R & $1.16 \mathrm{E}+01$ \\
\hline ENSBTAG00000014744 & $5.70 \mathrm{E}-10$ & $4.63 \mathrm{E}-11$ & TXNDC15 & $1.23 E+01$ \\
\hline ENSBTAG00000021041 & $3.15 \mathrm{E}-10$ & $2.55 \mathrm{E}-11$ & PRAM1 & $1.23 \mathrm{E}+01$ \\
\hline ENSBTAG00000020721 & $7.19 \mathrm{E}-10$ & 5.30E-11 & MRPL55 & $1.35 \mathrm{E}+01$ \\
\hline ENSBTAG00000004258 & $1.66 \mathrm{E}-10$ & $1.16 \mathrm{E}-11$ & EEF2 & $1.42 \mathrm{E}+01$ \\
\hline ENSBTAG00000021106 & $4.09 \mathrm{E}-10$ & $2.85 \mathrm{E}-11$ & CASP14 & $1.43 E+01$ \\
\hline ENSBTAG00000021160 & $5.91 \mathrm{E}-10$ & $3.85 \mathrm{E}-11$ & AP1M2 & $1.53 \mathrm{E}+01$ \\
\hline ENSBTAG00000016359 & $2.10 \mathrm{E}-10$ & $1.34 \mathrm{E}-11$ & $\mathrm{SLCO} 4 \mathrm{C} 1$ & $1.55 E+01$ \\
\hline ENSBTAG00000012762 & $2.36 \mathrm{E}-10$ & $1.41 \mathrm{E}-11$ & C5orf24 & $1.66 \mathrm{E}+01$ \\
\hline ENSBTAG00000031231 & $2.24 \mathrm{E}-10$ & $1.28 \mathrm{E}-11$ & IRF1 & $1.74 \mathrm{E}+01$ \\
\hline
\end{tabular}




\begin{tabular}{|c|c|c|c|c|}
\hline ENSBTAG00000046103 & $7.63 \mathrm{E}-10$ & 4.34E-11 & & $1.75 E+01$ \\
\hline ENSBTAG00000030711 & $1.01 \mathrm{E}-09$ & 4.84E-11 & LYPD8 & $2.08 \mathrm{E}+01$ \\
\hline ENSBTAG00000045539 & $1.02 \mathrm{E}-09$ & 4.39E-11 & PCDHA5 & $2.32 E+01$ \\
\hline ENSBTAG00000016783 & 5.77E-10 & $2.41 \mathrm{E}-11$ & PGLS & $2.39 \mathrm{E}+01$ \\
\hline ENSBTAG00000044077 & $5.12 \mathrm{E}-10$ & $1.37 \mathrm{E}-11$ & POLR3G & $3.71 E+01$ \\
\hline ENSBTAG00000046787 & $1.75 \mathrm{E}-09$ & 4.61E-11 & OR14C36 & $3.78 E+01$ \\
\hline ENSBTAG00000017123 & $2.57 \mathrm{E}-10$ & 4.15E-12 & ZNF354B & $6.06 E+01$ \\
\hline ENSBTAG00000016179 & $7.09 \mathrm{E}-10$ & $9.86 \mathrm{E}-12$ & CIB3 & $7.12 E+01$ \\
\hline ENSBTAG00000007737 & $1.37 \mathrm{E}-10$ & 0 & UBA52 & $1.38 \mathrm{E}+03$ \\
\hline ENSBTAG00000014579 & $6.54 \mathrm{E}-11$ & 0 & MRPL34 & $6.55 E+02$ \\
\hline ENSBTAG00000024397 & $2.30 \mathrm{E}-10$ & 0 & & $2.30 \mathrm{E}+03$ \\
\hline ENSBTAG00000017041 & $2.71 \mathrm{E}-10$ & 0 & PTGER1 & $2.71 E+03$ \\
\hline ENSBTAG00000017037 & $5.34 \mathrm{E}-10$ & 0 & PKN1 & $5.34 E+03$ \\
\hline ENSBTAG00000004085 & $5.27 \mathrm{E}-11$ & 0 & ASF1B & $5.28 E+02$ \\
\hline ENSBTAG00000016937 & $5.43 \mathrm{E}-11$ & 0 & C19orf67 & $5.44 \mathrm{E}+02$ \\
\hline ENSBTAG00000047132 & $1.35 \mathrm{E}-10$ & 0 & & $1.35 E+03$ \\
\hline ENSBTAG00000011825 & $1.34 \mathrm{E}-10$ & 0 & C19orf43 & $1.34 \mathrm{E}+03$ \\
\hline ENSBTAG00000006251 & 2.67E-10 & 0 & & $2.67 E+03$ \\
\hline ENSBTAG00000038714 & 8.10E-10 & 0 & & $8.11 E+03$ \\
\hline ENSBTAG00000008196 & $1.03 \mathrm{E}-10$ & 0 & SWSAP1 & $1.03 E+03$ \\
\hline ENSBTAG00000046266 & $4.51 \mathrm{E}-10$ & 0 & TNFSF9 & $4.51 E+03$ \\
\hline ENSBTAG00000031794 & 0 & 0 & PSPN & $1.00 E+00$ \\
\hline ENSBTAG00000025550 & $6.18 \mathrm{E}-11$ & 0 & PRR22 & $6.19 E+02$ \\
\hline ENSBTAG00000018655 & $1.24 \mathrm{E}-10$ & 0 & MYDGF & $1.24 \mathrm{E}+03$ \\
\hline ENSBTAG00000024041 & $5.77 \mathrm{E}-10$ & 0 & & $5.77 E+03$ \\
\hline ENSBTAG00000045755 & 0 & 0 & & $1.00 \mathrm{E}+00$ \\
\hline ENSBTAG00000001570 & $6.61 \mathrm{E}-10$ & 0 & CSF2 & $6.61 E+03$ \\
\hline ENSBTAG00000039886 & 0 & 0 & & $1.00 \mathrm{E}+00$ \\
\hline ENSBTAG00000001394 & $1.66 \mathrm{E}-10$ & 0 & TMED9 & $1.66 \mathrm{E}+03$ \\
\hline ENSBTAG00000040033 & $9.78 \mathrm{E}-10$ & 0 & OR2AJ1 & $9.78 \mathrm{E}+03$ \\
\hline ENSBTAG00000048122 & 0 & 0 & & $1.00 \mathrm{E}+00$ \\
\hline ENSBTAG00000037418 & $2.70 \mathrm{E}-11$ & 0 & KISS1R & $2.71 E+02$ \\
\hline ENSBTAG00000007480 & 0 & 0 & & $1.00 \mathrm{E}+00$ \\
\hline ENSBTAG00000018926 & 8.94E-11 & 0 & LEAP2 & $8.95 E+02$ \\
\hline ENSBTAG00000018290 & $4.41 \mathrm{E}-10$ & 0 & IL9 & $4.41 E+03$ \\
\hline ENSBTAG00000014192 & 8.13E-11 & 0 & LRRTM2 & $8.14 E+02$ \\
\hline ENSBTAG00000046926 & 0 & 0 & GPX4 & $1.00 \mathrm{E}+00$ \\
\hline ENSBTAG00000038337 & 7.87E-11 & 0 & MZB1 & $7.88 \mathrm{E}+02$ \\
\hline ENSBTAG00000002296 & $1.03 \mathrm{E}-10$ & 0 & TMEM173 & $1.03 E+03$ \\
\hline ENSBTAG00000012339 & $8.71 \mathrm{E}-11$ & 0 & & $8.72 \mathrm{E}+02$ \\
\hline ENSBTAG00000046227 & 2.33E-10 & 0 & & $2.33 \mathrm{E}+03$ \\
\hline ENSBTAG00000030204 & $2.82 \mathrm{E}-10$ & 0 & & $2.82 E+03$ \\
\hline ENSBTAG00000017725 & $1.69 \mathrm{E}-10$ & 0 & OR11L1 & $1.69 E+03$ \\
\hline ENSBTAG00000030725 & $2.63 \mathrm{E}-10$ & 0 & & $2.63 E+03$ \\
\hline ENSBTAG00000022690 & $6.14 \mathrm{E}-10$ & 0 & & $6.14 E+03$ \\
\hline ENSBTAG00000046950 & 0 & 0 & & $1.00 \mathrm{E}+00$ \\
\hline ENSBTAG00000030714 & $6.04 \mathrm{E}-10$ & 0 & & $6.04 E+03$ \\
\hline ENSBTAG00000047560 & $1.30 \mathrm{E}-10$ & 0 & $\mathrm{C} 2 \mathrm{CD} 4 \mathrm{C}$ & $1.30 \mathrm{E}+03$ \\
\hline ENSBTAG00000003331 & $3.31 \mathrm{E}-10$ & 0 & NEUROG1 & $3.31 E+03$ \\
\hline ENSBTAG00000016929 & 0 & 0 & HNRNPAO & $1.00 \mathrm{E}+00$ \\
\hline ENSBTAG00000030520 & $5.38 \mathrm{E}-11$ & 0 & PROB1 & $5.39 E+02$ \\
\hline ENSBTAG00000008881 & 0 & 0 & PURA & $1.00 \mathrm{E}+00$ \\
\hline ENSBTAG00000018736 & $7.98 \mathrm{E}-10$ & 0 & & $7.98 \mathrm{E}+03$ \\
\hline ENSBTAG00000046945 & 0 & 0 & & $1.00 \mathrm{E}+00$ \\
\hline ENSBTAG00000022663 & $1.71 \mathrm{E}-10$ & 0 & & $1.71 E+03$ \\
\hline ENSBTAG00000006649 & $9.96 \mathrm{E}-11$ & 0 & TSSK6 & $9.97 \mathrm{E}+02$ \\
\hline ENSBTAG00000027878 & 3.30E-10 & 0 & & $3.30 \mathrm{E}+03$ \\
\hline ENSBTAG00000025226 & 0 & 0 & IGIP & $1.00 \mathrm{E}+00$ \\
\hline ENSBTAG00000011945 & $1.10 \mathrm{E}-10$ & 0 & TAF7 & $1.10 \mathrm{E}+03$ \\
\hline ENSBTAG00000040473 & $3.19 \mathrm{E}-10$ & 0 & & $3.19 E+03$ \\
\hline ENSBTAG00000047946 & 0 & 0 & & $1.00 \mathrm{E}+00$ \\
\hline ENSBTAG00000016789 & 0 & 0 & & $1.00 E+00$ \\
\hline ENSBTAG00000045651 & 0 & 0 & & $1.00 \mathrm{E}+00$ \\
\hline ENSBTAG00000002144 & $4.58 \mathrm{E}-10$ & 0 & ADRB2 & $4.58 \mathrm{E}+03$ \\
\hline ENSBTAG00000046694 & $1.20 \mathrm{E}-10$ & 0 & FNDC9 & $1.20 \mathrm{E}+03$ \\
\hline ENSBTAG00000046080 & $1.63 \mathrm{E}-09$ & 0 & & $1.63 E+04$ \\
\hline ENSBTAG00000030274 & $1.16 \mathrm{E}-10$ & 0 & & $1.16 \mathrm{E}+03$ \\
\hline ENSBTAG00000035395 & 4.38E-10 & 0 & & $4.38 \mathrm{E}+03$ \\
\hline ENSBTAG00000045635 & 0 & 0 & & $1.00 \mathrm{E}+00$ \\
\hline ENSBTAG00000046336 & 0 & 0 & & $1.00 E+00$ \\
\hline
\end{tabular}




\begin{tabular}{|c|c|c|c|c|}
\hline ENSBTAG00000048269 & 0 & 0 & CTXN1 & $1.00 \mathrm{E}+00$ \\
\hline ENSBTAG00000045914 & $1.99 \mathrm{E}-10$ & 0 & & $2.00 E+03$ \\
\hline ENSBTAG00000005779 & $4.43 \mathrm{E}-10$ & 0 & FTMT & $4.43 E+03$ \\
\hline ENSBTAG00000024713 & $1.36 \mathrm{E}-09$ & 0 & & $1.36 \mathrm{E}+04$ \\
\hline ENSBTAG00000045852 & 0 & $9.89 \mathrm{E}-10$ & TAL2 & $1.01 \mathrm{E}-04$ \\
\hline ENSBTAG00000047836 & 0 & $3.45 \mathrm{E}-10$ & & 2.90E-04 \\
\hline ENSBTAG00000046803 & 0 & $9.97 \mathrm{E}-11$ & GAS1 & $1.00 \mathrm{E}-03$ \\
\hline ENSBTAG00000011442 & $6.47 \mathrm{E}-11$ & $5.31 \mathrm{E}-10$ & SPAG8 & $1.22 \mathrm{E}-01$ \\
\hline ENSBTAG00000001315 & $8.26 \mathrm{E}-11$ & $6.48 \mathrm{E}-10$ & DMRTA1 & $1.28 \mathrm{E}-01$ \\
\hline ENSBTAG00000011445 & 7.63E-11 & $5.19 \mathrm{E}-10$ & & 1.47E-01 \\
\hline ENSBTAG00000047598 & $5.87 \mathrm{E}-11$ & $2.97 \mathrm{E}-10$ & & $1.98 \mathrm{E}-01$ \\
\hline ENSBTAG00000039069 & $1.41 \mathrm{E}-10$ & $5.34 \mathrm{E}-10$ & IFNE & 2.64E-01 \\
\hline ENSBTAG00000011434 & $1.05 \mathrm{E}-10$ & $3.12 \mathrm{E}-10$ & & 3.37E-01 \\
\hline ENSBTAG00000016259 & $1.54 \mathrm{E}-10$ & $3.99 \mathrm{E}-10$ & KIAA1958 & 3.85E-01 \\
\hline ENSBTAG00000023462 & $4.93 \mathrm{E}-11$ & $1.24 \mathrm{E}-10$ & & $3.98 \mathrm{E}-01$ \\
\hline ENSBTAG00000012560 & $5.62 \mathrm{E}-11$ & $1.42 \mathrm{E}-10$ & SFTPC & 3.96E-01 \\
\hline ENSBTAG00000038777 & 8.57E-11 & $2.16 \mathrm{E}-10$ & MSMP & 3.97E-01 \\
\hline ENSBTAG00000021586 & $5.00 \mathrm{E}-11$ & $1.26 \mathrm{E}-10$ & FAM122A & 3.97E-01 \\
\hline ENSBTAG00000005222 & $1.33 \mathrm{E}-10$ & $3.13 \mathrm{E}-10$ & FAM221B & 4.24E-01 \\
\hline ENSBTAG00000011444 & $3.53 \mathrm{E}-10$ & $7.71 \mathrm{E}-10$ & HINT2 & 4.59E-01 \\
\hline ENSBTAG00000043983 & $5.86 \mathrm{E}-11$ & $1.23 \mathrm{E}-10$ & LRRC19 & 4.77E-01 \\
\hline ENSBTAG00000005017 & $3.64 \mathrm{E}-10$ & $7.24 \mathrm{E}-10$ & ZNF169 & 5.03E-01 \\
\hline ENSBTAG00000032983 & $1.22 \mathrm{E}-10$ & $2.31 \mathrm{E}-10$ & & 5.29E-01 \\
\hline ENSBTAG00000046824 & $5.77 \mathrm{E}-10$ & $1.09 \mathrm{E}-09$ & & 5.30E-01 \\
\hline ENSBTAG00000047748 & $2.79 \mathrm{E}-10$ & $5.28 \mathrm{E}-10$ & & 5.29E-01 \\
\hline ENSBTAG00000018841 & $3.14 \mathrm{E}-10$ & $5.89 \mathrm{E}-10$ & PTPN3 & 5.33E-01 \\
\hline ENSBTAG00000031517 & $2.52 \mathrm{E}-10$ & $4.23 \mathrm{E}-10$ & & $5.95 \mathrm{E}-01$ \\
\hline ENSBTAG00000016224 & $2.51 \mathrm{E}-10$ & $4.16 \mathrm{E}-10$ & RPS7 & $6.04 \mathrm{E}-01$ \\
\hline ENSBTAG00000003726 & $3.44 \mathrm{E}-10$ & $5.63 \mathrm{E}-10$ & MTMR9 & $6.12 \mathrm{E}-01$ \\
\hline ENSBTAG00000021235 & $1.62 \mathrm{E}-10$ & $2.62 \mathrm{E}-10$ & & $6.18 \mathrm{E}-01$ \\
\hline ENSBTAG00000011433 & $1.56 \mathrm{E}-10$ & $2.50 \mathrm{E}-10$ & RGP1 & $6.24 \mathrm{E}-01$ \\
\hline ENSBTAG00000021131 & $5.83 \mathrm{E}-11$ & $9.18 \mathrm{E}-11$ & HNRNPK & $6.35 \mathrm{E}-01$ \\
\hline ENSBTAG00000000885 & $2.21 \mathrm{E}-10$ & $3.49 \mathrm{E}-10$ & & 6.34E-01 \\
\hline ENSBTAG00000009504 & 8.17E-11 & $1.29 \mathrm{E}-10$ & REEP4 & $6.34 \mathrm{E}-01$ \\
\hline ENSBTAG00000004578 & $5.49 \mathrm{E}-11$ & 8.64E-11 & LGI3 & $6.35 \mathrm{E}-01$ \\
\hline ENSBTAG00000021696 & $1.68 \mathrm{E}-10$ & $2.65 \mathrm{E}-10$ & CCIN & $6.36 \mathrm{E}-01$ \\
\hline ENSBTAG00000004592 & $1.99 \mathrm{E}-10$ & $3.07 \mathrm{E}-10$ & SNAPC3 & $6.50 \mathrm{E}-01$ \\
\hline ENSBTAG00000012757 & $1.69 \mathrm{E}-10$ & $2.55 \mathrm{E}-10$ & GCNT1 & $6.62 \mathrm{E}-01$ \\
\hline ENSBTAG00000010036 & $7.83 \mathrm{E}-11$ & $1.15 \mathrm{E}-10$ & POLR3D & $6.81 E-01$ \\
\hline ENSBTAG00000004944 & $5.23 \mathrm{E}-10$ & $7.63 \mathrm{E}-10$ & ANXA10 & $6.85 \mathrm{E}-01$ \\
\hline ENSBTAG00000021991 & $2.75 \mathrm{E}-10$ & $3.93 \mathrm{E}-10$ & TMEFF1 & 7.00E-01 \\
\hline ENSBTAG00000010178 & $2.61 E-10$ & $3.70 \mathrm{E}-10$ & PTPRD & 7.06E-01 \\
\hline ENSBTAG00000034325 & $1.31 \mathrm{E}-09$ & $1.83 \mathrm{E}-09$ & & 7.15E-01 \\
\hline ENSBTAG00000006478 & $6.27 \mathrm{E}-11$ & $8.78 \mathrm{E}-11$ & C9orf64 & 7.14E-01 \\
\hline ENSBTAG00000005667 & $1.38 \mathrm{E}-10$ & $1.92 \mathrm{E}-10$ & & 7.21E-01 \\
\hline ENSBTAG00000025928 & $1.60 \mathrm{E}-09$ & $2.21 E-09$ & & 7.25E-01 \\
\hline ENSBTAG00000014430 & 8.07E-11 & $1.11 \mathrm{E}-10$ & $\mathrm{CDC} 26$ & 7.28E-01 \\
\hline ENSBTAG00000031682 & $4.90 \mathrm{E}-10$ & $6.69 \mathrm{E}-10$ & ZNF484 & 7.32E-01 \\
\hline ENSBTAG00000015027 & $5.66 \mathrm{E}-10$ & $7.73 \mathrm{E}-10$ & & 7.33E-01 \\
\hline ENSBTAG00000020749 & $1.44 \mathrm{E}-10$ & $1.94 \mathrm{E}-10$ & AAED1 & 7.40E-01 \\
\hline ENSBTAG00000016225 & $3.74 \mathrm{E}-10$ & $5.02 \mathrm{E}-10$ & COLEC11 & 7.44E-01 \\
\hline ENSBTAG00000009764 & $1.82 \mathrm{E}-10$ & $2.40 \mathrm{E}-10$ & & 7.59E-01 \\
\hline ENSBTAG00000005092 & $5.13 \mathrm{E}-10$ & $6.72 \mathrm{E}-10$ & ROR2 & 7.63E-01 \\
\hline ENSBTAG00000013838 & $2.75 \mathrm{E}-10$ & $3.60 \mathrm{E}-10$ & ALLC & 7.65E-01 \\
\hline ENSBTAG00000000250 & $1.46 \mathrm{E}-09$ & $1.91 \mathrm{E}-09$ & HDHD3 & 7.64E-01 \\
\hline ENSBTAG00000038489 & $6.72 \mathrm{E}-10$ & $8.71 E-10$ & DCDC2C & 7.72E-01 \\
\hline ENSBTAG00000014467 & $1.84 \mathrm{E}-10$ & $2.39 \mathrm{E}-10$ & & 7.72E-01 \\
\hline ENSBTAG00000047788 & 2.67E-10 & $3.44 \mathrm{E}-10$ & & 7.78E-01 \\
\hline ENSBTAG00000021573 & $1.75 \mathrm{E}-10$ & $2.26 \mathrm{E}-10$ & DCAF12 & 7.74E-01 \\
\hline ENSBTAG00000047551 & $1.28 \mathrm{E}-09$ & $1.63 \mathrm{E}-09$ & IFN-tau-c1 & 7.83E-01 \\
\hline ENSBTAG00000040034 & $5.40 \mathrm{E}-10$ & $6.88 \mathrm{E}-10$ & & 7.85E-01 \\
\hline ENSBTAG00000010247 & $1.89 \mathrm{E}-10$ & $2.40 \mathrm{E}-10$ & FKTN & 7.86E-01 \\
\hline ENSBTAG00000047195 & $1.13 \mathrm{E}-09$ & $1.42 \mathrm{E}-09$ & & 7.92E-01 \\
\hline ENSBTAG00000025954 & $1.88 \mathrm{E}-10$ & 2.37E-10 & & 7.95E-01 \\
\hline ENSBTAG00000045958 & $3.51 E-10$ & $4.42 \mathrm{E}-10$ & & 7.94E-01 \\
\hline ENSBTAG00000048007 & $1.89 \mathrm{E}-10$ & $2.39 \mathrm{E}-10$ & & 7.92E-01 \\
\hline ENSBTAG00000047237 & $3.50 \mathrm{E}-10$ & $4.41 \mathrm{E}-10$ & & 7.93E-01 \\
\hline ENSBTAG00000047209 & 5.77E-10 & 7.27E-10 & & 7.94E-01 \\
\hline ENSBTAG00000011874 & $1.38 \mathrm{E}-10$ & $1.74 \mathrm{E}-10$ & TMEM252 & 7.93E-01 \\
\hline ENSBTAG00000023412 & $2.56 \mathrm{E}-10$ & $3.23 \mathrm{E}-10$ & IZUMO3 & 7.94E-01 \\
\hline
\end{tabular}




\begin{tabular}{|c|c|c|c|c|}
\hline ENSBTAG00000001221 & $9.91 \mathrm{E}-11$ & $1.25 \mathrm{E}-10$ & NKX3-1 & 7.93E-01 \\
\hline ENSBTAG00000015804 & $3.01 \mathrm{E}-11$ & $3.80 \mathrm{E}-11$ & SIGMAR1 & $7.94 \mathrm{E}-01$ \\
\hline ENSBTAG00000045585 & $9.92 \mathrm{E}-11$ & $1.25 \mathrm{E}-10$ & & $7.93 \mathrm{E}-01$ \\
\hline ENSBTAG00000012684 & $8.58 \mathrm{E}-11$ & $1.08 \mathrm{E}-10$ & CCL19 & $7.95 \mathrm{E}-01$ \\
\hline ENSBTAG00000000699 & $1.11 \mathrm{E}-10$ & $1.40 \mathrm{E}-10$ & & $7.94 \mathrm{E}-01$ \\
\hline ENSBTAG00000025792 & $8.61 \mathrm{E}-11$ & $1.09 \mathrm{E}-10$ & ARID3C & $7.90 \mathrm{E}-01$ \\
\hline ENSBTAG00000020385 & $1.05 \mathrm{E}-10$ & $1.32 \mathrm{E}-10$ & ALDH1B1 & $7.94 \mathrm{E}-01$ \\
\hline ENSBTAG00000046456 & $4.26 \mathrm{E}-10$ & 5.37E-10 & & $7.94 \mathrm{E}-01$ \\
\hline ENSBTAG00000038308 & $2.56 \mathrm{E}-10$ & 3.23E-10 & & 7.94E-01 \\
\hline ENSBTAG00000000203 & $1.37 \mathrm{E}-10$ & $1.72 \mathrm{E}-10$ & ATP6V1G1 & 7.95E-01 \\
\hline ENSBTAG00000047782 & $4.32 \mathrm{E}-10$ & $5.45 \mathrm{E}-10$ & & $7.93 \mathrm{E}-01$ \\
\hline ENSBTAG00000038086 & $8.53 E-11$ & $1.07 \mathrm{E}-10$ & & 7.97E-01 \\
\hline ENSBTAG00000047982 & $7.00 \mathrm{E}-10$ & $8.83 \mathrm{E}-10$ & & $7.93 \mathrm{E}-01$ \\
\hline ENSBTAG00000046729 & $7.43 \mathrm{E}-11$ & $9.36 \mathrm{E}-11$ & & $7.94 \mathrm{E}-01$ \\
\hline ENSBTAG00000002454 & $1.31 \mathrm{E}-10$ & $1.65 \mathrm{E}-10$ & ALG2 & 7.96E-01 \\
\hline ENSBTAG00000015757 & $1.14 \mathrm{E}-10$ & $1.44 \mathrm{E}-10$ & PHYHIP & 7.92E-01 \\
\hline ENSBTAG00000009073 & 1.89E-09 & $2.38 \mathrm{E}-09$ & & $7.93 \mathrm{E}-01$ \\
\hline ENSBTAG00000045506 & $1.66 \mathrm{E}-09$ & 2.09E-09 & & 7.95E-01 \\
\hline ENSBTAG00000046638 & $1.16 \mathrm{E}-09$ & $1.46 \mathrm{E}-09$ & & 7.95E-01 \\
\hline ENSBTAG00000039530 & $1.15 \mathrm{E}-10$ & $1.45 \mathrm{E}-10$ & NEFM & $7.92 \mathrm{E}-01$ \\
\hline ENSBTAG00000034867 & $1.86 \mathrm{E}-10$ & $2.31 \mathrm{E}-10$ & TMEM38B & $8.05 \mathrm{E}-01$ \\
\hline ENSBTAG00000047103 & $6.61 \mathrm{E}-10$ & $8.12 \mathrm{E}-10$ & IDNK & $8.14 \mathrm{E}-01$ \\
\hline ENSBTAG00000025830 & $4.03 \mathrm{E}-10$ & 4.95E-10 & R3HCC1 & $8.15 \mathrm{E}-01$ \\
\hline ENSBTAG00000000804 & $3.51 \mathrm{E}-10$ & 4.29E-10 & МOB3B & $8.18 \mathrm{E}-01$ \\
\hline ENSBTAG00000000076 & $4.75 \mathrm{E}-10$ & $5.81 \mathrm{E}-10$ & RECK & $8.18 \mathrm{E}-01$ \\
\hline ENSBTAG00000012390 & $1.61 \mathrm{E}-10$ & $1.95 \mathrm{E}-10$ & ZNF483 & $8.24 \mathrm{E}-01$ \\
\hline ENSBTAG00000009087 & $5.05 \mathrm{E}-10$ & $6.02 \mathrm{E}-10$ & GNG10 & $8.39 \mathrm{E}-01$ \\
\hline ENSBTAG00000039171 & $4.47 \mathrm{E}-10$ & $5.30 \mathrm{E}-10$ & ADAM7 & $8.43 \mathrm{E}-01$ \\
\hline ENSBTAG00000009804 & $2.02 \mathrm{E}-10$ & $2.38 \mathrm{E}-10$ & ERP44 & $8.49 \mathrm{E}-01$ \\
\hline ENSBTAG00000001306 & $7.38 \mathrm{E}-10$ & $8.65 E-10$ & FXN & 8.53E-01 \\
\hline ENSBTAG00000009596 & $4.00 \mathrm{E}-10$ & $4.66 \mathrm{E}-10$ & C9orf43 & $8.58 \mathrm{E}-01$ \\
\hline ENSBTAG00000033396 & $5.36 \mathrm{E}-10$ & $6.22 \mathrm{E}-10$ & C9orf135 & $8.62 \mathrm{E}-01$ \\
\hline ENSBTAG00000009654 & 4.97E-10 & $5.75 \mathrm{E}-10$ & LPAR1 & 8.64E-01 \\
\hline ENSBTAG00000011321 & $7.91 \mathrm{E}-10$ & $9.11 \mathrm{E}-10$ & NUGGC & $8.68 \mathrm{E}-01$ \\
\hline ENSBTAG00000026123 & $9.58 \mathrm{E}-10$ & 1.10E-09 & & $8.71 E-01$ \\
\hline ENSBTAG00000013357 & $6.22 \mathrm{E}-10$ & 7.10E-10 & PUM3 & $8.76 \mathrm{E}-01$ \\
\hline ENSBTAG00000011204 & $2.18 \mathrm{E}-10$ & $2.48 \mathrm{E}-10$ & NINJ1 & $8.81 \mathrm{E}-01$ \\
\hline ENSBTAG00000038291 & $1.59 \mathrm{E}-10$ & $1.81 \mathrm{E}-10$ & & $8.81 \mathrm{E}-01$ \\
\hline ENSBTAG00000012991 & $5.47 \mathrm{E}-10$ & $6.18 \mathrm{E}-10$ & PRUNE2 & $8.85 \mathrm{E}-01$ \\
\hline ENSBTAG00000020788 & 5.17E-10 & $5.83 \mathrm{E}-10$ & SLC18A1 & 8.87E-01 \\
\hline ENSBTAG00000003124 & $2.00 \mathrm{E}-10$ & $2.26 \mathrm{E}-10$ & MFSD14B & 8.87E-01 \\
\hline ENSBTAG00000017063 & $1.70 \mathrm{E}-10$ & $1.90 \mathrm{E}-10$ & EPB41L4B & 8.93E-01 \\
\hline ENSBTAG00000034391 & $3.86 \mathrm{E}-10$ & 4.33E-10 & EQTN & $8.92 \mathrm{E}-01$ \\
\hline ENSBTAG00000020055 & $2.64 \mathrm{E}-10$ & $2.94 \mathrm{E}-10$ & CEP44 & $8.98 \mathrm{E}-01$ \\
\hline ENSBTAG00000045868 & $8.82 \mathrm{E}-10$ & $9.81 \mathrm{E}-10$ & ZFP37 & 8.99E-01 \\
\hline ENSBTAG00000037929 & $3.25 \mathrm{E}-10$ & $3.60 \mathrm{E}-10$ & ADAM28 & $9.02 \mathrm{E}-01$ \\
\hline ENSBTAG00000047350 & $9.51 \mathrm{E}-10$ & $1.05 \mathrm{E}-09$ & & $9.06 \mathrm{E}-01$ \\
\hline ENSBTAG00000018035 & $1.50 \mathrm{E}-10$ & $1.66 \mathrm{E}-10$ & TGFBR1 & $9.02 \mathrm{E}-01$ \\
\hline ENSBTAG00000043972 & $5.05 \mathrm{E}-10$ & $5.59 \mathrm{E}-10$ & SLC24A2 & $9.04 \mathrm{E}-01$ \\
\hline ENSBTAG00000026915 & $5.74 \mathrm{E}-10$ & $6.36 \mathrm{E}-10$ & NEK1 & $9.03 \mathrm{E}-01$ \\
\hline ENSBTAG00000010821 & $3.95 \mathrm{E}-10$ & $4.35 \mathrm{E}-10$ & ACER2 & 9.07E-01 \\
\hline ENSBTAG00000011413 & $2.60 \mathrm{E}-10$ & $2.86 \mathrm{E}-10$ & CCDC107 & $9.08 \mathrm{E}-01$ \\
\hline ENSBTAG00000011770 & $5.78 \mathrm{E}-10$ & $6.37 \mathrm{E}-10$ & TJP2 & $9.08 \mathrm{E}-01$ \\
\hline ENSBTAG00000019939 & $3.60 \mathrm{E}-10$ & $3.97 \mathrm{E}-10$ & SLC35D2 & 9.07E-01 \\
\hline ENSBTAG00000008836 & $7.18 \mathrm{E}-10$ & $7.90 \mathrm{E}-10$ & ZNF782 & $9.09 \mathrm{E}-01$ \\
\hline ENSBTAG00000020148 & $3.16 \mathrm{E}-10$ & $3.48 \mathrm{E}-10$ & TEK & $9.09 \mathrm{E}-01$ \\
\hline ENSBTAG00000017077 & $1.26 \mathrm{E}-10$ & $1.39 \mathrm{E}-10$ & CTSL2 & $9.04 \mathrm{E}-01$ \\
\hline ENSBTAG00000001322 & $3.44 \mathrm{E}-10$ & $3.78 \mathrm{E}-10$ & SAXO1 & $9.11 \mathrm{E}-01$ \\
\hline ENSBTAG00000043987 & $2.40 \mathrm{E}-10$ & $2.64 \mathrm{E}-10$ & & $9.11 \mathrm{E}-01$ \\
\hline ENSBTAG00000000251 & $8.31 E-10$ & $9.10 \mathrm{E}-10$ & ALAD & $9.13 \mathrm{E}-01$ \\
\hline ENSBTAG00000004499 & $3.24 \mathrm{E}-10$ & $3.55 \mathrm{E}-10$ & RGS3 & $9.14 \mathrm{E}-01$ \\
\hline ENSBTAG00000011586 & $1.34 \mathrm{E}-10$ & $1.46 \mathrm{E}-10$ & HABP4 & $9.17 \mathrm{E}-01$ \\
\hline ENSBTAG00000008101 & $2.74 \mathrm{E}-10$ & $2.98 \mathrm{E}-10$ & PCSK5 & $9.19 \mathrm{E}-01$ \\
\hline ENSBTAG00000002252 & $4.71 \mathrm{E}-10$ & $5.14 \mathrm{E}-10$ & CHRNA2 & $9.16 \mathrm{E}-01$ \\
\hline ENSBTAG00000039827 & 2.13E-09 & $2.32 \mathrm{E}-09$ & & $9.18 \mathrm{E}-01$ \\
\hline ENSBTAG00000006901 & $6.03 E-10$ & $6.58 \mathrm{E}-10$ & NIPSNAP3A & $9.17 \mathrm{E}-01$ \\
\hline ENSBTAG00000001125 & $3.29 \mathrm{E}-10$ & $3.58 \mathrm{E}-10$ & ADAMDEC1 & $9.20 \mathrm{E}-01$ \\
\hline ENSBTAG00000003532 & $2.91 \mathrm{E}-10$ & $3.16 \mathrm{E}-10$ & TLE4 & $9.19 \mathrm{E}-01$ \\
\hline ENSBTAG00000005574 & $6.26 \mathrm{E}-10$ & $6.80 \mathrm{E}-10$ & & $9.20 \mathrm{E}-01$ \\
\hline ENSBTAG00000033076 & $5.01 \mathrm{E}-10$ & $5.44 \mathrm{E}-10$ & TRPM6 & $9.21 \mathrm{E}-01$ \\
\hline
\end{tabular}




\begin{tabular}{|c|c|c|c|c|}
\hline ENSBTAG000000005403 & 7.57E-10 & $8.21 \mathrm{E}-10$ & ADI1 & $9.22 \mathrm{E}-01$ \\
\hline ENSBTAG000000002744 & $1.51 \mathrm{E}-10$ & $1.63 \mathrm{E}-10$ & MUSK & $9.27 \mathrm{E}-01$ \\
\hline ENSBTAG000000011420 & $1.88 \mathrm{E}-10$ & $2.03 \mathrm{E}-10$ & CA9 & $9.27 \mathrm{E}-01$ \\
\hline ENSBTAG000000032669 & $6.14 \mathrm{E}-10$ & $6.62 \mathrm{E}-10$ & & $9.27 \mathrm{E}-01$ \\
\hline ENSBTAG000000008844 & $3.32 \mathrm{E}-10$ & 3.57E-10 & EXTL3 & $9.29 \mathrm{E}-01$ \\
\hline ENSBTAG000000002457 & $1.97 \mathrm{E}-10$ & $2.11 \mathrm{E}-10$ & SEC61B & $9.36 \mathrm{E}-01$ \\
\hline ENSBTAG000000004813 & $3.71 \mathrm{E}-10$ & $3.95 \mathrm{E}-10$ & SUSD1 & $9.38 \mathrm{E}-01$ \\
\hline ENSBTAG000000020155 & $4.27 \mathrm{E}-10$ & $4.54 \mathrm{E}-10$ & RP1L1 & $9.41 \mathrm{E}-01$ \\
\hline ENSBTAG000000044070 & $4.08 \mathrm{E}-10$ & $4.31 \mathrm{E}-10$ & SNX30 & $9.46 \mathrm{E}-01$ \\
\hline ENSBTAG000000019843 & $1.48 \mathrm{E}-10$ & $1.56 \mathrm{E}-10$ & HAUS6 & $9.50 \mathrm{E}-01$ \\
\hline ENSBTAG00000033190 & $2.20 \mathrm{E}-10$ & $2.32 \mathrm{E}-10$ & PGM5 & $9.48 \mathrm{E}-01$ \\
\hline ENSBTAG00000018095 & $7.95 \mathrm{E}-10$ & 8.37E-10 & & $9.50 \mathrm{E}-01$ \\
\hline ENSBTAG00000019345 & $8.76 \mathrm{E}-11$ & $9.20 \mathrm{E}-11$ & RFK & $9.52 \mathrm{E}-01$ \\
\hline ENSBTAG000000021678 & 4.47E-10 & $4.69 \mathrm{E}-10$ & SLC31A1 & $9.53 \mathrm{E}-01$ \\
\hline ENSBTAG000000005466 & $1.91 \mathrm{E}-10$ & $2.01 \mathrm{E}-10$ & & $9.51 \mathrm{E}-01$ \\
\hline ENSBTAG000000046629 & $8.42 \mathrm{E}-10$ & $8.83 \mathrm{E}-10$ & & $9.53 \mathrm{E}-01$ \\
\hline ENSBTAG000000004996 & $1.59 \mathrm{E}-10$ & 1.67E-10 & FAM206A & $9.51 \mathrm{E}-01$ \\
\hline ENSBTAG000000004997 & $1.99 \mathrm{E}-10$ & $2.07 \mathrm{E}-10$ & CTNNAL1 & $9.59 \mathrm{E}-01$ \\
\hline ENSBTAG000000026748 & $6.30 \mathrm{E}-10$ & $6.57 \mathrm{E}-10$ & & $9.59 \mathrm{E}-01$ \\
\hline ENSBTAG000000001938 & $1.84 \mathrm{E}-10$ & $1.90 \mathrm{E}-10$ & CKS2 & $9.70 \mathrm{E}-01$ \\
\hline ENSBTAG00000039873 & $4.84 \mathrm{E}-10$ & $4.98 \mathrm{E}-10$ & & $9.71 \mathrm{E}-01$ \\
\hline ENSBTAG000000001142 & $5.14 \mathrm{E}-10$ & $5.29 \mathrm{E}-10$ & GNE & $9.72 \mathrm{E}-01$ \\
\hline ENSBTAG000000013670 & 4.65E-10 & 4.77E-10 & SLC46A2 & $9.75 \mathrm{E}-01$ \\
\hline ENSBTAG000000047649 & $7.74 \mathrm{E}-10$ & $7.95 \mathrm{E}-10$ & & $9.74 \mathrm{E}-01$ \\
\hline ENSBTAG00000011027 & $4.06 \mathrm{E}-10$ & 4.17E-10 & & $9.74 \mathrm{E}-01$ \\
\hline ENSBTAG000000038794 & $2.19 \mathrm{E}-10$ & $2.25 \mathrm{E}-10$ & TMEM245 & $9.73 \mathrm{E}-01$ \\
\hline ENSBTAG000000000917 & $9.14 \mathrm{E}-11$ & $9.34 \mathrm{E}-11$ & BMP1 & $9.78 \mathrm{E}-01$ \\
\hline ENSBTAG00000004769 & $5.29 \mathrm{E}-10$ & $5.36 \mathrm{E}-10$ & NEIL2 & 9.87E-01 \\
\hline ENSBTAG000000003240 & $1.56 \mathrm{E}-10$ & $1.57 \mathrm{E}-10$ & $\mathrm{C} 8 \mathrm{H} 9$ orf152 & $9.95 \mathrm{E}-01$ \\
\hline ENSBTAG000000013960 & $4.97 \mathrm{E}-10$ & $5.02 \mathrm{E}-10$ & PSAT1 & 9.91E-01 \\
\hline ENSBTAG000000005519 & $2.75 \mathrm{E}-10$ & 2.77E-10 & RNF38 & 9.91E-01 \\
\hline ENSBTAG000000036005 & $4.29 \mathrm{E}-10$ & 4.32E-10 & & 9.94E-01 \\
\hline ENSBTAG000000024233 & $5.29 \mathrm{E}-10$ & $5.32 \mathrm{E}-10$ & & $9.93 \mathrm{E}-01$ \\
\hline ENSBTAG000000027442 & $3.75 E-10$ & $3.77 \mathrm{E}-10$ & NFIB & $9.95 \mathrm{E}-01$ \\
\hline ENSBTAG000000045900 & $4.38 \mathrm{E}-10$ & $4.41 \mathrm{E}-10$ & & $9.92 \mathrm{E}-01$ \\
\hline ENSBTAG00000007494 & $2.01 \mathrm{E}-10$ & $2.02 \mathrm{E}-10$ & SMARCA2 & 9.93E-01 \\
\hline ENSBTAG00000018778 & $4.94 \mathrm{E}-10$ & $4.95 \mathrm{E}-10$ & TMC1 & $9.98 \mathrm{E}-01$ \\
\hline ENSBTAG00000031359 & $9.69 \mathrm{E}-10$ & $9.72 \mathrm{E}-10$ & & 9.97E-01 \\
\hline ENSBTAG00000006547 & $1.98 \mathrm{E}-10$ & $1.98 \mathrm{E}-10$ & CCDC25 & $1.00 \mathrm{E}+00$ \\
\hline ENSBTAG00000005396 & $2.23 \mathrm{E}-10$ & $2.23 \mathrm{E}-10$ & & $1.00 E+00$ \\
\hline ENSBTAG00000035709 & $1.22 \mathrm{E}-09$ & $1.21 \mathrm{E}-09$ & & $1.01 E+00$ \\
\hline ENSBTAG00000012855 & $5.21 \mathrm{E}-10$ & $5.19 \mathrm{E}-10$ & LPL & $1.00 \mathrm{E}+00$ \\
\hline ENSBTAG00000017800 & $1.59 \mathrm{E}-10$ & $1.58 \mathrm{E}-10$ & DMRT3 & $1.00 \mathrm{E}+00$ \\
\hline ENSBTAG00000043961 & 3.27E-10 & $3.25 \mathrm{E}-10$ & MPDZ & $1.01 E+00$ \\
\hline ENSBTAG00000035958 & $6.58 \mathrm{E}-10$ & $6.53 \mathrm{E}-10$ & & $1.01 E+00$ \\
\hline ENSBTAG00000001081 & $4.45 \mathrm{E}-10$ & 4.42E-10 & PALLD & $1.01 E+00$ \\
\hline ENSBTAG00000030960 & $2.62 \mathrm{E}-10$ & $2.59 \mathrm{E}-10$ & & $1.01 \mathrm{E}+00$ \\
\hline ENSBTAG00000020626 & $3.50 \mathrm{E}-10$ & $3.46 \mathrm{E}-10$ & ERCC6L2 & $1.01 E+00$ \\
\hline ENSBTAG00000010326 & $6.23 \mathrm{E}-10$ & $6.15 E-10$ & AADAT & $1.01 E+00$ \\
\hline ENSBTAG00000000500 & $4.66 \mathrm{E}-10$ & $4.61 \mathrm{E}-10$ & PINX1 & $1.01 E+00$ \\
\hline ENSBTAG00000007778 & $3.43 \mathrm{E}-10$ & $3.38 \mathrm{E}-10$ & TRPM3 & $1.02 E+00$ \\
\hline ENSBTAG00000019554 & 7.33E-10 & $7.21 \mathrm{E}-10$ & FBP2 & $1.02 \mathrm{E}+00$ \\
\hline ENSBTAG00000017369 & $3.21 \mathrm{E}-10$ & $3.16 \mathrm{E}-10$ & MAMDC2 & $1.02 \mathrm{E}+00$ \\
\hline ENSBTAG00000014471 & $6.63 \mathrm{E}-10$ & $6.52 \mathrm{E}-10$ & AKAP2 & $1.02 E+00$ \\
\hline ENSBTAG00000015348 & $6.48 \mathrm{E}-11$ & $6.35 \mathrm{E}-11$ & ZNF189 & $1.02 \mathrm{E}+00$ \\
\hline ENSBTAG00000022013 & $4.24 \mathrm{E}-10$ & $4.16 \mathrm{E}-10$ & CBR4 & $1.02 E+00$ \\
\hline ENSBTAG00000045622 & $1.06 \mathrm{E}-10$ & $1.04 \mathrm{E}-10$ & & $1.02 \mathrm{E}+00$ \\
\hline ENSBTAG00000008962 & $1.04 \mathrm{E}-10$ & $1.01 \mathrm{E}-10$ & MRPL50 & $1.03 E+00$ \\
\hline ENSBTAG00000015936 & $3.93 \mathrm{E}-10$ & $3.83 \mathrm{E}-10$ & BRINP1 & $1.03 E+00$ \\
\hline ENSBTAG00000019225 & $3.55 \mathrm{E}-10$ & $3.46 \mathrm{E}-10$ & SLC39A14 & $1.03 E+00$ \\
\hline ENSBTAG00000011424 & $2.45 \mathrm{E}-10$ & $2.38 \mathrm{E}-10$ & TPM2 & $1.03 E+00$ \\
\hline ENSBTAG00000000534 & $3.35 \mathrm{E}-10$ & $3.26 \mathrm{E}-10$ & PNOC & $1.03 E+00$ \\
\hline ENSBTAG00000006532 & $4.74 \mathrm{E}-10$ & $4.61 \mathrm{E}-10$ & CDK5RAP2 & $1.03 E+00$ \\
\hline ENSBTAG00000006551 & $3.70 \mathrm{E}-10$ & $3.60 \mathrm{E}-10$ & & $1.03 E+00$ \\
\hline ENSBTAG00000011397 & 4.57E-10 & 4.44E-10 & UNC13B & $1.03 E+00$ \\
\hline ENSBTAG00000003943 & $6.28 \mathrm{E}-10$ & $6.09 \mathrm{E}-10$ & ТТСЗ9В & $1.03 E+00$ \\
\hline ENSBTAG00000020665 & $2.56 \mathrm{E}-10$ & $2.48 \mathrm{E}-10$ & GFRA2 & $1.03 \mathrm{E}+00$ \\
\hline ENSBTAG00000031916 & 5.27E-10 & $5.09 \mathrm{E}-10$ & RHOBTB2 & $1.04 \mathrm{E}+00$ \\
\hline ENSBTAG00000009516 & $1.57 \mathrm{E}-10$ & $1.52 \mathrm{E}-10$ & RNF20 & $1.03 E+00$ \\
\hline ENSBTAG00000011374 & $5.35 \mathrm{E}-10$ & 5.17E-10 & S1PR3 & $1.04 \mathrm{E}+00$ \\
\hline
\end{tabular}




\begin{tabular}{|c|c|c|c|c|}
\hline ENSBTAG000000005115 & $4.21 \mathrm{E}-10$ & 4.06E-10 & SLC31A2 & $1.04 \mathrm{E}+00$ \\
\hline ENSBTAG00000016235 & $4.31 \mathrm{E}-10$ & 4.15E-10 & RNASEH1 & $1.04 \mathrm{E}+00$ \\
\hline ENSBTAG00000048213 & $1.40 \mathrm{E}-10$ & $1.35 \mathrm{E}-10$ & & $1.04 \mathrm{E}+00$ \\
\hline ENSBTAG000000009806 & $2.26 \mathrm{E}-10$ & 2.17E-10 & INVS & $1.04 \mathrm{E}+00$ \\
\hline ENSBTAG000000035768 & $5.81 \mathrm{E}-10$ & $5.57 \mathrm{E}-10$ & & $1.04 \mathrm{E}+00$ \\
\hline ENSBTAG000000044004 & $1.87 \mathrm{E}-10$ & $1.79 \mathrm{E}-10$ & C9orf84 & $1.05 E+00$ \\
\hline ENSBTAG000000005247 & $7.09 \mathrm{E}-10$ & $6.76 \mathrm{E}-10$ & FRMD3 & $1.05 E+00$ \\
\hline ENSBTAG000000001864 & $1.66 \mathrm{E}-10$ & $1.59 \mathrm{E}-10$ & NR4A3 & $1.05 E+00$ \\
\hline ENSBTAG00000021699 & $3.04 \mathrm{E}-10$ & $2.89 \mathrm{E}-10$ & RORB & $1.05 E+00$ \\
\hline ENSBTAG000000000738 & $6.21 \mathrm{E}-10$ & $5.89 \mathrm{E}-10$ & DAPK1 & $1.05 E+00$ \\
\hline ENSBTAG000000001257 & $5.01 \mathrm{E}-10$ & $4.74 \mathrm{E}-10$ & AGTPBP1 & $1.06 \mathrm{E}+00$ \\
\hline ENSBTAG000000045674 & 1.18E-09 & 1.11E-09 & & $1.06 \mathrm{E}+00$ \\
\hline ENSBTAG00000021127 & $2.87 \mathrm{E}-10$ & $2.72 \mathrm{E}-10$ & GNA14 & $1.06 \mathrm{E}+00$ \\
\hline ENSBTAG00000046969 & $6.35 \mathrm{E}-10$ & 5.99E-10 & & $1.06 \mathrm{E}+00$ \\
\hline ENSBTAG000000032668 & 1.63E-09 & $1.54 \mathrm{E}-09$ & & $1.06 \mathrm{E}+00$ \\
\hline ENSBTAG000000021686 & $3.01 E-10$ & $2.83 \mathrm{E}-10$ & MELK & $1.06 \mathrm{E}+00$ \\
\hline ENSBTAG000000006712 & $2.95 \mathrm{E}-10$ & $2.76 \mathrm{E}-10$ & KIAA0368 & $1.07 \mathrm{E}+00$ \\
\hline ENSBTAG000000017155 & $1.90 \mathrm{E}-10$ & $1.77 \mathrm{E}-10$ & TRIM32 & $1.07 \mathrm{E}+00$ \\
\hline ENSBTAG000000019636 & $6.27 \mathrm{E}-10$ & $5.82 \mathrm{E}-10$ & SCARA5 & $1.08 \mathrm{E}+00$ \\
\hline ENSBTAG000000020516 & $2.97 \mathrm{E}-10$ & $2.75 E-10$ & & $1.08 \mathrm{E}+00$ \\
\hline ENSBTAG000000013420 & $1.90 \mathrm{E}-10$ & $1.76 \mathrm{E}-10$ & FSD1L & $1.08 \mathrm{E}+00$ \\
\hline ENSBTAG000000047990 & $8.12 \mathrm{E}-10$ & $7.49 \mathrm{E}-10$ & & $1.08 \mathrm{E}+00$ \\
\hline ENSBTAG000000006715 & 3.39E-10 & $3.12 \mathrm{E}-10$ & FBXO8 & $1.09 \mathrm{E}+00$ \\
\hline ENSBTAG000000005133 & $2.63 \mathrm{E}-10$ & $2.41 \mathrm{E}-10$ & ANKS6 & $1.09 \mathrm{E}+00$ \\
\hline ENSBTAG000000015978 & $6.25 \mathrm{E}-10$ & $5.73 \mathrm{E}-10$ & ANXA1 & $1.09 \mathrm{E}+00$ \\
\hline ENSBTAG000000017147 & $2.32 \mathrm{E}-10$ & $2.12 \mathrm{E}-10$ & AK3 & $1.09 E+00$ \\
\hline ENSBTAG000000023523 & $1.93 \mathrm{E}-10$ & $1.76 \mathrm{E}-10$ & GKAP1 & $1.09 E+00$ \\
\hline ENSBTAG000000038698 & $4.05 \mathrm{E}-10$ & $3.68 \mathrm{E}-10$ & & $1.10 \mathrm{E}+00$ \\
\hline ENSBTAG00000018517 & $3.68 \mathrm{E}-10$ & 3.32E-10 & VLDLR & $1.11 \mathrm{E}+00$ \\
\hline ENSBTAG000000018387 & $5.95 \mathrm{E}-10$ & $5.35 \mathrm{E}-10$ & TSSC1 & $1.11 E+00$ \\
\hline ENSBTAG000000016412 & $2.34 \mathrm{E}-10$ & $2.10 \mathrm{E}-10$ & CDKN2B & $1.11 E+00$ \\
\hline ENSBTAG000000018667 & $6.15 \mathrm{E}-10$ & $5.53 \mathrm{E}-10$ & RCL1 & $1.11 \mathrm{E}+00$ \\
\hline ENSBTAG000000025760 & $6.40 \mathrm{E}-10$ & $5.75 \mathrm{E}-10$ & & $1.11 E+00$ \\
\hline ENSBTAG000000019732 & 4.05E-10 & $3.64 \mathrm{E}-10$ & GLRA3 & $1.11 E+00$ \\
\hline ENSBTAG000000046815 & $9.83 \mathrm{E}-10$ & $8.83 \mathrm{E}-10$ & & $1.11 E+00$ \\
\hline ENSBTAG00000039963 & $1.02 \mathrm{E}-09$ & $9.15 \mathrm{E}-10$ & & $1.11 \mathrm{E}+00$ \\
\hline ENSBTAG00000017734 & $1.32 \mathrm{E}-10$ & $1.18 \mathrm{E}-10$ & & $1.12 \mathrm{E}+00$ \\
\hline ENSBTAG00000005116 & $5.81 \mathrm{E}-10$ & 5.19E-10 & FKBP15 & $1.12 \mathrm{E}+00$ \\
\hline ENSBTAG00000000095 & $2.74 \mathrm{E}-10$ & $2.43 \mathrm{E}-10$ & CD274 & $1.13 \mathrm{E}+00$ \\
\hline ENSBTAG00000015416 & $3.34 \mathrm{E}-10$ & $2.96 \mathrm{E}-10$ & PEBP4 & $1.13 E+00$ \\
\hline ENSBTAG00000017845 & $1.98 \mathrm{E}-10$ & $1.75 \mathrm{E}-10$ & BAG1 & $1.13 \mathrm{E}+00$ \\
\hline ENSBTAG00000004571 & $2.93 \mathrm{E}-10$ & $2.59 \mathrm{E}-10$ & PRPF4 & $1.13 \mathrm{E}+00$ \\
\hline ENSBTAG00000002190 & $2.81 \mathrm{E}-10$ & $2.48 \mathrm{E}-10$ & DOCK8 & $1.14 \mathrm{E}+00$ \\
\hline ENSBTAG00000031295 & $4.97 \mathrm{E}-10$ & $4.38 \mathrm{E}-10$ & HSDL2 & $1.14 \mathrm{E}+00$ \\
\hline ENSBTAG00000047142 & $1.48 \mathrm{E}-09$ & 1.30E-09 & & $1.14 \mathrm{E}+00$ \\
\hline ENSBTAG00000016958 & $2.57 \mathrm{E}-10$ & $2.26 \mathrm{E}-10$ & PHF19 & $1.14 \mathrm{E}+00$ \\
\hline ENSBTAG00000038375 & $4.98 \mathrm{E}-10$ & $4.36 \mathrm{E}-10$ & STOM & $1.14 \mathrm{E}+00$ \\
\hline ENSBTAG00000015788 & $4.09 \mathrm{E}-10$ & 3.57E-10 & CCDC171 & $1.14 \mathrm{E}+00$ \\
\hline ENSBTAG00000025929 & $9.50 \mathrm{E}-11$ & $8.31 E-11$ & & $1.14 \mathrm{E}+00$ \\
\hline ENSBTAG00000000536 & $4.81 \mathrm{E}-10$ & $4.21 E-10$ & ZNF395 & $1.14 \mathrm{E}+00$ \\
\hline ENSBTAG00000011431 & $1.92 \mathrm{E}-10$ & $1.68 \mathrm{E}-10$ & GBA2 & $1.14 \mathrm{E}+00$ \\
\hline ENSBTAG00000020132 & $2.10 \mathrm{E}-10$ & $1.83 \mathrm{E}-10$ & HPF1 & $1.15 E+00$ \\
\hline ENSBTAG00000005469 & $2.30 \mathrm{E}-10$ & 2.00E-10 & CCAR2 & $1.15 E+00$ \\
\hline ENSBTAG00000006214 & $6.51 \mathrm{E}-10$ & $5.64 \mathrm{E}-10$ & LOXL2 & $1.15 \mathrm{E}+00$ \\
\hline ENSBTAG00000017803 & $2.62 \mathrm{E}-10$ & 2.27E-10 & RAB14 & $1.15 E+00$ \\
\hline ENSBTAG00000020436 & $1.59 \mathrm{E}-10$ & $1.37 \mathrm{E}-10$ & KIF27 & $1.16 \mathrm{E}+00$ \\
\hline ENSBTAG00000009733 & $2.45 \mathrm{E}-10$ & $2.11 E-10$ & FBP1 & $1.16 \mathrm{E}+00$ \\
\hline ENSBTAG00000001137 & $2.50 \mathrm{E}-10$ & $2.15 \mathrm{E}-10$ & CLTA & $1.16 \mathrm{E}+00$ \\
\hline ENSBTAG00000004010 & $2.15 \mathrm{E}-10$ & $1.84 \mathrm{E}-10$ & PAPPA & $1.17 \mathrm{E}+00$ \\
\hline ENSBTAG00000044155 & $7.96 \mathrm{E}-10$ & $6.79 \mathrm{E}-10$ & BSPRY & $1.17 E+00$ \\
\hline ENSBTAG00000007369 & $2.12 \mathrm{E}-10$ & $1.81 \mathrm{E}-10$ & & $1.17 \mathrm{E}+00$ \\
\hline ENSBTAG00000012432 & $5.07 \mathrm{E}-10$ & 4.30E-10 & FDFT1 & $1.18 \mathrm{E}+00$ \\
\hline ENSBTAG00000013706 & $3.71 E-10$ & $3.15 \mathrm{E}-10$ & MEGF9 & $1.18 \mathrm{E}+00$ \\
\hline ENSBTAG00000000819 & $1.76 \mathrm{E}-10$ & $1.49 \mathrm{E}-10$ & RFX3 & $1.18 \mathrm{E}+00$ \\
\hline ENSBTAG00000002730 & $8.14 \mathrm{E}-10$ & $6.90 \mathrm{E}-10$ & ELP3 & $1.18 \mathrm{E}+00$ \\
\hline ENSBTAG00000008877 & $2.33 \mathrm{E}-10$ & $1.97 \mathrm{E}-10$ & APBA1 & $1.18 \mathrm{E}+00$ \\
\hline ENSBTAG00000033122 & $8.56 \mathrm{E}-10$ & $7.23 \mathrm{E}-10$ & FOXD4L1 & $1.18 \mathrm{E}+00$ \\
\hline ENSBTAG00000010647 & $2.55 \mathrm{E}-10$ & $2.15 \mathrm{E}-10$ & NTRK2 & $1.18 \mathrm{E}+00$ \\
\hline ENSBTAG00000046348 & 4.07E-10 & $3.43 \mathrm{E}-10$ & ELAVL2 & $1.19 \mathrm{E}+00$ \\
\hline ENSBTAG00000010686 & $2.59 \mathrm{E}-10$ & 2.17E-10 & OR2K2 & $1.19 E+00$ \\
\hline
\end{tabular}




\begin{tabular}{|c|c|c|c|c|}
\hline ENSBTAG00000019143 & $1.11 \mathrm{E}-10$ & $9.33 \mathrm{E}-11$ & RPN1 & $1.19 \mathrm{E}+00$ \\
\hline ENSBTAG00000013528 & $3.08 \mathrm{E}-10$ & $2.58 \mathrm{E}-10$ & CHMP7 & $1.19 E+00$ \\
\hline ENSBTAG00000030953 & $3.59 \mathrm{E}-10$ & $3.01 \mathrm{E}-10$ & & $1.19 E+00$ \\
\hline ENSBTAG00000036008 & $2.57 \mathrm{E}-10$ & $2.16 \mathrm{E}-10$ & & $1.19 \mathrm{E}+00$ \\
\hline ENSBTAG00000032093 & $5.79 \mathrm{E}-10$ & $4.85 \mathrm{E}-10$ & SPINK4 & $1.19 E+00$ \\
\hline ENSBTAG00000021632 & $3.23 \mathrm{E}-10$ & $2.70 \mathrm{E}-10$ & MSRA & $1.20 \mathrm{E}+00$ \\
\hline ENSBTAG00000010976 & $2.56 \mathrm{E}-10$ & $2.13 \mathrm{E}-10$ & KANK1 & $1.20 \mathrm{E}+00$ \\
\hline ENSBTAG00000014523 & $8.95 \mathrm{E}-10$ & 7.47E-10 & DIRAS2 & $1.20 \mathrm{E}+00$ \\
\hline ENSBTAG00000009037 & $6.84 \mathrm{E}-10$ & $5.70 \mathrm{E}-10$ & GALNT12 & $1.20 \mathrm{E}+00$ \\
\hline ENSBTAG00000018437 & $3.11 \mathrm{E}-10$ & 2.60E-10 & SMC5 & $1.20 \mathrm{E}+00$ \\
\hline ENSBTAG00000007872 & $1.80 \mathrm{E}-10$ & $1.49 \mathrm{E}-10$ & PSIP1 & $1.21 \mathrm{E}+00$ \\
\hline ENSBTAG00000005718 & $2.09 \mathrm{E}-10$ & $1.73 \mathrm{E}-10$ & PLIN2 & $1.21 \mathrm{E}+00$ \\
\hline ENSBTAG00000047389 & $1.46 \mathrm{E}-09$ & $1.21 \mathrm{E}-09$ & & $1.21 \mathrm{E}+00$ \\
\hline ENSBTAG00000017741 & $7.64 \mathrm{E}-10$ & $6.26 \mathrm{E}-10$ & HACD4 & $1.22 \mathrm{E}+00$ \\
\hline ENSBTAG00000034626 & $6.11 \mathrm{E}-10$ & $5.00 \mathrm{E}-10$ & FBXO16 & $1.22 \mathrm{E}+00$ \\
\hline ENSBTAG00000013231 & $2.52 \mathrm{E}-10$ & $2.06 \mathrm{E}-10$ & C9orf40 & $1.22 \mathrm{E}+00$ \\
\hline ENSBTAG00000017884 & $2.56 \mathrm{E}-10$ & 2.07E-10 & KCNV2 & $1.24 \mathrm{E}+00$ \\
\hline ENSBTAG00000008845 & $3.91 \mathrm{E}-10$ & $3.16 \mathrm{E}-10$ & INTS9 & $1.24 \mathrm{E}+00$ \\
\hline ENSBTAG00000019568 & $1.76 \mathrm{E}-10$ & $1.42 \mathrm{E}-10$ & IFT74 & $1.24 \mathrm{E}+00$ \\
\hline ENSBTAG00000005826 & $2.23 \mathrm{E}-10$ & $1.80 \mathrm{E}-10$ & PTAR1 & $1.24 \mathrm{E}+00$ \\
\hline ENSBTAG00000046486 & 4.65E-10 & $3.74 \mathrm{E}-10$ & & $1.24 \mathrm{E}+00$ \\
\hline ENSBTAG00000018347 & 3.07E-10 & 2.47E-10 & IL33 & $1.24 \mathrm{E}+00$ \\
\hline ENSBTAG00000024081 & $3.56 \mathrm{E}-10$ & $2.85 \mathrm{E}-10$ & ECM2 & $1.25 \mathrm{E}+00$ \\
\hline ENSBTAG00000019915 & $6.62 \mathrm{E}-10$ & 5.30E-10 & & $1.25 \mathrm{E}+00$ \\
\hline ENSBTAG00000040038 & $2.37 \mathrm{E}-10$ & $1.90 \mathrm{E}-10$ & & $1.25 E+00$ \\
\hline ENSBTAG00000009903 & $2.13 \mathrm{E}-10$ & $1.69 \mathrm{E}-10$ & & $1.26 \mathrm{E}+00$ \\
\hline ENSBTAG00000025868 & $2.07 \mathrm{E}-10$ & $1.64 \mathrm{E}-10$ & TLN1 & $1.26 \mathrm{E}+00$ \\
\hline ENSBTAG00000007440 & $6.62 \mathrm{E}-10$ & $5.24 \mathrm{E}-10$ & MLANA & $1.26 \mathrm{E}+00$ \\
\hline ENSBTAG00000019539 & $2.81 \mathrm{E}-10$ & $2.22 \mathrm{E}-10$ & MFAP3L & $1.26 \mathrm{E}+00$ \\
\hline ENSBTAG00000005958 & $3.60 \mathrm{E}-10$ & $2.82 \mathrm{E}-10$ & PTK2B & $1.28 \mathrm{E}+00$ \\
\hline ENSBTAG00000002997 & 3.17E-10 & $2.49 \mathrm{E}-10$ & ADAMTSL1 & $1.27 \mathrm{E}+00$ \\
\hline ENSBTAG00000016229 & $1.95 \mathrm{E}-10$ & $1.52 \mathrm{E}-10$ & KLF9 & $1.28 \mathrm{E}+00$ \\
\hline ENSBTAG00000031793 & $3.17 \mathrm{E}-10$ & $2.47 \mathrm{E}-10$ & NUDT2 & $1.28 \mathrm{E}+00$ \\
\hline ENSBTAG00000035007 & $5.11 \mathrm{E}-10$ & $3.99 \mathrm{E}-10$ & GALNTL6 & $1.28 \mathrm{E}+00$ \\
\hline ENSBTAG00000006768 & 4.94E-10 & $3.85 \mathrm{E}-10$ & ENTPD4 & $1.28 \mathrm{E}+00$ \\
\hline ENSBTAG00000001687 & $1.85 \mathrm{E}-10$ & $1.43 \mathrm{E}-10$ & STC1 & $1.30 \mathrm{E}+00$ \\
\hline ENSBTAG00000011409 & $2.00 \mathrm{E}-10$ & $1.54 \mathrm{E}-10$ & CD72 & $1.30 \mathrm{E}+00$ \\
\hline ENSBTAG00000035084 & $4.18 \mathrm{E}-10$ & $3.21 \mathrm{E}-10$ & & $1.30 \mathrm{E}+00$ \\
\hline ENSBTAG00000015676 & $7.04 \mathrm{E}-10$ & $5.36 \mathrm{E}-10$ & AMBP & $1.31 E+00$ \\
\hline ENSBTAG00000003097 & $1.22 \mathrm{E}-10$ & $9.30 \mathrm{E}-11$ & DENND4C & $1.32 \mathrm{E}+00$ \\
\hline ENSBTAG00000021751 & $3.48 \mathrm{E}-10$ & $2.63 \mathrm{E}-10$ & RASEF & $1.32 \mathrm{E}+00$ \\
\hline ENSBTAG00000003137 & $6.12 \mathrm{E}-10$ & 4.62E-10 & PLPPR1 & $1.32 \mathrm{E}+00$ \\
\hline ENSBTAG00000033708 & $6.01 \mathrm{E}-10$ & $4.53 \mathrm{E}-10$ & & $1.33 \mathrm{E}+00$ \\
\hline ENSBTAG00000031632 & $3.98 \mathrm{E}-10$ & $3.01 \mathrm{E}-10$ & ADRA1A & $1.32 \mathrm{E}+00$ \\
\hline ENSBTAG00000008678 & $2.20 \mathrm{E}-10$ & $1.66 \mathrm{E}-10$ & & $1.32 \mathrm{E}+00$ \\
\hline ENSBTAG00000022787 & $1.66 \mathrm{E}-09$ & $1.25 \mathrm{E}-09$ & & $1.33 E+00$ \\
\hline ENSBTAG00000003669 & $3.22 \mathrm{E}-10$ & $2.42 \mathrm{E}-10$ & BNC2 & $1.33 \mathrm{E}+00$ \\
\hline ENSBTAG00000000695 & $2.16 \mathrm{E}-10$ & $1.62 \mathrm{E}-10$ & UBAP2 & $1.34 \mathrm{E}+00$ \\
\hline ENSBTAG00000020105 & $1.87 \mathrm{E}-10$ & $1.40 \mathrm{E}-10$ & & $1.33 \mathrm{E}+00$ \\
\hline ENSBTAG00000009917 & $2.20 \mathrm{E}-10$ & $1.64 \mathrm{E}-10$ & PIP5K1B & $1.34 \mathrm{E}+00$ \\
\hline ENSBTAG00000021992 & $4.12 \mathrm{E}-10$ & $3.06 \mathrm{E}-10$ & MURC & $1.34 \mathrm{E}+00$ \\
\hline ENSBTAG00000018280 & $5.23 \mathrm{E}-10$ & $3.86 \mathrm{E}-10$ & SLC28A3 & $1.35 \mathrm{E}+00$ \\
\hline ENSBTAG00000035707 & $1.01 \mathrm{E}-09$ & 7.47E-10 & & $1.35 \mathrm{E}+00$ \\
\hline ENSBTAG00000019187 & $1.73 \mathrm{E}-10$ & $1.27 \mathrm{E}-10$ & ZNF462 & $1.36 \mathrm{E}+00$ \\
\hline ENSBTAG00000047413 & $1.70 \mathrm{E}-09$ & $1.24 \mathrm{E}-09$ & & $1.37 \mathrm{E}+00$ \\
\hline ENSBTAG00000018481 & $1.04 \mathrm{E}-09$ & $7.51 \mathrm{E}-10$ & & $1.38 \mathrm{E}+00$ \\
\hline ENSBTAG00000005049 & $7.36 \mathrm{E}-10$ & $5.33 \mathrm{E}-10$ & BLK & $1.38 \mathrm{E}+00$ \\
\hline ENSBTAG00000011859 & $1.90 \mathrm{E}-10$ & $1.38 \mathrm{E}-10$ & IPPK & $1.38 \mathrm{E}+00$ \\
\hline ENSBTAG00000047442 & $6.43 \mathrm{E}-10$ & $4.65 \mathrm{E}-10$ & & $1.38 \mathrm{E}+00$ \\
\hline ENSBTAG00000011416 & $1.60 \mathrm{E}-10$ & $1.15 \mathrm{E}-10$ & ARHGEF39 & $1.39 E+00$ \\
\hline ENSBTAG00000009958 & $1.04 \mathrm{E}-09$ & 7.52E-10 & TRIM35 & $1.39 E+00$ \\
\hline ENSBTAG00000011429 & $1.32 \mathrm{E}-10$ & $9.48 \mathrm{E}-11$ & CREB3 & $1.39 E+00$ \\
\hline ENSBTAG00000008204 & $2.22 \mathrm{E}-10$ & $1.59 \mathrm{E}-10$ & CARNMT1 & $1.39 E+00$ \\
\hline ENSBTAG00000046292 & $6.02 \mathrm{E}-10$ & $4.32 \mathrm{E}-10$ & & $1.39 E+00$ \\
\hline ENSBTAG00000001219 & $8.91 \mathrm{E}-10$ & $6.39 \mathrm{E}-10$ & & $1.39 E+00$ \\
\hline ENSBTAG00000000164 & $1.51 \mathrm{E}-10$ & $1.09 \mathrm{E}-10$ & GNRH1 & $1.39 E+00$ \\
\hline ENSBTAG00000020661 & $2.78 \mathrm{E}-10$ & $1.99 \mathrm{E}-10$ & ABCA1 & $1.40 \mathrm{E}+00$ \\
\hline ENSBTAG00000012498 & $2.42 \mathrm{E}-10$ & $1.72 \mathrm{E}-10$ & PAX5 & $1.41 \mathrm{E}+00$ \\
\hline ENSBTAG00000016073 & $3.85 \mathrm{E}-10$ & $2.75 \mathrm{E}-10$ & UGCG & $1.40 \mathrm{E}+00$ \\
\hline ENSBTAG00000001283 & $2.50 \mathrm{E}-10$ & $1.77 \mathrm{E}-10$ & RIC1 & $1.41 E+00$ \\
\hline
\end{tabular}




\begin{tabular}{|c|c|c|c|c|}
\hline ENSBTAG00000017195 & $3.90 \mathrm{E}-10$ & $2.76 \mathrm{E}-10$ & FANCC & $1.41 \mathrm{E}+00$ \\
\hline ENSBTAG00000034665 & $1.87 \mathrm{E}-10$ & $1.32 \mathrm{E}-10$ & PRSS55 & $1.42 \mathrm{E}+00$ \\
\hline ENSBTAG00000014124 & $2.03 \mathrm{E}-10$ & $1.43 \mathrm{E}-10$ & MLLT3 & $1.42 \mathrm{E}+00$ \\
\hline ENSBTAG00000020151 & $9.47 \mathrm{E}-10$ & $6.67 \mathrm{E}-10$ & & $1.42 \mathrm{E}+00$ \\
\hline ENSBTAG00000012383 & $1.47 \mathrm{E}-10$ & $1.04 \mathrm{E}-10$ & CHMP5 & $1.41 E+00$ \\
\hline ENSBTAG00000015807 & $2.14 \mathrm{E}-10$ & $1.50 \mathrm{E}-10$ & GALT & $1.43 E+00$ \\
\hline ENSBTAG00000009789 & $3.56 \mathrm{E}-10$ & $2.49 \mathrm{E}-10$ & GNAQ & $1.43 \mathrm{E}+00$ \\
\hline ENSBTAG00000007659 & $4.40 \mathrm{E}-10$ & $3.08 \mathrm{E}-10$ & CORO2A & $1.43 E+00$ \\
\hline ENSBTAG00000047207 & $8.11 \mathrm{E}-10$ & $5.66 \mathrm{E}-10$ & & $1.43 \mathrm{E}+00$ \\
\hline ENSBTAG00000015361 & $1.34 \mathrm{E}-10$ & $9.38 \mathrm{E}-11$ & CNTFR & $1.43 E+00$ \\
\hline ENSBTAG00000016867 & $4.05 \mathrm{E}-10$ & $2.82 \mathrm{E}-10$ & HSD17B3 & $1.44 \mathrm{E}+00$ \\
\hline ENSBTAG00000001941 & $5.09 \mathrm{E}-10$ & $3.55 E-10$ & SEMA4D & $1.43 E+00$ \\
\hline ENSBTAG00000014103 & $4.93 \mathrm{E}-10$ & $3.42 \mathrm{E}-10$ & SH3GL2 & $1.44 \mathrm{E}+00$ \\
\hline ENSBTAG00000012210 & $4.85 \mathrm{E}-10$ & $3.36 \mathrm{E}-10$ & C5 & $1.44 \mathrm{E}+00$ \\
\hline ENSBTAG00000015685 & $8.66 \mathrm{E}-10$ & $5.99 \mathrm{E}-10$ & KIF12 & $1.45 E+00$ \\
\hline ENSBTAG00000020130 & $2.40 \mathrm{E}-10$ & $1.66 \mathrm{E}-10$ & CLCN3 & $1.45 E+00$ \\
\hline ENSBTAG00000000078 & $8.75 \mathrm{E}-10$ & $6.04 \mathrm{E}-10$ & GLIPR2 & $1.45 E+00$ \\
\hline ENSBTAG00000000256 & $2.29 \mathrm{E}-10$ & $1.58 \mathrm{E}-10$ & TLE1 & $1.45 \mathrm{E}+00$ \\
\hline ENSBTAG00000046602 & $7.62 \mathrm{E}-10$ & $5.25 \mathrm{E}-10$ & PALM2 & $1.45 \mathrm{E}+00$ \\
\hline ENSBTAG00000011394 & $2.29 \mathrm{E}-10$ & $1.58 \mathrm{E}-10$ & FAM214B & $1.45 E+00$ \\
\hline ENSBTAG00000019275 & $5.38 \mathrm{E}-10$ & $3.70 \mathrm{E}-10$ & INIP & $1.45 E+00$ \\
\hline ENSBTAG00000035708 & $8.35 \mathrm{E}-10$ & $5.70 \mathrm{E}-10$ & & $1.46 \mathrm{E}+00$ \\
\hline ENSBTAG00000016750 & $3.30 \mathrm{E}-10$ & $2.26 \mathrm{E}-10$ & РTBP3 & $1.46 \mathrm{E}+00$ \\
\hline ENSBTAG00000006197 & $2.29 \mathrm{E}-10$ & $1.56 \mathrm{E}-10$ & ZDHHC21 & $1.47 \mathrm{E}+00$ \\
\hline ENSBTAG00000008538 & $4.35 \mathrm{E}-10$ & $2.96 \mathrm{E}-10$ & DNAI1 & $1.47 \mathrm{E}+00$ \\
\hline ENSBTAG00000033352 & $9.22 \mathrm{E}-11$ & $6.27 \mathrm{E}-11$ & DMRT1 & $1.47 \mathrm{E}+00$ \\
\hline ENSBTAG00000010819 & $4.32 \mathrm{E}-10$ & $2.92 \mathrm{E}-10$ & HMBOX1 & $1.48 \mathrm{E}+00$ \\
\hline ENSBTAG00000001847 & $2.49 \mathrm{E}-10$ & $1.68 \mathrm{E}-10$ & CNTLN & $1.48 \mathrm{E}+00$ \\
\hline ENSBTAG00000011417 & $3.19 \mathrm{E}-10$ & $2.15 \mathrm{E}-10$ & CNTRL & $1.48 \mathrm{E}+00$ \\
\hline ENSBTAG00000006446 & $6.61 E-10$ & $4.45 E-10$ & & $1.48 \mathrm{E}+00$ \\
\hline ENSBTAG00000038982 & $2.09 \mathrm{E}-10$ & $1.40 \mathrm{E}-10$ & & $1.49 \mathrm{E}+00$ \\
\hline ENSBTAG00000016265 & $3.31 \mathrm{E}-10$ & $2.23 \mathrm{E}-10$ & DNAJA1 & $1.48 \mathrm{E}+00$ \\
\hline ENSBTAG00000000320 & $2.50 \mathrm{E}-10$ & $1.68 \mathrm{E}-10$ & UBAP1 & $1.49 E+00$ \\
\hline ENSBTAG00000010082 & $5.09 \mathrm{E}-10$ & $3.42 \mathrm{E}-10$ & COL15A1 & $1.49 E+00$ \\
\hline ENSBTAG00000000252 & $4.70 \mathrm{E}-10$ & $3.15 E-10$ & POLE3 & $1.49 \mathrm{E}+00$ \\
\hline ENSBTAG00000018445 & $3.26 \mathrm{E}-10$ & $2.18 \mathrm{E}-10$ & GRIN3A & $1.49 E+00$ \\
\hline ENSBTAG00000010107 & $1.76 \mathrm{E}-10$ & $1.17 \mathrm{E}-10$ & WHRN & $1.50 \mathrm{E}+00$ \\
\hline ENSBTAG00000025903 & $1.77 \mathrm{E}-10$ & $1.18 \mathrm{E}-10$ & GLDC & $1.50 E+00$ \\
\hline ENSBTAG00000015249 & $2.07 \mathrm{E}-10$ & $1.38 \mathrm{E}-10$ & B4GALT1 & $1.50 E+00$ \\
\hline ENSBTAG00000012809 & $2.43 \mathrm{E}-10$ & $1.61 \mathrm{E}-10$ & PHF24 & $1.51 E+00$ \\
\hline ENSBTAG00000017232 & $6.53 \mathrm{E}-10$ & $4.31 \mathrm{E}-10$ & KIF13B & $1.52 \mathrm{E}+00$ \\
\hline ENSBTAG00000019125 & $4.55 \mathrm{E}-10$ & $3.01 \mathrm{E}-10$ & SLC1A1 & $1.51 E+00$ \\
\hline ENSBTAG00000000693 & $2.27 \mathrm{E}-10$ & $1.50 \mathrm{E}-10$ & UBE2R2 & $1.51 \mathrm{E}+00$ \\
\hline ENSBTAG00000000720 & $2.99 \mathrm{E}-10$ & $1.97 \mathrm{E}-10$ & CTSL & $1.52 \mathrm{E}+00$ \\
\hline ENSBTAG00000015171 & $2.10 \mathrm{E}-10$ & $1.38 \mathrm{E}-10$ & CDK20 & $1.52 \mathrm{E}+00$ \\
\hline ENSBTAG00000005495 & $3.13 \mathrm{E}-10$ & $2.02 \mathrm{E}-10$ & & $1.55 \mathrm{E}+00$ \\
\hline ENSBTAG00000020752 & $1.10 \mathrm{E}-10$ & $7.09 \mathrm{E}-11$ & CEP78 & $1.55 E+00$ \\
\hline ENSBTAG00000047511 & $2.76 \mathrm{E}-10$ & $1.77 \mathrm{E}-10$ & & $1.56 \mathrm{E}+00$ \\
\hline ENSBTAG00000003719 & $1.78 \mathrm{E}-10$ & $1.15 \mathrm{E}-10$ & TDRD7 & $1.55 \mathrm{E}+00$ \\
\hline ENSBTAG00000018317 & $1.94 \mathrm{E}-10$ & $1.23 \mathrm{E}-10$ & PPP2R2A & $1.57 \mathrm{E}+00$ \\
\hline ENSBTAG00000000712 & $2.62 \mathrm{E}-10$ & $1.66 \mathrm{E}-10$ & FBXW2 & $1.58 \mathrm{E}+00$ \\
\hline ENSBTAG00000004468 & $1.64 \mathrm{E}-10$ & $1.03 \mathrm{E}-10$ & BARX1 & $1.59 \mathrm{E}+00$ \\
\hline ENSBTAG00000044123 & $1.49 \mathrm{E}-10$ & $9.35 \mathrm{E}-11$ & HAND2 & $1.59 \mathrm{E}+00$ \\
\hline ENSBTAG00000047745 & $2.83 \mathrm{E}-10$ & $1.78 \mathrm{E}-10$ & & $1.59 \mathrm{E}+00$ \\
\hline ENSBTAG00000034326 & $2.79 \mathrm{E}-10$ & $1.76 \mathrm{E}-10$ & & $1.59 E+00$ \\
\hline ENSBTAG00000001223 & $3.46 \mathrm{E}-10$ & $2.18 \mathrm{E}-10$ & CAAP1 & $1.59 \mathrm{E}+00$ \\
\hline ENSBTAG00000017137 & $3.76 \mathrm{E}-10$ & $2.37 \mathrm{E}-10$ & CDC37L1 & $1.59 E+00$ \\
\hline ENSBTAG00000047099 & $1.71 \mathrm{E}-10$ & $1.07 \mathrm{E}-10$ & & $1.60 \mathrm{E}+00$ \\
\hline ENSBTAG00000020140 & $1.19 \mathrm{E}-10$ & $7.52 \mathrm{E}-11$ & CER1 & $1.59 \mathrm{E}+00$ \\
\hline ENSBTAG00000016173 & $3.55 \mathrm{E}-10$ & $2.24 \mathrm{E}-10$ & & $1.59 E+00$ \\
\hline ENSBTAG00000047550 & $1.86 \mathrm{E}-10$ & $1.17 \mathrm{E}-10$ & & $1.59 \mathrm{E}+00$ \\
\hline ENSBTAG00000046431 & $8.90 \mathrm{E}-10$ & $5.59 \mathrm{E}-10$ & & $1.59 \mathrm{E}+00$ \\
\hline ENSBTAG00000006939 & $6.97 \mathrm{E}-10$ & $4.36 \mathrm{E}-10$ & FGD3 & $1.60 \mathrm{E}+00$ \\
\hline ENSBTAG00000006587 & $2.86 \mathrm{E}-10$ & $1.78 \mathrm{E}-10$ & ZNF367 & $1.61 E+00$ \\
\hline ENSBTAG00000014719 & $4.26 \mathrm{E}-10$ & $2.61 \mathrm{E}-10$ & TMOD1 & $1.63 E+00$ \\
\hline ENSBTAG00000005966 & $2.93 \mathrm{E}-10$ & $1.79 \mathrm{E}-10$ & & $1.64 E+00$ \\
\hline ENSBTAG00000003323 & $2.10 \mathrm{E}-10$ & $1.28 \mathrm{E}-10$ & NOL6 & $1.64 E+00$ \\
\hline ENSBTAG00000016957 & $5.48 \mathrm{E}-10$ & $3.33 \mathrm{E}-10$ & & $1.65 E+00$ \\
\hline ENSBTAG00000025942 & $3.74 \mathrm{E}-10$ & $2.27 \mathrm{E}-10$ & HPGD & $1.65 E+00$ \\
\hline ENSBTAG00000013011 & $4.41 \mathrm{E}-10$ & $2.68 \mathrm{E}-10$ & PLAA & $1.64 \mathrm{E}+00$ \\
\hline
\end{tabular}




\begin{tabular}{|c|c|c|c|c|}
\hline ENSBTAG000000019238 & 5.37E-10 & 3.23E-10 & TRMO & $1.66 \mathrm{E}+00$ \\
\hline ENSBTAG00000017627 & $3.12 \mathrm{E}-10$ & $1.88 \mathrm{E}-10$ & STMN4 & $1.66 \mathrm{E}+00$ \\
\hline ENSBTAG00000008376 & $4.91 \mathrm{E}-10$ & $2.94 \mathrm{E}-10$ & C9orf24 & $1.67 \mathrm{E}+00$ \\
\hline ENSBTAG000000038335 & $3.48 \mathrm{E}-10$ & $2.08 \mathrm{E}-10$ & & $1.68 \mathrm{E}+00$ \\
\hline ENSBTAG000000015241 & $7.72 \mathrm{E}-10$ & $4.55 \mathrm{E}-10$ & EPHX2 & $1.70 \mathrm{E}+00$ \\
\hline ENSBTAG00000011173 & $3.70 \mathrm{E}-10$ & $2.18 \mathrm{E}-10$ & FAM189A2 & $1.70 \mathrm{E}+00$ \\
\hline ENSBTAG000000000974 & $1.60 \mathrm{E}-10$ & $9.40 \mathrm{E}-11$ & IARS & $1.71 \mathrm{E}+00$ \\
\hline ENSBTAG000000005884 & $6.83 \mathrm{E}-10$ & 4.00E-10 & WDR31 & $1.71 E+00$ \\
\hline ENSBTAG000000047647 & $1.57 \mathrm{E}-09$ & $9.18 \mathrm{E}-10$ & & $1.71 E+00$ \\
\hline ENSBTAG000000006651 & $3.00 \mathrm{E}-10$ & $1.75 \mathrm{E}-10$ & INSL6 & $1.71 \mathrm{E}+00$ \\
\hline ENSBTAG000000000269 & $1.85 \mathrm{E}-10$ & $1.08 \mathrm{E}-10$ & & $1.72 \mathrm{E}+00$ \\
\hline ENSBTAG000000046097 & 1.84E-09 & $1.06 \mathrm{E}-09$ & & $1.74 \mathrm{E}+00$ \\
\hline ENSBTAG00000025756 & $2.18 \mathrm{E}-10$ & $1.25 \mathrm{E}-10$ & FBXW12 & $1.74 \mathrm{E}+00$ \\
\hline ENSBTAG000000014722 & $3.05 \mathrm{E}-10$ & $1.74 \mathrm{E}-10$ & TSTD2 & $1.75 \mathrm{E}+00$ \\
\hline ENSBTAG000000012047 & $2.98 \mathrm{E}-10$ & $1.71 \mathrm{E}-10$ & JAK2 & $1.74 \mathrm{E}+00$ \\
\hline ENSBTAG000000017530 & 3.35E-10 & $1.92 \mathrm{E}-10$ & PLGRKT & $1.74 \mathrm{E}+00$ \\
\hline ENSBTAG000000003341 & $3.92 \mathrm{E}-10$ & $2.24 \mathrm{E}-10$ & DOK2 & $1.75 E+00$ \\
\hline ENSBTAG00000012385 & $2.14 \mathrm{E}-10$ & $1.22 \mathrm{E}-10$ & NFX1 & $1.76 \mathrm{E}+00$ \\
\hline ENSBTAG000000018613 & $1.42 \mathrm{E}-10$ & $7.94 \mathrm{E}-11$ & NOL8 & $1.79 \mathrm{E}+00$ \\
\hline ENSBTAG000000008103 & $6.44 \mathrm{E}-10$ & $3.56 \mathrm{E}-10$ & ALDH1A1 & $1.81 E+00$ \\
\hline ENSBTAG000000008537 & $2.29 \mathrm{E}-10$ & $1.27 \mathrm{E}-10$ & FAM219A & $1.80 \mathrm{E}+00$ \\
\hline ENSBTAG000000015578 & 2.65E-10 & $1.46 \mathrm{E}-10$ & SAP30 & $1.82 \mathrm{E}+00$ \\
\hline ENSBTAG000000001939 & $2.95 \mathrm{E}-10$ & $1.62 \mathrm{E}-10$ & SECISBP2 & $1.82 \mathrm{E}+00$ \\
\hline ENSBTAG000000020141 & $3.11 E-10$ & $1.71 \mathrm{E}-10$ & FREM1 & $1.82 \mathrm{E}+00$ \\
\hline ENSBTAG000000020243 & $3.85 \mathrm{E}-10$ & $2.11 E-10$ & SVEP1 & $1.83 \mathrm{E}+00$ \\
\hline ENSBTAG000000017936 & $1.74 \mathrm{E}-10$ & $9.54 \mathrm{E}-11$ & FRRS1L & $1.83 \mathrm{E}+00$ \\
\hline ENSBTAG000000011202 & $6.24 \mathrm{E}-10$ & $3.41 E-10$ & CARD19 & $1.83 E+00$ \\
\hline ENSBTAG00000016952 & $3.42 \mathrm{E}-10$ & $1.86 \mathrm{E}-10$ & PSMD5 & $1.84 \mathrm{E}+00$ \\
\hline ENSBTAG000000038181 & $5.96 \mathrm{E}-10$ & 3.24E-10 & FOCAD & $1.84 \mathrm{E}+00$ \\
\hline ENSBTAG000000020355 & $1.41 \mathrm{E}-10$ & $7.63 \mathrm{E}-11$ & KLF4 & $1.85 E+00$ \\
\hline ENSBTAG000000008493 & $2.89 \mathrm{E}-10$ & $1.56 \mathrm{E}-10$ & AQP3 & $1.85 E+00$ \\
\hline ENSBTAG000000003033 & $3.72 \mathrm{E}-10$ & $2.01 \mathrm{E}-10$ & GADD45G & $1.85 \mathrm{E}+00$ \\
\hline ENSBTAG00000032509 & 2.77E-10 & $1.49 \mathrm{E}-10$ & FAM166B & $1.86 \mathrm{E}+00$ \\
\hline ENSBTAG000000000575 & $2.92 \mathrm{E}-10$ & 1.57E-10 & TNC & $1.86 \mathrm{E}+00$ \\
\hline ENSBTAG00000014008 & $2.58 \mathrm{E}-10$ & $1.39 \mathrm{E}-10$ & NKX2-6 & $1.86 \mathrm{E}+00$ \\
\hline ENSBTAG00000015358 & $3.22 \mathrm{E}-10$ & $1.72 \mathrm{E}-10$ & ALDOB & $1.87 \mathrm{E}+00$ \\
\hline ENSBTAG00000033695 & $2.08 \mathrm{E}-09$ & $1.11 E-09$ & & $1.88 \mathrm{E}+00$ \\
\hline ENSBTAG00000014401 & $1.10 \mathrm{E}-10$ & 5.87E-11 & SORBS3 & $1.87 \mathrm{E}+00$ \\
\hline ENSBTAG00000021069 & $3.71 \mathrm{E}-10$ & $1.97 \mathrm{E}-10$ & & $1.88 \mathrm{E}+00$ \\
\hline ENSBTAG00000011403 & $2.07 \mathrm{E}-10$ & $1.10 \mathrm{E}-10$ & RUSC2 & $1.88 \mathrm{E}+00$ \\
\hline ENSBTAG00000011814 & $1.95 \mathrm{E}-10$ & $1.04 \mathrm{E}-10$ & & $1.88 \mathrm{E}+00$ \\
\hline ENSBTAG00000012535 & $2.51 \mathrm{E}-10$ & $1.33 \mathrm{E}-10$ & SCRG1 & $1.89 \mathrm{E}+00$ \\
\hline ENSBTAG00000007163 & $3.66 \mathrm{E}-10$ & $1.94 \mathrm{E}-10$ & SLC44A1 & $1.89 \mathrm{E}+00$ \\
\hline ENSBTAG00000018373 & $2.80 \mathrm{E}-10$ & $1.45 \mathrm{E}-10$ & DPYSL2 & $1.93 E+00$ \\
\hline ENSBTAG00000001508 & $5.49 \mathrm{E}-10$ & $2.83 \mathrm{E}-10$ & SH3RF1 & $1.94 \mathrm{E}+00$ \\
\hline ENSBTAG00000033316 & $2.02 \mathrm{E}-10$ & $1.03 \mathrm{E}-10$ & PDCD1LG2 & $1.96 \mathrm{E}+00$ \\
\hline ENSBTAG00000011402 & $2.80 \mathrm{E}-10$ & $1.42 \mathrm{E}-10$ & & $1.97 \mathrm{E}+00$ \\
\hline ENSBTAG00000019564 & 4.20E-10 & $2.12 \mathrm{E}-10$ & SMU1 & $1.98 \mathrm{E}+00$ \\
\hline ENSBTAG00000002953 & $1.05 \mathrm{E}-10$ & $5.31 \mathrm{E}-11$ & TXN & $1.98 \mathrm{E}+00$ \\
\hline ENSBTAG00000037986 & $2.08 \mathrm{E}-10$ & $1.05 \mathrm{E}-10$ & PNMA2 & $1.98 \mathrm{E}+00$ \\
\hline ENSBTAG00000004572 & $3.79 \mathrm{E}-10$ & $1.91 \mathrm{E}-10$ & RNF183 & $1.99 \mathrm{E}+00$ \\
\hline ENSBTAG00000015963 & $4.64 \mathrm{E}-10$ & 2.33E-10 & TMEM261 & $1.99 \mathrm{E}+00$ \\
\hline ENSBTAG00000008301 & $5.81 \mathrm{E}-10$ & $2.92 \mathrm{E}-10$ & WNK2 & $1.99 \mathrm{E}+00$ \\
\hline ENSBTAG00000012922 & $2.01 \mathrm{E}-10$ & $1.01 \mathrm{E}-10$ & PHF2 & $1.99 \mathrm{E}+00$ \\
\hline ENSBTAG00000012168 & $1.23 \mathrm{E}-10$ & $6.22 \mathrm{E}-11$ & RANBP6 & $1.98 \mathrm{E}+00$ \\
\hline ENSBTAG00000015000 & $1.84 \mathrm{E}-10$ & $9.10 \mathrm{E}-11$ & VCP & $2.02 E+00$ \\
\hline ENSBTAG00000012921 & $2.53 \mathrm{E}-10$ & $1.23 \mathrm{E}-10$ & KIF24 & $2.05 E+00$ \\
\hline ENSBTAG00000008062 & $1.54 \mathrm{E}-10$ & 7.44E-11 & DMRT2 & $2.06 \mathrm{E}+00$ \\
\hline ENSBTAG00000040390 & $2.13 \mathrm{E}-10$ & $1.02 \mathrm{E}-10$ & EBF2 & $2.09 E+00$ \\
\hline ENSBTAG00000013810 & $4.59 \mathrm{E}-10$ & $2.18 \mathrm{E}-10$ & GABBR2 & $2.11 E+00$ \\
\hline ENSBTAG00000002756 & $3.50 \mathrm{E}-10$ & $1.66 \mathrm{E}-10$ & & $2.11 E+00$ \\
\hline ENSBTAG00000002220 & $7.52 \mathrm{E}-10$ & 3.53E-10 & SPTLC1 & $2.13 E+00$ \\
\hline ENSBTAG00000000204 & $3.10 \mathrm{E}-10$ & $1.45 \mathrm{E}-10$ & TMEM268 & $2.14 \mathrm{E}+00$ \\
\hline ENSBTAG00000011589 & 4.39E-10 & $2.05 E-10$ & CDC14B & $2.14 \mathrm{E}+00$ \\
\hline ENSBTAG00000003366 & $7.58 \mathrm{E}-10$ & 3.54E-10 & DDX58 & $2.14 \mathrm{E}+00$ \\
\hline ENSBTAG00000005470 & $2.32 \mathrm{E}-10$ & $1.06 \mathrm{E}-10$ & BIN3 & $2.19 E+00$ \\
\hline ENSBTAG00000019550 & $3.84 \mathrm{E}-10$ & $1.75 \mathrm{E}-10$ & TBC1D2 & $2.20 \mathrm{E}+00$ \\
\hline ENSBTAG00000047698 & $1.57 \mathrm{E}-09$ & 7.12E-10 & & $2.20 \mathrm{E}+00$ \\
\hline ENSBTAG00000020815 & 4.66E-10 & $2.11 \mathrm{E}-10$ & UHRF2 & $2.21 E+00$ \\
\hline ENSBTAG00000001926 & $6.36 \mathrm{E}-10$ & $2.87 \mathrm{E}-10$ & RAD23B & $2.22 \mathrm{E}+00$ \\
\hline
\end{tabular}




\begin{tabular}{|c|c|c|c|c|}
\hline ENSBTAG00000010059 & $1.60 \mathrm{E}-10$ & $7.22 \mathrm{E}-11$ & C9orf72 & $2.21 E+00$ \\
\hline ENSBTAG00000011375 & $6.78 \mathrm{E}-10$ & $3.05 \mathrm{E}-10$ & $\mathrm{SHC3}$ & $2.22 \mathrm{E}+00$ \\
\hline ENSBTAG00000001016 & $1.30 \mathrm{E}-10$ & $5.86 \mathrm{E}-11$ & NAA35 & $2.22 \mathrm{E}+00$ \\
\hline ENSBTAG00000004778 & $5.36 \mathrm{E}-10$ & $2.41 \mathrm{E}-10$ & & $2.22 \mathrm{E}+00$ \\
\hline ENSBTAG00000012382 & $2.72 \mathrm{E}-10$ & $1.22 \mathrm{E}-10$ & KCTD9 & $2.23 E+00$ \\
\hline ENSBTAG00000008685 & $7.29 \mathrm{E}-10$ & $3.26 \mathrm{E}-10$ & TRAPPC12 & $2.23 E+00$ \\
\hline ENSBTAG00000005907 & $8.37 \mathrm{E}-10$ & $3.69 \mathrm{E}-10$ & NDUFB6 & $2.27 \mathrm{E}+00$ \\
\hline ENSBTAG00000013947 & $4.91 \mathrm{E}-10$ & 2.17E-10 & APTX & $2.26 E+00$ \\
\hline ENSBTAG00000034220 & $3.97 \mathrm{E}-10$ & $1.75 \mathrm{E}-10$ & CDKN2A & $2.27 E+00$ \\
\hline ENSBTAG00000046526 & $3.25 \mathrm{E}-10$ & $1.42 \mathrm{E}-10$ & ISCA1 & $2.29 E+00$ \\
\hline ENSBTAG00000021307 & $2.67 \mathrm{E}-10$ & $1.15 \mathrm{E}-10$ & BNIP3L & $2.32 \mathrm{E}+00$ \\
\hline ENSBTAG00000014922 & $2.47 \mathrm{E}-10$ & $1.06 \mathrm{E}-10$ & SHB & $2.33 E+00$ \\
\hline ENSBTAG00000015835 & $4.06 \mathrm{E}-10$ & $1.73 \mathrm{E}-10$ & PIWIL2 & $2.35 \mathrm{E}+00$ \\
\hline ENSBTAG00000005905 & $4.88 \mathrm{E}-10$ & $2.08 \mathrm{E}-10$ & TOPORS & $2.35 \mathrm{E}+00$ \\
\hline ENSBTAG00000005425 & $2.41 \mathrm{E}-10$ & $1.02 \mathrm{E}-10$ & GATA4 & $2.36 \mathrm{E}+00$ \\
\hline ENSBTAG00000019066 & $6.23 \mathrm{E}-10$ & $2.65 \mathrm{E}-10$ & FAM167A & $2.35 E+00$ \\
\hline ENSBTAG00000019687 & $5.60 \mathrm{E}-10$ & $2.38 \mathrm{E}-10$ & NMRK1 & $2.35 E+00$ \\
\hline ENSBTAG00000033543 & $1.55 \mathrm{E}-10$ & $6.52 \mathrm{E}-11$ & & $2.38 \mathrm{E}+00$ \\
\hline ENSBTAG00000011406 & $1.00 \mathrm{E}-10$ & $4.21 \mathrm{E}-11$ & TESK1 & $2.38 \mathrm{E}+00$ \\
\hline ENSBTAG00000014760 & 4.39E-10 & $1.84 \mathrm{E}-10$ & FOXE1 & $2.39 E+00$ \\
\hline ENSBTAG00000016295 & $1.12 \mathrm{E}-10$ & $4.70 \mathrm{E}-11$ & RPP25L & $2.38 \mathrm{E}+00$ \\
\hline ENSBTAG00000019958 & $2.24 \mathrm{E}-10$ & $9.40 \mathrm{E}-11$ & NXNL2 & $2.38 \mathrm{E}+00$ \\
\hline ENSBTAG00000046427 & $4.32 \mathrm{E}-10$ & $1.81 \mathrm{E}-10$ & & $2.39 E+00$ \\
\hline ENSBTAG00000016845 & $4.66 \mathrm{E}-10$ & $1.95 \mathrm{E}-10$ & GALNT7 & $2.39 E+00$ \\
\hline ENSBTAG00000008696 & 4.19E-10 & $1.73 \mathrm{E}-10$ & FAM120A & $2.42 \mathrm{E}+00$ \\
\hline ENSBTAG00000017306 & $2.84 \mathrm{E}-10$ & 1.17E-10 & AKNA & $2.42 \mathrm{E}+00$ \\
\hline ENSBTAG00000018646 & $2.60 \mathrm{E}-10$ & 1.07E-10 & ATP6V1B2 & $2.43 E+00$ \\
\hline ENSBTAG00000025782 & $2.87 \mathrm{E}-10$ & 1.17E-10 & TNFSF8 & $2.46 \mathrm{E}+00$ \\
\hline ENSBTAG00000019023 & $6.39 \mathrm{E}-10$ & $2.57 \mathrm{E}-10$ & NANS & $2.49 E+00$ \\
\hline ENSBTAG00000048226 & 1.07E-09 & $4.21 \mathrm{E}-10$ & & $2.53 E+00$ \\
\hline ENSBTAG00000046594 & $2.24 \mathrm{E}-10$ & $8.80 \mathrm{E}-11$ & & $2.54 \mathrm{E}+00$ \\
\hline ENSBTAG00000011847 & $3.01 \mathrm{E}-10$ & $1.18 \mathrm{E}-10$ & ASPN & $2.55 E+00$ \\
\hline ENSBTAG00000018318 & $1.37 \mathrm{E}-10$ & 5.31E-11 & DNAJB5 & $2.58 \mathrm{E}+00$ \\
\hline ENSBTAG00000037971 & $3.73 E-10$ & $1.44 \mathrm{E}-10$ & & $2.59 E+00$ \\
\hline ENSBTAG00000046683 & $1.84 \mathrm{E}-09$ & $7.05 \mathrm{E}-10$ & & $2.61 \mathrm{E}+00$ \\
\hline ENSBTAG00000005803 & $5.99 \mathrm{E}-10$ & $2.28 \mathrm{E}-10$ & PTPDC1 & $2.63 E+00$ \\
\hline ENSBTAG00000003483 & 3.53E-10 & $1.33 \mathrm{E}-10$ & NUDT18 & $2.66 \mathrm{E}+00$ \\
\hline ENSBTAG00000011836 & $3.61 \mathrm{E}-10$ & $1.36 \mathrm{E}-10$ & OMD & $2.65 E+00$ \\
\hline ENSBTAG00000021367 & $2.39 \mathrm{E}-10$ & $8.70 \mathrm{E}-11$ & & $2.74 \mathrm{E}+00$ \\
\hline ENSBTAG00000021949 & $4.04 \mathrm{E}-10$ & $1.45 \mathrm{E}-10$ & NEFL & $2.78 \mathrm{E}+00$ \\
\hline ENSBTAG00000015608 & $3.54 \mathrm{E}-10$ & $1.27 \mathrm{E}-10$ & CYLC2 & $2.79 E+00$ \\
\hline ENSBTAG00000000181 & $6.57 \mathrm{E}-10$ & $2.35 \mathrm{E}-10$ & SUSD3 & $2.79 E+00$ \\
\hline ENSBTAG00000006831 & $2.68 \mathrm{E}-10$ & $9.41 \mathrm{E}-11$ & & $2.85 \mathrm{E}+00$ \\
\hline ENSBTAG00000011048 & $6.10 \mathrm{E}-10$ & $2.14 \mathrm{E}-10$ & SPATA6L & $2.85 \mathrm{E}+00$ \\
\hline ENSBTAG00000015378 & $2.44 \mathrm{E}-10$ & $8.50 \mathrm{E}-11$ & & $2.87 E+00$ \\
\hline ENSBTAG00000004134 & $7.01 \mathrm{E}-10$ & $2.41 \mathrm{E}-10$ & C8orf74 & $2.91 \mathrm{E}+00$ \\
\hline ENSBTAG00000015005 & $1.52 \mathrm{E}-10$ & $5.23 \mathrm{E}-11$ & FANCG & $2.91 E+00$ \\
\hline ENSBTAG00000008016 & $2.04 \mathrm{E}-10$ & $6.95 \mathrm{E}-11$ & STX17 & $2.93 E+00$ \\
\hline ENSBTAG00000020133 & $1.26 \mathrm{E}-10$ & $4.28 \mathrm{E}-11$ & РРРЗСС & $2.93 E+00$ \\
\hline ENSBTAG00000031569 & $3.02 \mathrm{E}-10$ & $1.02 \mathrm{E}-10$ & GOLM1 & $2.96 \mathrm{E}+00$ \\
\hline ENSBTAG00000014078 & $1.89 \mathrm{E}-10$ & $6.09 \mathrm{E}-11$ & IGFBPL1 & $3.10 \mathrm{E}+00$ \\
\hline ENSBTAG00000018883 & $2.34 \mathrm{E}-10$ & $7.35 \mathrm{E}-11$ & DCTN3 & $3.18 \mathrm{E}+00$ \\
\hline ENSBTAG00000011388 & $3.02 \mathrm{E}-10$ & $9.50 \mathrm{E}-11$ & STOML2 & $3.18 \mathrm{E}+00$ \\
\hline ENSBTAG00000010829 & $5.45 \mathrm{E}-10$ & $1.71 \mathrm{E}-10$ & & $3.19 \mathrm{E}+00$ \\
\hline ENSBTAG00000046598 & 1.13E-09 & $3.52 \mathrm{E}-10$ & & $3.20 E+00$ \\
\hline ENSBTAG00000000145 & $6.97 \mathrm{E}-10$ & $2.19 \mathrm{E}-10$ & & $3.18 \mathrm{E}+00$ \\
\hline ENSBTAG00000046967 & 1.19E-09 & $3.71 \mathrm{E}-10$ & & $3.20 \mathrm{E}+00$ \\
\hline ENSBTAG00000020844 & $4.25 \mathrm{E}-10$ & $1.32 \mathrm{E}-10$ & TMEM246 & $3.22 \mathrm{E}+00$ \\
\hline ENSBTAG00000009732 & $3.24 \mathrm{E}-10$ & $9.96 \mathrm{E}-11$ & NCBP1 & $3.25 E+00$ \\
\hline ENSBTAG00000025659 & 4.55E-10 & $1.34 \mathrm{E}-10$ & ZNF618 & $3.39 E+00$ \\
\hline ENSBTAG00000009734 & $3.77 \mathrm{E}-10$ & $1.09 \mathrm{E}-10$ & XPA & $3.46 \mathrm{E}+00$ \\
\hline ENSBTAG00000015513 & $3.63 \mathrm{E}-10$ & $1.02 \mathrm{E}-10$ & DOCK5 & $3.55 E+00$ \\
\hline ENSBTAG00000010535 & $4.52 \mathrm{E}-10$ & $1.27 \mathrm{E}-10$ & TXNDC8 & $3.55 E+00$ \\
\hline ENSBTAG00000004991 & $2.87 \mathrm{E}-10$ & $7.98 \mathrm{E}-11$ & IKBKAP & $3.60 E+00$ \\
\hline ENSBTAG00000019099 & 2.17E-10 & $5.92 \mathrm{E}-11$ & SLC25A37 & $3.67 \mathrm{E}+00$ \\
\hline ENSBTAG00000018069 & $1.14 \mathrm{E}-10$ & 3.07E-11 & TNFSF15 & $3.70 \mathrm{E}+00$ \\
\hline ENSBTAG00000003044 & $4.76 \mathrm{E}-10$ & $1.23 \mathrm{E}-10$ & SPIN1 & $3.86 \mathrm{E}+00$ \\
\hline ENSBTAG00000048272 & 3.07E-10 & $7.72 \mathrm{E}-11$ & & $3.97 E+00$ \\
\hline ENSBTAG00000046462 & $3.89 \mathrm{E}-10$ & $9.78 \mathrm{E}-11$ & & $3.98 \mathrm{E}+00$ \\
\hline ENSBTAG00000044135 & $1.85 \mathrm{E}-10$ & $4.65 E-11$ & PIGO & $3.97 E+00$ \\
\hline
\end{tabular}




\begin{tabular}{|c|c|c|c|c|}
\hline ENSBTAG00000039868 & $4.31 \mathrm{E}-10$ & $1.08 \mathrm{E}-10$ & & $3.98 \mathrm{E}+00$ \\
\hline ENSBTAG00000017763 & $2.21 \mathrm{E}-10$ & $5.55 \mathrm{E}-11$ & NFIL3 & $3.97 E+00$ \\
\hline ENSBTAG00000048063 & $7.00 \mathrm{E}-10$ & $1.76 \mathrm{E}-10$ & & $3.98 \mathrm{E}+00$ \\
\hline ENSBTAG00000046258 & 2.77E-10 & $6.54 \mathrm{E}-11$ & SOX7 & $4.24 \mathrm{E}+00$ \\
\hline ENSBTAG00000020985 & $5.97 \mathrm{E}-10$ & $1.40 \mathrm{E}-10$ & TYRP1 & $4.26 \mathrm{E}+00$ \\
\hline ENSBTAG00000012036 & $2.81 \mathrm{E}-10$ & $6.55 \mathrm{E}-11$ & $\mathrm{HR}$ & $4.29 E+00$ \\
\hline ENSBTAG00000000011 & 7.14E-10 & $1.63 \mathrm{E}-10$ & & $4.38 \mathrm{E}+00$ \\
\hline ENSBTAG00000013445 & $9.14 \mathrm{E}-10$ & $2.08 \mathrm{E}-10$ & & $4.39 E+00$ \\
\hline ENSBTAG00000000555 & $6.65 \mathrm{E}-10$ & $1.48 \mathrm{E}-10$ & ACO1 & $4.49 \mathrm{E}+00$ \\
\hline ENSBTAG00000010431 & $5.62 \mathrm{E}-10$ & $1.21 \mathrm{E}-10$ & LURAP1L & $4.64 E+00$ \\
\hline ENSBTAG00000002746 & $3.32 \mathrm{E}-10$ & $7.10 \mathrm{E}-11$ & OSTF1 & $4.67 E+00$ \\
\hline ENSBTAG00000014907 & 7.57E-11 & $1.59 \mathrm{E}-11$ & IL11RA & $4.74 \mathrm{E}+00$ \\
\hline ENSBTAG00000011050 & 4.30E-10 & $9.00 \mathrm{E}-11$ & PLPP6 & $4.77 \mathrm{E}+00$ \\
\hline ENSBTAG00000017294 & $1.42 \mathrm{E}-10$ & 2.97E-11 & & $4.76 \mathrm{E}+00$ \\
\hline ENSBTAG00000025667 & $2.86 \mathrm{E}-10$ & $5.82 \mathrm{E}-11$ & & $4.91 E+00$ \\
\hline ENSBTAG00000032206 & $5.87 \mathrm{E}-10$ & $1.18 \mathrm{E}-10$ & HEMGN & $4.97 E+00$ \\
\hline ENSBTAG00000001737 & $1.85 \mathrm{E}-10$ & $3.64 \mathrm{E}-11$ & ZCCHC6 & $5.07 E+00$ \\
\hline ENSBTAG00000007657 & $3.26 \mathrm{E}-10$ & $6.35 \mathrm{E}-11$ & SCARA3 & $5.13 \mathrm{E}+00$ \\
\hline ENSBTAG00000024851 & $3.84 \mathrm{E}-10$ & 7.42E-11 & TRIM14 & $5.17 \mathrm{E}+00$ \\
\hline ENSBTAG00000008539 & $5.65 \mathrm{E}-10$ & 1.09E-10 & $\mathrm{ENHO}$ & $5.18 \mathrm{E}+00$ \\
\hline ENSBTAG00000011824 & $6.18 \mathrm{E}-10$ & $1.18 \mathrm{E}-10$ & OGN & $5.24 \mathrm{E}+00$ \\
\hline ENSBTAG00000012442 & $6.40 \mathrm{E}-10$ & $1.22 \mathrm{E}-10$ & CTSB & $5.25 E+00$ \\
\hline ENSBTAG00000044003 & $6.38 \mathrm{E}-10$ & $1.20 \mathrm{E}-10$ & TEX10 & $5.31 \mathrm{E}+00$ \\
\hline ENSBTAG00000014827 & $5.09 \mathrm{E}-10$ & $9.52 \mathrm{E}-11$ & UBQLN1 & $5.34 \mathrm{E}+00$ \\
\hline ENSBTAG00000014361 & $4.29 \mathrm{E}-10$ & $7.89 \mathrm{E}-11$ & FAM160B2 & $5.43 \mathrm{E}+00$ \\
\hline ENSBTAG00000015101 & $2.46 \mathrm{E}-10$ & 4.42E-11 & HMGB2 & $5.55 E+00$ \\
\hline ENSBTAG00000046443 & $9.04 \mathrm{E}-10$ & $1.62 \mathrm{E}-10$ & & $5.58 \mathrm{E}+00$ \\
\hline ENSBTAG00000047628 & $3.88 \mathrm{E}-10$ & $6.66 \mathrm{E}-11$ & XPO7 & $5.81 E+00$ \\
\hline ENSBTAG00000011545 & $5.45 \mathrm{E}-10$ & $9.25 \mathrm{E}-11$ & GDA & $5.88 \mathrm{E}+00$ \\
\hline ENSBTAG00000006816 & 2.97E-10 & 4.94E-11 & ABHD17B & $6.01 E+00$ \\
\hline ENSBTAG00000000841 & 2.94E-10 & 4.62E-11 & LZTS1 & $6.36 \mathrm{E}+00$ \\
\hline ENSBTAG00000006240 & $5.60 \mathrm{E}-10$ & 8.43E-11 & TLR4 & $6.64 E+00$ \\
\hline ENSBTAG00000045887 & $4.13 \mathrm{E}-10$ & $6.04 \mathrm{E}-11$ & DMTN & $6.83 E+00$ \\
\hline ENSBTAG00000018925 & $1.57 \mathrm{E}-10$ & $2.20 \mathrm{E}-11$ & EXOSC3 & $7.13 E+00$ \\
\hline ENSBTAG00000015607 & $4.05 \mathrm{E}-10$ & $5.59 \mathrm{E}-11$ & ERMP1 & $7.24 \mathrm{E}+00$ \\
\hline ENSBTAG00000008772 & $3.91 \mathrm{E}-10$ & $5.31 \mathrm{E}-11$ & SMC2 & $7.35 E+00$ \\
\hline ENSBTAG00000007761 & $2.33 \mathrm{E}-10$ & $3.08 \mathrm{E}-11$ & ZBTB5 & $7.53 E+00$ \\
\hline ENSBTAG00000004767 & $4.43 \mathrm{E}-10$ & $5.84 \mathrm{E}-11$ & SYK & $7.58 \mathrm{E}+00$ \\
\hline ENSBTAG00000005090 & $6.41 \mathrm{E}-10$ & $8.28 \mathrm{E}-11$ & TMEM2 & $7.73 \mathrm{E}+00$ \\
\hline ENSBTAG00000003012 & $6.37 \mathrm{E}-10$ & 7.73E-11 & TRAF1 & $8.23 E+00$ \\
\hline ENSBTAG00000014934 & $1.89 \mathrm{E}-10$ & $2.22 \mathrm{E}-11$ & DCAF10 & $8.48 \mathrm{E}+00$ \\
\hline ENSBTAG00000046918 & $2.59 \mathrm{E}-10$ & $2.84 \mathrm{E}-11$ & NPM2 & $9.10 E+00$ \\
\hline ENSBTAG00000019299 & $3.78 \mathrm{E}-10$ & $3.48 \mathrm{E}-11$ & GRHPR & $1.08 \mathrm{E}+01$ \\
\hline ENSBTAG00000013974 & $4.58 \mathrm{E}-10$ & 3.83E-11 & TMEM215 & $1.19 \mathrm{E}+01$ \\
\hline ENSBTAG00000014936 & $1.51 \mathrm{E}-10$ & $1.12 \mathrm{E}-11$ & & $1.34 \mathrm{E}+01$ \\
\hline ENSBTAG00000009417 & $2.66 \mathrm{E}-10$ & $1.96 \mathrm{E}-11$ & ZFAND5 & $1.35 \mathrm{E}+01$ \\
\hline ENSBTAG00000046549 & $6.02 \mathrm{E}-10$ & 4.25E-11 & BICD2 & $1.41 \mathrm{E}+01$ \\
\hline ENSBTAG00000017338 & $2.68 \mathrm{E}-10$ & $1.87 \mathrm{E}-11$ & FAM205C & $1.43 \mathrm{E}+01$ \\
\hline ENSBTAG00000046951 & $3.06 \mathrm{E}-10$ & $2.13 \mathrm{E}-11$ & FGF17 & $1.43 \mathrm{E}+01$ \\
\hline ENSBTAG00000025859 & $3.20 \mathrm{E}-10$ & $1.68 \mathrm{E}-11$ & $\mathrm{ZCCHC7}$ & $1.89 E+01$ \\
\hline ENSBTAG00000012243 & $6.40 \mathrm{E}-10$ & $2.61 \mathrm{E}-11$ & POLR1E & $2.44 \mathrm{E}+01$ \\
\hline ENSBTAG00000046574 & $1.87 \mathrm{E}-10$ & $6.14 \mathrm{E}-12$ & FRMPD1 & $3.00 \mathrm{E}+01$ \\
\hline ENSBTAG00000012262 & $7.59 \mathrm{E}-10$ & $1.81 \mathrm{E}-11$ & FBXO10 & $4.17 E+01$ \\
\hline ENSBTAG00000019807 & $3.16 \mathrm{E}-10$ & $3.89 \mathrm{E}-12$ & COL27A1 & $7.92 \mathrm{E}+01$ \\
\hline ENSBTAG00000035293 & 0 & 0 & & $1.00 \mathrm{E}+00$ \\
\hline ENSBTAG00000006853 & $1.26 \mathrm{E}-10$ & 0 & IFNK & $1.27 \mathrm{E}+03$ \\
\hline ENSBTAG00000046119 & 1.17E-10 & 0 & & $1.17 \mathrm{E}+03$ \\
\hline ENSBTAG00000046782 & 0 & 0 & & $1.00 E+00$ \\
\hline ENSBTAG00000011411 & $5.28 \mathrm{E}-11$ & 0 & SIT1 & $5.29 E+02$ \\
\hline ENSBTAG00000032777 & $6.22 \mathrm{E}-10$ & 0 & & $6.22 \mathrm{E}+03$ \\
\hline ENSBTAG00000018922 & $1.18 \mathrm{E}-10$ & 0 & TRMT10B & $1.18 \mathrm{E}+03$ \\
\hline ENSBTAG00000019293 & 0 & 0 & EGR3 & $1.00 \mathrm{E}+00$ \\
\hline ENSBTAG00000014908 & 0 & 0 & CCL27 & $1.00 \mathrm{E}+00$ \\
\hline ENSBTAG00000010161 & $1.43 \mathrm{E}-10$ & 0 & CCL21 & $1.43 E+03$ \\
\hline ENSBTAG00000039021 & $4.46 \mathrm{E}-10$ & 0 & & $4.46 \mathrm{E}+03$ \\
\hline ENSBTAG00000038486 & $3.45 \mathrm{E}-10$ & 0 & & $3.45 E+03$ \\
\hline ENSBTAG00000036013 & $1.72 \mathrm{E}-10$ & 0 & OR13C8 & $1.72 \mathrm{E}+03$ \\
\hline ENSBTAG00000019467 & $2.92 \mathrm{E}-10$ & 0 & ACTL7B & $2.92 E+03$ \\
\hline ENSBTAG00000019468 & $1.62 \mathrm{E}-10$ & 0 & ACTL7A & $1.62 \mathrm{E}+03$ \\
\hline ENSBTAG00000045845 & $1.98 \mathrm{E}-09$ & 0 & & $1.98 \mathrm{E}+04$ \\
\hline
\end{tabular}




\begin{tabular}{|c|c|c|c|c|}
\hline ENSBTAG00000046849 & $2.93 \mathrm{E}-10$ & 0 & IFNB3 & $2.93 E+03$ \\
\hline ENSBTAG00000011160 & 0 & 0 & TPD52L3 & $1.00 \mathrm{E}+00$ \\
\hline ENSBTAG00000046687 & 0 & 0 & & $1.00 E+00$ \\
\hline ENSBTAG00000032670 & $2.56 \mathrm{E}-10$ & 0 & OR2S2 & $2.56 \mathrm{E}+03$ \\
\hline ENSBTAG00000039443 & $2.57 \mathrm{E}-10$ & 0 & & $2.57 \mathrm{E}+03$ \\
\hline ENSBTAG00000032671 & $8.56 \mathrm{E}-11$ & 0 & OR13C7 & $8.57 E+02$ \\
\hline ENSBTAG00000037600 & 0 & 0 & & $1.00 \mathrm{E}+00$ \\
\hline ENSBTAG00000048106 & $1.71 \mathrm{E}-10$ & 0 & & $1.71 E+03$ \\
\hline ENSBTAG00000036012 & $7.94 \mathrm{E}-11$ & 0 & OR13C3 & $7.95 E+02$ \\
\hline ENSBTAG00000016070 & $2.25 \mathrm{E}-10$ & 0 & LINGO2 & $2.25 E+03$ \\
\hline ENSBTAG00000008551 & 0 & 0 & TUSC1 & $1.00 \mathrm{E}+00$ \\
\hline ENSBTAG00000045554 & $5.60 \mathrm{E}-10$ & 0 & & $5.60 \mathrm{E}+03$ \\
\hline ENSBTAG00000012375 & $1.96 \mathrm{E}-10$ & 0 & KLHL9 & $1.96 \mathrm{E}+03$ \\
\hline ENSBTAG00000045552 & 4.19E-10 & 0 & & $4.19 E+03$ \\
\hline ENSBTAG00000047134 & $9.83 \mathrm{E}-10$ & 0 & & $9.83 E+03$ \\
\hline ENSBTAG00000045999 & $2.15 E-10$ & $5.43 \mathrm{E}-10$ & & 3.96E-01 \\
\hline ENSBTAG00000039615 & $5.10 \mathrm{E}-11$ & $1.29 \mathrm{E}-10$ & & 3.96E-01 \\
\hline ENSBTAG00000045583 & $1.11 \mathrm{E}-10$ & $2.80 \mathrm{E}-10$ & & 3.97E-01 \\
\hline ENSBTAG00000045655 & $4.83 \mathrm{E}-11$ & $1.22 \mathrm{E}-10$ & & 3.97E-01 \\
\hline ENSBTAG00000011315 & $6.95 \mathrm{E}-11$ & $1.75 \mathrm{E}-10$ & GJA10 & 3.97E-01 \\
\hline ENSBTAG00000003925 & $9.43 \mathrm{E}-11$ & $1.98 \mathrm{E}-10$ & C6orf120 & 4.77E-01 \\
\hline ENSBTAG00000009243 & $3.69 \mathrm{E}-10$ & $6.42 \mathrm{E}-10$ & MFSD4B & $5.75 \mathrm{E}-01$ \\
\hline ENSBTAG00000012074 & $1.07 \mathrm{E}-10$ & $1.84 \mathrm{E}-10$ & MYB & 5.80E-01 \\
\hline ENSBTAG00000017170 & $1.54 \mathrm{E}-10$ & $2.59 \mathrm{E}-10$ & & 5.94E-01 \\
\hline ENSBTAG00000005456 & $9.21 \mathrm{E}-11$ & $1.52 \mathrm{E}-10$ & TTK & $6.06 \mathrm{E}-01$ \\
\hline ENSBTAG00000008145 & $9.96 \mathrm{E}-11$ & $1.57 \mathrm{E}-10$ & SMPD2 & $6.35 \mathrm{E}-01$ \\
\hline ENSBTAG00000015911 & $4.94 \mathrm{E}-10$ & $7.62 \mathrm{E}-10$ & RWDD2A & $6.49 \mathrm{E}-01$ \\
\hline ENSBTAG00000039477 & $3.90 \mathrm{E}-10$ & $5.74 \mathrm{E}-10$ & TPBG & $6.80 \mathrm{E}-01$ \\
\hline ENSBTAG00000006367 & $1.77 \mathrm{E}-10$ & $2.55 \mathrm{E}-10$ & CTGF & $6.96 \mathrm{E}-01$ \\
\hline ENSBTAG00000007388 & $1.74 \mathrm{E}-10$ & $2.43 \mathrm{E}-10$ & ZC3H12D & 7.15E-01 \\
\hline ENSBTAG00000033367 & $3.03 E-10$ & $4.22 \mathrm{E}-10$ & DDO & 7.17E-01 \\
\hline ENSBTAG00000000240 & $3.22 \mathrm{E}-10$ & $4.43 \mathrm{E}-10$ & AKAP7 & 7.27E-01 \\
\hline ENSBTAG00000008147 & $1.02 \mathrm{E}-10$ & $1.38 \mathrm{E}-10$ & MICAL1 & 7.42E-01 \\
\hline ENSBTAG00000003929 & $9.42 \mathrm{E}-11$ & $1.26 \mathrm{E}-10$ & PHF10 & 7.48E-01 \\
\hline ENSBTAG00000035623 & $9.34 \mathrm{E}-10$ & $1.23 \mathrm{E}-09$ & & 7.60E-01 \\
\hline ENSBTAG00000002294 & $2.13 \mathrm{E}-10$ & $2.76 \mathrm{E}-10$ & SIM1 & $7.71 \mathrm{E}-01$ \\
\hline ENSBTAG00000010047 & $3.30 \mathrm{E}-10$ & 4.27E-10 & TIAM2 & 7.73E-01 \\
\hline ENSBTAG00000004099 & $4.22 \mathrm{E}-10$ & $5.44 \mathrm{E}-10$ & CCDC170 & $7.75 \mathrm{E}-01$ \\
\hline ENSBTAG00000001644 & $1.92 \mathrm{E}-10$ & $2.46 \mathrm{E}-10$ & MDN1 & 7.81E-01 \\
\hline ENSBTAG00000002058 & $1.27 \mathrm{E}-10$ & $1.61 \mathrm{E}-10$ & ASF1A & 7.92E-01 \\
\hline ENSBTAG00000011224 & $1.37 \mathrm{E}-10$ & $1.73 \mathrm{E}-10$ & CITED2 & 7.92E-01 \\
\hline ENSBTAG00000009127 & $2.20 \mathrm{E}-10$ & $2.78 \mathrm{E}-10$ & TSPYL4 & 7.93E-01 \\
\hline ENSBTAG00000039875 & $3.49 \mathrm{E}-10$ & $4.39 \mathrm{E}-10$ & & 7.94E-01 \\
\hline ENSBTAG00000017992 & $1.07 \mathrm{E}-10$ & $1.35 \mathrm{E}-10$ & CD164 & 7.92E-01 \\
\hline ENSBTAG00000047032 & $3.97 \mathrm{E}-10$ & $5.00 \mathrm{E}-10$ & & 7.94E-01 \\
\hline ENSBTAG00000035076 & $2.47 \mathrm{E}-10$ & $3.11 E-10$ & & 7.94E-01 \\
\hline ENSBTAG00000047235 & $1.79 \mathrm{E}-10$ & $2.25 \mathrm{E}-10$ & & 7.94E-01 \\
\hline ENSBTAG00000037429 & $1.40 \mathrm{E}-10$ & $1.76 \mathrm{E}-10$ & HTR1B & 7.96E-01 \\
\hline ENSBTAG00000021827 & $5.51 \mathrm{E}-11$ & $6.94 \mathrm{E}-11$ & TCF21 & 7.94E-01 \\
\hline ENSBTAG00000031532 & $4.05 E-10$ & $5.10 \mathrm{E}-10$ & DACT2 & 7.94E-01 \\
\hline ENSBTAG00000032382 & $9.63 \mathrm{E}-10$ & $1.21 \mathrm{E}-09$ & & 7.96E-01 \\
\hline ENSBTAG00000018681 & $3.70 \mathrm{E}-10$ & $4.66 \mathrm{E}-10$ & $\mathrm{~T}$ & 7.94E-01 \\
\hline ENSBTAG00000008749 & $1.00 \mathrm{E}-10$ & $1.26 \mathrm{E}-10$ & OLIG3 & 7.94E-01 \\
\hline ENSBTAG00000038761 & $6.14 \mathrm{E}-10$ & $7.74 \mathrm{E}-10$ & & 7.94E-01 \\
\hline ENSBTAG00000012534 & 8.57E-11 & $1.08 \mathrm{E}-10$ & BEND3 & 7.93E-01 \\
\hline ENSBTAG00000007989 & $7.58 \mathrm{E}-11$ & $9.55 \mathrm{E}-11$ & FUT9 & 7.94E-01 \\
\hline ENSBTAG00000048313 & $5.50 \mathrm{E}-10$ & $6.93 \mathrm{E}-10$ & TAAR9 & 7.94E-01 \\
\hline ENSBTAG00000005096 & 5.77E-10 & $7.26 \mathrm{E}-10$ & COX7A2 & 7.94E-01 \\
\hline ENSBTAG00000031724 & $1.68 \mathrm{E}-10$ & $2.11 E-10$ & MAS1 & 7.94E-01 \\
\hline ENSBTAG00000026543 & $3.18 \mathrm{E}-10$ & $4.01 E-10$ & & 7.93E-01 \\
\hline ENSBTAG00000024773 & $2.18 \mathrm{E}-10$ & $2.74 \mathrm{E}-10$ & & 7.95E-01 \\
\hline ENSBTAG00000046726 & $2.50 \mathrm{E}-10$ & $3.15 E-10$ & CLDN20 & 7.93E-01 \\
\hline ENSBTAG00000011236 & $2.85 \mathrm{E}-10$ & $3.59 \mathrm{E}-10$ & STX11 & 7.94E-01 \\
\hline ENSBTAG00000009784 & $3.95 \mathrm{E}-10$ & $4.84 \mathrm{E}-10$ & RAB32 & $8.15 \mathrm{E}-01$ \\
\hline ENSBTAG00000021663 & $1.58 \mathrm{E}-10$ & $1.90 \mathrm{E}-10$ & SMIM8 & $8.32 \mathrm{E}-01$ \\
\hline ENSBTAG00000011672 & $5.00 \mathrm{E}-10$ & $6.00 \mathrm{E}-10$ & GABRR1 & 8.33E-01 \\
\hline ENSBTAG00000012096 & $2.68 \mathrm{E}-10$ & $3.21 \mathrm{E}-10$ & $\mathrm{BCKDHB}$ & $8.36 \mathrm{E}-01$ \\
\hline ENSBTAG00000010507 & 4.09E-10 & 4.89E-10 & SLC22A16 & $8.36 \mathrm{E}-01$ \\
\hline ENSBTAG00000009620 & $1.96 \mathrm{E}-10$ & $2.35 \mathrm{E}-10$ & WDR27 & $8.35 \mathrm{E}-01$ \\
\hline ENSBTAG00000008543 & $2.92 \mathrm{E}-10$ & $3.46 \mathrm{E}-10$ & GTF2H5 & 8.44E-01 \\
\hline
\end{tabular}




\begin{tabular}{|c|c|c|c|c|}
\hline ENSBTAG000000018436 & $2.54 \mathrm{E}-10$ & $3.00 \mathrm{E}-10$ & UBE2J1 & 8.47E-01 \\
\hline ENSBTAG00000006523 & $9.54 \mathrm{E}-10$ & 1.12E-09 & & $8.52 \mathrm{E}-01$ \\
\hline ENSBTAG000000046954 & $4.70 \mathrm{E}-10$ & $5.53 \mathrm{E}-10$ & & 8.50E-01 \\
\hline ENSBTAG000000001360 & $1.26 \mathrm{E}-09$ & 1.47E-09 & & 8.60E-01 \\
\hline ENSBTAG000000002625 & $2.02 \mathrm{E}-10$ & $2.34 \mathrm{E}-10$ & MAP3K7 & 8.65E-01 \\
\hline ENSBTAG000000004394 & $6.07 \mathrm{E}-10$ & $7.00 \mathrm{E}-10$ & PKIB & 8.67E-01 \\
\hline ENSBTAG000000003743 & $2.38 \mathrm{E}-09$ & $2.75 \mathrm{E}-09$ & & 8.67E-01 \\
\hline ENSBTAG00000014498 & $9.34 \mathrm{E}-10$ & $1.08 \mathrm{E}-09$ & UNC93A & 8.65E-01 \\
\hline ENSBTAG000000033535 & $3.57 \mathrm{E}-10$ & $4.12 \mathrm{E}-10$ & & 8.67E-01 \\
\hline ENSBTAG00000021398 & $8.50 \mathrm{E}-11$ & $9.74 \mathrm{E}-11$ & C6orf203 & 8.73E-01 \\
\hline ENSBTAG00000021099 & $3.68 \mathrm{E}-10$ & 4.19E-10 & CLVS2 & 8.77E-01 \\
\hline ENSBTAG000000010113 & $4.41 \mathrm{E}-10$ & $5.03 \mathrm{E}-10$ & ZBTB24 & $8.78 \mathrm{E}-01$ \\
\hline ENSBTAG00000011313 & $1.25 \mathrm{E}-10$ & $1.42 \mathrm{E}-10$ & CASP8AP2 & $8.78 \mathrm{E}-01$ \\
\hline ENSBTAG00000043950 & $7.31 \mathrm{E}-10$ & 8.19E-10 & LCA5 & 8.93E-01 \\
\hline ENSBTAG000000036287 & $4.10 \mathrm{E}-10$ & 4.57E-10 & SAMD5 & 8.97E-01 \\
\hline ENSBTAG000000019139 & $1.42 \mathrm{E}-10$ & 1.57E-10 & PHF3 & $9.06 \mathrm{E}-01$ \\
\hline ENSBTAG00000015638 & $3.82 \mathrm{E}-10$ & $4.22 \mathrm{E}-10$ & IL20RA & $9.06 \mathrm{E}-01$ \\
\hline ENSBTAG000000012418 & $4.62 \mathrm{E}-10$ & $5.09 \mathrm{E}-10$ & MED23 & 9.07E-01 \\
\hline ENSBTAG000000016164 & $6.47 \mathrm{E}-10$ & $7.11 \mathrm{E}-10$ & LMBRD1 & $9.10 \mathrm{E}-01$ \\
\hline ENSBTAG000000005083 & $3.01 \mathrm{E}-10$ & 3.27E-10 & TULP4 & $9.19 \mathrm{E}-01$ \\
\hline ENSBTAG000000003384 & $1.03 \mathrm{E}-09$ & $1.12 \mathrm{E}-09$ & CEP162 & 9.23E-01 \\
\hline ENSBTAG000000009277 & $1.97 \mathrm{E}-10$ & $2.14 \mathrm{E}-10$ & RGS17 & $9.18 \mathrm{E}-01$ \\
\hline ENSBTAG000000039329 & $8.00 \mathrm{E}-10$ & 8.63E-10 & RAET1G & 9.27E-01 \\
\hline ENSBTAG000000021420 & $4.05 \mathrm{E}-10$ & $4.36 \mathrm{E}-10$ & EPHA7 & $9.29 \mathrm{E}-01$ \\
\hline ENSBTAG00000011911 & $3.83 \mathrm{E}-10$ & 4.12E-10 & & 9.30E-01 \\
\hline ENSBTAG000000038414 & $9.61 \mathrm{E}-10$ & $1.03 \mathrm{E}-09$ & TAAR2 & 9.33E-01 \\
\hline ENSBTAG000000011106 & 4.44E-10 & 4.74E-10 & PACRG & $9.36 \mathrm{E}-01$ \\
\hline ENSBTAG000000033365 & $3.28 \mathrm{E}-10$ & 3.50E-10 & METTL24 & $9.36 \mathrm{E}-01$ \\
\hline ENSBTAG000000034531 & $3.92 \mathrm{E}-10$ & 4.17E-10 & & 9.39E-01 \\
\hline ENSBTAG000000017000 & $5.11 \mathrm{E}-10$ & $5.42 \mathrm{E}-10$ & IL22RA2 & $9.43 \mathrm{E}-01$ \\
\hline ENSBTAG000000008540 & $2.38 \mathrm{E}-10$ & $2.53 \mathrm{E}-10$ & SLC22A1 & $9.42 \mathrm{E}-01$ \\
\hline ENSBTAG000000020079 & $1.82 \mathrm{E}-10$ & $1.92 \mathrm{E}-10$ & MAP3K4 & $9.48 \mathrm{E}-01$ \\
\hline ENSBTAG00000002993 & $1.12 \mathrm{E}-09$ & 1.17E-09 & & $9.54 \mathrm{E}-01$ \\
\hline ENSBTAG000000007758 & $6.41 E-10$ & $6.74 \mathrm{E}-10$ & PDE10A & $9.51 \mathrm{E}-01$ \\
\hline ENSBTAG000000040533 & $4.78 \mathrm{E}-10$ & $5.01 \mathrm{E}-10$ & & 9.54E-01 \\
\hline ENSBTAG00000012946 & $1.60 \mathrm{E}-10$ & $1.67 \mathrm{E}-10$ & HSF2 & 9.55E-01 \\
\hline ENSBTAG00000015166 & $7.89 \mathrm{E}-10$ & $8.25 \mathrm{E}-10$ & PNLDC1 & $9.56 \mathrm{E}-01$ \\
\hline ENSBTAG00000019719 & $4.88 \mathrm{E}-10$ & $5.10 \mathrm{E}-10$ & OPRM1 & 9.57E-01 \\
\hline ENSBTAG00000033961 & $5.68 \mathrm{E}-10$ & $5.93 \mathrm{E}-10$ & & $9.58 \mathrm{E}-01$ \\
\hline ENSBTAG00000009575 & $4.49 \mathrm{E}-10$ & 4.67E-10 & TBC1D32 & $9.62 \mathrm{E}-01$ \\
\hline ENSBTAG00000007288 & $3.18 \mathrm{E}-10$ & $3.30 \mathrm{E}-10$ & CDK19 & $9.64 \mathrm{E}-01$ \\
\hline ENSBTAG00000010334 & $7.92 \mathrm{E}-10$ & $8.21 E-10$ & SYTL3 & 9.65E-01 \\
\hline ENSBTAG00000012403 & $5.95 \mathrm{E}-10$ & $6.15 \mathrm{E}-10$ & ARG1 & $9.68 \mathrm{E}-01$ \\
\hline ENSBTAG00000018464 & 5.50E-10 & $5.68 \mathrm{E}-10$ & ZDHHC14 & $9.69 \mathrm{E}-01$ \\
\hline ENSBTAG00000020174 & $1.41 \mathrm{E}-10$ & $1.46 \mathrm{E}-10$ & HBS1L & 9.63E-01 \\
\hline ENSBTAG00000001146 & $2.45 \mathrm{E}-10$ & $2.51 E-10$ & HIVEP2 & $9.75 \mathrm{E}-01$ \\
\hline ENSBTAG00000016046 & $1.75 \mathrm{E}-10$ & $1.79 \mathrm{E}-10$ & GOPC & $9.80 \mathrm{E}-01$ \\
\hline ENSBTAG00000031917 & $2.98 \mathrm{E}-10$ & 3.02E-10 & SCAF8 & $9.86 \mathrm{E}-01$ \\
\hline ENSBTAG00000005827 & $3.63 \mathrm{E}-10$ & $3.66 \mathrm{E}-10$ & FIG4 & $9.91 \mathrm{E}-01$ \\
\hline ENSBTAG00000003078 & $1.23 \mathrm{E}-10$ & $1.24 \mathrm{E}-10$ & COL10A1 & $9.95 \mathrm{E}-01$ \\
\hline ENSBTAG00000007686 & $1.69 \mathrm{E}-10$ & $1.71 \mathrm{E}-10$ & & $9.90 \mathrm{E}-01$ \\
\hline ENSBTAG00000019110 & $2.03 \mathrm{E}-10$ & $2.05 \mathrm{E}-10$ & PNRC1 & $9.92 \mathrm{E}-01$ \\
\hline ENSBTAG00000017554 & $4.61 \mathrm{E}-10$ & 4.65E-10 & RTN4IP1 & $9.90 \mathrm{E}-01$ \\
\hline ENSBTAG00000018996 & $6.77 \mathrm{E}-10$ & $6.83 \mathrm{E}-10$ & PARK2 & $9.92 \mathrm{E}-01$ \\
\hline ENSBTAG00000012299 & $3.47 \mathrm{E}-10$ & $3.50 \mathrm{E}-10$ & REPS1 & $9.92 \mathrm{E}-01$ \\
\hline ENSBTAG00000026716 & $3.52 \mathrm{E}-10$ & $3.55 \mathrm{E}-10$ & IBTK & 9.93E-01 \\
\hline ENSBTAG00000001446 & $1.09 \mathrm{E}-10$ & $1.10 \mathrm{E}-10$ & ABRACL & $9.90 \mathrm{E}-01$ \\
\hline ENSBTAG00000027223 & $3.82 \mathrm{E}-10$ & $3.85 \mathrm{E}-10$ & & 9.93E-01 \\
\hline ENSBTAG00000014534 & $1.21 \mathrm{E}-10$ & $1.22 \mathrm{E}-10$ & & $9.89 \mathrm{E}-01$ \\
\hline ENSBTAG00000015498 & $1.50 \mathrm{E}-10$ & $1.49 \mathrm{E}-10$ & ELOVL4 & $1.01 \mathrm{E}+00$ \\
\hline ENSBTAG00000047902 & $1.02 \mathrm{E}-09$ & $1.02 \mathrm{E}-09$ & ULBP21 & $1.00 E+00$ \\
\hline ENSBTAG00000019002 & $4.79 \mathrm{E}-10$ & $4.76 \mathrm{E}-10$ & SLC2A12 & $1.01 E+00$ \\
\hline ENSBTAG00000015242 & $6.60 \mathrm{E}-10$ & $6.57 \mathrm{E}-10$ & SLC18B1 & $1.01 \mathrm{E}+00$ \\
\hline ENSBTAG00000020196 & $5.95 \mathrm{E}-10$ & $5.91 \mathrm{E}-10$ & ENPP3 & $1.01 \mathrm{E}+00$ \\
\hline ENSBTAG00000011667 & $8.81 \mathrm{E}-11$ & $8.72 \mathrm{E}-11$ & PM20D2 & $1.01 \mathrm{E}+00$ \\
\hline ENSBTAG00000002829 & $4.89 \mathrm{E}-10$ & 4.83E-10 & TCP1 & $1.01 \mathrm{E}+00$ \\
\hline ENSBTAG00000013837 & $5.76 \mathrm{E}-10$ & 5.67E-10 & TUBE1 & $1.02 \mathrm{E}+00$ \\
\hline ENSBTAG00000047985 & 7.31E-10 & 7.16E-10 & TAAR5 & $1.02 \mathrm{E}+00$ \\
\hline ENSBTAG00000033195 & $2.42 \mathrm{E}-10$ & 2.37E-10 & & $1.02 \mathrm{E}+00$ \\
\hline ENSBTAG00000002725 & $1.91 \mathrm{E}-10$ & $1.86 \mathrm{E}-10$ & RNGTT & $1.03 E+00$ \\
\hline
\end{tabular}




\begin{tabular}{|c|c|c|c|c|}
\hline ENSBTAG00000019418 & $2.01 \mathrm{E}-10$ & $1.94 \mathrm{E}-10$ & UBE3D & $1.04 \mathrm{E}+00$ \\
\hline ENSBTAG00000015888 & $1.64 \mathrm{E}-10$ & $1.58 \mathrm{E}-10$ & PDE7B & $1.04 \mathrm{E}+00$ \\
\hline ENSBTAG00000003336 & $6.09 \mathrm{E}-10$ & $5.86 \mathrm{E}-10$ & TPD52L1 & $1.04 \mathrm{E}+00$ \\
\hline ENSBTAG00000032007 & $6.10 \mathrm{E}-10$ & $5.86 \mathrm{E}-10$ & LRP11 & $1.04 \mathrm{E}+00$ \\
\hline ENSBTAG00000003076 & $2.30 \mathrm{E}-10$ & $2.20 \mathrm{E}-10$ & NT5DC1 & $1.04 \mathrm{E}+00$ \\
\hline ENSBTAG00000046235 & $1.22 \mathrm{E}-10$ & $1.16 \mathrm{E}-10$ & SRSF12 & $1.05 \mathrm{E}+00$ \\
\hline ENSBTAG00000013591 & $8.31 \mathrm{E}-10$ & 7.93E-10 & CYB5R4 & $1.05 E+00$ \\
\hline ENSBTAG00000005100 & 4.20E-10 & $4.00 \mathrm{E}-10$ & TMEM30A & $1.05 E+00$ \\
\hline ENSBTAG00000016562 & $3.17 \mathrm{E}-10$ & $2.99 \mathrm{E}-10$ & SEC63 & $1.06 \mathrm{E}+00$ \\
\hline ENSBTAG00000026704 & $5.04 \mathrm{E}-10$ & 4.77E-10 & CENPW & $1.06 \mathrm{E}+00$ \\
\hline ENSBTAG00000001271 & $3.60 \mathrm{E}-10$ & $3.41 \mathrm{E}-10$ & & $1.06 \mathrm{E}+00$ \\
\hline ENSBTAG00000039444 & $3.18 \mathrm{E}-10$ & $3.00 \mathrm{E}-10$ & & $1.06 \mathrm{E}+00$ \\
\hline ENSBTAG00000016582 & $9.55 \mathrm{E}-11$ & $9.02 \mathrm{E}-11$ & GTF3C6 & $1.06 \mathrm{E}+00$ \\
\hline ENSBTAG00000011509 & $1.85 \mathrm{E}-10$ & $1.74 \mathrm{E}-10$ & SERAC1 & $1.06 \mathrm{E}+00$ \\
\hline ENSBTAG00000033083 & 4.97E-10 & 4.69E-10 & & $1.06 \mathrm{E}+00$ \\
\hline ENSBTAG00000013400 & 1.15E-10 & $1.09 \mathrm{E}-10$ & VIP & $1.05 E+00$ \\
\hline ENSBTAG00000021175 & $5.94 \mathrm{E}-10$ & $5.57 \mathrm{E}-10$ & & $1.07 \mathrm{E}+00$ \\
\hline ENSBTAG00000006323 & $2.88 \mathrm{E}-10$ & $2.70 \mathrm{E}-10$ & SNX9 & $1.07 \mathrm{E}+00$ \\
\hline ENSBTAG00000003938 & $6.66 \mathrm{E}-10$ & $6.22 \mathrm{E}-10$ & FNDC1 & $1.07 \mathrm{E}+00$ \\
\hline ENSBTAG00000031481 & $2.14 \mathrm{E}-10$ & 1.99E-10 & TTLL2 & $1.08 \mathrm{E}+00$ \\
\hline ENSBTAG00000040354 & $8.16 \mathrm{E}-10$ & $7.58 \mathrm{E}-10$ & & $1.08 \mathrm{E}+00$ \\
\hline ENSBTAG00000000918 & $1.88 \mathrm{E}-10$ & $1.74 \mathrm{E}-10$ & ERMARD & $1.08 \mathrm{E}+00$ \\
\hline ENSBTAG00000007791 & 4.40E-10 & $4.08 \mathrm{E}-10$ & KCNQ5 & $1.08 \mathrm{E}+00$ \\
\hline ENSBTAG00000002171 & $2.17 \mathrm{E}-10$ & $1.99 \mathrm{E}-10$ & FAM135A & $1.09 \mathrm{E}+00$ \\
\hline ENSBTAG00000009583 & 4.37E-10 & 4.01E-10 & SLC22A2 & $1.09 \mathrm{E}+00$ \\
\hline ENSBTAG00000017139 & $6.36 \mathrm{E}-10$ & $5.83 \mathrm{E}-10$ & STX7 & $1.09 E+00$ \\
\hline ENSBTAG00000046232 & $2.60 \mathrm{E}-09$ & $2.39 \mathrm{E}-09$ & & $1.09 \mathrm{E}+00$ \\
\hline ENSBTAG00000010347 & $5.43 \mathrm{E}-10$ & $4.96 \mathrm{E}-10$ & EZR & $1.09 E+00$ \\
\hline ENSBTAG00000016839 & $1.59 \mathrm{E}-10$ & $1.46 \mathrm{E}-10$ & MTO1 & $1.09 \mathrm{E}+00$ \\
\hline ENSBTAG00000015485 & $3.34 \mathrm{E}-10$ & $3.05 \mathrm{E}-10$ & C6orf118 & $1.10 \mathrm{E}+00$ \\
\hline ENSBTAG00000000264 & $2.49 \mathrm{E}-10$ & $2.27 \mathrm{E}-10$ & RFX6 & $1.10 \mathrm{E}+00$ \\
\hline ENSBTAG00000000436 & $2.86 \mathrm{E}-10$ & $2.60 \mathrm{E}-10$ & TNFAIP3 & $1.10 \mathrm{E}+00$ \\
\hline ENSBTAG00000013991 & $1.16 \mathrm{E}-10$ & $1.06 \mathrm{E}-10$ & NR2E1 & $1.09 \mathrm{E}+00$ \\
\hline ENSBTAG00000001598 & $2.86 \mathrm{E}-10$ & $2.59 \mathrm{E}-10$ & MAP7 & $1.10 \mathrm{E}+00$ \\
\hline ENSBTAG00000011234 & $1.94 \mathrm{E}-10$ & $1.75 \mathrm{E}-10$ & FOXO3 & $1.11 \mathrm{E}+00$ \\
\hline ENSBTAG00000010116 & $5.10 \mathrm{E}-10$ & $4.61 \mathrm{E}-10$ & AK9 & $1.11 E+00$ \\
\hline ENSBTAG00000013222 & $5.99 \mathrm{E}-10$ & $5.39 \mathrm{E}-10$ & CD109 & $1.11 \mathrm{E}+00$ \\
\hline ENSBTAG00000047224 & $3.81 \mathrm{E}-10$ & $3.43 \mathrm{E}-10$ & RYK & $1.11 \mathrm{E}+00$ \\
\hline ENSBTAG00000016751 & $9.48 \mathrm{E}-10$ & $8.48 \mathrm{E}-10$ & MYO6 & $1.12 \mathrm{E}+00$ \\
\hline ENSBTAG00000020056 & $3.68 \mathrm{E}-10$ & $3.27 \mathrm{E}-10$ & COL12A1 & $1.13 \mathrm{E}+00$ \\
\hline ENSBTAG00000001422 & $5.58 \mathrm{E}-10$ & $4.96 \mathrm{E}-10$ & HMGN3 & $1.13 \mathrm{E}+00$ \\
\hline ENSBTAG00000017393 & $3.19 \mathrm{E}-10$ & $2.83 \mathrm{E}-10$ & L3MBTL3 & $1.13 E+00$ \\
\hline ENSBTAG00000010204 & $2.41 \mathrm{E}-10$ & $2.13 \mathrm{E}-10$ & PCMT1 & $1.13 \mathrm{E}+00$ \\
\hline ENSBTAG00000031476 & $1.06 \mathrm{E}-10$ & $9.36 \mathrm{E}-11$ & DLL1 & $1.13 \mathrm{E}+00$ \\
\hline ENSBTAG00000033657 & $6.01 \mathrm{E}-10$ & $5.26 \mathrm{E}-10$ & SLC35F1 & $1.14 \mathrm{E}+00$ \\
\hline ENSBTAG00000014057 & $1.61 \mathrm{E}-10$ & $1.40 \mathrm{E}-10$ & SLC35A1 & $1.15 \mathrm{E}+00$ \\
\hline ENSBTAG00000018801 & $1.48 \mathrm{E}-10$ & $1.29 \mathrm{E}-10$ & SNX3 & $1.14 \mathrm{E}+00$ \\
\hline ENSBTAG00000008112 & $2.38 \mathrm{E}-10$ & $2.08 \mathrm{E}-10$ & SHPRH & $1.14 \mathrm{E}+00$ \\
\hline ENSBTAG00000016791 & $2.02 \mathrm{E}-10$ & $1.76 \mathrm{E}-10$ & PEX7 & $1.15 \mathrm{E}+00$ \\
\hline ENSBTAG00000012544 & $3.50 \mathrm{E}-10$ & $3.05 E-10$ & IFNGR1 & $1.15 \mathrm{E}+00$ \\
\hline ENSBTAG00000047912 & $1.00 \mathrm{E}-09$ & $8.70 \mathrm{E}-10$ & & $1.15 \mathrm{E}+00$ \\
\hline ENSBTAG00000024751 & $1.03 \mathrm{E}-09$ & $8.97 \mathrm{E}-10$ & & $1.15 \mathrm{E}+00$ \\
\hline ENSBTAG00000000019 & $2.48 \mathrm{E}-10$ & $2.15 \mathrm{E}-10$ & SERINC1 & $1.15 \mathrm{E}+00$ \\
\hline ENSBTAG00000021361 & $2.32 \mathrm{E}-10$ & $2.01 \mathrm{E}-10$ & SAMD3 & $1.15 \mathrm{E}+00$ \\
\hline ENSBTAG00000016844 & $3.73 \mathrm{E}-10$ & $3.22 \mathrm{E}-10$ & $A D G B$ & $1.16 \mathrm{E}+00$ \\
\hline ENSBTAG00000017879 & $3.65 \mathrm{E}-10$ & $3.15 \mathrm{E}-10$ & C6orf163 & $1.16 \mathrm{E}+00$ \\
\hline ENSBTAG00000015335 & $4.79 \mathrm{E}-10$ & $4.13 \mathrm{E}-10$ & ADGRB3 & $1.16 \mathrm{E}+00$ \\
\hline ENSBTAG00000039574 & $1.98 \mathrm{E}-10$ & $1.71 \mathrm{E}-10$ & & $1.16 \mathrm{E}+00$ \\
\hline ENSBTAG00000011330 & $5.34 \mathrm{E}-10$ & 4.59E-10 & & $1.16 \mathrm{E}+00$ \\
\hline ENSBTAG00000011179 & $2.99 \mathrm{E}-10$ & $2.57 \mathrm{E}-10$ & PDCD2 & $1.17 \mathrm{E}+00$ \\
\hline ENSBTAG00000001533 & $2.23 \mathrm{E}-10$ & $1.91 \mathrm{E}-10$ & HACE1 & $1.17 \mathrm{E}+00$ \\
\hline ENSBTAG00000047338 & $4.66 \mathrm{E}-10$ & $3.99 \mathrm{E}-10$ & DCBLD1 & $1.17 \mathrm{E}+00$ \\
\hline ENSBTAG00000008144 & $1.59 \mathrm{E}-10$ & $1.36 \mathrm{E}-10$ & PPIL6 & $1.17 \mathrm{E}+00$ \\
\hline ENSBTAG00000026586 & $6.33 \mathrm{E}-10$ & $5.41 \mathrm{E}-10$ & PPP1R14C & $1.17 \mathrm{E}+00$ \\
\hline ENSBTAG00000047024 & $2.00 \mathrm{E}-10$ & $1.70 \mathrm{E}-10$ & & $1.18 \mathrm{E}+00$ \\
\hline ENSBTAG00000038849 & $5.45 \mathrm{E}-10$ & $4.63 \mathrm{E}-10$ & & $1.18 \mathrm{E}+00$ \\
\hline ENSBTAG00000017557 & $2.92 \mathrm{E}-10$ & $2.48 \mathrm{E}-10$ & QRSL1 & $1.18 \mathrm{E}+00$ \\
\hline ENSBTAG00000003455 & $3.00 \mathrm{E}-10$ & $2.54 \mathrm{E}-10$ & ANKRD6 & $1.18 \mathrm{E}+00$ \\
\hline ENSBTAG00000026672 & $6.89 \mathrm{E}-10$ & $5.82 \mathrm{E}-10$ & & $1.18 \mathrm{E}+00$ \\
\hline ENSBTAG00000002082 & $1.18 \mathrm{E}-10$ & $9.90 \mathrm{E}-11$ & MARCKS & $1.19 \mathrm{E}+00$ \\
\hline
\end{tabular}




\begin{tabular}{|c|c|c|c|c|}
\hline ENSBTAG00000000995 & $1.01 \mathrm{E}-10$ & $8.49 \mathrm{E}-11$ & FAM46A & $1.19 \mathrm{E}+00$ \\
\hline ENSBTAG00000011851 & $3.74 \mathrm{E}-10$ & $3.13 \mathrm{E}-10$ & FYN & $1.19 E+00$ \\
\hline ENSBTAG00000016391 & $1.70 \mathrm{E}-09$ & $1.42 \mathrm{E}-09$ & & $1.19 E+00$ \\
\hline ENSBTAG00000003496 & $2.21 \mathrm{E}-10$ & $1.84 \mathrm{E}-10$ & AIG1 & $1.20 \mathrm{E}+00$ \\
\hline ENSBTAG00000021970 & $2.42 \mathrm{E}-10$ & $2.00 \mathrm{E}-10$ & EYA4 & $1.21 \mathrm{E}+00$ \\
\hline ENSBTAG00000016969 & $1.16 \mathrm{E}-10$ & $9.53 \mathrm{E}-11$ & ORC3 & $1.22 \mathrm{E}+00$ \\
\hline ENSBTAG00000003340 & $3.08 \mathrm{E}-10$ & $2.52 \mathrm{E}-10$ & HDDC2 & $1.22 \mathrm{E}+00$ \\
\hline ENSBTAG00000038112 & $2.27 \mathrm{E}-10$ & $1.85 \mathrm{E}-10$ & & $1.23 \mathrm{E}+00$ \\
\hline ENSBTAG00000007799 & $1.50 \mathrm{E}-10$ & $1.23 \mathrm{E}-10$ & MTFR2 & $1.22 \mathrm{E}+00$ \\
\hline ENSBTAG00000017958 & 2.17E-10 & $1.76 \mathrm{E}-10$ & AHI1 & $1.23 E+00$ \\
\hline ENSBTAG00000015935 & $3.53 \mathrm{E}-10$ & $2.84 \mathrm{E}-10$ & IYD & $1.24 \mathrm{E}+00$ \\
\hline ENSBTAG00000018435 & $8.22 \mathrm{E}-11$ & $6.59 \mathrm{E}-11$ & GABRR2 & $1.25 \mathrm{E}+00$ \\
\hline ENSBTAG00000036061 & $4.73 \mathrm{E}-10$ & $3.78 \mathrm{E}-10$ & & $1.25 \mathrm{E}+00$ \\
\hline ENSBTAG00000020097 & $3.15 \mathrm{E}-10$ & $2.52 \mathrm{E}-10$ & & $1.25 E+00$ \\
\hline ENSBTAG00000044181 & $5.09 \mathrm{E}-10$ & $4.06 \mathrm{E}-10$ & GRM1 & $1.25 \mathrm{E}+00$ \\
\hline ENSBTAG00000018161 & $3.48 \mathrm{E}-10$ & $2.77 \mathrm{E}-10$ & TBX18 & $1.26 \mathrm{E}+00$ \\
\hline ENSBTAG00000012674 & $6.23 \mathrm{E}-10$ & 4.94E-10 & CNKSR3 & $1.26 \mathrm{E}+00$ \\
\hline ENSBTAG00000003887 & 4.07E-10 & 3.22E-10 & ECHDC1 & $1.26 \mathrm{E}+00$ \\
\hline ENSBTAG00000000188 & 7.53E-10 & $5.90 \mathrm{E}-10$ & HS3ST5 & $1.28 \mathrm{E}+00$ \\
\hline ENSBTAG00000027049 & $3.68 \mathrm{E}-10$ & $2.88 \mathrm{E}-10$ & SDHAF4 & $1.28 \mathrm{E}+00$ \\
\hline ENSBTAG00000003764 & $1.51 \mathrm{E}-10$ & 1.17E-10 & AKIRIN2 & $1.29 \mathrm{E}+00$ \\
\hline ENSBTAG00000004795 & $2.56 \mathrm{E}-10$ & $1.98 \mathrm{E}-10$ & RIPPLY2 & $1.29 \mathrm{E}+00$ \\
\hline ENSBTAG00000015467 & 7.24E-10 & $5.60 \mathrm{E}-10$ & FAM184A & $1.29 \mathrm{E}+00$ \\
\hline ENSBTAG00000026637 & $1.69 \mathrm{E}-10$ & $1.30 \mathrm{E}-10$ & & $1.30 E+00$ \\
\hline ENSBTAG00000007685 & $2.13 \mathrm{E}-10$ & $1.63 \mathrm{E}-10$ & PSMB1 & $1.31 E+00$ \\
\hline ENSBTAG00000021814 & $3.39 \mathrm{E}-10$ & $2.59 \mathrm{E}-10$ & DDX43 & $1.31 E+00$ \\
\hline ENSBTAG00000044053 & $5.06 \mathrm{E}-10$ & 3.85E-10 & SLC17A5 & $1.31 E+00$ \\
\hline ENSBTAG00000002275 & $1.04 \mathrm{E}-10$ & 7.87E-11 & PTP4A1 & $1.32 \mathrm{E}+00$ \\
\hline ENSBTAG00000016967 & $1.16 \mathrm{E}-10$ & $8.79 \mathrm{E}-11$ & RARS2 & $1.32 \mathrm{E}+00$ \\
\hline ENSBTAG00000009795 & $6.11 \mathrm{E}-10$ & $4.61 \mathrm{E}-10$ & SF3B5 & $1.33 E+00$ \\
\hline ENSBTAG00000046593 & 4.17E-10 & $3.15 \mathrm{E}-10$ & & $1.33 \mathrm{E}+00$ \\
\hline ENSBTAG00000046733 & $8.30 \mathrm{E}-10$ & $6.25 \mathrm{E}-10$ & & $1.33 E+00$ \\
\hline ENSBTAG00000020410 & $2.79 \mathrm{E}-10$ & $2.10 \mathrm{E}-10$ & UFL1 & $1.33 \mathrm{E}+00$ \\
\hline ENSBTAG00000001803 & $3.41 \mathrm{E}-10$ & $2.56 \mathrm{E}-10$ & FHL5 & $1.33 \mathrm{E}+00$ \\
\hline ENSBTAG00000016357 & $1.08 \mathrm{E}-09$ & 8.10E-10 & VNN2 & $1.33 \mathrm{E}+00$ \\
\hline ENSBTAG00000005869 & $1.85 \mathrm{E}-10$ & $1.39 \mathrm{E}-10$ & SENP6 & $1.33 E+00$ \\
\hline ENSBTAG00000000679 & $3.91 \mathrm{E}-10$ & $2.93 \mathrm{E}-10$ & PGM3 & $1.34 \mathrm{E}+00$ \\
\hline ENSBTAG00000009065 & $3.13 \mathrm{E}-10$ & $2.34 \mathrm{E}-10$ & MAP3K5 & $1.34 \mathrm{E}+00$ \\
\hline ENSBTAG00000000892 & $1.98 \mathrm{E}-10$ & $1.47 \mathrm{E}-10$ & MB21D1 & $1.34 \mathrm{E}+00$ \\
\hline ENSBTAG00000001956 & $1.33 \mathrm{E}-10$ & $9.86 \mathrm{E}-11$ & HINT3 & $1.34 \mathrm{E}+00$ \\
\hline ENSBTAG00000003919 & $6.69 \mathrm{E}-10$ & 4.97E-10 & KIAA0408 & $1.35 E+00$ \\
\hline ENSBTAG00000008121 & $2.03 \mathrm{E}-10$ & $1.51 \mathrm{E}-10$ & & $1.34 \mathrm{E}+00$ \\
\hline ENSBTAG00000039080 & $3.02 \mathrm{E}-10$ & $2.24 \mathrm{E}-10$ & SLC22A3 & $1.35 \mathrm{E}+00$ \\
\hline ENSBTAG00000021193 & $3.05 E-10$ & $2.27 \mathrm{E}-10$ & FBXO5 & $1.35 E+00$ \\
\hline ENSBTAG00000019175 & $1.75 \mathrm{E}-10$ & $1.30 \mathrm{E}-10$ & SOBP & $1.35 E+00$ \\
\hline ENSBTAG00000015094 & $3.98 \mathrm{E}-10$ & $2.95 \mathrm{E}-10$ & VNN1 & $1.35 E+00$ \\
\hline ENSBTAG00000007694 & $8.50 \mathrm{E}-10$ & $6.29 \mathrm{E}-10$ & & $1.35 E+00$ \\
\hline ENSBTAG00000020238 & $5.12 \mathrm{E}-10$ & $3.76 \mathrm{E}-10$ & RIMS1 & $1.36 \mathrm{E}+00$ \\
\hline ENSBTAG00000011593 & $2.24 \mathrm{E}-10$ & $1.65 \mathrm{E}-10$ & QKI & $1.36 \mathrm{E}+00$ \\
\hline ENSBTAG00000007159 & $3.81 \mathrm{E}-10$ & $2.79 \mathrm{E}-10$ & ESR1 & $1.37 \mathrm{E}+00$ \\
\hline ENSBTAG00000011849 & $2.26 \mathrm{E}-10$ & $1.65 \mathrm{E}-10$ & HDAC2 & $1.37 \mathrm{E}+00$ \\
\hline ENSBTAG00000021107 & $1.68 \mathrm{E}-10$ & $1.22 \mathrm{E}-10$ & STXBP5 & $1.38 \mathrm{E}+00$ \\
\hline ENSBTAG00000016980 & $3.16 \mathrm{E}-10$ & $2.29 \mathrm{E}-10$ & PPIL4 & $1.38 \mathrm{E}+00$ \\
\hline ENSBTAG00000010760 & $1.25 \mathrm{E}-10$ & $9.04 \mathrm{E}-11$ & REV3L & $1.38 \mathrm{E}+00$ \\
\hline ENSBTAG00000045772 & $1.25 \mathrm{E}-09$ & $8.93 \mathrm{E}-10$ & & $1.40 E+00$ \\
\hline ENSBTAG00000009362 & $5.67 \mathrm{E}-10$ & 4.03E-10 & SYNE1 & $1.41 E+00$ \\
\hline ENSBTAG00000045624 & $1.39 \mathrm{E}-09$ & $9.84 \mathrm{E}-10$ & & $1.41 \mathrm{E}+00$ \\
\hline ENSBTAG00000005557 & $4.42 \mathrm{E}-10$ & $3.12 \mathrm{E}-10$ & AFDN & $1.42 \mathrm{E}+00$ \\
\hline ENSBTAG00000015181 & $3.44 \mathrm{E}-10$ & $2.41 \mathrm{E}-10$ & EPM2A & $1.43 E+00$ \\
\hline ENSBTAG00000046809 & $1.57 \mathrm{E}-10$ & $1.10 \mathrm{E}-10$ & & $1.43 \mathrm{E}+00$ \\
\hline ENSBTAG00000017370 & $3.78 \mathrm{E}-10$ & 2.64E-10 & ARFGEF3 & $1.43 E+00$ \\
\hline ENSBTAG00000009239 & $2.23 \mathrm{E}-10$ & $1.55 \mathrm{E}-10$ & SLC16A10 & $1.44 \mathrm{E}+00$ \\
\hline ENSBTAG00000005376 & $1.24 \mathrm{E}-10$ & $8.71 E-11$ & FBXO30 & $1.43 E+00$ \\
\hline ENSBTAG00000024595 & $2.27 \mathrm{E}-10$ & $1.58 \mathrm{E}-10$ & HECA & $1.43 \mathrm{E}+00$ \\
\hline ENSBTAG00000020829 & $4.85 \mathrm{E}-10$ & 3.36E-10 & PTPRK & $1.44 \mathrm{E}+00$ \\
\hline ENSBTAG00000033153 & $3.25 \mathrm{E}-10$ & $2.25 \mathrm{E}-10$ & GRIK2 & $1.45 E+00$ \\
\hline ENSBTAG00000001289 & 4.74E-10 & $3.25 \mathrm{E}-10$ & MRPL18 & $1.46 \mathrm{E}+00$ \\
\hline ENSBTAG00000019469 & $5.46 \mathrm{E}-10$ & $3.74 \mathrm{E}-10$ & SPACA1 & $1.46 \mathrm{E}+00$ \\
\hline ENSBTAG00000038190 & $3.39 \mathrm{E}-10$ & $2.31 \mathrm{E}-10$ & SMAP1 & $1.47 \mathrm{E}+00$ \\
\hline ENSBTAG00000010838 & $3.78 \mathrm{E}-10$ & $2.58 \mathrm{E}-10$ & TMEM181 & $1.46 \mathrm{E}+00$ \\
\hline
\end{tabular}




\begin{tabular}{|c|c|c|c|c|}
\hline ENSBTAG00000002728 & $2.99 \mathrm{E}-10$ & $2.04 \mathrm{E}-10$ & ARID1B & $1.47 \mathrm{E}+00$ \\
\hline ENSBTAG00000018634 & $2.96 \mathrm{E}-10$ & $2.01 \mathrm{E}-10$ & SH3BGRL2 & $1.47 \mathrm{E}+00$ \\
\hline ENSBTAG00000026708 & $1.02 \mathrm{E}-09$ & $6.91 \mathrm{E}-10$ & PRSS35 & $1.47 \mathrm{E}+00$ \\
\hline ENSBTAG00000019410 & 2.03E-10 & $1.37 \mathrm{E}-10$ & CEP57L1 & $1.48 \mathrm{E}+00$ \\
\hline ENSBTAG00000005681 & $5.80 \mathrm{E}-10$ & $3.91 \mathrm{E}-10$ & ME1 & $1.48 \mathrm{E}+00$ \\
\hline ENSBTAG00000044061 & $2.52 \mathrm{E}-10$ & $1.70 \mathrm{E}-10$ & FAXC & $1.48 \mathrm{E}+00$ \\
\hline ENSBTAG00000011890 & $2.71 \mathrm{E}-10$ & $1.82 \mathrm{E}-10$ & RSPH4A & $1.49 E+00$ \\
\hline ENSBTAG00000002827 & $6.64 \mathrm{E}-10$ & 4.47E-10 & АСАT2 & $1.49 E+00$ \\
\hline ENSBTAG00000045976 & $5.74 \mathrm{E}-10$ & $3.85 \mathrm{E}-10$ & & $1.49 \mathrm{E}+00$ \\
\hline ENSBTAG00000000880 & $1.68 \mathrm{E}-10$ & $1.13 \mathrm{E}-10$ & GINM1 & $1.49 E+00$ \\
\hline ENSBTAG00000033554 & $3.65 \mathrm{E}-10$ & $2.41 \mathrm{E}-10$ & TRAPPC3L & $1.51 \mathrm{E}+00$ \\
\hline ENSBTAG00000018438 & $1.75 \mathrm{E}-10$ & $1.15 \mathrm{E}-10$ & RRAGD & $1.52 \mathrm{E}+00$ \\
\hline ENSBTAG00000009275 & $4.82 \mathrm{E}-10$ & $3.14 \mathrm{E}-10$ & MTRF1L & $1.53 \mathrm{E}+00$ \\
\hline ENSBTAG00000008817 & $3.86 \mathrm{E}-10$ & $2.51 \mathrm{E}-10$ & LAMA4 & $1.54 \mathrm{E}+00$ \\
\hline ENSBTAG00000009679 & $3.92 \mathrm{E}-10$ & $2.52 \mathrm{E}-10$ & PLEKHG1 & $1.56 \mathrm{E}+00$ \\
\hline ENSBTAG00000026133 & $9.92 \mathrm{E}-10$ & $6.38 \mathrm{E}-10$ & & $1.55 \mathrm{E}+00$ \\
\hline ENSBTAG00000000817 & $2.77 \mathrm{E}-10$ & $1.77 \mathrm{E}-10$ & SYNJ2 & $1.56 \mathrm{E}+00$ \\
\hline ENSBTAG00000000053 & 4.77E-10 & $3.06 \mathrm{E}-10$ & FILIP1 & $1.56 \mathrm{E}+00$ \\
\hline ENSBTAG00000026673 & $2.23 \mathrm{E}-10$ & $1.40 \mathrm{E}-10$ & KPNA5 & $1.59 \mathrm{E}+00$ \\
\hline ENSBTAG00000001835 & $8.81 \mathrm{E}-11$ & $5.55 \mathrm{E}-11$ & GJA1 & $1.59 E+00$ \\
\hline ENSBTAG00000033218 & $1.26 \mathrm{E}-10$ & 7.94E-11 & & $1.59 E+00$ \\
\hline ENSBTAG00000046006 & $3.47 \mathrm{E}-10$ & $2.18 \mathrm{E}-10$ & CNR1 & $1.59 E+00$ \\
\hline ENSBTAG00000010885 & $3.02 \mathrm{E}-10$ & $1.90 \mathrm{E}-10$ & TSPYL1 & $1.59 \mathrm{E}+00$ \\
\hline ENSBTAG00000014104 & 7.23E-10 & $4.54 \mathrm{E}-10$ & & $1.59 \mathrm{E}+00$ \\
\hline ENSBTAG00000038931 & $2.31 \mathrm{E}-10$ & $1.46 \mathrm{E}-10$ & & $1.58 \mathrm{E}+00$ \\
\hline ENSBTAG00000048114 & $2.40 \mathrm{E}-10$ & $1.51 \mathrm{E}-10$ & & $1.59 \mathrm{E}+00$ \\
\hline ENSBTAG00000015497 & $1.30 \mathrm{E}-10$ & $8.17 E-11$ & & $1.59 E+00$ \\
\hline ENSBTAG00000000676 & $4.51 \mathrm{E}-10$ & $2.83 \mathrm{E}-10$ & DOPEY1 & $1.59 E+00$ \\
\hline ENSBTAG00000015381 & $1.94 \mathrm{E}-10$ & $1.20 \mathrm{E}-10$ & ARHGAP18 & $1.62 E+00$ \\
\hline ENSBTAG00000002059 & $2.13 \mathrm{E}-10$ & $1.32 \mathrm{E}-10$ & MCM9 & $1.62 E+00$ \\
\hline ENSBTAG00000019730 & $1.84 \mathrm{E}-10$ & $1.12 \mathrm{E}-10$ & PNISR & $1.65 \mathrm{E}+00$ \\
\hline ENSBTAG00000032163 & $2.29 \mathrm{E}-10$ & $1.38 \mathrm{E}-10$ & & $1.66 \mathrm{E}+00$ \\
\hline ENSBTAG00000019460 & $9.76 \mathrm{E}-10$ & $5.86 \mathrm{E}-10$ & MOXD1 & $1.66 \mathrm{E}+00$ \\
\hline ENSBTAG00000007802 & $1.20 \mathrm{E}-10$ & $7.21 \mathrm{E}-11$ & BCLAF1 & $1.67 E+00$ \\
\hline ENSBTAG00000007668 & $3.92 \mathrm{E}-10$ & $2.33 \mathrm{E}-10$ & AGPAT4 & $1.68 \mathrm{E}+00$ \\
\hline ENSBTAG00000007974 & 3.73E-10 & 2.19E-10 & & $1.71 \mathrm{E}+00$ \\
\hline ENSBTAG00000003921 & $5.52 \mathrm{E}-10$ & $3.23 \mathrm{E}-10$ & & $1.71 \mathrm{E}+00$ \\
\hline ENSBTAG00000018810 & $2.90 \mathrm{E}-10$ & $1.68 \mathrm{E}-10$ & THBS2 & $1.73 E+00$ \\
\hline ENSBTAG00000020707 & $3.80 \mathrm{E}-10$ & $2.18 \mathrm{E}-10$ & ADGRG6 & $1.74 \mathrm{E}+00$ \\
\hline ENSBTAG00000016281 & $3.07 \mathrm{E}-10$ & $1.76 \mathrm{E}-10$ & PREP & $1.74 \mathrm{E}+00$ \\
\hline ENSBTAG00000021859 & $2.25 \mathrm{E}-10$ & $1.29 \mathrm{E}-10$ & PDSS2 & $1.74 \mathrm{E}+00$ \\
\hline ENSBTAG00000014062 & $3.78 \mathrm{E}-10$ & $2.15 \mathrm{E}-10$ & FRMD1 & $1.76 \mathrm{E}+00$ \\
\hline ENSBTAG00000018790 & $1.87 \mathrm{E}-10$ & $1.05 \mathrm{E}-10$ & BVES & $1.78 \mathrm{E}+00$ \\
\hline ENSBTAG00000038447 & $7.18 \mathrm{E}-10$ & $4.01 \mathrm{E}-10$ & & $1.79 \mathrm{E}+00$ \\
\hline ENSBTAG00000013551 & $6.90 \mathrm{E}-10$ & $3.81 \mathrm{E}-10$ & NOX3 & $1.81 \mathrm{E}+00$ \\
\hline ENSBTAG00000046612 & $1.65 \mathrm{E}-10$ & $9.13 \mathrm{E}-11$ & ZNF292 & $1.81 E+00$ \\
\hline ENSBTAG00000014592 & $1.55 \mathrm{E}-10$ & $8.43 \mathrm{E}-11$ & LACE1 & $1.84 \mathrm{E}+00$ \\
\hline ENSBTAG00000026519 & $1.35 \mathrm{E}-10$ & 7.36E-11 & ADAT2 & $1.84 \mathrm{E}+00$ \\
\hline ENSBTAG00000021667 & $4.93 \mathrm{E}-10$ & $2.65 \mathrm{E}-10$ & & $1.86 \mathrm{E}+00$ \\
\hline ENSBTAG00000005729 & $1.80 \mathrm{E}-10$ & $9.71 \mathrm{E}-11$ & FBXL4 & $1.86 \mathrm{E}+00$ \\
\hline ENSBTAG00000010229 & $4.45 \mathrm{E}-10$ & $2.39 \mathrm{E}-10$ & LAMA2 & $1.86 \mathrm{E}+00$ \\
\hline ENSBTAG00000005301 & $5.88 \mathrm{E}-10$ & $3.14 \mathrm{E}-10$ & KLHL32 & $1.87 \mathrm{E}+00$ \\
\hline ENSBTAG00000021587 & $1.22 \mathrm{E}-10$ & $6.52 \mathrm{E}-11$ & SMPDL3A & $1.88 \mathrm{E}+00$ \\
\hline ENSBTAG00000015744 & $2.64 \mathrm{E}-10$ & $1.40 \mathrm{E}-10$ & CCNC & $1.89 \mathrm{E}+00$ \\
\hline ENSBTAG00000001826 & $4.52 \mathrm{E}-10$ & $2.40 \mathrm{E}-10$ & SASH1 & $1.88 \mathrm{E}+00$ \\
\hline ENSBTAG00000007201 & $1.66 \mathrm{E}-10$ & $8.83 \mathrm{E}-11$ & TMEM242 & $1.88 \mathrm{E}+00$ \\
\hline ENSBTAG00000020991 & $6.42 \mathrm{E}-10$ & $3.36 \mathrm{E}-10$ & & $1.91 \mathrm{E}+00$ \\
\hline ENSBTAG00000043973 & $1.40 \mathrm{E}-10$ & 7.27E-11 & LIN28B & $1.93 \mathrm{E}+00$ \\
\hline ENSBTAG00000014054 & $1.55 \mathrm{E}-10$ & $8.03 E-11$ & & $1.93 \mathrm{E}+00$ \\
\hline ENSBTAG00000009665 & $5.85 \mathrm{E}-10$ & $3.02 \mathrm{E}-10$ & UTRN & $1.94 \mathrm{E}+00$ \\
\hline ENSBTAG00000033429 & 9.54E-10 & $4.91 \mathrm{E}-10$ & FAM229B & $1.94 \mathrm{E}+00$ \\
\hline ENSBTAG00000021830 & $6.74 \mathrm{E}-10$ & $3.45 \mathrm{E}-10$ & ENPP1 & $1.95 \mathrm{E}+00$ \\
\hline ENSBTAG00000047118 & $2.59 \mathrm{E}-10$ & $1.33 \mathrm{E}-10$ & & $1.95 \mathrm{E}+00$ \\
\hline ENSBTAG00000001957 & $1.81 \mathrm{E}-10$ & $9.19 \mathrm{E}-11$ & TRMT11 & $1.96 \mathrm{E}+00$ \\
\hline ENSBTAG00000024688 & $2.81 \mathrm{E}-10$ & $1.43 \mathrm{E}-10$ & PHIP & $1.96 \mathrm{E}+00$ \\
\hline ENSBTAG00000009846 & $6.22 \mathrm{E}-10$ & $3.15 \mathrm{E}-10$ & MTHFD1L & $1.97 \mathrm{E}+00$ \\
\hline ENSBTAG00000046017 & $2.96 \mathrm{E}-10$ & $1.49 \mathrm{E}-10$ & POPDC3 & $1.99 \mathrm{E}+00$ \\
\hline ENSBTAG00000037718 & 4.06E-10 & $2.04 \mathrm{E}-10$ & TAAR1 & $1.99 \mathrm{E}+00$ \\
\hline ENSBTAG00000003174 & $3.79 \mathrm{E}-10$ & $1.91 \mathrm{E}-10$ & NDUFAF4 & $1.99 \mathrm{E}+00$ \\
\hline ENSBTAG00000037598 & $2.50 \mathrm{E}-10$ & $1.26 \mathrm{E}-10$ & & $1.98 \mathrm{E}+00$ \\
\hline
\end{tabular}




\begin{tabular}{|c|c|c|c|c|}
\hline ENSBTAG00000015516 & $1.24 \mathrm{E}-10$ & $6.27 \mathrm{E}-11$ & CCDC28A & $1.98 \mathrm{E}+00$ \\
\hline ENSBTAG00000020018 & $2.74 \mathrm{E}-10$ & $1.36 \mathrm{E}-10$ & FRK & $2.01 E+00$ \\
\hline ENSBTAG00000020713 & $2.96 \mathrm{E}-10$ & 1.47E-10 & $\mathrm{BACH} 2$ & $2.01 E+00$ \\
\hline ENSBTAG00000018528 & $2.32 \mathrm{E}-10$ & $1.14 \mathrm{E}-10$ & USP45 & $2.03 E+00$ \\
\hline ENSBTAG00000017527 & $3.91 \mathrm{E}-10$ & $1.91 \mathrm{E}-10$ & AIM1 & $2.05 E+00$ \\
\hline ENSBTAG00000007975 & $2.49 \mathrm{E}-10$ & $1.21 \mathrm{E}-10$ & ALDH8A1 & $2.06 \mathrm{E}+00$ \\
\hline ENSBTAG00000000629 & $3.53 \mathrm{E}-10$ & $1.72 \mathrm{E}-10$ & MMS22L & $2.05 \mathrm{E}+00$ \\
\hline ENSBTAG00000019729 & $2.97 \mathrm{E}-10$ & $1.42 \mathrm{E}-10$ & COQ3 & $2.09 \mathrm{E}+00$ \\
\hline ENSBTAG00000005955 & $6.81 \mathrm{E}-10$ & $3.23 \mathrm{E}-10$ & $\mathrm{RSPH} 3$ & $2.11 E+00$ \\
\hline ENSBTAG00000020825 & $2.89 \mathrm{E}-10$ & $1.37 \mathrm{E}-10$ & IRAK1BP1 & $2.11 E+00$ \\
\hline ENSBTAG00000005960 & $4.49 \mathrm{E}-10$ & $2.12 \mathrm{E}-10$ & EPB41L2 & $2.12 \mathrm{E}+00$ \\
\hline ENSBTAG00000032024 & $1.50 \mathrm{E}-10$ & $6.89 \mathrm{E}-11$ & KATNA1 & $2.17 E+00$ \\
\hline ENSBTAG00000000916 & $2.39 \mathrm{E}-10$ & $1.09 \mathrm{E}-10$ & TCTE3 & $2.19 E+00$ \\
\hline ENSBTAG00000009355 & $3.20 \mathrm{E}-10$ & $1.46 \mathrm{E}-10$ & SNAP91 & $2.19 E+00$ \\
\hline ENSBTAG00000016584 & $7.39 \mathrm{E}-11$ & 3.39E-11 & RPF2 & $2.18 \mathrm{E}+00$ \\
\hline ENSBTAG00000020368 & $3.54 \mathrm{E}-10$ & $1.62 \mathrm{E}-10$ & & $2.18 \mathrm{E}+00$ \\
\hline ENSBTAG00000017205 & $8.33 \mathrm{E}-10$ & $3.73 \mathrm{E}-10$ & WISP3 & $2.23 E+00$ \\
\hline ENSBTAG00000031895 & $2.45 \mathrm{E}-10$ & $1.09 \mathrm{E}-10$ & TFB1M & $2.25 E+00$ \\
\hline ENSBTAG00000026548 & 1.31E-09 & $5.80 \mathrm{E}-10$ & & $2.27 \mathrm{E}+00$ \\
\hline ENSBTAG00000003489 & 4.19E-10 & $1.83 \mathrm{E}-10$ & IPCEF1 & $2.29 \mathrm{E}+00$ \\
\hline ENSBTAG00000016138 & $1.70 \mathrm{E}-10$ & 7.34E-11 & GPA1 & $2.32 \mathrm{E}+00$ \\
\hline ENSBTAG00000005205 & $3.84 \mathrm{E}-10$ & $1.64 \mathrm{E}-10$ & DSE & $2.34 \mathrm{E}+00$ \\
\hline ENSBTAG00000004472 & $5.91 \mathrm{E}-10$ & $2.51 \mathrm{E}-10$ & DYNLT1 & $2.35 \mathrm{E}+00$ \\
\hline ENSBTAG00000021741 & $9.36 \mathrm{E}-10$ & $3.95 \mathrm{E}-10$ & RPS6KA2 & $2.37 E+00$ \\
\hline ENSBTAG00000047309 & $2.12 \mathrm{E}-10$ & $8.87 \mathrm{E}-11$ & & $2.38 \mathrm{E}+00$ \\
\hline ENSBTAG00000006095 & $1.27 \mathrm{E}-10$ & $5.33 \mathrm{E}-11$ & PRDM13 & $2.38 \mathrm{E}+00$ \\
\hline ENSBTAG00000046219 & $2.28 \mathrm{E}-10$ & $9.58 \mathrm{E}-11$ & & $2.38 \mathrm{E}+00$ \\
\hline ENSBTAG00000013176 & $4.58 \mathrm{E}-10$ & $1.92 \mathrm{E}-10$ & SMOC2 & $2.38 \mathrm{E}+00$ \\
\hline ENSBTAG00000040543 & $5.01 \mathrm{E}-10$ & $2.10 \mathrm{E}-10$ & TMEM200A & $2.39 E+00$ \\
\hline ENSBTAG00000040489 & $2.34 \mathrm{E}-10$ & $9.80 \mathrm{E}-11$ & & $2.38 \mathrm{E}+00$ \\
\hline ENSBTAG00000012254 & $5.72 \mathrm{E}-10$ & $2.39 \mathrm{E}-10$ & & $2.39 E+00$ \\
\hline ENSBTAG00000005699 & 4.13E-10 & $1.72 \mathrm{E}-10$ & SCML4 & $2.40 E+00$ \\
\hline ENSBTAG00000020482 & $4.56 \mathrm{E}-10$ & $1.90 \mathrm{E}-10$ & $\mathrm{ASCC} 3$ & $2.40 \mathrm{E}+00$ \\
\hline ENSBTAG00000002402 & $3.14 \mathrm{E}-10$ & $1.28 \mathrm{E}-10$ & IGF2R & $2.45 E+00$ \\
\hline ENSBTAG00000027312 & $3.33 \mathrm{E}-10$ & $1.35 \mathrm{E}-10$ & ECT2L & $2.46 \mathrm{E}+00$ \\
\hline ENSBTAG00000016498 & $3.16 \mathrm{E}-10$ & $1.28 \mathrm{E}-10$ & PHACTR2 & $2.47 E+00$ \\
\hline ENSBTAG00000000919 & $1.61 \mathrm{E}-10$ & $6.46 \mathrm{E}-11$ & HEY2 & $2.49 E+00$ \\
\hline ENSBTAG00000016374 & $2.65 \mathrm{E}-10$ & $1.05 \mathrm{E}-10$ & OGFRL1 & $2.52 \mathrm{E}+00$ \\
\hline ENSBTAG00000020817 & $2.95 \mathrm{E}-10$ & $1.16 \mathrm{E}-10$ & B3GAT2 & $2.54 \mathrm{E}+00$ \\
\hline ENSBTAG00000026578 & $2.60 \mathrm{E}-10$ & $9.94 \mathrm{E}-11$ & & $2.61 E+00$ \\
\hline ENSBTAG00000005026 & $4.83 \mathrm{E}-10$ & $1.84 \mathrm{E}-10$ & MANEA & $2.62 E+00$ \\
\hline ENSBTAG00000014494 & $9.98 \mathrm{E}-10$ & $3.64 \mathrm{E}-10$ & & $2.74 \mathrm{E}+00$ \\
\hline ENSBTAG00000000816 & $3.83 \mathrm{E}-10$ & $1.39 \mathrm{E}-10$ & PRDM1 & $2.76 \mathrm{E}+00$ \\
\hline ENSBTAG00000046282 & $9.90 \mathrm{E}-11$ & $3.56 \mathrm{E}-11$ & VGLL2 & $2.78 \mathrm{E}+00$ \\
\hline ENSBTAG00000026604 & $2.77 \mathrm{E}-10$ & $9.94 \mathrm{E}-11$ & LYRM2 & $2.78 \mathrm{E}+00$ \\
\hline ENSBTAG00000038891 & $1.50 \mathrm{E}-09$ & $5.35 \mathrm{E}-10$ & & $2.81 E+00$ \\
\hline ENSBTAG00000047600 & $3.71 \mathrm{E}-10$ & $1.30 \mathrm{E}-10$ & ROS1 & $2.85 E+00$ \\
\hline ENSBTAG00000013454 & $7.45 \mathrm{E}-11$ & $2.61 E-11$ & TAB2 & $2.85 E+00$ \\
\hline ENSBTAG00000012931 & $1.98 \mathrm{E}-10$ & $6.71 \mathrm{E}-11$ & PLN & $2.95 \mathrm{E}+00$ \\
\hline ENSBTAG00000010134 & $2.37 \mathrm{E}-10$ & 7.94E-11 & OSTM1 & $2.98 \mathrm{E}+00$ \\
\hline ENSBTAG00000002964 & $4.93 \mathrm{E}-10$ & $1.58 \mathrm{E}-10$ & TXLNB & $3.12 E+00$ \\
\hline ENSBTAG00000036087 & $4.45 \mathrm{E}-10$ & $1.42 \mathrm{E}-10$ & ARMC2 & $3.13 E+00$ \\
\hline ENSBTAG00000004269 & $1.18 \mathrm{E}-10$ & $3.71 \mathrm{E}-11$ & SGK1 & $3.17 \mathrm{E}+00$ \\
\hline ENSBTAG00000036273 & $1.77 \mathrm{E}-10$ & $5.55 \mathrm{E}-11$ & & $3.18 \mathrm{E}+00$ \\
\hline ENSBTAG00000015076 & $1.90 \mathrm{E}-10$ & 5.97E-11 & LATS1 & $3.18 \mathrm{E}+00$ \\
\hline ENSBTAG00000003878 & $6.10 \mathrm{E}-10$ & $1.91 \mathrm{E}-10$ & ZUFSP & $3.19 E+00$ \\
\hline ENSBTAG00000027879 & $6.49 \mathrm{E}-10$ & $2.01 \mathrm{E}-10$ & & $3.23 E+00$ \\
\hline ENSBTAG00000005851 & $5.28 \mathrm{E}-10$ & $1.62 \mathrm{E}-10$ & VTA1 & $3.26 \mathrm{E}+00$ \\
\hline ENSBTAG00000005449 & $7.41 \mathrm{E}-10$ & $2.27 \mathrm{E}-10$ & MCHR2 & $3.26 \mathrm{E}+00$ \\
\hline ENSBTAG00000003342 & $9.61 \mathrm{E}-11$ & $2.90 \mathrm{E}-11$ & AMD1 & $3.31 E+00$ \\
\hline ENSBTAG00000009689 & 4.63E-10 & $1.40 \mathrm{E}-10$ & RWDD1 & $3.31 E+00$ \\
\hline ENSBTAG00000035054 & $5.96 \mathrm{E}-10$ & $1.76 \mathrm{E}-10$ & COL9A1 & $3.39 E+00$ \\
\hline ENSBTAG00000015615 & $3.55 \mathrm{E}-10$ & $1.03 \mathrm{E}-10$ & THEMIS & $3.44 \mathrm{E}+00$ \\
\hline ENSBTAG00000043975 & $5.51 \mathrm{E}-10$ & $1.60 \mathrm{E}-10$ & MYCT1 & $3.44 \mathrm{E}+00$ \\
\hline ENSBTAG00000033803 & $2.82 \mathrm{E}-10$ & $8.18 \mathrm{E}-11$ & FABP7 & $3.44 \mathrm{E}+00$ \\
\hline ENSBTAG00000033460 & $6.77 \mathrm{E}-10$ & $1.91 \mathrm{E}-10$ & FAM162B & $3.55 E+00$ \\
\hline ENSBTAG00000035370 & $1.14 \mathrm{E}-10$ & $3.14 \mathrm{E}-11$ & TBPL1 & $3.63 E+00$ \\
\hline ENSBTAG00000047479 & 2.03E-09 & $5.48 \mathrm{E}-10$ & & $3.70 E+00$ \\
\hline ENSBTAG00000003015 & $2.43 \mathrm{E}-10$ & $6.50 \mathrm{E}-11$ & SESN1 & $3.74 \mathrm{E}+00$ \\
\hline ENSBTAG00000004098 & $2.61 \mathrm{E}-10$ & $6.87 E-11$ & ARMT1 & $3.80 E+00$ \\
\hline
\end{tabular}




\begin{tabular}{|c|c|c|c|c|}
\hline ENSBTAG00000010196 & $1.55 \mathrm{E}-10$ & $4.04 \mathrm{E}-11$ & NUP43 & $3.83 E+00$ \\
\hline ENSBTAG00000012929 & $4.12 \mathrm{E}-10$ & $9.80 \mathrm{E}-11$ & CEP85L & $4.21 \mathrm{E}+00$ \\
\hline ENSBTAG00000000243 & $3.87 \mathrm{E}-10$ & $9.11 \mathrm{E}-11$ & HEBP2 & $4.24 E+00$ \\
\hline ENSBTAG00000046503 & $6.31 \mathrm{E}-10$ & $1.48 \mathrm{E}-10$ & & $4.26 \mathrm{E}+00$ \\
\hline ENSBTAG00000006549 & $3.96 \mathrm{E}-10$ & $9.03 \mathrm{E}-11$ & FAM26F & $4.38 \mathrm{E}+00$ \\
\hline ENSBTAG00000014495 & $6.43 \mathrm{E}-10$ & 1.47E-10 & FGFR1OP & $4.37 E+00$ \\
\hline ENSBTAG00000045879 & $3.24 \mathrm{E}-10$ & 7.19E-11 & FAM26E & $4.50 \mathrm{E}+00$ \\
\hline ENSBTAG00000000257 & $6.07 \mathrm{E}-10$ & $1.35 \mathrm{E}-10$ & MRAP2 & $4.49 E+00$ \\
\hline ENSBTAG00000003884 & $1.99 \mathrm{E}-10$ & $4.28 \mathrm{E}-11$ & FAM120B & $4.65 E+00$ \\
\hline ENSBTAG00000038483 & $4.78 \mathrm{E}-10$ & $1.00 \mathrm{E}-10$ & & $4.78 \mathrm{E}+00$ \\
\hline ENSBTAG00000005400 & $2.18 \mathrm{E}-10$ & $4.56 \mathrm{E}-11$ & & $4.76 \mathrm{E}+00$ \\
\hline ENSBTAG00000040509 & $4.26 \mathrm{E}-10$ & $8.92 \mathrm{E}-11$ & TAAR6 & $4.77 E+00$ \\
\hline ENSBTAG00000034925 & $7.85 \mathrm{E}-10$ & $1.64 \mathrm{E}-10$ & KHDC3L & $4.78 \mathrm{E}+00$ \\
\hline ENSBTAG00000031701 & $6.08 \mathrm{E}-10$ & $1.20 \mathrm{E}-10$ & TAGAP & $5.07 E+00$ \\
\hline ENSBTAG00000013224 & $3.52 \mathrm{E}-10$ & $6.61 \mathrm{E}-11$ & RMND1 & $5.33 E+00$ \\
\hline ENSBTAG00000000409 & 3.67E-10 & $6.87 \mathrm{E}-11$ & $\mathrm{CDC} 40$ & $5.34 \mathrm{E}+00$ \\
\hline ENSBTAG00000016627 & $1.80 \mathrm{E}-10$ & $3.36 \mathrm{E}-11$ & WASF1 & $5.35 E+00$ \\
\hline ENSBTAG00000006672 & $1.53 \mathrm{E}-10$ & $2.75 \mathrm{E}-11$ & SYNCRIP & $5.54 \mathrm{E}+00$ \\
\hline ENSBTAG00000008159 & $1.99 \mathrm{E}-10$ & $3.45 \mathrm{E}-11$ & $\mathrm{ZC} 2 \mathrm{HC} 1 \mathrm{~B}$ & $5.76 \mathrm{E}+00$ \\
\hline ENSBTAG00000018408 & $2.43 \mathrm{E}-10$ & 3.93E-11 & SNX14 & $6.17 E+00$ \\
\hline ENSBTAG00000045726 & $1.75 \mathrm{E}-10$ & $2.75 \mathrm{E}-11$ & & $6.35 E+00$ \\
\hline ENSBTAG00000000245 & $2.73 \mathrm{E}-10$ & $4.23 \mathrm{E}-11$ & NHSL1 & $6.45 E+00$ \\
\hline ENSBTAG00000043954 & $6.21 \mathrm{E}-10$ & $8.79 E-11$ & NMBR & $7.06 \mathrm{E}+00$ \\
\hline ENSBTAG00000002868 & $3.10 \mathrm{E}-10$ & $4.33 \mathrm{E}-11$ & GPR6 & $7.14 E+00$ \\
\hline ENSBTAG00000014790 & $3.31 \mathrm{E}-10$ & 4.54E-11 & ZBTB2 & $7.28 \mathrm{E}+00$ \\
\hline ENSBTAG00000014788 & 4.65E-10 & $6.26 \mathrm{E}-11$ & AKAP12 & $7.42 \mathrm{E}+00$ \\
\hline ENSBTAG00000024381 & $6.39 \mathrm{E}-10$ & 8.17E-11 & MAN1A1 & $7.82 \mathrm{E}+00$ \\
\hline ENSBTAG00000039058 & $7.94 \mathrm{E}-10$ & $9.94 \mathrm{E}-11$ & & $7.98 \mathrm{E}+00$ \\
\hline ENSBTAG00000014496 & $8.15 E-10$ & $9.27 \mathrm{E}-11$ & CCR6 & $8.78 \mathrm{E}+00$ \\
\hline ENSBTAG00000017166 & $6.43 \mathrm{E}-10$ & $6.40 \mathrm{E}-11$ & C6orf58 & $1.00 \mathrm{E}+01$ \\
\hline ENSBTAG00000008600 & $4.36 \mathrm{E}-10$ & $4.21 \mathrm{E}-11$ & TRAF3IP2 & $1.03 \mathrm{E}+01$ \\
\hline ENSBTAG00000001747 & $6.19 \mathrm{E}-10$ & 5.47E-11 & PEX3 & $1.13 \mathrm{E}+01$ \\
\hline ENSBTAG00000001752 & $6.07 \mathrm{E}-10$ & $3.19 \mathrm{E}-11$ & FUCA2 & $1.90 \mathrm{E}+01$ \\
\hline ENSBTAG00000047687 & $8.60 \mathrm{E}-11$ & 0 & & $8.61 E+02$ \\
\hline ENSBTAG00000019600 & $2.09 \mathrm{E}-10$ & 0 & OOEP & $2.09 \mathrm{E}+03$ \\
\hline ENSBTAG00000047004 & $1.65 \mathrm{E}-10$ & 0 & UMAD1 & $1.65 E+03$ \\
\hline ENSBTAG00000047280 & 0 & 0 & & $1.00 \mathrm{E}+00$ \\
\hline ENSBTAG00000004890 & 0 & 0 & & $1.00 \mathrm{E}+00$ \\
\hline ENSBTAG00000047011 & 0 & 0 & & $1.00 \mathrm{E}+00$ \\
\hline ENSBTAG00000046168 & 0 & 0 & & $1.00 \mathrm{E}+00$ \\
\hline ENSBTAG00000016793 & 0 & 0 & SLC35D3 & $1.00 \mathrm{E}+00$ \\
\hline ENSBTAG00000026580 & 0 & 0 & HTR1E & $1.00 \mathrm{E}+00$ \\
\hline ENSBTAG00000021446 & 0 & 0 & & $1.00 \mathrm{E}+00$ \\
\hline ENSBTAG00000047327 & $7.98 \mathrm{E}-11$ & 0 & & $7.99 \mathrm{E}+02$ \\
\hline ENSBTAG00000026523 & $1.34 \mathrm{E}-10$ & 0 & PLAGL1 & $1.34 \mathrm{E}+03$ \\
\hline ENSBTAG00000006398 & 0 & 0 & TOMM7 & $1.00 \mathrm{E}+00$ \\
\hline ENSBTAG00000016405 & 0 & 0 & & $1.00 \mathrm{E}+00$ \\
\hline ENSBTAG00000014497 & $2.62 \mathrm{E}-10$ & 0 & GPR31 & $2.62 E+03$ \\
\hline ENSBTAG00000011735 & $2.26 \mathrm{E}-10$ & 0 & & $2.26 \mathrm{E}+03$ \\
\hline ENSBTAG00000039456 & $1.56 \mathrm{E}-10$ & 0 & & $1.56 \mathrm{E}+03$ \\
\hline ENSBTAG00000045504 & 0 & 0 & & $1.00 \mathrm{E}+00$ \\
\hline ENSBTAG00000023792 & $3.75 E-10$ & 0 & RPS19 & $3.75 E+03$ \\
\hline ENSBTAG00000030490 & $1.59 \mathrm{E}-09$ & 0 & & $1.59 \mathrm{E}+04$ \\
\hline ENSBTAG00000002915 & $3.08 \mathrm{E}-10$ & 0 & GPR63 & $3.08 \mathrm{E}+03$ \\
\hline ENSBTAG00000047483 & 0 & $2.00 \mathrm{E}-10$ & & 5.00E-04 \\
\hline ENSBTAG00000038188 & 0 & 1.44E-09 & & 6.94E-05 \\
\hline ENSBTAG00000011985 & $1.94 \mathrm{E}-10$ & $1.78 \mathrm{E}-09$ & & 1.09E-01 \\
\hline ENSBTAG00000039353 & $4.51 \mathrm{E}-11$ & $3.42 \mathrm{E}-10$ & & $1.32 \mathrm{E}-01$ \\
\hline ENSBTAG00000005214 & $1.42 \mathrm{E}-10$ & $1.03 \mathrm{E}-09$ & LCTL & $1.38 \mathrm{E}-01$ \\
\hline ENSBTAG00000008792 & $1.10 \mathrm{E}-10$ & $6.24 \mathrm{E}-10$ & RNASE6 & $1.76 \mathrm{E}-01$ \\
\hline ENSBTAG00000009642 & $6.36 \mathrm{E}-11$ & $3.21 \mathrm{E}-10$ & AKAP5 & $1.98 \mathrm{E}-01$ \\
\hline ENSBTAG00000047566 & $1.75 \mathrm{E}-10$ & $6.62 \mathrm{E}-10$ & & 2.64E-01 \\
\hline ENSBTAG00000008793 & $5.18 \mathrm{E}-11$ & $1.96 \mathrm{E}-10$ & & 2.64E-01 \\
\hline ENSBTAG00000011987 & $1.24 \mathrm{E}-10$ & $4.46 \mathrm{E}-10$ & C14orf1 & $2.78 \mathrm{E}-01$ \\
\hline ENSBTAG00000011567 & $1.22 \mathrm{E}-10$ & 4.40E-10 & VPS18 & $2.78 \mathrm{E}-01$ \\
\hline ENSBTAG00000035758 & $3.51 \mathrm{E}-10$ & 1.11E-09 & OR4K15 & $3.16 \mathrm{E}-01$ \\
\hline ENSBTAG00000003835 & 4.39E-11 & $1.38 \mathrm{E}-10$ & BMP4 & 3.18E-01 \\
\hline ENSBTAG00000020327 & $1.60 \mathrm{E}-10$ & $4.69 \mathrm{E}-10$ & ZNF410 & $3.41 \mathrm{E}-01$ \\
\hline ENSBTAG00000017177 & $4.42 \mathrm{E}-11$ & $1.24 \mathrm{E}-10$ & RBM25 & 3.57E-01 \\
\hline ENSBTAG00000003747 & $1.24 \mathrm{E}-10$ & $3.32 \mathrm{E}-10$ & & 3.73E-01 \\
\hline
\end{tabular}




\begin{tabular}{|c|c|c|c|c|}
\hline ENSBTAG00000010239 & $2.36 \mathrm{E}-10$ & $6.30 \mathrm{E}-10$ & FAM71D & $3.74 \mathrm{E}-01$ \\
\hline ENSBTAG00000005213 & $1.30 \mathrm{E}-10$ & $3.27 \mathrm{E}-10$ & ZWILCH & $3.96 \mathrm{E}-01$ \\
\hline ENSBTAG00000013249 & $1.58 \mathrm{E}-11$ & 3.99E-11 & & $3.98 \mathrm{E}-01$ \\
\hline ENSBTAG00000044071 & $7.58 \mathrm{E}-11$ & $1.91 \mathrm{E}-10$ & $\mathrm{HRH} 2$ & 3.97E-01 \\
\hline ENSBTAG00000009271 & $3.09 \mathrm{E}-11$ & $7.78 \mathrm{E}-11$ & PNMA1 & $3.97 \mathrm{E}-01$ \\
\hline ENSBTAG00000001950 & 3.93E-11 & $9.90 \mathrm{E}-11$ & RDH11 & $3.97 \mathrm{E}-01$ \\
\hline ENSBTAG00000045849 & $1.45 \mathrm{E}-10$ & $3.66 \mathrm{E}-10$ & & $3.96 \mathrm{E}-01$ \\
\hline ENSBTAG00000045595 & $1.41 \mathrm{E}-10$ & $3.55 \mathrm{E}-10$ & & $3.96 \mathrm{E}-01$ \\
\hline ENSBTAG00000020480 & $2.68 \mathrm{E}-10$ & $6.11 \mathrm{E}-10$ & SPTLC2 & $4.38 \mathrm{E}-01$ \\
\hline ENSBTAG00000011608 & $1.52 \mathrm{E}-10$ & $3.46 \mathrm{E}-10$ & KIAA1191 & $4.40 \mathrm{E}-01$ \\
\hline ENSBTAG00000034140 & $2.63 \mathrm{E}-10$ & $5.91 \mathrm{E}-10$ & MEGF11 & 4.46E-01 \\
\hline ENSBTAG00000046009 & $3.76 \mathrm{E}-10$ & 7.90E-10 & & $4.75 \mathrm{E}-01$ \\
\hline ENSBTAG00000020201 & 2.44E-10 & 5.00E-10 & S100Z & $4.88 \mathrm{E}-01$ \\
\hline ENSBTAG00000009274 & $1.11 \mathrm{E}-09$ & $2.12 \mathrm{E}-09$ & & $5.25 \mathrm{E}-01$ \\
\hline ENSBTAG00000039950 & $1.94 \mathrm{E}-10$ & $3.66 \mathrm{E}-10$ & & $5.29 \mathrm{E}-01$ \\
\hline ENSBTAG00000025385 & $2.60 \mathrm{E}-10$ & $4.93 \mathrm{E}-10$ & & $5.28 \mathrm{E}-01$ \\
\hline ENSBTAG00000039707 & $9.75 \mathrm{E}-11$ & $1.84 \mathrm{E}-10$ & & 5.30E-01 \\
\hline ENSBTAG00000006416 & 4.66E-11 & $8.82 \mathrm{E}-11$ & EMC4 & $5.29 \mathrm{E}-01$ \\
\hline ENSBTAG00000047305 & $1.78 \mathrm{E}-10$ & 3.37E-10 & & $5.28 \mathrm{E}-01$ \\
\hline ENSBTAG00000011757 & $1.56 \mathrm{E}-10$ & $2.89 \mathrm{E}-10$ & PSEN1 & $5.41 \mathrm{E}-01$ \\
\hline ENSBTAG00000025856 & $1.57 \mathrm{E}-10$ & $2.81 \mathrm{E}-10$ & JMY & 5.60E-01 \\
\hline ENSBTAG00000046668 & $1.87 \mathrm{E}-10$ & 3.32E-10 & & $5.63 \mathrm{E}-01$ \\
\hline ENSBTAG00000004732 & $2.39 \mathrm{E}-10$ & $4.18 \mathrm{E}-10$ & SPTB & $5.71 \mathrm{E}-01$ \\
\hline ENSBTAG00000017683 & $6.94 \mathrm{E}-11$ & 1.19E-10 & LYSMD2 & $5.84 \mathrm{E}-01$ \\
\hline ENSBTAG00000012838 & $8.33 \mathrm{E}-11$ & $1.40 \mathrm{E}-10$ & $\mathrm{RDH} 12$ & $5.95 \mathrm{E}-01$ \\
\hline ENSBTAG00000013741 & $1.22 \mathrm{E}-10$ & 2.04E-10 & CPLX2 & 5.97E-01 \\
\hline ENSBTAG00000047239 & $2.39 \mathrm{E}-10$ & 4.02E-10 & & $5.94 \mathrm{E}-01$ \\
\hline ENSBTAG00000047719 & $1.47 \mathrm{E}-10$ & $2.31 \mathrm{E}-10$ & DRD1 & $6.36 \mathrm{E}-01$ \\
\hline ENSBTAG00000010665 & $2.13 \mathrm{E}-10$ & $3.36 \mathrm{E}-10$ & CBLN3 & $6.34 \mathrm{E}-01$ \\
\hline ENSBTAG00000045492 & $1.98 \mathrm{E}-10$ & $3.12 \mathrm{E}-10$ & & $6.34 \mathrm{E}-01$ \\
\hline ENSBTAG00000004821 & $7.18 \mathrm{E}-10$ & 1.13E-09 & OR4K14 & $6.35 \mathrm{E}-01$ \\
\hline ENSBTAG00000007029 & $7.00 \mathrm{E}-10$ & 1.10E-09 & OR4E2 & $6.36 \mathrm{E}-01$ \\
\hline ENSBTAG00000021392 & $1.24 \mathrm{E}-10$ & $1.96 \mathrm{E}-10$ & DCAF11 & $6.35 \mathrm{E}-01$ \\
\hline ENSBTAG00000000081 & $3.02 \mathrm{E}-10$ & 4.73E-10 & COL4A3BP & $6.40 \mathrm{E}-01$ \\
\hline ENSBTAG00000009983 & $3.16 \mathrm{E}-10$ & $4.80 \mathrm{E}-10$ & KIF23 & $6.58 \mathrm{E}-01$ \\
\hline ENSBTAG00000045863 & $8.59 \mathrm{E}-10$ & 1.30E-09 & TRAV18 & $6.61 \mathrm{E}-01$ \\
\hline ENSBTAG000000002576 & $2.94 \mathrm{E}-10$ & 4.30E-10 & GLDN & $6.85 \mathrm{E}-01$ \\
\hline ENSBTAG00000014948 & $1.56 \mathrm{E}-10$ & 2.27E-10 & NUTM1 & $6.85 \mathrm{E}-01$ \\
\hline ENSBTAG00000014334 & $1.85 \mathrm{E}-10$ & 2.65E-10 & ZFYVE26 & $6.99 \mathrm{E}-01$ \\
\hline ENSBTAG00000040151 & $2.77 \mathrm{E}-10$ & 3.97E-10 & $\mathrm{GCH} 1$ & $6.99 \mathrm{E}-01$ \\
\hline ENSBTAG00000011349 & $2.36 \mathrm{E}-10$ & 3.37E-10 & $\mathrm{CDH} 24$ & $7.02 \mathrm{E}-01$ \\
\hline ENSBTAG00000009911 & $6.27 \mathrm{E}-11$ & $8.83 \mathrm{E}-11$ & HDC & 7.11E-01 \\
\hline ENSBTAG00000006884 & $1.07 \mathrm{E}-10$ & $1.50 \mathrm{E}-10$ & & 7.15E-01 \\
\hline ENSBTAG00000014824 & $1.74 \mathrm{E}-10$ & $2.44 \mathrm{E}-10$ & MMP14 & 7.13E-01 \\
\hline ENSBTAG000000020040 & $2.14 \mathrm{E}-10$ & $2.98 \mathrm{E}-10$ & LPCAT4 & 7.18E-01 \\
\hline ENSBTAG00000005395 & $7.92 \mathrm{E}-10$ & 1.04E-09 & & 7.61E-01 \\
\hline ENSBTAG00000011396 & 7.33E-10 & $9.66 \mathrm{E}-10$ & & 7.59E-01 \\
\hline ENSBTAG00000019612 & 2.07E-10 & $2.71 \mathrm{E}-10$ & RNASE4 & 7.65E-01 \\
\hline ENSBTAG00000012330 & 7.15E-10 & 9.17E-10 & B2M & 7.80E-01 \\
\hline ENSBTAG00000018971 & $1.68 \mathrm{E}-10$ & $2.14 \mathrm{E}-10$ & RAD51B & 7.85E-01 \\
\hline ENSBTAG00000014536 & $6.60 \mathrm{E}-10$ & $8.43 \mathrm{E}-10$ & SFXN1 & 7.83E-01 \\
\hline ENSBTAG00000012726 & $2.53 \mathrm{E}-10$ & $3.18 \mathrm{E}-10$ & PSMB5 & 7.94E-01 \\
\hline ENSBTAG00000030439 & $1.73 \mathrm{E}-10$ & $2.18 \mathrm{E}-10$ & & 7.95E-01 \\
\hline ENSBTAG00000005774 & $3.89 \mathrm{E}-11$ & $4.90 \mathrm{E}-11$ & VRTN & 7.95E-01 \\
\hline ENSBTAG00000011925 & $3.49 \mathrm{E}-10$ & $4.39 \mathrm{E}-10$ & & 7.94E-01 \\
\hline ENSBTAG00000020381 & $5.50 \mathrm{E}-11$ & $6.93 \mathrm{E}-11$ & FCF1 & 7.93E-01 \\
\hline ENSBTAG00000009039 & $3.38 \mathrm{E}-10$ & 4.26E-10 & OR4K5 & 7.93E-01 \\
\hline ENSBTAG00000035309 & $3.41 \mathrm{E}-10$ & 4.30E-10 & OR4F15 & 7.93E-01 \\
\hline ENSBTAG000000033036 & $4.87 \mathrm{E}-10$ & $6.14 \mathrm{E}-10$ & & 7.94E-01 \\
\hline ENSBTAG00000018039 & $8.35 E-10$ & 1.05E-09 & & 7.95E-01 \\
\hline ENSBTAG00000046189 & $1.77 \mathrm{E}-10$ & $2.24 \mathrm{E}-10$ & RNASE13 & 7.92E-01 \\
\hline ENSBTAG00000032789 & $1.77 \mathrm{E}-10$ & $2.24 \mathrm{E}-10$ & OR4N2 & 7.92E-01 \\
\hline ENSBTAG00000032873 & $7.63 \mathrm{E}-10$ & $9.62 \mathrm{E}-10$ & & $7.93 \mathrm{E}-01$ \\
\hline ENSBTAG00000046426 & $5.31 \mathrm{E}-10$ & $6.69 \mathrm{E}-10$ & & 7.94E-01 \\
\hline ENSBTAG00000047222 & $2.09 \mathrm{E}-10$ & $2.64 \mathrm{E}-10$ & & 7.93E-01 \\
\hline ENSBTAG00000045866 & $5.33 \mathrm{E}-10$ & $6.72 \mathrm{E}-10$ & & 7.93E-01 \\
\hline ENSBTAG00000046530 & $1.61 \mathrm{E}-10$ & $2.03 \mathrm{E}-10$ & & 7.95E-01 \\
\hline ENSBTAG00000048058 & $8.92 \mathrm{E}-10$ & $1.12 \mathrm{E}-09$ & & 7.96E-01 \\
\hline ENSBTAG00000047306 & $2.05 \mathrm{E}-09$ & $2.58 \mathrm{E}-09$ & OR6J1 & 7.95E-01 \\
\hline ENSBTAG00000039370 & $9.73 \mathrm{E}-10$ & 1.23E-09 & & $7.91 \mathrm{E}-01$ \\
\hline
\end{tabular}




\begin{tabular}{|c|c|c|c|c|}
\hline ENSBTAG00000012724 & $1.39 \mathrm{E}-10$ & $1.75 \mathrm{E}-10$ & AJUBA & 7.94E-01 \\
\hline ENSBTAG00000019114 & 8.37E-11 & $1.06 \mathrm{E}-10$ & FOXB1 & 7.90E-01 \\
\hline ENSBTAG00000046052 & $7.15 \mathrm{E}-11$ & $9.00 \mathrm{E}-11$ & ANKRD63 & 7.94E-01 \\
\hline ENSBTAG00000030841 & 1.57E-10 & $1.98 \mathrm{E}-10$ & & 7.93E-01 \\
\hline ENSBTAG00000045591 & $3.41 \mathrm{E}-10$ & $4.29 \mathrm{E}-10$ & & $7.94 \mathrm{E}-01$ \\
\hline ENSBTAG00000012728 & $6.31 \mathrm{E}-10$ & 7.95E-10 & PSMB11 & 7.94E-01 \\
\hline ENSBTAG00000032686 & $5.08 \mathrm{E}-11$ & $6.40 \mathrm{E}-11$ & HOMEZ & 7.94E-01 \\
\hline ENSBTAG00000045634 & $3.86 \mathrm{E}-10$ & 4.87E-10 & & 7.94E-01 \\
\hline ENSBTAG00000016381 & $3.17 \mathrm{E}-10$ & 4.00E-10 & REM2 & 7.93E-01 \\
\hline ENSBTAG00000008135 & $6.15 \mathrm{E}-10$ & 7.75E-10 & & 7.93E-01 \\
\hline ENSBTAG00000003549 & $1.32 \mathrm{E}-09$ & 1.67E-09 & & $7.92 \mathrm{E}-01$ \\
\hline ENSBTAG00000039354 & $4.38 \mathrm{E}-10$ & $5.51 \mathrm{E}-10$ & & $7.94 \mathrm{E}-01$ \\
\hline ENSBTAG00000040564 & $4.28 \mathrm{E}-10$ & $5.39 \mathrm{E}-10$ & & $7.94 \mathrm{E}-01$ \\
\hline ENSBTAG00000039462 & $1.36 \mathrm{E}-10$ & $1.72 \mathrm{E}-10$ & & $7.94 \mathrm{E}-01$ \\
\hline ENSBTAG00000008218 & $1.25 \mathrm{E}-10$ & $1.56 \mathrm{E}-10$ & NPTN & $8.00 \mathrm{E}-01$ \\
\hline ENSBTAG00000011076 & $4.32 \mathrm{E}-10$ & $5.35 \mathrm{E}-10$ & KCNN2 & $8.08 \mathrm{E}-01$ \\
\hline ENSBTAG00000017677 & $1.24 \mathrm{E}-10$ & $1.53 \mathrm{E}-10$ & SCG3 & 8.08E-01 \\
\hline ENSBTAG00000019454 & $1.25 \mathrm{E}-10$ & $1.52 \mathrm{E}-10$ & ANGEL1 & $8.22 \mathrm{E}-01$ \\
\hline ENSBTAG00000006463 & $1.48 \mathrm{E}-10$ & $1.81 \mathrm{E}-10$ & DLST & $8.19 \mathrm{E}-01$ \\
\hline ENSBTAG00000014145 & $2.66 \mathrm{E}-10$ & $3.24 \mathrm{E}-10$ & SKOR1 & $8.20 \mathrm{E}-01$ \\
\hline ENSBTAG00000005016 & $4.00 \mathrm{E}-10$ & $4.86 \mathrm{E}-10$ & AP3B1 & $8.24 \mathrm{E}-01$ \\
\hline ENSBTAG00000039884 & 7.53E-10 & $9.07 \mathrm{E}-10$ & & $8.30 \mathrm{E}-01$ \\
\hline ENSBTAG00000007809 & $4.03 \mathrm{E}-10$ & $4.84 \mathrm{E}-10$ & PPP1R36 & $8.32 \mathrm{E}-01$ \\
\hline ENSBTAG00000038234 & $3.29 \mathrm{E}-10$ & 3.95E-10 & BHMT2 & $8.33 \mathrm{E}-01$ \\
\hline ENSBTAG00000012723 & $4.11 \mathrm{E}-10$ & 4.89E-10 & & $8.40 \mathrm{E}-01$ \\
\hline ENSBTAG00000015580 & $3.19 \mathrm{E}-10$ & $3.81 \mathrm{E}-10$ & TLE3 & $8.38 \mathrm{E}-01$ \\
\hline ENSBTAG00000016658 & $6.85 \mathrm{E}-10$ & $8.15 \mathrm{E}-10$ & ABHD4 & $8.41 \mathrm{E}-01$ \\
\hline ENSBTAG00000009030 & $1.02 \mathrm{E}-09$ & $1.22 \mathrm{E}-09$ & NOX5 & $8.38 \mathrm{E}-01$ \\
\hline ENSBTAG00000032799 & $1.43 \mathrm{E}-09$ & 1.69E-09 & & 8.47E-01 \\
\hline ENSBTAG00000011571 & $2.99 \mathrm{E}-10$ & $3.52 \mathrm{E}-10$ & ACIN1 & 8.49E-01 \\
\hline ENSBTAG00000018320 & $5.73 \mathrm{E}-10$ & $6.74 \mathrm{E}-10$ & RPLP1 & $8.51 \mathrm{E}-01$ \\
\hline ENSBTAG00000005694 & $4.09 \mathrm{E}-10$ & $4.76 \mathrm{E}-10$ & TMED10 & $8.58 \mathrm{E}-01$ \\
\hline ENSBTAG00000012737 & $4.72 \mathrm{E}-10$ & 5.50E-10 & GPR65 & 8.59E-01 \\
\hline ENSBTAG00000011644 & $5.65 \mathrm{E}-10$ & $6.56 \mathrm{E}-10$ & & $8.61 \mathrm{E}-01$ \\
\hline ENSBTAG00000013652 & $1.85 \mathrm{E}-10$ & $2.14 \mathrm{E}-10$ & GTF2A1 & 8.64E-01 \\
\hline ENSBTAG00000017489 & $2.47 \mathrm{E}-10$ & $2.84 \mathrm{E}-10$ & TSHR & $8.68 \mathrm{E}-01$ \\
\hline ENSBTAG00000019819 & 4.97E-10 & $5.72 \mathrm{E}-10$ & FUT8 & $8.68 \mathrm{E}-01$ \\
\hline ENSBTAG00000034998 & $1.46 \mathrm{E}-10$ & 1.67E-10 & SIMC1 & $8.72 \mathrm{E}-01$ \\
\hline ENSBTAG00000025803 & $5.31 \mathrm{E}-10$ & $6.10 \mathrm{E}-10$ & C15orf59 & 8.70E-01 \\
\hline ENSBTAG00000001558 & $5.45 \mathrm{E}-10$ & $6.25 \mathrm{E}-10$ & PATL2 & $8.72 \mathrm{E}-01$ \\
\hline ENSBTAG00000014822 & $1.84 \mathrm{E}-10$ & $2.10 \mathrm{E}-10$ & MRPL52 & $8.75 \mathrm{E}-01$ \\
\hline ENSBTAG00000047802 & 9.77E-10 & 1.12E-09 & OR4L1 & $8.72 \mathrm{E}-01$ \\
\hline ENSBTAG00000011680 & $2.10 \mathrm{E}-10$ & $2.41 \mathrm{E}-10$ & PAPLN & $8.71 \mathrm{E}-01$ \\
\hline ENSBTAG00000010944 & $5.23 \mathrm{E}-10$ & 5.99E-10 & LRRC49 & 8.73E-01 \\
\hline ENSBTAG00000002255 & $1.85 \mathrm{E}-10$ & $2.11 \mathrm{E}-10$ & BHMT & 8.77E-01 \\
\hline ENSBTAG00000039618 & $4.82 \mathrm{E}-10$ & $5.48 \mathrm{E}-10$ & & 8.79E-01 \\
\hline ENSBTAG00000010952 & 7.76E-10 & 8.80E-10 & & $8.82 \mathrm{E}-01$ \\
\hline ENSBTAG00000025434 & $1.49 \mathrm{E}-10$ & 1.69E-10 & ZFP36L1 & $8.84 \mathrm{E}-01$ \\
\hline ENSBTAG00000000431 & $1.09 \mathrm{E}-09$ & $1.23 \mathrm{E}-09$ & & $8.88 \mathrm{E}-01$ \\
\hline ENSBTAG00000009151 & $3.98 \mathrm{E}-10$ & $4.50 \mathrm{E}-10$ & PYGO1 & $8.85 \mathrm{E}-01$ \\
\hline ENSBTAG00000012365 & $3.97 \mathrm{E}-10$ & 4.47E-10 & & $8.88 \mathrm{E}-01$ \\
\hline ENSBTAG00000005429 & $3.68 \mathrm{E}-10$ & $4.14 \mathrm{E}-10$ & & $8.88 \mathrm{E}-01$ \\
\hline ENSBTAG00000044029 & $5.91 \mathrm{E}-10$ & $6.66 \mathrm{E}-10$ & AVEN & $8.88 \mathrm{E}-01$ \\
\hline ENSBTAG00000008136 & $5.85 \mathrm{E}-10$ & $6.59 \mathrm{E}-10$ & SNW1 & $8.88 \mathrm{E}-01$ \\
\hline ENSBTAG00000000843 & $5.10 \mathrm{E}-10$ & $5.73 \mathrm{E}-10$ & NDRG2 & $8.90 \mathrm{E}-01$ \\
\hline ENSBTAG00000010270 & 4.67E-10 & $5.23 \mathrm{E}-10$ & & $8.93 \mathrm{E}-01$ \\
\hline ENSBTAG00000039315 & $7.83 \mathrm{E}-10$ & 8.76E-10 & & 8.93E-01 \\
\hline ENSBTAG00000010890 & $3.84 \mathrm{E}-10$ & 4.29E-10 & PRMT5 & 8.94E-01 \\
\hline ENSBTAG00000032801 & $1.56 \mathrm{E}-09$ & $1.75 \mathrm{E}-09$ & OR11H7 & $8.92 \mathrm{E}-01$ \\
\hline ENSBTAG00000013688 & $3.76 \mathrm{E}-10$ & $4.18 \mathrm{E}-10$ & PGF & 8.99E-01 \\
\hline ENSBTAG00000001342 & $5.62 \mathrm{E}-10$ & $6.23 \mathrm{E}-10$ & RSL24D1 & $9.01 \mathrm{E}-01$ \\
\hline ENSBTAG00000011540 & $1.02 \mathrm{E}-10$ & $1.13 \mathrm{E}-10$ & SPG21 & $9.04 \mathrm{E}-01$ \\
\hline ENSBTAG00000019120 & $1.01 \mathrm{E}-10$ & $1.12 \mathrm{E}-10$ & WDHD1 & $9.02 \mathrm{E}-01$ \\
\hline ENSBTAG00000022801 & $1.06 \mathrm{E}-10$ & 1.17E-10 & & $9.08 \mathrm{E}-01$ \\
\hline ENSBTAG00000015930 & $5.94 \mathrm{E}-10$ & $6.56 \mathrm{E}-10$ & DNAL1 & 9.06E-01 \\
\hline ENSBTAG00000000362 & $4.13 \mathrm{E}-10$ & $4.56 \mathrm{E}-10$ & SPG11 & 9.07E-01 \\
\hline ENSBTAG00000039785 & $6.82 \mathrm{E}-10$ & $7.51 \mathrm{E}-10$ & OR11H6 & $9.08 \mathrm{E}-01$ \\
\hline ENSBTAG00000006267 & $7.74 \mathrm{E}-10$ & $8.52 \mathrm{E}-10$ & & $9.08 \mathrm{E}-01$ \\
\hline ENSBTAG00000048302 & $5.23 \mathrm{E}-10$ & $5.76 \mathrm{E}-10$ & & 9.07E-01 \\
\hline ENSBTAG00000007104 & $1.70 \mathrm{E}-10$ & $1.87 \mathrm{E}-10$ & JPH4 & $9.08 \mathrm{E}-01$ \\
\hline
\end{tabular}




\begin{tabular}{|c|c|c|c|c|}
\hline ENSBTAG00000038485 & $6.97 \mathrm{E}-10$ & $7.68 \mathrm{E}-10$ & & $9.08 \mathrm{E}-01$ \\
\hline ENSBTAG00000015385 & $2.40 \mathrm{E}-10$ & $2.64 \mathrm{E}-10$ & CTDSPL2 & $9.09 \mathrm{E}-01$ \\
\hline ENSBTAG00000013836 & $5.60 \mathrm{E}-10$ & $6.14 \mathrm{E}-10$ & & $9.12 \mathrm{E}-01$ \\
\hline ENSBTAG00000038333 & $2.46 \mathrm{E}-10$ & 2.69E-10 & RBM23 & $9.14 \mathrm{E}-01$ \\
\hline ENSBTAG00000015983 & $1.28 \mathrm{E}-09$ & $1.40 \mathrm{E}-09$ & & $9.16 \mathrm{E}-01$ \\
\hline ENSBTAG00000047101 & $2.74 \mathrm{E}-10$ & $2.96 \mathrm{E}-10$ & CCDC177 & $9.25 \mathrm{E}-01$ \\
\hline ENSBTAG00000010236 & 4.05E-10 & 4.37E-10 & CIDEB & 9.27E-01 \\
\hline ENSBTAG00000039789 & $6.05 \mathrm{E}-10$ & $6.53 \mathrm{E}-10$ & & $9.26 \mathrm{E}-01$ \\
\hline ENSBTAG00000008287 & $6.39 \mathrm{E}-11$ & $6.91 \mathrm{E}-11$ & YLPM1 & $9.25 \mathrm{E}-01$ \\
\hline ENSBTAG00000046906 & $6.26 \mathrm{E}-10$ & $6.75 \mathrm{E}-10$ & & $9.27 \mathrm{E}-01$ \\
\hline ENSBTAG00000006688 & $1.85 \mathrm{E}-10$ & $1.99 \mathrm{E}-10$ & RPS6KL1 & $9.27 \mathrm{E}-01$ \\
\hline ENSBTAG00000010235 & 3.87E-10 & $4.18 \mathrm{E}-10$ & NOP9 & $9.27 \mathrm{E}-01$ \\
\hline ENSBTAG00000010052 & 2.62E-10 & $2.82 \mathrm{E}-10$ & EXD2 & $9.27 \mathrm{E}-01$ \\
\hline ENSBTAG00000046814 & $1.60 \mathrm{E}-10$ & $1.73 \mathrm{E}-10$ & & $9.24 \mathrm{E}-01$ \\
\hline ENSBTAG00000019355 & $6.37 \mathrm{E}-10$ & $6.86 \mathrm{E}-10$ & KCNK10 & $9.29 \mathrm{E}-01$ \\
\hline ENSBTAG00000025405 & $1.02 \mathrm{E}-10$ & $1.10 \mathrm{E}-10$ & BATF & $9.29 \mathrm{E}-01$ \\
\hline ENSBTAG00000017525 & $4.01 \mathrm{E}-10$ & 4.29E-10 & ATG12 & $9.34 \mathrm{E}-01$ \\
\hline ENSBTAG00000020329 & $3.85 \mathrm{E}-10$ & $4.11 \mathrm{E}-10$ & FAM161B & $9.36 \mathrm{E}-01$ \\
\hline ENSBTAG00000004218 & $4.26 \mathrm{E}-10$ & 4.54E-10 & SYNDIGIL & $9.38 \mathrm{E}-01$ \\
\hline ENSBTAG00000018255 & $3.58 \mathrm{E}-10$ & $3.82 \mathrm{E}-10$ & ACTN1 & $9.36 \mathrm{E}-01$ \\
\hline ENSBTAG00000020379 & 7.49E-11 & $7.98 \mathrm{E}-11$ & AREL1 & $9.39 \mathrm{E}-01$ \\
\hline ENSBTAG00000030453 & $2.20 \mathrm{E}-10$ & $2.34 \mathrm{E}-10$ & ZC3H14 & $9.41 \mathrm{E}-01$ \\
\hline ENSBTAG00000013992 & $2.48 \mathrm{E}-10$ & $2.63 \mathrm{E}-10$ & ACYP1 & $9.43 \mathrm{E}-01$ \\
\hline ENSBTAG00000000372 & $3.54 \mathrm{E}-10$ & $3.74 \mathrm{E}-10$ & TIPIN & $9.46 \mathrm{E}-01$ \\
\hline ENSBTAG00000025308 & $4.53 \mathrm{E}-10$ & $4.79 \mathrm{E}-10$ & STON2 & $9.45 \mathrm{E}-01$ \\
\hline ENSBTAG00000004558 & $1.35 \mathrm{E}-10$ & $1.42 \mathrm{E}-10$ & C15orf48 & $9.50 \mathrm{E}-01$ \\
\hline ENSBTAG00000007053 & $1.92 \mathrm{E}-10$ & $2.01 \mathrm{E}-10$ & ZFHX2 & 9.53E-01 \\
\hline ENSBTAG00000022915 & $5.41 \mathrm{E}-10$ & $5.68 \mathrm{E}-10$ & OR4Q2 & $9.53 \mathrm{E}-01$ \\
\hline ENSBTAG00000011934 & $2.97 \mathrm{E}-10$ & $3.12 \mathrm{E}-10$ & PCK2 & $9.52 \mathrm{E}-01$ \\
\hline ENSBTAG00000011831 & $1.76 \mathrm{E}-10$ & $1.85 \mathrm{E}-10$ & SPPL2A & $9.51 \mathrm{E}-01$ \\
\hline ENSBTAG00000003469 & $6.83 \mathrm{E}-10$ & 7.14E-10 & CHURC1 & $9.56 \mathrm{E}-01$ \\
\hline ENSBTAG00000006392 & 4.17E-10 & $4.36 \mathrm{E}-10$ & TTC7B & $9.57 \mathrm{E}-01$ \\
\hline ENSBTAG00000008378 & $3.45 \mathrm{E}-10$ & $3.58 \mathrm{E}-10$ & FEM1B & $9.65 \mathrm{E}-01$ \\
\hline ENSBTAG00000039416 & 2.03E-09 & $2.11 \mathrm{E}-09$ & & $9.62 \mathrm{E}-01$ \\
\hline ENSBTAG00000017713 & $3.95 \mathrm{E}-10$ & 4.10E-10 & KTN1 & $9.64 \mathrm{E}-01$ \\
\hline ENSBTAG00000005217 & 3.04E-09 & $3.15 \mathrm{E}-09$ & & $9.66 \mathrm{E}-01$ \\
\hline ENSBTAG00000006703 & $4.28 \mathrm{E}-10$ & 4.42E-10 & PTGDR & $9.69 \mathrm{E}-01$ \\
\hline ENSBTAG00000018277 & $5.07 \mathrm{E}-10$ & $5.22 \mathrm{E}-10$ & TBPL2 & $9.71 \mathrm{E}-01$ \\
\hline ENSBTAG00000025068 & $9.34 \mathrm{E}-10$ & $9.62 \mathrm{E}-10$ & & $9.71 \mathrm{E}-01$ \\
\hline ENSBTAG00000033983 & $3.78 \mathrm{E}-10$ & $3.89 \mathrm{E}-10$ & MAP2K1 & $9.71 \mathrm{E}-01$ \\
\hline ENSBTAG00000020202 & $1.58 \mathrm{E}-10$ & $1.62 \mathrm{E}-10$ & $\mathrm{CRHBP}$ & $9.72 \mathrm{E}-01$ \\
\hline ENSBTAG00000004171 & $2.18 \mathrm{E}-10$ & $2.24 \mathrm{E}-10$ & GPHN & $9.73 \mathrm{E}-01$ \\
\hline ENSBTAG00000047981 & $1.58 \mathrm{E}-09$ & $1.62 \mathrm{E}-09$ & & $9.74 \mathrm{E}-01$ \\
\hline ENSBTAG00000002163 & $1.06 \mathrm{E}-10$ & $1.08 \mathrm{E}-10$ & PTGER2 & $9.81 \mathrm{E}-01$ \\
\hline ENSBTAG00000007374 & $7.88 \mathrm{E}-10$ & $8.06 \mathrm{E}-10$ & LHFPL2 & 9.77E-01 \\
\hline ENSBTAG00000035174 & $2.44 \mathrm{E}-10$ & $2.49 \mathrm{E}-10$ & THOC3 & $9.80 \mathrm{E}-01$ \\
\hline ENSBTAG00000011037 & $7.12 \mathrm{E}-10$ & 7.27E-10 & RBPMS2 & $9.79 \mathrm{E}-01$ \\
\hline ENSBTAG00000007800 & 4.77E-10 & $4.86 \mathrm{E}-10$ & TBC1D21 & $9.81 \mathrm{E}-01$ \\
\hline ENSBTAG00000019924 & $3.17 \mathrm{E}-10$ & $3.24 \mathrm{E}-10$ & DDHD1 & $9.79 \mathrm{E}-01$ \\
\hline ENSBTAG00000008771 & $1.22 \mathrm{E}-10$ & $1.23 \mathrm{E}-10$ & MYEF2 & $9.91 \mathrm{E}-01$ \\
\hline ENSBTAG00000008133 & 7.31E-10 & $7.42 \mathrm{E}-10$ & & $9.86 \mathrm{E}-01$ \\
\hline ENSBTAG00000016283 & $2.61 \mathrm{E}-10$ & $2.65 \mathrm{E}-10$ & TMEM63C & $9.86 \mathrm{E}-01$ \\
\hline ENSBTAG00000025663 & $2.65 \mathrm{E}-10$ & $2.67 \mathrm{E}-10$ & RAC1 & $9.94 \mathrm{E}-01$ \\
\hline ENSBTAG00000021988 & $1.18 \mathrm{E}-10$ & $1.19 \mathrm{E}-10$ & SOCS4 & $9.89 \mathrm{E}-01$ \\
\hline ENSBTAG00000020937 & $2.36 \mathrm{E}-10$ & $2.38 \mathrm{E}-10$ & SNAPC1 & $9.90 \mathrm{E}-01$ \\
\hline ENSBTAG00000040056 & $2.85 \mathrm{E}-10$ & $2.87 \mathrm{E}-10$ & LTB4R2 & $9.91 \mathrm{E}-01$ \\
\hline ENSBTAG00000027162 & $1.71 \mathrm{E}-10$ & $1.72 \mathrm{E}-10$ & TMCO5A & $9.94 \mathrm{E}-01$ \\
\hline ENSBTAG00000048109 & $4.35 \mathrm{E}-10$ & $4.38 \mathrm{E}-10$ & & 9.93E-01 \\
\hline ENSBTAG00000015202 & $1.70 \mathrm{E}-10$ & $1.71 \mathrm{E}-10$ & ATP5S & 9.93E-01 \\
\hline ENSBTAG00000018919 & 4.39E-10 & $4.42 \mathrm{E}-10$ & OR5AU1 & $9.93 \mathrm{E}-01$ \\
\hline ENSBTAG00000046157 & $8.80 \mathrm{E}-10$ & $8.85 \mathrm{E}-10$ & & $9.94 \mathrm{E}-01$ \\
\hline ENSBTAG00000040551 & $1.14 \mathrm{E}-10$ & $1.15 \mathrm{E}-10$ & ZBTB1 & $9.92 \mathrm{E}-01$ \\
\hline ENSBTAG00000017396 & $5.95 \mathrm{E}-10$ & $5.99 \mathrm{E}-10$ & & $9.93 \mathrm{E}-01$ \\
\hline ENSBTAG00000002710 & $1.67 \mathrm{E}-10$ & $1.69 \mathrm{E}-10$ & TSSK4 & $9.90 \mathrm{E}-01$ \\
\hline ENSBTAG00000000218 & $3.55 \mathrm{E}-10$ & $3.56 \mathrm{E}-10$ & MAP2K5 & 9.97E-01 \\
\hline ENSBTAG00000009643 & $2.39 \mathrm{E}-10$ & $2.39 \mathrm{E}-10$ & ZBTB25 & $9.99 \mathrm{E}-01$ \\
\hline ENSBTAG00000008083 & $3.87 \mathrm{E}-10$ & $3.88 \mathrm{E}-10$ & SEL1L & $9.98 \mathrm{E}-01$ \\
\hline ENSBTAG00000007385 & $6.27 \mathrm{E}-10$ & $6.29 \mathrm{E}-10$ & EMC7 & 9.97E-01 \\
\hline ENSBTAG00000014699 & $2.68 \mathrm{E}-10$ & $2.68 \mathrm{E}-10$ & FAM81A & $9.99 \mathrm{E}-01$ \\
\hline ENSBTAG00000005344 & $1.32 \mathrm{E}-10$ & $1.32 \mathrm{E}-10$ & GNPNAT1 & $1.00 \mathrm{E}+00$ \\
\hline
\end{tabular}




\begin{tabular}{|c|c|c|c|c|}
\hline ENSBTAG00000017442 & $1.40 \mathrm{E}-10$ & $1.39 \mathrm{E}-10$ & CDO1 & $1.01 E+00$ \\
\hline ENSBTAG00000047746 & $1.21 \mathrm{E}-09$ & $1.20 \mathrm{E}-09$ & & $1.01 \mathrm{E}+00$ \\
\hline ENSBTAG00000012408 & $2.70 \mathrm{E}-10$ & $2.67 \mathrm{E}-10$ & & $1.01 E+00$ \\
\hline ENSBTAG00000005426 & $8.91 \mathrm{E}-10$ & $8.81 \mathrm{E}-10$ & PSMC1 & $1.01 \mathrm{E}+00$ \\
\hline ENSBTAG00000012005 & 4.20E-10 & 4.15E-10 & IFT43 & $1.01 E+00$ \\
\hline ENSBTAG00000000737 & $6.94 \mathrm{E}-11$ & $6.86 \mathrm{E}-11$ & DMXL2 & $1.01 \mathrm{E}+00$ \\
\hline ENSBTAG00000019642 & $3.51 \mathrm{E}-10$ & $3.45 \mathrm{E}-10$ & ANKDD1A & $1.02 \mathrm{E}+00$ \\
\hline ENSBTAG00000025778 & $3.25 \mathrm{E}-10$ & $3.18 \mathrm{E}-10$ & EMC9 & $1.02 \mathrm{E}+00$ \\
\hline ENSBTAG00000046970 & 7.95E-10 & 7.78E-10 & & $1.02 \mathrm{E}+00$ \\
\hline ENSBTAG00000045618 & 7.93E-10 & 7.76E-10 & & $1.02 E+00$ \\
\hline ENSBTAG00000013138 & $5.58 \mathrm{E}-10$ & $5.44 \mathrm{E}-10$ & TDP1 & $1.03 E+00$ \\
\hline ENSBTAG00000044049 & $3.84 \mathrm{E}-10$ & $3.75 \mathrm{E}-10$ & ABHD12B & $1.02 \mathrm{E}+00$ \\
\hline ENSBTAG00000014493 & $2.28 \mathrm{E}-10$ & $2.21 \mathrm{E}-10$ & TRIP4 & $1.03 \mathrm{E}+00$ \\
\hline ENSBTAG00000030881 & $2.59 \mathrm{E}-10$ & $2.51 E-10$ & TMEM260 & $1.03 E+00$ \\
\hline ENSBTAG00000003474 & $6.52 \mathrm{E}-10$ & $6.33 \mathrm{E}-10$ & RAB15 & $1.03 \mathrm{E}+00$ \\
\hline ENSBTAG00000012681 & $5.13 \mathrm{E}-10$ & 4.97E-10 & $\mathrm{CORO} 2 \mathrm{~B}$ & $1.03 E+00$ \\
\hline ENSBTAG00000016612 & $1.65 \mathrm{E}-10$ & $1.60 \mathrm{E}-10$ & NEK9 & $1.03 E+00$ \\
\hline ENSBTAG00000030456 & $2.90 \mathrm{E}-10$ & $2.81 \mathrm{E}-10$ & SPATA7 & $1.03 E+00$ \\
\hline ENSBTAG00000012725 & $1.49 \mathrm{E}-10$ & $1.44 \mathrm{E}-10$ & C14orf93 & $1.03 E+00$ \\
\hline ENSBTAG00000003090 & $2.74 \mathrm{E}-10$ & $2.64 \mathrm{E}-10$ & LRRC9 & $1.04 \mathrm{E}+00$ \\
\hline ENSBTAG00000012804 & $3.70 \mathrm{E}-10$ & $3.55 \mathrm{E}-10$ & REEP5 & $1.04 \mathrm{E}+00$ \\
\hline ENSBTAG00000003762 & $5.23 \mathrm{E}-10$ & $5.03 \mathrm{E}-10$ & GNB5 & $1.04 \mathrm{E}+00$ \\
\hline ENSBTAG00000012106 & $6.17 \mathrm{E}-10$ & $5.91 \mathrm{E}-10$ & ADCK1 & $1.04 \mathrm{E}+00$ \\
\hline ENSBTAG00000014092 & $5.00 \mathrm{E}-10$ & 4.77E-10 & CELF6 & $1.05 \mathrm{E}+00$ \\
\hline ENSBTAG00000009641 & $2.00 \mathrm{E}-10$ & $1.91 \mathrm{E}-10$ & MTHFD1 & $1.05 \mathrm{E}+00$ \\
\hline ENSBTAG00000017507 & $9.60 \mathrm{E}-10$ & $9.12 \mathrm{E}-10$ & $\mathrm{SV} 2 \mathrm{C}$ & $1.05 E+00$ \\
\hline ENSBTAG00000005952 & $2.32 \mathrm{E}-10$ & $2.19 \mathrm{E}-10$ & CEBPE & $1.06 \mathrm{E}+00$ \\
\hline ENSBTAG00000008807 & $2.19 \mathrm{E}-10$ & 2.07E-10 & FBXL22 & $1.06 \mathrm{E}+00$ \\
\hline ENSBTAG00000048074 & $6.21 \mathrm{E}-10$ & $5.86 \mathrm{E}-10$ & & $1.06 \mathrm{E}+00$ \\
\hline ENSBTAG00000046897 & $5.87 \mathrm{E}-10$ & $5.54 \mathrm{E}-10$ & & $1.06 \mathrm{E}+00$ \\
\hline ENSBTAG00000001468 & $1.61 \mathrm{E}-10$ & $1.52 \mathrm{E}-10$ & SAMD4A & $1.06 \mathrm{E}+00$ \\
\hline ENSBTAG00000038227 & $7.02 \mathrm{E}-10$ & $6.62 \mathrm{E}-10$ & & $1.06 \mathrm{E}+00$ \\
\hline ENSBTAG00000002712 & $6.59 \mathrm{E}-10$ & $6.22 \mathrm{E}-10$ & & $1.06 \mathrm{E}+00$ \\
\hline ENSBTAG00000014181 & $2.45 \mathrm{E}-10$ & $2.30 \mathrm{E}-10$ & RNF111 & $1.06 \mathrm{E}+00$ \\
\hline ENSBTAG00000015155 & $3.02 \mathrm{E}-10$ & $2.83 \mathrm{E}-10$ & HACD3 & $1.07 \mathrm{E}+00$ \\
\hline ENSBTAG00000031433 & $6.98 \mathrm{E}-10$ & $6.56 \mathrm{E}-10$ & TEX9 & $1.06 \mathrm{E}+00$ \\
\hline ENSBTAG00000006514 & $1.45 \mathrm{E}-09$ & $1.36 \mathrm{E}-09$ & TRAV3 & $1.07 \mathrm{E}+00$ \\
\hline ENSBTAG00000014099 & $2.33 \mathrm{E}-10$ & $2.18 \mathrm{E}-10$ & YTHDC2 & $1.07 \mathrm{E}+00$ \\
\hline ENSBTAG00000003754 & $3.86 \mathrm{E}-10$ & $3.62 \mathrm{E}-10$ & & $1.07 \mathrm{E}+00$ \\
\hline ENSBTAG00000012900 & $1.64 \mathrm{E}-10$ & $1.53 \mathrm{E}-10$ & TMEM229B & $1.07 E+00$ \\
\hline ENSBTAG00000037932 & $3.77 \mathrm{E}-10$ & $3.52 \mathrm{E}-10$ & & $1.07 \mathrm{E}+00$ \\
\hline ENSBTAG00000015158 & $1.59 \mathrm{E}-10$ & $1.48 \mathrm{E}-10$ & VWA9 & $1.07 \mathrm{E}+00$ \\
\hline ENSBTAG00000035030 & 3.67E-10 & $3.41 \mathrm{E}-10$ & TMED7 & $1.08 \mathrm{E}+00$ \\
\hline ENSBTAG00000012803 & $1.52 \mathrm{E}-10$ & $1.40 \mathrm{E}-10$ & & $1.09 E+00$ \\
\hline ENSBTAG00000031981 & $2.30 \mathrm{E}-10$ & $2.13 \mathrm{E}-10$ & LRRC57 & $1.08 \mathrm{E}+00$ \\
\hline ENSBTAG00000048112 & $8.03 E-10$ & $7.42 \mathrm{E}-10$ & GPX2 & $1.08 \mathrm{E}+00$ \\
\hline ENSBTAG00000005697 & $4.18 \mathrm{E}-10$ & $3.84 \mathrm{E}-10$ & MDGA2 & $1.09 E+00$ \\
\hline ENSBTAG00000035662 & $2.28 \mathrm{E}-10$ & $2.10 \mathrm{E}-10$ & & $1.09 \mathrm{E}+00$ \\
\hline ENSBTAG00000037625 & 1.17E-09 & 1.07E-09 & & $1.09 \mathrm{E}+00$ \\
\hline ENSBTAG00000017095 & $9.48 \mathrm{E}-11$ & $8.68 \mathrm{E}-11$ & DENND4A & $1.09 \mathrm{E}+00$ \\
\hline ENSBTAG00000047260 & $3.62 \mathrm{E}-10$ & $3.31 \mathrm{E}-10$ & & $1.09 E+00$ \\
\hline ENSBTAG00000020331 & $4.20 \mathrm{E}-10$ & $3.83 \mathrm{E}-10$ & COQ6 & $1.10 \mathrm{E}+00$ \\
\hline ENSBTAG00000017798 & $6.06 \mathrm{E}-10$ & $5.52 \mathrm{E}-10$ & ERH & $1.10 \mathrm{E}+00$ \\
\hline ENSBTAG00000019496 & $2.64 \mathrm{E}-10$ & $2.40 \mathrm{E}-10$ & CDKN3 & $1.10 \mathrm{E}+00$ \\
\hline ENSBTAG00000002331 & $1.73 \mathrm{E}-10$ & $1.56 \mathrm{E}-10$ & DLGAP5 & $1.11 E+00$ \\
\hline ENSBTAG00000025324 & $5.28 \mathrm{E}-10$ & 4.77E-10 & & $1.11 E+00$ \\
\hline ENSBTAG00000002110 & $2.33 \mathrm{E}-10$ & $2.10 \mathrm{E}-10$ & DMGDH & $1.11 E+00$ \\
\hline ENSBTAG00000015146 & $1.12 \mathrm{E}-10$ & $1.01 \mathrm{E}-10$ & TOX4 & $1.11 E+00$ \\
\hline ENSBTAG00000047001 & 1.01E-09 & $9.07 \mathrm{E}-10$ & & $1.11 E+00$ \\
\hline ENSBTAG00000038544 & $1.06 \mathrm{E}-09$ & $9.47 \mathrm{E}-10$ & & $1.11 E+00$ \\
\hline ENSBTAG00000047974 & $1.43 \mathrm{E}-09$ & 1.27E-09 & & $1.12 \mathrm{E}+00$ \\
\hline ENSBTAG00000025403 & $2.25 \mathrm{E}-10$ & $2.02 \mathrm{E}-10$ & TTLL5 & $1.11 E+00$ \\
\hline ENSBTAG00000009788 & $3.96 \mathrm{E}-10$ & $3.53 \mathrm{E}-10$ & & $1.12 \mathrm{E}+00$ \\
\hline ENSBTAG00000005954 & $3.63 \mathrm{E}-10$ & $3.24 \mathrm{E}-10$ & TERB2 & $1.12 \mathrm{E}+00$ \\
\hline ENSBTAG00000025642 & 4.64E-10 & 4.12E-10 & RYR3 & $1.13 E+00$ \\
\hline ENSBTAG00000018852 & $2.72 \mathrm{E}-10$ & $2.41 \mathrm{E}-10$ & APC & $1.13 E+00$ \\
\hline ENSBTAG00000007114 & $6.80 \mathrm{E}-10$ & $6.01 \mathrm{E}-10$ & POC5 & $1.13 E+00$ \\
\hline ENSBTAG00000040384 & $2.28 \mathrm{E}-10$ & $2.01 \mathrm{E}-10$ & POLR2M & $1.13 E+00$ \\
\hline ENSBTAG00000002709 & 1.65E-10 & $1.45 \mathrm{E}-10$ & & $1.13 \mathrm{E}+00$ \\
\hline ENSBTAG00000021395 & $3.08 \mathrm{E}-10$ & $2.72 \mathrm{E}-10$ & PSME1 & $1.13 E+00$ \\
\hline
\end{tabular}




\begin{tabular}{|c|c|c|c|c|}
\hline ENSBTAG00000007415 & $2.02 \mathrm{E}-10$ & $1.78 \mathrm{E}-10$ & SLC7A8 & $1.14 \mathrm{E}+00$ \\
\hline ENSBTAG00000017665 & $6.28 \mathrm{E}-10$ & $5.52 \mathrm{E}-10$ & & $1.14 \mathrm{E}+00$ \\
\hline ENSBTAG00000039402 & $1.06 \mathrm{E}-09$ & $9.26 \mathrm{E}-10$ & & $1.15 \mathrm{E}+00$ \\
\hline ENSBTAG00000015806 & $2.54 \mathrm{E}-10$ & $2.21 \mathrm{E}-10$ & OAZ2 & $1.15 \mathrm{E}+00$ \\
\hline ENSBTAG00000020477 & $1.43 \mathrm{E}-10$ & $1.25 \mathrm{E}-10$ & AHSA1 & $1.14 \mathrm{E}+00$ \\
\hline ENSBTAG00000005897 & $5.68 \mathrm{E}-10$ & 4.94E-10 & & $1.15 E+00$ \\
\hline ENSBTAG00000006413 & $5.01 \mathrm{E}-10$ & 4.33E-10 & KATNBL1 & $1.16 \mathrm{E}+00$ \\
\hline ENSBTAG00000021872 & $2.22 \mathrm{E}-10$ & $1.92 \mathrm{E}-10$ & RASGRP1 & $1.16 \mathrm{E}+00$ \\
\hline ENSBTAG00000025853 & 4.60E-10 & $3.96 \mathrm{E}-10$ & HOMER1 & $1.16 \mathrm{E}+00$ \\
\hline ENSBTAG00000005141 & $5.21 \mathrm{E}-10$ & 4.49E-10 & PELI2 & $1.16 \mathrm{E}+00$ \\
\hline ENSBTAG00000038098 & $2.52 \mathrm{E}-09$ & 2.17E-09 & TRAV29DV5 & $1.16 \mathrm{E}+00$ \\
\hline ENSBTAG00000007518 & $3.24 \mathrm{E}-10$ & $2.77 \mathrm{E}-10$ & TRIM69 & $1.17 E+00$ \\
\hline ENSBTAG00000001948 & 2.25E-10 & $1.93 \mathrm{E}-10$ & VTI1B & $1.17 \mathrm{E}+00$ \\
\hline ENSBTAG00000045604 & $2.85 \mathrm{E}-10$ & $2.44 \mathrm{E}-10$ & TTC9 & $1.17 \mathrm{E}+00$ \\
\hline ENSBTAG00000013031 & $2.72 \mathrm{E}-10$ & $2.32 \mathrm{E}-10$ & TMEM62 & $1.17 \mathrm{E}+00$ \\
\hline ENSBTAG00000008735 & $1.14 \mathrm{E}-10$ & $9.75 E-11$ & VASH1 & $1.17 E+00$ \\
\hline ENSBTAG00000031165 & $1.89 \mathrm{E}-10$ & $1.61 \mathrm{E}-10$ & TRPM7 & $1.17 \mathrm{E}+00$ \\
\hline ENSBTAG00000020421 & $1.16 \mathrm{E}-10$ & $9.81 E-11$ & SUPT16H & $1.18 \mathrm{E}+00$ \\
\hline ENSBTAG00000005663 & $2.66 \mathrm{E}-10$ & $2.26 \mathrm{E}-10$ & HAUS2 & $1.18 \mathrm{E}+00$ \\
\hline ENSBTAG00000011767 & $1.11 \mathrm{E}-10$ & $9.43 \mathrm{E}-11$ & MPP5 & $1.18 \mathrm{E}+00$ \\
\hline ENSBTAG00000037511 & $2.62 \mathrm{E}-10$ & $2.20 \mathrm{E}-10$ & & $1.19 \mathrm{E}+00$ \\
\hline ENSBTAG00000027764 & $1.40 \mathrm{E}-10$ & $1.18 \mathrm{E}-10$ & & $1.19 E+00$ \\
\hline ENSBTAG00000007097 & $1.24 \mathrm{E}-10$ & $1.04 \mathrm{E}-10$ & THTPA & $1.19 \mathrm{E}+00$ \\
\hline ENSBTAG00000016285 & $3.21 \mathrm{E}-10$ & $2.69 \mathrm{E}-10$ & NGB & $1.19 \mathrm{E}+00$ \\
\hline ENSBTAG00000005816 & $2.50 \mathrm{E}-10$ & 2.10E-10 & IRF9 & $1.19 \mathrm{E}+00$ \\
\hline ENSBTAG00000032792 & $5.24 \mathrm{E}-10$ & $4.39 \mathrm{E}-10$ & OR4Q3 & $1.19 E+00$ \\
\hline ENSBTAG00000025644 & $3.12 \mathrm{E}-10$ & $2.62 \mathrm{E}-10$ & CALM1 & $1.19 E+00$ \\
\hline ENSBTAG00000023186 & $6.28 \mathrm{E}-10$ & 5.27E-10 & & $1.19 \mathrm{E}+00$ \\
\hline ENSBTAG00000008664 & $2.45 \mathrm{E}-10$ & $2.06 \mathrm{E}-10$ & EIF2B2 & $1.19 \mathrm{E}+00$ \\
\hline ENSBTAG00000047311 & $8.00 \mathrm{E}-10$ & $6.71 E-10$ & & $1.19 E+00$ \\
\hline ENSBTAG00000039546 & 1.07E-09 & $8.95 \mathrm{E}-10$ & & $1.19 \mathrm{E}+00$ \\
\hline ENSBTAG00000034823 & $4.09 \mathrm{E}-10$ & $3.42 \mathrm{E}-10$ & AGGF1 & $1.20 \mathrm{E}+00$ \\
\hline ENSBTAG00000039807 & $1.25 \mathrm{E}-09$ & $1.04 \mathrm{E}-09$ & & $1.20 \mathrm{E}+00$ \\
\hline ENSBTAG00000019279 & $1.93 \mathrm{E}-10$ & $1.60 \mathrm{E}-10$ & MLH3 & $1.20 \mathrm{E}+00$ \\
\hline ENSBTAG00000005427 & $8.88 \mathrm{E}-10$ & $7.39 \mathrm{E}-10$ & NRDE2 & $1.20 \mathrm{E}+00$ \\
\hline ENSBTAG00000019077 & $1.66 \mathrm{E}-10$ & 1.37E-10 & DAD1 & $1.21 E+00$ \\
\hline ENSBTAG00000024878 & $6.54 \mathrm{E}-10$ & $5.39 \mathrm{E}-10$ & & $1.21 E+00$ \\
\hline ENSBTAG00000005815 & $3.13 E-10$ & $2.57 \mathrm{E}-10$ & & $1.22 \mathrm{E}+00$ \\
\hline ENSBTAG00000046684 & $5.95 \mathrm{E}-10$ & $4.85 \mathrm{E}-10$ & & $1.23 \mathrm{E}+00$ \\
\hline ENSBTAG00000002326 & $1.71 \mathrm{E}-10$ & $1.39 \mathrm{E}-10$ & LGALS3 & $1.23 E+00$ \\
\hline ENSBTAG00000001539 & $1.76 \mathrm{E}-10$ & $1.43 \mathrm{E}-10$ & SAV1 & $1.23 E+00$ \\
\hline ENSBTAG00000007840 & $2.58 \mathrm{E}-10$ & $2.10 \mathrm{E}-10$ & HMGCR & $1.23 \mathrm{E}+00$ \\
\hline ENSBTAG00000001183 & $7.83 \mathrm{E}-10$ & $6.31 \mathrm{E}-10$ & KLHL33 & $1.24 \mathrm{E}+00$ \\
\hline ENSBTAG00000014633 & $1.84 \mathrm{E}-10$ & $1.48 \mathrm{E}-10$ & $A B C D 4$ & $1.24 \mathrm{E}+00$ \\
\hline ENSBTAG00000044207 & $2.25 \mathrm{E}-10$ & $1.81 \mathrm{E}-10$ & RAB2B & $1.24 \mathrm{E}+00$ \\
\hline ENSBTAG00000013616 & $1.49 \mathrm{E}-10$ & $1.20 \mathrm{E}-10$ & AP4E1 & $1.24 \mathrm{E}+00$ \\
\hline ENSBTAG00000008380 & $4.89 \mathrm{E}-10$ & $3.94 \mathrm{E}-10$ & ITGA11 & $1.24 \mathrm{E}+00$ \\
\hline ENSBTAG00000020422 & $1.26 \mathrm{E}-10$ & $1.01 E-10$ & $\mathrm{CHD} 8$ & $1.25 E+00$ \\
\hline ENSBTAG00000011146 & $3.12 \mathrm{E}-10$ & $2.51 E-10$ & RAB8B & $1.24 \mathrm{E}+00$ \\
\hline ENSBTAG00000032518 & $1.78 \mathrm{E}-10$ & $1.42 \mathrm{E}-10$ & SPRED1 & $1.25 \mathrm{E}+00$ \\
\hline ENSBTAG00000020199 & $4.61 \mathrm{E}-10$ & $3.69 \mathrm{E}-10$ & $\mathrm{~F} 2 \mathrm{R}$ & $1.25 \mathrm{E}+00$ \\
\hline ENSBTAG00000012998 & $1.57 \mathrm{E}-10$ & $1.26 \mathrm{E}-10$ & NAA30 & $1.24 \mathrm{E}+00$ \\
\hline ENSBTAG00000010298 & $1.83 \mathrm{E}-10$ & $1.46 \mathrm{E}-10$ & GTF2A2 & $1.25 E+00$ \\
\hline ENSBTAG00000047253 & $9.49 \mathrm{E}-10$ & $7.58 \mathrm{E}-10$ & & $1.25 \mathrm{E}+00$ \\
\hline ENSBTAG00000008805 & $3.44 \mathrm{E}-10$ & $2.75 \mathrm{E}-10$ & USP3 & $1.25 E+00$ \\
\hline ENSBTAG00000005268 & $6.03 \mathrm{E}-10$ & $4.80 \mathrm{E}-10$ & PLA2G4D & $1.26 \mathrm{E}+00$ \\
\hline ENSBTAG00000002842 & $2.29 \mathrm{E}-10$ & $1.83 \mathrm{E}-10$ & HEATR4 & $1.25 E+00$ \\
\hline ENSBTAG00000006613 & $2.32 \mathrm{E}-10$ & $1.83 \mathrm{E}-10$ & METTL17 & $1.27 \mathrm{E}+00$ \\
\hline ENSBTAG00000005814 & $2.01 \mathrm{E}-10$ & $1.58 \mathrm{E}-10$ & PSME2 & $1.27 \mathrm{E}+00$ \\
\hline ENSBTAG00000001423 & $2.24 \mathrm{E}-10$ & $1.76 \mathrm{E}-10$ & & $1.27 E+00$ \\
\hline ENSBTAG00000003737 & $1.24 \mathrm{E}-10$ & $9.74 \mathrm{E}-11$ & SLC51B & $1.27 \mathrm{E}+00$ \\
\hline ENSBTAG00000008411 & $6.01 \mathrm{E}-10$ & 4.73E-10 & PLEKHO2 & $1.27 \mathrm{E}+00$ \\
\hline ENSBTAG00000003763 & 4.10E-10 & 3.22E-10 & MYO5C & $1.27 \mathrm{E}+00$ \\
\hline ENSBTAG00000038060 & 1.33E-09 & 1.05E-09 & & $1.27 E+00$ \\
\hline ENSBTAG00000040504 & 1.19E-09 & 9.35E-10 & TRAV16 & $1.28 \mathrm{E}+00$ \\
\hline ENSBTAG00000033344 & $2.05 \mathrm{E}-10$ & $1.60 \mathrm{E}-10$ & & $1.28 \mathrm{E}+00$ \\
\hline ENSBTAG00000015208 & $5.02 \mathrm{E}-10$ & 3.92E-10 & CARMIL3 & $1.28 \mathrm{E}+00$ \\
\hline ENSBTAG00000003920 & $3.78 \mathrm{E}-10$ & $2.94 \mathrm{E}-10$ & TGM1 & $1.29 \mathrm{E}+00$ \\
\hline ENSBTAG00000032055 & $3.62 \mathrm{E}-10$ & $2.81 \mathrm{E}-10$ & VPS39 & $1.29 \mathrm{E}+00$ \\
\hline ENSBTAG00000000897 & $6.64 \mathrm{E}-10$ & $5.15 \mathrm{E}-10$ & IQGAP2 & $1.29 \mathrm{E}+00$ \\
\hline
\end{tabular}




\begin{tabular}{|c|c|c|c|c|}
\hline ENSBTAG00000005700 & $2.85 \mathrm{E}-10$ & $2.20 \mathrm{E}-10$ & МАРЗК 9 & $1.30 \mathrm{E}+00$ \\
\hline ENSBTAG00000017466 & 3.35E-10 & $2.58 \mathrm{E}-10$ & $\mathrm{HCN} 4$ & $1.30 \mathrm{E}+00$ \\
\hline ENSBTAG00000012004 & $6.10 \mathrm{E}-11$ & $4.69 \mathrm{E}-11$ & TGFB3 & $1.30 \mathrm{E}+00$ \\
\hline ENSBTAG00000013255 & 1.10E-09 & $8.46 E-10$ & & $1.29 \mathrm{E}+00$ \\
\hline ENSBTAG00000014821 & $1.07 \mathrm{E}-10$ & $8.20 \mathrm{E}-11$ & SLC7A7 & $1.30 \mathrm{E}+00$ \\
\hline ENSBTAG00000001898 & $3.96 \mathrm{E}-10$ & $3.04 \mathrm{E}-10$ & CCDC112 & $1.30 \mathrm{E}+00$ \\
\hline ENSBTAG00000014096 & $1.67 \mathrm{E}-10$ & $1.28 \mathrm{E}-10$ & PCNX4 & $1.30 \mathrm{E}+00$ \\
\hline ENSBTAG00000019474 & $2.32 \mathrm{E}-10$ & $1.78 \mathrm{E}-10$ & HERC1 & $1.30 \mathrm{E}+00$ \\
\hline ENSBTAG00000045679 & $5.70 \mathrm{E}-10$ & $4.35 \mathrm{E}-10$ & & $1.31 E+00$ \\
\hline ENSBTAG00000047348 & $1.59 \mathrm{E}-09$ & $1.21 \mathrm{E}-09$ & & $1.31 \mathrm{E}+00$ \\
\hline ENSBTAG00000014501 & $1.55 \mathrm{E}-10$ & $1.18 \mathrm{E}-10$ & FERMT2 & $1.31 E+00$ \\
\hline ENSBTAG00000017811 & $3.58 \mathrm{E}-10$ & $2.72 \mathrm{E}-10$ & SLC39A9 & $1.32 \mathrm{E}+00$ \\
\hline ENSBTAG00000000432 & $9.46 \mathrm{E}-10$ & $7.18 \mathrm{E}-10$ & TRAC & $1.32 \mathrm{E}+00$ \\
\hline ENSBTAG00000009994 & $2.38 \mathrm{E}-10$ & $1.80 \mathrm{E}-10$ & EML5 & $1.32 \mathrm{E}+00$ \\
\hline ENSBTAG00000005751 & $1.60 \mathrm{E}-10$ & $1.21 \mathrm{E}-10$ & CDAN1 & $1.32 \mathrm{E}+00$ \\
\hline ENSBTAG00000007099 & $1.47 \mathrm{E}-10$ & $1.11 \mathrm{E}-10$ & AP1G2 & $1.32 \mathrm{E}+00$ \\
\hline ENSBTAG00000009272 & $1.31 \mathrm{E}-10$ & $9.89 \mathrm{E}-11$ & ELMSAN1 & $1.32 \mathrm{E}+00$ \\
\hline ENSBTAG00000005479 & $2.84 \mathrm{E}-10$ & $2.12 \mathrm{E}-10$ & LIPC & $1.34 \mathrm{E}+00$ \\
\hline ENSBTAG00000025814 & $9.67 \mathrm{E}-10$ & $7.26 \mathrm{E}-10$ & TMEM202 & $1.33 E+00$ \\
\hline ENSBTAG00000001179 & $9.40 \mathrm{E}-10$ & 7.03E-10 & TEP1 & $1.34 \mathrm{E}+00$ \\
\hline ENSBTAG00000016311 & $1.28 \mathrm{E}-10$ & $9.58 \mathrm{E}-11$ & EIF2S1 & $1.34 \mathrm{E}+00$ \\
\hline ENSBTAG00000013638 & $3.99 \mathrm{E}-10$ & $2.98 \mathrm{E}-10$ & $\mathrm{IQCH}$ & $1.34 \mathrm{E}+00$ \\
\hline ENSBTAG00000021945 & $4.28 \mathrm{E}-10$ & $3.19 \mathrm{E}-10$ & NID2 & $1.34 \mathrm{E}+00$ \\
\hline ENSBTAG00000006341 & $2.14 \mathrm{E}-10$ & $1.58 \mathrm{E}-10$ & SLC35F4 & $1.35 E+00$ \\
\hline ENSBTAG00000020205 & $7.74 \mathrm{E}-10$ & $5.73 \mathrm{E}-10$ & PDE8B & $1.35 E+00$ \\
\hline ENSBTAG00000018422 & 3.37E-10 & $2.49 \mathrm{E}-10$ & RIPK3 & $1.35 \mathrm{E}+00$ \\
\hline ENSBTAG00000003043 & $5.26 \mathrm{E}-10$ & $3.88 \mathrm{E}-10$ & GNG2 & $1.35 E+00$ \\
\hline ENSBTAG00000013401 & $3.55 \mathrm{E}-10$ & $2.61 \mathrm{E}-10$ & ARHGEF40 & $1.36 \mathrm{E}+00$ \\
\hline ENSBTAG00000004498 & $2.64 \mathrm{E}-10$ & $1.94 \mathrm{E}-10$ & ESR2 & $1.36 \mathrm{E}+00$ \\
\hline ENSBTAG00000002718 & 3.37E-10 & $2.47 E-10$ & TINF2 & $1.36 \mathrm{E}+00$ \\
\hline ENSBTAG00000011683 & $1.55 \mathrm{E}-10$ & $1.14 \mathrm{E}-10$ & NUMB & $1.36 \mathrm{E}+00$ \\
\hline ENSBTAG00000011225 & 1.19E-10 & $8.67 E-11$ & TMX1 & $1.37 \mathrm{E}+00$ \\
\hline ENSBTAG00000016644 & $4.25 \mathrm{E}-10$ & $3.11 \mathrm{E}-10$ & LVRN & $1.37 \mathrm{E}+00$ \\
\hline ENSBTAG00000021019 & 4.49E-10 & $3.28 \mathrm{E}-10$ & SLC30A4 & $1.37 E+00$ \\
\hline ENSBTAG00000016309 & $9.89 \mathrm{E}-11$ & $7.21 \mathrm{E}-11$ & ATP6V1D & $1.37 \mathrm{E}+00$ \\
\hline ENSBTAG00000018596 & $7.43 \mathrm{E}-10$ & $5.41 \mathrm{E}-10$ & PTPN21 & $1.37 \mathrm{E}+00$ \\
\hline ENSBTAG00000013488 & $1.44 \mathrm{E}-10$ & $1.05 \mathrm{E}-10$ & PPP1R14D & $1.37 \mathrm{E}+00$ \\
\hline ENSBTAG00000004694 & $4.85 \mathrm{E}-10$ & $3.52 \mathrm{E}-10$ & RPGRIP1 & $1.38 \mathrm{E}+00$ \\
\hline ENSBTAG00000010906 & $3.97 \mathrm{E}-10$ & $2.88 \mathrm{E}-10$ & RPS6KA5 & $1.38 \mathrm{E}+00$ \\
\hline ENSBTAG00000039038 & $1.55 \mathrm{E}-09$ & $1.12 \mathrm{E}-09$ & & $1.39 E+00$ \\
\hline ENSBTAG00000014632 & $2.22 \mathrm{E}-10$ & $1.61 \mathrm{E}-10$ & VSX2 & $1.38 \mathrm{E}+00$ \\
\hline ENSBTAG00000017994 & $2.80 \mathrm{E}-10$ & $2.02 \mathrm{E}-10$ & MAX & $1.39 \mathrm{E}+00$ \\
\hline ENSBTAG00000046607 & $8.88 \mathrm{E}-11$ & $6.39 E-11$ & JMJD7 & $1.39 \mathrm{E}+00$ \\
\hline ENSBTAG00000039415 & $1.56 \mathrm{E}-10$ & $1.12 \mathrm{E}-10$ & CHMP4A & $1.39 \mathrm{E}+00$ \\
\hline ENSBTAG00000024752 & $6.12 \mathrm{E}-10$ & $4.39 \mathrm{E}-10$ & OR10G3 & $1.39 E+00$ \\
\hline ENSBTAG00000046061 & $1.21 \mathrm{E}-09$ & $8.67 \mathrm{E}-10$ & & $1.40 \mathrm{E}+00$ \\
\hline ENSBTAG00000004028 & $6.73 \mathrm{E}-10$ & $4.83 \mathrm{E}-10$ & POLK & $1.39 \mathrm{E}+00$ \\
\hline ENSBTAG00000046682 & $1.00 \mathrm{E}-09$ & 7.17E-10 & & $1.40 \mathrm{E}+00$ \\
\hline ENSBTAG00000014027 & $3.70 \mathrm{E}-10$ & $2.66 \mathrm{E}-10$ & TRIM36 & $1.39 \mathrm{E}+00$ \\
\hline ENSBTAG00000020729 & $1.79 \mathrm{E}-10$ & $1.28 \mathrm{E}-10$ & DHRS7 & $1.40 \mathrm{E}+00$ \\
\hline ENSBTAG00000009374 & $7.72 \mathrm{E}-10$ & $5.47 E-10$ & PARP2 & $1.41 E+00$ \\
\hline ENSBTAG00000015904 & $2.41 \mathrm{E}-10$ & $1.70 \mathrm{E}-10$ & RORA & $1.42 \mathrm{E}+00$ \\
\hline ENSBTAG00000005753 & $1.80 \mathrm{E}-10$ & $1.27 \mathrm{E}-10$ & PARP6 & $1.41 \mathrm{E}+00$ \\
\hline ENSBTAG00000016800 & $1.70 \mathrm{E}-10$ & $1.20 \mathrm{E}-10$ & SEMA6D & $1.41 \mathrm{E}+00$ \\
\hline ENSBTAG00000014312 & $1.12 \mathrm{E}-10$ & 7.79E-11 & ATL1 & $1.43 E+00$ \\
\hline ENSBTAG00000007371 & $4.70 \mathrm{E}-10$ & $3.29 \mathrm{E}-10$ & SCAMP1 & $1.43 E+00$ \\
\hline ENSBTAG00000018127 & $6.58 \mathrm{E}-11$ & $4.61 E-11$ & & $1.43 E+00$ \\
\hline ENSBTAG00000046962 & $1.33 \mathrm{E}-09$ & $9.25 \mathrm{E}-10$ & & $1.44 \mathrm{E}+00$ \\
\hline ENSBTAG00000000271 & $3.78 \mathrm{E}-10$ & $2.64 \mathrm{E}-10$ & MNS1 & $1.43 E+00$ \\
\hline ENSBTAG00000010666 & $2.56 \mathrm{E}-10$ & $1.79 \mathrm{E}-10$ & KHNYN & $1.43 E+00$ \\
\hline ENSBTAG00000021538 & $4.14 \mathrm{E}-10$ & $2.89 \mathrm{E}-10$ & MYO1E & $1.43 E+00$ \\
\hline ENSBTAG00000048236 & $1.48 \mathrm{E}-09$ & $1.03 E-09$ & & $1.44 \mathrm{E}+00$ \\
\hline ENSBTAG00000047893 & $8.31 \mathrm{E}-10$ & $5.80 \mathrm{E}-10$ & & $1.43 \mathrm{E}+00$ \\
\hline ENSBTAG00000044151 & $5.92 \mathrm{E}-10$ & 4.12E-10 & ANKDD1B & $1.44 \mathrm{E}+00$ \\
\hline ENSBTAG00000013136 & $3.28 \mathrm{E}-10$ & $2.28 \mathrm{E}-10$ & EFCAB11 & $1.44 \mathrm{E}+00$ \\
\hline ENSBTAG00000035858 & $4.87 \mathrm{E}-10$ & $3.39 \mathrm{E}-10$ & MCC & $1.44 E+00$ \\
\hline ENSBTAG00000004780 & 3.20E-10 & 2.23E-10 & CDKL1 & $1.44 E+00$ \\
\hline ENSBTAG00000045886 & $2.26 \mathrm{E}-10$ & $1.57 \mathrm{E}-10$ & STARD4 & $1.44 \mathrm{E}+00$ \\
\hline ENSBTAG00000005661 & $2.79 \mathrm{E}-10$ & $1.93 \mathrm{E}-10$ & SNAP23 & $1.44 \mathrm{E}+00$ \\
\hline ENSBTAG00000047398 & $8.08 \mathrm{E}-10$ & $5.53 \mathrm{E}-10$ & & $1.46 \mathrm{E}+00$ \\
\hline
\end{tabular}




\begin{tabular}{|c|c|c|c|c|}
\hline ENSBTAG000000000359 & $2.08 \mathrm{E}-10$ & $1.42 \mathrm{E}-10$ & EIF3J & $1.47 \mathrm{E}+00$ \\
\hline ENSBTAG00000018299 & $1.26 \mathrm{E}-10$ & $8.55 \mathrm{E}-11$ & PIGH & $1.47 \mathrm{E}+00$ \\
\hline ENSBTAG000000005269 & $2.27 \mathrm{E}-10$ & $1.53 \mathrm{E}-10$ & CCNB2 & $1.48 \mathrm{E}+00$ \\
\hline ENSBTAG000000025522 & $6.30 \mathrm{E}-10$ & $4.25 \mathrm{E}-10$ & UNC13C & $1.48 \mathrm{E}+00$ \\
\hline ENSBTAG000000015148 & $1.66 \mathrm{E}-10$ & $1.11 \mathrm{E}-10$ & METTL3 & $1.49 \mathrm{E}+00$ \\
\hline ENSBTAG000000002743 & $5.64 \mathrm{E}-10$ & $3.78 \mathrm{E}-10$ & OSGEP & $1.49 E+00$ \\
\hline ENSBTAG000000011379 & $5.00 \mathrm{E}-10$ & 3.35E-10 & & $1.49 E+00$ \\
\hline ENSBTAG000000047984 & $1.37 \mathrm{E}-09$ & 9.17E-10 & & $1.50 \mathrm{E}+00$ \\
\hline ENSBTAG000000005547 & $2.25 \mathrm{E}-10$ & $1.51 \mathrm{E}-10$ & VCPKMT & $1.49 \mathrm{E}+00$ \\
\hline ENSBTAG000000005848 & $1.24 \mathrm{E}-10$ & $8.30 \mathrm{E}-11$ & PRTG & $1.49 \mathrm{E}+00$ \\
\hline ENSBTAG000000010641 & $2.40 \mathrm{E}-10$ & $1.60 \mathrm{E}-10$ & APH1B & $1.50 \mathrm{E}+00$ \\
\hline ENSBTAG000000016236 & $1.55 \mathrm{E}-10$ & $1.03 \mathrm{E}-10$ & SLC12A6 & $1.50 \mathrm{E}+00$ \\
\hline ENSBTAG000000000936 & $5.79 \mathrm{E}-10$ & $3.85 \mathrm{E}-10$ & SLC39A2 & $1.50 \mathrm{E}+00$ \\
\hline ENSBTAG000000021986 & $1.57 \mathrm{E}-10$ & $1.04 \mathrm{E}-10$ & MAPK1IP1L & $1.51 E+00$ \\
\hline ENSBTAG000000014820 & $1.12 \mathrm{E}-10$ & $7.42 \mathrm{E}-11$ & OXA1L & $1.51 E+00$ \\
\hline ENSBTAG000000020334 & $3.19 E-10$ & $2.11 E-10$ & ENTPD5 & $1.51 E+00$ \\
\hline ENSBTAG00000015808 & $4.35 \mathrm{E}-10$ & $2.85 \mathrm{E}-10$ & ZNF609 & $1.53 E+00$ \\
\hline ENSBTAG000000019734 & $1.92 \mathrm{E}-10$ & $1.25 \mathrm{E}-10$ & CD276 & $1.53 E+00$ \\
\hline ENSBTAG000000011494 & $2.53 \mathrm{E}-10$ & $1.64 \mathrm{E}-10$ & PYGL & $1.54 \mathrm{E}+00$ \\
\hline ENSBTAG000000046956 & $1.21 \mathrm{E}-10$ & $7.83 \mathrm{E}-11$ & STYX & $1.55 E+00$ \\
\hline ENSBTAG000000006786 & $5.16 \mathrm{E}-10$ & 3.35E-10 & MED6 & $1.54 \mathrm{E}+00$ \\
\hline ENSBTAG000000014234 & $1.73 \mathrm{E}-10$ & $1.12 \mathrm{E}-10$ & LINC00238 & $1.54 \mathrm{E}+00$ \\
\hline ENSBTAG000000008576 & $1.65 \mathrm{E}-10$ & $1.07 \mathrm{E}-10$ & PLEKHD1 & $1.54 \mathrm{E}+00$ \\
\hline ENSBTAG000000003857 & $1.81 \mathrm{E}-10$ & 1.17E-10 & SUSD6 & $1.54 \mathrm{E}+00$ \\
\hline ENSBTAG000000004430 & $2.01 \mathrm{E}-10$ & $1.29 \mathrm{E}-10$ & SGPP1 & $1.55 E+00$ \\
\hline ENSBTAG000000025755 & $2.49 \mathrm{E}-10$ & $1.60 \mathrm{E}-10$ & RNF212B & $1.55 E+00$ \\
\hline ENSBTAG000000030792 & $3.03 E-10$ & $1.95 \mathrm{E}-10$ & & $1.55 E+00$ \\
\hline ENSBTAG000000002704 & $9.64 \mathrm{E}-11$ & $6.19 \mathrm{E}-11$ & РOMT2 & $1.56 \mathrm{E}+00$ \\
\hline ENSBTAG000000018467 & $2.61 \mathrm{E}-10$ & $1.68 \mathrm{E}-10$ & BBOF1 & $1.56 \mathrm{E}+00$ \\
\hline ENSBTAG000000002179 & $4.56 \mathrm{E}-10$ & $2.92 \mathrm{E}-10$ & C15orf41 & $1.56 \mathrm{E}+00$ \\
\hline ENSBTAG000000012545 & $3.43 \mathrm{E}-10$ & $2.18 \mathrm{E}-10$ & CASC4 & $1.58 \mathrm{E}+00$ \\
\hline ENSBTAG00000011936 & $4.90 \mathrm{E}-10$ & $3.11 E-10$ & ATP8B4 & $1.58 \mathrm{E}+00$ \\
\hline ENSBTAG000000008341 & $4.22 \mathrm{E}-10$ & 2.67E-10 & ARSB & $1.58 \mathrm{E}+00$ \\
\hline ENSBTAG000000025458 & $3.06 \mathrm{E}-10$ & $1.93 \mathrm{E}-10$ & DPF3 & $1.59 E+00$ \\
\hline ENSBTAG00000031697 & $1.58 \mathrm{E}-10$ & $9.98 \mathrm{E}-11$ & KLHDC1 & $1.59 \mathrm{E}+00$ \\
\hline ENSBTAG00000009443 & $3.20 \mathrm{E}-11$ & $2.01 \mathrm{E}-11$ & GCNT3 & $1.59 \mathrm{E}+00$ \\
\hline ENSBTAG00000021394 & $1.83 \mathrm{E}-10$ & $1.15 \mathrm{E}-10$ & FITM1 & $1.59 \mathrm{E}+00$ \\
\hline ENSBTAG00000006792 & $3.63 \mathrm{E}-10$ & $2.29 \mathrm{E}-10$ & EHD4 & $1.59 \mathrm{E}+00$ \\
\hline ENSBTAG00000046470 & $1.90 \mathrm{E}-10$ & $1.20 \mathrm{E}-10$ & ONECUT1 & $1.59 \mathrm{E}+00$ \\
\hline ENSBTAG00000047639 & $2.94 \mathrm{E}-10$ & $1.85 \mathrm{E}-10$ & & $1.59 E+00$ \\
\hline ENSBTAG00000020212 & $6.58 \mathrm{E}-10$ & 4.14E-10 & WDR41 & $1.59 E+00$ \\
\hline ENSBTAG00000038531 & $3.83 \mathrm{E}-10$ & $2.41 E-10$ & & $1.59 \mathrm{E}+00$ \\
\hline ENSBTAG00000009783 & $3.24 \mathrm{E}-10$ & $2.04 \mathrm{E}-10$ & & $1.59 \mathrm{E}+00$ \\
\hline ENSBTAG00000047156 & $2.60 \mathrm{E}-10$ & $1.63 \mathrm{E}-10$ & & $1.59 E+00$ \\
\hline ENSBTAG00000001405 & $1.23 \mathrm{E}-10$ & $7.73 \mathrm{E}-11$ & GLCE & $1.59 E+00$ \\
\hline ENSBTAG00000036202 & $2.83 \mathrm{E}-10$ & $1.78 \mathrm{E}-10$ & & $1.59 \mathrm{E}+00$ \\
\hline ENSBTAG00000002781 & $3.61 \mathrm{E}-10$ & 2.27E-10 & GCNT4 & $1.59 \mathrm{E}+00$ \\
\hline ENSBTAG00000021008 & $5.58 \mathrm{E}-10$ & $3.50 \mathrm{E}-10$ & ZNF219 & $1.59 E+00$ \\
\hline ENSBTAG00000045628 & $1.93 \mathrm{E}-10$ & $1.21 \mathrm{E}-10$ & C15orf61 & $1.59 E+00$ \\
\hline ENSBTAG00000046432 & $4.81 \mathrm{E}-10$ & 3.03E-10 & & $1.59 \mathrm{E}+00$ \\
\hline ENSBTAG00000040459 & 5.19E-10 & $3.26 \mathrm{E}-10$ & & $1.59 \mathrm{E}+00$ \\
\hline ENSBTAG00000009701 & $1.12 \mathrm{E}-10$ & $7.04 \mathrm{E}-11$ & IL25 & $1.59 \mathrm{E}+00$ \\
\hline ENSBTAG00000001870 & $5.53 \mathrm{E}-10$ & $3.47 \mathrm{E}-10$ & CPNE6 & $1.59 \mathrm{E}+00$ \\
\hline ENSBTAG00000046802 & $8.87 \mathrm{E}-10$ & 5.57E-10 & & $1.59 \mathrm{E}+00$ \\
\hline ENSBTAG00000037959 & $1.77 \mathrm{E}-10$ & $1.11 \mathrm{E}-10$ & & $1.59 E+00$ \\
\hline ENSBTAG00000045883 & $8.22 \mathrm{E}-10$ & $5.16 \mathrm{E}-10$ & & $1.59 \mathrm{E}+00$ \\
\hline ENSBTAG00000047909 & $1.18 \mathrm{E}-09$ & 7.37E-10 & & $1.60 \mathrm{E}+00$ \\
\hline ENSBTAG00000046478 & $9.98 \mathrm{E}-10$ & $6.26 \mathrm{E}-10$ & & $1.59 \mathrm{E}+00$ \\
\hline ENSBTAG00000025666 & $9.66 \mathrm{E}-10$ & $6.06 \mathrm{E}-10$ & RPS29 & $1.59 \mathrm{E}+00$ \\
\hline ENSBTAG00000047096 & 1.07E-09 & $6.70 \mathrm{E}-10$ & & $1.60 E+00$ \\
\hline ENSBTAG00000046643 & $1.42 \mathrm{E}-09$ & $8.90 \mathrm{E}-10$ & & $1.60 \mathrm{E}+00$ \\
\hline ENSBTAG00000014011 & $2.45 \mathrm{E}-10$ & $1.53 \mathrm{E}-10$ & TMOD2 & $1.60 \mathrm{E}+00$ \\
\hline ENSBTAG00000010119 & $2.87 \mathrm{E}-10$ & $1.79 \mathrm{E}-10$ & ALDH1A2 & $1.60 \mathrm{E}+00$ \\
\hline ENSBTAG00000005481 & $1.39 \mathrm{E}-10$ & $8.63 \mathrm{E}-11$ & ADAM10 & $1.61 E+00$ \\
\hline ENSBTAG00000022583 & 4.44E-10 & $2.74 \mathrm{E}-10$ & & $1.62 \mathrm{E}+00$ \\
\hline ENSBTAG00000046391 & $4.58 \mathrm{E}-10$ & $2.81 E-10$ & GRAMD2 & $1.63 E+00$ \\
\hline ENSBTAG00000025634 & $3.68 \mathrm{E}-10$ & $2.25 \mathrm{E}-10$ & & $1.63 E+00$ \\
\hline ENSBTAG00000011338 & $1.76 \mathrm{E}-10$ & $1.08 \mathrm{E}-10$ & & $1.63 E+00$ \\
\hline ENSBTAG00000015138 & $1.25 \mathrm{E}-10$ & 7.59E-11 & MGA & $1.65 \mathrm{E}+00$ \\
\hline ENSBTAG00000003276 & $4.59 \mathrm{E}-10$ & $2.77 \mathrm{E}-10$ & PRKCH & $1.66 \mathrm{E}+00$ \\
\hline
\end{tabular}




\begin{tabular}{|c|c|c|c|c|}
\hline ENSBTAG00000021876 & $4.34 \mathrm{E}-10$ & $2.62 \mathrm{E}-10$ & WDR72 & $1.66 \mathrm{E}+00$ \\
\hline ENSBTAG00000021225 & $1.16 \mathrm{E}-10$ & $7.01 \mathrm{E}-11$ & DCAF5 & $1.66 \mathrm{E}+00$ \\
\hline ENSBTAG00000003084 & $2.52 \mathrm{E}-10$ & $1.50 \mathrm{E}-10$ & GPATCH2L & $1.68 \mathrm{E}+00$ \\
\hline ENSBTAG00000003667 & $5.37 \mathrm{E}-10$ & 3.19E-10 & TLN2 & $1.68 \mathrm{E}+00$ \\
\hline ENSBTAG00000015805 & $3.89 \mathrm{E}-10$ & $2.29 \mathrm{E}-10$ & SIPA1L1 & $1.70 E+00$ \\
\hline ENSBTAG00000038920 & $4.74 \mathrm{E}-10$ & $2.80 \mathrm{E}-10$ & VPS13C & $1.69 \mathrm{E}+00$ \\
\hline ENSBTAG00000016255 & $1.86 \mathrm{E}-10$ & $1.10 \mathrm{E}-10$ & PLEK2 & $1.69 \mathrm{E}+00$ \\
\hline ENSBTAG00000008713 & $3.38 \mathrm{E}-10$ & $1.99 \mathrm{E}-10$ & DCAF4 & $1.70 \mathrm{E}+00$ \\
\hline ENSBTAG00000005373 & $2.38 \mathrm{E}-10$ & $1.39 \mathrm{E}-10$ & TPM1 & $1.71 E+00$ \\
\hline ENSBTAG00000030599 & $1.98 \mathrm{E}-10$ & $1.16 \mathrm{E}-10$ & SMOC1 & $1.71 \mathrm{E}+00$ \\
\hline ENSBTAG00000018947 & 4.07E-10 & $2.37 \mathrm{E}-10$ & SYT16 & $1.72 \mathrm{E}+00$ \\
\hline ENSBTAG00000014741 & $2.63 \mathrm{E}-10$ & $1.53 \mathrm{E}-10$ & OTX2 & $1.72 \mathrm{E}+00$ \\
\hline ENSBTAG00000039120 & $6.98 \mathrm{E}-10$ & $4.05 \mathrm{E}-10$ & & $1.72 \mathrm{E}+00$ \\
\hline ENSBTAG00000011022 & $1.27 \mathrm{E}-10$ & $7.36 \mathrm{E}-11$ & ARPP19 & $1.73 E+00$ \\
\hline ENSBTAG00000020469 & $2.50 \mathrm{E}-10$ & $1.44 \mathrm{E}-10$ & TMED8 & $1.73 E+00$ \\
\hline ENSBTAG00000001619 & $5.42 \mathrm{E}-10$ & $3.14 \mathrm{E}-10$ & SERINC5 & $1.73 E+00$ \\
\hline ENSBTAG00000004854 & $2.19 E-10$ & $1.26 \mathrm{E}-10$ & SLC24A5 & $1.74 \mathrm{E}+00$ \\
\hline ENSBTAG00000018297 & $2.79 \mathrm{E}-10$ & $1.61 \mathrm{E}-10$ & PLEKHH1 & $1.73 E+00$ \\
\hline ENSBTAG00000031388 & $1.01 \mathrm{E}-10$ & $5.83 \mathrm{E}-11$ & DYX1C1 & $1.73 E+00$ \\
\hline ENSBTAG00000016823 & $3.33 \mathrm{E}-10$ & $1.92 \mathrm{E}-10$ & CSNK1G1 & $1.73 \mathrm{E}+00$ \\
\hline ENSBTAG00000013043 & $2.29 \mathrm{E}-10$ & $1.31 \mathrm{E}-10$ & AP5M1 & $1.75 E+00$ \\
\hline ENSBTAG00000014792 & $2.25 \mathrm{E}-10$ & $1.29 \mathrm{E}-10$ & MAP4K5 & $1.74 \mathrm{E}+00$ \\
\hline ENSBTAG00000010361 & $9.93 \mathrm{E}-11$ & $5.68 \mathrm{E}-11$ & DLL4 & $1.75 \mathrm{E}+00$ \\
\hline ENSBTAG00000016378 & $3.18 \mathrm{E}-10$ & $1.82 \mathrm{E}-10$ & LRP10 & $1.75 \mathrm{E}+00$ \\
\hline ENSBTAG00000007638 & $2.73 \mathrm{E}-10$ & $1.56 \mathrm{E}-10$ & PIF1 & $1.75 \mathrm{E}+00$ \\
\hline ENSBTAG00000046500 & 1.30E-09 & 7.37E-10 & & $1.76 \mathrm{E}+00$ \\
\hline ENSBTAG00000037452 & 1.05E-09 & $5.95 \mathrm{E}-10$ & & $1.76 \mathrm{E}+00$ \\
\hline ENSBTAG00000009451 & 3.07E-10 & $1.75 \mathrm{E}-10$ & JDP2 & $1.76 \mathrm{E}+00$ \\
\hline ENSBTAG00000027080 & $3.87 \mathrm{E}-10$ & $2.19 \mathrm{E}-10$ & SLC8A3 & $1.77 \mathrm{E}+00$ \\
\hline ENSBTAG00000011439 & 4.13E-10 & $2.32 \mathrm{E}-10$ & EPB42 & $1.78 \mathrm{E}+00$ \\
\hline ENSBTAG00000014750 & $3.52 \mathrm{E}-10$ & $1.98 \mathrm{E}-10$ & EPB41L4A & $1.78 \mathrm{E}+00$ \\
\hline ENSBTAG00000009615 & $4.60 \mathrm{E}-10$ & $2.58 \mathrm{E}-10$ & ANXA2 & $1.78 \mathrm{E}+00$ \\
\hline ENSBTAG00000004926 & $2.04 \mathrm{E}-10$ & $1.14 \mathrm{E}-10$ & LRR1 & $1.79 \mathrm{E}+00$ \\
\hline ENSBTAG00000026909 & $3.44 \mathrm{E}-10$ & $1.92 \mathrm{E}-10$ & & $1.79 \mathrm{E}+00$ \\
\hline ENSBTAG00000046488 & $8.00 \mathrm{E}-10$ & $4.46 \mathrm{E}-10$ & OR4N5 & $1.79 \mathrm{E}+00$ \\
\hline ENSBTAG00000012981 & 8.13E-10 & $4.52 \mathrm{E}-10$ & & $1.80 \mathrm{E}+00$ \\
\hline ENSBTAG00000046993 & $1.42 \mathrm{E}-09$ & $7.90 \mathrm{E}-10$ & & $1.80 \mathrm{E}+00$ \\
\hline ENSBTAG00000045953 & 1.50E-09 & 8.32E-10 & & $1.80 \mathrm{E}+00$ \\
\hline ENSBTAG00000015142 & $1.85 \mathrm{E}-10$ & $1.03 \mathrm{E}-10$ & MAPKBP1 & $1.80 \mathrm{E}+00$ \\
\hline ENSBTAG00000024958 & $1.27 \mathrm{E}-10$ & 7.06E-11 & FAM214A & $1.79 E+00$ \\
\hline ENSBTAG00000002705 & 3.53E-10 & $1.95 \mathrm{E}-10$ & REC8 & $1.81 E+00$ \\
\hline ENSBTAG00000018796 & $3.20 \mathrm{E}-10$ & 1.77E-10 & RABGGTA & $1.80 \mathrm{E}+00$ \\
\hline ENSBTAG00000011820 & $6.46 \mathrm{E}-10$ & 3.57E-10 & DAPK2 & $1.81 E+00$ \\
\hline ENSBTAG00000032613 & $2.64 \mathrm{E}-10$ & $1.46 \mathrm{E}-10$ & SCG5 & $1.81 \mathrm{E}+00$ \\
\hline ENSBTAG00000004934 & $1.85 \mathrm{E}-10$ & $1.02 \mathrm{E}-10$ & NEMF & $1.81 E+00$ \\
\hline ENSBTAG00000002194 & $5.35 \mathrm{E}-10$ & $2.94 \mathrm{E}-10$ & MYZAP & $1.82 \mathrm{E}+00$ \\
\hline ENSBTAG00000011041 & $4.35 \mathrm{E}-11$ & $2.38 \mathrm{E}-11$ & ZFYVE1 & $1.82 \mathrm{E}+00$ \\
\hline ENSBTAG00000021924 & $3.74 \mathrm{E}-10$ & $2.05 \mathrm{E}-10$ & PLA2G4E & $1.83 \mathrm{E}+00$ \\
\hline ENSBTAG00000000898 & $8.46 \mathrm{E}-10$ & 4.63E-10 & F2RL2 & $1.83 \mathrm{E}+00$ \\
\hline ENSBTAG00000046370 & $2.25 \mathrm{E}-09$ & $1.23 \mathrm{E}-09$ & & $1.83 \mathrm{E}+00$ \\
\hline ENSBTAG00000010795 & $2.36 \mathrm{E}-10$ & $1.29 \mathrm{E}-10$ & ATG14 & $1.83 E+00$ \\
\hline ENSBTAG00000047000 & 1.33E-09 & $7.21 \mathrm{E}-10$ & & $1.84 \mathrm{E}+00$ \\
\hline ENSBTAG00000011998 & $2.64 \mathrm{E}-10$ & 1.44E-10 & & $1.83 \mathrm{E}+00$ \\
\hline ENSBTAG00000019692 & $1.18 \mathrm{E}-10$ & $6.37 \mathrm{E}-11$ & $\mathrm{BCL} 2 \mathrm{~L} 2$ & $1.85 \mathrm{E}+00$ \\
\hline ENSBTAG00000020935 & $2.07 \mathrm{E}-10$ & $1.12 \mathrm{E}-10$ & HIF1A & $1.85 \mathrm{E}+00$ \\
\hline ENSBTAG00000035530 & $8.14 \mathrm{E}-10$ & $4.38 \mathrm{E}-10$ & & $1.86 \mathrm{E}+00$ \\
\hline ENSBTAG00000046546 & $1.25 \mathrm{E}-09$ & $6.71 E-10$ & & $1.87 \mathrm{E}+00$ \\
\hline ENSBTAG00000013032 & $3.70 \mathrm{E}-10$ & $1.98 \mathrm{E}-10$ & CCNDBP1 & $1.87 E+00$ \\
\hline ENSBTAG00000000565 & $3.28 \mathrm{E}-10$ & $1.75 \mathrm{E}-10$ & RFX7 & $1.88 \mathrm{E}+00$ \\
\hline ENSBTAG00000025450 & 4.17E-10 & $2.22 \mathrm{E}-10$ & SYNE2 & $1.88 \mathrm{E}+00$ \\
\hline ENSBTAG00000021414 & $5.54 \mathrm{E}-10$ & $2.95 \mathrm{E}-10$ & & $1.88 \mathrm{E}+00$ \\
\hline ENSBTAG00000001551 & $1.96 \mathrm{E}-10$ & $1.04 \mathrm{E}-10$ & PLEKHG3 & $1.88 \mathrm{E}+00$ \\
\hline ENSBTAG00000011916 & $3.43 \mathrm{E}-10$ & $1.81 \mathrm{E}-10$ & USP8 & $1.90 \mathrm{E}+00$ \\
\hline ENSBTAG00000014189 & $4.31 \mathrm{E}-10$ & $2.27 \mathrm{E}-10$ & RGS6 & $1.90 E+00$ \\
\hline ENSBTAG00000001578 & $5.61 \mathrm{E}-10$ & $2.95 \mathrm{E}-10$ & ADPGK & $1.90 \mathrm{E}+00$ \\
\hline ENSBTAG00000021870 & 1.67E-10 & $8.81 E-11$ & FAM98B & $1.90 E+00$ \\
\hline ENSBTAG00000046390 & 1.70E-09 & $8.96 \mathrm{E}-10$ & & $1.90 E+00$ \\
\hline ENSBTAG00000001601 & $2.23 \mathrm{E}-10$ & 1.17E-10 & PKM & $1.91 E+00$ \\
\hline ENSBTAG00000030432 & $3.23 \mathrm{E}-10$ & $1.69 \mathrm{E}-10$ & TTC8 & $1.91 \mathrm{E}+00$ \\
\hline ENSBTAG00000005328 & $2.35 \mathrm{E}-10$ & $1.23 \mathrm{E}-10$ & ICE2 & $1.91 E+00$ \\
\hline
\end{tabular}




\begin{tabular}{|c|c|c|c|c|}
\hline ENSBTAG00000019784 & $3.10 \mathrm{E}-10$ & $1.62 \mathrm{E}-10$ & PPP2R5E & $1.91 E+00$ \\
\hline ENSBTAG00000038672 & 1.07E-09 & $5.53 \mathrm{E}-10$ & & $1.94 \mathrm{E}+00$ \\
\hline ENSBTAG00000009998 & $5.04 \mathrm{E}-10$ & $2.60 \mathrm{E}-10$ & GALNT16 & $1.94 \mathrm{E}+00$ \\
\hline ENSBTAG00000012285 & $3.54 \mathrm{E}-10$ & $1.81 \mathrm{E}-10$ & ESRRB & $1.95 \mathrm{E}+00$ \\
\hline ENSBTAG00000004086 & $1.89 \mathrm{E}-10$ & $9.66 \mathrm{E}-11$ & SPINT1 & $1.95 \mathrm{E}+00$ \\
\hline ENSBTAG00000004990 & $4.33 \mathrm{E}-10$ & $2.21 \mathrm{E}-10$ & NEO1 & $1.96 \mathrm{E}+00$ \\
\hline ENSBTAG00000019500 & $2.04 \mathrm{E}-10$ & $1.04 \mathrm{E}-10$ & CNIH1 & $1.96 \mathrm{E}+00$ \\
\hline ENSBTAG00000025664 & $2.08 \mathrm{E}-09$ & 1.06E-09 & & $1.96 \mathrm{E}+00$ \\
\hline ENSBTAG00000040368 & $5.18 \mathrm{E}-10$ & $2.62 \mathrm{E}-10$ & $A A G A B$ & $1.98 \mathrm{E}+00$ \\
\hline ENSBTAG00000013181 & $2.42 \mathrm{E}-10$ & $1.22 \mathrm{E}-10$ & LRRC74A & $1.98 \mathrm{E}+00$ \\
\hline ENSBTAG00000018419 & $1.94 \mathrm{E}-10$ & $9.74 \mathrm{E}-11$ & ADCY4 & $1.99 \mathrm{E}+00$ \\
\hline ENSBTAG00000039554 & $3.38 \mathrm{E}-10$ & $1.70 \mathrm{E}-10$ & & $1.99 \mathrm{E}+00$ \\
\hline ENSBTAG00000003715 & $1.83 \mathrm{E}-10$ & $9.20 \mathrm{E}-11$ & ADAM21 & $1.98 \mathrm{E}+00$ \\
\hline ENSBTAG00000047105 & $1.71 \mathrm{E}-10$ & 8.60E-11 & & $1.98 \mathrm{E}+00$ \\
\hline ENSBTAG00000002745 & $3.18 \mathrm{E}-10$ & $1.60 \mathrm{E}-10$ & APEX1 & $1.99 \mathrm{E}+00$ \\
\hline ENSBTAG00000004322 & $1.19 \mathrm{E}-10$ & $6.01 E-11$ & FOS & $1.98 \mathrm{E}+00$ \\
\hline ENSBTAG00000006823 & 5.17E-10 & $2.60 \mathrm{E}-10$ & CMYA5 & $1.99 E+00$ \\
\hline ENSBTAG00000022229 & $9.95 \mathrm{E}-10$ & 4.99E-10 & TRAV17 & $1.99 \mathrm{E}+00$ \\
\hline ENSBTAG00000007149 & 4.93E-10 & $2.44 \mathrm{E}-10$ & NYNRIN & $2.02 \mathrm{E}+00$ \\
\hline ENSBTAG00000017200 & $2.35 \mathrm{E}-10$ & $1.16 \mathrm{E}-10$ & RHOJ & $2.03 E+00$ \\
\hline ENSBTAG00000021708 & $3.48 \mathrm{E}-10$ & $1.71 \mathrm{E}-10$ & CEP128 & $2.03 E+00$ \\
\hline ENSBTAG00000001945 & $2.22 \mathrm{E}-10$ & $1.09 \mathrm{E}-10$ & ARG2 & $2.03 E+00$ \\
\hline ENSBTAG00000002707 & $2.78 \mathrm{E}-10$ & $1.35 \mathrm{E}-10$ & IPO4 & $2.06 \mathrm{E}+00$ \\
\hline ENSBTAG00000011929 & $3.76 \mathrm{E}-10$ & $1.83 \mathrm{E}-10$ & USP50 & $2.05 E+00$ \\
\hline ENSBTAG00000020352 & 7.97E-10 & $3.87 \mathrm{E}-10$ & PAQR5 & $2.06 \mathrm{E}+00$ \\
\hline ENSBTAG00000004931 & $1.80 \mathrm{E}-10$ & $8.75 E-11$ & POLE2 & $2.06 \mathrm{E}+00$ \\
\hline ENSBTAG00000012599 & $2.88 \mathrm{E}-10$ & $1.39 \mathrm{E}-10$ & SMAD3 & $2.07 E+00$ \\
\hline ENSBTAG00000046995 & $1.30 \mathrm{E}-09$ & $6.25 \mathrm{E}-10$ & & $2.08 \mathrm{E}+00$ \\
\hline ENSBTAG00000025311 & $1.23 \mathrm{E}-10$ & 5.92E-11 & GMFB & $2.08 \mathrm{E}+00$ \\
\hline ENSBTAG00000032798 & $1.68 \mathrm{E}-09$ & 7.99E-10 & & $2.10 E+00$ \\
\hline ENSBTAG00000013042 & $2.95 \mathrm{E}-10$ & $1.40 \mathrm{E}-10$ & EXOC5 & $2.11 E+00$ \\
\hline ENSBTAG00000004933 & $1.97 \mathrm{E}-10$ & $9.39 \mathrm{E}-11$ & KLHDC2 & $2.10 \mathrm{E}+00$ \\
\hline ENSBTAG00000014392 & $2.29 \mathrm{E}-10$ & $1.09 \mathrm{E}-10$ & ACTR10 & $2.10 \mathrm{E}+00$ \\
\hline ENSBTAG00000001895 & $3.39 \mathrm{E}-10$ & $1.61 \mathrm{E}-10$ & PGGT1B & $2.11 E+00$ \\
\hline ENSBTAG00000010365 & $3.08 \mathrm{E}-10$ & $1.46 \mathrm{E}-10$ & & $2.11 E+00$ \\
\hline ENSBTAG00000001364 & $2.31 \mathrm{E}-10$ & $1.09 \mathrm{E}-10$ & DPH6 & $2.12 \mathrm{E}+00$ \\
\hline ENSBTAG00000018279 & $1.61 \mathrm{E}-10$ & $7.59 \mathrm{E}-11$ & SPATA5L1 & $2.12 \mathrm{E}+00$ \\
\hline ENSBTAG00000013983 & $1.64 \mathrm{E}-10$ & $7.74 \mathrm{E}-11$ & $\mathrm{ZC} 2 \mathrm{HC} 1 \mathrm{C}$ & $2.12 \mathrm{E}+00$ \\
\hline ENSBTAG00000046361 & $1.43 \mathrm{E}-09$ & $6.69 \mathrm{E}-10$ & & $2.13 E+00$ \\
\hline ENSBTAG00000013830 & $1.41 \mathrm{E}-10$ & $6.58 \mathrm{E}-11$ & LEO1 & $2.14 E+00$ \\
\hline ENSBTAG00000011319 & $1.01 \mathrm{E}-10$ & $4.73 E-11$ & SLTM & $2.14 \mathrm{E}+00$ \\
\hline ENSBTAG00000031707 & $3.54 \mathrm{E}-10$ & $1.63 \mathrm{E}-10$ & FRMD6 & $2.17 E+00$ \\
\hline ENSBTAG00000021944 & $2.53 \mathrm{E}-10$ & $1.13 \mathrm{E}-10$ & C14orf166 & $2.24 \mathrm{E}+00$ \\
\hline ENSBTAG00000047672 & $1.26 \mathrm{E}-09$ & $5.62 \mathrm{E}-10$ & & $2.24 \mathrm{E}+00$ \\
\hline ENSBTAG00000010774 & $2.38 \mathrm{E}-10$ & $1.06 \mathrm{E}-10$ & NUSAP1 & $2.25 E+00$ \\
\hline ENSBTAG00000019076 & $4.92 \mathrm{E}-10$ & $2.18 \mathrm{E}-10$ & & $2.26 \mathrm{E}+00$ \\
\hline ENSBTAG00000000328 & $6.45 \mathrm{E}-10$ & $2.86 \mathrm{E}-10$ & TPPP2 & $2.25 \mathrm{E}+00$ \\
\hline ENSBTAG00000011661 & $6.62 \mathrm{E}-10$ & 2.93E-10 & CEP152 & $2.26 \mathrm{E}+00$ \\
\hline ENSBTAG00000000625 & $2.14 \mathrm{E}-10$ & $9.44 \mathrm{E}-11$ & SMAD6 & $2.27 \mathrm{E}+00$ \\
\hline ENSBTAG00000012740 & $1.43 \mathrm{E}-10$ & $6.32 \mathrm{E}-11$ & PIGB & $2.27 \mathrm{E}+00$ \\
\hline ENSBTAG00000010145 & $1.98 \mathrm{E}-10$ & $8.76 \mathrm{E}-11$ & SLC12A1 & $2.26 \mathrm{E}+00$ \\
\hline ENSBTAG00000003172 & $3.02 \mathrm{E}-10$ & $1.32 \mathrm{E}-10$ & MEIS2 & $2.28 \mathrm{E}+00$ \\
\hline ENSBTAG00000012391 & $2.10 \mathrm{E}-10$ & $9.20 \mathrm{E}-11$ & FAM63B & $2.28 \mathrm{E}+00$ \\
\hline ENSBTAG00000021087 & $2.96 \mathrm{E}-10$ & $1.29 \mathrm{E}-10$ & UBR1 & $2.29 \mathrm{E}+00$ \\
\hline ENSBTAG00000025826 & $1.94 \mathrm{E}-10$ & 8.37E-11 & SLC24A1 & $2.31 E+00$ \\
\hline ENSBTAG00000040078 & $3.82 \mathrm{E}-10$ & $1.64 \mathrm{E}-10$ & FLVCR2 & $2.33 E+00$ \\
\hline ENSBTAG00000010103 & $3.47 \mathrm{E}-10$ & $1.48 \mathrm{E}-10$ & TRIM9 & $2.34 \mathrm{E}+00$ \\
\hline ENSBTAG00000000371 & $3.68 \mathrm{E}-10$ & $1.57 \mathrm{E}-10$ & DIS3L & $2.35 E+00$ \\
\hline ENSBTAG00000025632 & $1.40 \mathrm{E}-10$ & $5.88 \mathrm{E}-11$ & NOP10 & $2.38 \mathrm{E}+00$ \\
\hline ENSBTAG00000032709 & $1.99 \mathrm{E}-10$ & $8.34 \mathrm{E}-11$ & CMTM5 & $2.38 \mathrm{E}+00$ \\
\hline ENSBTAG00000016467 & $8.71 \mathrm{E}-11$ & $3.66 \mathrm{E}-11$ & & $2.38 \mathrm{E}+00$ \\
\hline ENSBTAG00000005211 & $3.71 \mathrm{E}-10$ & $1.55 \mathrm{E}-10$ & & $2.39 E+00$ \\
\hline ENSBTAG00000046207 & $5.27 \mathrm{E}-10$ & $2.21 \mathrm{E}-10$ & & $2.38 \mathrm{E}+00$ \\
\hline ENSBTAG00000046305 & $5.26 \mathrm{E}-10$ & $2.20 \mathrm{E}-10$ & & $2.39 E+00$ \\
\hline ENSBTAG00000013493 & $3.76 \mathrm{E}-10$ & $1.58 \mathrm{E}-10$ & BNIP2 & $2.38 \mathrm{E}+00$ \\
\hline ENSBTAG00000033034 & $3.79 \mathrm{E}-10$ & $1.59 \mathrm{E}-10$ & & $2.38 \mathrm{E}+00$ \\
\hline ENSBTAG00000014662 & $5.11 \mathrm{E}-10$ & $2.14 \mathrm{E}-10$ & & $2.39 E+00$ \\
\hline ENSBTAG00000047624 & 4.90E-10 & $2.05 \mathrm{E}-10$ & & $2.39 E+00$ \\
\hline ENSBTAG00000047097 & $4.42 \mathrm{E}-10$ & $1.85 \mathrm{E}-10$ & & $2.39 E+00$ \\
\hline ENSBTAG00000047276 & $8.53 \mathrm{E}-10$ & $3.56 \mathrm{E}-10$ & & $2.40 E+00$ \\
\hline
\end{tabular}




\begin{tabular}{|c|c|c|c|c|}
\hline ENSBTAG00000047058 & 8.97E-10 & $3.75 \mathrm{E}-10$ & & $2.39 E+00$ \\
\hline ENSBTAG00000047041 & $9.20 \mathrm{E}-10$ & $3.84 \mathrm{E}-10$ & & $2.40 E+00$ \\
\hline ENSBTAG00000008575 & $6.57 \mathrm{E}-10$ & $2.74 \mathrm{E}-10$ & CGNL1 & $2.40 E+00$ \\
\hline ENSBTAG00000015716 & $6.80 \mathrm{E}-10$ & $2.82 \mathrm{E}-10$ & ERO1A & $2.41 \mathrm{E}+00$ \\
\hline ENSBTAG00000020475 & $2.05 \mathrm{E}-10$ & $8.46 \mathrm{E}-11$ & VIPAS39 & $2.42 \mathrm{E}+00$ \\
\hline ENSBTAG00000006751 & $2.43 \mathrm{E}-10$ & 9.97E-11 & PAPD4 & $2.44 \mathrm{E}+00$ \\
\hline ENSBTAG00000002843 & $6.27 \mathrm{E}-10$ & $2.56 \mathrm{E}-10$ & ACOT4 & $2.45 E+00$ \\
\hline ENSBTAG00000008699 & 2.07E-10 & $8.44 \mathrm{E}-11$ & FEM1C & $2.45 E+00$ \\
\hline ENSBTAG00000032068 & $1.93 \mathrm{E}-10$ & 7.87E-11 & PLA2G4F & $2.45 \mathrm{E}+00$ \\
\hline ENSBTAG00000001694 & $2.38 \mathrm{E}-10$ & $9.59 \mathrm{E}-11$ & TYRO3 & $2.48 \mathrm{E}+00$ \\
\hline ENSBTAG00000002715 & 4.30E-10 & $1.73 \mathrm{E}-10$ & & $2.48 \mathrm{E}+00$ \\
\hline ENSBTAG00000001881 & $2.15 \mathrm{E}-10$ & $8.56 \mathrm{E}-11$ & SLC10A1 & $2.51 \mathrm{E}+00$ \\
\hline ENSBTAG00000000552 & $3.69 \mathrm{E}-10$ & $1.45 \mathrm{E}-10$ & DISP2 & $2.54 \mathrm{E}+00$ \\
\hline ENSBTAG00000030595 & $4.86 \mathrm{E}-10$ & $1.91 \mathrm{E}-10$ & $\operatorname{cox} 16$ & $2.54 \mathrm{E}+00$ \\
\hline ENSBTAG00000034848 & $1.48 \mathrm{E}-10$ & $5.83 \mathrm{E}-11$ & F2RL1 & $2.54 E+00$ \\
\hline ENSBTAG00000004930 & $1.79 \mathrm{E}-10$ & 7.04E-11 & DNAAF2 & $2.54 \mathrm{E}+00$ \\
\hline ENSBTAG00000012866 & $5.92 \mathrm{E}-10$ & $2.32 \mathrm{E}-10$ & THBS4 & $2.55 \mathrm{E}+00$ \\
\hline ENSBTAG00000006784 & $1.41 \mathrm{E}-10$ & $5.53 \mathrm{E}-11$ & & $2.54 \mathrm{E}+00$ \\
\hline ENSBTAG00000013982 & $2.04 \mathrm{E}-10$ & $8.00 \mathrm{E}-11$ & UACA & $2.55 \mathrm{E}+00$ \\
\hline ENSBTAG00000015732 & $2.42 \mathrm{E}-10$ & $9.44 \mathrm{E}-11$ & PSMC6 & $2.56 \mathrm{E}+00$ \\
\hline ENSBTAG00000010238 & $5.28 \mathrm{E}-10$ & $2.04 \mathrm{E}-10$ & LTB4R & $2.59 E+00$ \\
\hline ENSBTAG00000047690 & 1.97E-09 & $7.46 \mathrm{E}-10$ & & $2.64 \mathrm{E}+00$ \\
\hline ENSBTAG00000006470 & $2.50 \mathrm{E}-10$ & $9.48 \mathrm{E}-11$ & TTBK2 & $2.63 E+00$ \\
\hline ENSBTAG00000046651 & $8.76 \mathrm{E}-10$ & $3.29 \mathrm{E}-10$ & & $2.66 \mathrm{E}+00$ \\
\hline ENSBTAG00000033045 & $1.48 \mathrm{E}-09$ & $5.54 \mathrm{E}-10$ & & $2.67 E+00$ \\
\hline ENSBTAG00000035785 & $7.72 \mathrm{E}-10$ & $2.89 \mathrm{E}-10$ & & $2.67 E+00$ \\
\hline ENSBTAG00000020880 & $3.70 \mathrm{E}-10$ & $1.39 \mathrm{E}-10$ & C14orf37 & $2.66 \mathrm{E}+00$ \\
\hline ENSBTAG00000006790 & $2.10 \mathrm{E}-10$ & 7.71E-11 & SPTBN5 & $2.73 E+00$ \\
\hline ENSBTAG00000011451 & $2.57 \mathrm{E}-10$ & $9.26 \mathrm{E}-11$ & RTN1 & $2.78 \mathrm{E}+00$ \\
\hline ENSBTAG00000002706 & $2.86 \mathrm{E}-10$ & $1.03 \mathrm{E}-10$ & GSTZ1 & $2.77 E+00$ \\
\hline ENSBTAG00000037679 & 4.56E-10 & $1.64 \mathrm{E}-10$ & PPP1R3E & $2.78 \mathrm{E}+00$ \\
\hline ENSBTAG00000047465 & $1.24 \mathrm{E}-09$ & 4.44E-10 & & $2.80 E+00$ \\
\hline ENSBTAG00000047522 & $9.97 \mathrm{E}-10$ & $3.56 \mathrm{E}-10$ & & $2.80 \mathrm{E}+00$ \\
\hline ENSBTAG00000046819 & $9.93 \mathrm{E}-10$ & $3.55 \mathrm{E}-10$ & & $2.80 \mathrm{E}+00$ \\
\hline ENSBTAG00000037924 & $1.47 \mathrm{E}-09$ & $5.23 \mathrm{E}-10$ & & $2.80 \mathrm{E}+00$ \\
\hline ENSBTAG00000020100 & $2.36 \mathrm{E}-10$ & $8.41 \mathrm{E}-11$ & ARHGAP11A & $2.80 E+00$ \\
\hline ENSBTAG00000047952 & $1.26 \mathrm{E}-09$ & 4.49E-10 & & $2.80 \mathrm{E}+00$ \\
\hline ENSBTAG00000007433 & $2.82 \mathrm{E}-10$ & $9.94 \mathrm{E}-11$ & & $2.84 E+00$ \\
\hline ENSBTAG00000012741 & $1.34 \mathrm{E}-10$ & 4.69E-11 & CCPG1 & $2.84 E+00$ \\
\hline ENSBTAG00000008868 & $2.27 \mathrm{E}-10$ & $7.96 \mathrm{E}-11$ & CAPN3 & $2.85 \mathrm{E}+00$ \\
\hline ENSBTAG00000001124 & $6.23 \mathrm{E}-10$ & $2.17 \mathrm{E}-10$ & GALC & $2.87 \mathrm{E}+00$ \\
\hline ENSBTAG00000015052 & $1.07 \mathrm{E}-10$ & 3.67E-11 & PROX2 & $2.90 \mathrm{E}+00$ \\
\hline ENSBTAG00000013774 & $3.00 \mathrm{E}-10$ & $1.03 \mathrm{E}-10$ & C15orf52 & $2.91 E+00$ \\
\hline ENSBTAG00000000767 & $3.93 \mathrm{E}-10$ & $1.34 \mathrm{E}-10$ & DCP2 & $2.93 E+00$ \\
\hline ENSBTAG00000013452 & $9.66 \mathrm{E}-10$ & $3.29 \mathrm{E}-10$ & AQP9 & $2.94 \mathrm{E}+00$ \\
\hline ENSBTAG00000002278 & $2.99 \mathrm{E}-10$ & $1.01 \mathrm{E}-10$ & FBN1 & $2.96 \mathrm{E}+00$ \\
\hline ENSBTAG00000037985 & 7.73E-10 & $2.58 \mathrm{E}-10$ & CHRM5 & $3.00 E+00$ \\
\hline ENSBTAG00000007237 & 4.17E-10 & $1.38 \mathrm{E}-10$ & BUB1B & $3.02 \mathrm{E}+00$ \\
\hline ENSBTAG00000006366 & $2.12 \mathrm{E}-10$ & 7.01E-11 & NFATC4 & $3.02 E+00$ \\
\hline ENSBTAG00000012565 & $5.60 \mathrm{E}-10$ & $1.84 \mathrm{E}-10$ & PCNX1 & $3.04 E+00$ \\
\hline ENSBTAG00000031396 & $1.53 \mathrm{E}-09$ & 4.97E-10 & & $3.08 \mathrm{E}+00$ \\
\hline ENSBTAG00000047312 & $2.13 \mathrm{E}-09$ & $6.89 \mathrm{E}-10$ & & $3.09 E+00$ \\
\hline ENSBTAG00000005565 & $5.79 \mathrm{E}-10$ & $1.86 \mathrm{E}-10$ & CLN6 & $3.11 \mathrm{E}+00$ \\
\hline ENSBTAG00000003493 & $4.55 \mathrm{E}-10$ & $1.46 \mathrm{E}-10$ & & $3.12 \mathrm{E}+00$ \\
\hline ENSBTAG00000040053 & $3.38 \mathrm{E}-10$ & $1.08 \mathrm{E}-10$ & MYH6 & $3.12 \mathrm{E}+00$ \\
\hline ENSBTAG00000005489 & $1.34 \mathrm{E}-10$ & 4.27E-11 & WDR36 & $3.13 E+00$ \\
\hline ENSBTAG00000005865 & $1.36 \mathrm{E}-10$ & $4.28 \mathrm{E}-11$ & MAPK6 & $3.18 \mathrm{E}+00$ \\
\hline ENSBTAG00000011596 & $1.91 \mathrm{E}-10$ & $6.00 \mathrm{E}-11$ & SRSF5 & $3.17 E+00$ \\
\hline ENSBTAG00000005210 & $2.94 \mathrm{E}-10$ & $9.25 \mathrm{E}-11$ & SNAPC5 & $3.18 \mathrm{E}+00$ \\
\hline ENSBTAG00000016713 & $2.94 \mathrm{E}-10$ & $9.25 \mathrm{E}-11$ & TMEMЗОВ & $3.18 \mathrm{E}+00$ \\
\hline ENSBTAG00000017471 & $2.38 \mathrm{E}-10$ & 7.49E-11 & KBTBD13 & $3.18 \mathrm{E}+00$ \\
\hline ENSBTAG00000012139 & $1.60 \mathrm{E}-10$ & $5.04 \mathrm{E}-11$ & SIX1 & $3.17 \mathrm{E}+00$ \\
\hline ENSBTAG00000047027 & $1.50 \mathrm{E}-10$ & 4.73E-11 & C14orf119 & $3.17 E+00$ \\
\hline ENSBTAG00000047015 & $5.64 \mathrm{E}-10$ & $1.77 \mathrm{E}-10$ & & $3.19 E+00$ \\
\hline ENSBTAG00000039077 & $5.91 \mathrm{E}-10$ & $1.85 \mathrm{E}-10$ & & $3.19 E+00$ \\
\hline ENSBTAG00000038377 & $1.48 \mathrm{E}-10$ & 4.66E-11 & ADAM20 & $3.17 E+00$ \\
\hline ENSBTAG00000037411 & $7.09 \mathrm{E}-10$ & $2.22 \mathrm{E}-10$ & & $3.19 E+00$ \\
\hline ENSBTAG00000004303 & $2.29 \mathrm{E}-10$ & 7.14E-11 & SLC27A2 & $3.20 E+00$ \\
\hline ENSBTAG00000015200 & $3.19 \mathrm{E}-10$ & $9.92 \mathrm{E}-11$ & L2HGDH & $3.21 \mathrm{E}+00$ \\
\hline ENSBTAG00000025822 & $6.10 \mathrm{E}-10$ & $1.88 \mathrm{E}-10$ & CALML4 & $3.24 \mathrm{E}+00$ \\
\hline
\end{tabular}




\begin{tabular}{|c|c|c|c|c|}
\hline ENSBTAG00000030557 & $1.14 \mathrm{E}-10$ & $3.48 \mathrm{E}-11$ & LIN52 & $3.27 E+00$ \\
\hline ENSBTAG00000005984 & $1.29 \mathrm{E}-10$ & 3.87E-11 & DAAM1 & $3.32 E+00$ \\
\hline ENSBTAG00000010399 & $3.92 \mathrm{E}-10$ & 1.17E-10 & TXNDC16 & $3.35 E+00$ \\
\hline ENSBTAG00000003915 & $1.20 \mathrm{E}-09$ & 3.57E-10 & LARP6 & $3.37 E+00$ \\
\hline ENSBTAG00000002748 & $3.03 \mathrm{E}-10$ & $8.80 \mathrm{E}-11$ & TMEM55B & $3.45 E+00$ \\
\hline ENSBTAG00000004631 & $2.89 \mathrm{E}-10$ & 8.37E-11 & KIAA0586 & $3.45 E+00$ \\
\hline ENSBTAG00000014807 & $1.98 \mathrm{E}-10$ & $5.71 \mathrm{E}-11$ & DPP8 & $3.47 \mathrm{E}+00$ \\
\hline ENSBTAG00000010766 & $1.66 \mathrm{E}-10$ & $4.64 \mathrm{E}-11$ & OIP5 & $3.57 E+00$ \\
\hline ENSBTAG00000025564 & $4.48 \mathrm{E}-10$ & $1.25 \mathrm{E}-10$ & RPL36A & $3.58 \mathrm{E}+00$ \\
\hline ENSBTAG00000038083 & $6.58 \mathrm{E}-10$ & $1.83 \mathrm{E}-10$ & & $3.59 E+00$ \\
\hline ENSBTAG00000007586 & $5.28 \mathrm{E}-10$ & $1.44 \mathrm{E}-10$ & SYNJ2BP & $3.66 \mathrm{E}+00$ \\
\hline ENSBTAG00000013485 & $1.61 \mathrm{E}-10$ & 4.35E-11 & ZFYVE19 & $3.70 \mathrm{E}+00$ \\
\hline ENSBTAG00000032908 & 4.07E-10 & 1.10E-10 & TMEM253 & $3.70 E+00$ \\
\hline ENSBTAG00000018898 & $3.23 \mathrm{E}-10$ & 8.57E-11 & ARID4A & $3.77 E+00$ \\
\hline ENSBTAG00000002357 & $3.59 \mathrm{E}-10$ & $9.49 \mathrm{E}-11$ & TICAM2 & $3.78 \mathrm{E}+00$ \\
\hline ENSBTAG00000009703 & $5.25 \mathrm{E}-10$ & $1.33 \mathrm{E}-10$ & MYH7 & $3.94 \mathrm{E}+00$ \\
\hline ENSBTAG00000010503 & $1.24 \mathrm{E}-10$ & $3.11 E-11$ & TIMM9 & $3.97 E+00$ \\
\hline ENSBTAG00000013477 & $1.09 \mathrm{E}-10$ & $2.74 \mathrm{E}-11$ & GCHFR & $3.96 \mathrm{E}+00$ \\
\hline ENSBTAG00000017641 & $5.52 \mathrm{E}-10$ & $1.39 \mathrm{E}-10$ & & $3.97 E+00$ \\
\hline ENSBTAG00000007614 & $4.83 \mathrm{E}-10$ & $1.21 \mathrm{E}-10$ & BBS4 & $3.99 E+00$ \\
\hline ENSBTAG00000008815 & $1.58 \mathrm{E}-10$ & $3.94 \mathrm{E}-11$ & WDR89 & $4.01 E+00$ \\
\hline ENSBTAG00000004013 & $3.10 \mathrm{E}-10$ & $7.59 \mathrm{E}-11$ & FGF7 & $4.08 \mathrm{E}+00$ \\
\hline ENSBTAG00000006489 & $2.71 \mathrm{E}-10$ & $6.52 \mathrm{E}-11$ & MYO5A & $4.15 E+00$ \\
\hline ENSBTAG00000013793 & 4.63E-10 & 1.09E-10 & NEDD4 & $4.24 E+00$ \\
\hline ENSBTAG00000005550 & $5.11 \mathrm{E}-10$ & $1.20 \mathrm{E}-10$ & sos2 & $4.25 E+00$ \\
\hline ENSBTAG00000019079 & $2.83 \mathrm{E}-10$ & $6.61 \mathrm{E}-11$ & PLCB2 & $4.28 \mathrm{E}+00$ \\
\hline ENSBTAG00000005564 & $3.41 \mathrm{E}-10$ & $7.89 \mathrm{E}-11$ & PIAS1 & $4.32 E+00$ \\
\hline ENSBTAG00000048064 & $5.88 \mathrm{E}-10$ & $1.36 \mathrm{E}-10$ & & $4.32 \mathrm{E}+00$ \\
\hline ENSBTAG00000006708 & $3.17 \mathrm{E}-10$ & 7.30E-11 & ARIH1 & $4.33 E+00$ \\
\hline ENSBTAG00000032340 & $4.09 \mathrm{E}-10$ & $9.43 \mathrm{E}-11$ & KNSTRN & $4.33 E+00$ \\
\hline ENSBTAG00000018469 & $2.32 \mathrm{E}-10$ & $5.24 \mathrm{E}-11$ & ALDH6A1 & $4.41 E+00$ \\
\hline ENSBTAG00000009862 & $5.22 \mathrm{E}-10$ & 1.17E-10 & C14orf105 & $4.46 \mathrm{E}+00$ \\
\hline ENSBTAG00000019601 & $1.13 \mathrm{E}-10$ & $2.52 \mathrm{E}-11$ & & $4.46 \mathrm{E}+00$ \\
\hline ENSBTAG00000020281 & $4.66 \mathrm{E}-10$ & $1.02 \mathrm{E}-10$ & NIN & $4.57 E+00$ \\
\hline ENSBTAG00000021630 & $4.88 \mathrm{E}-10$ & $1.06 \mathrm{E}-10$ & MTFMT & $4.60 E+00$ \\
\hline ENSBTAG00000012250 & $1.96 \mathrm{E}-10$ & 4.23E-11 & BLOC1S6 & $4.63 E+00$ \\
\hline ENSBTAG00000000062 & 4.11E-10 & $8.71 \mathrm{E}-11$ & RASL12 & $4.71 E+00$ \\
\hline ENSBTAG00000020471 & $2.97 \mathrm{E}-10$ & $6.23 \mathrm{E}-11$ & SAMD15 & $4.77 E+00$ \\
\hline ENSBTAG00000012352 & $1.94 \mathrm{E}-10$ & 4.07E-11 & PARP16 & $4.76 \mathrm{E}+00$ \\
\hline ENSBTAG00000039658 & $1.66 \mathrm{E}-10$ & $3.49 \mathrm{E}-11$ & PDCD7 & $4.76 \mathrm{E}+00$ \\
\hline ENSBTAG00000027251 & $2.53 \mathrm{E}-10$ & $5.30 \mathrm{E}-11$ & & $4.76 \mathrm{E}+00$ \\
\hline ENSBTAG00000012898 & $1.46 \mathrm{E}-10$ & $3.06 \mathrm{E}-11$ & & $4.76 \mathrm{E}+00$ \\
\hline ENSBTAG00000040609 & $5.11 \mathrm{E}-10$ & $1.07 \mathrm{E}-10$ & & $4.77 \mathrm{E}+00$ \\
\hline ENSBTAG00000038963 & $5.25 \mathrm{E}-10$ & $1.10 \mathrm{E}-10$ & & $4.77 E+00$ \\
\hline ENSBTAG00000047322 & $8.12 \mathrm{E}-10$ & $1.70 \mathrm{E}-10$ & & $4.78 \mathrm{E}+00$ \\
\hline ENSBTAG00000046482 & $1.05 \mathrm{E}-09$ & 2.19E-10 & & $4.81 E+00$ \\
\hline ENSBTAG00000004011 & $4.21 \mathrm{E}-10$ & 8.74E-11 & GALK2 & $4.81 E+00$ \\
\hline ENSBTAG00000014657 & $1.67 \mathrm{E}-10$ & $3.46 \mathrm{E}-11$ & C14orf39 & $4.81 E+00$ \\
\hline ENSBTAG00000008863 & $1.92 \mathrm{E}-10$ & $3.99 \mathrm{E}-11$ & TMEM87A & $4.81 \mathrm{E}+00$ \\
\hline ENSBTAG00000013132 & $4.49 \mathrm{E}-10$ & $9.21 \mathrm{E}-11$ & MTX3 & $4.88 \mathrm{E}+00$ \\
\hline ENSBTAG00000013480 & $1.15 \mathrm{E}-10$ & $2.36 \mathrm{E}-11$ & DNAJC17 & $4.85 E+00$ \\
\hline ENSBTAG00000012180 & $3.88 \mathrm{E}-10$ & $7.78 \mathrm{E}-11$ & BMF & $4.99 E+00$ \\
\hline ENSBTAG00000007931 & $4.13 \mathrm{E}-10$ & $8.26 \mathrm{E}-11$ & SLC38A6 & $5.00 E+00$ \\
\hline ENSBTAG00000038565 & $1.78 \mathrm{E}-09$ & $3.40 \mathrm{E}-10$ & & $5.23 E+00$ \\
\hline ENSBTAG00000003371 & $6.13 \mathrm{E}-10$ & 1.17E-10 & JKAMP & $5.24 E+00$ \\
\hline ENSBTAG00000009595 & $2.29 \mathrm{E}-10$ & $4.31 \mathrm{E}-11$ & NGDN & $5.29 E+00$ \\
\hline ENSBTAG00000016826 & $6.69 \mathrm{E}-10$ & $1.22 \mathrm{E}-10$ & & $5.48 \mathrm{E}+00$ \\
\hline ENSBTAG00000011448 & $2.22 \mathrm{E}-10$ & 4.03E-11 & CCDC175 & $5.50 E+00$ \\
\hline ENSBTAG00000002808 & $1.36 \mathrm{E}-10$ & $2.45 \mathrm{E}-11$ & PSMA3 & $5.54 \mathrm{E}+00$ \\
\hline ENSBTAG00000019421 & $2.44 \mathrm{E}-10$ & $4.38 \mathrm{E}-11$ & DACT1 & $5.56 \mathrm{E}+00$ \\
\hline ENSBTAG00000021393 & $2.90 \mathrm{E}-10$ & $5.20 \mathrm{E}-11$ & FLRT2 & $5.56 \mathrm{E}+00$ \\
\hline ENSBTAG00000020474 & $3.04 \mathrm{E}-10$ & $5.45 \mathrm{E}-11$ & NOXRED1 & $5.56 \mathrm{E}+00$ \\
\hline ENSBTAG00000004788 & $1.69 \mathrm{E}-10$ & $3.05 \mathrm{E}-11$ & SDR39U1 & $5.54 \mathrm{E}+00$ \\
\hline ENSBTAG00000006440 & $1.51 \mathrm{E}-10$ & $2.72 \mathrm{E}-11$ & IGDCC4 & $5.54 \mathrm{E}+00$ \\
\hline ENSBTAG00000014384 & $3.01 \mathrm{E}-10$ & $5.16 \mathrm{E}-11$ & ZDHHC22 & $5.82 \mathrm{E}+00$ \\
\hline ENSBTAG00000017141 & $8.06 \mathrm{E}-10$ & $1.36 \mathrm{E}-10$ & CGRRF1 & $5.92 \mathrm{E}+00$ \\
\hline ENSBTAG00000002586 & $4.86 \mathrm{E}-10$ & $8.19 \mathrm{E}-11$ & TCF12 & $5.92 \mathrm{E}+00$ \\
\hline ENSBTAG00000038733 & $1.29 \mathrm{E}-10$ & 2.17E-11 & EFS & $5.94 \mathrm{E}+00$ \\
\hline ENSBTAG00000038327 & $6.59 \mathrm{E}-10$ & 1.10E-10 & & $5.99 E+00$ \\
\hline ENSBTAG00000027173 & $1.50 \mathrm{E}-10$ & $2.50 \mathrm{E}-11$ & CHP1 & $5.98 \mathrm{E}+00$ \\
\hline
\end{tabular}




\begin{tabular}{|c|c|c|c|c|}
\hline ENSBTAG00000018959 & $1.66 \mathrm{E}-10$ & $2.68 \mathrm{E}-11$ & RAB11A & $6.18 \mathrm{E}+00$ \\
\hline ENSBTAG00000038868 & $6.44 \mathrm{E}-10$ & $1.01 \mathrm{E}-10$ & & $6.38 E+00$ \\
\hline ENSBTAG00000005994 & 7.03E-10 & $1.10 \mathrm{E}-10$ & SPESP1 & $6.38 \mathrm{E}+00$ \\
\hline ENSBTAG00000014890 & $1.89 \mathrm{E}-10$ & $2.81 \mathrm{E}-11$ & CYP19A1 & $6.71 \mathrm{E}+00$ \\
\hline ENSBTAG00000001675 & $1.67 \mathrm{E}-10$ & $2.47 \mathrm{E}-11$ & ITPKA & $6.74 \mathrm{E}+00$ \\
\hline ENSBTAG00000013425 & $4.58 \mathrm{E}-10$ & $6.72 \mathrm{E}-11$ & COPS2 & $6.81 E+00$ \\
\hline ENSBTAG00000046464 & $1.29 \mathrm{E}-09$ & $1.85 \mathrm{E}-10$ & & $6.95 E+00$ \\
\hline ENSBTAG00000004420 & $1.06 \mathrm{E}-10$ & $1.48 \mathrm{E}-11$ & BAHD1 & $7.13 E+00$ \\
\hline ENSBTAG00000038842 & 4.05E-10 & $5.65 E-11$ & NEDD8 & $7.15 E+00$ \\
\hline ENSBTAG00000045760 & $7.68 \mathrm{E}-10$ & $1.07 \mathrm{E}-10$ & & $7.17 E+00$ \\
\hline ENSBTAG00000021957 & $3.06 \mathrm{E}-10$ & 4.13E-11 & LTBP2 & $7.38 \mathrm{E}+00$ \\
\hline ENSBTAG00000014236 & 8.34E-10 & $1.10 \mathrm{E}-10$ & NR2E3 & $7.58 \mathrm{E}+00$ \\
\hline ENSBTAG00000017373 & $1.79 \mathrm{E}-10$ & $2.31 \mathrm{E}-11$ & LACTB & $7.71 \mathrm{E}+00$ \\
\hline ENSBTAG00000008466 & 7.15E-10 & $9.22 \mathrm{E}-11$ & CCNB1IP1 & $7.75 E+00$ \\
\hline ENSBTAG00000025593 & $1.74 \mathrm{E}-10$ & $2.18 \mathrm{E}-11$ & C15orf57 & $7.93 E+00$ \\
\hline ENSBTAG00000045758 & $1.49 \mathrm{E}-09$ & $1.85 \mathrm{E}-10$ & & $8.05 E+00$ \\
\hline ENSBTAG00000012015 & $6.18 \mathrm{E}-10$ & $7.51 \mathrm{E}-11$ & FSIP1 & $8.22 \mathrm{E}+00$ \\
\hline ENSBTAG00000005714 & $3.27 \mathrm{E}-10$ & $3.91 \mathrm{E}-11$ & ACTC1 & $8.34 \mathrm{E}+00$ \\
\hline ENSBTAG00000016821 & 7.30E-10 & $8.62 \mathrm{E}-11$ & SNX22 & $8.46 \mathrm{E}+00$ \\
\hline ENSBTAG00000004079 & $3.14 \mathrm{E}-10$ & $3.58 \mathrm{E}-11$ & ZNF106 & $8.74 \mathrm{E}+00$ \\
\hline ENSBTAG00000045993 & 1.57E-09 & $1.77 \mathrm{E}-10$ & & $8.85 \mathrm{E}+00$ \\
\hline ENSBTAG00000047091 & 2.03E-09 & $2.29 \mathrm{E}-10$ & & $8.86 E+00$ \\
\hline ENSBTAG00000046553 & $3.97 \mathrm{E}-10$ & $4.32 \mathrm{E}-11$ & SECISBP2L & $9.16 \mathrm{E}+00$ \\
\hline ENSBTAG00000008828 & $2.86 \mathrm{E}-10$ & $3.06 \mathrm{E}-11$ & IGDCC3 & $9.33 \mathrm{E}+00$ \\
\hline ENSBTAG00000014351 & $7.96 \mathrm{E}-10$ & $8.50 \mathrm{E}-11$ & EIF2AK4 & $9.35 \mathrm{E}+00$ \\
\hline ENSBTAG00000045799 & $4.79 \mathrm{E}-10$ & 4.87E-11 & & $9.82 \mathrm{E}+00$ \\
\hline ENSBTAG00000009923 & $1.99 \mathrm{E}-10$ & $1.97 \mathrm{E}-11$ & & $1.00 \mathrm{E}+01$ \\
\hline ENSBTAG00000019390 & $3.85 \mathrm{E}-10$ & $3.81 \mathrm{E}-11$ & CA12 & $1.01 E+01$ \\
\hline ENSBTAG00000046309 & $2.57 \mathrm{E}-10$ & $2.48 \mathrm{E}-11$ & & $1.03 E+01$ \\
\hline ENSBTAG00000021955 & $6.23 \mathrm{E}-10$ & $6.01 \mathrm{E}-11$ & NPC2 & $1.04 \mathrm{E}+01$ \\
\hline ENSBTAG00000002006 & $5.11 \mathrm{E}-10$ & 4.73E-11 & THBS1 & $1.08 \mathrm{E}+01$ \\
\hline ENSBTAG00000001486 & $2.69 \mathrm{E}-10$ & 2.43E-11 & ZNF280D & $1.10 \mathrm{E}+01$ \\
\hline ENSBTAG00000040167 & $1.47 \mathrm{E}-09$ & $1.30 \mathrm{E}-10$ & & $1.13 \mathrm{E}+01$ \\
\hline ENSBTAG00000010234 & $6.53 \mathrm{E}-10$ & $5.76 \mathrm{E}-11$ & DHRS1 & $1.13 E+01$ \\
\hline ENSBTAG00000005941 & 2.37E-09 & $2.09 \mathrm{E}-10$ & & $1.13 \mathrm{E}+01$ \\
\hline ENSBTAG00000030801 & $3.24 \mathrm{E}-10$ & $2.75 \mathrm{E}-11$ & MNAT1 & $1.17 \mathrm{E}+01$ \\
\hline ENSBTAG00000012317 & $7.81 \mathrm{E}-10$ & $6.63 \mathrm{E}-11$ & & $1.18 \mathrm{E}+01$ \\
\hline ENSBTAG00000000821 & $4.62 \mathrm{E}-10$ & $3.60 \mathrm{E}-11$ & GPR137C & $1.28 \mathrm{E}+01$ \\
\hline ENSBTAG00000001605 & $4.72 \mathrm{E}-10$ & $3.63 \mathrm{E}-11$ & & $1.30 \mathrm{E}+01$ \\
\hline ENSBTAG00000016822 & $6.79 \mathrm{E}-10$ & $5.21 \mathrm{E}-11$ & PPIB & $1.30 \mathrm{E}+01$ \\
\hline ENSBTAG00000002918 & $7.80 \mathrm{E}-11$ & $5.78 \mathrm{E}-12$ & RAD51 & $1.33 \mathrm{E}+01$ \\
\hline ENSBTAG00000002012 & $6.90 \mathrm{E}-10$ & 4.69E-11 & FAM96A & $1.47 \mathrm{E}+01$ \\
\hline ENSBTAG00000008866 & $3.65 \mathrm{E}-10$ & $2.44 \mathrm{E}-11$ & GANC & $1.49 E+01$ \\
\hline ENSBTAG00000010380 & $2.51 \mathrm{E}-10$ & $1.62 \mathrm{E}-11$ & INO80 & $1.54 \mathrm{E}+01$ \\
\hline ENSBTAG00000003263 & $2.09 \mathrm{E}-10$ & $1.33 \mathrm{E}-11$ & TBCA & $1.56 \mathrm{E}+01$ \\
\hline ENSBTAG00000001671 & $1.81 \mathrm{E}-10$ & $1.15 \mathrm{E}-11$ & RTF1 & $1.56 \mathrm{E}+01$ \\
\hline ENSBTAG00000008363 & $2.12 \mathrm{E}-10$ & $1.33 \mathrm{E}-11$ & EXD1 & $1.58 \mathrm{E}+01$ \\
\hline ENSBTAG00000016242 & $1.42 \mathrm{E}-10$ & $8.93 \mathrm{E}-12$ & DUOXA1 & $1.57 \mathrm{E}+01$ \\
\hline ENSBTAG00000006198 & $1.79 \mathrm{E}-09$ & $1.11 \mathrm{E}-10$ & & $1.61 \mathrm{E}+01$ \\
\hline ENSBTAG00000002014 & $5.02 \mathrm{E}-10$ & $3.08 \mathrm{E}-11$ & SNX1 & $1.63 \mathrm{E}+01$ \\
\hline ENSBTAG00000025496 & $5.38 \mathrm{E}-10$ & $3.01 \mathrm{E}-11$ & SORD & $1.78 \mathrm{E}+01$ \\
\hline ENSBTAG00000004950 & 1.17E-09 & $6.33 \mathrm{E}-11$ & & $1.84 \mathrm{E}+01$ \\
\hline ENSBTAG00000001693 & $4.81 \mathrm{E}-10$ & $2.59 \mathrm{E}-11$ & RPAP1 & $1.85 \mathrm{E}+01$ \\
\hline ENSBTAG00000016246 & $2.08 \mathrm{E}-10$ & $1.02 \mathrm{E}-11$ & DUOX1 & $2.02 \mathrm{E}+01$ \\
\hline ENSBTAG00000016234 & $2.41 \mathrm{E}-10$ & $1.17 \mathrm{E}-11$ & DUOX2 & $2.05 E+01$ \\
\hline ENSBTAG00000009372 & $9.24 \mathrm{E}-10$ & 4.42E-11 & TTC5 & $2.09 E+01$ \\
\hline ENSBTAG00000020934 & 3.77E-10 & $1.63 \mathrm{E}-11$ & SHF & $2.30 \mathrm{E}+01$ \\
\hline ENSBTAG00000016239 & $6.82 \mathrm{E}-10$ & $2.94 \mathrm{E}-11$ & DUOXA2 & $2.31 E+01$ \\
\hline ENSBTAG00000014353 & 7.57E-10 & $3.16 \mathrm{E}-11$ & SRP14 & $2.39 E+01$ \\
\hline ENSBTAG00000004409 & $2.23 \mathrm{E}-10$ & $8.76 \mathrm{E}-12$ & IVD & $2.52 \mathrm{E}+01$ \\
\hline ENSBTAG00000016327 & $5.14 \mathrm{E}-10$ & $1.58 \mathrm{E}-11$ & SLC28A2 & $3.23 \mathrm{E}+01$ \\
\hline ENSBTAG00000030824 & $5.36 \mathrm{E}-10$ & $1.64 \mathrm{E}-11$ & L3HYPDH & $3.25 \mathrm{E}+01$ \\
\hline ENSBTAG00000012712 & $6.14 \mathrm{E}-10$ & $1.76 \mathrm{E}-11$ & $\mathrm{AQR}$ & $3.47 \mathrm{E}+01$ \\
\hline ENSBTAG00000011796 & $4.80 \mathrm{E}-10$ & $9.72 \mathrm{E}-12$ & TRMT5 & $4.89 E+01$ \\
\hline ENSBTAG00000009249 & $1.12 \mathrm{E}-10$ & 0 & OTP & $1.12 \mathrm{E}+03$ \\
\hline ENSBTAG00000008386 & $1.62 \mathrm{E}-10$ & 0 & CLPX & $1.62 E+03$ \\
\hline ENSBTAG00000002821 & $3.35 \mathrm{E}-10$ & 0 & CILP & $3.35 E+03$ \\
\hline ENSBTAG00000001871 & $1.48 \mathrm{E}-10$ & 0 & NRL & $1.48 \mathrm{E}+03$ \\
\hline ENSBTAG00000047258 & $1.46 \mathrm{E}-10$ & 0 & & $1.46 \mathrm{E}+03$ \\
\hline ENSBTAG00000047444 & 1.46E-10 & 0 & & $1.46 \mathrm{E}+03$ \\
\hline
\end{tabular}




\begin{tabular}{|c|c|c|c|c|}
\hline ENSBTAG00000046477 & $1.46 \mathrm{E}-10$ & 0 & & $1.46 \mathrm{E}+03$ \\
\hline ENSBTAG00000047683 & $4.39 \mathrm{E}-10$ & 0 & & $4.40 E+03$ \\
\hline ENSBTAG00000046102 & $4.22 \mathrm{E}-10$ & 0 & & $4.22 \mathrm{E}+03$ \\
\hline ENSBTAG00000027170 & $1.54 \mathrm{E}-10$ & 0 & & $1.54 \mathrm{E}+03$ \\
\hline ENSBTAG00000040141 & 7.96E-10 & 0 & TRAV14DV4 & $7.96 \mathrm{E}+03$ \\
\hline ENSBTAG00000024659 & 0 & 0 & & $1.00 \mathrm{E}+00$ \\
\hline ENSBTAG00000047645 & $7.62 \mathrm{E}-11$ & 0 & & $7.63 E+02$ \\
\hline ENSBTAG00000021730 & $2.94 \mathrm{E}-10$ & 0 & GJD2 & $2.94 E+03$ \\
\hline ENSBTAG00000003193 & 8.17E-11 & 0 & RPUSD2 & $8.18 \mathrm{E}+02$ \\
\hline ENSBTAG00000002921 & $1.50 \mathrm{E}-10$ & 0 & RMDN3 & $1.50 \mathrm{E}+03$ \\
\hline ENSBTAG00000016291 & $2.21 \mathrm{E}-10$ & 0 & RHOV & $2.21 \mathrm{E}+03$ \\
\hline ENSBTAG00000010371 & $3.46 \mathrm{E}-10$ & 0 & $\mathrm{CHAC1}$ & $3.46 \mathrm{E}+03$ \\
\hline ENSBTAG00000038438 & $8.51 \mathrm{E}-10$ & 0 & SUMO1 & $8.51 \mathrm{E}+03$ \\
\hline ENSBTAG00000010778 & $9.93 \mathrm{E}-11$ & 0 & NDUFAF1 & $9.94 E+02$ \\
\hline ENSBTAG00000032894 & $2.72 \mathrm{E}-10$ & 0 & & $2.72 E+03$ \\
\hline ENSBTAG00000020814 & $2.22 \mathrm{E}-10$ & 0 & & $2.22 \mathrm{E}+03$ \\
\hline ENSBTAG00000047868 & $4.59 \mathrm{E}-11$ & 0 & & $4.60 E+02$ \\
\hline ENSBTAG00000006201 & $2.04 \mathrm{E}-10$ & 0 & DTWD1 & $2.04 E+03$ \\
\hline ENSBTAG00000046376 & $1.63 \mathrm{E}-10$ & 0 & & $1.63 \mathrm{E}+03$ \\
\hline ENSBTAG00000005586 & $1.54 \mathrm{E}-10$ & 0 & GATM & $1.54 \mathrm{E}+03$ \\
\hline ENSBTAG00000003370 & $1.10 \mathrm{E}-10$ & 0 & GPR135 & $1.10 E+03$ \\
\hline ENSBTAG00000013035 & $9.55 \mathrm{E}-11$ & 0 & SIX6 & $9.56 \mathrm{E}+02$ \\
\hline ENSBTAG00000046048 & 0 & 0 & & $1.00 \mathrm{E}+00$ \\
\hline ENSBTAG00000021956 & $6.11 \mathrm{E}-10$ & 0 & ISCA2 & $6.11 E+03$ \\
\hline ENSBTAG00000039241 & 2.47E-10 & 0 & OR6S1 & $2.47 E+03$ \\
\hline ENSBTAG00000046058 & 1.37E-10 & 0 & RNASE11 & $1.37 \mathrm{E}+03$ \\
\hline ENSBTAG00000003006 & 0 & 0 & RNASE9 & $1.00 \mathrm{E}+00$ \\
\hline ENSBTAG00000032869 & $2.58 \mathrm{E}-10$ & 0 & RNASE10 & $2.58 \mathrm{E}+03$ \\
\hline ENSBTAG00000038643 & $6.76 \mathrm{E}-10$ & 0 & OR11H4 & $6.76 \mathrm{E}+03$ \\
\hline ENSBTAG00000011510 & $8.53 \mathrm{E}-11$ & 0 & & $8.54 E+02$ \\
\hline ENSBTAG00000040208 & $2.56 \mathrm{E}-10$ & 0 & & $2.56 \mathrm{E}+03$ \\
\hline ENSBTAG00000037647 & $8.75 \mathrm{E}-11$ & 0 & & $8.76 E+02$ \\
\hline ENSBTAG00000046604 & $1.48 \mathrm{E}-10$ & 0 & GREM1 & $1.48 \mathrm{E}+03$ \\
\hline ENSBTAG00000040206 & $1.19 \mathrm{E}-10$ & 0 & ZNF770 & $1.19 E+03$ \\
\hline ENSBTAG00000004421 & $2.94 \mathrm{E}-10$ & 0 & CHST14 & $2.94 \mathrm{E}+03$ \\
\hline ENSBTAG00000047281 & 0 & 0 & C15orf62 & $1.00 E+00$ \\
\hline ENSBTAG00000024582 & $3.22 \mathrm{E}-10$ & 0 & RPL10L & $3.22 E+03$ \\
\hline ENSBTAG00000033891 & 0 & 0 & MGAT2 & $1.00 \mathrm{E}+00$ \\
\hline ENSBTAG00000045980 & 0 & 0 & ARF6 & $1.00 \mathrm{E}+00$ \\
\hline ENSBTAG00000010472 & 0 & 0 & TNFAIP8L3 & $1.00 \mathrm{E}+00$ \\
\hline ENSBTAG00000045847 & 0 & 0 & CTXN2 & $1.00 \mathrm{E}+00$ \\
\hline ENSBTAG00000047170 & $4.35 \mathrm{E}-10$ & 0 & & $4.35 E+03$ \\
\hline ENSBTAG00000020533 & 0 & 0 & $\mathrm{FBXO} 34$ & $1.00 \mathrm{E}+00$ \\
\hline ENSBTAG00000045619 & 0 & 0 & & $1.00 E+00$ \\
\hline ENSBTAG00000046952 & $3.63 \mathrm{E}-10$ & 0 & & $3.63 E+03$ \\
\hline ENSBTAG00000025372 & $1.31 \mathrm{E}-10$ & 0 & C14orf169 & $1.31 E+03$ \\
\hline ENSBTAG00000025329 & 0 & 0 & IRF2BPL & $1.00 E+00$ \\
\hline ENSBTAG00000030521 & 0 & $8.53 E-11$ & IER5L & 1.17E-03 \\
\hline ENSBTAG00000039113 & $1.41 \mathrm{E}-10$ & $8.92 \mathrm{E}-10$ & MSGN1 & $1.58 \mathrm{E}-01$ \\
\hline ENSBTAG00000016606 & 3.57E-11 & $1.35 \mathrm{E}-10$ & PCGF1 & $2.65 \mathrm{E}-01$ \\
\hline ENSBTAG00000023372 & $2.92 \mathrm{E}-11$ & $1.11 \mathrm{E}-10$ & & 2.64E-01 \\
\hline ENSBTAG00000006474 & $2.00 \mathrm{E}-10$ & $7.48 \mathrm{E}-10$ & EPCAM & 2.67E-01 \\
\hline ENSBTAG00000046939 & $1.00 \mathrm{E}-10$ & $3.28 \mathrm{E}-10$ & C9orf69 & 3.05E-01 \\
\hline ENSBTAG00000038183 & $1.66 \mathrm{E}-10$ & $5.24 \mathrm{E}-10$ & & 3.17E-01 \\
\hline ENSBTAG00000018426 & $9.80 \mathrm{E}-11$ & $2.81 \mathrm{E}-10$ & & 3.49E-01 \\
\hline ENSBTAG00000046018 & $8.61 \mathrm{E}-11$ & $2.17 \mathrm{E}-10$ & & 3.97E-01 \\
\hline ENSBTAG00000047475 & $4.91 \mathrm{E}-11$ & $1.24 \mathrm{E}-10$ & & 3.96E-01 \\
\hline ENSBTAG00000036099 & 3.39E-11 & $8.54 \mathrm{E}-11$ & & 3.97E-01 \\
\hline ENSBTAG00000047226 & $1.31 \mathrm{E}-10$ & $3.01 \mathrm{E}-10$ & & 4.37E-01 \\
\hline ENSBTAG00000047575 & $5.47 \mathrm{E}-11$ & $1.24 \mathrm{E}-10$ & & 4.42E-01 \\
\hline ENSBTAG00000015012 & $8.71 \mathrm{E}-11$ & $1.92 \mathrm{E}-10$ & PTRHD1 & 4.54E-01 \\
\hline ENSBTAG00000046416 & $2.54 \mathrm{E}-10$ & $5.61 \mathrm{E}-10$ & & $4.52 \mathrm{E}-01$ \\
\hline ENSBTAG00000012099 & $2.32 \mathrm{E}-10$ & 4.62E-10 & TMEM87B & $5.02 \mathrm{E}-01$ \\
\hline ENSBTAG00000015490 & 4.09E-10 & 8.10E-10 & HS1BP3 & 5.05E-01 \\
\hline ENSBTAG00000031731 & $1.94 \mathrm{E}-10$ & $3.66 \mathrm{E}-10$ & & 5.29E-01 \\
\hline ENSBTAG00000015014 & $9.81 \mathrm{E}-11$ & $1.79 \mathrm{E}-10$ & CENPO & $5.48 \mathrm{E}-01$ \\
\hline ENSBTAG00000009694 & $9.27 \mathrm{E}-11$ & $1.66 \mathrm{E}-10$ & PUS10 & 5.59E-01 \\
\hline ENSBTAG00000039531 & $3.42 \mathrm{E}-10$ & $6.04 \mathrm{E}-10$ & & $5.66 \mathrm{E}-01$ \\
\hline ENSBTAG00000046140 & $1.83 \mathrm{E}-10$ & $3.24 \mathrm{E}-10$ & NPDC1 & 5.66E-01 \\
\hline ENSBTAG00000015386 & $1.16 \mathrm{E}-10$ & $2.04 \mathrm{E}-10$ & REL & 5.69E-01 \\
\hline
\end{tabular}




\begin{tabular}{|c|c|c|c|c|}
\hline ENSBTAG00000038941 & $6.34 \mathrm{E}-10$ & $1.00 \mathrm{E}-09$ & & $6.34 \mathrm{E}-01$ \\
\hline ENSBTAG00000012736 & $1.73 \mathrm{E}-10$ & $2.73 \mathrm{E}-10$ & PTRH1 & $6.35 \mathrm{E}-01$ \\
\hline ENSBTAG00000010520 & $2.26 \mathrm{E}-10$ & $3.56 \mathrm{E}-10$ & C8G & $6.34 \mathrm{E}-01$ \\
\hline ENSBTAG00000018286 & $1.75 \mathrm{E}-10$ & $2.76 \mathrm{E}-10$ & RNF181 & $6.35 \mathrm{E}-01$ \\
\hline ENSBTAG00000017982 & $1.88 \mathrm{E}-10$ & $2.87 \mathrm{E}-10$ & FIBCD1 & $6.55 \mathrm{E}-01$ \\
\hline ENSBTAG00000010595 & $9.60 \mathrm{E}-11$ & $1.46 \mathrm{E}-10$ & WDR92 & $6.58 \mathrm{E}-01$ \\
\hline ENSBTAG00000000783 & 6.17E-11 & $9.19 E-11$ & TGFA & $6.71 \mathrm{E}-01$ \\
\hline ENSBTAG00000046484 & $1.67 \mathrm{E}-10$ & $2.35 \mathrm{E}-10$ & AIF1L & 7.11E-01 \\
\hline ENSBTAG00000012525 & $9.29 \mathrm{E}-10$ & 1.29E-09 & $\mathrm{ABO}$ & 7.20E-01 \\
\hline ENSBTAG00000008160 & $6.05 \mathrm{E}-10$ & $8.39 \mathrm{E}-10$ & MBOAT2 & $7.21 \mathrm{E}-01$ \\
\hline ENSBTAG00000032623 & $3.00 \mathrm{E}-10$ & 4.15E-10 & KCNG3 & 7.23E-01 \\
\hline ENSBTAG00000001424 & $1.23 \mathrm{E}-10$ & $1.70 \mathrm{E}-10$ & MSH6 & $7.24 \mathrm{E}-01$ \\
\hline ENSBTAG00000023928 & 4.35E-10 & $5.90 \mathrm{E}-10$ & RDH14 & 7.37E-01 \\
\hline ENSBTAG00000046218 & $1.07 \mathrm{E}-10$ & $1.45 \mathrm{E}-10$ & KLF11 & $7.38 \mathrm{E}-01$ \\
\hline ENSBTAG00000002034 & $2.70 \mathrm{E}-10$ & 3.57E-10 & MFSD2B & 7.57E-01 \\
\hline ENSBTAG00000040226 & 3.07E-10 & $4.04 \mathrm{E}-10$ & C2orf81 & $7.60 \mathrm{E}-01$ \\
\hline ENSBTAG00000004295 & 4.13E-10 & $5.37 \mathrm{E}-10$ & NDUFA8 & 7.69E-01 \\
\hline ENSBTAG00000019382 & $3.06 \mathrm{E}-10$ & $3.96 \mathrm{E}-10$ & PKDCC & 7.73E-01 \\
\hline ENSBTAG00000037856 & $1.30 \mathrm{E}-10$ & $1.64 \mathrm{E}-10$ & & 7.92E-01 \\
\hline ENSBTAG00000032651 & $1.36 \mathrm{E}-10$ & $1.71 \mathrm{E}-10$ & & 7.96E-01 \\
\hline ENSBTAG00000039138 & 1.27E-10 & $1.61 \mathrm{E}-10$ & MRPL41 & 7.92E-01 \\
\hline ENSBTAG00000039815 & $1.18 \mathrm{E}-10$ & $1.48 \mathrm{E}-10$ & ZBTB6 & 7.95E-01 \\
\hline ENSBTAG00000030676 & $2.59 \mathrm{E}-10$ & $3.26 \mathrm{E}-10$ & OR1K1 & 7.94E-01 \\
\hline ENSBTAG00000021935 & $2.34 \mathrm{E}-10$ & $2.95 \mathrm{E}-10$ & ELMOD3 & $7.94 \mathrm{E}-01$ \\
\hline ENSBTAG00000018579 & $5.69 \mathrm{E}-11$ & 7.17E-11 & DOK1 & 7.94E-01 \\
\hline ENSBTAG00000014667 & $1.75 \mathrm{E}-10$ & $2.21 \mathrm{E}-10$ & PAIP2B & 7.93E-01 \\
\hline ENSBTAG00000000726 & $9.64 \mathrm{E}-10$ & $1.21 \mathrm{E}-09$ & OR1J1 & 7.97E-01 \\
\hline ENSBTAG00000039708 & $8.39 \mathrm{E}-11$ & $1.06 \mathrm{E}-10$ & C9orf172 & 7.92E-01 \\
\hline ENSBTAG00000004159 & $5.08 \mathrm{E}-11$ & $6.40 \mathrm{E}-11$ & SIX2 & 7.94E-01 \\
\hline ENSBTAG00000046563 & $9.95 \mathrm{E}-10$ & $1.25 \mathrm{E}-09$ & TSPYL6 & 7.96E-01 \\
\hline ENSBTAG00000016607 & $5.84 \mathrm{E}-11$ & 7.35E-11 & TLX2 & 7.94E-01 \\
\hline ENSBTAG00000047693 & 2.63E-10 & $3.31 \mathrm{E}-10$ & & $7.94 \mathrm{E}-01$ \\
\hline ENSBTAG00000037767 & $9.70 \mathrm{E}-10$ & $1.22 \mathrm{E}-09$ & OR1N1 & 7.95E-01 \\
\hline ENSBTAG00000009033 & 7.90E-10 & $9.95 \mathrm{E}-10$ & TEX37 & 7.94E-01 \\
\hline ENSBTAG00000038610 & $6.02 \mathrm{E}-11$ & 7.59E-11 & ZBTB26 & 7.94E-01 \\
\hline ENSBTAG00000047503 & $4.02 \mathrm{E}-10$ & $5.06 \mathrm{E}-10$ & & $7.94 \mathrm{E}-01$ \\
\hline ENSBTAG00000045538 & 8.96E-10 & 1.13E-09 & & 7.93E-01 \\
\hline ENSBTAG00000047772 & $1.58 \mathrm{E}-10$ & $1.99 \mathrm{E}-10$ & FBXO48 & $7.94 \mathrm{E}-01$ \\
\hline ENSBTAG00000023787 & $6.71 \mathrm{E}-11$ & $8.45 \mathrm{E}-11$ & TOR4A & 7.94E-01 \\
\hline ENSBTAG00000047149 & $8.92 \mathrm{E}-11$ & $1.12 \mathrm{E}-10$ & & 7.96E-01 \\
\hline ENSBTAG00000007793 & $8.64 \mathrm{E}-11$ & $1.09 \mathrm{E}-10$ & SERTAD2 & 7.93E-01 \\
\hline ENSBTAG00000023997 & $4.40 \mathrm{E}-10$ & $5.54 \mathrm{E}-10$ & VAMP8 & 7.94E-01 \\
\hline ENSBTAG00000038257 & $6.29 \mathrm{E}-11$ & $7.92 \mathrm{E}-11$ & & 7.94E-01 \\
\hline ENSBTAG00000014150 & $2.41 \mathrm{E}-10$ & $3.04 \mathrm{E}-10$ & C9orf16 & 7.94E-01 \\
\hline ENSBTAG00000012484 & $1.10 \mathrm{E}-10$ & $1.39 \mathrm{E}-10$ & ENDOG & 7.91E-01 \\
\hline ENSBTAG00000047185 & 1.11E-09 & $1.40 \mathrm{E}-09$ & & 7.93E-01 \\
\hline ENSBTAG00000047907 & $1.60 \mathrm{E}-10$ & $2.02 \mathrm{E}-10$ & & 7.93E-01 \\
\hline ENSBTAG00000020123 & $2.70 \mathrm{E}-11$ & $3.40 \mathrm{E}-11$ & AUP1 & 7.95E-01 \\
\hline ENSBTAG00000019212 & $2.01 \mathrm{E}-10$ & $2.53 \mathrm{E}-10$ & KCNS3 & 7.93E-01 \\
\hline ENSBTAG00000005654 & $1.62 \mathrm{E}-10$ & $2.04 \mathrm{E}-10$ & TMSB10 & 7.95E-01 \\
\hline ENSBTAG00000039223 & $1.08 \mathrm{E}-10$ & $1.36 \mathrm{E}-10$ & WDR38 & $7.92 \mathrm{E}-01$ \\
\hline ENSBTAG00000012880 & $1.40 \mathrm{E}-10$ & $1.76 \mathrm{E}-10$ & DNLZ & 7.93E-01 \\
\hline ENSBTAG00000004562 & $1.16 \mathrm{E}-09$ & 1.43E-09 & CD207 & $8.10 \mathrm{E}-01$ \\
\hline ENSBTAG00000039851 & $2.36 \mathrm{E}-10$ & $2.91 \mathrm{E}-10$ & UBAC1 & 8.09E-01 \\
\hline ENSBTAG00000008905 & $1.19 \mathrm{E}-09$ & $1.47 \mathrm{E}-09$ & FIGLA & $8.10 \mathrm{E}-01$ \\
\hline ENSBTAG00000004297 & 4.70E-10 & $5.81 \mathrm{E}-10$ & ACOXL & 8.09E-01 \\
\hline ENSBTAG00000030246 & $6.22 \mathrm{E}-10$ & $7.61 \mathrm{E}-10$ & ENTPD8 & $8.18 \mathrm{E}-01$ \\
\hline ENSBTAG00000015007 & $1.12 \mathrm{E}-10$ & $1.35 \mathrm{E}-10$ & & $8.26 \mathrm{E}-01$ \\
\hline ENSBTAG00000001575 & $3.49 \mathrm{E}-10$ & $4.25 \mathrm{E}-10$ & & $8.21 \mathrm{E}-01$ \\
\hline ENSBTAG00000018401 & $4.86 \mathrm{E}-10$ & $5.90 \mathrm{E}-10$ & APLF & $8.24 \mathrm{E}-01$ \\
\hline ENSBTAG00000008997 & $1.76 \mathrm{E}-10$ & $2.13 \mathrm{E}-10$ & & 8.27E-01 \\
\hline ENSBTAG00000004664 & $5.72 \mathrm{E}-10$ & $6.91 \mathrm{E}-10$ & & $8.28 \mathrm{E}-01$ \\
\hline ENSBTAG00000017429 & $3.22 \mathrm{E}-10$ & $3.87 \mathrm{E}-10$ & PREPL & $8.31 \mathrm{E}-01$ \\
\hline ENSBTAG00000021934 & $3.46 \mathrm{E}-10$ & 4.14E-10 & RETSAT & $8.35 \mathrm{E}-01$ \\
\hline ENSBTAG00000014159 & $5.70 \mathrm{E}-10$ & $6.83 \mathrm{E}-10$ & TTL & $8.34 \mathrm{E}-01$ \\
\hline ENSBTAG00000011912 & $1.66 \mathrm{E}-10$ & $2.00 \mathrm{E}-10$ & SLC25A25 & $8.32 \mathrm{E}-01$ \\
\hline ENSBTAG00000004823 & $5.07 \mathrm{E}-10$ & $6.07 \mathrm{E}-10$ & MRPL35 & $8.36 \mathrm{E}-01$ \\
\hline ENSBTAG00000033801 & $2.90 \mathrm{E}-10$ & $3.48 \mathrm{E}-10$ & VRK2 & $8.34 \mathrm{E}-01$ \\
\hline ENSBTAG00000002085 & $8.03 \mathrm{E}-10$ & $9.53 \mathrm{E}-10$ & IL36G & $8.42 \mathrm{E}-01$ \\
\hline ENSBTAG00000000184 & $4.89 \mathrm{E}-10$ & $5.81 \mathrm{E}-10$ & EIF2AK3 & $8.42 \mathrm{E}-01$ \\
\hline
\end{tabular}




\begin{tabular}{|c|c|c|c|c|}
\hline ENSBTAG00000046303 & 3.24E-10 & $3.84 \mathrm{E}-10$ & & $8.44 \mathrm{E}-01$ \\
\hline ENSBTAG00000048218 & $1.44 \mathrm{E}-09$ & $1.71 \mathrm{E}-09$ & OR1L3 & 8.43E-01 \\
\hline ENSBTAG00000009246 & $2.01 \mathrm{E}-10$ & 2.37E-10 & DPY30 & $8.50 \mathrm{E}-01$ \\
\hline ENSBTAG00000044173 & 1.57E-10 & $1.85 \mathrm{E}-10$ & EHBP1 & $8.49 \mathrm{E}-01$ \\
\hline ENSBTAG00000004560 & 7.17E-10 & $8.41 \mathrm{E}-10$ & CLEC4F & $8.53 \mathrm{E}-01$ \\
\hline ENSBTAG00000009658 & 4.11E-10 & $4.82 \mathrm{E}-10$ & PLEK & $8.53 \mathrm{E}-01$ \\
\hline ENSBTAG00000000705 & $7.61 \mathrm{E}-10$ & $8.82 \mathrm{E}-10$ & COX7A2L & $8.62 \mathrm{E}-01$ \\
\hline ENSBTAG00000005704 & $5.54 \mathrm{E}-10$ & $6.41 \mathrm{E}-10$ & IL1RL2 & $8.65 \mathrm{E}-01$ \\
\hline ENSBTAG00000006572 & $2.79 \mathrm{E}-10$ & $3.22 \mathrm{E}-10$ & CARD9 & $8.66 \mathrm{E}-01$ \\
\hline ENSBTAG00000046126 & $1.05 \mathrm{E}-09$ & $1.21 \mathrm{E}-09$ & OR1J2 & $8.70 \mathrm{E}-01$ \\
\hline ENSBTAG00000005270 & $2.15 \mathrm{E}-10$ & $2.49 \mathrm{E}-10$ & & $8.65 \mathrm{E}-01$ \\
\hline ENSBTAG00000001140 & 8.36E-10 & $9.60 \mathrm{E}-10$ & IAH1 & $8.71 \mathrm{E}-01$ \\
\hline ENSBTAG00000016144 & $1.20 \mathrm{E}-09$ & $1.38 \mathrm{E}-09$ & LCN10 & $8.68 \mathrm{E}-01$ \\
\hline ENSBTAG00000045527 & 2.04E-09 & $2.34 \mathrm{E}-09$ & & $8.70 \mathrm{E}-01$ \\
\hline ENSBTAG00000038972 & $6.44 \mathrm{E}-10$ & 7.37E-10 & LCN9 & $8.73 \mathrm{E}-01$ \\
\hline ENSBTAG00000013580 & $2.26 \mathrm{E}-10$ & $2.58 \mathrm{E}-10$ & KDM3A & $8.74 \mathrm{E}-01$ \\
\hline ENSBTAG00000001949 & $1.67 \mathrm{E}-10$ & $1.91 \mathrm{E}-10$ & ZNF2 & $8.73 \mathrm{E}-01$ \\
\hline ENSBTAG00000003358 & $5.55 \mathrm{E}-10$ & $6.35 \mathrm{E}-10$ & MCFD2 & $8.74 \mathrm{E}-01$ \\
\hline ENSBTAG00000000899 & $2.25 \mathrm{E}-10$ & $2.57 \mathrm{E}-10$ & TMEM17 & $8.77 \mathrm{E}-01$ \\
\hline ENSBTAG00000012274 & $5.36 \mathrm{E}-10$ & $6.09 \mathrm{E}-10$ & CIZ1 & $8.80 \mathrm{E}-01$ \\
\hline ENSBTAG00000019697 & $5.29 \mathrm{E}-10$ & $6.01 E-10$ & NPAS2 & $8.81 \mathrm{E}-01$ \\
\hline ENSBTAG00000011553 & 7.27E-10 & $8.24 \mathrm{E}-10$ & & $8.82 \mathrm{E}-01$ \\
\hline ENSBTAG00000019254 & $6.14 \mathrm{E}-10$ & $6.95 \mathrm{E}-10$ & CFAP77 & $8.84 \mathrm{E}-01$ \\
\hline ENSBTAG00000004194 & $5.25 \mathrm{E}-10$ & $5.93 \mathrm{E}-10$ & RALGDS & $8.86 \mathrm{E}-01$ \\
\hline ENSBTAG00000046971 & $5.08 \mathrm{E}-10$ & $5.71 E-10$ & & $8.90 \mathrm{E}-01$ \\
\hline ENSBTAG00000013510 & $1.83 \mathrm{E}-10$ & $2.05 \mathrm{E}-10$ & TOR2A & $8.93 \mathrm{E}-01$ \\
\hline ENSBTAG00000032424 & $5.45 \mathrm{E}-10$ & $6.11 \mathrm{E}-10$ & FSHR & $8.92 \mathrm{E}-01$ \\
\hline ENSBTAG00000000703 & $4.09 \mathrm{E}-10$ & 4.57E-10 & ST6GAL2 & $8.94 \mathrm{E}-01$ \\
\hline ENSBTAG00000019735 & $6.19 \mathrm{E}-10$ & $6.93 \mathrm{E}-10$ & CPSF3 & 8.94E-01 \\
\hline ENSBTAG00000047715 & $1.09 \mathrm{E}-10$ & $1.22 \mathrm{E}-10$ & & $8.91 \mathrm{E}-01$ \\
\hline ENSBTAG00000001706 & $7.43 \mathrm{E}-10$ & $8.29 \mathrm{E}-10$ & SLC9A2 & $8.96 \mathrm{E}-01$ \\
\hline ENSBTAG00000021969 & $3.78 \mathrm{E}-10$ & $4.21 \mathrm{E}-10$ & KLHL29 & $8.98 \mathrm{E}-01$ \\
\hline ENSBTAG00000017199 & $7.20 \mathrm{E}-10$ & 8.00E-10 & GKN2 & 9.00E-01 \\
\hline ENSBTAG00000011129 & $2.05 \mathrm{E}-10$ & 2.27E-10 & QSOX2 & $9.02 \mathrm{E}-01$ \\
\hline ENSBTAG00000009076 & $4.01 \mathrm{E}-10$ & $4.42 \mathrm{E}-10$ & ADD2 & $9.08 \mathrm{E}-01$ \\
\hline ENSBTAG00000008048 & $5.53 \mathrm{E}-10$ & $6.08 \mathrm{E}-10$ & GCFC2 & $9.10 \mathrm{E}-01$ \\
\hline ENSBTAG00000004542 & $2.07 \mathrm{E}-10$ & $2.28 \mathrm{E}-10$ & FAM78A & $9.10 \mathrm{E}-01$ \\
\hline ENSBTAG00000014081 & $6.65 \mathrm{E}-10$ & $7.30 \mathrm{E}-10$ & NBAS & $9.12 \mathrm{E}-01$ \\
\hline ENSBTAG00000045545 & $1.26 \mathrm{E}-09$ & $1.38 \mathrm{E}-09$ & & $9.15 \mathrm{E}-01$ \\
\hline ENSBTAG00000005870 & $2.53 \mathrm{E}-10$ & $2.76 \mathrm{E}-10$ & FANCL & $9.16 \mathrm{E}-01$ \\
\hline ENSBTAG00000008994 & $3.74 \mathrm{E}-10$ & 4.07E-10 & FPGS & $9.19 \mathrm{E}-01$ \\
\hline ENSBTAG00000046775 & $5.52 \mathrm{E}-10$ & $6.00 \mathrm{E}-10$ & MAN1B1 & $9.20 \mathrm{E}-01$ \\
\hline ENSBTAG00000017039 & $4.20 \mathrm{E}-10$ & $4.56 \mathrm{E}-10$ & ARHGEF33 & $9.21 \mathrm{E}-01$ \\
\hline ENSBTAG00000011544 & $2.17 \mathrm{E}-10$ & $2.35 \mathrm{E}-10$ & GAPVD1 & 9.23E-01 \\
\hline ENSBTAG00000021120 & $5.37 \mathrm{E}-10$ & $5.80 \mathrm{E}-10$ & SMYD1 & $9.25 \mathrm{E}-01$ \\
\hline ENSBTAG00000001549 & $5.80 \mathrm{E}-10$ & $6.26 \mathrm{E}-10$ & OR1L1 & 9.27E-01 \\
\hline ENSBTAG00000004073 & $2.20 \mathrm{E}-10$ & $2.36 \mathrm{E}-10$ & MTA3 & $9.34 \mathrm{E}-01$ \\
\hline ENSBTAG00000015193 & $2.79 \mathrm{E}-10$ & $2.99 \mathrm{E}-10$ & BOLA3 & $9.32 \mathrm{E}-01$ \\
\hline ENSBTAG00000008216 & $3.06 \mathrm{E}-10$ & $3.28 \mathrm{E}-10$ & RRM2 & $9.33 \mathrm{E}-01$ \\
\hline ENSBTAG00000016987 & $2.27 \mathrm{E}-10$ & 2.44E-10 & WDCP & $9.32 \mathrm{E}-01$ \\
\hline ENSBTAG00000047297 & $1.80 \mathrm{E}-09$ & $1.93 \mathrm{E}-09$ & & 9.33E-01 \\
\hline ENSBTAG00000015553 & $1.73 \mathrm{E}-10$ & $1.85 \mathrm{E}-10$ & SCAI & $9.36 \mathrm{E}-01$ \\
\hline ENSBTAG00000014678 & $7.69 \mathrm{E}-10$ & $8.13 \mathrm{E}-10$ & PAEP & $9.45 \mathrm{E}-01$ \\
\hline ENSBTAG00000033010 & $2.84 \mathrm{E}-10$ & $3.01 E-10$ & TTC27 & $9.43 \mathrm{E}-01$ \\
\hline ENSBTAG00000018761 & 8.63E-10 & $9.11 \mathrm{E}-10$ & SLC35F6 & $9.47 \mathrm{E}-01$ \\
\hline ENSBTAG00000011554 & 4.50E-10 & $4.74 \mathrm{E}-10$ & SURF6 & $9.49 \mathrm{E}-01$ \\
\hline ENSBTAG00000030319 & $8.26 \mathrm{E}-10$ & 8.69E-10 & GBGT1 & $9.50 \mathrm{E}-01$ \\
\hline ENSBTAG00000007662 & $1.32 \mathrm{E}-10$ & $1.38 \mathrm{E}-10$ & & $9.54 \mathrm{E}-01$ \\
\hline ENSBTAG00000027015 & $7.22 \mathrm{E}-10$ & 7.57E-10 & & 9.53E-01 \\
\hline ENSBTAG00000002943 & $9.77 \mathrm{E}-10$ & $1.02 \mathrm{E}-09$ & & $9.58 \mathrm{E}-01$ \\
\hline ENSBTAG00000010497 & 7.30E-10 & $7.66 \mathrm{E}-10$ & TRAF2 & 9.53E-01 \\
\hline ENSBTAG00000023064 & $2.50 \mathrm{E}-10$ & $2.59 \mathrm{E}-10$ & CCDC187 & $9.66 \mathrm{E}-01$ \\
\hline ENSBTAG00000016366 & 4.11E-10 & 4.25E-10 & ABCG8 & $9.66 \mathrm{E}-01$ \\
\hline ENSBTAG00000038462 & 6.97E-10 & $7.20 \mathrm{E}-10$ & TMEM182 & $9.69 \mathrm{E}-01$ \\
\hline ENSBTAG00000002570 & 3.30E-10 & $3.40 \mathrm{E}-10$ & PPP1R21 & $9.70 \mathrm{E}-01$ \\
\hline ENSBTAG00000001425 & $1.21 \mathrm{E}-10$ & $1.24 \mathrm{E}-10$ & FBXO11 & $9.77 \mathrm{E}-01$ \\
\hline ENSBTAG00000007897 & 4.72E-10 & $4.84 \mathrm{E}-10$ & POMC & $9.76 \mathrm{E}-01$ \\
\hline ENSBTAG00000004722 & 1.22E-09 & $1.25 \mathrm{E}-09$ & THUMPD2 & $9.77 \mathrm{E}-01$ \\
\hline ENSBTAG00000004092 & $3.76 \mathrm{E}-10$ & $3.83 \mathrm{E}-10$ & AK8 & $9.81 \mathrm{E}-01$ \\
\hline ENSBTAG00000047031 & $3.67 \mathrm{E}-10$ & $3.74 \mathrm{E}-10$ & WDR5 & $9.82 \mathrm{E}-01$ \\
\hline
\end{tabular}




\begin{tabular}{|c|c|c|c|c|}
\hline ENSBTAG00000013063 & $6.44 \mathrm{E}-10$ & $6.55 \mathrm{E}-10$ & ITGB1BP1 & $9.83 \mathrm{E}-01$ \\
\hline ENSBTAG00000017005 & $1.35 \mathrm{E}-09$ & 1.36E-09 & LCN8 & $9.91 \mathrm{E}-01$ \\
\hline ENSBTAG00000003307 & $5.13 \mathrm{E}-10$ & $5.19 \mathrm{E}-10$ & IMMT & $9.88 \mathrm{E}-01$ \\
\hline ENSBTAG00000048091 & $1.04 \mathrm{E}-09$ & $1.05 \mathrm{E}-09$ & & $9.90 \mathrm{E}-01$ \\
\hline ENSBTAG00000004544 & $9.49 \mathrm{E}-10$ & $9.59 \mathrm{E}-10$ & TXNDC9 & $9.89 \mathrm{E}-01$ \\
\hline ENSBTAG00000021283 & $1.77 \mathrm{E}-10$ & $1.79 \mathrm{E}-10$ & KCNF1 & $9.91 \mathrm{E}-01$ \\
\hline ENSBTAG00000011727 & $1.22 \mathrm{E}-10$ & $1.23 \mathrm{E}-10$ & ETAA1 & $9.90 \mathrm{E}-01$ \\
\hline ENSBTAG00000007888 & $7.23 \mathrm{E}-10$ & $7.28 \mathrm{E}-10$ & EFR3B & $9.93 \mathrm{E}-01$ \\
\hline ENSBTAG00000045711 & 4.09E-10 & 4.12E-10 & CYSRT1 & $9.92 \mathrm{E}-01$ \\
\hline ENSBTAG00000012925 & 1.00E-09 & $1.00 \mathrm{E}-09$ & $\mathrm{NCAPH}$ & $1.00 \mathrm{E}+00$ \\
\hline ENSBTAG00000009599 & $1.18 \mathrm{E}-09$ & 1.19E-09 & LCN1 & 9.92E-01 \\
\hline ENSBTAG00000008505 & $3.12 \mathrm{E}-10$ & $3.12 \mathrm{E}-10$ & APOB & $1.00 \mathrm{E}+00$ \\
\hline ENSBTAG00000025612 & $2.35 \mathrm{E}-10$ & $2.34 \mathrm{E}-10$ & URM1 & $1.00 \mathrm{E}+00$ \\
\hline ENSBTAG00000016045 & $3.65 \mathrm{E}-10$ & $3.64 \mathrm{E}-10$ & TRIB2 & $1.00 \mathrm{E}+00$ \\
\hline ENSBTAG00000024822 & $3.35 \mathrm{E}-10$ & $3.33 \mathrm{E}-10$ & SETX & $1.01 \mathrm{E}+00$ \\
\hline ENSBTAG00000006259 & $1.99 \mathrm{E}-10$ & $1.97 \mathrm{E}-10$ & MOB1A & $1.01 E+00$ \\
\hline ENSBTAG00000003904 & $2.94 \mathrm{E}-10$ & $2.92 \mathrm{E}-10$ & GPN1 & $1.01 E+00$ \\
\hline ENSBTAG00000026111 & $2.48 \mathrm{E}-10$ & $2.46 \mathrm{E}-10$ & LBH & $1.01 \mathrm{E}+00$ \\
\hline ENSBTAG00000030302 & $2.94 \mathrm{E}-10$ & $2.91 \mathrm{E}-10$ & EDF1 & $1.01 E+00$ \\
\hline ENSBTAG00000011810 & $3.70 \mathrm{E}-10$ & $3.65 \mathrm{E}-10$ & ANGPTL2 & $1.01 \mathrm{E}+00$ \\
\hline ENSBTAG00000033041 & $2.38 \mathrm{E}-10$ & $2.35 \mathrm{E}-10$ & YIPF4 & $1.01 E+00$ \\
\hline ENSBTAG00000023999 & $3.02 \mathrm{E}-10$ & 2.97E-10 & GTF2A1L & $1.02 \mathrm{E}+00$ \\
\hline ENSBTAG00000003525 & $2.46 \mathrm{E}-10$ & $2.42 \mathrm{E}-10$ & STXBP1 & $1.01 \mathrm{E}+00$ \\
\hline ENSBTAG00000034586 & $5.22 \mathrm{E}-10$ & 5.13E-10 & & $1.02 \mathrm{E}+00$ \\
\hline ENSBTAG00000017421 & $6.05 \mathrm{E}-10$ & $5.95 \mathrm{E}-10$ & SLC3A1 & $1.02 \mathrm{E}+00$ \\
\hline ENSBTAG00000015436 & $2.95 \mathrm{E}-10$ & $2.90 \mathrm{E}-10$ & SLC27A4 & $1.02 \mathrm{E}+00$ \\
\hline ENSBTAG00000004352 & $3.41 \mathrm{E}-10$ & $3.35 \mathrm{E}-10$ & SLC5A7 & $1.02 E+00$ \\
\hline ENSBTAG00000016573 & $2.26 \mathrm{E}-10$ & $2.22 \mathrm{E}-10$ & & $1.02 \mathrm{E}+00$ \\
\hline ENSBTAG00000020313 & $3.86 \mathrm{E}-10$ & $3.78 \mathrm{E}-10$ & FNBP1 & $1.02 \mathrm{E}+00$ \\
\hline ENSBTAG00000009017 & $2.71 E-10$ & $2.66 \mathrm{E}-10$ & NR5A1 & $1.02 E+00$ \\
\hline ENSBTAG00000016534 & $2.58 \mathrm{E}-10$ & $2.53 \mathrm{E}-10$ & & $1.02 \mathrm{E}+00$ \\
\hline ENSBTAG00000046379 & $1.91 \mathrm{E}-10$ & 1.87E-10 & SLC34A3 & $1.02 \mathrm{E}+00$ \\
\hline ENSBTAG00000008223 & $3.45 \mathrm{E}-10$ & $3.37 \mathrm{E}-10$ & NLRC4 & $1.02 \mathrm{E}+00$ \\
\hline ENSBTAG00000007808 & 4.77E-10 & 4.65E-10 & ANTXR1 & $1.03 E+00$ \\
\hline ENSBTAG00000000933 & $4.96 \mathrm{E}-10$ & $4.84 \mathrm{E}-10$ & CCDC183 & $1.02 \mathrm{E}+00$ \\
\hline ENSBTAG00000016365 & $5.91 \mathrm{E}-10$ & $5.76 \mathrm{E}-10$ & ABCG5 & $1.03 \mathrm{E}+00$ \\
\hline ENSBTAG00000006025 & $2.13 \mathrm{E}-10$ & $2.08 \mathrm{E}-10$ & AHSA2 & $1.03 E+00$ \\
\hline ENSBTAG00000016218 & 3.37E-10 & 3.27E-10 & PTGES2 & $1.03 E+00$ \\
\hline ENSBTAG00000017318 & $2.68 \mathrm{E}-10$ & $2.60 \mathrm{E}-10$ & TMEM178A & $1.03 E+00$ \\
\hline ENSBTAG00000008703 & $3.73 \mathrm{E}-10$ & 3.62E-10 & EIF2AK2 & $1.03 E+00$ \\
\hline ENSBTAG00000012394 & $4.86 \mathrm{E}-10$ & $4.71 \mathrm{E}-10$ & CCDC85A & $1.03 E+00$ \\
\hline ENSBTAG00000003906 & $1.68 \mathrm{E}-10$ & $1.62 \mathrm{E}-10$ & SUPT7L & $1.03 E+00$ \\
\hline ENSBTAG00000025274 & $4.38 \mathrm{E}-10$ & 4.24E-10 & TUBB4B & $1.03 E+00$ \\
\hline ENSBTAG00000017473 & 4.64E-10 & 4.49E-10 & LAMC3 & $1.03 E+00$ \\
\hline ENSBTAG00000011317 & $1.34 \mathrm{E}-10$ & $1.30 \mathrm{E}-10$ & GOLGA2 & $1.03 E+00$ \\
\hline ENSBTAG00000005614 & $2.06 \mathrm{E}-10$ & $1.99 \mathrm{E}-10$ & UXS1 & $1.03 E+00$ \\
\hline ENSBTAG00000018705 & $1.65 \mathrm{E}-10$ & $1.60 \mathrm{E}-10$ & $A D C Y 3$ & $1.03 E+00$ \\
\hline ENSBTAG00000009649 & $3.24 \mathrm{E}-10$ & $3.11 \mathrm{E}-10$ & DRC1 & $1.04 E+00$ \\
\hline ENSBTAG00000003530 & $1.96 \mathrm{E}-10$ & $1.88 \mathrm{E}-10$ & DDX31 & $1.04 E+00$ \\
\hline ENSBTAG00000007734 & $1.26 \mathrm{E}-10$ & $1.21 \mathrm{E}-10$ & MEMO1 & $1.04 \mathrm{E}+00$ \\
\hline ENSBTAG00000015865 & $4.35 \mathrm{E}-10$ & $4.16 \mathrm{E}-10$ & CAPN14 & $1.04 \mathrm{E}+00$ \\
\hline ENSBTAG00000005257 & $2.06 \mathrm{E}-10$ & $1.97 \mathrm{E}-10$ & PEX13 & $1.04 \mathrm{E}+00$ \\
\hline ENSBTAG00000032519 & $4.43 \mathrm{E}-10$ & 4.22E-10 & CAMKMT & $1.05 E+00$ \\
\hline ENSBTAG00000008987 & $2.37 \mathrm{E}-10$ & $2.26 \mathrm{E}-10$ & SOCS5 & $1.05 E+00$ \\
\hline ENSBTAG00000039573 & $7.25 \mathrm{E}-10$ & $6.89 \mathrm{E}-10$ & RABL6 & $1.05 E+00$ \\
\hline ENSBTAG00000010935 & $2.05 \mathrm{E}-10$ & $1.95 \mathrm{E}-10$ & EML4 & $1.05 E+00$ \\
\hline ENSBTAG00000015037 & $3.04 \mathrm{E}-10$ & $2.89 \mathrm{E}-10$ & GAREM2 & $1.05 E+00$ \\
\hline ENSBTAG00000019979 & $1.94 \mathrm{E}-10$ & $1.83 \mathrm{E}-10$ & CMPK2 & $1.06 \mathrm{E}+00$ \\
\hline ENSBTAG00000019017 & 1.10E-09 & $1.04 \mathrm{E}-09$ & IFITM1 & $1.06 \mathrm{E}+00$ \\
\hline ENSBTAG00000014149 & $6.51 \mathrm{E}-10$ & $6.14 \mathrm{E}-10$ & LCN2 & $1.06 \mathrm{E}+00$ \\
\hline ENSBTAG00000008267 & $7.41 \mathrm{E}-10$ & $6.99 \mathrm{E}-10$ & CNOT11 & $1.06 \mathrm{E}+00$ \\
\hline ENSBTAG00000009510 & $7.36 \mathrm{E}-10$ & $6.94 \mathrm{E}-10$ & ADRA2B & $1.06 \mathrm{E}+00$ \\
\hline ENSBTAG00000001840 & $1.20 \mathrm{E}-10$ & $1.14 \mathrm{E}-10$ & & $1.05 E+00$ \\
\hline ENSBTAG00000047254 & $2.82 \mathrm{E}-10$ & $2.65 E-10$ & $A B C A 2$ & $1.07 E+00$ \\
\hline ENSBTAG00000034657 & $9.78 \mathrm{E}-10$ & $9.17 \mathrm{E}-10$ & MAL & $1.07 \mathrm{E}+00$ \\
\hline ENSBTAG00000009020 & $2.70 \mathrm{E}-10$ & $2.53 \mathrm{E}-10$ & CRIM1 & $1.07 E+00$ \\
\hline ENSBTAG00000002742 & $6.70 \mathrm{E}-10$ & $6.22 \mathrm{E}-10$ & MSH2 & $1.08 \mathrm{E}+00$ \\
\hline ENSBTAG00000012735 & $2.58 \mathrm{E}-10$ & $2.39 \mathrm{E}-10$ & CFAP157 & $1.08 \mathrm{E}+00$ \\
\hline ENSBTAG00000001321 & 3.67E-10 & $3.40 \mathrm{E}-10$ & IL1B & $1.08 \mathrm{E}+00$ \\
\hline ENSBTAG00000009021 & $6.31 \mathrm{E}-10$ & $5.82 \mathrm{E}-10$ & FEZ2 & $1.08 \mathrm{E}+00$ \\
\hline
\end{tabular}




\begin{tabular}{|c|c|c|c|c|}
\hline ENSBTAG00000012519 & $2.90 \mathrm{E}-10$ & 2.67E-10 & $\mathrm{XDH}$ & $1.08 \mathrm{E}+00$ \\
\hline ENSBTAG00000020947 & $2.68 \mathrm{E}-10$ & $2.46 \mathrm{E}-10$ & RNF103 & $1.09 \mathrm{E}+00$ \\
\hline ENSBTAG00000007379 & $3.38 \mathrm{E}-10$ & $3.11 \mathrm{E}-10$ & ALK & $1.09 \mathrm{E}+00$ \\
\hline ENSBTAG00000008548 & $1.71 \mathrm{E}-10$ & $1.57 \mathrm{E}-10$ & FAM98A & $1.09 \mathrm{E}+00$ \\
\hline ENSBTAG00000000111 & $1.62 \mathrm{E}-10$ & $1.49 \mathrm{E}-10$ & UGP2 & $1.09 \mathrm{E}+00$ \\
\hline ENSBTAG00000012637 & $4.46 \mathrm{E}-10$ & 4.07E-10 & C2orf40 & $1.10 \mathrm{E}+00$ \\
\hline ENSBTAG00000039225 & $1.01 \mathrm{E}-09$ & $9.20 \mathrm{E}-10$ & & $1.09 \mathrm{E}+00$ \\
\hline ENSBTAG00000023929 & $1.52 \mathrm{E}-10$ & $1.38 \mathrm{E}-10$ & FOSL2 & $1.10 \mathrm{E}+00$ \\
\hline ENSBTAG00000018975 & $2.98 \mathrm{E}-10$ & $2.71 \mathrm{E}-10$ & KCNT1 & $1.10 \mathrm{E}+00$ \\
\hline ENSBTAG00000014877 & 8.67E-10 & $7.88 \mathrm{E}-10$ & UNC50 & $1.10 \mathrm{E}+00$ \\
\hline ENSBTAG00000011307 & $1.36 \mathrm{E}-10$ & $1.23 \mathrm{E}-10$ & DNM1 & $1.10 E+00$ \\
\hline ENSBTAG00000012121 & $3.55 \mathrm{E}-10$ & $3.21 \mathrm{E}-10$ & NOXA1 & $1.11 \mathrm{E}+00$ \\
\hline ENSBTAG00000011643 & $2.09 \mathrm{E}-10$ & $1.88 \mathrm{E}-10$ & sos1 & $1.11 \mathrm{E}+00$ \\
\hline ENSBTAG00000013030 & $3.76 \mathrm{E}-10$ & $3.40 \mathrm{E}-10$ & LRRC8A & $1.10 \mathrm{E}+00$ \\
\hline ENSBTAG00000020311 & $3.16 \mathrm{E}-10$ & $2.85 \mathrm{E}-10$ & USP20 & $1.11 E+00$ \\
\hline ENSBTAG00000007508 & $2.52 \mathrm{E}-10$ & 2.27E-10 & SRBD1 & $1.11 E+00$ \\
\hline ENSBTAG00000037542 & $6.16 \mathrm{E}-10$ & $5.53 \mathrm{E}-10$ & & $1.11 \mathrm{E}+00$ \\
\hline ENSBTAG00000020297 & $1.33 \mathrm{E}-10$ & $1.20 \mathrm{E}-10$ & PQLC3 & $1.11 \mathrm{E}+00$ \\
\hline ENSBTAG00000001141 & $5.68 \mathrm{E}-10$ & $5.09 \mathrm{E}-10$ & ADAM17 & $1.12 \mathrm{E}+00$ \\
\hline ENSBTAG00000027932 & $2.10 \mathrm{E}-10$ & $1.87 \mathrm{E}-10$ & BIRC6 & $1.12 \mathrm{E}+00$ \\
\hline ENSBTAG00000010271 & $1.18 \mathrm{E}-10$ & $1.05 \mathrm{E}-10$ & MAPKAP1 & $1.12 \mathrm{E}+00$ \\
\hline ENSBTAG00000046536 & $1.23 \mathrm{E}-09$ & 1.11E-09 & & $1.11 \mathrm{E}+00$ \\
\hline ENSBTAG00000019295 & $3.28 \mathrm{E}-10$ & $2.93 \mathrm{E}-10$ & $\mathrm{MDH} 1$ & $1.12 \mathrm{E}+00$ \\
\hline ENSBTAG00000018153 & $1.57 \mathrm{E}-10$ & $1.40 \mathrm{E}-10$ & NRBP1 & $1.12 \mathrm{E}+00$ \\
\hline ENSBTAG00000016167 & $2.35 \mathrm{E}-10$ & 2.09E-10 & ATL2 & $1.13 \mathrm{E}+00$ \\
\hline ENSBTAG00000013861 & $5.25 \mathrm{E}-10$ & 4.67E-10 & SLC8A1 & $1.12 \mathrm{E}+00$ \\
\hline ENSBTAG00000009299 & $1.68 \mathrm{E}-10$ & $1.49 \mathrm{E}-10$ & AFTPH & $1.12 \mathrm{E}+00$ \\
\hline ENSBTAG00000006305 & $4.99 \mathrm{E}-10$ & $4.44 \mathrm{E}-10$ & AK1 & $1.12 \mathrm{E}+00$ \\
\hline ENSBTAG00000024042 & $1.70 \mathrm{E}-10$ & $1.51 \mathrm{E}-10$ & MORN2 & $1.12 \mathrm{E}+00$ \\
\hline ENSBTAG00000002288 & 1.14E-09 & $1.02 \mathrm{E}-09$ & NT5DC4 & $1.12 \mathrm{E}+00$ \\
\hline ENSBTAG00000018710 & $6.64 \mathrm{E}-10$ & $5.89 \mathrm{E}-10$ & TTF1 & $1.13 \mathrm{E}+00$ \\
\hline ENSBTAG00000020490 & $5.14 \mathrm{E}-10$ & $4.55 \mathrm{E}-10$ & NACC2 & $1.13 \mathrm{E}+00$ \\
\hline ENSBTAG00000014391 & $1.53 \mathrm{E}-10$ & $1.35 \mathrm{E}-10$ & CRB2 & $1.13 \mathrm{E}+00$ \\
\hline ENSBTAG00000017976 & $2.40 \mathrm{E}-10$ & $2.12 \mathrm{E}-10$ & ABL1 & $1.13 E+00$ \\
\hline ENSBTAG00000006716 & $6.94 \mathrm{E}-10$ & $6.11 \mathrm{E}-10$ & PTGS1 & $1.14 \mathrm{E}+00$ \\
\hline ENSBTAG00000010604 & $8.82 \mathrm{E}-11$ & $7.78 \mathrm{E}-11$ & PNO1 & $1.13 E+00$ \\
\hline ENSBTAG00000008674 & $1.84 \mathrm{E}-10$ & $1.63 \mathrm{E}-10$ & & $1.13 \mathrm{E}+00$ \\
\hline ENSBTAG00000021205 & $2.84 \mathrm{E}-10$ & $2.49 \mathrm{E}-10$ & PLPP7 & $1.14 \mathrm{E}+00$ \\
\hline ENSBTAG00000000054 & $2.09 \mathrm{E}-10$ & $1.84 \mathrm{E}-10$ & SNAPC4 & $1.14 \mathrm{E}+00$ \\
\hline ENSBTAG00000018193 & $2.59 \mathrm{E}-10$ & 2.27E-10 & KCNK3 & $1.14 \mathrm{E}+00$ \\
\hline ENSBTAG00000034255 & 4.77E-10 & $4.18 \mathrm{E}-10$ & EIF5B & $1.14 \mathrm{E}+00$ \\
\hline ENSBTAG00000020305 & $2.38 \mathrm{E}-10$ & $2.08 \mathrm{E}-10$ & TOR1A & $1.14 \mathrm{E}+00$ \\
\hline ENSBTAG00000003408 & 7.14E-10 & $6.23 \mathrm{E}-10$ & & $1.15 E+00$ \\
\hline ENSBTAG00000008973 & $2.04 \mathrm{E}-10$ & $1.78 \mathrm{E}-10$ & ASB6 & $1.15 E+00$ \\
\hline ENSBTAG00000015437 & $2.78 \mathrm{E}-10$ & $2.43 \mathrm{E}-10$ & NTMT1 & $1.15 E+00$ \\
\hline ENSBTAG00000020791 & $4.66 \mathrm{E}-10$ & 4.05E-10 & RAPGEF1 & $1.15 \mathrm{E}+00$ \\
\hline ENSBTAG00000015205 & 4.79E-10 & 4.16E-10 & NCK2 & $1.15 E+00$ \\
\hline ENSBTAG00000020249 & $2.92 \mathrm{E}-10$ & $2.54 \mathrm{E}-10$ & GLT6D1 & $1.15 \mathrm{E}+00$ \\
\hline ENSBTAG00000019867 & $6.17 \mathrm{E}-10$ & $5.34 \mathrm{E}-10$ & COMMD1 & $1.16 \mathrm{E}+00$ \\
\hline ENSBTAG00000005688 & $4.09 \mathrm{E}-10$ & $3.53 \mathrm{E}-10$ & MRPS2 & $1.16 \mathrm{E}+00$ \\
\hline ENSBTAG00000017955 & $5.60 \mathrm{E}-10$ & $4.86 \mathrm{E}-10$ & & $1.15 \mathrm{E}+00$ \\
\hline ENSBTAG00000014166 & $6.26 \mathrm{E}-10$ & $5.42 \mathrm{E}-10$ & POLR1B & $1.15 E+00$ \\
\hline ENSBTAG00000013509 & 4.05E-10 & $3.51 E-10$ & TTC16 & $1.16 \mathrm{E}+00$ \\
\hline ENSBTAG00000012090 & $3.51 \mathrm{E}-10$ & $3.04 \mathrm{E}-10$ & & $1.15 E+00$ \\
\hline ENSBTAG00000012156 & $5.40 \mathrm{E}-10$ & 4.67E-10 & NAIF1 & $1.16 \mathrm{E}+00$ \\
\hline ENSBTAG00000011559 & $3.96 \mathrm{E}-10$ & $3.43 \mathrm{E}-10$ & RPL7A & $1.15 E+00$ \\
\hline ENSBTAG00000016368 & $3.09 \mathrm{E}-10$ & 2.67E-10 & LRPPRC & $1.16 \mathrm{E}+00$ \\
\hline ENSBTAG00000016442 & 4.44E-10 & $3.84 \mathrm{E}-10$ & MAP4K3 & $1.16 \mathrm{E}+00$ \\
\hline ENSBTAG00000000115 & $2.30 \mathrm{E}-10$ & $1.98 \mathrm{E}-10$ & VPS54 & $1.16 \mathrm{E}+00$ \\
\hline ENSBTAG00000018680 & $1.84 \mathrm{E}-10$ & $1.59 \mathrm{E}-10$ & & $1.16 \mathrm{E}+00$ \\
\hline ENSBTAG00000019453 & $3.88 \mathrm{E}-10$ & $3.34 \mathrm{E}-10$ & PTGES & $1.16 \mathrm{E}+00$ \\
\hline ENSBTAG00000020646 & $2.09 \mathrm{E}-10$ & $1.80 \mathrm{E}-10$ & $\mathrm{ZC} 3 \mathrm{H} 6$ & $1.16 \mathrm{E}+00$ \\
\hline ENSBTAG00000000295 & $3.34 \mathrm{E}-10$ & $2.87 \mathrm{E}-10$ & $\mathrm{CLHC1}$ & $1.16 \mathrm{E}+00$ \\
\hline ENSBTAG00000038726 & 1.34E-09 & 1.16E-09 & & $1.16 \mathrm{E}+00$ \\
\hline ENSBTAG00000015723 & $1.66 \mathrm{E}-10$ & $1.42 \mathrm{E}-10$ & CRIPT & $1.17 \mathrm{E}+00$ \\
\hline ENSBTAG00000009290 & $4.20 \mathrm{E}-10$ & $3.60 \mathrm{E}-10$ & FAM161A & $1.17 E+00$ \\
\hline ENSBTAG00000002866 & $1.85 \mathrm{E}-10$ & $1.58 \mathrm{E}-10$ & RPIA & $1.17 E+00$ \\
\hline ENSBTAG00000001354 & $2.58 \mathrm{E}-10$ & $2.21 \mathrm{E}-10$ & INPP5E & $1.17 E+00$ \\
\hline ENSBTAG00000018054 & $3.01 \mathrm{E}-10$ & $2.57 \mathrm{E}-10$ & FABP1 & $1.17 \mathrm{E}+00$ \\
\hline ENSBTAG00000005736 & $2.21 \mathrm{E}-10$ & $1.89 \mathrm{E}-10$ & ADGRF3 & $1.17 \mathrm{E}+00$ \\
\hline
\end{tabular}




\begin{tabular}{|c|c|c|c|c|}
\hline ENSBTAG00000020059 & $1.98 \mathrm{E}-10$ & $1.68 \mathrm{E}-10$ & GEN1 & $1.18 \mathrm{E}+00$ \\
\hline ENSBTAG00000021861 & $3.98 \mathrm{E}-10$ & $3.38 \mathrm{E}-10$ & NELFB & $1.18 \mathrm{E}+00$ \\
\hline ENSBTAG00000017851 & $2.66 \mathrm{E}-10$ & $2.26 \mathrm{E}-10$ & RXRA & $1.18 \mathrm{E}+00$ \\
\hline ENSBTAG00000013923 & $2.19 \mathrm{E}-10$ & $1.85 \mathrm{E}-10$ & QPCT & $1.19 \mathrm{E}+00$ \\
\hline ENSBTAG00000016934 & $5.26 \mathrm{E}-10$ & $4.46 \mathrm{E}-10$ & C2orf71 & $1.18 \mathrm{E}+00$ \\
\hline ENSBTAG00000008033 & $3.81 \mathrm{E}-10$ & $3.22 \mathrm{E}-10$ & PPP6C & $1.18 \mathrm{E}+00$ \\
\hline ENSBTAG00000015481 & 4.07E-10 & $3.43 \mathrm{E}-10$ & MTIF2 & $1.19 \mathrm{E}+00$ \\
\hline ENSBTAG00000004507 & $1.86 \mathrm{E}-10$ & $1.56 \mathrm{E}-10$ & FAM163B & $1.19 \mathrm{E}+00$ \\
\hline ENSBTAG00000013734 & $2.16 \mathrm{E}-10$ & $1.81 \mathrm{E}-10$ & ATP6V1E2 & $1.19 \mathrm{E}+00$ \\
\hline ENSBTAG00000038249 & $2.59 \mathrm{E}-10$ & 2.17E-10 & OR5C1 & $1.19 E+00$ \\
\hline ENSBTAG00000006760 & $7.51 \mathrm{E}-11$ & $6.31 \mathrm{E}-11$ & OTX1 & $1.19 E+00$ \\
\hline ENSBTAG00000045659 & 4.65E-10 & $3.90 \mathrm{E}-10$ & & $1.19 E+00$ \\
\hline ENSBTAG00000016602 & $1.48 \mathrm{E}-10$ & $1.25 \mathrm{E}-10$ & LBX2 & $1.19 \mathrm{E}+00$ \\
\hline ENSBTAG00000015074 & $3.23 \mathrm{E}-10$ & $2.71 E-10$ & PTGDS & $1.19 E+00$ \\
\hline ENSBTAG00000000848 & 1.07E-09 & $8.96 \mathrm{E}-10$ & SNRNP200 & $1.19 \mathrm{E}+00$ \\
\hline ENSBTAG00000007895 & 2.02E-10 & $1.69 \mathrm{E}-10$ & SLC20A1 & $1.19 \mathrm{E}+00$ \\
\hline ENSBTAG00000000490 & $1.38 \mathrm{E}-10$ & $1.16 \mathrm{E}-10$ & RTKN & $1.19 E+00$ \\
\hline ENSBTAG00000023697 & $4.74 \mathrm{E}-10$ & $3.98 \mathrm{E}-10$ & & $1.19 E+00$ \\
\hline ENSBTAG00000022142 & $1.65 \mathrm{E}-10$ & $1.39 \mathrm{E}-10$ & & $1.19 E+00$ \\
\hline ENSBTAG00000047964 & $1.75 \mathrm{E}-10$ & $1.47 \mathrm{E}-10$ & TMEM210 & $1.19 E+00$ \\
\hline ENSBTAG00000004256 & $1.67 \mathrm{E}-10$ & $1.41 \mathrm{E}-10$ & & $1.19 E+00$ \\
\hline ENSBTAG00000048054 & $2.46 \mathrm{E}-10$ & 2.07E-10 & UAP1L1 & $1.19 E+00$ \\
\hline ENSBTAG00000008308 & $1.36 \mathrm{E}-10$ & $1.15 \mathrm{E}-10$ & & $1.19 \mathrm{E}+00$ \\
\hline ENSBTAG00000038477 & $2.00 \mathrm{E}-10$ & $1.68 \mathrm{E}-10$ & GGCX & $1.19 E+00$ \\
\hline ENSBTAG00000013889 & $9.74 \mathrm{E}-10$ & $8.16 \mathrm{E}-10$ & TMEM141 & $1.19 E+00$ \\
\hline ENSBTAG00000015575 & $4.91 \mathrm{E}-10$ & $4.10 \mathrm{E}-10$ & TACR1 & $1.20 \mathrm{E}+00$ \\
\hline ENSBTAG00000008241 & 1.11E-09 & $9.28 \mathrm{E}-10$ & MRPS5 & $1.20 \mathrm{E}+00$ \\
\hline ENSBTAG00000021731 & $8.78 \mathrm{E}-10$ & 7.33E-10 & TGFBRAP1 & $1.20 \mathrm{E}+00$ \\
\hline ENSBTAG00000031302 & 3.30E-10 & $2.75 \mathrm{E}-10$ & GCKR & $1.20 \mathrm{E}+00$ \\
\hline ENSBTAG00000005041 & $2.44 \mathrm{E}-10$ & 2.03E-10 & CIB4 & $1.20 E+00$ \\
\hline ENSBTAG00000024075 & $1.04 \mathrm{E}-09$ & $8.56 \mathrm{E}-10$ & SLC9A4 & $1.21 \mathrm{E}+00$ \\
\hline ENSBTAG00000006343 & $6.85 \mathrm{E}-10$ & $5.65 \mathrm{E}-10$ & IL1R2 & $1.21 \mathrm{E}+00$ \\
\hline ENSBTAG00000038584 & $6.06 \mathrm{E}-10$ & 5.00E-10 & OLFM1 & $1.21 \mathrm{E}+00$ \\
\hline ENSBTAG00000012847 & $2.23 \mathrm{E}-10$ & $1.84 \mathrm{E}-10$ & FAM129B & $1.21 \mathrm{E}+00$ \\
\hline ENSBTAG00000004887 & $2.19 \mathrm{E}-10$ & $1.80 \mathrm{E}-10$ & DCTN1 & $1.22 \mathrm{E}+00$ \\
\hline ENSBTAG00000006988 & $1.43 \mathrm{E}-10$ & $1.16 \mathrm{E}-10$ & M1AP & $1.23 E+00$ \\
\hline ENSBTAG00000021694 & $1.05 \mathrm{E}-10$ & $8.55 \mathrm{E}-11$ & SPAST & $1.23 E+00$ \\
\hline ENSBTAG00000021550 & $1.86 \mathrm{E}-10$ & $1.52 \mathrm{E}-10$ & MAMDC4 & $1.22 \mathrm{E}+00$ \\
\hline ENSBTAG00000002936 & $1.93 \mathrm{E}-10$ & $1.58 \mathrm{E}-10$ & & $1.22 \mathrm{E}+00$ \\
\hline ENSBTAG00000013254 & $1.43 \mathrm{E}-10$ & $1.16 \mathrm{E}-10$ & XPO1 & $1.24 \mathrm{E}+00$ \\
\hline ENSBTAG00000024000 & $3.74 \mathrm{E}-10$ & $3.04 \mathrm{E}-10$ & ATOH8 & $1.23 E+00$ \\
\hline ENSBTAG00000015402 & $4.82 \mathrm{E}-10$ & $3.91 \mathrm{E}-10$ & GREB1 & $1.23 E+00$ \\
\hline ENSBTAG00000023801 & $1.75 \mathrm{E}-10$ & $1.42 \mathrm{E}-10$ & BARHL1 & $1.23 \mathrm{E}+00$ \\
\hline ENSBTAG00000011558 & $2.02 \mathrm{E}-10$ & $1.63 \mathrm{E}-10$ & GPAT2 & $1.24 \mathrm{E}+00$ \\
\hline ENSBTAG00000001928 & $1.19 \mathrm{E}-10$ & $9.52 \mathrm{E}-11$ & PDIA6 & $1.25 E+00$ \\
\hline ENSBTAG00000000156 & $1.76 \mathrm{E}-10$ & $1.41 \mathrm{E}-10$ & LGALSL & $1.25 \mathrm{E}+00$ \\
\hline ENSBTAG00000017189 & $5.71 \mathrm{E}-10$ & $4.58 \mathrm{E}-10$ & POLE4 & $1.25 \mathrm{E}+00$ \\
\hline ENSBTAG00000012485 & $6.22 \mathrm{E}-10$ & $4.98 \mathrm{E}-10$ & SPOUT1 & $1.25 E+00$ \\
\hline ENSBTAG00000037793 & $1.05 \mathrm{E}-09$ & $8.38 \mathrm{E}-10$ & & $1.25 E+00$ \\
\hline ENSBTAG00000020614 & $3.07 \mathrm{E}-10$ & $2.46 \mathrm{E}-10$ & PRKCE & $1.25 \mathrm{E}+00$ \\
\hline ENSBTAG00000020893 & $1.71 \mathrm{E}-10$ & $1.36 \mathrm{E}-10$ & MATN3 & $1.26 \mathrm{E}+00$ \\
\hline ENSBTAG00000039172 & $2.04 \mathrm{E}-10$ & $1.62 \mathrm{E}-10$ & RABGAP1 & $1.26 \mathrm{E}+00$ \\
\hline ENSBTAG00000010605 & $1.60 \mathrm{E}-10$ & $1.27 \mathrm{E}-10$ & VAX2 & $1.26 \mathrm{E}+00$ \\
\hline ENSBTAG00000018578 & $1.74 \mathrm{E}-10$ & $1.38 \mathrm{E}-10$ & LOXL3 & $1.26 \mathrm{E}+00$ \\
\hline ENSBTAG00000001730 & $6.26 \mathrm{E}-10$ & $4.96 \mathrm{E}-10$ & ODF3 & $1.26 \mathrm{E}+00$ \\
\hline ENSBTAG00000007837 & $2.29 \mathrm{E}-10$ & $1.81 \mathrm{E}-10$ & SELENOI & $1.27 \mathrm{E}+00$ \\
\hline ENSBTAG00000022799 & $2.53 \mathrm{E}-10$ & $2.00 \mathrm{E}-10$ & NOTCH1 & $1.26 \mathrm{E}+00$ \\
\hline ENSBTAG00000038224 & $1.63 \mathrm{E}-10$ & $1.28 \mathrm{E}-10$ & GDF7 & $1.27 \mathrm{E}+00$ \\
\hline ENSBTAG00000039201 & $3.02 \mathrm{E}-10$ & 2.37E-10 & & $1.27 \mathrm{E}+00$ \\
\hline ENSBTAG00000019510 & $1.45 \mathrm{E}-10$ & $1.14 \mathrm{E}-10$ & EIF2B4 & $1.28 \mathrm{E}+00$ \\
\hline ENSBTAG00000000992 & $9.75 \mathrm{E}-11$ & $7.68 \mathrm{E}-11$ & BMP10 & $1.27 \mathrm{E}+00$ \\
\hline ENSBTAG00000030675 & $8.66 \mathrm{E}-11$ & $6.82 \mathrm{E}-11$ & PDCL & $1.27 \mathrm{E}+00$ \\
\hline ENSBTAG00000000046 & $1.65 \mathrm{E}-10$ & $1.29 \mathrm{E}-10$ & SURF2 & $1.28 \mathrm{E}+00$ \\
\hline ENSBTAG00000010582 & $3.28 \mathrm{E}-10$ & $2.58 \mathrm{E}-10$ & BRD3 & $1.27 \mathrm{E}+00$ \\
\hline ENSBTAG00000032051 & $6.86 \mathrm{E}-10$ & 5.39E-10 & & $1.27 \mathrm{E}+00$ \\
\hline ENSBTAG00000025898 & $3.87 \mathrm{E}-10$ & $3.03 \mathrm{E}-10$ & TBC1D8 & $1.28 \mathrm{E}+00$ \\
\hline ENSBTAG00000005151 & $2.31 \mathrm{E}-10$ & $1.81 \mathrm{E}-10$ & WDPCP & $1.27 \mathrm{E}+00$ \\
\hline ENSBTAG00000020865 & $3.69 \mathrm{E}-10$ & $2.89 \mathrm{E}-10$ & PELI1 & $1.28 \mathrm{E}+00$ \\
\hline ENSBTAG00000036101 & $4.79 \mathrm{E}-10$ & $3.75 \mathrm{E}-10$ & CCBL1 & $1.28 \mathrm{E}+00$ \\
\hline ENSBTAG00000039839 & $5.62 \mathrm{E}-10$ & $4.39 \mathrm{E}-10$ & IL36RN & $1.28 \mathrm{E}+00$ \\
\hline
\end{tabular}




\begin{tabular}{|c|c|c|c|c|}
\hline ENSBTAG00000023776 & $3.80 \mathrm{E}-10$ & $2.96 \mathrm{E}-10$ & C9orf116 & $1.28 \mathrm{E}+00$ \\
\hline ENSBTAG00000012126 & $2.25 \mathrm{E}-10$ & $1.75 \mathrm{E}-10$ & PNPLA7 & $1.28 \mathrm{E}+00$ \\
\hline ENSBTAG00000031669 & $5.18 \mathrm{E}-10$ & 4.03E-10 & & $1.28 \mathrm{E}+00$ \\
\hline ENSBTAG00000017576 & $6.86 \mathrm{E}-10$ & $5.29 \mathrm{E}-10$ & ADGRD2 & $1.30 \mathrm{E}+00$ \\
\hline ENSBTAG00000019354 & $5.61 \mathrm{E}-10$ & 4.33E-10 & PAX8 & $1.29 \mathrm{E}+00$ \\
\hline ENSBTAG00000012449 & $4.23 \mathrm{E}-10$ & $3.26 \mathrm{E}-10$ & & $1.30 \mathrm{E}+00$ \\
\hline ENSBTAG00000013072 & $5.91 \mathrm{E}-10$ & 4.54E-10 & FUBP3 & $1.30 \mathrm{E}+00$ \\
\hline ENSBTAG00000012844 & $2.30 \mathrm{E}-10$ & $1.77 \mathrm{E}-10$ & LRSAM1 & $1.30 \mathrm{E}+00$ \\
\hline ENSBTAG00000044064 & $2.90 \mathrm{E}-10$ & $2.22 \mathrm{E}-10$ & RNF144A & $1.30 \mathrm{E}+00$ \\
\hline ENSBTAG00000010111 & $1.33 \mathrm{E}-10$ & $1.02 \mathrm{E}-10$ & & $1.30 \mathrm{E}+00$ \\
\hline ENSBTAG00000044021 & $1.46 \mathrm{E}-10$ & $1.11 \mathrm{E}-10$ & FAM179A & $1.32 \mathrm{E}+00$ \\
\hline ENSBTAG00000046199 & 4.50E-10 & $3.42 \mathrm{E}-10$ & & $1.32 \mathrm{E}+00$ \\
\hline ENSBTAG00000038835 & 5.17E-10 & 3.93E-10 & & $1.32 \mathrm{E}+00$ \\
\hline ENSBTAG00000002716 & $4.46 \mathrm{E}-10$ & 3.39E-10 & NOL10 & $1.32 E+00$ \\
\hline ENSBTAG00000006948 & $3.73 \mathrm{E}-10$ & $2.83 \mathrm{E}-10$ & GARNL3 & $1.32 \mathrm{E}+00$ \\
\hline ENSBTAG00000017469 & $1.89 \mathrm{E}-10$ & $1.43 \mathrm{E}-10$ & CCDC88A & $1.32 \mathrm{E}+00$ \\
\hline ENSBTAG00000011124 & $1.22 \mathrm{E}-10$ & $9.21 \mathrm{E}-11$ & LHX3 & $1.32 \mathrm{E}+00$ \\
\hline ENSBTAG00000002542 & $9.45 \mathrm{E}-11$ & 7.14E-11 & KRCC1 & $1.32 \mathrm{E}+00$ \\
\hline ENSBTAG00000015038 & $1.55 \mathrm{E}-10$ & $1.17 \mathrm{E}-10$ & HADHA & $1.32 \mathrm{E}+00$ \\
\hline ENSBTAG00000039770 & $1.02 \mathrm{E}-10$ & 7.72E-11 & CEBPZ & $1.32 \mathrm{E}+00$ \\
\hline ENSBTAG00000009230 & 5.03E-10 & $3.80 \mathrm{E}-10$ & FBLN7 & $1.32 \mathrm{E}+00$ \\
\hline ENSBTAG00000039186 & 3.69E-10 & $2.79 \mathrm{E}-10$ & & $1.32 \mathrm{E}+00$ \\
\hline ENSBTAG00000046221 & $4.35 \mathrm{E}-10$ & $3.28 \mathrm{E}-10$ & OR1Q1 & $1.33 \mathrm{E}+00$ \\
\hline ENSBTAG00000034184 & $5.80 \mathrm{E}-10$ & $4.38 \mathrm{E}-10$ & NMS & $1.32 \mathrm{E}+00$ \\
\hline ENSBTAG00000001206 & $7.00 \mathrm{E}-10$ & $5.28 \mathrm{E}-10$ & LCN15 & $1.32 \mathrm{E}+00$ \\
\hline ENSBTAG00000037746 & $1.56 \mathrm{E}-10$ & $1.18 \mathrm{E}-10$ & SNRNP27 & $1.32 \mathrm{E}+00$ \\
\hline ENSBTAG00000008946 & $3.80 \mathrm{E}-10$ & $2.86 \mathrm{E}-10$ & CAPN13 & $1.33 \mathrm{E}+00$ \\
\hline ENSBTAG00000040585 & $1.33 \mathrm{E}-10$ & $9.98 \mathrm{E}-11$ & NR6A1 & $1.33 \mathrm{E}+00$ \\
\hline ENSBTAG00000030296 & $1.85 \mathrm{E}-10$ & $1.40 \mathrm{E}-10$ & LCNL1 & $1.32 E+00$ \\
\hline ENSBTAG00000046418 & $8.76 \mathrm{E}-10$ & $6.60 \mathrm{E}-10$ & RNF224 & $1.33 E+00$ \\
\hline ENSBTAG00000005432 & 1.10E-10 & $8.28 \mathrm{E}-11$ & CAPG & $1.32 \mathrm{E}+00$ \\
\hline ENSBTAG00000023867 & $1.38 \mathrm{E}-10$ & $1.03 \mathrm{E}-10$ & $\mathrm{RC} 3 \mathrm{H} 2$ & $1.34 \mathrm{E}+00$ \\
\hline ENSBTAG00000048155 & $7.84 \mathrm{E}-10$ & $5.87 \mathrm{E}-10$ & & $1.34 \mathrm{E}+00$ \\
\hline ENSBTAG00000018157 & $1.29 \mathrm{E}-10$ & $9.64 \mathrm{E}-11$ & IFT172 & $1.34 \mathrm{E}+00$ \\
\hline ENSBTAG00000005828 & 7.57E-10 & $5.64 \mathrm{E}-10$ & MERTK & $1.34 \mathrm{E}+00$ \\
\hline ENSBTAG00000012147 & 2.95E-10 & $2.20 \mathrm{E}-10$ & ARRDC1 & $1.34 \mathrm{E}+00$ \\
\hline ENSBTAG00000023788 & $3.33 \mathrm{E}-10$ & $2.48 \mathrm{E}-10$ & EXD3 & $1.34 \mathrm{E}+00$ \\
\hline ENSBTAG00000015965 & $8.80 \mathrm{E}-10$ & $6.54 \mathrm{E}-10$ & $\mathrm{ZC} 3 \mathrm{H} 8$ & $1.35 E+00$ \\
\hline ENSBTAG00000005273 & $6.06 \mathrm{E}-10$ & 4.49E-10 & IL1R1 & $1.35 \mathrm{E}+00$ \\
\hline ENSBTAG00000018799 & $3.77 \mathrm{E}-10$ & $2.79 \mathrm{E}-10$ & GPSM1 & $1.35 \mathrm{E}+00$ \\
\hline ENSBTAG00000010515 & $3.29 \mathrm{E}-10$ & $2.44 \mathrm{E}-10$ & FBXW5 & $1.35 \mathrm{E}+00$ \\
\hline ENSBTAG00000006027 & $1.71 \mathrm{E}-10$ & $1.26 \mathrm{E}-10$ & USP34 & $1.36 \mathrm{E}+00$ \\
\hline ENSBTAG00000013749 & $2.55 \mathrm{E}-10$ & $1.88 \mathrm{E}-10$ & RHOQ & $1.35 \mathrm{E}+00$ \\
\hline ENSBTAG00000045514 & 1.10E-09 & 8.13E-10 & & $1.36 \mathrm{E}+00$ \\
\hline ENSBTAG00000047061 & 2.77E-10 & $2.04 \mathrm{E}-10$ & & $1.36 \mathrm{E}+00$ \\
\hline ENSBTAG00000006075 & $5.72 \mathrm{E}-10$ & $4.20 \mathrm{E}-10$ & SUCLG1 & $1.36 \mathrm{E}+00$ \\
\hline ENSBTAG00000003902 & $3.28 \mathrm{E}-10$ & $2.41 \mathrm{E}-10$ & ZNF512 & $1.36 \mathrm{E}+00$ \\
\hline ENSBTAG00000030317 & $2.80 \mathrm{E}-10$ & $2.05 \mathrm{E}-10$ & PPP1R26 & $1.36 \mathrm{E}+00$ \\
\hline ENSBTAG00000010898 & $4.49 \mathrm{E}-10$ & $3.29 \mathrm{E}-10$ & SLC30A6 & $1.37 \mathrm{E}+00$ \\
\hline ENSBTAG00000024021 & $4.88 \mathrm{E}-10$ & $3.56 \mathrm{E}-10$ & & $1.37 \mathrm{E}+00$ \\
\hline ENSBTAG00000021102 & 4.27E-10 & $3.12 \mathrm{E}-10$ & GALM & $1.37 \mathrm{E}+00$ \\
\hline ENSBTAG00000024604 & $1.61 \mathrm{E}-10$ & $1.18 \mathrm{E}-10$ & & $1.36 \mathrm{E}+00$ \\
\hline ENSBTAG00000004168 & $1.81 \mathrm{E}-10$ & $1.32 \mathrm{E}-10$ & & $1.37 \mathrm{E}+00$ \\
\hline ENSBTAG00000007543 & $2.81 \mathrm{E}-10$ & $2.04 \mathrm{E}-10$ & TAF1B & $1.38 \mathrm{E}+00$ \\
\hline ENSBTAG00000001927 & $4.04 \mathrm{E}-10$ & $2.93 \mathrm{E}-10$ & ATP6V1C2 & $1.38 \mathrm{E}+00$ \\
\hline ENSBTAG00000004881 & $3.35 \mathrm{E}-10$ & $2.41 \mathrm{E}-10$ & MTHFD2 & $1.39 E+00$ \\
\hline ENSBTAG00000039878 & $7.02 \mathrm{E}-10$ & $5.06 \mathrm{E}-10$ & C2orf78 & $1.39 E+00$ \\
\hline ENSBTAG00000003610 & $2.29 \mathrm{E}-10$ & $1.65 E-10$ & DENND1A & $1.39 E+00$ \\
\hline ENSBTAG00000048125 & $2.09 \mathrm{E}-10$ & $1.50 \mathrm{E}-10$ & ENTPD2 & $1.39 E+00$ \\
\hline ENSBTAG00000003409 & $1.58 \mathrm{E}-10$ & $1.14 \mathrm{E}-10$ & FKBP1B & $1.39 E+00$ \\
\hline ENSBTAG00000047167 & $6.28 \mathrm{E}-10$ & 4.53E-10 & & $1.39 E+00$ \\
\hline ENSBTAG00000021072 & $2.45 \mathrm{E}-10$ & $1.76 \mathrm{E}-10$ & DTNB & $1.39 E+00$ \\
\hline ENSBTAG00000015278 & 7.97E-11 & $5.74 \mathrm{E}-11$ & ARPC5L & $1.39 E+00$ \\
\hline ENSBTAG00000000893 & $4.29 \mathrm{E}-10$ & $3.08 \mathrm{E}-10$ & & $1.39 E+00$ \\
\hline ENSBTAG00000048142 & $5.13 \mathrm{E}-10$ & $3.69 \mathrm{E}-10$ & GPR45 & $1.39 E+00$ \\
\hline ENSBTAG00000004695 & $1.29 \mathrm{E}-10$ & $9.28 \mathrm{E}-11$ & CDK9 & $1.39 E+00$ \\
\hline ENSBTAG00000046419 & 4.74E-10 & $3.40 \mathrm{E}-10$ & & $1.40 \mathrm{E}+00$ \\
\hline ENSBTAG00000023843 & $2.55 \mathrm{E}-10$ & $1.83 \mathrm{E}-10$ & RALGPS1 & $1.39 E+00$ \\
\hline ENSBTAG00000005014 & $3.83 \mathrm{E}-10$ & $2.73 \mathrm{E}-10$ & LCLAT1 & $1.40 \mathrm{E}+00$ \\
\hline ENSBTAG00000001114 & $1.99 \mathrm{E}-10$ & $1.42 \mathrm{E}-10$ & PRKD3 & $1.40 \mathrm{E}+00$ \\
\hline
\end{tabular}




\begin{tabular}{|c|c|c|c|c|}
\hline ENSBTAG000000015093 & $6.81 \mathrm{E}-10$ & $4.84 \mathrm{E}-10$ & ADAMTS13 & $1.41 \mathrm{E}+00$ \\
\hline ENSBTAG000000008113 & $2.66 \mathrm{E}-10$ & $1.89 \mathrm{E}-10$ & OSR1 & $1.41 E+00$ \\
\hline ENSBTAG000000017500 & $3.33 \mathrm{E}-10$ & $2.36 \mathrm{E}-10$ & KCNK12 & $1.41 E+00$ \\
\hline ENSBTAG000000038011 & $4.31 \mathrm{E}-10$ & $3.04 \mathrm{E}-10$ & SH3RF3 & $1.42 \mathrm{E}+00$ \\
\hline ENSBTAG000000019458 & $5.20 \mathrm{E}-10$ & $3.67 \mathrm{E}-10$ & & $1.42 \mathrm{E}+00$ \\
\hline ENSBTAG00000015286 & $1.44 \mathrm{E}-10$ & $1.02 \mathrm{E}-10$ & GOLGA1 & $1.41 E+00$ \\
\hline ENSBTAG000000009962 & $3.16 \mathrm{E}-10$ & $2.22 \mathrm{E}-10$ & PDCL3 & $1.42 \mathrm{E}+00$ \\
\hline ENSBTAG000000016722 & 5.37E-10 & $3.77 \mathrm{E}-10$ & PGGHG & $1.42 \mathrm{E}+00$ \\
\hline ENSBTAG00000016038 & $1.72 \mathrm{E}-10$ & $1.21 \mathrm{E}-10$ & $\mathrm{GCC} 2$ & $1.42 \mathrm{E}+00$ \\
\hline ENSBTAG000000021921 & $1.32 \mathrm{E}-10$ & $9.22 \mathrm{E}-11$ & STRBP & $1.43 \mathrm{E}+00$ \\
\hline ENSBTAG000000002329 & $7.79 \mathrm{E}-10$ & $5.47 \mathrm{E}-10$ & ASAP2 & $1.42 \mathrm{E}+00$ \\
\hline ENSBTAG000000048286 & $2.90 \mathrm{E}-10$ & $2.03 \mathrm{E}-10$ & E2F6 & $1.43 E+00$ \\
\hline ENSBTAG00000021187 & $3.20 \mathrm{E}-10$ & $2.24 \mathrm{E}-10$ & ID2 & $1.43 \mathrm{E}+00$ \\
\hline ENSBTAG000000000489 & $1.91 \mathrm{E}-10$ & $1.34 \mathrm{E}-10$ & WDR54 & $1.43 E+00$ \\
\hline ENSBTAG00000015151 & $1.37 \mathrm{E}-10$ & 9.57E-11 & FOXN2 & $1.44 \mathrm{E}+00$ \\
\hline ENSBTAG000000002033 & $5.01 \mathrm{E}-10$ & $3.47 \mathrm{E}-10$ & UBXN2A & $1.44 \mathrm{E}+00$ \\
\hline ENSBTAG000000001824 & $4.53 \mathrm{E}-10$ & $3.14 \mathrm{E}-10$ & SLC2A6 & $1.44 \mathrm{E}+00$ \\
\hline ENSBTAG000000015719 & $2.95 \mathrm{E}-10$ & $2.04 \mathrm{E}-10$ & PIGF & $1.45 \mathrm{E}+00$ \\
\hline ENSBTAG000000000985 & $1.06 \mathrm{E}-10$ & $7.30 \mathrm{E}-11$ & RAB10 & $1.45 E+00$ \\
\hline ENSBTAG000000017460 & $4.32 \mathrm{E}-10$ & $2.96 \mathrm{E}-10$ & & $1.46 \mathrm{E}+00$ \\
\hline ENSBTAG00000014891 & $3.31 \mathrm{E}-10$ & $2.27 \mathrm{E}-10$ & SRSF7 & $1.46 \mathrm{E}+00$ \\
\hline ENSBTAG00000047816 & $1.95 \mathrm{E}-09$ & 1.34E-09 & & $1.46 \mathrm{E}+00$ \\
\hline ENSBTAG000000003067 & 3.20E-10 & $2.18 \mathrm{E}-10$ & PSMB7 & $1.47 \mathrm{E}+00$ \\
\hline ENSBTAG000000004095 & $2.96 \mathrm{E}-10$ & $2.01 \mathrm{E}-10$ & FAM166A & $1.47 \mathrm{E}+00$ \\
\hline ENSBTAG000000004884 & $5.68 \mathrm{E}-10$ & $3.85 \mathrm{E}-10$ & SLC4A5 & $1.47 \mathrm{E}+00$ \\
\hline ENSBTAG000000004824 & 7.69E-10 & $5.21 \mathrm{E}-10$ & REEP1 & $1.48 \mathrm{E}+00$ \\
\hline ENSBTAG000000015085 & $2.40 \mathrm{E}-10$ & $1.63 \mathrm{E}-10$ & CLIC3 & $1.47 E+00$ \\
\hline ENSBTAG000000004948 & $3.85 \mathrm{E}-10$ & $2.58 \mathrm{E}-10$ & FAM84A & $1.49 E+00$ \\
\hline ENSBTAG00000035244 & $7.28 \mathrm{E}-10$ & $4.86 \mathrm{E}-10$ & ANKRD39 & $1.50 \mathrm{E}+00$ \\
\hline ENSBTAG000000018555 & 7.93E-10 & $5.29 \mathrm{E}-10$ & SFXN5 & $1.50 \mathrm{E}+00$ \\
\hline ENSBTAG000000065558 & $2.93 \mathrm{E}-10$ & $1.95 \mathrm{E}-10$ & SOHLH1 & $1.50 \mathrm{E}+00$ \\
\hline ENSBTAG000000038440 & 1.07E-09 & 7.12E-10 & & $1.51 E+00$ \\
\hline ENSBTAG00000015098 & 3.97E-10 & 2.64E-10 & RABEPK & $1.50 \mathrm{E}+00$ \\
\hline ENSBTAG000000003407 & $1.92 \mathrm{E}-10$ & 1.27E-10 & SF3B6 & $1.51 E+00$ \\
\hline ENSBTAG00000030247 & $2.71 \mathrm{E}-10$ & $1.78 \mathrm{E}-10$ & NSMF & $1.52 \mathrm{E}+00$ \\
\hline ENSBTAG00000017158 & 4.97E-10 & $3.25 \mathrm{E}-10$ & VSNL1 & $1.53 E+00$ \\
\hline ENSBTAG00000020307 & $2.79 \mathrm{E}-10$ & $1.82 \mathrm{E}-10$ & C9orf78 & $1.53 \mathrm{E}+00$ \\
\hline ENSBTAG00000018590 & 2.15E-10 & $1.40 \mathrm{E}-10$ & STRN & $1.54 \mathrm{E}+00$ \\
\hline ENSBTAG00000013290 & 3.27E-10 & $2.12 \mathrm{E}-10$ & DYSF & $1.54 \mathrm{E}+00$ \\
\hline ENSBTAG00000006019 & $7.54 \mathrm{E}-10$ & 4.87E-10 & FAM178B & $1.55 E+00$ \\
\hline ENSBTAG00000018046 & $1.93 \mathrm{E}-10$ & $1.25 \mathrm{E}-10$ & & $1.55 E+00$ \\
\hline ENSBTAG00000006852 & $7.03 \mathrm{E}-10$ & $4.51 \mathrm{E}-10$ & ACYP2 & $1.56 \mathrm{E}+00$ \\
\hline ENSBTAG00000004105 & 1.07E-09 & $6.84 \mathrm{E}-10$ & TMEM247 & $1.57 \mathrm{E}+00$ \\
\hline ENSBTAG00000015587 & $1.26 \mathrm{E}-10$ & 7.95E-11 & TTC32 & $1.59 E+00$ \\
\hline ENSBTAG00000000382 & $3.24 \mathrm{E}-10$ & $2.04 \mathrm{E}-10$ & MED27 & $1.59 E+00$ \\
\hline ENSBTAG00000001843 & $6.85 \mathrm{E}-11$ & 4.31E-11 & WBP1 & $1.59 \mathrm{E}+00$ \\
\hline ENSBTAG00000034995 & $9.82 \mathrm{E}-11$ & $6.18 \mathrm{E}-11$ & ITPRIPL1 & $1.59 \mathrm{E}+00$ \\
\hline ENSBTAG00000020873 & $9.84 \mathrm{E}-11$ & $6.20 \mathrm{E}-11$ & MAT2A & $1.59 E+00$ \\
\hline ENSBTAG00000046516 & $3.71 \mathrm{E}-10$ & $2.33 \mathrm{E}-10$ & FUT7 & $1.59 E+00$ \\
\hline ENSBTAG00000001352 & $3.67 \mathrm{E}-10$ & $2.31 \mathrm{E}-10$ & SDCCAG3 & $1.59 \mathrm{E}+00$ \\
\hline ENSBTAG00000015521 & $9.07 \mathrm{E}-11$ & $5.71 \mathrm{E}-11$ & GEMIN6 & $1.59 \mathrm{E}+00$ \\
\hline ENSBTAG00000020124 & $1.99 \mathrm{E}-10$ & $1.25 \mathrm{E}-10$ & HTRA2 & $1.59 \mathrm{E}+00$ \\
\hline ENSBTAG00000027017 & 4.05E-10 & $2.55 \mathrm{E}-10$ & SIX3 & $1.59 \mathrm{E}+00$ \\
\hline ENSBTAG00000001105 & $3.22 \mathrm{E}-10$ & $2.02 \mathrm{E}-10$ & ANXA4 & $1.59 \mathrm{E}+00$ \\
\hline ENSBTAG00000038309 & $5.24 \mathrm{E}-10$ & $3.29 \mathrm{E}-10$ & & $1.59 E+00$ \\
\hline ENSBTAG00000015097 & $3.11 \mathrm{E}-10$ & $1.95 \mathrm{E}-10$ & CACFD1 & $1.59 \mathrm{E}+00$ \\
\hline ENSBTAG00000046310 & $4.85 \mathrm{E}-10$ & $3.05 \mathrm{E}-10$ & & $1.59 \mathrm{E}+00$ \\
\hline ENSBTAG00000004663 & $1.82 \mathrm{E}-10$ & $1.15 \mathrm{E}-10$ & TRUB2 & $1.59 E+00$ \\
\hline ENSBTAG00000014679 & $2.81 \mathrm{E}-10$ & $1.77 \mathrm{E}-10$ & DHX57 & $1.59 \mathrm{E}+00$ \\
\hline ENSBTAG00000004091 & $1.94 \mathrm{E}-10$ & $1.22 \mathrm{E}-10$ & GTF3C4 & $1.59 E+00$ \\
\hline ENSBTAG00000020575 & $7.24 \mathrm{E}-10$ & 4.54E-10 & IFITM5 & $1.59 \mathrm{E}+00$ \\
\hline ENSBTAG00000021549 & $2.27 \mathrm{E}-10$ & $1.43 \mathrm{E}-10$ & PHPT1 & $1.59 \mathrm{E}+00$ \\
\hline ENSBTAG00000047728 & $5.22 \mathrm{E}-10$ & $3.28 \mathrm{E}-10$ & & $1.59 \mathrm{E}+00$ \\
\hline ENSBTAG00000030278 & $8.92 \mathrm{E}-10$ & $5.60 \mathrm{E}-10$ & & $1.59 E+00$ \\
\hline ENSBTAG00000020894 & $1.28 \mathrm{E}-10$ & 8.04E-11 & LAPTM4A & $1.59 \mathrm{E}+00$ \\
\hline ENSBTAG00000001108 & $2.04 \mathrm{E}-10$ & $1.28 \mathrm{E}-10$ & GMCL1 & $1.60 \mathrm{E}+00$ \\
\hline ENSBTAG00000004400 & $2.45 \mathrm{E}-10$ & $1.52 \mathrm{E}-10$ & PRRC2B & $1.61 E+00$ \\
\hline ENSBTAG00000009600 & $6.07 \mathrm{E}-10$ & $3.77 \mathrm{E}-10$ & STAMBP & $1.61 E+00$ \\
\hline ENSBTAG00000031332 & $1.71 \mathrm{E}-10$ & $1.06 \mathrm{E}-10$ & RBKS & $1.62 \mathrm{E}+00$ \\
\hline ENSBTAG00000003861 & $3.02 \mathrm{E}-10$ & $1.86 \mathrm{E}-10$ & GPATCH11 & $1.62 E+00$ \\
\hline
\end{tabular}




\begin{tabular}{|c|c|c|c|c|}
\hline ENSBTAG00000002803 & $9.74 \mathrm{E}-10$ & $6.01 \mathrm{E}-10$ & SULT1C3 & $1.62 \mathrm{E}+00$ \\
\hline ENSBTAG00000015659 & $5.58 \mathrm{E}-10$ & $3.43 \mathrm{E}-10$ & CIAO1 & $1.63 E+00$ \\
\hline ENSBTAG00000047449 & $9.09 \mathrm{E}-10$ & $5.50 \mathrm{E}-10$ & & $1.65 E+00$ \\
\hline ENSBTAG00000016061 & $1.28 \mathrm{E}-10$ & $7.75 \mathrm{E}-11$ & RSAD2 & $1.65 \mathrm{E}+00$ \\
\hline ENSBTAG00000018267 & $9.67 \mathrm{E}-11$ & $5.84 \mathrm{E}-11$ & TRIM54 & $1.65 \mathrm{E}+00$ \\
\hline ENSBTAG00000019839 & $3.51 \mathrm{E}-10$ & $2.12 \mathrm{E}-10$ & LTBP1 & $1.66 \mathrm{E}+00$ \\
\hline ENSBTAG00000001353 & $3.92 \mathrm{E}-10$ & $2.36 \mathrm{E}-10$ & PMPCA & $1.66 \mathrm{E}+00$ \\
\hline ENSBTAG00000047202 & $2.34 \mathrm{E}-10$ & $1.41 \mathrm{E}-10$ & GRIN1 & $1.66 \mathrm{E}+00$ \\
\hline ENSBTAG00000008068 & $3.57 \mathrm{E}-10$ & $2.14 \mathrm{E}-10$ & ERLEC1 & $1.67 E+00$ \\
\hline ENSBTAG00000014668 & $3.94 \mathrm{E}-10$ & $2.36 \mathrm{E}-10$ & NAGK & $1.67 E+00$ \\
\hline ENSBTAG00000027991 & $5.43 \mathrm{E}-10$ & $3.25 \mathrm{E}-10$ & ANAPC1 & $1.67 E+00$ \\
\hline ENSBTAG00000016148 & 8.97E-10 & $5.33 \mathrm{E}-10$ & & $1.68 \mathrm{E}+00$ \\
\hline ENSBTAG00000019072 & $9.32 \mathrm{E}-10$ & $5.53 \mathrm{E}-10$ & PSD4 & $1.69 \mathrm{E}+00$ \\
\hline ENSBTAG00000009343 & $2.32 \mathrm{E}-10$ & $1.37 \mathrm{E}-10$ & AAK1 & $1.69 E+00$ \\
\hline ENSBTAG00000021896 & $3.17 E-10$ & $1.86 \mathrm{E}-10$ & NUP214 & $1.70 \mathrm{E}+00$ \\
\hline ENSBTAG00000008232 & $2.61 \mathrm{E}-10$ & $1.53 \mathrm{E}-10$ & DAB2IP & $1.70 \mathrm{E}+00$ \\
\hline ENSBTAG00000004296 & $3.02 \mathrm{E}-10$ & $1.77 \mathrm{E}-10$ & MORN5 & $1.71 E+00$ \\
\hline ENSBTAG00000007675 & $2.90 \mathrm{E}-10$ & $1.69 \mathrm{E}-10$ & NPHP1 & $1.71 E+00$ \\
\hline ENSBTAG00000015667 & $6.35 \mathrm{E}-10$ & $3.70 \mathrm{E}-10$ & TMEM127 & $1.72 \mathrm{E}+00$ \\
\hline ENSBTAG00000021373 & $2.96 \mathrm{E}-10$ & $1.72 \mathrm{E}-10$ & GTF3C5 & $1.72 \mathrm{E}+00$ \\
\hline ENSBTAG00000003205 & $2.31 \mathrm{E}-10$ & $1.34 \mathrm{E}-10$ & & $1.72 \mathrm{E}+00$ \\
\hline ENSBTAG00000024980 & $4.37 \mathrm{E}-10$ & $2.54 \mathrm{E}-10$ & VIT & $1.72 \mathrm{E}+00$ \\
\hline ENSBTAG00000008707 & $2.04 \mathrm{E}-10$ & $1.18 \mathrm{E}-10$ & SULT6B1 & $1.73 \mathrm{E}+00$ \\
\hline ENSBTAG00000023885 & $1.39 \mathrm{E}-10$ & $8.05 \mathrm{E}-11$ & ZNF79 & $1.72 \mathrm{E}+00$ \\
\hline ENSBTAG00000006482 & $5.94 \mathrm{E}-10$ & $3.42 \mathrm{E}-10$ & PTCD3 & $1.74 \mathrm{E}+00$ \\
\hline ENSBTAG00000034384 & $7.81 \mathrm{E}-10$ & $4.49 \mathrm{E}-10$ & VWA3B & $1.74 \mathrm{E}+00$ \\
\hline ENSBTAG00000038116 & $1.92 \mathrm{E}-10$ & $1.10 \mathrm{E}-10$ & TGOLN2 & $1.74 \mathrm{E}+00$ \\
\hline ENSBTAG00000007492 & $2.90 \mathrm{E}-10$ & $1.66 \mathrm{E}-10$ & ADAMTSL2 & $1.75 E+00$ \\
\hline ENSBTAG00000019628 & $1.11 \mathrm{E}-10$ & $6.35 \mathrm{E}-11$ & EDAR & $1.75 \mathrm{E}+00$ \\
\hline ENSBTAG00000047807 & $1.87 \mathrm{E}-10$ & 1.07E-10 & SAPCD2 & $1.74 \mathrm{E}+00$ \\
\hline ENSBTAG00000023933 & $3.26 \mathrm{E}-10$ & $1.86 \mathrm{E}-10$ & SEC16A & $1.75 E+00$ \\
\hline ENSBTAG00000020630 & $2.51 \mathrm{E}-10$ & $1.43 \mathrm{E}-10$ & KCMF1 & $1.75 E+00$ \\
\hline ENSBTAG00000007689 & $2.45 \mathrm{E}-10$ & $1.39 \mathrm{E}-10$ & LPIN1 & $1.76 \mathrm{E}+00$ \\
\hline ENSBTAG00000020799 & $4.56 \mathrm{E}-10$ & $2.58 \mathrm{E}-10$ & EXOC6B & $1.77 \mathrm{E}+00$ \\
\hline ENSBTAG00000008269 & $3.76 \mathrm{E}-10$ & $2.12 \mathrm{E}-10$ & RNF149 & $1.77 \mathrm{E}+00$ \\
\hline ENSBTAG00000006861 & $7.31 \mathrm{E}-10$ & $4.12 \mathrm{E}-10$ & C2orf73 & $1.78 \mathrm{E}+00$ \\
\hline ENSBTAG00000001042 & $1.30 \mathrm{E}-10$ & $7.26 \mathrm{E}-11$ & MXD1 & $1.79 E+00$ \\
\hline ENSBTAG00000010531 & $1.34 \mathrm{E}-10$ & 7.49E-11 & CYP1B1 & $1.79 \mathrm{E}+00$ \\
\hline ENSBTAG00000012480 & $1.65 \mathrm{E}-10$ & $9.22 \mathrm{E}-11$ & TBC1D13 & $1.79 \mathrm{E}+00$ \\
\hline ENSBTAG00000019138 & $1.07 \mathrm{E}-10$ & $6.05 \mathrm{E}-11$ & KIF3C & $1.77 \mathrm{E}+00$ \\
\hline ENSBTAG00000019701 & $2.76 \mathrm{E}-10$ & $1.54 \mathrm{E}-10$ & & $1.79 E+00$ \\
\hline ENSBTAG00000012148 & $3.58 \mathrm{E}-10$ & $1.99 \mathrm{E}-10$ & EHMT1 & $1.80 \mathrm{E}+00$ \\
\hline ENSBTAG00000048041 & $7.45 \mathrm{E}-10$ & $4.15 \mathrm{E}-10$ & ACTR1B & $1.79 \mathrm{E}+00$ \\
\hline ENSBTAG00000003975 & $5.21 \mathrm{E}-10$ & $2.89 \mathrm{E}-10$ & LMAN2L & $1.80 \mathrm{E}+00$ \\
\hline ENSBTAG00000007963 & $3.36 \mathrm{E}-10$ & $1.86 \mathrm{E}-10$ & KIDINS220 & $1.81 E+00$ \\
\hline ENSBTAG00000003963 & $7.88 \mathrm{E}-10$ & 4.35E-10 & FER1L5 & $1.81 \mathrm{E}+00$ \\
\hline ENSBTAG00000002044 & $5.05 \mathrm{E}-10$ & $2.79 \mathrm{E}-10$ & SIRT3 & $1.81 E+00$ \\
\hline ENSBTAG00000044129 & $1.23 \mathrm{E}-10$ & $6.76 \mathrm{E}-11$ & ST6GALNAC6 & $1.82 \mathrm{E}+00$ \\
\hline ENSBTAG00000006008 & $1.94 \mathrm{E}-10$ & $1.06 \mathrm{E}-10$ & CAMSAP1 & $1.83 \mathrm{E}+00$ \\
\hline ENSBTAG00000005525 & $1.97 \mathrm{E}-10$ & $1.07 \mathrm{E}-10$ & LHX6 & $1.84 \mathrm{E}+00$ \\
\hline ENSBTAG00000020567 & $9.58 \mathrm{E}-11$ & $5.21 \mathrm{E}-11$ & HNRNPLL & $1.84 \mathrm{E}+00$ \\
\hline ENSBTAG00000005189 & $2.72 \mathrm{E}-10$ & $1.48 \mathrm{E}-10$ & SPACA9 & $1.84 \mathrm{E}+00$ \\
\hline ENSBTAG00000024044 & $6.19 E-10$ & $3.36 \mathrm{E}-10$ & CDKL4 & $1.84 \mathrm{E}+00$ \\
\hline ENSBTAG00000024058 & $2.32 \mathrm{E}-10$ & $1.25 \mathrm{E}-10$ & EGR4 & $1.85 E+00$ \\
\hline ENSBTAG00000047112 & $6.12 \mathrm{E}-10$ & $3.29 \mathrm{E}-10$ & & $1.86 \mathrm{E}+00$ \\
\hline ENSBTAG00000002965 & $2.60 \mathrm{E}-10$ & $1.40 \mathrm{E}-10$ & NEURL3 & $1.86 \mathrm{E}+00$ \\
\hline ENSBTAG00000011101 & $8.51 \mathrm{E}-10$ & $4.58 \mathrm{E}-10$ & EML6 & $1.86 \mathrm{E}+00$ \\
\hline ENSBTAG00000010388 & $4.73 \mathrm{E}-10$ & $2.54 \mathrm{E}-10$ & MGAT4A & $1.86 \mathrm{E}+00$ \\
\hline ENSBTAG00000024105 & $5.36 \mathrm{E}-10$ & $2.87 \mathrm{E}-10$ & BCL2L11 & $1.87 \mathrm{E}+00$ \\
\hline ENSBTAG00000003711 & $3.55 \mathrm{E}-10$ & $1.90 \mathrm{E}-10$ & EPAS1 & $1.87 \mathrm{E}+00$ \\
\hline ENSBTAG00000023797 & $2.46 \mathrm{E}-10$ & $1.32 \mathrm{E}-10$ & PRDM12 & $1.87 \mathrm{E}+00$ \\
\hline ENSBTAG00000011154 & $2.50 \mathrm{E}-10$ & $1.34 \mathrm{E}-10$ & SDC1 & $1.86 \mathrm{E}+00$ \\
\hline ENSBTAG00000007485 & $2.29 \mathrm{E}-10$ & $1.22 \mathrm{E}-10$ & GRHL1 & $1.87 \mathrm{E}+00$ \\
\hline ENSBTAG00000030384 & $1.93 \mathrm{E}-10$ & $1.03 \mathrm{E}-10$ & & $1.87 \mathrm{E}+00$ \\
\hline ENSBTAG00000019515 & $2.71 \mathrm{E}-10$ & $1.44 \mathrm{E}-10$ & SNX17 & $1.88 \mathrm{E}+00$ \\
\hline ENSBTAG00000005892 & $7.81 \mathrm{E}-10$ & 4.13E-10 & ZAP70 & $1.89 \mathrm{E}+00$ \\
\hline ENSBTAG00000021141 & $3.52 \mathrm{E}-10$ & $1.86 \mathrm{E}-10$ & CD8A & $1.89 \mathrm{E}+00$ \\
\hline ENSBTAG00000005190 & $3.03 \mathrm{E}-10$ & $1.60 \mathrm{E}-10$ & TSC1 & $1.89 \mathrm{E}+00$ \\
\hline ENSBTAG00000003108 & $2.79 \mathrm{E}-10$ & $1.47 \mathrm{E}-10$ & SRD5A2 & $1.90 \mathrm{E}+00$ \\
\hline ENSBTAG00000012212 & $3.47 \mathrm{E}-10$ & $1.82 \mathrm{E}-10$ & CYP26B1 & $1.90 \mathrm{E}+00$ \\
\hline
\end{tabular}




\begin{tabular}{|c|c|c|c|c|}
\hline ENSBTAG00000004511 & $9.75 \mathrm{E}-10$ & $5.09 \mathrm{E}-10$ & VAV2 & $1.92 \mathrm{E}+00$ \\
\hline ENSBTAG00000007235 & $4.41 \mathrm{E}-10$ & $2.30 \mathrm{E}-10$ & NTNG2 & $1.92 E+00$ \\
\hline ENSBTAG00000007486 & $2.68 \mathrm{E}-10$ & $1.40 \mathrm{E}-10$ & CEL & $1.92 \mathrm{E}+00$ \\
\hline ENSBTAG00000018269 & $1.50 \mathrm{E}-10$ & $7.78 \mathrm{E}-11$ & MPV17 & $1.93 \mathrm{E}+00$ \\
\hline ENSBTAG00000038691 & $3.73 \mathrm{E}-10$ & $1.94 \mathrm{E}-10$ & DPH7 & $1.92 \mathrm{E}+00$ \\
\hline ENSBTAG00000007763 & $2.52 \mathrm{E}-10$ & $1.31 \mathrm{E}-10$ & SLC1A4 & $1.92 \mathrm{E}+00$ \\
\hline ENSBTAG00000046548 & $1.97 \mathrm{E}-10$ & $1.01 \mathrm{E}-10$ & ST6GALNAC4 & $1.95 \mathrm{E}+00$ \\
\hline ENSBTAG00000006957 & $2.00 \mathrm{E}-10$ & $1.03 \mathrm{E}-10$ & SLC2A8 & $1.94 \mathrm{E}+00$ \\
\hline ENSBTAG00000010208 & $1.55 \mathrm{E}-10$ & 7.97E-11 & ZER1 & $1.95 \mathrm{E}+00$ \\
\hline ENSBTAG00000011104 & $2.98 \mathrm{E}-10$ & $1.51 \mathrm{E}-10$ & RTN4 & $1.97 E+00$ \\
\hline ENSBTAG00000021735 & $3.46 \mathrm{E}-10$ & $1.74 \mathrm{E}-10$ & GALNT14 & $1.99 \mathrm{E}+00$ \\
\hline ENSBTAG00000018262 & $1.28 \mathrm{E}-10$ & $6.45 \mathrm{E}-11$ & DNAJC5G & $1.98 \mathrm{E}+00$ \\
\hline ENSBTAG00000014072 & 5.33E-10 & $2.68 \mathrm{E}-10$ & & $1.99 \mathrm{E}+00$ \\
\hline ENSBTAG00000002545 & $4.51 \mathrm{E}-10$ & $2.26 \mathrm{E}-10$ & & $1.99 \mathrm{E}+00$ \\
\hline ENSBTAG00000001086 & $6.58 \mathrm{E}-10$ & $3.29 \mathrm{E}-10$ & FHL2 & $2.00 \mathrm{E}+00$ \\
\hline ENSBTAG00000001408 & $1.11 \mathrm{E}-10$ & $5.53 \mathrm{E}-11$ & CNRIP1 & $2.01 E+00$ \\
\hline ENSBTAG00000012779 & $6.79 \mathrm{E}-10$ & $3.38 \mathrm{E}-10$ & FAM49A & $2.01 E+00$ \\
\hline ENSBTAG00000010349 & $3.21 \mathrm{E}-10$ & $1.60 \mathrm{E}-10$ & IL1A & $2.01 E+00$ \\
\hline ENSBTAG00000000168 & 3.35E-09 & $1.66 \mathrm{E}-09$ & & $2.02 \mathrm{E}+00$ \\
\hline ENSBTAG00000001034 & $5.40 \mathrm{E}-10$ & 2.67E-10 & IL18R1 & $2.02 \mathrm{E}+00$ \\
\hline ENSBTAG00000013023 & 4.16E-10 & $2.04 \mathrm{E}-10$ & MAP4K4 & $2.04 \mathrm{E}+00$ \\
\hline ENSBTAG00000019513 & $1.97 \mathrm{E}-10$ & $9.66 \mathrm{E}-11$ & C9orf50 & $2.04 \mathrm{E}+00$ \\
\hline ENSBTAG00000018256 & $1.70 \mathrm{E}-10$ & $8.30 \mathrm{E}-11$ & SMC6 & $2.05 \mathrm{E}+00$ \\
\hline ENSBTAG00000018542 & $7.24 \mathrm{E}-10$ & $3.53 \mathrm{E}-10$ & & $2.05 E+00$ \\
\hline ENSBTAG00000018239 & $1.23 \mathrm{E}-10$ & $5.98 \mathrm{E}-11$ & DNAJC27 & $2.06 \mathrm{E}+00$ \\
\hline ENSBTAG00000017448 & $3.30 \mathrm{E}-10$ & $1.60 \mathrm{E}-10$ & EFEMP1 & $2.06 \mathrm{E}+00$ \\
\hline ENSBTAG00000038278 & 1.60E-09 & $7.78 \mathrm{E}-10$ & & $2.06 \mathrm{E}+00$ \\
\hline ENSBTAG00000011172 & $7.84 \mathrm{E}-10$ & $3.81 \mathrm{E}-10$ & ALMS1 & $2.06 \mathrm{E}+00$ \\
\hline ENSBTAG00000010228 & $1.78 \mathrm{E}-10$ & $8.64 \mathrm{E}-11$ & LMX1B & $2.06 \mathrm{E}+00$ \\
\hline ENSBTAG00000010083 & $1.95 \mathrm{E}-10$ & $9.40 \mathrm{E}-11$ & HADHB & $2.07 \mathrm{E}+00$ \\
\hline ENSBTAG00000004259 & $6.95 \mathrm{E}-10$ & $3.35 \mathrm{E}-10$ & HPCAL1 & $2.07 E+00$ \\
\hline ENSBTAG00000039263 & $6.58 \mathrm{E}-10$ & $3.18 \mathrm{E}-10$ & & $2.07 E+00$ \\
\hline ENSBTAG00000046223 & $2.83 \mathrm{E}-10$ & $1.36 \mathrm{E}-10$ & & $2.08 \mathrm{E}+00$ \\
\hline ENSBTAG00000003691 & $3.19 E-10$ & $1.52 \mathrm{E}-10$ & NTSR2 & $2.10 \mathrm{E}+00$ \\
\hline ENSBTAG00000031335 & $1.99 \mathrm{E}-10$ & $9.48 \mathrm{E}-11$ & BRE & $2.10 \mathrm{E}+00$ \\
\hline ENSBTAG00000001947 & $1.48 \mathrm{E}-10$ & $6.98 \mathrm{E}-11$ & ZNF514 & $2.12 E+00$ \\
\hline ENSBTAG00000046117 & $2.66 \mathrm{E}-10$ & $1.26 \mathrm{E}-10$ & & $2.11 E+00$ \\
\hline ENSBTAG00000010849 & 4.60E-10 & 2.17E-10 & ANKRD23 & $2.12 \mathrm{E}+00$ \\
\hline ENSBTAG00000012949 & $1.12 \mathrm{E}-10$ & $5.29 \mathrm{E}-11$ & GKN1 & $2.12 \mathrm{E}+00$ \\
\hline ENSBTAG00000010971 & $1.89 \mathrm{E}-10$ & 8.92E-11 & & $2.12 \mathrm{E}+00$ \\
\hline ENSBTAG00000003965 & 4.55E-10 & $2.15 E-10$ & LIPT1 & $2.12 \mathrm{E}+00$ \\
\hline ENSBTAG00000023163 & $1.91 \mathrm{E}-10$ & $9.00 \mathrm{E}-11$ & & $2.12 \mathrm{E}+00$ \\
\hline ENSBTAG00000021230 & 4.43E-10 & $2.08 \mathrm{E}-10$ & SFTPB & $2.13 E+00$ \\
\hline ENSBTAG00000009906 & $8.03 E-10$ & $3.75 \mathrm{E}-10$ & DDX1 & $2.14 \mathrm{E}+00$ \\
\hline ENSBTAG00000038369 & $1.06 \mathrm{E}-09$ & $4.96 \mathrm{E}-10$ & SULT1C2 & $2.14 \mathrm{E}+00$ \\
\hline ENSBTAG00000006989 & $1.48 \mathrm{E}-10$ & $6.87 \mathrm{E}-11$ & SEMA4F & $2.15 E+00$ \\
\hline ENSBTAG00000019456 & $1.64 \mathrm{E}-10$ & 7.63E-11 & SPRED2 & $2.15 E+00$ \\
\hline ENSBTAG00000013589 & $3.69 \mathrm{E}-10$ & $1.71 \mathrm{E}-10$ & & $2.16 \mathrm{E}+00$ \\
\hline ENSBTAG00000013242 & 4.17E-10 & $1.92 \mathrm{E}-10$ & TMEM $8 \mathrm{C}$ & $2.17 \mathrm{E}+00$ \\
\hline ENSBTAG00000015375 & $2.13 \mathrm{E}-10$ & $9.76 \mathrm{E}-11$ & $\mathrm{SH} 2 \mathrm{D} 3 \mathrm{C}$ & $2.18 \mathrm{E}+00$ \\
\hline ENSBTAG00000017283 & $7.08 \mathrm{E}-11$ & $3.24 \mathrm{E}-11$ & GTF3C2 & $2.18 \mathrm{E}+00$ \\
\hline ENSBTAG00000016600 & $1.42 \mathrm{E}-10$ & $6.51 \mathrm{E}-11$ & CCDC142 & $2.18 \mathrm{E}+00$ \\
\hline ENSBTAG00000005847 & $1.87 \mathrm{E}-10$ & $8.55 \mathrm{E}-11$ & ROCK2 & $2.18 \mathrm{E}+00$ \\
\hline ENSBTAG00000021161 & $7.55 \mathrm{E}-10$ & $3.43 \mathrm{E}-10$ & CREG2 & $2.20 \mathrm{E}+00$ \\
\hline ENSBTAG00000011601 & $3.24 \mathrm{E}-10$ & 1.47E-10 & ST3GAL5 & $2.21 E+00$ \\
\hline ENSBTAG00000016720 & $2.13 \mathrm{E}-10$ & $9.66 \mathrm{E}-11$ & RAB1A & $2.21 E+00$ \\
\hline ENSBTAG00000010990 & $1.29 \mathrm{E}-10$ & $5.75 \mathrm{E}-11$ & LHX2 & $2.24 \mathrm{E}+00$ \\
\hline ENSBTAG00000016270 & $1.59 \mathrm{E}-10$ & 7.13E-11 & & $2.22 \mathrm{E}+00$ \\
\hline ENSBTAG00000021143 & $1.65 \mathrm{E}-10$ & $7.40 \mathrm{E}-11$ & DNMT3A & $2.23 \mathrm{E}+00$ \\
\hline ENSBTAG00000004510 & $6.19 E-10$ & $2.75 \mathrm{E}-10$ & SARDH & $2.25 \mathrm{E}+00$ \\
\hline ENSBTAG00000002837 & $2.93 \mathrm{E}-10$ & $1.30 \mathrm{E}-10$ & TOR1B & $2.25 \mathrm{E}+00$ \\
\hline ENSBTAG00000019522 & $1.48 \mathrm{E}-10$ & $6.57 \mathrm{E}-11$ & PPM1G & $2.26 \mathrm{E}+00$ \\
\hline ENSBTAG00000003555 & $2.94 \mathrm{E}-10$ & $1.30 \mathrm{E}-10$ & THADA & $2.26 \mathrm{E}+00$ \\
\hline ENSBTAG00000024061 & $7.45 \mathrm{E}-10$ & $3.28 \mathrm{E}-10$ & NOTO & $2.27 E+00$ \\
\hline ENSBTAG00000030566 & $2.02 \mathrm{E}-10$ & $8.86 \mathrm{E}-11$ & GLE1 & $2.27 \mathrm{E}+00$ \\
\hline ENSBTAG00000006150 & $7.21 \mathrm{E}-10$ & $3.16 \mathrm{E}-10$ & MRPS9 & $2.28 \mathrm{E}+00$ \\
\hline ENSBTAG00000010623 & $3.98 \mathrm{E}-10$ & $1.74 \mathrm{E}-10$ & ANKRD53 & $2.29 \mathrm{E}+00$ \\
\hline ENSBTAG00000018669 & $2.68 \mathrm{E}-10$ & 1.17E-10 & PLB1 & $2.29 \mathrm{E}+00$ \\
\hline ENSBTAG00000000223 & $1.91 \mathrm{E}-10$ & $8.28 \mathrm{E}-11$ & PPM1B & $2.30 \mathrm{E}+00$ \\
\hline ENSBTAG00000001744 & $5.51 \mathrm{E}-10$ & $2.38 \mathrm{E}-10$ & BET1L & $2.31 E+00$ \\
\hline
\end{tabular}




\begin{tabular}{|c|c|c|c|c|}
\hline ENSBTAG00000021181 & $7.93 \mathrm{E}-10$ & $3.42 \mathrm{E}-10$ & BUB1 & $2.32 \mathrm{E}+00$ \\
\hline ENSBTAG00000004674 & $2.00 \mathrm{E}-10$ & $8.65 \mathrm{E}-11$ & HAAO & $2.32 E+00$ \\
\hline ENSBTAG00000018196 & $1.68 \mathrm{E}-10$ & $7.13 \mathrm{E}-11$ & WDR43 & $2.35 E+00$ \\
\hline ENSBTAG00000030567 & $2.06 \mathrm{E}-10$ & $8.76 \mathrm{E}-11$ & ODF2 & $2.35 \mathrm{E}+00$ \\
\hline ENSBTAG00000002108 & $2.72 \mathrm{E}-10$ & $1.15 \mathrm{E}-10$ & YWHAQ & $2.36 \mathrm{E}+00$ \\
\hline ENSBTAG00000011155 & $2.78 \mathrm{E}-10$ & $1.18 \mathrm{E}-10$ & PUM2 & $2.36 \mathrm{E}+00$ \\
\hline ENSBTAG00000004204 & $3.43 \mathrm{E}-10$ & $1.44 \mathrm{E}-10$ & GPR107 & $2.38 \mathrm{E}+00$ \\
\hline ENSBTAG00000023744 & $1.73 \mathrm{E}-10$ & 7.27E-11 & YPEL5 & $2.38 \mathrm{E}+00$ \\
\hline ENSBTAG00000008985 & $1.67 \mathrm{E}-10$ & $7.00 \mathrm{E}-11$ & PCBP1 & $2.38 \mathrm{E}+00$ \\
\hline ENSBTAG00000023860 & $2.62 \mathrm{E}-10$ & $1.10 \mathrm{E}-10$ & & $2.38 \mathrm{E}+00$ \\
\hline ENSBTAG00000018164 & $1.23 \mathrm{E}-10$ & $5.15 \mathrm{E}-11$ & FNDC4 & $2.38 \mathrm{E}+00$ \\
\hline ENSBTAG00000001903 & $1.64 \mathrm{E}-10$ & $6.90 \mathrm{E}-11$ & C2orf50 & $2.38 \mathrm{E}+00$ \\
\hline ENSBTAG00000019213 & 7.19E-10 & $3.01 \mathrm{E}-10$ & CNNM4 & $2.39 \mathrm{E}+00$ \\
\hline ENSBTAG00000025161 & $5.24 \mathrm{E}-10$ & $2.19 \mathrm{E}-10$ & AGPAT2 & $2.39 E+00$ \\
\hline ENSBTAG00000030834 & $4.80 \mathrm{E}-10$ & $2.01 E-10$ & & $2.39 \mathrm{E}+00$ \\
\hline ENSBTAG00000019518 & $1.42 \mathrm{E}-10$ & $5.95 \mathrm{E}-11$ & ZNF513 & $2.38 \mathrm{E}+00$ \\
\hline ENSBTAG00000048207 & $5.09 \mathrm{E}-10$ & $2.13 \mathrm{E}-10$ & C2orf15 & $2.39 E+00$ \\
\hline ENSBTAG00000038796 & $5.29 \mathrm{E}-10$ & $2.21 \mathrm{E}-10$ & & $2.39 E+00$ \\
\hline ENSBTAG00000010620 & $3.54 \mathrm{E}-10$ & $1.48 \mathrm{E}-10$ & ATP6V1B1 & $2.39 E+00$ \\
\hline ENSBTAG00000012772 & $6.08 \mathrm{E}-10$ & $2.54 \mathrm{E}-10$ & GFI1B & $2.39 \mathrm{E}+00$ \\
\hline ENSBTAG00000043999 & $5.61 \mathrm{E}-10$ & $2.34 \mathrm{E}-10$ & REV1 & $2.40 \mathrm{E}+00$ \\
\hline ENSBTAG00000001866 & $3.91 \mathrm{E}-10$ & $1.62 \mathrm{E}-10$ & EMX1 & $2.42 \mathrm{E}+00$ \\
\hline ENSBTAG00000020669 & $4.47 \mathrm{E}-10$ & $1.84 \mathrm{E}-10$ & PROKR1 & $2.43 \mathrm{E}+00$ \\
\hline ENSBTAG00000022381 & $4.49 \mathrm{E}-10$ & $1.85 \mathrm{E}-10$ & TET3 & $2.43 E+00$ \\
\hline ENSBTAG00000009761 & $1.03 \mathrm{E}-10$ & $4.23 \mathrm{E}-11$ & ACTR2 & $2.43 E+00$ \\
\hline ENSBTAG00000019459 & $4.49 \mathrm{E}-10$ & $1.85 \mathrm{E}-10$ & $\mathrm{CHAC2}$ & $2.43 E+00$ \\
\hline ENSBTAG00000012144 & 4.47E-10 & $1.83 \mathrm{E}-10$ & ZMYND19 & $2.44 \mathrm{E}+00$ \\
\hline ENSBTAG00000012827 & 5.67E-10 & $2.30 \mathrm{E}-10$ & & $2.47 E+00$ \\
\hline ENSBTAG00000021140 & $4.42 \mathrm{E}-10$ & $1.79 \mathrm{E}-10$ & RMND5A & $2.47 \mathrm{E}+00$ \\
\hline ENSBTAG00000019721 & $2.49 \mathrm{E}-10$ & $1.00 \mathrm{E}-10$ & REXO4 & $2.49 E+00$ \\
\hline ENSBTAG00000020262 & $4.01 \mathrm{E}-10$ & $1.62 \mathrm{E}-10$ & PSME4 & $2.48 \mathrm{E}+00$ \\
\hline ENSBTAG00000040093 & $4.68 \mathrm{E}-10$ & $1.88 \mathrm{E}-10$ & FOXI3 & $2.49 E+00$ \\
\hline ENSBTAG00000032521 & $3.62 \mathrm{E}-10$ & $1.45 \mathrm{E}-10$ & PLEKHH2 & $2.50 \mathrm{E}+00$ \\
\hline ENSBTAG00000002042 & $3.38 \mathrm{E}-10$ & $1.35 \mathrm{E}-10$ & RIC8A & $2.50 \mathrm{E}+00$ \\
\hline ENSBTAG00000007135 & $6.12 \mathrm{E}-10$ & $2.44 \mathrm{E}-10$ & & $2.51 \mathrm{E}+00$ \\
\hline ENSBTAG00000010846 & $9.22 \mathrm{E}-10$ & $3.64 \mathrm{E}-10$ & CNNM3 & $2.53 E+00$ \\
\hline ENSBTAG00000007882 & $3.91 \mathrm{E}-10$ & $1.54 \mathrm{E}-10$ & CACNA1B & $2.54 \mathrm{E}+00$ \\
\hline ENSBTAG00000016794 & $6.92 \mathrm{E}-10$ & $2.73 \mathrm{E}-10$ & SLC10A6 & $2.53 E+00$ \\
\hline ENSBTAG00000000152 & $1.74 \mathrm{E}-10$ & $6.85 \mathrm{E}-11$ & RANBP2 & $2.53 E+00$ \\
\hline ENSBTAG00000012332 & $6.07 \mathrm{E}-10$ & $2.38 \mathrm{E}-10$ & C2orf49 & $2.55 E+00$ \\
\hline ENSBTAG00000019983 & $1.99 \mathrm{E}-10$ & 7.77E-11 & & $2.56 \mathrm{E}+00$ \\
\hline ENSBTAG00000008956 & $3.79 \mathrm{E}-10$ & $1.46 \mathrm{E}-10$ & $\mathrm{CD} 8 \mathrm{~B}$ & $2.59 E+00$ \\
\hline ENSBTAG00000016032 & $4.39 \mathrm{E}-10$ & $1.70 \mathrm{E}-10$ & & $2.58 \mathrm{E}+00$ \\
\hline ENSBTAG00000006963 & $2.62 \mathrm{E}-10$ & $1.02 \mathrm{E}-10$ & & $2.57 E+00$ \\
\hline ENSBTAG00000010810 & $1.97 \mathrm{E}-10$ & $7.63 \mathrm{E}-11$ & UCK1 & $2.58 \mathrm{E}+00$ \\
\hline ENSBTAG00000017255 & $3.57 \mathrm{E}-10$ & 1.37E-10 & ATAD2B & $2.61 E+00$ \\
\hline ENSBTAG00000032905 & $5.13 \mathrm{E}-10$ & $1.97 \mathrm{E}-10$ & RMDN2 & $2.61 E+00$ \\
\hline ENSBTAG00000002319 & $3.60 \mathrm{E}-10$ & $1.38 \mathrm{E}-10$ & HMCN2 & $2.60 E+00$ \\
\hline ENSBTAG00000023976 & $2.94 \mathrm{E}-10$ & $1.12 \mathrm{E}-10$ & ARHGAP25 & $2.62 \mathrm{E}+00$ \\
\hline ENSBTAG00000019470 & $2.55 \mathrm{E}-10$ & $9.69 \mathrm{E}-11$ & NEK6 & $2.63 E+00$ \\
\hline ENSBTAG00000011176 & $2.45 \mathrm{E}-10$ & $9.24 \mathrm{E}-11$ & TPRKB & $2.65 E+00$ \\
\hline ENSBTAG00000021920 & $3.51 E-10$ & $1.31 \mathrm{E}-10$ & SEMA4C & $2.68 \mathrm{E}+00$ \\
\hline ENSBTAG00000008006 & $4.23 \mathrm{E}-10$ & $1.58 \mathrm{E}-10$ & RASGRP3 & $2.68 \mathrm{E}+00$ \\
\hline ENSBTAG00000011556 & 3.37E-10 & $1.26 \mathrm{E}-10$ & MED22 & $2.67 E+00$ \\
\hline ENSBTAG00000011381 & $1.64 \mathrm{E}-10$ & $6.06 \mathrm{E}-11$ & SLC30A3 & $2.70 \mathrm{E}+00$ \\
\hline ENSBTAG00000018289 & $3.03 E-10$ & $1.12 \mathrm{E}-10$ & TMEM150A & $2.71 E+00$ \\
\hline ENSBTAG00000004328 & $8.61 \mathrm{E}-10$ & 3.17E-10 & CHST10 & $2.72 \mathrm{E}+00$ \\
\hline ENSBTAG00000006744 & $3.80 \mathrm{E}-10$ & $1.39 \mathrm{E}-10$ & TMEM131 & $2.73 E+00$ \\
\hline ENSBTAG00000047998 & $4.96 \mathrm{E}-10$ & $1.79 \mathrm{E}-10$ & COL5A1 & $2.77 \mathrm{E}+00$ \\
\hline ENSBTAG00000001113 & $2.40 \mathrm{E}-10$ & $8.64 \mathrm{E}-11$ & NDUFAF7 & $2.78 \mathrm{E}+00$ \\
\hline ENSBTAG00000031014 & $3.86 \mathrm{E}-10$ & $1.37 \mathrm{E}-10$ & LDAH & $2.81 E+00$ \\
\hline ENSBTAG00000015327 & $2.12 \mathrm{E}-10$ & $7.53 \mathrm{E}-11$ & SPTAN1 & $2.81 E+00$ \\
\hline ENSBTAG00000021928 & $3.54 \mathrm{E}-10$ & $1.24 \mathrm{E}-10$ & FAM69B & $2.85 E+00$ \\
\hline ENSBTAG00000000102 & $4.31 \mathrm{E}-10$ & $1.50 \mathrm{E}-10$ & GPR75 & $2.87 E+00$ \\
\hline ENSBTAG00000016939 & $1.36 \mathrm{E}-10$ & $4.75 \mathrm{E}-11$ & C2orf42 & $2.87 \mathrm{E}+00$ \\
\hline ENSBTAG00000004848 & $3.00 \mathrm{E}-10$ & $1.04 \mathrm{E}-10$ & OLFML2A & $2.89 \mathrm{E}+00$ \\
\hline ENSBTAG00000018576 & $5.39 \mathrm{E}-10$ & $1.86 \mathrm{E}-10$ & DPYSL5 & $2.90 \mathrm{E}+00$ \\
\hline ENSBTAG00000032558 & $3.80 \mathrm{E}-10$ & $1.31 \mathrm{E}-10$ & TTC7A & $2.90 \mathrm{E}+00$ \\
\hline ENSBTAG00000002557 & 1.05E-09 & $3.59 \mathrm{E}-10$ & MRPL19 & $2.91 \mathrm{E}+00$ \\
\hline ENSBTAG00000024091 & $2.34 \mathrm{E}-10$ & 8.01E-11 & MALL & $2.92 \mathrm{E}+00$ \\
\hline
\end{tabular}




\begin{tabular}{|c|c|c|c|c|}
\hline ENSBTAG00000021961 & $8.22 \mathrm{E}-10$ & $2.80 \mathrm{E}-10$ & SPR & $2.93 \mathrm{E}+00$ \\
\hline ENSBTAG00000012759 & $2.81 \mathrm{E}-10$ & $9.54 \mathrm{E}-11$ & FBXO41 & $2.94 \mathrm{E}+00$ \\
\hline ENSBTAG00000006995 & $5.02 \mathrm{E}-10$ & $1.70 \mathrm{E}-10$ & SPTBN1 & $2.95 \mathrm{E}+00$ \\
\hline ENSBTAG00000004497 & $3.23 \mathrm{E}-10$ & $1.09 \mathrm{E}-10$ & KANSL3 & $2.96 \mathrm{E}+00$ \\
\hline ENSBTAG00000001714 & $5.53 \mathrm{E}-10$ & $1.86 \mathrm{E}-10$ & MFSD9 & $2.97 E+00$ \\
\hline ENSBTAG00000009650 & $4.01 \mathrm{E}-10$ & $1.34 \mathrm{E}-10$ & OTOF & $2.99 \mathrm{E}+00$ \\
\hline ENSBTAG00000016717 & $3.49 \mathrm{E}-10$ & $1.16 \mathrm{E}-10$ & CEP68 & $3.01 E+00$ \\
\hline ENSBTAG00000002582 & $6.25 \mathrm{E}-10$ & $2.08 \mathrm{E}-10$ & LYG2 & $3.01 E+00$ \\
\hline ENSBTAG00000006481 & $4.25 \mathrm{E}-10$ & $1.41 \mathrm{E}-10$ & POLR1A & $3.01 E+00$ \\
\hline ENSBTAG00000017894 & $7.25 \mathrm{E}-11$ & $2.40 \mathrm{E}-11$ & $C A D$ & $3.01 E+00$ \\
\hline ENSBTAG00000014310 & 4.37E-10 & $1.45 \mathrm{E}-10$ & HEATR5B & $3.01 E+00$ \\
\hline ENSBTAG00000000157 & $1.31 \mathrm{E}-10$ & $4.29 \mathrm{E}-11$ & CCDC138 & $3.04 \mathrm{E}+00$ \\
\hline ENSBTAG00000002046 & $7.19 E-10$ & $2.35 \mathrm{E}-10$ & & $3.06 \mathrm{E}+00$ \\
\hline ENSBTAG00000018372 & $4.04 \mathrm{E}-10$ & $1.31 \mathrm{E}-10$ & STARD7 & $3.08 E+00$ \\
\hline ENSBTAG00000010619 & $4.28 \mathrm{E}-10$ & $1.38 \mathrm{E}-10$ & PPP3R1 & $3.10 E+00$ \\
\hline ENSBTAG00000007378 & $5.11 \mathrm{E}-10$ & $1.64 \mathrm{E}-10$ & CLIP4 & $3.11 E+00$ \\
\hline ENSBTAG00000018291 & $2.77 \mathrm{E}-10$ & $8.71 E-11$ & C2orf68 & $3.18 \mathrm{E}+00$ \\
\hline ENSBTAG00000016599 & $1.06 \mathrm{E}-10$ & 3.34E-11 & MRPL53 & $3.17 E+00$ \\
\hline ENSBTAG00000010805 & $2.17 \mathrm{E}-10$ & $6.82 \mathrm{E}-11$ & POMT1 & $3.18 \mathrm{E}+00$ \\
\hline ENSBTAG00000047822 & $3.11 E-10$ & $9.77 \mathrm{E}-11$ & & $3.18 \mathrm{E}+00$ \\
\hline ENSBTAG00000047610 & 3.17E-10 & 9.97E-11 & & $3.18 \mathrm{E}+00$ \\
\hline ENSBTAG00000016609 & $1.47 \mathrm{E}-10$ & $4.63 \mathrm{E}-11$ & DQX1 & $3.17 E+00$ \\
\hline ENSBTAG00000015081 & $2.09 \mathrm{E}-10$ & $6.57 \mathrm{E}-11$ & C9orf142 & $3.18 \mathrm{E}+00$ \\
\hline ENSBTAG00000037739 & $6.88 \mathrm{E}-10$ & $2.16 \mathrm{E}-10$ & & $3.19 E+00$ \\
\hline ENSBTAG00000045528 & $6.88 \mathrm{E}-10$ & $2.16 \mathrm{E}-10$ & OR1B1 & $3.19 E+00$ \\
\hline ENSBTAG00000038665 & $7.00 \mathrm{E}-10$ & $2.19 \mathrm{E}-10$ & & $3.19 E+00$ \\
\hline ENSBTAG00000004364 & $5.91 \mathrm{E}-10$ & $1.84 \mathrm{E}-10$ & THNSL2 & $3.21 E+00$ \\
\hline ENSBTAG00000033998 & $7.22 \mathrm{E}-10$ & $2.24 \mathrm{E}-10$ & RFX8 & $3.22 \mathrm{E}+00$ \\
\hline ENSBTAG00000004531 & $1.02 \mathrm{E}-09$ & $3.11 \mathrm{E}-10$ & CCT4 & $3.28 \mathrm{E}+00$ \\
\hline ENSBTAG00000031347 & $1.57 \mathrm{E}-10$ & $4.73 \mathrm{E}-11$ & SPDYA & $3.31 E+00$ \\
\hline ENSBTAG00000020828 & $2.95 \mathrm{E}-10$ & $8.85 \mathrm{E}-11$ & & $3.34 \mathrm{E}+00$ \\
\hline ENSBTAG00000020747 & $4.93 \mathrm{E}-10$ & 1.47E-10 & ASS1 & $3.35 E+00$ \\
\hline ENSBTAG00000001952 & $6.93 \mathrm{E}-10$ & $2.05 \mathrm{E}-10$ & PROM2 & $3.38 \mathrm{E}+00$ \\
\hline ENSBTAG00000006162 & $5.66 \mathrm{E}-10$ & $1.66 \mathrm{E}-10$ & & $3.41 E+00$ \\
\hline ENSBTAG00000019182 & $1.46 \mathrm{E}-10$ & $4.25 \mathrm{E}-11$ & TIA1 & $3.42 \mathrm{E}+00$ \\
\hline ENSBTAG00000014873 & 1.07E-09 & $3.12 \mathrm{E}-10$ & COA5 & $3.44 E+00$ \\
\hline ENSBTAG00000033748 & $5.10 \mathrm{E}-10$ & $1.48 \mathrm{E}-10$ & IL18RAP & $3.45 E+00$ \\
\hline ENSBTAG00000004140 & $3.05 \mathrm{E}-10$ & $8.85 \mathrm{E}-11$ & STKLD1 & $3.44 \mathrm{E}+00$ \\
\hline ENSBTAG00000003970 & $4.55 \mathrm{E}-10$ & $1.30 \mathrm{E}-10$ & MITD1 & $3.50 \mathrm{E}+00$ \\
\hline ENSBTAG00000001076 & $7.78 \mathrm{E}-10$ & $2.21 \mathrm{E}-10$ & NLRP6 & $3.52 E+00$ \\
\hline ENSBTAG00000002045 & $9.19 \mathrm{E}-10$ & $2.57 \mathrm{E}-10$ & PSMD13 & $3.58 \mathrm{E}+00$ \\
\hline ENSBTAG00000009242 & $4.99 \mathrm{E}-10$ & $1.40 \mathrm{E}-10$ & ZNF638 & $3.56 \mathrm{E}+00$ \\
\hline ENSBTAG00000019018 & $6.95 \mathrm{E}-10$ & $1.94 \mathrm{E}-10$ & & $3.58 \mathrm{E}+00$ \\
\hline ENSBTAG00000017330 & 1.17E-09 & $3.26 \mathrm{E}-10$ & & $3.60 E+00$ \\
\hline ENSBTAG00000018571 & $5.61 \mathrm{E}-10$ & $1.54 \mathrm{E}-10$ & IL1RL1 & $3.64 E+00$ \\
\hline ENSBTAG00000008726 & $2.38 \mathrm{E}-10$ & $6.52 \mathrm{E}-11$ & NCS1 & $3.65 E+00$ \\
\hline ENSBTAG00000037812 & $2.89 \mathrm{E}-10$ & $7.91 E-11$ & RAD51AP2 & $3.65 E+00$ \\
\hline ENSBTAG00000001662 & $2.49 \mathrm{E}-10$ & 6.77E-11 & EHD3 & $3.68 \mathrm{E}+00$ \\
\hline ENSBTAG00000007414 & $1.92 \mathrm{E}-10$ & $5.16 \mathrm{E}-11$ & PAPOLG & $3.71 E+00$ \\
\hline ENSBTAG00000010195 & $1.64 \mathrm{E}-10$ & $4.41 E-11$ & PKN3 & $3.71 E+00$ \\
\hline ENSBTAG00000002105 & $6.93 \mathrm{E}-10$ & $1.87 \mathrm{E}-10$ & & $3.71 E+00$ \\
\hline ENSBTAG00000015343 & $2.58 \mathrm{E}-10$ & $6.92 \mathrm{E}-11$ & WDR34 & $3.73 E+00$ \\
\hline ENSBTAG00000002298 & $5.92 \mathrm{E}-10$ & $1.58 \mathrm{E}-10$ & CKAP2L & $3.75 E+00$ \\
\hline ENSBTAG00000046280 & $3.94 \mathrm{E}-10$ & $9.90 \mathrm{E}-11$ & C2orf16 & $3.98 \mathrm{E}+00$ \\
\hline ENSBTAG00000019665 & $5.30 \mathrm{E}-10$ & $1.33 \mathrm{E}-10$ & IL1RN & $3.98 \mathrm{E}+00$ \\
\hline ENSBTAG00000016367 & $3.46 \mathrm{E}-10$ & $8.58 \mathrm{E}-11$ & RBM18 & $4.02 E+00$ \\
\hline ENSBTAG00000033255 & $1.02 \mathrm{E}-09$ & $2.53 \mathrm{E}-10$ & DUSP11 & $4.03 E+00$ \\
\hline ENSBTAG00000000542 & $3.00 \mathrm{E}-10$ & 7.27E-11 & CFAP36 & $4.12 \mathrm{E}+00$ \\
\hline ENSBTAG00000013108 & $5.09 \mathrm{E}-10$ & $1.23 \mathrm{E}-10$ & HK2 & $4.13 E+00$ \\
\hline ENSBTAG00000002087 & $4.99 \mathrm{E}-10$ & $1.19 \mathrm{E}-10$ & IL36A & $4.19 E+00$ \\
\hline ENSBTAG00000030608 & $1.97 \mathrm{E}-10$ & $4.65 \mathrm{E}-11$ & SWI5 & $4.23 E+00$ \\
\hline ENSBTAG00000008448 & $1.40 \mathrm{E}-10$ & 3.27E-11 & MEIS1 & $4.29 E+00$ \\
\hline ENSBTAG00000023938 & 3.57E-10 & 8.30E-11 & & $4.29 E+00$ \\
\hline ENSBTAG00000009834 & $9.61 \mathrm{E}-10$ & $2.19 \mathrm{E}-10$ & CNGA3 & $4.38 \mathrm{E}+00$ \\
\hline ENSBTAG00000008909 & 3.75E-10 & $8.29 \mathrm{E}-11$ & PNPT1 & $4.52 \mathrm{E}+00$ \\
\hline ENSBTAG00000031150 & $5.15 \mathrm{E}-10$ & $1.12 \mathrm{E}-10$ & C2orf70 & $4.59 E+00$ \\
\hline ENSBTAG00000003438 & $1.66 \mathrm{E}-10$ & $3.56 \mathrm{E}-11$ & ZBTB43 & $4.66 \mathrm{E}+00$ \\
\hline ENSBTAG00000008764 & $5.35 \mathrm{E}-10$ & $1.13 \mathrm{E}-10$ & SMYD5 & $4.74 \mathrm{E}+00$ \\
\hline ENSBTAG00000003405 & $1.63 \mathrm{E}-10$ & $3.41 E-11$ & TP53I3 & $4.76 \mathrm{E}+00$ \\
\hline ENSBTAG00000030540 & $1.57 \mathrm{E}-10$ & $3.29 \mathrm{E}-11$ & ZDHHC12 & $4.75 E+00$ \\
\hline
\end{tabular}




\begin{tabular}{|c|c|c|c|c|}
\hline ENSBTAG00000046137 & $5.27 \mathrm{E}-10$ & $1.10 \mathrm{E}-10$ & & $4.79 E+00$ \\
\hline ENSBTAG00000015473 & $7.48 \mathrm{E}-10$ & $1.56 \mathrm{E}-10$ & RPS27A & $4.79 E+00$ \\
\hline ENSBTAG00000031828 & $7.55 \mathrm{E}-10$ & $1.58 \mathrm{E}-10$ & & $4.78 \mathrm{E}+00$ \\
\hline ENSBTAG00000014836 & 1.44E-09 & 2.99E-10 & & $4.81 \mathrm{E}+00$ \\
\hline ENSBTAG00000018285 & $5.97 \mathrm{E}-10$ & $1.21 \mathrm{E}-10$ & VAMP5 & $4.93 \mathrm{E}+00$ \\
\hline ENSBTAG00000014090 & 4.44E-10 & $8.84 \mathrm{E}-11$ & ARID5A & $5.02 \mathrm{E}+00$ \\
\hline ENSBTAG00000004814 & 7.30E-10 & $1.42 \mathrm{E}-10$ & INPP4A & $5.14 E+00$ \\
\hline ENSBTAG00000031185 & $2.47 \mathrm{E}-10$ & $4.78 \mathrm{E}-11$ & ATRAID & $5.16 \mathrm{E}+00$ \\
\hline ENSBTAG00000010691 & $6.13 \mathrm{E}-10$ & 1.19E-10 & USP39 & $5.15 E+00$ \\
\hline ENSBTAG00000038139 & $1.20 \mathrm{E}-10$ & $2.16 \mathrm{E}-11$ & CERCAM & $5.53 E+00$ \\
\hline ENSBTAG00000038949 & $9.04 \mathrm{E}-10$ & $1.62 \mathrm{E}-10$ & & $5.58 \mathrm{E}+00$ \\
\hline ENSBTAG00000000545 & 5.97E-10 & $1.02 \mathrm{E}-10$ & PPP4R3B & $5.85 \mathrm{E}+00$ \\
\hline ENSBTAG00000017626 & $3.41 \mathrm{E}-10$ & $5.79 \mathrm{E}-11$ & GFPT1 & $5.88 \mathrm{E}+00$ \\
\hline ENSBTAG00000020348 & $9.21 \mathrm{E}-10$ & $1.53 \mathrm{E}-10$ & ASTL & $6.01 E+00$ \\
\hline ENSBTAG00000004508 & 1.30E-09 & $2.09 \mathrm{E}-10$ & DBH & $6.21 E+00$ \\
\hline ENSBTAG00000002088 & $5.45 \mathrm{E}-10$ & $8.42 \mathrm{E}-11$ & IL36B & $6.47 E+00$ \\
\hline ENSBTAG00000006832 & $2.06 \mathrm{E}-10$ & $3.04 \mathrm{E}-11$ & SLC5A6 & $6.74 E+00$ \\
\hline ENSBTAG00000013676 & $3.23 \mathrm{E}-10$ & $4.60 \mathrm{E}-11$ & DYNC2LI1 & $7.01 \mathrm{E}+00$ \\
\hline ENSBTAG00000018012 & 4.57E-10 & $6.37 \mathrm{E}-11$ & IL1F10 & $7.16 \mathrm{E}+00$ \\
\hline ENSBTAG00000011325 & $2.97 \mathrm{E}-10$ & 4.06E-11 & KHK & $7.30 \mathrm{E}+00$ \\
\hline ENSBTAG00000013314 & $2.50 \mathrm{E}-10$ & $3.32 \mathrm{E}-11$ & PBX3 & $7.51 E+00$ \\
\hline ENSBTAG00000022461 & 3.76E-10 & 4.95E-11 & 10-Sep & $7.59 E+00$ \\
\hline ENSBTAG00000023172 & $7.31 \mathrm{E}-11$ & $9.56 \mathrm{E}-12$ & ITSN2 & $7.57 \mathrm{E}+00$ \\
\hline ENSBTAG00000023851 & $1.69 \mathrm{E}-10$ & $2.16 \mathrm{E}-11$ & FAM102A & $7.80 E+00$ \\
\hline ENSBTAG00000012447 & $1.55 \mathrm{E}-10$ & $1.98 \mathrm{E}-11$ & PPP1CB & $7.78 \mathrm{E}+00$ \\
\hline ENSBTAG00000004327 & $7.15 \mathrm{E}-10$ & $9.12 \mathrm{E}-11$ & LONRF2 & $7.84 \mathrm{E}+00$ \\
\hline ENSBTAG00000007077 & $1.18 \mathrm{E}-10$ & $1.48 \mathrm{E}-11$ & $\mathrm{ABHD} 1$ & $7.91 E+00$ \\
\hline ENSBTAG00000017423 & $2.06 \mathrm{E}-10$ & $2.46 \mathrm{E}-11$ & TMEM214 & $8.33 E+00$ \\
\hline ENSBTAG00000002783 & $7.83 \mathrm{E}-11$ & $8.96 \mathrm{E}-12$ & PCYOX1 & $8.65 E+00$ \\
\hline ENSBTAG00000048071 & $4.18 \mathrm{E}-10$ & 4.67E-11 & EXOSC2 & $8.94 \mathrm{E}+00$ \\
\hline ENSBTAG00000018991 & $4.61 \mathrm{E}-10$ & $4.95 \mathrm{E}-11$ & TSGA10 & $9.30 \mathrm{E}+00$ \\
\hline ENSBTAG00000012037 & $5.59 \mathrm{E}-10$ & $5.72 \mathrm{E}-11$ & DGUOK & $9.77 E+00$ \\
\hline ENSBTAG00000013675 & $6.18 \mathrm{E}-10$ & $6.14 \mathrm{E}-11$ & IL37 & $1.00 \mathrm{E}+01$ \\
\hline ENSBTAG00000001933 & $2.80 \mathrm{E}-10$ & $2.61 \mathrm{E}-11$ & PTPA & $1.07 \mathrm{E}+01$ \\
\hline ENSBTAG00000011314 & $1.23 \mathrm{E}-09$ & $1.13 \mathrm{E}-10$ & & $1.09 E+01$ \\
\hline ENSBTAG00000032775 & 2.12E-09 & $1.87 \mathrm{E}-10$ & & $1.13 E+01$ \\
\hline ENSBTAG00000037822 & 9.17E-10 & 7.16E-11 & & $1.28 \mathrm{E}+01$ \\
\hline ENSBTAG00000010613 & $1.57 \mathrm{E}-10$ & 1.19E-11 & NUP188 & $1.31 \mathrm{E}+01$ \\
\hline ENSBTAG00000018554 & $1.58 \mathrm{E}-09$ & $1.15 \mathrm{E}-10$ & GAPDH & $1.37 \mathrm{E}+01$ \\
\hline ENSBTAG00000024019 & $1.01 \mathrm{E}-09$ & 7.15E-11 & & $1.41 \mathrm{E}+01$ \\
\hline ENSBTAG00000038831 & $1.84 \mathrm{E}-10$ & $1.22 \mathrm{E}-11$ & PHYHD1 & $1.49 \mathrm{E}+01$ \\
\hline ENSBTAG00000012756 & 1.07E-09 & 7.04E-11 & $\mathrm{CCT7}$ & $1.52 \mathrm{E}+01$ \\
\hline ENSBTAG00000008884 & $3.73 \mathrm{E}-10$ & $2.42 \mathrm{E}-11$ & MAPRE3 & $1.53 \mathrm{E}+01$ \\
\hline ENSBTAG00000013403 & $1.82 \mathrm{E}-10$ & $1.18 \mathrm{E}-11$ & AGBL5 & $1.53 \mathrm{E}+01$ \\
\hline ENSBTAG00000008765 & $3.98 \mathrm{E}-10$ & $2.50 \mathrm{E}-11$ & PRADC1 & $1.59 \mathrm{E}+01$ \\
\hline ENSBTAG00000004567 & $2.69 \mathrm{E}-10$ & $1.66 \mathrm{E}-11$ & C1D & $1.61 E+01$ \\
\hline ENSBTAG00000000141 & $4.52 \mathrm{E}-10$ & $2.50 \mathrm{E}-11$ & NFU1 & $1.80 \mathrm{E}+01$ \\
\hline ENSBTAG00000010626 & $3.81 \mathrm{E}-10$ & $1.81 \mathrm{E}-11$ & SH3GLB2 & $2.09 E+01$ \\
\hline ENSBTAG00000011324 & $2.78 \mathrm{E}-10$ & $1.29 \mathrm{E}-11$ & EMILIN1 & $2.14 \mathrm{E}+01$ \\
\hline ENSBTAG00000010653 & $4.72 \mathrm{E}-10$ & $2.07 \mathrm{E}-11$ & MIGA2 & $2.27 \mathrm{E}+01$ \\
\hline ENSBTAG00000003387 & $3.09 \mathrm{E}-10$ & $1.18 \mathrm{E}-11$ & PIP5KL1 & $2.59 E+01$ \\
\hline ENSBTAG00000015441 & $4.82 \mathrm{E}-10$ & $6.95 \mathrm{E}-12$ & ACTG2 & $6.83 \mathrm{E}+01$ \\
\hline ENSBTAG00000020350 & $2.41 \mathrm{E}-10$ & 0 & DUSP2 & $2.42 \mathrm{E}+03$ \\
\hline ENSBTAG00000001844 & 0 & 0 & MOGS & $1.00 \mathrm{E}+00$ \\
\hline ENSBTAG00000047969 & $2.47 \mathrm{E}-10$ & 0 & NAT8 & $2.48 \mathrm{E}+03$ \\
\hline ENSBTAG00000012984 & $4.58 \mathrm{E}-11$ & 0 & & $4.59 E+02$ \\
\hline ENSBTAG00000003553 & $1.79 \mathrm{E}-10$ & 0 & ZFP36L2 & $1.79 E+03$ \\
\hline ENSBTAG00000039476 & 4.12E-10 & 0 & & $4.12 E+03$ \\
\hline ENSBTAG00000018156 & $9.93 \mathrm{E}-11$ & 0 & KRTCAP3 & $9.94 \mathrm{E}+02$ \\
\hline ENSBTAG00000014813 & $1.38 \mathrm{E}-10$ & 0 & TCF23 & $1.38 \mathrm{E}+03$ \\
\hline ENSBTAG00000007080 & 7.99E-11 & 0 & PREB & $8.00 E+02$ \\
\hline ENSBTAG00000011328 & $2.55 \mathrm{E}-10$ & 0 & CGREF1 & $2.55 E+03$ \\
\hline ENSBTAG00000000261 & $8.14 \mathrm{E}-11$ & 0 & & $8.15 E+02$ \\
\hline ENSBTAG00000030864 & $9.87 \mathrm{E}-11$ & 0 & & $9.88 \mathrm{E}+02$ \\
\hline ENSBTAG00000003404 & $1.27 \mathrm{E}-10$ & 0 & PFN4 & $1.27 \mathrm{E}+03$ \\
\hline ENSBTAG00000040296 & 7.50E-11 & 0 & GPR21 & $7.51 E+02$ \\
\hline ENSBTAG00000003388 & $1.61 \mathrm{E}-10$ & 0 & DPM2 & $1.61 E+03$ \\
\hline ENSBTAG00000020959 & $1.28 \mathrm{E}-10$ & 0 & SET & $1.29 \mathrm{E}+03$ \\
\hline ENSBTAG00000017922 & $3.16 \mathrm{E}-10$ & 0 & DOLPP1 & $3.16 \mathrm{E}+03$ \\
\hline ENSBTAG00000001932 & $1.85 \mathrm{E}-10$ & 0 & CRAT & $1.85 \mathrm{E}+03$ \\
\hline
\end{tabular}




\begin{tabular}{|c|c|c|c|c|}
\hline ENSBTAG00000046415 & $1.62 \mathrm{E}-10$ & 0 & SSNA1 & $1.62 \mathrm{E}+03$ \\
\hline ENSBTAG00000045734 & 0 & 0 & LRRC26 & $1.00 \mathrm{E}+00$ \\
\hline ENSBTAG00000033887 & $3.49 \mathrm{E}-10$ & 0 & RPL36A-HNRNPH2 & $3.49 E+03$ \\
\hline ENSBTAG00000023736 & $1.01 \mathrm{E}-10$ & 0 & CDC42EP3 & $1.01 \mathrm{E}+03$ \\
\hline ENSBTAG00000004076 & $6.91 \mathrm{E}-11$ & 0 & OXER1 & $6.92 \mathrm{E}+02$ \\
\hline ENSBTAG00000047029 & 0 & 0 & & $1.00 \mathrm{E}+00$ \\
\hline ENSBTAG00000000374 & $5.25 \mathrm{E}-11$ & 0 & LRRTM4 & $5.26 \mathrm{E}+02$ \\
\hline ENSBTAG00000018268 & 0 & 0 & UCN & $1.00 E+00$ \\
\hline ENSBTAG00000047596 & $6.84 \mathrm{E}-11$ & 0 & PRR30 & $6.85 E+02$ \\
\hline ENSBTAG00000046054 & $3.70 \mathrm{E}-11$ & 0 & RHOB & $3.71 E+02$ \\
\hline ENSBTAG00000048065 & 2.63E-10 & 0 & & $2.63 \mathrm{E}+03$ \\
\hline ENSBTAG00000020660 & 8.67E-11 & 0 & & $8.68 \mathrm{E}+02$ \\
\hline ENSBTAG00000038551 & $6.16 \mathrm{E}-10$ & 0 & & $6.16 \mathrm{E}+03$ \\
\hline ENSBTAG00000004667 & $1.98 \mathrm{E}-09$ & 0 & & $1.98 \mathrm{E}+04$ \\
\hline ENSBTAG00000030677 & $2.61 \mathrm{E}-10$ & 0 & & $2.61 E+03$ \\
\hline ENSBTAG00000039343 & $5.34 \mathrm{E}-11$ & 0 & ZBTB34 & $5.35 E+02$ \\
\hline ENSBTAG00000023823 & 0 & 0 & & $1.00 \mathrm{E}+00$ \\
\hline ENSBTAG00000010601 & $1.96 \mathrm{E}-10$ & 0 & DOLK & $1.96 \mathrm{E}+03$ \\
\hline ENSBTAG00000017980 & $6.10 \mathrm{E}-10$ & 0 & QRFP & $6.10 \mathrm{E}+03$ \\
\hline ENSBTAG00000046122 & 0 & 0 & NRARP & $1.00 \mathrm{E}+00$ \\
\hline ENSBTAG00000047357 & $1.02 \mathrm{E}-10$ & 0 & RNF208 & $1.02 E+03$ \\
\hline ENSBTAG00000046215 & 0 & 0 & TMEM203 & $1.00 \mathrm{E}+00$ \\
\hline ENSBTAG00000022484 & 0 & $1.44 \mathrm{E}-10$ & SOX21 & $6.94 \mathrm{E}-04$ \\
\hline ENSBTAG00000046889 & 0 & $2.13 \mathrm{E}-10$ & & 4.69E-04 \\
\hline ENSBTAG00000003595 & $2.89 \mathrm{E}-10$ & $9.39 \mathrm{E}-10$ & FAM216B & $3.08 \mathrm{E}-01$ \\
\hline ENSBTAG00000006759 & $1.78 \mathrm{E}-10$ & $5.58 \mathrm{E}-10$ & SUCLA2 & 3.19E-01 \\
\hline ENSBTAG00000038268 & $2.52 \mathrm{E}-10$ & $5.40 \mathrm{E}-10$ & & $4.68 \mathrm{E}-01$ \\
\hline ENSBTAG00000001365 & $1.25 \mathrm{E}-10$ & $2.62 \mathrm{E}-10$ & & 4.76E-01 \\
\hline ENSBTAG00000003511 & $3.32 \mathrm{E}-10$ & $6.64 \mathrm{E}-10$ & & 5.00E-01 \\
\hline ENSBTAG00000019645 & $1.96 \mathrm{E}-10$ & $3.77 \mathrm{E}-10$ & & 5.20E-01 \\
\hline ENSBTAG00000019963 & $1.99 \mathrm{E}-10$ & $3.62 \mathrm{E}-10$ & GRK1 & $5.51 \mathrm{E}-01$ \\
\hline ENSBTAG00000015132 & $5.35 \mathrm{E}-11$ & $9.45 \mathrm{E}-11$ & RXFP2 & 5.67E-01 \\
\hline ENSBTAG00000018846 & $1.99 \mathrm{E}-10$ & $3.04 \mathrm{E}-10$ & & $6.55 \mathrm{E}-01$ \\
\hline ENSBTAG00000019965 & $2.75 \mathrm{E}-10$ & $4.02 \mathrm{E}-10$ & TMEM255B & $6.85 \mathrm{E}-01$ \\
\hline ENSBTAG00000017573 & $1.08 \mathrm{E}-10$ & $1.53 \mathrm{E}-10$ & RBM26 & 7.04E-01 \\
\hline ENSBTAG00000046271 & $1.46 \mathrm{E}-10$ & $2.05 E-10$ & & 7.13E-01 \\
\hline ENSBTAG00000019961 & $2.38 \mathrm{E}-10$ & $3.23 \mathrm{E}-10$ & ATP4B & 7.37E-01 \\
\hline ENSBTAG00000019971 & $1.20 \mathrm{E}-10$ & $1.63 \mathrm{E}-10$ & CDC16 & 7.39E-01 \\
\hline ENSBTAG00000012849 & 3.32E-10 & 4.43E-10 & COL4A1 & 7.49E-01 \\
\hline ENSBTAG00000020403 & $8.30 \mathrm{E}-11$ & $1.08 \mathrm{E}-10$ & ZMYM2 & 7.69E-01 \\
\hline ENSBTAG00000001865 & $1.60 \mathrm{E}-10$ & $2.08 \mathrm{E}-10$ & SGCG & $7.68 \mathrm{E}-01$ \\
\hline ENSBTAG00000011862 & $1.91 \mathrm{E}-10$ & $2.40 \mathrm{E}-10$ & & 7.94E-01 \\
\hline ENSBTAG00000006645 & $2.22 \mathrm{E}-10$ & $2.79 \mathrm{E}-10$ & LPAR6 & 7.94E-01 \\
\hline ENSBTAG00000047881 & $1.04 \mathrm{E}-10$ & $1.31 \mathrm{E}-10$ & & 7.93E-01 \\
\hline ENSBTAG00000038272 & $7.38 \mathrm{E}-11$ & $9.30 \mathrm{E}-11$ & SOX1 & 7.93E-01 \\
\hline ENSBTAG00000010769 & $1.63 \mathrm{E}-10$ & $2.06 \mathrm{E}-10$ & GPR12 & 7.92E-01 \\
\hline ENSBTAG00000002137 & 4.07E-10 & $5.05 \mathrm{E}-10$ & SUGT1 & 8.06E-01 \\
\hline ENSBTAG00000018848 & $2.68 \mathrm{E}-10$ & $3.28 \mathrm{E}-10$ & FBXL3 & 8.16E-01 \\
\hline ENSBTAG00000007356 & $3.25 \mathrm{E}-10$ & $3.96 \mathrm{E}-10$ & ELF1 & $8.20 \mathrm{E}-01$ \\
\hline ENSBTAG00000034495 & $2.47 \mathrm{E}-10$ & $2.98 \mathrm{E}-10$ & GTF2F2 & 8.29E-01 \\
\hline ENSBTAG00000031962 & $2.36 \mathrm{E}-10$ & $2.80 \mathrm{E}-10$ & RAB20 & $8.42 \mathrm{E}-01$ \\
\hline ENSBTAG00000011726 & $2.21 \mathrm{E}-10$ & $2.62 \mathrm{E}-10$ & CRYL1 & 8.43E-01 \\
\hline ENSBTAG00000014619 & $4.35 \mathrm{E}-10$ & $5.13 \mathrm{E}-10$ & WASF3 & $8.48 \mathrm{E}-01$ \\
\hline ENSBTAG00000034785 & $5.21 \mathrm{E}-10$ & $6.13 \mathrm{E}-10$ & DNAJC15 & $8.50 \mathrm{E}-01$ \\
\hline ENSBTAG00000008647 & $6.02 \mathrm{E}-10$ & $6.93 \mathrm{E}-10$ & KLHL1 & $8.68 \mathrm{E}-01$ \\
\hline ENSBTAG00000014406 & $1.66 \mathrm{E}-10$ & $1.91 \mathrm{E}-10$ & LATS2 & 8.67E-01 \\
\hline ENSBTAG00000025246 & $1.63 \mathrm{E}-10$ & $1.87 \mathrm{E}-10$ & ZIC2 & $8.71 \mathrm{E}-01$ \\
\hline ENSBTAG00000012019 & $1.76 \mathrm{E}-10$ & $2.00 \mathrm{E}-10$ & IRS2 & 8.80E-01 \\
\hline ENSBTAG00000000446 & $3.86 \mathrm{E}-10$ & $4.35 E-10$ & ATP11A & 8.86E-01 \\
\hline ENSBTAG00000034753 & $4.04 \mathrm{E}-10$ & $4.52 \mathrm{E}-10$ & CCDC122 & 8.93E-01 \\
\hline ENSBTAG00000001648 & $2.20 \mathrm{E}-10$ & $2.46 \mathrm{E}-10$ & & 8.93E-01 \\
\hline ENSBTAG00000009912 & $9.61 \mathrm{E}-11$ & $1.07 \mathrm{E}-10$ & PAN3 & 8.98E-01 \\
\hline ENSBTAG00000025210 & $5.78 \mathrm{E}-10$ & $6.43 \mathrm{E}-10$ & COL4A2 & 8.99E-01 \\
\hline ENSBTAG00000010246 & $1.24 \mathrm{E}-10$ & $1.38 \mathrm{E}-10$ & GRTP1 & 8.97E-01 \\
\hline ENSBTAG00000000869 & $2.74 \mathrm{E}-10$ & $3.04 \mathrm{E}-10$ & RNF219 & $9.01 \mathrm{E}-01$ \\
\hline ENSBTAG00000047764 & $4.19 \mathrm{E}-10$ & $4.63 \mathrm{E}-10$ & & $9.04 \mathrm{E}-01$ \\
\hline ENSBTAG00000046664 & $2.73 \mathrm{E}-10$ & $3.01 \mathrm{E}-10$ & & $9.06 \mathrm{E}-01$ \\
\hline ENSBTAG00000045907 & $5.41 \mathrm{E}-10$ & $5.93 \mathrm{E}-10$ & & $9.12 \mathrm{E}-01$ \\
\hline ENSBTAG00000001178 & $8.83 \mathrm{E}-11$ & $9.67 \mathrm{E}-11$ & USPL1 & $9.13 \mathrm{E}-01$ \\
\hline ENSBTAG00000021815 & $2.00 \mathrm{E}-10$ & 2.17E-10 & STARD13 & $9.22 \mathrm{E}-01$ \\
\hline
\end{tabular}




\begin{tabular}{|c|c|c|c|c|}
\hline ENSBTAG00000007167 & $1.75 \mathrm{E}-10$ & $1.89 \mathrm{E}-10$ & & $9.28 \mathrm{E}-01$ \\
\hline ENSBTAG00000003934 & $1.81 \mathrm{E}-10$ & $1.96 \mathrm{E}-10$ & PSPC1 & $9.26 \mathrm{E}-01$ \\
\hline ENSBTAG00000012443 & 4.87E-10 & 5.19E-10 & DIAPH3 & 9.38E-01 \\
\hline ENSBTAG00000003710 & $1.36 \mathrm{E}-10$ & $1.45 \mathrm{E}-10$ & XPO4 & $9.38 \mathrm{E}-01$ \\
\hline ENSBTAG00000018577 & $1.81 \mathrm{E}-10$ & $1.92 \mathrm{E}-10$ & SLC7A1 & $9.40 \mathrm{E}-01$ \\
\hline ENSBTAG00000002959 & $3.65 \mathrm{E}-10$ & $3.86 \mathrm{E}-10$ & LRCH1 & 9.45E-01 \\
\hline ENSBTAG00000018237 & $3.29 \mathrm{E}-10$ & $3.46 \mathrm{E}-10$ & MYO16 & $9.50 \mathrm{E}-01$ \\
\hline ENSBTAG00000017179 & $1.34 \mathrm{E}-10$ & $1.40 \mathrm{E}-10$ & USP12 & $9.55 \mathrm{E}-01$ \\
\hline ENSBTAG00000018671 & $9.34 \mathrm{E}-11$ & $9.81 \mathrm{E}-11$ & DCUN1D2 & $9.52 \mathrm{E}-01$ \\
\hline ENSBTAG00000012170 & $3.01 \mathrm{E}-10$ & $3.16 \mathrm{E}-10$ & UBL3 & 9.54E-01 \\
\hline ENSBTAG00000015704 & $1.57 \mathrm{E}-10$ & $1.65 \mathrm{E}-10$ & TMCO3 & 9.50E-01 \\
\hline ENSBTAG00000008924 & 8.00E-10 & $8.34 \mathrm{E}-10$ & TNFSF11 & $9.60 \mathrm{E}-01$ \\
\hline ENSBTAG00000017907 & $1.93 \mathrm{E}-10$ & $2.02 \mathrm{E}-10$ & COG3 & 9.57E-01 \\
\hline ENSBTAG00000009405 & $5.10 \mathrm{E}-10$ & $5.24 \mathrm{E}-10$ & TRPC4 & 9.73E-01 \\
\hline ENSBTAG00000019964 & $3.33 \mathrm{E}-10$ & $3.43 \mathrm{E}-10$ & GAS6 & 9.70E-01 \\
\hline ENSBTAG00000013371 & $3.61 \mathrm{E}-10$ & $3.70 \mathrm{E}-10$ & RUBCNL & 9.76E-01 \\
\hline ENSBTAG00000003922 & $1.55 \mathrm{E}-10$ & $1.58 \mathrm{E}-10$ & MPHOSPH8 & 9.79E-01 \\
\hline ENSBTAG00000007067 & $2.76 \mathrm{E}-10$ & $2.81 \mathrm{E}-10$ & $\mathrm{ZC} 3 \mathrm{H} 13$ & $9.83 \mathrm{E}-01$ \\
\hline ENSBTAG00000003116 & $8.62 \mathrm{E}-11$ & $8.79 \mathrm{E}-11$ & MTMR6 & $9.80 \mathrm{E}-01$ \\
\hline ENSBTAG00000047383 & 1.15E-09 & 1.17E-09 & & 9.83E-01 \\
\hline ENSBTAG00000008880 & 4.49E-10 & $4.55 \mathrm{E}-10$ & KDELC1 & $9.87 \mathrm{E}-01$ \\
\hline ENSBTAG00000014043 & $4.64 \mathrm{E}-10$ & 4.67E-10 & & 9.93E-01 \\
\hline ENSBTAG00000015943 & $2.13 \mathrm{E}-10$ & $2.14 \mathrm{E}-10$ & MICU2 & 9.93E-01 \\
\hline ENSBTAG00000025428 & $4.65 \mathrm{E}-10$ & 4.66E-10 & & $9.98 \mathrm{E}-01$ \\
\hline ENSBTAG00000003415 & $2.50 \mathrm{E}-10$ & $2.51 E-10$ & ESD & 9.96E-01 \\
\hline ENSBTAG00000013201 & $2.41 \mathrm{E}-10$ & $2.40 \mathrm{E}-10$ & ALOX5AP & $1.00 \mathrm{E}+00$ \\
\hline ENSBTAG00000017289 & $3.28 \mathrm{E}-10$ & $3.27 \mathrm{E}-10$ & MCF2L & $1.00 \mathrm{E}+00$ \\
\hline ENSBTAG00000001094 & $4.78 \mathrm{E}-10$ & 4.77E-10 & MTUS2 & $1.00 \mathrm{E}+00$ \\
\hline ENSBTAG00000010395 & $4.81 \mathrm{E}-10$ & $4.78 \mathrm{E}-10$ & DOCK9 & $1.01 E+00$ \\
\hline ENSBTAG00000011466 & $6.45 \mathrm{E}-10$ & $6.41 \mathrm{E}-10$ & NAXD & $1.01 E+00$ \\
\hline ENSBTAG00000025502 & $3.37 \mathrm{E}-10$ & $3.35 \mathrm{E}-10$ & LECT1 & $1.01 E+00$ \\
\hline ENSBTAG00000015994 & $7.21 \mathrm{E}-10$ & $7.14 \mathrm{E}-10$ & LRRC63 & $1.01 E+00$ \\
\hline ENSBTAG00000010420 & $4.15 \mathrm{E}-10$ & 4.10E-10 & PCDH17 & $1.01 E+00$ \\
\hline ENSBTAG00000034885 & $3.87 \mathrm{E}-10$ & $3.79 \mathrm{E}-10$ & RGCC & $1.02 \mathrm{E}+00$ \\
\hline ENSBTAG00000010787 & $4.13 \mathrm{E}-10$ & $3.99 \mathrm{E}-10$ & RFC3 & $1.03 E+00$ \\
\hline ENSBTAG00000007298 & $1.38 \mathrm{E}-10$ & $1.33 \mathrm{E}-10$ & TEX29 & $1.04 \mathrm{E}+00$ \\
\hline ENSBTAG00000018580 & 4.07E-10 & $3.90 \mathrm{E}-10$ & SPERT & $1.04 \mathrm{E}+00$ \\
\hline ENSBTAG00000046041 & $7.06 \mathrm{E}-10$ & $6.72 \mathrm{E}-10$ & & $1.05 E+00$ \\
\hline ENSBTAG00000009340 & $2.35 \mathrm{E}-10$ & $2.23 \mathrm{E}-10$ & KATNAL1 & $1.05 \mathrm{E}+00$ \\
\hline ENSBTAG00000016052 & $3.76 \mathrm{E}-10$ & 3.57E-10 & & $1.05 \mathrm{E}+00$ \\
\hline ENSBTAG00000019886 & $5.39 \mathrm{E}-10$ & $5.11 \mathrm{E}-10$ & BORA & $1.06 \mathrm{E}+00$ \\
\hline ENSBTAG00000010680 & $2.70 \mathrm{E}-10$ & $2.55 \mathrm{E}-10$ & & $1.06 \mathrm{E}+00$ \\
\hline ENSBTAG00000020861 & $1.36 \mathrm{E}-10$ & $1.28 \mathrm{E}-10$ & CHAMP1 & $1.06 \mathrm{E}+00$ \\
\hline ENSBTAG00000019340 & $1.06 \mathrm{E}-10$ & $9.99 \mathrm{E}-11$ & $\mathrm{PCDH} 9$ & $1.06 \mathrm{E}+00$ \\
\hline ENSBTAG00000019502 & $2.95 \mathrm{E}-10$ & $2.78 \mathrm{E}-10$ & MED4 & $1.06 \mathrm{E}+00$ \\
\hline ENSBTAG00000009308 & $6.72 \mathrm{E}-10$ & $6.34 \mathrm{E}-10$ & TDRD3 & $1.06 \mathrm{E}+00$ \\
\hline ENSBTAG00000038168 & $1.57 \mathrm{E}-10$ & $1.48 \mathrm{E}-10$ & SLITRK1 & $1.06 \mathrm{E}+00$ \\
\hline ENSBTAG00000032727 & $8.66 \mathrm{E}-10$ & 8.17E-10 & & $1.06 \mathrm{E}+00$ \\
\hline ENSBTAG00000016691 & $2.08 \mathrm{E}-10$ & $1.94 \mathrm{E}-10$ & & $1.07 E+00$ \\
\hline ENSBTAG00000018851 & $1.73 \mathrm{E}-10$ & $1.60 \mathrm{E}-10$ & MYCBP2 & $1.08 \mathrm{E}+00$ \\
\hline ENSBTAG00000001867 & $1.80 \mathrm{E}-10$ & $1.67 \mathrm{E}-10$ & SACS & $1.08 \mathrm{E}+00$ \\
\hline ENSBTAG00000012409 & $2.16 \mathrm{E}-10$ & $2.00 \mathrm{E}-10$ & POSTN & $1.08 \mathrm{E}+00$ \\
\hline ENSBTAG00000020093 & $1.67 \mathrm{E}-10$ & $1.55 \mathrm{E}-10$ & CUL4A & $1.08 \mathrm{E}+00$ \\
\hline ENSBTAG00000000993 & $1.78 \mathrm{E}-10$ & $1.64 \mathrm{E}-10$ & N4BP2L1 & $1.08 \mathrm{E}+00$ \\
\hline ENSBTAG00000005284 & $4.36 \mathrm{E}-10$ & $4.04 \mathrm{E}-10$ & SERPINE3 & $1.08 \mathrm{E}+00$ \\
\hline ENSBTAG00000016637 & 3.02E-10 & $2.80 \mathrm{E}-10$ & WBP4 & $1.08 \mathrm{E}+00$ \\
\hline ENSBTAG00000004041 & $4.35 \mathrm{E}-10$ & 4.02E-10 & ANKRD10 & $1.08 \mathrm{E}+00$ \\
\hline ENSBTAG00000011471 & $3.22 \mathrm{E}-10$ & $2.97 \mathrm{E}-10$ & CARS2 & $1.08 \mathrm{E}+00$ \\
\hline ENSBTAG00000032234 & $5.53 \mathrm{E}-10$ & $5.09 \mathrm{E}-10$ & STK24 & $1.09 \mathrm{E}+00$ \\
\hline ENSBTAG00000047739 & $2.64 \mathrm{E}-10$ & $2.42 \mathrm{E}-10$ & & $1.09 E+00$ \\
\hline ENSBTAG00000005012 & $1.78 \mathrm{E}-10$ & $1.63 \mathrm{E}-10$ & $\mathrm{HSPH} 1$ & $1.09 \mathrm{E}+00$ \\
\hline ENSBTAG00000038251 & $2.43 \mathrm{E}-10$ & $2.20 \mathrm{E}-10$ & NAA16 & $1.10 \mathrm{E}+00$ \\
\hline ENSBTAG00000010672 & $2.59 \mathrm{E}-10$ & $2.34 \mathrm{E}-10$ & PCCA & $1.11 E+00$ \\
\hline ENSBTAG00000007066 & $3.94 \mathrm{E}-10$ & 3.57E-10 & TMTC4 & $1.10 \mathrm{E}+00$ \\
\hline ENSBTAG00000019967 & $6.33 \mathrm{E}-10$ & $5.68 \mathrm{E}-10$ & RASA3 & $1.12 \mathrm{E}+00$ \\
\hline ENSBTAG00000025400 & $1.45 \mathrm{E}-10$ & $1.29 \mathrm{E}-10$ & PARP4 & $1.13 E+00$ \\
\hline ENSBTAG00000005299 & $3.91 \mathrm{E}-10$ & $3.46 \mathrm{E}-10$ & EDNRB & $1.13 E+00$ \\
\hline ENSBTAG00000005309 & $8.76 \mathrm{E}-10$ & $7.74 \mathrm{E}-10$ & TGDS & $1.13 E+00$ \\
\hline ENSBTAG00000020149 & $2.71 \mathrm{E}-10$ & $2.39 \mathrm{E}-10$ & & $1.13 E+00$ \\
\hline ENSBTAG00000020858 & $1.44 \mathrm{E}-10$ & $1.26 \mathrm{E}-10$ & UPF3A & $1.14 \mathrm{E}+00$ \\
\hline
\end{tabular}




\begin{tabular}{|c|c|c|c|c|}
\hline ENSBTAG00000013498 & $5.34 \mathrm{E}-10$ & 4.67E-10 & HTR2A & $1.14 E+00$ \\
\hline ENSBTAG00000044184 & $4.29 \mathrm{E}-10$ & $3.75 \mathrm{E}-10$ & TUBGCP3 & $1.14 \mathrm{E}+00$ \\
\hline ENSBTAG00000007073 & $3.66 \mathrm{E}-10$ & $3.19 \mathrm{E}-10$ & CPB2 & $1.15 E+00$ \\
\hline ENSBTAG00000032084 & $2.69 \mathrm{E}-10$ & $2.33 \mathrm{E}-10$ & GPR183 & $1.15 E+00$ \\
\hline ENSBTAG00000015225 & $1.12 \mathrm{E}-10$ & $9.60 \mathrm{E}-11$ & NUP58 & $1.16 \mathrm{E}+00$ \\
\hline ENSBTAG00000000146 & $5.01 \mathrm{E}-10$ & 4.32E-10 & FARP1 & $1.16 \mathrm{E}+00$ \\
\hline ENSBTAG00000000278 & $1.09 \mathrm{E}-10$ & $9.29 \mathrm{E}-11$ & RNF17 & $1.17 \mathrm{E}+00$ \\
\hline ENSBTAG00000034222 & 7.07E-10 & $6.02 \mathrm{E}-10$ & CAB39L & $1.17 \mathrm{E}+00$ \\
\hline ENSBTAG00000010949 & $5.56 \mathrm{E}-10$ & 4.74E-10 & PROSER1 & $1.17 \mathrm{E}+00$ \\
\hline ENSBTAG00000018214 & $1.76 \mathrm{E}-10$ & $1.48 \mathrm{E}-10$ & SHISA2 & $1.19 E+00$ \\
\hline ENSBTAG00000008656 & $1.16 \mathrm{E}-10$ & $9.74 \mathrm{E}-11$ & KBTBD6 & $1.19 \mathrm{E}+00$ \\
\hline ENSBTAG00000007079 & $6.75 \mathrm{E}-10$ & $5.64 \mathrm{E}-10$ & LCP1 & $1.20 \mathrm{E}+00$ \\
\hline ENSBTAG00000045751 & $1.43 \mathrm{E}-09$ & $1.18 \mathrm{E}-09$ & & $1.21 \mathrm{E}+00$ \\
\hline ENSBTAG00000016737 & $1.05 \mathrm{E}-10$ & 8.70E-11 & CDK8 & $1.21 E+00$ \\
\hline ENSBTAG00000048237 & $1.52 \mathrm{E}-10$ & $1.26 \mathrm{E}-10$ & & $1.21 E+00$ \\
\hline ENSBTAG00000023279 & $1.26 \mathrm{E}-10$ & $1.04 \mathrm{E}-10$ & & $1.21 E+00$ \\
\hline ENSBTAG00000019054 & $4.78 \mathrm{E}-10$ & $3.92 \mathrm{E}-10$ & EPSTI1 & $1.22 \mathrm{E}+00$ \\
\hline ENSBTAG00000025221 & $4.21 \mathrm{E}-10$ & $3.43 \mathrm{E}-10$ & TEX30 & $1.23 E+00$ \\
\hline ENSBTAG00000038662 & $1.82 \mathrm{E}-10$ & $1.49 \mathrm{E}-10$ & GJB6 & $1.22 \mathrm{E}+00$ \\
\hline ENSBTAG00000017048 & $1.40 \mathrm{E}-10$ & $1.13 \mathrm{E}-10$ & CENPJ & $1.24 \mathrm{E}+00$ \\
\hline ENSBTAG00000002300 & $1.02 \mathrm{E}-09$ & $8.26 \mathrm{E}-10$ & DCT & $1.24 \mathrm{E}+00$ \\
\hline ENSBTAG00000008708 & $4.05 E-10$ & $3.25 \mathrm{E}-10$ & GPC5 & $1.24 \mathrm{E}+00$ \\
\hline ENSBTAG00000032821 & $3.35 \mathrm{E}-10$ & $2.66 \mathrm{E}-10$ & SCEL & $1.26 \mathrm{E}+00$ \\
\hline ENSBTAG00000020726 & $3.09 \mathrm{E}-10$ & $2.44 \mathrm{E}-10$ & ARHGEF7 & $1.27 \mathrm{E}+00$ \\
\hline ENSBTAG00000032984 & $4.95 \mathrm{E}-10$ & $3.89 \mathrm{E}-10$ & & $1.27 \mathrm{E}+00$ \\
\hline ENSBTAG00000003314 & $2.08 \mathrm{E}-10$ & $1.61 \mathrm{E}-10$ & SKA3 & $1.29 \mathrm{E}+00$ \\
\hline ENSBTAG00000016915 & $2.55 \mathrm{E}-10$ & $1.97 \mathrm{E}-10$ & FLT1 & $1.30 \mathrm{E}+00$ \\
\hline ENSBTAG00000002129 & $2.64 \mathrm{E}-10$ & $2.03 E-10$ & & $1.30 \mathrm{E}+00$ \\
\hline ENSBTAG00000020389 & $4.72 \mathrm{E}-10$ & $3.61 \mathrm{E}-10$ & TPP2 & $1.31 E+00$ \\
\hline ENSBTAG00000007589 & $5.65 E-10$ & $4.30 \mathrm{E}-10$ & SMAD9 & $1.31 E+00$ \\
\hline ENSBTAG00000003109 & $7.76 \mathrm{E}-10$ & $5.89 \mathrm{E}-10$ & ITM2B & $1.32 \mathrm{E}+00$ \\
\hline ENSBTAG00000017425 & $8.12 \mathrm{E}-11$ & $6.14 \mathrm{E}-11$ & GJB2 & $1.32 \mathrm{E}+00$ \\
\hline ENSBTAG00000012260 & $1.91 \mathrm{E}-10$ & $1.44 \mathrm{E}-10$ & MTRF1 & $1.32 \mathrm{E}+00$ \\
\hline ENSBTAG00000004440 & 7.70E-10 & $5.81 \mathrm{E}-10$ & SLC15A1 & $1.33 E+00$ \\
\hline ENSBTAG00000018103 & $1.66 \mathrm{E}-10$ & $1.26 \mathrm{E}-10$ & HMGB1 & $1.32 \mathrm{E}+00$ \\
\hline ENSBTAG00000012434 & $5.64 \mathrm{E}-10$ & 4.19E-10 & ENOX1 & $1.35 E+00$ \\
\hline ENSBTAG00000005925 & $1.09 \mathrm{E}-09$ & 8.02E-10 & SLC10A2 & $1.36 \mathrm{E}+00$ \\
\hline ENSBTAG00000001344 & $2.81 \mathrm{E}-10$ & $2.05 \mathrm{E}-10$ & & $1.37 E+00$ \\
\hline ENSBTAG00000044027 & $5.58 \mathrm{E}-10$ & $4.08 \mathrm{E}-10$ & ITGBL1 & $1.37 \mathrm{E}+00$ \\
\hline ENSBTAG00000019545 & $4.12 \mathrm{E}-10$ & $3.01 E-10$ & & $1.37 \mathrm{E}+00$ \\
\hline ENSBTAG00000013879 & $3.69 \mathrm{E}-10$ & $2.69 \mathrm{E}-10$ & DGKH & $1.37 \mathrm{E}+00$ \\
\hline ENSBTAG00000040347 & $6.29 \mathrm{E}-10$ & $4.56 \mathrm{E}-10$ & & $1.38 \mathrm{E}+00$ \\
\hline ENSBTAG00000005682 & $2.58 \mathrm{E}-10$ & $1.87 \mathrm{E}-10$ & UBAC2 & $1.38 \mathrm{E}+00$ \\
\hline ENSBTAG00000002240 & $1.53 \mathrm{E}-10$ & $1.10 \mathrm{E}-10$ & GPR18 & $1.39 E+00$ \\
\hline ENSBTAG00000008888 & $5.15 E-10$ & $3.68 \mathrm{E}-10$ & MIPEP & $1.40 \mathrm{E}+00$ \\
\hline ENSBTAG00000010242 & $1.57 \mathrm{E}-10$ & $1.12 \mathrm{E}-10$ & LAMP1 & $1.41 \mathrm{E}+00$ \\
\hline ENSBTAG00000034033 & $3.49 \mathrm{E}-10$ & $2.48 \mathrm{E}-10$ & LHFP & $1.41 E+00$ \\
\hline ENSBTAG00000000988 & $1.03 \mathrm{E}-10$ & $7.28 \mathrm{E}-11$ & BRCA2 & $1.41 E+00$ \\
\hline ENSBTAG00000018300 & $2.91 \mathrm{E}-10$ & $2.06 \mathrm{E}-10$ & & $1.41 E+00$ \\
\hline ENSBTAG00000017508 & $5.49 \mathrm{E}-10$ & 3.87E-10 & CYSLTR2 & $1.42 \mathrm{E}+00$ \\
\hline ENSBTAG00000016529 & $2.07 \mathrm{E}-10$ & $1.45 \mathrm{E}-10$ & SLC25A30 & $1.43 E+00$ \\
\hline ENSBTAG00000018572 & $2.10 \mathrm{E}-10$ & $1.47 \mathrm{E}-10$ & FLT3 & $1.43 E+00$ \\
\hline ENSBTAG00000048161 & $5.39 \mathrm{E}-10$ & 3.69E-10 & & $1.46 \mathrm{E}+00$ \\
\hline ENSBTAG00000011850 & $2.61 \mathrm{E}-10$ & $1.78 \mathrm{E}-10$ & ALG5 & $1.46 \mathrm{E}+00$ \\
\hline ENSBTAG00000009415 & $3.25 \mathrm{E}-10$ & $2.19 \mathrm{E}-10$ & NUFIP1 & $1.48 \mathrm{E}+00$ \\
\hline ENSBTAG00000015124 & $2.15 \mathrm{E}-10$ & $1.44 \mathrm{E}-10$ & ARGLU1 & $1.49 E+00$ \\
\hline ENSBTAG00000005760 & 5.23E-10 & 3.49E-10 & TBC1D4 & $1.50 \mathrm{E}+00$ \\
\hline ENSBTAG00000008686 & 7.72E-11 & $5.15 \mathrm{E}-11$ & SLC46A3 & $1.50 E+00$ \\
\hline ENSBTAG00000013159 & $4.92 \mathrm{E}-10$ & $3.26 \mathrm{E}-10$ & & $1.51 E+00$ \\
\hline ENSBTAG00000004165 & $2.77 \mathrm{E}-10$ & $1.83 \mathrm{E}-10$ & CLYBL & $1.51 E+00$ \\
\hline ENSBTAG00000026070 & $1.53 \mathrm{E}-09$ & 1.01E-09 & & $1.52 \mathrm{E}+00$ \\
\hline ENSBTAG00000008259 & $2.87 \mathrm{E}-10$ & $1.89 \mathrm{E}-10$ & ACOD1 & $1.52 E+00$ \\
\hline ENSBTAG00000019895 & $2.85 \mathrm{E}-10$ & $1.86 \mathrm{E}-10$ & PIBF1 & $1.53 E+00$ \\
\hline ENSBTAG00000003568 & $1.89 \mathrm{E}-09$ & $1.23 \mathrm{E}-09$ & CLDN10 & $1.53 E+00$ \\
\hline ENSBTAG00000025220 & $4.52 \mathrm{E}-10$ & $2.94 \mathrm{E}-10$ & FAM155A & $1.54 \mathrm{E}+00$ \\
\hline ENSBTAG00000020412 & $9.78 \mathrm{E}-10$ & $6.36 \mathrm{E}-10$ & RAP2A & $1.54 E+00$ \\
\hline ENSBTAG00000019440 & $2.70 \mathrm{E}-10$ & $1.75 \mathrm{E}-10$ & PROZ & $1.54 \mathrm{E}+00$ \\
\hline ENSBTAG00000015614 & $2.44 \mathrm{E}-10$ & $1.58 \mathrm{E}-10$ & LNX2 & $1.55 E+00$ \\
\hline ENSBTAG00000013984 & $2.03 \mathrm{E}-10$ & $1.30 \mathrm{E}-10$ & $\mathrm{KL}$ & $1.56 \mathrm{E}+00$ \\
\hline ENSBTAG00000037786 & $4.66 \mathrm{E}-10$ & $2.97 \mathrm{E}-10$ & NALCN & $1.57 \mathrm{E}+00$ \\
\hline
\end{tabular}




\begin{tabular}{|c|c|c|c|c|}
\hline ENSBTAG00000022779 & $7.10 \mathrm{E}-10$ & $4.50 \mathrm{E}-10$ & OLFM4 & $1.58 \mathrm{E}+00$ \\
\hline ENSBTAG00000039823 & $1.60 \mathrm{E}-10$ & $1.01 \mathrm{E}-10$ & KBTBD7 & $1.59 \mathrm{E}+00$ \\
\hline ENSBTAG00000025211 & $2.27 \mathrm{E}-10$ & $1.43 \mathrm{E}-10$ & & $1.59 \mathrm{E}+00$ \\
\hline ENSBTAG00000034069 & $6.76 \mathrm{E}-11$ & $4.26 \mathrm{E}-11$ & MAB21L1 & $1.58 \mathrm{E}+00$ \\
\hline ENSBTAG00000005244 & $2.21 \mathrm{E}-10$ & $1.39 \mathrm{E}-10$ & RASL11A & $1.59 \mathrm{E}+00$ \\
\hline ENSBTAG00000038732 & $2.64 \mathrm{E}-10$ & $1.66 \mathrm{E}-10$ & & $1.59 \mathrm{E}+00$ \\
\hline ENSBTAG00000014024 & 7.35E-11 & 4.63E-11 & POMP & $1.59 E+00$ \\
\hline ENSBTAG00000010690 & $9.88 \mathrm{E}-11$ & $6.22 \mathrm{E}-11$ & PDX1 & $1.59 E+00$ \\
\hline ENSBTAG00000020530 & $1.44 \mathrm{E}-10$ & $9.08 \mathrm{E}-11$ & MTIF3 & $1.59 \mathrm{E}+00$ \\
\hline ENSBTAG00000046942 & $3.28 \mathrm{E}-10$ & $2.06 \mathrm{E}-10$ & & $1.59 E+00$ \\
\hline ENSBTAG00000016998 & $5.33 \mathrm{E}-10$ & $3.35 \mathrm{E}-10$ & ARL11 & $1.59 \mathrm{E}+00$ \\
\hline ENSBTAG00000010156 & $2.31 \mathrm{E}-10$ & $1.46 \mathrm{E}-10$ & & $1.58 \mathrm{E}+00$ \\
\hline ENSBTAG00000026384 & $2.08 \mathrm{E}-10$ & $1.31 \mathrm{E}-10$ & & $1.59 \mathrm{E}+00$ \\
\hline ENSBTAG00000006177 & $2.60 \mathrm{E}-10$ & $1.61 \mathrm{E}-10$ & ZDHHC20 & $1.62 E+00$ \\
\hline ENSBTAG00000001589 & $6.37 \mathrm{E}-10$ & 3.93E-10 & TM9SF2 & $1.62 E+00$ \\
\hline ENSBTAG00000006640 & $4.89 \mathrm{E}-10$ & $2.92 \mathrm{E}-10$ & RB1 & $1.67 E+00$ \\
\hline ENSBTAG00000016385 & $3.72 \mathrm{E}-10$ & $2.23 \mathrm{E}-10$ & F10 & $1.67 E+00$ \\
\hline ENSBTAG00000038495 & $5.76 \mathrm{E}-10$ & $3.40 \mathrm{E}-10$ & & $1.69 \mathrm{E}+00$ \\
\hline ENSBTAG00000016991 & $2.43 \mathrm{E}-10$ & $1.43 \mathrm{E}-10$ & EFNB2 & $1.70 \mathrm{E}+00$ \\
\hline ENSBTAG00000020393 & $3.53 \mathrm{E}-10$ & $2.05 \mathrm{E}-10$ & METTL21C & $1.72 \mathrm{E}+00$ \\
\hline ENSBTAG00000014486 & $7.71 \mathrm{E}-11$ & 4.37E-11 & ATP12A & $1.76 \mathrm{E}+00$ \\
\hline ENSBTAG00000014752 & 3.23E-10 & $1.82 \mathrm{E}-10$ & AKAP11 & $1.77 \mathrm{E}+00$ \\
\hline ENSBTAG00000018631 & $2.83 \mathrm{E}-10$ & $1.58 \mathrm{E}-10$ & SAP18 & $1.79 \mathrm{E}+00$ \\
\hline ENSBTAG00000019889 & $2.96 \mathrm{E}-10$ & $1.63 \mathrm{E}-10$ & DIS3 & $1.82 \mathrm{E}+00$ \\
\hline ENSBTAG00000001133 & $7.19 \mathrm{E}-10$ & $3.95 \mathrm{E}-10$ & VWA8 & $1.82 \mathrm{E}+00$ \\
\hline ENSBTAG00000020681 & $2.01 \mathrm{E}-10$ & $1.10 \mathrm{E}-10$ & FNDC3A & $1.83 \mathrm{E}+00$ \\
\hline ENSBTAG00000019501 & $2.89 \mathrm{E}-10$ & $1.58 \mathrm{E}-10$ & NUDT15 & $1.83 \mathrm{E}+00$ \\
\hline ENSBTAG00000046408 & $6.82 \mathrm{E}-10$ & $3.71 \mathrm{E}-10$ & FAM124A & $1.84 \mathrm{E}+00$ \\
\hline ENSBTAG00000007284 & $3.84 \mathrm{E}-10$ & $2.08 \mathrm{E}-10$ & CCNA1 & $1.85 \mathrm{E}+00$ \\
\hline ENSBTAG00000017032 & $4.99 \mathrm{E}-10$ & $2.64 \mathrm{E}-10$ & FREM2 & $1.89 \mathrm{E}+00$ \\
\hline ENSBTAG00000025402 & 1.17E-10 & $5.95 \mathrm{E}-11$ & IL17D & $1.96 \mathrm{E}+00$ \\
\hline ENSBTAG00000011654 & 9.77E-10 & 4.99E-10 & & $1.96 \mathrm{E}+00$ \\
\hline ENSBTAG00000023187 & $2.16 \mathrm{E}-10$ & $1.09 \mathrm{E}-10$ & & $1.98 \mathrm{E}+00$ \\
\hline ENSBTAG00000018179 & $9.18 \mathrm{E}-11$ & 4.63E-11 & $\mathrm{PCDH} 8$ & $1.98 \mathrm{E}+00$ \\
\hline ENSBTAG00000010951 & $2.01 \mathrm{E}-10$ & $1.01 \mathrm{E}-10$ & NHLRC3 & $1.99 E+00$ \\
\hline ENSBTAG00000007457 & $2.79 \mathrm{E}-10$ & $1.40 \mathrm{E}-10$ & & $1.99 \mathrm{E}+00$ \\
\hline ENSBTAG00000003315 & $3.79 \mathrm{E}-10$ & $1.91 \mathrm{E}-10$ & MRPL57 & $1.98 \mathrm{E}+00$ \\
\hline ENSBTAG00000032878 & $1.14 \mathrm{E}-09$ & $5.70 \mathrm{E}-10$ & & $2.00 E+00$ \\
\hline ENSBTAG00000014286 & $1.37 \mathrm{E}-10$ & $6.81 \mathrm{E}-11$ & IFT88 & $2.02 \mathrm{E}+00$ \\
\hline ENSBTAG00000008642 & $3.77 \mathrm{E}-10$ & $1.85 \mathrm{E}-10$ & & $2.04 \mathrm{E}+00$ \\
\hline ENSBTAG00000008053 & $5.11 \mathrm{E}-10$ & $2.48 \mathrm{E}-10$ & WDFY2 & $2.06 \mathrm{E}+00$ \\
\hline ENSBTAG00000019529 & $3.21 \mathrm{E}-10$ & $1.56 \mathrm{E}-10$ & & $2.06 \mathrm{E}+00$ \\
\hline ENSBTAG00000016109 & $5.90 \mathrm{E}-10$ & $2.82 \mathrm{E}-10$ & DHRS12 & $2.09 E+00$ \\
\hline ENSBTAG00000020928 & $2.84 \mathrm{E}-10$ & $1.35 \mathrm{E}-10$ & ADPRHL1 & $2.10 E+00$ \\
\hline ENSBTAG00000010693 & $3.50 \mathrm{E}-10$ & $1.65 \mathrm{E}-10$ & LMO7 & $2.12 \mathrm{E}+00$ \\
\hline ENSBTAG00000039714 & $1.36 \mathrm{E}-09$ & $6.35 E-10$ & & $2.14 \mathrm{E}+00$ \\
\hline ENSBTAG00000018232 & $2.85 \mathrm{E}-10$ & $1.33 \mathrm{E}-10$ & STOML3 & $2.14 \mathrm{E}+00$ \\
\hline ENSBTAG00000033386 & 2.67E-10 & $1.24 \mathrm{E}-10$ & TEX26 & $2.15 \mathrm{E}+00$ \\
\hline ENSBTAG00000034269 & $5.13 \mathrm{E}-10$ & $2.37 \mathrm{E}-10$ & RCBTB1 & $2.17 \mathrm{E}+00$ \\
\hline ENSBTAG00000003569 & $1.63 \mathrm{E}-09$ & 7.39E-10 & DZIP1 & $2.21 \mathrm{E}+00$ \\
\hline ENSBTAG00000035926 & $8.31 \mathrm{E}-10$ & $3.72 \mathrm{E}-10$ & & $2.23 \mathrm{E}+00$ \\
\hline ENSBTAG00000044007 & $3.76 \mathrm{E}-10$ & $1.65 \mathrm{E}-10$ & KLF12 & $2.28 \mathrm{E}+00$ \\
\hline ENSBTAG00000011647 & 8.17E-10 & $3.51 \mathrm{E}-10$ & SLC25A15 & $2.33 \mathrm{E}+00$ \\
\hline ENSBTAG00000026861 & $2.27 \mathrm{E}-10$ & $9.51 \mathrm{E}-11$ & & $2.38 \mathrm{E}+00$ \\
\hline ENSBTAG00000047831 & $1.61 \mathrm{E}-10$ & $6.77 \mathrm{E}-11$ & & $2.38 \mathrm{E}+00$ \\
\hline ENSBTAG00000032711 & $3.22 \mathrm{E}-10$ & $1.35 \mathrm{E}-10$ & HTATSF1 & $2.39 E+00$ \\
\hline ENSBTAG00000002970 & $3.18 \mathrm{E}-10$ & $1.31 \mathrm{E}-10$ & INTS6 & $2.43 E+00$ \\
\hline ENSBTAG00000005351 & 4.77E-10 & $1.88 \mathrm{E}-10$ & RFXAP & $2.54 \mathrm{E}+00$ \\
\hline ENSBTAG00000006647 & $5.27 \mathrm{E}-10$ & $2.07 \mathrm{E}-10$ & RCBTB2 & $2.54 \mathrm{E}+00$ \\
\hline ENSBTAG00000017515 & $8.01 \mathrm{E}-10$ & $3.09 \mathrm{E}-10$ & & $2.59 E+00$ \\
\hline ENSBTAG00000008024 & $5.38 \mathrm{E}-10$ & $2.06 \mathrm{E}-10$ & UCHL3 & $2.61 E+00$ \\
\hline ENSBTAG00000002271 & $5.15 \mathrm{E}-10$ & $1.92 \mathrm{E}-10$ & CDADC1 & $2.68 \mathrm{E}+00$ \\
\hline ENSBTAG00000025028 & $6.35 \mathrm{E}-10$ & $2.31 \mathrm{E}-10$ & MZT1 & $2.75 \mathrm{E}+00$ \\
\hline ENSBTAG00000008296 & 2.17E-10 & 7.71E-11 & EEF1AKMT1 & $2.81 E+00$ \\
\hline ENSBTAG00000009416 & $6.73 \mathrm{E}-10$ & $2.30 \mathrm{E}-10$ & GPALPP1 & $2.92 \mathrm{E}+00$ \\
\hline ENSBTAG00000012600 & $2.14 \mathrm{E}-10$ & 7.24E-11 & GTF3A & $2.95 \mathrm{E}+00$ \\
\hline ENSBTAG00000014227 & $1.82 \mathrm{E}-10$ & $6.06 \mathrm{E}-11$ & NDFIP2 & $3.00 \mathrm{E}+00$ \\
\hline ENSBTAG00000022991 & $4.63 \mathrm{E}-10$ & $1.48 \mathrm{E}-10$ & NBEA & $3.13 E+00$ \\
\hline ENSBTAG00000006771 & $3.30 \mathrm{E}-10$ & $1.05 \mathrm{E}-10$ & FRY & $3.14 \mathrm{E}+00$ \\
\hline ENSBTAG00000005318 & 3.53E-11 & $1.11 \mathrm{E}-11$ & RNF6 & $3.16 \mathrm{E}+00$ \\
\hline
\end{tabular}




\begin{tabular}{|c|c|c|c|c|}
\hline ENSBTAG00000009166 & 2.63E-10 & 8.27E-11 & & $3.18 \mathrm{E}+00$ \\
\hline ENSBTAG00000021345 & $3.24 \mathrm{E}-10$ & $1.02 \mathrm{E}-10$ & OXGR1 & $3.17 E+00$ \\
\hline ENSBTAG00000018313 & $5.83 \mathrm{E}-10$ & $1.80 \mathrm{E}-10$ & MBNL2 & $3.23 E+00$ \\
\hline ENSBTAG00000007329 & $4.51 \mathrm{E}-10$ & $1.38 \mathrm{E}-10$ & SETDB2 & $3.26 \mathrm{E}+00$ \\
\hline ENSBTAG00000032603 & $1.34 \mathrm{E}-09$ & $3.95 \mathrm{E}-10$ & $A B C C 4$ & $3.38 \mathrm{E}+00$ \\
\hline ENSBTAG00000047181 & $8.24 \mathrm{E}-10$ & $2.43 \mathrm{E}-10$ & & $3.39 \mathrm{E}+00$ \\
\hline ENSBTAG00000033412 & $6.22 \mathrm{E}-10$ & $1.76 \mathrm{E}-10$ & B3GLCT & $3.53 \mathrm{E}+00$ \\
\hline ENSBTAG00000018829 & $3.09 \mathrm{E}-10$ & $8.70 \mathrm{E}-11$ & SPRYD7 & $3.55 E+00$ \\
\hline ENSBTAG00000011597 & $1.27 \mathrm{E}-10$ & $3.56 \mathrm{E}-11$ & PDS5B & $3.56 \mathrm{E}+00$ \\
\hline ENSBTAG00000015522 & $2.78 \mathrm{E}-10$ & 7.73E-11 & MRPS31 & $3.60 \mathrm{E}+00$ \\
\hline ENSBTAG00000003560 & $9.60 \mathrm{E}-10$ & $2.67 \mathrm{E}-10$ & & $3.59 \mathrm{E}+00$ \\
\hline ENSBTAG00000043985 & $4.71 \mathrm{E}-10$ & 1.27E-10 & & $3.70 \mathrm{E}+00$ \\
\hline ENSBTAG00000005310 & $7.23 \mathrm{E}-10$ & $1.94 \mathrm{E}-10$ & GPR180 & $3.72 E+00$ \\
\hline ENSBTAG00000007411 & $3.40 \mathrm{E}-10$ & $8.21 \mathrm{E}-11$ & F7 & $4.14 \mathrm{E}+00$ \\
\hline ENSBTAG00000008176 & $2.40 \mathrm{E}-10$ & $5.66 \mathrm{E}-11$ & KCNRG & $4.24 E+00$ \\
\hline ENSBTAG00000011860 & 3.67E-10 & $8.38 \mathrm{E}-11$ & SUPT2OH & $4.37 E+00$ \\
\hline ENSBTAG00000011563 & $4.50 \mathrm{E}-10$ & $9.82 \mathrm{E}-11$ & & $4.58 \mathrm{E}+00$ \\
\hline ENSBTAG00000025192 & $1.08 \mathrm{E}-09$ & $2.23 \mathrm{E}-10$ & & $4.84 \mathrm{E}+00$ \\
\hline ENSBTAG00000004850 & 3.87E-10 & 7.17E-11 & KPNA3 & $5.40 \mathrm{E}+00$ \\
\hline ENSBTAG00000018410 & 7.92E-10 & $1.45 \mathrm{E}-10$ & EBPL & $5.46 \mathrm{E}+00$ \\
\hline ENSBTAG00000010129 & $1.92 \mathrm{E}-10$ & $3.44 \mathrm{E}-11$ & SLITRK5 & $5.56 \mathrm{E}+00$ \\
\hline ENSBTAG00000015868 & $3.96 \mathrm{E}-10$ & $6.71 \mathrm{E}-11$ & LIG4 & $5.89 \mathrm{E}+00$ \\
\hline ENSBTAG00000021725 & $3.06 \mathrm{E}-10$ & $4.81 \mathrm{E}-11$ & PCID2 & $6.36 \mathrm{E}+00$ \\
\hline ENSBTAG00000023309 & $6.38 \mathrm{E}-10$ & $9.86 \mathrm{E}-11$ & & $6.47 E+00$ \\
\hline ENSBTAG00000004401 & $1.27 \mathrm{E}-09$ & $1.80 \mathrm{E}-10$ & UGGT2 & $7.03 E+00$ \\
\hline ENSBTAG00000019268 & $1.25 \mathrm{E}-09$ & $1.73 \mathrm{E}-10$ & & $7.21 \mathrm{E}+00$ \\
\hline ENSBTAG00000008271 & $8.36 \mathrm{E}-10$ & $1.08 \mathrm{E}-10$ & MEDAG & $7.73 E+00$ \\
\hline ENSBTAG00000008173 & $1.90 \mathrm{E}-10$ & $2.39 \mathrm{E}-11$ & TRIM13 & $7.91 E+00$ \\
\hline ENSBTAG00000001819 & $1.87 \mathrm{E}-10$ & $2.13 \mathrm{E}-11$ & & $8.72 E+00$ \\
\hline ENSBTAG00000039374 & $5.59 \mathrm{E}-10$ & $6.30 \mathrm{E}-11$ & IPO5 & $8.87 E+00$ \\
\hline ENSBTAG00000011855 & 4.35E-10 & 4.46E-11 & EXOSC8 & $9.74 E+00$ \\
\hline ENSBTAG00000010337 & $1.95 \mathrm{E}-10$ & $1.89 \mathrm{E}-11$ & ALG11 & $1.03 E+01$ \\
\hline ENSBTAG00000008040 & $5.05 E-10$ & 4.70E-11 & SPG20 & $1.07 E+01$ \\
\hline ENSBTAG00000044105 & $3.54 \mathrm{E}-10$ & $3.11 E-11$ & FOXO1 & $1.13 E+01$ \\
\hline ENSBTAG00000010475 & $6.56 \mathrm{E}-10$ & $5.14 \mathrm{E}-11$ & MLNR & $1.27 E+01$ \\
\hline ENSBTAG00000018955 & 4.69E-10 & $2.22 \mathrm{E}-11$ & & $2.10 \mathrm{E}+01$ \\
\hline ENSBTAG00000016635 & $2.66 \mathrm{E}-10$ & $1.13 \mathrm{E}-11$ & COG6 & $2.34 \mathrm{E}+01$ \\
\hline ENSBTAG00000013819 & 5.74E-10 & $2.25 \mathrm{E}-11$ & URAD & $2.54 \mathrm{E}+01$ \\
\hline ENSBTAG00000019863 & $1.21 \mathrm{E}-09$ & 4.50E-11 & CCDC70 & $2.68 \mathrm{E}+01$ \\
\hline ENSBTAG00000020374 & $4.31 \mathrm{E}-10$ & $1.48 \mathrm{E}-11$ & THSD1 & $2.89 \mathrm{E}+01$ \\
\hline ENSBTAG00000012065 & $1.40 \mathrm{E}-09$ & $4.75 \mathrm{E}-11$ & DNAJC3 & $2.93 \mathrm{E}+01$ \\
\hline ENSBTAG00000020612 & $5.62 \mathrm{E}-10$ & 1.77E-11 & NEK3 & $3.16 \mathrm{E}+01$ \\
\hline ENSBTAG00000019134 & $3.40 \mathrm{E}-10$ & $8.38 \mathrm{E}-12$ & NEK5 & $4.02 \mathrm{E}+01$ \\
\hline ENSBTAG00000004307 & 3.04E-10 & $6.54 \mathrm{E}-12$ & VPS36 & $4.59 \mathrm{E}+01$ \\
\hline ENSBTAG00000010353 & $9.49 \mathrm{E}-10$ & $6.48 \mathrm{E}-12$ & ATP7B & $1.44 \mathrm{E}+02$ \\
\hline ENSBTAG00000021162 & 4.67E-10 & 0 & CKAP2 & $4.67 E+03$ \\
\hline ENSBTAG00000014274 & $5.07 \mathrm{E}-11$ & 0 & & $5.08 \mathrm{E}+02$ \\
\hline ENSBTAG00000039065 & 3.77E-10 & 0 & & $3.77 E+03$ \\
\hline ENSBTAG00000046552 & $1.84 \mathrm{E}-10$ & 0 & GGACT & $1.84 \mathrm{E}+03$ \\
\hline ENSBTAG00000000059 & 0 & 0 & & $1.00 \mathrm{E}+00$ \\
\hline ENSBTAG00000019416 & $1.05 \mathrm{E}-10$ & 0 & KCTD4 & $1.05 E+03$ \\
\hline ENSBTAG00000046386 & 0 & 0 & SERTM1 & $1.00 \mathrm{E}+00$ \\
\hline ENSBTAG00000020400 & 0 & 0 & GJA3 & $1.00 \mathrm{E}+00$ \\
\hline ENSBTAG00000032979 & $3.69 \mathrm{E}-10$ & 0 & & $3.69 \mathrm{E}+03$ \\
\hline ENSBTAG00000001414 & 0 & 0 & KCTD12 & $1.00 \mathrm{E}+00$ \\
\hline ENSBTAG00000001774 & $8.65 \mathrm{E}-11$ & 0 & SPRY2 & $8.66 \mathrm{E}+02$ \\
\hline ENSBTAG00000004388 & $1.62 \mathrm{E}-10$ & 0 & SLITRK6 & $1.62 \mathrm{E}+03$ \\
\hline ENSBTAG00000045566 & $8.49 \mathrm{E}-10$ & 0 & & $8.49 E+03$ \\
\hline ENSBTAG00000002432 & $8.08 \mathrm{E}-11$ & 0 & ABHD13 & $8.09 \mathrm{E}+02$ \\
\hline ENSBTAG00000048089 & 0 & $2.24 \mathrm{E}-11$ & & 4.44E-03 \\
\hline ENSBTAG00000047779 & 0 & $1.50 \mathrm{E}-10$ & SRY & $6.66 \mathrm{E}-04$ \\
\hline ENSBTAG00000027412 & 0 & $1.26 \mathrm{E}-10$ & & 7.93E-04 \\
\hline ENSBTAG00000048009 & 0 & $8.23 \mathrm{E}-10$ & & $1.21 \mathrm{E}-04$ \\
\hline ENSBTAG00000016406 & $1.30 \mathrm{E}-10$ & $7.38 \mathrm{E}-10$ & MCM10 & $1.76 \mathrm{E}-01$ \\
\hline ENSBTAG00000047752 & $1.70 \mathrm{E}-10$ & $6.45 \mathrm{E}-10$ & OTUD1 & 2.64E-01 \\
\hline ENSBTAG00000016746 & $1.91 \mathrm{E}-10$ & 7.25E-10 & UBE2C & 2.64E-01 \\
\hline ENSBTAG00000013737 & $1.64 \mathrm{E}-10$ & $6.01 \mathrm{E}-10$ & $\mathrm{MROH} 8$ & 2.73E-01 \\
\hline ENSBTAG00000018812 & $1.56 \mathrm{E}-10$ & 5.53E-10 & & 2.83E-01 \\
\hline ENSBTAG00000008812 & $1.88 \mathrm{E}-10$ & 5.10E-10 & PFDN4 & 3.70E-01 \\
\hline ENSBTAG00000031625 & $3.93 \mathrm{E}-11$ & $9.90 \mathrm{E}-11$ & $\mathrm{HRH} 3$ & 3.97E-01 \\
\hline
\end{tabular}




\begin{tabular}{|c|c|c|c|c|}
\hline ENSBTAG00000011233 & $2.74 \mathrm{E}-10$ & $6.92 \mathrm{E}-10$ & GTSF1L & $3.96 \mathrm{E}-01$ \\
\hline ENSBTAG00000013899 & $2.01 \mathrm{E}-10$ & $4.85 \mathrm{E}-10$ & LARP4B & 4.15E-01 \\
\hline ENSBTAG00000047638 & $1.84 \mathrm{E}-11$ & $4.06 \mathrm{E}-11$ & & 4.55E-01 \\
\hline ENSBTAG00000046776 & $1.92 \mathrm{E}-11$ & 4.24E-11 & & 4.55E-01 \\
\hline ENSBTAG00000018813 & $1.65 \mathrm{E}-10$ & $3.55 \mathrm{E}-10$ & $\mathrm{IDH} 3 \mathrm{~B}$ & 4.66E-01 \\
\hline ENSBTAG00000018873 & $3.79 \mathrm{E}-10$ & 8.07E-10 & TTPAL & 4.69E-01 \\
\hline ENSBTAG00000044074 & $6.94 \mathrm{E}-11$ & $1.47 \mathrm{E}-10$ & MLLT10 & 4.72E-01 \\
\hline ENSBTAG00000047962 & $2.19 \mathrm{E}-11$ & 4.60E-11 & & $4.78 \mathrm{E}-01$ \\
\hline ENSBTAG00000047102 & $1.99 \mathrm{E}-11$ & $3.76 \mathrm{E}-11$ & & $5.31 \mathrm{E}-01$ \\
\hline ENSBTAG00000046716 & $2.22 \mathrm{E}-11$ & $4.20 \mathrm{E}-11$ & & $5.30 \mathrm{E}-01$ \\
\hline ENSBTAG00000047653 & $3.48 \mathrm{E}-11$ & $6.58 \mathrm{E}-11$ & & $5.29 \mathrm{E}-01$ \\
\hline ENSBTAG00000014555 & $1.28 \mathrm{E}-10$ & $2.40 \mathrm{E}-10$ & CASS4 & $5.32 \mathrm{E}-01$ \\
\hline ENSBTAG00000016743 & $2.99 \mathrm{E}-10$ & $5.59 \mathrm{E}-10$ & DNTTIP1 & $5.35 \mathrm{E}-01$ \\
\hline ENSBTAG00000018295 & $1.36 \mathrm{E}-10$ & $2.51 \mathrm{E}-10$ & SEC23B & 5.43E-01 \\
\hline ENSBTAG00000017938 & $3.55 \mathrm{E}-10$ & $6.48 \mathrm{E}-10$ & & $5.49 \mathrm{E}-01$ \\
\hline ENSBTAG00000006065 & $9.44 \mathrm{E}-11$ & $1.70 \mathrm{E}-10$ & PCNA & $5.56 \mathrm{E}-01$ \\
\hline ENSBTAG00000008105 & $5.13 \mathrm{E}-11$ & $8.62 \mathrm{E}-11$ & RBM38 & $5.96 \mathrm{E}-01$ \\
\hline ENSBTAG00000007658 & $3.29 \mathrm{E}-10$ & $5.48 \mathrm{E}-10$ & CTNNBL1 & $6.00 \mathrm{E}-01$ \\
\hline ENSBTAG00000009117 & $1.47 \mathrm{E}-10$ & $2.32 \mathrm{E}-10$ & THNSL1 & 6.34E-01 \\
\hline ENSBTAG00000040147 & 2.17E-10 & $3.40 \mathrm{E}-10$ & SYNDIG1 & $6.39 \mathrm{E}-01$ \\
\hline ENSBTAG00000011458 & $3.22 \mathrm{E}-10$ & $5.04 \mathrm{E}-10$ & CPXM1 & $6.39 \mathrm{E}-01$ \\
\hline ENSBTAG00000014179 & $7.64 \mathrm{E}-11$ & $1.18 \mathrm{E}-10$ & SLC12A5 & $6.48 \mathrm{E}-01$ \\
\hline ENSBTAG00000003639 & $1.40 \mathrm{E}-10$ & $2.15 \mathrm{E}-10$ & ELMO2 & $6.51 \mathrm{E}-01$ \\
\hline ENSBTAG00000039722 & $2.72 \mathrm{E}-10$ & 4.10E-10 & & $6.63 \mathrm{E}-01$ \\
\hline ENSBTAG00000014186 & $1.33 \mathrm{E}-10$ & $2.00 \mathrm{E}-10$ & NCOA5 & $6.66 \mathrm{E}-01$ \\
\hline ENSBTAG00000005275 & $3.79 \mathrm{E}-10$ & $5.55 \mathrm{E}-10$ & PKIG & $6.82 \mathrm{E}-01$ \\
\hline ENSBTAG00000009626 & $4.46 \mathrm{E}-10$ & $6.47 \mathrm{E}-10$ & SPINT4 & $6.90 \mathrm{E}-01$ \\
\hline ENSBTAG00000020736 & 5.07E-10 & 7.27E-10 & CD40 & $6.98 \mathrm{E}-01$ \\
\hline ENSBTAG00000000288 & $1.55 \mathrm{E}-10$ & $2.20 \mathrm{E}-10$ & UPF2 & 7.03E-01 \\
\hline ENSBTAG00000030800 & $5.68 \mathrm{E}-10$ & $8.06 \mathrm{E}-10$ & WFDC10B & 7.05E-01 \\
\hline ENSBTAG00000047315 & $4.69 \mathrm{E}-10$ & $6.65 \mathrm{E}-10$ & PTF1A & 7.05E-01 \\
\hline ENSBTAG00000005280 & $4.83 \mathrm{E}-10$ & $6.83 \mathrm{E}-10$ & $\mathrm{ADA}$ & 7.07E-01 \\
\hline ENSBTAG00000013167 & $1.15 \mathrm{E}-10$ & $1.63 \mathrm{E}-10$ & SIGLEC1 & 7.06E-01 \\
\hline ENSBTAG00000001015 & $9.34 \mathrm{E}-11$ & $1.31 \mathrm{E}-10$ & TM9SF4 & 7.14E-01 \\
\hline ENSBTAG00000012377 & $4.69 \mathrm{E}-10$ & $6.56 \mathrm{E}-10$ & ECHDC3 & 7.15E-01 \\
\hline ENSBTAG00000000309 & $4.84 \mathrm{E}-10$ & $6.77 \mathrm{E}-10$ & & 7.15E-01 \\
\hline ENSBTAG00000013160 & $1.74 \mathrm{E}-10$ & $2.43 \mathrm{E}-10$ & GFRA4 & 7.15E-01 \\
\hline ENSBTAG00000030798 & $5.52 \mathrm{E}-10$ & $7.45 \mathrm{E}-10$ & WFDC11 & $7.41 \mathrm{E}-01$ \\
\hline ENSBTAG00000019511 & $3.79 \mathrm{E}-10$ & $5.09 \mathrm{E}-10$ & CDC123 & 7.44E-01 \\
\hline ENSBTAG00000003901 & $1.94 \mathrm{E}-10$ & $2.59 \mathrm{E}-10$ & DNMT3B & 7.49E-01 \\
\hline ENSBTAG00000004281 & $2.57 \mathrm{E}-10$ & $3.40 \mathrm{E}-10$ & ACSS1 & 7.55E-01 \\
\hline ENSBTAG00000007783 & $1.26 \mathrm{E}-10$ & 1.67E-10 & MYBL2 & 7.57E-01 \\
\hline ENSBTAG00000004474 & $1.32 \mathrm{E}-10$ & $1.74 \mathrm{E}-10$ & BANF2 & 7.57E-01 \\
\hline ENSBTAG00000037942 & 1.43E-09 & $1.86 \mathrm{E}-09$ & & 7.69E-01 \\
\hline ENSBTAG00000035696 & $6.68 \mathrm{E}-10$ & 8.64E-10 & UCN3 & 7.74E-01 \\
\hline ENSBTAG00000016742 & $6.16 \mathrm{E}-10$ & $7.89 \mathrm{E}-10$ & & $7.81 E-01$ \\
\hline ENSBTAG00000019488 & $4.92 \mathrm{E}-10$ & $6.24 \mathrm{E}-10$ & USP6NL & 7.89E-01 \\
\hline ENSBTAG00000044011 & $2.07 \mathrm{E}-10$ & $2.60 \mathrm{E}-10$ & & 7.95E-01 \\
\hline ENSBTAG00000014917 & $1.84 \mathrm{E}-10$ & $2.32 \mathrm{E}-10$ & GTPBP4 & $7.94 \mathrm{E}-01$ \\
\hline ENSBTAG00000020371 & $3.02 \mathrm{E}-10$ & $3.80 \mathrm{E}-10$ & АСОT8 & 7.94E-01 \\
\hline ENSBTAG00000000959 & $3.54 \mathrm{E}-10$ & $4.46 \mathrm{E}-10$ & ZSWIM1 & 7.94E-01 \\
\hline ENSBTAG00000022534 & $4.14 \mathrm{E}-10$ & $5.21 \mathrm{E}-10$ & & 7.94E-01 \\
\hline ENSBTAG00000005202 & $5.79 \mathrm{E}-10$ & 7.30E-10 & SCP2D1 & $7.94 \mathrm{E}-01$ \\
\hline ENSBTAG00000039028 & $1.04 \mathrm{E}-09$ & $1.31 \mathrm{E}-09$ & & $7.91 \mathrm{E}-01$ \\
\hline ENSBTAG00000048291 & $5.56 \mathrm{E}-10$ & $7.00 \mathrm{E}-10$ & & 7.94E-01 \\
\hline ENSBTAG00000003589 & $3.43 \mathrm{E}-11$ & $4.32 \mathrm{E}-11$ & COMMD3 & 7.95E-01 \\
\hline ENSBTAG00000021805 & $3.10 \mathrm{E}-10$ & $3.90 \mathrm{E}-10$ & TGIF2-C20orf24 & $7.94 \mathrm{E}-01$ \\
\hline ENSBTAG00000020030 & $2.04 \mathrm{E}-10$ & $2.57 \mathrm{E}-10$ & FITM2 & 7.94E-01 \\
\hline ENSBTAG00000027397 & $9.10 \mathrm{E}-11$ & $1.15 \mathrm{E}-10$ & PPP1R3D & $7.92 \mathrm{E}-01$ \\
\hline ENSBTAG00000003937 & $3.13 \mathrm{E}-10$ & $3.94 \mathrm{E}-10$ & & $7.94 \mathrm{E}-01$ \\
\hline ENSBTAG00000027326 & $3.03 \mathrm{E}-10$ & $3.82 \mathrm{E}-10$ & TP53RK & $7.93 \mathrm{E}-01$ \\
\hline ENSBTAG00000000960 & $1.59 \mathrm{E}-10$ & $2.00 \mathrm{E}-10$ & SPATA25 & 7.93E-01 \\
\hline ENSBTAG00000010873 & $1.22 \mathrm{E}-10$ & $1.54 \mathrm{E}-10$ & BHLHE23 & 7.95E-01 \\
\hline ENSBTAG00000014623 & $1.89 \mathrm{E}-10$ & $2.35 \mathrm{E}-10$ & MCM8 & 8.03E-01 \\
\hline ENSBTAG00000031376 & $4.55 \mathrm{E}-10$ & $5.62 \mathrm{E}-10$ & & $8.09 \mathrm{E}-01$ \\
\hline ENSBTAG00000010130 & $5.48 \mathrm{E}-10$ & $6.74 \mathrm{E}-10$ & ZNF335 & $8.13 \mathrm{E}-01$ \\
\hline ENSBTAG00000003908 & $1.14 \mathrm{E}-10$ & $1.40 \mathrm{E}-10$ & MAPRE1 & 8.17E-01 \\
\hline ENSBTAG00000027383 & $1.06 \mathrm{E}-09$ & $1.28 \mathrm{E}-09$ & & $8.25 \mathrm{E}-01$ \\
\hline ENSBTAG00000019621 & $1.52 \mathrm{E}-10$ & $1.84 \mathrm{E}-10$ & NDRG3 & 8.27E-01 \\
\hline ENSBTAG00000008964 & $1.57 \mathrm{E}-10$ & $1.87 \mathrm{E}-10$ & DTD1 & $8.42 \mathrm{E}-01$ \\
\hline
\end{tabular}




\begin{tabular}{|c|c|c|c|c|}
\hline ENSBTAG00000003364 & $7.41 \mathrm{E}-10$ & $8.80 \mathrm{E}-10$ & & $8.42 \mathrm{E}-01$ \\
\hline ENSBTAG00000009881 & $3.72 \mathrm{E}-10$ & 4.40E-10 & ENTPD6 & 8.46E-01 \\
\hline ENSBTAG00000038260 & 8.99E-10 & $1.06 \mathrm{E}-09$ & AKR1E2 & $8.49 \mathrm{E}-01$ \\
\hline ENSBTAG00000016849 & $2.54 \mathrm{E}-10$ & $2.99 \mathrm{E}-10$ & PABPC1L & $8.51 \mathrm{E}-01$ \\
\hline ENSBTAG00000019508 & $1.55 \mathrm{E}-10$ & $1.82 \mathrm{E}-10$ & SEC61A2 & $8.53 \mathrm{E}-01$ \\
\hline ENSBTAG00000000958 & $6.58 \mathrm{E}-10$ & 7.72E-10 & ZSWIM3 & $8.52 \mathrm{E}-01$ \\
\hline ENSBTAG00000000961 & 4.13E-10 & $4.83 \mathrm{E}-10$ & NEURL2 & $8.55 \mathrm{E}-01$ \\
\hline ENSBTAG00000001376 & 4.69E-10 & $5.48 \mathrm{E}-10$ & RAD21L1 & $8.55 \mathrm{E}-01$ \\
\hline ENSBTAG00000031375 & 7.69E-10 & $8.93 \mathrm{E}-10$ & & $8.61 \mathrm{E}-01$ \\
\hline ENSBTAG00000001204 & $1.95 \mathrm{E}-10$ & $2.25 \mathrm{E}-10$ & KIAA1462 & $8.65 \mathrm{E}-01$ \\
\hline ENSBTAG00000032704 & 4.94E-10 & $5.73 \mathrm{E}-10$ & NAPB & $8.63 \mathrm{E}-01$ \\
\hline ENSBTAG00000027328 & $1.05 \mathrm{E}-09$ & $1.22 \mathrm{E}-09$ & & $8.61 \mathrm{E}-01$ \\
\hline ENSBTAG00000013545 & $1.48 \mathrm{E}-10$ & $1.69 \mathrm{E}-10$ & MAVS & $8.75 \mathrm{E}-01$ \\
\hline ENSBTAG00000034987 & $4.76 \mathrm{E}-10$ & $5.46 \mathrm{E}-10$ & MKKS & $8.72 \mathrm{E}-01$ \\
\hline ENSBTAG00000005316 & $1.83 \mathrm{E}-10$ & $2.09 \mathrm{E}-10$ & GDI2 & $8.76 \mathrm{E}-01$ \\
\hline ENSBTAG00000046276 & 7.16E-11 & $8.18 \mathrm{E}-11$ & & $8.76 \mathrm{E}-01$ \\
\hline ENSBTAG00000000291 & $1.69 \mathrm{E}-10$ & $1.92 \mathrm{E}-10$ & DHTKD1 & $8.80 \mathrm{E}-01$ \\
\hline ENSBTAG00000006670 & $5.79 \mathrm{E}-10$ & $6.60 \mathrm{E}-10$ & ERGIC3 & $8.78 \mathrm{E}-01$ \\
\hline ENSBTAG00000027384 & 8.57E-10 & $9.76 \mathrm{E}-10$ & & $8.78 \mathrm{E}-01$ \\
\hline ENSBTAG00000002942 & $6.52 \mathrm{E}-10$ & 7.43E-10 & SLC2A10 & $8.78 \mathrm{E}-01$ \\
\hline ENSBTAG00000019197 & $1.52 \mathrm{E}-10$ & $1.72 \mathrm{E}-10$ & FAM208B & $8.82 \mathrm{E}-01$ \\
\hline ENSBTAG00000020555 & 4.44E-10 & $5.05 \mathrm{E}-10$ & DEFB123 & $8.80 \mathrm{E}-01$ \\
\hline ENSBTAG00000010126 & $6.93 \mathrm{E}-10$ & 7.87E-10 & PCIF1 & $8.81 \mathrm{E}-01$ \\
\hline ENSBTAG00000010210 & $1.42 \mathrm{E}-10$ & $1.60 \mathrm{E}-10$ & SUV39H2 & 8.87E-01 \\
\hline ENSBTAG00000002856 & $5.20 \mathrm{E}-10$ & $5.86 \mathrm{E}-10$ & MEIG1 & 8.87E-01 \\
\hline ENSBTAG00000027937 & $2.58 \mathrm{E}-10$ & $2.91 \mathrm{E}-10$ & $\operatorname{PrP}$ & $8.88 \mathrm{E}-01$ \\
\hline ENSBTAG00000003608 & $2.50 \mathrm{E}-10$ & $2.81 \mathrm{E}-10$ & SYCP2 & $8.88 \mathrm{E}-01$ \\
\hline ENSBTAG00000030556 & $1.65 \mathrm{E}-10$ & $1.84 \mathrm{E}-10$ & ZNF217 & 8.95E-01 \\
\hline ENSBTAG00000047944 & $7.40 \mathrm{E}-11$ & $8.28 \mathrm{E}-11$ & GATA5 & 8.94E-01 \\
\hline ENSBTAG00000040607 & $4.68 \mathrm{E}-10$ & $5.24 \mathrm{E}-10$ & SLA2 & $8.94 \mathrm{E}-01$ \\
\hline ENSBTAG00000047336 & $3.56 \mathrm{E}-10$ & $3.98 \mathrm{E}-10$ & & $8.94 \mathrm{E}-01$ \\
\hline ENSBTAG00000000559 & $7.24 \mathrm{E}-10$ & 8.09E-10 & PLTP & 8.95E-01 \\
\hline ENSBTAG00000002578 & $1.86 \mathrm{E}-10$ & 2.07E-10 & ZMYND11 & 9.00E-01 \\
\hline ENSBTAG00000009144 & $9.66 \mathrm{E}-10$ & 1.06E-09 & & $9.11 \mathrm{E}-01$ \\
\hline ENSBTAG00000017188 & $4.01 \mathrm{E}-10$ & $4.38 \mathrm{E}-10$ & ISM1 & $9.15 \mathrm{E}-01$ \\
\hline ENSBTAG00000003335 & $1.43 \mathrm{E}-10$ & $1.56 \mathrm{E}-10$ & VSX1 & 9.17E-01 \\
\hline ENSBTAG00000022003 & $4.18 \mathrm{E}-10$ & $4.55 \mathrm{E}-10$ & NSUN6 & $9.19 \mathrm{E}-01$ \\
\hline ENSBTAG00000014345 & $9.33 \mathrm{E}-10$ & 1.01E-09 & & $9.23 \mathrm{E}-01$ \\
\hline ENSBTAG00000009675 & $6.53 \mathrm{E}-10$ & 7.09E-10 & & $9.21 \mathrm{E}-01$ \\
\hline ENSBTAG00000013336 & $3.58 \mathrm{E}-10$ & $3.88 \mathrm{E}-10$ & EYA2 & $9.23 \mathrm{E}-01$ \\
\hline ENSBTAG00000006043 & 3.37E-10 & $3.65 \mathrm{E}-10$ & SLC6A9 & $9.24 \mathrm{E}-01$ \\
\hline ENSBTAG00000033315 & $9.55 \mathrm{E}-11$ & $1.03 \mathrm{E}-10$ & DNAJC1 & 9.27E-01 \\
\hline ENSBTAG00000016873 & $3.84 \mathrm{E}-10$ & $4.11 \mathrm{E}-10$ & OSER1 & $9.33 \mathrm{E}-01$ \\
\hline ENSBTAG00000001512 & $6.20 \mathrm{E}-10$ & $6.63 \mathrm{E}-10$ & ZFP64 & $9.35 \mathrm{E}-01$ \\
\hline ENSBTAG00000021727 & $5.71 \mathrm{E}-10$ & $6.08 \mathrm{E}-10$ & NTSR1 & $9.39 \mathrm{E}-01$ \\
\hline ENSBTAG00000008908 & $3.71 \mathrm{E}-10$ & $3.95 \mathrm{E}-10$ & GZF1 & $9.38 \mathrm{E}-01$ \\
\hline ENSBTAG00000017354 & $3.05 \mathrm{E}-10$ & $3.24 \mathrm{E}-10$ & MPP7 & $9.40 \mathrm{E}-01$ \\
\hline ENSBTAG00000011782 & $7.11 \mathrm{E}-10$ & 7.57E-10 & CHGB & $9.40 \mathrm{E}-01$ \\
\hline ENSBTAG00000006037 & $2.18 \mathrm{E}-10$ & $2.31 \mathrm{E}-10$ & WISP2 & 9.44E-01 \\
\hline ENSBTAG00000031453 & $2.44 \mathrm{E}-10$ & $2.59 \mathrm{E}-10$ & CCM2L & $9.41 \mathrm{E}-01$ \\
\hline ENSBTAG00000006066 & $2.90 \mathrm{E}-10$ & 3.07E-10 & CDS2 & $9.44 \mathrm{E}-01$ \\
\hline ENSBTAG00000032893 & $9.78 \mathrm{E}-10$ & $1.04 \mathrm{E}-09$ & & $9.40 \mathrm{E}-01$ \\
\hline ENSBTAG00000015362 & $2.14 \mathrm{E}-10$ & $2.27 \mathrm{E}-10$ & BMP7 & $9.44 \mathrm{E}-01$ \\
\hline ENSBTAG00000000528 & $2.92 \mathrm{E}-10$ & $3.09 \mathrm{E}-10$ & MANBAL & $9.46 \mathrm{E}-01$ \\
\hline ENSBTAG00000007962 & $6.08 \mathrm{E}-10$ & $6.41 \mathrm{E}-10$ & ATP9A & $9.48 \mathrm{E}-01$ \\
\hline ENSBTAG00000023920 & $2.27 \mathrm{E}-10$ & $2.40 \mathrm{E}-10$ & GPR158 & $9.47 \mathrm{E}-01$ \\
\hline ENSBTAG00000038966 & $3.87 \mathrm{E}-10$ & 4.07E-10 & & $9.50 \mathrm{E}-01$ \\
\hline ENSBTAG00000003163 & $1.93 \mathrm{E}-10$ & $2.02 \mathrm{E}-10$ & RBBP8NL & $9.54 \mathrm{E}-01$ \\
\hline ENSBTAG00000015609 & $2.84 \mathrm{E}-10$ & $2.98 \mathrm{E}-10$ & PREX1 & $9.52 \mathrm{E}-01$ \\
\hline ENSBTAG00000009871 & $5.65 \mathrm{E}-10$ & $5.93 \mathrm{E}-10$ & RAB18 & $9.53 \mathrm{E}-01$ \\
\hline ENSBTAG00000003058 & 8.57E-11 & 8.99E-11 & ADRM1 & 9.53E-01 \\
\hline ENSBTAG00000047975 & $6.48 \mathrm{E}-10$ & $6.80 \mathrm{E}-10$ & & $9.53 \mathrm{E}-01$ \\
\hline ENSBTAG00000016427 & $2.27 \mathrm{E}-10$ & $2.39 \mathrm{E}-10$ & COMMD7 & $9.51 \mathrm{E}-01$ \\
\hline ENSBTAG00000007932 & $1.81 \mathrm{E}-10$ & $1.89 \mathrm{E}-10$ & $\mathrm{HCK}$ & $9.56 \mathrm{E}-01$ \\
\hline ENSBTAG00000003382 & $3.44 \mathrm{E}-10$ & $3.60 \mathrm{E}-10$ & RIMS4 & 9.55E-01 \\
\hline ENSBTAG00000012109 & $2.26 \mathrm{E}-10$ & $2.35 \mathrm{E}-10$ & EDN3 & $9.60 \mathrm{E}-01$ \\
\hline ENSBTAG00000038412 & $9.39 \mathrm{E}-10$ & $9.74 \mathrm{E}-10$ & BPIFB4 & $9.64 \mathrm{E}-01$ \\
\hline ENSBTAG00000006078 & 4.17E-10 & $4.32 \mathrm{E}-10$ & & 9.65E-01 \\
\hline ENSBTAG00000014648 & $2.33 \mathrm{E}-10$ & 2.42E-10 & RPN2 & $9.61 \mathrm{E}-01$ \\
\hline ENSBTAG00000016122 & $4.76 \mathrm{E}-10$ & 4.94E-10 & PIGT & $9.64 \mathrm{E}-01$ \\
\hline
\end{tabular}




\begin{tabular}{|c|c|c|c|c|}
\hline ENSBTAG00000003045 & $1.31 \mathrm{E}-10$ & $1.35 \mathrm{E}-10$ & BAMBI & $9.69 \mathrm{E}-01$ \\
\hline ENSBTAG00000004302 & $2.77 \mathrm{E}-10$ & $2.86 \mathrm{E}-10$ & NDUFAF5 & $9.68 \mathrm{E}-01$ \\
\hline ENSBTAG00000013950 & $2.31 \mathrm{E}-10$ & $2.38 \mathrm{E}-10$ & & $9.69 \mathrm{E}-01$ \\
\hline ENSBTAG00000013339 & $3.74 \mathrm{E}-10$ & $3.85 \mathrm{E}-10$ & NEBL & $9.70 \mathrm{E}-01$ \\
\hline ENSBTAG00000008293 & $3.07 \mathrm{E}-10$ & $3.13 \mathrm{E}-10$ & GPCPD1 & $9.80 \mathrm{E}-01$ \\
\hline ENSBTAG00000015581 & $1.45 \mathrm{E}-10$ & $1.48 \mathrm{E}-10$ & COL9A3 & $9.82 \mathrm{E}-01$ \\
\hline ENSBTAG00000047320 & $7.54 \mathrm{E}-11$ & $7.69 \mathrm{E}-11$ & & $9.80 \mathrm{E}-01$ \\
\hline ENSBTAG00000017524 & $3.60 \mathrm{E}-10$ & $3.66 \mathrm{E}-10$ & CBLN4 & $9.84 \mathrm{E}-01$ \\
\hline ENSBTAG00000009475 & $4.53 \mathrm{E}-10$ & $4.59 \mathrm{E}-10$ & PLXDC2 & $9.87 E-01$ \\
\hline ENSBTAG00000005672 & $1.34 \mathrm{E}-10$ & $1.35 \mathrm{E}-10$ & $\mathrm{CDH} 22$ & $9.93 \mathrm{E}-01$ \\
\hline ENSBTAG00000004750 & $4.65 \mathrm{E}-10$ & $4.70 \mathrm{E}-10$ & VSTM2L & $9.90 \mathrm{E}-01$ \\
\hline ENSBTAG00000020053 & $1.56 \mathrm{E}-10$ & $1.57 \mathrm{E}-10$ & ZEB1 & $9.91 \mathrm{E}-01$ \\
\hline ENSBTAG00000039356 & $4.89 \mathrm{E}-10$ & $4.92 \mathrm{E}-10$ & & $9.93 \mathrm{E}-01$ \\
\hline ENSBTAG00000007120 & $4.24 \mathrm{E}-10$ & 4.27E-10 & ARFGEF2 & $9.94 \mathrm{E}-01$ \\
\hline ENSBTAG00000004993 & $6.16 \mathrm{E}-10$ & $6.20 \mathrm{E}-10$ & NXT1 & $9.94 \mathrm{E}-01$ \\
\hline ENSBTAG00000002187 & $2.44 \mathrm{E}-10$ & $2.45 \mathrm{E}-10$ & SERINC3 & $9.96 \mathrm{E}-01$ \\
\hline ENSBTAG00000036222 & $5.61 \mathrm{E}-10$ & $5.62 \mathrm{E}-10$ & & $9.98 \mathrm{E}-01$ \\
\hline ENSBTAG00000010220 & $1.76 \mathrm{E}-10$ & $1.76 \mathrm{E}-10$ & DCLRE1C & $9.99 \mathrm{E}-01$ \\
\hline ENSBTAG00000044112 & 5.37E-10 & $5.35 \mathrm{E}-10$ & MSRB2 & $1.00 \mathrm{E}+00$ \\
\hline ENSBTAG00000007648 & $2.02 \mathrm{E}-10$ & $2.01 \mathrm{E}-10$ & NET1 & $1.00 \mathrm{E}+00$ \\
\hline ENSBTAG00000021601 & $7.13 \mathrm{E}-10$ & $7.11 \mathrm{E}-10$ & PARD6B & $1.00 \mathrm{E}+00$ \\
\hline ENSBTAG00000002765 & $3.01 \mathrm{E}-10$ & $3.00 \mathrm{E}-10$ & CYP24A1 & $1.00 \mathrm{E}+00$ \\
\hline ENSBTAG00000018463 & $1.45 \mathrm{E}-10$ & $1.43 \mathrm{E}-10$ & VIM & $1.01 E+00$ \\
\hline ENSBTAG00000027390 & $1.20 \mathrm{E}-10$ & $1.19 \mathrm{E}-10$ & RTFDC1 & $1.01 E+00$ \\
\hline ENSBTAG00000015188 & $1.04 \mathrm{E}-10$ & $1.02 \mathrm{E}-10$ & KLF6 & $1.02 \mathrm{E}+00$ \\
\hline ENSBTAG00000010277 & $2.82 \mathrm{E}-10$ & $2.76 \mathrm{E}-10$ & NKX2-2 & $1.02 \mathrm{E}+00$ \\
\hline ENSBTAG00000012276 & $7.20 \mathrm{E}-10$ & $7.04 \mathrm{E}-10$ & & $1.02 \mathrm{E}+00$ \\
\hline ENSBTAG00000019606 & $5.81 \mathrm{E}-10$ & $5.67 E-10$ & IFT52 & $1.03 E+00$ \\
\hline ENSBTAG00000008291 & $5.03 \mathrm{E}-10$ & $4.91 \mathrm{E}-10$ & PROCR & $1.03 E+00$ \\
\hline ENSBTAG00000013930 & $2.66 \mathrm{E}-10$ & $2.59 \mathrm{E}-10$ & ATP5C1 & $1.03 E+00$ \\
\hline ENSBTAG00000018270 & $3.33 \mathrm{E}-10$ & $3.23 E-10$ & NFATC2 & $1.03 E+00$ \\
\hline ENSBTAG00000000683 & $3.11 \mathrm{E}-10$ & $3.02 \mathrm{E}-10$ & WDR37 & $1.03 E+00$ \\
\hline ENSBTAG00000001552 & $1.64 \mathrm{E}-10$ & $1.59 \mathrm{E}-10$ & EPC1 & $1.03 E+00$ \\
\hline ENSBTAG00000022005 & $2.41 \mathrm{E}-10$ & $2.34 \mathrm{E}-10$ & TLDC2 & $1.03 E+00$ \\
\hline ENSBTAG00000017402 & $2.78 \mathrm{E}-10$ & $2.69 \mathrm{E}-10$ & OSBPL2 & $1.03 E+00$ \\
\hline ENSBTAG00000031254 & 7.73E-10 & $7.48 \mathrm{E}-10$ & DEFB124 & $1.03 E+00$ \\
\hline ENSBTAG00000013114 & $2.77 \mathrm{E}-10$ & $2.68 \mathrm{E}-10$ & ZMYND8 & $1.03 \mathrm{E}+00$ \\
\hline ENSBTAG00000000835 & $3.61 \mathrm{E}-10$ & $3.48 \mathrm{E}-10$ & BCAS1 & $1.04 \mathrm{E}+00$ \\
\hline ENSBTAG00000005186 & $3.17 \mathrm{E}-10$ & $3.05 E-10$ & DBNDD2 & $1.04 \mathrm{E}+00$ \\
\hline ENSBTAG00000001495 & $2.32 \mathrm{E}-10$ & $2.23 E-10$ & & $1.04 \mathrm{E}+00$ \\
\hline ENSBTAG00000008401 & $3.05 \mathrm{E}-10$ & $2.92 \mathrm{E}-10$ & PFKFB3 & $1.04 \mathrm{E}+00$ \\
\hline ENSBTAG00000018805 & $3.73 E-10$ & $3.57 \mathrm{E}-10$ & ASB13 & $1.04 \mathrm{E}+00$ \\
\hline ENSBTAG00000004278 & $6.01 \mathrm{E}-10$ & $5.75 \mathrm{E}-10$ & APMAP & $1.05 \mathrm{E}+00$ \\
\hline ENSBTAG00000017328 & $6.56 \mathrm{E}-10$ & $6.26 \mathrm{E}-10$ & & $1.05 \mathrm{E}+00$ \\
\hline ENSBTAG00000005733 & $5.10 \mathrm{E}-10$ & $4.86 \mathrm{E}-10$ & CHD6 & $1.05 E+00$ \\
\hline ENSBTAG00000027506 & $7.95 \mathrm{E}-10$ & $7.55 E-10$ & SNRPB2 & $1.05 \mathrm{E}+00$ \\
\hline ENSBTAG00000020465 & $3.86 \mathrm{E}-10$ & $3.68 \mathrm{E}-10$ & FERMT1 & $1.05 \mathrm{E}+00$ \\
\hline ENSBTAG00000005086 & $2.45 \mathrm{E}-10$ & $2.32 \mathrm{E}-10$ & RPRD1B & $1.06 \mathrm{E}+00$ \\
\hline ENSBTAG00000004429 & $1.74 \mathrm{E}-10$ & $1.65 \mathrm{E}-10$ & GDF5 & $1.06 \mathrm{E}+00$ \\
\hline ENSBTAG00000045509 & $2.88 \mathrm{E}-10$ & $2.72 \mathrm{E}-10$ & FAM217B & $1.06 \mathrm{E}+00$ \\
\hline ENSBTAG00000013220 & $2.88 \mathrm{E}-10$ & $2.72 \mathrm{E}-10$ & R3HDML & $1.06 \mathrm{E}+00$ \\
\hline ENSBTAG00000011010 & $3.91 \mathrm{E}-10$ & $3.69 \mathrm{E}-10$ & PRND & $1.06 \mathrm{E}+00$ \\
\hline ENSBTAG00000000340 & $3.58 \mathrm{E}-10$ & $3.38 \mathrm{E}-10$ & ADNP & $1.06 \mathrm{E}+00$ \\
\hline ENSBTAG00000005127 & $1.18 \mathrm{E}-10$ & $1.12 \mathrm{E}-10$ & PSMA7 & $1.05 \mathrm{E}+00$ \\
\hline ENSBTAG00000013925 & $2.65 \mathrm{E}-10$ & $2.49 \mathrm{E}-10$ & KIN & $1.06 \mathrm{E}+00$ \\
\hline ENSBTAG00000005136 & $3.08 \mathrm{E}-10$ & $2.90 \mathrm{E}-10$ & ABI1 & $1.06 \mathrm{E}+00$ \\
\hline ENSBTAG00000015569 & $2.82 \mathrm{E}-10$ & $2.63 \mathrm{E}-10$ & ENKUR & $1.07 \mathrm{E}+00$ \\
\hline ENSBTAG00000004387 & $4.32 \mathrm{E}-10$ & $4.04 \mathrm{E}-10$ & MTPAP & $1.07 \mathrm{E}+00$ \\
\hline ENSBTAG00000018418 & 4.67E-10 & $4.36 \mathrm{E}-10$ & TMEM74B & $1.07 \mathrm{E}+00$ \\
\hline ENSBTAG00000012710 & $2.38 \mathrm{E}-10$ & $2.22 \mathrm{E}-10$ & GHRH & $1.07 E+00$ \\
\hline ENSBTAG00000013163 & 1.17E-10 & $1.08 \mathrm{E}-10$ & ADAM33 & $1.08 \mathrm{E}+00$ \\
\hline ENSBTAG00000003061 & $1.51 \mathrm{E}-10$ & $1.39 \mathrm{E}-10$ & LAMA5 & $1.08 \mathrm{E}+00$ \\
\hline ENSBTAG00000039643 & $9.70 \mathrm{E}-10$ & $8.97 E-10$ & & $1.08 \mathrm{E}+00$ \\
\hline ENSBTAG00000021951 & $2.01 \mathrm{E}-10$ & $1.85 \mathrm{E}-10$ & RBM17 & $1.08 \mathrm{E}+00$ \\
\hline ENSBTAG00000002115 & $1.55 \mathrm{E}-10$ & $1.42 \mathrm{E}-10$ & C20orf96 & $1.09 \mathrm{E}+00$ \\
\hline ENSBTAG00000020120 & $6.33 \mathrm{E}-10$ & $5.81 \mathrm{E}-10$ & SEL1L2 & $1.09 \mathrm{E}+00$ \\
\hline ENSBTAG00000030976 & $3.50 \mathrm{E}-10$ & $3.21 \mathrm{E}-10$ & & $1.09 \mathrm{E}+00$ \\
\hline ENSBTAG00000013301 & 4.10E-10 & $3.75 \mathrm{E}-10$ & GGT7 & $1.09 \mathrm{E}+00$ \\
\hline ENSBTAG00000046614 & $1.37 \mathrm{E}-09$ & $1.25 \mathrm{E}-09$ & & $1.10 \mathrm{E}+00$ \\
\hline ENSBTAG00000037509 & $2.93 \mathrm{E}-10$ & $2.66 \mathrm{E}-10$ & & $1.10 \mathrm{E}+00$ \\
\hline
\end{tabular}




\begin{tabular}{|c|c|c|c|c|}
\hline ENSBTAG00000004039 & $6.52 \mathrm{E}-10$ & $5.91 \mathrm{E}-10$ & DHX35 & $1.10 \mathrm{E}+00$ \\
\hline ENSBTAG00000003848 & $3.00 \mathrm{E}-10$ & $2.72 \mathrm{E}-10$ & ATRN & $1.10 E+00$ \\
\hline ENSBTAG00000010383 & $6.19 \mathrm{E}-10$ & $5.59 \mathrm{E}-10$ & YTHDF1 & $1.11 E+00$ \\
\hline ENSBTAG00000004147 & $5.52 \mathrm{E}-10$ & $4.99 \mathrm{E}-10$ & FBXO18 & $1.11 E+00$ \\
\hline ENSBTAG00000008056 & $2.77 \mathrm{E}-10$ & $2.50 \mathrm{E}-10$ & GAD2 & $1.11 E+00$ \\
\hline ENSBTAG00000011638 & $2.71 E-09$ & $2.44 \mathrm{E}-09$ & & $1.11 E+00$ \\
\hline ENSBTAG00000018417 & $8.48 \mathrm{E}-10$ & $7.64 \mathrm{E}-10$ & PSMF1 & $1.11 E+00$ \\
\hline ENSBTAG00000011704 & $1.35 \mathrm{E}-09$ & $1.21 \mathrm{E}-09$ & & $1.11 E+00$ \\
\hline ENSBTAG00000030624 & $9.97 \mathrm{E}-10$ & $8.95 \mathrm{E}-10$ & WFDC5 & $1.11 E+00$ \\
\hline ENSBTAG00000007489 & $6.68 \mathrm{E}-10$ & $5.98 \mathrm{E}-10$ & NCOA3 & $1.12 \mathrm{E}+00$ \\
\hline ENSBTAG00000011600 & $1.62 \mathrm{E}-10$ & $1.45 \mathrm{E}-10$ & MAР3К8 & $1.12 \mathrm{E}+00$ \\
\hline ENSBTAG00000003398 & $5.32 \mathrm{E}-10$ & $4.75 \mathrm{E}-10$ & KCNG1 & $1.12 \mathrm{E}+00$ \\
\hline ENSBTAG00000019378 & $2.36 \mathrm{E}-10$ & 2.07E-10 & KIAA1755 & $1.14 \mathrm{E}+00$ \\
\hline ENSBTAG00000031718 & $1.08 \mathrm{E}-10$ & $9.50 \mathrm{E}-11$ & OGFR & $1.13 E+00$ \\
\hline ENSBTAG00000008439 & $1.93 \mathrm{E}-10$ & $1.70 \mathrm{E}-10$ & AP5S1 & $1.13 E+00$ \\
\hline ENSBTAG00000027337 & $6.91 \mathrm{E}-10$ & $6.06 \mathrm{E}-10$ & CTSA & $1.14 \mathrm{E}+00$ \\
\hline ENSBTAG00000031361 & 1.03E-09 & $9.05 \mathrm{E}-10$ & & $1.14 \mathrm{E}+00$ \\
\hline ENSBTAG00000017475 & $1.18 \mathrm{E}-10$ & $1.04 \mathrm{E}-10$ & & $1.14 \mathrm{E}+00$ \\
\hline ENSBTAG00000021885 & $6.31 \mathrm{E}-10$ & $5.53 \mathrm{E}-10$ & RSPO4 & $1.14 \mathrm{E}+00$ \\
\hline ENSBTAG00000039571 & $4.48 \mathrm{E}-10$ & $3.92 \mathrm{E}-10$ & PROSER2 & $1.14 \mathrm{E}+00$ \\
\hline ENSBTAG00000002065 & $1.97 \mathrm{E}-10$ & $1.73 \mathrm{E}-10$ & TFAP2C & $1.14 \mathrm{E}+00$ \\
\hline ENSBTAG00000021614 & $2.53 \mathrm{E}-10$ & $2.21 \mathrm{E}-10$ & TASP1 & $1.15 E+00$ \\
\hline ENSBTAG00000015872 & $2.91 \mathrm{E}-10$ & $2.54 \mathrm{E}-10$ & PROKR2 & $1.15 \mathrm{E}+00$ \\
\hline ENSBTAG00000002683 & $4.46 \mathrm{E}-10$ & 3.87E-10 & PFKP & $1.15 \mathrm{E}+00$ \\
\hline ENSBTAG00000034145 & 4.74E-10 & 4.11E-10 & & $1.15 \mathrm{E}+00$ \\
\hline ENSBTAG00000016864 & 7.17E-10 & $6.19 \mathrm{E}-10$ & LBP & $1.16 \mathrm{E}+00$ \\
\hline ENSBTAG00000017007 & 1.19E-10 & $1.03 \mathrm{E}-10$ & TRIB3 & $1.16 \mathrm{E}+00$ \\
\hline ENSBTAG00000002658 & $2.85 \mathrm{E}-10$ & $2.46 \mathrm{E}-10$ & STAM & $1.16 \mathrm{E}+00$ \\
\hline ENSBTAG00000015434 & $2.08 \mathrm{E}-10$ & $1.79 \mathrm{E}-10$ & DSTN & $1.16 \mathrm{E}+00$ \\
\hline ENSBTAG00000004100 & 4.57E-10 & 3.93E-10 & TTI1 & $1.16 \mathrm{E}+00$ \\
\hline ENSBTAG00000017002 & $1.05 \mathrm{E}-10$ & $9.02 \mathrm{E}-11$ & RBCK1 & $1.16 \mathrm{E}+00$ \\
\hline ENSBTAG00000000065 & $2.97 \mathrm{E}-10$ & $2.55 \mathrm{E}-10$ & CRLS1 & $1.16 \mathrm{E}+00$ \\
\hline ENSBTAG00000010112 & $4.66 \mathrm{E}-10$ & 4.00E-10 & BPIFB6 & $1.17 \mathrm{E}+00$ \\
\hline ENSBTAG00000005957 & $2.45 \mathrm{E}-10$ & $2.09 \mathrm{E}-10$ & CSE1L & $1.17 E+00$ \\
\hline ENSBTAG00000033089 & $2.20 \mathrm{E}-10$ & $1.88 \mathrm{E}-10$ & HACD1 & $1.17 \mathrm{E}+00$ \\
\hline ENSBTAG00000039446 & $1.90 \mathrm{E}-09$ & 1.63E-09 & & $1.17 \mathrm{E}+00$ \\
\hline ENSBTAG00000000512 & $2.88 \mathrm{E}-10$ & $2.46 \mathrm{E}-10$ & SNTA1 & $1.17 \mathrm{E}+00$ \\
\hline ENSBTAG00000017537 & $5.52 \mathrm{E}-10$ & 4.70E-10 & PTGIS & $1.18 \mathrm{E}+00$ \\
\hline ENSBTAG00000006021 & $1.94 \mathrm{E}-10$ & $1.64 \mathrm{E}-10$ & CEP250 & $1.18 \mathrm{E}+00$ \\
\hline ENSBTAG00000047223 & $1.08 \mathrm{E}-10$ & $9.10 \mathrm{E}-11$ & & $1.19 E+00$ \\
\hline ENSBTAG00000027327 & $2.45 \mathrm{E}-10$ & $2.06 \mathrm{E}-10$ & OCSTAMP & $1.19 \mathrm{E}+00$ \\
\hline ENSBTAG00000005111 & $8.73 E-11$ & $7.33 \mathrm{E}-11$ & BMP2 & $1.19 \mathrm{E}+00$ \\
\hline ENSBTAG00000003319 & $9.59 \mathrm{E}-11$ & $8.05 \mathrm{E}-11$ & FLRT3 & $1.19 \mathrm{E}+00$ \\
\hline ENSBTAG00000004075 & $2.36 \mathrm{E}-10$ & $1.98 \mathrm{E}-10$ & IDI1 & $1.19 \mathrm{E}+00$ \\
\hline ENSBTAG00000017191 & $3.07 \mathrm{E}-10$ & $2.58 \mathrm{E}-10$ & & $1.19 E+00$ \\
\hline ENSBTAG00000011312 & $2.17 \mathrm{E}-10$ & $1.82 \mathrm{E}-10$ & NKX2-4 & $1.19 \mathrm{E}+00$ \\
\hline ENSBTAG00000002685 & $6.02 \mathrm{E}-10$ & $5.02 \mathrm{E}-10$ & PITRM1 & $1.20 \mathrm{E}+00$ \\
\hline ENSBTAG00000021867 & $4.78 \mathrm{E}-10$ & 3.99E-10 & & $1.20 \mathrm{E}+00$ \\
\hline ENSBTAG00000006531 & $2.34 \mathrm{E}-10$ & $1.95 \mathrm{E}-10$ & DIP2C & $1.20 \mathrm{E}+00$ \\
\hline ENSBTAG00000001640 & $5.52 \mathrm{E}-10$ & 4.57E-10 & EPB41L1 & $1.21 \mathrm{E}+00$ \\
\hline ENSBTAG00000016401 & $2.66 \mathrm{E}-10$ & $2.21 \mathrm{E}-10$ & OPTN & $1.20 \mathrm{E}+00$ \\
\hline ENSBTAG00000014048 & $6.34 \mathrm{E}-10$ & $5.25 \mathrm{E}-10$ & C20orf196 & $1.21 E+00$ \\
\hline ENSBTAG00000014046 & $6.34 \mathrm{E}-10$ & $5.25 \mathrm{E}-10$ & BPI & $1.21 \mathrm{E}+00$ \\
\hline ENSBTAG00000004354 & 1.12E-09 & $9.17 \mathrm{E}-10$ & & $1.22 \mathrm{E}+00$ \\
\hline ENSBTAG00000008279 & $2.63 \mathrm{E}-10$ & 2.17E-10 & FRMD4A & $1.21 \mathrm{E}+00$ \\
\hline ENSBTAG00000016450 & $1.10 \mathrm{E}-10$ & $9.05 E-11$ & CDNF & $1.21 E+00$ \\
\hline ENSBTAG00000018783 & $2.81 \mathrm{E}-10$ & $2.30 \mathrm{E}-10$ & NELFCD & $1.22 \mathrm{E}+00$ \\
\hline ENSBTAG00000016348 & $1.70 \mathrm{E}-10$ & $1.39 \mathrm{E}-10$ & XKR7 & $1.23 \mathrm{E}+00$ \\
\hline ENSBTAG00000007130 & $1.69 \mathrm{E}-10$ & $1.38 \mathrm{E}-10$ & ESF1 & $1.22 \mathrm{E}+00$ \\
\hline ENSBTAG00000000873 & $3.95 \mathrm{E}-10$ & 3.22E-10 & SLC13A3 & $1.23 \mathrm{E}+00$ \\
\hline ENSBTAG00000012526 & $4.42 \mathrm{E}-10$ & $3.61 E-10$ & APBB1IP & $1.22 \mathrm{E}+00$ \\
\hline ENSBTAG00000046631 & $8.41 \mathrm{E}-11$ & $6.86 \mathrm{E}-11$ & & $1.23 \mathrm{E}+00$ \\
\hline ENSBTAG00000018122 & $4.12 \mathrm{E}-10$ & 3.35E-10 & & $1.23 \mathrm{E}+00$ \\
\hline ENSBTAG00000007702 & $2.37 \mathrm{E}-10$ & $1.93 \mathrm{E}-10$ & RPP38 & $1.23 \mathrm{E}+00$ \\
\hline ENSBTAG00000011425 & $2.02 \mathrm{E}-10$ & $1.64 \mathrm{E}-10$ & PTPRA & $1.23 \mathrm{E}+00$ \\
\hline ENSBTAG00000000066 & $2.43 \mathrm{E}-10$ & $1.97 \mathrm{E}-10$ & LRRN4 & $1.23 E+00$ \\
\hline ENSBTAG00000045928 & $3.81 E-10$ & $3.09 \mathrm{E}-10$ & & $1.23 \mathrm{E}+00$ \\
\hline ENSBTAG00000008870 & $7.68 \mathrm{E}-10$ & $6.23 \mathrm{E}-10$ & KCNS1 & $1.23 \mathrm{E}+00$ \\
\hline ENSBTAG00000009165 & $2.11 \mathrm{E}-10$ & $1.72 \mathrm{E}-10$ & LPIN3 & $1.23 \mathrm{E}+00$ \\
\hline ENSBTAG00000019866 & $2.62 \mathrm{E}-10$ & $2.12 \mathrm{E}-10$ & NRP1 & $1.24 \mathrm{E}+00$ \\
\hline
\end{tabular}




\begin{tabular}{|c|c|c|c|c|}
\hline ENSBTAG00000011366 & $2.13 \mathrm{E}-10$ & $1.71 \mathrm{E}-10$ & & $1.25 \mathrm{E}+00$ \\
\hline ENSBTAG00000027446 & $5.10 \mathrm{E}-10$ & $4.11 \mathrm{E}-10$ & RSU1 & $1.24 \mathrm{E}+00$ \\
\hline ENSBTAG00000003815 & $4.48 \mathrm{E}-10$ & $3.60 \mathrm{E}-10$ & EDEM2 & $1.24 \mathrm{E}+00$ \\
\hline ENSBTAG00000002236 & $2.88 \mathrm{E}-10$ & $2.31 \mathrm{E}-10$ & TRPC4AP & $1.24 \mathrm{E}+00$ \\
\hline ENSBTAG00000027387 & $1.41 \mathrm{E}-10$ & $1.13 \mathrm{E}-10$ & NRSN2 & $1.25 \mathrm{E}+00$ \\
\hline ENSBTAG00000003256 & $5.35 \mathrm{E}-10$ & 4.29E-10 & TOMM34 & $1.25 \mathrm{E}+00$ \\
\hline ENSBTAG00000015438 & $2.80 \mathrm{E}-10$ & $2.24 \mathrm{E}-10$ & & $1.25 \mathrm{E}+00$ \\
\hline ENSBTAG00000008410 & $2.56 \mathrm{E}-10$ & $2.04 \mathrm{E}-10$ & OVOL2 & $1.25 \mathrm{E}+00$ \\
\hline ENSBTAG00000010024 & $6.91 \mathrm{E}-10$ & $5.51 \mathrm{E}-10$ & MMP24 & $1.25 \mathrm{E}+00$ \\
\hline ENSBTAG00000008687 & $1.95 \mathrm{E}-10$ & $1.55 \mathrm{E}-10$ & PCSK2 & $1.26 \mathrm{E}+00$ \\
\hline ENSBTAG00000020546 & $6.65 \mathrm{E}-10$ & $5.30 \mathrm{E}-10$ & TRDMT1 & $1.25 \mathrm{E}+00$ \\
\hline ENSBTAG00000011882 & 4.12E-10 & $3.28 \mathrm{E}-10$ & SLC24A3 & $1.26 \mathrm{E}+00$ \\
\hline ENSBTAG00000014438 & $1.21 \mathrm{E}-10$ & $9.70 \mathrm{E}-11$ & SS18L1 & $1.25 \mathrm{E}+00$ \\
\hline ENSBTAG00000011541 & $1.60 \mathrm{E}-10$ & $1.27 \mathrm{E}-10$ & RBL1 & $1.26 \mathrm{E}+00$ \\
\hline ENSBTAG00000022570 & 7.15E-10 & $5.64 \mathrm{E}-10$ & & $1.27 \mathrm{E}+00$ \\
\hline ENSBTAG00000014178 & $6.73 \mathrm{E}-10$ & $5.30 \mathrm{E}-10$ & RALGAPA2 & $1.27 \mathrm{E}+00$ \\
\hline ENSBTAG00000010934 & $1.11 \mathrm{E}-10$ & $8.75 \mathrm{E}-11$ & RAE1 & $1.27 \mathrm{E}+00$ \\
\hline ENSBTAG00000020166 & $3.17 \mathrm{E}-10$ & $2.49 \mathrm{E}-10$ & ZNFX1 & $1.27 \mathrm{E}+00$ \\
\hline ENSBTAG00000016279 & $3.51 \mathrm{E}-10$ & $2.76 \mathrm{E}-10$ & EMILIN3 & $1.27 \mathrm{E}+00$ \\
\hline ENSBTAG00000020800 & $3.15 \mathrm{E}-10$ & $2.47 \mathrm{E}-10$ & BEND7 & $1.27 \mathrm{E}+00$ \\
\hline ENSBTAG00000021857 & $4.59 \mathrm{E}-10$ & $3.59 \mathrm{E}-10$ & ARHGAP21 & $1.28 \mathrm{E}+00$ \\
\hline ENSBTAG00000015724 & $6.27 \mathrm{E}-10$ & $4.89 \mathrm{E}-10$ & BPIFB1 & $1.28 \mathrm{E}+00$ \\
\hline ENSBTAG00000019752 & $1.16 \mathrm{E}-09$ & $9.01 \mathrm{E}-10$ & & $1.28 \mathrm{E}+00$ \\
\hline ENSBTAG00000012116 & $4.78 \mathrm{E}-10$ & $3.72 \mathrm{E}-10$ & HNF4A & $1.28 \mathrm{E}+00$ \\
\hline ENSBTAG00000016388 & $1.96 \mathrm{E}-10$ & $1.52 \mathrm{E}-10$ & SLCO4A1 & $1.29 \mathrm{E}+00$ \\
\hline ENSBTAG00000002123 & $1.53 \mathrm{E}-10$ & $1.20 \mathrm{E}-10$ & MYO3A & $1.28 \mathrm{E}+00$ \\
\hline ENSBTAG00000008288 & $5.67 \mathrm{E}-10$ & $4.40 \mathrm{E}-10$ & ANKRD26 & $1.29 E+00$ \\
\hline ENSBTAG00000020261 & $5.22 \mathrm{E}-10$ & 4.04E-10 & $\mathrm{CDH} 26$ & $1.29 E+00$ \\
\hline ENSBTAG00000022931 & $5.48 \mathrm{E}-10$ & 4.22E-10 & & $1.30 \mathrm{E}+00$ \\
\hline ENSBTAG00000006639 & $1.38 \mathrm{E}-10$ & $1.06 \mathrm{E}-10$ & CSTF1 & $1.30 \mathrm{E}+00$ \\
\hline ENSBTAG00000016846 & $1.91 \mathrm{E}-10$ & $1.46 \mathrm{E}-10$ & YWHAB & $1.31 \mathrm{E}+00$ \\
\hline ENSBTAG00000008938 & $2.43 \mathrm{E}-10$ & $1.86 \mathrm{E}-10$ & SRC & $1.31 \mathrm{E}+00$ \\
\hline ENSBTAG00000018784 & $2.42 \mathrm{E}-10$ & $1.85 \mathrm{E}-10$ & CTSZ & $1.31 \mathrm{E}+00$ \\
\hline ENSBTAG00000034441 & $6.34 \mathrm{E}-10$ & $4.84 \mathrm{E}-10$ & MACROD2 & $1.31 E+00$ \\
\hline ENSBTAG00000003422 & $1.59 \mathrm{E}-10$ & $1.21 \mathrm{E}-10$ & & $1.31 E+00$ \\
\hline ENSBTAG00000019509 & $2.72 \mathrm{E}-10$ & 2.07E-10 & NUDT5 & $1.31 E+00$ \\
\hline ENSBTAG00000001785 & $4.85 \mathrm{E}-10$ & $3.69 \mathrm{E}-10$ & TGM3 & $1.31 \mathrm{E}+00$ \\
\hline ENSBTAG00000020929 & $4.79 \mathrm{E}-10$ & $3.62 \mathrm{E}-10$ & ARMC3 & $1.32 \mathrm{E}+00$ \\
\hline ENSBTAG00000018535 & $1.26 \mathrm{E}-09$ & $9.54 \mathrm{E}-10$ & CDK5RAP1 & $1.33 \mathrm{E}+00$ \\
\hline ENSBTAG00000027407 & $2.72 \mathrm{E}-10$ & $2.05 \mathrm{E}-10$ & TNFRSF6B & $1.33 \mathrm{E}+00$ \\
\hline ENSBTAG00000003863 & $4.02 \mathrm{E}-10$ & $3.03 \mathrm{E}-10$ & PCED1A & $1.33 \mathrm{E}+00$ \\
\hline ENSBTAG00000045671 & 5.17E-10 & $3.90 \mathrm{E}-10$ & & $1.33 \mathrm{E}+00$ \\
\hline ENSBTAG00000003212 & $3.43 \mathrm{E}-10$ & $2.59 \mathrm{E}-10$ & NNAT & $1.33 \mathrm{E}+00$ \\
\hline ENSBTAG00000046623 & $9.49 \mathrm{E}-10$ & $7.15 \mathrm{E}-10$ & & $1.33 \mathrm{E}+00$ \\
\hline ENSBTAG00000032694 & $6.65 \mathrm{E}-10$ & $4.96 \mathrm{E}-10$ & CST8 & $1.34 \mathrm{E}+00$ \\
\hline ENSBTAG00000007213 & $1.41 \mathrm{E}-09$ & $1.05 \mathrm{E}-09$ & & $1.34 \mathrm{E}+00$ \\
\hline ENSBTAG00000021321 & $2.09 \mathrm{E}-10$ & $1.55 \mathrm{E}-10$ & SDCBP2 & $1.35 \mathrm{E}+00$ \\
\hline ENSBTAG00000045555 & $1.24 \mathrm{E}-10$ & $9.18 \mathrm{E}-11$ & & $1.35 \mathrm{E}+00$ \\
\hline ENSBTAG00000016127 & $4.78 \mathrm{E}-10$ & $3.54 \mathrm{E}-10$ & ELP & $1.35 \mathrm{E}+00$ \\
\hline ENSBTAG00000048047 & $1.26 \mathrm{E}-09$ & $9.31 \mathrm{E}-10$ & & $1.35 \mathrm{E}+00$ \\
\hline ENSBTAG00000001314 & $2.80 \mathrm{E}-10$ & $2.06 \mathrm{E}-10$ & TRMT6 & $1.36 \mathrm{E}+00$ \\
\hline ENSBTAG00000031402 & $1.44 \mathrm{E}-10$ & $1.05 \mathrm{E}-10$ & ASXL1 & $1.37 \mathrm{E}+00$ \\
\hline ENSBTAG00000020676 & $2.13 \mathrm{E}-10$ & $1.55 \mathrm{E}-10$ & MMP9 & $1.37 \mathrm{E}+00$ \\
\hline ENSBTAG00000004622 & $2.36 \mathrm{E}-10$ & $1.72 \mathrm{E}-10$ & DZANK1 & $1.37 \mathrm{E}+00$ \\
\hline ENSBTAG00000013009 & $1.79 \mathrm{E}-10$ & $1.30 \mathrm{E}-10$ & AURKA & $1.37 \mathrm{E}+00$ \\
\hline ENSBTAG00000010330 & $2.41 \mathrm{E}-10$ & $1.74 \mathrm{E}-10$ & & $1.38 \mathrm{E}+00$ \\
\hline ENSBTAG00000016060 & $1.41 \mathrm{E}-10$ & $1.02 \mathrm{E}-10$ & CREM & $1.38 \mathrm{E}+00$ \\
\hline ENSBTAG00000023216 & 4.42E-10 & $3.19 \mathrm{E}-10$ & & $1.38 \mathrm{E}+00$ \\
\hline ENSBTAG00000002758 & $1.58 \mathrm{E}-10$ & $1.14 \mathrm{E}-10$ & THBD & $1.38 \mathrm{E}+00$ \\
\hline ENSBTAG00000013079 & $2.88 \mathrm{E}-10$ & $2.06 \mathrm{E}-10$ & CTCFL & $1.40 \mathrm{E}+00$ \\
\hline ENSBTAG00000014991 & 4.14E-10 & $2.98 \mathrm{E}-10$ & PARD3 & $1.39 E+00$ \\
\hline ENSBTAG00000005571 & $1.09 \mathrm{E}-10$ & $7.81 \mathrm{E}-11$ & PHF20 & $1.40 \mathrm{E}+00$ \\
\hline ENSBTAG00000000308 & $3.25 \mathrm{E}-10$ & $2.33 \mathrm{E}-10$ & & $1.39 E+00$ \\
\hline ENSBTAG00000017243 & $1.97 \mathrm{E}-10$ & $1.41 \mathrm{E}-10$ & GATA3 & $1.39 E+00$ \\
\hline ENSBTAG00000043964 & $1.50 \mathrm{E}-10$ & $1.07 \mathrm{E}-10$ & ARL5B & $1.40 \mathrm{E}+00$ \\
\hline ENSBTAG00000002822 & $3.53 \mathrm{E}-10$ & $2.50 \mathrm{E}-10$ & CNBD2 & $1.41 \mathrm{E}+00$ \\
\hline ENSBTAG00000006616 & $1.34 \mathrm{E}-10$ & $9.35 \mathrm{E}-11$ & PTPN1 & $1.43 \mathrm{E}+00$ \\
\hline ENSBTAG00000038057 & 7.10E-10 & $4.96 \mathrm{E}-10$ & & $1.43 E+00$ \\
\hline ENSBTAG00000034077 & $3.19 \mathrm{E}-10$ & $2.21 \mathrm{E}-10$ & ASIP & $1.45 \mathrm{E}+00$ \\
\hline ENSBTAG00000008825 & $1.16 \mathrm{E}-10$ & $8.06 \mathrm{E}-11$ & POLR3F & $1.44 \mathrm{E}+00$ \\
\hline
\end{tabular}




\begin{tabular}{|c|c|c|c|c|}
\hline ENSBTAG00000047454 & $1.91 \mathrm{E}-10$ & $1.31 \mathrm{E}-10$ & OLAH & $1.46 \mathrm{E}+00$ \\
\hline ENSBTAG00000046074 & $3.38 \mathrm{E}-10$ & 2.32E-10 & & $1.46 \mathrm{E}+00$ \\
\hline ENSBTAG00000003635 & $1.22 \mathrm{E}-10$ & $8.41 \mathrm{E}-11$ & SLC35C2 & $1.46 \mathrm{E}+00$ \\
\hline ENSBTAG00000038687 & $8.19 \mathrm{E}-10$ & $5.60 \mathrm{E}-10$ & BPIFB3 & $1.46 \mathrm{E}+00$ \\
\hline ENSBTAG00000005072 & $5.14 \mathrm{E}-10$ & 3.51E-10 & CUBN & $1.46 \mathrm{E}+00$ \\
\hline ENSBTAG00000048262 & $6.57 \mathrm{E}-10$ & 4.47E-10 & ACTR5 & $1.47 E+00$ \\
\hline ENSBTAG00000011263 & $1.81 \mathrm{E}-10$ & $1.23 \mathrm{E}-10$ & & $1.47 \mathrm{E}+00$ \\
\hline ENSBTAG00000023169 & $8.98 \mathrm{E}-10$ & $6.11 \mathrm{E}-10$ & & $1.47 \mathrm{E}+00$ \\
\hline ENSBTAG00000008937 & $3.43 \mathrm{E}-10$ & $2.33 \mathrm{E}-10$ & CELF2 & $1.47 \mathrm{E}+00$ \\
\hline ENSBTAG00000003209 & $3.91 \mathrm{E}-10$ & $2.66 \mathrm{E}-10$ & BLCAP & $1.47 E+00$ \\
\hline ENSBTAG00000048087 & $1.20 \mathrm{E}-09$ & $8.11 \mathrm{E}-10$ & & $1.47 \mathrm{E}+00$ \\
\hline ENSBTAG00000001144 & $1.73 \mathrm{E}-10$ & 1.17E-10 & NAA20 & $1.47 E+00$ \\
\hline ENSBTAG00000004620 & 4.49E-10 & $3.04 \mathrm{E}-10$ & & $1.48 \mathrm{E}+00$ \\
\hline ENSBTAG00000019200 & $6.10 \mathrm{E}-10$ & 4.12E-10 & BPIFB2 & $1.48 \mathrm{E}+00$ \\
\hline ENSBTAG00000030990 & 3.06E-10 & $2.06 \mathrm{E}-10$ & UQCC1 & $1.48 \mathrm{E}+00$ \\
\hline ENSBTAG00000018063 & $3.36 \mathrm{E}-10$ & $2.26 \mathrm{E}-10$ & SPATA2 & $1.49 \mathrm{E}+00$ \\
\hline ENSBTAG00000016741 & $3.14 \mathrm{E}-10$ & $2.11 E-10$ & TOX2 & $1.49 E+00$ \\
\hline ENSBTAG00000001645 & $4.76 \mathrm{E}-10$ & 3.17E-10 & AAR2 & $1.50 \mathrm{E}+00$ \\
\hline ENSBTAG00000034991 & $3.93 \mathrm{E}-10$ & $2.60 \mathrm{E}-10$ & SLX4IP & $1.51 E+00$ \\
\hline ENSBTAG00000021685 & $2.24 \mathrm{E}-10$ & $1.48 \mathrm{E}-10$ & EEF1A2 & $1.51 E+00$ \\
\hline ENSBTAG00000039520 & $1.09 \mathrm{E}-09$ & 7.16E-10 & & $1.52 \mathrm{E}+00$ \\
\hline ENSBTAG00000011283 & $1.87 \mathrm{E}-10$ & $1.23 \mathrm{E}-10$ & RBPJL & $1.52 \mathrm{E}+00$ \\
\hline ENSBTAG00000003977 & $1.49 \mathrm{E}-10$ & $9.73 \mathrm{E}-11$ & SLC52A3 & $1.54 \mathrm{E}+00$ \\
\hline ENSBTAG00000002836 & $2.12 \mathrm{E}-10$ & $1.38 \mathrm{E}-10$ & TAF3 & $1.54 \mathrm{E}+00$ \\
\hline ENSBTAG00000000241 & $4.26 \mathrm{E}-10$ & $2.76 \mathrm{E}-10$ & STAU1 & $1.54 \mathrm{E}+00$ \\
\hline ENSBTAG00000013007 & $2.54 \mathrm{E}-10$ & $1.65 \mathrm{E}-10$ & FAM210B & $1.54 \mathrm{E}+00$ \\
\hline ENSBTAG00000046520 & $2.40 \mathrm{E}-10$ & $1.54 \mathrm{E}-10$ & & $1.56 \mathrm{E}+00$ \\
\hline ENSBTAG00000040490 & $5.56 \mathrm{E}-10$ & $3.55 \mathrm{E}-10$ & & $1.57 \mathrm{E}+00$ \\
\hline ENSBTAG00000005915 & $6.10 \mathrm{E}-10$ & $3.90 \mathrm{E}-10$ & SFMBT2 & $1.56 \mathrm{E}+00$ \\
\hline ENSBTAG00000022000 & $3.65 \mathrm{E}-10$ & $2.33 \mathrm{E}-10$ & CACNB2 & $1.57 \mathrm{E}+00$ \\
\hline ENSBTAG00000011940 & $3.63 \mathrm{E}-10$ & $2.30 \mathrm{E}-10$ & ZNF831 & $1.58 \mathrm{E}+00$ \\
\hline ENSBTAG00000014075 & $3.48 \mathrm{E}-10$ & $2.21 \mathrm{E}-10$ & ITIH2 & $1.58 \mathrm{E}+00$ \\
\hline ENSBTAG00000008323 & $5.64 \mathrm{E}-10$ & 3.57E-10 & SNAP25 & $1.58 \mathrm{E}+00$ \\
\hline ENSBTAG00000008638 & $1.67 \mathrm{E}-10$ & $1.05 \mathrm{E}-10$ & FAM110A & $1.59 E+00$ \\
\hline ENSBTAG00000013330 & $1.29 \mathrm{E}-10$ & $8.15 E-11$ & TBC1D20 & $1.59 E+00$ \\
\hline ENSBTAG00000044047 & $6.06 \mathrm{E}-11$ & 3.82E-11 & SKIDA1 & $1.59 E+00$ \\
\hline ENSBTAG00000045609 & $1.05 \mathrm{E}-10$ & $6.60 \mathrm{E}-11$ & & $1.59 E+00$ \\
\hline ENSBTAG00000031462 & $1.04 \mathrm{E}-10$ & $6.58 \mathrm{E}-11$ & & $1.59 E+00$ \\
\hline ENSBTAG00000008027 & $3.25 \mathrm{E}-10$ & 2.04E-10 & AVP & $1.59 E+00$ \\
\hline ENSBTAG00000016451 & $9.32 \mathrm{E}-11$ & 5.87E-11 & HSPA14 & $1.59 E+00$ \\
\hline ENSBTAG00000006243 & $2.23 \mathrm{E}-10$ & $1.41 \mathrm{E}-10$ & GCNT7 & $1.58 \mathrm{E}+00$ \\
\hline ENSBTAG00000020454 & $2.33 \mathrm{E}-10$ & $1.47 \mathrm{E}-10$ & LZTS3 & $1.58 \mathrm{E}+00$ \\
\hline ENSBTAG00000008826 & $1.52 \mathrm{E}-10$ & $9.54 \mathrm{E}-11$ & RBBP9 & $1.59 \mathrm{E}+00$ \\
\hline ENSBTAG00000013854 & $1.96 \mathrm{E}-10$ & $1.24 \mathrm{E}-10$ & CALML5 & $1.58 \mathrm{E}+00$ \\
\hline ENSBTAG00000039490 & $2.53 \mathrm{E}-10$ & $1.59 \mathrm{E}-10$ & WFDC8 & $1.59 E+00$ \\
\hline ENSBTAG00000017568 & $4.41 \mathrm{E}-10$ & 2.77E-10 & WFDC13 & $1.59 E+00$ \\
\hline ENSBTAG00000004148 & $2.14 \mathrm{E}-10$ & $1.35 \mathrm{E}-10$ & SLPI & $1.59 E+00$ \\
\hline ENSBTAG00000047894 & $3.71 \mathrm{E}-10$ & $2.34 \mathrm{E}-10$ & SLC32A1 & $1.59 E+00$ \\
\hline ENSBTAG00000012407 & $2.49 \mathrm{E}-10$ & $1.56 \mathrm{E}-10$ & FOXA2 & $1.59 E+00$ \\
\hline ENSBTAG00000006063 & $2.54 \mathrm{E}-10$ & $1.60 \mathrm{E}-10$ & & $1.59 \mathrm{E}+00$ \\
\hline ENSBTAG00000046725 & $1.47 \mathrm{E}-10$ & $9.26 \mathrm{E}-11$ & & $1.59 E+00$ \\
\hline ENSBTAG00000008338 & $6.89 \mathrm{E}-10$ & $4.31 \mathrm{E}-10$ & PLCB1 & $1.60 E+00$ \\
\hline ENSBTAG00000007922 & $4.58 \mathrm{E}-10$ & $2.85 \mathrm{E}-10$ & RNF24 & $1.61 E+00$ \\
\hline ENSBTAG00000021669 & $2.16 \mathrm{E}-10$ & $1.34 \mathrm{E}-10$ & SOGA1 & $1.61 E+00$ \\
\hline ENSBTAG00000017584 & $3.81 \mathrm{E}-10$ & $2.36 \mathrm{E}-10$ & PLCG1 & $1.62 E+00$ \\
\hline ENSBTAG00000018981 & $2.71 \mathrm{E}-10$ & $1.68 \mathrm{E}-10$ & TMEM236 & $1.61 E+00$ \\
\hline ENSBTAG00000022922 & $4.72 \mathrm{E}-10$ & $2.91 \mathrm{E}-10$ & & $1.62 E+00$ \\
\hline ENSBTAG00000003494 & $2.10 \mathrm{E}-10$ & $1.27 \mathrm{E}-10$ & C20orf194 & $1.65 E+00$ \\
\hline ENSBTAG00000004916 & $3.34 \mathrm{E}-10$ & $2.00 \mathrm{E}-10$ & ARFGAP1 & $1.67 E+00$ \\
\hline ENSBTAG00000003215 & $2.18 \mathrm{E}-10$ & $1.31 \mathrm{E}-10$ & KIF3B & $1.66 \mathrm{E}+00$ \\
\hline ENSBTAG00000018395 & $6.22 \mathrm{E}-10$ & $3.72 \mathrm{E}-10$ & KIAA1217 & $1.67 E+00$ \\
\hline ENSBTAG00000000939 & $4.57 \mathrm{E}-10$ & 2.73E-10 & KIF16B & $1.67 E+00$ \\
\hline ENSBTAG00000046375 & $1.05 \mathrm{E}-09$ & $6.26 \mathrm{E}-10$ & & $1.67 E+00$ \\
\hline ENSBTAG00000021558 & $2.07 \mathrm{E}-10$ & $1.23 \mathrm{E}-10$ & & $1.68 \mathrm{E}+00$ \\
\hline ENSBTAG00000000660 & $5.91 \mathrm{E}-10$ & $3.51 E-10$ & FAM83D & $1.68 \mathrm{E}+00$ \\
\hline ENSBTAG00000010023 & 4.01E-10 & 2.37E-10 & & $1.69 \mathrm{E}+00$ \\
\hline ENSBTAG00000012626 & $3.05 \mathrm{E}-10$ & $1.80 \mathrm{E}-10$ & B4GALT5 & $1.70 \mathrm{E}+00$ \\
\hline ENSBTAG00000031524 & $3.67 \mathrm{E}-10$ & $2.15 \mathrm{E}-10$ & PMEPA1 & $1.71 E+00$ \\
\hline ENSBTAG00000022564 & $7.63 \mathrm{E}-10$ & $4.48 \mathrm{E}-10$ & & $1.70 \mathrm{E}+00$ \\
\hline ENSBTAG00000012817 & $2.99 \mathrm{E}-10$ & $1.74 \mathrm{E}-10$ & JAG1 & $1.72 \mathrm{E}+00$ \\
\hline
\end{tabular}




\begin{tabular}{|c|c|c|c|c|}
\hline ENSBTAG00000003640 & $2.40 \mathrm{E}-10$ & $1.39 \mathrm{E}-10$ & GJD4 & $1.72 \mathrm{E}+00$ \\
\hline ENSBTAG00000013004 & $6.33 \mathrm{E}-10$ & 3.66E-10 & ITIH5 & $1.73 E+00$ \\
\hline ENSBTAG00000016926 & $1.92 \mathrm{E}-10$ & $1.11 \mathrm{E}-10$ & C20orf85 & $1.73 E+00$ \\
\hline ENSBTAG00000044210 & $6.26 \mathrm{E}-11$ & 3.59E-11 & SPO11 & $1.74 \mathrm{E}+00$ \\
\hline ENSBTAG00000020797 & $1.70 \mathrm{E}-10$ & $9.71 \mathrm{E}-11$ & CABLES2 & $1.75 \mathrm{E}+00$ \\
\hline ENSBTAG00000001936 & $2.95 \mathrm{E}-10$ & $1.69 \mathrm{E}-10$ & PCK1 & $1.74 \mathrm{E}+00$ \\
\hline ENSBTAG00000000653 & $6.27 \mathrm{E}-10$ & 3.57E-10 & PPP1R16B & $1.75 E+00$ \\
\hline ENSBTAG00000027317 & $3.74 \mathrm{E}-10$ & $2.13 \mathrm{E}-10$ & RNF114 & $1.75 E+00$ \\
\hline ENSBTAG00000015058 & $2.75 E-10$ & $1.56 \mathrm{E}-10$ & $\mathrm{CDH} 4$ & $1.76 \mathrm{E}+00$ \\
\hline ENSBTAG00000027420 & $3.79 \mathrm{E}-10$ & $2.15 \mathrm{E}-10$ & & $1.76 \mathrm{E}+00$ \\
\hline ENSBTAG00000008348 & $1.80 \mathrm{E}-10$ & $1.01 \mathrm{E}-10$ & & $1.78 \mathrm{E}+00$ \\
\hline ENSBTAG00000020892 & $2.06 \mathrm{E}-10$ & $1.16 \mathrm{E}-10$ & IL2RA & $1.78 \mathrm{E}+00$ \\
\hline ENSBTAG00000006002 & $3.50 \mathrm{E}-10$ & $1.97 \mathrm{E}-10$ & DIDO1 & $1.78 \mathrm{E}+00$ \\
\hline ENSBTAG00000009291 & $5.01 \mathrm{E}-10$ & $2.81 \mathrm{E}-10$ & CFAP61 & $1.78 \mathrm{E}+00$ \\
\hline ENSBTAG00000007133 & $2.90 \mathrm{E}-10$ & $1.62 \mathrm{E}-10$ & CUL2 & $1.79 \mathrm{E}+00$ \\
\hline ENSBTAG00000038929 & 4.22E-10 & $2.36 \mathrm{E}-10$ & BCAS4 & $1.79 \mathrm{E}+00$ \\
\hline ENSBTAG00000007700 & $2.05 \mathrm{E}-10$ & 1.14E-10 & & $1.80 \mathrm{E}+00$ \\
\hline ENSBTAG00000006278 & $3.66 \mathrm{E}-10$ & $2.02 \mathrm{E}-10$ & FAM83C & $1.81 \mathrm{E}+00$ \\
\hline ENSBTAG00000007960 & $2.34 \mathrm{E}-10$ & $1.29 \mathrm{E}-10$ & TOP1 & $1.81 \mathrm{E}+00$ \\
\hline ENSBTAG00000038974 & $1.35 \mathrm{E}-10$ & $7.41 \mathrm{E}-11$ & STMN3 & $1.81 \mathrm{E}+00$ \\
\hline ENSBTAG00000016294 & $2.65 \mathrm{E}-10$ & $1.44 \mathrm{E}-10$ & ZNF438 & $1.84 \mathrm{E}+00$ \\
\hline ENSBTAG00000038453 & $6.99 \mathrm{E}-10$ & $3.80 \mathrm{E}-10$ & & $1.84 \mathrm{E}+00$ \\
\hline ENSBTAG00000008787 & 3.64E-10 & $1.98 \mathrm{E}-10$ & FAM171A1 & $1.84 \mathrm{E}+00$ \\
\hline ENSBTAG00000001741 & $5.58 \mathrm{E}-10$ & $3.02 \mathrm{E}-10$ & DLGAP4 & $1.85 \mathrm{E}+00$ \\
\hline ENSBTAG00000013928 & $1.61 \mathrm{E}-10$ & $8.66 \mathrm{E}-11$ & WFDC2 & $1.85 \mathrm{E}+00$ \\
\hline ENSBTAG00000018033 & $3.85 \mathrm{E}-10$ & $2.08 \mathrm{E}-10$ & PIP4K2A & $1.85 \mathrm{E}+00$ \\
\hline ENSBTAG00000027405 & $2.21 \mathrm{E}-10$ & 1.19E-10 & FNDC11 & $1.86 \mathrm{E}+00$ \\
\hline ENSBTAG00000032156 & $4.00 \mathrm{E}-10$ & $2.16 \mathrm{E}-10$ & & $1.85 \mathrm{E}+00$ \\
\hline ENSBTAG00000003949 & $1.72 \mathrm{E}-10$ & $9.23 \mathrm{E}-11$ & RBM39 & $1.86 \mathrm{E}+00$ \\
\hline ENSBTAG00000047991 & $2.02 \mathrm{E}-10$ & $1.08 \mathrm{E}-10$ & & $1.87 \mathrm{E}+00$ \\
\hline ENSBTAG00000003504 & $3.45 \mathrm{E}-10$ & $1.84 \mathrm{E}-10$ & GSS & $1.87 \mathrm{E}+00$ \\
\hline ENSBTAG00000017740 & 4.60E-10 & $2.43 \mathrm{E}-10$ & & $1.89 E+00$ \\
\hline ENSBTAG00000002280 & 4.64E-10 & $2.45 \mathrm{E}-10$ & KIF5B & $1.89 \mathrm{E}+00$ \\
\hline ENSBTAG00000013116 & $6.30 \mathrm{E}-10$ & $3.33 \mathrm{E}-10$ & PLCB4 & $1.89 \mathrm{E}+00$ \\
\hline ENSBTAG00000045520 & $4.60 \mathrm{E}-10$ & $2.42 \mathrm{E}-10$ & PDYN & $1.90 \mathrm{E}+00$ \\
\hline ENSBTAG00000015080 & $3.42 \mathrm{E}-10$ & $1.79 \mathrm{E}-10$ & PHACTR3 & $1.91 \mathrm{E}+00$ \\
\hline ENSBTAG00000001320 & $3.36 \mathrm{E}-10$ & $1.77 \mathrm{E}-10$ & VPS16 & $1.90 \mathrm{E}+00$ \\
\hline ENSBTAG00000016677 & $1.45 \mathrm{E}-10$ & $7.60 \mathrm{E}-11$ & C1QL3 & $1.91 E+00$ \\
\hline ENSBTAG00000000654 & $5.82 \mathrm{E}-10$ & $2.99 \mathrm{E}-10$ & ARMC4 & $1.95 \mathrm{E}+00$ \\
\hline ENSBTAG00000005286 & $2.39 \mathrm{E}-10$ & $1.23 \mathrm{E}-10$ & TGIF2 & $1.94 \mathrm{E}+00$ \\
\hline ENSBTAG00000045630 & $6.26 \mathrm{E}-10$ & $3.21 \mathrm{E}-10$ & & $1.95 \mathrm{E}+00$ \\
\hline ENSBTAG00000015910 & $2.01 \mathrm{E}-10$ & $1.03 \mathrm{E}-10$ & ITGB1 & $1.95 \mathrm{E}+00$ \\
\hline ENSBTAG00000047869 & $1.06 \mathrm{E}-09$ & 5.40E-10 & & $1.96 \mathrm{E}+00$ \\
\hline ENSBTAG00000007602 & $5.75 \mathrm{E}-10$ & 2.92E-10 & ITGA8 & $1.97 \mathrm{E}+00$ \\
\hline ENSBTAG00000031188 & $4.84 \mathrm{E}-10$ & $2.46 \mathrm{E}-10$ & ADIG & $1.97 \mathrm{E}+00$ \\
\hline ENSBTAG00000012297 & $3.16 \mathrm{E}-10$ & 1.60E-10 & FAM65C & $1.97 \mathrm{E}+00$ \\
\hline ENSBTAG00000006578 & $3.45 \mathrm{E}-10$ & $1.73 \mathrm{E}-10$ & SPEF1 & $1.99 \mathrm{E}+00$ \\
\hline ENSBTAG00000031716 & $2.57 \mathrm{E}-10$ & $1.29 \mathrm{E}-10$ & MRGBP & $1.99 \mathrm{E}+00$ \\
\hline ENSBTAG00000011655 & $1.13 \mathrm{E}-10$ & $5.69 \mathrm{E}-11$ & SAMD10 & $1.98 \mathrm{E}+00$ \\
\hline ENSBTAG00000018334 & $1.24 \mathrm{E}-10$ & $6.27 \mathrm{E}-11$ & PLAGL2 & $1.98 \mathrm{E}+00$ \\
\hline ENSBTAG00000014194 & $3.63 \mathrm{E}-10$ & $1.80 \mathrm{E}-10$ & ADARB2 & $2.01 E+00$ \\
\hline ENSBTAG00000013056 & 7.35E-10 & $3.63 \mathrm{E}-10$ & SUN5 & $2.03 E+00$ \\
\hline ENSBTAG00000008821 & $1.98 \mathrm{E}-10$ & $9.74 \mathrm{E}-11$ & SEPHS1 & $2.03 E+00$ \\
\hline ENSBTAG00000012341 & $1.39 \mathrm{E}-10$ & $6.83 \mathrm{E}-11$ & CSNK2A1 & $2.03 E+00$ \\
\hline ENSBTAG00000006096 & $1.93 \mathrm{E}-10$ & $9.50 \mathrm{E}-11$ & & $2.03 E+00$ \\
\hline ENSBTAG00000008472 & $2.79 \mathrm{E}-10$ & 1.37E-10 & CRNKL1 & $2.03 E+00$ \\
\hline ENSBTAG00000017346 & $1.77 \mathrm{E}-10$ & $8.63 \mathrm{E}-11$ & RASSF2 & $2.05 E+00$ \\
\hline ENSBTAG00000010664 & $4.20 \mathrm{E}-10$ & $2.06 \mathrm{E}-10$ & PRKCQ & $2.04 E+00$ \\
\hline ENSBTAG00000017244 & $2.49 \mathrm{E}-10$ & $1.22 \mathrm{E}-10$ & & $2.04 E+00$ \\
\hline ENSBTAG00000033397 & 2.77E-10 & $1.35 \mathrm{E}-10$ & & $2.05 E+00$ \\
\hline ENSBTAG00000013574 & $3.83 \mathrm{E}-10$ & $1.87 \mathrm{E}-10$ & ANKRD60 & $2.05 \mathrm{E}+00$ \\
\hline ENSBTAG00000006529 & 3.75E-10 & $1.82 \mathrm{E}-10$ & PDSS1 & $2.06 \mathrm{E}+00$ \\
\hline ENSBTAG00000017424 & $3.15 \mathrm{E}-10$ & $1.52 \mathrm{E}-10$ & VAPB & $2.07 E+00$ \\
\hline ENSBTAG00000006533 & 3.67E-10 & $1.78 \mathrm{E}-10$ & NSFL1C & $2.06 \mathrm{E}+00$ \\
\hline ENSBTAG00000007498 & $2.82 \mathrm{E}-10$ & $1.36 \mathrm{E}-10$ & & $2.07 \mathrm{E}+00$ \\
\hline ENSBTAG00000016171 & 1.16E-09 & $5.48 \mathrm{E}-10$ & COX4I2 & $2.11 E+00$ \\
\hline ENSBTAG00000047150 & $2.70 \mathrm{E}-10$ & $1.27 \mathrm{E}-10$ & & $2.12 \mathrm{E}+00$ \\
\hline ENSBTAG00000003257 & $6.50 \mathrm{E}-10$ & 3.07E-10 & STK4 & $2.12 \mathrm{E}+00$ \\
\hline ENSBTAG00000016664 & $2.21 \mathrm{E}-10$ & $1.04 \mathrm{E}-10$ & SLC2A4RG & $2.13 \mathrm{E}+00$ \\
\hline ENSBTAG00000004402 & $3.03 \mathrm{E}-10$ & $1.43 \mathrm{E}-10$ & PRELID3B & $2.12 \mathrm{E}+00$ \\
\hline
\end{tabular}




\begin{tabular}{|c|c|c|c|c|}
\hline ENSBTAG00000008026 & $7.23 \mathrm{E}-10$ & $3.40 \mathrm{E}-10$ & OXT & $2.13 \mathrm{E}+00$ \\
\hline ENSBTAG00000001420 & $2.88 \mathrm{E}-10$ & $1.36 \mathrm{E}-10$ & ABHD12 & $2.12 \mathrm{E}+00$ \\
\hline ENSBTAG00000017012 & 4.00E-10 & $1.88 \mathrm{E}-10$ & & $2.13 E+00$ \\
\hline ENSBTAG00000027419 & $6.01 \mathrm{E}-10$ & $2.80 \mathrm{E}-10$ & & $2.14 \mathrm{E}+00$ \\
\hline ENSBTAG00000018732 & $2.51 \mathrm{E}-10$ & $1.16 \mathrm{E}-10$ & HSPA12B & $2.17 \mathrm{E}+00$ \\
\hline ENSBTAG00000021033 & $3.46 \mathrm{E}-10$ & $1.59 \mathrm{E}-10$ & SGK2 & $2.18 \mathrm{E}+00$ \\
\hline ENSBTAG00000039016 & 1.45E-09 & $6.63 \mathrm{E}-10$ & & $2.18 \mathrm{E}+00$ \\
\hline ENSBTAG00000027444 & $3.68 \mathrm{E}-10$ & $1.69 \mathrm{E}-10$ & & $2.17 E+00$ \\
\hline ENSBTAG00000046981 & $2.65 \mathrm{E}-10$ & $1.22 \mathrm{E}-10$ & & $2.17 E+00$ \\
\hline ENSBTAG00000003380 & $1.65 \mathrm{E}-10$ & $7.55 \mathrm{E}-11$ & KCNK15 & $2.18 \mathrm{E}+00$ \\
\hline ENSBTAG00000006962 & $1.58 \mathrm{E}-10$ & $7.24 \mathrm{E}-11$ & NFS1 & $2.18 \mathrm{E}+00$ \\
\hline ENSBTAG00000000598 & $2.30 \mathrm{E}-10$ & $1.05 \mathrm{E}-10$ & & $2.19 E+00$ \\
\hline ENSBTAG00000018785 & $1.82 \mathrm{E}-10$ & $8.31 \mathrm{E}-11$ & TUBB1 & $2.18 \mathrm{E}+00$ \\
\hline ENSBTAG00000015127 & $2.28 \mathrm{E}-10$ & $1.04 \mathrm{E}-10$ & SDC4 & $2.19 E+00$ \\
\hline ENSBTAG00000003512 & $1.22 \mathrm{E}-10$ & $5.56 \mathrm{E}-11$ & MYH7B & $2.20 E+00$ \\
\hline ENSBTAG00000022007 & $1.02 \mathrm{E}-10$ & $4.62 \mathrm{E}-11$ & SAMHD1 & $2.21 E+00$ \\
\hline ENSBTAG00000006539 & $1.91 \mathrm{E}-10$ & $8.59 \mathrm{E}-11$ & & $2.22 \mathrm{E}+00$ \\
\hline ENSBTAG00000008527 & $2.03 \mathrm{E}-10$ & $9.11 \mathrm{E}-11$ & SRSF6 & $2.22 \mathrm{E}+00$ \\
\hline ENSBTAG00000020382 & $6.33 \mathrm{E}-10$ & $2.83 \mathrm{E}-10$ & RIN2 & $2.24 \mathrm{E}+00$ \\
\hline ENSBTAG00000031354 & 5.17E-10 & $2.28 \mathrm{E}-10$ & & $2.27 \mathrm{E}+00$ \\
\hline ENSBTAG00000027320 & 4.16E-10 & $1.83 \mathrm{E}-10$ & KCNB1 & $2.27 \mathrm{E}+00$ \\
\hline ENSBTAG00000021096 & 2.99E-10 & $1.31 \mathrm{E}-10$ & & $2.28 \mathrm{E}+00$ \\
\hline ENSBTAG00000011304 & $4.39 \mathrm{E}-10$ & $1.92 \mathrm{E}-10$ & XRN2 & $2.29 \mathrm{E}+00$ \\
\hline ENSBTAG00000015525 & $2.59 \mathrm{E}-10$ & $1.13 \mathrm{E}-10$ & SLC39A12 & $2.29 \mathrm{E}+00$ \\
\hline ENSBTAG00000002593 & $2.59 \mathrm{E}-10$ & $1.13 \mathrm{E}-10$ & GDAP1L1 & $2.29 \mathrm{E}+00$ \\
\hline ENSBTAG00000005969 & $2.43 \mathrm{E}-10$ & $1.05 \mathrm{E}-10$ & EIF2S2 & $2.31 E+00$ \\
\hline ENSBTAG00000016676 & $5.90 \mathrm{E}-10$ & $2.56 \mathrm{E}-10$ & PTER & $2.31 E+00$ \\
\hline ENSBTAG00000003101 & 3.30E-10 & $1.43 \mathrm{E}-10$ & SALL4 & $2.31 E+00$ \\
\hline ENSBTAG00000007721 & 4.16E-10 & $1.78 \mathrm{E}-10$ & DPM1 & $2.33 E+00$ \\
\hline ENSBTAG00000019984 & $1.73 \mathrm{E}-09$ & $7.36 \mathrm{E}-10$ & GINS1 & $2.36 \mathrm{E}+00$ \\
\hline ENSBTAG00000001038 & $2.18 \mathrm{E}-10$ & $9.15 \mathrm{E}-11$ & SNRPB & $2.38 \mathrm{E}+00$ \\
\hline ENSBTAG00000005504 & $1.56 \mathrm{E}-10$ & $6.55 \mathrm{E}-11$ & TP53INP2 & $2.38 \mathrm{E}+00$ \\
\hline ENSBTAG00000048079 & $1.84 \mathrm{E}-10$ & $7.71 \mathrm{E}-11$ & & $2.38 \mathrm{E}+00$ \\
\hline ENSBTAG00000039208 & 3.34E-10 & $1.40 \mathrm{E}-10$ & & $2.38 \mathrm{E}+00$ \\
\hline ENSBTAG00000008436 & $2.56 \mathrm{E}-10$ & $1.08 \mathrm{E}-10$ & CDC25B & $2.37 \mathrm{E}+00$ \\
\hline ENSBTAG00000000686 & $1.50 \mathrm{E}-10$ & $6.31 \mathrm{E}-11$ & NANP & $2.38 \mathrm{E}+00$ \\
\hline ENSBTAG00000020795 & $1.95 \mathrm{E}-10$ & $8.19 \mathrm{E}-11$ & & $2.38 \mathrm{E}+00$ \\
\hline ENSBTAG00000002858 & $1.41 \mathrm{E}-10$ & $5.90 \mathrm{E}-11$ & ACBD7 & $2.38 \mathrm{E}+00$ \\
\hline ENSBTAG00000039071 & $5.79 \mathrm{E}-10$ & $2.40 \mathrm{E}-10$ & ANKRD16 & $2.41 \mathrm{E}+00$ \\
\hline ENSBTAG00000007678 & $3.38 \mathrm{E}-10$ & $1.40 \mathrm{E}-10$ & MKX & $2.41 E+00$ \\
\hline ENSBTAG00000007920 & $4.87 \mathrm{E}-10$ & $2.00 \mathrm{E}-10$ & PANK2 & $2.43 \mathrm{E}+00$ \\
\hline ENSBTAG00000021811 & $3.96 \mathrm{E}-10$ & $1.62 \mathrm{E}-10$ & ANGPT4 & $2.44 \mathrm{E}+00$ \\
\hline ENSBTAG00000004911 & $1.12 \mathrm{E}-09$ & $4.58 \mathrm{E}-10$ & BIRC7 & $2.45 \mathrm{E}+00$ \\
\hline ENSBTAG00000022571 & $3.68 \mathrm{E}-10$ & $1.50 \mathrm{E}-10$ & & $2.45 \mathrm{E}+00$ \\
\hline ENSBTAG00000015949 & $3.03 \mathrm{E}-10$ & $1.22 \mathrm{E}-10$ & & $2.48 \mathrm{E}+00$ \\
\hline ENSBTAG00000016724 & $1.30 \mathrm{E}-10$ & $5.25 \mathrm{E}-11$ & NPEPL1 & $2.48 \mathrm{E}+00$ \\
\hline ENSBTAG00000039289 & $8.00 \mathrm{E}-10$ & $3.20 \mathrm{E}-10$ & & $2.50 \mathrm{E}+00$ \\
\hline ENSBTAG00000013753 & 3.17E-10 & $1.26 \mathrm{E}-10$ & DDRGK1 & $2.51 \mathrm{E}+00$ \\
\hline ENSBTAG00000038048 & $3.30 \mathrm{E}-10$ & $1.30 \mathrm{E}-10$ & MRC1 & $2.54 \mathrm{E}+00$ \\
\hline ENSBTAG00000005492 & $1.82 \mathrm{E}-10$ & $7.15 \mathrm{E}-11$ & CCNY & $2.54 \mathrm{E}+00$ \\
\hline ENSBTAG00000044137 & $6.46 \mathrm{E}-10$ & $2.54 \mathrm{E}-10$ & & $2.54 \mathrm{E}+00$ \\
\hline ENSBTAG00000015379 & $3.04 \mathrm{E}-10$ & $1.19 \mathrm{E}-10$ & UBOX5 & $2.56 \mathrm{E}+00$ \\
\hline ENSBTAG00000018101 & 4.13E-10 & $1.58 \mathrm{E}-10$ & $\mathrm{AHCY}$ & $2.61 E+00$ \\
\hline ENSBTAG00000014176 & $3.87 \mathrm{E}-10$ & $1.47 \mathrm{E}-10$ & REM1 & $2.63 E+00$ \\
\hline ENSBTAG00000006134 & $5.99 \mathrm{E}-10$ & $2.27 \mathrm{E}-10$ & DYNLRB1 & $2.64 \mathrm{E}+00$ \\
\hline ENSBTAG00000018263 & $2.18 \mathrm{E}-10$ & $8.27 \mathrm{E}-11$ & WAC & $2.63 E+00$ \\
\hline ENSBTAG00000008650 & $7.09 \mathrm{E}-10$ & $2.65 \mathrm{E}-10$ & CAMK1D & $2.68 \mathrm{E}+00$ \\
\hline ENSBTAG00000014769 & $1.39 \mathrm{E}-10$ & $5.16 \mathrm{E}-11$ & GMEB2 & $2.70 E+00$ \\
\hline ENSBTAG00000016208 & $6.06 \mathrm{E}-10$ & $2.25 \mathrm{E}-10$ & TGM2 & $2.69 E+00$ \\
\hline ENSBTAG00000019726 & 7.95E-10 & $2.94 \mathrm{E}-10$ & ANKEF1 & $2.70 E+00$ \\
\hline ENSBTAG00000011279 & $1.98 \mathrm{E}-10$ & 7.34E-11 & MATN4 & $2.70 E+00$ \\
\hline ENSBTAG00000047302 & $4.08 \mathrm{E}-10$ & $1.50 \mathrm{E}-10$ & & $2.72 \mathrm{E}+00$ \\
\hline ENSBTAG00000014554 & $3.22 \mathrm{E}-10$ & $1.18 \mathrm{E}-10$ & SNAI1 & $2.72 \mathrm{E}+00$ \\
\hline ENSBTAG00000007497 & $3.19 \mathrm{E}-10$ & $1.17 \mathrm{E}-10$ & ARFRP1 & $2.73 E+00$ \\
\hline ENSBTAG00000027409 & $3.77 \mathrm{E}-10$ & $1.38 \mathrm{E}-10$ & SLC4A11 & $2.73 E+00$ \\
\hline ENSBTAG00000013776 & $4.69 \mathrm{E}-10$ & $1.69 \mathrm{E}-10$ & ITPA & $2.77 E+00$ \\
\hline ENSBTAG00000010131 & $3.87 \mathrm{E}-10$ & $1.40 \mathrm{E}-10$ & CBFA2T2 & $2.77 \mathrm{E}+00$ \\
\hline ENSBTAG00000007501 & $2.40 \mathrm{E}-10$ & $8.61 \mathrm{E}-11$ & & $2.78 \mathrm{E}+00$ \\
\hline ENSBTAG00000003425 & $3.40 \mathrm{E}-10$ & $1.22 \mathrm{E}-10$ & MGME1 & $2.79 \mathrm{E}+00$ \\
\hline ENSBTAG00000040046 & 3.34E-10 & $1.20 \mathrm{E}-10$ & & $2.78 \mathrm{E}+00$ \\
\hline
\end{tabular}




\begin{tabular}{|c|c|c|c|c|}
\hline ENSBTAG00000018811 & $5.33 \mathrm{E}-10$ & $1.91 \mathrm{E}-10$ & TMC2 & $2.79 \mathrm{E}+00$ \\
\hline ENSBTAG00000030564 & $9.25 \mathrm{E}-10$ & $3.31 \mathrm{E}-10$ & & $2.79 E+00$ \\
\hline ENSBTAG00000012677 & $3.21 \mathrm{E}-10$ & 1.14E-10 & ARHGAP12 & $2.82 \mathrm{E}+00$ \\
\hline ENSBTAG00000020397 & $3.56 \mathrm{E}-10$ & $1.26 \mathrm{E}-10$ & APCDD1L & $2.83 \mathrm{E}+00$ \\
\hline ENSBTAG00000007703 & $2.35 \mathrm{E}-10$ & 8.25E-11 & NMT2 & $2.85 \mathrm{E}+00$ \\
\hline ENSBTAG00000044091 & $2.34 \mathrm{E}-10$ & $8.18 \mathrm{E}-11$ & PTK6 & $2.86 \mathrm{E}+00$ \\
\hline ENSBTAG00000003872 & $1.69 \mathrm{E}-10$ & $5.89 \mathrm{E}-11$ & STX16-NPEPL1 & $2.87 \mathrm{E}+00$ \\
\hline ENSBTAG00000006802 & 8.57E-10 & $2.95 \mathrm{E}-10$ & BPIFA3 & $2.91 E+00$ \\
\hline ENSBTAG00000011646 & $1.62 \mathrm{E}-10$ & $5.56 \mathrm{E}-11$ & ZNF512B & $2.91 E+00$ \\
\hline ENSBTAG00000007917 & $2.94 \mathrm{E}-10$ & $1.01 \mathrm{E}-10$ & TSHZ2 & $2.91 E+00$ \\
\hline ENSBTAG00000004207 & $3.04 \mathrm{E}-10$ & $1.04 \mathrm{E}-10$ & CD93 & $2.92 E+00$ \\
\hline ENSBTAG00000014252 & $7.34 \mathrm{E}-10$ & $2.51 \mathrm{E}-10$ & SPTLC3 & $2.92 \mathrm{E}+00$ \\
\hline ENSBTAG00000001829 & $3.38 \mathrm{E}-10$ & $1.15 \mathrm{E}-10$ & SLC17A9 & $2.93 \mathrm{E}+00$ \\
\hline ENSBTAG00000006526 & $2.76 \mathrm{E}-10$ & $9.38 \mathrm{E}-11$ & BCL2L1 & $2.94 \mathrm{E}+00$ \\
\hline ENSBTAG00000037453 & $4.22 \mathrm{E}-10$ & $1.43 \mathrm{E}-10$ & & $2.95 E+00$ \\
\hline ENSBTAG00000013416 & $3.06 \mathrm{E}-10$ & $1.03 \mathrm{E}-10$ & & $2.97 E+00$ \\
\hline ENSBTAG00000006135 & $7.48 \mathrm{E}-10$ & $2.50 \mathrm{E}-10$ & MAP1LC3A & $2.99 \mathrm{E}+00$ \\
\hline ENSBTAG00000018978 & $4.58 \mathrm{E}-10$ & $1.53 \mathrm{E}-10$ & KAT14 & $3.00 \mathrm{E}+00$ \\
\hline ENSBTAG00000016445 & $3.22 \mathrm{E}-10$ & $1.08 \mathrm{E}-10$ & YME1L1 & $2.98 \mathrm{E}+00$ \\
\hline ENSBTAG00000003971 & $3.76 \mathrm{E}-10$ & $1.24 \mathrm{E}-10$ & E2F1 & $3.03 E+00$ \\
\hline ENSBTAG00000000516 & $5.98 \mathrm{E}-10$ & $1.98 \mathrm{E}-10$ & OTOR & $3.02 \mathrm{E}+00$ \\
\hline ENSBTAG00000020813 & $1.74 \mathrm{E}-09$ & $5.72 \mathrm{E}-10$ & & $3.04 E+00$ \\
\hline ENSBTAG00000003561 & $3.72 \mathrm{E}-10$ & $1.21 \mathrm{E}-10$ & STK35 & $3.07 \mathrm{E}+00$ \\
\hline ENSBTAG00000018053 & $4.05 \mathrm{E}-10$ & $1.31 \mathrm{E}-10$ & RAB22A & $3.09 E+00$ \\
\hline ENSBTAG00000039693 & $6.42 \mathrm{E}-10$ & 2.07E-10 & & $3.10 \mathrm{E}+00$ \\
\hline ENSBTAG00000008303 & $5.19 \mathrm{E}-10$ & $1.67 \mathrm{E}-10$ & & $3.11 E+00$ \\
\hline ENSBTAG00000003946 & $3.41 \mathrm{E}-10$ & $1.09 \mathrm{E}-10$ & NECAB3 & $3.13 E+00$ \\
\hline ENSBTAG00000015798 & $2.92 \mathrm{E}-10$ & $9.18 \mathrm{E}-11$ & UCKL1 & $3.18 \mathrm{E}+00$ \\
\hline ENSBTAG00000014442 & $1.45 \mathrm{E}-10$ & 4.57E-11 & MTG2 & $3.17 \mathrm{E}+00$ \\
\hline ENSBTAG00000001985 & $3.42 \mathrm{E}-10$ & 1.07E-10 & SRMS & $3.19 E+00$ \\
\hline ENSBTAG00000032687 & $3.66 \mathrm{E}-10$ & $1.15 \mathrm{E}-10$ & & $3.18 \mathrm{E}+00$ \\
\hline ENSBTAG00000027316 & 3.17E-10 & $9.97 \mathrm{E}-11$ & UBE2V1 & $3.18 \mathrm{E}+00$ \\
\hline ENSBTAG00000002675 & $1.34 \mathrm{E}-10$ & 4.23E-11 & CCDC7 & $3.17 \mathrm{E}+00$ \\
\hline ENSBTAG00000006671 & $3.24 \mathrm{E}-10$ & $1.02 \mathrm{E}-10$ & DDX27 & $3.18 \mathrm{E}+00$ \\
\hline ENSBTAG00000017594 & $3.48 \mathrm{E}-10$ & $1.09 \mathrm{E}-10$ & $\mathrm{ZHX3}$ & $3.19 E+00$ \\
\hline ENSBTAG00000005573 & $3.95 \mathrm{E}-10$ & $1.24 \mathrm{E}-10$ & SCAND1 & $3.18 \mathrm{E}+00$ \\
\hline ENSBTAG00000019811 & $3.47 \mathrm{E}-10$ & $1.08 \mathrm{E}-10$ & $\mathrm{HAO1}$ & $3.21 E+00$ \\
\hline ENSBTAG00000006352 & $4.32 \mathrm{E}-10$ & $1.32 \mathrm{E}-10$ & MYT1 & $3.27 E+00$ \\
\hline ENSBTAG00000013915 & $8.74 \mathrm{E}-11$ & $2.59 \mathrm{E}-11$ & DSN1 & $3.36 \mathrm{E}+00$ \\
\hline ENSBTAG00000011620 & $6.55 \mathrm{E}-10$ & $1.93 \mathrm{E}-10$ & PIGU & $3.39 E+00$ \\
\hline ENSBTAG00000004273 & 7.61E-10 & $2.23 \mathrm{E}-10$ & CST7 & $3.41 E+00$ \\
\hline ENSBTAG00000004625 & $9.15 \mathrm{E}-10$ & 2.67E-10 & PYGB & $3.43 E+00$ \\
\hline ENSBTAG00000047137 & 4.16E-10 & $1.21 \mathrm{E}-10$ & & $3.44 E+00$ \\
\hline ENSBTAG00000017278 & 5.19E-10 & $1.49 \mathrm{E}-10$ & ACBD5 & $3.48 \mathrm{E}+00$ \\
\hline ENSBTAG00000021837 & $3.23 \mathrm{E}-10$ & $9.23 \mathrm{E}-11$ & RGS19 & $3.50 E+00$ \\
\hline ENSBTAG00000031715 & $8.22 \mathrm{E}-10$ & $2.34 \mathrm{E}-10$ & PCMTD2 & $3.51 E+00$ \\
\hline ENSBTAG00000019504 & $6.28 \mathrm{E}-10$ & $1.75 \mathrm{E}-10$ & ADRA1D & $3.59 E+00$ \\
\hline ENSBTAG00000030796 & $6.36 \mathrm{E}-10$ & $1.77 \mathrm{E}-10$ & WFDC10A & $3.59 E+00$ \\
\hline ENSBTAG00000033291 & $1.35 \mathrm{E}-10$ & $3.71 E-11$ & SPAG6 & $3.64 E+00$ \\
\hline ENSBTAG00000003040 & $2.73 \mathrm{E}-10$ & $7.40 \mathrm{E}-11$ & RALGAPB & $3.69 E+00$ \\
\hline ENSBTAG00000017098 & $3.84 \mathrm{E}-10$ & $1.02 \mathrm{E}-10$ & BPIFA1 & $3.76 \mathrm{E}+00$ \\
\hline ENSBTAG00000001112 & $3.87 \mathrm{E}-10$ & $1.03 \mathrm{E}-10$ & SMOX & $3.76 \mathrm{E}+00$ \\
\hline ENSBTAG00000001827 & $5.06 \mathrm{E}-10$ & $1.34 \mathrm{E}-10$ & GID8 & $3.77 E+00$ \\
\hline ENSBTAG00000016231 & $1.24 \mathrm{E}-10$ & 3.17E-11 & POFUT1 & $3.90 E+00$ \\
\hline ENSBTAG00000030802 & $1.89 \mathrm{E}-10$ & $4.76 \mathrm{E}-11$ & & $3.97 \mathrm{E}+00$ \\
\hline ENSBTAG00000021423 & $2.26 \mathrm{E}-10$ & 5.67E-11 & LKAAEAR1 & $3.97 E+00$ \\
\hline ENSBTAG00000003962 & $2.66 \mathrm{E}-10$ & $6.69 \mathrm{E}-11$ & C20orf144 & $3.97 E+00$ \\
\hline ENSBTAG00000005750 & $2.06 \mathrm{E}-10$ & $5.18 \mathrm{E}-11$ & TCF15 & $3.97 E+00$ \\
\hline ENSBTAG00000007490 & $6.51 \mathrm{E}-10$ & $1.63 \mathrm{E}-10$ & SULF2 & $3.99 E+00$ \\
\hline ENSBTAG00000021817 & $7.08 \mathrm{E}-10$ & 1.77E-10 & & $4.00 E+00$ \\
\hline ENSBTAG00000021368 & $7.58 \mathrm{E}-10$ & $1.89 \mathrm{E}-10$ & & $4.01 E+00$ \\
\hline ENSBTAG00000031214 & $4.78 \mathrm{E}-10$ & $1.18 \mathrm{E}-10$ & & $4.05 E+00$ \\
\hline ENSBTAG00000011656 & $2.54 \mathrm{E}-10$ & $6.22 \mathrm{E}-11$ & PRPF6 & $4.08 \mathrm{E}+00$ \\
\hline ENSBTAG00000044015 & $3.53 \mathrm{E}-10$ & $8.32 \mathrm{E}-11$ & RBM12 & $4.24 \mathrm{E}+00$ \\
\hline ENSBTAG00000010913 & $4.28 \mathrm{E}-10$ & $9.96 \mathrm{E}-11$ & SRXN1 & $4.29 E+00$ \\
\hline ENSBTAG00000016667 & $2.85 \mathrm{E}-10$ & $6.58 \mathrm{E}-11$ & ZBTB46 & $4.33 E+00$ \\
\hline ENSBTAG00000006742 & 7.35E-10 & $1.69 \mathrm{E}-10$ & PAX1 & $4.35 E+00$ \\
\hline ENSBTAG00000026119 & $6.31 \mathrm{E}-10$ & $1.44 \mathrm{E}-10$ & & $4.38 \mathrm{E}+00$ \\
\hline ENSBTAG00000015796 & $2.66 \mathrm{E}-10$ & $6.05 \mathrm{E}-11$ & DNAJC5 & $4.39 E+00$ \\
\hline ENSBTAG00000039313 & $4.91 \mathrm{E}-10$ & $1.11 \mathrm{E}-10$ & ZNF341 & $4.42 \mathrm{E}+00$ \\
\hline
\end{tabular}




\begin{tabular}{|c|c|c|c|c|}
\hline ENSBTAG00000005937 & $6.15 \mathrm{E}-10$ & $1.39 \mathrm{E}-10$ & TTLL9 & $4.42 \mathrm{E}+00$ \\
\hline ENSBTAG00000031278 & $2.03 \mathrm{E}-10$ & $4.50 \mathrm{E}-11$ & SNPH & $4.50 E+00$ \\
\hline ENSBTAG00000004582 & $4.50 \mathrm{E}-10$ & $9.90 \mathrm{E}-11$ & COL20A1 & $4.55 E+00$ \\
\hline ENSBTAG00000009743 & $5.35 \mathrm{E}-10$ & $1.14 \mathrm{E}-10$ & PDRG1 & $4.69 \mathrm{E}+00$ \\
\hline ENSBTAG00000037757 & $7.90 \mathrm{E}-10$ & 1.67E-10 & EBF4 & $4.73 E+00$ \\
\hline ENSBTAG00000013387 & $5.96 \mathrm{E}-10$ & $1.23 \mathrm{E}-10$ & CHMP4B & $4.84 \mathrm{E}+00$ \\
\hline ENSBTAG00000017271 & $3.56 \mathrm{E}-10$ & $7.30 \mathrm{E}-11$ & MASTL & $4.87 \mathrm{E}+00$ \\
\hline ENSBTAG00000037464 & $5.64 \mathrm{E}-10$ & $1.15 \mathrm{E}-10$ & TGM6 & $4.90 \mathrm{E}+00$ \\
\hline ENSBTAG00000010135 & $4.52 \mathrm{E}-10$ & $9.19 \mathrm{E}-11$ & TMEM189 & $4.92 \mathrm{E}+00$ \\
\hline ENSBTAG00000007930 & $3.72 \mathrm{E}-10$ & 7.55E-11 & NCOA6 & $4.92 \mathrm{E}+00$ \\
\hline ENSBTAG00000000825 & $9.02 \mathrm{E}-10$ & $1.81 \mathrm{E}-10$ & MRPS26 & $4.98 \mathrm{E}+00$ \\
\hline ENSBTAG00000031373 & 1.07E-09 & $2.10 \mathrm{E}-10$ & & $5.09 \mathrm{E}+00$ \\
\hline ENSBTAG00000003030 & $5.84 \mathrm{E}-10$ & $1.14 \mathrm{E}-10$ & KCNQ2 & $5.12 \mathrm{E}+00$ \\
\hline ENSBTAG00000014930 & $4.01 \mathrm{E}-10$ & 7.79E-11 & MYLK2 & $5.14 \mathrm{E}+00$ \\
\hline ENSBTAG00000017587 & $8.32 \mathrm{E}-10$ & $1.60 \mathrm{E}-10$ & & $5.20 \mathrm{E}+00$ \\
\hline ENSBTAG00000006955 & 3.87E-10 & $7.18 \mathrm{E}-11$ & CPNE1 & $5.38 \mathrm{E}+00$ \\
\hline ENSBTAG00000011473 & $6.30 \mathrm{E}-10$ & 1.17E-10 & MYL9 & $5.38 \mathrm{E}+00$ \\
\hline ENSBTAG00000013303 & $3.58 \mathrm{E}-10$ & $6.61 \mathrm{E}-11$ & ACSS2 & $5.41 E+00$ \\
\hline ENSBTAG00000007722 & $9.60 \mathrm{E}-10$ & 1.77E-10 & MOCS3 & $5.42 \mathrm{E}+00$ \\
\hline ENSBTAG00000018735 & 4.35E-10 & $7.92 \mathrm{E}-11$ & C20orf27 & $5.49 E+00$ \\
\hline ENSBTAG00000010387 & $3.28 \mathrm{E}-10$ & $5.95 \mathrm{E}-11$ & FAM188A & $5.51 \mathrm{E}+00$ \\
\hline ENSBTAG00000016524 & $6.65 \mathrm{E}-10$ & $1.20 \mathrm{E}-10$ & RALY & $5.54 \mathrm{E}+00$ \\
\hline ENSBTAG00000021836 & $2.25 \mathrm{E}-10$ & 4.04E-11 & TCEA2 & $5.55 \mathrm{E}+00$ \\
\hline ENSBTAG00000014329 & $1.05 \mathrm{E}-09$ & $1.88 \mathrm{E}-10$ & & $5.59 E+00$ \\
\hline ENSBTAG00000023002 & $6.61 \mathrm{E}-10$ & $1.05 \mathrm{E}-10$ & $\mathrm{JPH} 2$ & $6.29 E+00$ \\
\hline ENSBTAG00000048288 & $3.02 \mathrm{E}-10$ & $4.75 E-11$ & DEFB129 & $6.36 \mathrm{E}+00$ \\
\hline ENSBTAG00000006949 & $4.19 \mathrm{E}-10$ & $6.58 \mathrm{E}-11$ & SPAG4 & $6.36 \mathrm{E}+00$ \\
\hline ENSBTAG00000020988 & 5.17E-10 & $7.75 \mathrm{E}-11$ & KIZ & $6.66 \mathrm{E}+00$ \\
\hline ENSBTAG00000013415 & $2.12 \mathrm{E}-10$ & $3.08 \mathrm{E}-11$ & CST11 & $6.86 \mathrm{E}+00$ \\
\hline ENSBTAG00000008969 & $5.52 \mathrm{E}-10$ & 7.97E-11 & SLC9A8 & $6.92 \mathrm{E}+00$ \\
\hline ENSBTAG00000021232 & $2.32 \mathrm{E}-10$ & $3.24 \mathrm{E}-11$ & TPD52L2 & $7.14 \mathrm{E}+00$ \\
\hline ENSBTAG00000017198 & $6.33 \mathrm{E}-10$ & $8.82 \mathrm{E}-11$ & CHRNA4 & $7.17 \mathrm{E}+00$ \\
\hline ENSBTAG00000005184 & $5.13 \mathrm{E}-10$ & $7.16 \mathrm{E}-11$ & TP53TG5 & $7.16 \mathrm{E}+00$ \\
\hline ENSBTAG00000007977 & $9.14 \mathrm{E}-10$ & $1.20 \mathrm{E}-10$ & LAMP5 & $7.61 E+00$ \\
\hline ENSBTAG00000008840 & 4.54E-10 & $5.83 \mathrm{E}-11$ & HM13 & $7.77 \mathrm{E}+00$ \\
\hline ENSBTAG00000032701 & $7.25 \mathrm{E}-10$ & $9.08 \mathrm{E}-11$ & CSTL1 & $7.98 \mathrm{E}+00$ \\
\hline ENSBTAG00000019304 & $3.01 \mathrm{E}-10$ & $3.60 \mathrm{E}-11$ & BTBD3 & $8.33 \mathrm{E}+00$ \\
\hline ENSBTAG00000018775 & $3.12 \mathrm{E}-10$ & 3.63E-11 & TPX2 & $8.57 E+00$ \\
\hline ENSBTAG00000038738 & $5.83 \mathrm{E}-10$ & $6.70 \mathrm{E}-11$ & PXMP4 & $8.69 E+00$ \\
\hline ENSBTAG00000017679 & $1.12 \mathrm{E}-09$ & $1.24 \mathrm{E}-10$ & PAK5 & $9.02 \mathrm{E}+00$ \\
\hline ENSBTAG00000032366 & $3.71 \mathrm{E}-10$ & 3.87E-11 & SLC23A2 & $9.57 \mathrm{E}+00$ \\
\hline ENSBTAG00000005181 & $3.04 \mathrm{E}-10$ & $2.94 \mathrm{E}-11$ & SYS1 & $1.03 E+01$ \\
\hline ENSBTAG00000025720 & $5.99 \mathrm{E}-10$ & $5.37 \mathrm{E}-11$ & & $1.11 \mathrm{E}+01$ \\
\hline ENSBTAG00000037969 & $9.75 \mathrm{E}-10$ & $7.40 \mathrm{E}-11$ & & $1.32 \mathrm{E}+01$ \\
\hline ENSBTAG00000012406 & $4.34 \mathrm{E}-10$ & $3.14 \mathrm{E}-11$ & ZBP1 & $1.38 \mathrm{E}+01$ \\
\hline ENSBTAG00000035323 & $3.43 \mathrm{E}-10$ & $1.22 \mathrm{E}-11$ & & $2.79 E+01$ \\
\hline ENSBTAG00000009005 & $4.48 \mathrm{E}-10$ & $1.22 \mathrm{E}-11$ & DUSP15 & $3.64 \mathrm{E}+01$ \\
\hline ENSBTAG00000024139 & $3.50 \mathrm{E}-10$ & 0 & OR4S2 & $3.50 E+03$ \\
\hline ENSBTAG00000015584 & 0 & 0 & & $1.00 \mathrm{E}+00$ \\
\hline ENSBTAG00000015072 & 0 & 0 & & $1.00 \mathrm{E}+00$ \\
\hline ENSBTAG00000045526 & 0 & 0 & INSM1 & $1.00 \mathrm{E}+00$ \\
\hline ENSBTAG00000035643 & $2.80 \mathrm{E}-10$ & 0 & & $2.80 \mathrm{E}+03$ \\
\hline ENSBTAG00000045753 & 0 & 0 & & $1.00 \mathrm{E}+00$ \\
\hline ENSBTAG00000045784 & $1.73 \mathrm{E}-10$ & 0 & & $1.74 \mathrm{E}+03$ \\
\hline ENSBTAG00000021816 & $2.39 \mathrm{E}-10$ & 0 & TMEM239 & $2.39 E+03$ \\
\hline ENSBTAG00000047704 & $2.71 \mathrm{E}-10$ & 0 & NPBWR2 & $2.71 E+03$ \\
\hline ENSBTAG00000045701 & $1.78 \mathrm{E}-10$ & 0 & OPRL1 & $1.78 \mathrm{E}+03$ \\
\hline ENSBTAG00000011662 & $1.15 \mathrm{E}-10$ & 0 & SOX18 & $1.15 E+03$ \\
\hline ENSBTAG00000031800 & $2.41 \mathrm{E}-10$ & 0 & PPDPF & $2.41 E+03$ \\
\hline ENSBTAG00000016169 & $2.12 \mathrm{E}-10$ & 0 & ID1 & $2.12 \mathrm{E}+03$ \\
\hline ENSBTAG00000027361 & $1.07 \mathrm{E}-10$ & 0 & ROMO1 & $1.07 \mathrm{E}+03$ \\
\hline ENSBTAG00000024891 & $1.08 \mathrm{E}-09$ & 0 & & $1.08 \mathrm{E}+04$ \\
\hline ENSBTAG00000035199 & $1.25 \mathrm{E}-09$ & 0 & MRPL33 & $1.25 E+04$ \\
\hline ENSBTAG00000045631 & $3.60 \mathrm{E}-10$ & 0 & & $3.60 E+03$ \\
\hline ENSBTAG00000027416 & $2.02 \mathrm{E}-10$ & 0 & CALML3 & $2.03 E+03$ \\
\hline ENSBTAG00000027221 & $3.53 \mathrm{E}-10$ & 0 & & $3.53 E+03$ \\
\hline ENSBTAG00000031906 & $1.46 \mathrm{E}-10$ & 0 & ABHD16B & $1.46 \mathrm{E}+03$ \\
\hline ENSBTAG00000024608 & 0 & 0 & & $1.00 E+00$ \\
\hline ENSBTAG00000004712 & $1.10 \mathrm{E}-09$ & 0 & MC3R & $1.10 E+04$ \\
\hline ENSBTAG00000002116 & $1.21 \mathrm{E}-10$ & 0 & ZCCHC3 & $1.21 E+03$ \\
\hline
\end{tabular}




\begin{tabular}{|c|c|c|c|c|}
\hline ENSBTAG00000009206 & $2.51 \mathrm{E}-10$ & 0 & FOXS1 & $2.51 E+03$ \\
\hline ENSBTAG00000047885 & $5.57 \mathrm{E}-10$ & 0 & ACTL10 & $5.57 \mathrm{E}+03$ \\
\hline ENSBTAG00000003396 & 0 & 0 & MAFB & $1.00 \mathrm{E}+00$ \\
\hline ENSBTAG00000046673 & 0 & 0 & & $1.00 \mathrm{E}+00$ \\
\hline ENSBTAG00000046704 & $1.38 \mathrm{E}-09$ & 0 & & $1.38 \mathrm{E}+04$ \\
\hline ENSBTAG00000013408 & 0 & $1.40 \mathrm{E}-10$ & RBM12B & 7.14E-04 \\
\hline ENSBTAG00000037399 & 0 & $1.98 \mathrm{E}-10$ & & $5.05 \mathrm{E}-04$ \\
\hline ENSBTAG00000000312 & $7.80 \mathrm{E}-11$ & $2.62 \mathrm{E}-10$ & GRINA & $2.98 \mathrm{E}-01$ \\
\hline ENSBTAG00000015717 & $1.41 \mathrm{E}-10$ & 4.29E-10 & HEY1 & 3.30E-01 \\
\hline ENSBTAG00000011922 & $1.30 \mathrm{E}-10$ & $3.75 \mathrm{E}-10$ & PLEC & 3.47E-01 \\
\hline ENSBTAG00000009677 & $9.82 \mathrm{E}-11$ & 2.63E-10 & PARP10 & 3.74E-01 \\
\hline ENSBTAG00000039141 & $8.92 \mathrm{E}-11$ & $2.25 \mathrm{E}-10$ & TMEM74 & 3.97E-01 \\
\hline ENSBTAG00000022539 & $1.43 \mathrm{E}-10$ & $3.61 E-10$ & POLR2K & 3.96E-01 \\
\hline ENSBTAG00000046121 & $4.54 \mathrm{E}-10$ & $9.01 \mathrm{E}-10$ & & $5.04 \mathrm{E}-01$ \\
\hline ENSBTAG00000033173 & $1.66 \mathrm{E}-10$ & $3.13 \mathrm{E}-10$ & BHLHE22 & $5.29 \mathrm{E}-01$ \\
\hline ENSBTAG00000021887 & $4.09 \mathrm{E}-10$ & $6.88 \mathrm{E}-10$ & DPYS & 5.95E-01 \\
\hline ENSBTAG00000004970 & $4.57 \mathrm{E}-11$ & $7.68 \mathrm{E}-11$ & LRRC24 & 5.96E-01 \\
\hline ENSBTAG00000002192 & $8.73 \mathrm{E}-11$ & $1.41 \mathrm{E}-10$ & C8orf46 & $6.19 \mathrm{E}-01$ \\
\hline ENSBTAG00000026320 & $6.75 \mathrm{E}-11$ & $1.06 \mathrm{E}-10$ & VPS28 & 6.37E-01 \\
\hline ENSBTAG00000004411 & $2.36 \mathrm{E}-10$ & 3.67E-10 & CRISPLD1 & $6.42 \mathrm{E}-01$ \\
\hline ENSBTAG00000046208 & $7.89 \mathrm{E}-11$ & 1.19E-10 & SCRT1 & 6.64E-01 \\
\hline ENSBTAG00000014691 & $4.31 \mathrm{E}-10$ & $6.38 \mathrm{E}-10$ & ARFGEF1 & $6.75 \mathrm{E}-01$ \\
\hline ENSBTAG00000021009 & 4.44E-10 & $6.43 \mathrm{E}-10$ & TRIM55 & $6.91 \mathrm{E}-01$ \\
\hline ENSBTAG00000047330 & $1.10 \mathrm{E}-10$ & $1.58 \mathrm{E}-10$ & FABP5 & $6.96 \mathrm{E}-01$ \\
\hline ENSBTAG00000020099 & $3.90 \mathrm{E}-10$ & $5.53 \mathrm{E}-10$ & STMN2 & 7.06E-01 \\
\hline ENSBTAG00000032548 & $5.18 \mathrm{E}-10$ & 7.32E-10 & SYBU & 7.08E-01 \\
\hline ENSBTAG00000016754 & $3.59 \mathrm{E}-10$ & $5.03 \mathrm{E}-10$ & KLHL38 & 7.13E-01 \\
\hline ENSBTAG00000010276 & $1.26 \mathrm{E}-10$ & $1.75 \mathrm{E}-10$ & RECQL4 & $7.22 \mathrm{E}-01$ \\
\hline ENSBTAG00000008355 & $1.08 \mathrm{E}-10$ & 1.50E-10 & CPSF1 & $7.18 \mathrm{E}-01$ \\
\hline ENSBTAG00000023218 & $2.07 \mathrm{E}-10$ & $2.81 \mathrm{E}-10$ & UBE2V2 & 7.36E-01 \\
\hline ENSBTAG00000037571 & $1.76 \mathrm{E}-10$ & $2.29 \mathrm{E}-10$ & EBAG9 & 7.67E-01 \\
\hline ENSBTAG00000040021 & $8.53 \mathrm{E}-10$ & 1.11E-09 & & $7.69 \mathrm{E}-01$ \\
\hline ENSBTAG00000036304 & $5.80 \mathrm{E}-10$ & 7.44E-10 & CNGB3 & $7.80 \mathrm{E}-01$ \\
\hline ENSBTAG00000020523 & $1.24 \mathrm{E}-10$ & 1.57E-10 & SLC10A5 & 7.93E-01 \\
\hline ENSBTAG00000021240 & $4.21 \mathrm{E}-10$ & $5.31 \mathrm{E}-10$ & DCSTAMP & 7.93E-01 \\
\hline ENSBTAG00000043978 & 2.17E-10 & $2.74 \mathrm{E}-10$ & & 7.93E-01 \\
\hline ENSBTAG00000026327 & $3.14 \mathrm{E}-11$ & $3.96 \mathrm{E}-11$ & & 7.94E-01 \\
\hline ENSBTAG00000014607 & $4.36 \mathrm{E}-11$ & 5.50E-11 & EXOSC4 & 7.93E-01 \\
\hline ENSBTAG00000048160 & $4.21 \mathrm{E}-11$ & $5.31 \mathrm{E}-11$ & TIGD5 & 7.93E-01 \\
\hline ENSBTAG00000032812 & $2.63 \mathrm{E}-10$ & $3.31 \mathrm{E}-10$ & & 7.94E-01 \\
\hline ENSBTAG00000046932 & $8.84 \mathrm{E}-10$ & $1.11 E-09$ & & 7.96E-01 \\
\hline ENSBTAG00000006213 & $3.95 \mathrm{E}-10$ & $4.98 \mathrm{E}-10$ & & 7.94E-01 \\
\hline ENSBTAG00000026344 & $7.75 \mathrm{E}-11$ & $9.77 \mathrm{E}-11$ & MAFA & 7.94E-01 \\
\hline ENSBTAG00000026309 & $9.76 \mathrm{E}-11$ & $1.23 \mathrm{E}-10$ & $\mathrm{ZHX} 2$ & 7.94E-01 \\
\hline ENSBTAG00000020226 & $1.52 \mathrm{E}-10$ & $1.91 \mathrm{E}-10$ & CHRAC1 & 7.94E-01 \\
\hline ENSBTAG00000007753 & $9.56 \mathrm{E}-11$ & $1.20 \mathrm{E}-10$ & KIFC2 & 7.97E-01 \\
\hline ENSBTAG00000046031 & $3.22 \mathrm{E}-11$ & $4.06 \mathrm{E}-11$ & C8orf82 & 7.95E-01 \\
\hline ENSBTAG00000002851 & $4.66 \mathrm{E}-10$ & $5.88 \mathrm{E}-10$ & & 7.93E-01 \\
\hline ENSBTAG00000045579 & $4.09 \mathrm{E}-10$ & $5.15 \mathrm{E}-10$ & & 7.94E-01 \\
\hline ENSBTAG00000017281 & $1.98 \mathrm{E}-10$ & $2.50 \mathrm{E}-10$ & OPLAH & 7.94E-01 \\
\hline ENSBTAG00000047607 & $1.46 \mathrm{E}-10$ & $1.84 \mathrm{E}-10$ & & 7.94E-01 \\
\hline ENSBTAG00000034261 & $1.44 \mathrm{E}-10$ & $1.81 \mathrm{E}-10$ & & 7.94E-01 \\
\hline ENSBTAG00000046358 & $3.75 \mathrm{E}-10$ & 4.55E-10 & PABPC1 & 8.24E-01 \\
\hline ENSBTAG00000046325 & $3.40 \mathrm{E}-10$ & 4.09E-10 & MCM4 & $8.31 \mathrm{E}-01$ \\
\hline ENSBTAG00000000948 & $4.78 \mathrm{E}-10$ & $5.74 \mathrm{E}-10$ & RAB2A & $8.32 \mathrm{E}-01$ \\
\hline ENSBTAG00000037526 & $3.93 \mathrm{E}-10$ & $4.71 \mathrm{E}-10$ & FABP4 & 8.34E-01 \\
\hline ENSBTAG00000003789 & $3.28 \mathrm{E}-10$ & $3.90 \mathrm{E}-10$ & KIAA1429 & $8.40 \mathrm{E}-01$ \\
\hline ENSBTAG00000020312 & $1.18 \mathrm{E}-10$ & $1.41 \mathrm{E}-10$ & NCOA2 & 8.39E-01 \\
\hline ENSBTAG00000039718 & $7.60 \mathrm{E}-10$ & $9.01 \mathrm{E}-10$ & FABP9 & 8.44E-01 \\
\hline ENSBTAG00000008958 & $5.80 \mathrm{E}-10$ & $6.79 \mathrm{E}-10$ & NSMAF & 8.54E-01 \\
\hline ENSBTAG00000026350 & $3.41 \mathrm{E}-10$ & $3.99 \mathrm{E}-10$ & SPATC1 & 8.53E-01 \\
\hline ENSBTAG00000007386 & $4.92 \mathrm{E}-10$ & $5.74 \mathrm{E}-10$ & NUDCD1 & 8.57E-01 \\
\hline ENSBTAG00000008285 & $2.18 \mathrm{E}-10$ & $2.54 \mathrm{E}-10$ & OXR1 & $8.58 \mathrm{E}-01$ \\
\hline ENSBTAG00000011205 & $3.35 \mathrm{E}-10$ & $3.89 \mathrm{E}-10$ & & $8.61 E-01$ \\
\hline ENSBTAG00000000199 & $2.25 \mathrm{E}-10$ & $2.59 \mathrm{E}-10$ & PDP1 & 8.69E-01 \\
\hline ENSBTAG00000026172 & $3.57 \mathrm{E}-10$ & 4.09E-10 & FAM84B & $8.72 \mathrm{E}-01$ \\
\hline ENSBTAG00000032544 & $3.16 \mathrm{E}-10$ & $3.62 \mathrm{E}-10$ & SPAG1 & 8.73E-01 \\
\hline ENSBTAG00000003339 & $1.20 \mathrm{E}-10$ & $1.38 \mathrm{E}-10$ & ZBTB10 & 8.73E-01 \\
\hline ENSBTAG00000044080 & $5.73 \mathrm{E}-10$ & $6.51 \mathrm{E}-10$ & CPA6 & $8.81 E-01$ \\
\hline ENSBTAG00000002062 & 4.17E-10 & 4.73E-10 & TRPA1 & 8.81E-01 \\
\hline
\end{tabular}




\begin{tabular}{|c|c|c|c|c|}
\hline ENSBTAG00000019864 & $2.86 \mathrm{E}-10$ & $3.25 \mathrm{E}-10$ & MAPK15 & $8.81 E-01$ \\
\hline ENSBTAG00000046435 & $7.30 \mathrm{E}-10$ & $8.28 \mathrm{E}-10$ & & $8.82 \mathrm{E}-01$ \\
\hline ENSBTAG00000006209 & $3.74 \mathrm{E}-10$ & $4.20 \mathrm{E}-10$ & EXT1 & 8.91E-01 \\
\hline ENSBTAG00000019910 & $2.22 \mathrm{E}-10$ & $2.49 \mathrm{E}-10$ & SDCBP & 8.91E-01 \\
\hline ENSBTAG00000000707 & $1.25 \mathrm{E}-10$ & $1.40 \mathrm{E}-10$ & WISP1 & 8.95E-01 \\
\hline ENSBTAG00000020143 & $1.78 \mathrm{E}-10$ & 1.99E-10 & RDH10 & $8.92 \mathrm{E}-01$ \\
\hline ENSBTAG00000000620 & $1.82 \mathrm{E}-10$ & $2.04 \mathrm{E}-10$ & MCMDC2 & $8.95 \mathrm{E}-01$ \\
\hline ENSBTAG00000004792 & $5.55 \mathrm{E}-10$ & $6.15 \mathrm{E}-10$ & CHMP4C & $9.03 \mathrm{E}-01$ \\
\hline ENSBTAG00000014689 & $6.08 \mathrm{E}-10$ & $6.72 \mathrm{E}-10$ & CSPP1 & $9.05 \mathrm{E}-01$ \\
\hline ENSBTAG00000019793 & $2.75 \mathrm{E}-10$ & 3.00E-10 & RGS22 & 9.17E-01 \\
\hline ENSBTAG00000006695 & $1.00 \mathrm{E}-10$ & $1.09 \mathrm{E}-10$ & VCPIP1 & $9.18 \mathrm{E}-01$ \\
\hline ENSBTAG00000020801 & $3.72 \mathrm{E}-10$ & $4.00 \mathrm{E}-10$ & FAM49B & $9.29 \mathrm{E}-01$ \\
\hline ENSBTAG00000003338 & $7.21 \mathrm{E}-10$ & $7.75 \mathrm{E}-10$ & LRRCC1 & $9.31 \mathrm{E}-01$ \\
\hline ENSBTAG00000015229 & $6.41 \mathrm{E}-10$ & $6.87 \mathrm{E}-10$ & DNAJC5B & $9.33 \mathrm{E}-01$ \\
\hline ENSBTAG00000018352 & $2.50 \mathrm{E}-10$ & 2.67E-10 & ABRA & 9.37E-01 \\
\hline ENSBTAG00000048317 & $6.22 \mathrm{E}-10$ & $6.63 \mathrm{E}-10$ & & $9.39 \mathrm{E}-01$ \\
\hline ENSBTAG00000005025 & $4.70 \mathrm{E}-10$ & 4.99E-10 & FAM91A1 & $9.42 \mathrm{E}-01$ \\
\hline ENSBTAG00000002080 & $1.86 \mathrm{E}-10$ & $1.97 \mathrm{E}-10$ & NOV & $9.44 \mathrm{E}-01$ \\
\hline ENSBTAG00000007731 & $3.59 \mathrm{E}-10$ & $3.78 \mathrm{E}-10$ & & $9.50 \mathrm{E}-01$ \\
\hline ENSBTAG00000011981 & $1.56 \mathrm{E}-10$ & $1.64 \mathrm{E}-10$ & MYBL1 & 9.50E-01 \\
\hline ENSBTAG00000047534 & $1.79 \mathrm{E}-10$ & $1.88 \mathrm{E}-10$ & & $9.52 \mathrm{E}-01$ \\
\hline ENSBTAG00000005762 & $4.70 \mathrm{E}-10$ & $4.93 \mathrm{E}-10$ & & $9.53 \mathrm{E}-01$ \\
\hline ENSBTAG00000033128 & $6.11 \mathrm{E}-10$ & $6.41 \mathrm{E}-10$ & $\mathrm{CRH}$ & $9.53 \mathrm{E}-01$ \\
\hline ENSBTAG00000017833 & $1.20 \mathrm{E}-10$ & $1.26 \mathrm{E}-10$ & RNF19A & $9.56 \mathrm{E}-01$ \\
\hline ENSBTAG00000013405 & $1.32 \mathrm{E}-10$ & $1.38 \mathrm{E}-10$ & & $9.59 \mathrm{E}-01$ \\
\hline ENSBTAG00000017529 & $2.24 \mathrm{E}-10$ & $2.33 \mathrm{E}-10$ & CA8 & $9.62 \mathrm{E}-01$ \\
\hline ENSBTAG00000017138 & 4.33E-10 & $4.50 \mathrm{E}-10$ & TMEM71 & $9.62 \mathrm{E}-01$ \\
\hline ENSBTAG00000011064 & $1.39 \mathrm{E}-10$ & $1.44 \mathrm{E}-10$ & ADCK5 & $9.63 \mathrm{E}-01$ \\
\hline ENSBTAG00000047834 & $3.48 \mathrm{E}-10$ & $3.62 \mathrm{E}-10$ & & $9.62 \mathrm{E}-01$ \\
\hline ENSBTAG00000008054 & $1.30 \mathrm{E}-10$ & $1.35 \mathrm{E}-10$ & ZNF706 & $9.66 \mathrm{E}-01$ \\
\hline ENSBTAG00000017733 & $1.70 \mathrm{E}-10$ & $1.75 \mathrm{E}-10$ & CA2 & $9.73 \mathrm{E}-01$ \\
\hline ENSBTAG00000019795 & $1.75 \mathrm{E}-10$ & $1.76 \mathrm{E}-10$ & FBXO43 & $9.92 \mathrm{E}-01$ \\
\hline ENSBTAG00000008150 & $5.41 \mathrm{E}-10$ & $5.45 \mathrm{E}-10$ & PKIA & $9.92 \mathrm{E}-01$ \\
\hline ENSBTAG00000036098 & $3.44 \mathrm{E}-10$ & $3.46 \mathrm{E}-10$ & & $9.94 \mathrm{E}-01$ \\
\hline ENSBTAG00000004969 & $1.18 \mathrm{E}-10$ & 1.19E-10 & LRRC14 & 9.94E-01 \\
\hline ENSBTAG00000019997 & $5.77 \mathrm{E}-10$ & 5.79E-10 & TRHR & 9.97E-01 \\
\hline ENSBTAG00000002784 & $5.42 \mathrm{E}-10$ & $5.44 \mathrm{E}-10$ & COPS5 & 9.97E-01 \\
\hline ENSBTAG00000000914 & $3.84 \mathrm{E}-10$ & 3.83E-10 & OPRK1 & $1.00 \mathrm{E}+00$ \\
\hline ENSBTAG00000001156 & 1.09E-09 & $1.08 \mathrm{E}-09$ & ST3GAL1 & $1.01 E+00$ \\
\hline ENSBTAG00000009378 & $3.87 \mathrm{E}-10$ & $3.85 \mathrm{E}-10$ & E2F5 & $1.01 E+00$ \\
\hline ENSBTAG00000000711 & $4.62 \mathrm{E}-10$ & 4.57E-10 & NDRG1 & $1.01 E+00$ \\
\hline ENSBTAG00000018902 & $3.60 \mathrm{E}-10$ & $3.55 \mathrm{E}-10$ & ZC2HC1A & $1.01 E+00$ \\
\hline ENSBTAG00000026233 & $2.09 \mathrm{E}-10$ & $2.06 \mathrm{E}-10$ & & $1.01 E+00$ \\
\hline ENSBTAG00000012920 & $5.60 \mathrm{E}-10$ & $5.48 \mathrm{E}-10$ & TMEM70 & $1.02 E+00$ \\
\hline ENSBTAG00000008629 & $4.08 \mathrm{E}-10$ & $3.98 \mathrm{E}-10$ & MTFR1 & $1.02 \mathrm{E}+00$ \\
\hline ENSBTAG00000015734 & $7.39 \mathrm{E}-10$ & $7.18 \mathrm{E}-10$ & RMDN1 & $1.03 E+00$ \\
\hline ENSBTAG00000015901 & $3.18 \mathrm{E}-10$ & 3.09E-10 & ARMC1 & $1.03 E+00$ \\
\hline ENSBTAG00000017249 & $6.16 \mathrm{E}-10$ & $5.95 \mathrm{E}-10$ & SBSPON & $1.03 E+00$ \\
\hline ENSBTAG00000009474 & $9.97 \mathrm{E}-10$ & $9.62 \mathrm{E}-10$ & & $1.04 E+00$ \\
\hline ENSBTAG00000034176 & $9.38 \mathrm{E}-10$ & $8.95 \mathrm{E}-10$ & & $1.05 E+00$ \\
\hline ENSBTAG00000001649 & $3.98 \mathrm{E}-10$ & $3.78 \mathrm{E}-10$ & ZFPM2 & $1.05 E+00$ \\
\hline ENSBTAG00000003098 & $2.88 \mathrm{E}-10$ & $2.73 \mathrm{E}-10$ & MTDH & $1.05 E+00$ \\
\hline ENSBTAG00000004954 & $1.88 \mathrm{E}-10$ & $1.78 \mathrm{E}-10$ & TOX & $1.06 \mathrm{E}+00$ \\
\hline ENSBTAG00000048040 & $3.76 \mathrm{E}-10$ & $3.55 \mathrm{E}-10$ & & $1.06 \mathrm{E}+00$ \\
\hline ENSBTAG00000016159 & 3.30E-10 & $3.11 \mathrm{E}-10$ & NPBWR1 & $1.06 \mathrm{E}+00$ \\
\hline ENSBTAG00000007302 & $6.04 \mathrm{E}-10$ & $5.69 \mathrm{E}-10$ & & $1.06 \mathrm{E}+00$ \\
\hline ENSBTAG00000013598 & 4.14E-10 & $3.89 \mathrm{E}-10$ & RSPO2 & $1.06 \mathrm{E}+00$ \\
\hline ENSBTAG00000013678 & $1.55 \mathrm{E}-10$ & $1.45 \mathrm{E}-10$ & OSGIN2 & $1.07 \mathrm{E}+00$ \\
\hline ENSBTAG00000016284 & $6.24 \mathrm{E}-10$ & $5.83 \mathrm{E}-10$ & IL7 & $1.07 E+00$ \\
\hline ENSBTAG00000011516 & $2.38 \mathrm{E}-10$ & $2.21 \mathrm{E}-10$ & LRP12 & $1.07 E+00$ \\
\hline ENSBTAG00000000071 & $6.73 \mathrm{E}-10$ & $6.25 \mathrm{E}-10$ & PMP2 & $1.08 \mathrm{E}+00$ \\
\hline ENSBTAG00000017717 & $6.52 \mathrm{E}-10$ & $6.04 \mathrm{E}-10$ & RALYL & $1.08 \mathrm{E}+00$ \\
\hline ENSBTAG00000007736 & $5.76 \mathrm{E}-10$ & 5.33E-10 & & $1.08 \mathrm{E}+00$ \\
\hline ENSBTAG00000035254 & $2.05 \mathrm{E}-10$ & $1.90 \mathrm{E}-10$ & CYHR1 & $1.08 \mathrm{E}+00$ \\
\hline ENSBTAG00000004476 & $1.77 \mathrm{E}-10$ & $1.63 \mathrm{E}-10$ & ADHFE1 & $1.08 \mathrm{E}+00$ \\
\hline ENSBTAG00000018493 & $5.44 \mathrm{E}-10$ & $5.01 \mathrm{E}-10$ & ERICH5 & $1.08 \mathrm{E}+00$ \\
\hline ENSBTAG00000015924 & $3.60 \mathrm{E}-10$ & 3.30E-10 & CALB1 & $1.09 E+00$ \\
\hline ENSBTAG00000026242 & $3.73 E-10$ & $3.40 \mathrm{E}-10$ & BAALC & $1.10 \mathrm{E}+00$ \\
\hline ENSBTAG00000018218 & $6.95 \mathrm{E}-10$ & $6.31 \mathrm{E}-10$ & & $1.10 \mathrm{E}+00$ \\
\hline ENSBTAG00000008512 & $1.88 \mathrm{E}-10$ & $1.69 \mathrm{E}-10$ & KCNV1 & $1.11 E+00$ \\
\hline
\end{tabular}




\begin{tabular}{|c|c|c|c|c|}
\hline ENSBTAG00000015985 & $5.30 \mathrm{E}-10$ & 4.76E-10 & GPR20 & $1.11 E+00$ \\
\hline ENSBTAG00000015338 & $4.05 \mathrm{E}-10$ & $3.61 \mathrm{E}-10$ & DSCC1 & $1.12 \mathrm{E}+00$ \\
\hline ENSBTAG00000008280 & 4.24E-10 & 3.77E-10 & HNF4G & $1.12 \mathrm{E}+00$ \\
\hline ENSBTAG00000032236 & $1.12 \mathrm{E}-09$ & $9.92 \mathrm{E}-10$ & & $1.13 E+00$ \\
\hline ENSBTAG00000047303 & $5.80 \mathrm{E}-10$ & $5.13 \mathrm{E}-10$ & & $1.13 \mathrm{E}+00$ \\
\hline ENSBTAG00000002181 & $2.32 \mathrm{E}-10$ & $2.05 E-10$ & KHDRBS3 & $1.13 E+00$ \\
\hline ENSBTAG00000004595 & $7.96 \mathrm{E}-10$ & $7.01 \mathrm{E}-10$ & GML & $1.14 \mathrm{E}+00$ \\
\hline ENSBTAG00000001808 & $1.42 \mathrm{E}-10$ & $1.25 \mathrm{E}-10$ & LACTB2 & $1.14 \mathrm{E}+00$ \\
\hline ENSBTAG00000014600 & $5.77 \mathrm{E}-10$ & $5.02 \mathrm{E}-10$ & $\mathrm{ADCY} 8$ & $1.15 \mathrm{E}+00$ \\
\hline ENSBTAG00000046739 & $8.00 \mathrm{E}-10$ & $6.96 \mathrm{E}-10$ & & $1.15 E+00$ \\
\hline ENSBTAG00000004596 & $6.70 \mathrm{E}-10$ & $5.76 \mathrm{E}-10$ & & $1.16 \mathrm{E}+00$ \\
\hline ENSBTAG00000021747 & $6.10 \mathrm{E}-10$ & $5.23 \mathrm{E}-10$ & & $1.17 \mathrm{E}+00$ \\
\hline ENSBTAG00000017165 & $4.85 \mathrm{E}-10$ & $4.11 \mathrm{E}-10$ & MATN2 & $1.18 \mathrm{E}+00$ \\
\hline ENSBTAG00000000632 & $3.44 \mathrm{E}-10$ & 2.92E-10 & & $1.18 \mathrm{E}+00$ \\
\hline ENSBTAG00000039978 & $1.07 \mathrm{E}-10$ & $9.01 \mathrm{E}-11$ & & $1.19 E+00$ \\
\hline ENSBTAG00000007562 & $2.06 \mathrm{E}-10$ & $1.73 \mathrm{E}-10$ & TRAM1 & $1.19 E+00$ \\
\hline ENSBTAG00000048001 & $1.98 \mathrm{E}-10$ & $1.66 \mathrm{E}-10$ & & $1.19 \mathrm{E}+00$ \\
\hline ENSBTAG00000038682 & $2.11 \mathrm{E}-10$ & $1.77 \mathrm{E}-10$ & FAM83H & $1.19 E+00$ \\
\hline ENSBTAG00000007835 & $8.18 \mathrm{E}-11$ & $6.87 \mathrm{E}-11$ & GPT & $1.19 E+00$ \\
\hline ENSBTAG00000018455 & $1.21 \mathrm{E}-10$ & $1.01 \mathrm{E}-10$ & COMMD5 & $1.19 \mathrm{E}+00$ \\
\hline ENSBTAG00000000856 & $8.22 \mathrm{E}-11$ & $6.91 \mathrm{E}-11$ & & $1.19 E+00$ \\
\hline ENSBTAG00000034613 & $4.88 \mathrm{E}-11$ & $4.10 \mathrm{E}-11$ & MROH6 & $1.19 E+00$ \\
\hline ENSBTAG00000017016 & $7.04 \mathrm{E}-10$ & $5.90 \mathrm{E}-10$ & & $1.19 E+00$ \\
\hline ENSBTAG00000015040 & $1.94 \mathrm{E}-10$ & 1.63E-10 & & $1.19 E+00$ \\
\hline ENSBTAG00000005748 & $1.36 \mathrm{E}-10$ & $1.15 \mathrm{E}-10$ & SOX17 & $1.19 \mathrm{E}+00$ \\
\hline ENSBTAG00000047205 & $1.82 \mathrm{E}-10$ & $1.53 \mathrm{E}-10$ & & $1.19 E+00$ \\
\hline ENSBTAG00000013284 & $1.51 \mathrm{E}-10$ & $1.26 \mathrm{E}-10$ & & $1.20 \mathrm{E}+00$ \\
\hline ENSBTAG00000003185 & $6.48 \mathrm{E}-10$ & 5.37E-10 & ASAP1 & $1.21 \mathrm{E}+00$ \\
\hline ENSBTAG00000002104 & $2.40 \mathrm{E}-10$ & $1.97 \mathrm{E}-10$ & RHPN1 & $1.22 \mathrm{E}+00$ \\
\hline ENSBTAG00000048138 & $1.18 \mathrm{E}-10$ & $9.78 \mathrm{E}-11$ & XKR9 & $1.21 E+00$ \\
\hline ENSBTAG00000034196 & $3.31 \mathrm{E}-10$ & $2.72 \mathrm{E}-10$ & HHLA1 & $1.22 \mathrm{E}+00$ \\
\hline ENSBTAG00000013369 & $6.55 \mathrm{E}-10$ & $5.34 \mathrm{E}-10$ & COL14A1 & $1.23 E+00$ \\
\hline ENSBTAG00000015341 & $7.05 \mathrm{E}-10$ & $5.72 \mathrm{E}-10$ & DEPTOR & $1.23 E+00$ \\
\hline ENSBTAG00000002076 & $2.65 \mathrm{E}-10$ & $2.14 \mathrm{E}-10$ & & $1.24 \mathrm{E}+00$ \\
\hline ENSBTAG00000011614 & $4.72 \mathrm{E}-10$ & $3.81 \mathrm{E}-10$ & PDE7A & $1.24 \mathrm{E}+00$ \\
\hline ENSBTAG00000001000 & $3.11 \mathrm{E}-10$ & $2.50 \mathrm{E}-10$ & CA13 & $1.24 \mathrm{E}+00$ \\
\hline ENSBTAG00000007749 & $1.64 \mathrm{E}-10$ & $1.31 \mathrm{E}-10$ & TONSL & $1.25 \mathrm{E}+00$ \\
\hline ENSBTAG00000017019 & $6.71 \mathrm{E}-10$ & $5.35 \mathrm{E}-10$ & PRKDC & $1.25 \mathrm{E}+00$ \\
\hline ENSBTAG00000000236 & $1.77 \mathrm{E}-10$ & $1.41 \mathrm{E}-10$ & YWHAZ & $1.26 \mathrm{E}+00$ \\
\hline ENSBTAG00000003827 & $2.94 \mathrm{E}-10$ & $2.34 \mathrm{E}-10$ & RIMS2 & $1.26 \mathrm{E}+00$ \\
\hline ENSBTAG00000019663 & $3.49 \mathrm{E}-10$ & $2.78 \mathrm{E}-10$ & DECR1 & $1.26 \mathrm{E}+00$ \\
\hline ENSBTAG00000006869 & $1.90 \mathrm{E}-10$ & $1.50 \mathrm{E}-10$ & PHF20L1 & $1.27 \mathrm{E}+00$ \\
\hline ENSBTAG00000018570 & $1.57 \mathrm{E}-10$ & $1.24 \mathrm{E}-10$ & SDR16C5 & $1.27 \mathrm{E}+00$ \\
\hline ENSBTAG00000015536 & $8.21 \mathrm{E}-10$ & 6.47E-10 & CPNE3 & $1.27 \mathrm{E}+00$ \\
\hline ENSBTAG00000013621 & $2.24 \mathrm{E}-10$ & $1.75 \mathrm{E}-10$ & DPY19L4 & $1.28 \mathrm{E}+00$ \\
\hline ENSBTAG00000018557 & $1.42 \mathrm{E}-10$ & $1.11 \mathrm{E}-10$ & YTHDF3 & $1.28 \mathrm{E}+00$ \\
\hline ENSBTAG00000026254 & $3.77 \mathrm{E}-10$ & $2.95 \mathrm{E}-10$ & MED30 & $1.28 \mathrm{E}+00$ \\
\hline ENSBTAG00000014051 & $3.65 \mathrm{E}-10$ & $2.83 \mathrm{E}-10$ & ANGPT1 & $1.29 E+00$ \\
\hline ENSBTAG00000007823 & $4.61 E-10$ & $3.56 \mathrm{E}-10$ & TG & $1.30 \mathrm{E}+00$ \\
\hline ENSBTAG00000043996 & $3.39 \mathrm{E}-10$ & $2.61 \mathrm{E}-10$ & SAMD12 & $1.30 \mathrm{E}+00$ \\
\hline ENSBTAG00000024984 & $3.16 \mathrm{E}-10$ & $2.43 \mathrm{E}-10$ & FSBP & $1.30 \mathrm{E}+00$ \\
\hline ENSBTAG00000003885 & $6.79 \mathrm{E}-10$ & $5.22 \mathrm{E}-10$ & PEX2 & $1.30 \mathrm{E}+00$ \\
\hline ENSBTAG00000008842 & $4.39 \mathrm{E}-10$ & 3.37E-10 & $\mathrm{JPH} 1$ & $1.30 \mathrm{E}+00$ \\
\hline ENSBTAG00000032227 & 4.37E-10 & $3.34 \mathrm{E}-10$ & & $1.31 E+00$ \\
\hline ENSBTAG00000004720 & $2.01 \mathrm{E}-10$ & $1.54 \mathrm{E}-10$ & SULF1 & $1.30 \mathrm{E}+00$ \\
\hline ENSBTAG00000034498 & $1.16 \mathrm{E}-09$ & 8.87E-10 & LY6D & $1.31 E+00$ \\
\hline ENSBTAG00000020139 & 1.17E-10 & $8.82 E-11$ & & $1.32 \mathrm{E}+00$ \\
\hline ENSBTAG00000011939 & $1.67 \mathrm{E}-10$ & $1.26 \mathrm{E}-10$ & LY6H & $1.33 \mathrm{E}+00$ \\
\hline ENSBTAG00000011709 & $2.56 \mathrm{E}-10$ & $1.94 \mathrm{E}-10$ & IMPA1 & $1.32 \mathrm{E}+00$ \\
\hline ENSBTAG00000001299 & $2.53 \mathrm{E}-10$ & $1.91 \mathrm{E}-10$ & CYP7B1 & $1.33 E+00$ \\
\hline ENSBTAG00000026963 & $6.92 \mathrm{E}-10$ & $5.21 \mathrm{E}-10$ & NCALD & $1.33 E+00$ \\
\hline ENSBTAG00000003460 & $2.63 \mathrm{E}-10$ & $1.96 \mathrm{E}-10$ & TCEA1 & $1.34 \mathrm{E}+00$ \\
\hline ENSBTAG00000011271 & $2.05 \mathrm{E}-10$ & $1.53 \mathrm{E}-10$ & SNX31 & $1.34 \mathrm{E}+00$ \\
\hline ENSBTAG00000004518 & $6.88 \mathrm{E}-10$ & $5.11 \mathrm{E}-10$ & GRHL2 & $1.35 E+00$ \\
\hline ENSBTAG00000015214 & 3.37E-10 & $2.48 \mathrm{E}-10$ & CA3 & $1.36 \mathrm{E}+00$ \\
\hline ENSBTAG00000007834 & $1.90 \mathrm{E}-10$ & $1.40 \mathrm{E}-10$ & PPP1R16A & $1.36 \mathrm{E}+00$ \\
\hline ENSBTAG00000009578 & $3.54 \mathrm{E}-10$ & 2.60E-10 & PTK2 & $1.36 \mathrm{E}+00$ \\
\hline ENSBTAG00000021838 & $1.48 \mathrm{E}-10$ & $1.08 \mathrm{E}-10$ & ESRP1 & $1.37 E+00$ \\
\hline ENSBTAG00000013225 & $1.99 \mathrm{E}-10$ & $1.45 \mathrm{E}-10$ & NBN & $1.37 \mathrm{E}+00$ \\
\hline ENSBTAG00000040422 & $2.15 \mathrm{E}-10$ & $1.57 \mathrm{E}-10$ & UTP23 & $1.37 \mathrm{E}+00$ \\
\hline
\end{tabular}




\begin{tabular}{|c|c|c|c|c|}
\hline ENSBTAG000000003588 & $2.50 \mathrm{E}-10$ & $1.82 \mathrm{E}-10$ & ZFAND1 & $1.37 \mathrm{E}+00$ \\
\hline ENSBTAG000000026247 & 8.94E-10 & $6.48 \mathrm{E}-10$ & & $1.38 \mathrm{E}+00$ \\
\hline ENSBTAG000000037824 & $8.36 \mathrm{E}-10$ & $6.06 \mathrm{E}-10$ & & $1.38 \mathrm{E}+00$ \\
\hline ENSBTAG000000000879 & $2.81 \mathrm{E}-10$ & $2.02 \mathrm{E}-10$ & C8orf33 & $1.39 E+00$ \\
\hline ENSBTAG000000014642 & $1.82 \mathrm{E}-10$ & $1.31 \mathrm{E}-10$ & NAPRT & $1.39 \mathrm{E}+00$ \\
\hline ENSBTAG00000012357 & $2.64 \mathrm{E}-10$ & $1.89 \mathrm{E}-10$ & & $1.40 \mathrm{E}+00$ \\
\hline ENSBTAG000000013537 & $6.55 \mathrm{E}-10$ & 4.65E-10 & FER1L6 & $1.41 E+00$ \\
\hline ENSBTAG000000007387 & $1.99 \mathrm{E}-10$ & $1.42 \mathrm{E}-10$ & ENY2 & $1.40 \mathrm{E}+00$ \\
\hline ENSBTAG000000006770 & $2.18 \mathrm{E}-10$ & $1.54 \mathrm{E}-10$ & MTBP & $1.42 \mathrm{E}+00$ \\
\hline ENSBTAG000000008421 & $2.45 \mathrm{E}-10$ & $1.72 \mathrm{E}-10$ & SCRIB & $1.42 \mathrm{E}+00$ \\
\hline ENSBTAG000000013165 & $1.67 \mathrm{E}-10$ & 1.17E-10 & ENPP2 & $1.42 \mathrm{E}+00$ \\
\hline ENSBTAG000000012353 & $2.16 \mathrm{E}-10$ & $1.51 \mathrm{E}-10$ & ZNF34 & $1.43 E+00$ \\
\hline ENSBTAG00000036116 & $9.62 \mathrm{E}-10$ & $6.70 \mathrm{E}-10$ & & $1.44 \mathrm{E}+00$ \\
\hline ENSBTAG000000007687 & $1.99 \mathrm{E}-10$ & $1.37 \mathrm{E}-10$ & GDF6 & $1.45 \mathrm{E}+00$ \\
\hline ENSBTAG000000018206 & $2.29 \mathrm{E}-10$ & $1.58 \mathrm{E}-10$ & NIPAL2 & $1.45 E+00$ \\
\hline ENSBTAG000000003445 & $2.69 \mathrm{E}-10$ & $1.86 \mathrm{E}-10$ & ODF1 & $1.44 \mathrm{E}+00$ \\
\hline ENSBTAG000000008409 & $1.74 \mathrm{E}-10$ & 1.19E-10 & MYC & $1.46 \mathrm{E}+00$ \\
\hline ENSBTAG000000018456 & $1.56 \mathrm{E}-10$ & $1.07 \mathrm{E}-10$ & ZNF7 & $1.46 \mathrm{E}+00$ \\
\hline ENSBTAG000000008864 & $2.57 \mathrm{E}-10$ & $1.76 \mathrm{E}-10$ & LY96 & $1.46 \mathrm{E}+00$ \\
\hline ENSBTAG000000005585 & $6.66 \mathrm{E}-10$ & $4.52 \mathrm{E}-10$ & ANXA13 & $1.47 E+00$ \\
\hline ENSBTAG00000015333 & $3.69 \mathrm{E}-10$ & $2.49 \mathrm{E}-10$ & TAF2 & $1.48 \mathrm{E}+00$ \\
\hline ENSBTAG000000040321 & $1.95 \mathrm{E}-10$ & $1.31 E-10$ & & $1.49 \mathrm{E}+00$ \\
\hline ENSBTAG000000004902 & 2.69E-10 & $1.80 \mathrm{E}-10$ & INTS8 & $1.49 E+00$ \\
\hline ENSBTAG000000014032 & $1.53 \mathrm{E}-10$ & $1.03 \mathrm{E}-10$ & EIF3H & $1.49 \mathrm{E}+00$ \\
\hline ENSBTAG00000009138 & $3.78 \mathrm{E}-10$ & $2.52 \mathrm{E}-10$ & UBXN2B & $1.50 \mathrm{E}+00$ \\
\hline ENSBTAG000000047212 & $2.94 \mathrm{E}-10$ & $1.96 \mathrm{E}-10$ & & $1.50 \mathrm{E}+00$ \\
\hline ENSBTAG000000038281 & $6.04 \mathrm{E}-10$ & $4.03 \mathrm{E}-10$ & CSMD3 & $1.50 \mathrm{E}+00$ \\
\hline ENSBTAG000000015374 & $6.88 \mathrm{E}-10$ & $4.58 \mathrm{E}-10$ & COL22A1 & $1.50 \mathrm{E}+00$ \\
\hline ENSBTAG00000047532 & $3.56 \mathrm{E}-10$ & $2.36 \mathrm{E}-10$ & TRIQK & $1.51 E+00$ \\
\hline ENSBTAG000000016203 & $3.81 \mathrm{E}-10$ & $2.53 \mathrm{E}-10$ & ZNF16 & $1.51 E+00$ \\
\hline ENSBTAG000000007570 & $2.85 \mathrm{E}-10$ & $1.88 \mathrm{E}-10$ & NDUFAF6 & $1.52 \mathrm{E}+00$ \\
\hline ENSBTAG00000032982 & $3.20 \mathrm{E}-10$ & $2.11 \mathrm{E}-10$ & TERF1 & $1.52 \mathrm{E}+00$ \\
\hline ENSBTAG00000044126 & $8.18 \mathrm{E}-10$ & $5.38 \mathrm{E}-10$ & SNTB1 & $1.52 \mathrm{E}+00$ \\
\hline ENSBTAG000000021449 & $1.39 \mathrm{E}-10$ & $9.14 \mathrm{E}-11$ & DCAF13 & $1.52 E+00$ \\
\hline ENSBTAG00000017478 & $4.80 \mathrm{E}-10$ & $3.15 \mathrm{E}-10$ & GSDMC & $1.52 \mathrm{E}+00$ \\
\hline ENSBTAG00000009811 & $2.03 \mathrm{E}-10$ & $1.33 \mathrm{E}-10$ & BOP1 & $1.52 \mathrm{E}+00$ \\
\hline ENSBTAG00000005877 & $7.04 \mathrm{E}-10$ & $4.61 E-10$ & FAM83A & $1.53 \mathrm{E}+00$ \\
\hline ENSBTAG00000007186 & $1.77 \mathrm{E}-10$ & $1.16 \mathrm{E}-10$ & ARHGAP39 & $1.53 E+00$ \\
\hline ENSBTAG00000012608 & $2.34 \mathrm{E}-10$ & $1.52 \mathrm{E}-10$ & GDAP1 & $1.54 \mathrm{E}+00$ \\
\hline ENSBTAG00000011779 & $1.73 \mathrm{E}-10$ & $1.12 \mathrm{E}-10$ & MAL2 & $1.54 \mathrm{E}+00$ \\
\hline ENSBTAG00000026234 & $3.81 \mathrm{E}-10$ & $2.46 \mathrm{E}-10$ & STK3 & $1.55 E+00$ \\
\hline ENSBTAG00000001579 & $3.27 \mathrm{E}-10$ & 2.07E-10 & AGO2 & $1.58 \mathrm{E}+00$ \\
\hline ENSBTAG00000020407 & $2.88 \mathrm{E}-10$ & $1.82 \mathrm{E}-10$ & MTSS1 & $1.58 \mathrm{E}+00$ \\
\hline ENSBTAG00000021208 & $4.02 \mathrm{E}-10$ & $2.54 \mathrm{E}-10$ & RRM2B & $1.58 \mathrm{E}+00$ \\
\hline ENSBTAG00000026232 & $1.02 \mathrm{E}-10$ & $6.41 \mathrm{E}-11$ & TP53INP1 & $1.59 E+00$ \\
\hline ENSBTAG00000016716 & $9.30 \mathrm{E}-11$ & $5.85 \mathrm{E}-11$ & RRS1 & $1.59 \mathrm{E}+00$ \\
\hline ENSBTAG00000038286 & $3.66 \mathrm{E}-10$ & 2.30E-10 & & $1.59 \mathrm{E}+00$ \\
\hline ENSBTAG00000004924 & $2.61 \mathrm{E}-10$ & $1.64 \mathrm{E}-10$ & PENK & $1.59 E+00$ \\
\hline ENSBTAG00000015271 & $2.21 \mathrm{E}-10$ & $1.39 \mathrm{E}-10$ & RIPK2 & $1.59 E+00$ \\
\hline ENSBTAG00000010813 & $5.68 \mathrm{E}-10$ & 3.57E-10 & & $1.59 \mathrm{E}+00$ \\
\hline ENSBTAG00000047661 & $2.09 \mathrm{E}-10$ & $1.32 \mathrm{E}-10$ & FABP12 & $1.58 \mathrm{E}+00$ \\
\hline ENSBTAG00000040496 & $6.45 \mathrm{E}-10$ & 4.05E-10 & KCNB2 & $1.59 \mathrm{E}+00$ \\
\hline ENSBTAG00000017169 & $1.11 \mathrm{E}-09$ & $6.97 \mathrm{E}-10$ & & $1.59 \mathrm{E}+00$ \\
\hline ENSBTAG00000002448 & $6.84 \mathrm{E}-10$ & $4.21 \mathrm{E}-10$ & SNTG1 & $1.62 E+00$ \\
\hline ENSBTAG00000002002 & $2.78 \mathrm{E}-10$ & $1.72 \mathrm{E}-10$ & ATAD2 & $1.62 E+00$ \\
\hline ENSBTAG00000033902 & $2.90 \mathrm{E}-10$ & $1.76 \mathrm{E}-10$ & SLC25A32 & $1.65 E+00$ \\
\hline ENSBTAG00000007943 & $1.82 \mathrm{E}-10$ & $1.10 \mathrm{E}-10$ & AZIN1 & $1.66 \mathrm{E}+00$ \\
\hline ENSBTAG00000003606 & $3.34 \mathrm{E}-10$ & $2.01 E-10$ & & $1.66 \mathrm{E}+00$ \\
\hline ENSBTAG00000032591 & $1.06 \mathrm{E}-10$ & $6.35 \mathrm{E}-11$ & CTHRC1 & $1.67 E+00$ \\
\hline ENSBTAG00000044106 & $3.21 \mathrm{E}-10$ & $1.92 \mathrm{E}-10$ & SPIDR & $1.67 E+00$ \\
\hline ENSBTAG00000032301 & 7.67E-10 & $4.54 \mathrm{E}-10$ & SLC26A7 & $1.69 E+00$ \\
\hline ENSBTAG00000046026 & $3.11 \mathrm{E}-10$ & $1.84 \mathrm{E}-10$ & SLC39A4 & $1.69 \mathrm{E}+00$ \\
\hline ENSBTAG00000015319 & $3.44 \mathrm{E}-10$ & $2.03 \mathrm{E}-10$ & & $1.70 \mathrm{E}+00$ \\
\hline ENSBTAG00000007303 & $2.36 \mathrm{E}-10$ & $1.38 \mathrm{E}-10$ & RAD21 & $1.71 E+00$ \\
\hline ENSBTAG00000021841 & $4.96 \mathrm{E}-10$ & $2.90 \mathrm{E}-10$ & CHD7 & $1.71 \mathrm{E}+00$ \\
\hline ENSBTAG00000010529 & $1.34 \mathrm{E}-10$ & $7.86 \mathrm{E}-11$ & FZD6 & $1.71 E+00$ \\
\hline ENSBTAG00000048078 & $4.76 \mathrm{E}-10$ & $2.77 \mathrm{E}-10$ & & $1.72 \mathrm{E}+00$ \\
\hline ENSBTAG00000014357 & 4.29E-10 & $2.49 \mathrm{E}-10$ & SDC2 & $1.72 \mathrm{E}+00$ \\
\hline ENSBTAG00000013513 & $2.77 \mathrm{E}-10$ & 1.60E-10 & ATP6V1C1 & $1.73 \mathrm{E}+00$ \\
\hline ENSBTAG00000017694 & $2.51 \mathrm{E}-10$ & $1.45 \mathrm{E}-10$ & TRPS1 & $1.73 E+00$ \\
\hline
\end{tabular}




\begin{tabular}{|c|c|c|c|c|}
\hline ENSBTAG00000013955 & $6.72 \mathrm{E}-10$ & $3.86 \mathrm{E}-10$ & TRAPPC9 & $1.74 \mathrm{E}+00$ \\
\hline ENSBTAG00000011268 & $2.69 \mathrm{E}-10$ & $1.54 \mathrm{E}-10$ & TMEM64 & $1.74 \mathrm{E}+00$ \\
\hline ENSBTAG00000006132 & $3.33 \mathrm{E}-10$ & $1.88 \mathrm{E}-10$ & DENND3 & $1.77 \mathrm{E}+00$ \\
\hline ENSBTAG00000011908 & 4.25E-10 & $2.39 \mathrm{E}-10$ & $\mathrm{CPQ}$ & $1.78 \mathrm{E}+00$ \\
\hline ENSBTAG00000032881 & $5.76 \mathrm{E}-10$ & $3.24 \mathrm{E}-10$ & SLCO5A1 & $1.78 \mathrm{E}+00$ \\
\hline ENSBTAG00000007423 & $1.89 \mathrm{E}-10$ & $1.06 E-10$ & TNFRSF11B & $1.78 \mathrm{E}+00$ \\
\hline ENSBTAG00000021743 & $2.18 \mathrm{E}-10$ & $1.22 \mathrm{E}-10$ & ZNF704 & $1.78 \mathrm{E}+00$ \\
\hline ENSBTAG00000006385 & $4.94 \mathrm{E}-10$ & $2.76 \mathrm{E}-10$ & ADGRB1 & $1.79 \mathrm{E}+00$ \\
\hline ENSBTAG00000005287 & $3.16 \mathrm{E}-10$ & $1.76 \mathrm{E}-10$ & CYP7A1 & $1.79 E+00$ \\
\hline ENSBTAG00000000857 & $2.81 \mathrm{E}-10$ & $1.57 \mathrm{E}-10$ & & $1.79 \mathrm{E}+00$ \\
\hline ENSBTAG00000022588 & $5.00 \mathrm{E}-10$ & $2.70 \mathrm{E}-10$ & C8orf34 & $1.85 \mathrm{E}+00$ \\
\hline ENSBTAG00000003454 & $3.25 \mathrm{E}-10$ & $1.76 \mathrm{E}-10$ & RGS20 & $1.85 \mathrm{E}+00$ \\
\hline ENSBTAG00000016194 & 4.07E-10 & $2.19 \mathrm{E}-10$ & $\mathrm{FBXO} 32$ & $1.86 \mathrm{E}+00$ \\
\hline ENSBTAG00000014130 & $2.15 \mathrm{E}-10$ & $1.14 \mathrm{E}-10$ & $\operatorname{cox} 6 \mathrm{C}$ & $1.88 \mathrm{E}+00$ \\
\hline ENSBTAG00000016959 & $5.79 \mathrm{E}-10$ & 3.07E-10 & LAPTM4B & $1.89 \mathrm{E}+00$ \\
\hline ENSBTAG00000007335 & $2.72 \mathrm{E}-10$ & $1.41 \mathrm{E}-10$ & TPD52 & $1.93 E+00$ \\
\hline ENSBTAG00000008260 & $2.33 \mathrm{E}-10$ & $1.20 \mathrm{E}-10$ & UBE2W & $1.94 \mathrm{E}+00$ \\
\hline ENSBTAG00000021964 & $4.55 \mathrm{E}-10$ & $2.33 \mathrm{E}-10$ & $\mathrm{CDH} 17$ & $1.95 \mathrm{E}+00$ \\
\hline ENSBTAG00000015720 & $7.30 \mathrm{E}-10$ & $3.71 \mathrm{E}-10$ & WWP1 & $1.97 \mathrm{E}+00$ \\
\hline ENSBTAG00000016810 & $2.73 \mathrm{E}-10$ & $1.37 \mathrm{E}-10$ & PYCRL & $1.99 \mathrm{E}+00$ \\
\hline ENSBTAG00000020667 & $3.76 \mathrm{E}-10$ & $1.87 \mathrm{E}-10$ & KCNQ3 & $2.01 E+00$ \\
\hline ENSBTAG00000009974 & $3.55 \mathrm{E}-10$ & $1.76 \mathrm{E}-10$ & TSNARE1 & $2.02 \mathrm{E}+00$ \\
\hline ENSBTAG00000009394 & $2.85 \mathrm{E}-10$ & $1.41 \mathrm{E}-10$ & NSMCE2 & $2.02 \mathrm{E}+00$ \\
\hline ENSBTAG00000001254 & $2.35 \mathrm{E}-10$ & $1.15 \mathrm{E}-10$ & ANKRD46 & $2.04 \mathrm{E}+00$ \\
\hline ENSBTAG00000020751 & $1.95 \mathrm{E}-10$ & $9.42 \mathrm{E}-11$ & HSF1 & $2.07 \mathrm{E}+00$ \\
\hline ENSBTAG00000006877 & $4.95 \mathrm{E}-10$ & $2.38 \mathrm{E}-10$ & MMP16 & $2.08 \mathrm{E}+00$ \\
\hline ENSBTAG00000047488 & $4.03 E-10$ & $1.90 \mathrm{E}-10$ & KCNK9 & $2.12 \mathrm{E}+00$ \\
\hline ENSBTAG00000005498 & $3.54 \mathrm{E}-10$ & 1.67E-10 & SQLE & $2.12 \mathrm{E}+00$ \\
\hline ENSBTAG00000008257 & $5.96 \mathrm{E}-10$ & $2.81 \mathrm{E}-10$ & & $2.12 \mathrm{E}+00$ \\
\hline ENSBTAG00000010548 & $4.20 \mathrm{E}-10$ & $1.96 \mathrm{E}-10$ & LRRC6 & $2.14 \mathrm{E}+00$ \\
\hline ENSBTAG00000021209 & $2.16 \mathrm{E}-10$ & $1.01 \mathrm{E}-10$ & UBR5 & $2.14 \mathrm{E}+00$ \\
\hline ENSBTAG00000033446 & $1.35 \mathrm{E}-10$ & $6.23 E-11$ & TATDN1 & $2.17 \mathrm{E}+00$ \\
\hline ENSBTAG00000005560 & $4.69 \mathrm{E}-10$ & $2.11 \mathrm{E}-10$ & ST18 & $2.22 \mathrm{E}+00$ \\
\hline ENSBTAG00000014458 & $1.85 \mathrm{E}-10$ & $8.29 \mathrm{E}-11$ & $\mathrm{MROH} 1$ & $2.22 \mathrm{E}+00$ \\
\hline ENSBTAG00000004906 & $2.71 \mathrm{E}-10$ & $1.21 \mathrm{E}-10$ & CCNE2 & $2.24 \mathrm{E}+00$ \\
\hline ENSBTAG00000039968 & $7.28 \mathrm{E}-10$ & $3.16 E-10$ & TMEM55A & $2.30 \mathrm{E}+00$ \\
\hline ENSBTAG00000044190 & $2.11 \mathrm{E}-10$ & $9.09 E-11$ & & $2.32 \mathrm{E}+00$ \\
\hline ENSBTAG00000006702 & $2.43 \mathrm{E}-10$ & $1.03 \mathrm{E}-10$ & EIF3E & $2.36 \mathrm{E}+00$ \\
\hline ENSBTAG00000019639 & $5.23 \mathrm{E}-10$ & $2.21 \mathrm{E}-10$ & SNX16 & $2.37 \mathrm{E}+00$ \\
\hline ENSBTAG00000007828 & $5.16 \mathrm{E}-10$ & $2.18 \mathrm{E}-10$ & SLA & $2.37 E+00$ \\
\hline ENSBTAG00000009816 & $1.26 \mathrm{E}-10$ & $5.30 \mathrm{E}-11$ & SCX & $2.38 \mathrm{E}+00$ \\
\hline ENSBTAG00000020399 & $2.27 \mathrm{E}-10$ & $9.52 \mathrm{E}-11$ & RNF139 & $2.38 \mathrm{E}+00$ \\
\hline ENSBTAG00000033284 & $1.67 \mathrm{E}-10$ & 7.00E-11 & $\mathrm{CHCHD7}$ & $2.38 \mathrm{E}+00$ \\
\hline ENSBTAG00000045791 & $1.49 \mathrm{E}-10$ & $6.24 \mathrm{E}-11$ & ZNF623 & $2.38 \mathrm{E}+00$ \\
\hline ENSBTAG00000039068 & $2.03 \mathrm{E}-10$ & $8.52 \mathrm{E}-11$ & & $2.38 \mathrm{E}+00$ \\
\hline ENSBTAG00000016278 & $5.09 \mathrm{E}-10$ & $2.13 \mathrm{E}-10$ & RPL30 & $2.39 E+00$ \\
\hline ENSBTAG00000000158 & $9.21 \mathrm{E}-10$ & $3.85 \mathrm{E}-10$ & LY6K & $2.39 E+00$ \\
\hline ENSBTAG00000008597 & $2.48 \mathrm{E}-10$ & $1.03 \mathrm{E}-10$ & SLC45A4 & $2.40 \mathrm{E}+00$ \\
\hline ENSBTAG00000001302 & $2.97 \mathrm{E}-10$ & $1.22 \mathrm{E}-10$ & MRPS28 & $2.44 \mathrm{E}+00$ \\
\hline ENSBTAG00000017040 & $5.55 \mathrm{E}-10$ & $2.23 \mathrm{E}-10$ & LY6E & $2.49 \mathrm{E}+00$ \\
\hline ENSBTAG00000043970 & $1.68 \mathrm{E}-10$ & $6.73 \mathrm{E}-11$ & C8orf37 & $2.49 E+00$ \\
\hline ENSBTAG00000010275 & $5.42 \mathrm{E}-10$ & $2.18 \mathrm{E}-10$ & KIAA0196 & $2.49 \mathrm{E}+00$ \\
\hline ENSBTAG00000013213 & $2.04 \mathrm{E}-10$ & 8.09E-11 & & $2.52 E+00$ \\
\hline ENSBTAG00000017731 & $4.40 \mathrm{E}-10$ & $1.74 \mathrm{E}-10$ & ZFAT & $2.53 \mathrm{E}+00$ \\
\hline ENSBTAG00000023179 & $2.92 \mathrm{E}-10$ & $1.14 \mathrm{E}-10$ & TRIB1 & $2.56 \mathrm{E}+00$ \\
\hline ENSBTAG00000001174 & $1.92 \mathrm{E}-10$ & 7.44E-11 & MRPL15 & $2.58 \mathrm{E}+00$ \\
\hline ENSBTAG00000034691 & $1.69 \mathrm{E}-10$ & $6.39 \mathrm{E}-11$ & TSTA3 & $2.65 E+00$ \\
\hline ENSBTAG00000021472 & $2.02 \mathrm{E}-10$ & $7.56 E-11$ & $\mathrm{ZC} 3 \mathrm{H} 3$ & $2.67 \mathrm{E}+00$ \\
\hline ENSBTAG00000007596 & $3.43 \mathrm{E}-10$ & $1.28 \mathrm{E}-10$ & GEM & $2.68 \mathrm{E}+00$ \\
\hline ENSBTAG00000038798 & $5.86 \mathrm{E}-10$ & 2.17E-10 & & $2.70 \mathrm{E}+00$ \\
\hline ENSBTAG00000017339 & $6.35 \mathrm{E}-10$ & $2.33 \mathrm{E}-10$ & RUNX1T1 & $2.72 \mathrm{E}+00$ \\
\hline ENSBTAG00000040461 & $6.91 \mathrm{E}-10$ & $2.51 E-10$ & & $2.75 E+00$ \\
\hline ENSBTAG00000026306 & $6.10 \mathrm{E}-10$ & $2.19 \mathrm{E}-10$ & & $2.78 \mathrm{E}+00$ \\
\hline ENSBTAG00000021092 & $6.69 \mathrm{E}-10$ & $2.39 \mathrm{E}-10$ & ATP6V0D2 & $2.80 \mathrm{E}+00$ \\
\hline ENSBTAG00000032432 & $1.23 \mathrm{E}-09$ & $4.39 \mathrm{E}-10$ & & $2.80 \mathrm{E}+00$ \\
\hline ENSBTAG00000011203 & $1.61 \mathrm{E}-10$ & $5.64 \mathrm{E}-11$ & RP1 & $2.86 \mathrm{E}+00$ \\
\hline ENSBTAG00000011298 & $3.19 \mathrm{E}-10$ & $1.11 \mathrm{E}-10$ & EYA1 & $2.88 \mathrm{E}+00$ \\
\hline ENSBTAG00000017492 & $2.48 \mathrm{E}-10$ & $8.56 E-11$ & PCMTD1 & $2.90 \mathrm{E}+00$ \\
\hline ENSBTAG00000044050 & $4.93 \mathrm{E}-10$ & $1.63 \mathrm{E}-10$ & XKR4 & $3.02 \mathrm{E}+00$ \\
\hline ENSBTAG00000022114 & 4.33E-10 & $1.42 \mathrm{E}-10$ & TMEM65 & $3.05 E+00$ \\
\hline
\end{tabular}




\begin{tabular}{|c|c|c|c|c|}
\hline ENSBTAG00000012596 & $2.45 \mathrm{E}-10$ & 7.87E-11 & POP1 & $3.10 \mathrm{E}+00$ \\
\hline ENSBTAG00000004243 & $3.17 \mathrm{E}-10$ & $1.01 \mathrm{E}-10$ & & $3.14 \mathrm{E}+00$ \\
\hline ENSBTAG00000014396 & $1.19 \mathrm{E}-10$ & $3.74 \mathrm{E}-11$ & KLF10 & $3.17 E+00$ \\
\hline ENSBTAG00000008693 & $2.39 \mathrm{E}-10$ & $7.50 \mathrm{E}-11$ & EFCAB1 & $3.18 \mathrm{E}+00$ \\
\hline ENSBTAG00000026356 & $2.49 \mathrm{E}-10$ & 7.84E-11 & DGAT1 & $3.18 \mathrm{E}+00$ \\
\hline ENSBTAG00000046836 & $5.40 \mathrm{E}-10$ & $1.70 \mathrm{E}-10$ & & $3.18 \mathrm{E}+00$ \\
\hline ENSBTAG00000032233 & $8.00 \mathrm{E}-10$ & $2.51 \mathrm{E}-10$ & & $3.19 E+00$ \\
\hline ENSBTAG00000015334 & $2.84 \mathrm{E}-10$ & $8.60 \mathrm{E}-11$ & $\mathrm{ZHX1}$ & $3.30 \mathrm{E}+00$ \\
\hline ENSBTAG00000002196 & $4.37 \mathrm{E}-10$ & $1.31 \mathrm{E}-10$ & TCEB1 & $3.34 \mathrm{E}+00$ \\
\hline ENSBTAG00000019581 & $6.79 \mathrm{E}-10$ & $2.02 \mathrm{E}-10$ & PLEKHF2 & $3.36 \mathrm{E}+00$ \\
\hline ENSBTAG00000000878 & $5.34 \mathrm{E}-10$ & $1.56 \mathrm{E}-10$ & RB1CC1 & $3.42 E+00$ \\
\hline ENSBTAG00000012242 & $3.52 \mathrm{E}-10$ & $1.02 \mathrm{E}-10$ & MAF1 & $3.45 E+00$ \\
\hline ENSBTAG00000006004 & $1.18 \mathrm{E}-09$ & $3.34 \mathrm{E}-10$ & LRRC69 & $3.55 E+00$ \\
\hline ENSBTAG00000003450 & $5.08 \mathrm{E}-10$ & $1.39 \mathrm{E}-10$ & ATP6V1H & $3.66 \mathrm{E}+00$ \\
\hline ENSBTAG00000021474 & $2.29 \mathrm{E}-10$ & $6.17 \mathrm{E}-11$ & GSDMD & $3.71 E+00$ \\
\hline ENSBTAG00000006767 & $6.48 \mathrm{E}-10$ & $1.73 \mathrm{E}-10$ & MRPL13 & $3.74 \mathrm{E}+00$ \\
\hline ENSBTAG00000032738 & $6.01 \mathrm{E}-10$ & 1.57E-10 & AARD & $3.82 \mathrm{E}+00$ \\
\hline ENSBTAG00000012595 & $7.24 \mathrm{E}-10$ & $1.89 \mathrm{E}-10$ & RIDA & $3.83 E+00$ \\
\hline ENSBTAG00000007838 & $1.98 \mathrm{E}-10$ & 4.97E-11 & MFSD3 & $3.97 E+00$ \\
\hline ENSBTAG00000026340 & $8.33 \mathrm{E}-10$ & $2.10 \mathrm{E}-10$ & & $3.97 \mathrm{E}+00$ \\
\hline ENSBTAG00000009072 & $5.96 \mathrm{E}-10$ & $1.45 \mathrm{E}-10$ & ZNF572 & $4.11 E+00$ \\
\hline ENSBTAG00000010006 & $1.79 \mathrm{E}-10$ & 4.14E-11 & EMC2 & $4.32 \mathrm{E}+00$ \\
\hline ENSBTAG00000020405 & $1.22 \mathrm{E}-10$ & $2.78 \mathrm{E}-11$ & NDUFB9 & $4.36 \mathrm{E}+00$ \\
\hline ENSBTAG00000020693 & $3.48 \mathrm{E}-10$ & 7.91E-11 & DERL1 & $4.39 E+00$ \\
\hline ENSBTAG00000037493 & $1.14 \mathrm{E}-10$ & $2.53 \mathrm{E}-11$ & PUF60 & $4.48 \mathrm{E}+00$ \\
\hline ENSBTAG00000022169 & $4.64 \mathrm{E}-10$ & $1.04 \mathrm{E}-10$ & PREX2 & $4.46 \mathrm{E}+00$ \\
\hline ENSBTAG00000026283 & 4.44E-10 & $9.58 \mathrm{E}-11$ & & $4.63 E+00$ \\
\hline ENSBTAG00000046467 & $3.75 \mathrm{E}-10$ & 8.07E-11 & PTP4A3 & $4.64 E+00$ \\
\hline ENSBTAG00000019892 & $2.74 \mathrm{E}-10$ & $5.81 \mathrm{E}-11$ & HAS2 & $4.72 \mathrm{E}+00$ \\
\hline ENSBTAG00000013901 & $2.25 \mathrm{E}-10$ & $4.75 E-11$ & PTDSS1 & $4.73 E+00$ \\
\hline ENSBTAG00000005063 & $2.83 \mathrm{E}-10$ & $5.94 \mathrm{E}-11$ & THEM6 & $4.76 \mathrm{E}+00$ \\
\hline ENSBTAG00000000060 & $1.60 \mathrm{E}-10$ & $3.36 \mathrm{E}-11$ & PRDM14 & $4.76 \mathrm{E}+00$ \\
\hline ENSBTAG00000009916 & $5.81 \mathrm{E}-10$ & $1.21 \mathrm{E}-10$ & WDYHV1 & $4.79 \mathrm{E}+00$ \\
\hline ENSBTAG00000019587 & $4.33 \mathrm{E}-10$ & $8.64 \mathrm{E}-11$ & PI15 & $5.01 E+00$ \\
\hline ENSBTAG00000014610 & $4.82 \mathrm{E}-10$ & $9.56 \mathrm{E}-11$ & GPAA1 & $5.04 \mathrm{E}+00$ \\
\hline ENSBTAG00000008079 & $1.51 \mathrm{E}-10$ & $2.93 \mathrm{E}-11$ & NRBP2 & $5.15 E+00$ \\
\hline ENSBTAG00000012235 & $1.32 \mathrm{E}-10$ & $2.38 \mathrm{E}-11$ & SHARPIN & $5.55 E+00$ \\
\hline ENSBTAG00000046166 & 3.17E-10 & $5.68 \mathrm{E}-11$ & & $5.56 \mathrm{E}+00$ \\
\hline ENSBTAG00000033627 & $5.02 \mathrm{E}-10$ & $9.00 \mathrm{E}-11$ & & $5.57 E+00$ \\
\hline ENSBTAG00000022471 & $2.27 \mathrm{E}-10$ & $3.98 \mathrm{E}-11$ & TTPA & $5.69 E+00$ \\
\hline ENSBTAG00000000658 & $2.86 \mathrm{E}-10$ & $4.79 \mathrm{E}-11$ & WDR97 & $5.95 E+00$ \\
\hline ENSBTAG00000015637 & $2.52 \mathrm{E}-10$ & 3.87E-11 & IMPAD1 & $6.49 E+00$ \\
\hline ENSBTAG00000016209 & $6.83 \mathrm{E}-10$ & $8.56 \mathrm{E}-11$ & SLURP1 & $7.98 \mathrm{E}+00$ \\
\hline ENSBTAG00000047355 & $9.28 \mathrm{E}-10$ & $1.16 \mathrm{E}-10$ & & $8.00 E+00$ \\
\hline ENSBTAG00000040351 & $3.73 \mathrm{E}-10$ & $3.90 \mathrm{E}-11$ & & $9.53 \mathrm{E}+00$ \\
\hline ENSBTAG00000017343 & $9.59 \mathrm{E}-10$ & $9.94 \mathrm{E}-11$ & COLEC10 & $9.64 \mathrm{E}+00$ \\
\hline ENSBTAG00000003481 & $5.86 \mathrm{E}-10$ & $5.82 \mathrm{E}-11$ & OTUD6B & $1.01 E+01$ \\
\hline ENSBTAG00000033268 & $2.43 \mathrm{E}-10$ & $2.20 \mathrm{E}-11$ & ZFHX4 & $1.10 \mathrm{E}+01$ \\
\hline ENSBTAG00000011572 & $9.99 \mathrm{E}-10$ & $8.92 \mathrm{E}-11$ & & $1.12 \mathrm{E}+01$ \\
\hline ENSBTAG00000001522 & $1.34 \mathrm{E}-10$ & $1.16 \mathrm{E}-11$ & MTERF3 & $1.15 \mathrm{E}+01$ \\
\hline ENSBTAG00000014643 & $1.59 \mathrm{E}-10$ & $1.18 \mathrm{E}-11$ & eef1d & $1.34 \mathrm{E}+01$ \\
\hline ENSBTAG00000014026 & $6.08 \mathrm{E}-10$ & $4.04 \mathrm{E}-11$ & PAG1 & $1.50 \mathrm{E}+01$ \\
\hline ENSBTAG00000021156 & $7.80 \mathrm{E}-10$ & $5.10 \mathrm{E}-11$ & TBC1D31 & $1.53 \mathrm{E}+01$ \\
\hline ENSBTAG00000007534 & $5.92 \mathrm{E}-10$ & $1.82 \mathrm{E}-11$ & GGH & $3.24 \mathrm{E}+01$ \\
\hline ENSBTAG00000005893 & $6.63 \mathrm{E}-10$ & $8.64 \mathrm{E}-12$ & TMEM68 & $7.58 \mathrm{E}+01$ \\
\hline ENSBTAG00000020034 & $3.34 \mathrm{E}-10$ & $1.40 \mathrm{E}-12$ & LYN & $2.23 E+02$ \\
\hline ENSBTAG00000004761 & $5.26 \mathrm{E}-11$ & 0 & FOXH1 & $5.27 E+02$ \\
\hline ENSBTAG00000035158 & $1.47 \mathrm{E}-10$ & 0 & TMEM249 & $1.47 E+03$ \\
\hline ENSBTAG00000039705 & $2.71 \mathrm{E}-10$ & 0 & $\mathrm{HGH} 1$ & $2.71 E+03$ \\
\hline ENSBTAG00000012232 & $2.05 \mathrm{E}-10$ & 0 & CYC1 & $2.06 \mathrm{E}+03$ \\
\hline ENSBTAG00000040360 & $1.85 \mathrm{E}-10$ & 0 & CCDC166 & $1.85 \mathrm{E}+03$ \\
\hline ENSBTAG00000046866 & 7.77E-11 & 0 & & $7.78 \mathrm{E}+02$ \\
\hline ENSBTAG00000016210 & 3.20E-10 & 0 & LYPD2 & $3.20 E+03$ \\
\hline ENSBTAG00000047022 & $2.10 \mathrm{E}-10$ & 0 & PSCA & $2.10 E+03$ \\
\hline ENSBTAG00000013227 & $6.82 \mathrm{E}-11$ & 0 & SNAI2 & $6.83 E+02$ \\
\hline ENSBTAG00000005898 & $3.51 \mathrm{E}-10$ & 0 & TGS1 & $3.51 E+03$ \\
\hline ENSBTAG00000019147 & $1.32 \mathrm{E}-10$ & 0 & RPS20 & $1.32 \mathrm{E}+03$ \\
\hline ENSBTAG00000004022 & $2.04 \mathrm{E}-10$ & 0 & PLAG1 & $2.04 \mathrm{E}+03$ \\
\hline ENSBTAG00000039031 & 0 & 0 & & $1.00 \mathrm{E}+00$ \\
\hline ENSBTAG00000011933 & $6.79 \mathrm{E}-11$ & 0 & FAM110B & $6.80 E+02$ \\
\hline
\end{tabular}




\begin{tabular}{|c|c|c|c|c|}
\hline ENSBTAG00000005404 & 4.07E-11 & 0 & MSC & $4.08 \mathrm{E}+02$ \\
\hline ENSBTAG00000046135 & 4.87E-10 & 0 & & $4.87 E+03$ \\
\hline ENSBTAG00000001521 & $1.39 \mathrm{E}-10$ & 0 & UQCRB & $1.39 E+03$ \\
\hline ENSBTAG00000045865 & 2.73E-10 & 0 & & $2.74 \mathrm{E}+03$ \\
\hline ENSBTAG00000021639 & $6.88 \mathrm{E}-11$ & 0 & ARC & $6.89 \mathrm{E}+02$ \\
\hline ENSBTAG00000020398 & $8.95 \mathrm{E}-11$ & 0 & TRMT12 & $8.96 \mathrm{E}+02$ \\
\hline ENSBTAG00000019145 & $2.46 \mathrm{E}-10$ & 0 & MOS & $2.46 \mathrm{E}+03$ \\
\hline ENSBTAG00000047136 & 0 & 0 & & $1.00 E+00$ \\
\hline ENSBTAG00000037470 & $1.44 \mathrm{E}-10$ & 0 & & $1.44 \mathrm{E}+03$ \\
\hline ENSBTAG00000032831 & $8.33 \mathrm{E}-10$ & 0 & MRPL33 & $8.33 E+03$ \\
\hline ENSBTAG00000039499 & $1.19 \mathrm{E}-10$ & 0 & & $1.19 E+03$ \\
\hline ENSBTAG00000007768 & $1.14 \mathrm{E}-10$ & 0 & KCNS2 & $1.14 \mathrm{E}+03$ \\
\hline ENSBTAG00000039134 & 0 & $1.08 \mathrm{E}-10$ & & $9.25 \mathrm{E}-04$ \\
\hline ENSBTAG00000024952 & 0 & $1.09 \mathrm{E}-10$ & OR5AK2 & $9.17 \mathrm{E}-04$ \\
\hline ENSBTAG00000032276 & 0 & 4.39E-10 & & $2.28 \mathrm{E}-04$ \\
\hline ENSBTAG00000032280 & 0 & $6.56 \mathrm{E}-10$ & & $1.52 \mathrm{E}-04$ \\
\hline ENSBTAG00000011873 & 0 & $3.45 \mathrm{E}-10$ & KCNE3 & $2.90 \mathrm{E}-04$ \\
\hline ENSBTAG00000000434 & $1.97 \mathrm{E}-10$ & 1.09E-09 & CRYAB & $1.81 \mathrm{E}-01$ \\
\hline ENSBTAG00000031119 & $1.77 \mathrm{E}-10$ & $7.83 \mathrm{E}-10$ & OR4S1 & $2.26 \mathrm{E}-01$ \\
\hline ENSBTAG00000001516 & 4.97E-11 & $1.88 \mathrm{E}-10$ & & $2.65 \mathrm{E}-01$ \\
\hline ENSBTAG00000003930 & $4.21 \mathrm{E}-11$ & $1.59 \mathrm{E}-10$ & & $2.65 \mathrm{E}-01$ \\
\hline ENSBTAG00000000440 & $1.23 \mathrm{E}-10$ & $3.88 \mathrm{E}-10$ & & 3.17E-01 \\
\hline ENSBTAG00000013989 & $2.11 \mathrm{E}-10$ & $5.37 \mathrm{E}-10$ & C11orf63 & 3.94E-01 \\
\hline ENSBTAG00000003199 & 5.87E-11 & $1.48 \mathrm{E}-10$ & SLC35C1 & 3.97E-01 \\
\hline ENSBTAG00000048023 & $2.16 \mathrm{E}-11$ & $5.45 \mathrm{E}-11$ & & $3.98 \mathrm{E}-01$ \\
\hline ENSBTAG00000024803 & $1.99 \mathrm{E}-10$ & 4.99E-10 & ENDOD1 & $3.98 \mathrm{E}-01$ \\
\hline ENSBTAG00000031915 & $4.21 \mathrm{E}-10$ & 8.87E-10 & & 4.75E-01 \\
\hline ENSBTAG00000017228 & $5.49 \mathrm{E}-10$ & $1.15 \mathrm{E}-09$ & & 4.77E-01 \\
\hline ENSBTAG00000005620 & $2.82 \mathrm{E}-10$ & $5.66 \mathrm{E}-10$ & RPS3 & 4.99E-01 \\
\hline ENSBTAG00000011800 & $9.64 \mathrm{E}-11$ & $1.86 \mathrm{E}-10$ & $\mathrm{ABCG} 4$ & $5.18 \mathrm{E}-01$ \\
\hline ENSBTAG00000018246 & $3.06 \mathrm{E}-10$ & $5.82 \mathrm{E}-10$ & RAB39A & $5.26 \mathrm{E}-01$ \\
\hline ENSBTAG00000000368 & $1.71 \mathrm{E}-10$ & $3.24 \mathrm{E}-10$ & OR56A1 & 5.29E-01 \\
\hline ENSBTAG00000037398 & $1.63 \mathrm{E}-10$ & $3.08 \mathrm{E}-10$ & OR51T1 & $5.28 \mathrm{E}-01$ \\
\hline ENSBTAG00000040293 & $1.57 \mathrm{E}-10$ & $2.97 \mathrm{E}-10$ & RAG1 & 5.29E-01 \\
\hline ENSBTAG00000027723 & $1.83 \mathrm{E}-10$ & $3.45 \mathrm{E}-10$ & & 5.29E-01 \\
\hline ENSBTAG00000005843 & $9.14 \mathrm{E}-11$ & $1.73 \mathrm{E}-10$ & REXO2 & 5.29E-01 \\
\hline ENSBTAG00000047384 & $1.71 \mathrm{E}-10$ & $3.23 \mathrm{E}-10$ & & 5.29E-01 \\
\hline ENSBTAG00000023125 & $1.71 \mathrm{E}-10$ & $3.23 \mathrm{E}-10$ & & 5.29E-01 \\
\hline ENSBTAG00000002258 & $1.74 \mathrm{E}-10$ & 3.29E-10 & APOA1 & 5.29E-01 \\
\hline ENSBTAG00000004861 & $6.08 \mathrm{E}-10$ & $1.12 \mathrm{E}-09$ & & 5.43E-01 \\
\hline ENSBTAG00000003565 & $6.36 \mathrm{E}-11$ & $1.12 \mathrm{E}-10$ & TRIM3 & 5.69E-01 \\
\hline ENSBTAG00000012057 & 4.79E-10 & $8.40 \mathrm{E}-10$ & GIF & 5.71E-01 \\
\hline ENSBTAG00000047406 & $3.52 \mathrm{E}-10$ & $6.14 \mathrm{E}-10$ & & 5.73E-01 \\
\hline ENSBTAG00000010322 & $1.30 \mathrm{E}-10$ & $2.25 \mathrm{E}-10$ & HYOU1 & $5.78 \mathrm{E}-01$ \\
\hline ENSBTAG00000019754 & $1.52 \mathrm{E}-10$ & $2.55 E-10$ & PRKCDBP & 5.96E-01 \\
\hline ENSBTAG00000039307 & $3.73 \mathrm{E}-10$ & $5.93 \mathrm{E}-10$ & & $6.29 \mathrm{E}-01$ \\
\hline ENSBTAG00000010071 & $1.79 \mathrm{E}-10$ & $2.85 \mathrm{E}-10$ & FBXO3 & 6.30E-01 \\
\hline ENSBTAG00000005349 & $2.34 \mathrm{E}-10$ & $3.69 \mathrm{E}-10$ & & $6.35 \mathrm{E}-01$ \\
\hline ENSBTAG00000046894 & $5.38 \mathrm{E}-10$ & $8.48 \mathrm{E}-10$ & & $6.34 \mathrm{E}-01$ \\
\hline ENSBTAG00000031907 & 4.94E-10 & $7.79 \mathrm{E}-10$ & & $6.34 \mathrm{E}-01$ \\
\hline ENSBTAG00000046167 & $2.25 \mathrm{E}-10$ & $3.55 E-10$ & OR5J2 & $6.35 \mathrm{E}-01$ \\
\hline ENSBTAG00000032262 & $3.17 \mathrm{E}-10$ & $5.00 \mathrm{E}-10$ & & $6.34 \mathrm{E}-01$ \\
\hline ENSBTAG00000000351 & 4.36E-10 & $6.60 \mathrm{E}-10$ & OR52E5 & $6.61 \mathrm{E}-01$ \\
\hline ENSBTAG00000021338 & $1.21 \mathrm{E}-10$ & $1.81 \mathrm{E}-10$ & OAF & $6.70 \mathrm{E}-01$ \\
\hline ENSBTAG00000000118 & $8.29 \mathrm{E}-10$ & $1.24 \mathrm{E}-09$ & & $6.68 \mathrm{E}-01$ \\
\hline ENSBTAG00000004951 & $9.38 \mathrm{E}-11$ & $1.40 \mathrm{E}-10$ & MGC134087 & $6.70 \mathrm{E}-01$ \\
\hline ENSBTAG00000047461 & 7.40E-10 & 1.10E-09 & & $6.73 \mathrm{E}-01$ \\
\hline ENSBTAG00000032274 & $5.31 \mathrm{E}-10$ & $7.81 \mathrm{E}-10$ & OR52E4 & $6.79 \mathrm{E}-01$ \\
\hline ENSBTAG00000046228 & $4.08 \mathrm{E}-10$ & $6.00 \mathrm{E}-10$ & & $6.79 \mathrm{E}-01$ \\
\hline ENSBTAG00000015087 & $3.93 \mathrm{E}-10$ & $5.75 E-10$ & KLHL35 & $6.83 \mathrm{E}-01$ \\
\hline ENSBTAG00000039306 & $6.16 \mathrm{E}-10$ & $8.87 \mathrm{E}-10$ & & $6.94 \mathrm{E}-01$ \\
\hline ENSBTAG00000009044 & $1.29 \mathrm{E}-09$ & $1.84 \mathrm{E}-09$ & OR52N1 & 6.99E-01 \\
\hline ENSBTAG00000020748 & $2.37 \mathrm{E}-10$ & $3.37 \mathrm{E}-10$ & FAM76B & 7.03E-01 \\
\hline ENSBTAG00000035986 & $8.22 \mathrm{E}-10$ & 1.15E-09 & & 7.15E-01 \\
\hline ENSBTAG00000038536 & $1.20 \mathrm{E}-09$ & $1.66 \mathrm{E}-09$ & & 7.23E-01 \\
\hline ENSBTAG00000015119 & $1.60 \mathrm{E}-10$ & $2.17 \mathrm{E}-10$ & SIK3 & 7.37E-01 \\
\hline ENSBTAG00000020277 & $2.42 \mathrm{E}-10$ & $3.25 E-10$ & PPP2R1B & 7.44E-01 \\
\hline ENSBTAG00000004949 & $5.43 \mathrm{E}-10$ & $7.30 \mathrm{E}-10$ & FDXACB1 & 7.44E-01 \\
\hline ENSBTAG00000015821 & $5.60 \mathrm{E}-10$ & $7.51 \mathrm{E}-10$ & AMPD3 & 7.46E-01 \\
\hline ENSBTAG00000016501 & 4.44E-10 & $5.90 \mathrm{E}-10$ & ARHGAP1 & 7.52E-01 \\
\hline
\end{tabular}




\begin{tabular}{|c|c|c|c|c|}
\hline ENSBTAG00000021858 & $1.76 \mathrm{E}-10$ & $2.32 \mathrm{E}-10$ & SLC5A12 & 7.58E-01 \\
\hline ENSBTAG00000021346 & $1.86 \mathrm{E}-10$ & $2.41 \mathrm{E}-10$ & $\mathrm{CCDC} 34$ & $7.72 \mathrm{E}-01$ \\
\hline ENSBTAG00000047176 & $2.20 \mathrm{E}-09$ & $2.85 \mathrm{E}-09$ & OR56B4 & $7.71 \mathrm{E}-01$ \\
\hline ENSBTAG00000005343 & $1.90 \mathrm{E}-10$ & $2.44 \mathrm{E}-10$ & TPH1 & $7.78 \mathrm{E}-01$ \\
\hline ENSBTAG00000000954 & $2.36 \mathrm{E}-10$ & $3.00 \mathrm{E}-10$ & DCDC1 & 7.87E-01 \\
\hline ENSBTAG00000006060 & 4.25E-10 & $5.40 \mathrm{E}-10$ & & 7.87E-01 \\
\hline ENSBTAG00000016266 & $1.45 \mathrm{E}-10$ & $1.82 \mathrm{E}-10$ & & $7.95 \mathrm{E}-01$ \\
\hline ENSBTAG00000038319 & $1.76 \mathrm{E}-10$ & $2.21 \mathrm{E}-10$ & & 7.95E-01 \\
\hline ENSBTAG00000021150 & $1.69 \mathrm{E}-10$ & $2.13 \mathrm{E}-10$ & & 7.94E-01 \\
\hline ENSBTAG00000045696 & $1.27 \mathrm{E}-09$ & $1.61 \mathrm{E}-09$ & & 7.91E-01 \\
\hline ENSBTAG00000039054 & $1.71 \mathrm{E}-10$ & $2.16 \mathrm{E}-10$ & & 7.93E-01 \\
\hline ENSBTAG00000038047 & $1.25 \mathrm{E}-10$ & $1.58 \mathrm{E}-10$ & H2AFX & $7.92 \mathrm{E}-01$ \\
\hline ENSBTAG00000008220 & $3.01 \mathrm{E}-10$ & $3.79 \mathrm{E}-10$ & C15H11orf71 & 7.93E-01 \\
\hline ENSBTAG00000004130 & $2.75 \mathrm{E}-10$ & $3.46 \mathrm{E}-10$ & C15H11orf87 & $7.94 \mathrm{E}-01$ \\
\hline ENSBTAG00000024639 & $3.04 \mathrm{E}-10$ & $3.83 \mathrm{E}-10$ & & 7.93E-01 \\
\hline ENSBTAG00000032259 & 5.27E-10 & $6.64 \mathrm{E}-10$ & & $7.94 \mathrm{E}-01$ \\
\hline ENSBTAG00000005156 & $2.84 \mathrm{E}-10$ & $3.58 \mathrm{E}-10$ & PIH1D2 & 7.95E-01 \\
\hline ENSBTAG00000038598 & $4.30 \mathrm{E}-10$ & $5.42 \mathrm{E}-10$ & & 7.93E-01 \\
\hline ENSBTAG00000036326 & $6.82 \mathrm{E}-10$ & $8.59 \mathrm{E}-10$ & & $7.94 \mathrm{E}-01$ \\
\hline ENSBTAG00000046853 & $8.72 \mathrm{E}-11$ & 1.10E-10 & & 7.93E-01 \\
\hline ENSBTAG00000047425 & $8.59 \mathrm{E}-10$ & $1.08 \mathrm{E}-09$ & & 7.96E-01 \\
\hline ENSBTAG00000046471 & 8.67E-11 & $1.09 \mathrm{E}-10$ & & 7.95E-01 \\
\hline ENSBTAG00000008075 & $8.91 \mathrm{E}-11$ & $1.12 \mathrm{E}-10$ & TRAPPC4 & 7.96E-01 \\
\hline ENSBTAG00000047178 & $3.18 \mathrm{E}-10$ & $4.01 \mathrm{E}-10$ & & 7.94E-01 \\
\hline ENSBTAG00000046354 & $8.21 \mathrm{E}-11$ & $1.03 \mathrm{E}-10$ & & 7.97E-01 \\
\hline ENSBTAG00000046378 & $1.69 \mathrm{E}-10$ & 2.13E-10 & & $7.93 \mathrm{E}-01$ \\
\hline ENSBTAG00000035485 & $8.61 \mathrm{E}-11$ & $1.09 \mathrm{E}-10$ & & 7.90E-01 \\
\hline ENSBTAG00000003567 & $1.89 \mathrm{E}-10$ & $2.38 \mathrm{E}-10$ & TIMM10B & 7.95E-01 \\
\hline ENSBTAG00000005087 & $8.25 \mathrm{E}-11$ & $1.04 \mathrm{E}-10$ & & 7.93E-01 \\
\hline ENSBTAG00000002216 & $1.86 \mathrm{E}-10$ & $2.34 \mathrm{E}-10$ & MYOD1 & 7.94E-01 \\
\hline ENSBTAG00000047246 & $2.60 \mathrm{E}-10$ & 3.27E-10 & & $7.94 \mathrm{E}-01$ \\
\hline ENSBTAG00000046913 & $4.35 \mathrm{E}-10$ & $5.48 \mathrm{E}-10$ & & 7.93E-01 \\
\hline ENSBTAG00000048003 & $1.06 \mathrm{E}-09$ & $1.34 \mathrm{E}-09$ & & 7.91E-01 \\
\hline ENSBTAG00000037474 & $8.76 \mathrm{E}-10$ & 1.10E-09 & & 7.96E-01 \\
\hline ENSBTAG00000045790 & $1.31 \mathrm{E}-10$ & $1.66 \mathrm{E}-10$ & & 7.92E-01 \\
\hline ENSBTAG00000048223 & 8.64E-11 & $1.09 \mathrm{E}-10$ & & 7.93E-01 \\
\hline ENSBTAG00000013503 & $5.22 \mathrm{E}-10$ & $6.58 \mathrm{E}-10$ & & 7.94E-01 \\
\hline ENSBTAG00000020952 & $1.74 \mathrm{E}-10$ & $2.19 \mathrm{E}-10$ & & 7.95E-01 \\
\hline ENSBTAG00000005228 & 5.07E-11 & $6.39 \mathrm{E}-11$ & CNGA4 & $7.94 \mathrm{E}-01$ \\
\hline ENSBTAG00000032255 & $5.12 \mathrm{E}-10$ & $6.45 \mathrm{E}-10$ & & $7.94 \mathrm{E}-01$ \\
\hline ENSBTAG00000035985 & $1.71 \mathrm{E}-10$ & $2.15 \mathrm{E}-10$ & & $7.94 \mathrm{E}-01$ \\
\hline ENSBTAG00000003566 & $1.12 \mathrm{E}-10$ & $1.41 \mathrm{E}-10$ & ARFIP2 & 7.95E-01 \\
\hline ENSBTAG00000039448 & $3.53 \mathrm{E}-10$ & $4.45 \mathrm{E}-10$ & & 7.94E-01 \\
\hline ENSBTAG00000001301 & $1.27 \mathrm{E}-10$ & $1.60 \mathrm{E}-10$ & LRRC32 & 7.94E-01 \\
\hline ENSBTAG00000002000 & $1.65 \mathrm{E}-10$ & $2.08 \mathrm{E}-10$ & TIMM8B & 7.94E-01 \\
\hline ENSBTAG00000016140 & $4.63 \mathrm{E}-10$ & $5.78 \mathrm{E}-10$ & PPFIBP2 & $8.01 \mathrm{E}-01$ \\
\hline ENSBTAG00000018213 & $1.98 \mathrm{E}-10$ & $2.48 \mathrm{E}-10$ & TBCEL & 8.00E-01 \\
\hline ENSBTAG00000008134 & $1.80 \mathrm{E}-10$ & $2.24 \mathrm{E}-10$ & BDNF & $8.05 \mathrm{E}-01$ \\
\hline ENSBTAG00000005193 & $4.69 \mathrm{E}-10$ & $5.80 \mathrm{E}-10$ & CRTAM & 8.09E-01 \\
\hline ENSBTAG00000020890 & $6.19 \mathrm{E}-10$ & 7.62E-10 & TRIM66 & $8.12 \mathrm{E}-01$ \\
\hline ENSBTAG00000027722 & $1.34 \mathrm{E}-10$ & $1.63 \mathrm{E}-10$ & IPO7 & 8.19E-01 \\
\hline ENSBTAG00000006564 & $4.91 \mathrm{E}-10$ & $5.95 \mathrm{E}-10$ & & 8.26E-01 \\
\hline ENSBTAG00000031309 & $2.22 \mathrm{E}-10$ & $2.66 \mathrm{E}-10$ & RAG2 & $8.33 \mathrm{E}-01$ \\
\hline ENSBTAG00000048141 & $1.13 \mathrm{E}-09$ & $1.36 \mathrm{E}-09$ & & $8.29 \mathrm{E}-01$ \\
\hline ENSBTAG00000020272 & $3.02 \mathrm{E}-10$ & $3.60 \mathrm{E}-10$ & SIK2 & $8.40 \mathrm{E}-01$ \\
\hline ENSBTAG00000008661 & $4.58 \mathrm{E}-10$ & $5.45 \mathrm{E}-10$ & C11orf52 & $8.41 \mathrm{E}-01$ \\
\hline ENSBTAG00000001999 & $2.66 \mathrm{E}-10$ & $3.16 \mathrm{E}-10$ & C11orf57 & 8.43E-01 \\
\hline ENSBTAG00000018483 & $3.09 \mathrm{E}-10$ & $3.66 \mathrm{E}-10$ & NDUFS3 & 8.44E-01 \\
\hline ENSBTAG00000015876 & $1.25 \mathrm{E}-09$ & 1.47E-09 & & 8.53E-01 \\
\hline ENSBTAG00000038650 & 1.11E-09 & 1.30E-09 & MS4A7 & $8.51 \mathrm{E}-01$ \\
\hline ENSBTAG00000047088 & 7.97E-10 & $9.31 \mathrm{E}-10$ & & 8.56E-01 \\
\hline ENSBTAG00000027126 & $4.79 \mathrm{E}-10$ & $5.56 \mathrm{E}-10$ & MUC15 & $8.61 \mathrm{E}-01$ \\
\hline ENSBTAG00000047129 & 1.75E-09 & 2.02E-09 & B3GNT6 & 8.67E-01 \\
\hline ENSBTAG00000046636 & $2.89 \mathrm{E}-10$ & $3.33 \mathrm{E}-10$ & & 8.69E-01 \\
\hline ENSBTAG00000039917 & $9.70 \mathrm{E}-10$ & 1.11E-09 & OR5B3 & $8.74 \mathrm{E}-01$ \\
\hline ENSBTAG00000046618 & $9.83 \mathrm{E}-10$ & 1.13E-09 & & $8.70 \mathrm{E}-01$ \\
\hline ENSBTAG00000008227 & $7.51 \mathrm{E}-10$ & $8.60 \mathrm{E}-10$ & LIPT2 & 8.73E-01 \\
\hline ENSBTAG00000021125 & 3.34E-10 & $3.81 \mathrm{E}-10$ & NUP160 & $8.76 \mathrm{E}-01$ \\
\hline ENSBTAG00000048290 & $1.89 \mathrm{E}-10$ & $2.16 \mathrm{E}-10$ & & $8.75 \mathrm{E}-01$ \\
\hline ENSBTAG00000046149 & $1.26 \mathrm{E}-09$ & 1.44E-09 & & $8.74 \mathrm{E}-01$ \\
\hline
\end{tabular}




\begin{tabular}{|c|c|c|c|c|}
\hline ENSBTAG00000003361 & $1.01 \mathrm{E}-10$ & $1.15 \mathrm{E}-10$ & METTL15 & $8.78 \mathrm{E}-01$ \\
\hline ENSBTAG00000016557 & 5.99E-10 & $6.81 \mathrm{E}-10$ & MTMR2 & 8.80E-01 \\
\hline ENSBTAG00000016320 & $4.44 \mathrm{E}-10$ & $5.04 \mathrm{E}-10$ & LMO1 & $8.81 \mathrm{E}-01$ \\
\hline ENSBTAG00000006835 & $1.06 \mathrm{E}-10$ & $1.20 \mathrm{E}-10$ & MCAM & $8.82 \mathrm{E}-01$ \\
\hline ENSBTAG00000001703 & $6.80 \mathrm{E}-10$ & $7.71 \mathrm{E}-10$ & SYT13 & 8.83E-01 \\
\hline ENSBTAG00000010820 & $1.59 \mathrm{E}-10$ & $1.79 \mathrm{E}-10$ & WNT11 & 8.86E-01 \\
\hline ENSBTAG00000032200 & $2.85 \mathrm{E}-10$ & $3.22 \mathrm{E}-10$ & DNAJC24 & $8.85 \mathrm{E}-01$ \\
\hline ENSBTAG00000010008 & $4.15 \mathrm{E}-10$ & $4.68 \mathrm{E}-10$ & TTC12 & $8.88 \mathrm{E}-01$ \\
\hline ENSBTAG00000015824 & $3.03 E-10$ & $3.39 \mathrm{E}-10$ & RNF141 & 8.93E-01 \\
\hline ENSBTAG00000018482 & $1.97 \mathrm{E}-10$ & $2.20 \mathrm{E}-10$ & KBTBD4 & 8.95E-01 \\
\hline ENSBTAG00000019544 & $7.88 \mathrm{E}-10$ & $8.81 \mathrm{E}-10$ & & 8.94E-01 \\
\hline ENSBTAG00000038977 & $7.78 \mathrm{E}-10$ & $8.70 \mathrm{E}-10$ & & 8.94E-01 \\
\hline ENSBTAG00000020466 & $6.29 \mathrm{E}-10$ & $7.00 \mathrm{E}-10$ & & $8.98 \mathrm{E}-01$ \\
\hline ENSBTAG00000001460 & $2.06 \mathrm{E}-10$ & $2.28 \mathrm{E}-10$ & LIN7C & $9.01 \mathrm{E}-01$ \\
\hline ENSBTAG00000037603 & $2.20 \mathrm{E}-09$ & $2.43 \mathrm{E}-09$ & & $9.04 \mathrm{E}-01$ \\
\hline ENSBTAG00000038479 & $8.41 \mathrm{E}-10$ & $9.27 \mathrm{E}-10$ & & 9.07E-01 \\
\hline ENSBTAG00000040252 & $6.97 \mathrm{E}-10$ & $7.68 \mathrm{E}-10$ & & $9.08 \mathrm{E}-01$ \\
\hline ENSBTAG00000005255 & $1.40 \mathrm{E}-09$ & $1.54 \mathrm{E}-09$ & & $9.09 \mathrm{E}-01$ \\
\hline ENSBTAG00000037580 & $5.85 \mathrm{E}-10$ & $6.41 E-10$ & MSANTD3 & $9.12 \mathrm{E}-01$ \\
\hline ENSBTAG00000047356 & $1.32 \mathrm{E}-09$ & $1.45 \mathrm{E}-09$ & & $9.11 \mathrm{E}-01$ \\
\hline ENSBTAG00000021611 & $3.51 \mathrm{E}-10$ & $3.85 \mathrm{E}-10$ & C11orf74 & $9.12 \mathrm{E}-01$ \\
\hline ENSBTAG00000017647 & $9.98 \mathrm{E}-10$ & 1.09E-09 & & $9.16 \mathrm{E}-01$ \\
\hline ENSBTAG00000047960 & $1.26 \mathrm{E}-09$ & $1.37 \mathrm{E}-09$ & & $9.20 \mathrm{E}-01$ \\
\hline ENSBTAG00000008253 & $6.32 \mathrm{E}-10$ & $6.88 \mathrm{E}-10$ & EXPH5 & $9.19 \mathrm{E}-01$ \\
\hline ENSBTAG00000020066 & $4.09 \mathrm{E}-10$ & $4.45 \mathrm{E}-10$ & ZW10 & 9.19E-01 \\
\hline ENSBTAG00000037921 & 4.34E-10 & $4.71 \mathrm{E}-10$ & $\mathrm{OVCH} 2$ & $9.22 \mathrm{E}-01$ \\
\hline ENSBTAG00000005424 & $3.75 \mathrm{E}-10$ & $4.06 \mathrm{E}-10$ & $\mathrm{KCNC1}$ & $9.24 \mathrm{E}-01$ \\
\hline ENSBTAG00000006556 & $4.21 \mathrm{E}-10$ & $4.55 E-10$ & COPB1 & 9.25E-01 \\
\hline ENSBTAG00000023511 & $6.02 \mathrm{E}-10$ & $6.50 \mathrm{E}-10$ & & $9.26 \mathrm{E}-01$ \\
\hline ENSBTAG00000038452 & $6.16 \mathrm{E}-10$ & $6.64 \mathrm{E}-10$ & & $9.27 \mathrm{E}-01$ \\
\hline ENSBTAG00000019045 & $2.65 \mathrm{E}-10$ & $2.86 \mathrm{E}-10$ & COMMD9 & $9.25 \mathrm{E}-01$ \\
\hline ENSBTAG00000010826 & $7.98 \mathrm{E}-11$ & $8.60 E-11$ & EMSY & $9.28 \mathrm{E}-01$ \\
\hline ENSBTAG00000035487 & $1.83 \mathrm{E}-09$ & 1.97E-09 & & 9.30E-01 \\
\hline ENSBTAG00000027557 & $4.07 \mathrm{E}-10$ & $4.37 \mathrm{E}-10$ & ZNF408 & $9.32 \mathrm{E}-01$ \\
\hline ENSBTAG00000019309 & 7.47E-11 & $8.02 \mathrm{E}-11$ & & $9.32 \mathrm{E}-01$ \\
\hline ENSBTAG00000006581 & $5.23 \mathrm{E}-10$ & $5.61 E-10$ & CCDC82 & 9.33E-01 \\
\hline ENSBTAG00000007971 & $1.13 \mathrm{E}-09$ & $1.20 \mathrm{E}-09$ & OR6Q1 & 9.39E-01 \\
\hline ENSBTAG00000020096 & $2.15 \mathrm{E}-10$ & $2.31 \mathrm{E}-10$ & MS4A2 & 9.33E-01 \\
\hline ENSBTAG00000001987 & $4.59 \mathrm{E}-10$ & $4.90 \mathrm{E}-10$ & SWAP70 & $9.36 \mathrm{E}-01$ \\
\hline ENSBTAG00000010642 & $3.39 \mathrm{E}-10$ & $3.61 E-10$ & ALKBH8 & 9.39E-01 \\
\hline ENSBTAG00000003692 & $8.29 E-10$ & $8.83 \mathrm{E}-10$ & UCP2 & 9.39E-01 \\
\hline ENSBTAG00000015357 & $9.93 \mathrm{E}-10$ & $1.06 \mathrm{E}-09$ & OR52B4 & 9.37E-01 \\
\hline ENSBTAG00000002071 & $1.15 E-09$ & $1.23 \mathrm{E}-09$ & & 9.37E-01 \\
\hline ENSBTAG00000013366 & $1.89 \mathrm{E}-10$ & $2.01 \mathrm{E}-10$ & NRIP3 & $9.41 E-01$ \\
\hline ENSBTAG00000040567 & $5.37 \mathrm{E}-10$ & $5.71 \mathrm{E}-10$ & MMP27 & $9.40 \mathrm{E}-01$ \\
\hline ENSBTAG00000000697 & 7.17E-10 & 7.57E-10 & RRP8 & $9.47 \mathrm{E}-01$ \\
\hline ENSBTAG00000016142 & $3.87 \mathrm{E}-10$ & $4.08 \mathrm{E}-10$ & CYB5R2 & $9.49 \mathrm{E}-01$ \\
\hline ENSBTAG00000038787 & $5.24 \mathrm{E}-10$ & $5.50 \mathrm{E}-10$ & & $9.52 \mathrm{E}-01$ \\
\hline ENSBTAG00000045723 & $5.24 \mathrm{E}-10$ & $5.50 \mathrm{E}-10$ & & $9.52 \mathrm{E}-01$ \\
\hline ENSBTAG00000046278 & $5.24 \mathrm{E}-10$ & $5.50 \mathrm{E}-10$ & & $9.52 \mathrm{E}-01$ \\
\hline ENSBTAG00000002691 & 4.64E-10 & $4.88 \mathrm{E}-10$ & ELMOD1 & $9.51 \mathrm{E}-01$ \\
\hline ENSBTAG00000024788 & $5.43 \mathrm{E}-10$ & $5.70 \mathrm{E}-10$ & & $9.52 \mathrm{E}-01$ \\
\hline ENSBTAG00000006676 & $2.65 \mathrm{E}-10$ & $2.78 \mathrm{E}-10$ & FIBIN & $9.53 \mathrm{E}-01$ \\
\hline ENSBTAG00000047472 & $5.08 \mathrm{E}-10$ & $5.32 \mathrm{E}-10$ & OR10V1 & $9.54 \mathrm{E}-01$ \\
\hline ENSBTAG00000045612 & 5.17E-10 & $5.43 \mathrm{E}-10$ & & $9.52 \mathrm{E}-01$ \\
\hline ENSBTAG00000035489 & $3.70 \mathrm{E}-10$ & $3.88 \mathrm{E}-10$ & & $9.53 \mathrm{E}-01$ \\
\hline ENSBTAG00000038679 & $1.58 \mathrm{E}-09$ & $1.65 \mathrm{E}-09$ & & $9.56 \mathrm{E}-01$ \\
\hline ENSBTAG00000000886 & $6.15 \mathrm{E}-10$ & $6.40 \mathrm{E}-10$ & & $9.60 \mathrm{E}-01$ \\
\hline ENSBTAG00000023648 & $1.94 \mathrm{E}-09$ & $2.02 \mathrm{E}-09$ & & $9.60 \mathrm{E}-01$ \\
\hline ENSBTAG00000009419 & $4.92 \mathrm{E}-10$ & $5.08 \mathrm{E}-10$ & TRIM29 & $9.68 \mathrm{E}-01$ \\
\hline ENSBTAG00000019080 & $3.56 \mathrm{E}-10$ & $3.67 \mathrm{E}-10$ & PTH & $9.70 \mathrm{E}-01$ \\
\hline ENSBTAG00000007129 & 4.14E-10 & $4.26 \mathrm{E}-10$ & MRVI1 & $9.71 \mathrm{E}-01$ \\
\hline ENSBTAG00000034711 & $9.52 \mathrm{E}-10$ & $9.80 \mathrm{E}-10$ & & $9.71 \mathrm{E}-01$ \\
\hline ENSBTAG00000003624 & $7.23 \mathrm{E}-10$ & $7.39 \mathrm{E}-10$ & CNTF & $9.78 \mathrm{E}-01$ \\
\hline ENSBTAG00000040285 & $5.97 \mathrm{E}-10$ & $6.09 \mathrm{E}-10$ & & $9.80 \mathrm{E}-01$ \\
\hline ENSBTAG00000024648 & $5.36 \mathrm{E}-10$ & $5.47 \mathrm{E}-10$ & & $9.80 \mathrm{E}-01$ \\
\hline ENSBTAG00000033519 & $1.21 \mathrm{E}-09$ & $1.24 \mathrm{E}-09$ & & $9.80 \mathrm{E}-01$ \\
\hline ENSBTAG00000004423 & $5.38 \mathrm{E}-10$ & $5.48 \mathrm{E}-10$ & ARHGAP42 & $9.81 \mathrm{E}-01$ \\
\hline ENSBTAG00000046732 & $1.20 \mathrm{E}-09$ & $1.23 \mathrm{E}-09$ & MMP8 & $9.78 \mathrm{E}-01$ \\
\hline ENSBTAG00000005308 & $2.20 \mathrm{E}-10$ & $2.23 \mathrm{E}-10$ & ANGPTL5 & 9.87E-01 \\
\hline
\end{tabular}




\begin{tabular}{|c|c|c|c|c|}
\hline ENSBTAG00000016747 & $6.40 \mathrm{E}-10$ & $6.48 \mathrm{E}-10$ & NAT10 & $9.88 \mathrm{E}-01$ \\
\hline ENSBTAG00000012750 & $2.18 \mathrm{E}-10$ & $2.20 \mathrm{E}-10$ & UPK2 & $9.93 \mathrm{E}-01$ \\
\hline ENSBTAG00000008429 & $2.74 \mathrm{E}-10$ & $2.76 \mathrm{E}-10$ & LRP4 & 9.92E-01 \\
\hline ENSBTAG00000048032 & $4.40 \mathrm{E}-10$ & 4.44E-10 & & $9.92 \mathrm{E}-01$ \\
\hline ENSBTAG00000011227 & $3.67 \mathrm{E}-10$ & $3.69 \mathrm{E}-10$ & TRPC6 & 9.94E-01 \\
\hline ENSBTAG00000031032 & 8.87E-10 & $8.93 \mathrm{E}-10$ & & 9.94E-01 \\
\hline ENSBTAG00000002887 & $3.98 \mathrm{E}-10$ & $4.01 \mathrm{E}-10$ & DDI1 & $9.93 \mathrm{E}-01$ \\
\hline ENSBTAG00000016548 & $5.47 \mathrm{E}-10$ & $5.51 \mathrm{E}-10$ & TEX12 & 9.93E-01 \\
\hline ENSBTAG00000037666 & $9.17 \mathrm{E}-10$ & $9.23 \mathrm{E}-10$ & & 9.94E-01 \\
\hline ENSBTAG00000005874 & $4.29 \mathrm{E}-10$ & $4.32 \mathrm{E}-10$ & & 9.94E-01 \\
\hline ENSBTAG00000039300 & $4.32 \mathrm{E}-10$ & $4.36 \mathrm{E}-10$ & OR5B2 & $9.92 \mathrm{E}-01$ \\
\hline ENSBTAG00000009440 & $4.96 \mathrm{E}-10$ & $5.00 \mathrm{E}-10$ & & $9.93 \mathrm{E}-01$ \\
\hline ENSBTAG00000036131 & $8.57 \mathrm{E}-10$ & $8.62 \mathrm{E}-10$ & & $9.94 \mathrm{E}-01$ \\
\hline ENSBTAG00000006610 & $4.15 \mathrm{E}-10$ & $4.16 \mathrm{E}-10$ & XRRA1 & $9.98 \mathrm{E}-01$ \\
\hline ENSBTAG00000031210 & $3.29 \mathrm{E}-10$ & $3.29 \mathrm{E}-10$ & & $1.00 \mathrm{E}+00$ \\
\hline ENSBTAG00000021855 & 3.77E-10 & 3.77E-10 & ANO3 & $9.99 \mathrm{E}-01$ \\
\hline ENSBTAG00000014910 & 8.19E-10 & 8.17E-10 & PIWIL4 & $1.00 \mathrm{E}+00$ \\
\hline ENSBTAG00000002450 & $8.06 \mathrm{E}-10$ & $7.96 \mathrm{E}-10$ & FAM111A & $1.01 E+00$ \\
\hline ENSBTAG00000036009 & $1.63 \mathrm{E}-10$ & $1.61 \mathrm{E}-10$ & TRAF6 & $1.01 E+00$ \\
\hline ENSBTAG00000008310 & $1.40 \mathrm{E}-10$ & $1.38 \mathrm{E}-10$ & TMEM9B & $1.02 \mathrm{E}+00$ \\
\hline ENSBTAG00000021484 & $4.26 \mathrm{E}-10$ & 4.20E-10 & TSPAN18 & $1.01 E+00$ \\
\hline ENSBTAG00000014399 & $5.38 \mathrm{E}-10$ & $5.28 \mathrm{E}-10$ & QSER1 & $1.02 \mathrm{E}+00$ \\
\hline ENSBTAG00000008312 & $1.32 \mathrm{E}-09$ & $1.29 \mathrm{E}-09$ & & $1.02 \mathrm{E}+00$ \\
\hline ENSBTAG00000007740 & $3.76 \mathrm{E}-10$ & $3.68 \mathrm{E}-10$ & MDK & $1.02 E+00$ \\
\hline ENSBTAG00000037505 & $7.90 \mathrm{E}-10$ & $7.73 \mathrm{E}-10$ & & $1.02 \mathrm{E}+00$ \\
\hline ENSBTAG00000004448 & $2.34 \mathrm{E}-10$ & $2.29 \mathrm{E}-10$ & CKAP5 & $1.02 \mathrm{E}+00$ \\
\hline ENSBTAG00000020279 & 4.07E-10 & 3.97E-10 & & $1.03 E+00$ \\
\hline ENSBTAG00000044144 & $6.47 \mathrm{E}-10$ & $6.30 \mathrm{E}-10$ & GUCY1A2 & $1.03 E+00$ \\
\hline ENSBTAG00000005710 & $3.22 \mathrm{E}-10$ & $3.13 \mathrm{E}-10$ & NCAM1 & $1.03 E+00$ \\
\hline ENSBTAG00000035735 & $1.49 \mathrm{E}-10$ & $1.44 \mathrm{E}-10$ & BIRC2 & $1.03 E+00$ \\
\hline ENSBTAG00000010089 & $1.94 \mathrm{E}-10$ & $1.87 \mathrm{E}-10$ & & $1.03 E+00$ \\
\hline ENSBTAG00000039893 & $2.30 \mathrm{E}-09$ & $2.22 \mathrm{E}-09$ & OR9I1 & $1.04 \mathrm{E}+00$ \\
\hline ENSBTAG00000004862 & $2.96 \mathrm{E}-10$ & $2.85 \mathrm{E}-10$ & TUB & $1.04 E+00$ \\
\hline ENSBTAG00000046380 & 1.51E-09 & $1.46 \mathrm{E}-09$ & & $1.04 \mathrm{E}+00$ \\
\hline ENSBTAG00000048300 & 1.15E-09 & $1.11 \mathrm{E}-09$ & & $1.03 E+00$ \\
\hline ENSBTAG00000021809 & $3.89 \mathrm{E}-10$ & $3.74 \mathrm{E}-10$ & DEPDC7 & $1.04 \mathrm{E}+00$ \\
\hline ENSBTAG00000006644 & $8.87 \mathrm{E}-10$ & $8.53 \mathrm{E}-10$ & SPCS2 & $1.04 \mathrm{E}+00$ \\
\hline ENSBTAG00000018742 & $2.79 \mathrm{E}-10$ & 2.69E-10 & $\mathrm{MTCH} 2$ & $1.04 E+00$ \\
\hline ENSBTAG00000019627 & $5.30 \mathrm{E}-10$ & $5.09 \mathrm{E}-10$ & THY1 & $1.04 \mathrm{E}+00$ \\
\hline ENSBTAG00000047268 & $3.61 \mathrm{E}-10$ & $3.47 \mathrm{E}-10$ & WT1 & $1.04 \mathrm{E}+00$ \\
\hline ENSBTAG00000017932 & $2.56 \mathrm{E}-10$ & $2.45 \mathrm{E}-10$ & CCDC84 & $1.05 E+00$ \\
\hline ENSBTAG00000014847 & $4.31 \mathrm{E}-10$ & $4.11 \mathrm{E}-10$ & BBOX1 & $1.05 E+00$ \\
\hline ENSBTAG00000006443 & $3.17 \mathrm{E}-10$ & $3.01 \mathrm{E}-10$ & & $1.05 E+00$ \\
\hline ENSBTAG00000009611 & $1.64 \mathrm{E}-10$ & $1.55 \mathrm{E}-10$ & PHF21A & $1.06 \mathrm{E}+00$ \\
\hline ENSBTAG00000027772 & $1.91 \mathrm{E}-10$ & $1.81 \mathrm{E}-10$ & & $1.06 \mathrm{E}+00$ \\
\hline ENSBTAG00000003564 & $6.84 \mathrm{E}-11$ & $6.46 \mathrm{E}-11$ & HPX & $1.06 \mathrm{E}+00$ \\
\hline ENSBTAG00000038245 & 3.39E-10 & 3.20E-10 & & $1.06 \mathrm{E}+00$ \\
\hline ENSBTAG00000022858 & $3.43 \mathrm{E}-10$ & 3.24E-10 & & $1.06 \mathrm{E}+00$ \\
\hline ENSBTAG00000036328 & $3.41 \mathrm{E}-10$ & $3.22 \mathrm{E}-10$ & & $1.06 \mathrm{E}+00$ \\
\hline ENSBTAG00000046387 & 1.51E-09 & $1.42 \mathrm{E}-09$ & & $1.06 \mathrm{E}+00$ \\
\hline ENSBTAG00000047344 & $6.88 \mathrm{E}-10$ & $6.50 \mathrm{E}-10$ & & $1.06 \mathrm{E}+00$ \\
\hline ENSBTAG00000032224 & $6.88 \mathrm{E}-10$ & $6.50 \mathrm{E}-10$ & & $1.06 \mathrm{E}+00$ \\
\hline ENSBTAG00000016684 & $6.43 \mathrm{E}-10$ & 6.07E-10 & ZNF202 & $1.06 \mathrm{E}+00$ \\
\hline ENSBTAG00000045658 & $3.50 \mathrm{E}-10$ & $3.31 \mathrm{E}-10$ & & $1.06 \mathrm{E}+00$ \\
\hline ENSBTAG00000027430 & $6.67 \mathrm{E}-10$ & $6.30 \mathrm{E}-10$ & & $1.06 \mathrm{E}+00$ \\
\hline ENSBTAG00000047714 & $6.95 \mathrm{E}-10$ & $6.56 \mathrm{E}-10$ & OR51A7 & $1.06 \mathrm{E}+00$ \\
\hline ENSBTAG00000030909 & $1.46 \mathrm{E}-09$ & $1.38 \mathrm{E}-09$ & & $1.06 \mathrm{E}+00$ \\
\hline ENSBTAG00000000025 & $5.72 \mathrm{E}-10$ & $5.35 \mathrm{E}-10$ & & $1.07 E+00$ \\
\hline ENSBTAG00000004594 & $2.50 \mathrm{E}-10$ & $2.34 \mathrm{E}-10$ & MMP7 & $1.07 \mathrm{E}+00$ \\
\hline ENSBTAG00000046320 & $5.49 \mathrm{E}-10$ & $5.13 \mathrm{E}-10$ & CXCR5 & $1.07 \mathrm{E}+00$ \\
\hline ENSBTAG00000005215 & $4.88 \mathrm{E}-10$ & 4.55E-10 & IL10RA & $1.07 E+00$ \\
\hline ENSBTAG00000005034 & $9.67 \mathrm{E}-10$ & $9.01 E-10$ & CAPN5 & $1.07 \mathrm{E}+00$ \\
\hline ENSBTAG00000000628 & $8.18 \mathrm{E}-10$ & $7.60 \mathrm{E}-10$ & & $1.08 \mathrm{E}+00$ \\
\hline ENSBTAG00000012628 & $1.92 \mathrm{E}-10$ & $1.77 \mathrm{E}-10$ & SLC1A2 & $1.08 \mathrm{E}+00$ \\
\hline ENSBTAG00000000317 & $3.06 \mathrm{E}-10$ & $2.83 \mathrm{E}-10$ & MAPK8IP1 & $1.08 \mathrm{E}+00$ \\
\hline ENSBTAG00000011578 & $3.40 \mathrm{E}-10$ & $3.14 \mathrm{E}-10$ & $\mathrm{CD} 44$ & $1.08 \mathrm{E}+00$ \\
\hline ENSBTAG00000021343 & $3.01 \mathrm{E}-10$ & $2.78 \mathrm{E}-10$ & ARHGEF12 & $1.08 \mathrm{E}+00$ \\
\hline ENSBTAG00000016074 & $1.39 \mathrm{E}-10$ & $1.28 \mathrm{E}-10$ & & $1.08 \mathrm{E}+00$ \\
\hline ENSBTAG00000027651 & $1.31 \mathrm{E}-09$ & $1.21 \mathrm{E}-09$ & OR51I2 & $1.09 \mathrm{E}+00$ \\
\hline ENSBTAG00000047025 & 3.07E-09 & 2.82E-09 & & $1.09 \mathrm{E}+00$ \\
\hline
\end{tabular}




\begin{tabular}{|c|c|c|c|c|}
\hline ENSBTAG00000039348 & $2.82 \mathrm{E}-09$ & $2.59 \mathrm{E}-09$ & & $1.09 \mathrm{E}+00$ \\
\hline ENSBTAG00000021483 & $1.01 \mathrm{E}-09$ & $9.31 \mathrm{E}-10$ & OLFML1 & $1.09 \mathrm{E}+00$ \\
\hline ENSBTAG00000012279 & $2.59 \mathrm{E}-10$ & $2.38 \mathrm{E}-10$ & & $1.09 E+00$ \\
\hline ENSBTAG00000020573 & $2.43 \mathrm{E}-10$ & $2.23 \mathrm{E}-10$ & SCUBE2 & $1.09 \mathrm{E}+00$ \\
\hline ENSBTAG00000005914 & $9.77 \mathrm{E}-10$ & $8.93 \mathrm{E}-10$ & & $1.09 \mathrm{E}+00$ \\
\hline ENSBTAG00000031027 & $9.77 \mathrm{E}-10$ & 8.93E-10 & & $1.09 \mathrm{E}+00$ \\
\hline ENSBTAG00000000987 & $3.18 \mathrm{E}-10$ & $2.90 \mathrm{E}-10$ & OTOG & $1.10 E+00$ \\
\hline ENSBTAG00000015405 & $5.44 \mathrm{E}-10$ & $4.96 \mathrm{E}-10$ & DCHS1 & $1.10 \mathrm{E}+00$ \\
\hline ENSBTAG00000007148 & 4.17E-10 & $3.81 \mathrm{E}-10$ & $\mathrm{~F} 2$ & $1.10 \mathrm{E}+00$ \\
\hline ENSBTAG00000038031 & 3.34E-09 & 3.04E-09 & & $1.10 E+00$ \\
\hline ENSBTAG00000027563 & $2.92 \mathrm{E}-10$ & $2.66 \mathrm{E}-10$ & & $1.10 E+00$ \\
\hline ENSBTAG00000027438 & $5.88 \mathrm{E}-10$ & $5.35 \mathrm{E}-10$ & & $1.10 \mathrm{E}+00$ \\
\hline ENSBTAG00000019644 & $2.48 \mathrm{E}-10$ & $2.25 \mathrm{E}-10$ & EXT2 & $1.10 \mathrm{E}+00$ \\
\hline ENSBTAG00000005236 & $1.36 \mathrm{E}-10$ & $1.24 \mathrm{E}-10$ & CCKBR & $1.10 \mathrm{E}+00$ \\
\hline ENSBTAG00000034346 & $9.31 \mathrm{E}-10$ & $8.43 \mathrm{E}-10$ & BTG4 & $1.10 \mathrm{E}+00$ \\
\hline ENSBTAG00000015403 & $3.86 \mathrm{E}-10$ & $3.49 E-10$ & TPP1 & $1.10 \mathrm{E}+00$ \\
\hline ENSBTAG00000013636 & $3.28 \mathrm{E}-10$ & $2.96 \mathrm{E}-10$ & DGKZ & $1.11 \mathrm{E}+00$ \\
\hline ENSBTAG00000006322 & $3.74 \mathrm{E}-10$ & $3.38 \mathrm{E}-10$ & & $1.11 \mathrm{E}+00$ \\
\hline ENSBTAG00000008077 & $1.11 \mathrm{E}-10$ & $1.00 \mathrm{E}-10$ & SLC37A4 & $1.11 \mathrm{E}+00$ \\
\hline ENSBTAG00000005609 & 1.17E-09 & $1.06 \mathrm{E}-09$ & & $1.11 E+00$ \\
\hline ENSBTAG00000005251 & $4.04 \mathrm{E}-10$ & $3.63 \mathrm{E}-10$ & & $1.11 \mathrm{E}+00$ \\
\hline ENSBTAG00000046811 & $6.14 \mathrm{E}-10$ & $5.51 \mathrm{E}-10$ & & $1.11 \mathrm{E}+00$ \\
\hline ENSBTAG00000035989 & $5.98 \mathrm{E}-10$ & $5.37 \mathrm{E}-10$ & & $1.11 \mathrm{E}+00$ \\
\hline ENSBTAG00000012460 & $5.25 \mathrm{E}-10$ & 4.70E-10 & ELF5 & $1.12 \mathrm{E}+00$ \\
\hline ENSBTAG00000046541 & $1.21 \mathrm{E}-09$ & $1.09 \mathrm{E}-09$ & OR52B6 & $1.11 \mathrm{E}+00$ \\
\hline ENSBTAG00000015309 & $2.25 \mathrm{E}-10$ & $2.02 \mathrm{E}-10$ & & $1.12 \mathrm{E}+00$ \\
\hline ENSBTAG00000032079 & 7.47E-10 & $6.68 \mathrm{E}-10$ & SYT9 & $1.12 \mathrm{E}+00$ \\
\hline ENSBTAG00000037768 & $6.47 \mathrm{E}-10$ & $5.78 \mathrm{E}-10$ & & $1.12 \mathrm{E}+00$ \\
\hline ENSBTAG00000014606 & $2.02 \mathrm{E}-10$ & $1.80 \mathrm{E}-10$ & FOXR1 & $1.12 \mathrm{E}+00$ \\
\hline ENSBTAG00000007332 & $1.71 \mathrm{E}-10$ & $1.52 \mathrm{E}-10$ & & $1.13 \mathrm{E}+00$ \\
\hline ENSBTAG00000004282 & $3.44 \mathrm{E}-10$ & $3.06 \mathrm{E}-10$ & AMBRA1 & $1.12 \mathrm{E}+00$ \\
\hline ENSBTAG00000026922 & $1.33 \mathrm{E}-09$ & $1.18 \mathrm{E}-09$ & & $1.13 \mathrm{E}+00$ \\
\hline ENSBTAG00000015818 & $6.97 \mathrm{E}-10$ & 6.17E-10 & & $1.13 \mathrm{E}+00$ \\
\hline ENSBTAG00000012885 & $3.70 \mathrm{E}-10$ & $3.27 \mathrm{E}-10$ & ACAT1 & $1.13 E+00$ \\
\hline ENSBTAG00000019144 & $1.47 \mathrm{E}-09$ & $1.30 \mathrm{E}-09$ & OR6A2 & $1.13 \mathrm{E}+00$ \\
\hline ENSBTAG00000016547 & $5.63 \mathrm{E}-10$ & $4.96 \mathrm{E}-10$ & CEP57 & $1.13 E+00$ \\
\hline ENSBTAG00000047652 & $5.26 \mathrm{E}-10$ & 4.64E-10 & & $1.13 \mathrm{E}+00$ \\
\hline ENSBTAG00000046801 & $8.73 \mathrm{E}-10$ & $7.68 \mathrm{E}-10$ & & $1.14 \mathrm{E}+00$ \\
\hline ENSBTAG00000036031 & $8.90 \mathrm{E}-10$ & $7.83 \mathrm{E}-10$ & & $1.14 \mathrm{E}+00$ \\
\hline ENSBTAG00000032151 & $3.92 \mathrm{E}-10$ & $3.45 \mathrm{E}-10$ & $\mathrm{CCDC73}$ & $1.14 \mathrm{E}+00$ \\
\hline ENSBTAG00000039592 & $8.70 \mathrm{E}-10$ & $7.66 \mathrm{E}-10$ & & $1.14 \mathrm{E}+00$ \\
\hline ENSBTAG00000002606 & $1.65 \mathrm{E}-10$ & $1.45 \mathrm{E}-10$ & LGR4 & $1.14 \mathrm{E}+00$ \\
\hline ENSBTAG00000011905 & $1.36 \mathrm{E}-10$ & $1.19 \mathrm{E}-10$ & RRAS2 & $1.14 \mathrm{E}+00$ \\
\hline ENSBTAG00000016494 & 4.34E-10 & $3.80 \mathrm{E}-10$ & RELT & $1.14 \mathrm{E}+00$ \\
\hline ENSBTAG00000004206 & $5.29 \mathrm{E}-10$ & $4.64 \mathrm{E}-10$ & LRRC55 & $1.14 \mathrm{E}+00$ \\
\hline ENSBTAG00000008564 & 7.61E-10 & $6.66 \mathrm{E}-10$ & & $1.14 \mathrm{E}+00$ \\
\hline ENSBTAG00000023202 & $3.75 \mathrm{E}-10$ & $3.27 \mathrm{E}-10$ & & $1.15 E+00$ \\
\hline ENSBTAG00000022368 & $1.13 \mathrm{E}-09$ & $9.86 \mathrm{E}-10$ & & $1.15 \mathrm{E}+00$ \\
\hline ENSBTAG00000038579 & $8.95 \mathrm{E}-10$ & $7.78 \mathrm{E}-10$ & & $1.15 \mathrm{E}+00$ \\
\hline ENSBTAG00000018257 & $4.74 \mathrm{E}-10$ & $4.12 \mathrm{E}-10$ & APIP & $1.15 \mathrm{E}+00$ \\
\hline ENSBTAG00000040463 & $1.24 \mathrm{E}-09$ & 1.07E-09 & & $1.16 \mathrm{E}+00$ \\
\hline ENSBTAG00000040119 & $1.13 \mathrm{E}-09$ & $9.77 \mathrm{E}-10$ & & $1.16 \mathrm{E}+00$ \\
\hline ENSBTAG00000037465 & $6.52 \mathrm{E}-10$ & $5.62 \mathrm{E}-10$ & & $1.16 \mathrm{E}+00$ \\
\hline ENSBTAG00000038075 & $8.86 \mathrm{E}-10$ & $7.62 \mathrm{E}-10$ & & $1.16 \mathrm{E}+00$ \\
\hline ENSBTAG00000040341 & $1.43 \mathrm{E}-09$ & $1.23 \mathrm{E}-09$ & & $1.16 \mathrm{E}+00$ \\
\hline ENSBTAG00000014354 & $2.44 \mathrm{E}-10$ & $2.09 \mathrm{E}-10$ & FXYD6 & $1.17 \mathrm{E}+00$ \\
\hline ENSBTAG00000004034 & $1.61 \mathrm{E}-10$ & $1.38 \mathrm{E}-10$ & SESN3 & $1.17 \mathrm{E}+00$ \\
\hline ENSBTAG00000000842 & $5.35 \mathrm{E}-10$ & $4.58 \mathrm{E}-10$ & UBASH3B & $1.17 \mathrm{E}+00$ \\
\hline ENSBTAG00000008665 & $5.59 \mathrm{E}-10$ & $4.78 \mathrm{E}-10$ & DIXDC1 & $1.17 \mathrm{E}+00$ \\
\hline ENSBTAG00000004863 & 4.24E-10 & $3.62 E-10$ & RIC3 & $1.17 \mathrm{E}+00$ \\
\hline ENSBTAG00000017325 & $3.46 \mathrm{E}-10$ & $2.94 \mathrm{E}-10$ & ATG13 & $1.18 \mathrm{E}+00$ \\
\hline ENSBTAG00000017218 & $1.52 \mathrm{E}-10$ & $1.29 \mathrm{E}-10$ & ACER3 & $1.18 \mathrm{E}+00$ \\
\hline ENSBTAG00000040320 & $1.66 \mathrm{E}-09$ & $1.40 \mathrm{E}-09$ & & $1.18 \mathrm{E}+00$ \\
\hline ENSBTAG00000001607 & $7.19 \mathrm{E}-10$ & $6.09 \mathrm{E}-10$ & TMEM41B & $1.18 \mathrm{E}+00$ \\
\hline ENSBTAG00000006817 & $1.89 \mathrm{E}-10$ & $1.60 \mathrm{E}-10$ & $\mathrm{CBL}$ & $1.18 \mathrm{E}+00$ \\
\hline ENSBTAG00000012505 & $4.28 \mathrm{E}-10$ & $3.62 \mathrm{E}-10$ & ARHGEF17 & $1.18 \mathrm{E}+00$ \\
\hline ENSBTAG00000015059 & $7.01 \mathrm{E}-10$ & $5.90 \mathrm{E}-10$ & MMP13 & $1.19 E+00$ \\
\hline ENSBTAG00000023283 & $4.45 \mathrm{E}-10$ & $3.74 \mathrm{E}-10$ & JAML & $1.19 \mathrm{E}+00$ \\
\hline ENSBTAG00000047850 & $2.62 \mathrm{E}-10$ & $2.20 \mathrm{E}-10$ & & $1.19 \mathrm{E}+00$ \\
\hline ENSBTAG00000020515 & $1.03 \mathrm{E}-10$ & 8.67E-11 & FAM160A2 & $1.19 E+00$ \\
\hline
\end{tabular}




\begin{tabular}{|c|c|c|c|c|}
\hline ENSBTAG00000001432 & $1.39 \mathrm{E}-09$ & 1.17E-09 & & $1.19 E+00$ \\
\hline ENSBTAG00000023912 & $5.08 \mathrm{E}-10$ & 4.26E-10 & & $1.19 E+00$ \\
\hline ENSBTAG00000026924 & $5.08 \mathrm{E}-10$ & $4.26 \mathrm{E}-10$ & & $1.19 E+00$ \\
\hline ENSBTAG00000046254 & $3.91 \mathrm{E}-10$ & $3.28 \mathrm{E}-10$ & & $1.19 E+00$ \\
\hline ENSBTAG00000011521 & $7.80 \mathrm{E}-10$ & $6.54 \mathrm{E}-10$ & & $1.19 \mathrm{E}+00$ \\
\hline ENSBTAG00000045639 & $6.15 E-10$ & $5.16 \mathrm{E}-10$ & & $1.19 E+00$ \\
\hline ENSBTAG00000039801 & $9.71 \mathrm{E}-10$ & 8.14E-10 & & $1.19 E+00$ \\
\hline ENSBTAG00000005070 & $9.71 \mathrm{E}-10$ & 8.14E-10 & & $1.19 E+00$ \\
\hline ENSBTAG00000047932 & $6.69 \mathrm{E}-10$ & $5.61 \mathrm{E}-10$ & & $1.19 E+00$ \\
\hline ENSBTAG00000014572 & $1.05 \mathrm{E}-09$ & $8.81 E-10$ & & $1.19 E+00$ \\
\hline ENSBTAG00000016090 & $1.05 \mathrm{E}-09$ & $8.79 \mathrm{E}-10$ & & $1.19 \mathrm{E}+00$ \\
\hline ENSBTAG00000039503 & $1.05 \mathrm{E}-09$ & $8.81 \mathrm{E}-10$ & & $1.19 E+00$ \\
\hline ENSBTAG00000015566 & $2.72 \mathrm{E}-10$ & $2.28 \mathrm{E}-10$ & CTR9 & $1.19 E+00$ \\
\hline ENSBTAG00000032050 & $2.32 \mathrm{E}-09$ & 1.93E-09 & & $1.20 E+00$ \\
\hline ENSBTAG00000010419 & $2.26 \mathrm{E}-10$ & $1.88 \mathrm{E}-10$ & CYP2R1 & $1.20 \mathrm{E}+00$ \\
\hline ENSBTAG00000046196 & $2.94 \mathrm{E}-09$ & 2.44E-09 & & $1.20 \mathrm{E}+00$ \\
\hline ENSBTAG00000009150 & 4.17E-10 & 3.47E-10 & SPON1 & $1.20 \mathrm{E}+00$ \\
\hline ENSBTAG00000020285 & $2.02 \mathrm{E}-09$ & $1.68 \mathrm{E}-09$ & & $1.20 \mathrm{E}+00$ \\
\hline ENSBTAG00000006143 & $3.83 \mathrm{E}-10$ & 3.17E-10 & CREB3L1 & $1.21 \mathrm{E}+00$ \\
\hline ENSBTAG00000002289 & 8.94E-10 & $7.40 \mathrm{E}-10$ & NLRP14 & $1.21 \mathrm{E}+00$ \\
\hline ENSBTAG00000018479 & $5.00 \mathrm{E}-10$ & 4.13E-10 & PTPMT1 & $1.21 \mathrm{E}+00$ \\
\hline ENSBTAG00000014884 & $1.99 \mathrm{E}-10$ & $1.64 \mathrm{E}-10$ & HIPK3 & $1.21 E+00$ \\
\hline ENSBTAG00000006176 & $2.28 \mathrm{E}-10$ & $1.88 \mathrm{E}-10$ & GRIA4 & $1.21 E+00$ \\
\hline ENSBTAG00000004672 & $5.36 \mathrm{E}-10$ & 4.42E-10 & RDX & $1.21 \mathrm{E}+00$ \\
\hline ENSBTAG00000023989 & $2.05 \mathrm{E}-10$ & $1.69 \mathrm{E}-10$ & MSANTD4 & $1.21 \mathrm{E}+00$ \\
\hline ENSBTAG00000009749 & $1.68 \mathrm{E}-10$ & $1.38 \mathrm{E}-10$ & USP2 & $1.22 \mathrm{E}+00$ \\
\hline ENSBTAG00000001154 & $5.06 \mathrm{E}-10$ & 4.14E-10 & DGAT2 & $1.22 \mathrm{E}+00$ \\
\hline ENSBTAG00000036016 & $4.71 \mathrm{E}-10$ & $3.85 \mathrm{E}-10$ & FAM111B & $1.22 \mathrm{E}+00$ \\
\hline ENSBTAG00000021188 & $3.87 \mathrm{E}-10$ & 3.17E-10 & MRPL16 & $1.22 \mathrm{E}+00$ \\
\hline ENSBTAG00000037657 & $6.80 \mathrm{E}-10$ & $5.55 \mathrm{E}-10$ & & $1.23 E+00$ \\
\hline ENSBTAG00000016497 & $2.94 \mathrm{E}-10$ & $2.40 \mathrm{E}-10$ & FAM168A & $1.23 E+00$ \\
\hline ENSBTAG00000034827 & $4.39 \mathrm{E}-10$ & 3.57E-10 & PDGFD & $1.23 E+00$ \\
\hline ENSBTAG00000033529 & $1.17 \mathrm{E}-10$ & $9.54 \mathrm{E}-11$ & $\mathrm{SCN} 2 \mathrm{~B}$ & $1.23 E+00$ \\
\hline ENSBTAG00000007913 & $2.70 \mathrm{E}-10$ & $2.19 \mathrm{E}-10$ & & $1.23 E+00$ \\
\hline ENSBTAG00000043981 & $3.39 \mathrm{E}-10$ & $2.74 \mathrm{E}-10$ & SBF2 & $1.24 \mathrm{E}+00$ \\
\hline ENSBTAG00000002007 & $1.26 \mathrm{E}-09$ & $1.02 \mathrm{E}-09$ & PRG3 & $1.24 \mathrm{E}+00$ \\
\hline ENSBTAG00000002323 & $1.92 \mathrm{E}-10$ & $1.54 \mathrm{E}-10$ & USP28 & $1.25 \mathrm{E}+00$ \\
\hline ENSBTAG00000012936 & 4.00E-10 & $3.20 \mathrm{E}-10$ & THYN1 & $1.25 \mathrm{E}+00$ \\
\hline ENSBTAG00000010286 & $4.09 \mathrm{E}-10$ & $3.27 \mathrm{E}-10$ & NPAT & $1.25 \mathrm{E}+00$ \\
\hline ENSBTAG00000031903 & $9.61 \mathrm{E}-10$ & $7.68 \mathrm{E}-10$ & & $1.25 \mathrm{E}+00$ \\
\hline ENSBTAG00000003682 & $6.63 \mathrm{E}-10$ & $5.28 \mathrm{E}-10$ & DNAJB13 & $1.26 \mathrm{E}+00$ \\
\hline ENSBTAG00000000802 & $3.45 \mathrm{E}-10$ & $2.75 \mathrm{E}-10$ & LYVE1 & $1.25 \mathrm{E}+00$ \\
\hline ENSBTAG00000000983 & $5.53 \mathrm{E}-10$ & 4.39E-10 & $A B C C 8$ & $1.26 \mathrm{E}+00$ \\
\hline ENSBTAG00000015596 & 4.46E-10 & $3.55 \mathrm{E}-10$ & SLCO2B1 & $1.26 \mathrm{E}+00$ \\
\hline ENSBTAG00000024657 & $4.91 \mathrm{E}-10$ & $3.89 \mathrm{E}-10$ & & $1.26 \mathrm{E}+00$ \\
\hline ENSBTAG00000023831 & $2.59 \mathrm{E}-10$ & 2.04E-10 & ELP4 & $1.27 \mathrm{E}+00$ \\
\hline ENSBTAG00000009576 & $2.23 \mathrm{E}-10$ & $1.75 \mathrm{E}-10$ & PACSIN3 & $1.27 \mathrm{E}+00$ \\
\hline ENSBTAG00000048176 & $7.06 \mathrm{E}-10$ & $5.55 \mathrm{E}-10$ & & $1.27 \mathrm{E}+00$ \\
\hline ENSBTAG00000024781 & $8.96 \mathrm{E}-10$ & 7.03E-10 & & $1.27 \mathrm{E}+00$ \\
\hline ENSBTAG00000004561 & $1.57 \mathrm{E}-10$ & $1.23 \mathrm{E}-10$ & PAX6 & $1.28 \mathrm{E}+00$ \\
\hline ENSBTAG00000008925 & $2.32 \mathrm{E}-10$ & $1.81 \mathrm{E}-10$ & MRE11 & $1.28 \mathrm{E}+00$ \\
\hline ENSBTAG00000009828 & $2.29 \mathrm{E}-10$ & $1.79 \mathrm{E}-10$ & FXYD2 & $1.28 \mathrm{E}+00$ \\
\hline ENSBTAG00000005392 & $3.07 \mathrm{E}-10$ & $2.38 \mathrm{E}-10$ & KBTBD3 & $1.29 E+00$ \\
\hline ENSBTAG00000026796 & $1.68 \mathrm{E}-09$ & $1.31 \mathrm{E}-09$ & & $1.29 \mathrm{E}+00$ \\
\hline ENSBTAG00000024715 & $7.05 \mathrm{E}-10$ & $5.46 \mathrm{E}-10$ & & $1.29 E+00$ \\
\hline ENSBTAG00000005060 & $8.78 \mathrm{E}-10$ & $6.79 \mathrm{E}-10$ & & $1.29 E+00$ \\
\hline ENSBTAG00000020911 & $4.41 \mathrm{E}-10$ & $3.40 \mathrm{E}-10$ & FNBP4 & $1.30 \mathrm{E}+00$ \\
\hline ENSBTAG00000038317 & $1.14 \mathrm{E}-09$ & $8.81 E-10$ & & $1.30 \mathrm{E}+00$ \\
\hline ENSBTAG00000019725 & $2.12 \mathrm{E}-10$ & $1.63 \mathrm{E}-10$ & UBE4A & $1.30 \mathrm{E}+00$ \\
\hline ENSBTAG00000025931 & $5.55 \mathrm{E}-10$ & 4.27E-10 & NEU3 & $1.30 \mathrm{E}+00$ \\
\hline ENSBTAG00000000926 & 1.16E-09 & $8.96 \mathrm{E}-10$ & & $1.29 E+00$ \\
\hline ENSBTAG00000037937 & $6.75 \mathrm{E}-10$ & $5.18 \mathrm{E}-10$ & & $1.30 \mathrm{E}+00$ \\
\hline ENSBTAG00000010860 & $1.45 \mathrm{E}-10$ & $1.11 \mathrm{E}-10$ & DRD2 & $1.31 E+00$ \\
\hline ENSBTAG00000015655 & $3.33 \mathrm{E}-10$ & $2.55 \mathrm{E}-10$ & APBB1 & $1.31 E+00$ \\
\hline ENSBTAG00000014474 & $3.06 \mathrm{E}-10$ & $2.34 \mathrm{E}-10$ & API5 & $1.31 E+00$ \\
\hline ENSBTAG00000002650 & $4.04 \mathrm{E}-10$ & 3.07E-10 & ZNF259 & $1.32 \mathrm{E}+00$ \\
\hline ENSBTAG00000031330 & $6.12 \mathrm{E}-10$ & 4.65E-10 & HTR3B & $1.32 \mathrm{E}+00$ \\
\hline ENSBTAG00000021518 & $3.41 \mathrm{E}-10$ & $2.58 \mathrm{E}-10$ & TMPRSS4 & $1.32 \mathrm{E}+00$ \\
\hline ENSBTAG00000000694 & $2.90 \mathrm{E}-10$ & $2.19 \mathrm{E}-10$ & TAF10 & $1.32 \mathrm{E}+00$ \\
\hline ENSBTAG00000006453 & $2.29 \mathrm{E}-10$ & $1.73 \mathrm{E}-10$ & CD3G & $1.32 \mathrm{E}+00$ \\
\hline
\end{tabular}




\begin{tabular}{|c|c|c|c|c|}
\hline ENSBTAG00000048088 & 4.27E-10 & 3.22E-10 & OR2AT4 & $1.32 \mathrm{E}+00$ \\
\hline ENSBTAG00000010855 & $1.69 \mathrm{E}-10$ & $1.27 \mathrm{E}-10$ & ANKK1 & $1.33 E+00$ \\
\hline ENSBTAG00000039467 & $8.65 \mathrm{E}-10$ & $6.52 \mathrm{E}-10$ & & $1.33 \mathrm{E}+00$ \\
\hline ENSBTAG00000046441 & $4.28 \mathrm{E}-10$ & 3.23E-10 & & $1.32 E+00$ \\
\hline ENSBTAG00000038641 & $4.38 \mathrm{E}-10$ & $3.30 \mathrm{E}-10$ & & $1.33 \mathrm{E}+00$ \\
\hline ENSBTAG00000047721 & $8.90 \mathrm{E}-10$ & $6.71 \mathrm{E}-10$ & & $1.33 \mathrm{E}+00$ \\
\hline ENSBTAG00000035988 & $8.76 \mathrm{E}-10$ & $6.60 \mathrm{E}-10$ & & $1.33 \mathrm{E}+00$ \\
\hline ENSBTAG00000018182 & $8.76 \mathrm{E}-10$ & $6.60 \mathrm{E}-10$ & & $1.33 E+00$ \\
\hline ENSBTAG00000010919 & $4.89 \mathrm{E}-10$ & $3.69 \mathrm{E}-10$ & USP47 & $1.33 \mathrm{E}+00$ \\
\hline ENSBTAG00000009067 & $1.11 \mathrm{E}-10$ & $8.29 E-11$ & DDX6 & $1.33 \mathrm{E}+00$ \\
\hline ENSBTAG00000015791 & $1.79 \mathrm{E}-09$ & $1.34 \mathrm{E}-09$ & & $1.34 \mathrm{E}+00$ \\
\hline ENSBTAG00000031025 & $1.34 \mathrm{E}-09$ & $1.01 \mathrm{E}-09$ & & $1.32 \mathrm{E}+00$ \\
\hline ENSBTAG00000000977 & $3.26 \mathrm{E}-10$ & $2.45 \mathrm{E}-10$ & CADM1 & $1.33 \mathrm{E}+00$ \\
\hline ENSBTAG00000037875 & 1.30E-09 & $9.80 \mathrm{E}-10$ & & $1.33 \mathrm{E}+00$ \\
\hline ENSBTAG00000037483 & $5.00 \mathrm{E}-10$ & $3.76 \mathrm{E}-10$ & & $1.33 E+00$ \\
\hline ENSBTAG00000031299 & $5.94 \mathrm{E}-10$ & $4.45 \mathrm{E}-10$ & TTC17 & $1.33 E+00$ \\
\hline ENSBTAG00000037962 & $4.76 \mathrm{E}-10$ & $3.55 \mathrm{E}-10$ & MAML2 & $1.34 \mathrm{E}+00$ \\
\hline ENSBTAG00000012630 & $2.12 \mathrm{E}-10$ & $1.57 \mathrm{E}-10$ & PAMR1 & $1.35 \mathrm{E}+00$ \\
\hline ENSBTAG00000008465 & $1.80 \mathrm{E}-10$ & $1.33 \mathrm{E}-10$ & ACCS & $1.35 E+00$ \\
\hline ENSBTAG00000032056 & $1.04 \mathrm{E}-09$ & $7.70 \mathrm{E}-10$ & OR10A4 & $1.36 \mathrm{E}+00$ \\
\hline ENSBTAG00000032052 & $1.90 \mathrm{E}-09$ & 1.40E-09 & & $1.35 \mathrm{E}+00$ \\
\hline ENSBTAG00000010060 & $1.42 \mathrm{E}-09$ & $1.04 \mathrm{E}-09$ & & $1.36 \mathrm{E}+00$ \\
\hline ENSBTAG00000040449 & 4.70E-10 & $3.45 \mathrm{E}-10$ & C11orf97 & $1.36 \mathrm{E}+00$ \\
\hline ENSBTAG00000047111 & $3.22 \mathrm{E}-10$ & 2.37E-10 & & $1.36 \mathrm{E}+00$ \\
\hline ENSBTAG00000013111 & $6.74 \mathrm{E}-10$ & 4.94E-10 & RRM1 & $1.36 \mathrm{E}+00$ \\
\hline ENSBTAG00000013029 & $2.30 \mathrm{E}-10$ & $1.68 \mathrm{E}-10$ & ARNTL & $1.37 \mathrm{E}+00$ \\
\hline ENSBTAG00000005338 & $2.92 \mathrm{E}-10$ & $2.13 \mathrm{E}-10$ & EIF3M & $1.37 E+00$ \\
\hline ENSBTAG00000038058 & 1.11E-09 & 8.10E-10 & & $1.37 \mathrm{E}+00$ \\
\hline ENSBTAG00000002302 & $2.90 \mathrm{E}-10$ & $2.11 \mathrm{E}-10$ & CD59 & $1.37 \mathrm{E}+00$ \\
\hline ENSBTAG00000026441 & 1.67E-09 & $1.21 \mathrm{E}-09$ & & $1.38 \mathrm{E}+00$ \\
\hline ENSBTAG00000048029 & $6.29 \mathrm{E}-10$ & $4.55 \mathrm{E}-10$ & & $1.38 \mathrm{E}+00$ \\
\hline ENSBTAG00000009255 & $5.84 \mathrm{E}-10$ & $4.21 \mathrm{E}-10$ & DNHD1 & $1.39 E+00$ \\
\hline ENSBTAG00000021700 & $1.67 \mathrm{E}-10$ & $1.21 \mathrm{E}-10$ & MADD & $1.38 \mathrm{E}+00$ \\
\hline ENSBTAG00000039760 & $4.48 \mathrm{E}-10$ & 3.22E-10 & & $1.39 E+00$ \\
\hline ENSBTAG00000000763 & $6.12 \mathrm{E}-10$ & 4.39E-10 & & $1.39 E+00$ \\
\hline ENSBTAG00000018259 & $6.16 \mathrm{E}-10$ & 4.42E-10 & & $1.39 E+00$ \\
\hline ENSBTAG00000014853 & $6.06 \mathrm{E}-10$ & $4.35 \mathrm{E}-10$ & & $1.39 E+00$ \\
\hline ENSBTAG00000014611 & $3.57 \mathrm{E}-10$ & $2.56 \mathrm{E}-10$ & SORL1 & $1.39 E+00$ \\
\hline ENSBTAG00000002907 & $5.43 \mathrm{E}-10$ & $3.88 \mathrm{E}-10$ & $\mathrm{C} 2 \mathrm{CD} 3$ & $1.40 \mathrm{E}+00$ \\
\hline ENSBTAG00000016855 & $1.93 \mathrm{E}-10$ & $1.38 \mathrm{E}-10$ & BUD13 & $1.40 E+00$ \\
\hline ENSBTAG00000011910 & $2.72 \mathrm{E}-10$ & $1.93 \mathrm{E}-10$ & STK33 & $1.41 \mathrm{E}+00$ \\
\hline ENSBTAG00000005218 & $1.93 \mathrm{E}-10$ & 1.37E-10 & PDE3B & $1.41 E+00$ \\
\hline ENSBTAG00000005356 & $3.53 \mathrm{E}-10$ & $2.51 \mathrm{E}-10$ & ST5 & $1.41 E+00$ \\
\hline ENSBTAG00000003661 & $1.49 \mathrm{E}-10$ & $1.05 \mathrm{E}-10$ & & $1.42 \mathrm{E}+00$ \\
\hline ENSBTAG00000016744 & $1.65 \mathrm{E}-10$ & 1.17E-10 & CAPRIN1 & $1.41 E+00$ \\
\hline ENSBTAG00000009748 & $1.38 \mathrm{E}-10$ & 9.67E-11 & MFRP & $1.43 E+00$ \\
\hline ENSBTAG00000001917 & $1.49 \mathrm{E}-10$ & $1.04 \mathrm{E}-10$ & C11orf16 & $1.43 E+00$ \\
\hline ENSBTAG00000040197 & $1.15 \mathrm{E}-09$ & $8.00 \mathrm{E}-10$ & OR10W1 & $1.43 E+00$ \\
\hline ENSBTAG00000004456 & $5.53 \mathrm{E}-10$ & 3.87E-10 & TECTA & $1.43 E+00$ \\
\hline ENSBTAG00000040150 & $7.61 \mathrm{E}-10$ & $5.31 \mathrm{E}-10$ & & $1.43 \mathrm{E}+00$ \\
\hline ENSBTAG00000030908 & $7.85 \mathrm{E}-10$ & $5.48 \mathrm{E}-10$ & OR9Q2 & $1.43 E+00$ \\
\hline ENSBTAG00000039405 & $7.85 \mathrm{E}-10$ & $5.48 \mathrm{E}-10$ & & $1.43 \mathrm{E}+00$ \\
\hline ENSBTAG00000012621 & $2.49 \mathrm{E}-10$ & $1.73 \mathrm{E}-10$ & RTN4RL2 & $1.44 E+00$ \\
\hline ENSBTAG00000008732 & $3.20 \mathrm{E}-10$ & $2.22 \mathrm{E}-10$ & $\mathrm{ZC} 3 \mathrm{H} 12 \mathrm{C}$ & $1.44 \mathrm{E}+00$ \\
\hline ENSBTAG00000040480 & $2.35 \mathrm{E}-09$ & 1.63E-09 & & $1.44 \mathrm{E}+00$ \\
\hline ENSBTAG00000008613 & $3.14 \mathrm{E}-10$ & 2.17E-10 & TCP11L1 & $1.45 E+00$ \\
\hline ENSBTAG00000008617 & $3.00 \mathrm{E}-10$ & 2.07E-10 & CSTF3 & $1.45 \mathrm{E}+00$ \\
\hline ENSBTAG00000040569 & $1.87 \mathrm{E}-10$ & $1.29 \mathrm{E}-10$ & NLRX1 & $1.45 \mathrm{E}+00$ \\
\hline ENSBTAG00000005340 & $4.99 \mathrm{E}-10$ & $3.42 \mathrm{E}-10$ & SERGEF & $1.46 \mathrm{E}+00$ \\
\hline ENSBTAG00000037751 & $6.41 \mathrm{E}-10$ & $4.40 \mathrm{E}-10$ & & $1.46 \mathrm{E}+00$ \\
\hline ENSBTAG00000013162 & $2.04 \mathrm{E}-10$ & $1.40 \mathrm{E}-10$ & HSPA8 & $1.45 E+00$ \\
\hline ENSBTAG00000038161 & $9.55 \mathrm{E}-10$ & $6.54 \mathrm{E}-10$ & & $1.46 \mathrm{E}+00$ \\
\hline ENSBTAG00000044185 & $3.65 E-10$ & $2.49 \mathrm{E}-10$ & sox6 & $1.47 \mathrm{E}+00$ \\
\hline ENSBTAG00000031943 & $1.14 \mathrm{E}-09$ & $7.68 \mathrm{E}-10$ & & $1.48 \mathrm{E}+00$ \\
\hline ENSBTAG00000018261 & $8.46 \mathrm{E}-10$ & $5.73 \mathrm{E}-10$ & PDHX & $1.48 \mathrm{E}+00$ \\
\hline ENSBTAG00000001280 & $7.24 \mathrm{E}-10$ & $4.90 \mathrm{E}-10$ & CLPB & $1.48 \mathrm{E}+00$ \\
\hline ENSBTAG00000016869 & $4.61 \mathrm{E}-10$ & $3.12 \mathrm{E}-10$ & POLD3 & $1.48 \mathrm{E}+00$ \\
\hline ENSBTAG00000027348 & $6.78 \mathrm{E}-10$ & 4.59E-10 & & $1.48 \mathrm{E}+00$ \\
\hline ENSBTAG00000037853 & 1.10E-09 & 7.42E-10 & & $1.48 \mathrm{E}+00$ \\
\hline ENSBTAG00000020095 & $3.02 \mathrm{E}-10$ & $2.04 \mathrm{E}-10$ & MS4A3 & $1.48 \mathrm{E}+00$ \\
\hline
\end{tabular}




\begin{tabular}{|c|c|c|c|c|}
\hline ENSBTAG00000008219 & $1.69 \mathrm{E}-10$ & $1.13 \mathrm{E}-10$ & RBM7 & $1.49 \mathrm{E}+00$ \\
\hline ENSBTAG00000037928 & $1.33 \mathrm{E}-09$ & 8.87E-10 & & $1.50 \mathrm{E}+00$ \\
\hline ENSBTAG00000002679 & $1.33 \mathrm{E}-09$ & $8.87 E-10$ & & $1.50 \mathrm{E}+00$ \\
\hline ENSBTAG00000020046 & 3.33E-10 & $2.22 \mathrm{E}-10$ & CLMP & $1.50 \mathrm{E}+00$ \\
\hline ENSBTAG00000021233 & $1.31 \mathrm{E}-09$ & $8.79 \mathrm{E}-10$ & OR5B17 & $1.50 \mathrm{E}+00$ \\
\hline ENSBTAG00000007196 & $2.65 \mathrm{E}-10$ & $1.77 \mathrm{E}-10$ & TAGLN & $1.50 \mathrm{E}+00$ \\
\hline ENSBTAG00000000696 & $1.88 \mathrm{E}-10$ & 1.25E-10 & ILK & $1.51 \mathrm{E}+00$ \\
\hline ENSBTAG00000032657 & $4.87 \mathrm{E}-10$ & 3.25E-10 & TEAD1 & $1.50 \mathrm{E}+00$ \\
\hline ENSBTAG00000038323 & $1.61 \mathrm{E}-09$ & 1.07E-09 & GLYAT & $1.50 \mathrm{E}+00$ \\
\hline ENSBTAG00000000475 & $1.96 \mathrm{E}-10$ & $1.30 \mathrm{E}-10$ & IMMP1L & $1.50 \mathrm{E}+00$ \\
\hline ENSBTAG00000014251 & $4.79 \mathrm{E}-10$ & $3.18 \mathrm{E}-10$ & MMP20 & $1.51 \mathrm{E}+00$ \\
\hline ENSBTAG00000039088 & $1.48 \mathrm{E}-09$ & $9.83 \mathrm{E}-10$ & & $1.51 \mathrm{E}+00$ \\
\hline ENSBTAG00000027759 & $6.97 \mathrm{E}-10$ & 4.61E-10 & & $1.51 \mathrm{E}+00$ \\
\hline ENSBTAG00000010681 & $2.41 \mathrm{E}-10$ & $1.59 \mathrm{E}-10$ & NR1H3 & $1.51 E+00$ \\
\hline ENSBTAG00000031252 & $3.36 \mathrm{E}-10$ & $2.21 \mathrm{E}-10$ & CD82 & $1.52 E+00$ \\
\hline ENSBTAG00000031362 & $1.85 \mathrm{E}-10$ & $1.22 \mathrm{E}-10$ & NXPE2 & $1.51 E+00$ \\
\hline ENSBTAG00000044059 & $1.53 \mathrm{E}-09$ & $1.00 \mathrm{E}-09$ & CHRNA10 & $1.53 E+00$ \\
\hline ENSBTAG00000023542 & $1.30 \mathrm{E}-09$ & $8.56 \mathrm{E}-10$ & & $1.52 \mathrm{E}+00$ \\
\hline ENSBTAG00000038737 & $3.40 \mathrm{E}-10$ & $2.23 \mathrm{E}-10$ & P2RY6 & $1.52 \mathrm{E}+00$ \\
\hline ENSBTAG00000001410 & $5.59 \mathrm{E}-10$ & $3.66 \mathrm{E}-10$ & GRAMD1B & $1.53 \mathrm{E}+00$ \\
\hline ENSBTAG00000011793 & 2.25E-10 & $1.47 \mathrm{E}-10$ & FDX1 & $1.53 \mathrm{E}+00$ \\
\hline ENSBTAG00000000087 & 2.69E-10 & $1.75 \mathrm{E}-10$ & HSD17B12 & $1.53 E+00$ \\
\hline ENSBTAG00000020580 & $7.32 \mathrm{E}-10$ & 4.73E-10 & TCN1 & $1.55 \mathrm{E}+00$ \\
\hline ENSBTAG00000012540 & $1.24 \mathrm{E}-09$ & $8.01 \mathrm{E}-10$ & & $1.55 E+00$ \\
\hline ENSBTAG00000003679 & $1.83 \mathrm{E}-10$ & $1.18 \mathrm{E}-10$ & & $1.55 \mathrm{E}+00$ \\
\hline ENSBTAG00000044158 & 4.34E-10 & $2.78 \mathrm{E}-10$ & LDLRAD3 & $1.56 \mathrm{E}+00$ \\
\hline ENSBTAG00000034193 & $2.52 \mathrm{E}-10$ & $1.62 \mathrm{E}-10$ & $\mathrm{BCO} 2$ & $1.56 \mathrm{E}+00$ \\
\hline ENSBTAG00000001233 & $1.36 \mathrm{E}-10$ & $8.76 \mathrm{E}-11$ & MPZL3 & $1.56 \mathrm{E}+00$ \\
\hline ENSBTAG00000013033 & $2.36 \mathrm{E}-10$ & $1.51 \mathrm{E}-10$ & BTBD10 & $1.56 \mathrm{E}+00$ \\
\hline ENSBTAG00000034338 & $2.03 \mathrm{E}-10$ & $1.29 \mathrm{E}-10$ & MGC133619 & $1.57 \mathrm{E}+00$ \\
\hline ENSBTAG00000002117 & $9.39 \mathrm{E}-11$ & $5.98 \mathrm{E}-11$ & KIF18A & $1.57 \mathrm{E}+00$ \\
\hline ENSBTAG00000021590 & $1.67 \mathrm{E}-10$ & $1.05 \mathrm{E}-10$ & OR52B2 & $1.59 \mathrm{E}+00$ \\
\hline ENSBTAG00000047489 & $1.73 \mathrm{E}-10$ & $1.09 \mathrm{E}-10$ & & $1.59 \mathrm{E}+00$ \\
\hline ENSBTAG00000047662 & $1.69 \mathrm{E}-10$ & $1.06 \mathrm{E}-10$ & & $1.60 \mathrm{E}+00$ \\
\hline ENSBTAG00000045558 & $1.71 \mathrm{E}-10$ & $1.08 \mathrm{E}-10$ & & $1.59 E+00$ \\
\hline ENSBTAG00000017794 & 7.74E-11 & 4.87E-11 & CCDC153 & $1.59 \mathrm{E}+00$ \\
\hline ENSBTAG00000047380 & $1.08 \mathrm{E}-10$ & $6.80 \mathrm{E}-11$ & CALCA & $1.59 \mathrm{E}+00$ \\
\hline ENSBTAG00000047083 & $3.34 \mathrm{E}-10$ & $2.10 \mathrm{E}-10$ & & $1.59 \mathrm{E}+00$ \\
\hline ENSBTAG00000005278 & $7.09 \mathrm{E}-11$ & 4.47E-11 & WEE1 & $1.59 \mathrm{E}+00$ \\
\hline ENSBTAG00000047498 & $3.50 \mathrm{E}-10$ & $2.20 \mathrm{E}-10$ & & $1.59 E+00$ \\
\hline ENSBTAG00000045939 & $3.85 \mathrm{E}-10$ & $2.42 \mathrm{E}-10$ & MPEG1 & $1.59 \mathrm{E}+00$ \\
\hline ENSBTAG00000027443 & $3.36 \mathrm{E}-10$ & $2.11 \mathrm{E}-10$ & & $1.59 E+00$ \\
\hline ENSBTAG00000024918 & $6.88 \mathrm{E}-11$ & $4.33 \mathrm{E}-11$ & BIRC3 & $1.59 E+00$ \\
\hline ENSBTAG00000015710 & $1.70 \mathrm{E}-10$ & $1.07 \mathrm{E}-10$ & CD3E & $1.59 \mathrm{E}+00$ \\
\hline ENSBTAG00000036130 & $1.76 \mathrm{E}-10$ & $1.11 \mathrm{E}-10$ & & $1.59 \mathrm{E}+00$ \\
\hline ENSBTAG00000027716 & $5.54 \mathrm{E}-10$ & $3.48 \mathrm{E}-10$ & & $1.59 E+00$ \\
\hline ENSBTAG00000023310 & $1.71 \mathrm{E}-10$ & $1.07 \mathrm{E}-10$ & & $1.60 \mathrm{E}+00$ \\
\hline ENSBTAG00000045959 & $1.71 \mathrm{E}-10$ & $1.07 \mathrm{E}-10$ & & $1.60 \mathrm{E}+00$ \\
\hline ENSBTAG00000001704 & $5.47 \mathrm{E}-10$ & $3.44 \mathrm{E}-10$ & & $1.59 \mathrm{E}+00$ \\
\hline ENSBTAG00000027577 & $1.69 \mathrm{E}-10$ & $1.06 \mathrm{E}-10$ & C11orf91 & $1.59 \mathrm{E}+00$ \\
\hline ENSBTAG00000020746 & 5.17E-10 & $3.25 \mathrm{E}-10$ & & $1.59 \mathrm{E}+00$ \\
\hline ENSBTAG00000027562 & $2.54 \mathrm{E}-10$ & $1.60 \mathrm{E}-10$ & C11orf94 & $1.59 \mathrm{E}+00$ \\
\hline ENSBTAG00000021375 & $6.86 \mathrm{E}-10$ & $4.31 \mathrm{E}-10$ & OR10Q1 & $1.59 \mathrm{E}+00$ \\
\hline ENSBTAG00000020673 & $6.86 \mathrm{E}-10$ & 4.31E-10 & & $1.59 E+00$ \\
\hline ENSBTAG00000048221 & $1.79 \mathrm{E}-10$ & $1.13 \mathrm{E}-10$ & & $1.59 \mathrm{E}+00$ \\
\hline ENSBTAG00000006452 & $1.97 \mathrm{E}-10$ & $1.24 \mathrm{E}-10$ & CD3D & $1.59 \mathrm{E}+00$ \\
\hline ENSBTAG00000038539 & $5.22 \mathrm{E}-10$ & $3.28 \mathrm{E}-10$ & & $1.59 E+00$ \\
\hline ENSBTAG00000026826 & $7.06 \mathrm{E}-10$ & 4.44E-10 & & $1.59 E+00$ \\
\hline ENSBTAG00000037644 & $7.86 \mathrm{E}-10$ & 4.93E-10 & HBG & $1.59 E+00$ \\
\hline ENSBTAG00000026929 & $5.09 \mathrm{E}-10$ & $3.20 \mathrm{E}-10$ & & $1.59 E+00$ \\
\hline ENSBTAG00000038637 & $7.00 \mathrm{E}-10$ & 4.39E-10 & & $1.59 \mathrm{E}+00$ \\
\hline ENSBTAG00000046274 & $1.77 \mathrm{E}-10$ & $1.11 \mathrm{E}-10$ & & $1.59 \mathrm{E}+00$ \\
\hline ENSBTAG00000046028 & $1.82 \mathrm{E}-10$ & $1.15 \mathrm{E}-10$ & & $1.58 \mathrm{E}+00$ \\
\hline ENSBTAG00000035006 & $6.97 \mathrm{E}-10$ & $4.38 \mathrm{E}-10$ & & $1.59 E+00$ \\
\hline ENSBTAG00000035990 & $6.95 \mathrm{E}-10$ & $4.36 \mathrm{E}-10$ & & $1.59 \mathrm{E}+00$ \\
\hline ENSBTAG00000011332 & $8.29 \mathrm{E}-10$ & $5.20 \mathrm{E}-10$ & TRIM68 & $1.59 E+00$ \\
\hline ENSBTAG00000038636 & 8.70E-10 & $5.46 \mathrm{E}-10$ & & $1.59 \mathrm{E}+00$ \\
\hline ENSBTAG00000039214 & $1.58 \mathrm{E}-09$ & $9.89 \mathrm{E}-10$ & & $1.60 E+00$ \\
\hline ENSBTAG00000031030 & $1.29 \mathrm{E}-09$ & $8.07 \mathrm{E}-10$ & & $1.60 \mathrm{E}+00$ \\
\hline ENSBTAG00000038267 & $1.92 \mathrm{E}-09$ & $1.20 \mathrm{E}-09$ & & $1.60 \mathrm{E}+00$ \\
\hline
\end{tabular}




\begin{tabular}{|c|c|c|c|c|}
\hline ENSBTAG00000019062 & $3.50 \mathrm{E}-10$ & 2.17E-10 & FCHSD2 & $1.61 E+00$ \\
\hline ENSBTAG00000002914 & $5.97 \mathrm{E}-10$ & $3.69 \mathrm{E}-10$ & GALNT18 & $1.62 E+00$ \\
\hline ENSBTAG00000021340 & $2.35 \mathrm{E}-10$ & $1.45 \mathrm{E}-10$ & $\mathrm{POU} 2 \mathrm{~F} 3$ & $1.62 E+00$ \\
\hline ENSBTAG00000011549 & $1.11 \mathrm{E}-09$ & $6.85 \mathrm{E}-10$ & & $1.62 E+00$ \\
\hline ENSBTAG00000017443 & $2.79 \mathrm{E}-10$ & $1.72 \mathrm{E}-10$ & & $1.62 \mathrm{E}+00$ \\
\hline ENSBTAG00000009518 & $3.28 \mathrm{E}-10$ & $2.01 E-10$ & ALKBH3 & $1.63 E+00$ \\
\hline ENSBTAG00000007192 & $2.07 \mathrm{E}-10$ & $1.27 \mathrm{E}-10$ & SIDT2 & $1.63 E+00$ \\
\hline ENSBTAG00000006951 & $1.89 \mathrm{E}-10$ & $1.15 \mathrm{E}-10$ & LMO2 & $1.64 \mathrm{E}+00$ \\
\hline ENSBTAG00000021002 & $3.05 \mathrm{E}-10$ & $1.85 \mathrm{E}-10$ & ACP2 & $1.65 \mathrm{E}+00$ \\
\hline ENSBTAG00000020956 & $3.30 \mathrm{E}-10$ & $2.00 \mathrm{E}-10$ & MPPED2 & $1.65 E+00$ \\
\hline ENSBTAG00000005138 & $1.95 \mathrm{E}-10$ & $1.18 \mathrm{E}-10$ & BCL9L & $1.65 \mathrm{E}+00$ \\
\hline ENSBTAG00000017146 & $3.73 \mathrm{E}-10$ & $2.25 \mathrm{E}-10$ & GRIK4 & $1.66 \mathrm{E}+00$ \\
\hline ENSBTAG00000004581 & $8.40 \mathrm{E}-10$ & $5.05 \mathrm{E}-10$ & MS4A14 & $1.66 \mathrm{E}+00$ \\
\hline ENSBTAG00000011266 & $3.37 \mathrm{E}-10$ & 2.02E-10 & ZBTB16 & $1.67 E+00$ \\
\hline ENSBTAG00000008804 & $3.73 \mathrm{E}-10$ & $2.24 \mathrm{E}-10$ & SAAL1 & $1.66 \mathrm{E}+00$ \\
\hline ENSBTAG00000018629 & $8.47 \mathrm{E}-10$ & $5.04 \mathrm{E}-10$ & CWF19L2 & $1.68 \mathrm{E}+00$ \\
\hline ENSBTAG00000046829 & $1.87 \mathrm{E}-09$ & $1.11 \mathrm{E}-09$ & & $1.68 \mathrm{E}+00$ \\
\hline ENSBTAG00000012846 & $3.88 \mathrm{E}-10$ & $2.30 \mathrm{E}-10$ & KIAA1549L & $1.69 E+00$ \\
\hline ENSBTAG00000017934 & $1.64 \mathrm{E}-09$ & $9.74 \mathrm{E}-10$ & & $1.69 E+00$ \\
\hline ENSBTAG00000039272 & $1.34 \mathrm{E}-09$ & $7.81 \mathrm{E}-10$ & & $1.71 \mathrm{E}+00$ \\
\hline ENSBTAG00000016355 & $2.64 \mathrm{E}-10$ & $1.53 \mathrm{E}-10$ & UVRAG & $1.73 \mathrm{E}+00$ \\
\hline ENSBTAG00000020332 & $4.93 \mathrm{E}-10$ & $2.86 \mathrm{E}-10$ & OSBP & $1.72 \mathrm{E}+00$ \\
\hline ENSBTAG00000046285 & $7.05 \mathrm{E}-10$ & 4.09E-10 & OR8I2 & $1.72 \mathrm{E}+00$ \\
\hline ENSBTAG00000038727 & $1.12 \mathrm{E}-09$ & $6.50 \mathrm{E}-10$ & & $1.73 E+00$ \\
\hline ENSBTAG00000010709 & $5.30 \mathrm{E}-10$ & $3.06 \mathrm{E}-10$ & & $1.73 \mathrm{E}+00$ \\
\hline ENSBTAG00000039735 & $1.14 \mathrm{E}-09$ & $6.60 \mathrm{E}-10$ & & $1.73 \mathrm{E}+00$ \\
\hline ENSBTAG00000003111 & $2.73 \mathrm{E}-10$ & 1.57E-10 & ATM & $1.74 \mathrm{E}+00$ \\
\hline ENSBTAG00000045556 & $1.85 \mathrm{E}-09$ & 1.07E-09 & & $1.73 E+00$ \\
\hline ENSBTAG00000012937 & $4.34 \mathrm{E}-10$ & 2.47E-10 & ACAD8 & $1.76 \mathrm{E}+00$ \\
\hline ENSBTAG00000016692 & $2.98 \mathrm{E}-10$ & $1.68 \mathrm{E}-10$ & DSCAML1 & $1.77 E+00$ \\
\hline ENSBTAG00000039340 & $5.55 \mathrm{E}-10$ & $3.12 \mathrm{E}-10$ & SCN4B & $1.78 \mathrm{E}+00$ \\
\hline ENSBTAG00000036260 & $4.33 \mathrm{E}-10$ & 2.44E-10 & LPXN & $1.77 \mathrm{E}+00$ \\
\hline ENSBTAG00000004046 & $1.13 \mathrm{E}-09$ & $6.30 \mathrm{E}-10$ & DTX4 & $1.79 E+00$ \\
\hline ENSBTAG00000009176 & $2.63 \mathrm{E}-10$ & 1.47E-10 & ANKRD49 & $1.79 E+00$ \\
\hline ENSBTAG00000020731 & $3.48 \mathrm{E}-10$ & $1.95 \mathrm{E}-10$ & PRRG4 & $1.79 E+00$ \\
\hline ENSBTAG00000040408 & $7.88 \mathrm{E}-10$ & 4.39E-10 & & $1.79 E+00$ \\
\hline ENSBTAG00000046710 & $8.06 \mathrm{E}-10$ & 4.49E-10 & & $1.79 E+00$ \\
\hline ENSBTAG00000009574 & $1.41 \mathrm{E}-10$ & $7.87 E-11$ & ARFGAP2 & $1.79 E+00$ \\
\hline ENSBTAG00000040330 & $7.78 \mathrm{E}-10$ & 4.34E-10 & OR5M11 & $1.79 E+00$ \\
\hline ENSBTAG00000006345 & $8.85 \mathrm{E}-10$ & 4.93E-10 & & $1.79 E+00$ \\
\hline ENSBTAG00000046489 & $7.98 \mathrm{E}-10$ & 4.45E-10 & & $1.79 \mathrm{E}+00$ \\
\hline ENSBTAG00000014309 & 8.19E-10 & 4.57E-10 & & $1.79 E+00$ \\
\hline ENSBTAG00000006690 & $1.23 \mathrm{E}-10$ & $6.83 \mathrm{E}-11$ & ARCN1 & $1.80 \mathrm{E}+00$ \\
\hline ENSBTAG00000000727 & $1.80 \mathrm{E}-10$ & $9.94 \mathrm{E}-11$ & RNF169 & $1.81 E+00$ \\
\hline ENSBTAG00000021147 & $2.45 \mathrm{E}-09$ & $1.35 \mathrm{E}-09$ & & $1.81 E+00$ \\
\hline ENSBTAG00000001027 & $3.29 \mathrm{E}-10$ & $1.82 \mathrm{E}-10$ & SERPINH1 & $1.81 \mathrm{E}+00$ \\
\hline ENSBTAG00000015628 & $2.94 \mathrm{E}-10$ & $1.62 \mathrm{E}-10$ & SMPD1 & $1.82 \mathrm{E}+00$ \\
\hline ENSBTAG00000015939 & $3.49 \mathrm{E}-10$ & $1.91 \mathrm{E}-10$ & PVRL1 & $1.83 \mathrm{E}+00$ \\
\hline ENSBTAG00000003770 & $6.26 \mathrm{E}-10$ & $3.42 \mathrm{E}-10$ & PTS & $1.83 \mathrm{E}+00$ \\
\hline ENSBTAG00000008709 & $2.34 \mathrm{E}-10$ & $1.28 \mathrm{E}-10$ & KDELC2 & $1.83 \mathrm{E}+00$ \\
\hline ENSBTAG00000021306 & $4.28 \mathrm{E}-10$ & $2.34 \mathrm{E}-10$ & CHRDL2 & $1.83 E+00$ \\
\hline ENSBTAG00000047026 & $1.43 \mathrm{E}-09$ & $7.81 E-10$ & & $1.83 \mathrm{E}+00$ \\
\hline ENSBTAG00000019436 & $1.73 \mathrm{E}-10$ & $9.34 \mathrm{E}-11$ & SLC39A13 & $1.85 \mathrm{E}+00$ \\
\hline ENSBTAG00000001028 & $3.41 \mathrm{E}-10$ & $1.84 \mathrm{E}-10$ & MAP6 & $1.85 \mathrm{E}+00$ \\
\hline ENSBTAG00000033510 & $1.63 \mathrm{E}-10$ & $8.81 E-11$ & MPZL2 & $1.85 \mathrm{E}+00$ \\
\hline ENSBTAG00000002043 & $6.12 \mathrm{E}-10$ & $3.29 \mathrm{E}-10$ & & $1.86 \mathrm{E}+00$ \\
\hline ENSBTAG00000048301 & $6.16 \mathrm{E}-10$ & $3.31 \mathrm{E}-10$ & & $1.86 \mathrm{E}+00$ \\
\hline ENSBTAG00000019768 & $6.20 \mathrm{E}-10$ & 3.33E-10 & OR5P3 & $1.86 \mathrm{E}+00$ \\
\hline ENSBTAG00000035059 & $6.08 \mathrm{E}-10$ & 3.27E-10 & & $1.86 \mathrm{E}+00$ \\
\hline ENSBTAG00000004583 & $7.78 \mathrm{E}-10$ & 4.17E-10 & MS4A5 & $1.87 \mathrm{E}+00$ \\
\hline ENSBTAG00000003087 & $2.14 \mathrm{E}-10$ & $1.15 \mathrm{E}-10$ & DCUN1D5 & $1.86 \mathrm{E}+00$ \\
\hline ENSBTAG00000046741 & $1.23 \mathrm{E}-09$ & $6.58 \mathrm{E}-10$ & & $1.86 \mathrm{E}+00$ \\
\hline ENSBTAG00000005923 & $6.21 \mathrm{E}-10$ & $3.30 \mathrm{E}-10$ & ABTB2 & $1.88 \mathrm{E}+00$ \\
\hline ENSBTAG00000039182 & $1.69 \mathrm{E}-09$ & $8.90 \mathrm{E}-10$ & OR5M3 & $1.90 \mathrm{E}+00$ \\
\hline ENSBTAG00000005357 & $1.92 \mathrm{E}-10$ & $1.01 \mathrm{E}-10$ & VPS11 & $1.90 \mathrm{E}+00$ \\
\hline ENSBTAG00000000333 & $2.40 \mathrm{E}-10$ & $1.26 \mathrm{E}-10$ & ARHGAP20 & $1.91 E+00$ \\
\hline ENSBTAG00000031026 & $1.07 \mathrm{E}-09$ & 5.57E-10 & OR4X1 & $1.91 E+00$ \\
\hline ENSBTAG00000047464 & $1.04 \mathrm{E}-09$ & $5.41 E-10$ & & $1.91 \mathrm{E}+00$ \\
\hline ENSBTAG00000037886 & $1.02 \mathrm{E}-09$ & 5.34E-10 & & $1.92 \mathrm{E}+00$ \\
\hline ENSBTAG00000012549 & $1.06 \mathrm{E}-09$ & $5.53 \mathrm{E}-10$ & OR4C3 & $1.92 \mathrm{E}+00$ \\
\hline
\end{tabular}




\begin{tabular}{|c|c|c|c|c|}
\hline ENSBTAG00000012229 & $5.50 \mathrm{E}-10$ & $2.87 \mathrm{E}-10$ & & $1.92 \mathrm{E}+00$ \\
\hline ENSBTAG00000032782 & $3.63 \mathrm{E}-10$ & $1.89 \mathrm{E}-10$ & INSC & $1.92 \mathrm{E}+00$ \\
\hline ENSBTAG00000021029 & $5.58 \mathrm{E}-10$ & $2.89 \mathrm{E}-10$ & PRR5L & $1.93 \mathrm{E}+00$ \\
\hline ENSBTAG00000015182 & $3.30 \mathrm{E}-10$ & $1.70 \mathrm{E}-10$ & STARD10 & $1.94 \mathrm{E}+00$ \\
\hline ENSBTAG00000009573 & $2.89 \mathrm{E}-10$ & $1.48 \mathrm{E}-10$ & C11orf49 & $1.95 E+00$ \\
\hline ENSBTAG00000013997 & $1.06 \mathrm{E}-09$ & $5.41 \mathrm{E}-10$ & & $1.95 E+00$ \\
\hline ENSBTAG00000027612 & $2.23 \mathrm{E}-10$ & $1.14 \mathrm{E}-10$ & PPME1 & $1.95 \mathrm{E}+00$ \\
\hline ENSBTAG00000012882 & $2.48 \mathrm{E}-10$ & $1.26 \mathrm{E}-10$ & CUL5 & $1.97 E+00$ \\
\hline ENSBTAG00000002411 & $4.80 \mathrm{E}-10$ & $2.44 \mathrm{E}-10$ & CTNND1 & $1.97 E+00$ \\
\hline ENSBTAG00000005627 & $1.90 \mathrm{E}-10$ & $9.63 \mathrm{E}-11$ & PAFAH1B2 & $1.97 \mathrm{E}+00$ \\
\hline ENSBTAG00000018945 & $2.18 \mathrm{E}-10$ & $1.10 \mathrm{E}-10$ & AMOTL1 & $1.98 \mathrm{E}+00$ \\
\hline ENSBTAG00000003126 & $7.24 \mathrm{E}-11$ & $3.65 E-11$ & PEX16 & $1.98 \mathrm{E}+00$ \\
\hline ENSBTAG00000010486 & $3.32 \mathrm{E}-10$ & $1.67 \mathrm{E}-10$ & OR9G1 & $1.98 \mathrm{E}+00$ \\
\hline ENSBTAG00000048166 & $4.27 \mathrm{E}-10$ & $2.14 \mathrm{E}-10$ & & $1.99 \mathrm{E}+00$ \\
\hline ENSBTAG00000019770 & $4.45 \mathrm{E}-10$ & $2.24 \mathrm{E}-10$ & APOA4 & $1.98 \mathrm{E}+00$ \\
\hline ENSBTAG00000047104 & $4.31 \mathrm{E}-10$ & $2.17 \mathrm{E}-10$ & & $1.98 \mathrm{E}+00$ \\
\hline ENSBTAG00000027949 & $4.25 \mathrm{E}-10$ & $2.14 \mathrm{E}-10$ & & $1.99 \mathrm{E}+00$ \\
\hline ENSBTAG00000032859 & $3.78 \mathrm{E}-10$ & $1.90 \mathrm{E}-10$ & & $1.99 \mathrm{E}+00$ \\
\hline ENSBTAG00000004765 & $4.29 \mathrm{E}-10$ & $2.16 \mathrm{E}-10$ & & $1.99 \mathrm{E}+00$ \\
\hline ENSBTAG00000027889 & $4.23 \mathrm{E}-10$ & $2.12 \mathrm{E}-10$ & OR52N4 & $1.99 \mathrm{E}+00$ \\
\hline ENSBTAG00000035675 & 3.35E-09 & $1.68 \mathrm{E}-09$ & & $1.99 \mathrm{E}+00$ \\
\hline ENSBTAG00000047945 & 8.67E-10 & $4.35 \mathrm{E}-10$ & & $1.99 \mathrm{E}+00$ \\
\hline ENSBTAG00000047803 & 1.85E-09 & $9.24 \mathrm{E}-10$ & & $2.00 \mathrm{E}+00$ \\
\hline ENSBTAG00000045488 & 1.73E-09 & $8.65 E-10$ & & $2.00 \mathrm{E}+00$ \\
\hline ENSBTAG00000024423 & $1.71 \mathrm{E}-09$ & $8.54 \mathrm{E}-10$ & & $2.00 \mathrm{E}+00$ \\
\hline ENSBTAG00000019059 & $3.07 \mathrm{E}-10$ & $1.53 \mathrm{E}-10$ & ATG16L2 & $2.00 \mathrm{E}+00$ \\
\hline ENSBTAG00000003582 & $7.40 \mathrm{E}-10$ & $3.65 E-10$ & OR51I1 & $2.03 E+00$ \\
\hline ENSBTAG00000004275 & $3.99 \mathrm{E}-10$ & $1.96 \mathrm{E}-10$ & DKK3 & $2.03 E+00$ \\
\hline ENSBTAG00000014137 & $2.36 \mathrm{E}-10$ & $1.16 \mathrm{E}-10$ & CEP164 & $2.03 E+00$ \\
\hline ENSBTAG00000021707 & $1.90 \mathrm{E}-10$ & $9.31 \mathrm{E}-11$ & MYBPC3 & $2.04 \mathrm{E}+00$ \\
\hline ENSBTAG00000018390 & $8.31 \mathrm{E}-10$ & $4.08 \mathrm{E}-10$ & GLB1L2 & $2.04 \mathrm{E}+00$ \\
\hline ENSBTAG00000016197 & $1.64 \mathrm{E}-10$ & $8.02 \mathrm{E}-11$ & PHLDB1 & $2.04 \mathrm{E}+00$ \\
\hline ENSBTAG00000012417 & $2.18 \mathrm{E}-10$ & $1.07 \mathrm{E}-10$ & ARL14EP & $2.04 \mathrm{E}+00$ \\
\hline ENSBTAG00000038756 & 7.37E-10 & $3.61 E-10$ & GLB1L3 & $2.04 E+00$ \\
\hline ENSBTAG00000014838 & $3.24 \mathrm{E}-10$ & $1.58 \mathrm{E}-10$ & VPS26B & $2.05 E+00$ \\
\hline ENSBTAG00000006579 & $4.68 \mathrm{E}-10$ & $2.27 \mathrm{E}-10$ & P4HA3 & $2.06 \mathrm{E}+00$ \\
\hline ENSBTAG00000012398 & $8.29 \mathrm{E}-10$ & $4.00 \mathrm{E}-10$ & ApoC3 & $2.07 \mathrm{E}+00$ \\
\hline ENSBTAG00000017964 & $6.62 \mathrm{E}-10$ & $3.18 \mathrm{E}-10$ & LRRC51 & $2.08 \mathrm{E}+00$ \\
\hline ENSBTAG00000015091 & $3.32 \mathrm{E}-10$ & $1.60 \mathrm{E}-10$ & & $2.08 \mathrm{E}+00$ \\
\hline ENSBTAG00000033509 & $1.15 \mathrm{E}-09$ & $5.53 \mathrm{E}-10$ & OR4D9 & $2.08 \mathrm{E}+00$ \\
\hline ENSBTAG00000020485 & $3.14 \mathrm{E}-10$ & $1.50 \mathrm{E}-10$ & ARRB1 & $2.09 E+00$ \\
\hline ENSBTAG00000037991 & $1.21 \mathrm{E}-09$ & $5.74 \mathrm{E}-10$ & & $2.10 \mathrm{E}+00$ \\
\hline ENSBTAG00000016202 & $2.21 \mathrm{E}-10$ & $1.04 \mathrm{E}-10$ & TREH & $2.13 \mathrm{E}+00$ \\
\hline ENSBTAG00000009607 & $2.51 \mathrm{E}-10$ & $1.18 \mathrm{E}-10$ & LARGE2 & $2.12 \mathrm{E}+00$ \\
\hline ENSBTAG00000046527 & $7.11 \mathrm{E}-10$ & $3.35 E-10$ & & $2.12 \mathrm{E}+00$ \\
\hline ENSBTAG00000027942 & $9.37 \mathrm{E}-10$ & $4.40 \mathrm{E}-10$ & & $2.13 \mathrm{E}+00$ \\
\hline ENSBTAG00000008923 & 2.17E-10 & $1.01 \mathrm{E}-10$ & GPR83 & $2.15 E+00$ \\
\hline ENSBTAG00000017650 & $9.36 \mathrm{E}-10$ & $4.37 \mathrm{E}-10$ & TMPRSS5 & $2.14 \mathrm{E}+00$ \\
\hline ENSBTAG00000020884 & $2.40 \mathrm{E}-10$ & $1.11 \mathrm{E}-10$ & CASP4 & $2.16 \mathrm{E}+00$ \\
\hline ENSBTAG00000046762 & 1.60E-09 & $7.27 \mathrm{E}-10$ & & $2.20 \mathrm{E}+00$ \\
\hline ENSBTAG00000031777 & $3.09 \mathrm{E}-10$ & $1.39 \mathrm{E}-10$ & GDPD5 & $2.22 \mathrm{E}+00$ \\
\hline ENSBTAG00000039110 & $1.21 \mathrm{E}-09$ & $5.39 \mathrm{E}-10$ & & $2.24 \mathrm{E}+00$ \\
\hline ENSBTAG00000047362 & $1.95 \mathrm{E}-10$ & $8.68 \mathrm{E}-11$ & RCN1 & $2.25 E+00$ \\
\hline ENSBTAG00000031029 & 1.55E-09 & $6.82 \mathrm{E}-10$ & & $2.27 \mathrm{E}+00$ \\
\hline ENSBTAG00000003001 & $5.96 \mathrm{E}-10$ & $2.60 \mathrm{E}-10$ & LAMTOR1 & $2.29 E+00$ \\
\hline ENSBTAG00000009061 & $2.26 \mathrm{E}-10$ & $9.75 E-11$ & FAR1 & $2.32 \mathrm{E}+00$ \\
\hline ENSBTAG00000018449 & $7.92 \mathrm{E}-10$ & $3.38 \mathrm{E}-10$ & NUMA1 & $2.34 \mathrm{E}+00$ \\
\hline ENSBTAG00000039821 & $1.72 \mathrm{E}-10$ & $7.23 \mathrm{E}-11$ & & $2.38 \mathrm{E}+00$ \\
\hline ENSBTAG00000038062 & $1.74 \mathrm{E}-10$ & $7.32 \mathrm{E}-11$ & FUT4 & $2.38 \mathrm{E}+00$ \\
\hline ENSBTAG00000012507 & $9.09 \mathrm{E}-11$ & $3.82 \mathrm{E}-11$ & PDZD3 & $2.38 \mathrm{E}+00$ \\
\hline ENSBTAG00000020032 & $3.56 \mathrm{E}-10$ & $1.49 \mathrm{E}-10$ & CEP126 & $2.39 E+00$ \\
\hline ENSBTAG00000005671 & $4.09 \mathrm{E}-10$ & $1.71 \mathrm{E}-10$ & UBQLNL & $2.39 E+00$ \\
\hline ENSBTAG00000047007 & $5.36 \mathrm{E}-10$ & $2.24 \mathrm{E}-10$ & & $2.39 E+00$ \\
\hline ENSBTAG00000037878 & $5.24 \mathrm{E}-10$ & $2.19 \mathrm{E}-10$ & & $2.39 E+00$ \\
\hline ENSBTAG00000040008 & $2.65 \mathrm{E}-10$ & $1.11 \mathrm{E}-10$ & & $2.38 \mathrm{E}+00$ \\
\hline ENSBTAG00000021048 & $1.08 \mathrm{E}-10$ & $4.52 \mathrm{E}-11$ & ADM & $2.38 \mathrm{E}+00$ \\
\hline ENSBTAG00000027610 & $5.92 \mathrm{E}-10$ & $2.48 \mathrm{E}-10$ & & $2.39 E+00$ \\
\hline ENSBTAG00000019622 & $3.62 \mathrm{E}-10$ & $1.52 \mathrm{E}-10$ & & $2.38 \mathrm{E}+00$ \\
\hline ENSBTAG00000035008 & $5.25 \mathrm{E}-10$ & $2.20 \mathrm{E}-10$ & & $2.39 E+00$ \\
\hline ENSBTAG00000031923 & $5.22 \mathrm{E}-10$ & $2.19 \mathrm{E}-10$ & & $2.38 \mathrm{E}+00$ \\
\hline
\end{tabular}




\begin{tabular}{|c|c|c|c|c|}
\hline ENSBTAG000000005945 & $1.16 \mathrm{E}-10$ & 4.87E-11 & NXPE4 & $2.38 \mathrm{E}+00$ \\
\hline ENSBTAG000000027637 & $7.78 \mathrm{E}-10$ & $3.25 \mathrm{E}-10$ & & $2.39 E+00$ \\
\hline ENSBTAG000000038049 & $1.89 \mathrm{E}-09$ & 7.83E-10 & & $2.41 E+00$ \\
\hline ENSBTAG000000046460 & 1.33E-09 & $5.55 \mathrm{E}-10$ & & $2.40 E+00$ \\
\hline ENSBTAG000000016267 & $9.84 \mathrm{E}-10$ & $4.08 \mathrm{E}-10$ & SERPING1 & $2.41 E+00$ \\
\hline ENSBTAG00000006755 & $6.33 \mathrm{E}-10$ & $2.61 \mathrm{E}-10$ & C11orf58 & $2.43 E+00$ \\
\hline ENSBTAG00000012941 & $1.80 \mathrm{E}-10$ & $7.32 \mathrm{E}-11$ & IFT46 & $2.46 \mathrm{E}+00$ \\
\hline ENSBTAG000000005259 & $7.54 \mathrm{E}-10$ & $3.06 \mathrm{E}-10$ & UCP3 & $2.47 E+00$ \\
\hline ENSBTAG00000015013 & $2.07 E-10$ & $8.34 \mathrm{E}-11$ & $\mathrm{C} 2 \mathrm{CD} 2 \mathrm{~L}$ & $2.48 \mathrm{E}+00$ \\
\hline ENSBTAG000000010791 & $3.01 \mathrm{E}-10$ & $1.21 \mathrm{E}-10$ & HTR3A & $2.48 \mathrm{E}+00$ \\
\hline ENSBTAG000000027516 & 5.07E-10 & $2.03 \mathrm{E}-10$ & APLNR & $2.50 \mathrm{E}+00$ \\
\hline ENSBTAG000000044177 & $9.86 \mathrm{E}-10$ & $3.93 \mathrm{E}-10$ & C11orf53 & $2.51 E+00$ \\
\hline ENSBTAG00000017468 & $8.05 \mathrm{E}-10$ & $3.20 \mathrm{E}-10$ & NUCB2 & $2.52 \mathrm{E}+00$ \\
\hline ENSBTAG00000020999 & $1.54 \mathrm{E}-10$ & $6.11 \mathrm{E}-11$ & DDB2 & $2.51 E+00$ \\
\hline ENSBTAG000000030920 & $9.97 \mathrm{E}-10$ & $3.94 \mathrm{E}-10$ & & $2.53 E+00$ \\
\hline ENSBTAG000000047556 & 1.40E-09 & $5.51 E-10$ & & $2.54 \mathrm{E}+00$ \\
\hline ENSBTAG000000021342 & $1.58 \mathrm{E}-10$ & $6.11 E-11$ & TMEM136 & $2.58 \mathrm{E}+00$ \\
\hline ENSBTAG000000002382 & $6.10 \mathrm{E}-10$ & $2.32 \mathrm{E}-10$ & DDX10 & $2.63 E+00$ \\
\hline ENSBTAG000000000986 & $6.83 \mathrm{E}-10$ & $2.57 \mathrm{E}-10$ & USH1C & $2.66 \mathrm{E}+00$ \\
\hline ENSBTAG000000039773 & $5.98 \mathrm{E}-10$ & $2.25 \mathrm{E}-10$ & & $2.66 \mathrm{E}+00$ \\
\hline ENSBTAG00000018093 & $1.61 \mathrm{E}-10$ & $6.03 \mathrm{E}-11$ & КМт2A & $2.66 \mathrm{E}+00$ \\
\hline ENSBTAG000000035027 & 8.59E-10 & 3.23E-10 & & $2.66 \mathrm{E}+00$ \\
\hline ENSBTAG000000040425 & $8.87 \mathrm{E}-10$ & 3.33E-10 & & $2.66 \mathrm{E}+00$ \\
\hline ENSBTAG000000013156 & $9.14 \mathrm{E}-10$ & $3.43 \mathrm{E}-10$ & & $2.66 \mathrm{E}+00$ \\
\hline ENSBTAG00000047021 & $8.38 \mathrm{E}-10$ & 3.15E-10 & & $2.66 \mathrm{E}+00$ \\
\hline ENSBTAG000000005913 & $8.03 E-10$ & $3.01 \mathrm{E}-10$ & PATL1 & $2.67 E+00$ \\
\hline ENSBTAG000000045878 & 2.19E-09 & 8.17E-10 & & $2.68 \mathrm{E}+00$ \\
\hline ENSBTAG00000021709 & $1.85 \mathrm{E}-10$ & $6.73 \mathrm{E}-11$ & SPI1 & $2.74 \mathrm{E}+00$ \\
\hline ENSBTAG000000004639 & $1.47 \mathrm{E}-10$ & $5.28 \mathrm{E}-11$ & HARBI1 & $2.78 \mathrm{E}+00$ \\
\hline ENSBTAG000000020793 & $2.31 \mathrm{E}-10$ & $8.30 \mathrm{E}-11$ & KCNA4 & $2.78 \mathrm{E}+00$ \\
\hline ENSBTAG000000040236 & $6.02 \mathrm{E}-10$ & $2.16 \mathrm{E}-10$ & & $2.79 E+00$ \\
\hline ENSBTAG000000035029 & $6.12 \mathrm{E}-10$ & 2.19E-10 & & $2.79 E+00$ \\
\hline ENSBTAG000000037384 & $6.16 \mathrm{E}-10$ & $2.21 \mathrm{E}-10$ & & $2.78 \mathrm{E}+00$ \\
\hline ENSBTAG000000039330 & $5.98 \mathrm{E}-10$ & $2.14 \mathrm{E}-10$ & & $2.79 E+00$ \\
\hline ENSBTAG00000008236 & $5.98 \mathrm{E}-10$ & $2.14 \mathrm{E}-10$ & OR51E2 & $2.79 \mathrm{E}+00$ \\
\hline ENSBTAG00000027490 & $1.22 \mathrm{E}-09$ & 4.36E-10 & OR5A1 & $2.80 \mathrm{E}+00$ \\
\hline ENSBTAG00000048037 & $1.26 \mathrm{E}-09$ & 4.49E-10 & & $2.80 \mathrm{E}+00$ \\
\hline ENSBTAG00000013109 & $8.38 \mathrm{E}-10$ & $2.97 \mathrm{E}-10$ & STIM1 & $2.82 \mathrm{E}+00$ \\
\hline ENSBTAG00000003052 & $3.98 \mathrm{E}-10$ & $1.39 \mathrm{E}-10$ & PCSK7 & $2.86 \mathrm{E}+00$ \\
\hline ENSBTAG00000006974 & $4.76 \mathrm{E}-10$ & $1.65 \mathrm{E}-10$ & PLEKHA7 & $2.89 E+00$ \\
\hline ENSBTAG00000027524 & $7.36 \mathrm{E}-10$ & $2.55 \mathrm{E}-10$ & SMTNL1 & $2.89 \mathrm{E}+00$ \\
\hline ENSBTAG00000016768 & $9.36 \mathrm{E}-10$ & $3.22 \mathrm{E}-10$ & SCN3B & $2.91 \mathrm{E}+00$ \\
\hline ENSBTAG00000001537 & $1.35 \mathrm{E}-10$ & $4.62 \mathrm{E}-11$ & TSKU & $2.91 E+00$ \\
\hline ENSBTAG00000002999 & $5.69 \mathrm{E}-10$ & $1.95 \mathrm{E}-10$ & ANAPC15 & $2.92 \mathrm{E}+00$ \\
\hline ENSBTAG00000000700 & $5.32 \mathrm{E}-10$ & $1.82 \mathrm{E}-10$ & PARVA & $2.92 \mathrm{E}+00$ \\
\hline ENSBTAG00000039050 & $5.51 \mathrm{E}-10$ & $1.89 \mathrm{E}-10$ & $\mathrm{P} 2 \mathrm{RY} 2$ & $2.92 \mathrm{E}+00$ \\
\hline ENSBTAG00000005364 & $1.24 \mathrm{E}-10$ & 4.24E-11 & HMBS & $2.91 E+00$ \\
\hline ENSBTAG00000015810 & $8.24 \mathrm{E}-10$ & $2.82 \mathrm{E}-10$ & PLET1 & $2.92 \mathrm{E}+00$ \\
\hline ENSBTAG00000033506 & $9.70 \mathrm{E}-10$ & $3.31 \mathrm{E}-10$ & & $2.93 E+00$ \\
\hline ENSBTAG00000009830 & $1.96 \mathrm{E}-10$ & $6.68 \mathrm{E}-11$ & PLEKHB1 & $2.94 \mathrm{E}+00$ \\
\hline ENSBTAG00000016146 & $1.96 \mathrm{E}-09$ & $6.64 \mathrm{E}-10$ & & $2.95 E+00$ \\
\hline ENSBTAG00000012989 & 8.75E-10 & $2.95 \mathrm{E}-10$ & UBE2L6 & $2.97 E+00$ \\
\hline ENSBTAG00000039331 & $1.22 \mathrm{E}-09$ & 4.07E-10 & & $3.00 E+00$ \\
\hline ENSBTAG00000020980 & $6.30 \mathrm{E}-10$ & 2.07E-10 & САT & $3.04 E+00$ \\
\hline ENSBTAG00000002404 & 4.73E-10 & $1.55 \mathrm{E}-10$ & TMX2 & $3.05 E+00$ \\
\hline ENSBTAG00000012820 & $9.15 \mathrm{E}-10$ & $2.99 \mathrm{E}-10$ & STX3 & $3.06 \mathrm{E}+00$ \\
\hline ENSBTAG00000002260 & 4.35E-10 & 1.40E-10 & NCAPD3 & $3.10 E+00$ \\
\hline ENSBTAG00000031017 & $7.50 \mathrm{E}-10$ & $2.40 \mathrm{E}-10$ & & $3.12 E+00$ \\
\hline ENSBTAG00000006282 & $1.67 \mathrm{E}-10$ & 5.37E-11 & POU2AF1 & $3.11 E+00$ \\
\hline ENSBTAG00000003955 & $6.20 \mathrm{E}-10$ & $1.96 \mathrm{E}-10$ & MYO7A & $3.16 \mathrm{E}+00$ \\
\hline ENSBTAG00000014594 & $3.12 \mathrm{E}-10$ & $9.82 \mathrm{E}-11$ & & $3.18 \mathrm{E}+00$ \\
\hline ENSBTAG00000040478 & $3.50 \mathrm{E}-10$ & $1.10 \mathrm{E}-10$ & & $3.18 \mathrm{E}+00$ \\
\hline ENSBTAG00000045850 & $3.48 \mathrm{E}-10$ & $1.09 \mathrm{E}-10$ & & $3.19 E+00$ \\
\hline ENSBTAG00000046624 & $3.98 \mathrm{E}-10$ & $1.25 \mathrm{E}-10$ & & $3.19 E+00$ \\
\hline ENSBTAG00000033699 & $4.66 \mathrm{E}-10$ & $1.46 \mathrm{E}-10$ & & $3.19 E+00$ \\
\hline ENSBTAG00000046845 & $7.00 \mathrm{E}-10$ & 2.19E-10 & & $3.19 E+00$ \\
\hline ENSBTAG00000027489 & $6.97 \mathrm{E}-10$ & $2.19 \mathrm{E}-10$ & OR4D6 & $3.18 \mathrm{E}+00$ \\
\hline ENSBTAG00000000770 & 4.85E-10 & $1.51 \mathrm{E}-10$ & PGM2L1 & $3.21 E+00$ \\
\hline ENSBTAG00000002520 & $1.78 \mathrm{E}-10$ & $5.55 \mathrm{E}-11$ & CELF1 & $3.21 E+00$ \\
\hline ENSBTAG00000019358 & $3.65 \mathrm{E}-10$ & $1.13 \mathrm{E}-10$ & RNF214 & $3.23 E+00$ \\
\hline
\end{tabular}




\begin{tabular}{|c|c|c|c|c|}
\hline ENSBTAG00000004580 & $7.56 \mathrm{E}-10$ & $2.33 \mathrm{E}-10$ & MS4A1 & $3.24 E+00$ \\
\hline ENSBTAG00000003721 & $5.58 \mathrm{E}-10$ & $1.68 \mathrm{E}-10$ & CHST1 & $3.32 E+00$ \\
\hline ENSBTAG00000030847 & $9.48 \mathrm{E}-10$ & $2.83 \mathrm{E}-10$ & GLYATL2 & $3.35 E+00$ \\
\hline ENSBTAG00000035686 & $1.78 \mathrm{E}-09$ & $5.26 \mathrm{E}-10$ & & $3.38 \mathrm{E}+00$ \\
\hline ENSBTAG00000019549 & $1.12 \mathrm{E}-09$ & $3.22 \mathrm{E}-10$ & OR51M1 & $3.47 E+00$ \\
\hline ENSBTAG00000046825 & $1.11 \mathrm{E}-09$ & $3.19 \mathrm{E}-10$ & & $3.47 E+00$ \\
\hline ENSBTAG00000039056 & $1.00 \mathrm{E}-09$ & $2.86 \mathrm{E}-10$ & & $3.51 E+00$ \\
\hline ENSBTAG00000019365 & $2.35 \mathrm{E}-10$ & $6.68 \mathrm{E}-11$ & BACE1 & $3.52 E+00$ \\
\hline ENSBTAG00000039236 & 7.97E-10 & $2.24 \mathrm{E}-10$ & & $3.56 E+00$ \\
\hline ENSBTAG00000020033 & $7.88 \mathrm{E}-10$ & $2.19 \mathrm{E}-10$ & & $3.60 \mathrm{E}+00$ \\
\hline ENSBTAG00000047477 & $7.90 \mathrm{E}-10$ & $2.20 \mathrm{E}-10$ & & $3.59 E+00$ \\
\hline ENSBTAG00000012053 & $4.48 \mathrm{E}-10$ & 1.23E-10 & ACCSL & $3.64 \mathrm{E}+00$ \\
\hline ENSBTAG00000002381 & $6.03 \mathrm{E}-10$ & $1.64 \mathrm{E}-10$ & ZDHHC5 & $3.68 \mathrm{E}+00$ \\
\hline ENSBTAG00000048316 & $2.84 \mathrm{E}-10$ & $7.70 \mathrm{E}-11$ & & $3.69 E+00$ \\
\hline ENSBTAG00000027275 & 2.07E-09 & $5.58 \mathrm{E}-10$ & & $3.71 E+00$ \\
\hline ENSBTAG00000021744 & $1.88 \mathrm{E}-10$ & $5.06 \mathrm{E}-11$ & PSMC3 & $3.70 E+00$ \\
\hline ENSBTAG00000030898 & $5.61 \mathrm{E}-10$ & $1.50 \mathrm{E}-10$ & MS4A13 & $3.74 \mathrm{E}+00$ \\
\hline ENSBTAG00000037538 & $1.68 \mathrm{E}-09$ & $4.41 \mathrm{E}-10$ & & $3.81 E+00$ \\
\hline ENSBTAG00000021223 & $7.36 \mathrm{E}-10$ & $1.93 \mathrm{E}-10$ & CRY2 & $3.81 E+00$ \\
\hline ENSBTAG00000008347 & $5.94 \mathrm{E}-10$ & $1.54 \mathrm{E}-10$ & MRPL48 & $3.85 E+00$ \\
\hline ENSBTAG00000027477 & 7.11E-10 & $1.83 \mathrm{E}-10$ & B3GAT1 & $3.88 \mathrm{E}+00$ \\
\hline ENSBTAG00000000277 & $3.37 \mathrm{E}-10$ & $8.64 \mathrm{E}-11$ & IL18 & $3.90 E+00$ \\
\hline ENSBTAG00000031609 & $6.47 \mathrm{E}-10$ & $1.65 E-10$ & THAP12 & $3.92 \mathrm{E}+00$ \\
\hline ENSBTAG00000012940 & $9.42 \mathrm{E}-11$ & $2.37 \mathrm{E}-11$ & TMEM25 & $3.96 \mathrm{E}+00$ \\
\hline ENSBTAG00000040023 & $3.91 \mathrm{E}-10$ & $9.84 \mathrm{E}-11$ & & $3.98 \mathrm{E}+00$ \\
\hline ENSBTAG00000047232 & $4.29 \mathrm{E}-10$ & $1.08 \mathrm{E}-10$ & & $3.97 E+00$ \\
\hline ENSBTAG00000007324 & $2.13 \mathrm{E}-10$ & $5.36 \mathrm{E}-11$ & LRRC4C & $3.97 E+00$ \\
\hline ENSBTAG00000038518 & 7.77E-10 & $1.95 \mathrm{E}-10$ & OR2D3 & $3.98 \mathrm{E}+00$ \\
\hline ENSBTAG00000045739 & $1.29 \mathrm{E}-09$ & $3.23 \mathrm{E}-10$ & OR5R1 & $4.01 E+00$ \\
\hline ENSBTAG00000017150 & $3.32 \mathrm{E}-10$ & $8.29 E-11$ & EHF & $4.00 E+00$ \\
\hline ENSBTAG00000037389 & $6.43 \mathrm{E}-10$ & $1.55 \mathrm{E}-10$ & TRIM44 & $4.15 E+00$ \\
\hline ENSBTAG00000010889 & $1.09 \mathrm{E}-09$ & $2.62 \mathrm{E}-10$ & FSHB & $4.16 \mathrm{E}+00$ \\
\hline ENSBTAG00000017901 & 5.37E-10 & $1.24 \mathrm{E}-10$ & LAYN & $4.33 E+00$ \\
\hline ENSBTAG00000037992 & $1.46 \mathrm{E}-09$ & $3.21 \mathrm{E}-10$ & OR51Q1 & $4.55 E+00$ \\
\hline ENSBTAG00000001922 & $6.56 \mathrm{E}-11$ & $1.38 \mathrm{E}-11$ & AKIP1 & $4.72 \mathrm{E}+00$ \\
\hline ENSBTAG00000006093 & $3.23 \mathrm{E}-10$ & $6.77 \mathrm{E}-11$ & & $4.77 E+00$ \\
\hline ENSBTAG00000035031 & $5.24 \mathrm{E}-10$ & $1.10 \mathrm{E}-10$ & & $4.76 \mathrm{E}+00$ \\
\hline ENSBTAG00000016119 & $5.20 \mathrm{E}-10$ & $1.09 \mathrm{E}-10$ & & $4.77 \mathrm{E}+00$ \\
\hline ENSBTAG00000018490 & $5.17 \mathrm{E}-10$ & $1.08 \mathrm{E}-10$ & OR2D2 & $4.78 \mathrm{E}+00$ \\
\hline ENSBTAG00000040017 & $5.22 \mathrm{E}-10$ & $1.09 \mathrm{E}-10$ & & $4.79 E+00$ \\
\hline ENSBTAG00000003023 & $5.19 \mathrm{E}-10$ & $1.09 \mathrm{E}-10$ & & $4.76 \mathrm{E}+00$ \\
\hline ENSBTAG00000047860 & $8.46 \mathrm{E}-10$ & $1.76 \mathrm{E}-10$ & & $4.80 E+00$ \\
\hline ENSBTAG00000006421 & $1.04 \mathrm{E}-09$ & $2.17 \mathrm{E}-10$ & OR5AR1 & $4.80 E+00$ \\
\hline ENSBTAG00000000232 & $1.02 \mathrm{E}-09$ & $2.12 \mathrm{E}-10$ & OR51D1 & $4.79 E+00$ \\
\hline ENSBTAG00000008342 & $1.03 \mathrm{E}-09$ & $2.12 \mathrm{E}-10$ & TMPRSS13 & $4.86 \mathrm{E}+00$ \\
\hline ENSBTAG00000000347 & $6.91 \mathrm{E}-10$ & $1.38 \mathrm{E}-10$ & RHOG & $5.01 E+00$ \\
\hline ENSBTAG00000019168 & $5.46 \mathrm{E}-10$ & $1.05 \mathrm{E}-10$ & $\mathrm{PHOX} 2 \mathrm{~A}$ & $5.20 \mathrm{E}+00$ \\
\hline ENSBTAG00000014167 & $8.53 \mathrm{E}-10$ & $1.64 \mathrm{E}-10$ & RNF121 & $5.20 \mathrm{E}+00$ \\
\hline ENSBTAG00000031937 & $1.98 \mathrm{E}-09$ & $3.63 \mathrm{E}-10$ & & $5.45 E+00$ \\
\hline ENSBTAG00000008419 & $6.18 \mathrm{E}-10$ & $1.12 \mathrm{E}-10$ & PDE2A & $5.51 E+00$ \\
\hline ENSBTAG00000048307 & $5.36 \mathrm{E}-10$ & $9.60 \mathrm{E}-11$ & & $5.57 \mathrm{E}+00$ \\
\hline ENSBTAG00000031924 & $6.04 \mathrm{E}-10$ & $1.08 \mathrm{E}-10$ & OR52M1 & $5.59 E+00$ \\
\hline ENSBTAG00000039253 & $6.00 \mathrm{E}-10$ & $1.07 \mathrm{E}-10$ & OR51F2 & $5.60 E+00$ \\
\hline ENSBTAG00000008432 & $5.22 \mathrm{E}-10$ & $9.27 \mathrm{E}-11$ & NUP98 & $5.63 E+00$ \\
\hline ENSBTAG00000000376 & $7.20 \mathrm{E}-10$ & $1.28 \mathrm{E}-10$ & $\mathrm{P} 2 \mathrm{R} X 3$ & $5.62 E+00$ \\
\hline ENSBTAG00000020308 & $1.82 \mathrm{E}-10$ & $2.99 \mathrm{E}-11$ & EIF4G2 & $6.07 E+00$ \\
\hline ENSBTAG00000039178 & $1.23 \mathrm{E}-09$ & $1.99 \mathrm{E}-10$ & & $6.16 E+00$ \\
\hline ENSBTAG00000003638 & $9.76 \mathrm{E}-10$ & $1.58 \mathrm{E}-10$ & ZNF215 & $6.17 E+00$ \\
\hline ENSBTAG00000038899 & $6.82 \mathrm{E}-10$ & $1.07 E-10$ & & $6.37 E+00$ \\
\hline ENSBTAG00000039298 & $6.86 \mathrm{E}-10$ & $1.07 \mathrm{E}-10$ & & $6.41 E+00$ \\
\hline ENSBTAG00000003000 & $5.63 \mathrm{E}-10$ & $8.82 \mathrm{E}-11$ & TOMT & $6.37 E+00$ \\
\hline ENSBTAG00000048214 & $7.18 \mathrm{E}-10$ & $1.12 \mathrm{E}-10$ & & $6.41 E+00$ \\
\hline ENSBTAG00000007944 & $6.97 \mathrm{E}-10$ & $1.09 \mathrm{E}-10$ & & $6.39 E+00$ \\
\hline ENSBTAG00000020007 & $9.61 \mathrm{E}-10$ & $1.50 \mathrm{E}-10$ & & $6.40 E+00$ \\
\hline ENSBTAG00000037815 & $1.48 \mathrm{E}-09$ & $2.24 \mathrm{E}-10$ & & $6.61 E+00$ \\
\hline ENSBTAG00000021745 & $2.28 \mathrm{E}-10$ & 3.37E-11 & RAPSN & $6.74 \mathrm{E}+00$ \\
\hline ENSBTAG00000047396 & $1.51 E-09$ & $2.21 \mathrm{E}-10$ & & $6.84 \mathrm{E}+00$ \\
\hline ENSBTAG00000025266 & 1.09E-09 & $1.56 \mathrm{E}-10$ & UBQLN3 & $7.01 E+00$ \\
\hline ENSBTAG00000047968 & $3.59 \mathrm{E}-10$ & $5.00 \mathrm{E}-11$ & BTBD18 & $7.16 \mathrm{E}+00$ \\
\hline ENSBTAG00000015015 & $1.68 \mathrm{E}-10$ & $2.35 \mathrm{E}-11$ & HINFP & $7.14 \mathrm{E}+00$ \\
\hline
\end{tabular}




\begin{tabular}{|c|c|c|c|c|}
\hline ENSBTAG00000008433 & $4.93 \mathrm{E}-10$ & $6.88 \mathrm{E}-11$ & PGAP2 & $7.16 \mathrm{E}+00$ \\
\hline ENSBTAG00000045709 & $7.88 \mathrm{E}-10$ & 1.10E-10 & & $7.15 E+00$ \\
\hline ENSBTAG00000036025 & $8.02 \mathrm{E}-10$ & $1.12 \mathrm{E}-10$ & & $7.16 \mathrm{E}+00$ \\
\hline ENSBTAG00000002389 & $6.97 \mathrm{E}-10$ & $8.99 \mathrm{E}-11$ & MED19 & $7.75 E+00$ \\
\hline ENSBTAG00000038748 & $5.01 \mathrm{E}-10$ & $6.28 \mathrm{E}-11$ & $\mathrm{HBB}$ & $7.96 \mathrm{E}+00$ \\
\hline ENSBTAG00000046923 & $1.23 \mathrm{E}-09$ & $1.49 \mathrm{E}-10$ & & $8.27 E+00$ \\
\hline ENSBTAG00000006493 & $2.91 \mathrm{E}-10$ & $3.32 \mathrm{E}-11$ & CLP1 & $8.73 E+00$ \\
\hline ENSBTAG00000031028 & $9.77 \mathrm{E}-10$ & $1.11 \mathrm{E}-10$ & & $8.79 E+00$ \\
\hline ENSBTAG00000048052 & $1.63 \mathrm{E}-09$ & $1.84 \mathrm{E}-10$ & & $8.85 E+00$ \\
\hline ENSBTAG00000040185 & $1.03 \mathrm{E}-09$ & 1.07E-10 & OR51V1 & $9.61 E+00$ \\
\hline ENSBTAG00000002051 & $6.84 \mathrm{E}-10$ & $6.98 \mathrm{E}-11$ & & $9.79 E+00$ \\
\hline ENSBTAG00000010005 & $1.03 \mathrm{E}-09$ & $9.86 \mathrm{E}-11$ & & $1.04 \mathrm{E}+01$ \\
\hline ENSBTAG00000037826 & 1.47E-09 & $1.41 \mathrm{E}-10$ & & $1.05 \mathrm{E}+01$ \\
\hline ENSBTAG00000027676 & $5.87 \mathrm{E}-10$ & $5.25 \mathrm{E}-11$ & IL18BP & $1.12 \mathrm{E}+01$ \\
\hline ENSBTAG00000020289 & $7.82 \mathrm{E}-10$ & $6.99 \mathrm{E}-11$ & ZNF214 & $1.12 \mathrm{E}+01$ \\
\hline ENSBTAG00000038532 & $8.66 \mathrm{E}-10$ & $7.56 \mathrm{E}-11$ & & $1.14 \mathrm{E}+01$ \\
\hline ENSBTAG00000019246 & $5.30 \mathrm{E}-10$ & $4.32 \mathrm{E}-11$ & & $1.22 \mathrm{E}+01$ \\
\hline ENSBTAG00000021007 & $9.39 \mathrm{E}-10$ & $7.51 \mathrm{E}-11$ & FOLR1 & $1.25 \mathrm{E}+01$ \\
\hline ENSBTAG00000016170 & 4.97E-10 & $3.89 \mathrm{E}-11$ & KCNJ11 & $1.27 \mathrm{E}+01$ \\
\hline ENSBTAG00000019764 & $6.15 \mathrm{E}-10$ & $4.82 \mathrm{E}-11$ & APOA5 & $1.27 \mathrm{E}+01$ \\
\hline ENSBTAG00000024701 & $1.05 \mathrm{E}-09$ & $7.96 \mathrm{E}-11$ & & $1.32 \mathrm{E}+01$ \\
\hline ENSBTAG00000004901 & $5.30 \mathrm{E}-10$ & $3.91 \mathrm{E}-11$ & & $1.35 \mathrm{E}+01$ \\
\hline ENSBTAG00000016807 & 2.06E-09 & $1.11 \mathrm{E}-10$ & & $1.85 \mathrm{E}+01$ \\
\hline ENSBTAG00000036042 & 2.05E-09 & $1.10 \mathrm{E}-10$ & & $1.86 \mathrm{E}+01$ \\
\hline ENSBTAG00000016469 & $9.06 \mathrm{E}-10$ & 4.39E-11 & TNKS1BP1 & $2.06 \mathrm{E}+01$ \\
\hline ENSBTAG00000019167 & 7.67E-10 & $3.66 \mathrm{E}-11$ & INPPL1 & $2.09 \mathrm{E}+01$ \\
\hline ENSBTAG00000000375 & $4.56 \mathrm{E}-10$ & $2.16 \mathrm{E}-11$ & SSRP1 & $2.10 \mathrm{E}+01$ \\
\hline ENSBTAG00000014855 & $4.82 \mathrm{E}-10$ & 2.17E-11 & MICAL2 & $2.21 E+01$ \\
\hline ENSBTAG00000008193 & $3.23 \mathrm{E}-10$ & 4.89E-12 & MICALCL & $6.48 \mathrm{E}+01$ \\
\hline ENSBTAG00000013900 & $6.19 \mathrm{E}-10$ & $9.23 \mathrm{E}-12$ & TRIM21 & $6.63 E+01$ \\
\hline ENSBTAG00000037488 & 0 & 0 & JRKL & $1.00 \mathrm{E}+00$ \\
\hline ENSBTAG00000045821 & 0 & 0 & & $1.00 \mathrm{E}+00$ \\
\hline ENSBTAG00000014899 & $2.04 \mathrm{E}-10$ & 0 & TTC36 & $2.04 E+03$ \\
\hline ENSBTAG00000005371 & $1.36 \mathrm{E}-10$ & 0 & DPAGT1 & $1.36 \mathrm{E}+03$ \\
\hline ENSBTAG00000027766 & 0 & 0 & C1QTNF5 & $1.00 \mathrm{E}+00$ \\
\hline ENSBTAG00000013994 & 4.44E-11 & 0 & $B S X$ & $4.45 E+02$ \\
\hline ENSBTAG00000015244 & $4.13 \mathrm{E}-10$ & 0 & & $4.13 E+03$ \\
\hline ENSBTAG00000040308 & $8.80 \mathrm{E}-10$ & 0 & & $8.80 E+03$ \\
\hline ENSBTAG00000011914 & 0 & 0 & RASSF10 & $1.00 \mathrm{E}+00$ \\
\hline ENSBTAG00000047961 & $1.39 \mathrm{E}-09$ & 0 & & $1.39 E+04$ \\
\hline ENSBTAG00000010568 & $3.41 \mathrm{E}-10$ & 0 & ZBED5 & $3.41 E+03$ \\
\hline ENSBTAG00000032339 & 0 & 0 & $\mathrm{ASCL3}$ & $1.00 \mathrm{E}+00$ \\
\hline ENSBTAG00000027642 & $1.20 \mathrm{E}-10$ & 0 & C11orf42 & $1.20 E+03$ \\
\hline ENSBTAG00000047847 & $2.57 \mathrm{E}-10$ & 0 & & $2.58 \mathrm{E}+03$ \\
\hline ENSBTAG00000012325 & $3.08 \mathrm{E}-10$ & 0 & & $3.08 \mathrm{E}+03$ \\
\hline ENSBTAG00000027684 & $8.80 \mathrm{E}-10$ & 0 & & $8.80 E+03$ \\
\hline ENSBTAG00000027578 & $2.09 \mathrm{E}-10$ & 0 & & $2.09 E+03$ \\
\hline ENSBTAG00000000487 & $6.46 \mathrm{E}-10$ & 0 & & $6.46 \mathrm{E}+03$ \\
\hline ENSBTAG00000047298 & $5.49 \mathrm{E}-11$ & 0 & & $5.50 \mathrm{E}+02$ \\
\hline ENSBTAG00000038160 & $2.00 \mathrm{E}-10$ & 0 & & $2.00 \mathrm{E}+03$ \\
\hline ENSBTAG00000016082 & $5.06 \mathrm{E}-11$ & 0 & & $5.07 \mathrm{E}+02$ \\
\hline ENSBTAG00000040339 & $5.16 \mathrm{E}-10$ & 0 & & $5.16 \mathrm{E}+03$ \\
\hline ENSBTAG00000046627 & $8.64 \mathrm{E}-11$ & 0 & OR51L1 & $8.65 E+02$ \\
\hline ENSBTAG00000046816 & 0 & 0 & OR51G1 & $1.00 E+00$ \\
\hline ENSBTAG00000038170 & 0 & 0 & & $1.00 \mathrm{E}+00$ \\
\hline ENSBTAG00000000233 & $8.58 \mathrm{E}-11$ & 0 & OR51E1 & $8.59 E+02$ \\
\hline ENSBTAG00000013683 & $4.33 \mathrm{E}-10$ & 0 & & $4.33 E+03$ \\
\hline ENSBTAG00000040075 & 5.17E-10 & 0 & & $5.17 E+03$ \\
\hline ENSBTAG00000034724 & $1.75 \mathrm{E}-09$ & 0 & & $1.75 E+04$ \\
\hline ENSBTAG00000039149 & $2.52 \mathrm{E}-10$ & 0 & CLDN25 & $2.52 E+03$ \\
\hline ENSBTAG00000012562 & $6.29 \mathrm{E}-11$ & 0 & RNF26 & $6.30 E+02$ \\
\hline ENSBTAG00000040051 & 0 & 0 & & $1.00 \mathrm{E}+00$ \\
\hline ENSBTAG00000045599 & $6.04 \mathrm{E}-10$ & 0 & & $6.04 E+03$ \\
\hline ENSBTAG00000004443 & $2.57 \mathrm{E}-10$ & 0 & & $2.57 E+03$ \\
\hline ENSBTAG00000008892 & $8.50 \mathrm{E}-11$ & 0 & OR52W1 & $8.51 E+02$ \\
\hline ENSBTAG00000032256 & $1.02 \mathrm{E}-10$ & 0 & & $1.02 E+03$ \\
\hline ENSBTAG00000012546 & 0 & 0 & & $1.00 \mathrm{E}+00$ \\
\hline ENSBTAG00000005624 & 1.37E-09 & 0 & & $1.37 E+04$ \\
\hline ENSBTAG00000003056 & $7.68 \mathrm{E}-10$ & 0 & & $7.68 \mathrm{E}+03$ \\
\hline ENSBTAG00000001291 & $9.67 \mathrm{E}-10$ & 0 & & $9.67 \mathrm{E}+03$ \\
\hline
\end{tabular}




\begin{tabular}{|c|c|c|c|c|}
\hline ENSBTAG00000046983 & $8.72 \mathrm{E}-11$ & 0 & & $8.73 E+02$ \\
\hline ENSBTAG00000046921 & $1.73 \mathrm{E}-10$ & 0 & & $1.74 E+03$ \\
\hline ENSBTAG00000039651 & $2.54 \mathrm{E}-10$ & 0 & & $2.54 E+03$ \\
\hline ENSBTAG00000037523 & 0 & $1.04 \mathrm{E}-10$ & B3GALT6 & $9.61 \mathrm{E}-04$ \\
\hline ENSBTAG00000018143 & $6.23 \mathrm{E}-11$ & $6.20 \mathrm{E}-10$ & C1orf116 & $1.01 \mathrm{E}-01$ \\
\hline ENSBTAG00000006692 & $7.59 \mathrm{E}-11$ & 7.11E-10 & IL19 & $1.07 \mathrm{E}-01$ \\
\hline ENSBTAG00000047603 & $1.97 \mathrm{E}-10$ & $9.96 \mathrm{E}-10$ & & $1.97 \mathrm{E}-01$ \\
\hline ENSBTAG00000033252 & $2.04 \mathrm{E}-10$ & 1.03E-09 & & $1.98 \mathrm{E}-01$ \\
\hline ENSBTAG00000002757 & $1.62 \mathrm{E}-10$ & $8.21 \mathrm{E}-10$ & IL20 & $1.98 \mathrm{E}-01$ \\
\hline ENSBTAG00000019799 & $1.63 \mathrm{E}-10$ & $5.40 \mathrm{E}-10$ & FCAMR & $3.02 \mathrm{E}-01$ \\
\hline ENSBTAG00000002126 & $9.69 \mathrm{E}-11$ & $2.98 \mathrm{E}-10$ & PFKFB2 & $3.26 \mathrm{E}-01$ \\
\hline ENSBTAG00000039764 & $2.52 \mathrm{E}-10$ & 7.44E-10 & IER5 & $3.39 \mathrm{E}-01$ \\
\hline ENSBTAG00000002854 & $2.46 \mathrm{E}-10$ & $6.92 \mathrm{E}-10$ & TMEM63A & $3.55 \mathrm{E}-01$ \\
\hline ENSBTAG00000014705 & 8.07E-11 & $2.03 \mathrm{E}-10$ & HES4 & $3.98 \mathrm{E}-01$ \\
\hline ENSBTAG00000020273 & $1.46 \mathrm{E}-10$ & $3.22 \mathrm{E}-10$ & YOD1 & 4.53E-01 \\
\hline ENSBTAG00000005835 & $3.52 \mathrm{E}-10$ & $7.32 \mathrm{E}-10$ & & 4.81E-01 \\
\hline ENSBTAG00000019339 & $1.03 \mathrm{E}-10$ & $2.08 \mathrm{E}-10$ & & 4.95E-01 \\
\hline ENSBTAG00000007997 & $5.03 \mathrm{E}-10$ & $9.98 \mathrm{E}-10$ & & $5.04 \mathrm{E}-01$ \\
\hline ENSBTAG00000048308 & $8.68 \mathrm{E}-11$ & $1.64 \mathrm{E}-10$ & ICMT & $5.29 \mathrm{E}-01$ \\
\hline ENSBTAG00000010803 & $1.68 \mathrm{E}-10$ & 3.17E-10 & CHML & $5.29 \mathrm{E}-01$ \\
\hline ENSBTAG00000004064 & $3.61 \mathrm{E}-10$ & $6.73 \mathrm{E}-10$ & BPNT1 & $5.36 \mathrm{E}-01$ \\
\hline ENSBTAG00000018723 & $2.38 \mathrm{E}-10$ & $4.15 E-10$ & SLC25A34 & 5.73E-01 \\
\hline ENSBTAG00000009618 & $2.32 \mathrm{E}-10$ & $4.06 \mathrm{E}-10$ & NEK2 & 5.73E-01 \\
\hline ENSBTAG00000000140 & $2.94 \mathrm{E}-10$ & $5.01 \mathrm{E}-10$ & EPHX1 & 5.87E-01 \\
\hline ENSBTAG00000000792 & $2.23 \mathrm{E}-10$ & 3.63E-10 & AJAP1 & $6.16 \mathrm{E}-01$ \\
\hline ENSBTAG00000000772 & $2.74 \mathrm{E}-10$ & 4.15E-10 & & $6.61 \mathrm{E}-01$ \\
\hline ENSBTAG00000045754 & $1.09 \mathrm{E}-10$ & $1.65 \mathrm{E}-10$ & SMIM1 & $6.60 \mathrm{E}-01$ \\
\hline ENSBTAG00000007606 & $9.49 \mathrm{E}-11$ & $1.41 \mathrm{E}-10$ & HNRNPU & $6.73 \mathrm{E}-01$ \\
\hline ENSBTAG00000000759 & $5.50 \mathrm{E}-10$ & 8.05E-10 & NENF & $6.84 \mathrm{E}-01$ \\
\hline ENSBTAG00000002266 & $3.60 \mathrm{E}-10$ & $5.23 \mathrm{E}-10$ & NPL & $6.88 \mathrm{E}-01$ \\
\hline ENSBTAG00000014059 & $1.59 \mathrm{E}-10$ & $2.28 \mathrm{E}-10$ & ATP2B4 & $6.95 \mathrm{E}-01$ \\
\hline ENSBTAG00000046655 & $3.33 \mathrm{E}-10$ & 4.80E-10 & & $6.94 \mathrm{E}-01$ \\
\hline ENSBTAG00000014319 & $1.54 \mathrm{E}-10$ & 2.19E-10 & & 7.04E-01 \\
\hline ENSBTAG00000012389 & $3.00 \mathrm{E}-10$ & 4.15E-10 & & 7.22E-01 \\
\hline ENSBTAG00000018722 & $3.40 \mathrm{E}-10$ & $4.68 \mathrm{E}-10$ & PLEKHM2 & 7.27E-01 \\
\hline ENSBTAG00000007851 & $7.46 \mathrm{E}-10$ & $1.02 \mathrm{E}-09$ & IL24 & 7.32E-01 \\
\hline ENSBTAG00000018725 & $1.44 \mathrm{E}-10$ & $1.95 \mathrm{E}-10$ & FBLIM1 & 7.37E-01 \\
\hline ENSBTAG00000010392 & 4.29E-10 & $5.63 \mathrm{E}-10$ & ESRRG & 7.61E-01 \\
\hline ENSBTAG00000030910 & $5.98 \mathrm{E}-10$ & $7.78 \mathrm{E}-10$ & & 7.69E-01 \\
\hline ENSBTAG00000007860 & $2.68 \mathrm{E}-10$ & 3.47E-10 & ASPM & $7.72 \mathrm{E}-01$ \\
\hline ENSBTAG00000013197 & $5.02 \mathrm{E}-10$ & $6.45 \mathrm{E}-10$ & PSEN2 & 7.79E-01 \\
\hline ENSBTAG00000024525 & $1.33 \mathrm{E}-10$ & $1.68 \mathrm{E}-10$ & HES3 & 7.92E-01 \\
\hline ENSBTAG00000011508 & $1.14 \mathrm{E}-10$ & $1.43 \mathrm{E}-10$ & & 7.95E-01 \\
\hline ENSBTAG00000037896 & $1.27 \mathrm{E}-10$ & $1.59 \mathrm{E}-10$ & & 7.96E-01 \\
\hline ENSBTAG00000006030 & $1.15 \mathrm{E}-10$ & $1.45 \mathrm{E}-10$ & MYOG & 7.96E-01 \\
\hline ENSBTAG00000015635 & $5.76 \mathrm{E}-11$ & $7.26 \mathrm{E}-11$ & TNFRSF4 & 7.94E-01 \\
\hline ENSBTAG00000034366 & $5.48 \mathrm{E}-10$ & $6.91 \mathrm{E}-10$ & RGS2 & 7.94E-01 \\
\hline ENSBTAG00000044081 & $9.52 \mathrm{E}-10$ & $1.20 \mathrm{E}-09$ & $\mathrm{AUH}$ & 7.94E-01 \\
\hline ENSBTAG00000024542 & $3.68 \mathrm{E}-10$ & 4.63E-10 & & 7.94E-01 \\
\hline ENSBTAG00000019630 & $4.06 \mathrm{E}-10$ & $5.12 \mathrm{E}-10$ & RGL1 & 7.93E-01 \\
\hline ENSBTAG00000004176 & $1.01 E-09$ & $1.27 \mathrm{E}-09$ & PDC & 7.92E-01 \\
\hline ENSBTAG00000039662 & $4.42 \mathrm{E}-11$ & $5.57 \mathrm{E}-11$ & RSC1A1 & 7.94E-01 \\
\hline ENSBTAG00000022942 & $1.86 \mathrm{E}-10$ & $2.34 \mathrm{E}-10$ & & 7.94E-01 \\
\hline ENSBTAG00000033322 & $1.38 \mathrm{E}-10$ & $1.74 \mathrm{E}-10$ & SRP9 & 7.92E-01 \\
\hline ENSBTAG00000010909 & $2.65 \mathrm{E}-11$ & 3.34E-11 & SLC30A1 & 7.94E-01 \\
\hline ENSBTAG00000000477 & $6.35 \mathrm{E}-11$ & $8.01 \mathrm{E}-11$ & TLR5 & 7.94E-01 \\
\hline ENSBTAG00000014127 & $1.40 \mathrm{E}-10$ & $1.76 \mathrm{E}-10$ & PTGS2 & 7.94E-01 \\
\hline ENSBTAG00000015632 & $1.83 \mathrm{E}-10$ & $2.31 E-10$ & TNFRSF18 & 7.94E-01 \\
\hline ENSBTAG00000002139 & $1.78 \mathrm{E}-10$ & $2.24 \mathrm{E}-10$ & MIXL1 & 7.95E-01 \\
\hline ENSBTAG00000009265 & $4.32 \mathrm{E}-10$ & $5.30 \mathrm{E}-10$ & NR5A2 & $8.15 \mathrm{E}-01$ \\
\hline ENSBTAG00000011340 & $4.12 \mathrm{E}-10$ & $5.02 \mathrm{E}-10$ & NSL1 & $8.21 \mathrm{E}-01$ \\
\hline ENSBTAG00000000070 & $9.25 \mathrm{E}-10$ & 1.13E-09 & F13B & $8.19 \mathrm{E}-01$ \\
\hline ENSBTAG00000009307 & $1.96 \mathrm{E}-10$ & 2.37E-10 & RER1 & 8.27E-01 \\
\hline ENSBTAG00000018724 & $3.68 \mathrm{E}-10$ & 4.42E-10 & TMEM82 & $8.33 \mathrm{E}-01$ \\
\hline ENSBTAG00000037661 & $3.71 \mathrm{E}-10$ & $4.43 \mathrm{E}-10$ & & $8.39 \mathrm{E}-01$ \\
\hline ENSBTAG00000010460 & $2.15 E-10$ & $2.54 \mathrm{E}-10$ & MARK1 & $8.45 \mathrm{E}-01$ \\
\hline ENSBTAG00000003697 & $2.08 \mathrm{E}-10$ & $2.43 \mathrm{E}-10$ & & $8.54 \mathrm{E}-01$ \\
\hline ENSBTAG00000003295 & 4.80E-10 & 5.59E-10 & GORAB & $8.58 \mathrm{E}-01$ \\
\hline ENSBTAG00000004738 & $2.88 \mathrm{E}-10$ & $3.35 \mathrm{E}-10$ & LRRC47 & 8.60E-01 \\
\hline ENSBTAG00000019471 & $1.38 \mathrm{E}-10$ & 1.60E-10 & CNIH4 & $8.64 \mathrm{E}-01$ \\
\hline
\end{tabular}




\begin{tabular}{|c|c|c|c|c|}
\hline ENSBTAG00000038000 & $2.79 \mathrm{E}-10$ & $3.22 \mathrm{E}-10$ & & 8.67E-01 \\
\hline ENSBTAG00000013483 & 7.90E-10 & $9.14 \mathrm{E}-10$ & TAF1A & 8.64E-01 \\
\hline ENSBTAG00000018223 & $6.67 \mathrm{E}-10$ & 7.68E-10 & CHI3L1 & $8.68 \mathrm{E}-01$ \\
\hline ENSBTAG00000018142 & $2.50 \mathrm{E}-10$ & $2.87 \mathrm{E}-10$ & DTL & $8.71 \mathrm{E}-01$ \\
\hline ENSBTAG00000010720 & $7.52 \mathrm{E}-11$ & $8.61 \mathrm{E}-11$ & MIB2 & $8.73 \mathrm{E}-01$ \\
\hline ENSBTAG00000024449 & $2.52 \mathrm{E}-10$ & $2.88 \mathrm{E}-10$ & CENPF & $8.76 \mathrm{E}-01$ \\
\hline ENSBTAG00000016685 & $8.89 \mathrm{E}-11$ & $1.01 \mathrm{E}-10$ & FMO4 & $8.80 \mathrm{E}-01$ \\
\hline ENSBTAG00000001729 & $2.89 \mathrm{E}-10$ & 3.27E-10 & DUSP10 & $8.85 \mathrm{E}-01$ \\
\hline ENSBTAG00000021298 & $1.73 \mathrm{E}-10$ & $1.95 \mathrm{E}-10$ & METTL11B & $8.88 \mathrm{E}-01$ \\
\hline ENSBTAG00000002291 & $5.30 \mathrm{E}-10$ & $5.95 \mathrm{E}-10$ & ZBTB41 & $8.90 \mathrm{E}-01$ \\
\hline ENSBTAG00000011982 & $1.66 \mathrm{E}-10$ & $1.86 \mathrm{E}-10$ & BATF3 & $8.94 \mathrm{E}-01$ \\
\hline ENSBTAG00000008453 & $5.13 \mathrm{E}-10$ & $5.72 \mathrm{E}-10$ & LBR & 8.97E-01 \\
\hline ENSBTAG00000033056 & $5.89 \mathrm{E}-10$ & $6.56 \mathrm{E}-10$ & PLD5 & $8.99 \mathrm{E}-01$ \\
\hline ENSBTAG00000048234 & 4.27E-10 & $4.74 \mathrm{E}-10$ & TMEM9 & $9.00 \mathrm{E}-01$ \\
\hline ENSBTAG00000047287 & $7.18 \mathrm{E}-10$ & 7.91E-10 & 01-Mar & $9.08 \mathrm{E}-01$ \\
\hline ENSBTAG00000012605 & $4.71 \mathrm{E}-10$ & 5.17E-10 & GPR157 & $9.10 \mathrm{E}-01$ \\
\hline ENSBTAG00000017721 & $2.72 \mathrm{E}-10$ & $2.98 \mathrm{E}-10$ & METTL13 & $9.14 \mathrm{E}-01$ \\
\hline ENSBTAG00000000837 & $3.29 \mathrm{E}-10$ & $3.60 \mathrm{E}-10$ & PARP1 & $9.15 \mathrm{E}-01$ \\
\hline ENSBTAG00000018548 & $3.35 \mathrm{E}-10$ & $3.65 \mathrm{E}-10$ & INTS7 & $9.17 \mathrm{E}-01$ \\
\hline ENSBTAG00000015642 & $1.68 \mathrm{E}-10$ & $1.84 \mathrm{E}-10$ & SDF4 & $9.16 \mathrm{E}-01$ \\
\hline ENSBTAG00000047766 & $6.34 \mathrm{E}-10$ & $6.84 \mathrm{E}-10$ & GOS2 & 9.27E-01 \\
\hline ENSBTAG00000047491 & $4.59 \mathrm{E}-10$ & 4.96E-10 & CACNA1C & $9.26 \mathrm{E}-01$ \\
\hline ENSBTAG00000002690 & $6.61 \mathrm{E}-10$ & 7.12E-10 & BLZF1 & $9.29 \mathrm{E}-01$ \\
\hline ENSBTAG00000009091 & $4.72 \mathrm{E}-10$ & $5.08 \mathrm{E}-10$ & RNASEL & $9.30 \mathrm{E}-01$ \\
\hline ENSBTAG00000020989 & $2.87 \mathrm{E}-10$ & 3.07E-10 & SUSD4 & $9.33 \mathrm{E}-01$ \\
\hline ENSBTAG00000035836 & $4.53 \mathrm{E}-10$ & $4.85 \mathrm{E}-10$ & COLGALT2 & $9.34 \mathrm{E}-01$ \\
\hline ENSBTAG00000021880 & $4.98 \mathrm{E}-10$ & 5.33E-10 & COQ8A & $9.34 \mathrm{E}-01$ \\
\hline ENSBTAG00000002408 & $3.56 \mathrm{E}-10$ & $3.80 \mathrm{E}-10$ & FHAD1 & $9.36 \mathrm{E}-01$ \\
\hline ENSBTAG00000020563 & $3.56 \mathrm{E}-10$ & $3.78 \mathrm{E}-10$ & DDX59 & $9.43 \mathrm{E}-01$ \\
\hline ENSBTAG00000033127 & $6.89 \mathrm{E}-10$ & 7.30E-10 & C1orf100 & $9.44 \mathrm{E}-01$ \\
\hline ENSBTAG00000021874 & $7.95 \mathrm{E}-10$ & $8.40 \mathrm{E}-10$ & KIF14 & $9.46 \mathrm{E}-01$ \\
\hline ENSBTAG00000039196 & $7.28 \mathrm{E}-10$ & $7.68 \mathrm{E}-10$ & & $9.48 \mathrm{E}-01$ \\
\hline ENSBTAG00000006188 & $5.65 E-10$ & $5.96 \mathrm{E}-10$ & USH2A & $9.48 \mathrm{E}-01$ \\
\hline ENSBTAG00000024496 & $3.05 \mathrm{E}-10$ & $3.21 \mathrm{E}-10$ & & $9.51 \mathrm{E}-01$ \\
\hline ENSBTAG00000038264 & $1.69 \mathrm{E}-10$ & 1.77E-10 & & 9.55E-01 \\
\hline ENSBTAG00000024509 & $2.54 \mathrm{E}-10$ & $2.66 \mathrm{E}-10$ & TMEM52 & 9.53E-01 \\
\hline ENSBTAG00000009386 & $1.95 \mathrm{E}-10$ & 2.04E-10 & C1orf167 & $9.54 \mathrm{E}-01$ \\
\hline ENSBTAG00000002874 & $3.94 \mathrm{E}-10$ & 4.14E-10 & TMEM183A & $9.51 \mathrm{E}-01$ \\
\hline ENSBTAG00000023784 & $1.49 \mathrm{E}-10$ & $1.56 \mathrm{E}-10$ & TMEM88B & $9.53 \mathrm{E}-01$ \\
\hline ENSBTAG00000013064 & $7.71 \mathrm{E}-10$ & 8.07E-10 & RGS18 & $9.56 \mathrm{E}-01$ \\
\hline ENSBTAG00000008153 & $3.57 \mathrm{E}-10$ & $3.72 \mathrm{E}-10$ & CAMSAP2 & $9.59 \mathrm{E}-01$ \\
\hline ENSBTAG00000015885 & 4.24E-10 & $4.40 \mathrm{E}-10$ & PPFIA4 & $9.64 \mathrm{E}-01$ \\
\hline ENSBTAG00000044171 & $5.33 \mathrm{E}-10$ & $5.51 \mathrm{E}-10$ & $\mathrm{CNIH} 3$ & 9.67E-01 \\
\hline ENSBTAG00000012462 & $6.28 \mathrm{E}-10$ & $6.50 \mathrm{E}-10$ & MAP1LC3C & 9.67E-01 \\
\hline ENSBTAG00000015086 & $4.18 \mathrm{E}-10$ & $4.33 \mathrm{E}-10$ & HSD11B1 & $9.66 \mathrm{E}-01$ \\
\hline ENSBTAG00000013128 & $5.11 \mathrm{E}-10$ & 5.27E-10 & ACBD3 & $9.69 \mathrm{E}-01$ \\
\hline ENSBTAG00000021166 & $4.06 \mathrm{E}-10$ & 4.19E-10 & FAM71A & $9.70 \mathrm{E}-01$ \\
\hline ENSBTAG00000008672 & $1.71 \mathrm{E}-10$ & $1.76 \mathrm{E}-10$ & GABRD & $9.71 \mathrm{E}-01$ \\
\hline ENSBTAG00000010719 & $2.41 \mathrm{E}-10$ & $2.48 \mathrm{E}-10$ & ANGPTL1 & $9.71 \mathrm{E}-01$ \\
\hline ENSBTAG00000015197 & $9.08 \mathrm{E}-10$ & $9.33 \mathrm{E}-10$ & KLHDC8A & $9.73 \mathrm{E}-01$ \\
\hline ENSBTAG00000018220 & $3.12 \mathrm{E}-10$ & $3.20 \mathrm{E}-10$ & RGSL1 & $9.75 \mathrm{E}-01$ \\
\hline ENSBTAG00000046007 & $1.43 \mathrm{E}-10$ & $1.47 \mathrm{E}-10$ & CCDC27 & $9.73 \mathrm{E}-01$ \\
\hline ENSBTAG00000009075 & $1.79 \mathrm{E}-10$ & $1.83 \mathrm{E}-10$ & RNF207 & $9.77 \mathrm{E}-01$ \\
\hline ENSBTAG00000019821 & $4.32 \mathrm{E}-10$ & 4.42E-10 & $\mathrm{DHX9}$ & $9.79 \mathrm{E}-01$ \\
\hline ENSBTAG00000040409 & $1.24 \mathrm{E}-09$ & $1.27 \mathrm{E}-09$ & & $9.73 \mathrm{E}-01$ \\
\hline ENSBTAG00000012715 & $4.91 \mathrm{E}-10$ & $5.01 \mathrm{E}-10$ & KIF26B & $9.79 \mathrm{E}-01$ \\
\hline ENSBTAG00000013999 & $9.29 \mathrm{E}-11$ & $9.46 \mathrm{E}-11$ & FAM20B & $9.82 \mathrm{E}-01$ \\
\hline ENSBTAG00000008545 & $3.93 \mathrm{E}-10$ & $3.99 \mathrm{E}-10$ & ATF3 & $9.86 \mathrm{E}-01$ \\
\hline ENSBTAG00000024555 & $3.32 \mathrm{E}-10$ & 3.37E-10 & EFCAB2 & $9.86 \mathrm{E}-01$ \\
\hline ENSBTAG00000007110 & $1.39 \mathrm{E}-10$ & $1.41 \mathrm{E}-10$ & RCOR3 & $9.86 \mathrm{E}-01$ \\
\hline ENSBTAG00000010850 & $2.33 \mathrm{E}-10$ & $2.35 \mathrm{E}-10$ & SERTAD4 & $9.93 \mathrm{E}-01$ \\
\hline ENSBTAG00000012705 & $1.00 \mathrm{E}-10$ & $1.01 \mathrm{E}-10$ & DEGS1 & $9.95 \mathrm{E}-01$ \\
\hline ENSBTAG00000021200 & $3.12 \mathrm{E}-10$ & $3.14 \mathrm{E}-10$ & KISS1 & $9.94 \mathrm{E}-01$ \\
\hline ENSBTAG00000046630 & $1.04 \mathrm{E}-10$ & $1.05 \mathrm{E}-10$ & & $9.91 \mathrm{E}-01$ \\
\hline ENSBTAG00000019859 & $5.37 \mathrm{E}-11$ & $5.41 \mathrm{E}-11$ & B3GALT2 & $9.93 \mathrm{E}-01$ \\
\hline ENSBTAG00000034611 & $9.71 \mathrm{E}-10$ & $9.77 \mathrm{E}-10$ & & $9.94 \mathrm{E}-01$ \\
\hline ENSBTAG00000011650 & $2.74 \mathrm{E}-10$ & $2.76 \mathrm{E}-10$ & & $9.93 \mathrm{E}-01$ \\
\hline ENSBTAG00000018028 & $2.19 \mathrm{E}-10$ & $2.20 \mathrm{E}-10$ & TTLL10 & 9.97E-01 \\
\hline ENSBTAG00000004570 & $2.65 \mathrm{E}-10$ & $2.66 \mathrm{E}-10$ & PRRX1 & $9.94 \mathrm{E}-01$ \\
\hline ENSBTAG00000002231 & $5.06 \mathrm{E}-10$ & 5.07E-10 & SLC9C2 & $9.98 \mathrm{E}-01$ \\
\hline
\end{tabular}




\begin{tabular}{|c|c|c|c|c|}
\hline ENSBTAG00000006408 & 4.19E-10 & 4.18E-10 & МROH9 & $1.00 \mathrm{E}+00$ \\
\hline ENSBTAG00000012585 & $2.84 \mathrm{E}-10$ & $2.83 \mathrm{E}-10$ & RAB3GAP2 & $1.00 \mathrm{E}+00$ \\
\hline ENSBTAG00000009306 & $1.70 \mathrm{E}-10$ & $1.69 \mathrm{E}-10$ & MORN1 & $1.00 \mathrm{E}+00$ \\
\hline ENSBTAG00000009876 & $5.47 \mathrm{E}-10$ & $5.44 \mathrm{E}-10$ & & $1.01 E+00$ \\
\hline ENSBTAG00000018718 & $1.33 \mathrm{E}-10$ & $1.33 \mathrm{E}-10$ & DDI2 & $1.00 \mathrm{E}+00$ \\
\hline ENSBTAG00000024482 & 4.64E-10 & 4.61E-10 & & $1.01 E+00$ \\
\hline ENSBTAG00000021211 & $1.91 \mathrm{E}-10$ & $1.90 \mathrm{E}-10$ & DPT & $1.01 E+00$ \\
\hline ENSBTAG00000006403 & $2.25 \mathrm{E}-10$ & $2.23 \mathrm{E}-10$ & Clorf115 & $1.01 E+00$ \\
\hline ENSBTAG00000040570 & $4.09 \mathrm{E}-10$ & 4.05E-10 & & $1.01 E+00$ \\
\hline ENSBTAG00000013885 & $5.30 \mathrm{E}-10$ & $5.22 \mathrm{E}-10$ & ADSS & $1.02 \mathrm{E}+00$ \\
\hline ENSBTAG00000010169 & $1.36 \mathrm{E}-10$ & $1.33 \mathrm{E}-10$ & SUCO & $1.02 \mathrm{E}+00$ \\
\hline ENSBTAG00000017714 & $6.58 \mathrm{E}-10$ & $6.44 \mathrm{E}-10$ & C4BPB & $1.02 \mathrm{E}+00$ \\
\hline ENSBTAG00000020015 & $2.80 \mathrm{E}-10$ & $2.74 \mathrm{E}-10$ & DFFB & $1.02 \mathrm{E}+00$ \\
\hline ENSBTAG00000016185 & 4.57E-10 & $4.46 \mathrm{E}-10$ & ENAH & $1.03 E+00$ \\
\hline ENSBTAG00000021408 & $3.94 \mathrm{E}-10$ & $3.83 \mathrm{E}-10$ & FMO1 & $1.03 E+00$ \\
\hline ENSBTAG00000014439 & $6.44 \mathrm{E}-10$ & $6.21 E-10$ & KMO & $1.04 \mathrm{E}+00$ \\
\hline ENSBTAG00000022777 & $3.32 \mathrm{E}-10$ & $3.21 \mathrm{E}-10$ & CDC42BPA & $1.04 \mathrm{E}+00$ \\
\hline ENSBTAG00000031238 & $5.28 \mathrm{E}-10$ & $5.09 \mathrm{E}-10$ & SHCBP1L & $1.04 \mathrm{E}+00$ \\
\hline ENSBTAG00000024497 & $2.02 \mathrm{E}-10$ & $1.95 \mathrm{E}-10$ & CTRC & $1.04 E+00$ \\
\hline ENSBTAG00000020698 & $3.46 \mathrm{E}-10$ & $3.31 \mathrm{E}-10$ & MTHFR & $1.05 \mathrm{E}+00$ \\
\hline ENSBTAG00000020264 & $3.56 \mathrm{E}-10$ & $3.39 \mathrm{E}-10$ & CCDC181 & $1.05 E+00$ \\
\hline ENSBTAG00000038171 & 7.77E-10 & 7.36E-10 & & $1.06 \mathrm{E}+00$ \\
\hline ENSBTAG00000038261 & $3.90 \mathrm{E}-10$ & $3.69 \mathrm{E}-10$ & & $1.06 \mathrm{E}+00$ \\
\hline ENSBTAG00000033136 & $1.98 \mathrm{E}-10$ & $1.87 \mathrm{E}-10$ & $\operatorname{cox} 20$ & $1.06 \mathrm{E}+00$ \\
\hline ENSBTAG00000013535 & $6.30 \mathrm{E}-11$ & $5.95 \mathrm{E}-11$ & & $1.06 \mathrm{E}+00$ \\
\hline ENSBTAG00000012349 & $4.08 \mathrm{E}-10$ & $3.85 \mathrm{E}-10$ & LAX1 & $1.06 \mathrm{E}+00$ \\
\hline ENSBTAG00000020597 & $3.27 \mathrm{E}-10$ & $3.08 \mathrm{E}-10$ & FMO3 & $1.06 \mathrm{E}+00$ \\
\hline ENSBTAG00000033267 & $9.65 \mathrm{E}-11$ & $9.08 \mathrm{E}-11$ & LIN9 & $1.06 \mathrm{E}+00$ \\
\hline ENSBTAG00000020014 & $3.84 \mathrm{E}-10$ & $3.61 \mathrm{E}-10$ & CEP104 & $1.06 \mathrm{E}+00$ \\
\hline ENSBTAG00000002167 & $6.44 \mathrm{E}-10$ & $6.04 \mathrm{E}-10$ & NPHS2 & $1.07 E+00$ \\
\hline ENSBTAG00000024561 & $2.70 \mathrm{E}-10$ & $2.52 \mathrm{E}-10$ & H3F3B & $1.07 E+00$ \\
\hline ENSBTAG00000004414 & $2.73 \mathrm{E}-10$ & $2.54 \mathrm{E}-10$ & SLC30A10 & $1.07 \mathrm{E}+00$ \\
\hline ENSBTAG00000006718 & $1.71 \mathrm{E}-10$ & $1.59 \mathrm{E}-10$ & NVL & $1.07 \mathrm{E}+00$ \\
\hline ENSBTAG00000002846 & $4.16 \mathrm{E}-10$ & $3.86 \mathrm{E}-10$ & TRAF3IP3 & $1.08 \mathrm{E}+00$ \\
\hline ENSBTAG00000020755 & $6.25 \mathrm{E}-10$ & $5.73 \mathrm{E}-10$ & SELP & $1.09 E+00$ \\
\hline ENSBTAG00000024485 & 3.67E-10 & 3.37E-10 & KLHL20 & $1.09 E+00$ \\
\hline ENSBTAG00000015373 & $1.25 \mathrm{E}-10$ & $1.15 \mathrm{E}-10$ & AADACL3 & $1.09 \mathrm{E}+00$ \\
\hline ENSBTAG00000002849 & $3.13 \mathrm{E}-10$ & $2.86 \mathrm{E}-10$ & IRF6 & $1.10 \mathrm{E}+00$ \\
\hline ENSBTAG00000033180 & $3.04 \mathrm{E}-10$ & 2.77E-10 & SMYD3 & $1.10 \mathrm{E}+00$ \\
\hline ENSBTAG00000010737 & $1.36 \mathrm{E}-10$ & $1.25 \mathrm{E}-10$ & & $1.09 \mathrm{E}+00$ \\
\hline ENSBTAG00000047972 & $5.52 \mathrm{E}-10$ & $5.04 \mathrm{E}-10$ & C1orf106 & $1.10 \mathrm{E}+00$ \\
\hline ENSBTAG00000012020 & $3.15 \mathrm{E}-10$ & $2.86 \mathrm{E}-10$ & TRAF5 & $1.10 \mathrm{E}+00$ \\
\hline ENSBTAG00000001574 & $6.27 \mathrm{E}-10$ & $5.68 \mathrm{E}-10$ & GPATCH2 & $1.10 \mathrm{E}+00$ \\
\hline ENSBTAG00000019818 & $1.46 \mathrm{E}-10$ & $1.31 \mathrm{E}-10$ & CASZ1 & $1.11 \mathrm{E}+00$ \\
\hline ENSBTAG00000013949 & $2.18 \mathrm{E}-10$ & $1.97 \mathrm{E}-10$ & AHCTF1 & $1.10 \mathrm{E}+00$ \\
\hline ENSBTAG00000017722 & 7.07E-10 & $6.38 \mathrm{E}-10$ & F5 & $1.11 \mathrm{E}+00$ \\
\hline ENSBTAG00000021210 & $2.31 \mathrm{E}-10$ & 2.07E-10 & HES2 & $1.11 \mathrm{E}+00$ \\
\hline ENSBTAG00000005359 & $2.59 \mathrm{E}-10$ & $2.32 \mathrm{E}-10$ & TGFB2 & $1.12 \mathrm{E}+00$ \\
\hline ENSBTAG00000008420 & $4.52 \mathrm{E}-10$ & $4.05 \mathrm{E}-10$ & BRINP3 & $1.12 \mathrm{E}+00$ \\
\hline ENSBTAG00000008449 & $2.92 \mathrm{E}-10$ & $2.59 \mathrm{E}-10$ & & $1.13 \mathrm{E}+00$ \\
\hline ENSBTAG00000013812 & $1.21 \mathrm{E}-10$ & 1.07E-10 & TROVE2 & $1.13 E+00$ \\
\hline ENSBTAG00000013499 & $2.80 \mathrm{E}-10$ & $2.48 \mathrm{E}-10$ & LHX9 & $1.13 \mathrm{E}+00$ \\
\hline ENSBTAG00000000072 & $2.86 \mathrm{E}-10$ & $2.53 \mathrm{E}-10$ & TFB2M & $1.13 E+00$ \\
\hline ENSBTAG00000017301 & $2.97 \mathrm{E}-10$ & $2.62 \mathrm{E}-10$ & AVPR1B & $1.13 \mathrm{E}+00$ \\
\hline ENSBTAG00000016542 & $6.63 \mathrm{E}-10$ & $5.85 \mathrm{E}-10$ & LAMB3 & $1.13 E+00$ \\
\hline ENSBTAG00000013538 & $1.52 \mathrm{E}-10$ & $1.33 \mathrm{E}-10$ & PEX14 & $1.14 \mathrm{E}+00$ \\
\hline ENSBTAG00000005397 & $2.92 \mathrm{E}-10$ & $2.55 \mathrm{E}-10$ & CD46 & $1.14 \mathrm{E}+00$ \\
\hline ENSBTAG00000020458 & $3.26 \mathrm{E}-10$ & $2.85 \mathrm{E}-10$ & STX6 & $1.14 \mathrm{E}+00$ \\
\hline ENSBTAG00000039995 & $1.45 \mathrm{E}-09$ & $1.27 \mathrm{E}-09$ & & $1.14 \mathrm{E}+00$ \\
\hline ENSBTAG00000016369 & $3.13 \mathrm{E}-10$ & $2.73 \mathrm{E}-10$ & TSEN15 & $1.15 E+00$ \\
\hline ENSBTAG00000010177 & $4.72 \mathrm{E}-10$ & $4.11 \mathrm{E}-10$ & GOLT1A & $1.15 E+00$ \\
\hline ENSBTAG00000004966 & $3.07 \mathrm{E}-10$ & 2.67E-10 & KIF1B & $1.15 \mathrm{E}+00$ \\
\hline ENSBTAG00000017788 & $5.05 \mathrm{E}-10$ & 4.37E-10 & AKT3 & $1.16 \mathrm{E}+00$ \\
\hline ENSBTAG00000006186 & $3.90 \mathrm{E}-10$ & 3.37E-10 & KCTD3 & $1.16 \mathrm{E}+00$ \\
\hline ENSBTAG00000012582 & $6.32 \mathrm{E}-10$ & $5.45 \mathrm{E}-10$ & IARS2 & $1.16 \mathrm{E}+00$ \\
\hline ENSBTAG00000017775 & 4.97E-10 & $4.28 \mathrm{E}-10$ & SDCCAG8 & $1.16 \mathrm{E}+00$ \\
\hline ENSBTAG00000015517 & $1.45 \mathrm{E}-09$ & $1.24 \mathrm{E}-09$ & & $1.17 \mathrm{E}+00$ \\
\hline ENSBTAG00000020227 & $6.89 \mathrm{E}-10$ & $5.83 \mathrm{E}-10$ & & $1.18 \mathrm{E}+00$ \\
\hline ENSBTAG00000002476 & $1.50 \mathrm{E}-10$ & $1.27 \mathrm{E}-10$ & DNAJC16 & $1.18 \mathrm{E}+00$ \\
\hline ENSBTAG00000024647 & $1.25 \mathrm{E}-09$ & $1.05 \mathrm{E}-09$ & & $1.19 E+00$ \\
\hline
\end{tabular}




\begin{tabular}{|c|c|c|c|c|}
\hline ENSBTAG00000009838 & $1.54 \mathrm{E}-10$ & $1.30 \mathrm{E}-10$ & & $1.19 E+00$ \\
\hline ENSBTAG00000013700 & $1.87 \mathrm{E}-10$ & 1.57E-10 & BECN2 & $1.19 E+00$ \\
\hline ENSBTAG00000045702 & $8.35 \mathrm{E}-11$ & $7.01 \mathrm{E}-11$ & ZBED6 & $1.19 E+00$ \\
\hline ENSBTAG00000015972 & 1.07E-10 & 8.97E-11 & GLRX2 & $1.19 E+00$ \\
\hline ENSBTAG00000009943 & $6.89 \mathrm{E}-11$ & $5.79 \mathrm{E}-11$ & & $1.19 \mathrm{E}+00$ \\
\hline ENSBTAG00000021432 & $2.73 \mathrm{E}-10$ & $2.29 \mathrm{E}-10$ & TEDDM1 & $1.19 E+00$ \\
\hline ENSBTAG00000024510 & $6.89 \mathrm{E}-11$ & 5.79E-11 & & $1.19 E+00$ \\
\hline ENSBTAG00000016541 & $1.98 \mathrm{E}-10$ & $1.66 \mathrm{E}-10$ & CAMK1G & $1.19 E+00$ \\
\hline ENSBTAG00000002478 & $5.57 \mathrm{E}-11$ & $4.68 \mathrm{E}-11$ & AGMAT & $1.19 E+00$ \\
\hline ENSBTAG00000004305 & $2.65 \mathrm{E}-10$ & $2.22 \mathrm{E}-10$ & RGS16 & $1.19 E+00$ \\
\hline ENSBTAG00000046243 & $1.45 \mathrm{E}-10$ & $1.22 \mathrm{E}-10$ & ZNF648 & $1.19 \mathrm{E}+00$ \\
\hline ENSBTAG00000006609 & $1.90 \mathrm{E}-10$ & $1.59 \mathrm{E}-10$ & ERRFI1 & $1.19 E+00$ \\
\hline ENSBTAG00000002590 & $2.92 \mathrm{E}-10$ & $2.44 \mathrm{E}-10$ & EXOSC10 & $1.20 E+00$ \\
\hline ENSBTAG00000000511 & $3.91 \mathrm{E}-10$ & $3.25 \mathrm{E}-10$ & CNST & $1.20 \mathrm{E}+00$ \\
\hline ENSBTAG00000001382 & 3.20E-10 & $2.65 \mathrm{E}-10$ & SLC26A9 & $1.21 \mathrm{E}+00$ \\
\hline ENSBTAG00000008944 & $6.45 \mathrm{E}-10$ & $5.35 \mathrm{E}-10$ & CRB1 & $1.21 \mathrm{E}+00$ \\
\hline ENSBTAG00000021202 & $3.19 \mathrm{E}-10$ & $2.64 \mathrm{E}-10$ & ACOT7 & $1.21 \mathrm{E}+00$ \\
\hline ENSBTAG00000024560 & $4.62 \mathrm{E}-10$ & $3.81 \mathrm{E}-10$ & STUM & $1.21 E+00$ \\
\hline ENSBTAG00000013527 & $4.02 \mathrm{E}-10$ & $3.30 \mathrm{E}-10$ & PGD & $1.22 \mathrm{E}+00$ \\
\hline ENSBTAG00000011461 & $2.72 \mathrm{E}-10$ & $2.23 \mathrm{E}-10$ & ADORA1 & $1.22 \mathrm{E}+00$ \\
\hline ENSBTAG00000007595 & $3.84 \mathrm{E}-10$ & $3.15 \mathrm{E}-10$ & BROX & $1.22 \mathrm{E}+00$ \\
\hline ENSBTAG00000008710 & $5.77 \mathrm{E}-10$ & $4.73 \mathrm{E}-10$ & KCNH1 & $1.22 \mathrm{E}+00$ \\
\hline ENSBTAG00000014793 & $3.14 \mathrm{E}-10$ & $2.56 \mathrm{E}-10$ & C1orf112 & $1.23 E+00$ \\
\hline ENSBTAG00000020266 & $3.64 \mathrm{E}-10$ & $2.96 \mathrm{E}-10$ & SLC19A2 & $1.23 \mathrm{E}+00$ \\
\hline ENSBTAG00000003701 & $1.77 \mathrm{E}-10$ & $1.43 \mathrm{E}-10$ & VASH2 & $1.24 \mathrm{E}+00$ \\
\hline ENSBTAG00000004361 & $2.09 \mathrm{E}-10$ & $1.68 \mathrm{E}-10$ & ZBTB37 & $1.24 \mathrm{E}+00$ \\
\hline ENSBTAG00000015974 & $2.86 \mathrm{E}-10$ & $2.31 \mathrm{E}-10$ & FLVCR1 & $1.24 \mathrm{E}+00$ \\
\hline ENSBTAG00000021672 & $2.20 \mathrm{E}-10$ & $1.76 \mathrm{E}-10$ & RGS1 & $1.25 \mathrm{E}+00$ \\
\hline ENSBTAG00000026068 & $1.40 \mathrm{E}-09$ & 1.12E-09 & & $1.25 \mathrm{E}+00$ \\
\hline ENSBTAG00000016703 & $1.59 \mathrm{E}-10$ & $1.27 \mathrm{E}-10$ & $\mathrm{CDC73}$ & $1.25 E+00$ \\
\hline ENSBTAG00000001642 & $2.96 \mathrm{E}-10$ & $2.36 \mathrm{E}-10$ & TTC34 & $1.25 \mathrm{E}+00$ \\
\hline ENSBTAG00000014560 & $2.74 \mathrm{E}-10$ & $2.18 \mathrm{E}-10$ & HLX & $1.26 \mathrm{E}+00$ \\
\hline ENSBTAG00000019538 & $4.09 \mathrm{E}-10$ & $3.24 \mathrm{E}-10$ & FBXO28 & $1.26 \mathrm{E}+00$ \\
\hline ENSBTAG00000019798 & $5.56 \mathrm{E}-10$ & $4.41 E-10$ & PIGR & $1.26 \mathrm{E}+00$ \\
\hline ENSBTAG00000014794 & $4.68 \mathrm{E}-10$ & $3.70 \mathrm{E}-10$ & SCYL3 & $1.26 \mathrm{E}+00$ \\
\hline ENSBTAG00000026481 & $7.38 \mathrm{E}-10$ & $5.83 \mathrm{E}-10$ & RGS8 & $1.27 \mathrm{E}+00$ \\
\hline ENSBTAG00000017561 & 7.32E-10 & $5.78 \mathrm{E}-10$ & HHIPL2 & $1.27 \mathrm{E}+00$ \\
\hline ENSBTAG00000006685 & $1.66 \mathrm{E}-10$ & $1.31 \mathrm{E}-10$ & IL10 & $1.27 \mathrm{E}+00$ \\
\hline ENSBTAG00000009384 & $2.70 \mathrm{E}-10$ & 2.12E-10 & AGTRAP & $1.27 \mathrm{E}+00$ \\
\hline ENSBTAG00000008513 & $1.87 \mathrm{E}-10$ & $1.47 \mathrm{E}-10$ & Clorf186 & $1.27 \mathrm{E}+00$ \\
\hline ENSBTAG00000017352 & $2.23 \mathrm{E}-10$ & $1.74 \mathrm{E}-10$ & & $1.28 \mathrm{E}+00$ \\
\hline ENSBTAG00000024493 & $2.60 \mathrm{E}-10$ & $2.04 \mathrm{E}-10$ & DHRS3 & $1.27 \mathrm{E}+00$ \\
\hline ENSBTAG00000001821 & $1.15 \mathrm{E}-10$ & $9.03 \mathrm{E}-11$ & PRRC2C & $1.28 \mathrm{E}+00$ \\
\hline ENSBTAG00000002407 & $3.45 \mathrm{E}-10$ & $2.65 \mathrm{E}-10$ & TDRD5 & $1.30 \mathrm{E}+00$ \\
\hline ENSBTAG00000046177 & $3.39 \mathrm{E}-10$ & $2.58 \mathrm{E}-10$ & IGFN1 & $1.31 E+00$ \\
\hline ENSBTAG00000013620 & $1.49 \mathrm{E}-10$ & $1.13 \mathrm{E}-10$ & UCHL5 & $1.31 \mathrm{E}+00$ \\
\hline ENSBTAG00000013166 & $6.33 \mathrm{E}-10$ & 4.80E-10 & SMYD2 & $1.32 \mathrm{E}+00$ \\
\hline ENSBTAG00000008125 & $1.76 \mathrm{E}-10$ & $1.33 \mathrm{E}-10$ & KCNAB2 & $1.32 \mathrm{E}+00$ \\
\hline ENSBTAG00000002848 & $1.82 \mathrm{E}-10$ & $1.37 \mathrm{E}-10$ & C1orf74 & $1.33 E+00$ \\
\hline ENSBTAG00000038189 & $1.89 \mathrm{E}-10$ & $1.43 \mathrm{E}-10$ & GPR52 & $1.32 \mathrm{E}+00$ \\
\hline ENSBTAG00000013726 & $5.85 \mathrm{E}-10$ & 4.39E-10 & RNPEP & $1.33 \mathrm{E}+00$ \\
\hline ENSBTAG00000006255 & $3.26 \mathrm{E}-10$ & $2.44 \mathrm{E}-10$ & MDM4 & $1.34 \mathrm{E}+00$ \\
\hline ENSBTAG00000020518 & $2.64 \mathrm{E}-10$ & $1.97 \mathrm{E}-10$ & PARK7 & $1.34 \mathrm{E}+00$ \\
\hline ENSBTAG00000031749 & $1.42 \mathrm{E}-10$ & $1.05 \mathrm{E}-10$ & & $1.36 \mathrm{E}+00$ \\
\hline ENSBTAG00000038222 & $1.58 \mathrm{E}-09$ & $1.16 \mathrm{E}-09$ & & $1.36 \mathrm{E}+00$ \\
\hline ENSBTAG00000005200 & $5.37 \mathrm{E}-10$ & $3.94 \mathrm{E}-10$ & WDR64 & $1.36 \mathrm{E}+00$ \\
\hline ENSBTAG00000005231 & $1.62 \mathrm{E}-10$ & 1.19E-10 & & $1.36 \mathrm{E}+00$ \\
\hline ENSBTAG00000002688 & $1.44 \mathrm{E}-10$ & $1.05 \mathrm{E}-10$ & ATP1B1 & $1.37 \mathrm{E}+00$ \\
\hline ENSBTAG00000047274 & $2.35 \mathrm{E}-10$ & $1.71 \mathrm{E}-10$ & KIF21B & $1.38 \mathrm{E}+00$ \\
\hline ENSBTAG00000004407 & $4.33 \mathrm{E}-10$ & $3.14 \mathrm{E}-10$ & KCNK2 & $1.38 \mathrm{E}+00$ \\
\hline ENSBTAG00000040477 & $1.81 \mathrm{E}-10$ & $1.32 \mathrm{E}-10$ & CHD5 & $1.37 \mathrm{E}+00$ \\
\hline ENSBTAG00000011217 & $1.58 \mathrm{E}-10$ & $1.14 \mathrm{E}-10$ & DNAJC11 & $1.39 E+00$ \\
\hline ENSBTAG00000005410 & $4.40 \mathrm{E}-10$ & $3.18 \mathrm{E}-10$ & RGS7 & $1.38 \mathrm{E}+00$ \\
\hline ENSBTAG00000018824 & $1.29 \mathrm{E}-10$ & $9.23 \mathrm{E}-11$ & MIA3 & $1.39 E+00$ \\
\hline ENSBTAG00000006704 & 2.77E-10 & $1.99 \mathrm{E}-10$ & CCDC185 & $1.39 E+00$ \\
\hline ENSBTAG00000021671 & $2.46 \mathrm{E}-10$ & $1.77 \mathrm{E}-10$ & METTL18 & $1.39 E+00$ \\
\hline ENSBTAG00000001478 & $4.96 \mathrm{E}-10$ & 3.55E-10 & DESI2 & $1.40 \mathrm{E}+00$ \\
\hline ENSBTAG00000002575 & $1.26 \mathrm{E}-10$ & $9.00 \mathrm{E}-11$ & & $1.40 \mathrm{E}+00$ \\
\hline ENSBTAG00000003702 & $1.04 \mathrm{E}-10$ & 7.43E-11 & ANGEL2 & $1.40 \mathrm{E}+00$ \\
\hline ENSBTAG00000008082 & $2.18 \mathrm{E}-10$ & $1.55 \mathrm{E}-10$ & CEP350 & $1.41 E+00$ \\
\hline
\end{tabular}




\begin{tabular}{|c|c|c|c|c|}
\hline ENSBTAG00000007307 & $2.53 \mathrm{E}-10$ & $1.79 \mathrm{E}-10$ & SELE & $1.41 E+00$ \\
\hline ENSBTAG00000016943 & $3.02 \mathrm{E}-10$ & $2.13 \mathrm{E}-10$ & NEK7 & $1.42 \mathrm{E}+00$ \\
\hline ENSBTAG00000018774 & $2.91 \mathrm{E}-10$ & $2.05 \mathrm{E}-10$ & & $1.42 \mathrm{E}+00$ \\
\hline ENSBTAG00000018965 & $3.09 \mathrm{E}-10$ & $2.17 \mathrm{E}-10$ & PRDM16 & $1.42 \mathrm{E}+00$ \\
\hline ENSBTAG00000013631 & $3.34 \mathrm{E}-10$ & $2.35 \mathrm{E}-10$ & GLUL & $1.42 \mathrm{E}+00$ \\
\hline ENSBTAG00000007566 & $2.20 \mathrm{E}-10$ & $1.55 \mathrm{E}-10$ & CEP170 & $1.42 \mathrm{E}+00$ \\
\hline ENSBTAG00000012158 & $1.62 \mathrm{E}-10$ & 1.13E-10 & C1orf158 & $1.43 \mathrm{E}+00$ \\
\hline ENSBTAG00000040419 & $2.47 \mathrm{E}-10$ & $1.73 \mathrm{E}-10$ & & $1.43 E+00$ \\
\hline ENSBTAG00000024520 & $1.24 \mathrm{E}-10$ & $8.65 \mathrm{E}-11$ & C1orf174 & $1.43 \mathrm{E}+00$ \\
\hline ENSBTAG00000001173 & $3.28 \mathrm{E}-10$ & $2.28 \mathrm{E}-10$ & PLXNA2 & $1.44 \mathrm{E}+00$ \\
\hline ENSBTAG00000013074 & $4.38 \mathrm{E}-10$ & $3.04 \mathrm{E}-10$ & PPP1R15B & $1.44 \mathrm{E}+00$ \\
\hline ENSBTAG00000014249 & $3.40 \mathrm{E}-10$ & $2.35 \mathrm{E}-10$ & IVNS1ABP & $1.45 \mathrm{E}+00$ \\
\hline ENSBTAG00000000793 & 4.09E-10 & $2.82 \mathrm{E}-10$ & LAMC2 & $1.45 \mathrm{E}+00$ \\
\hline ENSBTAG00000015026 & $1.40 \mathrm{E}-10$ & $9.44 \mathrm{E}-11$ & $A B L 2$ & $1.48 \mathrm{E}+00$ \\
\hline ENSBTAG00000010427 & $6.70 \mathrm{E}-10$ & 4.49E-10 & RASSF5 & $1.49 \mathrm{E}+00$ \\
\hline ENSBTAG00000014726 & $1.29 \mathrm{E}-10$ & $8.66 \mathrm{E}-11$ & VAMP3 & $1.49 \mathrm{E}+00$ \\
\hline ENSBTAG00000015235 & $5.26 \mathrm{E}-10$ & $3.52 \mathrm{E}-10$ & HMCN1 & $1.49 \mathrm{E}+00$ \\
\hline ENSBTAG00000019796 & $6.38 \mathrm{E}-10$ & 4.24E-10 & FCMR & $1.50 \mathrm{E}+00$ \\
\hline ENSBTAG00000014006 & $1.11 \mathrm{E}-10$ & $7.38 \mathrm{E}-11$ & TOR3A & $1.51 E+00$ \\
\hline ENSBTAG00000011214 & $2.77 \mathrm{E}-10$ & $1.84 \mathrm{E}-10$ & THAP3 & $1.51 E+00$ \\
\hline ENSBTAG00000002894 & $8.81 \mathrm{E}-11$ & $5.81 \mathrm{E}-11$ & TNFSF4 & $1.52 \mathrm{E}+00$ \\
\hline ENSBTAG00000018729 & $1.12 \mathrm{E}-10$ & 7.33E-11 & SPEN & $1.53 \mathrm{E}+00$ \\
\hline ENSBTAG00000010718 & $4.38 \mathrm{E}-10$ & $2.86 \mathrm{E}-10$ & RALGPS2 & $1.53 \mathrm{E}+00$ \\
\hline ENSBTAG00000039728 & $1.06 \mathrm{E}-10$ & $6.90 \mathrm{E}-11$ & & $1.54 \mathrm{E}+00$ \\
\hline ENSBTAG00000027809 & $2.03 \mathrm{E}-10$ & $1.29 \mathrm{E}-10$ & & $1.57 E+00$ \\
\hline ENSBTAG00000014119 & $2.80 \mathrm{E}-10$ & $1.78 \mathrm{E}-10$ & PRKCZ & $1.57 \mathrm{E}+00$ \\
\hline ENSBTAG00000000758 & $2.91 \mathrm{E}-10$ & $1.85 \mathrm{E}-10$ & TMEM206 & $1.57 \mathrm{E}+00$ \\
\hline ENSBTAG00000006898 & $1.58 \mathrm{E}-10$ & $1.00 \mathrm{E}-10$ & EPRS & $1.58 \mathrm{E}+00$ \\
\hline ENSBTAG00000009727 & $2.14 \mathrm{E}-10$ & $1.35 \mathrm{E}-10$ & ADIPOR1 & $1.59 \mathrm{E}+00$ \\
\hline ENSBTAG00000009856 & $5.27 \mathrm{E}-11$ & 3.32E-11 & ACAP3 & $1.59 \mathrm{E}+00$ \\
\hline ENSBTAG00000001401 & $6.01 \mathrm{E}-11$ & $3.78 \mathrm{E}-11$ & SLC45A1 & $1.59 E+00$ \\
\hline ENSBTAG00000039944 & $3.51 \mathrm{E}-10$ & $2.21 \mathrm{E}-10$ & & $1.59 E+00$ \\
\hline ENSBTAG00000025113 & $5.22 \mathrm{E}-10$ & $3.28 \mathrm{E}-10$ & & $1.59 E+00$ \\
\hline ENSBTAG00000047059 & $4.66 \mathrm{E}-10$ & $2.93 \mathrm{E}-10$ & TIMM17A & $1.59 \mathrm{E}+00$ \\
\hline ENSBTAG00000015384 & $2.37 \mathrm{E}-10$ & $1.49 \mathrm{E}-10$ & PHF13 & $1.59 E+00$ \\
\hline ENSBTAG00000034323 & $4.30 \mathrm{E}-10$ & $2.70 \mathrm{E}-10$ & & $1.59 E+00$ \\
\hline ENSBTAG00000008756 & 3.63E-10 & $2.28 \mathrm{E}-10$ & ELF3 & $1.59 E+00$ \\
\hline ENSBTAG00000031385 & $2.34 \mathrm{E}-10$ & $1.46 \mathrm{E}-10$ & RFWD2 & $1.60 \mathrm{E}+00$ \\
\hline ENSBTAG00000001788 & 3.07E-10 & $1.91 \mathrm{E}-10$ & PDPN & $1.61 E+00$ \\
\hline ENSBTAG00000003313 & $2.13 \mathrm{E}-10$ & $1.31 \mathrm{E}-10$ & TNFRSF9 & $1.63 E+00$ \\
\hline ENSBTAG00000012787 & $1.96 \mathrm{E}-10$ & $1.20 \mathrm{E}-10$ & SDE2 & $1.63 E+00$ \\
\hline ENSBTAG00000024633 & 4.15E-10 & $2.53 \mathrm{E}-10$ & PIK3C2B & $1.64 \mathrm{E}+00$ \\
\hline ENSBTAG00000009726 & $2.78 \mathrm{E}-10$ & $1.68 \mathrm{E}-10$ & KLHL12 & $1.66 \mathrm{E}+00$ \\
\hline ENSBTAG00000018509 & $2.41 E-10$ & $1.45 \mathrm{E}-10$ & ETNK2 & $1.66 \mathrm{E}+00$ \\
\hline ENSBTAG00000002974 & 5.17E-10 & $3.11 E-10$ & $\mathrm{FMO} 2$ & $1.66 \mathrm{E}+00$ \\
\hline ENSBTAG00000006984 & $1.93 \mathrm{E}-10$ & $1.16 \mathrm{E}-10$ & CD55 & $1.67 \mathrm{E}+00$ \\
\hline ENSBTAG00000011285 & $3.47 \mathrm{E}-10$ & $2.08 \mathrm{E}-10$ & TRMT1L & $1.67 E+00$ \\
\hline ENSBTAG00000037494 & $5.88 \mathrm{E}-10$ & $3.52 \mathrm{E}-10$ & RGS21 & $1.67 E+00$ \\
\hline ENSBTAG00000004641 & $1.87 \mathrm{E}-10$ & $1.12 \mathrm{E}-10$ & PLEKHA6 & $1.67 \mathrm{E}+00$ \\
\hline ENSBTAG00000013294 & $3.28 \mathrm{E}-10$ & $1.96 \mathrm{E}-10$ & DYRK3 & $1.68 \mathrm{E}+00$ \\
\hline ENSBTAG00000001181 & $3.42 \mathrm{E}-10$ & $2.04 \mathrm{E}-10$ & RPS6KC1 & $1.67 E+00$ \\
\hline ENSBTAG00000046350 & $3.12 \mathrm{E}-10$ & $1.85 \mathrm{E}-10$ & PKP1 & $1.69 \mathrm{E}+00$ \\
\hline ENSBTAG00000035230 & $2.90 \mathrm{E}-10$ & $1.72 \mathrm{E}-10$ & TOR1AIP2 & $1.68 \mathrm{E}+00$ \\
\hline ENSBTAG00000013463 & $2.31 \mathrm{E}-10$ & $1.35 \mathrm{E}-10$ & & $1.71 E+00$ \\
\hline ENSBTAG00000012433 & $5.87 \mathrm{E}-10$ & $3.40 \mathrm{E}-10$ & & $1.73 E+00$ \\
\hline ENSBTAG00000021333 & $2.00 \mathrm{E}-10$ & $1.15 \mathrm{E}-10$ & REN & $1.74 \mathrm{E}+00$ \\
\hline ENSBTAG00000020839 & $2.93 \mathrm{E}-10$ & $1.68 \mathrm{E}-10$ & MEGF6 & $1.75 \mathrm{E}+00$ \\
\hline ENSBTAG00000011515 & $3.00 \mathrm{E}-10$ & $1.72 \mathrm{E}-10$ & SELL & $1.74 \mathrm{E}+00$ \\
\hline ENSBTAG00000044066 & $5.20 \mathrm{E}-10$ & $2.96 \mathrm{E}-10$ & C1orf101 & $1.76 \mathrm{E}+00$ \\
\hline ENSBTAG00000046723 & 4.44E-10 & $2.51 E-10$ & & $1.77 \mathrm{E}+00$ \\
\hline ENSBTAG00000016729 & $5.41 \mathrm{E}-10$ & $3.02 \mathrm{E}-10$ & KCNT2 & $1.79 \mathrm{E}+00$ \\
\hline ENSBTAG00000024443 & $1.41 \mathrm{E}-10$ & $7.83 \mathrm{E}-11$ & TATDN3 & $1.80 \mathrm{E}+00$ \\
\hline ENSBTAG00000023177 & $1.12 \mathrm{E}-09$ & $6.19 \mathrm{E}-10$ & & $1.82 \mathrm{E}+00$ \\
\hline ENSBTAG00000047621 & 7.65E-10 & 4.22E-10 & & $1.81 \mathrm{E}+00$ \\
\hline ENSBTAG00000002689 & $3.78 \mathrm{E}-10$ & $2.07 \mathrm{E}-10$ & NME7 & $1.83 \mathrm{E}+00$ \\
\hline ENSBTAG00000006519 & $1.33 \mathrm{E}-10$ & $7.18 \mathrm{E}-11$ & TNFRSF25 & $1.85 \mathrm{E}+00$ \\
\hline ENSBTAG00000018272 & $1.70 \mathrm{E}-10$ & $9.23 \mathrm{E}-11$ & RERE & $1.85 \mathrm{E}+00$ \\
\hline ENSBTAG00000014548 & $3.81 \mathrm{E}-10$ & $2.06 \mathrm{E}-10$ & FAM129A & $1.85 \mathrm{E}+00$ \\
\hline ENSBTAG00000008004 & $4.81 \mathrm{E}-10$ & $2.59 \mathrm{E}-10$ & NCF2 & $1.86 \mathrm{E}+00$ \\
\hline ENSBTAG00000006574 & $1.82 \mathrm{E}-10$ & $9.80 \mathrm{E}-11$ & ARL8A & $1.85 \mathrm{E}+00$ \\
\hline
\end{tabular}




\begin{tabular}{|c|c|c|c|c|}
\hline ENSBTAG00000012808 & 4.44E-10 & $2.38 \mathrm{E}-10$ & MASP2 & $1.87 \mathrm{E}+00$ \\
\hline ENSBTAG00000013298 & 4.07E-10 & $2.18 \mathrm{E}-10$ & PLA2G4A & $1.87 \mathrm{E}+00$ \\
\hline ENSBTAG00000012774 & 7.97E-10 & 4.25E-10 & RAB7B & $1.88 \mathrm{E}+00$ \\
\hline ENSBTAG00000004362 & 2.01E-10 & $1.07 \mathrm{E}-10$ & SERPINC1 & $1.88 \mathrm{E}+00$ \\
\hline ENSBTAG00000003600 & $4.90 \mathrm{E}-10$ & $2.59 \mathrm{E}-10$ & CAPN8 & $1.89 \mathrm{E}+00$ \\
\hline ENSBTAG00000014912 & $3.92 \mathrm{E}-10$ & $2.05 \mathrm{E}-10$ & FMOD & $1.91 \mathrm{E}+00$ \\
\hline ENSBTAG00000035226 & $5.09 \mathrm{E}-10$ & $2.62 \mathrm{E}-10$ & TOR1AIP1 & $1.94 \mathrm{E}+00$ \\
\hline ENSBTAG00000003992 & $1.43 \mathrm{E}-10$ & $7.32 \mathrm{E}-11$ & LHX4 & $1.95 \mathrm{E}+00$ \\
\hline ENSBTAG00000011785 & $1.57 \mathrm{E}-10$ & 8.03E-11 & WDR26 & $1.95 \mathrm{E}+00$ \\
\hline ENSBTAG00000006175 & 2.37E-10 & $1.21 \mathrm{E}-10$ & KDM5B & $1.96 \mathrm{E}+00$ \\
\hline ENSBTAG00000016728 & $1.72 \mathrm{E}-10$ & $8.72 \mathrm{E}-11$ & PLEKHG5 & $1.97 \mathrm{E}+00$ \\
\hline ENSBTAG00000003016 & $2.73 \mathrm{E}-10$ & $1.37 \mathrm{E}-10$ & PTPN7 & $1.99 \mathrm{E}+00$ \\
\hline ENSBTAG00000004874 & $9.16 \mathrm{E}-11$ & 4.62E-11 & AURKAIP1 & $1.98 \mathrm{E}+00$ \\
\hline ENSBTAG00000024492 & $7.71 \mathrm{E}-11$ & $3.88 \mathrm{E}-11$ & & $1.98 \mathrm{E}+00$ \\
\hline ENSBTAG00000012765 & $4.98 \mathrm{E}-10$ & $2.50 \mathrm{E}-10$ & & $1.99 \mathrm{E}+00$ \\
\hline ENSBTAG00000015600 & $2.69 \mathrm{E}-10$ & $1.35 \mathrm{E}-10$ & & $1.99 \mathrm{E}+00$ \\
\hline ENSBTAG00000004492 & $4.58 \mathrm{E}-10$ & $2.30 \mathrm{E}-10$ & SWT1 & $1.99 \mathrm{E}+00$ \\
\hline ENSBTAG00000011932 & $7.49 \mathrm{E}-10$ & $3.74 \mathrm{E}-10$ & PRG4 & $2.00 \mathrm{E}+00$ \\
\hline ENSBTAG00000015534 & $3.04 \mathrm{E}-10$ & $1.51 \mathrm{E}-10$ & LYPLAL1 & $2.01 E+00$ \\
\hline ENSBTAG00000006515 & $1.88 \mathrm{E}-10$ & $9.29 \mathrm{E}-11$ & ESPN & $2.02 E+00$ \\
\hline ENSBTAG00000013764 & $3.27 \mathrm{E}-10$ & $1.60 \mathrm{E}-10$ & OPTC & $2.04 \mathrm{E}+00$ \\
\hline ENSBTAG00000020837 & $2.97 \mathrm{E}-10$ & $1.44 \mathrm{E}-10$ & ARHGEF16 & $2.07 E+00$ \\
\hline ENSBTAG00000014511 & 1.17E-10 & $5.67 \mathrm{E}-11$ & C1orf159 & $2.06 \mathrm{E}+00$ \\
\hline ENSBTAG00000039937 & $2.96 \mathrm{E}-10$ & $1.43 \mathrm{E}-10$ & TNFRSF8 & $2.07 E+00$ \\
\hline ENSBTAG00000011706 & $2.87 \mathrm{E}-10$ & $1.36 \mathrm{E}-10$ & TNR & $2.11 \mathrm{E}+00$ \\
\hline ENSBTAG00000045582 & $2.09 \mathrm{E}-10$ & $9.89 \mathrm{E}-11$ & ARPC5 & $2.12 \mathrm{E}+00$ \\
\hline ENSBTAG00000002855 & $2.30 \mathrm{E}-10$ & $1.09 \mathrm{E}-10$ & & $2.11 E+00$ \\
\hline ENSBTAG00000002361 & $2.99 \mathrm{E}-10$ & $1.41 \mathrm{E}-10$ & TMCC2 & $2.12 \mathrm{E}+00$ \\
\hline ENSBTAG00000002164 & 4.20E-10 & $1.97 \mathrm{E}-10$ & AXDND1 & $2.13 E+00$ \\
\hline ENSBTAG00000006260 & $9.02 \mathrm{E}-10$ & $4.22 \mathrm{E}-10$ & KIAA1614 & $2.14 \mathrm{E}+00$ \\
\hline ENSBTAG00000006925 & 3.19E-10 & $1.49 \mathrm{E}-10$ & CENPL & $2.14 \mathrm{E}+00$ \\
\hline ENSBTAG00000004059 & $2.70 \mathrm{E}-10$ & $1.25 \mathrm{E}-10$ & SOAT1 & $2.16 E+00$ \\
\hline ENSBTAG00000015650 & $2.65 \mathrm{E}-10$ & $1.23 \mathrm{E}-10$ & TNN & $2.15 \mathrm{E}+00$ \\
\hline ENSBTAG00000024928 & $1.69 \mathrm{E}-10$ & 7.70E-11 & TNFRSF1B & $2.19 E+00$ \\
\hline ENSBTAG00000010978 & $1.38 \mathrm{E}-10$ & $6.28 \mathrm{E}-11$ & PROX1 & $2.19 E+00$ \\
\hline ENSBTAG00000018730 & 1.03E-10 & 4.67E-11 & ZBTB17 & $2.20 \mathrm{E}+00$ \\
\hline ENSBTAG00000000754 & 2.13E-10 & $9.54 \mathrm{E}-11$ & PPP2R5A & $2.23 E+00$ \\
\hline ENSBTAG00000009048 & $2.45 \mathrm{E}-10$ & $1.08 \mathrm{E}-10$ & EFHD2 & $2.26 \mathrm{E}+00$ \\
\hline ENSBTAG00000014423 & $2.45 \mathrm{E}-10$ & $1.07 E-10$ & & $2.29 \mathrm{E}+00$ \\
\hline ENSBTAG00000008739 & $1.15 \mathrm{E}-10$ & $5.00 \mathrm{E}-11$ & SAMD11 & $2.29 E+00$ \\
\hline ENSBTAG00000004358 & 4.74E-10 & $2.05 \mathrm{E}-10$ & DARS2 & $2.31 \mathrm{E}+00$ \\
\hline ENSBTAG00000017727 & $1.38 \mathrm{E}-10$ & $5.94 \mathrm{E}-11$ & KIFAP3 & $2.32 \mathrm{E}+00$ \\
\hline ENSBTAG00000014602 & $1.82 \mathrm{E}-10$ & $7.81 \mathrm{E}-11$ & CAMTA1 & $2.33 \mathrm{E}+00$ \\
\hline ENSBTAG00000001361 & $5.54 \mathrm{E}-10$ & $2.38 \mathrm{E}-10$ & NMNAT1 & $2.33 E+00$ \\
\hline ENSBTAG00000021688 & $3.67 \mathrm{E}-10$ & $1.56 \mathrm{E}-10$ & $\mathrm{FH}$ & $2.35 \mathrm{E}+00$ \\
\hline ENSBTAG00000016735 & $1.82 \mathrm{E}-10$ & 7.64E-11 & ZBTB48 & $2.38 \mathrm{E}+00$ \\
\hline ENSBTAG00000009309 & $2.22 \mathrm{E}-10$ & $9.29 \mathrm{E}-11$ & PEX10 & $2.38 \mathrm{E}+00$ \\
\hline ENSBTAG00000004873 & $9.06 \mathrm{E}-11$ & $3.80 \mathrm{E}-11$ & CCNL2 & $2.38 \mathrm{E}+00$ \\
\hline ENSBTAG00000016528 & $1.04 \mathrm{E}-10$ & 4.35E-11 & NOC2L & $2.38 \mathrm{E}+00$ \\
\hline ENSBTAG00000013275 & $8.83 \mathrm{E}-11$ & $3.71 \mathrm{E}-11$ & MAD2L2 & $2.38 \mathrm{E}+00$ \\
\hline ENSBTAG00000006709 & $3.38 \mathrm{E}-10$ & $1.41 \mathrm{E}-10$ & NPPA & $2.39 E+00$ \\
\hline ENSBTAG00000047412 & $1.97 \mathrm{E}-10$ & $8.28 \mathrm{E}-11$ & TNFSF18 & $2.38 \mathrm{E}+00$ \\
\hline ENSBTAG00000005304 & $2.54 \mathrm{E}-10$ & $1.06 \mathrm{E}-10$ & SRM & $2.39 \mathrm{E}+00$ \\
\hline ENSBTAG00000005812 & $3.82 \mathrm{E}-10$ & $1.60 \mathrm{E}-10$ & TP73 & $2.39 E+00$ \\
\hline ENSBTAG00000013071 & $1.36 \mathrm{E}-10$ & $5.72 \mathrm{E}-11$ & GPR153 & $2.38 \mathrm{E}+00$ \\
\hline ENSBTAG00000017929 & $3.48 \mathrm{E}-10$ & $1.46 \mathrm{E}-10$ & ACTRT2 & $2.38 \mathrm{E}+00$ \\
\hline ENSBTAG00000046091 & $3.87 \mathrm{E}-10$ & $1.62 \mathrm{E}-10$ & & $2.39 E+00$ \\
\hline ENSBTAG00000002483 & $1.58 \mathrm{E}-10$ & $6.61 E-11$ & HES5 & $2.38 \mathrm{E}+00$ \\
\hline ENSBTAG00000002472 & $5.64 \mathrm{E}-10$ & $2.35 \mathrm{E}-10$ & CASP9 & $2.40 \mathrm{E}+00$ \\
\hline ENSBTAG00000020887 & $5.81 \mathrm{E}-10$ & $2.39 \mathrm{E}-10$ & CACNA1E & $2.43 E+00$ \\
\hline ENSBTAG00000038079 & 4.11E-10 & $1.68 \mathrm{E}-10$ & & $2.44 \mathrm{E}+00$ \\
\hline ENSBTAG00000038716 & $2.09 \mathrm{E}-10$ & $8.52 \mathrm{E}-11$ & SKI & $2.46 \mathrm{E}+00$ \\
\hline ENSBTAG00000015537 & $4.88 \mathrm{E}-10$ & $1.97 \mathrm{E}-10$ & RASAL2 & $2.47 \mathrm{E}+00$ \\
\hline ENSBTAG00000016277 & $6.03 \mathrm{E}-10$ & $2.42 \mathrm{E}-10$ & 02-Mar & $2.49 E+00$ \\
\hline ENSBTAG00000007620 & $1.51 \mathrm{E}-10$ & $6.01 \mathrm{E}-11$ & CPSF3L & $2.51 \mathrm{E}+00$ \\
\hline ENSBTAG00000009924 & $2.73 \mathrm{E}-10$ & $1.09 \mathrm{E}-10$ & MR1 & $2.50 \mathrm{E}+00$ \\
\hline ENSBTAG00000020591 & $1.94 \mathrm{E}-10$ & 7.70E-11 & VAMP4 & $2.51 E+00$ \\
\hline ENSBTAG00000047256 & $4.20 \mathrm{E}-10$ & $1.64 \mathrm{E}-10$ & & $2.56 \mathrm{E}+00$ \\
\hline ENSBTAG00000008350 & $1.81 \mathrm{E}-10$ & $7.08 \mathrm{E}-11$ & KLHL21 & $2.56 \mathrm{E}+00$ \\
\hline ENSBTAG00000024539 & $1.61 \mathrm{E}-10$ & $6.18 \mathrm{E}-11$ & SPSB1 & $2.61 E+00$ \\
\hline
\end{tabular}




\begin{tabular}{|c|c|c|c|c|}
\hline ENSBTAG00000015945 & $3.81 \mathrm{E}-10$ & $1.44 \mathrm{E}-10$ & TMEM81 & $2.65 E+00$ \\
\hline ENSBTAG00000002219 & $8.50 \mathrm{E}-11$ & $3.15 \mathrm{E}-11$ & & $2.69 \mathrm{E}+00$ \\
\hline ENSBTAG00000016080 & $2.53 \mathrm{E}-10$ & $9.34 \mathrm{E}-11$ & VPS13D & $2.71 E+00$ \\
\hline ENSBTAG00000010016 & $2.71 \mathrm{E}-10$ & $9.96 \mathrm{E}-11$ & SPATA45 & $2.72 E+00$ \\
\hline ENSBTAG00000009396 & $3.11 \mathrm{E}-10$ & 1.14E-10 & EXO1 & $2.72 E+00$ \\
\hline ENSBTAG00000015859 & $1.64 \mathrm{E}-10$ & $6.03 \mathrm{E}-11$ & WRAP73 & $2.72 \mathrm{E}+00$ \\
\hline ENSBTAG00000008003 & $2.75 \mathrm{E}-10$ & $9.95 \mathrm{E}-11$ & SMG7 & $2.76 \mathrm{E}+00$ \\
\hline ENSBTAG00000010696 & $1.26 \mathrm{E}-10$ & 4.53E-11 & DVL1 & $2.78 \mathrm{E}+00$ \\
\hline ENSBTAG00000021294 & $1.10 \mathrm{E}-10$ & $3.94 \mathrm{E}-11$ & VWA1 & $2.78 \mathrm{E}+00$ \\
\hline ENSBTAG00000011966 & $3.30 \mathrm{E}-10$ & $1.13 \mathrm{E}-10$ & LAMC1 & $2.92 \mathrm{E}+00$ \\
\hline ENSBTAG00000005137 & $1.31 \mathrm{E}-10$ & $4.44 \mathrm{E}-11$ & LPGAT1 & $2.94 \mathrm{E}+00$ \\
\hline ENSBTAG00000008734 & $5.03 \mathrm{E}-10$ & $1.69 \mathrm{E}-10$ & PM20D1 & $2.98 \mathrm{E}+00$ \\
\hline ENSBTAG00000004246 & $3.68 \mathrm{E}-10$ & $1.23 \mathrm{E}-10$ & H6PD & $2.99 \mathrm{E}+00$ \\
\hline ENSBTAG00000024663 & $4.36 \mathrm{E}-10$ & $1.45 \mathrm{E}-10$ & LAD1 & $3.00 E+00$ \\
\hline ENSBTAG00000016733 & $1.01 \mathrm{E}-10$ & $3.36 \mathrm{E}-11$ & NOL9 & $3.01 E+00$ \\
\hline ENSBTAG00000010401 & $2.44 \mathrm{E}-10$ & $8.06 \mathrm{E}-11$ & UBE4B & $3.03 E+00$ \\
\hline ENSBTAG00000002479 & $1.42 \mathrm{E}-10$ & 4.64E-11 & PANK4 & $3.06 \mathrm{E}+00$ \\
\hline ENSBTAG00000020700 & $1.65 \mathrm{E}-10$ & $5.36 \mathrm{E}-11$ & CLCN6 & $3.08 \mathrm{E}+00$ \\
\hline ENSBTAG00000011465 & $5.45 \mathrm{E}-10$ & $1.74 \mathrm{E}-10$ & MYBPH & $3.13 E+00$ \\
\hline ENSBTAG00000001483 & $5.02 \mathrm{E}-10$ & $1.58 \mathrm{E}-10$ & SRGAP2 & $3.18 \mathrm{E}+00$ \\
\hline ENSBTAG00000009111 & $2.17 \mathrm{E}-10$ & $6.82 \mathrm{E}-11$ & & $3.17 E+00$ \\
\hline ENSBTAG00000014707 & $3.16 \mathrm{E}-10$ & $9.93 \mathrm{E}-11$ & ISG15 & $3.18 \mathrm{E}+00$ \\
\hline ENSBTAG00000015857 & $1.17 \mathrm{E}-10$ & $3.69 \mathrm{E}-11$ & TPRG1L & $3.17 E+00$ \\
\hline ENSBTAG00000015946 & $4.62 \mathrm{E}-10$ & $1.45 \mathrm{E}-10$ & RBBP5 & $3.19 E+00$ \\
\hline ENSBTAG00000009169 & $2.83 \mathrm{E}-10$ & $8.73 \mathrm{E}-11$ & XPR1 & $3.24 \mathrm{E}+00$ \\
\hline ENSBTAG00000020342 & $2.61 \mathrm{E}-10$ & $8.02 \mathrm{E}-11$ & MYOC & $3.25 E+00$ \\
\hline ENSBTAG00000020552 & $2.94 \mathrm{E}-10$ & $9.02 \mathrm{E}-11$ & PRDM2 & $3.26 \mathrm{E}+00$ \\
\hline ENSBTAG00000017834 & $1.69 \mathrm{E}-10$ & $5.14 \mathrm{E}-11$ & PRELP & $3.29 E+00$ \\
\hline ENSBTAG00000047648 & 4.67E-10 & $1.41 \mathrm{E}-10$ & SYT2 & $3.31 E+00$ \\
\hline ENSBTAG00000015325 & $1.87 \mathrm{E}-10$ & 5.57E-11 & MTOR & $3.36 \mathrm{E}+00$ \\
\hline ENSBTAG00000015882 & $1.71 \mathrm{E}-10$ & $5.05 \mathrm{E}-11$ & UBE2J2 & $3.37 E+00$ \\
\hline ENSBTAG00000004953 & $3.51 \mathrm{E}-10$ & $1.03 \mathrm{E}-10$ & ELK4 & $3.41 E+00$ \\
\hline ENSBTAG00000015606 & $4.31 \mathrm{E}-10$ & $1.26 \mathrm{E}-10$ & KAZN & $3.42 \mathrm{E}+00$ \\
\hline ENSBTAG00000010408 & $6.98 \mathrm{E}-10$ & $2.02 \mathrm{E}-10$ & IKBKE & $3.45 E+00$ \\
\hline ENSBTAG00000037813 & 1.17E-10 & $3.34 \mathrm{E}-11$ & & $3.49 E+00$ \\
\hline ENSBTAG00000000795 & $3.82 \mathrm{E}-10$ & $1.09 \mathrm{E}-10$ & NMNAT2 & $3.50 \mathrm{E}+00$ \\
\hline ENSBTAG00000007593 & $3.15 \mathrm{E}-10$ & $8.99 \mathrm{E}-11$ & AIDA & $3.50 E+00$ \\
\hline ENSBTAG00000038496 & $3.47 \mathrm{E}-10$ & $9.84 \mathrm{E}-11$ & CR2 & $3.52 E+00$ \\
\hline ENSBTAG00000047727 & $2.78 \mathrm{E}-10$ & 7.77E-11 & & $3.57 E+00$ \\
\hline ENSBTAG00000008132 & $4.31 \mathrm{E}-10$ & $1.20 \mathrm{E}-10$ & SOX13 & $3.59 E+00$ \\
\hline ENSBTAG00000013560 & $5.74 \mathrm{E}-10$ & $1.60 \mathrm{E}-10$ & RABIF & $3.59 E+00$ \\
\hline ENSBTAG00000024453 & $7.73 E-10$ & $2.15 \mathrm{E}-10$ & RD3 & $3.59 E+00$ \\
\hline ENSBTAG00000004941 & $4.06 \mathrm{E}-10$ & 1.10E-10 & TEX35 & $3.69 E+00$ \\
\hline ENSBTAG00000016734 & $1.46 \mathrm{E}-10$ & 3.93E-11 & TAS1R1 & $3.70 E+00$ \\
\hline ENSBTAG00000019388 & $2.04 \mathrm{E}-10$ & $5.50 \mathrm{E}-11$ & KIAA2013 & $3.70 \mathrm{E}+00$ \\
\hline ENSBTAG00000046060 & $1.37 \mathrm{E}-09$ & $3.66 \mathrm{E}-10$ & & $3.74 E+00$ \\
\hline ENSBTAG00000032808 & $1.75 \mathrm{E}-10$ & 4.64E-11 & FASLG & $3.77 \mathrm{E}+00$ \\
\hline ENSBTAG00000012673 & $3.29 \mathrm{E}-10$ & $8.70 \mathrm{E}-11$ & CDK18 & $3.78 \mathrm{E}+00$ \\
\hline ENSBTAG00000016618 & 4.39E-10 & $1.14 \mathrm{E}-10$ & DSTYK & $3.85 E+00$ \\
\hline ENSBTAG00000010432 & $5.57 \mathrm{E}-10$ & $1.45 \mathrm{E}-10$ & EIF2D & $3.84 \mathrm{E}+00$ \\
\hline ENSBTAG00000012953 & 4.63E-10 & $1.20 \mathrm{E}-10$ & ASTN1 & $3.85 E+00$ \\
\hline ENSBTAG00000013849 & $5.02 \mathrm{E}-10$ & $1.29 \mathrm{E}-10$ & RGS13 & $3.89 E+00$ \\
\hline ENSBTAG00000000215 & $1.64 \mathrm{E}-10$ & $4.14 \mathrm{E}-11$ & GNB1 & $3.94 \mathrm{E}+00$ \\
\hline ENSBTAG00000047073 & $1.32 \mathrm{E}-10$ & $3.32 \mathrm{E}-11$ & & $3.97 E+00$ \\
\hline ENSBTAG00000019931 & $1.63 \mathrm{E}-10$ & 4.10E-11 & & $3.96 \mathrm{E}+00$ \\
\hline ENSBTAG00000004872 & $1.35 \mathrm{E}-10$ & $3.39 \mathrm{E}-11$ & MRPL20 & $3.97 E+00$ \\
\hline ENSBTAG00000032617 & $1.01 \mathrm{E}-10$ & $2.54 \mathrm{E}-11$ & $\mathrm{FBXO44}$ & $3.97 E+00$ \\
\hline ENSBTAG00000014862 & 5.67E-10 & $1.42 \mathrm{E}-10$ & & $3.99 E+00$ \\
\hline ENSBTAG00000017883 & 1.03E-09 & $2.54 \mathrm{E}-10$ & LRRC38 & $4.05 E+00$ \\
\hline ENSBTAG00000010562 & $2.70 \mathrm{E}-10$ & $6.61 E-11$ & CD34 & $4.08 \mathrm{E}+00$ \\
\hline ENSBTAG00000015533 & $1.99 \mathrm{E}-10$ & $4.83 \mathrm{E}-11$ & DISP3 & $4.10 \mathrm{E}+00$ \\
\hline ENSBTAG00000047231 & $1.78 \mathrm{E}-10$ & 4.27E-11 & TNNI1 & $4.17 E+00$ \\
\hline ENSBTAG00000000023 & $2.16 \mathrm{E}-10$ & 5.19E-11 & RNF2 & $4.16 \mathrm{E}+00$ \\
\hline ENSBTAG00000038107 & $8.52 E-10$ & 2.03E-10 & MAPKAPK2 & $4.19 E+00$ \\
\hline ENSBTAG00000046172 & $2.04 \mathrm{E}-10$ & 4.75E-11 & & $4.29 E+00$ \\
\hline ENSBTAG00000001109 & $4.36 \mathrm{E}-10$ & $1.01 \mathrm{E}-10$ & & $4.31 E+00$ \\
\hline ENSBTAG00000014014 & $9.13 E-11$ & $2.09 \mathrm{E}-11$ & UBIAD1 & $4.35 E+00$ \\
\hline ENSBTAG00000000212 & $1.52 \mathrm{E}-10$ & $3.38 \mathrm{E}-11$ & NADK & $4.49 E+00$ \\
\hline ENSBTAG00000007693 & $3.36 \mathrm{E}-10$ & $7.35 \mathrm{E}-11$ & SLC45A3 & $4.57 E+00$ \\
\hline ENSBTAG00000002464 & $2.02 \mathrm{E}-10$ & $4.31 \mathrm{E}-11$ & $\mathrm{PLCH} 2$ & $4.69 E+00$ \\
\hline
\end{tabular}




\begin{tabular}{|c|c|c|c|c|}
\hline ENSBTAG000000011943 & 7.49E-10 & $1.59 \mathrm{E}-10$ & TPR & $4.71 E+00$ \\
\hline ENSBTAG000000007623 & $1.27 \mathrm{E}-10$ & 2.67E-11 & CPTP & $4.76 \mathrm{E}+00$ \\
\hline ENSBTAG000000014537 & $1.36 \mathrm{E}-10$ & $2.85 \mathrm{E}-11$ & PLEKHN1 & $4.76 \mathrm{E}+00$ \\
\hline ENSBTAG000000014992 & $2.51 \mathrm{E}-10$ & 5.27E-11 & GPR37L1 & $4.76 \mathrm{E}+00$ \\
\hline ENSBTAG000000012247 & $1.13 \mathrm{E}-10$ & $2.37 \mathrm{E}-11$ & MXRA8 & $4.75 E+00$ \\
\hline ENSBTAG00000031507 & 5.37E-10 & $1.12 \mathrm{E}-10$ & & $4.79 E+00$ \\
\hline ENSBTAG000000014645 & $5.12 \mathrm{E}-10$ & 1.05E-10 & CNTN2 & $4.87 E+00$ \\
\hline ENSBTAG000000045895 & $6.78 \mathrm{E}-10$ & $1.29 \mathrm{E}-10$ & NFASC & $5.25 E+00$ \\
\hline ENSBTAG000000019556 & $3.53 \mathrm{E}-10$ & $6.49 \mathrm{E}-11$ & MIIP & $5.43 E+00$ \\
\hline ENSBTAG000000008126 & $7.38 \mathrm{E}-10$ & $1.35 \mathrm{E}-10$ & NPHP4 & $5.46 \mathrm{E}+00$ \\
\hline ENSBTAG000000011772 & $4.38 \mathrm{E}-10$ & $7.94 \mathrm{E}-11$ & PPP1R12B & $5.52 E+00$ \\
\hline ENSBTAG000000011946 & $8.58 \mathrm{E}-10$ & $1.55 \mathrm{E}-10$ & C1orf27 & $5.53 E+00$ \\
\hline ENSBTAG00000014136 & $1.24 \mathrm{E}-10$ & $2.23 \mathrm{E}-11$ & & $5.53 \mathrm{E}+00$ \\
\hline ENSBTAG00000045789 & $2.09 \mathrm{E}-10$ & $3.76 \mathrm{E}-11$ & & $5.55 E+00$ \\
\hline ENSBTAG000000015711 & $5.01 \mathrm{E}-10$ & $8.98 \mathrm{E}-11$ & BTG2 & $5.57 \mathrm{E}+00$ \\
\hline ENSBTAG000000004855 & $2.40 \mathrm{E}-10$ & $4.16 \mathrm{E}-11$ & PRDX6 & $5.76 \mathrm{E}+00$ \\
\hline ENSBTAG00000014191 & $3.81 \mathrm{E}-10$ & $6.48 \mathrm{E}-11$ & QSOX1 & $5.87 \mathrm{E}+00$ \\
\hline ENSBTAG000000004952 & $5.84 \mathrm{E}-10$ & $9.82 \mathrm{E}-11$ & MFSD4A & $5.94 \mathrm{E}+00$ \\
\hline ENSBTAG000000005379 & $2.90 \mathrm{E}-10$ & $4.86 \mathrm{E}-11$ & SLC41A1 & $5.95 E+00$ \\
\hline ENSBTAG000000021553 & $4.53 \mathrm{E}-10$ & $7.50 \mathrm{E}-11$ & PTPN14 & $6.03 E+00$ \\
\hline ENSBTAG000000001210 & $6.86 \mathrm{E}-10$ & $1.11 \mathrm{E}-10$ & & $6.17 E+00$ \\
\hline ENSBTAG000000009938 & $2.08 \mathrm{E}-10$ & 3.26E-11 & & $6.35 E+00$ \\
\hline ENSBTAG000000015340 & $2.16 \mathrm{E}-10$ & 3.39E-11 & ANGPTL7 & $6.34 E+00$ \\
\hline ENSBTAG00000013191 & $2.13 \mathrm{E}-10$ & $3.25 \mathrm{E}-11$ & AGRN & $6.53 E+00$ \\
\hline ENSBTAG000000000854 & $3.74 \mathrm{E}-10$ & $5.49 \mathrm{E}-11$ & SEC16B & $6.79 E+00$ \\
\hline ENSBTAG000000020886 & $6.39 \mathrm{E}-10$ & $9.19 \mathrm{E}-11$ & DIEXF & $6.94 E+00$ \\
\hline ENSBTAG000000004452 & $2.81 \mathrm{E}-10$ & $3.70 \mathrm{E}-11$ & IPO9 & $7.58 \mathrm{E}+00$ \\
\hline ENSBTAG000000019890 & $8.43 \mathrm{E}-10$ & $1.08 \mathrm{E}-10$ & ATP6V1G3 & $7.80 \mathrm{E}+00$ \\
\hline ENSBTAG000000009349 & $5.16 \mathrm{E}-10$ & $6.53 \mathrm{E}-11$ & MMEL1 & $7.89 \mathrm{E}+00$ \\
\hline ENSBTAG000000044117 & $2.52 \mathrm{E}-10$ & $3.16 \mathrm{E}-11$ & ANKRD45 & $7.94 \mathrm{E}+00$ \\
\hline ENSBTAG000000015574 & $3.19 E-10$ & $4.01 E-11$ & RBP7 & $7.94 \mathrm{E}+00$ \\
\hline ENSBTAG000000008001 & $4.20 \mathrm{E}-10$ & $5.24 \mathrm{E}-11$ & NUCKS1 & $8.01 E+00$ \\
\hline ENSBTAG00000004789 & $3.35 \mathrm{E}-10$ & 3.93E-11 & LGR6 & $8.51 E+00$ \\
\hline ENSBTAG000000047285 & $3.90 \mathrm{E}-10$ & $4.29 \mathrm{E}-11$ & & $9.07 E+00$ \\
\hline ENSBTAG00000001107 & $1.75 \mathrm{E}-10$ & $1.92 \mathrm{E}-11$ & САCYBP & $9.09 \mathrm{E}+00$ \\
\hline ENSBTAG00000048267 & $3.08 \mathrm{E}-10$ & 2.97E-11 & & $1.03 E+01$ \\
\hline ENSBTAG00000021576 & $3.51 \mathrm{E}-10$ & 3.39E-11 & LMOD1 & $1.03 E+01$ \\
\hline ENSBTAG00000016057 & $3.12 \mathrm{E}-10$ & $2.98 \mathrm{E}-11$ & CSRP1 & $1.05 E+01$ \\
\hline ENSBTAG00000018303 & $2.86 \mathrm{E}-10$ & $2.61 \mathrm{E}-11$ & PAPPA2 & $1.09 \mathrm{E}+01$ \\
\hline ENSBTAG00000003804 & $8.47 \mathrm{E}-10$ & 7.67E-11 & SCCPDH & $1.10 \mathrm{E}+01$ \\
\hline ENSBTAG00000014777 & $1.40 \mathrm{E}-10$ & 1.17E-11 & FBXO6 & $1.18 \mathrm{E}+01$ \\
\hline ENSBTAG00000005314 & 4.69E-10 & $3.74 \mathrm{E}-11$ & MFN2 & $1.25 \mathrm{E}+01$ \\
\hline ENSBTAG00000009515 & $6.00 \mathrm{E}-10$ & 4.57E-11 & CA6 & $1.31 \mathrm{E}+01$ \\
\hline ENSBTAG00000012778 & $4.66 \mathrm{E}-10$ & $3.36 \mathrm{E}-11$ & CAPN2 & $1.38 \mathrm{E}+01$ \\
\hline ENSBTAG00000002052 & $4.42 \mathrm{E}-10$ & $3.05 \mathrm{E}-11$ & PLOD1 & $1.45 E+01$ \\
\hline ENSBTAG00000021919 & $3.30 \mathrm{E}-10$ & 2.04E-11 & NAV1 & $1.61 \mathrm{E}+01$ \\
\hline ENSBTAG00000003180 & $3.13 \mathrm{E}-10$ & $1.90 \mathrm{E}-11$ & TMEM201 & $1.64 \mathrm{E}+01$ \\
\hline ENSBTAG00000034632 & $9.38 \mathrm{E}-10$ & 4.47E-11 & LEMD1 & $2.09 E+01$ \\
\hline ENSBTAG00000046502 & $5.10 \mathrm{E}-10$ & $2.42 \mathrm{E}-11$ & ACBD6 & $2.10 \mathrm{E}+01$ \\
\hline ENSBTAG00000003232 & $6.10 \mathrm{E}-10$ & $2.89 \mathrm{E}-11$ & RAB29 & $2.10 \mathrm{E}+01$ \\
\hline ENSBTAG00000013715 & $7.28 \mathrm{E}-10$ & 3.07E-11 & BRINP2 & $2.36 \mathrm{E}+01$ \\
\hline ENSBTAG00000024791 & $8.54 \mathrm{E}-10$ & 3.56E-11 & & $2.39 E+01$ \\
\hline ENSBTAG00000011823 & $2.72 \mathrm{E}-10$ & $1.08 \mathrm{E}-11$ & CLSTN1 & $2.49 E+01$ \\
\hline ENSBTAG00000018984 & $2.09 \mathrm{E}-10$ & $7.52 \mathrm{E}-12$ & PIK3CD & $2.75 E+01$ \\
\hline ENSBTAG00000021492 & $3.65 \mathrm{E}-10$ & $1.31 \mathrm{E}-11$ & FAM163A & $2.77 \mathrm{E}+01$ \\
\hline ENSBTAG00000012781 & $5.24 \mathrm{E}-10$ & $1.80 \mathrm{E}-11$ & ТР53BP2 & $2.90 \mathrm{E}+01$ \\
\hline ENSBTAG00000019264 & $3.11 \mathrm{E}-10$ & $7.48 \mathrm{E}-12$ & EDEM3 & $4.10 \mathrm{E}+01$ \\
\hline ENSBTAG00000013531 & $3.31 \mathrm{E}-10$ & $7.04 \mathrm{E}-12$ & CENPS & $4.63 E+01$ \\
\hline ENSBTAG00000014359 & $5.09 \mathrm{E}-10$ & $1.08 \mathrm{E}-11$ & DRAXIN & $4.68 \mathrm{E}+01$ \\
\hline ENSBTAG00000023144 & $6.92 \mathrm{E}-10$ & $8.82 \mathrm{E}-12$ & PTPRC & $7.76 \mathrm{E}+01$ \\
\hline ENSBTAG00000047233 & $3.46 \mathrm{E}-10$ & 0 & & $3.46 \mathrm{E}+03$ \\
\hline ENSBTAG00000000385 & $8.00 \mathrm{E}-11$ & 0 & ZBTB18 & $8.01 E+02$ \\
\hline ENSBTAG00000021739 & $2.36 \mathrm{E}-10$ & 0 & NPPB & $2.36 \mathrm{E}+03$ \\
\hline ENSBTAG00000007223 & 3.13E-10 & 0 & FBXO2 & $3.13 E+03$ \\
\hline ENSBTAG00000046494 & $1.02 \mathrm{E}-10$ & 0 & CORT & $1.03 E+03$ \\
\hline ENSBTAG00000005933 & 4.05E-10 & 0 & CTNNBIP1 & $4.05 E+03$ \\
\hline ENSBTAG00000003177 & $3.21 \mathrm{E}-10$ & 0 & SLC25A33 & $3.21 E+03$ \\
\hline ENSBTAG00000013411 & $2.61 \mathrm{E}-10$ & 0 & ENO1 & $2.61 E+03$ \\
\hline ENSBTAG00000030805 & $1.94 \mathrm{E}-10$ & 0 & SHISA4 & $1.94 \mathrm{E}+03$ \\
\hline ENSBTAG00000001727 & $1.58 \mathrm{E}-10$ & 0 & FAM213B & $1.58 \mathrm{E}+03$ \\
\hline
\end{tabular}




\begin{tabular}{|c|c|c|c|c|}
\hline ENSBTAG00000024508 & 0 & 0 & CALML6 & $1.00 \mathrm{E}+00$ \\
\hline ENSBTAG00000010732 & $3.51 \mathrm{E}-11$ & 0 & MMP23B & $3.52 \mathrm{E}+02$ \\
\hline ENSBTAG00000047755 & 0 & 0 & FNDC10 & $1.00 E+00$ \\
\hline ENSBTAG00000046073 & $9.14 \mathrm{E}-11$ & 0 & TMEM240 & $9.15 E+02$ \\
\hline ENSBTAG00000039055 & 8.37E-11 & 0 & ANKRD65 & $8.38 \mathrm{E}+02$ \\
\hline ENSBTAG00000031631 & $6.37 \mathrm{E}-11$ & 0 & FAM132A & $6.38 \mathrm{E}+02$ \\
\hline ENSBTAG00000007619 & $2.89 \mathrm{E}-11$ & 0 & PUSL1 & $2.90 \mathrm{E}+02$ \\
\hline ENSBTAG00000017674 & $1.47 \mathrm{E}-10$ & 0 & SCNN1D & $1.47 E+03$ \\
\hline ENSBTAG00000014540 & $2.78 \mathrm{E}-10$ & 0 & PERM1 & $2.78 \mathrm{E}+03$ \\
\hline ENSBTAG00000013813 & $1.16 \mathrm{E}-10$ & 0 & KLHL17 & $1.16 \mathrm{E}+03$ \\
\hline ENSBTAG00000005300 & $3.32 \mathrm{E}-10$ & 0 & TMEM51 & $3.32 \mathrm{E}+03$ \\
\hline ENSBTAG00000019927 & $2.89 \mathrm{E}-10$ & 0 & CYB5R1 & $2.89 \mathrm{E}+03$ \\
\hline ENSBTAG00000046799 & $1.73 \mathrm{E}-10$ & 0 & & $1.73 \mathrm{E}+03$ \\
\hline ENSBTAG00000004790 & $1.78 \mathrm{E}-10$ & 0 & UBE2T & $1.78 \mathrm{E}+03$ \\
\hline ENSBTAG00000035659 & $2.63 \mathrm{E}-10$ & 0 & & $2.63 E+03$ \\
\hline ENSBTAG00000016347 & 0 & 0 & & $1.00 \mathrm{E}+00$ \\
\hline ENSBTAG00000046062 & 0 & 0 & & $1.00 \mathrm{E}+00$ \\
\hline ENSBTAG00000046758 & $1.09 \mathrm{E}-10$ & 0 & RNF223 & $1.09 \mathrm{E}+03$ \\
\hline ENSBTAG00000038238 & $2.89 \mathrm{E}-10$ & 0 & & $2.90 \mathrm{E}+03$ \\
\hline ENSBTAG00000047395 & $8.22 \mathrm{E}-10$ & 0 & APOBEC4 & $8.22 E+03$ \\
\hline ENSBTAG00000045729 & 0 & 0 & & $1.00 \mathrm{E}+00$ \\
\hline ENSBTAG00000047559 & $2.15 E-10$ & 0 & & $2.15 E+03$ \\
\hline ENSBTAG00000046345 & $3.07 \mathrm{E}-10$ & 0 & & $3.07 E+03$ \\
\hline ENSBTAG00000047428 & $5.88 \mathrm{E}-11$ & 0 & & $5.89 \mathrm{E}+02$ \\
\hline ENSBTAG00000045738 & 0 & $3.88 \mathrm{E}-10$ & & $2.58 \mathrm{E}-04$ \\
\hline ENSBTAG00000046439 & 0 & $1.97 \mathrm{E}-10$ & & 5.07E-04 \\
\hline ENSBTAG00000033620 & $3.38 \mathrm{E}-11$ & $2.99 \mathrm{E}-10$ & & 1.13E-01 \\
\hline ENSBTAG00000047626 & $4.75 \mathrm{E}-11$ & $2.40 \mathrm{E}-10$ & & $1.98 \mathrm{E}-01$ \\
\hline ENSBTAG00000001255 & $1.48 \mathrm{E}-10$ & $6.24 \mathrm{E}-10$ & CRYBB3 & 2.37E-01 \\
\hline ENSBTAG00000046986 & $4.86 \mathrm{E}-11$ & $1.84 \mathrm{E}-10$ & & 2.64E-01 \\
\hline ENSBTAG00000035012 & $8.68 \mathrm{E}-10$ & $2.55 \mathrm{E}-09$ & & 3.40E-01 \\
\hline ENSBTAG00000011962 & $6.48 \mathrm{E}-11$ & $1.84 \mathrm{E}-10$ & PLA2G3 & 3.52E-01 \\
\hline ENSBTAG00000038258 & $1.09 \mathrm{E}-10$ & $2.76 \mathrm{E}-10$ & & $3.96 \mathrm{E}-01$ \\
\hline ENSBTAG00000039237 & $2.56 \mathrm{E}-10$ & $6.45 \mathrm{E}-10$ & & 3.96E-01 \\
\hline ENSBTAG00000005648 & $4.89 \mathrm{E}-11$ & $1.23 \mathrm{E}-10$ & POU4F2 & $3.98 \mathrm{E}-01$ \\
\hline ENSBTAG00000001594 & $3.18 \mathrm{E}-11$ & 8.00E-11 & ARL6IP4 & $3.98 \mathrm{E}-01$ \\
\hline ENSBTAG00000047526 & $1.37 \mathrm{E}-10$ & $3.45 \mathrm{E}-10$ & GSC2 & $3.96 \mathrm{E}-01$ \\
\hline ENSBTAG00000019280 & $2.45 \mathrm{E}-10$ & $6.11 E-10$ & CRYBB1 & 4.01E-01 \\
\hline ENSBTAG00000046302 & $6.08 \mathrm{E}-10$ & 1.40E-09 & & 4.34E-01 \\
\hline ENSBTAG00000040370 & $1.02 \mathrm{E}-09$ & 2.31E-09 & & 4.40E-01 \\
\hline ENSBTAG00000034761 & $9.95 \mathrm{E}-11$ & $2.09 \mathrm{E}-10$ & & 4.76E-01 \\
\hline ENSBTAG00000001593 & $6.11 \mathrm{E}-11$ & $1.28 \mathrm{E}-10$ & OGFOD2 & $4.78 \mathrm{E}-01$ \\
\hline ENSBTAG00000013001 & $5.14 \mathrm{E}-11$ & $1.08 \mathrm{E}-10$ & & 4.76E-01 \\
\hline ENSBTAG00000003100 & $2.23 \mathrm{E}-10$ & 4.62E-10 & SMTN & 4.82E-01 \\
\hline ENSBTAG00000046669 & $7.00 \mathrm{E}-10$ & $1.41 \mathrm{E}-09$ & PRAME & 4.97E-01 \\
\hline ENSBTAG00000033558 & $2.48 \mathrm{E}-10$ & $5.00 \mathrm{E}-10$ & & 4.95E-01 \\
\hline ENSBTAG00000005240 & 8.67E-11 & $1.72 \mathrm{E}-10$ & ZNF605 & 5.04E-01 \\
\hline ENSBTAG00000007139 & $1.99 \mathrm{E}-10$ & 3.80E-10 & WSB2 & 5.24E-01 \\
\hline ENSBTAG00000031468 & $3.61 E-10$ & $6.83 \mathrm{E}-10$ & & $5.28 \mathrm{E}-01$ \\
\hline ENSBTAG00000031993 & $6.39 \mathrm{E}-11$ & $1.21 \mathrm{E}-10$ & FAM222A & 5.29E-01 \\
\hline ENSBTAG00000047085 & $6.13 \mathrm{E}-10$ & 1.13E-09 & PRAME & 5.42E-01 \\
\hline ENSBTAG00000047139 & $5.10 \mathrm{E}-11$ & $8.36 \mathrm{E}-11$ & C12orf49 & $6.11 \mathrm{E}-01$ \\
\hline ENSBTAG00000015767 & $1.63 \mathrm{E}-10$ & $2.55 \mathrm{E}-10$ & RNF10 & $6.41 \mathrm{E}-01$ \\
\hline ENSBTAG00000046306 & $1.23 \mathrm{E}-09$ & $1.91 E-09$ & & 6.43E-01 \\
\hline ENSBTAG00000014464 & $3.63 \mathrm{E}-10$ & $5.60 \mathrm{E}-10$ & SEC14L3 & $6.48 \mathrm{E}-01$ \\
\hline ENSBTAG00000010812 & $3.71 \mathrm{E}-10$ & $5.64 \mathrm{E}-10$ & $\mathrm{P} 2 \mathrm{RX} 4$ & $6.58 \mathrm{E}-01$ \\
\hline ENSBTAG00000047597 & $8.54 \mathrm{E}-10$ & 1.27E-09 & & 6.73E-01 \\
\hline ENSBTAG00000006118 & $5.64 \mathrm{E}-11$ & $8.41 E-11$ & RSRC2 & $6.72 \mathrm{E}-01$ \\
\hline ENSBTAG00000016680 & $6.24 \mathrm{E}-10$ & $9.08 \mathrm{E}-10$ & PPID & $6.88 \mathrm{E}-01$ \\
\hline ENSBTAG00000031279 & $1.72 \mathrm{E}-10$ & $2.48 \mathrm{E}-10$ & SELENOM & 6.95E-01 \\
\hline ENSBTAG00000005435 & $4.96 \mathrm{E}-10$ & $7.14 \mathrm{E}-10$ & & 6.94E-01 \\
\hline ENSBTAG00000005478 & $1.41 \mathrm{E}-10$ & $2.01 E-10$ & PATZ1 & 7.00E-01 \\
\hline ENSBTAG00000006504 & $2.44 \mathrm{E}-10$ & $3.43 \mathrm{E}-10$ & $\mathrm{TCHP}$ & 7.11E-01 \\
\hline ENSBTAG00000019278 & $1.54 \mathrm{E}-10$ & $2.08 \mathrm{E}-10$ & KNTC1 & 7.39E-01 \\
\hline ENSBTAG00000024545 & $3.44 \mathrm{E}-10$ & $4.58 \mathrm{E}-10$ & DCHS2 & $7.51 \mathrm{E}-01$ \\
\hline ENSBTAG00000017459 & $2.95 \mathrm{E}-10$ & $3.90 \mathrm{E}-10$ & DUSP18 & 7.56E-01 \\
\hline ENSBTAG00000018647 & $4.73 \mathrm{E}-10$ & $6.24 \mathrm{E}-10$ & & $7.58 \mathrm{E}-01$ \\
\hline ENSBTAG00000017799 & $1.01 \mathrm{E}-10$ & $1.33 \mathrm{E}-10$ & PITPNB & 7.62E-01 \\
\hline ENSBTAG00000002770 & $3.03 \mathrm{E}-10$ & $3.95 \mathrm{E}-10$ & TCN2 & 7.67E-01 \\
\hline ENSBTAG00000039769 & $1.53 \mathrm{E}-09$ & $1.98 \mathrm{E}-09$ & & 7.73E-01 \\
\hline
\end{tabular}




\begin{tabular}{|c|c|c|c|c|}
\hline ENSBTAG00000006608 & $3.22 \mathrm{E}-10$ & $4.17 \mathrm{E}-10$ & GGT5 & 7.71E-01 \\
\hline ENSBTAG00000003297 & $3.60 \mathrm{E}-10$ & 4.66E-10 & OASL & $7.73 \mathrm{E}-01$ \\
\hline ENSBTAG00000012826 & $1.22 \mathrm{E}-10$ & $1.58 \mathrm{E}-10$ & SLC25A31 & $7.73 \mathrm{E}-01$ \\
\hline ENSBTAG00000044058 & $8.68 \mathrm{E}-11$ & $1.12 \mathrm{E}-10$ & MFSD8 & $7.75 \mathrm{E}-01$ \\
\hline ENSBTAG00000015692 & $1.18 \mathrm{E}-10$ & $1.51 \mathrm{E}-10$ & HSPA4L & 7.82E-01 \\
\hline ENSBTAG00000005951 & $1.09 \mathrm{E}-10$ & $1.38 \mathrm{E}-10$ & $\mathrm{HIC2}$ & $7.92 \mathrm{E}-01$ \\
\hline ENSBTAG00000033967 & $1.48 \mathrm{E}-10$ & $1.86 \mathrm{E}-10$ & & $7.94 \mathrm{E}-01$ \\
\hline ENSBTAG00000002122 & $2.52 \mathrm{E}-10$ & $3.18 \mathrm{E}-10$ & FAM109A & 7.94E-01 \\
\hline ENSBTAG00000021262 & $2.16 \mathrm{E}-10$ & $2.72 \mathrm{E}-10$ & SNRPD3 & 7.94E-01 \\
\hline ENSBTAG00000022030 & $6.45 \mathrm{E}-10$ & $8.12 \mathrm{E}-10$ & C22orf15 & 7.94E-01 \\
\hline ENSBTAG00000007375 & $5.18 \mathrm{E}-10$ & $6.53 \mathrm{E}-10$ & MIF & 7.94E-01 \\
\hline ENSBTAG00000039316 & $1.04 \mathrm{E}-10$ & $1.31 \mathrm{E}-10$ & ZNF268 & 7.95E-01 \\
\hline ENSBTAG00000005128 & $5.88 \mathrm{E}-10$ & $7.41 \mathrm{E}-10$ & VPREB3 & 7.93E-01 \\
\hline ENSBTAG00000018084 & $5.44 \mathrm{E}-11$ & $6.85 \mathrm{E}-11$ & GATSL3 & $7.94 \mathrm{E}-01$ \\
\hline ENSBTAG00000004197 & $7.54 \mathrm{E}-11$ & $9.50 \mathrm{E}-11$ & B3GNT4 & 7.94E-01 \\
\hline ENSBTAG00000046887 & $6.48 \mathrm{E}-10$ & 8.17E-10 & & 7.93E-01 \\
\hline ENSBTAG00000047924 & $7.58 \mathrm{E}-11$ & $9.55 E-11$ & MAB21L2 & 7.94E-01 \\
\hline ENSBTAG00000037415 & $3.58 \mathrm{E}-10$ & $4.51 \mathrm{E}-10$ & & $7.93 \mathrm{E}-01$ \\
\hline ENSBTAG00000045938 & $9.91 \mathrm{E}-11$ & $1.25 \mathrm{E}-10$ & & 7.93E-01 \\
\hline ENSBTAG00000022020 & $2.57 \mathrm{E}-10$ & $3.24 \mathrm{E}-10$ & CLDN5 & 7.94E-01 \\
\hline ENSBTAG00000003390 & $1.58 \mathrm{E}-10$ & $1.99 \mathrm{E}-10$ & SF3A1 & 7.94E-01 \\
\hline ENSBTAG00000012800 & $1.78 \mathrm{E}-10$ & $2.25 \mathrm{E}-10$ & MMAA & $7.92 \mathrm{E}-01$ \\
\hline ENSBTAG00000007424 & $1.14 \mathrm{E}-10$ & $1.44 \mathrm{E}-10$ & LIF & 7.92E-01 \\
\hline ENSBTAG00000014576 & $3.08 \mathrm{E}-10$ & $3.85 \mathrm{E}-10$ & GUCY1A3 & 7.99E-01 \\
\hline ENSBTAG00000001005 & $7.30 \mathrm{E}-10$ & $9.08 \mathrm{E}-10$ & & 8.04E-01 \\
\hline ENSBTAG00000016679 & $3.72 \mathrm{E}-10$ & $4.62 \mathrm{E}-10$ & ETFDH & 8.05E-01 \\
\hline ENSBTAG00000031351 & $5.46 \mathrm{E}-10$ & $6.72 \mathrm{E}-10$ & SEC14L4 & $8.13 \mathrm{E}-01$ \\
\hline ENSBTAG00000044022 & $4.92 \mathrm{E}-10$ & $6.01 \mathrm{E}-10$ & KIAA1671 & $8.19 \mathrm{E}-01$ \\
\hline ENSBTAG00000013150 & $2.62 \mathrm{E}-10$ & $3.17 \mathrm{E}-10$ & THOC5 & 8.27E-01 \\
\hline ENSBTAG00000004286 & $5.11 \mathrm{E}-10$ & $6.18 \mathrm{E}-10$ & CDC45 & $8.28 \mathrm{E}-01$ \\
\hline ENSBTAG00000040298 & $2.47 \mathrm{E}-10$ & $2.97 \mathrm{E}-10$ & & $8.30 \mathrm{E}-01$ \\
\hline ENSBTAG00000009797 & $3.02 \mathrm{E}-10$ & $3.64 \mathrm{E}-10$ & RIMBP2 & $8.29 \mathrm{E}-01$ \\
\hline ENSBTAG00000001638 & $2.67 \mathrm{E}-10$ & $3.21 \mathrm{E}-10$ & & $8.31 \mathrm{E}-01$ \\
\hline ENSBTAG00000001295 & $5.47 \mathrm{E}-10$ & $6.56 \mathrm{E}-10$ & DEPDC5 & 8.33E-01 \\
\hline ENSBTAG00000008948 & $2.34 \mathrm{E}-10$ & $2.80 \mathrm{E}-10$ & $\mathrm{P} 2 \mathrm{R} \times 7$ & $8.36 \mathrm{E}-01$ \\
\hline ENSBTAG00000014060 & $5.45 \mathrm{E}-10$ & $6.52 \mathrm{E}-10$ & LSM6 & $8.36 \mathrm{E}-01$ \\
\hline ENSBTAG00000016779 & $2.91 \mathrm{E}-10$ & $3.47 \mathrm{E}-10$ & CLIP1 & $8.38 \mathrm{E}-01$ \\
\hline ENSBTAG00000012193 & $1.34 \mathrm{E}-10$ & $1.59 \mathrm{E}-10$ & TTC28 & $8.42 \mathrm{E}-01$ \\
\hline ENSBTAG00000005183 & $7.48 \mathrm{E}-10$ & $8.87 \mathrm{E}-10$ & MVK & $8.43 \mathrm{E}-01$ \\
\hline ENSBTAG00000040336 & $8.24 \mathrm{E}-10$ & $9.76 \mathrm{E}-10$ & & $8.44 \mathrm{E}-01$ \\
\hline ENSBTAG00000047664 & $3.07 \mathrm{E}-10$ & $3.61 \mathrm{E}-10$ & GNAZ & $8.50 \mathrm{E}-01$ \\
\hline ENSBTAG00000007266 & $8.58 \mathrm{E}-10$ & $1.01 \mathrm{E}-09$ & DDX55 & $8.50 \mathrm{E}-01$ \\
\hline ENSBTAG00000005178 & $5.33 \mathrm{E}-10$ & $6.27 \mathrm{E}-10$ & MMAB & $8.50 \mathrm{E}-01$ \\
\hline ENSBTAG00000014269 & $3.38 \mathrm{E}-10$ & $3.96 \mathrm{E}-10$ & SCARB1 & $8.53 \mathrm{E}-01$ \\
\hline ENSBTAG00000020414 & $3.53 \mathrm{E}-10$ & $4.14 \mathrm{E}-10$ & DHX37 & 8.53E-01 \\
\hline ENSBTAG00000036183 & $4.31 \mathrm{E}-10$ & $5.05 E-10$ & FBXW8 & $8.54 \mathrm{E}-01$ \\
\hline ENSBTAG00000037993 & $9.61 \mathrm{E}-10$ & $1.12 \mathrm{E}-09$ & & $8.58 \mathrm{E}-01$ \\
\hline ENSBTAG00000009988 & $2.48 \mathrm{E}-10$ & $2.88 \mathrm{E}-10$ & SMARCB1 & $8.59 \mathrm{E}-01$ \\
\hline ENSBTAG00000000031 & $1.34 \mathrm{E}-10$ & $1.54 \mathrm{E}-10$ & TRPV4 & 8.70E-01 \\
\hline ENSBTAG00000012824 & $2.83 \mathrm{E}-10$ & $3.25 \mathrm{E}-10$ & INTU & $8.69 \mathrm{E}-01$ \\
\hline ENSBTAG00000032433 & $5.47 \mathrm{E}-10$ & $6.26 \mathrm{E}-10$ & & $8.75 \mathrm{E}-01$ \\
\hline ENSBTAG00000005644 & $4.21 \mathrm{E}-10$ & $4.80 \mathrm{E}-10$ & & 8.77E-01 \\
\hline ENSBTAG00000003107 & $1.87 \mathrm{E}-10$ & $2.13 \mathrm{E}-10$ & LHX5 & $8.78 \mathrm{E}-01$ \\
\hline ENSBTAG00000006594 & $1.09 \mathrm{E}-09$ & $1.24 \mathrm{E}-09$ & & $8.76 \mathrm{E}-01$ \\
\hline ENSBTAG00000004647 & $6.72 \mathrm{E}-10$ & $7.65 E-10$ & UCP1 & $8.78 \mathrm{E}-01$ \\
\hline ENSBTAG00000004179 & $2.34 \mathrm{E}-10$ & $2.66 \mathrm{E}-10$ & PSMD9 & $8.78 \mathrm{E}-01$ \\
\hline ENSBTAG00000012288 & $4.16 \mathrm{E}-10$ & $4.72 \mathrm{E}-10$ & TFIP11 & $8.81 \mathrm{E}-01$ \\
\hline ENSBTAG00000007804 & $2.19 \mathrm{E}-10$ & $2.49 \mathrm{E}-10$ & RILPL2 & $8.80 \mathrm{E}-01$ \\
\hline ENSBTAG00000019846 & $2.82 \mathrm{E}-10$ & $3.20 \mathrm{E}-10$ & ALKBH2 & $8.82 \mathrm{E}-01$ \\
\hline ENSBTAG00000002385 & $1.38 \mathrm{E}-10$ & $1.57 \mathrm{E}-10$ & SLC35E4 & $8.82 \mathrm{E}-01$ \\
\hline ENSBTAG00000026626 & $5.38 \mathrm{E}-10$ & $6.07 E-10$ & ANHX & 8.87E-01 \\
\hline ENSBTAG00000018086 & $1.50 \mathrm{E}-10$ & $1.69 \mathrm{E}-10$ & & $8.85 \mathrm{E}-01$ \\
\hline ENSBTAG00000012851 & $8.26 \mathrm{E}-10$ & $9.29 \mathrm{E}-10$ & SLC5A1 & 8.89E-01 \\
\hline ENSBTAG00000004633 & $8.85 \mathrm{E}-10$ & $9.96 \mathrm{E}-10$ & & 8.89E-01 \\
\hline ENSBTAG00000031723 & $1.50 \mathrm{E}-10$ & $1.68 \mathrm{E}-10$ & & 8.95E-01 \\
\hline ENSBTAG00000020050 & $3.61 \mathrm{E}-10$ & $4.03 E-10$ & MLEC & 8.95E-01 \\
\hline ENSBTAG00000007269 & $6.56 \mathrm{E}-10$ & $7.31 \mathrm{E}-10$ & EIF2B1 & $8.98 \mathrm{E}-01$ \\
\hline ENSBTAG00000001597 & $1.41 \mathrm{E}-10$ & $1.56 \mathrm{E}-10$ & PITPNM2 & $9.03 \mathrm{E}-01$ \\
\hline ENSBTAG00000002048 & $1.62 \mathrm{E}-10$ & $1.80 \mathrm{E}-10$ & PTPN11 & $9.01 \mathrm{E}-01$ \\
\hline ENSBTAG00000007484 & $2.33 \mathrm{E}-10$ & $2.57 \mathrm{E}-10$ & ACADS & 9.07E-01 \\
\hline
\end{tabular}




\begin{tabular}{|c|c|c|c|c|}
\hline ENSBTAG00000013152 & $3.37 \mathrm{E}-10$ & $3.71 \mathrm{E}-10$ & NIPSNAP1 & 9.07E-01 \\
\hline ENSBTAG00000017886 & 3.17E-10 & $3.49 \mathrm{E}-10$ & CABP7 & $9.08 \mathrm{E}-01$ \\
\hline ENSBTAG000000022027 & $2.45 \mathrm{E}-10$ & $2.71 \mathrm{E}-10$ & DDT & $9.06 \mathrm{E}-01$ \\
\hline ENSBTAG00000004284 & $5.31 \mathrm{E}-10$ & $5.84 \mathrm{E}-10$ & UFD1L & $9.10 \mathrm{E}-01$ \\
\hline ENSBTAG000000001398 & $1.38 \mathrm{E}-10$ & $1.51 \mathrm{E}-10$ & ATP2A2 & $9.12 \mathrm{E}-01$ \\
\hline ENSBTAG000000021795 & $4.82 \mathrm{E}-10$ & $5.26 \mathrm{E}-10$ & HNF1A & $9.16 \mathrm{E}-01$ \\
\hline ENSBTAG000000008996 & $6.15 \mathrm{E}-10$ & $6.70 \mathrm{E}-10$ & SFI1 & $9.17 \mathrm{E}-01$ \\
\hline ENSBTAG000000033486 & $3.82 \mathrm{E}-10$ & 4.17E-10 & C4orf46 & $9.16 \mathrm{E}-01$ \\
\hline ENSBTAG000000001580 & $2.24 \mathrm{E}-10$ & $2.43 \mathrm{E}-10$ & CLGN & $9.21 \mathrm{E}-01$ \\
\hline ENSBTAG000000034522 & $8.00 \mathrm{E}-10$ & 8.70E-10 & & $9.19 \mathrm{E}-01$ \\
\hline ENSBTAG00000045580 & $9.13 \mathrm{E}-10$ & $9.91 \mathrm{E}-10$ & & $9.21 \mathrm{E}-01$ \\
\hline ENSBTAG00000022058 & $3.78 \mathrm{E}-10$ & $4.11 \mathrm{E}-10$ & ACACB & $9.20 \mathrm{E}-01$ \\
\hline ENSBTAG000000002835 & $1.97 \mathrm{E}-10$ & $2.13 \mathrm{E}-10$ & SMAD1 & $9.25 \mathrm{E}-01$ \\
\hline ENSBTAG000000010843 & $1.55 \mathrm{E}-10$ & $1.68 \mathrm{E}-10$ & PGRMC2 & $9.25 \mathrm{E}-01$ \\
\hline ENSBTAG000000007496 & $1.62 \mathrm{E}-10$ & $1.75 \mathrm{E}-10$ & $\mathrm{P} 2 \mathrm{R} \times 2$ & $9.24 \mathrm{E}-01$ \\
\hline ENSBTAG000000018546 & $3.85 \mathrm{E}-10$ & 4.17E-10 & LRBA & $9.24 \mathrm{E}-01$ \\
\hline ENSBTAG000000046900 & $6.23 \mathrm{E}-10$ & $6.73 \mathrm{E}-10$ & & $9.25 \mathrm{E}-01$ \\
\hline ENSBTAG000000032517 & 3.65E-10 & 3.93E-10 & BCL7A & $9.28 \mathrm{E}-01$ \\
\hline ENSBTAG000000003393 & $1.89 \mathrm{E}-10$ & $2.04 \mathrm{E}-10$ & CCDC157 & $9.24 \mathrm{E}-01$ \\
\hline ENSBTAG000000018951 & $6.04 \mathrm{E}-10$ & $6.49 \mathrm{E}-10$ & TBC1D9 & $9.31 \mathrm{E}-01$ \\
\hline ENSBTAG000000010630 & $9.07 \mathrm{E}-11$ & $9.75 \mathrm{E}-11$ & ABHD18 & 9.30E-01 \\
\hline ENSBTAG000000008990 & $3.75 E-10$ & 4.03E-10 & DRG1 & 9.30E-01 \\
\hline ENSBTAG000000019848 & 4.15E-10 & 4.45E-10 & UNG & $9.32 \mathrm{E}-01$ \\
\hline ENSBTAG000000008945 & $4.70 \mathrm{E}-10$ & $5.04 \mathrm{E}-10$ & SDSL & $9.33 \mathrm{E}-01$ \\
\hline ENSBTAG000000022109 & $5.96 \mathrm{E}-10$ & $6.36 \mathrm{E}-10$ & $\mathrm{SCOC}$ & $9.36 \mathrm{E}-01$ \\
\hline ENSBTAG000000001696 & $3.48 \mathrm{E}-10$ & $3.71 \mathrm{E}-10$ & RBM46 & $9.38 \mathrm{E}-01$ \\
\hline ENSBTAG000000046730 & $1.97 \mathrm{E}-10$ & $2.09 \mathrm{E}-10$ & & $9.41 \mathrm{E}-01$ \\
\hline ENSBTAG00000022120 & $4.56 \mathrm{E}-10$ & $4.83 \mathrm{E}-10$ & FGB & $9.45 \mathrm{E}-01$ \\
\hline ENSBTAG000000002328 & $3.32 \mathrm{E}-10$ & $3.50 \mathrm{E}-10$ & KDM2B & 9.47E-01 \\
\hline ENSBTAG000000017438 & $2.12 \mathrm{E}-10$ & $2.23 \mathrm{E}-10$ & C5orf52 & $9.51 \mathrm{E}-01$ \\
\hline ENSBTAG000000030979 & $2.58 \mathrm{E}-10$ & $2.71 \mathrm{E}-10$ & & $9.52 \mathrm{E}-01$ \\
\hline ENSBTAG00000016813 & $4.53 \mathrm{E}-10$ & $4.76 \mathrm{E}-10$ & SH3D19 & 9.53E-01 \\
\hline ENSBTAG000000047084 & $5.74 \mathrm{E}-10$ & $6.02 \mathrm{E}-10$ & & 9.53E-01 \\
\hline ENSBTAG000000017219 & 7.33E-11 & $7.69 \mathrm{E}-11$ & DDX51 & 9.53E-01 \\
\hline ENSBTAG000000026437 & $1.74 \mathrm{E}-10$ & $1.83 \mathrm{E}-10$ & ULBP3 & 9.50E-01 \\
\hline ENSBTAG00000017305 & 5.17E-10 & 5.37E-10 & & $9.62 \mathrm{E}-01$ \\
\hline ENSBTAG00000010958 & $1.84 \mathrm{E}-10$ & $1.90 \mathrm{E}-10$ & & $9.70 \mathrm{E}-01$ \\
\hline ENSBTAG00000018831 & $3.63 \mathrm{E}-10$ & $3.75 E-10$ & DAO & 9.67E-01 \\
\hline ENSBTAG00000008464 & $2.91 \mathrm{E}-10$ & $3.01 E-10$ & $\mathrm{ABCB} 9$ & $9.66 \mathrm{E}-01$ \\
\hline ENSBTAG00000008998 & $3.11 \mathrm{E}-10$ & $3.20 \mathrm{E}-10$ & PISD & $9.72 \mathrm{E}-01$ \\
\hline ENSBTAG00000012791 & $1.39 \mathrm{E}-10$ & $1.43 \mathrm{E}-10$ & & $9.69 \mathrm{E}-01$ \\
\hline ENSBTAG00000025263 & $5.31 \mathrm{E}-10$ & $5.45 \mathrm{E}-10$ & FAM160A1 & $9.75 \mathrm{E}-01$ \\
\hline ENSBTAG00000008762 & $1.99 \mathrm{E}-10$ & $2.03 \mathrm{E}-10$ & & $9.83 \mathrm{E}-01$ \\
\hline ENSBTAG00000019845 & 4.93E-10 & 5.00E-10 & USP30 & $9.86 \mathrm{E}-01$ \\
\hline ENSBTAG00000020161 & $2.60 \mathrm{E}-10$ & $2.64 \mathrm{E}-10$ & KLHL22 & $9.86 \mathrm{E}-01$ \\
\hline ENSBTAG00000009800 & $2.91 \mathrm{E}-10$ & $2.95 \mathrm{E}-10$ & MSI1 & $9.85 \mathrm{E}-01$ \\
\hline ENSBTAG00000020346 & $6.09 \mathrm{E}-10$ & $6.17 \mathrm{E}-10$ & CCDC92 & 9.87E-01 \\
\hline ENSBTAG00000047805 & $3.97 \mathrm{E}-10$ & $4.00 \mathrm{E}-10$ & & $9.92 \mathrm{E}-01$ \\
\hline ENSBTAG00000021121 & $4.14 \mathrm{E}-10$ & 4.17E-10 & PPIL2 & $9.92 \mathrm{E}-01$ \\
\hline ENSBTAG00000020532 & $7.59 \mathrm{E}-10$ & 7.64E-10 & & $9.93 \mathrm{E}-01$ \\
\hline ENSBTAG00000017069 & $5.62 \mathrm{E}-10$ & $5.65 \mathrm{E}-10$ & FAM198B & 9.94E-01 \\
\hline ENSBTAG00000010662 & $4.01 \mathrm{E}-10$ & $4.01 E-10$ & LIMK2 & $1.00 \mathrm{E}+00$ \\
\hline ENSBTAG00000005998 & $1.90 \mathrm{E}-10$ & $1.88 \mathrm{E}-10$ & NOCT & $1.01 \mathrm{E}+00$ \\
\hline ENSBTAG00000002828 & $1.28 \mathrm{E}-10$ & $1.27 \mathrm{E}-10$ & RNF185 & $1.01 E+00$ \\
\hline ENSBTAG00000020049 & $3.25 \mathrm{E}-10$ & $3.21 \mathrm{E}-10$ & & $1.01 E+00$ \\
\hline ENSBTAG00000004088 & $2.80 \mathrm{E}-10$ & $2.76 \mathrm{E}-10$ & CRYBB2 & $1.01 \mathrm{E}+00$ \\
\hline ENSBTAG00000008761 & $2.06 \mathrm{E}-10$ & $2.01 E-10$ & TRAFD1 & $1.02 \mathrm{E}+00$ \\
\hline ENSBTAG00000015761 & $2.16 \mathrm{E}-10$ & $2.12 \mathrm{E}-10$ & COQ5 & $1.02 E+00$ \\
\hline ENSBTAG00000004458 & $8.42 \mathrm{E}-11$ & $8.25 \mathrm{E}-11$ & MORN3 & $1.02 \mathrm{E}+00$ \\
\hline ENSBTAG00000007137 & $1.38 \mathrm{E}-10$ & $1.35 \mathrm{E}-10$ & RFC5 & $1.02 \mathrm{E}+00$ \\
\hline ENSBTAG00000018200 & $1.39 \mathrm{E}-10$ & $1.36 \mathrm{E}-10$ & IL15 & $1.02 \mathrm{E}+00$ \\
\hline ENSBTAG00000044109 & $2.95 \mathrm{E}-10$ & $2.88 \mathrm{E}-10$ & ASIC5 & $1.02 \mathrm{E}+00$ \\
\hline ENSBTAG00000012135 & $1.96 \mathrm{E}-10$ & $1.91 \mathrm{E}-10$ & LARP1B & $1.02 \mathrm{E}+00$ \\
\hline ENSBTAG00000021764 & $4.61 \mathrm{E}-10$ & $4.50 \mathrm{E}-10$ & GLRB & $1.02 \mathrm{E}+00$ \\
\hline ENSBTAG00000003208 & $2.16 \mathrm{E}-10$ & 2.10E-10 & RAB33B & $1.03 E+00$ \\
\hline ENSBTAG00000020423 & 1.57E-10 & $1.52 \mathrm{E}-10$ & EP400 & $1.03 E+00$ \\
\hline ENSBTAG00000007953 & $4.93 \mathrm{E}-10$ & $4.78 \mathrm{E}-10$ & FBXW7 & $1.03 E+00$ \\
\hline ENSBTAG00000010667 & $6.70 \mathrm{E}-10$ & $6.50 \mathrm{E}-10$ & PIK3IP1 & $1.03 E+00$ \\
\hline ENSBTAG00000011384 & $2.26 \mathrm{E}-10$ & 2.19E-10 & TBX5 & $1.03 E+00$ \\
\hline ENSBTAG00000004956 & $2.78 \mathrm{E}-10$ & $2.69 \mathrm{E}-10$ & CHEK2 & $1.04 E+00$ \\
\hline
\end{tabular}




\begin{tabular}{|c|c|c|c|c|}
\hline ENSBTAG000000014007 & $2.63 \mathrm{E}-10$ & $2.53 \mathrm{E}-10$ & ELMOD2 & $1.04 \mathrm{E}+00$ \\
\hline ENSBTAG000000032353 & $8.01 \mathrm{E}-10$ & 7.70E-10 & CCDC63 & $1.04 \mathrm{E}+00$ \\
\hline ENSBTAG000000021779 & $4.66 \mathrm{E}-10$ & $4.48 \mathrm{E}-10$ & MGST2 & $1.04 \mathrm{E}+00$ \\
\hline ENSBTAG000000009167 & $3.10 \mathrm{E}-10$ & $2.96 \mathrm{E}-10$ & SLC15A4 & $1.05 E+00$ \\
\hline ENSBTAG000000017890 & $1.76 \mathrm{E}-10$ & $1.68 \mathrm{E}-10$ & ZMAT5 & $1.05 E+00$ \\
\hline ENSBTAG000000066569 & $4.83 \mathrm{E}-10$ & $4.59 \mathrm{E}-10$ & SEZ6L & $1.05 E+00$ \\
\hline ENSBTAG000000002023 & $3.81 \mathrm{E}-10$ & $3.62 \mathrm{E}-10$ & NOS1 & $1.05 E+00$ \\
\hline ENSBTAG000000008991 & $6.22 \mathrm{E}-10$ & $5.90 \mathrm{E}-10$ & EIF4ENIF1 & $1.05 E+00$ \\
\hline ENSBTAG00000011889 & $1.32 \mathrm{E}-10$ & $1.25 \mathrm{E}-10$ & NOC4L & $1.06 \mathrm{E}+00$ \\
\hline ENSBTAG000000039552 & $7.70 \mathrm{E}-11$ & $7.27 \mathrm{E}-11$ & PLK4 & $1.06 \mathrm{E}+00$ \\
\hline ENSBTAG000000036113 & 3.07E-10 & $2.91 \mathrm{E}-10$ & RSPH14 & $1.06 \mathrm{E}+00$ \\
\hline ENSBTAG000000046322 & 4.57E-10 & $4.32 \mathrm{E}-10$ & & $1.06 \mathrm{E}+00$ \\
\hline ENSBTAG00000018369 & $3.37 \mathrm{E}-10$ & $3.18 \mathrm{E}-10$ & MYL2 & $1.06 \mathrm{E}+00$ \\
\hline ENSBTAG000000013848 & $4.55 \mathrm{E}-10$ & 4.29E-10 & ADGRD1 & $1.06 \mathrm{E}+00$ \\
\hline ENSBTAG000000012790 & $1.42 \mathrm{E}-10$ & $1.34 \mathrm{E}-10$ & TRIAP1 & $1.06 \mathrm{E}+00$ \\
\hline ENSBTAG000000016163 & $1.13 \mathrm{E}-10$ & 1.07E-10 & OSM & $1.05 E+00$ \\
\hline ENSBTAG000000000297 & $3.56 \mathrm{E}-10$ & 3.35E-10 & & $1.06 \mathrm{E}+00$ \\
\hline ENSBTAG000000047595 & $4.43 \mathrm{E}-10$ & $4.15 \mathrm{E}-10$ & & $1.07 \mathrm{E}+00$ \\
\hline ENSBTAG000000047107 & $3.26 \mathrm{E}-10$ & $3.05 E-10$ & TNIP3 & $1.07 E+00$ \\
\hline ENSBTAG000000031018 & $4.21 \mathrm{E}-10$ & 3.93E-10 & & $1.07 E+00$ \\
\hline ENSBTAG00000019977 & $2.27 \mathrm{E}-10$ & $2.11 E-10$ & $\mathrm{PCDH} 10$ & $1.08 \mathrm{E}+00$ \\
\hline ENSBTAG000000008060 & 4.17E-10 & 3.87E-10 & SNRNP35 & $1.08 \mathrm{E}+00$ \\
\hline ENSBTAG000000019871 & $3.08 \mathrm{E}-10$ & $2.84 \mathrm{E}-10$ & TRMT2A & $1.08 \mathrm{E}+00$ \\
\hline ENSBTAG000000002599 & $1.58 \mathrm{E}-10$ & $1.45 \mathrm{E}-10$ & MGARP & $1.09 \mathrm{E}+00$ \\
\hline ENSBTAG00000013686 & $1.08 \mathrm{E}-09$ & $9.88 \mathrm{E}-10$ & WSCD2 & $1.10 E+00$ \\
\hline ENSBTAG000000008098 & $3.41 \mathrm{E}-10$ & $3.12 \mathrm{E}-10$ & DCLK2 & $1.09 E+00$ \\
\hline ENSBTAG000000014376 & $3.48 \mathrm{E}-10$ & $3.15 E-10$ & ANKRD13A & $1.11 E+00$ \\
\hline ENSBTAG000000000439 & $2.12 \mathrm{E}-10$ & $1.91 \mathrm{E}-10$ & SFSWAP & $1.11 \mathrm{E}+00$ \\
\hline ENSBTAG000000008117 & $4.82 \mathrm{E}-10$ & 4.35E-10 & SLC5A4 & $1.11 \mathrm{E}+00$ \\
\hline ENSBTAG000000011787 & $1.88 \mathrm{E}-10$ & $1.69 \mathrm{E}-10$ & ISCU & $1.11 E+00$ \\
\hline ENSBTAG000000010312 & 3.97E-10 & $3.55 \mathrm{E}-10$ & MAPK1 & $1.12 \mathrm{E}+00$ \\
\hline ENSBTAG000000033890 & $4.88 \mathrm{E}-10$ & 4.33E-10 & & $1.13 \mathrm{E}+00$ \\
\hline ENSBTAG00000031824 & $4.70 \mathrm{E}-10$ & 4.17E-10 & RBM19 & $1.13 \mathrm{E}+00$ \\
\hline ENSBTAG000000004192 & $1.97 \mathrm{E}-10$ & $1.74 \mathrm{E}-10$ & LRRC43 & $1.13 E+00$ \\
\hline ENSBTAG00000021595 & $2.80 \mathrm{E}-10$ & $2.48 \mathrm{E}-10$ & HIP1R & $1.13 \mathrm{E}+00$ \\
\hline ENSBTAG00000004189 & $3.32 \mathrm{E}-10$ & $2.94 \mathrm{E}-10$ & MLXIP & $1.13 E+00$ \\
\hline ENSBTAG00000000281 & $5.90 \mathrm{E}-10$ & $5.21 E-10$ & MND1 & $1.13 \mathrm{E}+00$ \\
\hline ENSBTAG00000005678 & $3.63 \mathrm{E}-10$ & $3.19 E-10$ & AACS & $1.14 E+00$ \\
\hline ENSBTAG00000046083 & $1.32 \mathrm{E}-09$ & 1.17E-09 & ZNF280A & $1.13 \mathrm{E}+00$ \\
\hline ENSBTAG00000001325 & $6.20 \mathrm{E}-10$ & 5.44E-10 & UPB1 & $1.14 \mathrm{E}+00$ \\
\hline ENSBTAG00000019282 & $3.02 \mathrm{E}-10$ & $2.65 \mathrm{E}-10$ & CRYBA4 & $1.14 \mathrm{E}+00$ \\
\hline ENSBTAG00000046657 & $1.88 \mathrm{E}-09$ & $1.65 \mathrm{E}-09$ & & $1.14 \mathrm{E}+00$ \\
\hline ENSBTAG00000008470 & $1.53 \mathrm{E}-10$ & $1.33 \mathrm{E}-10$ & MPHOSPH9 & $1.15 \mathrm{E}+00$ \\
\hline ENSBTAG00000018116 & $2.96 \mathrm{E}-10$ & $2.58 \mathrm{E}-10$ & MTFP1 & $1.15 \mathrm{E}+00$ \\
\hline ENSBTAG00000026732 & $7.20 \mathrm{E}-10$ & $6.27 \mathrm{E}-10$ & & $1.15 E+00$ \\
\hline ENSBTAG00000004745 & $2.41 \mathrm{E}-10$ & $2.08 \mathrm{E}-10$ & NAA15 & $1.16 \mathrm{E}+00$ \\
\hline ENSBTAG00000014646 & $3.10 \mathrm{E}-10$ & $2.68 \mathrm{E}-10$ & & $1.15 E+00$ \\
\hline ENSBTAG00000013153 & $3.75 \mathrm{E}-10$ & 3.24E-10 & NF2 & $1.16 \mathrm{E}+00$ \\
\hline ENSBTAG00000013877 & $2.41 \mathrm{E}-10$ & $2.08 \mathrm{E}-10$ & HORMAD2 & $1.16 \mathrm{E}+00$ \\
\hline ENSBTAG00000016514 & $6.87 \mathrm{E}-10$ & $5.92 \mathrm{E}-10$ & $\mathrm{CPE}$ & $1.16 \mathrm{E}+00$ \\
\hline ENSBTAG00000024603 & $1.87 \mathrm{E}-10$ & $1.61 \mathrm{E}-10$ & NCOR2 & $1.16 \mathrm{E}+00$ \\
\hline ENSBTAG00000039861 & $8.52 \mathrm{E}-10$ & 7.32E-10 & & $1.16 \mathrm{E}+00$ \\
\hline ENSBTAG00000019375 & $2.39 \mathrm{E}-10$ & $2.04 \mathrm{E}-10$ & SCARF2 & $1.17 E+00$ \\
\hline ENSBTAG00000020584 & $2.00 \mathrm{E}-10$ & $1.70 \mathrm{E}-10$ & IFT81 & $1.18 \mathrm{E}+00$ \\
\hline ENSBTAG00000020594 & $1.49 \mathrm{E}-10$ & $1.26 \mathrm{E}-10$ & ELF2 & $1.18 \mathrm{E}+00$ \\
\hline ENSBTAG00000047121 & $5.18 \mathrm{E}-10$ & $4.39 \mathrm{E}-10$ & & $1.18 \mathrm{E}+00$ \\
\hline ENSBTAG00000009722 & $1.37 \mathrm{E}-10$ & $1.15 \mathrm{E}-10$ & & $1.19 \mathrm{E}+00$ \\
\hline ENSBTAG00000024605 & $2.02 \mathrm{E}-10$ & $1.70 \mathrm{E}-10$ & & $1.19 \mathrm{E}+00$ \\
\hline ENSBTAG00000003696 & $2.07 \mathrm{E}-10$ & $1.74 \mathrm{E}-10$ & BICDL1 & $1.19 E+00$ \\
\hline ENSBTAG00000020760 & $2.37 \mathrm{E}-10$ & $1.99 \mathrm{E}-10$ & SNAP29 & $1.19 E+00$ \\
\hline ENSBTAG00000044198 & $1.08 \mathrm{E}-10$ & $9.06 \mathrm{E}-11$ & & $1.19 \mathrm{E}+00$ \\
\hline ENSBTAG00000004247 & $5.69 \mathrm{E}-10$ & $4.78 \mathrm{E}-10$ & $\mathrm{RPH} 3 \mathrm{~A}$ & $1.19 E+00$ \\
\hline ENSBTAG00000030927 & $1.77 \mathrm{E}-10$ & $1.49 \mathrm{E}-10$ & & $1.19 \mathrm{E}+00$ \\
\hline ENSBTAG00000008587 & 4.07E-10 & $3.42 \mathrm{E}-10$ & & $1.19 E+00$ \\
\hline ENSBTAG00000046677 & $1.73 \mathrm{E}-10$ & $1.45 \mathrm{E}-10$ & RTN4R & $1.19 \mathrm{E}+00$ \\
\hline ENSBTAG00000017379 & $2.06 \mathrm{E}-10$ & $1.73 \mathrm{E}-10$ & GCN1 & $1.19 \mathrm{E}+00$ \\
\hline ENSBTAG00000000913 & $2.24 \mathrm{E}-10$ & $1.88 \mathrm{E}-10$ & UQCR10 & $1.19 \mathrm{E}+00$ \\
\hline ENSBTAG00000009908 & $5.26 \mathrm{E}-10$ & $4.42 \mathrm{E}-10$ & & $1.19 \mathrm{E}+00$ \\
\hline ENSBTAG00000047986 & $7.06 \mathrm{E}-10$ & 5.92E-10 & & $1.19 \mathrm{E}+00$ \\
\hline ENSBTAG00000047904 & $2.27 \mathrm{E}-10$ & $1.91 \mathrm{E}-10$ & & $1.19 E+00$ \\
\hline
\end{tabular}




\begin{tabular}{|c|c|c|c|c|}
\hline ENSBTAG00000018825 & $2.49 \mathrm{E}-10$ & $2.09 \mathrm{E}-10$ & SSH1 & $1.19 \mathrm{E}+00$ \\
\hline ENSBTAG00000035606 & $6.30 \mathrm{E}-10$ & $5.28 \mathrm{E}-10$ & & $1.19 E+00$ \\
\hline ENSBTAG00000021168 & $2.27 \mathrm{E}-10$ & $1.91 \mathrm{E}-10$ & SIRT4 & $1.19 E+00$ \\
\hline ENSBTAG00000002452 & $2.92 \mathrm{E}-10$ & $2.44 \mathrm{E}-10$ & $\mathrm{ASCC} 2$ & $1.20 \mathrm{E}+00$ \\
\hline ENSBTAG00000006506 & $2.77 \mathrm{E}-10$ & $2.32 \mathrm{E}-10$ & GIT2 & $1.20 E+00$ \\
\hline ENSBTAG00000006468 & $4.00 \mathrm{E}-10$ & $3.34 \mathrm{E}-10$ & PIWIL1 & $1.20 \mathrm{E}+00$ \\
\hline ENSBTAG00000007270 & $1.01 \mathrm{E}-09$ & $8.44 \mathrm{E}-10$ & GTF2H3 & $1.20 \mathrm{E}+00$ \\
\hline ENSBTAG00000001543 & $6.59 \mathrm{E}-10$ & $5.49 \mathrm{E}-10$ & BRI3BP & $1.20 \mathrm{E}+00$ \\
\hline ENSBTAG00000010815 & $2.12 \mathrm{E}-10$ & $1.76 \mathrm{E}-10$ & CAMKK2 & $1.20 \mathrm{E}+00$ \\
\hline ENSBTAG00000004187 & 5.47E-10 & 4.54E-10 & WDR66 & $1.21 \mathrm{E}+00$ \\
\hline ENSBTAG00000012738 & $2.96 \mathrm{E}-10$ & $2.42 \mathrm{E}-10$ & ZNF827 & $1.22 \mathrm{E}+00$ \\
\hline ENSBTAG00000009006 & $1.60 \mathrm{E}-10$ & $1.30 \mathrm{E}-10$ & PRR14L & $1.23 \mathrm{E}+00$ \\
\hline ENSBTAG00000013971 & $3.65 \mathrm{E}-10$ & $2.99 \mathrm{E}-10$ & PI4KA & $1.22 \mathrm{E}+00$ \\
\hline ENSBTAG00000034089 & $5.05 \mathrm{E}-10$ & $4.11 \mathrm{E}-10$ & & $1.23 \mathrm{E}+00$ \\
\hline ENSBTAG00000006114 & $2.56 \mathrm{E}-10$ & $2.08 \mathrm{E}-10$ & $\mathrm{ZCCHC8}$ & $1.23 E+00$ \\
\hline ENSBTAG00000019146 & 8.07E-10 & $6.56 \mathrm{E}-10$ & OSBP2 & $1.23 \mathrm{E}+00$ \\
\hline ENSBTAG00000008061 & $1.77 \mathrm{E}-10$ & $1.44 \mathrm{E}-10$ & RILPL1 & $1.23 \mathrm{E}+00$ \\
\hline ENSBTAG00000009755 & $1.78 \mathrm{E}-10$ & $1.44 \mathrm{E}-10$ & & $1.24 \mathrm{E}+00$ \\
\hline ENSBTAG00000018048 & $3.65 \mathrm{E}-10$ & $2.93 \mathrm{E}-10$ & MED15 & $1.25 E+00$ \\
\hline ENSBTAG00000039630 & $2.28 \mathrm{E}-10$ & $1.83 \mathrm{E}-10$ & & $1.25 \mathrm{E}+00$ \\
\hline ENSBTAG00000044119 & $3.87 \mathrm{E}-10$ & $3.11 E-10$ & KSR2 & $1.25 \mathrm{E}+00$ \\
\hline ENSBTAG00000004203 & $1.44 \mathrm{E}-10$ & 1.15E-10 & & $1.25 \mathrm{E}+00$ \\
\hline ENSBTAG00000008571 & $4.28 \mathrm{E}-10$ & $3.42 \mathrm{E}-10$ & CUX2 & $1.25 \mathrm{E}+00$ \\
\hline ENSBTAG00000000429 & $3.55 \mathrm{E}-10$ & $2.84 \mathrm{E}-10$ & DGCR2 & $1.25 \mathrm{E}+00$ \\
\hline ENSBTAG00000002145 & $1.02 \mathrm{E}-10$ & $8.12 \mathrm{E}-11$ & MMP17 & $1.25 \mathrm{E}+00$ \\
\hline ENSBTAG00000001473 & $1.88 \mathrm{E}-10$ & $1.49 \mathrm{E}-10$ & ARVCF & $1.26 \mathrm{E}+00$ \\
\hline ENSBTAG00000003345 & $3.90 \mathrm{E}-10$ & $3.09 \mathrm{E}-10$ & FAT4 & $1.26 \mathrm{E}+00$ \\
\hline ENSBTAG00000048020 & $5.65 \mathrm{E}-10$ & $4.48 \mathrm{E}-10$ & & $1.26 \mathrm{E}+00$ \\
\hline ENSBTAG00000000139 & $1.86 \mathrm{E}-10$ & 1.47E-10 & KMT5A & $1.26 \mathrm{E}+00$ \\
\hline ENSBTAG00000003068 & $7.31 \mathrm{E}-10$ & 5.77E-10 & MSMO1 & $1.27 E+00$ \\
\hline ENSBTAG00000043976 & $1.43 \mathrm{E}-10$ & $1.13 \mathrm{E}-10$ & RNFT2 & $1.26 \mathrm{E}+00$ \\
\hline ENSBTAG00000007234 & $5.38 \mathrm{E}-10$ & 4.23E-10 & PLRG1 & $1.27 \mathrm{E}+00$ \\
\hline ENSBTAG00000007272 & $2.21 \mathrm{E}-10$ & $1.73 \mathrm{E}-10$ & ATP6V0A2 & $1.28 \mathrm{E}+00$ \\
\hline ENSBTAG00000017023 & $1.32 \mathrm{E}-10$ & $1.04 \mathrm{E}-10$ & & $1.27 \mathrm{E}+00$ \\
\hline ENSBTAG00000006659 & $1.98 \mathrm{E}-10$ & $1.55 \mathrm{E}-10$ & TMEM116 & $1.28 \mathrm{E}+00$ \\
\hline ENSBTAG00000010847 & $2.07 \mathrm{E}-10$ & $1.61 \mathrm{E}-10$ & FOXN4 & $1.28 \mathrm{E}+00$ \\
\hline ENSBTAG00000007993 & $2.02 \mathrm{E}-10$ & $1.58 \mathrm{E}-10$ & CORO1C & $1.28 \mathrm{E}+00$ \\
\hline ENSBTAG00000033339 & $2.24 \mathrm{E}-10$ & $1.75 \mathrm{E}-10$ & ANKLE2 & $1.28 \mathrm{E}+00$ \\
\hline ENSBTAG00000047538 & $2.25 \mathrm{E}-10$ & $1.74 \mathrm{E}-10$ & & $1.29 \mathrm{E}+00$ \\
\hline ENSBTAG00000004167 & $4.36 \mathrm{E}-10$ & 3.37E-10 & TTC29 & $1.29 E+00$ \\
\hline ENSBTAG00000014111 & 4.47E-10 & $3.45 \mathrm{E}-10$ & INPP4B & $1.30 \mathrm{E}+00$ \\
\hline ENSBTAG00000008438 & $9.05 \mathrm{E}-10$ & 6.97E-10 & ARFIP1 & $1.30 \mathrm{E}+00$ \\
\hline ENSBTAG00000022036 & $4.04 \mathrm{E}-10$ & $3.11 \mathrm{E}-10$ & ZNRF3 & $1.30 \mathrm{E}+00$ \\
\hline ENSBTAG00000044078 & $2.64 \mathrm{E}-10$ & 2.03E-10 & TESC & $1.30 \mathrm{E}+00$ \\
\hline ENSBTAG00000006626 & $6.34 \mathrm{E}-10$ & $4.85 \mathrm{E}-10$ & TMEM132B & $1.31 E+00$ \\
\hline ENSBTAG00000002315 & $1.70 \mathrm{E}-10$ & $1.30 \mathrm{E}-10$ & RNF34 & $1.31 E+00$ \\
\hline ENSBTAG00000031851 & $3.84 \mathrm{E}-10$ & $2.92 \mathrm{E}-10$ & SELPLG & $1.32 \mathrm{E}+00$ \\
\hline ENSBTAG00000012987 & $3.11 \mathrm{E}-10$ & $2.37 \mathrm{E}-10$ & ULK1 & $1.31 E+00$ \\
\hline ENSBTAG00000022069 & $7.71 \mathrm{E}-11$ & 5.87E-11 & ATXN2 & $1.31 E+00$ \\
\hline ENSBTAG00000007483 & $1.46 \mathrm{E}-10$ & $1.10 \mathrm{E}-10$ & UNC119B & $1.32 \mathrm{E}+00$ \\
\hline ENSBTAG00000000590 & $2.42 \mathrm{E}-10$ & $1.84 \mathrm{E}-10$ & POLE & $1.32 \mathrm{E}+00$ \\
\hline ENSBTAG00000001187 & $1.41 \mathrm{E}-10$ & $1.07 \mathrm{E}-10$ & RASL10A & $1.32 \mathrm{E}+00$ \\
\hline ENSBTAG00000002130 & $3.42 \mathrm{E}-10$ & $2.58 \mathrm{E}-10$ & SMPD4 & $1.33 \mathrm{E}+00$ \\
\hline ENSBTAG00000021796 & $4.85 \mathrm{E}-10$ & $3.66 \mathrm{E}-10$ & C12orf43 & $1.33 \mathrm{E}+00$ \\
\hline ENSBTAG00000022084 & 4.14E-10 & $3.13 \mathrm{E}-10$ & & $1.32 \mathrm{E}+00$ \\
\hline ENSBTAG00000008528 & $1.65 \mathrm{E}-10$ & $1.25 \mathrm{E}-10$ & SLC25A1 & $1.32 \mathrm{E}+00$ \\
\hline ENSBTAG00000013973 & $3.81 E-10$ & $2.88 \mathrm{E}-10$ & SERPIND1 & $1.32 \mathrm{E}+00$ \\
\hline ENSBTAG00000032436 & $9.02 \mathrm{E}-10$ & $6.80 \mathrm{E}-10$ & & $1.33 \mathrm{E}+00$ \\
\hline ENSBTAG00000011102 & $3.90 \mathrm{E}-10$ & $2.92 \mathrm{E}-10$ & TPCN1 & $1.34 \mathrm{E}+00$ \\
\hline ENSBTAG00000001030 & $3.44 \mathrm{E}-10$ & $2.55 \mathrm{E}-10$ & MTMR3 & $1.35 E+00$ \\
\hline ENSBTAG00000002531 & $5.47 \mathrm{E}-10$ & $4.05 \mathrm{E}-10$ & ARHGAP10 & $1.35 \mathrm{E}+00$ \\
\hline ENSBTAG00000003527 & $2.63 \mathrm{E}-10$ & $1.94 \mathrm{E}-10$ & CCDC62 & $1.36 \mathrm{E}+00$ \\
\hline ENSBTAG00000013611 & $3.64 \mathrm{E}-10$ & 2.69E-10 & SCLT1 & $1.35 \mathrm{E}+00$ \\
\hline ENSBTAG00000020505 & $1.44 \mathrm{E}-10$ & $1.06 \mathrm{E}-10$ & SBNO1 & $1.35 E+00$ \\
\hline ENSBTAG00000043959 & $5.62 \mathrm{E}-10$ & 4.13E-10 & PDGFC & $1.36 \mathrm{E}+00$ \\
\hline ENSBTAG00000012368 & $1.38 \mathrm{E}-10$ & $1.01 \mathrm{E}-10$ & IL21 & $1.36 \mathrm{E}+00$ \\
\hline ENSBTAG00000031814 & 3.63E-10 & $2.66 \mathrm{E}-10$ & SDS & $1.36 \mathrm{E}+00$ \\
\hline ENSBTAG00000047718 & $7.34 \mathrm{E}-10$ & $5.38 \mathrm{E}-10$ & ZNF70 & $1.36 \mathrm{E}+00$ \\
\hline ENSBTAG00000007510 & $2.54 \mathrm{E}-10$ & $1.85 \mathrm{E}-10$ & PXMP2 & $1.37 \mathrm{E}+00$ \\
\hline ENSBTAG00000015549 & $1.82 \mathrm{E}-10$ & $1.33 \mathrm{E}-10$ & PCDH18 & $1.37 E+00$ \\
\hline
\end{tabular}




\begin{tabular}{|c|c|c|c|c|}
\hline ENSBTAG00000013038 & $3.03 \mathrm{E}-10$ & $2.20 \mathrm{E}-10$ & UBE2L3 & $1.38 \mathrm{E}+00$ \\
\hline ENSBTAG00000037527 & $9.61 \mathrm{E}-10$ & $6.98 \mathrm{E}-10$ & OAS1X & $1.38 \mathrm{E}+00$ \\
\hline ENSBTAG00000007855 & $1.62 \mathrm{E}-10$ & $1.16 \mathrm{E}-10$ & VSIG10 & $1.39 E+00$ \\
\hline ENSBTAG00000019244 & $6.00 \mathrm{E}-10$ & $4.33 \mathrm{E}-10$ & P2RX6 & $1.39 \mathrm{E}+00$ \\
\hline ENSBTAG00000016523 & $5.11 \mathrm{E}-10$ & $3.68 \mathrm{E}-10$ & DNAH10 & $1.39 E+00$ \\
\hline ENSBTAG00000006753 & $3.53 \mathrm{E}-10$ & $2.54 \mathrm{E}-10$ & MBD3L1 & $1.39 \mathrm{E}+00$ \\
\hline ENSBTAG00000004775 & $2.88 \mathrm{E}-10$ & 2.07E-10 & GRIA2 & $1.39 \mathrm{E}+00$ \\
\hline ENSBTAG00000013914 & $3.64 \mathrm{E}-10$ & 2.60E-10 & GATB & $1.40 \mathrm{E}+00$ \\
\hline ENSBTAG00000000005 & $2.46 \mathrm{E}-10$ & $1.75 \mathrm{E}-10$ & GRK3 & $1.41 E+00$ \\
\hline ENSBTAG00000011970 & $2.52 \mathrm{E}-10$ & $1.79 \mathrm{E}-10$ & FNIP2 & $1.41 \mathrm{E}+00$ \\
\hline ENSBTAG00000020430 & $4.82 \mathrm{E}-10$ & $3.42 \mathrm{E}-10$ & GLT1D1 & $1.41 E+00$ \\
\hline ENSBTAG00000004175 & $2.18 \mathrm{E}-10$ & $1.54 \mathrm{E}-10$ & HPD & $1.41 \mathrm{E}+00$ \\
\hline ENSBTAG00000010306 & $7.89 \mathrm{E}-10$ & $5.55 \mathrm{E}-10$ & RXFP1 & $1.42 \mathrm{E}+00$ \\
\hline ENSBTAG00000011103 & $4.66 \mathrm{E}-10$ & $3.28 \mathrm{E}-10$ & SLC8B1 & $1.42 \mathrm{E}+00$ \\
\hline ENSBTAG00000012504 & $1.35 \mathrm{E}-10$ & $9.44 \mathrm{E}-11$ & GAS2L1 & $1.43 \mathrm{E}+00$ \\
\hline ENSBTAG00000031160 & $7.84 \mathrm{E}-10$ & $5.49 \mathrm{E}-10$ & & $1.43 E+00$ \\
\hline ENSBTAG00000022019 & $2.29 \mathrm{E}-10$ & $1.60 \mathrm{E}-10$ & & $1.43 \mathrm{E}+00$ \\
\hline ENSBTAG00000006666 & $1.43 \mathrm{E}-10$ & $9.98 \mathrm{E}-11$ & NAA25 & $1.43 \mathrm{E}+00$ \\
\hline ENSBTAG00000014232 & $5.60 \mathrm{E}-10$ & $3.91 \mathrm{E}-10$ & GAL3ST1 & $1.43 E+00$ \\
\hline ENSBTAG00000016944 & $2.23 \mathrm{E}-10$ & $1.55 \mathrm{E}-10$ & ADORA2A & $1.44 \mathrm{E}+00$ \\
\hline ENSBTAG00000047676 & $2.36 \mathrm{E}-10$ & $1.64 \mathrm{E}-10$ & & $1.44 \mathrm{E}+00$ \\
\hline ENSBTAG00000045910 & $2.28 \mathrm{E}-10$ & $1.58 \mathrm{E}-10$ & & $1.44 \mathrm{E}+00$ \\
\hline ENSBTAG00000009101 & $1.03 \mathrm{E}-10$ & $7.10 \mathrm{E}-11$ & $\mathrm{SH} 2 \mathrm{~B} 3$ & $1.45 \mathrm{E}+00$ \\
\hline ENSBTAG00000009963 & $2.44 \mathrm{E}-10$ & 1.67E-10 & MORC2 & $1.46 \mathrm{E}+00$ \\
\hline ENSBTAG00000000170 & $1.95 \mathrm{E}-10$ & $1.34 \mathrm{E}-10$ & & $1.46 \mathrm{E}+00$ \\
\hline ENSBTAG00000043581 & $2.89 \mathrm{E}-10$ & $1.98 \mathrm{E}-10$ & TXNRD2 & $1.46 \mathrm{E}+00$ \\
\hline ENSBTAG00000001806 & 3.30E-10 & $2.26 \mathrm{E}-10$ & IQCD & $1.46 \mathrm{E}+00$ \\
\hline ENSBTAG00000014628 & $8.98 \mathrm{E}-10$ & $6.13 \mathrm{E}-10$ & OAS2 & $1.47 \mathrm{E}+00$ \\
\hline ENSBTAG00000001189 & $2.99 \mathrm{E}-10$ & 2.03E-10 & AP1B1 & $1.47 \mathrm{E}+00$ \\
\hline ENSBTAG00000043963 & 7.13E-10 & $4.83 \mathrm{E}-10$ & FHDC1 & $1.48 \mathrm{E}+00$ \\
\hline ENSBTAG00000018108 & $3.10 \mathrm{E}-10$ & $2.09 \mathrm{E}-10$ & PXN & $1.48 \mathrm{E}+00$ \\
\hline ENSBTAG00000010368 & $4.84 \mathrm{E}-10$ & $3.26 \mathrm{E}-10$ & TPST2 & $1.49 \mathrm{E}+00$ \\
\hline ENSBTAG00000006108 & $3.03 \mathrm{E}-10$ & 2.03E-10 & MMP11 & $1.49 \mathrm{E}+00$ \\
\hline ENSBTAG00000020504 & $1.32 \mathrm{E}-10$ & $8.79 E-11$ & CDK2AP1 & $1.50 \mathrm{E}+00$ \\
\hline ENSBTAG00000001888 & $1.48 \mathrm{E}-10$ & $9.84 \mathrm{E}-11$ & MED13L & $1.50 \mathrm{E}+00$ \\
\hline ENSBTAG00000027182 & $3.98 \mathrm{E}-10$ & $2.64 \mathrm{E}-10$ & NR3C2 & $1.51 \mathrm{E}+00$ \\
\hline ENSBTAG00000005263 & $5.56 \mathrm{E}-10$ & $3.68 \mathrm{E}-10$ & KLHL2 & $1.51 E+00$ \\
\hline ENSBTAG00000017867 & $4.59 \mathrm{E}-10$ & $3.02 \mathrm{E}-10$ & YPEL1 & $1.52 \mathrm{E}+00$ \\
\hline ENSBTAG00000009602 & $2.08 \mathrm{E}-10$ & $1.36 \mathrm{E}-10$ & TMEM120B & $1.53 \mathrm{E}+00$ \\
\hline ENSBTAG00000004172 & $1.55 \mathrm{E}-10$ & $9.99 \mathrm{E}-11$ & SETD1B & $1.55 E+00$ \\
\hline ENSBTAG00000046191 & $1.37 \mathrm{E}-10$ & $8.82 \mathrm{E}-11$ & ADAD1 & $1.55 E+00$ \\
\hline ENSBTAG00000009346 & $3.12 \mathrm{E}-10$ & $1.99 \mathrm{E}-10$ & MAPKAPK5 & $1.57 \mathrm{E}+00$ \\
\hline ENSBTAG00000006745 & 2.07E-10 & $1.30 \mathrm{E}-10$ & FGG & $1.59 \mathrm{E}+00$ \\
\hline ENSBTAG00000017755 & $1.54 \mathrm{E}-10$ & $9.69 \mathrm{E}-11$ & EWSR1 & $1.59 \mathrm{E}+00$ \\
\hline ENSBTAG00000031134 & $2.19 \mathrm{E}-10$ & $1.38 \mathrm{E}-10$ & & $1.59 E+00$ \\
\hline ENSBTAG00000003394 & $2.50 \mathrm{E}-10$ & $1.58 \mathrm{E}-10$ & RNF215 & $1.58 \mathrm{E}+00$ \\
\hline ENSBTAG00000004199 & $2.70 \mathrm{E}-10$ & 1.70E-10 & DIABLO & $1.59 E+00$ \\
\hline ENSBTAG00000019891 & $2.61 \mathrm{E}-10$ & 1.64E-10 & MRPL40 & $1.59 E+00$ \\
\hline ENSBTAG00000012421 & $1.20 \mathrm{E}-10$ & $7.54 \mathrm{E}-11$ & & $1.59 E+00$ \\
\hline ENSBTAG00000036310 & $3.15 \mathrm{E}-10$ & $1.98 \mathrm{E}-10$ & & $1.59 \mathrm{E}+00$ \\
\hline ENSBTAG00000031693 & $1.35 \mathrm{E}-10$ & 8.47E-11 & ADAM1B & $1.59 E+00$ \\
\hline ENSBTAG00000009145 & $3.94 \mathrm{E}-10$ & $2.48 \mathrm{E}-10$ & SLC7A4 & $1.59 \mathrm{E}+00$ \\
\hline ENSBTAG00000047529 & $5.79 E-10$ & 3.64E-10 & & $1.59 E+00$ \\
\hline ENSBTAG00000005385 & $2.88 \mathrm{E}-10$ & $1.81 \mathrm{E}-10$ & POP5 & $1.59 \mathrm{E}+00$ \\
\hline ENSBTAG00000021706 & $2.30 \mathrm{E}-10$ & $1.45 \mathrm{E}-10$ & TBX3 & $1.59 \mathrm{E}+00$ \\
\hline ENSBTAG00000046584 & $9.18 \mathrm{E}-10$ & $5.76 \mathrm{E}-10$ & & $1.59 E+00$ \\
\hline ENSBTAG00000008816 & $6.68 \mathrm{E}-10$ & $4.11 \mathrm{E}-10$ & TRIM2 & $1.62 \mathrm{E}+00$ \\
\hline ENSBTAG00000032097 & $3.05 \mathrm{E}-10$ & $1.87 \mathrm{E}-10$ & FBXO21 & $1.63 E+00$ \\
\hline ENSBTAG00000011198 & $1.42 \mathrm{E}-10$ & $8.66 \mathrm{E}-11$ & PPP1CC & $1.64 \mathrm{E}+00$ \\
\hline ENSBTAG00000005968 & $6.45 \mathrm{E}-10$ & $3.92 \mathrm{E}-10$ & TLL1 & $1.64 \mathrm{E}+00$ \\
\hline ENSBTAG00000005691 & $2.54 \mathrm{E}-10$ & $1.53 \mathrm{E}-10$ & & $1.66 \mathrm{E}+00$ \\
\hline ENSBTAG00000017493 & $1.41 \mathrm{E}-10$ & $8.31 E-11$ & JADE1 & $1.70 \mathrm{E}+00$ \\
\hline ENSBTAG00000005695 & $1.48 \mathrm{E}-10$ & $8.71 E-11$ & NUDT6 & $1.70 \mathrm{E}+00$ \\
\hline ENSBTAG00000034075 & $6.00 \mathrm{E}-10$ & $3.50 \mathrm{E}-10$ & SLC7A11 & $1.71 E+00$ \\
\hline ENSBTAG00000019757 & $1.73 \mathrm{E}-10$ & $1.01 \mathrm{E}-10$ & SUDS3 & $1.72 \mathrm{E}+00$ \\
\hline ENSBTAG00000046256 & $4.56 \mathrm{E}-10$ & $2.61 \mathrm{E}-10$ & TMEM132C & $1.75 E+00$ \\
\hline ENSBTAG00000021656 & $4.24 \mathrm{E}-10$ & $2.42 \mathrm{E}-10$ & SPECC1L & $1.75 E+00$ \\
\hline ENSBTAG00000018833 & $6.91 \mathrm{E}-10$ & $3.91 \mathrm{E}-10$ & SVOP & $1.77 E+00$ \\
\hline ENSBTAG00000002695 & $6.43 \mathrm{E}-10$ & $3.63 \mathrm{E}-10$ & MYO1H & $1.77 \mathrm{E}+00$ \\
\hline ENSBTAG00000012892 & $2.38 \mathrm{E}-10$ & $1.33 \mathrm{E}-10$ & C22orf31 & $1.79 E+00$ \\
\hline
\end{tabular}




\begin{tabular}{|c|c|c|c|c|}
\hline ENSBTAG00000000698 & $3.35 \mathrm{E}-10$ & $1.86 \mathrm{E}-10$ & MYO18B & $1.80 \mathrm{E}+00$ \\
\hline ENSBTAG00000019021 & $3.43 \mathrm{E}-10$ & $1.91 \mathrm{E}-10$ & TRPC3 & $1.80 \mathrm{E}+00$ \\
\hline ENSBTAG00000013951 & $3.63 \mathrm{E}-10$ & $2.00 \mathrm{E}-10$ & CHFR & $1.82 \mathrm{E}+00$ \\
\hline ENSBTAG00000011373 & 4.67E-10 & $2.56 \mathrm{E}-10$ & MAML3 & $1.82 \mathrm{E}+00$ \\
\hline ENSBTAG00000010376 & $2.20 \mathrm{E}-10$ & $1.21 \mathrm{E}-10$ & TMEM184C & $1.82 \mathrm{E}+00$ \\
\hline ENSBTAG00000007728 & $3.31 \mathrm{E}-10$ & $1.81 \mathrm{E}-10$ & PES1 & $1.83 E+00$ \\
\hline ENSBTAG00000016338 & $3.37 \mathrm{E}-10$ & $1.83 \mathrm{E}-10$ & SPPL3 & $1.84 \mathrm{E}+00$ \\
\hline ENSBTAG00000012450 & $4.32 \mathrm{E}-10$ & $2.33 \mathrm{E}-10$ & RAPGEF2 & $1.85 \mathrm{E}+00$ \\
\hline ENSBTAG00000033547 & $5.85 \mathrm{E}-10$ & $3.16 \mathrm{E}-10$ & C4orf45 & $1.85 \mathrm{E}+00$ \\
\hline ENSBTAG00000001117 & $3.77 \mathrm{E}-10$ & $2.04 \mathrm{E}-10$ & ANKRD50 & $1.85 \mathrm{E}+00$ \\
\hline ENSBTAG00000019854 & $7.32 \mathrm{E}-10$ & $3.92 \mathrm{E}-10$ & $A B C E 1$ & $1.87 \mathrm{E}+00$ \\
\hline ENSBTAG00000020566 & $5.34 \mathrm{E}-10$ & $2.86 \mathrm{E}-10$ & $\mathrm{BCR}$ & $1.87 \mathrm{E}+00$ \\
\hline ENSBTAG00000000125 & $3.19 \mathrm{E}-10$ & $1.70 \mathrm{E}-10$ & SPATA5 & $1.87 E+00$ \\
\hline ENSBTAG00000019869 & $3.02 \mathrm{E}-10$ & $1.58 \mathrm{E}-10$ & DGCR8 & $1.91 E+00$ \\
\hline ENSBTAG00000002591 & $6.45 \mathrm{E}-10$ & $3.36 \mathrm{E}-10$ & GLTP & $1.92 \mathrm{E}+00$ \\
\hline ENSBTAG00000013147 & $3.91 \mathrm{E}-10$ & $2.02 \mathrm{E}-10$ & NEFH & $1.93 E+00$ \\
\hline ENSBTAG00000034281 & $2.80 \mathrm{E}-10$ & $1.43 \mathrm{E}-10$ & MGAT4D & $1.96 \mathrm{E}+00$ \\
\hline ENSBTAG00000011292 & $3.89 \mathrm{E}-10$ & $1.98 \mathrm{E}-10$ & LZTR1 & $1.97 \mathrm{E}+00$ \\
\hline ENSBTAG00000032534 & $9.16 \mathrm{E}-11$ & $4.62 \mathrm{E}-11$ & RHOF & $1.98 \mathrm{E}+00$ \\
\hline ENSBTAG00000039766 & $2.01 \mathrm{E}-10$ & $1.01 \mathrm{E}-10$ & FBRSL1 & $1.99 \mathrm{E}+00$ \\
\hline ENSBTAG00000039688 & $1.64 \mathrm{E}-10$ & $8.27 \mathrm{E}-11$ & FAM101A & $1.98 \mathrm{E}+00$ \\
\hline ENSBTAG00000002697 & $5.69 \mathrm{E}-10$ & $2.85 \mathrm{E}-10$ & KCTD10 & $2.00 E+00$ \\
\hline ENSBTAG00000011063 & $2.79 \mathrm{E}-10$ & $1.37 \mathrm{E}-10$ & CTSO & $2.04 E+00$ \\
\hline ENSBTAG00000019229 & $7.37 \mathrm{E}-10$ & $3.60 \mathrm{E}-10$ & TMEM144 & $2.05 E+00$ \\
\hline ENSBTAG00000038806 & $1.35 \mathrm{E}-10$ & $6.63 \mathrm{E}-11$ & ZNF891 & $2.04 E+00$ \\
\hline ENSBTAG00000016675 & $2.99 \mathrm{E}-10$ & $1.46 \mathrm{E}-10$ & & $2.05 E+00$ \\
\hline ENSBTAG00000022044 & $2.50 \mathrm{E}-10$ & $1.22 \mathrm{E}-10$ & RAB35 & $2.05 E+00$ \\
\hline ENSBTAG00000032047 & $3.74 \mathrm{E}-10$ & $1.81 \mathrm{E}-10$ & & $2.07 E+00$ \\
\hline ENSBTAG00000002698 & $4.13 \mathrm{E}-10$ & $1.99 \mathrm{E}-10$ & UBE3B & $2.08 \mathrm{E}+00$ \\
\hline ENSBTAG00000004693 & $5.45 \mathrm{E}-10$ & $2.61 E-10$ & CCDC60 & $2.09 E+00$ \\
\hline ENSBTAG00000004457 & $1.15 \mathrm{E}-10$ & $5.52 \mathrm{E}-11$ & ORAI1 & $2.08 \mathrm{E}+00$ \\
\hline ENSBTAG00000011062 & $3.01 \mathrm{E}-10$ & $1.43 \mathrm{E}-10$ & TDO2 & $2.10 \mathrm{E}+00$ \\
\hline ENSBTAG00000019426 & $8.65 \mathrm{E}-10$ & 4.07E-10 & RNF175 & $2.13 E+00$ \\
\hline ENSBTAG00000024708 & $4.07 \mathrm{E}-10$ & $1.90 \mathrm{E}-10$ & CABIN1 & $2.14 E+00$ \\
\hline ENSBTAG00000047484 & $3.66 \mathrm{E}-10$ & $1.71 \mathrm{E}-10$ & HRK & $2.14 \mathrm{E}+00$ \\
\hline ENSBTAG00000002950 & $7.72 \mathrm{E}-10$ & $3.58 \mathrm{E}-10$ & & $2.16 \mathrm{E}+00$ \\
\hline ENSBTAG00000018534 & $2.36 \mathrm{E}-10$ & $1.09 \mathrm{E}-10$ & DGCR14 & $2.16 \mathrm{E}+00$ \\
\hline ENSBTAG00000007271 & $3.83 \mathrm{E}-10$ & $1.78 \mathrm{E}-10$ & TCTN2 & $2.15 E+00$ \\
\hline ENSBTAG00000007513 & $2.04 \mathrm{E}-10$ & $9.45 \mathrm{E}-11$ & PGAM5 & $2.16 \mathrm{E}+00$ \\
\hline ENSBTAG00000009216 & $2.99 \mathrm{E}-10$ & $1.38 \mathrm{E}-10$ & HVCN1 & $2.17 E+00$ \\
\hline ENSBTAG00000047331 & $1.54 \mathrm{E}-09$ & $7.06 \mathrm{E}-10$ & & $2.18 \mathrm{E}+00$ \\
\hline ENSBTAG00000019894 & $4.22 \mathrm{E}-10$ & $1.93 \mathrm{E}-10$ & C22orf39 & $2.19 E+00$ \\
\hline ENSBTAG00000045548 & $4.21 \mathrm{E}-10$ & $1.92 \mathrm{E}-10$ & CRKL & $2.19 \mathrm{E}+00$ \\
\hline ENSBTAG00000016071 & $9.41 \mathrm{E}-10$ & $4.27 \mathrm{E}-10$ & HHIP & $2.20 \mathrm{E}+00$ \\
\hline ENSBTAG00000012990 & $7.63 \mathrm{E}-10$ & $3.43 \mathrm{E}-10$ & PUS1 & $2.22 \mathrm{E}+00$ \\
\hline ENSBTAG00000022032 & $6.20 \mathrm{E}-10$ & $2.78 \mathrm{E}-10$ & $\mathrm{CHCHD} 10$ & $2.23 \mathrm{E}+00$ \\
\hline ENSBTAG00000007988 & $2.42 \mathrm{E}-10$ & $1.08 \mathrm{E}-10$ & STX2 & $2.24 \mathrm{E}+00$ \\
\hline ENSBTAG00000003581 & $1.46 \mathrm{E}-10$ & $6.47 \mathrm{E}-11$ & SETD7 & $2.26 \mathrm{E}+00$ \\
\hline ENSBTAG00000006657 & $3.10 \mathrm{E}-10$ & $1.33 \mathrm{E}-10$ & ZDHHC8 & $2.33 E+00$ \\
\hline ENSBTAG00000008320 & $7.89 \mathrm{E}-11$ & $3.31 \mathrm{E}-11$ & 05-Sep & $2.38 \mathrm{E}+00$ \\
\hline ENSBTAG00000040072 & $7.99 \mathrm{E}-11$ & $3.36 \mathrm{E}-11$ & ZNF140 & $2.38 \mathrm{E}+00$ \\
\hline ENSBTAG00000006665 & $2.96 \mathrm{E}-10$ & $1.24 \mathrm{E}-10$ & ERP29 & $2.39 E+00$ \\
\hline ENSBTAG00000017389 & $1.83 \mathrm{E}-10$ & $7.67 \mathrm{E}-11$ & RPLPO & $2.38 \mathrm{E}+00$ \\
\hline ENSBTAG00000006173 & $8.19 \mathrm{E}-11$ & $3.44 \mathrm{E}-11$ & CCDC116 & $2.38 \mathrm{E}+00$ \\
\hline ENSBTAG00000013036 & $1.57 \mathrm{E}-10$ & $6.59 \mathrm{E}-11$ & YDJC & $2.38 \mathrm{E}+00$ \\
\hline ENSBTAG00000046676 & $4.43 \mathrm{E}-10$ & $1.86 \mathrm{E}-10$ & & $2.38 \mathrm{E}+00$ \\
\hline ENSBTAG00000006730 & $3.06 \mathrm{E}-10$ & $1.28 \mathrm{E}-10$ & SUSD2 & $2.39 E+00$ \\
\hline ENSBTAG00000039582 & $5.05 \mathrm{E}-10$ & $2.12 \mathrm{E}-10$ & NDUFC1 & $2.38 \mathrm{E}+00$ \\
\hline ENSBTAG00000022028 & $1.18 \mathrm{E}-10$ & $4.96 \mathrm{E}-11$ & DERL3 & $2.38 \mathrm{E}+00$ \\
\hline ENSBTAG00000046747 & $1.24 \mathrm{E}-09$ & $5.18 \mathrm{E}-10$ & & $2.40 E+00$ \\
\hline ENSBTAG00000023007 & $4.93 \mathrm{E}-10$ & $2.05 \mathrm{E}-10$ & & $2.40 \mathrm{E}+00$ \\
\hline ENSBTAG00000048030 & $1.38 \mathrm{E}-09$ & $5.73 \mathrm{E}-10$ & & $2.40 \mathrm{E}+00$ \\
\hline ENSBTAG00000006938 & $6.37 \mathrm{E}-10$ & $2.58 \mathrm{E}-10$ & PPM1F & $2.47 E+00$ \\
\hline ENSBTAG00000005347 & $2.16 \mathrm{E}-10$ & $8.52 E-11$ & GPN3 & $2.53 E+00$ \\
\hline ENSBTAG00000016147 & $2.57 \mathrm{E}-10$ & $9.96 \mathrm{E}-11$ & & $2.58 \mathrm{E}+00$ \\
\hline ENSBTAG00000017038 & $1.03 \mathrm{E}-09$ & $3.97 \mathrm{E}-10$ & HPS4 & $2.59 E+00$ \\
\hline ENSBTAG00000018563 & $3.74 \mathrm{E}-10$ & $1.44 \mathrm{E}-10$ & SFRP2 & $2.59 E+00$ \\
\hline ENSBTAG00000013674 & $5.52 \mathrm{E}-10$ & $2.12 \mathrm{E}-10$ & EDNRA & $2.60 \mathrm{E}+00$ \\
\hline ENSBTAG00000016739 & $3.69 \mathrm{E}-10$ & $1.42 \mathrm{E}-10$ & RASAL1 & $2.60 E+00$ \\
\hline ENSBTAG00000001164 & $1.92 \mathrm{E}-10$ & $7.35 \mathrm{E}-11$ & ACAD10 & $2.61 E+00$ \\
\hline
\end{tabular}




\begin{tabular}{|c|c|c|c|c|}
\hline ENSBTAG00000020883 & $1.72 \mathrm{E}-10$ & $6.51 \mathrm{E}-11$ & IL2 & $2.65 \mathrm{E}+00$ \\
\hline ENSBTAG00000031849 & $3.80 \mathrm{E}-10$ & $1.43 \mathrm{E}-10$ & TMEM119 & $2.65 E+00$ \\
\hline ENSBTAG00000015526 & $9.54 \mathrm{E}-10$ & $3.59 \mathrm{E}-10$ & PIWIL3 & $2.66 \mathrm{E}+00$ \\
\hline ENSBTAG00000005345 & $2.81 \mathrm{E}-10$ & $1.05 \mathrm{E}-10$ & ARPC3 & $2.67 \mathrm{E}+00$ \\
\hline ENSBTAG00000031846 & $5.62 \mathrm{E}-10$ & $2.08 \mathrm{E}-10$ & SART3 & $2.70 \mathrm{E}+00$ \\
\hline ENSBTAG00000008743 & $5.10 \mathrm{E}-10$ & $1.89 \mathrm{E}-10$ & & $2.70 E+00$ \\
\hline ENSBTAG00000000067 & $2.96 \mathrm{E}-10$ & $1.06 \mathrm{E}-10$ & SDF2L1 & $2.79 E+00$ \\
\hline ENSBTAG00000001303 & $3.72 \mathrm{E}-10$ & $1.32 \mathrm{E}-10$ & HSPB8 & $2.82 \mathrm{E}+00$ \\
\hline ENSBTAG00000008963 & $4.43 \mathrm{E}-10$ & $1.56 \mathrm{E}-10$ & CIT & $2.84 \mathrm{E}+00$ \\
\hline ENSBTAG00000006592 & $6.58 \mathrm{E}-10$ & $2.32 \mathrm{E}-10$ & SGSM1 & $2.83 \mathrm{E}+00$ \\
\hline ENSBTAG00000012550 & $1.29 \mathrm{E}-10$ & $4.42 \mathrm{E}-11$ & TAOK3 & $2.91 E+00$ \\
\hline ENSBTAG00000037832 & $6.96 \mathrm{E}-10$ & $2.38 \mathrm{E}-10$ & ASPHD2 & $2.92 \mathrm{E}+00$ \\
\hline ENSBTAG00000001168 & $2.19 \mathrm{E}-10$ & $7.33 \mathrm{E}-11$ & BRAP & $2.99 \mathrm{E}+00$ \\
\hline ENSBTAG00000019857 & 7.93E-10 & $2.64 \mathrm{E}-10$ & OTUD4 & $3.00 \mathrm{E}+00$ \\
\hline ENSBTAG00000012277 & $4.60 \mathrm{E}-10$ & $1.50 \mathrm{E}-10$ & SRRD & $3.06 \mathrm{E}+00$ \\
\hline ENSBTAG00000013326 & $7.02 \mathrm{E}-10$ & $2.28 \mathrm{E}-10$ & ANAPC10 & $3.08 \mathrm{E}+00$ \\
\hline ENSBTAG00000007047 & $1.68 \mathrm{E}-10$ & $5.27 \mathrm{E}-11$ & FZD10 & $3.18 \mathrm{E}+00$ \\
\hline ENSBTAG00000012788 & $1.75 \mathrm{E}-10$ & $5.50 \mathrm{E}-11$ & & $3.18 \mathrm{E}+00$ \\
\hline ENSBTAG00000016257 & $5.10 \mathrm{E}-10$ & $1.60 \mathrm{E}-10$ & ZNF74 & $3.19 E+00$ \\
\hline ENSBTAG00000015811 & $3.53 \mathrm{E}-10$ & $1.11 \mathrm{E}-10$ & & $3.18 \mathrm{E}+00$ \\
\hline ENSBTAG00000016625 & $5.16 \mathrm{E}-10$ & $1.57 \mathrm{E}-10$ & KIAA0922 & $3.29 \mathrm{E}+00$ \\
\hline ENSBTAG00000008008 & $8.79 \mathrm{E}-10$ & $2.56 \mathrm{E}-10$ & TLR2 & $3.43 \mathrm{E}+00$ \\
\hline ENSBTAG00000003840 & $3.99 \mathrm{E}-10$ & $1.14 \mathrm{E}-10$ & GUCY1B3 & $3.50 \mathrm{E}+00$ \\
\hline ENSBTAG00000021052 & $2.59 \mathrm{E}-10$ & 7.33E-11 & GNB1L & $3.54 E+00$ \\
\hline ENSBTAG00000035265 & $5.21 \mathrm{E}-10$ & $1.46 \mathrm{E}-10$ & TMEM192 & $3.56 \mathrm{E}+00$ \\
\hline ENSBTAG00000022022 & 1.19E-09 & $3.36 \mathrm{E}-10$ & GUCD1 & $3.55 E+00$ \\
\hline ENSBTAG00000010381 & $3.14 \mathrm{E}-10$ & $8.53 \mathrm{E}-11$ & PRMT9 & $3.68 \mathrm{E}+00$ \\
\hline ENSBTAG00000019888 & $2.84 \mathrm{E}-10$ & 7.63E-11 & HIRA & $3.72 \mathrm{E}+00$ \\
\hline ENSBTAG00000003092 & $3.63 \mathrm{E}-10$ & $9.71 \mathrm{E}-11$ & ANAPC5 & $3.73 E+00$ \\
\hline ENSBTAG00000039325 & $1.29 \mathrm{E}-09$ & $3.42 \mathrm{E}-10$ & & $3.77 \mathrm{E}+00$ \\
\hline ENSBTAG00000046005 & $1.40 \mathrm{E}-10$ & $3.53 \mathrm{E}-11$ & & $3.97 \mathrm{E}+00$ \\
\hline ENSBTAG00000012792 & $1.30 \mathrm{E}-10$ & $3.28 \mathrm{E}-11$ & SRSF9 & $3.97 E+00$ \\
\hline ENSBTAG00000016738 & $4.33 \mathrm{E}-10$ & $1.07 \mathrm{E}-10$ & DTX1 & $4.04 E+00$ \\
\hline ENSBTAG00000038132 & $2.12 \mathrm{E}-10$ & 4.77E-11 & AIFM3 & $4.45 E+00$ \\
\hline ENSBTAG00000017167 & $2.79 \mathrm{E}-10$ & $5.84 \mathrm{E}-11$ & LRAT & $4.76 \mathrm{E}+00$ \\
\hline ENSBTAG00000048129 & $7.41 \mathrm{E}-10$ & $1.55 \mathrm{E}-10$ & & $4.78 \mathrm{E}+00$ \\
\hline ENSBTAG00000017750 & $3.02 \mathrm{E}-10$ & 5.99E-11 & RHBDD3 & $5.03 E+00$ \\
\hline ENSBTAG00000018332 & $2.25 \mathrm{E}-10$ & $4.26 \mathrm{E}-11$ & PPTC7 & $5.27 E+00$ \\
\hline ENSBTAG00000002813 & $9.12 \mathrm{E}-10$ & $1.70 \mathrm{E}-10$ & GAB1 & $5.36 \mathrm{E}+00$ \\
\hline ENSBTAG00000011771 & $6.00 \mathrm{E}-10$ & $1.11 \mathrm{E}-10$ & FICD & $5.40 \mathrm{E}+00$ \\
\hline ENSBTAG00000003399 & $8.38 \mathrm{E}-10$ & $1.54 \mathrm{E}-10$ & SMARCA5 & $5.44 \mathrm{E}+00$ \\
\hline ENSBTAG00000019053 & $9.03 \mathrm{E}-10$ & $1.62 \mathrm{E}-10$ & MAP9 & $5.57 \mathrm{E}+00$ \\
\hline ENSBTAG00000019040 & $5.71 \mathrm{E}-10$ & $1.02 \mathrm{E}-10$ & PLBD2 & $5.59 \mathrm{E}+00$ \\
\hline ENSBTAG00000010464 & $2.16 \mathrm{E}-10$ & $3.78 \mathrm{E}-11$ & MN1 & $5.69 E+00$ \\
\hline ENSBTAG00000011930 & $9.54 \mathrm{E}-10$ & $1.59 \mathrm{E}-10$ & DDX54 & $6.00 \mathrm{E}+00$ \\
\hline ENSBTAG00000011937 & 8.07E-10 & $1.31 \mathrm{E}-10$ & RITA1 & $6.16 \mathrm{E}+00$ \\
\hline ENSBTAG00000005940 & $6.20 \mathrm{E}-10$ & $9.86 \mathrm{E}-11$ & PRKAB1 & $6.28 \mathrm{E}+00$ \\
\hline ENSBTAG00000022067 & $1.53 \mathrm{E}-10$ & $2.41 \mathrm{E}-11$ & FAM216A & $6.33 E+00$ \\
\hline ENSBTAG00000021245 & $1.59 \mathrm{E}-10$ & $2.50 \mathrm{E}-11$ & SPRY1 & $6.34 \mathrm{E}+00$ \\
\hline ENSBTAG00000005970 & $3.97 \mathrm{E}-10$ & $6.23 \mathrm{E}-11$ & XBP1 & $6.36 \mathrm{E}+00$ \\
\hline ENSBTAG00000017404 & $2.79 \mathrm{E}-10$ & $4.32 \mathrm{E}-11$ & & $6.44 E+00$ \\
\hline ENSBTAG00000017738 & $1.84 \mathrm{E}-10$ & $2.79 \mathrm{E}-11$ & EMID1 & $6.56 \mathrm{E}+00$ \\
\hline ENSBTAG00000018330 & $2.01 \mathrm{E}-10$ & $2.89 \mathrm{E}-11$ & RAD9B & $6.94 \mathrm{E}+00$ \\
\hline ENSBTAG00000016633 & $1.75 \mathrm{E}-10$ & $2.45 \mathrm{E}-11$ & NPY2R & $7.12 \mathrm{E}+00$ \\
\hline ENSBTAG00000039242 & $7.09 \mathrm{E}-10$ & $9.86 \mathrm{E}-11$ & ZNF10 & $7.18 \mathrm{E}+00$ \\
\hline ENSBTAG00000015075 & $5.61 \mathrm{E}-10$ & $7.60 \mathrm{E}-11$ & $\mathrm{HSCB}$ & $7.37 \mathrm{E}+00$ \\
\hline ENSBTAG00000001356 & 7.12E-10 & $9.24 \mathrm{E}-11$ & USP38 & $7.70 \mathrm{E}+00$ \\
\hline ENSBTAG00000017277 & $3.45 \mathrm{E}-10$ & 4.43E-11 & RAB36 & $7.78 \mathrm{E}+00$ \\
\hline ENSBTAG00000009773 & $1.23 \mathrm{E}-10$ & $1.55 \mathrm{E}-11$ & KREMEN1 & $7.88 \mathrm{E}+00$ \\
\hline ENSBTAG00000009161 & $5.34 \mathrm{E}-10$ & $6.70 \mathrm{E}-11$ & TIGD4 & $7.97 \mathrm{E}+00$ \\
\hline ENSBTAG00000008676 & $3.40 \mathrm{E}-10$ & $3.66 \mathrm{E}-11$ & SRRM4 & $9.25 \mathrm{E}+00$ \\
\hline ENSBTAG00000011924 & 7.04E-10 & 7.15E-11 & CFAP73 & $9.83 E+00$ \\
\hline ENSBTAG00000047299 & $2.59 \mathrm{E}-10$ & $2.51 E-11$ & & $1.03 E+01$ \\
\hline ENSBTAG00000003557 & $4.47 \mathrm{E}-10$ & $2.80 \mathrm{E}-11$ & CCDC117 & $1.59 \mathrm{E}+01$ \\
\hline ENSBTAG00000018115 & $5.81 \mathrm{E}-10$ & $2.28 \mathrm{E}-11$ & PEBP1 & $2.54 \mathrm{E}+01$ \\
\hline ENSBTAG00000044159 & $1.28 \mathrm{E}-09$ & $3.68 \mathrm{E}-11$ & C4orf33 & $3.48 \mathrm{E}+01$ \\
\hline ENSBTAG00000009208 & $2.42 \mathrm{E}-10$ & $3.75 \mathrm{E}-12$ & TCTN1 & $6.28 \mathrm{E}+01$ \\
\hline ENSBTAG00000019880 & $1.22 \mathrm{E}-10$ & 0 & & $1.22 \mathrm{E}+03$ \\
\hline ENSBTAG00000009110 & 0 & 0 & & $1.00 \mathrm{E}+00$ \\
\hline ENSBTAG00000020503 & $3.43 \mathrm{E}-11$ & 0 & C12orf65 & $3.44 \mathrm{E}+02$ \\
\hline
\end{tabular}




\begin{tabular}{|c|c|c|c|c|}
\hline ENSBTAG00000008417 & $2.55 \mathrm{E}-10$ & 0 & VPS29 & $2.55 E+03$ \\
\hline ENSBTAG00000023431 & $1.33 \mathrm{E}-10$ & 0 & & $1.33 E+03$ \\
\hline ENSBTAG00000039059 & $1.01 \mathrm{E}-10$ & 0 & GP1BB & $1.01 E+03$ \\
\hline ENSBTAG00000019516 & $1.53 \mathrm{E}-10$ & 0 & & $1.53 E+03$ \\
\hline ENSBTAG00000006656 & $3.33 \mathrm{E}-10$ & 0 & & $3.33 E+03$ \\
\hline ENSBTAG00000007564 & $1.16 \mathrm{E}-10$ & 0 & BBS12 & $1.16 \mathrm{E}+03$ \\
\hline ENSBTAG00000046833 & 0 & 0 & & $1.00 \mathrm{E}+00$ \\
\hline ENSBTAG00000039023 & $5.23 \mathrm{E}-10$ & 0 & ZNF664 & $5.23 E+03$ \\
\hline ENSBTAG00000000908 & $8.12 \mathrm{E}-11$ & 0 & HCAR1 & $8.13 E+02$ \\
\hline ENSBTAG00000022039 & $2.26 \mathrm{E}-10$ & 0 & CMKLR1 & $2.26 \mathrm{E}+03$ \\
\hline ENSBTAG00000046165 & $2.32 \mathrm{E}-10$ & 0 & RPL31 & $2.32 \mathrm{E}+03$ \\
\hline ENSBTAG00000045955 & $2.85 \mathrm{E}-10$ & 0 & TSSK1B & $2.85 E+03$ \\
\hline ENSBTAG00000048123 & $1.52 \mathrm{E}-10$ & 0 & TSSK2 & $1.52 \mathrm{E}+03$ \\
\hline ENSBTAG00000047896 & 0 & 0 & & $1.00 \mathrm{E}+00$ \\
\hline ENSBTAG00000045665 & 0 & 0 & & $1.00 \mathrm{E}+00$ \\
\hline ENSBTAG00000004743 & $1.72 \mathrm{E}-10$ & 0 & & $1.72 \mathrm{E}+03$ \\
\hline ENSBTAG00000009824 & $3.18 \mathrm{E}-10$ & 0 & COMMD6 & $3.18 \mathrm{E}+03$ \\
\hline ENSBTAG00000012102 & 0 & $8.62 \mathrm{E}-12$ & TCF25 & $1.15 \mathrm{E}-02$ \\
\hline ENSBTAG00000023617 & 0 & $4.34 \mathrm{E}-11$ & & $2.30 \mathrm{E}-03$ \\
\hline ENSBTAG00000008502 & 0 & $1.06 \mathrm{E}-10$ & & $9.43 \mathrm{E}-04$ \\
\hline ENSBTAG00000013283 & 0 & $3.29 \mathrm{E}-11$ & PRR19 & 3.03E-03 \\
\hline ENSBTAG00000047508 & 0 & $3.99 \mathrm{E}-10$ & ZNF580 & $2.51 \mathrm{E}-04$ \\
\hline ENSBTAG00000027711 & $7.29 \mathrm{E}-11$ & $3.91 E-10$ & YIF1B & 1.87E-01 \\
\hline ENSBTAG00000015004 & $1.47 \mathrm{E}-10$ & $7.43 \mathrm{E}-10$ & TBCB & $1.98 \mathrm{E}-01$ \\
\hline ENSBTAG00000009172 & $3.51 E-11$ & $1.62 \mathrm{E}-10$ & BCAT2 & 2.17E-01 \\
\hline ENSBTAG00000019461 & $1.16 \mathrm{E}-10$ & 4.30E-10 & NUMBL & 2.69E- 01 \\
\hline ENSBTAG00000030939 & $2.14 \mathrm{E}-10$ & $7.39 \mathrm{E}-10$ & ZNF575 & 2.90E-01 \\
\hline ENSBTAG00000023472 & $1.57 \mathrm{E}-10$ & $5.20 \mathrm{E}-10$ & PPP1R14A & $3.02 \mathrm{E}-01$ \\
\hline ENSBTAG00000001260 & $3.05 E-10$ & 1.00E-09 & PINLYP & 3.05E-01 \\
\hline ENSBTAG00000031648 & $1.96 \mathrm{E}-10$ & $6.19 \mathrm{E}-10$ & & 3.17E-01 \\
\hline ENSBTAG00000013337 & $1.20 \mathrm{E}-10$ & $3.77 \mathrm{E}-10$ & $\mathrm{DHDH}$ & 3.17E-01 \\
\hline ENSBTAG00000039691 & $2.75 E-11$ & 8.67E-11 & & $3.18 \mathrm{E}-01$ \\
\hline ENSBTAG00000009521 & $2.21 \mathrm{E}-10$ & $6.61 \mathrm{E}-10$ & ZNF565 & $3.34 \mathrm{E}-01$ \\
\hline ENSBTAG00000000182 & $2.66 \mathrm{E}-10$ & $7.26 \mathrm{E}-10$ & SPINT2 & 3.66E-01 \\
\hline ENSBTAG00000003465 & $1.32 \mathrm{E}-10$ & $3.32 \mathrm{E}-10$ & ZNF329 & 3.97E-01 \\
\hline ENSBTAG00000047423 & $3.81 \mathrm{E}-11$ & $9.60 \mathrm{E}-11$ & ERICH4 & 3.97E-01 \\
\hline ENSBTAG00000047405 & $9.79 \mathrm{E}-11$ & $2.47 \mathrm{E}-10$ & & 3.96E-01 \\
\hline ENSBTAG00000047815 & $2.40 \mathrm{E}-11$ & $6.06 \mathrm{E}-11$ & & $3.98 \mathrm{E}-01$ \\
\hline ENSBTAG00000019787 & $5.78 \mathrm{E}-11$ & $1.46 \mathrm{E}-10$ & PAFAH1B3 & $3.96 \mathrm{E}-01$ \\
\hline ENSBTAG00000027787 & $2.23 \mathrm{E}-10$ & $5.63 \mathrm{E}-10$ & & $3.96 \mathrm{E}-01$ \\
\hline ENSBTAG00000033563 & $1.89 \mathrm{E}-10$ & $4.00 \mathrm{E}-10$ & & 4.72E-01 \\
\hline ENSBTAG00000011963 & $9.78 \mathrm{E}-11$ & $2.06 \mathrm{E}-10$ & & 4.75E-01 \\
\hline ENSBTAG00000009077 & $1.21 \mathrm{E}-10$ & $2.48 \mathrm{E}-10$ & SNRPA & $4.88 \mathrm{E}-01$ \\
\hline ENSBTAG00000018882 & $2.49 \mathrm{E}-10$ & $5.08 \mathrm{E}-10$ & & 4.91E-01 \\
\hline ENSBTAG00000007841 & $2.79 \mathrm{E}-10$ & $5.54 \mathrm{E}-10$ & WTIP & $5.04 \mathrm{E}-01$ \\
\hline ENSBTAG00000046111 & $2.63 \mathrm{E}-10$ & $4.98 \mathrm{E}-10$ & ZNF444 & $5.29 \mathrm{E}-01$ \\
\hline ENSBTAG00000039015 & $3.34 \mathrm{E}-11$ & $6.31 E-11$ & TMEM145 & 5.30E-01 \\
\hline ENSBTAG00000003961 & $5.65 \mathrm{E}-11$ & $1.07 \mathrm{E}-10$ & SNX20 & $5.29 \mathrm{E}-01$ \\
\hline ENSBTAG00000002682 & $1.27 \mathrm{E}-10$ & $2.36 \mathrm{E}-10$ & MARK4 & $5.40 \mathrm{E}-01$ \\
\hline ENSBTAG00000004379 & $3.42 \mathrm{E}-10$ & $6.11 E-10$ & ETHE1 & 5.60E-01 \\
\hline ENSBTAG00000020756 & 4.40E-11 & $7.76 \mathrm{E}-11$ & & 5.67E-01 \\
\hline ENSBTAG00000006387 & 4.34E-11 & $7.66 \mathrm{E}-11$ & TSNAXIP1 & 5.67E- 01 \\
\hline ENSBTAG00000014878 & $1.58 \mathrm{E}-10$ & $2.66 \mathrm{E}-10$ & COX7A1 & 5.95E-01 \\
\hline ENSBTAG00000009079 & $5.65 \mathrm{E}-11$ & $9.49 \mathrm{E}-11$ & & $5.95 \mathrm{E}-01$ \\
\hline ENSBTAG00000013929 & 7.69E-11 & $1.29 \mathrm{E}-10$ & RRAD & 5.96E-01 \\
\hline ENSBTAG00000015599 & $3.19 \mathrm{E}-10$ & $5.17 \mathrm{E}-10$ & PRKD2 & 6.17E-01 \\
\hline ENSBTAG00000003886 & $2.71 \mathrm{E}-10$ & 4.35E-10 & & $6.24 \mathrm{E}-01$ \\
\hline ENSBTAG00000045572 & $1.86 \mathrm{E}-10$ & $2.93 \mathrm{E}-10$ & ZNF319 & $6.36 \mathrm{E}-01$ \\
\hline ENSBTAG00000037983 & $5.15 E-11$ & $8.12 \mathrm{E}-11$ & CEACAM16 & $6.35 \mathrm{E}-01$ \\
\hline ENSBTAG00000002639 & $3.54 \mathrm{E}-10$ & $5.52 \mathrm{E}-10$ & ZDHHC7 & $6.42 \mathrm{E}-01$ \\
\hline ENSBTAG00000019785 & $3.17 \mathrm{E}-11$ & $4.89 \mathrm{E}-11$ & & $6.50 \mathrm{E}-01$ \\
\hline ENSBTAG00000015758 & $1.50 \mathrm{E}-10$ & $2.29 \mathrm{E}-10$ & USB1 & $6.55 \mathrm{E}-01$ \\
\hline ENSBTAG00000003503 & $1.28 \mathrm{E}-10$ & $1.94 \mathrm{E}-10$ & C16orf87 & $6.62 \mathrm{E}-01$ \\
\hline ENSBTAG00000016268 & $8.20 \mathrm{E}-10$ & $1.23 \mathrm{E}-09$ & XRCC1 & $6.67 \mathrm{E}-01$ \\
\hline ENSBTAG00000000458 & $9.16 \mathrm{E}-11$ & $1.36 \mathrm{E}-10$ & DYNC1LI2 & $6.74 \mathrm{E}-01$ \\
\hline ENSBTAG00000013941 & $1.02 \mathrm{E}-10$ & $1.51 \mathrm{E}-10$ & TANGO6 & $6.78 \mathrm{E}-01$ \\
\hline ENSBTAG00000008500 & $1.06 \mathrm{E}-10$ & $1.55 \mathrm{E}-10$ & & $6.81 \mathrm{E}-01$ \\
\hline ENSBTAG00000008625 & $5.13 E-11$ & $7.55 \mathrm{E}-11$ & PLEKHF1 & $6.81 \mathrm{E}-01$ \\
\hline ENSBTAG00000039635 & $2.06 \mathrm{E}-10$ & $3.00 \mathrm{E}-10$ & CCDC97 & $6.88 \mathrm{E}-01$ \\
\hline ENSBTAG00000020943 & $2.23 \mathrm{E}-10$ & $3.24 \mathrm{E}-10$ & CTU2 & $6.89 \mathrm{E}-01$ \\
\hline
\end{tabular}




\begin{tabular}{|c|c|c|c|c|}
\hline ENSBTAG00000002378 & $2.81 \mathrm{E}-10$ & 4.06E-10 & NUCB1 & $6.93 \mathrm{E}-01$ \\
\hline ENSBTAG00000000080 & $1.89 \mathrm{E}-10$ & $2.73 \mathrm{E}-10$ & GRWD1 & $6.93 \mathrm{E}-01$ \\
\hline ENSBTAG00000039914 & $1.44 \mathrm{E}-10$ & $2.08 \mathrm{E}-10$ & & 6.94E-01 \\
\hline ENSBTAG00000017718 & $8.15 E-11$ & $1.16 \mathrm{E}-10$ & CCL22 & 7.03E-01 \\
\hline ENSBTAG00000014872 & $2.25 \mathrm{E}-10$ & $3.13 \mathrm{E}-10$ & CAPNS1 & 7.19E-01 \\
\hline ENSBTAG00000005830 & $2.42 \mathrm{E}-10$ & $3.35 \mathrm{E}-10$ & STRN4 & 7.24E-01 \\
\hline ENSBTAG00000038326 & $1.00 \mathrm{E}-10$ & $1.38 \mathrm{E}-10$ & & 7.27E-01 \\
\hline ENSBTAG00000002493 & $5.47 \mathrm{E}-10$ & 7.43E-10 & VPS35 & 7.37E-01 \\
\hline ENSBTAG00000007065 & $1.36 \mathrm{E}-10$ & $1.82 \mathrm{E}-10$ & TRAPPC6A & 7.49E-01 \\
\hline ENSBTAG00000015836 & $1.37 \mathrm{E}-10$ & $1.83 \mathrm{E}-10$ & & 7.48E-01 \\
\hline ENSBTAG00000006414 & $1.00 \mathrm{E}-10$ & $1.33 \mathrm{E}-10$ & NUTF2 & $7.53 \mathrm{E}-01$ \\
\hline ENSBTAG00000037981 & $5.42 \mathrm{E}-10$ & $7.20 \mathrm{E}-10$ & & 7.52E-01 \\
\hline ENSBTAG00000001783 & 4.70E-10 & $6.23 \mathrm{E}-10$ & FBXO17 & $7.54 \mathrm{E}-01$ \\
\hline ENSBTAG00000009174 & $1.56 \mathrm{E}-10$ & $2.07 \mathrm{E}-10$ & HSD17B14 & 7.55E-01 \\
\hline ENSBTAG00000030705 & $1.69 \mathrm{E}-10$ & $2.23 \mathrm{E}-10$ & DACT3 & 7.59E-01 \\
\hline ENSBTAG00000007821 & $2.12 \mathrm{E}-10$ & $2.78 \mathrm{E}-10$ & NPHS1 & 7.61E-01 \\
\hline ENSBTAG00000001262 & $6.91 \mathrm{E}-10$ & $9.03 \mathrm{E}-10$ & IRGQ & 7.65E-01 \\
\hline ENSBTAG00000006121 & 7.63E-10 & $9.92 \mathrm{E}-10$ & SBK2 & 7.69E-01 \\
\hline ENSBTAG00000038045 & $3.08 \mathrm{E}-10$ & $3.98 \mathrm{E}-10$ & FLT3LG & 7.73E-01 \\
\hline ENSBTAG00000017618 & $1.28 \mathrm{E}-10$ & $1.61 \mathrm{E}-10$ & MAP3K10 & 7.93E-01 \\
\hline ENSBTAG00000048127 & $1.09 \mathrm{E}-10$ & $1.38 \mathrm{E}-10$ & SPACA4 & 7.92E-01 \\
\hline ENSBTAG00000039426 & $1.47 \mathrm{E}-10$ & $1.85 \mathrm{E}-10$ & DPEP2 & 7.93E-01 \\
\hline ENSBTAG00000018040 & $3.21 \mathrm{E}-11$ & 4.04E-11 & & 7.95E-01 \\
\hline ENSBTAG00000037582 & $2.60 \mathrm{E}-10$ & $3.28 \mathrm{E}-10$ & & 7.93E-01 \\
\hline ENSBTAG00000012059 & $1.48 \mathrm{E}-10$ & $1.86 \mathrm{E}-10$ & MVD & 7.94E-01 \\
\hline ENSBTAG00000047691 & $2.52 \mathrm{E}-10$ & $3.18 \mathrm{E}-10$ & & 7.94E-01 \\
\hline ENSBTAG00000014556 & $1.09 \mathrm{E}-10$ & $1.37 \mathrm{E}-10$ & AGRP & 7.96E-01 \\
\hline ENSBTAG00000033604 & $2.56 \mathrm{E}-10$ & $3.22 \mathrm{E}-10$ & & 7.94E-01 \\
\hline ENSBTAG00000046690 & $3.70 \mathrm{E}-10$ & 4.66E-10 & & 7.94E-01 \\
\hline ENSBTAG00000001882 & $8.88 \mathrm{E}-11$ & $1.12 \mathrm{E}-10$ & CD79A & 7.93E-01 \\
\hline ENSBTAG00000004290 & $8.94 \mathrm{E}-11$ & $1.13 \mathrm{E}-10$ & $\mathrm{DD} 28$ & 7.91E-01 \\
\hline ENSBTAG00000013924 & $6.73 \mathrm{E}-11$ & $8.48 \mathrm{E}-11$ & RPS11 & 7.94E-01 \\
\hline ENSBTAG00000047277 & $7.68 \mathrm{E}-10$ & $9.68 \mathrm{E}-10$ & & 7.94E-01 \\
\hline ENSBTAG00000009078 & $5.72 \mathrm{E}-11$ & 7.20E-11 & & 7.94E-01 \\
\hline ENSBTAG00000000332 & $3.75 \mathrm{E}-11$ & $4.73 E-11$ & ERF & 7.93E-01 \\
\hline ENSBTAG00000017762 & $1.37 \mathrm{E}-10$ & $1.72 \mathrm{E}-10$ & GNG8 & 7.95E-01 \\
\hline ENSBTAG00000047128 & $8.13 \mathrm{E}-11$ & $1.02 \mathrm{E}-10$ & IL17C & 7.98E-01 \\
\hline ENSBTAG00000038735 & $8.40 \mathrm{E}-11$ & $1.06 \mathrm{E}-10$ & & 7.93E-01 \\
\hline ENSBTAG00000012063 & $1.36 \mathrm{E}-10$ & $1.71 \mathrm{E}-10$ & E2F4 & 7.94E-01 \\
\hline ENSBTAG00000047471 & $6.66 \mathrm{E}-11$ & 8.39E-11 & & 7.94E-01 \\
\hline ENSBTAG00000046666 & $7.00 \mathrm{E}-11$ & $8.82 \mathrm{E}-11$ & TTC9B & 7.94E-01 \\
\hline ENSBTAG00000047505 & $1.97 \mathrm{E}-10$ & $2.48 \mathrm{E}-10$ & NANOS2 & 7.93E-01 \\
\hline ENSBTAG00000023734 & 8.37E-11 & $1.05 \mathrm{E}-10$ & PARD6A & 7.97E-01 \\
\hline ENSBTAG00000011995 & $1.01 \mathrm{E}-10$ & $1.27 \mathrm{E}-10$ & & 7.92E-01 \\
\hline ENSBTAG00000023562 & $5.95 \mathrm{E}-11$ & $7.50 \mathrm{E}-11$ & KCNK6 & 7.94E-01 \\
\hline ENSBTAG00000015644 & $5.48 \mathrm{E}-11$ & $6.91 \mathrm{E}-11$ & & 7.93E-01 \\
\hline ENSBTAG00000020429 & $6.97 \mathrm{E}-11$ & $8.78 \mathrm{E}-11$ & MED29 & 7.94E-01 \\
\hline ENSBTAG00000039593 & $8.31 \mathrm{E}-11$ & $1.05 \mathrm{E}-10$ & FAM98C & 7.92E-01 \\
\hline ENSBTAG00000018398 & $4.70 \mathrm{E}-11$ & $5.92 \mathrm{E}-11$ & GPR4 & 7.94E-01 \\
\hline ENSBTAG00000046708 & $1.03 \mathrm{E}-10$ & $1.29 \mathrm{E}-10$ & PDP2 & 7.96E-01 \\
\hline ENSBTAG00000038711 & $1.05 \mathrm{E}-10$ & $1.33 \mathrm{E}-10$ & TMEM91 & 7.93E-01 \\
\hline ENSBTAG00000021789 & $9.96 \mathrm{E}-11$ & $1.26 \mathrm{E}-10$ & ZNF574 & 7.91E-01 \\
\hline ENSBTAG00000047196 & $1.48 \mathrm{E}-10$ & $1.87 \mathrm{E}-10$ & & 7.94E-01 \\
\hline ENSBTAG00000018242 & 8.47E-11 & 1.07E-10 & & 7.92E-01 \\
\hline ENSBTAG00000031687 & $6.56 \mathrm{E}-10$ & $8.26 \mathrm{E}-10$ & & 7.94E-01 \\
\hline ENSBTAG00000006411 & $4.89 \mathrm{E}-11$ & $6.16 \mathrm{E}-11$ & THAP11 & 7.94E-01 \\
\hline ENSBTAG00000021557 & $7.91 \mathrm{E}-11$ & 9.97E-11 & FUT2 & 7.94E-01 \\
\hline ENSBTAG00000011119 & $1.74 \mathrm{E}-10$ & 2.19E-10 & & 7.95E-01 \\
\hline ENSBTAG00000006404 & $2.07 \mathrm{E}-11$ & $2.61 E-11$ & CENPT & 7.94E-01 \\
\hline ENSBTAG00000018593 & $2.48 \mathrm{E}-10$ & $3.12 \mathrm{E}-10$ & & 7.94E-01 \\
\hline ENSBTAG00000013985 & $2.09 \mathrm{E}-10$ & 2.64E-10 & FKRP & 7.92E-01 \\
\hline ENSBTAG00000013921 & $1.13 \mathrm{E}-10$ & $1.43 \mathrm{E}-10$ & CKM & 7.91E-01 \\
\hline ENSBTAG00000017993 & $1.38 \mathrm{E}-09$ & 1.73E-09 & & 7.96E-01 \\
\hline ENSBTAG00000031001 & $1.39 \mathrm{E}-10$ & $1.75 \mathrm{E}-10$ & MEGF8 & 7.92E-01 \\
\hline ENSBTAG00000020781 & $1.56 \mathrm{E}-10$ & $1.96 \mathrm{E}-10$ & SETD6 & 7.93E-01 \\
\hline ENSBTAG00000037461 & $1.57 \mathrm{E}-10$ & 1.97E-10 & SDHAF1 & 7.95E-01 \\
\hline ENSBTAG00000013343 & $1.34 \mathrm{E}-10$ & $1.69 \mathrm{E}-10$ & & 7.94E-01 \\
\hline ENSBTAG00000020782 & $2.37 \mathrm{E}-10$ & 2.97E-10 & CNOT1 & 7.97E-01 \\
\hline ENSBTAG00000011844 & $9.97 \mathrm{E}-10$ & $1.24 \mathrm{E}-09$ & & 8.04E-01 \\
\hline ENSBTAG00000004805 & $4.40 \mathrm{E}-10$ & $5.48 \mathrm{E}-10$ & ITFG1 & 8.03E-01 \\
\hline
\end{tabular}




\begin{tabular}{|c|c|c|c|c|}
\hline ENSBTAG00000019266 & $5.50 \mathrm{E}-10$ & $6.80 \mathrm{E}-10$ & & $8.09 \mathrm{E}-01$ \\
\hline ENSBTAG00000000418 & $2.32 \mathrm{E}-10$ & $2.86 \mathrm{E}-10$ & TOX3 & $8.11 \mathrm{E}-01$ \\
\hline ENSBTAG00000047053 & $6.06 \mathrm{E}-10$ & 7.41E-10 & & $8.18 \mathrm{E}-01$ \\
\hline ENSBTAG00000018908 & $1.19 \mathrm{E}-10$ & $1.44 \mathrm{E}-10$ & GFOD2 & $8.25 \mathrm{E}-01$ \\
\hline ENSBTAG00000015428 & $5.75 \mathrm{E}-10$ & 7.01E-10 & & $8.21 \mathrm{E}-01$ \\
\hline ENSBTAG00000018240 & 4.00E-10 & $4.86 \mathrm{E}-10$ & CYP2S1 & $8.23 \mathrm{E}-01$ \\
\hline ENSBTAG00000005702 & $2.60 \mathrm{E}-10$ & $3.12 \mathrm{E}-10$ & CNGB1 & $8.32 \mathrm{E}-01$ \\
\hline ENSBTAG00000038067 & $9.17 \mathrm{E}-10$ & 1.10E-09 & & $8.34 \mathrm{E}-01$ \\
\hline ENSBTAG00000008556 & $1.43 \mathrm{E}-10$ & $1.71 \mathrm{E}-10$ & POU2F2 & $8.36 \mathrm{E}-01$ \\
\hline ENSBTAG00000023439 & $1.83 \mathrm{E}-09$ & $2.18 \mathrm{E}-09$ & & $8.40 \mathrm{E}-01$ \\
\hline ENSBTAG00000009337 & 7.63E-10 & $9.08 \mathrm{E}-10$ & PNMAL1 & $8.40 \mathrm{E}-01$ \\
\hline ENSBTAG00000010584 & $4.61 \mathrm{E}-10$ & $5.48 \mathrm{E}-10$ & AP2S1 & $8.40 \mathrm{E}-01$ \\
\hline ENSBTAG00000005615 & $6.08 \mathrm{E}-10$ & $7.22 \mathrm{E}-10$ & CEACAM1 & $8.42 \mathrm{E}-01$ \\
\hline ENSBTAG00000027431 & $7.62 \mathrm{E}-10$ & $9.05 \mathrm{E}-10$ & ZNF227 & $8.42 \mathrm{E}-01$ \\
\hline ENSBTAG00000013697 & $4.02 \mathrm{E}-10$ & 4.76E-10 & CLASRP & $8.44 \mathrm{E}-01$ \\
\hline ENSBTAG00000007826 & $3.32 \mathrm{E}-10$ & $3.94 \mathrm{E}-10$ & KIRREL2 & $8.43 \mathrm{E}-01$ \\
\hline ENSBTAG00000017752 & $1.38 \mathrm{E}-10$ & $1.63 \mathrm{E}-10$ & DYNLRB2 & $8.45 \mathrm{E}-01$ \\
\hline ENSBTAG00000000195 & $8.51 \mathrm{E}-10$ & $1.00 \mathrm{E}-09$ & & $8.51 \mathrm{E}-01$ \\
\hline ENSBTAG00000018394 & $6.05 \mathrm{E}-10$ & 7.13E-10 & SDR42E1 & $8.49 \mathrm{E}-01$ \\
\hline ENSBTAG00000000868 & $6.75 \mathrm{E}-10$ & $7.95 \mathrm{E}-10$ & ZFP1 & $8.49 \mathrm{E}-01$ \\
\hline ENSBTAG00000008084 & $4.85 \mathrm{E}-10$ & 5.70E-10 & ZNF382 & 8.50E-01 \\
\hline ENSBTAG00000002976 & $5.37 \mathrm{E}-10$ & $6.29 \mathrm{E}-10$ & CD177 & 8.54E-01 \\
\hline ENSBTAG00000003168 & $2.13 \mathrm{E}-10$ & $2.50 \mathrm{E}-10$ & HNRNPUL1 & 8.53E-01 \\
\hline ENSBTAG00000013577 & $1.92 \mathrm{E}-10$ & $2.24 \mathrm{E}-10$ & SLC1A5 & $8.56 \mathrm{E}-01$ \\
\hline ENSBTAG00000003261 & $2.82 \mathrm{E}-10$ & 3.29E-10 & & $8.56 \mathrm{E}-01$ \\
\hline ENSBTAG00000012590 & 8.13E-10 & $9.48 \mathrm{E}-10$ & ACP7 & $8.58 \mathrm{E}-01$ \\
\hline ENSBTAG00000022783 & $2.81 \mathrm{E}-10$ & $3.28 \mathrm{E}-10$ & & 8.57E-01 \\
\hline ENSBTAG00000002904 & $2.91 \mathrm{E}-10$ & $3.40 \mathrm{E}-10$ & ZNF787 & 8.57E-01 \\
\hline ENSBTAG00000006961 & 1.07E-09 & $1.24 \mathrm{E}-09$ & NLRP13 & 8.60E-01 \\
\hline ENSBTAG00000045507 & $1.49 \mathrm{E}-10$ & $1.72 \mathrm{E}-10$ & ZNF469 & $8.64 \mathrm{E}-01$ \\
\hline ENSBTAG00000020434 & $1.69 \mathrm{E}-10$ & $1.96 \mathrm{E}-10$ & & $8.65 \mathrm{E}-01$ \\
\hline ENSBTAG00000014996 & $6.25 \mathrm{E}-10$ & 7.22E-10 & WDR62 & 8.65E-01 \\
\hline ENSBTAG00000030667 & $2.93 \mathrm{E}-10$ & $3.40 \mathrm{E}-10$ & CCDC114 & 8.63E-01 \\
\hline ENSBTAG00000030933 & $3.93 \mathrm{E}-10$ & 4.54E-10 & ZNF576 & 8.66E-01 \\
\hline ENSBTAG00000017872 & $4.25 \mathrm{E}-10$ & $4.91 \mathrm{E}-10$ & ZNF304 & 8.66E-01 \\
\hline ENSBTAG00000015677 & $5.54 \mathrm{E}-10$ & 6.37E-10 & KARS & 8.69E-01 \\
\hline ENSBTAG00000007036 & 4.24E-10 & $4.88 \mathrm{E}-10$ & SLC17A7 & 8.69E-01 \\
\hline ENSBTAG00000015432 & $4.32 \mathrm{E}-10$ & $4.94 \mathrm{E}-10$ & & $8.74 \mathrm{E}-01$ \\
\hline ENSBTAG00000019884 & $5.96 \mathrm{E}-10$ & $6.82 \mathrm{E}-10$ & PKD1L3 & $8.74 \mathrm{E}-01$ \\
\hline ENSBTAG00000011617 & $1.76 \mathrm{E}-10$ & $2.02 \mathrm{E}-10$ & MAMSTR & $8.72 \mathrm{E}-01$ \\
\hline ENSBTAG00000047307 & $1.33 \mathrm{E}-09$ & $1.53 \mathrm{E}-09$ & IFNL3 & $8.72 \mathrm{E}-01$ \\
\hline ENSBTAG00000015543 & $3.19 \mathrm{E}-10$ & $3.65 \mathrm{E}-10$ & ARHGAP35 & $8.74 \mathrm{E}-01$ \\
\hline ENSBTAG00000020944 & $3.37 \mathrm{E}-10$ & $3.84 \mathrm{E}-10$ & PIEZO1 & 8.79E-01 \\
\hline ENSBTAG00000003166 & $2.61 E-10$ & 2.97E-10 & AXL & $8.80 \mathrm{E}-01$ \\
\hline ENSBTAG00000015427 & $4.28 \mathrm{E}-10$ & $4.85 \mathrm{E}-10$ & CFDP1 & $8.82 \mathrm{E}-01$ \\
\hline ENSBTAG00000030348 & $4.31 \mathrm{E}-10$ & $4.88 \mathrm{E}-10$ & & 8.83E-01 \\
\hline ENSBTAG00000012915 & $3.57 \mathrm{E}-10$ & 4.04E-10 & ZNF404 & $8.82 \mathrm{E}-01$ \\
\hline ENSBTAG00000000621 & 2.87E-10 & $3.24 \mathrm{E}-10$ & CATSPERG & $8.85 \mathrm{E}-01$ \\
\hline ENSBTAG00000014818 & $6.99 \mathrm{E}-10$ & $7.89 \mathrm{E}-10$ & MYLK3 & $8.85 \mathrm{E}-01$ \\
\hline ENSBTAG00000011164 & $7.78 \mathrm{E}-10$ & $8.76 \mathrm{E}-10$ & CES5A & $8.88 \mathrm{E}-01$ \\
\hline ENSBTAG00000019462 & $1.71 \mathrm{E}-10$ & $1.92 \mathrm{E}-10$ & COQ8B & 8.93E-01 \\
\hline ENSBTAG00000009460 & $6.76 \mathrm{E}-10$ & $7.59 \mathrm{E}-10$ & ZNF550 & $8.90 \mathrm{E}-01$ \\
\hline ENSBTAG00000030882 & $3.26 \mathrm{E}-10$ & $3.65 \mathrm{E}-10$ & & 8.93E-01 \\
\hline ENSBTAG00000039453 & $1.71 \mathrm{E}-10$ & $1.91 \mathrm{E}-10$ & & $8.95 \mathrm{E}-01$ \\
\hline ENSBTAG00000000336 & 2.57E-09 & $2.88 \mathrm{E}-09$ & & $8.93 \mathrm{E}-01$ \\
\hline ENSBTAG00000020457 & $1.49 \mathrm{E}-10$ & $1.66 \mathrm{E}-10$ & TGFB1 & 8.97E-01 \\
\hline ENSBTAG00000002573 & $1.84 \mathrm{E}-10$ & $2.05 \mathrm{E}-10$ & UBA2 & 8.99E-01 \\
\hline ENSBTAG00000005170 & $4.61 \mathrm{E}-10$ & $5.15 \mathrm{E}-10$ & ADGRG5 & 8.96E-01 \\
\hline ENSBTAG00000010480 & $4.05 E-10$ & $4.52 \mathrm{E}-10$ & TIMM50 & 8.96E-01 \\
\hline ENSBTAG00000007170 & $1.22 \mathrm{E}-10$ & $1.36 \mathrm{E}-10$ & SLC38A7 & $8.98 \mathrm{E}-01$ \\
\hline ENSBTAG00000040318 & $3.38 \mathrm{E}-10$ & $3.74 \mathrm{E}-10$ & & 9.03E-01 \\
\hline ENSBTAG00000006999 & $4.28 \mathrm{E}-10$ & $4.74 \mathrm{E}-10$ & RYR1 & $9.04 \mathrm{E}-01$ \\
\hline ENSBTAG00000001872 & $1.57 \mathrm{E}-10$ & $1.74 \mathrm{E}-10$ & ORC6 & $9.05 \mathrm{E}-01$ \\
\hline ENSBTAG00000009805 & $4.54 \mathrm{E}-10$ & $5.02 \mathrm{E}-10$ & & $9.04 \mathrm{E}-01$ \\
\hline ENSBTAG00000021570 & $7.48 \mathrm{E}-11$ & $8.25 \mathrm{E}-11$ & PLEKHG4 & 9.07E-01 \\
\hline ENSBTAG00000013702 & $1.76 \mathrm{E}-10$ & $1.94 \mathrm{E}-10$ & ZNF296 & 9.09E-01 \\
\hline ENSBTAG00000009872 & $1.81 \mathrm{E}-10$ & $1.99 \mathrm{E}-10$ & SPHK2 & $9.08 \mathrm{E}-01$ \\
\hline ENSBTAG00000032763 & $1.07 \mathrm{E}-10$ & $1.18 \mathrm{E}-10$ & RANBP10 & 9.09E-01 \\
\hline ENSBTAG00000019272 & $3.63 \mathrm{E}-10$ & 4.00E-10 & LPCAT2 & $9.08 \mathrm{E}-01$ \\
\hline ENSBTAG00000012131 & 8.67E-10 & $9.55 \mathrm{E}-10$ & & $9.08 \mathrm{E}-01$ \\
\hline
\end{tabular}




\begin{tabular}{|c|c|c|c|c|}
\hline ENSBTAG00000018771 & $5.98 \mathrm{E}-10$ & $6.59 \mathrm{E}-10$ & DNAAF1 & 9.07E-01 \\
\hline ENSBTAG00000011036 & $3.36 \mathrm{E}-10$ & 3.70E-10 & CEACAM20 & $9.09 \mathrm{E}-01$ \\
\hline ENSBTAG00000013175 & $2.58 \mathrm{E}-10$ & $2.84 \mathrm{E}-10$ & KIAA0355 & $9.10 \mathrm{E}-01$ \\
\hline ENSBTAG00000010467 & $4.61 \mathrm{E}-10$ & $5.06 \mathrm{E}-10$ & SUPT5H & $9.11 \mathrm{E}-01$ \\
\hline ENSBTAG00000031686 & $1.29 \mathrm{E}-10$ & $1.41 \mathrm{E}-10$ & ZNF566 & $9.15 \mathrm{E}-01$ \\
\hline ENSBTAG00000038706 & $6.08 \mathrm{E}-10$ & $6.63 \mathrm{E}-10$ & & $9.17 \mathrm{E}-01$ \\
\hline ENSBTAG00000018599 & $5.95 \mathrm{E}-10$ & $6.49 \mathrm{E}-10$ & & $9.16 \mathrm{E}-01$ \\
\hline ENSBTAG00000015001 & $6.63 \mathrm{E}-10$ & 7.23E-10 & OVOL3 & $9.17 \mathrm{E}-01$ \\
\hline ENSBTAG00000032576 & 7.07E-10 & 7.70E-10 & CMTM2 & $9.19 \mathrm{E}-01$ \\
\hline ENSBTAG00000011619 & $1.75 \mathrm{E}-10$ & $1.90 \mathrm{E}-10$ & RASIP1 & $9.19 \mathrm{E}-01$ \\
\hline ENSBTAG00000038134 & $1.74 \mathrm{E}-10$ & $1.90 \mathrm{E}-10$ & ZDHHC1 & $9.18 \mathrm{E}-01$ \\
\hline ENSBTAG00000020074 & $2.35 \mathrm{E}-10$ & $2.56 \mathrm{E}-10$ & NKPD1 & $9.18 \mathrm{E}-01$ \\
\hline ENSBTAG00000009428 & $8.14 \mathrm{E}-10$ & $8.85 \mathrm{E}-10$ & GAN & $9.20 \mathrm{E}-01$ \\
\hline ENSBTAG00000010868 & $3.62 \mathrm{E}-10$ & $3.94 \mathrm{E}-10$ & SIAH1 & $9.19 \mathrm{E}-01$ \\
\hline ENSBTAG00000039946 & $3.10 \mathrm{E}-10$ & 3.37E-10 & & $9.19 \mathrm{E}-01$ \\
\hline ENSBTAG00000021185 & 4.07E-10 & 4.42E-10 & CRX & $9.20 \mathrm{E}-01$ \\
\hline ENSBTAG00000019273 & $1.08 \mathrm{E}-10$ & 1.17E-10 & DMRTC2 & $9.26 \mathrm{E}-01$ \\
\hline ENSBTAG00000021799 & $1.36 \mathrm{E}-10$ & 1.47E-10 & RCN3 & $9.26 \mathrm{E}-01$ \\
\hline ENSBTAG00000001834 & $1.16 \mathrm{E}-10$ & $1.25 \mathrm{E}-10$ & PNKP & $9.25 \mathrm{E}-01$ \\
\hline ENSBTAG00000002635 & $2.78 \mathrm{E}-10$ & $3.00 \mathrm{E}-10$ & PGLYRP1 & $9.28 \mathrm{E}-01$ \\
\hline ENSBTAG00000018770 & $3.42 \mathrm{E}-10$ & $3.69 \mathrm{E}-10$ & HSDL1 & $9.27 \mathrm{E}-01$ \\
\hline ENSBTAG00000032018 & $1.65 \mathrm{E}-10$ & 1.77E-10 & & $9.31 \mathrm{E}-01$ \\
\hline ENSBTAG00000001286 & $1.27 \mathrm{E}-10$ & 1.37E-10 & ELMO3 & $9.26 \mathrm{E}-01$ \\
\hline ENSBTAG00000003894 & $2.42 \mathrm{E}-10$ & $2.60 \mathrm{E}-10$ & NDRG4 & $9.29 \mathrm{E}-01$ \\
\hline ENSBTAG00000002398 & $6.43 \mathrm{E}-10$ & $6.91 \mathrm{E}-10$ & ZNF567 & $9.31 \mathrm{E}-01$ \\
\hline ENSBTAG00000015686 & $3.40 \mathrm{E}-10$ & $3.65 \mathrm{E}-10$ & TERF2IP & $9.31 \mathrm{E}-01$ \\
\hline ENSBTAG00000017009 & $2.72 \mathrm{E}-10$ & $2.92 \mathrm{E}-10$ & & $9.33 \mathrm{E}-01$ \\
\hline ENSBTAG00000009305 & $3.39 \mathrm{E}-10$ & $3.64 \mathrm{E}-10$ & ANKRD27 & $9.32 \mathrm{E}-01$ \\
\hline ENSBTAG00000038322 & $5.64 \mathrm{E}-10$ & $6.01 \mathrm{E}-10$ & & $9.39 \mathrm{E}-01$ \\
\hline ENSBTAG00000004806 & 3.37E-10 & $3.58 \mathrm{E}-10$ & PHKB & $9.40 \mathrm{E}-01$ \\
\hline ENSBTAG00000001595 & $7.96 \mathrm{E}-10$ & $8.47 \mathrm{E}-10$ & & $9.39 \mathrm{E}-01$ \\
\hline ENSBTAG00000001400 & $1.49 \mathrm{E}-10$ & $1.59 \mathrm{E}-10$ & AKT2 & $9.40 \mathrm{E}-01$ \\
\hline ENSBTAG00000007544 & $1.06 \mathrm{E}-10$ & $1.12 \mathrm{E}-10$ & MARVELD3 & 9.47E-01 \\
\hline ENSBTAG00000012064 & $7.02 \mathrm{E}-10$ & $7.45 \mathrm{E}-10$ & ZNF473 & $9.42 \mathrm{E}-01$ \\
\hline ENSBTAG00000048021 & $8.02 \mathrm{E}-10$ & $8.50 \mathrm{E}-10$ & & $9.44 \mathrm{E}-01$ \\
\hline ENSBTAG00000009177 & $1.75 \mathrm{E}-10$ & $1.85 \mathrm{E}-10$ & PLEKHA4 & $9.45 \mathrm{E}-01$ \\
\hline ENSBTAG00000003895 & $2.94 \mathrm{E}-10$ & $3.11 \mathrm{E}-10$ & CYBA & $9.47 \mathrm{E}-01$ \\
\hline ENSBTAG00000023453 & $3.78 \mathrm{E}-10$ & $3.99 \mathrm{E}-10$ & C19orf54 & $9.48 \mathrm{E}-01$ \\
\hline ENSBTAG00000018425 & $3.19 \mathrm{E}-10$ & $3.37 \mathrm{E}-10$ & PSMD7 & $9.46 \mathrm{E}-01$ \\
\hline ENSBTAG00000003353 & $3.06 \mathrm{E}-10$ & $3.23 \mathrm{E}-10$ & SLC6A2 & $9.49 \mathrm{E}-01$ \\
\hline ENSBTAG00000026263 & $3.97 \mathrm{E}-10$ & 4.19E-10 & ZNF45 & $9.48 \mathrm{E}-01$ \\
\hline ENSBTAG00000045803 & $1.08 \mathrm{E}-09$ & $1.14 \mathrm{E}-09$ & & $9.51 \mathrm{E}-01$ \\
\hline ENSBTAG00000047473 & $9.03 \mathrm{E}-10$ & $9.49 \mathrm{E}-10$ & & $9.51 \mathrm{E}-01$ \\
\hline ENSBTAG00000000639 & $1.78 \mathrm{E}-10$ & $1.86 \mathrm{E}-10$ & APRT & $9.55 \mathrm{E}-01$ \\
\hline ENSBTAG00000010483 & $2.77 \mathrm{E}-10$ & $2.91 \mathrm{E}-10$ & DLL3 & $9.52 \mathrm{E}-01$ \\
\hline ENSBTAG00000007942 & $2.97 \mathrm{E}-10$ & $3.12 \mathrm{E}-10$ & & $9.53 \mathrm{E}-01$ \\
\hline ENSBTAG00000005685 & $8.61 \mathrm{E}-11$ & $9.04 \mathrm{E}-11$ & HSD11B2 & $9.53 \mathrm{E}-01$ \\
\hline ENSBTAG00000044192 & 4.35E-10 & $4.56 \mathrm{E}-10$ & MAF & $9.53 \mathrm{E}-01$ \\
\hline ENSBTAG00000012002 & $2.05 \mathrm{E}-10$ & $2.15 \mathrm{E}-10$ & BCAR1 & $9.53 \mathrm{E}-01$ \\
\hline ENSBTAG00000018507 & $2.19 \mathrm{E}-10$ & $2.30 \mathrm{E}-10$ & & $9.51 \mathrm{E}-01$ \\
\hline ENSBTAG00000015984 & $1.08 \mathrm{E}-10$ & $1.13 \mathrm{E}-10$ & CADM4 & $9.52 \mathrm{E}-01$ \\
\hline ENSBTAG00000045985 & $6.63 \mathrm{E}-10$ & $6.92 \mathrm{E}-10$ & & $9.58 \mathrm{E}-01$ \\
\hline ENSBTAG00000004138 & $2.79 \mathrm{E}-10$ & $2.91 \mathrm{E}-10$ & GLTSCR1 & $9.58 \mathrm{E}-01$ \\
\hline ENSBTAG00000003272 & $2.67 \mathrm{E}-10$ & $2.78 \mathrm{E}-10$ & & $9.59 \mathrm{E}-01$ \\
\hline ENSBTAG00000013247 & $6.78 \mathrm{E}-10$ & 7.07E-10 & NLRP5 & $9.59 \mathrm{E}-01$ \\
\hline ENSBTAG00000008720 & $3.64 \mathrm{E}-10$ & $3.79 \mathrm{E}-10$ & $\mathrm{C} 18 \mathrm{H} 16$ orf78 & $9.62 \mathrm{E}-01$ \\
\hline ENSBTAG00000016079 & $2.31 \mathrm{E}-10$ & $2.40 \mathrm{E}-10$ & COX4I1 & $9.62 \mathrm{E}-01$ \\
\hline ENSBTAG00000004017 & $1.36 \mathrm{E}-10$ & $1.41 \mathrm{E}-10$ & PLEKHG2 & $9.64 \mathrm{E}-01$ \\
\hline ENSBTAG00000006965 & $8.51 \mathrm{E}-10$ & $8.84 \mathrm{E}-10$ & NLRP8 & $9.62 \mathrm{E}-01$ \\
\hline ENSBTAG00000031641 & $5.49 \mathrm{E}-10$ & $5.70 \mathrm{E}-10$ & PSMD8 & $9.64 \mathrm{E}-01$ \\
\hline ENSBTAG00000013353 & $4.02 \mathrm{E}-10$ & 4.17E-10 & ZNF274 & $9.64 \mathrm{E}-01$ \\
\hline ENSBTAG00000005058 & $2.02 \mathrm{E}-10$ & $2.10 \mathrm{E}-10$ & C16orf70 & $9.60 \mathrm{E}-01$ \\
\hline ENSBTAG00000010858 & $4.22 \mathrm{E}-10$ & 4.36E-10 & ABCC11 & $9.68 \mathrm{E}-01$ \\
\hline ENSBTAG00000037581 & $1.98 \mathrm{E}-10$ & $2.04 \mathrm{E}-10$ & MZF1 & $9.71 \mathrm{E}-01$ \\
\hline ENSBTAG00000000462 & $3.39 \mathrm{E}-10$ & 3.50E-10 & CCDC102A & 9.67E-01 \\
\hline ENSBTAG00000009889 & $1.44 \mathrm{E}-10$ & $1.49 \mathrm{E}-10$ & & $9.69 \mathrm{E}-01$ \\
\hline ENSBTAG00000016313 & $3.95 \mathrm{E}-10$ & 4.07E-10 & $\mathrm{ECH} 1$ & $9.71 \mathrm{E}-01$ \\
\hline ENSBTAG00000039081 & $6.09 \mathrm{E}-10$ & $6.27 \mathrm{E}-10$ & & $9.72 \mathrm{E}-01$ \\
\hline ENSBTAG00000016103 & $3.06 \mathrm{E}-10$ & $3.14 \mathrm{E}-10$ & CBFB & $9.73 \mathrm{E}-01$ \\
\hline ENSBTAG00000018834 & $1.28 \mathrm{E}-10$ & $1.32 \mathrm{E}-10$ & PPP1R37 & $9.73 \mathrm{E}-01$ \\
\hline
\end{tabular}




\begin{tabular}{|c|c|c|c|c|}
\hline ENSBTAG00000016526 & $3.81 \mathrm{E}-10$ & $3.91 \mathrm{E}-10$ & PIH1D1 & $9.75 \mathrm{E}-01$ \\
\hline ENSBTAG00000016997 & $6.45 \mathrm{E}-10$ & $6.60 \mathrm{E}-10$ & & $9.77 \mathrm{E}-01$ \\
\hline ENSBTAG00000015915 & $7.62 \mathrm{E}-10$ & $7.81 \mathrm{E}-10$ & SBSN & $9.76 \mathrm{E}-01$ \\
\hline ENSBTAG00000013125 & $4.95 \mathrm{E}-10$ & $5.06 \mathrm{E}-10$ & PLAUR & $9.78 \mathrm{E}-01$ \\
\hline ENSBTAG00000010123 & $4.89 \mathrm{E}-10$ & $5.01 \mathrm{E}-10$ & APOE & 9.77E-01 \\
\hline ENSBTAG00000046961 & $1.66 \mathrm{E}-09$ & $1.69 \mathrm{E}-09$ & & $9.79 \mathrm{E}-01$ \\
\hline ENSBTAG00000012919 & $3.88 \mathrm{E}-10$ & $3.96 \mathrm{E}-10$ & MMP15 & $9.79 \mathrm{E}-01$ \\
\hline ENSBTAG00000046749 & 4.17E-10 & 4.25E-10 & & $9.81 \mathrm{E}-01$ \\
\hline ENSBTAG00000010878 & $3.29 \mathrm{E}-10$ & $3.33 \mathrm{E}-10$ & HYDIN & 9.87E-01 \\
\hline ENSBTAG00000038951 & 7.44E-10 & $7.54 \mathrm{E}-10$ & ZNF461 & 9.87E-01 \\
\hline ENSBTAG00000012744 & $1.29 \mathrm{E}-10$ & $1.30 \mathrm{E}-10$ & UBE2M & $9.90 \mathrm{E}-01$ \\
\hline ENSBTAG00000003514 & $1.73 \mathrm{E}-10$ & $1.74 \mathrm{E}-10$ & HSF4 & $9.91 \mathrm{E}-01$ \\
\hline ENSBTAG00000001666 & $1.48 \mathrm{E}-10$ & $1.49 \mathrm{E}-10$ & NIP7 & $9.92 \mathrm{E}-01$ \\
\hline ENSBTAG00000001665 & $1.19 \mathrm{E}-10$ & $1.20 \mathrm{E}-10$ & & $9.90 \mathrm{E}-01$ \\
\hline ENSBTAG00000008614 & $3.96 \mathrm{E}-10$ & 4.00E-10 & CCDC155 & $9.90 \mathrm{E}-01$ \\
\hline ENSBTAG00000007336 & 3.17E-10 & $3.19 \mathrm{E}-10$ & HCST & $9.94 \mathrm{E}-01$ \\
\hline ENSBTAG00000018013 & $1.89 \mathrm{E}-10$ & $1.90 \mathrm{E}-10$ & EMP3 & $9.94 \mathrm{E}-01$ \\
\hline ENSBTAG00000006779 & $1.57 \mathrm{E}-10$ & $1.58 \mathrm{E}-10$ & LDHD & $9.93 \mathrm{E}-01$ \\
\hline ENSBTAG00000005296 & $2.33 \mathrm{E}-10$ & $2.34 \mathrm{E}-10$ & & $9.94 \mathrm{E}-01$ \\
\hline ENSBTAG00000003234 & $3.45 \mathrm{E}-10$ & $3.47 \mathrm{E}-10$ & HSD17B2 & $9.95 \mathrm{E}-01$ \\
\hline ENSBTAG00000014585 & $1.86 \mathrm{E}-10$ & $1.87 \mathrm{E}-10$ & PTGIR & $9.92 \mathrm{E}-01$ \\
\hline ENSBTAG00000018906 & $2.28 \mathrm{E}-10$ & $2.30 \mathrm{E}-10$ & $\mathrm{C} 18 \mathrm{H} 16$ orf 86 & $9.92 \mathrm{E}-01$ \\
\hline ENSBTAG00000007488 & 3.99E-10 & 4.00E-10 & ZFP90 & 9.97E-01 \\
\hline ENSBTAG00000012985 & 4.95E-10 & 4.97E-10 & FUK & 9.97E-01 \\
\hline ENSBTAG00000010151 & $3.77 \mathrm{E}-10$ & $3.78 \mathrm{E}-10$ & CA5A & $9.99 \mathrm{E}-01$ \\
\hline ENSBTAG00000005819 & $5.40 \mathrm{E}-10$ & $5.42 \mathrm{E}-10$ & CCDC61 & 9.97E-01 \\
\hline ENSBTAG00000007262 & $4.08 \mathrm{E}-10$ & 4.09E-10 & ZNF536 & 9.97E-01 \\
\hline ENSBTAG00000009304 & $4.76 \mathrm{E}-10$ & $4.76 \mathrm{E}-10$ & PDCD5 & $1.00 E+00$ \\
\hline ENSBTAG00000018037 & $1.51 \mathrm{E}-10$ & $1.51 \mathrm{E}-10$ & PSKH1 & $1.00 E+00$ \\
\hline ENSBTAG00000003456 & $7.56 \mathrm{E}-10$ & $7.54 \mathrm{E}-10$ & ZNF606 & $1.00 E+00$ \\
\hline ENSBTAG00000023780 & $7.52 \mathrm{E}-10$ & $7.46 \mathrm{E}-10$ & SYCE1L & $1.01 \mathrm{E}+00$ \\
\hline ENSBTAG00000040000 & $6.80 \mathrm{E}-10$ & $6.74 \mathrm{E}-10$ & & $1.01 E+00$ \\
\hline ENSBTAG00000037537 & 8.77E-10 & $8.70 \mathrm{E}-10$ & & $1.01 \mathrm{E}+00$ \\
\hline ENSBTAG00000031772 & 1.17E-09 & $1.16 \mathrm{E}-09$ & $\mathrm{PRODH} 2$ & $1.01 E+00$ \\
\hline ENSBTAG00000039932 & $5.40 \mathrm{E}-10$ & $5.34 \mathrm{E}-10$ & & $1.01 E+00$ \\
\hline ENSBTAG00000032092 & $2.43 \mathrm{E}-10$ & $2.40 \mathrm{E}-10$ & $\mathrm{CDH} 11$ & $1.01 E+00$ \\
\hline ENSBTAG00000019678 & 4.57E-10 & $4.50 \mathrm{E}-10$ & ALKBH6 & $1.02 E+00$ \\
\hline ENSBTAG00000019520 & $1.56 \mathrm{E}-10$ & $1.53 \mathrm{E}-10$ & $\mathrm{ZC} 3 \mathrm{H} 18$ & $1.02 \mathrm{E}+00$ \\
\hline ENSBTAG00000002233 & $2.17 \mathrm{E}-10$ & $2.13 \mathrm{E}-10$ & CPNE2 & $1.02 \mathrm{E}+00$ \\
\hline ENSBTAG00000019674 & $1.53 \mathrm{E}-10$ & $1.51 \mathrm{E}-10$ & CLIP3 & $1.02 \mathrm{E}+00$ \\
\hline ENSBTAG00000037375 & $6.22 \mathrm{E}-10$ & $6.10 \mathrm{E}-10$ & & $1.02 \mathrm{E}+00$ \\
\hline ENSBTAG00000013340 & $1.77 \mathrm{E}-10$ & $1.73 \mathrm{E}-10$ & BAX & $1.02 \mathrm{E}+00$ \\
\hline ENSBTAG00000020889 & $8.16 \mathrm{E}-11$ & 7.99E-11 & CA7 & $1.02 \mathrm{E}+00$ \\
\hline ENSBTAG00000005761 & $9.71 \mathrm{E}-11$ & $9.51 \mathrm{E}-11$ & DEDD2 & $1.02 \mathrm{E}+00$ \\
\hline ENSBTAG00000020872 & $2.16 \mathrm{E}-10$ & $2.12 \mathrm{E}-10$ & C5AR1 & $1.02 \mathrm{E}+00$ \\
\hline ENSBTAG00000046851 & $9.31 \mathrm{E}-10$ & $9.11 \mathrm{E}-10$ & & $1.02 \mathrm{E}+00$ \\
\hline ENSBTAG00000019938 & $2.63 \mathrm{E}-10$ & $2.57 \mathrm{E}-10$ & N4BP1 & $1.02 \mathrm{E}+00$ \\
\hline ENSBTAG00000018225 & 3.37E-10 & $3.28 \mathrm{E}-10$ & SIPA1L3 & $1.03 E+00$ \\
\hline ENSBTAG00000007714 & $1.08 \mathrm{E}-09$ & $1.05 E-09$ & ZNF235 & $1.03 E+00$ \\
\hline ENSBTAG00000008385 & $4.01 \mathrm{E}-10$ & $3.90 \mathrm{E}-10$ & KLHL36 & $1.03 E+00$ \\
\hline ENSBTAG00000008902 & $5.01 \mathrm{E}-10$ & $4.86 \mathrm{E}-10$ & ZNF850 & $1.03 E+00$ \\
\hline ENSBTAG00000020942 & $3.84 \mathrm{E}-10$ & $3.72 \mathrm{E}-10$ & RNF166 & $1.03 E+00$ \\
\hline ENSBTAG00000013157 & $2.50 \mathrm{E}-10$ & $2.41 \mathrm{E}-10$ & NOVA2 & $1.04 \mathrm{E}+00$ \\
\hline ENSBTAG00000014923 & 4.47E-10 & $4.31 \mathrm{E}-10$ & BBS2 & $1.04 E+00$ \\
\hline ENSBTAG00000006550 & $2.44 \mathrm{E}-10$ & $2.36 \mathrm{E}-10$ & GABARAPL2 & $1.04 E+00$ \\
\hline ENSBTAG00000023452 & $1.84 \mathrm{E}-10$ & 1.77E-10 & B9D2 & $1.04 E+00$ \\
\hline ENSBTAG00000017824 & $2.88 \mathrm{E}-10$ & $2.77 \mathrm{E}-10$ & IRF8 & $1.04 \mathrm{E}+00$ \\
\hline ENSBTAG00000038715 & $4.18 \mathrm{E}-10$ & $4.02 \mathrm{E}-10$ & & $1.04 E+00$ \\
\hline ENSBTAG00000005527 & $1.69 \mathrm{E}-10$ & $1.62 \mathrm{E}-10$ & GIPR & $1.04 E+00$ \\
\hline ENSBTAG00000021176 & $1.38 \mathrm{E}-10$ & $1.32 \mathrm{E}-10$ & CRISPLD2 & $1.05 E+00$ \\
\hline ENSBTAG00000001010 & $5.07 \mathrm{E}-10$ & $4.83 \mathrm{E}-10$ & ADAMTS18 & $1.05 E+00$ \\
\hline ENSBTAG00000002571 & $1.48 \mathrm{E}-10$ & $1.42 \mathrm{E}-10$ & & $1.04 E+00$ \\
\hline ENSBTAG00000011815 & $1.14 \mathrm{E}-09$ & $1.09 \mathrm{E}-09$ & SMG9 & $1.04 E+00$ \\
\hline ENSBTAG00000009171 & $1.84 \mathrm{E}-09$ & $1.75 \mathrm{E}-09$ & & $1.05 E+00$ \\
\hline ENSBTAG00000001851 & $3.41 \mathrm{E}-10$ & $3.24 \mathrm{E}-10$ & & $1.05 E+00$ \\
\hline ENSBTAG00000006396 & $1.90 \mathrm{E}-10$ & $1.80 \mathrm{E}-10$ & GPI & $1.05 E+00$ \\
\hline ENSBTAG00000012508 & $6.28 \mathrm{E}-10$ & $5.95 \mathrm{E}-10$ & OSGIN1 & $1.06 \mathrm{E}+00$ \\
\hline ENSBTAG00000021093 & $1.33 \mathrm{E}-10$ & $1.26 \mathrm{E}-10$ & RPS16 & $1.05 E+00$ \\
\hline ENSBTAG00000006422 & $1.61 \mathrm{E}-10$ & $1.52 \mathrm{E}-10$ & TRIM28 & $1.06 \mathrm{E}+00$ \\
\hline ENSBTAG00000009785 & $1.08 \mathrm{E}-10$ & $1.02 \mathrm{E}-10$ & SAMD4B & $1.06 \mathrm{E}+00$ \\
\hline
\end{tabular}




\begin{tabular}{|c|c|c|c|c|}
\hline ENSBTAG00000034185 & $1.49 \mathrm{E}-10$ & $1.40 \mathrm{E}-10$ & & $1.06 \mathrm{E}+00$ \\
\hline ENSBTAG00000006757 & $5.23 \mathrm{E}-11$ & 4.94E-11 & CA11 & $1.06 \mathrm{E}+00$ \\
\hline ENSBTAG00000001838 & $1.70 \mathrm{E}-10$ & $1.61 \mathrm{E}-10$ & LRRC36 & $1.06 \mathrm{E}+00$ \\
\hline ENSBTAG00000038947 & 4.50E-10 & 4.25E-10 & ZSCAN4 & $1.06 \mathrm{E}+00$ \\
\hline ENSBTAG00000017678 & 5.77E-11 & $5.45 \mathrm{E}-11$ & SPRED3 & $1.06 \mathrm{E}+00$ \\
\hline ENSBTAG00000019822 & $9.16 \mathrm{E}-11$ & $8.65 \mathrm{E}-11$ & TPPP3 & $1.06 \mathrm{E}+00$ \\
\hline ENSBTAG00000006998 & $9.59 \mathrm{E}-11$ & $9.06 \mathrm{E}-11$ & RASGRP4 & $1.06 \mathrm{E}+00$ \\
\hline ENSBTAG00000003267 & $1.80 \mathrm{E}-10$ & $1.70 \mathrm{E}-10$ & ZNF132 & $1.06 \mathrm{E}+00$ \\
\hline ENSBTAG00000007559 & $2.63 \mathrm{E}-10$ & $2.48 \mathrm{E}-10$ & URI1 & $1.06 \mathrm{E}+00$ \\
\hline ENSBTAG00000048177 & $1.42 \mathrm{E}-10$ & $1.34 \mathrm{E}-10$ & & $1.06 \mathrm{E}+00$ \\
\hline ENSBTAG00000014553 & $1.34 \mathrm{E}-10$ & $1.27 \mathrm{E}-10$ & ATP6V0D1 & $1.06 \mathrm{E}+00$ \\
\hline ENSBTAG00000023349 & $1.08 \mathrm{E}-09$ & $1.02 \mathrm{E}-09$ & & $1.06 \mathrm{E}+00$ \\
\hline ENSBTAG00000018421 & 2.77E-10 & $2.60 \mathrm{E}-10$ & WWP2 & $1.07 E+00$ \\
\hline ENSBTAG00000030403 & 4.42E-10 & 4.14E-10 & ZNF579 & $1.07 E+00$ \\
\hline ENSBTAG00000008932 & $4.83 \mathrm{E}-10$ & $4.52 \mathrm{E}-10$ & SHISA7 & $1.07 E+00$ \\
\hline ENSBTAG00000017397 & 4.29E-10 & $3.98 \mathrm{E}-10$ & ZNF423 & $1.08 \mathrm{E}+00$ \\
\hline ENSBTAG00000047616 & $1.08 \mathrm{E}-09$ & $1.00 \mathrm{E}-09$ & ZNF114 & $1.08 \mathrm{E}+00$ \\
\hline ENSBTAG00000020415 & $4.14 \mathrm{E}-10$ & $3.84 \mathrm{E}-10$ & KLK4 & $1.08 \mathrm{E}+00$ \\
\hline ENSBTAG00000034373 & $5.66 \mathrm{E}-10$ & $5.24 \mathrm{E}-10$ & $\mathrm{CDH} 13$ & $1.08 \mathrm{E}+00$ \\
\hline ENSBTAG00000037735 & $2.59 \mathrm{E}-10$ & $2.40 \mathrm{E}-10$ & C5AR2 & $1.08 \mathrm{E}+00$ \\
\hline ENSBTAG00000006353 & $3.33 \mathrm{E}-10$ & 3.07E-10 & TXNL4B & $1.08 \mathrm{E}+00$ \\
\hline ENSBTAG00000012785 & $2.97 \mathrm{E}-10$ & $2.73 \mathrm{E}-10$ & TEPP & $1.09 E+00$ \\
\hline ENSBTAG00000007181 & $6.14 \mathrm{E}-10$ & $5.65 \mathrm{E}-10$ & KIFC3 & $1.09 E+00$ \\
\hline ENSBTAG00000032396 & $1.66 \mathrm{E}-10$ & $1.53 \mathrm{E}-10$ & KCTD19 & $1.09 \mathrm{E}+00$ \\
\hline ENSBTAG00000033642 & $6.27 \mathrm{E}-10$ & $5.76 \mathrm{E}-10$ & & $1.09 \mathrm{E}+00$ \\
\hline ENSBTAG00000004864 & $4.39 \mathrm{E}-10$ & 4.03E-10 & CEP89 & $1.09 \mathrm{E}+00$ \\
\hline ENSBTAG00000020714 & $9.21 \mathrm{E}-10$ & $8.45 \mathrm{E}-10$ & DDX19B & $1.09 \mathrm{E}+00$ \\
\hline ENSBTAG00000009895 & $1.89 \mathrm{E}-10$ & $1.73 \mathrm{E}-10$ & ZNF599 & $1.09 E+00$ \\
\hline ENSBTAG00000023434 & $2.02 \mathrm{E}-10$ & $1.85 \mathrm{E}-10$ & & $1.09 E+00$ \\
\hline ENSBTAG00000046087 & $3.30 \mathrm{E}-10$ & $3.02 \mathrm{E}-10$ & & $1.09 E+00$ \\
\hline ENSBTAG00000015950 & $6.17 \mathrm{E}-10$ & $5.66 \mathrm{E}-10$ & ISOC2 & $1.09 E+00$ \\
\hline ENSBTAG00000014123 & $2.01 \mathrm{E}-10$ & $1.84 \mathrm{E}-10$ & ZNF507 & $1.09 \mathrm{E}+00$ \\
\hline ENSBTAG00000046323 & $1.39 \mathrm{E}-09$ & 1.27E-09 & & $1.09 E+00$ \\
\hline ENSBTAG00000002303 & $7.82 \mathrm{E}-10$ & 7.13E-10 & GLG1 & $1.10 E+00$ \\
\hline ENSBTAG00000013524 & $5.14 \mathrm{E}-10$ & 4.69E-10 & CMIP & $1.10 \mathrm{E}+00$ \\
\hline ENSBTAG00000010207 & $3.65 \mathrm{E}-10$ & 3.33E-10 & NUDT21 & $1.10 \mathrm{E}+00$ \\
\hline ENSBTAG00000003462 & 8.54E-10 & 7.77E-10 & & $1.10 \mathrm{E}+00$ \\
\hline ENSBTAG00000021192 & $1.71 \mathrm{E}-10$ & $1.55 \mathrm{E}-10$ & GLTSCR2 & $1.10 E+00$ \\
\hline ENSBTAG00000019087 & $2.49 \mathrm{E}-10$ & $2.26 \mathrm{E}-10$ & & $1.10 \mathrm{E}+00$ \\
\hline ENSBTAG00000038926 & $5.29 \mathrm{E}-10$ & $4.79 \mathrm{E}-10$ & & $1.10 \mathrm{E}+00$ \\
\hline ENSBTAG00000019465 & $3.32 \mathrm{E}-10$ & $3.00 \mathrm{E}-10$ & ITPKC & $1.11 \mathrm{E}+00$ \\
\hline ENSBTAG00000007172 & $1.11 \mathrm{E}-10$ & $1.00 \mathrm{E}-10$ & GOT2 & $1.11 E+00$ \\
\hline ENSBTAG00000002980 & $8.95 \mathrm{E}-10$ & 8.08E-10 & GPT2 & $1.11 E+00$ \\
\hline ENSBTAG00000048005 & $3.04 \mathrm{E}-10$ & $2.75 \mathrm{E}-10$ & & $1.11 E+00$ \\
\hline ENSBTAG00000001287 & $8.70 \mathrm{E}-11$ & $7.83 \mathrm{E}-11$ & & $1.11 E+00$ \\
\hline ENSBTAG00000008573 & $8.29 \mathrm{E}-11$ & $7.46 \mathrm{E}-11$ & ZFP36 & $1.11 \mathrm{E}+00$ \\
\hline ENSBTAG00000030454 & $4.18 \mathrm{E}-10$ & $3.76 \mathrm{E}-10$ & & $1.11 E+00$ \\
\hline ENSBTAG00000001854 & $8.98 \mathrm{E}-11$ & $8.08 \mathrm{E}-11$ & CIAPIN1 & $1.11 E+00$ \\
\hline ENSBTAG00000006754 & $7.91 \mathrm{E}-11$ & $7.12 \mathrm{E}-11$ & DBP & $1.11 E+00$ \\
\hline ENSBTAG00000039190 & $9.41 \mathrm{E}-11$ & 8.47E-11 & SLC9A5 & $1.11 \mathrm{E}+00$ \\
\hline ENSBTAG00000047602 & $1.33 \mathrm{E}-09$ & 1.19E-09 & & $1.12 \mathrm{E}+00$ \\
\hline ENSBTAG00000018904 & $1.67 \mathrm{E}-10$ & $1.50 \mathrm{E}-10$ & ENKD1 & $1.11 E+00$ \\
\hline ENSBTAG00000002214 & $2.66 \mathrm{E}-10$ & $2.39 \mathrm{E}-10$ & TAT & $1.11 E+00$ \\
\hline ENSBTAG00000003519 & $3.19 \mathrm{E}-10$ & 2.87E-10 & NOL3 & $1.11 \mathrm{E}+00$ \\
\hline ENSBTAG00000014597 & $2.87 \mathrm{E}-10$ & $2.57 \mathrm{E}-10$ & C19orf68 & $1.12 \mathrm{E}+00$ \\
\hline ENSBTAG00000018652 & $8.62 \mathrm{E}-10$ & 7.69E-10 & CXCL17 & $1.12 E+00$ \\
\hline ENSBTAG00000030578 & $1.84 \mathrm{E}-10$ & $1.64 \mathrm{E}-10$ & PTOV1 & $1.12 \mathrm{E}+00$ \\
\hline ENSBTAG00000001852 & $2.60 \mathrm{E}-10$ & $2.31 E-10$ & & $1.13 E+00$ \\
\hline ENSBTAG00000009211 & $3.59 \mathrm{E}-10$ & $3.19 \mathrm{E}-10$ & UBE2S & $1.13 E+00$ \\
\hline ENSBTAG00000046451 & $3.69 \mathrm{E}-10$ & $3.28 \mathrm{E}-10$ & & $1.13 E+00$ \\
\hline ENSBTAG00000040086 & $1.64 \mathrm{E}-10$ & $1.44 \mathrm{E}-10$ & SLC38A8 & $1.14 \mathrm{E}+00$ \\
\hline ENSBTAG00000010046 & $9.41 \mathrm{E}-10$ & $8.32 \mathrm{E}-10$ & & $1.13 E+00$ \\
\hline ENSBTAG00000003570 & $9.10 \mathrm{E}-10$ & 8.04E-10 & DDX19A & $1.13 E+00$ \\
\hline ENSBTAG00000023632 & 2.07E-10 & $1.83 \mathrm{E}-10$ & & $1.13 E+00$ \\
\hline ENSBTAG00000011215 & $2.22 \mathrm{E}-10$ & $1.96 \mathrm{E}-10$ & ACTN4 & $1.13 \mathrm{E}+00$ \\
\hline ENSBTAG00000040356 & $1.63 \mathrm{E}-10$ & $1.44 \mathrm{E}-10$ & SPIRE2 & $1.13 E+00$ \\
\hline ENSBTAG00000018903 & $1.40 \mathrm{E}-10$ & $1.23 \mathrm{E}-10$ & $A C D$ & $1.13 E+00$ \\
\hline ENSBTAG00000034283 & $5.66 \mathrm{E}-10$ & 4.97E-10 & ADAD2 & $1.14 \mathrm{E}+00$ \\
\hline ENSBTAG00000046786 & $7.41 \mathrm{E}-10$ & $6.50 \mathrm{E}-10$ & UQCRFS1 & $1.14 \mathrm{E}+00$ \\
\hline ENSBTAG00000047236 & 1.13E-09 & $9.90 \mathrm{E}-10$ & & $1.15 \mathrm{E}+00$ \\
\hline
\end{tabular}




\begin{tabular}{|c|c|c|c|c|}
\hline ENSBTAG000000021575 & $2.15 \mathrm{E}-10$ & $1.89 \mathrm{E}-10$ & BRD7 & $1.14 \mathrm{E}+00$ \\
\hline ENSBTAG00000038797 & 1.09E-09 & $9.51 \mathrm{E}-10$ & & $1.14 \mathrm{E}+00$ \\
\hline ENSBTAG000000044132 & $1.89 \mathrm{E}-10$ & $1.65 \mathrm{E}-10$ & NTN5 & $1.14 \mathrm{E}+00$ \\
\hline ENSBTAG000000015669 & $2.66 \mathrm{E}-10$ & $2.32 \mathrm{E}-10$ & ADAT1 & $1.15 E+00$ \\
\hline ENSBTAG000000012642 & $1.79 \mathrm{E}-10$ & $1.56 \mathrm{E}-10$ & TRADD & $1.15 E+00$ \\
\hline ENSBTAG000000014995 & $1.69 \mathrm{E}-10$ & $1.47 \mathrm{E}-10$ & THAP8 & $1.15 E+00$ \\
\hline ENSBTAG000000021191 & $2.11 \mathrm{E}-10$ & $1.83 \mathrm{E}-10$ & EHD2 & $1.15 \mathrm{E}+00$ \\
\hline ENSBTAG000000004377 & $8.01 \mathrm{E}-10$ & $6.95 \mathrm{E}-10$ & PHLDB3 & $1.15 E+00$ \\
\hline ENSBTAG000000015576 & $3.15 \mathrm{E}-10$ & $2.73 \mathrm{E}-10$ & NPAS1 & $1.15 E+00$ \\
\hline ENSBTAG000000014644 & $1.23 \mathrm{E}-10$ & $1.06 \mathrm{E}-10$ & DUS2 & $1.16 \mathrm{E}+00$ \\
\hline ENSBTAG000000007512 & $9.50 \mathrm{E}-11$ & $8.22 \mathrm{E}-11$ & PLA2G15 & $1.16 \mathrm{E}+00$ \\
\hline ENSBTAG000000017112 & $4.40 \mathrm{E}-10$ & $3.81 \mathrm{E}-10$ & CDYL2 & $1.15 E+00$ \\
\hline ENSBTAG00000046291 & $1.31 \mathrm{E}-10$ & $1.14 \mathrm{E}-10$ & FAM83E & $1.15 \mathrm{E}+00$ \\
\hline ENSBTAG000000026080 & 1.19E-09 & 1.03E-09 & & $1.16 \mathrm{E}+00$ \\
\hline ENSBTAG000000010132 & $1.20 \mathrm{E}-10$ & $1.03 \mathrm{E}-10$ & NAE1 & $1.16 \mathrm{E}+00$ \\
\hline ENSBTAG000000004588 & $6.09 \mathrm{E}-10$ & $5.26 \mathrm{E}-10$ & KCNN4 & $1.16 \mathrm{E}+00$ \\
\hline ENSBTAG000000040063 & $2.11 E-10$ & $1.82 \mathrm{E}-10$ & ZNF585B & $1.16 \mathrm{E}+00$ \\
\hline ENSBTAG000000009714 & $2.68 \mathrm{E}-10$ & $2.31 \mathrm{E}-10$ & ARHGAP33 & $1.16 \mathrm{E}+00$ \\
\hline ENSBTAG000000045581 & $9.30 \mathrm{E}-10$ & 8.01E-10 & & $1.16 \mathrm{E}+00$ \\
\hline ENSBTAG000000012446 & $1.31 \mathrm{E}-10$ & $1.12 \mathrm{E}-10$ & MTHFSD & $1.17 E+00$ \\
\hline ENSBTAG000000006221 & $2.95 \mathrm{E}-10$ & $2.52 \mathrm{E}-10$ & ADGRG3 & $1.17 \mathrm{E}+00$ \\
\hline ENSBTAG00000023419 & $3.03 \mathrm{E}-10$ & $2.58 \mathrm{E}-10$ & & $1.17 \mathrm{E}+00$ \\
\hline ENSBTAG000000006611 & $1.48 \mathrm{E}-10$ & $1.25 \mathrm{E}-10$ & NUP93 & $1.18 \mathrm{E}+00$ \\
\hline ENSBTAG000000014328 & 1.33E-09 & 1.13E-09 & & $1.18 \mathrm{E}+00$ \\
\hline ENSBTAG00000011621 & $1.66 \mathrm{E}-10$ & $1.39 \mathrm{E}-10$ & IZUMO1 & $1.19 \mathrm{E}+00$ \\
\hline ENSBTAG000000005363 & $1.67 \mathrm{E}-10$ & $1.40 \mathrm{E}-10$ & GGN & $1.19 E+00$ \\
\hline ENSBTAG000000010668 & $7.16 \mathrm{E}-11$ & $6.01 E-11$ & GEMIN7 & $1.19 E+00$ \\
\hline ENSBTAG000000038986 & $2.59 \mathrm{E}-10$ & 2.17E-10 & & $1.19 \mathrm{E}+00$ \\
\hline ENSBTAG00000009449 & $1.02 \mathrm{E}-10$ & $8.53 \mathrm{E}-11$ & ATP5SL & $1.19 \mathrm{E}+00$ \\
\hline ENSBTAG000000047367 & $3.22 \mathrm{E}-10$ & $2.70 \mathrm{E}-10$ & CMTR2 & $1.19 E+00$ \\
\hline ENSBTAG000000020835 & $1.45 \mathrm{E}-10$ & $1.22 \mathrm{E}-10$ & KIAA0513 & $1.19 \mathrm{E}+00$ \\
\hline ENSBTAG000000037595 & $1.93 \mathrm{E}-10$ & $1.62 \mathrm{E}-10$ & & $1.19 \mathrm{E}+00$ \\
\hline ENSBTAG000000001663 & 2.67E-10 & $2.24 \mathrm{E}-10$ & PDF & $1.19 \mathrm{E}+00$ \\
\hline ENSBTAG000000010403 & $2.61 E-10$ & $2.19 \mathrm{E}-10$ & FAAP24 & $1.19 E+00$ \\
\hline ENSBTAG00000047937 & $3.14 \mathrm{E}-10$ & $2.64 \mathrm{E}-10$ & & $1.19 \mathrm{E}+00$ \\
\hline ENSBTAG00000040067 & $2.56 \mathrm{E}-10$ & $2.15 \mathrm{E}-10$ & HIPK4 & $1.19 \mathrm{E}+00$ \\
\hline ENSBTAG00000039969 & $2.12 \mathrm{E}-10$ & $1.78 \mathrm{E}-10$ & & $1.19 \mathrm{E}+00$ \\
\hline ENSBTAG00000013338 & $7.61 \mathrm{E}-10$ & $6.38 \mathrm{E}-10$ & SLC7A9 & $1.19 \mathrm{E}+00$ \\
\hline ENSBTAG00000000688 & 1.70E-09 & $1.42 \mathrm{E}-09$ & & $1.20 \mathrm{E}+00$ \\
\hline ENSBTAG00000008777 & 1.13E-09 & $9.46 \mathrm{E}-10$ & & $1.19 E+00$ \\
\hline ENSBTAG00000038141 & $5.71 \mathrm{E}-10$ & 4.74E-10 & & $1.20 \mathrm{E}+00$ \\
\hline ENSBTAG00000012016 & $1.87 \mathrm{E}-10$ & $1.55 \mathrm{E}-10$ & LMTK3 & $1.21 \mathrm{E}+00$ \\
\hline ENSBTAG00000016243 & $1.92 \mathrm{E}-10$ & 1.59E-10 & RBL2 & $1.21 \mathrm{E}+00$ \\
\hline ENSBTAG00000040209 & $2.17 \mathrm{E}-10$ & $1.78 \mathrm{E}-10$ & ZNF112 & $1.22 \mathrm{E}+00$ \\
\hline ENSBTAG00000036215 & $8.56 \mathrm{E}-10$ & $7.03 \mathrm{E}-10$ & & $1.22 \mathrm{E}+00$ \\
\hline ENSBTAG00000012522 & $5.43 \mathrm{E}-10$ & $4.46 \mathrm{E}-10$ & ZNF283 & $1.22 \mathrm{E}+00$ \\
\hline ENSBTAG00000032427 & $1.32 \mathrm{E}-10$ & $1.09 \mathrm{E}-10$ & FHOD1 & $1.21 \mathrm{E}+00$ \\
\hline ENSBTAG00000015866 & $9.43 \mathrm{E}-10$ & 7.73E-10 & ZNF211 & $1.22 \mathrm{E}+00$ \\
\hline ENSBTAG00000016777 & $5.25 \mathrm{E}-10$ & $4.29 \mathrm{E}-10$ & TEX101 & $1.22 \mathrm{E}+00$ \\
\hline ENSBTAG00000006859 & $1.51 \mathrm{E}-09$ & $1.23 \mathrm{E}-09$ & & $1.22 \mathrm{E}+00$ \\
\hline ENSBTAG00000013784 & $2.39 \mathrm{E}-10$ & $1.94 \mathrm{E}-10$ & CSNK2A2 & $1.23 E+00$ \\
\hline ENSBTAG00000012101 & $7.56 \mathrm{E}-10$ & $6.13 \mathrm{E}-10$ & SYMPK & $1.23 E+00$ \\
\hline ENSBTAG00000018912 & $3.70 \mathrm{E}-10$ & 3.00E-10 & ARHGEF1 & $1.23 \mathrm{E}+00$ \\
\hline ENSBTAG00000013106 & $6.71 \mathrm{E}-10$ & $5.44 \mathrm{E}-10$ & C19orf81 & $1.23 \mathrm{E}+00$ \\
\hline ENSBTAG00000003264 & $1.73 \mathrm{E}-10$ & $1.40 \mathrm{E}-10$ & $\mathrm{NR} 1 \mathrm{H} 2$ & $1.23 E+00$ \\
\hline ENSBTAG00000010501 & $1.85 \mathrm{E}-10$ & $1.48 \mathrm{E}-10$ & SELENOV & $1.25 E+00$ \\
\hline ENSBTAG00000013265 & $4.37 \mathrm{E}-10$ & 3.50E-10 & $\mathrm{DHX38}$ & $1.25 \mathrm{E}+00$ \\
\hline ENSBTAG00000007107 & $3.41 \mathrm{E}-10$ & 2.73E-10 & TMEM143 & $1.25 \mathrm{E}+00$ \\
\hline ENSBTAG00000011421 & $5.33 \mathrm{E}-10$ & $4.26 \mathrm{E}-10$ & CD37 & $1.25 \mathrm{E}+00$ \\
\hline ENSBTAG00000016695 & $3.38 \mathrm{E}-10$ & $2.70 \mathrm{E}-10$ & KCNG4 & $1.25 E+00$ \\
\hline ENSBTAG00000013345 & 1.65E-09 & $1.31 \mathrm{E}-09$ & & $1.26 \mathrm{E}+00$ \\
\hline ENSBTAG00000046996 & $6.12 \mathrm{E}-10$ & 4.87E-10 & EPN1 & $1.26 \mathrm{E}+00$ \\
\hline ENSBTAG00000004043 & $5.10 \mathrm{E}-10$ & $4.05 \mathrm{E}-10$ & & $1.26 \mathrm{E}+00$ \\
\hline ENSBTAG00000023464 & $3.46 \mathrm{E}-10$ & $2.75 E-10$ & & $1.26 \mathrm{E}+00$ \\
\hline ENSBTAG00000003457 & $1.64 \mathrm{E}-10$ & $1.29 \mathrm{E}-10$ & ATF5 & $1.27 \mathrm{E}+00$ \\
\hline ENSBTAG00000015388 & $1.74 \mathrm{E}-10$ & $1.37 \mathrm{E}-10$ & RPL18 & $1.27 \mathrm{E}+00$ \\
\hline ENSBTAG00000012501 & $3.68 \mathrm{E}-10$ & $2.90 \mathrm{E}-10$ & FTO & $1.27 \mathrm{E}+00$ \\
\hline ENSBTAG00000013958 & 2.25E-10 & $1.77 \mathrm{E}-10$ & PAK4 & $1.27 \mathrm{E}+00$ \\
\hline ENSBTAG00000045571 & $5.48 \mathrm{E}-10$ & $4.31 \mathrm{E}-10$ & & $1.27 \mathrm{E}+00$ \\
\hline ENSBTAG00000001857 & $1.67 \mathrm{E}-10$ & $1.32 \mathrm{E}-10$ & DOK4 & $1.27 \mathrm{E}+00$ \\
\hline
\end{tabular}




\begin{tabular}{|c|c|c|c|c|}
\hline ENSBTAG00000037710 & $1.01 \mathrm{E}-09$ & $7.92 \mathrm{E}-10$ & & $1.27 \mathrm{E}+00$ \\
\hline ENSBTAG00000018045 & $7.88 \mathrm{E}-11$ & $6.14 \mathrm{E}-11$ & SLC12A4 & $1.28 \mathrm{E}+00$ \\
\hline ENSBTAG00000000472 & $2.59 \mathrm{E}-10$ & $2.01 \mathrm{E}-10$ & ZNF570 & $1.29 E+00$ \\
\hline ENSBTAG00000010170 & $1.39 \mathrm{E}-10$ & $1.08 \mathrm{E}-10$ & MBTPS1 & $1.29 \mathrm{E}+00$ \\
\hline ENSBTAG00000013239 & $3.18 \mathrm{E}-10$ & 2.47E-10 & CAPN12 & $1.29 \mathrm{E}+00$ \\
\hline ENSBTAG00000005413 & $3.55 \mathrm{E}-10$ & $2.75 \mathrm{E}-10$ & NLRC5 & $1.29 E+00$ \\
\hline ENSBTAG00000014296 & $2.38 \mathrm{E}-10$ & $1.84 \mathrm{E}-10$ & NCCRP1 & $1.29 \mathrm{E}+00$ \\
\hline ENSBTAG00000019184 & $2.71 \mathrm{E}-10$ & 2.10E-10 & TRAPPC2L & $1.29 E+00$ \\
\hline ENSBTAG00000012295 & $2.46 \mathrm{E}-10$ & $1.91 \mathrm{E}-10$ & NKD1 & $1.29 E+00$ \\
\hline ENSBTAG00000016075 & $1.25 \mathrm{E}-10$ & $9.65 \mathrm{E}-11$ & EMC8 & $1.30 \mathrm{E}+00$ \\
\hline ENSBTAG00000015894 & $4.75 \mathrm{E}-10$ & $3.66 \mathrm{E}-10$ & WWOX & $1.30 \mathrm{E}+00$ \\
\hline ENSBTAG00000016312 & $5.80 \mathrm{E}-10$ & 4.47E-10 & LGALS4 & $1.30 \mathrm{E}+00$ \\
\hline ENSBTAG00000019547 & $1.45 \mathrm{E}-09$ & 1.11E-09 & & $1.30 \mathrm{E}+00$ \\
\hline ENSBTAG00000012988 & $6.92 \mathrm{E}-10$ & $5.32 \mathrm{E}-10$ & COG4 & $1.30 \mathrm{E}+00$ \\
\hline ENSBTAG00000006780 & $4.02 \mathrm{E}-10$ & $3.09 \mathrm{E}-10$ & NAPSA & $1.30 \mathrm{E}+00$ \\
\hline ENSBTAG00000045731 & $5.12 \mathrm{E}-10$ & $3.94 \mathrm{E}-10$ & & $1.30 \mathrm{E}+00$ \\
\hline ENSBTAG00000000153 & $2.75 \mathrm{E}-10$ & $2.10 \mathrm{E}-10$ & LRFN3 & $1.31 E+00$ \\
\hline ENSBTAG00000008518 & $2.41 \mathrm{E}-10$ & $1.85 \mathrm{E}-10$ & MED25 & $1.30 \mathrm{E}+00$ \\
\hline ENSBTAG00000014514 & 1.74E-10 & $1.33 \mathrm{E}-10$ & & $1.31 E+00$ \\
\hline ENSBTAG00000039215 & 1.47E-09 & $1.12 \mathrm{E}-09$ & & $1.31 \mathrm{E}+00$ \\
\hline ENSBTAG00000008005 & 4.15E-10 & 3.17E-10 & DRC7 & $1.31 E+00$ \\
\hline ENSBTAG00000045598 & $7.49 \mathrm{E}-10$ & $5.72 \mathrm{E}-10$ & & $1.31 E+00$ \\
\hline ENSBTAG00000003089 & $3.72 \mathrm{E}-10$ & $2.83 \mathrm{E}-10$ & RHPN2 & $1.31 E+00$ \\
\hline ENSBTAG00000021742 & $2.12 \mathrm{E}-10$ & $1.61 \mathrm{E}-10$ & ZNF568 & $1.32 \mathrm{E}+00$ \\
\hline ENSBTAG00000006805 & $2.33 \mathrm{E}-10$ & $1.77 \mathrm{E}-10$ & ZNF180 & $1.32 \mathrm{E}+00$ \\
\hline ENSBTAG00000021657 & $8.70 \mathrm{E}-10$ & $6.62 \mathrm{E}-10$ & CACNG6 & $1.31 \mathrm{E}+00$ \\
\hline ENSBTAG00000013791 & $8.16 \mathrm{E}-10$ & $6.19 \mathrm{E}-10$ & & $1.32 \mathrm{E}+00$ \\
\hline ENSBTAG00000003231 & $1.05 \mathrm{E}-09$ & 7.97E-10 & LIM2 & $1.32 \mathrm{E}+00$ \\
\hline ENSBTAG00000017528 & $1.46 \mathrm{E}-10$ & 1.10E-10 & SNAI3 & $1.32 \mathrm{E}+00$ \\
\hline ENSBTAG00000031004 & $1.82 \mathrm{E}-10$ & $1.37 \mathrm{E}-10$ & CNFN & $1.33 E+00$ \\
\hline ENSBTAG00000003521 & $2.14 \mathrm{E}-10$ & $1.62 \mathrm{E}-10$ & KIAA0895L & $1.32 \mathrm{E}+00$ \\
\hline ENSBTAG00000001855 & $1.72 \mathrm{E}-10$ & $1.30 \mathrm{E}-10$ & COQ9 & $1.32 \mathrm{E}+00$ \\
\hline ENSBTAG00000001075 & $2.07 \mathrm{E}-10$ & $1.57 \mathrm{E}-10$ & CALB2 & $1.32 \mathrm{E}+00$ \\
\hline ENSBTAG00000017996 & $5.32 \mathrm{E}-11$ & $4.02 \mathrm{E}-11$ & & $1.32 \mathrm{E}+00$ \\
\hline ENSBTAG00000000009 & $1.38 \mathrm{E}-10$ & $1.04 \mathrm{E}-10$ & FOXF1 & $1.32 \mathrm{E}+00$ \\
\hline ENSBTAG00000007216 & $9.35 \mathrm{E}-11$ & $7.06 \mathrm{E}-11$ & DPF1 & $1.32 \mathrm{E}+00$ \\
\hline ENSBTAG00000023659 & 4.24E-10 & $3.20 \mathrm{E}-10$ & & $1.33 \mathrm{E}+00$ \\
\hline ENSBTAG00000013874 & $2.79 \mathrm{E}-10$ & $2.11 E-10$ & CFAP20 & $1.32 \mathrm{E}+00$ \\
\hline ENSBTAG00000048261 & $4.45 \mathrm{E}-10$ & $3.36 \mathrm{E}-10$ & & $1.32 \mathrm{E}+00$ \\
\hline ENSBTAG00000018598 & $3.26 \mathrm{E}-10$ & $2.46 \mathrm{E}-10$ & HSPB6 & $1.32 \mathrm{E}+00$ \\
\hline ENSBTAG00000038125 & $1.22 \mathrm{E}-09$ & $9.19 \mathrm{E}-10$ & & $1.33 \mathrm{E}+00$ \\
\hline ENSBTAG00000018594 & $4.16 \mathrm{E}-10$ & $3.14 \mathrm{E}-10$ & LIN37 & $1.33 \mathrm{E}+00$ \\
\hline ENSBTAG00000045737 & $1.89 \mathrm{E}-10$ & $1.43 \mathrm{E}-10$ & & $1.32 \mathrm{E}+00$ \\
\hline ENSBTAG00000040550 & $9.11 \mathrm{E}-10$ & $6.86 \mathrm{E}-10$ & & $1.33 \mathrm{E}+00$ \\
\hline ENSBTAG00000040169 & $9.31 \mathrm{E}-10$ & $6.98 \mathrm{E}-10$ & & $1.33 \mathrm{E}+00$ \\
\hline ENSBTAG00000019227 & $1.59 \mathrm{E}-09$ & 1.19E-09 & & $1.34 \mathrm{E}+00$ \\
\hline ENSBTAG00000004568 & $2.27 \mathrm{E}-10$ & $1.70 \mathrm{E}-10$ & GALNS & $1.34 \mathrm{E}+00$ \\
\hline ENSBTAG00000002103 & $4.73 \mathrm{E}-10$ & $3.54 \mathrm{E}-10$ & PLCG2 & $1.34 \mathrm{E}+00$ \\
\hline ENSBTAG00000019883 & $3.13 \mathrm{E}-10$ & $2.33 \mathrm{E}-10$ & IST1 & $1.34 \mathrm{E}+00$ \\
\hline ENSBTAG00000023369 & $2.84 \mathrm{E}-10$ & $2.11 \mathrm{E}-10$ & GRIN2D & $1.34 \mathrm{E}+00$ \\
\hline ENSBTAG00000034090 & $7.38 \mathrm{E}-10$ & $5.48 \mathrm{E}-10$ & & $1.35 E+00$ \\
\hline ENSBTAG00000015917 & 4.07E-10 & 3.03E-10 & GAPDHS & $1.34 \mathrm{E}+00$ \\
\hline ENSBTAG00000023745 & $2.46 \mathrm{E}-10$ & $1.82 \mathrm{E}-10$ & BANP & $1.35 E+00$ \\
\hline ENSBTAG00000039413 & $2.12 \mathrm{E}-09$ & 1.57E-09 & & $1.35 \mathrm{E}+00$ \\
\hline ENSBTAG00000023374 & $3.49 \mathrm{E}-10$ & $2.59 \mathrm{E}-10$ & FUT1 & $1.35 E+00$ \\
\hline ENSBTAG00000023611 & $3.85 \mathrm{E}-10$ & $2.85 \mathrm{E}-10$ & ZBTB32 & $1.35 E+00$ \\
\hline ENSBTAG00000047670 & $4.16 \mathrm{E}-10$ & 3.07E-10 & CHST8 & $1.36 \mathrm{E}+00$ \\
\hline ENSBTAG00000030885 & 5.57E-10 & 4.10E-10 & SSC5D & $1.36 \mathrm{E}+00$ \\
\hline ENSBTAG00000000330 & $3.58 \mathrm{E}-10$ & $2.63 \mathrm{E}-10$ & ALDH16A1 & $1.36 \mathrm{E}+00$ \\
\hline ENSBTAG00000019605 & $3.24 \mathrm{E}-10$ & 2.37E-10 & OPA3 & $1.36 \mathrm{E}+00$ \\
\hline ENSBTAG00000008213 & $2.68 \mathrm{E}-10$ & $1.96 \mathrm{E}-10$ & ATMIN & $1.37 \mathrm{E}+00$ \\
\hline ENSBTAG00000001780 & $1.72 \mathrm{E}-10$ & $1.25 \mathrm{E}-10$ & & $1.38 \mathrm{E}+00$ \\
\hline ENSBTAG00000006731 & $4.91 \mathrm{E}-10$ & $3.59 \mathrm{E}-10$ & SLC7A5 & $1.37 E+00$ \\
\hline ENSBTAG00000015766 & $9.20 \mathrm{E}-11$ & $6.71 \mathrm{E}-11$ & ZFPM1 & $1.37 E+00$ \\
\hline ENSBTAG00000039958 & $1.87 \mathrm{E}-10$ & $1.36 \mathrm{E}-10$ & GYS1 & $1.38 \mathrm{E}+00$ \\
\hline ENSBTAG00000039892 & $4.54 \mathrm{E}-10$ & $3.31 \mathrm{E}-10$ & & $1.37 E+00$ \\
\hline ENSBTAG00000002287 & $1.67 \mathrm{E}-10$ & $1.21 \mathrm{E}-10$ & CHD9 & $1.38 \mathrm{E}+00$ \\
\hline ENSBTAG00000014144 & $2.98 \mathrm{E}-10$ & 2.17E-10 & CCDC113 & $1.37 E+00$ \\
\hline ENSBTAG00000001848 & $3.58 \mathrm{E}-10$ & $2.60 \mathrm{E}-10$ & KATNB1 & $1.38 \mathrm{E}+00$ \\
\hline ENSBTAG00000036224 & $1.68 \mathrm{E}-09$ & $1.22 \mathrm{E}-09$ & & $1.37 E+00$ \\
\hline
\end{tabular}




\begin{tabular}{|c|c|c|c|c|}
\hline ENSBTAG00000006848 & $2.54 \mathrm{E}-10$ & $1.84 \mathrm{E}-10$ & DHX34 & $1.38 \mathrm{E}+00$ \\
\hline ENSBTAG00000003871 & $1.59 \mathrm{E}-10$ & $1.15 \mathrm{E}-10$ & CYP2B6 & $1.38 \mathrm{E}+00$ \\
\hline ENSBTAG00000030470 & $7.58 \mathrm{E}-10$ & $5.48 \mathrm{E}-10$ & & $1.38 \mathrm{E}+00$ \\
\hline ENSBTAG00000013926 & $1.86 \mathrm{E}-10$ & $1.34 \mathrm{E}-10$ & FCGRT & $1.39 E+00$ \\
\hline ENSBTAG00000038534 & $1.35 \mathrm{E}-10$ & $9.70 \mathrm{E}-11$ & ZNF446 & $1.39 E+00$ \\
\hline ENSBTAG00000038022 & $4.23 \mathrm{E}-10$ & 3.04E-10 & & $1.39 E+00$ \\
\hline ENSBTAG00000020556 & $4.84 \mathrm{E}-10$ & $3.48 \mathrm{E}-10$ & APOC4 & $1.39 E+00$ \\
\hline ENSBTAG00000015579 & $2.35 \mathrm{E}-10$ & $1.69 \mathrm{E}-10$ & TMEM160 & $1.39 E+00$ \\
\hline ENSBTAG00000011262 & $1.10 \mathrm{E}-10$ & $7.89 \mathrm{E}-11$ & ZNF548 & $1.39 E+00$ \\
\hline ENSBTAG00000013105 & $9.17 \mathrm{E}-10$ & $6.55 \mathrm{E}-10$ & SYT3 & $1.40 E+00$ \\
\hline ENSBTAG00000006208 & $3.40 \mathrm{E}-10$ & $2.42 \mathrm{E}-10$ & ADCY7 & $1.40 \mathrm{E}+00$ \\
\hline ENSBTAG00000005152 & $2.78 \mathrm{E}-10$ & $1.98 \mathrm{E}-10$ & TK2 & $1.40 \mathrm{E}+00$ \\
\hline ENSBTAG00000016640 & $4.83 \mathrm{E}-10$ & $3.43 \mathrm{E}-10$ & HEATR3 & $1.41 \mathrm{E}+00$ \\
\hline ENSBTAG00000006417 & $1.19 \mathrm{E}-10$ & $8.41 E-11$ & EDC4 & $1.41 E+00$ \\
\hline ENSBTAG00000004757 & $9.09 \mathrm{E}-11$ & $6.44 \mathrm{E}-11$ & LTBP4 & $1.41 E+00$ \\
\hline ENSBTAG00000007334 & $3.17 \mathrm{E}-10$ & $2.24 \mathrm{E}-10$ & NFKBID & $1.41 E+00$ \\
\hline ENSBTAG00000020236 & $4.01 \mathrm{E}-10$ & $2.83 \mathrm{E}-10$ & NECAB2 & $1.42 \mathrm{E}+00$ \\
\hline ENSBTAG00000045708 & $2.71 \mathrm{E}-10$ & $1.91 \mathrm{E}-10$ & MYPOP & $1.42 \mathrm{E}+00$ \\
\hline ENSBTAG00000011635 & $1.15 \mathrm{E}-10$ & 8.03E-11 & CENPN & $1.43 E+00$ \\
\hline ENSBTAG00000004375 & $1.24 \mathrm{E}-10$ & $8.68 \mathrm{E}-11$ & ESRP2 & $1.43 E+00$ \\
\hline ENSBTAG00000004751 & $2.03 \mathrm{E}-10$ & $1.42 \mathrm{E}-10$ & SPTBN4 & $1.43 \mathrm{E}+00$ \\
\hline ENSBTAG00000006840 & $3.32 \mathrm{E}-10$ & $2.32 \mathrm{E}-10$ & ASPDH & $1.43 E+00$ \\
\hline ENSBTAG00000044006 & $1.53 \mathrm{E}-10$ & $1.08 \mathrm{E}-10$ & GINS2 & $1.42 \mathrm{E}+00$ \\
\hline ENSBTAG00000017956 & $1.95 \mathrm{E}-10$ & 1.37E-10 & KCTD15 & $1.43 \mathrm{E}+00$ \\
\hline ENSBTAG00000030360 & $8.16 \mathrm{E}-10$ & 5.70E-10 & & $1.43 \mathrm{E}+00$ \\
\hline ENSBTAG00000004766 & $7.32 \mathrm{E}-10$ & $5.11 \mathrm{E}-10$ & & $1.43 \mathrm{E}+00$ \\
\hline ENSBTAG00000009638 & $1.26 \mathrm{E}-10$ & 8.79E-11 & PRMT7 & $1.43 \mathrm{E}+00$ \\
\hline ENSBTAG00000009357 & $5.60 \mathrm{E}-10$ & $3.90 \mathrm{E}-10$ & IL34 & $1.44 \mathrm{E}+00$ \\
\hline ENSBTAG00000030505 & $1.54 \mathrm{E}-10$ & $1.06 \mathrm{E}-10$ & & $1.45 \mathrm{E}+00$ \\
\hline ENSBTAG00000019604 & $1.96 \mathrm{E}-10$ & $1.35 \mathrm{E}-10$ & VASP & $1.45 E+00$ \\
\hline ENSBTAG00000012177 & $5.76 \mathrm{E}-10$ & $3.98 \mathrm{E}-10$ & SNRPD2 & $1.45 E+00$ \\
\hline ENSBTAG00000006638 & $1.28 \mathrm{E}-10$ & 8.77E-11 & BCL2L12 & $1.45 \mathrm{E}+00$ \\
\hline ENSBTAG00000018077 & $2.04 \mathrm{E}-10$ & 1.40E-10 & LYPD3 & $1.46 \mathrm{E}+00$ \\
\hline ENSBTAG00000037699 & 1.99E-09 & 1.37E-09 & & $1.45 E+00$ \\
\hline ENSBTAG00000009639 & $1.40 \mathrm{E}-10$ & $9.62 \mathrm{E}-11$ & SMPD3 & $1.45 \mathrm{E}+00$ \\
\hline ENSBTAG00000046101 & $8.62 \mathrm{E}-10$ & 5.93E-10 & & $1.45 E+00$ \\
\hline ENSBTAG00000002596 & $1.04 \mathrm{E}-09$ & 7.11E-10 & VSTM1 & $1.46 \mathrm{E}+00$ \\
\hline ENSBTAG00000020633 & $3.24 \mathrm{E}-10$ & $2.22 \mathrm{E}-10$ & NOB1 & $1.46 \mathrm{E}+00$ \\
\hline ENSBTAG00000020632 & $4.07 \mathrm{E}-10$ & $2.79 \mathrm{E}-10$ & NQO1 & $1.46 \mathrm{E}+00$ \\
\hline ENSBTAG00000047724 & $6.58 \mathrm{E}-10$ & $4.51 \mathrm{E}-10$ & & $1.46 \mathrm{E}+00$ \\
\hline ENSBTAG00000013107 & $5.07 \mathrm{E}-10$ & $3.47 \mathrm{E}-10$ & SHANK1 & $1.46 \mathrm{E}+00$ \\
\hline ENSBTAG00000018146 & $7.95 \mathrm{E}-10$ & $5.43 \mathrm{E}-10$ & VAT1L & $1.46 \mathrm{E}+00$ \\
\hline ENSBTAG00000001151 & $1.83 \mathrm{E}-10$ & $1.25 \mathrm{E}-10$ & APLP1 & $1.46 \mathrm{E}+00$ \\
\hline ENSBTAG00000011083 & $4.89 \mathrm{E}-10$ & 3.34E-10 & POLD1 & $1.46 \mathrm{E}+00$ \\
\hline ENSBTAG00000012097 & $5.43 \mathrm{E}-10$ & $3.69 \mathrm{E}-10$ & RSPH6A & $1.47 \mathrm{E}+00$ \\
\hline ENSBTAG00000001996 & $1.36 \mathrm{E}-10$ & $9.29 \mathrm{E}-11$ & SNRNP70 & $1.47 \mathrm{E}+00$ \\
\hline ENSBTAG00000015991 & $3.77 \mathrm{E}-10$ & $2.56 \mathrm{E}-10$ & $\mathrm{CDH} 1$ & $1.47 \mathrm{E}+00$ \\
\hline ENSBTAG00000012216 & $8.36 \mathrm{E}-10$ & $5.68 \mathrm{E}-10$ & MLKL & $1.47 \mathrm{E}+00$ \\
\hline ENSBTAG00000014450 & $1.78 \mathrm{E}-10$ & $1.20 \mathrm{E}-10$ & NOSIP & $1.48 \mathrm{E}+00$ \\
\hline ENSBTAG00000016315 & $4.12 \mathrm{E}-10$ & $2.80 \mathrm{E}-10$ & COTL1 & $1.47 \mathrm{E}+00$ \\
\hline ENSBTAG00000016438 & 7.74E-11 & $5.25 \mathrm{E}-11$ & TERB1 & $1.47 \mathrm{E}+00$ \\
\hline ENSBTAG00000023343 & $3.91 \mathrm{E}-10$ & 2.65E-10 & RPL28 & $1.48 \mathrm{E}+00$ \\
\hline ENSBTAG00000006862 & $1.95 \mathrm{E}-10$ & $1.32 \mathrm{E}-10$ & MEIS3 & $1.48 \mathrm{E}+00$ \\
\hline ENSBTAG00000023726 & $1.46 \mathrm{E}-10$ & $9.90 \mathrm{E}-11$ & & $1.47 E+00$ \\
\hline ENSBTAG00000012796 & $2.36 \mathrm{E}-10$ & $1.60 \mathrm{E}-10$ & ZNF428 & $1.47 \mathrm{E}+00$ \\
\hline ENSBTAG00000015139 & $7.87 \mathrm{E}-10$ & $5.32 \mathrm{E}-10$ & & $1.48 \mathrm{E}+00$ \\
\hline ENSBTAG00000001781 & $2.02 \mathrm{E}-10$ & $1.36 \mathrm{E}-10$ & Tom40A & $1.48 \mathrm{E}+00$ \\
\hline ENSBTAG00000014953 & $1.32 \mathrm{E}-09$ & 8.93E-10 & & $1.48 \mathrm{E}+00$ \\
\hline ENSBTAG00000030543 & $3.10 \mathrm{E}-10$ & $2.09 \mathrm{E}-10$ & SLC6A16 & $1.49 E+00$ \\
\hline ENSBTAG00000018900 & $1.56 \mathrm{E}-10$ & $1.05 \mathrm{E}-10$ & CARMIL2 & $1.49 E+00$ \\
\hline ENSBTAG00000006646 & $2.41 \mathrm{E}-10$ & $1.62 \mathrm{E}-10$ & & $1.49 E+00$ \\
\hline ENSBTAG00000034689 & $4.10 \mathrm{E}-10$ & $2.75 \mathrm{E}-10$ & ZNRF1 & $1.49 E+00$ \\
\hline ENSBTAG00000008860 & $1.65 \mathrm{E}-10$ & $1.11 \mathrm{E}-10$ & & $1.48 \mathrm{E}+00$ \\
\hline ENSBTAG00000013766 & $3.15 \mathrm{E}-10$ & $2.12 \mathrm{E}-10$ & IRGC & $1.49 E+00$ \\
\hline ENSBTAG00000004925 & $4.81 \mathrm{E}-10$ & $3.22 \mathrm{E}-10$ & & $1.49 E+00$ \\
\hline ENSBTAG00000037440 & $8.10 \mathrm{E}-10$ & $5.41 E-10$ & & $1.50 \mathrm{E}+00$ \\
\hline ENSBTAG00000018772 & 4.44E-10 & $2.95 \mathrm{E}-10$ & TAF1C & $1.51 E+00$ \\
\hline ENSBTAG00000039075 & $7.04 \mathrm{E}-10$ & 4.67E-10 & ZNF527 & $1.51 E+00$ \\
\hline ENSBTAG00000001659 & $1.45 \mathrm{E}-10$ & $9.62 \mathrm{E}-11$ & & $1.51 \mathrm{E}+00$ \\
\hline ENSBTAG00000030444 & $9.90 \mathrm{E}-10$ & $6.55 \mathrm{E}-10$ & & $1.51 \mathrm{E}+00$ \\
\hline
\end{tabular}




\begin{tabular}{|c|c|c|c|c|}
\hline ENSBTAG00000038869 & $2.80 \mathrm{E}-10$ & $1.84 \mathrm{E}-10$ & & $1.52 \mathrm{E}+00$ \\
\hline ENSBTAG00000017044 & $8.55 \mathrm{E}-10$ & $5.62 \mathrm{E}-10$ & MAG & $1.52 E+00$ \\
\hline ENSBTAG00000004368 & $1.18 \mathrm{E}-10$ & $7.78 \mathrm{E}-11$ & NFATC3 & $1.52 \mathrm{E}+00$ \\
\hline ENSBTAG00000012222 & $1.01 \mathrm{E}-09$ & $6.60 \mathrm{E}-10$ & $\mathrm{FA} 2 \mathrm{H}$ & $1.53 \mathrm{E}+00$ \\
\hline ENSBTAG00000012112 & $1.09 \mathrm{E}-10$ & 7.06E-11 & SLC7A6 & $1.55 \mathrm{E}+00$ \\
\hline ENSBTAG00000004526 & $6.16 \mathrm{E}-10$ & $3.98 \mathrm{E}-10$ & & $1.55 \mathrm{E}+00$ \\
\hline ENSBTAG00000001171 & $5.09 \mathrm{E}-10$ & $3.28 \mathrm{E}-10$ & OGFOD1 & $1.55 \mathrm{E}+00$ \\
\hline ENSBTAG00000000630 & $3.04 \mathrm{E}-10$ & $1.95 \mathrm{E}-10$ & LSM14A & $1.56 \mathrm{E}+00$ \\
\hline ENSBTAG00000020936 & $1.31 \mathrm{E}-10$ & $8.42 E-11$ & CARD15 & $1.56 \mathrm{E}+00$ \\
\hline ENSBTAG00000019348 & $7.02 \mathrm{E}-10$ & $4.48 \mathrm{E}-10$ & & $1.57 E+00$ \\
\hline ENSBTAG00000012693 & 4.43E-10 & $2.83 \mathrm{E}-10$ & & $1.56 \mathrm{E}+00$ \\
\hline ENSBTAG00000038149 & $1.29 \mathrm{E}-09$ & $8.14 \mathrm{E}-10$ & NLRP12 & $1.58 \mathrm{E}+00$ \\
\hline ENSBTAG00000002830 & $6.03 \mathrm{E}-10$ & $3.83 \mathrm{E}-10$ & U2AF2 & $1.58 \mathrm{E}+00$ \\
\hline ENSBTAG00000014595 & $3.22 \mathrm{E}-10$ & $2.05 \mathrm{E}-10$ & LIG1 & $1.57 E+00$ \\
\hline ENSBTAG00000023601 & $1.27 \mathrm{E}-10$ & 7.97E-11 & CD3EAP & $1.59 E+00$ \\
\hline ENSBTAG00000019183 & $2.01 \mathrm{E}-10$ & $1.27 \mathrm{E}-10$ & FUZ & $1.59 E+00$ \\
\hline ENSBTAG00000008182 & $2.56 \mathrm{E}-11$ & $1.61 \mathrm{E}-11$ & FOSB & $1.58 \mathrm{E}+00$ \\
\hline ENSBTAG00000020251 & $1.37 \mathrm{E}-10$ & $8.62 \mathrm{E}-11$ & LYPD4 & $1.59 E+00$ \\
\hline ENSBTAG00000005763 & 1.97E-10 & $1.24 \mathrm{E}-10$ & AKT1S1 & $1.59 E+00$ \\
\hline ENSBTAG00000004838 & $5.09 \mathrm{E}-11$ & $3.20 \mathrm{E}-11$ & IRX5 & $1.59 \mathrm{E}+00$ \\
\hline ENSBTAG00000019051 & $2.73 \mathrm{E}-10$ & $1.72 \mathrm{E}-10$ & NAT14 & $1.59 E+00$ \\
\hline ENSBTAG00000024869 & $9.08 \mathrm{E}-11$ & $5.72 \mathrm{E}-11$ & CX3CL1 & $1.59 E+00$ \\
\hline ENSBTAG00000019679 & $3.97 \mathrm{E}-10$ & $2.50 \mathrm{E}-10$ & SYNE4 & $1.59 E+00$ \\
\hline ENSBTAG00000001856 & $3.94 \mathrm{E}-11$ & $2.48 \mathrm{E}-11$ & POLR2C & $1.59 \mathrm{E}+00$ \\
\hline ENSBTAG00000002327 & 7.57E-11 & 4.77E-11 & TMEM208 & $1.59 \mathrm{E}+00$ \\
\hline ENSBTAG00000013931 & $1.76 \mathrm{E}-10$ & $1.11 \mathrm{E}-10$ & FAM96B & $1.59 \mathrm{E}+00$ \\
\hline ENSBTAG00000039441 & $2.30 \mathrm{E}-10$ & $1.45 \mathrm{E}-10$ & RGS9BP & $1.58 \mathrm{E}+00$ \\
\hline ENSBTAG00000005390 & $1.33 \mathrm{E}-10$ & $8.40 \mathrm{E}-11$ & GMFG & $1.59 E+00$ \\
\hline ENSBTAG00000014147 & $4.59 \mathrm{E}-10$ & $2.88 \mathrm{E}-10$ & PRSS54 & $1.59 E+00$ \\
\hline ENSBTAG00000012035 & $1.77 \mathrm{E}-10$ & $1.12 \mathrm{E}-10$ & EXOC3L1 & $1.58 \mathrm{E}+00$ \\
\hline ENSBTAG00000038050 & $2.00 \mathrm{E}-10$ & $1.26 \mathrm{E}-10$ & ZNF571 & $1.58 \mathrm{E}+00$ \\
\hline ENSBTAG00000016886 & $2.26 \mathrm{E}-10$ & $1.42 \mathrm{E}-10$ & MT3 & $1.59 E+00$ \\
\hline ENSBTAG00000047568 & $3.39 \mathrm{E}-10$ & $2.13 \mathrm{E}-10$ & VN1R1 & $1.59 E+00$ \\
\hline ENSBTAG00000016299 & $7.59 \mathrm{E}-10$ & 4.77E-10 & ZNF784 & $1.59 E+00$ \\
\hline ENSBTAG00000021647 & $7.81 \mathrm{E}-10$ & $4.90 \mathrm{E}-10$ & FCAR & $1.59 E+00$ \\
\hline ENSBTAG00000023998 & $4.81 \mathrm{E}-10$ & 3.03E-10 & & $1.59 E+00$ \\
\hline ENSBTAG00000046365 & $2.21 \mathrm{E}-10$ & $1.39 \mathrm{E}-10$ & & $1.59 E+00$ \\
\hline ENSBTAG00000037881 & $1.47 \mathrm{E}-09$ & $9.19 \mathrm{E}-10$ & & $1.60 E+00$ \\
\hline ENSBTAG00000021057 & $1.57 \mathrm{E}-10$ & $9.86 \mathrm{E}-11$ & TMEM190 & $1.59 E+00$ \\
\hline ENSBTAG00000010927 & $1.54 \mathrm{E}-10$ & $9.60 \mathrm{E}-11$ & CBFA2T3 & $1.60 E+00$ \\
\hline ENSBTAG00000000946 & $8.56 \mathrm{E}-10$ & $5.33 \mathrm{E}-10$ & TLDC1 & $1.61 \mathrm{E}+00$ \\
\hline ENSBTAG00000019810 & $9.08 \mathrm{E}-10$ & $5.64 \mathrm{E}-10$ & AARS & $1.61 E+00$ \\
\hline ENSBTAG00000021433 & $5.31 \mathrm{E}-10$ & $3.29 \mathrm{E}-10$ & & $1.61 E+00$ \\
\hline ENSBTAG00000015912 & $4.88 \mathrm{E}-10$ & 3.02E-10 & DMKN & $1.61 E+00$ \\
\hline ENSBTAG00000005841 & 1.37E-09 & 8.47E-10 & & $1.61 E+00$ \\
\hline ENSBTAG00000040358 & $2.52 \mathrm{E}-10$ & $1.56 \mathrm{E}-10$ & ZNF772 & $1.62 \mathrm{E}+00$ \\
\hline ENSBTAG00000000721 & $8.75 \mathrm{E}-10$ & $5.41 \mathrm{E}-10$ & GPATCH1 & $1.62 E+00$ \\
\hline ENSBTAG00000000945 & $3.37 \mathrm{E}-10$ & $2.08 \mathrm{E}-10$ & ATP2C2 & $1.62 E+00$ \\
\hline ENSBTAG00000002412 & $1.39 \mathrm{E}-10$ & $8.53 \mathrm{E}-11$ & CYB5B & $1.63 E+00$ \\
\hline ENSBTAG00000039935 & $2.99 \mathrm{E}-10$ & $1.84 \mathrm{E}-10$ & KCNC3 & $1.62 \mathrm{E}+00$ \\
\hline ENSBTAG00000019179 & $2.11 \mathrm{E}-10$ & $1.30 \mathrm{E}-10$ & TSKS & $1.63 E+00$ \\
\hline ENSBTAG00000030440 & $6.78 \mathrm{E}-10$ & 4.15E-10 & & $1.63 E+00$ \\
\hline ENSBTAG00000011689 & $6.04 \mathrm{E}-10$ & 3.69E-10 & LENG8 & $1.64 E+00$ \\
\hline ENSBTAG00000047301 & $1.73 \mathrm{E}-09$ & $1.06 \mathrm{E}-09$ & & $1.63 E+00$ \\
\hline ENSBTAG00000012176 & $4.58 \mathrm{E}-10$ & $2.79 \mathrm{E}-10$ & QPCTL & $1.64 \mathrm{E}+00$ \\
\hline ENSBTAG00000006795 & $1.87 \mathrm{E}-10$ & $1.14 \mathrm{E}-10$ & & $1.64 \mathrm{E}+00$ \\
\hline ENSBTAG00000040442 & $2.09 \mathrm{E}-10$ & $1.26 \mathrm{E}-10$ & & $1.66 \mathrm{E}+00$ \\
\hline ENSBTAG00000047175 & $1.59 \mathrm{E}-09$ & $9.63 \mathrm{E}-10$ & & $1.65 E+00$ \\
\hline ENSBTAG00000000027 & $7.62 \mathrm{E}-10$ & 4.60E-10 & IGFL1 & $1.66 \mathrm{E}+00$ \\
\hline ENSBTAG00000011530 & $1.08 \mathrm{E}-10$ & $6.52 E-11$ & $\mathrm{CDH} 15$ & $1.65 E+00$ \\
\hline ENSBTAG00000046156 & $7.25 \mathrm{E}-10$ & $4.38 \mathrm{E}-10$ & ST3GAL2 & $1.66 \mathrm{E}+00$ \\
\hline ENSBTAG00000031538 & $9.92 \mathrm{E}-10$ & $5.99 \mathrm{E}-10$ & & $1.66 \mathrm{E}+00$ \\
\hline ENSBTAG00000004608 & $8.14 \mathrm{E}-10$ & $4.89 \mathrm{E}-10$ & & $1.66 \mathrm{E}+00$ \\
\hline ENSBTAG00000004755 & $1.40 \mathrm{E}-10$ & $8.41 E-11$ & SHKBP1 & $1.67 E+00$ \\
\hline ENSBTAG00000003826 & $3.32 \mathrm{E}-10$ & $1.98 \mathrm{E}-10$ & SCN1B & $1.67 \mathrm{E}+00$ \\
\hline ENSBTAG00000012205 & $1.73 \mathrm{E}-10$ & $1.04 \mathrm{E}-10$ & CPT1C & $1.66 \mathrm{E}+00$ \\
\hline ENSBTAG00000044191 & $6.22 \mathrm{E}-10$ & $3.71 E-10$ & $\mathrm{ABCC} 12$ & $1.68 \mathrm{E}+00$ \\
\hline ENSBTAG00000011021 & $1.72 \mathrm{E}-10$ & $1.03 \mathrm{E}-10$ & CES1 & $1.67 E+00$ \\
\hline ENSBTAG00000038635 & $9.89 \mathrm{E}-10$ & $5.89 \mathrm{E}-10$ & & $1.68 \mathrm{E}+00$ \\
\hline ENSBTAG00000040005 & $5.32 \mathrm{E}-10$ & 3.17E-10 & & $1.68 \mathrm{E}+00$ \\
\hline
\end{tabular}




\begin{tabular}{|c|c|c|c|c|}
\hline ENSBTAG00000001055 & $1.18 \mathrm{E}-10$ & $6.97 \mathrm{E}-11$ & & $1.69 E+00$ \\
\hline ENSBTAG00000006139 & $9.39 \mathrm{E}-11$ & $5.55 \mathrm{E}-11$ & TRPM4 & $1.69 \mathrm{E}+00$ \\
\hline ENSBTAG00000031941 & $9.34 \mathrm{E}-10$ & $5.49 \mathrm{E}-10$ & PEPD & $1.70 \mathrm{E}+00$ \\
\hline ENSBTAG00000010627 & $2.53 \mathrm{E}-10$ & 1.49E-10 & SF3B3 & $1.70 \mathrm{E}+00$ \\
\hline ENSBTAG00000038024 & $1.23 \mathrm{E}-09$ & $7.20 \mathrm{E}-10$ & SIGLECL1 & $1.71 \mathrm{E}+00$ \\
\hline ENSBTAG00000006291 & $2.28 \mathrm{E}-10$ & $1.33 \mathrm{E}-10$ & CYLD & $1.71 \mathrm{E}+00$ \\
\hline ENSBTAG00000030769 & $3.75 \mathrm{E}-10$ & $2.18 \mathrm{E}-10$ & KPTN & $1.72 \mathrm{E}+00$ \\
\hline ENSBTAG00000009286 & $2.04 \mathrm{E}-10$ & $1.19 \mathrm{E}-10$ & $\mathrm{ZC} 3 \mathrm{H} 4$ & $1.72 \mathrm{E}+00$ \\
\hline ENSBTAG00000002072 & $1.34 \mathrm{E}-10$ & $7.81 \mathrm{E}-11$ & ERCC2 & $1.72 \mathrm{E}+00$ \\
\hline ENSBTAG00000020543 & $2.63 \mathrm{E}-10$ & $1.53 \mathrm{E}-10$ & UPK1A & $1.72 \mathrm{E}+00$ \\
\hline ENSBTAG00000019180 & $1.94 \mathrm{E}-10$ & $1.11 \mathrm{E}-10$ & AP2A1 & $1.74 \mathrm{E}+00$ \\
\hline ENSBTAG00000016250 & $7.92 \mathrm{E}-11$ & 4.53E-11 & AKTIP & $1.75 \mathrm{E}+00$ \\
\hline ENSBTAG00000045529 & 4.18E-10 & $2.39 \mathrm{E}-10$ & NCR1 & $1.75 \mathrm{E}+00$ \\
\hline ENSBTAG00000015899 & $4.65 \mathrm{E}-10$ & $2.66 \mathrm{E}-10$ & & $1.75 \mathrm{E}+00$ \\
\hline ENSBTAG00000045795 & $9.86 \mathrm{E}-10$ & $5.63 \mathrm{E}-10$ & KIR2DS1 & $1.75 E+00$ \\
\hline ENSBTAG00000039971 & $3.03 \mathrm{E}-10$ & $1.73 \mathrm{E}-10$ & & $1.75 \mathrm{E}+00$ \\
\hline ENSBTAG00000038911 & $9.67 \mathrm{E}-10$ & $5.51 \mathrm{E}-10$ & & $1.76 \mathrm{E}+00$ \\
\hline ENSBTAG00000048204 & $2.67 \mathrm{E}-10$ & $1.52 \mathrm{E}-10$ & SPACA6 & $1.76 \mathrm{E}+00$ \\
\hline ENSBTAG00000003470 & $9.95 \mathrm{E}-10$ & $5.66 \mathrm{E}-10$ & TTYH1 & $1.76 \mathrm{E}+00$ \\
\hline ENSBTAG00000040413 & $1.28 \mathrm{E}-10$ & 7.27E-11 & CES3 & $1.76 \mathrm{E}+00$ \\
\hline ENSBTAG00000038702 & 7.44E-10 & $4.21 \mathrm{E}-10$ & ZNF23 & $1.77 \mathrm{E}+00$ \\
\hline ENSBTAG00000003447 & 9.63E-10 & $5.41 \mathrm{E}-10$ & & $1.78 \mathrm{E}+00$ \\
\hline ENSBTAG00000038858 & $4.35 \mathrm{E}-10$ & $2.44 \mathrm{E}-10$ & CKLF & $1.78 \mathrm{E}+00$ \\
\hline ENSBTAG00000023417 & $1.72 \mathrm{E}-10$ & $9.65 \mathrm{E}-11$ & RUVBL2 & $1.79 E+00$ \\
\hline ENSBTAG00000013412 & $1.80 \mathrm{E}-10$ & $1.01 \mathrm{E}-10$ & NFAT5 & $1.78 \mathrm{E}+00$ \\
\hline ENSBTAG00000000638 & $1.73 \mathrm{E}-10$ & $9.69 \mathrm{E}-11$ & CDT1 & $1.79 E+00$ \\
\hline ENSBTAG00000040108 & $6.93 \mathrm{E}-10$ & $3.87 \mathrm{E}-10$ & ZNF829 & $1.79 E+00$ \\
\hline ENSBTAG00000004725 & $1.06 \mathrm{E}-09$ & $5.87 \mathrm{E}-10$ & NLRP9 & $1.80 \mathrm{E}+00$ \\
\hline ENSBTAG00000021438 & $1.20 \mathrm{E}-10$ & $6.65 \mathrm{E}-11$ & ZNF821 & $1.80 \mathrm{E}+00$ \\
\hline ENSBTAG00000016037 & $1.85 \mathrm{E}-10$ & $1.03 \mathrm{E}-10$ & BCKDHA & $1.80 \mathrm{E}+00$ \\
\hline ENSBTAG00000026004 & 7.57E-10 & 4.17E-10 & CNTNAP4 & $1.81 \mathrm{E}+00$ \\
\hline ENSBTAG00000023338 & $3.99 \mathrm{E}-10$ & $2.19 \mathrm{E}-10$ & & $1.82 \mathrm{E}+00$ \\
\hline ENSBTAG00000007545 & $1.58 \mathrm{E}-10$ & 8.67E-11 & PHLPP2 & $1.82 \mathrm{E}+00$ \\
\hline ENSBTAG00000014900 & $8.22 \mathrm{E}-10$ & $4.50 \mathrm{E}-10$ & CNOT3 & $1.83 E+00$ \\
\hline ENSBTAG00000011887 & $3.46 \mathrm{E}-10$ & $1.89 \mathrm{E}-10$ & CD22 & $1.83 \mathrm{E}+00$ \\
\hline ENSBTAG00000017847 & 8.06E-10 & 4.37E-10 & PPP1R12C & $1.84 \mathrm{E}+00$ \\
\hline ENSBTAG00000038755 & 8.39E-10 & 4.54E-10 & & $1.85 \mathrm{E}+00$ \\
\hline ENSBTAG00000002824 & $1.36 \mathrm{E}-10$ & $7.35 \mathrm{E}-11$ & DPEP3 & $1.85 \mathrm{E}+00$ \\
\hline ENSBTAG00000012499 & $2.36 \mathrm{E}-10$ & $1.27 \mathrm{E}-10$ & RPGRIP1L & $1.86 \mathrm{E}+00$ \\
\hline ENSBTAG00000033248 & 2.07E-10 & $1.12 \mathrm{E}-10$ & $\mathrm{CDH} 3$ & $1.85 \mathrm{E}+00$ \\
\hline ENSBTAG00000006841 & $4.71 \mathrm{E}-10$ & $2.54 \mathrm{E}-10$ & LRRC4B & $1.85 \mathrm{E}+00$ \\
\hline ENSBTAG00000017816 & $5.36 \mathrm{E}-10$ & $2.89 \mathrm{E}-10$ & FXYD1 & $1.85 \mathrm{E}+00$ \\
\hline ENSBTAG00000038890 & $1.94 \mathrm{E}-09$ & $1.04 \mathrm{E}-09$ & & $1.86 \mathrm{E}+00$ \\
\hline ENSBTAG00000045498 & $6.12 \mathrm{E}-10$ & $3.26 \mathrm{E}-10$ & SULT2B1 & $1.88 \mathrm{E}+00$ \\
\hline ENSBTAG00000008185 & $1.15 \mathrm{E}-10$ & $6.05 \mathrm{E}-11$ & RTN2 & $1.90 \mathrm{E}+00$ \\
\hline ENSBTAG00000048283 & $4.61 \mathrm{E}-10$ & $2.42 \mathrm{E}-10$ & & $1.91 \mathrm{E}+00$ \\
\hline ENSBTAG00000002055 & $7.66 \mathrm{E}-10$ & $3.99 \mathrm{E}-10$ & ZFP28 & $1.92 \mathrm{E}+00$ \\
\hline ENSBTAG00000040411 & $6.50 \mathrm{E}-10$ & $3.38 \mathrm{E}-10$ & & $1.92 \mathrm{E}+00$ \\
\hline ENSBTAG00000038416 & $1.12 \mathrm{E}-09$ & $5.83 \mathrm{E}-10$ & & $1.92 \mathrm{E}+00$ \\
\hline ENSBTAG00000009636 & $1.74 \mathrm{E}-10$ & $9.04 \mathrm{E}-11$ & SLC7A6OS & $1.93 \mathrm{E}+00$ \\
\hline ENSBTAG00000009137 & $8.69 \mathrm{E}-10$ & $4.51 \mathrm{E}-10$ & NKG7 & $1.93 \mathrm{E}+00$ \\
\hline ENSBTAG00000014117 & $4.31 \mathrm{E}-10$ & $2.23 \mathrm{E}-10$ & CCDC9 & $1.93 \mathrm{E}+00$ \\
\hline ENSBTAG00000038674 & $3.91 \mathrm{E}-10$ & $2.01 \mathrm{E}-10$ & ZNF677 & $1.95 \mathrm{E}+00$ \\
\hline ENSBTAG00000009762 & $2.76 \mathrm{E}-10$ & $1.42 \mathrm{E}-10$ & BEAN1 & $1.94 \mathrm{E}+00$ \\
\hline ENSBTAG00000033078 & $3.86 \mathrm{E}-10$ & $1.97 \mathrm{E}-10$ & CNEP1R1 & $1.96 \mathrm{E}+00$ \\
\hline ENSBTAG00000008546 & $3.43 \mathrm{E}-10$ & $1.75 \mathrm{E}-10$ & SYNGR4 & $1.96 \mathrm{E}+00$ \\
\hline ENSBTAG00000014636 & 3.37E-10 & $1.72 \mathrm{E}-10$ & ZFHX3 & $1.96 \mathrm{E}+00$ \\
\hline ENSBTAG00000011052 & 5.30E-10 & $2.69 \mathrm{E}-10$ & & $1.97 \mathrm{E}+00$ \\
\hline ENSBTAG00000010867 & $2.34 \mathrm{E}-10$ & $1.18 \mathrm{E}-10$ & LONP2 & $1.98 \mathrm{E}+00$ \\
\hline ENSBTAG00000020558 & $1.83 \mathrm{E}-10$ & $9.21 \mathrm{E}-11$ & & $1.98 \mathrm{E}+00$ \\
\hline ENSBTAG00000020689 & $8.31 \mathrm{E}-11$ & 4.19E-11 & PPP1R13L & $1.98 \mathrm{E}+00$ \\
\hline ENSBTAG00000013757 & $1.92 \mathrm{E}-10$ & $9.58 \mathrm{E}-11$ & $\mathrm{CTCF}$ & $2.01 E+00$ \\
\hline ENSBTAG00000015987 & $1.68 \mathrm{E}-09$ & $8.35 \mathrm{E}-10$ & & $2.01 E+00$ \\
\hline ENSBTAG00000030600 & $4.55 \mathrm{E}-10$ & $2.25 \mathrm{E}-10$ & & $2.02 E+00$ \\
\hline ENSBTAG00000045510 & $5.19 \mathrm{E}-10$ & $2.54 \mathrm{E}-10$ & EMC10 & $2.04 \mathrm{E}+00$ \\
\hline ENSBTAG00000047035 & $2.95 \mathrm{E}-10$ & $1.44 \mathrm{E}-10$ & TBC1D17 & $2.05 E+00$ \\
\hline ENSBTAG00000006899 & $6.01 \mathrm{E}-10$ & $2.93 \mathrm{E}-10$ & JPH3 & $2.05 E+00$ \\
\hline ENSBTAG00000039628 & $9.66 \mathrm{E}-10$ & $4.71 \mathrm{E}-10$ & & $2.05 E+00$ \\
\hline ENSBTAG00000015908 & $1.13 \mathrm{E}-09$ & $5.41 \mathrm{E}-10$ & MBOAT7 & $2.08 \mathrm{E}+00$ \\
\hline ENSBTAG00000003794 & $4.40 \mathrm{E}-10$ & $2.12 \mathrm{E}-10$ & GNAO1 & $2.07 E+00$ \\
\hline
\end{tabular}




\begin{tabular}{|c|c|c|c|c|}
\hline ENSBTAG00000010502 & $1.95 \mathrm{E}-10$ & $9.21 \mathrm{E}-11$ & SERTAD3 & $2.12 \mathrm{E}+00$ \\
\hline ENSBTAG00000009414 & $2.04 \mathrm{E}-10$ & $9.50 \mathrm{E}-11$ & PSMC4 & $2.15 E+00$ \\
\hline ENSBTAG00000005330 & $6.48 \mathrm{E}-10$ & $3.01 \mathrm{E}-10$ & KRTDAP & $2.15 E+00$ \\
\hline ENSBTAG00000013084 & 4.75E-10 & $2.19 \mathrm{E}-10$ & NAPA & $2.17 \mathrm{E}+00$ \\
\hline ENSBTAG00000011723 & 3.27E-10 & $1.51 \mathrm{E}-10$ & GRIK5 & $2.17 \mathrm{E}+00$ \\
\hline ENSBTAG00000038321 & $4.33 \mathrm{E}-10$ & 2.00E-10 & LIPE & $2.17 E+00$ \\
\hline ENSBTAG00000005576 & $3.92 \mathrm{E}-10$ & $1.79 \mathrm{E}-10$ & CLEC11A & $2.19 E+00$ \\
\hline ENSBTAG00000001498 & $4.03 \mathrm{E}-10$ & $1.84 \mathrm{E}-10$ & CYTH2 & $2.19 E+00$ \\
\hline ENSBTAG00000018859 & $1.76 \mathrm{E}-10$ & 8.06E-11 & C19orf47 & $2.18 \mathrm{E}+00$ \\
\hline ENSBTAG00000018635 & $2.46 \mathrm{E}-10$ & $1.12 \mathrm{E}-10$ & ATP1A3 & $2.19 E+00$ \\
\hline ENSBTAG00000001104 & $3.40 \mathrm{E}-10$ & $1.54 \mathrm{E}-10$ & CMTM4 & $2.21 E+00$ \\
\hline ENSBTAG00000019267 & $2.46 \mathrm{E}-10$ & $1.11 \mathrm{E}-10$ & MMP2 & $2.21 E+00$ \\
\hline ENSBTAG00000020062 & $7.04 \mathrm{E}-10$ & $3.17 \mathrm{E}-10$ & TMC4 & $2.22 \mathrm{E}+00$ \\
\hline ENSBTAG00000011700 & $2.13 \mathrm{E}-10$ & 9.57E-11 & PLLP & $2.22 \mathrm{E}+00$ \\
\hline ENSBTAG00000017355 & $7.87 \mathrm{E}-11$ & 3.54E-11 & ERCC1 & $2.22 \mathrm{E}+00$ \\
\hline ENSBTAG00000031810 & $3.87 \mathrm{E}-10$ & $1.73 \mathrm{E}-10$ & WDR88 & $2.24 \mathrm{E}+00$ \\
\hline ENSBTAG00000009495 & $2.25 \mathrm{E}-10$ & $1.00 \mathrm{E}-10$ & BCAM & $2.25 \mathrm{E}+00$ \\
\hline ENSBTAG00000020836 & $2.15 \mathrm{E}-10$ & $9.59 \mathrm{E}-11$ & FAM92B & $2.24 \mathrm{E}+00$ \\
\hline ENSBTAG00000021157 & $5.70 \mathrm{E}-10$ & $2.54 \mathrm{E}-10$ & PPP6R1 & $2.24 \mathrm{E}+00$ \\
\hline ENSBTAG00000009446 & $3.10 \mathrm{E}-10$ & 1.37E-10 & USP10 & $2.26 \mathrm{E}+00$ \\
\hline ENSBTAG00000011664 & $5.91 \mathrm{E}-10$ & $2.60 \mathrm{E}-10$ & & $2.27 \mathrm{E}+00$ \\
\hline ENSBTAG00000011926 & $4.87 \mathrm{E}-10$ & $2.14 \mathrm{E}-10$ & & $2.28 \mathrm{E}+00$ \\
\hline ENSBTAG00000006630 & $2.27 \mathrm{E}-10$ & $9.95 \mathrm{E}-11$ & SLC12A3 & $2.28 \mathrm{E}+00$ \\
\hline ENSBTAG00000003820 & $4.83 \mathrm{E}-10$ & $2.12 \mathrm{E}-10$ & GRAMD1A & $2.28 \mathrm{E}+00$ \\
\hline ENSBTAG00000038428 & $3.20 \mathrm{E}-10$ & $1.40 \mathrm{E}-10$ & RELB & $2.29 E+00$ \\
\hline ENSBTAG00000017560 & $2.71 \mathrm{E}-10$ & $1.19 \mathrm{E}-10$ & CEBPG & $2.28 \mathrm{E}+00$ \\
\hline ENSBTAG00000002763 & $1.88 \mathrm{E}-10$ & $8.23 \mathrm{E}-11$ & КМT2B & $2.28 \mathrm{E}+00$ \\
\hline ENSBTAG00000007184 & $9.54 \mathrm{E}-10$ & 4.12E-10 & NUDT7 & $2.31 E+00$ \\
\hline ENSBTAG00000038240 & $2.93 \mathrm{E}-10$ & $1.26 \mathrm{E}-10$ & ZNF134 & $2.33 \mathrm{E}+00$ \\
\hline ENSBTAG00000046772 & $4.30 \mathrm{E}-10$ & $1.84 \mathrm{E}-10$ & CLEC3A & $2.33 \mathrm{E}+00$ \\
\hline ENSBTAG00000022813 & $6.31 \mathrm{E}-10$ & $2.69 \mathrm{E}-10$ & TMEM150B & $2.34 \mathrm{E}+00$ \\
\hline ENSBTAG00000004460 & $5.72 \mathrm{E}-10$ & $2.43 \mathrm{E}-10$ & RFWD3 & $2.35 E+00$ \\
\hline ENSBTAG00000015764 & $4.44 \mathrm{E}-10$ & $1.89 \mathrm{E}-10$ & EML2 & $2.35 E+00$ \\
\hline ENSBTAG00000002769 & $1.16 \mathrm{E}-10$ & $4.91 \mathrm{E}-11$ & AP1G1 & $2.36 \mathrm{E}+00$ \\
\hline ENSBTAG00000037882 & $2.11 \mathrm{E}-10$ & $8.85 \mathrm{E}-11$ & ZNF584 & $2.38 \mathrm{E}+00$ \\
\hline ENSBTAG00000001698 & $6.29 \mathrm{E}-11$ & $2.64 \mathrm{E}-11$ & CHTF8 & $2.38 \mathrm{E}+00$ \\
\hline ENSBTAG00000007992 & $8.29 E-11$ & $3.48 \mathrm{E}-11$ & HAS3 & $2.38 \mathrm{E}+00$ \\
\hline ENSBTAG00000015084 & $1.07 \mathrm{E}-10$ & 4.47E-11 & MT4 & $2.38 \mathrm{E}+00$ \\
\hline ENSBTAG00000006633 & $8.81 \mathrm{E}-11$ & $3.70 \mathrm{E}-11$ & IRF3 & $2.38 \mathrm{E}+00$ \\
\hline ENSBTAG00000012186 & $3.12 \mathrm{E}-10$ & $1.31 \mathrm{E}-10$ & DKKL1 & $2.38 \mathrm{E}+00$ \\
\hline ENSBTAG00000033334 & $6.74 \mathrm{E}-11$ & $2.83 \mathrm{E}-11$ & SPATA2L & $2.38 \mathrm{E}+00$ \\
\hline ENSBTAG00000007200 & $1.65 \mathrm{E}-10$ & $6.91 \mathrm{E}-11$ & COX6B2 & $2.38 \mathrm{E}+00$ \\
\hline ENSBTAG00000047400 & $8.02 \mathrm{E}-11$ & 3.37E-11 & IL11 & $2.38 \mathrm{E}+00$ \\
\hline ENSBTAG00000001191 & $8.50 \mathrm{E}-11$ & 3.57E-11 & CCL17 & $2.38 \mathrm{E}+00$ \\
\hline ENSBTAG00000012465 & $4.12 \mathrm{E}-10$ & $1.72 \mathrm{E}-10$ & FOXC2 & $2.39 E+00$ \\
\hline ENSBTAG00000020428 & $1.14 \mathrm{E}-10$ & 4.79E-11 & PAF1 & $2.38 \mathrm{E}+00$ \\
\hline ENSBTAG00000038034 & $3.41 \mathrm{E}-10$ & $1.43 \mathrm{E}-10$ & ZNF628 & $2.38 \mathrm{E}+00$ \\
\hline ENSBTAG00000006839 & $4.75 \mathrm{E}-10$ & $1.99 \mathrm{E}-10$ & JOSD2 & $2.38 \mathrm{E}+00$ \\
\hline ENSBTAG00000002370 & $3.49 \mathrm{E}-10$ & $1.46 \mathrm{E}-10$ & ZNF792 & $2.39 \mathrm{E}+00$ \\
\hline ENSBTAG00000047571 & $4.82 \mathrm{E}-10$ & $2.02 \mathrm{E}-10$ & & $2.38 \mathrm{E}+00$ \\
\hline ENSBTAG00000007617 & $2.39 \mathrm{E}-10$ & $1.00 \mathrm{E}-10$ & TERF2 & $2.39 E+00$ \\
\hline ENSBTAG00000018181 & $5.65 \mathrm{E}-10$ & $2.36 \mathrm{E}-10$ & $\mathrm{CCDC} 8$ & $2.39 \mathrm{E}+00$ \\
\hline ENSBTAG00000039086 & $7.41 \mathrm{E}-10$ & $3.10 \mathrm{E}-10$ & & $2.39 \mathrm{E}+00$ \\
\hline ENSBTAG00000002579 & $2.28 \mathrm{E}-10$ & $9.50 \mathrm{E}-11$ & FBL & $2.40 \mathrm{E}+00$ \\
\hline ENSBTAG00000040294 & 1.39E-09 & $5.79 \mathrm{E}-10$ & & $2.40 \mathrm{E}+00$ \\
\hline ENSBTAG00000038325 & $2.78 \mathrm{E}-10$ & $1.14 \mathrm{E}-10$ & CES4A & $2.44 \mathrm{E}+00$ \\
\hline ENSBTAG00000030922 & $7.02 \mathrm{E}-10$ & $2.87 \mathrm{E}-10$ & & $2.44 \mathrm{E}+00$ \\
\hline ENSBTAG00000019851 & $6.71 \mathrm{E}-10$ & $2.74 \mathrm{E}-10$ & PPP2R1A & $2.45 \mathrm{E}+00$ \\
\hline ENSBTAG00000016471 & $7.99 \mathrm{E}-10$ & $3.26 \mathrm{E}-10$ & PDPR & $2.45 E+00$ \\
\hline ENSBTAG00000014087 & $4.24 \mathrm{E}-10$ & $1.72 \mathrm{E}-10$ & KMT5C & $2.46 \mathrm{E}+00$ \\
\hline ENSBTAG00000003829 & $2.42 \mathrm{E}-10$ & $9.81 \mathrm{E}-11$ & HPN & $2.47 E+00$ \\
\hline ENSBTAG00000040559 & $8.72 \mathrm{E}-10$ & $3.53 \mathrm{E}-10$ & & $2.47 E+00$ \\
\hline ENSBTAG00000044157 & 5.67E-10 & $2.26 \mathrm{E}-10$ & & $2.51 E+00$ \\
\hline ENSBTAG00000013347 & $1.75 \mathrm{E}-10$ & $6.94 \mathrm{E}-11$ & DMPK & $2.51 E+00$ \\
\hline ENSBTAG00000002676 & $1.75 \mathrm{E}-10$ & $6.96 \mathrm{E}-11$ & SAE1 & $2.51 E+00$ \\
\hline ENSBTAG00000037906 & $6.52 \mathrm{E}-10$ & $2.59 \mathrm{E}-10$ & & $2.51 E+00$ \\
\hline ENSBTAG00000039849 & $7.04 \mathrm{E}-10$ & $2.79 \mathrm{E}-10$ & & $2.52 \mathrm{E}+00$ \\
\hline ENSBTAG00000017672 & $3.49 \mathrm{E}-10$ & 1.37E-10 & HAS1 & $2.54 \mathrm{E}+00$ \\
\hline ENSBTAG00000016513 & $6.62 \mathrm{E}-10$ & $2.61 \mathrm{E}-10$ & ZNF331 & $2.54 \mathrm{E}+00$ \\
\hline ENSBTAG00000023795 & $4.32 \mathrm{E}-10$ & $1.69 \mathrm{E}-10$ & VAC14 & $2.55 E+00$ \\
\hline
\end{tabular}




\begin{tabular}{|c|c|c|c|c|}
\hline ENSBTAG000000004463 & $2.61 \mathrm{E}-10$ & $1.02 \mathrm{E}-10$ & KDELR1 & $2.56 \mathrm{E}+00$ \\
\hline ENSBTAG00000009358 & $3.28 \mathrm{E}-10$ & $1.28 \mathrm{E}-10$ & MTSS1L & $2.56 \mathrm{E}+00$ \\
\hline ENSBTAG000000012104 & $1.63 \mathrm{E}-10$ & $6.31 \mathrm{E}-11$ & FOXA3 & $2.58 \mathrm{E}+00$ \\
\hline ENSBTAG000000017613 & $1.45 \mathrm{E}-10$ & $5.63 \mathrm{E}-11$ & & $2.58 \mathrm{E}+00$ \\
\hline ENSBTAG000000021145 & $1.33 \mathrm{E}-10$ & $5.11 \mathrm{E}-11$ & MAP4K1 & $2.61 E+00$ \\
\hline ENSBTAG00000048075 & 7.70E-10 & $2.93 \mathrm{E}-10$ & & $2.63 E+00$ \\
\hline ENSBTAG000000015968 & $1.82 \mathrm{E}-10$ & $6.94 \mathrm{E}-11$ & ACSF3 & $2.63 E+00$ \\
\hline ENSBTAG000000047062 & $3.63 \mathrm{E}-10$ & $1.38 \mathrm{E}-10$ & LRP3 & $2.63 E+00$ \\
\hline ENSBTAG000000024889 & $2.60 \mathrm{E}-10$ & $9.81 E-11$ & HSBP1 & $2.65 E+00$ \\
\hline ENSBTAG000000018244 & $2.61 \mathrm{E}-10$ & $9.85 \mathrm{E}-11$ & VSTM2B & $2.65 E+00$ \\
\hline ENSBTAG000000015164 & $8.69 \mathrm{E}-11$ & $3.28 \mathrm{E}-11$ & SLC27A5 & $2.64 E+00$ \\
\hline ENSBTAG000000038903 & $3.99 \mathrm{E}-10$ & $1.50 \mathrm{E}-10$ & & $2.66 \mathrm{E}+00$ \\
\hline ENSBTAG00000015920 & $4.35 \mathrm{E}-10$ & $1.64 \mathrm{E}-10$ & TMEM147 & $2.65 \mathrm{E}+00$ \\
\hline ENSBTAG000000011517 & 4.63E-10 & $1.74 \mathrm{E}-10$ & CCDC106 & $2.66 \mathrm{E}+00$ \\
\hline ENSBTAG000000011693 & $5.30 \mathrm{E}-10$ & $2.00 \mathrm{E}-10$ & LENG9 & $2.65 E+00$ \\
\hline ENSBTAG000000040603 & $9.21 \mathrm{E}-10$ & 3.47E-10 & ZNF175 & $2.65 E+00$ \\
\hline ENSBTAG000000040392 & $1.85 \mathrm{E}-09$ & $6.96 \mathrm{E}-10$ & & $2.66 \mathrm{E}+00$ \\
\hline ENSBTAG000000026944 & $9.53 \mathrm{E}-10$ & $3.58 \mathrm{E}-10$ & & $2.66 \mathrm{E}+00$ \\
\hline ENSBTAG000000030344 & 2.47E-09 & $9.19 \mathrm{E}-10$ & & $2.69 E+00$ \\
\hline ENSBTAG000000016896 & $1.34 \mathrm{E}-10$ & $4.96 \mathrm{E}-11$ & HERPUD1 & $2.70 \mathrm{E}+00$ \\
\hline ENSBTAG000000002368 & $1.70 \mathrm{E}-10$ & $6.31 \mathrm{E}-11$ & TULP2 & $2.70 \mathrm{E}+00$ \\
\hline ENSBTAG000000014267 & $2.16 \mathrm{E}-10$ & $8.05 E-11$ & $\mathrm{ZCCHC14}$ & $2.69 E+00$ \\
\hline ENSBTAG000000016114 & 3.97E-10 & $1.47 \mathrm{E}-10$ & CABP5 & $2.70 E+00$ \\
\hline ENSBTAG000000007421 & $4.39 \mathrm{E}-10$ & $1.63 \mathrm{E}-10$ & $\mathrm{CDH} 5$ & $2.69 \mathrm{E}+00$ \\
\hline ENSBTAG000000014583 & $1.45 \mathrm{E}-10$ & $5.36 \mathrm{E}-11$ & & $2.70 \mathrm{E}+00$ \\
\hline ENSBTAG000000038487 & $5.91 \mathrm{E}-10$ & $2.17 \mathrm{E}-10$ & ZNF613 & $2.72 \mathrm{E}+00$ \\
\hline ENSBTAG000000009585 & $5.78 \mathrm{E}-11$ & $2.08 \mathrm{E}-11$ & $\mathrm{CDH} 16$ & $2.77 E+00$ \\
\hline ENSBTAG000000005093 & $9.45 \mathrm{E}-11$ & $3.40 \mathrm{E}-11$ & CES2 & $2.77 \mathrm{E}+00$ \\
\hline ENSBTAG000000016349 & $1.62 \mathrm{E}-10$ & $5.83 \mathrm{E}-11$ & TEAD2 & $2.78 \mathrm{E}+00$ \\
\hline ENSBTAG000000020450 & $4.48 \mathrm{E}-10$ & $1.61 \mathrm{E}-10$ & & $2.78 \mathrm{E}+00$ \\
\hline ENSBTAG000000000801 & $3.47 \mathrm{E}-10$ & $1.23 \mathrm{E}-10$ & ZNF583 & $2.82 \mathrm{E}+00$ \\
\hline ENSBTAG000000015226 & $8.64 \mathrm{E}-10$ & $3.04 \mathrm{E}-10$ & BRSK1 & $2.84 \mathrm{E}+00$ \\
\hline ENSBTAG00000016968 & 2.17E-10 & 7.57E-11 & CHST4 & $2.86 \mathrm{E}+00$ \\
\hline ENSBTAG000000006354 & $4.80 \mathrm{E}-10$ & $1.68 \mathrm{E}-10$ & & $2.86 \mathrm{E}+00$ \\
\hline ENSBTAG00000030502 & $1.83 \mathrm{E}-10$ & $6.36 \mathrm{E}-11$ & & $2.87 \mathrm{E}+00$ \\
\hline ENSBTAG00000014187 & $1.68 \mathrm{E}-10$ & $5.78 \mathrm{E}-11$ & PPM1N & $2.91 E+00$ \\
\hline ENSBTAG00000000082 & $2.62 \mathrm{E}-10$ & $8.99 \mathrm{E}-11$ & KCNJ14 & $2.91 E+00$ \\
\hline ENSBTAG00000015194 & $3.47 \mathrm{E}-10$ & 1.19E-10 & CBLN1 & $2.91 E+00$ \\
\hline ENSBTAG00000001776 & $3.19 \mathrm{E}-10$ & 1.09E-10 & SIRT2 & $2.92 \mathrm{E}+00$ \\
\hline ENSBTAG00000006905 & 7.39E-10 & $2.52 \mathrm{E}-10$ & KLHDC4 & $2.93 E+00$ \\
\hline ENSBTAG00000001026 & $3.47 \mathrm{E}-10$ & 1.17E-10 & $\mathrm{BCO} 1$ & $2.96 \mathrm{E}+00$ \\
\hline ENSBTAG00000011632 & $1.60 \mathrm{E}-10$ & $5.42 \mathrm{E}-11$ & & $2.95 \mathrm{E}+00$ \\
\hline ENSBTAG00000043953 & $2.37 \mathrm{E}-10$ & $7.95 \mathrm{E}-11$ & & $2.98 \mathrm{E}+00$ \\
\hline ENSBTAG00000004939 & $3.96 \mathrm{E}-10$ & $1.31 \mathrm{E}-10$ & ZNF569 & $3.02 E+00$ \\
\hline ENSBTAG00000015694 & $8.10 \mathrm{E}-10$ & $2.68 \mathrm{E}-10$ & MON1B & $3.02 E+00$ \\
\hline ENSBTAG00000006486 & $4.82 \mathrm{E}-10$ & $1.58 \mathrm{E}-10$ & TSEN34 & $3.05 E+00$ \\
\hline ENSBTAG00000038088 & $6.92 \mathrm{E}-10$ & $2.23 \mathrm{E}-10$ & & $3.10 E+00$ \\
\hline ENSBTAG00000016407 & $4.82 \mathrm{E}-10$ & $1.56 \mathrm{E}-10$ & IRX6 & $3.09 E+00$ \\
\hline ENSBTAG00000005367 & $3.35 \mathrm{E}-10$ & $1.07 \mathrm{E}-10$ & VRK3 & $3.13 E+00$ \\
\hline ENSBTAG00000014370 & $4.98 \mathrm{E}-10$ & $1.60 \mathrm{E}-10$ & NETO2 & $3.11 E+00$ \\
\hline ENSBTAG00000047761 & $1.06 \mathrm{E}-09$ & 3.37E-10 & & $3.15 E+00$ \\
\hline ENSBTAG00000001294 & $1.93 \mathrm{E}-10$ & $6.06 \mathrm{E}-11$ & PPP1R15A & $3.18 \mathrm{E}+00$ \\
\hline ENSBTAG00000007616 & $1.25 \mathrm{E}-10$ & $3.94 \mathrm{E}-11$ & & $3.17 E+00$ \\
\hline ENSBTAG00000046626 & $9.70 \mathrm{E}-11$ & $3.05 \mathrm{E}-11$ & CNTD2 & $3.17 E+00$ \\
\hline ENSBTAG00000039111 & $2.47 \mathrm{E}-10$ & $7.76 \mathrm{E}-11$ & & $3.18 \mathrm{E}+00$ \\
\hline ENSBTAG00000006954 & $5.23 \mathrm{E}-10$ & $1.64 \mathrm{E}-10$ & ZNF432 & $3.19 E+00$ \\
\hline ENSBTAG00000002515 & $4.30 \mathrm{E}-10$ & $1.35 \mathrm{E}-10$ & & $3.18 \mathrm{E}+00$ \\
\hline ENSBTAG00000001051 & $4.81 \mathrm{E}-10$ & $1.51 E-10$ & OSCAR & $3.18 \mathrm{E}+00$ \\
\hline ENSBTAG00000048145 & $7.78 \mathrm{E}-10$ & $2.44 \mathrm{E}-10$ & & $3.19 E+00$ \\
\hline ENSBTAG00000046510 & $6.97 \mathrm{E}-10$ & $2.19 \mathrm{E}-10$ & & $3.18 \mathrm{E}+00$ \\
\hline ENSBTAG00000015260 & $7.18 \mathrm{E}-10$ & $2.25 \mathrm{E}-10$ & & $3.19 E+00$ \\
\hline ENSBTAG00000045854 & $9.01 \mathrm{E}-10$ & $2.82 \mathrm{E}-10$ & & $3.19 E+00$ \\
\hline ENSBTAG00000047030 & $1.21 \mathrm{E}-09$ & $3.78 \mathrm{E}-10$ & & $3.20 \mathrm{E}+00$ \\
\hline ENSBTAG00000047791 & 1.04E-09 & $3.24 \mathrm{E}-10$ & & $3.20 \mathrm{E}+00$ \\
\hline ENSBTAG00000046947 & $1.02 \mathrm{E}-09$ & 3.19E-10 & & $3.20 \mathrm{E}+00$ \\
\hline ENSBTAG00000020449 & $7.21 \mathrm{E}-10$ & $2.22 \mathrm{E}-10$ & ETFB & $3.25 E+00$ \\
\hline ENSBTAG00000003644 & $6.00 \mathrm{E}-10$ & $1.83 \mathrm{E}-10$ & HSPBP1 & $3.28 \mathrm{E}+00$ \\
\hline ENSBTAG00000006612 & $2.22 \mathrm{E}-10$ & $6.75 E-11$ & SCAF1 & $3.29 E+00$ \\
\hline ENSBTAG00000002853 & $4.56 \mathrm{E}-10$ & $1.38 \mathrm{E}-10$ & $\mathrm{HRC}$ & $3.31 E+00$ \\
\hline ENSBTAG00000031441 & $6.97 \mathrm{E}-10$ & $2.09 \mathrm{E}-10$ & FXYD5 & $3.33 E+00$ \\
\hline
\end{tabular}




\begin{tabular}{|c|c|c|c|c|}
\hline ENSBTAG00000001775 & $2.02 \mathrm{E}-10$ & $6.06 \mathrm{E}-11$ & RINL & $3.33 \mathrm{E}+00$ \\
\hline ENSBTAG00000002522 & $7.38 \mathrm{E}-10$ & $2.19 \mathrm{E}-10$ & SYT5 & $3.37 E+00$ \\
\hline ENSBTAG00000045880 & $1.01 \mathrm{E}-09$ & $3.00 \mathrm{E}-10$ & & $3.37 E+00$ \\
\hline ENSBTAG00000002844 & $8.69 \mathrm{E}-11$ & $2.57 \mathrm{E}-11$ & PPFIA3 & $3.37 \mathrm{E}+00$ \\
\hline ENSBTAG00000012606 & $6.45 \mathrm{E}-10$ & $1.90 \mathrm{E}-10$ & ZNF541 & $3.39 \mathrm{E}+00$ \\
\hline ENSBTAG00000017033 & $2.74 \mathrm{E}-10$ & 7.95E-11 & & $3.44 E+00$ \\
\hline ENSBTAG00000005766 & $4.21 \mathrm{E}-10$ & $1.21 \mathrm{E}-10$ & IL4I1 & $3.47 \mathrm{E}+00$ \\
\hline ENSBTAG00000014454 & $1.93 \mathrm{E}-10$ & $5.51 \mathrm{E}-11$ & PRR12 & $3.49 E+00$ \\
\hline ENSBTAG00000047219 & $2.99 \mathrm{E}-10$ & $8.54 \mathrm{E}-11$ & ZNF793 & $3.50 E+00$ \\
\hline ENSBTAG00000018948 & $6.55 \mathrm{E}-10$ & $1.87 \mathrm{E}-10$ & HIF3A & $3.50 \mathrm{E}+00$ \\
\hline ENSBTAG00000010856 & $2.84 \mathrm{E}-10$ & $8.02 \mathrm{E}-11$ & SLC8A2 & $3.54 E+00$ \\
\hline ENSBTAG00000011212 & $1.14 \mathrm{E}-10$ & $3.20 \mathrm{E}-11$ & EIF3K & $3.57 E+00$ \\
\hline ENSBTAG00000007757 & $4.70 \mathrm{E}-10$ & $1.31 \mathrm{E}-10$ & PRPF31 & $3.59 \mathrm{E}+00$ \\
\hline ENSBTAG00000000930 & $6.80 \mathrm{E}-10$ & $1.89 \mathrm{E}-10$ & & $3.59 E+00$ \\
\hline ENSBTAG00000047345 & $3.83 \mathrm{E}-10$ & $1.06 \mathrm{E}-10$ & & $3.61 E+00$ \\
\hline ENSBTAG00000015165 & $3.25 \mathrm{E}-10$ & $9.00 \mathrm{E}-11$ & DPY19L3 & $3.60 \mathrm{E}+00$ \\
\hline ENSBTAG00000017814 & $4.69 \mathrm{E}-10$ & $1.29 \mathrm{E}-10$ & LGI4 & $3.63 E+00$ \\
\hline ENSBTAG00000004248 & $2.93 \mathrm{E}-10$ & $8.00 \mathrm{E}-11$ & & $3.66 \mathrm{E}+00$ \\
\hline ENSBTAG00000006419 & $6.95 \mathrm{E}-10$ & $1.87 \mathrm{E}-10$ & TNNT1 & $3.71 E+00$ \\
\hline ENSBTAG00000015318 & $3.94 \mathrm{E}-10$ & $1.05 \mathrm{E}-10$ & NECTIN2 & $3.75 E+00$ \\
\hline ENSBTAG00000048184 & $1.59 \mathrm{E}-09$ & $4.22 \mathrm{E}-10$ & & $3.76 \mathrm{E}+00$ \\
\hline ENSBTAG00000008014 & $6.65 \mathrm{E}-10$ & $1.76 \mathrm{E}-10$ & PPP5C & $3.78 \mathrm{E}+00$ \\
\hline ENSBTAG00000020560 & $2.49 \mathrm{E}-10$ & $6.59 \mathrm{E}-11$ & CLPTM1 & $3.78 \mathrm{E}+00$ \\
\hline ENSBTAG00000013440 & $2.65 \mathrm{E}-10$ & $6.94 \mathrm{E}-11$ & RBM42 & $3.82 E+00$ \\
\hline ENSBTAG00000013266 & $4.55 \mathrm{E}-10$ & $1.19 \mathrm{E}-10$ & PMFBP1 & $3.82 \mathrm{E}+00$ \\
\hline ENSBTAG00000045861 & $8.22 \mathrm{E}-10$ & $2.14 \mathrm{E}-10$ & TARM1 & $3.84 E+00$ \\
\hline ENSBTAG00000016125 & $1.51 \mathrm{E}-10$ & $3.80 \mathrm{E}-11$ & KCNA7 & $3.97 \mathrm{E}+00$ \\
\hline ENSBTAG00000023752 & $1.54 \mathrm{E}-10$ & $3.88 \mathrm{E}-11$ & PABPN1L & $3.97 \mathrm{E}+00$ \\
\hline ENSBTAG00000008544 & $2.03 \mathrm{E}-10$ & $5.12 \mathrm{E}-11$ & SALL1 & $3.96 \mathrm{E}+00$ \\
\hline ENSBTAG00000013882 & $1.52 \mathrm{E}-10$ & $3.82 \mathrm{E}-11$ & CHMP2A & $3.97 \mathrm{E}+00$ \\
\hline ENSBTAG00000033331 & $5.99 \mathrm{E}-11$ & $1.51 \mathrm{E}-11$ & CHMP1A & $3.95 E+00$ \\
\hline ENSBTAG00000020983 & $1.02 \mathrm{E}-10$ & $2.56 \mathrm{E}-11$ & RRAS & $3.96 \mathrm{E}+00$ \\
\hline ENSBTAG00000021165 & $4.36 \mathrm{E}-11$ & 1.10E-11 & VPS9D1 & $3.93 E+00$ \\
\hline ENSBTAG00000023765 & $1.72 \mathrm{E}-10$ & 4.33E-11 & C16orf46 & $3.96 \mathrm{E}+00$ \\
\hline ENSBTAG00000007509 & $8.62 \mathrm{E}-10$ & $2.16 \mathrm{E}-10$ & CACNG8 & $3.99 E+00$ \\
\hline ENSBTAG00000046367 & $5.88 \mathrm{E}-10$ & $1.44 \mathrm{E}-10$ & & $4.08 \mathrm{E}+00$ \\
\hline ENSBTAG00000005261 & $4.28 \mathrm{E}-10$ & $1.02 \mathrm{E}-10$ & & $4.19 E+00$ \\
\hline ENSBTAG00000017651 & $6.02 \mathrm{E}-10$ & $1.42 \mathrm{E}-10$ & ZNF665 & $4.24 \mathrm{E}+00$ \\
\hline ENSBTAG00000004735 & $1.37 \mathrm{E}-10$ & $3.24 \mathrm{E}-11$ & CCNE1 & $4.23 E+00$ \\
\hline ENSBTAG00000012189 & $6.90 \mathrm{E}-10$ & $1.62 \mathrm{E}-10$ & TMEM86B & $4.26 \mathrm{E}+00$ \\
\hline ENSBTAG00000009364 & $1.57 \mathrm{E}-09$ & $3.68 \mathrm{E}-10$ & & $4.27 E+00$ \\
\hline ENSBTAG00000009746 & $4.89 \mathrm{E}-10$ & $1.14 \mathrm{E}-10$ & MPHOSPH6 & $4.29 E+00$ \\
\hline ENSBTAG00000009918 & $2.55 \mathrm{E}-10$ & 5.67E-11 & GSE1 & $4.50 \mathrm{E}+00$ \\
\hline ENSBTAG00000006487 & $1.35 \mathrm{E}-09$ & $2.97 \mathrm{E}-10$ & RPS9 & $4.54 \mathrm{E}+00$ \\
\hline ENSBTAG00000015989 & $3.38 \mathrm{E}-10$ & $7.38 \mathrm{E}-11$ & RPS5 & $4.57 E+00$ \\
\hline ENSBTAG00000046295 & $2.00 \mathrm{E}-10$ & $4.20 \mathrm{E}-11$ & CCER2 & $4.76 \mathrm{E}+00$ \\
\hline ENSBTAG00000001906 & $5.25 \mathrm{E}-11$ & 1.10E-11 & FANCA & $4.74 E+00$ \\
\hline ENSBTAG00000033326 & $8.28 \mathrm{E}-11$ & $1.74 \mathrm{E}-11$ & DPEP1 & $4.74 \mathrm{E}+00$ \\
\hline ENSBTAG00000002687 & $3.05 \mathrm{E}-10$ & $6.38 \mathrm{E}-11$ & TSHZ3 & $4.77 \mathrm{E}+00$ \\
\hline ENSBTAG00000038442 & $3.54 \mathrm{E}-10$ & 7.41E-11 & WDR87 & $4.77 \mathrm{E}+00$ \\
\hline ENSBTAG00000007506 & $1.12 \mathrm{E}-09$ & $2.35 \mathrm{E}-10$ & CACNG7 & $4.75 E+00$ \\
\hline ENSBTAG00000039341 & $4.68 \mathrm{E}-10$ & $9.80 \mathrm{E}-11$ & & $4.77 \mathrm{E}+00$ \\
\hline ENSBTAG00000018152 & $5.30 \mathrm{E}-10$ & $1.11 \mathrm{E}-10$ & & $4.77 \mathrm{E}+00$ \\
\hline ENSBTAG00000001062 & $6.19 \mathrm{E}-10$ & $1.29 \mathrm{E}-10$ & PTH2 & $4.79 E+00$ \\
\hline ENSBTAG00000023614 & $5.44 \mathrm{E}-10$ & $1.14 \mathrm{E}-10$ & NUDT19 & $4.76 \mathrm{E}+00$ \\
\hline ENSBTAG00000007754 & $7.38 \mathrm{E}-10$ & $1.54 \mathrm{E}-10$ & & $4.79 E+00$ \\
\hline ENSBTAG00000020080 & $6.30 \mathrm{E}-10$ & $1.29 \mathrm{E}-10$ & MYBPC2 & $4.88 \mathrm{E}+00$ \\
\hline ENSBTAG00000020447 & $7.03 \mathrm{E}-10$ & $1.44 \mathrm{E}-10$ & VSIG10L & $4.88 \mathrm{E}+00$ \\
\hline ENSBTAG00000007756 & $6.68 \mathrm{E}-10$ & $1.35 \mathrm{E}-10$ & TFPT & $4.94 \mathrm{E}+00$ \\
\hline ENSBTAG00000001654 & $3.61 \mathrm{E}-10$ & $7.26 \mathrm{E}-11$ & SNTB2 & $4.96 \mathrm{E}+00$ \\
\hline ENSBTAG00000014593 & $5.75 \mathrm{E}-10$ & $1.15 \mathrm{E}-10$ & & $4.99 E+00$ \\
\hline ENSBTAG00000008203 & $3.58 \mathrm{E}-10$ & $7.09 \mathrm{E}-11$ & SELENOW & $5.04 E+00$ \\
\hline ENSBTAG00000001778 & $4.48 \mathrm{E}-10$ & 8.73E-11 & NFKBIB & $5.13 \mathrm{E}+00$ \\
\hline ENSBTAG00000002242 & $3.72 \mathrm{E}-10$ & $7.14 \mathrm{E}-11$ & FAM192A & $5.20 \mathrm{E}+00$ \\
\hline ENSBTAG00000016878 & 7.11E-10 & $1.36 \mathrm{E}-10$ & WDR59 & $5.22 \mathrm{E}+00$ \\
\hline ENSBTAG00000010508 & $3.47 \mathrm{E}-10$ & $6.66 \mathrm{E}-11$ & BLVRB & $5.20 \mathrm{E}+00$ \\
\hline ENSBTAG00000016113 & 1.13E-09 & 2.17E-10 & ELSPBP1 & $5.23 \mathrm{E}+00$ \\
\hline ENSBTAG00000045692 & $8.87 \mathrm{E}-10$ & $1.69 \mathrm{E}-10$ & & $5.24 \mathrm{E}+00$ \\
\hline ENSBTAG00000021417 & $2.38 \mathrm{E}-10$ & 4.49E-11 & DMWD & $5.29 \mathrm{E}+00$ \\
\hline ENSBTAG00000009363 & $5.08 \mathrm{E}-10$ & 9.57E-11 & GINS3 & $5.31 E+00$ \\
\hline
\end{tabular}




\begin{tabular}{|c|c|c|c|c|}
\hline ENSBTAG000000037830 & 7.45E-10 & $1.40 \mathrm{E}-10$ & & $5.32 \mathrm{E}+00$ \\
\hline ENSBTAG00000018326 & $4.28 \mathrm{E}-10$ & $8.00 \mathrm{E}-11$ & FXYD7 & $5.34 \mathrm{E}+00$ \\
\hline ENSBTAG00000023333 & $6.76 \mathrm{E}-10$ & $1.25 \mathrm{E}-10$ & LENG1 & $5.40 \mathrm{E}+00$ \\
\hline ENSBTAG000000030393 & $1.28 \mathrm{E}-09$ & 2.37E-10 & $\mathrm{RDH} 13$ & $5.39 E+00$ \\
\hline ENSBTAG000000020082 & $6.16 \mathrm{E}-10$ & $1.13 \mathrm{E}-10$ & FAM71E1 & $5.45 E+00$ \\
\hline ENSBTAG000000007096 & $1.69 \mathrm{E}-10$ & 3.03E-11 & & $5.56 \mathrm{E}+00$ \\
\hline ENSBTAG000000009494 & $1.10 \mathrm{E}-10$ & $1.98 \mathrm{E}-11$ & CBLC & $5.53 E+00$ \\
\hline ENSBTAG000000018633 & $1.99 \mathrm{E}-10$ & $3.58 \mathrm{E}-11$ & RABAC1 & $5.56 \mathrm{E}+00$ \\
\hline ENSBTAG000000006566 & $5.32 \mathrm{E}-10$ & $9.54 \mathrm{E}-11$ & FFAR2 & $5.57 \mathrm{E}+00$ \\
\hline ENSBTAG000000024895 & $1.76 \mathrm{E}-09$ & 3.13E-10 & & $5.64 \mathrm{E}+00$ \\
\hline ENSBTAG000000009512 & $9.49 \mathrm{E}-10$ & $1.68 \mathrm{E}-10$ & EPS8L1 & $5.65 E+00$ \\
\hline ENSBTAG000000003798 & $6.92 \mathrm{E}-10$ & $1.22 \mathrm{E}-10$ & AMFR & $5.67 E+00$ \\
\hline ENSBTAG00000037888 & $5.19 \mathrm{E}-10$ & $8.89 \mathrm{E}-11$ & LYPD5 & $5.84 \mathrm{E}+00$ \\
\hline ENSBTAG000000009493 & $1.99 \mathrm{E}-10$ & $3.42 \mathrm{E}-11$ & $\mathrm{BCL3}$ & $5.81 E+00$ \\
\hline ENSBTAG000000023365 & $6.53 \mathrm{E}-10$ & $1.12 \mathrm{E}-10$ & & $5.83 E+00$ \\
\hline ENSBTAG000000039491 & $6.59 \mathrm{E}-10$ & $1.10 \mathrm{E}-10$ & & $5.99 E+00$ \\
\hline ENSBTAG000000047617 & 1.31E-09 & 2.17E-10 & & $6.02 \mathrm{E}+00$ \\
\hline ENSBTAG000000002580 & 7.65E-10 & $1.23 \mathrm{E}-10$ & MYH14 & $6.22 \mathrm{E}+00$ \\
\hline ENSBTAG000000006424 & $8.52 E-10$ & $1.37 \mathrm{E}-10$ & TNNI3 & $6.21 E+00$ \\
\hline ENSBTAG000000019887 & $4.83 \mathrm{E}-10$ & $7.68 \mathrm{E}-11$ & & $6.29 E+00$ \\
\hline ENSBTAG000000023415 & $1.81 \mathrm{E}-10$ & $2.84 \mathrm{E}-11$ & LIN7B & $6.35 E+00$ \\
\hline ENSBTAG00000019150 & $6.97 \mathrm{E}-11$ & $1.10 \mathrm{E}-11$ & PLD3 & $6.29 E+00$ \\
\hline ENSBTAG000000002070 & $8.01 \mathrm{E}-11$ & $1.26 \mathrm{E}-11$ & $\mathrm{KLC3}$ & $6.32 E+00$ \\
\hline ENSBTAG000000039014 & $5.29 \mathrm{E}-11$ & $8.32 \mathrm{E}-12$ & DEF8 & $6.29 E+00$ \\
\hline ENSBTAG000000015061 & $3.31 \mathrm{E}-10$ & $5.20 \mathrm{E}-11$ & & $6.36 \mathrm{E}+00$ \\
\hline ENSBTAG000000006347 & $4.85 \mathrm{E}-10$ & $7.61 \mathrm{E}-11$ & UTP4 & $6.37 E+00$ \\
\hline ENSBTAG000000045806 & $7.30 \mathrm{E}-10$ & $1.14 \mathrm{E}-10$ & FFAR1 & $6.40 \mathrm{E}+00$ \\
\hline ENSBTAG000000002226 & $5.21 \mathrm{E}-10$ & $8.10 \mathrm{E}-11$ & & $6.42 \mathrm{E}+00$ \\
\hline ENSBTAG00000013436 & $9.71 \mathrm{E}-10$ & $1.50 \mathrm{E}-10$ & HAUS5 & $6.47 E+00$ \\
\hline ENSBTAG000000046805 & $2.89 \mathrm{E}-10$ & $4.36 \mathrm{E}-11$ & CYP2F1 & $6.62 E+00$ \\
\hline ENSBTAG00000019473 & $9.15 \mathrm{E}-10$ & $1.36 \mathrm{E}-10$ & IGLON5 & $6.72 \mathrm{E}+00$ \\
\hline ENSBTAG000000003541 & $5.47 \mathrm{E}-10$ & 8.07E-11 & ZNF614 & $6.77 \mathrm{E}+00$ \\
\hline ENSBTAG00000039212 & $7.62 \mathrm{E}-10$ & $1.11 \mathrm{E}-10$ & & $6.86 \mathrm{E}+00$ \\
\hline ENSBTAG000000047953 & $9.11 E-10$ & $1.32 \mathrm{E}-10$ & & $6.90 \mathrm{E}+00$ \\
\hline ENSBTAG000000001994 & $6.90 \mathrm{E}-10$ & $9.83 \mathrm{E}-11$ & FAM187B & $7.01 E+00$ \\
\hline ENSBTAG00000013346 & $2.08 \mathrm{E}-10$ & $2.91 \mathrm{E}-11$ & SIX5 & $7.14 \mathrm{E}+00$ \\
\hline ENSBTAG00000047158 & $1.23 \mathrm{E}-10$ & $1.71 \mathrm{E}-11$ & LRFN1 & $7.13 E+00$ \\
\hline ENSBTAG00000012538 & $7.46 \mathrm{E}-10$ & $1.04 \mathrm{E}-10$ & KLK14 & $7.17 E+00$ \\
\hline ENSBTAG00000046908 & 1.30E-09 & $1.80 \mathrm{E}-10$ & & $7.21 \mathrm{E}+00$ \\
\hline ENSBTAG00000018162 & $1.65 \mathrm{E}-09$ & $2.28 \mathrm{E}-10$ & VN1R4 & $7.24 E+00$ \\
\hline ENSBTAG00000004842 & $1.83 \mathrm{E}-10$ & $2.50 \mathrm{E}-11$ & RSPRY1 & $7.30 E+00$ \\
\hline ENSBTAG00000000803 & $6.13 \mathrm{E}-10$ & $8.33 \mathrm{E}-11$ & ZNF667 & $7.35 \mathrm{E}+00$ \\
\hline ENSBTAG00000012509 & $2.05 \mathrm{E}-10$ & $2.71 E-11$ & DYRK1B & $7.54 \mathrm{E}+00$ \\
\hline ENSBTAG00000017333 & $1.03 \mathrm{E}-10$ & $1.30 \mathrm{E}-11$ & PRX & $7.87 E+00$ \\
\hline ENSBTAG00000047654 & $4.01 \mathrm{E}-10$ & $5.04 \mathrm{E}-11$ & СMTM3 & $7.95 E+00$ \\
\hline ENSBTAG00000046425 & $6.71 \mathrm{E}-10$ & $8.41 E-11$ & LEUTX & $7.97 \mathrm{E}+00$ \\
\hline ENSBTAG00000003757 & 4.33E-10 & 5.35E-11 & DNAJA2 & $8.09 E+00$ \\
\hline ENSBTAG00000008852 & $8.16 \mathrm{E}-10$ & $1.01 \mathrm{E}-10$ & SIGLEC10 & $8.07 E+00$ \\
\hline ENSBTAG00000013550 & $6.65 \mathrm{E}-10$ & 8.01E-11 & PRKCG & $8.29 E+00$ \\
\hline ENSBTAG00000012215 & $1.43 \mathrm{E}-10$ & $1.71 E-11$ & CPNE7 & $8.30 \mathrm{E}+00$ \\
\hline ENSBTAG00000002764 & $3.44 \mathrm{E}-10$ & $3.93 \mathrm{E}-11$ & IGFLR1 & $8.74 \mathrm{E}+00$ \\
\hline ENSBTAG00000033441 & $3.02 \mathrm{E}-10$ & 3.32E-11 & SHCBP1 & $9.06 \mathrm{E}+00$ \\
\hline ENSBTAG00000046544 & $5.94 \mathrm{E}-10$ & $6.42 \mathrm{E}-11$ & SLC7A10 & $9.25 E+00$ \\
\hline ENSBTAG00000016034 & $1.14 \mathrm{E}-10$ & $1.19 \mathrm{E}-11$ & EXOSC5 & $9.47 \mathrm{E}+00$ \\
\hline ENSBTAG00000017035 & 3.07E-10 & $3.21 \mathrm{E}-11$ & USF2 & $9.54 E+00$ \\
\hline ENSBTAG00000025288 & $1.06 \mathrm{E}-09$ & $1.10 \mathrm{E}-10$ & & $9.59 E+00$ \\
\hline ENSBTAG00000004611 & $8.17 \mathrm{E}-10$ & $8.18 \mathrm{E}-11$ & CEACAM18 & $9.98 \mathrm{E}+00$ \\
\hline ENSBTAG00000025023 & $9.28 \mathrm{E}-10$ & $9.28 \mathrm{E}-11$ & & $9.99 \mathrm{E}+00$ \\
\hline ENSBTAG00000030563 & $1.91 \mathrm{E}-10$ & $1.84 \mathrm{E}-11$ & PRRG2 & $1.03 E+01$ \\
\hline ENSBTAG00000038051 & $5.20 \mathrm{E}-11$ & $5.04 \mathrm{E}-12$ & & $1.01 E+01$ \\
\hline ENSBTAG00000009735 & $5.34 \mathrm{E}-10$ & $4.96 \mathrm{E}-11$ & A1BG & $1.07 \mathrm{E}+01$ \\
\hline ENSBTAG00000023610 & $4.98 \mathrm{E}-10$ & 4.46E-11 & & $1.11 \mathrm{E}+01$ \\
\hline ENSBTAG00000016006 & $1.53 \mathrm{E}-10$ & $1.28 \mathrm{E}-11$ & ANKRD11 & $1.18 \mathrm{E}+01$ \\
\hline ENSBTAG00000015115 & $4.80 \mathrm{E}-10$ & $4.02 \mathrm{E}-11$ & $\mathrm{ACPT}$ & $1.19 \mathrm{E}+01$ \\
\hline ENSBTAG00000046472 & $1.34 \mathrm{E}-09$ & $1.11 \mathrm{E}-10$ & & $1.20 \mathrm{E}+01$ \\
\hline ENSBTAG00000012041 & $2.35 \mathrm{E}-10$ & $1.94 \mathrm{E}-11$ & SPG7 & $1.21 \mathrm{E}+01$ \\
\hline ENSBTAG00000045989 & 1.00E-09 & $8.23 \mathrm{E}-11$ & CDC42EP5 & $1.22 \mathrm{E}+01$ \\
\hline ENSBTAG00000002223 & $1.42 \mathrm{E}-10$ & $1.12 \mathrm{E}-11$ & POP4 & $1.26 \mathrm{E}+01$ \\
\hline ENSBTAG00000018539 & $5.17 \mathrm{E}-10$ & $4.06 \mathrm{E}-11$ & KLK1 & $1.27 \mathrm{E}+01$ \\
\hline ENSBTAG00000030484 & $6.88 \mathrm{E}-10$ & $5.22 \mathrm{E}-11$ & KLK12 & $1.32 \mathrm{E}+01$ \\
\hline
\end{tabular}




\begin{tabular}{|c|c|c|c|c|}
\hline ENSBTAG00000002290 & $6.45 \mathrm{E}-10$ & $4.85 \mathrm{E}-11$ & & $1.33 E+01$ \\
\hline ENSBTAG00000044113 & $7.84 \mathrm{E}-10$ & $5.01 \mathrm{E}-11$ & IZUMO2 & $1.56 \mathrm{E}+01$ \\
\hline ENSBTAG00000009382 & $9.21 \mathrm{E}-10$ & $5.42 \mathrm{E}-11$ & KLK13 & $1.70 \mathrm{E}+01$ \\
\hline ENSBTAG00000011079 & $6.14 \mathrm{E}-10$ & $3.21 \mathrm{E}-11$ & & $1.91 E+01$ \\
\hline ENSBTAG00000018545 & 4.57E-10 & $2.12 \mathrm{E}-11$ & KLK15 & $2.15 \mathrm{E}+01$ \\
\hline ENSBTAG00000040177 & 7.30E-10 & 3.39E-11 & & $2.15 E+01$ \\
\hline ENSBTAG00000038526 & $1.26 \mathrm{E}-09$ & $5.33 \mathrm{E}-11$ & & $2.35 \mathrm{E}+01$ \\
\hline ENSBTAG00000015313 & $2.81 \mathrm{E}-10$ & $1.12 \mathrm{E}-11$ & CEACAM19 & $2.49 \mathrm{E}+01$ \\
\hline ENSBTAG00000015129 & $9.12 \mathrm{E}-10$ & $3.45 \mathrm{E}-11$ & KLK10 & $2.64 \mathrm{E}+01$ \\
\hline ENSBTAG00000015128 & 7.47E-10 & $1.99 \mathrm{E}-11$ & KLK8 & $3.74 \mathrm{E}+01$ \\
\hline ENSBTAG00000035868 & $1.06 \mathrm{E}-09$ & $2.59 \mathrm{E}-11$ & & $4.06 \mathrm{E}+01$ \\
\hline ENSBTAG00000047675 & 1.57E-09 & 3.57E-11 & & $4.39 \mathrm{E}+01$ \\
\hline ENSBTAG00000008851 & $1.01 \mathrm{E}-09$ & $2.26 \mathrm{E}-11$ & & 4.47E+01 \\
\hline ENSBTAG00000030482 & 7.84E-10 & $1.21 \mathrm{E}-11$ & KLK6 & $6.43 E+01$ \\
\hline ENSBTAG00000015125 & 7.17E-10 & $1.08 \mathrm{E}-11$ & KLK5 & $6.58 \mathrm{E}+01$ \\
\hline ENSBTAG00000011533 & $5.38 \mathrm{E}-11$ & 0 & & $5.39 E+02$ \\
\hline ENSBTAG00000012044 & $1.03 \mathrm{E}-10$ & 0 & RPL13 & $1.03 E+03$ \\
\hline ENSBTAG00000033333 & $4.59 \mathrm{E}-11$ & 0 & CDK10 & $4.60 E+02$ \\
\hline ENSBTAG00000001904 & $7.40 \mathrm{E}-11$ & 0 & ZNF276 & $7.41 E+02$ \\
\hline ENSBTAG00000023730 & $9.46 \mathrm{E}-12$ & 0 & TUBB3 & $9.56 \mathrm{E}+01$ \\
\hline ENSBTAG00000020318 & $4.15 \mathrm{E}-11$ & 0 & & $4.16 \mathrm{E}+02$ \\
\hline ENSBTAG00000012083 & $1.32 \mathrm{E}-10$ & 0 & IRX3 & $1.32 \mathrm{E}+03$ \\
\hline ENSBTAG00000004844 & $1.58 \mathrm{E}-10$ & 0 & ARL2BP & $1.58 \mathrm{E}+03$ \\
\hline ENSBTAG00000018043 & $2.52 \mathrm{E}-11$ & 0 & LCAT & $2.53 \mathrm{E}+02$ \\
\hline ENSBTAG00000017042 & $5.12 \mathrm{E}-10$ & 0 & HAMP & $5.12 E+03$ \\
\hline ENSBTAG00000031420 & $2.86 \mathrm{E}-10$ & 0 & & $2.86 \mathrm{E}+03$ \\
\hline ENSBTAG00000013444 & $1.05 \mathrm{E}-10$ & 0 & ETV2 & $1.05 \mathrm{E}+03$ \\
\hline ENSBTAG00000007338 & $2.43 \mathrm{E}-10$ & 0 & TYROBP & $2.43 E+03$ \\
\hline ENSBTAG00000015002 & $1.32 \mathrm{E}-10$ & 0 & POLR2I & $1.32 \mathrm{E}+03$ \\
\hline ENSBTAG00000001782 & $8.65 E-11$ & 0 & MRPS12 & $8.66 E+02$ \\
\hline ENSBTAG00000010493 & $2.27 \mathrm{E}-10$ & 0 & SERTAD1 & $2.27 E+03$ \\
\hline ENSBTAG00000016041 & $9.11 \mathrm{E}-11$ & 0 & B3GNT8 & $9.12 \mathrm{E}+02$ \\
\hline ENSBTAG00000020754 & $4.95 \mathrm{E}-11$ & 0 & ZNF526 & $4.96 E+02$ \\
\hline ENSBTAG00000040054 & $1.04 \mathrm{E}-10$ & 0 & & $1.04 \mathrm{E}+03$ \\
\hline ENSBTAG00000011624 & $5.61 \mathrm{E}-11$ & 0 & FGF21 & $5.62 \mathrm{E}+02$ \\
\hline ENSBTAG00000047078 & 0 & 0 & NTF4 & $1.00 \mathrm{E}+00$ \\
\hline ENSBTAG00000030483 & $7.58 \mathrm{E}-10$ & 0 & KLK7 & $7.58 \mathrm{E}+03$ \\
\hline ENSBTAG00000002257 & 7.26E-10 & 0 & KLK11 & $7.26 \mathrm{E}+03$ \\
\hline ENSBTAG00000037721 & $2.94 \mathrm{E}-10$ & 0 & & $2.94 \mathrm{E}+03$ \\
\hline ENSBTAG00000046987 & $1.83 \mathrm{E}-10$ & 0 & CENPBD1 & $1.83 E+03$ \\
\hline ENSBTAG00000040605 & 0 & 0 & FOXL1 & $1.00 \mathrm{E}+00$ \\
\hline ENSBTAG00000023731 & 0 & 0 & MC1R & $1.00 \mathrm{E}+00$ \\
\hline ENSBTAG00000046255 & $7.94 \mathrm{E}-11$ & 0 & ATXN1L & $7.95 E+02$ \\
\hline ENSBTAG00000047787 & 0 & 0 & & $1.00 E+00$ \\
\hline ENSBTAG00000045656 & $1.52 \mathrm{E}-09$ & 0 & & $1.52 E+04$ \\
\hline ENSBTAG00000045930 & 0 & 0 & SYCN & $1.00 \mathrm{E}+00$ \\
\hline ENSBTAG00000001350 & $2.97 \mathrm{E}-10$ & 0 & & $2.97 E+03$ \\
\hline ENSBTAG00000007070 & 0 & 0 & & $1.00 \mathrm{E}+00$ \\
\hline ENSBTAG00000045864 & $1.37 \mathrm{E}-10$ & 0 & ZNF581 & $1.37 \mathrm{E}+03$ \\
\hline ENSBTAG00000044492 & $4.35 \mathrm{E}-10$ & 0 & & $4.35 E+03$ \\
\hline ENSBTAG00000034302 & $2.53 \mathrm{E}-10$ & 0 & & $2.53 E+03$ \\
\hline ENSBTAG00000017221 & 0 & $1.72 \mathrm{E}-10$ & & 5.81E-04 \\
\hline ENSBTAG00000013898 & 0 & $4.61 E-10$ & NPB & 2.17E-04 \\
\hline ENSBTAG00000030186 & 0 & $3.52 \mathrm{E}-10$ & & $2.84 \mathrm{E}-04$ \\
\hline ENSBTAG00000048260 & 7.63E-11 & $5.79 \mathrm{E}-10$ & & $1.32 \mathrm{E}-01$ \\
\hline ENSBTAG00000000666 & $8.75 \mathrm{E}-11$ & $6.08 \mathrm{E}-10$ & GHDC & 1.44E-01 \\
\hline ENSBTAG00000015298 & $2.43 \mathrm{E}-11$ & $1.53 \mathrm{E}-10$ & TNFRSF13B & $1.60 \mathrm{E}-01$ \\
\hline ENSBTAG00000006777 & $6.73 \mathrm{E}-11$ & $2.55 \mathrm{E}-10$ & TLCD1 & 2.64E-01 \\
\hline ENSBTAG00000007603 & $6.02 \mathrm{E}-11$ & $2.28 \mathrm{E}-10$ & HOXB1 & $2.64 \mathrm{E}-01$ \\
\hline ENSBTAG00000018897 & $5.32 \mathrm{E}-11$ & $1.73 \mathrm{E}-10$ & PSMD11 & $3.08 \mathrm{E}-01$ \\
\hline ENSBTAG00000010576 & $9.19 \mathrm{E}-11$ & $2.72 \mathrm{E}-10$ & & $3.38 \mathrm{E}-01$ \\
\hline ENSBTAG00000015951 & $1.93 \mathrm{E}-10$ & $5.60 \mathrm{E}-10$ & NOL11 & $3.45 \mathrm{E}-01$ \\
\hline ENSBTAG00000006982 & $1.13 \mathrm{E}-10$ & 3.26E-10 & & $3.46 \mathrm{E}-01$ \\
\hline ENSBTAG00000047262 & $1.61 \mathrm{E}-10$ & $4.58 \mathrm{E}-10$ & & $3.52 \mathrm{E}-01$ \\
\hline ENSBTAG00000017969 & $1.58 \mathrm{E}-10$ & 4.39E-10 & CA4 & $3.60 \mathrm{E}-01$ \\
\hline ENSBTAG00000008978 & $4.89 \mathrm{E}-11$ & $1.34 \mathrm{E}-10$ & USP22 & 3.65E-01 \\
\hline ENSBTAG00000001195 & $1.97 \mathrm{E}-10$ & $5.44 \mathrm{E}-10$ & $\mathrm{KCNH} 4$ & $3.62 \mathrm{E}-01$ \\
\hline ENSBTAG00000006806 & 2.13E-10 & $5.65 \mathrm{E}-10$ & KRT17 & $3.77 \mathrm{E}-01$ \\
\hline ENSBTAG00000014663 & 8.99E-11 & 2.27E-10 & HOXB13 & $3.96 \mathrm{E}-01$ \\
\hline ENSBTAG00000045824 & $4.39 \mathrm{E}-11$ & $1.11 \mathrm{E}-10$ & & $3.96 \mathrm{E}-01$ \\
\hline
\end{tabular}




\begin{tabular}{|c|c|c|c|c|}
\hline ENSBTAG00000010317 & $1.19 \mathrm{E}-10$ & $2.99 \mathrm{E}-10$ & SPPL2C & 3.97E-01 \\
\hline ENSBTAG00000025121 & $9.64 \mathrm{E}-11$ & $2.43 \mathrm{E}-10$ & EMC6 & 3.97E-01 \\
\hline ENSBTAG00000000665 & $6.57 \mathrm{E}-11$ & $1.66 \mathrm{E}-10$ & HCRT & $3.96 \mathrm{E}-01$ \\
\hline ENSBTAG00000018221 & $8.48 \mathrm{E}-11$ & $2.14 \mathrm{E}-10$ & & 3.96E-01 \\
\hline ENSBTAG00000020590 & 4.60E-11 & $1.16 \mathrm{E}-10$ & FZD2 & 3.97E-01 \\
\hline ENSBTAG00000014278 & $1.79 \mathrm{E}-10$ & $4.40 \mathrm{E}-10$ & TBX2 & $4.08 \mathrm{E}-01$ \\
\hline ENSBTAG00000004680 & $1.44 \mathrm{E}-10$ & $3.18 \mathrm{E}-10$ & SLC13A5 & 4.52E-01 \\
\hline ENSBTAG00000009635 & $2.89 \mathrm{E}-10$ & $6.35 \mathrm{E}-10$ & HEATR6 & 4.55E-01 \\
\hline ENSBTAG00000011456 & $2.98 \mathrm{E}-10$ & $6.45 \mathrm{E}-10$ & NT5C3B & 4.62E-01 \\
\hline ENSBTAG00000033298 & $2.87 \mathrm{E}-10$ & $6.03 \mathrm{E}-10$ & & $4.75 \mathrm{E}-01$ \\
\hline ENSBTAG00000013906 & $1.76 \mathrm{E}-10$ & $3.71 \mathrm{E}-10$ & & $4.75 \mathrm{E}-01$ \\
\hline ENSBTAG00000009864 & $2.78 \mathrm{E}-10$ & $5.72 \mathrm{E}-10$ & B4GALNT2 & 4.86E-01 \\
\hline ENSBTAG00000015739 & $2.89 \mathrm{E}-10$ & $5.92 \mathrm{E}-10$ & MRC2 & 4.89E-01 \\
\hline ENSBTAG00000016344 & $2.52 \mathrm{E}-10$ & $5.12 \mathrm{E}-10$ & PIK3R6 & 4.92E-01 \\
\hline ENSBTAG00000003699 & $1.04 \mathrm{E}-10$ & $2.04 \mathrm{E}-10$ & ZFP3 & 5.09E-01 \\
\hline ENSBTAG00000047186 & $3.72 \mathrm{E}-10$ & 7.20E-10 & & 5.17E-01 \\
\hline ENSBTAG00000021720 & $1.02 \mathrm{E}-10$ & $1.92 \mathrm{E}-10$ & MRPL27 & 5.30E-01 \\
\hline ENSBTAG00000021427 & 4.44E-11 & $8.39 \mathrm{E}-11$ & HOXB3 & 5.29E-01 \\
\hline ENSBTAG00000003733 & $7.77 \mathrm{E}-11$ & 1.47E-10 & TM4SF5 & 5.29E-01 \\
\hline ENSBTAG00000046283 & $1.77 \mathrm{E}-10$ & 3.35E-10 & & $5.28 \mathrm{E}-01$ \\
\hline ENSBTAG00000018416 & $2.82 \mathrm{E}-10$ & 5.17E-10 & CCDC42 & 5.45E-01 \\
\hline ENSBTAG00000002282 & $2.24 \mathrm{E}-10$ & 4.04E-10 & EIF1 & 5.56E-01 \\
\hline ENSBTAG00000033766 & $2.67 \mathrm{E}-10$ & $4.81 \mathrm{E}-10$ & KRT16 & 5.55E-01 \\
\hline ENSBTAG00000016133 & $1.27 \mathrm{E}-10$ & $2.23 \mathrm{E}-10$ & MRPS7 & $5.68 \mathrm{E}-01$ \\
\hline ENSBTAG00000039012 & $1.90 \mathrm{E}-10$ & $3.36 \mathrm{E}-10$ & & $5.66 \mathrm{E}-01$ \\
\hline ENSBTAG00000020115 & $2.82 \mathrm{E}-10$ & $4.90 \mathrm{E}-10$ & UBE2O & $5.76 \mathrm{E}-01$ \\
\hline ENSBTAG00000046966 & $9.06 \mathrm{E}-11$ & $1.52 \mathrm{E}-10$ & NATD1 & $5.96 \mathrm{E}-01$ \\
\hline ENSBTAG00000021302 & 5.97E-11 & $1.00 \mathrm{E}-10$ & $\mathrm{CHAD}$ & $5.98 \mathrm{E}-01$ \\
\hline ENSBTAG00000046595 & $3.59 \mathrm{E}-10$ & $6.01 E-10$ & & 5.97E-01 \\
\hline ENSBTAG00000002654 & $1.98 \mathrm{E}-10$ & $3.22 \mathrm{E}-10$ & DNAJC7 & 6.15E-01 \\
\hline ENSBTAG00000047689 & $6.16 \mathrm{E}-10$ & $9.99 \mathrm{E}-10$ & & $6.16 \mathrm{E}-01$ \\
\hline ENSBTAG00000009407 & $1.47 \mathrm{E}-10$ & $2.39 \mathrm{E}-10$ & MBTD1 & $6.16 \mathrm{E}-01$ \\
\hline ENSBTAG00000034396 & $3.80 \mathrm{E}-10$ & 6.17E-10 & PIGW & $6.16 \mathrm{E}-01$ \\
\hline ENSBTAG00000039119 & $2.65 E-10$ & $4.18 \mathrm{E}-10$ & & 6.35E-01 \\
\hline ENSBTAG00000045667 & $3.48 \mathrm{E}-10$ & $5.48 \mathrm{E}-10$ & & $6.34 \mathrm{E}-01$ \\
\hline ENSBTAG00000008940 & $5.12 \mathrm{E}-11$ & 8.07E-11 & NPTX1 & $6.35 \mathrm{E}-01$ \\
\hline ENSBTAG00000001615 & $1.03 \mathrm{E}-10$ & $1.61 \mathrm{E}-10$ & RUNDC3A & 6.37E-01 \\
\hline ENSBTAG00000045964 & $1.22 \mathrm{E}-10$ & $1.92 \mathrm{E}-10$ & & $6.34 \mathrm{E}-01$ \\
\hline ENSBTAG00000012040 & $1.13 \mathrm{E}-10$ & $1.78 \mathrm{E}-10$ & GIT1 & $6.34 \mathrm{E}-01$ \\
\hline ENSBTAG00000037665 & $1.04 \mathrm{E}-09$ & 1.57E-09 & & 6.60E-01 \\
\hline ENSBTAG00000010566 & $8.81 \mathrm{E}-11$ & $1.33 \mathrm{E}-10$ & TMEM11 & $6.62 \mathrm{E}-01$ \\
\hline ENSBTAG00000015518 & $2.40 \mathrm{E}-10$ & $3.59 \mathrm{E}-10$ & KRT12 & $6.68 \mathrm{E}-01$ \\
\hline ENSBTAG00000046372 & $2.12 \mathrm{E}-10$ & $3.14 \mathrm{E}-10$ & & $6.75 \mathrm{E}-01$ \\
\hline ENSBTAG00000047760 & $1.63 \mathrm{E}-10$ & $2.39 \mathrm{E}-10$ & DUS1L & $6.81 \mathrm{E}-01$ \\
\hline ENSBTAG00000015535 & $9.46 \mathrm{E}-11$ & $1.39 \mathrm{E}-10$ & NEK8 & $6.81 \mathrm{E}-01$ \\
\hline ENSBTAG00000020321 & $5.77 \mathrm{E}-11$ & $8.48 \mathrm{E}-11$ & SNX11 & $6.80 \mathrm{E}-01$ \\
\hline ENSBTAG00000010125 & $2.48 \mathrm{E}-10$ & $3.63 \mathrm{E}-10$ & STAT5B & $6.82 \mathrm{E}-01$ \\
\hline ENSBTAG00000005978 & $3.04 \mathrm{E}-10$ & 4.44E-10 & PIK3R5 & $6.85 \mathrm{E}-01$ \\
\hline ENSBTAG00000021301 & $2.27 \mathrm{E}-10$ & $3.30 \mathrm{E}-10$ & ACSF2 & $6.89 \mathrm{E}-01$ \\
\hline ENSBTAG00000047313 & $1.23 \mathrm{E}-09$ & $1.78 \mathrm{E}-09$ & & 6.93E-01 \\
\hline ENSBTAG00000037811 & $3.07 \mathrm{E}-10$ & $4.42 \mathrm{E}-10$ & $\mathrm{CCL} 2$ & $6.94 \mathrm{E}-01$ \\
\hline ENSBTAG00000000546 & $1.84 \mathrm{E}-10$ & 2.65E-10 & TOB1 & $6.94 \mathrm{E}-01$ \\
\hline ENSBTAG00000000202 & $2.02 \mathrm{E}-10$ & $2.91 \mathrm{E}-10$ & SLC25A19 & 6.93E-01 \\
\hline ENSBTAG00000012103 & $9.62 \mathrm{E}-11$ & $1.38 \mathrm{E}-10$ & MAP2K4 & 6.97E-01 \\
\hline ENSBTAG00000013645 & $2.08 \mathrm{E}-10$ & $3.00 \mathrm{E}-10$ & RFFL & 6.93E-01 \\
\hline ENSBTAG00000010559 & $1.32 \mathrm{E}-10$ & $1.89 \mathrm{E}-10$ & DHRS7B & 7.00E-01 \\
\hline ENSBTAG00000048099 & $6.82 \mathrm{E}-10$ & $9.68 \mathrm{E}-10$ & & 7.05E-01 \\
\hline ENSBTAG00000019853 & $1.64 \mathrm{E}-10$ & $2.32 \mathrm{E}-10$ & SMURF2 & 7.06E-01 \\
\hline ENSBTAG00000044019 & $2.13 \mathrm{E}-10$ & $3.00 \mathrm{E}-10$ & КАT2A & 7.10E-01 \\
\hline ENSBTAG00000046580 & $1.34 \mathrm{E}-10$ & $1.89 \mathrm{E}-10$ & DHX58 & 7.10E-01 \\
\hline ENSBTAG00000046652 & $8.70 \mathrm{E}-10$ & $1.21 \mathrm{E}-09$ & & 7.19E-01 \\
\hline ENSBTAG00000012344 & $3.35 \mathrm{E}-10$ & 4.65E-10 & RPL26 & 7.21E-01 \\
\hline ENSBTAG00000020061 & $1.44 \mathrm{E}-10$ & $1.99 \mathrm{E}-10$ & & 7.25E-01 \\
\hline ENSBTAG00000019379 & $1.89 \mathrm{E}-10$ & $2.60 \mathrm{E}-10$ & TUSC5 & 7.25E-01 \\
\hline ENSBTAG00000017461 & $1.80 \mathrm{E}-10$ & $2.44 \mathrm{E}-10$ & SLC16A3 & 7.36E-01 \\
\hline ENSBTAG00000006072 & $9.06 \mathrm{E}-11$ & $1.23 \mathrm{E}-10$ & LRRC59 & 7.37E-01 \\
\hline ENSBTAG00000025167 & $2.17 \mathrm{E}-10$ & $2.94 \mathrm{E}-10$ & ABHD15 & 7.37E-01 \\
\hline ENSBTAG00000001290 & $3.12 \mathrm{E}-10$ & 4.19E-10 & TUBD1 & 7.45E-01 \\
\hline ENSBTAG00000007946 & $6.52 \mathrm{E}-10$ & $8.75 \mathrm{E}-10$ & PIPOX & 7.45E-01 \\
\hline ENSBTAG00000019391 & 1.39E-09 & 1.86E-09 & & $7.48 \mathrm{E}-01$ \\
\hline
\end{tabular}




\begin{tabular}{|c|c|c|c|c|}
\hline ENSBTAG00000010290 & $2.71 \mathrm{E}-10$ & 3.57E-10 & GGNBP2 & $7.59 \mathrm{E}-01$ \\
\hline ENSBTAG00000012672 & $5.68 \mathrm{E}-10$ & $7.31 \mathrm{E}-10$ & ZNHIT3 & $7.78 \mathrm{E}-01$ \\
\hline ENSBTAG00000008883 & $3.19 \mathrm{E}-10$ & 4.09E-10 & ZNF207 & $7.80 \mathrm{E}-01$ \\
\hline ENSBTAG00000015763 & $1.13 \mathrm{E}-09$ & $1.45 \mathrm{E}-09$ & SPATA22 & $7.78 \mathrm{E}-01$ \\
\hline ENSBTAG00000047992 & $1.07 \mathrm{E}-10$ & $1.35 \mathrm{E}-10$ & C17orf107 & 7.95E-01 \\
\hline ENSBTAG00000008481 & $1.08 \mathrm{E}-09$ & $1.36 \mathrm{E}-09$ & & $7.96 \mathrm{E}-01$ \\
\hline ENSBTAG00000020340 & $1.41 \mathrm{E}-10$ & $1.78 \mathrm{E}-10$ & ZNF830 & $7.94 \mathrm{E}-01$ \\
\hline ENSBTAG00000039018 & 2.14E-09 & 2.70E-09 & OR1G1 & 7.93E-01 \\
\hline ENSBTAG00000003280 & $1.50 \mathrm{E}-10$ & $1.89 \mathrm{E}-10$ & & 7.93E-01 \\
\hline ENSBTAG00000033841 & $3.03 \mathrm{E}-10$ & $3.82 \mathrm{E}-10$ & & $7.93 \mathrm{E}-01$ \\
\hline ENSBTAG00000046458 & $1.85 \mathrm{E}-10$ & $2.33 \mathrm{E}-10$ & & 7.95E-01 \\
\hline ENSBTAG00000001825 & $2.91 \mathrm{E}-10$ & 3.67E-10 & SP6 & 7.93E-01 \\
\hline ENSBTAG00000017663 & $3.55 \mathrm{E}-10$ & 4.47E-10 & GAST & 7.94E-01 \\
\hline ENSBTAG00000037660 & $1.89 \mathrm{E}-10$ & $2.39 \mathrm{E}-10$ & & $7.92 \mathrm{E}-01$ \\
\hline ENSBTAG00000002441 & $4.50 \mathrm{E}-10$ & 5.67E-10 & PPY & 7.94E-01 \\
\hline ENSBTAG00000016253 & $1.39 \mathrm{E}-10$ & $1.75 \mathrm{E}-10$ & G6PC3 & 7.95E-01 \\
\hline ENSBTAG00000047870 & $5.27 \mathrm{E}-10$ & $6.64 \mathrm{E}-10$ & & 7.94E-01 \\
\hline ENSBTAG00000032467 & $3.48 \mathrm{E}-10$ & $4.38 \mathrm{E}-10$ & & $7.93 \mathrm{E}-01$ \\
\hline ENSBTAG00000012854 & 4.14E-10 & $5.21 \mathrm{E}-10$ & GSDMB & 7.94E-01 \\
\hline ENSBTAG00000021802 & $5.95 \mathrm{E}-11$ & $7.50 \mathrm{E}-11$ & MIEN1 & 7.94E-01 \\
\hline ENSBTAG00000004690 & $3.50 \mathrm{E}-10$ & $4.40 \mathrm{E}-10$ & CD300E & 7.94E-01 \\
\hline ENSBTAG00000014355 & $6.15 E-11$ & $7.74 \mathrm{E}-11$ & MYCBPAP & 7.94E-01 \\
\hline ENSBTAG00000045946 & $8.01 E-11$ & $1.01 \mathrm{E}-10$ & VMO1 & 7.93E-01 \\
\hline ENSBTAG00000047325 & $5.61 \mathrm{E}-11$ & 7.07E-11 & GNGT2 & 7.94E-01 \\
\hline ENSBTAG00000017081 & $8.56 \mathrm{E}-11$ & $1.08 \mathrm{E}-10$ & MIEF2 & 7.93E-01 \\
\hline ENSBTAG00000035001 & $8.75 E-11$ & $1.10 \mathrm{E}-10$ & & 7.96E-01 \\
\hline ENSBTAG00000009979 & $7.24 \mathrm{E}-11$ & $9.13 \mathrm{E}-11$ & HOXB2 & 7.94E-01 \\
\hline ENSBTAG00000011575 & $1.94 \mathrm{E}-10$ & $2.45 \mathrm{E}-10$ & RFNG & 7.94E-01 \\
\hline ENSBTAG00000039633 & $1.70 \mathrm{E}-09$ & $2.14 \mathrm{E}-09$ & & $7.94 \mathrm{E}-01$ \\
\hline ENSBTAG00000019349 & $4.11 \mathrm{E}-10$ & $5.18 \mathrm{E}-10$ & SLC6A4 & 7.94E-01 \\
\hline ENSBTAG00000015299 & $7.75 \mathrm{E}-11$ & $9.77 \mathrm{E}-11$ & & 7.94E-01 \\
\hline ENSBTAG00000025310 & $1.50 \mathrm{E}-10$ & $1.88 \mathrm{E}-10$ & & 7.96E-01 \\
\hline ENSBTAG00000000042 & $8.78 \mathrm{E}-11$ & $1.11 \mathrm{E}-10$ & PYCR1 & $7.91 \mathrm{E}-01$ \\
\hline ENSBTAG00000007436 & $2.55 \mathrm{E}-10$ & 3.22E-10 & GGT6 & 7.93E-01 \\
\hline ENSBTAG00000025183 & $1.74 \mathrm{E}-10$ & $2.19 \mathrm{E}-10$ & & 7.95E-01 \\
\hline ENSBTAG00000037604 & $1.69 \mathrm{E}-10$ & $2.13 \mathrm{E}-10$ & EFNB3 & $7.92 \mathrm{E}-01$ \\
\hline ENSBTAG00000047858 & 7.10E-10 & $8.95 \mathrm{E}-10$ & & 7.93E-01 \\
\hline ENSBTAG00000002664 & $1.69 \mathrm{E}-10$ & $2.13 \mathrm{E}-10$ & WNT9B & 7.92E-01 \\
\hline ENSBTAG00000046960 & $6.10 \mathrm{E}-10$ & $7.68 \mathrm{E}-10$ & & $7.94 \mathrm{E}-01$ \\
\hline ENSBTAG00000011454 & 4.26E-10 & 5.37E-10 & FKBP10 & 7.93E-01 \\
\hline ENSBTAG00000010073 & $3.30 \mathrm{E}-10$ & 4.15E-10 & FLOT2 & 7.95E-01 \\
\hline ENSBTAG00000047515 & $2.76 \mathrm{E}-10$ & $3.48 \mathrm{E}-10$ & & 7.94E-01 \\
\hline ENSBTAG00000015293 & $1.16 \mathrm{E}-10$ & $1.46 \mathrm{E}-10$ & $\mathrm{NXPH3}$ & 7.96E-01 \\
\hline ENSBTAG00000018176 & $1.49 \mathrm{E}-10$ & $1.88 \mathrm{E}-10$ & C17orf62 & $7.94 \mathrm{E}-01$ \\
\hline ENSBTAG00000024929 & $1.12 \mathrm{E}-10$ & $1.41 \mathrm{E}-10$ & PPP1R27 & 7.94E-01 \\
\hline ENSBTAG00000022927 & $1.08 \mathrm{E}-10$ & $1.36 \mathrm{E}-10$ & RAC3 & $7.92 \mathrm{E}-01$ \\
\hline ENSBTAG00000011460 & $1.80 \mathrm{E}-10$ & 2.27E-10 & & $7.93 \mathrm{E}-01$ \\
\hline ENSBTAG00000048216 & $1.21 \mathrm{E}-10$ & $1.53 \mathrm{E}-10$ & BHLHA9 & 7.93E-01 \\
\hline ENSBTAG00000012675 & $9.05 \mathrm{E}-10$ & 1.13E-09 & MYO19 & 8.01E-01 \\
\hline ENSBTAG00000021303 & $6.14 \mathrm{E}-10$ & 7.63E-10 & RSAD1 & $8.05 \mathrm{E}-01$ \\
\hline ENSBTAG00000018381 & $3.70 \mathrm{E}-10$ & 4.59E-10 & CRLF3 & $8.06 \mathrm{E}-01$ \\
\hline ENSBTAG00000016740 & $2.87 \mathrm{E}-10$ & $3.55 \mathrm{E}-10$ & ACLY & 8.09E-01 \\
\hline ENSBTAG00000024926 & $6.82 \mathrm{E}-10$ & $8.40 \mathrm{E}-10$ & TMEM235 & $8.12 \mathrm{E}-01$ \\
\hline ENSBTAG00000030546 & $5.62 \mathrm{E}-10$ & $6.91 E-10$ & KRT26 & $8.14 \mathrm{E}-01$ \\
\hline ENSBTAG00000010311 & $9.58 \mathrm{E}-10$ & 1.17E-09 & MRM1 & $8.19 \mathrm{E}-01$ \\
\hline ENSBTAG00000010297 & $2.57 \mathrm{E}-10$ & $3.13 \mathrm{E}-10$ & DHRS11 & $8.21 \mathrm{E}-01$ \\
\hline ENSBTAG00000006807 & $5.36 \mathrm{E}-10$ & $6.51 E-10$ & KRT35 & 8.23E-01 \\
\hline ENSBTAG00000030519 & $6.86 \mathrm{E}-10$ & $8.32 \mathrm{E}-10$ & & $8.24 \mathrm{E}-01$ \\
\hline ENSBTAG00000011459 & 3.27E-10 & $3.96 \mathrm{E}-10$ & KLHL10 & $8.26 \mathrm{E}-01$ \\
\hline ENSBTAG00000000831 & $4.03 E-10$ & $4.88 \mathrm{E}-10$ & CTNS & 8.27E-01 \\
\hline ENSBTAG00000004129 & $6.53 \mathrm{E}-10$ & 7.87E-10 & CCL11 & $8.30 \mathrm{E}-01$ \\
\hline ENSBTAG00000019317 & $4.23 \mathrm{E}-10$ & $5.09 \mathrm{E}-10$ & GPS1 & $8.31 \mathrm{E}-01$ \\
\hline ENSBTAG00000000589 & $5.47 \mathrm{E}-10$ & $6.56 \mathrm{E}-10$ & CACNG4 & 8.33E-01 \\
\hline ENSBTAG00000019321 & $1.75 \mathrm{E}-10$ & $2.10 \mathrm{E}-10$ & CCDC57 & $8.32 \mathrm{E}-01$ \\
\hline ENSBTAG00000008744 & $1.92 \mathrm{E}-10$ & $2.29 \mathrm{E}-10$ & PDK2 & 8.37E-01 \\
\hline ENSBTAG00000037553 & $5.71 \mathrm{E}-10$ & $6.80 \mathrm{E}-10$ & & $8.40 \mathrm{E}-01$ \\
\hline ENSBTAG00000007169 & $5.16 \mathrm{E}-10$ & $6.14 \mathrm{E}-10$ & $\mathrm{P} 2 \mathrm{R} \times 1$ & $8.40 \mathrm{E}-01$ \\
\hline ENSBTAG00000037560 & $6.51 \mathrm{E}-10$ & $7.73 \mathrm{E}-10$ & & $8.42 \mathrm{E}-01$ \\
\hline ENSBTAG00000004675 & $1.16 \mathrm{E}-10$ & $1.37 \mathrm{E}-10$ & ZNF18 & $8.45 \mathrm{E}-01$ \\
\hline ENSBTAG00000025313 & $3.74 \mathrm{E}-10$ & 4.43E-10 & DYNLL2 & $8.44 \mathrm{E}-01$ \\
\hline
\end{tabular}




\begin{tabular}{|c|c|c|c|c|}
\hline ENSBTAG00000003334 & $4.33 \mathrm{E}-10$ & 5.13E-10 & ADAP2 & $8.45 \mathrm{E}-01$ \\
\hline ENSBTAG00000000731 & 4.21E-10 & $4.95 \mathrm{E}-10$ & WFIKKN2 & $8.50 \mathrm{E}-01$ \\
\hline ENSBTAG00000044204 & 4.16E-10 & $4.89 \mathrm{E}-10$ & CD79B & $8.51 \mathrm{E}-01$ \\
\hline ENSBTAG00000010001 & $4.89 \mathrm{E}-10$ & $5.75 \mathrm{E}-10$ & RHOT1 & 8.50E-01 \\
\hline ENSBTAG00000019498 & $5.06 \mathrm{E}-10$ & $5.93 \mathrm{E}-10$ & ITGA3 & 8.53E-01 \\
\hline ENSBTAG00000020992 & 4.67E-10 & $5.45 \mathrm{E}-10$ & RHBDL3 & 8.56E-01 \\
\hline ENSBTAG00000009332 & $3.67 \mathrm{E}-10$ & 4.29E-10 & TTLL6 & 8.56E-01 \\
\hline ENSBTAG00000016799 & $3.52 \mathrm{E}-10$ & $4.11 \mathrm{E}-10$ & ERN1 & 8.57E-01 \\
\hline ENSBTAG00000002267 & $2.82 \mathrm{E}-10$ & $3.28 \mathrm{E}-10$ & ANKRD40 & 8.59E-01 \\
\hline ENSBTAG00000016384 & $9.05 \mathrm{E}-10$ & $1.05 \mathrm{E}-09$ & KRT32 & 8.62E-01 \\
\hline ENSBTAG00000013100 & $2.12 \mathrm{E}-10$ & $2.45 \mathrm{E}-10$ & SPAG5 & 8.65E-01 \\
\hline ENSBTAG00000004723 & $5.87 \mathrm{E}-10$ & $6.81 \mathrm{E}-10$ & TEX2 & $8.62 \mathrm{E}-01$ \\
\hline ENSBTAG00000025009 & 2.49E-10 & $2.88 \mathrm{E}-10$ & HOXB9 & $8.65 \mathrm{E}-01$ \\
\hline ENSBTAG00000013698 & $4.06 \mathrm{E}-10$ & $4.68 \mathrm{E}-10$ & TTC25 & 8.67E-01 \\
\hline ENSBTAG00000014687 & $4.44 \mathrm{E}-10$ & $5.11 \mathrm{E}-10$ & TP53I13 & 8.69E-01 \\
\hline ENSBTAG00000000356 & $8.32 \mathrm{E}-10$ & $9.56 \mathrm{E}-10$ & CCDC137 & $8.70 \mathrm{E}-01$ \\
\hline ENSBTAG00000002279 & $1.78 \mathrm{E}-10$ & $2.03 \mathrm{E}-10$ & LUC7L3 & $8.76 \mathrm{E}-01$ \\
\hline ENSBTAG00000007238 & $5.29 \mathrm{E}-10$ & $6.06 \mathrm{E}-10$ & & 8.73E-01 \\
\hline ENSBTAG00000040362 & $9.74 \mathrm{E}-10$ & 1.11E-09 & & 8.77E-01 \\
\hline ENSBTAG00000004746 & $1.99 \mathrm{E}-10$ & $2.28 \mathrm{E}-10$ & HELZ & $8.74 \mathrm{E}-01$ \\
\hline ENSBTAG00000017225 & $3.98 \mathrm{E}-10$ & $4.52 \mathrm{E}-10$ & RNF135 & $8.80 \mathrm{E}-01$ \\
\hline ENSBTAG00000038990 & $1.35 \mathrm{E}-10$ & $1.53 \mathrm{E}-10$ & TRAF4 & $8.81 \mathrm{E}-01$ \\
\hline ENSBTAG00000038823 & $4.10 \mathrm{E}-10$ & 4.66E-10 & & $8.81 \mathrm{E}-01$ \\
\hline ENSBTAG00000020994 & $1.40 \mathrm{E}-10$ & $1.59 \mathrm{E}-10$ & C17orf75 & $8.81 \mathrm{E}-01$ \\
\hline ENSBTAG00000046559 & 2.19E-09 & $2.48 \mathrm{E}-09$ & & $8.84 \mathrm{E}-01$ \\
\hline ENSBTAG00000000033 & $3.70 \mathrm{E}-10$ & 4.20E-10 & PHOSPHO1 & $8.82 \mathrm{E}-01$ \\
\hline ENSBTAG00000013792 & $2.80 \mathrm{E}-10$ & 3.17E-10 & UBALD2 & $8.82 \mathrm{E}-01$ \\
\hline ENSBTAG00000013501 & $3.36 \mathrm{E}-10$ & $3.81 \mathrm{E}-10$ & DHRS13 & $8.82 \mathrm{E}-01$ \\
\hline ENSBTAG00000018699 & $5.54 \mathrm{E}-10$ & $6.26 \mathrm{E}-10$ & KAT7 & 8.85E-01 \\
\hline ENSBTAG00000017380 & $3.29 \mathrm{E}-10$ & $3.72 \mathrm{E}-10$ & MYO18A & 8.83E-01 \\
\hline ENSBTAG00000000355 & $6.76 \mathrm{E}-10$ & $7.62 \mathrm{E}-10$ & OXLD1 & 8.87E-01 \\
\hline ENSBTAG00000046162 & $1.88 \mathrm{E}-10$ & $2.11 \mathrm{E}-10$ & ZNF385C & $8.90 \mathrm{E}-01$ \\
\hline ENSBTAG00000040111 & $1.22 \mathrm{E}-09$ & 1.37E-09 & OTOP3 & 8.93E-01 \\
\hline ENSBTAG00000000417 & $6.86 \mathrm{E}-10$ & 7.69E-10 & & $8.92 \mathrm{E}-01$ \\
\hline ENSBTAG00000012293 & $2.41 \mathrm{E}-10$ & $2.69 \mathrm{E}-10$ & SPAG9 & 8.95E-01 \\
\hline ENSBTAG00000001937 & $1.42 \mathrm{E}-10$ & $1.59 \mathrm{E}-10$ & & 8.94E-01 \\
\hline ENSBTAG00000039954 & $8.08 \mathrm{E}-11$ & $9.05 \mathrm{E}-11$ & COPZ2 & 8.93E-01 \\
\hline ENSBTAG00000020070 & $2.02 \mathrm{E}-10$ & $2.26 \mathrm{E}-10$ & $\mathrm{ABCC} 3$ & $8.92 \mathrm{E}-01$ \\
\hline ENSBTAG00000001119 & $2.23 \mathrm{E}-10$ & $2.49 \mathrm{E}-10$ & ANKRD13B & 8.95E-01 \\
\hline ENSBTAG00000007191 & $1.02 \mathrm{E}-09$ & 1.14E-09 & CCL5 & 8.95E-01 \\
\hline ENSBTAG00000011988 & $2.18 \mathrm{E}-10$ & $2.42 \mathrm{E}-10$ & KIAA0100 & 8.99E-01 \\
\hline ENSBTAG00000004406 & $3.67 E-10$ & $4.08 \mathrm{E}-10$ & & $9.00 \mathrm{E}-01$ \\
\hline ENSBTAG00000006048 & $4.62 \mathrm{E}-10$ & $5.12 \mathrm{E}-10$ & DCAKD & $9.02 \mathrm{E}-01$ \\
\hline ENSBTAG00000030523 & $2.12 \mathrm{E}-10$ & $2.34 \mathrm{E}-10$ & & $9.04 \mathrm{E}-01$ \\
\hline ENSBTAG00000021916 & $2.54 \mathrm{E}-10$ & $2.81 \mathrm{E}-10$ & MYL4 & 9.06E-01 \\
\hline ENSBTAG00000018172 & $1.36 \mathrm{E}-10$ & $1.50 \mathrm{E}-10$ & OGFOD3 & 9.07E-01 \\
\hline ENSBTAG00000030166 & $6.82 \mathrm{E}-10$ & 7.54E-10 & BTBD17 & 9.05E-01 \\
\hline ENSBTAG00000036115 & $4.90 \mathrm{E}-10$ & $5.42 \mathrm{E}-10$ & & $9.04 \mathrm{E}-01$ \\
\hline ENSBTAG00000021523 & $5.01 \mathrm{E}-10$ & $5.53 \mathrm{E}-10$ & STAT3 & $9.06 \mathrm{E}-01$ \\
\hline ENSBTAG00000007547 & $8.61 \mathrm{E}-11$ & $9.49 \mathrm{E}-11$ & CACNG1 & 9.07E-01 \\
\hline ENSBTAG00000019443 & $6.30 \mathrm{E}-10$ & $6.92 \mathrm{E}-10$ & CTDNEP1 & $9.11 \mathrm{E}-01$ \\
\hline ENSBTAG00000040521 & $1.85 \mathrm{E}-10$ & $2.03 \mathrm{E}-10$ & SAMD14 & $9.13 \mathrm{E}-01$ \\
\hline ENSBTAG00000011447 & $1.23 \mathrm{E}-10$ & $1.34 \mathrm{E}-10$ & FAM171A2 & $9.19 \mathrm{E}-01$ \\
\hline ENSBTAG00000013098 & $4.26 \mathrm{E}-10$ & $4.66 \mathrm{E}-10$ & PIGS & $9.14 \mathrm{E}-01$ \\
\hline ENSBTAG00000048282 & $1.98 \mathrm{E}-09$ & 2.17E-09 & & $9.15 \mathrm{E}-01$ \\
\hline ENSBTAG00000020338 & $2.91 \mathrm{E}-10$ & 3.17E-10 & ССТ6В & $9.17 \mathrm{E}-01$ \\
\hline ENSBTAG00000013095 & $3.64 \mathrm{E}-10$ & $3.96 \mathrm{E}-10$ & FOXN1 & $9.18 \mathrm{E}-01$ \\
\hline ENSBTAG00000018752 & $2.10 \mathrm{E}-10$ & $2.28 \mathrm{E}-10$ & SUZ12 & $9.19 \mathrm{E}-01$ \\
\hline ENSBTAG00000031933 & $7.47 \mathrm{E}-10$ & $8.11 E-10$ & & $9.21 \mathrm{E}-01$ \\
\hline ENSBTAG00000030548 & $6.28 \mathrm{E}-10$ & $6.81 \mathrm{E}-10$ & KRT27 & $9.23 \mathrm{E}-01$ \\
\hline ENSBTAG00000019115 & $2.16 \mathrm{E}-10$ & $2.34 \mathrm{E}-10$ & CDC42EP4 & $9.24 \mathrm{E}-01$ \\
\hline ENSBTAG00000004155 & $1.43 \mathrm{E}-10$ & $1.55 \mathrm{E}-10$ & SPATA20 & $9.26 \mathrm{E}-01$ \\
\hline ENSBTAG00000021681 & $6.08 \mathrm{E}-10$ & $6.58 \mathrm{E}-10$ & PRR11 & $9.25 \mathrm{E}-01$ \\
\hline ENSBTAG00000004036 & $5.31 \mathrm{E}-10$ & $5.74 \mathrm{E}-10$ & GJC1 & $9.24 \mathrm{E}-01$ \\
\hline ENSBTAG00000046394 & $1.98 \mathrm{E}-10$ & 2.13E-10 & & $9.28 \mathrm{E}-01$ \\
\hline ENSBTAG00000007910 & $7.61 \mathrm{E}-10$ & $8.22 \mathrm{E}-10$ & EXOC7 & $9.26 \mathrm{E}-01$ \\
\hline ENSBTAG00000012739 & $6.37 \mathrm{E}-10$ & $6.87 \mathrm{E}-10$ & C1QBP & 9.27E-01 \\
\hline ENSBTAG00000046022 & 4.64E-10 & $5.00 \mathrm{E}-10$ & PYY & 9.27E-01 \\
\hline ENSBTAG00000047043 & $2.95 \mathrm{E}-10$ & $3.18 \mathrm{E}-10$ & & 9.27E-01 \\
\hline ENSBTAG00000030168 & $1.59 \mathrm{E}-10$ & $1.72 \mathrm{E}-10$ & MIF4GD & $9.25 \mathrm{E}-01$ \\
\hline
\end{tabular}




\begin{tabular}{|c|c|c|c|c|}
\hline ENSBTAG00000045805 & $1.07 \mathrm{E}-10$ & $1.16 \mathrm{E}-10$ & & $9.26 \mathrm{E}-01$ \\
\hline ENSBTAG00000015527 & $5.15 \mathrm{E}-10$ & $5.52 \mathrm{E}-10$ & MYO1D & $9.33 \mathrm{E}-01$ \\
\hline ENSBTAG00000009376 & $2.65 \mathrm{E}-10$ & $2.83 \mathrm{E}-10$ & DPH1 & $9.37 \mathrm{E}-01$ \\
\hline ENSBTAG00000016128 & $3.15 \mathrm{E}-10$ & $3.37 \mathrm{E}-10$ & GGA3 & 9.34E-01 \\
\hline ENSBTAG00000039411 & 1.15E-09 & $1.22 \mathrm{E}-09$ & & $9.39 \mathrm{E}-01$ \\
\hline ENSBTAG00000021292 & $3.41 \mathrm{E}-10$ & $3.63 \mathrm{E}-10$ & ANKFN1 & $9.40 \mathrm{E}-01$ \\
\hline ENSBTAG00000000560 & $8.18 \mathrm{E}-10$ & $8.71 \mathrm{E}-10$ & & $9.39 \mathrm{E}-01$ \\
\hline ENSBTAG00000017685 & $4.43 \mathrm{E}-10$ & $4.72 \mathrm{E}-10$ & JUP & 9.39E-01 \\
\hline ENSBTAG00000009496 & $2.80 \mathrm{E}-10$ & $2.98 \mathrm{E}-10$ & STAT5A & $9.41 \mathrm{E}-01$ \\
\hline ENSBTAG00000020907 & $3.33 \mathrm{E}-10$ & $3.54 \mathrm{E}-10$ & PSMC3IP & $9.42 \mathrm{E}-01$ \\
\hline ENSBTAG00000001885 & $3.34 \mathrm{E}-10$ & $3.55 E-10$ & COPRS & $9.42 \mathrm{E}-01$ \\
\hline ENSBTAG00000020629 & $4.19 \mathrm{E}-10$ & $4.45 E-10$ & UTP18 & $9.42 \mathrm{E}-01$ \\
\hline ENSBTAG00000013096 & $2.76 \mathrm{E}-10$ & $2.93 \mathrm{E}-10$ & UNC119 & $9.43 \mathrm{E}-01$ \\
\hline ENSBTAG00000009298 & $1.17 \mathrm{E}-10$ & $1.24 \mathrm{E}-10$ & XYLT2 & $9.45 \mathrm{E}-01$ \\
\hline ENSBTAG00000009345 & $3.25 \mathrm{E}-10$ & $3.43 \mathrm{E}-10$ & AMZ2 & $9.48 \mathrm{E}-01$ \\
\hline ENSBTAG00000000833 & $4.00 \mathrm{E}-10$ & $4.23 \mathrm{E}-10$ & TAX1BP3 & $9.46 \mathrm{E}-01$ \\
\hline ENSBTAG00000009835 & $4.04 \mathrm{E}-10$ & $4.27 \mathrm{E}-10$ & CACNA1G & $9.46 \mathrm{E}-01$ \\
\hline ENSBTAG00000040460 & $9.80 \mathrm{E}-10$ & $1.03 \mathrm{E}-09$ & KRT23 & $9.51 \mathrm{E}-01$ \\
\hline ENSBTAG00000013855 & $4.83 \mathrm{E}-10$ & $5.09 \mathrm{E}-10$ & ORMDL3 & $9.48 \mathrm{E}-01$ \\
\hline ENSBTAG00000020252 & $1.80 \mathrm{E}-10$ & $1.89 \mathrm{E}-10$ & NLGN2 & $9.52 \mathrm{E}-01$ \\
\hline ENSBTAG00000019986 & $2.20 \mathrm{E}-10$ & $2.31 \mathrm{E}-10$ & CSNK1D & 9.53E-01 \\
\hline ENSBTAG00000046174 & $3.90 \mathrm{E}-10$ & $4.10 \mathrm{E}-10$ & GNA13 & $9.51 \mathrm{E}-01$ \\
\hline ENSBTAG00000002726 & $1.80 \mathrm{E}-10$ & $1.89 \mathrm{E}-10$ & $\mathrm{CDC} 27$ & $9.51 \mathrm{E}-01$ \\
\hline ENSBTAG00000032372 & $6.63 \mathrm{E}-10$ & $6.95 \mathrm{E}-10$ & GSG2 & 9.54E-01 \\
\hline ENSBTAG00000008747 & $7.48 \mathrm{E}-10$ & $7.85 \mathrm{E}-10$ & DCXR & 9.53E-01 \\
\hline ENSBTAG00000015593 & $2.78 \mathrm{E}-10$ & $2.93 \mathrm{E}-10$ & KIAA0753 & 9.50E-01 \\
\hline ENSBTAG00000014682 & $3.98 \mathrm{E}-10$ & $4.18 \mathrm{E}-10$ & & 9.53E-01 \\
\hline ENSBTAG00000021931 & $1.77 \mathrm{E}-10$ & $1.86 \mathrm{E}-10$ & & 9.53E-01 \\
\hline ENSBTAG00000011731 & $5.85 \mathrm{E}-10$ & $6.14 \mathrm{E}-10$ & PNMT & 9.53E-01 \\
\hline ENSBTAG00000013395 & $4.11 \mathrm{E}-10$ & $4.31 \mathrm{E}-10$ & & $9.54 \mathrm{E}-01$ \\
\hline ENSBTAG00000016012 & $1.84 \mathrm{E}-10$ & $1.93 \mathrm{E}-10$ & WNT3 & $9.51 \mathrm{E}-01$ \\
\hline ENSBTAG00000011623 & $2.68 \mathrm{E}-10$ & $2.81 \mathrm{E}-10$ & VMP1 & 9.55E-01 \\
\hline ENSBTAG00000010738 & $9.25 \mathrm{E}-10$ & $9.70 \mathrm{E}-10$ & CCL14 & 9.53E-01 \\
\hline ENSBTAG00000007429 & $6.15 E-10$ & $6.43 E-10$ & & 9.56E-01 \\
\hline ENSBTAG00000002773 & $8.11 \mathrm{E}-10$ & $8.48 \mathrm{E}-10$ & & $9.56 \mathrm{E}-01$ \\
\hline ENSBTAG00000017823 & $6.65 \mathrm{E}-10$ & $6.95 \mathrm{E}-10$ & CFAP52 & 9.57E-01 \\
\hline ENSBTAG00000044138 & $4.79 \mathrm{E}-10$ & $4.99 \mathrm{E}-10$ & METTL23 & $9.60 \mathrm{E}-01$ \\
\hline ENSBTAG00000010292 & $3.53 \mathrm{E}-10$ & $3.68 \mathrm{E}-10$ & SYNRG & $9.60 \mathrm{E}-01$ \\
\hline ENSBTAG00000013573 & $5.20 \mathrm{E}-10$ & $5.40 \mathrm{E}-10$ & BIRC5 & $9.62 \mathrm{E}-01$ \\
\hline ENSBTAG00000025140 & $3.96 \mathrm{E}-10$ & $4.11 \mathrm{E}-10$ & $\mathrm{DOC} 2 \mathrm{~B}$ & 9.63E-01 \\
\hline ENSBTAG00000037424 & $9.91 \mathrm{E}-10$ & $1.03 \mathrm{E}-09$ & & $9.63 \mathrm{E}-01$ \\
\hline ENSBTAG00000009711 & $6.19 \mathrm{E}-10$ & $6.44 \mathrm{E}-10$ & RPA1 & $9.62 \mathrm{E}-01$ \\
\hline ENSBTAG00000006881 & $2.04 \mathrm{E}-10$ & $2.12 \mathrm{E}-10$ & SLC35B1 & $9.63 \mathrm{E}-01$ \\
\hline ENSBTAG00000025242 & $4.57 \mathrm{E}-10$ & $4.72 \mathrm{E}-10$ & C17orf78 & $9.69 \mathrm{E}-01$ \\
\hline ENSBTAG00000021257 & $8.33 \mathrm{E}-10$ & $8.60 \mathrm{E}-10$ & OTOP2 & $9.69 \mathrm{E}-01$ \\
\hline ENSBTAG00000004651 & $4.39 \mathrm{E}-10$ & $4.53 \mathrm{E}-10$ & & $9.70 \mathrm{E}-01$ \\
\hline ENSBTAG00000017567 & $3.86 \mathrm{E}-10$ & $3.98 \mathrm{E}-10$ & ACACA & $9.71 \mathrm{E}-01$ \\
\hline ENSBTAG00000046075 & $1.18 \mathrm{E}-09$ & $1.21 \mathrm{E}-09$ & & $9.72 \mathrm{E}-01$ \\
\hline ENSBTAG00000002816 & $2.86 \mathrm{E}-10$ & $2.95 \mathrm{E}-10$ & SARM1 & $9.69 \mathrm{E}-01$ \\
\hline ENSBTAG00000048152 & $5.61 \mathrm{E}-10$ & $5.73 \mathrm{E}-10$ & & $9.78 \mathrm{E}-01$ \\
\hline ENSBTAG00000012366 & $2.63 \mathrm{E}-10$ & $2.70 \mathrm{E}-10$ & MRPS23 & $9.76 \mathrm{E}-01$ \\
\hline ENSBTAG00000003695 & $1.64 \mathrm{E}-10$ & $1.68 \mathrm{E}-10$ & WDR45B & 9.77E-01 \\
\hline ENSBTAG00000008339 & $3.57 \mathrm{E}-10$ & $3.63 \mathrm{E}-10$ & $\mathrm{DHX8}$ & $9.83 \mathrm{E}-01$ \\
\hline ENSBTAG00000018391 & $1.53 \mathrm{E}-10$ & $1.56 \mathrm{E}-10$ & C17orf64 & $9.83 \mathrm{E}-01$ \\
\hline ENSBTAG00000010313 & $2.43 \mathrm{E}-10$ & $2.46 \mathrm{E}-10$ & DDX52 & 9.87E-01 \\
\hline ENSBTAG00000030175 & $6.95 \mathrm{E}-10$ & $7.05 E-10$ & FBF1 & $9.85 \mathrm{E}-01$ \\
\hline ENSBTAG00000001621 & $6.16 \mathrm{E}-10$ & $6.24 \mathrm{E}-10$ & & 9.87E-01 \\
\hline ENSBTAG00000007794 & $3.74 \mathrm{E}-10$ & $3.79 \mathrm{E}-10$ & KRT20 & $9.88 \mathrm{E}-01$ \\
\hline ENSBTAG00000013099 & $1.17 \mathrm{E}-10$ & $1.18 \mathrm{E}-10$ & ALDOC & $9.91 \mathrm{E}-01$ \\
\hline ENSBTAG00000004489 & $3.36 \mathrm{E}-10$ & $3.39 \mathrm{E}-10$ & APPBP2 & $9.90 \mathrm{E}-01$ \\
\hline ENSBTAG00000021865 & $1.46 \mathrm{E}-10$ & $1.47 \mathrm{E}-10$ & MED31 & $9.91 \mathrm{E}-01$ \\
\hline ENSBTAG00000035706 & $3.72 \mathrm{E}-10$ & $3.75 \mathrm{E}-10$ & MYOCD & $9.92 \mathrm{E}-01$ \\
\hline ENSBTAG00000011984 & $1.50 \mathrm{E}-10$ & $1.51 \mathrm{E}-10$ & & $9.92 \mathrm{E}-01$ \\
\hline ENSBTAG00000030209 & $1.02 \mathrm{E}-10$ & $1.03 \mathrm{E}-10$ & ARHGDIA & $9.90 \mathrm{E}-01$ \\
\hline ENSBTAG00000017409 & $7.91 \mathrm{E}-11$ & 7.97E-11 & DLX3 & $9.93 \mathrm{E}-01$ \\
\hline ENSBTAG00000019903 & $2.22 \mathrm{E}-10$ & $2.24 \mathrm{E}-10$ & RAMP2 & $9.91 \mathrm{E}-01$ \\
\hline ENSBTAG00000022006 & $2.30 \mathrm{E}-10$ & $2.32 \mathrm{E}-10$ & TRIM65 & 9.93E-01 \\
\hline ENSBTAG00000046907 & $2.66 \mathrm{E}-09$ & 2.67E-09 & & 9.97E-01 \\
\hline ENSBTAG00000025200 & $5.47 \mathrm{E}-10$ & $5.47 \mathrm{E}-10$ & ASIC2 & $1.00 E+00$ \\
\hline ENSBTAG00000002680 & $3.05 \mathrm{E}-10$ & $3.05 E-10$ & TMEM132E & $1.00 \mathrm{E}+00$ \\
\hline
\end{tabular}




\begin{tabular}{|c|c|c|c|c|}
\hline ENSBTAG00000020433 & $5.85 \mathrm{E}-10$ & $5.83 E-10$ & NLRP1 & $1.00 \mathrm{E}+00$ \\
\hline ENSBTAG00000000267 & $2.28 \mathrm{E}-10$ & $2.27 \mathrm{E}-10$ & SMG6 & $1.00 \mathrm{E}+00$ \\
\hline ENSBTAG00000006846 & $4.73 \mathrm{E}-10$ & $4.69 \mathrm{E}-10$ & & $1.01 \mathrm{E}+00$ \\
\hline ENSBTAG00000037794 & $7.14 \mathrm{E}-10$ & $7.08 \mathrm{E}-10$ & MYH4 & $1.01 E+00$ \\
\hline ENSBTAG00000025752 & $5.44 \mathrm{E}-10$ & $5.38 \mathrm{E}-10$ & $\mathrm{P} 3 \mathrm{H} 4$ & $1.01 E+00$ \\
\hline ENSBTAG00000015406 & $2.15 \mathrm{E}-10$ & $2.12 \mathrm{E}-10$ & ZNF750 & $1.01 E+00$ \\
\hline ENSBTAG00000012137 & $2.74 \mathrm{E}-10$ & $2.70 \mathrm{E}-10$ & NTN1 & $1.01 E+00$ \\
\hline ENSBTAG00000004907 & $3.63 \mathrm{E}-10$ & $3.58 \mathrm{E}-10$ & MINK1 & $1.01 E+00$ \\
\hline ENSBTAG00000014806 & $5.18 \mathrm{E}-10$ & $5.10 \mathrm{E}-10$ & ATP2A3 & $1.02 E+00$ \\
\hline ENSBTAG00000006086 & $1.79 \mathrm{E}-10$ & $1.76 \mathrm{E}-10$ & MMP28 & $1.02 \mathrm{E}+00$ \\
\hline ENSBTAG00000000039 & $1.19 \mathrm{E}-10$ & 1.17E-10 & SIRT7 & $1.02 \mathrm{E}+00$ \\
\hline ENSBTAG00000006894 & $8.05 \mathrm{E}-10$ & $7.91 \mathrm{E}-10$ & NOS2 & $1.02 \mathrm{E}+00$ \\
\hline ENSBTAG00000015893 & $1.56 \mathrm{E}-10$ & $1.52 \mathrm{E}-10$ & EPN3 & $1.02 \mathrm{E}+00$ \\
\hline ENSBTAG00000022509 & $4.10 \mathrm{E}-10$ & $4.01 \mathrm{E}-10$ & DNAH9 & $1.02 E+00$ \\
\hline ENSBTAG00000015036 & $2.89 \mathrm{E}-10$ & $2.82 \mathrm{E}-10$ & RAD51C & $1.02 \mathrm{E}+00$ \\
\hline ENSBTAG00000014433 & $2.72 \mathrm{E}-10$ & $2.64 \mathrm{E}-10$ & UBE2G1 & $1.03 E+00$ \\
\hline ENSBTAG00000024950 & $9.01 \mathrm{E}-11$ & $8.77 E-11$ & ACE & $1.03 E+00$ \\
\hline ENSBTAG00000032493 & $3.81 \mathrm{E}-10$ & $3.71 \mathrm{E}-10$ & FAM134C & $1.03 E+00$ \\
\hline ENSBTAG00000047956 & $1.53 \mathrm{E}-10$ & $1.48 \mathrm{E}-10$ & NAGLU & $1.03 E+00$ \\
\hline ENSBTAG00000021927 & $2.29 \mathrm{E}-10$ & $2.21 \mathrm{E}-10$ & AATF & $1.04 \mathrm{E}+00$ \\
\hline ENSBTAG00000012071 & $3.95 \mathrm{E}-10$ & $3.80 \mathrm{E}-10$ & ASGR1 & $1.04 \mathrm{E}+00$ \\
\hline ENSBTAG00000018420 & $1.73 \mathrm{E}-10$ & $1.67 \mathrm{E}-10$ & POLG2 & $1.04 E+00$ \\
\hline ENSBTAG00000014799 & $2.08 \mathrm{E}-10$ & $1.99 \mathrm{E}-10$ & ARMC7 & $1.04 \mathrm{E}+00$ \\
\hline ENSBTAG00000017268 & $1.85 \mathrm{E}-10$ & $1.78 \mathrm{E}-10$ & PROCA1 & $1.04 \mathrm{E}+00$ \\
\hline ENSBTAG00000046919 & $2.46 \mathrm{E}-10$ & $2.36 \mathrm{E}-10$ & ADPRM & $1.04 \mathrm{E}+00$ \\
\hline ENSBTAG00000024490 & $9.54 \mathrm{E}-10$ & $9.12 \mathrm{E}-10$ & ULBP27 & $1.05 \mathrm{E}+00$ \\
\hline ENSBTAG00000033077 & $2.21 \mathrm{E}-10$ & $2.11 \mathrm{E}-10$ & NUFIP2 & $1.05 E+00$ \\
\hline ENSBTAG00000018383 & 3.37E-10 & $3.22 \mathrm{E}-10$ & ATAD5 & $1.05 E+00$ \\
\hline ENSBTAG00000009427 & $1.36 \mathrm{E}-10$ & $1.29 \mathrm{E}-10$ & PPM1D & $1.05 E+00$ \\
\hline ENSBTAG00000015980 & $1.97 \mathrm{E}-10$ & $1.88 \mathrm{E}-10$ & FASN & $1.05 E+00$ \\
\hline ENSBTAG00000030539 & $5.12 \mathrm{E}-10$ & $4.86 \mathrm{E}-10$ & & $1.05 E+00$ \\
\hline ENSBTAG00000020089 & $2.70 \mathrm{E}-10$ & $2.57 \mathrm{E}-10$ & & $1.05 E+00$ \\
\hline ENSBTAG00000047717 & $1.57 \mathrm{E}-10$ & $1.49 \mathrm{E}-10$ & FAM222B & $1.05 E+00$ \\
\hline ENSBTAG00000011990 & $1.15 \mathrm{E}-10$ & $1.08 \mathrm{E}-10$ & ALOX15 & $1.06 \mathrm{E}+00$ \\
\hline ENSBTAG00000004787 & $1.78 \mathrm{E}-10$ & $1.68 \mathrm{E}-10$ & METRNL & $1.06 \mathrm{E}+00$ \\
\hline ENSBTAG00000000920 & $5.68 \mathrm{E}-10$ & $5.38 \mathrm{E}-10$ & DNAH17 & $1.06 \mathrm{E}+00$ \\
\hline ENSBTAG00000013653 & $2.69 \mathrm{E}-11$ & $2.55 E-11$ & NFE2L1 & $1.06 \mathrm{E}+00$ \\
\hline ENSBTAG00000047926 & $2.35 \mathrm{E}-10$ & $2.22 \mathrm{E}-10$ & OVCA2 & $1.06 \mathrm{E}+00$ \\
\hline ENSBTAG00000000354 & $3.81 \mathrm{E}-10$ & $3.60 \mathrm{E}-10$ & PDE6G & $1.06 \mathrm{E}+00$ \\
\hline ENSBTAG00000019070 & $9.23 \mathrm{E}-10$ & $8.73 \mathrm{E}-10$ & PMP22 & $1.06 \mathrm{E}+00$ \\
\hline ENSBTAG00000046129 & $9.67 \mathrm{E}-11$ & $9.14 \mathrm{E}-11$ & RAB34 & $1.06 \mathrm{E}+00$ \\
\hline ENSBTAG00000018258 & $4.28 \mathrm{E}-10$ & $4.04 \mathrm{E}-10$ & & $1.06 \mathrm{E}+00$ \\
\hline ENSBTAG00000019914 & $9.66 \mathrm{E}-11$ & $9.13 E-11$ & BECN1 & $1.06 \mathrm{E}+00$ \\
\hline ENSBTAG00000008009 & $2.35 \mathrm{E}-10$ & $2.22 \mathrm{E}-10$ & PLD6 & $1.06 \mathrm{E}+00$ \\
\hline ENSBTAG00000018823 & $9.38 \mathrm{E}-11$ & $8.86 E-11$ & GRN & $1.06 \mathrm{E}+00$ \\
\hline ENSBTAG00000008498 & $8.66 \mathrm{E}-11$ & $8.18 \mathrm{E}-11$ & ALYREF & $1.06 \mathrm{E}+00$ \\
\hline ENSBTAG00000016736 & $2.50 \mathrm{E}-10$ & $2.36 \mathrm{E}-10$ & HIGD1B & $1.06 \mathrm{E}+00$ \\
\hline ENSBTAG00000047604 & $1.31 \mathrm{E}-10$ & $1.24 \mathrm{E}-10$ & HSD17B1 & $1.06 \mathrm{E}+00$ \\
\hline ENSBTAG00000004108 & $2.71 \mathrm{E}-10$ & $2.56 \mathrm{E}-10$ & CENPX & $1.06 \mathrm{E}+00$ \\
\hline ENSBTAG00000016126 & $2.70 \mathrm{E}-10$ & $2.55 E-10$ & NUP85 & $1.06 \mathrm{E}+00$ \\
\hline ENSBTAG00000019463 & $7.21 \mathrm{E}-11$ & $6.81 \mathrm{E}-11$ & SLC25A39 & $1.06 \mathrm{E}+00$ \\
\hline ENSBTAG00000016847 & $5.15 \mathrm{E}-10$ & $4.86 \mathrm{E}-10$ & ARL4D & $1.06 \mathrm{E}+00$ \\
\hline ENSBTAG00000008621 & $3.75 \mathrm{E}-10$ & $3.54 \mathrm{E}-10$ & PRKAR1A & $1.06 \mathrm{E}+00$ \\
\hline ENSBTAG00000039587 & $5.22 \mathrm{E}-10$ & $4.93 \mathrm{E}-10$ & & $1.06 \mathrm{E}+00$ \\
\hline ENSBTAG00000038059 & $6.97 \mathrm{E}-10$ & $6.58 \mathrm{E}-10$ & & $1.06 \mathrm{E}+00$ \\
\hline ENSBTAG00000046124 & $8.27 \mathrm{E}-10$ & $7.80 \mathrm{E}-10$ & KRT36 & $1.06 \mathrm{E}+00$ \\
\hline ENSBTAG00000001120 & $1.19 \mathrm{E}-10$ & $1.13 \mathrm{E}-10$ & CORO6 & $1.06 \mathrm{E}+00$ \\
\hline ENSBTAG00000014002 & $1.88 \mathrm{E}-10$ & $1.77 \mathrm{E}-10$ & CEP95 & $1.06 \mathrm{E}+00$ \\
\hline ENSBTAG00000032457 & $2.48 \mathrm{E}-09$ & 2.33E-09 & & $1.06 \mathrm{E}+00$ \\
\hline ENSBTAG00000008109 & $8.96 \mathrm{E}-10$ & $8.41 \mathrm{E}-10$ & TMEM97 & $1.07 E+00$ \\
\hline ENSBTAG00000016972 & $4.11 \mathrm{E}-10$ & $3.84 \mathrm{E}-10$ & RAB11FIP4 & $1.07 \mathrm{E}+00$ \\
\hline ENSBTAG00000009708 & $2.70 \mathrm{E}-10$ & $2.53 \mathrm{E}-10$ & SMYD4 & $1.07 \mathrm{E}+00$ \\
\hline ENSBTAG00000004449 & $3.88 \mathrm{E}-10$ & $3.62 \mathrm{E}-10$ & DGKE & $1.07 \mathrm{E}+00$ \\
\hline ENSBTAG00000003958 & $2.37 \mathrm{E}-10$ & $2.21 \mathrm{E}-10$ & TMEM220 & $1.07 E+00$ \\
\hline ENSBTAG00000007193 & $4.74 \mathrm{E}-10$ & $4.41 \mathrm{E}-10$ & CCL16 & $1.07 \mathrm{E}+00$ \\
\hline ENSBTAG00000008110 & $5.46 \mathrm{E}-10$ & $5.08 \mathrm{E}-10$ & IFT20 & $1.08 \mathrm{E}+00$ \\
\hline ENSBTAG00000011736 & $2.69 \mathrm{E}-10$ & $2.49 \mathrm{E}-10$ & IGF2BP1 & $1.08 \mathrm{E}+00$ \\
\hline ENSBTAG00000004617 & $8.91 \mathrm{E}-10$ & $8.25 \mathrm{E}-10$ & KIF19 & $1.08 \mathrm{E}+00$ \\
\hline ENSBTAG00000030172 & $4.32 \mathrm{E}-10$ & $3.99 \mathrm{E}-10$ & PRPSAP1 & $1.08 \mathrm{E}+00$ \\
\hline ENSBTAG00000045606 & $1.69 \mathrm{E}-09$ & $1.56 \mathrm{E}-09$ & & $1.08 \mathrm{E}+00$ \\
\hline
\end{tabular}




\begin{tabular}{|c|c|c|c|c|}
\hline ENSBTAG00000006943 & $3.84 \mathrm{E}-10$ & $3.54 \mathrm{E}-10$ & EFCAB3 & $1.08 \mathrm{E}+00$ \\
\hline ENSBTAG00000001279 & $2.65 \mathrm{E}-10$ & $2.45 \mathrm{E}-10$ & MPP2 & $1.08 \mathrm{E}+00$ \\
\hline ENSBTAG00000015979 & 4.17E-10 & $3.85 \mathrm{E}-10$ & SEZ6 & $1.08 \mathrm{E}+00$ \\
\hline ENSBTAG00000009029 & $4.42 \mathrm{E}-10$ & $4.08 \mathrm{E}-10$ & & $1.08 \mathrm{E}+00$ \\
\hline ENSBTAG00000018345 & 8.57E-10 & $7.89 \mathrm{E}-10$ & FBXO39 & $1.09 E+00$ \\
\hline ENSBTAG00000009948 & $6.93 \mathrm{E}-10$ & $6.38 \mathrm{E}-10$ & TRIM25 & $1.09 E+00$ \\
\hline ENSBTAG00000030164 & $5.30 \mathrm{E}-10$ & $4.88 \mathrm{E}-10$ & RPL38 & $1.09 \mathrm{E}+00$ \\
\hline ENSBTAG00000030173 & 3.67E-10 & 3.37E-10 & QRICH2 & $1.09 \mathrm{E}+00$ \\
\hline ENSBTAG00000011717 & 4.27E-10 & $3.92 \mathrm{E}-10$ & SAP30BP & $1.09 \mathrm{E}+00$ \\
\hline ENSBTAG00000021723 & $1.41 \mathrm{E}-10$ & $1.30 \mathrm{E}-10$ & EME1 & $1.09 \mathrm{E}+00$ \\
\hline ENSBTAG00000008294 & $2.95 \mathrm{E}-10$ & $2.71 \mathrm{E}-10$ & KCNJ2 & $1.09 \mathrm{E}+00$ \\
\hline ENSBTAG00000001836 & $1.01 \mathrm{E}-09$ & $9.29 \mathrm{E}-10$ & & $1.09 E+00$ \\
\hline ENSBTAG00000006051 & $2.26 \mathrm{E}-10$ & $2.08 \mathrm{E}-10$ & NMT1 & $1.09 \mathrm{E}+00$ \\
\hline ENSBTAG00000002898 & $9.93 \mathrm{E}-10$ & $9.11 \mathrm{E}-10$ & UNC45B & $1.09 \mathrm{E}+00$ \\
\hline ENSBTAG00000001672 & $1.20 \mathrm{E}-09$ & 1.10E-09 & & $1.09 \mathrm{E}+00$ \\
\hline ENSBTAG00000010155 & $2.87 \mathrm{E}-10$ & 2.63E-10 & & $1.09 \mathrm{E}+00$ \\
\hline ENSBTAG00000018386 & $6.04 \mathrm{E}-10$ & $5.53 \mathrm{E}-10$ & TEFM & $1.09 \mathrm{E}+00$ \\
\hline ENSBTAG00000004632 & $1.51 \mathrm{E}-10$ & $1.39 \mathrm{E}-10$ & & $1.09 \mathrm{E}+00$ \\
\hline ENSBTAG00000017242 & $9.12 \mathrm{E}-10$ & $8.34 \mathrm{E}-10$ & FADS6 & $1.09 E+00$ \\
\hline ENSBTAG00000025149 & $4.68 \mathrm{E}-10$ & 4.27E-10 & RPAIN & $1.10 \mathrm{E}+00$ \\
\hline ENSBTAG00000001919 & $1.79 \mathrm{E}-10$ & $1.63 \mathrm{E}-10$ & ZNF652 & $1.10 \mathrm{E}+00$ \\
\hline ENSBTAG00000016708 & $2.85 \mathrm{E}-10$ & 2.60E-10 & CLTC & $1.10 \mathrm{E}+00$ \\
\hline ENSBTAG00000011616 & 7.23E-10 & $6.58 \mathrm{E}-10$ & MPO & $1.10 \mathrm{E}+00$ \\
\hline ENSBTAG00000013103 & $1.22 \mathrm{E}-10$ & $1.11 \mathrm{E}-10$ & COL1A1 & $1.10 \mathrm{E}+00$ \\
\hline ENSBTAG00000017751 & 4.67E-10 & 4.24E-10 & RGS9 & $1.10 \mathrm{E}+00$ \\
\hline ENSBTAG00000016710 & $3.63 \mathrm{E}-10$ & $3.28 \mathrm{E}-10$ & PTRH2 & $1.11 E+00$ \\
\hline ENSBTAG00000016851 & $2.95 \mathrm{E}-10$ & 2.67E-10 & RPS6KB1 & $1.10 \mathrm{E}+00$ \\
\hline ENSBTAG00000002982 & $5.99 \mathrm{E}-10$ & $5.40 \mathrm{E}-10$ & PITPNM3 & $1.11 E+00$ \\
\hline ENSBTAG00000020316 & $2.85 \mathrm{E}-10$ & $2.57 \mathrm{E}-10$ & & $1.11 E+00$ \\
\hline ENSBTAG00000039321 & $1.95 \mathrm{E}-10$ & $1.75 \mathrm{E}-10$ & & $1.11 E+00$ \\
\hline ENSBTAG00000026645 & $1.82 \mathrm{E}-10$ & $1.63 \mathrm{E}-10$ & & $1.11 \mathrm{E}+00$ \\
\hline ENSBTAG00000005352 & $2.48 \mathrm{E}-10$ & $2.23 \mathrm{E}-10$ & CRYBA1 & $1.11 \mathrm{E}+00$ \\
\hline ENSBTAG00000001711 & $3.14 \mathrm{E}-10$ & $2.82 \mathrm{E}-10$ & & $1.11 \mathrm{E}+00$ \\
\hline ENSBTAG00000030334 & $1.21 \mathrm{E}-10$ & $1.09 \mathrm{E}-10$ & $\mathrm{AOC2}$ & $1.11 E+00$ \\
\hline ENSBTAG00000047510 & $4.04 \mathrm{E}-10$ & $3.63 \mathrm{E}-10$ & SMIM6 & $1.11 E+00$ \\
\hline ENSBTAG00000006276 & $1.75 \mathrm{E}-10$ & $1.57 \mathrm{E}-10$ & C1QTNF1 & $1.11 \mathrm{E}+00$ \\
\hline ENSBTAG00000020520 & $3.61 \mathrm{E}-10$ & $3.24 \mathrm{E}-10$ & RASD1 & $1.11 E+00$ \\
\hline ENSBTAG00000004915 & $1.73 \mathrm{E}-10$ & $1.55 \mathrm{E}-10$ & & $1.11 \mathrm{E}+00$ \\
\hline ENSBTAG00000015368 & $3.98 \mathrm{E}-10$ & $3.56 \mathrm{E}-10$ & 04-Sep & $1.12 \mathrm{E}+00$ \\
\hline ENSBTAG00000021231 & $2.14 \mathrm{E}-10$ & $1.92 \mathrm{E}-10$ & KCTD2 & $1.12 \mathrm{E}+00$ \\
\hline ENSBTAG00000002749 & $2.75 \mathrm{E}-10$ & $2.45 \mathrm{E}-10$ & CRHR1 & $1.12 \mathrm{E}+00$ \\
\hline ENSBTAG00000001998 & $1.05 \mathrm{E}-09$ & $9.39 \mathrm{E}-10$ & ST6GALNAC1 & $1.12 \mathrm{E}+00$ \\
\hline ENSBTAG00000009228 & $2.95 \mathrm{E}-10$ & 2.63E-10 & SRP68 & $1.12 \mathrm{E}+00$ \\
\hline ENSBTAG00000007906 & $3.31 \mathrm{E}-10$ & $2.94 \mathrm{E}-10$ & ZACN & $1.13 E+00$ \\
\hline ENSBTAG00000016432 & $4.54 \mathrm{E}-10$ & $4.03 \mathrm{E}-10$ & PEMT & $1.13 E+00$ \\
\hline ENSBTAG00000020688 & $2.64 \mathrm{E}-10$ & $2.34 \mathrm{E}-10$ & ARHGEF15 & $1.13 E+00$ \\
\hline ENSBTAG00000018518 & $1.28 \mathrm{E}-10$ & $1.13 \mathrm{E}-10$ & RASL10B & $1.14 \mathrm{E}+00$ \\
\hline ENSBTAG00000011483 & $3.29 \mathrm{E}-10$ & $2.90 \mathrm{E}-10$ & SCARF1 & $1.14 \mathrm{E}+00$ \\
\hline ENSBTAG00000000571 & $3.75 \mathrm{E}-10$ & $3.31 \mathrm{E}-10$ & ARHGAP27 & $1.13 E+00$ \\
\hline ENSBTAG00000019090 & $9.72 \mathrm{E}-11$ & 8.57E-11 & PLEKHH3 & $1.13 \mathrm{E}+00$ \\
\hline ENSBTAG00000015339 & $2.05 \mathrm{E}-10$ & $1.79 \mathrm{E}-10$ & ENDOV & $1.14 \mathrm{E}+00$ \\
\hline ENSBTAG00000008634 & $2.63 \mathrm{E}-10$ & $2.31 \mathrm{E}-10$ & WBP2 & $1.14 \mathrm{E}+00$ \\
\hline ENSBTAG00000003074 & $4.85 \mathrm{E}-10$ & $4.24 \mathrm{E}-10$ & SLC16A6 & $1.14 \mathrm{E}+00$ \\
\hline ENSBTAG00000047165 & $4.45 \mathrm{E}-10$ & $3.88 \mathrm{E}-10$ & KRT9 & $1.15 E+00$ \\
\hline ENSBTAG00000020517 & $2.10 \mathrm{E}-10$ & $1.83 \mathrm{E}-10$ & MED9 & $1.15 E+00$ \\
\hline ENSBTAG00000007437 & $4.07 \mathrm{E}-10$ & $3.55 \mathrm{E}-10$ & TEKT1 & $1.15 E+00$ \\
\hline ENSBTAG00000018658 & $4.12 \mathrm{E}-10$ & $3.59 \mathrm{E}-10$ & TNRC6C & $1.15 E+00$ \\
\hline ENSBTAG00000012500 & $2.84 \mathrm{E}-10$ & $2.48 \mathrm{E}-10$ & RARA & $1.15 \mathrm{E}+00$ \\
\hline ENSBTAG00000046944 & $6.97 \mathrm{E}-10$ & $6.06 \mathrm{E}-10$ & & $1.15 E+00$ \\
\hline ENSBTAG00000018175 & $1.49 \mathrm{E}-10$ & $1.29 \mathrm{E}-10$ & HEXDC & $1.15 E+00$ \\
\hline ENSBTAG00000024983 & $2.10 \mathrm{E}-10$ & $1.83 \mathrm{E}-10$ & FBXO47 & $1.15 E+00$ \\
\hline ENSBTAG00000004908 & $2.86 \mathrm{E}-10$ & 2.47E-10 & CHRNE & $1.16 \mathrm{E}+00$ \\
\hline ENSBTAG00000030555 & $3.30 \mathrm{E}-10$ & $2.86 \mathrm{E}-10$ & KRT28 & $1.16 \mathrm{E}+00$ \\
\hline ENSBTAG00000009036 & $1.16 \mathrm{E}-10$ & $1.01 \mathrm{E}-10$ & SUPT6H & $1.15 E+00$ \\
\hline ENSBTAG00000006916 & $2.81 \mathrm{E}-10$ & $2.42 \mathrm{E}-10$ & TAF15 & $1.16 \mathrm{E}+00$ \\
\hline ENSBTAG00000006886 & $1.04 \mathrm{E}-10$ & $9.01 \mathrm{E}-11$ & FAM117A & $1.16 \mathrm{E}+00$ \\
\hline ENSBTAG00000020000 & 7.57E-10 & $6.53 \mathrm{E}-10$ & RAP1GAP2 & $1.16 \mathrm{E}+00$ \\
\hline ENSBTAG00000003687 & $5.26 \mathrm{E}-10$ & $4.52 \mathrm{E}-10$ & FOXK2 & $1.16 \mathrm{E}+00$ \\
\hline ENSBTAG00000015133 & $2.80 \mathrm{E}-10$ & $2.41 \mathrm{E}-10$ & CCR7 & $1.16 \mathrm{E}+00$ \\
\hline ENSBTAG00000011007 & $1.08 \mathrm{E}-09$ & $9.27 \mathrm{E}-10$ & TTYH2 & $1.17 \mathrm{E}+00$ \\
\hline
\end{tabular}




\begin{tabular}{|c|c|c|c|c|}
\hline ENSBTAG00000047973 & $2.19 \mathrm{E}-10$ & $1.88 \mathrm{E}-10$ & & $1.17 \mathrm{E}+00$ \\
\hline ENSBTAG00000021780 & $2.92 \mathrm{E}-10$ & $2.50 \mathrm{E}-10$ & $\mathrm{SCO} 1$ & $1.17 \mathrm{E}+00$ \\
\hline ENSBTAG00000008120 & 4.77E-10 & 4.07E-10 & TMEM199 & $1.17 \mathrm{E}+00$ \\
\hline ENSBTAG00000031725 & $6.19 \mathrm{E}-10$ & $5.28 \mathrm{E}-10$ & TNFSF12 & $1.17 \mathrm{E}+00$ \\
\hline ENSBTAG00000021060 & $2.67 \mathrm{E}-10$ & $2.28 \mathrm{E}-10$ & FTSJ3 & $1.17 \mathrm{E}+00$ \\
\hline ENSBTAG00000025337 & $5.82 \mathrm{E}-10$ & 4.96E-10 & MYH13 & $1.17 \mathrm{E}+00$ \\
\hline ENSBTAG00000008092 & $4.36 \mathrm{E}-10$ & $3.69 \mathrm{E}-10$ & RAB37 & $1.18 \mathrm{E}+00$ \\
\hline ENSBTAG00000045732 & $3.59 \mathrm{E}-10$ & $3.03 \mathrm{E}-10$ & C17orf105 & $1.19 E+00$ \\
\hline ENSBTAG00000007935 & $2.01 \mathrm{E}-10$ & $1.71 \mathrm{E}-10$ & CALCOCO2 & $1.18 \mathrm{E}+00$ \\
\hline ENSBTAG00000017815 & 2.97E-10 & $2.51 E-10$ & STX8 & $1.19 E+00$ \\
\hline ENSBTAG00000008154 & $1.32 \mathrm{E}-10$ & $1.11 \mathrm{E}-10$ & LIMD2 & $1.19 \mathrm{E}+00$ \\
\hline ENSBTAG00000005052 & $1.06 \mathrm{E}-09$ & $8.93 \mathrm{E}-10$ & GLP2R & $1.19 E+00$ \\
\hline ENSBTAG00000011527 & $1.15 \mathrm{E}-10$ & $9.70 \mathrm{E}-11$ & NT5C & $1.19 E+00$ \\
\hline ENSBTAG00000008165 & $7.52 \mathrm{E}-11$ & $6.32 \mathrm{E}-11$ & ITGA2B & $1.19 E+00$ \\
\hline ENSBTAG00000024910 & $9.98 \mathrm{E}-11$ & $8.38 \mathrm{E}-11$ & GALR2 & $1.19 E+00$ \\
\hline ENSBTAG00000014766 & $6.59 \mathrm{E}-11$ & $5.53 \mathrm{E}-11$ & SRSF1 & $1.19 E+00$ \\
\hline ENSBTAG00000008788 & $1.12 \mathrm{E}-10$ & $9.41 \mathrm{E}-11$ & SLC13A2 & $1.19 \mathrm{E}+00$ \\
\hline ENSBTAG00000039440 & $4.73 \mathrm{E}-10$ & 3.97E-10 & & $1.19 E+00$ \\
\hline ENSBTAG00000001868 & $1.72 \mathrm{E}-10$ & $1.44 \mathrm{E}-10$ & PCYT2 & $1.19 E+00$ \\
\hline ENSBTAG00000020605 & 2.05E-10 & $1.72 \mathrm{E}-10$ & SMTNL2 & $1.19 \mathrm{E}+00$ \\
\hline ENSBTAG00000039880 & $7.49 \mathrm{E}-10$ & $6.28 \mathrm{E}-10$ & & $1.19 E+00$ \\
\hline ENSBTAG00000047777 & $4.36 \mathrm{E}-10$ & $3.65 \mathrm{E}-10$ & & $1.19 E+00$ \\
\hline ENSBTAG00000001832 & $2.11 \mathrm{E}-10$ & 1.77E-10 & MRPL10 & $1.19 E+00$ \\
\hline ENSBTAG00000045769 & 7.93E-10 & $6.64 \mathrm{E}-10$ & & $1.19 E+00$ \\
\hline ENSBTAG00000002663 & $4.48 \mathrm{E}-10$ & $3.75 \mathrm{E}-10$ & BCAS3 & $1.19 \mathrm{E}+00$ \\
\hline ENSBTAG00000000032 & $1.86 \mathrm{E}-10$ & 1.57E-10 & ABI3 & $1.19 E+00$ \\
\hline ENSBTAG00000018920 & $1.11 \mathrm{E}-10$ & $9.30 \mathrm{E}-11$ & TRIM47 & $1.19 E+00$ \\
\hline ENSBTAG00000021864 & $1.13 \mathrm{E}-10$ & $9.47 \mathrm{E}-11$ & TXNDC17 & $1.19 E+00$ \\
\hline ENSBTAG00000012780 & 7.67E-10 & $6.41 \mathrm{E}-10$ & LPO & $1.20 \mathrm{E}+00$ \\
\hline ENSBTAG00000004223 & $2.66 \mathrm{E}-10$ & $2.21 \mathrm{E}-10$ & SRR & $1.20 \mathrm{E}+00$ \\
\hline ENSBTAG00000000827 & $1.37 \mathrm{E}-10$ & $1.15 \mathrm{E}-10$ & TAOK1 & $1.19 E+00$ \\
\hline ENSBTAG00000018126 & $3.35 \mathrm{E}-10$ & $2.78 \mathrm{E}-10$ & VPS53 & $1.21 \mathrm{E}+00$ \\
\hline ENSBTAG00000015958 & $6.81 \mathrm{E}-10$ & $5.64 \mathrm{E}-10$ & & $1.21 \mathrm{E}+00$ \\
\hline ENSBTAG00000002817 & $4.11 \mathrm{E}-10$ & $3.41 E-10$ & SLC46A1 & $1.21 E+00$ \\
\hline ENSBTAG00000040042 & $2.42 \mathrm{E}-10$ & $2.00 \mathrm{E}-10$ & GRIN2C & $1.21 \mathrm{E}+00$ \\
\hline ENSBTAG00000010899 & 4.94E-10 & $4.08 \mathrm{E}-10$ & TIMP2 & $1.21 E+00$ \\
\hline ENSBTAG00000047055 & $5.69 \mathrm{E}-10$ & 4.69E-10 & & $1.21 \mathrm{E}+00$ \\
\hline ENSBTAG00000017512 & $3.52 \mathrm{E}-10$ & $2.90 \mathrm{E}-10$ & & $1.21 \mathrm{E}+00$ \\
\hline ENSBTAG00000005556 & $2.18 \mathrm{E}-10$ & 1.79E-10 & CYGB & $1.22 \mathrm{E}+00$ \\
\hline ENSBTAG00000004111 & $1.26 \mathrm{E}-10$ & $1.03 \mathrm{E}-10$ & LRRC45 & $1.22 \mathrm{E}+00$ \\
\hline ENSBTAG00000021938 & $2.01 \mathrm{E}-10$ & $1.65 \mathrm{E}-10$ & ARHGAP44 & $1.22 \mathrm{E}+00$ \\
\hline ENSBTAG00000007958 & $4.73 \mathrm{E}-10$ & $3.88 \mathrm{E}-10$ & TNS4 & $1.22 \mathrm{E}+00$ \\
\hline ENSBTAG00000030587 & $4.01 \mathrm{E}-10$ & $3.28 \mathrm{E}-10$ & LASP1 & $1.22 \mathrm{E}+00$ \\
\hline ENSBTAG00000044065 & $2.64 \mathrm{E}-10$ & $2.14 \mathrm{E}-10$ & & $1.23 E+00$ \\
\hline ENSBTAG00000000855 & $3.04 \mathrm{E}-10$ & $2.46 \mathrm{E}-10$ & NXN & $1.23 E+00$ \\
\hline ENSBTAG00000032083 & 8.17E-10 & $6.62 \mathrm{E}-10$ & DHX33 & $1.23 E+00$ \\
\hline ENSBTAG00000004225 & $1.26 \mathrm{E}-10$ & $1.02 \mathrm{E}-10$ & TSR1 & $1.24 \mathrm{E}+00$ \\
\hline ENSBTAG00000008832 & $3.72 \mathrm{E}-10$ & $3.01 E-10$ & CCL1 & $1.24 \mathrm{E}+00$ \\
\hline ENSBTAG00000008913 & $1.61 \mathrm{E}-10$ & $1.30 \mathrm{E}-10$ & TMEM98 & $1.24 \mathrm{E}+00$ \\
\hline ENSBTAG00000007220 & $5.62 \mathrm{E}-10$ & $4.52 \mathrm{E}-10$ & CASKIN2 & $1.24 \mathrm{E}+00$ \\
\hline ENSBTAG00000002850 & $6.75 \mathrm{E}-10$ & $5.43 \mathrm{E}-10$ & HEATR9 & $1.24 \mathrm{E}+00$ \\
\hline ENSBTAG00000016316 & $5.12 \mathrm{E}-10$ & 4.11E-10 & ZZEF1 & $1.25 E+00$ \\
\hline ENSBTAG00000021677 & $2.71 \mathrm{E}-10$ & $2.18 \mathrm{E}-10$ & TRIM37 & $1.24 E+00$ \\
\hline ENSBTAG00000038889 & $2.99 \mathrm{E}-10$ & $2.40 \mathrm{E}-10$ & RILP & $1.25 \mathrm{E}+00$ \\
\hline ENSBTAG00000039504 & $4.80 \mathrm{E}-10$ & $3.84 \mathrm{E}-10$ & & $1.25 E+00$ \\
\hline ENSBTAG00000030162 & $4.85 \mathrm{E}-10$ & 3.87E-10 & AXIN2 & $1.25 E+00$ \\
\hline ENSBTAG00000006618 & $6.53 \mathrm{E}-10$ & $5.20 \mathrm{E}-10$ & HLF & $1.25 \mathrm{E}+00$ \\
\hline ENSBTAG00000046670 & $2.19 \mathrm{E}-10$ & $1.75 \mathrm{E}-10$ & MLX & $1.25 \mathrm{E}+00$ \\
\hline ENSBTAG00000015612 & $1.96 \mathrm{E}-10$ & $1.56 \mathrm{E}-10$ & UTP6 & $1.26 \mathrm{E}+00$ \\
\hline ENSBTAG00000016017 & $3.13 \mathrm{E}-10$ & $2.49 \mathrm{E}-10$ & $\mathrm{CCDC} 40$ & $1.26 \mathrm{E}+00$ \\
\hline ENSBTAG00000044195 & $4.89 \mathrm{E}-10$ & $3.88 \mathrm{E}-10$ & SDK2 & $1.26 \mathrm{E}+00$ \\
\hline ENSBTAG00000007430 & 4.30E-10 & $3.42 \mathrm{E}-10$ & MYBBP1A & $1.26 \mathrm{E}+00$ \\
\hline ENSBTAG00000015294 & $4.55 \mathrm{E}-10$ & $3.61 \mathrm{E}-10$ & $\operatorname{cox} 10$ & $1.26 \mathrm{E}+00$ \\
\hline ENSBTAG00000021939 & $4.35 \mathrm{E}-10$ & $3.44 \mathrm{E}-10$ & ELAC2 & $1.26 \mathrm{E}+00$ \\
\hline ENSBTAG00000019912 & $1.48 \mathrm{E}-10$ & $1.16 \mathrm{E}-10$ & CNTD1 & $1.27 \mathrm{E}+00$ \\
\hline ENSBTAG00000015387 & $5.02 \mathrm{E}-10$ & $3.96 \mathrm{E}-10$ & & $1.27 E+00$ \\
\hline ENSBTAG00000040316 & $2.24 \mathrm{E}-10$ & $1.77 \mathrm{E}-10$ & TMEM92 & $1.27 \mathrm{E}+00$ \\
\hline ENSBTAG00000017220 & $1.93 \mathrm{E}-10$ & $1.52 \mathrm{E}-10$ & $\mathrm{GH}$ & $1.27 \mathrm{E}+00$ \\
\hline ENSBTAG00000021227 & $1.38 \mathrm{E}-10$ & $1.08 \mathrm{E}-10$ & & $1.27 \mathrm{E}+00$ \\
\hline ENSBTAG00000003303 & $1.86 \mathrm{E}-10$ & $1.46 \mathrm{E}-10$ & MRPL58 & $1.27 \mathrm{E}+00$ \\
\hline
\end{tabular}




\begin{tabular}{|c|c|c|c|c|}
\hline ENSBTAG00000038316 & $1.46 \mathrm{E}-10$ & $1.13 \mathrm{E}-10$ & GPATCH8 & $1.29 \mathrm{E}+00$ \\
\hline ENSBTAG00000016319 & $2.98 \mathrm{E}-10$ & $2.32 \mathrm{E}-10$ & CYB5D2 & $1.29 \mathrm{E}+00$ \\
\hline ENSBTAG00000016254 & $2.78 \mathrm{E}-10$ & $2.16 \mathrm{E}-10$ & HDAC5 & $1.28 \mathrm{E}+00$ \\
\hline ENSBTAG00000015414 & $4.92 \mathrm{E}-10$ & $3.82 \mathrm{E}-10$ & TBCD & $1.29 \mathrm{E}+00$ \\
\hline ENSBTAG00000038306 & $1.69 \mathrm{E}-10$ & $1.31 \mathrm{E}-10$ & CBX2 & $1.29 \mathrm{E}+00$ \\
\hline ENSBTAG00000008590 & $2.57 \mathrm{E}-10$ & $1.99 \mathrm{E}-10$ & SPAG7 & $1.29 \mathrm{E}+00$ \\
\hline ENSBTAG00000011359 & $3.13 \mathrm{E}-10$ & $2.42 \mathrm{E}-10$ & CD7 & $1.29 \mathrm{E}+00$ \\
\hline ENSBTAG00000015308 & $3.39 \mathrm{E}-10$ & $2.61 \mathrm{E}-10$ & SMARCE1 & $1.30 \mathrm{E}+00$ \\
\hline ENSBTAG00000015261 & $6.15 \mathrm{E}-10$ & 4.74E-10 & CARD14 & $1.30 \mathrm{E}+00$ \\
\hline ENSBTAG00000018271 & $2.86 \mathrm{E}-10$ & $2.20 \mathrm{E}-10$ & SLC38A10 & $1.30 \mathrm{E}+00$ \\
\hline ENSBTAG00000005824 & $1.59 \mathrm{E}-10$ & $1.23 \mathrm{E}-10$ & SPOP & $1.29 \mathrm{E}+00$ \\
\hline ENSBTAG00000018169 & $7.20 \mathrm{E}-10$ & $5.53 \mathrm{E}-10$ & ITGB4 & $1.30 \mathrm{E}+00$ \\
\hline ENSBTAG00000019105 & $3.58 \mathrm{E}-10$ & $2.75 \mathrm{E}-10$ & NPLOC4 & $1.30 \mathrm{E}+00$ \\
\hline ENSBTAG00000011003 & $1.94 \mathrm{E}-10$ & $1.49 \mathrm{E}-10$ & IKZF3 & $1.30 \mathrm{E}+00$ \\
\hline ENSBTAG00000019107 & 4.07E-10 & $3.12 \mathrm{E}-10$ & GAS7 & $1.31 E+00$ \\
\hline ENSBTAG00000014443 & $3.75 \mathrm{E}-10$ & $2.87 \mathrm{E}-10$ & & $1.31 E+00$ \\
\hline ENSBTAG00000025078 & $5.79 \mathrm{E}-10$ & $4.42 \mathrm{E}-10$ & LRRC75A & $1.31 \mathrm{E}+00$ \\
\hline ENSBTAG00000007866 & $5.40 \mathrm{E}-10$ & $4.12 \mathrm{E}-10$ & & $1.31 \mathrm{E}+00$ \\
\hline ENSBTAG00000017120 & $2.94 \mathrm{E}-10$ & $2.24 \mathrm{E}-10$ & PHB & $1.31 E+00$ \\
\hline ENSBTAG00000014598 & $3.08 \mathrm{E}-10$ & $2.34 \mathrm{E}-10$ & BPTF & $1.31 \mathrm{E}+00$ \\
\hline ENSBTAG00000045500 & $1.68 \mathrm{E}-10$ & $1.27 \mathrm{E}-10$ & SGSM2 & $1.32 \mathrm{E}+00$ \\
\hline ENSBTAG00000000044 & $1.31 \mathrm{E}-10$ & $9.87 \mathrm{E}-11$ & MYADML2 & $1.32 \mathrm{E}+00$ \\
\hline ENSBTAG00000018840 & $1.59 \mathrm{E}-10$ & $1.20 \mathrm{E}-10$ & & $1.33 \mathrm{E}+00$ \\
\hline ENSBTAG00000008324 & $2.88 \mathrm{E}-10$ & 2.17E-10 & & $1.33 \mathrm{E}+00$ \\
\hline ENSBTAG00000009705 & $1.05 \mathrm{E}-10$ & 7.95E-11 & SERPINF1 & $1.32 \mathrm{E}+00$ \\
\hline ENSBTAG00000000540 & $2.04 \mathrm{E}-10$ & $1.54 \mathrm{E}-10$ & RNF112 & $1.33 E+00$ \\
\hline ENSBTAG00000019660 & $1.04 \mathrm{E}-10$ & $7.84 \mathrm{E}-11$ & TMUB2 & $1.32 \mathrm{E}+00$ \\
\hline ENSBTAG00000006663 & $2.13 \mathrm{E}-10$ & $1.61 \mathrm{E}-10$ & CYTH1 & $1.32 \mathrm{E}+00$ \\
\hline ENSBTAG00000039599 & $1.45 \mathrm{E}-10$ & $1.10 \mathrm{E}-10$ & HOXB4 & $1.32 \mathrm{E}+00$ \\
\hline ENSBTAG00000002633 & $5.83 \mathrm{E}-10$ & $4.38 \mathrm{E}-10$ & & $1.33 E+00$ \\
\hline ENSBTAG00000011480 & $2.54 \mathrm{E}-10$ & $1.91 \mathrm{E}-10$ & PITPNA & $1.33 \mathrm{E}+00$ \\
\hline ENSBTAG00000007147 & $3.26 \mathrm{E}-10$ & $2.44 \mathrm{E}-10$ & CUEDC1 & $1.34 \mathrm{E}+00$ \\
\hline ENSBTAG00000021680 & $1.78 \mathrm{E}-10$ & $1.33 \mathrm{E}-10$ & SKA2 & $1.34 \mathrm{E}+00$ \\
\hline ENSBTAG00000019907 & $1.22 \mathrm{E}-10$ & $9.10 \mathrm{E}-11$ & WNK4 & $1.34 \mathrm{E}+00$ \\
\hline ENSBTAG00000008333 & 1.19E-10 & $8.85 \mathrm{E}-11$ & ETV4 & $1.34 \mathrm{E}+00$ \\
\hline ENSBTAG00000003629 & 7.45E-10 & $5.56 \mathrm{E}-10$ & ASPA & $1.34 \mathrm{E}+00$ \\
\hline ENSBTAG00000015976 & $1.56 \mathrm{E}-10$ & $1.16 \mathrm{E}-10$ & PHF12 & $1.35 E+00$ \\
\hline ENSBTAG00000030174 & $1.71 \mathrm{E}-10$ & $1.28 \mathrm{E}-10$ & ACOX1 & $1.34 \mathrm{E}+00$ \\
\hline ENSBTAG00000003956 & $2.01 \mathrm{E}-10$ & $1.50 \mathrm{E}-10$ & SGCA & $1.34 \mathrm{E}+00$ \\
\hline ENSBTAG00000006785 & $2.62 \mathrm{E}-10$ & $1.95 \mathrm{E}-10$ & & $1.34 \mathrm{E}+00$ \\
\hline ENSBTAG00000020359 & $5.58 \mathrm{E}-10$ & 4.13E-10 & PLEKHM1 & $1.35 \mathrm{E}+00$ \\
\hline ENSBTAG00000018315 & $1.48 \mathrm{E}-10$ & $1.10 \mathrm{E}-10$ & PELP1 & $1.35 E+00$ \\
\hline ENSBTAG00000044038 & $5.90 \mathrm{E}-10$ & 4.37E-10 & TEN1 & $1.35 \mathrm{E}+00$ \\
\hline ENSBTAG00000003075 & $3.23 \mathrm{E}-10$ & $2.38 \mathrm{E}-10$ & DVL2 & $1.36 \mathrm{E}+00$ \\
\hline ENSBTAG00000012845 & $1.35 \mathrm{E}-10$ & $9.90 \mathrm{E}-11$ & SDF2 & $1.36 \mathrm{E}+00$ \\
\hline ENSBTAG00000003229 & $2.26 \mathrm{E}-10$ & $1.66 \mathrm{E}-10$ & & $1.36 \mathrm{E}+00$ \\
\hline ENSBTAG00000016021 & $2.87 \mathrm{E}-10$ & $2.10 \mathrm{E}-10$ & GAA & $1.37 \mathrm{E}+00$ \\
\hline ENSBTAG00000011715 & 4.59E-10 & $3.36 \mathrm{E}-10$ & RECQL5 & $1.37 E+00$ \\
\hline ENSBTAG00000007583 & 3.57E-10 & $2.60 \mathrm{E}-10$ & KRT14 & $1.37 \mathrm{E}+00$ \\
\hline ENSBTAG00000025129 & $2.00 \mathrm{E}-10$ & $1.45 \mathrm{E}-10$ & MNT & $1.38 \mathrm{E}+00$ \\
\hline ENSBTAG00000010637 & 4.64E-10 & 3.37E-10 & & $1.38 \mathrm{E}+00$ \\
\hline ENSBTAG00000001061 & $6.10 \mathrm{E}-10$ & $4.42 \mathrm{E}-10$ & PRKCA & $1.38 \mathrm{E}+00$ \\
\hline ENSBTAG00000033680 & $4.61 \mathrm{E}-10$ & $3.33 \mathrm{E}-10$ & CEP112 & $1.38 \mathrm{E}+00$ \\
\hline ENSBTAG00000011786 & $1.39 \mathrm{E}-10$ & $1.00 \mathrm{E}-10$ & CLUH & $1.39 E+00$ \\
\hline ENSBTAG00000003365 & $2.28 \mathrm{E}-10$ & $1.64 \mathrm{E}-10$ & SMG8 & $1.39 \mathrm{E}+00$ \\
\hline ENSBTAG00000019122 & $4.85 \mathrm{E}-10$ & $3.50 \mathrm{E}-10$ & EFCAB5 & $1.39 E+00$ \\
\hline ENSBTAG00000018689 & $6.64 \mathrm{E}-10$ & $4.78 \mathrm{E}-10$ & LIG3 & $1.39 E+00$ \\
\hline ENSBTAG00000019082 & $1.06 \mathrm{E}-09$ & 7.63E-10 & RAD51D & $1.39 \mathrm{E}+00$ \\
\hline ENSBTAG00000025250 & $5.70 \mathrm{E}-10$ & $4.09 \mathrm{E}-10$ & & $1.39 E+00$ \\
\hline ENSBTAG00000007176 & $3.03 E-10$ & 2.17E-10 & GDPD1 & $1.40 E+00$ \\
\hline ENSBTAG00000034963 & $5.96 \mathrm{E}-10$ & 4.27E-10 & MKS1 & $1.39 E+00$ \\
\hline ENSBTAG00000046693 & $1.14 \mathrm{E}-09$ & $8.17 \mathrm{E}-10$ & & $1.40 \mathrm{E}+00$ \\
\hline ENSBTAG00000006937 & 1.15E-09 & $8.16 \mathrm{E}-10$ & ABCA10 & $1.41 \mathrm{E}+00$ \\
\hline ENSBTAG00000018706 & $9.01 \mathrm{E}-10$ & $6.43 \mathrm{E}-10$ & & $1.40 E+00$ \\
\hline ENSBTAG00000009987 & 4.29E-10 & $3.05 \mathrm{E}-10$ & & $1.41 \mathrm{E}+00$ \\
\hline ENSBTAG00000022009 & $2.89 \mathrm{E}-10$ & $2.05 \mathrm{E}-10$ & MRPL38 & $1.41 E+00$ \\
\hline ENSBTAG00000018661 & $6.89 \mathrm{E}-10$ & $4.88 \mathrm{E}-10$ & TMC6 & $1.41 E+00$ \\
\hline ENSBTAG00000013685 & $3.46 \mathrm{E}-10$ & $2.45 \mathrm{E}-10$ & & $1.41 E+00$ \\
\hline ENSBTAG00000012070 & $2.08 \mathrm{E}-10$ & 1.47E-10 & INTS2 & $1.42 \mathrm{E}+00$ \\
\hline ENSBTAG00000001610 & $1.26 \mathrm{E}-10$ & 8.92E-11 & SLC4A1 & $1.42 \mathrm{E}+00$ \\
\hline
\end{tabular}




\begin{tabular}{|c|c|c|c|c|}
\hline ENSBTAG000000010809 & $8.10 \mathrm{E}-10$ & $5.73 \mathrm{E}-10$ & HID1 & $1.41 \mathrm{E}+00$ \\
\hline ENSBTAG00000006606 & $2.23 \mathrm{E}-10$ & $1.58 \mathrm{E}-10$ & RFLNB & $1.41 E+00$ \\
\hline ENSBTAG000000000029 & $1.74 \mathrm{E}-10$ & $1.23 \mathrm{E}-10$ & LYZL6 & $1.41 E+00$ \\
\hline ENSBTAG000000007125 & $3.91 \mathrm{E}-10$ & $2.76 \mathrm{E}-10$ & AFMID & $1.42 \mathrm{E}+00$ \\
\hline ENSBTAG000000030170 & $2.35 \mathrm{E}-10$ & $1.66 \mathrm{E}-10$ & HN1 & $1.41 E+00$ \\
\hline ENSBTAG00000031509 & $2.70 \mathrm{E}-10$ & $1.89 \mathrm{E}-10$ & PFAS & $1.43 E+00$ \\
\hline ENSBTAG000000025260 & $1.56 \mathrm{E}-09$ & 1.10E-09 & & $1.42 \mathrm{E}+00$ \\
\hline ENSBTAG000000019448 & $3.32 \mathrm{E}-10$ & $2.32 \mathrm{E}-10$ & CLDN7 & $1.43 E+00$ \\
\hline ENSBTAG000000040279 & $6.97 \mathrm{E}-10$ & $4.87 \mathrm{E}-10$ & KRT24 & $1.43 \mathrm{E}+00$ \\
\hline ENSBTAG000000018316 & $1.45 \mathrm{E}-10$ & $1.01 \mathrm{E}-10$ & ZMYND15 & $1.43 \mathrm{E}+00$ \\
\hline ENSBTAG000000004193 & $3.31 \mathrm{E}-10$ & $2.30 \mathrm{E}-10$ & VEZF1 & $1.44 \mathrm{E}+00$ \\
\hline ENSBTAG000000000120 & $6.72 \mathrm{E}-10$ & $4.66 \mathrm{E}-10$ & USP43 & $1.44 \mathrm{E}+00$ \\
\hline ENSBTAG00000008442 & $1.65 \mathrm{E}-10$ & $1.14 \mathrm{E}-10$ & NSF & $1.45 \mathrm{E}+00$ \\
\hline ENSBTAG000000004710 & $1.52 \mathrm{E}-10$ & $1.05 \mathrm{E}-10$ & HNF1B & $1.45 \mathrm{E}+00$ \\
\hline ENSBTAG000000011829 & $2.59 \mathrm{E}-10$ & $1.78 \mathrm{E}-10$ & NF1 & $1.45 E+00$ \\
\hline ENSBTAG000000038745 & $4.74 \mathrm{E}-10$ & $3.26 \mathrm{E}-10$ & CHMP6 & $1.45 E+00$ \\
\hline ENSBTAG000000040323 & 1.01E-09 & $6.94 \mathrm{E}-10$ & & $1.46 \mathrm{E}+00$ \\
\hline ENSBTAG00000017868 & $4.28 \mathrm{E}-10$ & $2.92 \mathrm{E}-10$ & $\mathrm{DHX} 40$ & $1.46 \mathrm{E}+00$ \\
\hline ENSBTAG000000014677 & $2.00 \mathrm{E}-10$ & $1.36 \mathrm{E}-10$ & TADA2A & $1.47 \mathrm{E}+00$ \\
\hline ENSBTAG000000011721 & $1.97 \mathrm{E}-10$ & $1.34 \mathrm{E}-10$ & MED13 & $1.47 E+00$ \\
\hline ENSBTAG000000006280 & $4.12 \mathrm{E}-10$ & $2.79 \mathrm{E}-10$ & RBFOX3 & $1.48 \mathrm{E}+00$ \\
\hline ENSBTAG00000034681 & $5.07 \mathrm{E}-10$ & $3.43 \mathrm{E}-10$ & CA10 & $1.48 \mathrm{E}+00$ \\
\hline ENSBTAG000000011387 & $2.24 \mathrm{E}-10$ & $1.52 \mathrm{E}-10$ & NAT9 & $1.47 \mathrm{E}+00$ \\
\hline ENSBTAG000000030542 & $6.89 \mathrm{E}-10$ & $4.66 \mathrm{E}-10$ & KRT25 & $1.48 \mathrm{E}+00$ \\
\hline ENSBTAG000000016621 & $3.11 \mathrm{E}-10$ & $2.10 \mathrm{E}-10$ & NCBP3 & $1.48 \mathrm{E}+00$ \\
\hline ENSBTAG00000000056 & $1.67 \mathrm{E}-10$ & $1.13 \mathrm{E}-10$ & STRADA & $1.48 \mathrm{E}+00$ \\
\hline ENSBTAG000000035147 & $1.21 \mathrm{E}-09$ & $8.22 \mathrm{E}-10$ & C17orf67 & $1.48 \mathrm{E}+00$ \\
\hline ENSBTAG00000019162 & $5.21 \mathrm{E}-10$ & $3.51 \mathrm{E}-10$ & MRM3 & $1.48 \mathrm{E}+00$ \\
\hline ENSBTAG00000033562 & $2.12 \mathrm{E}-10$ & $1.42 \mathrm{E}-10$ & LHX1 & $1.49 \mathrm{E}+00$ \\
\hline ENSBTAG000000038138 & $2.22 \mathrm{E}-10$ & $1.49 \mathrm{E}-10$ & & $1.49 E+00$ \\
\hline ENSBTAG000000018260 & $7.55 \mathrm{E}-10$ & 5.07E-10 & MFSD11 & $1.49 \mathrm{E}+00$ \\
\hline ENSBTAG00000012068 & $3.50 \mathrm{E}-10$ & $2.34 \mathrm{E}-10$ & BRIP1 & $1.49 \mathrm{E}+00$ \\
\hline ENSBTAG000000001735 & $2.25 \mathrm{E}-10$ & $1.50 \mathrm{E}-10$ & ADAM11 & $1.50 \mathrm{E}+00$ \\
\hline ENSBTAG000000006277 & $1.60 \mathrm{E}-10$ & $1.06 \mathrm{E}-10$ & ENGASE & $1.51 E+00$ \\
\hline ENSBTAG000000038126 & $4.28 \mathrm{E}-10$ & $2.86 \mathrm{E}-10$ & WIPF2 & $1.50 \mathrm{E}+00$ \\
\hline ENSBTAG00000015264 & $6.49 \mathrm{E}-10$ & 4.32E-10 & ITGAE & $1.50 \mathrm{E}+00$ \\
\hline ENSBTAG00000014199 & $1.18 \mathrm{E}-10$ & $7.88 \mathrm{E}-11$ & TBX21 & $1.50 \mathrm{E}+00$ \\
\hline ENSBTAG00000019432 & 7.05E-10 & $4.68 \mathrm{E}-10$ & ICAM2 & $1.51 E+00$ \\
\hline ENSBTAG00000006045 & $1.39 \mathrm{E}-10$ & $9.30 \mathrm{E}-11$ & $\mathrm{P} 4 \mathrm{HB}$ & $1.50 \mathrm{E}+00$ \\
\hline ENSBTAG00000013392 & $2.53 \mathrm{E}-10$ & $1.68 \mathrm{E}-10$ & PLD2 & $1.51 E+00$ \\
\hline ENSBTAG00000019044 & $5.48 \mathrm{E}-10$ & $3.63 \mathrm{E}-10$ & BAIAP2 & $1.51 E+00$ \\
\hline ENSBTAG00000008151 & $2.42 \mathrm{E}-10$ & $1.61 \mathrm{E}-10$ & МАРЗКЗ & $1.50 \mathrm{E}+00$ \\
\hline ENSBTAG00000001368 & $4.73 \mathrm{E}-10$ & 3.13E-10 & LGALS3BP & $1.51 \mathrm{E}+00$ \\
\hline ENSBTAG00000004736 & $3.16 \mathrm{E}-10$ & $2.09 \mathrm{E}-10$ & GRB2 & $1.51 E+00$ \\
\hline ENSBTAG00000014825 & $2.56 \mathrm{E}-10$ & $1.68 \mathrm{E}-10$ & NLK & $1.52 \mathrm{E}+00$ \\
\hline ENSBTAG00000039556 & $6.45 \mathrm{E}-10$ & 4.24E-10 & WIPI1 & $1.52 \mathrm{E}+00$ \\
\hline ENSBTAG00000015352 & 3.87E-10 & $2.54 \mathrm{E}-10$ & MTMR4 & $1.52 \mathrm{E}+00$ \\
\hline ENSBTAG00000012371 & $1.37 \mathrm{E}-10$ & $8.98 \mathrm{E}-11$ & CPD & $1.53 E+00$ \\
\hline ENSBTAG00000001804 & $1.45 \mathrm{E}-10$ & $9.45 \mathrm{E}-11$ & GPR179 & $1.53 E+00$ \\
\hline ENSBTAG00000017252 & $5.50 \mathrm{E}-10$ & 3.59E-10 & USH1G & $1.53 \mathrm{E}+00$ \\
\hline ENSBTAG00000000030 & $2.17 \mathrm{E}-10$ & $1.41 \mathrm{E}-10$ & RDM1 & $1.54 \mathrm{E}+00$ \\
\hline ENSBTAG00000016240 & 3.60E-10 & $2.35 \mathrm{E}-10$ & RNF157 & $1.53 E+00$ \\
\hline ENSBTAG00000039765 & $7.78 \mathrm{E}-10$ & $5.07 \mathrm{E}-10$ & & $1.53 \mathrm{E}+00$ \\
\hline ENSBTAG00000006052 & $2.35 \mathrm{E}-10$ & 1.53E-10 & PLCD3 & $1.54 \mathrm{E}+00$ \\
\hline ENSBTAG00000008116 & $6.77 \mathrm{E}-10$ & $4.39 \mathrm{E}-10$ & POLDIP2 & $1.54 \mathrm{E}+00$ \\
\hline ENSBTAG00000024915 & $7.53 \mathrm{E}-10$ & $4.86 \mathrm{E}-10$ & & $1.55 E+00$ \\
\hline ENSBTAG00000008115 & $3.31 \mathrm{E}-10$ & $2.14 \mathrm{E}-10$ & TNFAIP1 & $1.55 E+00$ \\
\hline ENSBTAG00000002747 & $9.19 \mathrm{E}-10$ & 5.93E-10 & ABCA5 & $1.55 E+00$ \\
\hline ENSBTAG00000039563 & $5.81 \mathrm{E}-10$ & $3.74 \mathrm{E}-10$ & & $1.55 E+00$ \\
\hline ENSBTAG00000007893 & 1.16E-09 & $7.49 \mathrm{E}-10$ & SCPEP1 & $1.55 E+00$ \\
\hline ENSBTAG00000011479 & $2.21 \mathrm{E}-10$ & $1.42 \mathrm{E}-10$ & INPP5K & $1.56 \mathrm{E}+00$ \\
\hline ENSBTAG00000010534 & $2.28 \mathrm{E}-10$ & $1.46 \mathrm{E}-10$ & MPRIP & $1.56 \mathrm{E}+00$ \\
\hline ENSBTAG00000039819 & $5.71 \mathrm{E}-10$ & $3.63 \mathrm{E}-10$ & RPH3AL & $1.57 \mathrm{E}+00$ \\
\hline ENSBTAG00000004249 & $3.59 \mathrm{E}-10$ & 2.27E-10 & TANC2 & $1.58 \mathrm{E}+00$ \\
\hline ENSBTAG00000023970 & $1.07 \mathrm{E}-10$ & $6.76 \mathrm{E}-11$ & & $1.59 \mathrm{E}+00$ \\
\hline ENSBTAG00000006601 & 4.09E-10 & $2.58 \mathrm{E}-10$ & KSR1 & $1.58 \mathrm{E}+00$ \\
\hline ENSBTAG00000048085 & $1.40 \mathrm{E}-10$ & $8.83 \mathrm{E}-11$ & & $1.59 \mathrm{E}+00$ \\
\hline ENSBTAG00000006056 & $1.71 \mathrm{E}-10$ & $1.08 \mathrm{E}-10$ & HEXIM1 & $1.59 \mathrm{E}+00$ \\
\hline ENSBTAG00000046746 & $2.01 \mathrm{E}-10$ & $1.27 \mathrm{E}-10$ & COASY & $1.58 \mathrm{E}+00$ \\
\hline ENSBTAG00000014964 & $2.26 \mathrm{E}-10$ & $1.42 \mathrm{E}-10$ & GALK1 & $1.59 \mathrm{E}+00$ \\
\hline
\end{tabular}




\begin{tabular}{|c|c|c|c|c|}
\hline ENSBTAG00000000824 & $2.78 \mathrm{E}-10$ & $1.75 \mathrm{E}-10$ & TSEN54 & $1.59 E+00$ \\
\hline ENSBTAG00000018339 & 7.72E-11 & $4.86 E-11$ & ATP5G1 & $1.59 E+00$ \\
\hline ENSBTAG00000024995 & $3.49 \mathrm{E}-10$ & $2.19 E-10$ & & $1.59 E+00$ \\
\hline ENSBTAG00000048034 & $1.25 \mathrm{E}-10$ & 7.87E-11 & GLTPD2 & $1.59 E+00$ \\
\hline ENSBTAG00000004905 & $8.54 \mathrm{E}-11$ & $5.38 \mathrm{E}-11$ & KRT19 & $1.59 \mathrm{E}+00$ \\
\hline ENSBTAG00000013532 & $3.89 \mathrm{E}-10$ & $2.45 \mathrm{E}-10$ & & $1.59 E+00$ \\
\hline ENSBTAG00000000040 & $5.90 \mathrm{E}-11$ & $3.72 \mathrm{E}-11$ & MAFG & $1.59 E+00$ \\
\hline ENSBTAG00000005462 & $8.75 E-11$ & $5.51 \mathrm{E}-11$ & FXR2 & $1.59 E+00$ \\
\hline ENSBTAG00000020859 & $8.61 \mathrm{E}-11$ & $5.42 \mathrm{E}-11$ & SERPINF2 & $1.59 E+00$ \\
\hline ENSBTAG00000013523 & $2.10 \mathrm{E}-10$ & $1.32 \mathrm{E}-10$ & OSBPL7 & $1.59 E+00$ \\
\hline ENSBTAG00000011730 & $2.71 \mathrm{E}-10$ & $1.71 \mathrm{E}-10$ & TCAP & $1.59 \mathrm{E}+00$ \\
\hline ENSBTAG00000018808 & $1.25 \mathrm{E}-10$ & 7.87E-11 & SLC25A35 & $1.59 \mathrm{E}+00$ \\
\hline ENSBTAG00000002657 & $3.13 \mathrm{E}-10$ & $1.97 \mathrm{E}-10$ & NKIRAS2 & $1.59 E+00$ \\
\hline ENSBTAG00000017739 & $1.73 \mathrm{E}-10$ & $1.09 \mathrm{E}-10$ & TNK1 & $1.59 E+00$ \\
\hline ENSBTAG00000001828 & $1.49 \mathrm{E}-10$ & $9.41 \mathrm{E}-11$ & SCRN2 & $1.59 E+00$ \\
\hline ENSBTAG00000014881 & $2.26 \mathrm{E}-10$ & $1.42 \mathrm{E}-10$ & PHF23 & $1.59 E+00$ \\
\hline ENSBTAG00000018837 & $9.07 \mathrm{E}-10$ & $5.70 \mathrm{E}-10$ & SLC39A11 & $1.59 \mathrm{E}+00$ \\
\hline ENSBTAG00000005461 & $1.81 \mathrm{E}-10$ & 1.14E-10 & SOX15 & $1.59 E+00$ \\
\hline ENSBTAG00000004909 & $6.75 E-10$ & 4.24E-10 & GP1BA & $1.59 E+00$ \\
\hline ENSBTAG00000024942 & $2.30 \mathrm{E}-10$ & $1.45 \mathrm{E}-10$ & RPRML & $1.59 \mathrm{E}+00$ \\
\hline ENSBTAG00000003786 & $1.20 \mathrm{E}-10$ & $7.58 \mathrm{E}-11$ & GCGR & $1.59 E+00$ \\
\hline ENSBTAG00000019906 & $2.07 \mathrm{E}-10$ & $1.30 \mathrm{E}-10$ & VPS25 & $1.59 E+00$ \\
\hline ENSBTAG00000047049 & $1.20 \mathrm{E}-09$ & $7.51 \mathrm{E}-10$ & & $1.60 E+00$ \\
\hline ENSBTAG00000016732 & $8.22 \mathrm{E}-10$ & $5.16 \mathrm{E}-10$ & DNAI2 & $1.59 E+00$ \\
\hline ENSBTAG00000000134 & $1.45 \mathrm{E}-10$ & $9.11 \mathrm{E}-11$ & MPDU1 & $1.59 E+00$ \\
\hline ENSBTAG00000021151 & $3.89 \mathrm{E}-10$ & $2.43 \mathrm{E}-10$ & MYH10 & $1.60 \mathrm{E}+00$ \\
\hline ENSBTAG00000016806 & $1.28 \mathrm{E}-10$ & $8.00 \mathrm{E}-11$ & PAFAH1B1 & $1.60 \mathrm{E}+00$ \\
\hline ENSBTAG00000019160 & $1.20 \mathrm{E}-09$ & 7.50E-10 & GLOD4 & $1.60 E+00$ \\
\hline ENSBTAG00000016613 & $2.20 \mathrm{E}-10$ & $1.36 \mathrm{E}-10$ & $\mathrm{KCNH} 6$ & $1.61 E+00$ \\
\hline ENSBTAG00000011343 & $4.31 \mathrm{E}-10$ & 2.67E-10 & XAF1 & $1.61 E+00$ \\
\hline ENSBTAG00000018178 & $2.16 \mathrm{E}-10$ & $1.33 \mathrm{E}-10$ & NARF & $1.63 E+00$ \\
\hline ENSBTAG00000013469 & $2.33 \mathrm{E}-10$ & $1.44 \mathrm{E}-10$ & SRCIN1 & $1.62 \mathrm{E}+00$ \\
\hline ENSBTAG00000038770 & 4.94E-10 & $3.04 \mathrm{E}-10$ & & $1.62 \mathrm{E}+00$ \\
\hline ENSBTAG00000015255 & $5.46 \mathrm{E}-10$ & $3.35 \mathrm{E}-10$ & ST6GALNAC2 & $1.63 E+00$ \\
\hline ENSBTAG00000009702 & $2.39 \mathrm{E}-10$ & $1.47 \mathrm{E}-10$ & MYH8 & $1.62 E+00$ \\
\hline ENSBTAG00000017440 & $1.66 \mathrm{E}-10$ & $1.01 \mathrm{E}-10$ & & $1.64 \mathrm{E}+00$ \\
\hline ENSBTAG00000013677 & 7.02E-10 & $4.28 \mathrm{E}-10$ & SEC14L1 & $1.64 \mathrm{E}+00$ \\
\hline ENSBTAG00000016776 & $1.79 \mathrm{E}-10$ & $1.08 \mathrm{E}-10$ & BAHCC1 & $1.66 \mathrm{E}+00$ \\
\hline ENSBTAG00000020432 & $4.65 \mathrm{E}-10$ & $2.81 \mathrm{E}-10$ & MIS12 & $1.65 \mathrm{E}+00$ \\
\hline ENSBTAG00000005665 & $1.02 \mathrm{E}-10$ & $6.18 \mathrm{E}-11$ & CRK & $1.65 E+00$ \\
\hline ENSBTAG00000008591 & $2.94 \mathrm{E}-10$ & $1.77 \mathrm{E}-10$ & CAMTA2 & $1.66 \mathrm{E}+00$ \\
\hline ENSBTAG00000016252 & $1.91 \mathrm{E}-10$ & $1.15 \mathrm{E}-10$ & LSM12 & $1.66 \mathrm{E}+00$ \\
\hline ENSBTAG00000016078 & $7.96 \mathrm{E}-10$ & 4.77E-10 & & $1.67 \mathrm{E}+00$ \\
\hline ENSBTAG00000008010 & $2.25 \mathrm{E}-10$ & $1.35 \mathrm{E}-10$ & & $1.67 E+00$ \\
\hline ENSBTAG00000006525 & $9.48 \mathrm{E}-10$ & $5.68 \mathrm{E}-10$ & FDXR & $1.67 E+00$ \\
\hline ENSBTAG00000017408 & $2.42 \mathrm{E}-10$ & $1.45 \mathrm{E}-10$ & KRT40 & $1.67 E+00$ \\
\hline ENSBTAG00000021505 & 4.17E-10 & $2.49 E-10$ & USP36 & $1.67 \mathrm{E}+00$ \\
\hline ENSBTAG00000002564 & $2.26 \mathrm{E}-10$ & $1.35 \mathrm{E}-10$ & DLG4 & $1.67 E+00$ \\
\hline ENSBTAG00000007121 & $1.16 \mathrm{E}-10$ & $6.94 \mathrm{E}-11$ & TK1 & $1.67 E+00$ \\
\hline ENSBTAG00000019446 & $2.74 \mathrm{E}-10$ & $1.63 E-10$ & ELP5 & $1.68 \mathrm{E}+00$ \\
\hline ENSBTAG00000016287 & $3.54 \mathrm{E}-10$ & $2.10 \mathrm{E}-10$ & UNK & $1.68 \mathrm{E}+00$ \\
\hline ENSBTAG00000015639 & $3.09 \mathrm{E}-10$ & $1.83 \mathrm{E}-10$ & ERAL1 & $1.69 E+00$ \\
\hline ENSBTAG00000001110 & $1.47 \mathrm{E}-10$ & $8.73 E-11$ & SLC16A5 & $1.69 E+00$ \\
\hline ENSBTAG00000011382 & $2.34 \mathrm{E}-10$ & $1.38 \mathrm{E}-10$ & SLC9A3R1 & $1.70 E+00$ \\
\hline ENSBTAG00000008327 & $1.18 \mathrm{E}-09$ & $6.92 \mathrm{E}-10$ & CD300LB & $1.70 \mathrm{E}+00$ \\
\hline ENSBTAG00000008611 & $2.12 \mathrm{E}-10$ & $1.25 \mathrm{E}-10$ & IGFBP4 & $1.70 E+00$ \\
\hline ENSBTAG00000012478 & $1.79 \mathrm{E}-10$ & $1.05 \mathrm{E}-10$ & ALOXE3 & $1.70 \mathrm{E}+00$ \\
\hline ENSBTAG00000047770 & $1.33 \mathrm{E}-09$ & $7.76 \mathrm{E}-10$ & & $1.71 E+00$ \\
\hline ENSBTAG00000001915 & $2.63 \mathrm{E}-10$ & $1.53 \mathrm{E}-10$ & $\mathrm{APOH}$ & $1.72 \mathrm{E}+00$ \\
\hline ENSBTAG00000005534 & $2.07 \mathrm{E}-10$ & $1.20 \mathrm{E}-10$ & ENO3 & $1.72 \mathrm{E}+00$ \\
\hline ENSBTAG00000019218 & $1.23 \mathrm{E}-10$ & 7.13E-11 & ATP6V0A1 & $1.72 \mathrm{E}+00$ \\
\hline ENSBTAG00000001332 & $1.26 \mathrm{E}-10$ & 7.23E-11 & MYO1C & $1.73 E+00$ \\
\hline ENSBTAG00000000829 & $3.43 \mathrm{E}-10$ & $1.98 \mathrm{E}-10$ & & $1.73 E+00$ \\
\hline ENSBTAG00000024974 & $1.30 \mathrm{E}-10$ & 7.42E-11 & HEXIM2 & $1.75 E+00$ \\
\hline ENSBTAG00000009782 & $1.15 \mathrm{E}-10$ & $6.56 \mathrm{E}-11$ & CBX4 & $1.75 \mathrm{E}+00$ \\
\hline ENSBTAG00000021091 & $5.26 \mathrm{E}-10$ & $3.01 \mathrm{E}-10$ & ANKFY1 & $1.75 E+00$ \\
\hline ENSBTAG00000013740 & $2.65 \mathrm{E}-10$ & $1.52 \mathrm{E}-10$ & SP2 & $1.75 \mathrm{E}+00$ \\
\hline ENSBTAG00000020078 & $2.73 \mathrm{E}-10$ & $1.56 \mathrm{E}-10$ & KRT39 & $1.75 E+00$ \\
\hline ENSBTAG00000010674 & $1.67 \mathrm{E}-09$ & $9.47 \mathrm{E}-10$ & & $1.76 \mathrm{E}+00$ \\
\hline ENSBTAG00000024901 & $1.81 \mathrm{E}-09$ & $1.02 \mathrm{E}-09$ & & $1.77 \mathrm{E}+00$ \\
\hline
\end{tabular}




\begin{tabular}{|c|c|c|c|c|}
\hline ENSBTAG00000019341 & $2.19 \mathrm{E}-10$ & $1.23 \mathrm{E}-10$ & NSRP1 & $1.78 \mathrm{E}+00$ \\
\hline ENSBTAG00000011011 & $1.90 \mathrm{E}-10$ & $1.07 E-10$ & $\mathrm{SSH} 2$ & $1.77 \mathrm{E}+00$ \\
\hline ENSBTAG00000010519 & $6.23 \mathrm{E}-11$ & 3.49E-11 & & $1.78 \mathrm{E}+00$ \\
\hline ENSBTAG00000007409 & $3.41 \mathrm{E}-10$ & $1.91 \mathrm{E}-10$ & MILR1 & $1.78 \mathrm{E}+00$ \\
\hline ENSBTAG00000008773 & $1.53 \mathrm{E}-10$ & $8.55 \mathrm{E}-11$ & GUCY2D & $1.79 \mathrm{E}+00$ \\
\hline ENSBTAG00000020431 & 4.34E-10 & $2.42 \mathrm{E}-10$ & DERL2 & $1.79 \mathrm{E}+00$ \\
\hline ENSBTAG00000004913 & 3.13E-10 & $1.75 \mathrm{E}-10$ & & $1.79 E+00$ \\
\hline ENSBTAG00000045899 & $1.58 \mathrm{E}-09$ & 8.79E-10 & & $1.80 \mathrm{E}+00$ \\
\hline ENSBTAG00000004637 & $4.39 \mathrm{E}-10$ & $2.44 \mathrm{E}-10$ & 10-Mar & $1.80 \mathrm{E}+00$ \\
\hline ENSBTAG00000010375 & $2.09 \mathrm{E}-10$ & $1.16 \mathrm{E}-10$ & & $1.80 \mathrm{E}+00$ \\
\hline ENSBTAG00000019104 & 3.05E-10 & $1.69 \mathrm{E}-10$ & FAAP100 & $1.80 \mathrm{E}+00$ \\
\hline ENSBTAG00000025320 & $2.40 \mathrm{E}-10$ & $1.33 \mathrm{E}-10$ & AKAP1 & $1.80 \mathrm{E}+00$ \\
\hline ENSBTAG00000019153 & 3.53E-10 & $1.95 \mathrm{E}-10$ & JMJD6 & $1.81 \mathrm{E}+00$ \\
\hline ENSBTAG00000011389 & $2.40 \mathrm{E}-10$ & $1.32 \mathrm{E}-10$ & TMEM104 & $1.82 \mathrm{E}+00$ \\
\hline ENSBTAG00000038910 & 4.40E-10 & $2.41 \mathrm{E}-10$ & & $1.83 \mathrm{E}+00$ \\
\hline ENSBTAG00000024903 & $6.57 \mathrm{E}-10$ & $3.59 \mathrm{E}-10$ & KCNJ16 & $1.83 \mathrm{E}+00$ \\
\hline ENSBTAG00000013236 & $1.19 \mathrm{E}-10$ & $6.47 \mathrm{E}-11$ & MED1 & $1.83 \mathrm{E}+00$ \\
\hline ENSBTAG00000007090 & $1.47 \mathrm{E}-10$ & $7.92 \mathrm{E}-11$ & & $1.85 \mathrm{E}+00$ \\
\hline ENSBTAG00000018973 & $2.93 \mathrm{E}-10$ & $1.59 \mathrm{E}-10$ & COPS3 & $1.84 \mathrm{E}+00$ \\
\hline ENSBTAG00000021468 & $3.74 \mathrm{E}-10$ & $2.02 \mathrm{E}-10$ & MED24 & $1.85 \mathrm{E}+00$ \\
\hline ENSBTAG00000010517 & $4.62 \mathrm{E}-10$ & $2.50 \mathrm{E}-10$ & EVPL & $1.85 \mathrm{E}+00$ \\
\hline ENSBTAG00000025130 & $1.49 \mathrm{E}-10$ & $8.02 \mathrm{E}-11$ & HIC1 & $1.85 \mathrm{E}+00$ \\
\hline ENSBTAG00000009747 & $5.44 \mathrm{E}-11$ & $2.94 \mathrm{E}-11$ & RNFT1 & $1.85 \mathrm{E}+00$ \\
\hline ENSBTAG00000019662 & 7.14E-11 & $3.86 \mathrm{E}-11$ & ATXN7L3 & $1.85 \mathrm{E}+00$ \\
\hline ENSBTAG00000010384 & $1.57 \mathrm{E}-10$ & $8.46 \mathrm{E}-11$ & CDC6 & $1.85 \mathrm{E}+00$ \\
\hline ENSBTAG00000008507 & $2.71 \mathrm{E}-10$ & $1.46 \mathrm{E}-10$ & SPHK1 & $1.86 \mathrm{E}+00$ \\
\hline ENSBTAG00000000573 & 7.70E-10 & 4.10E-10 & & $1.88 \mathrm{E}+00$ \\
\hline ENSBTAG00000031107 & $4.61 \mathrm{E}-10$ & $2.45 \mathrm{E}-10$ & HS3ST3A1 & $1.88 \mathrm{E}+00$ \\
\hline ENSBTAG00000003889 & $2.82 \mathrm{E}-10$ & $1.49 \mathrm{E}-10$ & PER1 & $1.89 \mathrm{E}+00$ \\
\hline ENSBTAG00000005104 & $5.95 \mathrm{E}-10$ & $3.14 \mathrm{E}-10$ & MGAT5B & $1.89 \mathrm{E}+00$ \\
\hline ENSBTAG00000040229 & 1.57E-09 & $8.26 \mathrm{E}-10$ & & $1.90 \mathrm{E}+00$ \\
\hline ENSBTAG00000009126 & $1.64 \mathrm{E}-10$ & $8.59 \mathrm{E}-11$ & YBX2 & $1.90 \mathrm{E}+00$ \\
\hline ENSBTAG00000013526 & $4.91 \mathrm{E}-10$ & $2.57 \mathrm{E}-10$ & EFTUD2 & $1.91 \mathrm{E}+00$ \\
\hline ENSBTAG00000005067 & $8.60 \mathrm{E}-11$ & $4.51 \mathrm{E}-11$ & UBTF & $1.90 \mathrm{E}+00$ \\
\hline ENSBTAG00000003289 & $6.71 \mathrm{E}-10$ & $3.51 \mathrm{E}-10$ & & $1.91 E+00$ \\
\hline ENSBTAG00000008328 & $9.55 \mathrm{E}-10$ & 4.96E-10 & & $1.92 \mathrm{E}+00$ \\
\hline ENSBTAG00000015656 & $5.84 \mathrm{E}-10$ & $3.03 \mathrm{E}-10$ & WSCD1 & $1.93 \mathrm{E}+00$ \\
\hline ENSBTAG00000040573 & $3.52 \mathrm{E}-10$ & $1.83 \mathrm{E}-10$ & TSPAN10 & $1.93 E+00$ \\
\hline ENSBTAG00000021933 & $2.12 \mathrm{E}-10$ & $1.10 \mathrm{E}-10$ & ALOX12 & $1.93 \mathrm{E}+00$ \\
\hline ENSBTAG00000027274 & $2.49 \mathrm{E}-10$ & $1.29 \mathrm{E}-10$ & & $1.93 \mathrm{E}+00$ \\
\hline ENSBTAG00000021370 & $1.24 \mathrm{E}-10$ & $6.39 \mathrm{E}-11$ & BLMH & $1.94 \mathrm{E}+00$ \\
\hline ENSBTAG00000007166 & $4.02 \mathrm{E}-10$ & $2.07 \mathrm{E}-10$ & CAMKK1 & $1.94 \mathrm{E}+00$ \\
\hline ENSBTAG00000019806 & $2.06 \mathrm{E}-10$ & $1.06 \mathrm{E}-10$ & YPEL2 & $1.95 \mathrm{E}+00$ \\
\hline ENSBTAG00000001071 & $1.87 \mathrm{E}-10$ & $9.61 \mathrm{E}-11$ & WRAP53 & $1.95 \mathrm{E}+00$ \\
\hline ENSBTAG00000000641 & $1.57 \mathrm{E}-10$ & $8.02 \mathrm{E}-11$ & SKAP1 & $1.96 \mathrm{E}+00$ \\
\hline ENSBTAG00000000020 & $6.69 \mathrm{E}-10$ & $3.38 \mathrm{E}-10$ & TRPV3 & $1.98 \mathrm{E}+00$ \\
\hline ENSBTAG00000013390 & $1.85 \mathrm{E}-10$ & $9.32 \mathrm{E}-11$ & PSMB6 & $1.99 \mathrm{E}+00$ \\
\hline ENSBTAG00000002949 & $6.43 \mathrm{E}-11$ & $3.24 \mathrm{E}-11$ & CBX1 & $1.98 \mathrm{E}+00$ \\
\hline ENSBTAG00000013959 & $2.21 \mathrm{E}-10$ & $1.11 \mathrm{E}-10$ & GPR142 & $1.99 \mathrm{E}+00$ \\
\hline ENSBTAG00000001041 & $2.06 \mathrm{E}-10$ & $1.04 \mathrm{E}-10$ & & $1.98 \mathrm{E}+00$ \\
\hline ENSBTAG00000025257 & $2.63 \mathrm{E}-10$ & $1.32 \mathrm{E}-10$ & & $1.99 \mathrm{E}+00$ \\
\hline ENSBTAG00000017345 & $5.52 \mathrm{E}-10$ & $2.77 \mathrm{E}-10$ & KIF2B & $1.99 \mathrm{E}+00$ \\
\hline ENSBTAG00000019812 & $1.72 \mathrm{E}-10$ & $8.64 \mathrm{E}-11$ & MCRIP1 & $1.99 \mathrm{E}+00$ \\
\hline ENSBTAG00000019658 & $1.63 \mathrm{E}-10$ & $8.21 \mathrm{E}-11$ & ASB16 & $1.99 \mathrm{E}+00$ \\
\hline ENSBTAG00000039933 & $3.69 \mathrm{E}-10$ & $1.86 \mathrm{E}-10$ & LRRC3C & $1.98 \mathrm{E}+00$ \\
\hline ENSBTAG00000004496 & $3.92 \mathrm{E}-10$ & $1.97 \mathrm{E}-10$ & TVP23B & $1.99 \mathrm{E}+00$ \\
\hline ENSBTAG00000003072 & $2.37 \mathrm{E}-10$ & $1.19 \mathrm{E}-10$ & ACADVL & $1.99 \mathrm{E}+00$ \\
\hline ENSBTAG00000047458 & $1.32 \mathrm{E}-09$ & $6.60 \mathrm{E}-10$ & & $2.00 E+00$ \\
\hline ENSBTAG00000013273 & 7.12E-10 & 3.54E-10 & PIGL & $2.01 E+00$ \\
\hline ENSBTAG00000002605 & 7.99E-10 & $3.98 \mathrm{E}-10$ & & $2.01 E+00$ \\
\hline ENSBTAG00000007389 & $2.27 \mathrm{E}-10$ & $1.13 \mathrm{E}-10$ & IFI35 & $2.00 E+00$ \\
\hline ENSBTAG00000007013 & $6.37 \mathrm{E}-10$ & $3.14 \mathrm{E}-10$ & TOM1L1 & $2.03 E+00$ \\
\hline ENSBTAG00000001609 & $8.92 \mathrm{E}-10$ & 4.39E-10 & MAP2K6 & $2.03 E+00$ \\
\hline ENSBTAG00000003966 & $3.08 \mathrm{E}-10$ & $1.52 \mathrm{E}-10$ & DUSP3 & $2.03 E+00$ \\
\hline ENSBTAG00000004196 & $2.01 \mathrm{E}-10$ & $9.88 \mathrm{E}-11$ & FMNL1 & $2.03 E+00$ \\
\hline ENSBTAG00000024934 & $9.07 \mathrm{E}-10$ & $4.46 \mathrm{E}-10$ & & $2.03 E+00$ \\
\hline ENSBTAG00000030333 & $6.71 \mathrm{E}-10$ & $3.30 \mathrm{E}-10$ & & $2.03 E+00$ \\
\hline ENSBTAG00000003192 & $2.49 \mathrm{E}-10$ & $1.22 \mathrm{E}-10$ & TBC1D16 & $2.04 \mathrm{E}+00$ \\
\hline ENSBTAG00000019723 & $3.50 \mathrm{E}-10$ & $1.71 \mathrm{E}-10$ & PEX12 & $2.05 \mathrm{E}+00$ \\
\hline ENSBTAG00000006054 & $2.24 \mathrm{E}-10$ & $1.10 \mathrm{E}-10$ & ACBD4 & $2.04 \mathrm{E}+00$ \\
\hline
\end{tabular}




\begin{tabular}{|c|c|c|c|c|}
\hline ENSBTAG00000002883 & $3.54 \mathrm{E}-10$ & $1.73 \mathrm{E}-10$ & RPTOR & $2.04 E+00$ \\
\hline ENSBTAG00000022520 & $1.42 \mathrm{E}-10$ & $6.89 \mathrm{E}-11$ & BRCA1 & $2.05 E+00$ \\
\hline ENSBTAG00000016544 & $4.43 \mathrm{E}-10$ & $2.16 \mathrm{E}-10$ & SLC47A1 & $2.05 E+00$ \\
\hline ENSBTAG00000002981 & $2.65 \mathrm{E}-10$ & $1.28 \mathrm{E}-10$ & FAM64A & $2.07 E+00$ \\
\hline ENSBTAG00000000675 & $3.79 \mathrm{E}-10$ & $1.83 \mathrm{E}-10$ & PGS1 & $2.07 E+00$ \\
\hline ENSBTAG00000045621 & $1.87 \mathrm{E}-10$ & 8.85E-11 & C17orf50 & $2.12 \mathrm{E}+00$ \\
\hline ENSBTAG00000018880 & $5.20 \mathrm{E}-10$ & $2.45 \mathrm{E}-10$ & TRPV1 & $2.12 \mathrm{E}+00$ \\
\hline ENSBTAG00000020853 & $1.88 \mathrm{E}-10$ & $8.86 \mathrm{E}-11$ & SLC16A13 & $2.12 \mathrm{E}+00$ \\
\hline ENSBTAG00000018170 & $5.70 \mathrm{E}-10$ & $2.68 \mathrm{E}-10$ & UTS2R & $2.13 E+00$ \\
\hline ENSBTAG00000016023 & $2.79 \mathrm{E}-10$ & $1.32 \mathrm{E}-10$ & EIF4A3 & $2.12 \mathrm{E}+00$ \\
\hline ENSBTAG00000039161 & $4.68 \mathrm{E}-10$ & $2.20 \mathrm{E}-10$ & ALDH3A2 & $2.13 \mathrm{E}+00$ \\
\hline ENSBTAG00000011532 & 2.03E-10 & $9.43 \mathrm{E}-11$ & MLLT6 & $2.15 \mathrm{E}+00$ \\
\hline ENSBTAG00000004770 & $7.86 \mathrm{E}-10$ & $3.64 \mathrm{E}-10$ & SCN4A & $2.16 \mathrm{E}+00$ \\
\hline ENSBTAG00000011505 & $2.48 \mathrm{E}-10$ & $1.15 \mathrm{E}-10$ & RABEP1 & $2.16 \mathrm{E}+00$ \\
\hline ENSBTAG00000008424 & $5.11 \mathrm{E}-10$ & $2.35 \mathrm{E}-10$ & $A B R$ & $2.17 E+00$ \\
\hline ENSBTAG00000016175 & $4.92 \mathrm{E}-10$ & $2.25 \mathrm{E}-10$ & SPNS3 & $2.19 E+00$ \\
\hline ENSBTAG00000001085 & $8.86 \mathrm{E}-10$ & $4.04 \mathrm{E}-10$ & FAM20A & $2.19 E+00$ \\
\hline ENSBTAG00000006088 & $6.30 \mathrm{E}-10$ & $2.87 \mathrm{E}-10$ & FAM104A & $2.20 \mathrm{E}+00$ \\
\hline ENSBTAG00000037943 & $1.52 \mathrm{E}-09$ & $6.91 \mathrm{E}-10$ & & $2.20 E+00$ \\
\hline ENSBTAG00000008371 & $2.38 \mathrm{E}-10$ & $1.08 \mathrm{E}-10$ & FBXL20 & $2.20 \mathrm{E}+00$ \\
\hline ENSBTAG00000001069 & $2.39 \mathrm{E}-10$ & $1.09 \mathrm{E}-10$ & TP53 & $2.19 E+00$ \\
\hline ENSBTAG00000030820 & $4.90 \mathrm{E}-10$ & $2.21 \mathrm{E}-10$ & NT5M & $2.21 E+00$ \\
\hline ENSBTAG00000015258 & $4.25 \mathrm{E}-10$ & $1.92 \mathrm{E}-10$ & & $2.21 E+00$ \\
\hline ENSBTAG00000021798 & $2.30 \mathrm{E}-10$ & 1.03E-10 & ERBB2 & $2.23 E+00$ \\
\hline ENSBTAG00000020067 & $5.77 \mathrm{E}-10$ & 2.60E-10 & LLGL2 & $2.22 \mathrm{E}+00$ \\
\hline ENSBTAG00000021062 & $1.35 \mathrm{E}-10$ & $6.09 \mathrm{E}-11$ & SMARCD2 & $2.22 \mathrm{E}+00$ \\
\hline ENSBTAG00000004961 & $2.43 \mathrm{E}-10$ & $1.08 \mathrm{E}-10$ & GOSR1 & $2.25 \mathrm{E}+00$ \\
\hline ENSBTAG00000019097 & $9.69 \mathrm{E}-11$ & $4.31 \mathrm{E}-11$ & CNTNAP1 & $2.25 \mathrm{E}+00$ \\
\hline ENSBTAG00000003436 & $3.06 \mathrm{E}-10$ & $1.36 \mathrm{E}-10$ & RHBDF2 & $2.25 \mathrm{E}+00$ \\
\hline ENSBTAG00000001166 & $5.24 \mathrm{E}-10$ & $2.32 \mathrm{E}-10$ & HSF5 & $2.26 \mathrm{E}+00$ \\
\hline ENSBTAG00000004768 & $2.90 \mathrm{E}-10$ & $1.28 \mathrm{E}-10$ & RAI1 & $2.27 E+00$ \\
\hline ENSBTAG00000046765 & $3.97 \mathrm{E}-10$ & $1.74 \mathrm{E}-10$ & POLR2A & $2.28 \mathrm{E}+00$ \\
\hline ENSBTAG00000003802 & $5.58 \mathrm{E}-10$ & $2.41 \mathrm{E}-10$ & TEKT3 & $2.31 E+00$ \\
\hline ENSBTAG00000006087 & $6.46 \mathrm{E}-10$ & $2.79 \mathrm{E}-10$ & COG1 & $2.32 E+00$ \\
\hline ENSBTAG00000000411 & $4.76 \mathrm{E}-10$ & $2.05 \mathrm{E}-10$ & HGS & $2.32 \mathrm{E}+00$ \\
\hline ENSBTAG00000020119 & $5.56 \mathrm{E}-10$ & $2.39 \mathrm{E}-10$ & RNF43 & $2.33 E+00$ \\
\hline ENSBTAG00000031084 & $3.66 \mathrm{E}-10$ & $1.55 \mathrm{E}-10$ & CDRT4 & $2.36 \mathrm{E}+00$ \\
\hline ENSBTAG00000018519 & $1.40 \mathrm{E}-10$ & $5.89 \mathrm{E}-11$ & GAS2L2 & $2.38 \mathrm{E}+00$ \\
\hline ENSBTAG00000020253 & $1.92 \mathrm{E}-10$ & 8.04E-11 & SPEM1 & $2.38 \mathrm{E}+00$ \\
\hline ENSBTAG00000006189 & $8.63 \mathrm{E}-11$ & 3.62E-11 & & $2.38 \mathrm{E}+00$ \\
\hline ENSBTAG00000000133 & $1.19 \mathrm{E}-10$ & $4.98 \mathrm{E}-11$ & CD68 & $2.38 \mathrm{E}+00$ \\
\hline ENSBTAG00000012249 & $9.00 \mathrm{E}-11$ & $3.78 \mathrm{E}-11$ & PRR15L & $2.38 \mathrm{E}+00$ \\
\hline ENSBTAG00000007916 & $1.55 \mathrm{E}-10$ & $6.50 \mathrm{E}-11$ & FOXJ1 & $2.38 \mathrm{E}+00$ \\
\hline ENSBTAG00000003700 & $2.99 \mathrm{E}-10$ & $1.26 \mathrm{E}-10$ & SLC5A10 & $2.38 \mathrm{E}+00$ \\
\hline ENSBTAG00000002060 & $1.40 \mathrm{E}-10$ & 5.87E-11 & RPL19 & $2.38 \mathrm{E}+00$ \\
\hline ENSBTAG00000024909 & $4.15 \mathrm{E}-10$ & $1.74 \mathrm{E}-10$ & & $2.38 \mathrm{E}+00$ \\
\hline ENSBTAG00000033270 & $2.54 \mathrm{E}-10$ & $1.07 \mathrm{E}-10$ & SEBOX & $2.37 \mathrm{E}+00$ \\
\hline ENSBTAG00000044056 & $2.88 \mathrm{E}-10$ & $1.21 \mathrm{E}-10$ & C17orf98 & $2.38 \mathrm{E}+00$ \\
\hline ENSBTAG00000013680 & $1.54 \mathrm{E}-10$ & $6.45 \mathrm{E}-11$ & ATP1B2 & $2.38 \mathrm{E}+00$ \\
\hline ENSBTAG00000004222 & $1.48 \mathrm{E}-10$ & $6.22 \mathrm{E}-11$ & SAT2 & $2.38 \mathrm{E}+00$ \\
\hline ENSBTAG00000012302 & $1.83 \mathrm{E}-10$ & $7.69 \mathrm{E}-11$ & RTN4RL1 & $2.38 \mathrm{E}+00$ \\
\hline ENSBTAG00000039644 & $6.97 \mathrm{E}-10$ & $2.91 E-10$ & & $2.39 E+00$ \\
\hline ENSBTAG00000014940 & $1.84 \mathrm{E}-10$ & 7.73E-11 & KCNAB3 & $2.38 \mathrm{E}+00$ \\
\hline ENSBTAG00000040237 & $6.21 \mathrm{E}-10$ & 2.60E-10 & & $2.39 E+00$ \\
\hline ENSBTAG00000045837 & $6.48 \mathrm{E}-10$ & $2.71 E-10$ & & $2.39 E+00$ \\
\hline ENSBTAG00000010788 & $3.41 \mathrm{E}-10$ & $1.41 \mathrm{E}-10$ & PPM1E & $2.42 \mathrm{E}+00$ \\
\hline ENSBTAG00000039466 & $2.63 \mathrm{E}-10$ & 1.07E-10 & CDR2L & $2.45 \mathrm{E}+00$ \\
\hline ENSBTAG00000013534 & $2.21 \mathrm{E}-10$ & $8.89 \mathrm{E}-11$ & GFAP & $2.48 \mathrm{E}+00$ \\
\hline ENSBTAG00000009968 & $6.05 E-10$ & $2.42 \mathrm{E}-10$ & TBX4 & $2.50 \mathrm{E}+00$ \\
\hline ENSBTAG00000048278 & $8.95 \mathrm{E}-10$ & $3.59 \mathrm{E}-10$ & C17orf47 & $2.49 E+00$ \\
\hline ENSBTAG00000011507 & $1.55 \mathrm{E}-10$ & $6.19 \mathrm{E}-11$ & NUP88 & $2.50 E+00$ \\
\hline ENSBTAG00000046975 & $3.96 \mathrm{E}-10$ & $1.58 \mathrm{E}-10$ & & $2.51 E+00$ \\
\hline ENSBTAG00000025762 & $2.67 \mathrm{E}-10$ & $1.06 \mathrm{E}-10$ & CNP & $2.52 E+00$ \\
\hline ENSBTAG00000013556 & $3.58 \mathrm{E}-10$ & $1.41 \mathrm{E}-10$ & UNC13D & $2.54 \mathrm{E}+00$ \\
\hline ENSBTAG00000017366 & $1.50 \mathrm{E}-10$ & 5.91E-11 & GRB7 & $2.54 \mathrm{E}+00$ \\
\hline ENSBTAG00000015140 & $1.65 \mathrm{E}-10$ & $6.49 \mathrm{E}-11$ & RUNDC1 & $2.54 E+00$ \\
\hline ENSBTAG00000003705 & $3.12 \mathrm{E}-10$ & $1.22 \mathrm{E}-10$ & FAM83G & $2.55 E+00$ \\
\hline ENSBTAG00000000943 & $1.61 \mathrm{E}-10$ & $6.30 \mathrm{E}-11$ & & $2.56 \mathrm{E}+00$ \\
\hline ENSBTAG00000007581 & $3.36 \mathrm{E}-10$ & $1.31 \mathrm{E}-10$ & ADORA2B & $2.56 \mathrm{E}+00$ \\
\hline ENSBTAG00000006896 & 1.19E-09 & $4.61 \mathrm{E}-10$ & ABCA9 & $2.57 \mathrm{E}+00$ \\
\hline
\end{tabular}




\begin{tabular}{|c|c|c|c|c|}
\hline ENSBTAG000000004910 & $3.91 \mathrm{E}-10$ & $1.51 \mathrm{E}-10$ & SLC25A11 & $2.59 \mathrm{E}+00$ \\
\hline ENSBTAG00000007353 & $1.91 \mathrm{E}-10$ & $7.39 \mathrm{E}-11$ & DLX4 & $2.58 \mathrm{E}+00$ \\
\hline ENSBTAG00000003707 & $5.49 \mathrm{E}-10$ & $2.12 \mathrm{E}-10$ & & $2.59 \mathrm{E}+00$ \\
\hline ENSBTAG00000007217 & 4.55E-10 & $1.75 \mathrm{E}-10$ & TMEM94 & $2.60 \mathrm{E}+00$ \\
\hline ENSBTAG00000011280 & $1.01 \mathrm{E}-10$ & $3.83 \mathrm{E}-11$ & TBKBP1 & $2.64 \mathrm{E}+00$ \\
\hline ENSBTAG00000001632 & $1.83 \mathrm{E}-10$ & $6.90 \mathrm{E}-11$ & NBR1 & $2.65 E+00$ \\
\hline ENSBTAG00000007390 & $1.10 \mathrm{E}-10$ & 4.14E-11 & VAT1 & $2.65 \mathrm{E}+00$ \\
\hline ENSBTAG00000038386 & $7.63 \mathrm{E}-10$ & 2.87E-10 & & $2.66 \mathrm{E}+00$ \\
\hline ENSBTAG00000011834 & $2.77 \mathrm{E}-10$ & $1.04 \mathrm{E}-10$ & CCDC47 & $2.66 \mathrm{E}+00$ \\
\hline ENSBTAG000000010279 & $2.27 \mathrm{E}-10$ & $8.46 \mathrm{E}-11$ & DUSP14 & $2.69 \mathrm{E}+00$ \\
\hline ENSBTAG00000012564 & $1.82 \mathrm{E}-10$ & $6.74 \mathrm{E}-11$ & KANSL1 & $2.69 \mathrm{E}+00$ \\
\hline ENSBTAG000000011482 & $3.08 \mathrm{E}-10$ & $1.13 \mathrm{E}-10$ & SLC43A2 & $2.72 \mathrm{E}+00$ \\
\hline ENSBTAG00000019347 & $5.02 \mathrm{E}-10$ & $1.83 \mathrm{E}-10$ & PLXDC1 & $2.74 \mathrm{E}+00$ \\
\hline ENSBTAG00000010070 & $5.73 \mathrm{E}-10$ & $2.07 \mathrm{E}-10$ & GSDMA & $2.77 E+00$ \\
\hline ENSBTAG000000000131 & $1.44 \mathrm{E}-10$ & 5.19E-11 & & $2.78 \mathrm{E}+00$ \\
\hline ENSBTAG000000012225 & $2.92 \mathrm{E}-10$ & $1.05 \mathrm{E}-10$ & KPNA2 & $2.78 \mathrm{E}+00$ \\
\hline ENSBTAG000000021061 & $1.25 \mathrm{E}-10$ & $4.50 \mathrm{E}-11$ & PSMC5 & $2.77 E+00$ \\
\hline ENSBTAG00000011491 & $6.21 \mathrm{E}-11$ & $2.24 \mathrm{E}-11$ & & $2.77 \mathrm{E}+00$ \\
\hline ENSBTAG000000039607 & $2.03 \mathrm{E}-10$ & $7.29 \mathrm{E}-11$ & PRCD & $2.78 \mathrm{E}+00$ \\
\hline ENSBTAG000000015390 & $3.32 \mathrm{E}-10$ & 1.17E-10 & FN3KRP & $2.83 E+00$ \\
\hline ENSBTAG000000010167 & $4.33 \mathrm{E}-10$ & $1.52 \mathrm{E}-10$ & TEX14 & $2.85 E+00$ \\
\hline ENSBTAG00000002423 & $2.62 \mathrm{E}-10$ & $9.12 \mathrm{E}-11$ & PSMD12 & $2.87 E+00$ \\
\hline ENSBTAG00000020979 & $2.91 \mathrm{E}-10$ & $1.01 \mathrm{E}-10$ & NGFR & $2.88 \mathrm{E}+00$ \\
\hline ENSBTAG00000004635 & $2.82 \mathrm{E}-10$ & $9.74 \mathrm{E}-11$ & LLGL1 & $2.90 \mathrm{E}+00$ \\
\hline ENSBTAG00000022489 & $3.15 \mathrm{E}-10$ & $1.09 \mathrm{E}-10$ & & $2.89 \mathrm{E}+00$ \\
\hline ENSBTAG000000010527 & $1.55 \mathrm{E}-10$ & 5.31E-11 & ACAP1 & $2.91 E+00$ \\
\hline ENSBTAG000000017275 & $5.45 \mathrm{E}-10$ & $1.86 \mathrm{E}-10$ & COIL & $2.93 E+00$ \\
\hline ENSBTAG000000000875 & $4.90 \mathrm{E}-10$ & $1.67 \mathrm{E}-10$ & CEP131 & $2.93 E+00$ \\
\hline ENSBTAG000000003983 & $3.18 \mathrm{E}-10$ & $1.08 \mathrm{E}-10$ & TOM1L2 & $2.95 E+00$ \\
\hline ENSBTAG000000007015 & $3.28 \mathrm{E}-10$ & $1.11 \mathrm{E}-10$ & cox11 & $2.96 \mathrm{E}+00$ \\
\hline ENSBTAG000000066921 & 1.01E-09 & $3.41 \mathrm{E}-10$ & ABCA6 & $2.96 \mathrm{E}+00$ \\
\hline ENSBTAG00000015713 & $1.97 \mathrm{E}-10$ & $6.61 \mathrm{E}-11$ & TLK2 & $2.97 \mathrm{E}+00$ \\
\hline ENSBTAG000000001717 & $2.54 \mathrm{E}-10$ & $8.52 \mathrm{E}-11$ & AURKB & $2.98 \mathrm{E}+00$ \\
\hline ENSBTAG000000013274 & $7.53 \mathrm{E}-10$ & $2.52 \mathrm{E}-10$ & CENPV & $2.99 \mathrm{E}+00$ \\
\hline ENSBTAG00000021461 & $3.98 \mathrm{E}-10$ & $1.33 \mathrm{E}-10$ & PSMD3 & $2.99 \mathrm{E}+00$ \\
\hline ENSBTAG00000010509 & $3.63 \mathrm{E}-10$ & $1.20 \mathrm{E}-10$ & & $3.02 \mathrm{E}+00$ \\
\hline ENSBTAG00000031071 & $4.36 \mathrm{E}-10$ & $1.41 \mathrm{E}-10$ & ZNF624 & $3.09 E+00$ \\
\hline ENSBTAG00000011488 & $4.31 \mathrm{E}-10$ & $1.39 \mathrm{E}-10$ & PRPF8 & $3.10 \mathrm{E}+00$ \\
\hline ENSBTAG00000001830 & 7.40E-11 & $2.33 \mathrm{E}-11$ & LRRC46 & $3.17 \mathrm{E}+00$ \\
\hline ENSBTAG00000016251 & $9.36 \mathrm{E}-11$ & $2.95 \mathrm{E}-11$ & TMEM101 & $3.17 E+00$ \\
\hline ENSBTAG00000039684 & $2.09 \mathrm{E}-10$ & $6.56 \mathrm{E}-11$ & PTRF & $3.18 \mathrm{E}+00$ \\
\hline ENSBTAG00000005546 & $2.71 E-10$ & 8.51E-11 & HOXB7 & $3.18 \mathrm{E}+00$ \\
\hline ENSBTAG00000031998 & $9.18 \mathrm{E}-11$ & $2.89 \mathrm{E}-11$ & CXCL16 & $3.17 E+00$ \\
\hline ENSBTAG00000003891 & $2.68 \mathrm{E}-10$ & $8.44 \mathrm{E}-11$ & VAMP2 & $3.18 \mathrm{E}+00$ \\
\hline ENSBTAG00000020854 & $2.47 \mathrm{E}-10$ & $7.76 \mathrm{E}-11$ & BCL6B & $3.18 \mathrm{E}+00$ \\
\hline ENSBTAG00000008368 & $4.01 \mathrm{E}-10$ & $1.26 \mathrm{E}-10$ & & $3.18 \mathrm{E}+00$ \\
\hline ENSBTAG00000010745 & $3.18 \mathrm{E}-10$ & $1.00 \mathrm{E}-10$ & THRA & $3.18 \mathrm{E}+00$ \\
\hline ENSBTAG00000037529 & $3.17 \mathrm{E}-10$ & $9.97 \mathrm{E}-11$ & & $3.18 \mathrm{E}+00$ \\
\hline ENSBTAG00000039299 & $4.98 \mathrm{E}-10$ & $1.56 \mathrm{E}-10$ & RNF222 & $3.19 E+00$ \\
\hline ENSBTAG00000016161 & $3.11 E-10$ & $9.76 \mathrm{E}-11$ & FLII & $3.18 \mathrm{E}+00$ \\
\hline ENSBTAG00000011994 & $3.99 \mathrm{E}-10$ & $1.25 \mathrm{E}-10$ & NDEL1 & $3.19 E+00$ \\
\hline ENSBTAG00000009991 & $4.41 \mathrm{E}-10$ & $1.39 \mathrm{E}-10$ & CANT1 & $3.17 E+00$ \\
\hline ENSBTAG00000012259 & $2.00 \mathrm{E}-10$ & $6.30 \mathrm{E}-11$ & PNPO & $3.18 \mathrm{E}+00$ \\
\hline ENSBTAG00000048062 & $1.61 \mathrm{E}-10$ & $5.06 \mathrm{E}-11$ & KDM6B & $3.18 \mathrm{E}+00$ \\
\hline ENSBTAG00000020810 & $1.58 \mathrm{E}-10$ & $4.96 \mathrm{E}-11$ & CYB561 & $3.18 \mathrm{E}+00$ \\
\hline ENSBTAG00000009190 & $1.41 \mathrm{E}-10$ & $4.42 \mathrm{E}-11$ & SLC2A4 & $3.18 \mathrm{E}+00$ \\
\hline ENSBTAG00000045921 & $7.09 \mathrm{E}-10$ & $2.22 \mathrm{E}-10$ & & $3.19 E+00$ \\
\hline ENSBTAG00000013271 & $3.90 \mathrm{E}-10$ & $1.18 \mathrm{E}-10$ & NCOR1 & $3.31 E+00$ \\
\hline ENSBTAG00000017790 & $3.74 \mathrm{E}-10$ & $1.11 \mathrm{E}-10$ & B9D1 & $3.37 E+00$ \\
\hline ENSBTAG00000012311 & $7.73 \mathrm{E}-10$ & $2.29 \mathrm{E}-10$ & CACNG5 & $3.38 \mathrm{E}+00$ \\
\hline ENSBTAG00000005008 & $2.94 \mathrm{E}-10$ & $8.71 E-11$ & WSB1 & $3.38 \mathrm{E}+00$ \\
\hline ENSBTAG00000021058 & $2.61 \mathrm{E}-10$ & $7.69 \mathrm{E}-11$ & DDX42 & $3.39 E+00$ \\
\hline ENSBTAG00000003014 & $6.82 \mathrm{E}-10$ & $2.01 E-10$ & TRPV2 & $3.39 E+00$ \\
\hline ENSBTAG00000006520 & $3.68 \mathrm{E}-10$ & $1.08 \mathrm{E}-10$ & MYO15A & $3.41 E+00$ \\
\hline ENSBTAG00000035975 & $2.86 \mathrm{E}-10$ & 8.17E-11 & SCIMP & $3.50 \mathrm{E}+00$ \\
\hline ENSBTAG00000006187 & 7.15E-10 & $2.04 \mathrm{E}-10$ & MFAP4 & $3.50 E+00$ \\
\hline ENSBTAG00000020824 & $5.86 \mathrm{E}-10$ & $1.66 \mathrm{E}-10$ & KRT10 & $3.53 E+00$ \\
\hline ENSBTAG00000046321 & $4.71 \mathrm{E}-10$ & $1.31 \mathrm{E}-10$ & TSPOAP1 & $3.59 E+00$ \\
\hline ENSBTAG00000038548 & $4.49 \mathrm{E}-10$ & $1.25 \mathrm{E}-10$ & SLC47A2 & $3.59 E+00$ \\
\hline ENSBTAG00000013270 & $3.83 \mathrm{E}-10$ & $1.06 \mathrm{E}-10$ & TTC19 & $3.61 E+00$ \\
\hline
\end{tabular}




\begin{tabular}{|c|c|c|c|c|}
\hline ENSBTAG00000019262 & 4.86E-10 & $1.33 \mathrm{E}-10$ & TOP2A & $3.65 \mathrm{E}+00$ \\
\hline ENSBTAG00000002036 & $1.57 \mathrm{E}-10$ & 4.24E-11 & CACNB1 & $3.70 E+00$ \\
\hline ENSBTAG00000025101 & $4.61 \mathrm{E}-10$ & $1.24 \mathrm{E}-10$ & ASGR2 & $3.72 \mathrm{E}+00$ \\
\hline ENSBTAG00000007884 & $3.12 \mathrm{E}-10$ & $8.36 \mathrm{E}-11$ & SREBF1 & $3.73 \mathrm{E}+00$ \\
\hline ENSBTAG00000019049 & $4.51 \mathrm{E}-10$ & $1.20 \mathrm{E}-10$ & AATK & $3.76 \mathrm{E}+00$ \\
\hline ENSBTAG00000005696 & 4.55E-10 & $1.21 \mathrm{E}-10$ & DBF4B & $3.76 \mathrm{E}+00$ \\
\hline ENSBTAG00000015394 & $5.28 \mathrm{E}-10$ & $1.39 \mathrm{E}-10$ & FN3K & $3.79 E+00$ \\
\hline ENSBTAG00000019094 & $1.25 \mathrm{E}-09$ & 3.30E-10 & NLE1 & $3.80 \mathrm{E}+00$ \\
\hline ENSBTAG00000030591 & $3.00 \mathrm{E}-10$ & $7.86 \mathrm{E}-11$ & CWC25 & $3.81 E+00$ \\
\hline ENSBTAG00000024932 & $3.40 \mathrm{E}-10$ & 8.91E-11 & FSCN2 & $3.82 \mathrm{E}+00$ \\
\hline ENSBTAG00000007117 & $4.54 \mathrm{E}-10$ & 1.19E-10 & CTC1 & $3.81 E+00$ \\
\hline ENSBTAG00000046264 & $5.30 \mathrm{E}-10$ & $1.38 \mathrm{E}-10$ & & $3.84 \mathrm{E}+00$ \\
\hline ENSBTAG00000018204 & $2.32 \mathrm{E}-10$ & $6.01 \mathrm{E}-11$ & MYH1 & $3.86 \mathrm{E}+00$ \\
\hline ENSBTAG00000008605 & $2.77 \mathrm{E}-10$ & 7.10E-11 & DNAH2 & $3.90 \mathrm{E}+00$ \\
\hline ENSBTAG00000001349 & $4.93 \mathrm{E}-10$ & $1.27 \mathrm{E}-10$ & KIF1C & $3.88 \mathrm{E}+00$ \\
\hline ENSBTAG00000006250 & $6.53 \mathrm{E}-10$ & $1.66 \mathrm{E}-10$ & CASC3 & $3.93 E+00$ \\
\hline ENSBTAG00000008289 & $1.10 \mathrm{E}-10$ & $2.78 \mathrm{E}-11$ & ZBTB4 & $3.96 \mathrm{E}+00$ \\
\hline ENSBTAG00000010184 & $1.52 \mathrm{E}-10$ & $3.82 \mathrm{E}-11$ & G6PC & $3.97 E+00$ \\
\hline ENSBTAG00000045892 & $1.38 \mathrm{E}-10$ & 3.47E-11 & & $3.97 E+00$ \\
\hline ENSBTAG00000018423 & $6.23 \mathrm{E}-11$ & 1.57E-11 & DDX5 & $3.95 E+00$ \\
\hline ENSBTAG00000010513 & $1.56 \mathrm{E}-10$ & $3.93 \mathrm{E}-11$ & GPS2 & $3.97 \mathrm{E}+00$ \\
\hline ENSBTAG00000006801 & $2.40 \mathrm{E}-10$ & $6.03 \mathrm{E}-11$ & TMEM106A & $3.97 \mathrm{E}+00$ \\
\hline ENSBTAG00000030347 & 8.34E-11 & $2.10 \mathrm{E}-11$ & TUBG2 & $3.96 \mathrm{E}+00$ \\
\hline ENSBTAG00000035587 & $9.78 \mathrm{E}-11$ & $2.46 \mathrm{E}-11$ & ARRB2 & $3.96 \mathrm{E}+00$ \\
\hline ENSBTAG00000019911 & $3.70 \mathrm{E}-10$ & $9.30 \mathrm{E}-11$ & $\mathrm{COA} 3$ & $3.97 E+00$ \\
\hline ENSBTAG00000004464 & $1.33 \mathrm{E}-10$ & 3.36E-11 & C17orf53 & $3.96 \mathrm{E}+00$ \\
\hline ENSBTAG00000005693 & $2.64 \mathrm{E}-10$ & $6.63 \mathrm{E}-11$ & $\mathrm{CCDC} 43$ & $3.97 \mathrm{E}+00$ \\
\hline ENSBTAG00000025126 & 7.63E-10 & $1.91 \mathrm{E}-10$ & & $3.99 E+00$ \\
\hline ENSBTAG00000017136 & 7.44E-10 & $1.87 \mathrm{E}-10$ & SSTR2 & $3.98 \mathrm{E}+00$ \\
\hline ENSBTAG00000021113 & $4.91 \mathrm{E}-10$ & $1.20 \mathrm{E}-10$ & ALDH3A1 & $4.09 E+00$ \\
\hline ENSBTAG00000008798 & $7.42 \mathrm{E}-10$ & $1.80 \mathrm{E}-10$ & DRC3 & $4.12 \mathrm{E}+00$ \\
\hline ENSBTAG00000025258 & $1.07 \mathrm{E}-09$ & $2.59 \mathrm{E}-10$ & & $4.13 E+00$ \\
\hline ENSBTAG00000017246 & $1.35 \mathrm{E}-09$ & $3.27 \mathrm{E}-10$ & & $4.14 E+00$ \\
\hline ENSBTAG00000007084 & $2.73 \mathrm{E}-10$ & $6.56 \mathrm{E}-11$ & MAP3К14 & $4.15 E+00$ \\
\hline ENSBTAG00000018663 & $4.92 \mathrm{E}-10$ & 1.17E-10 & GPRC5C & $4.21 E+00$ \\
\hline ENSBTAG00000011803 & $3.96 \mathrm{E}-10$ & $9.37 E-11$ & MYH3 & $4.23 E+00$ \\
\hline ENSBTAG00000000928 & $3.39 \mathrm{E}-10$ & 7.94E-11 & SPECC1 & $4.26 \mathrm{E}+00$ \\
\hline ENSBTAG00000007083 & $1.71 \mathrm{E}-10$ & $3.98 \mathrm{E}-11$ & SPATA32 & $4.28 \mathrm{E}+00$ \\
\hline ENSBTAG00000019437 & $5.48 \mathrm{E}-10$ & $1.27 \mathrm{E}-10$ & & $4.31 E+00$ \\
\hline ENSBTAG00000006586 & $1.42 \mathrm{E}-10$ & 3.30E-11 & KPNB1 & $4.30 \mathrm{E}+00$ \\
\hline ENSBTAG00000017536 & $2.20 \mathrm{E}-10$ & $5.08 \mathrm{E}-11$ & MPP3 & $4.33 E+00$ \\
\hline ENSBTAG00000009354 & $2.21 \mathrm{E}-10$ & $5.06 \mathrm{E}-11$ & & $4.36 \mathrm{E}+00$ \\
\hline ENSBTAG00000046273 & $3.59 \mathrm{E}-10$ & $8.20 \mathrm{E}-11$ & FAM57A & $4.37 \mathrm{E}+00$ \\
\hline ENSBTAG00000013134 & $5.54 \mathrm{E}-10$ & $1.26 \mathrm{E}-10$ & SOST & $4.40 \mathrm{E}+00$ \\
\hline ENSBTAG00000046217 & $9.64 \mathrm{E}-10$ & $2.19 \mathrm{E}-10$ & & $4.40 \mathrm{E}+00$ \\
\hline ENSBTAG00000017771 & $5.21 \mathrm{E}-10$ & $1.19 \mathrm{E}-10$ & EPN2 & $4.38 \mathrm{E}+00$ \\
\hline ENSBTAG00000044099 & $3.33 \mathrm{E}-10$ & $7.51 \mathrm{E}-11$ & ZSWIM7 & $4.43 E+00$ \\
\hline ENSBTAG00000019972 & $8.31 \mathrm{E}-10$ & $1.86 \mathrm{E}-10$ & TEPSIN & $4.47 E+00$ \\
\hline ENSBTAG00000013238 & $1.87 \mathrm{E}-10$ & 4.05E-11 & CDK12 & $4.61 \mathrm{E}+00$ \\
\hline ENSBTAG00000019241 & $1.23 \mathrm{E}-10$ & $2.59 \mathrm{E}-11$ & FGF11 & $4.74 \mathrm{E}+00$ \\
\hline ENSBTAG00000008593 & $2.21 \mathrm{E}-10$ & $4.62 \mathrm{E}-11$ & INCA1 & $4.77 \mathrm{E}+00$ \\
\hline ENSBTAG00000002609 & $7.52 \mathrm{E}-11$ & $1.58 \mathrm{E}-11$ & TMIGD1 & $4.74 \mathrm{E}+00$ \\
\hline ENSBTAG00000005664 & $1.70 \mathrm{E}-10$ & $3.56 \mathrm{E}-11$ & YWHAE & $4.75 \mathrm{E}+00$ \\
\hline ENSBTAG00000014883 & $2.55 \mathrm{E}-10$ & $5.34 \mathrm{E}-11$ & GABARAP & $4.77 \mathrm{E}+00$ \\
\hline ENSBTAG00000014113 & $7.29 \mathrm{E}-10$ & $1.52 \mathrm{E}-10$ & CCL8 & $4.79 E+00$ \\
\hline ENSBTAG00000008802 & $6.58 \mathrm{E}-10$ & $1.37 \mathrm{E}-10$ & GID4 & $4.80 \mathrm{E}+00$ \\
\hline ENSBTAG00000018802 & $3.30 \mathrm{E}-10$ & $6.80 \mathrm{E}-11$ & UBE2Z & $4.85 E+00$ \\
\hline ENSBTAG00000015136 & $1.70 \mathrm{E}-10$ & $3.48 \mathrm{E}-11$ & PTGES3L-AARSD1 & $4.88 \mathrm{E}+00$ \\
\hline ENSBTAG00000008801 & $5.66 \mathrm{E}-10$ & $1.15 \mathrm{E}-10$ & ATPAF2 & $4.92 \mathrm{E}+00$ \\
\hline ENSBTAG00000001965 & 7.17E-10 & $1.44 \mathrm{E}-10$ & ENPP7 & $4.98 \mathrm{E}+00$ \\
\hline ENSBTAG00000030169 & $2.01 \mathrm{E}-10$ & $4.00 \mathrm{E}-11$ & SUMO2 & $5.02 \mathrm{E}+00$ \\
\hline ENSBTAG00000019092 & $6.61 \mathrm{E}-10$ & $1.31 \mathrm{E}-10$ & FNDC8 & $5.04 E+00$ \\
\hline ENSBTAG00000012066 & $2.70 \mathrm{E}-10$ & $5.28 \mathrm{E}-11$ & PECAM1 & $5.11 E+00$ \\
\hline ENSBTAG00000008779 & 4.24E-10 & $8.28 \mathrm{E}-11$ & ALOX15B & $5.11 E+00$ \\
\hline ENSBTAG00000000205 & $6.05 \mathrm{E}-10$ & $1.16 \mathrm{E}-10$ & MMD & $5.21 E+00$ \\
\hline ENSBTAG00000006089 & $4.54 \mathrm{E}-10$ & 8.55E-11 & C17orf80 & $5.30 \mathrm{E}+00$ \\
\hline ENSBTAG00000008088 & $3.31 \mathrm{E}-10$ & $6.21 \mathrm{E}-11$ & KIF18B & $5.32 \mathrm{E}+00$ \\
\hline ENSBTAG00000011732 & 4.37E-10 & $8.15 \mathrm{E}-11$ & PGAP3 & $5.35 E+00$ \\
\hline ENSBTAG00000015751 & $1.48 \mathrm{E}-10$ & $2.74 \mathrm{E}-11$ & MEOX1 & $5.39 \mathrm{E}+00$ \\
\hline ENSBTAG00000009885 & 7.33E-10 & $1.35 \mathrm{E}-10$ & TACO1 & $5.42 \mathrm{E}+00$ \\
\hline
\end{tabular}




\begin{tabular}{|c|c|c|c|c|}
\hline ENSBTAG00000003500 & $6.65 \mathrm{E}-10$ & $1.20 \mathrm{E}-10$ & DCAF7 & $5.54 \mathrm{E}+00$ \\
\hline ENSBTAG00000002018 & $1.18 \mathrm{E}-10$ & $2.12 \mathrm{E}-11$ & EIF5A & $5.53 \mathrm{E}+00$ \\
\hline ENSBTAG00000009292 & $2.35 \mathrm{E}-10$ & $4.21 \mathrm{E}-11$ & TMEM100 & $5.56 \mathrm{E}+00$ \\
\hline ENSBTAG00000001014 & 4.56E-10 & 8.17E-11 & MAPK7 & $5.57 \mathrm{E}+00$ \\
\hline ENSBTAG00000013529 & $7.11 \mathrm{E}-10$ & $1.27 \mathrm{E}-10$ & & $5.59 E+00$ \\
\hline ENSBTAG00000006517 & $8.53 \mathrm{E}-10$ & $1.52 \mathrm{E}-10$ & DRG2 & $5.61 E+00$ \\
\hline ENSBTAG00000014858 & $2.27 \mathrm{E}-10$ & $4.00 \mathrm{E}-11$ & PRPSAP2 & $5.65 \mathrm{E}+00$ \\
\hline ENSBTAG00000025001 & $2.13 \mathrm{E}-10$ & $3.75 E-11$ & MRPL45 & $5.66 \mathrm{E}+00$ \\
\hline ENSBTAG00000009353 & $4.78 \mathrm{E}-10$ & $8.39 E-11$ & EVI2B & $5.69 E+00$ \\
\hline ENSBTAG00000047756 & $3.40 \mathrm{E}-10$ & $5.89 \mathrm{E}-11$ & CNTROB & $5.76 \mathrm{E}+00$ \\
\hline ENSBTAG00000010758 & $2.60 \mathrm{E}-10$ & $4.45 \mathrm{E}-11$ & SMIM5 & $5.83 E+00$ \\
\hline ENSBTAG00000015232 & $3.46 \mathrm{E}-10$ & $5.85 E-11$ & RAPGEFL1 & $5.90 \mathrm{E}+00$ \\
\hline ENSBTAG00000008318 & $7.69 \mathrm{E}-10$ & $1.29 \mathrm{E}-10$ & AIPL1 & $5.96 \mathrm{E}+00$ \\
\hline ENSBTAG00000016249 & $2.36 \mathrm{E}-10$ & $3.95 \mathrm{E}-11$ & NAGS & $5.95 E+00$ \\
\hline ENSBTAG00000012178 & $2.58 \mathrm{E}-10$ & $4.24 \mathrm{E}-11$ & NR1D1 & $6.08 \mathrm{E}+00$ \\
\hline ENSBTAG00000016151 & $6.46 \mathrm{E}-10$ & $1.06 \mathrm{E}-10$ & VTN & $6.09 E+00$ \\
\hline ENSBTAG00000017233 & $4.96 \mathrm{E}-10$ & $8.06 \mathrm{E}-11$ & & $6.15 E+00$ \\
\hline ENSBTAG00000019069 & $1.74 \mathrm{E}-10$ & $2.73 \mathrm{E}-11$ & SYNGR2 & $6.34 \mathrm{E}+00$ \\
\hline ENSBTAG00000024947 & $5.52 \mathrm{E}-10$ & $8.65 \mathrm{E}-11$ & & $6.37 E+00$ \\
\hline ENSBTAG00000025046 & $3.74 \mathrm{E}-10$ & $5.79 E-11$ & ALKBH5 & $6.44 E+00$ \\
\hline ENSBTAG00000005048 & 8.67E-10 & $1.32 \mathrm{E}-10$ & DHRS7C & $6.56 \mathrm{E}+00$ \\
\hline ENSBTAG00000039485 & $1.52 \mathrm{E}-10$ & $2.30 \mathrm{E}-11$ & MEIOC & $6.59 E+00$ \\
\hline ENSBTAG00000014377 & $2.98 \mathrm{E}-10$ & $4.44 \mathrm{E}-11$ & $\mathrm{CHD} 3$ & $6.70 \mathrm{E}+00$ \\
\hline ENSBTAG00000025088 & $8.03 E-10$ & $1.16 \mathrm{E}-10$ & RCVRN & $6.92 \mathrm{E}+00$ \\
\hline ENSBTAG00000030977 & $3.74 \mathrm{E}-10$ & $5.36 \mathrm{E}-11$ & ULK2 & $6.96 \mathrm{E}+00$ \\
\hline ENSBTAG00000030200 & $3.15 \mathrm{E}-10$ & $4.40 \mathrm{E}-11$ & ARL16 & $7.15 E+00$ \\
\hline ENSBTAG00000003386 & $6.42 \mathrm{E}-10$ & $8.95 E-11$ & SUPT4H1 & $7.17 E+00$ \\
\hline ENSBTAG00000024899 & $8.71 \mathrm{E}-10$ & $1.20 \mathrm{E}-10$ & & $7.25 E+00$ \\
\hline ENSBTAG00000021918 & $2.34 \mathrm{E}-10$ & $3.19 \mathrm{E}-11$ & EZH1 & $7.31 E+00$ \\
\hline ENSBTAG00000039206 & $4.18 \mathrm{E}-10$ & $5.53 \mathrm{E}-11$ & SLFN14 & $7.55 E+00$ \\
\hline ENSBTAG00000011729 & $7.59 \mathrm{E}-10$ & $1.00 \mathrm{E}-10$ & STARD3 & $7.58 \mathrm{E}+00$ \\
\hline ENSBTAG00000007434 & $7.65 \mathrm{E}-10$ & $9.75 E-11$ & CD300LG & $7.84 \mathrm{E}+00$ \\
\hline ENSBTAG00000000344 & $2.74 \mathrm{E}-10$ & $3.44 \mathrm{E}-11$ & & $7.94 \mathrm{E}+00$ \\
\hline ENSBTAG00000040118 & $1.38 \mathrm{E}-09$ & $1.72 \mathrm{E}-10$ & & $8.03 E+00$ \\
\hline ENSBTAG00000009458 & $3.74 \mathrm{E}-10$ & $4.61 \mathrm{E}-11$ & & $8.10 \mathrm{E}+00$ \\
\hline ENSBTAG00000003439 & $5.02 \mathrm{E}-10$ & $5.96 \mathrm{E}-11$ & AKAP10 & $8.40 E+00$ \\
\hline ENSBTAG00000012475 & $2.35 \mathrm{E}-10$ & $2.69 \mathrm{E}-11$ & ALOX12B & $8.72 \mathrm{E}+00$ \\
\hline ENSBTAG00000000132 & $1.64 \mathrm{E}-10$ & $1.72 \mathrm{E}-11$ & EIF4A1 & $9.47 E+00$ \\
\hline ENSBTAG00000019242 & $1.07 \mathrm{E}-10$ & $1.12 \mathrm{E}-11$ & CHRNB1 & $9.46 \mathrm{E}+00$ \\
\hline ENSBTAG00000006035 & $3.46 \mathrm{E}-10$ & $3.62 \mathrm{E}-11$ & PPP1R1B & $9.53 \mathrm{E}+00$ \\
\hline ENSBTAG00000017087 & $2.97 \mathrm{E}-10$ & $3.03 E-11$ & TOP3A & $9.79 E+00$ \\
\hline ENSBTAG00000017090 & $4.31 \mathrm{E}-10$ & $4.39 \mathrm{E}-11$ & SMCR8 & $9.80 \mathrm{E}+00$ \\
\hline ENSBTAG00000005051 & $7.70 \mathrm{E}-10$ & $7.81 \mathrm{E}-11$ & GSG1L2 & $9.85 E+00$ \\
\hline ENSBTAG00000046148 & $3.70 \mathrm{E}-10$ & $3.58 \mathrm{E}-11$ & & $1.03 E+01$ \\
\hline ENSBTAG00000008366 & $2.83 \mathrm{E}-10$ & $2.60 \mathrm{E}-11$ & STAC2 & $1.08 \mathrm{E}+01$ \\
\hline ENSBTAG00000015276 & $3.21 \mathrm{E}-10$ & $2.92 \mathrm{E}-11$ & SLC26A11 & $1.10 \mathrm{E}+01$ \\
\hline ENSBTAG00000005045 & 7.07E-10 & $5.90 \mathrm{E}-11$ & GIP & $1.20 \mathrm{E}+01$ \\
\hline ENSBTAG00000017895 & $2.71 \mathrm{E}-10$ & $2.13 \mathrm{E}-11$ & CLEC10A & $1.26 \mathrm{E}+01$ \\
\hline ENSBTAG00000021462 & $5.70 \mathrm{E}-10$ & 4.47E-11 & CSF3 & $1.27 \mathrm{E}+01$ \\
\hline ENSBTAG00000011713 & 4.39E-10 & $3.34 \mathrm{E}-11$ & & $1.31 E+01$ \\
\hline ENSBTAG00000004903 & $8.62 \mathrm{E}-10$ & $6.47 \mathrm{E}-11$ & KRT15 & $1.33 \mathrm{E}+01$ \\
\hline ENSBTAG00000011435 & $1.36 \mathrm{E}-10$ & $9.90 \mathrm{E}-12$ & NPEPPS & $1.36 \mathrm{E}+01$ \\
\hline ENSBTAG00000014202 & 4.63E-10 & $3.29 \mathrm{E}-11$ & CDK5RAP3 & $1.40 \mathrm{E}+01$ \\
\hline ENSBTAG00000008423 & $5.00 \mathrm{E}-10$ & $3.39 \mathrm{E}-11$ & TIMM22 & $1.47 \mathrm{E}+01$ \\
\hline ENSBTAG00000018803 & $3.58 \mathrm{E}-10$ & $2.31 \mathrm{E}-11$ & SNF8 & $1.54 \mathrm{E}+01$ \\
\hline ENSBTAG00000008535 & $1.99 \mathrm{E}-10$ & $1.25 \mathrm{E}-11$ & socs7 & $1.58 \mathrm{E}+01$ \\
\hline ENSBTAG00000016911 & $1.97 \mathrm{E}-10$ & $1.10 \mathrm{E}-11$ & ARHGAP23 & $1.77 \mathrm{E}+01$ \\
\hline ENSBTAG00000002035 & $3.62 \mathrm{E}-10$ & $1.75 \mathrm{E}-11$ & ARL5C & $2.06 \mathrm{E}+01$ \\
\hline ENSBTAG00000015267 & $3.33 \mathrm{E}-10$ & $1.44 \mathrm{E}-11$ & SGSH & $2.30 E+01$ \\
\hline ENSBTAG00000019067 & $5.41 \mathrm{E}-10$ & $2.30 \mathrm{E}-11$ & TMC8 & $2.34 \mathrm{E}+01$ \\
\hline ENSBTAG00000006499 & $3.31 \mathrm{E}-10$ & $9.24 \mathrm{E}-12$ & PIP4K2B & $3.55 E+01$ \\
\hline ENSBTAG00000000812 & $9.06 \mathrm{E}-10$ & $1.21 \mathrm{E}-11$ & & $7.43 E+01$ \\
\hline ENSBTAG00000045560 & 0 & 0 & & $1.00 \mathrm{E}+00$ \\
\hline ENSBTAG00000025213 & $1.56 \mathrm{E}-10$ & 0 & OMG & $1.56 \mathrm{E}+03$ \\
\hline ENSBTAG00000011490 & $9.82 \mathrm{E}-11$ & 0 & TLCD2 & $9.83 E+02$ \\
\hline ENSBTAG00000018314 & 4.47E-11 & 0 & MED11 & $4.48 \mathrm{E}+02$ \\
\hline ENSBTAG00000021932 & $2.86 \mathrm{E}-10$ & 0 & RNASEK & $2.86 \mathrm{E}+03$ \\
\hline ENSBTAG00000020852 & $1.68 \mathrm{E}-10$ & 0 & SLC16A11 & $1.68 \mathrm{E}+03$ \\
\hline ENSBTAG00000017747 & $1.08 \mathrm{E}-10$ & 0 & TMEM256-PLSCR3 & $1.08 \mathrm{E}+03$ \\
\hline ENSBTAG00000031752 & 7.13E-11 & 0 & & $7.14 E+02$ \\
\hline
\end{tabular}




\begin{tabular}{|c|c|c|c|c|}
\hline ENSBTAG00000031737 & $3.95 \mathrm{E}-11$ & 0 & TMEM102 & $3.96 \mathrm{E}+02$ \\
\hline ENSBTAG00000000130 & $1.72 \mathrm{E}-10$ & 0 & TNFSF13 & $1.72 E+03$ \\
\hline ENSBTAG00000004226 & $1.45 \mathrm{E}-10$ & 0 & SHBG & $1.45 E+03$ \\
\hline ENSBTAG00000014693 & 0 & 0 & TMEM88 & $1.00 \mathrm{E}+00$ \\
\hline ENSBTAG00000014695 & $6.68 \mathrm{E}-11$ & 0 & NAA38 & $6.69 \mathrm{E}+02$ \\
\hline ENSBTAG00000014696 & 0 & 0 & CYB5D1 & $1.00 \mathrm{E}+00$ \\
\hline ENSBTAG00000047214 & 0 & 0 & TRAPPC1 & $1.00 \mathrm{E}+00$ \\
\hline ENSBTAG00000012436 & $3.31 \mathrm{E}-11$ & 0 & HES7 & $3.32 \mathrm{E}+02$ \\
\hline ENSBTAG00000001702 & $3.66 \mathrm{E}-11$ & 0 & TMEM107 & $3.67 E+02$ \\
\hline ENSBTAG00000018809 & $1.16 \mathrm{E}-10$ & 0 & RANGRF & $1.16 \mathrm{E}+03$ \\
\hline ENSBTAG00000017094 & 4.73E-10 & 0 & SHMT1 & $4.73 E+03$ \\
\hline ENSBTAG00000045835 & $1.16 \mathrm{E}-10$ & 0 & HOXB5 & $1.16 \mathrm{E}+03$ \\
\hline ENSBTAG00000046569 & $1.71 \mathrm{E}-10$ & 0 & CISD3 & $1.71 E+03$ \\
\hline ENSBTAG00000011539 & $2.77 \mathrm{E}-10$ & 0 & PCGF2 & $2.77 E+03$ \\
\hline ENSBTAG00000003830 & $2.08 \mathrm{E}-10$ & 0 & PSMB3 & $2.08 \mathrm{E}+03$ \\
\hline ENSBTAG00000019095 & 4.16E-11 & 0 & CCR10 & $4.17 E+02$ \\
\hline ENSBTAG00000019918 & $1.66 \mathrm{E}-11$ & 0 & PSME3 & $1.67 E+02$ \\
\hline ENSBTAG00000017441 & $1.80 \mathrm{E}-10$ & 0 & & $1.80 \mathrm{E}+03$ \\
\hline ENSBTAG00000007393 & $6.52 E-11$ & 0 & RND2 & $6.53 E+02$ \\
\hline ENSBTAG00000045652 & 0 & 0 & & $1.00 E+00$ \\
\hline ENSBTAG00000011759 & $1.36 \mathrm{E}-10$ & 0 & C1QL1 & $1.36 \mathrm{E}+03$ \\
\hline ENSBTAG00000024975 & $8.32 \mathrm{E}-11$ & 0 & & $8.33 E+02$ \\
\hline ENSBTAG00000047667 & 0 & 0 & & $1.00 \mathrm{E}+00$ \\
\hline ENSBTAG00000009107 & $1.34 \mathrm{E}-10$ & 0 & CBX8 & $1.34 \mathrm{E}+03$ \\
\hline ENSBTAG00000040282 & 0 & 0 & NOG & $1.00 \mathrm{E}+00$ \\
\hline ENSBTAG00000047272 & $5.34 \mathrm{E}-10$ & 0 & CCDC182 & $5.34 E+03$ \\
\hline ENSBTAG00000034506 & 0 & 0 & SNRPG & $1.00 E+00$ \\
\hline ENSBTAG00000034433 & $1.30 \mathrm{E}-10$ & 0 & & $1.30 E+03$ \\
\hline ENSBTAG00000025219 & 0 & 0 & & $1.00 E+00$ \\
\hline ENSBTAG00000004475 & 0 & 0 & CDK5R1 & $1.00 E+00$ \\
\hline ENSBTAG00000024979 & 0 & 0 & HSPB9 & $1.00 E+00$ \\
\hline ENSBTAG00000030305 & 0 & 0 & & $1.00 \mathrm{E}+00$ \\
\hline ENSBTAG00000046260 & 0 & 0 & & $1.00 E+00$ \\
\hline ENSBTAG00000047866 & 0 & 0 & & $1.00 E+00$ \\
\hline ENSBTAG00000008441 & $1.17 \mathrm{E}-10$ & 0 & socs3 & $1.17 \mathrm{E}+03$ \\
\hline ENSBTAG00000017248 & 0 & 0 & & $1.00 \mathrm{E}+00$ \\
\hline ENSBTAG00000013393 & $2.58 \mathrm{E}-10$ & 0 & GEMIN4 & $2.58 \mathrm{E}+03$ \\
\hline ENSBTAG00000026859 & $2.60 \mathrm{E}-10$ & 0 & & $2.60 E+03$ \\
\hline ENSBTAG00000010532 & $1.49 \mathrm{E}-10$ & 0 & KCTD11 & $1.49 \mathrm{E}+03$ \\
\hline ENSBTAG00000046290 & $3.97 \mathrm{E}-10$ & 0 & PIRT & $3.97 E+03$ \\
\hline ENSBTAG00000043979 & 0 & 0 & HILS1 & $1.00 E+00$ \\
\hline ENSBTAG00000046615 & $3.65 E-10$ & 0 & & $3.65 E+03$ \\
\hline ENSBTAG00000048067 & 0 & 0 & C17orf96 & $1.00 \mathrm{E}+00$ \\
\hline ENSBTAG00000004119 & 0 & 0 & NEUROD2 & $1.00 E+00$ \\
\hline ENSBTAG00000046225 & 0 & 0 & & $1.00 E+00$ \\
\hline ENSBTAG00000048083 & 0 & 0 & KRTAP3-1 & $1.00 E+00$ \\
\hline ENSBTAG00000030559 & $1.72 \mathrm{E}-10$ & 0 & & $1.72 E+03$ \\
\hline ENSBTAG00000038759 & $3.65 \mathrm{E}-10$ & 0 & & $3.65 E+03$ \\
\hline ENSBTAG00000024839 & $8.18 \mathrm{E}-10$ & 0 & & $8.18 \mathrm{E}+03$ \\
\hline ENSBTAG00000047906 & 0 & $8.83 \mathrm{E}-11$ & MRPL36 & $1.13 \mathrm{E}-03$ \\
\hline ENSBTAG00000034577 & 0 & $6.58 \mathrm{E}-11$ & & $1.52 \mathrm{E}-03$ \\
\hline ENSBTAG00000016441 & $1.02 \mathrm{E}-10$ & $7.48 \mathrm{E}-10$ & ZNF622 & $1.36 \mathrm{E}-01$ \\
\hline ENSBTAG00000032508 & $9.19 \mathrm{E}-11$ & $3.48 \mathrm{E}-10$ & TAS2R1 & $2.64 \mathrm{E}-01$ \\
\hline ENSBTAG00000048105 & $8.04 \mathrm{E}-11$ & $2.03 \mathrm{E}-10$ & & $3.97 \mathrm{E}-01$ \\
\hline ENSBTAG00000027865 & $6.12 \mathrm{E}-11$ & $1.25 \mathrm{E}-10$ & & $4.90 \mathrm{E}-01$ \\
\hline ENSBTAG00000047499 & $1.68 \mathrm{E}-10$ & $3.03 \mathrm{E}-10$ & & 5.54E-01 \\
\hline ENSBTAG00000017013 & $2.31 \mathrm{E}-10$ & 4.15E-10 & SMIM23 & $5.56 \mathrm{E}-01$ \\
\hline ENSBTAG00000017885 & $1.75 \mathrm{E}-10$ & $3.14 \mathrm{E}-10$ & FAM173B & $5.57 \mathrm{E}-01$ \\
\hline ENSBTAG00000047116 & $2.23 \mathrm{E}-10$ & $3.56 \mathrm{E}-10$ & TPPP & $6.26 \mathrm{E}-01$ \\
\hline ENSBTAG00000047780 & 7.35E-11 & $1.12 \mathrm{E}-10$ & CCDC127 & $6.56 \mathrm{E}-01$ \\
\hline ENSBTAG00000016444 & $4.68 \mathrm{E}-10$ & $7.14 \mathrm{E}-10$ & FAM134B & $6.55 \mathrm{E}-01$ \\
\hline ENSBTAG00000046493 & $7.76 \mathrm{E}-11$ & $1.17 \mathrm{E}-10$ & PDCD6 & $6.64 \mathrm{E}-01$ \\
\hline ENSBTAG00000005743 & $1.77 \mathrm{E}-10$ & $2.57 \mathrm{E}-10$ & & $6.90 \mathrm{E}-01$ \\
\hline ENSBTAG00000015316 & $1.75 \mathrm{E}-10$ & $2.53 \mathrm{E}-10$ & & 6.91E-01 \\
\hline ENSBTAG00000003539 & $1.32 \mathrm{E}-10$ & $1.88 \mathrm{E}-10$ & ROPN1L & 7.04E-01 \\
\hline ENSBTAG00000006346 & $7.32 \mathrm{E}-10$ & $1.04 \mathrm{E}-09$ & & 7.04E-01 \\
\hline ENSBTAG00000044175 & $5.57 \mathrm{E}-11$ & $7.86 \mathrm{E}-11$ & CENPK & 7.09E-01 \\
\hline ENSBTAG00000011405 & $5.16 \mathrm{E}-10$ & $7.08 \mathrm{E}-10$ & CEP72 & $7.28 \mathrm{E}-01$ \\
\hline ENSBTAG00000010423 & $3.04 \mathrm{E}-10$ & 4.10E-10 & LIFR & 7.41E-01 \\
\hline ENSBTAG00000018235 & $2.98 \mathrm{E}-10$ & $3.89 \mathrm{E}-10$ & SLC45A2 & $7.66 \mathrm{E}-01$ \\
\hline
\end{tabular}




\begin{tabular}{|c|c|c|c|c|}
\hline ENSBTAG00000015478 & $2.13 \mathrm{E}-10$ & $2.76 \mathrm{E}-10$ & SRD5A1 & 7.70E-01 \\
\hline ENSBTAG00000005021 & $5.23 \mathrm{E}-10$ & $6.80 \mathrm{E}-10$ & SEMA5A & $7.69 \mathrm{E}-01$ \\
\hline ENSBTAG00000044160 & $3.44 \mathrm{E}-10$ & 4.44E-10 & MCCC2 & $7.75 \mathrm{E}-01$ \\
\hline ENSBTAG00000032442 & 7.10E-10 & $9.08 \mathrm{E}-10$ & & 7.82E-01 \\
\hline ENSBTAG00000038364 & $1.50 \mathrm{E}-10$ & $1.89 \mathrm{E}-10$ & & 7.94E-01 \\
\hline ENSBTAG00000034170 & $3.04 \mathrm{E}-10$ & 3.83E-10 & DYNLL1 & 7.94E-01 \\
\hline ENSBTAG00000008876 & $2.97 \mathrm{E}-10$ & $3.75 \mathrm{E}-10$ & EFCAB9 & 7.93E-01 \\
\hline ENSBTAG00000046810 & $1.69 \mathrm{E}-10$ & $2.13 \mathrm{E}-10$ & & 7.93E-01 \\
\hline ENSBTAG00000003329 & $1.97 \mathrm{E}-10$ & $2.48 \mathrm{E}-10$ & FST & 7.94E-01 \\
\hline ENSBTAG00000008606 & 7.62E-11 & $9.60 \mathrm{E}-11$ & EXOC3 & 7.94E-01 \\
\hline ENSBTAG00000013863 & $1.06 \mathrm{E}-10$ & $1.34 \mathrm{E}-10$ & DUSP1 & 7.94E-01 \\
\hline ENSBTAG00000046019 & $7.71 E-11$ & $9.72 \mathrm{E}-11$ & SDHA & 7.93E-01 \\
\hline ENSBTAG00000011045 & 8.03E-11 & $1.01 \mathrm{E}-10$ & MRPS36 & 7.95E-01 \\
\hline ENSBTAG00000022714 & $2.41 \mathrm{E}-10$ & $3.04 \mathrm{E}-10$ & HSPB3 & 7.94E-01 \\
\hline ENSBTAG00000012775 & $3.43 \mathrm{E}-10$ & $4.33 \mathrm{E}-10$ & & 7.93E-01 \\
\hline ENSBTAG00000009381 & $3.55 \mathrm{E}-10$ & 4.39E-10 & LCP2 & $8.08 \mathrm{E}-01$ \\
\hline ENSBTAG00000001001 & $3.69 \mathrm{E}-10$ & $4.53 \mathrm{E}-10$ & ANKRD55 & $8.14 \mathrm{E}-01$ \\
\hline ENSBTAG00000017222 & $5.56 \mathrm{E}-10$ & $6.82 \mathrm{E}-10$ & CTNND2 & 8.16E-01 \\
\hline ENSBTAG00000012558 & $4.41 \mathrm{E}-10$ & $5.38 \mathrm{E}-10$ & ADAMTS12 & $8.20 \mathrm{E}-01$ \\
\hline ENSBTAG00000015466 & $2.06 \mathrm{E}-10$ & $2.50 \mathrm{E}-10$ & NSUN2 & $8.22 \mathrm{E}-01$ \\
\hline ENSBTAG00000021960 & 4.79E-10 & $5.82 \mathrm{E}-10$ & GPX8 & 8.23E-01 \\
\hline ENSBTAG00000010801 & $4.04 \mathrm{E}-10$ & $4.89 \mathrm{E}-10$ & $\mathrm{CMBL}$ & 8.27E-01 \\
\hline ENSBTAG00000012992 & $2.63 \mathrm{E}-10$ & 3.17E-10 & $\mathrm{CDH} 6$ & $8.28 \mathrm{E}-01$ \\
\hline ENSBTAG00000016002 & $4.25 \mathrm{E}-10$ & 5.11E-10 & FAM169A & 8.31E-01 \\
\hline ENSBTAG00000002134 & $8.53 \mathrm{E}-10$ & $1.02 \mathrm{E}-09$ & FAM196B & 8.37E-01 \\
\hline ENSBTAG00000009400 & $5.50 \mathrm{E}-10$ & $6.57 \mathrm{E}-10$ & FASTKD3 & 8.37E-01 \\
\hline ENSBTAG00000016903 & $1.34 \mathrm{E}-10$ & $1.59 \mathrm{E}-10$ & NLN & $8.41 \mathrm{E}-01$ \\
\hline ENSBTAG00000001997 & $7.45 \mathrm{E}-10$ & $8.75 \mathrm{E}-10$ & $\mathrm{MROH} 2 \mathrm{~B}$ & $8.52 \mathrm{E}-01$ \\
\hline ENSBTAG00000016963 & $9.47 \mathrm{E}-11$ & 1.10E-10 & ADAMTS6 & 8.61E-01 \\
\hline ENSBTAG00000011895 & $3.69 \mathrm{E}-10$ & 4.27E-10 & PANK3 & $8.64 \mathrm{E}-01$ \\
\hline ENSBTAG00000000579 & $1.04 \mathrm{E}-10$ & $1.20 \mathrm{E}-10$ & NIPBL & $8.68 \mathrm{E}-01$ \\
\hline ENSBTAG00000010822 & $4.10 \mathrm{E}-10$ & 4.73E-10 & PLCXD3 & 8.67E-01 \\
\hline ENSBTAG00000046449 & $1.96 \mathrm{E}-10$ & $2.27 \mathrm{E}-10$ & & 8.65E-01 \\
\hline ENSBTAG00000020142 & $1.62 \mathrm{E}-10$ & $1.86 \mathrm{E}-10$ & LMBRD2 & 8.69E-01 \\
\hline ENSBTAG00000019210 & $6.95 \mathrm{E}-10$ & $8.01 \mathrm{E}-10$ & $\mathrm{ADCY} 2$ & $8.68 \mathrm{E}-01$ \\
\hline ENSBTAG00000003536 & $1.24 \mathrm{E}-10$ & $1.43 \mathrm{E}-10$ & 06-Mar & $8.70 \mathrm{E}-01$ \\
\hline ENSBTAG00000019209 & $7.51 \mathrm{E}-10$ & $8.62 \mathrm{E}-10$ & RAD1 & $8.71 \mathrm{E}-01$ \\
\hline ENSBTAG00000024593 & $5.28 \mathrm{E}-10$ & $6.05 \mathrm{E}-10$ & SPZ1 & $8.73 \mathrm{E}-01$ \\
\hline ENSBTAG00000002458 & $1.30 \mathrm{E}-10$ & $1.47 \mathrm{E}-10$ & NUP155 & $8.86 \mathrm{E}-01$ \\
\hline ENSBTAG00000000809 & $1.64 \mathrm{E}-10$ & $1.86 \mathrm{E}-10$ & IRX1 & $8.82 \mathrm{E}-01$ \\
\hline ENSBTAG00000000084 & $1.67 \mathrm{E}-10$ & $1.89 \mathrm{E}-10$ & MRPS30 & $8.86 \mathrm{E}-01$ \\
\hline ENSBTAG00000032705 & $6.38 \mathrm{E}-10$ & 7.14E-10 & CALM2 & $8.94 \mathrm{E}-01$ \\
\hline ENSBTAG00000015519 & $5.86 \mathrm{E}-10$ & $6.51 \mathrm{E}-10$ & GFM2 & $9.01 \mathrm{E}-01$ \\
\hline ENSBTAG00000015099 & $7.55 \mathrm{E}-10$ & $8.34 \mathrm{E}-10$ & RPL26L1 & 9.06E-01 \\
\hline ENSBTAG00000013562 & $1.75 \mathrm{E}-10$ & $1.93 \mathrm{E}-10$ & & 9.09E-01 \\
\hline ENSBTAG00000039929 & 4.67E-10 & 5.15E-10 & RXFP3 & 9.07E-01 \\
\hline ENSBTAG00000021224 & $1.74 \mathrm{E}-10$ & $1.91 \mathrm{E}-10$ & ZSWIM6 & $9.14 \mathrm{E}-01$ \\
\hline ENSBTAG00000015648 & $5.41 \mathrm{E}-10$ & 5.93E-10 & & $9.13 \mathrm{E}-01$ \\
\hline ENSBTAG00000005169 & $1.28 \mathrm{E}-10$ & $1.39 \mathrm{E}-10$ & RGS7BP & $9.18 \mathrm{E}-01$ \\
\hline ENSBTAG00000017569 & $6.32 \mathrm{E}-10$ & $6.90 \mathrm{E}-10$ & C5orf22 & $9.16 \mathrm{E}-01$ \\
\hline ENSBTAG00000007071 & $3.19 \mathrm{E}-10$ & $3.48 \mathrm{E}-10$ & RAI14 & $9.18 \mathrm{E}-01$ \\
\hline ENSBTAG00000016900 & $2.89 \mathrm{E}-10$ & $3.14 \mathrm{E}-10$ & TRAPPC13 & $9.21 \mathrm{E}-01$ \\
\hline ENSBTAG00000014612 & $5.31 \mathrm{E}-10$ & $5.75 \mathrm{E}-10$ & & $9.24 \mathrm{E}-01$ \\
\hline ENSBTAG00000034659 & $1.79 \mathrm{E}-10$ & $1.94 \mathrm{E}-10$ & UBTD2 & $9.25 \mathrm{E}-01$ \\
\hline ENSBTAG00000001429 & $2.18 \mathrm{E}-10$ & $2.36 \mathrm{E}-10$ & NEURL1B & $9.24 \mathrm{E}-01$ \\
\hline ENSBTAG00000017071 & $5.89 \mathrm{E}-10$ & $6.36 \mathrm{E}-10$ & & $9.26 \mathrm{E}-01$ \\
\hline ENSBTAG00000033107 & $5.11 \mathrm{E}-10$ & $5.50 \mathrm{E}-10$ & OSMR & $9.29 \mathrm{E}-01$ \\
\hline ENSBTAG00000011766 & $6.13 \mathrm{E}-10$ & $6.58 \mathrm{E}-10$ & $\mathrm{C7}$ & $9.32 \mathrm{E}-01$ \\
\hline ENSBTAG00000014177 & $6.35 \mathrm{E}-10$ & $6.76 \mathrm{E}-10$ & $\mathrm{C} 6$ & $9.39 \mathrm{E}-01$ \\
\hline ENSBTAG00000019211 & $4.16 \mathrm{E}-10$ & $4.42 \mathrm{E}-10$ & BRIX1 & $9.41 \mathrm{E}-01$ \\
\hline ENSBTAG00000003066 & $1.49 \mathrm{E}-10$ & $1.59 \mathrm{E}-10$ & NSA2 & $9.38 \mathrm{E}-01$ \\
\hline ENSBTAG00000009401 & $6.11 \mathrm{E}-10$ & $6.46 \mathrm{E}-10$ & MTRR & $9.46 \mathrm{E}-01$ \\
\hline ENSBTAG00000021959 & $3.00 \mathrm{E}-10$ & 3.17E-10 & CDC20B & $9.47 \mathrm{E}-01$ \\
\hline ENSBTAG00000015907 & $1.24 \mathrm{E}-10$ & $1.30 \mathrm{E}-10$ & IRX4 & $9.54 \mathrm{E}-01$ \\
\hline ENSBTAG00000000672 & $2.33 \mathrm{E}-10$ & $2.46 \mathrm{E}-10$ & FAM105A & $9.49 \mathrm{E}-01$ \\
\hline ENSBTAG00000047486 & $2.36 \mathrm{E}-10$ & $2.48 \mathrm{E}-10$ & CARTPT & $9.52 \mathrm{E}-01$ \\
\hline ENSBTAG00000008180 & $1.16 \mathrm{E}-10$ & $1.22 \mathrm{E}-10$ & SPDL1 & 9.54E-01 \\
\hline ENSBTAG00000002845 & 5.70E-10 & 5.93E-10 & $\mathrm{CDH} 10$ & $9.61 \mathrm{E}-01$ \\
\hline ENSBTAG00000039409 & $3.40 \mathrm{E}-10$ & $3.54 \mathrm{E}-10$ & & $9.61 \mathrm{E}-01$ \\
\hline ENSBTAG00000008130 & $3.41 \mathrm{E}-10$ & $3.53 \mathrm{E}-10$ & MED10 & $9.65 \mathrm{E}-01$ \\
\hline
\end{tabular}




\begin{tabular}{|c|c|c|c|c|}
\hline ENSBTAG00000013391 & $4.91 \mathrm{E}-10$ & 5.07E-10 & ANKH & $9.68 \mathrm{E}-01$ \\
\hline ENSBTAG00000000363 & $3.36 \mathrm{E}-10$ & $3.45 \mathrm{E}-10$ & BDP1 & $9.73 \mathrm{E}-01$ \\
\hline ENSBTAG00000008871 & $5.38 \mathrm{E}-10$ & $5.51 \mathrm{E}-10$ & DDX4 & $9.76 \mathrm{E}-01$ \\
\hline ENSBTAG00000022684 & $3.35 \mathrm{E}-10$ & $3.38 \mathrm{E}-10$ & & 9.92E-01 \\
\hline ENSBTAG00000020126 & $4.36 \mathrm{E}-10$ & 4.39E-10 & MYO10 & 9.92E-01 \\
\hline ENSBTAG00000014620 & $6.97 \mathrm{E}-10$ & $6.95 \mathrm{E}-10$ & FOXI1 & $1.00 \mathrm{E}+00$ \\
\hline ENSBTAG00000005514 & $5.56 \mathrm{E}-10$ & $5.45 \mathrm{E}-10$ & TRIO & $1.02 \mathrm{E}+00$ \\
\hline ENSBTAG00000001962 & $2.79 \mathrm{E}-10$ & $2.73 \mathrm{E}-10$ & MRPS27 & $1.02 \mathrm{E}+00$ \\
\hline ENSBTAG00000037844 & $5.59 \mathrm{E}-10$ & $5.45 \mathrm{E}-10$ & $\mathrm{CDH} 18$ & $1.03 \mathrm{E}+00$ \\
\hline ENSBTAG00000000617 & $8.02 \mathrm{E}-10$ & $7.76 \mathrm{E}-10$ & & $1.03 E+00$ \\
\hline ENSBTAG00000033313 & $1.66 \mathrm{E}-10$ & $1.60 \mathrm{E}-10$ & SLC38A9 & $1.04 \mathrm{E}+00$ \\
\hline ENSBTAG00000006972 & $3.19 E-10$ & 3.07E-10 & TRIP13 & $1.04 \mathrm{E}+00$ \\
\hline ENSBTAG00000006971 & $6.71 \mathrm{E}-10$ & $6.43 \mathrm{E}-10$ & BRD9 & $1.04 \mathrm{E}+00$ \\
\hline ENSBTAG00000007461 & $1.50 \mathrm{E}-10$ & $1.42 \mathrm{E}-10$ & C5orf34 & $1.05 \mathrm{E}+00$ \\
\hline ENSBTAG00000015504 & $1.00 \mathrm{E}-10$ & $9.45 \mathrm{E}-11$ & ZNF131 & $1.06 \mathrm{E}+00$ \\
\hline ENSBTAG00000047208 & $6.86 \mathrm{E}-10$ & $6.48 \mathrm{E}-10$ & & $1.06 \mathrm{E}+00$ \\
\hline ENSBTAG00000012688 & $4.49 \mathrm{E}-10$ & $4.24 \mathrm{E}-10$ & CAPSL & $1.06 \mathrm{E}+00$ \\
\hline ENSBTAG00000001457 & $3.27 \mathrm{E}-10$ & $3.08 \mathrm{E}-10$ & TMEM267 & $1.06 \mathrm{E}+00$ \\
\hline ENSBTAG00000017026 & $4.33 \mathrm{E}-10$ & 4.07E-10 & DEPDC1B & $1.06 \mathrm{E}+00$ \\
\hline ENSBTAG00000017746 & $5.21 \mathrm{E}-10$ & $4.85 \mathrm{E}-10$ & SLIT3 & $1.07 \mathrm{E}+00$ \\
\hline ENSBTAG00000004066 & $3.84 \mathrm{E}-10$ & $3.55 \mathrm{E}-10$ & PARP8 & $1.08 \mathrm{E}+00$ \\
\hline ENSBTAG00000004629 & $1.80 \mathrm{E}-10$ & $1.66 \mathrm{E}-10$ & SLC9A3 & $1.08 \mathrm{E}+00$ \\
\hline ENSBTAG00000011334 & $1.74 \mathrm{E}-10$ & $1.60 \mathrm{E}-10$ & NADK2 & $1.09 \mathrm{E}+00$ \\
\hline ENSBTAG00000027980 & $8.65 \mathrm{E}-10$ & 7.94E-10 & TAF9 & $1.09 \mathrm{E}+00$ \\
\hline ENSBTAG00000007665 & 4.14E-10 & 3.80E-10 & NPR3 & $1.09 \mathrm{E}+00$ \\
\hline ENSBTAG00000001961 & $2.54 \mathrm{E}-10$ & $2.30 \mathrm{E}-10$ & MAP1B & $1.10 \mathrm{E}+00$ \\
\hline ENSBTAG00000016981 & $1.34 \mathrm{E}-10$ & $1.20 \mathrm{E}-10$ & FBXO4 & $1.12 \mathrm{E}+00$ \\
\hline ENSBTAG00000005633 & $5.18 \mathrm{E}-10$ & $4.65 \mathrm{E}-10$ & ARHGEF28 & $1.11 E+00$ \\
\hline ENSBTAG00000033187 & $7.58 \mathrm{E}-10$ & $6.81 \mathrm{E}-10$ & & $1.11 \mathrm{E}+00$ \\
\hline ENSBTAG00000024801 & $2.29 \mathrm{E}-10$ & $2.04 \mathrm{E}-10$ & RANBP17 & $1.12 \mathrm{E}+00$ \\
\hline ENSBTAG00000017889 & $4.40 \mathrm{E}-10$ & $3.92 \mathrm{E}-10$ & CCT5 & $1.12 \mathrm{E}+00$ \\
\hline ENSBTAG00000009514 & $7.40 \mathrm{E}-11$ & $6.52 \mathrm{E}-11$ & PPWD1 & $1.13 \mathrm{E}+00$ \\
\hline ENSBTAG00000034360 & $2.17 \mathrm{E}-10$ & $1.91 \mathrm{E}-10$ & & $1.14 \mathrm{E}+00$ \\
\hline ENSBTAG00000001492 & $2.22 \mathrm{E}-10$ & $1.95 \mathrm{E}-10$ & FYB & $1.14 E+00$ \\
\hline ENSBTAG00000043948 & $3.95 \mathrm{E}-10$ & $3.45 \mathrm{E}-10$ & HCN1 & $1.15 E+00$ \\
\hline ENSBTAG00000006697 & $1.45 \mathrm{E}-10$ & $1.26 \mathrm{E}-10$ & RICTOR & $1.15 \mathrm{E}+00$ \\
\hline ENSBTAG00000021813 & $2.53 \mathrm{E}-10$ & $2.19 \mathrm{E}-10$ & AMACR & $1.15 E+00$ \\
\hline ENSBTAG00000009519 & $1.23 \mathrm{E}-10$ & $1.03 \mathrm{E}-10$ & TRIM23 & $1.19 \mathrm{E}+00$ \\
\hline ENSBTAG00000047828 & $1.89 \mathrm{E}-10$ & $1.58 \mathrm{E}-10$ & & $1.19 \mathrm{E}+00$ \\
\hline ENSBTAG00000008430 & $1.28 \mathrm{E}-10$ & $1.07 \mathrm{E}-10$ & RANBP3L & $1.20 \mathrm{E}+00$ \\
\hline ENSBTAG00000019595 & $6.84 \mathrm{E}-10$ & $5.72 \mathrm{E}-10$ & EGFLAM & $1.20 \mathrm{E}+00$ \\
\hline ENSBTAG00000010989 & $3.99 \mathrm{E}-10$ & $3.33 \mathrm{E}-10$ & PIK3R1 & $1.20 \mathrm{E}+00$ \\
\hline ENSBTAG00000020376 & $1.41 \mathrm{E}-10$ & 1.17E-10 & & $1.20 \mathrm{E}+00$ \\
\hline ENSBTAG00000021972 & 7.61E-10 & $6.29 \mathrm{E}-10$ & DNAH5 & $1.21 E+00$ \\
\hline ENSBTAG00000011885 & $1.53 \mathrm{E}-10$ & $1.26 \mathrm{E}-10$ & NNT & $1.22 \mathrm{E}+00$ \\
\hline ENSBTAG00000000012 & $5.27 \mathrm{E}-10$ & $4.36 \mathrm{E}-10$ & TTC33 & $1.21 \mathrm{E}+00$ \\
\hline ENSBTAG00000019289 & $3.71 \mathrm{E}-10$ & 3.05E-10 & ITGA2 & $1.21 E+00$ \\
\hline ENSBTAG00000010526 & $1.18 \mathrm{E}-10$ & $9.74 \mathrm{E}-11$ & PLPP1 & $1.21 E+00$ \\
\hline ENSBTAG00000018245 & $3.89 \mathrm{E}-10$ & 3.17E-10 & SLC1A3 & $1.23 \mathrm{E}+00$ \\
\hline ENSBTAG00000027983 & $1.42 \mathrm{E}-10$ & $1.16 \mathrm{E}-10$ & GTF2H2 & $1.22 \mathrm{E}+00$ \\
\hline ENSBTAG00000000586 & $5.42 \mathrm{E}-10$ & 4.39E-10 & & $1.24 \mathrm{E}+00$ \\
\hline ENSBTAG00000001329 & $4.58 \mathrm{E}-10$ & 3.67E-10 & CLPTM1L & $1.25 \mathrm{E}+00$ \\
\hline ENSBTAG00000033095 & $2.46 \mathrm{E}-10$ & $1.95 \mathrm{E}-10$ & WDR70 & $1.26 \mathrm{E}+00$ \\
\hline ENSBTAG00000004221 & $1.48 \mathrm{E}-10$ & 1.17E-10 & ESM1 & $1.27 \mathrm{E}+00$ \\
\hline ENSBTAG00000003381 & 3.77E-10 & $2.96 \mathrm{E}-10$ & PAPD7 & $1.27 \mathrm{E}+00$ \\
\hline ENSBTAG00000003326 & $1.22 \mathrm{E}-10$ & $9.52 \mathrm{E}-11$ & NAIP & $1.28 \mathrm{E}+00$ \\
\hline ENSBTAG00000004351 & $2.90 \mathrm{E}-10$ & $2.26 \mathrm{E}-10$ & IL31RA & $1.28 \mathrm{E}+00$ \\
\hline ENSBTAG00000002021 & $1.80 \mathrm{E}-10$ & $1.41 \mathrm{E}-10$ & BNIP1 & $1.28 \mathrm{E}+00$ \\
\hline ENSBTAG00000004929 & $2.53 \mathrm{E}-10$ & $1.95 \mathrm{E}-10$ & C5orf51 & $1.30 \mathrm{E}+00$ \\
\hline ENSBTAG00000007690 & $4.66 \mathrm{E}-10$ & $3.58 \mathrm{E}-10$ & AGXT2 & $1.30 \mathrm{E}+00$ \\
\hline ENSBTAG00000001557 & $3.42 \mathrm{E}-10$ & $2.63 \mathrm{E}-10$ & CCL28 & $1.30 \mathrm{E}+00$ \\
\hline ENSBTAG00000048135 & $1.68 \mathrm{E}-09$ & $1.29 \mathrm{E}-09$ & & $1.30 \mathrm{E}+00$ \\
\hline ENSBTAG00000002985 & $1.36 \mathrm{E}-10$ & $1.05 \mathrm{E}-10$ & KCNMB1 & $1.30 \mathrm{E}+00$ \\
\hline ENSBTAG00000021607 & $5.34 \mathrm{E}-10$ & 4.05E-10 & NDUFAF2 & $1.32 \mathrm{E}+00$ \\
\hline ENSBTAG00000009914 & $1.55 \mathrm{E}-10$ & 1.17E-10 & NDUFS6 & $1.33 \mathrm{E}+00$ \\
\hline ENSBTAG00000047087 & $6.55 \mathrm{E}-11$ & $4.95 \mathrm{E}-11$ & MCIDAS & $1.32 E+00$ \\
\hline ENSBTAG00000016525 & $1.84 \mathrm{E}-10$ & $1.39 \mathrm{E}-10$ & ITGA1 & $1.32 \mathrm{E}+00$ \\
\hline ENSBTAG00000005142 & $6.33 \mathrm{E}-10$ & 4.77E-10 & RPL37 & $1.33 E+00$ \\
\hline ENSBTAG00000015512 & $5.34 \mathrm{E}-10$ & 4.03E-10 & & $1.32 \mathrm{E}+00$ \\
\hline ENSBTAG00000032848 & $2.08 \mathrm{E}-10$ & $1.57 \mathrm{E}-10$ & GOLPH3 & $1.33 E+00$ \\
\hline
\end{tabular}




\begin{tabular}{|c|c|c|c|c|}
\hline ENSBTAG000000047548 & $9.30 \mathrm{E}-10$ & $7.00 \mathrm{E}-10$ & & $1.33 \mathrm{E}+00$ \\
\hline ENSBTAG00000010510 & $4.71 \mathrm{E}-10$ & $3.55 \mathrm{E}-10$ & DHX29 & $1.33 \mathrm{E}+00$ \\
\hline ENSBTAG000000002701 & $3.05 \mathrm{E}-10$ & $2.30 \mathrm{E}-10$ & & $1.33 E+00$ \\
\hline ENSBTAG000000010518 & $1.58 \mathrm{E}-10$ & $1.18 \mathrm{E}-10$ & SKIV2L2 & $1.34 \mathrm{E}+00$ \\
\hline ENSBTAG000000012278 & $1.89 \mathrm{E}-10$ & $1.41 \mathrm{E}-10$ & MTMR12 & $1.34 \mathrm{E}+00$ \\
\hline ENSBTAG000000009019 & $3.70 \mathrm{E}-10$ & $2.73 \mathrm{E}-10$ & SH3PXD2B & $1.35 E+00$ \\
\hline ENSBTAG000000044010 & $7.43 \mathrm{E}-10$ & $5.47 \mathrm{E}-10$ & EMB & $1.36 \mathrm{E}+00$ \\
\hline ENSBTAG000000014972 & 4.39E-10 & 3.23E-10 & PTGER4 & $1.36 \mathrm{E}+00$ \\
\hline ENSBTAG000000003200 & 4.65E-10 & $3.41 \mathrm{E}-10$ & & $1.36 \mathrm{E}+00$ \\
\hline ENSBTAG000000018460 & $2.04 \mathrm{E}-10$ & $1.50 \mathrm{E}-10$ & ADAMTS16 & $1.36 \mathrm{E}+00$ \\
\hline ENSBTAG000000000128 & $3.05 \mathrm{E}-10$ & $2.24 \mathrm{E}-10$ & FGF18 & $1.36 \mathrm{E}+00$ \\
\hline ENSBTAG000000000037 & $1.41 \mathrm{E}-10$ & $1.03 \mathrm{E}-10$ & CWC27 & $1.37 \mathrm{E}+00$ \\
\hline ENSBTAG00000014455 & $1.97 \mathrm{E}-10$ & $1.43 \mathrm{E}-10$ & PDZD2 & $1.38 \mathrm{E}+00$ \\
\hline ENSBTAG000000025893 & $5.23 \mathrm{E}-10$ & $3.80 \mathrm{E}-10$ & $\mathrm{CDH} 12$ & $1.38 \mathrm{E}+00$ \\
\hline ENSBTAG000000037576 & $3.42 \mathrm{E}-10$ & $2.42 \mathrm{E}-10$ & 11-Mar & $1.41 E+00$ \\
\hline ENSBTAG000000012567 & $5.64 \mathrm{E}-10$ & $3.98 \mathrm{E}-10$ & TERT & $1.42 \mathrm{E}+00$ \\
\hline ENSBTAG000000011046 & $2.74 \mathrm{E}-10$ & $1.91 \mathrm{E}-10$ & CDK7 & $1.43 E+00$ \\
\hline ENSBTAG000000005176 & $1.92 \mathrm{E}-10$ & $1.33 \mathrm{E}-10$ & GDNF & $1.45 \mathrm{E}+00$ \\
\hline ENSBTAG000000017545 & $2.76 \mathrm{E}-10$ & $1.91 \mathrm{E}-10$ & $\mathrm{FCHO} 2$ & $1.45 E+00$ \\
\hline ENSBTAG000000021606 & $4.40 \mathrm{E}-10$ & $3.00 \mathrm{E}-10$ & ERCC8 & $1.47 E+00$ \\
\hline ENSBTAG000000002865 & $5.86 \mathrm{E}-10$ & $3.92 \mathrm{E}-10$ & KCNIP1 & $1.49 \mathrm{E}+00$ \\
\hline ENSBTAG00000017457 & $3.01 \mathrm{E}-10$ & $2.00 \mathrm{E}-10$ & STK10 & $1.50 \mathrm{E}+00$ \\
\hline ENSBTAG000000017551 & $4.83 \mathrm{E}-10$ & $3.22 \mathrm{E}-10$ & DROSHA & $1.50 \mathrm{E}+00$ \\
\hline ENSBTAG000000002020 & $1.27 \mathrm{E}-10$ & $8.45 \mathrm{E}-11$ & CREBRF & $1.50 \mathrm{E}+00$ \\
\hline ENSBTAG00000003728 & $4.92 \mathrm{E}-10$ & $3.26 \mathrm{E}-10$ & NDUFS4 & $1.51 E+00$ \\
\hline ENSBTAG000000003219 & $1.48 \mathrm{E}-10$ & $9.81 \mathrm{E}-11$ & FBXL7 & $1.51 E+00$ \\
\hline ENSBTAG000000021965 & $2.66 \mathrm{E}-10$ & $1.74 \mathrm{E}-10$ & SUB1 & $1.53 E+00$ \\
\hline ENSBTAG000000027924 & $3.75 \mathrm{E}-10$ & $2.44 \mathrm{E}-10$ & SNX18 & $1.54 \mathrm{E}+00$ \\
\hline ENSBTAG000000011337 & $4.61 \mathrm{E}-10$ & $2.95 \mathrm{E}-10$ & ANKRD33B & $1.56 \mathrm{E}+00$ \\
\hline ENSBTAG000000007306 & $5.09 \mathrm{E}-10$ & $3.25 \mathrm{E}-10$ & RAB3C & $1.56 \mathrm{E}+00$ \\
\hline ENSBTAG000000023529 & $1.66 \mathrm{E}-10$ & $1.06 \mathrm{E}-10$ & SPEF2 & $1.57 \mathrm{E}+00$ \\
\hline ENSBTAG000000047594 & $1.50 \mathrm{E}-10$ & $9.43 \mathrm{E}-11$ & CCNO & $1.59 \mathrm{E}+00$ \\
\hline ENSBTAG000000005380 & $2.70 \mathrm{E}-10$ & $1.70 \mathrm{E}-10$ & MOCS2 & $1.59 E+00$ \\
\hline ENSBTAG000000034431 & $4.80 \mathrm{E}-10$ & $3.02 \mathrm{E}-10$ & & $1.59 E+00$ \\
\hline ENSBTAG00000043992 & $3.78 \mathrm{E}-10$ & $2.38 \mathrm{E}-10$ & RNF180 & $1.59 \mathrm{E}+00$ \\
\hline ENSBTAG00000005229 & $4.29 \mathrm{E}-10$ & $2.70 \mathrm{E}-10$ & & $1.59 \mathrm{E}+00$ \\
\hline ENSBTAG00000010003 & $1.51 \mathrm{E}-10$ & $9.52 \mathrm{E}-11$ & TLX3 & $1.59 \mathrm{E}+00$ \\
\hline ENSBTAG00000034138 & $5.70 \mathrm{E}-10$ & $3.56 \mathrm{E}-10$ & & $1.60 E+00$ \\
\hline ENSBTAG00000025035 & 3.03E-10 & $1.85 \mathrm{E}-10$ & PRLR & $1.64 \mathrm{E}+00$ \\
\hline ENSBTAG00000000561 & $4.41 \mathrm{E}-10$ & $2.66 \mathrm{E}-10$ & OCLN & $1.66 \mathrm{E}+00$ \\
\hline ENSBTAG00000016902 & $1.93 \mathrm{E}-10$ & $1.16 \mathrm{E}-10$ & SGTB & $1.66 \mathrm{E}+00$ \\
\hline ENSBTAG00000026369 & $5.34 \mathrm{E}-10$ & $3.10 \mathrm{E}-10$ & ENC1 & $1.72 \mathrm{E}+00$ \\
\hline ENSBTAG00000007704 & $2.48 \mathrm{E}-10$ & $1.43 \mathrm{E}-10$ & ELOVL7 & $1.74 \mathrm{E}+00$ \\
\hline ENSBTAG00000009468 & $1.58 \mathrm{E}-10$ & $9.07 \mathrm{E}-11$ & FAM159B & $1.75 \mathrm{E}+00$ \\
\hline ENSBTAG00000045954 & $1.84 \mathrm{E}-10$ & $1.05 \mathrm{E}-10$ & LRRC14B & $1.75 \mathrm{E}+00$ \\
\hline ENSBTAG00000001013 & $1.49 \mathrm{E}-10$ & $8.39 \mathrm{E}-11$ & SLC30A5 & $1.77 \mathrm{E}+00$ \\
\hline ENSBTAG00000011839 & $1.69 \mathrm{E}-10$ & $9.48 \mathrm{E}-11$ & HMGCS1 & $1.78 \mathrm{E}+00$ \\
\hline ENSBTAG00000019834 & $3.45 \mathrm{E}-10$ & $1.92 \mathrm{E}-10$ & ARL15 & $1.80 \mathrm{E}+00$ \\
\hline ENSBTAG00000014675 & $6.08 \mathrm{E}-10$ & $3.38 \mathrm{E}-10$ & PTCD2 & $1.80 \mathrm{E}+00$ \\
\hline ENSBTAG00000000013 & $2.37 \mathrm{E}-10$ & $1.31 \mathrm{E}-10$ & PRKAA1 & $1.81 \mathrm{E}+00$ \\
\hline ENSBTAG00000005164 & $2.37 \mathrm{E}-10$ & $1.29 \mathrm{E}-10$ & GZMK & $1.83 \mathrm{E}+00$ \\
\hline ENSBTAG00000003186 & $4.41 \mathrm{E}-10$ & $2.38 \mathrm{E}-10$ & OTULIN & $1.85 E+00$ \\
\hline ENSBTAG00000021958 & $6.39 \mathrm{E}-11$ & $3.45 \mathrm{E}-11$ & GZMA & $1.85 \mathrm{E}+00$ \\
\hline ENSBTAG00000014261 & $1.33 \mathrm{E}-10$ & $7.04 \mathrm{E}-11$ & TARS & $1.89 \mathrm{E}+00$ \\
\hline ENSBTAG00000045695 & $5.15 \mathrm{E}-10$ & $2.73 \mathrm{E}-10$ & SLC6A19 & $1.88 \mathrm{E}+00$ \\
\hline ENSBTAG00000001241 & $1.95 \mathrm{E}-10$ & $1.02 \mathrm{E}-10$ & ISL1 & $1.91 E+00$ \\
\hline ENSBTAG00000014246 & $2.05 \mathrm{E}-10$ & $1.04 \mathrm{E}-10$ & CENPH & $1.97 \mathrm{E}+00$ \\
\hline ENSBTAG00000046181 & $6.17 \mathrm{E}-10$ & $3.10 \mathrm{E}-10$ & & $1.99 \mathrm{E}+00$ \\
\hline ENSBTAG00000046192 & $6.51 \mathrm{E}-10$ & $3.21 \mathrm{E}-10$ & AK6 & $2.03 E+00$ \\
\hline ENSBTAG00000001335 & $4.83 \mathrm{E}-10$ & $2.37 \mathrm{E}-10$ & GHR & $2.04 E+00$ \\
\hline ENSBTAG00000007979 & $2.32 \mathrm{E}-10$ & $1.14 \mathrm{E}-10$ & TNPO1 & $2.03 E+00$ \\
\hline ENSBTAG00000018234 & $4.63 \mathrm{E}-10$ & $2.22 \mathrm{E}-10$ & $\mathrm{CDH} 9$ & $2.08 \mathrm{E}+00$ \\
\hline ENSBTAG00000012559 & $3.62 \mathrm{E}-10$ & $1.72 \mathrm{E}-10$ & SLC6A18 & $2.10 \mathrm{E}+00$ \\
\hline ENSBTAG00000025964 & $5.93 \mathrm{E}-10$ & $2.78 \mathrm{E}-10$ & MAST4 & $2.13 E+00$ \\
\hline ENSBTAG00000019975 & $2.26 \mathrm{E}-10$ & $1.04 \mathrm{E}-10$ & IL7R & $2.18 \mathrm{E}+00$ \\
\hline ENSBTAG00000016149 & $2.90 \mathrm{E}-10$ & $1.33 \mathrm{E}-10$ & $\mathrm{C9}$ & $2.18 \mathrm{E}+00$ \\
\hline ENSBTAG00000015100 & $3.14 \mathrm{E}-10$ & $1.41 \mathrm{E}-10$ & ATP6V0E1 & $2.23 E+00$ \\
\hline ENSBTAG00000014374 & $4.92 \mathrm{E}-10$ & $2.18 \mathrm{E}-10$ & CARD6 & $2.26 \mathrm{E}+00$ \\
\hline ENSBTAG00000033186 & $3.94 \mathrm{E}-10$ & $1.72 \mathrm{E}-10$ & OXCT1 & $2.29 E+00$ \\
\hline ENSBTAG00000020601 & $3.06 \mathrm{E}-10$ & $1.34 \mathrm{E}-10$ & ZNF366 & $2.28 \mathrm{E}+00$ \\
\hline
\end{tabular}




\begin{tabular}{|c|c|c|c|c|}
\hline ENSBTAG00000032933 & $4.63 \mathrm{E}-10$ & $2.02 \mathrm{E}-10$ & TTC23L & $2.29 \mathrm{E}+00$ \\
\hline ENSBTAG00000017745 & $1.82 \mathrm{E}-10$ & 7.87E-11 & IL6ST & $2.31 E+00$ \\
\hline ENSBTAG00000008414 & $1.50 \mathrm{E}-10$ & $6.43 \mathrm{E}-11$ & ZFR & $2.34 \mathrm{E}+00$ \\
\hline ENSBTAG00000026527 & $3.38 \mathrm{E}-10$ & $1.39 \mathrm{E}-10$ & AHRR & $2.43 \mathrm{E}+00$ \\
\hline ENSBTAG00000008494 & $2.18 \mathrm{E}-10$ & $8.82 \mathrm{E}-11$ & TMEM171 & $2.47 \mathrm{E}+00$ \\
\hline ENSBTAG00000016771 & $2.61 \mathrm{E}-10$ & $1.04 \mathrm{E}-10$ & PLK2 & $2.51 \mathrm{E}+00$ \\
\hline ENSBTAG00000015955 & $4.78 \mathrm{E}-10$ & $1.85 \mathrm{E}-10$ & ERGIC1 & $2.58 \mathrm{E}+00$ \\
\hline ENSBTAG00000039486 & $4.83 \mathrm{E}-10$ & $1.85 \mathrm{E}-10$ & & $2.61 \mathrm{E}+00$ \\
\hline ENSBTAG00000034435 & $4.05 \mathrm{E}-10$ & $1.55 \mathrm{E}-10$ & NKD2 & $2.61 E+00$ \\
\hline ENSBTAG00000040001 & $6.70 \mathrm{E}-10$ & $2.55 \mathrm{E}-10$ & MARVELD2 & $2.63 \mathrm{E}+00$ \\
\hline ENSBTAG00000015022 & $1.15 \mathrm{E}-10$ & 4.30E-11 & KIF2A & $2.67 E+00$ \\
\hline ENSBTAG00000002779 & $6.05 \mathrm{E}-10$ & $2.25 \mathrm{E}-10$ & GABRP & $2.69 E+00$ \\
\hline ENSBTAG00000010012 & 7.05E-10 & $2.53 \mathrm{E}-10$ & BTF3 & $2.79 \mathrm{E}+00$ \\
\hline ENSBTAG00000018501 & $6.55 \mathrm{E}-10$ & $2.28 \mathrm{E}-10$ & ICE1 & $2.87 E+00$ \\
\hline ENSBTAG00000016152 & $3.31 \mathrm{E}-10$ & $1.13 \mathrm{E}-10$ & DAB2 & $2.93 E+00$ \\
\hline ENSBTAG00000006843 & $4.16 \mathrm{E}-10$ & $1.38 \mathrm{E}-10$ & UTP15 & $3.01 E+00$ \\
\hline ENSBTAG00000002073 & $5.58 \mathrm{E}-10$ & $1.83 \mathrm{E}-10$ & RAD17 & $3.05 E+00$ \\
\hline ENSBTAG00000013790 & $5.29 \mathrm{E}-10$ & $1.69 \mathrm{E}-10$ & MAP3K1 & $3.13 E+00$ \\
\hline ENSBTAG00000009469 & $2.91 \mathrm{E}-10$ & $9.30 \mathrm{E}-11$ & SREK1IP1 & $3.13 E+00$ \\
\hline ENSBTAG00000018616 & $3.82 \mathrm{E}-10$ & $1.21 \mathrm{E}-10$ & IPO11 & $3.16 \mathrm{E}+00$ \\
\hline ENSBTAG00000007321 & $1.61 \mathrm{E}-10$ & $5.06 \mathrm{E}-11$ & & $3.17 E+00$ \\
\hline ENSBTAG00000047333 & $3.76 \mathrm{E}-10$ & $1.18 \mathrm{E}-10$ & & $3.19 E+00$ \\
\hline ENSBTAG00000017011 & $5.87 \mathrm{E}-10$ & $1.74 \mathrm{E}-10$ & CCDC125 & $3.37 E+00$ \\
\hline ENSBTAG00000020568 & $2.40 \mathrm{E}-10$ & $6.70 \mathrm{E}-11$ & NKX2-5 & $3.58 \mathrm{E}+00$ \\
\hline ENSBTAG00000034598 & 4.64E-10 & $1.18 \mathrm{E}-10$ & & $3.93 E+00$ \\
\hline ENSBTAG00000014239 & $8.47 \mathrm{E}-11$ & $2.13 \mathrm{E}-11$ & CCNB1 & $3.96 \mathrm{E}+00$ \\
\hline ENSBTAG00000003268 & $2.10 \mathrm{E}-10$ & $5.28 \mathrm{E}-11$ & PELO & $3.97 \mathrm{E}+00$ \\
\hline ENSBTAG00000003377 & $4.46 \mathrm{E}-10$ & $1.12 \mathrm{E}-10$ & TMEM174 & $3.98 \mathrm{E}+00$ \\
\hline ENSBTAG00000015376 & $2.04 \mathrm{E}-10$ & 4.67E-11 & FBXW11 & $4.36 \mathrm{E}+00$ \\
\hline ENSBTAG00000038537 & $3.90 \mathrm{E}-10$ & 8.91E-11 & & $4.37 \mathrm{E}+00$ \\
\hline ENSBTAG00000011812 & $1.33 \mathrm{E}-10$ & $2.96 \mathrm{E}-11$ & DIMT1 & $4.49 E+00$ \\
\hline ENSBTAG00000013873 & $1.01 \mathrm{E}-10$ & $2.11 \mathrm{E}-11$ & MSX2 & $4.75 E+00$ \\
\hline ENSBTAG00000004920 & $1.11 \mathrm{E}-09$ & $2.31 \mathrm{E}-10$ & COX8A & $4.79 E+00$ \\
\hline ENSBTAG00000006775 & $5.51 \mathrm{E}-10$ & $1.11 \mathrm{E}-10$ & SLC12A7 & $4.96 \mathrm{E}+00$ \\
\hline ENSBTAG00000014248 & $4.68 \mathrm{E}-10$ & $9.36 \mathrm{E}-11$ & MIER3 & $4.99 E+00$ \\
\hline ENSBTAG00000000944 & $7.91 \mathrm{E}-10$ & $1.54 \mathrm{E}-10$ & LPCAT1 & $5.13 E+00$ \\
\hline ENSBTAG00000016770 & $7.44 \mathrm{E}-10$ & $1.42 \mathrm{E}-10$ & GAPT & $5.24 \mathrm{E}+00$ \\
\hline ENSBTAG00000006233 & $3.00 \mathrm{E}-10$ & $5.62 \mathrm{E}-11$ & NIM1K & $5.34 \mathrm{E}+00$ \\
\hline ENSBTAG00000006360 & $4.49 \mathrm{E}-10$ & $7.85 \mathrm{E}-11$ & CD180 & $5.72 E+00$ \\
\hline ENSBTAG00000000494 & $3.29 \mathrm{E}-10$ & $5.62 \mathrm{E}-11$ & PDE4D & $5.84 \mathrm{E}+00$ \\
\hline ENSBTAG00000009995 & $2.55 \mathrm{E}-10$ & $4.28 \mathrm{E}-11$ & CPEB4 & $5.95 E+00$ \\
\hline ENSBTAG00000000415 & $2.71 \mathrm{E}-10$ & $4.28 \mathrm{E}-11$ & ERBIN & $6.32 \mathrm{E}+00$ \\
\hline ENSBTAG00000015843 & $7.38 \mathrm{E}-10$ & $1.05 \mathrm{E}-10$ & SLC6A3 & $7.02 \mathrm{E}+00$ \\
\hline ENSBTAG00000013426 & $6.29 \mathrm{E}-10$ & $8.63 \mathrm{E}-11$ & SETD9 & $7.29 E+00$ \\
\hline ENSBTAG00000033221 & $6.69 \mathrm{E}-10$ & $8.55 \mathrm{E}-11$ & CCDC152 & $7.81 E+00$ \\
\hline ENSBTAG00000001823 & $2.39 \mathrm{E}-10$ & $2.90 \mathrm{E}-11$ & STC2 & $8.20 \mathrm{E}+00$ \\
\hline ENSBTAG00000000564 & $5.79 \mathrm{E}-10$ & $6.34 \mathrm{E}-11$ & & $9.12 \mathrm{E}+00$ \\
\hline ENSBTAG00000012124 & $6.00 \mathrm{E}-10$ & $5.79 \mathrm{E}-11$ & GPBP1 & $1.03 E+01$ \\
\hline ENSBTAG00000018452 & $5.27 \mathrm{E}-10$ & $2.00 \mathrm{E}-11$ & ANKRA2 & $2.62 \mathrm{E}+01$ \\
\hline ENSBTAG00000046997 & $1.56 \mathrm{E}-10$ & 0 & & $1.56 \mathrm{E}+03$ \\
\hline ENSBTAG00000046575 & 0 & 0 & & $1.00 \mathrm{E}+00$ \\
\hline ENSBTAG00000011657 & $3.47 \mathrm{E}-10$ & 0 & & $3.47 E+03$ \\
\hline ENSBTAG00000038985 & 5.97E-11 & 0 & & $5.98 \mathrm{E}+02$ \\
\hline ENSBTAG00000045813 & 0 & 0 & & $1.00 \mathrm{E}+00$ \\
\hline ENSBTAG00000022809 & 0 & 0 & & $1.00 \mathrm{E}+00$ \\
\hline ENSBTAG00000040439 & $6.45 \mathrm{E}-11$ & 0 & HTR1A & $6.46 \mathrm{E}+02$ \\
\hline ENSBTAG00000026505 & $2.16 \mathrm{E}-09$ & 0 & & $2.16 \mathrm{E}+04$ \\
\hline ENSBTAG00000012971 & $1.34 \mathrm{E}-09$ & 0 & RNF167 & $1.34 \mathrm{E}+04$ \\
\hline ENSBTAG00000005491 & $1.31 \mathrm{E}-10$ & 0 & & $1.31 E+03$ \\
\hline ENSBTAG00000012833 & $1.00 \mathrm{E}-10$ & $5.41 \mathrm{E}-10$ & ATG2B & $1.85 \mathrm{E}-01$ \\
\hline ENSBTAG00000018400 & $1.43 \mathrm{E}-10$ & $5.61 \mathrm{E}-10$ & & $2.55 \mathrm{E}-01$ \\
\hline ENSBTAG00000001021 & $2.85 E-11$ & $1.08 \mathrm{E}-10$ & CYP1A1 & 2.64E-01 \\
\hline ENSBTAG00000013423 & $2.15 \mathrm{E}-10$ & $7.00 \mathrm{E}-10$ & GLRX5 & 3.07E-01 \\
\hline ENSBTAG00000002415 & $2.08 \mathrm{E}-10$ & $5.34 \mathrm{E}-10$ & TSPAN3 & $3.90 \mathrm{E}-01$ \\
\hline ENSBTAG00000032477 & $6.02 \mathrm{E}-11$ & $1.53 \mathrm{E}-10$ & HECTD1 & 3.94E-01 \\
\hline ENSBTAG00000005846 & 4.10E-11 & $1.03 \mathrm{E}-10$ & FAM219B & $3.98 \mathrm{E}-01$ \\
\hline ENSBTAG00000035184 & $2.19 \mathrm{E}-10$ & $5.52 \mathrm{E}-10$ & & $3.96 \mathrm{E}-01$ \\
\hline ENSBTAG00000012351 & $2.63 \mathrm{E}-10$ & $6.20 \mathrm{E}-10$ & & $4.25 \mathrm{E}-01$ \\
\hline ENSBTAG00000005845 & $9.78 \mathrm{E}-11$ & $2.16 \mathrm{E}-10$ & MPI & 4.53E-01 \\
\hline ENSBTAG00000046990 & 1.67E-10 & $3.69 \mathrm{E}-10$ & GON7 & 4.54E-01 \\
\hline
\end{tabular}




\begin{tabular}{|c|c|c|c|c|}
\hline ENSBTAG00000003419 & $1.63 \mathrm{E}-10$ & $3.08 \mathrm{E}-10$ & EAPP & $5.28 \mathrm{E}-01$ \\
\hline ENSBTAG00000016683 & 7.24E-11 & $1.22 \mathrm{E}-10$ & NFKBIA & $5.94 \mathrm{E}-01$ \\
\hline ENSBTAG00000007346 & $1.34 \mathrm{E}-10$ & $2.26 \mathrm{E}-10$ & COMMD4 & $5.94 \mathrm{E}-01$ \\
\hline ENSBTAG00000012751 & $1.15 \mathrm{E}-10$ & $1.93 \mathrm{E}-10$ & BTBD6 & 5.96E-01 \\
\hline ENSBTAG00000016282 & $6.15 \mathrm{E}-11$ & 9.97E-11 & EIF5 & $6.18 \mathrm{E}-01$ \\
\hline ENSBTAG00000015780 & $2.12 \mathrm{E}-10$ & $3.42 \mathrm{E}-10$ & RCN2 & $6.21 \mathrm{E}-01$ \\
\hline ENSBTAG00000047229 & $2.17 \mathrm{E}-10$ & $3.42 \mathrm{E}-10$ & & 6.35E-01 \\
\hline ENSBTAG00000021293 & $1.15 \mathrm{E}-10$ & $1.75 \mathrm{E}-10$ & SNUPN & 6.55E-01 \\
\hline ENSBTAG00000012219 & $8.94 \mathrm{E}-11$ & 1.27E-10 & CSPG4 & 7.04E-01 \\
\hline ENSBTAG00000017096 & $1.48 \mathrm{E}-10$ & $2.03 \mathrm{E}-10$ & ERICH1 & 7.28E-01 \\
\hline ENSBTAG00000046364 & $4.28 \mathrm{E}-10$ & 5.87E-10 & & 7.30E-01 \\
\hline ENSBTAG00000047166 & $1.15 \mathrm{E}-10$ & $1.58 \mathrm{E}-10$ & $\mathrm{SH} 2 \mathrm{D} 7$ & 7.27E-01 \\
\hline ENSBTAG00000040134 & $6.49 \mathrm{E}-10$ & $8.84 \mathrm{E}-10$ & & $7.34 \mathrm{E}-01$ \\
\hline ENSBTAG00000018007 & $9.06 \mathrm{E}-11$ & $1.22 \mathrm{E}-10$ & NR2F2 & 7.43E-01 \\
\hline ENSBTAG00000022989 & $1.71 \mathrm{E}-10$ & $2.29 \mathrm{E}-10$ & FAM174B & 7.49E-01 \\
\hline ENSBTAG00000021425 & $1.74 \mathrm{E}-10$ & $2.28 \mathrm{E}-10$ & LMAN1L & 7.64E-01 \\
\hline ENSBTAG00000008495 & $1.62 \mathrm{E}-10$ & $2.13 \mathrm{E}-10$ & АР3B2 & 7.61E-01 \\
\hline ENSBTAG00000001407 & $1.93 \mathrm{E}-10$ & $2.53 \mathrm{E}-10$ & AP3S2 & 7.63E-01 \\
\hline ENSBTAG00000000623 & $3.44 \mathrm{E}-10$ & 4.46E-10 & CPEB1 & $7.72 \mathrm{E}-01$ \\
\hline ENSBTAG00000021144 & $6.69 \mathrm{E}-10$ & $8.55 \mathrm{E}-10$ & PSTPIP1 & 7.82E-01 \\
\hline ENSBTAG00000018637 & $1.32 \mathrm{E}-10$ & $1.66 \mathrm{E}-10$ & HDDC3 & 7.93E-01 \\
\hline ENSBTAG00000047240 & $3.04 \mathrm{E}-10$ & 3.83E-10 & & 7.94E-01 \\
\hline ENSBTAG00000047130 & $1.63 \mathrm{E}-10$ & 2.05E-10 & & 7.93E-01 \\
\hline ENSBTAG00000002661 & $1.45 \mathrm{E}-10$ & $1.83 \mathrm{E}-10$ & ISLR2 & 7.95E-01 \\
\hline ENSBTAG00000047970 & $3.08 \mathrm{E}-10$ & $3.88 \mathrm{E}-10$ & & 7.94E-01 \\
\hline ENSBTAG00000047606 & $5.85 \mathrm{E}-10$ & 7.37E-10 & MTA1 & 7.94E-01 \\
\hline ENSBTAG00000020195 & 7.17E-10 & $9.04 \mathrm{E}-10$ & ANKRD34C & 7.93E-01 \\
\hline ENSBTAG00000021426 & $1.20 \mathrm{E}-10$ & $1.51 \mathrm{E}-10$ & CPLX3 & 7.93E-01 \\
\hline ENSBTAG00000005838 & $8.16 \mathrm{E}-11$ & 1.03E-10 & ULK3 & 7.93E-01 \\
\hline ENSBTAG00000004272 & $5.77 \mathrm{E}-10$ & 7.27E-10 & & 7.93E-01 \\
\hline ENSBTAG00000046186 & $1.47 \mathrm{E}-10$ & $1.86 \mathrm{E}-10$ & & 7.92E-01 \\
\hline ENSBTAG00000046377 & $9.13 \mathrm{E}-11$ & 1.15E-10 & & 7.94E-01 \\
\hline ENSBTAG00000008624 & $4.18 \mathrm{E}-11$ & 5.27E-11 & MESDC1 & 7.94E-01 \\
\hline ENSBTAG00000027075 & $1.76 \mathrm{E}-10$ & $2.22 \mathrm{E}-10$ & & 7.92E-01 \\
\hline ENSBTAG00000006868 & $4.11 \mathrm{E}-10$ & 5.17E-10 & OTUD7A & 7.95E-01 \\
\hline ENSBTAG00000048010 & $1.50 \mathrm{E}-10$ & $1.89 \mathrm{E}-10$ & NUDT14 & 7.95E-01 \\
\hline ENSBTAG00000012753 & $4.12 \mathrm{E}-10$ & $5.06 \mathrm{E}-10$ & BRF1 & $8.15 \mathrm{E}-01$ \\
\hline ENSBTAG00000048049 & $5.67 \mathrm{E}-10$ & $6.89 \mathrm{E}-10$ & & 8.23E-01 \\
\hline ENSBTAG00000021845 & $1.72 \mathrm{E}-10$ & $2.08 \mathrm{E}-10$ & STRN3 & 8.29E-01 \\
\hline ENSBTAG00000002440 & $2.43 \mathrm{E}-10$ & $2.92 \mathrm{E}-10$ & KIF7 & 8.33E-01 \\
\hline ENSBTAG00000009836 & $7.25 \mathrm{E}-10$ & 8.67E-10 & $\mathrm{CHGA}$ & $8.36 \mathrm{E}-01$ \\
\hline ENSBTAG00000013981 & $1.46 \mathrm{E}-10$ & $1.73 \mathrm{E}-10$ & SPTSSA & $8.42 \mathrm{E}-01$ \\
\hline ENSBTAG00000046161 & $6.15 \mathrm{E}-10$ & $7.26 \mathrm{E}-10$ & & 8.47E-01 \\
\hline ENSBTAG00000019506 & $3.11 \mathrm{E}-10$ & $3.66 \mathrm{E}-10$ & ODF3L1 & 8.50E-01 \\
\hline ENSBTAG00000038020 & $7.73 \mathrm{E}-10$ & $9.06 \mathrm{E}-10$ & & 8.53E-01 \\
\hline ENSBTAG00000000735 & $2.12 \mathrm{E}-10$ & $2.46 \mathrm{E}-10$ & & 8.63E-01 \\
\hline ENSBTAG00000000010 & 7.37E-11 & $8.51 \mathrm{E}-11$ & UBL7 & 8.66E-01 \\
\hline ENSBTAG00000016651 & 1.59E-10 & $1.82 \mathrm{E}-10$ & ISL2 & 8.73E-01 \\
\hline ENSBTAG00000032951 & $2.88 \mathrm{E}-10$ & $3.27 \mathrm{E}-10$ & ABHD17C & $8.81 \mathrm{E}-01$ \\
\hline ENSBTAG00000007368 & $3.41 \mathrm{E}-10$ & $3.84 \mathrm{E}-10$ & MAN2C1 & 8.87E-01 \\
\hline ENSBTAG00000011213 & $4.93 \mathrm{E}-10$ & $5.53 \mathrm{E}-10$ & & $8.91 \mathrm{E}-01$ \\
\hline ENSBTAG00000010964 & $1.71 \mathrm{E}-10$ & $1.91 \mathrm{E}-10$ & STARD5 & 8.94E-01 \\
\hline ENSBTAG00000017565 & $3.69 \mathrm{E}-10$ & 4.10E-10 & SCFD1 & 9.00E-01 \\
\hline ENSBTAG00000006738 & $4.41 \mathrm{E}-10$ & $4.90 \mathrm{E}-10$ & GPR68 & $9.01 \mathrm{E}-01$ \\
\hline ENSBTAG00000011446 & $1.41 \mathrm{E}-10$ & 1.57E-10 & SEMA7A & 8.96E-01 \\
\hline ENSBTAG00000002418 & $3.46 \mathrm{E}-10$ & $3.84 \mathrm{E}-10$ & TICRR & $9.00 \mathrm{E}-01$ \\
\hline ENSBTAG00000006673 & $2.84 \mathrm{E}-10$ & $3.14 \mathrm{E}-10$ & TMEM179 & $9.04 \mathrm{E}-01$ \\
\hline ENSBTAG00000037938 & $6.13 \mathrm{E}-10$ & $6.76 \mathrm{E}-10$ & & 9.07E-01 \\
\hline ENSBTAG00000048314 & $2.19 \mathrm{E}-10$ & $2.41 \mathrm{E}-10$ & & 9.07E-01 \\
\hline ENSBTAG00000045986 & $1.05 E-09$ & 1.15E-09 & MIA2 & $9.10 \mathrm{E}-01$ \\
\hline ENSBTAG00000001565 & $2.84 \mathrm{E}-10$ & $3.08 \mathrm{E}-10$ & MESDC2 & $9.22 \mathrm{E}-01$ \\
\hline ENSBTAG00000007651 & $3.35 \mathrm{E}-10$ & 3.64E-10 & TARSL2 & $9.21 \mathrm{E}-01$ \\
\hline ENSBTAG00000000622 & $1.67 \mathrm{E}-10$ & $1.80 \mathrm{E}-10$ & RPS17 & $9.26 \mathrm{E}-01$ \\
\hline ENSBTAG00000007308 & $2.25 \mathrm{E}-10$ & $2.42 \mathrm{E}-10$ & EDC3 & $9.28 \mathrm{E}-01$ \\
\hline ENSBTAG00000022775 & $9.46 \mathrm{E}-10$ & $1.02 \mathrm{E}-09$ & C14orf180 & 9.27E-01 \\
\hline ENSBTAG00000014089 & $2.73 \mathrm{E}-10$ & $2.94 \mathrm{E}-10$ & PPCDC & $9.30 \mathrm{E}-01$ \\
\hline ENSBTAG00000021318 & $7.73 \mathrm{E}-11$ & $8.28 \mathrm{E}-11$ & SCAMP5 & $9.34 \mathrm{E}-01$ \\
\hline ENSBTAG00000021527 & $2.66 \mathrm{E}-10$ & $2.84 \mathrm{E}-10$ & IGF1R & $9.36 \mathrm{E}-01$ \\
\hline ENSBTAG00000009985 & $1.55 \mathrm{E}-10$ & $1.65 \mathrm{E}-10$ & SIN3A & $9.41 \mathrm{E}-01$ \\
\hline ENSBTAG00000008172 & $3.93 \mathrm{E}-10$ & 4.17E-10 & EGLN3 & $9.44 \mathrm{E}-01$ \\
\hline
\end{tabular}




\begin{tabular}{|c|c|c|c|c|}
\hline ENSBTAG00000001616 & $2.50 \mathrm{E}-10$ & 2.64E-10 & ZNF592 & $9.47 \mathrm{E}-01$ \\
\hline ENSBTAG00000001387 & $3.41 \mathrm{E}-10$ & $3.60 \mathrm{E}-10$ & WDR73 & $9.48 \mathrm{E}-01$ \\
\hline ENSBTAG00000007348 & $2.63 \mathrm{E}-10$ & 2.77E-10 & STRA6 & $9.48 \mathrm{E}-01$ \\
\hline ENSBTAG00000005844 & $1.58 \mathrm{E}-10$ & $1.66 \mathrm{E}-10$ & SCAMP2 & $9.50 \mathrm{E}-01$ \\
\hline ENSBTAG00000007304 & $2.11 \mathrm{E}-10$ & $2.22 \mathrm{E}-10$ & CLK3 & $9.52 \mathrm{E}-01$ \\
\hline ENSBTAG00000000541 & $1.30 \mathrm{E}-10$ & $1.37 \mathrm{E}-10$ & NKX2-1 & $9.50 \mathrm{E}-01$ \\
\hline ENSBTAG00000013422 & $1.68 \mathrm{E}-10$ & $1.76 \mathrm{E}-10$ & GABRB3 & $9.56 \mathrm{E}-01$ \\
\hline ENSBTAG00000017267 & $1.26 \mathrm{E}-10$ & $1.31 \mathrm{E}-10$ & $\operatorname{cox} 5 \mathrm{~A}$ & $9.65 \mathrm{E}-01$ \\
\hline ENSBTAG00000022847 & $2.89 \mathrm{E}-10$ & $3.00 \mathrm{E}-10$ & HEATR5A & $9.63 \mathrm{E}-01$ \\
\hline ENSBTAG00000001385 & $8.03 \mathrm{E}-10$ & $8.32 \mathrm{E}-10$ & ZSCAN2 & $9.65 \mathrm{E}-01$ \\
\hline ENSBTAG00000007649 & $2.30 \mathrm{E}-10$ & $2.39 \mathrm{E}-10$ & TM2D3 & $9.64 \mathrm{E}-01$ \\
\hline ENSBTAG00000044121 & $6.42 \mathrm{E}-10$ & $6.63 \mathrm{E}-10$ & NUBPL & $9.68 \mathrm{E}-01$ \\
\hline ENSBTAG00000003423 & $5.44 \mathrm{E}-10$ & $5.62 \mathrm{E}-10$ & DDX24 & $9.69 \mathrm{E}-01$ \\
\hline ENSBTAG00000017212 & $7.22 \mathrm{E}-10$ & 7.43E-10 & HYPK & $9.71 \mathrm{E}-01$ \\
\hline ENSBTAG00000007319 & $1.57 \mathrm{E}-10$ & $1.62 \mathrm{E}-10$ & JAG2 & $9.69 \mathrm{E}-01$ \\
\hline ENSBTAG00000038080 & $1.08 \mathrm{E}-09$ & 1.11E-09 & & $9.76 \mathrm{E}-01$ \\
\hline ENSBTAG00000003392 & $1.82 \mathrm{E}-10$ & $1.87 \mathrm{E}-10$ & GABRA5 & $9.75 \mathrm{E}-01$ \\
\hline ENSBTAG00000021424 & $1.20 \mathrm{E}-10$ & $1.24 \mathrm{E}-10$ & CSK & $9.71 \mathrm{E}-01$ \\
\hline ENSBTAG00000006912 & $2.27 \mathrm{E}-10$ & $2.32 \mathrm{E}-10$ & CCDC33 & $9.76 \mathrm{E}-01$ \\
\hline ENSBTAG00000001617 & $2.64 \mathrm{E}-10$ & $2.69 \mathrm{E}-10$ & G2E3 & $9.80 \mathrm{E}-01$ \\
\hline ENSBTAG00000037899 & $9.88 \mathrm{E}-11$ & $9.96 \mathrm{E}-11$ & DLK1 & $9.92 \mathrm{E}-01$ \\
\hline ENSBTAG00000017299 & $4.23 \mathrm{E}-10$ & 4.24E-10 & & $9.97 \mathrm{E}-01$ \\
\hline ENSBTAG00000001639 & $2.14 \mathrm{E}-10$ & $2.11 E-10$ & TRPM1 & $1.02 \mathrm{E}+00$ \\
\hline ENSBTAG00000019836 & $3.06 \mathrm{E}-10$ & $3.01 E-10$ & GSKIP & $1.02 \mathrm{E}+00$ \\
\hline ENSBTAG00000017776 & $8.61 \mathrm{E}-11$ & $8.41 E-11$ & CHD2 & $1.02 \mathrm{E}+00$ \\
\hline ENSBTAG00000002941 & $1.57 \mathrm{E}-10$ & $1.53 \mathrm{E}-10$ & FES & $1.03 E+00$ \\
\hline ENSBTAG00000012490 & $2.25 \mathrm{E}-10$ & 2.19E-10 & ETFA & $1.03 E+00$ \\
\hline ENSBTAG00000001388 & $3.23 \mathrm{E}-10$ & $3.13 \mathrm{E}-10$ & NMB & $1.03 E+00$ \\
\hline ENSBTAG00000044101 & $5.13 \mathrm{E}-10$ & $4.94 \mathrm{E}-10$ & C14orf159 & $1.04 \mathrm{E}+00$ \\
\hline ENSBTAG00000004598 & $7.46 \mathrm{E}-10$ & $7.18 \mathrm{E}-10$ & GOLGA5 & $1.04 \mathrm{E}+00$ \\
\hline ENSBTAG00000012873 & $2.60 \mathrm{E}-10$ & $2.50 \mathrm{E}-10$ & SNX6 & $1.04 \mathrm{E}+00$ \\
\hline ENSBTAG00000031885 & $6.07 \mathrm{E}-10$ & $5.85 \mathrm{E}-10$ & TMEM251 & $1.04 \mathrm{E}+00$ \\
\hline ENSBTAG00000018310 & $3.57 \mathrm{E}-10$ & $3.43 \mathrm{E}-10$ & SETD3 & $1.04 \mathrm{E}+00$ \\
\hline ENSBTAG00000020042 & $8.24 \mathrm{E}-10$ & $7.90 \mathrm{E}-10$ & KLHL28 & $1.04 \mathrm{E}+00$ \\
\hline ENSBTAG00000007357 & $2.00 \mathrm{E}-10$ & $1.91 \mathrm{E}-10$ & CHSY1 & $1.05 E+00$ \\
\hline ENSBTAG00000020250 & $5.78 \mathrm{E}-10$ & $5.50 \mathrm{E}-10$ & SLC28A1 & $1.05 \mathrm{E}+00$ \\
\hline ENSBTAG00000017896 & $5.24 \mathrm{E}-10$ & $4.98 \mathrm{E}-10$ & ST8SIA2 & $1.05 \mathrm{E}+00$ \\
\hline ENSBTAG00000006024 & $2.10 \mathrm{E}-10$ & $1.98 \mathrm{E}-10$ & ISLR & $1.06 \mathrm{E}+00$ \\
\hline ENSBTAG00000039634 & $2.16 \mathrm{E}-10$ & $2.04 \mathrm{E}-10$ & NSMCE3 & $1.06 \mathrm{E}+00$ \\
\hline ENSBTAG00000009894 & $3.37 \mathrm{E}-10$ & $3.16 \mathrm{E}-10$ & SERPINA10 & $1.07 \mathrm{E}+00$ \\
\hline ENSBTAG00000003414 & $1.75 \mathrm{E}-10$ & $1.64 \mathrm{E}-10$ & SAXO2 & $1.07 E+00$ \\
\hline ENSBTAG00000009086 & $2.72 \mathrm{E}-10$ & $2.54 \mathrm{E}-10$ & LOXL1 & $1.07 E+00$ \\
\hline ENSBTAG00000021531 & $1.91 \mathrm{E}-10$ & $1.78 \mathrm{E}-10$ & PGPEP1L & $1.07 \mathrm{E}+00$ \\
\hline ENSBTAG00000000807 & $1.43 \mathrm{E}-10$ & $1.33 \mathrm{E}-10$ & TMED3 & $1.08 \mathrm{E}+00$ \\
\hline ENSBTAG00000001282 & $2.16 \mathrm{E}-10$ & $2.00 \mathrm{E}-10$ & RALGAPA1 & $1.08 \mathrm{E}+00$ \\
\hline ENSBTAG00000031834 & $8.04 \mathrm{E}-10$ & 7.39E-10 & & $1.09 \mathrm{E}+00$ \\
\hline ENSBTAG00000046346 & $5.69 \mathrm{E}-10$ & $5.22 \mathrm{E}-10$ & PACS2 & $1.09 \mathrm{E}+00$ \\
\hline ENSBTAG00000015398 & $1.33 \mathrm{E}-10$ & $1.21 \mathrm{E}-10$ & TJP1 & $1.10 \mathrm{E}+00$ \\
\hline ENSBTAG00000048048 & $1.84 \mathrm{E}-09$ & $1.68 \mathrm{E}-09$ & & $1.10 \mathrm{E}+00$ \\
\hline ENSBTAG00000006227 & $1.26 \mathrm{E}-10$ & $1.14 \mathrm{E}-10$ & $\mathrm{IDH} 3 \mathrm{~A}$ & $1.11 \mathrm{E}+00$ \\
\hline ENSBTAG00000003708 & $2.97 \mathrm{E}-10$ & $2.69 \mathrm{E}-10$ & SEC23A & $1.10 \mathrm{E}+00$ \\
\hline ENSBTAG00000046857 & $5.39 \mathrm{E}-10$ & 4.87E-10 & & $1.11 E+00$ \\
\hline ENSBTAG00000002504 & $1.83 \mathrm{E}-10$ & $1.65 \mathrm{E}-10$ & IREB2 & $1.11 E+00$ \\
\hline ENSBTAG00000003132 & $2.30 \mathrm{E}-10$ & $2.08 \mathrm{E}-10$ & CHRNB4 & $1.11 E+00$ \\
\hline ENSBTAG00000046611 & $1.06 \mathrm{E}-09$ & $9.50 \mathrm{E}-10$ & & $1.11 E+00$ \\
\hline ENSBTAG00000001652 & $5.55 \mathrm{E}-10$ & $4.95 \mathrm{E}-10$ & SLCO3A1 & $1.12 \mathrm{E}+00$ \\
\hline ENSBTAG00000047002 & $9.05 \mathrm{E}-10$ & $8.06 \mathrm{E}-10$ & & $1.12 \mathrm{E}+00$ \\
\hline ENSBTAG00000047955 & $4.24 \mathrm{E}-10$ & 3.77E-10 & ZNF774 & $1.12 \mathrm{E}+00$ \\
\hline ENSBTAG00000004024 & $9.42 \mathrm{E}-11$ & $8.36 \mathrm{E}-11$ & UBE2Q2 & $1.13 \mathrm{E}+00$ \\
\hline ENSBTAG00000003152 & $5.85 \mathrm{E}-10$ & $5.18 \mathrm{E}-10$ & & $1.13 E+00$ \\
\hline ENSBTAG00000003375 & $2.07 \mathrm{E}-10$ & $1.82 \mathrm{E}-10$ & TC2N & $1.13 \mathrm{E}+00$ \\
\hline ENSBTAG00000017213 & $6.30 \mathrm{E}-10$ & $5.56 \mathrm{E}-10$ & MFAP1 & $1.13 \mathrm{E}+00$ \\
\hline ENSBTAG00000011239 & $2.38 \mathrm{E}-10$ & $2.06 \mathrm{E}-10$ & SEC11A & $1.16 \mathrm{E}+00$ \\
\hline ENSBTAG00000018603 & $1.46 \mathrm{E}-10$ & $1.27 \mathrm{E}-10$ & RGMA & $1.15 \mathrm{E}+00$ \\
\hline ENSBTAG00000035333 & $9.35 \mathrm{E}-10$ & $8.08 \mathrm{E}-10$ & & $1.16 \mathrm{E}+00$ \\
\hline ENSBTAG00000013055 & $1.03 \mathrm{E}-09$ & $8.79 \mathrm{E}-10$ & & $1.17 E+00$ \\
\hline ENSBTAG00000009293 & $4.35 \mathrm{E}-10$ & $3.71 E-10$ & EVL & $1.17 \mathrm{E}+00$ \\
\hline ENSBTAG00000015909 & $1.96 \mathrm{E}-10$ & $1.68 \mathrm{E}-10$ & PDE8A & $1.17 \mathrm{E}+00$ \\
\hline ENSBTAG00000013060 & $7.44 \mathrm{E}-10$ & $6.32 \mathrm{E}-10$ & IQGAP1 & $1.18 \mathrm{E}+00$ \\
\hline ENSBTAG00000013410 & $1.92 \mathrm{E}-10$ & 1.62E-10 & MARK3 & $1.18 \mathrm{E}+00$ \\
\hline
\end{tabular}




\begin{tabular}{|c|c|c|c|c|}
\hline ENSBTAG00000019350 & $3.37 \mathrm{E}-10$ & $2.85 \mathrm{E}-10$ & SLC25A21 & $1.18 \mathrm{E}+00$ \\
\hline ENSBTAG00000009653 & $3.38 \mathrm{E}-10$ & $2.84 \mathrm{E}-10$ & TCL1B & $1.19 \mathrm{E}+00$ \\
\hline ENSBTAG00000032631 & $3.77 \mathrm{E}-10$ & $3.16 \mathrm{E}-10$ & & $1.19 \mathrm{E}+00$ \\
\hline ENSBTAG00000013352 & $1.52 \mathrm{E}-10$ & $1.28 \mathrm{E}-10$ & NKX2-8 & $1.19 \mathrm{E}+00$ \\
\hline ENSBTAG00000006620 & $6.80 \mathrm{E}-10$ & $5.65 E-10$ & SLC24A4 & $1.20 \mathrm{E}+00$ \\
\hline ENSBTAG00000000655 & $5.61 \mathrm{E}-10$ & $4.65 \mathrm{E}-10$ & & $1.21 E+00$ \\
\hline ENSBTAG00000007187 & $3.50 \mathrm{E}-10$ & $2.88 \mathrm{E}-10$ & INF2 & $1.21 \mathrm{E}+00$ \\
\hline ENSBTAG00000026913 & $2.94 \mathrm{E}-10$ & $2.41 \mathrm{E}-10$ & HHIPL1 & $1.22 \mathrm{E}+00$ \\
\hline ENSBTAG00000046076 & $8.25 \mathrm{E}-10$ & $6.76 \mathrm{E}-10$ & & $1.22 \mathrm{E}+00$ \\
\hline ENSBTAG00000016062 & $1.59 \mathrm{E}-10$ & $1.30 \mathrm{E}-10$ & C14orf28 & $1.22 \mathrm{E}+00$ \\
\hline ENSBTAG00000002613 & $7.43 \mathrm{E}-10$ & $6.04 \mathrm{E}-10$ & MIS18BP1 & $1.23 E+00$ \\
\hline ENSBTAG00000048268 & $9.82 \mathrm{E}-10$ & $7.98 \mathrm{E}-10$ & & $1.23 \mathrm{E}+00$ \\
\hline ENSBTAG00000040580 & 1.10E-09 & $8.87 E-10$ & & $1.24 \mathrm{E}+00$ \\
\hline ENSBTAG00000047905 & $3.02 \mathrm{E}-10$ & $2.43 \mathrm{E}-10$ & & $1.24 \mathrm{E}+00$ \\
\hline ENSBTAG00000016091 & $1.08 \mathrm{E}-09$ & $8.68 \mathrm{E}-10$ & KLHL25 & $1.25 E+00$ \\
\hline ENSBTAG00000009854 & $1.46 \mathrm{E}-10$ & 1.17E-10 & MPHOSPH10 & $1.25 E+00$ \\
\hline ENSBTAG00000010416 & $3.61 \mathrm{E}-10$ & $2.87 \mathrm{E}-10$ & RIN3 & $1.26 \mathrm{E}+00$ \\
\hline ENSBTAG00000003130 & $1.21 \mathrm{E}-10$ & $9.66 \mathrm{E}-11$ & CHRNA3 & $1.26 \mathrm{E}+00$ \\
\hline ENSBTAG00000012338 & $5.88 \mathrm{E}-10$ & $4.66 \mathrm{E}-10$ & $\mathrm{FAH}$ & $1.26 \mathrm{E}+00$ \\
\hline ENSBTAG00000014440 & $2.83 \mathrm{E}-10$ & $2.24 \mathrm{E}-10$ & PSMA4 & $1.26 \mathrm{E}+00$ \\
\hline ENSBTAG00000035995 & $1.95 \mathrm{E}-09$ & $1.53 \mathrm{E}-09$ & TNFAIP2 & $1.27 \mathrm{E}+00$ \\
\hline ENSBTAG00000013567 & 1.10E-09 & $8.64 \mathrm{E}-10$ & OR5D13 & $1.27 \mathrm{E}+00$ \\
\hline ENSBTAG00000021199 & $1.02 \mathrm{E}-09$ & $8.02 \mathrm{E}-10$ & APOPT1 & $1.28 \mathrm{E}+00$ \\
\hline ENSBTAG00000000539 & $1.66 \mathrm{E}-10$ & $1.31 \mathrm{E}-10$ & LRRC28 & $1.27 \mathrm{E}+00$ \\
\hline ENSBTAG00000018709 & $4.52 \mathrm{E}-10$ & $3.53 \mathrm{E}-10$ & CATSPERB & $1.28 \mathrm{E}+00$ \\
\hline ENSBTAG00000017194 & $3.97 \mathrm{E}-10$ & $3.09 \mathrm{E}-10$ & ASPG & $1.28 \mathrm{E}+00$ \\
\hline ENSBTAG00000014476 & $8.98 \mathrm{E}-10$ & $6.91 \mathrm{E}-10$ & ADAL & $1.30 \mathrm{E}+00$ \\
\hline ENSBTAG00000016984 & $1.82 \mathrm{E}-10$ & $1.40 \mathrm{E}-10$ & PTPN9 & $1.30 \mathrm{E}+00$ \\
\hline ENSBTAG00000048042 & $3.45 \mathrm{E}-10$ & $2.64 \mathrm{E}-10$ & & $1.31 \mathrm{E}+00$ \\
\hline ENSBTAG00000020402 & $6.37 \mathrm{E}-10$ & $4.81 \mathrm{E}-10$ & TDRD9 & $1.32 \mathrm{E}+00$ \\
\hline ENSBTAG00000004054 & $1.59 \mathrm{E}-10$ & $1.20 \mathrm{E}-10$ & PAPOLA & $1.32 \mathrm{E}+00$ \\
\hline ENSBTAG00000009855 & $1.57 \mathrm{E}-10$ & 1.17E-10 & FAN1 & $1.35 \mathrm{E}+00$ \\
\hline ENSBTAG00000039813 & 1.07E-09 & 7.97E-10 & GZMB & $1.34 \mathrm{E}+00$ \\
\hline ENSBTAG00000020514 & $4.68 \mathrm{E}-10$ & $3.49 \mathrm{E}-10$ & & $1.34 \mathrm{E}+00$ \\
\hline ENSBTAG00000018527 & $2.68 \mathrm{E}-10$ & $2.00 \mathrm{E}-10$ & HDGFRP3 & $1.34 \mathrm{E}+00$ \\
\hline ENSBTAG00000017716 & $2.77 \mathrm{E}-10$ & $2.06 \mathrm{E}-10$ & BEGAIN & $1.34 \mathrm{E}+00$ \\
\hline ENSBTAG00000009941 & $1.69 \mathrm{E}-10$ & $1.26 \mathrm{E}-10$ & MBIP & $1.34 \mathrm{E}+00$ \\
\hline ENSBTAG00000026995 & $2.31 \mathrm{E}-10$ & $1.71 \mathrm{E}-10$ & PNN & $1.35 E+00$ \\
\hline ENSBTAG00000045929 & $6.36 \mathrm{E}-10$ & $4.70 \mathrm{E}-10$ & & $1.35 \mathrm{E}+00$ \\
\hline ENSBTAG00000007365 & $3.82 \mathrm{E}-10$ & $2.82 \mathrm{E}-10$ & NEIL1 & $1.36 \mathrm{E}+00$ \\
\hline ENSBTAG00000012321 & $2.80 \mathrm{E}-10$ & $2.05 \mathrm{E}-10$ & & $1.37 E+00$ \\
\hline ENSBTAG00000010981 & $1.61 \mathrm{E}-10$ & $1.18 \mathrm{E}-10$ & CIB2 & $1.36 \mathrm{E}+00$ \\
\hline ENSBTAG00000001308 & $1.14 \mathrm{E}-09$ & $8.24 \mathrm{E}-10$ & & $1.38 \mathrm{E}+00$ \\
\hline ENSBTAG00000018642 & $9.88 \mathrm{E}-11$ & $7.11 \mathrm{E}-11$ & RCCD1 & $1.39 \mathrm{E}+00$ \\
\hline ENSBTAG00000046401 & $4.61 \mathrm{E}-10$ & $3.32 \mathrm{E}-10$ & $\mathrm{RD} 3 \mathrm{~L}$ & $1.39 E+00$ \\
\hline ENSBTAG00000000026 & $1.64 \mathrm{E}-10$ & 1.17E-10 & VPS33B & $1.40 \mathrm{E}+00$ \\
\hline ENSBTAG00000047729 & $1.29 \mathrm{E}-09$ & $9.15 \mathrm{E}-10$ & & $1.41 E+00$ \\
\hline ENSBTAG00000018123 & $2.59 \mathrm{E}-10$ & $1.84 \mathrm{E}-10$ & FBLN5 & $1.41 E+00$ \\
\hline ENSBTAG00000009891 & $3.36 \mathrm{E}-10$ & $2.37 \mathrm{E}-10$ & PPP4R4 & $1.42 \mathrm{E}+00$ \\
\hline ENSBTAG00000007502 & $2.59 \mathrm{E}-10$ & $1.83 \mathrm{E}-10$ & & $1.42 \mathrm{E}+00$ \\
\hline ENSBTAG00000015750 & 4.57E-10 & $3.22 \mathrm{E}-10$ & AMN & $1.42 \mathrm{E}+00$ \\
\hline ENSBTAG00000017719 & $3.40 \mathrm{E}-10$ & $2.38 \mathrm{E}-10$ & AKAP6 & $1.43 \mathrm{E}+00$ \\
\hline ENSBTAG00000007109 & $5.36 \mathrm{E}-10$ & $3.75 \mathrm{E}-10$ & ASB2 & $1.43 \mathrm{E}+00$ \\
\hline ENSBTAG00000002833 & $1.20 \mathrm{E}-10$ & $8.35 \mathrm{E}-11$ & $\mathrm{FBXO} 22$ & $1.44 \mathrm{E}+00$ \\
\hline ENSBTAG00000026893 & $1.26 \mathrm{E}-09$ & $8.72 \mathrm{E}-10$ & EXOC3L4 & $1.44 \mathrm{E}+00$ \\
\hline ENSBTAG00000003155 & $1.13 \mathrm{E}-09$ & $7.79 \mathrm{E}-10$ & & $1.45 \mathrm{E}+00$ \\
\hline ENSBTAG00000018643 & $1.16 \mathrm{E}-10$ & $7.98 \mathrm{E}-11$ & PRC1 & $1.45 E+00$ \\
\hline ENSBTAG00000037383 & $5.28 \mathrm{E}-10$ & $3.65 E-10$ & AKAP13 & $1.45 \mathrm{E}+00$ \\
\hline ENSBTAG00000011828 & $1.42 \mathrm{E}-10$ & $9.82 \mathrm{E}-11$ & ARID3B & $1.44 E+00$ \\
\hline ENSBTAG00000009845 & $3.47 \mathrm{E}-10$ & $2.40 \mathrm{E}-10$ & ITPK1 & $1.45 E+00$ \\
\hline ENSBTAG00000011752 & $1.74 \mathrm{E}-10$ & $1.20 \mathrm{E}-10$ & SYNM & $1.45 E+00$ \\
\hline ENSBTAG00000015772 & $2.18 \mathrm{E}-10$ & $1.50 \mathrm{E}-10$ & STOML1 & $1.45 E+00$ \\
\hline ENSBTAG00000017054 & $9.34 \mathrm{E}-10$ & $6.39 \mathrm{E}-10$ & & $1.46 \mathrm{E}+00$ \\
\hline ENSBTAG00000046828 & $3.76 \mathrm{E}-10$ & $2.56 \mathrm{E}-10$ & & $1.47 E+00$ \\
\hline ENSBTAG00000010828 & $9.92 \mathrm{E}-10$ & $6.75 E-10$ & & $1.47 \mathrm{E}+00$ \\
\hline ENSBTAG00000013689 & $5.52 \mathrm{E}-10$ & $3.75 \mathrm{E}-10$ & MCTP2 & $1.47 \mathrm{E}+00$ \\
\hline ENSBTAG00000011185 & $1.26 \mathrm{E}-10$ & $8.55 \mathrm{E}-11$ & TTC23 & $1.47 \mathrm{E}+00$ \\
\hline ENSBTAG00000006270 & $4.01 \mathrm{E}-10$ & $2.71 \mathrm{E}-10$ & HSP90AA1 & $1.48 \mathrm{E}+00$ \\
\hline ENSBTAG00000006231 & $1.61 \mathrm{E}-10$ & $1.08 \mathrm{E}-10$ & ACSBG1 & $1.49 \mathrm{E}+00$ \\
\hline ENSBTAG00000010992 & $3.41 \mathrm{E}-10$ & $2.29 \mathrm{E}-10$ & $\mathrm{CTSH}$ & $1.49 \mathrm{E}+00$ \\
\hline
\end{tabular}




\begin{tabular}{|c|c|c|c|c|}
\hline ENSBTAG000000010370 & $2.35 \mathrm{E}-10$ & $1.58 \mathrm{E}-10$ & C14orf79 & $1.49 \mathrm{E}+00$ \\
\hline ENSBTAG00000018639 & $2.51 \mathrm{E}-10$ & $1.69 \mathrm{E}-10$ & UNC45A & $1.49 E+00$ \\
\hline ENSBTAG000000017196 & $2.29 \mathrm{E}-10$ & $1.53 \mathrm{E}-10$ & PDIA3 & $1.50 \mathrm{E}+00$ \\
\hline ENSBTAG000000014981 & $9.26 \mathrm{E}-10$ & $6.20 \mathrm{E}-10$ & OTUB2 & $1.49 \mathrm{E}+00$ \\
\hline ENSBTAG000000012326 & $1.52 \mathrm{E}-09$ & 1.01E-09 & & $1.51 E+00$ \\
\hline ENSBTAG000000015779 & $2.49 \mathrm{E}-10$ & $1.66 \mathrm{E}-10$ & PML & $1.50 \mathrm{E}+00$ \\
\hline ENSBTAG000000015018 & 7.19E-10 & 4.79E-10 & FSD2 & $1.50 \mathrm{E}+00$ \\
\hline ENSBTAG000000008384 & $8.66 \mathrm{E}-10$ & $5.73 \mathrm{E}-10$ & SLC25A47 & $1.51 E+00$ \\
\hline ENSBTAG000000027059 & $2.04 \mathrm{E}-10$ & $1.35 \mathrm{E}-10$ & & $1.51 E+00$ \\
\hline ENSBTAG000000000085 & $2.57 \mathrm{E}-10$ & $1.69 \mathrm{E}-10$ & CYP1A2 & $1.52 \mathrm{E}+00$ \\
\hline ENSBTAG000000017201 & $1.98 \mathrm{E}-10$ & $1.30 \mathrm{E}-10$ & AP4S1 & $1.52 E+00$ \\
\hline ENSBTAG000000012789 & $2.68 \mathrm{E}-10$ & $1.76 \mathrm{E}-10$ & PRKD1 & $1.53 E+00$ \\
\hline ENSBTAG00000012926 & $4.21 \mathrm{E}-10$ & 2.77E-10 & TM6SF1 & $1.52 \mathrm{E}+00$ \\
\hline ENSBTAG000000021341 & $1.35 \mathrm{E}-10$ & $8.82 \mathrm{E}-11$ & BTBD1 & $1.53 E+00$ \\
\hline ENSBTAG000000027033 & $7.54 \mathrm{E}-10$ & $4.92 \mathrm{E}-10$ & & $1.53 E+00$ \\
\hline ENSBTAG000000009294 & $6.10 \mathrm{E}-10$ & $3.97 \mathrm{E}-10$ & DEGS2 & $1.54 \mathrm{E}+00$ \\
\hline ENSBTAG00000019831 & $6.77 \mathrm{E}-10$ & $4.40 \mathrm{E}-10$ & MRPS11 & $1.54 \mathrm{E}+00$ \\
\hline ENSBTAG000000002939 & $1.81 \mathrm{E}-10$ & $1.14 \mathrm{E}-10$ & FURIN & $1.59 \mathrm{E}+00$ \\
\hline ENSBTAG000000046590 & $1.68 \mathrm{E}-10$ & $1.06 \mathrm{E}-10$ & FAM181A & $1.59 E+00$ \\
\hline ENSBTAG000000006130 & $2.60 \mathrm{E}-10$ & $1.64 \mathrm{E}-10$ & CLEC14A & $1.58 \mathrm{E}+00$ \\
\hline ENSBTAG000000010536 & $1.60 \mathrm{E}-10$ & $1.01 \mathrm{E}-10$ & GPR33 & $1.59 \mathrm{E}+00$ \\
\hline ENSBTAG00000015350 & $7.09 \mathrm{E}-11$ & $4.46 \mathrm{E}-11$ & PLIN1 & $1.59 E+00$ \\
\hline ENSBTAG000000004310 & $2.04 \mathrm{E}-10$ & $1.28 \mathrm{E}-10$ & UBR7 & $1.59 \mathrm{E}+00$ \\
\hline ENSBTAG000000018905 & $1.61 \mathrm{E}-10$ & $1.02 \mathrm{E}-10$ & MAN2A2 & $1.58 \mathrm{E}+00$ \\
\hline ENSBTAG00000018252 & $1.95 \mathrm{E}-10$ & $1.23 \mathrm{E}-10$ & ARRDC4 & $1.59 \mathrm{E}+00$ \\
\hline ENSBTAG000000045497 & $4.94 \mathrm{E}-10$ & $3.10 \mathrm{E}-10$ & & $1.59 E+00$ \\
\hline ENSBTAG000000015462 & $2.87 \mathrm{E}-10$ & $1.81 \mathrm{E}-10$ & BNC1 & $1.59 E+00$ \\
\hline ENSBTAG000000003556 & $2.86 \mathrm{E}-10$ & $1.80 \mathrm{E}-10$ & ZFYVE21 & $1.59 \mathrm{E}+00$ \\
\hline ENSBTAG000000047319 & $2.72 \mathrm{E}-10$ & $1.71 \mathrm{E}-10$ & & $1.59 E+00$ \\
\hline ENSBTAG000000018019 & $2.13 \mathrm{E}-10$ & $1.33 \mathrm{E}-10$ & BCL11B & $1.60 \mathrm{E}+00$ \\
\hline ENSBTAG00000013491 & $6.58 \mathrm{E}-10$ & $4.09 \mathrm{E}-10$ & EML1 & $1.61 E+00$ \\
\hline ENSBTAG000000002786 & $1.26 \mathrm{E}-09$ & $7.79 \mathrm{E}-10$ & & $1.61 E+00$ \\
\hline ENSBTAG00000012489 & 1.64E-10 & $1.00 \mathrm{E}-10$ & TMEM266 & $1.64 \mathrm{E}+00$ \\
\hline ENSBTAG000000020023 & $3.61 E-10$ & $2.21 \mathrm{E}-10$ & & $1.63 E+00$ \\
\hline ENSBTAG000000008017 & $3.24 \mathrm{E}-10$ & $1.97 \mathrm{E}-10$ & UNC79 & $1.64 \mathrm{E}+00$ \\
\hline ENSBTAG00000021844 & $1.92 \mathrm{E}-10$ & 1.17E-10 & $\mathrm{COCH}$ & $1.64 \mathrm{E}+00$ \\
\hline ENSBTAG00000003492 & $6.72 \mathrm{E}-10$ & $4.08 \mathrm{E}-10$ & & $1.65 E+00$ \\
\hline ENSBTAG00000015775 & $1.50 \mathrm{E}-10$ & $8.98 \mathrm{E}-11$ & & $1.67 E+00$ \\
\hline ENSBTAG00000014230 & $1.01 \mathrm{E}-10$ & $6.05 \mathrm{E}-11$ & VRK1 & $1.67 \mathrm{E}+00$ \\
\hline ENSBTAG00000013475 & $7.32 \mathrm{E}-10$ & 4.36E-10 & TRAF3 & $1.68 \mathrm{E}+00$ \\
\hline ENSBTAG00000017636 & $3.40 \mathrm{E}-10$ & $2.02 \mathrm{E}-10$ & AKT1 & $1.68 \mathrm{E}+00$ \\
\hline ENSBTAG00000019580 & 3.13E-10 & $1.86 \mathrm{E}-10$ & TCL1A & $1.68 \mathrm{E}+00$ \\
\hline ENSBTAG00000010649 & $3.89 \mathrm{E}-10$ & $2.30 \mathrm{E}-10$ & MEF2A & $1.69 \mathrm{E}+00$ \\
\hline ENSBTAG00000019823 & $4.75 \mathrm{E}-10$ & $2.81 \mathrm{E}-10$ & ADAMTS17 & $1.69 E+00$ \\
\hline ENSBTAG00000017215 & $6.21 \mathrm{E}-10$ & $3.65 \mathrm{E}-10$ & WDR76 & $1.70 \mathrm{E}+00$ \\
\hline ENSBTAG00000014422 & $9.61 \mathrm{E}-11$ & $5.63 \mathrm{E}-11$ & MTMR10 & $1.70 \mathrm{E}+00$ \\
\hline ENSBTAG00000021273 & $1.72 \mathrm{E}-10$ & $1.01 \mathrm{E}-10$ & SEMA4B & $1.71 E+00$ \\
\hline ENSBTAG00000021904 & $7.05 \mathrm{E}-10$ & $4.10 \mathrm{E}-10$ & KIF26A & $1.72 \mathrm{E}+00$ \\
\hline ENSBTAG00000004679 & $6.36 \mathrm{E}-10$ & $3.70 \mathrm{E}-10$ & WARS & $1.72 \mathrm{E}+00$ \\
\hline ENSBTAG00000019916 & $8.23 \mathrm{E}-10$ & $4.72 \mathrm{E}-10$ & CLMN & $1.74 \mathrm{E}+00$ \\
\hline ENSBTAG00000003526 & $1.02 \mathrm{E}-09$ & $5.85 \mathrm{E}-10$ & SYNE3 & $1.75 \mathrm{E}+00$ \\
\hline ENSBTAG00000017622 & $4.12 \mathrm{E}-10$ & $2.35 \mathrm{E}-10$ & SIVA1 & $1.75 E+00$ \\
\hline ENSBTAG00000035998 & $2.79 \mathrm{E}-10$ & $1.59 \mathrm{E}-10$ & CKB & $1.75 E+00$ \\
\hline ENSBTAG00000017211 & $3.52 \mathrm{E}-10$ & $1.99 \mathrm{E}-10$ & SERINC4 & $1.77 \mathrm{E}+00$ \\
\hline ENSBTAG00000015160 & $3.00 \mathrm{E}-10$ & $1.70 \mathrm{E}-10$ & PLD4 & $1.77 \mathrm{E}+00$ \\
\hline ENSBTAG00000018636 & $2.67 \mathrm{E}-10$ & $1.51 \mathrm{E}-10$ & CCNK & $1.76 \mathrm{E}+00$ \\
\hline ENSBTAG00000018843 & $4.56 \mathrm{E}-10$ & $2.54 \mathrm{E}-10$ & SERPINA1 & $1.80 \mathrm{E}+00$ \\
\hline ENSBTAG00000012166 & $2.30 \mathrm{E}-10$ & $1.27 \mathrm{E}-10$ & TMC3 & $1.81 E+00$ \\
\hline ENSBTAG00000009186 & $1.47 \mathrm{E}-10$ & $8.14 \mathrm{E}-11$ & PAX9 & $1.80 \mathrm{E}+00$ \\
\hline ENSBTAG00000006141 & $1.67 \mathrm{E}-10$ & $9.19 \mathrm{E}-11$ & WDR93 & $1.82 \mathrm{E}+00$ \\
\hline ENSBTAG00000027024 & $1.34 \mathrm{E}-10$ & $7.40 \mathrm{E}-11$ & ARHGAP5 & $1.82 \mathrm{E}+00$ \\
\hline ENSBTAG00000000838 & $6.05 \mathrm{E}-10$ & $3.29 \mathrm{E}-10$ & CCDC88C & $1.84 \mathrm{E}+00$ \\
\hline ENSBTAG00000003410 & $4.61 \mathrm{E}-10$ & $2.48 \mathrm{E}-10$ & EFL1 & $1.86 \mathrm{E}+00$ \\
\hline ENSBTAG00000013657 & $2.50 \mathrm{E}-10$ & $1.33 \mathrm{E}-10$ & CHRNA5 & $1.88 \mathrm{E}+00$ \\
\hline ENSBTAG00000006819 & $1.93 \mathrm{E}-10$ & $1.02 \mathrm{E}-10$ & & $1.89 \mathrm{E}+00$ \\
\hline ENSBTAG00000015880 & $9.21 \mathrm{E}-10$ & $4.85 \mathrm{E}-10$ & PRIMA1 & $1.90 \mathrm{E}+00$ \\
\hline ENSBTAG00000011861 & $1.81 \mathrm{E}-10$ & $9.47 \mathrm{E}-11$ & CFAP161 & $1.91 \mathrm{E}+00$ \\
\hline ENSBTAG00000007431 & $2.03 \mathrm{E}-10$ & $1.06 \mathrm{E}-10$ & CEMIP & $1.91 \mathrm{E}+00$ \\
\hline ENSBTAG00000046979 & $2.32 \mathrm{E}-10$ & $1.21 \mathrm{E}-10$ & LGMN & $1.92 \mathrm{E}+00$ \\
\hline ENSBTAG00000020301 & $2.56 \mathrm{E}-10$ & $1.33 \mathrm{E}-10$ & BLM & $1.92 \mathrm{E}+00$ \\
\hline
\end{tabular}




\begin{tabular}{|c|c|c|c|c|}
\hline ENSBTAG000000016522 & 3.77E-10 & $1.96 \mathrm{E}-10$ & C15orf40 & $1.92 \mathrm{E}+00$ \\
\hline ENSBTAG00000003559 & $3.87 \mathrm{E}-10$ & $2.00 \mathrm{E}-10$ & PPP1R13B & $1.93 \mathrm{E}+00$ \\
\hline ENSBTAG00000001396 & $3.00 \mathrm{E}-10$ & $1.54 \mathrm{E}-10$ & ADAMTS7 & $1.95 \mathrm{E}+00$ \\
\hline ENSBTAG000000018004 & 4.20E-10 & $2.16 \mathrm{E}-10$ & SH3GL3 & $1.94 \mathrm{E}+00$ \\
\hline ENSBTAG000000009966 & $2.94 \mathrm{E}-10$ & $1.51 \mathrm{E}-10$ & XRCC3 & $1.95 E+00$ \\
\hline ENSBTAG000000007503 & $2.80 \mathrm{E}-10$ & $1.43 \mathrm{E}-10$ & STRC & $1.96 \mathrm{E}+00$ \\
\hline ENSBTAG000000004645 & $2.81 \mathrm{E}-10$ & $1.43 \mathrm{E}-10$ & ADAMTSL3 & $1.96 \mathrm{E}+00$ \\
\hline ENSBTAG000000021291 & $6.37 \mathrm{E}-10$ & 3.23E-10 & STXBP6 & $1.97 E+00$ \\
\hline ENSBTAG000000007052 & $8.43 \mathrm{E}-10$ & 4.25E-10 & BDKRB1 & $1.98 \mathrm{E}+00$ \\
\hline ENSBTAG000000007007 & $3.60 \mathrm{E}-10$ & $1.81 \mathrm{E}-10$ & WDR20 & $1.99 \mathrm{E}+00$ \\
\hline ENSBTAG000000001786 & 4.03E-10 & $2.03 \mathrm{E}-10$ & HYKK & $1.99 \mathrm{E}+00$ \\
\hline ENSBTAG000000037578 & $5.93 \mathrm{E}-10$ & $2.98 \mathrm{E}-10$ & & $1.99 \mathrm{E}+00$ \\
\hline ENSBTAG00000007094 & $1.24 \mathrm{E}-10$ & $6.22 \mathrm{E}-11$ & KIAA1024 & $1.98 \mathrm{E}+00$ \\
\hline ENSBTAG000000002612 & $5.91 \mathrm{E}-10$ & $2.94 \mathrm{E}-10$ & & $2.01 E+00$ \\
\hline ENSBTAG000000020192 & $2.48 \mathrm{E}-10$ & $1.23 \mathrm{E}-10$ & PPP2R5C & $2.02 \mathrm{E}+00$ \\
\hline ENSBTAG000000025181 & $6.82 \mathrm{E}-10$ & 3.35E-10 & CCDC85C & $2.03 E+00$ \\
\hline ENSBTAG000000013234 & $7.58 \mathrm{E}-10$ & $3.72 \mathrm{E}-10$ & & $2.04 \mathrm{E}+00$ \\
\hline ENSBTAG000000009097 & $2.45 \mathrm{E}-10$ & $1.19 \mathrm{E}-10$ & FANCI & $2.05 E+00$ \\
\hline ENSBTAG000000003957 & $2.60 \mathrm{E}-10$ & $1.26 \mathrm{E}-10$ & & $2.06 \mathrm{E}+00$ \\
\hline ENSBTAG000000039808 & $3.58 \mathrm{E}-10$ & $1.72 \mathrm{E}-10$ & SERPINA6 & $2.08 \mathrm{E}+00$ \\
\hline ENSBTAG000000010915 & $2.16 \mathrm{E}-10$ & $1.03 \mathrm{E}-10$ & CRTC3 & $2.10 \mathrm{E}+00$ \\
\hline ENSBTAG00000021717 & $6.17 \mathrm{E}-10$ & $2.93 \mathrm{E}-10$ & BDKRB2 & $2.11 E+00$ \\
\hline ENSBTAG000000032130 & $4.22 \mathrm{E}-10$ & 2.00E-10 & CATSPER2 & $2.11 E+00$ \\
\hline ENSBTAG000000017715 & $4.51 \mathrm{E}-10$ & $2.13 \mathrm{E}-10$ & HOMER2 & $2.12 \mathrm{E}+00$ \\
\hline ENSBTAG000000031327 & $2.42 \mathrm{E}-10$ & $1.12 \mathrm{E}-10$ & TRMT61A & $2.16 \mathrm{E}+00$ \\
\hline ENSBTAG000000005040 & $1.70 \mathrm{E}-10$ & $7.83 \mathrm{E}-11$ & PPP4R3A & $2.17 E+00$ \\
\hline ENSBTAG000000002170 & $3.78 \mathrm{E}-10$ & $1.73 \mathrm{E}-10$ & NOVA1 & $2.18 \mathrm{E}+00$ \\
\hline ENSBTAG000000001567 & $5.55 \mathrm{E}-10$ & $2.47 \mathrm{E}-10$ & LRRK1 & $2.25 E+00$ \\
\hline ENSBTAG000000017216 & $6.34 \mathrm{E}-10$ & $2.79 \mathrm{E}-10$ & FRMD5 & $2.27 \mathrm{E}+00$ \\
\hline ENSBTAG000000006934 & $2.23 \mathrm{E}-10$ & $9.66 \mathrm{E}-11$ & CYP11A1 & $2.30 \mathrm{E}+00$ \\
\hline ENSBTAG000000007382 & $3.05 \mathrm{E}-10$ & $1.31 \mathrm{E}-10$ & SCAPER & $2.33 \mathrm{E}+00$ \\
\hline ENSBTAG000000004802 & $2.89 \mathrm{E}-10$ & $1.23 \mathrm{E}-10$ & CEP170B & $2.35 \mathrm{E}+00$ \\
\hline ENSBTAG00000010407 & 1.57E-10 & $6.60 \mathrm{E}-11$ & GSC & $2.38 \mathrm{E}+00$ \\
\hline ENSBTAG000000010057 & $7.25 \mathrm{E}-11$ & $3.04 \mathrm{E}-11$ & GZMB & $2.38 \mathrm{E}+00$ \\
\hline ENSBTAG00000015944 & $9.02 \mathrm{E}-11$ & $3.79 \mathrm{E}-11$ & WDR61 & $2.38 \mathrm{E}+00$ \\
\hline ENSBTAG00000006582 & $1.98 \mathrm{E}-10$ & $8.29 \mathrm{E}-11$ & SSTR1 & $2.38 \mathrm{E}+00$ \\
\hline ENSBTAG00000015095 & $4.25 \mathrm{E}-10$ & $1.77 \mathrm{E}-10$ & TTC6 & $2.40 \mathrm{E}+00$ \\
\hline ENSBTAG00000016881 & 3.67E-10 & $1.52 \mathrm{E}-10$ & ANPEP & $2.41 E+00$ \\
\hline ENSBTAG00000002610 & $6.57 \mathrm{E}-10$ & $2.71 E-10$ & FKBP3 & $2.42 \mathrm{E}+00$ \\
\hline ENSBTAG00000000967 & $9.59 \mathrm{E}-10$ & $3.95 \mathrm{E}-10$ & DET1 & $2.43 E+00$ \\
\hline ENSBTAG00000004063 & $3.35 \mathrm{E}-10$ & $1.35 \mathrm{E}-10$ & SERPINA5 & $2.48 \mathrm{E}+00$ \\
\hline ENSBTAG00000003435 & $5.40 \mathrm{E}-10$ & $2.16 \mathrm{E}-10$ & FAM189A1 & $2.50 \mathrm{E}+00$ \\
\hline ENSBTAG00000019940 & 2.19E-10 & $8.75 E-11$ & RASGRF1 & $2.50 \mathrm{E}+00$ \\
\hline ENSBTAG00000010514 & $3.84 \mathrm{E}-10$ & $1.53 \mathrm{E}-10$ & ASB7 & $2.51 E+00$ \\
\hline ENSBTAG00000023079 & $7.31 \mathrm{E}-10$ & $2.90 \mathrm{E}-10$ & & $2.52 E+00$ \\
\hline ENSBTAG00000014762 & 1.10E-09 & 4.35E-10 & ISG20 & $2.54 \mathrm{E}+00$ \\
\hline ENSBTAG00000012909 & $1.82 \mathrm{E}-10$ & 7.17E-11 & CRABP1 & $2.54 \mathrm{E}+00$ \\
\hline ENSBTAG00000026886 & $4.91 \mathrm{E}-10$ & $1.93 \mathrm{E}-10$ & C14orf2 & $2.54 \mathrm{E}+00$ \\
\hline ENSBTAG00000035247 & $1.33 \mathrm{E}-10$ & $5.13 \mathrm{E}-11$ & MCEE & $2.58 \mathrm{E}+00$ \\
\hline ENSBTAG00000003300 & $9.10 \mathrm{E}-10$ & 3.50E-10 & MFGE8 & $2.60 \mathrm{E}+00$ \\
\hline ENSBTAG00000021064 & $6.01 \mathrm{E}-10$ & $2.29 \mathrm{E}-10$ & & $2.62 \mathrm{E}+00$ \\
\hline ENSBTAG00000007233 & $5.68 \mathrm{E}-10$ & 2.17E-10 & WDR25 & $2.62 E+00$ \\
\hline ENSBTAG00000019085 & 4.35E-10 & $1.64 \mathrm{E}-10$ & SRP54 & $2.65 E+00$ \\
\hline ENSBTAG00000001740 & $1.00 \mathrm{E}-09$ & $3.73 \mathrm{E}-10$ & HAPLN3 & $2.69 \mathrm{E}+00$ \\
\hline ENSBTAG00000038159 & $4.78 \mathrm{E}-10$ & $1.78 \mathrm{E}-10$ & & $2.68 \mathrm{E}+00$ \\
\hline ENSBTAG00000038494 & 2.20E-09 & $8.11 E-10$ & & $2.72 E+00$ \\
\hline ENSBTAG00000003684 & $3.52 E-10$ & $1.29 \mathrm{E}-10$ & & $2.72 \mathrm{E}+00$ \\
\hline ENSBTAG00000047040 & 4.55E-10 & 1.67E-10 & & $2.72 \mathrm{E}+00$ \\
\hline ENSBTAG00000009680 & $3.51 \mathrm{E}-10$ & $1.28 \mathrm{E}-10$ & FAM177A1 & $2.74 \mathrm{E}+00$ \\
\hline ENSBTAG00000013666 & $4.28 \mathrm{E}-10$ & $1.56 \mathrm{E}-10$ & SLC25A29 & $2.74 \mathrm{E}+00$ \\
\hline ENSBTAG00000010882 & $4.99 \mathrm{E}-10$ & $1.79 \mathrm{E}-10$ & ANKRD9 & $2.79 E+00$ \\
\hline ENSBTAG00000009681 & $2.10 \mathrm{E}-10$ & $7.22 \mathrm{E}-11$ & PPP2R3C & $2.91 E+00$ \\
\hline ENSBTAG00000033721 & $3.16 \mathrm{E}-10$ & $1.08 \mathrm{E}-10$ & RLBP1 & $2.92 \mathrm{E}+00$ \\
\hline ENSBTAG00000032132 & $2.69 \mathrm{E}-10$ & $9.23 \mathrm{E}-11$ & ELL3 & $2.91 E+00$ \\
\hline ENSBTAG00000012022 & $3.82 \mathrm{E}-10$ & $1.27 \mathrm{E}-10$ & TBC1D2B & $3.01 \mathrm{E}+00$ \\
\hline ENSBTAG00000002603 & $5.70 \mathrm{E}-10$ & $1.88 \mathrm{E}-10$ & PRPF39 & $3.03 E+00$ \\
\hline ENSBTAG00000009125 & $3.42 \mathrm{E}-10$ & $1.12 \mathrm{E}-10$ & ALDH1A3 & $3.05 E+00$ \\
\hline ENSBTAG00000004462 & $3.72 \mathrm{E}-10$ & $1.22 \mathrm{E}-10$ & NPAS3 & $3.05 E+00$ \\
\hline ENSBTAG00000027081 & $6.86 \mathrm{E}-10$ & $2.25 \mathrm{E}-10$ & ATP10A & $3.05 E+00$ \\
\hline ENSBTAG00000017711 & 4.69E-10 & $1.53 \mathrm{E}-10$ & WHAMM & $3.07 E+00$ \\
\hline
\end{tabular}




\begin{tabular}{|c|c|c|c|c|}
\hline ENSBTAG00000010988 & $2.59 \mathrm{E}-10$ & $8.28 \mathrm{E}-11$ & & $3.12 \mathrm{E}+00$ \\
\hline ENSBTAG00000017361 & $1.13 \mathrm{E}-10$ & 3.57E-11 & BAG5 & $3.17 E+00$ \\
\hline ENSBTAG00000006140 & $4.62 \mathrm{E}-11$ & $1.46 \mathrm{E}-11$ & & $3.15 E+00$ \\
\hline ENSBTAG00000031934 & $3.42 \mathrm{E}-10$ & $1.08 \mathrm{E}-10$ & FSCB & $3.17 E+00$ \\
\hline ENSBTAG00000005174 & $1.42 \mathrm{E}-10$ & 4.46E-11 & GDPGP1 & $3.18 \mathrm{E}+00$ \\
\hline ENSBTAG00000015053 & $2.47 \mathrm{E}-10$ & $7.78 \mathrm{E}-11$ & CFL2 & $3.18 \mathrm{E}+00$ \\
\hline ENSBTAG00000005145 & 4.45E-10 & $1.39 \mathrm{E}-10$ & PEAK1 & $3.20 E+00$ \\
\hline ENSBTAG00000047587 & $5.84 \mathrm{E}-10$ & $1.83 \mathrm{E}-10$ & & $3.19 E+00$ \\
\hline ENSBTAG00000017632 & $7.12 \mathrm{E}-10$ & $2.23 \mathrm{E}-10$ & AEN & $3.19 E+00$ \\
\hline ENSBTAG00000046185 & $2.48 \mathrm{E}-10$ & $7.70 \mathrm{E}-11$ & BTBD7 & $3.22 \mathrm{E}+00$ \\
\hline ENSBTAG00000017616 & $3.64 \mathrm{E}-10$ & $1.10 \mathrm{E}-10$ & ADSSL1 & $3.31 \mathrm{E}+00$ \\
\hline ENSBTAG00000009098 & $3.10 \mathrm{E}-10$ & $9.30 \mathrm{E}-11$ & POLG & $3.33 \mathrm{E}+00$ \\
\hline ENSBTAG00000015942 & $2.96 \mathrm{E}-10$ & $8.78 \mathrm{E}-11$ & DNAJA4 & $3.36 \mathrm{E}+00$ \\
\hline ENSBTAG00000006675 & $3.82 \mathrm{E}-10$ & $1.11 \mathrm{E}-10$ & PCSK6 & $3.44 \mathrm{E}+00$ \\
\hline ENSBTAG00000001526 & 2.97E-09 & 8.56E-10 & & $3.47 E+00$ \\
\hline ENSBTAG00000020819 & 4.16E-10 & 1.19E-10 & YY1 & $3.50 \mathrm{E}+00$ \\
\hline ENSBTAG00000046540 & $6.87 \mathrm{E}-10$ & $1.96 \mathrm{E}-10$ & & $3.51 E+00$ \\
\hline ENSBTAG00000014005 & 4.15E-10 & $1.17 \mathrm{E}-10$ & PPIP5K1 & $3.54 \mathrm{E}+00$ \\
\hline ENSBTAG00000012852 & $4.96 \mathrm{E}-10$ & $1.39 \mathrm{E}-10$ & DICER1 & $3.57 E+00$ \\
\hline ENSBTAG00000019954 & $9.01 \mathrm{E}-10$ & $2.53 \mathrm{E}-10$ & ABHD2 & $3.56 \mathrm{E}+00$ \\
\hline ENSBTAG00000016603 & $5.88 \mathrm{E}-10$ & $1.62 \mathrm{E}-10$ & & $3.63 E+00$ \\
\hline ENSBTAG00000009682 & $2.29 \mathrm{E}-10$ & $6.25 \mathrm{E}-11$ & & $3.66 \mathrm{E}+00$ \\
\hline ENSBTAG00000014479 & $7.98 \mathrm{E}-10$ & $2.18 \mathrm{E}-10$ & TUBGCP4 & $3.66 \mathrm{E}+00$ \\
\hline ENSBTAG00000014505 & $3.11 \mathrm{E}-10$ & $8.50 \mathrm{E}-11$ & NGRN & $3.66 \mathrm{E}+00$ \\
\hline ENSBTAG00000002336 & $4.63 \mathrm{E}-10$ & $1.26 \mathrm{E}-10$ & RCOR1 & $3.67 E+00$ \\
\hline ENSBTAG00000016112 & $6.38 \mathrm{E}-10$ & $1.74 \mathrm{E}-10$ & LINC00521 & $3.67 E+00$ \\
\hline ENSBTAG00000039864 & $9.37 \mathrm{E}-10$ & $2.53 \mathrm{E}-10$ & SERPINA4 & $3.70 E+00$ \\
\hline ENSBTAG00000016854 & $6.24 \mathrm{E}-10$ & $1.65 \mathrm{E}-10$ & AK7 & $3.78 \mathrm{E}+00$ \\
\hline ENSBTAG00000048094 & $4.62 \mathrm{E}-10$ & $1.22 \mathrm{E}-10$ & & $3.79 E+00$ \\
\hline ENSBTAG00000007392 & 4.77E-10 & $1.26 \mathrm{E}-10$ & & $3.78 \mathrm{E}+00$ \\
\hline ENSBTAG00000004339 & $9.09 \mathrm{E}-10$ & $2.39 \mathrm{E}-10$ & & $3.80 \mathrm{E}+00$ \\
\hline ENSBTAG00000014001 & 4.06E-10 & $1.06 \mathrm{E}-10$ & MAP1A & $3.83 E+00$ \\
\hline ENSBTAG00000019830 & $7.82 \mathrm{E}-10$ & $2.00 \mathrm{E}-10$ & MRPL46 & $3.91 E+00$ \\
\hline ENSBTAG00000016456 & $5.30 \mathrm{E}-10$ & $1.35 \mathrm{E}-10$ & CDC42BPB & $3.92 \mathrm{E}+00$ \\
\hline ENSBTAG00000021275 & $1.29 \mathrm{E}-10$ & $3.25 \mathrm{E}-11$ & CIB1 & $3.96 \mathrm{E}+00$ \\
\hline ENSBTAG00000015023 & $6.79 \mathrm{E}-10$ & $1.68 \mathrm{E}-10$ & TRAPPC6B & $4.04 \mathrm{E}+00$ \\
\hline ENSBTAG00000010963 & $1.78 \mathrm{E}-10$ & $4.31 \mathrm{E}-11$ & IL16 & $4.13 E+00$ \\
\hline ENSBTAG00000016158 & 7.37E-10 & $1.67 \mathrm{E}-10$ & ACAN & $4.41 E+00$ \\
\hline ENSBTAG00000027074 & $4.61 \mathrm{E}-10$ & $1.02 \mathrm{E}-10$ & SV2B & $4.52 \mathrm{E}+00$ \\
\hline ENSBTAG00000016598 & $3.40 \mathrm{E}-10$ & $7.45 \mathrm{E}-11$ & DYNC1H1 & $4.56 \mathrm{E}+00$ \\
\hline ENSBTAG00000035544 & 1.17E-09 & $2.45 \mathrm{E}-10$ & CYP46A1 & $4.76 \mathrm{E}+00$ \\
\hline ENSBTAG00000027058 & $2.21 \mathrm{E}-10$ & 4.62E-11 & MESP2 & $4.77 E+00$ \\
\hline ENSBTAG00000047694 & $2.84 \mathrm{E}-10$ & $5.96 \mathrm{E}-11$ & & $4.77 \mathrm{E}+00$ \\
\hline ENSBTAG00000036082 & 1.35E-09 & $2.82 \mathrm{E}-10$ & & $4.80 \mathrm{E}+00$ \\
\hline ENSBTAG00000010995 & $2.92 \mathrm{E}-10$ & $5.76 \mathrm{E}-11$ & CINP & $5.06 \mathrm{E}+00$ \\
\hline ENSBTAG00000005471 & $3.97 \mathrm{E}-10$ & $7.84 \mathrm{E}-11$ & RHCG & $5.06 \mathrm{E}+00$ \\
\hline ENSBTAG00000048096 & $1.85 \mathrm{E}-09$ & $3.63 \mathrm{E}-10$ & & $5.10 \mathrm{E}+00$ \\
\hline ENSBTAG00000038361 & $6.99 \mathrm{E}-10$ & $1.36 \mathrm{E}-10$ & SERPINA11 & $5.14 \mathrm{E}+00$ \\
\hline ENSBTAG00000020441 & $3.30 \mathrm{E}-10$ & $6.34 \mathrm{E}-11$ & HMG20A & $5.21 \mathrm{E}+00$ \\
\hline ENSBTAG00000007043 & $6.68 \mathrm{E}-10$ & $1.23 \mathrm{E}-10$ & & $5.43 E+00$ \\
\hline ENSBTAG00000013593 & $6.06 \mathrm{E}-10$ & $1.11 \mathrm{E}-10$ & SNRPA1 & $5.46 \mathrm{E}+00$ \\
\hline ENSBTAG00000039117 & $4.55 \mathrm{E}-10$ & $8.24 \mathrm{E}-11$ & FAM179B & $5.52 \mathrm{E}+00$ \\
\hline ENSBTAG00000012206 & $2.79 \mathrm{E}-10$ & $5.01 \mathrm{E}-11$ & SNX33 & $5.56 \mathrm{E}+00$ \\
\hline ENSBTAG00000047710 & $7.38 \mathrm{E}-10$ & $1.32 \mathrm{E}-10$ & & $5.58 \mathrm{E}+00$ \\
\hline ENSBTAG00000033335 & $1.22 \mathrm{E}-09$ & $2.18 \mathrm{E}-10$ & & $5.60 \mathrm{E}+00$ \\
\hline ENSBTAG00000005786 & $3.31 \mathrm{E}-10$ & $5.89 \mathrm{E}-11$ & ATXN3 & $5.60 E+00$ \\
\hline ENSBTAG00000021304 & $6.16 \mathrm{E}-10$ & $1.09 \mathrm{E}-10$ & TP53BP1 & $5.64 \mathrm{E}+00$ \\
\hline ENSBTAG00000001618 & $3.06 \mathrm{E}-10$ & $5.33 \mathrm{E}-11$ & ALPK3 & $5.74 \mathrm{E}+00$ \\
\hline ENSBTAG00000020164 & $2.06 \mathrm{E}-10$ & 3.50E-11 & BAZ1A & $5.88 \mathrm{E}+00$ \\
\hline ENSBTAG00000027069 & $3.53 \mathrm{E}-10$ & $5.78 \mathrm{E}-11$ & FAM103A1 & $6.09 E+00$ \\
\hline ENSBTAG00000026953 & 3.03E-10 & 4.71E-11 & & $6.42 E+00$ \\
\hline ENSBTAG00000020930 & $5.54 \mathrm{E}-10$ & $8.63 \mathrm{E}-11$ & GEMIN2 & $6.42 \mathrm{E}+00$ \\
\hline ENSBTAG00000007041 & $5.14 \mathrm{E}-10$ & 7.79E-11 & & $6.60 E+00$ \\
\hline ENSBTAG00000016495 & $1.10 \mathrm{E}-10$ & $1.54 \mathrm{E}-11$ & LINS1 & $7.13 E+00$ \\
\hline ENSBTAG00000014478 & 4.27E-10 & $5.96 \mathrm{E}-11$ & ZSCAN29 & $7.16 \mathrm{E}+00$ \\
\hline ENSBTAG00000017924 & $9.10 \mathrm{E}-10$ & $1.20 \mathrm{E}-10$ & ODC1 & $7.57 E+00$ \\
\hline ENSBTAG00000006191 & $7.75 \mathrm{E}-10$ & $1.00 \mathrm{E}-10$ & SERPINA12 & $7.74 \mathrm{E}+00$ \\
\hline ENSBTAG00000032059 & $4.68 \mathrm{E}-10$ & $5.79 \mathrm{E}-11$ & TGM5 & $8.07 E+00$ \\
\hline ENSBTAG00000010993 & $5.12 \mathrm{E}-10$ & $6.17 \mathrm{E}-11$ & & $8.28 \mathrm{E}+00$ \\
\hline ENSBTAG00000026917 & $6.57 \mathrm{E}-10$ & 7.72E-11 & & $8.50 E+00$ \\
\hline
\end{tabular}




\begin{tabular}{|c|c|c|c|c|}
\hline ENSBTAG00000047366 & $1.23 \mathrm{E}-09$ & $1.40 \mathrm{E}-10$ & & $8.80 E+00$ \\
\hline ENSBTAG00000014093 & $3.93 \mathrm{E}-10$ & $4.43 \mathrm{E}-11$ & $\mathrm{IDH} 2$ & $8.85 E+00$ \\
\hline ENSBTAG00000047632 & $6.80 \mathrm{E}-10$ & $6.55 \mathrm{E}-11$ & IGHE & $1.04 \mathrm{E}+01$ \\
\hline ENSBTAG00000012143 & $5.52 \mathrm{E}-10$ & $5.27 \mathrm{E}-11$ & TECPR2 & $1.05 \mathrm{E}+01$ \\
\hline ENSBTAG00000020413 & 4.57E-10 & 4.27E-11 & APBA2 & $1.07 \mathrm{E}+01$ \\
\hline ENSBTAG00000006836 & $7.86 \mathrm{E}-10$ & 7.34E-11 & FBXO33 & $1.07 \mathrm{E}+01$ \\
\hline ENSBTAG00000006648 & $2.39 \mathrm{E}-10$ & $2.14 \mathrm{E}-11$ & & $1.11 \mathrm{E}+01$ \\
\hline ENSBTAG00000006621 & $1.44 \mathrm{E}-10$ & $1.25 \mathrm{E}-11$ & & $1.14 \mathrm{E}+01$ \\
\hline ENSBTAG00000021021 & $2.36 \mathrm{E}-10$ & $1.98 \mathrm{E}-11$ & C15orf39 & $1.19 E+01$ \\
\hline ENSBTAG00000010227 & $3.39 \mathrm{E}-10$ & $2.80 \mathrm{E}-11$ & CPSF2 & $1.21 \mathrm{E}+01$ \\
\hline ENSBTAG00000015156 & 3.67E-10 & $2.98 \mathrm{E}-11$ & & $1.23 \mathrm{E}+01$ \\
\hline ENSBTAG00000019174 & $6.99 \mathrm{E}-10$ & $5.41 \mathrm{E}-11$ & ZNF710 & $1.29 \mathrm{E}+01$ \\
\hline ENSBTAG00000047543 & 1.06E-09 & $7.92 \mathrm{E}-11$ & & $1.33 \mathrm{E}+01$ \\
\hline ENSBTAG00000009683 & $5.36 \mathrm{E}-10$ & $3.91 \mathrm{E}-11$ & PSMA6 & $1.37 E+01$ \\
\hline ENSBTAG00000006987 & $3.43 \mathrm{E}-10$ & 2.47E-11 & CERS3 & $1.38 \mathrm{E}+01$ \\
\hline ENSBTAG00000018708 & $5.55 \mathrm{E}-10$ & 3.87E-11 & & $1.43 \mathrm{E}+01$ \\
\hline ENSBTAG00000015412 & $9.30 \mathrm{E}-10$ & $6.30 \mathrm{E}-11$ & TGM7 & $1.47 \mathrm{E}+01$ \\
\hline ENSBTAG00000008251 & $2.03 \mathrm{E}-10$ & $1.32 \mathrm{E}-11$ & SNRPN & $1.53 \mathrm{E}+01$ \\
\hline ENSBTAG00000002487 & $1.31 \mathrm{E}-10$ & 8.13E-12 & UBE3A & $1.59 \mathrm{E}+01$ \\
\hline ENSBTAG00000000640 & $6.37 \mathrm{E}-10$ & $2.25 \mathrm{E}-11$ & SELENOS & $2.82 \mathrm{E}+01$ \\
\hline ENSBTAG00000007008 & $6.63 \mathrm{E}-10$ & $6.91 \mathrm{E}-12$ & MOK & $9.45 \mathrm{E}+01$ \\
\hline ENSBTAG00000016630 & $2.19 \mathrm{E}-10$ & 0 & MEX3B & $2.19 E+03$ \\
\hline ENSBTAG00000047935 & $4.52 \mathrm{E}-10$ & 0 & & $4.52 E+03$ \\
\hline ENSBTAG00000012199 & 8.03E-10 & 0 & IMP3 & $8.03 E+03$ \\
\hline ENSBTAG00000013888 & $1.30 \mathrm{E}-10$ & 0 & FOXA1 & $1.30 \mathrm{E}+03$ \\
\hline ENSBTAG00000009980 & $7.52 \mathrm{E}-11$ & 0 & & $7.53 \mathrm{E}+02$ \\
\hline ENSBTAG00000007009 & $2.75 \mathrm{E}-10$ & 0 & & $2.75 \mathrm{E}+03$ \\
\hline ENSBTAG00000047700 & $1.40 \mathrm{E}-09$ & 0 & & $1.40 E+04$ \\
\hline ENSBTAG00000002186 & $5.09 \mathrm{E}-11$ & 0 & NDN & $5.10 E+02$ \\
\hline ENSBTAG00000045998 & $9.22 \mathrm{E}-11$ & 0 & MAGEL2 & $9.23 E+02$ \\
\hline ENSBTAG00000010192 & $3.18 \mathrm{E}-10$ & 0 & & $3.18 \mathrm{E}+03$ \\
\hline ENSBTAG00000046412 & 0 & 0 & & $1.00 E+00$ \\
\hline ENSBTAG00000023147 & $2.67 \mathrm{E}-10$ & 0 & LINGO1 & $2.67 E+03$ \\
\hline ENSBTAG00000021693 & 0 & 0 & RPP25 & $1.00 \mathrm{E}+00$ \\
\hline ENSBTAG00000031558 & 0 & 0 & & $1.00 \mathrm{E}+00$ \\
\hline ENSBTAG00000014472 & $6.00 \mathrm{E}-10$ & 0 & LCMT2 & $6.00 E+03$ \\
\hline ENSBTAG00000000425 & 0 & 0 & & $1.00 \mathrm{E}+00$ \\
\hline ENSBTAG00000024311 & 0 & 0 & & $1.00 \mathrm{E}+00$ \\
\hline ENSBTAG00000046585 & $1.44 \mathrm{E}-10$ & 0 & RTL1 & $1.44 \mathrm{E}+03$ \\
\hline ENSBTAG00000043578 & 0 & 0 & & $1.00 \mathrm{E}+00$ \\
\hline ENSBTAG00000037977 & 0 & 0 & & $1.00 \mathrm{E}+00$ \\
\hline ENSBTAG00000005038 & $6.64 \mathrm{E}-11$ & 0 & ZBTB42 & $6.65 E+02$ \\
\hline ENSBTAG00000031242 & $2.45 \mathrm{E}-10$ & 0 & CDCA4 & $2.45 E+03$ \\
\hline ENSBTAG00000005647 & $1.46 \mathrm{E}-10$ & 0 & GPR132 & $1.46 \mathrm{E}+03$ \\
\hline ENSBTAG00000045594 & $8.56 \mathrm{E}-11$ & 0 & TMEM121 & $8.57 E+02$ \\
\hline ENSBTAG00000046763 & $1.90 \mathrm{E}-10$ & $6.73 \mathrm{E}-10$ & FAM107A & 2.82E-01 \\
\hline ENSBTAG00000016615 & $2.04 \mathrm{E}-10$ & $6.33 \mathrm{E}-10$ & ABHD6 & 3.22E-01 \\
\hline ENSBTAG00000022656 & $1.35 \mathrm{E}-10$ & 4.10E-10 & KCTD6 & 3.30E-01 \\
\hline ENSBTAG00000010916 & $3.66 \mathrm{E}-11$ & $1.08 \mathrm{E}-10$ & LHFPL4 & $3.40 \mathrm{E}-01$ \\
\hline ENSBTAG00000019078 & $2.42 \mathrm{E}-10$ & $6.11 \mathrm{E}-10$ & UCN2 & 3.96E-01 \\
\hline ENSBTAG00000020962 & 4.67E-11 & $1.18 \mathrm{E}-10$ & TUSC2 & 3.96E-01 \\
\hline ENSBTAG00000008244 & $5.35 \mathrm{E}-11$ & $1.35 \mathrm{E}-10$ & & 3.97E-01 \\
\hline ENSBTAG00000006500 & $2.90 \mathrm{E}-11$ & $7.32 \mathrm{E}-11$ & RBM15B & 3.97E-01 \\
\hline ENSBTAG00000021508 & $1.67 \mathrm{E}-10$ & $3.45 \mathrm{E}-10$ & LMOD3 & 4.85E-01 \\
\hline ENSBTAG00000040574 & $8.65 \mathrm{E}-11$ & $1.64 \mathrm{E}-10$ & IQCF5 & $5.28 \mathrm{E}-01$ \\
\hline ENSBTAG00000046011 & $9.72 \mathrm{E}-11$ & $1.84 \mathrm{E}-10$ & & 5.29E-01 \\
\hline ENSBTAG00000032026 & $6.88 \mathrm{E}-11$ & 1.30E-10 & GMPPB & 5.29E-01 \\
\hline ENSBTAG00000004878 & $1.42 \mathrm{E}-10$ & 2.60E-10 & CAMKV & 5.47E-01 \\
\hline ENSBTAG00000047135 & $1.89 \mathrm{E}-10$ & $3.21 \mathrm{E}-10$ & GORASP1 & 5.89E-01 \\
\hline ENSBTAG00000031287 & $3.05 \mathrm{E}-10$ & $5.13 \mathrm{E}-10$ & TMEM158 & 5.94E-01 \\
\hline ENSBTAG00000031447 & $9.61 \mathrm{E}-11$ & $1.62 \mathrm{E}-10$ & PRSS45 & 5.94E-01 \\
\hline ENSBTAG00000001067 & $1.26 \mathrm{E}-10$ & 2.07E-10 & GRM2 & 6.07E-01 \\
\hline ENSBTAG00000002147 & $1.05 \mathrm{E}-10$ & $1.71 \mathrm{E}-10$ & PPP4R2 & $6.14 \mathrm{E}-01$ \\
\hline ENSBTAG00000013716 & $2.08 \mathrm{E}-10$ & $3.32 \mathrm{E}-10$ & NR2C2 & $6.26 \mathrm{E}-01$ \\
\hline ENSBTAG00000003622 & $2.43 \mathrm{E}-10$ & $3.69 \mathrm{E}-10$ & ZKSCAN7 & $6.60 \mathrm{E}-01$ \\
\hline ENSBTAG00000011585 & $8.35 E-11$ & $1.26 \mathrm{E}-10$ & MST1 & $6.63 \mathrm{E}-01$ \\
\hline ENSBTAG00000022598 & $1.36 \mathrm{E}-10$ & $2.00 \mathrm{E}-10$ & & $6.81 \mathrm{E}-01$ \\
\hline ENSBTAG00000016013 & $4.78 \mathrm{E}-10$ & $6.94 \mathrm{E}-10$ & ASB14 & $6.88 \mathrm{E}-01$ \\
\hline ENSBTAG00000011088 & $1.79 \mathrm{E}-10$ & $2.61 \mathrm{E}-10$ & SLC6A6 & $6.86 \mathrm{E}-01$ \\
\hline ENSBTAG00000016101 & $1.18 \mathrm{E}-10$ & 1.69E-10 & VHLL & $6.96 \mathrm{E}-01$ \\
\hline
\end{tabular}




\begin{tabular}{|c|c|c|c|c|}
\hline ENSBTAG00000018622 & $6.58 \mathrm{E}-11$ & $9.33 \mathrm{E}-11$ & PCBP4 & $7.06 \mathrm{E}-01$ \\
\hline ENSBTAG00000004512 & $4.40 \mathrm{E}-10$ & $6.22 \mathrm{E}-10$ & MKRN2 & 7.08E-01 \\
\hline ENSBTAG00000022681 & $2.11 \mathrm{E}-10$ & $2.89 \mathrm{E}-10$ & EOGT & 7.31E-01 \\
\hline ENSBTAG00000038640 & $1.80 \mathrm{E}-10$ & $2.43 \mathrm{E}-10$ & & $7.42 \mathrm{E}-01$ \\
\hline ENSBTAG00000013725 & $1.39 \mathrm{E}-10$ & $1.86 \mathrm{E}-10$ & RBSN & 7.46E-01 \\
\hline ENSBTAG00000022575 & $3.78 \mathrm{E}-10$ & $5.00 \mathrm{E}-10$ & TPRA1 & $7.55 \mathrm{E}-01$ \\
\hline ENSBTAG00000003110 & $4.29 \mathrm{E}-10$ & $5.60 \mathrm{E}-10$ & & $7.66 \mathrm{E}-01$ \\
\hline ENSBTAG00000010477 & $1.56 \mathrm{E}-10$ & $1.99 \mathrm{E}-10$ & TTC21A & $7.82 \mathrm{E}-01$ \\
\hline ENSBTAG00000001874 & $2.06 \mathrm{E}-10$ & $2.62 \mathrm{E}-10$ & DOCK3 & 7.87E-01 \\
\hline ENSBTAG00000006501 & $1.13 \mathrm{E}-10$ & $1.43 \mathrm{E}-10$ & DCAF1 & $7.89 \mathrm{E}-01$ \\
\hline ENSBTAG00000013356 & $1.15 \mathrm{E}-10$ & $1.45 \mathrm{E}-10$ & & $7.92 \mathrm{E}-01$ \\
\hline ENSBTAG00000019163 & $1.12 \mathrm{E}-10$ & $1.42 \mathrm{E}-10$ & & $7.92 \mathrm{E}-01$ \\
\hline ENSBTAG00000006179 & $1.18 \mathrm{E}-10$ & $1.48 \mathrm{E}-10$ & DNAJB8 & $7.95 \mathrm{E}-01$ \\
\hline ENSBTAG00000039727 & $2.16 \mathrm{E}-10$ & $2.72 \mathrm{E}-10$ & DCLK3 & 7.95E-01 \\
\hline ENSBTAG00000022733 & $4.48 \mathrm{E}-11$ & $5.65 \mathrm{E}-11$ & EPM2AIP1 & $7.93 \mathrm{E}-01$ \\
\hline ENSBTAG00000004747 & $4.96 \mathrm{E}-11$ & $6.25 \mathrm{E}-11$ & & $7.94 \mathrm{E}-01$ \\
\hline ENSBTAG00000047771 & 3.37E-10 & 4.24E-10 & & 7.94E-01 \\
\hline ENSBTAG00000039133 & $6.18 \mathrm{E}-10$ & $7.79 \mathrm{E}-10$ & C3orf49 & 7.93E-01 \\
\hline ENSBTAG00000020965 & $3.86 \mathrm{E}-11$ & $4.86 \mathrm{E}-11$ & ZMYND10 & 7.94E-01 \\
\hline ENSBTAG00000045666 & $2.58 \mathrm{E}-10$ & $3.25 \mathrm{E}-10$ & & $7.93 \mathrm{E}-01$ \\
\hline ENSBTAG00000013932 & $8.89 \mathrm{E}-11$ & 1.12E-10 & CCDC71 & $7.94 \mathrm{E}-01$ \\
\hline ENSBTAG00000010835 & $6.04 \mathrm{E}-11$ & $7.61 \mathrm{E}-11$ & CRELD1 & $7.94 \mathrm{E}-01$ \\
\hline ENSBTAG00000019161 & $4.98 \mathrm{E}-11$ & $6.28 \mathrm{E}-11$ & NPRL2 & $7.94 \mathrm{E}-01$ \\
\hline ENSBTAG00000044079 & $2.55 \mathrm{E}-11$ & $3.21 \mathrm{E}-11$ & SMIM4 & 7.95E-01 \\
\hline ENSBTAG00000005728 & $1.10 \mathrm{E}-10$ & $1.38 \mathrm{E}-10$ & CDHR4 & 7.97E-01 \\
\hline ENSBTAG00000022632 & $2.84 \mathrm{E}-11$ & $3.58 \mathrm{E}-11$ & TCTA & 7.93E-01 \\
\hline ENSBTAG00000031797 & $2.13 \mathrm{E}-11$ & $2.69 \mathrm{E}-11$ & MANF & $7.93 \mathrm{E}-01$ \\
\hline ENSBTAG00000019832 & $2.68 \mathrm{E}-10$ & $3.35 \mathrm{E}-10$ & TGFBR2 & $8.00 \mathrm{E}-01$ \\
\hline ENSBTAG00000005857 & 8.97E-10 & 1.09E-09 & SLC6A1 & 8.23E-01 \\
\hline ENSBTAG00000019987 & $5.20 \mathrm{E}-10$ & $6.27 \mathrm{E}-10$ & RPP14 & $8.30 \mathrm{E}-01$ \\
\hline ENSBTAG00000004178 & $1.89 \mathrm{E}-10$ & $2.28 \mathrm{E}-10$ & ACOX2 & $8.29 \mathrm{E}-01$ \\
\hline ENSBTAG00000008778 & $2.61 \mathrm{E}-10$ & $3.14 \mathrm{E}-10$ & CHST13 & $8.30 \mathrm{E}-01$ \\
\hline ENSBTAG00000013124 & $3.85 \mathrm{E}-10$ & $4.62 \mathrm{E}-10$ & LRTM1 & 8.32E- 01 \\
\hline ENSBTAG00000038298 & $3.46 \mathrm{E}-10$ & $4.12 \mathrm{E}-10$ & CCDC174 & $8.40 \mathrm{E}-01$ \\
\hline ENSBTAG00000047174 & 4.20E-10 & 4.97E-10 & & $8.44 \mathrm{E}-01$ \\
\hline ENSBTAG00000032374 & $5.30 \mathrm{E}-10$ & $6.27 \mathrm{E}-10$ & SELENOK & $8.45 \mathrm{E}-01$ \\
\hline ENSBTAG00000046301 & $2.87 \mathrm{E}-10$ & $3.37 E-10$ & ZNF445 & 8.51E-01 \\
\hline ENSBTAG00000005321 & $3.42 \mathrm{E}-10$ & $3.99 \mathrm{E}-10$ & CADPS & 8.57E-01 \\
\hline ENSBTAG00000000982 & $1.23 \mathrm{E}-10$ & $1.42 \mathrm{E}-10$ & C3orf84 & $8.66 \mathrm{E}-01$ \\
\hline ENSBTAG00000019536 & $6.27 \mathrm{E}-10$ & 7.23E-10 & CRBN & $8.68 \mathrm{E}-01$ \\
\hline ENSBTAG00000011178 & $6.86 \mathrm{E}-10$ & $7.90 \mathrm{E}-10$ & IL17RB & $8.68 \mathrm{E}-01$ \\
\hline ENSBTAG00000048050 & $1.08 \mathrm{E}-10$ & $1.24 \mathrm{E}-10$ & & 8.67E-01 \\
\hline ENSBTAG00000032872 & $5.75 \mathrm{E}-10$ & $6.54 \mathrm{E}-10$ & & $8.80 \mathrm{E}-01$ \\
\hline ENSBTAG00000021911 & $3.66 \mathrm{E}-10$ & $4.14 \mathrm{E}-10$ & PTPRG & $8.84 \mathrm{E}-01$ \\
\hline ENSBTAG00000014547 & $3.14 \mathrm{E}-10$ & $3.51 \mathrm{E}-10$ & PGAM2 & $8.94 \mathrm{E}-01$ \\
\hline ENSBTAG00000002321 & $1.70 \mathrm{E}-10$ & $1.91 \mathrm{E}-10$ & AMT & 8.93E-01 \\
\hline ENSBTAG00000004014 & $6.52 \mathrm{E}-10$ & $7.26 \mathrm{E}-10$ & FBLN2 & 8.97E-01 \\
\hline ENSBTAG00000015046 & $3.13 E-10$ & $3.47 \mathrm{E}-10$ & MST1R & $9.03 \mathrm{E}-01$ \\
\hline ENSBTAG00000007131 & $4.48 \mathrm{E}-10$ & 4.96E-10 & GADL1 & $9.02 \mathrm{E}-01$ \\
\hline ENSBTAG00000014550 & 1.67E-10 & $1.85 \mathrm{E}-10$ & GLT8D1 & $9.04 \mathrm{E}-01$ \\
\hline ENSBTAG00000019193 & $5.96 \mathrm{E}-11$ & $6.57 \mathrm{E}-11$ & SETD5 & $9.08 \mathrm{E}-01$ \\
\hline ENSBTAG00000002923 & $9.40 \mathrm{E}-11$ & $1.04 \mathrm{E}-10$ & CX3CR1 & $9.04 \mathrm{E}-01$ \\
\hline ENSBTAG00000021643 & $8.64 \mathrm{E}-10$ & $9.48 \mathrm{E}-10$ & EFCAB12 & $9.12 \mathrm{E}-01$ \\
\hline ENSBTAG00000014784 & $2.07 \mathrm{E}-10$ & $2.27 \mathrm{E}-10$ & NT5DC2 & $9.11 \mathrm{E}-01$ \\
\hline ENSBTAG00000018198 & $2.84 \mathrm{E}-10$ & $3.10 \mathrm{E}-10$ & & $9.17 \mathrm{E}-01$ \\
\hline ENSBTAG00000014091 & $4.33 \mathrm{E}-10$ & $4.72 \mathrm{E}-10$ & ARHGEF3 & $9.17 \mathrm{E}-01$ \\
\hline ENSBTAG00000015708 & $2.30 \mathrm{E}-10$ & $2.51 \mathrm{E}-10$ & CXCR6 & $9.17 \mathrm{E}-01$ \\
\hline ENSBTAG00000020260 & $2.27 \mathrm{E}-10$ & $2.48 \mathrm{E}-10$ & SYN2 & $9.16 \mathrm{E}-01$ \\
\hline ENSBTAG00000005431 & $3.38 \mathrm{E}-10$ & $3.66 \mathrm{E}-10$ & LMCD1 & $9.24 \mathrm{E}-01$ \\
\hline ENSBTAG00000019096 & $3.28 \mathrm{E}-10$ & $3.54 \mathrm{E}-10$ & UQCRC1 & 9.27E-01 \\
\hline ENSBTAG00000004749 & $1.03 \mathrm{E}-10$ & $1.11 \mathrm{E}-10$ & NEK4 & $9.28 \mathrm{E}-01$ \\
\hline ENSBTAG00000008406 & $3.06 \mathrm{E}-10$ & $3.31 \mathrm{E}-10$ & TREX1 & $9.26 \mathrm{E}-01$ \\
\hline ENSBTAG00000031348 & $6.07 \mathrm{E}-10$ & $6.55 \mathrm{E}-10$ & CCR9 & $9.27 \mathrm{E}-01$ \\
\hline ENSBTAG00000014782 & $1.49 \mathrm{E}-10$ & $1.59 \mathrm{E}-10$ & STAB1 & $9.36 \mathrm{E}-01$ \\
\hline ENSBTAG00000015554 & $8.90 \mathrm{E}-11$ & $9.51 \mathrm{E}-11$ & TMF1 & $9.36 \mathrm{E}-01$ \\
\hline ENSBTAG00000001292 & $6.07 \mathrm{E}-10$ & $6.49 \mathrm{E}-10$ & LF & $9.35 \mathrm{E}-01$ \\
\hline ENSBTAG00000040133 & $3.61 \mathrm{E}-10$ & $3.85 \mathrm{E}-10$ & C3orf20 & 9.37E-01 \\
\hline ENSBTAG00000019534 & $7.86 \mathrm{E}-10$ & 8.31E-10 & TRNT1 & $9.46 \mathrm{E}-01$ \\
\hline ENSBTAG00000017547 & $6.07 \mathrm{E}-10$ & $6.41 \mathrm{E}-10$ & DBNL & 9.47E-01 \\
\hline ENSBTAG00000021835 & $5.46 \mathrm{E}-10$ & $5.74 \mathrm{E}-10$ & PRICKLE2 & $9.51 \mathrm{E}-01$ \\
\hline
\end{tabular}




\begin{tabular}{|c|c|c|c|c|}
\hline ENSBTAG00000010094 & $1.51 \mathrm{E}-10$ & $1.58 \mathrm{E}-10$ & FANCD2OS & $9.54 \mathrm{E}-01$ \\
\hline ENSBTAG00000009863 & $1.74 \mathrm{E}-10$ & $1.83 \mathrm{E}-10$ & BHLHE40 & 9.52E-01 \\
\hline ENSBTAG00000047200 & $6.96 \mathrm{E}-10$ & 7.30E-10 & & 9.53E-01 \\
\hline ENSBTAG00000010026 & 3.27E-10 & $3.43 \mathrm{E}-10$ & CACNA1D & 9.52E-01 \\
\hline ENSBTAG00000005922 & $5.80 \mathrm{E}-10$ & $6.05 \mathrm{E}-10$ & $\mathrm{EFCC1}$ & $9.59 \mathrm{E}-01$ \\
\hline ENSBTAG00000003237 & $3.05 \mathrm{E}-10$ & 3.17E-10 & IQSEC1 & $9.61 \mathrm{E}-01$ \\
\hline ENSBTAG00000018921 & $1.23 \mathrm{E}-10$ & $1.27 \mathrm{E}-10$ & USP19 & 9.65E-01 \\
\hline ENSBTAG00000013413 & $2.48 \mathrm{E}-10$ & $2.57 \mathrm{E}-10$ & CCDC51 & 9.67E-01 \\
\hline ENSBTAG00000006735 & $5.40 \mathrm{E}-10$ & $5.58 \mathrm{E}-10$ & STAC & $9.68 \mathrm{E}-01$ \\
\hline ENSBTAG00000004118 & $4.50 \mathrm{E}-10$ & 4.64E-10 & ALAS1 & $9.70 \mathrm{E}-01$ \\
\hline ENSBTAG00000011894 & $2.64 \mathrm{E}-10$ & $2.72 \mathrm{E}-10$ & C3orf62 & $9.69 \mathrm{E}-01$ \\
\hline ENSBTAG00000018767 & $3.33 \mathrm{E}-10$ & $3.43 \mathrm{E}-10$ & RTP3 & $9.70 \mathrm{E}-01$ \\
\hline ENSBTAG00000047883 & $2.74 \mathrm{E}-10$ & $2.81 \mathrm{E}-10$ & & $9.74 \mathrm{E}-01$ \\
\hline ENSBTAG00000006824 & $1.74 \mathrm{E}-10$ & $1.79 \mathrm{E}-10$ & APPL1 & $9.73 \mathrm{E}-01$ \\
\hline ENSBTAG00000006193 & $2.71 \mathrm{E}-10$ & $2.79 \mathrm{E}-10$ & IRAK2 & $9.71 \mathrm{E}-01$ \\
\hline ENSBTAG00000038962 & $3.57 \mathrm{E}-10$ & $3.66 \mathrm{E}-10$ & SLC6A11 & $9.76 \mathrm{E}-01$ \\
\hline ENSBTAG00000007964 & $1.43 \mathrm{E}-10$ & 1.47E-10 & ARPC4 & $9.75 \mathrm{E}-01$ \\
\hline ENSBTAG00000030855 & $2.65 \mathrm{E}-10$ & $2.70 \mathrm{E}-10$ & $\mathrm{CHCHD} 6$ & $9.80 \mathrm{E}-01$ \\
\hline ENSBTAG00000001333 & $2.25 \mathrm{E}-10$ & $2.28 \mathrm{E}-10$ & PPARG & $9.86 \mathrm{E}-01$ \\
\hline ENSBTAG00000034871 & $8.15 \mathrm{E}-10$ & $8.29 \mathrm{E}-10$ & & $9.84 \mathrm{E}-01$ \\
\hline ENSBTAG00000016725 & $7.34 \mathrm{E}-10$ & $7.43 \mathrm{E}-10$ & KIAA1143 & 9.87E-01 \\
\hline ENSBTAG00000008099 & $1.29 \mathrm{E}-10$ & $1.30 \mathrm{E}-10$ & CCR5 & $9.96 \mathrm{E}-01$ \\
\hline ENSBTAG00000021645 & $9.67 \mathrm{E}-10$ & $9.75 E-10$ & MBD4 & $9.92 \mathrm{E}-01$ \\
\hline ENSBTAG00000003112 & $6.34 \mathrm{E}-11$ & $6.39 \mathrm{E}-11$ & THUMPD3 & $9.92 \mathrm{E}-01$ \\
\hline ENSBTAG00000004117 & $4.90 \mathrm{E}-10$ & 4.92E-10 & AZI2 & $9.95 \mathrm{E}-01$ \\
\hline ENSBTAG00000018928 & $1.12 \mathrm{E}-10$ & $1.13 \mathrm{E}-10$ & QARS & $9.91 \mathrm{E}-01$ \\
\hline ENSBTAG00000003328 & $5.23 \mathrm{E}-10$ & $5.25 \mathrm{E}-10$ & & $9.95 \mathrm{E}-01$ \\
\hline ENSBTAG00000008509 & $3.84 \mathrm{E}-10$ & $3.85 \mathrm{E}-10$ & SLC38A3 & $9.98 \mathrm{E}-01$ \\
\hline ENSBTAG00000045757 & $1.42 \mathrm{E}-10$ & $1.43 \mathrm{E}-10$ & TNNC1 & $9.91 \mathrm{E}-01$ \\
\hline ENSBTAG00000014971 & $9.47 \mathrm{E}-10$ & $9.50 \mathrm{E}-10$ & SEC61G & 9.97E-01 \\
\hline ENSBTAG00000004877 & $2.71 \mathrm{E}-10$ & $2.70 \mathrm{E}-10$ & TRAIP & $1.00 E+00$ \\
\hline ENSBTAG00000018307 & $2.89 \mathrm{E}-10$ & $2.88 \mathrm{E}-10$ & SEMA3F & $1.00 E+00$ \\
\hline ENSBTAG00000016532 & 7.00E-11 & $6.98 \mathrm{E}-11$ & MAPKAPK3 & $1.00 \mathrm{E}+00$ \\
\hline ENSBTAG00000007363 & $4.89 \mathrm{E}-10$ & 4.87E-10 & LSM3 & $1.00 \mathrm{E}+00$ \\
\hline ENSBTAG00000001889 & $2.95 \mathrm{E}-10$ & $2.93 \mathrm{E}-10$ & C3orf14 & $1.01 E+00$ \\
\hline ENSBTAG00000003501 & $7.18 \mathrm{E}-10$ & 7.13E-10 & SACM1L & $1.01 E+00$ \\
\hline ENSBTAG00000018026 & $6.21 \mathrm{E}-10$ & $6.16 \mathrm{E}-10$ & LANCL2 & $1.01 E+00$ \\
\hline ENSBTAG00000014418 & $5.30 \mathrm{E}-10$ & $5.25 \mathrm{E}-10$ & FHIT & $1.01 E+00$ \\
\hline ENSBTAG00000001305 & $4.02 \mathrm{E}-10$ & $3.96 \mathrm{E}-10$ & ATP2B2 & $1.02 \mathrm{E}+00$ \\
\hline ENSBTAG00000016076 & $2.61 \mathrm{E}-10$ & $2.57 \mathrm{E}-10$ & TRAK1 & $1.01 E+00$ \\
\hline ENSBTAG00000016533 & $1.90 \mathrm{E}-10$ & $1.87 \mathrm{E}-10$ & FOXP1 & $1.02 \mathrm{E}+00$ \\
\hline ENSBTAG00000015556 & 1.19E-10 & 1.17E-10 & UBA3 & $1.02 \mathrm{E}+00$ \\
\hline ENSBTAG00000034796 & $3.00 \mathrm{E}-10$ & $2.95 \mathrm{E}-10$ & TEX264 & $1.02 \mathrm{E}+00$ \\
\hline ENSBTAG00000004937 & $9.16 \mathrm{E}-10$ & $9.00 \mathrm{E}-10$ & SEC61A1 & $1.02 \mathrm{E}+00$ \\
\hline ENSBTAG00000007615 & $7.32 \mathrm{E}-10$ & 7.19E-10 & CNTN3 & $1.02 \mathrm{E}+00$ \\
\hline ENSBTAG00000040083 & $2.04 \mathrm{E}-10$ & 2.00E-10 & & $1.02 \mathrm{E}+00$ \\
\hline ENSBTAG00000016731 & $2.12 \mathrm{E}-10$ & 2.07E-10 & TMEM42 & $1.02 \mathrm{E}+00$ \\
\hline ENSBTAG00000019989 & $2.75 \mathrm{E}-10$ & $2.70 \mathrm{E}-10$ & PXK & $1.02 \mathrm{E}+00$ \\
\hline ENSBTAG00000008416 & $3.82 \mathrm{E}-10$ & $3.74 \mathrm{E}-10$ & ABHD5 & $1.02 \mathrm{E}+00$ \\
\hline ENSBTAG00000019479 & $3.29 \mathrm{E}-10$ & $3.20 \mathrm{E}-10$ & & $1.03 E+00$ \\
\hline ENSBTAG00000007208 & $8.21 \mathrm{E}-10$ & $7.96 \mathrm{E}-10$ & HDAC11 & $1.03 E+00$ \\
\hline ENSBTAG00000020047 & $1.63 \mathrm{E}-10$ & $1.59 \mathrm{E}-10$ & MON1A & $1.02 \mathrm{E}+00$ \\
\hline ENSBTAG00000047611 & 1.10E-09 & $1.07 \mathrm{E}-09$ & & $1.03 E+00$ \\
\hline ENSBTAG00000011899 & $1.89 \mathrm{E}-10$ & $1.83 \mathrm{E}-10$ & USP4 & $1.03 E+00$ \\
\hline ENSBTAG00000006789 & 3.33E-10 & 3.23E-10 & FGD5 & $1.03 E+00$ \\
\hline ENSBTAG00000018031 & $3.38 \mathrm{E}-10$ & $3.28 \mathrm{E}-10$ & PODXL2 & $1.03 E+00$ \\
\hline ENSBTAG00000001712 & $6.27 \mathrm{E}-10$ & $6.02 \mathrm{E}-10$ & CMTM7 & $1.04 E+00$ \\
\hline ENSBTAG00000015112 & $3.44 \mathrm{E}-10$ & $3.28 \mathrm{E}-10$ & PSMD6 & $1.05 E+00$ \\
\hline ENSBTAG00000031612 & $1.25 \mathrm{E}-09$ & 1.19E-09 & SPINK8 & $1.05 E+00$ \\
\hline ENSBTAG00000012335 & $1.08 \mathrm{E}-10$ & $1.02 \mathrm{E}-10$ & UBA7 & $1.06 \mathrm{E}+00$ \\
\hline ENSBTAG00000013442 & $1.55 \mathrm{E}-10$ & 1.47E-10 & GPR62 & $1.06 \mathrm{E}+00$ \\
\hline ENSBTAG00000030930 & $6.41 \mathrm{E}-10$ & $6.07 \mathrm{E}-10$ & HMCES & $1.06 \mathrm{E}+00$ \\
\hline ENSBTAG00000008399 & $1.87 \mathrm{E}-10$ & $1.77 \mathrm{E}-10$ & & $1.06 \mathrm{E}+00$ \\
\hline ENSBTAG00000011583 & $2.32 \mathrm{E}-10$ & $2.19 \mathrm{E}-10$ & APEH & $1.06 \mathrm{E}+00$ \\
\hline ENSBTAG00000015483 & $3.10 \mathrm{E}-10$ & $2.93 \mathrm{E}-10$ & CCR8 & $1.06 \mathrm{E}+00$ \\
\hline ENSBTAG00000009183 & $1.79 \mathrm{E}-10$ & $1.70 \mathrm{E}-10$ & SHISA5 & $1.06 \mathrm{E}+00$ \\
\hline ENSBTAG00000020263 & $1.43 \mathrm{E}-10$ & $1.35 \mathrm{E}-10$ & TIMP4 & $1.06 \mathrm{E}+00$ \\
\hline ENSBTAG00000020019 & $3.28 \mathrm{E}-10$ & $3.09 \mathrm{E}-10$ & XCR1 & $1.06 \mathrm{E}+00$ \\
\hline ENSBTAG00000004120 & $1.46 \mathrm{E}-10$ & $1.35 \mathrm{E}-10$ & HEMK1 & $1.08 \mathrm{E}+00$ \\
\hline ENSBTAG00000005306 & $6.71 \mathrm{E}-10$ & $6.24 \mathrm{E}-10$ & TRH & $1.07 E+00$ \\
\hline
\end{tabular}




\begin{tabular}{|c|c|c|c|c|}
\hline ENSBTAG00000034106 & $4.77 \mathrm{E}-10$ & 4.43E-10 & & $1.08 \mathrm{E}+00$ \\
\hline ENSBTAG00000005617 & $4.08 \mathrm{E}-10$ & $3.78 \mathrm{E}-10$ & SLC41A3 & $1.08 \mathrm{E}+00$ \\
\hline ENSBTAG00000009453 & $4.53 \mathrm{E}-10$ & 4.16E-10 & URGCP & $1.09 \mathrm{E}+00$ \\
\hline ENSBTAG00000032304 & $1.33 \mathrm{E}-10$ & $1.21 \mathrm{E}-10$ & & $1.10 \mathrm{E}+00$ \\
\hline ENSBTAG00000019748 & $2.27 \mathrm{E}-10$ & $2.08 \mathrm{E}-10$ & FAM208A & $1.09 \mathrm{E}+00$ \\
\hline ENSBTAG00000016760 & $1.51 \mathrm{E}-10$ & $1.38 \mathrm{E}-10$ & LRRFIP2 & $1.10 \mathrm{E}+00$ \\
\hline ENSBTAG00000011896 & $3.16 \mathrm{E}-10$ & $2.86 \mathrm{E}-10$ & GRIP2 & $1.10 \mathrm{E}+00$ \\
\hline ENSBTAG00000019772 & $2.72 \mathrm{E}-10$ & $2.46 \mathrm{E}-10$ & OXTR & $1.10 \mathrm{E}+00$ \\
\hline ENSBTAG00000035286 & $3.58 \mathrm{E}-10$ & $3.24 \mathrm{E}-10$ & CMC1 & $1.10 \mathrm{E}+00$ \\
\hline ENSBTAG00000006330 & $1.32 \mathrm{E}-10$ & $1.20 \mathrm{E}-10$ & RBM5 & $1.10 \mathrm{E}+00$ \\
\hline ENSBTAG00000011333 & $1.67 \mathrm{E}-10$ & $1.52 \mathrm{E}-10$ & CLASP2 & $1.10 \mathrm{E}+00$ \\
\hline ENSBTAG00000002634 & $1.21 \mathrm{E}-10$ & $1.09 \mathrm{E}-10$ & TMEM115 & $1.11 \mathrm{E}+00$ \\
\hline ENSBTAG00000003025 & $2.55 \mathrm{E}-10$ & $2.29 \mathrm{E}-10$ & NME6 & $1.11 \mathrm{E}+00$ \\
\hline ENSBTAG00000030533 & $1.39 \mathrm{E}-09$ & $1.25 \mathrm{E}-09$ & & $1.11 E+00$ \\
\hline ENSBTAG00000001668 & $4.13 \mathrm{E}-10$ & $3.71 \mathrm{E}-10$ & WNT7A & $1.11 E+00$ \\
\hline ENSBTAG00000019746 & $2.17 \mathrm{E}-10$ & $1.95 \mathrm{E}-10$ & CCDC66 & $1.12 \mathrm{E}+00$ \\
\hline ENSBTAG00000037726 & $3.96 \mathrm{E}-10$ & $3.53 \mathrm{E}-10$ & PLCD1 & $1.12 \mathrm{E}+00$ \\
\hline ENSBTAG00000011547 & $6.42 \mathrm{E}-10$ & $5.73 \mathrm{E}-10$ & KBTBD12 & $1.12 \mathrm{E}+00$ \\
\hline ENSBTAG00000035827 & $4.72 \mathrm{E}-10$ & $4.21 \mathrm{E}-10$ & ATG7 & $1.12 \mathrm{E}+00$ \\
\hline ENSBTAG00000006614 & 1.16E-10 & $1.03 \mathrm{E}-10$ & TMCC1 & $1.13 \mathrm{E}+00$ \\
\hline ENSBTAG00000020481 & $2.26 \mathrm{E}-10$ & $1.98 \mathrm{E}-10$ & GXYLT2 & $1.14 \mathrm{E}+00$ \\
\hline ENSBTAG00000011595 & $8.49 \mathrm{E}-11$ & $7.43 \mathrm{E}-11$ & IP6K1 & $1.14 \mathrm{E}+00$ \\
\hline ENSBTAG00000010138 & $1.68 \mathrm{E}-10$ & $1.46 \mathrm{E}-10$ & SEMA3B & $1.15 \mathrm{E}+00$ \\
\hline ENSBTAG00000018924 & $1.63 \mathrm{E}-10$ & $1.42 \mathrm{E}-10$ & QRICH1 & $1.15 \mathrm{E}+00$ \\
\hline ENSBTAG00000044167 & $2.38 \mathrm{E}-10$ & $2.05 \mathrm{E}-10$ & TOPAZ1 & $1.16 \mathrm{E}+00$ \\
\hline ENSBTAG00000021506 & $1.34 \mathrm{E}-10$ & $1.15 \mathrm{E}-10$ & ARL6IP5 & $1.16 \mathrm{E}+00$ \\
\hline ENSBTAG00000018248 & $6.87 \mathrm{E}-10$ & 5.94E-10 & MGLL & $1.16 \mathrm{E}+00$ \\
\hline ENSBTAG00000012142 & $1.89 \mathrm{E}-10$ & $1.63 \mathrm{E}-10$ & RAD18 & $1.16 \mathrm{E}+00$ \\
\hline ENSBTAG00000047794 & $1.02 \mathrm{E}-10$ & 8.79E-11 & DNAH1 & $1.16 \mathrm{E}+00$ \\
\hline ENSBTAG00000008719 & $5.31 \mathrm{E}-10$ & 4.57E-10 & PRKCD & $1.16 \mathrm{E}+00$ \\
\hline ENSBTAG00000018600 & $3.58 \mathrm{E}-10$ & $3.08 \mathrm{E}-10$ & VGLL4 & $1.16 \mathrm{E}+00$ \\
\hline ENSBTAG00000011588 & $2.11 \mathrm{E}-10$ & $1.82 \mathrm{E}-10$ & RNF123 & $1.16 \mathrm{E}+00$ \\
\hline ENSBTAG00000005436 & $2.10 \mathrm{E}-10$ & $1.81 \mathrm{E}-10$ & SHQ1 & $1.16 \mathrm{E}+00$ \\
\hline ENSBTAG00000002471 & $3.68 \mathrm{E}-10$ & $3.14 \mathrm{E}-10$ & CHL1 & $1.17 E+00$ \\
\hline ENSBTAG00000013015 & $2.50 \mathrm{E}-10$ & $2.13 \mathrm{E}-10$ & CCDC13 & $1.17 \mathrm{E}+00$ \\
\hline ENSBTAG00000011581 & $3.23 \mathrm{E}-10$ & $2.75 \mathrm{E}-10$ & BSN & $1.18 \mathrm{E}+00$ \\
\hline ENSBTAG00000021442 & $1.38 \mathrm{E}-09$ & 1.17E-09 & CRTAP & $1.18 \mathrm{E}+00$ \\
\hline ENSBTAG00000001814 & $3.10 \mathrm{E}-10$ & $2.62 \mathrm{E}-10$ & PLXND1 & $1.18 \mathrm{E}+00$ \\
\hline ENSBTAG00000018910 & $1.47 \mathrm{E}-10$ & $1.23 \mathrm{E}-10$ & WDR6 & $1.19 E+00$ \\
\hline ENSBTAG00000037377 & $1.35 \mathrm{E}-10$ & $1.13 \mathrm{E}-10$ & ABHD14B & $1.19 E+00$ \\
\hline ENSBTAG00000018913 & $1.53 \mathrm{E}-10$ & $1.28 \mathrm{E}-10$ & DALRD3 & $1.20 \mathrm{E}+00$ \\
\hline ENSBTAG00000020302 & $5.25 \mathrm{E}-11$ & $4.41 \mathrm{E}-11$ & FEZF2 & $1.19 E+00$ \\
\hline ENSBTAG00000038084 & $2.60 \mathrm{E}-10$ & $2.18 \mathrm{E}-10$ & NAT6 & $1.19 E+00$ \\
\hline ENSBTAG00000006155 & $5.19 \mathrm{E}-10$ & $4.36 \mathrm{E}-10$ & CCRL2 & $1.19 E+00$ \\
\hline ENSBTAG00000000563 & $2.34 \mathrm{E}-10$ & $1.96 \mathrm{E}-10$ & MyD88 & $1.19 E+00$ \\
\hline ENSBTAG00000010077 & $1.80 \mathrm{E}-10$ & $1.51 \mathrm{E}-10$ & FANCD2 & $1.19 \mathrm{E}+00$ \\
\hline ENSBTAG00000001447 & $6.69 \mathrm{E}-10$ & $5.61 \mathrm{E}-10$ & $\mathrm{HRH} 1$ & $1.19 E+00$ \\
\hline ENSBTAG00000031065 & $1.30 \mathrm{E}-09$ & 1.09E-09 & & $1.20 \mathrm{E}+00$ \\
\hline ENSBTAG00000031115 & 7.07E-10 & $5.91 \mathrm{E}-10$ & & $1.20 \mathrm{E}+00$ \\
\hline ENSBTAG00000044062 & $3.58 \mathrm{E}-10$ & $3.00 \mathrm{E}-10$ & RBMS3 & $1.19 \mathrm{E}+00$ \\
\hline ENSBTAG00000016622 & $2.50 \mathrm{E}-10$ & $2.08 \mathrm{E}-10$ & TCAIM & $1.20 \mathrm{E}+00$ \\
\hline ENSBTAG00000037803 & $1.45 \mathrm{E}-10$ & $1.20 \mathrm{E}-10$ & ZNF197 & $1.21 \mathrm{E}+00$ \\
\hline ENSBTAG00000037821 & $2.59 \mathrm{E}-10$ & $2.14 \mathrm{E}-10$ & & $1.21 \mathrm{E}+00$ \\
\hline ENSBTAG00000000161 & 8.07E-10 & $6.64 \mathrm{E}-10$ & TMEM40 & $1.22 \mathrm{E}+00$ \\
\hline ENSBTAG00000003165 & $2.36 \mathrm{E}-10$ & $1.94 \mathrm{E}-10$ & ADAMTS9 & $1.21 E+00$ \\
\hline ENSBTAG00000000480 & $2.07 \mathrm{E}-10$ & $1.68 \mathrm{E}-10$ & IFRD2 & $1.23 E+00$ \\
\hline ENSBTAG00000033225 & $5.70 \mathrm{E}-10$ & 4.65E-10 & CNTN4 & $1.22 \mathrm{E}+00$ \\
\hline ENSBTAG00000031567 & $2.62 \mathrm{E}-10$ & $2.13 \mathrm{E}-10$ & SMARCC1 & $1.23 E+00$ \\
\hline ENSBTAG00000014779 & $1.33 \mathrm{E}-10$ & $1.09 \mathrm{E}-10$ & $\mathrm{NISCH}$ & $1.22 \mathrm{E}+00$ \\
\hline ENSBTAG00000010029 & $2.23 \mathrm{E}-10$ & $1.79 \mathrm{E}-10$ & ERC2 & $1.25 E+00$ \\
\hline ENSBTAG00000010193 & $4.34 \mathrm{E}-10$ & $3.48 \mathrm{E}-10$ & RAB7A & $1.25 \mathrm{E}+00$ \\
\hline ENSBTAG00000007776 & $6.94 \mathrm{E}-11$ & $5.56 \mathrm{E}-11$ & BRPF1 & $1.25 E+00$ \\
\hline ENSBTAG00000019121 & $6.55 \mathrm{E}-10$ & $5.23 \mathrm{E}-10$ & IFT122 & $1.25 E+00$ \\
\hline ENSBTAG00000013047 & $6.40 \mathrm{E}-10$ & $5.10 \mathrm{E}-10$ & GRM7 & $1.25 E+00$ \\
\hline ENSBTAG00000009489 & $2.39 \mathrm{E}-10$ & $1.91 \mathrm{E}-10$ & CACNA2D2 & $1.25 \mathrm{E}+00$ \\
\hline ENSBTAG00000005490 & $1.80 \mathrm{E}-10$ & $1.44 \mathrm{E}-10$ & P4HTM & $1.25 E+00$ \\
\hline ENSBTAG00000006567 & $2.57 \mathrm{E}-10$ & $2.04 \mathrm{E}-10$ & VIPR1 & $1.26 \mathrm{E}+00$ \\
\hline ENSBTAG00000019041 & $4.42 \mathrm{E}-10$ & 3.50E-10 & FAM19A1 & $1.26 \mathrm{E}+00$ \\
\hline ENSBTAG00000013402 & $3.61 \mathrm{E}-10$ & $2.85 \mathrm{E}-10$ & CTDSPL & $1.27 \mathrm{E}+00$ \\
\hline ENSBTAG00000004514 & $4.08 \mathrm{E}-10$ & $3.22 \mathrm{E}-10$ & & $1.27 \mathrm{E}+00$ \\
\hline
\end{tabular}




\begin{tabular}{|c|c|c|c|c|}
\hline ENSBTAG00000019841 & $1.38 \mathrm{E}-10$ & $1.09 \mathrm{E}-10$ & KIF9 & $1.26 \mathrm{E}+00$ \\
\hline ENSBTAG00000006573 & $3.20 \mathrm{E}-10$ & $2.52 \mathrm{E}-10$ & KLHL18 & $1.27 E+00$ \\
\hline ENSBTAG00000000220 & $5.70 \mathrm{E}-10$ & $4.48 \mathrm{E}-10$ & & $1.27 E+00$ \\
\hline ENSBTAG00000008567 & $3.12 \mathrm{E}-10$ & $2.43 \mathrm{E}-10$ & DLEC1 & $1.28 \mathrm{E}+00$ \\
\hline ENSBTAG00000014927 & $2.01 \mathrm{E}-10$ & $1.56 \mathrm{E}-10$ & OXSR1 & $1.29 \mathrm{E}+00$ \\
\hline ENSBTAG00000038381 & $1.98 \mathrm{E}-10$ & $1.53 \mathrm{E}-10$ & SLC26A6 & $1.29 \mathrm{E}+00$ \\
\hline ENSBTAG00000005488 & $2.03 \mathrm{E}-10$ & $1.57 \mathrm{E}-10$ & ARIH2 & $1.30 E+00$ \\
\hline ENSBTAG00000009371 & $2.27 \mathrm{E}-10$ & $1.75 \mathrm{E}-10$ & CCDC36 & $1.29 \mathrm{E}+00$ \\
\hline ENSBTAG00000047658 & $1.15 \mathrm{E}-10$ & $8.81 \mathrm{E}-11$ & PHF7 & $1.30 \mathrm{E}+00$ \\
\hline ENSBTAG00000020998 & $4.41 \mathrm{E}-10$ & $3.40 \mathrm{E}-10$ & RUVBL1 & $1.30 \mathrm{E}+00$ \\
\hline ENSBTAG00000016069 & $1.80 \mathrm{E}-10$ & $1.38 \mathrm{E}-10$ & ARF4 & $1.30 \mathrm{E}+00$ \\
\hline ENSBTAG00000013212 & $3.31 \mathrm{E}-10$ & $2.52 \mathrm{E}-10$ & COPG1 & $1.31 \mathrm{E}+00$ \\
\hline ENSBTAG00000013723 & $4.77 \mathrm{E}-10$ & $3.63 \mathrm{E}-10$ & MRPS25 & $1.31 \mathrm{E}+00$ \\
\hline ENSBTAG00000003325 & $3.64 \mathrm{E}-10$ & $2.76 \mathrm{E}-10$ & SNRK & $1.32 \mathrm{E}+00$ \\
\hline ENSBTAG00000019707 & $1.62 \mathrm{E}-10$ & $1.22 \mathrm{E}-10$ & GATA2 & $1.33 E+00$ \\
\hline ENSBTAG00000005433 & $3.84 \mathrm{E}-10$ & $2.91 \mathrm{E}-10$ & SSUH2 & $1.32 \mathrm{E}+00$ \\
\hline ENSBTAG00000011344 & $5.25 \mathrm{E}-10$ & 3.97E-10 & & $1.32 \mathrm{E}+00$ \\
\hline ENSBTAG00000000478 & $2.91 \mathrm{E}-10$ & $2.20 \mathrm{E}-10$ & LSMEM2 & $1.32 \mathrm{E}+00$ \\
\hline ENSBTAG00000030494 & $2.43 \mathrm{E}-10$ & $1.83 \mathrm{E}-10$ & & $1.33 \mathrm{E}+00$ \\
\hline ENSBTAG00000009159 & $1.98 \mathrm{E}-10$ & $1.48 \mathrm{E}-10$ & PLXNA1 & $1.34 \mathrm{E}+00$ \\
\hline ENSBTAG00000004279 & $1.61 \mathrm{E}-10$ & $1.21 \mathrm{E}-10$ & $\mathrm{RHOA}$ & $1.33 \mathrm{E}+00$ \\
\hline ENSBTAG00000015529 & $8.31 \mathrm{E}-10$ & $6.23 \mathrm{E}-10$ & $\mathrm{CHCHD} 4$ & $1.33 \mathrm{E}+00$ \\
\hline ENSBTAG00000014162 & $3.51 \mathrm{E}-10$ & 2.62E-10 & ACKR2 & $1.34 \mathrm{E}+00$ \\
\hline ENSBTAG00000011180 & $6.67 \mathrm{E}-10$ & 4.93E-10 & ACTR8 & $1.35 \mathrm{E}+00$ \\
\hline ENSBTAG00000016563 & $6.85 \mathrm{E}-11$ & 5.07E-11 & GOLGA4 & $1.35 \mathrm{E}+00$ \\
\hline ENSBTAG00000003592 & $3.44 \mathrm{E}-10$ & $2.54 \mathrm{E}-10$ & CNTN6 & $1.35 E+00$ \\
\hline ENSBTAG00000022622 & $1.82 \mathrm{E}-10$ & $1.33 \mathrm{E}-10$ & $\mathrm{CISH}$ & $1.37 E+00$ \\
\hline ENSBTAG00000014205 & $1.88 \mathrm{E}-10$ & $1.38 \mathrm{E}-10$ & PRKAR2A & $1.36 \mathrm{E}+00$ \\
\hline ENSBTAG00000016420 & $2.03 \mathrm{E}-10$ & $1.48 \mathrm{E}-10$ & CTNNB1 & $1.37 \mathrm{E}+00$ \\
\hline ENSBTAG00000010863 & $4.01 E-10$ & $2.91 \mathrm{E}-10$ & DCP1A & $1.38 \mathrm{E}+00$ \\
\hline ENSBTAG00000000484 & $1.12 \mathrm{E}-10$ & 8.03E-11 & HYAL2 & $1.39 \mathrm{E}+00$ \\
\hline ENSBTAG00000007966 & $1.55 \mathrm{E}-10$ & $1.12 \mathrm{E}-10$ & & $1.39 \mathrm{E}+00$ \\
\hline ENSBTAG00000030962 & $6.45 \mathrm{E}-10$ & 4.64E-10 & EEFSEC & $1.39 E+00$ \\
\hline ENSBTAG00000000387 & $2.71 \mathrm{E}-10$ & $1.95 \mathrm{E}-10$ & & $1.39 E+00$ \\
\hline ENSBTAG00000016920 & $1.14 \mathrm{E}-10$ & $8.20 \mathrm{E}-11$ & EOMES & $1.39 \mathrm{E}+00$ \\
\hline ENSBTAG00000013117 & $3.56 \mathrm{E}-10$ & $2.56 \mathrm{E}-10$ & CACNA2D3 & $1.39 \mathrm{E}+00$ \\
\hline ENSBTAG00000009155 & $5.04 \mathrm{E}-10$ & $3.59 \mathrm{E}-10$ & SCN5A & $1.40 \mathrm{E}+00$ \\
\hline ENSBTAG00000020455 & $3.89 \mathrm{E}-10$ & $2.78 \mathrm{E}-10$ & ITPR1 & $1.40 \mathrm{E}+00$ \\
\hline ENSBTAG00000015254 & $4.72 \mathrm{E}-10$ & $3.35 \mathrm{E}-10$ & GLB1 & $1.41 \mathrm{E}+00$ \\
\hline ENSBTAG00000023954 & $4.79 \mathrm{E}-10$ & $3.40 \mathrm{E}-10$ & & $1.41 E+00$ \\
\hline ENSBTAG00000026097 & $5.33 \mathrm{E}-10$ & $3.77 \mathrm{E}-10$ & LIMD1 & $1.41 E+00$ \\
\hline ENSBTAG00000008313 & 4.07E-10 & $2.88 \mathrm{E}-10$ & KLF15 & $1.41 E+00$ \\
\hline ENSBTAG00000011628 & $4.43 \mathrm{E}-10$ & $3.12 \mathrm{E}-10$ & EGFR & $1.42 \mathrm{E}+00$ \\
\hline ENSBTAG00000003845 & $2.25 \mathrm{E}-10$ & $1.58 \mathrm{E}-10$ & CSRNP1 & $1.42 \mathrm{E}+00$ \\
\hline ENSBTAG00000022699 & $3.97 \mathrm{E}-10$ & $2.79 \mathrm{E}-10$ & CAV3 & $1.42 \mathrm{E}+00$ \\
\hline ENSBTAG00000016758 & $2.21 \mathrm{E}-10$ & $1.55 \mathrm{E}-10$ & MLH1 & $1.43 E+00$ \\
\hline ENSBTAG00000007398 & $2.88 \mathrm{E}-10$ & $2.02 \mathrm{E}-10$ & MRPS24 & $1.43 E+00$ \\
\hline ENSBTAG00000018644 & $4.83 \mathrm{E}-10$ & $3.35 \mathrm{E}-10$ & PDZRN3 & $1.44 E+00$ \\
\hline ENSBTAG00000021164 & 2.65E-10 & $1.83 \mathrm{E}-10$ & SLMAP & $1.45 E+00$ \\
\hline ENSBTAG00000007315 & $4.80 \mathrm{E}-10$ & $3.31 \mathrm{E}-10$ & LARS2 & $1.45 \mathrm{E}+00$ \\
\hline ENSBTAG00000007969 & $2.12 \mathrm{E}-10$ & $1.45 \mathrm{E}-10$ & CIDEC & $1.46 \mathrm{E}+00$ \\
\hline ENSBTAG00000003745 & $2.40 \mathrm{E}-10$ & $1.63 \mathrm{E}-10$ & WDR48 & $1.47 \mathrm{E}+00$ \\
\hline ENSBTAG00000004232 & 4.05E-10 & $2.75 \mathrm{E}-10$ & TMIE & $1.47 \mathrm{E}+00$ \\
\hline ENSBTAG00000009430 & $2.70 \mathrm{E}-10$ & $1.83 \mathrm{E}-10$ & MYRIP & $1.48 \mathrm{E}+00$ \\
\hline ENSBTAG00000001710 & 8.07E-10 & $5.46 \mathrm{E}-10$ & СMTM8 & $1.48 \mathrm{E}+00$ \\
\hline ENSBTAG00000020802 & $5.51 \mathrm{E}-10$ & $3.72 \mathrm{E}-10$ & & $1.48 \mathrm{E}+00$ \\
\hline ENSBTAG00000013407 & $6.61 E-10$ & 4.42E-10 & PLXNB1 & $1.49 \mathrm{E}+00$ \\
\hline ENSBTAG00000007624 & $2.13 \mathrm{E}-10$ & $1.42 \mathrm{E}-10$ & FRMD4B & $1.50 \mathrm{E}+00$ \\
\hline ENSBTAG00000015698 & $5.31 \mathrm{E}-10$ & $3.53 \mathrm{E}-10$ & FYCO1 & $1.51 E+00$ \\
\hline ENSBTAG00000037972 & $2.06 \mathrm{E}-10$ & 1.37E-10 & SLC25A26 & $1.50 E+00$ \\
\hline ENSBTAG00000008463 & $2.82 \mathrm{E}-10$ & $1.86 \mathrm{E}-10$ & SLC22A14 & $1.52 \mathrm{E}+00$ \\
\hline ENSBTAG00000006570 & $1.57 \mathrm{E}-10$ & $1.02 \mathrm{E}-10$ & SEC22C & $1.54 \mathrm{E}+00$ \\
\hline ENSBTAG00000009874 & $4.08 \mathrm{E}-10$ & $2.64 \mathrm{E}-10$ & ZXDC & $1.55 \mathrm{E}+00$ \\
\hline ENSBTAG00000014380 & $4.02 \mathrm{E}-10$ & $2.56 \mathrm{E}-10$ & MCM2 & $1.57 E+00$ \\
\hline ENSBTAG00000021695 & $1.31 \mathrm{E}-10$ & $8.24 \mathrm{E}-11$ & GLYCTK & $1.59 \mathrm{E}+00$ \\
\hline ENSBTAG00000003242 & $5.94 \mathrm{E}-10$ & $3.75 \mathrm{E}-10$ & ACAD9 & $1.58 \mathrm{E}+00$ \\
\hline ENSBTAG00000007961 & $1.70 \mathrm{E}-10$ & 1.07E-10 & TADA3 & $1.59 E+00$ \\
\hline ENSBTAG00000021390 & $1.85 \mathrm{E}-10$ & $1.16 \mathrm{E}-10$ & TMEM89 & $1.59 E+00$ \\
\hline ENSBTAG00000007020 & $1.36 \mathrm{E}-10$ & $8.55 \mathrm{E}-11$ & ZNF502 & $1.59 \mathrm{E}+00$ \\
\hline ENSBTAG00000013027 & $8.76 \mathrm{E}-11$ & $5.52 \mathrm{E}-11$ & CCK & $1.59 \mathrm{E}+00$ \\
\hline
\end{tabular}




\begin{tabular}{|c|c|c|c|c|}
\hline ENSBTAG00000018628 & $1.51 \mathrm{E}-10$ & $9.49 \mathrm{E}-11$ & RPL29 & $1.59 \mathrm{E}+00$ \\
\hline ENSBTAG00000008394 & $1.13 \mathrm{E}-10$ & $7.13 \mathrm{E}-11$ & MYL3 & $1.59 E+00$ \\
\hline ENSBTAG00000047965 & $1.14 \mathrm{E}-10$ & $7.20 \mathrm{E}-11$ & & $1.59 E+00$ \\
\hline ENSBTAG00000000483 & $1.25 \mathrm{E}-10$ & 7.87E-11 & & $1.59 \mathrm{E}+00$ \\
\hline ENSBTAG00000012159 & $9.39 \mathrm{E}-11$ & $5.91 \mathrm{E}-11$ & CNBP & $1.59 \mathrm{E}+00$ \\
\hline ENSBTAG00000032954 & 4.19E-10 & $2.64 \mathrm{E}-10$ & & $1.59 \mathrm{E}+00$ \\
\hline ENSBTAG00000046634 & $2.02 \mathrm{E}-10$ & $1.27 \mathrm{E}-10$ & & $1.59 \mathrm{E}+00$ \\
\hline ENSBTAG00000000459 & $2.05 \mathrm{E}-10$ & $1.29 \mathrm{E}-10$ & POMGNT2 & $1.59 \mathrm{E}+00$ \\
\hline ENSBTAG00000021912 & $1.51 \mathrm{E}-10$ & $9.47 \mathrm{E}-11$ & DUSP7 & $1.59 \mathrm{E}+00$ \\
\hline ENSBTAG00000013586 & $1.47 \mathrm{E}-10$ & $9.27 E-11$ & CCR2 & $1.59 \mathrm{E}+00$ \\
\hline ENSBTAG00000032279 & 3.27E-10 & $2.05 E-10$ & IQCF2 & $1.59 \mathrm{E}+00$ \\
\hline ENSBTAG00000006752 & $3.47 \mathrm{E}-10$ & $2.18 \mathrm{E}-10$ & PFKFB4 & $1.59 \mathrm{E}+00$ \\
\hline ENSBTAG00000014549 & $1.30 \mathrm{E}-10$ & $8.18 \mathrm{E}-11$ & GNL3 & $1.59 \mathrm{E}+00$ \\
\hline ENSBTAG00000000981 & $1.36 \mathrm{E}-10$ & $8.58 \mathrm{E}-11$ & KLHDC8B & $1.59 \mathrm{E}+00$ \\
\hline ENSBTAG00000001812 & $5.60 \mathrm{E}-10$ & $3.50 \mathrm{E}-10$ & H1FOO & $1.60 \mathrm{E}+00$ \\
\hline ENSBTAG00000015839 & $3.42 \mathrm{E}-10$ & $2.13 \mathrm{E}-10$ & MAP4 & $1.60 E+00$ \\
\hline ENSBTAG00000006328 & $1.73 \mathrm{E}-10$ & $1.08 \mathrm{E}-10$ & RBM6 & $1.60 \mathrm{E}+00$ \\
\hline ENSBTAG00000009586 & $2.09 \mathrm{E}-10$ & $1.29 \mathrm{E}-10$ & CDC25A & $1.62 \mathrm{E}+00$ \\
\hline ENSBTAG00000000265 & $3.18 \mathrm{E}-10$ & $1.95 \mathrm{E}-10$ & SYNPR & $1.63 E+00$ \\
\hline ENSBTAG00000031340 & $3.68 \mathrm{E}-10$ & $2.25 \mathrm{E}-10$ & SLC6A20 & $1.64 \mathrm{E}+00$ \\
\hline ENSBTAG00000046512 & $2.58 \mathrm{E}-10$ & $1.57 \mathrm{E}-10$ & XIRP1 & $1.64 \mathrm{E}+00$ \\
\hline ENSBTAG00000018433 & $1.44 \mathrm{E}-10$ & $8.72 \mathrm{E}-11$ & DENND6A & $1.65 E+00$ \\
\hline ENSBTAG00000024991 & $2.99 \mathrm{E}-10$ & $1.80 \mathrm{E}-10$ & MKRN2OS & $1.66 \mathrm{E}+00$ \\
\hline ENSBTAG00000000191 & $1.61 \mathrm{E}-10$ & $9.71 \mathrm{E}-11$ & SLC25A20 & $1.66 \mathrm{E}+00$ \\
\hline ENSBTAG00000021452 & $3.11 \mathrm{E}-10$ & $1.87 \mathrm{E}-10$ & TRANK1 & $1.66 \mathrm{E}+00$ \\
\hline ENSBTAG00000018283 & $2.34 \mathrm{E}-10$ & $1.41 \mathrm{E}-10$ & ACAA1 & $1.66 \mathrm{E}+00$ \\
\hline ENSBTAG00000037980 & $8.36 \mathrm{E}-10$ & $5.02 \mathrm{E}-10$ & TSEN2 & $1.66 \mathrm{E}+00$ \\
\hline ENSBTAG00000017174 & $6.00 \mathrm{E}-10$ & $3.59 \mathrm{E}-10$ & SCN11A & $1.67 E+00$ \\
\hline ENSBTAG00000046924 & $1.69 \mathrm{E}-10$ & $1.01 \mathrm{E}-10$ & SEMA3G & $1.67 \mathrm{E}+00$ \\
\hline ENSBTAG00000018238 & 3.35E-10 & $2.00 \mathrm{E}-10$ & ABTB1 & $1.68 \mathrm{E}+00$ \\
\hline ENSBTAG00000005327 & $4.91 \mathrm{E}-10$ & $2.93 E-10$ & SUSD5 & $1.68 \mathrm{E}+00$ \\
\hline ENSBTAG00000004685 & $2.38 \mathrm{E}-10$ & $1.42 \mathrm{E}-10$ & SRGAP3 & $1.67 \mathrm{E}+00$ \\
\hline ENSBTAG00000039310 & $1.92 \mathrm{E}-10$ & $1.14 \mathrm{E}-10$ & SLC22A13 & $1.68 \mathrm{E}+00$ \\
\hline ENSBTAG00000031454 & $3.63 \mathrm{E}-10$ & $2.16 \mathrm{E}-10$ & PRSS42 & $1.68 \mathrm{E}+00$ \\
\hline ENSBTAG00000023207 & $6.25 \mathrm{E}-10$ & $3.72 \mathrm{E}-10$ & FAM3D & $1.68 \mathrm{E}+00$ \\
\hline ENSBTAG00000031837 & $2.89 \mathrm{E}-10$ & $1.71 \mathrm{E}-10$ & IMPDH2 & $1.69 \mathrm{E}+00$ \\
\hline ENSBTAG00000021853 & $2.38 \mathrm{E}-10$ & $1.39 \mathrm{E}-10$ & IP6K2 & $1.71 E+00$ \\
\hline ENSBTAG00000045817 & $1.49 \mathrm{E}-10$ & $8.64 \mathrm{E}-11$ & BAP1 & $1.72 \mathrm{E}+00$ \\
\hline ENSBTAG00000010165 & $1.26 \mathrm{E}-10$ & $7.33 \mathrm{E}-11$ & C3orf18 & $1.72 \mathrm{E}+00$ \\
\hline ENSBTAG00000022751 & $7.59 \mathrm{E}-10$ & $4.41 \mathrm{E}-10$ & & $1.72 \mathrm{E}+00$ \\
\hline ENSBTAG00000020221 & $1.85 \mathrm{E}-10$ & $1.07 E-10$ & WNT5A & $1.72 \mathrm{E}+00$ \\
\hline ENSBTAG00000002774 & $2.92 \mathrm{E}-10$ & $1.68 \mathrm{E}-10$ & PTPN23 & $1.74 \mathrm{E}+00$ \\
\hline ENSBTAG00000033669 & $1.42 \mathrm{E}-10$ & $8.04 \mathrm{E}-11$ & IL17RC & $1.76 \mathrm{E}+00$ \\
\hline ENSBTAG00000011287 & $2.09 \mathrm{E}-10$ & $1.18 \mathrm{E}-10$ & ATXN7 & $1.77 \mathrm{E}+00$ \\
\hline ENSBTAG00000003882 & $2.17 \mathrm{E}-10$ & $1.22 \mathrm{E}-10$ & EMC3 & $1.78 \mathrm{E}+00$ \\
\hline ENSBTAG00000048044 & 4.37E-10 & $2.44 \mathrm{E}-10$ & IQCF3 & $1.79 \mathrm{E}+00$ \\
\hline ENSBTAG00000031519 & $1.81 \mathrm{E}-10$ & $1.01 \mathrm{E}-10$ & & $1.79 E+00$ \\
\hline ENSBTAG00000007780 & $1.39 \mathrm{E}-10$ & 7.80E-11 & CAMK1 & $1.79 E+00$ \\
\hline ENSBTAG00000021846 & 2.19E-10 & $1.22 \mathrm{E}-10$ & CELSR3 & $1.80 \mathrm{E}+00$ \\
\hline ENSBTAG00000008013 & $4.52 \mathrm{E}-10$ & $2.49 \mathrm{E}-10$ & LRRC2 & $1.81 \mathrm{E}+00$ \\
\hline ENSBTAG00000047509 & $5.94 \mathrm{E}-10$ & $3.26 \mathrm{E}-10$ & KIAA1257 & $1.82 \mathrm{E}+00$ \\
\hline ENSBTAG00000003952 & $5.01 \mathrm{E}-10$ & $2.75 \mathrm{E}-10$ & FBXL2 & $1.82 \mathrm{E}+00$ \\
\hline ENSBTAG00000016566 & $3.94 \mathrm{E}-10$ & $2.14 \mathrm{E}-10$ & ITGA9 & $1.84 \mathrm{E}+00$ \\
\hline ENSBTAG00000018601 & $1.18 \mathrm{E}-10$ & $6.37 \mathrm{E}-11$ & LYZL4 & $1.85 \mathrm{E}+00$ \\
\hline ENSBTAG00000016098 & $1.52 \mathrm{E}-10$ & $8.20 \mathrm{E}-11$ & BRK1 & $1.85 \mathrm{E}+00$ \\
\hline ENSBTAG00000007843 & $8.05 E-11$ & 4.34E-11 & ITIH1 & $1.85 E+00$ \\
\hline ENSBTAG00000021724 & $3.18 \mathrm{E}-10$ & $1.72 \mathrm{E}-10$ & PDHB & $1.85 \mathrm{E}+00$ \\
\hline ENSBTAG00000010833 & $1.75 \mathrm{E}-10$ & $9.43 E-11$ & IL17RE & $1.85 \mathrm{E}+00$ \\
\hline ENSBTAG00000007732 & $4.21 \mathrm{E}-10$ & $2.26 \mathrm{E}-10$ & ARPP21 & $1.86 \mathrm{E}+00$ \\
\hline ENSBTAG00000010027 & $3.05 \mathrm{E}-10$ & $1.63 \mathrm{E}-10$ & $\mathrm{CHDH}$ & $1.87 E+00$ \\
\hline ENSBTAG00000007438 & $2.88 \mathrm{E}-10$ & $1.54 \mathrm{E}-10$ & STT3B & $1.87 \mathrm{E}+00$ \\
\hline ENSBTAG00000011081 & $1.56 \mathrm{E}-10$ & $8.29 \mathrm{E}-11$ & KBTBD8 & $1.89 \mathrm{E}+00$ \\
\hline ENSBTAG00000006679 & $2.33 \mathrm{E}-10$ & $1.23 \mathrm{E}-10$ & MITF & $1.89 \mathrm{E}+00$ \\
\hline ENSBTAG00000017426 & $7.15 \mathrm{E}-10$ & $3.78 \mathrm{E}-10$ & PDCD6IP & $1.89 E+00$ \\
\hline ENSBTAG00000011551 & $1.20 \mathrm{E}-10$ & $6.36 \mathrm{E}-11$ & CPNE9 & $1.89 \mathrm{E}+00$ \\
\hline ENSBTAG00000012902 & $3.00 \mathrm{E}-10$ & $1.56 \mathrm{E}-10$ & ARL8B & $1.92 \mathrm{E}+00$ \\
\hline ENSBTAG00000022635 & $2.36 \mathrm{E}-10$ & $1.23 \mathrm{E}-10$ & LAMB2 & $1.92 \mathrm{E}+00$ \\
\hline ENSBTAG00000039194 & 1.14E-09 & $5.96 \mathrm{E}-10$ & C3orf22 & $1.91 E+00$ \\
\hline ENSBTAG00000014786 & $1.46 \mathrm{E}-10$ & 7.40E-11 & PBRM1 & $1.97 \mathrm{E}+00$ \\
\hline ENSBTAG00000007362 & $8.24 \mathrm{E}-10$ & 4.15E-10 & XPC & $1.98 \mathrm{E}+00$ \\
\hline
\end{tabular}




\begin{tabular}{|c|c|c|c|c|}
\hline ENSBTAG000000005019 & $2.38 \mathrm{E}-10$ & $1.20 \mathrm{E}-10$ & PRSS50 & $1.98 \mathrm{E}+00$ \\
\hline ENSBTAG00000018057 & $2.13 \mathrm{E}-10$ & $1.07 \mathrm{E}-10$ & & $1.99 \mathrm{E}+00$ \\
\hline ENSBTAG000000002795 & $2.38 \mathrm{E}-10$ & $1.19 \mathrm{E}-10$ & NKTR & $2.00 E+00$ \\
\hline ENSBTAG000000016726 & $5.85 \mathrm{E}-10$ & $2.92 \mathrm{E}-10$ & KIF15 & $2.00 E+00$ \\
\hline ENSBTAG000000011484 & $2.49 \mathrm{E}-10$ & $1.24 \mathrm{E}-10$ & & $2.01 E+00$ \\
\hline ENSBTAG000000002948 & $2.41 \mathrm{E}-10$ & $1.19 \mathrm{E}-10$ & SETD2 & $2.02 \mathrm{E}+00$ \\
\hline ENSBTAG000000011580 & $4.93 \mathrm{E}-10$ & $2.43 \mathrm{E}-10$ & DAG1 & $2.03 E+00$ \\
\hline ENSBTAG00000001471 & $1.89 \mathrm{E}-10$ & $9.32 \mathrm{E}-11$ & POC1A & $2.02 \mathrm{E}+00$ \\
\hline ENSBTAG000000034883 & $3.82 \mathrm{E}-10$ & $1.88 \mathrm{E}-10$ & CCR4 & $2.03 E+00$ \\
\hline ENSBTAG000000009765 & $1.95 \mathrm{E}-10$ & $9.49 \mathrm{E}-11$ & MTMR14 & $2.05 E+00$ \\
\hline ENSBTAG000000001484 & $3.76 \mathrm{E}-10$ & $1.83 \mathrm{E}-10$ & LZTFL1 & $2.05 E+00$ \\
\hline ENSBTAG000000012482 & $5.62 \mathrm{E}-10$ & $2.72 \mathrm{E}-10$ & $\mathrm{CDCP} 1$ & $2.07 E+00$ \\
\hline ENSBTAG00000010999 & $3.73 \mathrm{E}-10$ & $1.80 \mathrm{E}-10$ & IL17RD & $2.07 E+00$ \\
\hline ENSBTAG000000017825 & $6.51 \mathrm{E}-10$ & $3.14 \mathrm{E}-10$ & SEC13 & $2.07 E+00$ \\
\hline ENSBTAG000000002960 & $1.86 \mathrm{E}-10$ & $8.96 \mathrm{E}-11$ & & $2.08 \mathrm{E}+00$ \\
\hline ENSBTAG000000021850 & $2.76 \mathrm{E}-10$ & $1.32 \mathrm{E}-10$ & NCKIPSD & $2.09 E+00$ \\
\hline ENSBTAG000000003665 & $8.82 \mathrm{E}-10$ & $4.19 \mathrm{E}-10$ & NUP210 & $2.10 \mathrm{E}+00$ \\
\hline ENSBTAG000000013026 & $3.06 \mathrm{E}-10$ & $1.45 \mathrm{E}-10$ & SNTN & $2.11 E+00$ \\
\hline ENSBTAG000000018331 & $2.15 \mathrm{E}-10$ & $1.01 \mathrm{E}-10$ & CLEC3B & $2.13 E+00$ \\
\hline ENSBTAG000000001310 & $3.16 \mathrm{E}-10$ & 1.47E-10 & $\mathrm{RHO}$ & $2.15 E+00$ \\
\hline ENSBTAG000000032253 & $2.07 \mathrm{E}-10$ & $9.52 \mathrm{E}-11$ & RAD54L2 & $2.18 \mathrm{E}+00$ \\
\hline ENSBTAG000000009757 & $2.26 \mathrm{E}-10$ & $1.04 \mathrm{E}-10$ & RPSA & $2.18 \mathrm{E}+00$ \\
\hline ENSBTAG000000009194 & $6.34 \mathrm{E}-10$ & $2.89 \mathrm{E}-10$ & OSBPL10 & $2.19 E+00$ \\
\hline ENSBTAG000000007360 & $4.75 \mathrm{E}-10$ & 2.17E-10 & TMEM43 & $2.19 E+00$ \\
\hline ENSBTAG00000009541 & 3.37E-10 & $1.51 \mathrm{E}-10$ & SUCLG2 & $2.23 \mathrm{E}+00$ \\
\hline ENSBTAG000000006563 & $1.90 \mathrm{E}-10$ & $8.45 E-11$ & KLHL40 & $2.25 E+00$ \\
\hline ENSBTAG000000019330 & $3.03 E-10$ & $1.35 \mathrm{E}-10$ & PROK2 & $2.24 \mathrm{E}+00$ \\
\hline ENSBTAG000000020373 & $6.11 \mathrm{E}-10$ & 2.69E-10 & C3orf67 & $2.27 \mathrm{E}+00$ \\
\hline ENSBTAG000000008893 & $2.32 \mathrm{E}-10$ & $1.02 \mathrm{E}-10$ & ANO10 & $2.27 \mathrm{E}+00$ \\
\hline ENSBTAG000000022004 & $4.56 \mathrm{E}-10$ & $2.01 \mathrm{E}-10$ & FLNB & $2.27 E+00$ \\
\hline ENSBTAG00000012073 & $5.39 \mathrm{E}-10$ & $2.36 \mathrm{E}-10$ & VOPP1 & $2.28 \mathrm{E}+00$ \\
\hline ENSBTAG000000010360 & $3.12 \mathrm{E}-10$ & $1.36 \mathrm{E}-10$ & LRIG1 & $2.29 \mathrm{E}+00$ \\
\hline ENSBTAG00000002309 & $2.16 \mathrm{E}-10$ & $9.33 \mathrm{E}-11$ & CSPG5 & $2.31 E+00$ \\
\hline ENSBTAG000000007846 & $3.47 \mathrm{E}-10$ & $1.48 \mathrm{E}-10$ & ITIH3 & $2.35 E+00$ \\
\hline ENSBTAG00000018227 & $4.51 \mathrm{E}-10$ & $1.90 \mathrm{E}-10$ & SLC4A7 & $2.37 \mathrm{E}+00$ \\
\hline ENSBTAG00000007777 & $1.31 \mathrm{E}-10$ & $5.52 \mathrm{E}-11$ & OGG1 & $2.38 \mathrm{E}+00$ \\
\hline ENSBTAG00000001439 & $1.46 \mathrm{E}-10$ & $6.14 \mathrm{E}-11$ & HESX1 & $2.38 \mathrm{E}+00$ \\
\hline ENSBTAG00000015563 & $1.33 \mathrm{E}-10$ & $5.56 \mathrm{E}-11$ & PDE12 & $2.38 \mathrm{E}+00$ \\
\hline ENSBTAG00000015833 & $1.15 \mathrm{E}-10$ & $4.84 \mathrm{E}-11$ & DHX30 & $2.38 \mathrm{E}+00$ \\
\hline ENSBTAG00000032531 & $2.15 \mathrm{E}-10$ & $9.00 \mathrm{E}-11$ & & $2.38 \mathrm{E}+00$ \\
\hline ENSBTAG00000020645 & $2.44 \mathrm{E}-10$ & $1.02 \mathrm{E}-10$ & GNAI2 & $2.39 E+00$ \\
\hline ENSBTAG00000023918 & $4.42 \mathrm{E}-10$ & $1.85 \mathrm{E}-10$ & & $2.39 E+00$ \\
\hline ENSBTAG00000005191 & $9.00 \mathrm{E}-10$ & $3.63 \mathrm{E}-10$ & & $2.48 \mathrm{E}+00$ \\
\hline ENSBTAG00000018862 & $2.26 \mathrm{E}-10$ & 8.97E-11 & EIF4E3 & $2.52 E+00$ \\
\hline ENSBTAG00000003244 & $5.34 \mathrm{E}-10$ & $2.11 \mathrm{E}-10$ & & $2.53 \mathrm{E}+00$ \\
\hline ENSBTAG00000011795 & $6.86 \mathrm{E}-10$ & $2.68 \mathrm{E}-10$ & TXNRD3 & $2.56 \mathrm{E}+00$ \\
\hline ENSBTAG00000019526 & $5.21 \mathrm{E}-10$ & $2.01 \mathrm{E}-10$ & CMTM6 & $2.59 E+00$ \\
\hline ENSBTAG00000019402 & $9.10 \mathrm{E}-10$ & $3.48 \mathrm{E}-10$ & CFAP100 & $2.61 E+00$ \\
\hline ENSBTAG00000030915 & $6.05 \mathrm{E}-10$ & $2.28 \mathrm{E}-10$ & & $2.65 E+00$ \\
\hline ENSBTAG00000015283 & $8.11 E-10$ & $3.02 \mathrm{E}-10$ & & $2.68 \mathrm{E}+00$ \\
\hline ENSBTAG00000000273 & 7.07E-10 & $2.58 \mathrm{E}-10$ & IL5RA & $2.74 \mathrm{E}+00$ \\
\hline ENSBTAG00000018105 & $4.75 \mathrm{E}-10$ & $1.73 \mathrm{E}-10$ & ACVR2B & $2.74 E+00$ \\
\hline ENSBTAG00000039855 & $4.06 \mathrm{E}-10$ & $1.47 \mathrm{E}-10$ & SUMF1 & $2.76 \mathrm{E}+00$ \\
\hline ENSBTAG00000016948 & $1.34 \mathrm{E}-10$ & $4.76 \mathrm{E}-11$ & PTH1R & $2.82 \mathrm{E}+00$ \\
\hline ENSBTAG00000014470 & $6.55 \mathrm{E}-10$ & $2.27 \mathrm{E}-10$ & ALDH1L1 & $2.89 \mathrm{E}+00$ \\
\hline ENSBTAG00000003997 & $3.61 \mathrm{E}-10$ & $1.25 \mathrm{E}-10$ & UBE2D4 & $2.89 E+00$ \\
\hline ENSBTAG00000011702 & $1.04 \mathrm{E}-10$ & $3.35 \mathrm{E}-11$ & ENTPD3 & $3.09 \mathrm{E}+00$ \\
\hline ENSBTAG00000033422 & $1.58 \mathrm{E}-10$ & 4.96E-11 & HHATL & $3.17 E+00$ \\
\hline ENSBTAG00000020973 & $7.42 \mathrm{E}-10$ & $2.34 \mathrm{E}-10$ & RFT1 & $3.17 E+00$ \\
\hline ENSBTAG00000018020 & $2.62 \mathrm{E}-10$ & $8.23 \mathrm{E}-11$ & GNAT1 & $3.18 \mathrm{E}+00$ \\
\hline ENSBTAG00000024852 & $4.37 \mathrm{E}-10$ & $1.37 \mathrm{E}-10$ & & $3.19 E+00$ \\
\hline ENSBTAG00000007968 & $2.06 \mathrm{E}-10$ & $6.47 \mathrm{E}-11$ & RPUSD3 & $3.18 \mathrm{E}+00$ \\
\hline ENSBTAG00000019081 & $2.33 \mathrm{E}-10$ & $7.15 E-11$ & COL7A1 & $3.25 E+00$ \\
\hline ENSBTAG00000019305 & $7.20 \mathrm{E}-10$ & $2.15 E-10$ & EXOG & $3.35 E+00$ \\
\hline ENSBTAG00000000670 & $1.77 \mathrm{E}-10$ & $5.23 \mathrm{E}-11$ & RRP9 & $3.37 \mathrm{E}+00$ \\
\hline ENSBTAG00000018294 & $2.71 \mathrm{E}-10$ & 7.97E-11 & DNASE1L3 & $3.39 E+00$ \\
\hline ENSBTAG00000012904 & $4.66 \mathrm{E}-10$ & $1.36 \mathrm{E}-10$ & EDEM1 & $3.42 E+00$ \\
\hline ENSBTAG00000017365 & $1.89 \mathrm{E}-10$ & $5.48 \mathrm{E}-11$ & ZBTB47 & $3.44 E+00$ \\
\hline ENSBTAG00000010581 & 4.13E-10 & $1.18 \mathrm{E}-10$ & MAGI1 & $3.50 E+00$ \\
\hline ENSBTAG00000003758 & $5.94 \mathrm{E}-10$ & $1.58 \mathrm{E}-10$ & TKT & $3.76 \mathrm{E}+00$ \\
\hline
\end{tabular}




\begin{tabular}{|c|c|c|c|c|}
\hline ENSBTAG00000038616 & 3.12E-10 & $8.12 \mathrm{E}-11$ & & $3.83 E+00$ \\
\hline ENSBTAG00000034005 & $1.95 \mathrm{E}-10$ & $4.91 \mathrm{E}-11$ & ZNF35 & $3.96 \mathrm{E}+00$ \\
\hline ENSBTAG00000010836 & $1.32 \mathrm{E}-10$ & $3.31 \mathrm{E}-11$ & PRRT3 & $3.97 E+00$ \\
\hline ENSBTAG00000020963 & $6.70 \mathrm{E}-11$ & $1.69 \mathrm{E}-11$ & RASSF1 & $3.95 E+00$ \\
\hline ENSBTAG00000010832 & $1.46 \mathrm{E}-10$ & $3.68 \mathrm{E}-11$ & JAGN1 & $3.97 E+00$ \\
\hline ENSBTAG00000039879 & $7.22 \mathrm{E}-10$ & $1.81 \mathrm{E}-10$ & & $3.99 \mathrm{E}+00$ \\
\hline ENSBTAG00000008774 & $2.63 \mathrm{E}-10$ & $6.47 \mathrm{E}-11$ & UROC1 & $4.07 E+00$ \\
\hline ENSBTAG00000017812 & $3.86 \mathrm{E}-10$ & $9.32 \mathrm{E}-11$ & $\mathrm{ALS} 2 \mathrm{CL}$ & $4.13 E+00$ \\
\hline ENSBTAG00000011345 & $4.04 \mathrm{E}-10$ & $9.76 \mathrm{E}-11$ & SFMBT1 & $4.13 E+00$ \\
\hline ENSBTAG00000045748 & 4.76E-10 & $1.15 \mathrm{E}-10$ & & $4.14 E+00$ \\
\hline ENSBTAG00000014700 & 7.14E-10 & $1.71 \mathrm{E}-10$ & DYNC1LI1 & $4.18 \mathrm{E}+00$ \\
\hline ENSBTAG00000000671 & $4.85 \mathrm{E}-10$ & $1.14 \mathrm{E}-10$ & PARP3 & $4.26 \mathrm{E}+00$ \\
\hline ENSBTAG00000004834 & $6.94 \mathrm{E}-10$ & $1.61 \mathrm{E}-10$ & CNOT10 & $4.31 \mathrm{E}+00$ \\
\hline ENSBTAG00000003968 & $2.71 \mathrm{E}-10$ & $6.30 \mathrm{E}-11$ & UBP1 & $4.30 \mathrm{E}+00$ \\
\hline ENSBTAG00000002962 & $1.69 \mathrm{E}-10$ & 3.87E-11 & & $4.36 \mathrm{E}+00$ \\
\hline ENSBTAG00000015273 & $5.01 \mathrm{E}-10$ & $1.11 \mathrm{E}-10$ & CAND2 & $4.51 E+00$ \\
\hline ENSBTAG00000007850 & $3.83 \mathrm{E}-10$ & $8.26 \mathrm{E}-11$ & ITIH4 & $4.64 E+00$ \\
\hline ENSBTAG00000031355 & $4.38 \mathrm{E}-10$ & $9.43 \mathrm{E}-11$ & & $4.64 \mathrm{E}+00$ \\
\hline ENSBTAG00000001176 & $2.29 \mathrm{E}-10$ & $4.80 \mathrm{E}-11$ & LRRN1 & $4.76 \mathrm{E}+00$ \\
\hline ENSBTAG00000031594 & $9.08 \mathrm{E}-10$ & $1.89 \mathrm{E}-10$ & & $4.80 \mathrm{E}+00$ \\
\hline ENSBTAG00000009826 & $6.63 \mathrm{E}-10$ & $1.38 \mathrm{E}-10$ & GPD1L & $4.80 \mathrm{E}+00$ \\
\hline ENSBTAG00000012963 & $1.03 \mathrm{E}-09$ & $2.12 \mathrm{E}-10$ & VILL & $4.83 E+00$ \\
\hline ENSBTAG00000022689 & $1.93 \mathrm{E}-10$ & $3.95 \mathrm{E}-11$ & & $4.88 \mathrm{E}+00$ \\
\hline ENSBTAG00000018935 & $6.08 \mathrm{E}-10$ & $1.24 \mathrm{E}-10$ & & $4.90 \mathrm{E}+00$ \\
\hline ENSBTAG00000009774 & $3.06 \mathrm{E}-10$ & $5.82 \mathrm{E}-11$ & & $5.25 E+00$ \\
\hline ENSBTAG00000022744 & $5.84 \mathrm{E}-10$ & $1.09 \mathrm{E}-10$ & TRIM71 & $5.35 \mathrm{E}+00$ \\
\hline ENSBTAG00000012328 & $4.96 \mathrm{E}-10$ & $9.23 \mathrm{E}-11$ & GHRL & $5.37 \mathrm{E}+00$ \\
\hline ENSBTAG00000018329 & $3.16 \mathrm{E}-10$ & $5.73 \mathrm{E}-11$ & EXOSC7 & $5.51 \mathrm{E}+00$ \\
\hline ENSBTAG00000011311 & $4.43 \mathrm{E}-10$ & $7.66 \mathrm{E}-11$ & CCDC12 & $5.78 \mathrm{E}+00$ \\
\hline ENSBTAG00000011278 & $7.35 E-10$ & $1.13 \mathrm{E}-10$ & $X Y L B$ & $6.50 \mathrm{E}+00$ \\
\hline ENSBTAG00000003022 & 3.77E-10 & $5.26 \mathrm{E}-11$ & & $7.15 E+00$ \\
\hline ENSBTAG00000002038 & $2.16 \mathrm{E}-10$ & 3.02E-11 & RPL14 & $7.14 E+00$ \\
\hline ENSBTAG00000015493 & $5.92 \mathrm{E}-10$ & 8.06E-11 & & $7.33 E+00$ \\
\hline ENSBTAG00000031599 & $7.55 E-10$ & $9.45 \mathrm{E}-11$ & & $7.98 \mathrm{E}+00$ \\
\hline ENSBTAG00000046911 & $3.57 \mathrm{E}-10$ & $4.31 \mathrm{E}-11$ & SCN10A & $8.27 E+00$ \\
\hline ENSBTAG00000015782 & $3.00 \mathrm{E}-10$ & $3.51 E-11$ & SCAP & $8.53 E+00$ \\
\hline ENSBTAG00000009760 & $1.95 \mathrm{E}-10$ & $1.80 \mathrm{E}-11$ & & $1.08 \mathrm{E}+01$ \\
\hline ENSBTAG00000022721 & 4.00E-10 & $3.44 \mathrm{E}-11$ & SLC25A38 & $1.16 \mathrm{E}+01$ \\
\hline ENSBTAG00000011478 & $4.23 \mathrm{E}-10$ & 3.37E-11 & ELP6 & $1.25 \mathrm{E}+01$ \\
\hline ENSBTAG00000019428 & $5.85 \mathrm{E}-10$ & $3.41 \mathrm{E}-11$ & & $1.71 E+01$ \\
\hline ENSBTAG00000020077 & $1.02 \mathrm{E}-09$ & $5.33 \mathrm{E}-11$ & & $1.92 \mathrm{E}+01$ \\
\hline ENSBTAG00000021119 & 7.83E-10 & $2.23 \mathrm{E}-11$ & & $3.49 \mathrm{E}+01$ \\
\hline ENSBTAG00000019106 & $1.66 \mathrm{E}-10$ & 0 & EIF1B & $1.66 \mathrm{E}+03$ \\
\hline ENSBTAG00000046330 & 0 & 0 & & $1.00 \mathrm{E}+00$ \\
\hline ENSBTAG00000008534 & $2.30 \mathrm{E}-10$ & 0 & & $2.30 \mathrm{E}+03$ \\
\hline ENSBTAG00000000805 & $2.74 \mathrm{E}-10$ & 0 & IQCF1 & $2.74 E+03$ \\
\hline ENSBTAG00000037410 & $2.05 E-11$ & 0 & & $2.06 \mathrm{E}+02$ \\
\hline ENSBTAG00000012322 & $4.66 \mathrm{E}-11$ & 0 & FAM212A & $4.67 E+02$ \\
\hline ENSBTAG00000018918 & 0 & 0 & NDUFAF3 & $1.00 \mathrm{E}+00$ \\
\hline ENSBTAG00000016153 & $5.68 \mathrm{E}-10$ & 0 & & $5.68 \mathrm{E}+03$ \\
\hline ENSBTAG00000020076 & $3.78 \mathrm{E}-10$ & 0 & & $3.78 \mathrm{E}+03$ \\
\hline ENSBTAG00000017173 & $1.85 \mathrm{E}-10$ & 0 & & $1.85 E+03$ \\
\hline ENSBTAG00000025595 & 0 & 0 & & $1.00 \mathrm{E}+00$ \\
\hline ENSBTAG00000034496 & 0 & 0 & SHFM1 & $1.00 \mathrm{E}+00$ \\
\hline ENSBTAG00000047851 & 0 & 0 & & $1.00 \mathrm{E}+00$ \\
\hline ENSBTAG00000011633 & $2.46 \mathrm{E}-10$ & 0 & CHCHD5 & $2.46 \mathrm{E}+03$ \\
\hline ENSBTAG00000045942 & 0 & 0 & IQCF6 & $1.00 \mathrm{E}+00$ \\
\hline ENSBTAG00000045547 & $5.33 \mathrm{E}-11$ & 0 & AMIGO3 & $5.34 E+02$ \\
\hline ENSBTAG00000001338 & $7.60 \mathrm{E}-11$ & 0 & CCR3 & $7.61 E+02$ \\
\hline ENSBTAG00000026650 & 1.27E-09 & 0 & & $1.27 \mathrm{E}+04$ \\
\hline ENSBTAG00000014666 & 0 & 0 & GP9 & $1.00 \mathrm{E}+00$ \\
\hline ENSBTAG00000040116 & 0 & 0 & H1FX & $1.00 \mathrm{E}+00$ \\
\hline ENSBTAG00000030941 & 0 & 0 & & $1.00 \mathrm{E}+00$ \\
\hline ENSBTAG00000018884 & 0 & $9.98 \mathrm{E}-11$ & RING1 & $1.00 \mathrm{E}-03$ \\
\hline ENSBTAG00000002643 & $8.38 \mathrm{E}-11$ & 8.73E-10 & VPS52 & $9.61 \mathrm{E}-02$ \\
\hline ENSBTAG00000038128 & $2.91 \mathrm{E}-10$ & 1.62E-09 & & $1.79 \mathrm{E}-01$ \\
\hline ENSBTAG00000003352 & $2.35 \mathrm{E}-10$ & $1.29 \mathrm{E}-09$ & & $1.82 \mathrm{E}-01$ \\
\hline ENSBTAG00000046556 & 5.17E-11 & $1.96 \mathrm{E}-10$ & SOX4 & 2.64E-01 \\
\hline ENSBTAG00000008191 & $3.64 \mathrm{E}-11$ & $1.38 \mathrm{E}-10$ & SLC39A7 & 2.65E-01 \\
\hline ENSBTAG00000038783 & $4.59 \mathrm{E}-11$ & $1.74 \mathrm{E}-10$ & & 2.64E-01 \\
\hline
\end{tabular}




\begin{tabular}{|c|c|c|c|c|}
\hline ENSBTAG000000021077 & $4.62 \mathrm{E}-10$ & $1.18 \mathrm{E}-09$ & & $3.91 \mathrm{E}-01$ \\
\hline ENSBTAG00000021381 & $1.79 \mathrm{E}-10$ & $3.47 \mathrm{E}-10$ & DAAM2 & 5.17E-01 \\
\hline ENSBTAG00000020559 & $2.44 \mathrm{E}-10$ & 4.30E-10 & STMND1 & $5.68 \mathrm{E}-01$ \\
\hline ENSBTAG00000031249 & 4.57E-10 & $7.92 \mathrm{E}-10$ & PSMG4 & $5.77 \mathrm{E}-01$ \\
\hline ENSBTAG00000032247 & $5.01 \mathrm{E}-10$ & $8.66 \mathrm{E}-10$ & PPP1R11 & $5.79 \mathrm{E}-01$ \\
\hline ENSBTAG00000026163 & $3.39 \mathrm{E}-10$ & $5.70 \mathrm{E}-10$ & & $5.94 \mathrm{E}-01$ \\
\hline ENSBTAG00000019913 & $1.03 \mathrm{E}-10$ & $1.74 \mathrm{E}-10$ & DNPH1 & $5.94 \mathrm{E}-01$ \\
\hline ENSBTAG00000047009 & $2.01 E-10$ & $2.98 \mathrm{E}-10$ & ANKRD66 & $6.75 \mathrm{E}-01$ \\
\hline ENSBTAG00000017533 & $3.87 \mathrm{E}-10$ & 5.71E-10 & LRRC1 & $6.78 \mathrm{E}-01$ \\
\hline ENSBTAG00000018879 & $1.65 \mathrm{E}-10$ & $2.36 \mathrm{E}-10$ & ZNF451 & $7.01 \mathrm{E}-01$ \\
\hline ENSBTAG00000006170 & $2.82 \mathrm{E}-10$ & $3.98 \mathrm{E}-10$ & ZNF76 & 7.09E-01 \\
\hline ENSBTAG00000047516 & $6.12 \mathrm{E}-10$ & 8.49E-10 & & $7.21 \mathrm{E}-01$ \\
\hline ENSBTAG00000012652 & $4.89 \mathrm{E}-10$ & $6.71 \mathrm{E}-10$ & TINAG & 7.28E-01 \\
\hline ENSBTAG00000040187 & $9.66 \mathrm{E}-10$ & 1.31E-09 & & 7.38E-01 \\
\hline ENSBTAG00000036106 & $4.27 \mathrm{E}-10$ & $5.76 \mathrm{E}-10$ & & 7.42E-01 \\
\hline ENSBTAG000000020425 & $5.55 \mathrm{E}-10$ & 7.43E-10 & TFAP2D & 7.47E-01 \\
\hline ENSBTAG000000002934 & $1.67 \mathrm{E}-10$ & $2.18 \mathrm{E}-10$ & ADGRF4 & 7.64E-01 \\
\hline ENSBTAG00000001475 & $4.38 \mathrm{E}-10$ & $5.69 \mathrm{E}-10$ & MCUR1 & 7.70E-01 \\
\hline ENSBTAG000000021556 & $4.84 \mathrm{E}-10$ & $6.23 \mathrm{E}-10$ & RANBP9 & $7.78 \mathrm{E}-01$ \\
\hline ENSBTAG000000025314 & $6.05 \mathrm{E}-10$ & 7.73E-10 & & 7.83E-01 \\
\hline ENSBTAG00000010256 & $5.16 \mathrm{E}-10$ & $6.58 \mathrm{E}-10$ & TMEM170B & 7.84E-01 \\
\hline ENSBTAG00000013711 & $1.25 \mathrm{E}-10$ & $1.58 \mathrm{E}-10$ & & 7.94E-01 \\
\hline ENSBTAG00000006485 & $1.11 \mathrm{E}-10$ & $1.40 \mathrm{E}-10$ & TREML1 & 7.96E-01 \\
\hline ENSBTAG00000024180 & $1.69 \mathrm{E}-09$ & 2.13E-09 & HIST1H2AD & 7.94E-01 \\
\hline ENSBTAG00000046711 & $1.50 \mathrm{E}-10$ & $1.89 \mathrm{E}-10$ & DEFB112 & 7.93E-01 \\
\hline ENSBTAG00000046057 & $1.58 \mathrm{E}-10$ & $2.00 \mathrm{E}-10$ & PPP1R3G & 7.92E-01 \\
\hline ENSBTAG00000024178 & $2.10 \mathrm{E}-10$ & 2.64E-10 & & $7.94 \mathrm{E}-01$ \\
\hline ENSBTAG00000007140 & $4.19 \mathrm{E}-10$ & $5.28 \mathrm{E}-10$ & & 7.94E-01 \\
\hline ENSBTAG00000039492 & $4.18 \mathrm{E}-10$ & $5.27 \mathrm{E}-10$ & & 7.93E-01 \\
\hline ENSBTAG000000002534 & $2.07 \mathrm{E}-10$ & $2.61 E-10$ & & 7.95E-01 \\
\hline ENSBTAG00000010723 & $2.61 \mathrm{E}-10$ & $3.29 \mathrm{E}-10$ & PFDN6 & 7.93E-01 \\
\hline ENSBTAG00000017227 & $6.14 \mathrm{E}-10$ & 7.73E-10 & & 7.94E-01 \\
\hline ENSBTAG00000008517 & $5.96 \mathrm{E}-10$ & $7.51 \mathrm{E}-10$ & & 7.94E-01 \\
\hline ENSBTAG00000047812 & $5.59 \mathrm{E}-11$ & 7.04E-11 & & 7.94E-01 \\
\hline ENSBTAG00000020927 & 7.74E-11 & $9.75 \mathrm{E}-11$ & HSD17B8 & 7.94E-01 \\
\hline ENSBTAG00000024177 & $5.08 \mathrm{E}-10$ & $6.39 \mathrm{E}-10$ & & 7.94E-01 \\
\hline ENSBTAG00000045923 & $8.90 \mathrm{E}-10$ & 1.12E-09 & & 7.95E-01 \\
\hline ENSBTAG00000015130 & 8.23E-11 & $1.04 \mathrm{E}-10$ & HDGFL1 & $7.92 \mathrm{E}-01$ \\
\hline ENSBTAG00000037687 & $4.58 \mathrm{E}-10$ & 5.77E-10 & ADGRF2 & 7.94E-01 \\
\hline ENSBTAG00000000602 & $1.10 \mathrm{E}-10$ & $1.38 \mathrm{E}-10$ & RXRB & 7.96E-01 \\
\hline ENSBTAG00000019672 & $1.66 \mathrm{E}-10$ & $2.09 \mathrm{E}-10$ & & 7.96E-01 \\
\hline ENSBTAG00000008085 & $6.55 \mathrm{E}-10$ & $8.18 \mathrm{E}-10$ & CRISP1 & $8.00 \mathrm{E}-01$ \\
\hline ENSBTAG00000006015 & $1.06 \mathrm{E}-09$ & 1.31E-09 & POLH & 8.10E-01 \\
\hline ENSBTAG00000021359 & $4.02 \mathrm{E}-10$ & $4.95 \mathrm{E}-10$ & & $8.11 \mathrm{E}-01$ \\
\hline ENSBTAG00000016302 & $3.20 \mathrm{E}-10$ & $3.94 \mathrm{E}-10$ & SLC25A27 & 8.13E-01 \\
\hline ENSBTAG00000025304 & $5.25 \mathrm{E}-10$ & $6.42 \mathrm{E}-10$ & & 8.17E-01 \\
\hline ENSBTAG000000007275 & $5.51 \mathrm{E}-10$ & $6.71 E-10$ & TREM2 & $8.21 \mathrm{E}-01$ \\
\hline ENSBTAG00000004115 & $3.74 \mathrm{E}-10$ & $4.56 \mathrm{E}-10$ & MYLIP & 8.19E-01 \\
\hline ENSBTAG00000031444 & $4.84 \mathrm{E}-10$ & $5.89 \mathrm{E}-10$ & LY86 & $8.21 \mathrm{E}-01$ \\
\hline ENSBTAG00000017949 & $4.89 \mathrm{E}-10$ & 5.93E-10 & & $8.24 \mathrm{E}-01$ \\
\hline ENSBTAG00000047707 & $4.28 \mathrm{E}-10$ & $5.16 \mathrm{E}-10$ & HLA-DOB & 8.29E-01 \\
\hline ENSBTAG000000015565 & $8.90 \mathrm{E}-10$ & 1.07E-09 & & $8.31 \mathrm{E}-01$ \\
\hline ENSBTAG00000031430 & $6.97 \mathrm{E}-10$ & $8.42 \mathrm{E}-10$ & CD83 & $8.28 \mathrm{E}-01$ \\
\hline ENSBTAG00000005248 & $3.40 \mathrm{E}-10$ & $4.06 \mathrm{E}-10$ & OFCC1 & $8.38 \mathrm{E}-01$ \\
\hline ENSBTAG00000017617 & $3.03 \mathrm{E}-10$ & 3.57E-10 & C6orf222 & $8.48 \mathrm{E}-01$ \\
\hline ENSBTAG000000025280 & $6.46 \mathrm{E}-10$ & $7.62 \mathrm{E}-10$ & SERPINB6 & $8.48 \mathrm{E}-01$ \\
\hline ENSBTAG00000004979 & $4.06 \mathrm{E}-10$ & $4.71 \mathrm{E}-10$ & MRS2 & $8.62 \mathrm{E}-01$ \\
\hline ENSBTAG00000040457 & 1.07E-09 & 1.24E-09 & & 8.66E-01 \\
\hline ENSBTAG00000012451 & $7.34 \mathrm{E}-10$ & $8.49 \mathrm{E}-10$ & & 8.65E-01 \\
\hline ENSBTAG00000009549 & $4.34 \mathrm{E}-10$ & $5.00 \mathrm{E}-10$ & $\mathrm{RSPH} 9$ & 8.69E-01 \\
\hline ENSBTAG00000014272 & 4.93E-10 & 5.67E-10 & MUT & $8.70 \mathrm{E}-01$ \\
\hline ENSBTAG00000017483 & $6.23 \mathrm{E}-10$ & 7.14E-10 & & $8.72 \mathrm{E}-01$ \\
\hline ENSBTAG000000001443 & $1.25 \mathrm{E}-10$ & $1.43 \mathrm{E}-10$ & & $8.72 \mathrm{E}-01$ \\
\hline ENSBTAG00000015707 & $4.92 \mathrm{E}-10$ & 5.55E-10 & TREML2 & $8.86 \mathrm{E}-01$ \\
\hline ENSBTAG00000027547 & $1.29 \mathrm{E}-09$ & 1.45E-09 & & $8.92 \mathrm{E}-01$ \\
\hline ENSBTAG00000031640 & $6.48 \mathrm{E}-10$ & 7.27E-10 & & $8.91 \mathrm{E}-01$ \\
\hline ENSBTAG00000019217 & $3.83 \mathrm{E}-10$ & $4.31 \mathrm{E}-10$ & KIF13A & 8.89E-01 \\
\hline ENSBTAG000000025571 & 4.64E-10 & $5.20 \mathrm{E}-10$ & MRPS18A & $8.91 \mathrm{E}-01$ \\
\hline ENSBTAG00000038928 & $1.28 \mathrm{E}-09$ & 1.44E-09 & & $8.92 \mathrm{E}-01$ \\
\hline ENSBTAG00000015301 & $3.20 \mathrm{E}-10$ & $3.58 \mathrm{E}-10$ & MRPS10 & $8.94 \mathrm{E}-01$ \\
\hline
\end{tabular}




\begin{tabular}{|c|c|c|c|c|}
\hline ENSBTAG00000015176 & $1.64 \mathrm{E}-10$ & $1.84 \mathrm{E}-10$ & & $8.92 \mathrm{E}-01$ \\
\hline ENSBTAG00000037381 & 1.77E-09 & $1.95 \mathrm{E}-09$ & TRIM40 & $9.06 \mathrm{E}-01$ \\
\hline ENSBTAG00000005888 & $5.59 \mathrm{E}-10$ & $6.12 \mathrm{E}-10$ & MDGA1 & $9.13 \mathrm{E}-01$ \\
\hline ENSBTAG00000025617 & 2.34E-10 & $2.56 \mathrm{E}-10$ & & $9.16 \mathrm{E}-01$ \\
\hline ENSBTAG00000015829 & $3.95 \mathrm{E}-10$ & $4.31 \mathrm{E}-10$ & ENPP5 & $9.16 \mathrm{E}-01$ \\
\hline ENSBTAG00000012755 & $1.28 \mathrm{E}-10$ & $1.39 \mathrm{E}-10$ & KLC4 & $9.22 \mathrm{E}-01$ \\
\hline ENSBTAG00000016828 & 4.25E-10 & 4.60E-10 & TAPBP & $9.23 \mathrm{E}-01$ \\
\hline ENSBTAG00000019386 & $1.20 \mathrm{E}-09$ & $1.30 \mathrm{E}-09$ & BOLA-NC1 & $9.21 \mathrm{E}-01$ \\
\hline ENSBTAG00000008321 & $2.79 \mathrm{E}-10$ & $3.01 \mathrm{E}-10$ & RNF8 & $9.26 \mathrm{E}-01$ \\
\hline ENSBTAG00000047485 & $6.12 \mathrm{E}-10$ & $6.60 \mathrm{E}-10$ & & $9.27 \mathrm{E}-01$ \\
\hline ENSBTAG00000015175 & $5.98 \mathrm{E}-10$ & $6.45 \mathrm{E}-10$ & & $9.27 \mathrm{E}-01$ \\
\hline ENSBTAG00000039562 & $1.80 \mathrm{E}-09$ & $1.94 \mathrm{E}-09$ & OR12D3 & $9.30 \mathrm{E}-01$ \\
\hline ENSBTAG00000027197 & $6.98 \mathrm{E}-10$ & $7.48 \mathrm{E}-10$ & & $9.33 \mathrm{E}-01$ \\
\hline ENSBTAG00000009157 & $8.58 \mathrm{E}-10$ & $9.16 \mathrm{E}-10$ & SLC35B3 & $9.36 \mathrm{E}-01$ \\
\hline ENSBTAG00000025398 & $2.19 \mathrm{E}-09$ & $2.33 \mathrm{E}-09$ & & $9.41 \mathrm{E}-01$ \\
\hline ENSBTAG00000015550 & 2.83E-10 & $3.01 \mathrm{E}-10$ & MLN & $9.39 \mathrm{E}-01$ \\
\hline ENSBTAG00000009798 & $5.80 \mathrm{E}-10$ & $6.14 \mathrm{E}-10$ & DCDC2 & $9.45 \mathrm{E}-01$ \\
\hline ENSBTAG00000011112 & $7.43 \mathrm{E}-10$ & $7.82 \mathrm{E}-10$ & KCNK5 & $9.51 \mathrm{E}-01$ \\
\hline ENSBTAG00000006357 & $1.73 \mathrm{E}-10$ & $1.82 \mathrm{E}-10$ & CRIP3 & $9.50 \mathrm{E}-01$ \\
\hline ENSBTAG00000045888 & $2.96 \mathrm{E}-10$ & $3.10 \mathrm{E}-10$ & & $9.54 \mathrm{E}-01$ \\
\hline ENSBTAG00000026896 & $1.28 \mathrm{E}-10$ & $1.34 \mathrm{E}-10$ & FOXF2 & $9.55 \mathrm{E}-01$ \\
\hline ENSBTAG00000016058 & $5.88 \mathrm{E}-10$ & $6.15 \mathrm{E}-10$ & FAM83B & 9.55E-01 \\
\hline ENSBTAG00000046193 & $1.01 \mathrm{E}-09$ & 1.06E-09 & & $9.55 \mathrm{E}-01$ \\
\hline ENSBTAG00000047561 & $3.80 \mathrm{E}-10$ & $3.94 \mathrm{E}-10$ & & $9.64 \mathrm{E}-01$ \\
\hline ENSBTAG00000016827 & $2.56 \mathrm{E}-10$ & $2.63 \mathrm{E}-10$ & RGL2 & $9.72 \mathrm{E}-01$ \\
\hline ENSBTAG00000020783 & $2.66 \mathrm{E}-10$ & $2.74 \mathrm{E}-10$ & MAPK14 & $9.73 \mathrm{E}-01$ \\
\hline ENSBTAG00000019588 & $2.35 \mathrm{E}-09$ & $2.42 \mathrm{E}-09$ & & $9.71 \mathrm{E}-01$ \\
\hline ENSBTAG00000005974 & 3.17E-10 & $3.26 \mathrm{E}-10$ & APOBEC2 & $9.74 \mathrm{E}-01$ \\
\hline ENSBTAG00000018970 & $3.26 \mathrm{E}-10$ & $3.36 \mathrm{E}-10$ & XPO5 & $9.71 \mathrm{E}-01$ \\
\hline ENSBTAG00000031753 & $1.04 \mathrm{E}-09$ & $1.06 \mathrm{E}-09$ & & $9.78 \mathrm{E}-01$ \\
\hline ENSBTAG00000010943 & $3.85 \mathrm{E}-10$ & $3.92 \mathrm{E}-10$ & & $9.82 \mathrm{E}-01$ \\
\hline ENSBTAG00000004982 & 8.83E-10 & 8.91E-10 & GPLD1 & $9.91 \mathrm{E}-01$ \\
\hline ENSBTAG00000016829 & $2.15 \mathrm{E}-10$ & $2.17 \mathrm{E}-10$ & ZBTB22 & $9.92 \mathrm{E}-01$ \\
\hline ENSBTAG00000000940 & $1.56 \mathrm{E}-10$ & $1.57 \mathrm{E}-10$ & TBCC & $9.95 \mathrm{E}-01$ \\
\hline ENSBTAG00000024187 & $1.05 \mathrm{E}-09$ & $1.06 \mathrm{E}-09$ & & $9.92 \mathrm{E}-01$ \\
\hline ENSBTAG00000038888 & 1.45E-10 & $1.46 \mathrm{E}-10$ & ZВTB9 & $9.93 \mathrm{E}-01$ \\
\hline ENSBTAG00000007530 & $1.49 \mathrm{E}-09$ & $1.49 \mathrm{E}-09$ & TRIM10 & $9.98 \mathrm{E}-01$ \\
\hline ENSBTAG00000037605 & 2.81E-09 & 2.82E-09 & & $9.96 \mathrm{E}-01$ \\
\hline ENSBTAG00000009873 & $2.56 \mathrm{E}-10$ & $2.56 \mathrm{E}-10$ & NRN1 & $1.00 \mathrm{E}+00$ \\
\hline ENSBTAG00000002919 & 4.01E-10 & $3.98 \mathrm{E}-10$ & UNC5CL & $1.01 E+00$ \\
\hline ENSBTAG00000020595 & $2.05 \mathrm{E}-10$ & $2.04 \mathrm{E}-10$ & KCTD20 & $1.00 \mathrm{E}+00$ \\
\hline ENSBTAG00000012039 & $6.45 \mathrm{E}-10$ & $6.37 \mathrm{E}-10$ & PHACTR1 & $1.01 E+00$ \\
\hline ENSBTAG00000007075 & 2.04E-09 & $2.02 \mathrm{E}-09$ & & $1.01 E+00$ \\
\hline ENSBTAG00000040026 & 1.70E-09 & $1.68 \mathrm{E}-09$ & & $1.01 \mathrm{E}+00$ \\
\hline ENSBTAG00000047671 & $3.86 \mathrm{E}-10$ & $3.81 \mathrm{E}-10$ & PRP2 & $1.01 \mathrm{E}+00$ \\
\hline ENSBTAG00000038444 & $1.58 \mathrm{E}-09$ & $1.56 \mathrm{E}-09$ & & $1.01 E+00$ \\
\hline ENSBTAG00000011126 & $1.71 \mathrm{E}-10$ & $1.68 \mathrm{E}-10$ & STK38 & $1.02 \mathrm{E}+00$ \\
\hline ENSBTAG00000045551 & $2.83 \mathrm{E}-10$ & $2.77 \mathrm{E}-10$ & & $1.02 \mathrm{E}+00$ \\
\hline ENSBTAG00000019234 & $3.21 \mathrm{E}-10$ & $3.15 \mathrm{E}-10$ & BMP6 & $1.02 \mathrm{E}+00$ \\
\hline ENSBTAG00000037597 & $1.58 \mathrm{E}-09$ & $1.55 \mathrm{E}-09$ & & $1.02 E+00$ \\
\hline ENSBTAG00000048304 & $2.26 \mathrm{E}-09$ & $2.20 \mathrm{E}-09$ & & $1.03 E+00$ \\
\hline ENSBTAG00000005362 & $2.06 \mathrm{E}-10$ & $2.00 \mathrm{E}-10$ & RAB23 & $1.03 E+00$ \\
\hline ENSBTAG00000024549 & $9.94 \mathrm{E}-11$ & $9.63 \mathrm{E}-11$ & SNRPC & $1.03 \mathrm{E}+00$ \\
\hline ENSBTAG00000021902 & $1.06 \mathrm{E}-09$ & $1.03 E-09$ & ALDH5A1 & $1.03 E+00$ \\
\hline ENSBTAG00000011719 & $5.01 \mathrm{E}-10$ & $4.82 \mathrm{E}-10$ & HCRTR2 & $1.04 E+00$ \\
\hline ENSBTAG00000006016 & $2.11 \mathrm{E}-10$ & $2.03 \mathrm{E}-10$ & GTPBP2 & $1.04 E+00$ \\
\hline ENSBTAG00000003632 & $3.36 \mathrm{E}-10$ & $3.22 \mathrm{E}-10$ & CYP39A1 & $1.04 \mathrm{E}+00$ \\
\hline ENSBTAG00000026919 & $5.01 \mathrm{E}-10$ & $4.79 E-10$ & PXDC1 & $1.05 E+00$ \\
\hline ENSBTAG00000022731 & $3.58 \mathrm{E}-10$ & $3.42 \mathrm{E}-10$ & SSR1 & $1.05 E+00$ \\
\hline ENSBTAG00000016891 & 3.33E-10 & $3.17 \mathrm{E}-10$ & TCP11 & $1.05 E+00$ \\
\hline ENSBTAG00000046886 & $3.15 \mathrm{E}-10$ & $2.98 \mathrm{E}-10$ & TUBB2A & $1.06 \mathrm{E}+00$ \\
\hline ENSBTAG00000003499 & $2.64 \mathrm{E}-10$ & $2.49 \mathrm{E}-10$ & ENPP4 & $1.06 \mathrm{E}+00$ \\
\hline ENSBTAG00000031889 & $8.66 \mathrm{E}-10$ & $8.17 \mathrm{E}-10$ & & $1.06 \mathrm{E}+00$ \\
\hline ENSBTAG00000016890 & $3.14 \mathrm{E}-10$ & $2.95 \mathrm{E}-10$ & ANKS1A & $1.06 \mathrm{E}+00$ \\
\hline ENSBTAG00000034493 & $2.22 \mathrm{E}-10$ & $2.08 \mathrm{E}-10$ & C6orf106 & $1.07 E+00$ \\
\hline ENSBTAG00000033513 & $3.26 \mathrm{E}-10$ & $3.06 \mathrm{E}-10$ & & $1.07 E+00$ \\
\hline ENSBTAG00000002086 & $1.98 \mathrm{E}-10$ & $1.86 \mathrm{E}-10$ & PRPF4B & $1.07 E+00$ \\
\hline ENSBTAG00000000786 & $1.43 \mathrm{E}-10$ & $1.33 \mathrm{E}-10$ & NQO2 & $1.08 \mathrm{E}+00$ \\
\hline ENSBTAG00000002497 & $3.02 \mathrm{E}-10$ & $2.81 \mathrm{E}-10$ & ELOVL2 & $1.08 \mathrm{E}+00$ \\
\hline ENSBTAG00000046023 & $2.06 \mathrm{E}-09$ & 1.91E-09 & & $1.08 \mathrm{E}+00$ \\
\hline
\end{tabular}




\begin{tabular}{|c|c|c|c|c|}
\hline ENSBTAG00000033453 & $4.11 \mathrm{E}-10$ & $3.80 \mathrm{E}-10$ & & $1.08 \mathrm{E}+00$ \\
\hline ENSBTAG00000007268 & 7.99E-10 & 7.38E-10 & $\mathrm{F} 13 \mathrm{~A} 1$ & $1.08 \mathrm{E}+00$ \\
\hline ENSBTAG00000006287 & $3.36 \mathrm{E}-10$ & $3.10 \mathrm{E}-10$ & NEDD9 & $1.08 \mathrm{E}+00$ \\
\hline ENSBTAG00000005182 & $1.67 \mathrm{E}-09$ & $1.54 \mathrm{E}-09$ & BoLA & $1.08 \mathrm{E}+00$ \\
\hline ENSBTAG00000002581 & $6.78 \mathrm{E}-10$ & $6.24 \mathrm{E}-10$ & & $1.09 \mathrm{E}+00$ \\
\hline ENSBTAG00000013654 & $1.29 \mathrm{E}-09$ & 1.19E-09 & OR14J1 & $1.08 \mathrm{E}+00$ \\
\hline ENSBTAG00000012439 & $8.69 \mathrm{E}-10$ & $7.96 \mathrm{E}-10$ & GUCA1B & $1.09 E+00$ \\
\hline ENSBTAG00000032887 & $1.61 \mathrm{E}-10$ & $1.48 \mathrm{E}-10$ & & $1.09 E+00$ \\
\hline ENSBTAG00000011975 & $1.72 \mathrm{E}-10$ & 1.57E-10 & & $1.09 \mathrm{E}+00$ \\
\hline ENSBTAG00000033515 & $5.20 \mathrm{E}-10$ & $4.74 \mathrm{E}-10$ & & $1.10 \mathrm{E}+00$ \\
\hline ENSBTAG00000012973 & 8.04E-10 & $7.32 \mathrm{E}-10$ & GFRAL & $1.10 \mathrm{E}+00$ \\
\hline ENSBTAG00000014063 & $5.45 \mathrm{E}-10$ & $4.95 \mathrm{E}-10$ & DNAH8 & $1.10 \mathrm{E}+00$ \\
\hline ENSBTAG00000031835 & $1.56 \mathrm{E}-09$ & $1.42 \mathrm{E}-09$ & & $1.10 \mathrm{E}+00$ \\
\hline ENSBTAG00000034529 & $2.02 \mathrm{E}-10$ & $1.83 \mathrm{E}-10$ & HMGA1 & $1.10 \mathrm{E}+00$ \\
\hline ENSBTAG00000005885 & $1.60 \mathrm{E}-09$ & 1.45E-09 & & $1.10 \mathrm{E}+00$ \\
\hline ENSBTAG00000031778 & $5.86 \mathrm{E}-10$ & 5.27E-10 & HIST1H2BD & $1.11 E+00$ \\
\hline ENSBTAG00000010730 & $5.66 \mathrm{E}-10$ & $5.09 \mathrm{E}-10$ & & $1.11 \mathrm{E}+00$ \\
\hline ENSBTAG00000039901 & $6.24 \mathrm{E}-10$ & $5.60 \mathrm{E}-10$ & & $1.11 \mathrm{E}+00$ \\
\hline ENSBTAG00000024176 & 1.19E-09 & $1.06 \mathrm{E}-09$ & & $1.12 \mathrm{E}+00$ \\
\hline ENSBTAG00000009978 & 2.02E-10 & $1.81 \mathrm{E}-10$ & TDRD6 & $1.11 \mathrm{E}+00$ \\
\hline ENSBTAG00000021111 & $8.25 \mathrm{E}-10$ & 7.37E-10 & & $1.12 \mathrm{E}+00$ \\
\hline ENSBTAG00000001123 & $3.15 E-10$ & $2.82 \mathrm{E}-10$ & SNRNP48 & $1.12 \mathrm{E}+00$ \\
\hline ENSBTAG00000011189 & $1.53 \mathrm{E}-10$ & 1.37E-10 & TJAP1 & $1.12 \mathrm{E}+00$ \\
\hline ENSBTAG00000015106 & $6.41 \mathrm{E}-10$ & $5.72 \mathrm{E}-10$ & DSP & $1.12 \mathrm{E}+00$ \\
\hline ENSBTAG00000021721 & $1.58 \mathrm{E}-09$ & $1.41 \mathrm{E}-09$ & CDSN & $1.12 \mathrm{E}+00$ \\
\hline ENSBTAG00000023563 & 2.14E-09 & $1.91 \mathrm{E}-09$ & & $1.12 \mathrm{E}+00$ \\
\hline ENSBTAG00000007532 & $1.91 \mathrm{E}-09$ & $1.70 \mathrm{E}-09$ & TRIM15 & $1.13 \mathrm{E}+00$ \\
\hline ENSBTAG00000002092 & $3.50 \mathrm{E}-10$ & $3.11 \mathrm{E}-10$ & PI16 & $1.13 E+00$ \\
\hline ENSBTAG00000012208 & $9.23 \mathrm{E}-10$ & 8.19E-10 & & $1.13 \mathrm{E}+00$ \\
\hline ENSBTAG00000009267 & $2.14 \mathrm{E}-10$ & $1.89 \mathrm{E}-10$ & UHRF1BP1 & $1.13 E+00$ \\
\hline ENSBTAG00000009055 & $3.86 \mathrm{E}-10$ & $3.42 \mathrm{E}-10$ & RNF144B & $1.13 E+00$ \\
\hline ENSBTAG00000037577 & $1.49 \mathrm{E}-09$ & $1.32 \mathrm{E}-09$ & & $1.13 E+00$ \\
\hline ENSBTAG00000008959 & 2.04E-09 & 1.80E-09 & & $1.14 \mathrm{E}+00$ \\
\hline ENSBTAG00000018674 & $2.82 \mathrm{E}-10$ & $2.49 \mathrm{E}-10$ & PAK1IP1 & $1.13 E+00$ \\
\hline ENSBTAG00000018787 & $1.34 \mathrm{E}-10$ & 1.19E-10 & GCM2 & $1.13 \mathrm{E}+00$ \\
\hline ENSBTAG00000003250 & $8.81 \mathrm{E}-10$ & $7.76 \mathrm{E}-10$ & $\mathrm{OR} 2 \mathrm{H} 2$ & $1.14 \mathrm{E}+00$ \\
\hline ENSBTAG00000018969 & $1.19 \mathrm{E}-10$ & $1.05 \mathrm{E}-10$ & POLR1C & $1.14 \mathrm{E}+00$ \\
\hline ENSBTAG00000003037 & $6.83 \mathrm{E}-10$ & $5.99 \mathrm{E}-10$ & & $1.14 E+00$ \\
\hline ENSBTAG00000031874 & $1.10 \mathrm{E}-09$ & $9.64 \mathrm{E}-10$ & ZNF165 & $1.15 \mathrm{E}+00$ \\
\hline ENSBTAG00000025317 & $2.40 \mathrm{E}-10$ & $2.10 \mathrm{E}-10$ & & $1.14 \mathrm{E}+00$ \\
\hline ENSBTAG00000021415 & $5.75 \mathrm{E}-10$ & $5.02 \mathrm{E}-10$ & PTCHD4 & $1.15 \mathrm{E}+00$ \\
\hline ENSBTAG00000016887 & $2.79 \mathrm{E}-10$ & $2.43 \mathrm{E}-10$ & TAF11 & $1.15 \mathrm{E}+00$ \\
\hline ENSBTAG00000025531 & $1.50 \mathrm{E}-09$ & 1.30E-09 & PSORS1C2 & $1.15 \mathrm{E}+00$ \\
\hline ENSBTAG00000038472 & $1.08 \mathrm{E}-09$ & $9.38 \mathrm{E}-10$ & OR5V1 & $1.15 \mathrm{E}+00$ \\
\hline ENSBTAG00000011300 & $5.19 \mathrm{E}-10$ & $4.50 \mathrm{E}-10$ & RHAG & $1.15 E+00$ \\
\hline ENSBTAG00000002376 & $1.53 \mathrm{E}-10$ & $1.32 \mathrm{E}-10$ & PPIL1 & $1.16 \mathrm{E}+00$ \\
\hline ENSBTAG00000003892 & $6.41 \mathrm{E}-10$ & $5.53 \mathrm{E}-10$ & & $1.16 \mathrm{E}+00$ \\
\hline ENSBTAG00000007937 & $4.70 \mathrm{E}-10$ & $4.06 \mathrm{E}-10$ & PRIM2 & $1.16 \mathrm{E}+00$ \\
\hline ENSBTAG00000004345 & $6.83 \mathrm{E}-10$ & $5.88 \mathrm{E}-10$ & MEP1A & $1.16 \mathrm{E}+00$ \\
\hline ENSBTAG00000039326 & $4.52 \mathrm{E}-10$ & $3.89 \mathrm{E}-10$ & GCNT2 & $1.16 \mathrm{E}+00$ \\
\hline ENSBTAG00000039695 & 7.37E-10 & $6.34 \mathrm{E}-10$ & PPP1R18 & $1.16 \mathrm{E}+00$ \\
\hline ENSBTAG00000014587 & $4.72 \mathrm{E}-10$ & 4.05E-10 & CAGE1 & $1.17 E+00$ \\
\hline ENSBTAG00000032680 & 4.27E-10 & 3.64E-10 & TMEM63B & $1.17 \mathrm{E}+00$ \\
\hline ENSBTAG00000015598 & $3.99 \mathrm{E}-10$ & $3.39 \mathrm{E}-10$ & & $1.18 \mathrm{E}+00$ \\
\hline ENSBTAG00000012069 & $2.11 \mathrm{E}-10$ & $1.79 \mathrm{E}-10$ & & $1.18 \mathrm{E}+00$ \\
\hline ENSBTAG00000027246 & $1.32 \mathrm{E}-09$ & 1.12E-09 & UBD & $1.18 \mathrm{E}+00$ \\
\hline ENSBTAG00000012079 & $3.06 \mathrm{E}-10$ & $2.59 \mathrm{E}-10$ & MYLK4 & $1.18 \mathrm{E}+00$ \\
\hline ENSBTAG00000035744 & 1.79E-09 & $1.52 \mathrm{E}-09$ & TRIM26 & $1.18 \mathrm{E}+00$ \\
\hline ENSBTAG00000012870 & $5.43 \mathrm{E}-10$ & 4.57E-10 & BMP5 & $1.19 E+00$ \\
\hline ENSBTAG00000013705 & $1.00 \mathrm{E}-10$ & $8.41 E-11$ & NFKBIE & $1.19 E+00$ \\
\hline ENSBTAG00000006761 & $4.41 \mathrm{E}-10$ & $3.70 \mathrm{E}-10$ & & $1.19 E+00$ \\
\hline ENSBTAG00000024186 & $6.01 \mathrm{E}-10$ & $5.04 \mathrm{E}-10$ & & $1.19 E+00$ \\
\hline ENSBTAG00000000214 & $5.17 \mathrm{E}-10$ & $4.34 \mathrm{E}-10$ & & $1.19 E+00$ \\
\hline ENSBTAG00000008349 & $8.05 \mathrm{E}-10$ & $6.74 \mathrm{E}-10$ & ZNF311 & $1.19 E+00$ \\
\hline ENSBTAG00000010197 & $7.09 \mathrm{E}-10$ & $5.95 \mathrm{E}-10$ & TSPO2 & $1.19 E+00$ \\
\hline ENSBTAG00000024179 & 7.93E-10 & $6.64 \mathrm{E}-10$ & & $1.19 E+00$ \\
\hline ENSBTAG00000001635 & $1.77 \mathrm{E}-10$ & $1.49 \mathrm{E}-10$ & CUTA & $1.19 E+00$ \\
\hline ENSBTAG00000009548 & $1.79 \mathrm{E}-10$ & $1.50 \mathrm{E}-10$ & MAD2L1BP & $1.19 E+00$ \\
\hline ENSBTAG00000034582 & 1.97E-09 & $1.64 \mathrm{E}-09$ & & $1.20 \mathrm{E}+00$ \\
\hline ENSBTAG00000047644 & 2.07E-09 & $1.73 \mathrm{E}-09$ & & $1.20 \mathrm{E}+00$ \\
\hline
\end{tabular}




\begin{tabular}{|c|c|c|c|c|}
\hline ENSBTAG00000014581 & $6.72 \mathrm{E}-10$ & $5.60 \mathrm{E}-10$ & MLIP & $1.20 \mathrm{E}+00$ \\
\hline ENSBTAG00000016924 & $3.46 \mathrm{E}-10$ & $2.87 \mathrm{E}-10$ & CAP2 & $1.21 E+00$ \\
\hline ENSBTAG00000017128 & $3.31 \mathrm{E}-10$ & $2.74 \mathrm{E}-10$ & NUP153 & $1.21 E+00$ \\
\hline ENSBTAG00000031792 & $1.28 \mathrm{E}-09$ & $1.06 \mathrm{E}-09$ & ZNRD1 & $1.21 E+00$ \\
\hline ENSBTAG00000018072 & $5.03 \mathrm{E}-10$ & 4.15E-10 & FARS2 & $1.21 E+00$ \\
\hline ENSBTAG00000000527 & $4.33 \mathrm{E}-10$ & $3.56 \mathrm{E}-10$ & ZFAND3 & $1.22 \mathrm{E}+00$ \\
\hline ENSBTAG00000031871 & $5.91 \mathrm{E}-10$ & $4.86 \mathrm{E}-10$ & NKAPL & $1.22 \mathrm{E}+00$ \\
\hline ENSBTAG00000016519 & $6.86 \mathrm{E}-10$ & $5.62 \mathrm{E}-10$ & MBOAT1 & $1.22 \mathrm{E}+00$ \\
\hline ENSBTAG00000009905 & $2.15 \mathrm{E}-10$ & $1.76 \mathrm{E}-10$ & NFYA & $1.22 \mathrm{E}+00$ \\
\hline ENSBTAG00000010101 & $3.00 \mathrm{E}-10$ & $2.44 \mathrm{E}-10$ & BYSL & $1.23 \mathrm{E}+00$ \\
\hline ENSBTAG00000012865 & $2.20 \mathrm{E}-10$ & $1.79 \mathrm{E}-10$ & DEK & $1.23 \mathrm{E}+00$ \\
\hline ENSBTAG00000013722 & $1.84 \mathrm{E}-10$ & $1.50 \mathrm{E}-10$ & AARS2 & $1.22 \mathrm{E}+00$ \\
\hline ENSBTAG00000032642 & 1.39E-09 & 1.13E-09 & & $1.23 \mathrm{E}+00$ \\
\hline ENSBTAG00000015900 & $1.70 \mathrm{E}-10$ & $1.39 \mathrm{E}-10$ & CNPY3 & $1.23 \mathrm{E}+00$ \\
\hline ENSBTAG00000038810 & $1.41 \mathrm{E}-09$ & 1.14E-09 & SFTA2 & $1.24 \mathrm{E}+00$ \\
\hline ENSBTAG00000010877 & $2.04 \mathrm{E}-10$ & $1.64 \mathrm{E}-10$ & ARMC12 & $1.24 \mathrm{E}+00$ \\
\hline ENSBTAG00000021237 & $7.34 \mathrm{E}-10$ & $5.90 \mathrm{E}-10$ & DST & $1.24 \mathrm{E}+00$ \\
\hline ENSBTAG00000010007 & $2.15 \mathrm{E}-10$ & $1.72 \mathrm{E}-10$ & MAPK13 & $1.25 \mathrm{E}+00$ \\
\hline ENSBTAG00000015321 & $3.28 \mathrm{E}-10$ & $2.63 \mathrm{E}-10$ & TRERF1 & $1.25 E+00$ \\
\hline ENSBTAG00000021609 & $7.01 \mathrm{E}-10$ & $5.59 \mathrm{E}-10$ & ADGRF1 & $1.25 \mathrm{E}+00$ \\
\hline ENSBTAG00000004490 & $1.08 \mathrm{E}-09$ & $8.58 \mathrm{E}-10$ & TRIM31 & $1.25 \mathrm{E}+00$ \\
\hline ENSBTAG00000017329 & $1.38 \mathrm{E}-09$ & 1.10E-09 & GMNN & $1.25 \mathrm{E}+00$ \\
\hline ENSBTAG00000021993 & $2.18 \mathrm{E}-10$ & $1.73 \mathrm{E}-10$ & SPDEF & $1.26 \mathrm{E}+00$ \\
\hline ENSBTAG00000001476 & 1.57E-09 & $1.24 \mathrm{E}-09$ & & $1.26 \mathrm{E}+00$ \\
\hline ENSBTAG00000016397 & $5.93 \mathrm{E}-10$ & $4.68 \mathrm{E}-10$ & CDYL & $1.27 \mathrm{E}+00$ \\
\hline ENSBTAG00000020710 & $8.14 \mathrm{E}-10$ & $6.40 \mathrm{E}-10$ & CENPQ & $1.27 \mathrm{E}+00$ \\
\hline ENSBTAG00000005971 & $2.32 \mathrm{E}-10$ & $1.82 \mathrm{E}-10$ & $\mathrm{PRPH} 2$ & $1.27 E+00$ \\
\hline ENSBTAG00000017542 & $2.56 \mathrm{E}-10$ & $2.02 \mathrm{E}-10$ & PPARD & $1.27 \mathrm{E}+00$ \\
\hline ENSBTAG00000019300 & $3.32 \mathrm{E}-10$ & $2.60 \mathrm{E}-10$ & TPMT & $1.28 \mathrm{E}+00$ \\
\hline ENSBTAG00000038908 & 1.41E-09 & 1.11E-09 & & $1.27 E+00$ \\
\hline ENSBTAG00000033993 & $3.99 \mathrm{E}-10$ & $3.13 \mathrm{E}-10$ & PRP14 & $1.28 \mathrm{E}+00$ \\
\hline ENSBTAG00000014434 & $1.28 \mathrm{E}-09$ & 1.00E-09 & CCHCR1 & $1.28 \mathrm{E}+00$ \\
\hline ENSBTAG00000009656 & $1.00 \mathrm{E}-09$ & $7.86 \mathrm{E}-10$ & & $1.28 \mathrm{E}+00$ \\
\hline ENSBTAG00000014492 & $1.16 \mathrm{E}-09$ & $9.11 E-10$ & NFKBIL1 & $1.28 \mathrm{E}+00$ \\
\hline ENSBTAG00000017160 & $2.53 \mathrm{E}-10$ & $1.97 \mathrm{E}-10$ & TBC1D7 & $1.28 \mathrm{E}+00$ \\
\hline ENSBTAG00000003575 & $8.23 \mathrm{E}-10$ & $6.43 \mathrm{E}-10$ & ZKSCAN4 & $1.28 \mathrm{E}+00$ \\
\hline ENSBTAG00000013919 & 1.65E-09 & $1.28 \mathrm{E}-09$ & & $1.29 \mathrm{E}+00$ \\
\hline ENSBTAG00000010166 & 1.74E-09 & $1.36 \mathrm{E}-09$ & & $1.28 \mathrm{E}+00$ \\
\hline ENSBTAG00000039657 & $7.85 \mathrm{E}-10$ & $6.10 \mathrm{E}-10$ & HIST1H2AC & $1.29 \mathrm{E}+00$ \\
\hline ENSBTAG00000015274 & $4.96 \mathrm{E}-10$ & 3.84E-10 & PRL & $1.29 E+00$ \\
\hline ENSBTAG00000017239 & $9.93 \mathrm{E}-10$ & $7.68 \mathrm{E}-10$ & GABBR1 & $1.29 \mathrm{E}+00$ \\
\hline ENSBTAG00000001634 & $1.99 \mathrm{E}-10$ & $1.55 \mathrm{E}-10$ & PHF1 & $1.29 \mathrm{E}+00$ \\
\hline ENSBTAG00000048194 & 3.09E-09 & $2.38 \mathrm{E}-09$ & & $1.30 \mathrm{E}+00$ \\
\hline ENSBTAG00000005850 & $3.47 \mathrm{E}-10$ & 2.67E-10 & DLK2 & $1.30 \mathrm{E}+00$ \\
\hline ENSBTAG00000025718 & $4.74 \mathrm{E}-10$ & $3.65 \mathrm{E}-10$ & HMGCLL1 & $1.30 \mathrm{E}+00$ \\
\hline ENSBTAG00000027245 & $2.32 \mathrm{E}-09$ & $1.78 \mathrm{E}-09$ & $\mathrm{OR} 2 \mathrm{H} 1$ & $1.30 \mathrm{E}+00$ \\
\hline ENSBTAG00000047763 & $1.61 \mathrm{E}-09$ & 1.23E-09 & & $1.31 E+00$ \\
\hline ENSBTAG00000006933 & $5.63 \mathrm{E}-10$ & 4.30E-10 & PPP1R10 & $1.31 E+00$ \\
\hline ENSBTAG00000017818 & 1.13E-09 & 8.60E-10 & MOG & $1.31 E+00$ \\
\hline ENSBTAG00000040006 & $1.91 \mathrm{E}-10$ & $1.44 \mathrm{E}-10$ & SRSF3 & $1.32 \mathrm{E}+00$ \\
\hline ENSBTAG00000001631 & $1.93 \mathrm{E}-10$ & $1.46 \mathrm{E}-10$ & KIFC1 & $1.32 \mathrm{E}+00$ \\
\hline ENSBTAG00000040454 & 1.33E-09 & $9.99 \mathrm{E}-10$ & & $1.33 \mathrm{E}+00$ \\
\hline ENSBTAG00000047502 & $1.36 \mathrm{E}-10$ & $1.02 \mathrm{E}-10$ & FKBP5 & $1.33 \mathrm{E}+00$ \\
\hline ENSBTAG00000012761 & $2.80 \mathrm{E}-10$ & $2.10 \mathrm{E}-10$ & PTK7 & $1.33 \mathrm{E}+00$ \\
\hline ENSBTAG00000037738 & $7.86 \mathrm{E}-10$ & $5.89 \mathrm{E}-10$ & SLC17A4 & $1.33 \mathrm{E}+00$ \\
\hline ENSBTAG00000013821 & $7.01 \mathrm{E}-10$ & $5.25 \mathrm{E}-10$ & RIOK1 & $1.34 \mathrm{E}+00$ \\
\hline ENSBTAG00000014490 & 1.00E-09 & $7.50 \mathrm{E}-10$ & DDX39B & $1.34 \mathrm{E}+00$ \\
\hline ENSBTAG00000005674 & $1.08 \mathrm{E}-09$ & $8.12 \mathrm{E}-10$ & NEU1 & $1.34 \mathrm{E}+00$ \\
\hline ENSBTAG00000001250 & $1.82 \mathrm{E}-10$ & $1.34 \mathrm{E}-10$ & TFAP2A & $1.36 \mathrm{E}+00$ \\
\hline ENSBTAG00000040171 & 1.51E-09 & 1.11E-09 & & $1.36 \mathrm{E}+00$ \\
\hline ENSBTAG00000011074 & $2.75 \mathrm{E}-10$ & $2.02 \mathrm{E}-10$ & BRD2 & $1.36 \mathrm{E}+00$ \\
\hline ENSBTAG00000003818 & $5.94 \mathrm{E}-10$ & $4.36 \mathrm{E}-10$ & PGC & $1.36 \mathrm{E}+00$ \\
\hline ENSBTAG00000033603 & 4.17E-10 & $3.06 \mathrm{E}-10$ & IP6K3 & $1.36 \mathrm{E}+00$ \\
\hline ENSBTAG00000005339 & $2.10 \mathrm{E}-10$ & $1.54 \mathrm{E}-10$ & VEGFA & $1.36 \mathrm{E}+00$ \\
\hline ENSBTAG00000000228 & $1.05 \mathrm{E}-09$ & $7.66 \mathrm{E}-10$ & & $1.37 \mathrm{E}+00$ \\
\hline ENSBTAG00000015827 & $4.56 \mathrm{E}-10$ & $3.34 \mathrm{E}-10$ & & $1.36 \mathrm{E}+00$ \\
\hline ENSBTAG00000011339 & $2.58 \mathrm{E}-10$ & $1.89 \mathrm{E}-10$ & TAF8 & $1.37 E+00$ \\
\hline ENSBTAG00000018224 & $3.11 E-10$ & $2.26 \mathrm{E}-10$ & GLP1R & $1.38 \mathrm{E}+00$ \\
\hline ENSBTAG00000006379 & $2.86 \mathrm{E}-10$ & $2.08 \mathrm{E}-10$ & BPHL & $1.38 \mathrm{E}+00$ \\
\hline ENSBTAG00000014608 & $1.93 \mathrm{E}-10$ & $1.39 \mathrm{E}-10$ & KDM1B & $1.39 E+00$ \\
\hline
\end{tabular}




\begin{tabular}{|c|c|c|c|c|}
\hline ENSBTAG00000031747 & $7.54 \mathrm{E}-10$ & $5.45 \mathrm{E}-10$ & & $1.38 \mathrm{E}+00$ \\
\hline ENSBTAG00000008228 & $6.98 \mathrm{E}-10$ & $5.04 \mathrm{E}-10$ & BEND6 & $1.38 \mathrm{E}+00$ \\
\hline ENSBTAG00000005980 & $2.65 \mathrm{E}-10$ & $1.91 \mathrm{E}-10$ & RREB1 & $1.39 \mathrm{E}+00$ \\
\hline ENSBTAG00000038397 & $2.15 \mathrm{E}-09$ & $1.54 \mathrm{E}-09$ & & $1.40 \mathrm{E}+00$ \\
\hline ENSBTAG00000018967 & $1.34 \mathrm{E}-10$ & 9.67E-11 & YIPF3 & $1.39 E+00$ \\
\hline ENSBTAG00000015396 & $2.41 \mathrm{E}-10$ & $1.72 \mathrm{E}-10$ & RBM24 & $1.40 E+00$ \\
\hline ENSBTAG00000016124 & $4.11 \mathrm{E}-10$ & $2.94 \mathrm{E}-10$ & PACSIN1 & $1.40 \mathrm{E}+00$ \\
\hline ENSBTAG00000009302 & $5.64 \mathrm{E}-10$ & $4.01 \mathrm{E}-10$ & RCAN2 & $1.41 \mathrm{E}+00$ \\
\hline ENSBTAG00000003359 & $1.21 \mathrm{E}-09$ & $8.66 \mathrm{E}-10$ & ELOVL5 & $1.40 \mathrm{E}+00$ \\
\hline ENSBTAG00000004347 & $6.09 \mathrm{E}-10$ & $4.32 \mathrm{E}-10$ & ADGRF5 & $1.41 E+00$ \\
\hline ENSBTAG00000019908 & $1.46 \mathrm{E}-10$ & $1.04 \mathrm{E}-10$ & CUL9 & $1.41 \mathrm{E}+00$ \\
\hline ENSBTAG00000040280 & $1.29 \mathrm{E}-09$ & $9.06 \mathrm{E}-10$ & & $1.42 \mathrm{E}+00$ \\
\hline ENSBTAG00000025302 & $8.56 \mathrm{E}-10$ & $6.05 \mathrm{E}-10$ & & $1.41 \mathrm{E}+00$ \\
\hline ENSBTAG00000021066 & $2.68 \mathrm{E}-10$ & $1.88 \mathrm{E}-10$ & CAPN11 & $1.42 \mathrm{E}+00$ \\
\hline ENSBTAG00000005355 & $2.78 \mathrm{E}-10$ & $1.95 \mathrm{E}-10$ & BAG2 & $1.42 \mathrm{E}+00$ \\
\hline ENSBTAG00000006350 & $1.14 \mathrm{E}-10$ & $7.94 \mathrm{E}-11$ & SLC22A7 & $1.43 \mathrm{E}+00$ \\
\hline ENSBTAG00000004288 & $1.32 \mathrm{E}-09$ & $9.21 \mathrm{E}-10$ & GSTA4 & $1.43 \mathrm{E}+00$ \\
\hline ENSBTAG00000005146 & $1.01 E-09$ & 7.04E-10 & & $1.43 E+00$ \\
\hline ENSBTAG00000048019 & $2.66 \mathrm{E}-09$ & $1.86 \mathrm{E}-09$ & & $1.43 E+00$ \\
\hline ENSBTAG00000019492 & $3.24 \mathrm{E}-10$ & $2.26 \mathrm{E}-10$ & FANCE & $1.43 \mathrm{E}+00$ \\
\hline ENSBTAG00000015730 & $2.73 \mathrm{E}-10$ & $1.91 \mathrm{E}-10$ & HLA-DMA & $1.43 \mathrm{E}+00$ \\
\hline ENSBTAG00000039513 & $3.39 \mathrm{E}-10$ & 2.37E-10 & & $1.43 \mathrm{E}+00$ \\
\hline ENSBTAG00000031832 & $7.90 \mathrm{E}-10$ & $5.51 \mathrm{E}-10$ & & $1.43 \mathrm{E}+00$ \\
\hline ENSBTAG00000031873 & $9.35 \mathrm{E}-10$ & $6.50 \mathrm{E}-10$ & ZSCAN16 & $1.44 \mathrm{E}+00$ \\
\hline ENSBTAG00000024188 & 1.97E-09 & 1.37E-09 & & $1.44 \mathrm{E}+00$ \\
\hline ENSBTAG00000031905 & 1.71E-09 & 1.19E-09 & & $1.44 \mathrm{E}+00$ \\
\hline ENSBTAG00000017836 & $1.04 \mathrm{E}-09$ & $7.24 \mathrm{E}-10$ & ZFP57 & $1.44 \mathrm{E}+00$ \\
\hline ENSBTAG00000031432 & $8.15 \mathrm{E}-10$ & $5.66 \mathrm{E}-10$ & LYRM4 & $1.44 \mathrm{E}+00$ \\
\hline ENSBTAG00000005808 & $2.45 \mathrm{E}-10$ & $1.70 \mathrm{E}-10$ & & $1.44 \mathrm{E}+00$ \\
\hline ENSBTAG00000011237 & $8.71 \mathrm{E}-10$ & $6.03 \mathrm{E}-10$ & PKHD1 & $1.44 \mathrm{E}+00$ \\
\hline ENSBTAG00000031267 & $1.82 \mathrm{E}-10$ & $1.26 \mathrm{E}-10$ & SERPINB9 & $1.45 \mathrm{E}+00$ \\
\hline ENSBTAG00000016838 & $3.48 \mathrm{E}-10$ & 2.40E-10 & SRPK1 & $1.45 E+00$ \\
\hline ENSBTAG00000000016 & $1.06 \mathrm{E}-09$ & 7.29E-10 & LTA & $1.45 \mathrm{E}+00$ \\
\hline ENSBTAG00000006864 & $1.53 \mathrm{E}-09$ & $1.05 \mathrm{E}-09$ & & $1.45 \mathrm{E}+00$ \\
\hline ENSBTAG00000014172 & $7.28 \mathrm{E}-10$ & $5.00 \mathrm{E}-10$ & FGD2 & $1.46 \mathrm{E}+00$ \\
\hline ENSBTAG00000012823 & $5.16 \mathrm{E}-10$ & $3.54 \mathrm{E}-10$ & GUCA1A & $1.46 \mathrm{E}+00$ \\
\hline ENSBTAG00000009180 & $7.96 \mathrm{E}-10$ & $5.45 \mathrm{E}-10$ & NRSN1 & $1.46 \mathrm{E}+00$ \\
\hline ENSBTAG00000046116 & $1.04 \mathrm{E}-09$ & 7.14E-10 & & $1.46 \mathrm{E}+00$ \\
\hline ENSBTAG00000002150 & $1.03 \mathrm{E}-09$ & 7.06E-10 & IL17 & $1.46 \mathrm{E}+00$ \\
\hline ENSBTAG00000008096 & $4.12 \mathrm{E}-10$ & $2.81 E-10$ & EDN1 & $1.47 \mathrm{E}+00$ \\
\hline ENSBTAG00000015743 & $8.59 \mathrm{E}-10$ & $5.87 \mathrm{E}-10$ & GMPR & $1.46 \mathrm{E}+00$ \\
\hline ENSBTAG00000005967 & $1.83 \mathrm{E}-10$ & $1.25 \mathrm{E}-10$ & UBR2 & $1.46 \mathrm{E}+00$ \\
\hline ENSBTAG00000015178 & $2.35 \mathrm{E}-10$ & $1.61 \mathrm{E}-10$ & $\mathrm{ECI} 2$ & $1.46 \mathrm{E}+00$ \\
\hline ENSBTAG00000040168 & 1.17E-09 & $7.95 \mathrm{E}-10$ & ZSCAN9 & $1.47 \mathrm{E}+00$ \\
\hline ENSBTAG00000015131 & $1.93 \mathrm{E}-10$ & $1.32 \mathrm{E}-10$ & SLC29A1 & $1.46 \mathrm{E}+00$ \\
\hline ENSBTAG00000046573 & $7.55 \mathrm{E}-10$ & $5.13 \mathrm{E}-10$ & PGBD1 & $1.47 \mathrm{E}+00$ \\
\hline ENSBTAG00000008148 & $1.55 \mathrm{E}-09$ & $1.05 \mathrm{E}-09$ & GCM1 & $1.48 \mathrm{E}+00$ \\
\hline ENSBTAG00000037421 & $1.35 \mathrm{E}-09$ & $9.08 \mathrm{E}-10$ & & $1.49 E+00$ \\
\hline ENSBTAG00000016499 & $6.98 \mathrm{E}-10$ & $4.69 \mathrm{E}-10$ & OPN5 & $1.49 E+00$ \\
\hline ENSBTAG00000020975 & $1.76 \mathrm{E}-10$ & $1.18 \mathrm{E}-10$ & SYNGAP1 & $1.49 \mathrm{E}+00$ \\
\hline ENSBTAG00000008954 & $7.83 \mathrm{E}-10$ & $5.23 \mathrm{E}-10$ & PSMB9 & $1.50 E+00$ \\
\hline ENSBTAG00000046160 & $2.54 \mathrm{E}-10$ & $1.70 \mathrm{E}-10$ & SMIM13 & $1.50 \mathrm{E}+00$ \\
\hline ENSBTAG00000046777 & $1.78 \mathrm{E}-09$ & $1.18 \mathrm{E}-09$ & & $1.50 \mathrm{E}+00$ \\
\hline ENSBTAG00000047014 & $1.55 \mathrm{E}-09$ & 1.03E-09 & & $1.51 E+00$ \\
\hline ENSBTAG00000006966 & $5.15 \mathrm{E}-10$ & $3.41 \mathrm{E}-10$ & NRM & $1.51 E+00$ \\
\hline ENSBTAG00000019675 & $2.65 \mathrm{E}-10$ & $1.75 \mathrm{E}-10$ & ATXN1 & $1.51 E+00$ \\
\hline ENSBTAG00000010682 & 1.17E-09 & $7.75 E-10$ & DDR1 & $1.51 E+00$ \\
\hline ENSBTAG00000005842 & $2.59 \mathrm{E}-10$ & $1.70 \mathrm{E}-10$ & & $1.52 \mathrm{E}+00$ \\
\hline ENSBTAG00000000580 & $6.47 \mathrm{E}-10$ & $4.24 \mathrm{E}-10$ & & $1.53 E+00$ \\
\hline ENSBTAG00000040188 & $2.15 \mathrm{E}-09$ & 1.41E-09 & & $1.52 \mathrm{E}+00$ \\
\hline ENSBTAG00000000578 & $9.68 \mathrm{E}-10$ & $6.33 \mathrm{E}-10$ & ABHD16A & $1.53 E+00$ \\
\hline ENSBTAG00000021516 & $1.52 \mathrm{E}-09$ & $9.86 \mathrm{E}-10$ & & $1.54 \mathrm{E}+00$ \\
\hline ENSBTAG00000014435 & $6.82 \mathrm{E}-10$ & $4.42 \mathrm{E}-10$ & TCF19 & $1.54 \mathrm{E}+00$ \\
\hline ENSBTAG00000006927 & $9.74 \mathrm{E}-10$ & $6.28 \mathrm{E}-10$ & ABCF1 & $1.55 E+00$ \\
\hline ENSBTAG00000002089 & $5.35 \mathrm{E}-10$ & $3.45 \mathrm{E}-10$ & FAM217A & $1.55 \mathrm{E}+00$ \\
\hline ENSBTAG00000020012 & $7.10 \mathrm{E}-10$ & $4.58 \mathrm{E}-10$ & CDC5L & $1.55 E+00$ \\
\hline ENSBTAG00000005532 & $2.81 \mathrm{E}-10$ & $1.81 \mathrm{E}-10$ & PEX6 & $1.55 \mathrm{E}+00$ \\
\hline ENSBTAG00000002393 & $2.31 \mathrm{E}-10$ & $1.48 \mathrm{E}-10$ & C6orf89 & $1.56 \mathrm{E}+00$ \\
\hline ENSBTAG00000025535 & $3.59 \mathrm{E}-10$ & $2.30 \mathrm{E}-10$ & GLYATL3 & $1.56 \mathrm{E}+00$ \\
\hline ENSBTAG00000015590 & $1.36 \mathrm{E}-09$ & $8.69 \mathrm{E}-10$ & ICK & $1.56 \mathrm{E}+00$ \\
\hline
\end{tabular}




\begin{tabular}{|c|c|c|c|c|}
\hline ENSBTAG00000015118 & $2.31 \mathrm{E}-10$ & $1.47 \mathrm{E}-10$ & PNPLA1 & $1.57 \mathrm{E}+00$ \\
\hline ENSBTAG00000006546 & $1.50 \mathrm{E}-09$ & $9.49 \mathrm{E}-10$ & & $1.58 \mathrm{E}+00$ \\
\hline ENSBTAG00000016835 & $1.93 \mathrm{E}-09$ & $1.23 \mathrm{E}-09$ & IL17F & $1.57 \mathrm{E}+00$ \\
\hline ENSBTAG00000010106 & $1.20 \mathrm{E}-10$ & 7.57E-11 & CCND3 & $1.59 E+00$ \\
\hline ENSBTAG00000037949 & $1.22 \mathrm{E}-09$ & 7.77E-10 & & $1.58 \mathrm{E}+00$ \\
\hline ENSBTAG00000010217 & $9.73 \mathrm{E}-11$ & $6.12 \mathrm{E}-11$ & ZNF318 & $1.59 E+00$ \\
\hline ENSBTAG00000010100 & $2.12 \mathrm{E}-10$ & $1.33 \mathrm{E}-10$ & MED20 & $1.59 E+00$ \\
\hline ENSBTAG00000001074 & $2.69 \mathrm{E}-10$ & $1.69 \mathrm{E}-10$ & SAYSD1 & $1.59 E+00$ \\
\hline ENSBTAG00000031721 & $4.00 \mathrm{E}-10$ & $2.51 \mathrm{E}-10$ & & $1.59 E+00$ \\
\hline ENSBTAG00000031761 & 4.00E-10 & $2.51 E-10$ & HIST1H3J & $1.59 E+00$ \\
\hline ENSBTAG00000005975 & $2.39 \mathrm{E}-10$ & $1.50 \mathrm{E}-10$ & OARD1 & $1.59 \mathrm{E}+00$ \\
\hline ENSBTAG00000012384 & $1.25 \mathrm{E}-10$ & $7.86 \mathrm{E}-11$ & TFEB & $1.59 \mathrm{E}+00$ \\
\hline ENSBTAG00000025669 & $2.01 \mathrm{E}-10$ & $1.26 \mathrm{E}-10$ & B3GALT4 & $1.59 E+00$ \\
\hline ENSBTAG00000031776 & $4.31 \mathrm{E}-10$ & $2.71 E-10$ & HIST1H2BE & $1.59 E+00$ \\
\hline ENSBTAG00000012087 & $2.74 \mathrm{E}-10$ & $1.72 \mathrm{E}-10$ & NHLRC1 & $1.59 E+00$ \\
\hline ENSBTAG00000021903 & $1.05 \mathrm{E}-09$ & $6.59 \mathrm{E}-10$ & KIAA0319 & $1.59 E+00$ \\
\hline ENSBTAG00000047206 & $7.53 \mathrm{E}-10$ & $4.72 \mathrm{E}-10$ & HIST1H1E & $1.59 \mathrm{E}+00$ \\
\hline ENSBTAG00000006941 & $4.76 \mathrm{E}-10$ & $2.99 \mathrm{E}-10$ & ATAT1 & $1.59 E+00$ \\
\hline ENSBTAG00000031768 & $1.26 \mathrm{E}-09$ & $7.92 \mathrm{E}-10$ & & $1.60 E+00$ \\
\hline ENSBTAG00000040307 & $1.24 \mathrm{E}-09$ & $7.78 \mathrm{E}-10$ & & $1.60 E+00$ \\
\hline ENSBTAG00000039274 & $1.40 \mathrm{E}-09$ & 8.79E-10 & & $1.60 \mathrm{E}+00$ \\
\hline ENSBTAG00000038983 & $8.87 \mathrm{E}-10$ & 5.57E-10 & & $1.59 E+00$ \\
\hline ENSBTAG00000009960 & $9.00 \mathrm{E}-10$ & $5.65 \mathrm{E}-10$ & FLOT1 & $1.59 E+00$ \\
\hline ENSBTAG00000025554 & 1.17E-10 & 7.37E-11 & LRRC73 & $1.59 E+00$ \\
\hline ENSBTAG00000018165 & $8.78 \mathrm{E}-10$ & $5.51 \mathrm{E}-10$ & & $1.59 E+00$ \\
\hline ENSBTAG00000027955 & $1.23 \mathrm{E}-09$ & 7.71E-10 & & $1.60 \mathrm{E}+00$ \\
\hline ENSBTAG00000031773 & $1.21 \mathrm{E}-09$ & 7.57E-10 & & $1.60 \mathrm{E}+00$ \\
\hline ENSBTAG00000048235 & $1.29 \mathrm{E}-09$ & 8.10E-10 & HIST1H2BA & $1.60 E+00$ \\
\hline ENSBTAG00000024185 & $1.06 \mathrm{E}-09$ & $6.64 \mathrm{E}-10$ & & $1.60 E+00$ \\
\hline ENSBTAG00000039116 & $1.36 \mathrm{E}-09$ & $8.49 \mathrm{E}-10$ & & $1.60 E+00$ \\
\hline ENSBTAG00000047558 & $1.59 E-09$ & $9.96 \mathrm{E}-10$ & & $1.60 \mathrm{E}+00$ \\
\hline ENSBTAG00000047829 & $1.61 \mathrm{E}-09$ & 1.01E-09 & & $1.60 \mathrm{E}+00$ \\
\hline ENSBTAG00000014904 & $4.78 \mathrm{E}-10$ & $2.96 \mathrm{E}-10$ & SIRT5 & $1.61 E+00$ \\
\hline ENSBTAG00000017593 & $2.99 \mathrm{E}-10$ & $1.84 \mathrm{E}-10$ & TREM1 & $1.62 E+00$ \\
\hline ENSBTAG00000019315 & $6.56 \mathrm{E}-10$ & 4.04E-10 & PLA2G7 & $1.62 \mathrm{E}+00$ \\
\hline ENSBTAG00000000322 & $6.87 \mathrm{E}-10$ & 4.23E-10 & CD2AP & $1.62 E+00$ \\
\hline ENSBTAG00000010852 & $8.06 \mathrm{E}-10$ & 4.93E-10 & & $1.63 E+00$ \\
\hline ENSBTAG00000007514 & $4.34 \mathrm{E}-10$ & 2.65E-10 & CPNE5 & $1.64 E+00$ \\
\hline ENSBTAG00000019533 & $6.61 \mathrm{E}-10$ & 4.03E-10 & ADTRP & $1.64 \mathrm{E}+00$ \\
\hline ENSBTAG00000020054 & $6.27 \mathrm{E}-10$ & 3.82E-10 & TNFRSF21 & $1.64 \mathrm{E}+00$ \\
\hline ENSBTAG00000006936 & $3.88 \mathrm{E}-10$ & $2.36 \mathrm{E}-10$ & MRPS18B & $1.64 \mathrm{E}+00$ \\
\hline ENSBTAG00000012461 & 8.30E-10 & $5.05 \mathrm{E}-10$ & PRSS16 & $1.64 \mathrm{E}+00$ \\
\hline ENSBTAG00000001252 & $5.23 \mathrm{E}-10$ & $3.18 \mathrm{E}-10$ & HIVEP1 & $1.65 E+00$ \\
\hline ENSBTAG00000009861 & $2.14 \mathrm{E}-10$ & $1.29 \mathrm{E}-10$ & FRS3 & $1.66 \mathrm{E}+00$ \\
\hline ENSBTAG00000012938 & $3.59 \mathrm{E}-10$ & 2.17E-10 & JARID2 & $1.65 E+00$ \\
\hline ENSBTAG00000012058 & $3.37 \mathrm{E}-10$ & 2.04E-10 & & $1.65 E+00$ \\
\hline ENSBTAG00000002069 & $5.86 \mathrm{E}-10$ & $3.55 \mathrm{E}-10$ & BOLA & $1.65 E+00$ \\
\hline ENSBTAG00000037988 & $9.97 \mathrm{E}-10$ & $5.96 \mathrm{E}-10$ & ZSCAN31 & $1.67 E+00$ \\
\hline ENSBTAG00000039677 & $2.22 \mathrm{E}-09$ & $1.33 \mathrm{E}-09$ & OR2B6 & $1.67 E+00$ \\
\hline ENSBTAG00000037533 & 7.14E-10 & $4.26 \mathrm{E}-10$ & & $1.68 \mathrm{E}+00$ \\
\hline ENSBTAG00000046892 & $2.05 \mathrm{E}-09$ & $1.22 \mathrm{E}-09$ & & $1.68 \mathrm{E}+00$ \\
\hline ENSBTAG00000005634 & $9.50 \mathrm{E}-10$ & $5.64 \mathrm{E}-10$ & LSM2 & $1.68 \mathrm{E}+00$ \\
\hline ENSBTAG00000006960 & $1.14 \mathrm{E}-09$ & $6.69 \mathrm{E}-10$ & DHX16 & $1.70 \mathrm{E}+00$ \\
\hline ENSBTAG00000039812 & $9.31 \mathrm{E}-10$ & $5.50 \mathrm{E}-10$ & & $1.69 \mathrm{E}+00$ \\
\hline ENSBTAG00000021045 & $7.16 \mathrm{E}-10$ & $4.21 \mathrm{E}-10$ & E2F3 & $1.70 \mathrm{E}+00$ \\
\hline ENSBTAG00000031825 & $2.59 \mathrm{E}-09$ & $1.51 \mathrm{E}-09$ & & $1.71 E+00$ \\
\hline ENSBTAG00000022590 & $5.16 \mathrm{E}-10$ & $3.01 E-10$ & & $1.71 \mathrm{E}+00$ \\
\hline ENSBTAG00000016549 & $9.71 \mathrm{E}-10$ & $5.58 \mathrm{E}-10$ & CARMIL1 & $1.74 \mathrm{E}+00$ \\
\hline ENSBTAG00000018523 & $5.19 \mathrm{E}-10$ & $2.98 \mathrm{E}-10$ & TRIM38 & $1.74 \mathrm{E}+00$ \\
\hline ENSBTAG00000016830 & $2.06 \mathrm{E}-10$ & $1.18 \mathrm{E}-10$ & DAXX & $1.75 E+00$ \\
\hline ENSBTAG00000040136 & $3.16 \mathrm{E}-10$ & $1.80 \mathrm{E}-10$ & & $1.75 E+00$ \\
\hline ENSBTAG00000034939 & $1.25 \mathrm{E}-09$ & 7.15E-10 & & $1.75 E+00$ \\
\hline ENSBTAG00000010244 & $9.43 \mathrm{E}-10$ & $5.39 \mathrm{E}-10$ & CLIC5 & $1.75 E+00$ \\
\hline ENSBTAG00000017091 & $2.64 \mathrm{E}-10$ & $1.51 \mathrm{E}-10$ & CMTR1 & $1.75 E+00$ \\
\hline ENSBTAG00000000365 & $8.23 \mathrm{E}-10$ & $4.69 \mathrm{E}-10$ & TDP2 & $1.75 E+00$ \\
\hline ENSBTAG00000019684 & $1.88 \mathrm{E}-10$ & $1.07 \mathrm{E}-10$ & NUDT3 & $1.75 E+00$ \\
\hline ENSBTAG00000023541 & $1.63 \mathrm{E}-09$ & $9.26 \mathrm{E}-10$ & C6orf10 & $1.77 \mathrm{E}+00$ \\
\hline ENSBTAG00000006969 & $4.11 \mathrm{E}-10$ & $2.30 \mathrm{E}-10$ & TUBB & $1.79 E+00$ \\
\hline ENSBTAG00000038562 & $5.83 \mathrm{E}-10$ & $3.25 \mathrm{E}-10$ & & $1.79 E+00$ \\
\hline ENSBTAG00000037917 & $6.57 \mathrm{E}-10$ & 3.67E-10 & SLC17A1 & $1.79 \mathrm{E}+00$ \\
\hline
\end{tabular}




\begin{tabular}{|c|c|c|c|c|}
\hline ENSBTAG00000003038 & $5.53 \mathrm{E}-10$ & $3.08 \mathrm{E}-10$ & TAP2 & $1.80 \mathrm{E}+00$ \\
\hline ENSBTAG00000034945 & $1.52 \mathrm{E}-09$ & $8.43 \mathrm{E}-10$ & & $1.80 \mathrm{E}+00$ \\
\hline ENSBTAG00000014253 & $3.97 \mathrm{E}-10$ & $2.19 \mathrm{E}-10$ & TBC1D22B & $1.81 E+00$ \\
\hline ENSBTAG00000017097 & $2.45 \mathrm{E}-10$ & $1.35 \mathrm{E}-10$ & PTCRA & $1.82 \mathrm{E}+00$ \\
\hline ENSBTAG00000000582 & $1.06 \mathrm{E}-09$ & $5.86 \mathrm{E}-10$ & LY6G6E & $1.81 \mathrm{E}+00$ \\
\hline ENSBTAG00000038608 & $1.31 E-09$ & 7.15E-10 & & $1.83 \mathrm{E}+00$ \\
\hline ENSBTAG00000014491 & $8.30 \mathrm{E}-10$ & 4.53E-10 & & $1.83 \mathrm{E}+00$ \\
\hline ENSBTAG00000031869 & $9.85 \mathrm{E}-10$ & 5.37E-10 & ZSCAN26 & $1.83 \mathrm{E}+00$ \\
\hline ENSBTAG00000033979 & 1.63E-09 & $8.83 \mathrm{E}-10$ & & $1.84 \mathrm{E}+00$ \\
\hline ENSBTAG00000025622 & $1.54 \mathrm{E}-10$ & $8.31 \mathrm{E}-11$ & DEF6 & $1.85 \mathrm{E}+00$ \\
\hline ENSBTAG00000004436 & 9.57E-10 & $5.16 \mathrm{E}-10$ & PPT2 & $1.85 \mathrm{E}+00$ \\
\hline ENSBTAG00000038718 & $2.23 \mathrm{E}-10$ & $1.20 \mathrm{E}-10$ & & $1.86 \mathrm{E}+00$ \\
\hline ENSBTAG00000007450 & $1.12 \mathrm{E}-09$ & $6.05 \mathrm{E}-10$ & C2 & $1.85 \mathrm{E}+00$ \\
\hline ENSBTAG00000031377 & 4.06E-10 & 2.19E-10 & SYCP2L & $1.85 E+00$ \\
\hline ENSBTAG00000046621 & $8.83 \mathrm{E}-10$ & $4.75 \mathrm{E}-10$ & HIST1H1A & $1.86 \mathrm{E}+00$ \\
\hline ENSBTAG00000012956 & $1.21 \mathrm{E}-09$ & $6.48 \mathrm{E}-10$ & & $1.86 \mathrm{E}+00$ \\
\hline ENSBTAG00000015538 & $5.93 \mathrm{E}-10$ & $3.16 \mathrm{E}-10$ & LEMD2 & $1.88 \mathrm{E}+00$ \\
\hline ENSBTAG00000038619 & 1.13E-09 & $6.02 \mathrm{E}-10$ & & $1.87 \mathrm{E}+00$ \\
\hline ENSBTAG00000001444 & $1.12 \mathrm{E}-09$ & $5.93 \mathrm{E}-10$ & TNXB & $1.88 \mathrm{E}+00$ \\
\hline ENSBTAG00000045577 & $1.95 \mathrm{E}-09$ & 1.03E-09 & MCCD1 & $1.89 \mathrm{E}+00$ \\
\hline ENSBTAG00000007643 & 8.74E-10 & $4.60 \mathrm{E}-10$ & TRIM39-RPP21 & $1.90 \mathrm{E}+00$ \\
\hline ENSBTAG00000039683 & $2.35 \mathrm{E}-09$ & $1.23 \mathrm{E}-09$ & & $1.91 \mathrm{E}+00$ \\
\hline ENSBTAG00000025526 & $7.49 \mathrm{E}-10$ & $3.91 \mathrm{E}-10$ & MDC1 & $1.91 \mathrm{E}+00$ \\
\hline ENSBTAG00000025441 & $1.03 \mathrm{E}-09$ & 5.37E-10 & & $1.92 \mathrm{E}+00$ \\
\hline ENSBTAG00000025424 & $1.04 \mathrm{E}-09$ & $5.41 \mathrm{E}-10$ & $\mathrm{NOTCH} 4$ & $1.92 \mathrm{E}+00$ \\
\hline ENSBTAG00000003332 & $4.88 \mathrm{E}-10$ & $2.54 \mathrm{E}-10$ & RPP40 & $1.92 \mathrm{E}+00$ \\
\hline ENSBTAG00000015083 & $1.43 \mathrm{E}-09$ & $7.43 \mathrm{E}-10$ & FBXO9 & $1.93 E+00$ \\
\hline ENSBTAG00000010645 & $1.32 \mathrm{E}-09$ & $6.83 \mathrm{E}-10$ & BoLA DR-ALPHA & $1.93 E+00$ \\
\hline ENSBTAG00000000601 & $3.38 \mathrm{E}-10$ & $1.75 \mathrm{E}-10$ & COL11A2 & $1.93 E+00$ \\
\hline ENSBTAG00000031741 & $7.44 \mathrm{E}-10$ & $3.82 \mathrm{E}-10$ & & $1.95 \mathrm{E}+00$ \\
\hline ENSBTAG00000006914 & $8.91 \mathrm{E}-10$ & $4.57 \mathrm{E}-10$ & PRR3 & $1.95 \mathrm{E}+00$ \\
\hline ENSBTAG00000046839 & $2.88 \mathrm{E}-10$ & $1.48 \mathrm{E}-10$ & & $1.95 \mathrm{E}+00$ \\
\hline ENSBTAG00000019343 & $1.77 \mathrm{E}-10$ & $9.06 \mathrm{E}-11$ & MDFI & $1.96 \mathrm{E}+00$ \\
\hline ENSBTAG00000025485 & $1.32 \mathrm{E}-09$ & $6.66 \mathrm{E}-10$ & & $1.98 \mathrm{E}+00$ \\
\hline ENSBTAG00000025516 & 1.11E-09 & $5.61 \mathrm{E}-10$ & GNL1 & $1.97 \mathrm{E}+00$ \\
\hline ENSBTAG00000012752 & $9.73 \mathrm{E}-11$ & $4.90 \mathrm{E}-11$ & MRPL2 & $1.98 \mathrm{E}+00$ \\
\hline ENSBTAG00000004093 & $2.94 \mathrm{E}-10$ & $1.48 \mathrm{E}-10$ & TUBB2B & $1.99 \mathrm{E}+00$ \\
\hline ENSBTAG00000008353 & $1.48 \mathrm{E}-10$ & 7.47E-11 & CDKN1A & $1.99 E+00$ \\
\hline ENSBTAG00000013384 & $4.36 \mathrm{E}-10$ & $2.19 \mathrm{E}-10$ & & $1.99 \mathrm{E}+00$ \\
\hline ENSBTAG00000008076 & $1.11 \mathrm{E}-10$ & $5.58 \mathrm{E}-11$ & SLC35B2 & $1.98 \mathrm{E}+00$ \\
\hline ENSBTAG00000047039 & $7.05 \mathrm{E}-10$ & $3.54 \mathrm{E}-10$ & & $1.99 E+00$ \\
\hline ENSBTAG00000012939 & $4.55 \mathrm{E}-10$ & $2.28 \mathrm{E}-10$ & DTNBP1 & $1.99 E+00$ \\
\hline ENSBTAG00000047086 & 8.93E-10 & $4.48 \mathrm{E}-10$ & & $1.99 E+00$ \\
\hline ENSBTAG00000031763 & $1.33 \mathrm{E}-09$ & $6.64 \mathrm{E}-10$ & HIST1H4K & $2.00 \mathrm{E}+00$ \\
\hline ENSBTAG00000020674 & $1.03 \mathrm{E}-09$ & $5.15 \mathrm{E}-10$ & LTB & $2.00 E+00$ \\
\hline ENSBTAG00000031766 & $1.00 \mathrm{E}-09$ & $5.04 \mathrm{E}-10$ & & $1.99 \mathrm{E}+00$ \\
\hline ENSBTAG00000024184 & $1.00 \mathrm{E}-09$ & $5.04 \mathrm{E}-10$ & & $1.99 \mathrm{E}+00$ \\
\hline ENSBTAG00000025494 & 1.47E-09 & $7.36 \mathrm{E}-10$ & PAQR8 & $2.00 E+00$ \\
\hline ENSBTAG00000010352 & 4.33E-10 & $2.14 \mathrm{E}-10$ & KCNK16 & $2.02 E+00$ \\
\hline ENSBTAG00000017810 & $1.85 \mathrm{E}-09$ & $9.12 \mathrm{E}-10$ & EFHC1 & $2.03 E+00$ \\
\hline ENSBTAG00000031843 & $1.60 \mathrm{E}-09$ & $7.76 \mathrm{E}-10$ & & $2.06 \mathrm{E}+00$ \\
\hline ENSBTAG00000040378 & $1.39 \mathrm{E}-09$ & $6.70 \mathrm{E}-10$ & HIST1H2AI & $2.08 \mathrm{E}+00$ \\
\hline ENSBTAG00000014211 & $4.08 \mathrm{E}-10$ & $1.96 \mathrm{E}-10$ & FOXP4 & $2.08 \mathrm{E}+00$ \\
\hline ENSBTAG00000046934 & $2.21 \mathrm{E}-09$ & $1.06 \mathrm{E}-09$ & & $2.09 E+00$ \\
\hline ENSBTAG00000048230 & $3.91 \mathrm{E}-10$ & $1.86 \mathrm{E}-10$ & C6orf132 & $2.10 E+00$ \\
\hline ENSBTAG00000013316 & $1.72 \mathrm{E}-10$ & $8.13 \mathrm{E}-11$ & BRPF3 & $2.12 \mathrm{E}+00$ \\
\hline ENSBTAG00000012749 & $9.39 \mathrm{E}-11$ & 4.44E-11 & CUL7 & $2.11 E+00$ \\
\hline ENSBTAG00000000529 & $3.49 \mathrm{E}-10$ & $1.64 \mathrm{E}-10$ & & $2.13 E+00$ \\
\hline ENSBTAG00000005206 & $1.61 E-09$ & $7.50 \mathrm{E}-10$ & TMEM14A & $2.15 E+00$ \\
\hline ENSBTAG00000009112 & $1.39 \mathrm{E}-09$ & $6.45 \mathrm{E}-10$ & TRAM2 & $2.15 E+00$ \\
\hline ENSBTAG00000011760 & $2.69 \mathrm{E}-10$ & $1.23 \mathrm{E}-10$ & MRPL14 & $2.19 E+00$ \\
\hline ENSBTAG00000038904 & $5.35 \mathrm{E}-10$ & 2.44E-10 & PRRT1 & $2.19 E+00$ \\
\hline ENSBTAG00000023628 & $5.51 \mathrm{E}-10$ & $2.52 \mathrm{E}-10$ & C6orf47 & $2.19 E+00$ \\
\hline ENSBTAG00000039740 & $1.23 \mathrm{E}-09$ & $5.55 \mathrm{E}-10$ & & $2.22 \mathrm{E}+00$ \\
\hline ENSBTAG00000005530 & $3.36 \mathrm{E}-10$ & $1.51 \mathrm{E}-10$ & GNMT & $2.22 \mathrm{E}+00$ \\
\hline ENSBTAG00000038955 & 1.61E-09 & $7.25 \mathrm{E}-10$ & LY6G6D & $2.22 \mathrm{E}+00$ \\
\hline ENSBTAG00000007074 & $9.31 \mathrm{E}-10$ & $4.18 \mathrm{E}-10$ & & $2.23 \mathrm{E}+00$ \\
\hline ENSBTAG00000006490 & $6.77 \mathrm{E}-10$ & $3.03 \mathrm{E}-10$ & BOLA-DOA & $2.23 \mathrm{E}+00$ \\
\hline ENSBTAG00000043990 & $6.20 \mathrm{E}-10$ & $2.76 \mathrm{E}-10$ & KHDRBS2 & $2.24 \mathrm{E}+00$ \\
\hline ENSBTAG00000016824 & $5.46 \mathrm{E}-10$ & $2.43 \mathrm{E}-10$ & WDR46 & $2.24 \mathrm{E}+00$ \\
\hline
\end{tabular}




\begin{tabular}{|c|c|c|c|c|}
\hline ENSBTAG00000006166 & $4.09 \mathrm{E}-10$ & $1.81 \mathrm{E}-10$ & WRNIP1 & $2.26 \mathrm{E}+00$ \\
\hline ENSBTAG00000014421 & 8.05E-10 & $3.56 \mathrm{E}-10$ & PBX2 & $2.26 \mathrm{E}+00$ \\
\hline ENSBTAG00000006958 & $5.76 \mathrm{E}-10$ & $2.50 \mathrm{E}-10$ & & $2.30 E+00$ \\
\hline ENSBTAG00000010698 & $8.80 \mathrm{E}-10$ & $3.82 \mathrm{E}-10$ & VARS2 & $2.30 \mathrm{E}+00$ \\
\hline ENSBTAG00000014053 & 4.26E-10 & $1.84 \mathrm{E}-10$ & MAK & $2.31 E+00$ \\
\hline ENSBTAG00000004104 & $1.80 \mathrm{E}-10$ & 7.79E-11 & RUNX2 & $2.31 E+00$ \\
\hline ENSBTAG00000008943 & $1.18 \mathrm{E}-09$ & $5.01 \mathrm{E}-10$ & ZSCAN12 & $2.36 \mathrm{E}+00$ \\
\hline ENSBTAG00000038277 & $1.08 \mathrm{E}-09$ & 4.56E-10 & HIST1H2BJ & $2.37 E+00$ \\
\hline ENSBTAG00000002648 & $3.70 \mathrm{E}-10$ & $1.55 \mathrm{E}-10$ & RPS18 & $2.39 \mathrm{E}+00$ \\
\hline ENSBTAG00000045499 & $2.12 \mathrm{E}-10$ & $8.88 \mathrm{E}-11$ & & $2.38 \mathrm{E}+00$ \\
\hline ENSBTAG00000008977 & $1.83 \mathrm{E}-10$ & $7.66 \mathrm{E}-11$ & & $2.38 \mathrm{E}+00$ \\
\hline ENSBTAG00000047585 & $2.69 \mathrm{E}-10$ & $1.13 \mathrm{E}-10$ & & $2.38 \mathrm{E}+00$ \\
\hline ENSBTAG00000038173 & $3.65 \mathrm{E}-10$ & $1.53 \mathrm{E}-10$ & $\mathrm{H} 2 \mathrm{BFS}$ & $2.38 \mathrm{E}+00$ \\
\hline ENSBTAG00000024183 & $6.28 \mathrm{E}-10$ & $2.63 \mathrm{E}-10$ & & $2.39 E+00$ \\
\hline ENSBTAG00000025442 & $6.20 \mathrm{E}-10$ & $2.59 \mathrm{E}-10$ & HSPA1L & $2.39 E+00$ \\
\hline ENSBTAG00000040277 & $6.24 \mathrm{E}-10$ & $2.61 E-10$ & & $2.39 \mathrm{E}+00$ \\
\hline ENSBTAG00000031719 & $7.93 \mathrm{E}-10$ & $3.31 \mathrm{E}-10$ & & $2.39 E+00$ \\
\hline ENSBTAG00000039145 & $8.03 \mathrm{E}-10$ & $3.36 \mathrm{E}-10$ & & $2.39 E+00$ \\
\hline ENSBTAG00000007453 & $8.62 \mathrm{E}-10$ & $3.60 \mathrm{E}-10$ & NELFE & $2.39 E+00$ \\
\hline ENSBTAG00000039534 & $1.06 \mathrm{E}-09$ & $4.42 \mathrm{E}-10$ & & $2.40 E+00$ \\
\hline ENSBTAG00000004539 & $1.48 \mathrm{E}-09$ & 6.17E-10 & C6orf15 & $2.40 \mathrm{E}+00$ \\
\hline ENSBTAG00000019237 & $3.21 \mathrm{E}-10$ & $1.32 \mathrm{E}-10$ & & $2.43 E+00$ \\
\hline ENSBTAG00000008837 & 1.27E-09 & $5.22 \mathrm{E}-10$ & Csnk2b-Ly6g5b & $2.44 \mathrm{E}+00$ \\
\hline ENSBTAG00000017118 & $3.65 \mathrm{E}-10$ & $1.49 \mathrm{E}-10$ & FAM8A1 & $2.45 \mathrm{E}+00$ \\
\hline ENSBTAG00000013245 & $2.36 \mathrm{E}-10$ & $9.50 \mathrm{E}-11$ & ITPR3 & $2.48 \mathrm{E}+00$ \\
\hline ENSBTAG00000008833 & $9.80 \mathrm{E}-10$ & $3.93 \mathrm{E}-10$ & APOM & $2.49 E+00$ \\
\hline ENSBTAG00000025471 & $6.55 \mathrm{E}-10$ & $2.62 \mathrm{E}-10$ & TNF & $2.50 E+00$ \\
\hline ENSBTAG00000019682 & $6.98 \mathrm{E}-10$ & $2.79 \mathrm{E}-10$ & PRRC2A & $2.50 E+00$ \\
\hline ENSBTAG00000021554 & $7.39 \mathrm{E}-10$ & $2.93 \mathrm{E}-10$ & NOL7 & $2.52 \mathrm{E}+00$ \\
\hline ENSBTAG00000039732 & $5.46 \mathrm{E}-10$ & $2.15 E-10$ & BLOC1S5-TXNDC5 & $2.54 \mathrm{E}+00$ \\
\hline ENSBTAG00000045586 & $1.62 \mathrm{E}-09$ & $6.38 \mathrm{E}-10$ & OR10C1 & $2.54 \mathrm{E}+00$ \\
\hline ENSBTAG00000004442 & 7.05E-10 & $2.76 \mathrm{E}-10$ & AGPAT1 & $2.55 \mathrm{E}+00$ \\
\hline ENSBTAG00000038597 & $1.41 \mathrm{E}-09$ & 5.50E-10 & & $2.56 \mathrm{E}+00$ \\
\hline ENSBTAG00000018167 & $3.36 \mathrm{E}-10$ & $1.30 \mathrm{E}-10$ & KLHL31 & $2.58 \mathrm{E}+00$ \\
\hline ENSBTAG00000031886 & $5.03 \mathrm{E}-10$ & $1.94 \mathrm{E}-10$ & HIST1H2BN & $2.59 E+00$ \\
\hline ENSBTAG00000020215 & $3.44 \mathrm{E}-10$ & $1.31 \mathrm{E}-10$ & EXOC2 & $2.62 \mathrm{E}+00$ \\
\hline ENSBTAG00000010784 & $8.38 \mathrm{E}-10$ & $3.15 \mathrm{E}-10$ & ABT1 & $2.66 \mathrm{E}+00$ \\
\hline ENSBTAG00000039390 & $1.80 \mathrm{E}-09$ & $6.71 \mathrm{E}-10$ & & $2.68 \mathrm{E}+00$ \\
\hline ENSBTAG00000017971 & $6.40 \mathrm{E}-10$ & $2.39 \mathrm{E}-10$ & LRFN2 & $2.68 \mathrm{E}+00$ \\
\hline ENSBTAG00000013525 & $9.45 \mathrm{E}-10$ & $3.52 \mathrm{E}-10$ & C6orf25 & $2.68 \mathrm{E}+00$ \\
\hline ENSBTAG00000025413 & $8.56 \mathrm{E}-10$ & $3.13 \mathrm{E}-10$ & RNF5 & $2.73 E+00$ \\
\hline ENSBTAG00000012777 & $8.43 \mathrm{E}-11$ & $3.03 \mathrm{E}-11$ & SRF & $2.78 \mathrm{E}+00$ \\
\hline ENSBTAG00000011358 & 4.54E-10 & 1.63E-10 & IER3 & $2.78 \mathrm{E}+00$ \\
\hline ENSBTAG00000010728 & $6.31 \mathrm{E}-10$ & $2.26 \mathrm{E}-10$ & & $2.79 E+00$ \\
\hline ENSBTAG00000025449 & 1.07E-09 & $3.82 \mathrm{E}-10$ & LY6G5C & $2.79 E+00$ \\
\hline ENSBTAG00000015571 & $2.36 \mathrm{E}-10$ & $8.44 \mathrm{E}-11$ & GCLC & $2.79 E+00$ \\
\hline ENSBTAG00000018478 & $4.56 \mathrm{E}-10$ & $1.60 \mathrm{E}-10$ & RPL7L1 & $2.85 \mathrm{E}+00$ \\
\hline ENSBTAG00000000585 & 7.70E-10 & $2.70 \mathrm{E}-10$ & LY6G6C & $2.85 \mathrm{E}+00$ \\
\hline ENSBTAG00000012703 & $2.64 \mathrm{E}-10$ & $9.21 \mathrm{E}-11$ & GLO1 & $2.87 \mathrm{E}+00$ \\
\hline ENSBTAG00000040064 & $1.78 \mathrm{E}-09$ & $6.20 \mathrm{E}-10$ & & $2.87 \mathrm{E}+00$ \\
\hline ENSBTAG00000047736 & $9.67 \mathrm{E}-10$ & 3.30E-10 & & $2.93 E+00$ \\
\hline ENSBTAG00000019685 & $8.75 \mathrm{E}-10$ & $2.95 \mathrm{E}-10$ & BAG6 & $2.97 \mathrm{E}+00$ \\
\hline ENSBTAG00000031850 & $1.32 \mathrm{E}-09$ & $4.39 \mathrm{E}-10$ & & $3.00 \mathrm{E}+00$ \\
\hline ENSBTAG00000012746 & $1.71 \mathrm{E}-10$ & $5.62 \mathrm{E}-11$ & KLHDC3 & $3.04 E+00$ \\
\hline ENSBTAG00000017251 & $4.41 \mathrm{E}-10$ & $1.43 \mathrm{E}-10$ & SLC26A8 & $3.09 E+00$ \\
\hline ENSBTAG00000002641 & $1.23 \mathrm{E}-09$ & $3.98 \mathrm{E}-10$ & & $3.08 \mathrm{E}+00$ \\
\hline ENSBTAG00000013632 & $5.67 \mathrm{E}-10$ & $1.81 \mathrm{E}-10$ & GRM4 & $3.13 E+00$ \\
\hline ENSBTAG00000008981 & $1.28 \mathrm{E}-10$ & 4.04E-11 & USP49 & $3.17 E+00$ \\
\hline ENSBTAG00000005536 & $2.00 \mathrm{E}-10$ & $6.29 \mathrm{E}-11$ & & $3.18 \mathrm{E}+00$ \\
\hline ENSBTAG00000034519 & $1.20 \mathrm{E}-10$ & $3.79 \mathrm{E}-11$ & & $3.17 E+00$ \\
\hline ENSBTAG00000024175 & $8.02 \mathrm{E}-10$ & $2.51 \mathrm{E}-10$ & & $3.20 E+00$ \\
\hline ENSBTAG00000005588 & $9.12 \mathrm{E}-10$ & $2.86 \mathrm{E}-10$ & DXO & $3.19 E+00$ \\
\hline ENSBTAG00000031881 & $1.69 \mathrm{E}-09$ & $5.26 \mathrm{E}-10$ & & $3.21 E+00$ \\
\hline ENSBTAG00000006339 & $1.20 \mathrm{E}-09$ & $3.76 \mathrm{E}-10$ & & $3.20 E+00$ \\
\hline ENSBTAG00000008835 & $1.23 \mathrm{E}-09$ & $3.76 \mathrm{E}-10$ & GPANK1 & $3.27 E+00$ \\
\hline ENSBTAG00000014420 & $9.81 \mathrm{E}-10$ & $2.98 \mathrm{E}-10$ & AGER & $3.29 E+00$ \\
\hline ENSBTAG00000007618 & $1.48 \mathrm{E}-09$ & $4.52 \mathrm{E}-10$ & & $3.28 \mathrm{E}+00$ \\
\hline ENSBTAG00000025589 & $5.84 \mathrm{E}-10$ & $1.75 \mathrm{E}-10$ & CDKAL1 & $3.34 \mathrm{E}+00$ \\
\hline ENSBTAG00000010328 & $4.09 \mathrm{E}-10$ & $1.22 \mathrm{E}-10$ & KCNK17 & $3.35 E+00$ \\
\hline ENSBTAG00000020554 & $7.61 E-10$ & $2.27 \mathrm{E}-10$ & AIF1 & $3.35 E+00$ \\
\hline
\end{tabular}




\begin{tabular}{|c|c|c|c|c|}
\hline ENSBTAG00000039052 & $1.48 \mathrm{E}-09$ & $4.35 \mathrm{E}-10$ & & $3.41 \mathrm{E}+00$ \\
\hline ENSBTAG00000031780 & $1.06 \mathrm{E}-09$ & 3.07E-10 & HIST1H2BM & $3.46 \mathrm{E}+00$ \\
\hline ENSBTAG00000005630 & $8.84 \mathrm{E}-10$ & $2.50 \mathrm{E}-10$ & VWA7 & $3.53 E+00$ \\
\hline ENSBTAG00000011057 & $1.44 \mathrm{E}-10$ & $4.02 \mathrm{E}-11$ & & $3.57 E+00$ \\
\hline ENSBTAG00000000428 & $2.47 \mathrm{E}-10$ & $6.89 \mathrm{E}-11$ & BAK1 & $3.57 E+00$ \\
\hline ENSBTAG00000037781 & $4.68 \mathrm{E}-10$ & $1.31 \mathrm{E}-10$ & FKBPL & $3.57 \mathrm{E}+00$ \\
\hline ENSBTAG00000006378 & 2.87E-10 & $8.02 \mathrm{E}-11$ & RIPK1 & $3.58 \mathrm{E}+00$ \\
\hline ENSBTAG00000039620 & 7.16E-10 & $1.99 \mathrm{E}-10$ & & $3.59 E+00$ \\
\hline ENSBTAG00000040582 & $7.88 \mathrm{E}-10$ & $2.19 \mathrm{E}-10$ & & $3.60 \mathrm{E}+00$ \\
\hline ENSBTAG00000046158 & $7.66 \mathrm{E}-10$ & $2.06 \mathrm{E}-10$ & & $3.72 E+00$ \\
\hline ENSBTAG00000008794 & $1.26 \mathrm{E}-09$ & 3.35E-10 & ATF6B & $3.76 \mathrm{E}+00$ \\
\hline ENSBTAG00000019790 & $7.95 \mathrm{E}-10$ & $2.09 \mathrm{E}-10$ & MSH5 & $3.80 \mathrm{E}+00$ \\
\hline ENSBTAG00000005675 & $1.23 \mathrm{E}-09$ & $3.17 \mathrm{E}-10$ & SLC44A4 & $3.89 \mathrm{E}+00$ \\
\hline ENSBTAG00000038916 & $1.73 \mathrm{E}-10$ & 4.34E-11 & & $3.97 E+00$ \\
\hline ENSBTAG00000000778 & 7.27E-11 & $1.83 \mathrm{E}-11$ & HSP90AB1 & $3.96 \mathrm{E}+00$ \\
\hline ENSBTAG00000005533 & $2.47 \mathrm{E}-10$ & $6.20 \mathrm{E}-11$ & PPP2R5D & $3.97 E+00$ \\
\hline ENSBTAG00000031769 & $7.25 \mathrm{E}-10$ & $1.82 \mathrm{E}-10$ & & $3.98 \mathrm{E}+00$ \\
\hline ENSBTAG00000039079 & $1.86 \mathrm{E}-09$ & $4.61 \mathrm{E}-10$ & & $4.03 E+00$ \\
\hline ENSBTAG00000013533 & $9.74 \mathrm{E}-10$ & $2.40 \mathrm{E}-10$ & CLIC1 & $4.06 \mathrm{E}+00$ \\
\hline ENSBTAG00000000871 & $9.33 \mathrm{E}-10$ & $2.23 \mathrm{E}-10$ & TRIM27 & $4.18 \mathrm{E}+00$ \\
\hline ENSBTAG00000013530 & $9.85 \mathrm{E}-10$ & $2.35 \mathrm{E}-10$ & DDAH2 & $4.19 E+00$ \\
\hline ENSBTAG00000010721 & $1.81 \mathrm{E}-09$ & $4.29 \mathrm{E}-10$ & MCM3 & $4.21 \mathrm{E}+00$ \\
\hline ENSBTAG00000040583 & $1.86 \mathrm{E}-09$ & $4.41 \mathrm{E}-10$ & & $4.22 \mathrm{E}+00$ \\
\hline ENSBTAG00000037628 & 2.03E-09 & $4.81 \mathrm{E}-10$ & & $4.23 E+00$ \\
\hline ENSBTAG00000046107 & $2.03 \mathrm{E}-10$ & $4.78 \mathrm{E}-11$ & & $4.23 E+00$ \\
\hline ENSBTAG00000031774 & $8.25 \mathrm{E}-10$ & $1.94 \mathrm{E}-10$ & & $4.25 E+00$ \\
\hline ENSBTAG00000033117 & $8.18 \mathrm{E}-10$ & $1.92 \mathrm{E}-10$ & & $4.26 \mathrm{E}+00$ \\
\hline ENSBTAG00000005631 & 1.10E-09 & $2.56 \mathrm{E}-10$ & VARS & $4.30 \mathrm{E}+00$ \\
\hline ENSBTAG00000039163 & $6.07 \mathrm{E}-10$ & $1.41 \mathrm{E}-10$ & HIST1H1D & $4.30 E+00$ \\
\hline ENSBTAG00000005589 & $1.12 \mathrm{E}-09$ & $2.57 \mathrm{E}-10$ & STK19 & $4.35 E+00$ \\
\hline ENSBTAG00000019495 & $2.69 \mathrm{E}-10$ & $6.07 \mathrm{E}-11$ & TEAD3 & $4.42 \mathrm{E}+00$ \\
\hline ENSBTAG00000013065 & 7.30E-10 & $1.60 \mathrm{E}-10$ & HFE & $4.56 \mathrm{E}+00$ \\
\hline ENSBTAG00000018343 & $1.37 \mathrm{E}-09$ & $2.91 \mathrm{E}-10$ & NCR3 & $4.70 \mathrm{E}+00$ \\
\hline ENSBTAG00000031785 & $7.32 \mathrm{E}-10$ & $1.56 \mathrm{E}-10$ & & $4.69 E+00$ \\
\hline ENSBTAG00000031717 & $1.21 \mathrm{E}-09$ & $2.51 \mathrm{E}-10$ & HIST1H3G & $4.81 E+00$ \\
\hline ENSBTAG00000031757 & $1.30 \mathrm{E}-09$ & $2.71 \mathrm{E}-10$ & & $4.81 \mathrm{E}+00$ \\
\hline ENSBTAG00000031738 & 1.14E-09 & 2.37E-10 & & $4.80 \mathrm{E}+00$ \\
\hline ENSBTAG00000020426 & $4.36 \mathrm{E}-10$ & 8.92E-11 & TFAP2B & $4.88 \mathrm{E}+00$ \\
\hline ENSBTAG00000010449 & $3.95 \mathrm{E}-10$ & $8.08 \mathrm{E}-11$ & & $4.89 E+00$ \\
\hline ENSBTAG00000020496 & $5.01 \mathrm{E}-10$ & $1.02 \mathrm{E}-10$ & C6orf62 & $4.91 E+00$ \\
\hline ENSBTAG00000002369 & $5.81 \mathrm{E}-10$ & 1.10E-10 & TMEM217 & $5.28 \mathrm{E}+00$ \\
\hline ENSBTAG00000047591 & $6.22 \mathrm{E}-10$ & $1.18 \mathrm{E}-10$ & C6orf141 & $5.26 \mathrm{E}+00$ \\
\hline ENSBTAG00000046757 & $9.14 \mathrm{E}-10$ & $1.72 \mathrm{E}-10$ & GTF2H4 & $5.31 E+00$ \\
\hline ENSBTAG00000009226 & $2.74 \mathrm{E}-10$ & $5.11 \mathrm{E}-11$ & SCUBE3 & $5.36 \mathrm{E}+00$ \\
\hline ENSBTAG00000005676 & $8.84 \mathrm{E}-10$ & $1.61 \mathrm{E}-10$ & EHMT2 & $5.49 E+00$ \\
\hline ENSBTAG00000027375 & $4.22 \mathrm{E}-10$ & $7.69 \mathrm{E}-11$ & DUSP22 & $5.48 \mathrm{E}+00$ \\
\hline ENSBTAG00000019494 & $2.30 \mathrm{E}-10$ & $4.14 \mathrm{E}-11$ & RPL10A & $5.56 \mathrm{E}+00$ \\
\hline ENSBTAG00000005587 & 1.16E-09 & 2.07E-10 & SKIV2L & $5.59 E+00$ \\
\hline ENSBTAG00000014814 & $1.76 \mathrm{E}-10$ & $2.97 \mathrm{E}-11$ & GLTSCR1L & $5.91 E+00$ \\
\hline ENSBTAG00000034287 & $2.16 \mathrm{E}-10$ & $3.58 \mathrm{E}-11$ & TULP1 & $6.03 E+00$ \\
\hline ENSBTAG00000016337 & $7.18 \mathrm{E}-10$ & $9.99 \mathrm{E}-11$ & АСОT13 & $7.18 \mathrm{E}+00$ \\
\hline ENSBTAG00000045936 & $7.43 \mathrm{E}-10$ & $1.03 \mathrm{E}-10$ & & $7.20 \mathrm{E}+00$ \\
\hline ENSBTAG00000031758 & $1.62 \mathrm{E}-09$ & 2.24E-10 & HIST1H2AL & $7.24 \mathrm{E}+00$ \\
\hline ENSBTAG00000016834 & $6.33 \mathrm{E}-10$ & $8.63 \mathrm{E}-11$ & LHFPL5 & $7.33 E+00$ \\
\hline ENSBTAG00000000396 & $4.03 \mathrm{E}-10$ & $5.33 \mathrm{E}-11$ & PIM1 & $7.55 E+00$ \\
\hline ENSBTAG00000002166 & $8.74 \mathrm{E}-10$ & $1.15 \mathrm{E}-10$ & CRISP2 & $7.59 E+00$ \\
\hline ENSBTAG00000025391 & $7.49 \mathrm{E}-10$ & $9.80 \mathrm{E}-11$ & & $7.64 \mathrm{E}+00$ \\
\hline ENSBTAG00000003797 & $6.68 \mathrm{E}-10$ & $8.68 \mathrm{E}-11$ & SLC17A3 & $7.69 E+00$ \\
\hline ENSBTAG00000002642 & $5.91 \mathrm{E}-10$ & $7.36 \mathrm{E}-11$ & SLC17A2 & $8.02 \mathrm{E}+00$ \\
\hline ENSBTAG00000031888 & $1.84 \mathrm{E}-09$ & $2.28 \mathrm{E}-10$ & HIST1H2AJ & $8.06 \mathrm{E}+00$ \\
\hline ENSBTAG00000008953 & $9.03 \mathrm{E}-10$ & $1.04 \mathrm{E}-10$ & TAP1 & $8.67 E+00$ \\
\hline ENSBTAG00000024169 & 7.87E-10 & $8.95 \mathrm{E}-11$ & POM121L2 & $8.78 \mathrm{E}+00$ \\
\hline ENSBTAG00000016833 & $1.18 \mathrm{E}-09$ & $1.26 \mathrm{E}-10$ & CLPS & $9.34 \mathrm{E}+00$ \\
\hline ENSBTAG00000010640 & $1.22 \mathrm{E}-09$ & 1.17E-10 & & $1.04 \mathrm{E}+01$ \\
\hline ENSBTAG00000012918 & 7.19E-10 & $6.81 \mathrm{E}-11$ & CRISP3 & $1.05 \mathrm{E}+01$ \\
\hline ENSBTAG00000002929 & $5.55 \mathrm{E}-10$ & $5.18 \mathrm{E}-11$ & IRF4 & $1.07 \mathrm{E}+01$ \\
\hline ENSBTAG00000019926 & $1.08 \mathrm{E}-09$ & $9.85 \mathrm{E}-11$ & SCGN & $1.09 \mathrm{E}+01$ \\
\hline ENSBTAG00000009152 & $6.49 \mathrm{E}-10$ & 5.89E-11 & ZNF391 & $1.10 \mathrm{E}+01$ \\
\hline ENSBTAG00000025410 & $1.12 \mathrm{E}-09$ & $7.56 \mathrm{E}-11$ & EGFL8 & $1.48 \mathrm{E}+01$ \\
\hline ENSBTAG00000011498 & $8.88 \mathrm{E}-10$ & $2.57 \mathrm{E}-11$ & ZNF184 & $3.44 \mathrm{E}+01$ \\
\hline
\end{tabular}




\begin{tabular}{|c|c|c|c|c|}
\hline ENSBTAG00000003039 & $1.45 \mathrm{E}-09$ & 3.06E-11 & PSMB8 & $4.73 E+01$ \\
\hline ENSBTAG00000020116 & $2.23 \mathrm{E}-11$ & 0 & JSP.1 & $2.24 \mathrm{E}+02$ \\
\hline ENSBTAG00000031770 & $8.66 \mathrm{E}-10$ & 0 & & $8.66 \mathrm{E}+03$ \\
\hline ENSBTAG00000031771 & $1.05 \mathrm{E}-09$ & 0 & & $1.05 E+04$ \\
\hline ENSBTAG00000011677 & $9.00 \mathrm{E}-10$ & 0 & HIST1H1C & $9.00 \mathrm{E}+03$ \\
\hline ENSBTAG00000025882 & $2.14 \mathrm{E}-10$ & 0 & & $2.14 \mathrm{E}+03$ \\
\hline ENSBTAG00000046992 & $1.71 \mathrm{E}-09$ & 0 & & $1.71 \mathrm{E}+04$ \\
\hline ENSBTAG00000045884 & $4.38 \mathrm{E}-10$ & 0 & RNF182 & $4.38 E+03$ \\
\hline ENSBTAG00000038782 & $9.42 \mathrm{E}-10$ & 0 & & $9.42 \mathrm{E}+03$ \\
\hline ENSBTAG00000024182 & $4.31 \mathrm{E}-10$ & 0 & & $4.31 E+03$ \\
\hline ENSBTAG00000046096 & $8.33 \mathrm{E}-10$ & 0 & & $8.33 E+03$ \\
\hline ENSBTAG00000031756 & 7.93E-10 & 0 & & $7.93 E+03$ \\
\hline ENSBTAG00000031759 & 2.24E-09 & 0 & & $2.24 \mathrm{E}+04$ \\
\hline ENSBTAG00000031760 & $1.30 \mathrm{E}-09$ & 0 & HIST1H2BI & $1.30 E+04$ \\
\hline ENSBTAG00000031762 & $1.06 \mathrm{E}-09$ & 0 & HIST4H4 & $1.06 \mathrm{E}+04$ \\
\hline ENSBTAG00000004219 & 0 & 0 & RPL27 & $1.00 \mathrm{E}+00$ \\
\hline ENSBTAG00000012822 & 0 & 0 & MGC137036 & $1.00 \mathrm{E}+00$ \\
\hline ENSBTAG00000032031 & $5.93 \mathrm{E}-11$ & 0 & ZBTB12 & $5.94 \mathrm{E}+02$ \\
\hline ENSBTAG00000034656 & 0 & $2.63 \mathrm{E}-10$ & & $3.80 \mathrm{E}-04$ \\
\hline ENSBTAG00000045511 & $1.03 \mathrm{E}-10$ & $2.16 \mathrm{E}-10$ & & 4.75E-01 \\
\hline ENSBTAG00000009687 & $2.01 \mathrm{E}-10$ & $3.80 \mathrm{E}-10$ & TIMM21 & 5.29E-01 \\
\hline ENSBTAG00000014886 & $1.20 \mathrm{E}-10$ & 2.19E-10 & MEX3С & 5.49E-01 \\
\hline ENSBTAG00000019455 & $4.18 \mathrm{E}-10$ & $6.93 \mathrm{E}-10$ & MYO5B & 6.03E-01 \\
\hline ENSBTAG00000010547 & $1.18 \mathrm{E}-10$ & $1.90 \mathrm{E}-10$ & CEP76 & $6.23 \mathrm{E}-01$ \\
\hline ENSBTAG00000039248 & $1.32 \mathrm{E}-09$ & 2.09E-09 & & 6.33E-01 \\
\hline ENSBTAG00000016462 & $1.51 \mathrm{E}-10$ & $2.35 \mathrm{E}-10$ & TCF4 & $6.44 \mathrm{E}-01$ \\
\hline ENSBTAG00000018954 & $2.03 \mathrm{E}-10$ & $3.12 \mathrm{E}-10$ & ELP2 & $6.50 \mathrm{E}-01$ \\
\hline ENSBTAG00000001801 & $3.69 \mathrm{E}-10$ & $5.50 \mathrm{E}-10$ & SYT4 & 6.71E-01 \\
\hline ENSBTAG00000021334 & $1.66 \mathrm{E}-10$ & $2.48 \mathrm{E}-10$ & C18orf32 & $6.71 \mathrm{E}-01$ \\
\hline ENSBTAG00000044212 & $2.75 \mathrm{E}-10$ & $3.85 \mathrm{E}-10$ & & 7.14E-01 \\
\hline ENSBTAG00000014259 & $3.01 \mathrm{E}-10$ & $3.93 \mathrm{E}-10$ & KCTD1 & 7.65E-01 \\
\hline ENSBTAG00000016620 & $9.70 \mathrm{E}-10$ & $1.26 \mathrm{E}-09$ & SIGLEC15 & 7.70E-01 \\
\hline ENSBTAG00000006393 & $3.84 \mathrm{E}-10$ & $4.99 \mathrm{E}-10$ & $\mathrm{FECH}$ & 7.70E-01 \\
\hline ENSBTAG00000009266 & $4.75 \mathrm{E}-10$ & $6.06 \mathrm{E}-10$ & CD226 & 7.84E-01 \\
\hline ENSBTAG00000026266 & $2.29 \mathrm{E}-10$ & 2.90E-10 & MYL12B & 7.90E-01 \\
\hline ENSBTAG00000022902 & $1.80 \mathrm{E}-10$ & $2.26 \mathrm{E}-10$ & & 7.95E-01 \\
\hline ENSBTAG00000002507 & $4.71 \mathrm{E}-10$ & 5.93E-10 & ATP5A1 & 7.94E-01 \\
\hline ENSBTAG00000018216 & $1.30 \mathrm{E}-10$ & $1.64 \mathrm{E}-10$ & SKA1 & 7.96E-01 \\
\hline ENSBTAG00000046170 & $2.12 \mathrm{E}-10$ & $2.67 \mathrm{E}-10$ & & 7.93E-01 \\
\hline ENSBTAG00000015315 & $6.06 \mathrm{E}-11$ & 7.64E-11 & & 7.94E-01 \\
\hline ENSBTAG00000022826 & $1.39 \mathrm{E}-10$ & $1.75 \mathrm{E}-10$ & RAX & 7.94E-01 \\
\hline ENSBTAG00000001724 & $8.83 \mathrm{E}-11$ & $1.11 \mathrm{E}-10$ & $\mathrm{ZADH} 2$ & 7.96E-01 \\
\hline ENSBTAG00000047013 & $1.71 \mathrm{E}-10$ & $2.15 \mathrm{E}-10$ & & 7.94E-01 \\
\hline ENSBTAG00000000422 & $2.28 \mathrm{E}-10$ & $2.88 \mathrm{E}-10$ & TSHZ1 & 7.92E-01 \\
\hline ENSBTAG00000010542 & $1.36 \mathrm{E}-10$ & $1.68 \mathrm{E}-10$ & SPIRE1 & $8.12 \mathrm{E}-01$ \\
\hline ENSBTAG00000013306 & $1.74 \mathrm{E}-10$ & $2.12 \mathrm{E}-10$ & ZBTB7C & $8.21 \mathrm{E}-01$ \\
\hline ENSBTAG00000019585 & $2.86 \mathrm{E}-10$ & $3.47 \mathrm{E}-10$ & MYOM1 & 8.24E-01 \\
\hline ENSBTAG00000007567 & $7.68 \mathrm{E}-10$ & $9.26 \mathrm{E}-10$ & TMX3 & 8.30E-01 \\
\hline ENSBTAG00000015731 & $5.29 \mathrm{E}-10$ & $6.30 \mathrm{E}-10$ & TXNL1 & $8.39 \mathrm{E}-01$ \\
\hline ENSBTAG00000000106 & $6.04 \mathrm{E}-10$ & 7.14E-10 & FAM69C & 8.46E-01 \\
\hline ENSBTAG00000022895 & $5.31 \mathrm{E}-10$ & $6.26 \mathrm{E}-10$ & PQLC1 & 8.49E-01 \\
\hline ENSBTAG00000009459 & 4.09E-10 & $4.78 \mathrm{E}-10$ & MTCL1 & 8.56E-01 \\
\hline ENSBTAG00000004257 & $5.23 \mathrm{E}-10$ & $6.09 \mathrm{E}-10$ & TAF4B & $8.58 \mathrm{E}-01$ \\
\hline ENSBTAG00000018901 & $3.33 \mathrm{E}-10$ & $3.86 \mathrm{E}-10$ & CTIF & $8.62 \mathrm{E}-01$ \\
\hline ENSBTAG00000005154 & $2.17 \mathrm{E}-10$ & $2.51 \mathrm{E}-10$ & APCDD1 & 8.63E-01 \\
\hline ENSBTAG00000019271 & $1.77 \mathrm{E}-10$ & $2.04 \mathrm{E}-10$ & & 8.66E-01 \\
\hline ENSBTAG00000023198 & $7.00 \mathrm{E}-10$ & 8.06E-10 & & $8.69 \mathrm{E}-01$ \\
\hline ENSBTAG00000012060 & $2.18 \mathrm{E}-10$ & $2.50 \mathrm{E}-10$ & LPIN2 & $8.72 \mathrm{E}-01$ \\
\hline ENSBTAG00000005985 & $7.86 \mathrm{E}-10$ & $8.99 \mathrm{E}-10$ & CBLN2 & $8.75 \mathrm{E}-01$ \\
\hline ENSBTAG00000006919 & $1.70 \mathrm{E}-10$ & $1.94 \mathrm{E}-10$ & SMAD4 & 8.74E-01 \\
\hline ENSBTAG00000023026 & $7.65 \mathrm{E}-10$ & 8.67E-10 & & $8.82 \mathrm{E}-01$ \\
\hline ENSBTAG00000017698 & $3.80 \mathrm{E}-10$ & 4.29E-10 & PSMA8 & $8.85 \mathrm{E}-01$ \\
\hline ENSBTAG00000001739 & $7.93 \mathrm{E}-10$ & $8.75 \mathrm{E}-10$ & $\mathrm{CDH} 2 \mathrm{O}$ & 9.07E-01 \\
\hline ENSBTAG00000014012 & $4.61 \mathrm{E}-10$ & 5.09E-10 & CLUL1 & $9.06 \mathrm{E}-01$ \\
\hline ENSBTAG00000012067 & $4.48 \mathrm{E}-10$ & $4.90 \mathrm{E}-10$ & EPG5 & $9.14 \mathrm{E}-01$ \\
\hline ENSBTAG00000011171 & $2.30 \mathrm{E}-10$ & $2.51 E-10$ & PIEZO2 & $9.16 \mathrm{E}-01$ \\
\hline ENSBTAG00000002128 & $9.55 \mathrm{E}-11$ & $1.04 \mathrm{E}-10$ & MPPE1 & $9.18 \mathrm{E}-01$ \\
\hline ENSBTAG00000012889 & $2.79 \mathrm{E}-10$ & 3.03E-10 & NETO1 & $9.20 \mathrm{E}-01$ \\
\hline ENSBTAG00000005738 & $2.64 \mathrm{E}-10$ & $2.86 \mathrm{E}-10$ & ATP8B1 & $9.24 \mathrm{E}-01$ \\
\hline ENSBTAG00000001128 & $6.93 \mathrm{E}-10$ & $7.50 \mathrm{E}-10$ & RNF165 & $9.24 \mathrm{E}-01$ \\
\hline
\end{tabular}




\begin{tabular}{|c|c|c|c|c|}
\hline ENSBTAG00000046396 & $3.24 \mathrm{E}-10$ & $3.49 \mathrm{E}-10$ & TMEM200C & $9.28 \mathrm{E}-01$ \\
\hline ENSBTAG00000035182 & $9.18 \mathrm{E}-10$ & $9.91 \mathrm{E}-10$ & SERPINB13 & $9.27 \mathrm{E}-01$ \\
\hline ENSBTAG00000001770 & $6.04 \mathrm{E}-10$ & $6.46 \mathrm{E}-10$ & ST8SIA5 & $9.35 \mathrm{E}-01$ \\
\hline ENSBTAG00000002755 & $2.46 \mathrm{E}-10$ & $2.63 \mathrm{E}-10$ & ANKRD12 & 9.34E-01 \\
\hline ENSBTAG00000013587 & $9.71 \mathrm{E}-11$ & $1.04 \mathrm{E}-10$ & TXNL4A & 9.34E-01 \\
\hline ENSBTAG00000015210 & $5.41 \mathrm{E}-10$ & $5.72 \mathrm{E}-10$ & & $9.46 \mathrm{E}-01$ \\
\hline ENSBTAG00000032961 & $5.65 \mathrm{E}-10$ & $5.97 \mathrm{E}-10$ & & $9.46 \mathrm{E}-01$ \\
\hline ENSBTAG00000017983 & $1.32 \mathrm{E}-10$ & $1.38 \mathrm{E}-10$ & PRELID3A & $9.54 \mathrm{E}-01$ \\
\hline ENSBTAG00000015238 & $5.66 \mathrm{E}-10$ & $5.95 \mathrm{E}-10$ & DSC3 & $9.51 E-01$ \\
\hline ENSBTAG00000027181 & $5.50 \mathrm{E}-10$ & $5.77 \mathrm{E}-10$ & LAMA3 & 9.53E-01 \\
\hline ENSBTAG00000008158 & $3.98 \mathrm{E}-10$ & $4.16 \mathrm{E}-10$ & CCDC68 & $9.58 \mathrm{E}-01$ \\
\hline ENSBTAG00000046533 & $2.59 \mathrm{E}-10$ & 2.67E-10 & RAB31 & $9.70 \mathrm{E}-01$ \\
\hline ENSBTAG00000002660 & $2.09 \mathrm{E}-10$ & $2.15 \mathrm{E}-10$ & CFAP53 & $9.70 \mathrm{E}-01$ \\
\hline ENSBTAG00000002019 & $1.21 \mathrm{E}-09$ & $1.24 \mathrm{E}-09$ & SERPINB5 & $9.73 \mathrm{E}-01$ \\
\hline ENSBTAG00000021190 & $4.71 \mathrm{E}-10$ & $4.83 \mathrm{E}-10$ & $\mathrm{CDH} 2$ & $9.75 \mathrm{E}-01$ \\
\hline ENSBTAG00000016269 & $5.23 \mathrm{E}-10$ & $5.30 \mathrm{E}-10$ & ME2 & 9.87E-01 \\
\hline ENSBTAG00000002624 & $2.80 \mathrm{E}-10$ & $2.83 \mathrm{E}-10$ & PSTPIP2 & $9.89 \mathrm{E}-01$ \\
\hline ENSBTAG00000016024 & $3.54 \mathrm{E}-10$ & $3.54 \mathrm{E}-10$ & MYL12A & $1.00 \mathrm{E}+00$ \\
\hline ENSBTAG00000011622 & $8.39 \mathrm{E}-10$ & $8.34 \mathrm{E}-10$ & C18orf21 & $1.01 E+00$ \\
\hline ENSBTAG00000004871 & $2.21 \mathrm{E}-10$ & $2.19 \mathrm{E}-10$ & NDUFV2 & $1.01 \mathrm{E}+00$ \\
\hline ENSBTAG00000037907 & $3.16 \mathrm{E}-10$ & $3.12 \mathrm{E}-10$ & DSEL & $1.01 \mathrm{E}+00$ \\
\hline ENSBTAG00000012252 & $8.50 \mathrm{E}-10$ & $8.38 \mathrm{E}-10$ & MOCOS & $1.01 \mathrm{E}+00$ \\
\hline ENSBTAG00000011531 & $1.81 \mathrm{E}-10$ & $1.78 \mathrm{E}-10$ & SS18 & $1.02 \mathrm{E}+00$ \\
\hline ENSBTAG00000009686 & $9.16 \mathrm{E}-10$ & $8.95 \mathrm{E}-10$ & FBXO15 & $1.02 E+00$ \\
\hline ENSBTAG00000008705 & $3.90 \mathrm{E}-10$ & $3.79 \mathrm{E}-10$ & & $1.03 E+00$ \\
\hline ENSBTAG00000036297 & $1.42 \mathrm{E}-10$ & $1.38 \mathrm{E}-10$ & RBFA & $1.03 E+00$ \\
\hline ENSBTAG00000005683 & $1.15 \mathrm{E}-10$ & $1.12 \mathrm{E}-10$ & ELAC1 & $1.03 E+00$ \\
\hline ENSBTAG00000005638 & $1.43 \mathrm{E}-10$ & $1.39 \mathrm{E}-10$ & CABYR & $1.03 E+00$ \\
\hline ENSBTAG00000040584 & $7.57 \mathrm{E}-10$ & $7.31 \mathrm{E}-10$ & $\mathrm{DSC2}$ & $1.04 \mathrm{E}+00$ \\
\hline ENSBTAG00000005917 & $1.50 \mathrm{E}-10$ & $1.44 \mathrm{E}-10$ & PARD6G & $1.04 \mathrm{E}+00$ \\
\hline ENSBTAG00000010563 & $1.43 \mathrm{E}-10$ & $1.38 \mathrm{E}-10$ & PTPN2 & $1.04 \mathrm{E}+00$ \\
\hline ENSBTAG00000001523 & $4.50 \mathrm{E}-10$ & 4.34E-10 & YES1 & $1.04 \mathrm{E}+00$ \\
\hline ENSBTAG00000018088 & $2.45 \mathrm{E}-10$ & $2.34 \mathrm{E}-10$ & SETBP1 & $1.05 E+00$ \\
\hline ENSBTAG00000013665 & $3.88 \mathrm{E}-10$ & $3.71 \mathrm{E}-10$ & KCNG2 & $1.04 \mathrm{E}+00$ \\
\hline ENSBTAG00000000998 & $3.64 \mathrm{E}-10$ & 3.47E-10 & SLC39A6 & $1.05 E+00$ \\
\hline ENSBTAG00000001207 & $8.70 \mathrm{E}-10$ & $8.29 \mathrm{E}-10$ & SERPINB8 & $1.05 E+00$ \\
\hline ENSBTAG00000003880 & $2.84 \mathrm{E}-10$ & $2.69 \mathrm{E}-10$ & EMILIN2 & $1.05 E+00$ \\
\hline ENSBTAG00000005412 & $2.65 \mathrm{E}-10$ & $2.50 \mathrm{E}-10$ & NEDD4L & $1.06 \mathrm{E}+00$ \\
\hline ENSBTAG00000021668 & 5.19E-10 & $4.82 \mathrm{E}-10$ & METTL4 & $1.08 \mathrm{E}+00$ \\
\hline ENSBTAG00000000517 & 8.07E-10 & $7.48 \mathrm{E}-10$ & ANKRD29 & $1.08 \mathrm{E}+00$ \\
\hline ENSBTAG00000032964 & $1.61 \mathrm{E}-10$ & $1.48 \mathrm{E}-10$ & & $1.09 E+00$ \\
\hline ENSBTAG00000006726 & $9.19 \mathrm{E}-10$ & $8.49 \mathrm{E}-10$ & CCDC102B & $1.08 \mathrm{E}+00$ \\
\hline ENSBTAG00000014284 & $4.64 \mathrm{E}-10$ & $4.28 \mathrm{E}-10$ & ALPK2 & $1.09 E+00$ \\
\hline ENSBTAG00000003113 & $4.98 \mathrm{E}-10$ & 4.60E-10 & RNF125 & $1.08 \mathrm{E}+00$ \\
\hline ENSBTAG00000001805 & $1.70 \mathrm{E}-10$ & $1.57 \mathrm{E}-10$ & TWSG1 & $1.09 \mathrm{E}+00$ \\
\hline ENSBTAG00000024015 & $3.96 \mathrm{E}-10$ & 3.64E-10 & PTPRM & $1.09 \mathrm{E}+00$ \\
\hline ENSBTAG00000017597 & $2.30 \mathrm{E}-10$ & $2.10 \mathrm{E}-10$ & ESCO1 & $1.10 \mathrm{E}+00$ \\
\hline ENSBTAG00000021884 & $1.66 \mathrm{E}-10$ & $1.52 \mathrm{E}-10$ & $\mathrm{CXXC1}$ & $1.09 \mathrm{E}+00$ \\
\hline ENSBTAG00000013871 & $4.31 \mathrm{E}-10$ & $3.95 \mathrm{E}-10$ & & $1.09 \mathrm{E}+00$ \\
\hline ENSBTAG00000017279 & $2.23 \mathrm{E}-10$ & $2.04 \mathrm{E}-10$ & VAPA & $1.10 \mathrm{E}+00$ \\
\hline ENSBTAG00000007705 & $4.25 \mathrm{E}-10$ & 3.87E-10 & COLEC12 & $1.10 \mathrm{E}+00$ \\
\hline ENSBTAG00000012012 & $6.05 \mathrm{E}-10$ & $5.51 \mathrm{E}-10$ & CYB5A & $1.10 \mathrm{E}+00$ \\
\hline ENSBTAG00000019251 & $4.96 \mathrm{E}-10$ & 4.49E-10 & EPB41L3 & $1.11 \mathrm{E}+00$ \\
\hline ENSBTAG00000006833 & $4.00 \mathrm{E}-10$ & $3.61 \mathrm{E}-10$ & LMAN1 & $1.11 E+00$ \\
\hline ENSBTAG00000032962 & $2.98 \mathrm{E}-10$ & 2.69E-10 & SKOR2 & $1.11 E+00$ \\
\hline ENSBTAG00000011038 & $1.39 \mathrm{E}-10$ & $1.24 \mathrm{E}-10$ & $\mathrm{MC} 2 \mathrm{R}$ & $1.12 \mathrm{E}+00$ \\
\hline ENSBTAG00000001102 & $1.06 \mathrm{E}-09$ & $9.39 \mathrm{E}-10$ & SERPINB10 & $1.13 \mathrm{E}+00$ \\
\hline ENSBTAG00000019036 & $6.35 \mathrm{E}-10$ & $5.61 \mathrm{E}-10$ & ARHGAP28 & $1.13 E+00$ \\
\hline ENSBTAG00000021428 & $2.17 \mathrm{E}-10$ & $1.92 \mathrm{E}-10$ & CNDP1 & $1.13 \mathrm{E}+00$ \\
\hline ENSBTAG00000011250 & $1.42 \mathrm{E}-10$ & $1.25 \mathrm{E}-10$ & AFG3L2 & $1.14 \mathrm{E}+00$ \\
\hline ENSBTAG00000046046 & $3.79 \mathrm{E}-10$ & 3.30E-10 & LRRC30 & $1.15 E+00$ \\
\hline ENSBTAG00000011893 & $2.02 \mathrm{E}-10$ & $1.74 \mathrm{E}-10$ & & $1.16 \mathrm{E}+00$ \\
\hline ENSBTAG00000021003 & $2.96 \mathrm{E}-10$ & $2.54 \mathrm{E}-10$ & GALR1 & $1.16 \mathrm{E}+00$ \\
\hline ENSBTAG00000019870 & $6.27 \mathrm{E}-10$ & $5.34 \mathrm{E}-10$ & SLC14A1 & $1.17 \mathrm{E}+00$ \\
\hline ENSBTAG00000035171 & $9.59 \mathrm{E}-10$ & 8.17E-10 & SERPINB12 & $1.17 \mathrm{E}+00$ \\
\hline ENSBTAG00000005324 & $6.65 \mathrm{E}-10$ & $5.66 \mathrm{E}-10$ & & $1.17 \mathrm{E}+00$ \\
\hline ENSBTAG00000022890 & $6.98 \mathrm{E}-10$ & $5.91 \mathrm{E}-10$ & & $1.18 \mathrm{E}+00$ \\
\hline ENSBTAG00000014082 & $4.56 \mathrm{E}-10$ & $3.83 \mathrm{E}-10$ & CHST9 & $1.19 \mathrm{E}+00$ \\
\hline ENSBTAG00000020650 & $2.62 \mathrm{E}-10$ & $2.20 \mathrm{E}-10$ & ADCYAP1 & $1.19 \mathrm{E}+00$ \\
\hline ENSBTAG00000005372 & $3.90 \mathrm{E}-10$ & 3.24E-10 & DLGAP1 & $1.20 \mathrm{E}+00$ \\
\hline
\end{tabular}




\begin{tabular}{|c|c|c|c|c|}
\hline ENSBTAG00000047809 & $3.78 \mathrm{E}-10$ & $3.11 \mathrm{E}-10$ & & $1.21 \mathrm{E}+00$ \\
\hline ENSBTAG00000005916 & $1.03 \mathrm{E}-10$ & $8.50 \mathrm{E}-11$ & ADNP2 & $1.21 \mathrm{E}+00$ \\
\hline ENSBTAG00000010492 & $4.53 \mathrm{E}-10$ & $3.72 \mathrm{E}-10$ & VPS4B & $1.22 \mathrm{E}+00$ \\
\hline ENSBTAG00000016666 & $2.92 \mathrm{E}-10$ & $2.38 \mathrm{E}-10$ & KIAA1468 & $1.23 \mathrm{E}+00$ \\
\hline ENSBTAG00000010552 & $1.15 \mathrm{E}-10$ & $9.34 \mathrm{E}-11$ & PSMG2 & $1.23 \mathrm{E}+00$ \\
\hline ENSBTAG00000023270 & $5.85 \mathrm{E}-10$ & 4.68E-10 & $\mathrm{CDH} 19$ & $1.25 \mathrm{E}+00$ \\
\hline ENSBTAG00000005497 & 3.19E-10 & $2.51 \mathrm{E}-10$ & RPRD1A & $1.27 \mathrm{E}+00$ \\
\hline ENSBTAG00000048303 & 1.30E-10 & $1.02 \mathrm{E}-10$ & & $1.27 \mathrm{E}+00$ \\
\hline ENSBTAG00000036298 & $1.27 \mathrm{E}-10$ & $9.88 \mathrm{E}-11$ & & $1.28 \mathrm{E}+00$ \\
\hline ENSBTAG00000046957 & $5.25 \mathrm{E}-10$ & $4.11 \mathrm{E}-10$ & DOK6 & $1.28 \mathrm{E}+00$ \\
\hline ENSBTAG00000022829 & 7.67E-10 & $5.91 \mathrm{E}-10$ & & $1.30 \mathrm{E}+00$ \\
\hline ENSBTAG00000000024 & $1.54 \mathrm{E}-10$ & $1.18 \mathrm{E}-10$ & DYM & $1.30 \mathrm{E}+00$ \\
\hline ENSBTAG00000022466 & $3.76 \mathrm{E}-10$ & $2.88 \mathrm{E}-10$ & C18orf63 & $1.30 \mathrm{E}+00$ \\
\hline ENSBTAG00000040586 & $7.32 \mathrm{E}-10$ & $5.59 \mathrm{E}-10$ & & $1.31 E+00$ \\
\hline ENSBTAG00000005158 & $2.91 \mathrm{E}-10$ & $2.21 \mathrm{E}-10$ & NAPG & $1.32 \mathrm{E}+00$ \\
\hline ENSBTAG00000009142 & 1.57E-10 & 1.19E-10 & RNMT & $1.32 \mathrm{E}+00$ \\
\hline ENSBTAG00000007696 & $3.31 \mathrm{E}-10$ & $2.52 \mathrm{E}-10$ & & $1.31 E+00$ \\
\hline ENSBTAG00000016663 & 4.07E-10 & $3.09 \mathrm{E}-10$ & PIGN & $1.32 \mathrm{E}+00$ \\
\hline ENSBTAG00000007833 & $1.76 \mathrm{E}-10$ & $1.32 \mathrm{E}-10$ & PIAS2 & $1.33 \mathrm{E}+00$ \\
\hline ENSBTAG00000004650 & $2.50 \mathrm{E}-10$ & $1.86 \mathrm{E}-10$ & ZNF516 & $1.34 \mathrm{E}+00$ \\
\hline ENSBTAG00000010299 & $5.98 \mathrm{E}-10$ & $4.45 \mathrm{E}-10$ & NOL4 & $1.34 \mathrm{E}+00$ \\
\hline ENSBTAG00000003801 & $1.86 \mathrm{E}-10$ & $1.38 \mathrm{E}-10$ & MBD1 & $1.35 \mathrm{E}+00$ \\
\hline ENSBTAG00000010255 & $2.66 \mathrm{E}-10$ & $1.96 \mathrm{E}-10$ & & $1.36 \mathrm{E}+00$ \\
\hline ENSBTAG00000006743 & $2.04 \mathrm{E}-10$ & $1.50 \mathrm{E}-10$ & RBBP8 & $1.36 \mathrm{E}+00$ \\
\hline ENSBTAG00000007467 & $1.89 \mathrm{E}-10$ & $1.38 \mathrm{E}-10$ & MIB1 & $1.37 \mathrm{E}+00$ \\
\hline ENSBTAG00000010356 & $1.69 \mathrm{E}-10$ & $1.23 \mathrm{E}-10$ & RAB12 & $1.37 \mathrm{E}+00$ \\
\hline ENSBTAG00000002863 & $3.15 \mathrm{E}-10$ & $2.26 \mathrm{E}-10$ & ACAA2 & $1.39 E+00$ \\
\hline ENSBTAG00000010037 & $6.03 \mathrm{E}-10$ & 4.33E-10 & TMEM241 & $1.39 E+00$ \\
\hline ENSBTAG00000005734 & $2.00 \mathrm{E}-10$ & $1.44 \mathrm{E}-10$ & & $1.39 E+00$ \\
\hline ENSBTAG00000023259 & $4.42 \mathrm{E}-10$ & $3.17 \mathrm{E}-10$ & OSBPL1A & $1.40 \mathrm{E}+00$ \\
\hline ENSBTAG00000013588 & 2.97E-10 & $2.12 \mathrm{E}-10$ & ZNF532 & $1.40 \mathrm{E}+00$ \\
\hline ENSBTAG00000007003 & $3.01 \mathrm{E}-10$ & $2.14 \mathrm{E}-10$ & TYMS & $1.41 \mathrm{E}+00$ \\
\hline ENSBTAG00000010792 & $1.49 \mathrm{E}-10$ & $1.04 \mathrm{E}-10$ & SEH1L & $1.43 \mathrm{E}+00$ \\
\hline ENSBTAG00000000531 & $6.12 \mathrm{E}-10$ & $4.28 \mathrm{E}-10$ & & $1.43 E+00$ \\
\hline ENSBTAG00000022819 & $2.68 \mathrm{E}-10$ & $1.86 \mathrm{E}-10$ & ONECUT2 & $1.44 \mathrm{E}+00$ \\
\hline ENSBTAG00000004940 & 3.02E-10 & $2.08 \mathrm{E}-10$ & CELF4 & $1.45 \mathrm{E}+00$ \\
\hline ENSBTAG00000047003 & $3.52 \mathrm{E}-10$ & $2.40 \mathrm{E}-10$ & ASXL3 & $1.47 \mathrm{E}+00$ \\
\hline ENSBTAG00000015195 & $4.85 \mathrm{E}-10$ & $3.29 \mathrm{E}-10$ & NPC1 & $1.47 E+00$ \\
\hline ENSBTAG00000012426 & $6.09 \mathrm{E}-10$ & 4.07E-10 & & $1.50 \mathrm{E}+00$ \\
\hline ENSBTAG00000019199 & $1.87 \mathrm{E}-10$ & $1.25 \mathrm{E}-10$ & & $1.50 \mathrm{E}+00$ \\
\hline ENSBTAG00000002099 & $2.29 \mathrm{E}-10$ & $1.51 \mathrm{E}-10$ & SLC14A2 & $1.52 \mathrm{E}+00$ \\
\hline ENSBTAG00000007347 & $1.90 \mathrm{E}-10$ & $1.26 \mathrm{E}-10$ & ST8SIA3 & $1.50 \mathrm{E}+00$ \\
\hline ENSBTAG00000012307 & $5.42 \mathrm{E}-10$ & $3.55 \mathrm{E}-10$ & DTNA & $1.53 E+00$ \\
\hline ENSBTAG00000002788 & $9.59 \mathrm{E}-10$ & $6.23 \mathrm{E}-10$ & RAB27B & $1.54 \mathrm{E}+00$ \\
\hline ENSBTAG00000021581 & $3.73 \mathrm{E}-10$ & $2.39 \mathrm{E}-10$ & FHOD3 & $1.56 \mathrm{E}+00$ \\
\hline ENSBTAG00000022887 & $3.83 \mathrm{E}-10$ & $2.45 \mathrm{E}-10$ & ZNF407 & $1.56 \mathrm{E}+00$ \\
\hline ENSBTAG00000008723 & 4.04E-10 & $2.57 \mathrm{E}-10$ & CCBE1 & $1.57 \mathrm{E}+00$ \\
\hline ENSBTAG00000027916 & $3.10 \mathrm{E}-10$ & $1.97 \mathrm{E}-10$ & MALT1 & $1.57 \mathrm{E}+00$ \\
\hline ENSBTAG00000013221 & $3.07 \mathrm{E}-10$ & $1.94 \mathrm{E}-10$ & RTTN & $1.58 \mathrm{E}+00$ \\
\hline ENSBTAG00000019676 & $1.89 \mathrm{E}-10$ & $1.19 \mathrm{E}-10$ & MC4R & $1.58 \mathrm{E}+00$ \\
\hline ENSBTAG00000044087 & $1.78 \mathrm{E}-10$ & $1.12 \mathrm{E}-10$ & & $1.59 E+00$ \\
\hline ENSBTAG00000007718 & $1.25 \mathrm{E}-10$ & $7.88 \mathrm{E}-11$ & & $1.59 \mathrm{E}+00$ \\
\hline ENSBTAG00000043951 & $1.20 \mathrm{E}-10$ & $7.54 \mathrm{E}-11$ & IMPA2 & $1.59 \mathrm{E}+00$ \\
\hline ENSBTAG00000017412 & $1.98 \mathrm{E}-10$ & $1.24 \mathrm{E}-10$ & socs6 & $1.59 \mathrm{E}+00$ \\
\hline ENSBTAG00000013809 & $5.24 \mathrm{E}-11$ & $3.30 \mathrm{E}-11$ & & $1.59 \mathrm{E}+00$ \\
\hline ENSBTAG00000047569 & $3.66 \mathrm{E}-10$ & $2.30 \mathrm{E}-10$ & & $1.59 \mathrm{E}+00$ \\
\hline ENSBTAG00000008292 & $2.97 \mathrm{E}-10$ & $1.87 \mathrm{E}-10$ & SNRPD1 & $1.59 E+00$ \\
\hline ENSBTAG00000013380 & $1.61 \mathrm{E}-10$ & $1.01 \mathrm{E}-10$ & CEP192 & $1.60 E+00$ \\
\hline ENSBTAG00000009143 & $5.04 \mathrm{E}-10$ & $3.17 \mathrm{E}-10$ & MC5R & $1.59 \mathrm{E}+00$ \\
\hline ENSBTAG00000047113 & $1.60 \mathrm{E}-10$ & $1.01 \mathrm{E}-10$ & & $1.58 \mathrm{E}+00$ \\
\hline ENSBTAG00000047609 & $1.27 \mathrm{E}-10$ & 7.99E-11 & & $1.59 \mathrm{E}+00$ \\
\hline ENSBTAG00000019037 & 2.77E-10 & $1.70 \mathrm{E}-10$ & AQP4 & $1.63 E+00$ \\
\hline ENSBTAG00000020391 & $2.70 \mathrm{E}-10$ & $1.60 \mathrm{E}-10$ & MEP1B & $1.69 E+00$ \\
\hline ENSBTAG00000039916 & $1.69 \mathrm{E}-10$ & $9.91 \mathrm{E}-11$ & SMAD2 & $1.70 \mathrm{E}+00$ \\
\hline ENSBTAG00000039037 & $1.06 \mathrm{E}-09$ & $6.21 \mathrm{E}-10$ & & $1.71 \mathrm{E}+00$ \\
\hline ENSBTAG00000014488 & $5.43 \mathrm{E}-10$ & $3.18 \mathrm{E}-10$ & $\mathrm{CDH7}$ & $1.71 E+00$ \\
\hline ENSBTAG00000007569 & $5.77 \mathrm{E}-10$ & $3.35 \mathrm{E}-10$ & TNFRSF11A & $1.72 \mathrm{E}+00$ \\
\hline ENSBTAG00000009139 & $2.04 \mathrm{E}-10$ & $1.18 \mathrm{E}-10$ & LDLRAD4 & $1.73 E+00$ \\
\hline ENSBTAG00000009907 & $2.55 \mathrm{E}-10$ & $1.47 \mathrm{E}-10$ & MAPK4 & $1.73 \mathrm{E}+00$ \\
\hline ENSBTAG00000039850 & 2.07E-09 & 1.13E-09 & & $1.83 \mathrm{E}+00$ \\
\hline
\end{tabular}




\begin{tabular}{|c|c|c|c|c|}
\hline ENSBTAG00000004005 & $4.42 \mathrm{E}-10$ & $2.37 \mathrm{E}-10$ & WDR7 & $1.86 \mathrm{E}+00$ \\
\hline ENSBTAG00000008403 & $3.05 \mathrm{E}-10$ & $1.60 \mathrm{E}-10$ & ROCK1 & $1.91 \mathrm{E}+00$ \\
\hline ENSBTAG00000019215 & $5.90 \mathrm{E}-10$ & $3.10 \mathrm{E}-10$ & THOC1 & $1.90 E+00$ \\
\hline ENSBTAG00000020003 & $5.10 \mathrm{E}-10$ & $2.68 \mathrm{E}-10$ & KLHL14 & $1.90 \mathrm{E}+00$ \\
\hline ENSBTAG00000037608 & $7.96 \mathrm{E}-10$ & $4.14 \mathrm{E}-10$ & & $1.92 \mathrm{E}+00$ \\
\hline ENSBTAG00000009841 & $9.85 \mathrm{E}-10$ & $5.11 \mathrm{E}-10$ & CNDP2 & $1.93 \mathrm{E}+00$ \\
\hline ENSBTAG00000019302 & $5.01 \mathrm{E}-10$ & $2.58 \mathrm{E}-10$ & $\mathrm{BCL} 2$ & $1.94 \mathrm{E}+00$ \\
\hline ENSBTAG00000035224 & $9.16 \mathrm{E}-10$ & 4.70E-10 & & $1.95 \mathrm{E}+00$ \\
\hline ENSBTAG00000010252 & $2.72 \mathrm{E}-10$ & $1.40 \mathrm{E}-10$ & & $1.94 \mathrm{E}+00$ \\
\hline ENSBTAG00000005709 & $2.92 \mathrm{E}-10$ & $1.47 \mathrm{E}-10$ & ABHD3 & $1.99 \mathrm{E}+00$ \\
\hline ENSBTAG00000002512 & $7.12 \mathrm{E}-10$ & $3.54 \mathrm{E}-10$ & HAUS1 & $2.01 E+00$ \\
\hline ENSBTAG00000037773 & $7.56 \mathrm{E}-10$ & $3.72 \mathrm{E}-10$ & SERPINB7 & $2.03 E+00$ \\
\hline ENSBTAG00000004796 & 7.17E-10 & $3.52 \mathrm{E}-10$ & GRP & $2.04 \mathrm{E}+00$ \\
\hline ENSBTAG00000007520 & $6.46 \mathrm{E}-10$ & $3.14 \mathrm{E}-10$ & MAPRE2 & $2.06 \mathrm{E}+00$ \\
\hline ENSBTAG00000047635 & $4.05 \mathrm{E}-10$ & $1.96 \mathrm{E}-10$ & MRO & $2.07 E+00$ \\
\hline ENSBTAG00000019331 & $3.17 \mathrm{E}-10$ & $1.53 \mathrm{E}-10$ & KATNAL2 & $2.07 E+00$ \\
\hline ENSBTAG00000045832 & $5.79 \mathrm{E}-10$ & $2.74 \mathrm{E}-10$ & PHLPP1 & $2.11 E+00$ \\
\hline ENSBTAG00000017517 & $3.22 \mathrm{E}-10$ & $1.44 \mathrm{E}-10$ & ZNF236 & $2.23 \mathrm{E}+00$ \\
\hline ENSBTAG00000007000 & $4.22 \mathrm{E}-10$ & $1.88 \mathrm{E}-10$ & ENOSF1 & $2.25 \mathrm{E}+00$ \\
\hline ENSBTAG00000038739 & $5.48 \mathrm{E}-10$ & $2.41 \mathrm{E}-10$ & CPLX4 & $2.27 E+00$ \\
\hline ENSBTAG00000021923 & $1.20 \mathrm{E}-09$ & $5.22 \mathrm{E}-10$ & DSG2 & $2.30 E+00$ \\
\hline ENSBTAG00000007383 & $3.78 \mathrm{E}-10$ & $1.63 \mathrm{E}-10$ & ZNF521 & $2.32 \mathrm{E}+00$ \\
\hline ENSBTAG00000007723 & $5.31 \mathrm{E}-10$ & $2.22 \mathrm{E}-10$ & KDSR & $2.39 \mathrm{E}+00$ \\
\hline ENSBTAG00000018160 & $5.62 \mathrm{E}-10$ & $2.29 \mathrm{E}-10$ & LAMA1 & $2.45 \mathrm{E}+00$ \\
\hline ENSBTAG00000011206 & $5.06 \mathrm{E}-10$ & 2.07E-10 & GALNT1 & $2.45 \mathrm{E}+00$ \\
\hline ENSBTAG00000009129 & $6.07 \mathrm{E}-10$ & $2.45 \mathrm{E}-10$ & POLI & $2.48 \mathrm{E}+00$ \\
\hline ENSBTAG00000009141 & $1.61 \mathrm{E}-10$ & $6.51 \mathrm{E}-11$ & FAM210A & $2.47 E+00$ \\
\hline ENSBTAG00000037554 & $6.49 \mathrm{E}-10$ & $2.60 \mathrm{E}-10$ & & $2.50 \mathrm{E}+00$ \\
\hline ENSBTAG00000015190 & $5.17 \mathrm{E}-10$ & $2.06 \mathrm{E}-10$ & $\mathrm{C} 24 \mathrm{H} 18$ orf8 & $2.51 \mathrm{E}+00$ \\
\hline ENSBTAG00000008275 & $3.40 \mathrm{E}-10$ & $1.31 \mathrm{E}-10$ & GREB1L & $2.60 \mathrm{E}+00$ \\
\hline ENSBTAG00000037457 & $4.76 \mathrm{E}-10$ & $1.80 \mathrm{E}-10$ & & $2.64 \mathrm{E}+00$ \\
\hline ENSBTAG00000020286 & $2.78 \mathrm{E}-10$ & $1.05 \mathrm{E}-10$ & GAREM1 & $2.64 \mathrm{E}+00$ \\
\hline ENSBTAG00000020160 & $2.78 \mathrm{E}-10$ & $1.01 \mathrm{E}-10$ & RNF138 & $2.75 \mathrm{E}+00$ \\
\hline ENSBTAG00000026290 & $5.73 E-10$ & $2.06 \mathrm{E}-10$ & PIK3C3 & $2.78 \mathrm{E}+00$ \\
\hline ENSBTAG00000040126 & $8.96 \mathrm{E}-10$ & $3.11 \mathrm{E}-10$ & DSG4 & $2.88 \mathrm{E}+00$ \\
\hline ENSBTAG00000013831 & $1.02 \mathrm{E}-09$ & $3.53 \mathrm{E}-10$ & DSG1 & $2.89 \mathrm{E}+00$ \\
\hline ENSBTAG00000000138 & $2.24 \mathrm{E}-10$ & $7.46 \mathrm{E}-11$ & CCDC178 & $3.00 E+00$ \\
\hline ENSBTAG00000010991 & $8.00 \mathrm{E}-10$ & $2.56 \mathrm{E}-10$ & TTR & $3.12 \mathrm{E}+00$ \\
\hline ENSBTAG00000039296 & $3.91 \mathrm{E}-10$ & $1.24 \mathrm{E}-10$ & & $3.15 E+00$ \\
\hline ENSBTAG00000005635 & $3.14 \mathrm{E}-10$ & $9.54 \mathrm{E}-11$ & TTC39C & $3.28 \mathrm{E}+00$ \\
\hline ENSBTAG00000003035 & $9.14 \mathrm{E}-10$ & $2.79 \mathrm{E}-10$ & IMPACT & $3.28 \mathrm{E}+00$ \\
\hline ENSBTAG00000000656 & $3.92 \mathrm{E}-10$ & $1.19 \mathrm{E}-10$ & NFATC1 & $3.29 \mathrm{E}+00$ \\
\hline ENSBTAG00000010042 & $3.69 \mathrm{E}-10$ & $1.11 \mathrm{E}-10$ & RIOK3 & $3.32 \mathrm{E}+00$ \\
\hline ENSBTAG00000009822 & $2.76 \mathrm{E}-10$ & $8.23 \mathrm{E}-11$ & PPP4R1 & $3.35 \mathrm{E}+00$ \\
\hline ENSBTAG00000046547 & $1.99 \mathrm{E}-10$ & $5.89 \mathrm{E}-11$ & & $3.37 E+00$ \\
\hline ENSBTAG00000021583 & $1.77 \mathrm{E}-10$ & 5.19E-11 & TPGS2 & $3.41 E+00$ \\
\hline ENSBTAG00000021673 & $2.91 \mathrm{E}-10$ & $8.43 \mathrm{E}-11$ & NDC80 & $3.45 E+00$ \\
\hline ENSBTAG00000011954 & $6.09 \mathrm{E}-10$ & $1.75 \mathrm{E}-10$ & SEC11C & $3.48 \mathrm{E}+00$ \\
\hline ENSBTAG00000001224 & $6.12 \mathrm{E}-10$ & $1.65 \mathrm{E}-10$ & ATP9B & $3.71 E+00$ \\
\hline ENSBTAG00000017276 & $1.24 \mathrm{E}-09$ & $3.29 E-10$ & & $3.77 \mathrm{E}+00$ \\
\hline ENSBTAG00000044063 & $6.11 \mathrm{E}-10$ & $1.59 \mathrm{E}-10$ & B4GALT6 & $3.84 \mathrm{E}+00$ \\
\hline ENSBTAG00000008168 & $6.84 \mathrm{E}-10$ & $1.42 \mathrm{E}-10$ & STARD6 & $4.82 \mathrm{E}+00$ \\
\hline ENSBTAG00000019214 & $5.77 \mathrm{E}-10$ & $1.08 \mathrm{E}-10$ & USP14 & $5.34 \mathrm{E}+00$ \\
\hline ENSBTAG00000002125 & $6.51 \mathrm{E}-10$ & $1.11 \mathrm{E}-10$ & GNAL & $5.86 \mathrm{E}+00$ \\
\hline ENSBTAG00000020467 & $6.52 \mathrm{E}-10$ & $1.06 \mathrm{E}-10$ & $\mathrm{HRH} 4$ & $6.15 \mathrm{E}+00$ \\
\hline ENSBTAG00000046901 & $1.08 \mathrm{E}-09$ & $1.75 \mathrm{E}-10$ & & $6.14 \mathrm{E}+00$ \\
\hline ENSBTAG00000038970 & $4.88 \mathrm{E}-10$ & $7.66 \mathrm{E}-11$ & ZBTB14 & $6.37 E+00$ \\
\hline ENSBTAG00000019876 & $9.71 \mathrm{E}-10$ & $1.46 \mathrm{E}-10$ & DSC1 & $6.65 \mathrm{E}+00$ \\
\hline ENSBTAG00000021250 & $4.32 \mathrm{E}-10$ & $5.31 \mathrm{E}-11$ & RALBP1 & $8.12 \mathrm{E}+00$ \\
\hline ENSBTAG00000012441 & $3.75 \mathrm{E}-10$ & $4.11 \mathrm{E}-11$ & SMAD7 & $9.11 \mathrm{E}+00$ \\
\hline ENSBTAG00000008169 & $7.66 \mathrm{E}-10$ & $8.12 \mathrm{E}-11$ & C18orf54 & $9.42 \mathrm{E}+00$ \\
\hline ENSBTAG00000003354 & $4.29 E-10$ & $2.78 \mathrm{E}-11$ & SMCHD1 & $1.54 \mathrm{E}+01$ \\
\hline ENSBTAG00000039483 & $7.19 \mathrm{E}-10$ & $9.85 \mathrm{E}-12$ & DSG3 & $7.23 \mathrm{E}+01$ \\
\hline ENSBTAG00000034676 & $1.20 \mathrm{E}-10$ & 0 & & $1.20 \mathrm{E}+03$ \\
\hline ENSBTAG00000046269 & $2.16 \mathrm{E}-10$ & 0 & & $2.16 \mathrm{E}+03$ \\
\hline ENSBTAG00000034555 & $9.58 \mathrm{E}-11$ & 0 & AP3S1 & $9.59 \mathrm{E}+02$ \\
\hline ENSBTAG00000012320 & $9.24 \mathrm{E}-11$ & 0 & CETN1 & $9.25 E+02$ \\
\hline ENSBTAG00000046337 & $1.54 \mathrm{E}-10$ & 0 & TUBB6 & $1.54 \mathrm{E}+03$ \\
\hline ENSBTAG00000002367 & 0 & $7.66 \mathrm{E}-11$ & HCFC1R1 & $1.30 \mathrm{E}-03$ \\
\hline ENSBTAG00000032643 & 0 & 4.12E-10 & & $2.43 \mathrm{E}-04$ \\
\hline
\end{tabular}




\begin{tabular}{|c|c|c|c|c|}
\hline ENSBTAG00000019743 & $1.32 \mathrm{E}-10$ & $5.38 \mathrm{E}-10$ & CHTF18 & 2.45E-01 \\
\hline ENSBTAG00000034238 & $1.01 \mathrm{E}-10$ & $3.06 \mathrm{E}-10$ & ZSCAN10 & $3.30 \mathrm{E}-01$ \\
\hline ENSBTAG00000009842 & $2.68 \mathrm{E}-10$ & $8.06 \mathrm{E}-10$ & CRYM & 3.33E-01 \\
\hline ENSBTAG00000009928 & $2.13 \mathrm{E}-10$ & $5.92 \mathrm{E}-10$ & MLST8 & 3.60E-01 \\
\hline ENSBTAG00000032198 & $2.02 \mathrm{E}-10$ & $5.11 \mathrm{E}-10$ & & 3.96E-01 \\
\hline ENSBTAG00000017697 & $2.32 \mathrm{E}-11$ & $5.85 \mathrm{E}-11$ & CTF1 & $3.98 \mathrm{E}-01$ \\
\hline ENSBTAG00000018000 & $3.31 \mathrm{E}-10$ & 7.93E-10 & & 4.17E-01 \\
\hline ENSBTAG00000005572 & $3.04 \mathrm{E}-10$ & $6.97 \mathrm{E}-10$ & ZNF205 & 4.37E-01 \\
\hline ENSBTAG00000020734 & $1.52 \mathrm{E}-10$ & $2.88 \mathrm{E}-10$ & ARL6IP1 & $5.28 \mathrm{E}-01$ \\
\hline ENSBTAG00000032880 & $2.20 \mathrm{E}-10$ & 4.17E-10 & & 5.29E-01 \\
\hline ENSBTAG00000009868 & $1.19 \mathrm{E}-10$ & $2.24 \mathrm{E}-10$ & VGF & $5.30 \mathrm{E}-01$ \\
\hline ENSBTAG00000002935 & $5.59 \mathrm{E}-11$ & $1.06 \mathrm{E}-10$ & TMEM248 & $5.28 \mathrm{E}-01$ \\
\hline ENSBTAG00000019123 & $3.82 \mathrm{E}-10$ & $6.90 \mathrm{E}-10$ & MEFV & $5.54 \mathrm{E}-01$ \\
\hline ENSBTAG00000000179 & $1.79 \mathrm{E}-10$ & $2.91 \mathrm{E}-10$ & RPUSD1 & 6.16E-01 \\
\hline ENSBTAG00000026408 & $4.12 \mathrm{E}-10$ & $6.58 \mathrm{E}-10$ & ZNF213 & 6.26E-01 \\
\hline ENSBTAG00000035373 & $1.02 \mathrm{E}-10$ & $1.61 \mathrm{E}-10$ & & 6.33E-01 \\
\hline ENSBTAG00000009945 & $2.29 \mathrm{E}-10$ & $3.61 E-10$ & BRICD5 & 6.35E-01 \\
\hline ENSBTAG00000011162 & $3.45 \mathrm{E}-10$ & $5.41 \mathrm{E}-10$ & ZKSCAN2 & 6.37E-01 \\
\hline ENSBTAG00000003607 & $3.49 \mathrm{E}-10$ & $5.41 \mathrm{E}-10$ & FAM20C & 6.46E-01 \\
\hline ENSBTAG00000018011 & $5.56 \mathrm{E}-10$ & $8.57 \mathrm{E}-10$ & APOBR & 6.49E-01 \\
\hline ENSBTAG00000007402 & $1.89 \mathrm{E}-10$ & $2.91 \mathrm{E}-10$ & ZFAND2A & $6.50 \mathrm{E}-01$ \\
\hline ENSBTAG00000006593 & $1.82 \mathrm{E}-10$ & $2.75 \mathrm{E}-10$ & ANKS3 & $6.62 \mathrm{E}-01$ \\
\hline ENSBTAG00000021407 & $4.42 \mathrm{E}-10$ & $6.57 \mathrm{E}-10$ & & $6.73 \mathrm{E}-01$ \\
\hline ENSBTAG00000004875 & $8.16 \mathrm{E}-11$ & $1.21 \mathrm{E}-10$ & NAT15 & $6.75 \mathrm{E}-01$ \\
\hline ENSBTAG00000034368 & $1.77 \mathrm{E}-10$ & $2.60 \mathrm{E}-10$ & PRSS33 & $6.81 \mathrm{E}-01$ \\
\hline ENSBTAG00000033174 & $8.79 \mathrm{E}-11$ & $1.28 \mathrm{E}-10$ & TFAP4 & 6.87E-01 \\
\hline ENSBTAG00000002470 & $1.66 \mathrm{E}-10$ & $2.40 \mathrm{E}-10$ & CCDC78 & $6.94 \mathrm{E}-01$ \\
\hline ENSBTAG00000015045 & $4.98 \mathrm{E}-10$ & $6.95 \mathrm{E}-10$ & PRSS21 & 7.16E-01 \\
\hline ENSBTAG00000005195 & $5.49 \mathrm{E}-10$ & $7.57 \mathrm{E}-10$ & ZNF200 & 7.26E-01 \\
\hline ENSBTAG00000026194 & $4.70 \mathrm{E}-10$ & $6.36 \mathrm{E}-10$ & AMZ1 & 7.39E-01 \\
\hline ENSBTAG00000016330 & $1.77 \mathrm{E}-10$ & $2.36 \mathrm{E}-10$ & 12-Sep & $7.48 \mathrm{E}-01$ \\
\hline ENSBTAG00000026428 & 2.44E-10 & 3.27E-10 & ATP6VOC & 7.47E-01 \\
\hline ENSBTAG00000035782 & $3.40 \mathrm{E}-10$ & $4.50 \mathrm{E}-10$ & METTL22 & 7.55E-01 \\
\hline ENSBTAG00000008190 & 5.17E-10 & $6.81 E-10$ & SUMF2 & 7.59E-01 \\
\hline ENSBTAG00000013858 & $2.47 \mathrm{E}-10$ & $3.15 \mathrm{E}-10$ & C16orf72 & 7.84E-01 \\
\hline ENSBTAG00000045625 & $1.25 \mathrm{E}-10$ & $1.58 \mathrm{E}-10$ & NTN3 & 7.94E-01 \\
\hline ENSBTAG00000040432 & $1.35 \mathrm{E}-10$ & $1.70 \mathrm{E}-10$ & CLDN3 & 7.94E-01 \\
\hline ENSBTAG00000018016 & $1.23 \mathrm{E}-10$ & $1.55 \mathrm{E}-10$ & NUPR1 & 7.95E-01 \\
\hline ENSBTAG00000004386 & 2.44E-10 & $3.08 \mathrm{E}-10$ & SOCS1 & 7.93E-01 \\
\hline ENSBTAG00000016567 & $1.12 \mathrm{E}-10$ & $1.42 \mathrm{E}-10$ & HBQ1 & 7.91E-01 \\
\hline ENSBTAG00000008487 & $1.72 \mathrm{E}-10$ & $2.17 \mathrm{E}-10$ & VPS37D & 7.94E-01 \\
\hline ENSBTAG00000047634 & $3.50 \mathrm{E}-10$ & $4.41 \mathrm{E}-10$ & & 7.93E-01 \\
\hline ENSBTAG00000006595 & $1.71 \mathrm{E}-10$ & $2.16 \mathrm{E}-10$ & C16orf71 & $7.92 \mathrm{E}-01$ \\
\hline ENSBTAG00000048231 & 8.87E-10 & 1.12E-09 & & 7.92E-01 \\
\hline ENSBTAG00000026417 & $1.07 \mathrm{E}-10$ & $1.34 \mathrm{E}-10$ & & 7.96E-01 \\
\hline ENSBTAG00000019136 & $3.47 \mathrm{E}-11$ & $4.38 \mathrm{E}-11$ & STUB1 & 7.93E-01 \\
\hline ENSBTAG00000018625 & $5.35 \mathrm{E}-10$ & $6.75 \mathrm{E}-10$ & ZNF263 & 7.93E-01 \\
\hline ENSBTAG00000020209 & $9.69 \mathrm{E}-10$ & $1.22 \mathrm{E}-09$ & TIGD7 & 7.94E-01 \\
\hline ENSBTAG00000033580 & $6.76 \mathrm{E}-10$ & $8.52 \mathrm{E}-10$ & MSLNL & 7.94E-01 \\
\hline ENSBTAG00000002467 & $5.44 \mathrm{E}-11$ & $6.85 \mathrm{E}-11$ & FAM173A & 7.94E-01 \\
\hline ENSBTAG00000039962 & 4.47E-11 & $5.64 \mathrm{E}-11$ & DCTPP1 & 7.94E-01 \\
\hline ENSBTAG00000005757 & $2.70 \mathrm{E}-10$ & $3.40 \mathrm{E}-10$ & FUS & 7.95E-01 \\
\hline ENSBTAG00000038330 & $2.36 \mathrm{E}-10$ & $2.97 \mathrm{E}-10$ & & 7.95E-01 \\
\hline ENSBTAG00000001078 & $3.22 \mathrm{E}-10$ & $4.06 \mathrm{E}-10$ & & 7.94E-01 \\
\hline ENSBTAG00000016581 & $3.20 \mathrm{E}-11$ & $4.04 \mathrm{E}-11$ & MRPL28 & 7.93E-01 \\
\hline ENSBTAG00000033526 & $4.29 \mathrm{E}-10$ & $5.41 \mathrm{E}-10$ & GNG13 & 7.93E-01 \\
\hline ENSBTAG00000019804 & $3.80 \mathrm{E}-11$ & $4.79 \mathrm{E}-11$ & SNRNP25 & 7.93E-01 \\
\hline ENSBTAG00000010851 & $1.81 \mathrm{E}-10$ & $2.29 \mathrm{E}-10$ & SEPHS2 & 7.92E-01 \\
\hline ENSBTAG00000005446 & $1.69 \mathrm{E}-10$ & $2.12 \mathrm{E}-10$ & & 7.95E-01 \\
\hline ENSBTAG00000020896 & $1.36 \mathrm{E}-10$ & $1.72 \mathrm{E}-10$ & GRIFIN & 7.93E-01 \\
\hline ENSBTAG00000040381 & $6.39 \mathrm{E}-10$ & 7.97E-10 & ZNF75A & $8.02 \mathrm{E}-01$ \\
\hline ENSBTAG00000022825 & $9.11 \mathrm{E}-10$ & 1.11E-09 & & $8.20 \mathrm{E}-01$ \\
\hline ENSBTAG00000002484 & $3.90 \mathrm{E}-10$ & $4.76 \mathrm{E}-10$ & NARFL & $8.20 \mathrm{E}-01$ \\
\hline ENSBTAG00000000177 & $5.74 \mathrm{E}-10$ & $6.94 \mathrm{E}-10$ & & 8.27E-01 \\
\hline ENSBTAG00000048279 & 1.14E-09 & $1.38 \mathrm{E}-09$ & & $8.25 \mathrm{E}-01$ \\
\hline ENSBTAG00000008446 & $3.60 \mathrm{E}-10$ & $4.34 \mathrm{E}-10$ & TYW1 & $8.31 \mathrm{E}-01$ \\
\hline ENSBTAG00000009961 & $3.24 \mathrm{E}-10$ & $3.87 \mathrm{E}-10$ & E4F1 & 8.39E-01 \\
\hline ENSBTAG00000046098 & $5.28 \mathrm{E}-10$ & $6.28 \mathrm{E}-10$ & ACSM4 & $8.41 \mathrm{E}-01$ \\
\hline ENSBTAG00000017015 & $6.10 \mathrm{E}-10$ & $7.25 \mathrm{E}-10$ & TEKT5 & $8.41 \mathrm{E}-01$ \\
\hline ENSBTAG00000046142 & $2.22 \mathrm{E}-10$ & $2.62 \mathrm{E}-10$ & & 8.46E-01 \\
\hline
\end{tabular}




\begin{tabular}{|c|c|c|c|c|}
\hline ENSBTAG00000040575 & $4.10 \mathrm{E}-10$ & $4.82 \mathrm{E}-10$ & KCTD5 & 8.51E-01 \\
\hline ENSBTAG00000019593 & $9.28 \mathrm{E}-10$ & $1.09 \mathrm{E}-09$ & KNOP1 & $8.52 \mathrm{E}-01$ \\
\hline ENSBTAG00000002101 & $6.61 \mathrm{E}-10$ & $7.69 \mathrm{E}-10$ & GDE1 & 8.59E-01 \\
\hline ENSBTAG00000026278 & $6.93 \mathrm{E}-10$ & $8.05 \mathrm{E}-10$ & CLDN4 & $8.60 \mathrm{E}-01$ \\
\hline ENSBTAG00000014541 & $1.91 \mathrm{E}-10$ & $2.22 \mathrm{E}-10$ & PDGFA & $8.61 \mathrm{E}-01$ \\
\hline ENSBTAG00000018250 & $2.75 \mathrm{E}-10$ & $3.17 \mathrm{E}-10$ & CLEC19A & $8.68 \mathrm{E}-01$ \\
\hline ENSBTAG00000002352 & $7.31 \mathrm{E}-10$ & $8.39 \mathrm{E}-10$ & GGA2 & $8.71 \mathrm{E}-01$ \\
\hline ENSBTAG00000020198 & $3.89 \mathrm{E}-10$ & 4.41E-10 & METRN & $8.82 \mathrm{E}-01$ \\
\hline ENSBTAG00000016588 & $1.73 \mathrm{E}-10$ & $1.95 \mathrm{E}-10$ & TMEM8A & $8.89 \mathrm{E}-01$ \\
\hline ENSBTAG00000003960 & $4.18 \mathrm{E}-10$ & $4.71 \mathrm{E}-10$ & CCP110 & 8.87E-01 \\
\hline ENSBTAG00000004676 & $2.25 \mathrm{E}-10$ & $2.54 \mathrm{E}-10$ & TMEM184A & $8.86 \mathrm{E}-01$ \\
\hline ENSBTAG00000009964 & $4.84 \mathrm{E}-10$ & $5.42 \mathrm{E}-10$ & DNASE1L2 & 8.94E-01 \\
\hline ENSBTAG00000032373 & $3.31 \mathrm{E}-10$ & $3.69 \mathrm{E}-10$ & & 8.97E-01 \\
\hline ENSBTAG00000014826 & $4.01 \mathrm{E}-10$ & $4.46 \mathrm{E}-10$ & ZC3H7A & $9.00 \mathrm{E}-01$ \\
\hline ENSBTAG00000001374 & $5.12 \mathrm{E}-10$ & $5.67 \mathrm{E}-10$ & WBSCR27 & 9.03E-01 \\
\hline ENSBTAG00000005458 & $1.46 \mathrm{E}-10$ & $1.62 \mathrm{E}-10$ & TBC1D24 & $9.02 \mathrm{E}-01$ \\
\hline ENSBTAG00000026273 & $3.09 E-10$ & $3.40 \mathrm{E}-10$ & MYL10 & $9.08 \mathrm{E}-01$ \\
\hline ENSBTAG00000047520 & $4.47 \mathrm{E}-10$ & $4.88 \mathrm{E}-10$ & & $9.16 \mathrm{E}-01$ \\
\hline ENSBTAG00000010163 & $3.41 \mathrm{E}-10$ & $3.70 \mathrm{E}-10$ & SCNN1G & $9.22 \mathrm{E}-01$ \\
\hline ENSBTAG00000012928 & 7.07E-11 & 7.64E-11 & PPP4C & $9.26 \mathrm{E}-01$ \\
\hline ENSBTAG00000024884 & $1.35 \mathrm{E}-10$ & $1.45 \mathrm{E}-10$ & C25H16orf59 & $9.29 \mathrm{E}-01$ \\
\hline ENSBTAG00000047781 & $8.06 \mathrm{E}-10$ & 8.70E-10 & & 9.27E-01 \\
\hline ENSBTAG00000020387 & $2.77 \mathrm{E}-10$ & $2.99 \mathrm{E}-10$ & KIAA0430 & $9.28 \mathrm{E}-01$ \\
\hline ENSBTAG00000020898 & $1.79 \mathrm{E}-10$ & $1.91 \mathrm{E}-10$ & CHST12 & $9.35 \mathrm{E}-01$ \\
\hline ENSBTAG00000007900 & 4.50E-10 & $4.81 \mathrm{E}-10$ & FIS1 & $9.35 \mathrm{E}-01$ \\
\hline ENSBTAG00000046014 & $2.12 \mathrm{E}-10$ & $2.26 \mathrm{E}-10$ & GPR146 & $9.38 \mathrm{E}-01$ \\
\hline ENSBTAG00000019249 & $3.58 \mathrm{E}-10$ & $3.82 \mathrm{E}-10$ & HS3ST6 & $9.38 \mathrm{E}-01$ \\
\hline ENSBTAG00000016331 & $1.85 \mathrm{E}-10$ & 1.97E-10 & ROGDI & $9.38 \mathrm{E}-01$ \\
\hline ENSBTAG00000015986 & $5.03 \mathrm{E}-10$ & $5.36 \mathrm{E}-10$ & NDE1 & $9.39 \mathrm{E}-01$ \\
\hline ENSBTAG00000002178 & $4.32 \mathrm{E}-10$ & 4.57E-10 & PPL & $9.46 \mathrm{E}-01$ \\
\hline ENSBTAG00000020109 & $1.43 \mathrm{E}-10$ & $1.51 \mathrm{E}-10$ & TRAP1 & $9.49 \mathrm{E}-01$ \\
\hline ENSBTAG00000034643 & 3.57E-10 & $3.75 \mathrm{E}-10$ & & $9.51 \mathrm{E}-01$ \\
\hline ENSBTAG00000019920 & $1.30 \mathrm{E}-10$ & $1.37 \mathrm{E}-10$ & $\mathrm{BICDL} 2$ & $9.52 \mathrm{E}-01$ \\
\hline ENSBTAG00000019435 & 3.25E-10 & $3.41 \mathrm{E}-10$ & & $9.52 \mathrm{E}-01$ \\
\hline ENSBTAG00000003191 & $3.64 \mathrm{E}-10$ & $3.82 \mathrm{E}-10$ & FSCN1 & $9.53 \mathrm{E}-01$ \\
\hline ENSBTAG00000032223 & $6.67 \mathrm{E}-10$ & $6.99 \mathrm{E}-10$ & COG7 & $9.54 \mathrm{E}-01$ \\
\hline ENSBTAG00000031806 & $1.13 \mathrm{E}-10$ & $1.18 \mathrm{E}-10$ & & $9.56 \mathrm{E}-01$ \\
\hline ENSBTAG00000004502 & $2.43 \mathrm{E}-10$ & $2.53 \mathrm{E}-10$ & CLUAP1 & $9.59 \mathrm{E}-01$ \\
\hline ENSBTAG00000020184 & $2.83 \mathrm{E}-10$ & $2.95 \mathrm{E}-10$ & AQP8 & $9.58 \mathrm{E}-01$ \\
\hline ENSBTAG00000038115 & $2.22 \mathrm{E}-10$ & $2.31 \mathrm{E}-10$ & FLYWCH2 & $9.63 \mathrm{E}-01$ \\
\hline ENSBTAG00000026381 & 4.19E-10 & $4.35 \mathrm{E}-10$ & TMEM114 & $9.63 \mathrm{E}-01$ \\
\hline ENSBTAG00000040438 & 1.07E-09 & $1.12 \mathrm{E}-09$ & & $9.59 \mathrm{E}-01$ \\
\hline ENSBTAG00000019745 & $3.21 \mathrm{E}-10$ & $3.31 \mathrm{E}-10$ & LMF1 & $9.69 \mathrm{E}-01$ \\
\hline ENSBTAG00000011744 & $5.02 \mathrm{E}-10$ & 5.19E-10 & USP31 & $9.68 \mathrm{E}-01$ \\
\hline ENSBTAG00000026403 & $1.78 \mathrm{E}-10$ & $1.84 \mathrm{E}-10$ & CREBBP & $9.70 \mathrm{E}-01$ \\
\hline ENSBTAG00000039782 & $1.91 \mathrm{E}-10$ & $1.97 \mathrm{E}-10$ & ZNF12 & $9.71 \mathrm{E}-01$ \\
\hline ENSBTAG00000010309 & $1.15 \mathrm{E}-10$ & 1.17E-10 & XPO6 & $9.81 \mathrm{E}-01$ \\
\hline ENSBTAG00000019596 & $6.48 \mathrm{E}-10$ & $6.59 \mathrm{E}-10$ & GPRC5B & $9.83 \mathrm{E}-01$ \\
\hline ENSBTAG00000021522 & 4.07E-10 & 4.14E-10 & PLA2G10 & $9.84 \mathrm{E}-01$ \\
\hline ENSBTAG00000018050 & $1.88 \mathrm{E}-10$ & $1.90 \mathrm{E}-10$ & CDR2 & $9.91 \mathrm{E}-01$ \\
\hline ENSBTAG00000004804 & $1.40 \mathrm{E}-10$ & $1.42 \mathrm{E}-10$ & RRN3 & $9.84 \mathrm{E}-01$ \\
\hline ENSBTAG00000044092 & $4.05 \mathrm{E}-10$ & $4.09 \mathrm{E}-10$ & IQCK & 9.91E-01 \\
\hline ENSBTAG00000038921 & $3.57 \mathrm{E}-10$ & $3.60 \mathrm{E}-10$ & & $9.92 \mathrm{E}-01$ \\
\hline ENSBTAG00000032503 & $2.56 \mathrm{E}-10$ & $2.58 \mathrm{E}-10$ & ITPRIPL2 & $9.94 \mathrm{E}-01$ \\
\hline ENSBTAG00000008632 & $6.07 \mathrm{E}-10$ & $6.11 \mathrm{E}-10$ & & $9.93 \mathrm{E}-01$ \\
\hline ENSBTAG00000010339 & $1.71 \mathrm{E}-10$ & $1.72 \mathrm{E}-10$ & ABHD11 & $9.92 \mathrm{E}-01$ \\
\hline ENSBTAG00000026415 & $3.28 \mathrm{E}-10$ & $3.30 \mathrm{E}-10$ & MRPS34 & 9.93E-01 \\
\hline ENSBTAG00000001404 & $4.95 \mathrm{E}-10$ & 4.97E-10 & CACNG3 & $9.95 \mathrm{E}-01$ \\
\hline ENSBTAG00000021651 & $7.91 \mathrm{E}-10$ & $7.96 \mathrm{E}-10$ & UQCRC2 & $9.94 \mathrm{E}-01$ \\
\hline ENSBTAG00000015009 & $1.24 \mathrm{E}-10$ & $1.24 \mathrm{E}-10$ & LAMTOR4 & $9.96 \mathrm{E}-01$ \\
\hline ENSBTAG00000003842 & $1.08 \mathrm{E}-10$ & $1.08 \mathrm{E}-10$ & RABGEF1 & $1.00 \mathrm{E}+00$ \\
\hline ENSBTAG00000007880 & $2.35 \mathrm{E}-10$ & $2.35 \mathrm{E}-10$ & RAB $40 C$ & $1.00 \mathrm{E}+00$ \\
\hline ENSBTAG00000020157 & $1.02 \mathrm{E}-09$ & $1.02 \mathrm{E}-09$ & & $9.98 \mathrm{E}-01$ \\
\hline ENSBTAG00000017435 & $2.19 E-10$ & 2.17E-10 & KCTD7 & $1.01 E+00$ \\
\hline ENSBTAG00000008195 & $6.90 \mathrm{E}-10$ & $6.81 \mathrm{E}-10$ & PHKG1 & $1.01 \mathrm{E}+00$ \\
\hline ENSBTAG00000040394 & $3.82 \mathrm{E}-10$ & $3.75 \mathrm{E}-10$ & PRSS27 & $1.02 E+00$ \\
\hline ENSBTAG00000047673 & 4.44E-10 & 4.37E-10 & THUMPD1 & $1.02 E+00$ \\
\hline ENSBTAG00000002097 & $3.21 \mathrm{E}-10$ & $3.16 \mathrm{E}-10$ & TMC5 & $1.02 \mathrm{E}+00$ \\
\hline ENSBTAG00000004051 & $6.77 \mathrm{E}-10$ & $6.62 \mathrm{E}-10$ & SBDS & $1.02 \mathrm{E}+00$ \\
\hline ENSBTAG00000034436 & $5.24 \mathrm{E}-10$ & $5.12 \mathrm{E}-10$ & PDPK1 & $1.02 \mathrm{E}+00$ \\
\hline
\end{tabular}




\begin{tabular}{|c|c|c|c|c|}
\hline ENSBTAG00000008222 & $1.02 \mathrm{E}-09$ & $9.93 \mathrm{E}-10$ & & $1.02 \mathrm{E}+00$ \\
\hline ENSBTAG00000043969 & $2.46 \mathrm{E}-10$ & 2.40E-10 & CALN1 & $1.03 E+00$ \\
\hline ENSBTAG00000006447 & $5.62 \mathrm{E}-10$ & 5.47E-10 & ACSM3 & $1.03 E+00$ \\
\hline ENSBTAG00000009969 & $2.00 \mathrm{E}-10$ & $1.95 \mathrm{E}-10$ & RNPS1 & $1.03 \mathrm{E}+00$ \\
\hline ENSBTAG00000003577 & $1.89 \mathrm{E}-10$ & $1.83 \mathrm{E}-10$ & GSPT1 & $1.03 \mathrm{E}+00$ \\
\hline ENSBTAG00000047767 & $1.32 \mathrm{E}-09$ & 1.27E-09 & & $1.04 \mathrm{E}+00$ \\
\hline ENSBTAG00000001022 & $2.09 \mathrm{E}-10$ & $2.01 \mathrm{E}-10$ & AMDHD2 & $1.04 \mathrm{E}+00$ \\
\hline ENSBTAG00000026192 & $1.96 \mathrm{E}-10$ & $1.87 \mathrm{E}-10$ & C7orf50 & $1.05 E+00$ \\
\hline ENSBTAG00000012290 & $2.34 \mathrm{E}-10$ & $2.24 \mathrm{E}-10$ & SCNN1B & $1.04 \mathrm{E}+00$ \\
\hline ENSBTAG00000017067 & $2.38 \mathrm{E}-10$ & 2.27E-10 & WBSCR22 & $1.05 E+00$ \\
\hline ENSBTAG00000003467 & 5.77E-10 & $5.50 \mathrm{E}-10$ & SLC5A11 & $1.05 \mathrm{E}+00$ \\
\hline ENSBTAG00000004801 & $1.90 \mathrm{E}-10$ & $1.81 \mathrm{E}-10$ & PDXDC1 & $1.05 \mathrm{E}+00$ \\
\hline ENSBTAG00000006591 & $2.27 \mathrm{E}-10$ & $2.16 \mathrm{E}-10$ & CCNF & $1.05 \mathrm{E}+00$ \\
\hline ENSBTAG00000018870 & $2.56 \mathrm{E}-10$ & $2.43 \mathrm{E}-10$ & OTOA & $1.05 \mathrm{E}+00$ \\
\hline ENSBTAG00000009199 & $1.48 \mathrm{E}-10$ & $1.40 \mathrm{E}-10$ & GLIS2 & $1.06 \mathrm{E}+00$ \\
\hline ENSBTAG00000020742 & $1.85 \mathrm{E}-10$ & $1.75 \mathrm{E}-10$ & FZD9 & $1.06 \mathrm{E}+00$ \\
\hline ENSBTAG00000009965 & $5.79 \mathrm{E}-10$ & $5.48 \mathrm{E}-10$ & ECI1 & $1.06 \mathrm{E}+00$ \\
\hline ENSBTAG00000006542 & $1.34 \mathrm{E}-10$ & $1.26 \mathrm{E}-10$ & RABEP2 & $1.06 \mathrm{E}+00$ \\
\hline ENSBTAG00000006590 & $2.56 \mathrm{E}-10$ & $2.42 \mathrm{E}-10$ & NUDT16L1 & $1.06 \mathrm{E}+00$ \\
\hline ENSBTAG00000048253 & 2.33E-10 & $2.20 \mathrm{E}-10$ & & $1.06 \mathrm{E}+00$ \\
\hline ENSBTAG00000031789 & $9.03 \mathrm{E}-11$ & $8.53 \mathrm{E}-11$ & FBRS & $1.06 \mathrm{E}+00$ \\
\hline ENSBTAG00000002481 & $1.37 \mathrm{E}-10$ & $1.29 \mathrm{E}-10$ & HAGHL & $1.06 \mathrm{E}+00$ \\
\hline ENSBTAG00000026248 & $2.93 \mathrm{E}-10$ & 2.77E-10 & POP7 & $1.06 \mathrm{E}+00$ \\
\hline ENSBTAG00000046132 & $3.28 \mathrm{E}-10$ & $3.10 \mathrm{E}-10$ & & $1.06 \mathrm{E}+00$ \\
\hline ENSBTAG00000018869 & $1.41 \mathrm{E}-10$ & $1.33 \mathrm{E}-10$ & IGSF6 & $1.06 \mathrm{E}+00$ \\
\hline ENSBTAG00000020107 & $2.08 \mathrm{E}-10$ & $1.96 \mathrm{E}-10$ & DNASE1 & $1.06 \mathrm{E}+00$ \\
\hline ENSBTAG00000003305 & $3.48 \mathrm{E}-10$ & $3.27 \mathrm{E}-10$ & NCF1 & $1.06 \mathrm{E}+00$ \\
\hline ENSBTAG00000018868 & 1.17E-10 & $1.09 \mathrm{E}-10$ & METTL9 & $1.07 \mathrm{E}+00$ \\
\hline ENSBTAG00000015988 & $6.54 \mathrm{E}-10$ & $6.12 \mathrm{E}-10$ & MYH11 & $1.07 \mathrm{E}+00$ \\
\hline ENSBTAG00000018938 & $2.72 \mathrm{E}-10$ & $2.55 \mathrm{E}-10$ & C16orf5 & $1.07 E+00$ \\
\hline ENSBTAG00000031598 & $5.82 \mathrm{E}-10$ & $5.43 \mathrm{E}-10$ & GBAS & $1.07 E+00$ \\
\hline ENSBTAG00000005679 & $6.55 \mathrm{E}-10$ & $6.11 \mathrm{E}-10$ & TMEM130 & $1.07 E+00$ \\
\hline ENSBTAG00000004509 & $8.43 \mathrm{E}-11$ & $7.83 \mathrm{E}-11$ & SLX4 & $1.08 \mathrm{E}+00$ \\
\hline ENSBTAG00000018560 & $8.09 \mathrm{E}-10$ & 7.50E-10 & DNAH3 & $1.08 \mathrm{E}+00$ \\
\hline ENSBTAG00000005455 & $2.81 \mathrm{E}-10$ & $2.59 \mathrm{E}-10$ & USP7 & $1.08 \mathrm{E}+00$ \\
\hline ENSBTAG00000007896 & $3.86 \mathrm{E}-10$ & $3.56 \mathrm{E}-10$ & NAGPA & $1.08 \mathrm{E}+00$ \\
\hline ENSBTAG00000010015 & $5.18 \mathrm{E}-10$ & $4.76 \mathrm{E}-10$ & NMRAL1 & $1.09 E+00$ \\
\hline ENSBTAG00000020043 & $3.84 \mathrm{E}-10$ & $3.51 E-10$ & ARHGAP17 & $1.09 \mathrm{E}+00$ \\
\hline ENSBTAG00000018937 & $2.01 \mathrm{E}-10$ & $1.83 \mathrm{E}-10$ & HMOX2 & $1.10 \mathrm{E}+00$ \\
\hline ENSBTAG00000017662 & $3.74 \mathrm{E}-10$ & $3.41 E-10$ & EEF2K & $1.10 \mathrm{E}+00$ \\
\hline ENSBTAG00000005934 & $5.23 \mathrm{E}-10$ & 4.77E-10 & TTYH3 & $1.10 \mathrm{E}+00$ \\
\hline ENSBTAG00000019181 & $4.69 \mathrm{E}-10$ & 4.27E-10 & BAIAP2L1 & $1.10 \mathrm{E}+00$ \\
\hline ENSBTAG00000000390 & $2.36 \mathrm{E}-10$ & $2.14 \mathrm{E}-10$ & TPST1 & $1.10 \mathrm{E}+00$ \\
\hline ENSBTAG00000032122 & $2.49 \mathrm{E}-10$ & 2.27E-10 & CD19 & $1.10 \mathrm{E}+00$ \\
\hline ENSBTAG00000016571 & $2.47 \mathrm{E}-10$ & $2.24 \mathrm{E}-10$ & & $1.10 \mathrm{E}+00$ \\
\hline ENSBTAG00000046498 & $1.96 \mathrm{E}-10$ & $1.78 \mathrm{E}-10$ & & $1.10 \mathrm{E}+00$ \\
\hline ENSBTAG00000026307 & $2.24 \mathrm{E}-10$ & 2.03E-10 & ZNF629 & $1.11 E+00$ \\
\hline ENSBTAG00000006239 & $3.98 \mathrm{E}-10$ & $3.59 \mathrm{E}-10$ & & $1.11 E+00$ \\
\hline ENSBTAG00000016594 & $2.08 \mathrm{E}-10$ & $1.87 \mathrm{E}-10$ & PIGQ & $1.11 E+00$ \\
\hline ENSBTAG00000032884 & $3.74 \mathrm{E}-10$ & $3.36 \mathrm{E}-10$ & Tnp2 & $1.11 \mathrm{E}+00$ \\
\hline ENSBTAG00000020535 & $4.32 \mathrm{E}-10$ & $3.88 \mathrm{E}-10$ & & $1.11 E+00$ \\
\hline ENSBTAG00000004505 & $1.41 \mathrm{E}-10$ & $1.27 \mathrm{E}-10$ & NLRC3 & $1.11 E+00$ \\
\hline ENSBTAG00000046326 & $6.10 \mathrm{E}-10$ & $5.48 \mathrm{E}-10$ & & $1.11 E+00$ \\
\hline ENSBTAG00000000345 & $5.66 \mathrm{E}-10$ & $5.06 \mathrm{E}-10$ & CLDN15 & $1.12 \mathrm{E}+00$ \\
\hline ENSBTAG00000001606 & $1.23 \mathrm{E}-09$ & $1.11 \mathrm{E}-09$ & $\mathrm{OR} 2 \mathrm{C} 1$ & $1.11 E+00$ \\
\hline ENSBTAG00000009153 & $2.18 \mathrm{E}-10$ & $1.94 \mathrm{E}-10$ & MLXIPL & $1.12 \mathrm{E}+00$ \\
\hline ENSBTAG00000008175 & $3.59 \mathrm{E}-10$ & $3.20 \mathrm{E}-10$ & ZNF713 & $1.12 \mathrm{E}+00$ \\
\hline ENSBTAG00000005908 & 4.24E-10 & 3.77E-10 & & $1.12 \mathrm{E}+00$ \\
\hline ENSBTAG00000007887 & $2.11 \mathrm{E}-10$ & $1.86 \mathrm{E}-10$ & GRIN2A & $1.13 E+00$ \\
\hline ENSBTAG00000003446 & $6.02 \mathrm{E}-10$ & $5.31 \mathrm{E}-10$ & EPHB4 & $1.13 E+00$ \\
\hline ENSBTAG00000021521 & $6.35 \mathrm{E}-10$ & $5.60 \mathrm{E}-10$ & BFAR & $1.13 E+00$ \\
\hline ENSBTAG00000003432 & $4.31 \mathrm{E}-10$ & 3.80E-10 & ZAN & $1.13 E+00$ \\
\hline ENSBTAG00000009547 & $7.36 \mathrm{E}-10$ & $6.49 \mathrm{E}-10$ & ZDHHC4 & $1.13 E+00$ \\
\hline ENSBTAG00000020051 & $8.02 \mathrm{E}-10$ & 7.05E-10 & C16orf82 & $1.14 \mathrm{E}+00$ \\
\hline ENSBTAG00000002346 & $3.07 \mathrm{E}-10$ & 2.67E-10 & HSD3B7 & $1.15 \mathrm{E}+00$ \\
\hline ENSBTAG00000002176 & $3.95 \mathrm{E}-10$ & $3.42 \mathrm{E}-10$ & NSMCE1 & $1.15 E+00$ \\
\hline ENSBTAG00000001417 & $5.63 \mathrm{E}-10$ & 4.87E-10 & ACSM1 & $1.16 \mathrm{E}+00$ \\
\hline ENSBTAG00000014503 & $3.48 \mathrm{E}-10$ & $2.98 \mathrm{E}-10$ & IQCE & $1.17 E+00$ \\
\hline ENSBTAG00000017759 & 7.30E-10 & $6.24 \mathrm{E}-10$ & & $1.17 \mathrm{E}+00$ \\
\hline ENSBTAG00000045896 & $5.72 \mathrm{E}-10$ & $4.89 \mathrm{E}-10$ & NPTX2 & $1.17 \mathrm{E}+00$ \\
\hline
\end{tabular}




\begin{tabular}{|c|c|c|c|c|}
\hline ENSBTAG00000010264 & $5.14 \mathrm{E}-10$ & $4.39 \mathrm{E}-10$ & FBXL18 & $1.17 \mathrm{E}+00$ \\
\hline ENSBTAG00000011504 & $3.90 \mathrm{E}-10$ & 3.33E-10 & ZP2 & $1.17 \mathrm{E}+00$ \\
\hline ENSBTAG00000044040 & 4.47E-10 & $3.80 \mathrm{E}-10$ & PDAP1 & $1.18 \mathrm{E}+00$ \\
\hline ENSBTAG00000009560 & $2.39 \mathrm{E}-10$ & $2.02 \mathrm{E}-10$ & NUBP1 & $1.18 \mathrm{E}+00$ \\
\hline ENSBTAG00000012201 & $8.94 \mathrm{E}-10$ & $7.58 \mathrm{E}-10$ & & $1.18 \mathrm{E}+00$ \\
\hline ENSBTAG00000007113 & 5.34E-10 & $4.52 \mathrm{E}-10$ & TRRAP & $1.18 \mathrm{E}+00$ \\
\hline ENSBTAG00000008718 & 5.77E-10 & $4.88 \mathrm{E}-10$ & WBSCR17 & $1.18 \mathrm{E}+00$ \\
\hline ENSBTAG00000018094 & $1.16 \mathrm{E}-10$ & $9.70 \mathrm{E}-11$ & ZNF689 & $1.19 E+00$ \\
\hline ENSBTAG00000001614 & $2.61 \mathrm{E}-10$ & $2.19 \mathrm{E}-10$ & ZNF174 & $1.19 \mathrm{E}+00$ \\
\hline ENSBTAG00000047266 & $6.86 \mathrm{E}-10$ & $5.75 \mathrm{E}-10$ & & $1.19 \mathrm{E}+00$ \\
\hline ENSBTAG00000017075 & $1.25 \mathrm{E}-10$ & $1.05 \mathrm{E}-10$ & STX1A & $1.19 \mathrm{E}+00$ \\
\hline ENSBTAG00000026325 & $1.78 \mathrm{E}-10$ & $1.49 \mathrm{E}-10$ & ZG16 & $1.19 \mathrm{E}+00$ \\
\hline ENSBTAG00000010521 & 7.10E-10 & $5.95 E-10$ & PVRIG & $1.19 E+00$ \\
\hline ENSBTAG00000040319 & $7.75 \mathrm{E}-10$ & $6.50 \mathrm{E}-10$ & & $1.19 \mathrm{E}+00$ \\
\hline ENSBTAG00000046943 & $1.16 \mathrm{E}-10$ & $9.74 \mathrm{E}-11$ & & $1.19 E+00$ \\
\hline ENSBTAG00000008728 & $1.74 \mathrm{E}-10$ & $1.45 \mathrm{E}-10$ & MKL2 & $1.20 \mathrm{E}+00$ \\
\hline ENSBTAG00000005622 & $2.29 \mathrm{E}-10$ & $1.91 \mathrm{E}-10$ & LITAF & $1.20 \mathrm{E}+00$ \\
\hline ENSBTAG00000046248 & $3.65 \mathrm{E}-10$ & $3.04 \mathrm{E}-10$ & ARPC1B & $1.20 \mathrm{E}+00$ \\
\hline ENSBTAG00000013917 & $1.52 \mathrm{E}-10$ & $1.25 \mathrm{E}-10$ & & $1.22 \mathrm{E}+00$ \\
\hline ENSBTAG00000004607 & $4.66 \mathrm{E}-10$ & $3.84 \mathrm{E}-10$ & GSG1L & $1.21 E+00$ \\
\hline ENSBTAG00000002189 & $5.66 \mathrm{E}-10$ & $4.66 \mathrm{E}-10$ & & $1.21 \mathrm{E}+00$ \\
\hline ENSBTAG00000012541 & $6.26 \mathrm{E}-10$ & $5.15 \mathrm{E}-10$ & CHP2 & $1.22 \mathrm{E}+00$ \\
\hline ENSBTAG00000001731 & $1.03 \mathrm{E}-10$ & $8.44 \mathrm{E}-11$ & GLYR1 & $1.22 \mathrm{E}+00$ \\
\hline ENSBTAG00000013169 & $2.94 \mathrm{E}-10$ & $2.40 \mathrm{E}-10$ & LCMT1 & $1.22 \mathrm{E}+00$ \\
\hline ENSBTAG00000009201 & $2.29 \mathrm{E}-10$ & $1.87 \mathrm{E}-10$ & & $1.22 \mathrm{E}+00$ \\
\hline ENSBTAG00000002477 & $1.02 \mathrm{E}-09$ & $8.30 \mathrm{E}-10$ & MRM2 & $1.23 \mathrm{E}+00$ \\
\hline ENSBTAG00000020582 & $3.24 \mathrm{E}-10$ & $2.63 \mathrm{E}-10$ & PRSS22 & $1.23 E+00$ \\
\hline ENSBTAG00000038568 & $7.34 \mathrm{E}-10$ & $5.97 \mathrm{E}-10$ & & $1.23 E+00$ \\
\hline ENSBTAG00000007210 & $3.09 \mathrm{E}-10$ & $2.51 \mathrm{E}-10$ & RBFOX1 & $1.23 \mathrm{E}+00$ \\
\hline ENSBTAG00000020440 & $2.20 \mathrm{E}-10$ & $1.78 \mathrm{E}-10$ & & $1.23 E+00$ \\
\hline ENSBTAG00000016591 & $2.79 \mathrm{E}-10$ & $2.25 \mathrm{E}-10$ & RAB11FIP3 & $1.24 \mathrm{E}+00$ \\
\hline ENSBTAG00000015253 & $1.61 \mathrm{E}-10$ & $1.29 \mathrm{E}-10$ & Cox19 & $1.25 \mathrm{E}+00$ \\
\hline ENSBTAG00000037449 & $7.15 \mathrm{E}-10$ & $5.72 \mathrm{E}-10$ & ACSM5 & $1.25 \mathrm{E}+00$ \\
\hline ENSBTAG00000014502 & $4.11 E-10$ & $3.29 \mathrm{E}-10$ & BRAT1 & $1.25 E+00$ \\
\hline ENSBTAG00000047264 & $5.01 \mathrm{E}-10$ & $4.00 \mathrm{E}-10$ & & $1.25 E+00$ \\
\hline ENSBTAG00000007142 & 8.67E-10 & $6.93 \mathrm{E}-10$ & CPPED1 & $1.25 \mathrm{E}+00$ \\
\hline ENSBTAG00000010013 & $3.52 \mathrm{E}-10$ & $2.81 \mathrm{E}-10$ & DNAJA3 & $1.25 \mathrm{E}+00$ \\
\hline ENSBTAG00000015952 & $2.92 \mathrm{E}-10$ & $2.32 \mathrm{E}-10$ & ADAP1 & $1.26 \mathrm{E}+00$ \\
\hline ENSBTAG00000046840 & $6.89 \mathrm{E}-10$ & $5.47 \mathrm{E}-10$ & DEXI & $1.26 \mathrm{E}+00$ \\
\hline ENSBTAG00000000704 & $3.71 \mathrm{E}-10$ & $2.92 \mathrm{E}-10$ & GUSB & $1.27 \mathrm{E}+00$ \\
\hline ENSBTAG00000037397 & $1.57 \mathrm{E}-10$ & $1.24 \mathrm{E}-10$ & PRSS36 & $1.27 \mathrm{E}+00$ \\
\hline ENSBTAG00000004035 & $5.12 \mathrm{E}-10$ & $4.03 E-10$ & SDK1 & $1.27 \mathrm{E}+00$ \\
\hline ENSBTAG00000046339 & $2.46 \mathrm{E}-10$ & $1.93 \mathrm{E}-10$ & VASN & $1.27 \mathrm{E}+00$ \\
\hline ENSBTAG00000046399 & $2.76 \mathrm{E}-10$ & $2.18 \mathrm{E}-10$ & & $1.27 \mathrm{E}+00$ \\
\hline ENSBTAG00000018999 & $2.84 \mathrm{E}-10$ & $2.22 \mathrm{E}-10$ & & $1.28 \mathrm{E}+00$ \\
\hline ENSBTAG00000019008 & $1.84 \mathrm{E}-10$ & $1.44 \mathrm{E}-10$ & FBXL16 & $1.28 \mathrm{E}+00$ \\
\hline ENSBTAG00000001736 & $1.73 \mathrm{E}-10$ & $1.35 \mathrm{E}-10$ & UBN1 & $1.28 \mathrm{E}+00$ \\
\hline ENSBTAG00000011692 & $6.57 \mathrm{E}-10$ & $5.08 \mathrm{E}-10$ & C16orf45 & $1.29 \mathrm{E}+00$ \\
\hline ENSBTAG00000009327 & $7.99 \mathrm{E}-10$ & $6.12 \mathrm{E}-10$ & 14-Sep & $1.30 \mathrm{E}+00$ \\
\hline ENSBTAG00000007118 & $5.96 \mathrm{E}-10$ & $4.55 E-10$ & SMURF1 & $1.31 \mathrm{E}+00$ \\
\hline ENSBTAG00000014823 & $7.50 \mathrm{E}-10$ & $5.74 \mathrm{E}-10$ & TXNDC11 & $1.31 \mathrm{E}+00$ \\
\hline ENSBTAG00000007772 & $2.18 \mathrm{E}-10$ & $1.66 \mathrm{E}-10$ & SLC29A4 & $1.31 E+00$ \\
\hline ENSBTAG00000026371 & $8.54 \mathrm{E}-10$ & $6.51 \mathrm{E}-10$ & SNX29 & $1.31 E+00$ \\
\hline ENSBTAG00000010496 & $5.58 \mathrm{E}-10$ & $4.24 \mathrm{E}-10$ & STAG3 & $1.32 \mathrm{E}+00$ \\
\hline ENSBTAG00000016590 & $1.30 \mathrm{E}-10$ & $9.80 \mathrm{E}-11$ & NME4 & $1.32 \mathrm{E}+00$ \\
\hline ENSBTAG00000045593 & $2.24 \mathrm{E}-10$ & $1.70 \mathrm{E}-10$ & EARS2 & $1.32 \mathrm{E}+00$ \\
\hline ENSBTAG00000030757 & $1.70 \mathrm{E}-10$ & $1.29 \mathrm{E}-10$ & ELFN1 & $1.32 \mathrm{E}+00$ \\
\hline ENSBTAG00000016568 & $1.39 \mathrm{E}-10$ & $1.05 \mathrm{E}-10$ & LUC7L & $1.33 \mathrm{E}+00$ \\
\hline ENSBTAG00000019219 & $1.37 \mathrm{E}-10$ & $1.04 \mathrm{E}-10$ & $\mathrm{SH} 2 \mathrm{~B} 1$ & $1.32 \mathrm{E}+00$ \\
\hline ENSBTAG00000016592 & $7.29 \mathrm{E}-11$ & $5.51 \mathrm{E}-11$ & CAPN15 & $1.32 \mathrm{E}+00$ \\
\hline ENSBTAG00000001139 & $1.82 \mathrm{E}-10$ & $1.37 \mathrm{E}-10$ & ACHE & $1.33 \mathrm{E}+00$ \\
\hline ENSBTAG00000010904 & $5.44 \mathrm{E}-11$ & $4.11 \mathrm{E}-11$ & & $1.32 \mathrm{E}+00$ \\
\hline ENSBTAG00000007372 & $3.24 \mathrm{E}-10$ & $2.45 \mathrm{E}-10$ & ANKRD61 & $1.32 \mathrm{E}+00$ \\
\hline ENSBTAG00000003421 & $1.10 \mathrm{E}-10$ & $8.30 \mathrm{E}-11$ & RNF40 & $1.32 \mathrm{E}+00$ \\
\hline ENSBTAG00000018097 & $4.83 \mathrm{E}-10$ & $3.64 \mathrm{E}-10$ & PARN & $1.33 \mathrm{E}+00$ \\
\hline ENSBTAG00000017871 & $6.98 \mathrm{E}-10$ & $5.27 \mathrm{E}-10$ & GNA12 & $1.32 \mathrm{E}+00$ \\
\hline ENSBTAG00000000728 & $1.98 \mathrm{E}-10$ & $1.50 \mathrm{E}-10$ & ZNF597 & $1.32 \mathrm{E}+00$ \\
\hline ENSBTAG00000047572 & $1.51 \mathrm{E}-10$ & $1.14 \mathrm{E}-10$ & ZNF789 & $1.33 E+00$ \\
\hline ENSBTAG00000037400 & $2.78 \mathrm{E}-10$ & $2.09 \mathrm{E}-10$ & TNRC18 & $1.33 \mathrm{E}+00$ \\
\hline ENSBTAG00000018044 & $3.14 \mathrm{E}-10$ & $2.36 \mathrm{E}-10$ & POLR3E & $1.33 \mathrm{E}+00$ \\
\hline
\end{tabular}




\begin{tabular}{|c|c|c|c|c|}
\hline ENSBTAG000000007370 & $2.71 \mathrm{E}-10$ & 2.03E-10 & EIF2AK1 & $1.34 \mathrm{E}+00$ \\
\hline ENSBTAG00000014456 & 5.55E-10 & $4.11 \mathrm{E}-10$ & ERN2 & $1.35 \mathrm{E}+00$ \\
\hline ENSBTAG000000000343 & $6.60 \mathrm{E}-10$ & $4.88 \mathrm{E}-10$ & ZNHIT1 & $1.35 \mathrm{E}+00$ \\
\hline ENSBTAG000000015737 & $9.98 \mathrm{E}-10$ & 7.33E-10 & KPNA7 & $1.36 \mathrm{E}+00$ \\
\hline ENSBTAG000000016553 & $3.48 \mathrm{E}-10$ & $2.56 \mathrm{E}-10$ & EME2 & $1.36 \mathrm{E}+00$ \\
\hline ENSBTAG00000017999 & 1.67E-10 & $1.22 \mathrm{E}-10$ & TNRC6A & $1.37 \mathrm{E}+00$ \\
\hline ENSBTAG00000014468 & $3.23 \mathrm{E}-10$ & $2.36 \mathrm{E}-10$ & AP1S1 & $1.37 \mathrm{E}+00$ \\
\hline ENSBTAG000000016564 & $2.45 \mathrm{E}-10$ & $1.78 \mathrm{E}-10$ & NPRL3 & $1.38 \mathrm{E}+00$ \\
\hline ENSBTAG000000016424 & $3.23 \mathrm{E}-10$ & $2.34 \mathrm{E}-10$ & MMP25 & $1.38 \mathrm{E}+00$ \\
\hline ENSBTAG00000045857 & $1.13 \mathrm{E}-10$ & $8.10 \mathrm{E}-11$ & MCRIP2 & $1.39 \mathrm{E}+00$ \\
\hline ENSBTAG000000002391 & $9.33 \mathrm{E}-11$ & $6.72 \mathrm{E}-11$ & TGFB1I1 & $1.39 E+00$ \\
\hline ENSBTAG000000033299 & $3.13 E-10$ & $2.25 \mathrm{E}-10$ & IGFALS & $1.39 \mathrm{E}+00$ \\
\hline ENSBTAG00000017701 & $1.39 \mathrm{E}-10$ & $1.00 \mathrm{E}-10$ & CYP2W1 & $1.39 \mathrm{E}+00$ \\
\hline ENSBTAG000000031765 & $9.70 \mathrm{E}-11$ & $6.98 \mathrm{E}-11$ & CCDC189 & $1.39 E+00$ \\
\hline ENSBTAG000000019412 & $7.03 E-10$ & $5.06 \mathrm{E}-10$ & C16orf62 & $1.39 \mathrm{E}+00$ \\
\hline ENSBTAG000000021222 & $6.84 \mathrm{E}-11$ & $4.93 \mathrm{E}-11$ & ZNF771 & $1.39 E+00$ \\
\hline ENSBTAG000000008633 & $5.15 \mathrm{E}-10$ & $3.70 \mathrm{E}-10$ & & $1.39 \mathrm{E}+00$ \\
\hline ENSBTAG000000010659 & 4.14E-10 & $2.94 \mathrm{E}-10$ & CUX1 & $1.41 E+00$ \\
\hline ENSBTAG000000064448 & $2.96 \mathrm{E}-10$ & $2.10 \mathrm{E}-10$ & ERI2 & $1.41 E+00$ \\
\hline ENSBTAG000000007474 & $2.48 \mathrm{E}-10$ & $1.76 \mathrm{E}-10$ & EIF3B & $1.41 E+00$ \\
\hline ENSBTAG000000020735 & $1.82 \mathrm{E}-10$ & $1.29 \mathrm{E}-10$ & SMG1 & $1.41 E+00$ \\
\hline ENSBTAG000000002737 & $2.91 \mathrm{E}-10$ & $2.04 \mathrm{E}-10$ & WFIKKN1 & $1.43 E+00$ \\
\hline ENSBTAG000000005208 & $3.85 \mathrm{E}-10$ & 2.69E-10 & CLEC16A & $1.43 E+00$ \\
\hline ENSBTAG000000015017 & $4.21 \mathrm{E}-10$ & $2.92 \mathrm{E}-10$ & GAL3ST4 & $1.44 \mathrm{E}+00$ \\
\hline ENSBTAG00000007177 & $8.49 \mathrm{E}-10$ & $5.86 \mathrm{E}-10$ & EMP2 & $1.45 \mathrm{E}+00$ \\
\hline ENSBTAG000000002474 & $6.20 \mathrm{E}-10$ & $4.26 \mathrm{E}-10$ & MAD1L1 & $1.45 E+00$ \\
\hline ENSBTAG000000037462 & 1.71E-09 & 1.17E-09 & & $1.46 \mathrm{E}+00$ \\
\hline ENSBTAG000000015738 & $4.05 \mathrm{E}-10$ & $2.78 \mathrm{E}-10$ & GIGYF1 & $1.46 \mathrm{E}+00$ \\
\hline ENSBTAG000000020439 & $2.27 \mathrm{E}-10$ & $1.56 \mathrm{E}-10$ & BUD31 & $1.46 \mathrm{E}+00$ \\
\hline ENSBTAG000000032087 & $1.81 \mathrm{E}-10$ & $1.23 \mathrm{E}-10$ & ATXN2L & $1.47 E+00$ \\
\hline ENSBTAG000000026461 & $5.22 \mathrm{E}-10$ & 3.57E-10 & CACNA1H & $1.46 \mathrm{E}+00$ \\
\hline ENSBTAG000000002083 & $7.64 \mathrm{E}-10$ & $5.22 \mathrm{E}-10$ & AUTS2 & $1.46 \mathrm{E}+00$ \\
\hline ENSBTAG00000037945 & $2.86 \mathrm{E}-10$ & $1.95 \mathrm{E}-10$ & CASKIN1 & $1.47 \mathrm{E}+00$ \\
\hline ENSBTAG000000006457 & $3.06 \mathrm{E}-10$ & 2.07E-10 & AHSP & $1.48 \mathrm{E}+00$ \\
\hline ENSBTAG00000013708 & $7.90 \mathrm{E}-10$ & $5.34 \mathrm{E}-10$ & SNX8 & $1.48 \mathrm{E}+00$ \\
\hline ENSBTAG00000019357 & $4.43 \mathrm{E}-10$ & 2.97E-10 & ZNF688 & $1.49 E+00$ \\
\hline ENSBTAG00000026429 & $1.97 \mathrm{E}-10$ & $1.30 \mathrm{E}-10$ & DECR2 & $1.51 E+00$ \\
\hline ENSBTAG00000000781 & $2.99 \mathrm{E}-10$ & $1.96 \mathrm{E}-10$ & HIP1 & $1.53 E+00$ \\
\hline ENSBTAG00000007890 & $3.04 \mathrm{E}-10$ & $1.98 \mathrm{E}-10$ & SEC14L5 & $1.53 \mathrm{E}+00$ \\
\hline ENSBTAG00000003049 & $3.45 \mathrm{E}-10$ & $2.25 \mathrm{E}-10$ & MICALL2 & $1.53 E+00$ \\
\hline ENSBTAG00000014698 & $4.31 \mathrm{E}-10$ & $2.80 \mathrm{E}-10$ & CARD11 & $1.54 \mathrm{E}+00$ \\
\hline ENSBTAG00000006129 & $7.09 \mathrm{E}-10$ & $4.61 E-10$ & KIAA0556 & $1.54 \mathrm{E}+00$ \\
\hline ENSBTAG00000017175 & $1.98 \mathrm{E}-10$ & $1.28 \mathrm{E}-10$ & RNF216 & $1.54 \mathrm{E}+00$ \\
\hline ENSBTAG00000047808 & $5.70 \mathrm{E}-10$ & $3.68 \mathrm{E}-10$ & & $1.55 E+00$ \\
\hline ENSBTAG00000019517 & $4.84 \mathrm{E}-10$ & $3.12 \mathrm{E}-10$ & ELN & $1.55 E+00$ \\
\hline ENSBTAG00000005197 & $1.80 \mathrm{E}-10$ & $1.16 \mathrm{E}-10$ & BAZ1B & $1.56 \mathrm{E}+00$ \\
\hline ENSBTAG00000005448 & $3.38 \mathrm{E}-10$ & 2.17E-10 & & $1.56 \mathrm{E}+00$ \\
\hline ENSBTAG00000016577 & $3.20 \mathrm{E}-10$ & $2.06 \mathrm{E}-10$ & AXIN1 & $1.55 E+00$ \\
\hline ENSBTAG00000047368 & $2.38 \mathrm{E}-09$ & $1.51 \mathrm{E}-09$ & & $1.57 E+00$ \\
\hline ENSBTAG00000021913 & $7.04 \mathrm{E}-10$ & $4.46 \mathrm{E}-10$ & MMD2 & $1.58 \mathrm{E}+00$ \\
\hline ENSBTAG00000016552 & $1.38 \mathrm{E}-10$ & 8.70E-11 & NME3 & $1.59 \mathrm{E}+00$ \\
\hline ENSBTAG00000035915 & $8.41 \mathrm{E}-10$ & $5.31 E-10$ & & $1.58 \mathrm{E}+00$ \\
\hline ENSBTAG00000008412 & $1.43 \mathrm{E}-10$ & $9.03 \mathrm{E}-11$ & & $1.59 \mathrm{E}+00$ \\
\hline ENSBTAG00000014453 & $2.33 \mathrm{E}-10$ & $1.47 \mathrm{E}-10$ & PLK1 & $1.59 \mathrm{E}+00$ \\
\hline ENSBTAG00000009780 & $2.84 \mathrm{E}-10$ & $1.79 \mathrm{E}-10$ & GTF2I & $1.58 \mathrm{E}+00$ \\
\hline ENSBTAG00000012927 & $3.47 \mathrm{E}-11$ & $2.19 \mathrm{E}-11$ & ALDOA & $1.58 \mathrm{E}+00$ \\
\hline ENSBTAG00000037566 & $8.66 \mathrm{E}-11$ & $5.45 \mathrm{E}-11$ & ZNF500 & $1.59 \mathrm{E}+00$ \\
\hline ENSBTAG00000017284 & $9.81 \mathrm{E}-11$ & $6.18 \mathrm{E}-11$ & RHBDL1 & $1.59 \mathrm{E}+00$ \\
\hline ENSBTAG00000009099 & $1.62 \mathrm{E}-10$ & $1.02 \mathrm{E}-10$ & PRR14 & $1.59 \mathrm{E}+00$ \\
\hline ENSBTAG00000019530 & $3.04 \mathrm{E}-10$ & $1.91 \mathrm{E}-10$ & KDM8 & $1.59 E+00$ \\
\hline ENSBTAG00000026199 & 3.37E-10 & $2.12 \mathrm{E}-10$ & ACTB & $1.59 \mathrm{E}+00$ \\
\hline ENSBTAG00000021218 & $6.65 \mathrm{E}-11$ & 4.19E-11 & MYLPF & $1.59 \mathrm{E}+00$ \\
\hline ENSBTAG00000020737 & $1.88 \mathrm{E}-10$ & $1.18 \mathrm{E}-10$ & 50x8 & $1.59 \mathrm{E}+00$ \\
\hline ENSBTAG00000009046 & $4.08 \mathrm{E}-11$ & $2.57 \mathrm{E}-11$ & TBX6 & $1.58 \mathrm{E}+00$ \\
\hline ENSBTAG00000048251 & $4.00 \mathrm{E}-10$ & $2.51 \mathrm{E}-10$ & NHLRC4 & $1.59 \mathrm{E}+00$ \\
\hline ENSBTAG00000004242 & $3.90 \mathrm{E}-10$ & $2.45 \mathrm{E}-10$ & ARPC1A & $1.59 \mathrm{E}+00$ \\
\hline ENSBTAG00000000050 & $1.90 \mathrm{E}-10$ & $1.20 \mathrm{E}-10$ & PSMG3 & $1.58 \mathrm{E}+00$ \\
\hline ENSBTAG00000033739 & $2.48 \mathrm{E}-10$ & $1.56 \mathrm{E}-10$ & PRR35 & $1.59 \mathrm{E}+00$ \\
\hline ENSBTAG00000046517 & $6.08 \mathrm{E}-10$ & $3.82 \mathrm{E}-10$ & & $1.59 \mathrm{E}+00$ \\
\hline ENSBTAG00000009691 & $5.10 \mathrm{E}-10$ & $3.21 \mathrm{E}-10$ & $\mathrm{SH} 2 \mathrm{~B} 2$ & $1.59 \mathrm{E}+00$ \\
\hline
\end{tabular}




\begin{tabular}{|c|c|c|c|c|}
\hline ENSBTAG00000017941 & $2.02 \mathrm{E}-10$ & $1.27 \mathrm{E}-10$ & NSUN5 & $1.59 \mathrm{E}+00$ \\
\hline ENSBTAG00000014101 & $6.73 E-10$ & 4.23E-10 & TSR3 & $1.59 E+00$ \\
\hline ENSBTAG00000011753 & $2.45 \mathrm{E}-10$ & $1.52 \mathrm{E}-10$ & $\mathrm{RCC1L}$ & $1.61 E+00$ \\
\hline ENSBTAG00000038978 & $2.48 \mathrm{E}-10$ & $1.53 \mathrm{E}-10$ & & $1.62 \mathrm{E}+00$ \\
\hline ENSBTAG00000038866 & $4.80 \mathrm{E}-10$ & $2.94 \mathrm{E}-10$ & UBE2I & $1.63 \mathrm{E}+00$ \\
\hline ENSBTAG00000009441 & $2.79 \mathrm{E}-10$ & $1.71 \mathrm{E}-10$ & RBBP6 & $1.63 E+00$ \\
\hline ENSBTAG00000020921 & 4.76E-10 & $2.90 \mathrm{E}-10$ & PRKCB & $1.64 \mathrm{E}+00$ \\
\hline ENSBTAG00000001503 & $6.14 \mathrm{E}-10$ & 3.73E-10 & TRIM72 & $1.65 \mathrm{E}+00$ \\
\hline ENSBTAG00000026422 & $3.06 \mathrm{E}-10$ & $1.85 \mathrm{E}-10$ & MPG & $1.65 \mathrm{E}+00$ \\
\hline ENSBTAG00000009233 & $4.46 \mathrm{E}-10$ & $2.69 \mathrm{E}-10$ & RAC1 & $1.66 \mathrm{E}+00$ \\
\hline ENSBTAG00000019524 & $7.26 \mathrm{E}-10$ & 4.37E-10 & ITGAD & $1.66 \mathrm{E}+00$ \\
\hline ENSBTAG00000046033 & $2.99 \mathrm{E}-10$ & $1.80 \mathrm{E}-10$ & UBFD1 & $1.66 \mathrm{E}+00$ \\
\hline ENSBTAG00000009200 & $2.59 \mathrm{E}-10$ & $1.54 \mathrm{E}-10$ & PAM16 & $1.68 \mathrm{E}+00$ \\
\hline ENSBTAG00000047734 & $3.33 \mathrm{E}-10$ & $1.98 \mathrm{E}-10$ & C16orf58 & $1.68 \mathrm{E}+00$ \\
\hline ENSBTAG00000014118 & $8.99 E-10$ & $5.36 \mathrm{E}-10$ & GNPTG & $1.68 \mathrm{E}+00$ \\
\hline ENSBTAG00000032640 & $1.49 \mathrm{E}-10$ & $8.81 \mathrm{E}-11$ & FOPNL & $1.69 \mathrm{E}+00$ \\
\hline ENSBTAG00000009236 & 4.19E-10 & $2.48 \mathrm{E}-10$ & DAGLB & $1.69 E+00$ \\
\hline ENSBTAG00000048140 & $4.70 \mathrm{E}-10$ & $2.77 \mathrm{E}-10$ & DNAAF5 & $1.70 \mathrm{E}+00$ \\
\hline ENSBTAG00000001602 & $4.24 \mathrm{E}-10$ & $2.50 \mathrm{E}-10$ & IL4R & $1.70 \mathrm{E}+00$ \\
\hline ENSBTAG00000003417 & $1.33 \mathrm{E}-10$ & $7.80 \mathrm{E}-11$ & PHKG2 & $1.70 \mathrm{E}+00$ \\
\hline ENSBTAG00000008869 & $2.26 \mathrm{E}-10$ & $1.33 \mathrm{E}-10$ & GPC2 & $1.70 \mathrm{E}+00$ \\
\hline ENSBTAG00000003617 & $3.09 \mathrm{E}-10$ & $1.80 \mathrm{E}-10$ & ALKBH4 & $1.71 \mathrm{E}+00$ \\
\hline ENSBTAG00000003953 & $4.08 \mathrm{E}-10$ & $2.36 \mathrm{E}-10$ & ZNF316 & $1.73 \mathrm{E}+00$ \\
\hline ENSBTAG00000018562 & $3.52 \mathrm{E}-10$ & $2.03 \mathrm{E}-10$ & TMEM159 & $1.73 E+00$ \\
\hline ENSBTAG00000014448 & 3.23E-10 & $1.85 \mathrm{E}-10$ & & $1.75 E+00$ \\
\hline ENSBTAG00000016629 & $1.01 \mathrm{E}-10$ & $5.78 \mathrm{E}-11$ & ADCY9 & $1.75 E+00$ \\
\hline ENSBTAG00000018082 & $5.93 \mathrm{E}-10$ & $3.37 \mathrm{E}-10$ & & $1.76 \mathrm{E}+00$ \\
\hline ENSBTAG00000019103 & $2.12 \mathrm{E}-09$ & $1.20 \mathrm{E}-09$ & & $1.77 \mathrm{E}+00$ \\
\hline ENSBTAG00000015191 & $3.38 \mathrm{E}-10$ & $1.92 \mathrm{E}-10$ & $A B C C 6$ & $1.76 \mathrm{E}+00$ \\
\hline ENSBTAG00000014126 & 1.04E-09 & $5.89 \mathrm{E}-10$ & & $1.77 \mathrm{E}+00$ \\
\hline ENSBTAG00000006212 & $8.00 \mathrm{E}-10$ & $4.52 \mathrm{E}-10$ & GTF2IRD1 & $1.77 \mathrm{E}+00$ \\
\hline ENSBTAG00000004038 & $1.88 \mathrm{E}-10$ & $1.06 \mathrm{E}-10$ & ABAT & $1.77 \mathrm{E}+00$ \\
\hline ENSBTAG00000044083 & 7.33E-10 & $4.12 \mathrm{E}-10$ & LIMK1 & $1.78 \mathrm{E}+00$ \\
\hline ENSBTAG00000016883 & $1.15 \mathrm{E}-10$ & $6.42 \mathrm{E}-11$ & & $1.78 \mathrm{E}+00$ \\
\hline ENSBTAG00000026394 & $1.46 \mathrm{E}-10$ & 8.19E-11 & UBALD1 & $1.79 \mathrm{E}+00$ \\
\hline ENSBTAG00000001131 & $4.04 \mathrm{E}-10$ & $2.26 \mathrm{E}-10$ & SLC12A9 & $1.79 E+00$ \\
\hline ENSBTAG00000001134 & $3.44 \mathrm{E}-10$ & $1.90 \mathrm{E}-10$ & SRRT & $1.81 E+00$ \\
\hline ENSBTAG00000004135 & $2.93 \mathrm{E}-10$ & $1.61 \mathrm{E}-10$ & LMTK2 & $1.82 \mathrm{E}+00$ \\
\hline ENSBTAG00000019216 & $3.32 \mathrm{E}-10$ & $1.83 \mathrm{E}-10$ & TUFM & $1.81 E+00$ \\
\hline ENSBTAG00000016505 & $4.25 \mathrm{E}-10$ & $2.32 \mathrm{E}-10$ & TMC7 & $1.83 \mathrm{E}+00$ \\
\hline ENSBTAG00000019006 & $1.11 \mathrm{E}-10$ & $6.01 E-11$ & WDR24 & $1.85 E+00$ \\
\hline ENSBTAG00000006543 & $1.42 \mathrm{E}-10$ & 7.67E-11 & & $1.85 E+00$ \\
\hline ENSBTAG00000046965 & $3.65 \mathrm{E}-10$ & 1.97E-10 & & $1.85 \mathrm{E}+00$ \\
\hline ENSBTAG00000038577 & $3.38 \mathrm{E}-10$ & $1.82 \mathrm{E}-10$ & & $1.86 \mathrm{E}+00$ \\
\hline ENSBTAG00000007523 & $1.98 \mathrm{E}-10$ & 1.07E-10 & STX4 & $1.85 E+00$ \\
\hline ENSBTAG00000026286 & $3.45 \mathrm{E}-10$ & $1.85 \mathrm{E}-10$ & & $1.86 \mathrm{E}+00$ \\
\hline ENSBTAG00000009461 & $6.52 \mathrm{E}-10$ & $3.47 \mathrm{E}-10$ & STYXL1 & $1.88 \mathrm{E}+00$ \\
\hline ENSBTAG00000047379 & $5.68 \mathrm{E}-10$ & $3.02 \mathrm{E}-10$ & & $1.88 \mathrm{E}+00$ \\
\hline ENSBTAG00000002374 & $2.91 \mathrm{E}-10$ & $1.55 \mathrm{E}-10$ & ARMC5 & $1.88 \mathrm{E}+00$ \\
\hline ENSBTAG00000014834 & $4.78 \mathrm{E}-10$ & $2.54 \mathrm{E}-10$ & & $1.88 \mathrm{E}+00$ \\
\hline ENSBTAG00000016244 & $8.32 \mathrm{E}-10$ & $4.39 \mathrm{E}-10$ & VWA3A & $1.89 \mathrm{E}+00$ \\
\hline ENSBTAG00000033677 & $1.74 \mathrm{E}-10$ & $9.18 \mathrm{E}-11$ & WDR90 & $1.89 \mathrm{E}+00$ \\
\hline ENSBTAG00000026375 & $4.74 \mathrm{E}-10$ & $2.50 \mathrm{E}-10$ & RMI2 & $1.90 \mathrm{E}+00$ \\
\hline ENSBTAG00000004077 & $3.97 \mathrm{E}-10$ & $2.08 \mathrm{E}-10$ & YWHAG & $1.91 E+00$ \\
\hline ENSBTAG00000007879 & $5.96 \mathrm{E}-10$ & $3.12 \mathrm{E}-10$ & SRRM3 & $1.91 E+00$ \\
\hline ENSBTAG00000006717 & $6.86 \mathrm{E}-10$ & $3.59 \mathrm{E}-10$ & & $1.91 \mathrm{E}+00$ \\
\hline ENSBTAG00000040361 & $2.79 \mathrm{E}-10$ & $1.45 \mathrm{E}-10$ & LFNG & $1.92 \mathrm{E}+00$ \\
\hline ENSBTAG00000031247 & $5.05 \mathrm{E}-10$ & $2.61 \mathrm{E}-10$ & & $1.94 \mathrm{E}+00$ \\
\hline ENSBTAG00000047125 & $6.49 \mathrm{E}-10$ & $3.34 \mathrm{E}-10$ & & $1.94 \mathrm{E}+00$ \\
\hline ENSBTAG00000018015 & $3.24 \mathrm{E}-10$ & 1.67E-10 & IL27 & $1.94 \mathrm{E}+00$ \\
\hline ENSBTAG00000005523 & $3.88 \mathrm{E}-10$ & $1.99 \mathrm{E}-10$ & XYLT1 & $1.95 E+00$ \\
\hline ENSBTAG00000030690 & $5.15 \mathrm{E}-10$ & $2.64 \mathrm{E}-10$ & RADIL & $1.95 E+00$ \\
\hline ENSBTAG00000018589 & $5.34 \mathrm{E}-10$ & $2.73 \mathrm{E}-10$ & $\mathrm{RFC2}$ & $1.96 \mathrm{E}+00$ \\
\hline ENSBTAG00000020544 & $2.40 \mathrm{E}-10$ & $1.22 \mathrm{E}-10$ & BCL7B & $1.97 \mathrm{E}+00$ \\
\hline ENSBTAG00000000342 & $4.87 \mathrm{E}-10$ & $2.47 \mathrm{E}-10$ & PLOD3 & $1.97 \mathrm{E}+00$ \\
\hline ENSBTAG00000021773 & $3.76 \mathrm{E}-10$ & $1.90 \mathrm{E}-10$ & ERCC4 & $1.98 \mathrm{E}+00$ \\
\hline ENSBTAG00000005031 & $7.48 \mathrm{E}-11$ & 3.77E-11 & $\mathrm{DOC} 2 \mathrm{~A}$ & $1.98 \mathrm{E}+00$ \\
\hline ENSBTAG00000019303 & 3.37E-10 & $1.70 \mathrm{E}-10$ & MAFK & $1.98 \mathrm{E}+00$ \\
\hline ENSBTAG00000006541 & $2.27 \mathrm{E}-10$ & $1.15 \mathrm{E}-10$ & ATP2A1 & $1.98 \mathrm{E}+00$ \\
\hline ENSBTAG00000001138 & $4.06 \mathrm{E}-10$ & $2.04 \mathrm{E}-10$ & UFSP1 & $1.99 \mathrm{E}+00$ \\
\hline
\end{tabular}




\begin{tabular}{|c|c|c|c|c|}
\hline ENSBTAG00000001415 & $1.49 \mathrm{E}-10$ & $7.52 \mathrm{E}-11$ & THOC6 & $1.98 \mathrm{E}+00$ \\
\hline ENSBTAG00000002094 & $1.61 \mathrm{E}-10$ & 8.10E-11 & ATP5J2 & $1.98 \mathrm{E}+00$ \\
\hline ENSBTAG00000018559 & $3.52 \mathrm{E}-10$ & $1.75 \mathrm{E}-10$ & LYRM1 & $2.01 E+00$ \\
\hline ENSBTAG00000021248 & $2.79 \mathrm{E}-10$ & $1.38 \mathrm{E}-10$ & C7orf26 & $2.02 \mathrm{E}+00$ \\
\hline ENSBTAG00000040568 & $2.98 \mathrm{E}-10$ & $1.46 \mathrm{E}-10$ & & $2.04 \mathrm{E}+00$ \\
\hline ENSBTAG00000014343 & $6.81 \mathrm{E}-10$ & $3.33 \mathrm{E}-10$ & IFT22 & $2.04 \mathrm{E}+00$ \\
\hline ENSBTAG00000008295 & $2.92 \mathrm{E}-10$ & $1.43 \mathrm{E}-10$ & ACTL6B & $2.04 E+00$ \\
\hline ENSBTAG00000013669 & $7.19 \mathrm{E}-11$ & $3.48 \mathrm{E}-11$ & KIF22 & $2.06 \mathrm{E}+00$ \\
\hline ENSBTAG00000024772 & $2.78 \mathrm{E}-10$ & $1.34 \mathrm{E}-10$ & TRIP6 & $2.07 \mathrm{E}+00$ \\
\hline ENSBTAG00000016520 & $5.76 \mathrm{E}-10$ & $2.78 \mathrm{E}-10$ & & $2.07 E+00$ \\
\hline ENSBTAG00000014742 & $2.77 \mathrm{E}-10$ & $1.33 \mathrm{E}-10$ & LRWD1 & $2.08 \mathrm{E}+00$ \\
\hline ENSBTAG00000011470 & $5.13 \mathrm{E}-10$ & $2.45 \mathrm{E}-10$ & & $2.09 E+00$ \\
\hline ENSBTAG00000000321 & $2.49 \mathrm{E}-10$ & 1.17E-10 & TBL2 & $2.13 E+00$ \\
\hline ENSBTAG00000004551 & $1.35 \mathrm{E}-10$ & $6.39 \mathrm{E}-11$ & RHOT2 & $2.12 \mathrm{E}+00$ \\
\hline ENSBTAG00000003160 & $4.83 \mathrm{E}-10$ & $2.25 \mathrm{E}-10$ & $\mathrm{LRCH} 4$ & $2.15 E+00$ \\
\hline ENSBTAG00000005450 & $3.70 \mathrm{E}-10$ & $1.72 \mathrm{E}-10$ & & $2.15 E+00$ \\
\hline ENSBTAG00000047249 & $5.00 \mathrm{E}-10$ & $2.30 \mathrm{E}-10$ & $\mathrm{EIF} 4 \mathrm{H}$ & $2.17 E+00$ \\
\hline ENSBTAG00000006449 & $2.68 \mathrm{E}-10$ & $1.23 \mathrm{E}-10$ & & $2.18 \mathrm{E}+00$ \\
\hline ENSBTAG00000040295 & $3.16 \mathrm{E}-10$ & $1.44 \mathrm{E}-10$ & $\mathrm{CHCHD2}$ & $2.19 E+00$ \\
\hline ENSBTAG00000047448 & $4.28 \mathrm{E}-10$ & $1.92 \mathrm{E}-10$ & C16orf54 & $2.23 E+00$ \\
\hline ENSBTAG00000016561 & $4.40 \mathrm{E}-10$ & $1.98 \mathrm{E}-10$ & NUBP2 & $2.22 \mathrm{E}+00$ \\
\hline ENSBTAG00000007364 & $2.12 \mathrm{E}-10$ & $9.55 \mathrm{E}-11$ & PMS2 & $2.22 \mathrm{E}+00$ \\
\hline ENSBTAG00000019805 & $2.15 \mathrm{E}-10$ & $9.56 \mathrm{E}-11$ & RHBDF1 & $2.24 \mathrm{E}+00$ \\
\hline ENSBTAG00000040555 & $2.86 \mathrm{E}-10$ & $1.27 \mathrm{E}-10$ & FBXL19 & $2.25 E+00$ \\
\hline ENSBTAG00000019310 & 4.80E-10 & $2.12 \mathrm{E}-10$ & FOXK1 & $2.26 \mathrm{E}+00$ \\
\hline ENSBTAG00000010096 & $5.06 \mathrm{E}-10$ & $2.22 \mathrm{E}-10$ & SLC5A2 & $2.28 \mathrm{E}+00$ \\
\hline ENSBTAG00000009462 & $9.09 \mathrm{E}-10$ & $3.99 \mathrm{E}-10$ & $\mathrm{MDH} 2$ & $2.28 \mathrm{E}+00$ \\
\hline ENSBTAG00000005798 & $2.55 \mathrm{E}-10$ & $1.11 \mathrm{E}-10$ & & $2.29 E+00$ \\
\hline ENSBTAG00000004803 & $1.48 \mathrm{E}-10$ & $6.43 \mathrm{E}-11$ & NTAN1 & $2.29 \mathrm{E}+00$ \\
\hline ENSBTAG00000007367 & $2.63 \mathrm{E}-10$ & $1.14 \mathrm{E}-10$ & AIMP2 & $2.30 \mathrm{E}+00$ \\
\hline ENSBTAG00000026236 & $5.57 \mathrm{E}-10$ & $2.41 \mathrm{E}-10$ & AZGP1 & $2.31 E+00$ \\
\hline ENSBTAG00000032260 & $2.69 \mathrm{E}-10$ & $1.15 \mathrm{E}-10$ & DCUN1D3 & $2.34 \mathrm{E}+00$ \\
\hline ENSBTAG00000010524 & $1.17 \mathrm{E}-10$ & $4.90 \mathrm{E}-11$ & BCKDK & $2.38 \mathrm{E}+00$ \\
\hline ENSBTAG00000003020 & $2.88 \mathrm{E}-10$ & $1.21 \mathrm{E}-10$ & SSC4D & $2.38 \mathrm{E}+00$ \\
\hline ENSBTAG00000047316 & $3.10 \mathrm{E}-10$ & $1.30 \mathrm{E}-10$ & & $2.38 \mathrm{E}+00$ \\
\hline ENSBTAG00000004727 & $2.12 \mathrm{E}-10$ & $8.88 \mathrm{E}-11$ & PAQR4 & $2.38 \mathrm{E}+00$ \\
\hline ENSBTAG00000016558 & $2.65 \mathrm{E}-10$ & $1.11 \mathrm{E}-10$ & SPSB3 & $2.39 \mathrm{E}+00$ \\
\hline ENSBTAG00000037549 & $3.58 \mathrm{E}-10$ & $1.50 \mathrm{E}-10$ & MBLAC1 & $2.38 \mathrm{E}+00$ \\
\hline ENSBTAG00000020733 & $2.11 \mathrm{E}-10$ & $8.86 \mathrm{E}-11$ & & $2.38 \mathrm{E}+00$ \\
\hline ENSBTAG00000012038 & $4.35 \mathrm{E}-10$ & $1.82 \mathrm{E}-10$ & TRIM56 & $2.39 E+00$ \\
\hline ENSBTAG00000014465 & $5.05 \mathrm{E}-10$ & $2.12 \mathrm{E}-10$ & SERPINE1 & $2.38 \mathrm{E}+00$ \\
\hline ENSBTAG00000020524 & $4.77 \mathrm{E}-10$ & $2.00 \mathrm{E}-10$ & & $2.39 \mathrm{E}+00$ \\
\hline ENSBTAG00000007952 & $1.26 \mathrm{E}-10$ & $5.27 \mathrm{E}-11$ & MVP & $2.38 \mathrm{E}+00$ \\
\hline ENSBTAG00000045680 & $9.60 \mathrm{E}-10$ & $4.01 \mathrm{E}-10$ & & $2.39 \mathrm{E}+00$ \\
\hline ENSBTAG00000047238 & $3.69 \mathrm{E}-10$ & $1.54 \mathrm{E}-10$ & ITGAM & $2.40 \mathrm{E}+00$ \\
\hline ENSBTAG00000017391 & $2.59 \mathrm{E}-10$ & $1.08 \mathrm{E}-10$ & HS3ST2 & $2.40 \mathrm{E}+00$ \\
\hline ENSBTAG00000002480 & 1.10E-09 & $4.54 \mathrm{E}-10$ & NUDT1 & $2.43 E+00$ \\
\hline ENSBTAG00000012683 & $1.43 \mathrm{E}-10$ & $5.79 \mathrm{E}-11$ & SRRM2 & $2.46 \mathrm{E}+00$ \\
\hline ENSBTAG00000017422 & $1.21 \mathrm{E}-10$ & $4.87 \mathrm{E}-11$ & TAOK2 & $2.48 \mathrm{E}+00$ \\
\hline ENSBTAG00000026191 & $2.61 \mathrm{E}-10$ & $1.05 \mathrm{E}-10$ & GET4 & $2.49 \mathrm{E}+00$ \\
\hline ENSBTAG00000001066 & $6.71 \mathrm{E}-10$ & $2.69 \mathrm{E}-10$ & & $2.50 \mathrm{E}+00$ \\
\hline ENSBTAG00000013479 & $3.70 \mathrm{E}-10$ & $1.48 \mathrm{E}-10$ & SLC9A3R2 & $2.50 \mathrm{E}+00$ \\
\hline ENSBTAG00000005148 & 4.67E-10 & $1.85 \mathrm{E}-10$ & PTX4 & $2.52 \mathrm{E}+00$ \\
\hline ENSBTAG00000037489 & $4.72 \mathrm{E}-10$ & $1.87 \mathrm{E}-10$ & CRCP & $2.52 \mathrm{E}+00$ \\
\hline ENSBTAG00000020617 & $2.41 \mathrm{E}-10$ & $9.55 E-11$ & $\mathrm{TSC} 2$ & $2.53 E+00$ \\
\hline ENSBTAG00000016190 & $4.63 \mathrm{E}-10$ & $1.83 \mathrm{E}-10$ & ZNF394 & $2.53 E+00$ \\
\hline ENSBTAG00000007558 & $3.99 \mathrm{E}-10$ & $1.58 \mathrm{E}-10$ & IL9R & $2.52 E+00$ \\
\hline ENSBTAG00000039512 & $2.00 \mathrm{E}-10$ & $7.85 \mathrm{E}-11$ & ZSCAN25 & $2.54 \mathrm{E}+00$ \\
\hline ENSBTAG00000009536 & $2.64 \mathrm{E}-10$ & $1.02 \mathrm{E}-10$ & RNF151 & $2.59 E+00$ \\
\hline ENSBTAG00000020649 & $7.87 \mathrm{E}-10$ & $3.02 \mathrm{E}-10$ & CIITA & $2.60 E+00$ \\
\hline ENSBTAG00000015011 & $2.29 \mathrm{E}-10$ & $8.63 E-11$ & C7orf43 & $2.65 E+00$ \\
\hline ENSBTAG00000002871 & $2.97 \mathrm{E}-10$ & $1.12 \mathrm{E}-10$ & COPS6 & $2.65 E+00$ \\
\hline ENSBTAG00000030951 & $1.68 \mathrm{E}-10$ & $6.35 \mathrm{E}-11$ & & $2.65 \mathrm{E}+00$ \\
\hline ENSBTAG00000013305 & $7.55 \mathrm{E}-10$ & $2.83 \mathrm{E}-10$ & & $2.67 E+00$ \\
\hline ENSBTAG00000017456 & $2.29 \mathrm{E}-10$ & $8.49 \mathrm{E}-11$ & NYAP1 & $2.70 \mathrm{E}+00$ \\
\hline ENSBTAG00000014588 & $5.54 \mathrm{E}-10$ & $2.04 \mathrm{E}-10$ & RSL1D1 & $2.72 E+00$ \\
\hline ENSBTAG00000001441 & $2.23 \mathrm{E}-10$ & $8.14 \mathrm{E}-11$ & CARHSP1 & $2.73 E+00$ \\
\hline ENSBTAG00000019073 & $2.36 \mathrm{E}-10$ & $8.60 \mathrm{E}-11$ & TRAF7 & $2.74 \mathrm{E}+00$ \\
\hline ENSBTAG00000006391 & $2.86 \mathrm{E}-10$ & $1.04 \mathrm{E}-10$ & NDUFAB1 & $2.75 \mathrm{E}+00$ \\
\hline ENSBTAG00000008631 & $1.06 \mathrm{E}-10$ & $3.80 \mathrm{E}-11$ & CORO1A & $2.78 \mathrm{E}+00$ \\
\hline
\end{tabular}




\begin{tabular}{|c|c|c|c|c|}
\hline ENSBTAG00000032140 & $1.48 \mathrm{E}-10$ & $5.34 \mathrm{E}-11$ & SBK1 & $2.78 \mathrm{E}+00$ \\
\hline ENSBTAG00000006272 & $3.03 \mathrm{E}-10$ & $1.09 \mathrm{E}-10$ & NTHL1 & $2.78 \mathrm{E}+00$ \\
\hline ENSBTAG00000014903 & $5.66 \mathrm{E}-10$ & $2.03 \mathrm{E}-10$ & ABCA3 & $2.79 E+00$ \\
\hline ENSBTAG00000021652 & $5.20 \mathrm{E}-10$ & $1.85 \mathrm{E}-10$ & PDZD9 & $2.81 E+00$ \\
\hline ENSBTAG00000031852 & $1.49 \mathrm{E}-10$ & $5.21 \mathrm{E}-11$ & TBC1D10B & $2.86 \mathrm{E}+00$ \\
\hline ENSBTAG00000012049 & $6.22 \mathrm{E}-10$ & $2.18 \mathrm{E}-10$ & WIPI2 & $2.85 E+00$ \\
\hline ENSBTAG00000001440 & $3.13 \mathrm{E}-10$ & $1.10 \mathrm{E}-10$ & PMM2 & $2.85 \mathrm{E}+00$ \\
\hline ENSBTAG00000016574 & $6.39 \mathrm{E}-10$ & 2.23E-10 & RGS11 & $2.86 \mathrm{E}+00$ \\
\hline ENSBTAG00000007245 & 4.50E-10 & $1.55 \mathrm{E}-10$ & IFT140 & $2.90 \mathrm{E}+00$ \\
\hline ENSBTAG00000039720 & $2.28 \mathrm{E}-10$ & $7.81 \mathrm{E}-11$ & & $2.91 E+00$ \\
\hline ENSBTAG00000002188 & $6.07 \mathrm{E}-10$ & $2.09 \mathrm{E}-10$ & C16orf89 & $2.91 E+00$ \\
\hline ENSBTAG00000013081 & $5.36 \mathrm{E}-10$ & $1.82 \mathrm{E}-10$ & PSPH & $2.94 \mathrm{E}+00$ \\
\hline ENSBTAG00000012050 & $3.16 \mathrm{E}-10$ & $1.08 \mathrm{E}-10$ & ORAI2 & $2.92 \mathrm{E}+00$ \\
\hline ENSBTAG00000007246 & $5.09 \mathrm{E}-10$ & $1.72 \mathrm{E}-10$ & TMEM204 & $2.96 \mathrm{E}+00$ \\
\hline ENSBTAG00000011818 & $6.01 \mathrm{E}-10$ & $2.02 \mathrm{E}-10$ & COL26A1 & $2.97 E+00$ \\
\hline ENSBTAG00000010198 & $3.02 \mathrm{E}-10$ & $1.01 \mathrm{E}-10$ & ATF7IP2 & $2.99 \mathrm{E}+00$ \\
\hline ENSBTAG00000003308 & $5.73 \mathrm{E}-10$ & $1.90 \mathrm{E}-10$ & GRID2IP & $3.02 \mathrm{E}+00$ \\
\hline ENSBTAG00000007934 & $3.72 \mathrm{E}-10$ & $1.23 \mathrm{E}-10$ & & $3.02 E+00$ \\
\hline ENSBTAG00000008188 & $5.79 \mathrm{E}-10$ & $1.91 \mathrm{E}-10$ & C16orf52 & $3.03 E+00$ \\
\hline ENSBTAG00000038074 & $2.82 \mathrm{E}-10$ & $9.26 \mathrm{E}-11$ & GJC3 & $3.05 E+00$ \\
\hline ENSBTAG00000002209 & $4.56 \mathrm{E}-10$ & 1.47E-10 & HN1L & $3.10 \mathrm{E}+00$ \\
\hline ENSBTAG00000002090 & $2.61 \mathrm{E}-10$ & $8.41 \mathrm{E}-11$ & CPSF4 & $3.10 E+00$ \\
\hline ENSBTAG00000021242 & 1.17E-09 & $3.78 \mathrm{E}-10$ & COQ7 & $3.10 \mathrm{E}+00$ \\
\hline ENSBTAG00000020619 & $4.46 \mathrm{E}-10$ & $1.44 \mathrm{E}-10$ & PKD1 & $3.10 \mathrm{E}+00$ \\
\hline ENSBTAG00000017939 & $2.14 \mathrm{E}-10$ & $6.90 \mathrm{E}-11$ & & $3.10 \mathrm{E}+00$ \\
\hline ENSBTAG00000002202 & $4.93 \mathrm{E}-10$ & $1.57 \mathrm{E}-10$ & CRAMP1 & $3.14 E+00$ \\
\hline ENSBTAG00000019803 & $1.20 \mathrm{E}-10$ & $3.78 \mathrm{E}-11$ & POLR3K & $3.17 E+00$ \\
\hline ENSBTAG00000012082 & $2.25 \mathrm{E}-10$ & 7.07E-11 & TNFRSF12A & $3.17 E+00$ \\
\hline ENSBTAG00000015982 & $7.42 \mathrm{E}-11$ & 2.34E-11 & KCTD13 & $3.16 \mathrm{E}+00$ \\
\hline ENSBTAG00000000611 & $1.85 \mathrm{E}-10$ & $5.81 \mathrm{E}-11$ & C16orf90 & $3.18 \mathrm{E}+00$ \\
\hline ENSBTAG00000022535 & 4.17E-10 & $1.31 \mathrm{E}-10$ & GPR139 & $3.18 \mathrm{E}+00$ \\
\hline ENSBTAG00000026365 & $2.61 \mathrm{E}-10$ & $8.22 \mathrm{E}-11$ & & $3.18 \mathrm{E}+00$ \\
\hline ENSBTAG00000016139 & $4.82 \mathrm{E}-10$ & $1.51 \mathrm{E}-10$ & TMEM120A & $3.19 \mathrm{E}+00$ \\
\hline ENSBTAG00000014810 & $8.02 \mathrm{E}-10$ & $2.51 \mathrm{E}-10$ & WBSCR28 & $3.20 E+00$ \\
\hline ENSBTAG00000015889 & $6.82 \mathrm{E}-10$ & $2.11 \mathrm{E}-10$ & CLCN7 & $3.23 E+00$ \\
\hline ENSBTAG00000008184 & $3.84 \mathrm{E}-10$ & 1.17E-10 & ССТ6A & $3.28 \mathrm{E}+00$ \\
\hline ENSBTAG00000015272 & $2.94 \mathrm{E}-10$ & $8.95 \mathrm{E}-11$ & TSC22D4 & $3.28 \mathrm{E}+00$ \\
\hline ENSBTAG00000011875 & $9.36 \mathrm{E}-10$ & $2.82 \mathrm{E}-10$ & CLIP2 & $3.32 \mathrm{E}+00$ \\
\hline ENSBTAG00000017082 & $5.75 \mathrm{E}-10$ & $1.72 \mathrm{E}-10$ & POR & $3.34 \mathrm{E}+00$ \\
\hline ENSBTAG00000026326 & 4.65E-10 & $1.39 \mathrm{E}-10$ & SPN & $3.34 E+00$ \\
\hline ENSBTAG00000006117 & $2.51 \mathrm{E}-10$ & $7.50 \mathrm{E}-11$ & & $3.34 \mathrm{E}+00$ \\
\hline ENSBTAG00000002349 & $1.77 \mathrm{E}-10$ & $5.24 \mathrm{E}-11$ & STX1B & $3.37 E+00$ \\
\hline ENSBTAG00000002211 & 5.37E-10 & 1.57E-10 & MAPK8IP3 & $3.42 \mathrm{E}+00$ \\
\hline ENSBTAG00000019086 & $5.32 \mathrm{E}-10$ & $1.56 \mathrm{E}-10$ & TELO2 & $3.41 E+00$ \\
\hline ENSBTAG00000000568 & $5.03 \mathrm{E}-10$ & $1.43 \mathrm{E}-10$ & CCDC154 & $3.51 E+00$ \\
\hline ENSBTAG00000026376 & $4.40 \mathrm{E}-10$ & $1.21 \mathrm{E}-10$ & SNN & $3.63 E+00$ \\
\hline ENSBTAG00000003169 & 5.17E-10 & $1.41 \mathrm{E}-10$ & $\mathrm{FBXO} 24$ & $3.66 \mathrm{E}+00$ \\
\hline ENSBTAG00000002345 & $2.58 \mathrm{E}-10$ & 7.07E-11 & SETD1A & $3.65 E+00$ \\
\hline ENSBTAG00000016575 & 7.07E-10 & $1.93 \mathrm{E}-10$ & PDIA2 & $3.66 \mathrm{E}+00$ \\
\hline ENSBTAG00000020366 & $5.02 \mathrm{E}-10$ & $1.36 \mathrm{E}-10$ & TBL3 & $3.69 E+00$ \\
\hline ENSBTAG00000016360 & $2.14 \mathrm{E}-10$ & $5.76 \mathrm{E}-11$ & TVP23A & $3.70 E+00$ \\
\hline ENSBTAG00000004106 & $6.37 \mathrm{E}-10$ & $1.70 \mathrm{E}-10$ & PRKRIP1 & $3.75 E+00$ \\
\hline ENSBTAG00000002253 & $2.68 \mathrm{E}-10$ & $7.01 \mathrm{E}-11$ & FKBP6 & $3.81 E+00$ \\
\hline ENSBTAG00000005089 & $4.99 \mathrm{E}-10$ & $1.28 \mathrm{E}-10$ & RHBDD2 & $3.89 E+00$ \\
\hline ENSBTAG00000019543 & $2.06 \mathrm{E}-10$ & 5.17E-11 & & $3.97 \mathrm{E}+00$ \\
\hline ENSBTAG00000005028 & $1.14 \mathrm{E}-10$ & $2.87 \mathrm{E}-11$ & HIRIP3 & $3.96 \mathrm{E}+00$ \\
\hline ENSBTAG00000027854 & $3.31 \mathrm{E}-10$ & $8.31 \mathrm{E}-11$ & ARHGDIG & $3.98 \mathrm{E}+00$ \\
\hline ENSBTAG00000026246 & $4.53 \mathrm{E}-10$ & $1.14 \mathrm{E}-10$ & MOSPD3 & $3.97 E+00$ \\
\hline ENSBTAG00000006263 & $5.98 \mathrm{E}-10$ & $1.49 \mathrm{E}-10$ & GP2 & $4.01 E+00$ \\
\hline ENSBTAG00000009237 & $2.46 \mathrm{E}-10$ & $6.04 \mathrm{E}-11$ & KDELR2 & $4.06 \mathrm{E}+00$ \\
\hline ENSBTAG00000009837 & $7.46 \mathrm{E}-10$ & $1.81 \mathrm{E}-10$ & ANKS4B & $4.12 \mathrm{E}+00$ \\
\hline ENSBTAG00000012601 & $8.53 \mathrm{E}-10$ & $2.07 \mathrm{E}-10$ & TEKT4 & $4.12 \mathrm{E}+00$ \\
\hline ENSBTAG00000006495 & $4.12 \mathrm{E}-10$ & $9.96 \mathrm{E}-11$ & GNB2 & $4.14 \mathrm{E}+00$ \\
\hline ENSBTAG00000045767 & $7.24 \mathrm{E}-10$ & $1.73 \mathrm{E}-10$ & LAT2 & $4.18 \mathrm{E}+00$ \\
\hline ENSBTAG00000014100 & $5.61 \mathrm{E}-10$ & $1.34 \mathrm{E}-10$ & BAIAP3 & $4.18 \mathrm{E}+00$ \\
\hline ENSBTAG00000026383 & $7.42 \mathrm{E}-10$ & $1.77 \mathrm{E}-10$ & & $4.19 E+00$ \\
\hline ENSBTAG00000008504 & 3.13E-10 & 7.37E-11 & SYNGR3 & $4.24 \mathrm{E}+00$ \\
\hline ENSBTAG00000019567 & $3.59 \mathrm{E}-10$ & $8.38 \mathrm{E}-11$ & IL21R & $4.28 \mathrm{E}+00$ \\
\hline ENSBTAG00000021090 & $4.06 \mathrm{E}-10$ & $9.47 \mathrm{E}-11$ & $A B C C 1$ & $4.28 \mathrm{E}+00$ \\
\hline ENSBTAG00000020190 & $1.84 \mathrm{E}-10$ & $4.22 \mathrm{E}-11$ & KREMEN2 & $4.36 E+00$ \\
\hline
\end{tabular}




\begin{tabular}{|c|c|c|c|c|}
\hline ENSBTAG00000015314 & $2.06 \mathrm{E}-10$ & 4.70E-11 & & $4.37 E+00$ \\
\hline ENSBTAG00000020528 & $1.80 \mathrm{E}-10$ & $4.11 \mathrm{E}-11$ & PCOLCE & $4.36 \mathrm{E}+00$ \\
\hline ENSBTAG00000003430 & $3.79 \mathrm{E}-10$ & $8.65 \mathrm{E}-11$ & EPO & $4.37 E+00$ \\
\hline ENSBTAG00000014229 & $6.24 \mathrm{E}-10$ & $1.40 \mathrm{E}-10$ & SYT17 & $4.46 \mathrm{E}+00$ \\
\hline ENSBTAG00000000279 & $6.91 \mathrm{E}-10$ & $1.53 \mathrm{E}-10$ & RAB26 & $4.51 \mathrm{E}+00$ \\
\hline ENSBTAG00000010511 & $2.45 \mathrm{E}-10$ & $5.32 \mathrm{E}-11$ & KAT8 & $4.60 E+00$ \\
\hline ENSBTAG00000013052 & 9.27E-10 & 1.95E-10 & SDR42E2 & $4.75 E+00$ \\
\hline ENSBTAG00000005033 & $8.59 \mathrm{E}-11$ & $1.80 \mathrm{E}-11$ & & $4.75 E+00$ \\
\hline ENSBTAG00000026319 & $1.48 \mathrm{E}-10$ & 3.09E-11 & ASPHD1 & $4.76 \mathrm{E}+00$ \\
\hline ENSBTAG00000016155 & $9.20 \mathrm{E}-11$ & $1.93 \mathrm{E}-11$ & GDPD3 & $4.75 E+00$ \\
\hline ENSBTAG00000011969 & $3.73 \mathrm{E}-10$ & $7.82 \mathrm{E}-11$ & HSPB1 & $4.77 \mathrm{E}+00$ \\
\hline ENSBTAG00000004729 & $3.46 \mathrm{E}-10$ & $7.25 \mathrm{E}-11$ & PKMYT1 & $4.77 E+00$ \\
\hline ENSBTAG00000034024 & 4.26E-10 & $8.92 \mathrm{E}-11$ & & $4.77 \mathrm{E}+00$ \\
\hline ENSBTAG00000003288 & $3.50 \mathrm{E}-10$ & 7.12E-11 & & $4.91 E+00$ \\
\hline ENSBTAG00000006126 & 3.19E-10 & $6.43 \mathrm{E}-11$ & GTF3C1 & $4.96 E+00$ \\
\hline ENSBTAG00000006410 & $5.47 \mathrm{E}-10$ & $1.07 \mathrm{E}-10$ & DCTN5 & $5.11 \mathrm{E}+00$ \\
\hline ENSBTAG00000007955 & $1.05 \mathrm{E}-10$ & $2.04 \mathrm{E}-11$ & SEZ6L2 & $5.14 E+00$ \\
\hline ENSBTAG00000009534 & $4.01 \mathrm{E}-10$ & $7.75 \mathrm{E}-11$ & NDUFB10 & $5.17 \mathrm{E}+00$ \\
\hline ENSBTAG00000007016 & $2.46 \mathrm{E}-10$ & 4.77E-11 & CNPY4 & $5.16 \mathrm{E}+00$ \\
\hline ENSBTAG00000012197 & $7.26 \mathrm{E}-10$ & $1.40 \mathrm{E}-10$ & AP5Z1 & $5.18 \mathrm{E}+00$ \\
\hline ENSBTAG00000020001 & 3.54E-10 & $6.75 \mathrm{E}-11$ & MEIOB & $5.24 \mathrm{E}+00$ \\
\hline ENSBTAG00000000725 & 4.56E-10 & $8.59 \mathrm{E}-11$ & USP42 & $5.30 E+00$ \\
\hline ENSBTAG00000006405 & $3.40 \mathrm{E}-10$ & $6.29 \mathrm{E}-11$ & PALB2 & $5.40 \mathrm{E}+00$ \\
\hline ENSBTAG00000014417 & $7.93 \mathrm{E}-10$ & $1.42 \mathrm{E}-10$ & & $5.58 \mathrm{E}+00$ \\
\hline ENSBTAG00000019423 & $4.58 \mathrm{E}-10$ & $8.06 \mathrm{E}-11$ & & $5.68 \mathrm{E}+00$ \\
\hline ENSBTAG00000012668 & $3.53 \mathrm{E}-10$ & $6.09 \mathrm{E}-11$ & & $5.79 E+00$ \\
\hline ENSBTAG00000020369 & $6.29 \mathrm{E}-10$ & $1.05 \mathrm{E}-10$ & NOXO1 & $5.99 E+00$ \\
\hline ENSBTAG00000009533 & 2.77E-10 & 4.54E-11 & RPL3L & $6.09 E+00$ \\
\hline ENSBTAG00000000642 & $7.93 \mathrm{E}-10$ & $1.26 \mathrm{E}-10$ & INTS1 & $6.29 E+00$ \\
\hline ENSBTAG00000014650 & $1.26 \mathrm{E}-10$ & $1.99 \mathrm{E}-11$ & NFATC2IP & $6.32 E+00$ \\
\hline ENSBTAG00000011738 & 4.10E-10 & $6.43 \mathrm{E}-11$ & TFR2 & $6.36 \mathrm{E}+00$ \\
\hline ENSBTAG00000007295 & $4.78 \mathrm{E}-10$ & $7.50 \mathrm{E}-11$ & & $6.36 \mathrm{E}+00$ \\
\hline ENSBTAG00000039974 & $5.95 \mathrm{E}-10$ & $9.32 \mathrm{E}-11$ & SSTR5 & $6.37 \mathrm{E}+00$ \\
\hline ENSBTAG00000001023 & $4.55 \mathrm{E}-10$ & 7.01E-11 & ZNF598 & $6.48 \mathrm{E}+00$ \\
\hline ENSBTAG00000001068 & $3.61 \mathrm{E}-10$ & $5.18 \mathrm{E}-11$ & ZCWPW1 & $6.95 E+00$ \\
\hline ENSBTAG00000016973 & 1.70E-10 & $2.38 \mathrm{E}-11$ & PRSS8 & $7.13 E+00$ \\
\hline ENSBTAG00000026275 & $2.22 \mathrm{E}-10$ & 3.11E-11 & CCL24 & $7.13 E+00$ \\
\hline ENSBTAG00000005030 & $8.62 \mathrm{E}-11$ & $1.21 \mathrm{E}-11$ & INO80E & $7.07 E+00$ \\
\hline ENSBTAG00000002344 & $1.46 \mathrm{E}-10$ & $2.04 \mathrm{E}-11$ & ORAI3 & $7.13 E+00$ \\
\hline ENSBTAG00000002881 & $5.76 \mathrm{E}-10$ & $8.02 \mathrm{E}-11$ & AP4M1 & $7.17 \mathrm{E}+00$ \\
\hline ENSBTAG00000001470 & $4.93 \mathrm{E}-10$ & $6.81 \mathrm{E}-11$ & DTX2 & $7.23 E+00$ \\
\hline ENSBTAG00000006650 & $1.72 \mathrm{E}-10$ & $2.28 \mathrm{E}-11$ & ZNF668 & $7.53 E+00$ \\
\hline ENSBTAG00000048229 & $4.72 \mathrm{E}-10$ & $6.14 \mathrm{E}-11$ & & $7.67 \mathrm{E}+00$ \\
\hline ENSBTAG00000016687 & $2.15 \mathrm{E}-10$ & $2.70 \mathrm{E}-11$ & & $7.94 \mathrm{E}+00$ \\
\hline ENSBTAG00000046722 & $4.68 \mathrm{E}-10$ & $5.87 \mathrm{E}-11$ & & $7.95 \mathrm{E}+00$ \\
\hline ENSBTAG00000019998 & $6.45 \mathrm{E}-10$ & 7.74E-11 & $\mathrm{HAGH}$ & $8.32 E+00$ \\
\hline ENSBTAG00000030966 & $3.60 \mathrm{E}-10$ & 4.23E-11 & TAF6 & $8.48 \mathrm{E}+00$ \\
\hline ENSBTAG00000033481 & $6.66 \mathrm{E}-10$ & $7.59 \mathrm{E}-11$ & C1QTNF8 & $8.76 \mathrm{E}+00$ \\
\hline ENSBTAG00000007325 & $5.31 \mathrm{E}-10$ & $6.05 \mathrm{E}-11$ & TPSB2 & $8.76 \mathrm{E}+00$ \\
\hline ENSBTAG00000009840 & $1.92 \mathrm{E}-09$ & $2.17 \mathrm{E}-10$ & OR2AE1 & $8.85 E+00$ \\
\hline ENSBTAG00000021785 & $1.15 \mathrm{E}-10$ & $1.25 \mathrm{E}-11$ & TMEM219 & $9.10 \mathrm{E}+00$ \\
\hline ENSBTAG00000030956 & $3.91 \mathrm{E}-10$ & $3.98 \mathrm{E}-11$ & ZSCAN21 & $9.81 \mathrm{E}+00$ \\
\hline ENSBTAG00000006264 & $7.58 \mathrm{E}-10$ & $7.72 \mathrm{E}-11$ & UMOD & $9.81 \mathrm{E}+00$ \\
\hline ENSBTAG00000034372 & $1.72 \mathrm{E}-09$ & $1.59 \mathrm{E}-10$ & & $1.08 \mathrm{E}+01$ \\
\hline ENSBTAG00000016765 & $3.60 \mathrm{E}-10$ & $3.23 \mathrm{E}-11$ & C7orf61 & $1.11 \mathrm{E}+01$ \\
\hline ENSBTAG00000016757 & $5.62 \mathrm{E}-10$ & $5.03 \mathrm{E}-11$ & ZKSCAN1 & $1.12 \mathrm{E}+01$ \\
\hline ENSBTAG00000030965 & $4.87 \mathrm{E}-10$ & 4.17E-11 & MCM7 & $1.17 \mathrm{E}+01$ \\
\hline ENSBTAG00000007103 & 4.00E-10 & 3.42E-11 & ITGAL & $1.17 E+01$ \\
\hline ENSBTAG00000016434 & $4.80 \mathrm{E}-10$ & $3.87 \mathrm{E}-11$ & PDILT & $1.24 \mathrm{E}+01$ \\
\hline ENSBTAG00000017631 & $6.73 \mathrm{E}-10$ & 4.43E-11 & GFER & $1.52 \mathrm{E}+01$ \\
\hline ENSBTAG00000007384 & $3.58 \mathrm{E}-10$ & 7.36E-12 & CYTH3 & $4.80 E+01$ \\
\hline ENSBTAG00000000713 & $4.04 \mathrm{E}-10$ & $5.34 \mathrm{E}-12$ & AGFG2 & $7.44 \mathrm{E}+01$ \\
\hline ENSBTAG00000038757 & $1.05 \mathrm{E}-10$ & 0 & HBM & $1.05 E+03$ \\
\hline ENSBTAG00000026418 & 0 & 0 & & $1.00 \mathrm{E}+00$ \\
\hline ENSBTAG00000002735 & 4.60E-11 & 0 & C25H16ORF13 & $4.61 E+02$ \\
\hline ENSBTAG00000033672 & 0 & 0 & JMJD8 & $1.00 \mathrm{E}+00$ \\
\hline ENSBTAG00000033395 & 4.27E-10 & 0 & CCSMST1 & $4.28 \mathrm{E}+03$ \\
\hline ENSBTAG00000009535 & $2.26 \mathrm{E}-10$ & 0 & RPS2 & $2.26 \mathrm{E}+03$ \\
\hline ENSBTAG00000009951 & $1.43 \mathrm{E}-10$ & 0 & PGP & $1.43 \mathrm{E}+03$ \\
\hline ENSBTAG00000045901 & $8.65 \mathrm{E}-11$ & 0 & CLDN6 & $8.66 \mathrm{E}+02$ \\
\hline
\end{tabular}




\begin{tabular}{|c|c|c|c|c|}
\hline ENSBTAG00000022530 & $3.15 \mathrm{E}-11$ & 0 & TMEM186 & $3.16 \mathrm{E}+02$ \\
\hline ENSBTAG00000021493 & 0 & 0 & PRM1 & $1.00 \mathrm{E}+00$ \\
\hline ENSBTAG00000046216 & $1.00 \mathrm{E}-09$ & 0 & & $1.00 \mathrm{E}+04$ \\
\hline ENSBTAG00000018195 & $1.06 \mathrm{E}-10$ & 0 & & $1.06 \mathrm{E}+03$ \\
\hline ENSBTAG00000021249 & $3.20 \mathrm{E}-10$ & 0 & LAT & $3.20 \mathrm{E}+03$ \\
\hline ENSBTAG00000014653 & $1.25 \mathrm{E}-10$ & 0 & SPNS1 & $1.26 \mathrm{E}+03$ \\
\hline ENSBTAG00000008635 & $1.28 \mathrm{E}-10$ & 0 & & $1.28 \mathrm{E}+03$ \\
\hline ENSBTAG00000016156 & 1.16E-10 & 0 & MAPK3 & $1.16 \mathrm{E}+03$ \\
\hline ENSBTAG00000009047 & $7.31 \mathrm{E}-11$ & 0 & YPEL3 & $7.32 E+02$ \\
\hline ENSBTAG00000007954 & $4.78 \mathrm{E}-11$ & 0 & CDIPT & $4.79 E+02$ \\
\hline ENSBTAG00000046752 & $3.38 \mathrm{E}-11$ & 0 & & $3.39 \mathrm{E}+02$ \\
\hline ENSBTAG00000015486 & 0 & 0 & CD2BP2 & $1.00 \mathrm{E}+00$ \\
\hline ENSBTAG00000046855 & $1.36 \mathrm{E}-10$ & 0 & & $1.36 \mathrm{E}+03$ \\
\hline ENSBTAG00000021219 & $4.53 \mathrm{E}-11$ & 0 & 01-Sep & $4.54 E+02$ \\
\hline ENSBTAG00000043974 & $3.92 \mathrm{E}-11$ & 0 & & $3.93 E+02$ \\
\hline ENSBTAG00000031551 & $2.45 \mathrm{E}-10$ & 0 & PRSS53 & $2.45 E+03$ \\
\hline ENSBTAG00000000405 & $1.83 \mathrm{E}-10$ & 0 & & $1.83 E+03$ \\
\hline ENSBTAG00000019521 & $2.63 \mathrm{E}-10$ & 0 & COX6A2 & $2.63 E+03$ \\
\hline ENSBTAG00000031583 & $1.82 \mathrm{E}-10$ & 0 & NUPR2 & $1.82 \mathrm{E}+03$ \\
\hline ENSBTAG00000021430 & $3.26 \mathrm{E}-10$ & 0 & UPK3B & $3.26 \mathrm{E}+03$ \\
\hline ENSBTAG00000039601 & $4.69 \mathrm{E}-10$ & 0 & SAP25 & $4.69 E+03$ \\
\hline ENSBTAG00000046753 & 4.77E-10 & 0 & & $4.77 E+03$ \\
\hline ENSBTAG00000026243 & $2.65 \mathrm{E}-10$ & 0 & PPP1R35 & $2.66 \mathrm{E}+03$ \\
\hline ENSBTAG00000016763 & $2.89 \mathrm{E}-10$ & 0 & & $2.89 E+03$ \\
\hline ENSBTAG00000007012 & $3.60 \mathrm{E}-10$ & 0 & ZNF3 & $3.60 \mathrm{E}+03$ \\
\hline ENSBTAG00000038613 & $1.97 \mathrm{E}-10$ & 0 & & $1.97 \mathrm{E}+03$ \\
\hline ENSBTAG00000040014 & $1.25 \mathrm{E}-10$ & 0 & & $1.25 \mathrm{E}+03$ \\
\hline ENSBTAG00000010291 & 7.44E-10 & 0 & RBAK & $7.44 E+03$ \\
\hline ENSBTAG00000038627 & $8.94 \mathrm{E}-10$ & 0 & & $8.95 E+03$ \\
\hline ENSBTAG00000030721 & $7.02 \mathrm{E}-11$ & 0 & UNCX & $7.03 E+02$ \\
\hline ENSBTAG00000033015 & $3.03 \mathrm{E}-10$ & 0 & FAHD1 & $3.03 E+03$ \\
\hline ENSBTAG00000046703 & $4.43 \mathrm{E}-10$ & 0 & NPW & $4.43 E+03$ \\
\hline ENSBTAG00000047563 & $1.25 \mathrm{E}-10$ & 0 & CLDN9 & $1.25 E+03$ \\
\hline ENSBTAG00000013051 & 0 & 0 & PRM3 & $1.00 \mathrm{E}+00$ \\
\hline ENSBTAG00000031582 & 0 & 0 & & $1.00 \mathrm{E}+00$ \\
\hline ENSBTAG00000047342 & 0 & 0 & & $1.00 E+00$ \\
\hline ENSBTAG00000009022 & 0 & 0 & DNAJC30 & $1.00 \mathrm{E}+00$ \\
\hline ENSBTAG00000046010 & $1.45 \mathrm{E}-10$ & 0 & BHLHA15 & $1.45 \mathrm{E}+03$ \\
\hline ENSBTAG00000005600 & $3.37 \mathrm{E}-10$ & 0 & & $3.37 E+03$ \\
\hline ENSBTAG00000048154 & 0 & 0 & GPER1 & $1.00 E+00$ \\
\hline ENSBTAG00000003690 & $1.76 \mathrm{E}-10$ & $7.67 \mathrm{E}-10$ & NHLRC2 & 2.29E-01 \\
\hline ENSBTAG00000020193 & $1.84 \mathrm{E}-10$ & 4.74E-10 & DCLRE1A & 3.89E-01 \\
\hline ENSBTAG00000021118 & $2.29 \mathrm{E}-11$ & $5.77 \mathrm{E}-11$ & CYP26A1 & 3.98E-01 \\
\hline ENSBTAG00000007049 & $1.79 \mathrm{E}-10$ & $3.00 \mathrm{E}-10$ & MBL2 & 5.95E-01 \\
\hline ENSBTAG00000006218 & $1.53 \mathrm{E}-10$ & $2.56 \mathrm{E}-10$ & FOXI2 & 5.96E-01 \\
\hline ENSBTAG00000013763 & $2.33 \mathrm{E}-10$ & $3.88 \mathrm{E}-10$ & & $6.02 \mathrm{E}-01$ \\
\hline ENSBTAG00000002191 & $2.14 \mathrm{E}-10$ & $3.45 \mathrm{E}-10$ & RRP12 & 6.19E-01 \\
\hline ENSBTAG00000011917 & $1.05 \mathrm{E}-10$ & $1.68 \mathrm{E}-10$ & GPAM & $6.23 \mathrm{E}-01$ \\
\hline ENSBTAG00000000437 & $2.36 \mathrm{E}-10$ & $3.73 \mathrm{E}-10$ & FFAR4 & $6.33 \mathrm{E}-01$ \\
\hline ENSBTAG00000034597 & $1.71 \mathrm{E}-10$ & $2.70 \mathrm{E}-10$ & & $6.34 \mathrm{E}-01$ \\
\hline ENSBTAG00000013820 & $3.80 \mathrm{E}-10$ & $5.75 \mathrm{E}-10$ & & $6.61 \mathrm{E}-01$ \\
\hline ENSBTAG00000000551 & $3.40 \mathrm{E}-10$ & $4.69 \mathrm{E}-10$ & SMNDC1 & 7.26E-01 \\
\hline ENSBTAG00000002655 & $7.80 \mathrm{E}-10$ & $1.05 \mathrm{E}-09$ & & 7.43E-01 \\
\hline ENSBTAG00000011734 & $1.31 \mathrm{E}-10$ & $1.76 \mathrm{E}-10$ & ANKRD1 & 7.45E-01 \\
\hline ENSBTAG00000048118 & $5.63 \mathrm{E}-10$ & $7.39 \mathrm{E}-10$ & & 7.62E-01 \\
\hline ENSBTAG00000046239 & $1.09 \mathrm{E}-10$ & $1.38 \mathrm{E}-10$ & LCOR & 7.93E-01 \\
\hline ENSBTAG00000003027 & 1.57E-10 & $1.98 \mathrm{E}-10$ & EMX2 & 7.95E-01 \\
\hline ENSBTAG00000047164 & $2.72 \mathrm{E}-11$ & $3.42 \mathrm{E}-11$ & & 7.95E-01 \\
\hline ENSBTAG00000001242 & $5.83 \mathrm{E}-11$ & $7.35 E-11$ & ADRB1 & 7.94E-01 \\
\hline ENSBTAG00000007881 & $2.31 \mathrm{E}-10$ & $2.91 \mathrm{E}-10$ & IFIT1 & 7.93E-01 \\
\hline ENSBTAG00000034918 & $1.18 \mathrm{E}-10$ & $1.49 \mathrm{E}-10$ & IFIT2 & 7.95E-01 \\
\hline ENSBTAG00000010461 & $2.10 \mathrm{E}-10$ & $2.65 E-10$ & & 7.93E-01 \\
\hline ENSBTAG00000047474 & $1.90 \mathrm{E}-10$ & $2.39 \mathrm{E}-10$ & SPRN & 7.94E-01 \\
\hline ENSBTAG00000005791 & $1.08 \mathrm{E}-09$ & 1.33E-09 & UROS & $8.15 E-01$ \\
\hline ENSBTAG00000012416 & $9.80 \mathrm{E}-10$ & $1.20 \mathrm{E}-09$ & & 8.16E-01 \\
\hline ENSBTAG00000038063 & $8.42 \mathrm{E}-10$ & 1.03E-09 & LIPJ & 8.17E-01 \\
\hline ENSBTAG00000005792 & $9.42 \mathrm{E}-10$ & 1.15E-09 & BCCIP & 8.19E-01 \\
\hline ENSBTAG00000006928 & $1.23 \mathrm{E}-10$ & $1.50 \mathrm{E}-10$ & OAT & $8.20 \mathrm{E}-01$ \\
\hline ENSBTAG00000020233 & $1.43 \mathrm{E}-10$ & $1.74 \mathrm{E}-10$ & CCDC186 & $8.21 \mathrm{E}-01$ \\
\hline ENSBTAG00000006689 & $8.76 \mathrm{E}-11$ & $1.05 \mathrm{E}-10$ & TECTB & 8.35E-01 \\
\hline
\end{tabular}




\begin{tabular}{|c|c|c|c|c|}
\hline ENSBTAG000000008730 & $2.98 \mathrm{E}-10$ & $3.56 \mathrm{E}-10$ & SFXN4 & 8.37E-01 \\
\hline ENSBTAG000000007578 & $3.28 \mathrm{E}-10$ & 3.92E-10 & & 8.37E-01 \\
\hline ENSBTAG000000032299 & $4.24 \mathrm{E}-10$ & 4.97E-10 & CCDC172 & 8.54E-01 \\
\hline ENSBTAG00000014218 & $3.99 \mathrm{E}-10$ & $4.64 \mathrm{E}-10$ & DKK1 & $8.59 \mathrm{E}-01$ \\
\hline ENSBTAG00000009768 & $7.72 \mathrm{E}-10$ & 8.97E-10 & IFIT3 & $8.60 \mathrm{E}-01$ \\
\hline ENSBTAG000000046499 & $8.81 \mathrm{E}-10$ & $1.02 \mathrm{E}-09$ & & 8.63E-01 \\
\hline ENSBTAG000000010786 & $5.23 \mathrm{E}-10$ & $6.06 \mathrm{E}-10$ & TACC2 & 8.62E-01 \\
\hline ENSBTAG000000011197 & $1.15 \mathrm{E}-10$ & $1.32 \mathrm{E}-10$ & IPMK & $8.68 \mathrm{E}-01$ \\
\hline ENSBTAG000000011487 & $6.63 \mathrm{E}-10$ & $7.58 \mathrm{E}-10$ & PLEKHS1 & $8.74 \mathrm{E}-01$ \\
\hline ENSBTAG000000008783 & 3.33E-10 & $3.81 \mathrm{E}-10$ & HTR7 & $8.75 \mathrm{E}-01$ \\
\hline ENSBTAG00000013779 & $5.87 \mathrm{E}-10$ & $6.70 \mathrm{E}-10$ & HSPA12A & $8.76 \mathrm{E}-01$ \\
\hline ENSBTAG00000014335 & 1.33E-09 & $1.50 \mathrm{E}-09$ & CYP17A1 & 8.87E-01 \\
\hline ENSBTAG000000005027 & $2.26 \mathrm{E}-10$ & $2.55 \mathrm{E}-10$ & GLRX3 & 8.87E-01 \\
\hline ENSBTAG000000005129 & $2.97 \mathrm{E}-10$ & 3.34E-10 & CEP55 & $8.90 \mathrm{E}-01$ \\
\hline ENSBTAG000000022808 & $1.95 \mathrm{E}-10$ & $2.18 \mathrm{E}-10$ & CACUL1 & $8.94 \mathrm{E}-01$ \\
\hline ENSBTAG000000018941 & $3.96 \mathrm{E}-10$ & $4.45 \mathrm{E}-10$ & CPXM2 & $8.91 \mathrm{E}-01$ \\
\hline ENSBTAG000000008729 & $2.99 \mathrm{E}-10$ & 3.35E-10 & FAM45A & 8.93E-01 \\
\hline ENSBTAG000000013875 & $1.52 \mathrm{E}-10$ & $1.70 \mathrm{E}-10$ & VAX1 & 8.93E-01 \\
\hline ENSBTAG000000000442 & $2.38 \mathrm{E}-10$ & 2.67E-10 & RBP4 & $8.92 \mathrm{E}-01$ \\
\hline ENSBTAG000000031955 & $6.50 \mathrm{E}-10$ & $7.16 \mathrm{E}-10$ & GPR26 & $9.08 \mathrm{E}-01$ \\
\hline ENSBTAG000000004612 & $6.58 \mathrm{E}-10$ & $7.24 \mathrm{E}-10$ & & $9.08 \mathrm{E}-01$ \\
\hline ENSBTAG000000026185 & 5.19E-10 & $5.66 \mathrm{E}-10$ & & 9.17E-01 \\
\hline ENSBTAG000000019322 & $6.69 \mathrm{E}-10$ & 7.22E-10 & HABP2 & $9.26 \mathrm{E}-01$ \\
\hline ENSBTAG000000015062 & $9.23 \mathrm{E}-10$ & $9.83 \mathrm{E}-10$ & KCNK18 & $9.39 \mathrm{E}-01$ \\
\hline ENSBTAG00000006615 & $3.29 \mathrm{E}-10$ & $3.47 \mathrm{E}-10$ & CASP7 & 9.47E-01 \\
\hline ENSBTAG000000020918 & $1.46 \mathrm{E}-10$ & $1.53 \mathrm{E}-10$ & NKX1-2 & 9.53E-01 \\
\hline ENSBTAG00000019919 & 4.54E-10 & 4.77E-10 & & $9.52 \mathrm{E}-01$ \\
\hline ENSBTAG00000006855 & $6.89 \mathrm{E}-10$ & $7.22 \mathrm{E}-10$ & TDRD1 & 9.54E-01 \\
\hline ENSBTAG000000017913 & $1.16 \mathrm{E}-10$ & $1.20 \mathrm{E}-10$ & BUB3 & $9.64 \mathrm{E}-01$ \\
\hline ENSBTAG000000045905 & $7.15 \mathrm{E}-10$ & 7.37E-10 & PCDH15 & $9.70 \mathrm{E}-01$ \\
\hline ENSBTAG000000045728 & $3.29 \mathrm{E}-10$ & $3.38 \mathrm{E}-10$ & & $9.75 \mathrm{E}-01$ \\
\hline ENSBTAG00000019690 & $5.29 \mathrm{E}-10$ & 5.37E-10 & PNLIPRP3 & $9.86 \mathrm{E}-01$ \\
\hline ENSBTAG000000003395 & $7.74 \mathrm{E}-11$ & $7.84 \mathrm{E}-11$ & ZRANB1 & 9.87E-01 \\
\hline ENSBTAG000000001006 & $2.40 \mathrm{E}-10$ & $2.42 \mathrm{E}-10$ & HMX3 & $9.91 \mathrm{E}-01$ \\
\hline ENSBTAG000000002435 & 3.37E-10 & $3.40 \mathrm{E}-10$ & PTPRE & $9.92 \mathrm{E}-01$ \\
\hline ENSBTAG00000011401 & $2.81 \mathrm{E}-10$ & $2.81 E-10$ & PDZD8 & $1.00 E+00$ \\
\hline ENSBTAG00000015651 & $3.34 \mathrm{E}-10$ & 3.35E-10 & & 9.97E-01 \\
\hline ENSBTAG00000003397 & $2.82 \mathrm{E}-10$ & $2.82 \mathrm{E}-10$ & СТВР2 & $9.99 \mathrm{E}-01$ \\
\hline ENSBTAG00000033685 & 4.12E-10 & 4.03E-10 & CCNJ & $1.02 \mathrm{E}+00$ \\
\hline ENSBTAG00000008272 & $2.45 \mathrm{E}-10$ & $2.41 E-10$ & EBF3 & $1.02 \mathrm{E}+00$ \\
\hline ENSBTAG00000020283 & $1.82 \mathrm{E}-10$ & $1.76 \mathrm{E}-10$ & DUSP5 & $1.03 E+00$ \\
\hline ENSBTAG00000008389 & $7.61 \mathrm{E}-10$ & $7.36 \mathrm{E}-10$ & HTRA1 & $1.03 E+00$ \\
\hline ENSBTAG00000002444 & $4.39 \mathrm{E}-10$ & $4.21 \mathrm{E}-10$ & MKI67 & $1.04 E+00$ \\
\hline ENSBTAG00000023549 & $3.09 \mathrm{E}-10$ & $2.95 \mathrm{E}-10$ & & $1.05 E+00$ \\
\hline ENSBTAG00000007594 & $1.24 \mathrm{E}-10$ & $1.18 \mathrm{E}-10$ & CWF19L1 & $1.05 E+00$ \\
\hline ENSBTAG00000010796 & $5.96 \mathrm{E}-10$ & 5.67E-10 & SPADH1 & $1.05 E+00$ \\
\hline ENSBTAG00000032237 & 7.17E-10 & $6.82 \mathrm{E}-10$ & PNLIP & $1.05 E+00$ \\
\hline ENSBTAG00000007044 & $1.89 \mathrm{E}-10$ & $1.78 \mathrm{E}-10$ & CALHM1 & $1.06 \mathrm{E}+00$ \\
\hline ENSBTAG00000021856 & $5.79 \mathrm{E}-10$ & $5.48 \mathrm{E}-10$ & R3HCC1L & $1.06 \mathrm{E}+00$ \\
\hline ENSBTAG00000010785 & $4.97 \mathrm{E}-10$ & 4.70E-10 & FAS & $1.06 \mathrm{E}+00$ \\
\hline ENSBTAG00000014336 & 3.93E-10 & $3.66 \mathrm{E}-10$ & WBP1L & $1.07 E+00$ \\
\hline ENSBTAG00000039132 & $6.76 \mathrm{E}-10$ & $6.27 \mathrm{E}-10$ & & $1.08 \mathrm{E}+00$ \\
\hline ENSBTAG00000019519 & $2.91 \mathrm{E}-10$ & 2.70E-10 & PWWP2B & $1.08 \mathrm{E}+00$ \\
\hline ENSBTAG00000007981 & $2.67 \mathrm{E}-10$ & 2.47E-10 & GRK5 & $1.08 \mathrm{E}+00$ \\
\hline ENSBTAG00000031890 & $5.63 \mathrm{E}-10$ & $5.19 \mathrm{E}-10$ & DOCK1 & $1.08 \mathrm{E}+00$ \\
\hline ENSBTAG00000006860 & $3.53 \mathrm{E}-10$ & $3.26 \mathrm{E}-10$ & VWA2 & $1.08 \mathrm{E}+00$ \\
\hline ENSBTAG00000021397 & $2.54 \mathrm{E}-10$ & $2.33 \mathrm{E}-10$ & NKX2-3 & $1.09 E+00$ \\
\hline ENSBTAG00000047450 & $2.24 \mathrm{E}-10$ & $2.05 \mathrm{E}-10$ & SFR1 & $1.09 E+00$ \\
\hline ENSBTAG00000018404 & $5.12 \mathrm{E}-10$ & $4.68 \mathrm{E}-10$ & PRKG1 & $1.09 E+00$ \\
\hline ENSBTAG00000001298 & 4.35E-10 & 3.97E-10 & STAMBPL1 & $1.09 E+00$ \\
\hline ENSBTAG00000003989 & $6.66 \mathrm{E}-10$ & $6.03 \mathrm{E}-10$ & & $1.10 E+00$ \\
\hline ENSBTAG00000005979 & $1.97 \mathrm{E}-10$ & $1.78 \mathrm{E}-10$ & HELLS & $1.10 \mathrm{E}+00$ \\
\hline ENSBTAG00000011740 & 1.03E-09 & $9.30 \mathrm{E}-10$ & OPALIN & $1.11 \mathrm{E}+00$ \\
\hline ENSBTAG00000003636 & $3.99 \mathrm{E}-10$ & $3.58 \mathrm{E}-10$ & LIPA & $1.11 \mathrm{E}+00$ \\
\hline ENSBTAG00000003312 & $5.41 \mathrm{E}-10$ & 4.84E-10 & CHST15 & $1.12 \mathrm{E}+00$ \\
\hline ENSBTAG00000021015 & 4.22E-10 & $3.78 \mathrm{E}-10$ & FAM196A & $1.12 \mathrm{E}+00$ \\
\hline ENSBTAG00000009181 & $3.47 \mathrm{E}-10$ & $3.09 \mathrm{E}-10$ & INPP5A & $1.12 \mathrm{E}+00$ \\
\hline ENSBTAG00000011562 & 5.55E-10 & 4.94E-10 & STK32C & $1.12 \mathrm{E}+00$ \\
\hline ENSBTAG00000012107 & $3.51 \mathrm{E}-10$ & $3.11 \mathrm{E}-10$ & SLC25A28 & $1.13 \mathrm{E}+00$ \\
\hline ENSBTAG00000003178 & $2.45 \mathrm{E}-10$ & $2.15 E-10$ & ATE1 & $1.14 \mathrm{E}+00$ \\
\hline
\end{tabular}




\begin{tabular}{|c|c|c|c|c|}
\hline ENSBTAG00000011832 & $1.97 \mathrm{E}-10$ & $1.73 \mathrm{E}-10$ & ALDH18A1 & $1.14 \mathrm{E}+00$ \\
\hline ENSBTAG00000003265 & 4.45E-10 & $3.90 \mathrm{E}-10$ & ADD3 & $1.14 \mathrm{E}+00$ \\
\hline ENSBTAG00000006395 & $9.20 \mathrm{E}-10$ & $8.04 \mathrm{E}-10$ & TUBGCP2 & $1.14 \mathrm{E}+00$ \\
\hline ENSBTAG00000023840 & $2.75 \mathrm{E}-10$ & 2.40E-10 & & $1.15 \mathrm{E}+00$ \\
\hline ENSBTAG00000023891 & 4.35E-10 & $3.77 \mathrm{E}-10$ & RBM20 & $1.15 \mathrm{E}+00$ \\
\hline ENSBTAG00000010957 & $7.45 \mathrm{E}-10$ & $6.45 \mathrm{E}-10$ & LHPP & $1.16 \mathrm{E}+00$ \\
\hline ENSBTAG00000004895 & $2.53 \mathrm{E}-10$ & $2.18 \mathrm{E}-10$ & AFAP1L2 & $1.16 \mathrm{E}+00$ \\
\hline ENSBTAG00000013364 & $6.65 \mathrm{E}-10$ & $5.69 \mathrm{E}-10$ & LIPM & $1.17 \mathrm{E}+00$ \\
\hline ENSBTAG00000013905 & $1.83 \mathrm{E}-10$ & $1.55 \mathrm{E}-10$ & SMC3 & $1.18 \mathrm{E}+00$ \\
\hline ENSBTAG00000002528 & $1.40 \mathrm{E}-10$ & $1.18 \mathrm{E}-10$ & EIF3A & $1.18 \mathrm{E}+00$ \\
\hline ENSBTAG00000004029 & 7.37E-11 & $6.19 \mathrm{E}-11$ & & $1.19 \mathrm{E}+00$ \\
\hline ENSBTAG00000023845 & $3.50 \mathrm{E}-10$ & $2.94 \mathrm{E}-10$ & & $1.19 E+00$ \\
\hline ENSBTAG00000008731 & $3.16 \mathrm{E}-10$ & $2.65 \mathrm{E}-10$ & PRDX3 & $1.19 \mathrm{E}+00$ \\
\hline ENSBTAG00000017804 & $5.53 \mathrm{E}-10$ & 4.64E-10 & BNIP3 & $1.19 E+00$ \\
\hline ENSBTAG00000007588 & $2.24 \mathrm{E}-10$ & $1.88 \mathrm{E}-10$ & ERLIN1 & $1.19 E+00$ \\
\hline ENSBTAG00000003062 & $5.52 \mathrm{E}-10$ & $4.61 \mathrm{E}-10$ & FAM160B1 & $1.20 E+00$ \\
\hline ENSBTAG00000010105 & $3.45 \mathrm{E}-10$ & $2.88 \mathrm{E}-10$ & & $1.20 \mathrm{E}+00$ \\
\hline ENSBTAG00000019297 & 4.94E-10 & 4.12E-10 & GFRA1 & $1.20 \mathrm{E}+00$ \\
\hline ENSBTAG00000004636 & $2.43 \mathrm{E}-10$ & $2.02 \mathrm{E}-10$ & PLEKHA1 & $1.20 \mathrm{E}+00$ \\
\hline ENSBTAG00000011137 & $5.91 \mathrm{E}-10$ & $4.88 \mathrm{E}-10$ & MORN4 & $1.21 \mathrm{E}+00$ \\
\hline ENSBTAG00000000447 & $3.74 \mathrm{E}-10$ & $3.01 \mathrm{E}-10$ & FRA10AC1 & $1.24 \mathrm{E}+00$ \\
\hline ENSBTAG00000011368 & $3.80 \mathrm{E}-10$ & 3.03E-10 & NOC3L & $1.25 \mathrm{E}+00$ \\
\hline ENSBTAG00000004899 & $5.30 \mathrm{E}-10$ & 4.19E-10 & ABLIM1 & $1.26 \mathrm{E}+00$ \\
\hline ENSBTAG00000024700 & $5.25 \mathrm{E}-10$ & $4.11 \mathrm{E}-10$ & & $1.28 \mathrm{E}+00$ \\
\hline ENSBTAG00000012444 & $4.59 \mathrm{E}-10$ & $3.60 \mathrm{E}-10$ & ADAM12 & $1.28 \mathrm{E}+00$ \\
\hline ENSBTAG00000036127 & $1.39 \mathrm{E}-10$ & $1.09 \mathrm{E}-10$ & & $1.27 \mathrm{E}+00$ \\
\hline ENSBTAG00000005015 & $2.46 \mathrm{E}-10$ & $1.91 \mathrm{E}-10$ & SFXN3 & $1.29 E+00$ \\
\hline ENSBTAG00000040290 & $2.78 \mathrm{E}-10$ & $2.16 \mathrm{E}-10$ & GSTO2 & $1.29 E+00$ \\
\hline ENSBTAG00000002647 & $4.61 \mathrm{E}-10$ & $3.58 \mathrm{E}-10$ & RGS10 & $1.29 \mathrm{E}+00$ \\
\hline ENSBTAG00000019327 & $6.94 \mathrm{E}-10$ & $5.37 \mathrm{E}-10$ & NRAP & $1.29 E+00$ \\
\hline ENSBTAG00000014217 & $4.38 \mathrm{E}-10$ & $3.38 \mathrm{E}-10$ & HHEX & $1.30 \mathrm{E}+00$ \\
\hline ENSBTAG00000039755 & $1.35 \mathrm{E}-09$ & 1.03E-09 & & $1.31 \mathrm{E}+00$ \\
\hline ENSBTAG00000037559 & $3.00 \mathrm{E}-10$ & $2.30 \mathrm{E}-10$ & CYP2C18 & $1.30 \mathrm{E}+00$ \\
\hline ENSBTAG00000012857 & $6.00 \mathrm{E}-10$ & $4.58 \mathrm{E}-10$ & CNNM2 & $1.31 E+00$ \\
\hline ENSBTAG00000021357 & $9.20 \mathrm{E}-11$ & $6.96 \mathrm{E}-11$ & ZNF518A & $1.32 \mathrm{E}+00$ \\
\hline ENSBTAG00000046727 & 4.31E-10 & $3.25 \mathrm{E}-10$ & & $1.32 \mathrm{E}+00$ \\
\hline ENSBTAG00000021540 & 2.69E-10 & $2.02 \mathrm{E}-10$ & WDR11 & $1.33 \mathrm{E}+00$ \\
\hline ENSBTAG00000002973 & $1.70 \mathrm{E}-10$ & $1.26 \mathrm{E}-10$ & RPP30 & $1.35 E+00$ \\
\hline ENSBTAG00000043991 & $2.25 \mathrm{E}-10$ & $1.64 \mathrm{E}-10$ & SLIT1 & $1.37 \mathrm{E}+00$ \\
\hline ENSBTAG00000009439 & $1.74 \mathrm{E}-10$ & $1.27 \mathrm{E}-10$ & EDRF1 & $1.37 \mathrm{E}+00$ \\
\hline ENSBTAG00000038540 & $5.39 \mathrm{E}-10$ & $3.89 \mathrm{E}-10$ & & $1.38 \mathrm{E}+00$ \\
\hline ENSBTAG00000012720 & $1.29 \mathrm{E}-10$ & $9.28 \mathrm{E}-11$ & ANKRD2 & $1.39 E+00$ \\
\hline ENSBTAG00000014831 & $1.17 \mathrm{E}-10$ & $8.43 \mathrm{E}-11$ & PPP1R3C & $1.39 E+00$ \\
\hline ENSBTAG00000032106 & $2.53 \mathrm{E}-10$ & $1.81 \mathrm{E}-10$ & PLPP4 & $1.40 \mathrm{E}+00$ \\
\hline ENSBTAG00000002880 & $3.99 \mathrm{E}-10$ & $2.85 \mathrm{E}-10$ & & $1.40 \mathrm{E}+00$ \\
\hline ENSBTAG00000006714 & $3.34 \mathrm{E}-10$ & $2.37 \mathrm{E}-10$ & & $1.41 E+00$ \\
\hline ENSBTAG00000023832 & $5.32 \mathrm{E}-10$ & $3.78 \mathrm{E}-10$ & ADAM8 & $1.41 \mathrm{E}+00$ \\
\hline ENSBTAG00000007948 & $5.51 \mathrm{E}-10$ & $3.91 \mathrm{E}-10$ & SORCS1 & $1.41 \mathrm{E}+00$ \\
\hline ENSBTAG00000004080 & $2.03 \mathrm{E}-10$ & $1.43 \mathrm{E}-10$ & TIAL1 & $1.42 \mathrm{E}+00$ \\
\hline ENSBTAG00000021801 & $1.11 \mathrm{E}-10$ & $7.79 \mathrm{E}-11$ & & $1.43 \mathrm{E}+00$ \\
\hline ENSBTAG00000007011 & $7.18 \mathrm{E}-10$ & $5.03 \mathrm{E}-10$ & TEX36 & $1.43 \mathrm{E}+00$ \\
\hline ENSBTAG00000047077 & $3.36 \mathrm{E}-10$ & $2.36 \mathrm{E}-10$ & ENTPD7 & $1.43 \mathrm{E}+00$ \\
\hline ENSBTAG00000007709 & $1.79 \mathrm{E}-10$ & $1.25 \mathrm{E}-10$ & SHOC2 & $1.43 \mathrm{E}+00$ \\
\hline ENSBTAG00000016298 & $1.57 \mathrm{E}-10$ & $1.08 \mathrm{E}-10$ & TM9SF3 & $1.45 \mathrm{E}+00$ \\
\hline ENSBTAG00000011182 & $7.60 \mathrm{E}-10$ & $5.22 \mathrm{E}-10$ & PDLIM1 & $1.46 \mathrm{E}+00$ \\
\hline ENSBTAG00000004318 & $3.85 \mathrm{E}-10$ & $2.64 \mathrm{E}-10$ & ARL3 & $1.46 \mathrm{E}+00$ \\
\hline ENSBTAG00000019434 & $2.00 \mathrm{E}-10$ & $1.36 \mathrm{E}-10$ & PDCD4 & $1.47 \mathrm{E}+00$ \\
\hline ENSBTAG00000005965 & $3.89 \mathrm{E}-10$ & $2.63 \mathrm{E}-10$ & ADGRA1 & $1.48 \mathrm{E}+00$ \\
\hline ENSBTAG00000022917 & $5.96 \mathrm{E}-10$ & $4.01 \mathrm{E}-10$ & FANK1 & $1.49 E+00$ \\
\hline ENSBTAG00000019166 & 8.94E-11 & $6.00 \mathrm{E}-11$ & NSMCE4A & $1.49 E+00$ \\
\hline ENSBTAG00000006445 & $1.04 \mathrm{E}-09$ & $6.92 \mathrm{E}-10$ & & $1.50 \mathrm{E}+00$ \\
\hline ENSBTAG00000008939 & $6.63 \mathrm{E}-10$ & $4.42 \mathrm{E}-10$ & DNMBP & $1.50 \mathrm{E}+00$ \\
\hline ENSBTAG00000008102 & $4.82 \mathrm{E}-10$ & $3.21 \mathrm{E}-10$ & CRTAC1 & $1.50 \mathrm{E}+00$ \\
\hline ENSBTAG00000017780 & $3.25 \mathrm{E}-10$ & $2.15 \mathrm{E}-10$ & DPYSL4 & $1.51 E+00$ \\
\hline ENSBTAG00000002971 & $1.74 \mathrm{E}-10$ & $1.14 \mathrm{E}-10$ & CUTC & $1.52 \mathrm{E}+00$ \\
\hline ENSBTAG00000011841 & $1.95 \mathrm{E}-10$ & $1.28 \mathrm{E}-10$ & TCTN3 & $1.52 \mathrm{E}+00$ \\
\hline ENSBTAG00000000092 & $2.44 \mathrm{E}-10$ & $1.59 \mathrm{E}-10$ & HIF1AN & $1.53 \mathrm{E}+00$ \\
\hline ENSBTAG00000006713 & $1.59 \mathrm{E}-10$ & $1.04 \mathrm{E}-10$ & ZDHHC6 & $1.53 E+00$ \\
\hline ENSBTAG00000024911 & $5.17 \mathrm{E}-10$ & $3.37 \mathrm{E}-10$ & LIPN & $1.53 \mathrm{E}+00$ \\
\hline ENSBTAG00000047270 & 2.92E-10 & $1.90 \mathrm{E}-10$ & & $1.54 \mathrm{E}+00$ \\
\hline
\end{tabular}




\begin{tabular}{|c|c|c|c|c|}
\hline ENSBTAG00000000806 & $2.14 \mathrm{E}-10$ & $1.39 \mathrm{E}-10$ & ATAD1 & $1.54 \mathrm{E}+00$ \\
\hline ENSBTAG00000009693 & $1.43 \mathrm{E}-10$ & $9.17 E-11$ & TBC1D12 & $1.56 E+00$ \\
\hline ENSBTAG00000013641 & $2.20 \mathrm{E}-10$ & $1.41 \mathrm{E}-10$ & BAG3 & $1.56 \mathrm{E}+00$ \\
\hline ENSBTAG00000007956 & $1.98 \mathrm{E}-10$ & $1.25 \mathrm{E}-10$ & FRAT1 & $1.58 \mathrm{E}+00$ \\
\hline ENSBTAG00000040222 & $3.46 \mathrm{E}-11$ & $2.18 \mathrm{E}-11$ & & $1.58 \mathrm{E}+00$ \\
\hline ENSBTAG00000039324 & $4.25 \mathrm{E}-10$ & 2.67E-10 & FGFBP3 & $1.59 \mathrm{E}+00$ \\
\hline ENSBTAG00000003196 & $3.71 \mathrm{E}-10$ & $2.33 \mathrm{E}-10$ & PAPSS2 & $1.59 E+00$ \\
\hline ENSBTAG00000002717 & $4.33 \mathrm{E}-10$ & $2.72 \mathrm{E}-10$ & INA & $1.59 E+00$ \\
\hline ENSBTAG00000015450 & $3.64 \mathrm{E}-10$ & $2.26 \mathrm{E}-10$ & CPEB3 & $1.61 \mathrm{E}+00$ \\
\hline ENSBTAG00000023846 & $5.63 \mathrm{E}-10$ & $3.48 \mathrm{E}-10$ & & $1.62 \mathrm{E}+00$ \\
\hline ENSBTAG00000005057 & $1.98 \mathrm{E}-10$ & $1.22 \mathrm{E}-10$ & FAM204A & $1.62 \mathrm{E}+00$ \\
\hline ENSBTAG00000020589 & $3.08 \mathrm{E}-10$ & $1.90 \mathrm{E}-10$ & & $1.62 \mathrm{E}+00$ \\
\hline ENSBTAG00000012858 & 1.57E-10 & $9.65 \mathrm{E}-11$ & NT5C2 & $1.63 \mathrm{E}+00$ \\
\hline ENSBTAG00000016928 & $1.95 \mathrm{E}-10$ & $1.20 \mathrm{E}-10$ & & $1.62 E+00$ \\
\hline ENSBTAG00000023939 & $1.76 \mathrm{E}-10$ & $1.07 \mathrm{E}-10$ & & $1.65 E+00$ \\
\hline ENSBTAG00000021358 & $5.80 \mathrm{E}-10$ & $3.52 \mathrm{E}-10$ & BLNK & $1.65 E+00$ \\
\hline ENSBTAG00000007441 & $5.39 \mathrm{E}-10$ & $3.27 \mathrm{E}-10$ & SEC23IP & $1.65 E+00$ \\
\hline ENSBTAG00000014614 & $1.65 \mathrm{E}-10$ & $1.00 \mathrm{E}-10$ & ACTA2 & $1.65 \mathrm{E}+00$ \\
\hline ENSBTAG00000012970 & $3.16 \mathrm{E}-10$ & $1.91 \mathrm{E}-10$ & FAM53B & $1.65 \mathrm{E}+00$ \\
\hline ENSBTAG00000018966 & $5.55 \mathrm{E}-10$ & $3.34 \mathrm{E}-10$ & PLCE1 & $1.66 \mathrm{E}+00$ \\
\hline ENSBTAG00000004662 & $2.54 \mathrm{E}-10$ & $1.52 \mathrm{E}-10$ & SLC16A12 & $1.67 \mathrm{E}+00$ \\
\hline ENSBTAG00000020981 & $1.87 \mathrm{E}-10$ & $1.12 \mathrm{E}-10$ & 05-Mar & $1.67 \mathrm{E}+00$ \\
\hline ENSBTAG00000009877 & $2.60 \mathrm{E}-10$ & $1.56 \mathrm{E}-10$ & MCMBP & $1.67 \mathrm{E}+00$ \\
\hline ENSBTAG00000008029 & 8.64E-10 & $5.12 \mathrm{E}-10$ & & $1.69 \mathrm{E}+00$ \\
\hline ENSBTAG00000020304 & $3.47 \mathrm{E}-10$ & $2.05 \mathrm{E}-10$ & & $1.69 \mathrm{E}+00$ \\
\hline ENSBTAG00000018406 & $4.46 \mathrm{E}-10$ & $2.59 \mathrm{E}-10$ & CSTF2T & $1.72 \mathrm{E}+00$ \\
\hline ENSBTAG00000040427 & $2.50 \mathrm{E}-10$ & $1.45 \mathrm{E}-10$ & & $1.72 \mathrm{E}+00$ \\
\hline ENSBTAG00000021566 & $2.80 \mathrm{E}-10$ & $1.62 \mathrm{E}-10$ & PAX2 & $1.73 E+00$ \\
\hline ENSBTAG00000010442 & $2.58 \mathrm{E}-10$ & $1.48 \mathrm{E}-10$ & PANK1 & $1.74 \mathrm{E}+00$ \\
\hline ENSBTAG00000018041 & $4.91 \mathrm{E}-10$ & $2.81 \mathrm{E}-10$ & ACADSB & $1.75 E+00$ \\
\hline ENSBTAG00000012719 & $2.46 \mathrm{E}-10$ & $1.39 \mathrm{E}-10$ & UBTD1 & $1.77 \mathrm{E}+00$ \\
\hline ENSBTAG00000004964 & 2.47E-10 & $1.39 \mathrm{E}-10$ & PCGF5 & $1.78 \mathrm{E}+00$ \\
\hline ENSBTAG00000009498 & $1.72 \mathrm{E}-10$ & $9.62 \mathrm{E}-11$ & PTEN & $1.79 \mathrm{E}+00$ \\
\hline ENSBTAG00000008100 & $4.42 \mathrm{E}-10$ & $2.46 \mathrm{E}-10$ & GOLGA7B & $1.80 \mathrm{E}+00$ \\
\hline ENSBTAG00000007476 & $2.67 \mathrm{E}-10$ & $1.48 \mathrm{E}-10$ & BTRC & $1.81 \mathrm{E}+00$ \\
\hline ENSBTAG00000020375 & $6.47 \mathrm{E}-10$ & $3.52 \mathrm{E}-10$ & & $1.84 \mathrm{E}+00$ \\
\hline ENSBTAG00000005928 & $2.59 \mathrm{E}-10$ & $1.40 \mathrm{E}-10$ & MINPP1 & $1.85 \mathrm{E}+00$ \\
\hline ENSBTAG00000011651 & $4.48 \mathrm{E}-10$ & $2.43 \mathrm{E}-10$ & HECTD2 & $1.84 \mathrm{E}+00$ \\
\hline ENSBTAG00000003294 & 7.64E-11 & $4.12 \mathrm{E}-11$ & MRPL43 & $1.85 \mathrm{E}+00$ \\
\hline ENSBTAG00000020895 & $2.75 \mathrm{E}-10$ & $1.48 \mathrm{E}-10$ & & $1.85 \mathrm{E}+00$ \\
\hline ENSBTAG00000021116 & $1.36 \mathrm{E}-10$ & $7.29 \mathrm{E}-11$ & EXOC6 & $1.86 \mathrm{E}+00$ \\
\hline ENSBTAG00000007591 & $3.65 \mathrm{E}-10$ & $1.95 \mathrm{E}-10$ & CHUK & $1.87 \mathrm{E}+00$ \\
\hline ENSBTAG00000032761 & $5.73 \mathrm{E}-10$ & $3.06 \mathrm{E}-10$ & CALHM3 & $1.87 \mathrm{E}+00$ \\
\hline ENSBTAG00000005708 & $2.26 \mathrm{E}-10$ & $1.20 \mathrm{E}-10$ & & $1.89 \mathrm{E}+00$ \\
\hline ENSBTAG00000005795 & $1.05 \mathrm{E}-09$ & $5.58 \mathrm{E}-10$ & DHX32 & $1.88 \mathrm{E}+00$ \\
\hline ENSBTAG00000020427 & $8.21 \mathrm{E}-10$ & $4.33 \mathrm{E}-10$ & DNTT & $1.90 \mathrm{E}+00$ \\
\hline ENSBTAG00000021574 & $3.58 \mathrm{E}-10$ & $1.88 \mathrm{E}-10$ & & $1.90 \mathrm{E}+00$ \\
\hline ENSBTAG00000035072 & $1.98 \mathrm{E}-10$ & $1.03 \mathrm{E}-10$ & SYCE1 & $1.93 \mathrm{E}+00$ \\
\hline ENSBTAG00000014064 & $1.65 \mathrm{E}-10$ & $8.52 \mathrm{E}-11$ & FGFR2 & $1.93 \mathrm{E}+00$ \\
\hline ENSBTAG00000011587 & $3.50 \mathrm{E}-10$ & $1.81 \mathrm{E}-10$ & C10orf90 & $1.94 \mathrm{E}+00$ \\
\hline ENSBTAG00000008819 & $3.84 \mathrm{E}-10$ & $1.98 \mathrm{E}-10$ & BTBD16 & $1.94 \mathrm{E}+00$ \\
\hline ENSBTAG00000021246 & $9.66 \mathrm{E}-11$ & 4.87E-11 & & $1.98 \mathrm{E}+00$ \\
\hline ENSBTAG00000003529 & $5.11 \mathrm{E}-10$ & $2.55 \mathrm{E}-10$ & & $2.00 \mathrm{E}+00$ \\
\hline ENSBTAG00000019759 & $3.91 \mathrm{E}-10$ & $1.95 \mathrm{E}-10$ & IDE & $2.00 E+00$ \\
\hline ENSBTAG00000007243 & $7.76 \mathrm{E}-10$ & $3.84 \mathrm{E}-10$ & CFAP58 & $2.02 \mathrm{E}+00$ \\
\hline ENSBTAG00000039973 & $1.84 \mathrm{E}-10$ & $9.02 \mathrm{E}-11$ & CYP26C1 & $2.04 \mathrm{E}+00$ \\
\hline ENSBTAG00000022715 & $9.01 \mathrm{E}-10$ & $4.38 \mathrm{E}-10$ & & $2.06 \mathrm{E}+00$ \\
\hline ENSBTAG00000005106 & $2.53 \mathrm{E}-10$ & $1.22 \mathrm{E}-10$ & & $2.07 E+00$ \\
\hline ENSBTAG00000017709 & $7.09 \mathrm{E}-10$ & $3.40 \mathrm{E}-10$ & ATRNL1 & $2.08 \mathrm{E}+00$ \\
\hline ENSBTAG00000004739 & $9.28 \mathrm{E}-10$ & $4.40 \mathrm{E}-10$ & SLC18A2 & $2.11 E+00$ \\
\hline ENSBTAG00000012914 & $1.65 \mathrm{E}-10$ & 7.77E-11 & WNT8B & $2.12 \mathrm{E}+00$ \\
\hline ENSBTAG00000007460 & $1.63 \mathrm{E}-10$ & $7.71 \mathrm{E}-11$ & RAB11FIP2 & $2.12 \mathrm{E}+00$ \\
\hline ENSBTAG00000046362 & $1.54 \mathrm{E}-10$ & 7.26E-11 & & $2.12 \mathrm{E}+00$ \\
\hline ENSBTAG00000007248 & 7.02E-10 & $3.30 \mathrm{E}-10$ & & $2.13 E+00$ \\
\hline ENSBTAG00000014574 & $3.67 \mathrm{E}-10$ & $1.72 \mathrm{E}-10$ & CFAP43 & $2.13 E+00$ \\
\hline ENSBTAG00000009279 & $3.60 \mathrm{E}-10$ & $1.67 \mathrm{E}-10$ & TNKS2 & $2.16 \mathrm{E}+00$ \\
\hline ENSBTAG00000000445 & $5.88 \mathrm{E}-10$ & $2.70 \mathrm{E}-10$ & PDE6C & $2.18 \mathrm{E}+00$ \\
\hline ENSBTAG00000009879 & $2.35 \mathrm{E}-10$ & $1.08 \mathrm{E}-10$ & & $2.18 \mathrm{E}+00$ \\
\hline ENSBTAG00000003800 & $3.55 \mathrm{E}-10$ & $1.62 \mathrm{E}-10$ & LRRC27 & $2.19 \mathrm{E}+00$ \\
\hline ENSBTAG00000009383 & 2.30E-10 & $1.05 \mathrm{E}-10$ & KIF11 & $2.18 \mathrm{E}+00$ \\
\hline
\end{tabular}




\begin{tabular}{|c|c|c|c|c|}
\hline ENSBTAG000000013368 & $2.63 \mathrm{E}-10$ & 1.19E-10 & ANKRD22 & $2.21 E+00$ \\
\hline ENSBTAG00000012702 & $2.11 \mathrm{E}-10$ & $9.46 \mathrm{E}-11$ & ZDHHC16 & $2.22 \mathrm{E}+00$ \\
\hline ENSBTAG000000010797 & $4.66 \mathrm{E}-10$ & 2.07E-10 & C10orf120 & $2.25 \mathrm{E}+00$ \\
\hline ENSBTAG000000010522 & $3.71 E-10$ & $1.64 \mathrm{E}-10$ & & $2.26 \mathrm{E}+00$ \\
\hline ENSBTAG000000023847 & $2.33 \mathrm{E}-10$ & $1.02 \mathrm{E}-10$ & IKZF5 & $2.28 \mathrm{E}+00$ \\
\hline ENSBTAG00000012077 & $1.99 \mathrm{E}-10$ & $8.51 \mathrm{E}-11$ & SLF2 & $2.33 E+00$ \\
\hline ENSBTAG00000018604 & $1.08 \mathrm{E}-10$ & 4.54E-11 & SEMA4G & $2.38 \mathrm{E}+00$ \\
\hline ENSBTAG000000003296 & $1.83 \mathrm{E}-10$ & $7.68 \mathrm{E}-11$ & TWNK & $2.38 \mathrm{E}+00$ \\
\hline ENSBTAG000000047842 & $2.21 \mathrm{E}-10$ & $9.27 \mathrm{E}-11$ & PRLHR & $2.38 \mathrm{E}+00$ \\
\hline ENSBTAG000000017710 & $1.46 \mathrm{E}-09$ & $6.13 \mathrm{E}-10$ & ECHS1 & $2.39 E+00$ \\
\hline ENSBTAG000000048256 & 4.07E-10 & $1.70 \mathrm{E}-10$ & & $2.39 E+00$ \\
\hline ENSBTAG000000011960 & $1.14 \mathrm{E}-10$ & $4.66 \mathrm{E}-11$ & GOT1 & $2.45 E+00$ \\
\hline ENSBTAG00000011748 & $5.52 \mathrm{E}-10$ & $2.25 \mathrm{E}-10$ & SGMS1 & $2.45 \mathrm{E}+00$ \\
\hline ENSBTAG000000018321 & 4.63E-10 & $1.88 \mathrm{E}-10$ & PAOX & $2.46 \mathrm{E}+00$ \\
\hline ENSBTAG000000014028 & $3.50 \mathrm{E}-10$ & $1.42 \mathrm{E}-10$ & PSTK & $2.46 \mathrm{E}+00$ \\
\hline ENSBTAG000000018365 & $3.61 E-10$ & $1.45 \mathrm{E}-10$ & CYP2E1 & $2.49 E+00$ \\
\hline ENSBTAG000000001017 & $3.02 \mathrm{E}-10$ & $1.21 \mathrm{E}-10$ & SLK & $2.49 E+00$ \\
\hline ENSBTAG000000013478 & $2.03 \mathrm{E}-10$ & $8.08 \mathrm{E}-11$ & MARVELD1 & $2.52 E+00$ \\
\hline ENSBTAG000000011092 & $6.07 \mathrm{E}-10$ & $2.41 \mathrm{E}-10$ & KNDC1 & $2.52 E+00$ \\
\hline ENSBTAG000000023955 & 4.30E-10 & $1.70 \mathrm{E}-10$ & & $2.53 E+00$ \\
\hline ENSBTAG000000002010 & $3.17 E-10$ & $1.24 \mathrm{E}-10$ & & $2.55 E+00$ \\
\hline ENSBTAG000000017367 & $3.21 \mathrm{E}-10$ & $1.24 \mathrm{E}-10$ & IFIT5 & $2.59 \mathrm{E}+00$ \\
\hline ENSBTAG000000011743 & $3.22 \mathrm{E}-10$ & $1.23 \mathrm{E}-10$ & TLL2 & $2.62 E+00$ \\
\hline ENSBTAG000000006707 & $3.00 \mathrm{E}-10$ & $1.15 \mathrm{E}-10$ & ACSL5 & $2.61 E+00$ \\
\hline ENSBTAG000000012721 & $5.54 \mathrm{E}-10$ & $2.11 E-10$ & HOGA1 & $2.63 E+00$ \\
\hline ENSBTAG000000004330 & $1.30 \mathrm{E}-10$ & $4.96 \mathrm{E}-11$ & ENO4 & $2.62 E+00$ \\
\hline ENSBTAG000000021195 & $3.72 \mathrm{E}-10$ & $1.38 \mathrm{E}-10$ & $\mathrm{~A} 1 \mathrm{CF}$ & $2.69 E+00$ \\
\hline ENSBTAG000000003990 & $3.67 \mathrm{E}-10$ & $1.29 \mathrm{E}-10$ & ITPRIP & $2.84 \mathrm{E}+00$ \\
\hline ENSBTAG000000000951 & $5.16 \mathrm{E}-10$ & $1.80 \mathrm{E}-10$ & JAKMIP3 & $2.87 E+00$ \\
\hline ENSBTAG000000009715 & $3.98 \mathrm{E}-10$ & $1.35 \mathrm{E}-10$ & PDCD11 & $2.95 E+00$ \\
\hline ENSBTAG000000045703 & $1.25 \mathrm{E}-10$ & $4.24 \mathrm{E}-11$ & cox15 & $2.94 \mathrm{E}+00$ \\
\hline ENSBTAG000000017263 & $3.16 \mathrm{E}-10$ & $1.06 \mathrm{E}-10$ & MXI1 & $2.98 \mathrm{E}+00$ \\
\hline ENSBTAG00000011115 & $6.00 \mathrm{E}-10$ & $2.01 \mathrm{E}-10$ & $\mathrm{CH} 25 \mathrm{H}$ & $2.98 \mathrm{E}+00$ \\
\hline ENSBTAG000000044153 & $2.70 \mathrm{E}-10$ & $8.95 \mathrm{E}-11$ & SH3PXD2A & $3.02 E+00$ \\
\hline ENSBTAG00000000765 & 5.19E-10 & $1.69 \mathrm{E}-10$ & PNLIPRP2 & $3.07 \mathrm{E}+00$ \\
\hline ENSBTAG00000021068 & $2.10 \mathrm{E}-10$ & $6.74 \mathrm{E}-11$ & SUFU & $3.12 \mathrm{E}+00$ \\
\hline ENSBTAG00000004438 & $4.82 \mathrm{E}-10$ & $1.52 \mathrm{E}-10$ & XPNPEP1 & $3.17 E+00$ \\
\hline ENSBTAG00000047957 & 2.69E-10 & $8.46 \mathrm{E}-11$ & & $3.18 \mathrm{E}+00$ \\
\hline ENSBTAG00000002261 & $1.73 \mathrm{E}-10$ & 5.44E-11 & LBX1 & $3.17 \mathrm{E}+00$ \\
\hline ENSBTAG00000009713 & $2.49 \mathrm{E}-10$ & $7.81 \mathrm{E}-11$ & & $3.18 \mathrm{E}+00$ \\
\hline ENSBTAG00000005715 & $6.87 \mathrm{E}-10$ & 2.07E-10 & FUOM & $3.32 E+00$ \\
\hline ENSBTAG00000013717 & $1.06 \mathrm{E}-09$ & $3.18 \mathrm{E}-10$ & PRAP1 & $3.35 \mathrm{E}+00$ \\
\hline ENSBTAG00000011956 & $1.79 \mathrm{E}-10$ & $5.28 \mathrm{E}-11$ & CNNM1 & $3.39 E+00$ \\
\hline ENSBTAG00000016104 & $1.64 \mathrm{E}-10$ & 4.82E-11 & BTAF1 & $3.41 E+00$ \\
\hline ENSBTAG00000008936 & $6.60 \mathrm{E}-10$ & $1.89 \mathrm{E}-10$ & $A B C C 2$ & $3.49 E+00$ \\
\hline ENSBTAG00000003634 & $2.39 \mathrm{E}-10$ & $6.81 E-11$ & CISD1 & $3.51 E+00$ \\
\hline ENSBTAG00000012955 & $4.26 \mathrm{E}-10$ & $1.21 \mathrm{E}-10$ & TRUB1 & $3.52 \mathrm{E}+00$ \\
\hline ENSBTAG00000039834 & $4.11 \mathrm{E}-10$ & $1.15 \mathrm{E}-10$ & & $3.57 E+00$ \\
\hline ENSBTAG00000010739 & $2.46 \mathrm{E}-10$ & $6.41 \mathrm{E}-11$ & BLOC1S2 & $3.84 \mathrm{E}+00$ \\
\hline ENSBTAG00000003741 & $3.68 \mathrm{E}-10$ & $9.56 \mathrm{E}-11$ & NEURL1 & $3.85 E+00$ \\
\hline ENSBTAG00000039234 & $8.14 \mathrm{E}-10$ & 2.03E-10 & LIPK & $4.01 E+00$ \\
\hline ENSBTAG00000037795 & $3.58 \mathrm{E}-10$ & $8.82 \mathrm{E}-11$ & & $4.05 E+00$ \\
\hline ENSBTAG00000012704 & $1.70 \mathrm{E}-10$ & $4.04 \mathrm{E}-11$ & MMS19 & $4.19 E+00$ \\
\hline ENSBTAG00000003298 & $1.26 \mathrm{E}-10$ & $2.89 \mathrm{E}-11$ & LZTS2 & $4.36 \mathrm{E}+00$ \\
\hline ENSBTAG00000020796 & $1.01 \mathrm{E}-10$ & $2.26 \mathrm{E}-11$ & UBE2D1 & $4.45 E+00$ \\
\hline ENSBTAG00000015019 & $4.25 \mathrm{E}-10$ & $9.08 \mathrm{E}-11$ & STN1 & $4.67 E+00$ \\
\hline ENSBTAG00000000791 & $5.35 \mathrm{E}-10$ & $1.13 \mathrm{E}-10$ & CALY & $4.73 E+00$ \\
\hline ENSBTAG00000016918 & $6.09 \mathrm{E}-10$ & $1.29 \mathrm{E}-10$ & MYOF & $4.72 E+00$ \\
\hline ENSBTAG00000012697 & $7.55 \mathrm{E}-11$ & $1.58 \mathrm{E}-11$ & & $4.75 E+00$ \\
\hline ENSBTAG00000011080 & $1.88 \mathrm{E}-10$ & $3.94 \mathrm{E}-11$ & TLX1 & $4.76 \mathrm{E}+00$ \\
\hline ENSBTAG00000047324 & $6.56 \mathrm{E}-10$ & $1.36 \mathrm{E}-10$ & & $4.82 \mathrm{E}+00$ \\
\hline ENSBTAG00000012699 & $1.61 \mathrm{E}-10$ & $3.11 E-11$ & EXOSC1 & $5.15 E+00$ \\
\hline ENSBTAG00000023941 & $1.91 \mathrm{E}-10$ & $3.69 \mathrm{E}-11$ & CPN1 & $5.16 \mathrm{E}+00$ \\
\hline ENSBTAG00000003580 & $1.68 \mathrm{E}-10$ & 3.25E-11 & & $5.16 \mathrm{E}+00$ \\
\hline ENSBTAG00000019872 & $2.61 \mathrm{E}-10$ & 4.94E-11 & PIK3AP1 & $5.27 \mathrm{E}+00$ \\
\hline ENSBTAG00000021071 & $2.63 \mathrm{E}-10$ & 4.97E-11 & TRIM8 & $5.29 E+00$ \\
\hline ENSBTAG00000018566 & $4.61 \mathrm{E}-10$ & 8.57E-11 & SFRP5 & $5.37 \mathrm{E}+00$ \\
\hline ENSBTAG00000000091 & 3.19E-10 & $5.73 \mathrm{E}-11$ & NDUFB8 & $5.56 \mathrm{E}+00$ \\
\hline ENSBTAG00000009709 & $1.65 \mathrm{E}-10$ & $2.97 \mathrm{E}-11$ & TAF5 & $5.55 \mathrm{E}+00$ \\
\hline ENSBTAG00000047054 & $1.16 \mathrm{E}-09$ & $2.08 \mathrm{E}-10$ & CLRN3 & $5.57 E+00$ \\
\hline
\end{tabular}




\begin{tabular}{|c|c|c|c|c|}
\hline ENSBTAG00000000090 & $3.50 \mathrm{E}-10$ & $6.19 \mathrm{E}-11$ & SEC31B & $5.64 \mathrm{E}+00$ \\
\hline ENSBTAG00000046514 & $8.98 \mathrm{E}-10$ & $1.48 \mathrm{E}-10$ & ASMTL & $6.07 E+00$ \\
\hline ENSBTAG00000046848 & $2.76 \mathrm{E}-10$ & 4.34E-11 & & $6.35 E+00$ \\
\hline ENSBTAG00000010742 & $3.11 \mathrm{E}-10$ & $4.89 \mathrm{E}-11$ & PKD2L1 & $6.36 \mathrm{E}+00$ \\
\hline ENSBTAG00000007435 & 8.37E-11 & 1.17E-11 & NOLC1 & $7.10 \mathrm{E}+00$ \\
\hline ENSBTAG00000018564 & $6.76 \mathrm{E}-10$ & $8.70 \mathrm{E}-11$ & ZFYVE27 & $7.76 \mathrm{E}+00$ \\
\hline ENSBTAG00000023947 & $1.01 \mathrm{E}-10$ & $1.27 \mathrm{E}-11$ & AVPI1 & $7.91 E+00$ \\
\hline ENSBTAG00000009719 & $4.92 \mathrm{E}-10$ & $5.78 \mathrm{E}-11$ & CALHM2 & $8.49 E+00$ \\
\hline ENSBTAG00000002430 & $2.80 \mathrm{E}-10$ & $2.83 \mathrm{E}-11$ & COL17A1 & $9.87 \mathrm{E}+00$ \\
\hline ENSBTAG00000021067 & $2.06 \mathrm{E}-10$ & $1.89 \mathrm{E}-11$ & ACTR1B & $1.08 \mathrm{E}+01$ \\
\hline ENSBTAG00000038930 & $5.48 \mathrm{E}-11$ & $4.06 \mathrm{E}-12$ & & $1.32 \mathrm{E}+01$ \\
\hline ENSBTAG00000038684 & 1.36E-09 & $9.64 \mathrm{E}-11$ & & $1.41 \mathrm{E}+01$ \\
\hline ENSBTAG00000021065 & $2.04 \mathrm{E}-10$ & $1.39 \mathrm{E}-11$ & MFSD13A & $1.46 \mathrm{E}+01$ \\
\hline ENSBTAG00000015702 & $1.31 \mathrm{E}-10$ & $8.66 \mathrm{E}-12$ & PITX3 & $1.49 E+01$ \\
\hline ENSBTAG00000013120 & $2.40 \mathrm{E}-10$ & $1.53 \mathrm{E}-11$ & C10orf76 & $1.56 \mathrm{E}+01$ \\
\hline ENSBTAG00000001530 & $2.83 \mathrm{E}-10$ & $1.78 \mathrm{E}-11$ & FGF8 & $1.58 \mathrm{E}+01$ \\
\hline ENSBTAG00000006014 & 1.57E-10 & $9.25 \mathrm{E}-12$ & GBF1 & $1.68 \mathrm{E}+01$ \\
\hline ENSBTAG00000004321 & $3.89 \mathrm{E}-10$ & $2.24 \mathrm{E}-11$ & SFXN2 & $1.73 E+01$ \\
\hline ENSBTAG00000007427 & $1.41 \mathrm{E}-10$ & $7.42 \mathrm{E}-12$ & PPRC1 & $1.88 \mathrm{E}+01$ \\
\hline ENSBTAG00000003579 & $1.73 \mathrm{E}-10$ & 7.47E-12 & FBXW4 & $2.29 E+01$ \\
\hline ENSBTAG00000016343 & $1.46 \mathrm{E}-10$ & $5.57 \mathrm{E}-12$ & KCNIP2 & $2.58 \mathrm{E}+01$ \\
\hline ENSBTAG00000016336 & $2.14 \mathrm{E}-10$ & 7.69E-12 & MGEA5 & $2.75 E+01$ \\
\hline ENSBTAG00000014314 & $3.73 \mathrm{E}-10$ & $1.22 \mathrm{E}-11$ & HPS1 & $3.03 E+01$ \\
\hline ENSBTAG00000003578 & $1.52 \mathrm{E}-10$ & 4.67E-12 & DPCD & $3.19 E+01$ \\
\hline ENSBTAG00000014313 & $5.64 \mathrm{E}-10$ & $4.06 \mathrm{E}-12$ & PYROXD2 & $1.36 \mathrm{E}+02$ \\
\hline ENSBTAG00000008152 & 0 & 0 & & $1.00 E+00$ \\
\hline ENSBTAG00000023629 & 0 & 0 & & $1.00 \mathrm{E}+00$ \\
\hline ENSBTAG00000011489 & $1.04 \mathrm{E}-10$ & 0 & KAZALD1 & $1.05 E+03$ \\
\hline ENSBTAG00000003576 & 1.47E-10 & 0 & POLL & $1.47 E+03$ \\
\hline ENSBTAG00000016335 & $1.12 \mathrm{E}-10$ & 0 & NPM3 & $1.12 \mathrm{E}+03$ \\
\hline ENSBTAG00000005780 & $8.27 \mathrm{E}-11$ & 0 & LDB1 & $8.28 \mathrm{E}+02$ \\
\hline ENSBTAG00000015700 & $5.59 \mathrm{E}-11$ & 0 & ELOVL3 & $5.60 E+02$ \\
\hline ENSBTAG00000006017 & $8.27 \mathrm{E}-11$ & 0 & NFKB2 & $8.28 \mathrm{E}+02$ \\
\hline ENSBTAG00000002500 & $1.20 \mathrm{E}-10$ & 0 & FBXL15 & $1.20 E+03$ \\
\hline ENSBTAG00000002501 & $9.60 \mathrm{E}-11$ & 0 & CUEDC2 & $9.61 E+02$ \\
\hline ENSBTAG00000038993 & $6.59 \mathrm{E}-10$ & 0 & & $6.59 E+03$ \\
\hline ENSBTAG00000024999 & $1.75 \mathrm{E}-10$ & 0 & UTF1 & $1.75 E+03$ \\
\hline ENSBTAG00000017911 & $9.28 \mathrm{E}-11$ & 0 & HMX2 & $9.29 \mathrm{E}+02$ \\
\hline ENSBTAG00000035282 & 0 & 0 & & $1.00 \mathrm{E}+00$ \\
\hline ENSBTAG00000046109 & 0 & 0 & & $1.00 \mathrm{E}+00$ \\
\hline ENSBTAG00000046701 & 0 & 0 & FRAT2 & $1.00 \mathrm{E}+00$ \\
\hline ENSBTAG00000021942 & $9.38 \mathrm{E}-11$ & 0 & HPS6 & $9.39 E+02$ \\
\hline ENSBTAG00000047642 & 0 & 0 & & $1.00 E+00$ \\
\hline ENSBTAG00000005267 & $1.74 \mathrm{E}-10$ & 0 & & $1.74 \mathrm{E}+03$ \\
\hline ENSBTAG00000015913 & 0 & $3.63 \mathrm{E}-11$ & MFHAS1 & $2.75 \mathrm{E}-03$ \\
\hline ENSBTAG00000033140 & 0 & 2.65E-10 & LRRC3B & 3.77E-04 \\
\hline ENSBTAG00000010606 & 0 & $1.21 \mathrm{E}-10$ & PPP1R3B & $8.26 \mathrm{E}-04$ \\
\hline ENSBTAG00000020468 & 1.17E-10 & $3.89 \mathrm{E}-10$ & MICU3 & $3.01 \mathrm{E}-01$ \\
\hline ENSBTAG00000020084 & $1.52 \mathrm{E}-10$ & $5.01 \mathrm{E}-10$ & LONRF1 & $3.03 \mathrm{E}-01$ \\
\hline ENSBTAG00000044043 & $9.28 \mathrm{E}-11$ & $2.77 \mathrm{E}-10$ & FGF20 & 3.35E-01 \\
\hline ENSBTAG00000014737 & $1.67 \mathrm{E}-10$ & $4.53 \mathrm{E}-10$ & VEGFC & $3.70 \mathrm{E}-01$ \\
\hline ENSBTAG00000033304 & $6.29 \mathrm{E}-11$ & $1.59 \mathrm{E}-10$ & C8orf4 & $3.96 \mathrm{E}-01$ \\
\hline ENSBTAG00000033381 & $6.12 \mathrm{E}-11$ & $1.54 \mathrm{E}-10$ & & $3.98 \mathrm{E}-01$ \\
\hline ENSBTAG00000017183 & $1.10 \mathrm{E}-10$ & $2.63 \mathrm{E}-10$ & PDLIM3 & 4.17E-01 \\
\hline ENSBTAG00000004238 & $2.38 \mathrm{E}-10$ & $5.23 \mathrm{E}-10$ & TACC1 & 4.56E-01 \\
\hline ENSBTAG00000019532 & $2.45 \mathrm{E}-10$ & $4.86 \mathrm{E}-10$ & HTRA4 & $5.04 \mathrm{E}-01$ \\
\hline ENSBTAG00000018036 & $1.03 \mathrm{E}-10$ & $1.99 \mathrm{E}-10$ & CNOT7 & 5.20E-01 \\
\hline ENSBTAG00000010372 & $1.72 \mathrm{E}-10$ & 3.17E-10 & MTMR7 & 5.43E-01 \\
\hline ENSBTAG00000003081 & 2.17E-10 & $3.91 \mathrm{E}-10$ & RWDD4 & $5.54 \mathrm{E}-01$ \\
\hline ENSBTAG00000003509 & $3.78 \mathrm{E}-10$ & $5.60 \mathrm{E}-10$ & PLEKHA2 & $6.75 \mathrm{E}-01$ \\
\hline ENSBTAG00000007031 & $1.69 \mathrm{E}-10$ & $2.44 \mathrm{E}-10$ & OXSM & 6.94E-01 \\
\hline ENSBTAG00000048171 & $6.33 \mathrm{E}-10$ & $9.04 \mathrm{E}-10$ & & 7.00E-01 \\
\hline ENSBTAG00000044208 & $1.98 \mathrm{E}-10$ & $2.81 \mathrm{E}-10$ & DUSP4 & 7.04E-01 \\
\hline ENSBTAG00000010355 & $3.02 \mathrm{E}-10$ & 4.30E-10 & VPS37A & 7.03E-01 \\
\hline ENSBTAG00000018713 & $5.02 \mathrm{E}-10$ & $6.96 \mathrm{E}-10$ & & 7.21E-01 \\
\hline ENSBTAG00000047706 & $2.53 \mathrm{E}-10$ & $3.48 \mathrm{E}-10$ & ING2 & $7.28 \mathrm{E}-01$ \\
\hline ENSBTAG00000021263 & $2.25 \mathrm{E}-10$ & $2.96 \mathrm{E}-10$ & CYP4V2 & 7.60E-01 \\
\hline ENSBTAG00000000743 & $2.64 \mathrm{E}-10$ & $3.44 \mathrm{E}-10$ & MBOAT4 & 7.67E-01 \\
\hline ENSBTAG00000045990 & $2.54 \mathrm{E}-10$ & 3.19E-10 & & 7.95E-01 \\
\hline ENSBTAG00000017981 & $2.61 \mathrm{E}-10$ & $3.29 \mathrm{E}-10$ & ADRB3 & 7.93E-01 \\
\hline
\end{tabular}




\begin{tabular}{|c|c|c|c|c|}
\hline ENSBTAG00000045626 & 2.77E-10 & $3.49 \mathrm{E}-10$ & & 7.93E-01 \\
\hline ENSBTAG00000033345 & $9.93 \mathrm{E}-11$ & $1.25 \mathrm{E}-10$ & STAR & 7.95E-01 \\
\hline ENSBTAG00000026515 & $6.35 \mathrm{E}-10$ & $8.00 \mathrm{E}-10$ & & 7.94E-01 \\
\hline ENSBTAG00000045531 & $5.14 \mathrm{E}-11$ & $6.48 \mathrm{E}-11$ & & 7.93E-01 \\
\hline ENSBTAG00000038504 & $9.55 \mathrm{E}-11$ & $1.20 \mathrm{E}-10$ & & 7.96E-01 \\
\hline ENSBTAG00000039328 & $1.56 \mathrm{E}-10$ & $1.96 \mathrm{E}-10$ & PURG & 7.94E-01 \\
\hline ENSBTAG00000027665 & $7.81 \mathrm{E}-10$ & $9.60 \mathrm{E}-10$ & BRF2 & 8.14E-01 \\
\hline ENSBTAG00000010351 & $5.01 \mathrm{E}-10$ & $6.11 \mathrm{E}-10$ & SNX25 & 8.20E-01 \\
\hline ENSBTAG00000012191 & $2.27 \mathrm{E}-10$ & $2.76 \mathrm{E}-10$ & WWC2 & $8.21 \mathrm{E}-01$ \\
\hline ENSBTAG00000009685 & $2.93 \mathrm{E}-10$ & $3.56 \mathrm{E}-10$ & & $8.22 \mathrm{E}-01$ \\
\hline ENSBTAG00000014056 & $5.20 \mathrm{E}-10$ & $6.32 \mathrm{E}-10$ & CHRNA6 & 8.23E-01 \\
\hline ENSBTAG00000005094 & $8.39 \mathrm{E}-10$ & $1.01 \mathrm{E}-09$ & LRP2BP & $8.30 \mathrm{E}-01$ \\
\hline ENSBTAG00000000744 & $4.32 \mathrm{E}-10$ & $5.21 \mathrm{E}-10$ & DCTN6 & $8.30 \mathrm{E}-01$ \\
\hline ENSBTAG00000004593 & $4.91 \mathrm{E}-10$ & $5.81 \mathrm{E}-10$ & TOP2B & $8.45 \mathrm{E}-01$ \\
\hline ENSBTAG00000034952 & $4.31 \mathrm{E}-10$ & $5.02 \mathrm{E}-10$ & & $8.59 \mathrm{E}-01$ \\
\hline ENSBTAG00000003572 & $5.50 \mathrm{E}-10$ & $6.27 \mathrm{E}-10$ & F11 & $8.77 \mathrm{E}-01$ \\
\hline ENSBTAG00000007473 & $8.13 \mathrm{E}-10$ & $9.27 \mathrm{E}-10$ & XKR5 & $8.77 \mathrm{E}-01$ \\
\hline ENSBTAG00000013579 & $3.08 \mathrm{E}-10$ & $3.47 \mathrm{E}-10$ & SARAF & $8.88 \mathrm{E}-01$ \\
\hline ENSBTAG00000017021 & $5.40 \mathrm{E}-10$ & $6.02 \mathrm{E}-10$ & & 8.97E-01 \\
\hline ENSBTAG00000014141 & $8.56 \mathrm{E}-10$ & $9.50 \mathrm{E}-10$ & SPATA4 & $9.01 \mathrm{E}-01$ \\
\hline ENSBTAG00000010085 & $2.66 \mathrm{E}-10$ & $2.94 \mathrm{E}-10$ & SLC7A2 & $9.05 \mathrm{E}-01$ \\
\hline ENSBTAG00000001463 & $6.31 \mathrm{E}-10$ & $6.96 \mathrm{E}-10$ & TNKS & 9.07E-01 \\
\hline ENSBTAG00000003793 & 2.37E-10 & $2.61 \mathrm{E}-10$ & NGLY1 & $9.07 \mathrm{E}-01$ \\
\hline ENSBTAG00000034954 & 3.64E-10 & $4.01 \mathrm{E}-10$ & & 9.07E-01 \\
\hline ENSBTAG00000013208 & $1.63 \mathrm{E}-10$ & $1.80 \mathrm{E}-10$ & SLC25A4 & 9.07E-01 \\
\hline ENSBTAG00000008682 & $5.34 \mathrm{E}-10$ & $5.87 \mathrm{E}-10$ & TLR3 & 9.09E-01 \\
\hline ENSBTAG00000014135 & $8.38 \mathrm{E}-10$ & $9.19 \mathrm{E}-10$ & WDR17 & $9.11 \mathrm{E}-01$ \\
\hline ENSBTAG00000017133 & $2.44 \mathrm{E}-10$ & $2.67 \mathrm{E}-10$ & GINS4 & $9.12 \mathrm{E}-01$ \\
\hline ENSBTAG00000008950 & $2.74 \mathrm{E}-10$ & $2.99 \mathrm{E}-10$ & KIAA1456 & $9.16 \mathrm{E}-01$ \\
\hline ENSBTAG00000008585 & $8.76 \mathrm{E}-10$ & $9.53 \mathrm{E}-10$ & ARHGEF10 & $9.20 \mathrm{E}-01$ \\
\hline ENSBTAG00000047363 & $1.56 \mathrm{E}-09$ & $1.68 \mathrm{E}-09$ & & $9.26 \mathrm{E}-01$ \\
\hline ENSBTAG00000046937 & $2.91 \mathrm{E}-10$ & $3.14 \mathrm{E}-10$ & & $9.28 \mathrm{E}-01$ \\
\hline ENSBTAG00000005825 & $6.82 \mathrm{E}-10$ & 7.31E-10 & NEIL3 & $9.33 \mathrm{E}-01$ \\
\hline ENSBTAG00000005725 & $8.41 \mathrm{E}-10$ & $8.98 \mathrm{E}-10$ & IDO2 & 9.37E-01 \\
\hline ENSBTAG00000011518 & $4.27 \mathrm{E}-10$ & $4.50 \mathrm{E}-10$ & RARB & $9.48 \mathrm{E}-01$ \\
\hline ENSBTAG00000027625 & $3.58 \mathrm{E}-10$ & $3.77 \mathrm{E}-10$ & SFRP1 & $9.50 \mathrm{E}-01$ \\
\hline ENSBTAG00000000226 & $2.82 \mathrm{E}-10$ & $2.96 \mathrm{E}-10$ & DKK4 & $9.53 \mathrm{E}-01$ \\
\hline ENSBTAG00000007422 & $3.29 \mathrm{E}-10$ & $3.45 \mathrm{E}-10$ & TRAPPC11 & 9.54E-01 \\
\hline ENSBTAG00000004344 & $1.97 \mathrm{E}-10$ & $2.05 \mathrm{E}-10$ & ACSL1 & $9.62 \mathrm{E}-01$ \\
\hline ENSBTAG00000034430 & $7.24 \mathrm{E}-10$ & $7.55 \mathrm{E}-10$ & C4orf47 & 9.59E-01 \\
\hline ENSBTAG00000013145 & $1.24 \mathrm{E}-10$ & $1.29 \mathrm{E}-10$ & DDHD2 & $9.60 \mathrm{E}-01$ \\
\hline ENSBTAG00000048249 & 7.31E-10 & $7.58 \mathrm{E}-10$ & & $9.65 \mathrm{E}-01$ \\
\hline ENSBTAG00000000357 & $2.74 \mathrm{E}-10$ & $2.80 \mathrm{E}-10$ & & $9.78 \mathrm{E}-01$ \\
\hline ENSBTAG00000001186 & 7.23E-10 & $7.40 \mathrm{E}-10$ & SH2D4A & $9.77 \mathrm{E}-01$ \\
\hline ENSBTAG00000020282 & 7.37E-10 & $7.45 \mathrm{E}-10$ & DCTD & $9.89 \mathrm{E}-01$ \\
\hline ENSBTAG00000013211 & $4.65 \mathrm{E}-10$ & 4.69E-10 & CFAP97 & $9.91 \mathrm{E}-01$ \\
\hline ENSBTAG00000013581 & $1.67 \mathrm{E}-10$ & $1.68 \mathrm{E}-10$ & LEPROTL1 & 9.93E-01 \\
\hline ENSBTAG00000009408 & 3.37E-10 & $3.39 \mathrm{E}-10$ & ADAM2 & 9.95E-01 \\
\hline ENSBTAG00000001966 & $6.60 \mathrm{E}-10$ & $6.63 \mathrm{E}-10$ & PDGFRL & $9.95 \mathrm{E}-01$ \\
\hline ENSBTAG00000034393 & $3.58 \mathrm{E}-10$ & $3.59 \mathrm{E}-10$ & & $9.98 \mathrm{E}-01$ \\
\hline ENSBTAG00000016473 & $1.08 \mathrm{E}-09$ & 1.07E-09 & NAT1 & $1.01 E+00$ \\
\hline ENSBTAG00000014861 & $5.19 \mathrm{E}-10$ & $5.09 \mathrm{E}-10$ & SLC20A2 & $1.02 \mathrm{E}+00$ \\
\hline ENSBTAG00000011034 & $2.12 \mathrm{E}-10$ & $2.05 \mathrm{E}-10$ & ANGPT2 & $1.04 E+00$ \\
\hline ENSBTAG00000002885 & $3.61 \mathrm{E}-10$ & $3.48 \mathrm{E}-10$ & MSR1 & $1.04 E+00$ \\
\hline ENSBTAG00000027654 & 4.74E-10 & $4.55 \mathrm{E}-10$ & EIF4EBP1 & $1.04 E+00$ \\
\hline ENSBTAG00000015463 & $1.83 \mathrm{E}-10$ & $1.75 \mathrm{E}-10$ & TTI2 & $1.04 E+00$ \\
\hline ENSBTAG00000027629 & $5.31 \mathrm{E}-10$ & $5.06 \mathrm{E}-10$ & & $1.05 E+00$ \\
\hline ENSBTAG00000015541 & $2.75 \mathrm{E}-10$ & $2.61 \mathrm{E}-10$ & DLC1 & $1.05 E+00$ \\
\hline ENSBTAG00000009501 & $2.06 \mathrm{E}-10$ & $1.94 \mathrm{E}-10$ & KLKB1 & $1.06 \mathrm{E}+00$ \\
\hline ENSBTAG00000013943 & $1.19 \mathrm{E}-10$ & $1.12 \mathrm{E}-10$ & ZNF703 & $1.06 \mathrm{E}+00$ \\
\hline ENSBTAG00000005730 & $2.10 \mathrm{E}-10$ & $1.97 \mathrm{E}-10$ & GPAT4 & $1.07 E+00$ \\
\hline ENSBTAG00000018495 & $2.71 \mathrm{E}-10$ & $2.50 \mathrm{E}-10$ & & $1.08 \mathrm{E}+00$ \\
\hline ENSBTAG00000039682 & $5.46 \mathrm{E}-10$ & $5.04 \mathrm{E}-10$ & MTUS1 & $1.08 \mathrm{E}+00$ \\
\hline ENSBTAG00000011573 & $2.85 \mathrm{E}-10$ & $2.63 \mathrm{E}-10$ & ENPP6 & $1.08 \mathrm{E}+00$ \\
\hline ENSBTAG00000040445 & 4.12E-10 & $3.79 \mathrm{E}-10$ & & $1.09 \mathrm{E}+00$ \\
\hline ENSBTAG00000007630 & $3.53 \mathrm{E}-10$ & $3.23 \mathrm{E}-10$ & RNF170 & $1.09 \mathrm{E}+00$ \\
\hline ENSBTAG00000027118 & $6.11 \mathrm{E}-10$ & $5.56 \mathrm{E}-10$ & KCNU1 & $1.10 \mathrm{E}+00$ \\
\hline ENSBTAG00000004922 & $3.41 \mathrm{E}-10$ & $3.10 \mathrm{E}-10$ & AGPAT5 & $1.10 \mathrm{E}+00$ \\
\hline ENSBTAG00000047749 & $2.63 \mathrm{E}-10$ & $2.39 \mathrm{E}-10$ & & $1.10 \mathrm{E}+00$ \\
\hline ENSBTAG00000020657 & $3.51 \mathrm{E}-10$ & $3.19 \mathrm{E}-10$ & FAT1 & $1.10 \mathrm{E}+00$ \\
\hline
\end{tabular}




\begin{tabular}{|c|c|c|c|c|}
\hline ENSBTAG00000017831 & $2.06 \mathrm{E}-10$ & $1.87 \mathrm{E}-10$ & ERLIN2 & $1.10 \mathrm{E}+00$ \\
\hline ENSBTAG00000018454 & $2.10 \mathrm{E}-10$ & $1.87 \mathrm{E}-10$ & UFSP2 & $1.12 \mathrm{E}+00$ \\
\hline ENSBTAG00000033504 & $1.62 \mathrm{E}-10$ & $1.43 \mathrm{E}-10$ & MAK16 & $1.14 \mathrm{E}+00$ \\
\hline ENSBTAG00000010241 & $5.20 \mathrm{E}-10$ & $4.52 \mathrm{E}-10$ & UNC5D & $1.15 \mathrm{E}+00$ \\
\hline ENSBTAG00000014146 & $3.47 \mathrm{E}-10$ & $3.01 \mathrm{E}-10$ & SPCS3 & $1.15 \mathrm{E}+00$ \\
\hline ENSBTAG00000007634 & $7.22 \mathrm{E}-10$ & $6.21 \mathrm{E}-10$ & HOOK3 & $1.16 \mathrm{E}+00$ \\
\hline ENSBTAG00000046938 & $4.31 \mathrm{E}-10$ & $3.69 \mathrm{E}-10$ & MTNR1A & $1.17 \mathrm{E}+00$ \\
\hline ENSBTAG00000012691 & $3.50 \mathrm{E}-10$ & $2.99 \mathrm{E}-10$ & GTF2E2 & $1.17 E+00$ \\
\hline ENSBTAG00000007051 & $2.79 \mathrm{E}-10$ & $2.34 \mathrm{E}-10$ & CLDN23 & $1.19 \mathrm{E}+00$ \\
\hline ENSBTAG00000016411 & $3.32 \mathrm{E}-10$ & $2.79 \mathrm{E}-10$ & RNF122 & $1.19 \mathrm{E}+00$ \\
\hline ENSBTAG00000047159 & $2.69 \mathrm{E}-10$ & $2.26 \mathrm{E}-10$ & & $1.19 E+00$ \\
\hline ENSBTAG00000008639 & $1.42 \mathrm{E}-10$ & $1.19 \mathrm{E}-10$ & TRIML1 & $1.19 \mathrm{E}+00$ \\
\hline ENSBTAG00000004231 & $3.31 \mathrm{E}-10$ & 2.77E-10 & GPM6A & $1.20 \mathrm{E}+00$ \\
\hline ENSBTAG00000015457 & $2.29 \mathrm{E}-10$ & $1.92 \mathrm{E}-10$ & FGFR1 & $1.20 \mathrm{E}+00$ \\
\hline ENSBTAG00000001529 & $1.39 \mathrm{E}-10$ & $1.16 \mathrm{E}-10$ & WHSC1L1 & $1.19 \mathrm{E}+00$ \\
\hline ENSBTAG00000009739 & $6.76 \mathrm{E}-10$ & $5.62 \mathrm{E}-10$ & CSMD1 & $1.20 \mathrm{E}+00$ \\
\hline ENSBTAG00000000979 & $2.87 \mathrm{E}-10$ & $2.39 \mathrm{E}-10$ & SMIM19 & $1.20 \mathrm{E}+00$ \\
\hline ENSBTAG00000008584 & $5.47 \mathrm{E}-10$ & $4.50 \mathrm{E}-10$ & & $1.22 \mathrm{E}+00$ \\
\hline ENSBTAG00000001243 & $2.51 \mathrm{E}-10$ & $2.05 \mathrm{E}-10$ & AP3M2 & $1.23 \mathrm{E}+00$ \\
\hline ENSBTAG00000046144 & $6.11 \mathrm{E}-10$ & $4.89 \mathrm{E}-10$ & DEFB7 & $1.25 \mathrm{E}+00$ \\
\hline ENSBTAG00000023157 & $1.38 \mathrm{E}-09$ & 1.09E-09 & & $1.26 \mathrm{E}+00$ \\
\hline ENSBTAG00000021592 & $6.14 \mathrm{E}-10$ & 4.86E-10 & WRN & $1.26 \mathrm{E}+00$ \\
\hline ENSBTAG00000033727 & $2.72 \mathrm{E}-10$ & $2.15 \mathrm{E}-10$ & RBPMS & $1.27 \mathrm{E}+00$ \\
\hline ENSBTAG00000027225 & $3.52 \mathrm{E}-10$ & $2.77 \mathrm{E}-10$ & & $1.27 \mathrm{E}+00$ \\
\hline ENSBTAG00000045830 & $6.50 \mathrm{E}-10$ & $5.11 \mathrm{E}-10$ & & $1.27 \mathrm{E}+00$ \\
\hline ENSBTAG00000008814 & $4.60 \mathrm{E}-10$ & $3.56 \mathrm{E}-10$ & ADGRA2 & $1.29 \mathrm{E}+00$ \\
\hline ENSBTAG00000000225 & $3.41 \mathrm{E}-10$ & $2.58 \mathrm{E}-10$ & POLB & $1.32 \mathrm{E}+00$ \\
\hline ENSBTAG00000000957 & $1.15 \mathrm{E}-10$ & 8.71E-11 & & $1.32 \mathrm{E}+00$ \\
\hline ENSBTAG00000018453 & $1.57 \mathrm{E}-10$ & $1.19 \mathrm{E}-10$ & ANKRD37 & $1.32 \mathrm{E}+00$ \\
\hline ENSBTAG00000047421 & $2.39 \mathrm{E}-10$ & $1.81 \mathrm{E}-10$ & & $1.32 \mathrm{E}+00$ \\
\hline ENSBTAG00000045649 & $1.02 \mathrm{E}-09$ & $7.66 \mathrm{E}-10$ & & $1.33 \mathrm{E}+00$ \\
\hline ENSBTAG00000027635 & $2.47 \mathrm{E}-10$ & $1.82 \mathrm{E}-10$ & PLPP5 & $1.36 \mathrm{E}+00$ \\
\hline ENSBTAG00000017083 & $1.26 \mathrm{E}-10$ & $9.26 \mathrm{E}-11$ & TEX15 & $1.36 \mathrm{E}+00$ \\
\hline ENSBTAG00000033137 & $6.69 \mathrm{E}-10$ & $4.90 \mathrm{E}-10$ & PSD3 & $1.37 \mathrm{E}+00$ \\
\hline ENSBTAG00000005454 & $6.60 \mathrm{E}-10$ & $4.82 \mathrm{E}-10$ & FUT10 & $1.37 \mathrm{E}+00$ \\
\hline ENSBTAG00000016446 & $5.58 \mathrm{E}-10$ & $4.06 \mathrm{E}-10$ & GOT1L1 & $1.37 E+00$ \\
\hline ENSBTAG00000004613 & $6.28 \mathrm{E}-10$ & 4.57E-10 & CSGALNACT1 & $1.37 E+00$ \\
\hline ENSBTAG00000009191 & 7.85E-10 & $5.68 \mathrm{E}-10$ & TUSC3 & $1.38 \mathrm{E}+00$ \\
\hline ENSBTAG00000001244 & $2.02 \mathrm{E}-10$ & $1.46 \mathrm{E}-10$ & PLAT & $1.38 \mathrm{E}+00$ \\
\hline ENSBTAG00000011684 & $4.81 \mathrm{E}-10$ & $3.43 \mathrm{E}-10$ & RAB11FIP1 & $1.40 \mathrm{E}+00$ \\
\hline ENSBTAG00000021133 & $6.42 \mathrm{E}-10$ & 4.49E-10 & TENM3 & $1.43 E+00$ \\
\hline ENSBTAG00000046405 & $7.61 \mathrm{E}-10$ & $5.31 \mathrm{E}-10$ & & $1.43 E+00$ \\
\hline ENSBTAG00000011075 & $3.01 \mathrm{E}-10$ & $2.06 \mathrm{E}-10$ & PROSC & $1.46 \mathrm{E}+00$ \\
\hline ENSBTAG00000008637 & $5.76 \mathrm{E}-10$ & $3.89 \mathrm{E}-10$ & TRIML2 & $1.48 \mathrm{E}+00$ \\
\hline ENSBTAG00000016486 & $4.33 \mathrm{E}-10$ & $2.89 \mathrm{E}-10$ & & $1.50 \mathrm{E}+00$ \\
\hline ENSBTAG00000033312 & $7.50 \mathrm{E}-10$ & $5.00 \mathrm{E}-10$ & & $1.50 \mathrm{E}+00$ \\
\hline ENSBTAG00000011032 & 2.77E-10 & $1.82 \mathrm{E}-10$ & MCPH1 & $1.52 \mathrm{E}+00$ \\
\hline ENSBTAG00000007089 & $4.21 \mathrm{E}-10$ & $2.76 \mathrm{E}-10$ & FRG1 & $1.52 E+00$ \\
\hline ENSBTAG00000027626 & $1.63 \mathrm{E}-10$ & $1.05 \mathrm{E}-10$ & GOLGA7 & $1.55 \mathrm{E}+00$ \\
\hline ENSBTAG00000000978 & $1.27 \mathrm{E}-10$ & $8.18 \mathrm{E}-11$ & $\mathrm{ASH} 2 \mathrm{~L}$ & $1.55 E+00$ \\
\hline ENSBTAG00000016177 & $2.13 \mathrm{E}-10$ & $1.37 \mathrm{E}-10$ & FGL1 & $1.56 \mathrm{E}+00$ \\
\hline ENSBTAG00000039856 & $8.55 \mathrm{E}-10$ & $5.45 \mathrm{E}-10$ & & $1.57 \mathrm{E}+00$ \\
\hline ENSBTAG00000015444 & $2.01 \mathrm{E}-10$ & $1.27 \mathrm{E}-10$ & LETM2 & $1.59 \mathrm{E}+00$ \\
\hline ENSBTAG00000018284 & $1.70 \mathrm{E}-10$ & $1.07 \mathrm{E}-10$ & KBTBD11 & $1.59 \mathrm{E}+00$ \\
\hline ENSBTAG00000047740 & $3.97 \mathrm{E}-10$ & $2.50 \mathrm{E}-10$ & & $1.59 E+00$ \\
\hline ENSBTAG00000024526 & $1.73 \mathrm{E}-10$ & $1.09 \mathrm{E}-10$ & & $1.58 \mathrm{E}+00$ \\
\hline ENSBTAG00000007629 & $1.87 \mathrm{E}-10$ & 1.17E-10 & THAP1 & $1.59 \mathrm{E}+00$ \\
\hline ENSBTAG00000012207 & $2.47 \mathrm{E}-10$ & $1.56 \mathrm{E}-10$ & & $1.59 \mathrm{E}+00$ \\
\hline ENSBTAG00000008519 & $4.72 \mathrm{E}-10$ & $2.97 \mathrm{E}-10$ & & $1.59 \mathrm{E}+00$ \\
\hline ENSBTAG00000047284 & $7.72 \mathrm{E}-10$ & $4.85 \mathrm{E}-10$ & & $1.59 \mathrm{E}+00$ \\
\hline ENSBTAG00000022767 & $1.44 \mathrm{E}-09$ & $9.01 \mathrm{E}-10$ & & $1.60 \mathrm{E}+00$ \\
\hline ENSBTAG00000009387 & $5.24 \mathrm{E}-10$ & 3.17E-10 & MYOM2 & $1.65 E+00$ \\
\hline ENSBTAG00000014143 & $6.29 \mathrm{E}-10$ & $3.70 \mathrm{E}-10$ & ASB5 & $1.70 \mathrm{E}+00$ \\
\hline ENSBTAG00000017420 & $1.05 \mathrm{E}-09$ & $6.13 \mathrm{E}-10$ & ZMAT4 & $1.71 E+00$ \\
\hline ENSBTAG00000007115 & $5.09 \mathrm{E}-10$ & $2.96 \mathrm{E}-10$ & GSR & $1.72 \mathrm{E}+00$ \\
\hline ENSBTAG00000008886 & $3.72 \mathrm{E}-10$ & $2.12 \mathrm{E}-10$ & STOX2 & $1.75 E+00$ \\
\hline ENSBTAG00000007599 & $6.20 \mathrm{E}-10$ & $3.48 \mathrm{E}-10$ & IKBKB & $1.78 \mathrm{E}+00$ \\
\hline ENSBTAG00000007477 & $7.04 \mathrm{E}-10$ & $3.86 \mathrm{E}-10$ & ZNF596 & $1.82 \mathrm{E}+00$ \\
\hline ENSBTAG00000044194 & $5.01 \mathrm{E}-10$ & $2.73 \mathrm{E}-10$ & $\mathrm{ZDHHC2}$ & $1.84 \mathrm{E}+00$ \\
\hline ENSBTAG00000046204 & $1.85 \mathrm{E}-10$ & 9.97E-11 & ZNF705A & $1.85 E+00$ \\
\hline
\end{tabular}




\begin{tabular}{|c|c|c|c|c|}
\hline ENSBTAG00000014068 & $3.63 \mathrm{E}-10$ & $1.90 \mathrm{E}-10$ & & $1.91 \mathrm{E}+00$ \\
\hline ENSBTAG00000033545 & $6.75 E-10$ & 3.43E-10 & & $1.97 \mathrm{E}+00$ \\
\hline ENSBTAG00000004830 & $6.12 \mathrm{E}-10$ & 3.09E-10 & ADAM18 & $1.98 \mathrm{E}+00$ \\
\hline ENSBTAG00000007236 & $1.52 \mathrm{E}-10$ & 7.66E-11 & PCM1 & $1.98 \mathrm{E}+00$ \\
\hline ENSBTAG00000009406 & $2.86 \mathrm{E}-10$ & $1.44 \mathrm{E}-10$ & & $1.99 \mathrm{E}+00$ \\
\hline ENSBTAG00000003275 & $7.99 \mathrm{E}-10$ & $4.01 \mathrm{E}-10$ & & $1.99 \mathrm{E}+00$ \\
\hline ENSBTAG00000016413 & $3.61 E-10$ & $1.72 \mathrm{E}-10$ & DUSP26 & $2.10 \mathrm{E}+00$ \\
\hline ENSBTAG00000023146 & $5.94 \mathrm{E}-10$ & $2.83 \mathrm{E}-10$ & ADAM32 & $2.10 \mathrm{E}+00$ \\
\hline ENSBTAG00000025003 & $4.59 \mathrm{E}-10$ & $2.16 \mathrm{E}-10$ & INTS10 & $2.13 \mathrm{E}+00$ \\
\hline ENSBTAG00000017455 & $4.34 \mathrm{E}-10$ & $2.03 \mathrm{E}-10$ & ADAM9 & $2.14 E+00$ \\
\hline ENSBTAG00000020602 & $4.52 \mathrm{E}-10$ & $2.08 \mathrm{E}-10$ & IDO1 & $2.17 \mathrm{E}+00$ \\
\hline ENSBTAG00000033182 & $1.12 \mathrm{E}-09$ & $5.14 \mathrm{E}-10$ & HGSNAT & $2.19 \mathrm{E}+00$ \\
\hline ENSBTAG00000046071 & $4.18 \mathrm{E}-10$ & $1.87 \mathrm{E}-10$ & & $2.23 \mathrm{E}+00$ \\
\hline ENSBTAG00000013143 & $2.38 \mathrm{E}-10$ & $1.04 \mathrm{E}-10$ & BAG4 & $2.29 \mathrm{E}+00$ \\
\hline ENSBTAG00000044139 & 2.77E-10 & $1.16 \mathrm{E}-10$ & ZFP42 & $2.39 \mathrm{E}+00$ \\
\hline ENSBTAG00000017157 & $5.81 E-10$ & $2.40 \mathrm{E}-10$ & CCDC110 & $2.42 \mathrm{E}+00$ \\
\hline ENSBTAG00000007340 & $9.11 \mathrm{E}-10$ & $3.49 \mathrm{E}-10$ & & $2.61 \mathrm{E}+00$ \\
\hline ENSBTAG00000010002 & $4.05 \mathrm{E}-10$ & $1.53 \mathrm{E}-10$ & IRF2 & $2.64 E+00$ \\
\hline ENSBTAG00000017085 & $3.73 \mathrm{E}-10$ & $1.38 \mathrm{E}-10$ & AGA & $2.70 \mathrm{E}+00$ \\
\hline ENSBTAG00000011257 & $3.31 \mathrm{E}-10$ & $1.20 \mathrm{E}-10$ & ASAH1 & $2.76 \mathrm{E}+00$ \\
\hline ENSBTAG00000047930 & $6.84 \mathrm{E}-10$ & $2.23 \mathrm{E}-10$ & & $3.07 E+00$ \\
\hline ENSBTAG00000018863 & $5.65 \mathrm{E}-10$ & $1.81 \mathrm{E}-10$ & PRIMPOL & $3.12 \mathrm{E}+00$ \\
\hline ENSBTAG00000047857 & 4.30E-10 & 1.37E-10 & & $3.14 E+00$ \\
\hline ENSBTAG00000006680 & $6.54 \mathrm{E}-10$ & $2.04 \mathrm{E}-10$ & NKIRAS1 & $3.21 \mathrm{E}+00$ \\
\hline ENSBTAG00000018024 & $4.40 \mathrm{E}-10$ & $1.34 \mathrm{E}-10$ & NR1D2 & $3.28 \mathrm{E}+00$ \\
\hline ENSBTAG00000017802 & $7.28 \mathrm{E}-10$ & $2.20 \mathrm{E}-10$ & THRB & $3.31 \mathrm{E}+00$ \\
\hline ENSBTAG00000004015 & $3.63 \mathrm{E}-10$ & $1.06 \mathrm{E}-10$ & KAT6A & $3.42 \mathrm{E}+00$ \\
\hline ENSBTAG00000015874 & $4.84 \mathrm{E}-10$ & $1.35 \mathrm{E}-10$ & CASP3 & $3.58 \mathrm{E}+00$ \\
\hline ENSBTAG00000004150 & $2.28 \mathrm{E}-10$ & $6.34 \mathrm{E}-11$ & NRG1 & $3.60 \mathrm{E}+00$ \\
\hline ENSBTAG00000004173 & $3.49 \mathrm{E}-10$ & $8.89 \mathrm{E}-11$ & UBXN8 & $3.92 \mathrm{E}+00$ \\
\hline ENSBTAG00000003649 & $7.55 \mathrm{E}-10$ & $1.78 \mathrm{E}-10$ & POMK & $4.24 \mathrm{E}+00$ \\
\hline ENSBTAG00000009245 & $4.78 \mathrm{E}-10$ & $1.10 \mathrm{E}-10$ & PPP2CB & $4.34 E+00$ \\
\hline ENSBTAG00000045690 & $2.42 \mathrm{E}-09$ & $5.43 \mathrm{E}-10$ & & $4.45 E+00$ \\
\hline ENSBTAG00000003201 & $7.52 \mathrm{E}-10$ & $1.69 \mathrm{E}-10$ & FNTA & $4.45 E+00$ \\
\hline ENSBTAG00000003516 & $9.23 \mathrm{E}-10$ & $2.04 \mathrm{E}-10$ & TM2D2 & $4.52 \mathrm{E}+00$ \\
\hline ENSBTAG00000018864 & $8.94 \mathrm{E}-10$ & $1.96 \mathrm{E}-10$ & CENPU & $4.56 \mathrm{E}+00$ \\
\hline ENSBTAG00000035183 & $5.89 \mathrm{E}-10$ & $1.23 \mathrm{E}-10$ & & $4.78 \mathrm{E}+00$ \\
\hline ENSBTAG00000012209 & $7.56 \mathrm{E}-10$ & $1.58 \mathrm{E}-10$ & & $4.78 \mathrm{E}+00$ \\
\hline ENSBTAG00000045505 & $1.66 \mathrm{E}-09$ & $2.82 \mathrm{E}-10$ & & $5.89 \mathrm{E}+00$ \\
\hline ENSBTAG00000021882 & $5.03 \mathrm{E}-10$ & $6.31 \mathrm{E}-11$ & & $7.96 \mathrm{E}+00$ \\
\hline ENSBTAG00000014050 & $6.99 \mathrm{E}-10$ & $7.96 \mathrm{E}-11$ & CHRNB3 & $8.77 E+00$ \\
\hline ENSBTAG00000033080 & $5.84 \mathrm{E}-10$ & $5.23 \mathrm{E}-11$ & RPL15 & $1.11 E+01$ \\
\hline ENSBTAG00000047643 & $1.18 \mathrm{E}-09$ & $6.98 \mathrm{E}-11$ & C8orf48 & $1.68 \mathrm{E}+01$ \\
\hline ENSBTAG00000001612 & $6.42 \mathrm{E}-10$ & $2.40 \mathrm{E}-11$ & LSM1 & $2.66 \mathrm{E}+01$ \\
\hline ENSBTAG00000035013 & $3.38 \mathrm{E}-10$ & 0 & & $3.38 \mathrm{E}+03$ \\
\hline ENSBTAG00000010248 & $1.72 \mathrm{E}-10$ & 0 & & $1.72 E+03$ \\
\hline ENSBTAG00000014704 & 4.57E-11 & 0 & HELT & $4.58 \mathrm{E}+02$ \\
\hline ENSBTAG00000023987 & $3.00 \mathrm{E}-10$ & $1.35 \mathrm{E}-09$ & LRIT2 & 2.22E-01 \\
\hline ENSBTAG00000047666 & $2.08 \mathrm{E}-10$ & 7.00E-10 & & 2.97E-01 \\
\hline ENSBTAG00000046409 & $1.34 \mathrm{E}-10$ & $4.06 \mathrm{E}-10$ & EGR2 & 3.30E-01 \\
\hline ENSBTAG00000047323 & $3.79 \mathrm{E}-10$ & 1.05E-09 & & $3.61 \mathrm{E}-01$ \\
\hline ENSBTAG00000045493 & $4.84 \mathrm{E}-10$ & $1.23 \mathrm{E}-09$ & & 3.94E-01 \\
\hline ENSBTAG00000026085 & $2.14 \mathrm{E}-10$ & $5.27 \mathrm{E}-10$ & ZP4 & 4.07E-01 \\
\hline ENSBTAG00000021728 & $3.29 \mathrm{E}-10$ & 4.60E-10 & OGDHL & 7.15E-01 \\
\hline ENSBTAG00000021132 & $1.10 \mathrm{E}-10$ & $1.54 \mathrm{E}-10$ & & 7.14E-01 \\
\hline ENSBTAG00000009348 & $4.92 \mathrm{E}-10$ & $6.70 \mathrm{E}-10$ & OIT3 & 7.34E-01 \\
\hline ENSBTAG00000017611 & $1.97 \mathrm{E}-10$ & 2.63E-10 & CCSER2 & 7.50E-01 \\
\hline ENSBTAG00000039837 & 7.03E-10 & $9.35 \mathrm{E}-10$ & & $7.52 \mathrm{E}-01$ \\
\hline ENSBTAG00000013068 & $1.59 \mathrm{E}-10$ & $2.09 \mathrm{E}-10$ & GGPS1 & 7.60E-01 \\
\hline ENSBTAG00000007878 & $2.08 \mathrm{E}-10$ & $2.70 \mathrm{E}-10$ & ARHGAP22 & 7.69E-01 \\
\hline ENSBTAG00000007876 & $2.15 \mathrm{E}-10$ & $2.80 \mathrm{E}-10$ & MAPK8 & 7.69E-01 \\
\hline ENSBTAG00000011694 & $5.98 \mathrm{E}-10$ & 7.69E-10 & & $7.78 \mathrm{E}-01$ \\
\hline ENSBTAG00000046421 & $7.21 \mathrm{E}-10$ & $9.19 \mathrm{E}-10$ & & 7.85E-01 \\
\hline ENSBTAG00000019565 & $3.75 \mathrm{E}-10$ & 4.77E-10 & NCOA4 & 7.86E-01 \\
\hline ENSBTAG00000017521 & $1.79 \mathrm{E}-10$ & $2.25 \mathrm{E}-10$ & ATOH7 & 7.94E-01 \\
\hline ENSBTAG00000039845 & $2.51 \mathrm{E}-10$ & 3.17E-10 & & 7.93E-01 \\
\hline ENSBTAG00000012566 & $5.88 \mathrm{E}-11$ & 7.41E-11 & MAP10 & 7.93E-01 \\
\hline ENSBTAG00000013264 & $2.33 \mathrm{E}-10$ & $2.94 \mathrm{E}-10$ & & $7.92 \mathrm{E}-01$ \\
\hline ENSBTAG00000017946 & $8.26 \mathrm{E}-11$ & $1.04 \mathrm{E}-10$ & & 7.94E-01 \\
\hline ENSBTAG00000001008 & $6.71 \mathrm{E}-11$ & $8.45 \mathrm{E}-11$ & PRF1 & 7.94E-01 \\
\hline
\end{tabular}




\begin{tabular}{|c|c|c|c|c|}
\hline ENSBTAG00000008853 & $8.81 \mathrm{E}-11$ & $1.11 \mathrm{E}-10$ & & 7.94E-01 \\
\hline ENSBTAG00000011707 & $2.51 \mathrm{E}-10$ & $3.16 \mathrm{E}-10$ & OR5AS1 & 7.94E-01 \\
\hline ENSBTAG00000032655 & $2.08 \mathrm{E}-10$ & $2.62 \mathrm{E}-10$ & & 7.94E-01 \\
\hline ENSBTAG00000019039 & $2.56 \mathrm{E}-10$ & $3.22 \mathrm{E}-10$ & ZNF239 & 7.95E-01 \\
\hline ENSBTAG00000013544 & $5.45 \mathrm{E}-10$ & $6.76 \mathrm{E}-10$ & LGALS8 & $8.06 \mathrm{E}-01$ \\
\hline ENSBTAG00000015681 & $9.34 \mathrm{E}-10$ & 1.15E-09 & RGR & $8.12 \mathrm{E}-01$ \\
\hline ENSBTAG00000003515 & $6.49 \mathrm{E}-10$ & $7.95 \mathrm{E}-10$ & SNCG & $8.17 \mathrm{E}-01$ \\
\hline ENSBTAG00000001165 & $1.65 \mathrm{E}-10$ & $2.02 \mathrm{E}-10$ & AIFM2 & $8.17 \mathrm{E}-01$ \\
\hline ENSBTAG00000010932 & $4.32 \mathrm{E}-10$ & $5.22 \mathrm{E}-10$ & DNAJC12 & $8.28 \mathrm{E}-01$ \\
\hline ENSBTAG00000000315 & $4.56 \mathrm{E}-10$ & $5.46 \mathrm{E}-10$ & ECD & $8.35 \mathrm{E}-01$ \\
\hline ENSBTAG00000005666 & 5.57E-10 & $6.62 \mathrm{E}-10$ & LRRC20 & 8.42E-01 \\
\hline ENSBTAG00000017053 & $1.75 \mathrm{E}-10$ & $2.08 \mathrm{E}-10$ & ABCB10 & $8.42 \mathrm{E}-01$ \\
\hline ENSBTAG00000012664 & $2.04 \mathrm{E}-10$ & $2.38 \mathrm{E}-10$ & & $8.55 \mathrm{E}-01$ \\
\hline ENSBTAG00000033008 & $1.84 \mathrm{E}-10$ & 2.14E-10 & MYOZ1 & $8.60 \mathrm{E}-01$ \\
\hline ENSBTAG00000003510 & $3.40 \mathrm{E}-10$ & $3.94 \mathrm{E}-10$ & MMRN2 & $8.63 \mathrm{E}-01$ \\
\hline ENSBTAG00000004965 & $5.03 \mathrm{E}-10$ & $5.78 \mathrm{E}-10$ & NUP133 & 8.69E-01 \\
\hline ENSBTAG00000016191 & $4.25 \mathrm{E}-10$ & $4.89 \mathrm{E}-10$ & ZNF32 & $8.70 \mathrm{E}-01$ \\
\hline ENSBTAG00000021416 & $9.71 \mathrm{E}-10$ & 1.11E-09 & FAM213A & $8.75 \mathrm{E}-01$ \\
\hline ENSBTAG00000001231 & $5.80 \mathrm{E}-10$ & $6.62 \mathrm{E}-10$ & CDHR1 & $8.75 \mathrm{E}-01$ \\
\hline ENSBTAG00000000221 & $3.79 \mathrm{E}-10$ & 4.29E-10 & RBM34 & $8.83 \mathrm{E}-01$ \\
\hline ENSBTAG00000000949 & $1.75 \mathrm{E}-10$ & $1.96 \mathrm{E}-10$ & DNAJC9 & $8.95 \mathrm{E}-01$ \\
\hline ENSBTAG00000000222 & $1.37 \mathrm{E}-10$ & $1.53 \mathrm{E}-10$ & ARID4B & 8.93E-01 \\
\hline ENSBTAG00000013090 & $1.04 \mathrm{E}-10$ & $1.16 \mathrm{E}-10$ & NODAL & $8.96 \mathrm{E}-01$ \\
\hline ENSBTAG00000024811 & $5.40 \mathrm{E}-10$ & $6.05 E-10$ & & 8.93E-01 \\
\hline ENSBTAG00000003442 & $3.45 \mathrm{E}-10$ & 3.80E-10 & LRIT1 & 9.09E-01 \\
\hline ENSBTAG00000004702 & $3.02 \mathrm{E}-10$ & $3.33 \mathrm{E}-10$ & & 9.07E-01 \\
\hline ENSBTAG00000004517 & $5.67 \mathrm{E}-10$ & 6.17E-10 & TARBP1 & 9.19E-01 \\
\hline ENSBTAG00000019313 & $3.58 \mathrm{E}-10$ & $3.86 \mathrm{E}-10$ & ZMIZ1 & $9.29 \mathrm{E}-01$ \\
\hline ENSBTAG00000048036 & $1.72 \mathrm{E}-09$ & $1.85 \mathrm{E}-09$ & & $9.28 \mathrm{E}-01$ \\
\hline ENSBTAG00000018356 & $2.82 \mathrm{E}-10$ & $3.02 \mathrm{E}-10$ & & $9.33 \mathrm{E}-01$ \\
\hline ENSBTAG00000011108 & $1.89 \mathrm{E}-10$ & 2.02E-10 & USP54 & $9.36 \mathrm{E}-01$ \\
\hline ENSBTAG00000001573 & $3.48 \mathrm{E}-10$ & 3.67E-10 & JMJD1C & $9.49 \mathrm{E}-01$ \\
\hline ENSBTAG00000027205 & $1.23 \mathrm{E}-09$ & $1.29 \mathrm{E}-09$ & PGBD5 & 9.53E-01 \\
\hline ENSBTAG00000016814 & $6.15 \mathrm{E}-10$ & $6.45 \mathrm{E}-10$ & CHAT & 9.54E-01 \\
\hline ENSBTAG00000005963 & $1.29 \mathrm{E}-09$ & $1.35 \mathrm{E}-09$ & MAT1A & $9.55 \mathrm{E}-01$ \\
\hline ENSBTAG00000032829 & $4.29 \mathrm{E}-10$ & 4.47E-10 & GHITM & $9.59 \mathrm{E}-01$ \\
\hline ENSBTAG00000019537 & $4.32 \mathrm{E}-10$ & 4.49E-10 & & $9.62 \mathrm{E}-01$ \\
\hline ENSBTAG00000021499 & $9.62 \mathrm{E}-10$ & $9.92 \mathrm{E}-10$ & PSAP & $9.70 \mathrm{E}-01$ \\
\hline ENSBTAG00000003910 & $6.11 \mathrm{E}-10$ & $6.26 \mathrm{E}-10$ & $\mathrm{SH} 2 \mathrm{D} 4 \mathrm{~B}$ & $9.77 \mathrm{E}-01$ \\
\hline ENSBTAG00000044197 & $6.05 E-10$ & $6.13 \mathrm{E}-10$ & EDARADD & 9.87E-01 \\
\hline ENSBTAG00000018707 & $2.65 \mathrm{E}-10$ & $2.68 \mathrm{E}-10$ & LDB3 & $9.88 \mathrm{E}-01$ \\
\hline ENSBTAG00000010765 & $2.63 \mathrm{E}-10$ & 2.64E-10 & Clorf131 & 9.97E-01 \\
\hline ENSBTAG00000016711 & $1.13 \mathrm{E}-09$ & 1.13E-09 & PPIF & $9.96 \mathrm{E}-01$ \\
\hline ENSBTAG00000012657 & $2.00 \mathrm{E}-10$ & $2.00 \mathrm{E}-10$ & ZSWIM8 & $9.98 \mathrm{E}-01$ \\
\hline ENSBTAG00000005568 & $3.53 \mathrm{E}-10$ & $3.52 \mathrm{E}-10$ & FRMPD2 & $1.00 \mathrm{E}+00$ \\
\hline ENSBTAG00000016527 & $5.41 \mathrm{E}-10$ & $5.38 \mathrm{E}-10$ & C10orf35 & $1.01 E+00$ \\
\hline ENSBTAG00000001163 & $3.10 \mathrm{E}-10$ & 3.07E-10 & H2AFY2 & $1.01 E+00$ \\
\hline ENSBTAG00000019164 & $5.08 \mathrm{E}-10$ & $5.02 \mathrm{E}-10$ & RHOBTB1 & $1.01 E+00$ \\
\hline ENSBTAG00000001019 & $5.16 \mathrm{E}-10$ & 5.10E-10 & GDF10 & $1.01 E+00$ \\
\hline ENSBTAG00000010180 & $6.68 \mathrm{E}-10$ & $6.58 \mathrm{E}-10$ & URB2 & $1.01 \mathrm{E}+00$ \\
\hline ENSBTAG00000047537 & $2.61 \mathrm{E}-10$ & $2.57 \mathrm{E}-10$ & & $1.02 \mathrm{E}+00$ \\
\hline ENSBTAG00000006536 & $3.08 \mathrm{E}-10$ & $3.02 \mathrm{E}-10$ & & $1.02 \mathrm{E}+00$ \\
\hline ENSBTAG00000040143 & $7.51 \mathrm{E}-10$ & 7.35E-10 & OR13A1 & $1.02 \mathrm{E}+00$ \\
\hline ENSBTAG00000023074 & $4.06 \mathrm{E}-10$ & $3.96 \mathrm{E}-10$ & Clorf198 & $1.03 E+00$ \\
\hline ENSBTAG00000016882 & 1.17E-10 & $1.15 \mathrm{E}-10$ & VPS26A & $1.02 \mathrm{E}+00$ \\
\hline ENSBTAG00000019130 & $1.64 \mathrm{E}-10$ & $1.60 \mathrm{E}-10$ & FAM149B1 & $1.03 E+00$ \\
\hline ENSBTAG00000014315 & $2.62 \mathrm{E}-10$ & $2.53 \mathrm{E}-10$ & PBLD & $1.04 \mathrm{E}+00$ \\
\hline ENSBTAG00000004870 & $6.25 E-10$ & $6.03 \mathrm{E}-10$ & GALNT2 & $1.04 E+00$ \\
\hline ENSBTAG00000000079 & $9.28 \mathrm{E}-10$ & $8.93 \mathrm{E}-10$ & CCSAP & $1.04 \mathrm{E}+00$ \\
\hline ENSBTAG00000022886 & $4.87 \mathrm{E}-10$ & $4.68 \mathrm{E}-10$ & RYR2 & $1.04 \mathrm{E}+00$ \\
\hline ENSBTAG00000010591 & $6.03 \mathrm{E}-10$ & $5.76 \mathrm{E}-10$ & ZNF365 & $1.05 E+00$ \\
\hline ENSBTAG00000047227 & $3.08 \mathrm{E}-10$ & $2.91 \mathrm{E}-10$ & & $1.06 \mathrm{E}+00$ \\
\hline ENSBTAG00000000224 & $5.50 \mathrm{E}-10$ & $5.16 \mathrm{E}-10$ & TSPAN15 & $1.07 \mathrm{E}+00$ \\
\hline ENSBTAG00000023039 & $2.24 \mathrm{E}-10$ & $2.09 \mathrm{E}-10$ & & $1.07 \mathrm{E}+00$ \\
\hline ENSBTAG00000020319 & 4.15E-10 & $3.89 \mathrm{E}-10$ & ALOX5 & $1.07 \mathrm{E}+00$ \\
\hline ENSBTAG00000023073 & $1.96 \mathrm{E}-10$ & $1.84 \mathrm{E}-10$ & FAM89A & $1.07 E+00$ \\
\hline ENSBTAG00000012380 & $5.05 \mathrm{E}-10$ & $4.66 \mathrm{E}-10$ & HK1 & $1.08 \mathrm{E}+00$ \\
\hline ENSBTAG00000000942 & $4.72 \mathrm{E}-10$ & $4.34 \mathrm{E}-10$ & SIPA1L2 & $1.09 \mathrm{E}+00$ \\
\hline ENSBTAG00000013259 & $2.40 \mathrm{E}-10$ & $2.18 \mathrm{E}-10$ & POLR3A & $1.10 \mathrm{E}+00$ \\
\hline ENSBTAG00000018915 & $7.90 \mathrm{E}-10$ & $7.16 \mathrm{E}-10$ & FAM21A & $1.10 \mathrm{E}+00$ \\
\hline
\end{tabular}




\begin{tabular}{|c|c|c|c|c|}
\hline ENSBTAG00000001044 & $7.30 \mathrm{E}-10$ & $6.58 \mathrm{E}-10$ & SUPV3L1 & $1.11 E+00$ \\
\hline ENSBTAG00000012654 & $4.51 \mathrm{E}-10$ & 4.05E-10 & CHCHD1 & $1.11 E+00$ \\
\hline ENSBTAG00000020361 & $5.34 \mathrm{E}-10$ & 4.80E-10 & SLC35F3 & $1.11 \mathrm{E}+00$ \\
\hline ENSBTAG00000011741 & $5.29 \mathrm{E}-10$ & $4.72 \mathrm{E}-10$ & COL13A1 & $1.12 \mathrm{E}+00$ \\
\hline ENSBTAG00000017540 & $4.55 \mathrm{E}-10$ & 4.05E-10 & FAM13C & $1.12 \mathrm{E}+00$ \\
\hline ENSBTAG00000019623 & $7.69 \mathrm{E}-10$ & $6.82 \mathrm{E}-10$ & GRID1 & $1.13 E+00$ \\
\hline ENSBTAG00000000162 & $4.32 \mathrm{E}-10$ & $3.83 \mathrm{E}-10$ & ANAPC16 & $1.13 \mathrm{E}+00$ \\
\hline ENSBTAG00000011660 & $8.47 \mathrm{E}-10$ & 7.46E-10 & MSMB & $1.13 E+00$ \\
\hline ENSBTAG00000013735 & $4.45 \mathrm{E}-10$ & $3.89 \mathrm{E}-10$ & TBCE & $1.14 \mathrm{E}+00$ \\
\hline ENSBTAG00000025021 & $3.15 \mathrm{E}-10$ & $2.75 \mathrm{E}-10$ & & $1.15 E+00$ \\
\hline ENSBTAG00000012251 & 4.60E-10 & $4.02 \mathrm{E}-10$ & MTR & $1.15 \mathrm{E}+00$ \\
\hline ENSBTAG00000021883 & $1.42 \mathrm{E}-10$ & $1.24 \mathrm{E}-10$ & AP3M1 & $1.14 \mathrm{E}+00$ \\
\hline ENSBTAG00000009350 & $6.68 \mathrm{E}-10$ & $5.82 \mathrm{E}-10$ & PLA2G12B & $1.15 \mathrm{E}+00$ \\
\hline ENSBTAG00000013592 & $1.06 \mathrm{E}-10$ & $9.25 \mathrm{E}-11$ & ZNF248 & $1.15 \mathrm{E}+00$ \\
\hline ENSBTAG00000007244 & $3.67 \mathrm{E}-10$ & $3.15 \mathrm{E}-10$ & NID1 & $1.16 \mathrm{E}+00$ \\
\hline ENSBTAG00000012476 & $1.77 \mathrm{E}-10$ & $1.52 \mathrm{E}-10$ & NRBF2 & $1.17 E+00$ \\
\hline ENSBTAG00000018691 & $2.15 \mathrm{E}-10$ & $1.84 \mathrm{E}-10$ & RHOU & $1.17 \mathrm{E}+00$ \\
\hline ENSBTAG00000023018 & $2.93 \mathrm{E}-10$ & $2.50 \mathrm{E}-10$ & PARG & $1.17 \mathrm{E}+00$ \\
\hline ENSBTAG00000008583 & $3.34 \mathrm{E}-10$ & $2.86 \mathrm{E}-10$ & PALD1 & $1.17 E+00$ \\
\hline ENSBTAG00000000535 & $4.68 \mathrm{E}-10$ & $4.00 \mathrm{E}-10$ & PCNX2 & $1.17 \mathrm{E}+00$ \\
\hline ENSBTAG00000044100 & $2.55 \mathrm{E}-10$ & $2.18 \mathrm{E}-10$ & HEATR1 & $1.17 \mathrm{E}+00$ \\
\hline ENSBTAG00000019525 & $1.65 \mathrm{E}-10$ & $1.40 \mathrm{E}-10$ & & $1.18 \mathrm{E}+00$ \\
\hline ENSBTAG00000021180 & $3.50 \mathrm{E}-10$ & $2.98 \mathrm{E}-10$ & TBATA & $1.17 \mathrm{E}+00$ \\
\hline ENSBTAG00000006729 & $2.48 \mathrm{E}-10$ & $2.11 E-10$ & ARID5B & $1.18 \mathrm{E}+00$ \\
\hline ENSBTAG00000045699 & $5.42 \mathrm{E}-10$ & 4.59E-10 & CTNNA3 & $1.18 \mathrm{E}+00$ \\
\hline ENSBTAG00000047119 & $7.69 \mathrm{E}-10$ & $6.50 \mathrm{E}-10$ & & $1.18 \mathrm{E}+00$ \\
\hline ENSBTAG00000010367 & $3.07 \mathrm{E}-10$ & $2.58 \mathrm{E}-10$ & TTC13 & $1.19 E+00$ \\
\hline ENSBTAG00000021183 & $5.52 \mathrm{E}-10$ & $4.65 \mathrm{E}-10$ & & $1.19 E+00$ \\
\hline ENSBTAG00000038418 & $1.36 \mathrm{E}-10$ & $1.14 \mathrm{E}-10$ & & $1.19 E+00$ \\
\hline ENSBTAG00000012977 & $4.75 \mathrm{E}-10$ & $3.98 \mathrm{E}-10$ & COMTD1 & $1.19 E+00$ \\
\hline ENSBTAG00000033457 & $2.37 \mathrm{E}-10$ & $1.99 \mathrm{E}-10$ & & $1.19 E+00$ \\
\hline ENSBTAG00000046332 & $2.68 \mathrm{E}-10$ & $2.25 \mathrm{E}-10$ & ACTA1 & $1.19 \mathrm{E}+00$ \\
\hline ENSBTAG00000010773 & $2.88 \mathrm{E}-10$ & 2.40E-10 & GNPAT & $1.20 \mathrm{E}+00$ \\
\hline ENSBTAG00000012975 & $2.47 \mathrm{E}-10$ & 2.03E-10 & VDAC2 & $1.22 \mathrm{E}+00$ \\
\hline ENSBTAG00000014938 & $9.21 \mathrm{E}-10$ & $7.54 \mathrm{E}-10$ & PTPN20 & $1.22 \mathrm{E}+00$ \\
\hline ENSBTAG00000002791 & $4.16 \mathrm{E}-10$ & 3.37E-10 & SEC24C & $1.24 \mathrm{E}+00$ \\
\hline ENSBTAG00000010930 & $1.82 \mathrm{E}-10$ & $1.48 \mathrm{E}-10$ & NPFFR1 & $1.23 E+00$ \\
\hline ENSBTAG00000023054 & $1.48 \mathrm{E}-10$ & 1.19E-10 & SLC25A16 & $1.24 \mathrm{E}+00$ \\
\hline ENSBTAG00000000392 & $9.72 \mathrm{E}-10$ & 7.83E-10 & $\mathrm{ASCC} 1$ & $1.24 \mathrm{E}+00$ \\
\hline ENSBTAG00000012667 & $3.73 \mathrm{E}-10$ & 3.00E-10 & CAMK2G & $1.24 \mathrm{E}+00$ \\
\hline ENSBTAG00000004515 & $8.25 \mathrm{E}-10$ & $6.62 \mathrm{E}-10$ & KCNK1 & $1.25 \mathrm{E}+00$ \\
\hline ENSBTAG00000037756 & $2.09 \mathrm{E}-10$ & $1.67 \mathrm{E}-10$ & TET1 & $1.25 \mathrm{E}+00$ \\
\hline ENSBTAG00000047877 & $4.35 \mathrm{E}-10$ & $3.46 \mathrm{E}-10$ & TRIM67 & $1.26 \mathrm{E}+00$ \\
\hline ENSBTAG00000014023 & $1.76 \mathrm{E}-10$ & $1.40 \mathrm{E}-10$ & SIRT1 & $1.26 \mathrm{E}+00$ \\
\hline ENSBTAG00000021822 & $9.57 \mathrm{E}-11$ & $7.59 \mathrm{E}-11$ & ARV1 & $1.26 \mathrm{E}+00$ \\
\hline ENSBTAG00000011991 & $4.66 \mathrm{E}-10$ & $3.69 \mathrm{E}-10$ & DRGX & $1.26 \mathrm{E}+00$ \\
\hline ENSBTAG00000021177 & $4.68 \mathrm{E}-10$ & 3.70E-10 & ADAMTS14 & $1.27 \mathrm{E}+00$ \\
\hline ENSBTAG00000019052 & $3.69 \mathrm{E}-10$ & $2.91 \mathrm{E}-10$ & ANK3 & $1.27 \mathrm{E}+00$ \\
\hline ENSBTAG00000009763 & $5.73 \mathrm{E}-10$ & $4.50 \mathrm{E}-10$ & & $1.27 \mathrm{E}+00$ \\
\hline ENSBTAG00000008619 & 4.16E-10 & 3.23E-10 & SAMD8 & $1.29 E+00$ \\
\hline ENSBTAG00000023032 & $4.13 \mathrm{E}-10$ & $3.19 \mathrm{E}-10$ & SFTPA1 & $1.30 \mathrm{E}+00$ \\
\hline ENSBTAG00000002908 & $1.21 \mathrm{E}-09$ & $9.31 E-10$ & GNG4 & $1.30 \mathrm{E}+00$ \\
\hline ENSBTAG00000007540 & $3.79 \mathrm{E}-10$ & $2.92 \mathrm{E}-10$ & GLUD1 & $1.30 \mathrm{E}+00$ \\
\hline ENSBTAG00000033398 & $7.35 \mathrm{E}-10$ & $5.60 \mathrm{E}-10$ & HKDC1 & $1.31 E+00$ \\
\hline ENSBTAG00000006719 & $2.17 \mathrm{E}-10$ & $1.65 \mathrm{E}-10$ & ZFAND4 & $1.32 \mathrm{E}+00$ \\
\hline ENSBTAG00000013187 & $2.92 \mathrm{E}-10$ & $2.22 \mathrm{E}-10$ & DLG5 & $1.32 \mathrm{E}+00$ \\
\hline ENSBTAG00000034225 & $3.27 \mathrm{E}-10$ & $2.48 \mathrm{E}-10$ & TAF5L & $1.32 \mathrm{E}+00$ \\
\hline ENSBTAG00000000950 & $2.24 \mathrm{E}-10$ & $1.69 \mathrm{E}-10$ & MRPS16 & $1.32 \mathrm{E}+00$ \\
\hline ENSBTAG00000002669 & $3.67 \mathrm{E}-10$ & $2.76 \mathrm{E}-10$ & RASSF4 & $1.33 E+00$ \\
\hline ENSBTAG00000002792 & $3.87 \mathrm{E}-10$ & $2.89 \mathrm{E}-10$ & FUT11 & $1.34 \mathrm{E}+00$ \\
\hline ENSBTAG00000000570 & $7.29 \mathrm{E}-10$ & $5.44 \mathrm{E}-10$ & RET & $1.34 \mathrm{E}+00$ \\
\hline ENSBTAG00000012213 & $1.36 \mathrm{E}-10$ & $1.01 \mathrm{E}-10$ & WAPL & $1.34 \mathrm{E}+00$ \\
\hline ENSBTAG00000001656 & $7.63 \mathrm{E}-10$ & $5.68 \mathrm{E}-10$ & TMEM254 & $1.34 \mathrm{E}+00$ \\
\hline ENSBTAG00000013300 & $4.93 \mathrm{E}-10$ & $3.61 \mathrm{E}-10$ & KCNMA1 & $1.37 E+00$ \\
\hline ENSBTAG00000021879 & $2.82 \mathrm{E}-10$ & 2.07E-10 & VCL & $1.36 \mathrm{E}+00$ \\
\hline ENSBTAG00000023028 & $6.12 \mathrm{E}-10$ & $4.38 \mathrm{E}-10$ & WDFY4 & $1.40 E+00$ \\
\hline ENSBTAG00000032527 & $7.17 \mathrm{E}-10$ & 5.10E-10 & ERCC6 & $1.41 E+00$ \\
\hline ENSBTAG00000048082 & $5.81 \mathrm{E}-10$ & $4.08 \mathrm{E}-10$ & $\mathrm{CL} 46$ & $1.42 \mathrm{E}+00$ \\
\hline ENSBTAG00000010967 & $1.30 \mathrm{E}-10$ & $8.95 \mathrm{E}-11$ & & $1.45 \mathrm{E}+00$ \\
\hline ENSBTAG00000002614 & $2.16 \mathrm{E}-10$ & $1.48 \mathrm{E}-10$ & RUFY2 & $1.46 \mathrm{E}+00$ \\
\hline
\end{tabular}




\begin{tabular}{|c|c|c|c|c|}
\hline ENSBTAG00000010947 & $5.34 \mathrm{E}-10$ & $3.62 \mathrm{E}-10$ & PHYHIPL & $1.47 \mathrm{E}+00$ \\
\hline ENSBTAG00000010888 & $7.14 \mathrm{E}-10$ & 4.83E-10 & C10orf54 & $1.48 \mathrm{E}+00$ \\
\hline ENSBTAG00000016885 & $5.80 \mathrm{E}-10$ & $3.93 \mathrm{E}-10$ & LRRTM3 & $1.48 \mathrm{E}+00$ \\
\hline ENSBTAG00000046024 & $1.09 \mathrm{E}-10$ & $7.38 \mathrm{E}-11$ & & $1.47 \mathrm{E}+00$ \\
\hline ENSBTAG00000014561 & $4.34 \mathrm{E}-10$ & $2.93 \mathrm{E}-10$ & KIF1BP & $1.48 \mathrm{E}+00$ \\
\hline ENSBTAG00000008827 & $6.94 \mathrm{E}-10$ & 4.67E-10 & SPOCK2 & $1.49 E+00$ \\
\hline ENSBTAG00000003546 & $9.07 \mathrm{E}-10$ & $6.08 \mathrm{E}-10$ & TFAM & $1.49 E+00$ \\
\hline ENSBTAG00000003877 & $7.42 \mathrm{E}-10$ & $4.92 \mathrm{E}-10$ & $\mathrm{ZCCHC24}$ & $1.51 E+00$ \\
\hline ENSBTAG00000023023 & $7.22 \mathrm{E}-10$ & $4.76 \mathrm{E}-10$ & ANTXRL & $1.52 \mathrm{E}+00$ \\
\hline ENSBTAG00000021497 & $8.08 \mathrm{E}-10$ & $5.27 \mathrm{E}-10$ & $\mathrm{CDH} 23$ & $1.53 \mathrm{E}+00$ \\
\hline ENSBTAG00000013651 & $3.69 \mathrm{E}-10$ & $2.39 \mathrm{E}-10$ & DNAJB12 & $1.54 \mathrm{E}+00$ \\
\hline ENSBTAG00000016858 & $4.95 \mathrm{E}-10$ & 3.20E-10 & SAR1A & $1.55 \mathrm{E}+00$ \\
\hline ENSBTAG00000013750 & $8.16 \mathrm{E}-10$ & $5.25 E-10$ & B3GALNT2 & $1.55 E+00$ \\
\hline ENSBTAG00000019194 & $5.69 \mathrm{E}-10$ & $3.63 \mathrm{E}-10$ & FAM35A & $1.57 \mathrm{E}+00$ \\
\hline ENSBTAG00000018857 & $6.00 \mathrm{E}-10$ & $3.82 \mathrm{E}-10$ & RAB4A & $1.57 \mathrm{E}+00$ \\
\hline ENSBTAG00000047340 & $1.46 \mathrm{E}-10$ & $9.17 E-11$ & & $1.59 E+00$ \\
\hline ENSBTAG00000014270 & $5.84 \mathrm{E}-10$ & $3.69 \mathrm{E}-10$ & UNC5B & $1.58 \mathrm{E}+00$ \\
\hline ENSBTAG00000006042 & $2.00 \mathrm{E}-10$ & $1.26 \mathrm{E}-10$ & C10orf128 & $1.59 \mathrm{E}+00$ \\
\hline ENSBTAG00000000163 & $8.16 \mathrm{E}-11$ & $5.14 \mathrm{E}-11$ & DDIT4 & $1.59 E+00$ \\
\hline ENSBTAG00000014506 & $1.03 \mathrm{E}-10$ & $6.51 E-11$ & ZNF503 & $1.59 E+00$ \\
\hline ENSBTAG00000005165 & $3.03 \mathrm{E}-10$ & $1.90 \mathrm{E}-10$ & CSGALNACT2 & $1.59 E+00$ \\
\hline ENSBTAG00000018960 & $2.36 \mathrm{E}-10$ & $1.48 \mathrm{E}-10$ & & $1.59 E+00$ \\
\hline ENSBTAG00000011072 & $4.94 \mathrm{E}-10$ & $3.10 \mathrm{E}-10$ & & $1.59 E+00$ \\
\hline ENSBTAG00000002670 & $1.15 \mathrm{E}-10$ & $7.24 \mathrm{E}-11$ & C10orf10 & $1.59 \mathrm{E}+00$ \\
\hline ENSBTAG00000009696 & $2.61 \mathrm{E}-10$ & $1.62 \mathrm{E}-10$ & ACTN2 & $1.61 \mathrm{E}+00$ \\
\hline ENSBTAG00000019124 & $2.05 E-10$ & $1.26 \mathrm{E}-10$ & EIF4EBP2 & $1.62 E+00$ \\
\hline ENSBTAG00000024585 & $1.70 \mathrm{E}-10$ & $1.05 \mathrm{E}-10$ & ZNF33B & $1.62 E+00$ \\
\hline ENSBTAG00000000313 & $3.59 \mathrm{E}-10$ & $2.17 \mathrm{E}-10$ & NUDT13 & $1.66 \mathrm{E}+00$ \\
\hline ENSBTAG00000014020 & $2.49 \mathrm{E}-10$ & $1.50 \mathrm{E}-10$ & RTKN2 & $1.66 \mathrm{E}+00$ \\
\hline ENSBTAG00000006288 & $4.25 \mathrm{E}-10$ & $2.55 E-10$ & VSTM4 & $1.67 E+00$ \\
\hline ENSBTAG00000014316 & $2.10 \mathrm{E}-10$ & $1.22 \mathrm{E}-10$ & HNRNPH3 & $1.72 \mathrm{E}+00$ \\
\hline ENSBTAG00000047317 & $5.94 \mathrm{E}-10$ & $3.45 E-10$ & & $1.72 \mathrm{E}+00$ \\
\hline ENSBTAG00000019028 & $1.98 \mathrm{E}-10$ & $1.14 \mathrm{E}-10$ & STOX1 & $1.73 E+00$ \\
\hline ENSBTAG00000021664 & $5.19 \mathrm{E}-10$ & $2.98 \mathrm{E}-10$ & TACR2 & $1.74 \mathrm{E}+00$ \\
\hline ENSBTAG00000018499 & $1.04 \mathrm{E}-09$ & $5.92 \mathrm{E}-10$ & & $1.75 E+00$ \\
\hline ENSBTAG00000038845 & $2.40 \mathrm{E}-10$ & $1.36 \mathrm{E}-10$ & TYSND1 & $1.76 \mathrm{E}+00$ \\
\hline ENSBTAG00000000830 & $5.54 \mathrm{E}-10$ & $3.14 \mathrm{E}-10$ & GPR137B & $1.76 \mathrm{E}+00$ \\
\hline ENSBTAG00000032996 & $3.92 \mathrm{E}-10$ & $2.21 \mathrm{E}-10$ & P4HA1 & $1.77 \mathrm{E}+00$ \\
\hline ENSBTAG00000017509 & $1.61 \mathrm{E}-10$ & $9.09 \mathrm{E}-11$ & MYPN & $1.77 \mathrm{E}+00$ \\
\hline ENSBTAG00000047402 & $1.93 \mathrm{E}-10$ & $1.08 \mathrm{E}-10$ & FAM170B & $1.79 E+00$ \\
\hline ENSBTAG00000020218 & $3.90 \mathrm{E}-10$ & $2.17 \mathrm{E}-10$ & ANXA7 & $1.79 \mathrm{E}+00$ \\
\hline ENSBTAG00000014484 & $6.24 \mathrm{E}-10$ & $3.44 \mathrm{E}-10$ & TMEM26 & $1.81 \mathrm{E}+00$ \\
\hline ENSBTAG00000006870 & $3.92 \mathrm{E}-10$ & $2.13 \mathrm{E}-10$ & RASGEF1A & $1.84 \mathrm{E}+00$ \\
\hline ENSBTAG00000009984 & $2.17 \mathrm{E}-10$ & $1.16 \mathrm{E}-10$ & SGPL1 & $1.87 \mathrm{E}+00$ \\
\hline ENSBTAG00000000562 & $2.62 \mathrm{E}-10$ & $1.40 \mathrm{E}-10$ & & $1.87 \mathrm{E}+00$ \\
\hline ENSBTAG00000046355 & $1.75 \mathrm{E}-09$ & $9.35 E-10$ & & $1.87 \mathrm{E}+00$ \\
\hline ENSBTAG00000010500 & $2.09 \mathrm{E}-10$ & $1.11 \mathrm{E}-10$ & & $1.88 \mathrm{E}+00$ \\
\hline ENSBTAG00000020219 & $9.44 \mathrm{E}-11$ & $4.95 \mathrm{E}-11$ & MSS51 & $1.91 E+00$ \\
\hline ENSBTAG00000032774 & $1.83 \mathrm{E}-10$ & $9.20 \mathrm{E}-11$ & ADIRF & $1.98 \mathrm{E}+00$ \\
\hline ENSBTAG00000011866 & 1.14E-09 & $5.46 \mathrm{E}-10$ & PCBD1 & $2.09 \mathrm{E}+00$ \\
\hline ENSBTAG00000019755 & $3.08 \mathrm{E}-10$ & $1.46 \mathrm{E}-10$ & REEP3 & $2.11 E+00$ \\
\hline ENSBTAG00000020939 & $4.28 \mathrm{E}-10$ & $2.02 \mathrm{E}-10$ & PLAC9 & $2.12 \mathrm{E}+00$ \\
\hline ENSBTAG00000007836 & 4.27E-10 & $1.99 \mathrm{E}-10$ & PPA1 & $2.14 E+00$ \\
\hline ENSBTAG00000009587 & $6.26 \mathrm{E}-10$ & $2.92 \mathrm{E}-10$ & & $2.14 \mathrm{E}+00$ \\
\hline ENSBTAG00000032858 & $6.16 \mathrm{E}-10$ & $2.87 \mathrm{E}-10$ & DYDC1 & $2.15 E+00$ \\
\hline ENSBTAG00000003876 & $5.67 \mathrm{E}-10$ & $2.62 \mathrm{E}-10$ & $\mathrm{MCU}$ & $2.16 \mathrm{E}+00$ \\
\hline ENSBTAG00000002920 & $2.78 \mathrm{E}-10$ & $1.28 \mathrm{E}-10$ & KAT6B & $2.17 E+00$ \\
\hline ENSBTAG00000020940 & $7.38 \mathrm{E}-10$ & $3.36 \mathrm{E}-10$ & ANXA11 & $2.20 \mathrm{E}+00$ \\
\hline ENSBTAG00000005003 & $4.82 \mathrm{E}-10$ & $2.16 \mathrm{E}-10$ & RBP3 & $2.23 E+00$ \\
\hline ENSBTAG00000002955 & $1.31 \mathrm{E}-10$ & $5.76 \mathrm{E}-11$ & & $2.27 E+00$ \\
\hline ENSBTAG00000005077 & $2.32 \mathrm{E}-10$ & $1.02 \mathrm{E}-10$ & & $2.28 \mathrm{E}+00$ \\
\hline ENSBTAG00000014029 & $1.98 \mathrm{E}-10$ & $8.65 \mathrm{E}-11$ & HERC4 & $2.29 E+00$ \\
\hline ENSBTAG00000017863 & $1.24 \mathrm{E}-10$ & $5.22 \mathrm{E}-11$ & SRGN & $2.38 \mathrm{E}+00$ \\
\hline ENSBTAG00000038178 & $6.00 \mathrm{E}-11$ & $2.52 E-11$ & ZNF25 & $2.38 \mathrm{E}+00$ \\
\hline ENSBTAG00000006111 & $9.98 \mathrm{E}-11$ & $4.19 E-11$ & EXOC8 & $2.38 \mathrm{E}+00$ \\
\hline ENSBTAG00000004760 & $2.17 \mathrm{E}-10$ & $9.10 \mathrm{E}-11$ & & $2.38 \mathrm{E}+00$ \\
\hline ENSBTAG00000000231 & $2.67 \mathrm{E}-10$ & $1.08 \mathrm{E}-10$ & BMPR1A & $2.47 \mathrm{E}+00$ \\
\hline ENSBTAG00000003189 & $2.82 \mathrm{E}-10$ & $1.14 \mathrm{E}-10$ & CCDC6 & $2.47 E+00$ \\
\hline ENSBTAG00000046357 & $3.02 \mathrm{E}-10$ & $1.20 \mathrm{E}-10$ & & $2.52 \mathrm{E}+00$ \\
\hline ENSBTAG00000019792 & $3.66 \mathrm{E}-10$ & $1.43 \mathrm{E}-10$ & SLC16A9 & $2.56 \mathrm{E}+00$ \\
\hline
\end{tabular}




\begin{tabular}{|c|c|c|c|c|}
\hline ENSBTAG00000013935 & $9.27 \mathrm{E}-10$ & $3.21 \mathrm{E}-10$ & NTPCR & $2.89 E+00$ \\
\hline ENSBTAG00000006112 & $1.55 \mathrm{E}-10$ & $5.31 \mathrm{E}-11$ & SPRTN & $2.91 E+00$ \\
\hline ENSBTAG00000047434 & $5.74 \mathrm{E}-10$ & $1.85 \mathrm{E}-10$ & TSNAX & $3.10 E+00$ \\
\hline ENSBTAG00000048311 & $6.82 \mathrm{E}-10$ & $2.14 \mathrm{E}-10$ & & $3.19 E+00$ \\
\hline ENSBTAG00000045788 & $9.30 \mathrm{E}-10$ & $2.91 \mathrm{E}-10$ & & $3.20 E+00$ \\
\hline ENSBTAG00000002620 & $3.69 \mathrm{E}-10$ & $1.11 \mathrm{E}-10$ & DNA2 & $3.32 \mathrm{E}+00$ \\
\hline ENSBTAG00000010694 & $4.26 \mathrm{E}-10$ & $1.21 \mathrm{E}-10$ & BICC1 & $3.52 E+00$ \\
\hline ENSBTAG00000048153 & $9.16 \mathrm{E}-10$ & $2.55 \mathrm{E}-10$ & & $3.59 E+00$ \\
\hline ENSBTAG00000010109 & $4.63 \mathrm{E}-10$ & 1.13E-10 & CDK1 & $4.10 E+00$ \\
\hline ENSBTAG00000003907 & $8.04 \mathrm{E}-10$ & $1.96 \mathrm{E}-10$ & TSPAN14 & $4.10 \mathrm{E}+00$ \\
\hline ENSBTAG00000002924 & 4.37E-10 & $1.01 \mathrm{E}-10$ & DUPD1 & $4.33 E+00$ \\
\hline ENSBTAG00000034633 & $3.47 \mathrm{E}-10$ & $7.88 \mathrm{E}-11$ & ERO1B & $4.39 E+00$ \\
\hline ENSBTAG00000047535 & $1.10 \mathrm{E}-09$ & $2.49 \mathrm{E}-10$ & & $4.40 \mathrm{E}+00$ \\
\hline ENSBTAG00000045949 & $5.40 \mathrm{E}-10$ & $1.18 \mathrm{E}-10$ & & $4.57 E+00$ \\
\hline ENSBTAG00000032800 & $2.40 \mathrm{E}-10$ & $5.20 E-11$ & OPN4 & $4.61 E+00$ \\
\hline ENSBTAG00000005807 & $7.75 E-10$ & $1.58 \mathrm{E}-10$ & MICU1 & $4.90 E+00$ \\
\hline ENSBTAG00000021220 & $1.01 \mathrm{E}-09$ & 2.03E-10 & TMEM72 & $4.97 E+00$ \\
\hline ENSBTAG00000033413 & 4.67E-10 & $9.05 E-11$ & DDX50 & $5.16 \mathrm{E}+00$ \\
\hline ENSBTAG00000005004 & $5.59 \mathrm{E}-10$ & $1.00 \mathrm{E}-10$ & GDF2 & $5.59 E+00$ \\
\hline ENSBTAG00000005947 & $4.08 \mathrm{E}-10$ & $6.82 E-11$ & PLAU & $5.97 E+00$ \\
\hline ENSBTAG00000047155 & $3.24 \mathrm{E}-10$ & 4.79E-11 & C10orf71 & $6.75 E+00$ \\
\hline ENSBTAG00000014558 & 8.03E-10 & $1.15 \mathrm{E}-10$ & & $6.98 \mathrm{E}+00$ \\
\hline ENSBTAG00000045932 & 8.17E-10 & $1.06 \mathrm{E}-10$ & & $7.70 E+00$ \\
\hline ENSBTAG00000005996 & $9.90 \mathrm{E}-10$ & $1.21 \mathrm{E}-10$ & LRRC18 & $8.17 E+00$ \\
\hline ENSBTAG00000046597 & $7.26 \mathrm{E}-10$ & 7.57E-11 & GPRIN2 & $9.58 \mathrm{E}+00$ \\
\hline ENSBTAG00000032862 & $1.09 \mathrm{E}-09$ & $1.11 \mathrm{E}-10$ & DYDC2 & $9.78 \mathrm{E}+00$ \\
\hline ENSBTAG00000011014 & $7.35 \mathrm{E}-10$ & $7.08 \mathrm{E}-11$ & CHST3 & $1.04 \mathrm{E}+01$ \\
\hline ENSBTAG00000020314 & $1.04 \mathrm{E}-09$ & $7.31 \mathrm{E}-11$ & COG2 & $1.42 \mathrm{E}+01$ \\
\hline ENSBTAG00000016804 & $5.65 \mathrm{E}-10$ & 2.79E-11 & LYST & $2.02 E+01$ \\
\hline ENSBTAG00000012393 & $5.12 \mathrm{E}-10$ & 2.33E-11 & AGT & $2.19 E+01$ \\
\hline ENSBTAG00000012878 & $7.44 \mathrm{E}-10$ & 2.03E-11 & BMS1 & $3.65 E+01$ \\
\hline ENSBTAG00000004217 & $6.38 \mathrm{E}-11$ & 0 & & $6.39 E+02$ \\
\hline ENSBTAG00000026065 & $4.26 \mathrm{E}-10$ & 0 & & $4.26 E+03$ \\
\hline ENSBTAG00000045608 & $1.92 \mathrm{E}-10$ & 0 & & $1.93 E+03$ \\
\hline ENSBTAG00000046841 & $7.92 \mathrm{E}-11$ & 0 & IRF2BP2 & $7.93 E+02$ \\
\hline ENSBTAG00000033555 & $1.74 \mathrm{E}-10$ & 0 & & $1.74 E+03$ \\
\hline ENSBTAG00000019352 & 0 & 0 & OR5D14 & $1.00 E+00$ \\
\hline ENSBTAG00000004624 & $1.23 \mathrm{E}-10$ & 0 & & $1.23 E+03$ \\
\hline ENSBTAG00000008059 & 0 & 0 & CHRM3 & $1.00 \mathrm{E}+00$ \\
\hline ENSBTAG00000045717 & $2.02 \mathrm{E}-10$ & 0 & ADO & $2.02 E+03$ \\
\hline ENSBTAG00000006431 & $2.53 \mathrm{E}-10$ & 0 & NEUROG3 & $2.53 E+03$ \\
\hline ENSBTAG00000045826 & $2.20 \mathrm{E}-10$ & 0 & C10orf105 & $2.21 \mathrm{E}+03$ \\
\hline ENSBTAG00000013585 & $4.86 \mathrm{E}-10$ & 0 & & $4.86 \mathrm{E}+03$ \\
\hline ENSBTAG00000014990 & $5.20 \mathrm{E}-11$ & 0 & SLC18A3 & $5.21 E+02$ \\
\hline ENSBTAG00000038590 & 0 & 7.14E-11 & & $1.40 \mathrm{E}-03$ \\
\hline ENSBTAG00000031194 & 0 & 9.77E-11 & PHLDA2 & $1.02 \mathrm{E}-03$ \\
\hline ENSBTAG00000047349 & 0 & $3.75 \mathrm{E}-10$ & & 2.67E-04 \\
\hline ENSBTAG00000031875 & $1.69 \mathrm{E}-10$ & $5.32 \mathrm{E}-10$ & BANF1 & 3.17E-01 \\
\hline ENSBTAG00000013398 & $1.19 \mathrm{E}-10$ & $3.76 \mathrm{E}-10$ & & 3.17E-01 \\
\hline ENSBTAG00000006195 & $1.11 \mathrm{E}-10$ & $2.79 \mathrm{E}-10$ & C11orf68 & 3.97E-01 \\
\hline ENSBTAG00000015248 & $3.99 \mathrm{E}-10$ & 7.99E-10 & & 4.99E-01 \\
\hline ENSBTAG00000014339 & $6.62 \mathrm{E}-11$ & $1.25 \mathrm{E}-10$ & PUS3 & 5.30E-01 \\
\hline ENSBTAG00000018022 & $1.90 \mathrm{E}-10$ & $3.54 \mathrm{E}-10$ & C11orf84 & $5.36 \mathrm{E}-01$ \\
\hline ENSBTAG00000047090 & $2.63 \mathrm{E}-10$ & 4.42E-10 & & 5.95E-01 \\
\hline ENSBTAG00000010470 & $2.78 \mathrm{E}-10$ & $4.68 \mathrm{E}-10$ & UQCC3 & 5.95E-01 \\
\hline ENSBTAG00000039511 & $4.81 \mathrm{E}-10$ & 8.02E-10 & & $6.00 \mathrm{E}-01$ \\
\hline ENSBTAG00000005147 & $2.58 \mathrm{E}-10$ & $3.96 \mathrm{E}-10$ & HPS5 & $6.50 \mathrm{E}-01$ \\
\hline ENSBTAG00000016465 & 4.57E-10 & $6.83 E-10$ & DHCR7 & $6.69 \mathrm{E}-01$ \\
\hline ENSBTAG00000046644 & $1.69 \mathrm{E}-10$ & $2.48 \mathrm{E}-10$ & HRAS & $6.81 \mathrm{E}-01$ \\
\hline ENSBTAG00000039036 & $2.19 \mathrm{E}-10$ & $3.21 \mathrm{E}-10$ & & $6.81 \mathrm{E}-01$ \\
\hline ENSBTAG00000001902 & $1.07 \mathrm{E}-10$ & $1.54 \mathrm{E}-10$ & CEP295 & $6.95 \mathrm{E}-01$ \\
\hline ENSBTAG00000034921 & $6.43 \mathrm{E}-10$ & $9.01 \mathrm{E}-10$ & & 7.14E-01 \\
\hline ENSBTAG00000047758 & 7.47E-10 & $1.03 E-09$ & & $7.25 \mathrm{E}-01$ \\
\hline ENSBTAG00000000284 & $7.59 \mathrm{E}-10$ & $1.04 \mathrm{E}-09$ & NAALAD2 & 7.30E-01 \\
\hline ENSBTAG00000017602 & 4.06E-10 & $5.52 \mathrm{E}-10$ & TMEM45B & 7.36E-01 \\
\hline ENSBTAG00000012169 & 4.47E-10 & $6.06 \mathrm{E}-10$ & & 7.37E-01 \\
\hline ENSBTAG00000045802 & $1.97 \mathrm{E}-10$ & $2.65 \mathrm{E}-10$ & & 7.43E-01 \\
\hline ENSBTAG00000018762 & $1.62 \mathrm{E}-10$ & 2.19E-10 & SLC22A12 & $7.40 \mathrm{E}-01$ \\
\hline ENSBTAG00000002488 & $2.36 \mathrm{E}-10$ & $3.10 \mathrm{E}-10$ & LDHC & 7.62E-01 \\
\hline ENSBTAG00000039825 & $2.54 \mathrm{E}-10$ & $3.31 \mathrm{E}-10$ & & 7.67E-01 \\
\hline
\end{tabular}




\begin{tabular}{|c|c|c|c|c|}
\hline ENSBTAG00000005577 & $1.39 \mathrm{E}-10$ & $1.76 \mathrm{E}-10$ & RNASEH2C & 7.92E-01 \\
\hline ENSBTAG00000047811 & $1.31 \mathrm{E}-10$ & $1.65 \mathrm{E}-10$ & & $7.92 \mathrm{E}-01$ \\
\hline ENSBTAG00000038802 & $1.69 \mathrm{E}-10$ & $2.13 \mathrm{E}-10$ & OR8D4 & 7.94E-01 \\
\hline ENSBTAG00000002245 & $2.40 \mathrm{E}-10$ & $3.02 \mathrm{E}-10$ & MRGPRX2 & 7.94E-01 \\
\hline ENSBTAG00000002751 & $2.25 \mathrm{E}-10$ & $2.83 \mathrm{E}-10$ & VSTM5 & 7.94E-01 \\
\hline ENSBTAG00000020807 & $3.84 \mathrm{E}-10$ & $4.84 \mathrm{E}-10$ & & 7.93E-01 \\
\hline ENSBTAG00000046410 & $3.51 \mathrm{E}-10$ & $4.42 \mathrm{E}-10$ & & 7.94E-01 \\
\hline ENSBTAG00000047916 & $2.66 \mathrm{E}-10$ & $3.36 \mathrm{E}-10$ & & 7.93E-01 \\
\hline ENSBTAG00000006740 & $3.57 \mathrm{E}-10$ & $4.50 \mathrm{E}-10$ & SCYL1 & 7.94E-01 \\
\hline ENSBTAG00000018274 & $3.41 \mathrm{E}-10$ & $4.30 \mathrm{E}-10$ & FADD & 7.94E-01 \\
\hline ENSBTAG00000006629 & $3.36 \mathrm{E}-10$ & $4.23 \mathrm{E}-10$ & & $7.94 \mathrm{E}-01$ \\
\hline ENSBTAG00000046090 & $3.40 \mathrm{E}-10$ & $4.28 \mathrm{E}-10$ & & $7.94 \mathrm{E}-01$ \\
\hline ENSBTAG00000048298 & $2.28 \mathrm{E}-10$ & $2.87 \mathrm{E}-10$ & & 7.93E-01 \\
\hline ENSBTAG00000021329 & $2.13 \mathrm{E}-10$ & $2.68 \mathrm{E}-10$ & ZBTB3 & 7.94E-01 \\
\hline ENSBTAG00000033377 & $6.30 \mathrm{E}-10$ & $7.94 \mathrm{E}-10$ & & 7.94E-01 \\
\hline ENSBTAG00000039970 & $4.83 \mathrm{E}-10$ & $6.09 \mathrm{E}-10$ & PAG16 & 7.93E-01 \\
\hline ENSBTAG00000019015 & $6.23 \mathrm{E}-10$ & $7.85 \mathrm{E}-10$ & IFITM3 & 7.94E-01 \\
\hline ENSBTAG00000014718 & $6.61 \mathrm{E}-11$ & 8.33E-11 & & 7.94E-01 \\
\hline ENSBTAG00000020203 & $6.13 \mathrm{E}-11$ & $7.73 \mathrm{E}-11$ & TMEM151A & 7.94E-01 \\
\hline ENSBTAG00000047614 & $8.09 \mathrm{E}-10$ & $1.02 \mathrm{E}-09$ & & 7.93E-01 \\
\hline ENSBTAG00000031184 & $8.19 E-11$ & $1.03 \mathrm{E}-10$ & CDKN1C & 7.96E-01 \\
\hline ENSBTAG00000033621 & $8.15 \mathrm{E}-10$ & 1.03E-09 & SMCO4 & 7.91E-01 \\
\hline ENSBTAG00000040340 & $6.62 \mathrm{E}-10$ & $8.34 \mathrm{E}-10$ & & 7.94E-01 \\
\hline ENSBTAG00000047436 & $9.91 \mathrm{E}-10$ & $1.25 \mathrm{E}-09$ & & 7.93E-01 \\
\hline ENSBTAG00000046647 & $8.33 \mathrm{E}-10$ & $1.05 \mathrm{E}-09$ & & 7.93E-01 \\
\hline ENSBTAG00000003713 & $6.50 \mathrm{E}-11$ & $8.19 E-11$ & SSSCA1 & 7.94E-01 \\
\hline ENSBTAG00000018650 & $5.07 \mathrm{E}-10$ & $6.39 \mathrm{E}-10$ & HEPACAM & $7.94 \mathrm{E}-01$ \\
\hline ENSBTAG00000048059 & $6.59 \mathrm{E}-11$ & $8.30 \mathrm{E}-11$ & TMEM86A & 7.94E-01 \\
\hline ENSBTAG00000017735 & $2.58 \mathrm{E}-10$ & $3.26 \mathrm{E}-10$ & & 7.93E-01 \\
\hline ENSBTAG00000008530 & $5.17 \mathrm{E}-10$ & $6.51 \mathrm{E}-10$ & SLC36A4 & 7.94E-01 \\
\hline ENSBTAG00000033656 & $1.43 \mathrm{E}-10$ & $1.80 \mathrm{E}-10$ & & 7.94E-01 \\
\hline ENSBTAG00000007361 & $1.40 \mathrm{E}-10$ & $1.76 \mathrm{E}-10$ & EHD1 & 7.94E-01 \\
\hline ENSBTAG00000022293 & $1.13 \mathrm{E}-10$ & $1.42 \mathrm{E}-10$ & METTL12 & 7.95E-01 \\
\hline ENSBTAG00000009584 & $1.65 \mathrm{E}-10$ & $2.06 \mathrm{E}-10$ & PPP6R3 & $8.01 \mathrm{E}-01$ \\
\hline ENSBTAG00000002879 & $4.34 \mathrm{E}-10$ & $5.35 \mathrm{E}-10$ & DCPS & $8.12 \mathrm{E}-01$ \\
\hline ENSBTAG00000011869 & $2.42 \mathrm{E}-10$ & $2.95 \mathrm{E}-10$ & CSRP3 & $8.20 \mathrm{E}-01$ \\
\hline ENSBTAG00000021456 & $4.73 \mathrm{E}-10$ & $5.78 \mathrm{E}-10$ & MUS81 & $8.18 \mathrm{E}-01$ \\
\hline ENSBTAG00000036172 & $2.61 \mathrm{E}-10$ & 3.17E-10 & & $8.22 \mathrm{E}-01$ \\
\hline ENSBTAG00000032730 & $5.92 \mathrm{E}-10$ & 7.19E-10 & & $8.24 \mathrm{E}-01$ \\
\hline ENSBTAG00000000244 & $1.19 \mathrm{E}-10$ & $1.44 \mathrm{E}-10$ & NRXN2 & $8.27 \mathrm{E}-01$ \\
\hline ENSBTAG00000008568 & $8.71 \mathrm{E}-10$ & 1.05E-09 & SLC25A45 & $8.30 \mathrm{E}-01$ \\
\hline ENSBTAG00000002039 & $4.41 \mathrm{E}-10$ & $5.31 \mathrm{E}-10$ & FRMD8 & $8.30 \mathrm{E}-01$ \\
\hline ENSBTAG00000039463 & $7.69 \mathrm{E}-10$ & $9.27 \mathrm{E}-10$ & & $8.29 \mathrm{E}-01$ \\
\hline ENSBTAG00000013615 & 4.20E-10 & $5.05 \mathrm{E}-10$ & CHORDC1 & $8.31 \mathrm{E}-01$ \\
\hline ENSBTAG00000038169 & $2.93 \mathrm{E}-10$ & $3.52 \mathrm{E}-10$ & & 8.33E-01 \\
\hline ENSBTAG00000010230 & $4.08 \mathrm{E}-10$ & $4.90 \mathrm{E}-10$ & CAPN1 & 8.33E-01 \\
\hline ENSBTAG00000005903 & $5.16 \mathrm{E}-10$ & $6.15 \mathrm{E}-10$ & LRP5 & 8.39E-01 \\
\hline ENSBTAG00000001734 & $4.32 \mathrm{E}-10$ & $5.13 \mathrm{E}-10$ & MS4A10 & $8.41 \mathrm{E}-01$ \\
\hline ENSBTAG00000014215 & $9.18 \mathrm{E}-10$ & $1.08 \mathrm{E}-09$ & & 8.50E-01 \\
\hline ENSBTAG00000022960 & $3.67 \mathrm{E}-10$ & $4.28 \mathrm{E}-10$ & HEPHL1 & $8.58 \mathrm{E}-01$ \\
\hline ENSBTAG00000017495 & $1.06 \mathrm{E}-09$ & $1.22 \mathrm{E}-09$ & & 8.66E-01 \\
\hline ENSBTAG00000035110 & 4.22E-10 & $4.83 \mathrm{E}-10$ & DEUP1 & $8.74 \mathrm{E}-01$ \\
\hline ENSBTAG00000047753 & $5.84 \mathrm{E}-10$ & $6.69 \mathrm{E}-10$ & & $8.74 \mathrm{E}-01$ \\
\hline ENSBTAG00000009412 & $5.29 \mathrm{E}-10$ & $6.00 \mathrm{E}-10$ & SIAE & $8.82 \mathrm{E}-01$ \\
\hline ENSBTAG00000012054 & $3.66 \mathrm{E}-10$ & 4.15E-10 & ALDH3B2 & $8.82 \mathrm{E}-01$ \\
\hline ENSBTAG00000047145 & $1.07 E-09$ & $1.21 \mathrm{E}-09$ & & $8.84 \mathrm{E}-01$ \\
\hline ENSBTAG00000016293 & $2.28 \mathrm{E}-10$ & $2.57 \mathrm{E}-10$ & TRIM77 & $8.86 \mathrm{E}-01$ \\
\hline ENSBTAG00000009315 & $6.85 \mathrm{E}-10$ & $7.66 \mathrm{E}-10$ & CDON & 8.95E-01 \\
\hline ENSBTAG00000048073 & $4.14 \mathrm{E}-10$ & 4.64E-10 & & $8.92 \mathrm{E}-01$ \\
\hline ENSBTAG00000012656 & $2.60 \mathrm{E}-10$ & $2.90 \mathrm{E}-10$ & OVOL1 & 8.97E-01 \\
\hline ENSBTAG00000004356 & $7.79 \mathrm{E}-10$ & 8.67E-10 & $\mathrm{ROBO} 4$ & $8.98 \mathrm{E}-01$ \\
\hline ENSBTAG00000020900 & $6.05 E-10$ & $6.73 \mathrm{E}-10$ & RPUSD4 & $8.98 \mathrm{E}-01$ \\
\hline ENSBTAG00000003236 & $8.21 \mathrm{E}-10$ & $9.13 \mathrm{E}-10$ & & 8.99E-01 \\
\hline ENSBTAG00000007847 & $2.15 \mathrm{E}-10$ & $2.37 \mathrm{E}-10$ & EED & 9.07E-01 \\
\hline ENSBTAG00000004212 & $4.96 \mathrm{E}-10$ & $5.47 \mathrm{E}-10$ & & 9.07E-01 \\
\hline ENSBTAG00000006747 & $4.14 \mathrm{E}-10$ & $4.55 \mathrm{E}-10$ & LTBP3 & $9.11 \mathrm{E}-01$ \\
\hline ENSBTAG00000005076 & $5.40 \mathrm{E}-10$ & $5.92 \mathrm{E}-10$ & SYVN1 & $9.11 \mathrm{E}-01$ \\
\hline ENSBTAG00000019712 & $5.97 E-10$ & $6.51 E-10$ & ST14 & 9.17E-01 \\
\hline ENSBTAG00000015177 & $5.64 \mathrm{E}-10$ & $6.14 \mathrm{E}-10$ & PRSS23 & $9.19 \mathrm{E}-01$ \\
\hline ENSBTAG00000007203 & $3.41 \mathrm{E}-10$ & $3.71 E-10$ & STT3A & $9.19 \mathrm{E}-01$ \\
\hline
\end{tabular}




\begin{tabular}{|c|c|c|c|c|}
\hline ENSBTAG000000010880 & $6.97 \mathrm{E}-10$ & $7.58 \mathrm{E}-10$ & TNNI2 & $9.19 \mathrm{E}-01$ \\
\hline ENSBTAG00000022185 & $2.04 \mathrm{E}-10$ & $2.22 \mathrm{E}-10$ & IGHMBP2 & $9.18 \mathrm{E}-01$ \\
\hline ENSBTAG00000037908 & $3.08 \mathrm{E}-10$ & $3.32 \mathrm{E}-10$ & & $9.28 \mathrm{E}-01$ \\
\hline ENSBTAG000000047100 & $6.12 \mathrm{E}-10$ & $6.60 \mathrm{E}-10$ & & 9.27E-01 \\
\hline ENSBTAG000000020009 & $6.19 E-10$ & $6.68 \mathrm{E}-10$ & CCDC81 & 9.27E-01 \\
\hline ENSBTAG00000046497 & $2.84 \mathrm{E}-10$ & 3.07E-10 & & $9.25 \mathrm{E}-01$ \\
\hline ENSBTAG000000047989 & $5.87 \mathrm{E}-10$ & $6.32 \mathrm{E}-10$ & & $9.29 \mathrm{E}-01$ \\
\hline ENSBTAG000000001035 & $2.48 \mathrm{E}-10$ & 2.67E-10 & SF1 & $9.28 \mathrm{E}-01$ \\
\hline ENSBTAG000000007825 & $2.73 \mathrm{E}-10$ & $2.93 \mathrm{E}-10$ & MARK2 & 9.32E-01 \\
\hline ENSBTAG000000001043 & $4.25 \mathrm{E}-10$ & $4.56 \mathrm{E}-10$ & LUZP2 & $9.32 \mathrm{E}-01$ \\
\hline ENSBTAG000000011467 & $5.61 \mathrm{E}-10$ & $6.01 \mathrm{E}-10$ & BATF2 & 9.34E-01 \\
\hline ENSBTAG000000005578 & $2.83 \mathrm{E}-10$ & $3.02 \mathrm{E}-10$ & KAT5 & $9.38 \mathrm{E}-01$ \\
\hline ENSBTAG00000017610 & $5.15 \mathrm{E}-10$ & $5.49 \mathrm{E}-10$ & NFRKB & $9.38 \mathrm{E}-01$ \\
\hline ENSBTAG000000037784 & $9.41 \mathrm{E}-10$ & 1.00E-09 & & $9.41 \mathrm{E}-01$ \\
\hline ENSBTAG000000014366 & $3.33 \mathrm{E}-10$ & $3.55 \mathrm{E}-10$ & PRPF19 & $9.38 \mathrm{E}-01$ \\
\hline ENSBTAG000000038209 & 1.65E-09 & 1.75E-09 & & $9.45 \mathrm{E}-01$ \\
\hline ENSBTAG00000015793 & $3.64 \mathrm{E}-10$ & $3.83 \mathrm{E}-10$ & RAB38 & $9.51 \mathrm{E}-01$ \\
\hline ENSBTAG000000040257 & $7.86 \mathrm{E}-10$ & $8.22 \mathrm{E}-10$ & & 9.56E-01 \\
\hline ENSBTAG000000027868 & $3.98 \mathrm{E}-10$ & 4.17E-10 & & 9.55E-01 \\
\hline ENSBTAG000000039461 & $7.78 \mathrm{E}-10$ & $8.12 \mathrm{E}-10$ & MS4A15 & $9.59 \mathrm{E}-01$ \\
\hline ENSBTAG000000009210 & $1.90 \mathrm{E}-10$ & $1.98 \mathrm{E}-10$ & ZBTB44 & $9.59 \mathrm{E}-01$ \\
\hline ENSBTAG000000013563 & $2.78 \mathrm{E}-10$ & $2.88 \mathrm{E}-10$ & TSG101 & $9.66 \mathrm{E}-01$ \\
\hline ENSBTAG000000046299 & $1.00 \mathrm{E}-09$ & $1.04 \mathrm{E}-09$ & & 9.65E-01 \\
\hline ENSBTAG000000047914 & $5.71 \mathrm{E}-10$ & $5.91 \mathrm{E}-10$ & CDHR5 & $9.66 \mathrm{E}-01$ \\
\hline ENSBTAG00000019995 & $2.07 \mathrm{E}-10$ & $2.13 \mathrm{E}-10$ & HIKESHI & $9.70 \mathrm{E}-01$ \\
\hline ENSBTAG000000021504 & $1.87 \mathrm{E}-10$ & $1.93 \mathrm{E}-10$ & TIRAP & $9.71 \mathrm{E}-01$ \\
\hline ENSBTAG000000035631 & 1.51E-09 & 1.57E-09 & & 9.63E-01 \\
\hline ENSBTAG000000046130 & 2.09E-09 & $2.16 \mathrm{E}-09$ & OR8D2 & $9.69 \mathrm{E}-01$ \\
\hline ENSBTAG00000022396 & $2.40 \mathrm{E}-10$ & $2.48 \mathrm{E}-10$ & & $9.69 \mathrm{E}-01$ \\
\hline ENSBTAG000000010233 & $6.80 \mathrm{E}-10$ & 7.00E-10 & & $9.72 \mathrm{E}-01$ \\
\hline ENSBTAG000000006804 & $3.86 \mathrm{E}-10$ & $3.96 \mathrm{E}-10$ & KIRREL3 & $9.74 \mathrm{E}-01$ \\
\hline ENSBTAG000000046398 & $3.92 \mathrm{E}-10$ & $4.02 \mathrm{E}-10$ & & $9.76 \mathrm{E}-01$ \\
\hline ENSBTAG000000001660 & $4.03 \mathrm{E}-10$ & $4.11 \mathrm{E}-10$ & SPTY2D1 & $9.80 \mathrm{E}-01$ \\
\hline ENSBTAG000000021517 & $3.96 \mathrm{E}-10$ & $4.04 \mathrm{E}-10$ & TMEM216 & $9.81 \mathrm{E}-01$ \\
\hline ENSBTAG000000047934 & 2.33E-09 & $2.36 \mathrm{E}-09$ & & $9.87 \mathrm{E}-01$ \\
\hline ENSBTAG00000046686 & $9.99 \mathrm{E}-10$ & $1.01 E-09$ & & $9.89 \mathrm{E}-01$ \\
\hline ENSBTAG00000006812 & $2.22 \mathrm{E}-10$ & $2.23 \mathrm{E}-10$ & FAM181B & 9.94E-01 \\
\hline ENSBTAG00000005075 & $6.34 \mathrm{E}-10$ & $6.38 \mathrm{E}-10$ & MRPL49 & $9.93 \mathrm{E}-01$ \\
\hline ENSBTAG00000021999 & $5.78 \mathrm{E}-10$ & $5.81 \mathrm{E}-10$ & CPT1A & $9.95 \mathrm{E}-01$ \\
\hline ENSBTAG00000033464 & 7.03E-10 & 7.06E-10 & SPA17 & $9.95 \mathrm{E}-01$ \\
\hline ENSBTAG00000018431 & 4.69E-10 & $4.71 \mathrm{E}-10$ & NAV2 & $9.96 \mathrm{E}-01$ \\
\hline ENSBTAG00000014300 & $5.07 \mathrm{E}-10$ & $5.07 \mathrm{E}-10$ & SLC6A5 & $1.00 \mathrm{E}+00$ \\
\hline ENSBTAG00000001029 & $1.60 \mathrm{E}-10$ & 1.59E-10 & RASGRP2 & $1.01 E+00$ \\
\hline ENSBTAG00000011019 & $9.76 \mathrm{E}-10$ & $9.67 \mathrm{E}-10$ & & $1.01 E+00$ \\
\hline ENSBTAG00000009410 & $6.60 \mathrm{E}-10$ & $6.54 \mathrm{E}-10$ & TBRG1 & $1.01 E+00$ \\
\hline ENSBTAG00000040103 & $4.81 \mathrm{E}-10$ & $4.76 \mathrm{E}-10$ & SYT8 & $1.01 E+00$ \\
\hline ENSBTAG00000002341 & $3.36 \mathrm{E}-10$ & $3.31 E-10$ & ETS1 & $1.02 \mathrm{E}+00$ \\
\hline ENSBTAG00000006304 & $6.69 \mathrm{E}-10$ & $6.53 \mathrm{E}-10$ & & $1.02 \mathrm{E}+00$ \\
\hline ENSBTAG00000003176 & $3.73 E-10$ & $3.63 \mathrm{E}-10$ & JAM3 & $1.03 E+00$ \\
\hline ENSBTAG00000007622 & $1.01 \mathrm{E}-09$ & $9.80 \mathrm{E}-10$ & CTSD & $1.03 E+00$ \\
\hline ENSBTAG00000038918 & $8.60 \mathrm{E}-10$ & $8.36 \mathrm{E}-10$ & & $1.03 E+00$ \\
\hline ENSBTAG00000012072 & $2.84 \mathrm{E}-10$ & $2.75 \mathrm{E}-10$ & NDUFS8 & $1.03 E+00$ \\
\hline ENSBTAG00000009490 & $3.26 \mathrm{E}-10$ & 3.15E-10 & STX5 & $1.04 \mathrm{E}+00$ \\
\hline ENSBTAG00000016704 & $1.77 \mathrm{E}-10$ & $1.71 \mathrm{E}-10$ & SLC37A2 & $1.04 E+00$ \\
\hline ENSBTAG00000020257 & $1.92 \mathrm{E}-10$ & $1.85 \mathrm{E}-10$ & PTPN5 & $1.04 E+00$ \\
\hline ENSBTAG00000013568 & $4.24 \mathrm{E}-10$ & $4.08 \mathrm{E}-10$ & UEVLD & $1.04 E+00$ \\
\hline ENSBTAG00000011813 & $4.44 \mathrm{E}-10$ & $4.26 \mathrm{E}-10$ & TYR & $1.04 E+00$ \\
\hline ENSBTAG00000006902 & $5.15 \mathrm{E}-10$ & 4.95E-10 & KCNJ5 & $1.04 E+00$ \\
\hline ENSBTAG00000004114 & $1.24 \mathrm{E}-10$ & $1.18 \mathrm{E}-10$ & MAP3K11 & $1.05 E+00$ \\
\hline ENSBTAG00000014105 & $4.46 \mathrm{E}-10$ & $4.21 \mathrm{E}-10$ & SRPRA & $1.06 \mathrm{E}+00$ \\
\hline ENSBTAG00000045971 & $1.03 \mathrm{E}-10$ & $9.76 \mathrm{E}-11$ & & $1.06 \mathrm{E}+00$ \\
\hline ENSBTAG00000037404 & $2.61 \mathrm{E}-10$ & $2.46 \mathrm{E}-10$ & LRFN4 & $1.06 \mathrm{E}+00$ \\
\hline ENSBTAG00000005199 & 1.05E-09 & $9.89 \mathrm{E}-10$ & & $1.06 \mathrm{E}+00$ \\
\hline ENSBTAG00000032557 & $1.76 \mathrm{E}-10$ & $1.66 \mathrm{E}-10$ & TMEM258 & $1.06 \mathrm{E}+00$ \\
\hline ENSBTAG00000047463 & $7.46 \mathrm{E}-10$ & $7.04 \mathrm{E}-10$ & & $1.06 \mathrm{E}+00$ \\
\hline ENSBTAG00000007108 & $5.34 \mathrm{E}-10$ & $5.01 E-10$ & LGALS12 & $1.07 E+00$ \\
\hline ENSBTAG00000015183 & $8.88 \mathrm{E}-10$ & $8.32 \mathrm{E}-10$ & TPCN2 & $1.07 E+00$ \\
\hline ENSBTAG00000022394 & 8.39E-10 & 7.83E-10 & & $1.07 E+00$ \\
\hline ENSBTAG00000019001 & $4.52 \mathrm{E}-10$ & $4.21 \mathrm{E}-10$ & PRDM10 & $1.07 E+00$ \\
\hline ENSBTAG00000022382 & $5.25 \mathrm{E}-10$ & $4.89 \mathrm{E}-10$ & TMEM218 & $1.07 E+00$ \\
\hline
\end{tabular}




\begin{tabular}{|c|c|c|c|c|}
\hline ENSBTAG00000001032 & $1.32 \mathrm{E}-10$ & $1.22 \mathrm{E}-10$ & PYGM & $1.08 \mathrm{E}+00$ \\
\hline ENSBTAG00000033096 & $3.28 \mathrm{E}-10$ & 3.05E-10 & & $1.08 \mathrm{E}+00$ \\
\hline ENSBTAG00000046127 & $1.78 \mathrm{E}-10$ & $1.65 \mathrm{E}-10$ & PAG5 & $1.08 \mathrm{E}+00$ \\
\hline ENSBTAG00000009491 & $3.51 \mathrm{E}-10$ & 3.25E-10 & WDR74 & $1.08 \mathrm{E}+00$ \\
\hline ENSBTAG00000004783 & $2.23 \mathrm{E}-10$ & $2.06 \mathrm{E}-10$ & CORO1B & $1.08 \mathrm{E}+00$ \\
\hline ENSBTAG00000031590 & $2.77 \mathrm{E}-10$ & $2.55 \mathrm{E}-10$ & $\mathrm{SSH} 3$ & $1.09 E+00$ \\
\hline ENSBTAG00000047892 & 7.72E-10 & 7.11E-10 & & $1.09 E+00$ \\
\hline ENSBTAG00000038262 & $6.46 \mathrm{E}-10$ & $5.88 \mathrm{E}-10$ & & $1.10 \mathrm{E}+00$ \\
\hline ENSBTAG00000047020 & $1.62 \mathrm{E}-09$ & 1.47E-09 & & $1.10 \mathrm{E}+00$ \\
\hline ENSBTAG00000048061 & 4.67E-10 & 4.23E-10 & & $1.10 \mathrm{E}+00$ \\
\hline ENSBTAG00000020642 & $2.54 \mathrm{E}-10$ & $2.28 \mathrm{E}-10$ & ZDHHC13 & $1.11 \mathrm{E}+00$ \\
\hline ENSBTAG00000018792 & $1.65 \mathrm{E}-10$ & $1.48 \mathrm{E}-10$ & SLC22A11 & $1.11 \mathrm{E}+00$ \\
\hline ENSBTAG00000009393 & $2.97 \mathrm{E}-10$ & 2.67E-10 & GAL & $1.11 \mathrm{E}+00$ \\
\hline ENSBTAG00000046665 & $1.32 \mathrm{E}-09$ & 1.19E-09 & & $1.11 E+00$ \\
\hline ENSBTAG00000002963 & $4.10 \mathrm{E}-10$ & $3.66 \mathrm{E}-10$ & SAA4 & $1.12 \mathrm{E}+00$ \\
\hline ENSBTAG00000021457 & $4.64 \mathrm{E}-10$ & $4.13 \mathrm{E}-10$ & EFEMP2 & $1.12 \mathrm{E}+00$ \\
\hline ENSBTAG00000022209 & $1.72 \mathrm{E}-10$ & $1.52 \mathrm{E}-10$ & CABP2 & $1.13 E+00$ \\
\hline ENSBTAG00000004829 & $2.63 \mathrm{E}-10$ & $2.29 \mathrm{E}-10$ & YIF1A & $1.15 E+00$ \\
\hline ENSBTAG00000019173 & $2.58 \mathrm{E}-10$ & $2.23 \mathrm{E}-10$ & & $1.16 \mathrm{E}+00$ \\
\hline ENSBTAG00000004266 & $1.99 \mathrm{E}-10$ & $1.72 \mathrm{E}-10$ & DDX25 & $1.16 \mathrm{E}+00$ \\
\hline ENSBTAG00000048137 & $5.54 \mathrm{E}-10$ & $4.78 \mathrm{E}-10$ & & $1.16 \mathrm{E}+00$ \\
\hline ENSBTAG00000047172 & $3.30 \mathrm{E}-10$ & $2.84 \mathrm{E}-10$ & & $1.16 \mathrm{E}+00$ \\
\hline ENSBTAG00000011865 & $2.15 \mathrm{E}-10$ & $1.84 \mathrm{E}-10$ & MTA2 & $1.17 \mathrm{E}+00$ \\
\hline ENSBTAG00000001185 & $2.76 \mathrm{E}-10$ & $2.36 \mathrm{E}-10$ & КMT5B & $1.17 E+00$ \\
\hline ENSBTAG00000015277 & $1.78 \mathrm{E}-10$ & $1.53 \mathrm{E}-10$ & PCF11 & $1.17 \mathrm{E}+00$ \\
\hline ENSBTAG00000037623 & $1.75 \mathrm{E}-09$ & 1.50E-09 & & $1.17 \mathrm{E}+00$ \\
\hline ENSBTAG00000005160 & $1.63 \mathrm{E}-10$ & $1.39 \mathrm{E}-10$ & GTF2H1 & $1.17 \mathrm{E}+00$ \\
\hline ENSBTAG00000000052 & $6.38 \mathrm{E}-10$ & $5.42 \mathrm{E}-10$ & PKNOX2 & $1.18 \mathrm{E}+00$ \\
\hline ENSBTAG00000013066 & $2.16 \mathrm{E}-10$ & $1.83 \mathrm{E}-10$ & IGF2 & $1.18 \mathrm{E}+00$ \\
\hline ENSBTAG00000013214 & $4.30 \mathrm{E}-10$ & $3.64 \mathrm{E}-10$ & & $1.18 \mathrm{E}+00$ \\
\hline ENSBTAG00000003545 & $2.07 \mathrm{E}-10$ & $1.73 \mathrm{E}-10$ & TAF1D & $1.19 E+00$ \\
\hline ENSBTAG00000001474 & $2.81 \mathrm{E}-10$ & $2.36 \mathrm{E}-10$ & NRGN & $1.19 \mathrm{E}+00$ \\
\hline ENSBTAG00000018362 & $3.37 \mathrm{E}-10$ & $2.83 \mathrm{E}-10$ & TMEM109 & $1.19 E+00$ \\
\hline ENSBTAG00000005073 & $3.98 \mathrm{E}-10$ & $3.34 \mathrm{E}-10$ & ZNHIT2 & $1.19 E+00$ \\
\hline ENSBTAG00000001004 & $3.39 \mathrm{E}-10$ & $2.84 \mathrm{E}-10$ & ESAM & $1.19 E+00$ \\
\hline ENSBTAG00000021455 & 4.37E-10 & 3.67E-10 & CFL1 & $1.19 E+00$ \\
\hline ENSBTAG00000047530 & 7.63E-10 & $6.39 \mathrm{E}-10$ & & $1.19 E+00$ \\
\hline ENSBTAG00000033237 & $4.59 \mathrm{E}-10$ & $3.85 \mathrm{E}-10$ & & $1.19 E+00$ \\
\hline ENSBTAG00000003079 & $3.48 \mathrm{E}-10$ & $2.92 \mathrm{E}-10$ & TIGD3 & $1.19 E+00$ \\
\hline ENSBTAG00000047304 & $1.19 \mathrm{E}-09$ & $9.92 \mathrm{E}-10$ & & $1.20 E+00$ \\
\hline ENSBTAG00000000773 & 7.37E-10 & $6.10 \mathrm{E}-10$ & & $1.21 E+00$ \\
\hline ENSBTAG00000011898 & $3.87 \mathrm{E}-10$ & $3.19 \mathrm{E}-10$ & ORAOV1 & $1.21 \mathrm{E}+00$ \\
\hline ENSBTAG00000038347 & $2.33 \mathrm{E}-10$ & $1.91 \mathrm{E}-10$ & SLC17A6 & $1.22 \mathrm{E}+00$ \\
\hline ENSBTAG00000022160 & $2.31 E-10$ & $1.90 \mathrm{E}-10$ & NAP1L4 & $1.21 \mathrm{E}+00$ \\
\hline ENSBTAG00000000292 & $1.83 \mathrm{E}-10$ & $1.50 \mathrm{E}-10$ & TCIRG1 & $1.22 \mathrm{E}+00$ \\
\hline ENSBTAG00000035736 & $5.74 \mathrm{E}-10$ & 4.70E-10 & & $1.22 \mathrm{E}+00$ \\
\hline ENSBTAG00000046202 & $7.21 \mathrm{E}-10$ & $5.88 \mathrm{E}-10$ & & $1.23 E+00$ \\
\hline ENSBTAG00000011186 & $2.83 \mathrm{E}-10$ & $2.31 E-10$ & BEST1 & $1.23 E+00$ \\
\hline ENSBTAG00000033476 & $8.50 \mathrm{E}-10$ & $6.91 \mathrm{E}-10$ & & $1.23 E+00$ \\
\hline ENSBTAG00000003073 & $2.16 \mathrm{E}-10$ & $1.75 \mathrm{E}-10$ & NAA40 & $1.23 \mathrm{E}+00$ \\
\hline ENSBTAG00000040202 & $7.56 \mathrm{E}-10$ & $6.14 \mathrm{E}-10$ & & $1.23 E+00$ \\
\hline ENSBTAG00000014651 & $2.07 \mathrm{E}-10$ & $1.68 \mathrm{E}-10$ & SPATA19 & $1.23 E+00$ \\
\hline ENSBTAG00000010481 & $4.36 \mathrm{E}-10$ & 3.52E-10 & UBXN1 & $1.24 E+00$ \\
\hline ENSBTAG00000000064 & $2.39 \mathrm{E}-10$ & $1.93 \mathrm{E}-10$ & FEN1 & $1.24 \mathrm{E}+00$ \\
\hline ENSBTAG00000040140 & $7.23 \mathrm{E}-10$ & $5.82 \mathrm{E}-10$ & & $1.24 \mathrm{E}+00$ \\
\hline ENSBTAG00000002702 & $4.60 \mathrm{E}-10$ & $3.70 \mathrm{E}-10$ & TSPAN32 & $1.24 \mathrm{E}+00$ \\
\hline ENSBTAG00000022294 & $3.02 \mathrm{E}-10$ & $2.42 \mathrm{E}-10$ & FADS1 & $1.25 \mathrm{E}+00$ \\
\hline ENSBTAG00000011184 & $3.47 \mathrm{E}-10$ & $2.78 \mathrm{E}-10$ & FTH1 & $1.25 \mathrm{E}+00$ \\
\hline ENSBTAG00000003985 & $1.37 \mathrm{E}-10$ & $1.10 \mathrm{E}-10$ & CCDC15 & $1.25 E+00$ \\
\hline ENSBTAG00000022759 & $6.56 \mathrm{E}-10$ & $5.22 \mathrm{E}-10$ & & $1.26 \mathrm{E}+00$ \\
\hline ENSBTAG00000009736 & $2.55 \mathrm{E}-10$ & $2.03 \mathrm{E}-10$ & CDC42BPG & $1.26 \mathrm{E}+00$ \\
\hline ENSBTAG00000011455 & 4.30E-10 & $3.42 \mathrm{E}-10$ & POLA2 & $1.26 \mathrm{E}+00$ \\
\hline ENSBTAG00000011132 & $2.72 \mathrm{E}-10$ & $2.16 \mathrm{E}-10$ & CCDC83 & $1.26 \mathrm{E}+00$ \\
\hline ENSBTAG00000022379 & 4.27E-10 & $3.38 \mathrm{E}-10$ & VSIG2 & $1.26 \mathrm{E}+00$ \\
\hline ENSBTAG00000003077 & $2.27 \mathrm{E}-10$ & $1.79 \mathrm{E}-10$ & DPF2 & $1.27 \mathrm{E}+00$ \\
\hline ENSBTAG00000024769 & $6.80 \mathrm{E}-10$ & $5.34 \mathrm{E}-10$ & & $1.27 E+00$ \\
\hline ENSBTAG00000045915 & $5.25 \mathrm{E}-10$ & 4.13E-10 & SCT & $1.27 \mathrm{E}+00$ \\
\hline ENSBTAG00000016502 & $5.24 \mathrm{E}-10$ & $4.12 \mathrm{E}-10$ & & $1.27 \mathrm{E}+00$ \\
\hline ENSBTAG00000022158 & $2.39 \mathrm{E}-10$ & $1.87 \mathrm{E}-10$ & TNNT3 & $1.28 \mathrm{E}+00$ \\
\hline ENSBTAG00000004081 & $6.57 \mathrm{E}-10$ & $5.16 \mathrm{E}-10$ & FAT3 & $1.27 \mathrm{E}+00$ \\
\hline
\end{tabular}




\begin{tabular}{|c|c|c|c|c|}
\hline ENSBTAG00000031688 & $3.08 \mathrm{E}-10$ & $2.41 E-10$ & & $1.28 \mathrm{E}+00$ \\
\hline ENSBTAG00000014224 & $2.49 \mathrm{E}-10$ & $1.94 \mathrm{E}-10$ & IGSF9B & $1.28 \mathrm{E}+00$ \\
\hline ENSBTAG00000039943 & $3.53 \mathrm{E}-10$ & $2.74 \mathrm{E}-10$ & NARS2 & $1.29 E+00$ \\
\hline ENSBTAG00000047680 & $3.44 \mathrm{E}-10$ & $2.66 \mathrm{E}-10$ & IRF7 & $1.29 \mathrm{E}+00$ \\
\hline ENSBTAG00000000246 & $9.90 \mathrm{E}-10$ & 7.65E-10 & ME3 & $1.29 \mathrm{E}+00$ \\
\hline ENSBTAG00000046359 & $6.83 \mathrm{E}-10$ & $5.24 \mathrm{E}-10$ & PHRF1 & $1.30 \mathrm{E}+00$ \\
\hline ENSBTAG00000046085 & $6.83 \mathrm{E}-10$ & $5.24 \mathrm{E}-10$ & & $1.30 \mathrm{E}+00$ \\
\hline ENSBTAG00000012010 & $2.86 \mathrm{E}-10$ & 2.19E-10 & PANX1 & $1.31 E+00$ \\
\hline ENSBTAG00000048202 & 2.77E-10 & $2.09 \mathrm{E}-10$ & & $1.32 \mathrm{E}+00$ \\
\hline ENSBTAG00000047840 & 4.03E-10 & $3.05 \mathrm{E}-10$ & & $1.32 \mathrm{E}+00$ \\
\hline ENSBTAG00000016168 & $4.91 \mathrm{E}-10$ & $3.70 \mathrm{E}-10$ & APLP2 & $1.33 \mathrm{E}+00$ \\
\hline ENSBTAG00000011872 & $1.54 \mathrm{E}-10$ & $1.14 \mathrm{E}-10$ & EML3 & $1.35 \mathrm{E}+00$ \\
\hline ENSBTAG00000008683 & $1.33 \mathrm{E}-10$ & 9.87E-11 & LDHA & $1.35 \mathrm{E}+00$ \\
\hline ENSBTAG00000009124 & 8.13E-10 & $6.00 \mathrm{E}-10$ & FEZ1 & $1.35 \mathrm{E}+00$ \\
\hline ENSBTAG00000018364 & $2.84 \mathrm{E}-10$ & $2.09 \mathrm{E}-10$ & TMEM132A & $1.36 \mathrm{E}+00$ \\
\hline ENSBTAG00000047110 & $1.84 \mathrm{E}-10$ & $1.35 \mathrm{E}-10$ & & $1.36 \mathrm{E}+00$ \\
\hline ENSBTAG00000047151 & $1.03 \mathrm{E}-09$ & $7.56 \mathrm{E}-10$ & & $1.37 \mathrm{E}+00$ \\
\hline ENSBTAG00000007397 & $8.58 \mathrm{E}-10$ & $6.27 \mathrm{E}-10$ & FOLH1 & $1.37 E+00$ \\
\hline ENSBTAG00000017514 & $1.65 \mathrm{E}-10$ & $1.20 \mathrm{E}-10$ & CCND1 & $1.37 \mathrm{E}+00$ \\
\hline ENSBTAG00000001037 & $2.00 \mathrm{E}-10$ & $1.46 \mathrm{E}-10$ & MAP4K2 & $1.37 \mathrm{E}+00$ \\
\hline ENSBTAG00000010478 & $2.57 \mathrm{E}-10$ & $1.87 \mathrm{E}-10$ & AIP & $1.38 \mathrm{E}+00$ \\
\hline ENSBTAG00000005069 & $4.57 \mathrm{E}-10$ & $3.32 \mathrm{E}-10$ & TM7SF2 & $1.38 \mathrm{E}+00$ \\
\hline ENSBTAG00000003759 & $1.22 \mathrm{E}-10$ & $8.79 \mathrm{E}-11$ & PCNX3 & $1.39 E+00$ \\
\hline ENSBTAG00000004355 & $1.58 \mathrm{E}-10$ & $1.14 \mathrm{E}-10$ & $\mathrm{ROBO} 3$ & $1.39 E+00$ \\
\hline ENSBTAG00000005772 & 2.97E-10 & $2.14 \mathrm{E}-10$ & SPDYC & $1.39 \mathrm{E}+00$ \\
\hline ENSBTAG00000040090 & $5.94 \mathrm{E}-10$ & 4.27E-10 & & $1.39 \mathrm{E}+00$ \\
\hline ENSBTAG00000039652 & $4.71 \mathrm{E}-10$ & $3.39 \mathrm{E}-10$ & PTGDR2 & $1.39 E+00$ \\
\hline ENSBTAG00000004348 & $4.05 \mathrm{E}-10$ & $2.91 \mathrm{E}-10$ & MRPL21 & $1.39 E+00$ \\
\hline ENSBTAG00000047730 & $4.20 \mathrm{E}-10$ & 3.02E-10 & & $1.39 E+00$ \\
\hline ENSBTAG00000009997 & $3.64 \mathrm{E}-10$ & $2.62 \mathrm{E}-10$ & ANO1 & $1.39 E+00$ \\
\hline ENSBTAG00000004342 & $2.23 \mathrm{E}-10$ & $1.60 \mathrm{E}-10$ & CCDC87 & $1.39 E+00$ \\
\hline ENSBTAG00000046247 & $5.32 \mathrm{E}-10$ & $3.82 \mathrm{E}-10$ & & $1.39 \mathrm{E}+00$ \\
\hline ENSBTAG00000007827 & $1.45 \mathrm{E}-10$ & $1.04 \mathrm{E}-10$ & RCOR2 & $1.39 E+00$ \\
\hline ENSBTAG00000046745 & $1.24 \mathrm{E}-09$ & 8.87E-10 & & $1.40 E+00$ \\
\hline ENSBTAG00000034891 & $4.87 \mathrm{E}-10$ & $3.45 \mathrm{E}-10$ & & $1.41 \mathrm{E}+00$ \\
\hline ENSBTAG00000015266 & $1.38 \mathrm{E}-10$ & $9.75 \mathrm{E}-11$ & SDHAF2 & $1.41 E+00$ \\
\hline ENSBTAG00000000103 & $2.71 \mathrm{E}-10$ & $1.92 \mathrm{E}-10$ & & $1.41 E+00$ \\
\hline ENSBTAG00000008274 & $6.55 \mathrm{E}-10$ & $4.62 \mathrm{E}-10$ & MUC5B & $1.42 \mathrm{E}+00$ \\
\hline ENSBTAG00000018366 & $3.63 \mathrm{E}-10$ & $2.56 \mathrm{E}-10$ & SLC15A3 & $1.42 \mathrm{E}+00$ \\
\hline ENSBTAG00000023301 & $1.23 \mathrm{E}-09$ & 8.65E-10 & & $1.42 \mathrm{E}+00$ \\
\hline ENSBTAG00000046875 & $2.56 \mathrm{E}-10$ & $1.79 \mathrm{E}-10$ & AP5B1 & $1.43 \mathrm{E}+00$ \\
\hline ENSBTAG00000001777 & $3.46 \mathrm{E}-10$ & $2.42 \mathrm{E}-10$ & & $1.43 E+00$ \\
\hline ENSBTAG00000008237 & $4.25 \mathrm{E}-10$ & $2.97 \mathrm{E}-10$ & TOLLIP & $1.43 E+00$ \\
\hline ENSBTAG00000044073 & $6.36 \mathrm{E}-10$ & 4.44E-10 & CD248 & $1.43 \mathrm{E}+00$ \\
\hline ENSBTAG00000015263 & $1.93 \mathrm{E}-10$ & $1.34 \mathrm{E}-10$ & CPSF7 & $1.44 \mathrm{E}+00$ \\
\hline ENSBTAG00000000423 & $3.36 \mathrm{E}-10$ & $2.34 \mathrm{E}-10$ & RBM4B & $1.44 \mathrm{E}+00$ \\
\hline ENSBTAG00000010447 & $3.48 \mathrm{E}-10$ & $2.42 \mathrm{E}-10$ & LSP1 & $1.44 \mathrm{E}+00$ \\
\hline ENSBTAG00000015511 & $3.19 \mathrm{E}-10$ & $2.22 \mathrm{E}-10$ & FADS3 & $1.44 \mathrm{E}+00$ \\
\hline ENSBTAG00000026768 & $3.71 \mathrm{E}-10$ & $2.56 \mathrm{E}-10$ & $\mathrm{TH}$ & $1.45 E+00$ \\
\hline ENSBTAG00000004786 & $1.84 \mathrm{E}-10$ & $1.26 \mathrm{E}-10$ & TMEM134 & $1.46 \mathrm{E}+00$ \\
\hline ENSBTAG00000030999 & $6.50 \mathrm{E}-10$ & 4.47E-10 & DUSP8 & $1.45 \mathrm{E}+00$ \\
\hline ENSBTAG00000002508 & $3.45 \mathrm{E}-10$ & 2.37E-10 & GNG3 & $1.46 \mathrm{E}+00$ \\
\hline ENSBTAG00000010032 & $6.06 \mathrm{E}-10$ & 4.16E-10 & NTM & $1.46 \mathrm{E}+00$ \\
\hline ENSBTAG00000003552 & $1.86 \mathrm{E}-10$ & $1.28 \mathrm{E}-10$ & MED17 & $1.45 \mathrm{E}+00$ \\
\hline ENSBTAG00000001657 & $1.29 \mathrm{E}-10$ & $8.79 \mathrm{E}-11$ & PICALM & $1.46 \mathrm{E}+00$ \\
\hline ENSBTAG00000015505 & $4.53 \mathrm{E}-10$ & $3.10 \mathrm{E}-10$ & FADS2 & $1.46 \mathrm{E}+00$ \\
\hline ENSBTAG00000015499 & $1.66 \mathrm{E}-10$ & 1.13E-10 & VPS51 & $1.47 \mathrm{E}+00$ \\
\hline ENSBTAG00000007202 & $2.22 \mathrm{E}-10$ & $1.50 \mathrm{E}-10$ & EI24 & $1.48 \mathrm{E}+00$ \\
\hline ENSBTAG00000010859 & $1.76 \mathrm{E}-10$ & $1.19 \mathrm{E}-10$ & HYLS1 & $1.48 \mathrm{E}+00$ \\
\hline ENSBTAG00000017446 & $2.70 \mathrm{E}-10$ & $1.83 \mathrm{E}-10$ & E2F8 & $1.48 \mathrm{E}+00$ \\
\hline ENSBTAG00000046870 & $2.41 \mathrm{E}-10$ & $1.62 \mathrm{E}-10$ & & $1.49 E+00$ \\
\hline ENSBTAG00000047557 & $1.08 \mathrm{E}-09$ & 7.27E-10 & & $1.48 \mathrm{E}+00$ \\
\hline ENSBTAG00000003967 & $1.68 \mathrm{E}-10$ & $1.13 \mathrm{E}-10$ & OTUB1 & $1.48 \mathrm{E}+00$ \\
\hline ENSBTAG00000016470 & $6.96 \mathrm{E}-10$ & 4.65E-10 & NADSYN1 & $1.50 E+00$ \\
\hline ENSBTAG00000020242 & 7.14E-10 & 4.74E-10 & CRLF2 & $1.51 E+00$ \\
\hline ENSBTAG00000009484 & $2.12 \mathrm{E}-10$ & $1.40 \mathrm{E}-10$ & TAF6L & $1.51 E+00$ \\
\hline ENSBTAG00000013093 & $3.30 \mathrm{E}-10$ & $2.18 \mathrm{E}-10$ & ALDH3B1 & $1.51 E+00$ \\
\hline ENSBTAG00000017181 & $1.80 \mathrm{E}-10$ & $1.20 \mathrm{E}-10$ & MACROD1 & $1.50 \mathrm{E}+00$ \\
\hline ENSBTAG00000037645 & $6.75 \mathrm{E}-10$ & $4.31 E-10$ & & $1.57 \mathrm{E}+00$ \\
\hline ENSBTAG00000010304 & $1.82 \mathrm{E}-10$ & $1.16 \mathrm{E}-10$ & CHKA & $1.57 E+00$ \\
\hline
\end{tabular}




\begin{tabular}{|c|c|c|c|c|}
\hline ENSBTAG000000019204 & $3.21 \mathrm{E}-10$ & $2.05 \mathrm{E}-10$ & RTN3 & $1.57 \mathrm{E}+00$ \\
\hline ENSBTAG00000013240 & 4.50E-10 & $2.86 \mathrm{E}-10$ & SLC3A2 & $1.57 \mathrm{E}+00$ \\
\hline ENSBTAG000000046740 & $1.42 \mathrm{E}-10$ & $8.91 \mathrm{E}-11$ & & $1.59 E+00$ \\
\hline ENSBTAG000000038054 & $1.78 \mathrm{E}-10$ & $1.12 \mathrm{E}-10$ & & $1.59 E+00$ \\
\hline ENSBTAG000000040128 & $4.34 \mathrm{E}-11$ & $2.73 \mathrm{E}-11$ & FZD4 & $1.59 \mathrm{E}+00$ \\
\hline ENSBTAG000000005382 & $2.56 \mathrm{E}-10$ & $1.61 \mathrm{E}-10$ & CCDC85B & $1.59 \mathrm{E}+00$ \\
\hline ENSBTAG00000013003 & $1.41 \mathrm{E}-10$ & $8.88 \mathrm{E}-11$ & INS & $1.59 \mathrm{E}+00$ \\
\hline ENSBTAG000000001229 & $3.21 \mathrm{E}-10$ & $2.02 \mathrm{E}-10$ & SLC25A22 & $1.59 E+00$ \\
\hline ENSBTAG00000046789 & $3.33 \mathrm{E}-10$ & 2.09E-10 & & $1.59 \mathrm{E}+00$ \\
\hline ENSBTAG000000008477 & $3.28 \mathrm{E}-10$ & $2.06 \mathrm{E}-10$ & & $1.59 E+00$ \\
\hline ENSBTAG000000003712 & $2.01 \mathrm{E}-10$ & $1.26 \mathrm{E}-10$ & FAM89B & $1.59 E+00$ \\
\hline ENSBTAG000000021569 & $2.06 \mathrm{E}-10$ & $1.30 \mathrm{E}-10$ & DDB1 & $1.58 \mathrm{E}+00$ \\
\hline ENSBTAG00000033030 & $5.27 \mathrm{E}-10$ & $3.31 \mathrm{E}-10$ & HIST1H4J & $1.59 \mathrm{E}+00$ \\
\hline ENSBTAG000000014830 & $2.88 \mathrm{E}-10$ & $1.81 \mathrm{E}-10$ & PPP1R32 & $1.59 E+00$ \\
\hline ENSBTAG000000046728 & $1.20 \mathrm{E}-10$ & 7.57E-11 & GPR152 & $1.59 \mathrm{E}+00$ \\
\hline ENSBTAG000000022395 & $9.90 \mathrm{E}-10$ & $6.21 \mathrm{E}-10$ & & $1.59 E+00$ \\
\hline ENSBTAG000000037467 & 1.33E-09 & 8.35E-10 & & $1.60 \mathrm{E}+00$ \\
\hline ENSBTAG000000007439 & $7.62 \mathrm{E}-10$ & $4.75 \mathrm{E}-10$ & TENM4 & $1.60 E+00$ \\
\hline ENSBTAG000000019689 & $3.90 \mathrm{E}-10$ & $2.43 \mathrm{E}-10$ & & $1.61 E+00$ \\
\hline ENSBTAG000000047419 & $5.52 \mathrm{E}-10$ & $3.40 \mathrm{E}-10$ & & $1.62 E+00$ \\
\hline ENSBTAG000000010433 & $8.48 \mathrm{E}-10$ & $5.19 \mathrm{E}-10$ & & $1.63 E+00$ \\
\hline ENSBTAG000000010777 & $8.71 \mathrm{E}-10$ & 5.33E-10 & CARS & $1.63 E+00$ \\
\hline ENSBTAG000000013468 & $5.83 \mathrm{E}-10$ & 3.55E-10 & AHNAK & $1.64 E+00$ \\
\hline ENSBTAG000000013419 & $1.68 \mathrm{E}-10$ & $1.02 \mathrm{E}-10$ & HTATIP2 & $1.65 E+00$ \\
\hline ENSBTAG000000038655 & $1.96 \mathrm{E}-10$ & 1.19E-10 & & $1.65 E+00$ \\
\hline ENSBTAG000000005929 & $5.44 \mathrm{E}-10$ & $3.29 \mathrm{E}-10$ & SPTBN2 & $1.65 E+00$ \\
\hline ENSBTAG000000010482 & $1.65 \mathrm{E}-10$ & $9.95 \mathrm{E}-11$ & PITPNM1 & $1.65 E+00$ \\
\hline ENSBTAG000000026016 & $7.21 \mathrm{E}-10$ & 4.36E-10 & & $1.65 \mathrm{E}+00$ \\
\hline ENSBTAG00000012742 & $2.80 \mathrm{E}-10$ & $1.68 \mathrm{E}-10$ & SLC22A18 & $1.66 \mathrm{E}+00$ \\
\hline ENSBTAG000000008623 & $2.44 \mathrm{E}-10$ & $1.46 \mathrm{E}-10$ & FGF3 & $1.67 E+00$ \\
\hline ENSBTAG00000017805 & $9.74 \mathrm{E}-10$ & $5.82 \mathrm{E}-10$ & CRACR2B & $1.67 E+00$ \\
\hline ENSBTAG000000001193 & $1.55 \mathrm{E}-10$ & $9.24 \mathrm{E}-11$ & UNC93B1 & $1.68 \mathrm{E}+00$ \\
\hline ENSBTAG00000024611 & $4.98 \mathrm{E}-10$ & $2.96 \mathrm{E}-10$ & & $1.68 \mathrm{E}+00$ \\
\hline ENSBTAG000000017285 & $3.66 \mathrm{E}-10$ & 2.17E-10 & FGF19 & $1.69 E+00$ \\
\hline ENSBTAG000000022188 & $5.68 \mathrm{E}-10$ & $3.35 \mathrm{E}-10$ & PPFIA1 & $1.69 \mathrm{E}+00$ \\
\hline ENSBTAG00000000460 & 4.19E-10 & 2.47E-10 & SYTL2 & $1.69 E+00$ \\
\hline ENSBTAG00000001225 & $1.34 \mathrm{E}-10$ & $7.85 \mathrm{E}-11$ & RBM14 & $1.70 \mathrm{E}+00$ \\
\hline ENSBTAG00000010986 & $3.45 \mathrm{E}-10$ & $2.01 E-10$ & & $1.72 \mathrm{E}+00$ \\
\hline ENSBTAG00000038506 & $6.81 \mathrm{E}-10$ & $3.96 \mathrm{E}-10$ & & $1.72 \mathrm{E}+00$ \\
\hline ENSBTAG00000039153 & $5.43 \mathrm{E}-10$ & $3.15 \mathrm{E}-10$ & RPS6KA4 & $1.72 \mathrm{E}+00$ \\
\hline ENSBTAG00000013884 & $1.15 \mathrm{E}-10$ & $6.68 \mathrm{E}-11$ & SIPA1 & $1.72 \mathrm{E}+00$ \\
\hline ENSBTAG00000003550 & $1.92 \mathrm{E}-10$ & $1.10 \mathrm{E}-10$ & C11orf54 & $1.75 \mathrm{E}+00$ \\
\hline ENSBTAG00000038345 & $9.58 \mathrm{E}-10$ & $5.46 \mathrm{E}-10$ & OR8B8 & $1.75 \mathrm{E}+00$ \\
\hline ENSBTAG00000015745 & $2.35 \mathrm{E}-10$ & $1.34 \mathrm{E}-10$ & RAB3IL1 & $1.75 \mathrm{E}+00$ \\
\hline ENSBTAG00000021568 & $3.08 \mathrm{E}-10$ & $1.75 \mathrm{E}-10$ & VWCE & $1.76 \mathrm{E}+00$ \\
\hline ENSBTAG00000009488 & $1.45 \mathrm{E}-10$ & $8.21 \mathrm{E}-11$ & NXF1 & $1.76 \mathrm{E}+00$ \\
\hline ENSBTAG00000021654 & 4.74E-10 & $2.66 \mathrm{E}-10$ & GAS2 & $1.78 \mathrm{E}+00$ \\
\hline ENSBTAG00000039045 & $2.21 \mathrm{E}-10$ & $1.24 \mathrm{E}-10$ & & $1.79 E+00$ \\
\hline ENSBTAG00000002733 & $1.53 \mathrm{E}-10$ & $8.56 \mathrm{E}-11$ & DBX1 & $1.79 \mathrm{E}+00$ \\
\hline ENSBTAG00000023300 & $5.49 \mathrm{E}-10$ & $3.06 \mathrm{E}-10$ & & $1.79 \mathrm{E}+00$ \\
\hline ENSBTAG00000003171 & $8.64 \mathrm{E}-10$ & $4.79 \mathrm{E}-10$ & SHANK2 & $1.80 \mathrm{E}+00$ \\
\hline ENSBTAG00000009554 & $1.32 \mathrm{E}-10$ & 7.34E-11 & EHBP1L1 & $1.80 \mathrm{E}+00$ \\
\hline ENSBTAG00000013421 & $2.79 \mathrm{E}-10$ & $1.54 \mathrm{E}-10$ & PRMT3 & $1.81 \mathrm{E}+00$ \\
\hline ENSBTAG00000016950 & $2.84 \mathrm{E}-10$ & $1.56 \mathrm{E}-10$ & B3GAT3 & $1.82 \mathrm{E}+00$ \\
\hline ENSBTAG00000014584 & 4.73E-10 & $2.57 \mathrm{E}-10$ & & $1.84 \mathrm{E}+00$ \\
\hline ENSBTAG00000014122 & $6.44 \mathrm{E}-10$ & $3.51 \mathrm{E}-10$ & FOXRED1 & $1.84 \mathrm{E}+00$ \\
\hline ENSBTAG00000010455 & $1.24 \mathrm{E}-10$ & $6.70 \mathrm{E}-11$ & INTS5 & $1.85 E+00$ \\
\hline ENSBTAG00000018201 & $1.91 \mathrm{E}-10$ & $1.03 \mathrm{E}-10$ & TKFC & $1.85 \mathrm{E}+00$ \\
\hline ENSBTAG00000017951 & $3.22 \mathrm{E}-10$ & $1.74 \mathrm{E}-10$ & & $1.85 \mathrm{E}+00$ \\
\hline ENSBTAG00000009486 & $2.61 \mathrm{E}-10$ & $1.40 \mathrm{E}-10$ & TMEM179B & $1.86 \mathrm{E}+00$ \\
\hline ENSBTAG00000033477 & $5.91 \mathrm{E}-10$ & $3.18 \mathrm{E}-10$ & & $1.86 \mathrm{E}+00$ \\
\hline ENSBTAG00000013730 & $1.60 \mathrm{E}-10$ & $8.62 E-11$ & CD5 & $1.85 \mathrm{E}+00$ \\
\hline ENSBTAG00000018540 & $7.86 \mathrm{E}-10$ & $4.22 \mathrm{E}-10$ & NOX4 & $1.86 \mathrm{E}+00$ \\
\hline ENSBTAG00000048133 & $5.66 \mathrm{E}-10$ & 3.03E-10 & & $1.87 E+00$ \\
\hline ENSBTAG00000020769 & $7.25 \mathrm{E}-10$ & 3.87E-10 & OPCML & $1.87 \mathrm{E}+00$ \\
\hline ENSBTAG00000006990 & 1.60E-10 & $8.50 \mathrm{E}-11$ & MYRF & $1.89 \mathrm{E}+00$ \\
\hline ENSBTAG00000009197 & $4.74 \mathrm{E}-10$ & $2.51 \mathrm{E}-10$ & TRIM43 & $1.89 \mathrm{E}+00$ \\
\hline ENSBTAG00000048098 & 4.53E-10 & $2.40 \mathrm{E}-10$ & PKP3 & $1.89 \mathrm{E}+00$ \\
\hline ENSBTAG00000034244 & $1.70 \mathrm{E}-10$ & $8.99 \mathrm{E}-11$ & & $1.89 \mathrm{E}+00$ \\
\hline ENSBTAG00000006199 & 4.64E-10 & $2.43 \mathrm{E}-10$ & DRAP1 & $1.91 E+00$ \\
\hline
\end{tabular}




\begin{tabular}{|c|c|c|c|c|}
\hline ENSBTAG00000040250 & $1.06 \mathrm{E}-09$ & $5.55 \mathrm{E}-10$ & & $1.92 \mathrm{E}+00$ \\
\hline ENSBTAG00000002503 & $3.31 \mathrm{E}-10$ & $1.72 \mathrm{E}-10$ & BSCL2 & $1.93 E+00$ \\
\hline ENSBTAG00000046485 & $5.41 \mathrm{E}-10$ & $2.81 \mathrm{E}-10$ & RAB1B & $1.93 \mathrm{E}+00$ \\
\hline ENSBTAG00000022147 & $8.87 \mathrm{E}-10$ & $4.53 \mathrm{E}-10$ & EPS8L2 & $1.96 \mathrm{E}+00$ \\
\hline ENSBTAG00000021215 & $7.86 \mathrm{E}-10$ & $3.95 \mathrm{E}-10$ & VWA5A & $1.99 \mathrm{E}+00$ \\
\hline ENSBTAG00000022378 & $4.42 \mathrm{E}-10$ & $2.22 \mathrm{E}-10$ & & $1.99 E+00$ \\
\hline ENSBTAG00000009483 & $1.26 \mathrm{E}-10$ & 6.33E-11 & POLR2G & $1.98 \mathrm{E}+00$ \\
\hline ENSBTAG00000034536 & $5.12 \mathrm{E}-10$ & $2.55 E-10$ & & $2.01 E+00$ \\
\hline ENSBTAG00000019394 & $2.91 \mathrm{E}-10$ & $1.43 \mathrm{E}-10$ & ANO5 & $2.04 E+00$ \\
\hline ENSBTAG00000011843 & $2.65 \mathrm{E}-10$ & $1.29 \mathrm{E}-10$ & EEF1G & $2.05 E+00$ \\
\hline ENSBTAG00000010348 & $9.04 \mathrm{E}-10$ & $4.37 \mathrm{E}-10$ & PDDC1 & $2.07 \mathrm{E}+00$ \\
\hline ENSBTAG00000021604 & $3.63 \mathrm{E}-10$ & $1.76 \mathrm{E}-10$ & TMEM138 & $2.06 \mathrm{E}+00$ \\
\hline ENSBTAG00000004785 & $1.88 \mathrm{E}-10$ & $8.86 \mathrm{E}-11$ & CABP4 & $2.12 \mathrm{E}+00$ \\
\hline ENSBTAG00000024814 & $6.14 \mathrm{E}-10$ & $2.89 \mathrm{E}-10$ & B4GALNT4 & $2.12 \mathrm{E}+00$ \\
\hline ENSBTAG00000010336 & $8.96 \mathrm{E}-10$ & $4.21 \mathrm{E}-10$ & TALDO1 & $2.13 E+00$ \\
\hline ENSBTAG00000048190 & $7.83 \mathrm{E}-10$ & $3.67 E-10$ & & $2.13 E+00$ \\
\hline ENSBTAG00000019334 & $4.96 \mathrm{E}-10$ & $2.32 \mathrm{E}-10$ & $\mathrm{KLC2}$ & $2.14 \mathrm{E}+00$ \\
\hline ENSBTAG00000018202 & $2.74 \mathrm{E}-10$ & $1.27 \mathrm{E}-10$ & CYB561A3 & $2.16 \mathrm{E}+00$ \\
\hline ENSBTAG00000000008 & $6.00 \mathrm{E}-10$ & $2.76 \mathrm{E}-10$ & KCNJ1 & $2.18 \mathrm{E}+00$ \\
\hline ENSBTAG00000017582 & $5.26 \mathrm{E}-10$ & $2.41 \mathrm{E}-10$ & CHEK1 & $2.18 \mathrm{E}+00$ \\
\hline ENSBTAG00000016506 & $5.35 \mathrm{E}-10$ & $2.43 \mathrm{E}-10$ & ST3GAL4 & $2.20 \mathrm{E}+00$ \\
\hline ENSBTAG00000034940 & $2.85 \mathrm{E}-10$ & $1.28 \mathrm{E}-10$ & & $2.22 \mathrm{E}+00$ \\
\hline ENSBTAG00000039129 & $7.38 \mathrm{E}-10$ & $3.32 \mathrm{E}-10$ & & $2.22 \mathrm{E}+00$ \\
\hline ENSBTAG00000005930 & $3.13 \mathrm{E}-10$ & $1.39 \mathrm{E}-10$ & & $2.25 E+00$ \\
\hline ENSBTAG00000002417 & $5.15 \mathrm{E}-10$ & $2.28 \mathrm{E}-10$ & RAB30 & $2.26 \mathrm{E}+00$ \\
\hline ENSBTAG00000009026 & $6.98 \mathrm{E}-10$ & $3.08 \mathrm{E}-10$ & PACS1 & $2.27 E+00$ \\
\hline ENSBTAG00000032089 & $2.82 \mathrm{E}-10$ & $1.24 \mathrm{E}-10$ & CDC42EP2 & $2.28 \mathrm{E}+00$ \\
\hline ENSBTAG00000016857 & $3.42 \mathrm{E}-10$ & $1.49 \mathrm{E}-10$ & ADAMTS15 & $2.29 E+00$ \\
\hline ENSBTAG00000013942 & $3.69 \mathrm{E}-10$ & $1.57 \mathrm{E}-10$ & DAGLA & $2.35 \mathrm{E}+00$ \\
\hline ENSBTAG00000010484 & $9.27 \mathrm{E}-11$ & $3.89 \mathrm{E}-11$ & LRRN4CL & $2.38 \mathrm{E}+00$ \\
\hline ENSBTAG00000046110 & $1.07 \mathrm{E}-10$ & 4.47E-11 & CLCF1 & $2.38 \mathrm{E}+00$ \\
\hline ENSBTAG00000004782 & $3.42 \mathrm{E}-10$ & $1.43 \mathrm{E}-10$ & PTPRCAP & $2.39 E+00$ \\
\hline ENSBTAG00000010802 & $2.65 \mathrm{E}-10$ & $1.11 \mathrm{E}-10$ & KCNK7 & $2.39 E+00$ \\
\hline ENSBTAG00000010465 & $2.18 \mathrm{E}-10$ & $9.14 \mathrm{E}-11$ & & $2.38 \mathrm{E}+00$ \\
\hline ENSBTAG00000012141 & $3.33 \mathrm{E}-10$ & $1.40 \mathrm{E}-10$ & RAD9A & $2.38 \mathrm{E}+00$ \\
\hline ENSBTAG00000045834 & $3.87 \mathrm{E}-10$ & $1.62 \mathrm{E}-10$ & RASSF7 & $2.39 E+00$ \\
\hline ENSBTAG00000021454 & $3.02 \mathrm{E}-10$ & $1.27 \mathrm{E}-10$ & SNX32 & $2.38 \mathrm{E}+00$ \\
\hline ENSBTAG00000026769 & $5.26 \mathrm{E}-10$ & $2.20 \mathrm{E}-10$ & ASCL2 & $2.39 E+00$ \\
\hline ENSBTAG00000031271 & $5.67 \mathrm{E}-10$ & $2.37 \mathrm{E}-10$ & MRGPRG & $2.39 E+00$ \\
\hline ENSBTAG00000001228 & $5.86 \mathrm{E}-10$ & $2.45 \mathrm{E}-10$ & CEND1 & $2.39 E+00$ \\
\hline ENSBTAG00000032918 & $4.90 \mathrm{E}-10$ & $2.05 \mathrm{E}-10$ & SCGB1A1 & $2.39 \mathrm{E}+00$ \\
\hline ENSBTAG00000040239 & $1.06 \mathrm{E}-09$ & $4.44 \mathrm{E}-10$ & & $2.39 E+00$ \\
\hline ENSBTAG00000006634 & $1.06 \mathrm{E}-09$ & $4.41 \mathrm{E}-10$ & OR4D5 & $2.39 E+00$ \\
\hline ENSBTAG00000006910 & $1.89 \mathrm{E}-10$ & $7.73 \mathrm{E}-11$ & ASRGL1 & $2.44 \mathrm{E}+00$ \\
\hline ENSBTAG00000011636 & $6.04 \mathrm{E}-10$ & $2.46 \mathrm{E}-10$ & DDIAS & $2.45 E+00$ \\
\hline ENSBTAG00000020268 & $3.79 \mathrm{E}-10$ & $1.55 \mathrm{E}-10$ & RSF1 & $2.45 \mathrm{E}+00$ \\
\hline ENSBTAG00000008283 & $3.93 \mathrm{E}-10$ & $1.60 \mathrm{E}-10$ & FLI1 & $2.46 \mathrm{E}+00$ \\
\hline ENSBTAG00000019700 & $5.27 \mathrm{E}-10$ & $2.14 \mathrm{E}-10$ & PC & $2.46 \mathrm{E}+00$ \\
\hline ENSBTAG00000040190 & $4.94 \mathrm{E}-10$ & $2.00 \mathrm{E}-10$ & CCDC88B & $2.47 \mathrm{E}+00$ \\
\hline ENSBTAG00000019634 & 4.34E-10 & $1.75 \mathrm{E}-10$ & PIDD1 & $2.48 \mathrm{E}+00$ \\
\hline ENSBTAG00000003094 & $4.42 \mathrm{E}-10$ & $1.78 \mathrm{E}-10$ & SART1 & $2.48 \mathrm{E}+00$ \\
\hline ENSBTAG00000047495 & $3.62 \mathrm{E}-10$ & $1.46 \mathrm{E}-10$ & $\mathrm{CD} 81$ & $2.48 \mathrm{E}+00$ \\
\hline ENSBTAG00000039517 & $7.64 \mathrm{E}-10$ & $2.95 \mathrm{E}-10$ & & $2.59 E+00$ \\
\hline ENSBTAG00000016956 & $4.02 \mathrm{E}-10$ & $1.55 \mathrm{E}-10$ & GANAB & $2.59 \mathrm{E}+00$ \\
\hline ENSBTAG00000006194 & $3.47 \mathrm{E}-10$ & $1.33 \mathrm{E}-10$ & FOSL1 & $2.61 E+00$ \\
\hline ENSBTAG00000009409 & $9.32 \mathrm{E}-10$ & $3.57 E-10$ & PANX3 & $2.61 E+00$ \\
\hline ENSBTAG00000011248 & $6.79 \mathrm{E}-10$ & $2.54 \mathrm{E}-10$ & MSANTD2 & $2.67 E+00$ \\
\hline ENSBTAG00000017861 & $4.89 \mathrm{E}-10$ & $1.83 \mathrm{E}-10$ & BRSK2 & $2.67 E+00$ \\
\hline ENSBTAG00000010191 & $4.72 \mathrm{E}-10$ & $1.75 \mathrm{E}-10$ & PAK1 & $2.70 \mathrm{E}+00$ \\
\hline ENSBTAG00000002772 & $5.55 \mathrm{E}-10$ & $2.03 E-10$ & GAL3ST3 & $2.73 E+00$ \\
\hline ENSBTAG00000012512 & $1.86 \mathrm{E}-10$ & $6.70 \mathrm{E}-11$ & GPR137 & $2.78 \mathrm{E}+00$ \\
\hline ENSBTAG00000012024 & $2.34 \mathrm{E}-10$ & $8.42 \mathrm{E}-11$ & SLC29A2 & $2.78 \mathrm{E}+00$ \\
\hline ENSBTAG00000019579 & $7.20 \mathrm{E}-10$ & $2.58 \mathrm{E}-10$ & & $2.79 E+00$ \\
\hline ENSBTAG00000047697 & $6.34 \mathrm{E}-10$ & $2.26 \mathrm{E}-10$ & & $2.80 E+00$ \\
\hline ENSBTAG00000016647 & $1.23 \mathrm{E}-09$ & $4.41 \mathrm{E}-10$ & OR10D3 & $2.80 \mathrm{E}+00$ \\
\hline ENSBTAG00000046662 & $3.10 \mathrm{E}-10$ & $1.09 \mathrm{E}-10$ & TRPM5 & $2.84 \mathrm{E}+00$ \\
\hline ENSBTAG00000015940 & $2.64 \mathrm{E}-10$ & $9.06 \mathrm{E}-11$ & & $2.91 E+00$ \\
\hline ENSBTAG00000017681 & $5.10 \mathrm{E}-10$ & $1.75 \mathrm{E}-10$ & RIN1 & $2.91 E+00$ \\
\hline ENSBTAG00000016774 & $9.46 \mathrm{E}-10$ & $3.23 \mathrm{E}-10$ & & $2.93 \mathrm{E}+00$ \\
\hline ENSBTAG00000047716 & $9.70 \mathrm{E}-10$ & $3.31 \mathrm{E}-10$ & & $2.93 E+00$ \\
\hline
\end{tabular}




\begin{tabular}{|c|c|c|c|c|}
\hline ENSBTAG00000015185 & 5.07E-10 & $1.73 \mathrm{E}-10$ & MRGPRF & $2.93 \mathrm{E}+00$ \\
\hline ENSBTAG00000015905 & $5.16 \mathrm{E}-10$ & $1.73 \mathrm{E}-10$ & ARHGAP32 & $2.98 \mathrm{E}+00$ \\
\hline ENSBTAG00000003369 & $4.27 \mathrm{E}-10$ & $1.41 \mathrm{E}-10$ & SYT12 & $3.03 E+00$ \\
\hline ENSBTAG00000019947 & $1.64 \mathrm{E}-10$ & $5.37 E-11$ & SYT7 & $3.04 E+00$ \\
\hline ENSBTAG00000020775 & 4.67E-10 & $1.53 \mathrm{E}-10$ & FIBP & $3.05 E+00$ \\
\hline ENSBTAG00000011846 & $2.70 \mathrm{E}-10$ & $8.77 E-11$ & & $3.08 \mathrm{E}+00$ \\
\hline ENSBTAG00000002121 & $1.82 \mathrm{E}-10$ & $5.72 \mathrm{E}-11$ & CDK2AP2 & $3.17 \mathrm{E}+00$ \\
\hline ENSBTAG00000019696 & $1.06 \mathrm{E}-10$ & $3.35 E-11$ & RCE1 & $3.17 \mathrm{E}+00$ \\
\hline ENSBTAG00000048192 & $3.31 \mathrm{E}-10$ & $1.04 \mathrm{E}-10$ & & $3.18 \mathrm{E}+00$ \\
\hline ENSBTAG00000019226 & $9.58 \mathrm{E}-11$ & $3.02 \mathrm{E}-11$ & & $3.16 \mathrm{E}+00$ \\
\hline ENSBTAG00000033375 & $2.53 \mathrm{E}-10$ & 7.97E-11 & PATE2 & $3.18 \mathrm{E}+00$ \\
\hline ENSBTAG00000046150 & $2.83 \mathrm{E}-10$ & $8.89 \mathrm{E}-11$ & & $3.18 \mathrm{E}+00$ \\
\hline ENSBTAG00000014761 & $1.89 \mathrm{E}-10$ & 5.93E-11 & & $3.18 \mathrm{E}+00$ \\
\hline ENSBTAG00000047267 & 1.06E-09 & $3.32 \mathrm{E}-10$ & & $3.20 E+00$ \\
\hline ENSBTAG00000036277 & $4.03 \mathrm{E}-10$ & $1.24 \mathrm{E}-10$ & & $3.25 E+00$ \\
\hline ENSBTAG00000013644 & $3.38 \mathrm{E}-10$ & $1.04 \mathrm{E}-10$ & SLC22A8 & $3.25 E+00$ \\
\hline ENSBTAG00000002922 & $7.69 \mathrm{E}-10$ & $2.34 \mathrm{E}-10$ & GAB4 & $3.28 \mathrm{E}+00$ \\
\hline ENSBTAG00000014720 & $8.33 \mathrm{E}-10$ & $2.48 \mathrm{E}-10$ & CATSPER1 & $3.36 \mathrm{E}+00$ \\
\hline ENSBTAG00000022384 & 2.24E-09 & $6.66 \mathrm{E}-10$ & & $3.36 \mathrm{E}+00$ \\
\hline ENSBTAG00000007061 & $1.57 \mathrm{E}-10$ & 4.66E-11 & PPP2R5B & $3.37 E+00$ \\
\hline ENSBTAG00000020186 & $4.53 \mathrm{E}-10$ & $1.34 \mathrm{E}-10$ & EIF1AD & $3.38 \mathrm{E}+00$ \\
\hline ENSBTAG00000004343 & $3.07 \mathrm{E}-10$ & $9.09 E-11$ & CCS & $3.38 \mathrm{E}+00$ \\
\hline ENSBTAG00000011100 & $1.96 \mathrm{E}-10$ & $5.70 \mathrm{E}-11$ & CTSC & $3.43 E+00$ \\
\hline ENSBTAG00000013076 & $5.50 \mathrm{E}-10$ & $1.60 \mathrm{E}-10$ & SNX19 & $3.43 E+00$ \\
\hline ENSBTAG00000006071 & $4.80 \mathrm{E}-10$ & $1.37 \mathrm{E}-10$ & CTTN & $3.50 E+00$ \\
\hline ENSBTAG00000033731 & $6.02 \mathrm{E}-10$ & $1.72 \mathrm{E}-10$ & PRCP & $3.50 \mathrm{E}+00$ \\
\hline ENSBTAG00000019569 & $8.83 \mathrm{E}-10$ & $2.50 \mathrm{E}-10$ & CD151 & $3.53 E+00$ \\
\hline ENSBTAG00000032178 & $2.34 \mathrm{E}-10$ & $6.53 E-11$ & MAJIN & $3.57 E+00$ \\
\hline ENSBTAG00000007058 & 3.77E-10 & $1.03 \mathrm{E}-10$ & & $3.65 \mathrm{E}+00$ \\
\hline ENSBTAG00000011249 & $7.50 \mathrm{E}-10$ & $2.02 \mathrm{E}-10$ & MOB2 & $3.71 E+00$ \\
\hline ENSBTAG00000046013 & $9.10 \mathrm{E}-10$ & $2.44 \mathrm{E}-10$ & & $3.73 E+00$ \\
\hline ENSBTAG00000046360 & $1.92 \mathrm{E}-09$ & $5.12 \mathrm{E}-10$ & & $3.75 E+00$ \\
\hline ENSBTAG00000012944 & $6.38 \mathrm{E}-10$ & $1.68 \mathrm{E}-10$ & USP35 & $3.80 \mathrm{E}+00$ \\
\hline ENSBTAG00000011666 & $4.72 \mathrm{E}-10$ & $1.22 \mathrm{E}-10$ & THRSP & $3.87 E+00$ \\
\hline ENSBTAG00000002778 & $2.35 \mathrm{E}-10$ & $6.06 E-11$ & SF3B2 & $3.87 E+00$ \\
\hline ENSBTAG00000002095 & $6.51 \mathrm{E}-11$ & $1.64 \mathrm{E}-11$ & MEN1 & $3.95 E+00$ \\
\hline ENSBTAG00000002249 & $2.02 \mathrm{E}-10$ & $5.09 E-11$ & NAALADL1 & $3.96 \mathrm{E}+00$ \\
\hline ENSBTAG00000022238 & $1.82 \mathrm{E}-10$ & $4.59 \mathrm{E}-11$ & B4GAT1 & $3.96 \mathrm{E}+00$ \\
\hline ENSBTAG00000034850 & $3.15 \mathrm{E}-10$ & $7.91 \mathrm{E}-11$ & & $3.97 E+00$ \\
\hline ENSBTAG00000046587 & $6.19 \mathrm{E}-10$ & $1.55 \mathrm{E}-10$ & & $3.99 \mathrm{E}+00$ \\
\hline ENSBTAG00000020643 & $7.39 \mathrm{E}-10$ & $1.85 \mathrm{E}-10$ & BARX2 & $3.99 E+00$ \\
\hline ENSBTAG00000007479 & $5.16 \mathrm{E}-10$ & $1.29 \mathrm{E}-10$ & CCDC90B & $4.00 \mathrm{E}+00$ \\
\hline ENSBTAG00000047713 & $5.58 \mathrm{E}-10$ & $1.36 \mathrm{E}-10$ & LMNTD2 & $4.10 \mathrm{E}+00$ \\
\hline ENSBTAG00000017298 & $5.81 \mathrm{E}-10$ & $1.41 \mathrm{E}-10$ & AAMDC & $4.12 \mathrm{E}+00$ \\
\hline ENSBTAG00000031061 & $1.42 \mathrm{E}-09$ & $3.46 \mathrm{E}-10$ & POLR2L & $4.10 \mathrm{E}+00$ \\
\hline ENSBTAG00000018192 & $5.84 \mathrm{E}-10$ & $1.40 \mathrm{E}-10$ & ALG8 & $4.17 \mathrm{E}+00$ \\
\hline ENSBTAG00000047996 & $7.54 \mathrm{E}-10$ & $1.80 \mathrm{E}-10$ & IFITM10 & $4.19 E+00$ \\
\hline ENSBTAG00000020773 & $5.08 \mathrm{E}-10$ & $1.21 \mathrm{E}-10$ & CTSW & $4.19 E+00$ \\
\hline ENSBTAG00000010505 & $6.87 \mathrm{E}-10$ & $1.64 \mathrm{E}-10$ & INTS4 & $4.19 E+00$ \\
\hline ENSBTAG00000003330 & $2.81 \mathrm{E}-10$ & $6.71 \mathrm{E}-11$ & ATL3 & $4.18 \mathrm{E}+00$ \\
\hline ENSBTAG00000008644 & $1.90 \mathrm{E}-10$ & $4.48 \mathrm{E}-11$ & KCNK4 & $4.23 E+00$ \\
\hline ENSBTAG00000009032 & $8.99 \mathrm{E}-10$ & $2.11 \mathrm{E}-10$ & TMEM80 & $4.26 \mathrm{E}+00$ \\
\hline ENSBTAG00000047141 & $9.26 \mathrm{E}-10$ & 2.17E-10 & & $4.27 E+00$ \\
\hline ENSBTAG00000046038 & $5.86 \mathrm{E}-10$ & $1.36 \mathrm{E}-10$ & & $4.31 E+00$ \\
\hline ENSBTAG00000018367 & $5.80 \mathrm{E}-10$ & $1.34 \mathrm{E}-10$ & CD6 & $4.32 E+00$ \\
\hline ENSBTAG00000009289 & $3.36 \mathrm{E}-10$ & 7.69E-11 & MS4A8 & $4.37 E+00$ \\
\hline ENSBTAG00000047204 & $9.96 \mathrm{E}-10$ & $2.26 \mathrm{E}-10$ & & $4.41 E+00$ \\
\hline ENSBTAG00000037485 & $8.76 \mathrm{E}-10$ & $1.99 \mathrm{E}-10$ & & $4.40 \mathrm{E}+00$ \\
\hline ENSBTAG00000033502 & $8.44 \mathrm{E}-10$ & $1.87 \mathrm{E}-10$ & TMEM225 & $4.51 E+00$ \\
\hline ENSBTAG00000004116 & $3.89 \mathrm{E}-10$ & $8.55 E-11$ & & $4.55 E+00$ \\
\hline ENSBTAG00000005482 & $4.03 E-10$ & $8.74 \mathrm{E}-11$ & & $4.60 \mathrm{E}+00$ \\
\hline ENSBTAG00000013606 & $1.69 \mathrm{E}-10$ & $3.55 E-11$ & FKBP2 & $4.76 \mathrm{E}+00$ \\
\hline ENSBTAG00000005832 & $1.92 \mathrm{E}-10$ & 4.03E-11 & GRK2 & $4.76 \mathrm{E}+00$ \\
\hline ENSBTAG00000009915 & $9.19 E-10$ & $1.89 \mathrm{E}-10$ & $\mathrm{AP} 2 \mathrm{~A} 2$ & $4.86 \mathrm{E}+00$ \\
\hline ENSBTAG00000011149 & $3.45 \mathrm{E}-10$ & $6.93 E-11$ & SIGIRR & $4.97 E+00$ \\
\hline ENSBTAG00000026102 & 5.19E-10 & $1.03 \mathrm{E}-10$ & & $5.04 E+00$ \\
\hline ENSBTAG00000005144 & $2.23 \mathrm{E}-10$ & $4.32 \mathrm{E}-11$ & PNPLA2 & $5.16 E+00$ \\
\hline ENSBTAG00000040070 & $7.64 \mathrm{E}-10$ & $1.47 \mathrm{E}-10$ & & $5.19 E+00$ \\
\hline ENSBTAG00000013642 & $2.09 \mathrm{E}-10$ & $3.95 E-11$ & SLC22A6 & $5.28 \mathrm{E}+00$ \\
\hline ENSBTAG00000000451 & 7.13E-10 & $1.33 \mathrm{E}-10$ & & $5.36 \mathrm{E}+00$ \\
\hline
\end{tabular}




\begin{tabular}{|c|c|c|c|c|}
\hline ENSBTAG00000004392 & $2.83 \mathrm{E}-10$ & $5.23 \mathrm{E}-11$ & C11orf24 & $5.40 \mathrm{E}+00$ \\
\hline ENSBTAG00000009175 & $6.36 \mathrm{E}-10$ & $1.16 \mathrm{E}-10$ & RPS6KB2 & $5.48 \mathrm{E}+00$ \\
\hline ENSBTAG00000008645 & $1.77 \mathrm{E}-10$ & 3.19E-11 & ESRRA & $5.55 E+00$ \\
\hline ENSBTAG00000016551 & $2.19 \mathrm{E}-10$ & $3.93 \mathrm{E}-11$ & & $5.56 \mathrm{E}+00$ \\
\hline ENSBTAG00000001188 & $2.87 \mathrm{E}-10$ & 5.15E-11 & ROM1 & $5.56 \mathrm{E}+00$ \\
\hline ENSBTAG00000002238 & $1.47 \mathrm{E}-10$ & $2.64 \mathrm{E}-11$ & ARL2 & $5.55 E+00$ \\
\hline ENSBTAG00000013133 & $3.11 \mathrm{E}-10$ & 5.59E-11 & TBC1D10C & $5.56 \mathrm{E}+00$ \\
\hline ENSBTAG00000020596 & 4.36E-10 & $7.81 \mathrm{E}-11$ & TMEM126B & $5.57 E+00$ \\
\hline ENSBTAG00000001077 & 4.79E-10 & $8.54 \mathrm{E}-11$ & OSBPL5 & $5.60 \mathrm{E}+00$ \\
\hline ENSBTAG00000048240 & $1.24 \mathrm{E}-09$ & $2.21 \mathrm{E}-10$ & & $5.60 \mathrm{E}+00$ \\
\hline ENSBTAG00000002510 & $3.89 \mathrm{E}-10$ & $6.82 \mathrm{E}-11$ & & $5.70 \mathrm{E}+00$ \\
\hline ENSBTAG00000026971 & $2.98 \mathrm{E}-10$ & $4.91 \mathrm{E}-11$ & & $6.06 \mathrm{E}+00$ \\
\hline ENSBTAG00000008064 & $5.73 \mathrm{E}-10$ & $9.39 \mathrm{E}-11$ & VPS37C & $6.10 \mathrm{E}+00$ \\
\hline ENSBTAG00000003093 & $4.76 \mathrm{E}-10$ & $7.75 \mathrm{E}-11$ & TSGA10IP & $6.14 \mathrm{E}+00$ \\
\hline ENSBTAG00000033480 & $6.97 \mathrm{E}-10$ & $1.09 \mathrm{E}-10$ & OR8B4 & $6.39 \mathrm{E}+00$ \\
\hline ENSBTAG00000017689 & $7.49 \mathrm{E}-10$ & 1.17E-10 & BRMS1 & $6.40 E+00$ \\
\hline ENSBTAG00000005112 & 1.40E-09 & 2.19E-10 & & $6.41 E+00$ \\
\hline ENSBTAG00000019930 & $3.93 \mathrm{E}-10$ & $6.04 \mathrm{E}-11$ & CLNS1A & $6.49 \mathrm{E}+00$ \\
\hline ENSBTAG00000033384 & 7.60E-10 & $1.11 \mathrm{E}-10$ & ACRV1 & $6.84 \mathrm{E}+00$ \\
\hline ENSBTAG00000019928 & $4.90 \mathrm{E}-10$ & $7.12 \mathrm{E}-11$ & AQP11 & $6.87 E+00$ \\
\hline ENSBTAG00000033508 & $8.00 \mathrm{E}-10$ & $1.11 \mathrm{E}-10$ & OR10G6 & $7.21 E+00$ \\
\hline ENSBTAG00000011816 & $6.56 \mathrm{E}-10$ & $8.81 E-11$ & TBX10 & $7.44 E+00$ \\
\hline ENSBTAG00000038312 & $2.64 \mathrm{E}-10$ & $3.50 \mathrm{E}-11$ & MS4A18 & $7.53 \mathrm{E}+00$ \\
\hline ENSBTAG00000013895 & $3.71 \mathrm{E}-10$ & 4.77E-11 & RELA & $7.75 \mathrm{E}+00$ \\
\hline ENSBTAG00000000518 & 7.15E-10 & $9.15 \mathrm{E}-11$ & & $7.81 E+00$ \\
\hline ENSBTAG00000047793 & $2.25 \mathrm{E}-10$ & $2.83 \mathrm{E}-11$ & TSSC4 & $7.93 E+00$ \\
\hline ENSBTAG00000020600 & $6.47 \mathrm{E}-10$ & 7.67E-11 & RHOD & $8.42 E+00$ \\
\hline ENSBTAG00000020147 & $1.82 \mathrm{E}-10$ & $2.15 \mathrm{E}-11$ & & $8.43 E+00$ \\
\hline ENSBTAG00000000455 & 1.67E-10 & $1.91 \mathrm{E}-11$ & CREBZF & $8.69 E+00$ \\
\hline ENSBTAG00000008648 & $2.90 \mathrm{E}-10$ & $3.32 \mathrm{E}-11$ & PRDX5 & $8.72 E+00$ \\
\hline ENSBTAG00000019369 & $9.45 \mathrm{E}-10$ & $1.04 \mathrm{E}-10$ & TSPAN4 & $9.08 \mathrm{E}+00$ \\
\hline ENSBTAG00000021776 & 3.17E-10 & $3.46 \mathrm{E}-11$ & NDUFV1 & $9.13 E+00$ \\
\hline ENSBTAG00000015006 & $5.65 \mathrm{E}-10$ & $6.16 \mathrm{E}-11$ & MRPL11 & $9.16 \mathrm{E}+00$ \\
\hline ENSBTAG00000018188 & $6.94 \mathrm{E}-10$ & $7.56 \mathrm{E}-11$ & & $9.17 E+00$ \\
\hline ENSBTAG00000009899 & 1.40E-09 & $1.41 \mathrm{E}-10$ & CHID1 & $9.95 E+00$ \\
\hline ENSBTAG00000037570 & $5.10 \mathrm{E}-10$ & $5.01 \mathrm{E}-11$ & & $1.02 \mathrm{E}+01$ \\
\hline ENSBTAG00000002243 & $6.51 \mathrm{E}-10$ & $6.28 \mathrm{E}-11$ & SNX15 & $1.04 \mathrm{E}+01$ \\
\hline ENSBTAG00000048018 & $9.30 \mathrm{E}-10$ & 8.94E-11 & & $1.04 \mathrm{E}+01$ \\
\hline ENSBTAG00000021777 & $5.09 \mathrm{E}-10$ & $4.73 \mathrm{E}-11$ & $\mathrm{DOC} 2 \mathrm{G}$ & $1.07 \mathrm{E}+01$ \\
\hline ENSBTAG00000014925 & $2.35 \mathrm{E}-10$ & $2.11 \mathrm{E}-11$ & NPAS4 & $1.11 E+01$ \\
\hline ENSBTAG00000017690 & $3.38 \mathrm{E}-10$ & $3.03 \mathrm{E}-11$ & CARNS1 & $1.11 E+01$ \\
\hline ENSBTAG00000022348 & 3.97E-10 & $3.48 \mathrm{E}-11$ & PAG3 & $1.14 \mathrm{E}+01$ \\
\hline ENSBTAG00000021097 & $3.96 \mathrm{E}-10$ & $3.32 \mathrm{E}-11$ & PELI3 & $1.19 \mathrm{E}+01$ \\
\hline ENSBTAG00000048043 & 1.33E-09 & $1.10 \mathrm{E}-10$ & & $1.21 \mathrm{E}+01$ \\
\hline ENSBTAG00000048263 & $5.97 \mathrm{E}-10$ & $4.83 \mathrm{E}-11$ & LRRC56 & $1.23 \mathrm{E}+01$ \\
\hline ENSBTAG00000010411 & $2.28 \mathrm{E}-10$ & $1.76 \mathrm{E}-11$ & ADAMTS8 & $1.29 \mathrm{E}+01$ \\
\hline ENSBTAG00000007849 & $1.24 \mathrm{E}-09$ & $8.61 E-11$ & & $1.44 \mathrm{E}+01$ \\
\hline ENSBTAG00000044032 & 4.49E-10 & $3.05 E-11$ & KDM2A & $1.47 E+01$ \\
\hline ENSBTAG00000022242 & $5.26 \mathrm{E}-10$ & 3.56E-11 & DPP3 & $1.47 \mathrm{E}+01$ \\
\hline ENSBTAG00000022244 & $4.11 \mathrm{E}-10$ & 2.67E-11 & ACTN3 & $1.53 \mathrm{E}+01$ \\
\hline ENSBTAG00000012200 & $3.24 \mathrm{E}-10$ & $1.89 \mathrm{E}-11$ & ANKRD13D & $1.70 \mathrm{E}+01$ \\
\hline ENSBTAG00000015663 & $7.35 \mathrm{E}-10$ & $4.11 \mathrm{E}-11$ & STIP1 & $1.78 \mathrm{E}+01$ \\
\hline ENSBTAG00000005756 & $3.78 \mathrm{E}-10$ & $1.70 \mathrm{E}-11$ & ZDHHC24 & $2.21 E+01$ \\
\hline ENSBTAG00000045862 & $4.81 \mathrm{E}-10$ & $1.92 \mathrm{E}-11$ & & $2.49 E+01$ \\
\hline ENSBTAG00000012510 & 4.35E-10 & $1.64 \mathrm{E}-11$ & PLCB3 & $2.64 \mathrm{E}+01$ \\
\hline ENSBTAG00000045670 & 1.40E-09 & $5.21 \mathrm{E}-11$ & & $2.68 \mathrm{E}+01$ \\
\hline ENSBTAG00000001391 & $1.91 \mathrm{E}-10$ & $5.58 \mathrm{E}-12$ & & $3.36 \mathrm{E}+01$ \\
\hline ENSBTAG00000010970 & $5.11 \mathrm{E}-10$ & 0 & & $5.11 E+03$ \\
\hline ENSBTAG00000039757 & $3.13 \mathrm{E}-10$ & 0 & & $3.13 E+03$ \\
\hline ENSBTAG00000039676 & $5.55 \mathrm{E}-10$ & 0 & & $5.55 E+03$ \\
\hline ENSBTAG00000038182 & $1.46 \mathrm{E}-09$ & 0 & & $1.46 \mathrm{E}+04$ \\
\hline ENSBTAG00000009487 & 7.15E-11 & 0 & TMEM223 & $7.16 \mathrm{E}+02$ \\
\hline ENSBTAG00000011579 & $8.93 \mathrm{E}-11$ & 0 & CHRM1 & $8.94 \mathrm{E}+02$ \\
\hline ENSBTAG00000019208 & $5.52 E-11$ & 0 & C11orf95 & $5.53 E+02$ \\
\hline ENSBTAG00000006386 & $1.77 \mathrm{E}-10$ & 0 & & $1.77 \mathrm{E}+03$ \\
\hline ENSBTAG00000016560 & $2.25 \mathrm{E}-10$ & 0 & DNAJC4 & $2.25 E+03$ \\
\hline ENSBTAG00000016559 & $9.43 \mathrm{E}-11$ & 0 & NUDT22 & $9.44 \mathrm{E}+02$ \\
\hline ENSBTAG00000016555 & $3.24 \mathrm{E}-10$ & 0 & TRPT1 & $3.24 \mathrm{E}+03$ \\
\hline ENSBTAG00000047567 & $2.24 \mathrm{E}-10$ & 0 & VEGFB & $2.24 \mathrm{E}+03$ \\
\hline ENSBTAG00000047265 & 0 & 0 & & $1.00 E+00$ \\
\hline
\end{tabular}




\begin{tabular}{|c|c|c|c|c|}
\hline ENSBTAG00000012511 & $2.65 \mathrm{E}-10$ & 0 & BAD & $2.65 E+03$ \\
\hline ENSBTAG00000008646 & $1.84 \mathrm{E}-10$ & 0 & & $1.84 \mathrm{E}+03$ \\
\hline ENSBTAG00000007063 & 0 & 0 & GPHA2 & $1.00 \mathrm{E}+00$ \\
\hline ENSBTAG00000002248 & $3.32 \mathrm{E}-10$ & 0 & SAC3D1 & $3.33 E+03$ \\
\hline ENSBTAG00000015479 & $1.84 \mathrm{E}-10$ & 0 & ZFPL1 & $1.84 \mathrm{E}+03$ \\
\hline ENSBTAG00000010994 & $4.28 \mathrm{E}-10$ & 0 & CTSF & $4.28 \mathrm{E}+03$ \\
\hline ENSBTAG00000022246 & $2.21 \mathrm{E}-10$ & 0 & C11orf86 & $2.21 E+03$ \\
\hline ENSBTAG00000012146 & 5.57E-11 & 0 & & $5.58 \mathrm{E}+02$ \\
\hline ENSBTAG00000003548 & $6.90 \mathrm{E}-10$ & 0 & GSTP1 & $6.90 E+03$ \\
\hline ENSBTAG00000011809 & $2.91 \mathrm{E}-10$ & 0 & NUDT8 & $2.91 E+03$ \\
\hline ENSBTAG00000012563 & $5.64 \mathrm{E}-10$ & 0 & & $5.64 \mathrm{E}+03$ \\
\hline ENSBTAG00000000776 & 4.33E-10 & 0 & & $4.33 \mathrm{E}+03$ \\
\hline ENSBTAG00000048222 & $1.75 \mathrm{E}-10$ & 0 & & $1.75 \mathrm{E}+03$ \\
\hline ENSBTAG00000046459 & 1.15E-09 & 0 & & $1.15 E+04$ \\
\hline ENSBTAG00000039964 & $4.32 \mathrm{E}-10$ & 0 & & $4.32 E+03$ \\
\hline ENSBTAG00000046086 & 0 & 0 & & $1.00 \mathrm{E}+00$ \\
\hline ENSBTAG00000047310 & $9.74 \mathrm{E}-10$ & 0 & & $9.74 E+03$ \\
\hline ENSBTAG00000014305 & $1.73 \mathrm{E}-10$ & 0 & & $1.73 \mathrm{E}+03$ \\
\hline ENSBTAG00000022376 & $1.88 \mathrm{E}-09$ & 0 & & $1.88 \mathrm{E}+04$ \\
\hline ENSBTAG00000047786 & $3.80 \mathrm{E}-11$ & 0 & LRRC10B & $3.81 E+02$ \\
\hline ENSBTAG00000048264 & $4.02 \mathrm{E}-11$ & 0 & FLRT1 & $4.03 E+02$ \\
\hline ENSBTAG00000034845 & $2.15 \mathrm{E}-10$ & 0 & & $2.15 E+03$ \\
\hline ENSBTAG00000000457 & $3.48 \mathrm{E}-10$ & 0 & CCDC89 & $3.48 \mathrm{E}+03$ \\
\hline ENSBTAG00000022427 & 1.10E-10 & 0 & & $1.10 E+03$ \\
\hline ENSBTAG00000045601 & $3.14 \mathrm{E}-10$ & 0 & KCTD21 & $3.15 E+03$ \\
\hline ENSBTAG00000011668 & $5.79 \mathrm{E}-10$ & 0 & KCTD14 & $5.79 E+03$ \\
\hline ENSBTAG00000007230 & $6.82 \mathrm{E}-11$ & 0 & FANCF & $6.83 E+02$ \\
\hline ENSBTAG00000008684 & 0 & 0 & & $1.00 \mathrm{E}+00$ \\
\hline ENSBTAG00000000185 & $1.87 \mathrm{E}-10$ & $8.61 \mathrm{E}-11$ & SLC6A14 & $2.18 \mathrm{E}+00$ \\
\hline ENSBTAG00000005219 & $2.47 \mathrm{E}-10$ & $2.75 \mathrm{E}-10$ & KLHL4 & 8.99E-01 \\
\hline ENSBTAG00000047332 & 8.13E-10 & $1.27 \mathrm{E}-10$ & & $6.40 E+00$ \\
\hline ENSBTAG00000000501 & $2.62 \mathrm{E}-10$ & $1.12 \mathrm{E}-10$ & KLHL13 & $2.34 \mathrm{E}+00$ \\
\hline ENSBTAG00000046978 & $2.81 \mathrm{E}-10$ & $2.66 \mathrm{E}-10$ & & $1.06 \mathrm{E}+00$ \\
\hline ENSBTAG00000018697 & $1.75 \mathrm{E}-10$ & $1.91 \mathrm{E}-10$ & WDR44 & 9.17E-01 \\
\hline ENSBTAG00000006439 & $2.76 \mathrm{E}-10$ & $2.42 \mathrm{E}-10$ & DOCK11 & $1.14 \mathrm{E}+00$ \\
\hline ENSBTAG00000019338 & $1.42 \mathrm{E}-10$ & 7.38E-11 & IL13RA1 & $1.92 \mathrm{E}+00$ \\
\hline ENSBTAG00000035018 & $1.56 \mathrm{E}-10$ & $1.97 \mathrm{E}-10$ & & 7.94E-01 \\
\hline ENSBTAG00000002615 & $1.34 \mathrm{E}-10$ & 8.16E-11 & LONRF3 & $1.64 E+00$ \\
\hline ENSBTAG00000046288 & $1.91 \mathrm{E}-10$ & $2.00 \mathrm{E}-10$ & & 9.54E-01 \\
\hline ENSBTAG00000010782 & $2.01 \mathrm{E}-10$ & $1.16 \mathrm{E}-10$ & KIAA1210 & $1.73 \mathrm{E}+00$ \\
\hline ENSBTAG00000019552 & $1.76 \mathrm{E}-10$ & $2.96 \mathrm{E}-10$ & PGRMC1 & 5.95E-01 \\
\hline ENSBTAG00000019553 & $2.92 \mathrm{E}-10$ & $1.54 \mathrm{E}-10$ & & $1.90 \mathrm{E}+00$ \\
\hline ENSBTAG00000047757 & $1.75 \mathrm{E}-10$ & 1.47E-10 & & $1.19 E+00$ \\
\hline ENSBTAG00000001007 & $1.43 \mathrm{E}-10$ & $1.18 \mathrm{E}-10$ & SLC25A43 & $1.21 \mathrm{E}+00$ \\
\hline ENSBTAG00000046037 & $2.87 \mathrm{E}-10$ & $9.63 \mathrm{E}-11$ & SLC25A5 & $2.98 \mathrm{E}+00$ \\
\hline ENSBTAG00000047169 & $2.99 \mathrm{E}-10$ & $2.26 \mathrm{E}-10$ & CXorf56 & $1.32 \mathrm{E}+00$ \\
\hline ENSBTAG00000045779 & $2.00 \mathrm{E}-10$ & $1.38 \mathrm{E}-10$ & & $1.45 \mathrm{E}+00$ \\
\hline ENSBTAG00000045744 & $1.22 \mathrm{E}-10$ & $9.79 \mathrm{E}-11$ & NKRF & $1.25 \mathrm{E}+00$ \\
\hline ENSBTAG00000004291 & $2.41 \mathrm{E}-10$ & $1.52 \mathrm{E}-10$ & 06-Sep & $1.58 \mathrm{E}+00$ \\
\hline ENSBTAG00000035615 & $3.86 \mathrm{E}-11$ & $6.08 \mathrm{E}-12$ & & $6.27 E+00$ \\
\hline ENSBTAG00000006033 & $1.82 \mathrm{E}-10$ & $9.80 \mathrm{E}-11$ & NDUFA1 & $1.85 \mathrm{E}+00$ \\
\hline ENSBTAG00000010881 & $1.81 \mathrm{E}-10$ & $1.78 \mathrm{E}-10$ & AKAP14 & $1.02 \mathrm{E}+00$ \\
\hline ENSBTAG00000010884 & $9.30 \mathrm{E}-11$ & $8.46 \mathrm{E}-11$ & NKAP & $1.10 \mathrm{E}+00$ \\
\hline ENSBTAG00000046452 & $2.61 \mathrm{E}-10$ & $2.82 \mathrm{E}-10$ & & $9.25 \mathrm{E}-01$ \\
\hline ENSBTAG00000038497 & $5.39 \mathrm{E}-10$ & $3.94 \mathrm{E}-10$ & & $1.37 \mathrm{E}+00$ \\
\hline ENSBTAG00000048187 & $5.40 \mathrm{E}-10$ & $3.08 \mathrm{E}-10$ & & $1.75 \mathrm{E}+00$ \\
\hline ENSBTAG00000021410 & $1.62 \mathrm{E}-10$ & $1.78 \mathrm{E}-10$ & & $9.10 \mathrm{E}-01$ \\
\hline ENSBTAG00000034992 & $8.34 \mathrm{E}-11$ & $1.97 \mathrm{E}-10$ & MCTS1 & 4.24E-01 \\
\hline ENSBTAG00000018569 & $2.99 \mathrm{E}-10$ & $1.23 \mathrm{E}-10$ & CUL4B & $2.43 E+00$ \\
\hline ENSBTAG00000007399 & $9.64 \mathrm{E}-11$ & $3.73 \mathrm{E}-11$ & LAMP2 & $2.58 \mathrm{E}+00$ \\
\hline ENSBTAG00000008920 & $1.22 \mathrm{E}-10$ & $5.95 \mathrm{E}-11$ & ATP1B4 & $2.05 \mathrm{E}+00$ \\
\hline ENSBTAG00000017187 & $2.29 \mathrm{E}-10$ & $6.70 \mathrm{E}-11$ & TMEM255A & $3.41 E+00$ \\
\hline ENSBTAG00000017184 & $1.72 \mathrm{E}-10$ & $2.71 E-11$ & ZBTB33 & $6.34 \mathrm{E}+00$ \\
\hline ENSBTAG00000011833 & $1.84 \mathrm{E}-10$ & $1.61 \mathrm{E}-10$ & GRIA3 & $1.14 \mathrm{E}+00$ \\
\hline ENSBTAG00000008388 & $1.33 \mathrm{E}-10$ & $1.19 \mathrm{E}-10$ & THOC2 & $1.12 \mathrm{E}+00$ \\
\hline ENSBTAG00000040350 & $1.13 \mathrm{E}-10$ & $1.22 \mathrm{E}-10$ & & $9.24 \mathrm{E}-01$ \\
\hline ENSBTAG00000009121 & $1.24 \mathrm{E}-10$ & 1.39E-10 & STAG2 & 8.92E-01 \\
\hline ENSBTAG00000026067 & $1.13 \mathrm{E}-10$ & $1.52 \mathrm{E}-10$ & SH2D1A & 7.43E-01 \\
\hline ENSBTAG00000018819 & $2.20 \mathrm{E}-10$ & $2.38 \mathrm{E}-10$ & TENM1 & $9.24 \mathrm{E}-01$ \\
\hline ENSBTAG00000046796 & $2.92 \mathrm{E}-10$ & $1.38 \mathrm{E}-10$ & & $2.12 \mathrm{E}+00$ \\
\hline
\end{tabular}




\begin{tabular}{|c|c|c|c|c|}
\hline ENSBTAG00000008763 & $1.71 \mathrm{E}-10$ & 5.39E-11 & PRR32 & $3.17 E+00$ \\
\hline ENSBTAG00000048188 & $3.43 \mathrm{E}-10$ & $3.38 \mathrm{E}-10$ & & $1.01 E+00$ \\
\hline ENSBTAG00000002292 & 8.19E-11 & 1.10E-11 & SMARCA1 & $7.39 E+00$ \\
\hline ENSBTAG00000018247 & $2.13 \mathrm{E}-10$ & $3.18 \mathrm{E}-11$ & OCRL & $6.67 E+00$ \\
\hline ENSBTAG00000019993 & 4.23E-11 & 3.55E-11 & APLN & $1.19 E+00$ \\
\hline ENSBTAG00000010345 & 4.74E-11 & $1.51 \mathrm{E}-10$ & XPNPEP2 & $3.14 \mathrm{E}-01$ \\
\hline ENSBTAG00000002041 & $4.56 \mathrm{E}-11$ & $4.31 \mathrm{E}-11$ & SASH3 & $1.06 \mathrm{E}+00$ \\
\hline ENSBTAG00000015354 & $2.06 \mathrm{E}-10$ & 1.07E-10 & ZDHHC9 & $1.93 \mathrm{E}+00$ \\
\hline ENSBTAG00000013671 & $1.62 \mathrm{E}-10$ & 5.57E-11 & & $2.91 \mathrm{E}+00$ \\
\hline ENSBTAG00000004962 & $9.51 \mathrm{E}-11$ & $9.64 \mathrm{E}-11$ & BCORL1 & $9.86 \mathrm{E}-01$ \\
\hline ENSBTAG00000019061 & $7.52 \mathrm{E}-11$ & $7.58 \mathrm{E}-11$ & ELF4 & 9.92E-01 \\
\hline ENSBTAG00000006838 & $2.56 \mathrm{E}-10$ & $2.92 \mathrm{E}-10$ & AIFM1 & 8.76E-01 \\
\hline ENSBTAG00000034712 & $5.51 \mathrm{E}-11$ & $5.95 \mathrm{E}-11$ & RAB33A & $9.26 \mathrm{E}-01$ \\
\hline ENSBTAG00000019885 & $9.82 \mathrm{E}-11$ & $8.10 \mathrm{E}-11$ & ZNF280C & $1.21 E+00$ \\
\hline ENSBTAG00000016263 & $1.47 \mathrm{E}-10$ & $1.11 \mathrm{E}-10$ & SLC25A14 & $1.32 \mathrm{E}+00$ \\
\hline ENSBTAG00000014771 & $6.34 \mathrm{E}-10$ & $6.68 \mathrm{E}-10$ & RBMX2 & 9.49E-01 \\
\hline ENSBTAG00000010463 & $5.29 \mathrm{E}-10$ & $4.43 \mathrm{E}-10$ & & $1.19 E+00$ \\
\hline ENSBTAG00000021189 & $1.39 \mathrm{E}-10$ & $7.49 \mathrm{E}-11$ & ARHGAP36 & $1.85 \mathrm{E}+00$ \\
\hline ENSBTAG00000046476 & $1.07 \mathrm{E}-10$ & $9.41 \mathrm{E}-11$ & IGSF1 & $1.13 E+00$ \\
\hline ENSBTAG00000007647 & $1.66 \mathrm{E}-10$ & $1.04 \mathrm{E}-10$ & OR13H1 & $1.59 \mathrm{E}+00$ \\
\hline ENSBTAG00000019652 & $1.81 \mathrm{E}-10$ & 2.17E-10 & STK26 & $8.32 \mathrm{E}-01$ \\
\hline ENSBTAG00000007344 & $2.10 \mathrm{E}-10$ & $1.68 \mathrm{E}-10$ & FRMD7 & $1.25 \mathrm{E}+00$ \\
\hline ENSBTAG00000004131 & $1.60 \mathrm{E}-10$ & $1.62 \mathrm{E}-10$ & RAP2C & 9.91E-01 \\
\hline ENSBTAG00000014088 & $8.81 \mathrm{E}-11$ & $1.02 \mathrm{E}-10$ & MBNL3 & 8.64E-01 \\
\hline ENSBTAG00000020644 & $9.37 \mathrm{E}-11$ & 1.59E-10 & GPC4 & 5.89E-01 \\
\hline ENSBTAG00000020406 & $1.57 \mathrm{E}-10$ & $1.38 \mathrm{E}-10$ & GPC3 & $1.14 \mathrm{E}+00$ \\
\hline ENSBTAG00000030507 & $2.12 \mathrm{E}-10$ & $8.89 \mathrm{E}-11$ & & $2.38 \mathrm{E}+00$ \\
\hline ENSBTAG00000036349 & 1.17E-10 & $1.60 \mathrm{E}-10$ & CCDC160 & 7.33E-01 \\
\hline ENSBTAG00000015196 & $2.06 \mathrm{E}-10$ & $1.53 \mathrm{E}-10$ & PHF6 & $1.34 \mathrm{E}+00$ \\
\hline ENSBTAG00000014685 & $1.05 \mathrm{E}-10$ & $1.06 \mathrm{E}-10$ & & 9.91E-01 \\
\hline ENSBTAG00000006293 & $9.82 \mathrm{E}-11$ & $4.78 \mathrm{E}-11$ & PLAC1 & $2.05 E+00$ \\
\hline ENSBTAG00000001886 & $2.73 \mathrm{E}-10$ & $2.86 \mathrm{E}-10$ & FAM122B & $9.54 \mathrm{E}-01$ \\
\hline ENSBTAG00000013922 & $9.96 \mathrm{E}-11$ & $8.36 \mathrm{E}-12$ & MOSPD1 & $1.18 \mathrm{E}+01$ \\
\hline ENSBTAG00000039434 & $4.99 \mathrm{E}-10$ & $3.76 \mathrm{E}-10$ & & $1.33 E+00$ \\
\hline ENSBTAG00000019546 & $8.42 \mathrm{E}-11$ & 1.14E-10 & CT55 & 7.39E-01 \\
\hline ENSBTAG00000022837 & $6.05 \mathrm{E}-10$ & $5.42 \mathrm{E}-10$ & & $1.12 \mathrm{E}+00$ \\
\hline ENSBTAG00000046047 & $3.41 \mathrm{E}-10$ & 3.27E-10 & & $1.04 \mathrm{E}+00$ \\
\hline ENSBTAG00000047954 & $2.06 \mathrm{E}-10$ & $1.70 \mathrm{E}-10$ & & $1.21 \mathrm{E}+00$ \\
\hline ENSBTAG00000018090 & $4.11 \mathrm{E}-11$ & $1.29 \mathrm{E}-11$ & ZNF449 & $3.17 \mathrm{E}+00$ \\
\hline ENSBTAG00000047939 & $4.50 \mathrm{E}-10$ & $4.08 \mathrm{E}-10$ & & $1.10 \mathrm{E}+00$ \\
\hline ENSBTAG00000008993 & $7.60 \mathrm{E}-11$ & $3.68 \mathrm{E}-12$ & INTS6L & $2.01 E+01$ \\
\hline ENSBTAG00000047290 & $2.32 \mathrm{E}-10$ & $2.34 \mathrm{E}-10$ & & $9.93 \mathrm{E}-01$ \\
\hline ENSBTAG00000025297 & $8.04 \mathrm{E}-11$ & $1.35 \mathrm{E}-10$ & MMGT1 & $5.96 \mathrm{E}-01$ \\
\hline ENSBTAG00000009523 & $8.90 \mathrm{E}-11$ & $1.45 \mathrm{E}-10$ & SLC9A6 & $6.14 \mathrm{E}-01$ \\
\hline ENSBTAG00000018513 & $1.46 \mathrm{E}-10$ & $1.48 \mathrm{E}-10$ & FHL1 & $9.88 \mathrm{E}-01$ \\
\hline ENSBTAG00000014170 & $1.60 \mathrm{E}-10$ & $1.71 \mathrm{E}-10$ & MAP7D3 & 9.37E-01 \\
\hline ENSBTAG00000005616 & $1.54 \mathrm{E}-10$ & $2.18 \mathrm{E}-10$ & BRS3 & 7.05E-01 \\
\hline ENSBTAG00000001880 & $2.33 \mathrm{E}-10$ & 2.44E-10 & VGLL1 & $9.56 \mathrm{E}-01$ \\
\hline ENSBTAG00000017843 & $1.25 \mathrm{E}-10$ & 8.73E-11 & CD40LG & $1.43 \mathrm{E}+00$ \\
\hline ENSBTAG00000017850 & $1.42 \mathrm{E}-10$ & $1.66 \mathrm{E}-10$ & ARHGEF6 & $8.55 \mathrm{E}-01$ \\
\hline ENSBTAG00000013308 & $9.73 \mathrm{E}-11$ & $1.63 \mathrm{E}-10$ & & 5.97E-01 \\
\hline ENSBTAG00000022373 & $1.48 \mathrm{E}-10$ & $9.46 \mathrm{E}-11$ & & $1.57 \mathrm{E}+00$ \\
\hline ENSBTAG00000021413 & $1.22 \mathrm{E}-10$ & $9.23 \mathrm{E}-11$ & ZIC3 & $1.32 \mathrm{E}+00$ \\
\hline ENSBTAG00000018323 & $2.23 \mathrm{E}-10$ & $1.84 \mathrm{E}-10$ & & $1.21 \mathrm{E}+00$ \\
\hline ENSBTAG00000020194 & $1.07 \mathrm{E}-10$ & $9.48 \mathrm{E}-11$ & & $1.13 E+00$ \\
\hline ENSBTAG00000020022 & $1.35 \mathrm{E}-10$ & $1.12 \mathrm{E}-10$ & IL13RA2 & $1.21 E+00$ \\
\hline ENSBTAG00000004003 & $1.02 \mathrm{E}-10$ & $1.01 \mathrm{E}-10$ & F9 & $1.01 E+00$ \\
\hline ENSBTAG00000004007 & $1.03 \mathrm{E}-10$ & $9.33 \mathrm{E}-11$ & MCF2 & $1.10 \mathrm{E}+00$ \\
\hline ENSBTAG00000016484 & $6.99 \mathrm{E}-11$ & $4.40 \mathrm{E}-11$ & ATP11C & $1.59 E+00$ \\
\hline ENSBTAG00000048281 & $9.56 \mathrm{E}-10$ & $1.33 \mathrm{E}-10$ & & $7.19 E+00$ \\
\hline ENSBTAG00000009753 & $9.29 \mathrm{E}-10$ & $1.06 \mathrm{E}-10$ & & $8.76 \mathrm{E}+00$ \\
\hline ENSBTAG00000008226 & $1.47 \mathrm{E}-10$ & $1.11 \mathrm{E}-10$ & & $1.32 \mathrm{E}+00$ \\
\hline ENSBTAG00000045940 & $1.73 \mathrm{E}-09$ & 1.77E-09 & MGC148328 & $9.79 \mathrm{E}-01$ \\
\hline ENSBTAG00000039870 & $4.82 \mathrm{E}-10$ & $4.04 \mathrm{E}-10$ & & $1.19 \mathrm{E}+00$ \\
\hline ENSBTAG00000016940 & $6.90 \mathrm{E}-11$ & $2.90 \mathrm{E}-11$ & & $2.37 E+00$ \\
\hline ENSBTAG00000017628 & $5.23 \mathrm{E}-11$ & $4.22 \mathrm{E}-11$ & MAP7D2 & $1.24 \mathrm{E}+00$ \\
\hline ENSBTAG00000021962 & $6.60 \mathrm{E}-11$ & $5.26 \mathrm{E}-11$ & CXorf23 & $1.25 \mathrm{E}+00$ \\
\hline ENSBTAG00000012552 & $1.23 \mathrm{E}-10$ & 5.77E-11 & FMR1 & $2.13 E+00$ \\
\hline ENSBTAG00000034045 & $8.91 \mathrm{E}-11$ & $8.62 \mathrm{E}-11$ & FMR1NB & $1.03 E+00$ \\
\hline ENSBTAG00000020750 & $1.79 \mathrm{E}-10$ & $1.01 \mathrm{E}-10$ & AFF2 & $1.77 \mathrm{E}+00$ \\
\hline
\end{tabular}




\begin{tabular}{|c|c|c|c|c|}
\hline ENSBTAG00000011056 & $7.41 \mathrm{E}-11$ & $6.67 \mathrm{E}-11$ & IDS & $1.11 \mathrm{E}+00$ \\
\hline ENSBTAG00000045540 & 6.67E-11 & $8.41 \mathrm{E}-11$ & & 7.94E-01 \\
\hline ENSBTAG00000033874 & 0 & $5.66 \mathrm{E}-11$ & & $1.76 \mathrm{E}-03$ \\
\hline ENSBTAG00000048215 & 0 & 4.04E-11 & & $2.47 \mathrm{E}-03$ \\
\hline ENSBTAG00000046653 & $1.28 \mathrm{E}-10$ & $2.43 \mathrm{E}-10$ & & $5.28 \mathrm{E}-01$ \\
\hline ENSBTAG00000046035 & $1.15 \mathrm{E}-10$ & $7.25 \mathrm{E}-11$ & & $1.59 E+00$ \\
\hline ENSBTAG00000006818 & $6.93 \mathrm{E}-11$ & $1.11 \mathrm{E}-10$ & TMEM185A & $6.25 \mathrm{E}-01$ \\
\hline ENSBTAG00000021713 & $4.62 \mathrm{E}-11$ & 3.09E-11 & MAMLD1 & $1.49 \mathrm{E}+00$ \\
\hline ENSBTAG00000014138 & $2.17 \mathrm{E}-11$ & $6.83 \mathrm{E}-12$ & MTM1 & $3.14 \mathrm{E}+00$ \\
\hline ENSBTAG00000009657 & $1.27 \mathrm{E}-10$ & $1.38 \mathrm{E}-10$ & MTMR1 & $9.17 \mathrm{E}-01$ \\
\hline ENSBTAG00000047708 & $1.98 \mathrm{E}-10$ & $1.66 \mathrm{E}-10$ & & $1.19 \mathrm{E}+00$ \\
\hline ENSBTAG00000005150 & $4.21 \mathrm{E}-10$ & $1.96 \mathrm{E}-10$ & HMGB3 & $2.15 E+00$ \\
\hline ENSBTAG00000005950 & $1.43 \mathrm{E}-10$ & $1.80 \mathrm{E}-10$ & GPR50 & 7.93E-01 \\
\hline ENSBTAG00000009775 & $2.05 E-10$ & $6.45 \mathrm{E}-11$ & PRRG3 & $3.18 \mathrm{E}+00$ \\
\hline ENSBTAG00000006820 & $1.02 \mathrm{E}-10$ & $8.00 \mathrm{E}-11$ & FATE1 & $1.27 \mathrm{E}+00$ \\
\hline ENSBTAG00000020725 & $2.85 \mathrm{E}-10$ & $6.18 \mathrm{E}-11$ & CNGA2 & $4.61 E+00$ \\
\hline ENSBTAG00000013548 & $8.90 \mathrm{E}-11$ & $5.98 \mathrm{E}-11$ & GABRE & $1.49 \mathrm{E}+00$ \\
\hline ENSBTAG00000006691 & $1.53 \mathrm{E}-10$ & $9.65 \mathrm{E}-11$ & GABRA3 & $1.58 \mathrm{E}+00$ \\
\hline ENSBTAG00000013002 & $5.06 \mathrm{E}-11$ & $7.79 \mathrm{E}-11$ & GABRQ & $6.50 \mathrm{E}-01$ \\
\hline ENSBTAG00000007844 & 4.06E-11 & $2.56 \mathrm{E}-11$ & CETN2 & $1.58 \mathrm{E}+00$ \\
\hline ENSBTAG00000009231 & $3.46 \mathrm{E}-11$ & 3.57E-11 & NSDHL & 9.70E-01 \\
\hline ENSBTAG00000005776 & $5.10 \mathrm{E}-11$ & $2.95 \mathrm{E}-11$ & ZNF185 & $1.73 E+00$ \\
\hline ENSBTAG00000047518 & 0 & $8.78 \mathrm{E}-11$ & & $1.14 \mathrm{E}-03$ \\
\hline ENSBTAG00000045777 & $6.48 \mathrm{E}-10$ & $6.12 \mathrm{E}-10$ & & $1.06 \mathrm{E}+00$ \\
\hline ENSBTAG00000047971 & $7.25 \mathrm{E}-10$ & $8.93 \mathrm{E}-10$ & KIR3DL1 & $8.12 \mathrm{E}-01$ \\
\hline ENSBTAG00000047804 & 0 & $8.12 \mathrm{E}-11$ & & $1.23 \mathrm{E}-03$ \\
\hline ENSBTAG00000048199 & $2.75 \mathrm{E}-10$ & $2.42 \mathrm{E}-10$ & & $1.14 \mathrm{E}+00$ \\
\hline ENSBTAG00000033445 & $9.34 \mathrm{E}-10$ & $8.65 \mathrm{E}-10$ & & $1.08 \mathrm{E}+00$ \\
\hline ENSBTAG00000046143 & $2.43 \mathrm{E}-10$ & $1.75 \mathrm{E}-10$ & & $1.39 E+00$ \\
\hline ENSBTAG00000046442 & $9.21 \mathrm{E}-11$ & $1.16 \mathrm{E}-10$ & & 7.94E-01 \\
\hline ENSBTAG00000011648 & $5.33 \mathrm{E}-11$ & $6.72 \mathrm{E}-11$ & TMLHE & 7.94E-01 \\
\hline ENSBTAG00000010948 & $1.31 \mathrm{E}-11$ & 8.27E-12 & CLIC2 & $1.58 \mathrm{E}+00$ \\
\hline ENSBTAG00000017265 & $1.33 \mathrm{E}-11$ & $1.26 \mathrm{E}-11$ & VBP1 & $1.05 E+00$ \\
\hline ENSBTAG00000011144 & 0 & 3.83E-11 & & 2.60E-03 \\
\hline ENSBTAG00000007817 & $1.36 \mathrm{E}-11$ & $1.84 \mathrm{E}-11$ & $\mathrm{BRCC} 3$ & 7.38E-01 \\
\hline ENSBTAG00000016977 & $4.92 \mathrm{E}-11$ & $8.85 \mathrm{E}-12$ & FUNDC2 & $5.51 E+00$ \\
\hline ENSBTAG00000010726 & $6.77 \mathrm{E}-11$ & $3.08 \mathrm{E}-11$ & F8 & $2.20 E+00$ \\
\hline ENSBTAG00000033167 & 0 & $1.54 \mathrm{E}-10$ & & $6.49 \mathrm{E}-04$ \\
\hline ENSBTAG00000013046 & $2.99 \mathrm{E}-11$ & $9.43 \mathrm{E}-12$ & MPP1 & $3.15 E+00$ \\
\hline ENSBTAG00000013045 & $6.91 \mathrm{E}-12$ & $1.74 \mathrm{E}-11$ & DKC1 & 4.01E-01 \\
\hline ENSBTAG00000013044 & $5.19 E-11$ & $1.36 \mathrm{E}-11$ & GAB3 & $3.79 \mathrm{E}+00$ \\
\hline ENSBTAG00000022807 & $9.56 \mathrm{E}-10$ & 8.67E-10 & KIR3DS1 & $1.10 \mathrm{E}+00$ \\
\hline ENSBTAG00000045951 & $1.26 \mathrm{E}-09$ & 1.14E-09 & & $1.11 \mathrm{E}+00$ \\
\hline ENSBTAG00000046709 & $9.20 \mathrm{E}-10$ & 1.00E-09 & & $9.20 \mathrm{E}-01$ \\
\hline ENSBTAG00000038288 & $6.12 \mathrm{E}-10$ & $5.71 \mathrm{E}-10$ & & $1.07 E+00$ \\
\hline ENSBTAG00000038933 & 1.19E-09 & $1.23 \mathrm{E}-09$ & & $9.64 \mathrm{E}-01$ \\
\hline ENSBTAG00000021439 & $2.12 \mathrm{E}-10$ & $3.53 \mathrm{E}-10$ & & $6.01 \mathrm{E}-01$ \\
\hline ENSBTAG00000047577 & $7.29 \mathrm{E}-10$ & 7.70E-10 & & $9.46 \mathrm{E}-01$ \\
\hline ENSBTAG00000045675 & $2.40 \mathrm{E}-10$ & $3.27 \mathrm{E}-10$ & & 7.33E-01 \\
\hline ENSBTAG00000020185 & $5.12 \mathrm{E}-11$ & $1.16 \mathrm{E}-11$ & & $4.39 E+00$ \\
\hline ENSBTAG00000014095 & $2.41 \mathrm{E}-11$ & $6.06 \mathrm{E}-12$ & HAUS7 & $3.92 E+00$ \\
\hline ENSBTAG00000005250 & $5.35 E-11$ & $5.99 \mathrm{E}-11$ & BGN & 8.93E-01 \\
\hline ENSBTAG00000011350 & $1.72 \mathrm{E}-11$ & 2.17E-11 & FAM58A & 7.94E-01 \\
\hline ENSBTAG00000017430 & $2.49 \mathrm{E}-11$ & $1.25 \mathrm{E}-10$ & PNCK & $1.99 \mathrm{E}-01$ \\
\hline ENSBTAG00000009959 & $2.24 \mathrm{E}-11$ & $1.41 \mathrm{E}-11$ & & $1.59 E+00$ \\
\hline ENSBTAG00000020547 & $1.14 \mathrm{E}-11$ & $2.15 \mathrm{E}-11$ & BCAP31 & 5.31E-01 \\
\hline ENSBTAG00000020551 & $5.69 \mathrm{E}-11$ & $1.96 \mathrm{E}-11$ & $A B C D 1$ & $2.90 \mathrm{E}+00$ \\
\hline ENSBTAG00000010146 & $6.78 \mathrm{E}-12$ & $8.54 \mathrm{E}-12$ & PLXNB3 & 7.96E-01 \\
\hline ENSBTAG00000001058 & 0 & $2.24 \mathrm{E}-11$ & SRPK3 & 4.44E-03 \\
\hline ENSBTAG00000001059 & $5.74 \mathrm{E}-11$ & $2.41 E-11$ & IDH3G & $2.37 E+00$ \\
\hline ENSBTAG00000021421 & $2.18 \mathrm{E}-11$ & $2.75 \mathrm{E}-11$ & SSR4 & 7.93E-01 \\
\hline ENSBTAG00000013462 & $4.04 \mathrm{E}-11$ & 7.27E-11 & L1CAM & $5.56 \mathrm{E}-01$ \\
\hline ENSBTAG00000047138 & $1.02 \mathrm{E}-10$ & $1.72 \mathrm{E}-10$ & AVPR2 & 5.95E-01 \\
\hline ENSBTAG00000047531 & $6.77 \mathrm{E}-11$ & $1.05 \mathrm{E}-10$ & ARHGAP4 & $6.45 \mathrm{E}-01$ \\
\hline ENSBTAG00000047702 & $3.48 \mathrm{E}-11$ & $6.58 \mathrm{E}-11$ & & 5.30E-01 \\
\hline ENSBTAG00000047827 & $5.62 \mathrm{E}-11$ & $5.66 \mathrm{E}-11$ & RENBP & 9.93E-01 \\
\hline ENSBTAG00000011904 & $8.26 \mathrm{E}-11$ & $8.85 \mathrm{E}-11$ & HCFC1 & $9.34 \mathrm{E}-01$ \\
\hline ENSBTAG00000008278 & $1.81 \mathrm{E}-11$ & $6.85 \mathrm{E}-11$ & TMEM187 & $2.66 \mathrm{E}-01$ \\
\hline ENSBTAG00000016085 & 0 & $1.13 \mathrm{E}-10$ & IRAK1 & 8.84E-04 \\
\hline ENSBTAG00000047855 & $2.35 E-11$ & 1.17E-10 & MECP2 & $2.02 \mathrm{E}-01$ \\
\hline
\end{tabular}




\begin{tabular}{|c|c|c|c|c|}
\hline ENSBTAG00000020299 & $1.33 \mathrm{E}-11$ & $1.01 \mathrm{E}-10$ & & 1.33E-01 \\
\hline ENSBTAG00000020300 & $1.11 \mathrm{E}-11$ & 8.36E-11 & TEX28 & 1.33E-01 \\
\hline ENSBTAG00000020127 & $2.88 \mathrm{E}-11$ & $5.80 \mathrm{E}-11$ & TKTL1 & 4.97E-01 \\
\hline ENSBTAG00000011190 & $2.24 \mathrm{E}-11$ & $5.64 \mathrm{E}-11$ & FLNA & $3.98 \mathrm{E}-01$ \\
\hline ENSBTAG00000007455 & 0 & $1.55 \mathrm{E}-10$ & DNASE1L1 & $6.45 \mathrm{E}-04$ \\
\hline ENSBTAG00000007456 & 2.97E-11 & 4.98E-11 & TAZ & $5.96 \mathrm{E}-01$ \\
\hline ENSBTAG00000012117 & $5.26 \mathrm{E}-11$ & $5.30 \mathrm{E}-11$ & ATP6AP1 & 9.93E-01 \\
\hline ENSBTAG00000012125 & $1.42 \mathrm{E}-11$ & 5.37E-11 & GDI1 & 2.66E-01 \\
\hline ENSBTAG00000033032 & 0 & 1.79E-11 & FAM50A & $5.56 \mathrm{E}-03$ \\
\hline ENSBTAG00000008552 & $1.89 \mathrm{E}-11$ & $3.57 \mathrm{E}-11$ & & 5.30E-01 \\
\hline ENSBTAG00000014331 & $3.11 \mathrm{E}-11$ & $3.92 \mathrm{E}-11$ & & 7.95E-01 \\
\hline ENSBTAG00000014333 & 0 & $3.00 \mathrm{E}-11$ & SLC10A3 & 3.32E-03 \\
\hline ENSBTAG00000012747 & $2.94 \mathrm{E}-11$ & $3.71 \mathrm{E}-11$ & FAM3A & 7.94E-01 \\
\hline ENSBTAG00000019512 & $5.07 \mathrm{E}-12$ & $2.56 \mathrm{E}-11$ & G6PD & $2.01 \mathrm{E}-01$ \\
\hline ENSBTAG00000006268 & $2.83 \mathrm{E}-11$ & 2.97E-11 & IKBKG & $9.53 \mathrm{E}-01$ \\
\hline ENSBTAG00000012084 & $2.81 \mathrm{E}-11$ & $6.07 E-11$ & & 4.64E-01 \\
\hline ENSBTAG00000047120 & 0 & $1.25 \mathrm{E}-10$ & & 7.99E-04 \\
\hline ENSBTAG00000045853 & 0 & $5.84 \mathrm{E}-11$ & & $1.71 \mathrm{E}-03$ \\
\hline ENSBTAG00000019942 & $4.05 \mathrm{E}-11$ & $5.10 \mathrm{E}-11$ & PABPC5 & 7.94E-01 \\
\hline ENSBTAG00000001911 & $1.14 \mathrm{E}-10$ & $8.28 \mathrm{E}-11$ & & $1.38 \mathrm{E}+00$ \\
\hline ENSBTAG00000038815 & $2.56 \mathrm{E}-11$ & 3.77E-11 & & $6.81 \mathrm{E}-01$ \\
\hline ENSBTAG00000047213 & $5.64 \mathrm{E}-11$ & 3.55E-11 & & $1.59 E+00$ \\
\hline ENSBTAG00000048265 & 7.03E-11 & 8.86E-11 & & 7.94E-01 \\
\hline ENSBTAG00000019705 & $1.89 \mathrm{E}-11$ & $1.19 \mathrm{E}-11$ & & $1.58 \mathrm{E}+00$ \\
\hline ENSBTAG00000040255 & 7.97E-11 & $1.00 \mathrm{E}-10$ & & 7.97E-01 \\
\hline ENSBTAG00000037686 & $4.03 \mathrm{E}-10$ & 4.79E-10 & SRPX2 & $8.41 \mathrm{E}-01$ \\
\hline ENSBTAG00000045550 & $2.69 \mathrm{E}-10$ & $2.81 \mathrm{E}-10$ & TSPAN6 & $9.58 \mathrm{E}-01$ \\
\hline ENSBTAG00000021059 & $1.64 \mathrm{E}-10$ & $1.44 \mathrm{E}-11$ & TNMD & $1.13 \mathrm{E}+01$ \\
\hline ENSBTAG00000014169 & $3.00 \mathrm{E}-11$ & $8.83 \mathrm{E}-12$ & PCDH19 & $3.37 E+00$ \\
\hline ENSBTAG00000044025 & $6.73 \mathrm{E}-11$ & $3.85 \mathrm{E}-11$ & & $1.75 E+00$ \\
\hline ENSBTAG00000004836 & $2.11 \mathrm{E}-11$ & $7.58 \mathrm{E}-12$ & & $2.76 E+00$ \\
\hline ENSBTAG00000046095 & $1.55 \mathrm{E}-11$ & $9.74 \mathrm{E}-12$ & & $1.58 \mathrm{E}+00$ \\
\hline ENSBTAG00000047655 & $6.01 \mathrm{E}-11$ & $9.46 \mathrm{E}-11$ & SERPINA7 & $6.35 \mathrm{E}-01$ \\
\hline ENSBTAG00000046210 & $9.29 \mathrm{E}-10$ & 4.79E-10 & & $1.94 \mathrm{E}+00$ \\
\hline ENSBTAG00000005724 & $4.30 \mathrm{E}-11$ & $1.24 \mathrm{E}-10$ & SYTL4 & $3.48 \mathrm{E}-01$ \\
\hline ENSBTAG00000003547 & $5.48 \mathrm{E}-11$ & $6.31 \mathrm{E}-11$ & CSTF2 & 8.69E-01 \\
\hline ENSBTAG00000016761 & 7.43E-11 & 1.19E-10 & NOX1 & 6.25E-01 \\
\hline ENSBTAG00000005378 & $1.38 \mathrm{E}-11$ & $8.69 \mathrm{E}-12$ & XKRX & $1.58 \mathrm{E}+00$ \\
\hline ENSBTAG00000020390 & $9.25 \mathrm{E}-11$ & $1.06 \mathrm{E}-11$ & ARL13A & $8.65 E+00$ \\
\hline ENSBTAG00000021252 & $1.05 \mathrm{E}-10$ & 1.65E-11 & TMEM35A & $6.31 E+00$ \\
\hline ENSBTAG00000021254 & $1.05 \mathrm{E}-10$ & $3.44 \mathrm{E}-11$ & CENPI & $3.03 E+00$ \\
\hline ENSBTAG00000001975 & $6.53 \mathrm{E}-11$ & $1.07 \mathrm{E}-11$ & DRP2 & $6.05 E+00$ \\
\hline ENSBTAG00000019417 & $5.54 \mathrm{E}-11$ & 4.65E-11 & ARMCX2 & $1.19 \mathrm{E}+00$ \\
\hline ENSBTAG00000005319 & $4.00 \mathrm{E}-11$ & 4.20E-11 & & $9.53 \mathrm{E}-01$ \\
\hline ENSBTAG00000003601 & $5.96 \mathrm{E}-11$ & $5.00 \mathrm{E}-12$ & ZMAT1 & $1.17 \mathrm{E}+01$ \\
\hline ENSBTAG00000014670 & $3.58 \mathrm{E}-11$ & $4.51 \mathrm{E}-11$ & BEX5 & 7.94E-01 \\
\hline ENSBTAG00000027978 & $3.09 \mathrm{E}-11$ & $3.89 \mathrm{E}-11$ & & 7.94E-01 \\
\hline ENSBTAG00000020522 & $7.08 \mathrm{E}-11$ & 4.12E-11 & TCP11X2 & $1.72 \mathrm{E}+00$ \\
\hline ENSBTAG00000038449 & $2.25 \mathrm{E}-10$ & $1.13 \mathrm{E}-10$ & & $1.99 \mathrm{E}+00$ \\
\hline ENSBTAG00000022501 & $3.02 \mathrm{E}-10$ & $2.24 \mathrm{E}-10$ & & $1.35 \mathrm{E}+00$ \\
\hline ENSBTAG00000032787 & $2.02 \mathrm{E}-10$ & $1.60 \mathrm{E}-10$ & & $1.26 \mathrm{E}+00$ \\
\hline ENSBTAG00000046314 & $2.35 \mathrm{E}-10$ & $1.12 \mathrm{E}-10$ & & $2.09 E+00$ \\
\hline ENSBTAG00000022920 & $1.20 \mathrm{E}-10$ & $5.87 E-11$ & RNF128 & $2.04 \mathrm{E}+00$ \\
\hline ENSBTAG00000003668 & $1.85 \mathrm{E}-10$ & $1.26 \mathrm{E}-10$ & CXorf57 & $1.46 \mathrm{E}+00$ \\
\hline ENSBTAG00000047601 & 0 & $1.94 \mathrm{E}-10$ & & 5.15E-04 \\
\hline ENSBTAG00000047439 & $1.74 \mathrm{E}-10$ & $1.59 \mathrm{E}-10$ & & $1.09 \mathrm{E}+00$ \\
\hline ENSBTAG00000036028 & $2.07 \mathrm{E}-10$ & $1.96 \mathrm{E}-10$ & & $1.06 \mathrm{E}+00$ \\
\hline ENSBTAG00000006026 & $1.64 \mathrm{E}-10$ & $1.37 \mathrm{E}-10$ & BEX3 & $1.19 \mathrm{E}+00$ \\
\hline ENSBTAG00000022502 & $6.08 \mathrm{E}-10$ & $2.91 \mathrm{E}-10$ & & $2.09 E+00$ \\
\hline ENSBTAG00000027843 & $2.54 \mathrm{E}-10$ & $3.21 \mathrm{E}-10$ & TCEAL8 & 7.93E-01 \\
\hline ENSBTAG00000001311 & $5.40 \mathrm{E}-11$ & $8.50 \mathrm{E}-11$ & MORF4L2 & 6.35E-01 \\
\hline ENSBTAG00000003320 & $1.09 \mathrm{E}-10$ & $1.08 \mathrm{E}-10$ & GLRA4 & $1.01 \mathrm{E}+00$ \\
\hline ENSBTAG00000006977 & $1.40 \mathrm{E}-10$ & 7.83E-11 & PLP1 & $1.79 \mathrm{E}+00$ \\
\hline ENSBTAG00000019820 & $5.59 \mathrm{E}-11$ & $5.64 \mathrm{E}-11$ & RAB9B & 9.92E-01 \\
\hline ENSBTAG00000047211 & $3.56 \mathrm{E}-10$ & $3.59 \mathrm{E}-10$ & & $9.92 \mathrm{E}-01$ \\
\hline ENSBTAG00000047168 & $1.73 \mathrm{E}-10$ & 3.27E-10 & & 5.29E-01 \\
\hline ENSBTAG00000040363 & $3.96 \mathrm{E}-10$ & $5.62 \mathrm{E}-10$ & & 7.05E-01 \\
\hline ENSBTAG00000048051 & $6.31 \mathrm{E}-10$ & $1.22 \mathrm{E}-09$ & & 5.17E-01 \\
\hline ENSBTAG00000000441 & 8.23E-11 & $4.60 \mathrm{E}-11$ & SLC25A53 & $1.79 \mathrm{E}+00$ \\
\hline ENSBTAG00000004008 & $6.67 \mathrm{E}-11$ & $9.67 \mathrm{E}-11$ & FAM199X & $6.91 \mathrm{E}-01$ \\
\hline
\end{tabular}




\begin{tabular}{|c|c|c|c|c|}
\hline ENSBTAG000000005953 & $6.47 \mathrm{E}-11$ & $4.89 \mathrm{E}-11$ & & $1.32 \mathrm{E}+00$ \\
\hline ENSBTAG00000034644 & $2.68 \mathrm{E}-10$ & $2.45 \mathrm{E}-10$ & & $1.09 \mathrm{E}+00$ \\
\hline ENSBTAG000000045877 & $5.88 \mathrm{E}-11$ & $1.14 \mathrm{E}-10$ & TSC22D3 & $5.16 \mathrm{E}-01$ \\
\hline ENSBTAG000000019703 & $8.03 E-11$ & $1.24 \mathrm{E}-10$ & PRPS1 & $6.48 \mathrm{E}-01$ \\
\hline ENSBTAG000000003121 & $2.88 \mathrm{E}-11$ & $1.20 \mathrm{E}-10$ & FRMPD3 & 2.41E-01 \\
\hline ENSBTAG000000045876 & $2.03 \mathrm{E}-10$ & $1.72 \mathrm{E}-10$ & & $1.18 \mathrm{E}+00$ \\
\hline ENSBTAG000000005294 & $6.28 \mathrm{E}-11$ & $8.57 \mathrm{E}-11$ & & 7.33E-01 \\
\hline ENSBTAG000000006568 & $2.30 \mathrm{E}-10$ & $9.43 \mathrm{E}-11$ & & $2.44 E+00$ \\
\hline ENSBTAG000000003321 & $1.26 \mathrm{E}-10$ & $7.48 \mathrm{E}-11$ & PIH1D3 & $1.68 \mathrm{E}+00$ \\
\hline ENSBTAG000000000955 & $7.23 \mathrm{E}-11$ & $6.76 \mathrm{E}-11$ & RBM41 & $1.07 E+00$ \\
\hline ENSBTAG000000021968 & $8.98 \mathrm{E}-11$ & $6.20 \mathrm{E}-11$ & & $1.45 E+00$ \\
\hline ENSBTAG00000024943 & $3.13 \mathrm{E}-11$ & $3.94 \mathrm{E}-11$ & & 7.95E-01 \\
\hline ENSBTAG00000048086 & $5.18 \mathrm{E}-11$ & $1.30 \mathrm{E}-10$ & & 3.99E-01 \\
\hline ENSBTAG000000007859 & 7.34E-11 & $5.24 \mathrm{E}-11$ & VSIG1 & $1.40 \mathrm{E}+00$ \\
\hline ENSBTAG000000013721 & $3.36 \mathrm{E}-11$ & $2.83 \mathrm{E}-11$ & PSMD10 & $1.19 E+00$ \\
\hline ENSBTAG000000013724 & $1.01 \mathrm{E}-10$ & $6.93 \mathrm{E}-11$ & ATG4A & $1.45 E+00$ \\
\hline ENSBTAG000000013760 & $1.41 \mathrm{E}-10$ & $1.21 \mathrm{E}-10$ & COL4A6 & $1.16 \mathrm{E}+00$ \\
\hline ENSBTAG000000014575 & $1.37 \mathrm{E}-10$ & $1.21 \mathrm{E}-10$ & COL4A5 & $1.14 \mathrm{E}+00$ \\
\hline ENSBTAG000000026003 & $1.44 \mathrm{E}-10$ & $1.21 \mathrm{E}-10$ & GUCY2F & $1.19 E+00$ \\
\hline ENSBTAG000000020739 & $1.24 \mathrm{E}-11$ & 1.57E-11 & NXT2 & 7.94E-01 \\
\hline ENSBTAG00000018986 & $8.09 E-11$ & $5.92 \mathrm{E}-11$ & ACSL4 & $1.37 \mathrm{E}+00$ \\
\hline ENSBTAG000000037413 & 1.59E-10 & $1.07 \mathrm{E}-10$ & TMEM164 & $1.49 \mathrm{E}+00$ \\
\hline ENSBTAG000000022288 & $6.34 \mathrm{E}-11$ & $6.83 \mathrm{E}-11$ & AMMECR1 & $9.28 \mathrm{E}-01$ \\
\hline ENSBTAG000000037650 & $1.75 \mathrm{E}-11$ & $2.21 \mathrm{E}-11$ & RGAG1 & 7.94E-01 \\
\hline ENSBTAG000000004432 & $1.14 \mathrm{E}-10$ & $6.76 \mathrm{E}-11$ & CHRDL1 & $1.69 \mathrm{E}+00$ \\
\hline ENSBTAG000000048080 & $9.32 \mathrm{E}-10$ & $3.33 \mathrm{E}-10$ & & $2.80 \mathrm{E}+00$ \\
\hline ENSBTAG000000015670 & 4.33E-10 & $4.01 \mathrm{E}-10$ & PAK3 & $1.08 \mathrm{E}+00$ \\
\hline ENSBTAG000000000828 & $8.64 \mathrm{E}-11$ & $1.13 \mathrm{E}-10$ & CAPN6 & 7.64E-01 \\
\hline ENSBTAG00000019591 & $1.55 \mathrm{E}-10$ & $1.47 \mathrm{E}-10$ & DCX & $1.05 E+00$ \\
\hline ENSBTAG000000004586 & $1.32 \mathrm{E}-10$ & 1.47E-10 & & 8.96E-01 \\
\hline ENSBTAG00000015251 & $6.51 \mathrm{E}-11$ & 7.91E-11 & & $8.23 \mathrm{E}-01$ \\
\hline ENSBTAG000000010065 & 1.67E-10 & $1.65 \mathrm{E}-10$ & TRPC5 & $1.01 E+00$ \\
\hline ENSBTAG00000021624 & $2.32 \mathrm{E}-10$ & $3.65 \mathrm{E}-11$ & & $6.34 \mathrm{E}+00$ \\
\hline ENSBTAG000000006252 & $4.61 \mathrm{E}-10$ & $3.00 \mathrm{E}-10$ & & $1.54 \mathrm{E}+00$ \\
\hline ENSBTAG00000032485 & $2.09 \mathrm{E}-10$ & $2.50 \mathrm{E}-10$ & LHFPL1 & $8.36 \mathrm{E}-01$ \\
\hline ENSBTAG00000016221 & $1.52 \mathrm{E}-10$ & $1.38 \mathrm{E}-10$ & AMOT & $1.10 \mathrm{E}+00$ \\
\hline ENSBTAG00000002015 & $6.18 \mathrm{E}-11$ & $2.60 \mathrm{E}-11$ & RPS6KA6 & $2.37 E+00$ \\
\hline ENSBTAG00000047417 & $1.67 \mathrm{E}-10$ & 7.24E-11 & CYLC1 & $2.30 \mathrm{E}+00$ \\
\hline ENSBTAG00000010587 & $7.49 \mathrm{E}-11$ & $4.51 \mathrm{E}-11$ & SH3BGRL & $1.66 \mathrm{E}+00$ \\
\hline ENSBTAG00000001877 & $2.70 \mathrm{E}-11$ & $3.40 \mathrm{E}-11$ & & 7.95E-01 \\
\hline ENSBTAG00000016230 & $4.12 \mathrm{E}-11$ & $2.19 \mathrm{E}-11$ & BRWD3 & $1.88 \mathrm{E}+00$ \\
\hline ENSBTAG00000011613 & $1.23 \mathrm{E}-10$ & 8.97E-11 & PLS3 & $1.37 \mathrm{E}+00$ \\
\hline ENSBTAG00000047385 & $2.01 \mathrm{E}-10$ & $1.13 \mathrm{E}-10$ & & $1.78 \mathrm{E}+00$ \\
\hline ENSBTAG00000002001 & $1.13 \mathrm{E}-10$ & $6.81 E-11$ & & $1.66 \mathrm{E}+00$ \\
\hline ENSBTAG00000044037 & $8.02 E-11$ & $1.01 \mathrm{E}-11$ & & $7.88 \mathrm{E}+00$ \\
\hline ENSBTAG00000009598 & $2.04 \mathrm{E}-10$ & $1.59 \mathrm{E}-11$ & $\mathrm{CHM}$ & $1.28 \mathrm{E}+01$ \\
\hline ENSBTAG00000014485 & $6.26 \mathrm{E}-11$ & $1.37 \mathrm{E}-11$ & POF1B & $4.54 \mathrm{E}+00$ \\
\hline ENSBTAG00000021790 & $6.03 \mathrm{E}-11$ & $1.08 \mathrm{E}-11$ & APOOL & $5.54 \mathrm{E}+00$ \\
\hline ENSBTAG00000027867 & $8.78 \mathrm{E}-11$ & $1.63 \mathrm{E}-11$ & & $5.36 \mathrm{E}+00$ \\
\hline ENSBTAG00000045653 & $6.44 \mathrm{E}-11$ & $1.71 \mathrm{E}-11$ & FAM46D & $3.75 E+00$ \\
\hline ENSBTAG00000048060 & $3.38 \mathrm{E}-11$ & $1.06 \mathrm{E}-11$ & TBX22 & $3.17 E+00$ \\
\hline ENSBTAG00000046159 & $8.58 \mathrm{E}-11$ & $2.70 \mathrm{E}-11$ & ITM2A & $3.17 E+00$ \\
\hline ENSBTAG00000008555 & $3.06 \mathrm{E}-11$ & $2.76 \mathrm{E}-11$ & P2RY10 & $1.11 E+00$ \\
\hline ENSBTAG00000026025 & $8.54 \mathrm{E}-11$ & $7.39 \mathrm{E}-11$ & & $1.16 \mathrm{E}+00$ \\
\hline ENSBTAG00000000895 & $9.55 \mathrm{E}-11$ & $8.02 \mathrm{E}-11$ & TAF9B & $1.19 \mathrm{E}+00$ \\
\hline ENSBTAG00000000894 & $1.88 \mathrm{E}-10$ & $6.37 \mathrm{E}-11$ & PGK1 & $2.95 E+00$ \\
\hline ENSBTAG00000010018 & $1.21 \mathrm{E}-10$ & $8.96 \mathrm{E}-11$ & ATP7A & $1.35 \mathrm{E}+00$ \\
\hline ENSBTAG00000047768 & $6.19 \mathrm{E}-11$ & 5.20E-11 & & $1.19 \mathrm{E}+00$ \\
\hline ENSBTAG00000045931 & $9.11 E-11$ & $4.11 \mathrm{E}-11$ & MAGT1 & $2.21 E+00$ \\
\hline ENSBTAG00000038434 & $5.23 \mathrm{E}-11$ & $3.89 E-11$ & ATRX & $1.34 \mathrm{E}+00$ \\
\hline ENSBTAG00000006722 & $2.04 \mathrm{E}-11$ & $1.29 \mathrm{E}-11$ & FGF16 & $1.58 \mathrm{E}+00$ \\
\hline ENSBTAG00000046806 & $1.53 \mathrm{E}-10$ & $4.81 \mathrm{E}-11$ & & $3.17 E+00$ \\
\hline ENSBTAG00000008047 & $1.49 \mathrm{E}-10$ & $1.30 \mathrm{E}-10$ & ZDHHC15 & $1.14 \mathrm{E}+00$ \\
\hline ENSBTAG00000004549 & $1.22 \mathrm{E}-10$ & $1.03 \mathrm{E}-10$ & UPRT & $1.19 E+00$ \\
\hline ENSBTAG00000011028 & $2.05 \mathrm{E}-10$ & $1.59 \mathrm{E}-10$ & $A B C B 7$ & $1.29 \mathrm{E}+00$ \\
\hline ENSBTAG00000000300 & $4.21 \mathrm{E}-11$ & $1.77 \mathrm{E}-11$ & KIAA2022 & $2.37 E+00$ \\
\hline ENSBTAG00000015630 & $1.32 \mathrm{E}-10$ & $1.55 \mathrm{E}-10$ & RLIM & $8.50 \mathrm{E}-01$ \\
\hline ENSBTAG00000002585 & $1.00 \mathrm{E}-10$ & $6.32 \mathrm{E}-12$ & SLC16A2 & $1.56 \mathrm{E}+01$ \\
\hline ENSBTAG00000020826 & $1.31 \mathrm{E}-10$ & $2.79 \mathrm{E}-11$ & $\mathrm{CHIC1}$ & $4.69 E+00$ \\
\hline ENSBTAG00000032014 & $1.98 \mathrm{E}-10$ & $1.29 \mathrm{E}-10$ & & $1.53 E+00$ \\
\hline
\end{tabular}




\begin{tabular}{|c|c|c|c|c|}
\hline ENSBTAG00000015848 & $3.14 \mathrm{E}-10$ & $1.42 \mathrm{E}-10$ & PHKA1 & $2.21 \mathrm{E}+00$ \\
\hline ENSBTAG00000046092 & $8.70 \mathrm{E}-11$ & $2.38 \mathrm{E}-11$ & HDAC8 & $3.64 E+00$ \\
\hline ENSBTAG00000045925 & $5.43 \mathrm{E}-11$ & $5.13 \mathrm{E}-11$ & CITED1 & $1.06 \mathrm{E}+00$ \\
\hline ENSBTAG00000018800 & $9.19 \mathrm{E}-11$ & $1.16 \mathrm{E}-10$ & RPS4X & 7.93E-01 \\
\hline ENSBTAG00000005607 & $2.36 \mathrm{E}-10$ & $2.06 \mathrm{E}-10$ & ERCC6L & $1.14 \mathrm{E}+00$ \\
\hline ENSBTAG00000047376 & 2.44E-10 & $2.72 \mathrm{E}-10$ & PIN4 & $8.98 \mathrm{E}-01$ \\
\hline ENSBTAG00000045565 & $4.58 \mathrm{E}-11$ & $1.92 \mathrm{E}-11$ & NHSL2 & $2.38 \mathrm{E}+00$ \\
\hline ENSBTAG00000045642 & $8.95 \mathrm{E}-10$ & $8.83 \mathrm{E}-10$ & & $1.01 \mathrm{E}+00$ \\
\hline ENSBTAG00000048245 & $4.45 \mathrm{E}-10$ & 4.46E-10 & & $9.98 \mathrm{E}-01$ \\
\hline ENSBTAG00000000902 & $1.63 \mathrm{E}-10$ & 4.75E-11 & OGT & $3.43 E+00$ \\
\hline ENSBTAG00000006878 & $2.01 \mathrm{E}-10$ & $1.58 \mathrm{E}-10$ & & $1.27 \mathrm{E}+00$ \\
\hline ENSBTAG00000012163 & $6.86 \mathrm{E}-11$ & $2.88 \mathrm{E}-11$ & ITGB1BP2 & $2.38 \mathrm{E}+00$ \\
\hline ENSBTAG00000012152 & $1.43 \mathrm{E}-10$ & 7.07E-11 & NONO & $2.03 E+00$ \\
\hline ENSBTAG00000008492 & $1.25 \mathrm{E}-10$ & $4.31 \mathrm{E}-11$ & ZMYM3 & $2.91 E+00$ \\
\hline ENSBTAG00000020512 & $1.44 \mathrm{E}-10$ & $2.78 \mathrm{E}-11$ & GJB1 & $5.16 \mathrm{E}+00$ \\
\hline ENSBTAG00000007708 & $1.46 \mathrm{E}-10$ & 3.67E-11 & NLGN3 & $3.96 \mathrm{E}+00$ \\
\hline ENSBTAG00000021351 & $1.14 \mathrm{E}-10$ & 2.32E-11 & MED12 & $4.90 E+00$ \\
\hline ENSBTAG00000007626 & $1.80 \mathrm{E}-10$ & $1.42 \mathrm{E}-10$ & IL2RG & $1.27 \mathrm{E}+00$ \\
\hline ENSBTAG00000032209 & $2.36 \mathrm{E}-10$ & $9.91 \mathrm{E}-11$ & CXorf65 & $2.38 \mathrm{E}+00$ \\
\hline ENSBTAG00000004879 & 7.43E-11 & $4.68 \mathrm{E}-11$ & FOXO4 & $1.59 \mathrm{E}+00$ \\
\hline ENSBTAG00000007871 & $1.68 \mathrm{E}-10$ & $1.06 \mathrm{E}-10$ & SNX12 & $1.59 E+00$ \\
\hline ENSBTAG00000007403 & $1.20 \mathrm{E}-10$ & $9.45 \mathrm{E}-11$ & SLC7A3 & $1.27 \mathrm{E}+00$ \\
\hline ENSBTAG00000016272 & $1.61 \mathrm{E}-10$ & $2.65 \mathrm{E}-11$ & TEX11 & $6.04 \mathrm{E}+00$ \\
\hline ENSBTAG00000011291 & $1.33 \mathrm{E}-10$ & $3.27 \mathrm{E}-11$ & DLG3 & $4.06 \mathrm{E}+00$ \\
\hline ENSBTAG00000012863 & $1.92 \mathrm{E}-10$ & $5.48 \mathrm{E}-11$ & GDPD2 & $3.49 E+00$ \\
\hline ENSBTAG00000012861 & 5.17E-10 & $4.68 \mathrm{E}-11$ & KIF4A & $1.10 \mathrm{E}+01$ \\
\hline ENSBTAG00000012856 & $4.93 \mathrm{E}-10$ & $3.49 \mathrm{E}-11$ & ARR3 & $1.41 \mathrm{E}+01$ \\
\hline ENSBTAG00000018839 & $6.94 \mathrm{E}-10$ & $3.10 \mathrm{E}-10$ & AWAT1 & $2.24 E+00$ \\
\hline ENSBTAG00000007798 & $2.35 \mathrm{E}-10$ & $4.36 \mathrm{E}-11$ & DGAT2L6 & $5.37 E+00$ \\
\hline ENSBTAG00000007797 & $3.02 \mathrm{E}-10$ & $1.32 \mathrm{E}-11$ & & $2.27 \mathrm{E}+01$ \\
\hline ENSBTAG00000012281 & $5.51 \mathrm{E}-10$ & $1.54 \mathrm{E}-10$ & AWAT2 & $3.57 E+00$ \\
\hline ENSBTAG00000012543 & $3.52 \mathrm{E}-10$ & $1.38 \mathrm{E}-10$ & EDA & $2.55 E+00$ \\
\hline ENSBTAG00000011310 & $2.79 \mathrm{E}-10$ & $1.97 \mathrm{E}-10$ & FAM155B & $1.42 \mathrm{E}+00$ \\
\hline ENSBTAG00000015801 & $1.42 \mathrm{E}-10$ & $1.02 \mathrm{E}-10$ & EFNB1 & $1.39 E+00$ \\
\hline ENSBTAG00000033702 & $2.77 \mathrm{E}-10$ & $6.70 \mathrm{E}-11$ & STARD8 & $4.13 E+00$ \\
\hline ENSBTAG00000001977 & 8.99E-11 & 8.09E-12 & YIPF6 & $1.10 \mathrm{E}+01$ \\
\hline ENSBTAG00000022255 & $1.91 \mathrm{E}-10$ & $1.52 \mathrm{E}-11$ & $A R$ & $1.25 \mathrm{E}+01$ \\
\hline ENSBTAG00000047326 & $1.37 \mathrm{E}-10$ & $2.88 \mathrm{E}-11$ & EDA2R & $4.76 \mathrm{E}+00$ \\
\hline ENSBTAG00000047411 & $5.56 \mathrm{E}-10$ & $5.44 \mathrm{E}-10$ & & $1.02 \mathrm{E}+00$ \\
\hline ENSBTAG00000007654 & $1.79 \mathrm{E}-10$ & $1.02 \mathrm{E}-11$ & CHST7 & $1.74 \mathrm{E}+01$ \\
\hline ENSBTAG00000003290 & $2.43 \mathrm{E}-10$ & $8.62 \mathrm{E}-12$ & SLC9A7 & $2.79 E+01$ \\
\hline ENSBTAG00000005496 & $1.65 \mathrm{E}-10$ & $1.09 \mathrm{E}-10$ & RP2 & $1.51 E+00$ \\
\hline ENSBTAG00000005124 & $1.81 \mathrm{E}-10$ & $1.06 \mathrm{E}-10$ & JADE3 & $1.71 \mathrm{E}+00$ \\
\hline ENSBTAG00000046155 & $1.61 \mathrm{E}-10$ & $2.79 \mathrm{E}-11$ & RGN & $5.74 \mathrm{E}+00$ \\
\hline ENSBTAG00000002890 & $1.36 \mathrm{E}-10$ & $8.14 \mathrm{E}-11$ & RBM10 & $1.66 \mathrm{E}+00$ \\
\hline ENSBTAG00000016764 & $1.58 \mathrm{E}-10$ & $3.32 \mathrm{E}-11$ & UBA1 & $4.76 \mathrm{E}+00$ \\
\hline ENSBTAG00000016769 & $6.46 \mathrm{E}-11$ & $1.81 \mathrm{E}-11$ & CDK16 & $3.56 \mathrm{E}+00$ \\
\hline ENSBTAG00000016772 & $1.35 \mathrm{E}-10$ & $3.53 \mathrm{E}-11$ & USP11 & $3.81 E+00$ \\
\hline ENSBTAG00000038589 & 3.34E-10 & $1.12 \mathrm{E}-10$ & ZNF157 & $2.98 \mathrm{E}+00$ \\
\hline ENSBTAG00000002113 & $3.36 \mathrm{E}-10$ & $8.92 \mathrm{E}-11$ & & $3.76 \mathrm{E}+00$ \\
\hline ENSBTAG00000005039 & $7.06 \mathrm{E}-11$ & $1.97 \mathrm{E}-11$ & ARAF & $3.57 E+00$ \\
\hline ENSBTAG00000005042 & $1.38 \mathrm{E}-10$ & $1.49 \mathrm{E}-10$ & & $9.26 \mathrm{E}-01$ \\
\hline ENSBTAG00000005043 & $1.28 \mathrm{E}-10$ & $1.08 \mathrm{E}-10$ & TIMP1 & $1.19 E+00$ \\
\hline ENSBTAG00000005866 & $6.33 \mathrm{E}-11$ & $5.20 \mathrm{E}-11$ & ZNF81 & $1.22 \mathrm{E}+00$ \\
\hline ENSBTAG00000015817 & $3.88 \mathrm{E}-11$ & $2.09 \mathrm{E}-11$ & ELK1 & $1.85 \mathrm{E}+00$ \\
\hline ENSBTAG00000039794 & $1.04 \mathrm{E}-10$ & $4.35 \mathrm{E}-11$ & & $2.38 \mathrm{E}+00$ \\
\hline ENSBTAG00000031845 & $3.39 \mathrm{E}-10$ & $4.89 \mathrm{E}-10$ & SSX5 & 6.94E-01 \\
\hline ENSBTAG00000031829 & $4.49 \mathrm{E}-11$ & $2.26 \mathrm{E}-11$ & & $1.98 \mathrm{E}+00$ \\
\hline ENSBTAG00000009282 & $1.01 \mathrm{E}-10$ & $9.74 \mathrm{E}-11$ & PORCN & $1.03 E+00$ \\
\hline ENSBTAG00000009287 & $8.10 \mathrm{E}-11$ & $7.65 \mathrm{E}-11$ & EBP & $1.06 \mathrm{E}+00$ \\
\hline ENSBTAG00000009288 & $1.06 \mathrm{E}-10$ & 8.64E-11 & TBC1D25 & $1.23 \mathrm{E}+00$ \\
\hline ENSBTAG00000025848 & $5.94 \mathrm{E}-11$ & $7.48 \mathrm{E}-11$ & RBM3 & 7.94E-01 \\
\hline ENSBTAG00000018060 & $1.75 \mathrm{E}-10$ & $1.62 \mathrm{E}-10$ & WDR13 & $1.08 \mathrm{E}+00$ \\
\hline ENSBTAG00000046555 & $8.50 \mathrm{E}-11$ & $1.48 \mathrm{E}-10$ & WAS & 5.74E-01 \\
\hline ENSBTAG00000004706 & $8.88 \mathrm{E}-11$ & $1.04 \mathrm{E}-10$ & SUV39H1 & 8.54E-01 \\
\hline ENSBTAG00000003184 & $2.79 \mathrm{E}-11$ & 7.03E-11 & GATA1 & $3.98 \mathrm{E}-01$ \\
\hline ENSBTAG00000013244 & $3.46 \mathrm{E}-11$ & $6.54 \mathrm{E}-11$ & HDAC6 & 5.30E-01 \\
\hline ENSBTAG00000013250 & 3.63E-11 & $6.85 \mathrm{E}-11$ & PCSK1N & 5.30E-01 \\
\hline ENSBTAG00000018496 & 0 & $2.72 \mathrm{E}-11$ & TIMM17B & 3.66E-03 \\
\hline ENSBTAG00000018498 & $1.43 \mathrm{E}-11$ & $1.80 \mathrm{E}-11$ & PQBP1 & 7.93E-01 \\
\hline
\end{tabular}




\begin{tabular}{|c|c|c|c|c|}
\hline ENSBTAG00000002768 & $2.23 \mathrm{E}-11$ & $1.40 \mathrm{E}-11$ & SLC35A2 & $1.59 E+00$ \\
\hline ENSBTAG00000004597 & $1.46 \mathrm{E}-11$ & $1.84 \mathrm{E}-11$ & PIM2 & 7.95E-01 \\
\hline ENSBTAG00000004600 & $1.18 \mathrm{E}-10$ & 7.81E-11 & OTUD5 & $1.51 E+00$ \\
\hline ENSBTAG00000009024 & $2.05 \mathrm{E}-10$ & $8.61 \mathrm{E}-11$ & KCND1 & $2.38 \mathrm{E}+00$ \\
\hline ENSBTAG00000007788 & $1.72 \mathrm{E}-10$ & $2.88 \mathrm{E}-11$ & GRIPAP1 & $5.95 \mathrm{E}+00$ \\
\hline ENSBTAG00000008523 & $7.10 \mathrm{E}-11$ & $2.68 \mathrm{E}-11$ & TFE3 & $2.64 \mathrm{E}+00$ \\
\hline ENSBTAG00000002561 & $4.54 \mathrm{E}-11$ & $7.15 \mathrm{E}-11$ & CCDC120 & $6.35 \mathrm{E}-01$ \\
\hline ENSBTAG00000002563 & $4.72 \mathrm{E}-11$ & $3.96 \mathrm{E}-11$ & WDR45 & $1.19 E+00$ \\
\hline ENSBTAG00000002568 & 4.56E-11 & $5.75 \mathrm{E}-11$ & GPKOW & 7.94E-01 \\
\hline ENSBTAG00000013278 & $8.68 \mathrm{E}-11$ & $1.09 \mathrm{E}-10$ & MAGIX & 7.96E-01 \\
\hline ENSBTAG00000016093 & $5.39 \mathrm{E}-11$ & 3.39E-11 & & $1.59 \mathrm{E}+00$ \\
\hline ENSBTAG00000016095 & 3.64E-11 & $4.59 \mathrm{E}-11$ & PRICKLE3 & 7.94E-01 \\
\hline ENSBTAG00000016096 & $2.77 \mathrm{E}-11$ & $3.50 \mathrm{E}-11$ & SYP & 7.93E-01 \\
\hline ENSBTAG00000016097 & $7.24 \mathrm{E}-11$ & $3.19 \mathrm{E}-11$ & CACNA1F & $2.27 \mathrm{E}+00$ \\
\hline ENSBTAG00000013277 & $1.63 \mathrm{E}-11$ & $2.05 \mathrm{E}-11$ & CCDC22 & 7.95E-01 \\
\hline ENSBTAG00000013279 & $1.03 \mathrm{E}-10$ & $6.49 \mathrm{E}-11$ & FOXP3 & $1.59 E+00$ \\
\hline ENSBTAG00000021100 & $3.02 \mathrm{E}-11$ & $4.44 \mathrm{E}-11$ & PPP1R3F & $6.81 \mathrm{E}-01$ \\
\hline ENSBTAG00000037728 & $1.96 \mathrm{E}-10$ & $2.46 \mathrm{E}-10$ & PAGE4 & 7.95E-01 \\
\hline ENSBTAG00000046069 & $3.50 \mathrm{E}-11$ & $4.41 \mathrm{E}-11$ & LYZE & 7.94E-01 \\
\hline ENSBTAG00000046774 & $7.86 \mathrm{E}-11$ & $5.40 \mathrm{E}-11$ & ZNF182 & $1.45 E+00$ \\
\hline ENSBTAG00000001507 & $5.23 \mathrm{E}-11$ & $3.18 \mathrm{E}-11$ & CLCN5 & $1.64 \mathrm{E}+00$ \\
\hline ENSBTAG00000016399 & $1.50 \mathrm{E}-10$ & $5.72 \mathrm{E}-12$ & AKAP4 & $2.58 \mathrm{E}+01$ \\
\hline ENSBTAG00000046286 & $1.15 \mathrm{E}-10$ & $2.04 \mathrm{E}-11$ & CCNB3 & $5.63 \mathrm{E}+00$ \\
\hline ENSBTAG00000022962 & $1.99 \mathrm{E}-10$ & 6.67E-11 & DGKK & $2.98 \mathrm{E}+00$ \\
\hline ENSBTAG00000002996 & $2.02 \mathrm{E}-10$ & $6.60 \mathrm{E}-11$ & SHROOM4 & $3.05 E+00$ \\
\hline ENSBTAG00000045782 & $2.72 \mathrm{E}-10$ & $9.34 \mathrm{E}-11$ & BMP15 & $2.91 E+00$ \\
\hline ENSBTAG00000005252 & $1.76 \mathrm{E}-10$ & $6.05 \mathrm{E}-11$ & NUDT10 & $2.91 E+00$ \\
\hline ENSBTAG00000048077 & $1.42 \mathrm{E}-10$ & $1.52 \mathrm{E}-10$ & MAGED4B & 9.37E-01 \\
\hline ENSBTAG00000008733 & $1.56 \mathrm{E}-10$ & 9.07E-11 & MAGED1 & $1.72 \mathrm{E}+00$ \\
\hline ENSBTAG00000017767 & $1.34 \mathrm{E}-10$ & $1.59 \mathrm{E}-10$ & GPR173 & $8.42 \mathrm{E}-01$ \\
\hline ENSBTAG00000017783 & $4.75 \mathrm{E}-11$ & $5.98 \mathrm{E}-11$ & TSPYL2 & 7.94E-01 \\
\hline ENSBTAG00000014943 & $5.32 \mathrm{E}-11$ & 7.03E-11 & KDM5C & 7.57E-01 \\
\hline ENSBTAG00000014945 & $4.73 \mathrm{E}-11$ & $5.15 \mathrm{E}-11$ & IQSEC2 & $9.19 \mathrm{E}-01$ \\
\hline ENSBTAG00000017761 & $1.42 \mathrm{E}-11$ & $2.98 \mathrm{E}-11$ & SMC1A & $4.78 \mathrm{E}-01$ \\
\hline ENSBTAG00000017769 & $1.77 \mathrm{E}-11$ & $2.23 \mathrm{E}-11$ & RIBC1 & 7.96E-01 \\
\hline ENSBTAG00000006122 & $3.38 \mathrm{E}-11$ & $1.18 \mathrm{E}-11$ & HUWE1 & $2.85 E+00$ \\
\hline ENSBTAG00000013289 & $6.40 \mathrm{E}-11$ & $1.74 \mathrm{E}-11$ & PHF8 & $3.67 E+00$ \\
\hline ENSBTAG00000003855 & 4.97E-11 & 2.87E-11 & FAM120C & $1.73 \mathrm{E}+00$ \\
\hline ENSBTAG00000023182 & $7.90 \mathrm{E}-11$ & $4.56 \mathrm{E}-11$ & WNK3 & $1.73 \mathrm{E}+00$ \\
\hline ENSBTAG00000015858 & $3.30 \mathrm{E}-11$ & $4.16 \mathrm{E}-11$ & & 7.95E-01 \\
\hline ENSBTAG00000015860 & $3.26 \mathrm{E}-11$ & $2.46 \mathrm{E}-11$ & FGD1 & $1.32 \mathrm{E}+00$ \\
\hline ENSBTAG00000031564 & $1.30 \mathrm{E}-10$ & $6.92 \mathrm{E}-11$ & GNL3L & $1.88 \mathrm{E}+00$ \\
\hline ENSBTAG00000037931 & $1.94 \mathrm{E}-10$ & $2.44 \mathrm{E}-10$ & RPL39 & 7.94E-01 \\
\hline ENSBTAG00000000172 & $6.16 \mathrm{E}-11$ & $3.78 \mathrm{E}-11$ & PFKFB1 & $1.63 E+00$ \\
\hline ENSBTAG00000039888 & $5.63 \mathrm{E}-11$ & $4.26 \mathrm{E}-11$ & MAGED2 & $1.32 \mathrm{E}+00$ \\
\hline ENSBTAG00000013177 & $1.05 \mathrm{E}-11$ & $1.32 \mathrm{E}-11$ & APEX2 & 7.97E-01 \\
\hline ENSBTAG00000013178 & $3.01 \mathrm{E}-11$ & $2.28 \mathrm{E}-11$ & ALAS2 & $1.32 \mathrm{E}+00$ \\
\hline ENSBTAG00000012980 & $5.04 \mathrm{E}-11$ & $2.54 \mathrm{E}-12$ & RRAGB & $1.91 E+01$ \\
\hline ENSBTAG00000005852 & $5.31 \mathrm{E}-11$ & $1.27 \mathrm{E}-11$ & KLF8 & $4.16 \mathrm{E}+00$ \\
\hline ENSBTAG00000038387 & $3.51 \mathrm{E}-11$ & $1.47 \mathrm{E}-11$ & & $2.38 \mathrm{E}+00$ \\
\hline ENSBTAG00000047416 & $6.87 \mathrm{E}-11$ & $3.41 \mathrm{E}-11$ & HEPH & $2.01 E+00$ \\
\hline ENSBTAG00000015769 & $8.59 \mathrm{E}-11$ & $4.75 \mathrm{E}-11$ & VSIG4 & $1.81 E+00$ \\
\hline ENSBTAG00000003418 & $1.92 \mathrm{E}-10$ & $1.46 \mathrm{E}-10$ & MSN & $1.32 \mathrm{E}+00$ \\
\hline ENSBTAG00000002210 & 5.35E-11 & $1.04 \mathrm{E}-11$ & LAS1L & $5.11 E+00$ \\
\hline ENSBTAG00000002206 & $7.52 \mathrm{E}-11$ & $3.79 \mathrm{E}-11$ & $\mathrm{ZC} 3 \mathrm{H} 12 \mathrm{~B}$ & $1.98 \mathrm{E}+00$ \\
\hline ENSBTAG00000003779 & $1.21 \mathrm{E}-10$ & $5.05 \mathrm{E}-11$ & $\mathrm{ZC} 4 \mathrm{H} 2$ & $2.40 \mathrm{E}+00$ \\
\hline ENSBTAG00000022529 & $2.20 \mathrm{E}-10$ & $9.24 \mathrm{E}-11$ & & $2.38 \mathrm{E}+00$ \\
\hline ENSBTAG00000035705 & $9.77 \mathrm{E}-11$ & $4.55 \mathrm{E}-11$ & MTMR8 & $2.14 \mathrm{E}+00$ \\
\hline ENSBTAG00000007211 & $9.80 \mathrm{E}-11$ & $6.17 \mathrm{E}-11$ & ASB12 & $1.59 \mathrm{E}+00$ \\
\hline ENSBTAG00000012729 & $8.73 E-11$ & $3.13 \mathrm{E}-11$ & ARHGEF9 & $2.78 \mathrm{E}+00$ \\
\hline ENSBTAG00000016186 & $9.16 \mathrm{E}-11$ & 5.77E-11 & & $1.59 \mathrm{E}+00$ \\
\hline ENSBTAG00000002956 & $4.55 E-11$ & $9.04 \mathrm{E}-12$ & ZNF674 & $4.99 E+00$ \\
\hline ENSBTAG00000020717 & $2.18 \mathrm{E}-10$ & 2.60E-10 & CXorf36 & 8.37E-01 \\
\hline ENSBTAG00000003740 & $8.45 \mathrm{E}-11$ & $8.69 \mathrm{E}-11$ & KDM6A & $9.72 \mathrm{E}-01$ \\
\hline ENSBTAG00000001637 & $2.82 \mathrm{E}-10$ & $1.86 \mathrm{E}-11$ & FUNDC1 & $1.51 \mathrm{E}+01$ \\
\hline ENSBTAG00000046948 & $6.51 \mathrm{E}-11$ & $8.20 \mathrm{E}-11$ & & 7.94E-01 \\
\hline ENSBTAG00000012623 & 1.17E-10 & $1.75 \mathrm{E}-10$ & NDP & $6.72 \mathrm{E}-01$ \\
\hline ENSBTAG00000001288 & $1.85 \mathrm{E}-10$ & $1.58 \mathrm{E}-10$ & MAOB & $1.17 \mathrm{E}+00$ \\
\hline ENSBTAG00000016206 & $1.44 \mathrm{E}-10$ & $9.39 \mathrm{E}-11$ & MAOA & $1.54 \mathrm{E}+00$ \\
\hline ENSBTAG00000020136 & $1.12 \mathrm{E}-10$ & 8.67E-11 & CASK & $1.29 \mathrm{E}+00$ \\
\hline
\end{tabular}




\begin{tabular}{|c|c|c|c|c|}
\hline ENSBTAG00000035907 & $1.51 \mathrm{E}-10$ & $4.74 \mathrm{E}-11$ & DDX3X & $3.17 E+00$ \\
\hline ENSBTAG00000006242 & $1.36 \mathrm{E}-10$ & 8.19E-11 & USP9Y & $1.66 \mathrm{E}+00$ \\
\hline ENSBTAG00000011790 & $2.48 \mathrm{E}-10$ & $1.03 \mathrm{E}-11$ & MED14 & $2.38 \mathrm{E}+01$ \\
\hline ENSBTAG00000011788 & $3.05 \mathrm{E}-10$ & $1.31 \mathrm{E}-10$ & CXorf38 & $2.33 E+00$ \\
\hline ENSBTAG00000017801 & $5.05 \mathrm{E}-10$ & 4.13E-10 & ATP6AP2 & $1.22 \mathrm{E}+00$ \\
\hline ENSBTAG00000047339 & $2.42 \mathrm{E}-10$ & $6.08 \mathrm{E}-11$ & BCOR & $3.97 E+00$ \\
\hline ENSBTAG00000008966 & $1.59 \mathrm{E}-10$ & $7.71 \mathrm{E}-11$ & TSPAN7 & $2.06 \mathrm{E}+00$ \\
\hline ENSBTAG00000046998 & 0 & $1.22 \mathrm{E}-10$ & & 8.19E-04 \\
\hline ENSBTAG00000001488 & $2.21 \mathrm{E}-10$ & 2.10E-10 & ОтС & $1.05 \mathrm{E}+00$ \\
\hline ENSBTAG00000003893 & $1.43 \mathrm{E}-10$ & $1.71 \mathrm{E}-10$ & & $8.38 \mathrm{E}-01$ \\
\hline ENSBTAG00000045867 & $1.62 \mathrm{E}-10$ & $1.46 \mathrm{E}-10$ & & $1.11 \mathrm{E}+00$ \\
\hline ENSBTAG00000004280 & $1.48 \mathrm{E}-10$ & $1.32 \mathrm{E}-10$ & SRPX & $1.12 \mathrm{E}+00$ \\
\hline ENSBTAG00000004955 & $4.11 \mathrm{E}-10$ & $3.45 \mathrm{E}-10$ & SYTL5 & $1.19 E+00$ \\
\hline ENSBTAG00000038156 & $2.48 \mathrm{E}-10$ & 2.30E-10 & DYNLT3 & $1.08 \mathrm{E}+00$ \\
\hline ENSBTAG00000019953 & $1.44 \mathrm{E}-10$ & $9.09 \mathrm{E}-11$ & CYBB & $1.59 E+00$ \\
\hline ENSBTAG00000012718 & $1.25 \mathrm{E}-10$ & $2.92 \mathrm{E}-11$ & $\mathrm{XK}$ & $4.26 E+00$ \\
\hline ENSBTAG00000003088 & $1.65 \mathrm{E}-10$ & $1.76 \mathrm{E}-11$ & LANCL3 & $9.32 \mathrm{E}+00$ \\
\hline ENSBTAG00000047536 & $8.66 \mathrm{E}-11$ & $3.64 \mathrm{E}-11$ & PRRG1 & $2.38 E+00$ \\
\hline ENSBTAG00000005749 & 5.17E-10 & 2.07E-10 & & $2.50 \mathrm{E}+00$ \\
\hline ENSBTAG00000024662 & $2.18 \mathrm{E}-10$ & $2.13 \mathrm{E}-10$ & & $1.02 \mathrm{E}+00$ \\
\hline ENSBTAG00000047410 & $1.95 \mathrm{E}-10$ & $1.73 \mathrm{E}-10$ & & $1.13 \mathrm{E}+00$ \\
\hline ENSBTAG00000009140 & $2.44 \mathrm{E}-10$ & $4.35 \mathrm{E}-11$ & CFAP47 & $5.59 E+00$ \\
\hline ENSBTAG00000038595 & $2.34 \mathrm{E}-10$ & $6.24 \mathrm{E}-11$ & & $3.74 E+00$ \\
\hline ENSBTAG00000014304 & $2.03 \mathrm{E}-10$ & $1.86 \mathrm{E}-10$ & TMEM47 & $1.09 \mathrm{E}+00$ \\
\hline ENSBTAG00000040357 & $3.93 \mathrm{E}-10$ & $2.86 \mathrm{E}-10$ & & $1.37 \mathrm{E}+00$ \\
\hline ENSBTAG00000004324 & $1.62 \mathrm{E}-10$ & $2.34 \mathrm{E}-10$ & & $6.93 \mathrm{E}-01$ \\
\hline ENSBTAG00000046838 & $1.09 \mathrm{E}-10$ & $1.53 \mathrm{E}-10$ & & 7.13E-01 \\
\hline ENSBTAG00000039904 & $4.29 \mathrm{E}-10$ & $4.86 \mathrm{E}-10$ & & 8.83E-01 \\
\hline ENSBTAG00000008248 & $1.58 \mathrm{E}-10$ & $1.08 \mathrm{E}-10$ & & $1.47 \mathrm{E}+00$ \\
\hline ENSBTAG00000048159 & $1.75 \mathrm{E}-10$ & $4.22 \mathrm{E}-11$ & & $4.15 E+00$ \\
\hline ENSBTAG00000008124 & $1.51 \mathrm{E}-10$ & 2.63E-11 & & $5.72 \mathrm{E}+00$ \\
\hline ENSBTAG00000015532 & $2.54 \mathrm{E}-10$ & 7.10E-11 & NROB1 & $3.58 \mathrm{E}+00$ \\
\hline ENSBTAG00000034064 & 4.42E-10 & 3.34E-10 & MGC133764 & $1.32 \mathrm{E}+00$ \\
\hline ENSBTAG00000035745 & $6.55 \mathrm{E}-10$ & 4.37E-10 & & $1.50 E+00$ \\
\hline ENSBTAG00000004563 & $1.16 \mathrm{E}-09$ & $7.56 \mathrm{E}-10$ & & $1.54 \mathrm{E}+00$ \\
\hline ENSBTAG00000045537 & $1.33 \mathrm{E}-09$ & 1.77E-09 & & 7.49E-01 \\
\hline ENSBTAG00000046252 & $1.73 \mathrm{E}-10$ & $2.18 \mathrm{E}-10$ & & 7.94E-01 \\
\hline ENSBTAG00000014457 & 7.99E-11 & $1.01 \mathrm{E}-10$ & & 7.91E-01 \\
\hline ENSBTAG00000032895 & $1.81 \mathrm{E}-10$ & 3.19E-10 & & 5.67E-01 \\
\hline ENSBTAG00000037496 & $7.82 \mathrm{E}-10$ & 3.77E-10 & & $2.07 E+00$ \\
\hline ENSBTAG00000045890 & $5.32 \mathrm{E}-11$ & $2.01 \mathrm{E}-10$ & & 2.65E-01 \\
\hline ENSBTAG00000011750 & $5.13 \mathrm{E}-10$ & $2.53 \mathrm{E}-10$ & & $2.03 E+00$ \\
\hline ENSBTAG00000047709 & $6.76 \mathrm{E}-10$ & 5.37E-10 & & $1.26 \mathrm{E}+00$ \\
\hline ENSBTAG00000037469 & $2.29 \mathrm{E}-10$ & $5.76 \mathrm{E}-11$ & & $3.97 E+00$ \\
\hline ENSBTAG00000033894 & $1.43 \mathrm{E}-10$ & $1.80 \mathrm{E}-10$ & & 7.94E-01 \\
\hline ENSBTAG00000038876 & $1.74 \mathrm{E}-10$ & $1.31 \mathrm{E}-10$ & & $1.32 \mathrm{E}+00$ \\
\hline ENSBTAG00000008170 & $8.14 \mathrm{E}-11$ & 4.06E-11 & POLA1 & $2.00 E+00$ \\
\hline ENSBTAG00000016107 & $1.70 \mathrm{E}-10$ & $1.37 \mathrm{E}-10$ & PCYT1B & $1.24 \mathrm{E}+00$ \\
\hline ENSBTAG00000017832 & $2.27 \mathrm{E}-10$ & $2.45 \mathrm{E}-10$ & PDK3 & $9.27 \mathrm{E}-01$ \\
\hline ENSBTAG00000006384 & $6.76 \mathrm{E}-11$ & $2.56 \mathrm{E}-10$ & & $2.65 \mathrm{E}-01$ \\
\hline ENSBTAG00000006380 & $2.63 \mathrm{E}-10$ & $1.99 \mathrm{E}-10$ & & $1.32 \mathrm{E}+00$ \\
\hline ENSBTAG00000007730 & $2.97 \mathrm{E}-10$ & $6.07 \mathrm{E}-11$ & ZFY & $4.89 E+00$ \\
\hline ENSBTAG00000014337 & $7.15 \mathrm{E}-11$ & $9.70 \mathrm{E}-11$ & & 7.37E-01 \\
\hline ENSBTAG00000020561 & $9.33 \mathrm{E}-11$ & $5.56 \mathrm{E}-11$ & KLHL15 & $1.68 \mathrm{E}+00$ \\
\hline ENSBTAG00000010939 & $1.36 \mathrm{E}-10$ & $4.43 \mathrm{E}-11$ & CXorf58 & $3.07 E+00$ \\
\hline ENSBTAG00000010937 & $3.36 \mathrm{E}-11$ & $2.16 \mathrm{E}-10$ & APOO & $1.56 \mathrm{E}-01$ \\
\hline ENSBTAG00000017363 & $2.69 \mathrm{E}-11$ & $3.38 \mathrm{E}-11$ & SSAT-1 & 7.95E-01 \\
\hline ENSBTAG00000011258 & $5.61 \mathrm{E}-11$ & $1.20 \mathrm{E}-10$ & АСОT9 & 4.68E-01 \\
\hline ENSBTAG00000006165 & $1.14 \mathrm{E}-10$ & $3.98 \mathrm{E}-11$ & PRDX4 & $2.86 \mathrm{E}+00$ \\
\hline ENSBTAG00000020987 & $6.99 \mathrm{E}-11$ & $6.70 \mathrm{E}-11$ & PTCHD1 & $1.04 \mathrm{E}+00$ \\
\hline ENSBTAG00000046635 & $7.22 \mathrm{E}-10$ & $6.53 \mathrm{E}-10$ & DDX53 & $1.11 E+00$ \\
\hline ENSBTAG00000016653 & $2.69 \mathrm{E}-10$ & $3.39 \mathrm{E}-10$ & & 7.93E-01 \\
\hline ENSBTAG00000036343 & 0 & $8.52 \mathrm{E}-11$ & & 1.17E-03 \\
\hline ENSBTAG00000000419 & $1.99 \mathrm{E}-10$ & $1.32 \mathrm{E}-10$ & PHEX & $1.51 E+00$ \\
\hline ENSBTAG00000018382 & $1.69 \mathrm{E}-10$ & $7.23 \mathrm{E}-11$ & SMS & $2.34 \mathrm{E}+00$ \\
\hline ENSBTAG00000008183 & $9.26 \mathrm{E}-11$ & $2.57 \mathrm{E}-11$ & MBTPS2 & $3.59 E+00$ \\
\hline ENSBTAG00000015204 & 8.00E-11 & 7.37E-11 & SMPX & $1.09 E+00$ \\
\hline ENSBTAG00000044202 & $8.90 \mathrm{E}-11$ & 5.63E-11 & CNKSR2 & $1.58 \mathrm{E}+00$ \\
\hline ENSBTAG00000047699 & $1.08 \mathrm{E}-09$ & 4.19E-10 & & $2.58 \mathrm{E}+00$ \\
\hline ENSBTAG00000017639 & $9.22 \mathrm{E}-11$ & $6.58 \mathrm{E}-11$ & RPS6KA3 & $1.40 \mathrm{E}+00$ \\
\hline
\end{tabular}




\begin{tabular}{|c|c|c|c|c|}
\hline ENSBTAG000000017633 & $5.79 \mathrm{E}-11$ & 3.13E-11 & EIF1AX & $1.85 E+00$ \\
\hline ENSBTAG000000003727 & $4.86 \mathrm{E}-11$ & $9.80 \mathrm{E}-12$ & SH3KBP1 & $4.92 E+00$ \\
\hline ENSBTAG000000019855 & $8.22 \mathrm{E}-11$ & $1.70 \mathrm{E}-11$ & MAP3K15 & $4.82 E+00$ \\
\hline ENSBTAG000000019852 & 4.65E-11 & $4.50 \mathrm{E}-12$ & PDHA1 & $1.01 E+01$ \\
\hline ENSBTAG000000038660 & $3.42 \mathrm{E}-10$ & $2.62 \mathrm{E}-10$ & MGC134232 & $1.31 E+00$ \\
\hline ENSBTAG000000000576 & $1.34 \mathrm{E}-10$ & $1.42 \mathrm{E}-10$ & ADGRG2 & $9.42 \mathrm{E}-01$ \\
\hline ENSBTAG000000015355 & $1.70 \mathrm{E}-10$ & $1.72 \mathrm{E}-10$ & PHKA2 & $9.91 \mathrm{E}-01$ \\
\hline ENSBTAG000000009545 & $1.29 \mathrm{E}-10$ & $3.89 \mathrm{E}-10$ & PPEF1 & $3.31 \mathrm{E}-01$ \\
\hline ENSBTAG000000031145 & $9.43 \mathrm{E}-11$ & $1.25 \mathrm{E}-10$ & RS1 & $7.55 \mathrm{E}-01$ \\
\hline ENSBTAG000000007428 & $6.52 E-11$ & $1.92 \mathrm{E}-10$ & CDKL5 & 3.40E-01 \\
\hline ENSBTAG000000009420 & $9.36 \mathrm{E}-11$ & $8.71 E-11$ & SCML2 & $1.07 E+00$ \\
\hline ENSBTAG000000031198 & $9.47 \mathrm{E}-11$ & $8.48 \mathrm{E}-11$ & BEND2 & $1.12 \mathrm{E}+00$ \\
\hline ENSBTAG000000007022 & 7.87E-11 & $9.91 \mathrm{E}-11$ & & 7.94E-01 \\
\hline ENSBTAG000000017649 & $9.55 \mathrm{E}-11$ & $5.06 \mathrm{E}-11$ & SCML1 & $1.89 E+00$ \\
\hline ENSBTAG000000039195 & $9.68 \mathrm{E}-11$ & $1.30 \mathrm{E}-10$ & NHS & 7.45E-01 \\
\hline ENSBTAG000000031261 & $1.44 \mathrm{E}-10$ & $5.98 \mathrm{E}-11$ & REPS2 & $2.41 E+00$ \\
\hline ENSBTAG000000002820 & $8.81 E-11$ & $4.98 \mathrm{E}-11$ & RBBP7 & $1.77 \mathrm{E}+00$ \\
\hline ENSBTAG000000001768 & $3.59 \mathrm{E}-11$ & 2.77E-11 & TXLNG & $1.30 \mathrm{E}+00$ \\
\hline ENSBTAG000000003316 & 4.64E-11 & $3.25 \mathrm{E}-11$ & SYAP1 & $1.43 E+00$ \\
\hline ENSBTAG000000017017 & $1.13 \mathrm{E}-10$ & $1.09 \mathrm{E}-10$ & CTPS2 & $1.03 E+00$ \\
\hline ENSBTAG00000045809 & 0 & $7.42 \mathrm{E}-10$ & & $1.35 \mathrm{E}-04$ \\
\hline ENSBTAG000000021126 & $3.64 \mathrm{E}-10$ & $6.32 \mathrm{E}-10$ & & 5.77E-01 \\
\hline ENSBTAG000000007092 & $1.61 \mathrm{E}-10$ & $2.31 E-10$ & GRPR & 6.99E-01 \\
\hline ENSBTAG000000020420 & $9.58 \mathrm{E}-11$ & $6.03 \mathrm{E}-11$ & AP1S2 & $1.59 \mathrm{E}+00$ \\
\hline ENSBTAG00000020419 & $2.15 \mathrm{E}-10$ & $7.98 \mathrm{E}-11$ & & $2.69 E+00$ \\
\hline ENSBTAG000000020418 & $1.29 \mathrm{E}-10$ & $1.58 \mathrm{E}-10$ & CA5B & $8.14 \mathrm{E}-01$ \\
\hline ENSBTAG000000048296 & $2.35 \mathrm{E}-10$ & $2.51 E-10$ & & 9.37E-01 \\
\hline ENSBTAG000000047861 & $2.22 \mathrm{E}-10$ & 4.60E-10 & & 4.82E-01 \\
\hline ENSBTAG000000034402 & 2.63E-10 & $1.58 \mathrm{E}-10$ & ACE2 & $1.66 \mathrm{E}+00$ \\
\hline ENSBTAG000000000377 & $1.33 \mathrm{E}-10$ & $1.23 \mathrm{E}-10$ & BMX & $1.08 \mathrm{E}+00$ \\
\hline ENSBTAG000000009477 & $1.91 \mathrm{E}-10$ & $1.41 \mathrm{E}-10$ & PIR & $1.36 \mathrm{E}+00$ \\
\hline ENSBTAG000000009476 & $2.02 \mathrm{E}-10$ & $1.95 \mathrm{E}-10$ & VEGFD & $1.04 \mathrm{E}+00$ \\
\hline ENSBTAG000000007646 & $1.21 \mathrm{E}-10$ & $5.44 \mathrm{E}-11$ & PIGA & $2.22 \mathrm{E}+00$ \\
\hline ENSBTAG000000001514 & $1.47 \mathrm{E}-10$ & $6.41 E-11$ & ASB11 & $2.29 E+00$ \\
\hline ENSBTAG00000009198 & $3.38 \mathrm{E}-10$ & $2.92 \mathrm{E}-10$ & ASB9 & $1.16 \mathrm{E}+00$ \\
\hline ENSBTAG00000018292 & $1.79 \mathrm{E}-10$ & $1.45 \mathrm{E}-10$ & & $1.24 \mathrm{E}+00$ \\
\hline ENSBTAG00000016433 & $9.99 \mathrm{E}-11$ & $2.16 \mathrm{E}-10$ & FANCB & 4.63E-01 \\
\hline ENSBTAG00000017209 & $3.50 \mathrm{E}-10$ & $1.38 \mathrm{E}-10$ & GLRA2 & $2.54 \mathrm{E}+00$ \\
\hline ENSBTAG00000010289 & $1.08 \mathrm{E}-10$ & $5.92 \mathrm{E}-12$ & GEMIN8 & $1.80 \mathrm{E}+01$ \\
\hline ENSBTAG00000009260 & $1.69 \mathrm{E}-10$ & $1.12 \mathrm{E}-10$ & GPM6B & $1.51 E+00$ \\
\hline ENSBTAG00000004461 & $8.27 \mathrm{E}-11$ & 4.87E-11 & OFD1 & $1.70 \mathrm{E}+00$ \\
\hline ENSBTAG00000010922 & $1.15 \mathrm{E}-10$ & 5.97E-11 & & $1.93 \mathrm{E}+00$ \\
\hline ENSBTAG00000010923 & $9.68 \mathrm{E}-11$ & $4.24 \mathrm{E}-11$ & RAB9A & $2.28 \mathrm{E}+00$ \\
\hline ENSBTAG00000017945 & $3.11 \mathrm{E}-10$ & $3.72 \mathrm{E}-11$ & & $8.33 E+00$ \\
\hline ENSBTAG00000017927 & $4.47 \mathrm{E}-10$ & $3.00 \mathrm{E}-10$ & EGFL6 & $1.49 E+00$ \\
\hline ENSBTAG00000032833 & $6.49 \mathrm{E}-10$ & $7.43 \mathrm{E}-10$ & & $8.73 \mathrm{E}-01$ \\
\hline ENSBTAG00000012089 & $1.35 \mathrm{E}-10$ & $1.18 \mathrm{E}-10$ & & $1.15 E+00$ \\
\hline ENSBTAG00000002626 & $1.57 \mathrm{E}-10$ & $1.05 \mathrm{E}-10$ & ARHGAP6 & $1.50 \mathrm{E}+00$ \\
\hline ENSBTAG00000012113 & $2.07 \mathrm{E}-10$ & $1.18 \mathrm{E}-11$ & $\mathrm{HCCS}$ & $1.74 \mathrm{E}+01$ \\
\hline ENSBTAG00000014367 & $1.14 \mathrm{E}-09$ & $6.13 \mathrm{E}-10$ & PRKX & $1.86 \mathrm{E}+00$ \\
\hline ENSBTAG00000022150 & $4.28 \mathrm{E}-10$ & $3.02 \mathrm{E}-10$ & MXRA5 & $1.42 \mathrm{E}+00$ \\
\hline ENSBTAG00000047161 & $9.99 \mathrm{E}-10$ & $9.58 \mathrm{E}-10$ & ARSH & $1.04 E+00$ \\
\hline ENSBTAG00000014932 & $6.03 \mathrm{E}-10$ & $5.52 \mathrm{E}-10$ & ARSE & $1.09 \mathrm{E}+00$ \\
\hline ENSBTAG00000010152 & $2.16 \mathrm{E}-10$ & $1.88 \mathrm{E}-10$ & MID1 & $1.15 \mathrm{E}+00$ \\
\hline ENSBTAG00000013341 & $7.92 \mathrm{E}-10$ & 4.77E-10 & GYG2 & $1.66 \mathrm{E}+00$ \\
\hline ENSBTAG00000046328 & $7.40 \mathrm{E}-10$ & $6.44 \mathrm{E}-10$ & $X G$ & $1.15 E+00$ \\
\hline ENSBTAG00000008114 & $9.60 \mathrm{E}-10$ & $5.80 \mathrm{E}-10$ & CD99 & $1.66 \mathrm{E}+00$ \\
\hline ENSBTAG00000048287 & 7.15E-10 & $6.09 \mathrm{E}-10$ & & $1.17 E+00$ \\
\hline ENSBTAG00000038488 & $7.93 \mathrm{E}-11$ & $4.99 \mathrm{E}-11$ & TMSB4Y & $1.59 \mathrm{E}+00$ \\
\hline ENSBTAG00000022161 & $8.09 E-11$ & 6.97E-11 & TLR7 & $1.16 \mathrm{E}+00$ \\
\hline ENSBTAG00000047997 & $9.80 \mathrm{E}-11$ & $7.20 \mathrm{E}-11$ & PRPS2 & $1.36 \mathrm{E}+00$ \\
\hline ENSBTAG00000020803 & $1.31 \mathrm{E}-10$ & $5.52 E-11$ & & $2.38 \mathrm{E}+00$ \\
\hline ENSBTAG00000017785 & $1.37 \mathrm{E}-10$ & $1.01 \mathrm{E}-10$ & FRMPD4 & $1.36 \mathrm{E}+00$ \\
\hline ENSBTAG00000009278 & $2.22 \mathrm{E}-10$ & $6.66 \mathrm{E}-11$ & MSL3 & $3.33 E+00$ \\
\hline ENSBTAG00000048172 & $1.30 \mathrm{E}-11$ & 2.13E-10 & & $6.15 \mathrm{E}-02$ \\
\hline ENSBTAG00000011121 & $1.86 \mathrm{E}-10$ & $1.43 \mathrm{E}-10$ & CLCN4 & $1.30 \mathrm{E}+00$ \\
\hline ENSBTAG00000013824 & $1.74 \mathrm{E}-10$ & $1.37 \mathrm{E}-10$ & WWC3 & $1.27 \mathrm{E}+00$ \\
\hline ENSBTAG00000009932 & $5.72 \mathrm{E}-11$ & $1.01 \mathrm{E}-10$ & & $5.66 \mathrm{E}-01$ \\
\hline ENSBTAG00000046123 & $3.32 \mathrm{E}-11$ & $2.08 \mathrm{E}-10$ & UTY & $1.60 \mathrm{E}-01$ \\
\hline ENSBTAG00000045750 & 7.55E-12 & $2.29 \mathrm{E}-10$ & DDX3Y & $3.34 \mathrm{E}-02$ \\
\hline
\end{tabular}




\begin{tabular}{|c|c|c|c|c|}
\hline ENSBTAG00000047068 & $2.06 \mathrm{E}-10$ & $1.01 \mathrm{E}-10$ & & $2.03 E+00$ \\
\hline ENSBTAG00000002158 & $3.53 \mathrm{E}-10$ & $3.31 \mathrm{E}-10$ & & $1.07 E+00$ \\
\hline ENSBTAG00000035438 & $3.12 \mathrm{E}-10$ & $6.04 \mathrm{E}-10$ & & 5.17E-01 \\
\hline ENSBTAG00000046131 & $2.40 \mathrm{E}-10$ & $5.19 E-10$ & & 4.63E-01 \\
\hline ENSBTAG00000035122 & $2.25 \mathrm{E}-10$ & $8.63 \mathrm{E}-11$ & & $2.61 \mathrm{E}+00$ \\
\hline ENSBTAG00000048102 & $1.02 \mathrm{E}-11$ & $1.64 \mathrm{E}-10$ & & $6.27 \mathrm{E}-02$ \\
\hline ENSBTAG00000000211 & $7.95 \mathrm{E}-10$ & $2.00 \mathrm{E}-10$ & & $3.97 E+00$ \\
\hline ENSBTAG00000006472 & $6.27 \mathrm{E}-10$ & 9.77E-11 & GPR143 & $6.41 E+00$ \\
\hline ENSBTAG00000047774 & 0 & $1.20 \mathrm{E}-10$ & & 8.33E-04 \\
\hline ENSBTAG00000046201 & $4.26 \mathrm{E}-11$ & $2.30 \mathrm{E}-10$ & & $1.86 \mathrm{E}-01$ \\
\hline ENSBTAG00000045544 & $5.99 \mathrm{E}-11$ & $2.39 \mathrm{E}-10$ & & $2.51 \mathrm{E}-01$ \\
\hline ENSBTAG00000018112 & 7.97E-10 & $4.29 \mathrm{E}-10$ & TBL1Y & $1.86 \mathrm{E}+00$ \\
\hline ENSBTAG00000001049 & $9.98 \mathrm{E}-10$ & $6.90 \mathrm{E}-10$ & ANOS1 & $1.45 \mathrm{E}+00$ \\
\hline ENSBTAG00000015849 & 1.55E-09 & 1.10E-09 & PNPLA4 & $1.41 E+00$ \\
\hline ENSBTAG00000020789 & $5.74 \mathrm{E}-10$ & $7.00 \mathrm{E}-10$ & STS & 8.19E-01 \\
\hline ENSBTAG00000000207 & $7.64 \mathrm{E}-10$ & $6.31 \mathrm{E}-10$ & PUDP & $1.21 \mathrm{E}+00$ \\
\hline ENSBTAG00000027841 & $8.72 \mathrm{E}-10$ & $5.58 \mathrm{E}-10$ & & $1.56 \mathrm{E}+00$ \\
\hline ENSBTAG00000046084 & $2.15 E-10$ & $1.65 \mathrm{E}-10$ & & $1.30 \mathrm{E}+00$ \\
\hline ENSBTAG00000016820 & $7.74 \mathrm{E}-10$ & $1.12 \mathrm{E}-10$ & & $6.91 E+00$ \\
\hline ENSBTAG00000012491 & $6.71 \mathrm{E}-10$ & $7.27 \mathrm{E}-10$ & & $9.23 \mathrm{E}-01$ \\
\hline ENSBTAG00000025951 & $3.72 \mathrm{E}-10$ & $1.89 \mathrm{E}-10$ & & $1.97 \mathrm{E}+00$ \\
\hline ENSBTAG00000016989 & $5.09 \mathrm{E}-10$ & $5.68 \mathrm{E}-10$ & & 8.96E-01 \\
\hline ENSBTAG00000025952 & $2.89 \mathrm{E}-10$ & $3.11 \mathrm{E}-10$ & & $9.28 \mathrm{E}-01$ \\
\hline ENSBTAG00000039391 & $5.58 \mathrm{E}-11$ & 7.03E-11 & USP27X & 7.94E-01 \\
\hline ENSBTAG00000048163 & $6.48 \mathrm{E}-10$ & $8.17 E-10$ & & 7.93E-01 \\
\hline ENSBTAG00000047547 & $1.38 \mathrm{E}-09$ & $1.74 \mathrm{E}-09$ & & 7.96E-01 \\
\hline ENSBTAG00000046531 & $4.72 \mathrm{E}-10$ & $5.95 \mathrm{E}-10$ & & 7.94E-01 \\
\hline ENSBTAG00000008839 & 0 & $3.07 E-11$ & AMER1 & $3.25 \mathrm{E}-03$ \\
\hline ENSBTAG00000017948 & $2.19 \mathrm{E}-10$ & $1.38 \mathrm{E}-10$ & SPIN4 & $1.58 \mathrm{E}+00$ \\
\hline ENSBTAG00000008911 & $4.76 \mathrm{E}-10$ & $1.28 \mathrm{E}-10$ & & $3.72 E+00$ \\
\hline ENSBTAG00000031523 & $1.01 \mathrm{E}-10$ & 4.22E-11 & & $2.38 \mathrm{E}+00$ \\
\hline ENSBTAG00000046407 & $5.70 \mathrm{E}-11$ & 7.18E-11 & NYX & 7.94E-01 \\
\hline ENSBTAG00000046448 & $2.97 \mathrm{E}-10$ & $3.75 E-10$ & & 7.93E-01 \\
\hline ENSBTAG00000047493 & $5.18 \mathrm{E}-10$ & $1.30 \mathrm{E}-10$ & & $3.99 E+00$ \\
\hline ENSBTAG00000012533 & $2.97 \mathrm{E}-10$ & $3.75 \mathrm{E}-10$ & & 7.93E-01 \\
\hline ENSBTAG00000034437 & $6.55 E-10$ & $6.18 \mathrm{E}-10$ & & $1.06 \mathrm{E}+00$ \\
\hline ENSBTAG00000013995 & $1.58 \mathrm{E}-10$ & $1.99 \mathrm{E}-10$ & & 7.94E-01 \\
\hline ENSBTAG00000037704 & $7.85 \mathrm{E}-10$ & $6.58 \mathrm{E}-10$ & & $1.19 E+00$ \\
\hline ENSBTAG00000031320 & $3.92 \mathrm{E}-10$ & $3.95 \mathrm{E}-10$ & & 9.93E-01 \\
\hline ENSBTAG00000008511 & 3.53E-11 & $4.45 \mathrm{E}-11$ & PPP4R3CP & 7.94E-01 \\
\hline ENSBTAG00000047457 & $3.98 \mathrm{E}-10$ & $5.01 \mathrm{E}-10$ & & 7.94E-01 \\
\hline ENSBTAG00000046171 & $3.18 \mathrm{E}-10$ & 2.00E-10 & & $1.59 E+00$ \\
\hline ENSBTAG00000047665 & $2.56 \mathrm{E}-10$ & $1.07 E-10$ & & $2.39 E+00$ \\
\hline ENSBTAG00000038751 & $8.01 \mathrm{E}-11$ & $1.01 \mathrm{E}-10$ & MAGEB18 & 7.93E-01 \\
\hline ENSBTAG00000006383 & $2.06 \mathrm{E}-10$ & $2.60 \mathrm{E}-10$ & & 7.93E-01 \\
\hline ENSBTAG00000047581 & $4.78 \mathrm{E}-10$ & 4.01E-10 & & $1.19 E+00$ \\
\hline ENSBTAG00000011277 & $1.68 \mathrm{E}-10$ & $2.11 \mathrm{E}-10$ & & 7.94E-01 \\
\hline ENSBTAG00000016019 & $8.94 \mathrm{E}-10$ & $8.44 \mathrm{E}-10$ & & $1.06 \mathrm{E}+00$ \\
\hline ENSBTAG00000045756 & $3.04 \mathrm{E}-10$ & $3.83 E-10$ & & 7.94E-01 \\
\hline ENSBTAG00000040043 & $4.30 \mathrm{E}-11$ & $5.42 \mathrm{E}-11$ & ARMCX6 & 7.94E-01 \\
\hline ENSBTAG00000032806 & 0 & $1.88 \mathrm{E}-10$ & & $5.32 \mathrm{E}-04$ \\
\hline ENSBTAG00000014287 & $5.27 \mathrm{E}-10$ & $5.31 \mathrm{E}-10$ & & $9.93 \mathrm{E}-01$ \\
\hline ENSBTAG00000005290 & $6.13 \mathrm{E}-11$ & $3.86 \mathrm{E}-11$ & CLDN2 & $1.59 E+00$ \\
\hline ENSBTAG00000006535 & $2.17 \mathrm{E}-11$ & $2.74 \mathrm{E}-11$ & IRS4 & 7.94E-01 \\
\hline ENSBTAG00000046820 & $4.38 \mathrm{E}-10$ & $2.76 \mathrm{E}-10$ & & $1.59 \mathrm{E}+00$ \\
\hline ENSBTAG00000032696 & 3.36E-10 & $1.41 \mathrm{E}-10$ & & $2.38 \mathrm{E}+00$ \\
\hline ENSBTAG00000012319 & $5.86 \mathrm{E}-11$ & $7.38 \mathrm{E}-11$ & KCNE5 & 7.94E-01 \\
\hline ENSBTAG00000047218 & $3.08 \mathrm{E}-09$ & $1.81 \mathrm{E}-09$ & & $1.70 E+00$ \\
\hline ENSBTAG00000045614 & $3.58 \mathrm{E}-10$ & $2.25 \mathrm{E}-10$ & & $1.59 E+00$ \\
\hline ENSBTAG00000045678 & $1.21 \mathrm{E}-10$ & $1.52 \mathrm{E}-10$ & & 7.96E-01 \\
\hline ENSBTAG00000046678 & $1.36 \mathrm{E}-10$ & $1.71 \mathrm{E}-10$ & & 7.95E-01 \\
\hline ENSBTAG00000040541 & $7.51 \mathrm{E}-10$ & $9.40 \mathrm{E}-11$ & P2RY4 & $7.98 \mathrm{E}+00$ \\
\hline ENSBTAG00000047404 & $3.45 \mathrm{E}-10$ & $3.26 \mathrm{E}-10$ & & $1.06 \mathrm{E}+00$ \\
\hline ENSBTAG00000003343 & $7.54 \mathrm{E}-10$ & 4.20E-10 & & $1.79 E+00$ \\
\hline ENSBTAG00000024641 & $3.53 \mathrm{E}-10$ & $4.45 \mathrm{E}-10$ & ZBED1 & 7.93E-01 \\
\hline ENSBTAG00000021684 & $2.89 \mathrm{E}-10$ & $2.73 \mathrm{E}-10$ & & $1.06 \mathrm{E}+00$ \\
\hline ENSBTAG00000047462 & 0 & $5.34 \mathrm{E}-10$ & & 1.87E-04 \\
\hline ENSBTAG00000047839 & $2.31 \mathrm{E}-10$ & $2.91 \mathrm{E}-10$ & P2RY8 & 7.94E-01 \\
\hline ENSBTAG00000006034 & $6.40 \mathrm{E}-11$ & $8.06 \mathrm{E}-11$ & & 7.94E-01 \\
\hline ENSBTAG00000000154 & $9.96 \mathrm{E}-10$ & $1.25 \mathrm{E}-09$ & IRF2BP1 & 7.97E-01 \\
\hline
\end{tabular}




\begin{tabular}{|c|c|c|c|c|}
\hline ENSBTAG00000046395 & $1.28 \mathrm{E}-10$ & $1.61 \mathrm{E}-10$ & CCDC169 & 7.93E-01 \\
\hline ENSBTAG00000047994 & $1.66 \mathrm{E}-10$ & $2.09 \mathrm{E}-10$ & & $7.92 \mathrm{E}-01$ \\
\hline ENSBTAG00000024210 & $8.20 \mathrm{E}-11$ & $1.03 E-10$ & & 7.96E-01 \\
\hline ENSBTAG00000021344 & $6.09 \mathrm{E}-11$ & $1.53 \mathrm{E}-10$ & USP26 & $3.98 \mathrm{E}-01$ \\
\hline ENSBTAG00000048173 & $2.22 \mathrm{E}-10$ & $2.80 \mathrm{E}-10$ & & 7.93E-01 \\
\hline ENSBTAG00000038228 & $1.38 \mathrm{E}-10$ & $1.74 \mathrm{E}-10$ & & 7.94E-01 \\
\hline ENSBTAG00000036257 & $1.76 \mathrm{E}-10$ & 4.45E-10 & & $3.96 \mathrm{E}-01$ \\
\hline ENSBTAG00000045569 & $4.53 \mathrm{E}-10$ & $3.26 \mathrm{E}-10$ & & $1.39 \mathrm{E}+00$ \\
\hline ENSBTAG00000011780 & $1.30 \mathrm{E}-10$ & $1.23 \mathrm{E}-10$ & SLITRK4 & $1.06 \mathrm{E}+00$ \\
\hline ENSBTAG00000019009 & $3.21 \mathrm{E}-10$ & $3.03 \mathrm{E}-10$ & & $1.06 \mathrm{E}+00$ \\
\hline ENSBTAG00000039377 & $2.26 \mathrm{E}-10$ & $2.44 \mathrm{E}-10$ & SLITRK2 & $9.28 \mathrm{E}-01$ \\
\hline ENSBTAG00000036060 & $3.16 \mathrm{E}-10$ & $2.65 \mathrm{E}-10$ & & $1.19 \mathrm{E}+00$ \\
\hline ENSBTAG00000045798 & $2.15 \mathrm{E}-10$ & $2.71 \mathrm{E}-10$ & & 7.94E-01 \\
\hline ENSBTAG00000002805 & $2.88 \mathrm{E}-10$ & $9.05 \mathrm{E}-11$ & & $3.18 \mathrm{E}+00$ \\
\hline ENSBTAG00000012748 & 7.96E-11 & $2.01 \mathrm{E}-10$ & & $3.96 \mathrm{E}-01$ \\
\hline ENSBTAG00000023978 & $5.91 \mathrm{E}-10$ & 1.03E-09 & & $5.74 \mathrm{E}-01$ \\
\hline ENSBTAG00000047711 & 0 & 0 & & $1.00 \mathrm{E}+00$ \\
\hline ENSBTAG00000047392 & $5.16 \mathrm{E}-10$ & 0 & & $5.16 \mathrm{E}+03$ \\
\hline ENSBTAG00000046835 & 0 & 0 & & $1.00 \mathrm{E}+00$ \\
\hline ENSBTAG00000025651 & 0 & 0 & & $1.00 \mathrm{E}+00$ \\
\hline ENSBTAG00000019221 & $2.44 \mathrm{E}-10$ & 0 & & $2.45 E+03$ \\
\hline ENSBTAG00000000567 & $1.42 \mathrm{E}-10$ & 0 & & $1.42 E+03$ \\
\hline ENSBTAG00000036144 & $1.86 \mathrm{E}-10$ & 0 & & $1.86 \mathrm{E}+03$ \\
\hline ENSBTAG00000047819 & 3.57E-10 & 0 & & $3.57 E+03$ \\
\hline ENSBTAG00000014397 & 0 & 0 & & $1.00 \mathrm{E}+00$ \\
\hline ENSBTAG00000045773 & $1.14 \mathrm{E}-10$ & 0 & & $1.14 E+03$ \\
\hline ENSBTAG00000036058 & $2.18 \mathrm{E}-11$ & 0 & & $2.19 E+02$ \\
\hline ENSBTAG00000046565 & $2.11 \mathrm{E}-10$ & 0 & & $2.12 E+03$ \\
\hline ENSBTAG00000006296 & $1.74 \mathrm{E}-10$ & 0 & VMA21 & $1.74 \mathrm{E}+03$ \\
\hline ENSBTAG00000046340 & $3.20 \mathrm{E}-11$ & 0 & & $3.21 E+02$ \\
\hline ENSBTAG00000047230 & $1.56 \mathrm{E}-10$ & 0 & & $1.56 \mathrm{E}+03$ \\
\hline ENSBTAG00000019779 & 0 & 0 & RAB39B & $1.00 \mathrm{E}+00$ \\
\hline ENSBTAG00000007813 & $1.42 \mathrm{E}-11$ & 0 & CMC4 & $1.43 E+02$ \\
\hline ENSBTAG00000045697 & 0 & 0 & MTCP1 & $1.00 \mathrm{E}+00$ \\
\hline ENSBTAG00000047917 & $3.78 \mathrm{E}-10$ & 0 & & $3.79 E+03$ \\
\hline ENSBTAG00000000520 & $7.54 \mathrm{E}-12$ & 0 & ATP2B3 & $7.64 E+01$ \\
\hline ENSBTAG00000008319 & 0 & 0 & DUSP9 & $1.00 \mathrm{E}+00$ \\
\hline ENSBTAG00000021422 & 3.34E-11 & 0 & PDZD4 & $3.35 E+02$ \\
\hline ENSBTAG00000045947 & 0 & 0 & & $1.00 \mathrm{E}+00$ \\
\hline ENSBTAG00000022314 & 3.55E-11 & 0 & EMD & $3.56 \mathrm{E}+02$ \\
\hline ENSBTAG00000007454 & 0 & 0 & & $1.00 \mathrm{E}+00$ \\
\hline ENSBTAG00000034444 & 0 & 0 & & $1.00 \mathrm{E}+00$ \\
\hline ENSBTAG00000019407 & 0 & 0 & & $1.00 \mathrm{E}+00$ \\
\hline ENSBTAG00000022339 & $6.84 \mathrm{E}-11$ & 0 & & $6.85 E+02$ \\
\hline ENSBTAG00000010114 & 0 & 0 & ARMCX5 & $1.00 \mathrm{E}+00$ \\
\hline ENSBTAG00000032875 & 0 & 0 & & $1.00 \mathrm{E}+00$ \\
\hline ENSBTAG00000027052 & $2.47 \mathrm{E}-10$ & 0 & & $2.47 E+03$ \\
\hline ENSBTAG00000009457 & 0 & 0 & & $1.00 \mathrm{E}+00$ \\
\hline ENSBTAG00000001979 & $1.86 \mathrm{E}-11$ & 0 & TAF7L & $1.87 E+02$ \\
\hline ENSBTAG00000022292 & 0 & 0 & TIMM8A & $1.00 \mathrm{E}+00$ \\
\hline ENSBTAG00000019250 & $1.93 \mathrm{E}-11$ & 0 & BTK & $1.94 E+02$ \\
\hline ENSBTAG00000019253 & 0 & 0 & & $1.00 \mathrm{E}+00$ \\
\hline ENSBTAG00000019256 & $7.68 \mathrm{E}-12$ & 0 & GLA & $7.78 \mathrm{E}+01$ \\
\hline ENSBTAG00000007499 & $2.53 \mathrm{E}-11$ & 0 & HNRNPH2 & $2.54 E+02$ \\
\hline ENSBTAG00000026141 & $5.60 \mathrm{E}-11$ & 0 & & $5.61 \mathrm{E}+02$ \\
\hline ENSBTAG00000038204 & 0 & 0 & & $1.00 \mathrm{E}+00$ \\
\hline ENSBTAG00000037996 & $1.69 \mathrm{E}-11$ & 0 & ARMCX3 & $1.70 \mathrm{E}+02$ \\
\hline ENSBTAG00000047867 & 0 & 0 & & $1.00 \mathrm{E}+00$ \\
\hline ENSBTAG00000045706 & $1.00 \mathrm{E}-10$ & 0 & & $1.00 \mathrm{E}+03$ \\
\hline ENSBTAG00000048143 & 0 & 0 & TCEAL9 & $1.00 \mathrm{E}+00$ \\
\hline ENSBTAG00000047295 & 0 & 0 & & $1.00 \mathrm{E}+00$ \\
\hline ENSBTAG00000014223 & $4.18 \mathrm{E}-11$ & 0 & RAN & $4.19 E+02$ \\
\hline ENSBTAG00000008485 & 0 & 0 & TCEAL1 & $1.00 \mathrm{E}+00$ \\
\hline ENSBTAG00000046436 & $1.21 \mathrm{E}-10$ & 0 & & $1.21 \mathrm{E}+03$ \\
\hline ENSBTAG00000046030 & 0 & 0 & & $1.00 \mathrm{E}+00$ \\
\hline ENSBTAG00000044115 & $5.33 \mathrm{E}-11$ & 0 & RIPPLY1 & $5.34 E+02$ \\
\hline ENSBTAG00000046327 & 0 & 0 & & $1.00 \mathrm{E}+00$ \\
\hline ENSBTAG00000002668 & $2.59 \mathrm{E}-11$ & 0 & ZNF711 & $2.60 \mathrm{E}+02$ \\
\hline ENSBTAG00000013077 & 0 & 0 & & $1.00 \mathrm{E}+00$ \\
\hline ENSBTAG00000003299 & $7.38 \mathrm{E}-12$ & 0 & LPAR4 & $7.48 \mathrm{E}+01$ \\
\hline
\end{tabular}




\begin{tabular}{|c|c|c|c|c|}
\hline ENSBTAG00000008326 & 0 & 0 & ZCCHC5 & $1.00 \mathrm{E}+00$ \\
\hline ENSBTAG00000024841 & 0 & 0 & & $1.00 \mathrm{E}+00$ \\
\hline ENSBTAG00000003609 & 0 & 0 & PBDC1 & $1.00 \mathrm{E}+00$ \\
\hline ENSBTAG00000001726 & $1.26 \mathrm{E}-10$ & 0 & CDX4 & $1.26 \mathrm{E}+03$ \\
\hline ENSBTAG00000014798 & $2.34 \mathrm{E}-10$ & 0 & CXCR3 & $2.34 \mathrm{E}+03$ \\
\hline ENSBTAG00000020513 & $5.51 \mathrm{E}-10$ & 0 & HNRNPF & $5.51 E+03$ \\
\hline ENSBTAG00000012860 & $2.26 \mathrm{E}-10$ & 0 & PDZD11 & $2.26 \mathrm{E}+03$ \\
\hline ENSBTAG00000047278 & 7.26E-11 & 0 & NDUFB11 & $7.27 E+02$ \\
\hline ENSBTAG00000017660 & $7.52 \mathrm{E}-11$ & 0 & & $7.53 E+02$ \\
\hline ENSBTAG00000047194 & 0 & 0 & & $1.00 \mathrm{E}+00$ \\
\hline ENSBTAG00000015815 & 0 & 0 & CFP & $1.00 \mathrm{E}+00$ \\
\hline ENSBTAG00000015820 & $2.55 \mathrm{E}-11$ & 0 & UXT & $2.56 \mathrm{E}+02$ \\
\hline ENSBTAG00000011854 & $2.02 \mathrm{E}-11$ & 0 & SLC38A5 & $2.03 E+02$ \\
\hline ENSBTAG00000002562 & 0 & 0 & PRAF2 & $1.00 \mathrm{E}+00$ \\
\hline ENSBTAG00000007493 & 0 & 0 & & $1.00 E+00$ \\
\hline ENSBTAG00000017779 & 0 & 0 & HSD17B10 & $1.00 \mathrm{E}+00$ \\
\hline ENSBTAG00000013260 & 0 & 0 & & $1.00 E+00$ \\
\hline ENSBTAG00000046769 & $4.62 \mathrm{E}-10$ & 0 & & $4.62 E+03$ \\
\hline ENSBTAG00000045616 & 0 & 0 & & $1.00 \mathrm{E}+00$ \\
\hline ENSBTAG00000047279 & 0 & 0 & & $1.00 \mathrm{E}+00$ \\
\hline ENSBTAG00000047580 & $3.14 \mathrm{E}-10$ & 0 & & $3.15 E+03$ \\
\hline ENSBTAG00000004151 & $7.69 E-11$ & 0 & & $7.70 \mathrm{E}+02$ \\
\hline ENSBTAG00000008603 & $3.85 \mathrm{E}-11$ & 0 & CXorf21 & $3.86 \mathrm{E}+02$ \\
\hline ENSBTAG00000046027 & 0 & 0 & & $1.00 \mathrm{E}+00$ \\
\hline ENSBTAG00000017020 & $2.32 \mathrm{E}-11$ & 0 & S100G & $2.33 E+02$ \\
\hline ENSBTAG00000047092 & $6.88 \mathrm{E}-11$ & 0 & & $6.89 \mathrm{E}+02$ \\
\hline ENSBTAG00000002565 & 0 & 0 & & $1.00 \mathrm{E}+00$ \\
\hline ENSBTAG00000005098 & 0 & 0 & & $1.00 \mathrm{E}+00$ \\
\hline ENSBTAG00000019370 & 0 & 0 & RAB21 & $1.00 E+00$ \\
\hline ENSBTAG00000039090 & 0 & 0 & MAGEH1 & $1.00 \mathrm{E}+00$ \\
\hline ENSBTAG00000046659 & $8.61 \mathrm{E}-11$ & 0 & FOXR2 & $8.62 \mathrm{E}+02$ \\
\hline ENSBTAG00000021843 & 0 & 0 & UBQLN2 & $1.00 E+00$ \\
\hline ENSBTAG00000045736 & 0 & 0 & & $1.00 \mathrm{E}+00$ \\
\hline ENSBTAG00000001809 & 0 & 0 & DUSP21 & $1.00 \mathrm{E}+00$ \\
\hline ENSBTAG00000037883 & 0 & 0 & PPP1R2P9 & $1.00 \mathrm{E}+00$ \\
\hline ENSBTAG00000045934 & 0 & 0 & GPR82 & $1.00 E+00$ \\
\hline ENSBTAG00000020144 & 0 & 0 & GPR34 & $1.00 \mathrm{E}+00$ \\
\hline ENSBTAG00000011783 & $2.36 \mathrm{E}-10$ & 0 & MPC1L & $2.36 \mathrm{E}+03$ \\
\hline ENSBTAG00000011463 & $4.60 \mathrm{E}-11$ & 0 & MID1IP1 & $4.61 E+02$ \\
\hline ENSBTAG00000045660 & $1.48 \mathrm{E}-10$ & 0 & & $1.49 E+03$ \\
\hline ENSBTAG00000046582 & 0 & 0 & & $1.00 \mathrm{E}+00$ \\
\hline ENSBTAG00000037416 & 0 & 0 & & $1.00 \mathrm{E}+00$ \\
\hline ENSBTAG00000032896 & 0 & 0 & & $1.00 \mathrm{E}+00$ \\
\hline ENSBTAG00000045924 & 0 & 0 & MAGEB10 & $1.00 E+00$ \\
\hline ENSBTAG00000048092 & $1.59 \mathrm{E}-10$ & 0 & & $1.59 E+03$ \\
\hline ENSBTAG00000038630 & 0 & 0 & KLHL34 & $1.00 \mathrm{E}+00$ \\
\hline ENSBTAG00000013625 & 0 & 0 & & $1.00 E+00$ \\
\hline ENSBTAG00000023820 & 0 & 0 & & $1.00 \mathrm{E}+00$ \\
\hline ENSBTAG00000038379 & $1.62 \mathrm{E}-10$ & 0 & & $1.62 \mathrm{E}+03$ \\
\hline ENSBTAG00000046539 & 0 & 0 & & $1.00 \mathrm{E}+00$ \\
\hline ENSBTAG00000032923 & 0 & 0 & & $1.00 E+00$ \\
\hline ENSBTAG00000046229 & 0 & 0 & & $1.00 \mathrm{E}+00$ \\
\hline ENSBTAG00000032852 & 0 & 0 & GPRASP2 & $1.00 E+00$ \\
\hline ENSBTAG00000040132 & 0 & 0 & MUM1L1 & $1.00 \mathrm{E}+00$ \\
\hline ENSBTAG00000026139 & 0 & 0 & ARMCX1 & $1.00 \mathrm{E}+00$ \\
\hline ENSBTAG00000046707 & $2.73 \mathrm{E}-10$ & 0 & TCEAL7 & $2.74 \mathrm{E}+03$ \\
\hline ENSBTAG00000046972 & 0 & 0 & & $1.00 \mathrm{E}+00$ \\
\hline ENSBTAG00000032450 & 0 & 0 & PPP1R14B & $1.00 \mathrm{E}+00$ \\
\hline ENSBTAG00000022204 & 0 & 0 & & $1.00 E+00$ \\
\hline ENSBTAG00000017978 & $2.20 \mathrm{E}-10$ & 0 & & $2.21 E+03$ \\
\hline ENSBTAG00000048206 & 0 & 0 & & $1.00 E+00$ \\
\hline ENSBTAG00000015712 & 0 & 0 & POU3F4 & $1.00 E+00$ \\
\hline ENSBTAG00000039066 & $2.97 \mathrm{E}-10$ & 0 & SNRPE & $2.97 E+03$ \\
\hline ENSBTAG00000021437 & 0 & 0 & GPR174 & $1.00 E+00$ \\
\hline ENSBTAG00000024675 & $8.01 \mathrm{E}-11$ & 0 & & $8.02 \mathrm{E}+02$ \\
\hline ENSBTAG00000025062 & $3.65 E-11$ & 0 & MAGEE2 & $3.66 \mathrm{E}+02$ \\
\hline ENSBTAG00000032003 & $1.55 \mathrm{E}-10$ & 0 & ZCCHC13 & $1.55 E+03$ \\
\hline ENSBTAG00000045694 & 0 & 0 & & $1.00 \mathrm{E}+00$ \\
\hline ENSBTAG00000047545 & 0 & 0 & RGAG4 & $1.00 \mathrm{E}+00$ \\
\hline ENSBTAG00000004110 & $1.42 \mathrm{E}-10$ & 0 & & $1.42 \mathrm{E}+03$ \\
\hline
\end{tabular}




\begin{tabular}{|c|c|c|c|c|}
\hline ENSBTAG00000007839 & $2.81 \mathrm{E}-10$ & 0 & OTUD6A & $2.81 E+03$ \\
\hline ENSBTAG00000022278 & 0 & 0 & & $1.00 \mathrm{E}+00$ \\
\hline ENSBTAG00000012954 & 0 & 0 & ERAS & $1.00 \mathrm{E}+00$ \\
\hline ENSBTAG00000037550 & 0 & 0 & & $1.00 \mathrm{E}+00$ \\
\hline ENSBTAG00000039049 & 0 & 0 & RAI2 & $1.00 \mathrm{E}+00$ \\
\hline ENSBTAG00000031256 & 0 & 0 & & $1.00 \mathrm{E}+00$ \\
\hline ENSBTAG00000034174 & 3.34E-10 & 0 & & $3.34 \mathrm{E}+03$ \\
\hline ENSBTAG00000046518 & 0 & 0 & & $1.00 \mathrm{E}+00$ \\
\hline ENSBTAG00000046366 & 0 & 0 & CLDN34 & $1.00 \mathrm{E}+00$ \\
\hline ENSBTAG00000040386 & 7.52E-11 & 0 & AGTR2 & $7.53 E+02$ \\
\hline ENSBTAG00000046012 & $8.50 \mathrm{E}-11$ & 0 & SOWAHD & $8.51 E+02$ \\
\hline ENSBTAG00000047701 & $7.22 \mathrm{E}-11$ & 0 & ACTRT1 & $7.23 \mathrm{E}+02$ \\
\hline ENSBTAG00000014770 & $8.12 \mathrm{E}-11$ & 0 & GPR119 & $8.13 E+02$ \\
\hline ENSBTAG00000048126 & 0 & 0 & & $1.00 \mathrm{E}+00$ \\
\hline ENSBTAG00000039890 & 0 & 0 & & $1.00 \mathrm{E}+00$ \\
\hline ENSBTAG00000019548 & $5.42 \mathrm{E}-10$ & 0 & & $5.42 \mathrm{E}+03$ \\
\hline ENSBTAG00000039388 & $5.41 \mathrm{E}-11$ & 0 & GPR101 & $5.42 \mathrm{E}+02$ \\
\hline ENSBTAG00000035442 & 0 & 0 & & $1.00 E+00$ \\
\hline ENSBTAG00000018311 & 0 & 0 & & $1.00 \mathrm{E}+00$ \\
\hline ENSBTAG00000046045 & 0 & 0 & & $1.00 \mathrm{E}+00$ \\
\hline ENSBTAG00000010245 & 0 & 0 & SPRY3 & $1.00 \mathrm{E}+00$ \\
\hline
\end{tabular}

The columns show the substitution rates in Hanwoo and Holstein and the Hanwoo/Holstein substitution rate ratio. The pseudocount for calculating the ratios was $10^{-13}$. 